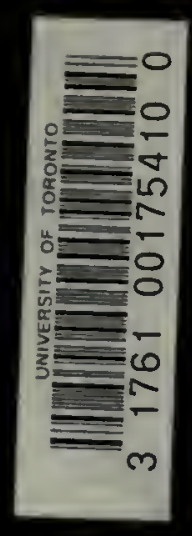



Digitized by the Internet Archive in 2007 with funding from Microsoft Corporation 





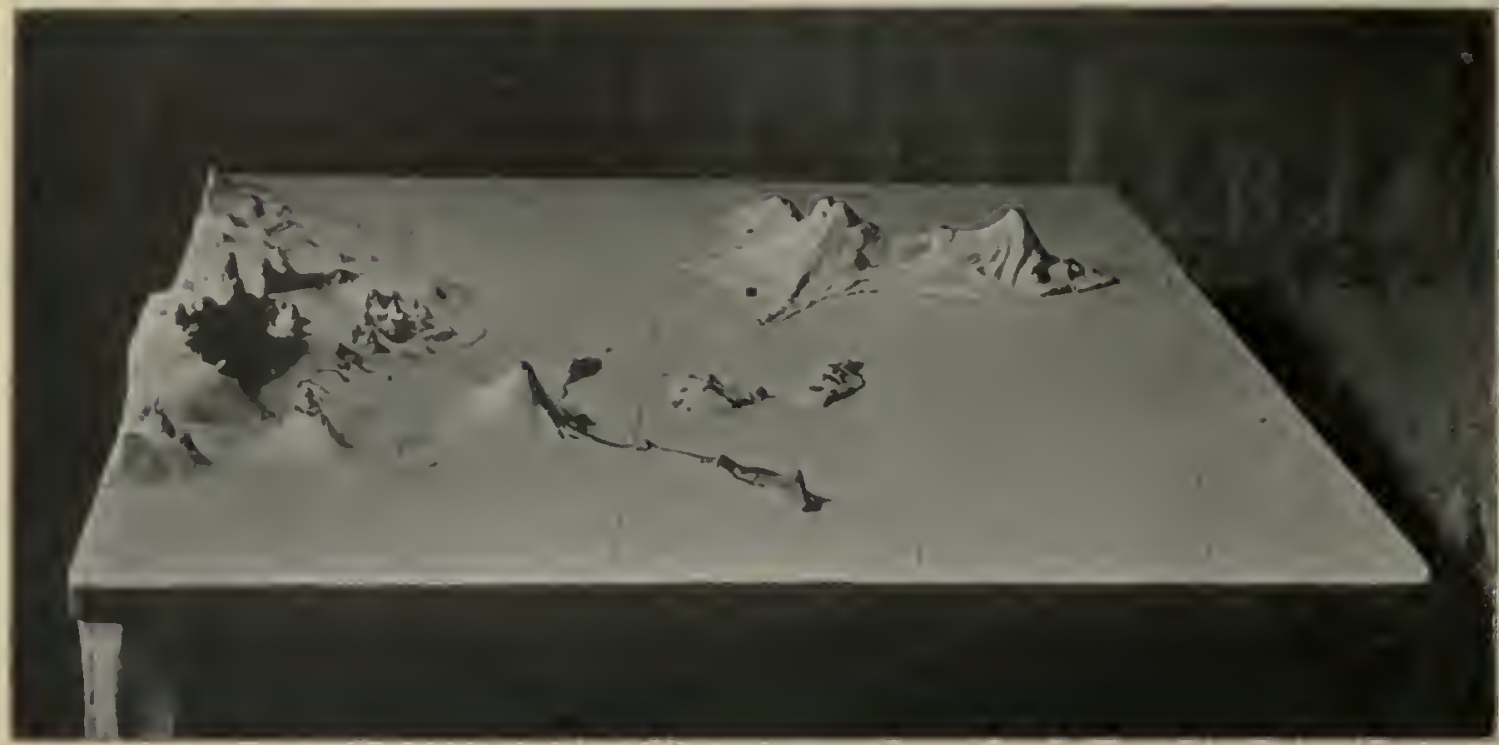

Looking Nortliward.

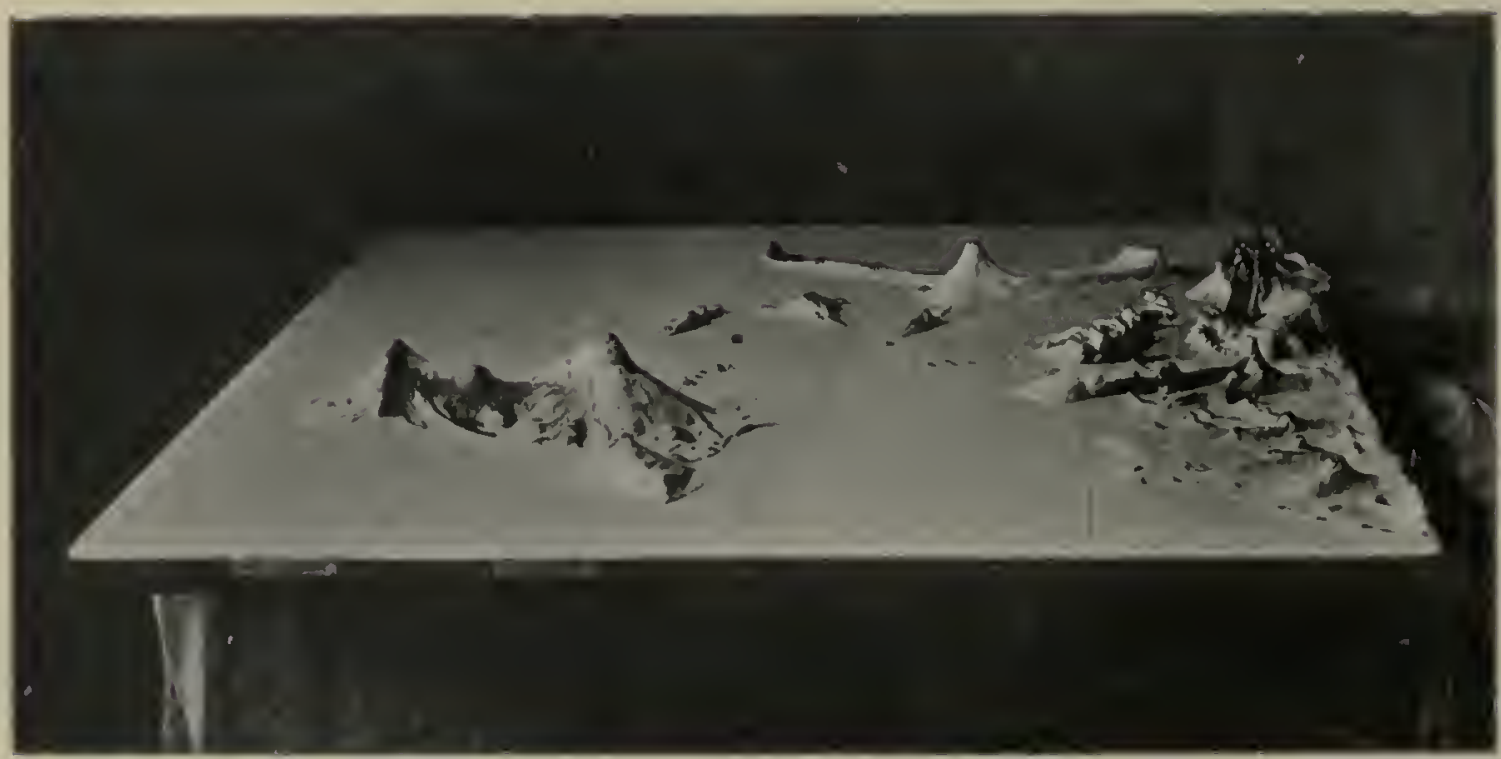

Looking Southward.

Protograph of a Modelo of the Reglon surrousdivg the Wixter Quarters of the "Discovery."

Vertical scale eight times the horizontal.

The straight lines are melidians. The wavy line slows the ice-crack between the barrier and the temporary ice. The position of the Winter Quarters is show'n by a vertical standard carrying a small square flag. 'The island ou the left of the lower photograph is Ross Island, with Mounts Erebus and Terror.

The three small islands just beyond the Winter Quarters are White Island, Black Island, and Brown Island; the prominent cone in the middle distance is Mount Discovery, with Minua Bluff on the left, and the rauge Royal Society Mountains with its glaciers on the right. In the background is the continuation of the Great Ice liarrier. 


\section{NATIONAL ANTARCTIC EXPEDITION 1901-1904}

\section{METEOROLOGY}

\section{PART I}

OBSERVATIONS AT WINTER QUARTERS AND ON SLEDGE JOURNEYS

WITH

DISCUSSIONS BY VARIOUS AUTHORS

PREPARED UNDER THE SUPERINTENDENCE OF THE DIRECTOR OF THE MÉTEOROLOGICAL OFFICE WITH THE CO-OPERATION OF

A COMMITTEE OF THE ROYAL SOCIETY

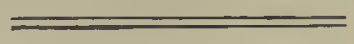

LONDON :

PUBLISHED BY THE ROYAL SOCIETY

$$
1908
$$




$$
\begin{aligned}
& Q \\
& 115 \\
& N_{32} \\
& p t .1
\end{aligned}
$$




\section{CONTENTS.}

Frontispiece. Photographs of a Model of the Region of the Winter Quarters of the "Diseovery."

Preface

I. Explanatory Notes regarding the Tables of Observations pp. 17-272 . . . . . . . . 1

II. Notes on the Meteorologieal Instruments and their Exposures, by Lientenant C. RoyDs, with Illustrations of Solar Phenomena and Cloud Observations, by Dr. E. A. WilsoN

Report, by Dr. J. A. HARKER, on the Temperature of Solidifieation of the Mereury in the Thermometer No. M.O. 30. . . . . . . . . . . . . . . . 15

III. Meteorological Journal of the "Diseovery" . . . . . . . . . . . . . . . . 17

IV. Journal of Observations on Sledge Journeys :-

I. Observations at Erebus Island Camp . . . . . . . . . . . . . . . 274

II. Mr. Bernacch to 15 miles South of Ship . . . . . . . . . . . . . . . 284

III. Capt. Scotr to lay out Depdt . . . . . . . . . . . . . . . . . . . . 284

IV. Lieut. Roxns' First Journey to Cape Crozier . . . . . . . . . . . . . . 285

V. Lieut. Armitage to bring in Depot . . . . . . . . . . . . . . . . 287

VI. Lieut. Roy DS to the Soutli-west . . . . . . . . . . . . . . . . . . . 288

VII. Lient. Arimtage to the West. . . . . . . . . . . . . . . . . . . 290

VIII. Dr. KoetTLITz round Blaek Island . . . . . . . . . . . . . . . . . . 291

IX. Lieut. Roy Ds' Seeond Journey to Cape Crozier . . . . . . . . . . . . . . 292

X. Dr. Koetruitz to Erebus Bay . . . . . . . . . . . . . . . . . . 294

XI. Liout. Roy Ds' Third Journey to Cape Crozier . . . . . . . . . . . . 295

XII. Dr. Koztruitz to the Western Mountains. . . . . . . . . . . . . . . . 297

XIIT. Mr. Sketron aud Lieut. Armitage's First Journey to the West . . . . . . . 298

XIV. Capt. SCOTT and Lieut. SHAChLetor to the South . . . . . . . . . . . . 308

XV. Dr. Koetruitz to Brown Island . . . . . . . . . . . . . . . 320

XVI, Dr. Koettritz to the Bluff . . . . . . . . . . . . . . . . . . 321

XVII. Lieut. Roy Ds' Fourth Journey to Capo Crozier . . . . . . . . . . . . . . 323

XVIII. Mr. SkeitToN's Seeond Journey to the West . . . . . . . . . . . . . . . 325

XIX. Lieut. BArNe to the South . . . . . . . . . . . . . . . . . 327

XX. Mr. Skrltox's Third Journey to the West . . . . . . . . . . . . . . 328

XXI. Dr. Wilson to Capo Crozior . . . . . . . . . . . . . . . . 330

XXII. Lieut. BARNe to the Soutli-west . . . . . . . . . . . . . . . 333

XXIII. Capt. Scott and Mr. Skerton up the Western Monntains . . . . . . . . . . 343

XXIV. Lieut. Royds and Mr. Crark to the South-east across the Iee-barrier . . . . . 352 
V. Tables of Two-hourly Rearlings at Winter Quarters . . . . . . . . . . 365

VI. Summary Tables of Olservations of the "Discovery" Fxpedition and other Antaretic Fxpeditions :-

1. Monthly and Fearly Sunuaries for the Winter Quarters of the "Discovery" (February, 1902-February, 190t) . . . . . . . . . . . . . . .

2. Monthly and Yearly Summaries for the Winter Quarters of the "Gauss" (Mareh, 1902February, 1903) . . . . . . . . . . . . . . . . 409

3. Wind Frequency at Wiuter Quarters of the "Gauss" (Mareh, 1902-February, 1903) . . . 410

4. Monthly Summaries for the Swedish Antaretie Expedition (Nareh, 1902-October, 1903) • . 411

5. Summary Tables for the Relief Slip "Morning" between $67^{\circ}$ and $77^{\circ}$ S. Latitude (1902-3) . 412

6. Summary 'Iables for Captain Scort's Sledge Journey to Furthest South, with Corresponding Valnes at Winter Quarters $(1902-3)$. . . . . . . . . . . . . . 413

7. Abstrnet of Ilourly Obserrations of the Scottish National Antaretio Expedition in the Weddell Sea and at Laurie Island, South Orkneys (February, 1903-February, 1904) . .

8. Abstract of Hourly Observations of the Seottish National Antaretic Expedition in the Weddell Sea and nt Lnurie Island, South Orkneys (February-April, 1904) . . . . .

VIt. Climatology of Sonth Victoria Land and the Neighbouring Seas, by M. W. Canpbet. Hepworth, C.B., Commander R.N.R. . . . . . . . . . . . . . . . . 417

VIII. Notes on the Observations of Tenperature at the Winter Quarters of the "Discovery," by

Dr. Charles Chree, F.R.S. . . . . . . . . . . . . . . . .

IX. Notes on Open Air Temperature, Screen Temperature, and on Terrestrial Radiation, hy W. H. DINES, F.R.S. . . . . . . . . . . . . . . . . . . . . . .

X. Notes on the Readings of the Aspiration Psychrometer and of the Dry and Wet Bulb Thermometers, and on the Observations of Evaporation and Precipitation, and of the Evaporation of Ice, by W. H. Dines, F.R.S. . . . . . . . . . . . . . .

XI. Notes on the Observations of Barometric Pressure, by R. H. Curtis. . . . . . . . 476

XII. Discussion of the Observations of the Direction and Foree of the Wind at Winter Quarters and on the Sledge Journeys, by R. H. CurTis . . . . . . . . . . . 483

XIII. Notes on the Records of Sunshine and on the Observations of Clond, by R. H. CuRTis. . 512

XIV. Discrission of the Observations of Atmospheric Electricity, by C. T. R. W'uson, F.R.S. . 519 Map of South Polar Regions.

Map, in Sections, of Sonth Victoria Land, showing the Tracks of the Sledge Journeys. 


\title{
PREFACE.
}

\author{
BY
}

THE DIRECTOR OF THE METEOROLOGICAL OFFICE.

THus volume contains the chief part of the results of the Meteorological Observations made in connexion with the voyage of the ship "Discovery" of the National Antaretic Expedition, which left Cowes muder the command of Commander I. F. ScotT, R.N., on the 6th Angust, 1901, and returued to Spithead on the 10 th September, 1904.

There still remain for publication the discussions of the special features of the traces of the barographs, thermographs and hygrographs obtained at Winter Quarters, and of about 1000 synchronous charts of the regions south of $30^{\circ} \mathrm{S}$. latitude, which have already been prepared from the daily observations at Greenwich noon contributed by international co-operation.

To the results now presented many persons have contributed, and in various ways.

\section{Programe and Equipment.}

The 'Antaretic Manual,' edited by Georac Murray, F.R.S., Director of the Civilian Scientific Staff of the Expedition 1ntil July, 1901, the guide to the scientific work of the Expedition, contains scetions on Antaretic Climate, and on Meteorological Instruments contributed by R. H. ScotT, F.R.S., and J. Y. Buchanan, F.R.S., respectively.

The ordinary meteorological equipment of the Expedition was supplied through the Meteorological Office at the expense partly of the Office and partly of the Admiralty. A number of additional instruments were also provided at the cost of the funds of the Expedition.

Sir Whlliam Wharton, F.R.S., Hydrographer of the Navy, and member, ex officio, of the Meteorological Council, took an active part in selecting the equipment. Dr. ScotT was also on the Advisory Comnittee, and in the later stages of the preparation I was associated with them, and the duty of working out the details of special construction and arranging for the mamufacture and supply devolved upon ne.

Ultimately the meteorological equipment supplied by the Office was made up as follows:-

2 marine barometers.

2 station barometers.

3 large aneroids.

6 watch aneroids.

3 barographs.

2 portable hypsometers.

12 mercurial thermometers, $0^{\circ}$ to $100^{\circ} \mathrm{F}$.

12 mercurial thermometers, $-40^{\circ}$ to $90^{\circ} \mathrm{F}$.

12 low-range spirit thermometers $+52^{\circ}$ to $-80^{\circ} \mathrm{F}$.

6 sling thermometers.

3 maximum thermometers $-30^{\circ}$ to $90^{\circ} \mathrm{F}$.

2 8-inch rain ganges, with duplicate receivers, cases and glasses.

2 special thermometer screens.

2 sumshine recorders (for 24 hours) with special cards.
1 DINES' self-1'egistering pressure-tube anemometer.

1 Drnes' sight-indicating anemomerer.

1 Rominson anemometer.

1 Assulaxis's psychrometer.

1 EXNER's elcctrometer.

3 thermographs (Ricuard type).

2 hygrographs (RICIIARD type).

8 solar radiation thermometers.

2 earth thermometers.

2 terrestrial radiation thermometers.

1 AITKEN's dust-comnter.

1 standard spirit thermometer.

5 Prue's evaporntion gauges.

6 lyydrometers.

12 spare tubes for anemometers.

The Meteorological Office also provided books of forms for the entry of obscrvations which were prepared in consultation with Sir W. WHARTON. 


\section{Mrscarmiage of Ixstructions.}

Care ws tuken to draw up special instructions for working the self-recording instruments in order to secure correct tining and neenrate recorls, ancl opportunity wis also providerl for members of the ship's staff to practise the working of the instruncuts beforehand. Unfortunately, in consequence of changes in the staff, these well-intentioner efforts failed altogether, and the meteorological observers under Lieutenant C. W. R. liovis, R.N., had to do the best they could with the instruments which they found at their disposal.

This failure of communication between those responsible for supplying the instruments and those using them accounts for some of the peculiarities in the procedure witl regard to instruments. The presence of an "index" in the low-temperature thermometers led the observers to set them apart for reading the ninimum and to use almost exclusively, for ordinary temperature readings, the mercury thernometers which were inclucled in the equipnent as auxiliaries. It was never contemplated that the station barometers would, as a rule, be so cxposed as to fail because the attached thermometers were not grarluater below $0^{\circ} \mathrm{F}$. The Dines self-recording anemograph was sent out with the irlea that the recording part would be kept in the living room and that the conducting pipes would be lashed to the stove-pipe. It seems doubtful, from what Lientenant Royds says, whether even that provision would have got over the difficnlty of the pipes filling up with ice needles. The difficulties experieneed witl the sunshine recorder, of which the recorls, sometimes practically continuous for 72 hours, are so interesting a fenture of the results, would not have occurred if the observer had had any previours experience with the instruments, and a number of questions arise as to temperatme differences, indicated by adjacent thermometers, which cannot be answered by the information at our disposal. They must be left for an observer in some future expedition who may be on the look-out for such differences when they occur.

In spite of the disadrantages at which they were placed by the want of rehearsal, Lieutenant RoyDs and his staff of observers must be congratulated upon their suecess in devising and carrying ont arrangements for the exposure of the instruments, ancl their devotion in maintaining the readings in such inhospitable conlitions. Many of the instruments used were of a new type, as applied in regions of intense cold, and the notes which Lientenant Royds has made will scrve as a valuable guide to the equipment of future expeditions.

\section{Arrangemfats for tile Discussion and Publication of the Observations. Material,}

\section{AVAILABLE.}

When the Expedition left England no provision had been made for the discussion of the observations which they were expected to bring back. Some preliminary observations, taken on the voyage to New Zealand, which were sent home from Lyttelton, were forwarded to the Metcorological Office by the Committec of the Expedition (26th March, 1902), with a request from the Presidents of the Royal and Royal Gcographical Societies for reports upon them.

Later on (21st October, 1903) documents began to be received in response to a request for international co-operation in the collection of daily observations at noon G.M.T. on land and sea beyond $30^{\circ}$ S. latiturle during the periol of the Expedition's stay in the Antaretic. The marine observations for which special forms had been prepared and sent out to the ships of the Navy and the Mercantile Marine eame to the Meteorological Office, while records from certain observatories were sent to the Royal Society and were forwarded thence to the Office.

This led to correspondenec as to the means of dealing with the data which were in process of collection and which were ultimately summariscd as follows:-

\section{Observations of the "J)iscovery" Expedition.}

The Meteonologicas Register of the "Discovery" comprising two-hourly readings from November 29, 1901, to December 10, 1903, and thereafter daily observations only, with supplementary observations, remarks, and also daily obscrvations of temperature at Cape Armitage.

The Rougn Books from which the registers had been compiled.

Mrteorological ReGisters (with Rougu Buoks) for the sledge journeys. 


\section{[ vii ]}

The TRACEs of the following self-reeording instruments:-

Barograph.-From Deeember 2, 1901, till March 28, 1904, conplete, generally in duplieate.

Thermograph.-From Deeember 2, 1901, till Fubruary 29, 1904, complete, generally in duplieate.

Ilygrograph. - Nearly complete, sometimes in duplieate, but triees of ten unsatisfactory.

Sunshine liecorder.-Some days missing.

Dines' Anemograph. - Ineomplete and of doubtful value.

\section{Auxiliary Daily Observations at Noon G.MI.I,}

(a) Land Observations. - Transcripts, daily charts, and traces of self-recording instruments from the Argentine Republic, Cape of Good Hope (Cape Town), Nauritius, Australia (Perth and Sydney), New Zealand (Wellington), Falkland Isles (Cape Pembroke), colleeted in response to a circular of the International Antaretic Committee and of the Royal Society.

(b) Marine Observations. - 30,200 days' readings between 1st Oetober, 1901, and 31st Mareh, 1904, in special or ordinary ship's logs. Collected by the Meteorological Office.

For the explanation of local circumstances and conditions we were dependent upon Iientenant Royns' 'Notes' and the Narrative of the Expedition, publisherl by Sunith, Ekler, and Co. for Captain SсотT. An oecasional visit from one or other of the members of the Expedition supplemented to some extent the information from other sources.

The Meteorologieal Council, whose staff' was already overloaded with material collecterl in the ordinary course of office business, wore not prepared to accept responsibilty for this additional volume of data. Undoubtedly, had circumstances permitted, it would have heen advisable to detail a special staff for the work under the control of a competent meteorologist, who would have been responsible for the issue of a complete report upon the observations. There were two objections to this obviously desirable course. First, competent meteorologists in this country were all too fully occupied to be able to devote the necessary time to the supervision; and, secondly, a meteorologieal staff requires some training and experience for which, in this case, no provision was available.

It was eventually decided that the work should be done in the Mleteorological Offiec under my general supervision, the office staff being increased for the time being by a few additional clerks in order to set free experienced tabulators and computers for the work of critical examination. The expense was charged against a guarantee of the Royal Geographieal Society, but ultimately placed upon the funds of the Expedition.

Scientific control was provided for by a Committee appointed by the Royal Society, consisting of Mr. J. Y. Buchanan, Dr. C. Chree, Mr. W. H. Dines, Admiral Field, Dr. Glazebrook, Sir J. Murray, Dr. W. N. Síaw, Captain Tizard, Mr. C. T. R. Wilson, Mr. H. N. Dickson, Mr. Ferrar, Mr. A. J. Herbertson, Dr. H. R. Mill, and Lieutenant Royds.

\section{Presentation of the Results.}

The data of the observations at Winter Quarters and on the sledge journeys have been earefully examined and are printed in extenso, pp. 17 to 364, with maps prepared by Lieutenant HULOcK for the Royal Geographical Society to illustrate the geographical positions.

The two-hourly values have also been reproduced, arranged in the form adopted hy international agreement for the publication of results from stations of the first order, pp. 365 to 406 ; and a summary of monthly results in the form usual for stations of the second order is given on p. 408 . Readers who wish to obtain a conspcctus of the salient meteorological features of the Winter Quarters of the "Discovery" will find it in this table. Tables of results for other expeditions are given for the purpose of comparison.

The introduction, which gives an account of the exposure of the instruments, is by Lieutenant C. W. R. Royds, R.N., who was in charge of the metcorological work of the Expedition. Various members of the 
staff of the Expedition have heen consulted as to different points arising in the course of the examination of the observations.

The preparation of a report upon the climatology of Sontl Victoria Land and the neighbouring seas was entrusted to Captain CAsprisl. Hepworti, Marine Superintendent of the Mleteorological Office, and notes on various aspects of the collected ohservations have been written by Mr. R. H. CurTis, Superintendent of Instruments, Dr, C. Cures, F.R.S., and Mr. W. H. DiNes, F.R.S. Mr. C. T. R. WiLsoN, F.R.S., has contributed the report upon the observations of atmospheric electrieity.

Observations were male with ozone papers (p. 4), but it has not been found practicable to formulate the results. Lientenaut ROYDs' note that the eoloration of the papers was most marked during heavy southerly gales should be remembered in connexion with the occurrence of abnormally high electrical potentials in drifting snow.

The division of the responsibility for the discussion of the observations between a number of different persons is a method of procedure which is subject to obvious disadvantages, but in the present case it was ineritable. The task of dealing with observations in the absence of the olservers themselves is always a lifficult one, especially so in the case of the "Discovery" observations, bccause there was no opportumity for preliminary understanding as to the course to be pursued and the precautions to be taken to ensure accuracy and avoid ambiguities. In preparing the observations for press, careful eomparison was made between the original entries in the rough books and the final records in the register and the curves of the self-recording instruments. .A number of doubtful points were thereby resolved. While this work was going on, the anxiliary observations from ships and land stations south of latitude $30^{\circ} \mathrm{S}$. were examined, transcribed on to separate sheets for each day, and plotted on eharts.

It is essential that a primary discussion of observations thus obtained should be undertaken by those who have conducted the examination and seen the evidence for the readings adopted in cases of doubt; otherwise the conclusions drawn may be weakened, if not invalidated, by stress being laid on uncertain values. The programme that was approved by the Committee included a primary cliscussion of the land observations by Mr. R. H. CURTIs, who was in charge of the documents from Winter Quarters, and of the auxiliary marine and land observations in relation to the observations at Winter Quarters by Captain Hepwonti. The points referred to Dr. Chree and Mr. Dines were special points eoncerning the instruments employed, or observations which were outside the ordinary routine. It was my purpose to complete the examination and printing of the observations forming the Journal as soon as possible and to distribute the eopies to those who were engaged upon the discussions, so that the whole of the material might be at their service. I then hoped to gather up the threads and deal with the general metcorological bearings of the results.

But this programme has not been fully realiserl. The task of examining and editing the observations has been a very long and difficult one. The plan of supplementing the staff of the Office by persons appointed temporarily, and therefore subject to frequent changes, proved unworkable, and when the special funds were exhausted the duty was taken over by the Office staff. In the meantime special transcripts of data were supplied to Dr. CineE and Mr. Dines, and their notes were completed before the printing of the Journal was accomplished. Moreover, the various authors used a permissible latitude in interpreting their terms of reference, and travelled, to a certain extent, over each other's ground. The titles of their contributions have been adjusted accordingly.

While this work has been in progress the Office itself has passed through many changes. As Director I succeeded in 1905 to the responsibilities of the Council for the administration of the internal affairs of the Office, and therewith presumably to the undertaking with the Royal Society to earry out the preparation of the Antarctic observations for publication; but the various duties incidental to the changes in administration have left me less opportunity for taking a personal share in the discussion of the observations than I anticipated.

The pressure of Office husiness has also postponed the preparation of a paper on "The Sequence of Weather Changes as inclicated by the Antiretic Records." This was entrusted to Mr. R. G. K. LEMPFERT, who joined me in the authorship of "The Life-History of Sulface Air-Currents," a strictly cognate subject. 
As Superintendent of Statistics, Mr. LAMMPFEr has been in charge of the revision of the weekly reports and other publications which have demanderl the whole of his personal attention. I trust that he will be in a position to take up the interesting question of the sequence of weather changes in the Antaretic before the close of the current year.

\section{Meteorological Results.}

Although not strictly in accordance with the original progranme, and inadequate as regards the observations of temperature, the papers published in this volume represent the primary discussion of the meteorological results of the Expelition.

It may be useful if I give in a fow sentences what appear to be the salient features of the elimate according to the two years' experience of the explorers.

Althongh the Winter Quarters were regarded as an exceptionally warm position in relation to others in the locality, temperature ranged from a maximum of only $42^{\circ} \mathrm{F}$. to a minimum of $-58^{\circ} \cdot 5 \mathrm{~F}$. Fluctuations are extraordinarily rapid and violent at all seasons. They are sometines, but not always, associated with changes of wind direction. Polar winds of ten bring with them an increase of temperature.

The daily range is large in consequence of the sulden fluctuations, but the mean diurnal variation, as represented by the averages of the two-hourly readings, amounts to only $3^{\circ}$ in midsummer and $1^{\circ}$ in midwinter. The time of maximum as regards the latter is rather dubious, but there is evidence of a diunal and semichurnal oscillation of temperature even in the Antarctic night.

The summers were remarkably cold. Very few days gave a mean temperature above the freezing-point. The highest mean temperature for any month was $26^{\circ} \cdot 2 \mathrm{~F}$, the lowest $-21^{\circ} \cdot 0 \mathrm{~F}$.

The air is remarkably dry and transparent, fog is comparatively infrequent; precipitation is slight, but there may be deep drifts of snow. Sunshine is remarkably abundant; the total for 1903 equalled that for Scilly, and in December of that year an average of 16 hours per day was registered. The sun's rays are intensely hot and terrestrial radiation extromely vigorous.

Pressure ranged between $30 \cdot 181$ and $28 \cdot 140$ inches; the two extromes occurring in consecutive months of 1902. The barometer stands high as compared with the values obtained for other positions in the Antarctic. The sequence of pressure changes as shown on the barographic records presents no very noteworthy peculiarities, and the use of the barometer for anticipating coning weather is less effective than in this country.

The pressure observations show the common semicliurnal oscillation. It amounts to 0.002 inch, with maxima at about 10 a.m. and 10 p.m. at all seasons of the year. The variation of twenty-four hours' period has, on the average, an amplitude of twice that amount, but the timos of maximum are different at different seasons.

The changes of pressure in high southern latitudes may be regarded as associatcd with the passage of cyclonic depressions much in the same way, mutatis mutumdis, as elsewhere.

The surface winds are chiefly easterly. Northerly and southerly winds occur, but winds from between south-west and north-west are almost unknown. The chief characteristic of the wind is its gustiness. Gales are experienced from every quarter between north-enst round to south-west on the eistern side, but, on the whole, they are not so frequent as in the British Isles.

The direction of motion of the lower clouds may be from any point of the compass. The resultant is a moderatc frequency from the south-east; there is a marked deficiency as recyards the west.

The resultant motion of smoke from Mount Erebus is from south of west and that of the upper clouds from the west.

Subject to some uncertainty on account of the state of the instrument, the mean electrical potontial is rather lower than at Kew, but abnormally high in drifting snow, or ice-erystals. A diurnal variation is shown in sunmer, with a maximum in the middle of the day and a minimum in the early morning hours. The seasonal variation has a maximum in summer and a ninimum in winter. No relation is shown between potential and aurora.

I add a few comments upon some of the points which have been raised in the course of the discussion. 
Temperatures.

Captain Hepwortir has called attention to the comparative warmth of southerly, i.e. polar, winds, which was also a special feature of the observations at Cape Adare by the "Southeru Cross" expedition. Mr. DLNes has dealt with the extraordinarily low sumuer temperatures in relation to the dryness of the atmosphere; he has also referred to the locally ligh temperature of the station at the Winter Quarters of the "Discovery," and has suggested an explanation. Dr. ChrFe has dealt with the diurnal inequalities and the extremes of temperature. There are other points which may be noted. The great range of temperature during the winter months is very remarkable; the range in June, July, and August, 1903, during the Antarctic night was $64^{\circ} \mathrm{F}$. $66 \cdot 2^{\circ} \mathrm{F}$, and $65^{\circ} \mathrm{F}$, respectively, and closcly connected with this point is the extraordinarily rapid fluctuation of temperature as shown by the thermograph. A specinen of the thermograph traces, giving examples of these rapid fluctuations of temperature, is reproduced here (fig. 1, p. xi.).

There is additional eridence of the existence of such rapid fluctuations. In default of any instructions to the contrary, an alcohol minimum thermometer, mounted in the screen beside the mercury thermometers, was rearl and set every two hours. Mr. Curtis has called my attention to the fact that the minimum reading for the interval is in nenly every case below the mercury readings at the beginning and end.

Such would certainly not occur in any ordinary climate. So persistent is this occurrence of a minimum every two hours that Mr. CuRTIS is disposed to doubt the accuracy of the readings as not being always horne out by the thermograph curves, but if there be an error of reading it is clifficult to say which of the two thermometers is liable to the error, and in very many cases a depression of temperature within the two-hour interval is shown on the thermograms.

In connexion with these extraordinary results for temperature it must be remembered that when the physical process going on at the surface is a transference of heat from the air to the ground, cooled by radiation or otherwise, very eapricious results are likely, because the eoolerl air remains at the surface. In any locality the fluctuations of ninimum temperature on a clear night are, for that reason, far greater than the fluctuations of maximum temperature on a cloudless day. This property of cooled air must be borne in mind in considering all questions of Antarctic surface temperatures. The coldest temperatures are likely to be found in stagnant air, and any wind that disturbs stagnant air will be a warm wind. Every fluctuation in the strength of the wind will, similarly, cause an alteration of the conditions governing the temperature. From this point of view it is to be regretted that the variation of temperature with height, within such moderate compass as can be reached on the ship, up to 50 or 60 feet, was not made the subject of experiment or observation at comparatively short horizontal distances apart.

On p. 429, and elsewhere, Captain Hepwortil suggests that the experience of relatively warm winds from the polar quarter may be attributed to the circulation of warm air in cyclonic systems, by which the eurrent brought to any point from the southward may be traced to some equatorial quarter. It is difficult to know how far such an explanation may be carried. Withont some limitation it would enable one to assign any desired characteristic as regards temperature to any part of the storn area. The detailed work upon the paths of air in travelling storms of temperate northern latitudes in "The Life-History of Surface Air-Currents" tends to show that it is difficult to give any general rule for the ultimate origin of the air supply for any particular part of a depression. In our experience in these latitudes, winds from equatorial quarters are generally warmer than those from polar quarters. But there is so little information about the mechanism of a travelling depression in the Antarctic regions that no final opinion is possible with regard to the question of the association of temperature and wind clirection.

\section{Pressure und II inds.}

The question of the prevailing wind at Winter Quarters and its relation to the distribution of pressure is another interesting point which is not entirely disposed of. The idea of an anticyclone, possibly of no great rertical heiglit, but spreading over the surface of the Sonth Polar continent, has found expression in many forms in meteorological literature; the references to HAxx and Supdx given by SCOTT in the 


\section{[ $\mathrm{xi}]$}

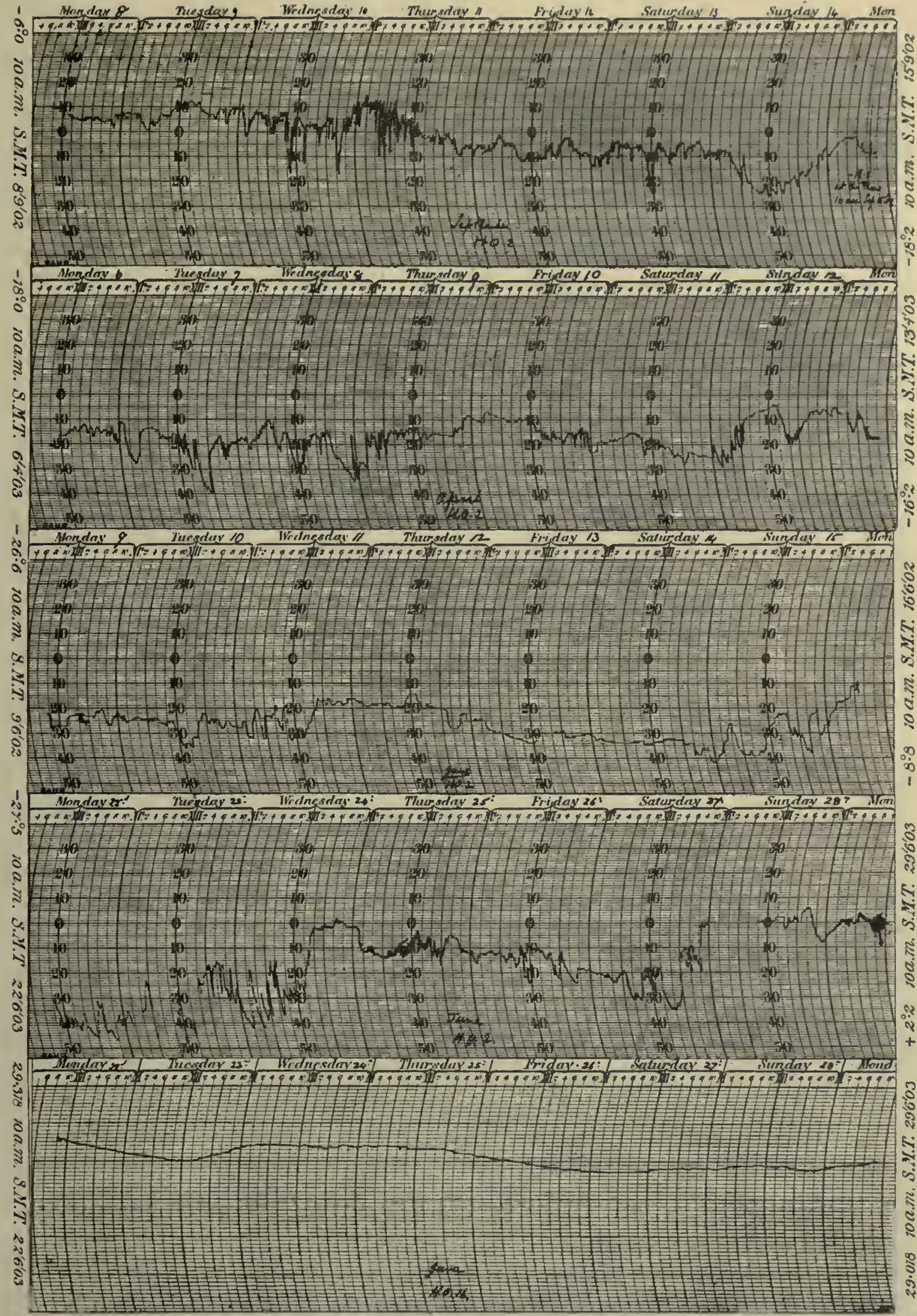

Fig. 1.

Facsimile reproductions of five traees of the self-recording instruments at Winter Quarters, representing rapid fluctuations of temperature and one associated barometric trace.

No. 1. Thermograph record of temperatures above and below zero. Septomber 8 to 14, 1902.

, 2.

"3.

"4.

" betwcen $-50^{\circ} \mathrm{F}$. alud $5^{\circ} \mathrm{F}$. Jume 22 to $28,1903$.

5. Barograph record for comparison, showing the absence of any noteworthy clange of pressure to correspond with chinges of temperature. below zcro $1:$ A pril 6 to 12,1903 .

below $-10^{\circ} \mathbf{F}$ Jume 9 to 15,1502

(1) 
Antaretic Manual' bear out this statement, and the idea was also put forwarl by Sir J. MUrRAY and Dr. Buclis, and was referred to by Mr. Bersaccin in his account of the observations at Cape Adare. I gave very precise expression to the idea in the 'Proceedings of the Royal Society,' rol. 74, p. 20, 1904, and shored that an cast to west circulation at the surface, with a superposed west to east circulation in tho upper air was to bo expected.

Such were the conditions actually found by the "Discovery" at Winter Quarter's, as well as by the "Gauss" in a far distant part of the Antarctic. Mr. CurTis has set out very clearly the full data on the question, and it seems difficult not to regard the observations as confirming the inference from general conditions, about which there can be no rloulst. But Captain ScoTT and other members of the Expedition brought back the general impression that the winds at Winter Quarters were a local phenomenon due to local configuration (see p. 422), and that as soon as an "open" situation was reached the prevailing wind was really from south-west. South-westerly winds were certainly noted at Cape Crozier on several occasions, though some of the Cape Crozier observations were at an altitude of nearly 1000 feet; and southerly winds helpel to bring back Captain SCOTT from farthest sonth, aeross the barrier ice. In spite of these facts it is difficult to imagine any cause for the existence of an easterly wind as a "local wind " at Winter Quarters or Cape Arnitage and the practical absence of observations of westerly or south-westerly winds at these points. In order to place those who were discussing these questions in as favourable a position as possible for realising the local conditions, I had a model in clay of Ross Island and the region round the Winter Qnarters, prepared in the office ly Miss Humpireys, who used all the indications that could be collected from inaps and photographs to give a true represcntation of the locality. The inodel was exhibited at the Royal Society in 1907, and was seen by various members of the Expedition, who used it to recall their experience in considering points put to them. It was approved as an accurate representation. Two photographs of it are reproduced here, to slow the general orographical conditions in regard to the point in question.

It is curions that endeavours to reach, by two separate crucial tests, a definite conclnsion upon this interesting point, as to whether the easterly wind at Winter Quarters is a local wind or a true gencral wind implying a high pressure to the sonth, fail through very slight omissions in the observations or the records.

The first test is the experience of Lieutenant RoYDs ou his journey across the ice barrier, November 10 to December 10, 1903 (p. 352), which should have been very favourable for extending the reach of the observers beyond the open position of Cape Armitage. Mr. RoYDs' observations are nearly all printed S. W., but it is curious that the deviation of that direction from the casterly direction olserved at Winter Quarters and Cape Armitage is actually the deviation of the compass in that locality. There is no entry - in the registers to show how the direction was obtained, nor whether the original entries were magnetic or true. Cross-examination of the members of the Expedition who visited the Ottice led to varying opinions, but finally Mr. Royds wrote (2nd May, 1907), "I believe on some other occasions wind lias been entered as magnetic, but I do not remember doing it on that journey; in fact I am sure I did not." They are therefore printed as they stand.

It is clear that when the magnetic variation is so great as to make the difference between a southwesterly and an easterly direction of the wind, special care should be taken to distinguish between the two specifications of direction. Indeed, anyone who has to deal with the nncertainties arising in a question of this kind will agree that our geographical linowledge has now become too extensive for the continued use of the symibol $\mathrm{N}$. to mean true or magnetic north according to circumstances, and that some other name for magnetic north ouglit to be devised when the point intended to be defined may be within twenty-five legrees of due south.

The second crucial test consists in endeavouring to identify the increase of pressure polewards that must correspond witl a normal wind from the east in south-polar regions. This also must be considered to have failed, for Captain HerworTi and Mr. CurTis lave both attempted to use it and have felt themselves jnstified in adopting different conclusions. The failure arises from the fact that the explorers brought back no certain information about the amount of slope of the barrier surface towards the sea. The fact that there is a definite "ice-fall" between White Island and Black Island leads one to infer that, in that 


\section{[ $\mathrm{xiii}]$}

part at any late, the surface was rising slightly from the sea-level at Winter Quarters, lut how much is very donbtful. Captrin Herworti (p. 439), using all average height for the barrier, is of opinion that the olservations give no conclusive indication of an increase of pressure southward, and eonjectures an isobar runing nearly north and south. Mr. CuRTis slows that the rise of surface necessary to aecount for the olserved diminution of pressure sonthward is so slight that more might well be allowerl, and infers that the sea-level pressure increased to the southwarl.

\section{The Antaretic Anticyclone and Local Winds.}

In this connexion it may be desirable to reconsider the general aspect of the question. First of all it is necessary to distinguish the average conditions and the instantaneous conditions on any particular occusion. No doubt the average is made up of the aggregate of individual occasions, and no process of averaging can convert a persistent south-westerly wind into an easterly one, or vice vcrsa. Actual averages are only available for a few points, and the true relation of even a considerable number of isolated observations in different localities to the average conditions is not easily estimated. The Antaretic anticyclone, if it exists, is a comparatively superficial effect attributable to the surface cold. But to give an easterly wind there must be sufficient thickness of cold air to reverse the gradient of the upper air, which, as shown by the smoke of Erebus, by cloud observations, and by the observations at high levels, is poleward; 5000 feet is probably an ample allowance for the thickness of the cold surface cap which has an east to west rotation. In order to give a resultant gradient for easterly winds, the gradient of the cold surface layer must exceed that of the westward moving layer up alove. One requires, therefore, a rapid change of pressure in the surface layer, and with that we should expect to find a rapul temperature gradient poleucurl.*

There seems 110 reason to suppose that when once the permanent ice is reacher there is very rapid diminution of temperature further to the south. We may suppose, on the contrary, that whell the transition from open water to ice takes place there will be a transition of air temperatire, and a pressure grarlient associated therewith, but once upon the permanent ice continent the clianges of general temperature and their influence upon surface pressure will be slight and possibly local.

The pressure conditions to be anticipated over the Antarctic may, perhaps, be described rather as a platean with a steep fringe along the line of transition from ice to water than as a region of continually increasing elevation. The shape of the platear will follow, roughly speaking, the shape of the permanent ice continent, and the average isobars will therefore ronghly follow the coast line. Captain HepworTr, on p. 440, has drawn attention to the tendency of isobars to conform to the coast line iu other regions. What local variations there may be within the area of the plateau is altogether undetermined.

The number of calms observed in the Antarctic shows that the surface giadients are often very slight. The rapid fluctuations of temperature which must be associated with change of surface pressure, extending perhaps to no great height and therefore not recorded on the barograph, show that changes must be local and spasmodic, and we get the idea of the polar region within the line of ninimum pressure as a region with a fringe of gradient for easterly winds where the rapid transition of temperature takes place from sea to ice, and within that belt little regular change of pressure, frequent calms with spasmodic changes of temperature and wind not closely associated with the general surges shown on the barograph.

Attention may also be called here to Mr. Dines' conchusion (p. 475) that the air of the Antarctic is cxceptionally dry, and that the dryness may be attributed to the descending air of an anticyclonic region. This suggestion olvviates to some extent the difficulty of the divergence of observation as regards wind

* It may be noted that, in order to account for the transition from the surface gradient for casterly winds through au intermediate stage of southerly airs to a grudient for westerls winds at the level of the upper elonds, we require a temperature distribution with a fall of temperature towards the east of south in the first stage and towards the west of south in the seeond stage. Regarding these distributions as a full of temporature towards the south in both stages, with a west to enst gradient of temperature in the lower stage and one from enst to west in the upper stage, we get a eombinution of conditions which is by no means improbable, in view of the faet that the temperature fell to the south along the surface, and in the upper layer the ligh land surfaee to the west would imply lower temperature there that at the same level in the free air over the sea of cold air lying on the barrier surface. 


\section{[ xiv ]}

direetion and the exceptional surface temperature. The descending air represents a snpply to some neighlonring lnw-pressure aren, and the air so supplied will be sulbject to a certain though unknown amount of dyuamical warming.

It is in necord with these ideas that the Antarctic anticyclone seems to be such an elusive object of pursuit. Fvery Antarctie expedition gets almost within touch of it, but none actually grasps it. Mr. BERxAccu inferred it from the observations at Cape Adare; the inference is confirmed, although not much strengthened, by the observations at Ross Island, 400 miles further south, but, be it renarked, along the coast line. The "Gauss," in the same way, got to the easterly winds in a much lower lertitnde than the "Discovery." The "Discovery" found easterly winds when coasting along the ice harrier to King Edward VII. land. The "Scotia" fonnd an increased barometric reading at her furthest south (p. 451). It appears indeed to be possible that the limit of the low-pressure area defines the linit that ean be reached hy a ship, and that wherever and whenever a ship is brought to a standstill by permanent ice on its voyage south, the zone of lowest pressure is passed and the pressure has begun to show an indication of anticylonic conclitions. The easterly wind met with in these circumstances may well he a general accompaniment of the pressure gradient associated with the transition from water to ice, and therefore only local in so far as it is confined to a narrow belt just beyond the line of ninimum pressure. But whenever the limit has been passed, the temperature gradient ceases, and therewith the pressure gradient; and beyond the Winter Quarters of the ship, on the line of nearest approach to the pole, wherever it may be, the pressure is generally uniform, with spasmodie gradients.

It must be allowed that these are merely suggestions not fully worked out. There is material in the observations now published for pursuing these ideas somewhat further.

The whole question of local winds is one of great meteorological interest and importance, which has long been noted for discussion in the Meteorological Offiee as soon as opportunity offers.

I had hoped to have the opportunity of eompleting the discussion as regards Antarctic winds before the publication of this volume, but, in the circumstances, it has not been possible, and to delay the publication of the volume on that account would serve no useful purpose.

W. N. SHaW. 


\section{METEOROLOGY.}

\section{EXPLANATORY NOTES REGARDING THE TABLES OF OBSERVATIONS}

(Pp. 17-272).

TuE data eontained in the tables of Meteorologieal Observations have been arranged as follows:-

Colnmn 1. Hour.-The hour at which the observations given in the suceeeding columns were made.

Column 2. Barometer.-The barometer was hung on board the "Discovery," where its cistern was 9 feet above the level of the sea. The readings have been corrected for instrumental error and redueed to sea-level and to $32^{\circ} \mathrm{F}$, but have not been corrected for gravity. The mean of the bi-hourly readings made each day is given at the foot of the column.

Column 3. Cape Armitage. - The temperature of the air in degrees Fahrenleit observed at a point off Cape Armitage abont 3000 yards south-south-east of the "Discovery." The thermometer nsed was a spirit mininum thermometer, supported upon a light wooden stand at a height of 4 feet ahove the snow, and freely exposed to the air. Throughout the summer months the supporting frame was covered by a wooden awning to protect the thermometer from the sun's rays, but during the winter months the awning was removed and the bulb left open to the sky. The realing of the thermometer at the time of observittion as well as the minimun temperature shown by the index was recorded at each observation.

Column 4. Spirit Minimum.-The minimun temperature of the air recorded by a spirit minimum thermometer placed in a screen about 120 yards from the ship. The thermometer was read and set every two hours. The following corrections lave been applied to the readings of this thermometer:-

$$
\begin{aligned}
& \text { When the temperature was above }-5^{\circ} \mathbf{F} \text {. . . . . . . . . } 0^{\circ} \cdot 0
\end{aligned}
$$

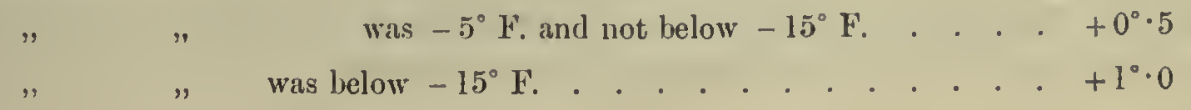

Column 5. Dry.-The temperature of the air in clegrees Fahrenheit, obtained from a mereurial thermometer exposed in the same thermometer sereen as the minimum thermometer, at a height of 4 feet above the snow. No eorrection for instrumental error has been applied to the readings of this thermo- 
meter. I) ring the winter months the thermometer sometimes gave rearliugs below the freezing-point of mercury*; but it was vccusionally frozen, and when this was the ease the temperature shown ly the spirit ninimum thermometer at the time of observation is entered in this column as the temperatnre of the air'. The mean of the bi-hourly olservations is given each day at the foot of the column.

Column 6. Wet-Temperature of the "wet" bulb in degrees Fahrenheit, oltained from another mercurial thermometer placed ly the side of the alry bulb, and at the same height above the suow. Throughout the stay of the "Discovery" in her winter qunters, watel in the opcn nir was invariahly frozen. The mushin with which the bulb of the thermometer was covered was therefore allowed to hang a little below the bull, and was kept coated wilh ice, the rule being to pnt a few drops of water upon it from a rubber syringe at ench time of observation, immediately after the thermometer had been read, care being taken, however, to keep the coating of ice as thin as possible. 'The mean of onch day's wet-bulh observations is given at the foot of the columu. Occusionally the wet-bulb thermometer indicated a higher temperatmre than the dry-bullh. In snch cases, if the difference did not exceed one degree, the rearliug is given, hut when the difference was greater thau one degree the wet-hnlb realing has been omitted.

Column 7. Unscreened Thermometer.-The temperature in legrees Fahrenheit by an ordinary mercurial thermometer placed in an upright position above the screen in which the dry-and wet-lulb thermometers were hung. Up to March, 1903, the thermometer was attached by india-rubber bands to one of the posts to which the sereen itself was fixed, lut from that date onwards it was supported vertically by strips of brass fastened to the top of the screen, the height of the hulb above the screen being 2 inches, and its distance back from the front edge of the screen 4 inches. The thermometer was therefore fully exposed to the air, and to the sun's rays whenever the sun was shining. The screen was painted white.

Colnmm 8. Wind Direction and Force.-The direction of the wind was obtained from a vane erected upon a post forming one of the supports of the thermometer screen. The height of the vane above the snow varied with the lepth of snow between 15 feet and 8 feet. All the wind directions given for Winter Quarters have been corrected for magnetic variation, and are therefore true. The force of the wind was estimated hy BEAUfOrT's Scale of 'Thirteen Points (0 to 12), in which 0 indicates a calm and 12 a hurricane.

Colımn 9. Wind Velocity by Cup Anemometer.-The mean velocity of the wind in statute miles per hour as recorded by a small cnp anemometer, the eups of which were 3 inches in clianeter and the arms $6 \frac{1}{2}$ inches long. This instrument was exposed upon another of the upright posts carrying the thermometer screens, the cups being at the same level as the vame. The dials were read every two hours, and the reading has been divided by 2 to get the average hourly run of the cups in the interval. Before it left England the anemometer was tested at the Kew Observatory of the National Physical Laboratory, and found to give approximately the same result as the large, "Kew pattern," anemometer. The rearlings of that instrument have to be correeted for the factor, and rednced in the ratio 2.2 to 3.0 to get results in statute miles per hour, and therefore the readings of the small instrument have been similarly dealt with.

Column 10. Wind Velocity by Pressure Tube Anemometer.-The actual velocity of the wind in statute niles per hour at the 'time of observation as indicated by a portable "sight-indicating" pressuretube anemometer. This instrument was attacherl to the side of another of the posts carrying the thernometer screens, and was connecter by 5 feet of composition tnbing with a vane placed at the top of the post 10 feet above the snow level. It was read every two hours when the other olsservations were made, and the oscillation of the fluid in the tube, denoting the variation in the wind force, was observed dnring a short interval. Thus the entry " 15.20 " indicates that whilst the observation was being made the velocity of the wind varied between 15 iniles and 20 miles per hour.

Column 11. Weather.-The weather at the time of observation indicated by the Beaufort notation, or by the Intermational weather symbols, as follows:-

* Sec Note on (liis subject by Dr. J. A. HAlKER, p. 15 , 


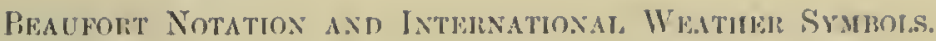

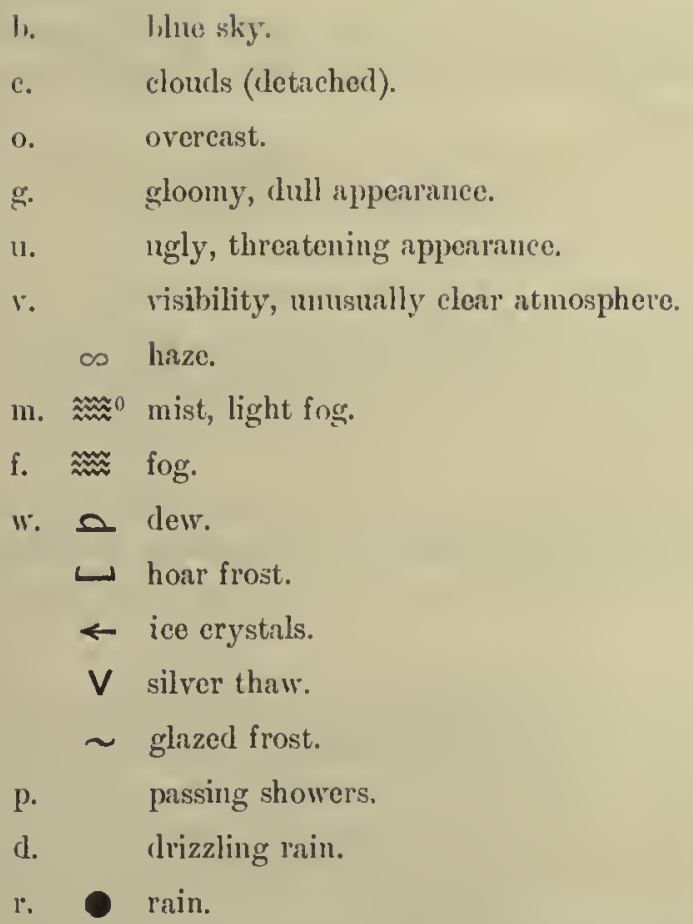

s.

f. snow irift.

\# snow lying (more than lialf the surround-

ing country covered with suow).

h. $\Delta$ hail.

$\Delta$ soft lail.

t. $T$ thumder.

l. $<$ lightuing.

R thuuderstorm.

w strong wind.

q. squally.

O solar coroni.

$\oplus$ solar halo.

(U) lunar coroni.

(1) lunar halo.

$\frown$ rainbow.

घy aurora.

A bar (-) under a letter indientes persistency, and a dot (.) intensity. The figure ${ }^{0}$ attached to a symbol indicates very slight, whilst the figure 2 indicates strong or henvy: thus ${ }^{0}=$ slight rain, $2^{2}=$ henvy rain.

Columı 12. Sunshine. - The cluration of sunshine is shown by the number of two-hour spaces covered by the vertical black line within the column. The record was obtained by a Campbell-Stokes sunshine recorder specially designed for use in high latitudes where the sun may he above the horizon throughout the twenty-four hours. The instrunent was placed on Hut Point, a conical mass of rock 50 feet high and about 300 yarks to the west of the ship (see map, p. 16). The base of the recorder was about 2 feet above the ground. The only possible olstruction to the free horizon of the instrument was Crater Hill, 1,000 feet high and about 2 miles to the eastward, which may possibly have cut off soune of the morning sumshine in November and February.

Columms 13 and 14. Clonds : Amount and Movement-The form, mmomnt, and hircetion of morement of the upper and lower clouds are given in these columns. As regards form, the clissification and nounenclature adopted is that of the International Cloud Atlas. The amount is given by a seale of 11 points (0 to 10) in which " 0 " signifies a clondless sky, and " 10 " a sky completely corered with cloud. The direction of movement is that from which the clond appeared to be travelling, and in common with all the olservations made at Winter Quarters, the bearings given are true and not magnetic.

Column 15. Temperatures by Assmann's Aspirator.-This apparatus was suspender from an iron arm attacherl to one of the posts earrying the thelmometer sereens, and was 3 feet above the level of the snow. 'The upper of the two realings taken at ench olservation is that of the dry hulb and the lower that of the wet bull,.

At the foot of the Columns. Maximum Temperature. Solar Radiation. Evaporation. Precipitation.Observations of the maximum temperature were made twice daily, at $8 \mathrm{~nm}$. and $8 \mathrm{p} . \mathrm{m}$., from a mereurial maximum thermometer placerl in the same sereen as the dry-and wet-hull, thelmometers. 
The olservatious of solar radiation were made on Hut loint, near the position occupied by the sunshine recorder. Both black-lull, and bright-lulb thermometers in vacuo were used, and were arrangenl horizontally upon a stand $3 \frac{1}{2}$ fect above the snow with their lulbs pointing to the north. Thermometers with large and small vacum bulbs were employed, lut, unless otherwise stated, the readings given are always those from the instruments having the larger bulbs.

The daily amonnt of evaporation was obtained lyg weighing a small tin vessel contrining ice, which was kept exposed upon the top of one of the thermometer screens. Generally two, and occasionally three, such vessels were weighed at each olservation.

The amount of precipitation was got from a rain gange placel on the ice near the thermometer screens. It was measured only when the sun fell withont much wind; at other times the drift made it impossible to use either the rain gange or the indications of the snow gauges, which were graduated stakes plared upright in the snow.

Remarks and Notes.-The observations given in the columns have occasionally heen amplified hy notes, amcl they have also heen supplemented ly descriptious of phenomena whieh it was not possible to tahulate. The following olservations made at more or less irregular intervals as occasion offered are always enteren in these notes:-

(A) The direction of drift of smoke from Mount Frebus as observed from a point a short distance to the westward of the "Discovery." The hearings have been corrected for variation and are therefore true.

(B) The temperature of the air olsscrved off Cape Armitage at times other than those specified in the table.

(c) The temperature of the air at the High-Level Station on the summit of Crater Hill, to the eastwarcl of the ship and 1000 feet above sea-level. The thermometer used was a spirit minimum thermometer, which was placed in a ventilated box proteeted lyy stones placed agaiust it.

(D) Olservations of the amount of ozone present in the atmosphere, deternined by the amount of discoloration of a strip of prepared paper exposed to the action of a current of air. The amount of diseoloration was estimated by a scale of 11 points, in which " 0 " indicated complete alosence of ozone and " 10 " the presence of the maximum amount.

All compass bearings given in the notes relating to olservations marle at Winter Qnarters have loeeu correeted for variation and are therefore true. 


\title{
II. NOTES ON THE METEOROLOGICAL INSTRUMENTS AND THEIR EXPOSURE.
}

$\mathrm{BY}$

LIEUTENANT C. ROYDS, R.N.

\section{WITH ILLUSTRATIONS OF SOLAR PHENOMENA AND CLOUD OBSERVATIONS.}

BY

\author{
DR. E. A. WILSON.
}

\section{Methon of Taking Orservatioss at Sia.}

ON the port side of the Upper Deck, under the bridge and in a small alley way, throngh which there was free circulation of air, was hung the meteorological screen, in which were placed the Dry- and Wet-Bull Thermometer and the Maximnm and Mininum Thermometers. The Barometer was hung in the loblyy leading down into the Ward Room.

Observations were taken by the Oflicer of the Watch every two hours, when possible, and always at the end of the watch, and were entered in the rough book supplied for the purpose. Water temperatures (surface) were also taken, the water being drawn by means of a canvas bucket from the bridge. The thermometer was immersed in it, left for a few moments, and then read.

Winds and direction of cloud-movement were taken by the Compass and are entered as magnetic. The latter observation at sea is difficult owing to the motion of the ship. The Maxinum and Minimum Thermometers are also affected by the same canse, and, when the motion is excessive, erroneous readings mist ocenr.

\section{Method of Taking Observations in Winter Quarters.}

Until the sea was frozen over, after our arrival in winter quarters, the same system of observing was nsed as at sea. $\Lambda$ s soon as the sea was frozen over, a meteorological screen was set np on the ice, abont 120 yards astern of the ship, so as to be well elcar from any effects which the ship might caluse, except in westerly winds, which did not occur (figs. 1 and 2).

Facing the E.N.E. was placed a screen enclosing the Thermograph and Hygrograph. The screen with the Dry- and Wet-Bulb and the Maximum and Minimum Thermometers was hung facing W.S.W., about 4 feet from the ground. There was also another screen in which another Thermograph and Hygrograph were placed.

$\Lambda$ wind vane was made on board the ship and placed about 12 feet from the ground, being set up true; all winds taken in winter quarters are logged as True Winds. The Rolinson Anemometer was set in about 15 feet from the ground; the instrument we called "I)ines" small Anemometer was set up with its head abont 10 feet aloove the level and with abont $\overline{5}$ feet of tubing to the recording part. Observations were taken, with hardly a break, every two hours, night and day by the Officers, assisted at times by the fonr Quarter-masters and by the assistant Cook, from April, 1902, to November, 1903. It is due to the eonscientions eare shown by each observer, and the nnselfish interest they all took in the work, that the reeord is as complete as it is.

The question of a lamp for taking the observations at night, and when the Sun is below the horizon during the Winter, is one for very carefnl consileration before the ship leaves the home port. For a time we nsed a small electrie lamp, but this nccessitaterl taking ont a heavy battery, so a candle lamp was made which worked well and seldom blew out, even in the strongest winds. 
The olservations were written down at the screens on a piece of paper, printerl for the purpose, seenred to a board, and on the arrival of the observer on hoard were eopied into the rough look.

The time of olservations was every two hours, local mean time, and every care was taken that the ulserver should leave the ship punetually. He was instructed to tako the barometer first, then to read

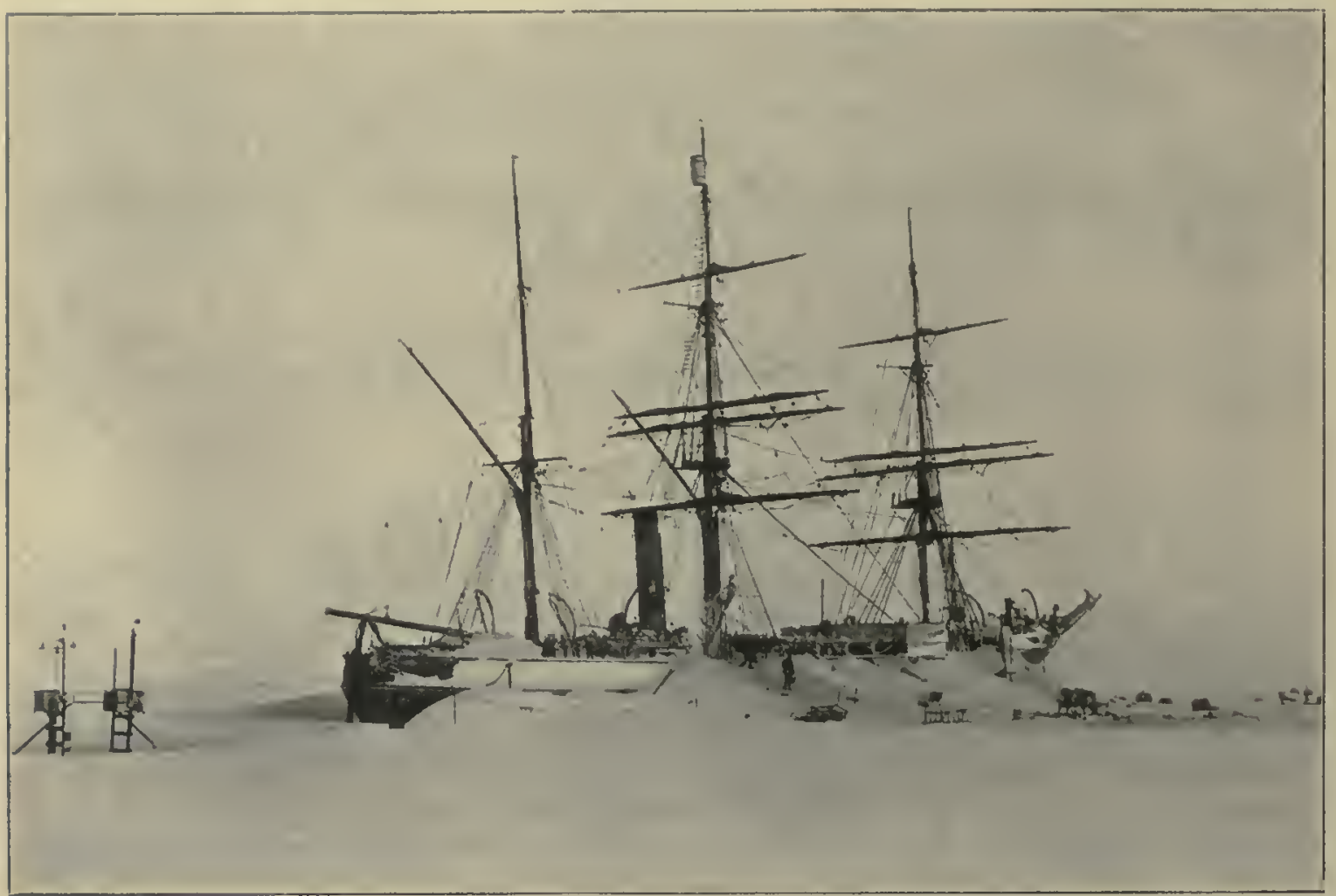

Fig. 1. "Discovery" in Winter Quarters, looking Sonth.

the dry- and wet-lull and minimnm thermometers, resetting the hatter; next to ascertain the direction and force of the wind by Beaufort scale, realing the Rohinson anemometer and the "small Dines," aurl, lastly, to olsserve the clouds and to make any general remarks that might be callerl for.

As the work of Meteorological Ohservations camnot depend wholly on one person, my thanks are dre to one and all for their very great assistance.

\section{Barometer.}

Thronghont the royage from England until we got into Winter Quarters in Latitude $77^{\circ} 50^{\prime} \mathrm{S}$. the barometers were hung in the entrance to the Ward-room loblby; the cistern loeing about 9 feet alove the sea-level.

Before our arrival at Cape Town, barometer No. 592 was broken and was replaced by No. 2244, kindly given $n$ by the Cape Town Observatory, and always nsed at sea. When we reached Winter Quarters the three barometers No. 915, No. 2244, No. 612 were hung in the Magnetic Honsc, which was sitnated on the upper deck, below the bridge and alove the mess-reek.

Two-hourly observations were taken with No. 915 and attached thermometer and at noon, and thronghont the first year at other times. Comparison olservations were male with the other two larometers, but owing to the attached thermometers on these instruments not registering below zero, many observations were taken withont the attached thermometer.

It will he noticed that there are large fluctuations of the attaehed thermometer on No. 915. This rariation was due to the door having been at times left open between the olservations, and when it was at 
length closed the heat from the mess-deck below warmed the Hagnetie House. Again, the snow-drift on the upper deek used often to block the door, ind prevent it from leing closed tightly.

The height of the cisterns of these barometers was about the same as when hung in the lobly, viz., 9 feet. The instruments were in an illeal place, except for the temperatures leing low at tinues. Fixcept the observer, no one nserl the Magnetic House, so that the barometers were free from any rongln or unnecessary handling.

On the jommey back to Fingland the barometer was still kept in the Nagnetie Iinom, but, owing to the observations for "Dip," the cistern heing made of iron, the instrument had to be moved each day during the times that the magnetic observations were being taken.

\section{Dry-isuld: 'L'Hermonkthe.}

This instrument, placed in the screen with the Wet-Bulh and with the Maximum and Ninimum Thermometers, was read every two hours. If the temperature at the time was below $-40^{\circ}$, the readings of the dry-bulb thermometer, which was a mercurial one, were not taken, but the present time readings of the spirit maximum as well as the minimum were read instead. Sometimes the nercury was not frozen until below $-40^{\circ}$.*

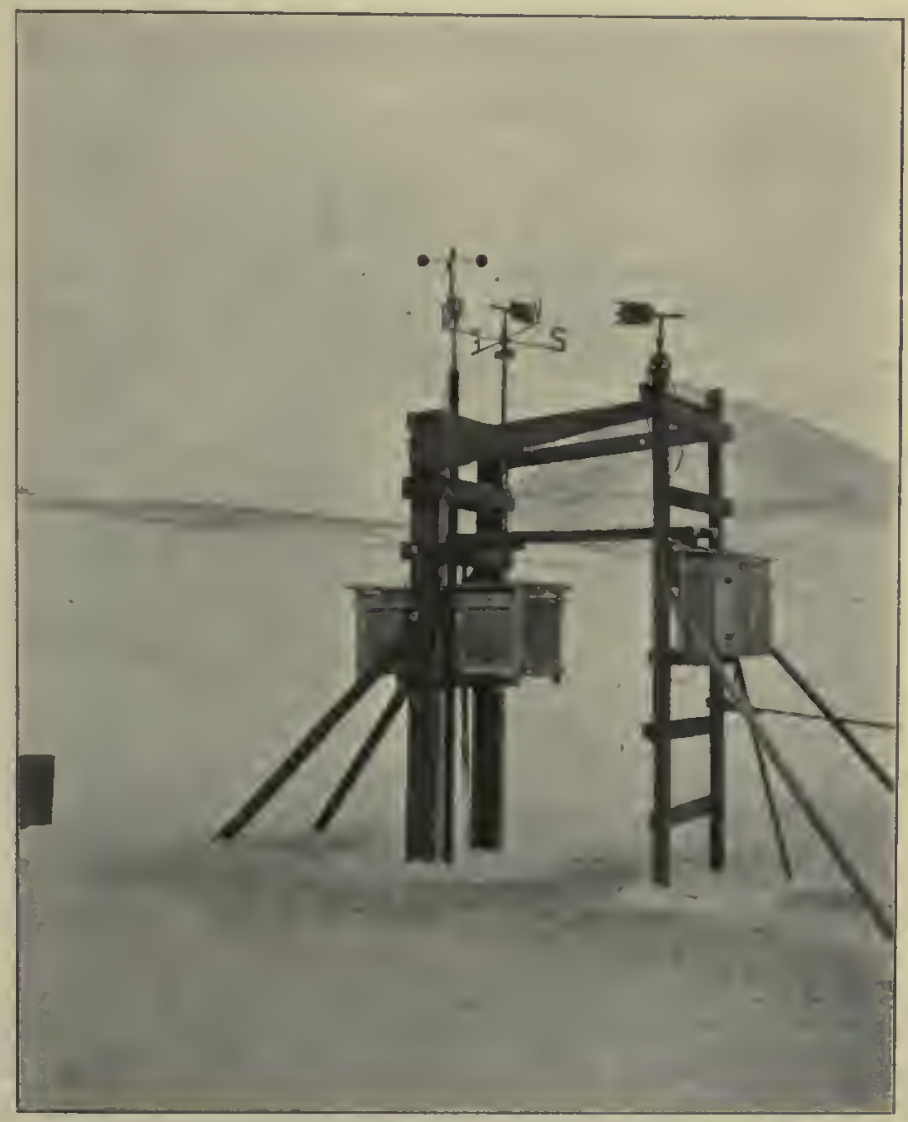

Fig. 2. Thermometer screens and anemometers.

It was noticed that the present reading of the spirit minimum often showed a lower reading than the mercurial, and at one time the two were recorded and entered in the register for the information of the authorities at home. The readings of the thermometer exposed outside and of the dry bull in the screen were seldom similar, and at one time I thonght the sereen seemed to retain the heat from the sunshine during the day and more especially on calm nights, when the sun was below the horizon. Radiation would, of course, affect the outside thermometer, and would account for the difference. 


\section{WHT-B"LB THEMOMETER.}

This instrunent, placed in the same screen with the dry-bull) and the maximum and minimum thermoneters, was read every two hours. Throughout the first year in winter quarters it dir not work at all satisfactorily, probably owing to the accumulation of too much ice on the lutlb. Throughout the second year, when I was careful to see that this accumulation did not take place, it worked much better.

It wis by neans uncommon for the reading of the wet-bulb thermometer to be higher than that of the dry hull. The fact of the nuslin being round the bull, with a coating of ice on it, however thin, seens to me to indicate that in any quick change of temperature, this instrunent shoulıl necessarily be more slnggish than where the bulb is free. On nore than one occasion, when the temperatures were very low, the mereurial dry bulb was observed to be frozen whilst the wet bulb was not.

\section{Maxinum Themometer.}

This instrument was placed in the screen with the dry- and wet-bulb thermometers and with the minimun thermometer, and was read and reset at 8 a.m. and 8 p.m. When the mercury was frozen, 10 maxinum temperature conld be read, unless the temperature hald been above the freezing-point of mercury during the time of observation. At sea this instrument is liable to show incorrect readings through the motion of the ship.

\section{Minimum Thermoneter.}

This instrument was placed in the screen with the dry-and wet-bulb and maximum thermometers, and was read every two hours and reset. When the temperature of the air was low enough to frecze the mereurial thermometers, the present time reading as well as the minimum was read and entered in the book. Also, as it was observed that there was a difference between the present time reading and the mercurial dry-bulb readings, a series of observations was taken for a short time. No defect in either thermometer was apparent.

These instruments gave considerable tronble on account of the column breaking, and a large number of them were broken in the vain attempts to restore them to working order.

\section{Radiation Thernometeis.}

\section{Black and Bright Bulbs.}

These were placed on a stand at Hut Point, 5 feet above snow-level, and about 30 feet above sei-level (fig. 3). They were read and reset at 8 p.m. daily. During our second year three thermoneters were in use, a large and small black bulb and a small bright bulb. During the winter these thermometers were not kept in position, but were placed as soon as possible ifter the sun returned.

\section{ReCORDing INSTRUMENTs.}

Thernographs.-Two of these instruments were placed in sercens on the Meteorologieal stand; one wais with large dimensions, and the other with small dimensions on the scale. The disadvantages that belong to this instrument are the same for all instruments of the same nature. The screens themselves are, of course, not drift-tight, and gradually get absolutely full of hard-packed drift snow, which surrounds the working part of the instrument and absolutely stops all working. Then, again, the drift gets inside and around the elock, and on and over the pen: consequently, after a blizzarl, it was necessary to clein the instruments and get them into working order again. Tine after time the record for the week bas been spoilt by the snow gotting on the pen, and either causing a big thick line or else drying up the ink and preventing it from marking altogether.

Hygrograph.-The same remarks apply to this instrunent, of which we had two in nse during the greater part of tho time. Another disadvantage of the Hygrograph is that snow, getting on the hairs, effectually prevents any accuracy. In all these recording instruments the clock was found to give at great deal of 
tronlile in the very eoll temperatures, and every neatus was tried to orercome this defect, lint with no satisfact ory result.

During the time we were at seat, the recording instruments were kept at Greenwich nean time, lut on the start of observations from the sereen on the ice, Ship apparent time was used, and the records were shifted on Mondays at $10 \mathrm{a} . \mathrm{m}$. The pain caused to the observers of these instruments is really great at times, for as it was nearly impossible to shift the papers with mits on, they had to be removed, and handling the metal meant frost-hitten fingers.

Biarographs.-Two and sometimes three of these were kept going on board, down in the living space. They always seemed to work well, and there was no reason why they should not. If found to be very different from the present time reading of the Mercurial Barometer, they were set to that reading.

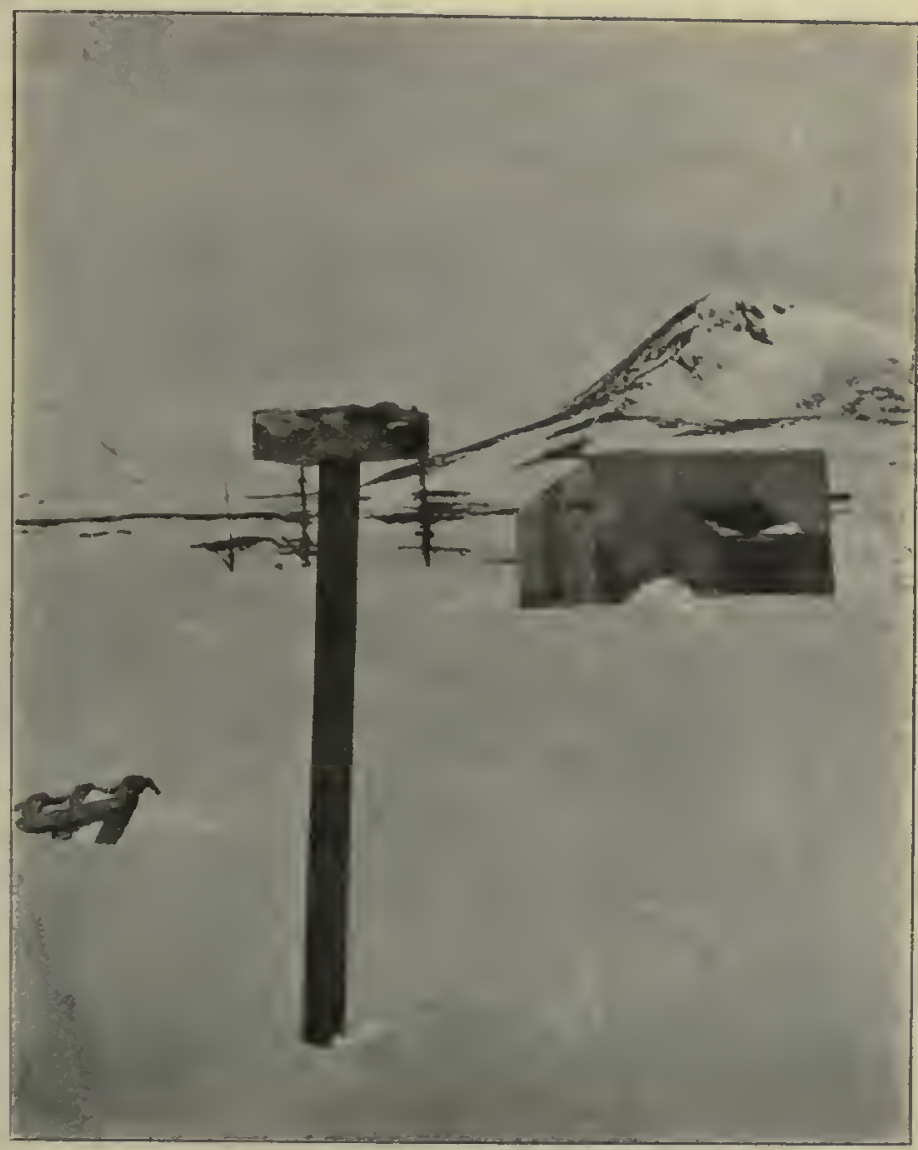

Fig. 3. Solar radiation thermometers.

Assulax's Aspirator:-For a considerable time during our second year this instrument was in use, and worked most satisfactorily. It was kept ont at the sereen, and was wound up on arrival of the observer at the screen. He then proceeded to take the various other observations and read the aspirator last. 'The observations, therefore, eannot be quite comparable with those of the dry-and wet-luulb thermometers, as the changes of temperature were very rapid, instances of its falling having been observed whilst actually reading the dry bulb.

I think very highly of this instrument, although it is a most difficult one to nse, its graduations being so small, and there being so much metal connected with it. Moreover, the winding of it up with mits on is by no means an easy task. Then, again, the wetting of the wet bulb means the unscrewing of the protecting part, which, in our experience, necessitated removing one's mits. Taking it all in all, the disadvantages of the instrument are great, but these, I think, could be overcome with a little trouble before leaving home, and the readings which it affords represent, in my opinion, much more the true air-tenperature that those taken liy the thermometer in the sereen. 
It was unfortunate that during a blizzard our instrument was broken. It should always be taken in before a heavy wind, because the dome for the fan will always get bally choked with drift snow. I do not know whether the speed which the fan reaches would in any way affect the readings. If so, this is another matter for consideration.

\section{Cape armitagf Thernometer.}

This was a minimum thermometer, placed on a stand about 4 feet 6 inches above the snow-level and well clear of all land (see map, p. 16, for position). It was read always once a day, weather permitting, and very often twice and three times, as any Officer going out wonld make this an excuse for a walk, and would read and reset the thermometer, taking the time lie did so. The differences between the readings of this thermometer and those of the minimum in the screen for the same interval were very marked.

During the Winter months the thermometer was open to the sky, but during the Summer a shade was placed to protect it from the sun's rays (fig. 4). Dr. KoetTliTz was the originator of this series of observations, and it is mainly due to him that the series remained as unbroken as it is. During the second year Licutenant MULOCK assisted Dr. KokTTLITZ in taking the observations, and generally recorded two observations in the day.

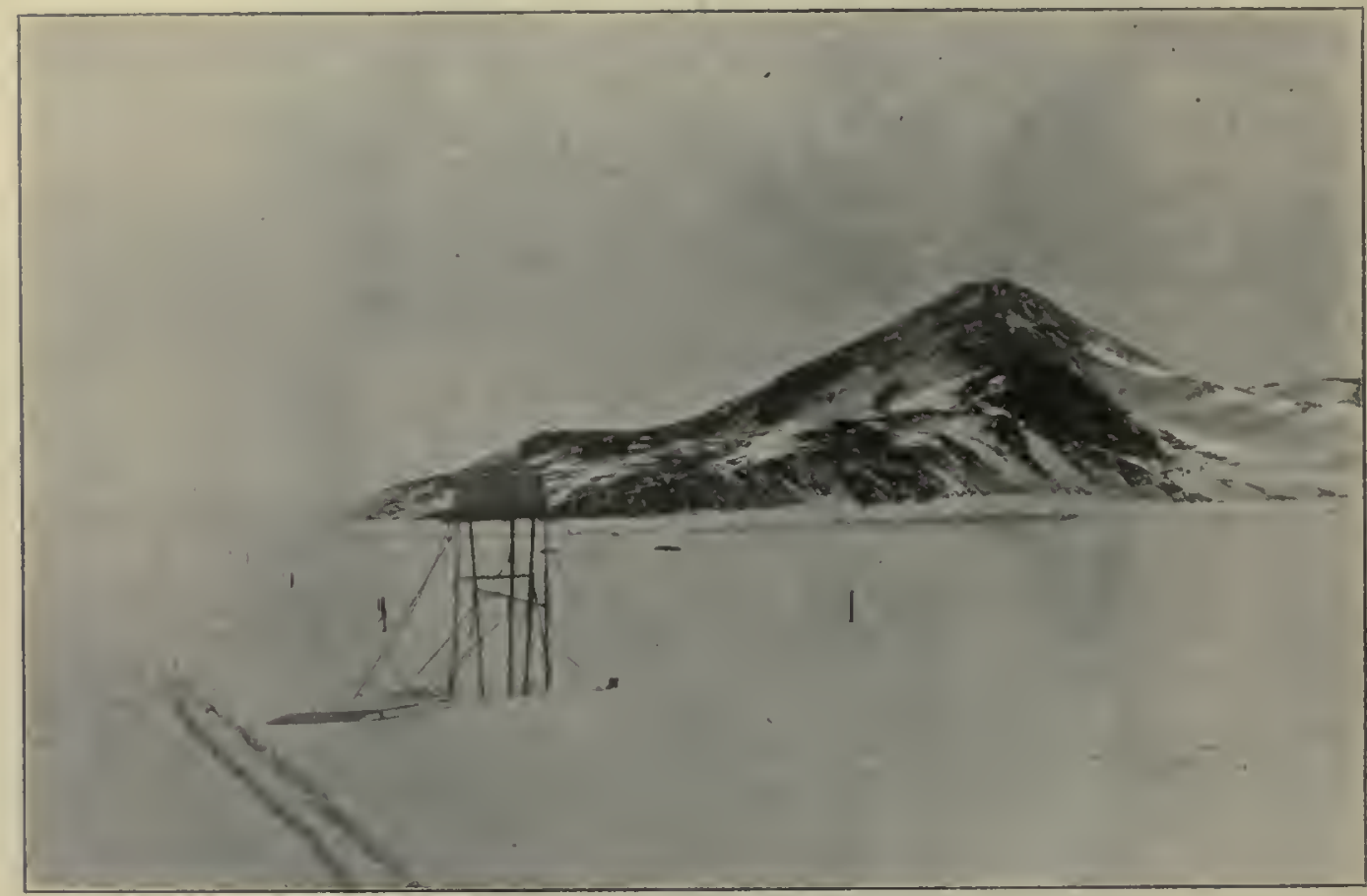

Fig. 4. Thermometers at Cape Armitage, looking North (showing the sereen placed over the thermometer during the Summer).

\section{DiNes SMall ANemoneter.}

This instrument was set up on the screen, the head being about 10 feet above the level, and the connecting pipes about 5 feet long. During the heavy snow drifts which always accompanied a southerly gale the open head of this instrument was invariably choked, and necessitated clearing every two hours, before observations were taken. The highest and lowest readings were recorded during the time the observer was looking at the instrument. Snow was found not only to collect in the head, but also in the pipes, and the only way to clear them was to take them on hoard and thaw them ont. A leak was observed at the lower glass joint, and the Engineer soldered all round it and so more or less stopped the leak. Pure alcohol was used, coloured by staining matter, and the evaporation of the alcohol appeared to be rapid. The observations taken with the instrument seemed to agree more or less with those of the Robinson, and more especially the estimates on the Beaufort scale. 


\section{- Robinson's ANemometer.}

The instrument was fixed to the end of two long pipes and was secured to the sereen at abont 15 feet above the level (fig. 2). Observations were taken of the miles run every two hours. This instrument worked well, but the credit of its performance is due to Engineer-Lientenant SkFı.Tox, who always repaired the instrunent after any heavy blizzard. No spare parts having been provided, the observations had to cease while the repairs were being effected. Snow was observed to collect in tlie cups, and this necessitated their being cleared at every observation when snow was drifting or falling. Also the glass face is by no means drift-tight, and unless made so, by gunming paper all round the edges, the working parts inside would very soon be completely covered.

\section{Jarge Dines Recording AnFMometer.}

The head of this instrument was secured on the mizen crosstrees, about 34 feet above the cistern, and the pipes were led down the T'g't. back-stay, and through the top of the signal locker to the cistern in the locker. During the strong drifts which invariably accompanied the southerly blizzards this head lecame choked, and was cleared, to the best of the observer's ahility, by his going aloft and knocking out the snow collected in the heal. Owing to its position the whole could not be taken down, and so the pipes were never touchel. It was found that the snow remaining in and about the hend would be melted in Summer by the heat of the sun, and then freezing again would stop the head from revolving. On some of the anemograms this can be seen to have been the case by the line working low down and, on the head being freed, shooting up to a higher level.

This instrument, to my mind, always worked too low, and thus under-rated the strength of the wind. When it was first set up, the liquid supplied was used, but when the colder weather came on, the piston stuck fast. On opening it up, I found ice on the top of the liquid, then the spirit, and below that a thick substance like condensed milk, which I presumed was the glycerine, which had separated. I then took all this out and filled it with spirit containing only a small amount of glycerine. In the very low temperatures and calms I always found that the piston was inclined to work in thick liquid and had to stir the whole lot up. Then, again, whilst shifting the anemogram, one's breath used to collect on the piston rod and prevent it working ensily throngh the collar. The clock gave a great deal of tronble, as it would not work in the very cold weather, and the ink also was very unsatisfactory; it was often found frozen and not marking.

'Taking it on the whole, this instrument has many disadvantages in the antaretic climate. Conld one only keep the recorling part down below in the warmth, it would be very much simplified, bnt if this were clone the cold wonld be conducted down the pipes and collect all over the cistem and piston rod, and efiectually stop all working. This was the ease with the unused stove, the whole of the stove in the Mess being covered with a thick coating of rime. Even if the pipes led up throngh the working store fummel, the head itself wonll become blockerl, as around the tops of the fumnels ice and snow collected to some extent. I see no way to get orer the fact that the orifice in the head always gets blocked.

For a recording wind instrument I wonld snggest a Robinson, attached to the mast heal, and lecl down to the deck, and even down to the living space, care being taken that the connecting rods are within easy reach. Such an instrument has, I own, many disadvantages, of which the height is the greatest. Should the observing party be living in hut, this objection could be easily orereome, and records of direction as well as force could be obtained.

\section{Evaporation Experiments.}

Alont the middle of our first Winter, the question of Evaporation being discussed among the Officers, it was decided to try an experinent snggested loy Captain ScoTT. Two open shallow dishes, 3 inehes by 4 inches, filled witl water which was allowed to freeze, were placed on the top of the meteorological screen. These were weighed before being taken out, and 24 hours later were weighed again, the clifference in 
grammes being the aunount lost in the interval, which conld only be lyy evaporation. Sometimes the dishes weigherl a trifle more than the day hefore, and this 1 put down to the fact of a fall of very minute ice erystals adhering to the ice surface. Any loose drift or suow was brusherl off before the dishes were weighed.

It will be noticed that the differences are seldom actually the same for each dish, and this is rather singular, secing that they were placerl side ly side, and presumally what affected one dish should also hare aftected the other. In summer it was fonnd impossible to continue these olservations, for the ice in the dishes was then melted, and it was most difficult to weigh them without spilling the water.

\section{liais or Snow Gatge.}

This instrument was placed on the snow at a little «listance from the Meteorological Screen (fig. 2). During the ordinary snow-fall it worked well, and the annount of snow was recorded at the actinal time it was taken. During the sontherly gales, which invariably brought with them a heavy snow-fall as well as dense drift, this instrument was useless, as any other instrument of a similar kind would have been under those circumstances. Very often after one of these gales it becane exceedingly difficult to find the instrument at all, since it might be buried under three and four feet of suow, and it was only because we knew its exact position and dng down to it that we did not lose it more than once. For some time the snow-fall was so small that it was measured in cubic centimetres and entered in the fair register in that scale. The bay in which we were situated formed a regilar cone for the full strength of the sontherly gales, and the drift which accmunlated all ronud and alont the ship, or any other obstacle, was enormons.

\section{Suxsune Recorder.}

This instrument was placed on a box on the summit of Hut Point abont 40 feet above the sea-level. The cards were shifted at Noon. Only since the return of the experlition have I ascertained that the cards were placed in the instrument inaccurately, which is the reason of the mark of the burn not going straight and at times jumping np or down on the card.

In the early Spring some few hours of sumlight could not be recorded, becanse the rays were cut of from 11 s log hills in the vicinity-Observation Hill, Crater Hill, Arrival Heights. This can be seen from the map of our Winter Quarters (p. 16).

The manipnlation of small screws, \&ec, connected with the sunshine recorder, and the very exposed position of the instrmment, made it ly no means a pleasing duty to shift the cards, and often inrolved frost-bitten fingers. The cards were not kept shipped during the first Winter, lont rluring the second Winter a carl was in the whole time ancl took the first recorl.

\section{Ozone Oeservations.}

For a loug time during our crnise no attempt was made to olserve ozone; it was such a very douluful observation. Towards the end of our seconcl Winter, however, I decided to try and see what effect would be produced on the papers supplied. One of these was placed in a small tea-strainer and hung in one of the meteorological screens, and when taken out was carried on board in a glass tulhe and placerl in water. The depth of tint obtained was then compared with the scale supplied.

To test whether the fact of doing this in the ward room affected the result, one of the papers was lumng in the mess all night, aurl on being placed in the water next morning, it was found to remain clean white. Also at times a paper was taken direct from the tin box in which they were kept, ind was placed in water, but it always showed clean white, very different from the tint produced ly exposure in the open air. The papers seemed most affected during heary southerly gales, and were quite hrown before being put into water. The length of time the paper was exposed was always noted, ind also the colour of the paper before being immersed in water. 


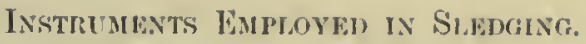

The instruments generally taken on a sledge jouncy were a minimum thermoneter and an imeruid. Some parties carried a hypsometer, and the realing was taken once daily at the same time as the aneroirl was nsed. I would suggest, for future guidance, that some indication should be given of what is wanted to be done at these secondary stations, which must necessarily be moving stations, and that instruments shonld be supplied for sledging purposes ouly; an aspirator wonld, I think, be a good and nsefnl instrument, aud also a minimum thermometer.

I strougly recommend the hypsometer, provided the olsservations are useful. The work of boiling anr getting a reading is small, and can easily be done whilst the olsserver is waiting for the pot to boil at the meal hour's. The question of weight has to le considered, as it is a most inportant factor in the work; and the sum total of instruments forms alrealy a large proportion of the equipment in sledge jonrueys.

The work of olserving is ensily performed, as the whole set of observations does not take more than a few minutes. 'T'wo-hourly readings can ensily be olstained.

\section{NOTES TO ILLUSTRATIONS OF SOLAR AND CLOUD PHENOMENA,}

\author{
ISY
}

\section{EDWARD A, WILSON, M.B.}

Plate I. Solar phenomenon observed on the Great Ice Barrier, November 29, 1902.-4 P.si., appt. time. S. lat. $80^{\circ} 30^{\prime}$, F. long. $165^{\circ}$. Wind S.S.W., force 1, driving showers of ice crystals. Blue sky with cirrostratus rapidly crossing to the N.E. A white fog-lank lay on the horizon persisting up to $30^{\circ}$ of altitude. A complete ring or band of white light ran horizontally through the Sum (altitude $27^{\circ}$ ). In this ring, dividing its circunference into three equal parts, appear the Sun and two Inminons white mock-suns. Round the Sun was a Solar halo of prismatic colonr with small ares of intense prismatic colour outside it, on a level with the Sun. The blue of the sky within the halo was considerably darker than the blue ontside. Immediately below the Sun-on the horizon-was a luminous white mock-sun. In a vertical line above the Sun-close to the zenith, and forming part of a small circle with zenith for eentre-was an exceedingly brilliant are of prismatic colour, set in the deep blite of the sky. The following measurements were taken by Captain SCOTT :-Altitnde of Moek-suns, Sun, and white band of light $27^{\circ}$ above the horizon. Bearing of "White Nock-suns" $120^{\circ}$ from one another. Diameter of Solar halo 54 . Rarlius of upper prismatic are (centre $=$ zenith), $7^{\circ}$. Fr.-cir.-str. rapidly eleared away as phenonenon appeared, leaving clear blue sky overheal down to within $30^{\circ}$ of horizon, where white fog-bank or low stratns persisted.

Plate II. Solar phenomenon olsserved on the Great Ice Barrier, December 16, 1902.-1 A.M. S. lat. $80^{\circ} 45^{\prime}$, E. long. $162^{\circ}$. Driving showers of ice crystals. Sontherly breeze, force 1-2. Low driving stratus elouds in patches, breaking occasionally to expose clear blue sky and fracto-cirro-stratus. The white mock-suus seen on November 29th were oceasioually visible, but were not connected by the horizontal band of white light. There was much nore drifting clond this day than on November $29 t$ l, and much less blue sky visible. The difference in depth of blue colour between the sky inclosed by the Solar halo and that outside it was not noticeable, owing to the fact that a white nistincss olssenred the blue of the sky almost all over the Sun's quarter (S). Z, zenith; a, broarl prismatic are; b, narrower ditto.

Plate III. Solar phenomenon observed on the Great Ice Barrier, December 29, 1902.-2 Pי.x. S. lat. $82^{\circ} 17^{\prime}$. The most brilliant and the liroadest bands of prismatic colour were those at $c$ and at the four points on the Sun's completed halo. The halo itself was neitler broad nor brilliant. The hortu-shaped band was at times very bright and moved round the halo with a change in the position of observer's eyes. The white mock-suns on the north, sonth-east, and south-westerly horizon were large and loright and dome-shaped. The zenith-centred sualler white circle and the detached white ares in the sonth inpeared only for a few moments and were never very pronounced. The sky was lolue and all the drift 
seemed low-ice erystals-passing in low showers rapidly overhead with sonth-westerly wind. Sun visible all the time; low fog-banks and drift all romd horizon. Wind sonth-westerly. Temperature, $+15^{\circ} \mathrm{F}$.

Plate IV. Febrnary 9, 1903.-5 P.M. Me.Iurdo Sound. Drawn from a sketch by Lieutenant C. R. Rorvs, R.N. The prismatic halo round Sun is ineomplete in this ease, as is also the zenith-eentred halo.

Plate V. Solar phenomenon observed on February 12, 1903, in MeNurdo Sound. Drawn from a sketch by Lientenant C. R. Rorps, R.N. No moek-sun was visible. Lower part of outer ring hidden by land. A very faint white beam erossed the inner and outer ring.

Plite VI. A fog bow frequently seen on the Great Ice Barrier when one stood, back to the Sun, in the morning honrs, shortly after midnight. It was always white, and brightest at the lower ends of the limhs. A good example was observed on December 17, 1902, and another well-ntarked instance oceurred on November 15. 1902.

Plate VIl. A remarkable shadow which appeared eonstantly in the sonthern sky when the Sun was helow, or low clown on, the northem horizon. It gave rise to many conjectures as to its canse, the most probable being that it was the shadow of Mount Erebus and Mount Terror on a thin alto-stratus. Varions notes as to its appearanee will be fonnd in the Remarks of the Register, and also in the notes on clouds. As the Sun mored apparently to the west, this shadow moved to the east, and was always visible in the forenoons in clear weather, while the Sun was suffieiently low on the horizon.

Plate VIII. A not nneommon type of cirrus clond, or fracto-eirro-stratus, which often accompanied very magnifieent eolonr effects in spring and antumn when the Sun was low on the horizon for hours at a time.

Plate IX. An unnsual appearanee at the summit of Mount Erebus, namely the differentiation of several columns of steam issuing from distinet rents. This was possible only when there was a flat calm at 13,000 feet above sea level. The colleetion and eondensation of the steam at a still higher level to form a heavy eloud was less unusual.

Plate X. represents the condensed vapour rising from the crater of Monnt Frebus and acting as a wind vane at a height of 13,000 feet abore sea level.

Plato XI. shows another phase in which the presence of a flat calm is demonstrated between about 13,000 feet and 14,000 feet, while above this level the pennant of "smoke" clearly shows the presence and clireetion of an npper enrrent.

Plate XII., fig. 1, from a photograph taken at Sea, Mareh 2, 1904, looking to the north over an extent of open ice-free water. The island, known as Buckle Island, is one of the Balleny gronp. Its summit is hidden by the heary stratus cloud which lies low over the water and forms a typical open-water sky, with very little reflected light in it.

Fig. 2, from a photograph taken in Wood Bay, February 21, 190t, looking westward to Mount Melbourne. The cloul here represented, a fracto-eirro-stratus, is very much higher than that shown in fig. 1, much higher even than the summit of Monnt Melhourne, which is 8337 feet above sea level.

Plate XIII., fig. 1, from a photograph taken in Robertson Bay, Jamuary 9, 1902, looking in a southerly direction over a stretch of open water, above which hangs a typieal low cumnlo-stratus clond. In the distance are the ice-eovered inland mountains of South Victoria Land, over which the sky is free from clond.

Fig. 2, from a photograph taken looking south-west from Cape Adare, Felornary 24, 190t. In the distance is a elear sky over the inland iec-corered monntains of Sonth Victoria Land; the open water of Robertson Bay, on the other hand, is overhung by a heavy stratus, whose edge coincides with the eoast line which lies beneath it.

Plate XIV., fig. 1, from a photograph taken looking north, at Cape Adare, February 24, 1904. In the far distanee is seen the well-defined border of a low and extensive stratus overlanging the open sea.

Fig. 2, from a photograph taken looking westward to Mount Melbonrue. Small patches of clond which lave formed at the summit of the higher peaks, from 8000 to 12,000 feet above sea level, are seen to be floating off into an otherwise cloudless sky. Mount Melbourne is not known to have any activity as a voleano, and the cloud patches must therefore be distinguished from such as are represented in Plate IX. as forming round a nucleus of steam emitted from the active crater of Mlount Erebus. 


\section{REPORT}

BY

DR. J. A. HARKER

ON OBSERVATIONS MADE AT THE NATIONAL PHYSICAL LABORATORY

UPON TIE

TEMPERATURE OF SOLIDIFICATION OF THE MERCURY IN THERMOMETER NO. M.O. 30.

The thermometer was eompared with a standard under the most favourable eonditions obtainable with the present apparatus, in a vaeuum vessel in aeetone, eooled by addling solid $\mathrm{CO}_{2}$.

The stirring was found to be absolutely essentual when readings to any eonsiderable accuraey were required.

As our own mereury standard Tonnelot only reads to $-40^{\circ} \mathrm{C}$., and eomparisons were wanted to the aetnal freezing-point of the mercury in the thermometer to be tested, the standard chosen was a toluene Tomnelot thermometer.

For this instrument it was not eonvenient to have eomplete immersion, so an emergent stem correetion, amounting to $\cdot 2^{\circ} \mathrm{F}$, has been applied when necessary.

The thermometer MI.O. 30 was tested down to the point at whieh its mereury solidified.

This point was determined six times. The lowest temperature reaehed before solidification was $-45 \cdot 5^{\circ} \mathrm{F}$, on its own seale, and the highest about $-44^{\circ} \mathrm{F}$. It was elanped independently of the stirrer, so as to be affected as little as possible by its vibration. During the whole time solid $\mathrm{CO}_{2}$ was addled in small quantities to prevent rise of temperature during the rendings.

The eorrections, as determined under these eonditions, were as follows, the tolnene readiugs being reduced to the hydrogen seale :-

Correction.

$\begin{array}{rrr}\text { At }-38^{\circ} \mathrm{F} . & 0.0 & \text { Freezing-point } 32^{\circ} \cdot 3 \mathrm{~F} . \\ -40^{\circ} \mathrm{F} . & -0.1 & \\ -41^{\circ} \mathrm{F} . & -0.3 & \\ -45^{\circ} \mathrm{F} . & -0.4 & \end{array}$

The readings eould not be made by teleseope, and the divisions are only about 1.3 millims. to $1^{\circ} \mathrm{F}$., and the thermometer is divided into whole degrees.

No pereeptible differenee appeared in the indication at the freezing-point of mereury $\left(-38^{\circ} \mathrm{F}\right.$.) of the thermometer before and after its mercury had been frozen.

After the freezing of the mereury the thermoneter was taken out of the liquid as quiekly as possible, and, on the last oeeasion, the thawing of the mercury in the bulb took place probably before the eapillary neck had been quite freed from solid, and the bull) was broken, thus rendering impossible the observations eontemplated at the higher ranges.

The freezing-point of mereury as dedueed from--

$$
\begin{array}{ll}
\text { Mereury Tonnelot } 11562=-37 \cdot 98^{\circ} \mathrm{F} . \\
\text { Toluene } 71042=-37 \cdot 96^{\circ} \mathrm{F} .
\end{array}
$$




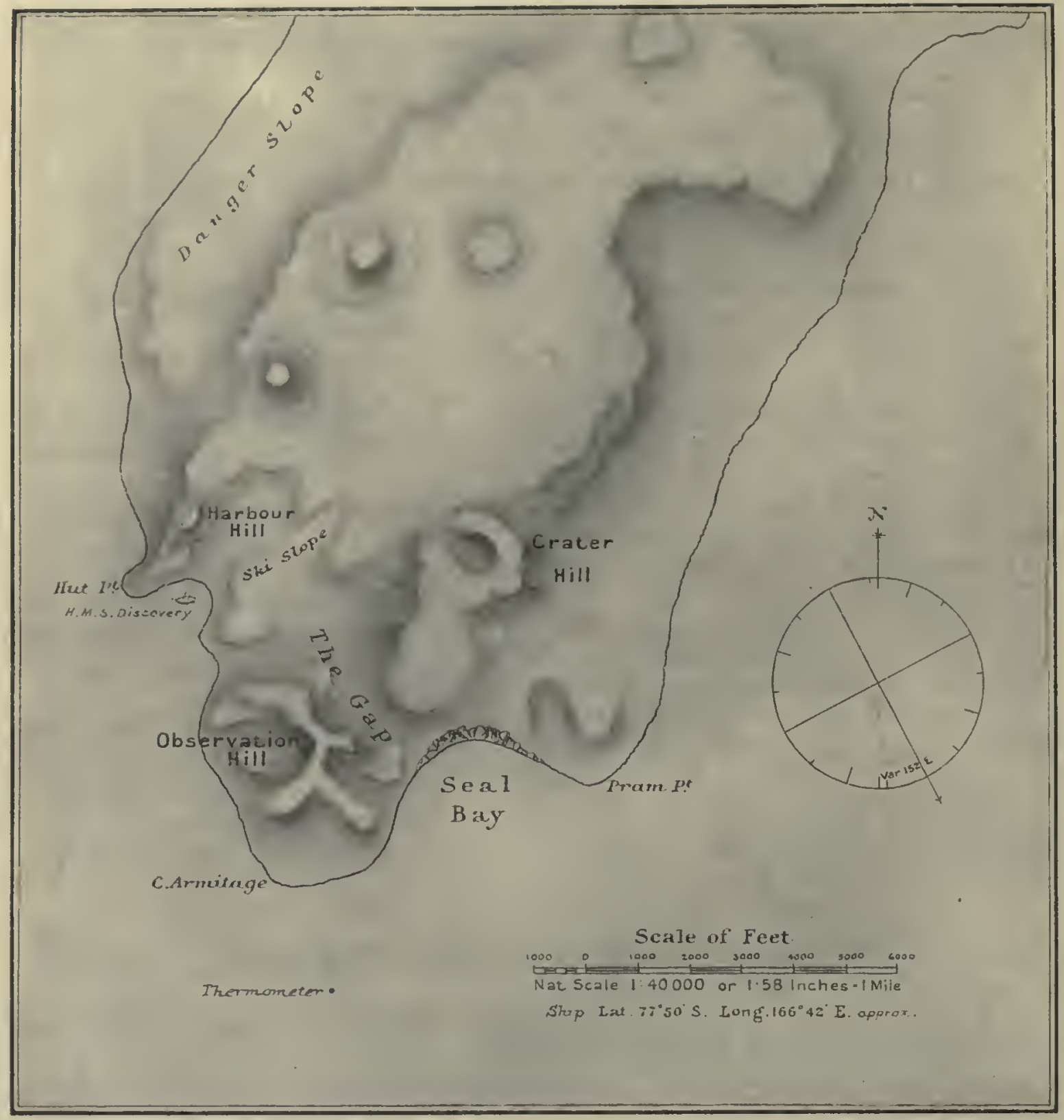

Map of soutll-western extremity of Ross Island, showing Winter Quarters of H.M.S. "Discovery."

Position of ship at Winter Quarters as given on Lieutenant MuLock's final chart :Latitude $77^{\circ} 50^{\prime} 50^{\prime \prime} \mathrm{S}$.

Longitude $166^{\circ} 44^{\prime} 45^{\prime \prime} \mathrm{E}$. 


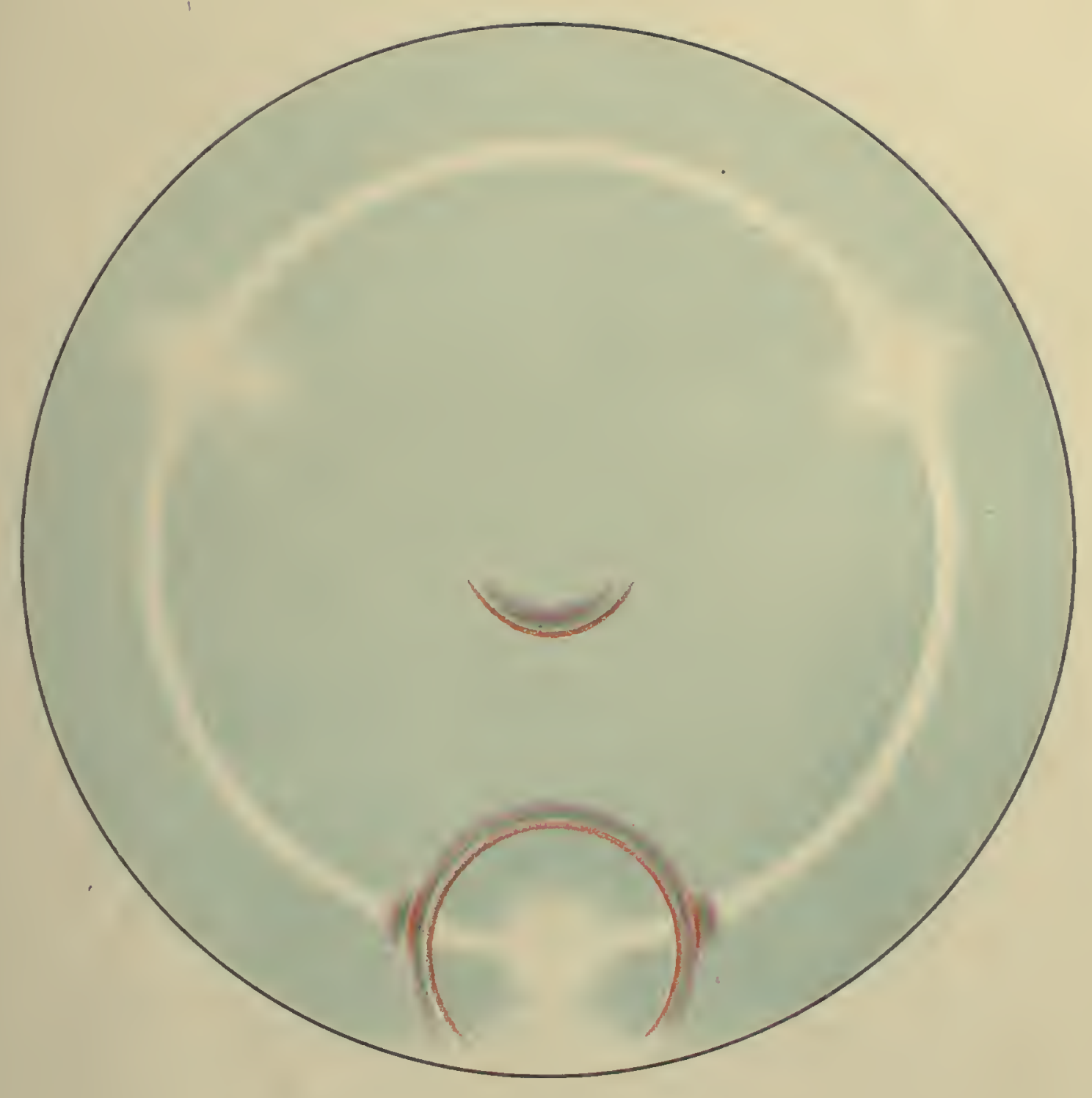

Seen November 29 th 1902. 



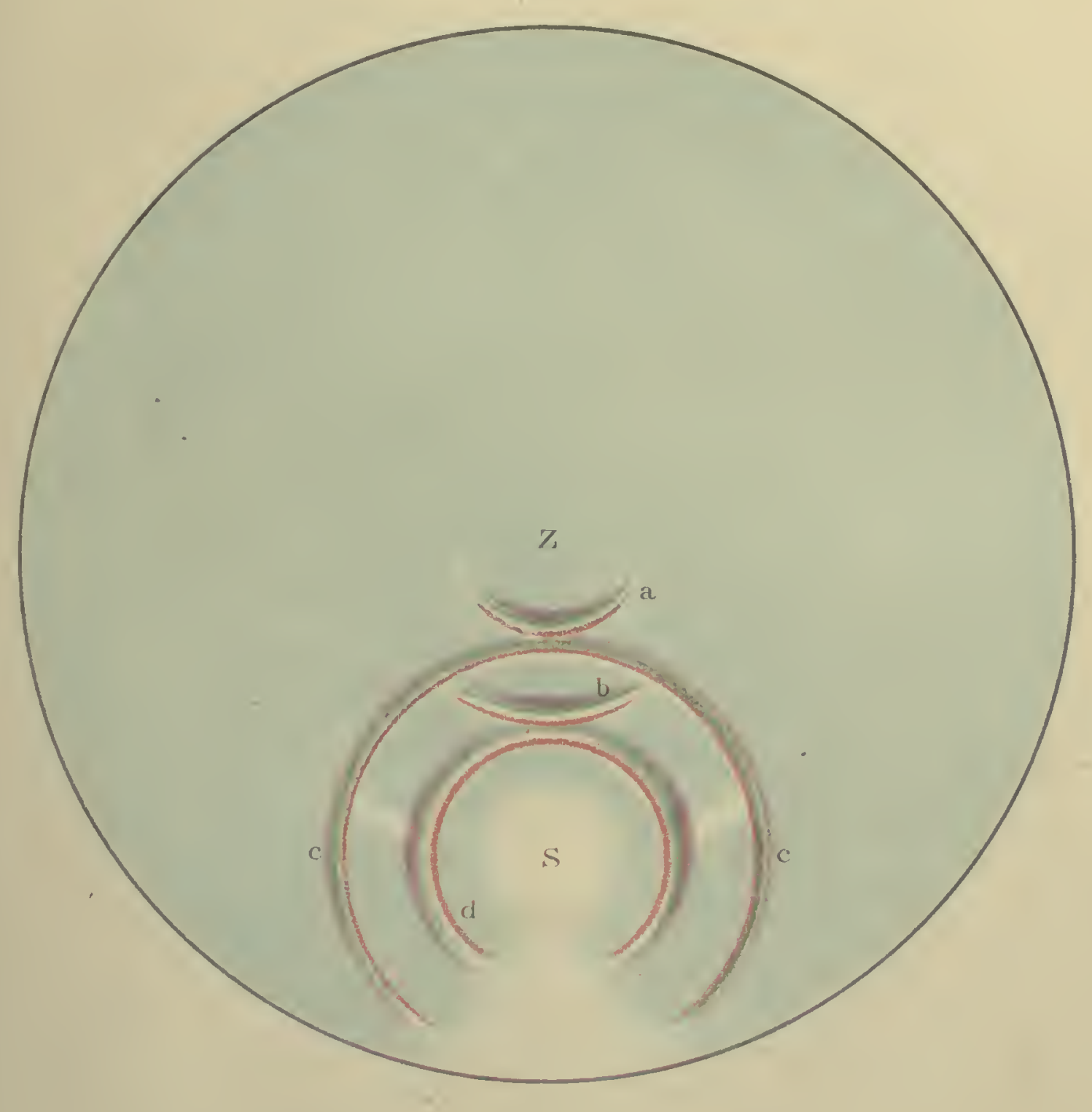

Seen December 16 th 1902. 



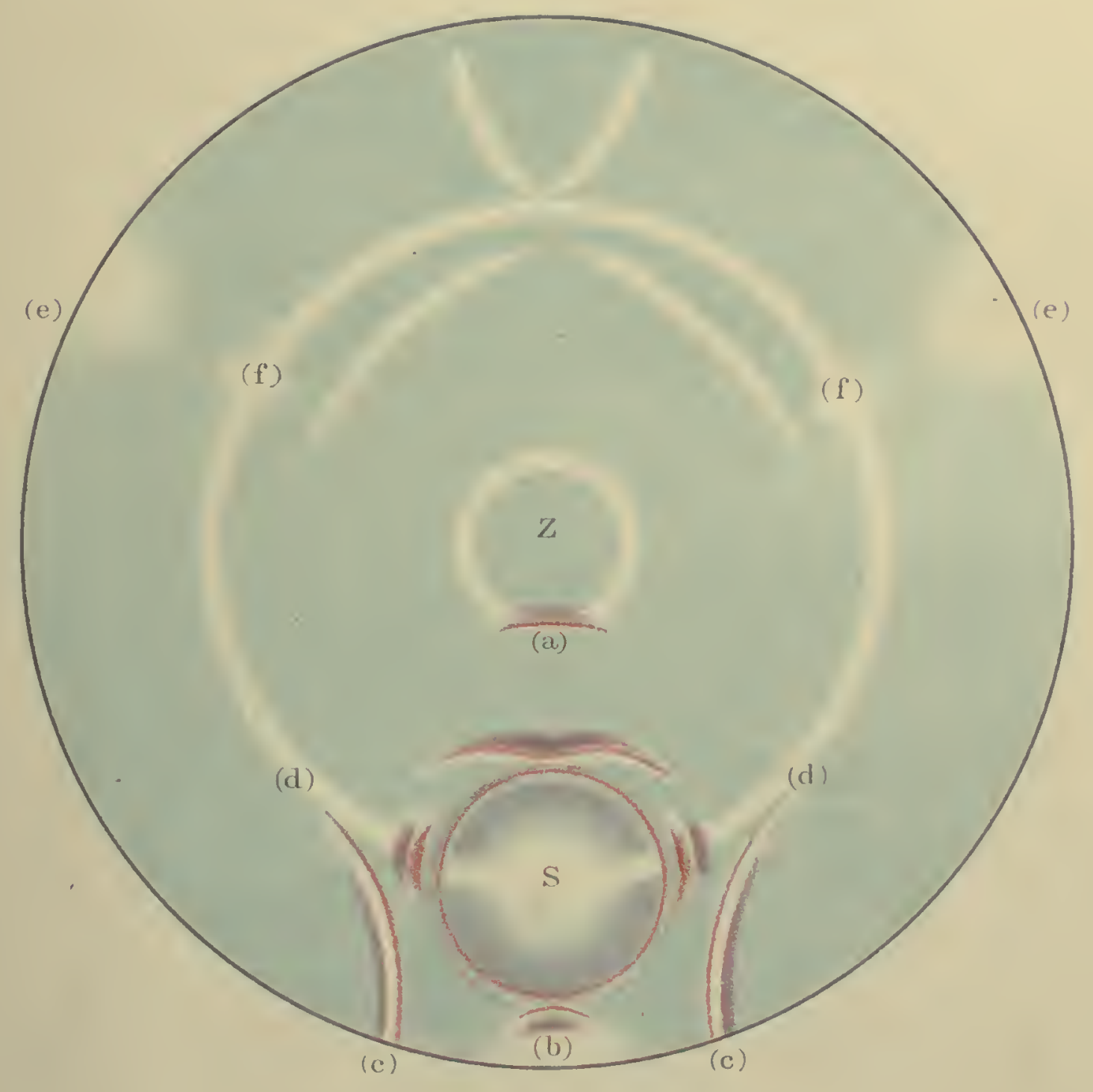

Seen December 29tr 1902. 



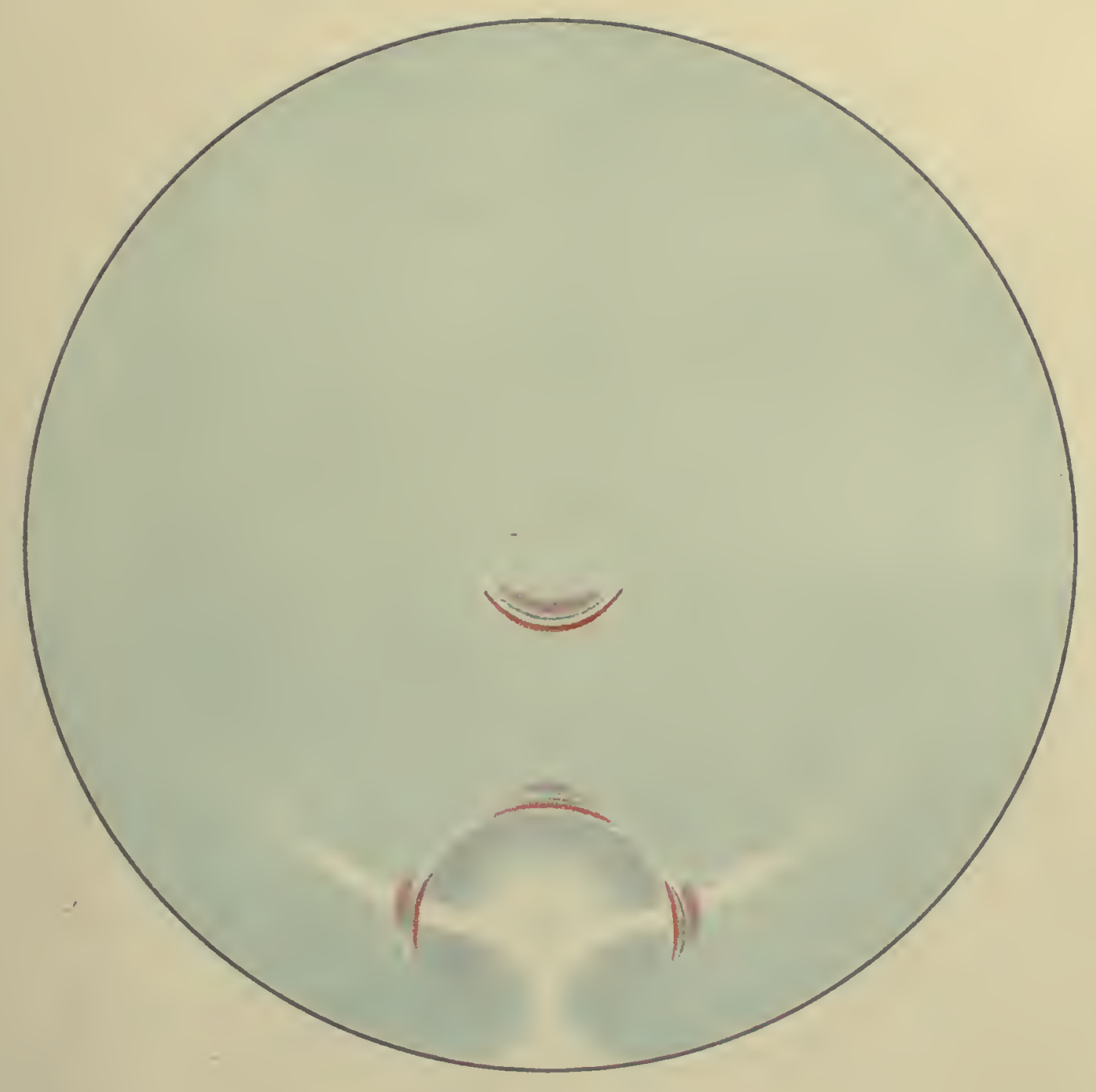

Seen February 9tr 1903. 


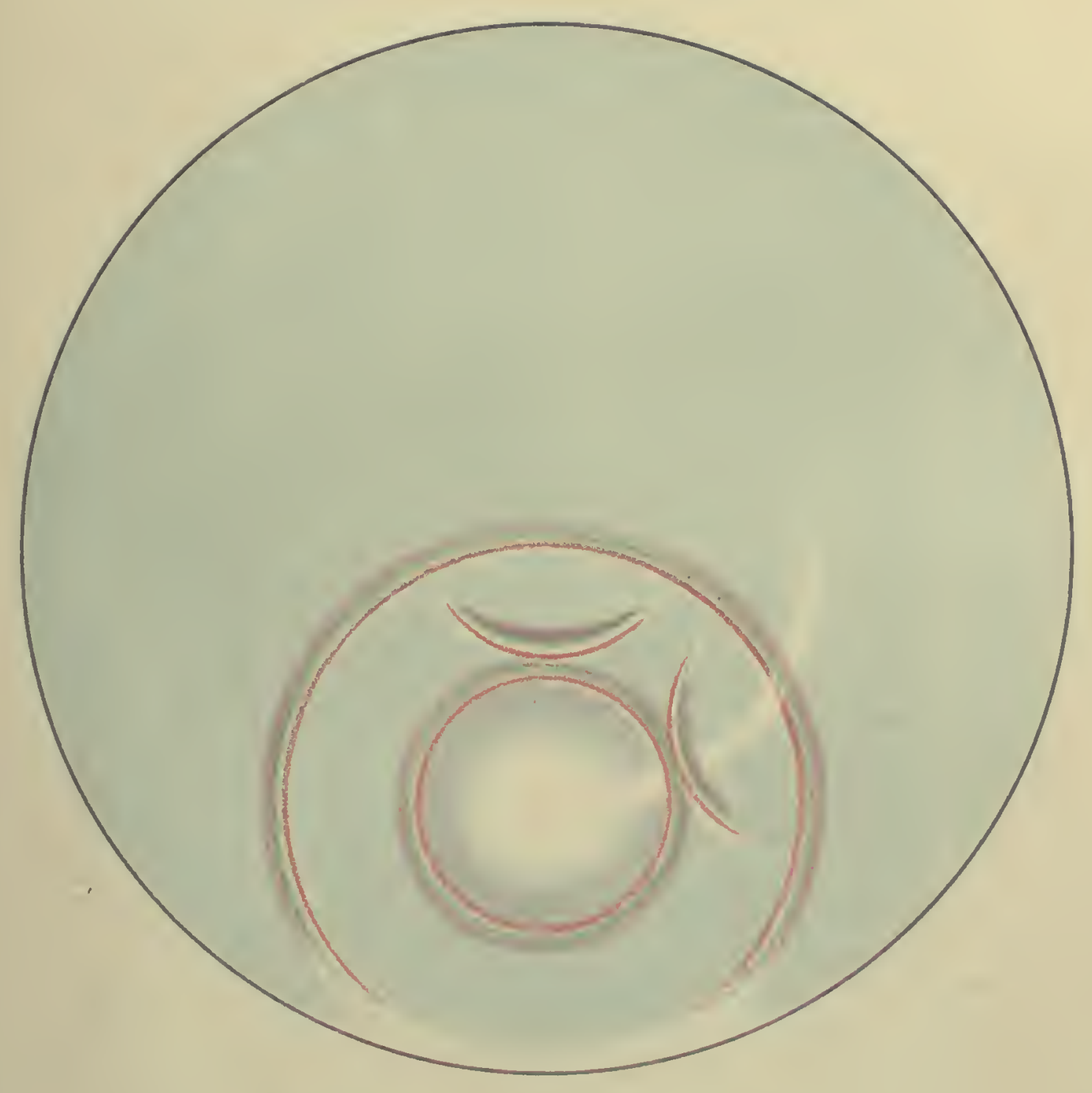

Seen Feoruary 12 1903. 


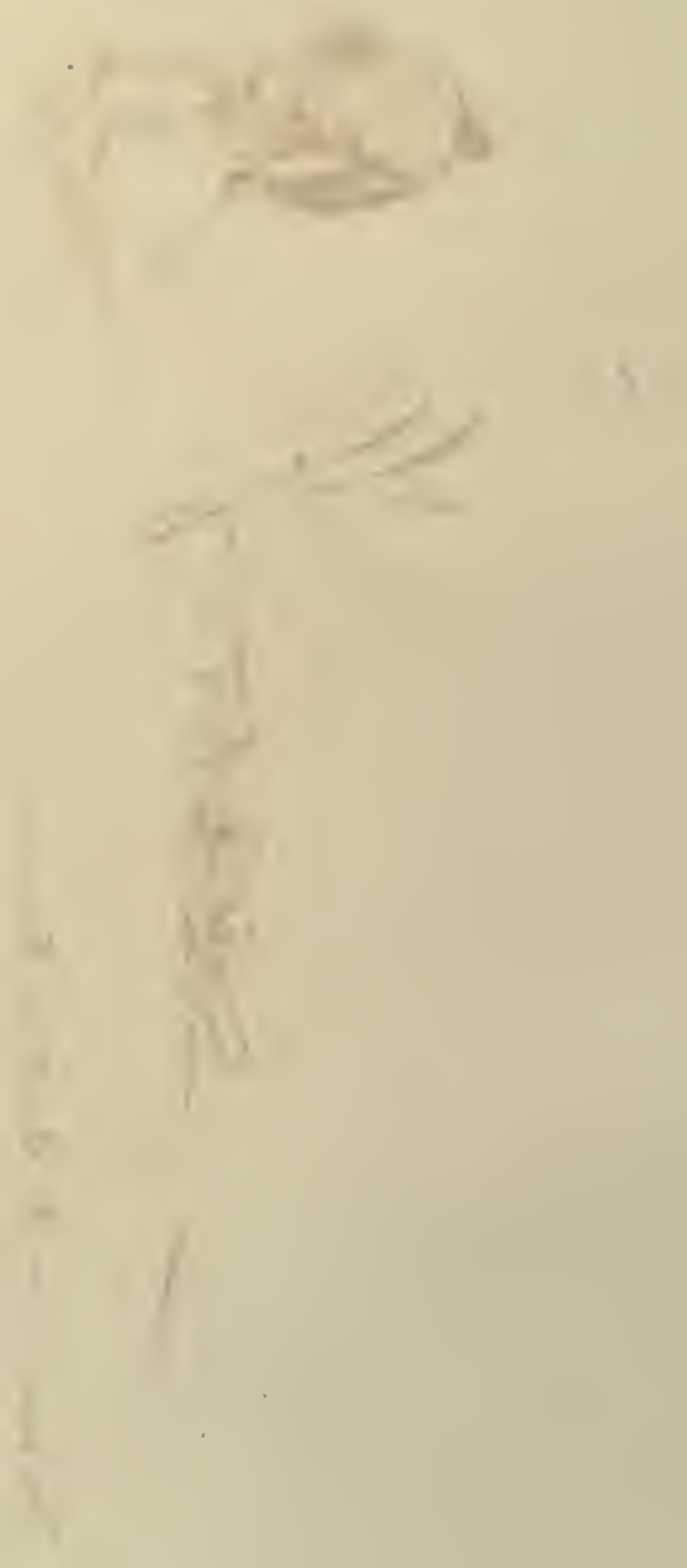




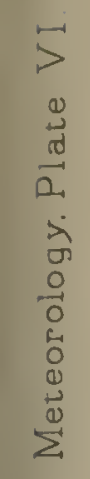

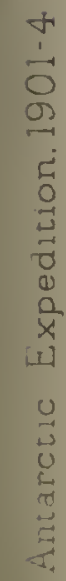
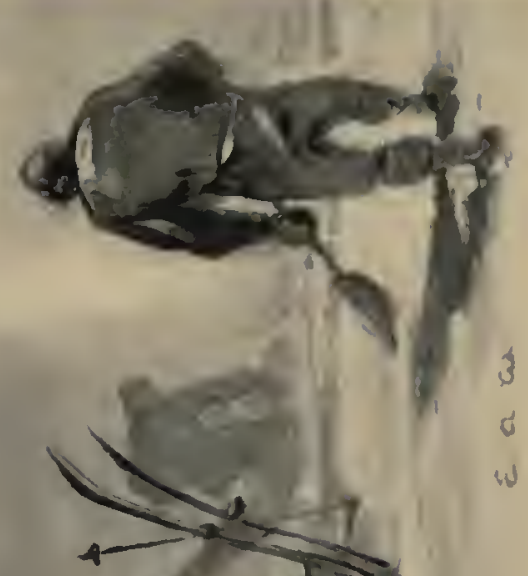

(1) 


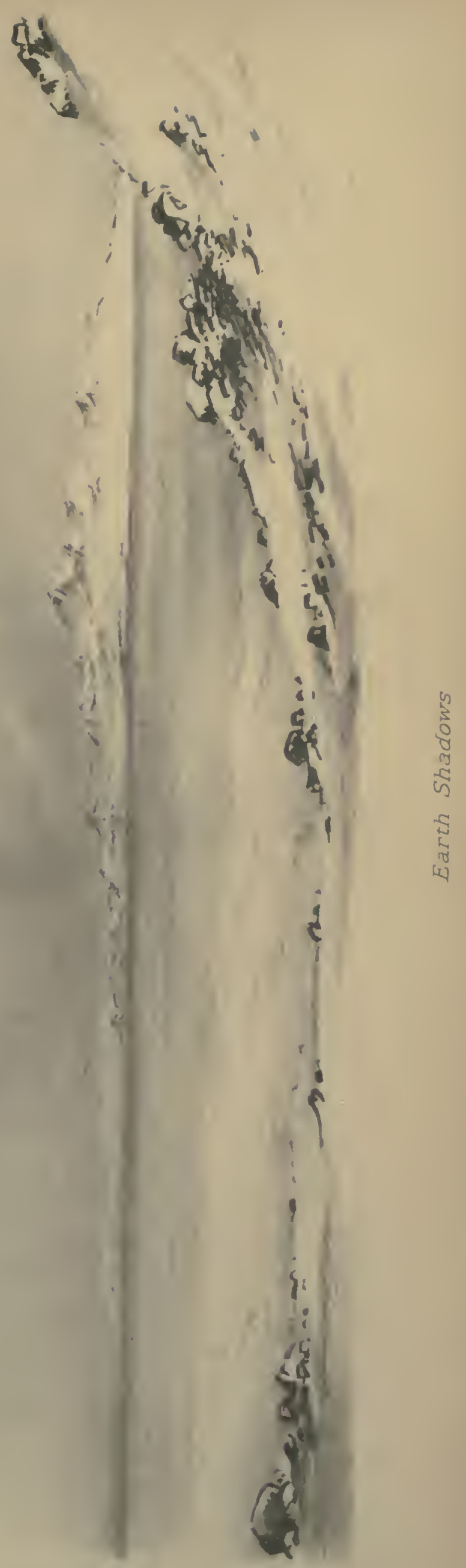




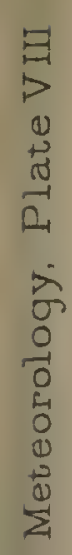





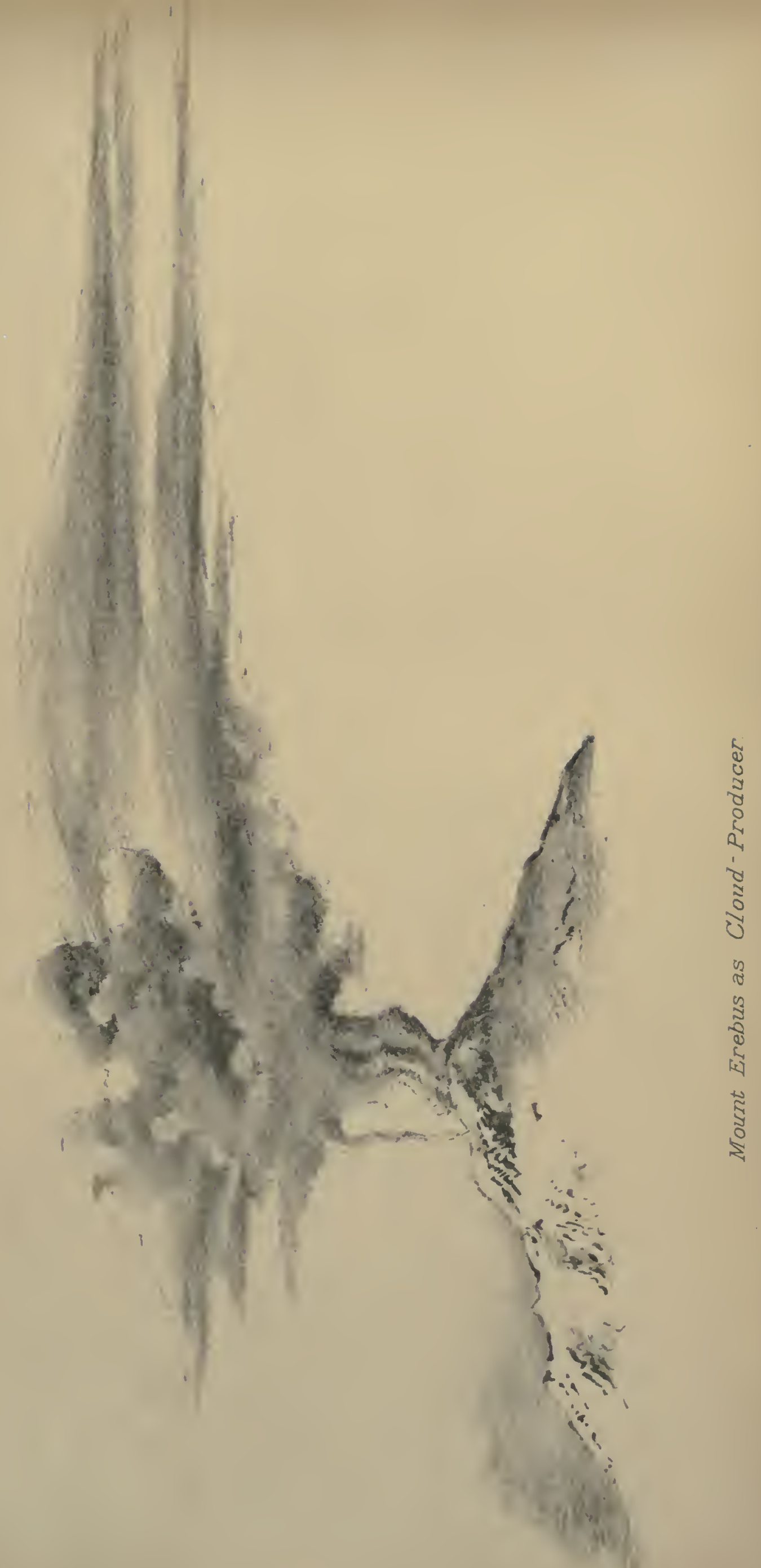




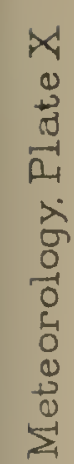

$\stackrel{2}{\infty}$

年

s

a

है

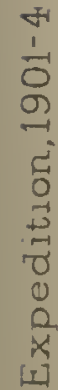

il a

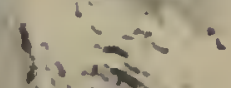

$1,2 x$

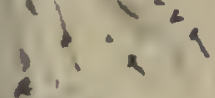

$\rightarrow$ igec

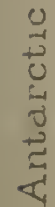

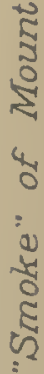




\section{.}

5 


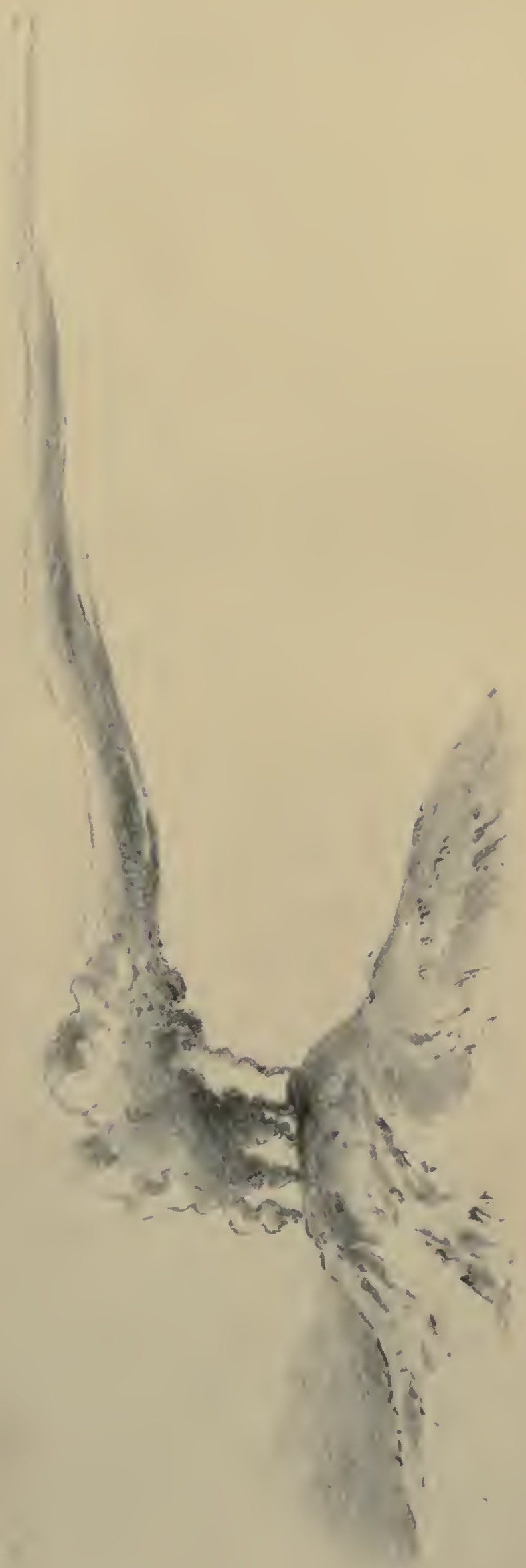

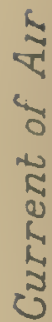

है

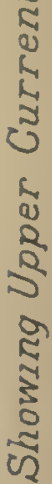

ปี

$1+1$

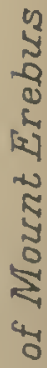

"๘) 
Antarctic Choun Forms.

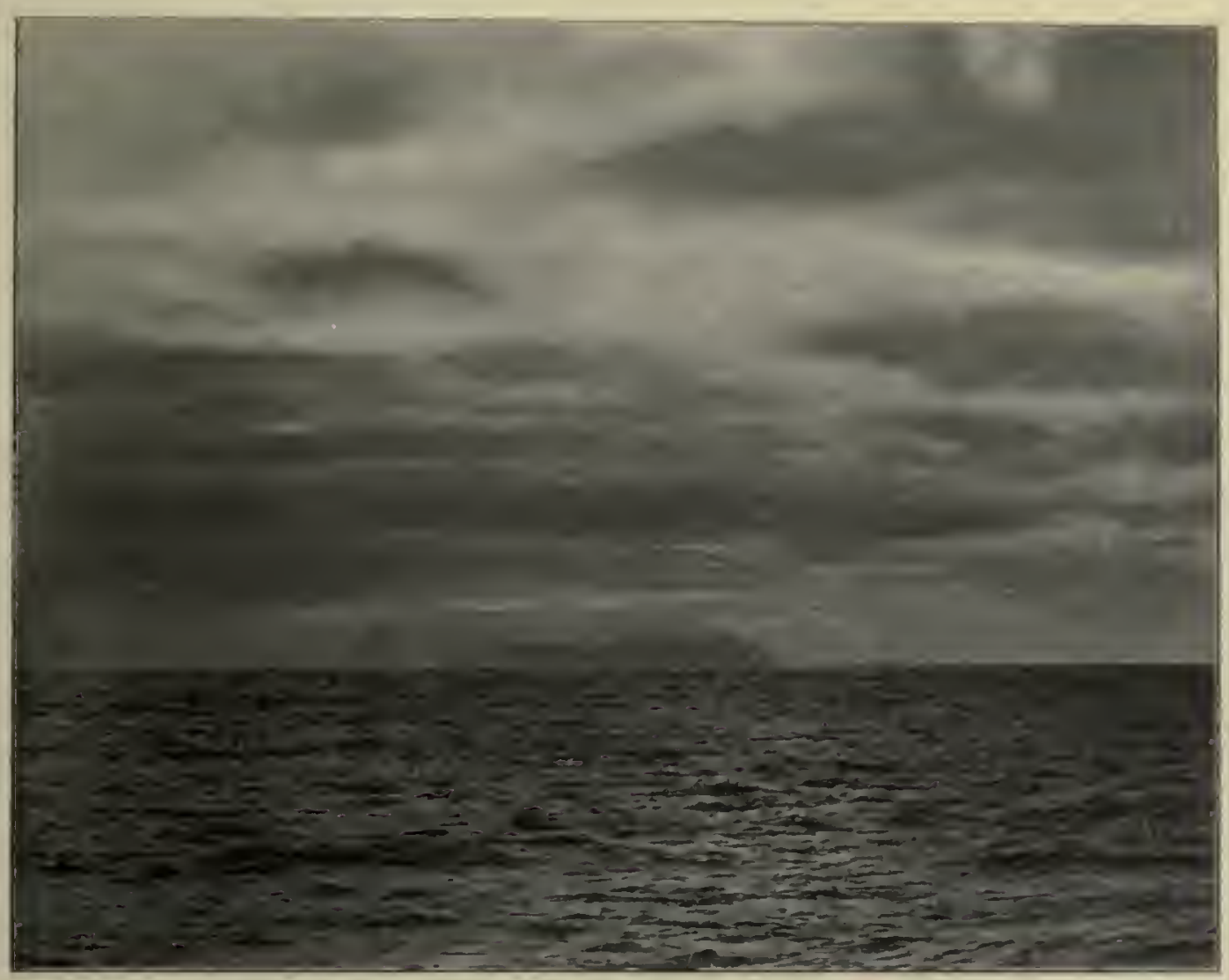

Fig. 1. Buckle Island, one of the Balleny Group ; from the South.

Showing a form of stratus cloud.

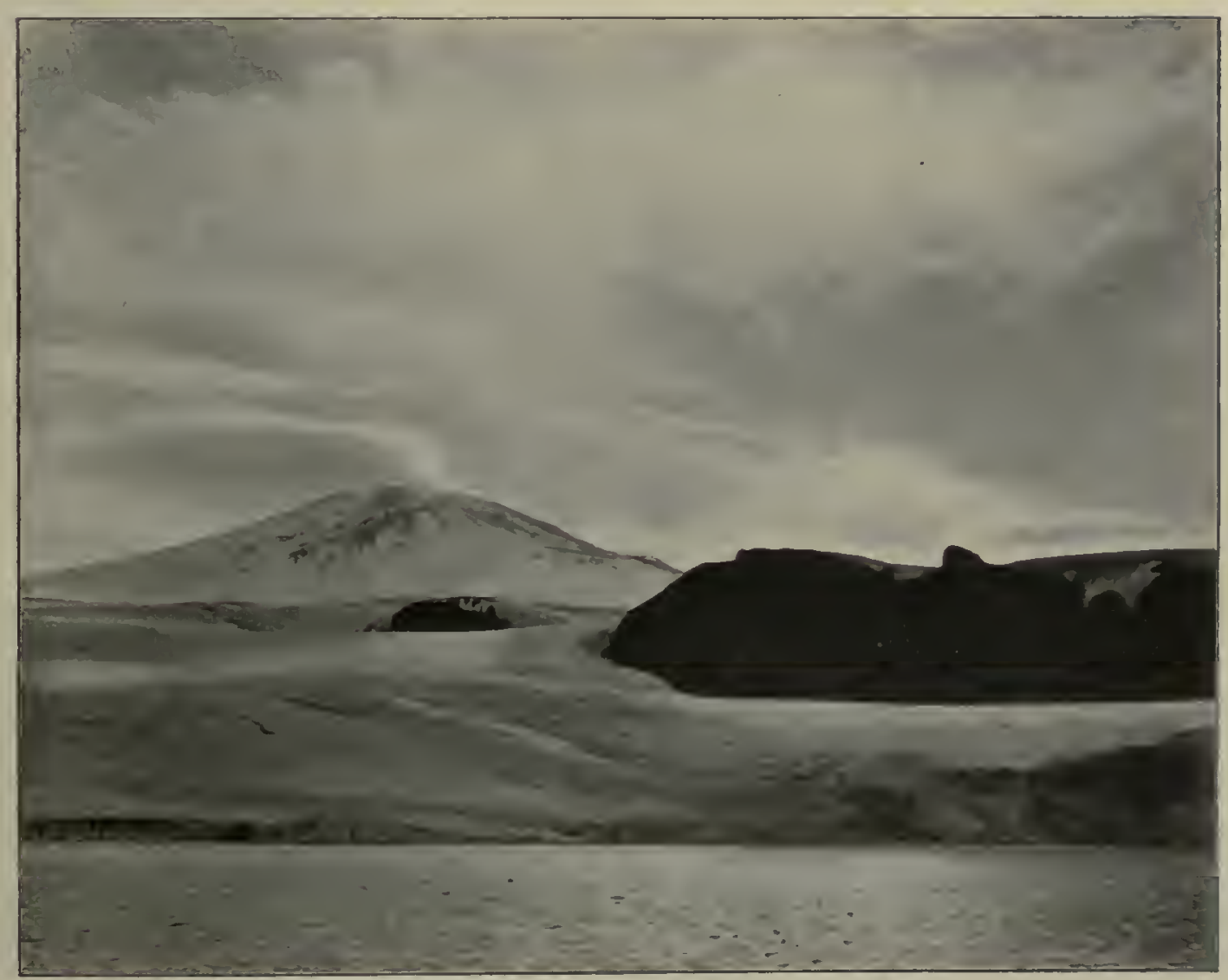

Fig. 2. Mount Melbourne from Wood liay; looking West.

Showiug cirro-stratus clouds. 

Axtarctic Cioun Foras.

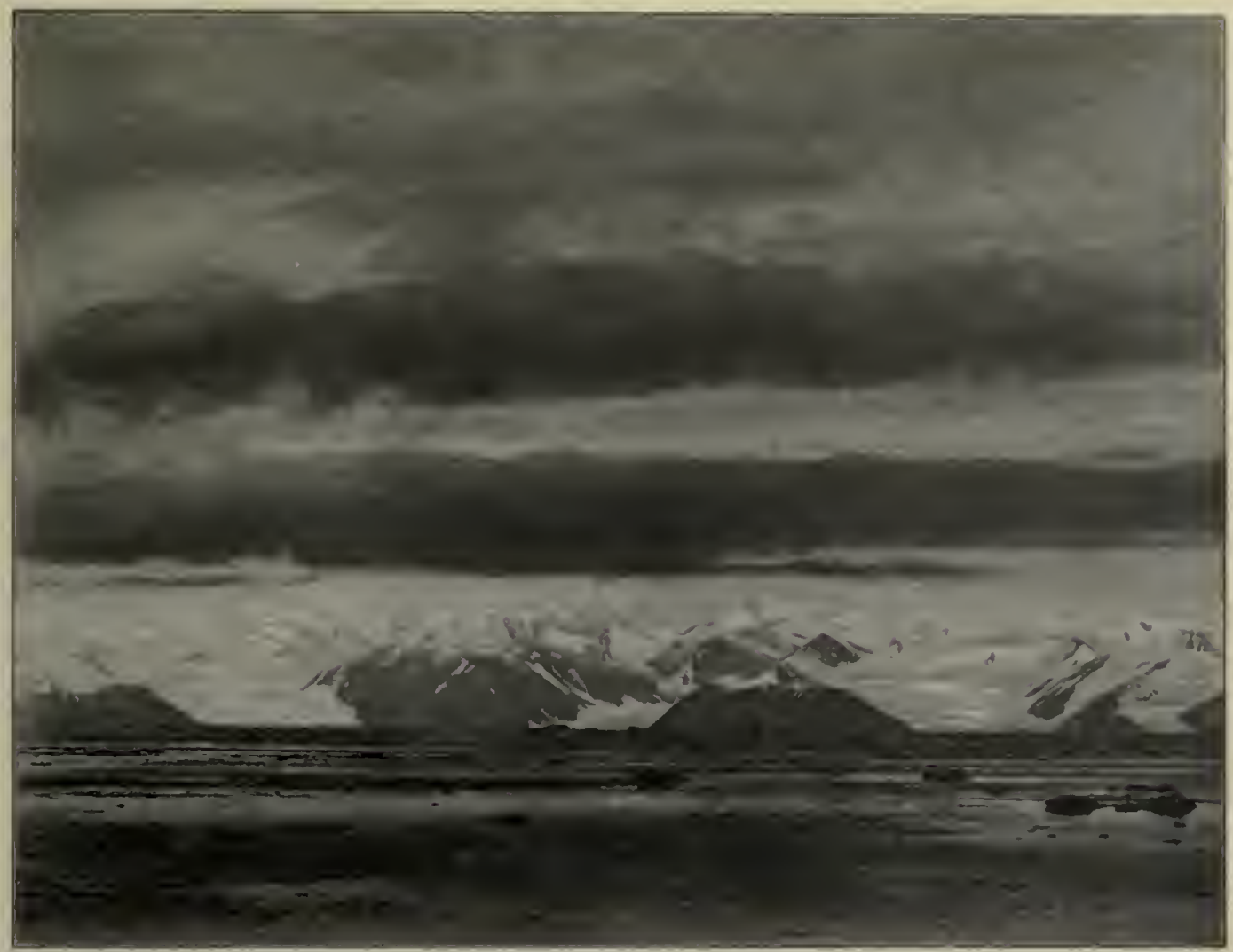

Fig. 1. Looking South to Mount Sabine from Cape Adare.

Showing typical cumulo-stratus clouds.

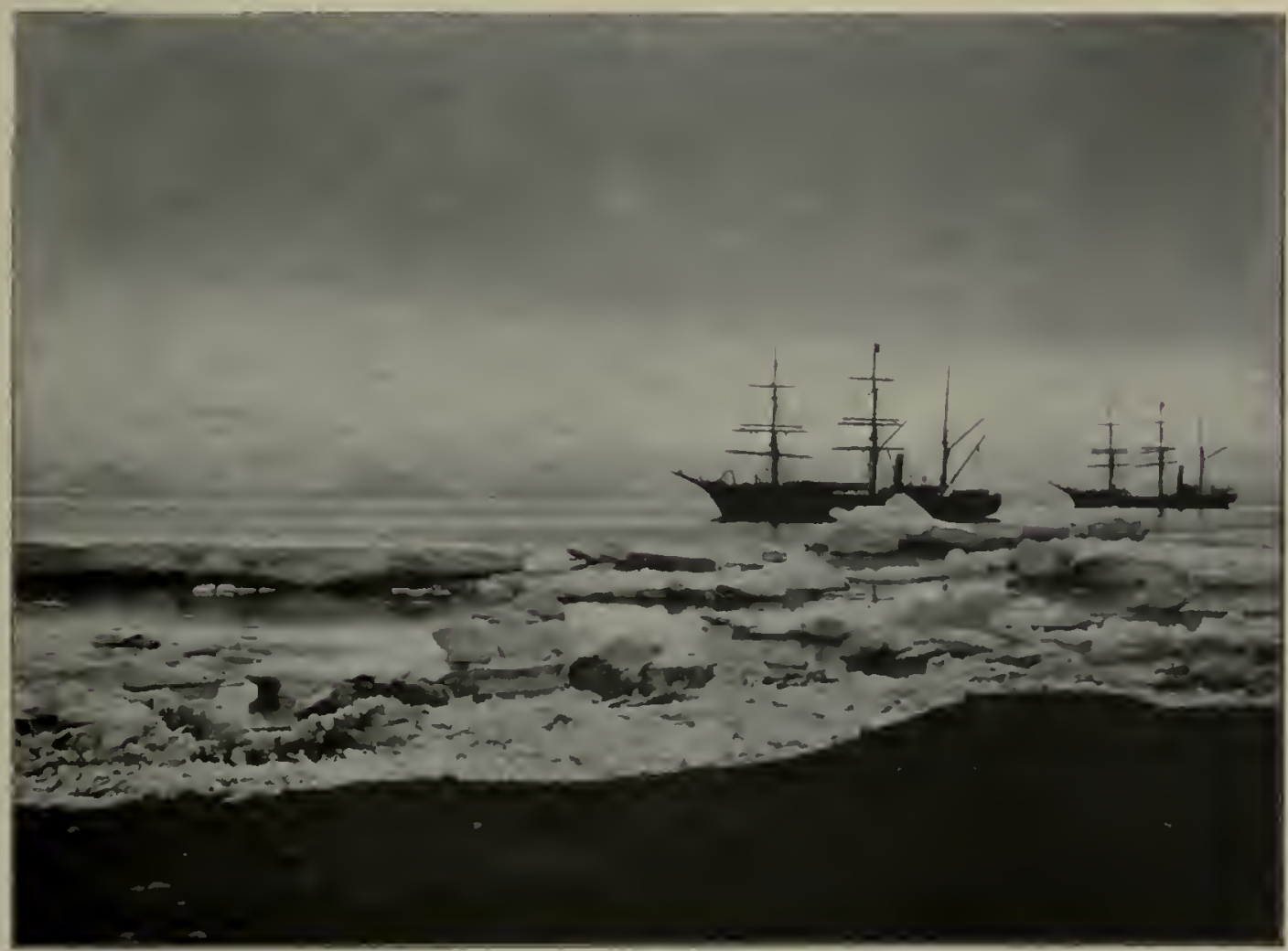

Fig. 2. Looking South-west from Cape Arlare.

Showing a common type of heavy stratus with defined edge, beneath which the sky is clear. 

Axtanctic Croun Folsus.

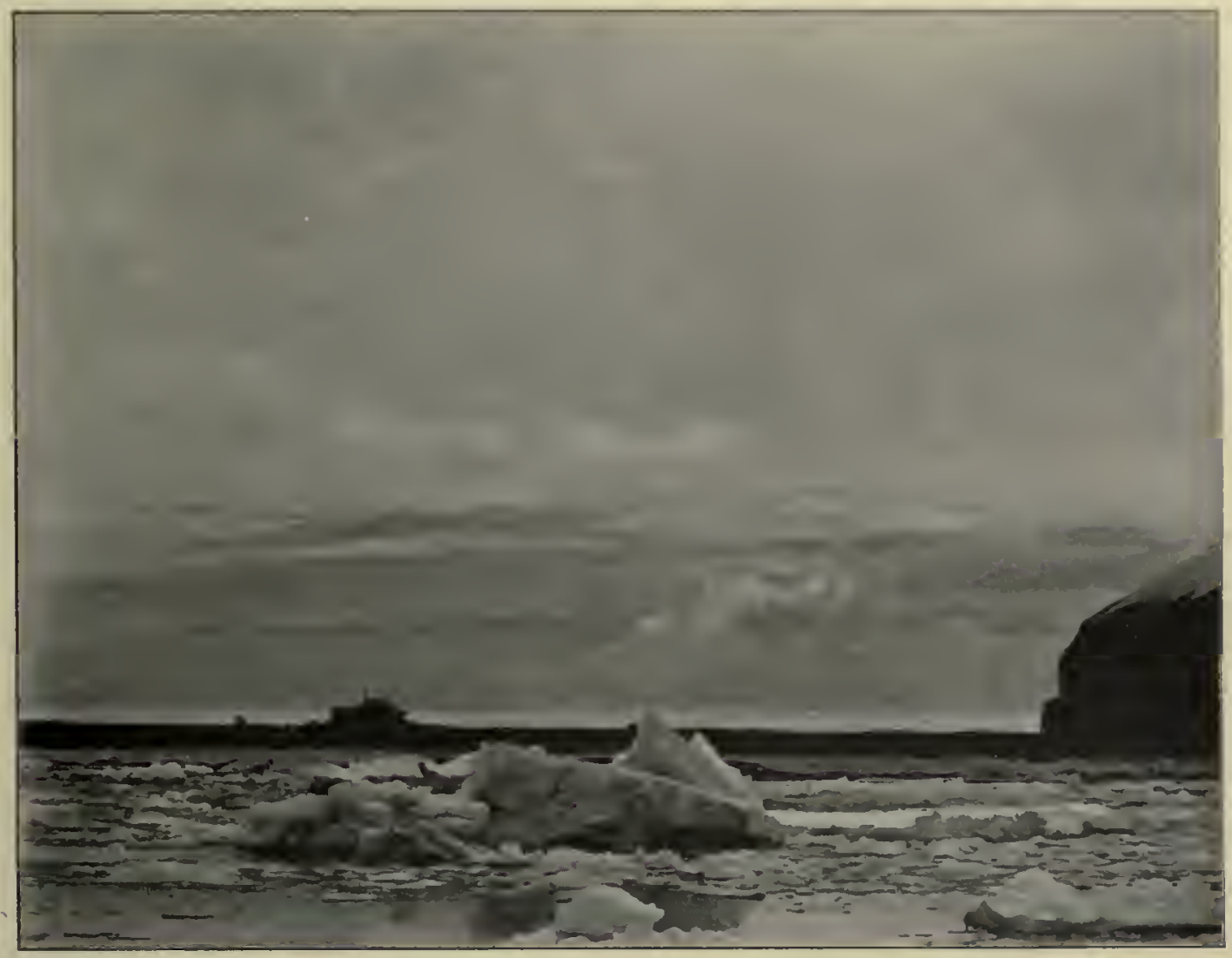

Fig. 1. Looking North from Cape Arlare.

Showing stratus.

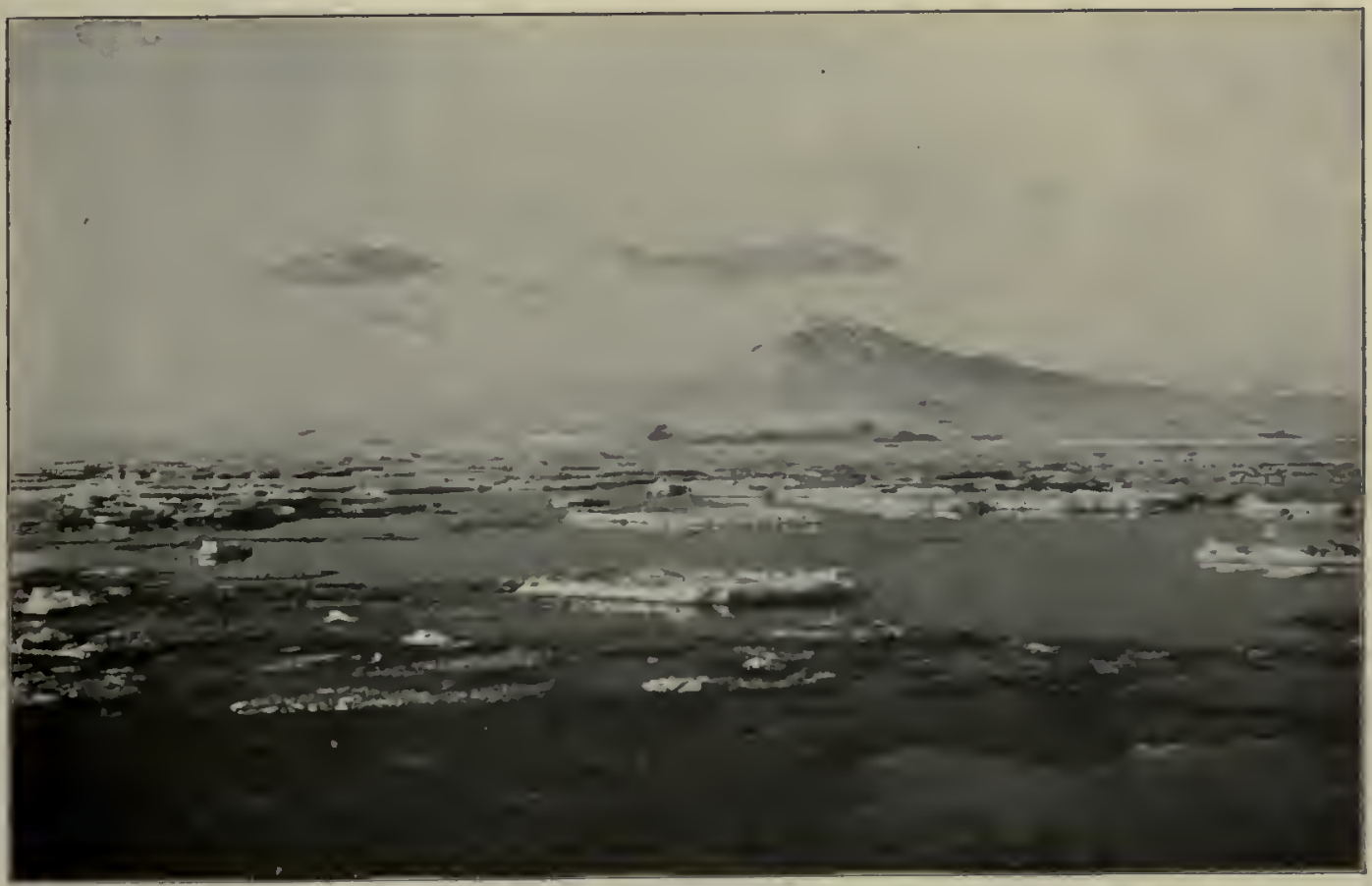

Fig. 2. Mount Melbourue from the East.

With examples of the clond patches which form over high peaks in an otherwise cloudless sky. 



\section{METEOROLOGICAL JOURNAL OF THE "DISCOVERY."}

[1902-February.

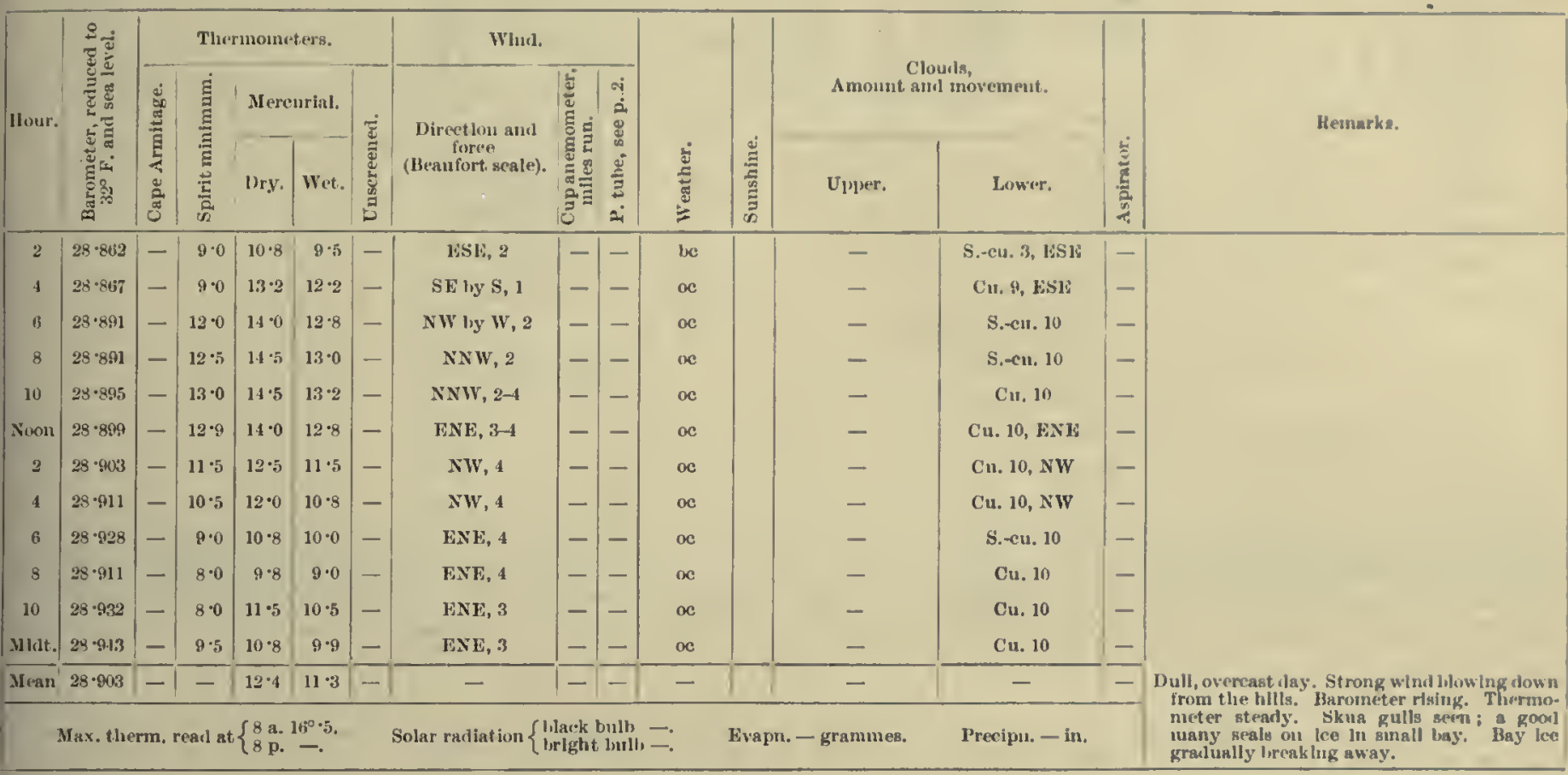

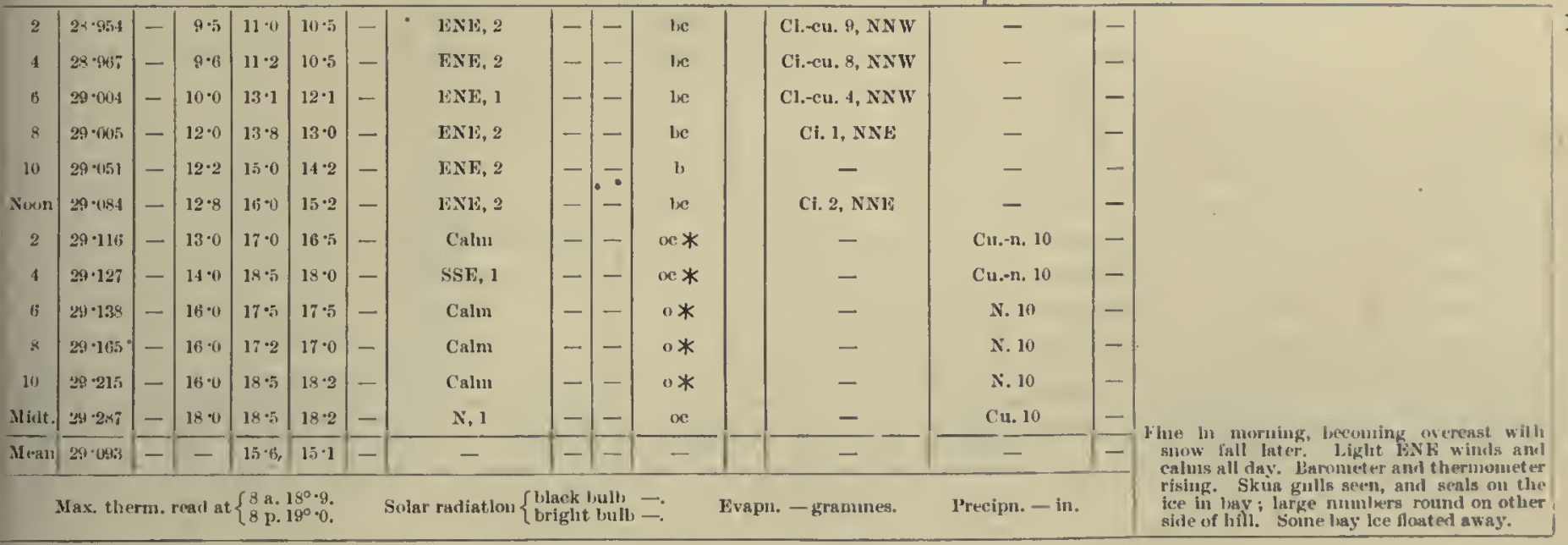

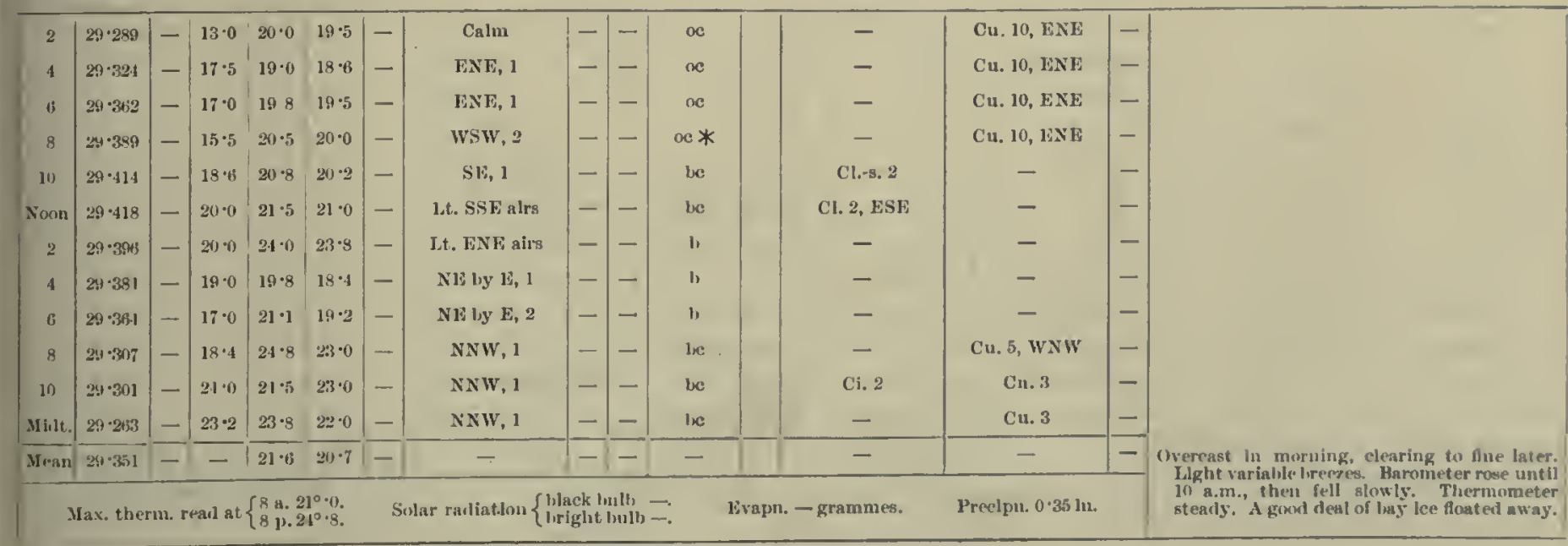


1902 -February.]

Meteorological, Jovrai, of tue "Discovery."

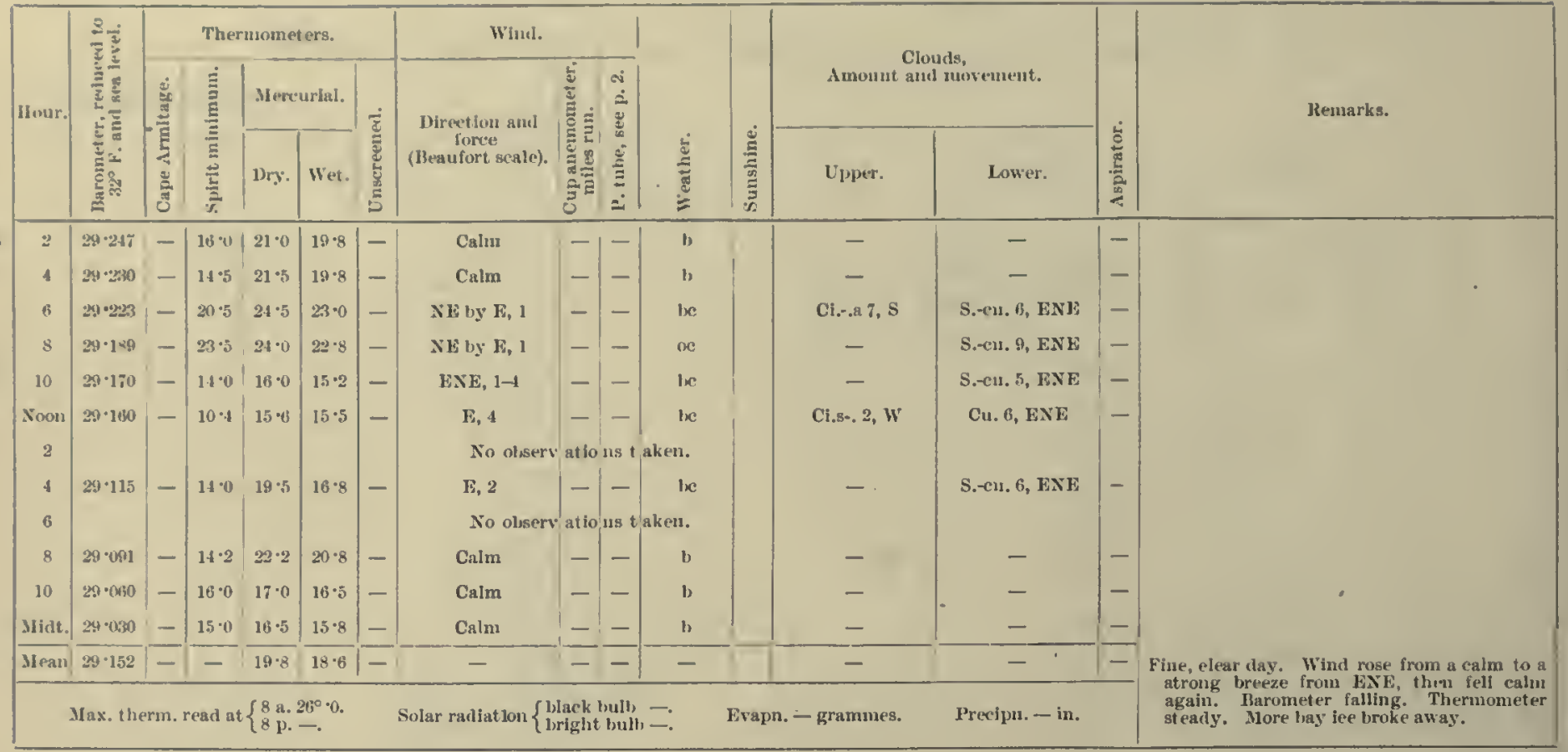

13 th.

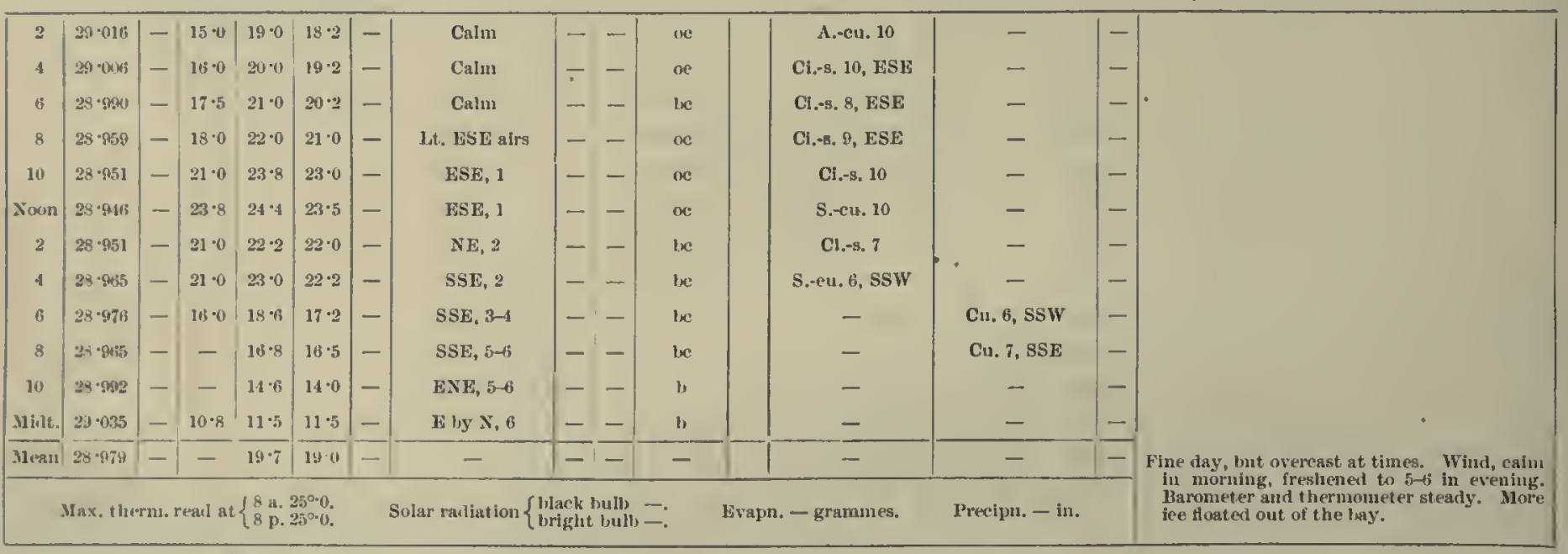

14th.

\begin{tabular}{|c|c|c|c|c|c|c|c|c|c|c|c|c|c|c|}
\hline 2 & $29 \cdot 044$ & -1 & $8 \cdot 0$ & 10.5 & 10.4 & -1 & BNE, 5 & -1 & - & b & - & - & - & \multirow{11}{*}{$\begin{array}{l}\text { - } \\
\text { Fine greater part of day. Wind strong from } \\
\text { EN } 1 \text {. laroueter rising. Thernoometer } \\
\text { steady. More bay lee floated out. }\end{array}$} \\
\hline 1 & $29) \cdot(m i 9$ & - & 10.5 & $11 \cdot 2$ & $11 \cdot 0$ & - & ENE, 5 & - & - & b & - & - & - & \\
\hline is & $29 \cdot 1145$ & - & $9 \cdot 0$ & $11 \cdot 2$ & $11 \cdot 0$ & - & ENE, 5 & - & - & b & - & - & - & \\
\hline 10 & $29 \cdot 133$ & - & $10 \cdot 0$ & $15 \cdot 0$ & $15 \cdot 0$ & - & BNE, 5 & - & - & b & - & - & - & \\
\hline Xoons & $29 \cdot 137$ & - & $13 \cdot 0$ & $17 \cdot 0$ & $15 \cdot 2$ & - & ENk, 5 & - & - & be & Ci.-eu. 6, WNW & Cu. 2, ENE & - & \\
\hline 2 & $29 \cdot 15$ & - & $17 \cdot 0$ & $17 \cdot i$ & $15 \cdot 8$ & -1 & RNE, 5 & - & - & oe & - & S. 10 & - & \\
\hline 8 & $29-159$ & - & $16 \cdot 0$ & $17 \cdot 1)$ & $15 \cdot 8$ & - & RNE, 3-4 & - & - & $1 \times:$ & Ci. 5, Ssw & - & - & \\
\hline 10 & $2 y \cdot 179$ & - & $13 \cdot 0$ & $14 \cdot 8$ & $13 \cdot 6$ & - & FNE, $3-4$ & - & - & lice & - & S.-eu. 6, NNF & - & \\
\hline Midi. & $28 \cdot 159$ & - & $13 \cdot 0$ & $11 \cdot 0$ & $13 \cdot 2$ & -1 & FN 6,3 & $|-|$ & - & the & $\mathrm{Cl}_{-}-\mathrm{s}, 4, \mathrm{ENJ}$ & - & - & \\
\hline IIean & $2 x \cdot 123$ & $\overline{-}$ & - & $14 \cdot 6$ & $13 \cdot 8$ & -1 & - & - & -1 & - & - & - & - & \\
\hline & 6. the & & & & & & radiation & & & & - grammes. & I'recipu. - in. & & \\
\hline
\end{tabular}


METEOLOLOGICAT, JOULXAL OF THF "DISCOVF,Y."

[1902-February.

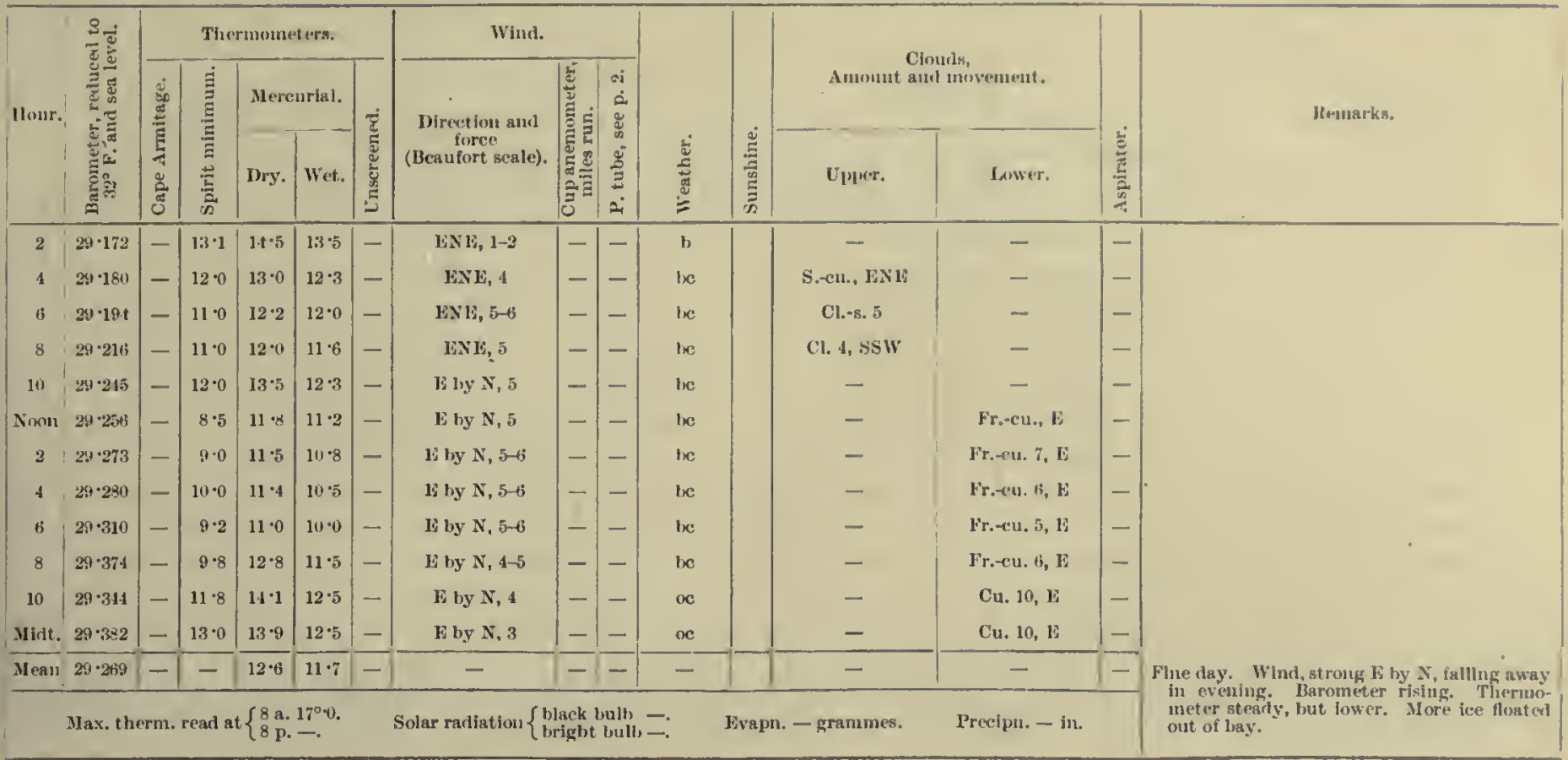

15 th.

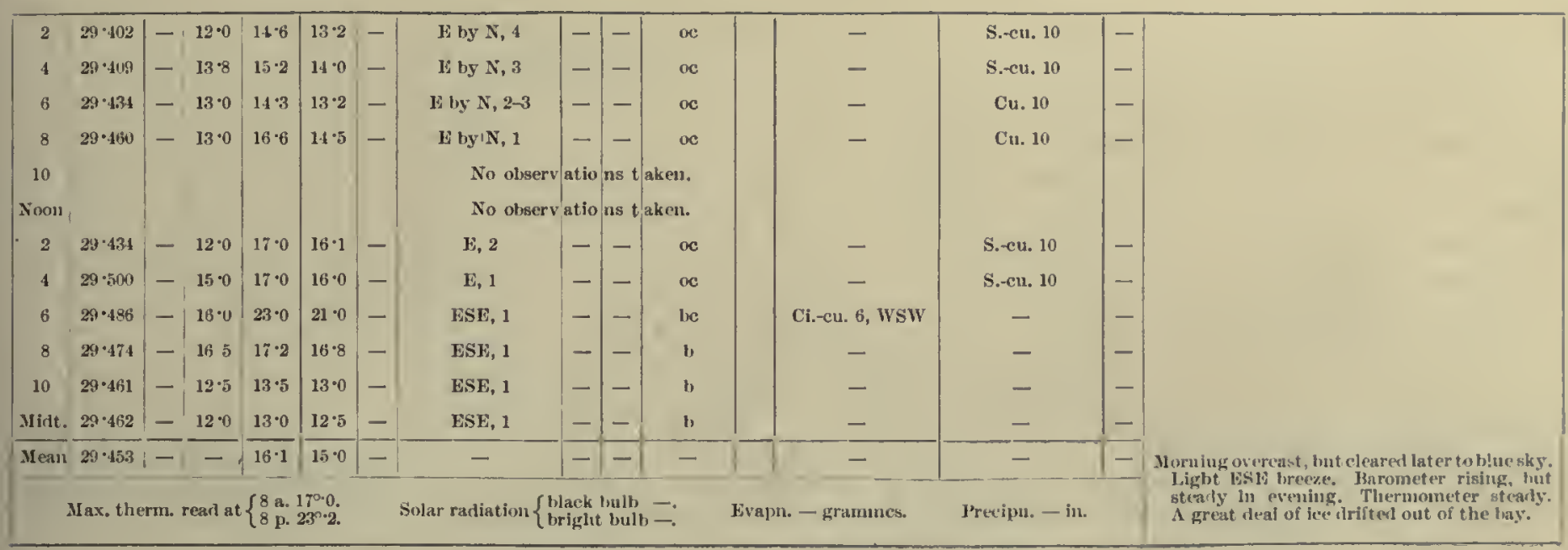

16 th.

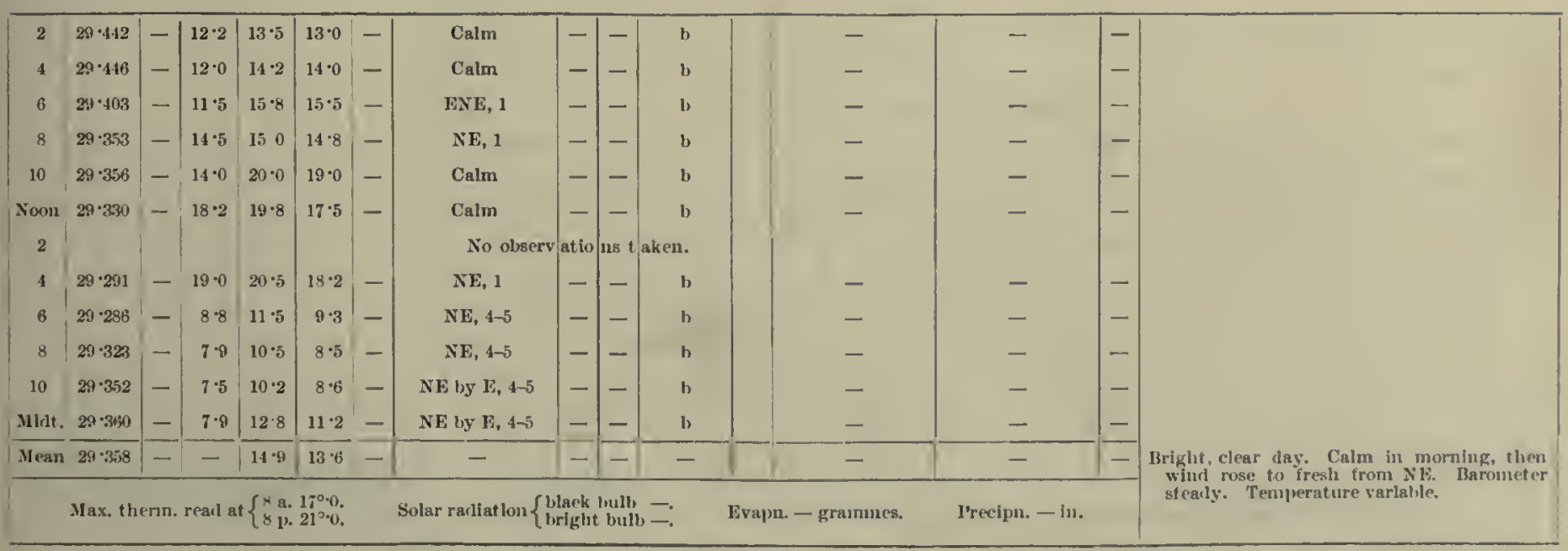

17 th. 
1902-February.]

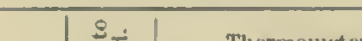

M etrolbological, Joursal of the "Discoveliy."

\begin{tabular}{|c|c|c|c|c|c|c|c|c|c|c|c|c|c|c|}
\hline \multirow{3}{*}{ nour. } & \multirow{3}{*}{ 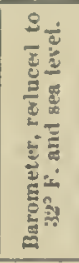 } & \multicolumn{5}{|c|}{ Thermometers. } & \multicolumn{2}{|l|}{ Wlud. } & \multirow[b]{3}{*}{ 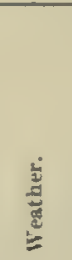 } & \multirow[b]{3}{*}{$\mid$} & \multirow{2}{*}{\multicolumn{2}{|c|}{$\begin{array}{l}\text { Clonds, } \\
\text { Amount aull noventent. }\end{array}$}} & & \multirow{3}{*}{ licmarks. } \\
\hline & & \multirow{2}{*}{ | } & \multirow{2}{*}{ 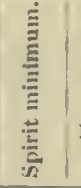 } & \multicolumn{2}{|c|}{ Mereurial. } & \multirow{2}{*}{ 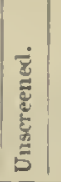 } & \multirow{2}{*}{$\begin{array}{c}\text { Direttlon and } \\
\text { furce } \\
\text { (Beafort scenle). }\end{array}$} & \multirow{2}{*}{ 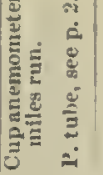 } & & & & & & \\
\hline & & & & Dry. & wet. & & & & & & Upper. & Lower. & & \\
\hline 3 & & & & & & & Xo whserv & atlo us t: & ken. & & & & & \\
\hline 4 & & & & & & & xo observ & ations t. & ken. & & & & & \\
\hline 6 & 2939 & - & 1.5 & $3 \cdot 1$ & 3.8 & - & ENE, $5-6$ & -- & b & & - & - & - & \\
\hline 8 & $29 \cdot 421$ & - & 0.8 & $2 \cdot 8$ & $2 \cdot 0$ & - & ENE, 13 & -1 & b & & - & - & - & \\
\hline 10 & $29 \cdot 4+12$ & - & 0.8 & $9 \cdot 2$ & 8.1 & 1- & ENE, 4 & -1 & b & & - & - & - & \\
\hline Noon & $20 \cdot 354$ & - & $8 \cdot 0$ & 170 & $15 \div$ & $1-$ & Calun & --1 & b & & - & - & - & \\
\hline 2 & $29 \cdot 2 ; n$ & -1 & 158 & 18.4 & $16 \cdot 6$ & - & Calm & $-1-$ & Ine & & - & Cu. 8, w & - & \\
\hline+ & 29:-2014 & -1 & 170 & $19 \cdot 5$ & $18 \cdot 0$ & - & $\mathrm{N}, 1$ & -1 & ow & & - & Cu. $4, N$ & - & \\
\hline 6 & 29161 & -1 & $17 \cdot 8$ & $23 \cdot 2$ & $21 \cdot 4$ & - & $\mathrm{N}, 4$ & $-1-$ & be & & - & Cu. $8, \mathrm{~N}$ & - & \\
\hline 8 & $29 \cdot 079$ & $1-1$ & $17 \cdot 8$ & $25 \cdot 0$ & $23 \cdot 0$ & -1 & NNW, 5-6 & $-1-$ & we & & $T$ & Cn. 5, XNW & - & \\
\hline 10 & 290018 & -2 & $24 \cdot 0$ & $21 \cdot 8$ & $23 \cdot 0$ & - & $\mathrm{X}$ by $\mathbf{W}, 7-\mathrm{s}$ & -1 & reg & & - & Cu. 4, XWw & - & \\
\hline Mlidt.] & $23 \cdot 4: 3$ & -12 & $22 \cdot 5$ & $23 \cdot 0$ & $21 \cdot 5$ & - & $\mathrm{N}$ by $\mathrm{W}, 85$ & -- & we & & - & Cu. 7, NXW & -1 & \\
\hline Mean: & $29 \cdot 229$ & -1 & - & $16 \cdot 6$ & $\overline{15 \cdot 2}$ & -1 & - & $-\mid-1$ & - & & - & - & - & 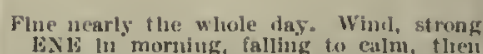 \\
\hline & Ifax, the & rum, rea & ad at \{ & $\left\{\begin{array}{l}8 \mathrm{~s} \text { a. } \\
8 \mathrm{p} \text {. }\end{array}\right.$ & & & tar radiation & $\begin{array}{l}\text { gen but } \\
\text { ght but }\end{array}$ & & & rammes. & Precipn. - - In. & & 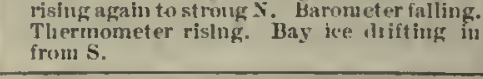 \\
\hline
\end{tabular}

18th.

19 th.

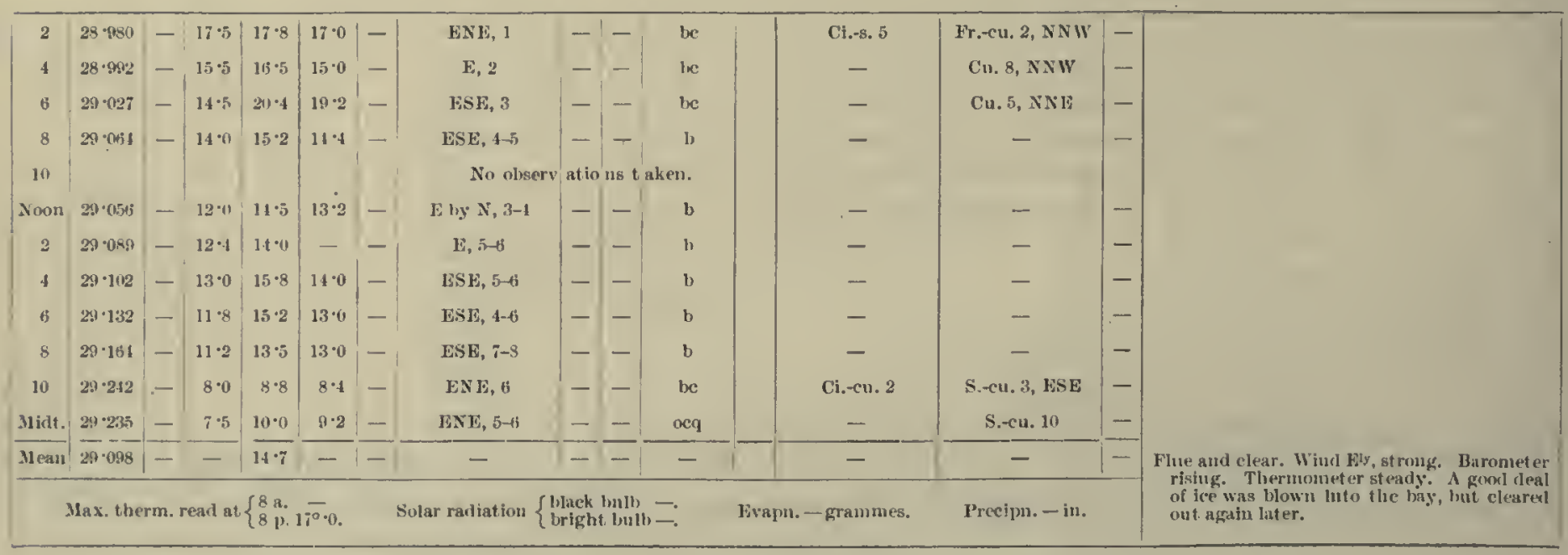

20 th.

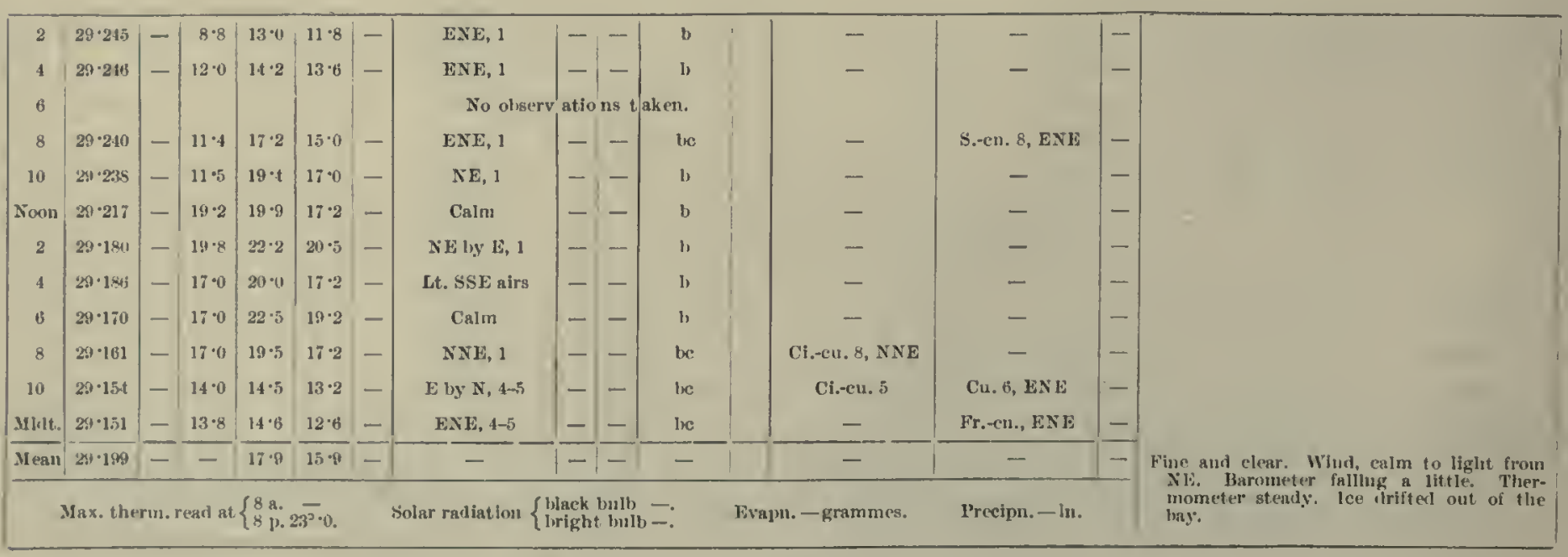




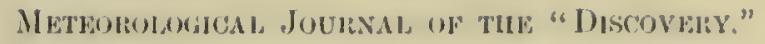

[1902-February.

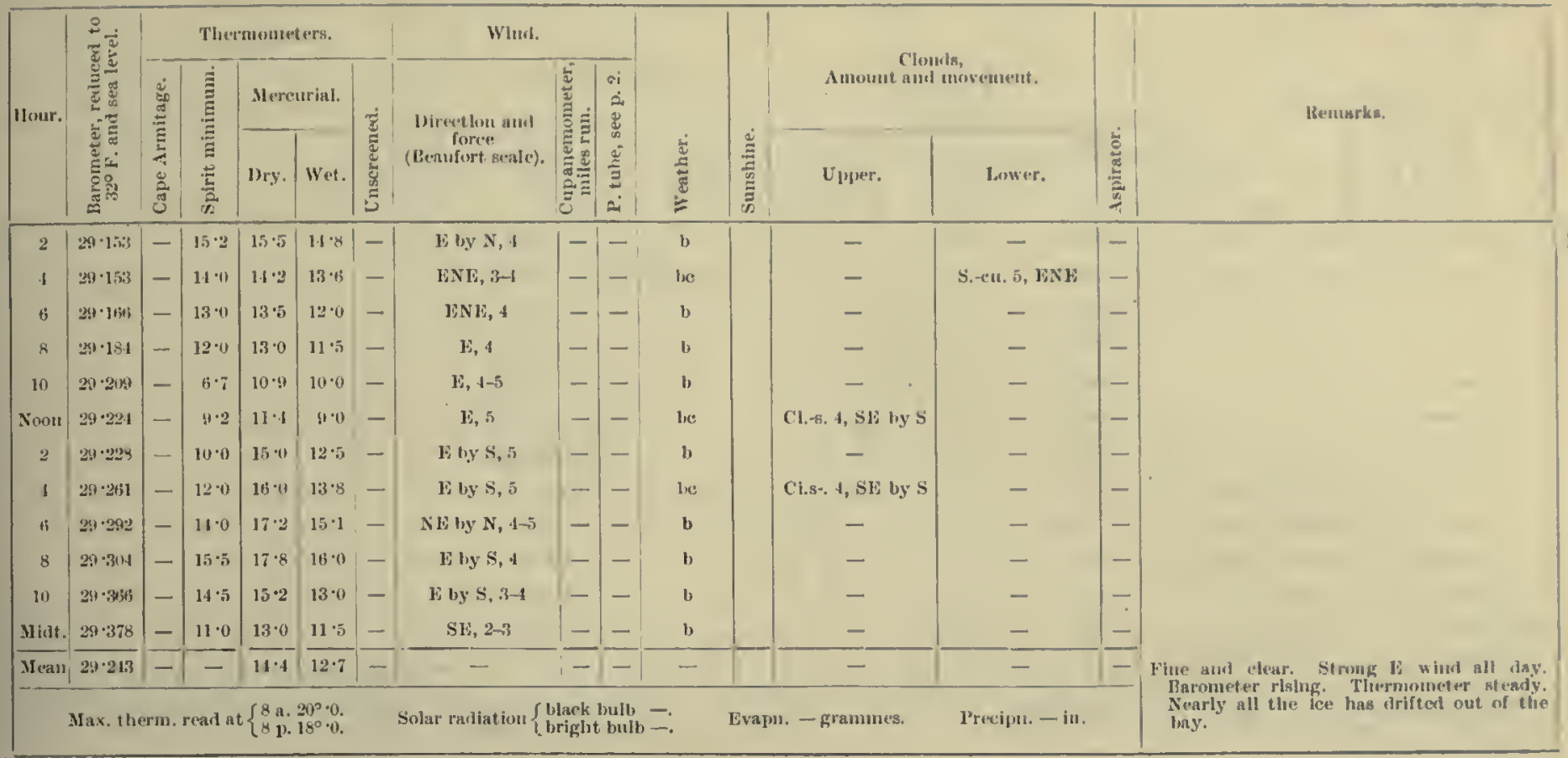

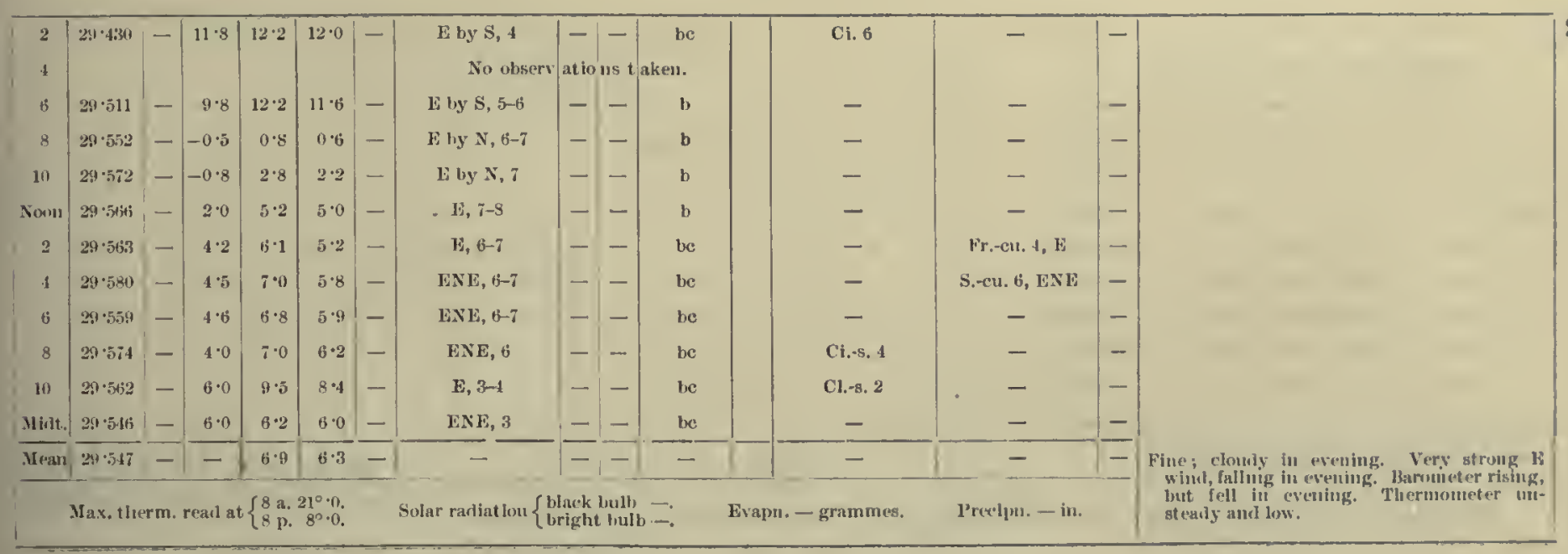

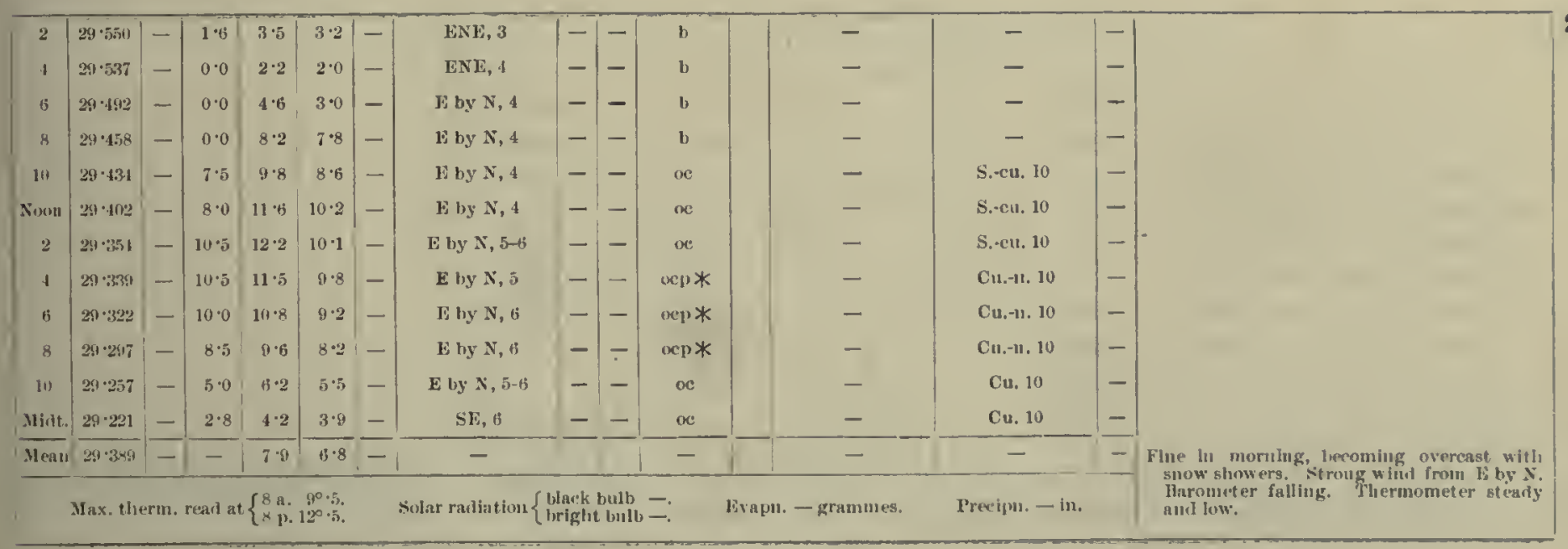


1902-February.]

Metrorological, Jounsal, of the "Discortery."

\begin{tabular}{|c|c|c|c|c|c|c|c|c|c|c|c|c|c|c|c|}
\hline \multirow{3}{*}{ II mur. } & \multirow{3}{*}{ 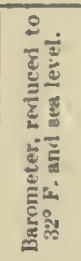 } & \multicolumn{5}{|c|}{ Thernzometers. } & \multicolumn{3}{|l|}{ Wind. } & \multirow[b]{3}{*}{ 离 } & \multirow[b]{3}{*}{ 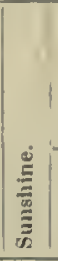 } & \multirow{2}{*}{\multicolumn{2}{|c|}{$\begin{array}{l}\text { Clourds, } \\
\text { Amutumt anul movement. }\end{array}$}} & \multirow[b]{3}{*}{$\frac{\dot{5}}{\frac{5}{2}}$} & \multirow{3}{*}{ Remarks. } \\
\hline & & \multirow{2}{*}{ 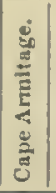 } & \multirow{2}{*}{ 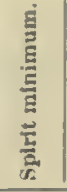 } & \multicolumn{2}{|c|}{ Mercurial. } & \multirow[b]{2}{*}{ 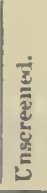 } & \multirow{2}{*}{$\begin{array}{l}\text { Dirediou aut } \\
\text { force } \\
\text { (Beatufort seale). }\end{array}$} & \multirow{2}{*}{ 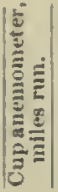 } & \multirow{2}{*}{ 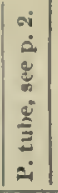 } & & & & & & \\
\hline & & & & 1)ry. & Wet. & & & & & & & Uрiнеr. & Lower. & & \\
\hline 2 & $293 \cdot 213$ & -1 & $-0 \cdot 6$ & $3 \cdot 0$ & 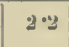 & -1 & JSE, 4 & - & -1 & oc & & - & S.cu. 10 & -1 & \\
\hline 4 & $2 x-200$ & - & $1 \cdot 0$ & $5 \cdot 0$ & $4 \cdot 2$ & - & ISL 4 & - & - & oc & & A.-fr.-cul, \&, NNW & Cul. 9, vsi: & - & \\
\hline 6 & 20201 & - & $3 \cdot 5$ & $5 \cdot 5$ & $5 \cdot 0$ & - & ESE $1,3-1$ & -1 & - & he & & - & Cu. 8 & - & \\
\hline 8 & $29 \cdot 214$ & - & 5.0 & 6.5 & $6 \cdot 1$ & - & $\mathrm{JSE}, 3$ & - & - & $\infty *$ & & - & Cu.-nl. 10, ESE & - & \\
\hline 10 & $20 \cdot 217$ & - & $5 \cdot 8$ & $7 \cdot 4$ & $7 \cdot 2$ & - & ESE: 3-4 & - & - & $\infty *$ & & - & $\mathrm{Cu}-11.10, \mathrm{E} 8 \mathrm{i}$ & -1 & \\
\hline soon & $29 \cdot 214$ & - & $6 \cdot 5$ & $8 \cdot 3$ & $8 \cdot 2$ & - & BSE, $4 \rightarrow 3$ & - & - & oc $*$ & & - & C11.-11. 10, WSI & - & \\
\hline 2 & $20 \cdot 252$ & - & 6.5 & $9 \cdot 0$ & $8 \cdot 6$ & - & $\mathrm{ESE}, 5-6$ & - & - & oc $*$ & & - & C11.-11. 10, ESL & - & \\
\hline 4 & $20 \cdot 277$ & - & $8 \cdot 2$ & $10 \cdot 0$ & $9 \cdot 8$ & - & $\mathbf{F}, \mathrm{i}-\mathrm{B}$ & - & - & $n \in *$ & & - & Cu.-11. 10 & - & \\
\hline 6 & $29 \cdot 306$ & - & 4.0 & $10 \cdot 0$ & $9 \cdot 6$ & - & E, $5-6$ & - & - & $\omega c$ & & - & Cu. 10 & - & \\
\hline 8 & $29 \cdot 318$ & - & $9 \cdot 0$ & 110 & $10 \cdot 2$ & - & E, 5-6 & - & - & $\infty$ & & - & Cu. 10 & - & \\
\hline 10 & $29 \cdot 316$ & - & $9 \cdot 9$ & $11 \cdot 0$ & $10 \cdot 1$ & - & E, $5-6$ & - & - & $\infty$ & & - & Cu. 10 & - & \\
\hline Mist. & $29 \cdot 857$ & -1 & $11 \cdot 0$ & $13 \cdot 2$ & $12 \cdot 3$ & -1 & $E, 6$ & -1 & - & oc & & - & $\mathrm{Cu} .10$ & - & \\
\hline Meau & $29 \cdot 260$ & -1 & - & 8.3 & $7 \cdot 8$ & -1 & - & - & -1 & - & & - & 7 & - & Overcast and clondy, light anow slowers. \\
\hline \multicolumn{2}{|c|}{$\begin{array}{l}\text { Max. } \\
\text { rer }\end{array}$} & $\left\{\begin{array}{l}8 \mathrm{a} \\
8 \mathrm{I}\end{array}\right.$ & $\begin{array}{l}n \\
.11^{\circ}\end{array}$ & & Solar & rar & on $\left\{\begin{array}{l}\text { blacek luall } \\
\text { bright bull }\end{array}\right.$ & 二: & & pur. & & $\begin{array}{c}\text { Pree } \\
\text { inerasi }\end{array}$ & pul. $\left\{\begin{array}{c}10 \text { a. } 0.30 \mathrm{ir} \\
8 \mathrm{p} \cdot 0 \cdot 25 \text { in }\end{array}\right.$ & & $\begin{array}{l}\text { Barnmeter rising. Tliermometer low and } \\
\text { rising. }\end{array}$ \\
\hline
\end{tabular}

24 th.

25 th.

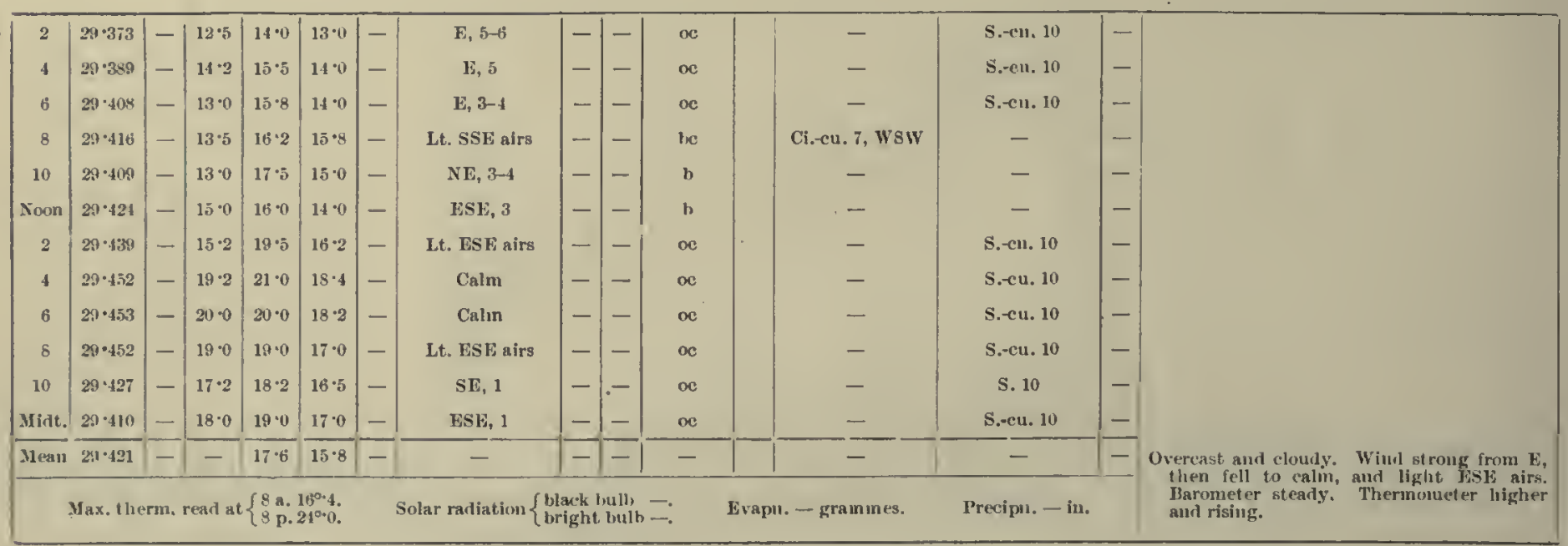

26 th.

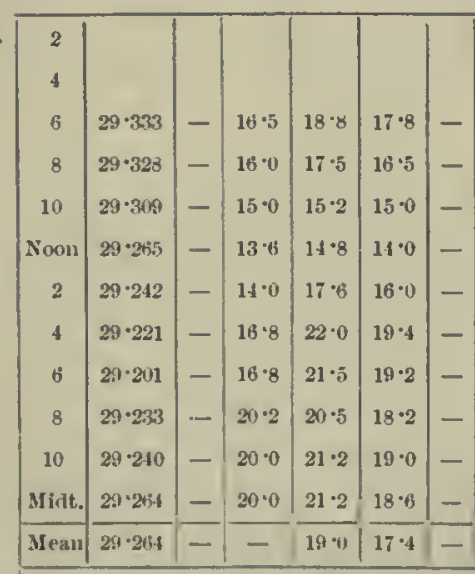

Max. therm, real at $\left\{\begin{array}{l}8 \text { a. } 20^{\circ} 0 . \\ 8 \mathrm{p} .21^{\circ} 0 .\end{array}\right.$

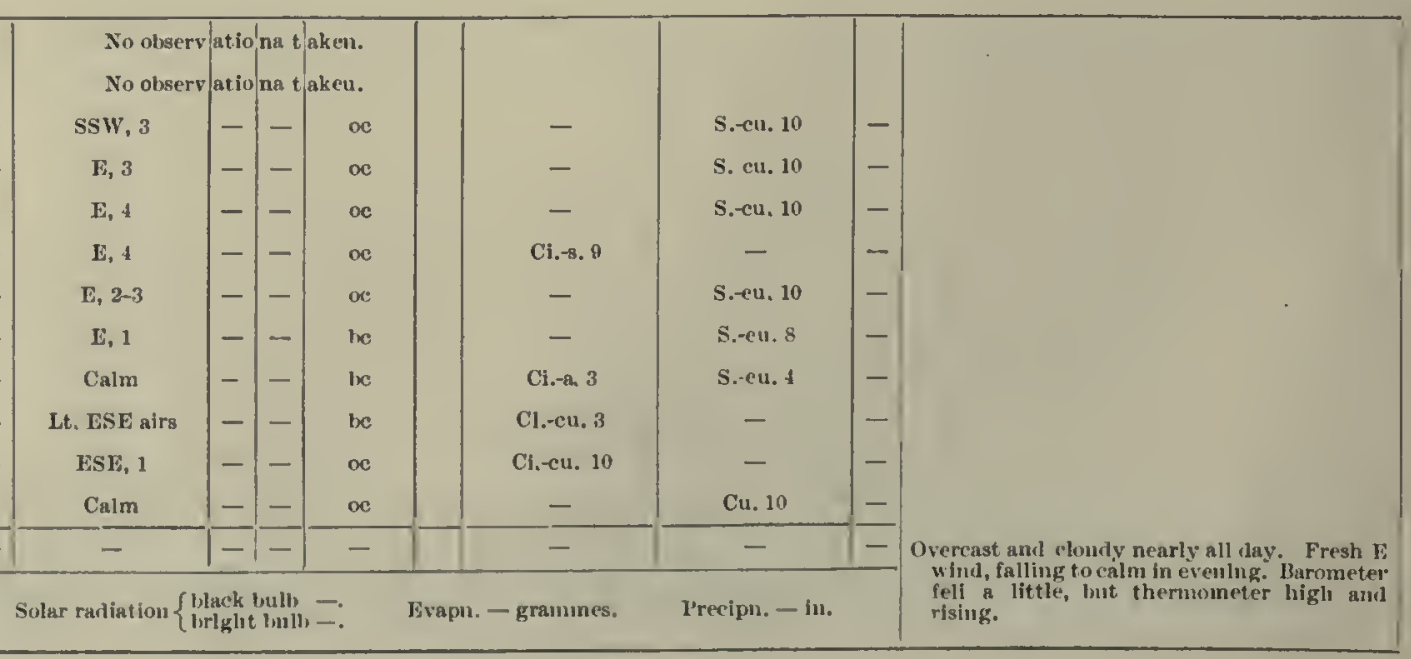


Metrorological, Journal, of thik "Discovery."

[1902-February.

\begin{tabular}{|c|c|c|c|c|c|c|c|c|c|c|c|c|c|c|c|}
\hline \multirow{3}{*}{ Hัน: } & \multirow{3}{*}{ 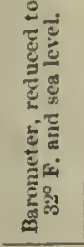 } & \multicolumn{5}{|c|}{ Thirmoneters. } & \multicolumn{3}{|l|}{ Wimul. } & \multirow[b]{3}{*}{ 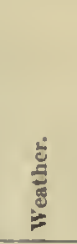 } & \multirow[b]{3}{*}{ 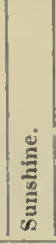 } & \multirow{2}{*}{\multicolumn{2}{|c|}{$\begin{array}{l}\text { Clouds, } \\
\text { Anount and unovement. }\end{array}$}} & \multirow[b]{3}{*}{$\frac{\frac{2}{3}}{\frac{5}{2}}$} & \multirow{3}{*}{ Nemarks. } \\
\hline & & \multirow{2}{*}{ 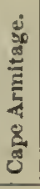 } & \multirow{2}{*}{ 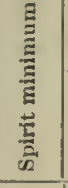 } & \multicolumn{2}{|c|}{ Mereurlal. } & \multirow{2}{*}{ 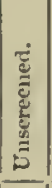 } & \multirow{2}{*}{$\begin{array}{c}\text { Dircetion au1 } \\
\text { force } \\
\text { (Bea fort scale). }\end{array}$} & \multirow{2}{*}{ 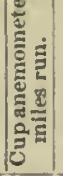 } & \multirow{2}{*}{ 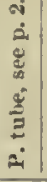 } & & & & & & \\
\hline & & & & Dry: & Wet. & & & & & & & Ü'twer. & Lower. & & \\
\hline 2 & & & & & & & No obser & utlo & tha $t$ & kent. & & & & & \multirow{5}{*}{ Muel lee form Ink In lay. } \\
\hline 4 & $29 \cdot 276$ & - & $20 \cdot 0$ & $22 \cdot 2$ & $21 \cdot 0$ & - & Calm & - & - & ne * & & - & Cu.-11. 10 & - & \\
\hline 6 & $29 \cdot 274$ & - & $21 \cdot 0$ & $23 \cdot 0$ & $21 \cdot 6$ & - & Calm & - & - & $\infty *$ & & - & Cu.tn. 10 & - & \\
\hline 8 & $29 \cdot 278$ & - & $21 \cdot 5$ & $24 \cdot 0$ & $22 \cdot 5$ & - & $\mathbf{E}, \mathbf{l}$ & -1 & - & oc & & - & Cu.--11. 10 & - & \\
\hline 10 & $20 \cdot 305$ & - & $23 \cdot 4$ & $2 B \cdot 0$ & $24 \cdot 2$ & - & $1 ;, 1$ & - & - & 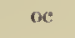 & & - & Cu.n. 10 & - & \\
\hline Nuon & $29 \cdot 326$ & - & $25 \cdot 0$ & $25 \cdot 2$ & $24 \cdot 8$ & - & SSW, 5 & - & - & $o$ & & - & S.ecu. 10 & - & \multirow{2}{*}{$\begin{array}{l}0.30 \text { p.m. Whod shlfted mudslenly to SSL, } \\
\text { force s. }\end{array}$} \\
\hline 2 & $29 \cdot 338$ & - & $24 \cdot 8$ & $26 \cdot 5$ & $25 \cdot 5$ & - & SW by $S, 5-6$ & -1 & $\rightarrow$ & $o c$ & & - & S.cu. 10 & - & \\
\hline 4 & $29 \cdot 375$ & - & $24 \cdot 5$ & $26 \cdot 8$ & $25 \cdot 8$ & - & SW by $S, 3-1$ & -1 & - & $o c$ & & Cl.-ell. 7 & S.-cu. 6, SSW & - & \multirow{5}{*}{$\begin{array}{l}\text { Lower elouls novlug fast. } \\
\text { Lower clowels nooving fast. } \\
\text { Lower elouis moving fast. Bay full of the lee. }\end{array}$} \\
\hline 6 & $29 \cdot 362$ & - & $26^{\circ} 0$ & $27 \cdot 9$ & $25 \cdot 8$ & - & SSW, 4-5 & - & - & be & & Fr.eu, 6, SSW & S.cu. 5, SSW & - & \\
\hline 8 & $29 \cdot 387$ & - & $25 \cdot 6$ & $26 \cdot 2$ & $24 \cdot 5$ & - & SW by $S, 5-6$ & -1 & - & $b c$ & & Ci.-cu. 8, SSW & Cu. 3, SSW & - & \\
\hline 10 & $29 \cdot 382$ & - & $20 \cdot 8$ & $22 \cdot 5$ & $20 \cdot 8$ & - & SSW, 3-4 & - & - & $o c$ & & - & Cu. 10, SSW & - & \\
\hline Midt. & $29 \cdot 394$ & - & $19 \cdot 0$ & $21 \cdot 0$ & $20 \cdot 0$ & - & SW by $S, 2-6$ & - & - & ocq & & - & s.-cu, 10, SSW & - & \\
\hline M ean! & $29 \cdot 336$ & - & - & $21 \cdot 7$ & $23 \cdot 3$ & - & 一 & - & - & - & & - & 一 & - & \multirow{2}{*}{ 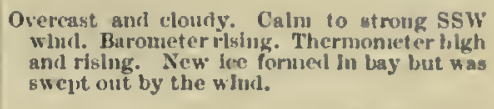 } \\
\hline \multicolumn{7}{|c|}{ Max. thern. read at $\left\{\begin{array}{l}8 \text { a. } 21^{\circ} \cdot 0 \\
8 \text { p. }-\end{array}\right.$} & \multicolumn{4}{|c|}{ Solar radiation $\left\{\begin{array}{l}\text { black bulb }- \text {. } \\
\text { bright lulb- }\end{array}\right.$} & \multicolumn{2}{|c|}{ Evaju. - gramues. } & \multicolumn{2}{|l|}{ P'recipur. 一 in. } & \\
\hline
\end{tabular}

27 th.

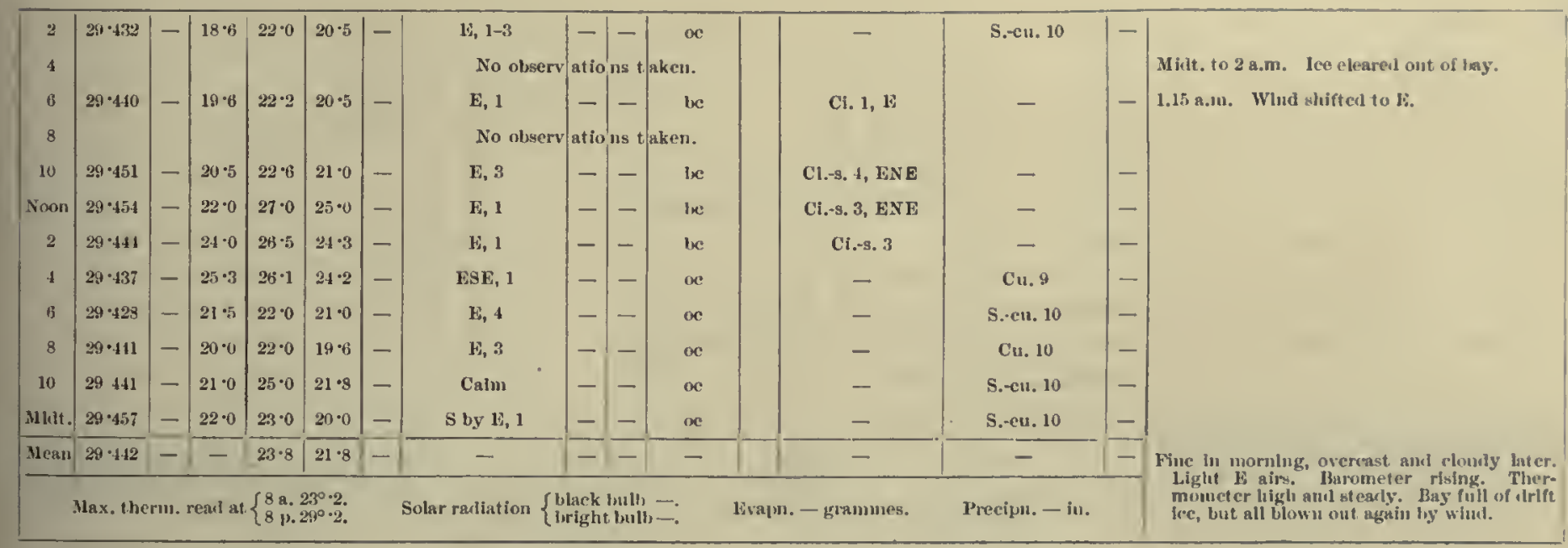




\begin{tabular}{|c|c|c|c|c|c|c|c|c|c|c|c|c|c|c|c|}
\hline \multirow{3}{*}{ Hour. } & \multirow{3}{*}{ 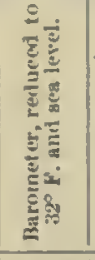 } & \multicolumn{5}{|c|}{ Therumometers. } & \multicolumn{3}{|l|}{ WInil. } & \multirow[b]{3}{*}{ 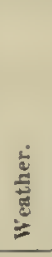 } & \multirow[b]{3}{*}{ 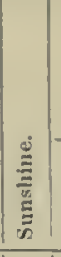 } & \multirow{2}{*}{\multicolumn{2}{|c|}{$\begin{array}{l}\text { Clouils, } \\
\text { Amount aud movenuent. }\end{array}$}} & \multirow[b]{3}{*}{ 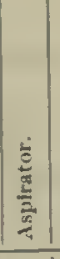 } & \multirow{3}{*}{ Remarks. } \\
\hline & & \multirow{2}{*}{ 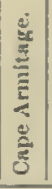 } & \multirow{2}{*}{ 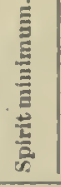 } & \multicolumn{2}{|c|}{ Mercurlal. } & \multirow{2}{*}{ 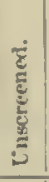 } & \multirow{2}{*}{$\begin{array}{c}\text { Dl rectlou aud } \\
\text { foree } \\
\text { (Beaufort scale). }\end{array}$} & \multirow{2}{*}{ 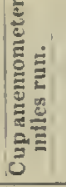 } & & & & & & & \\
\hline & & & & Dry. & Wet. & & & & & & & Upper. & Lower. & & \\
\hline 2 & $29 \cdot 463$ & -1 & $19 \cdot 1$ & $21 \cdot 0$ & $18 \cdot 6$ & -1 & S1: ty 5,1 & -1 & - & $\kappa$ & & - & C'u. Io, Fisl: & -1 & \\
\hline 4 & $29 \cdot 445$ & - & $20 \cdot 0$ & $21 \cdot 8$ & $19 \cdot 1$ & - & SK, 2 & - & - & $I^{\circ}$ & & $C i_{.}-(01,6,15 S E$ & S.-c1. 5, lisili & -1 & \\
\hline 6 & $29 \cdot 451$ & - & $20 \cdot 0$ & $23 \cdot 0$ & $20 \cdot 2$ & - & $S$ by $F, 2$ & - & - & $o c$ & & - & S. en. 10 & -1 & \\
\hline 8 & $20 \cdot 4.18$ & - & $22 \cdot 2$ & $21 \cdot 0$ & $21 \cdot 8$ & - & $\mathbf{E}, 2$ & - & - & or & & C1. -8.6 & S. 11 & - & Very thin stratus. \\
\hline 10 & $29 \cdot 456$ & - & $21 \cdot 8$ & $22 \cdot 8$ & $21 \cdot 2$ & - & SSE, 3 & - & - & $o e$ & & Ci.-en. 9 & - & -1 & \\
\hline X(ко) & $23 \cdot 4.51$ & - & $21 \cdot 0$ & $22 \cdot 0$ & $20 \cdot 6$ & - & $\operatorname{SS1}, 4-5$ & - & - & $n$ & & 一 & S. 9 & - & Stmatus, lrigh and tluln. \\
\hline 2 & $29 \cdot 419$ & - & $20 \cdot 0$ & $21 \cdot 2$ & $20 \cdot 0$ & - & Sk $1, y, 3,-4$ & - & - & oc & & 一 & S.-111. 10 & - & \\
\hline 4 & $29 \cdot 419$ & - & $19 \cdot 8$ & $21 \cdot 0$ & $20 \cdot 2$ & - & SE ly S, 3 & - & - & $\mathrm{bc}$ & & Ci.-9. 10 & - & - & \\
\hline 6 & $29 \cdot 454$ & - & $19 \cdot 5$ & $21 \cdot 8$ & $20 \cdot 2$ & - & F by $\mathrm{N}, 4$ & - & - & Ine & & CI.-8. 10 & - & - & IIills looking very near. \\
\hline 8 & $29 \cdot 417$ & - & $20 \cdot 8$ & $23 \cdot 0$ & $21 \cdot 8$ & - & E l,y $N, 3^{\circ}$ & - & - & be & & Cl.-ett. 5 & - & - & \\
\hline 10 & $29 \cdot 421$ & - & $21 \cdot 0$ & $22 \cdot 0$ & $20 \cdot 8$ & - & E l,y $N, 3-1$ & - & - & lse & & 一 & S.еи. 8 & - & \\
\hline Milt. & $29 \cdot 410$ & - & $19 \cdot 6$ & $22 \cdot 1$ & $21 \cdot 2$ & - & E by $\mathbf{X}, 4$ & - & 二 & Ix & & Ci. $-8,4$ & S. - & -1 & \\
\hline Mean & $29 \cdot 44 t$ & -1 & - & $22 \cdot 1$ & 21) $\cdot 1$ & -1 & - & -1 & -1 & - & & - & $\dot{-}$ & - & $\begin{array}{l}\text { Fine day, thin st rat us remaining lighl all day. } \\
\text { Strong variable E wind. Burometer falling. }\end{array}$ \\
\hline & Max. the & I1. & a & $\left\{\begin{array}{l}8 \text { a. } 2 \\
81,2\end{array}\right.$ & $5^{\circ} \cdot 0$ & & Solar radiation $\left\{\begin{array}{l}1 \\
1\end{array}\right.$ & $\begin{array}{l}\text { Ulack } \\
\text { uright }\end{array}$ & $\begin{array}{l}\text { bult } \\
\text { bull }\end{array}$ & & val & - granmes. & Previjn. - in. & & $\begin{array}{l}\text { Thermometer ligh anl stearly. Yomng ice } \\
\text { forming in the bay. }\end{array}$ \\
\hline
\end{tabular}

2nd.

\begin{tabular}{|c|c|c|c|c|c|c|c|c|c|c|c|c|c|c|}
\hline 2 & $29 \cdot 116$ & -1 & $19: 5$ & $20 \cdot 0$ & $19 \cdot 2$ & -1 & $\mathbf{N} \operatorname{ly} \mathbf{N}, 2$ & - & -1 & b & - & - & - & \multirow{4}{*}{$\begin{array}{l}7.30 \text { a.m. Wind rose and blew up strong very } \\
\text { quickly. }\end{array}$} \\
\hline 4 & $29 \cdot 100$ & - & $14 \cdot 2$ & $1 \leqslant \cdot 8$ & $15 \cdot 0$ & - & Caln & - & - & $\mathrm{b}$ & - & - & - & \\
\hline 6 & $29 \cdot 386$ & - & $14 \cdot 0$ & $14 \cdot 2$ & $13 \%$ & - & Calm & - & - & 1 & - & - & - & \\
\hline 8 & $29 \cdot 361$ & - & $12 \cdot 0$ & $13 \cdot 0$ & $12 \cdot 8$ & - & TSNE, 5-6 & - & - & $\mathrm{b}$ & - & - & - & \\
\hline 10 & & & & & & & No olsery & atio & $n s t_{a}^{a}$ & en. & & & & \\
\hline Ninon & $29 \cdot 352$ & - & $13 \cdot 2$ & $15 \cdot 5$ & $13 \cdot 6$ & - & ENE, 5 & - & - & the & C1.-s. 8, ENE & - & - & \\
\hline 2 & $28 \cdot 350$ & - & $12 \cdot 0$ & 15.5 & $14 \cdot 0$ & - & ENE, 6 & - & - & b & - & - & - & \\
\hline 6 & & & & & & & No nhserv & ation & ns t a & en. & & & & \\
\hline 8 & $29 \cdot 343$ & - & $13 \cdot 4$ & $15 \cdot 2$ & $14 \cdot 0$ & - & INE, 3 & - & - & hic & Ci. 8 , JiNE & - & - & \\
\hline 10 & & & & & & & No olserv & atio ? & ns $t_{\text {a }}$ & en. & & & & First star seen to-night. \\
\hline Minlt. & $29 \cdot 33.1$ & -1 & $12 \cdot 0$ & $12 \cdot 8$ & $12 \cdot 0$ & -1 & BNE, 3 & - & - & b) & - & $一$ & -1 & \\
\hline Nean, & $29 \cdot 348$ & $\overline{-1}$ & - & $15 \cdot 2$ & $1 \cdot 1 \cdot 3$ & -1 & - & -1 & - & - & - & - & $1-$ & 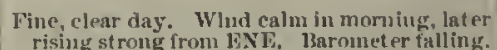 \\
\hline & Max. the & n. & d at & $\begin{cases}8 \mathrm{~s} .2 \\
8 \mathrm{p} .1\end{cases}$ & 1 & & ar radiation $\left\{\begin{array}{l}b \\
1, r\end{array}\right.$ & $\begin{array}{ll}\text { acek b } \\
\text { ightit }\end{array}$ & $\begin{array}{l}\text { ulit } \\
\text { bults. }\end{array}$ & & Wvapn. - grammes. & Precipm. - in. & & $\begin{array}{l}\text { Thermometer falling. Young ice is the } \\
\text { llay, but belng lilow out. }\end{array}$ \\
\hline
\end{tabular}

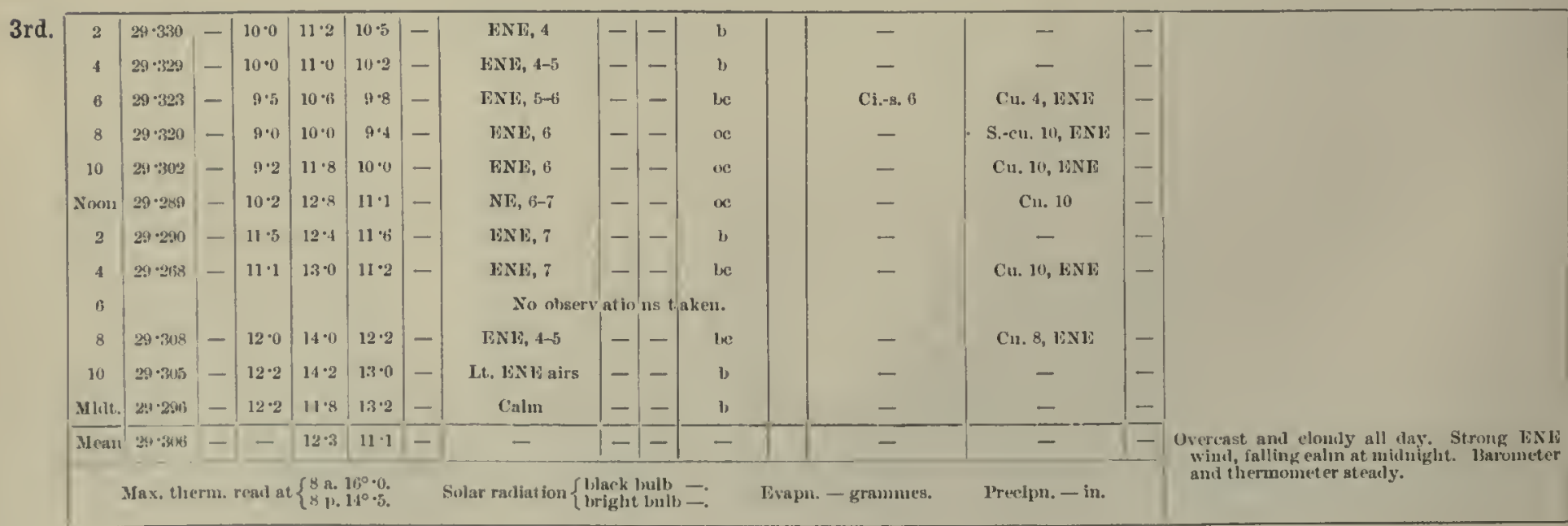


Mhteorolofical Journal of the "Discovery."

[1902-March.

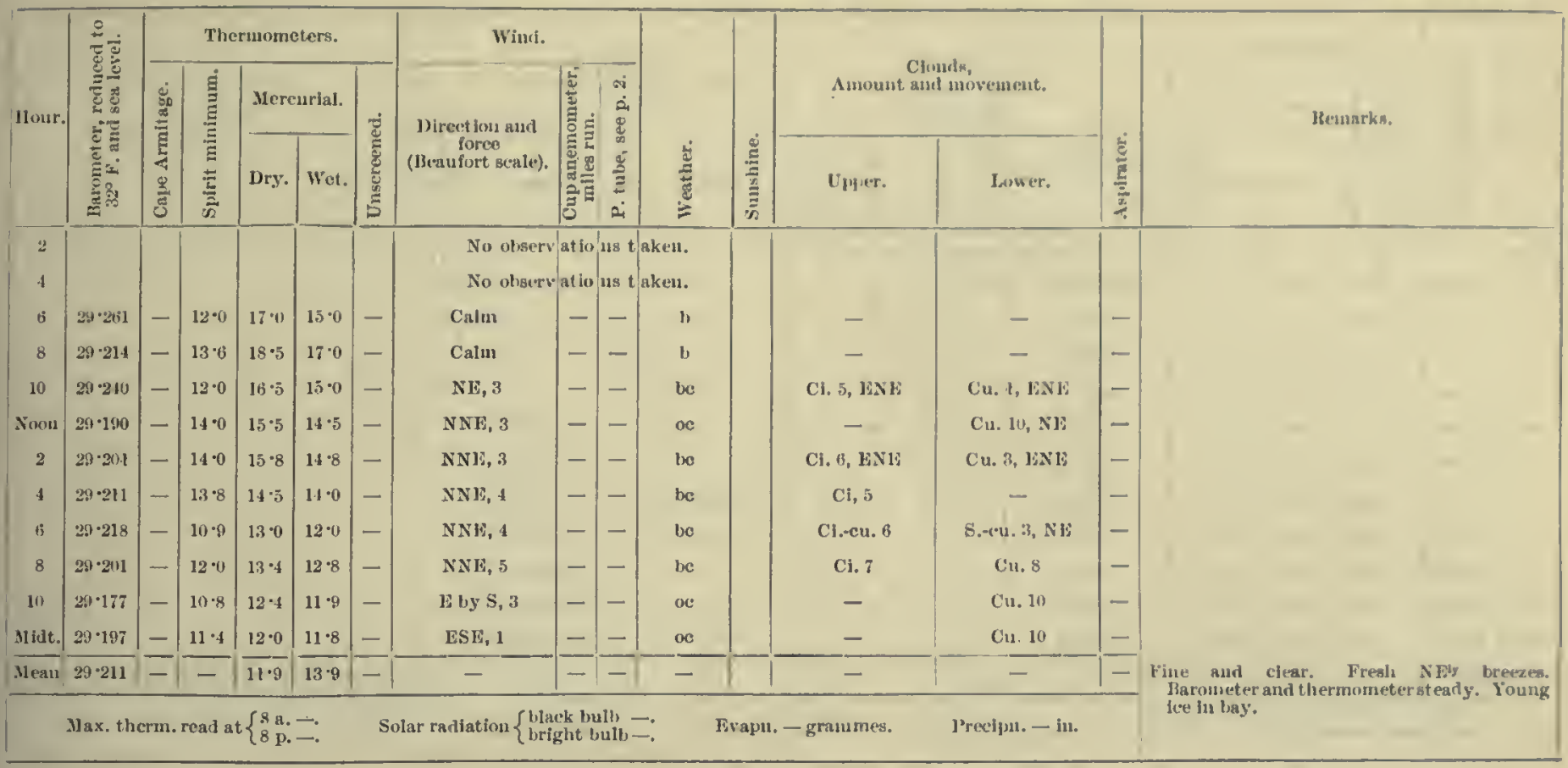

4 th.

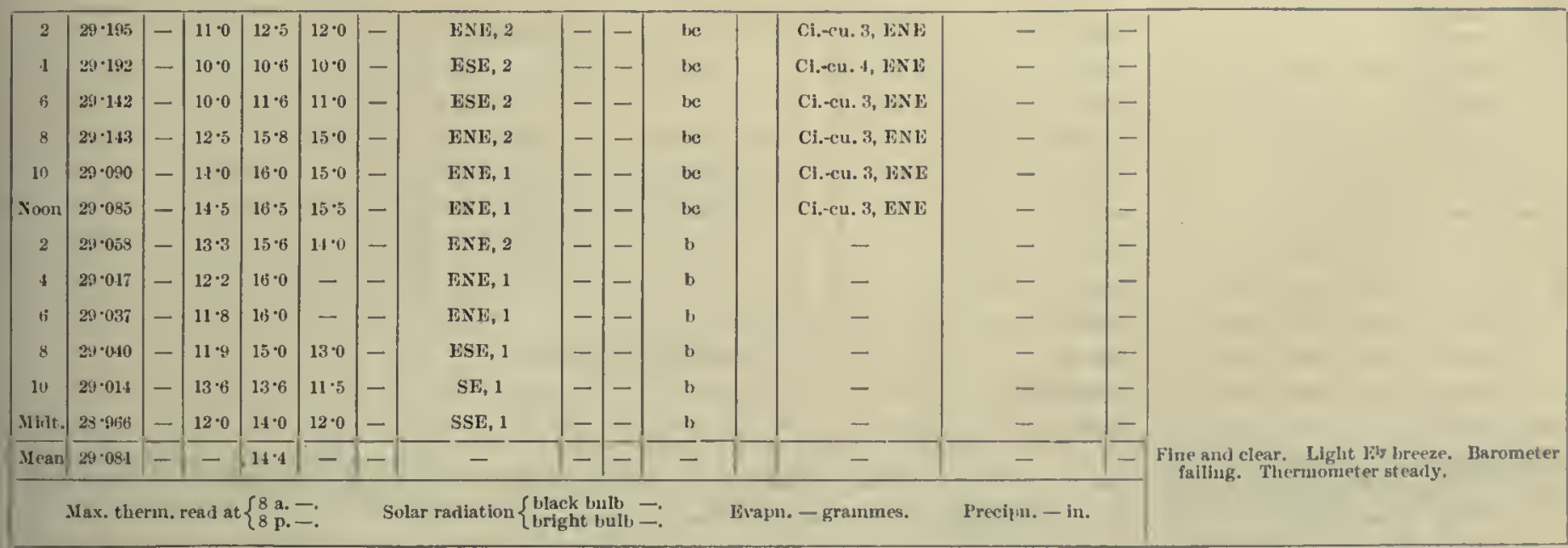

5 th.

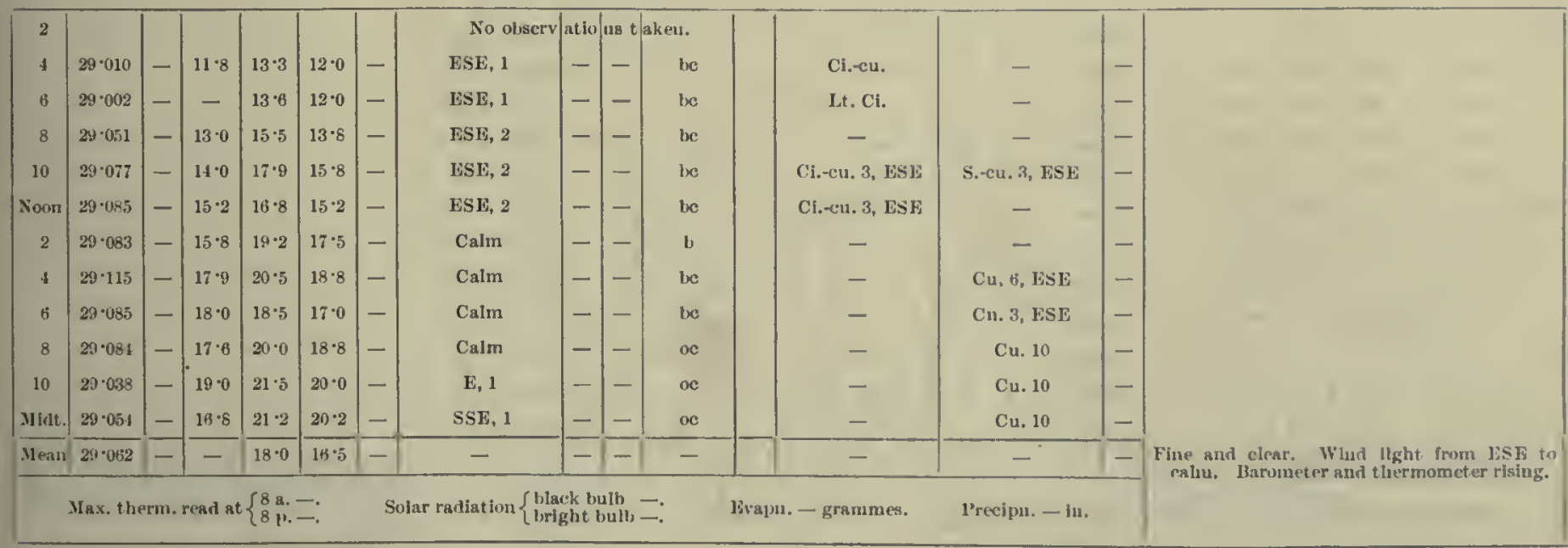




\begin{tabular}{|c|c|c|c|c|c|c|c|c|c|}
\hline \multirow{3}{*}{ our. } & \multirow{3}{*}{ 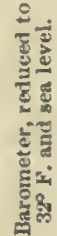 } & \multicolumn{5}{|c|}{ Thermometers. } & \multicolumn{3}{|l|}{ Wind. } \\
\hline & & \multirow{2}{*}{ 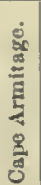 } & \multirow{2}{*}{ 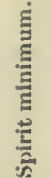 } & \multicolumn{2}{|c|}{ Mercurlal. } & \multirow{2}{*}{ 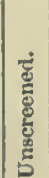 } & \multirow{2}{*}{$\begin{array}{c}\text { Direction and } \\
\text { foree } \\
\text { (Bcaufort scale). }\end{array}$} & \multirow{2}{*}{ 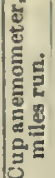 } & \\
\hline & & & & Dry. & Wet. & & & & $\frac{\frac{g}{3}}{2}$ \\
\hline
\end{tabular}

7 th.

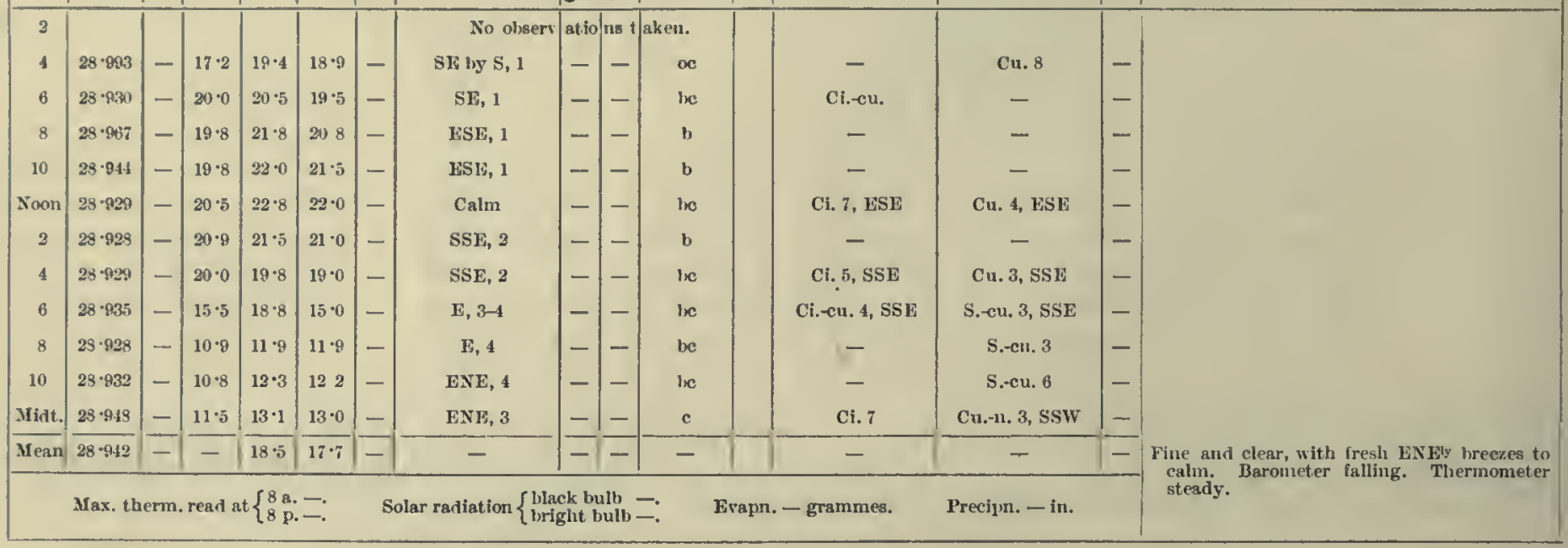

8th.

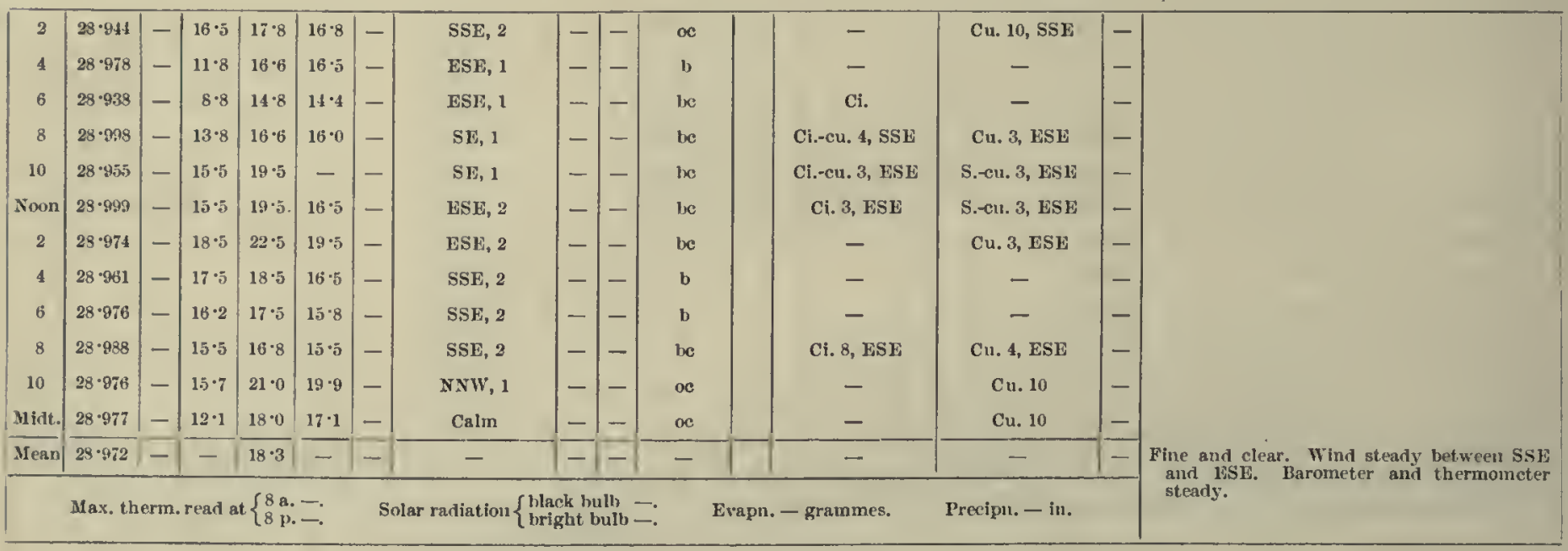

9 th.

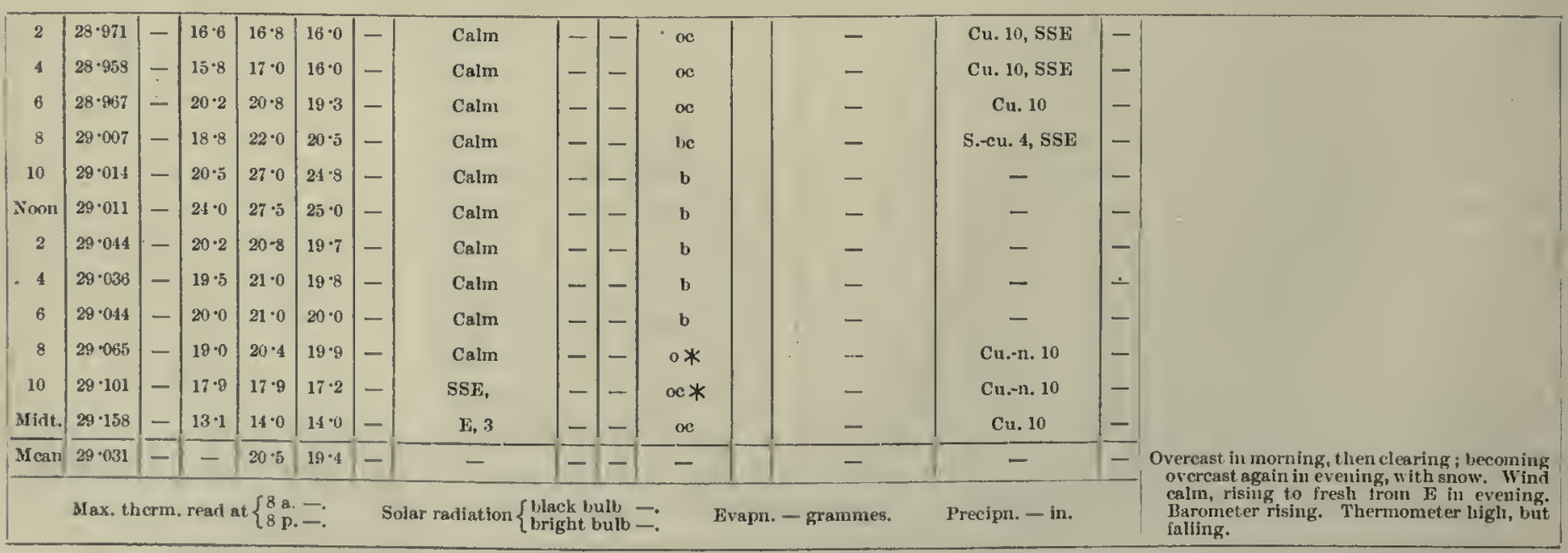


Meteonolonicat Journal of tJie "Discovery."

[1902-March.

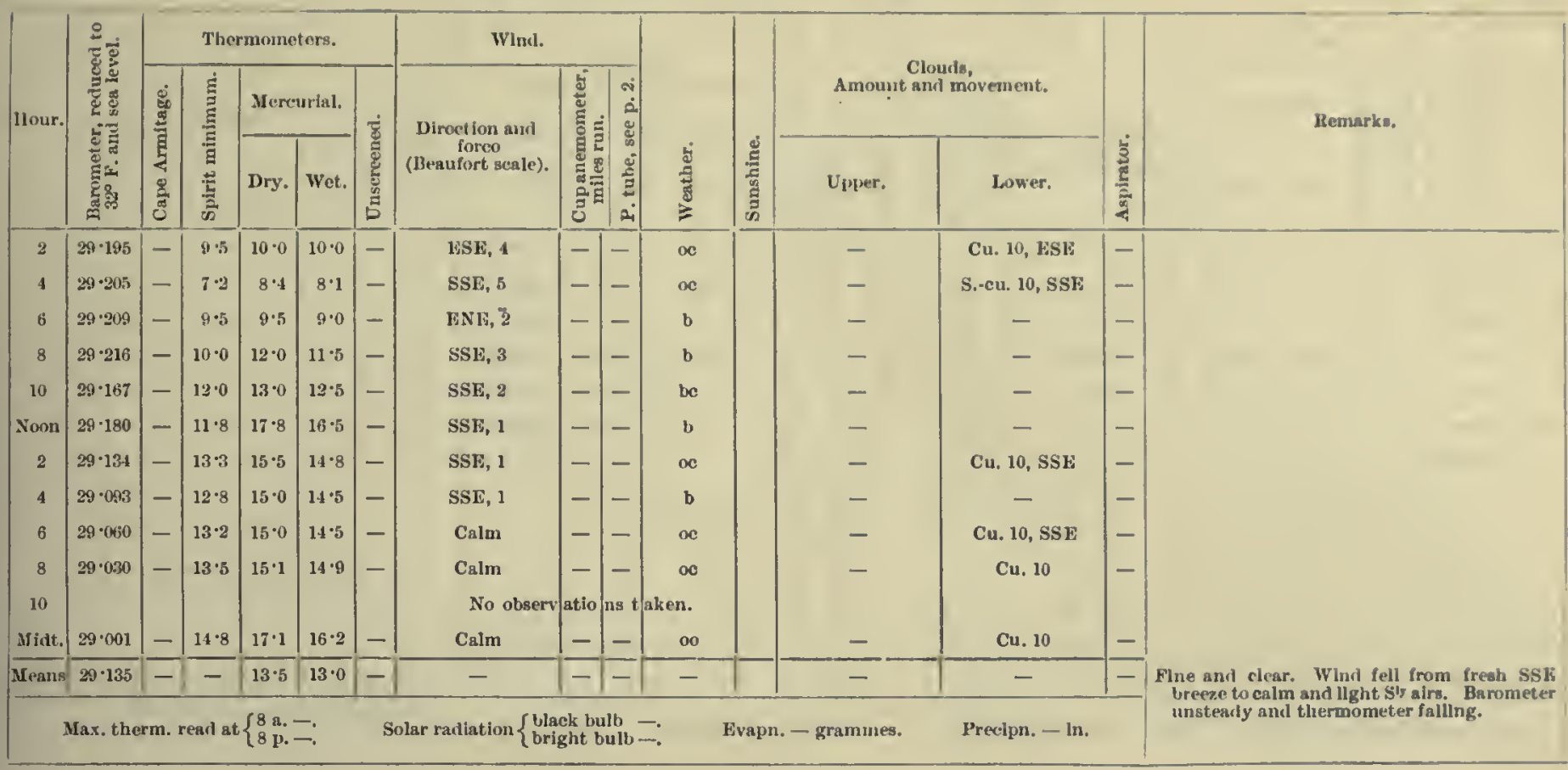

10 th.

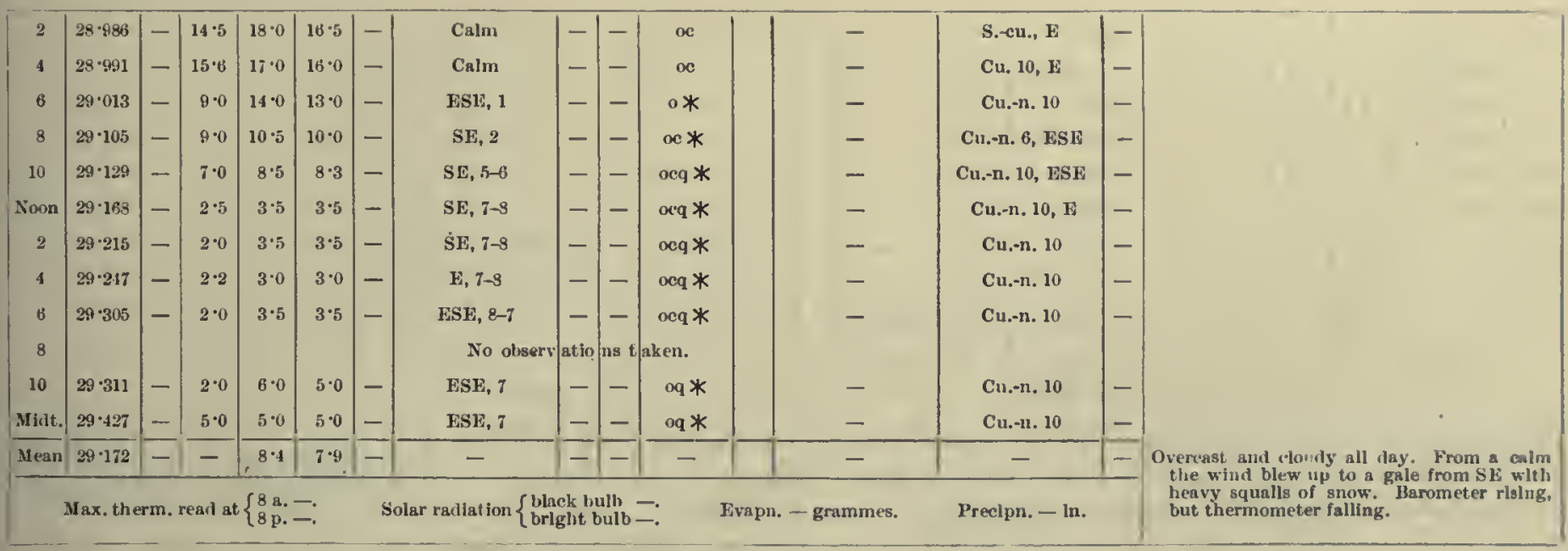

11 th.

\begin{tabular}{|c|c|c|c|c|c|c|c|c|c|c|c|c|c|c|}
\hline 2 & $29-392$ & - & $3 \cdot 5$ & $8 \cdot 0$ & 9.0 & -1 & $\mathbf{E}, 7$ & $1-$ & - & oq $*$ & - & Cu, -n. 10 & - & \\
\hline 4 & $29 \cdot 415$ & - & 2.5 & $3 \cdot 5$ & $3 \cdot 5$ & - & $\mathrm{E}, 6$ & - & - & $\infty$ & - & $\mathrm{Cu}, 10$ & - & - \\
\hline 6 & $23 \cdot 500$ & - & $3 \cdot 2$ & $4 \cdot 0$ & $3 \cdot 6$ & -1 & E by $N, 5-6$ & - & - & oc & - & Cu. 10 & - & . \\
\hline 8 & $29 \cdot 511$ & - & $4 \cdot 5$ & $5 \cdot 0$ & $5 \cdot 0$ & - & E, 5 & - & - & oe & - & Cir. 10 & - & \\
\hline 10 & $29 \cdot 496$ & - & $4 \cdot 0$ & 4.6 & $4 \cdot 6$ & - & $\mathrm{E}, 5-6$ & - & - & $\infty$ & - & Cu. 10 & - & \\
\hline Noon & $22 \cdot 473$ & - & $4 \cdot 0$ & $4 \cdot 6$ & $4 \cdot 6$ & - & $\mathrm{E}, \mathrm{B}$ & - & - & oc & - & Cu. 10 & - & \\
\hline 2 & $29 \cdot 572$ & - & $3 \cdot 8$ & $4 \cdot 5$ & $4 \cdot 5$ & - & $\mathrm{E}, 6$ & - & - & $o c$ & - & Cu. 10 & - & \\
\hline 4 & $29 \cdot 595$ & - & $3 \cdot 8$ & 5.5 & $5 \cdot 5$ & - & E, $7-3$ & - & - & oeq & - & Cu. 10 & - & \\
\hline$\theta$ & $29 \cdot 612$ & - & $3 \cdot 5$ & $4 \cdot 0$ & $4 \cdot 2$ & - & E, 7-8 & - & - & $o c q$ & - & Cu. 10 & - & \\
\hline 8 & $29 \cdot 669$ & - & 1.5 & $2 \cdot 2$ & $2 \cdot 2$ & - & $\mathrm{E}, 7$ & - & - & $o$ & - & Cu. 10 & - & \\
\hline 10 & $29 \cdot 677$ & - & 0.5 & $1 \cdot 2$ & $1 \cdot 0$ & - & E, 8 & - & - & $\boldsymbol{o c}$ & - & Cu. 10 & - & \\
\hline Midt. & $29 \cdot 691$ & - & 0.0 & 1.0 & 0.8 & -1 & E, 7 & - & - & $\infty$ & - & Cu. 10 & -1 & \\
\hline I ean & $29 \cdot 550$ & - & - & $4 \cdot 1$ & $4 \cdot 0$ & - & - & - & - & - & - & - & - & $\begin{array}{l}\text { Overeast and cloudy all day. Galo from } \\
\text { with heary squalls. Barometer rising, but }\end{array}$ \\
\hline
\end{tabular}


Methorological Journal of THe "Discovery."

\begin{tabular}{|c|c|c|c|c|c|c|c|c|c|c|c|c|c|c|}
\hline \multirow{3}{*}{ Hour. } & \multirow{3}{*}{ 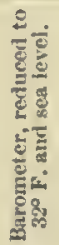 } & \multicolumn{5}{|c|}{ Thermometers. } & \multicolumn{3}{|l|}{ Wind. } & \multirow[b]{3}{*}{ 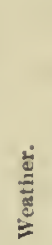 } & \multirow[b]{3}{*}{ 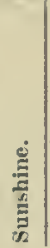 } & \multirow{2}{*}{\multicolumn{2}{|c|}{$\begin{array}{l}\text { Clouds, } \\
\text { Amount and movement. }\end{array}$}} & \\
\hline & & \multirow{2}{*}{ 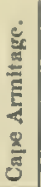 } & \multirow{2}{*}{ 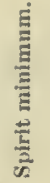 } & \multicolumn{2}{|c|}{ Mereurial. } & \multirow{2}{*}{ 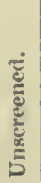 } & \multirow{2}{*}{$\begin{array}{l}\text { Direction and } \\
\text { force } \\
\text { (Heatufort seale). }\end{array}$} & \multirow{2}{*}{ 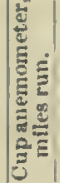 } & \multirow{2}{*}{ 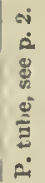 } & & & & & \multirow[b]{2}{*}{ 总 } \\
\hline & & & & Dry. & Wet. & & & & & & & Uprer. & Lower. & \\
\hline
\end{tabular}

13 th.

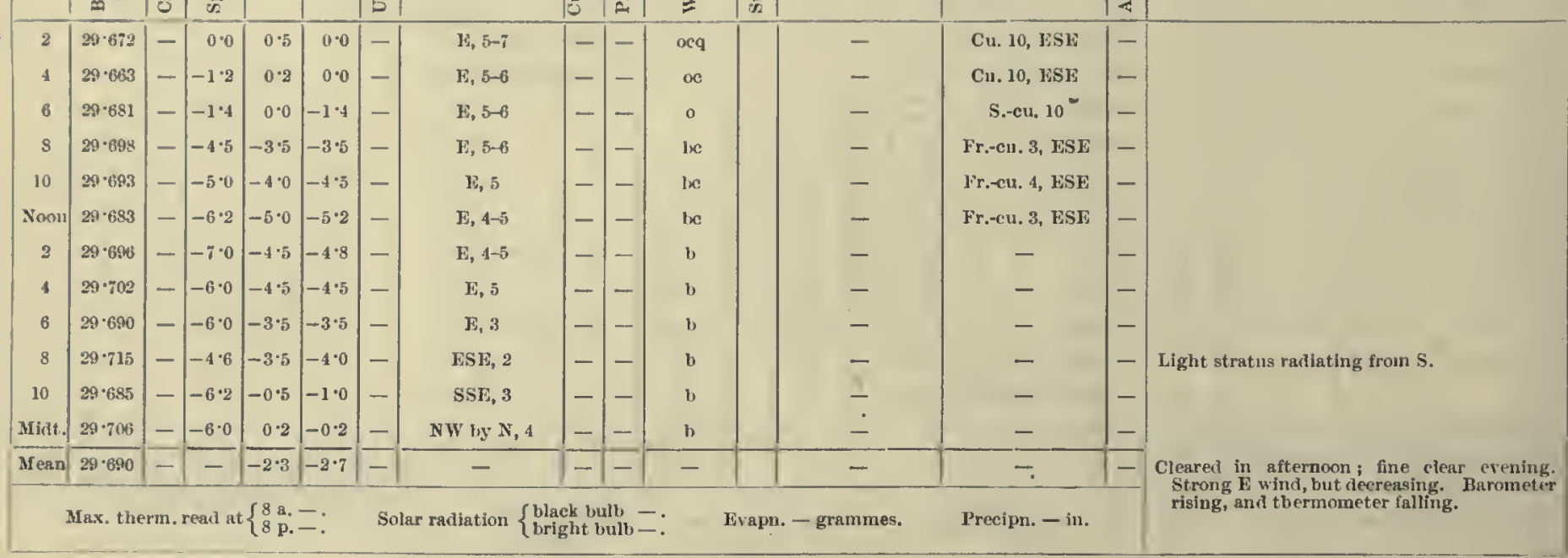

14th.

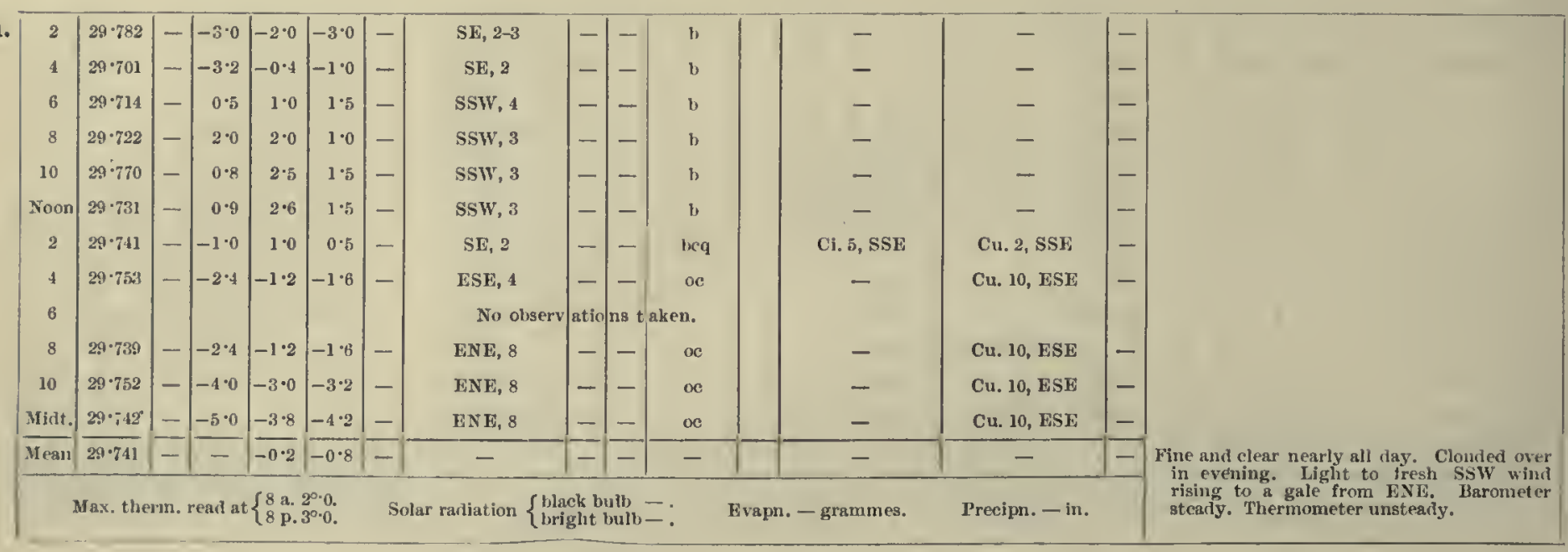

15 th.

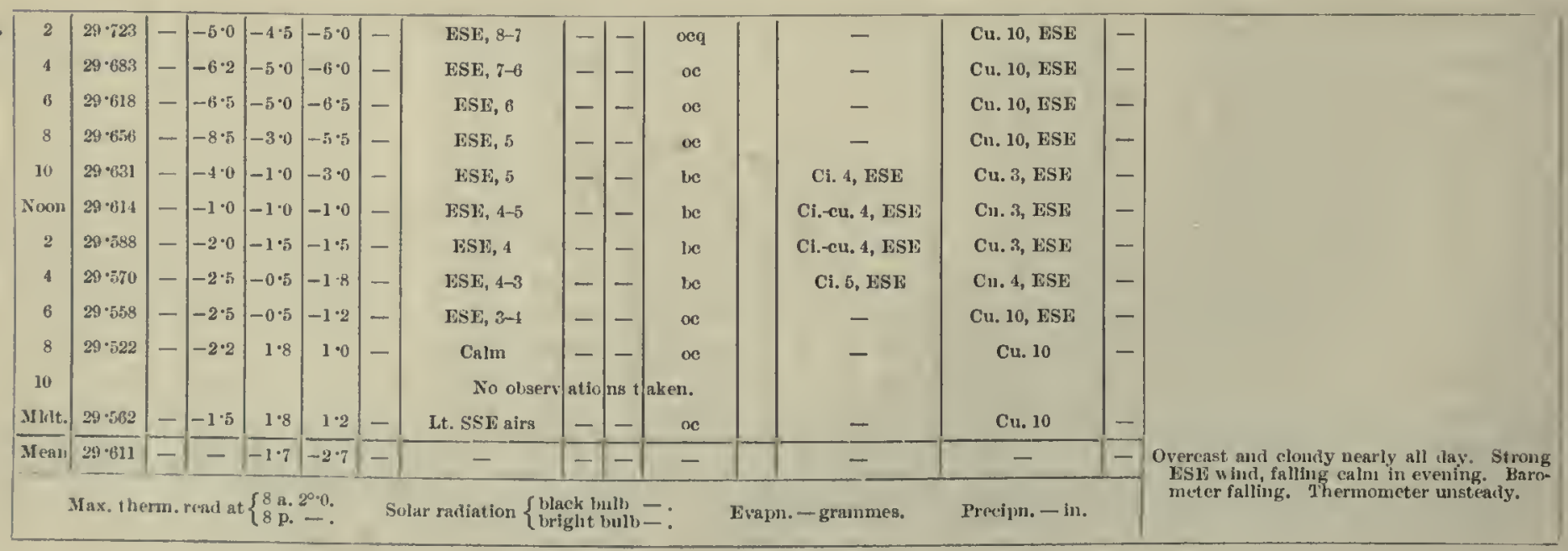


Methorologicat, Joursat, of tile "Discoveiz,"

[1902-March.

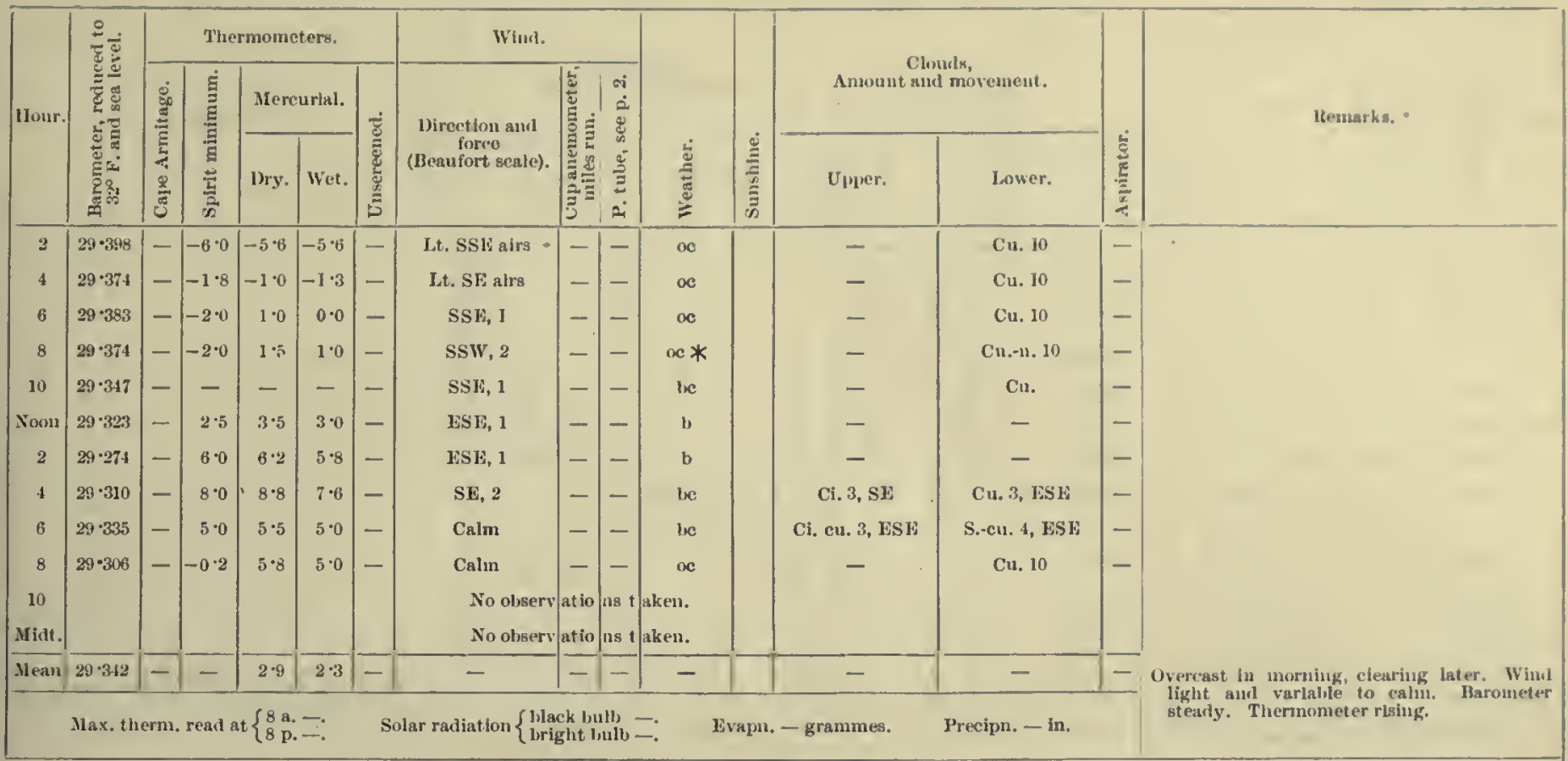

16 th

\begin{tabular}{|c|c|c|c|c|c|c|c|c|c|c|c|c|c|c|}
\hline 2 & $29 \cdot 323$ & $1-1$ & $7 \%$ & $11 \% 5$ & $\mid 11 \cdot 0$ & -1 & NNR, 1 & - & - & be & - & Cu. 5, NNT & \multirow{10}{*}{\multicolumn{2}{|c|}{ 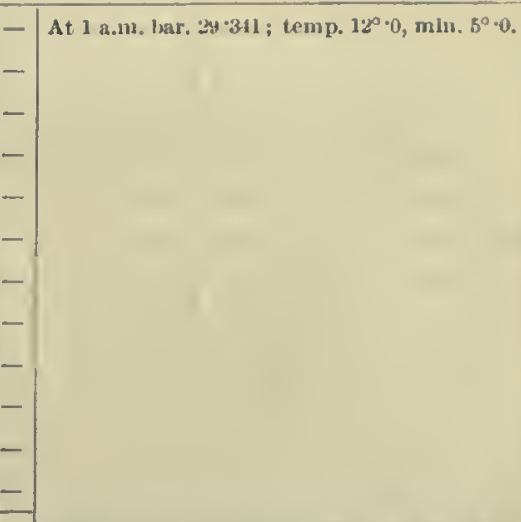 }} \\
\hline 4 & 29301 & - & $7 \cdot 0$ & $11 \cdot 0$ & $10 \cdot 5$ & - & NNE, 1 & - & - & $\infty$ & - & $\mathrm{Cu}, 10, \mathrm{~N}$. & & \\
\hline 6 & $29 \cdot 326$ & - & $7 \cdot 6$ & $8 \cdot n$ & $7 \cdot 5$ & - & $\mathrm{NNE}_{2}, 1$ & - & - & be & - & Cu. & & \\
\hline 8 & $29 \cdot 426$ & - & $7 \cdot 5$ & $10 \cdot 8$ & $10^{\circ} 0$ & - & NNE, 2 & - & - & in & - & Cn. 5, ENE & & \\
\hline 10 & $29 \cdot 160$ & - & $12 \cdot 0$ & $14 \cdot 5$ & $12 \cdot 8$ & - & NNE, I & - & - & be & - & Cu. 6, NNE & & \\
\hline Noon & $29 \cdot 490$ & - & $12 \cdot 8$ & $14 \cdot 0$ & $13 \cdot 8$ & -1 & $\mathrm{SST}, 2$ & - & - & $\mathbf{c}$ & - & Cu. 10, SSE & & \\
\hline 2 & $29 \cdot 405$ & - & $12 \cdot 0$ & $14 \cdot 0$ & $13 \div$ & - & SSE, $6-5$ & - & - & ne & - & $\mathrm{Cu}, 10, \mathrm{SSE}$ & & \\
\hline 8 & $29 \cdot 596$ & - & $13 \cdot 5$ & $16 \cdot 0$ & $15 \cdot 0$ & - & SSW, 8 & - & - & $\mathrm{oc}$ & - & Cu. 10 & & \\
\hline 10 & $29 \cdot 601$ & - & $14 \cdot 6$ & $17 \cdot 8$ & $14 \cdot 8$ & - & Ssw, 8 & - & - & $\propto c$ & - & Cu. 10 & & \\
\hline Miilt. & $29 \cdot 607$ & $=$ & $15 \cdot 8$ & $17 \cdot 2$ & 16.8 & - & Ssw, 9 & - & - & oc & - & Cu. 10 & & \\
\hline Nean & $29 \cdot 479$ & 二 & - & $13 \cdot \overline{7}$ & $12 \cdot 9$ & - & - & -1 & -1 & $\cdot-$ & - & - & - & \multirow{2}{*}{$\begin{array}{l}\text { Overcast nearly all day. Wind light NXE, } \\
\text { rising to a gale from sSW, fores } 9 \text {. Baro- } \\
\text { neter aud thernometer rising. }\end{array}$} \\
\hline & $\mathrm{HI}$ & & ant & & & & & & & & nes. & Precipn. - in. & & \\
\hline
\end{tabular}

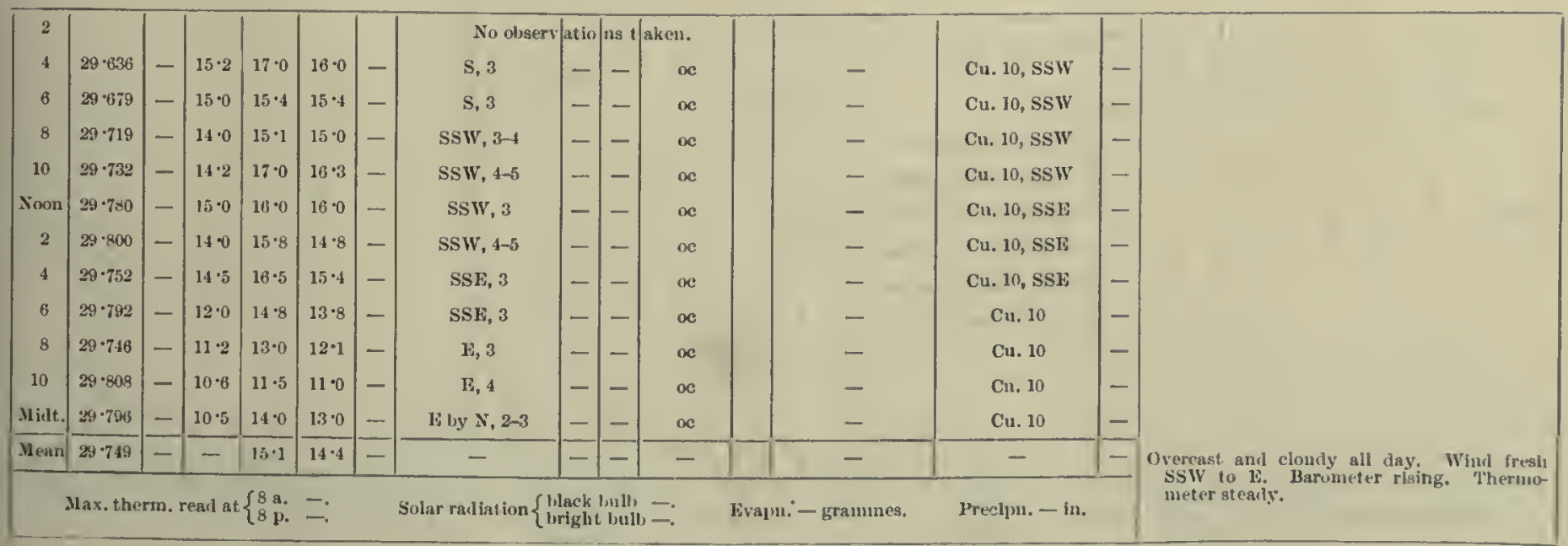

18 th. 
1902 -March.]

Metworological Journat, of tie "Discovery."

19 th.

\begin{tabular}{|c|c|c|c|c|c|c|c|c|c|c|c|c|c|c|c|}
\hline \multirow{3}{*}{ Hour. } & \multirow{3}{*}{ 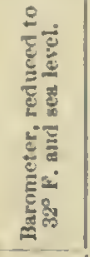 } & \multicolumn{5}{|c|}{ Thermomoters. } & \multicolumn{3}{|l|}{ Wiucl. } & \multirow[b]{3}{*}{ 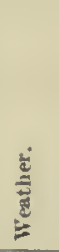 } & \multirow{3}{*}{ 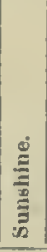 } & \multirow{2}{*}{\multicolumn{2}{|c|}{$\begin{array}{l}\text { Clouds, } \\
\text { Amount and novement. }\end{array}$}} & \multirow[b]{3}{*}{ 遏 } & \multirow{3}{*}{ Lemarks. } \\
\hline & & \multirow{2}{*}{ 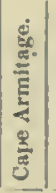 } & \multirow{2}{*}{ 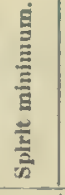 } & \multicolumn{2}{|c|}{ Mercurlal. } & \multirow{2}{*}{ 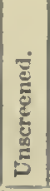 } & \multirow{2}{*}{$\begin{array}{c}\text { Direetion and } \\
\text { force } \\
\text { (Beaufort scale). }\end{array}$} & \multirow{2}{*}{ 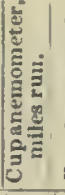 } & \multirow{2}{*}{ 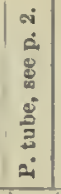 } & & & & & & \\
\hline & & & & Dry. & Wet. & & & & & & & Uper. & Lower. & & \\
\hline 2 & & & & & & & No olserv & atio & met & ens. & & & - & & \\
\hline 4 & $29 \cdot 720$ & - & $13 \cdot 6$ & $14 \cdot 0$ & $14 \cdot 5$ & - & SSW, 1 & - & - & lic & & Ci. & - & - & \\
\hline 6 & $29 \cdot 761$ & - & $12 \cdot 3$ & $14 \cdot 3$ & $13 \cdot 7$ & - & L.t. SSli airs & - & - & b & & - & - & - & \\
\hline 8 & $29-735$ & - & $13 \cdot 3$ & $17 \cdot 0$ & $16 \cdot 0$ & - & Calm & - & - & $b$ & & - & - & - & \\
\hline 10 & $29 \cdot 735$ & - & $13 \cdot 8$ & $18 \cdot 5$ & $17 \div 5$ & - & SSE, 2 & - & - & be & & - & Cu. 9, SSE & - & \\
\hline Xoon & $29 \cdot 612$ & - & 16.5 & $18 \cdot 0$ & $17 \cdot 0$ & - & $\operatorname{SSK}, 2-3$ & - & - & $\infty$ & & - & C11, 10 & - & \\
\hline 2 & $29 \cdot 67^{2}$ & - & $13 \cdot 5$ & $14 \cdot 8$ & $14 \cdot 0$ & - & ISE, 3 & -1 & - & $\infty$ & & - & Cu. 10, 1isL: & - & \\
\hline $\begin{array}{l}4 \\
6\end{array}$ & $29 \cdot 62$ & - & $11 \cdot 0$ & $21 \cdot 8$ & $19 \cdot 8$ & - & $\begin{array}{l}\text { LSE, } 1 \\
\text { No observ }\end{array}$ & - & - & be & & Ci.-ell. 3 , ESIE & - & - & - \\
\hline 8 & $29 \cdot 714$ & - & $12 \cdot 0$ & $18 \cdot 5$ & $18^{\circ} 0$ & - & SSE, $0-1$ & - & - & $\alpha$ & & T & C11. 10 & - & \\
\hline 10 & $29 \cdot 705$ & - & $18 \cdot 0$ & $20 \cdot 3$ & $19 \cdot 8$ & - & SSE, 1 & - & - & $\infty$ & & - & C11. 10 & - & \\
\hline Midt. & $29 \cdot 696$ & - & $19 \cdot 5$ & $22 \cdot 8$ & $22 \cdot 4$ & - & SW by $\mathrm{S}, 3$ & - & - & c & & - & Ci1. 10 & - & \\
\hline Mean & $29 \cdot 705$ & - & - & $18 \cdot 0$ & $17 \cdot 3$ & - & - & - & -1 & - & & - & 一. & - & $\begin{array}{l}\text { Fine in morning, clouding over in cvening. } \\
\text { Wiud light and variable. Barometer steady. }\end{array}$ \\
\hline & Max. the & rm. & read at & $\begin{cases}8 & \mathrm{a} . \\
8 & \mathrm{p} .\end{cases}$ & & & lar radiation $\left\{\begin{array}{l}\text { bla } \\
\text { brig }\end{array}\right.$ & ight bu & alb - . & & & -grammes. & I'recipn. - in. & & eter \\
\hline
\end{tabular}

20 th.

\begin{tabular}{|c|c|c|c|c|c|c|c|c|c|c|c|c|c|c|}
\hline 2 & $29 \cdot 696$ & $1-$ & $19 \cdot 7$ & $20 \cdot 4$ & $20 \cdot 0$ & $1-$ & SSW, 2-4 & -1 & -1 & ee & - & S.-eu, 9, SSW & - & . \\
\hline 4 & $29 \cdot 672$ & - & $19 \cdot 0$ & $21 \cdot 2$ & $21 \cdot 2$ & - & $\mathrm{SW}, 1$ & - & - & $a$ & - & S.cu. 10 & - & \\
\hline 6 & $29 \cdot 607$ & - & $19 \cdot 1$ & $19 \cdot 5$ & $19 \cdot 3$ & - & SSE, 1 & - & - & bc & Ci.ecu. 6 & - & - & \\
\hline 8 & 29.674 & - & $18 \cdot 5$ & $21 \cdot 8$ & $21 \cdot 5$ & - & S, 2-3 & - & - & oc & - & Fr.cu. 10, s & - & . \\
\hline Noon & $29 \cdot 531$ & - & $18 \cdot 5$ & $21 \cdot 5$ & $21 \cdot 2$ & - & $S, 1$ & -1 & - & be & Ci. 5,5 & Cu. 3,5 & - & \\
\hline 2 & $29 \cdot 544$ & - & $19 \cdot 5$ & $20 \cdot 5$ & $20 \cdot 2$ & - & S, 2 & - & - & be & Ci. $5, \mathrm{~s}$ & 8.cu. $3, \mathrm{~s}$ & - & \\
\hline 8 & $29 \cdot 632$ & - & $12 \cdot 0$ & $14 \cdot 0$ & $13 \cdot 9$ & - & E, 1-2 & - & - & $\mathrm{c}$ & - & Cu. 10 & - & \\
\hline 10 & $29 \cdot 601$ & - & $12 \cdot 0$ & $17 \cdot 0$ & $17 \cdot 0$ & - & SST, 6-8 & - & - & oc & - & S.-cu. 10 & - & \\
\hline Mliat. & $29 \cdot 636$ & - & $16 \cdot 0$ & $18 \cdot 2$ & $18 \cdot 0$ & - & $\mathrm{SSW}, 2-3$ & - & - & $\infty$ & - & S.cu. 10 & - & \\
\hline$\overline{\text { Mean }}$ & $29 \cdot 612$ & $\overline{-}$ & - & $19 \cdot 1$ & $18 \cdot 8$ & - & - & - & - & - & - & - & \multirow{2}{*}{\multicolumn{2}{|c|}{$\begin{array}{l}\text { Generally fine, overcast. at intervals. Strong } \\
\text { SSW nud S Wind. Rarouneter falling, but } \\
\text { thermometer steady. }\end{array}$}} \\
\hline \multicolumn{7}{|c|}{ Max, therm. read at $\left\{\begin{array}{l}8 \mathrm{a} . \\
8 \mathrm{p} . 二\end{array}\right.$} & \multicolumn{4}{|c|}{ Solar radiation $\left\{\begin{array}{l}\text { blaek bull, } \\
\text { bright bulb- }\end{array}\right.$} & mmes. & Precipn. - in. & & \\
\hline
\end{tabular}

21 st.

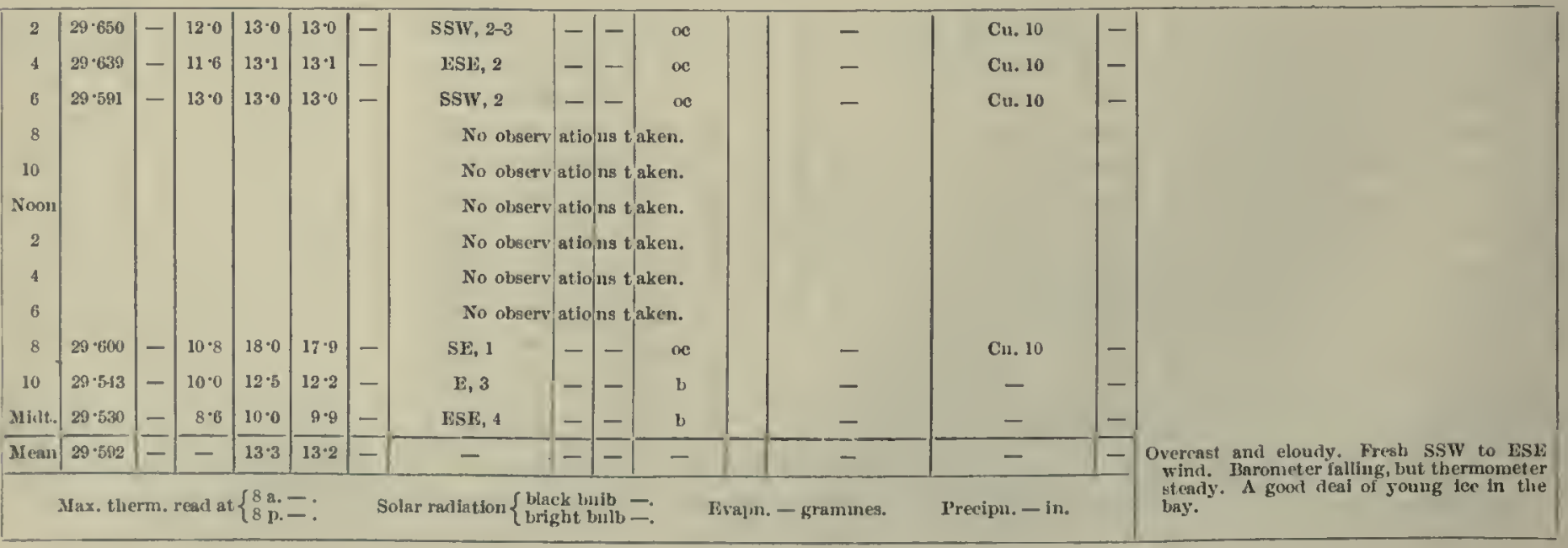


Methonological Journal of tim "Discovery."

[1902-March.

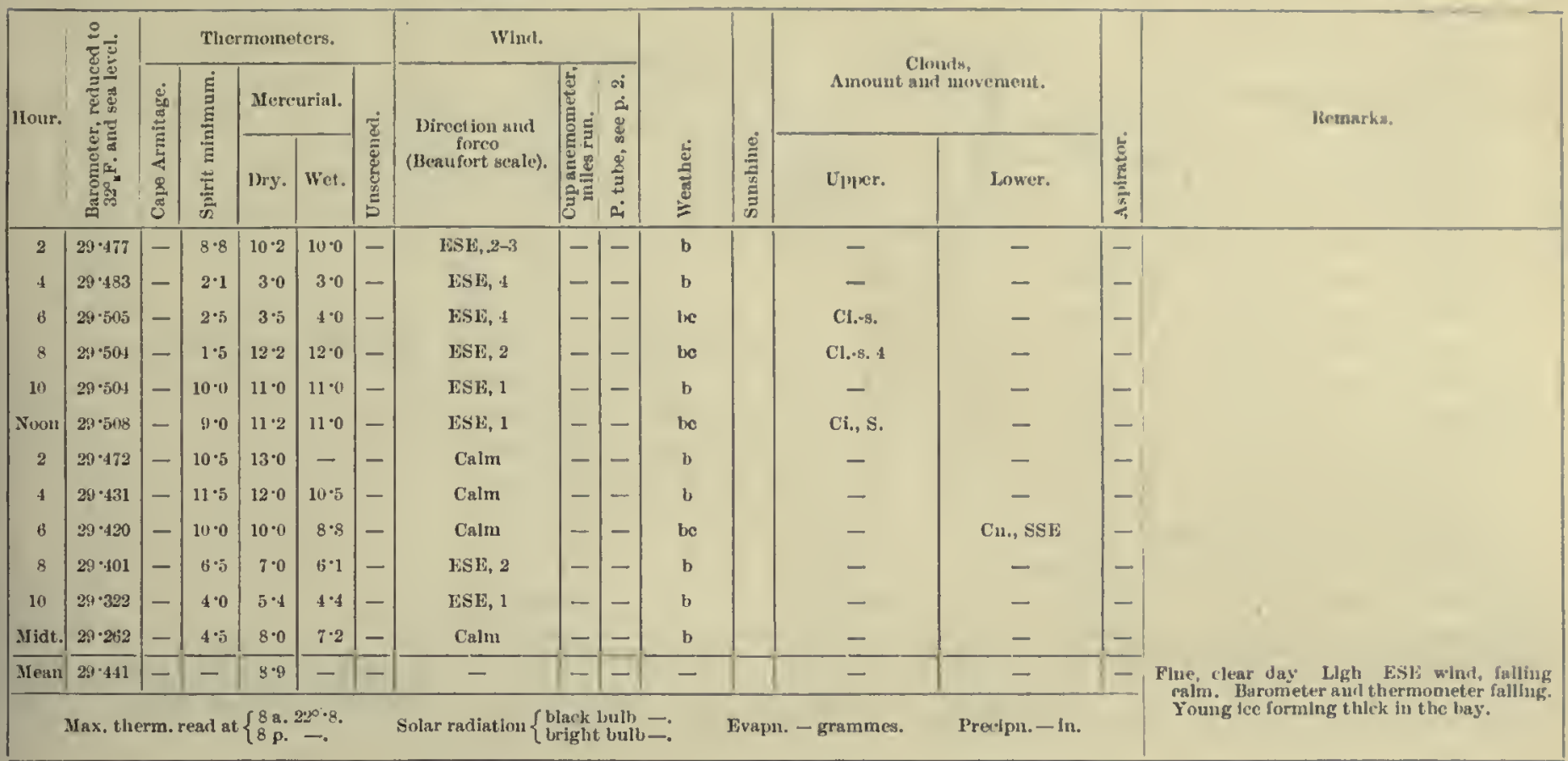

22nd.

\begin{tabular}{|c|c|c|c|c|c|c|c|c|c|c|c|c|c|c|}
\hline 2 & $29 \cdot 184$ & - & $5 \cdot 0$ & $6 \cdot 8$ & $6 \cdot 5$ & -1 & ESE, 2 & - & - & b & - & - & $1-$ & \multirow{8}{*}{ Strong mirage at the foot of all lund to the $\mathrm{S}$. } \\
\hline 4 & $29 \cdot 132$ & - & $5 \cdot 9$ & $6 \%$ & $5 \cdot 5$ & - & ESP, 2 & - & - & bc & - & S.Cu. & - & \\
\hline 6 & $29 \cdot 113$ & - & -0.5 & 0.8 & $0 \cdot 0$ & -1 & $\mathrm{NE}, 2$ & - & - & b & - & - & - & \\
\hline 8 & $29 \cdot 065$ & - & $0 \cdot 0$ & $4 \cdot 2$ & $3 \cdot 0$ & - & E by $N, 3$ & - & - & bc & - & Cu. 8, NNW & - & \\
\hline$x_{\text {๓⿴囗十 }}$ & $29 \cdot 032$ & - & -0.8 & $0 \cdot 0$ & $-0 \cdot 5$ & - & E by N, 3-4 & - & - & oc & - & Cu. 9, Nพ & - & \\
\hline 2 & $29 \cdot 018$ & - & $-1 \cdot 0$ & 0.0 & -0.8 & - & N by $\mathbf{N}, 4$ & - & - & oc- & - & Cu. 10 & - & \\
\hline 8 & $29 \cdot 082$ & - & $-5 \cdot 0$ & $-4 \cdot 2$ & $-4 \cdot 0$ & - & ESE, 8 & - & - & $a c+p^{2}$ & - & Cu. 10 & - & \\
\hline 10 & $29 \cdot 169$ & - & $-5 \cdot 0$ & $-3 \cdot 2$ & $-3 \cdot 2$ & - & ESE, 8 & - & - & $o c+t^{2}$ & - & Cu. 10 & - & \\
\hline Midt. & $29 \cdot 150$ & - & $-4 \cdot 0$ & $-1 \cdot 0$ & $-1 \cdot 5$ & -1 & ESE, 4 & - & - & oc* $*$ & - & Cu.-n. 10 & - & \multirow{2}{*}{ 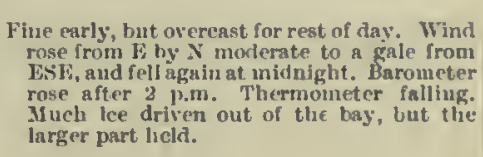 } \\
\hline Nean & $29 \cdot 082$ & - & - & 0.4 & $-0 \div 3$ & - & - & - & -1 & - & - & - & $1=$ & \\
\hline
\end{tabular}

$23 r d$.

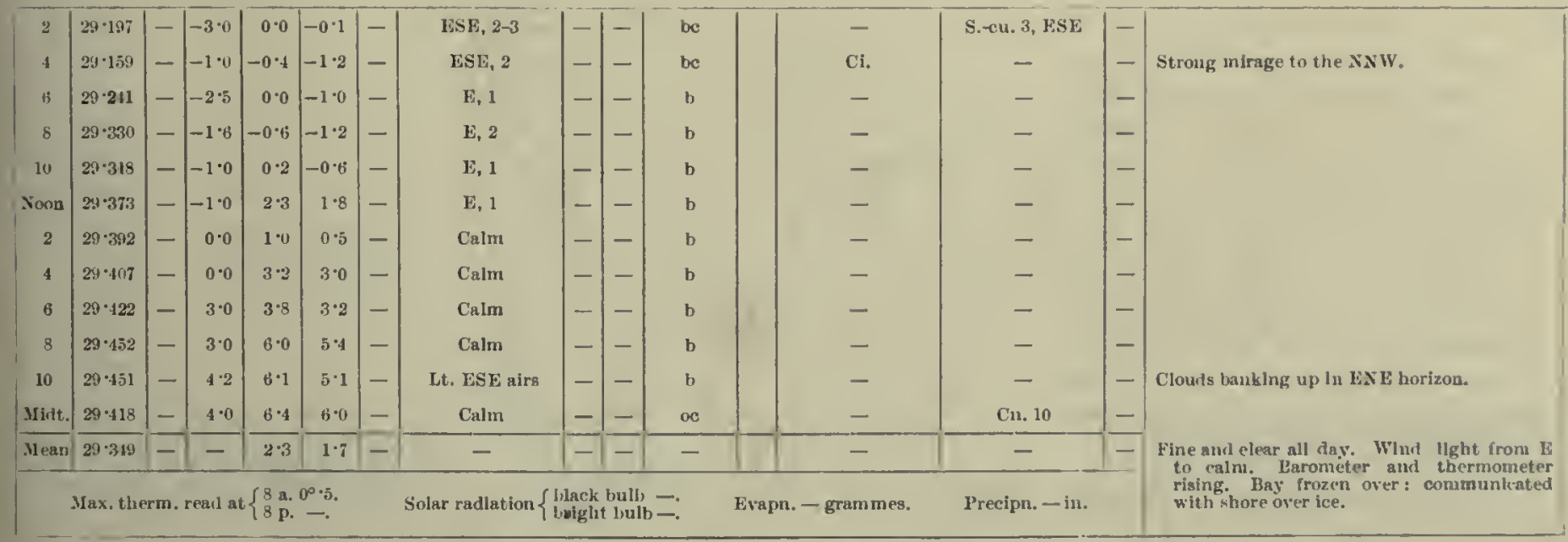

24th. 
1902 -March.]

Meteorologigal. Jouknal of thr "Discoveky."

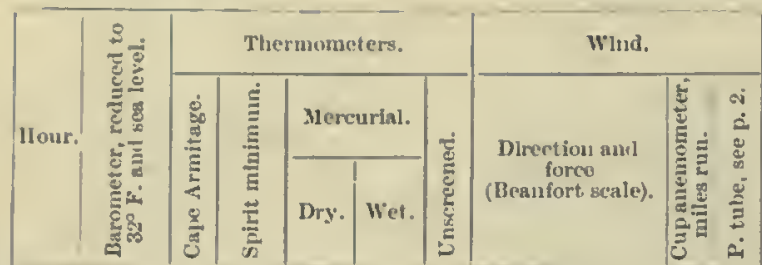

25th.

\begin{tabular}{|c|c|c|c|c|c|c|c|c|c|c|c|c|c|c|}
\hline 3 & $29 \cdot 418$ & - & $6 \cdot 0$ & $10 \cdot 0$ & $9 \cdot 8$ & -1 & 1:SF, a-1 & - & -1 & $\infty$ & - & Cu, 10, ISE & - & \multirow{14}{*}{ 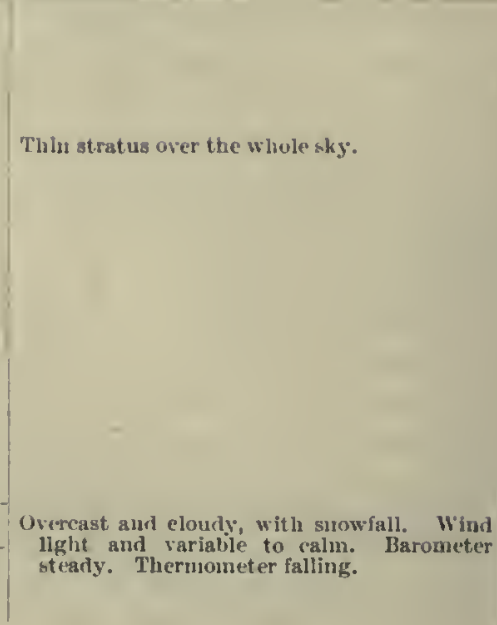 } \\
\hline 4 & 2989 & - & $9 \cdot 0$ & $0 \cdot 6$ & $9 \cdot 0$ & - & Calm & - & -1 & oe & - & Cu. 10 & - & \\
\hline b & $29 \cdot 412$ & - & $n \cdot 1$ & $11 \cdot 2$ & $10 \cdot 8$ & - & NNE, 1 & - & - & oc & - & Cu. 10 & - & \\
\hline 8 & $29 \cdot 395$ & - & $10 \cdot 2$ & $11 \cdot 2$ & $10 \cdot 8$ & - & $x, 1$ & - & - & be & Ci.-s. 5, N.XW & S.-cu. $6, \mathrm{NXl}$ & - & \\
\hline 10 & $29 \cdot 391$ & - & $10 \cdot 8$ & $11 \cdot 3$ & $11 \cdot 0$ & - & $x, 1-2$ & - & - & oc $*$ & - & Cu.-11. 10, NNE & - & \\
\hline Noon & $29 \cdot 370$ & - & $11 \cdot 0$ & $12 \cdot 2$ & $11 \cdot 6$ & - & Calm & - & - & le* $*$ & Cl. 5, NNW & Cu.-n. 7, NNE & - & \\
\hline 2 & $29 \cdot 336$ & - & $10 \cdot 0$ & $10 \cdot 2$ & $10 \cdot 0$ & - & Calm & - & - & be $*$ & Ci. $5, \mathrm{NNW}$ & C11.-n. 6, NNE & - & \\
\hline 4 & $20 \cdot 299$ & - & $8 \cdot 0$ & $9 \cdot 0$ & $9 \cdot 0$ & - & Ev, 2 & - & - & $\infty *$ & - & Cu.-n. 10, NN1i & - & \\
\hline$b$ & $29 \cdot 210$ & $-\cdot$ & $4 \cdot 6$ & $6 \cdot 0$ & $5 \cdot 8$ & - & Calm & - & - & oc $*$ & - & Cu.-n. in & - & \\
\hline 8 & $29 \cdot 2566$ & - & 40 & $5 \cdot 0$ & $4 \cdot 9$ & - & Lt. KNE alrs & - & - & $\propto *$ & $1-$ & Cu. -11.10 & - & \\
\hline 10 & $29 \cdot 3 \mu$ & - & $4 \cdot 0$ & 1.5 & $4 \cdot 2$ & - & $\mathrm{KSE}, 1$ & - & - & $\infty *$ & - & Cu.-11. 10 & - & \\
\hline Mlitt. & $20 \cdot 331$ & -1 & 0.5 & $1 \cdot 8$ & $1 \cdot 6$ & - & RSE, 3-4 & - & - & $\operatorname{\alpha cq} *$ & - & Cu.-n. 10 & - & \\
\hline 31 ean & $29 \cdot 3: 66$ & - & - & $8 \cdot 5$ & $8 \cdot 2$ & -1 & - & -1 & - & - & - & $\leadsto$ & - & \\
\hline & & & & & & & adiatlou. & $\begin{array}{l}\text { ek } \\
\text { ght }\end{array}$ & $\begin{array}{l}\text { bulh } \\
\text { bulb }\end{array}$ & & & Precipn. - in & & \\
\hline
\end{tabular}

26th.

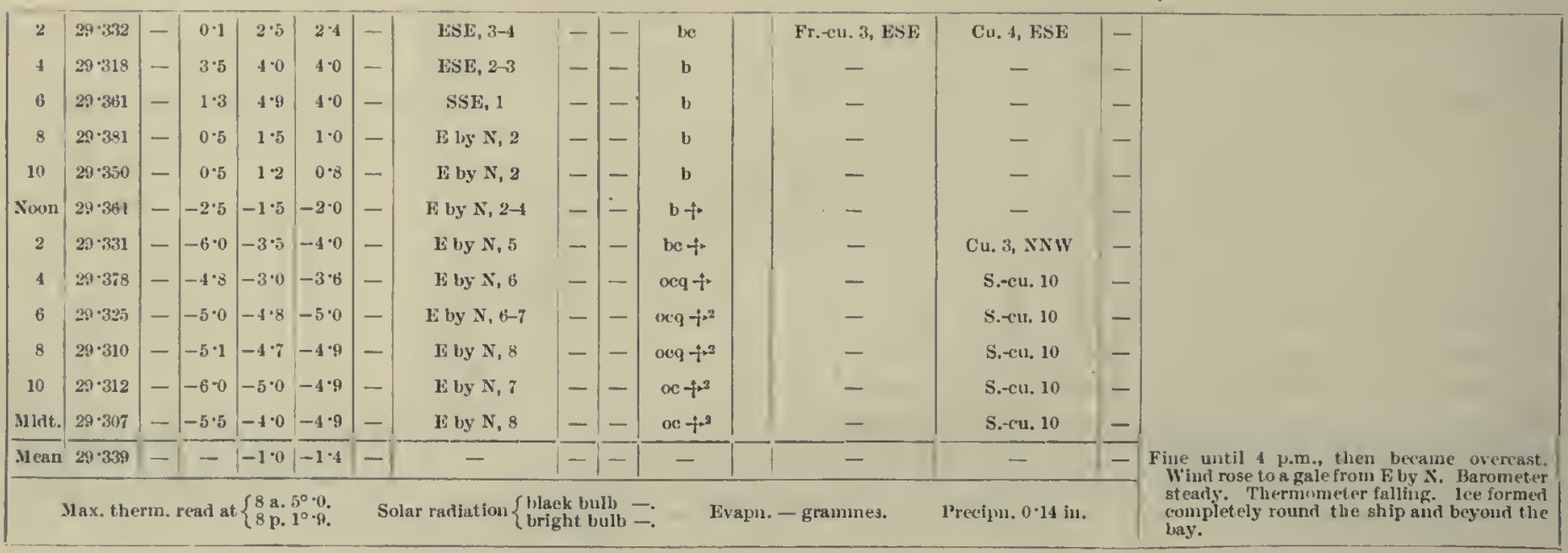

27th.

\begin{tabular}{|c|c|c|c|c|c|c|c|c|c|c|c|c|c|c|}
\hline 2 & $29 \cdot 287$ & $1-$ & $-6 \cdot 2$ & $-5 \cdot 0$ & $-5 \cdot 0$ & - & $S E, 7-8$ & - & $1-1$ & o $*$ & - & Cu.-11. 10, ESE & - & \multirow{10}{*}{$\begin{array}{l}\text { 2.30. Ouservel parhellon. } \\
\text { Parhelion vlsible. }\end{array}$} \\
\hline 4 & $29 \cdot 23 \pi$ & - & $-5 \cdot 5$ & $-5 \cdot 3$ & $-5 \cdot 8$ & - & SE, B & - & - & $\alpha * *$ & - & Cn1.-11. 10 & - & \\
\hline 6 & $29 \cdot 277$ & - & $-8 \cdot 2$ & $-7 \cdot 0$ & $-7 \cdot 0$ & - & ESE, 3 & - & - & oc* & - & C11.n. 10 & - & \\
\hline 8 & $29 \cdot 294$ & - & $-9 \cdot 0$ & $-7 \cdot 0$ & $-7 \cdot 0$ & - & ESE, $2-3$ & - & - & oef $*$ & - & S.-cu. 10 & - & \\
\hline 10 & $29 \cdot 290$ & - & $-8 \cdot 0$ & $-7 \cdot 5$ & $-7 \cdot 5$ & - & F by $\mathrm{S}, 2$ & - & - & oef & - & S.-cu. 10 & - & \\
\hline Nom & $29 \cdot 304$ & - & $-9 \cdot 0$ & $-7 \cdot 0$ & $-7 \cdot 0$ & - & v by $8,2-3$ & - & - & oef $*$ & - & S.cu. 10 & - & \\
\hline 2 & $29 \cdot 306$ & - & $-i \cdot 0$ & $-7 \cdot 0$ & $-7 \cdot 0$ & - & E, 1-2 & - & - & $b$ & - & - & - & \\
\hline 8 & $29 \cdot 302$ & - & $-6 \cdot 5$ & $-6 \cdot 5$ & -6.5 & - & Calm & - & - & b & - & - & - & \\
\hline 10 & $29 \cdot 300$ & - & $-8 \cdot 0$ & -8.0 & $-8 \cdot 0$ & - & Calm & - & - & b & - & - & - & \\
\hline slidt. & $29-300$ & - & $-9 \cdot 8$ & -8.8 & $-8 \cdot 9$ & -1 & Calm & - & -1 & b & - & - & $1-$ & \\
\hline Mean & $23 \cdot 292$ & - & - & $1-6.8$ & $-7 \cdot 0$ & -1 & - & - & -1 & - & - & - & $1-$ & \multirow{2}{*}{$\begin{array}{l}\text { Overcast and foggy until noon, then fine and } \\
\text { clear. Wind fell from a gale to calm. } \\
\text { Burometer rose a little. Thermometer } \\
\text { fallng. }\end{array}$} \\
\hline & ax, th & & ad at & $\left\{\begin{array}{l}8 \mathrm{a} . \\
8 \mathrm{p} .\end{array}\right.$ & $-\overline{6^{\circ} \cdot 5}$ & & Solar radiatlon & $\begin{array}{l}\text { lack } \\
\text { righ }\end{array}$ & & & 11. - grammes. & 1'reclyn. $0.58 \mathrm{in}$. & & \\
\hline
\end{tabular}

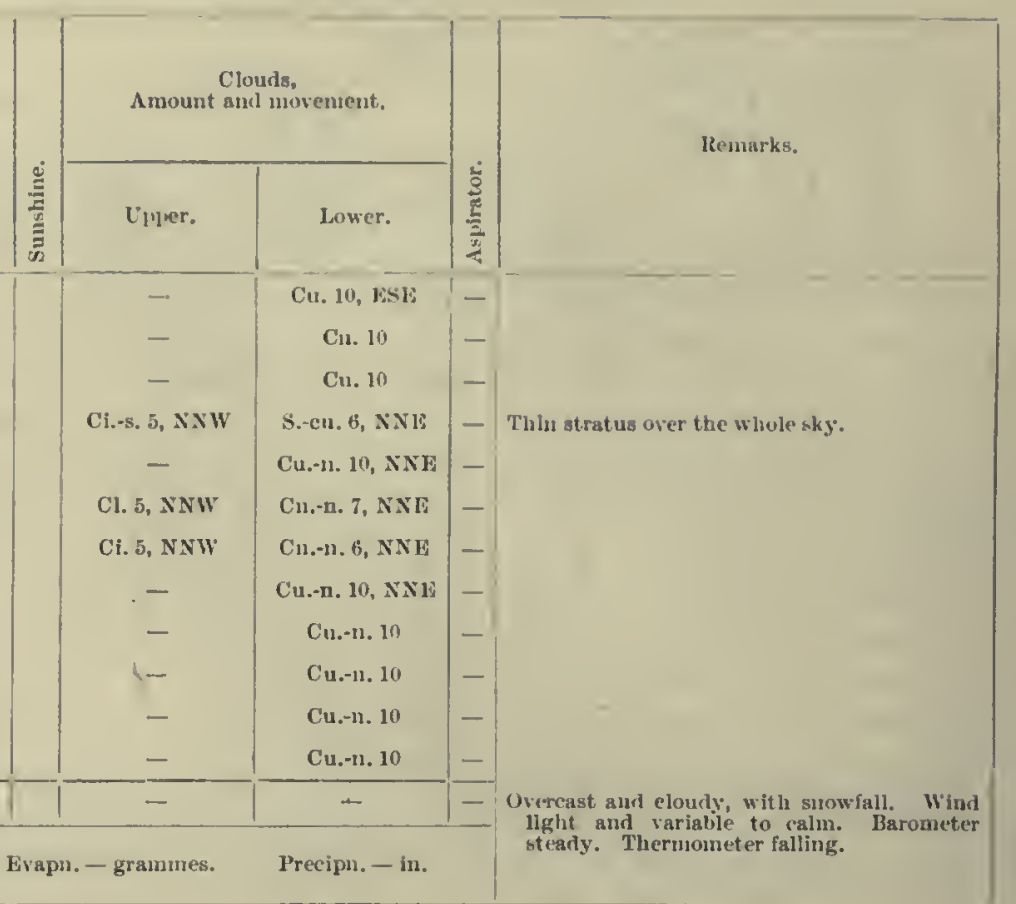
way. completely round the shipand beyour the 
Metworologicat, Journat, of the "I)iscovery."

[1902-March.
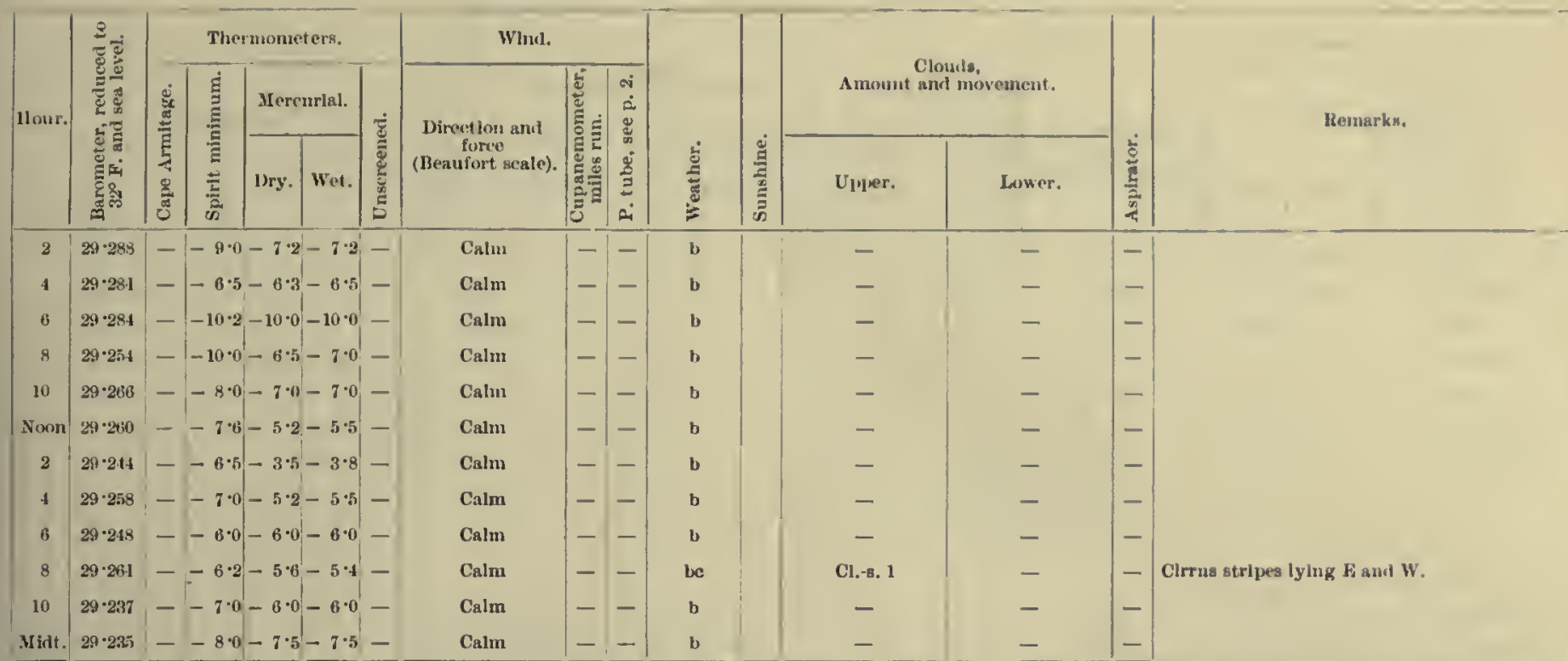

\begin{tabular}{l|l|l} 
Calm & - & - \\
Calm & - & - \\
Calm & - & - \\
Calm & - & - \\
Calmu & - & - \\
Calm & - & - \\
Calm & - & - \\
Calm & - & - \\
Calm & - & - \\
Calm & - & - \\
Calm & - & - \\
Calm & - \\
\hdashline- & - & -
\end{tabular}

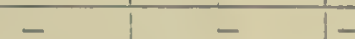

28th.

Mean $\overline{29 \cdot 260} \overline{-} \overline{-6 \cdot 3-6 \cdot 5}$

Max. therm. reat at $\left\{\begin{array}{l}8 \mathrm{a},-5^{\circ} \cdot 5 . \\ 8 \mathrm{p},-6\end{array}\right.$ Solar radiatlon $\left\{\begin{array}{l}\text { black bulb } \\ \text { bright bulb }- \text {. }\end{array}\right.$

Evapn. - grammes.

Precipn. - in.

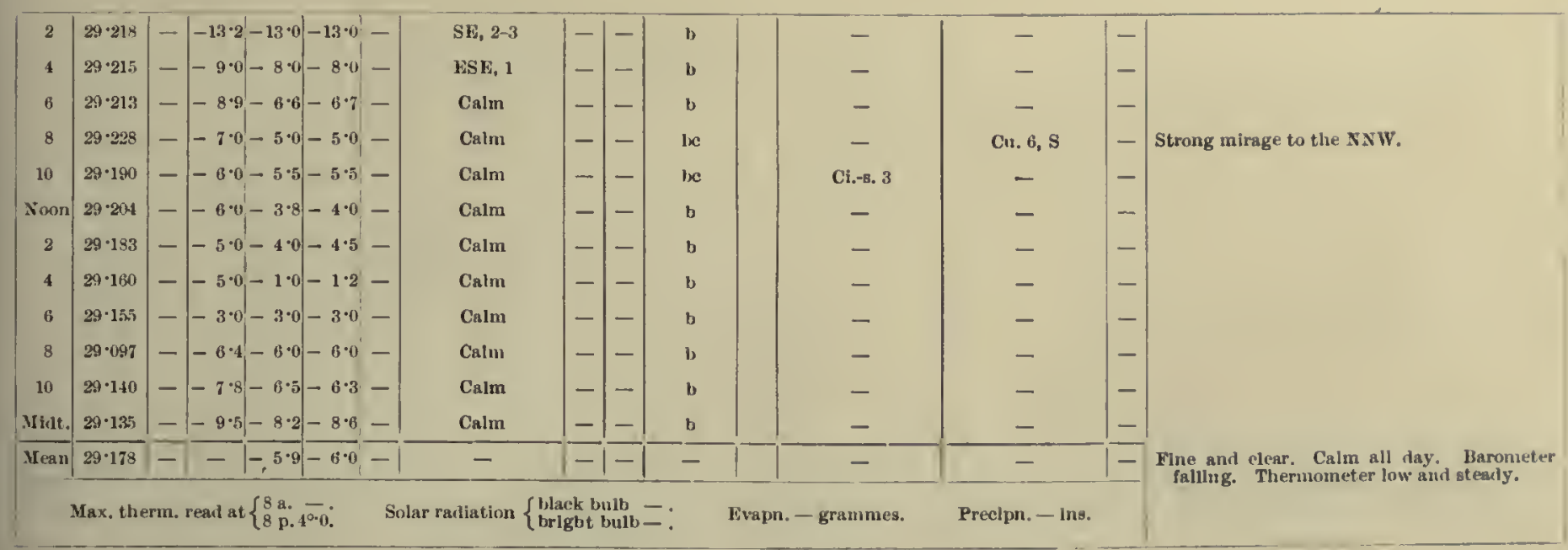

29th.

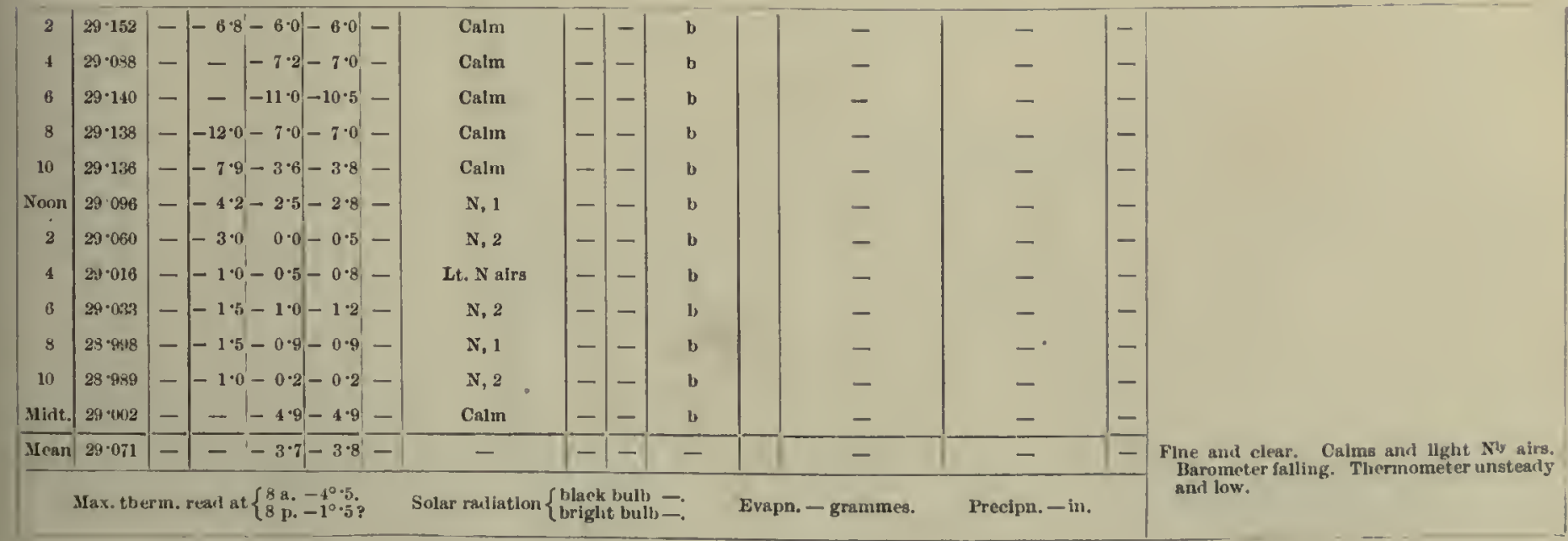

30th. 
Metworological Jourkat, of the "Discovery."

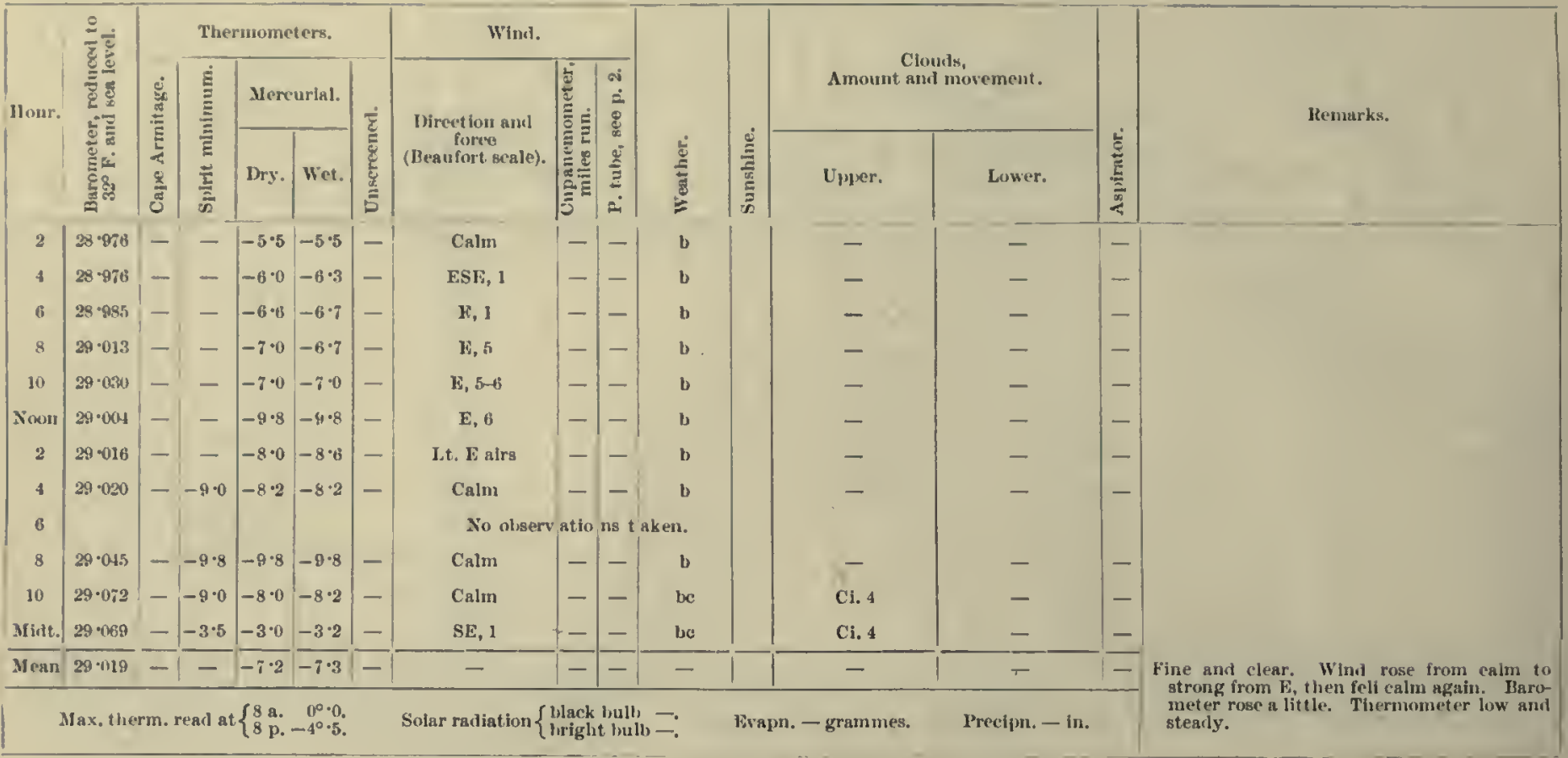


Mrthoroiogical JoURNAT, OF TILE "DISCOVERY."

[1902 - April.

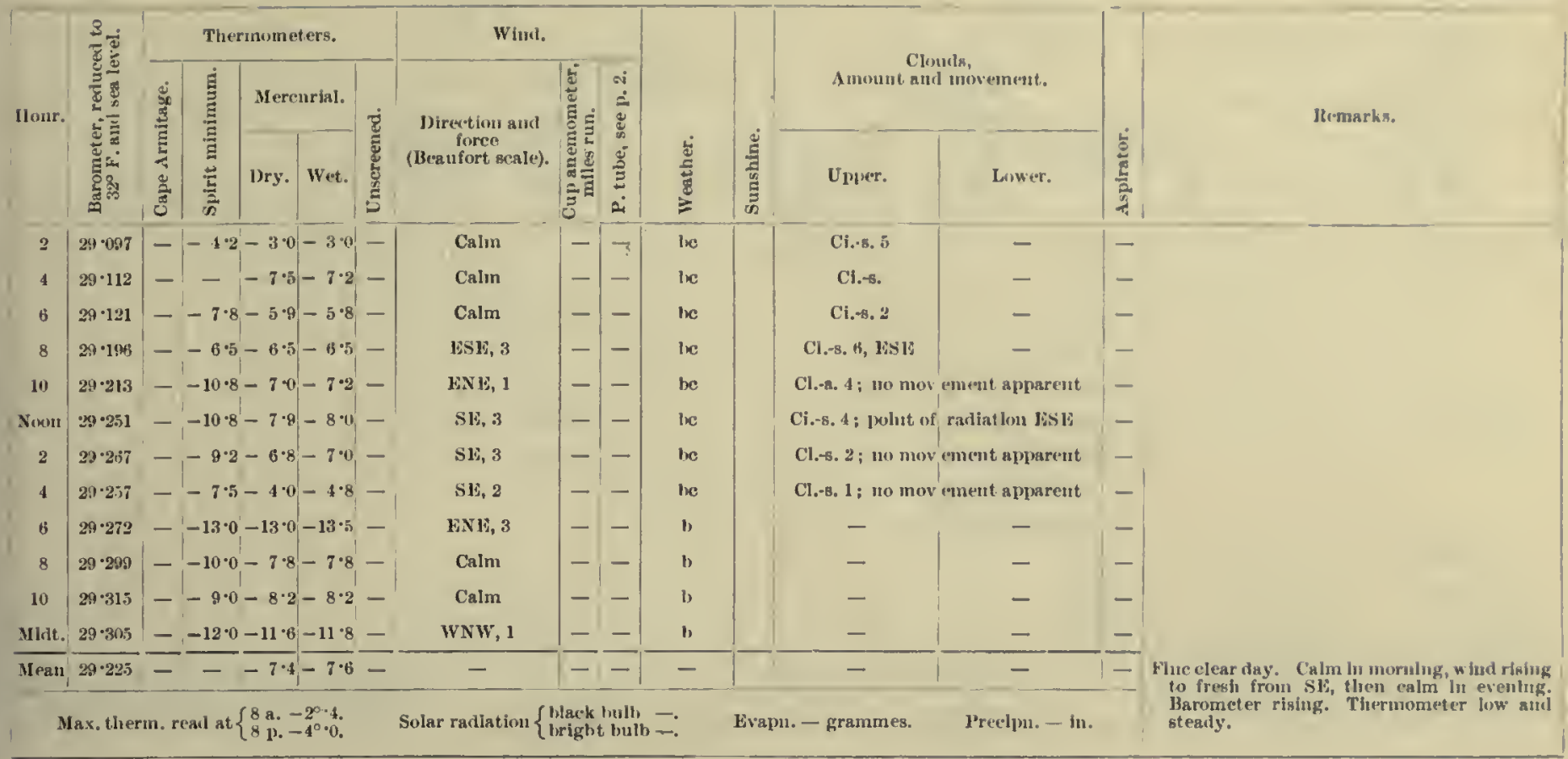

$18 t$.

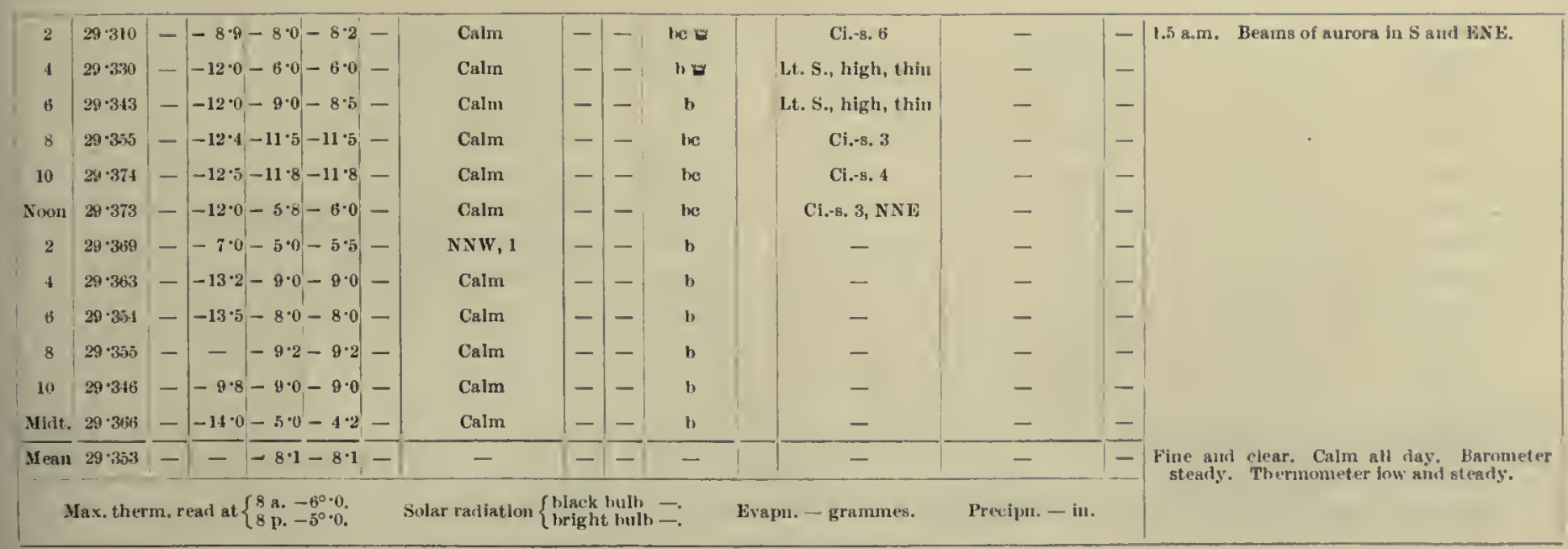

2nd.

\begin{tabular}{|c|c|c|c|c|c|c|c|c|c|c|c|c|}
\hline 2 & $29 \cdot 322$ & -1 & - & $-11 \cdot 8-11 \cdot 8-$ & Calm & -1 & -1 & the & Ci.-8. 3 & - & - & Very faint a urora beam in $\mathrm{SSE}$, ast. $6^{\circ}$ : \\
\hline 4 & $29 \cdot 313$ & - & $-14 \cdot 2$ & $-11 \cdot 0-11 \cdot 2-$ & Calm & - & - & b & - & - & - & \\
\hline 6 & $29 \cdot 2943$ & - & $-13 \cdot 8$ & $-13 \cdot 0-13 \cdot 2-$ & Calm & - & - & be & Ci.-cu. 3 & - & - & \\
\hline 8 & $29 \cdot 236$ & - & $-13 \cdot 5$ & $-12 \cdot 0=13 \cdot 0-$ & ESE, 3 & - & - & be & Cl.-cu. 3, ESE & S.-eu. 2, ESE & - & \\
\hline 10 & $29 \cdot 273$ & - & $-13 \cdot 8$ & $-11 \cdot 8-12 \cdot 0-$ & ESE, 3 & - & - & tic & - & Cu. 2, Niw & - & \\
\hline Noon & $29 \cdot 247$ & - & $-12 \cdot 0$ & $-8 \cdot 5-8 \cdot 8-$ & ESE, 2 & - & - & $\infty$ & - & S.cu. 10 & - & \\
\hline 2 & $29 \cdot 227$ & - & -9.0 & $-7 \cdot 8-8 \cdot 0-$ & Calm & - & - & $\propto$ & - & S.cur. 10 & - & \\
\hline 4 & $29 \cdot 212$ & - & $-12 \cdot 0$ & $-8 \cdot 5-8 \cdot 8-$ & Calm & - & - & $\infty$ & - & S.cur. 10 & - & \\
\hline 6 & $29 \cdot 198$ & - & $-9 \cdot 0$ & $-9 \cdot 0-9.0-$ & Calm & - & - & $\infty$ & - & S.cu. 10 & - & \\
\hline 8 & $29 \cdot 175$ & - & $-10 \cdot 0$ & $-9 \cdot 0-9 \cdot 2-$ & Caim & - & - & $\infty$ & - & S.cu. 10 & - & \\
\hline 10 & $29 \cdot 153$ & - & $-13 \cdot 0$ & $-8 \cdot 0-0.0-$ & Caim & - & - & h & - & - & - & \\
\hline Midt. & $29 \cdot 154$ & - & $-10 \cdot 0$ & $-9.4-9.4-$ & Calm & $=$ & - & $\mathrm{b}$ & - & - & $1-$ & \\
\hline$\overline{\text { Mean }}$ & $29 \cdot 233$ & - & - & $-10 \cdot 1-10 \cdot 3-$ & - & I- & $\overline{1}$ & $二$ & - & $\dot{-}$ & $1-$ & $\begin{array}{l}\text { Generally fime; overcast in afternoon. Calm } \\
\text { neariy all day. Baromet er falling. Ther- }\end{array}$ \\
\hline & & & & $\left\{\begin{array}{l}8 \text { a. } \\
8 \text { p. }-6^{\circ} \cdot 0 .\end{array}\right.$ & & & & & rammes. & Precipn. - i & & \\
\hline
\end{tabular}


1902-April.]

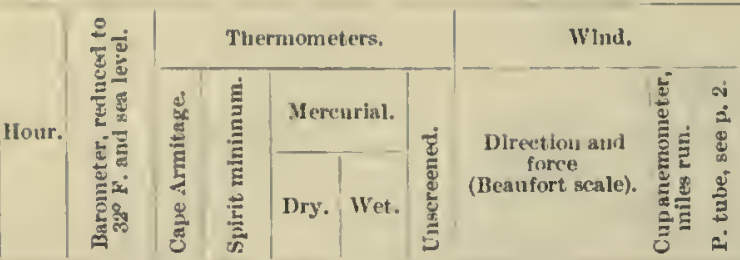

Metrorological Journal of the "Discovery."

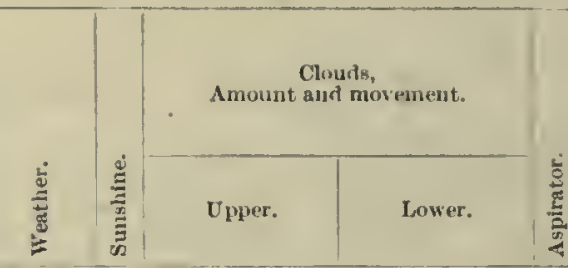

4th. $2|39 \cdot 163|-|-|-13 \cdot 6-13 \cdot 6|-| \quad$ Calm

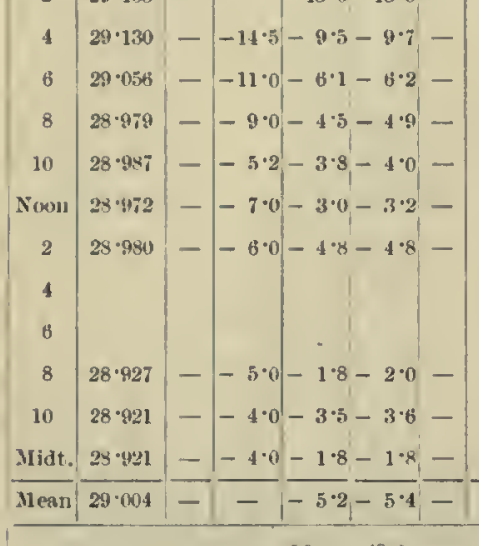

11ax, therm, read at $\left\{\begin{array}{l}8 \mathrm{a},-4^{0} \cdot 0 . \\ 8 \mathrm{p},-\end{array}\right.$

\begin{tabular}{|c|c|c|c|}
\hline Calm & - & - & b) \\
\hline Caln & - & - & by \\
\hline ESE, 2 & - & - & oe \\
\hline E. 2 & - & - & be \\
\hline Lt. $\mathrm{E}$ airs & - & - & oc \\
\hline Lt, $\mathrm{F}$ airs & - & - & oc \\
\hline Calm & - & - & $o *$ \\
\hline No ob & atio & $113 \mathrm{t}$ & aken. \\
\hline No ob & atlo & ns t & aken. \\
\hline ESE, 2 & - & - & b \\
\hline ESE, 2 & - & - & b \\
\hline $\mathrm{NW}, \mathbf{A}$ & - & - & oc $*$ \\
\hline 一 & - & - & - \\
\hline
\end{tabular}

Solar railiation $\left\{\begin{array}{l}\text { black bulb }- \text {. } \\ \text { bright bulb }- \text {. }\end{array}\right.$
Evapn. - grammes.

\begin{tabular}{|c|c|c|}
\hline - & - & - \\
\hline- & - & - \\
\hline- & S.-en. 10 & - \\
\hline- & Cu. & - \\
\hline- & S.-cu. 10 & - \\
\hline- & S. cu. 10 & - \\
\hline- & C11.-11. 10 & - \\
\hline- & - & - \\
\hline- & - & - \\
\hline- & Cu. -n. 10 & - \\
\hline- & - & - \\
\hline
\end{tabular}

Precipn. - in. lienuarks.

Dull and cloudy most of day, with snow showers. Caim, but wind rising in eveniug.
Barometer falling. Thermoneter rising.
5 th

\begin{tabular}{|c|c|c|c|c|c|c|c|c|c|}
\hline 2 & 23.887 & $-\begin{array}{lll}-2.2 & 1.0 & 0.2\end{array}$ & Lt. FNE airs & -- & oc & - & Cu. 10 & -1 & \multirow{12}{*}{$\begin{array}{l}\text { In the afternoou an awning was spreas over } \\
\text { the after part of the ship. }\end{array}$} \\
\hline 4 & $28 \cdot 898$ & $--\mid-3.5-3.3-$ & Lt. WNE airs & $-1-$ & $\infty$ & - & Cu. 10 & - & \\
\hline 6 & 28.850 & $--5 \cdot 0-5 \cdot 0-5 \cdot 1-$ & FNE, 1-2 & -1 & oc & - & S.cu. 10 & - & \\
\hline 8 & $23 \cdot 855$ & $--4.9-0.1-0.4-$ & St, 1 & - & oc & - & S.cu. 10 & - & \\
\hline 10 & $28 \cdot 852$ & $--1.0 \quad 0.0-0.5-$ & Calm & -- & oc & - & S.-cu. 10 & - & \\
\hline Noon? & 28.887 & $--5 \cdot 0-5 \cdot 0-5 \cdot 0-$ & S, 3 & - & $\infty$ & - & S.cu. 10 & $1-$ & \\
\hline 2 & 23.863 & $--5.2-4.2-4.5-$ & $\mathrm{s}, 3$ & - & oc & - & S.cu. 10 & $1-$ & \\
\hline 4 & $28 \cdot 834$ & $--8.0-8.0-8.0-$ & S, 3-4 & -1 & be & Ci.-s. 5, NNE & $\mathrm{Cu}, 3$, ssw & - & \\
\hline B & $2 s^{\prime}: 556$ & $--8 \cdot 0-5 \cdot 0-5 \cdot 2-$ & ENE, 1 & -- & b & - & - & - & \\
\hline 8 & $28 \cdot 869$ & $--6.00 .0-0.2-$ & Calm & - & b & - & - & - & \\
\hline 10 & $28 \cdot 86.3$ & $-\begin{array}{lll}-2.8 & 0.8 & 0.2\end{array}$ & Calin & -- & ocm & - & S. 10 & - & \\
\hline Mirt. & $23 \cdot 894$ & $--40-0.7-0.2-$ & wNw, 2 & -- & $\infty$ & - & S.ecu. 10 & - & \\
\hline Mean] & $28 \div 659$ & $--2 \cdot 5-2 \cdot 7-$ & - & $-1-$ & - & - & - & $\overline{-}$ & \multirow[t]{2}{*}{ Overeast and clourly all day ; wind variable. } \\
\hline & Max.t & 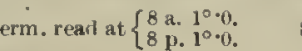 & ra liation $\left\{\begin{array}{l}t \\
!\end{array}\right.$ & $\begin{array}{l}\text { ack bu } \\
\text { ight t) }\end{array}$ & & grammes. & Precipn. - in, & & \\
\hline
\end{tabular}

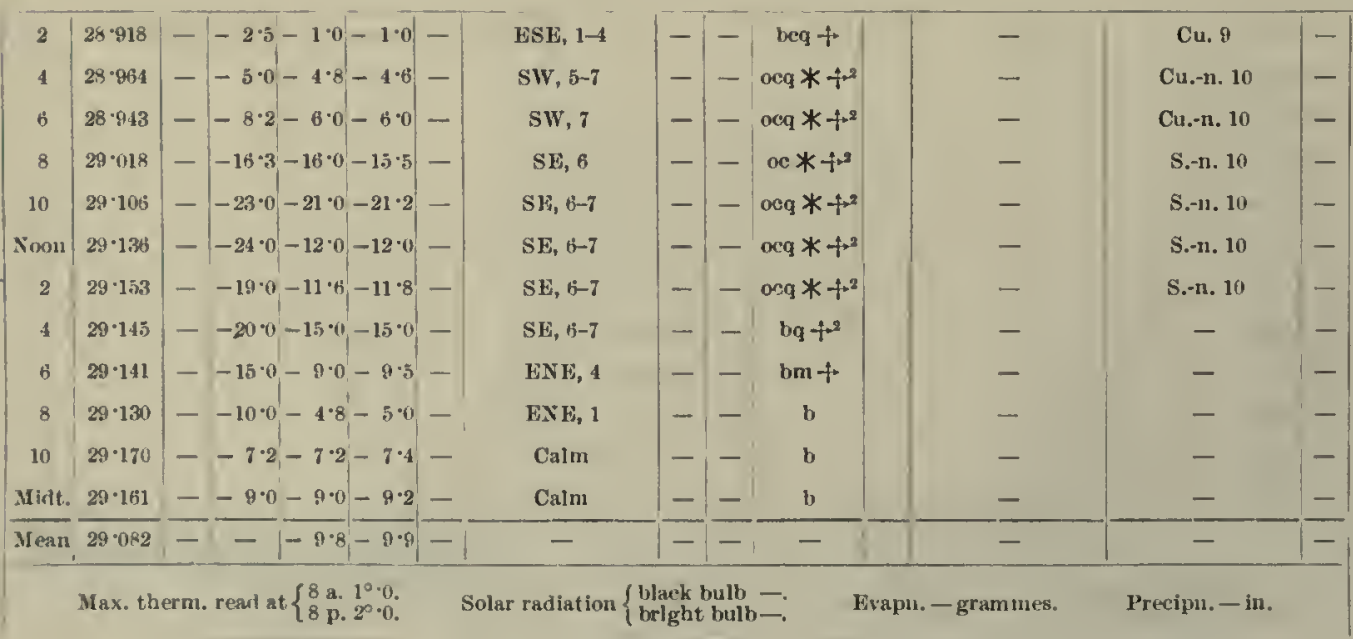

Overcast and cloudy, with heavy snow squalls. Wind rose to strong from $\mathrm{SE}$, but fell again in eveuing. Harometer rising. Ther- 
Methorolonilal Journal, OF the "Discovery."

[1902-April.

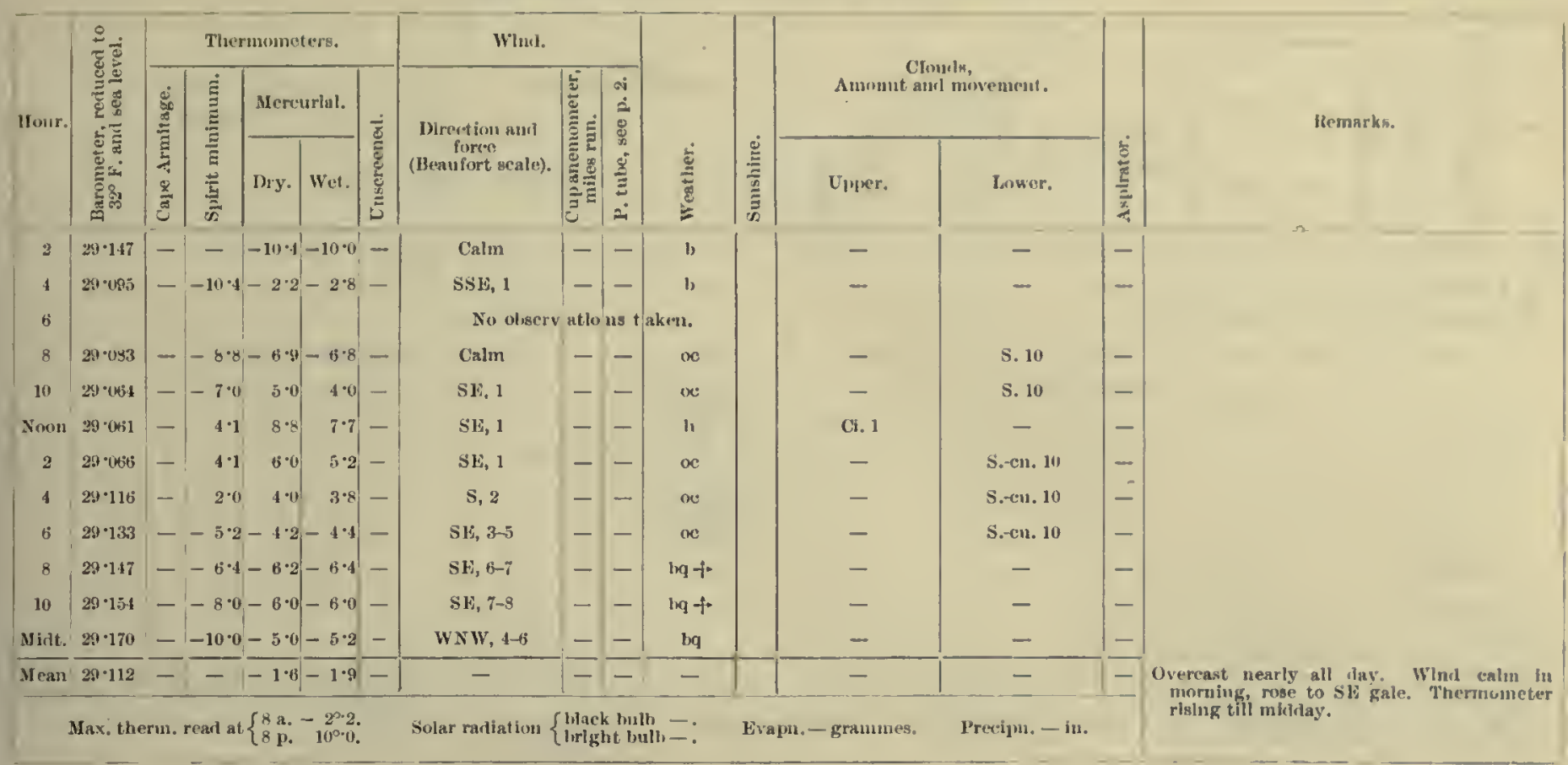

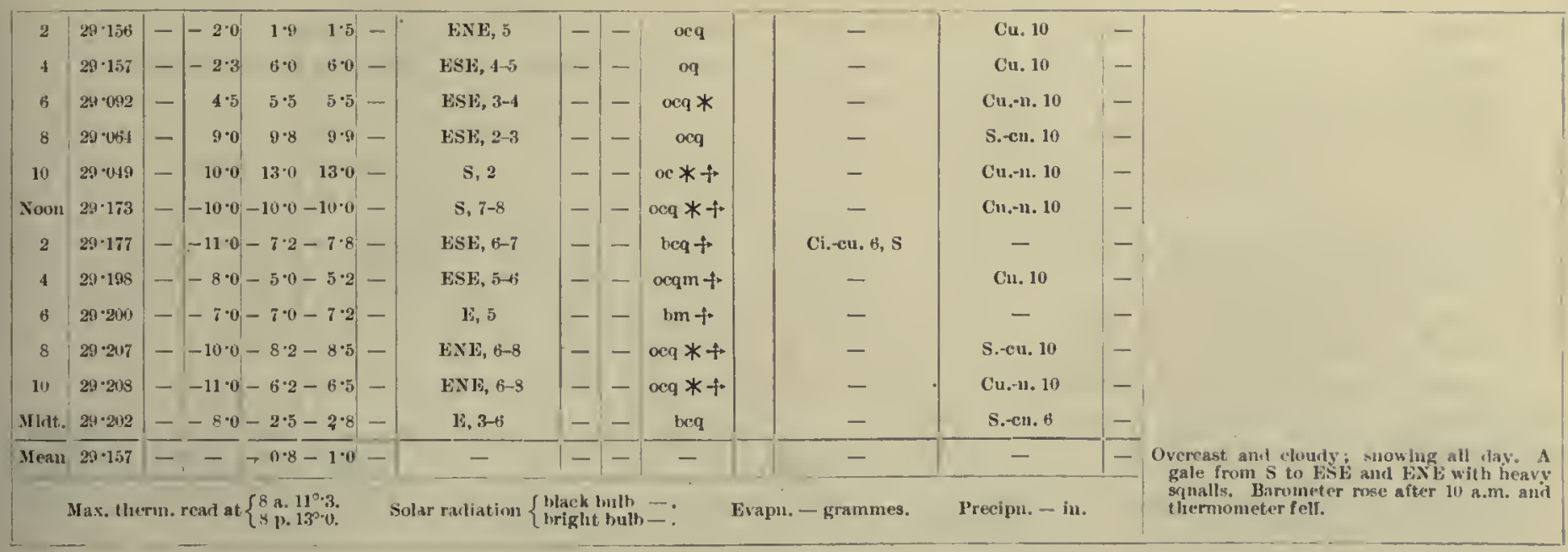

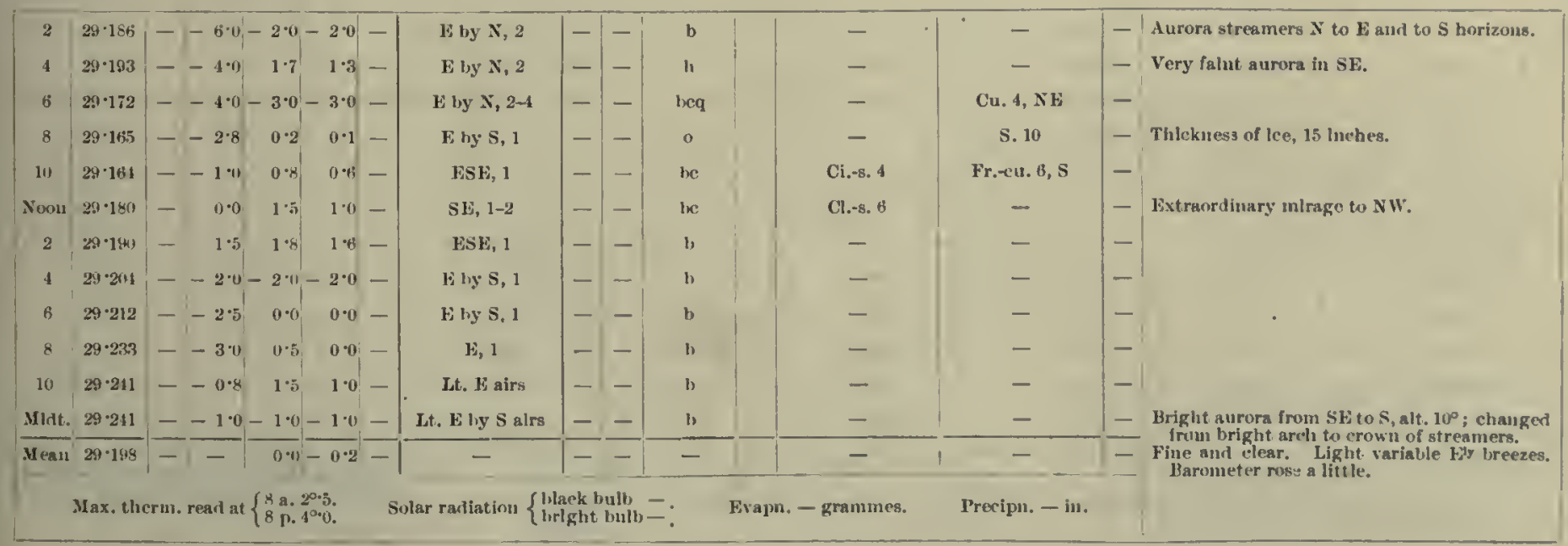


1902 -April.]

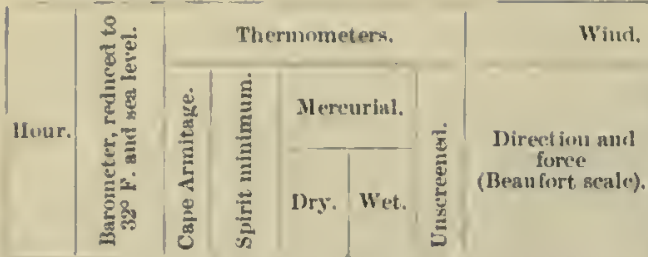

10 th.

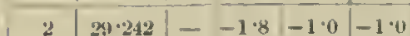

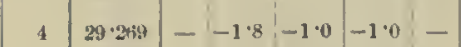

6 29.21र $--2.0-1 \cdot 0-1.0-$

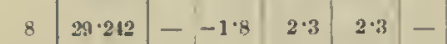

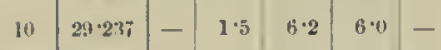

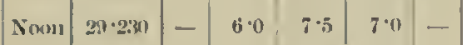

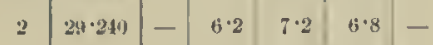

$4 \quad 29.242-0.5 \quad 5 \cdot 0 \quad 4.0-$

$6 \quad 24 \cdot 231-3.0 \quad 4 \cdot 2 \quad 3.8$ -

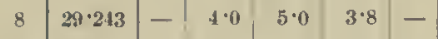

$10 \quad 29 \cdot 233-4 \cdot 0 \quad 7 \cdot 0 \quad 6.0$ -

$\frac{\text { Mlut. }}{\text { Meau }} \frac{29 \cdot 217}{29 \cdot 237}-\frac{6 \cdot 0}{-}-\frac{8 \cdot 0}{4 \cdot 1} \frac{7 \cdot 0}{3 \cdot 6}-$

Max, therm. read at $\left\{\begin{array}{l}8 \text { a. } \\ 8 \text { p. } \\ 8 \cdot 5 \\ 9\end{array}\right.$

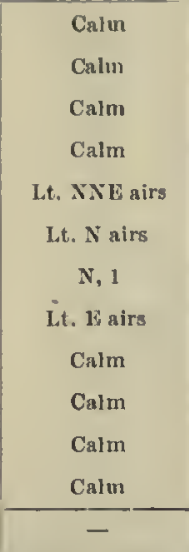

MFtEOROLOGICAL, JOURNAL OF THE "DISCOVKKY."

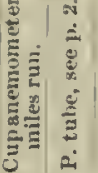

Clonds,
Amount nud inovement.

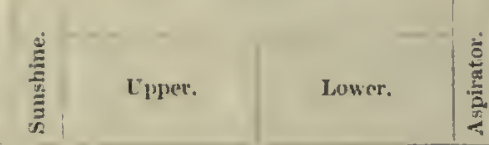

liemarks.

竞
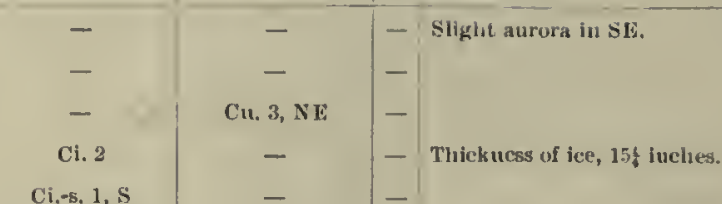

Ci,-s, 1, S

Ci.-s. 2, s

(very slow)
Ci.-s. 2 (thick s tratus over all)

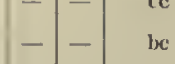

-1 be

Ci.-5. $2, s$

(quicker)

S. 10

\begin{tabular}{c|c}
- & 0 \\
- & be
\end{tabular}

- bm

$-\frac{-1}{-}+\frac{b m}{-}$

$-1-$

Solar raliation $\left\{\begin{array}{l}\text { black bulb } \\ \text { bright bulb }- \text {. }\end{array}\right.$

Evapu. - gramutes.

Precipu. - iu.

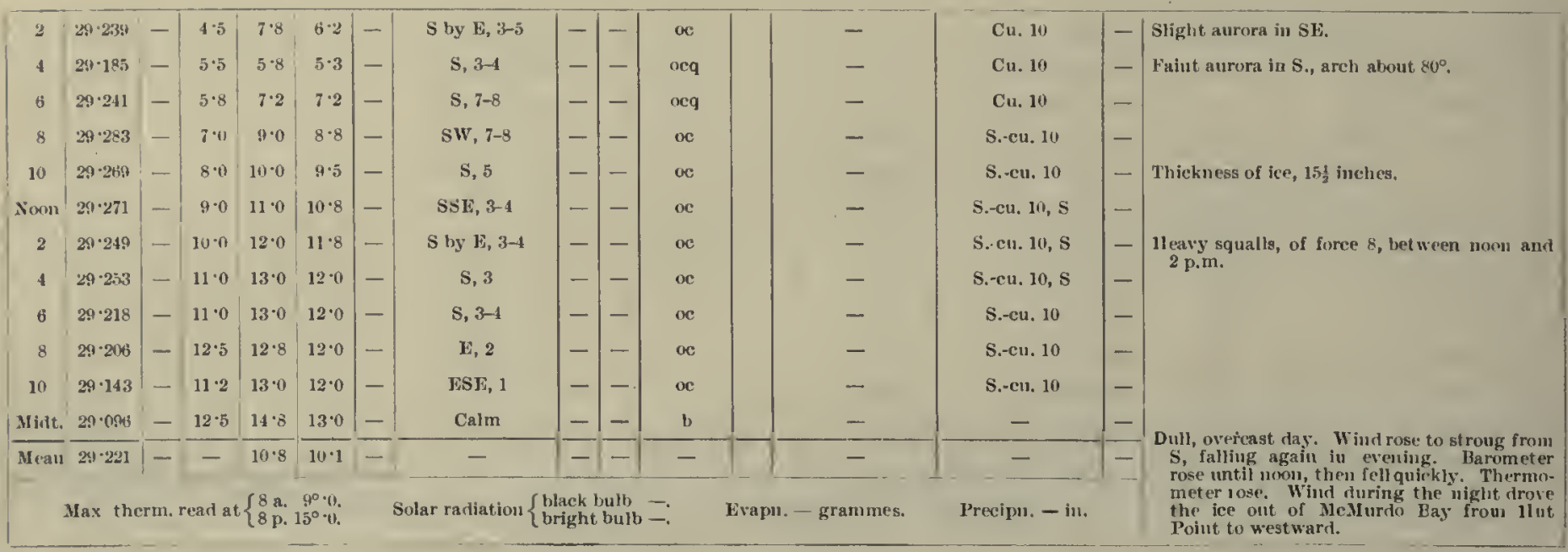

12th.

\begin{tabular}{|c|c|c|c|c|c|c|c|c|c|c|c|c|c|c|}
\hline 2 & $29 \cdot 111$ & -1 & $11 \cdot 2$ & $11 \cdot 8$ & $11 \cdot 2$ & - & SE, 3 & -1 & -1 & oc & - & $\mathrm{Cu}, 10, \mathrm{SE}$ & - & \multirow{10}{*}{ Thickness of ice to surface of water, 15$\rceil$ inches. } \\
\hline 4 & $29 \cdot 087$ & - & $11 \cdot 5$ & $12 \cdot 0$ & 11.8 & - & ESE, 3 & - & - & oq & - & Cu. 10, SE & - & \\
\hline 6 & $29 \cdot 083$ & - & $11 \cdot 0$ & $11 \cdot 8$ & $11 \cdot 0$ & - & ESE, 5-8 & - & -1 & oq $*$ & - & Cu.-11. 10 & - & \\
\hline 8 & $29 \cdot 119$ & - & $9 \cdot 4$ & $10 \cdot 6$ & $10 \cdot 2$ & - & ESł, 1 & - & - & o & - & s. 10 & - & \\
\hline 10 & $29 \cdot 121$ & - & $10 \cdot 0$ & $11 \cdot 8$ & $11 \cdot 1$ & - & SE, 1 & - & - & o & - & S. 10 & - & \\
\hline Nixon & $29 \cdot 110$ & - & $11 \cdot 0$ & $12 \cdot 2$ & $12 \cdot 0$ & - & S1E, 2 & - & - & o & - & S. 10 & - & \\
\hline 2 & $29 \cdot 131$ & - & $10^{\circ} 0$ & 10.8 & $10 \cdot 0$ & - & SE. 3 & - & -1 & oc & - & S.cu. 10. SE & - & \\
\hline 8 & $29 \cdot 215$ & - & $-2 \cdot 4$ & 0.1 & -0.1 & - & SE, 4-5 & - & - & oeq + & - & S.-cn. 10 & - & \\
\hline 10 & $29 \cdot 218$ & - & $-3 \cdot 2$ & 0.0 & -0.2 & - & SE, 4-6 & - & - & bq & - & - & - & \\
\hline Midt. & $29 \cdot 185$ & 1 & -0.6 & $5 \cdot 0$ & 4.9 & - & $\mathrm{SE}_{1,0-1}$ & - & - & bqum & - & - & - & \\
\hline Mean & $29 \cdot 147$ & $=$ & - & $8 \cdot 4$ & $7 \cdot 4$ & -1 & - & - & - & - & - & - & - & \multirow{2}{*}{$\begin{array}{l}\text { Orercast and cloudy, with some suow: Wind } \\
\text { steady in } \mathrm{SE} \text {, squally in morning, falliug iu } \\
\text { ereutug. Harometer rose aiter noou. } \\
\text { Thernometer fell towards eventing. }\end{array}$} \\
\hline & Nax. the & m & all at & & & & radiation & & $\begin{array}{l}\text { ulb } \\
\text { bulb }\end{array}$ & & ammes. & Preclpn. - in. & & \\
\hline
\end{tabular}


Methorofogical, Journat, of the "Discovery."

Ц1902-April.

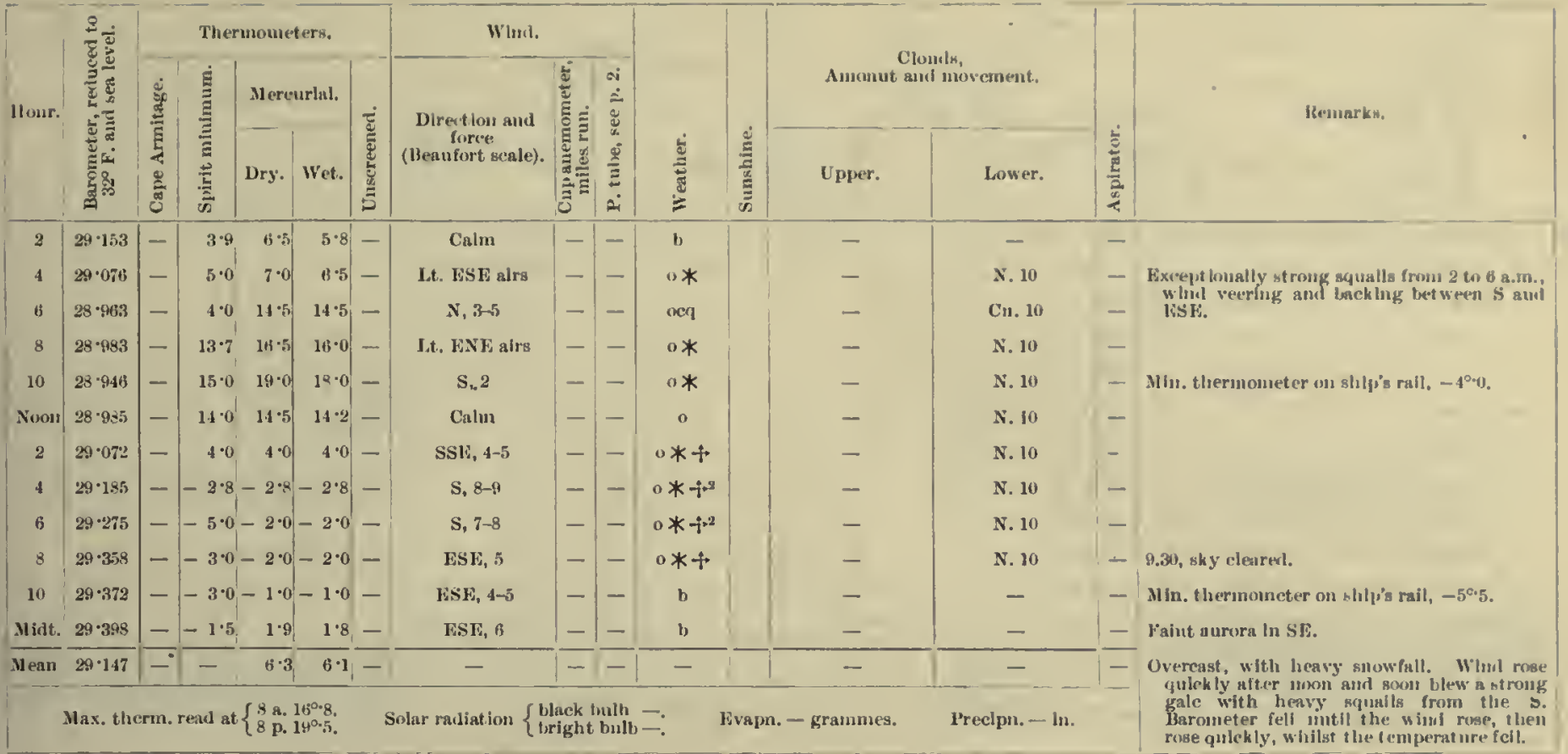

13 th.

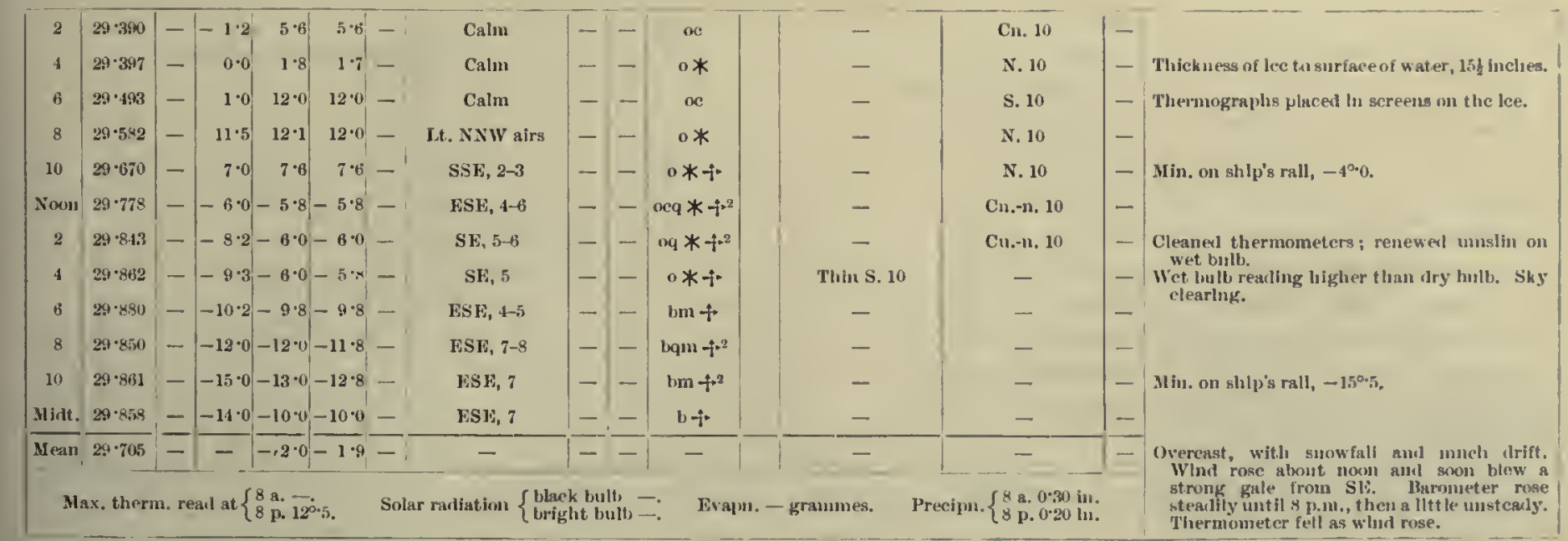

14th.

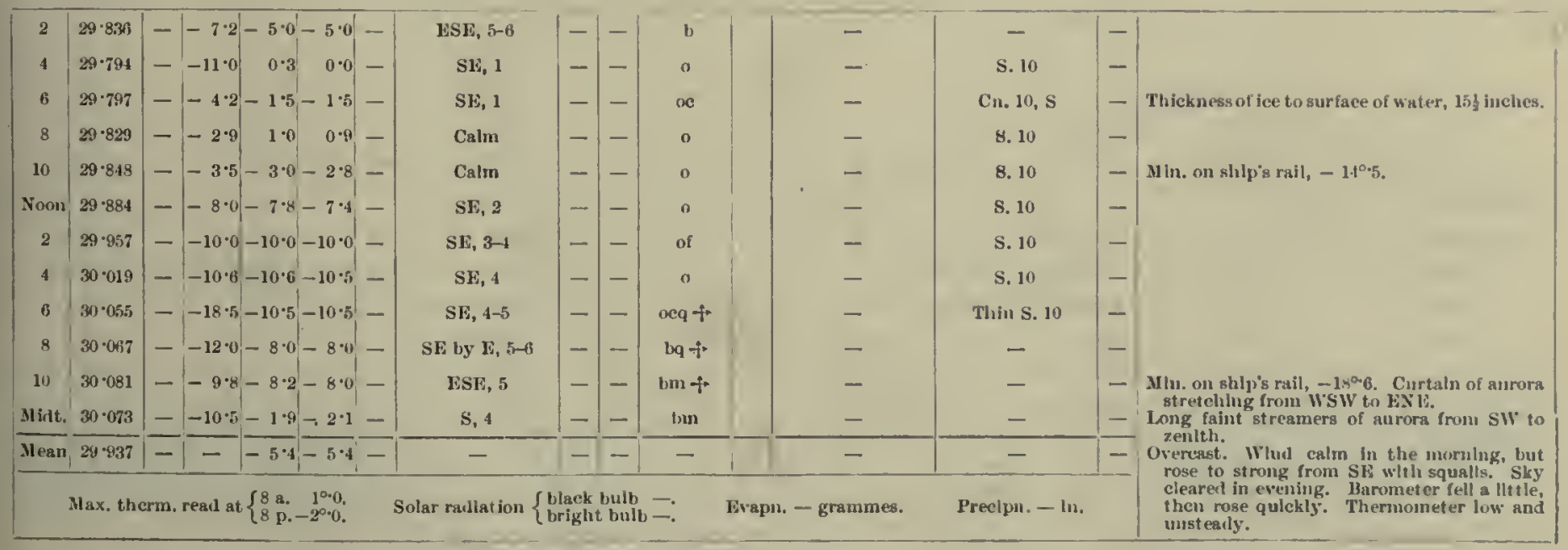


1902 -April.]

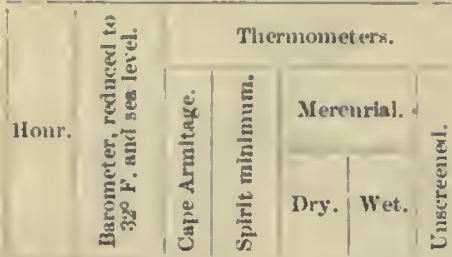

Meteolological, Journat, of the "Discovery."

\begin{tabular}{|c|c|c|c|c|c|c|c|c|}
\hline \multicolumn{3}{|c|}{ Whus. } & \multirow[b]{2}{*}{ 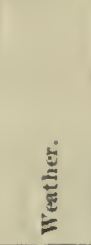 } & \multirow[b]{2}{*}{ 竧 } & \multicolumn{2}{|c|}{$\begin{array}{l}\text { Clouds, } \\
\text { Amount and movenent. }\end{array}$} & \multirow[b]{2}{*}{$\frac{5}{\frac{5}{2}}$} & \multirow{2}{*}{ Remiarks. } \\
\hline $\begin{array}{c}\text { Dirertion and } \\
\text { f(rrece } \\
\text { (Beafort sealc). }\end{array}$ & 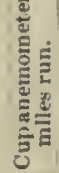 & 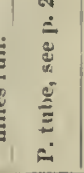 & & & Upper. & Lower. & & \\
\hline S li,y $E_{2}, 0-6$ & -1 & - & $x+$ & & - & Cn. 10 & $1-$ & \\
\hline $\mathrm{s}, 3-5$ & -1 & - & oq & & - & S. 10 & - & \\
\hline SSIE, 3- & - & - & ๓сq & & - & Cu. 10 & - & \\
\hline SSF, 2-5 & - & - & o:q & & - & Cn. 10 & $1-$ & \\
\hline $\operatorname{ssc}, 3-6$ & -1 & $15-20$ & q & & - & 8. 10 & - & Mint on shly's rail, $-11^{\circ} \cdot 0$. Show surface \\
\hline s, 2-5 & 12 & $15-20$ & 风⿻ - & & - & S. 10 & - & Thiofness of the to surface of water, 151 \\
\hline S1, 2-5 & 10 & 15 & oq $*$ & & - & x. 10 & - & \\
\hline $1: S 1: 2-3$ & 15 & $15-20 \mid$ & 0 & & - & N. 10 & - & Min. thermometer $($ M. $0 \cdot 45,11.0 \cdot 10)$ taken \\
\hline $\mathrm{s}, 2-3$ & 10 & $|10-15|$ & op * & & - & N. 10 & - & \\
\hline SE by 1, 3 & - & - & o* & & - & N. 10 & - & Min. on shlp's rail, $-5^{\circ} \cdot 0$. \\
\hline s, 3 & - & - & om & & - & N. 10 & - & \\
\hline $\mathrm{s}, 3$ & - & - & $\operatorname{limp} *$ & & - & $=$ & - & \\
\hline- & - & - & - & & - & - & - & 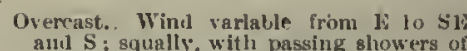 \\
\hline Solur radiatio & & $\begin{array}{l}\text { wall } \\
\text { bults. }\end{array}$ & & & ammes. & eipn. - in & & $\begin{array}{l}\text { anow. Barouiter feli steadily all day. } \\
\text { Thermoneter low and steadly. }\end{array}$ \\
\hline
\end{tabular}

17 th.

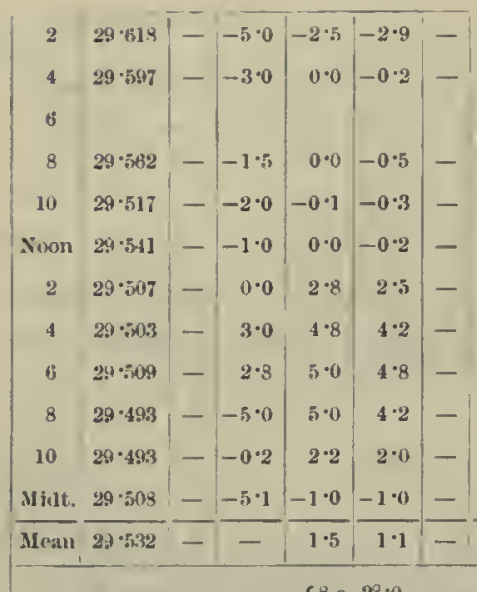

Max. therm. real at $\left\{\begin{array}{l}8 \text { a. } \\ 8 \text { p. } 6^{\circ} \cdot 0 . \\ 8.6^{\circ} \cdot 2 .\end{array}\right.$

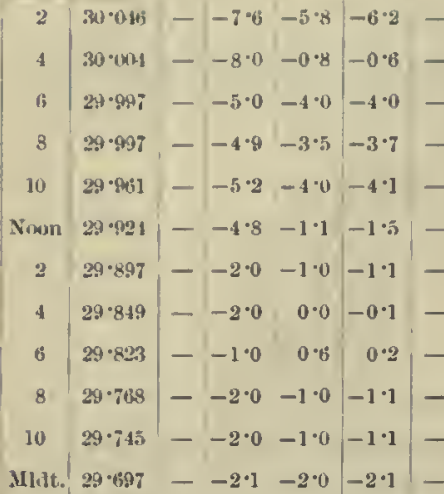

MLew1 $\frac{29 \cdot 697}{29 \cdot 592}=\frac{-2 \cdot 1}{-} \frac{-2 \cdot 0}{-2 \cdot 0} \frac{-2 \cdot 1}{-2 \cdot 1}=$

Max. therm. real at $\left\{\begin{array}{l}8 \text { a. } \\ 8 \text { p. }\end{array} 1^{\circ} \cdot \dot{0}\right.$.

\begin{tabular}{|c|c|c|c|c|}
\hline Ssli, 3 & -1 & - & $\propto$ & - \\
\hline SN, 3-4 & - & - & $b$ & - \\
\hline \multicolumn{5}{|c|}{ No ohsery al io ns tak en. } \\
\hline Lt. Sly airs & - & - & $b$ & $\mathrm{Ci}_{-}-\mathrm{R}_{2} \mathbf{1}$ \\
\hline Lt. NE airs & - & 5 & lic & Cl. $3, \mathrm{SW}$ \\
\hline Caln & 3 & 0 & $b$ & - \\
\hline NNE, 1 & 7 & 5 & $\infty$ & - \\
\hline SF, 2 & 6 & 10 & $\infty$ & - \\
\hline $13,1-2$ & 9 & 10 & 0 & - \\
\hline ESE, 1-2 & 12 & $10-15$ & o & - \\
\hline ESTE, 2 & 10 & $10-15$ & 0 & - \\
\hline SK, 2 & 10 & 15 & o & - \\
\hline- & -1 & - & - & - \\
\hline
\end{tabular}

Solar radiation $\left\{\begin{array}{l}\text { laack bulb } \\ \text { briglit bulls - }\end{array}\right.$
Evapm. - grammes.

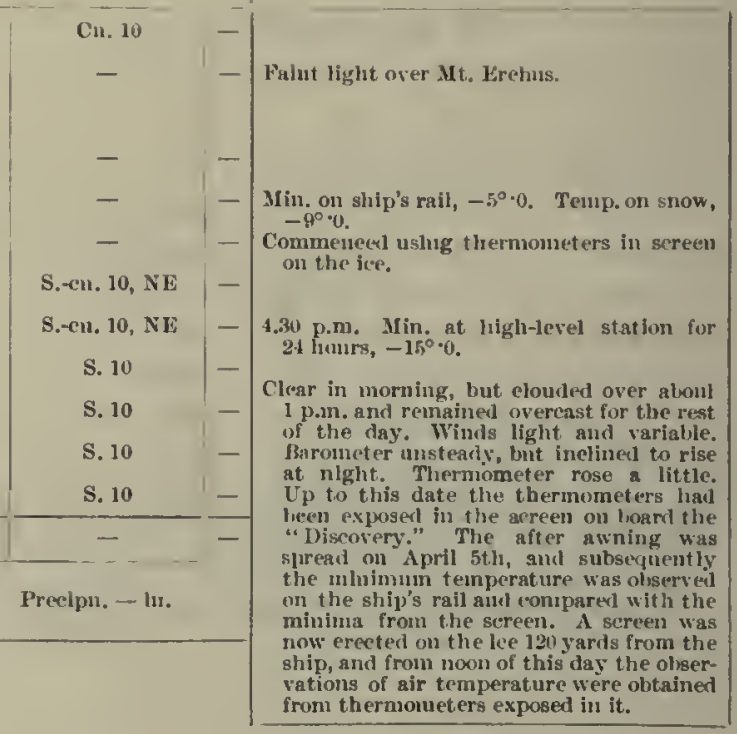

18 th.

\begin{tabular}{|c|c|c|c|c|c|c|c|c|c|c|c|c|c|}
\hline 2 & $29 \div 13$ & $--1 \cdot 2$ & $-1 \cdot 1$ & $-1 \cdot 1$ & - & S1E, 3 & 8 & $15-20$ & 0 & - & S. 10 & - & \multirow{7}{*}{ Min. on ship's rall, $-4^{\circ} 0$. } \\
\hline 4 & $29 \cdot 513$ & $--1 \cdot 0$ & $1 \cdot 0$ & $3 \cdot 8$ & - & SE, 2-3 & 8 & 10 & 0 & - & S. 10 & - & \\
\hline 0 & $29 \cdot 544$ & --1.8 & 6.5 & 6.0 & - & E, 1-2 & 8 & 10 & 0 & - & S. 10 & - & \\
\hline 8 & $29 \cdot 571$ & $--4 \cdot 0$ & $-3 \cdot 1$ & $-4 \cdot 0$ & - & KSE, 2 & 8 & $15-20$ & o & - & S. 10 & - & \\
\hline 10 & $29 \div 58$ & $--4 \cdot 0$ & $2 \cdot 0$ & $2 \cdot 0$ & - & $\mathrm{SE}, 2-3$ & 11 & $10-15$ & oc & - & S.-en. 10, NE & - & \\
\hline Nom & $29 \cdot 593$ & $--3 \cdot 5$ & $2 \cdot 2$ & $2 \cdot 0$ & - & E, 2-3 & 5 & $10-15$ & $\infty$ & - & $\mathrm{Fr} .8 .8$ & - & \\
\hline 2 & $29 \cdot 599$ & $--6 \cdot 8$ & $-3 \cdot 9$ & $-4 \cdot 0$ & - & Sle, 2-3 & 11 & $10-15$ & nc & - & 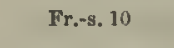 & - & \\
\hline 8 & $29 \cdot 55 \times 3$ & $--7 \cdot 0$ & $-6 \cdot 0$ & $-6 \cdot 0$ & - & SE, $3-4$ & 12 & 15 & $\circ *$ & - & N. 10 & - & $\begin{array}{l}\text { Min at lughrlevel station for } 24 \text { hours, } \\
-12^{\circ} 0 \text {. Tempo, at } 4 \text { p.m., }-11^{\circ} 0 \text {. }\end{array}$ \\
\hline 10 & 2969 & $--7 \cdot 0$ & $-3 \cdot 6$ & $-3 \cdot 0$ & - & N1E, 2-3 & 11 & $15-18$ & $o *$ & - & N. 10 & - & \multirow{4}{*}{ 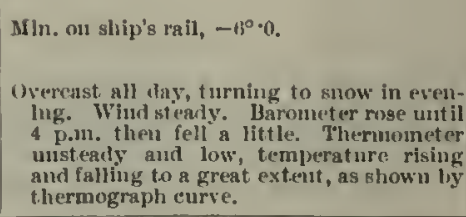 } \\
\hline Milt. & $29 \div 5 \pi^{2}$ & $-4 \cdot 0$ & $-2 \cdot 8$ & $-2 \cdot 8$ & - & $N H, 2-3$ & 14 & 22 & o* + & - & N. 10 & - & \\
\hline Mean & $28=-5 x^{2}+5$ & $-1-$ & $-1 \%$ & $-1 \cdot 2$ & - & - & - & - & - & - & - & $1-$ & \\
\hline & $\mathrm{Ma}$ & a & at $\left\{\begin{array}{l}8 \\
8\end{array}\right.$ & $\begin{array}{l}7^{\circ} \cdot 3 . \\
6^{\circ} \cdot 0.0\end{array}$ & & Solar radiatio & rlght & $\begin{array}{l}\text { melb } \\
t \text { bult }\end{array}$ & & ammes. & Precipu. - iı. & & \\
\hline
\end{tabular}


Meteorological Journal of tue "Discovery."

[1902-April.

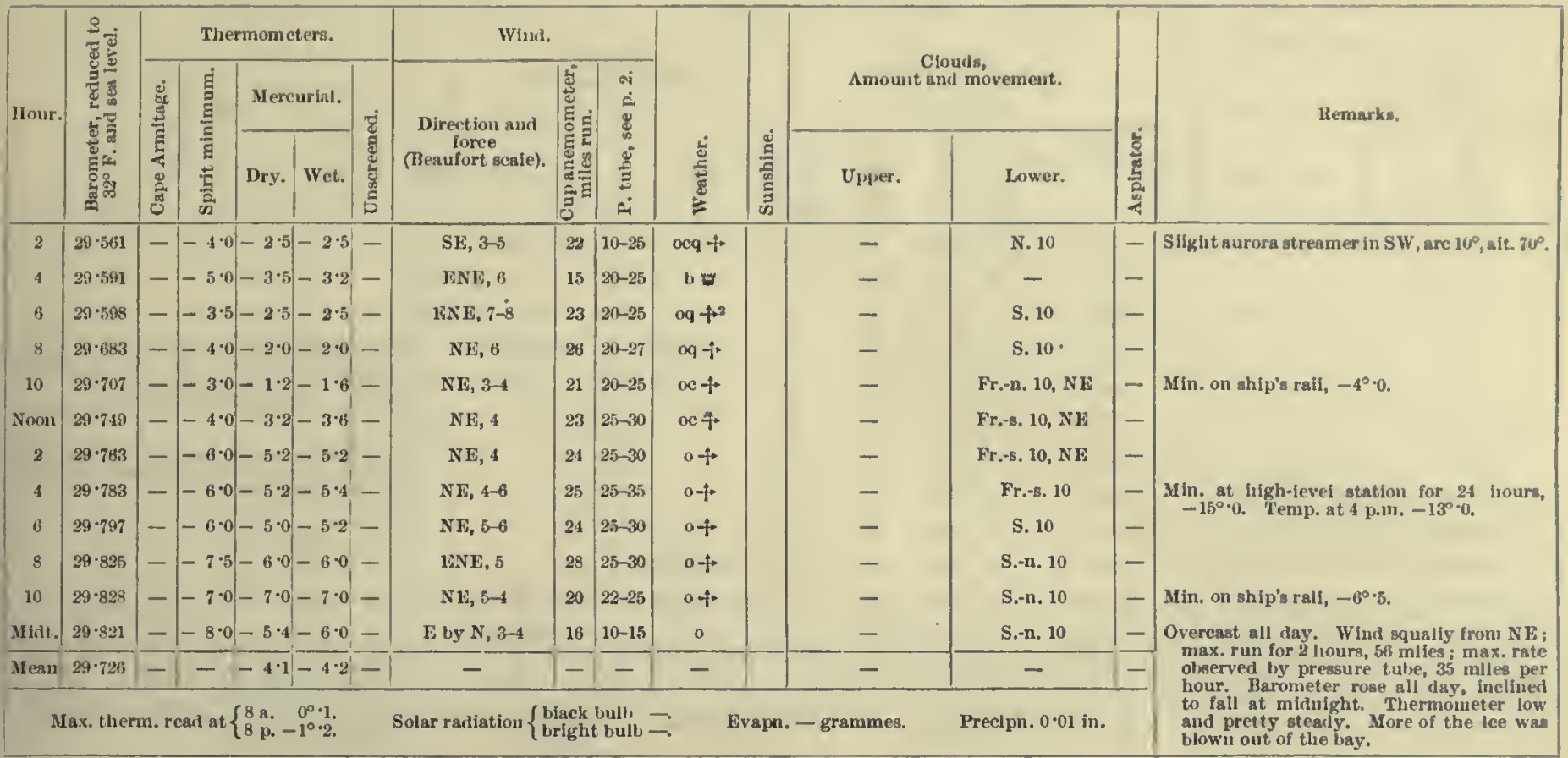

19 th.

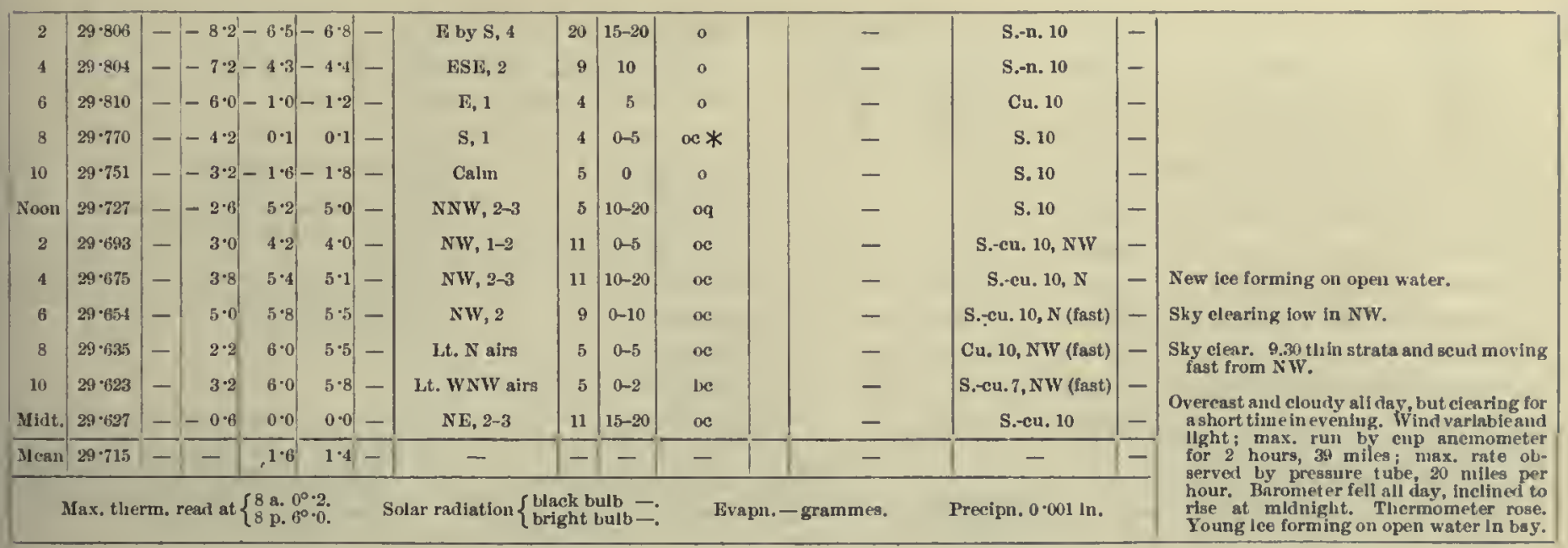

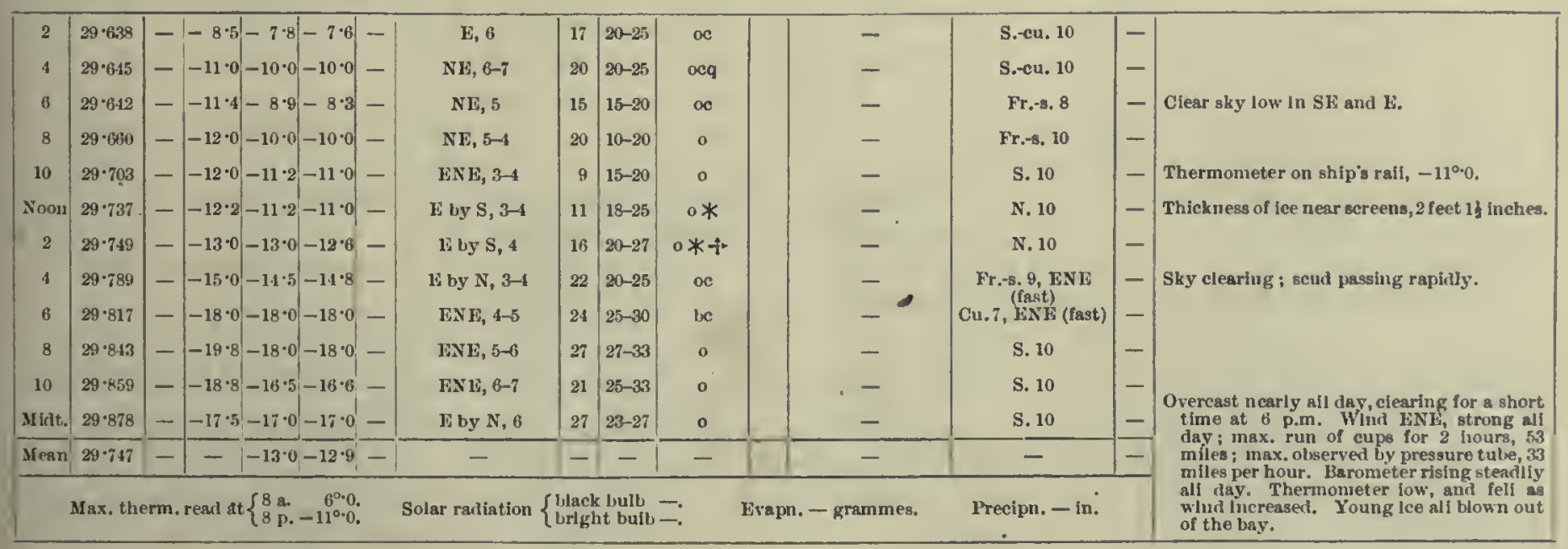


1902-April.]

Meteorological Journal of the "Discovery."
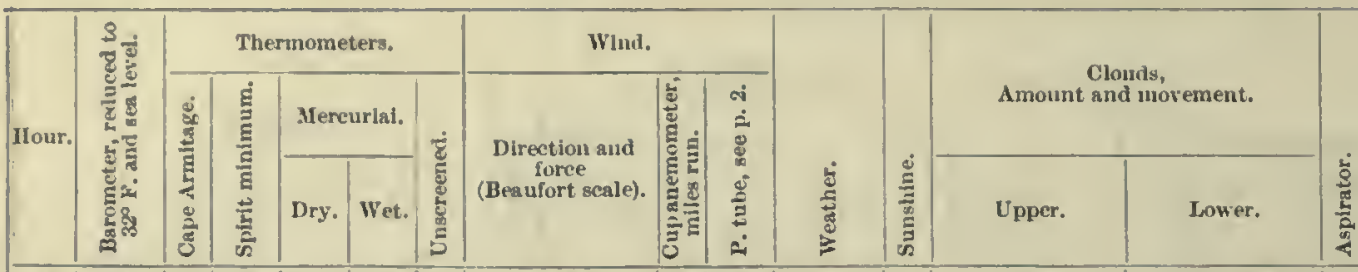

22nd.

\begin{tabular}{|c|c|c|c|c|c|c|}
\hline 2 & $29 \cdot 892$ & - & $-18 \cdot 8$ & $-18 \cdot 5$ & $-18 \cdot 6$ & - \\
4 & $29 \cdot 907$ & - & $-19 \cdot 8$ & $-17 \cdot 8$ & $-17 \cdot 5$ & - \\
6 & $29 \cdot 972$ & - & $-22 \cdot 5$ & $-20 \cdot 0$ & $-19 \cdot 8$ & - \\
8 & $29 \cdot 954$ & - & $-23 \cdot 0$ & $-18 \cdot 1$ & $-18 \cdot 0$ & - \\
10 & $29 \cdot 900$ & - & $-20 \cdot 0$ & $-17 \cdot 0$ & $-16 \cdot 8$ & - \\
Noon & $29 \cdot 895$ & - & $-21 \cdot 5$ & $-19 \cdot 8$ & $-18 \cdot 2$ & - \\
2 & $29 \cdot 901$ & - & $-21 \cdot 0$ & $-18 \cdot 1$ & $-18 \cdot 0$ & - \\
4 & $29 \cdot 897$ & - & $-25 \cdot 0$ & $-24 \cdot 1$ & $-23 \cdot 8$ & - \\
6 & $29 \cdot 903$ & - & $-25 \cdot 6$ & $-23 \cdot 5$ & $-23 \cdot 2$ & - \\
8 & $29 \cdot 905$ & - & $-24 \cdot 8$ & $-21 \cdot 2$ & $-21 \cdot 0$ & - \\
10 & $29 \cdot 891$ & - & $-21 \cdot 5$ & $-23 \cdot 8$ & $-23 \cdot 2$ & -
\end{tabular}

FNF, 6

k.NF, 6-7

FNE, 6-8

13X K, 2-3

21 20-25

be

1

NE, 4-6

NI, 5

EX' $\mathbf{E}, 4-5$

FNE, 5

$20 \quad 28$

$2425-30$ b

$\begin{array}{lll}15 & 15-20 & \end{array}$

$\begin{array}{lll}28 & 25-30 \quad \text { oq }\end{array}$

Ey $\mathrm{N}, 4-5$

$27 \quad 28-32$

$24 \quad 20-25$

o

\section{ENE, 3}

$23 \quad 25-30$ b

15-25 : b

$\begin{array}{llll}\mathrm{E} \text { by N, } 3 & 20 & 22-24 & \text { be }\end{array}$

Midt. $29 \cdot 891-|-25 \cdot 1|-21 \cdot 0-20 \cdot 8-$

$\mathbf{N F}, 4$

Miean $29 \cdot 900-\overline{-20 \cdot 2-20 \cdot 0}-$

Max, therm. read at $\left\{\begin{array}{l}8 a_{0}-15^{\circ} \cdot 6 . \\ 8 \mathrm{p},-15^{\circ} \cdot 8 .\end{array}\right.$

Solar radiation $\left\{\begin{array}{l}\text { black bulb } \\ \text { bright bulb -. }\end{array}\right.$

Evapn. - grammes.

Precipn. - la.

S.-cu. 6, N E

Ci. 2, SE (mod.)

C1,-s, 3, SE (slow) S.-cu, 4, Rinod.)

Min. on ship's rail, $-24^{\circ} \cdot 5$.

Fine day ; cloudy at intervals. Wind strong from Nik; max. run of eups for 2 hours, 543 miles; max. rate observed by pressure tube, 32 miles per hour. Barometer steady after 10 a.m.
and steady ali day.

$23 r d$.

\begin{tabular}{|c|c|c|c|c|c|c|c|c|c|c|c|c|}
\hline 2 & $29 \cdot 889$ & - & $|-22 \cdot 0|-21 \cdot 0-20 \cdot 2 \mid$ & -1 & E by $S, 3$ & 22 & $16-18$ & $\mathscr{H}$ & Fr.cio-s & - & - & \multirow{7}{*}{$\begin{array}{l}\text { Min. on slılp's rail, }-24^{\circ} \cdot 5 \text {. } \\
\text { Moon shows an irregular, flattened, oval } \\
\text { shape from excesslve nulrage. }\end{array}$} \\
\hline 4 & $29 \cdot 890$ & - & $-28 \cdot 5 \mid-18 \cdot 0-17 \cdot 5$ & - & E thy $N, 5$ & 17 & $20-25$ & $o c$ & - & Cu. $10, \mathbf{w}$ & - & \\
\hline 6 & $29 \cdot 875$ & - & $-22 \cdot 8-21 \cdot 0-21 \cdot 0$ & - & $N E, 4$ & 17 & $22-24$ & og & - & S. 10 & - & \\
\hline 8 & $29 \cdot 881$ & - & $-24 \cdot 5 \mid-24 \cdot 0-23 \cdot 0$ & - & NE, 3-4 & 22 & $20-25$ & oc & $\mathrm{Ci}_{.8 \mathrm{~s} .} 7$ & S. 3 & - & \\
\hline 10 & $29 \cdot 879$ & - & $-25 \cdot 0 \mid-17 \cdot 5-17 \cdot 5$ & - & NE, $2-3$ & 14 & 10 & be & Ci.-8. 6, SE & - & - & \\
\hline Noon & $29 \cdot 863$ & - & $-31 \cdot 5 \mid-31 \cdot 1-30 \cdot 5$ & - & ISE, 3-4 & 15 & $20-25$ & be & Cl, $1, \mathrm{SE}$ & - & - & \\
\hline 2 & $29 \cdot 854$ & - & \begin{tabular}{|l|l|}
$-31 \cdot 6$ & $-19 \cdot 0-19 \cdot 0$ \\
\end{tabular} & - & ENE, 2-3 & 12 & $10-15$ & be $\Psi$ & Ci.-8. 5, NE & Fr.-8. 2, Sk & - & \\
\hline 4 & $29 \cdot 842$ & - & $-24 \cdot 0-22 \cdot 5-22 \cdot 0$ & $\rightarrow$ & NE, 3 & 16 & $15-20$ & be & C1.-8. 6, N & - & - & \multirow{4}{*}{$\begin{array}{l}\text { Min. at high-level station for } 96 \text { hours, } \\
-32^{\circ}+. \text { Then at } 4 \text { p.m. }-30^{\circ} \cdot 0 \text {. Cl.-8. } \\
\text { radiating N. }\end{array}$} \\
\hline 0 & $29 \cdot 823$ & - & $-23 \cdot 8-23 \cdot 0-22 \cdot 8$ & - & $\mathrm{NE}, 4-5$ & 20 & $25-30$ & be & Cl.-5. 5, N & - & - & \\
\hline 8 & $29 \cdot 810$ & - & $|-25 \cdot 1|-24 \cdot 3-24 \cdot 1$ & - & $\mathrm{NE}, 5$ & 24 & $25-30$ & be & $\mathrm{Ci}_{0} \cdot \mathrm{s}, \mathrm{0}, \mathrm{NE}$ & - & - & \\
\hline 10 & $29 \cdot 804$ & - & $-24 \cdot 6 \mid-24 \cdot 2-24 \cdot 0$ & - & NE, 5 & 23 & $20-25$ & be & $\mathrm{Cl}_{.}-8.6, \mathrm{~N}$ & - & - & \\
\hline Milat. & $29 \cdot 789$ & -1 & $-25 \cdot 2-24 \cdot 2-23 \cdot 8$ & - & ENF, 4 & 18 & $18-24$ & ev & 1High S. 8 (Dir.?) & - & - & \multirow{3}{*}{$\begin{array}{l}\text { Fine clear weather. Cloud direction not } \\
\text { discernible. } \\
\text { Flne day. Fresh NE breeze; max. ruu of } \\
\text { cups for } 2 \text { hours, } 48 \text { miles; max, rate } \\
\text { observed by pressure tulle, } 30 \text { miles per } \\
\text { hour. Barometer falling slightly all day. } \\
\text { Thermometer lower and steady. }\end{array}$} \\
\hline Mean & $29 \cdot 850$ & - & $-|-22 \cdot 5|-22 \cdot 1$ & -1 & - & - & - & - & - & - & - & \\
\hline & Max. t t & $\mathrm{rm}$ & $\left\{\begin{array}{l}8 \text { a. }-16^{\circ} \cdot 0 \\
8 \text { p. }-16^{\circ} \cdot 0\end{array}\right.$ & & ar radia & & hu & & grammes. & Precipn. - In & & \\
\hline
\end{tabular}

24th.

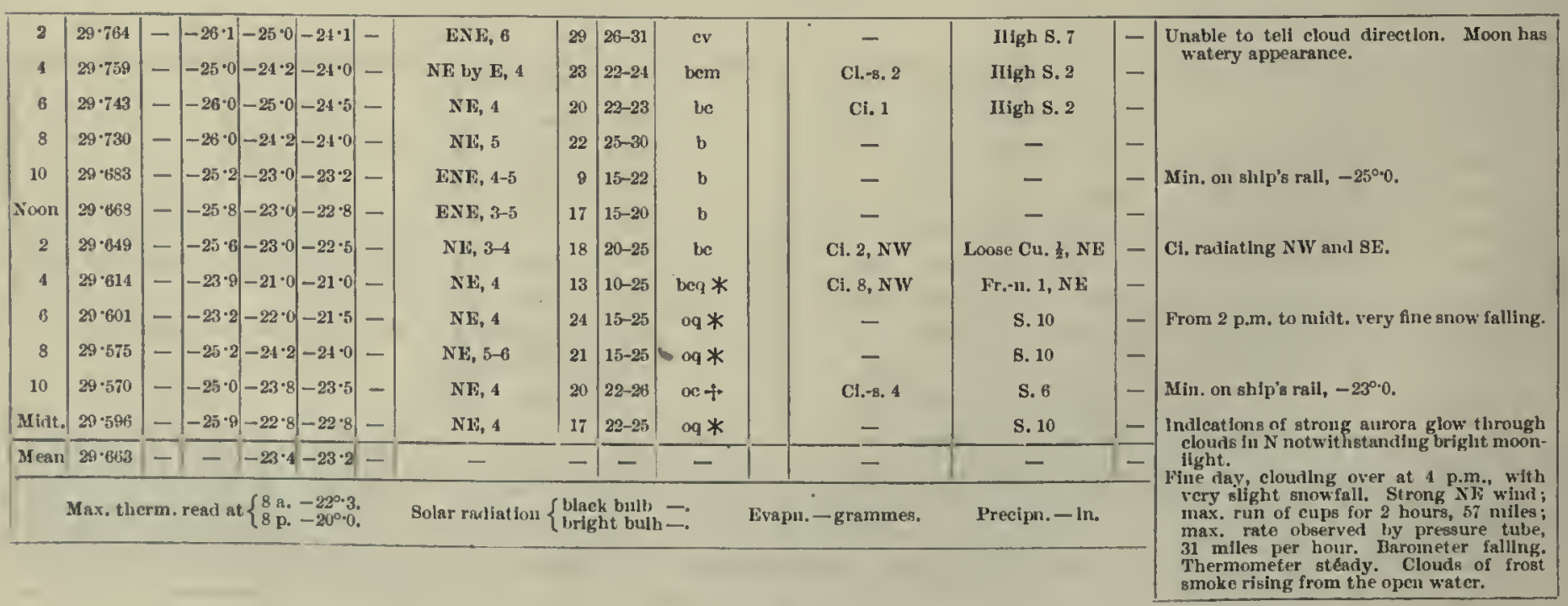


Meteorological Journal of the "Discovery."

[1902-April.

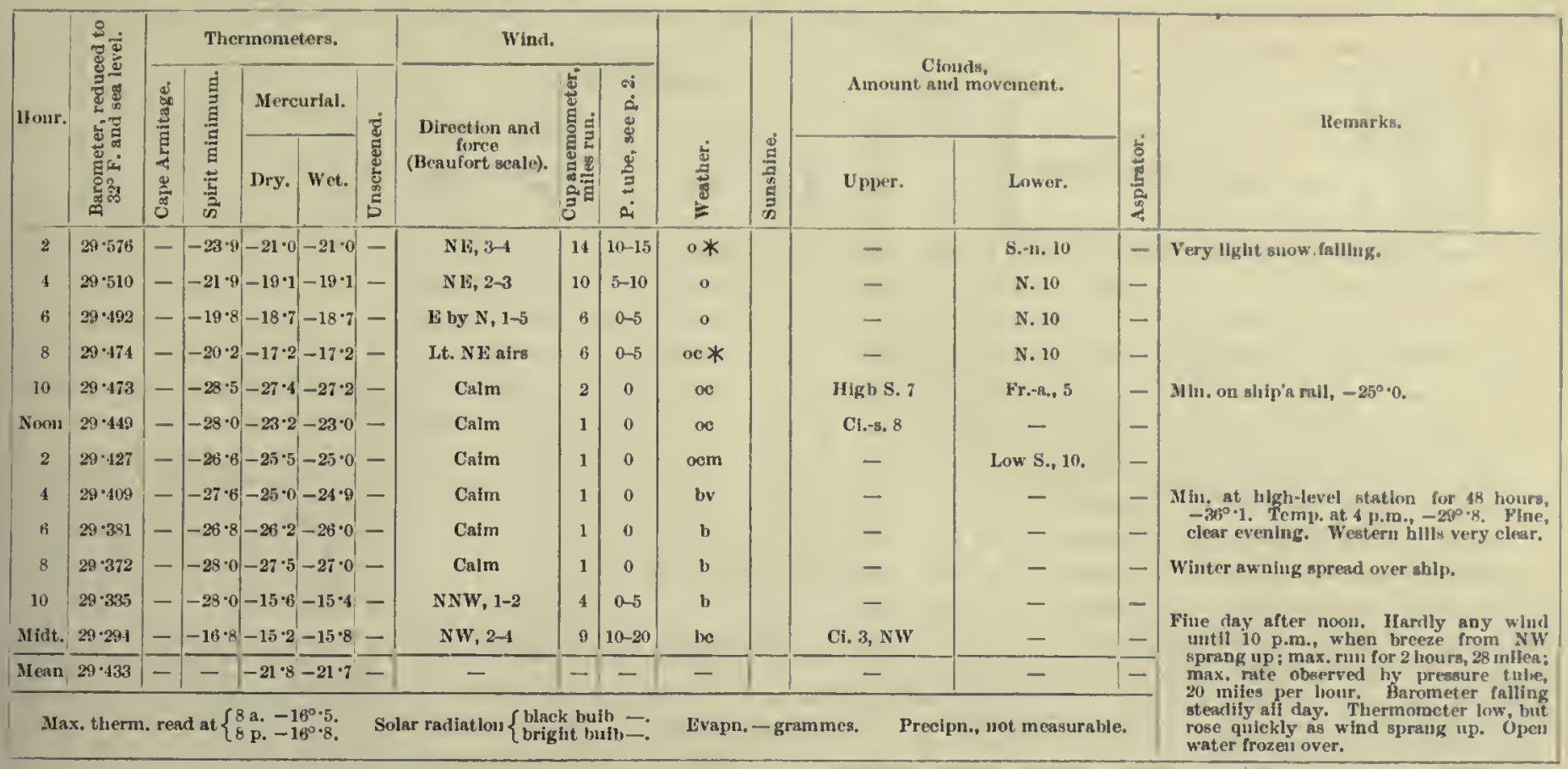

25th.

\begin{tabular}{|c|c|c|c|c|c|c|c|c|c|c|}
\hline 2 & $29-279$ & $-|-16 \cdot 6|-15 \cdot 0|-15 \cdot 0|-$ & NW hy $N, 2-4$ & 11 & $\mid 15-20$ & to & $\mathrm{Cl} .-8.4, \mathrm{NW}$ & - & -1 & \multirow{14}{*}{ 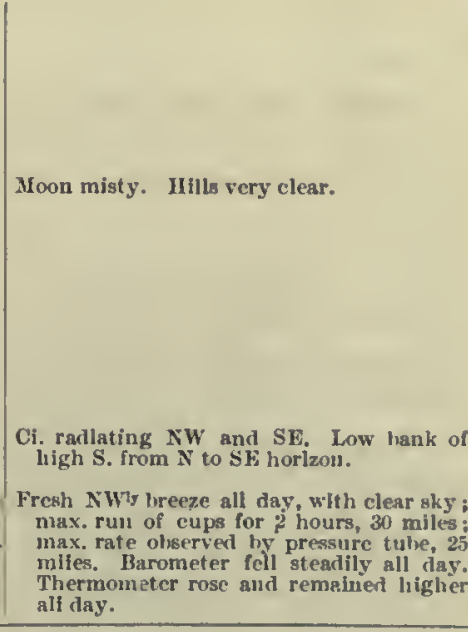 } \\
\hline 4 & $29 \cdot 227$ & $-|-16 \cdot 2-14 \cdot 1-14 \cdot 1|-$ & $N$ by $\mathbf{W}, 2-5$ & 12 & $|14-18|$ & beq & Cl. $3, \mathrm{NW}$ & - & - & \\
\hline 6 & $29-204$ & $-|-15 \cdot 0|-12 \cdot 8|-12 \cdot 8|-$ & $N \lg w, 1-5$ & 13 & $5-23$ & beq $\mathbb{D}$ & Ci.-8. $8, \mathrm{~N}$ hy W & - & - & \\
\hline 8 & $29 \cdot 247$ & $-|-13 \cdot 8|-11 \cdot 0|-11 \cdot 2|-$ & NNW, $2-3$ & 15 & $\mid 15-25$ & beq & Cl. 8 & - & - & \\
\hline 10 & $29 \cdot 233$ & $-|-12 \cdot 0|-8 \cdot 8|-8 \cdot 8|-$ & NNW, $2-3$ & 14 & $10-15$ & b & - & - & - & \\
\hline soon & $29 \cdot 206$ & $-|-9 \cdot 8-7 \cdot 0-7 \cdot 2|-$ & $N w, 3$ & 12 & $10-17$ & b & - & -. & - & \\
\hline 2 & $29 \cdot 190$ & $-|-9.8-9.0-9.0|-$ & $N, 3-4$ & 11 & $\mid 15-20$ & $b+$ & - & - & - & \\
\hline 4 & $29 \cdot 174$ & $-|-10.0|-6.0|-6.0|-$ & $\mathrm{NW}, 2-3$ & 12 & $|10-22|$ & b & - & - & - & \\
\hline 8 & $29 \cdot 159$ & $-|-7 \cdot 0|-6 \cdot 2|-6 \cdot 3|-$ & NNW, 2-3 & 11 & $\mid 10-15$ & b & - & - & - & \\
\hline 8 & $29 \cdot 158$ & $-|-9 \cdot 0|-8 \cdot 2|-8 \cdot 2|-$ & $\mathrm{NW}, 2-3$ & 12 & $15-20$ & h & - & - & - & \\
\hline 10 & $29 \cdot 150$ & $-|-10 \cdot 5|-9 \cdot 2|-9 \cdot 2|-$ & $N W, 2-3$ & 12 & $5-12$ & be $U$ & Ci. 2 & - & - & \\
\hline Miat. & $29 \cdot 151$ & $-|-10.6-10.0|-10.0-$ & NW lyy $N, 2-3$ & 10 & $5-14$ & be & Ci. 2 & - & - & \\
\hline Sean & $29 \cdot 198$ & $-1--9.8-9 \cdot 8-$ & - & - & -1 & - & - & - & 1 & \\
\hline \multicolumn{3}{|c|}{ 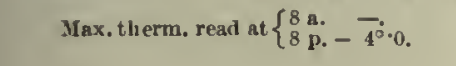 } & Solar radiatic & & & & grammes. & ipn. $-\ln$. & & \\
\hline
\end{tabular}

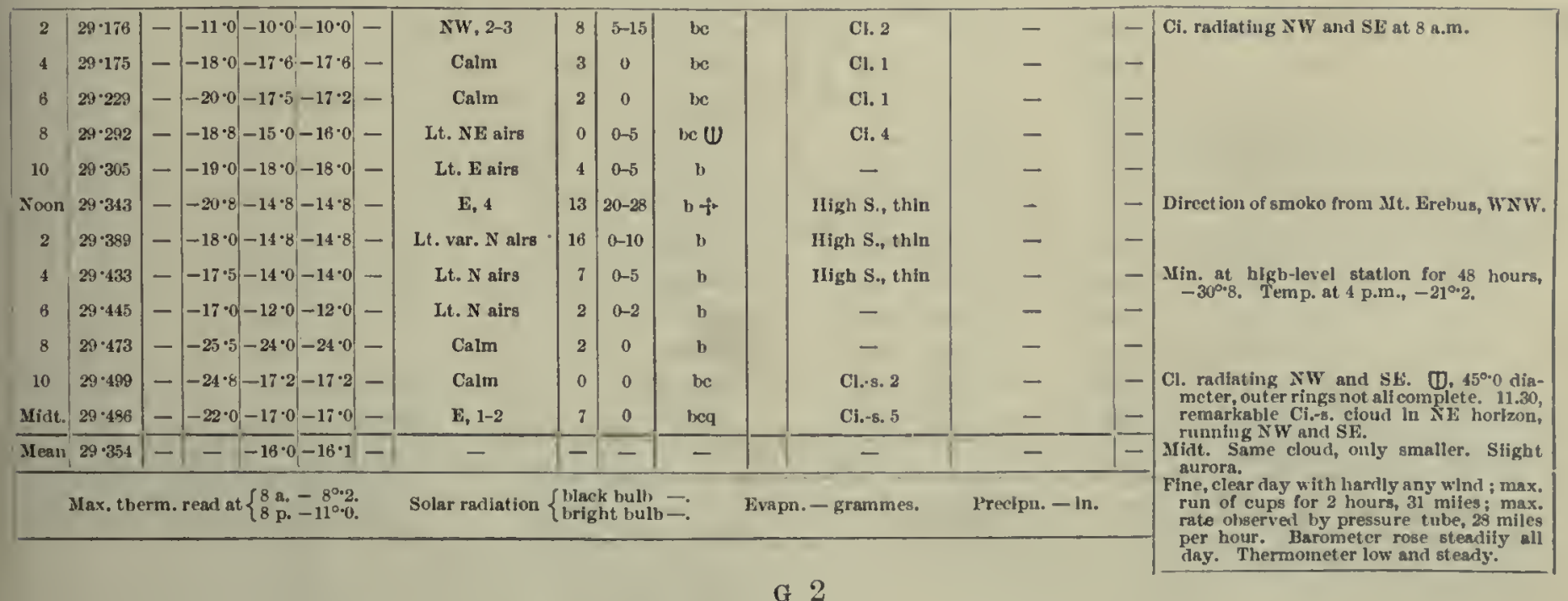


1902-April.]

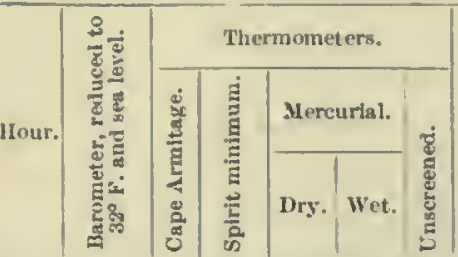

Miteorological Journal of the "Discovery."

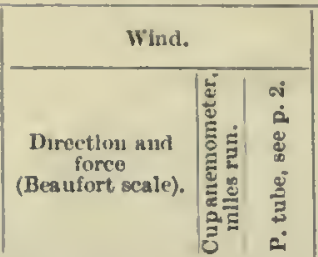

28th.

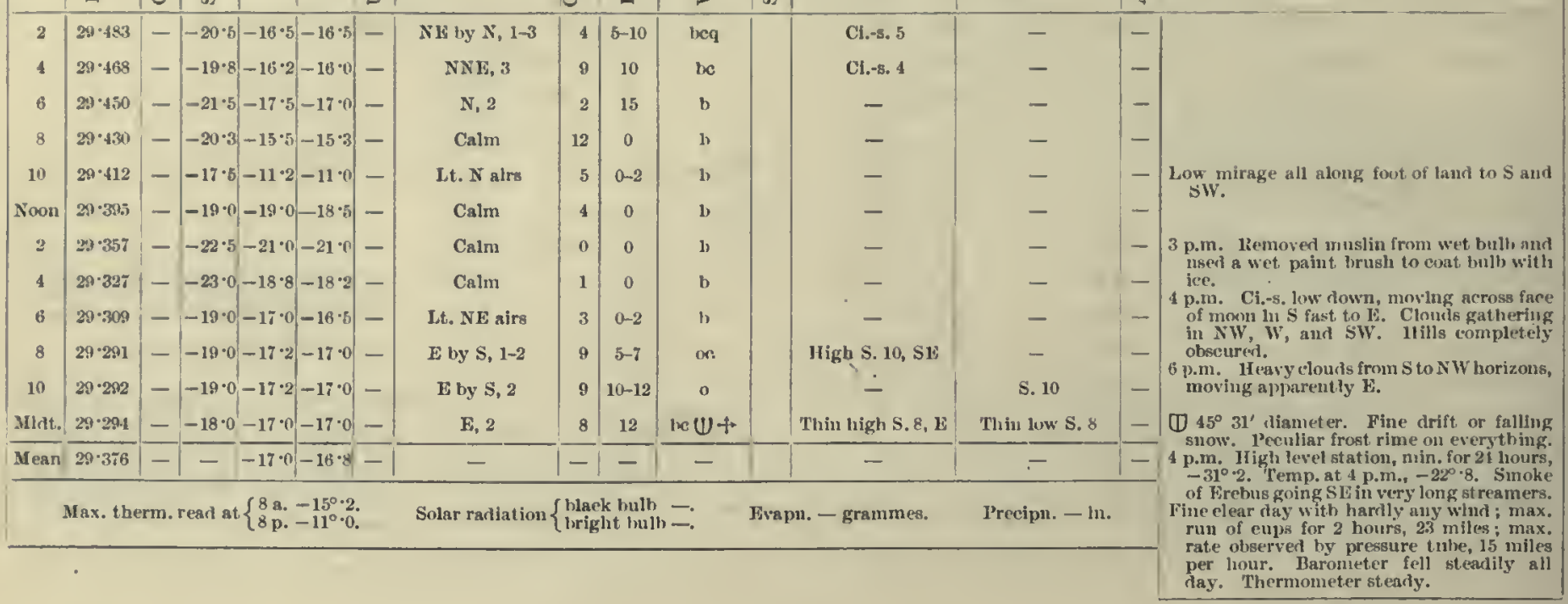

29 th

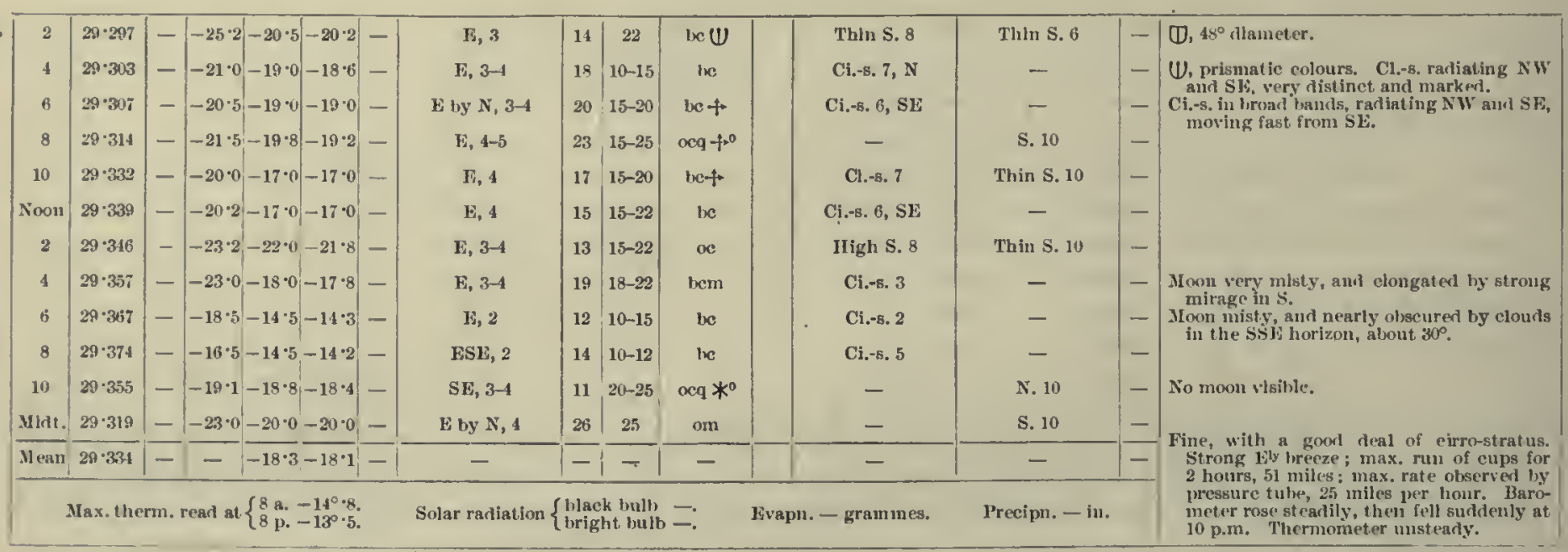

30 th.

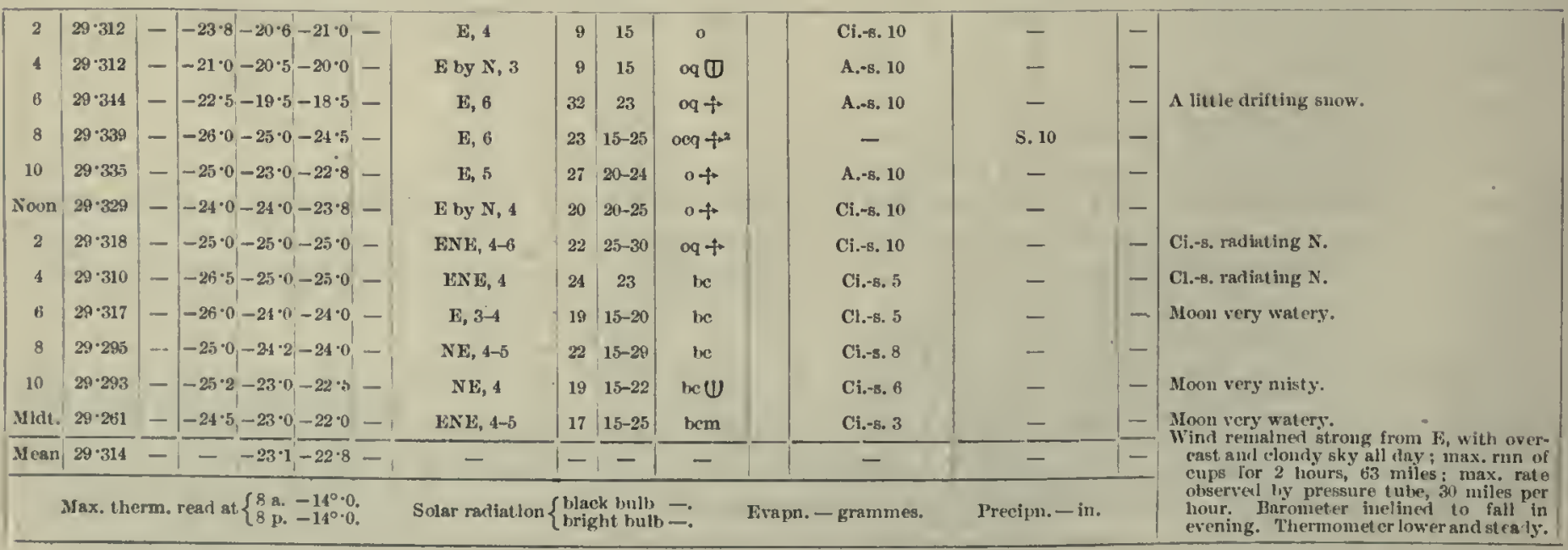




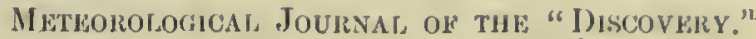

[1902-May.

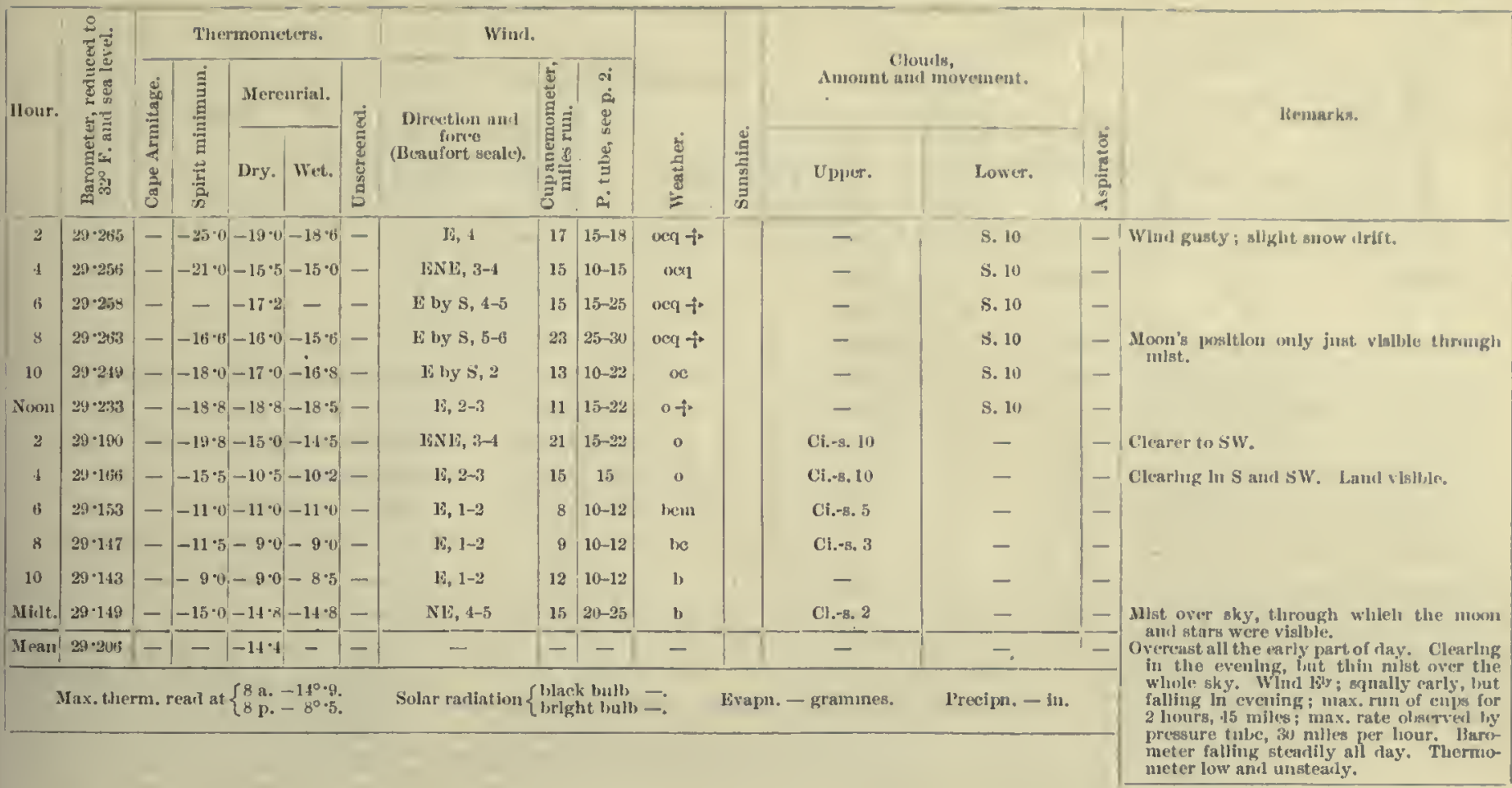

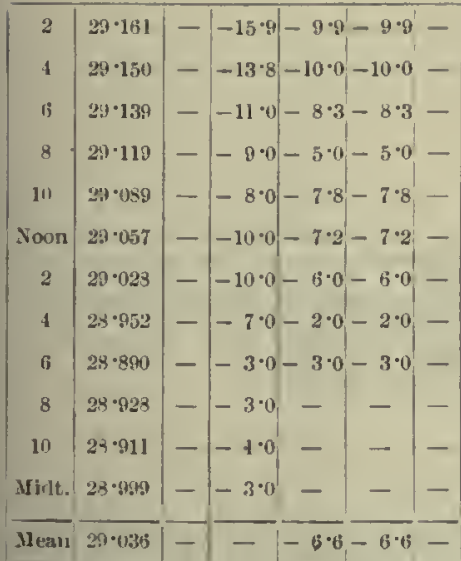

Max. thern. read at $\left\{\begin{array}{l}8 \mathrm{a},-5^{\circ} 0 . \\ 8 \mathrm{p}, \text { no observat ion. }\end{array}\right.$

\begin{tabular}{|c|c|c|c|}
\hline $\mathbf{E}$ by $N, 4$ & 14 & $15-20$ & b \\
\hline Nli, 4-5 & 15 & $20-25$ & bin \\
\hline N li, 5 & 18 & $20-25$ & bm \\
\hline SE, 2-3 & 17 & $15-20$ & $\operatorname{seq} 10$ \\
\hline $\mathrm{KSE}, 3-1$ & 17 & $15-22$ & $n+$ \\
\hline $\operatorname{lis} \mathrm{E}, 5-6$ & 23 & $20-38$ & ocq $f$ \\
\hline E by $\mathrm{S}, 4-5$ & 20 & $20-25$ & oq $t^{2}$ \\
\hline$S, 7-9$ & - & $25-40$ & $\operatorname{og}+r^{2}$ \\
\hline S by $W, 7-9$ & - & $35-15$ & $09+2$ \\
\hline$S$ by $W, 8-10$ & 34 & $45-52$ & og $f^{2}$ \\
\hline SW, 8-9 & 39 & ? & $\operatorname{og} f^{2}$ \\
\hline S by W, 10 & 39 & $?$ & og $\div^{2}$ \\
\hline & - & - & \\
\hline
\end{tabular}

Solar radiation $\left\{\begin{array}{l}\text { black bulh } \\ \text { lright bulb }- \text {. }\end{array}\right.$

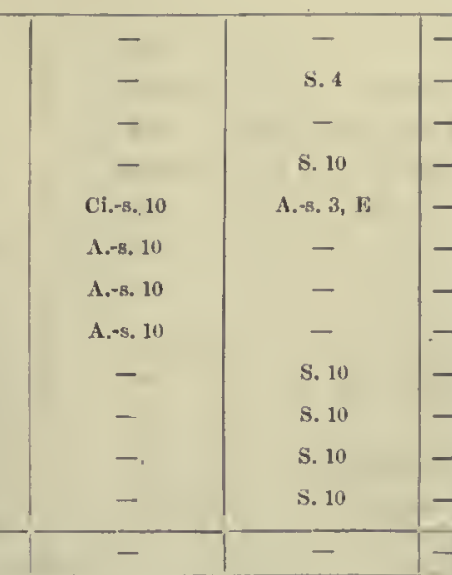

Fvapu. - gramnes.

Precipn. - in. - Mlsty W, rather faint.

2nd.

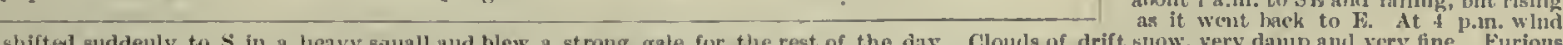

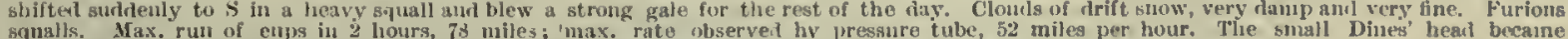

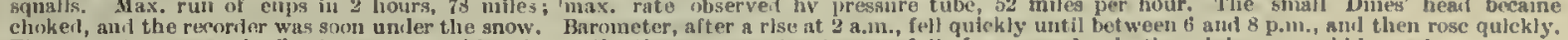
Thermometer rose as the S galc came on. After 6 p.m. the thermometer box beeame full of snow, and ouly the minimun could be real.

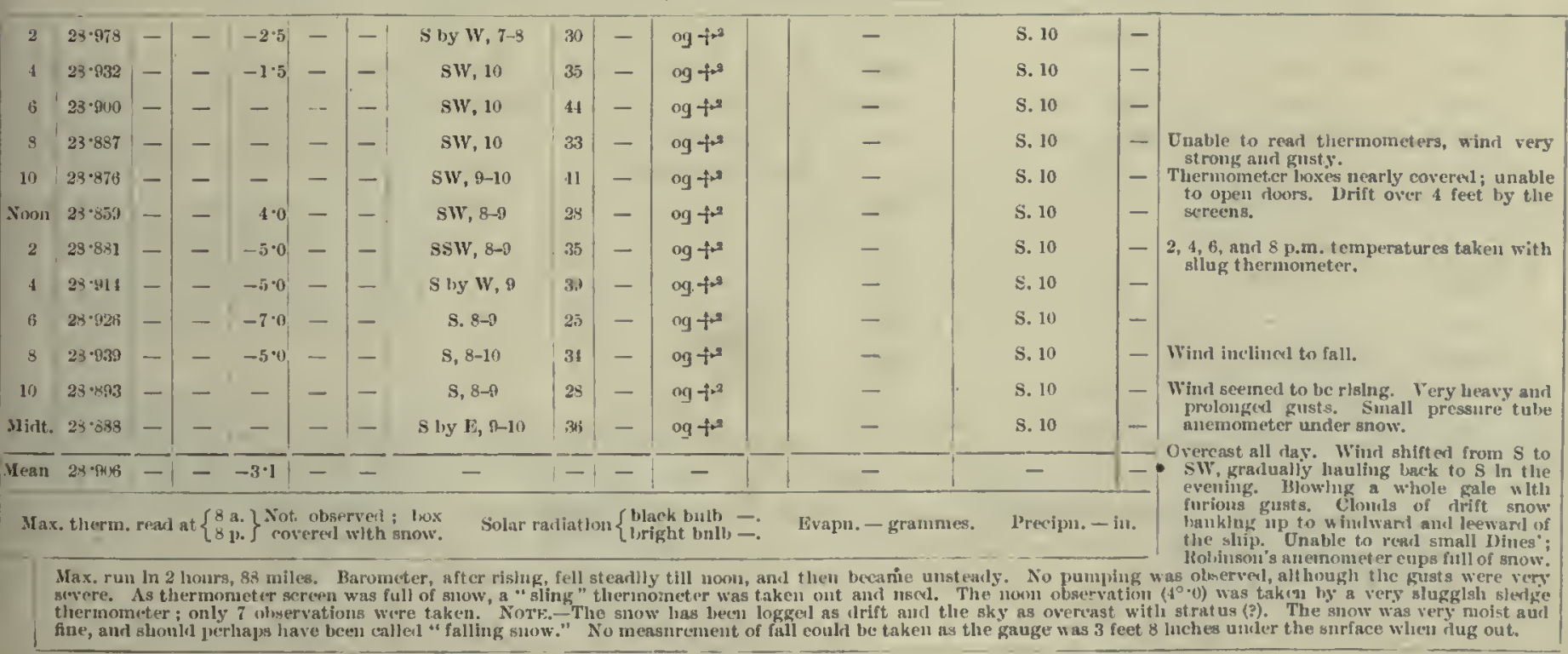


1902-May.]
Meteorological Journat of THE "Discovery."

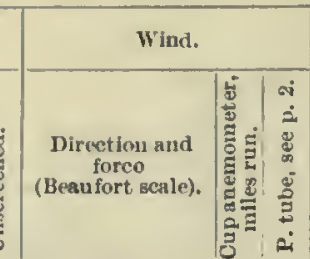

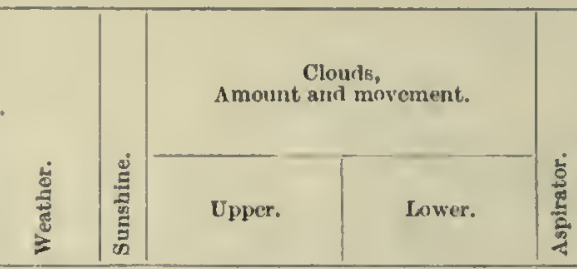

4th.

\begin{tabular}{|c|c|c|c|c|c|c|}
\hline \multirow{3}{*}{ liour. } & \multirow{3}{*}{ 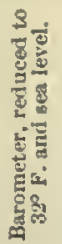 } & \multicolumn{5}{|c|}{ Thermometers. } \\
\hline & & $\ddot{x}$ & घू & Merc & arial. & \\
\hline & & 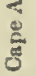 & है & Dry. & Wet. & 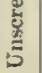 \\
\hline
\end{tabular}

\begin{tabular}{|c|c|c|c|c|c|c|c|c|c|c|}
\hline 2 & $28 \cdot 919$ & - & - & $10^{\circ}$ & - & - & $S \mathrm{~s} v \mathrm{k}, 9-10$ & 36 & - & $a+2$ \\
\hline 4 & $28 \cdot 948$ & - & - & $12 \cdot 0$ & - & - & S, 9-10 & 35 & - & oq $\uparrow^{2}$ \\
\hline 6 & $28 \cdot 974$ & - & - & $18 \cdot 0$ & - & - & $8,7-9$ & 35 & - & og +2 \\
\hline 8 & $28 \cdot 978$ & - & - & $19 \cdot 0$ & - & - & SE, 4-5 & 30 & - & oeq $t^{2}$ \\
\hline 10 & $28: \$ 11$ & - & - & $11 \cdot 0$ & - & - & SE, 4-5 & 22 & - & $\operatorname{seq} f^{2}$ \\
\hline Vom & $28 \cdot 969$ & - & - & $8 \cdot 0$ & - & - & $\mathrm{SE}, 5-6$ & 18 & - & $\operatorname{og} \dagger^{2}$ \\
\hline 2 & $25 \cdot 981$ & - & - & $7 \cdot 0$ & - & - & $\mathrm{SE}, 6$ & 17 & - & $\operatorname{og}+2^{2}$ \\
\hline 4 & $28 \cdot 993$ & - & - & $7 \cdot 0$ & - & - & SE, $5-7$ & 23 & - & $\alpha q+t^{2}$ \\
\hline 6 & $29 \cdot 099$ & - & - & $7 \cdot 0$ & - & - & SE, 5-7 & 22 & 一 & $0 \mathrm{q}+\varphi^{2}$ \\
\hline 8 & $29 \cdot 113$ & - & - & $6 \cdot 0$ & - & - & SE, 4-6 & 24 & - & $o \underline{q}+x^{2}$ \\
\hline 10 & $29 \cdot 145$ & - & - & $6 \cdot 0$ & - & - & E hy $S, 4-5$ & 22 & - & $\operatorname{og}+2$ \\
\hline Midt. & $29 \cdot 157$ & - & - & $8 \cdot 0$ & - & - & E, 3-4 & 17 & - & og $7^{+}$ \\
\hline Mean & •019 & - & - & 0.0 & - & - & - & - & - & - \\
\hline
\end{tabular}

Max. thcrm. read at $\left\{\begin{array}{l}8 \mathrm{a} . \\ 8 \mathrm{p} .\end{array}\right\}^{\text {Not ohserved: bo }}$ full of snow.

Solar radiation $\left\{\begin{array}{l}\text { black hulb } \\ \text { bright bulb }- \text {. }\end{array}\right.$

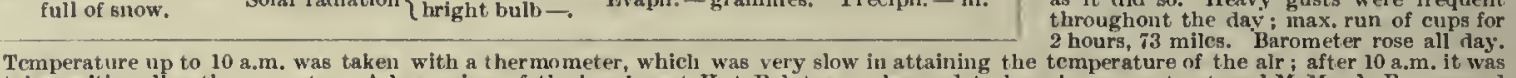
taken with a sling thermometer. A large picce of the ice abrenst Hut Polit was ohserved to have bcen swept out, and MirMurio Bay. It was clear of ice. Cleared the cups of Robitson's anemometer, which were filled witl snow. The heads of botit pressure-tube anemometers are completely choked.

5th.

\begin{tabular}{|r|r|r|r|r|r|r|}
\hline 2 & $29 \cdot 198$ & - & - & $7 \cdot 0$ & - & - \\
4 & $29 \cdot 181$ & - & - & $10 \cdot 7$ & - & - \\
6 & $29 \cdot 199$ & - & - & $10 \cdot 8$ & - & - \\
8 & $29 \cdot 191$ & - & - & $12 \cdot 0$ & - & - \\
10 & $29 \cdot 196$ & - & - & $11 \cdot 0$ & - & - \\
Noon & $29 \cdot 190$ & - & $9 \cdot 5$ & $11 \cdot 2$ & $11 \cdot 5$ & - \\
2 & $29 \cdot 174$ & - & $11 \cdot 0$ & $12 \cdot 0$ & $12 \cdot 0$ & - \\
4 & $29 \cdot 133$ & - & $9 \cdot 8$ & $10 \cdot 2$ & $10 \cdot 5$ & - \\
6 & $29 \cdot 120$ & - & $7 \cdot 0$ & $9 \cdot 0$ & $9 \cdot 0$ & - \\
8 & $29 \cdot 097$ & - & $5 \cdot 0$ & $7 \cdot 5$ & $7 \cdot 5$ & - \\
10 & $29 \cdot 060$ & - & $3 \cdot 0$ & $5 \cdot 0$ & $5 \cdot 0$ & - \\
Midt. & $29 \cdot 024$ & $4 \cdot 8$ & $7 \cdot 0$ & $7 \cdot 0$ & - \\
\hline
\end{tabular}

\begin{tabular}{c|c|c|c|} 
E, 4-5 & 26 & - & $0 q+$ \\
E hy S, 4-5 & 18 & - & bmq + \\
S hy E, 4-5 & 23 & - & $o q+$ \\
SE, 3-4 & 19 & - & $o c q+$ \\
E, 3-4 & 18 & - & $c c q$ \\
E hy N, 3-4 & 15 & - & $o c+$ \\
E, 2-3 & - & - & $a c$ \\
ESE, 2-3 & - & - & $c+4$ \\
E by S, 2-3 & - & - & $b c$ \\
E, 3 & - & - & $b c$ \\
E by N, 3-4 & - & - & $b c$ \\
ENE, 4 & - & - & b \\
\hline
\end{tabular}

\begin{tabular}{|c|c|}
- & S. 10 \\
- & - \\
- & S. 10 \\
- & S. 10 \\
- & S. 10 \\
A.--6. 10, E. & - \\
A.-6. 10, E. & - \\
A.-s. 10, E. & - \\
Thin 8. 6 & - \\
Thin 8. 5 & - \\
Thin S. 3 & - \\
- & - \\
\hline- & - \\
\hline
\end{tabular}
Max. therm. rear at $\left\{\begin{array}{l}8 \mathrm{a} . \text { not observed. } \\ 8 \mathrm{p} .12^{\circ} \cdot 0 .\end{array}\right.$ Solar radiation $\left\{\begin{array}{l}\text { black bulh }- \text {. } \\ \text { bright bulb }- \text {. }\end{array}\right.$

Erapn. - grammes.

Precipn. - in
Remarks.

S. 1

S. 10

S. 10

S. 10

S. 10

S. 10

S. 10

S. 10

S. 10

S. 10

- Wind had shifter, and falling.

- Wind falling, hut heavy drift continues,

- Wind Increasing, squalls lieavier.

- Heary squalls.

- Heavy squalls, mueh drift.

- Wind falling, squalls fewer and not so lueayy.

- Wind squally.

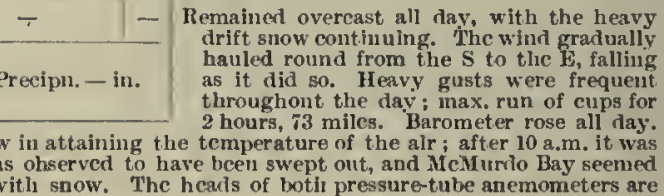

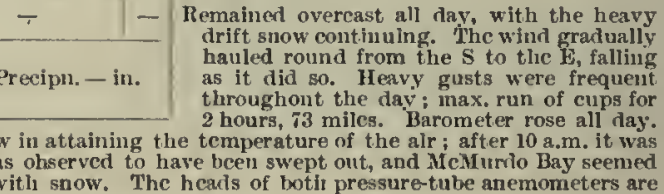

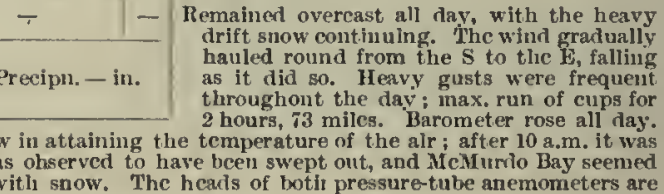

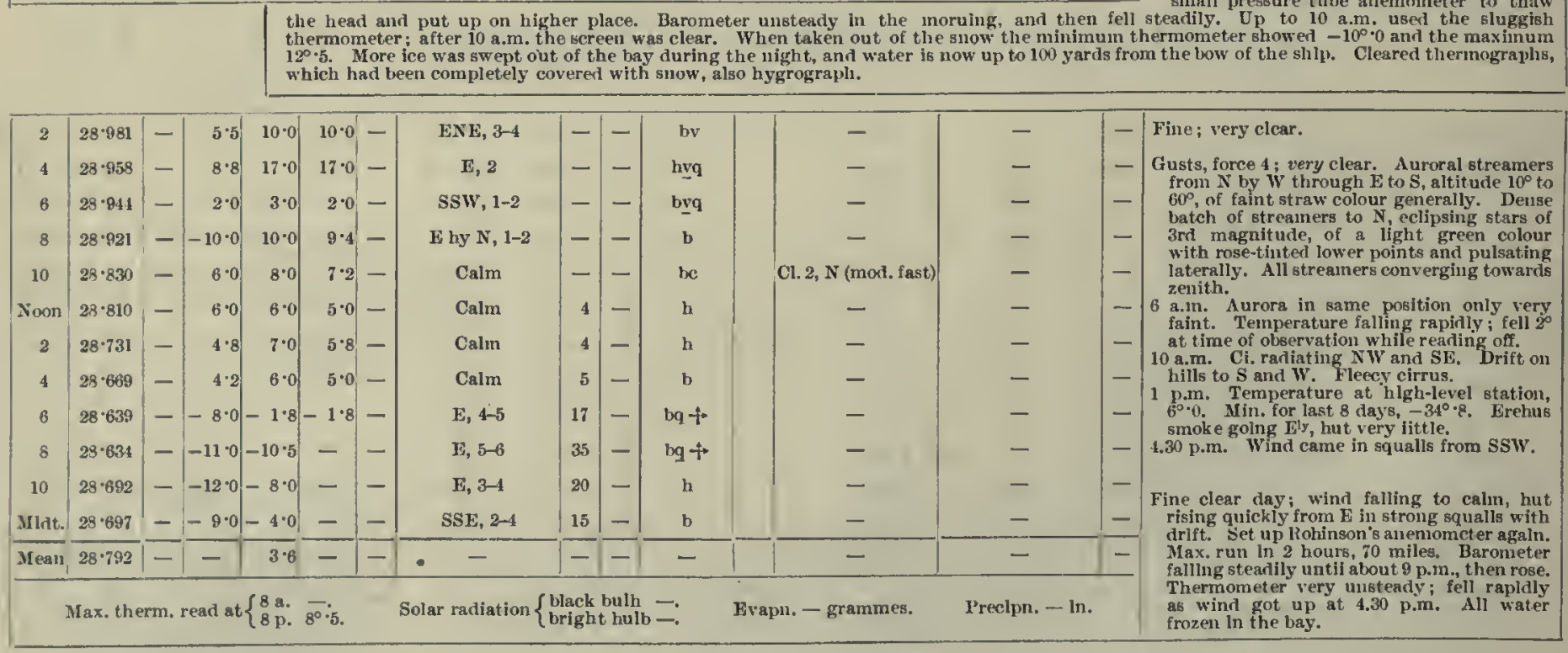
\begin{tabular}{l|l|}
- & Wind decreasing. \\
- & Lighter overhead. 4.15 a.m. heavy gusts. \\
- & \\
- & \\
- & \\
- & p.m. Took cup an emometer down. Cleared \\
thermoneter scrcens. Took down pressure \\
tube. Stratus in short dark lines lying E and \\
2 p.m. Strat \\
W. Clear to SW and W. \\
4 p.m. Wind rising ln gusts. Very dark to S \\
and SE. \\
6 p.m. Clearing overhead. \\
- \\
- \\
Ciearer overhead; thick low down. \\
- & Fine and clear.
\end{tabular}

Cleared slowly towards evening. Wind was strong and shifty, with a good deal of snow drift at times. Max. run of cups for and cups of alseinometer. Also took dowis small pressure tube anemometer to thaw mometer showed $-10^{\circ} 0$ and the maximum thermometer; after 10 a.m. the screen was clear. When taken out of the snow the minimum thermometer showed $-10^{\circ} \cdot 0$ and the maxinum
$12^{\circ} \cdot 5$. More ice was swept out of the bay during the night, and water is now up to 100 yards from the bow of the shlp. Cleared thermograplis,
which had been completely covered with snow, also hygrograpl.

6th. 
Meteorological Journal of tire "Discovery."

\begin{tabular}{|c|c|c|c|c|c|c|c|c|c|c|c|c|c|c|c|}
\hline \multirow{3}{*}{11 our. } & \multirow{3}{*}{ 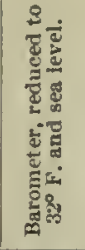 } & \multicolumn{5}{|c|}{ Thermometers. } & \multicolumn{3}{|l|}{ Wind. } & \multirow[b]{3}{*}{ 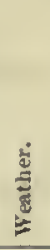 } & \multirow[b]{3}{*}{ 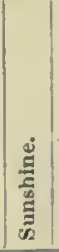 } & \multirow{2}{*}{\multicolumn{2}{|c|}{$\begin{array}{l}\text { Clouds, } \\
\text { Amount and movernent. }\end{array}$}} & \multirow[b]{3}{*}{$\frac{5}{\frac{\mathrm{g}}{2}}$} & \multirow{3}{*}{ Hemarka. } \\
\hline & & \multirow{2}{*}{ 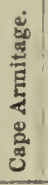 } & \multirow{2}{*}{ 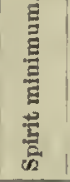 } & \multicolumn{2}{|c|}{ Mereurial. } & \multirow{2}{*}{ 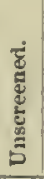 } & \multirow{2}{*}{$\begin{array}{c}\text { Direction and } \\
\text { force } \\
\text { (Beaufort scale). }\end{array}$} & \multirow{2}{*}{ 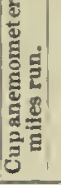 } & \multirow{2}{*}{ 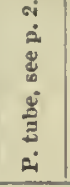 } & & & & & & \\
\hline & & & & Dry. & Wet. & & & & & & & Upper. & Lower. & & \\
\hline 2 & $28 \cdot 698$ & - & $-5 \cdot 0$ & $-3 \cdot 2$ & - & - & L by $S, 1-2$ & 9 & - & be & & Cl. 1, lis & - & -1 & \multirow{14}{*}{ 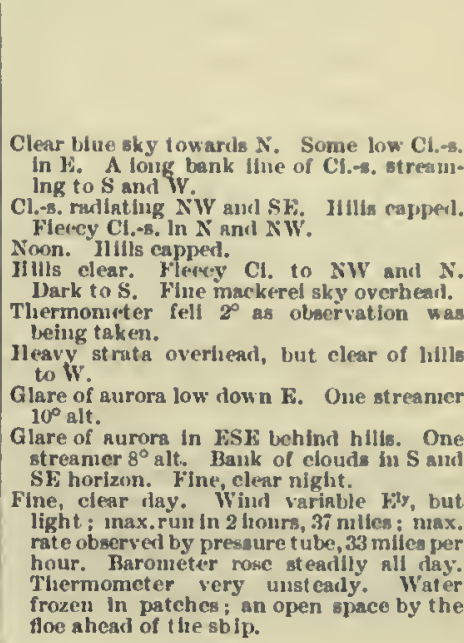 } \\
\hline 4 & $28 \cdot 715$ & - & $-3 \cdot 0$ & -2.8 & - & - & F by $\mathrm{N}, 1-2$ & 5 & - & b & & - & - & - & \\
\hline 6 & $28 \cdot 708$ & - & $-3 \cdot 1$ & $3 \cdot 3$ & - & - & $\mathrm{N}$ by $\mathbf{E}, 1-2$ & 6 & - & b & & - & - & - & \\
\hline 8 & $28 \cdot 759$ & - & $-6 \cdot 0$ & $4 \cdot 8$ & - & - & NW, 0-1 & 3 & - & be & & C1.-8. 6 & - & - & \\
\hline 10 & $28 \cdot 760$ & - & 4.0 & $8 \cdot 0$ & - & - & $\mathbf{N}, 2-3$ & 14 & - & be & & C1. -6.4 & - & - & \\
\hline Noon & $28 \cdot 780$ & - & $6 \cdot 2$ & $8 \cdot 5$ & - & - & Nh, 2-3 & 14 & - & be & & $\mathrm{Cl}_{.}-8,5, \mathrm{SW}$ & - & - & \\
\hline 2 & $28 \cdot 765$ & - & $6 \cdot 5$ & $8 \cdot 0$ & - & - & $N, 1-2$ & 12 & $10-15$ & be & & Ci.en. 6 & - & - & \\
\hline 4 & $28 \cdot 789$ & - & $2 \cdot 0$ & $2 \cdot 0$ & - & - & NE, 2-3 & 8 & $20-22$ & beq & & Cl.-cll. 5, SW & - & - & \\
\hline 6 & $28 \cdot 811$ & - & $-4 \cdot 0$ & -3.5 & - & - & NE, 2-3 & 16 & $20-25$ & b & & - & - & - & \\
\hline 8 & $28 \cdot 845$ & - & $-6 \cdot 0$ & $-6 \cdot 0$ & - & - & NF, 3 & 19 & $30-33$ & b & & - & - & - & \\
\hline 10 & $28 \cdot 874$ & - & $-\pi \cdot 0$ & $-4 \cdot 2$ & - & - & NE, 2-3 & 18 & 20 & b & & - & - & - & \\
\hline Midt. & $28 \cdot 880$ & - & $-6 \cdot 5$ & $-3 \cdot 7$ & - & -1 & F by $\mathrm{N}, 2-3$ & 14 & $24-26$ & 0 & & Ci.-s. 4 & - & - & \\
\hline Mean & $28 \cdot 780$ & - & - & 0.9 & - & -1 & - & -1 & - & - & & - & - & - & \\
\hline \multicolumn{7}{|c|}{ Max. therm. read at $\left\{\begin{array}{l}8 \text { a, } 13^{\circ} \cdot 5 . \\
8 \text { p. } 8^{\circ} 0 .\end{array}\right.$} & \multicolumn{4}{|c|}{ Solar radiation $\left\{\begin{array}{l}\text { black bulb } \\
\text { bright bulb }- \text {. }\end{array}\right.$} & Fvapn & grammes. & \multicolumn{2}{|l|}{ Preeipn. - lis. } & \\
\hline
\end{tabular}

7 th.

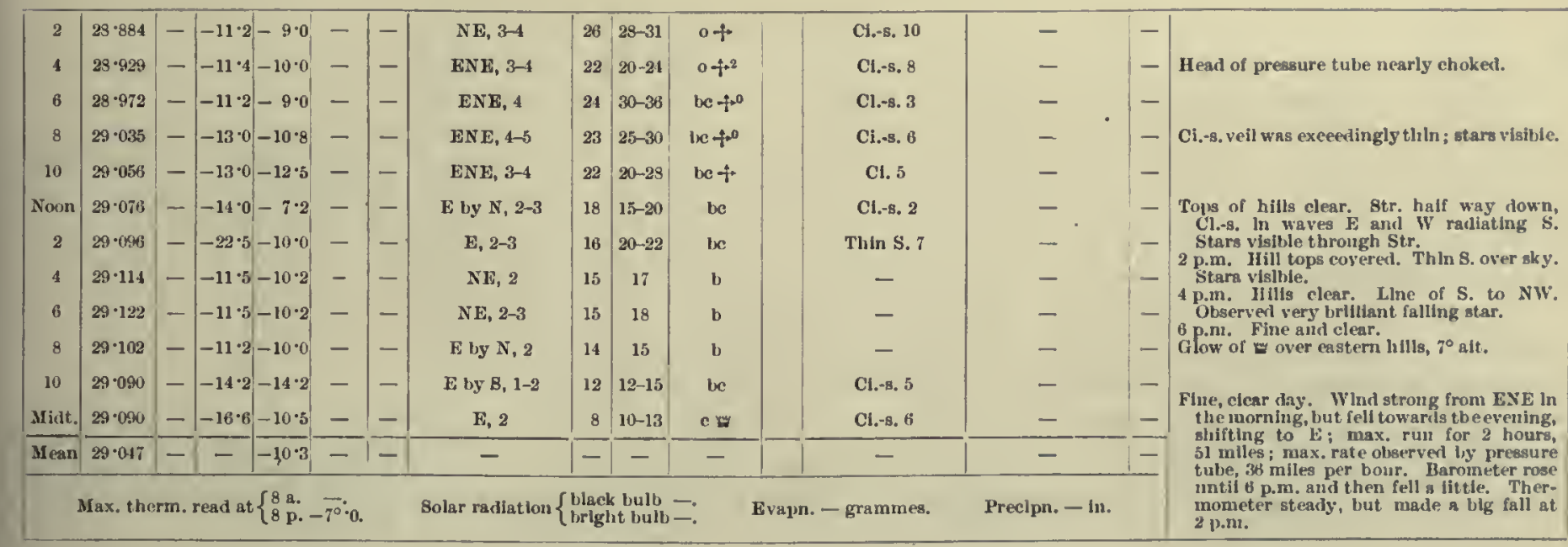

8th.

\begin{tabular}{|c|c|c|c|c|c|c|c|c|c|c|c|c|c|}
\hline 2 & $29 \cdot 100$ & - & $|-15 \cdot 0-12 \cdot 5|$ & - & -1 & ESE, 3-4 & 13 & $20-24$ & c & C.. 7 & - & $1-1$ & 2, 4, and 6 a.m. Slight aurors over hills \\
\hline 4 & $29 \cdot 116$ & - & $-18 \cdot 2-17 \cdot 5$ & - & - & E by $N, 4$ & 14 & $23-2 i$ & $b e+$ & C1.-8. 2 & - & - & \\
\hline 6 & $29 \cdot 150$ & - & $|-19 \cdot 5|-17 \cdot 0 \mid$ & - & - & E by N, 4-5 & 23 & $25-28$ & the & $\mathrm{Cl} . \mathrm{s.} 3$ & - & - & \\
\hline 8 & $29 \cdot 132$ & - & $-20 \cdot 2-18 \cdot 0$ & - & - & NE, 5-7 & 24 & $30-35$ & beq tro & Cl.s. 4 & - & - & Ci.-s. very thin, but massed in places. \\
\hline 10 & $29 \cdot 206$ & - & $-19 \cdot 8-18 \cdot 0$ & - & - & ENE, 3-4 & 22 & $22-25$ & $\mathrm{bm}$ & - & - & - & Hills covered and all land hldden to $\mathrm{s}$ \\
\hline Noon & $29: 208$ & - & $-20 \cdot 0-18.5$ & - & - & ENE,'2 & 15 & $18-20$ & be & Cl. $2, \mathrm{~N}$ & - & - & Hill tope clear. Line of stratus one-third \\
\hline 2 & $29 \cdot 212$ & - & $-19 \cdot 0-18 \cdot 6$ & - & - & FNE, 3-4 & 15 & 25 & $\alpha+$ & Fr. $\rightarrow .8,8$ (nod.) & - & - & Hills covereit. \\
\hline 4 & $29 \cdot 212$ & - & $-19 \cdot 0 \mid-12 \cdot 0$ & - & - & E by N, 2-3 & 18 & $15-20$ & $0 \rightarrow$ & A.s. $10, \mathrm{~W}$ & - & - & 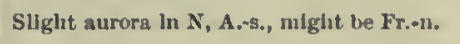 \\
\hline 6 & $29 \cdot 220$ & - & $-14 \cdot 0 \mid-10 \cdot 0$ & - & - & Calm & 11 & 0 & b & - & - & - & \\
\hline 8 & $29 \cdot 216$ & - & $-27 \cdot 0 \mid-27 \cdot 0$ & - & - & Calm & 2 & 0 & b & - & - & - & Temperature rose $2^{\circ}$ whilst observing. \\
\hline 10 & $29 \cdot 209$ & - & $-2 i \cdot 0-21 \cdot 2$ & - & - & Calm & 0 & 0 & 1) & - & - & - & curtain, and streaners. \\
\hline Midt. & $29 \cdot 202$ & - & $-24 \cdot 0-6 \cdot 5$ & - & -1 & $\mathrm{x}, 1-2$ & 5 & $10-15$ & be & - & - & - & 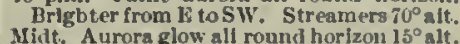 \\
\hline Mean & $29 \cdot 186$ & - & $--16 \cdot 6$ & -1 & $|-|$ & - & -1 & - & - & - & - & - & $\begin{array}{l}\text { Bright over Woservatlon Iillt. } \\
\text { Fine dav. Wind came up strons i }\end{array}$ \\
\hline \multicolumn{6}{|c|}{ Max, therm. read at $\left\{\begin{array}{l}8 \text { a. } \\
8 \mathrm{p} .\end{array}\right.$} & \multicolumn{4}{|c|}{ Solar radiation $\left\{\begin{array}{l}\text { biack bullb } \\
\text { lright, bulb }\end{array}\right.$} & . & \multirow{2}{*}{\multicolumn{2}{|c|}{ pn. - in. }} & 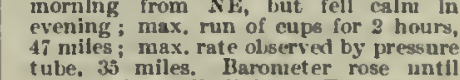 \\
\hline & & & & & & & & & & & & & $4 \mathrm{spll}$ \\
\hline
\end{tabular}


1902-May.]

Meteorologicat Journal of the "Discovery."

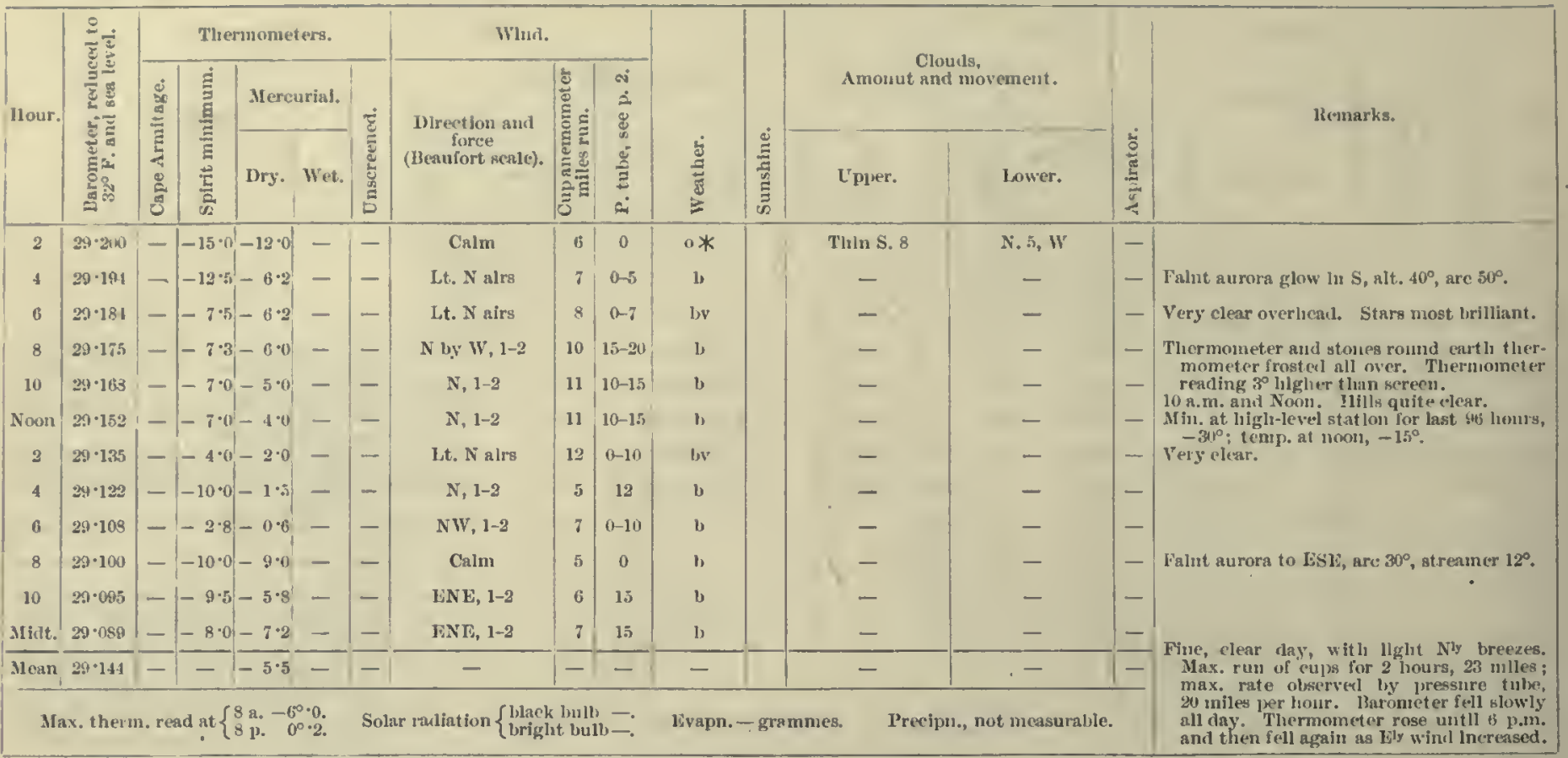

11 th.

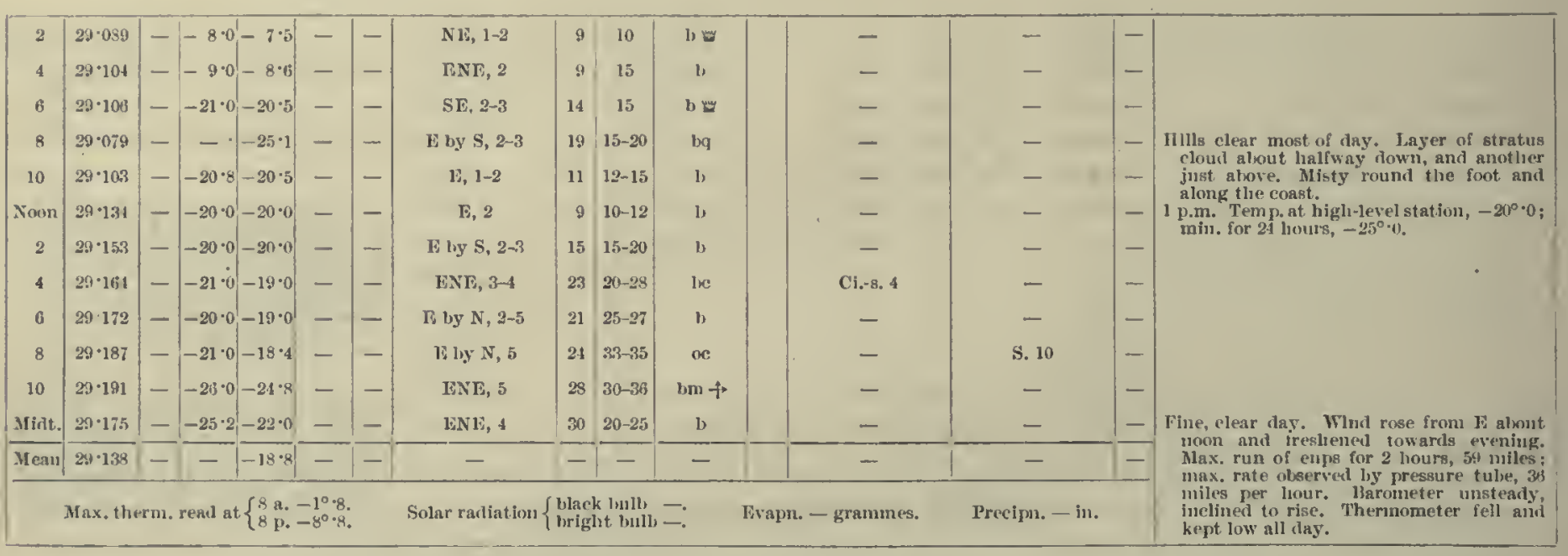

12 th.

\begin{tabular}{|c|c|c|c|c|c|c|c|c|c|c|c|c|c|}
\hline 2 & $29 \cdot 173$ & - & $-26 \cdot 4-25 \cdot 3$ & - & - & ENE, 4 & 25 & $20-25$ & $b m+$ & - & - & - & \multirow{4}{*}{$\begin{array}{l}\text { Stars faintly visille through mist. Therno- } \\
\text { neter oul grounl, }-13^{\circ} 0 . \\
\text { Very light snow. }\end{array}$} \\
\hline 4 & $29 \cdot 178$ & -1 & $-23 \cdot 6-19 \cdot 8$ & - & - & ENE, 3-4 & 19 & $150-20$ & $b+$ & - & - & - & \\
\hline 6 & $29 \cdot 178$ & - & $\mid-22 \cdot 5-19 \cdot 5$ & - & - & ENE, 4 & 19 & 20 & $\operatorname{lom}+$ & - & - & - & \\
\hline 10 & $29 \cdot 285$ & - & $-19 \cdot 0-15 \cdot 0$ & - & - & Calm & 8 & 0 & $o *$ & - & Fr. $n, 10$ & - & \\
\hline Noon & $29 \cdot 2.49$ & - & $-19 \cdot 0-16 \cdot 2$ & - & - & Calm & 3 & 0 & o* & - & Fr.-n, 8 & - & \multirow{3}{*}{$\begin{array}{l}\text { Very light and dry snow. } \\
\text { Very clear. Frost on glass in front of } \\
\text { anemumeter. } \\
\text { Tempernture fesl suddenly. }\end{array}$} \\
\hline 2 & $29 \cdot 270$ & - & $-17 \cdot 0-14 \cdot 0$. & . & - & Calm & 3 & 0 & om $*$ & - & Fr. $-1,10$ & - & \\
\hline 8 & $29 \cdot 335$ & - & $-37 \cdot 0 \mid-24 \cdot 5$ & - & - & Calm & 4 & 0 & b & - & - & - & \\
\hline 10 & $29 \cdot 304$ & - & $-22 \cdot 2-17 \cdot 0$ & - & - & Calın & 6 & 0 & b & - & - & - & \multirow{4}{*}{ 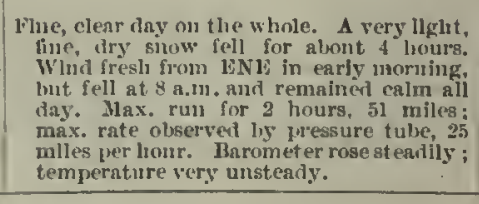 } \\
\hline Mint. & $29 \cdot 3.1$ & -1 & $-26 \cdot 7-21 \cdot 8$ & - & - & Calra & 0 & 0 & b & - & - & 二 & \\
\hline Mean & $29 \cdot 263$ & - & $--_{-20 \cdot 4}$ & - & -1 & $=$ & - & - & - & - & - & - & \\
\hline & Max. the & & read at $\left\{\begin{array}{l}8 \text { a } \\
8 \mathrm{p}\end{array}\right.$ & $\begin{array}{l}-11 \cdot 1 . \\
-14 \cdot 0 .\end{array}$ & & Solar radiatior & Wrigh & at $1,1,1$ & & ramanes. & Precipn. - in. & & \\
\hline
\end{tabular}


M ETEOROLOGICAL JOURNA, OF THL "DISCOVHRY."

[1902-May.

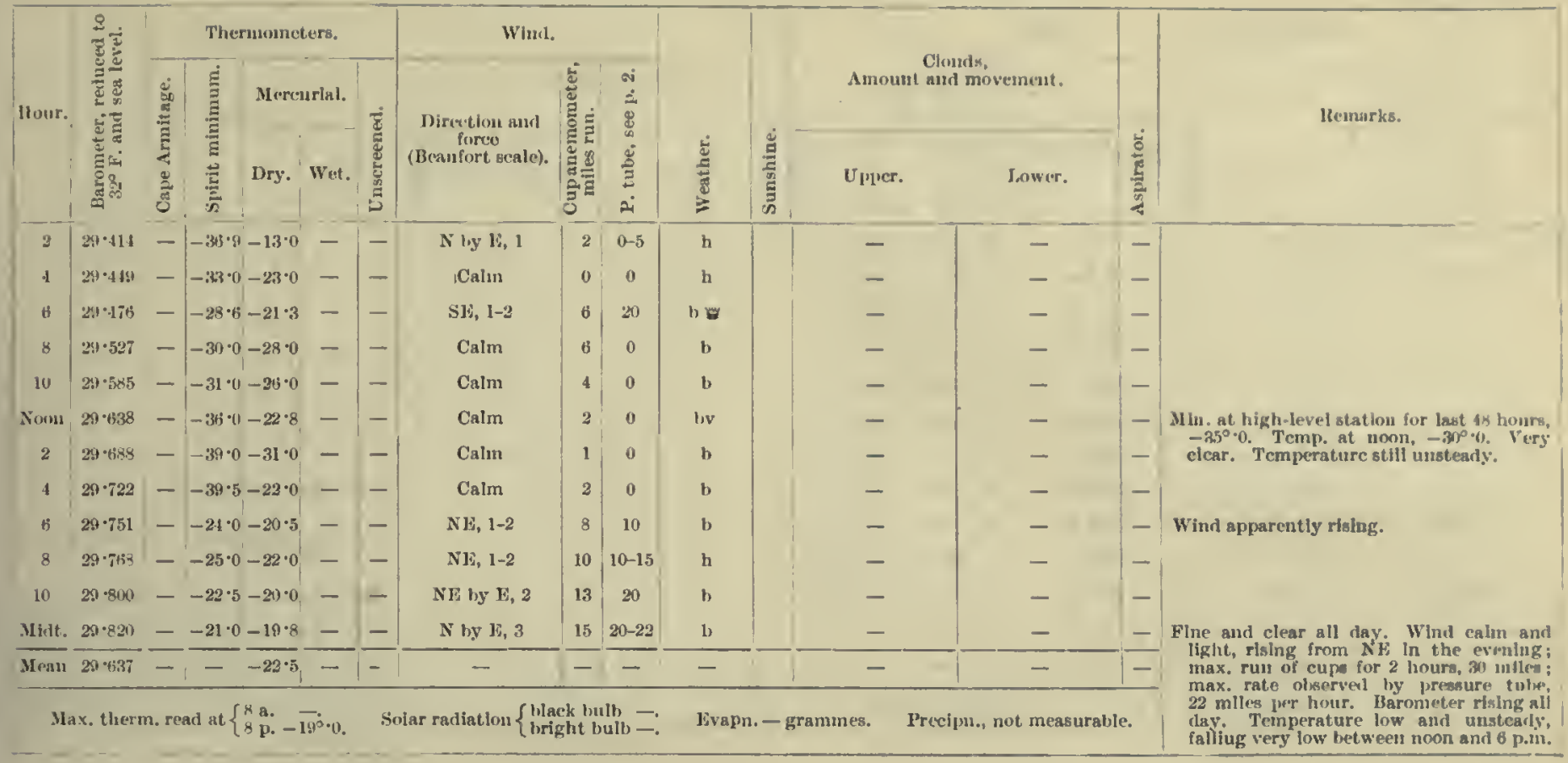

13 th.

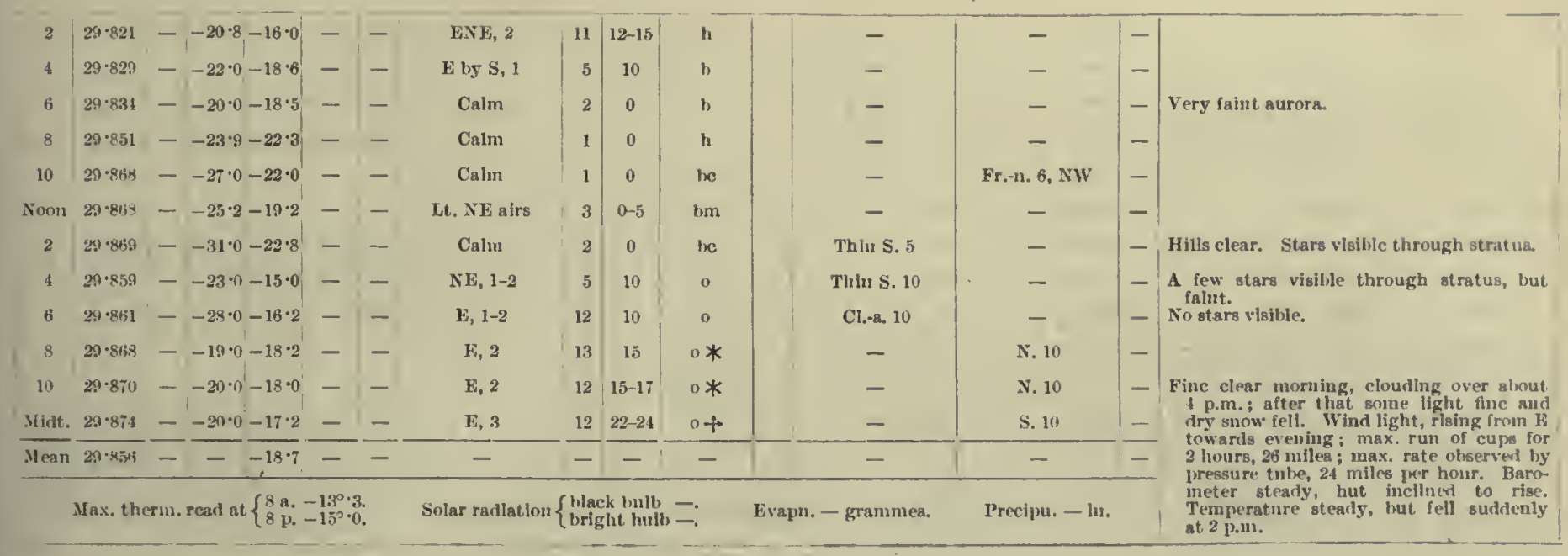

14 th.

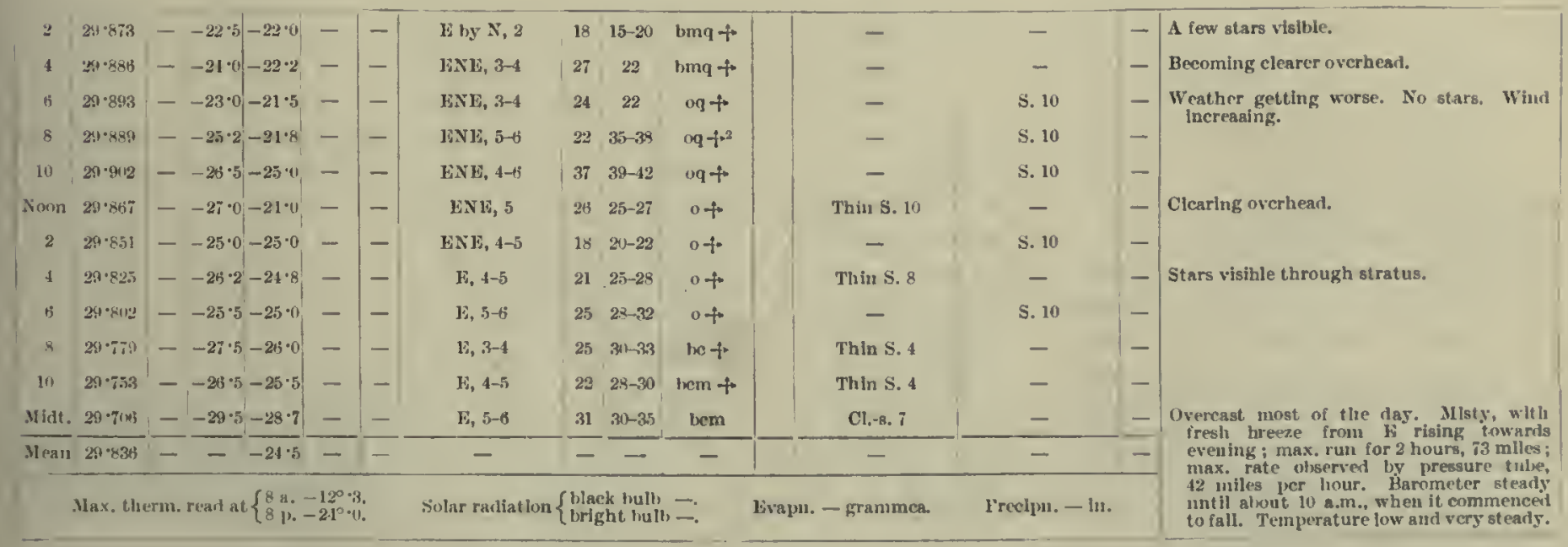




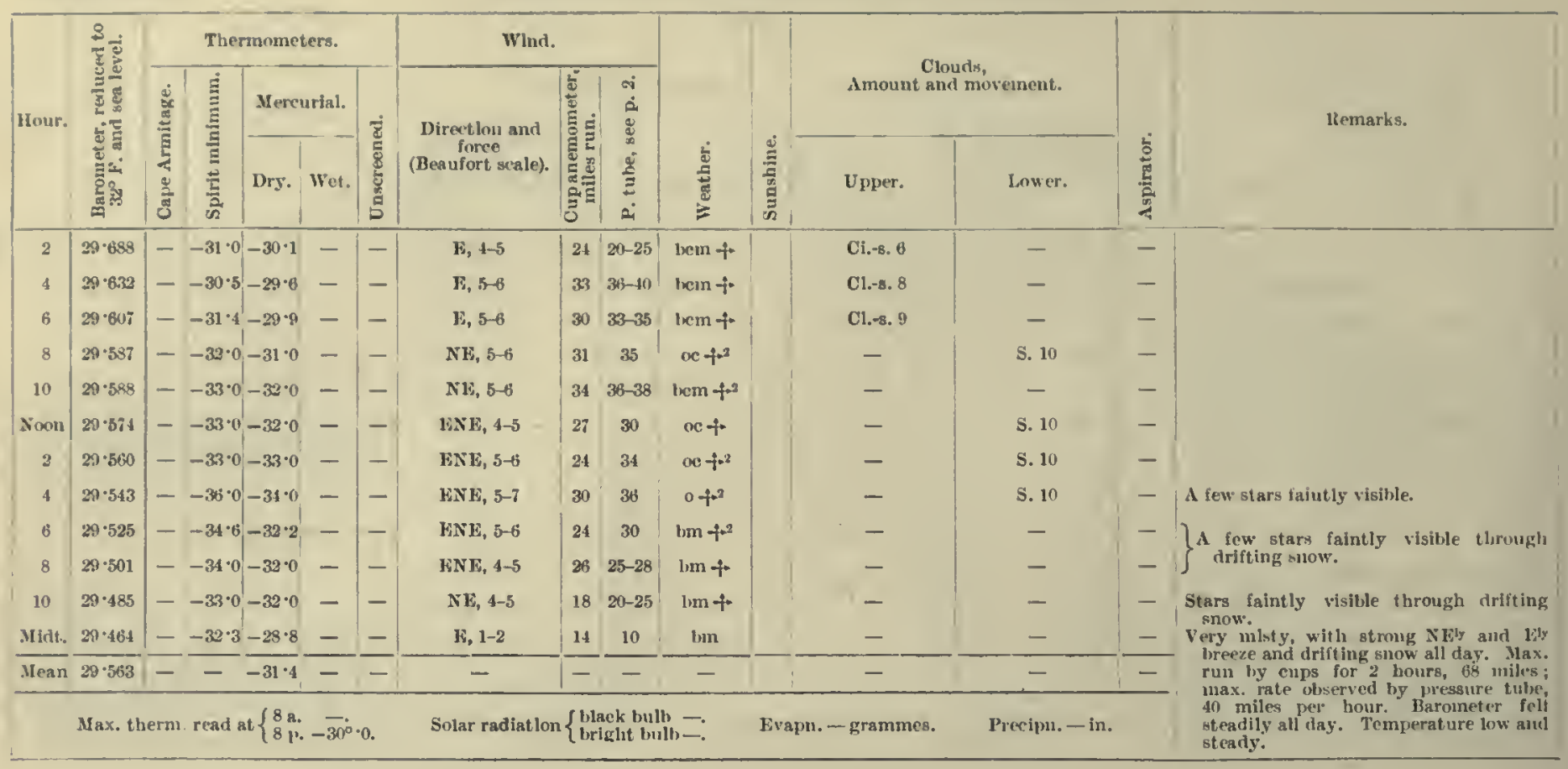

17 th.

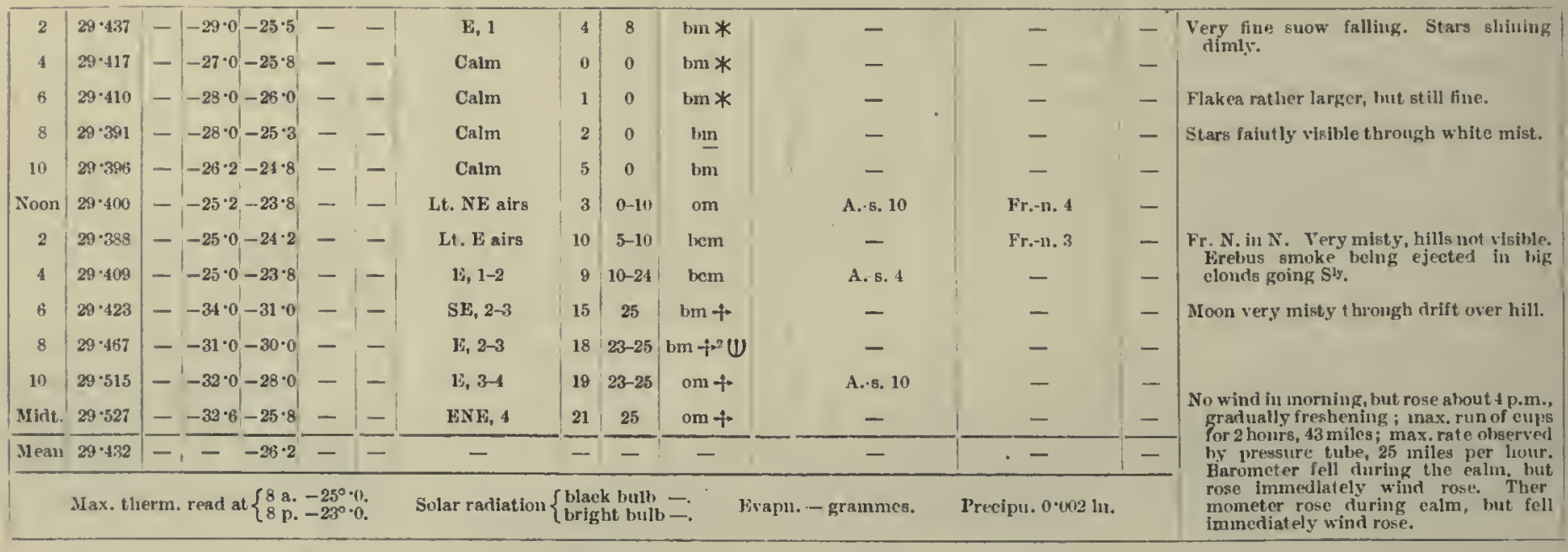

18th.

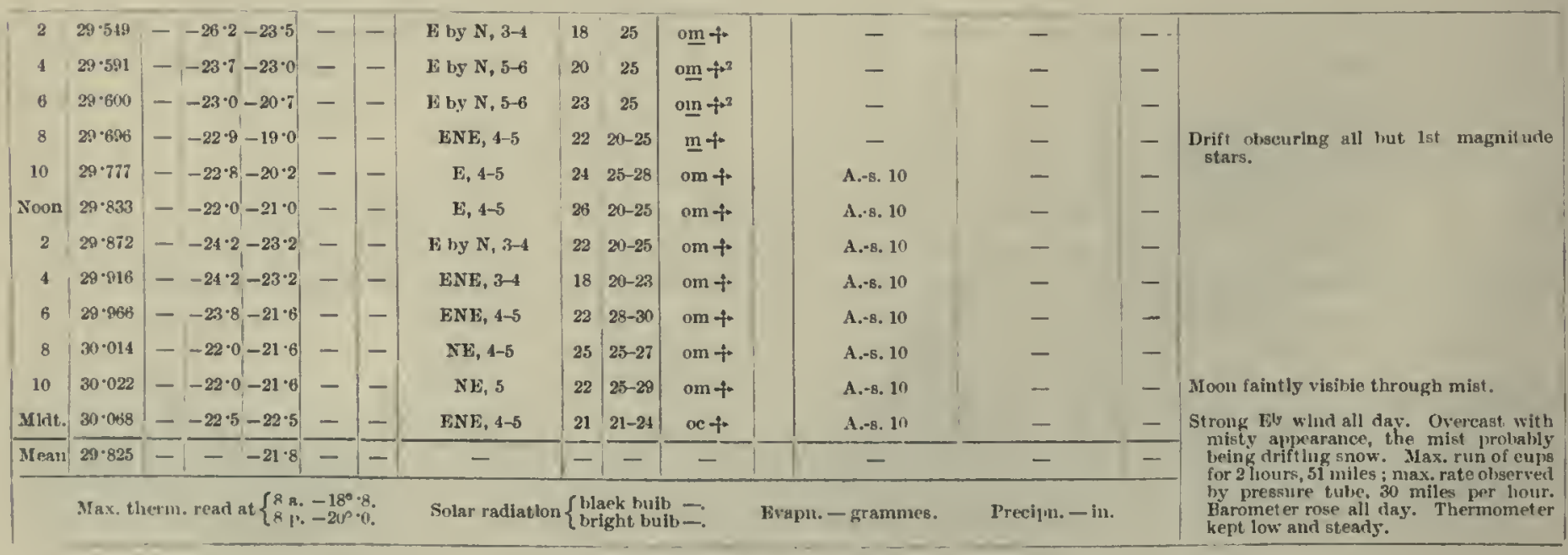


Meteorological Journat of tile "Discovery."

[1902-May.

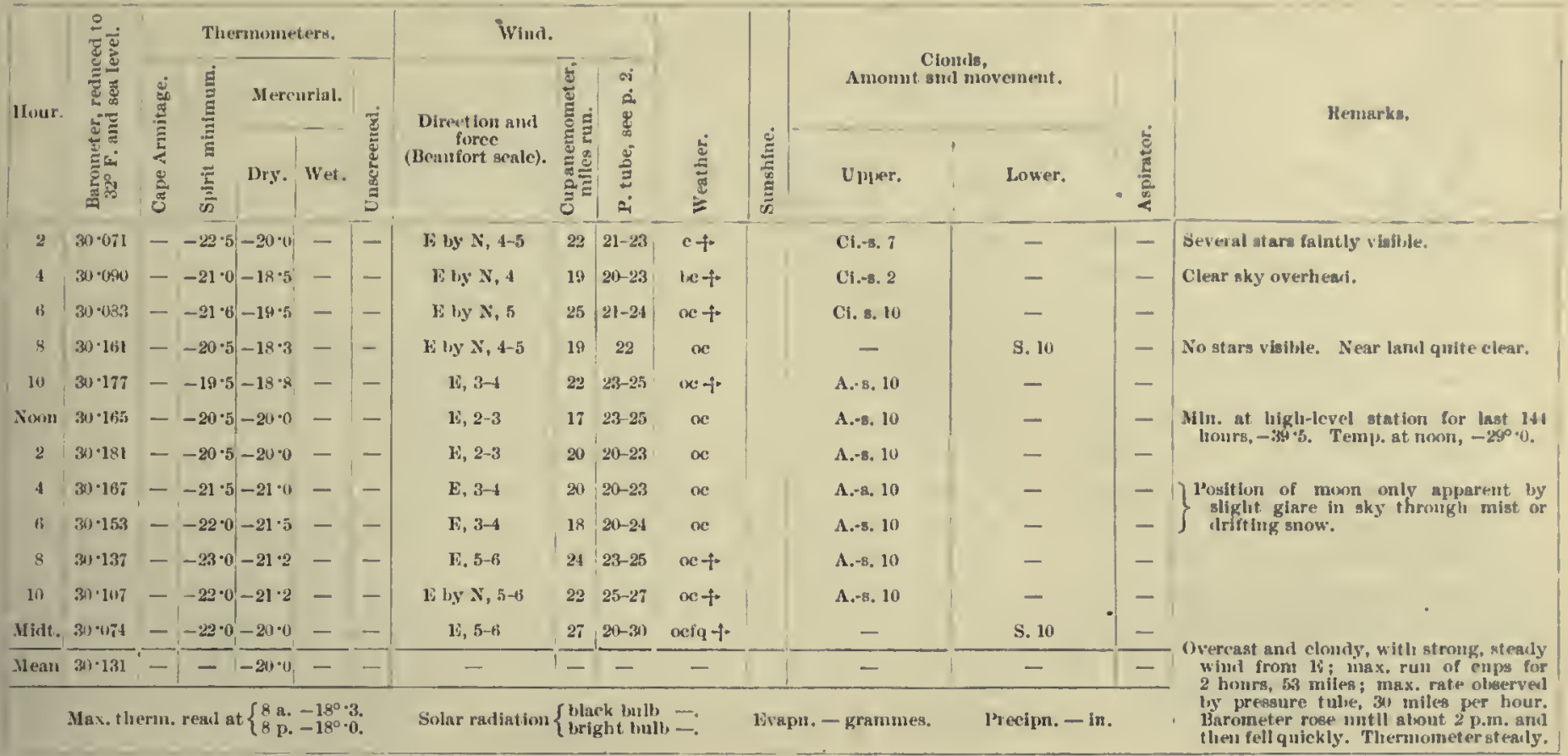

\begin{tabular}{|c|c|c|c|c|c|c|c|c|c|c|c|}
\hline 2 & 30.044 & - & $-21 \cdot 5-20 \cdot 5$ & - & E I I, N $N, 4-5$ & 32 & 20-21 xelt & - & S. 10 & - & \multirow{9}{*}{$\begin{array}{l}\text { Snow of a very fine character, dry and } \\
\text { laru. }\end{array}$} \\
\hline 4 & $29 \cdot 991$ & - & $|-21 \cdot v-18 \cdot 6|$ & - & W ly $\mathrm{N}, 5-8$ & 20 & $25-23 \sec * f$ & - & N. 10 & - & \\
\hline is & $29 \cdot 917$ & - & $\mid-21 \cdot 6-13 \cdot 2$ & - & NNE, 5-6; & 30 & 23-25 sef*-1. & - & N. 10 & - & \\
\hline 8 & $29 \cdot 921$ & - & $|-19 \cdot 14-14 \cdot 8|$ & - & bi, 3-4 & 23 & $20-22 \propto c *$ & - & N. 10 & - & \\
\hline 10 & 291910 & - & $|-15 \cdot 0-14 \cdot 8|$ & - & $\mathrm{SE}, 2-3$ & 16 & $20-2 i$ of $*$ & - & N. 10 & - & \\
\hline Sion & $29 \cdot 8 \times 8$ & - & $-16 \cdot 3-15 \cdot 2$ & -1 & SE, 3-t & 14 & $20-25$ om $\mathrm{f}$ & - & S. 10 & - & \\
\hline 2 & $29 \cdot 874$ & - & $-16 \cdot 5-16 \cdot v$ & $-1-$ & SSE, 3-4 & 18 & $25-23$ om $f$ & - & S. 10 & - & \\
\hline 4 & $29 \cdot \times 33$ & - & $-17 \cdot 5-17 \cdot 0$ & - & $\mathrm{F}, 2$ & 21 & on + to $^{\circ}$ & - & S. 10 & - & \\
\hline "s & $29 \cdot 793$ & - & $-19 \cdot 5-19 \cdot 1$ & - & $18,3-4$ & 13 & 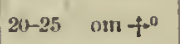 & - & S. 10 & - & \\
\hline 8 & $29 \cdot 732$ & - & $-19 \cdot 7-18 \cdot 3$ & $-1-$ & ENE, 5 & 28 & 25 on $* 4^{\circ} 0^{\circ}$ & - & N. 10 & - & \multirow{5}{*}{ 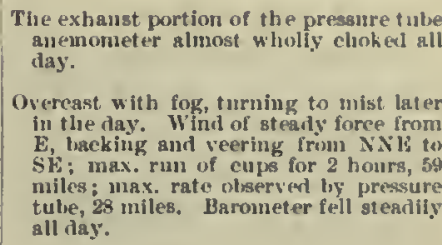 } \\
\hline 10 & $29 \cdot 6: 95$ & - & $-19 \cdot 3-18 \cdot 1$ & -- & 1:Ne, 5 & 21 & 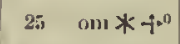 & - & N. 10 & - & \\
\hline Midt. & $29 \cdot 647$ & - & $-18 \cdot 5-18 \cdot 0$ & $-0-$ & $B, 3-4$ & 18 & 31-23 onf $\mathrm{f}^{-}$ & - & S. 10 & - & \\
\hline Nean & $29 \cdot 856$ & - & $--17 \cdot 4$ & --1 & - & -1 & - & - & - & $1-$ & \\
\hline & $M_{i}$ & & 1 at $\left\{\begin{array}{l}8 \mathrm{R} . \\
8 \mathrm{p} .\end{array}\right.$ & & Solar ra & & 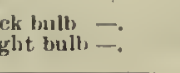 & ranme & 1'recipn. - & & \\
\hline
\end{tabular}

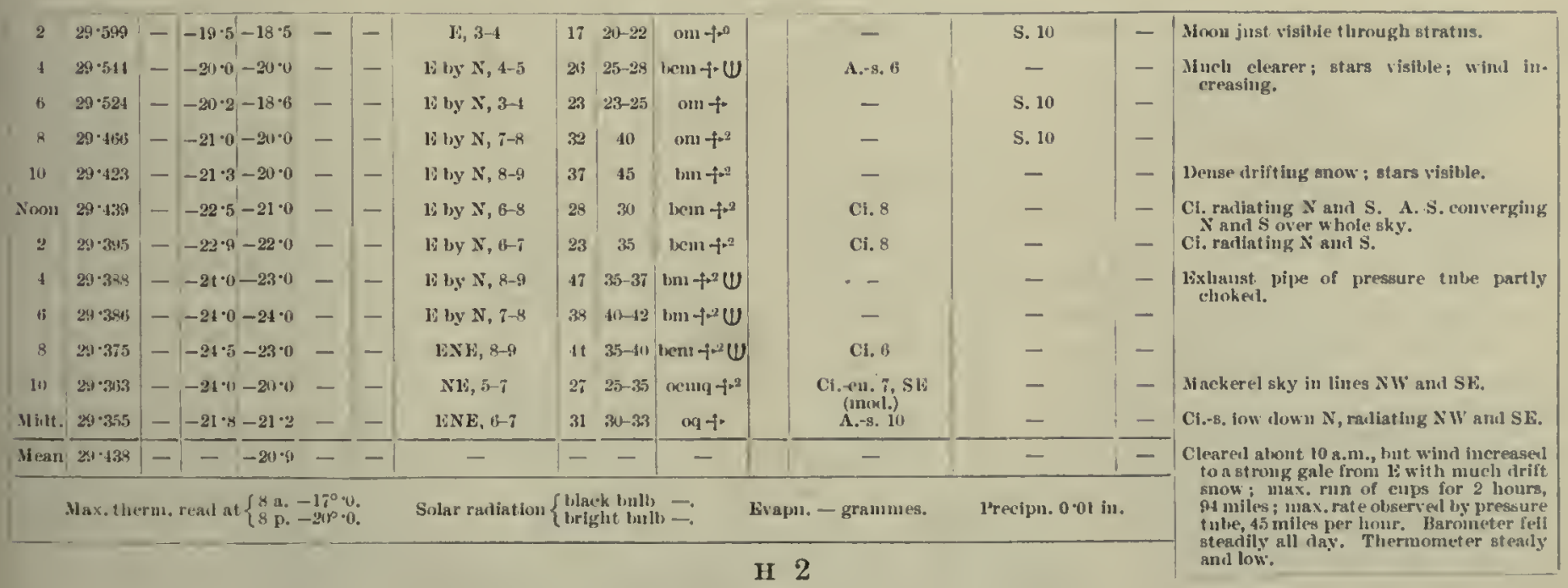


1902-May.]

Therinometers.

Meteorological Journat of the "Discoyery."

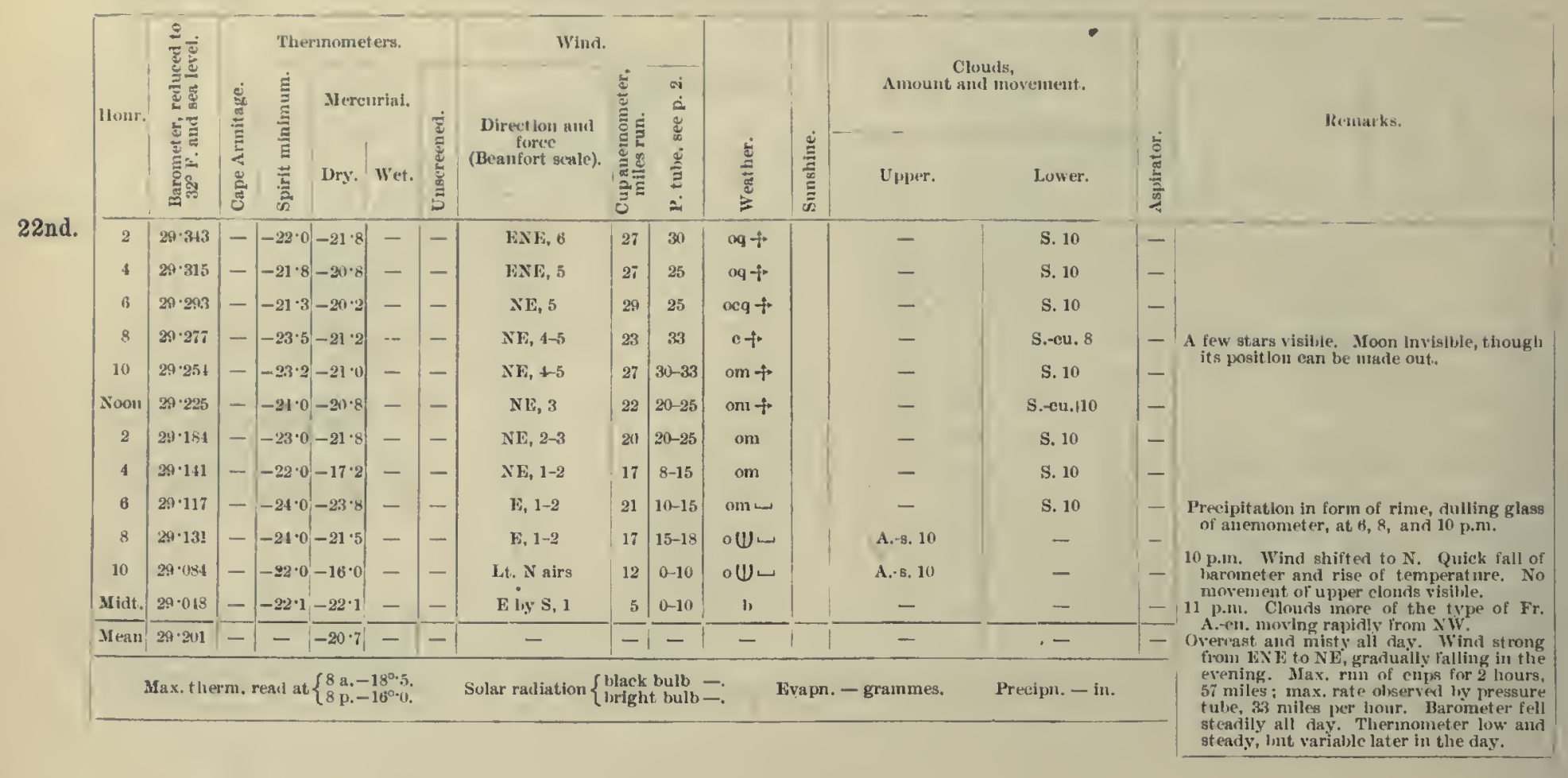

23rd.

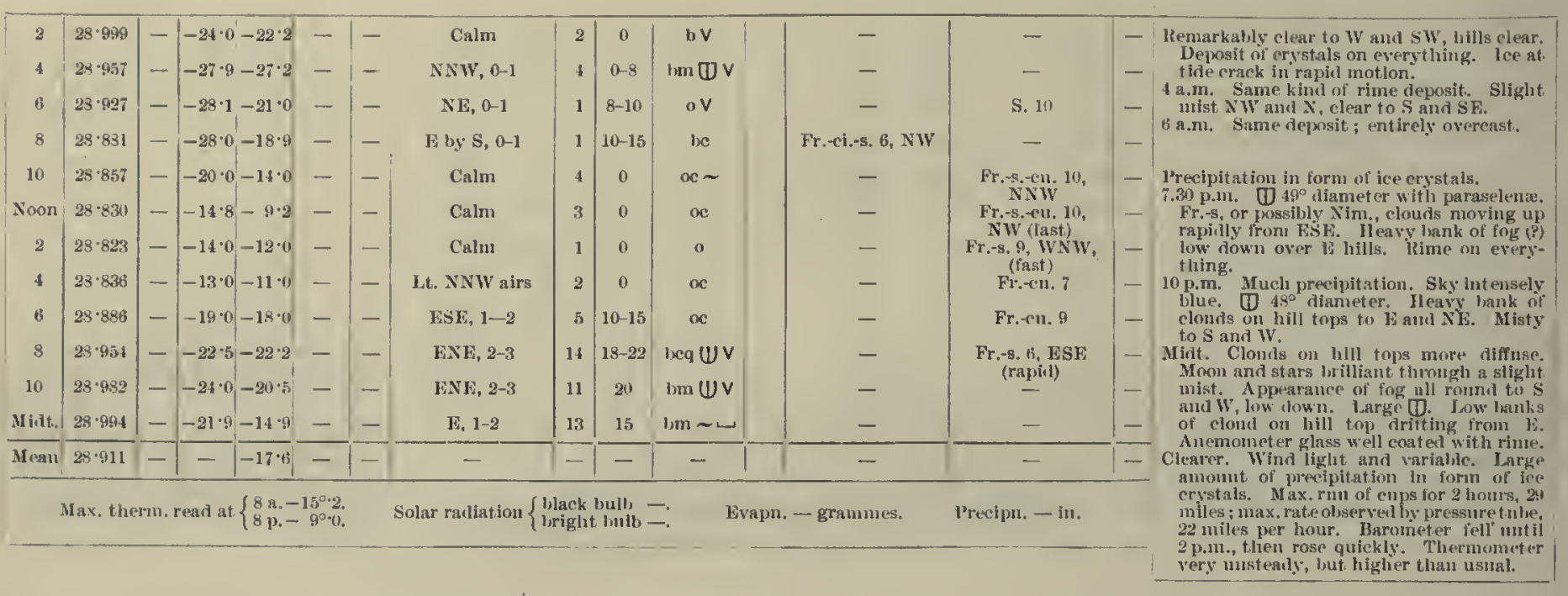

24th.

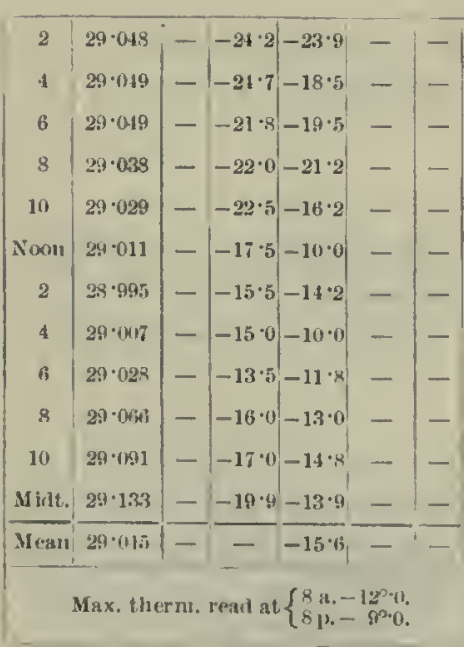

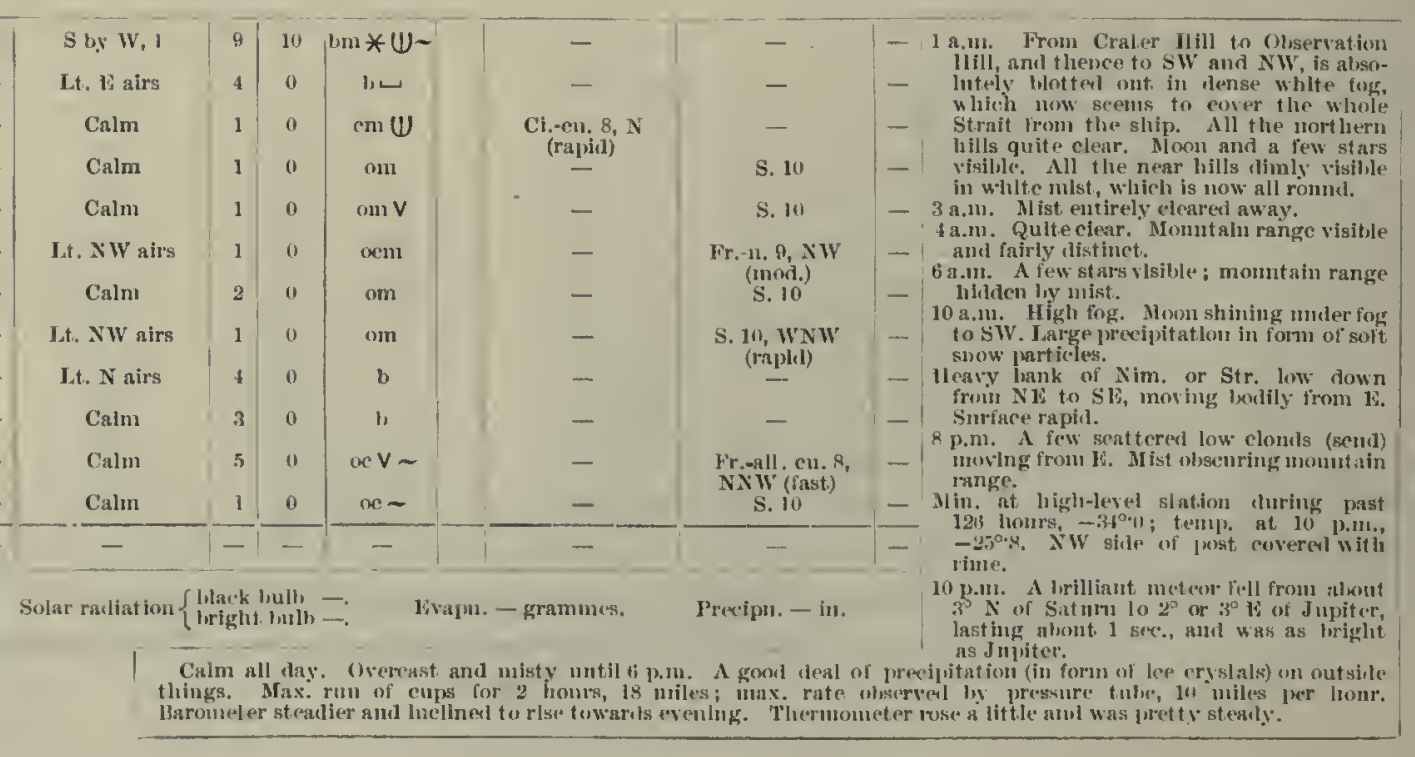


Metrorolonical, Journat, of tuF "IIscovirzy"

$[1902-$ May.

\begin{tabular}{|c|c|c|c|c|c|c|c|c|c|c|c|c|c|c|c|}
\hline Hour. & 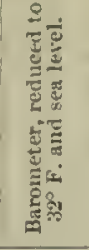 & 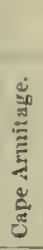 & 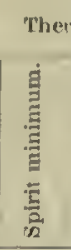 & $\begin{array}{l}\text { Mereu } \\
\text { Dry. }\end{array}$ & urlal. & 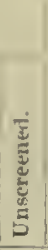 & $\begin{array}{l}\text { Directlon and } \\
\text { furce } \\
\text { (Bean for' ganle). }\end{array}$ & 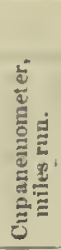 & 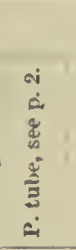 & 䢘 & 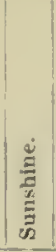 & $\begin{aligned} \text { Cl } \\
\text { Aluount ant }\end{aligned}$ & vecment. & 这 & lleutarks. \\
\hline 2 & $29 \cdot 17 i$ & - & $-20 \cdot 6$ & $-15 \cdot$ & - & - & $1 \mathrm{ssl} \mathrm{H}_{1} 1$ & 3 & $0-8$ & oc & & - & S. 9 & - & \multirow{5}{*}{$\begin{array}{l}\text { Only low land vilble; low bank of mist all } \\
\text { rounil. }\end{array}$} \\
\hline I & $29 \cdot 222$ & - & $-21 \cdot 0$ & $-17 \cdot 2$ & - & - & WSF, 1 & 5 & $0-10$ & oe & & - & S. 3 & - & \\
\hline is & $29 \cdot 273$ & - & $-23 \cdot 4$ & $-20 \cdot 7$ & - & - & lisk, 2 & 10 & $10-15$ & oc & & - & S. 4 & - & \\
\hline 8 & $29 \cdot 317$ & - & $-21 \cdot 5$ & $-19 \cdot 0$ & 一 & - & ESE, 1 & 10) & $5-10$ & $\ln U$ & & Cl.-cu. 5, NW & - & - & \\
\hline 10) & $29 \cdot 3113$ & - & $-22 \cdot 0$ & $-21 \cdot 0$ & - & - & Calm & 5 & 0 & b & & 一 & - & - & \\
\hline Noon & $29-35.53$ & - & $-26 \cdot 8$ & $-2 t \cdot 5$ & - & - & Calm & 3 & 0 & ' & & - & - & - & \multirow{5}{*}{ 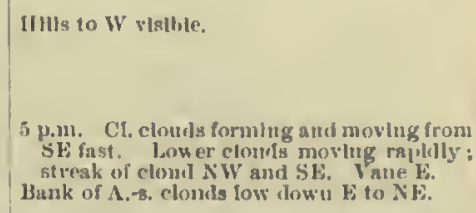 } \\
\hline 2 & $29 \cdot 3 i$ & - & $-32 \cdot 0$ & $-22 \cdot 5$ & - & - & Lt. IE ail's & 2 & $0-5$ & " & & - & - & - & \\
\hline 1 & $29 \cdot 362$ & - & $-23 \cdot 0$ & $-19 \cdot 5$ & - & - & Lt. Fi airs & $\theta$ & $0-5$ & b & & - & - & - & \\
\hline is & $\begin{array}{l}29 \cdot 3643 \\
29 \cdot 392\end{array}$ & - & $\begin{array}{l}-23 \cdot 0 \\
-29 \cdot 2\end{array}$ & $\begin{array}{l}-17 \cdot 0 \\
-28 \cdot 0\end{array}$ & - & - & Lt. var, airs & 6 & $0-5$ & h & & - & - & - & \\
\hline 3 & 20758 & - & -202 & -2.50 & - & - & Culun & 1 & 0 & 1) & & - & - & - & \\
\hline 10 & $29 \cdot 377$ & - & $-2 ; \cdot 0$ & $-23 \cdot 2$ & - & - & Calm & 1 & ") & $b U$ & & - & 一 & - & \multirow{4}{*}{ 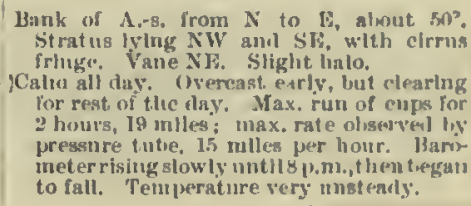 } \\
\hline Miilt. & $29 \cdot 373$ & - & $-3 i) \cdot 8$ & $-23 \cdot 6$ & 一 & - & Calıt & 1 & 0 & b & & 一 & 一 & 一 & \\
\hline Mean & $29 \cdot 324$ & - & 一 & $-21 \cdot 2$ & - & - & - & 一 & - & - & & - & - & - & \\
\hline & 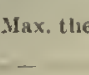 & & ad & $\left\{\begin{array}{l}8 \mathrm{a} \\
8 \mathrm{p} .\end{array}\right.$ & -129 & & Solar raflation & 的 & 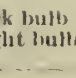 & 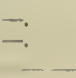 & 1 & n, - gramuиes. & Preclju. - In. & & \\
\hline
\end{tabular}

25 th.

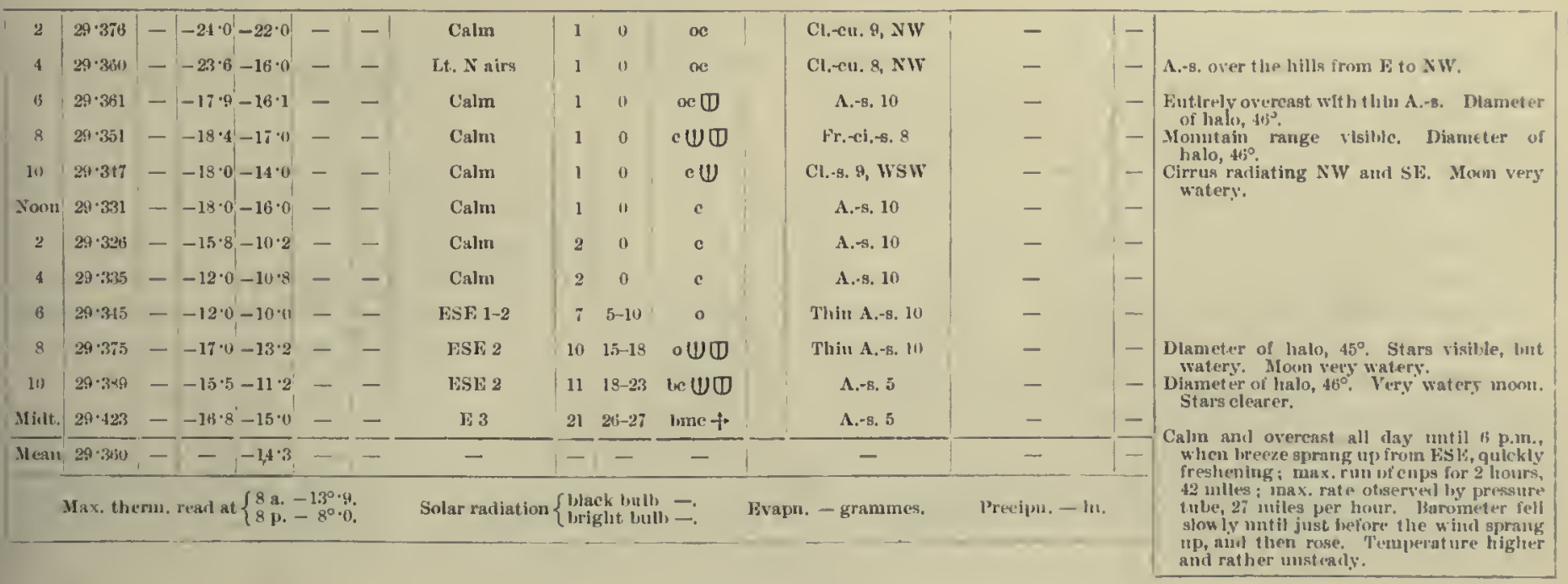

26 th.

\begin{tabular}{|c|c|c|c|c|c|c|c|c|c|c|c|c|c|}
\hline 2 & $29 \cdot 415$ & - & $-17 \cdot 0^{\prime}-14 \cdot 5$ & - & - & F by $N, 6$ & 22 & $23-30 \mid$ & keqv $U$ & Cl. $1, \mathrm{~N}$ & - & - & \multirow{5}{*}{ 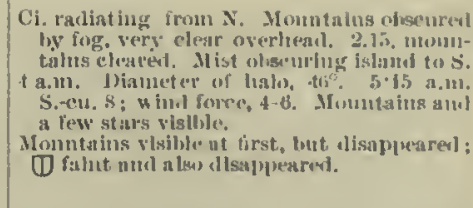 } \\
\hline$t$ & $29 \cdot 413$ & - & $-17 \cdot 0-15 \cdot 0$ & - & - & $F \log N_{1} 1$ & 25 & 21 & Iem (1) & Ci-s, $1, N$ & - & - & \\
\hline is & $2: 1 \cdot 123$ & - & $-18 \cdot 4-11 \cdot 2$ & - & - & E, 4 & 18 & $20-231$ & c & S. $-211,10, W$ & - & - & \\
\hline 8 & $21 \cdot 421$ & - & $-18 \cdot 9-14 \cdot 5$ & - & -1 & E hy $N, 3-1$ & 1.4 & $15-201$ & oe & - & S. 10 & - & \\
\hline 10 & 2,119 & - & $-18 \cdot 6-1.5 \cdot 5$ & - & - & E l ly $N, 3-4$ & 21 & $22-25$ & ic & Th/u A.-8, 8 & - & - & \\
\hline Xnon & $24 \cdot 379$ & - & $-16 \cdot 8-16 \cdot 5$ & - & - & $N \mathbb{B}_{1} 4$ & 243 & $25-28$ & 0 & A. - s, 10 & - & - & \multirow{3}{*}{$\begin{array}{l}\text { From nom to t p.m., sky ovemast : clear. } \\
\text { fing as wind tureasent. }\end{array}$} \\
\hline 2 & $2 x \cdot 3 \times 3$ & $\rightarrow$ & $-17 \cdot 11-11 \cdot 0$ & - & - & HNF, 2-3 & 21 & $20-22$ & 0 & Thin A.- 5.10 & - & - & \\
\hline 4 & $20 \cdot 38.3$ & - & $-15 \cdot 0)-11 \cdot 8$ & - & - & WNI?, 3-4 & 22 & $18-22$ & o & Thin $\Lambda .-8.10$ & - & - & \\
\hline 6 & $29 \cdot+19$ & - & $-11 \cdot 5-12 \cdot 1$ & - & - & S13, $2-3$ & 1.5 & $18-211$ & lic & Thh A.-s. 5 & - & - & \multirow{6}{*}{ 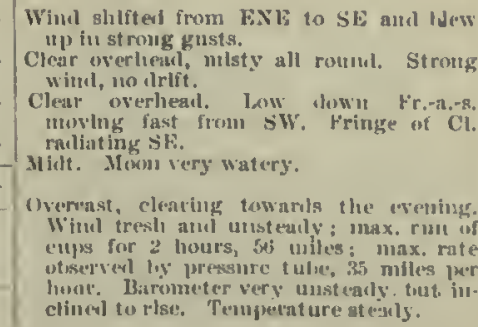 } \\
\hline \& & $29 \cdot+25$ & - & $-13 \cdot 8-9 \cdot 8$ & - & - & Sls, t-5 & 28 & $25-35$ & lmag & - & 一 & - & \\
\hline 10 & $29 \cdot 13$ & - & $-15 \cdot 01-15 \cdot 0$ & - & - & E. $2-3$ & 20) & $2(1-22)$ & b) & - & - & - & \\
\hline Mhit. & $20 \cdot 118$ & - & $-2(1 \cdot 1)-21 \cdot 11$ & - & - & $1 \mathrm{l}, \mathrm{x}, 5$ & 25 & $25-2 x$ & le $-i$ - & A. -8.6 & 一 & - & \\
\hline Mean & 124.1165 & - & $-\quad-11.1$ & - & - & - & - & - & - & - & - & - & \\
\hline & Max, t & & evul at $\left\{\begin{array}{l}8 \mathrm{a} . \\
8 \mathrm{p} .\end{array}\right.$ & $-11^{\circ}$ & & Solur ratiatis & & lus & & кари. - grammies. & p'recipu. - iи. & & \\
\hline
\end{tabular}


1902-May.]

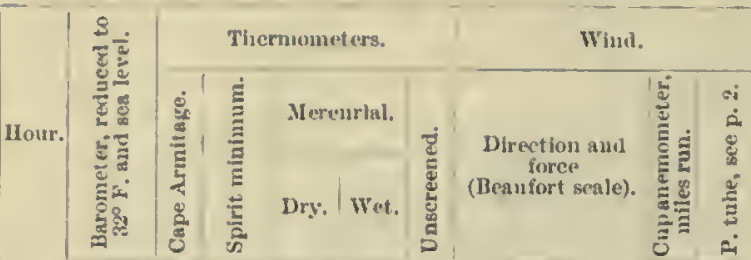

28th.

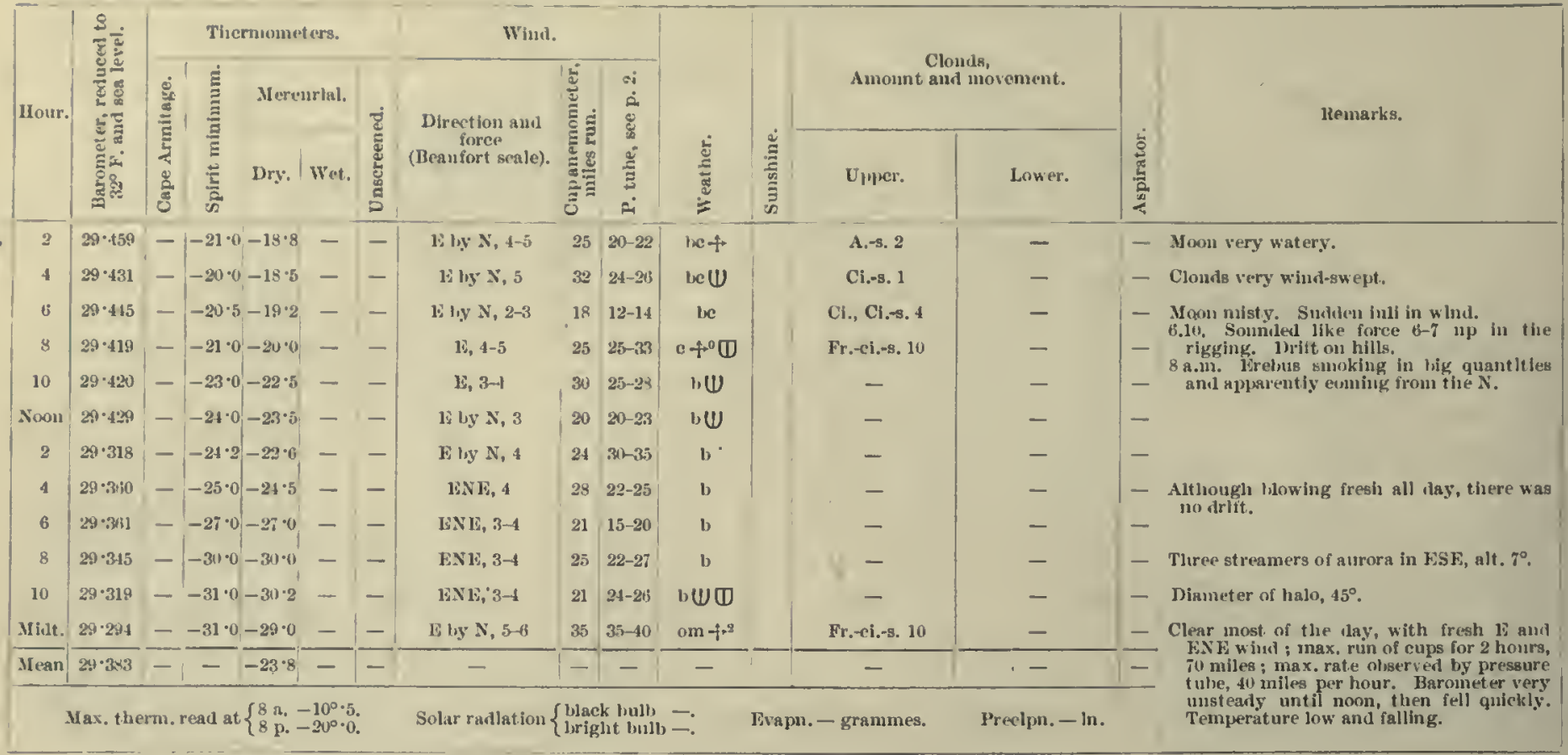

Meteorological Journal of the "Discovery."
29 th

\begin{tabular}{|c|c|c|c|c|c|c|c|c|c|c|c|c|}
\hline 2 & $29 \cdot 246^{\prime}$ & $--29 \cdot 0-25 \cdot 2$ & - & -1 & E lig $N, 4$ & 22 & 30 & $m m+0$ & Fr. $-8.10, F$ & - & - & \multirow{4}{*}{$\begin{array}{l}\text { At } 2,4 \text {, and } 6 \text { a.m., the Fr.-s. might possibly: } \\
\text { lave leen conisaratlvely ligh triving } \\
\text { snow (?) }\end{array}$} \\
\hline 4 & $29 \cdot 239$ & $--25 \cdot 2-25 \cdot \theta$ & - & - & F hy $\mathbf{x}, 4-5$ & 31 & 33 & $0 \mathrm{~m}+\mathrm{r}^{2}$ & Fr.-s. 10, E & - & - & \\
\hline 6 & $29 \cdot 252$ & $--2 i \cdot 8-25 \cdot 2$ & - & - & E l,y $\mathrm{N}, 5$ & 33 & $28-36$ & $\mathrm{omm}+\mathrm{i}^{3}$ & $F_{r}-\mathbf{B}, 10, \mathrm{E}$ & - & - & \\
\hline 8 & $29 \cdot 275$ & $--27 \cdot 0-16 \cdot 0$ & - & - & $12,3-5$ & 27 & $20-25$ & $0 \cap+0$ & - & S. 10 & - & \\
\hline 10 & $29 \cdot 283$ & $--17 \cdot 0-11 \cdot 2$ & - & - & IES12, 1-4 & 18 & $8-15$ & $o q$ & - & S. 10 & - & \multirow{3}{*}{$\begin{array}{l}\text { Shome thernnometer reading } 1^{\circ} \cdot 5 \text { litgher } \\
\text { than sereen. }\end{array}$} \\
\hline Noon & $29 \cdot 274$ & $--11 \cdot 5-9 \cdot 0$ & - & - & SN, 3-6 & 23 & $30-35$ & $\mathrm{oqt}$ & - & S. 10 & - & \\
\hline 2 & $29 \cdot 250$ & $--13 \cdot 0-9 \cdot 0$ & - & - & T.ST, 2-4 & 25 & $10-20$ & $\rho q+$ & - & Fr.-s. 10 & - & \\
\hline 8 & $29 \cdot 104$ & $--4 \cdot 5-1.8$ & - & - & $\mathbf{E}, 2-\mathbf{4}$ & 18 & $20-25$ & $n q+$ & A.-B. 10 & - & - & \multirow{3}{*}{ 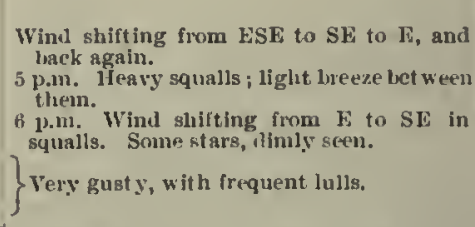 } \\
\hline 10 & $29 \cdot 1213$ & $-3 \cdot 5-1 \cdot 2$ & - & - & $\mathrm{ST}, \mathrm{Z}-5$ & 24 & $25-3 t$ & $n q+$ & A. $:$ s. 10 & - & - & \\
\hline stillt. & $28 \cdot 926$ & $-\begin{array}{ll}-3.4 & 1.0\end{array}$ & - & - & SE, 2-4 & 21 & $21-25$ & oq $++^{0}$ & A. - - .10 & - & - & \\
\hline Mean & $29 \cdot 192$ & $-\quad--10.4$ & - & -1 & - & - & - & - & - & - & - & \multirow{2}{*}{ 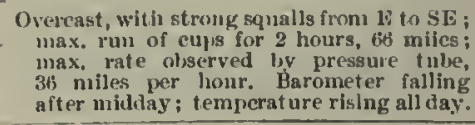 } \\
\hline & $a x+4$ & nn. read at $\left\{\begin{array}{l}8 \text { a. } \\
8 \text { 1. }\end{array}\right.$ & & & s & & & & nmes. & Preeipn. - in. & & \\
\hline
\end{tabular}

30 th.

\begin{tabular}{|c|c|c|c|c|c|c|c|c|c|c|c|c|c|c|}
\hline 2 & $28 \cdot 8415$ & - & 0.0 & $5 \cdot 0$ & - & - & S by k, 1-5 & 26 & $15-33$ & $\alpha q-i$ & A. -8.10 & - & - & \multirow{8}{*}{$\begin{array}{l}\text { Outiook very limited, caunot eveu see tise } \\
\text { shore. }\end{array}$} \\
\hline 4 & $23 \cdot 731$ & - & $4 \cdot 2$ & $6 \cdot 0$ & - & - & S, 1-5 & 22 & $15-36$ & oq $\dot{q}^{2}$. & A.-5. 10 & - & - & \\
\hline 6 & $28 \cdot 633$ & - & $5 \cdot 0$ & 9.0 & - & - & $\mathrm{S}, 3-6$ & 23 & $25-39^{\prime}$ & $0 q \psi^{2}$ & A. $-\mathrm{s}, 10$ & - & - & \\
\hline 8 & $28 * 526$ & - & $8 \cdot 0$ & $9 \cdot 6$ & - & - & $\sin 1,2-3$ & 22 & $10-15$ & oq $*$ & - & N. 10 & - & \\
\hline 10 & $28 \cdot 5.85$ & - & $8 \cdot 2$ & $10 \cdot 8$ & - & - & 1SE, 1-3 & 13 & $15-20$ & $\rho q$ & - & N. 10 & - & \\
\hline Noon & $28 \cdot 524$ & - & $10 \cdot 0$ & 13.0 & $13^{\circ} 0$ & -1 & 1AS1, 1-4 & 19 & $15-20$ & $m \rightarrow$ & - & N. 10 & - & \\
\hline 2 & $28 \cdot 405$ & - & $12 \cdot 0$ & $13 \cdot 9$ & $13 \cdot 8$ & - & S, 2-3 & 16 & $20-25$ & $a q+$ & - & N. 10 & - & \\
\hline 8 & $24 \cdot 521$ & - & $12 \cdot 5$ & $13 \cdot 8$ & $13 \cdot 8$ & - & 1SS1, 1-2 & 19 & $10-15$ & ive & A.-8. 2 & - & - & \\
\hline 10 & $29 \cdot 635$ & - & $12 \cdot 0$ & $13 \cdot 8$ & $13 \cdot 0$ & - & $\operatorname{ssl} 1,1-2$ & 9 & 10-1s & ne & A.-8. 8 & - & - & \multirow{4}{*}{ 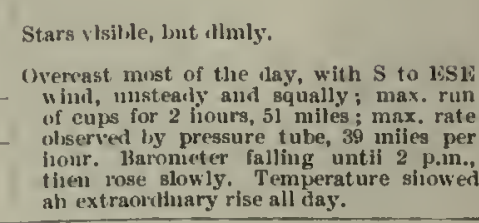 } \\
\hline Nidt. & $28 \cdot 579$ & - & $12 \cdot 5$ & $14 \cdot 5$ & $11 \cdot 0$ & - & 5514,3 & 11 & $15-21)$ & $\infty$ & A.-s. 10 & - & - & \\
\hline$\stackrel{M \text { ean }}{ }$ & 28.5817 & - & - & $11 \cdot 3$ & - & - & - & - & - & - & - & - & - & \\
\hline & as & & & & $n^{\circ}$ & & wiatic & & milt & & mแนเร. & precipm. - in. & & \\
\hline
\end{tabular}


Mhthorological JuURNat, of the "Discoviey."

[1902-May.

\begin{tabular}{|c|c|c|c|c|c|c|c|c|c|c|c|c|c|c|c|}
\hline llour. & 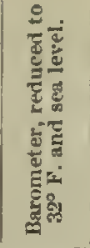 & 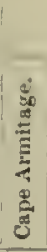 & 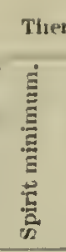 & Merc & Wet. & 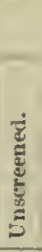 & $\begin{array}{l}\text { Direction nurl } \\
\text { force } \\
\text { (Beanfort scale). }\end{array}$ & 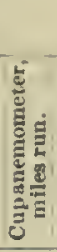 & 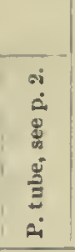 & 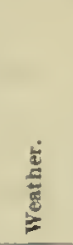 & $\frac{\dot{\Xi}}{\vec{E}}$ & \multicolumn{2}{|c|}{$\begin{array}{l}\text { Clouils, } \\
\text { Amonut and movement. }\end{array}$} & $\frac{\frac{5}{5}}{\frac{E}{2}}$ & Renuarks. \\
\hline 2 & $28 \cdot 633$ & - & $13 \cdot 2$ & $14 \cdot 2$ & $14 \cdot 0$ & - & $S$ by $18,1-2$ & 13 & $3-8$ & oc & & A. -8.10 & - & - & \\
\hline 4 & $28 \cdot 686$ & - & $13 \cdot 0$ & $11 \cdot 5$ & $14 \cdot 2$ & - & s' by w, 2-3 & 10 & $10-12$ & $\boldsymbol{\alpha}$ & & Ci. 10 & - & - & 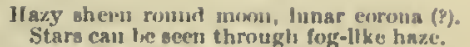 \\
\hline 6 & $28 \cdot 739$ & - & $12 \cdot \theta$ & $14 \cdot 2$ & $13 \cdot 8$ & - & HSL, 2-3 & 10 & $10-12$ & $\propto *$ & & Ci. 7 & N. 3 & - & Two patclies of aurora to $\mathrm{KE}$. \\
\hline 8 & $28 \cdot: 85$ & - & $12 \cdot 0$ & $13 \cdot 2$ & $13 \cdot 0$ & - & Lt. S airs & 7 & 0 & $\operatorname{con} *$ & & - & Thin N. 10 & - & $\begin{array}{l}\text { Mountain range not visible. Moon ani stars } \\
\text { all misty. }\end{array}$ \\
\hline 10 & $28 \cdot 815$ & - & $11 \cdot 0$ & $11 \cdot 2$ & $11 \cdot 0$ & - & Lt. $N$ alrs & 4 & 0 & lic & & A.e.11. 3, W & 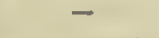 & - & \\
\hline Хัкоп & $23 \cdot 858$ & - & $7 \cdot 5$ & $11 \cdot 2$ & $11 \cdot 0$ & - & Calin & 6 & 0 & oc & & Ci. cul. 5, NW & A. cu. 4 & - & \\
\hline 2 & $28 \cdot 876$ & - & $8 \cdot 5$ & $13 \cdot 2$ & $12 \cdot 8$ & - & Calın & 5 & 0 & le & & Cl.-er. 4 & - & - & \\
\hline 4 & $28 \cdot 890$ & - & $8 \cdot 5$ & $11 \cdot 2$ & $10 \cdot 8$ & - & NE, 1-2 & a & $10-15$ & b & & - & - & - & \\
\hline 6 & $28 \cdot 939$ & - & $5 \cdot 5$ & $6 \cdot 0$ & $5 \cdot 5$ & - & $\mathrm{Niv}, 2$ & 14 & $10-20$ & $\omega$ & & A.*8. 10 & - & - & \\
\hline 8 & $28 \cdot 987$ & - & $2 \cdot 5$ & $4 \cdot 2$ & $3 \cdot 8$ & - & $\mathbf{E}, 2$ & 24 & $20-25$ & b) + & & - & - & - & \\
\hline 10 & $29 \cdot 028$ & - & $3 \cdot 0$ & $3 \cdot 0$ & $2 \cdot 8$ & - & NE, 2-3 & 16 & $25-28$ & $a c+$ & & Thin A. 8.10 & - & - & Finedar on the whole, Wind light \\
\hline Mist. & $29 \cdot 035$ & $\underline{-}$ & $1 \cdot 0$ & $3 \cdot 0$ & $2 \cdot 8$ & - & NE, 2-3 & 17 & $20-23$ & o & & Thin A.-5. 10 & - & - & day, rising from NF alwut i p.m, and \\
\hline Meau & Max. the & erin. & read & $\left\{\begin{array}{l}8 \mathrm{a} \\
8 \mathrm{p}\end{array}\right.$ & $\begin{array}{l}15^{\circ} \cdot 3 \\
14^{\circ} \cdot 0\end{array}$ & - & Solar radiation \{ & ingig & oulh & - & Fivap & grammes. & l'recipus. - in. & - & 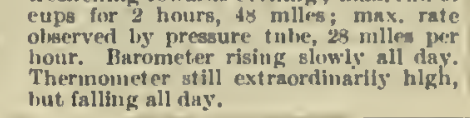 \\
\hline
\end{tabular}


1902 -June.]

Metronological Journal of the "1)iscovery."

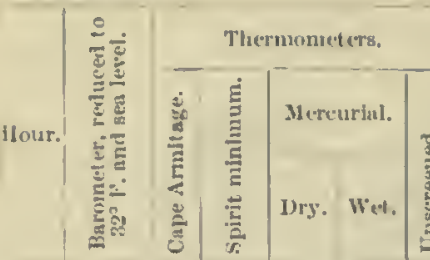
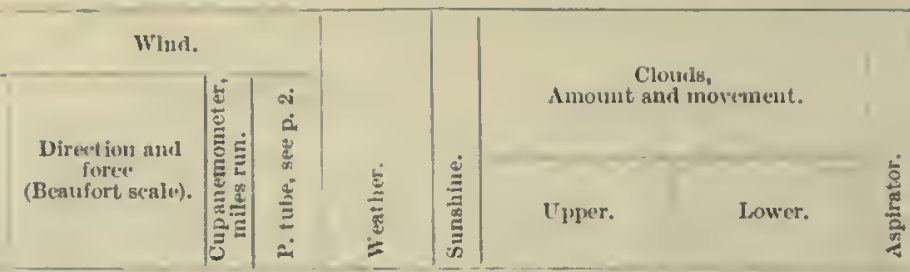

1 st.

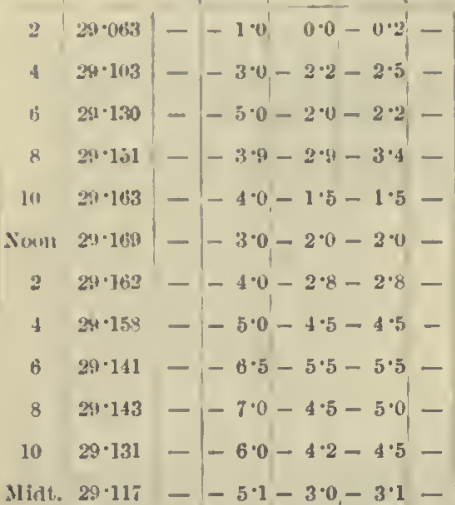

E $\ln x, 2-3$

$20 \quad 25-28$

Very thin A.-s. 10

-

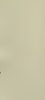

liemarks.

E, 2 20, 20-2

Ii, 2-3

$18 \quad 20-27$

$\mathrm{NH}_{1-2}$ 18 $10-20$ c

Exis, I-2 $11 \quad 15 \quad$ b

kX], 2-3 $11 \quad 20-23$ b-

NE, 2-3 $17 \quad 25 \quad h \sim$

NE, 2-3 $19 \quad 25-28 \quad$ b

Fx 1 , 3 23: $28-33$ b

$\mathrm{NE}, 3 \quad 21 \quad 28-33)$ be

L by $N, 3$

$\frac{18}{-} \frac{25}{-}$

IIcan $\overline{29 \cdot 136}-\overline{-2 \cdot 9-3 \cdot 1-}$

Max. therm. reas at $\left\{\begin{array}{l}8 \text { a. } 7^{\circ} \cdot 5 . \\ 8 \text { p. }-1^{\circ} \cdot 5 .\end{array}\right.$

Solar radiation $\left\{\begin{array}{l}\text { black bull, } \\ \text { bright lull }- \text {. }\end{array}\right.$

Evapn. - grammes. Precipn. - in.

$-$

Cl.-cu. 4, Nw

(very slow

Ci.-cil. 8

A.cus 3

- Stars dimly vilite. Gluw of aurora, alt. Heavy lank of clouds low down to $\mathrm{N}$ and $\mathrm{F}$. "Mackercl sky," movement extremely slow.

- Four rays of aurora rose to $30^{\circ}$ In NE and disapuearedi.

- $\quad$ - $\quad$ _ Low stratus all round covering hills to Nis

-
- $\quad$ - $\quad$ Low stratus over hillo to XE and li. Slight

- $\quad$ - $\quad$ - Same precipltation.

$\begin{array}{llll}- & - & -\end{array}$ Same preclpltation.

- $\quad$ - $\quad$ - $\quad$ Precipitation had apparently stopped.

Thin A.-s. 5

A.s. 10

-

On the whole a clear day, with light io fresh NF brcczes ; max. run of cups for 2 liwurs, Its miles ; max. rate observer by pressure until noon, and thent fell slowly. Temperature renains ligh and steady.

2nd.

\begin{tabular}{|c|c|c|c|c|c|c|c|c|c|c|}
\hline 2 & $28 \cdot 105$ & $--4 \cdot 0-1.8-2 \cdot 0-$ & $\mathrm{NE}, 3$ & 16 & 22 & $o$ & - & S. 10 & - & \multirow{5}{*}{ Faint aurora heain in NE. } \\
\hline 4 & $29 \cdot 091$ & $--2.8 \quad 0.0-0.2-$ & E hy $\mathrm{S}, 1$ & 8 & 8 & 0 & - & S. 10 & - & \\
\hline 6 & $29 \cdot 090$ & $--4 \cdot 0-2 \cdot 9-3 \cdot 0-$ & lis loy $\mathrm{S}, 1$ & 7 & 0 & l, & - & - & - & \\
\hline 8 & $29 \cdot 078$ & $--7 \cdot 2-2 \cdot 5-2 \cdot 5-$ & NE, 2 & 4 & $10-15$ & c & - & S. 8 & - - & \\
\hline 10 & $28 \cdot 049$ & $--10 \cdot 2-8 \cdot 5-8 \cdot 5-$ & $\mathrm{NE}, 2$ & 17 & $20-25$ & be & Ci.-s. 5 & - & - & \\
\hline Noon & $29 \cdot 025$ & $--10 \cdot 0-7 \cdot 0-7.0-$ & $\mathrm{NE}, 1-2$ & 11 & $10-15$ & b & Ci. 1, NW & Fr.-nt. $4, W N W$ & - & \multirow{3}{*}{$\begin{array}{l}\text { Min. at high-tevel station furing lrast } 21 \% \\
\text { hours, }-41^{\circ} \cdot 0 . \text { Temp. at } 1 \text { p.m., }-210^{\circ}(1) \\
\text { Brebus smoke lu big quantily going to } \\
\text { XE. }\end{array}$} \\
\hline 2 & $29 \cdot 041$ & $--17 \cdot 2,-14 \cdot 8,-14 \cdot 8-$ & S1.. 3-4 & 10 & $2 n-25$ & b & - & - & - & \\
\hline 8 & $29 \cdot 136$ & $--18 \cdot 0-18 \cdot 0--$ & v, 3-4 & 30 & $\mid 35-38$ & $\mathrm{~b}$ & - & - & -1 & \\
\hline 10 & $29 \cdot 221$ & $--18 \cdot 0-15 \cdot 0--$ & ENE, 1-2 & 24 & $20-23$ & b & - & - & - & \multirow{4}{*}{ 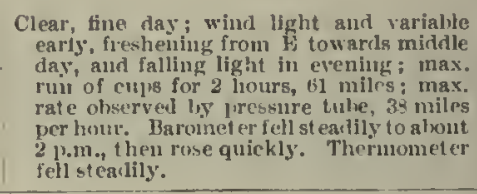 } \\
\hline Minlt. & $291 \cdot 282$ & $--16.8-10.0--$ & F, 1-2 & 11 & $10-15$ & b & - & - & - & \\
\hline Mean & $29 \cdot 15463$ & $---8.7-1=$ & - & - & - & - & - & - & - & \\
\hline & Max. thr & $\operatorname{crm}$. read at $\begin{cases}8 \mathrm{a}, & 2^{5} \cdot 0 . \\
81 \cdots, & -3^{\circ} \cdots\end{cases}$ & Solar radiation & $\begin{array}{l}\text { Ulack } \\
\text { lrigl }\end{array}$ & $\begin{array}{l}\text { k bulb } \\
\text { hit butl }\end{array}$ & & Evapu. _ grammes. & Preclpn. - in. & & \\
\hline
\end{tabular}

$3 r d$

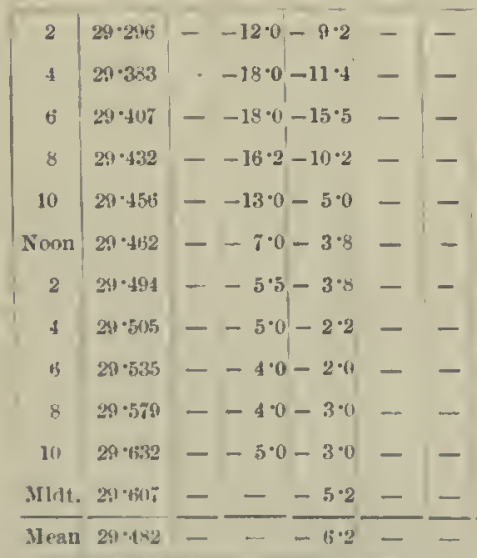

Max. therm, rear at $\left\{\begin{array}{l}8 \mu_{0}-8^{\circ} \cdot 2 . \\ 8 p_{0}-1^{\circ} \cdot 0 .\end{array}\right.$

\section{ESE, 1-2}

Chim

Calm

Calm

Lt. SW airs

SE, 1-2

E by $N, 1-2$

S, 2-4

SE var, 2-4

L, 2-3

SE var., 2-3

HSE, 1-2

\begin{tabular}{|c|cc}
11 & $10-15$ & $b$ \\
3 & $5-10$ & $0 c$ \\
1 & $0-10$ & $b$ \\
3 & 0 & $0 c$ \\
5 & 0 & tc \\
3 & $8-10$ & 0 \\
12 & $0-8$ & 0 \\
16 & $20-25$ & $0+t$ \\
21 & $15-25$ & $09+$ \\
22 & $15-20$ & 0 \\
17 & $15-18$ & 0 \\
11 & $10-15$ & $b$ \\
\hline- & - & -
\end{tabular}

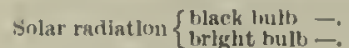

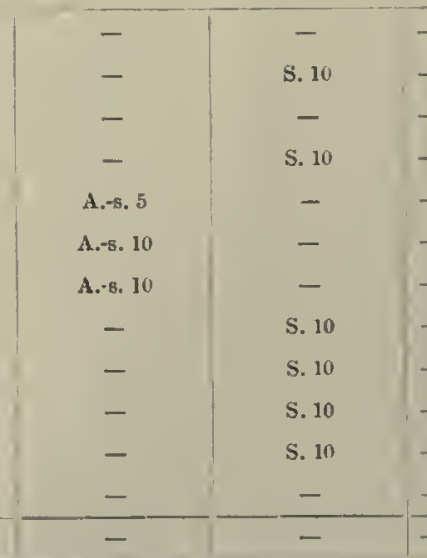

Nvaju. - grammes.
Precipn. - in
Stars dimly visible. Stratus may be high or low (?)

Wind coming from Ohservatory Hill : very varlathe, lis $\mathrm{l}, \mathrm{S}$ to $\mathrm{S}$ by $\mathrm{b}$.

- Wiud coming frou Otservatory Hill; very variable, Iis $\mathrm{E}$ to SS1\%, Clear sky. Mist apparent to the $\mathrm{B}$. vercast nearly all day, with wiud of variable force; nux. ruu of enils for 2 hourg, 43 miles; max. rate onservers liy pressure tibe, 25 miles ner hour. Baro

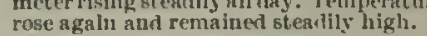


Mrtwolrolonical, Joulinal, of th: "Discoviliy."

[1902-June,

\begin{tabular}{|c|c|c|c|c|c|c|c|c|c|c|c|c|c|c|c|}
\hline \multirow{3}{*}{ Hour. } & \multirow{3}{*}{ 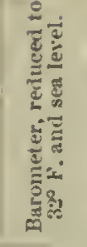 } & \multicolumn{5}{|c|}{ Thermomet tars. } & \multicolumn{3}{|l|}{ Wluwl. } & \multirow[b]{3}{*}{ 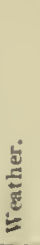 } & \multirow[b]{3}{*}{ 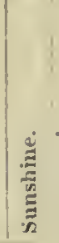 } & \multirow{2}{*}{\multicolumn{2}{|c|}{$\begin{array}{l}\text { Clouds, } \\
\text { Anwment aud uovenent. }\end{array}$}} & \multirow[b]{3}{*}{ 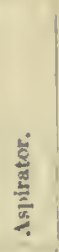 } & \multirow{3}{*}{ licmarks. } \\
\hline & & \multirow{2}{*}{ 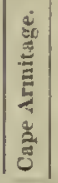 } & \multirow{2}{*}{ 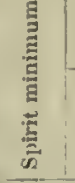 } & \multicolumn{2}{|c|}{ Mlercurial. } & \multirow{2}{*}{ 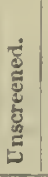 } & \multirow{2}{*}{$\begin{array}{c}\text { Direstion and } \\
\text { foret: } \\
\text { (Beanfort } 8 \text { (n) }\end{array}$} & \multirow{2}{*}{ 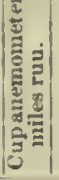 } & \multirow{2}{*}{ 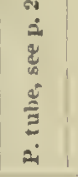 } & & & & & & \\
\hline & & & & Dry. & Wet. & & & & & & & Uiper. & Lower. & & \\
\hline 2 & $2 y \cdot 601$ & - & $-10 \cdot 1$ & $-8 \cdot 0 \mid$ & - & - & $15 \mathrm{~s} E, 1$ & 7 & 10 & l) & & - & - & - & 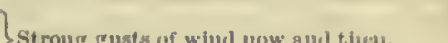 \\
\hline i & $2 n \cdot 541$ & - & $-10 \cdot 1$ & $-9 \cdot 8$ & - & - & FNI 1 , 2-il & 7 & $20-25$ & 1) & & - & - & - & (N) \\
\hline 6 & $2 x+5$ tio & -1 & $-11 \cdot 0$ & $-7 \cdot 1)$ & - & - & F l,y N, 2-3 & 24 & $15-25$ & 1) & & - & - & - & \\
\hline 8 & $29 \cdot 536$ & - & $-9 \cdot 0$ & $-8 \cdot 0$ & - & - & E. 1-5 & 20 & $15-20$ & b' & & - & - & - & \\
\hline 10 & $29 \cdot 533$ & - & $-11 \cdot 0$ & $-8 \cdot 0$ & - & - & $1 ;, 2$ & $1 !$ & $15-22$ & $1 \mathrm{ke}$ & & - & S. $2, \mathrm{NNW}$ & - & Hesvy bank of str. or Nim. covering \\
\hline Nown & $29 \cdot 501$ & - & $-10 \cdot 0$ & $-9 \cdot 2$ & - & - & FiNli, 3 & 22 & $23-27$ & $\infty$ & & - & S. 1, NNW & - & quickiy imm NNW. \\
\hline 2 & $2 ! 1 \cdot 49 !$ & - & $-10 \cdot x_{1}$ & $-7 \cdot x$ & - & - & $\mathrm{Nl}_{4}, 1-3$ & 17 & $15-23$ & 에 & & - & Fr.-B. $10, N$ & - & D. passing rapllily from NNW. \\
\hline$t$ & $2: 517$ & - & $-11 \cdot 8$ & $-10 \cdot 8$ & - & - & Nis, 1-2 & 18 & $15-20$ & $\ln$ & & - & Pr.-8. 5, N & - & \\
\hline 8 & $29 \cdot 5.33$ & - & $-12 \cdot 2$ & $-9 \cdot n$ & - & - & Nis, 1-2 & 12 & $10-15$ & $\ln$ & & - & Fr.-8. 4, N & - & Clear ta SW and silw. \\
\hline 8 & $29 \cdot 6 i(i i$ & - & $-11 \cdot 0$ & $-8 \cdot 2$ & - & - & KNL, 1-3 & 14 & $12-15$ & h & & - & - & - & \\
\hline 10) & $29 \cdot 545$ & -1 & $-12 \cdot 0$ & $-11 \cdot 5$ & - & - & NE, 1-2 & & 12 & bv & & - & - & - & 10 on the wolus \\
\hline Milt. & $29 \cdot 601$ & 一 & $-18 \cdot 0$ & $-11 \cdot 5$ & - & -1 & $N F_{1}, 3-4$ & 21 & $20-25$ & b & & - & - & - & $\begin{array}{l}\text { enrly, falling duriug thes day, and } \\
\text { freshening agaln fromul }\end{array}$ \\
\hline Mran & $29 \cdot 5 i 3$ & - & - & $-9 \cdot 6$ & - & -1 & - & - & - & - & • & - & - & $\therefore$ & $\begin{array}{l}\text { of cups for } 2 \text { honrs, } 48 \text { miles } \\
\text { ohserverl liy pressure tube, } 27 \text { miles pere }\end{array}$ \\
\hline & Max, the & rm. $r$ & read at & $\left\{\begin{array}{l}\mathrm{a} a \cdot-2 \\
8 \mathrm{p} .-2\end{array}\right.$ & $\begin{array}{l}-2^{2} \cdot 0 \\
-2^{\circ} \cdot 0\end{array}$ & & Solar radiation \{ & ack & luilb - & & apm. & ammes. & l'recipn. - in. & & 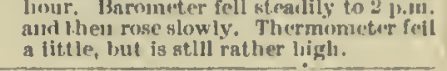 \\
\hline
\end{tabular}

4th.

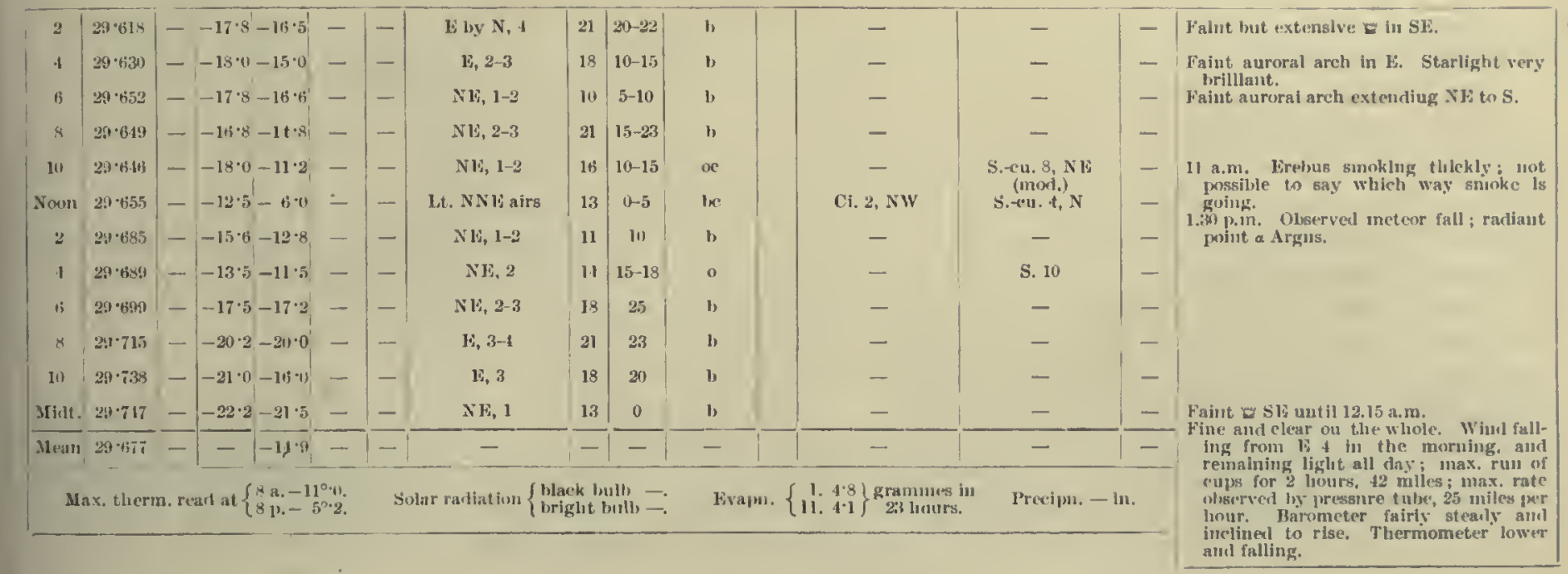

5 th.

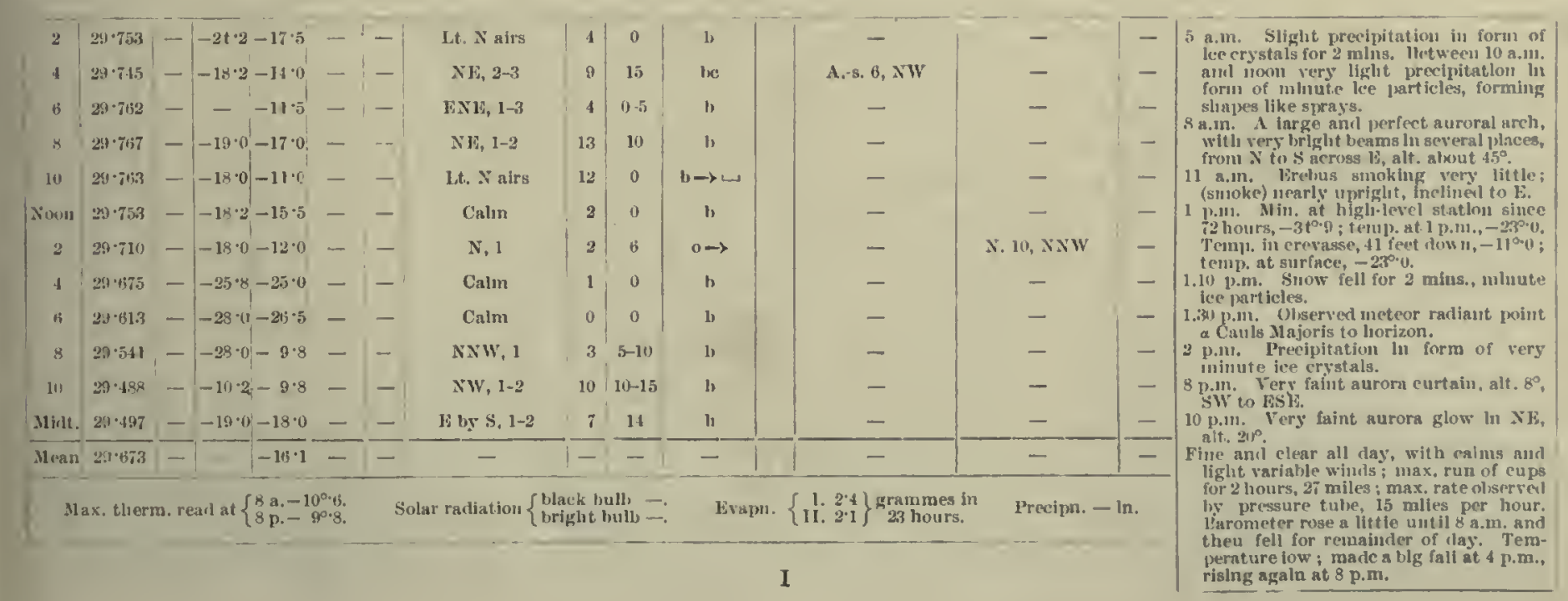

6 th. 


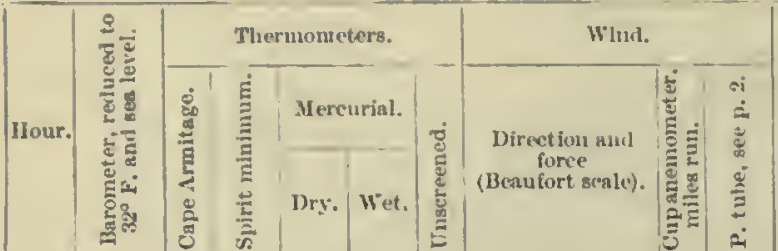

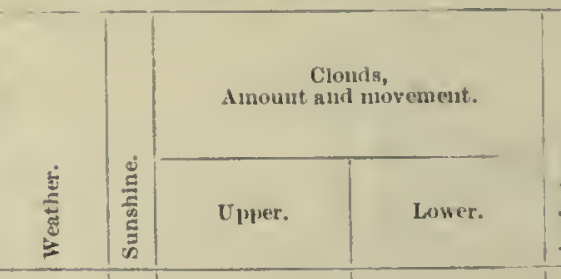

7 th.

\begin{tabular}{|c|c|c|c|c|c|c|c|c|c|c|c|}
\hline 2 & $29 \cdot 515$ & -1 & $-19 \cdot 0-16 \cdot 2$ & - & -1 & Lt. lis airs & 5 & 0 & b & - & - \\
\hline 1 & $29 \cdot 475$ & - & $-19 \cdot 5-17 \cdot 0$ & - & -1 & Lt. NE a Irs & 7 & 0 & b & - & - \\
\hline 6 & $29 \cdot 451$ & - & $-19 \cdot 0-15 \cdot 0$ & - & - & $N \mathrm{~N}, \mathrm{I}-2$ & 10 & 10 & $b x$ & - & - \\
\hline 8 & $29 \cdot 419$ & - & $-18 \cdot 1-17 \cdot 0$ & - & -1 & B hy $\mathrm{N}, 1$ & 5 & $0-10$ & b & - & - \\
\hline 10 & $29 \cdot 1 \cdot 19$ & - & $-26 \cdot 2-25 \cdot 5$ & - & -1 & E by $x, 1$ & 18 & $25-27$ & b & - & - \\
\hline Noon & $29 \cdot 463$ & - & $-30 \cdot 5-30 \cdot 0 \mid$ & - & -1 & $12,3-4$ & 23 & $30-32$ & b & - & - \\
\hline 2 & 29.510 & - & $-32 \cdot 5-29 \cdot 5$ & - & - & E, 3-4 & 24 & $23-25$ & b) & - & - \\
\hline 1 & $29 \cdot 575$ & - & $-30 \cdot 8-20^{\circ} \cdot 2$ & - & - & E, 3-1 & 26 & $25-27$ & b & - & - \\
\hline 6 & $29 \cdot 647$ & - & $-29 \cdot 0-25 \cdot 2$ & - & -1 & E, 2 & 18 & 15 & $\mathrm{bm}$ & - & - \\
\hline$s$ & $29 \cdot 706$ & - & $-20 \cdot 0-22 \cdot 0$ & - & - & E ly S, 1-2 & 12 & 12 & b & - & - \\
\hline 10 & $29 \cdot 696$ & - & $-23 \cdot 2-16 \cdot 8$ & - & - & NE, 1-2 & 3 & $10-12$ & b & - & - \\
\hline slint. & $29 \cdot 701$ & - & $-22 \cdot 0-21 \cdot 0$ & - & - & $\mathrm{x}, 1$ & 10 & 0 & $\mathrm{hm}$ & - & - \\
\hline Mean! & $29 \cdot 553$ & - . & $-|-22 \cdot 0|$ & - & -1 & - & - & - & - & - & - - \\
\hline
\end{tabular}

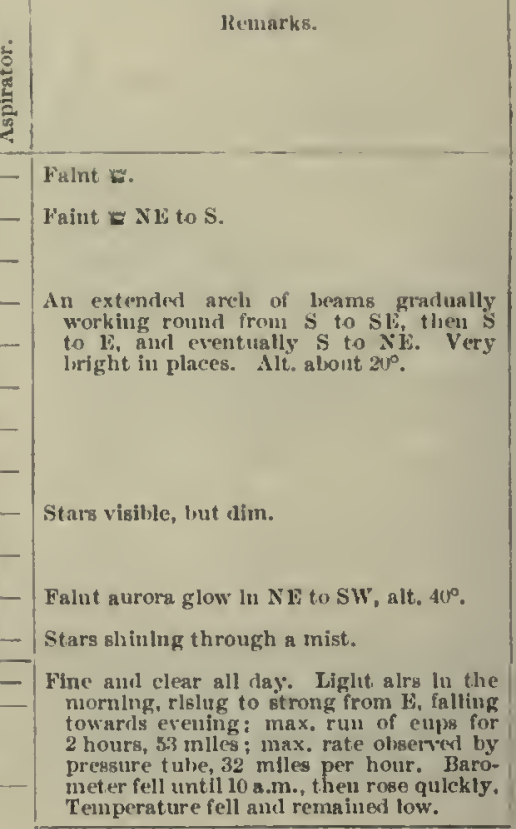

8th.

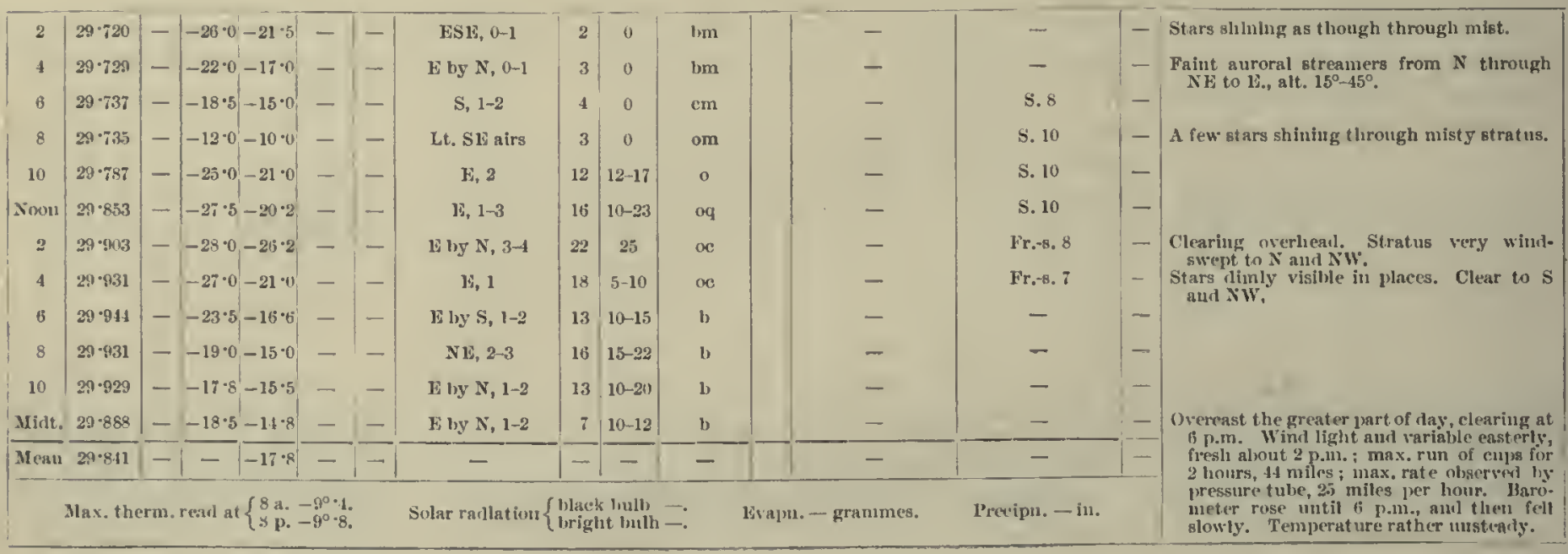

gth.

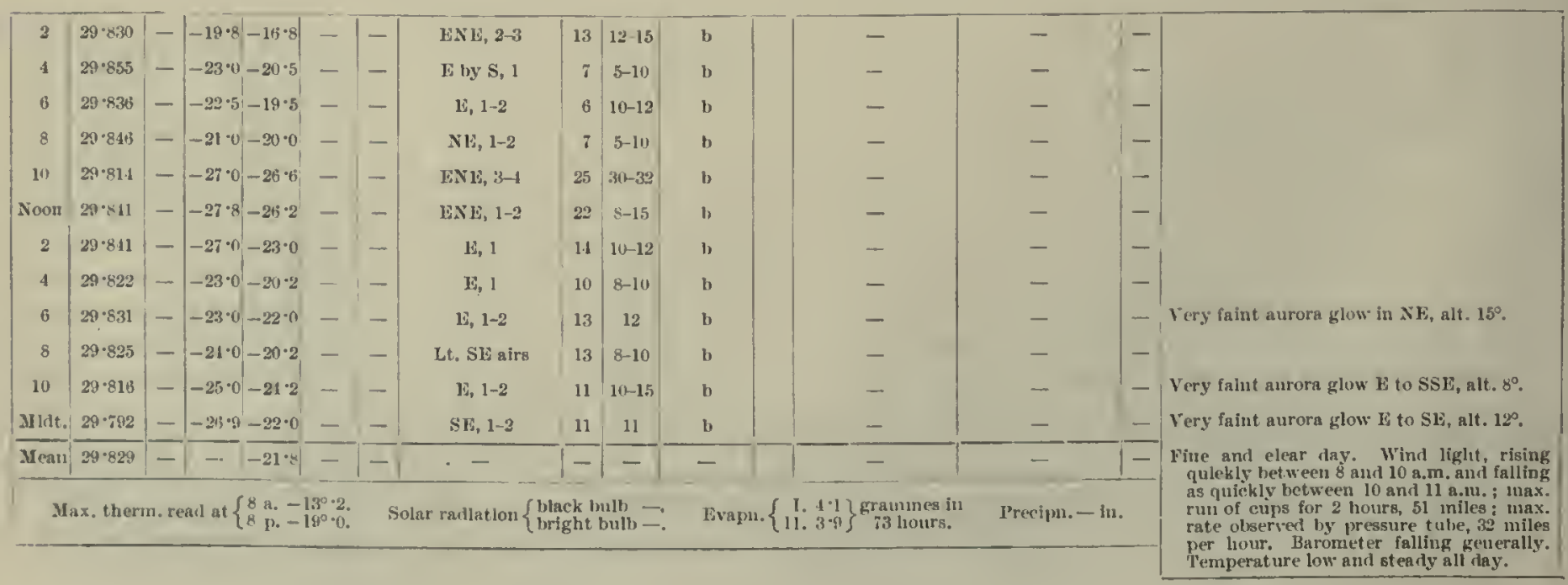


Meteorological, Journat, of Thl "Discovily."

[1902-June.

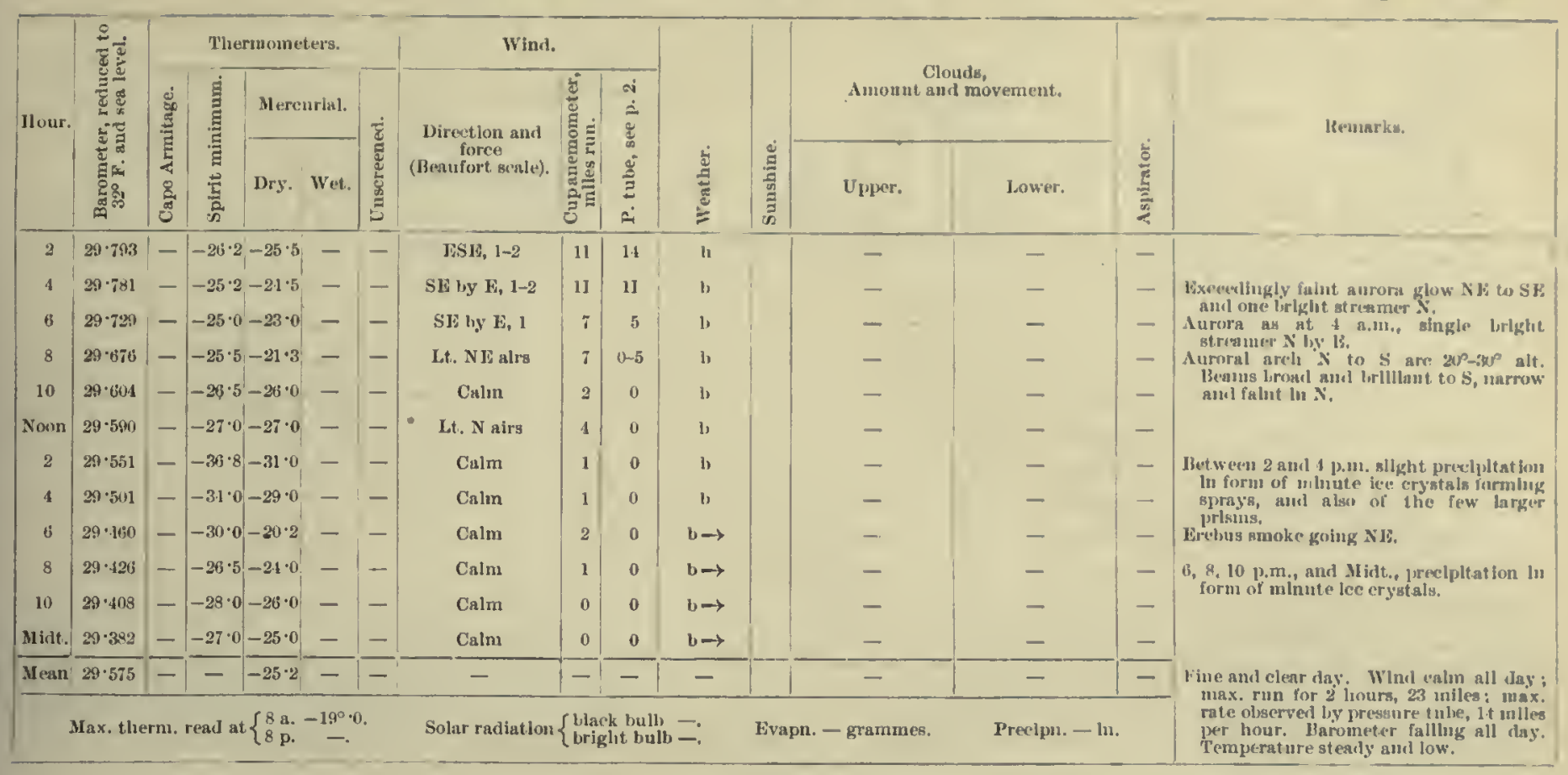

\begin{tabular}{|c|c|c|c|c|c|c|c|c|c|c|c|c|c|}
\hline 2 & $29 \cdot 363$ & - & $-27 \cdot 5 \mid-24 \cdot 5$ & - & -1 & Calm & 1 & 0 & $1, \rightarrow$ & - & - & - & \multirow{2}{*}{$\begin{array}{l}2,4,6, \text { and } 10 \text { a.m., precipitation in form } \\
\text { of minute oblong riglit-angled crystals. } \\
\text { Fine rime on anemoneter glass. }\end{array}$} \\
\hline 4 & $29 \cdot 39$ & - & $-33 \cdot 6-26 \cdot 0$ & - & - & Calm & 0 & 0 & $b \rightarrow$ & - & - & - & \\
\hline 8 & $29 \cdot 332$ & - & $-31 \cdot 5-30 \cdot 5$ & - & - & Calm & 1 & 0 & $b \rightarrow$ & - & - & - & Faint arch of streamers In S. \\
\hline 8 & $29 \cdot 317$ & - & $-32 \cdot 0-22 \cdot 0$ & - & - & Calm & 1 & 0 & b & - & - & - & \multirow{2}{*}{ Auroral arch of streamers $S$ to E., alt. $20^{\circ}$. } \\
\hline 10 & $29 \cdot 333$ & - & $-30 \cdot 5-28 \cdot 5$ & - & - & Calm & 0 & 0 & $b \rightarrow$ & - & - & - & \\
\hline Noon & $29 \cdot 314$ & - & $-31 \cdot 2-30 \cdot 8$ & - & - & Calm & 10 & 0 & bu & - & - & - & \multirow{5}{*}{ 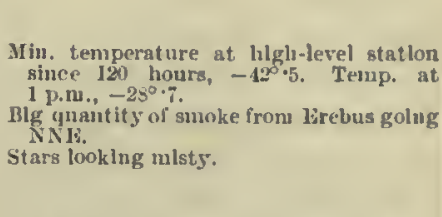 } \\
\hline 2 & $29 \cdot 395$ & - & $-31 \cdot 8-30 \cdot 2$ & - & - & Calm & 0 & 0 & 1, & - & - & - & \\
\hline 1 & $29 \cdot 495$ & - & $-31 \cdot 0-21 \cdot 0$ & - & - & Calm & 0 & 0 & $b \rightarrow \sqcup$ & - & - & - & \\
\hline 6 & $29 \cdot 552$ & - & $-21 \cdot 2-15 \cdot 8$ & - & - & $\mathrm{X}, 1-2$ & 2 & $\mid 10-12$ & $b *$ & - & - & - & \\
\hline 8 & $29 \cdot 603$ & - & $-15 \cdot 0-15 \cdot 8$ & - & - & FSE, 1-2 & 19 & 10 & $o *$ & - & N. 10 & - & \\
\hline 10 & $29 \cdot 647$ & - & $-20 \cdot 0-17 \cdot 0$ & - & - & $\mathrm{S}, 1$ & 15 & $111-12$ & of $*$ & $\rightarrow$ & N.-s. 10 & - & \multirow{4}{*}{ 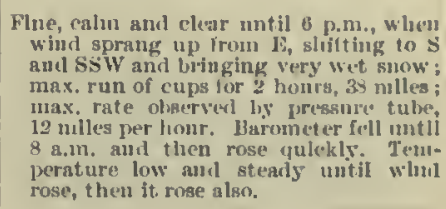 } \\
\hline Midit. & $29 \cdot 698$ & - & $-20 \cdot 0-16 \cdot 0$ & - & - & Ssw, 1-2 & 13 & $10-12$ & $\circ *$ & - & N. 10 & - & \\
\hline Mean & $29 \cdot 45.3$ & - & $-\quad-23 \cdot 4$ & -1 & -1 & - & - & - & - & - & - & - & \\
\hline & & & at $\left\{\begin{array}{l}8 \mathrm{a} . \\
8 \mathrm{p} .\end{array}\right.$ & & & 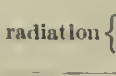 & & titb & & 48 & I'recil & & \\
\hline
\end{tabular}

11 th.

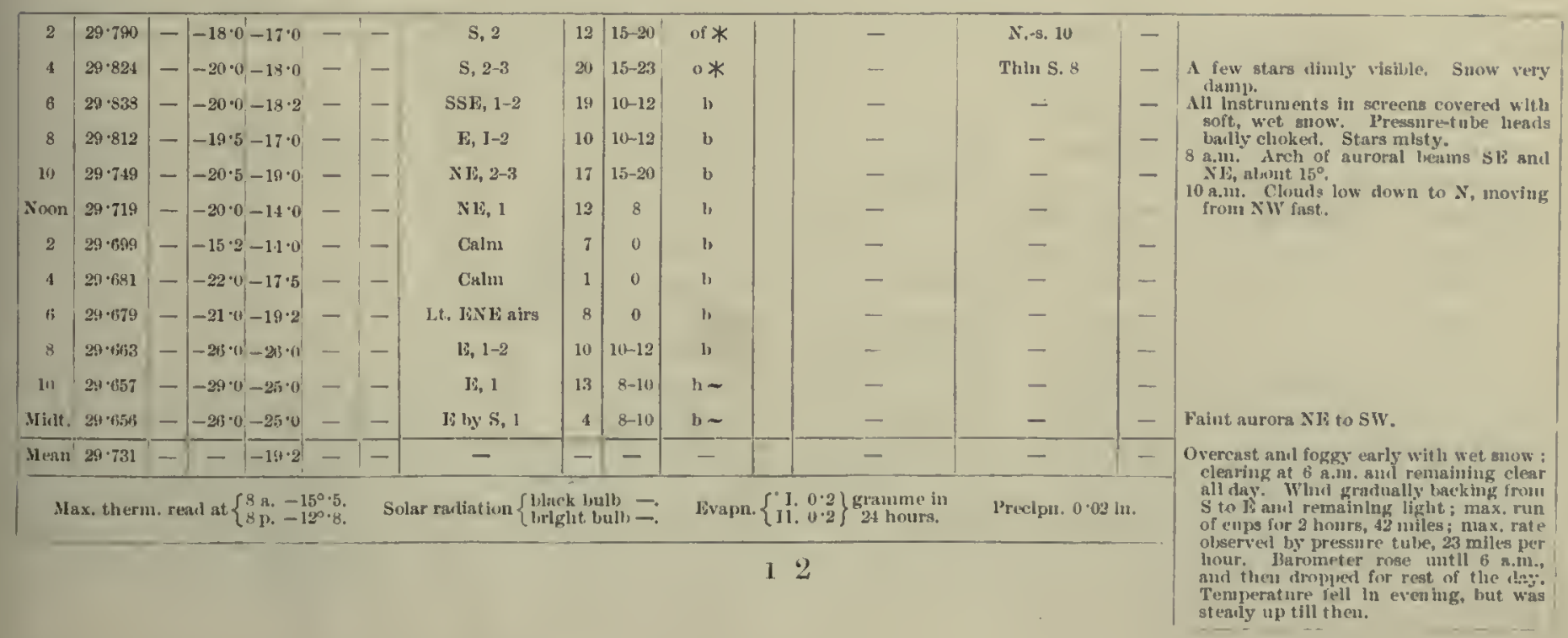

12 th. 
1902 -June.]

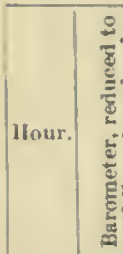

13th.

Meteorological Journal, of the "Discovely."

\begin{tabular}{|c|c|c|c|c|c|c|c|}
\hline \multicolumn{5}{|c|}{ Therntomet ers. } & \multicolumn{3}{|l|}{ WHard. } \\
\hline \multirow{2}{*}{ 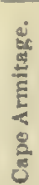 } & \multirow{2}{*}{$\begin{array}{l}\text { E } \\
\text { E } \\
\text { E } \\
\text { E } \\
\frac{\mathrm{E}}{\mathrm{E}}\end{array}$} & \multicolumn{2}{|c|}{ Mercurial. } & \multirow{2}{*}{ 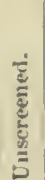 } & \multirow{2}{*}{$\begin{array}{l}\text { Direction ami } \\
\text { foree } \\
\text { (lieaufort seale). }\end{array}$} & \multirow{2}{*}{ 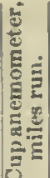 } & \multirow[b]{2}{*}{$\therefore$} \\
\hline & & Dry: & Wet. & & & & \\
\hline
\end{tabular}

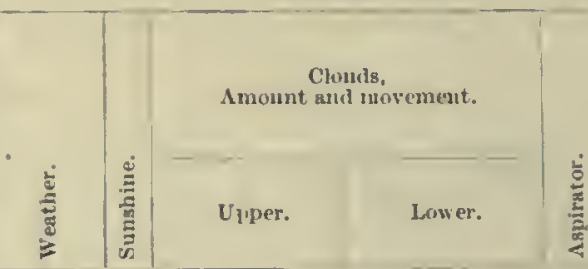

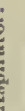

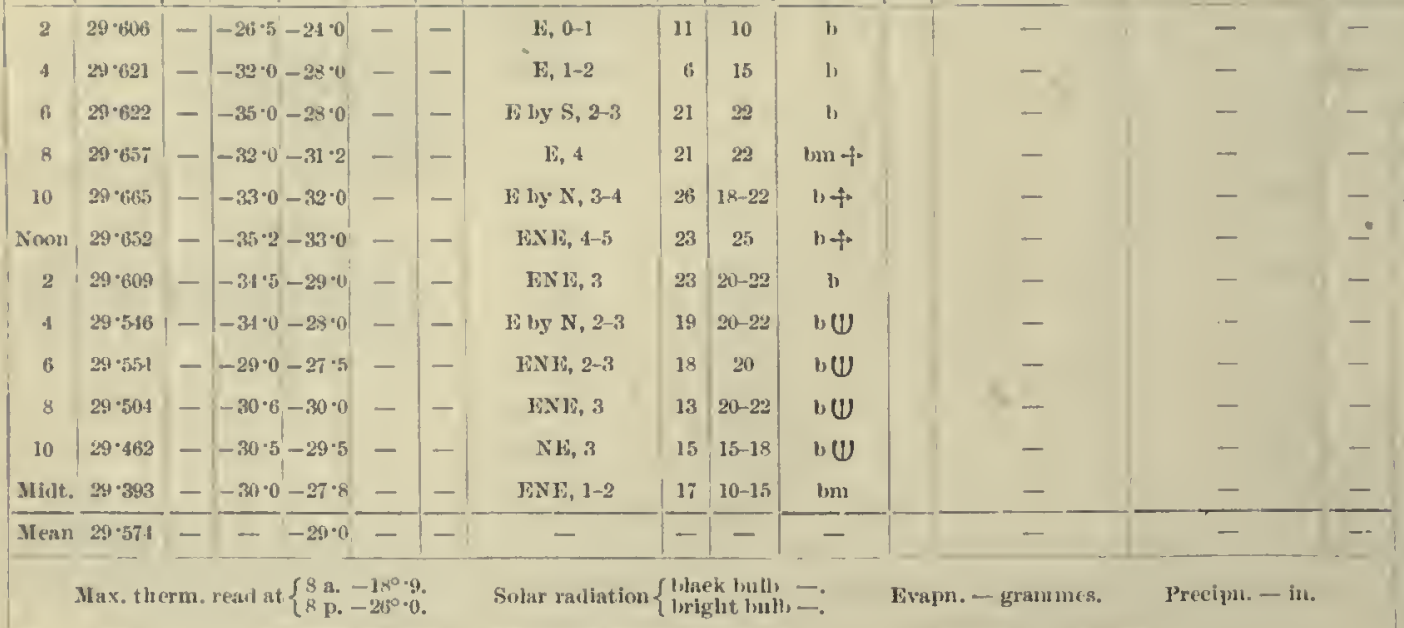

Faint aurres all night.

Faint aurura, alt. $30^{\circ}$ to $40^{\circ}$, in S to Sl.

Anemoneter glass found covered with lime or glazal

Chear, whit strong Wy hreezes: max. run of ellps for 2 hours, 51 inlles; max. rate lour, liarmeter rose aj to 10 a.m. and $\left[l_{1}+1\right.$ dropuled for rest of day. Timperature low and steady.

14th.

\begin{tabular}{|c|c|c|c|c|c|c|c|c|c|c|c|c|c|}
\hline 2 & $29 \cdot 336$ & - & $-31 \cdot 2-31 \cdot 2$ & - & -1 & LNI, 1-2 & 12 & 15 & $\operatorname{lm}$ & - & - & - & Very slight aurora glow in SW. \\
\hline 4 & $29 \cdot 296$ & - & $-32 \cdot 9-31 \cdot 8$ & - & -1 & $\mathrm{ENY}_{4}, 2-3$ & 11 & $15-20$ & b & - & - & - & Slight atrora arch Nis to Sls, alt. $45^{\circ}$. \\
\hline 6 & $29 \cdot 247$ & - & $-23 \cdot \mathrm{s}-32 \cdot 5$ & - & - & INN I, 2-3 & 15 & $15-20$ & b & - & - & - & \multirow{10}{*}{ 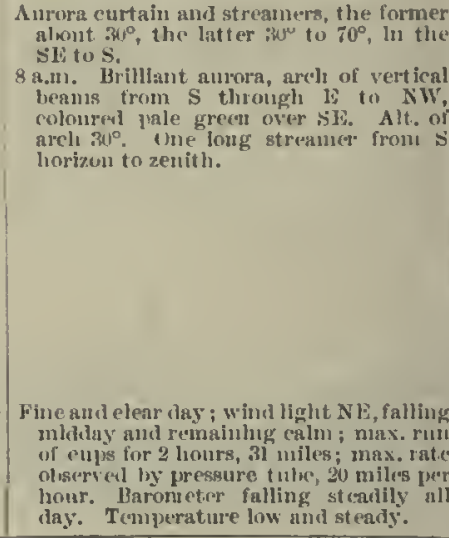 } \\
\hline 8 & $29-159$ & $1-$ & $-31 \cdot 0-32 \cdot 0$ & - & - & NE, 1-2 & 13 & 15 & b & - & - & - & \\
\hline 10 & $29 \cdot 122$ & - & $-33 \cdot 0-31 \cdot 5$ & - & - & $\mathrm{N}, 1$ & 12 & 10 & 1, & - & - & - & \\
\hline Nion & $29 \cdot 068$ & - & $-43 \cdot 0-32 \cdot 0$ & - & - & Caim & is & 0 & $11 \mathrm{x}$ & - & - & - & \\
\hline 2 & $20 \cdot 012$ & - & $-33 \cdot 0-31 \cdot 3$ & - & - & Calm & 2 & 6 & b & - & - & - & \\
\hline 8 & $28 \cdot 918$ & $1-$ & $-33 \cdot(1-30 \cdot 0$ & - & $1-$ & $N E_{1}, 1$ & 8 & 8 & b & - & - & - & \\
\hline 10 & $28 \cdot 913$ & - & $-40-2-36 \cdot 2$ & - & - & Calmı & 2 & 0 & lw & - & - & - & \\
\hline Mkilt. & $28 \cdot 921$ & - & $-38 \cdot 0-32 \cdot 2$ & - & - & Calm & 3 & 0 & b & - & - & - & \\
\hline \multirow[t]{2}{*}{ Misan } & $29 \cdot 074$ & - & $--31 \cdot 6$ & - & - & - & - & - & - & - & - & \multirow[t]{2}{*}{-} & \\
\hline & Max, $t$ & & read at $\left\{\begin{array}{l}8 \mathrm{a} \\
8 \mathrm{p} .\end{array}\right.$ & $-29^{\circ} \cdot 3$ & & Solar radiatio & & $i n$ & & apm. $-g r$ & Precipn. - in, & & \\
\hline
\end{tabular}

15 th.

\begin{tabular}{|c|c|c|c|c|c|c|c|c|c|c|c|c|}
\hline 2 & $28 \cdot 921$ & - & $-42 \cdot 0-33 \cdot 8$ & - & -1 & Calm & 1 & 0 & b) & - & - & - \\
\hline 1 & $28 \cdot 921$ & - & $-39 \cdot 2-31 \cdot 5$ & - & - & NW, 1 & 1 & 0 & b) & - & - & - \\
\hline 6 & $28 \cdot 916$ & - & $-35 \cdot 0-24 \cdot 8$ & - & - & $N, 1 m 2$ & 7 & o & b & - & - & - \\
\hline 8 & $28 \cdot 922$ & - & $-37-36 \cdot 6$ & - & - & Calm & 2 & 0 & b & - & - & - \\
\hline 10 & $29-939$ & - & $-37 \cdot 0-37 \cdot 0$ & - & - & Calm & 1 & 0 & b) & - & - & - \\
\hline Noon & $28-958$ & - & $-37 \cdot 5-31 \cdot 0$ & - & - & Lt. Sw alrs & 0 & 0 & b) & - & - & - \\
\hline 2 & $28 \cdot 972$ & - & $-34 \cdot 5-25 \cdot 0$ & - & - & Lt. Sls airs & 3 & 8 & lice & A.-s. 5 & - & - \\
\hline 4 & $29 \cdot 013$ & - & $-25 \cdot 5-20 \cdot 5$ & - & - & $\mathrm{SE}_{1} \mathrm{I}$ & 4 & $8-10$ & $\circ *$ & - & N. 10 & - \\
\hline is & $29 \cdot 047$ & - & $-26 \cdot 0-23 \cdot 5$ & - & - & $\mathrm{S}], \mathrm{y} \mathrm{S}, 2$ & 16 & 10-12 & bศे & - & - & - \\
\hline g & $29 \cdot 048$ & - & $-30 \cdot 5-28 \cdot 0$ & - & - & E $1, y, N, 3-5$ & 26 & $25-30$ & $h+$ & - & - & - \\
\hline 10 & $29 \cdot 054$ & - & $-35 \cdot 5-35 \cdot 2$ & - & - & INE & 24 & $80-32$ & $x \in$ & Ci.-8, 3, NW & - & - \\
\hline Mbas. & $29 \cdot 011$ & - & $-33 \cdot 0-22 \cdot 9 \mid$ & - & - & $\mathrm{N} F, 3-4$ & 26 & $15-20$ & e & Very tlin $\mathrm{Cl}_{1}-\mathrm{s}, 10$ & - & - \\
\hline Mean, & $28 \cdot 973$ & - & $--30 \cdot 2$ & - & - & - & $\overline{-}$ & - & - & - & - & - \\
\hline & & & & & & aullatio & & thb & & $\left.\begin{array}{r}1.0 .9 \\
11.1\end{array}\right\} \begin{array}{c}\text { gramme } \\
72 \text { hou1 }\end{array}$ & Preet & \\
\hline
\end{tabular}

Mereury in thy mult prolably frozen.

Aurora nrel of heants from $S$ to $X$ thromgh 1), allt. $20^{\circ}$ to $30^{\circ}$.

CI.-a., ratlinting $\mathrm{NW}$ and $\mathrm{SF}$ 11 p.M1. $1017^{\circ}$ aldunet

Fine aud generally eforr slay; ; wind light. ine aud generally eferer slay; wind light.
and culin up to 4 j.ml, then a strong

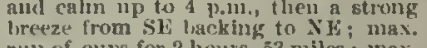
lireeze from si liscking to Na; max.
run of cups for 2 hours, 33 miles: 2 max. rate ohserved hy' pressure tube, 32 iniles per hour, Baronet er rose nutil wind per hener, and thent felt. Thernounter
tow and rising. 
Meteorological Journal of the "Iiscovery."

[1902-June.

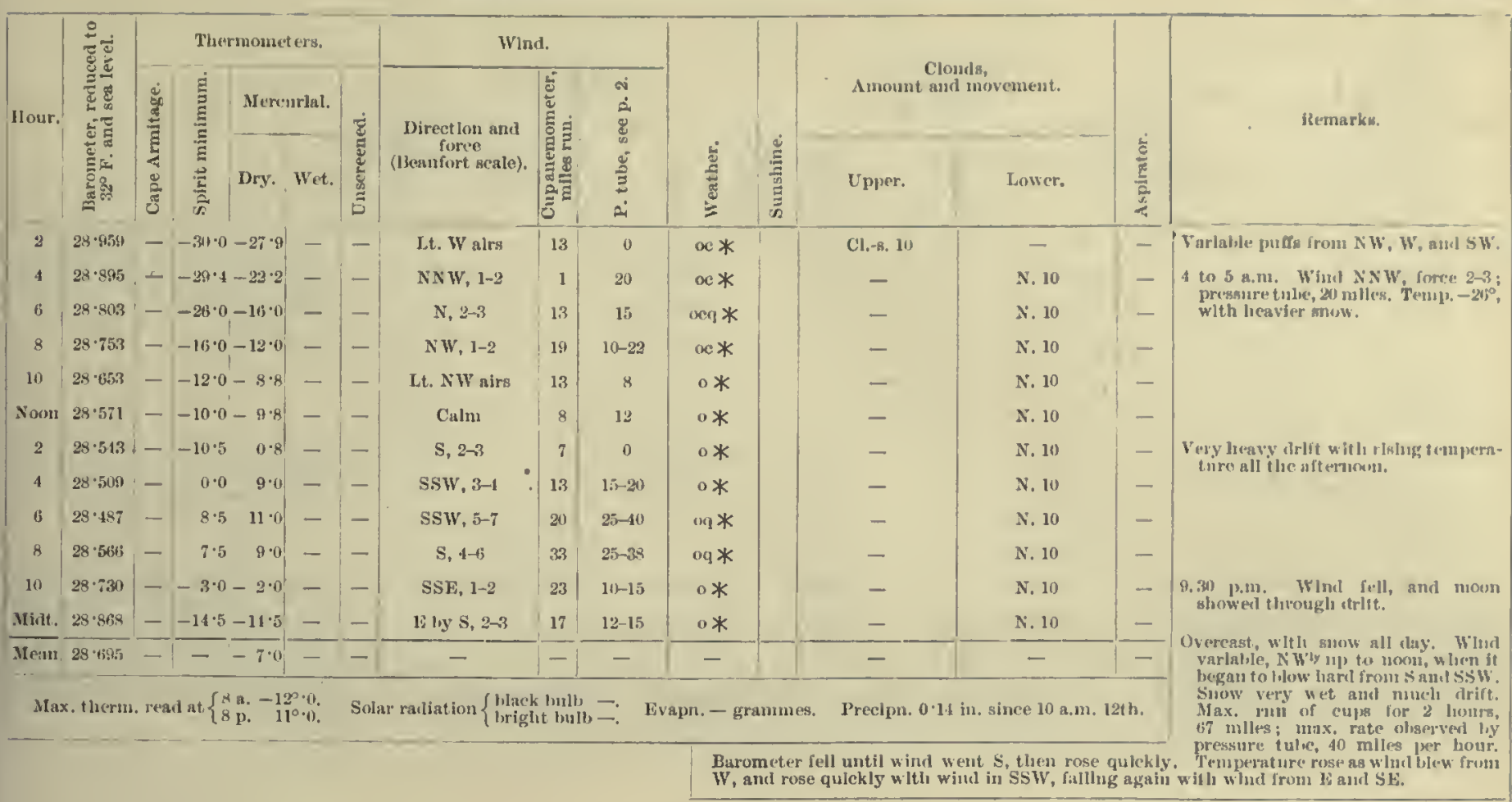

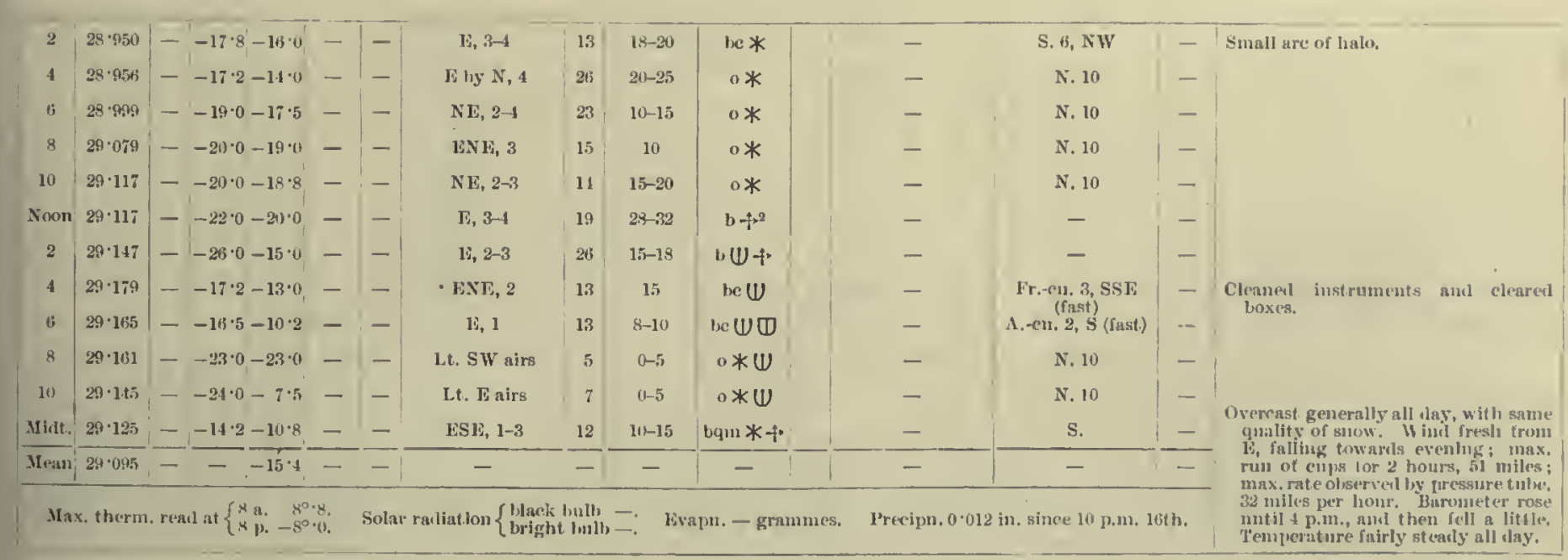

\begin{tabular}{|c|c|c|c|c|c|c|c|c|c|c|c|c|}
\hline 2 & $29 \cdot 111$ & $--15 \cdot 9-12 \cdot 0$ & - & - & ENE, 1-3 & 15 & 11 & be $t^{\prime}$ & $A,-s, 1$ & N. 1 & - & \multirow{4}{*}{$\begin{array}{l}\text { A.-8, radiating NE and SW, Nim. } \\
\text { NE and SW. }\end{array}$} \\
\hline 4 & $2 x \cdot 126$ & $--1.5-13.0$ & - & - & El ly $N, 1-3$ & 18 & 10 & $\mathrm{lnm}$ & - & Fr.-s. 5, NW & - & \\
\hline 6 & $29 \cdot 107$ & $--113 \cdot 0-13 \cdot 0$ & - & - & WN1, 1-3 & 16 & $10-14$ & $=$ bmeq $t^{2}{ }^{2}$ & - & S. 3 & - & \\
\hline 8 & $29 \cdot 139$ & $--15 \cdot 8-12 \cdot 2$ & - & - & $\mathrm{NE}, 3-4$ & 22 & 10 & $b e \rightarrow$ & Ci.-s. 3 & - & - & \\
\hline 10 & $29 \cdot 157$ & $--11 \cdot \bar{\jmath}-13 \cdot 8$ & - & - & 11,3 & 18 & 10 & 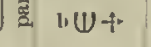 & - & - & - & \multirow{8}{*}{$\begin{array}{l}\text { 10.2u1 a.nu. } 4 f^{\circ} 2 v^{\circ} \text { diameter. } \\
\text { selenc visitle. }\end{array}$} \\
\hline Xoon & $29 \cdot 191$ & $--16 \cdot 2-15 \cdot 5$ & - & - & E., 2 & 20 & $10-15$ & b & - & - & - & \\
\hline 2 & $29 \cdot 223$ & $--19 \cdot 0-17 \cdot 8$ & - & - & $\mathrm{F}, 1-2$ & 14 & $10-1,5$ & b & - & - & - & \\
\hline 4 & $29 \cdot 217$ & $--20 \cdot 0-18 \cdot 11$ & - & - & E, $2-3$ & 18 & $15-20$ & $b U+$ & - & - & - & \\
\hline 6 & $29 \cdot 2 \pi 7$ & $--20 \cdot 0-10 \cdot 0$ & - & - & ExE, 2-3 & 21 & $10-15$ & bU & - & $\longrightarrow$ & - & \\
\hline 8 & $29 \cdot 3$ & $--18 \cdot 0-12 \cdot 0$ & - & - & Ni, 1-2 & $1 s$ & $111-15$ & $h U$ & - & - & - & \\
\hline 10 & $29 \cdot 319$ & $--13 \cdot 4-10 \cdot 0$ & - & - & S1:, 1-2 & 9 & $10-12$ & $1, U$ & - & - & - & \\
\hline Mlist. & $24 \cdot 335$ & $--13 \cdot 5-13 \cdot 0$ & - & - & SS15, 2 & il & $12-1.1$ & lev & $\mathrm{Cl}_{1}-\mathrm{so} .2$ & - & - & \\
\hline Neans & $23 \cdot 211$ & $-\quad--13 \cdot 9$ & - & - & - & - & - & - & - & - & - & \multirow{2}{*}{ 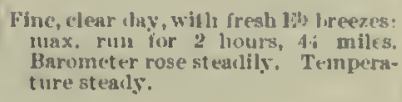 } \\
\hline $\operatorname{Max}$ & x. therm & . reari at $\left\{\begin{array}{l}8 \text { a. }- \\
8 \text { p. }-1\end{array}\right.$ & . & sol & diation $\{1\}$ & & ib =: & apm. $\left\{\begin{array}{l}1 \\
11\end{array}\right.$ & es for & Previpn. & & \\
\hline
\end{tabular}


1902-June.]

Meteorological Journal of the "Discovery."

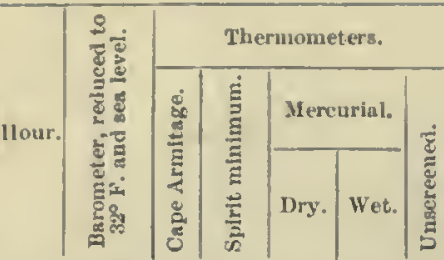

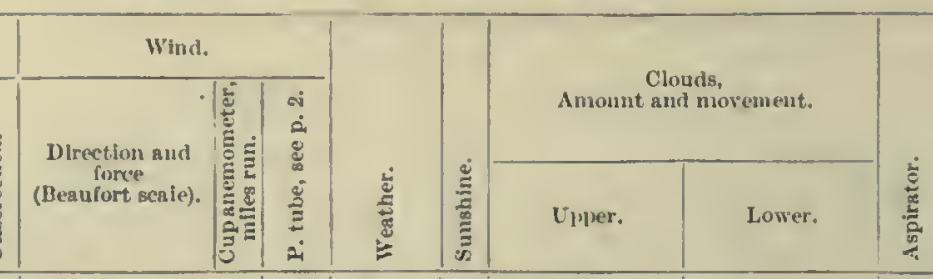

19 th.

\begin{tabular}{|c|c|c|c|c|c|c|c|c|c|c|}
\hline 2 & $29 \cdot 356$ & - & $-16 \cdot 0$ & $-15 \cdot 2$ & - & - & -3 & 15 & 15 & berc 0 \\
\hline 4 & $29 \cdot 350$ & - & 8 & $8 \cdot 0$ & - & - & liy $N, 7-8$ & 34 & $27-30$ & 1xet \\
\hline 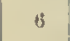 & $29 \cdot 373$ & - & $19 \cdot 5$ & $-18 \cdot 5$ & - & - & $\mathrm{NF}, 4-5$ & 22 & $20-22$ & $t$ \\
\hline 8 & $29 \cdot 3 \overline{2}$ & - & $-20 \cdot 0$ & $-16 \cdot 8$ & - & - & $18 \log x, 3-4$ & 13 & $10-15$ & me \\
\hline 10 & $29 \cdot 380$ & - & $-18 \cdot 0$ & $-17 \cdot 0$ & - & - & $\mathrm{NE}, 2$ & 14 & $10-15$ & \\
\hline on & $29 \cdot 353$ & - & $8 \cdot 0$ & $\mid-12 \cdot 8$ & - & - & NE, 1-2 & 11 & $8-10$ & \\
\hline 2 & $28 \cdot 341$ & - & 22. & $-16 \cdot 2$ & - & - & Caln & 7 & 0 & se * \\
\hline 4 & $29 \cdot 321$ & - & $-18 \cdot 0$ & $-18 \cdot 0$ & - & - & Calm & 1 & 0 & * \\
\hline is & $29 \cdot 237$ & - & $-18 \cdot 5$ & -10.8 & - & - & Lt. NW a irs & 1 & $0-8$ & $a x$ \\
\hline 8 & $29 \cdot 284$ & - & $-15 \cdot 5$ & $-14 \cdot 0$ & - & - & Lt. NNE alrs & 1 & $0-8$ & * * \\
\hline 10 & $29 \cdot 292$ & - & $-16 \cdot 8$ & $-14 \cdot 6$ & - & - & Calm & 2 & 0 & o* \\
\hline & 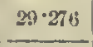 & - & - & $-15 \cdot 5$ & - & - & Calm & 0 & 0 & * \\
\hline & & & & & & - & & - & - & \\
\hline
\end{tabular}

\begin{tabular}{|c|c|}
\hline $\mathrm{Ci},-\mathrm{s}, 1$ & - \\
\hline$C_{-}{ }_{-8 .} 2$ & - \\
\hline Ci.-8. 3 & - \\
\hline Ci.-s. 6 & - \\
\hline - & - \\
\hline $\mathrm{Ci}_{.}-\mathrm{B} .6$ & - \\
\hline Cl.-8. $\overline{7}, \mathrm{NW}$ (slow) & - \\
\hline Cl.-8. 10 & - \\
\hline - & S. $10, N W$ (fast) \\
\hline- & N. 10 \\
\hline Cl.-8. 6 & A.-s. 6, SW (moll.) \\
\hline C1.s. 8 & - \\
\hline - & ' - \\
\hline
\end{tabular}

- 2 a.m., lunar halo, radius $27^{\circ} 49^{\prime}$, alt. $233^{\circ} 20^{\prime}$,

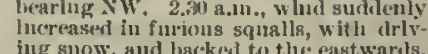
ing snow, and backet to the eastwarts. 4 a.111. Jlalo denser. 6.un. Very clear and distluet lialo:
prismat ie eolouring.

Remarks.

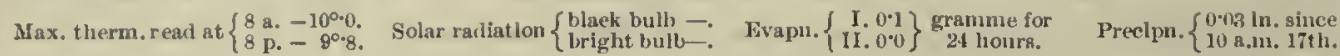
with very fine snowfall, alout \& p.m. ally lalling a gale from to calm; max. ruu of ally lalling away to calm; max. ruu of
cups for 2 hours, 138 mlles: max. rate observed by pressure tube, 331 miles per fell, and then fell slowly. Temperature steady.
ste

20 th.

\begin{tabular}{|c|c|c|c|c|c|c|c|c|c|c|c|c|c|}
\hline 2 & $29 \cdot 280$ & - & $|-15 \cdot 5|-12 \cdot 5 \mid$ & - & - & Calm & 2 & 0 & $\circ * \mathbb{T}$ & A.-8. 10 & - & - & \multirow{11}{*}{ 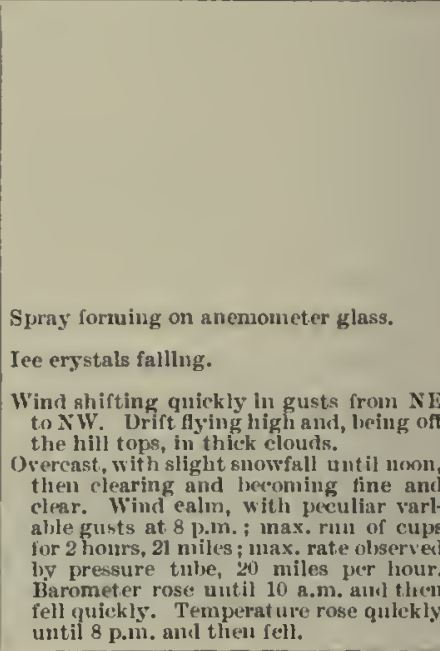 } \\
\hline 4 & $29 \cdot 297$ & - & $|-21 \cdot 5|-17 \cdot 5$ & - & - & Calm & 2 & 0 & $\circ * \mathbb{T}$ & $A_{0}-\mathrm{s}, 10$ & - & - & \\
\hline 6 & $29 \cdot 311$ & - & $|-17 \cdot 5|-12 \cdot 5$ & - & - & Calm & 2 & 0 & oe $*$ & $\mathrm{Ci} .-8.4$ & S. 2, SE & - & \\
\hline 8 & $29 \cdot 354$ & - & $-19 \cdot 7 \mid-10 \cdot 5$ & - & - & $\mathrm{N}$ by $\mathrm{F}_{1} 1$ & 2 & 2 & on & - & - & - & \\
\hline 10 & $29 \cdot 335$ & - & $|-11 \cdot 2|-7 \cdot 2$ & - & - & Lt. $\mathbf{N}$ alrs & 7 & 0 & oc & Ci.-s. 8 & - & - & \\
\hline Noon & $29 \cdot 373$ & - & $-8 \cdot 0-6 \cdot 8$ & - & - & $N, 1-2$ & 7 & 10 & $\operatorname{oc} U$ & Ci.-s. 8 & - & - & \\
\hline 2 & $29 \cdot 353$ & - & $-8 \cdot 0-6 \cdot 0$ & - & - & $x, 1-2$ & 10 & $10-12$ & be $U$ & C1.-8. 5 & - & - & \\
\hline 8 & $29 \cdot 225$ & - & $-16 \cdot 0 \mid \quad 5 \cdot 0$ & - & - & Var. $\mathbf{N}, 0-3$ & $\tau$ & $0-20$ & $b+4$ & - & - & - & \\
\hline 10 & $29 \cdot 194$ & - & $-11 \cdot 0-8 \cdot 8$ & - & - & Lt. Why $\mathrm{X}$ airs & 10 & $0-8$ & b & - & - & - & \\
\hline Midit. & $29 \cdot 134$ & . & $|-15 \cdot 0|-11 \cdot 7 \mid$ & - & - & Calm & 2 & 0 & bv & - & - & - & \\
\hline Mean & $29 \cdot 281$ & - & $--9 \cdot 5$ & - & $1-$ & - & - & - & - & - & - & - & \\
\hline
\end{tabular}

21st.

\begin{tabular}{|c|c|c|c|c|c|c|c|c|c|c|c|c|}
\hline 2 & $29 \cdot 164$ & -1 & $|-19 \cdot 6-13 \cdot 5|$ & - & - & NE hy $E, 1-2$ & 10 & $\mid 12-15$ & by & - & - & - \\
\hline 4 & $29 \cdot 177$ & - & $-18 \cdot 7-16 \cdot 4$ & - & - & $1,3-4$ & 19 & $25-31$ & bv $f$ & - & - & - \\
\hline 6 & $29 \cdot 145$ & - & $-18 \cdot 2-11 \cdot 5$ & - & - & E, 3-4 & 21 & $25-31$ & $b \underline{v}+$ & - & - & - \\
\hline 8 & $29 \cdot 175$ & - & $-20 \cdot 5-18 \cdot 0$ & - & - & $\mathbf{F}, 5$ & 22 & 25 & bqv + & - & - & - \\
\hline 10 & $29 \cdot 189$ & - & $-21 \cdot 8-20 \cdot 2$ & - & - & E, 4-5 & 24. & $25-31$ & int $t^{2}$ & - & - & - \\
\hline Noon & $29 \cdot 191$ & - & $-21 \cdot 2-17 \cdot 5$ & - & - & 10 by $N, 3-4$ & 22 & $20-25$ & 1, & - & - & - \\
\hline 2 & $29 \cdot 177$ & - & $-20 \cdot 0-19 \cdot 0$ & - & - & $\mathbf{E}, 2$ & 19 & $10-15$ & b & - & - & - \\
\hline 4 & $29 \cdot 157$ & - & $-19 \cdot 8-15 \cdot 8$ & - & - & F, 1-2 & 11 & $8-12$ & b & - & - & - \\
\hline 6 & $29 \cdot 165$ & - & $-29 \cdot 0-19 \cdot 0$ & - & - & Calm & 11 & 0 & b & - & - & - \\
\hline 8 & $2: 3 \cdot 110$ & - & $-26 \cdot 0-25 \cdot 5$ & - & - & Calm & 3 & 0 & b & - & - & - \\
\hline 111 & $29 \cdot 110$ & - & $-33 \cdot 0-21 \cdot 0$ & - & - & Calm & 1 & 0 & 1) & - & - & - \\
\hline Miclt. & $29 \cdot 143$ & - & $-36 \cdot 0-35 \cdot 0$ & - & - & Calm & 0 & 0 & wW & - & - & - \\
\hline Mean & $29 \cdot 157$ & - & $--19 \cdot 4$ & - & - & - & - & - & - & - & - & - \\
\hline
\end{tabular}

p.m. Olserrea very large meteor or starting al of $-y^{\circ}$ and al yellow colour $10^{\circ}$ of alt.

6 p.n. Frost sprays fornulug oul anemo meter glass.

9 p.m. Nins. at, hlygh-level station since 48 liours, $-30^{\circ} 8$; teinl , at 9 p.m., $-25^{\circ} \cdot 0$, lireluns smoke going $\mathrm{NE}$, witl ilist thet red glure on lower part of smoke. Ine. clear day. W'nd froshenlug froun s. until nown and then falling to calme: max. run of enps for 2 lours, 48 milles; max. rate ohserveyl by pressure tunte, 31 Temperature fell and was unsteady. 
Meteorologicat Journat of thie "I Discoverty."

[1902-June.

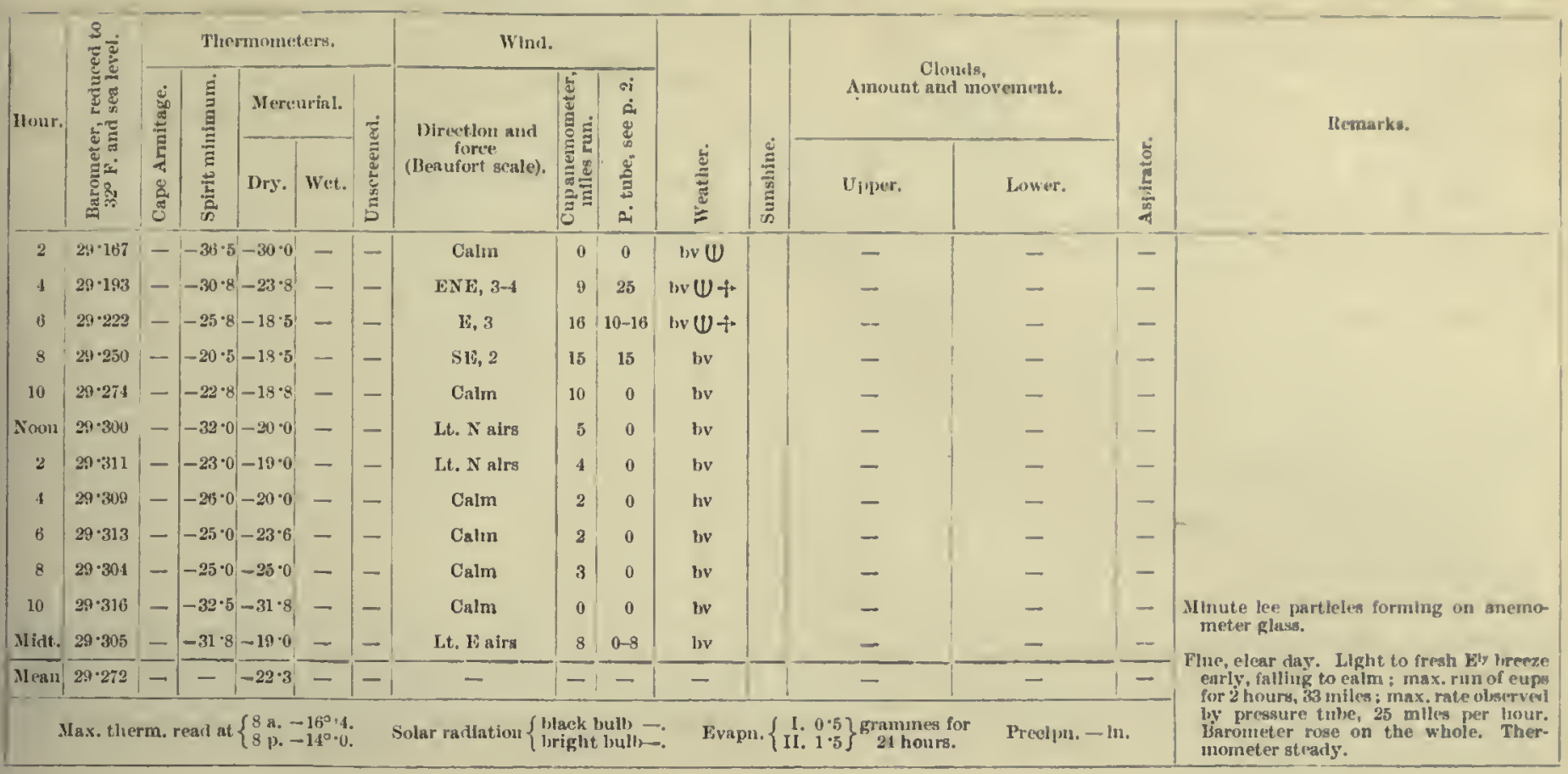

22nd.

\begin{tabular}{|c|c|c|c|c|c|c|c|c|c|c|c|c|c|}
\hline 2 & $29 \cdot 287$ & -1 & $|-23 \cdot 0|-18 \cdot 8 \mid$ & - & $1-$ & E, 1 & 7 & $5-10$ & bv & - & - & - & \multirow{14}{*}{ 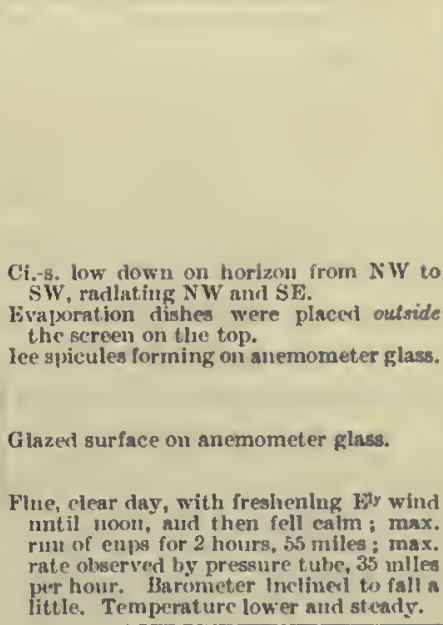 } \\
\hline 4 & $29 \cdot 265$ & - & $-27 \cdot 0-22 \cdot 0$ & - & - & Calm & 4 & 0 & bv & - & - & - & \\
\hline 6 & $29 \cdot 233$ & - & $-26 \cdot 0-20 \cdot 0$ & - & - & E, 1-2 & 4 & $10-15$ & bv & - & - & - & \\
\hline 8 & $29 \cdot 181$ & - & $-27 \cdot 0-26 \cdot 0$ & - & - & $\mathrm{NE}, 5$ & 25 & $30-35$ & $b+$ & - & - & - & \\
\hline 10 & $29 \cdot 156$ & - & $-28 \cdot 0-26 \cdot 0$ & - & - & E by $N, 3-5$ & 28 & $25-30$ & $b+$ & - & - & - & \\
\hline Noon & $29 \cdot 143$ & - & $-27 \cdot 6 \cdot-25 \cdot 8$ & - & - & NE, 4-5 & 21 & 28-33 & $b+$ & - & - & - & \\
\hline 2 & $29 \cdot 121$ & - & $-27 \cdot 0-23 \cdot 2$ & - & - & Lt. NE airs & 18 & $0-8$ & b & - & - & - & \\
\hline 4 & $29 \cdot 097$ & - & $-25 \cdot 0-20^{\circ} 0$ & - & - & Lt. NE airs & 7 & $a-5$ & Is & - & - & - & \\
\hline 0 & $29 \cdot 055$ & -- & $-33 \cdot 0-29 \cdot 2$ & - & - & Calm & 7 & 0 & bv & - & - & - & \\
\hline 8 & $29^{\circ} 021$ & - & $-40 \cdot 0-33 \cdot 0$ & - & - & Calm & 0 & 0 & bv & - & - & - & \\
\hline 10 & $28 \cdot 987$ & - & $-35 \cdot 2-30 \cdot 8$ & - & - & Calm & 4 & 0 & IN & - & - & - & \\
\hline Midt. & $28 \cdot 943$ & - & $-31 \cdot 8-27 \cdot 8$ & - & - & Lt. NE airs & 4 & $0-8$ & b & - & - & - & \\
\hline$\sqrt{M e \text { en }}$ & $29 \cdot \overline{121}$ & $\overline{-}$ & $--25 \cdot 2$ & - & $\overline{-}$ & - & $\overline{1}$ & - & - & - & - & - & \\
\hline rea & $\begin{array}{l}\text { therm. } \\
\text { arl at }\end{array}$ & & $\begin{array}{l}-18^{\circ} \cdot 0 . \\
-10^{\circ} \cdot 0 . \\
\mathrm{rad}\end{array}$ & tion & & - Ev & $\{1\}$ & 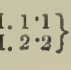 & & $A_{1}$ & & - In. & \\
\hline
\end{tabular}

$23 r d$.

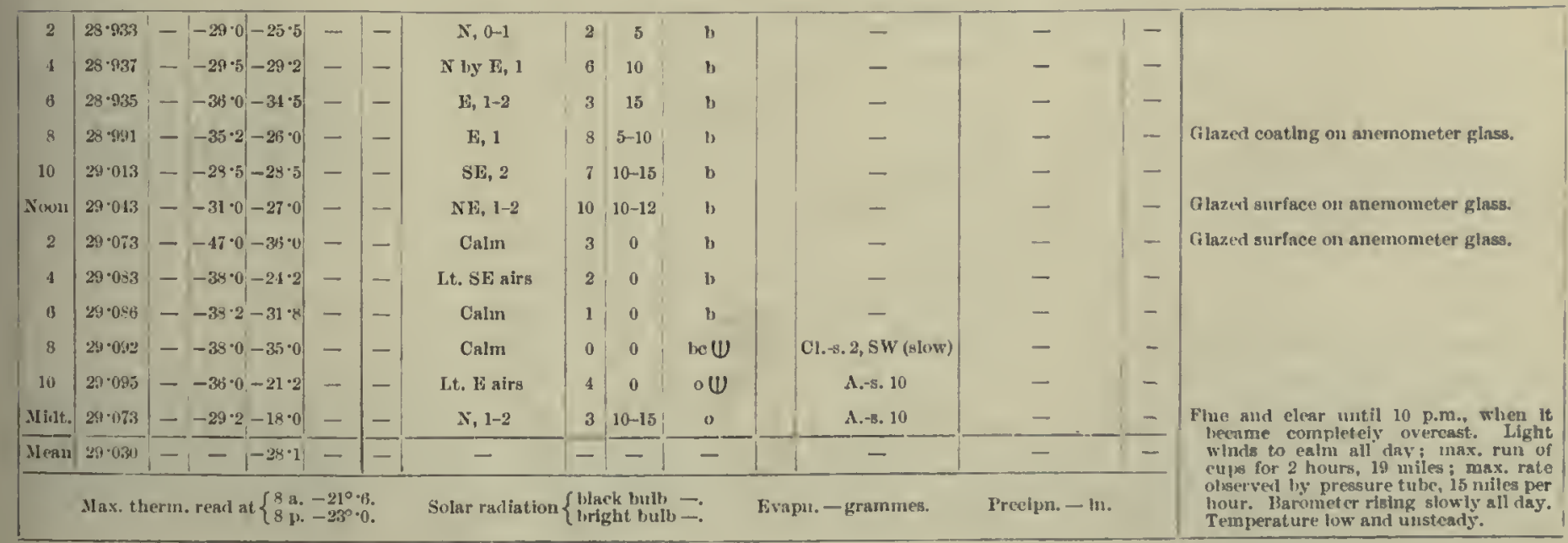


25 th.

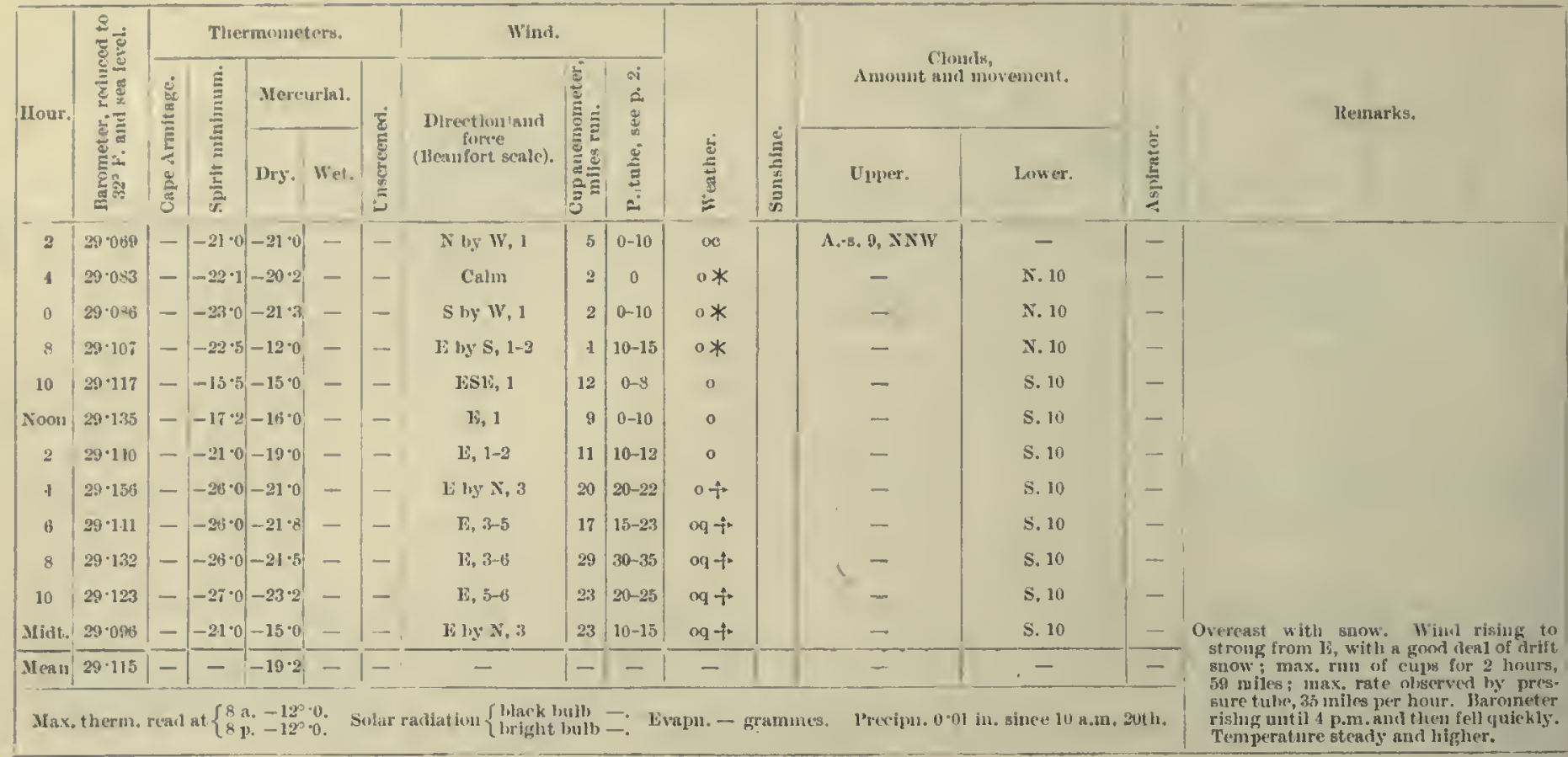

26 th.

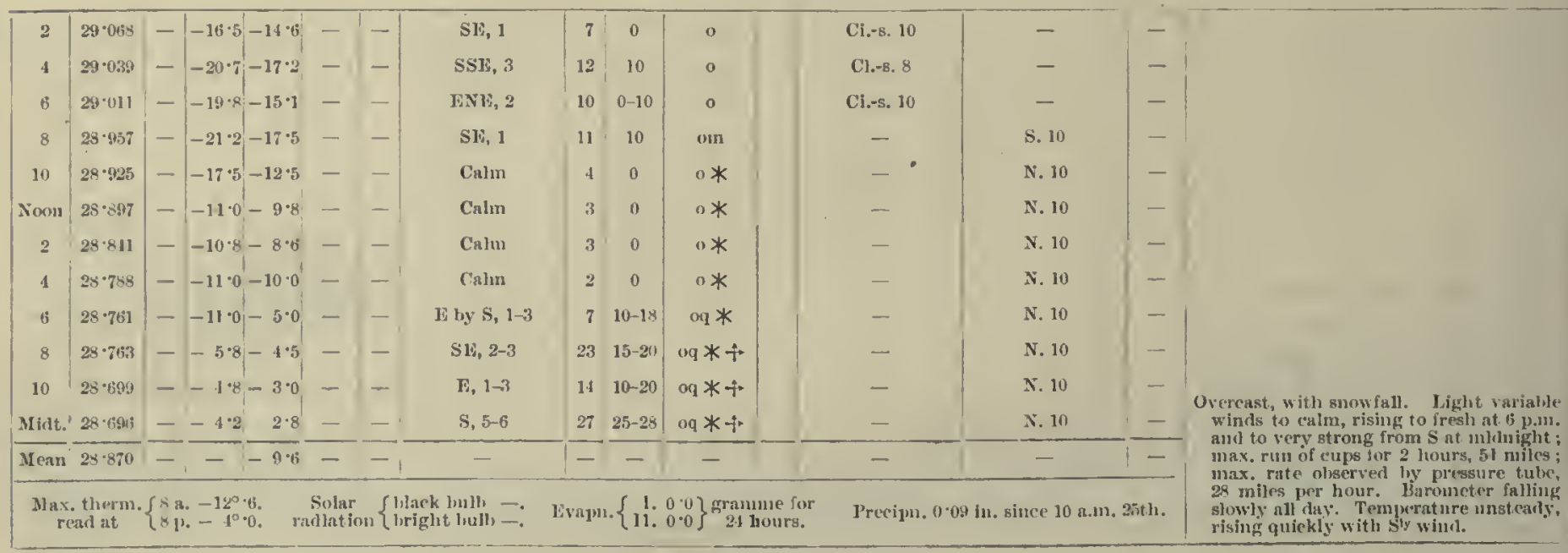

27th.

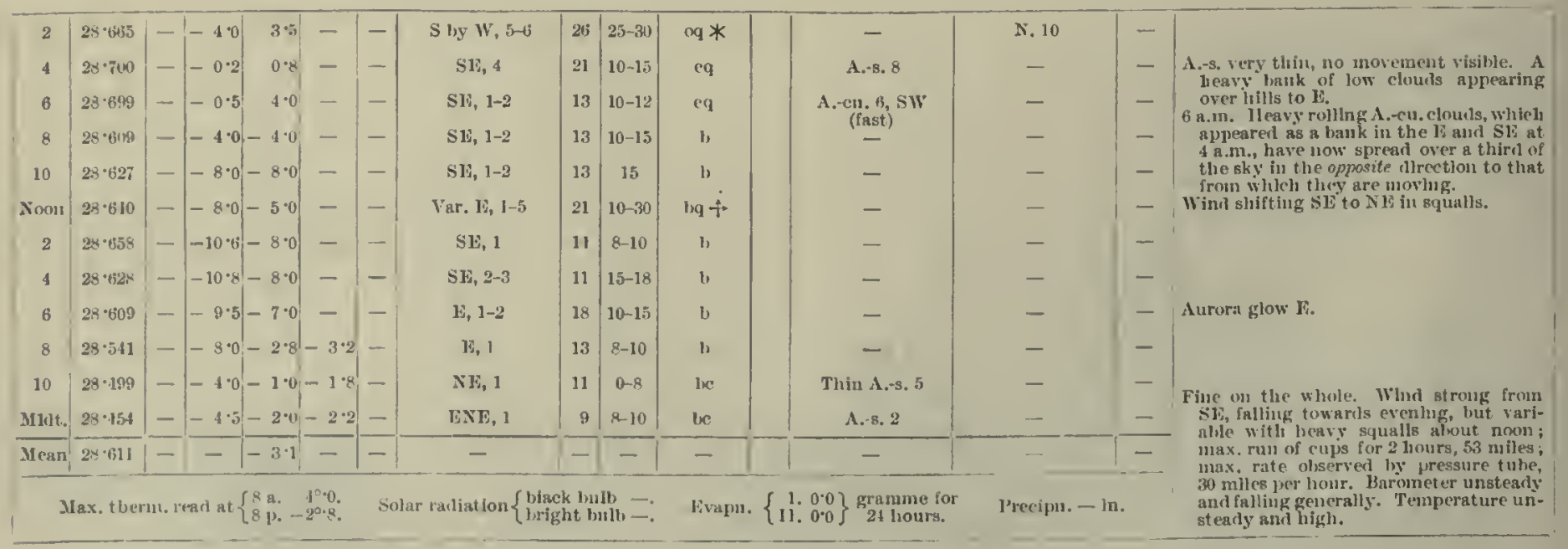


Meteorological Journal, of tII: " Discovi:ky."

[1902-June.

\begin{tabular}{|c|c|c|c|c|c|c|c|c|c|c|c|c|c|c|c|}
\hline \multirow{3}{*}{ Gour. } & \multirow{3}{*}{ 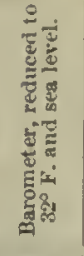 } & \multicolumn{5}{|c|}{ Thermomet ers. } & \multicolumn{3}{|l|}{ Wlund. } & \multirow[b]{3}{*}{ 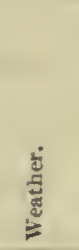 } & \multirow[b]{3}{*}{ 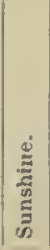 } & \multirow{2}{*}{\multicolumn{2}{|c|}{$\begin{array}{l}\text { Clourls, } \\
\text { Amount and movement. }\end{array}$}} & \multirow[b]{3}{*}{$\mid$} & \multirow{3}{*}{ Memarhs. } \\
\hline & & \multirow{2}{*}{ 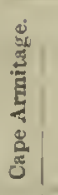 } & \multirow{2}{*}{ 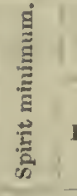 } & \multicolumn{2}{|c|}{ Mercurial. } & \multirow{2}{*}{ 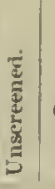 } & \multirow{2}{*}{$\begin{array}{l}\text { Dircet fon anul } \\
\text { forre } \\
\text { (Heatifort segle). }\end{array}$} & \multirow{2}{*}{ 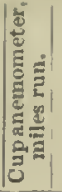 } & \multirow{2}{*}{ 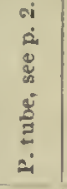 } & & & & & & \\
\hline & & & & Dry. & Wet. & & & & & & & Upiper. & Lower. & & \\
\hline 2 & $28 \cdot 439$ & - & $-4 \cdot 5-$ & $-1 \cdot 9$ & $-1 \cdot$ & - & is by $S, 1-2$ & 2 & $10-12$ & $\infty$ & & A. $\rightarrow$ s. 111 & - & -1 & \\
\hline 4 & $28: 357$ & - & $-2 \cdot 5$ & $3 \cdot 0$ & $3 \cdot 0$ & - & N1S, 1-3 & 11) & $12-15$ & we & & A.ะ8. $7, \mathrm{NE}$ & - & - & \\
\hline 6 & $28 \cdot 2918$ & - & $0 \cdot 5$ & $4 \cdot 1$ & $1 \cdot 2$ & - & Calm & 8 & 0 & $\infty$ & & A.-s. 10 & - & - & \\
\hline 8 & $28 \cdot 2 \cdot 19$ & - & $-0 \cdot 6$ & $4 \cdot 5$ & - & - & Calun & 2 & 0 & $\propto *$ & & A.-s. 10 & - & - & \\
\hline 10 & $28 \cdot 1: 2$ & - & $3 \cdot 0$ & 6.0 & tio & - & I.t. Nlíars & 3 & 0 & o & & A.8. 10 & - & - & Clerened fustramerus and hoxes, \\
\hline Noon & $28 \cdot 1 \cdot 10$ & - & $4: 5$ & $5 \cdot 2$ & $5 \cdot(1)$ & - & Caim & 7 & 0 & 0 & & A.s. 10 & - & - & 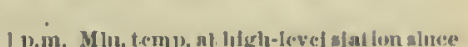 \\
\hline $\begin{array}{l}2 \\
4\end{array}$ & $\begin{array}{l}28 \cdot 2005 \\
28 \cdot 197\end{array}$ & - & $\begin{array}{l}3.5 \\
5.5\end{array}$ & $\begin{array}{cc}7 \cdot 6 \\
9 \cdot 0\end{array}$ & $\begin{array}{l}6 \cdot 2 \\
8 \cdot 2\end{array}$ & - & $\begin{array}{l}\text { Calm } \\
\text { Calm }\end{array}$ & 2 & 0 & 0 & & A.-×. 10 & - & - & 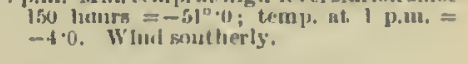 \\
\hline "s & $28 \cdot 282$ & - & $8 \cdot 0$ & 8.5 & $8 \cdot 2$ & - & $\begin{array}{ll}\text { Calm } \\
\text { S, 1-2 }\end{array}$ & $\begin{array}{c}2 \\
13\end{array}$ & $\begin{array}{c}0 \\
10-12\end{array}$ & 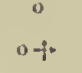 & & $\begin{array}{c}\text { A.-B, } 10 \\
-\end{array}$ & s. 11 & $\begin{array}{l}- \\
-\end{array}$ & $\begin{array}{l}\text { 4. } 20 \text { p.w. Whal rose frous } s \text { in hesvy } \\
\text { gusts. }\end{array}$ \\
\hline 8 & $28 \cdot 406 i$ & - & $0 \cdot 0$ & $0 \cdot 0$ & 0.0 & - & S, 3-5 & 29 & $15-25$ & oq + & & -. & S. 10 & $-\cdots$ & \\
\hline 10 & $28 \cdot 558$ & - & $-3 \cdot 0-$ & $-3 \cdot 0$ & $-2 \cdot v$ & - & SW, 1-3 & 16 & 10-15 & $o q-$ & & - & S. 10 & - & \\
\hline Minilt. & $28 \cdot 632$ & - & $-3 \cdot 9-$ & $-2 \cdot 0-$ & $-2 \cdot 0$ & - & SW by S, 0-1 & 15 & 0 & o * * & & $\because$ & S. 10 & -1 & l'ufs of wlal from S, fine snow falling. \\
\hline Menu & $2 x-3330$ & - & - & $3: 5$ & - & -1 & - & $1-$ & - & - & & - & - & & $\begin{array}{l}\text { Overeast and clondy with some smow, } \\
\text { WInd went to s' ait gralually thew wip }\end{array}$ \\
\hline & ax. Hoest & a. rear & $4 \mathrm{at}\left\{\begin{array}{l}8 \\
\mathrm{y}\end{array}\right.$ & 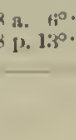 & & Solar & ir ralliation $\left\{\begin{array}{l}\text { hag } \\
\text { lurit }\end{array}\right.$ & linlls, & in $=$. & Livapm & & $\begin{array}{l}\text { gramum for } \\
\text { 24 hours. }\end{array}$ & Precipu, - in & & 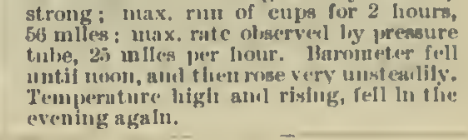 \\
\hline
\end{tabular}

28 th.

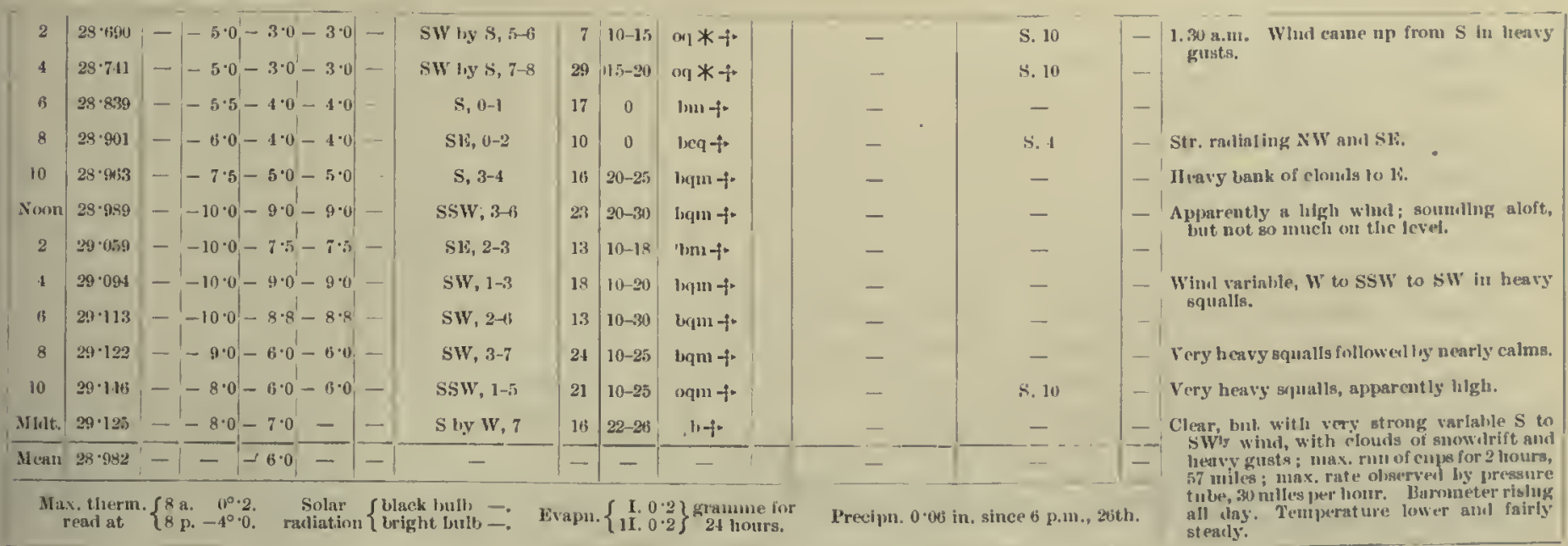

29 th.

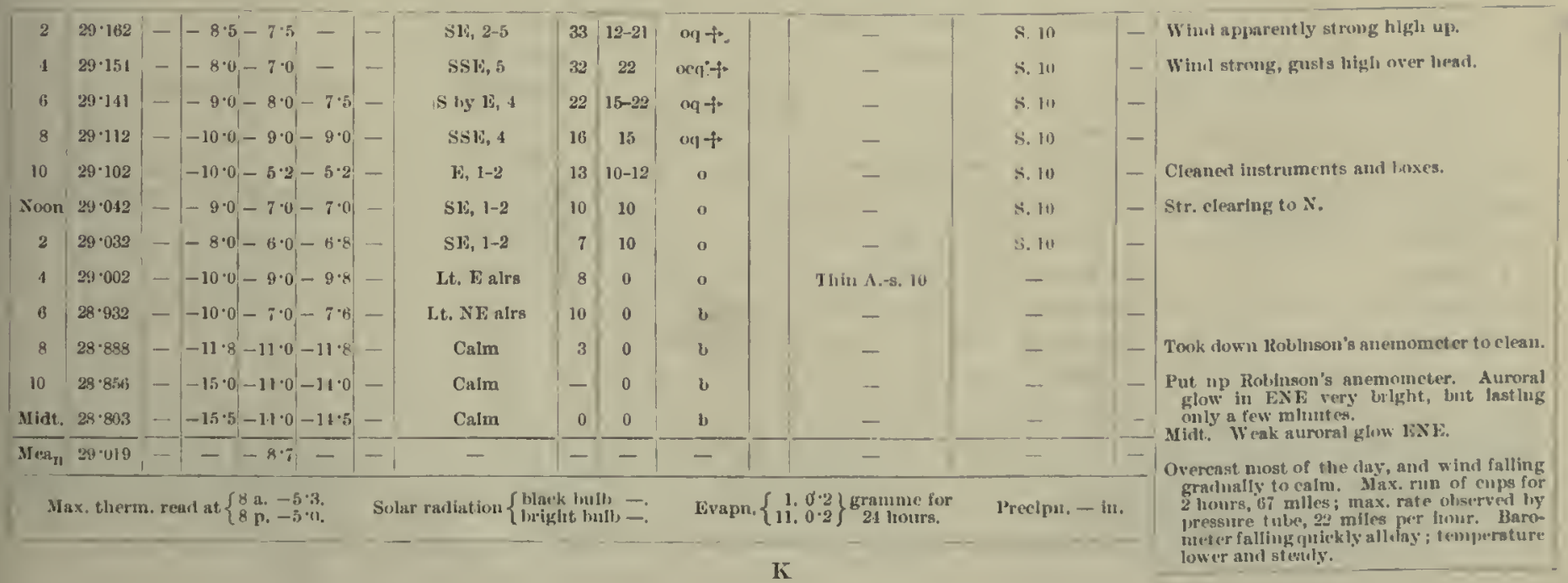

30 th. 


\begin{tabular}{|c|c|c|c|c|c|c|c|c|}
\hline${ }_{\tau}^{S}$ & & & rmome & ters. & & Wind. & & \\
\hline zั & s. & 音 & Mere & urial. & & & & ¿i \\
\hline 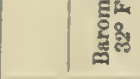 & 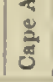 & $\frac{\vec{L}}{\frac{5}{a}}$ & Dry. & wet. & है & (Beaulort & हैं & ن. \\
\hline
\end{tabular}

1st.

\begin{tabular}{|c|c|c|c|c|c|}
\hline 2 & $28 \cdot 759$ & - & $|-19 \cdot 6|-15 \cdot 0 \mid$ & - & - \\
\hline & $28 \cdot 722$ & - & $|-17 \cdot 4|-13 \cdot 0$ & - & - \\
\hline & $28 \cdot 729$ & - & $\mid-22 \cdot-4-22 \cdot 0$ & - & - \\
\hline 8 & 28.714 & - & $-2 x \cdot 6-23 \cdot 0$ & - & \\
\hline 10 & $28 \cdot 714$ & - & -8.0 & - & - \\
\hline on & $28 \cdot 709$ & - & & $-3 \cdot 0$ & 1. \\
\hline 2 & $28 \cdot 709$ & - & 10 & -1.8 & 1 \\
\hline 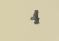 & $28 \cdot \pi 33$ & - & $5 \cdot 0$ & - & - \\
\hline 6 & $28 \cdot 746^{\circ}$ & - & $1 \cdot 0$ & - & \\
\hline$\xi$ & $28 \cdot 792$ & - & $-4 \cdot 0$ & - & - \\
\hline 10 & $28 \cdot 808$ & - & $-5 \cdot 8$ & $-6 \cdot 0$ & - \\
\hline & $28 \cdot 756$ & - & $-7 \cdot 8-4 \cdot 5$ & - & - \\
\hline & $8 \cdot 741$ & - & $-8 \cdot 6$ & - & 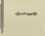 \\
\hline
\end{tabular}

\begin{tabular}{|c|c|c|}
\hline Calm & 0 & 0 \\
Lt. $\mathrm{N}$ uy $\mathrm{E}$ alrs & 0 & 0 \\
Calm & 0 & 0 \\
Calm & 0 & 0 \\
Calm & 0 & 0 \\
L.t. $N$ alrs & 6 & $0-5$ \\
L.t. $N$ alrs & 4 & $0-5$ \\
Calm & 4 & 0 \\
Calm & 4 & 0 \\
I.t. SE alrs & 4 & 0 \\
Lt. $\mathbb{E}$ alrs & 7 & 0 \\
IS by N, $1-2$ & 9 & $0-8$ \\
\hline - & - & - \\
\hline
\end{tabular}

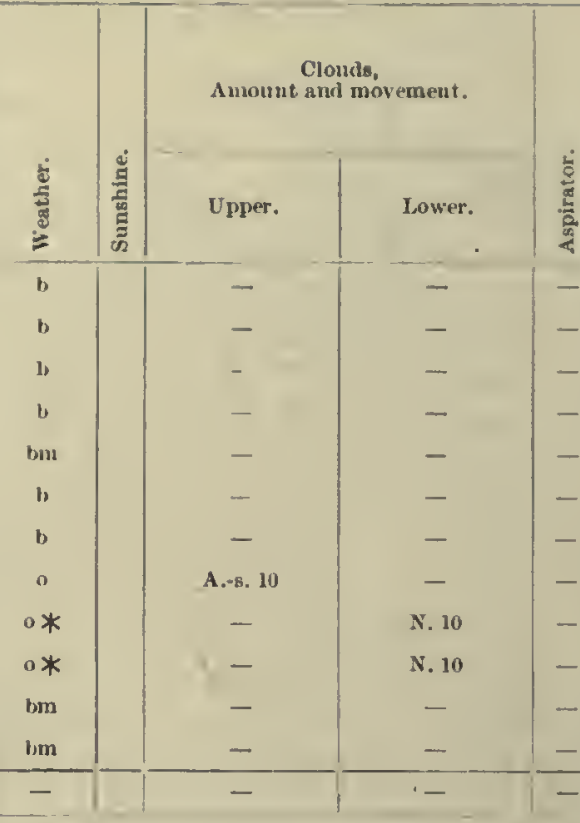

Max, thermu. read at $\left\{\begin{array}{l}8 \mathrm{a} .-10^{\circ} \circ \\ 8 \mathrm{p} .\end{array} 1^{\circ} 0\right.$.

Solar radlation $\left\{\begin{array}{l}\text { black bulb }- \text {. } \\ \text { briglit lunlb }- \text {. }\end{array}\right.$

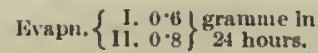

Precipn. - in.

IRemarka.
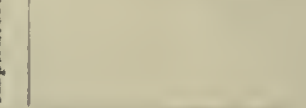

Anrora curtaln with streamers 1 WNE in Jistlnct double aurora eurtain ln SSl Stresuers to $40^{\circ}$ alt. Falnt anrora glow $\mathbb{N}$ to N $\mathrm{k}$.

Faint auroral areh $\mathrm{NE}$, NSE; alt. $30^{\circ}$.

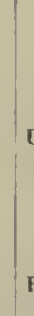

Fine day on the whole, with a little snow iall. Wind, ealu w light variable airs; max. run of clups for 2 hours, 18 mitles; max, rate olsersed by pressire tulue, 8 miles per hour. Barometer low sur stealy, inellued to rise. Tomperature ruse quickly and remalned ligh and
steady.

2nd.

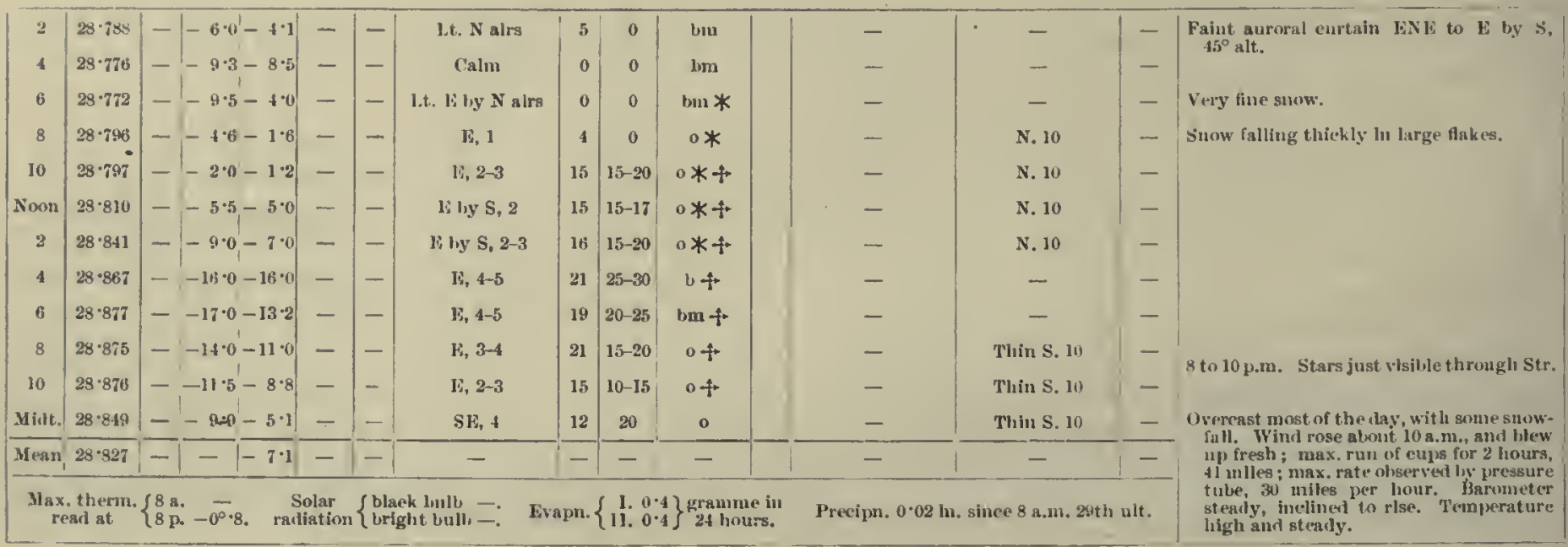

$3 r d$,

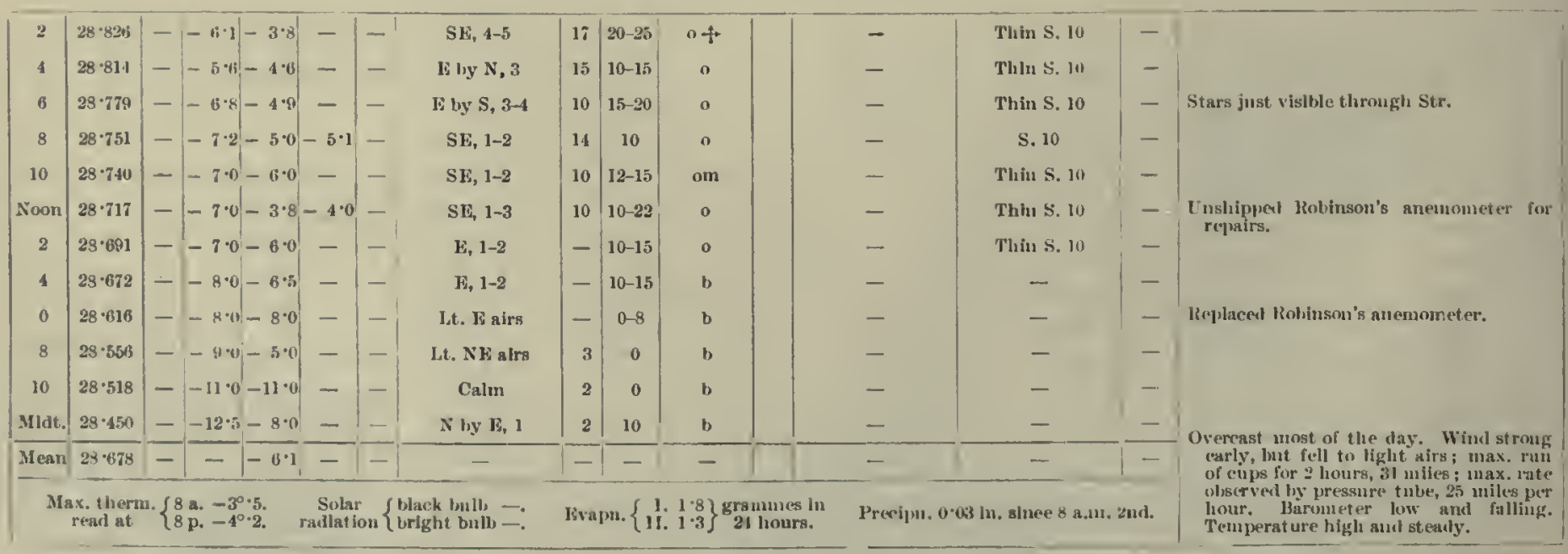


Mhtwokological Journat, of tile: "Discovfiry."

$[1902-J u l y$.

\begin{tabular}{|c|c|c|c|c|c|c|c|c|c|c|c|c|c|c|c|}
\hline \multirow{3}{*}{ IIour. } & \multirow{3}{*}{ 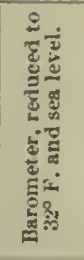 } & \multicolumn{5}{|c|}{ Thermomelers. } & \multicolumn{3}{|l|}{ Whind. } & \multirow[b]{3}{*}{ 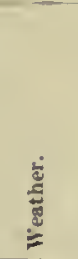 } & \multirow{3}{*}{ 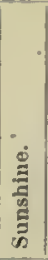 } & \multirow{2}{*}{\multicolumn{2}{|c|}{$\begin{array}{l}\text { Clours, } \\
\text { Amount and movernent. }\end{array}$}} & \multirow[b]{3}{*}{$\begin{array}{l}\text { के } \\
\frac{3}{2} \\
\frac{2}{2}\end{array}$} & \multirow{3}{*}{ lemurks. } \\
\hline & & \multirow{2}{*}{ 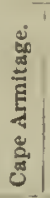 } & \multirow{2}{*}{ 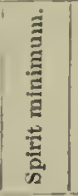 } & \multicolumn{2}{|c|}{ Mereurial. } & \multirow{2}{*}{ 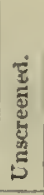 } & \multirow{2}{*}{$\begin{array}{l}\text { Direction and } \\
\text { force } \\
\text { (Beatufort scale). }\end{array}$} & \multirow{2}{*}{ 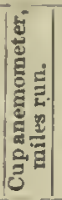 } & \multirow{2}{*}{ 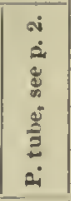 } & & & & & & \\
\hline & & & & Dry. & Wet. & & & & & & & Upper. & Lower. & & \\
\hline 2 & $25 \cdot 401$ & - & $-11 \cdot 5$ & $-7 \cdot 0$ & - & - & $\mathbf{N}, 0-1$ & 7 & 8 & h & & - & - & -1 & Aurnera patches lis and Nis. \\
\hline 4 & $28 \cdot 331$ & - & $-12 \cdot 0$ & $-9 \cdot 2$ & - & - & NNE, 0-I & 0 & 3 & b & & - & - & - & Bands of otrenun+rs $\mathbf{E}$ and $\mathrm{Nl}$ \%, rarlatiug \\
\hline bi & $28 \cdot 296$ & - & $-12 \cdot 5$ & $-9 \cdot 0$ & - & - & Calm & 2 & 0 & b & & - & - & - & $\begin{array}{l}\text { Arch baind (Interrinptel) of streamers SW to } \\
\text { NN k. }\end{array}$ \\
\hline 8 & $28: 304$ & - & $-11 \cdot 1$ & $-8 \cdot 0$ & - & - & Lt. N li airs & 1 & 5 & b & & - & - & - & Falut anch of vertleal leams from $S$ to $\mathrm{K}$. \\
\hline 10 & $28 \cdot 338$ & - & $-24 \cdot 0$ & $-11 \cdot 0$ & - & - & NE, 1 & 10 & $10-12$ & " & & - & - & - & \\
\hline Neon & $28: 343$ & - & $-14 \cdot 6$ & $-9 \cdot 0$ & - & - & $\mathbf{k}, 1$ & 12 & 10 & b & & - & - & - & Aurora streamer in zpulth showlug falut \\
\hline 2 & $28 \cdot 353$ & - & $-10 \cdot 2$ & $-6 \cdot 0$ & - & - & Lt. SW aira & $?$ & 0 & b & & - & - & - & $\begin{array}{l}\text { level station sluce } 13 \text { days }=-22.3 \text {. } \\
\text { lirelua smoke golng to the } \mathrm{N} \text {. }\end{array}$ \\
\hline 4 & $2 x \cdot 291$ & - & $-11 \cdot 6$ & $-11 \cdot 0$ & - & - & $N \mathrm{E}, \mathrm{I}$ & $\boldsymbol{7}$ & 0 & b & & - & - & - & \\
\hline b & $28 \cdot 257$ & - & $-13 \cdot 0$ & $-7 \cdot 0$ & - & - & Lt. NE airs & 7 & 0 & o & & - & N. 10 & - & \\
\hline 8 & $28 \cdot 226$ & - & $-12 \cdot 8$ & $-12 \cdot 0$ & - & - & Calm & 5 & 0 & ตทแ $*$ & & - & - & - & Stars vlsible lut mlsty. Big hakes of tiry \\
\hline 10 & $28 \cdot 17 \pi$ & - & $-12 \cdot 5$ & $-7 \cdot 0$ & - & - & Lt. $\mathbf{N}$ airs & 2 & 0 & b & & - & - & - & \\
\hline Mrialt. & $28 \cdot 167$ & - & $-8 \cdot 2$ & $-6 \cdot 0$ & - & - & $\mathrm{N} \mathbf{1 B}, 1$ & i & 10 & b) & & - & - & - & Falnt arch In SSl:. \\
\hline Mean & $28 \cdot 291$ & - & -1 & $-8 \cdot 8$ & - & -1 & - & -1 & - & - & & - & - & - & 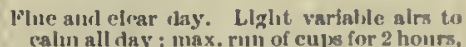 \\
\hline & $\mathrm{x}, \mathrm{th}$ & $r$ & at & $8 \mathrm{a},-5$ & & & radiation $\left\{\begin{array}{l}\mathrm{b} \\
\mathrm{br}\end{array}\right.$ & In & - & $1 \% \mathrm{v}$ & & $\left.\begin{array}{l}8 \\
9\end{array}\right\} \begin{array}{l}\text { gramme in } \\
24 \text { hours. }\end{array}$ & Precipn. - i & & $\begin{array}{l}12 \text { mlles ; inax. rate ohsorvesl hy preasure } \\
\text { unbe, } 12 \text { milus per hour. Baromner low } \\
\text { and falling; temperature high and st ewly. }\end{array}$ \\
\hline
\end{tabular}

4th.

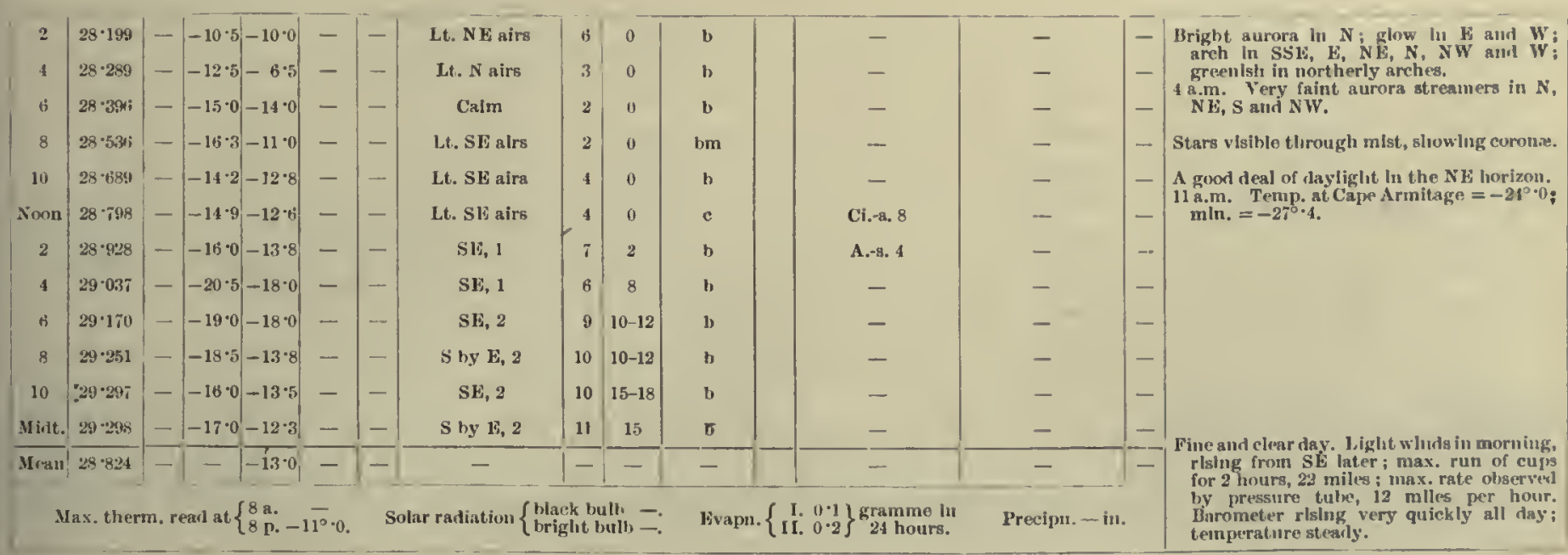

5th.

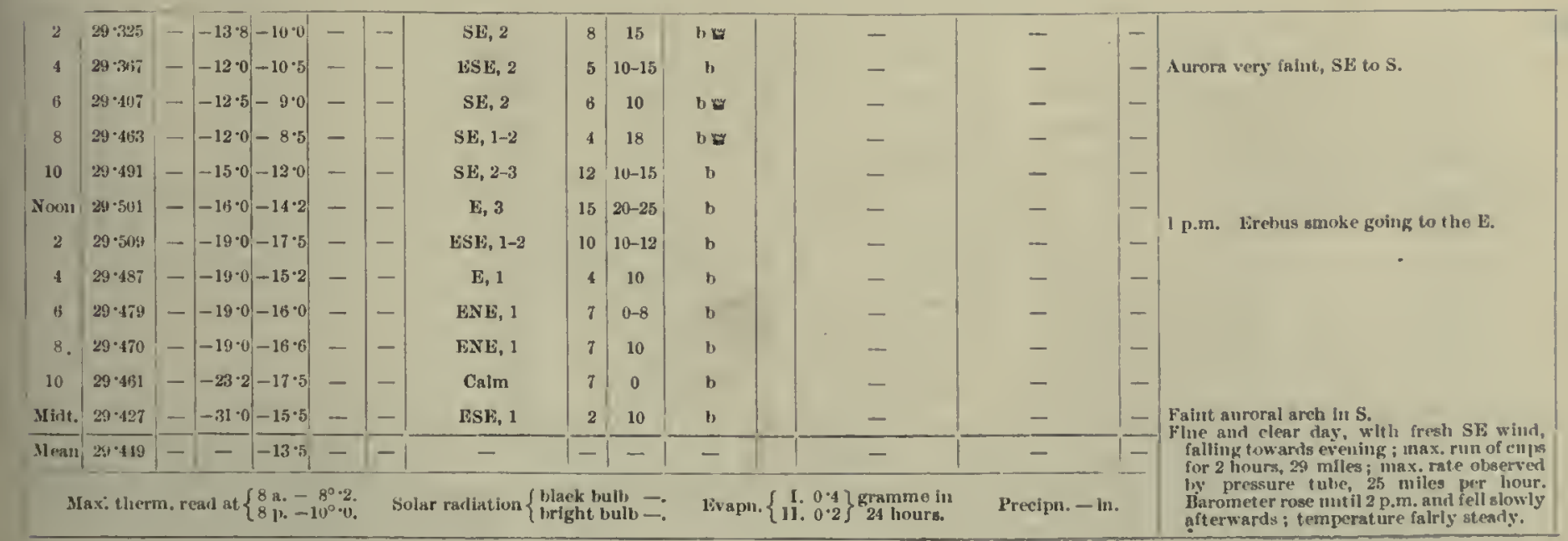

6th. 
$1902-J u l y$.

Metworological Jouknal of the "Discovfry."

\begin{tabular}{|c|c|c|c|c|c|c|c|c|c|c|c|c|}
\hline \multirow[b]{3}{*}{ है } & \multicolumn{5}{|c|}{ Thermometers. } & \multicolumn{3}{|l|}{ Wint. } & \multirow[b]{3}{*}{ 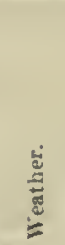 } & \multirow[b]{3}{*}{ 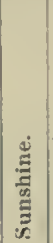 } & \multirow{2}{*}{\multicolumn{2}{|c|}{$\begin{array}{l}\text { Clonds, } \\
\text { Amomt and novement. }\end{array}$}} \\
\hline & \multirow{2}{*}{ 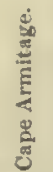 } & \multirow{2}{*}{ 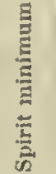 } & \multicolumn{2}{|c|}{ Merenrlal. } & \multirow{2}{*}{ 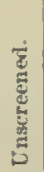 } & \multirow{2}{*}{$\begin{array}{c}\text { Directiou surd } \\
\text { force } \\
\text { (Benufort scale). }\end{array}$} & \multirow{2}{*}{ 爮 } & \multirow{2}{*}{ 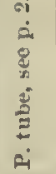 } & & & & \\
\hline & & & Dry. & $\mathrm{Wet}$. & & & & & & & pper. & ver. \\
\hline
\end{tabular}

7 th.

\begin{tabular}{|c|c|c|c|c|c|c|c|c|c|c|c|c|c|}
\hline 2 & $29 \cdot 442$ & - & $|-20 \cdot 0|-1 B \cdot 2 \mid$ & - & -1 & lin $\mathrm{S}, 1$ & 4 & 10 & li & - & - & - & Faiut auroral heans in NE. \\
\hline 4 & $29 \cdot 123$ & - & $-20 \cdot 0-13 \cdot 8$ & - & - & Ding $N, 1-2$ & 4 & $10-15$ & $\mathrm{bm}=$ & - & - & - & \\
\hline 6 & $29 \cdot 397$ & - & $-30 \cdot 5|-1: 5|$ & - & - & Lt, $S$ airs & 6 & $0-10$ & $\cdot b$ & - & - & - & Faiut auroral curtain lisle to $\mathrm{S}$. \\
\hline 8 & $29 \cdot 3: 77$ & - & $-2 \pi \cdot 0 \mid-24 \cdot 0$ & - & - & Calm & 0 & 0 & b) & - & - & - & Falnt anroral arcl in $\mathrm{E}$ and SE to $30^{\circ}$ alt. \\
\hline 10 & $29 \cdot 371$ & - & $-21 \cdot 8 \mid-19 \cdot 0$ & - & - & Caln & 0 & 0 & b) & - & - & - & Took down Rothinson's anemomet er. \\
\hline$x(0) 1$ & $29 \cdot 316$ & $-31 \cdot 2$ & $-20 \cdot 0|-15 \cdot 6|$ & - & - & Calra & - & 0 & b & - & - & - & Nlm. temp. at Cape Armitage since (?) \\
\hline 2 & $29 \cdot 313$ & - & $|-23 \cdot 2|-23 \cdot 2 \mid$ & - & - & $\mathrm{Ca} \mathbf{m}$ & - & 0 & b) & - & - & - & 1 p.m. \\
\hline 4 & $29 \cdot 317$ & - & $-23 \cdot 8 \mid-13 \cdot 8$ & - & - & 15,1 & - & 10 & b) & - & - & - & Wrelous sinoke guing $\mathrm{NE}$. \\
\hline 6 & $29 \cdot 277$ & - & $-17 \cdot 2 \mid-17 \cdot 2$ & - & - & E, 1-2 & 12 & 10 & " & - & - & - & $\begin{array}{l}4 \text { p.m. Put up Jotminson s ancmometer } \\
\text { in place. }\end{array}$ \\
\hline 8 & $20 \cdot 215$ & - & $-21 \cdot 0 \mid-14 \cdot 5$ & -. & - & $\mathrm{E}, 1-2$ & 15 & 15 & b & - & - & - & 6 p.n. Very lorlght aurora, glare in SE. \\
\hline 10 & $29 \cdot 234$ & - & $-17 \cdot 0|-11 \cdot 6|$ & - & - & Lt. $\mathbf{E}$ airs & 10 & $5-10$ & $b=$ & - & - & - & \multirow{3}{*}{ 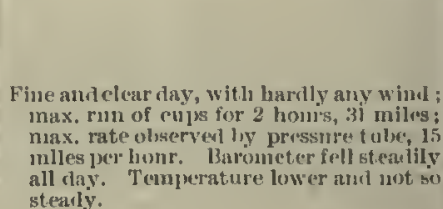 } \\
\hline Midt. & $29 \cdot 218$ & - & $-32 \cdot 0 \mid-19 \cdot 0$ & - & -1 & Calm & 4 & 0 & b & - & - & - & \\
\hline Ienn & $29 \cdot 334$ & - & $--17 \cdot 3$ & - & -1 & - & $\overline{-1}$ & -- & $\overline{-}$ & - & - & - & \\
\hline
\end{tabular}

8th.

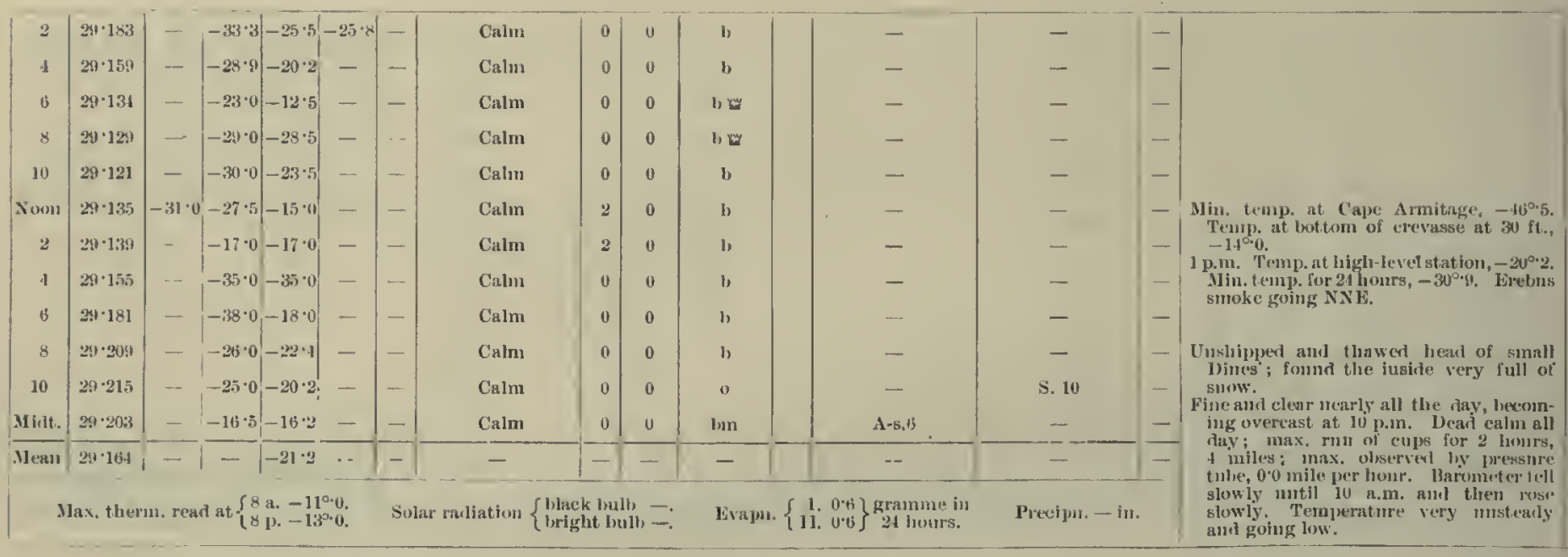

9th.

\begin{tabular}{|c|c|c|c|c|c|c|c|c|c|}
\hline 2 & $29 \cdot 1 \times 3$ & - & $|-16 \cdot 5|$ & $4 \cdot 9$ & $4: 9$ & - & $S$ l,y $\mathrm{E}, 0-2$ & 4 & $10 \cdot 1.5$ \\
\hline 4 & $29 \cdot 2066$ & - & 1.2 & 3.0 & $2 * i$ & -1 & $\mathrm{E}, 0-1$ & 7 & $10-12$ \\
\hline 6 & $29 \cdot 231$ & - & 0.5 & $2 \cdot 0$ & 1.8 & - & $\mathrm{E}, 1-3$ & 1.1 & 21-27 \\
\hline 8 & $29 \cdot 261$ & - & -0.1 & $3 \cdot 0$ & $3 \cdot 0$ & - & SE, 2-3 & 15 & 25 \\
\hline 10 & $29 \cdot 2 \cdot 15$ & - & 0.0 & $2 \cdot 5$ & $2 \cdot 3$ & - & SE, 1-3 & 18 & $15-23$ \\
\hline Noon & $29 \cdot 213$ & - & $2 \cdot 0$ & $4 \cdot 0$ & $3 \cdot 2$ & - & E by S, 1-2 & 12 & 15 \\
\hline 2 & $29 \cdot 150$ & - & $1 \cdot 0$ & $1 \cdot 8$ & $1 \cdot 0$ & - & $3,1-3$ & 12 & $10-20$ \\
\hline 4 & $29 \cdot 156$ & · & $1 \cdot 0$ & $3 \cdot 0$ & $2 \cdot 0$ & - & Var. $\mathbb{E}, 0-3$ & 13 & $0-20$ \\
\hline B & $29 \cdot 135$ & - & $1 \cdot 8$ & $3 \cdot 0$ & $2 \cdot 0$ & - & $1 \mathrm{lis} 1 \mathrm{~B}, 1-2$ & 8 & $10-14$ \\
\hline 8 & $2: 0 \leq 0$ & - & 0.0 & $2 \cdot 2$ & $2 \cdot 0$ & - & ENE, 0-1 & 5 & $10-12$ \\
\hline 10 & $29 \cdot 029$ & - & $-3 \cdot 0$ & $3 \cdot 0$ & $2 \cdot 0$ & - & Lt. W airs & 2 & 0 \\
\hline Mhat. | & $28 \cdot 4,186$ & - & -1.5 & -0.5 & -0.3 & -1 & Calın & 1 & 0 \\
\hline in & $29 \cdot 159$ & - & - & $2 \cdot 7$ & $2 \cdot 1$ & -1 & - & - & - \\
\hline
\end{tabular}

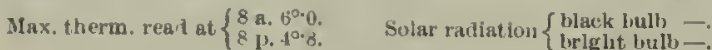

\section{Evaps. $\left\{\begin{array}{l}\text { I. } 4 \cdot \not \\ 11.4 \\ 4 \cdot 3\end{array}\right\}$ grammes in 24 lrours.}

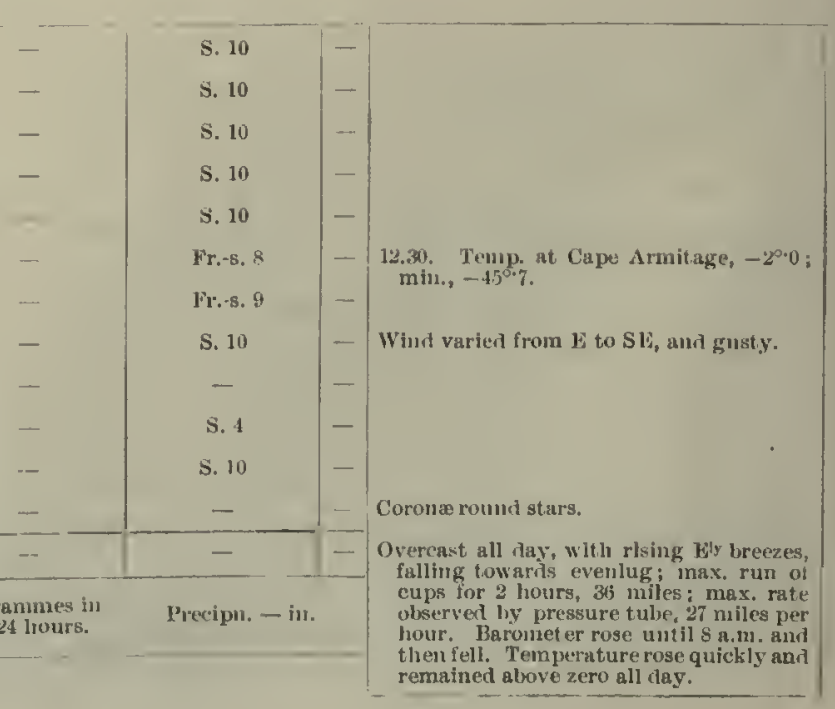


Mhteorologicat Journal of the "Discovini."

[1902-July.

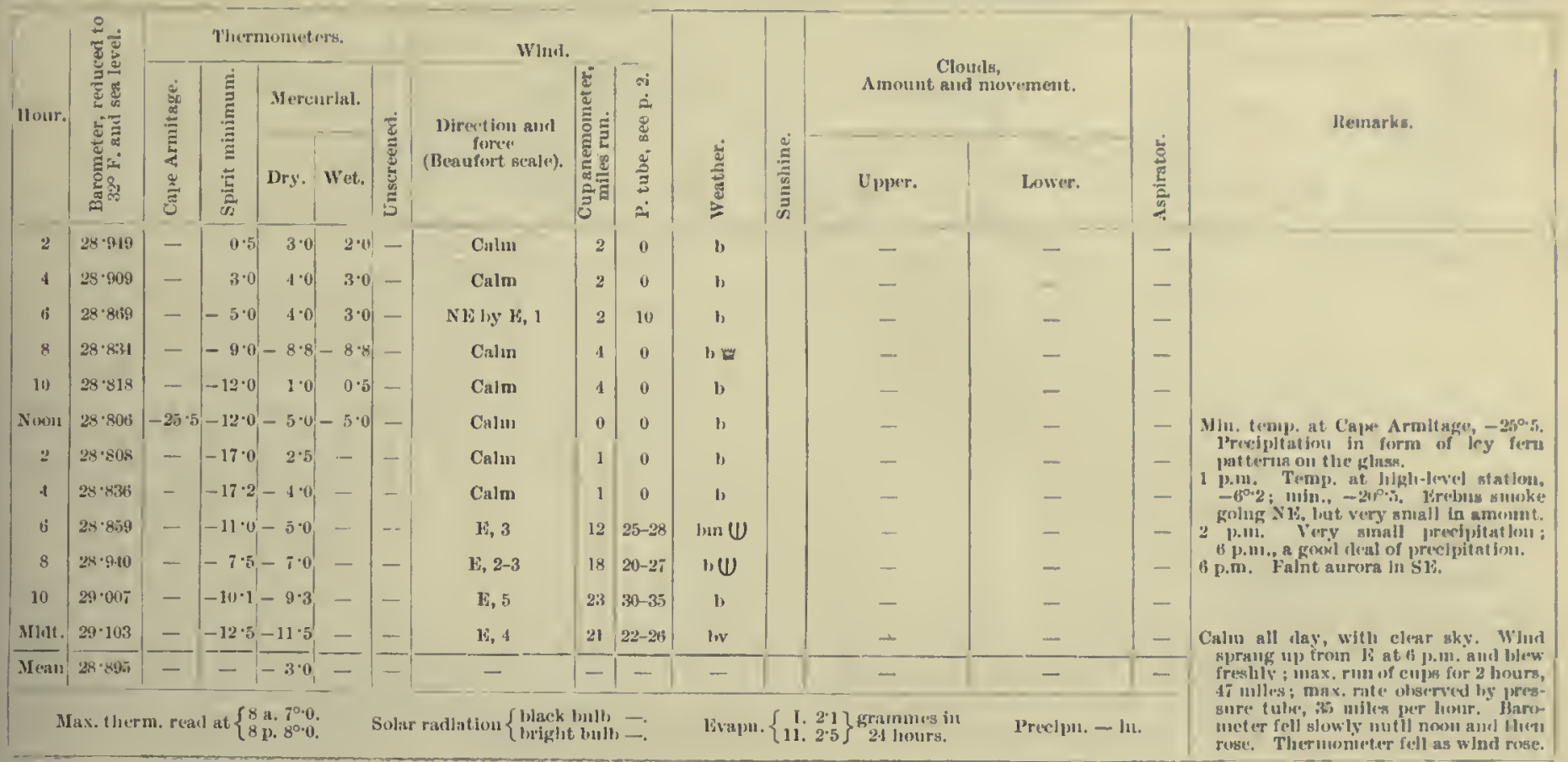

10 th.

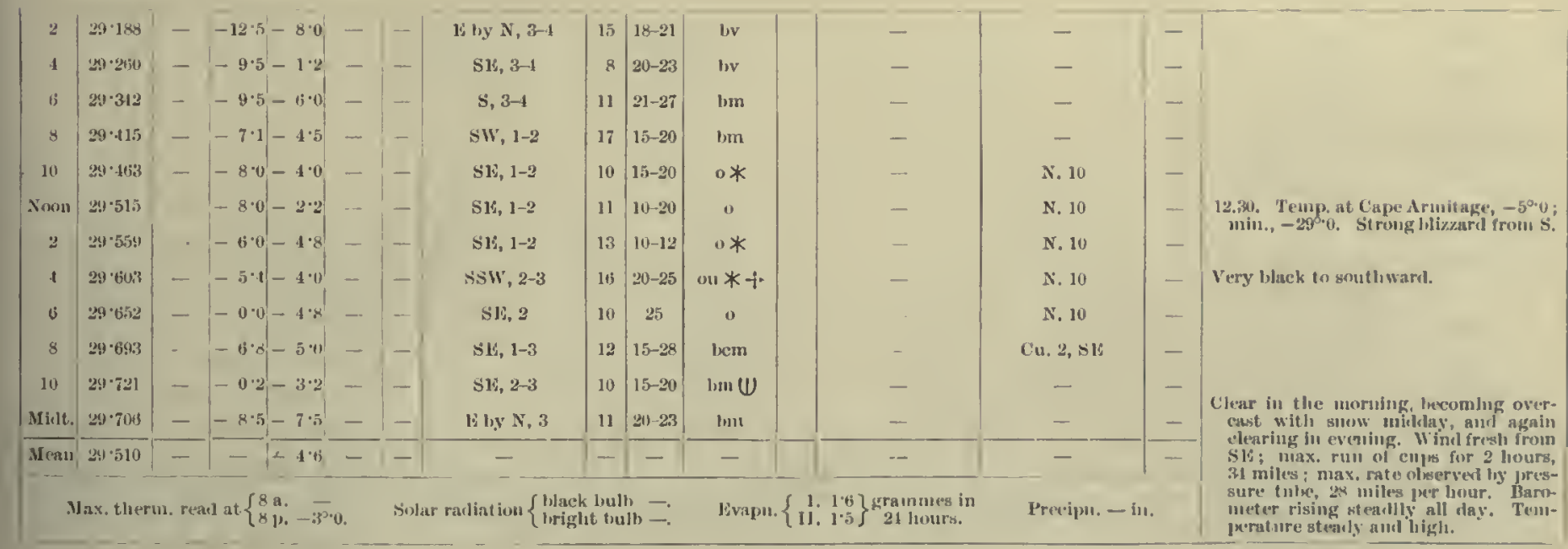

11th.

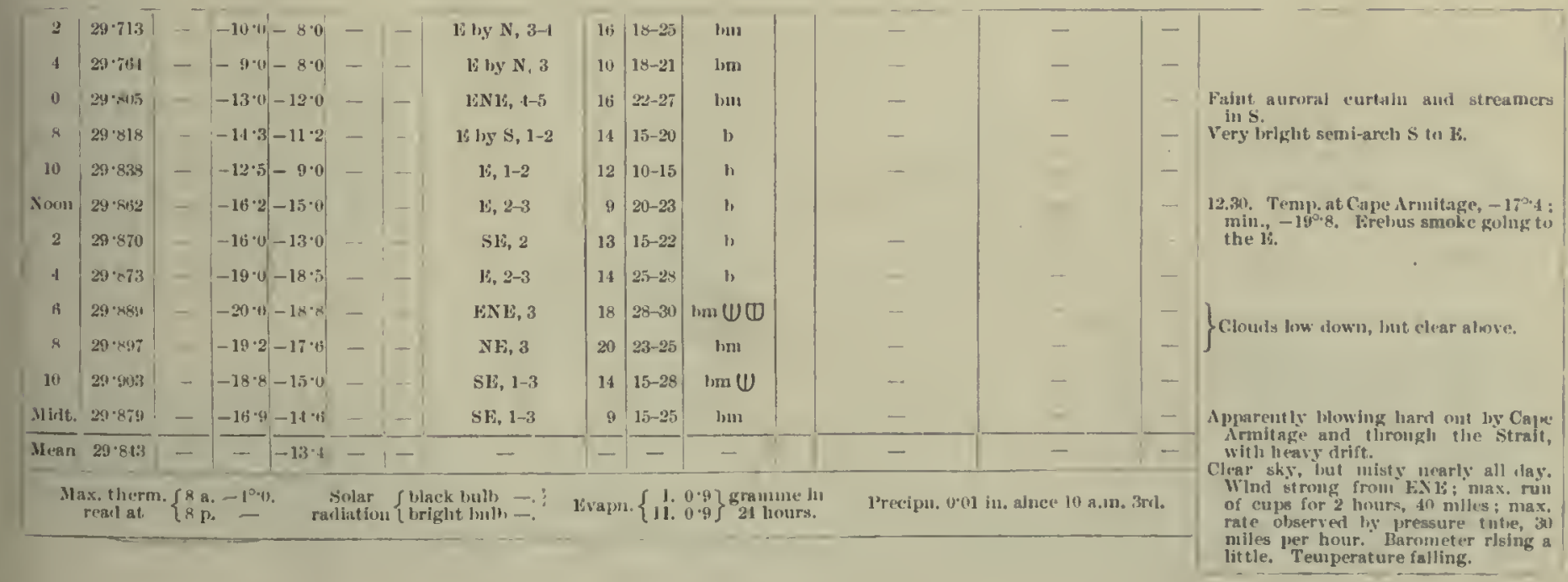


1902 -Jnly.]

Meteorological Jourxat, of thl; "Discoveriy."

13 th.

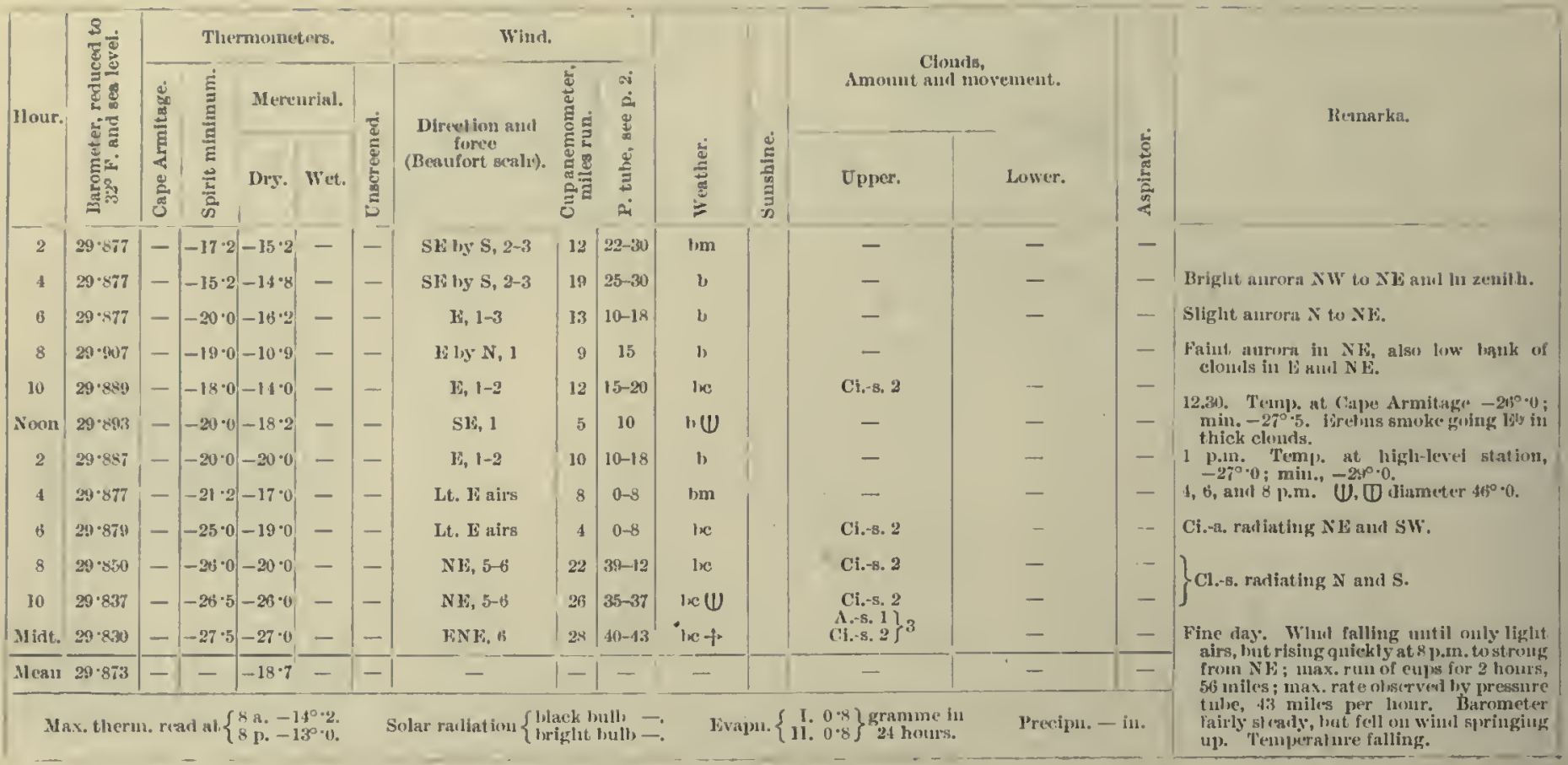

14 th.

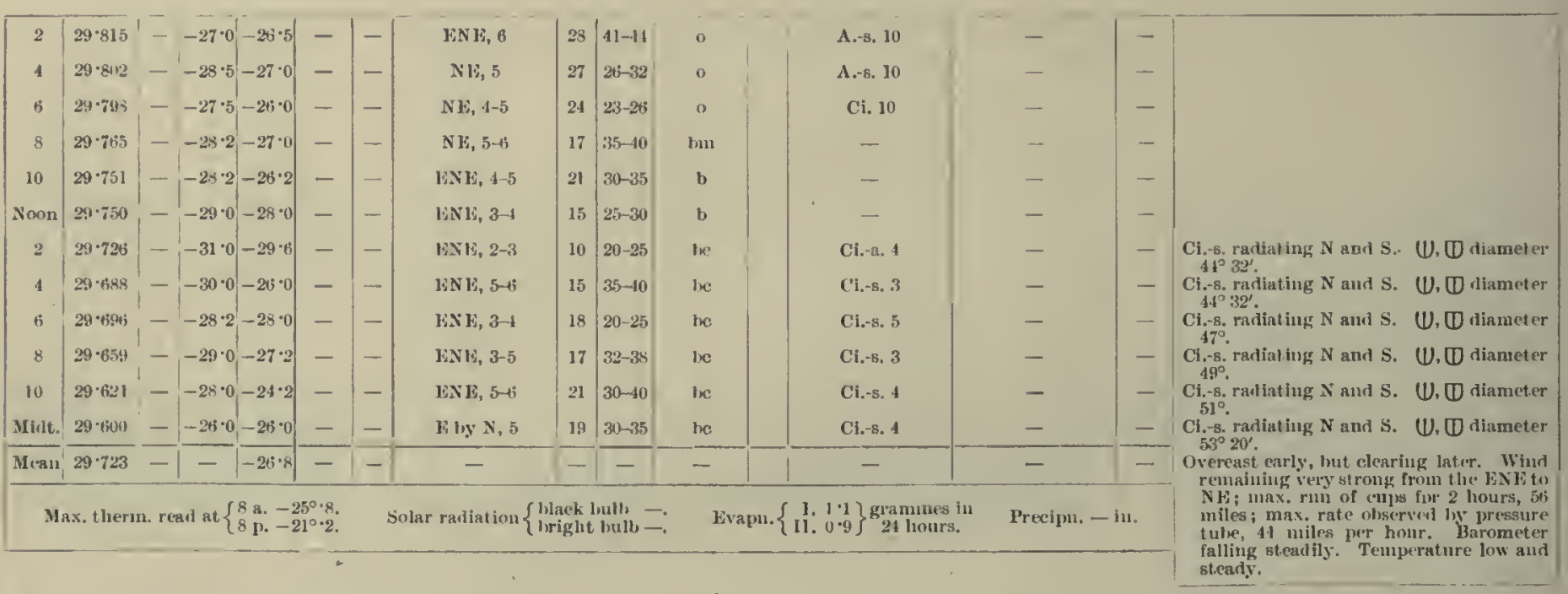

15th.

\begin{tabular}{|c|c|c|c|c|c|c|c|c|c|}
\hline 2 & $29 \cdot 3 \times 6 \mid$ & - & $-26 \cdot 2-25 \cdot 2$ & - & - & ENE, 4-5 & 20 & $23-35$ & ix \\
\hline 4 & $29 \cdot 592$ & & $-26 \cdot 0-23 \cdot 8$ & - & - & LNE, 3-4 & 15 & 23 & IאU \\
\hline 6 & $29 \div 576$ & - & $-24 \cdot 2-22 \cdot 2$ & - & - & F, 4-5 & 15 & $25-30$ & be \\
\hline 8 & $29 \cdot 531$ & - & $-23 \cdot 0-21 \cdot 8$ & - & - & NE, 5-6 & 21 & $30-35$ & be \\
\hline 10 & $29 \cdot 523$ & - & $-23 \cdot 5-20 \cdot 5$ & - & - & E loy $N, 4-5$ & 18 & $30-33$ & c \\
\hline nn & $29 \cdot 513$ & - & $-22 \cdot 0-19 \cdot 5$ & - & - & E by $\mathbf{N}, 2$ & 13 & $18-23$ & c \\
\hline 2 & $29 \cdot 54 \mathrm{mi}$ & - & $-19 \cdot 7-13 \cdot 5$ & - & - & SE, 1-2 & 6 & $15-20$ & c \\
\hline 4 & $29 \cdot 510$ & - & $-17 \cdot x-14 \cdot 2$ & - & - & $\mathrm{SL}_{1}, 1-2$ & 2 & $10-15$ & $\operatorname{oc} U$ \\
\hline 6 & $29 \cdot 504$ & - & $-16 \cdot 0-14 \cdot 2$ & - & - & ESE, 1 & 4 & 10 & oc \\
\hline 8 & $29 \cdot 487$ & - & $-16 \cdot 0-14 \cdot 2$ & - & - & WNE, 4-5 & 4 & $30-33$ & $\infty$ \\
\hline 10 & $29 \cdot 468$ & - & $-19 \cdot 0-17 \cdot 0$ & - & - & E log $N, 4-5$ & 13 & $30-35$ & be \\
\hline Milit. & $29 \cdot 446$ & - & $-21 \cdot 0-20 \cdot 5$ & - & - & E ly, $N, 5-6$ & 18 & $35-40$ & b) \\
\hline ran & $20 \cdot 622$ & - & $-\mid-18 \cdot 8$ & - & - & - & - & - & - \\
\hline
\end{tabular}

Max, therin, read at $\left\{\begin{array}{l}8 \mathrm{a} . \\ 8 \mathrm{p},-13^{\circ} 0\end{array}\right.$

\begin{tabular}{c|c} 
Ci.-s. 4 & - \\
Ci.-8. 6 & - \\
Ci.-8. 5 & - \\
Ci.-8. 6 & - \\
Ci.-8. 10 & - \\
Ci.-8. 10 & - \\
Ci.-8. 10 & - \\
$\begin{array}{c}\text { A.-8. 10, NW } \\
\text { (rapid) }\end{array}$ & - \\
- & S. 10 \\
A.-8. 6 & S. 10 \\
- & -
\end{tabular}


Meteorological, Journat, of tue "Discovate."

[1902-July.

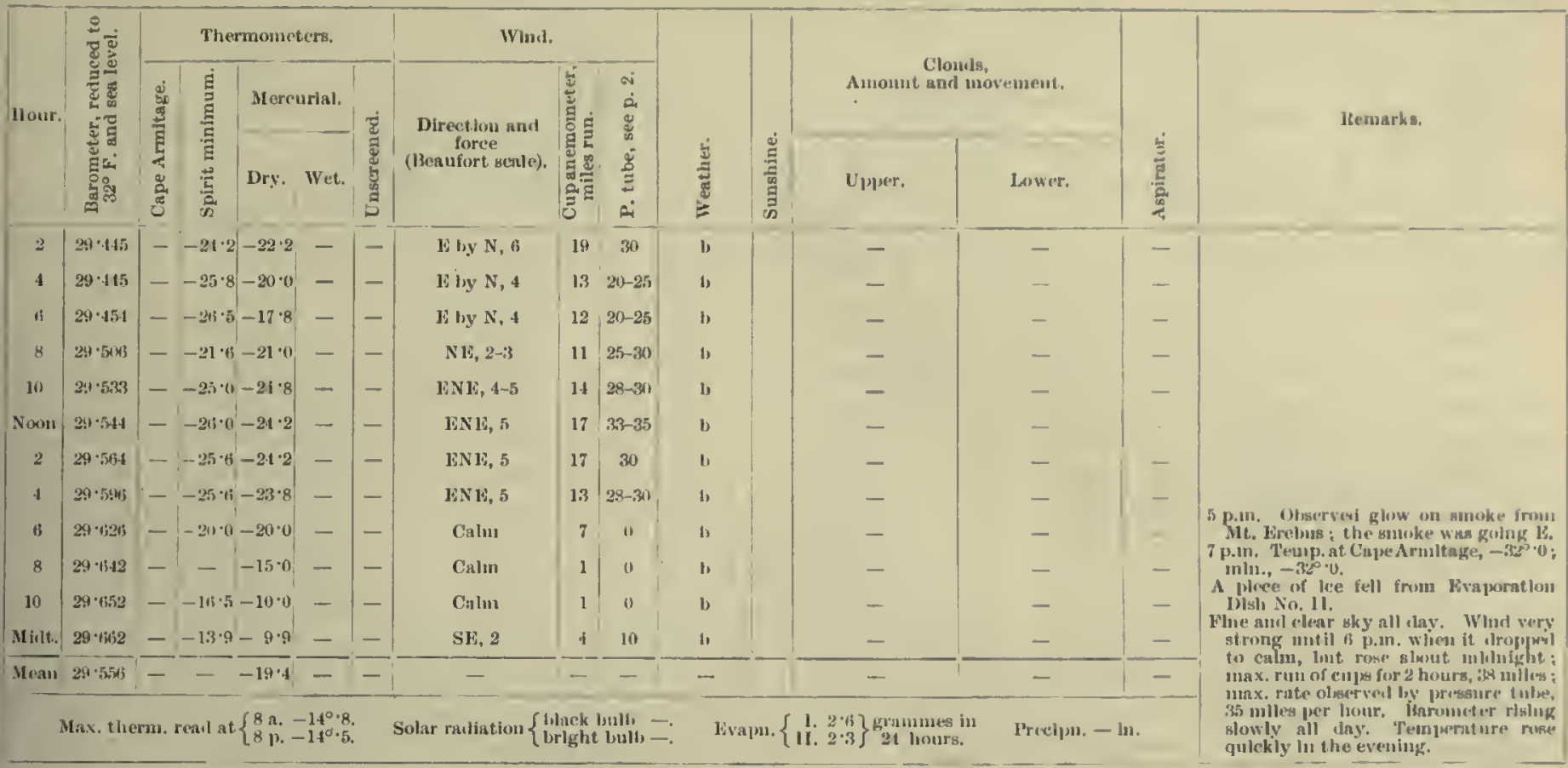

16 th.

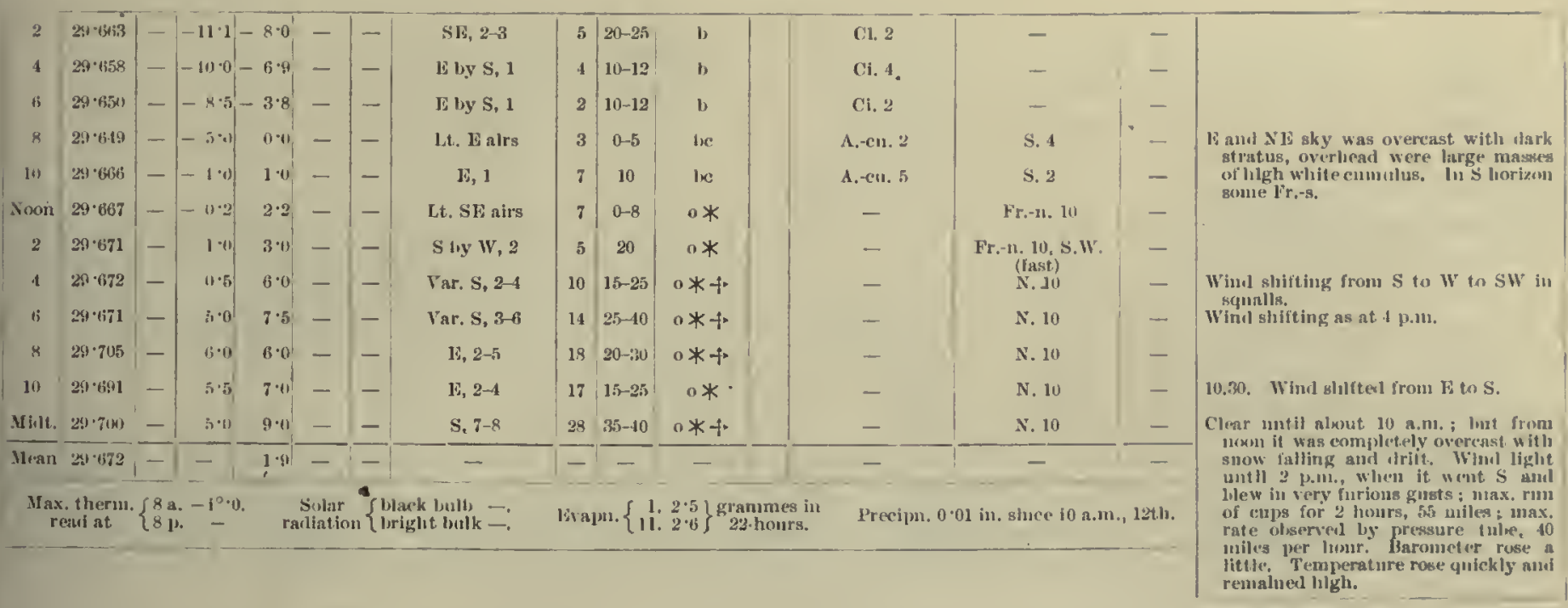

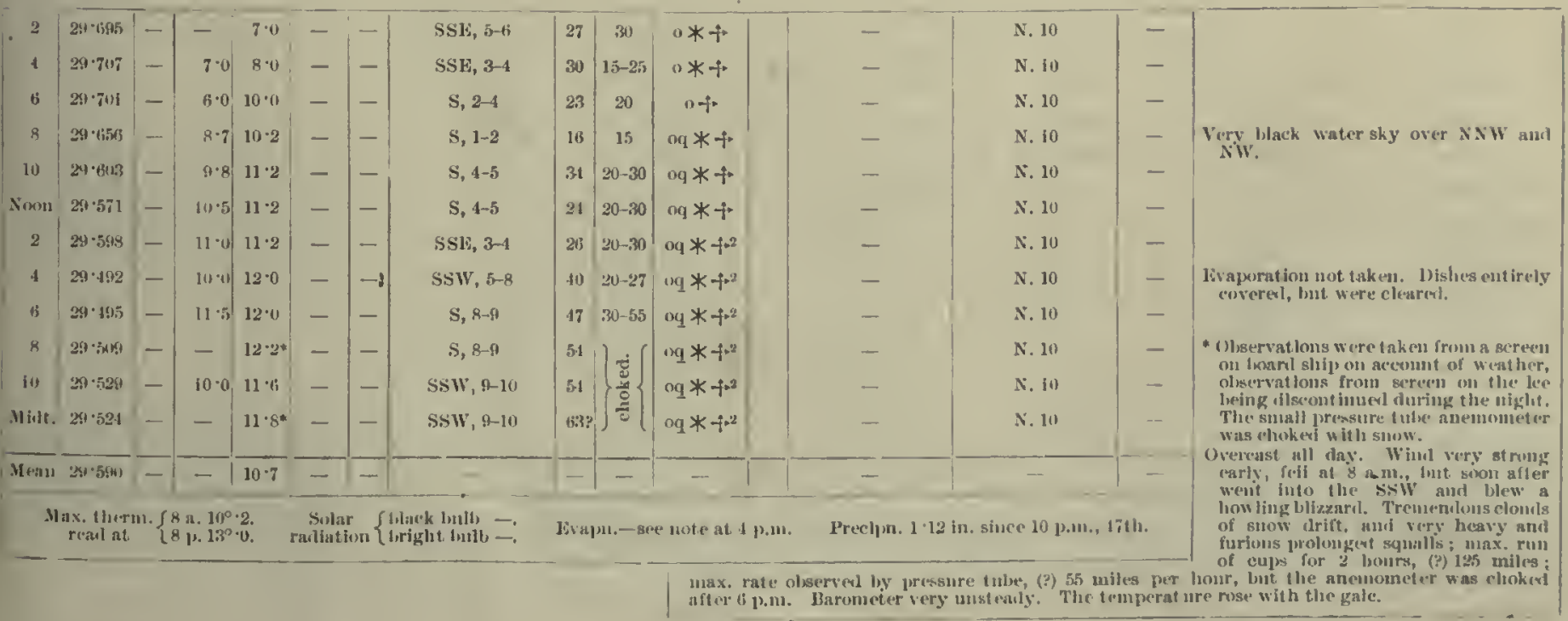


1902-July.]

\begin{tabular}{|c|c|c|c|c|c|}
\hline \multirow{3}{*}{11 our. } & \multirow{3}{*}{ 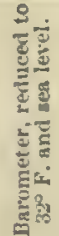 } & \multicolumn{4}{|c|}{ Thermoneters. } \\
\hline & & $\dot{\tilde{g}}$ & $\dot{E}$ & Mlerr & intint. \\
\hline & & $\sum_{\frac{\tilde{z}}{\tilde{z}}}$ & 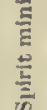 & I)ry. & Wet. \\
\hline
\end{tabular}

19 th.

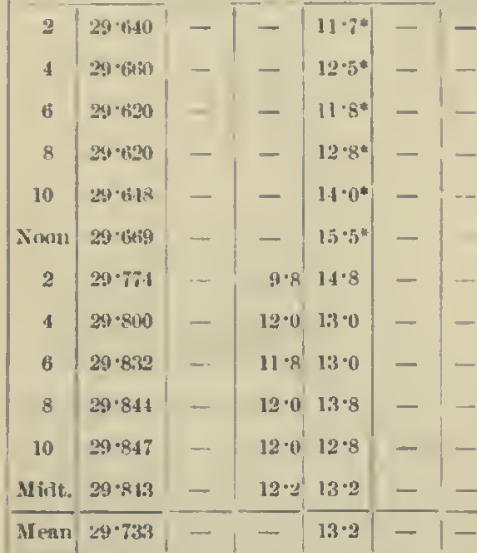

Meteorological Jourial, of the "Discoverey"

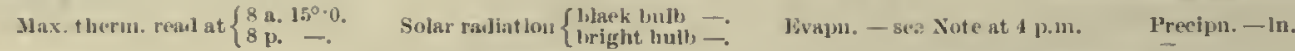

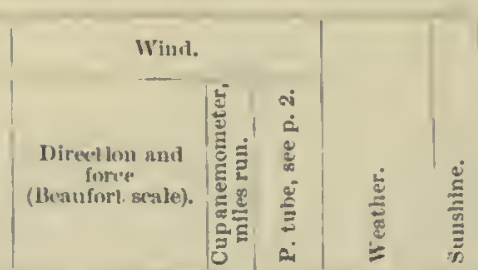

$5 \overline{\operatorname{lig}} \mathrm{W}, 8-10$

S lig $W, 10$

$S \operatorname{ly} 1 \mathrm{~W}, 10$

\$, 10-11

S, $9-10$

S, 8-9

S1: $7-8$

ST, $b-7$

ESW, 6

S1: 6

ES E, 3-5

JST, 3-5

\section{I}

og* $*$

og $* \div$

어 $*-$

$\mathrm{m}_{1} *$ -

on $*+$

oq $*+$

4i. $(10 \mathrm{og} *$

$35^{\prime} 3 u-35$ oq $*$ +

29 25-32 $\circ * \div$

3) 20-25 -i.

21) हु $\int_{\text {की }}$

$\left.\begin{array}{l}21 \\ 19\end{array}\right\}$ 造\{ $x$
Clomis,

Amount and inos cment.

\begin{tabular}{l|l|l|} 
Hiper. & Lower. \\
\hline \multirow{2}{*}{}
\end{tabular}

\begin{tabular}{|c|c|c|}
\hline- & N. II & - \\
\hline- & N. 10 & - \\
\hline- & N. 10 & $\cdots$ \\
\hline - & N. 111 & - \\
\hline- & N. 10 & - \\
\hline- & x. 10 & - \\
\hline & x. 10 & - \\
\hline- & N. 1i) & - \\
\hline- & N. 10 & - \\
\hline$V$ & Fr.-11. 11) & - \\
\hline A. (cu, : $4, \mathrm{~S}$ (fast) & - & - \\
\hline A.-(') $8, S$ (finst) & - & - \\
\hline- & - & 一 \\
\hline
\end{tabular}

Blowing laarilar than ever.

11 ta 11.45 a.า. A lull. Whal drew 10 Wsw, hit weoll. lmek fo s liy Wr am bilew st rong again In houvy gusts. he tenlykernture $15^{\circ} 5$ wits ollserved monceler sluwiel the sullue as the one cill the fee whilst wind was strong. p.m. It wus not possllyle to rigist show lall, as the gange was completely coverel with merumblaterl irift.

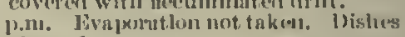

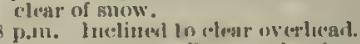

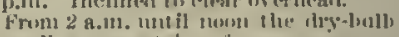
resuliugs w tuatrol slip).

Prereast all dis:. Wilnd remanual in the some cluarice und with the stunt velexity mitil alnut mom, when it

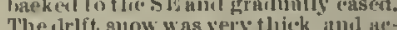
The drlit sucw was very thick and acecommlited in ligh drifl s mumbl the ship. Througlasit the night the lintrinsoli -
20 th.

\begin{tabular}{|c|c|c|c|c|c|c|}
\hline 2 & $29 \cdot 839$ & - & $12 \cdot 2$ & $12 \cdot 8^{\prime}$ & $12 \cdot 9$ & - \\
\hline 4 & $29 \cdot 827$ & - & $12 \cdot 5$ & 13.0 & 1.3 .1 & - \\
\hline 6 & $29 \cdot 805$ & - & $9 \cdot 2$ & $9 \cdot 5$ & $8 \cdot x$ & - \\
\hline 8 & $29 \cdot 779$ & - & $6 \cdot 2$ & $7 \cdot 0$ & $6 \cdot 5$ & - \\
\hline 10 & $29 \cdot 758$ & - & $4 \cdot 0$ & $5 \cdot 0$ & $4 \% 5$ & - \\
\hline Noon & $2 x_{1} \cdot 691$ & -15 & $2 \cdot 1$ & $3 \cdot 0$ & $3 \cdot 0$ & - \\
\hline 2 & $24 \cdot 639$ & - & $3 \cdot 0$ & $4 \cdot 8^{3}$ & $4 \cdot 2$ & - \\
\hline 4 & $29 \cdot 683$ & - & 80 & $2 \cdot 0$ & $2 \cdot 0$ & 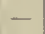 \\
\hline 6 & $29 \cdot 51.3$ & - & 8. & $-7 \cdot 0$ & $-7 \cdot 0$ & - \\
\hline 8 & $29-528$ & - & $-12 \cdot 0$ & $-11 \cdot 5$ & - & -1 \\
\hline 10 & $29 \cdot 493$ & - & $-12 \cdot 0$ & $-10 \cdot 0$ & - & - \\
\hline Mlldt. & $29 \cdot 4,95$ & - & $-11 \cdot 0$ & $-7 \cdot 5$ & - & -1 \\
\hline Nean & $29-6 i t 4$ & - & - & $1 \cdot 8$ & - & - \\
\hline
\end{tabular}

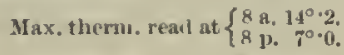

\begin{tabular}{|c|c|c|c|}
\hline $\mathrm{F}, 2-3$ & 17 & & nev \\
\hline $\mathrm{F} \operatorname{ly} \mathrm{N}, \mathrm{A}$ & 14 & 苋 & mev \\
\hline lity $\mathbf{N}, 1-5$ & $: 2$ & ( & oe \\
\hline $1 ;, 4$ & $2+i$ & $15-20$ & lne \\
\hline Ji, 4 & $26 i$ & $10-15$ & i) $U$ \\
\hline $\mathrm{NL}_{1} 4$ & 21 & $10-15$ & $\ln U$ \\
\hline $\mathrm{N}] \mathrm{E}, \mathrm{A}$ & 25 & $15-20$ & $\alpha:$ \\
\hline $\mathrm{Nl}$, 4-5 & 21 & $312-23$ & $\cup \uplus$ \\
\hline $\mathrm{J}, \mathrm{t}-7$ & 43 & $25-35$ & $1(\omega)-{ }^{-1}$ \\
\hline $\mathrm{E}, 6$ & $2 n$ & $35-38$ & () \\
\hline $13,6-7$ & 30 & $35-40$ & o \\
\hline $\mathrm{F}, 4$ & $2 x$ & 20 & 0 \\
\hline - & - & - & - \\
\hline
\end{tabular}

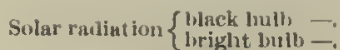

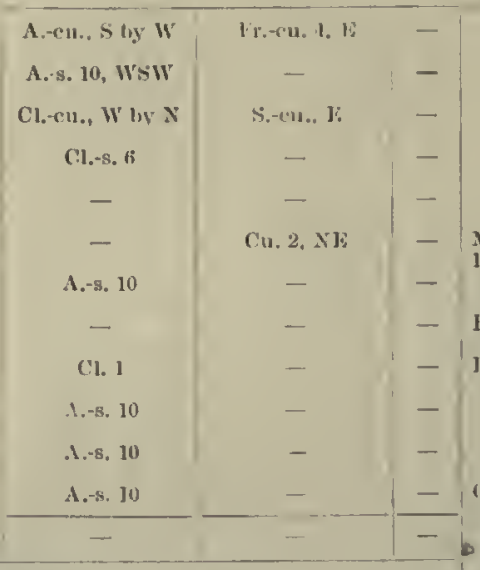

Preeipu. - in.
Min, temp. at Cape Armitage, $-5^{\circ} \cdot 0$.

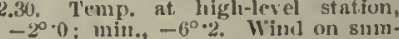
-2 , 1 ,

leavy lauk of so-eus, to Ji, low down, moving raphlly from Nl. (avy lank of $\mathrm{A}$. - c11, to li, low dow 11 , moving raplelly from $\mathbf{N} \mathbf{l}$.

vircast most of the day. clearing a

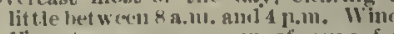
15ty, strong: max. run of colps for 2 lioms, 85 milis; max. rate olservert If pressure tule, fo miles per lour. lell stendily sll day.

\section{1 st}

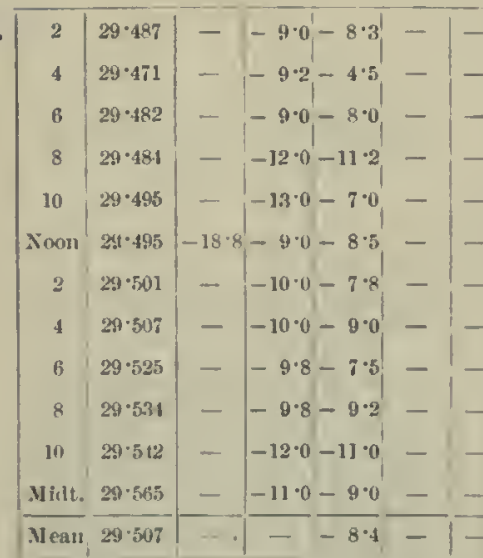

\begin{tabular}{|c|c|c|}
\hline 14,3 & 15 & 15 \\
\hline 14,4 & 10 & $10-15$ \\
\hline E by $N, 4-5$ & 12 & $20-22$ \\
\hline$N E, 2-3$ & 17 & $10-15$ \\
\hline $\mathrm{SE}, 1$ & $i$ & 10 \\
\hline 1,1 & 9 & 10 \\
\hline $1 \mathrm{H}, 1$ & 9 & 10 \\
\hline Lt. E airs & 8 & 0 \\
\hline Calm & s & 0 \\
\hline 816,1 & 8 & 10 \\
\hline Lt. E alrs & 8 & 0 \\
\hline SLE, 2 & 8 & $10-15$ \\
\hline
\end{tabular}

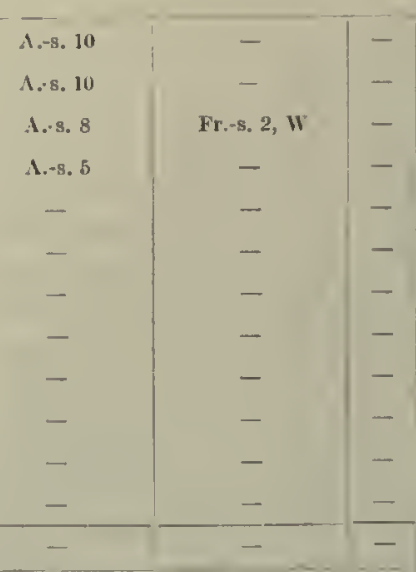

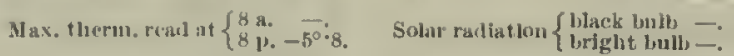

Western range of hills very dist inct. Cleaneel instrumeuts and screens.

Min. temp. at Cape Armitage, $-22^{\circ} \cdot 8$, Erebus suloke going $\mathrm{I}$, very small amomt. Ny treere at Cope Armitage

Ovireast carly, lant clearcul, and remainer tinc and clear all day. Wind strong early, lut tell to light airs in the evening : max. ruu of cups for 2 hours, 34 nuiles: max. rate olsserver!

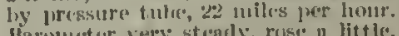

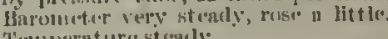
T'enlperntures sicwly. 
Metgoliolagical Journat, or tue "Discovery."

[1902-July.

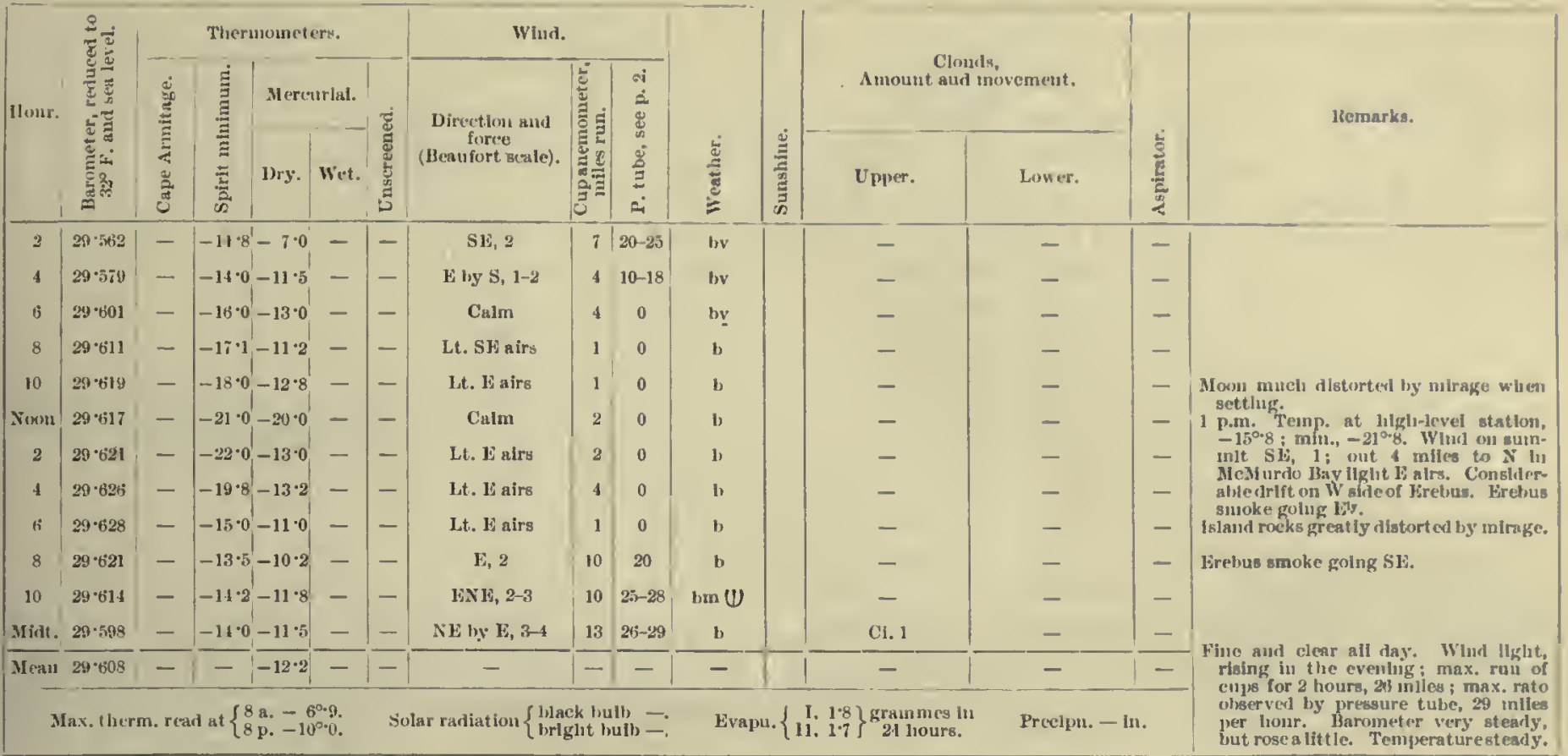

22nd.

\begin{tabular}{|c|c|c|c|c|c|c|c|c|c|c|c|c|c|}
\hline 2 & $29 \cdot 543$ & - & $-13.0-10.5 \mid$ & - & - & NE hy $\mathbf{E}, 2-3$ & 13 & $20-22$ & 1) & - & - & - & \multirow{13}{*}{ 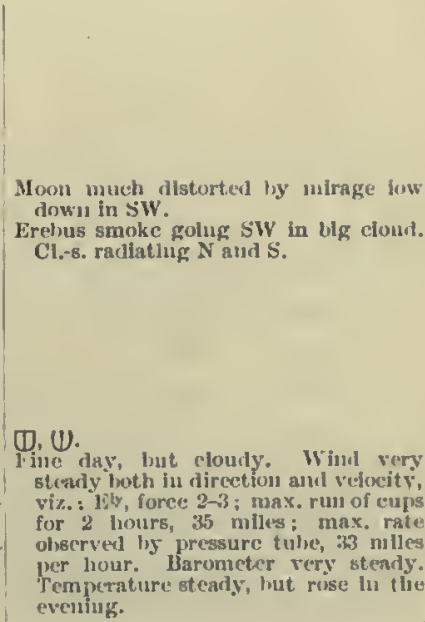 } \\
\hline 4 & $29 \cdot 583$ & - & $-11 \cdot 5-7 \cdot 5$ & - & - & LNE, 2 & 10 & $10-12$ & b) & Cl.-s. 2, E & - & - & \\
\hline is & $29 \cdot 5 \times 11^{\circ}$ & - & $-21 \cdot 5-8 \cdot 5$ & - & - & NH hy $\mathbf{E}, 2-4$ & 17 & $20-30$ & $b$ & CI.-s. 4, E & - & - & \\
\hline 8 & $29 \cdot 621$ & - & $-15 \cdot 2-10 \cdot 0$ & - & -1 & L by $\mathrm{N}, 2$ & 4 & $20-25$ & be & Fr.-cl.-s. b & - & - & \\
\hline 111 & $29 \cdot 611$ & - & $-11 \cdot 0-10 \cdot 0$ & - & - & E, 2-3 & 12 & $25-39$ & b & - & - & - & \\
\hline Nom & 29 thus & $-19 \cdot 5$ & $5-16 \cdot 2-15 \cdot 0$ & - & - & E, 2-4 & 15 & $25-33$ & te & $\mathrm{Ci}_{.-8.3} 3$ & - & - & \\
\hline 2 & $29 \cdot 5 ! 13$ & - & $-16 \cdot 5-12 \cdot 0$ & - & - & L, 2-3 & 17 & $25-30$ & he & Ci.-s. 5 & - & - & \\
\hline 4 & $29-591$ & - & $-15 \cdot 0-11 \cdot 2$ & - & - & ENE, 3 & 15 & $23-27$ & ine & Ci.-s. 4 & - & - & \\
\hline 6 & $29 \cdot 583$ & - & $-11 \cdot 0-10 \cdot 2$ & - & - & $1:, 2-3$ & 14 & 23-25 & lice & Cl.-5. 4 & - & - & \\
\hline 8 & $29 \cdot 579$ & - & $-12 \cdot 0-5 \cdot 0$ & - & - & E, 2 & 18 & $20-22$ & ins & CI.-8. 3 & - & - & \\
\hline 10 & $29 \div 5 \%$ & - & $-12 \cdot 0-3 \cdot 2$ & - & - & E. 2 & 15 & 20 & lise & Ci.-s. 3 & - & - & \\
\hline Midt. & $29 \cdot 571$ & - & $-5 \cdot 8-4 \cdot 5$ & - & - & If, $2-3$ & 17 & $20-27$ & the & Ci,-5. $2, \mathrm{~N}$ & - & - & \\
\hline 11 en & $24: 391$ & - & --0.3 & - & - & - & - & - & - & - & - & - & \\
\hline
\end{tabular}

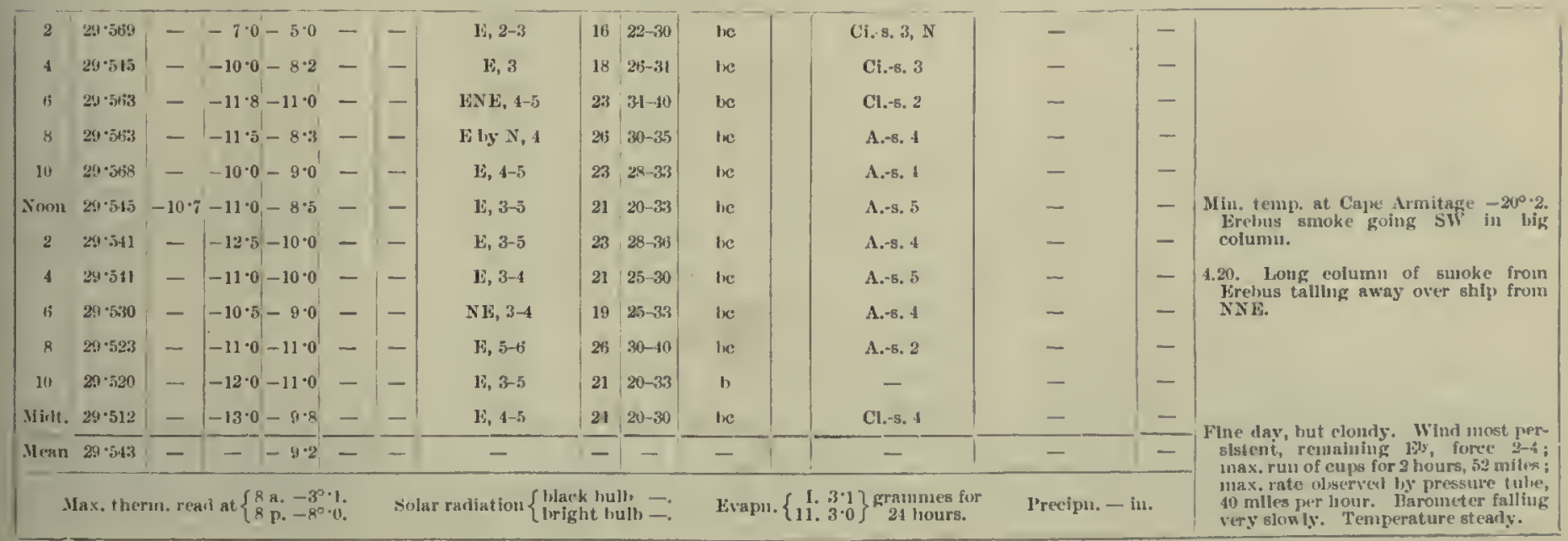


$1902-J u l y$.]

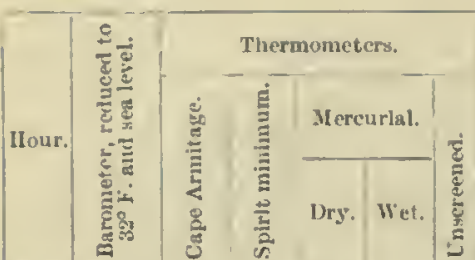

25th.

\begin{tabular}{|c|c|c|c|c|c|c|c|c|}
\hline 2 & $29 \cdot 514$ & - & $-11 \cdot 3^{1}-8 \cdot 0$ & - & - & $15,3-4$ & 20 & 20 \\
\hline 4 & $29 \cdot 523$ & - & $-10 \cdot 7-8 \cdot 3$ & - & - & $E, 4-5$ & $1 \pi$ & $20-35$ \\
\hline 6 & $29 \cdot 577$ & - & $-10 \cdot 1-0.0$ & - & - & E, 3-4 & 15 & $20-25$ \\
\hline 8 & $29 \cdot 513$ & - & $-10 \cdot 8-6 \cdot 1$ & - & - & E, 1-2 & 22 & 10-15 \\
\hline 10 & $29 \cdot 522$ & - & $-10 \cdot 0-8.0$ & - & - & E, 2-3 & 10 & $15-23$ \\
\hline Noon & $29 \cdot 5 \pi 3$ & $-12 \cdot 5$ & $-11 \cdot 0-8 \cdot 0$ & - & - & $1,1-2$ & 7 & $10-12$ \\
\hline 2 & $29 \cdot 570$ & - & $-10 \cdot 2-8 \cdot 2$ & - & - & E, 2-3 & 7 & $15-20$ \\
\hline 4 & $29 \cdot 519$ & - & $-8 \cdot 0-7 \cdot 0$ & - & - & F, 1 & 8 & 10-12. \\
\hline 6 & $29 \cdot 510$ & - & $-10 \cdot 0-6 \cdot 0$ & - & - & 1,1 & 7 & $10-12$ \\
\hline 8 & $29 \cdot 506$ & - & $-7 \cdot 2-6 \cdot 0$ & - & - & $\mathrm{SE}, 2-3$ & 8 & $20-25$ \\
\hline 10 & $29 \cdot 512$ & - & $-9 \cdot 0-7 \cdot 0$ & - & - & Lt. SSE airs & 8 & $0-5$ \\
\hline
\end{tabular}

Meteorological Joursal of the "Discovetiv."

\begin{tabular}{|c|c|c|c|c|c|c|c|c|}
\hline \multicolumn{3}{|l|}{ Wind. } & \multirow[b]{3}{*}{ 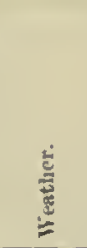 } & \multirow[b]{3}{*}{ 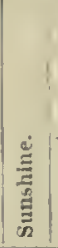 } & \multirow{2}{*}{\multicolumn{2}{|c|}{$\begin{array}{l}\text { Clourds, } \\
\text { Annount and movemont. }\end{array}$}} & \multirow[b]{3}{*}{$\frac{1}{2}$} & \multirow{3}{*}{ lemarks. } \\
\hline \multirow{2}{*}{$\begin{array}{l}\text { Direvtlon and } \\
\text { force } \\
\text { (Beaufort acale). }\end{array}$} & \multirow{2}{*}{ : } & \multirow{2}{*}{ 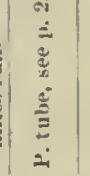 } & & & & & & \\
\hline & & & & & Upper. & Lower. & & \\
\hline $1,3-4$ & 20 & 20 & $1 x+4$ & & Ci.-ซ. 7 & - & |- & \multirow{12}{*}{ 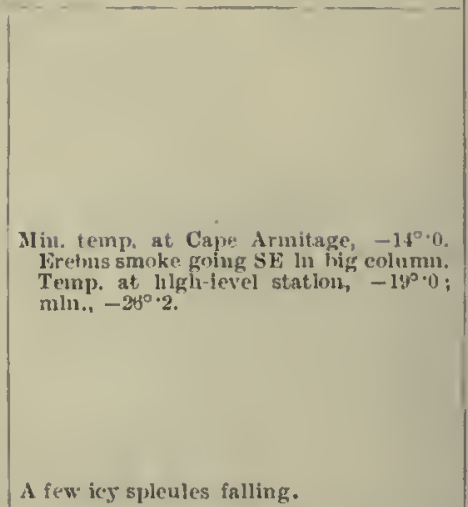 } \\
\hline$E, 4-5$ & 17 & $20-35$ & . & & Ci.-s. 8 & - & - & \\
\hline E, 3-4 & 15 & $20-25$ & o & & $\mathrm{Ci}_{1}-\mathrm{A}_{\mathrm{a}}, 10$ & - & - & \\
\hline E, 1-2 & 22 & $10-15$ & $\infty$ & & - & S. 10 & - & \\
\hline E, $2-3$ & 10 & $15-23$ & o & & A.-s. 10 & - & - & \\
\hline E, $1-2$ & 7 & |10-12 & o & & A.-s. 10 & - & - & \\
\hline$E, 2-3$ & 7 & $15-20$ & o & & A.-5. 10 & - & - & \\
\hline$F_{1}, 1$ & 8 & $10-12$ & bin & & - & - & -1 & \\
\hline$E_{0} 1$ & 7 & $10-12$ & $\mathrm{bm}$ & & - & - & - & \\
\hline SE, 2-3 & 8 & $20-25$ & ln & & - & - & - & \\
\hline Lt. SSE airs & 8 & $0-5$ & $\mathrm{bm}$ & & - & - & - & \\
\hline lise, $0-1$ & 9 & $0-8$ & $\ln n \rightarrow$ & & - & - & - & \\
\hline- & - & - & - & & - & - & - & $\begin{array}{l}\text { Overeast all the morning, clearing alout } \\
4 \text { p.m., but still remaining misty over- }\end{array}$ \\
\hline \multicolumn{3}{|c|}{ lar radiatiou $\left\{\begin{array}{l}\text { blaek hinllh } \\
\text { lright bulb }=\text {. }\end{array}\right.$} & \multicolumn{3}{|c|}{ 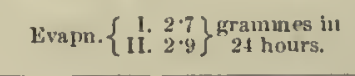 } & \multicolumn{2}{|c|}{ Precipm. - in. } & 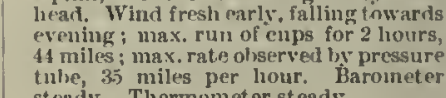 \\
\hline
\end{tabular}

26th.

\begin{tabular}{|c|c|c|c|c|c|c|c|c|c|c|c|}
\hline 2 & $29 \cdot 522$ & - & $-10.8-10.0$ & -1 & 15 by N, 2-3 & 2 & $20-23$ & burq & - & - & - \\
\hline 4 & $29 \cdot 509$ & - & $-10 \cdot 5-8 \cdot 5-$ & - & ENA $2-3$ & 8 & $15-25$ & limg & - & - & $1-$ \\
\hline 6 & $29 \cdot 504$ & - & $-10.5-7.8-$ & - & E by S, 2 & 9 & $15-24$ & bunq & - & - & - \\
\hline 8 & $29 \cdot 490$ & - & $-100-9 \cdot 0-$ & - & $E \operatorname{lng} 5,1$ & $1 \mathrm{i}$ & $15-22$ & 1. & - & - & - \\
\hline 10 & $29 \cdot 498$ & - & $-10.8-8.2-$ & - & E, 2 & 12 & $20-23$ & b) & - & - & - \\
\hline Noon & $29 \cdot 490$ & - & $-100-7.0-$ & - & SE, 2-3 & 13 & $20-25$ & b & - & - & - \\
\hline 2 & $29 \cdot 489$ & - & $-8.0-7.0-$ & - & Lt. SE airs & 7 & $0-8$ & o & - & S. 10 & $\begin{array}{lll}- & & -\end{array}$ \\
\hline 4 & $29 \cdot 482$ & - & $-8.5-4.8-$ & - & Lt. SF airs & 7 & $0-8$ & " & - & S. 10 & - \\
\hline 6 & $29 \cdot 467$ & - & $-6.5-5.0-$ & - & L.t. SE alrs & 3 & $0-5$ & 0 & - & S. 10 & - \\
\hline 8 & $29 \cdot 459$ & - & $|-5 \cdot 2-3 \cdot 0|-$ & - & $\mathrm{SE}_{0,1}$ & 6 & 10 & o & - & S. 10 & - \\
\hline 10 & $29 \cdot 411$ & - & $-6 \cdot 4-5 \cdot 0-$ & - & Lt. SE a irs & 3 & $0-5$ & 0 & - & S. 10 & - \\
\hline Mirit. & $29 \cdot 42 \cdot$ & - & $-6 \cdot 0-3 \cdot 3-$ & - & 1.t. Sliz airs & 2 & $0-5$ & o & - & s. 10 & - \\
\hline Mesui, & $29 \cdot 182$ & - & $|--86|-$ & - & - & 1 - & - & - & - & - & - \\
\hline & & & $\left\{\begin{array}{l}8 \mathrm{a} \text {. } \\
8 \mathrm{p}\end{array}\right.$ & So & ition. & & & & $\begin{array}{l}\text { Ylammess int } \\
24 \text { hours. }\end{array}$ & receipn. & \\
\hline
\end{tabular}

9.45 p.m. Temp. at Cape Armitage. Fiue aud elear until alout 2 p.m., when it breceme overeast, and remained so for the rest of the day. Wild was lyg. to licht airs: inax. rum of . to light ats 2 hours, 26 miles : max. rate olserved iw pressure tulie, 25 miles per lour.
Barometer fell slowly all day. Temperature ligh and steady.

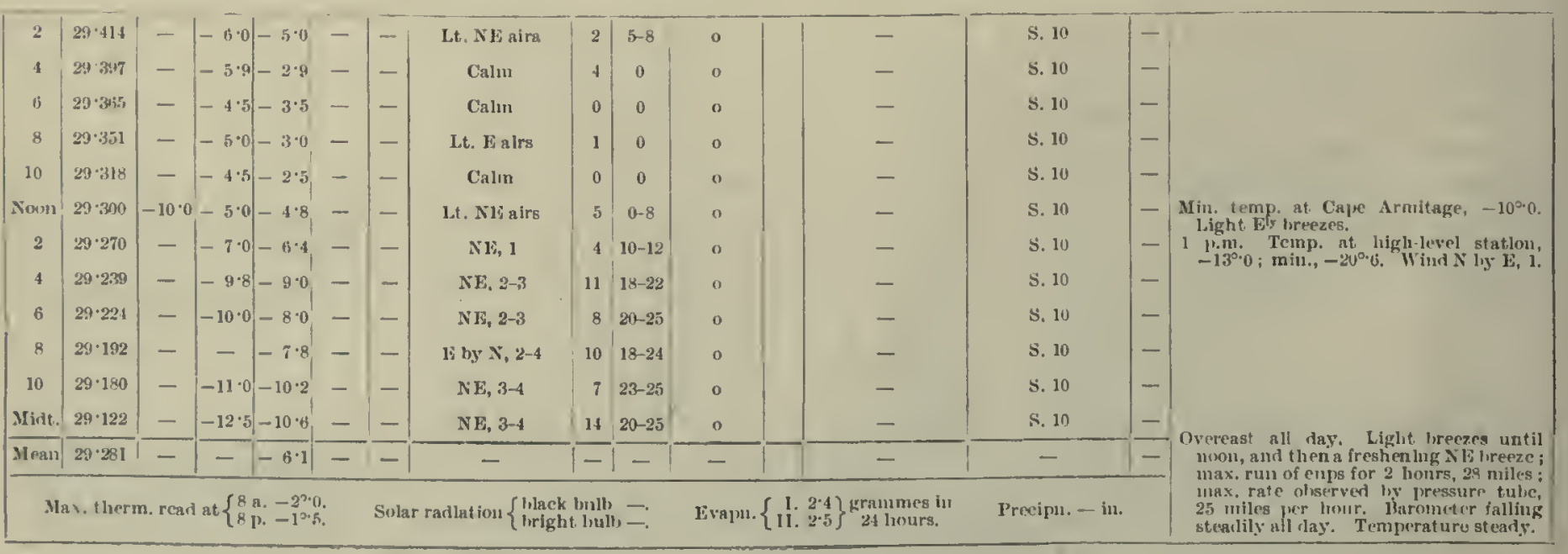


Miteorological Journat of the: "Discoverey."

[1902-July.

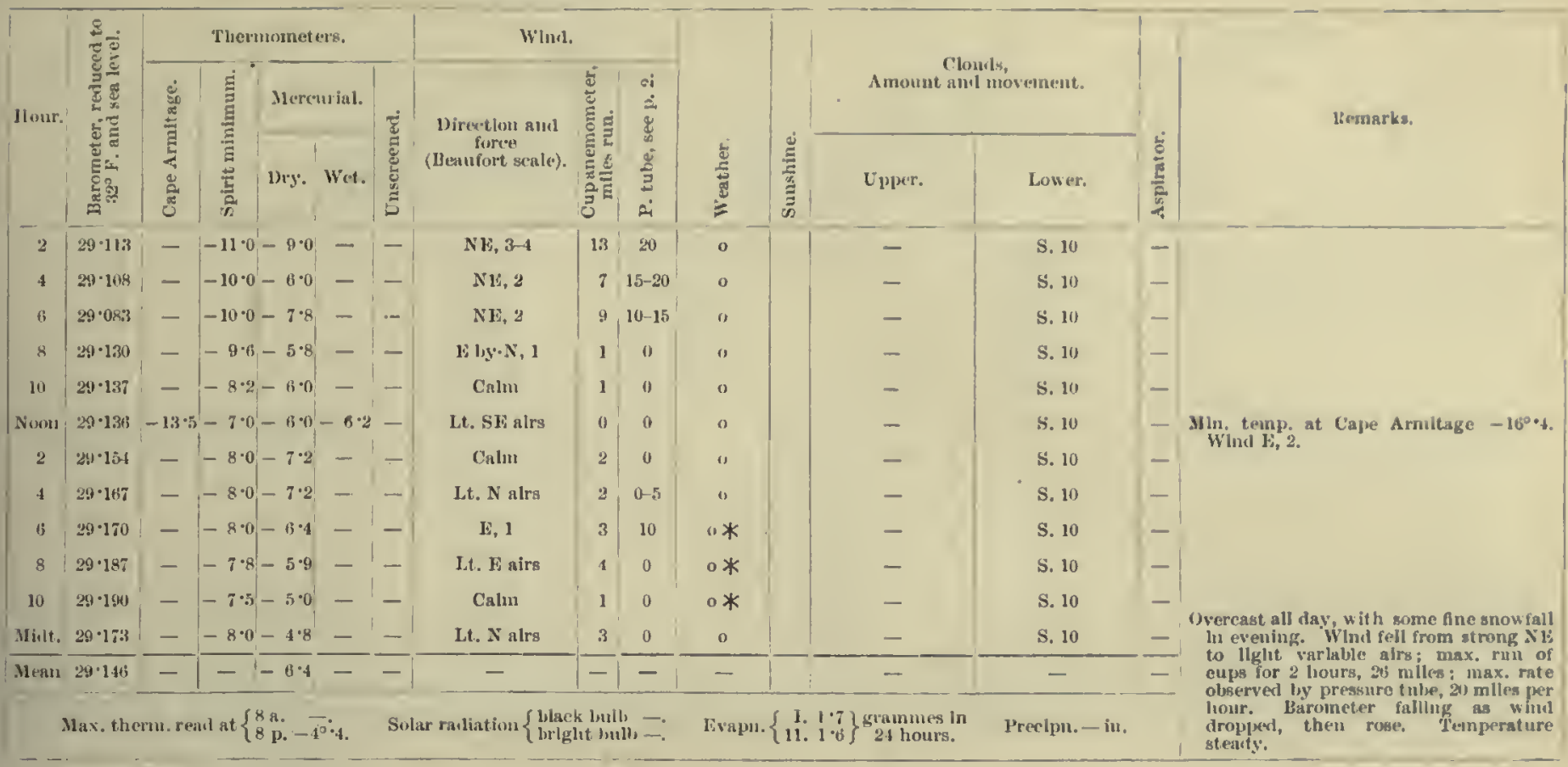

28th.

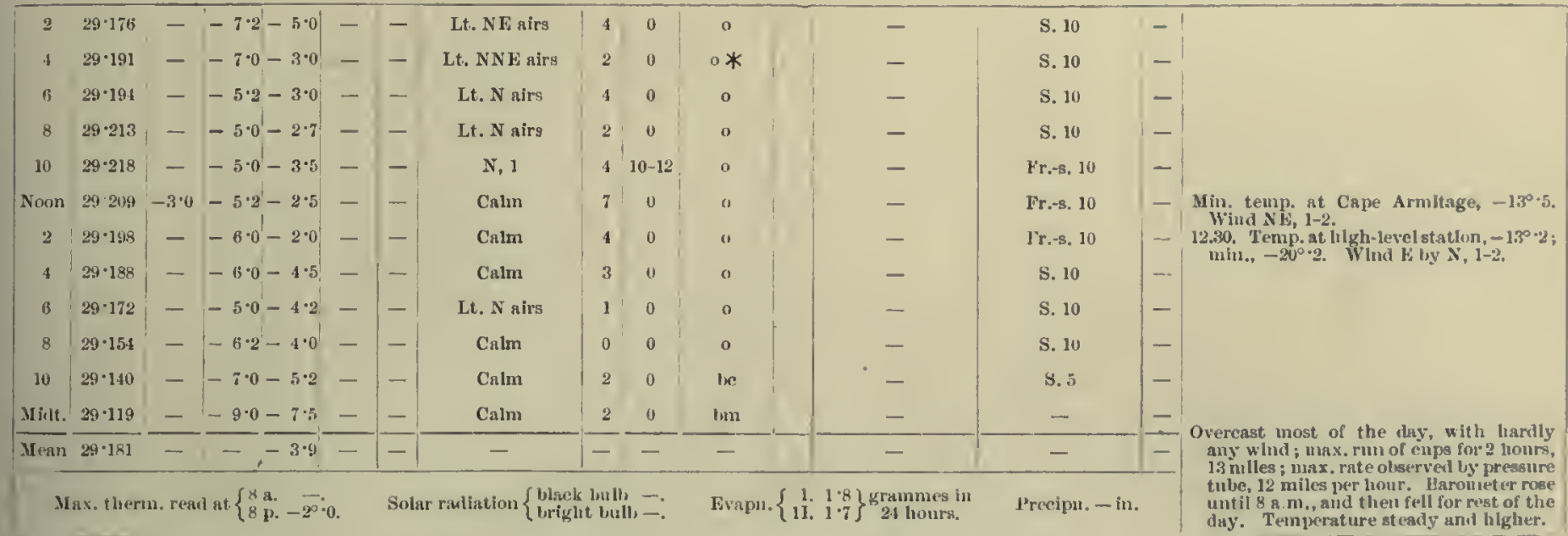

29th.

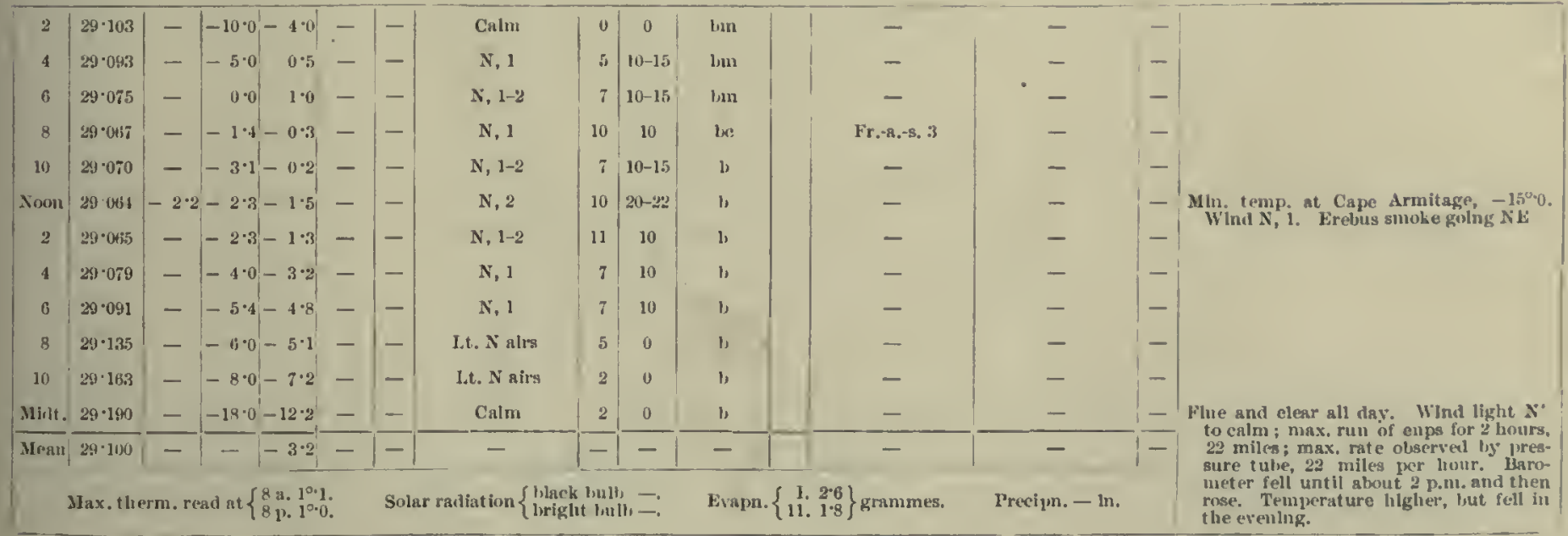

30th. 
1902-July.]

Mlitkorological Journal of tiff "Discovery."

31st.

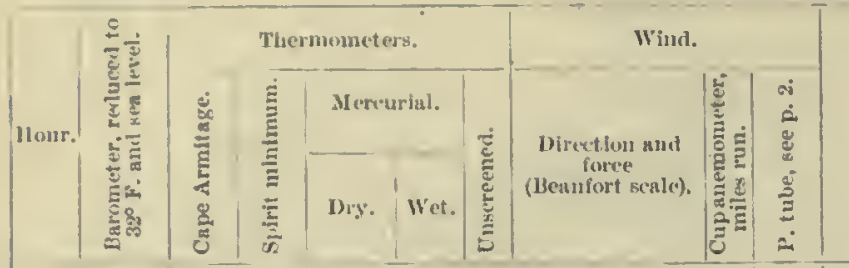

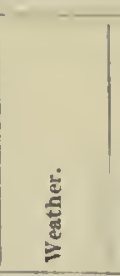

\begin{tabular}{|c|c|c|c|c|c|c|c|c|c|c|c|c|c|c|}
\hline \multirow{2}{*}{ Ilonr! } & \multirow{2}{*}{ 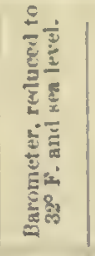 } & \multirow{2}{*}{ 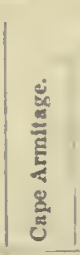 } & \multicolumn{4}{|c|}{ Thermometers. } & \multicolumn{3}{|l|}{ Wind. } & \multirow[b]{2}{*}{ 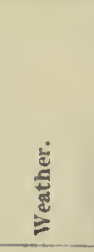 } & \multirow[b]{2}{*}{ 造 } & \multicolumn{2}{|c|}{$\begin{array}{l}\text { Clonds, } \\
\text { Amount aud movemacut. }\end{array}$} & \multirow[b]{2}{*}{ 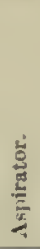 } \\
\hline & & & 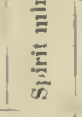 & Dry. & Wet. & 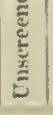 & $\begin{array}{c}\text { force } \\
\text { (Beanfort scalc). }\end{array}$ & 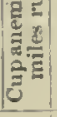 & $\begin{array}{l}3 \\
\Xi \\
2\end{array}$ & & & Ujper. & Lower. & \\
\hline 2 & $2 * 204$ & - & $-16 \cdot 2$ & $-12 \cdot 9$ & - & - & Lt. $\mathrm{N}$ airs & 1 & 0 & be & & A.-s. 10 & - & - \\
\hline 4 & $29 \cdot 207$ & - & $-15 \cdot 5$ & $-11 \cdot 0$ & - & - & Lt. NF alrs & 0 & 0 & ine & & A.-s. 10 & - & - \\
\hline a & $: ? \cdot 212$ & - & $-11 \cdot 8$ & $-8 \cdot 2$ & - & -1 & Lt. E lỵ S airs & 1 & 0 & txe & & A.-8. 10 & - & - \\
\hline 8 & $29 \cdot 189$ & - & $-11 \cdot 4$ & $-5 \cdot 0$ & - & - & $I_{3}, 1-2$ & 7 & $10-20$ & o & & - & S. 10 & - \\
\hline 10 & $29 \cdot 19 k$ & -1 & $-11 \cdot 1$ & $-9 \cdot 0$ & - & - & $\mathbf{I}, 2-3$ & 13 & $15-20$ & $0 * 4$ & & - & N. 10 & - \\
\hline Simon & $2(1) \cdot 178$ & $-11 \cdot 3$ & $-10 \%$ & $-7 \cdot 0$ & - & - & $\mathrm{E}, 3$ & 12 & 20 & $0 * 4$ & & - & N. 10 & 一 \\
\hline 2 & $28 \cdot 112$ & - & $-8 \cdot 0$ & $-1: 5$ & - & - & $\mathrm{E},: 3-4$ & 18 & $20-35$ & $0 *+$ & & - & N. 10 & - \\
\hline 4 & $29 \cdot 1.30$ & - & $-7 \cdot 5$ & $-3 \cdot 6$ & - & - & $\mathrm{SH}_{4}, 5-6^{\circ}$ & 18 & $35-44$ & $n * \div 2$ & & - & N. 10 & - \\
\hline 6 & $29 \cdot 126$ & - & -5.6 & $-4 \cdot 2$ & - & - & SE, 4-5 & in & $25-30$ & $0 * 42$ & & - & N. 10 & - \\
\hline 8 & $29 \cdot 112$ & - & -8.5 & $-8^{\circ} 0$ & - & - & SW, 4-5 & 24 & $28-32$ & $0 *+*^{2}$ & & - & N. 10 & - \\
\hline 10 & $29 \cdot I 10$ & - & - & $-655^{*} \mid$ & - & - & SW, $5-6$ & - & - & $0 * f^{2}$ & & - & N. 10 & - \\
\hline Mislt! & $29 \cdot 142$ & -1 & $1-$ & $-4 \cdot 8^{*}$ & - & - & $\mathrm{SW}, \mathrm{T}^{-}$ & - & - & $a *+2$ & & - & N. 10 & 二 \\
\hline I enu & $29 \cdot 16^{\circ} 2$ & -1 & $1-$ & $-7 \cdot 2$ & - & -1 & - & $1-1$ & - & - & & - & - & - \\
\hline
\end{tabular}

Min. temp, at Cape Armitage, $-20^{\circ} \cdot 5$. Wind li, 4, wh th unwh arlit snow.

10 p.m. and Mikt. Observatlons of temperature were taken from the screen on board.

Overeast and elondy most of the day. Wind strong from $\mathbf{b}$, cliangIng and increasing nintll it blew a sirmig gale from SW, Barmenter very unsteacy, but. falling grmeraily. remperatine stcadier than is usual with a SW wind. 
MFtrolotogical, Journat, OF THE "Discovery."

[1902-August.

\begin{tabular}{|c|c|c|c|c|c|c|c|c|c|c|c|c|c|c|c|}
\hline \multirow{3}{*}{ 11our. } & \multirow{3}{*}{ 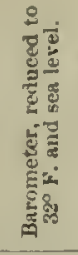 } & \multicolumn{5}{|c|}{ Theruometers. } & \multicolumn{3}{|l|}{ Whind. } & \multirow[b]{3}{*}{ 总 } & \multirow[b]{3}{*}{ 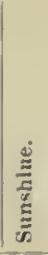 } & \multirow{2}{*}{\multicolumn{2}{|c|}{$\begin{array}{l}\text { Clsuls, } \\
\text { Amsunt mul novenurent. }\end{array}$}} & \multirow[b]{3}{*}{ 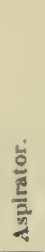 } & \multirow{3}{*}{ llemarks. } \\
\hline & & \multirow{2}{*}{ 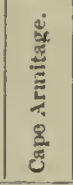 } & \multirow{2}{*}{ 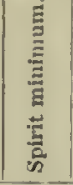 } & \multicolumn{2}{|c|}{ Mrreurlut. } & \multirow{2}{*}{ 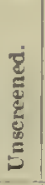 } & \multirow{2}{*}{$\begin{array}{c}\text { Directlom and } \\
\text { force } \\
\text { (Beasifort scale). }\end{array}$} & \multirow{2}{*}{ 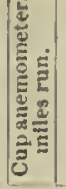 } & \multirow{2}{*}{. } & & & & & & \\
\hline & & & & Dry. & Wet. & & & & & & & Upper. & Lower. & & \\
\hline 2 & $29 \cdot 128$ & - & - & $-*+\cdot \theta$ & - & - & $S E, 6$ & - & - & $0 * \div 3$ & & - & N. 10 & - & \\
\hline 4 & $29 \cdot 136$ & - & - & $-7 \cdot 0$ & - & - & $\mathrm{S}, 8$ & - & - & $0 *+$ & & - & N. 10 & - & - 2, 4, ani bia.m. Olmervatlonis of tem- \\
\hline 6 & $29 \cdot 106$ & - & - & $-4 \cdot 0$ & - & - & Sw, 8 & - & - & (nq $* f+3$ & & - & N. 10 & - & $\begin{array}{l}\text { pes inoart. } \\
\text { ous }\end{array}$ \\
\hline 8 & $39 \cdot 132$ & - & $-9 \cdot 5$ & $-4 \cdot 8$ & - & - & $S_{1}, 2-3$ & - & $10-30$ & $\operatorname{sq} *+$ & & - & N. 10 & - & \\
\hline 10 & $29 \cdot 179$ & - & $-6 \cdot 2$ & $-5 \cdot 8$ & - & - & $\mathrm{ESF}_{2}, 2-3$ & 15 & $15-210$ & $n q+$ & & - & Fr.-11. 10 & - & \\
\hline Nom & $29 \cdot 171$ & $-11 \cdot 5$ & $-8 \cdot 5$ & $-6 \cdot 2$ & - & - & SSE, 1-2 & 15 & $10-13$ & bv & & - & - & - & Mu, temp, at Cape Armitage, $-11^{\circ} 5$. \\
\hline 2 & $29 \cdot 173$ & - & $-13+0$ & $-9 \cdot 8$ & - & - & liSE, 1-2 & 10 & $10-12$ & b & & 一 & - & - & Eretuss smoke golng to N Is. \\
\hline 4 & $29 \cdot 155$ & - & $-11 \cdot 8$ & $-10 \cdot 8$ & - & - & liNK, 1-3 & 10 & $15-18$ & b & & - & - & - & \\
\hline 6 & $29 \cdot 117$ & - & $-12 \cdot 8$ & $-11 \cdot 0$ & - & - & Nli, 2-3 & 13 & 10 & th & & - & - & - & \\
\hline 8 & $29 \cdot 091$ & - & $-12 \cdot 6$ & $-8 \cdot 8$ & - & - & $\mathrm{NF}, 2-3$ & 13 & $10-23$ & $\operatorname{ling}$ & & - & - & - & \\
\hline 10 & $29 \cdot 0.55$ & - & $-10 \cdot 2$ & -58 & - & - & Nis, 0-1 & 1.3 & - & le & & $A .-5$ & - & - & $\begin{array}{l}\text { Overcast mutll } 10 \text { a.uu., then elearing } \\
\text { sud renalnlug elear untll in lunlglit. }\end{array}$ \\
\hline Nlint. & $28 \cdot 9: 00$ & - & $-10 \cdot 0$ & $-8 \cdot 8$ & 一 & $\underline{-}$ & $\mathrm{N}$ hy $\mathbf{W}, 2$ & 5 & - & c & & - & S. 10 & - & When it agaln fiecame overeast. If hil \\
\hline Mean & $29 \cdot 119$ & - & $:-$ & $-7 \cdot 1$ & - & - & - & $1-1$ & 一 & - & & - & - & - & $\begin{array}{l}\text { heavy drift aul suow fall : max, ruis } \\
\text { of clins for } 2 \text { liours, } 46 \text { miles. Baro- }\end{array}$ \\
\hline & the & rea & $\{8$ & a. - & So & & ttion $\left\{\begin{array}{l}\text { black bul } \\
\text { bright bu }\end{array}\right.$ & - & & n. $\{1$ & & ie in 24 hours. & Preclpn. & & 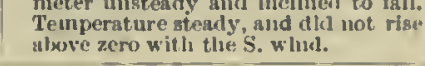 \\
\hline
\end{tabular}

\begin{tabular}{|c|c|c|c|c|c|c|c|c|c|c|c|c|c|}
\hline 2 & $28 \cdot 908$ & - & $|-10 \cdot 5-7 \cdot 8|$ & - & $1-$ & N by W, 1-2 & 2 & - & 0 & - & S.cu. 10 & - & \multirow{4}{*}{ N'Ine, damp вnow fallung. } \\
\hline 4 & $28 \cdot 824$ & - & $-8 \cdot 5-4 \cdot 0$ & - & - & $S F, 0-1$ & 2 & - . & uq $*$ & - & N. 10 & - & \\
\hline c & 28.775 & - & $-5 \cdot 0 \quad 2 \cdot 0$ & - & - & $\mathrm{N}$ by $\mathrm{W}, 2$ & 9 & - & $\operatorname{ogq} *$ & - & N. 9 & - & \\
\hline 8 & $28 \cdot 732$ & - & $-1 \cdot 3-0.5$ & - & - & Lt. W alrs & 6) & - & o* & - & N. 10 & - & \\
\hline 10 & $28 \cdot 746$ & - & $-2 \cdot 6 \quad 1 \cdot 2$ & - & - & NW, 1 & 4 & - & $\circ *$ & - & N. 10 & - & \multirow{7}{*}{$\begin{array}{l}\text { Very fine snow falling. } \\
\text { Min. temp. at Cape Armitige, }-20^{\circ} \cdot 0 \text {; } \\
\text { temperature falling quiekly. Wini } \\
\text { LSli, 2. Air very thlck witli Buow. }\end{array}$} \\
\hline Noon & $28 \cdot 669$ & $-18 \cdot 0$ & $\begin{array}{ll}-1.2 & 0.8\end{array}$ & - & - & Lt. NE airs & 4 & - & o* & - & N. 10 & - & \\
\hline 2 & $28 \cdot 634$ & - & $-8 \cdot 6-3 \cdot 6$ & - & $1-$ & Lt. $\mathrm{N}$ airs & 6 & - & o* & - & N. 10 & - & \\
\hline 4 & $2 A \cdot 59 k$ & - & $-8 \cdot 5-6 \cdot 2$ & - & - & Filyy N, 1-2 & 7 & - & o* & - & N. 10 & - & \\
\hline 6 & $28 \cdot 592$ & - & $-13 \cdot 0-11 \cdot 5$ & - & - & $\mathrm{NF}_{0}$ :-4 & 16 & $10-22$ & $0 *+$ & - & N. 10 & - & \\
\hline 8 & $28 \cdot 597$ & - & $-17 \cdot 0-16 \cdot 2$ & - & - & E, $2-3$ & 19 & $10-15$ & $\ln \rightarrow$ & - & - & - & \\
\hline 10 & $28 \cdot 613$ & - & $-17 \cdot 0-11 \cdot 0$ & - & - & $15,2-4$ & 19 & $12-20$ & o & - & $A_{a}=8.10$ & - & \\
\hline Mislt. & $28 \cdot 612$ & - & $-21 \cdot 1-20 \cdot 0$ & - & - & F by N, 5 & 23 & - & $0 \div$ & - & A.-s. 10 & - & \multirow{3}{*}{ 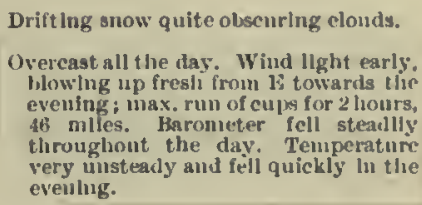 } \\
\hline Meaul & $2 k \cdot 692$ & - & $\overline{-}|\overline{-6 \cdot 4}|$ & - & $\overline{1}$ & - & 1 & - & - & - & - & - & \\
\hline & Mnx. & herm. & read at $\left\{\begin{array}{l}s \mathrm{a}, \\
8 \mathrm{p} .\end{array}\right.$ & & & Solar rauliatlon & ack & $\begin{array}{l}\text { ulb } \\
\text { bult }\end{array}$ & - . & lvмри. - grauйаs. & Precipu. - & & \\
\hline
\end{tabular}

2nd.

\begin{tabular}{|c|c|c|c|c|c|c|c|c|c|c|c|c|}
\hline 2 & $28 \cdot 6+0$ & - & $-21 \cdot 8-20 \cdot 8$ & - & - & Fi lyy N, 4-5 & 20 & $15-25$ & $0+$ & Cl. -8.10 & - & - \\
\hline 4 & $2 x \cdot(i 23)$ & - & $-22 \cdot 0-21 \cdot 0$ & - & - & $\mathrm{E}, \mathrm{G}-\bar{\tau}$ & 30 & $25-35$ & 0 & - & - & - \\
\hline$b^{\circ}$ & $28 \cdot \sin 2$ & - & $-23 \cdot 1-22 \cdot 0$ & - & - & E liy $N$, fi- & 10 & $25-35$ & $0+$ & - & - & - \\
\hline 8 & $28 \cdot 741$ & - & $-24 \cdot 3-22 \cdot 8$ & - & - & $\mathrm{Nl}, 6$ & 38 & $20-30$ & $0+$ & - & - & - \\
\hline 10 & $28 \cdot 76.5$ & - & $-24 \cdot 2-23 \cdot 0$ & - & - & ENY?, 4-5 & 16 & $25-30$ & $\mathrm{oq} \div$ & - & S. 10 & - \\
\hline Niron, & $28 \cdot 813$ & - & $-26 \cdot 0 \mid-25 \cdot 2$ & - & - & N1:, 3-5 & 18 & $25-34$ & $\mathrm{nq}+$ & - & S. 10 & - \\
\hline 2 & $28 \cdot 858$ & - & $-2 n \cdot 2-25 \cdot 0$ & - & - & F by $\mathrm{N}, 3-\overline{3}$ & 42 & $25-38$ & $\operatorname{sq}+$ & - & S. 10 & - \\
\hline 4 & $29 \cdot 880$ & - & $-25 \cdot 8-21 \cdot 6$ & - & - & N $1, y, 3$ & 24 & 20-27 & oq & - & S. 10 & - \\
\hline 6 & $28 \cdot 879$ & - & $-23 \cdot 8-19 \cdot 0$ & - & - & E., 1-2 & 15 & - & $o q$ & - & S. 10 & - \\
\hline 8 & $28 \cdot 849$ & - & $-26 \cdot 6-15 \cdot 2$ & - & - & FNK, 0-1 & 10 & - & 0 & - & S. 10 & - \\
\hline 1) & $28 \cdot 804$ & - & $-25 \cdot 0-22 \cdot 8$ & - & - & $\mathrm{NNF}_{4}, 1$ & 5 & - & 0 & - & S. 10 & - \\
\hline Mlint. & $2: 3 \cdot 720$ & - & $-25 \cdot 0-14 \cdot 0$ & - & - & Calm & b & - & 0 & - & S. 10 & - \\
\hline Mean & $28 \cdot \pi \pi 1$ & 二 & $--21 \cdot 0$ & - & - & - & -1 & - & - & - & - & - \\
\hline
\end{tabular}

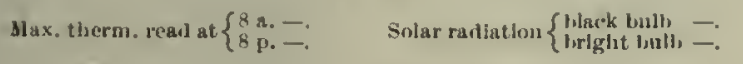

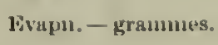

Preclpn. - In 3rd.

veresst all day. Wind stroug from the 1:, fallug light lu evening; max. Bammeter rose steallly unt il 6 p.m. then fell quickly. Temperature low and steary. 
1902 - A ugust.]

Mfetforological Jovinal of the "Discoviry."

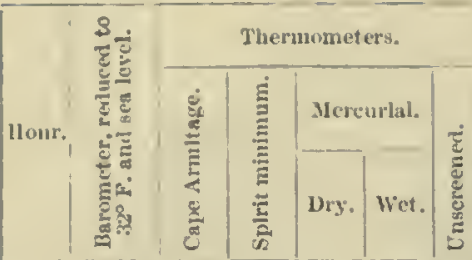

\begin{tabular}{|c|c|c|c|}
\hline Wind. & & & \\
\hline $\begin{array}{c}\text { Direction and } \\
\text { forre } \\
\text { (Beanfort senie). }\end{array}$ & 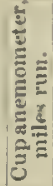 & 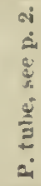 & 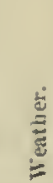 \\
\hline
\end{tabular}

4 th.

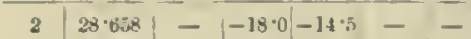

$428 \cdot 605--16 \cdot 0-12 \cdot 5-$

$622 \cdot 1 \% 3--13 \cdot 5-12.5-$

$828 \cdot 403--23 \cdot 0-22 \cdot 1-$

$10 \mid 28 \cdot 521--24 \cdot 5-26 \cdot 2-$

$x_{0011} 28 \cdot 580--2 k \cdot 8-25 \cdot 11--$

$228 \cdot 579--23 \cdot 5-25 \cdot 4-1$ -

$4 \quad 28 \cdot 639--28 \cdot 0-27 \cdot 0-1-$

$628 \cdot 683--28 \cdot 5-24 \cdot 0-1-$

$828 \cdot 714--23 \cdot 0-25 \cdot 5-1-$

$10|2 x \cdot 803|-\mid-26 \cdot 0,-23 \cdot 4-$ -

Mistt. $28 \cdot 805-20^{\circ} 0-22^{\circ} 0-$

Мени $28 \cdot 636,-\overline{-2103}-$

Max. therm. read at $\left\{\begin{array}{l}8 \text { a. }-12^{\circ} \cdot 2 . \\ 8,-20^{\circ} \cdot 0\end{array}\right.$

\begin{abstract}
Calm, $00-10$
NF, 1-2

lis ly N, 2

Fi ly $\mathrm{N}, 2-3$

12N พ, 5-6

20 :

$18-16 y+$

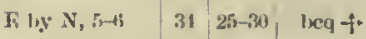

E ly $\mathrm{N}, 4-5$, 22 15-25 lec + +

E $\lg N, 5-6 \quad 23 \quad 30-355$ og $f$

F. lig $N, 2-3$

Fi, $\mathrm{S}, 1$

$$
21-\text { oq }
$$
\end{abstract}

F. ly. N, 1-2

\begin{abstract}
$10:-$
\end{abstract}
Solar radiaxion $\left\{\begin{array}{l}\text { biack bull, } \\ \text { liriglit bull, }- \text {. }\end{array}\right.$

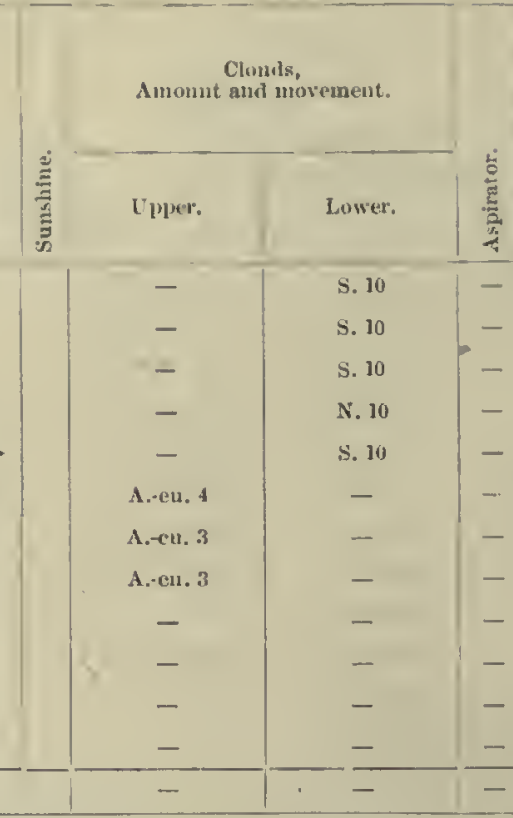

livapu. - grammes.
Precipn. - In. Ohservat lons taken without a lamp.
1.10 p.m. Tronp. at Cape Arultage $-25^{\circ} \cdot 5$, min., $-28^{\circ} \cdot 5$.

Reonarks.

J'ressure-tul,e anemometer not working properly.

Overcast, with little snow; (denrlug lu the evenlng. Wind rose irom calm to strong Iily at nonw, fallting again in evening: max. rum of cups tor 2 hours, inelined to rlse. Temperature low and steady. 5th.

\begin{tabular}{|c|c|c|c|c|c|c|}
\hline 2 & $28 \cdot 827$ & - & $-26 \cdot 0$ & $-24 \cdot 5$ & - & - \\
4 & $28 \cdot 818$ & - & $-25 \cdot 2-24 \cdot 8$ & - & - \\
6 & $28 \cdot 788$ & - & $-29 \cdot 6$ & $-29 \cdot 0$ & - & - \\
8 & $28 \cdot 77$ & - & $-29 \cdot 8-28 \cdot 0$ & - & - \\
10 & $28 \cdot 757$ & - & $-30 \cdot 1$ & $-28 \cdot 9$ & - & - \\
Noon & $28 \cdot 721$ & $-29 \cdot 4$ & $-29 \cdot 4$ & $-26 \cdot 8$ & - & - \\
2 & $28 \cdot 645$ & - & $-29 \cdot 0-28 \cdot 2$ & - & - \\
4 & $28 \cdot 685$ & - & $-30 \cdot 8-30 \cdot 0$ & - & - \\
6 & $28 \cdot 681$ & - & $-31 \cdot 6-30 \cdot 5$ & - & - \\
8 & $28 \cdot 679$ & - & $-32 \cdot 8-31 \cdot 5$ & - & - \\
10 & $28 \cdot 677$ & - & $-33 \cdot 5-30 \cdot 2$ & - & - \\
Mid1. & $28 \cdot 659$ & - & $-31 \cdot 5$ & $-30 \cdot 5$ & - & - \\
\hline Mean & $28 \cdot 731$ & - & - & $-28 \cdot 6$ & - & -
\end{tabular}

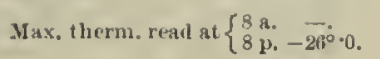

\begin{tabular}{|c|c|c|c|c|c|}
\hline SSIE, 1-2 & 11 & - & $b$ & - & - \\
\hline SSF, 2 & 10 & - & b) & - & - \\
\hline ENE, 4-5 & 18 & - & b) & - & - \\
\hline NE, 5 & 25 & $10-23$ & b & - & - \\
\hline $\mathrm{NE}, \mathrm{B}$ & 23 & $20-28$ & oq & - & S. 10 \\
\hline NE, 6 & 21 & $15-20$ & $n q$ & - & 5. 10 \\
\hline NF, 3-4 & 18 & $15-20$ & leq & A.eu. 6 & S. 4 \\
\hline E: lig $N, 3-4$ & 19 & $10-17$ & $o q$ & - & S. 11 \\
\hline $\mathrm{K}, 3$ & 17 & $13-15$ & o & - & s. 10 \\
\hline NE, 2-3 & 17 & $10-16$ & b & - & - \\
\hline $\mathrm{NL}, 2-3$ & 15 & $10-13$ & b) & - & - \\
\hline ENE, 3 & 13 & $0-20$ & b $y$ & - & - \\
\hline- & - & - & - & $\overline{-}$ & - \\
\hline
\end{tabular}

Faint ammo stramer In SSW.

Clear most of the day. Wind rislug till noon and fallng in ereuhng: max. Barometer falling slightis: all day. Temperature lower and fitling. 6th.

\begin{tabular}{|c|c|c|c|c|c|c|}
\hline 2 & $28 \cdot 6 \cdot 97$ & - & $-31 \cdot 0$ & $-32 \cdot 5$ & - & - \\
4 & $28 \cdot 669$ & - & $-31 \cdot 0$ & $-31 \cdot 5$ & - & - \\
6 & $28 \cdot 663$ & - & $-37 \cdot 0$ & $-34 \cdot 5$ & - & - \\
8 & $28 \cdot 66 \cdot 2$ & - & $-38 \cdot 9$ & $-38 \cdot 0$ & - & - \\
10 & $28 \cdot 671$ & - & $-39 \cdot 2$ & $-37 \cdot 5$ & - & - \\
Noon & $28 \cdot 653$ & - & $-38 \cdot 4$ & $-38 \cdot 6$ & - & - \\
2 & $28 \cdot 639$ & - & $-39 \cdot 0$ & $-38 \cdot 5$ & - & - \\
4 & $28 \cdot 635$ & - & $-40 \cdot 5$ & $-39 \cdot 0$ & - & - \\
6 & $28 \cdot 622$ & - & $-41 \cdot 5$ & $-40 \cdot 0$ & - & - \\
8 & $28 \cdot 608$ & - & $-43 \cdot 0$ & $-39 \cdot 8$ & - & - \\
10 & $28 \cdot 609$ & - & $-40 \cdot 8$ & $-33 \cdot 8$ & - & - \\
Midt. & $28 \cdot 577$ & - & $-36 \cdot 0$ & $-31 \cdot 0$ & - & - \\
\hline Mean & $28 \cdot 640$ & - & - & $-343 \cdot 3$ & - & - \\
\hline
\end{tabular}

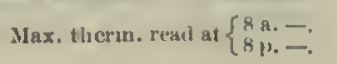

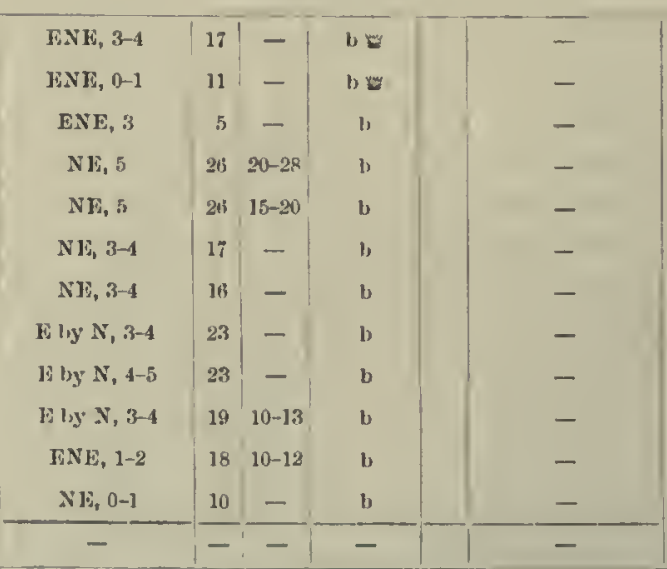

Solar maliat lon $\left\{\begin{array}{l}\text { black linll } \\ \text { bright thilb }- \text {. }\end{array}\right.$
Bvapณ. - yrammes.

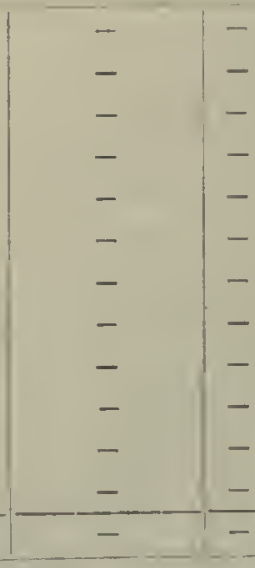

l'revil.n. - In. Long shatlow arches of auroral streamers
issning from dark elonds from liNli 4 a.m. (idd streamers in Nl:

$-$

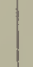

$-$

Dines. pressure-tulue ancmomcter not working properly.

Fine and clear all day. Wind wy, strong; max. run of cupss for 2 honrs, 54 miles. Barometer stendler, but falling sllghtly. Temperatnre lower and talling. Making ohservations is far from behig a pleasant task lin this temperature. 


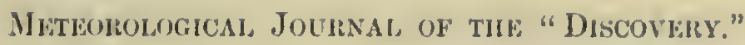

[1902- August.

\begin{tabular}{|c|c|c|c|c|c|c|c|c|c|c|c|c|c|c|c|}
\hline \multirow{3}{*}{ Hentr. } & \multirow{3}{*}{ 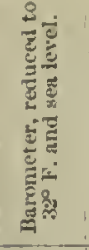 } & \multicolumn{5}{|c|}{ Thermometers. } & \multicolumn{3}{|l|}{ Whit. } & \multirow[b]{3}{*}{ 选 } & \multirow[b]{3}{*}{ 岂 } & \multirow{2}{*}{\multicolumn{2}{|c|}{$\begin{array}{l}\text { Clouds, } \\
\text { Amount and novement. }\end{array}$}} & \multirow[b]{3}{*}{$\frac{\sum^{2}}{2}$} & \multirow{3}{*}{ Itemarks. } \\
\hline & & \multirow{2}{*}{ 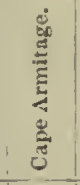 } & \multirow{2}{*}{ 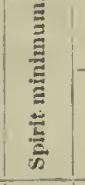 } & \multicolumn{2}{|c|}{ Mercurial. } & \multirow{2}{*}{ 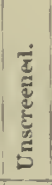 } & \multirow{2}{*}{$\begin{array}{c}\text { Direstion and } \\
\text { forte } \\
\text { (Beafort scnle). }\end{array}$} & \multirow{2}{*}{ 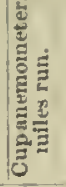 } & \multirow{2}{*}{ 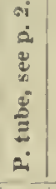 } & & & & & & \\
\hline & & & & Dry. & Wet. & & & & & & & C'pper. & lower. & & \\
\hline 2 & $28 \cdot 596$ & - & $-34 \cdot 2$ & $-3.4 \cdot 0$ & - & - & Calm & 1 & - & $\ln$ & & - & - & - & \\
\hline 4 & $28 \cdot 813$ & - & $-31 \cdot 5$ & $-32 \cdot 9$ & - & - & Culm & 0 & - & b. & & - & - & - & Faint curtain of aurora hit $\mathrm{N}$, wlt. $10^{\circ}$, \\
\hline 6 & $28 \cdot 619$ & - & $-35 \cdot 6$ & $-29 \cdot 1$ & - & - & Calın & 0 & - & $1,2-$ & & - & - & - & $\begin{array}{l}\text { Fahit sinnl-arch of iveams lu s to } \mathrm{sl} \text {, } \\
\text { alt. } 30^{\circ} \text {. }\end{array}$ \\
\hline 8 & $28 \cdot 648$ & - & $-44 \cdot 0$ & $-42 \cdot 5 *$ & 一 & - & Calm & 0 & $\rightarrow$ & $\ln n$ & & - & - & - & 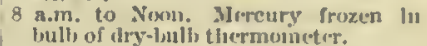 \\
\hline 10 & $28 \cdot 668$ & - & $-49 \cdot 5$ & $-47 \cdot 0^{*}$ & - & - & Calm & 0 & - & b & & - & - & - & \\
\hline Nonn & $28 \cdot 704$ & $-5 i \cdot 5 \mid$ & $-50 \cdot 5$ & $-48 \cdot 5 *$ & - & - & Calm & 0 & - & $\mathrm{lm}-$ & & - & - & - & White fog. Min.tenp. nt Cape Arml. \\
\hline 2 & $28 \cdot 723$ & - & $-50 \cdot 2$ & $-31 \cdot 0$ & - & - & NE, 1 & 1 & - & $\ln$ & & - & - & - & 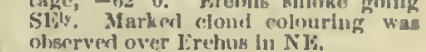 \\
\hline 4 & $28 \cdot 745$ & $-52 \cdot 4$ & $-33 \cdot 8$ & $-33 \cdot 2$ & - & - & E ly $N, 1-2$ & 6 & - & $1, U$ & & - & - & - & Min. temp. at Cape Armltage, $-57^{\circ} \cdot 8$. \\
\hline 6 & $28 \cdot 762$ & - & $-33 \cdot 8$ & $-32 \cdot 0$ & - & - & ENE, 1 & b & $\rightarrow$ & h & & - & - & - & \\
\hline 8 & $28 \cdot 790$ & - & $-35 \cdot 5$ & $-35 \cdot 0$ & - & - & FNI, 1 & 6 & - & 1, & & - & - & 一 & - learliugs of splrit thimnometer nt \\
\hline 10 & $28 \cdot 807$ & - & $-37 \cdot 0$ & $-33 \cdot 2$ & - & - & Nli, I & 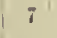 & - & $\mathrm{bm}$ & & - & - & - & \\
\hline Mint. & $28 \cdot 801$ & - & $-3 \pi \cdot 8$ & $-3 i \cdot 4$ & 一 & - & $\mathrm{N} 1 ;, 1$ & 10 & - & $1 \mathrm{~m}$ & & - & 一 & - & \multirow{3}{*}{ 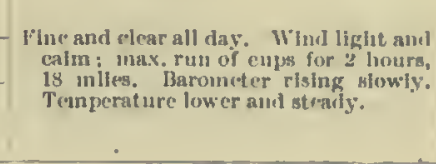 } \\
\hline \multirow[t]{2}{*}{ Mean } & $28 \cdot 70 \mathrm{Hi}$ & - & $1-$ & $-36 \div 5$ & - & -1 & - & $1-1$ & - & - & & - & - & - & \\
\hline & \multicolumn{5}{|c|}{ Iax. thems, read at $\left\{\begin{array}{l}8 \mathrm{a} . \\
8 \mathrm{p} .\end{array}\right.$} & \multicolumn{4}{|c|}{ Solar rarliation $\left\{\begin{array}{l}\text { black bulh } \\
\text { briglit bull, }- \text {. }\end{array}\right.$} & \multicolumn{3}{|c|}{ Evapn. - grammes, } & \multicolumn{2}{|l|}{ P'reilll. - in. } & \\
\hline
\end{tabular}

7 th.

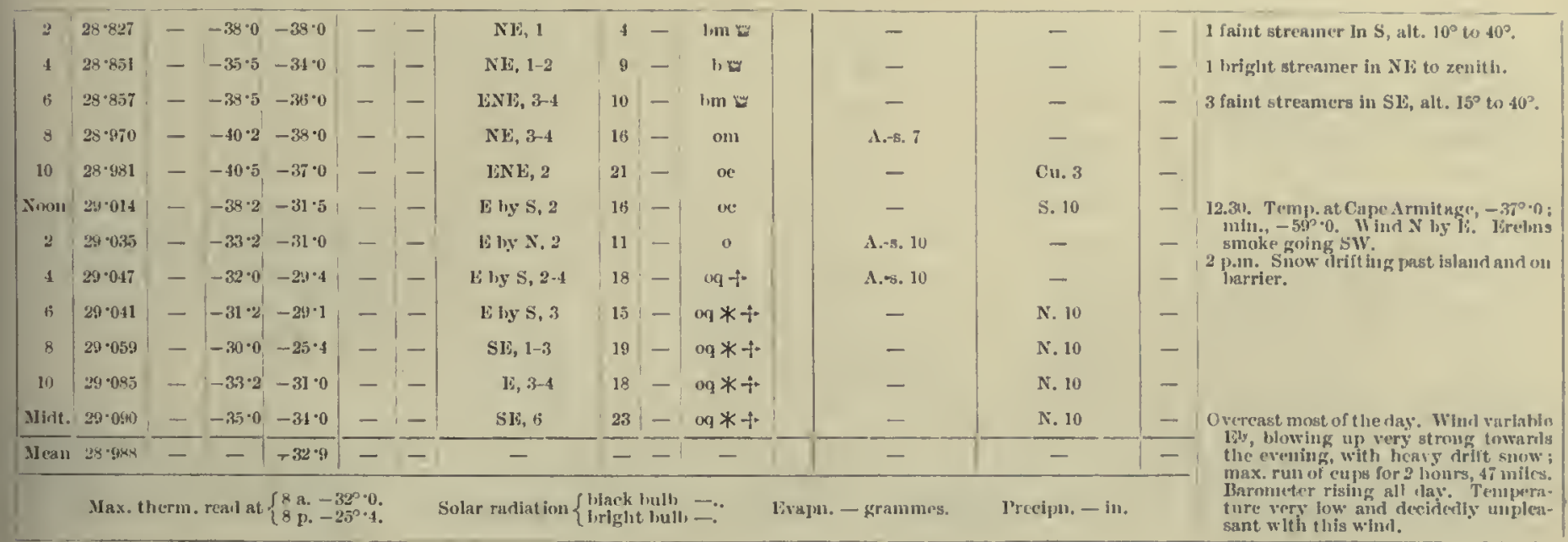

8th.

\begin{tabular}{|c|c|c|c|c|c|c|c|c|c|c|c|c|c|}
\hline 2 & $24 \cdot 113$ & - & $-34 \cdot 4$ & $-29 \cdot 0$ & - & - & S, 7-8 & 26 & $-\log * t^{2}$ & - & N. 10 & - & ind slifted to lo aud dropped, \\
\hline 4 & 2406 & - & $\mid-35 \cdot 1$ & $-29 \cdot 8$ & - & - & E $1, y$ S, 3-5 & 15 & $-b q+$ & - & - & - & \multirow{13}{*}{ 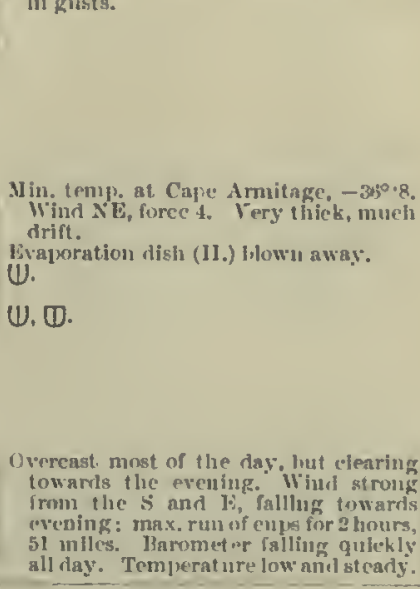 } \\
\hline is & $2 \div \cdot 018$ & - & -30.2 & -270 & - & - & $16,3-5$ & 18 & $1,9+$ & - & - & - & \\
\hline 8 & $28 \cdot 8 \times$ & - & $-29 \cdot 3$ & $-27 \cdot 5$ & - & - & $F, 1, y s, 3-4$ & 15 & $m+$ & - & s. 10 & - & \\
\hline 10 & $24.9+10$ & - & -30045 & $-30 \cdot 5$ & - & - & E. I, S S, 3-4 & 18 & $-\infty * i$ & - & N. 10 & - & \\
\hline Noon & $24 \cdot 876$ & $-34 \cdot 0$ & $-32 * 4$ & $-32 \cdot 0$ & - & - & E $1, y, 3,3-4$ & 23 & $-\log *+$ & - & N. 10 & - & \\
\hline 2 & $28 \cdot 84 i 1$ & - & $-31 \cdot 2 \mid$ & $-34 \cdot 0$ & - & - & $N \mathrm{~N}_{0}, 3-4$ & 22 & - о о $* \neq$ & - & N. 10 & - & \\
\hline 4 & $23 \cdot 838$ & - & $-31 \cdot 3$ & $-32 \cdot 0$ & - & - & $x E, 2-3$ & 18 & - Imact & C1.-s, 5 & - & - & \\
\hline B & $28: 807$ & - & $|-32 \cdot 4|$ & -30.5 & - & - & $\mathrm{N1}, 2$ & 11 & - hine to & Cl.s. 2 & - & - & \\
\hline 8 & 28.781 & - & $|-31 \cdot 3|$ & $-29 \cdot 0$ & - & - & $\mathrm{NIV}, 1-2$ & 13 & - & - & - & - & \\
\hline 1" & $28 \cdot 7638$ & - & $|-32 \cdot 0|$ & $-28 \cdot 2$ & - & - & l:NRE, 2 & 14 & $\mathrm{~cm}$ & - & 5. 8 & - & \\
\hline Misit. & $23 \cdot 728$ & - & $-3 n \cdot 0$ & $-24 \cdot 9$ & - & $=$ & $\mathrm{NE}, 0-1$ & i & him & $=$ & - & - & \\
\hline Ilean & $28 \cdot 8989$ & - & - & $-28 \cdot 5$ & - & - & - & $1-1$ & - & - & - & $1-$ & \\
\hline & .3 & & . & & & & 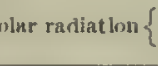 & reglit & & & n. & & \\
\hline
\end{tabular}


1902 - Angust.]

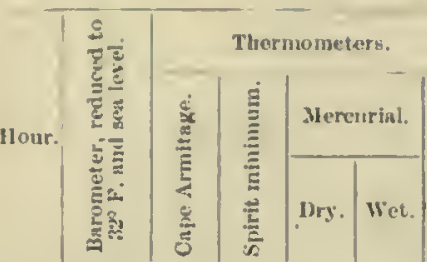

10 th.

\begin{tabular}{|c|c|c|c|c|c|c|}
\hline 2 & $2 x \cdot 733$ & - & $|-25 \cdot 7|$ & $|-23 \cdot 3|$ & - & - \\
\hline 4 & 28.725 & - & $-32 \cdot 6 \mid$ & $-28 \cdot 6$ & - & - \\
\hline 6 & $28 \cdot 725$ & - & -3014 & $-24 \cdot 5$ & - & - \\
\hline 8 & $2 \times-718$ & - & $-3 \div 0$ & $-31 \cdot 4 \mid$ & - & - \\
\hline 10 & $28.62 \times$ & - & $-37+4$ & -340 & - & - \\
\hline Noous & 28.72 & $-50 \cdot 1$ & $-35 \cdot 8$ & $-35 \cdot 0 \mid$ & - & - \\
\hline 2 & $2 x \cdot \pi 24$ & - & $-36 \cdot 2 \mid$ & $-32 \cdot 2$ & - & - \\
\hline 4 & 28.694 & - & -32 & $-27 \cdot 2$ & - & - \\
\hline 6 & $28 \cdot 712$ & - & $-24 \cdot 5$ & $-32 \cdot 8$ & - & $1-$ \\
\hline 8 & $28 \cdot 750$ & - & $-35 \cdot 0$ & $-30 \cdot 0$ & - & - \\
\hline 10 & 28.817 & - & $-30 \cdot 3$ & -28.8 & - & - \\
\hline Midt. & 28.868 & - & $-39 \cdot$ & $-3 k \cdot 8$ & - & $1-$ \\
\hline & 744 & - & - & $80 \cdot 8$ & - & - \\
\hline
\end{tabular}

Mlax. thenu, read at $\begin{cases}8 \text { a. } & -230 \cdot 0 . \\ 8 \text { p. } & -25^{\circ} 0 .\end{cases}$

Mhtholological, Jour.ya of tif "Discovery."

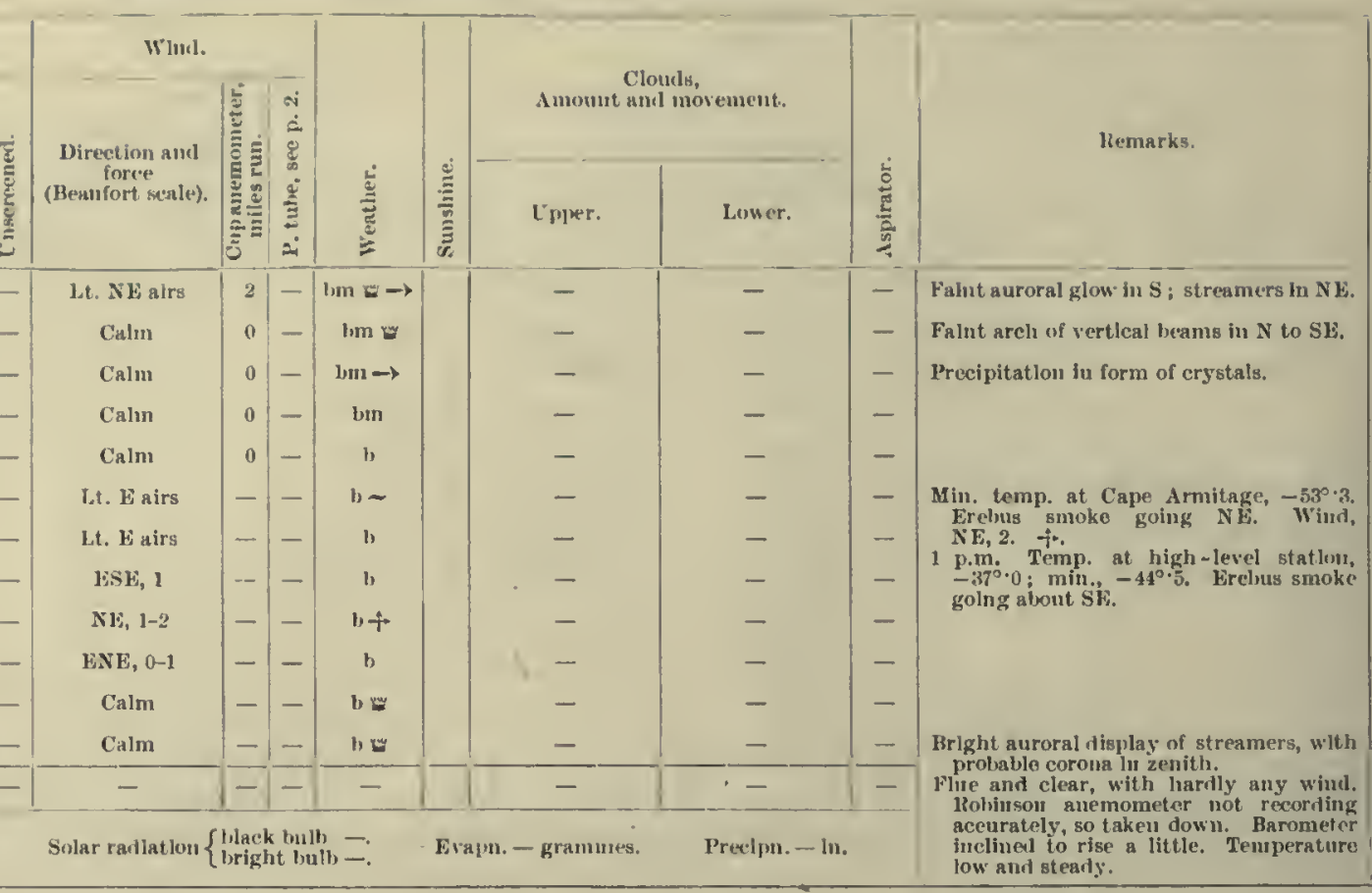

11 th

\begin{tabular}{|c|c|c|c|c|c|c|c|c|c|c|c|c|c|c|}
\hline 2 & $28 \cdot 893$ & - & $-33 \cdot 9$ & $|-29 \cdot 6|$ & - & -1 & NE, 2-3 & - & -1 & $115 \div$ & - & - & - & \multirow{14}{*}{ 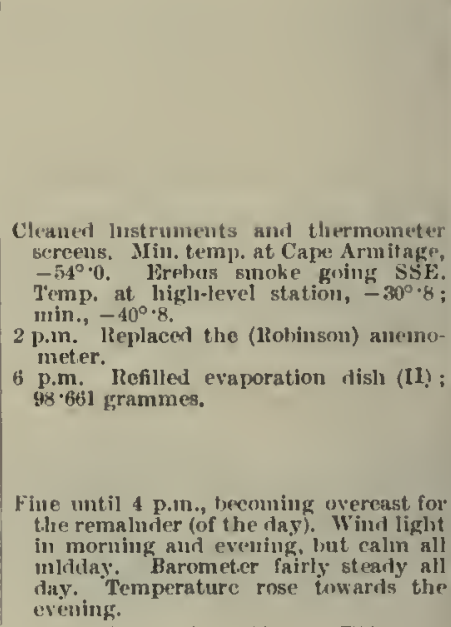 } \\
\hline 4 & $23 \cdot 911$ & - & $-30 \cdot 0$ & $-24 \cdot 2$ & - & - & N1: $1-2$ & - & - & $h=$ & - & - & - & \\
\hline is & $2 * 9.05$ & - & $-34 \cdot 2$ & $-33 \cdot 1 \mid$ & - & - & Calm & - & - & bm & - & - & - & \\
\hline 8 & $28 \cdot 912$ & - & $-37 \cdot 8$ & $|-20 \cdot 2|$ & - & - & N1:, 1-2 & - & - & b) & - & - & - & \\
\hline wu & $28 \cdot 912$ & - & $-30 \cdot 7$ & $|-29 \cdot 9|$ & - & - & Calun & - & - & $b \varphi$ & A. $\cdot 3,2$ & - & - & \\
\hline Noon & $28 \cdot 898$ & $|-3 B \cdot 0|$ & $-35 \cdot 2$ & $-31 \cdot 2$ & - & - & Calm & - & - & b) & A. 8.2 & - & - & \\
\hline 2 & $28 \cdot 8 i^{2}$ & - & $-34 \cdot 0$ & $-27 \cdot 6$ & - & - & Calm & - & - & inc & A.-3. 4 & - & - & \\
\hline 4 & $28 \cdot 860$ & - & $-3 ; 1 \cdot 0$ & $-26 \cdot 0$ & - & - & Calun & 0 & - & of $U$ & A.-s. 10 & - & - & \\
\hline is & $28 \cdot 847$ & - & $-27 \cdot 5$ & $-21 \cdot 2$ & - & - & Lt. Sli airs & 0 & - & of & A.-8. I10 & - & - & \\
\hline 8 & $28 \cdot 805$ & - & $-22 \cdot 5$ & $-18 \cdot 0$ & - & - & lis by s, 1-2 & 10 & - & $0-i$ & - & s. 10 & - & \\
\hline 10 & 28.800 & - & $-20 \cdot 0$ & $-17 \cdot k$ & - & - & vily $S, 1-2$ & 10 & - & a*+ & - & x. 10 & - & \\
\hline Minit & $2 x \cdot 81 x$ & - & $-25 \%$ & $-20 \cdot 0$ & - & - & E $1, y, 2-3$ & 19 & - & $\operatorname{seq} *$ & - & N. 10 & - & \\
\hline Mean & $2 x \cdot 866^{4}$ & - & - & $-24 \cdot x$ & - & - & - & - & -1 & - & - & - & - & \\
\hline \multicolumn{7}{|c|}{ Max, therm. reat at $\left\{\begin{array}{l}8 \mathrm{a}, \\
8 \mathrm{p} .\end{array}-20^{2} \cdot 0\right.}$. & Solar ratiation & rigl & & & Evapu. - grammes. & \multicolumn{2}{|l|}{ Precipu, - in. } & \\
\hline
\end{tabular}

12 th.

\begin{tabular}{|c|c|c|c|c|c|c|c|c|c|c|c|c|c|}
\hline 2 & $2 x \cdot 8014$ & - & $t-\mid-18 \cdot 0^{\prime}$ & - & $1-$ & $K \operatorname{lng} N, 2-3$ & 20 & - & oc $*+$ & - & N. 10 & - & \multirow{5}{*}{ 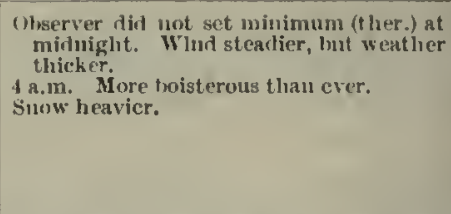 } \\
\hline 4 & $28 \cdot 7 \times 5$ & - & $-20 \cdot 2-18 \cdot 1$ & - & - & EN 1,4 & 23 & - & oc $*-1$ & - & N. 10 & - & \\
\hline b) & $2 x \cdot 821$ & - & $-19 \cdot 0-12 \cdot 5$ & - & $1-$ & $\mathrm{sk}, 2$ & 22 & - & $0 *+$ & - & N. 10 & - & \\
\hline 8 & $28 \cdot 811$ & - & $-14 \cdot 3-10 \cdot 2$ & - & - & lity $5,2-3$ & 21 & - & $0 * 42^{2}$ & - & N. 10 & - & \\
\hline 10 & $28 \cdot 857$ & - & $-14 \cdot 8-12 \cdot 5$ & -- & $1-$ & S12, 4-6 & 32 & - & oq $* * 2$ & - & N. 10 & - & \\
\hline Nowu & $2 x \cdot 815$ & - & $-14 \cdot 0-13 \cdot 2$ & - & - & S hy W, 5-6 & - & - & $\operatorname{og} *+$ & - & N. 10 & - & \multirow{4}{*}{$\begin{array}{l}\text { Snow got inside anemometer and made } \\
\text { Its indleations nureliable. }\end{array}$} \\
\hline 2 & $2 k \cdot 455$ & - & $-15 \cdot 4-11 \cdot 2$ & - & - & SSE, $6-7$ & - & - & on $*+$ & - & N. 10 & - & \\
\hline 8 & $28 \cdot 891$ & - & $-16 \cdot 2,-14 \cdot 0$ & - & - & Sk, 2-3 & - & - & $n+$ & - & N. 10 & - & \\
\hline 10 & $28 \cdot 875$ & - & $-15 \cdot 0-12 \cdot 5$ & - & -1 & ES15, 1-2 & - & - & $a+$ & - & N. 10 & - & \\
\hline Mislt. & $28 \cdot 8+11$ & - & $-15 \cdot(1-14 \cdot 1)$ & - & - & $8 n, 4$ & - & - & oq $* \div$ & - & N. 10 & - & \multirow{3}{*}{$\begin{array}{l}\text { Overcast all day. Wind very strong: } \\
\text { much snow drift. Warometer fairly } \\
\text { steady all day. Temperatere rlsing } \\
\text { stowly. }\end{array}$} \\
\hline Mean & $2 x \cdot 850$ & - & $-\overline{-14 \cdot \theta}$ & - & -1 & - & - & -1 & - & - & - & - & \\
\hline & $\begin{array}{l}\text { Inx. ther } \\
\text { rwal at }\end{array}$ & & & $\begin{array}{l}\text { Sula } \\
\text { rasliatl }\end{array}$ & & $\begin{array}{l}\text { k bull, } \\
\text { ght lonll 二. }\end{array}$ & & & tan & $\begin{array}{l}\text { urs. (i) plece } \\
\text { ff dish.) }\end{array}$ & Prexipu & & \\
\hline
\end{tabular}


Mateolological Joutinal of the "Discovery."

[1902-August.

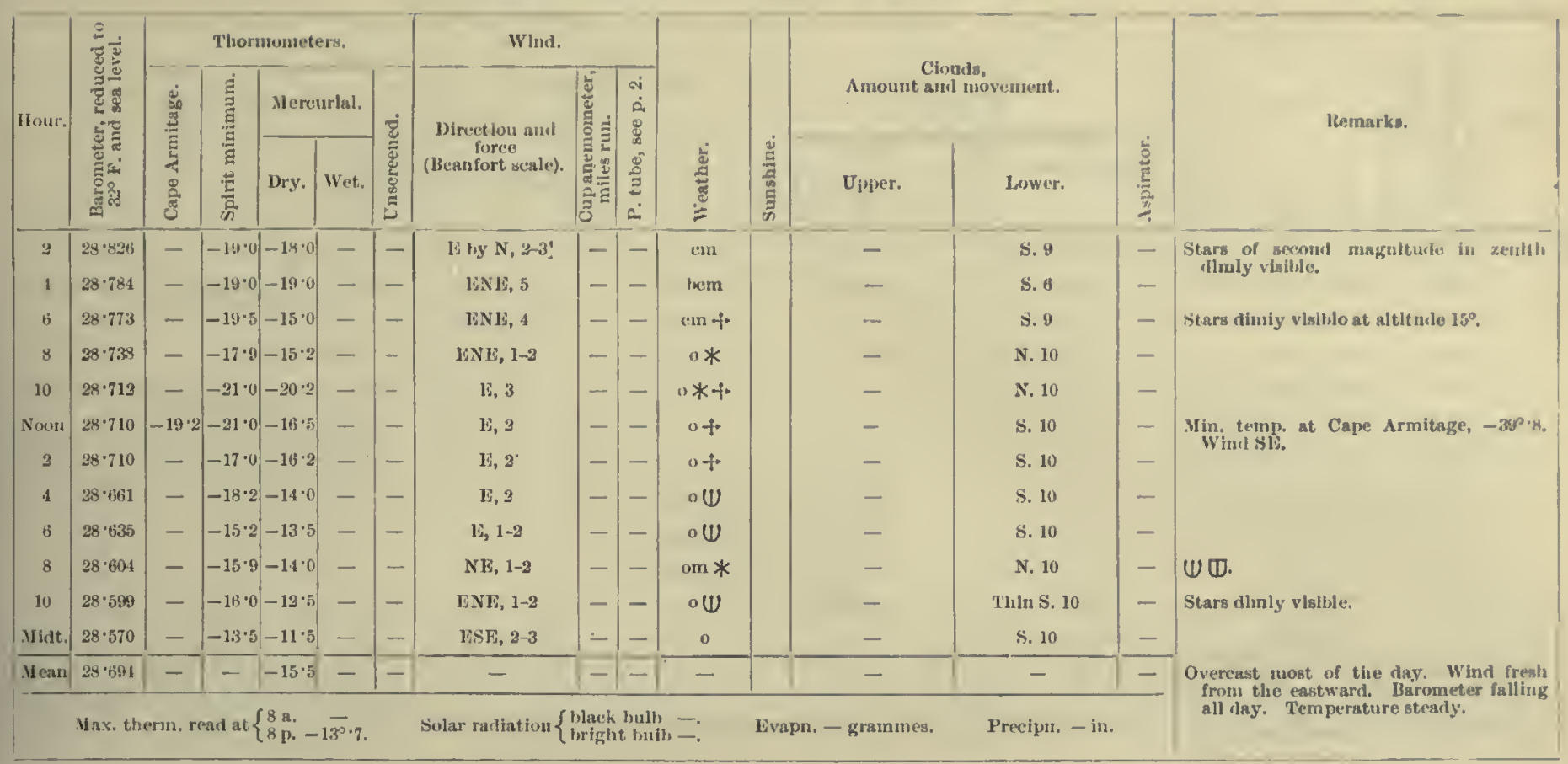

13 th.

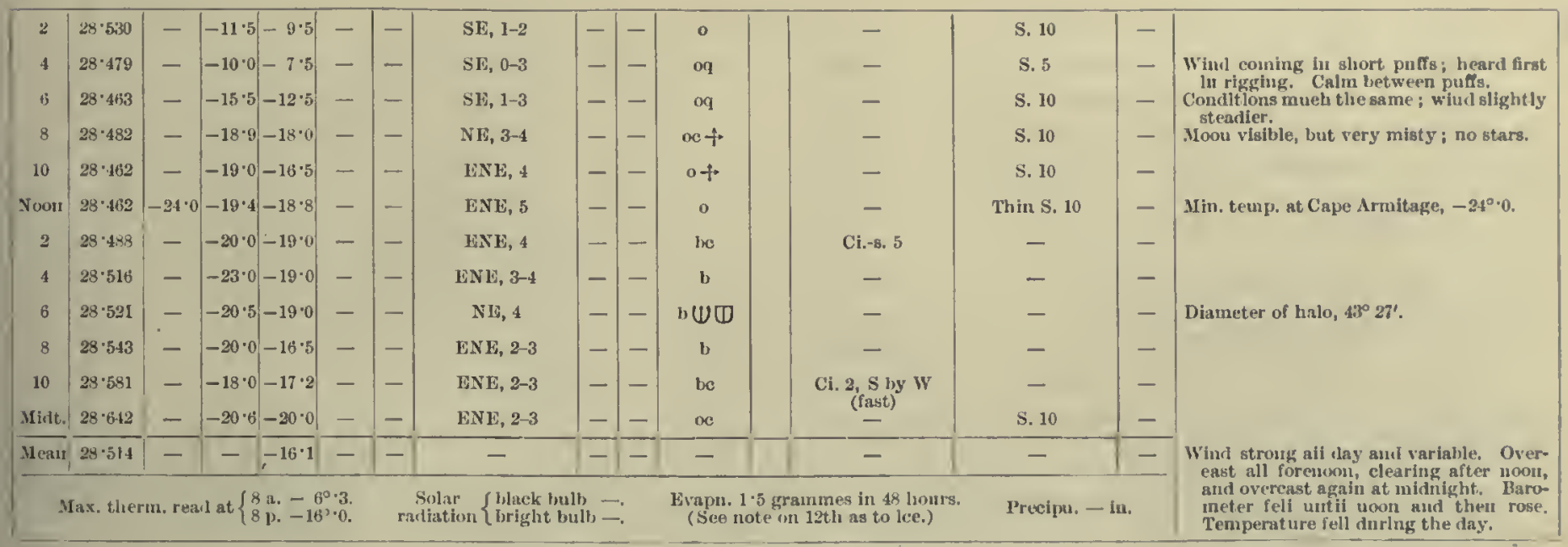

\begin{tabular}{|c|c|c|c|c|c|c|c|c|c|c|c|c|}
\hline 2 & $28 \cdot 704$ & - & $|-21 \cdot 7|-20 \cdot 2$ & $-\quad-1$ & ENE, 3-4 & - & -1 & oqm & - & S. 10 & - & \\
\hline 4 & 28.790 & - & $-22 \cdot 2-16 \cdot 8$ & $-\quad-1$ & ESL, 1-2 & - & - & $b \underline{y}$ & - & - & - & \\
\hline 6 & $28 \cdot 808$ & - & $-23 \cdot 8-18 \cdot 3$ & $-\quad-$ & EN1, 2-3 & - & - & b & - & $\cdots$ & - & \\
\hline 8 & 28.838 & - & $-21 \cdot 1-16 \cdot 1$ & $-\quad-$ & Lt. E sirs & - & - & b & - & - & - & \\
\hline 10 & $28 \cdot 83.3$ & - & $-22 \cdot 0-21 \cdot 2$ & -1 & $\mathrm{Culm}$ & - & - & b & - & - & - & \\
\hline Noon & $25 \cdot 883$ & $-37 \cdot 5$ & $-28.5-20 \cdot 0$ & $-\quad-$ & Lt. $\mathbf{E}$ alrs & - & - & 0 & A.-8. 10 & - & - & \multirow{9}{*}{ 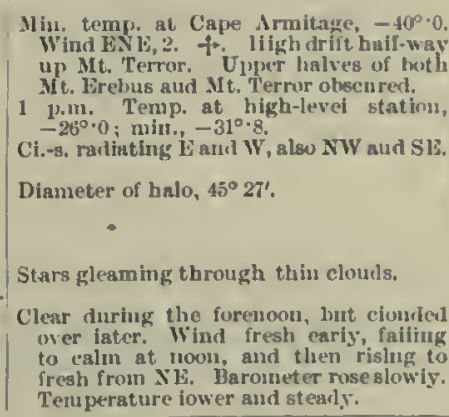 } \\
\hline 2 & $28 \cdot 8 \div 0$ & - & $|-21 \cdot 0-21 \cdot 0|$ & $-\quad-$ & $\mathrm{Nl}: 1-2$ & - & - & 0 & A.'8. 10 & - & - & \\
\hline 4 & $23 \cdot 889$ & - & $-27 \cdot 0-25 \cdot 2$ & $-\quad-$ & NL, 2-3 & - & - & o & A.-s. 10 & - & - & \\
\hline is & $2 x \cdot 893$ & - & $-26 \cdot 2-24 \cdot 5 \mid$ & $-\quad-$ & $\mathrm{NE}, 3$ & - & - & ix: $U$ & Cl.-8. 3 & - & - & \\
\hline 8 & $28 \cdot 882$ & - & $-26 \cdot 6-26^{\circ} 0$ & $-\quad-$ & $\mathrm{NI}, 4$ & - & - & ผ $U \Phi$ & A.-8. 4 & - & - & \\
\hline 10 & $28 \cdot 897$ & - & $-28 \cdot 5-27 \cdot 0 \mid$ & $-1-$ & ENE, 5 & - & - & $o c+$ & A.-6. 10 & - & - & \\
\hline Mirt. & $2 \times \cdot 893$ & - & $-28 \cdot 5-25 \cdot 5$ & -1 & $\mathrm{k}, 4$ & - & - & 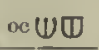 & A.-s. 10 & - & - & \\
\hline Siean & $2 x \cdot 853$ & - & $-\sqrt{-21 \cdot 9}$ & $-1-1$ & - & - & -1 & - & - & - & - & \\
\hline & a & & 1. - & 0. & & & & bis & mes in 24 & Precipn & & \\
\hline
\end{tabular}


1902-August.]

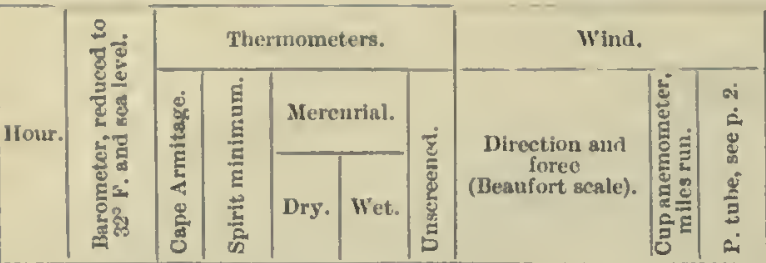

Metrokological Jounai of the “Discoveliy."

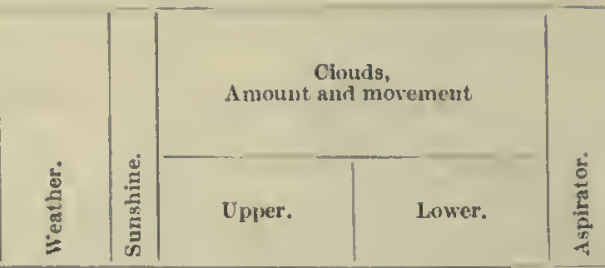

16 th.

\begin{tabular}{|c|c|c|c|c|c|c|c|c|c|c|c|c|c|}
\hline 2 & $28 \cdot 893$ & -1 & $-27 \cdot 8$ & -25.5 & - & -1 & E by $\mathbf{N}, 5$ & -1 & - & $o c+$ & A.-s. 10 & - & - \\
\hline 4 & $28 \cdot 882$ & - & $-26 \cdot 8$ & $-25 \cdot 2$ & - & - & $N E, 4-5$ & - & - & $\infty+U$ & A.-5. 10 & - & - \\
\hline 6 & $28 \cdot 85 i$ & - & $-2 B \cdot 2$ & $-2.5 \cdot$ & - & - & NE, 3-4 & - & - & inc & A. -8.3 & - & - \\
\hline 8 & $28 \cdot 854$ & - & $-26 \cdot 6$ & $-23 \cdot 8$ & - & - & $\mathrm{NE}_{\mathrm{N}} 1$ & - & - & b & - & - & - \\
\hline 10 & $28 \cdot 849$ & - & $-26 \cdot 0$ & $-25 \cdot 0$ & - & - & Calın & - & - & b & - & - & - \\
\hline Noou & $28 \cdot 851$ & - & $-26 \cdot 8$ & -22.8 & - & - & Caln & - & - & b & - & - & - \\
\hline 2 & $28 \cdot 848$ & - & $-27 \cdot 0$ & $-24 \cdot 0$ & - & - & Calm & - & - & D $U$ & - . & - & - \\
\hline 4 & $28 \cdot 851$ & - & $-31 \cdot 0$ & $-31 \cdot 0$ & - & - & Calm & - & - & be $U \rightarrow$ & Ci.-8. 5 & - & - \\
\hline 6 & $28 \cdot 830$ & - & $-32 \cdot 0$ & $-21 \cdot 0$ & - & - & Calm & - & - & $\circ U \rightarrow$ & Thin A.-8. 10 & - & - \\
\hline 8 & $28 \cdot 831$ & - & $-31 \cdot 5$ & $-25 \cdot 8$ & - & - & ESE, 2-3 & - & - & $\cdot U$ & Thin A.-s. 10 & - & - \\
\hline 10 & $28 \cdot 854$ & - & $-29 \cdot 0$ & $-25 \cdot 2$ & - & - & BSE, 2 & - & - & he $U \mathbb{0}$ & Thin A.-s. 5 & - & - \\
\hline Mint. & $28 \cdot 877$ & - & $-26 \cdot 3$ & $-22 \cdot 0$ & - & - & E by $5,3-4$ & - & - & 0 & - & S. 10 & - \\
\hline II ean & $28 \cdot 856$ & - & - & $-24 \cdot 7$ & - & - & - & - & - & - & - & - & - \\
\hline
\end{tabular}

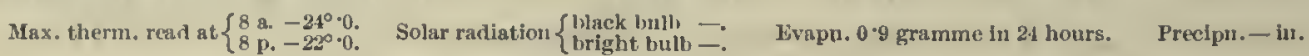

Remarks

U. ().

Bronglit thermometer sereens on hoard whitist digging out frame.

Very dark and ugly looking towards $\mathrm{S}$ and SW, with heavy stralus.

2.5. Temp; at Cape Armitage, $-45^{\circ} \cdot 2$; smoke going by 20. Temp. at Cape Armitage, $-45^{\circ} \cdot 2$; win., $-45^{\circ} \cdot 2$.

)iameter of lialo, $43^{\circ} 20^{\prime}$.

Overeast ln morning, fine midray, and again overcast in evening. Wind strong from NE early, falling to calm from $10 \mathrm{a} . \mathrm{m}$. to 6 p.m., and then rising to fresil from $\mathrm{S}$ of $\mathbf{1}$. Barometer fairly steaily all day. Temperature low and stealy:

17 th.

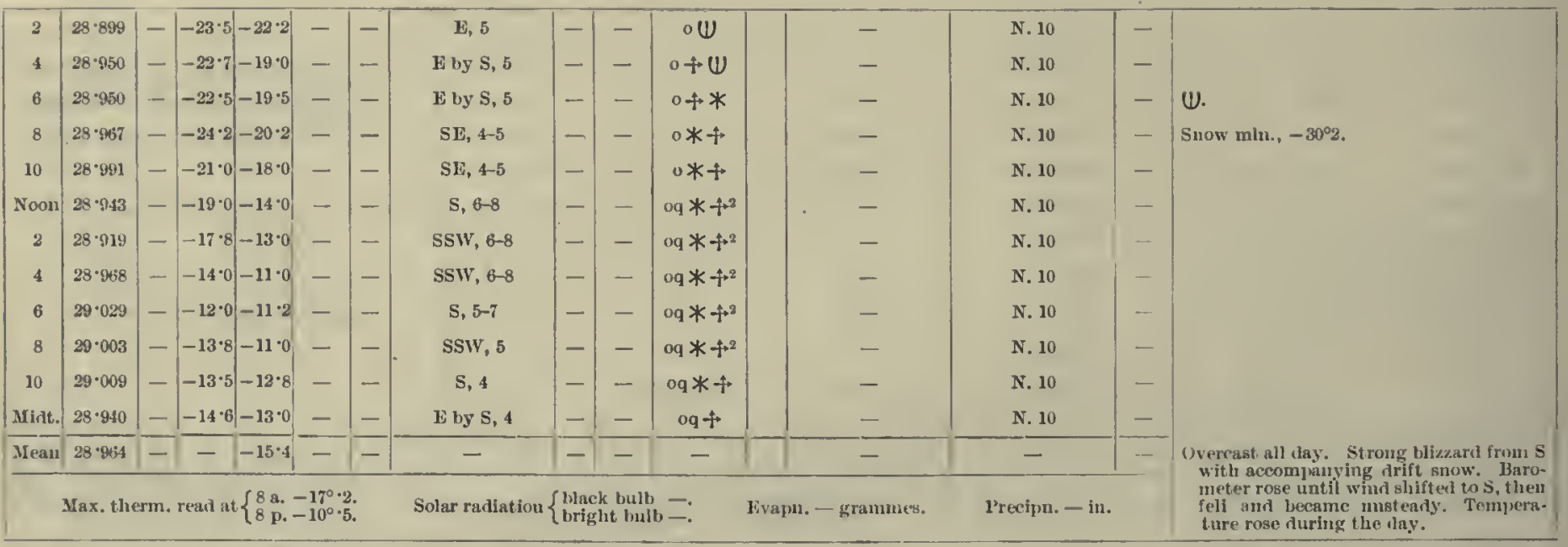

18 th.

\begin{tabular}{|c|c|c|c|c|c|c|c|c|c|c|c|c|c|}
\hline 2 & $28 \cdot 933$ & -1 & $|-14 \cdot 8|-13 \cdot 0 \mid$ & - & - & ESE, 3-4 & - & - & oq & - & N. 10 & - & \multirow{7}{*}{ 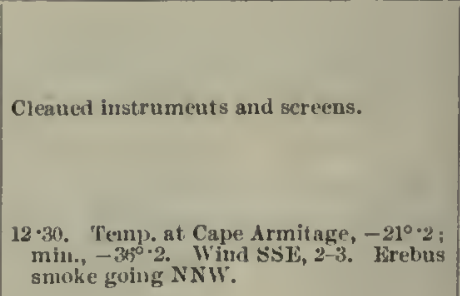 } \\
\hline 4 & $28 \cdot 880$ & - & $-13 \cdot 2-12 \cdot 0$ & - & - & S by $1,1-3$ & - & - & oq & - & N. 10 & - & \\
\hline 6 & $28 \cdot 785$ & - & $-13 \cdot 0-10 \cdot 2$ & - & - & $\mathrm{E}, 2-3$ & - & - & beq & - & Fr.-s. 10 & - & \\
\hline 8 & $28 \cdot 817$ & - & $-16 \cdot 9-14 \cdot 5$ & - & - & SE, 2-5 & - & - & beq & Fr.-ci.-s. 3 & - & - & \\
\hline 10 & $28 \cdot 789$ & - & $-18 \cdot 8-14 \cdot 0$ & - & - & $\mathrm{E}, 3-4$ & - & - & $1 x$ & Ci.-s. 2 & - & - & \\
\hline Noon & $28 \cdot 793$ & - & $-18 \cdot 0 \mid-15 \cdot 0$ & - & - & SSE, 2-3 & - & - & be & Fr.-ci.-s. 3 & - & - & \\
\hline 2 & $28 \cdot 781$ & - & $-19 \cdot 8-18 \cdot 0$ & - & - & $S E, 1$ & - & - & be & Ci.-s. 4 & - & - & \\
\hline 4 & $28 \cdot 716$ & - & $-20 \cdot 2-14 \cdot 8$ & - & - & $\mathrm{E}, 2$ & - & - & b & - & - & - & \multirow{7}{*}{ 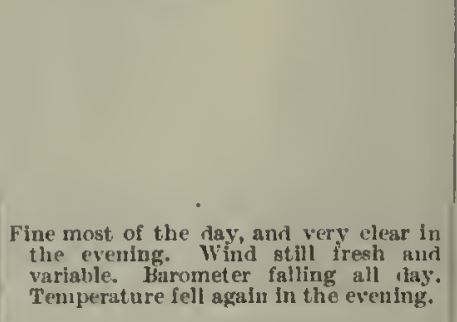 } \\
\hline 6 & $28 \cdot 700$ & - & \begin{tabular}{|l|l|}
$-23 \cdot 8$ & $-20 \cdot 0$ \\
\end{tabular} & - & - & E, 1-2 & - & - & b & - & - & - & \\
\hline 8 & $28 \cdot 624$ & - & $-23 \cdot 0-22 \cdot 2$ & - & - & ENE, 4 & - & - & bv $U$ & - & - & - & \\
\hline 10 & $28 \cdot 580$ & - & \begin{tabular}{|l|l|}
$-30 \cdot 0$ & $-29 \cdot 2$ \\
\end{tabular} & - & - & NE, 5 & - & - & $\operatorname{liv} U$ & - & - & - & \\
\hline Mide. & $28 \cdot 565$ & - & $-30 \cdot 0-24 \cdot 0$ & - & - & $\operatorname{EsE}, 2$ & - & - & $1, \mathbb{W}$ & - & - & - & \\
\hline Mean & $28 \cdot 747$ & - & $--17 \cdot 2$ & - & - & - & - & - & - & - & - & - & \\
\hline & ix. the & & $\{8$ a. - & $\begin{array}{l}9^{\circ} \cdot 8 . \\
2^{\circ} \cdot 0 .\end{array}$ & & Aiation \{ & & 二. & Eva & mumes in 48 & \multicolumn{2}{|c|}{ Precipn. - in. } & \\
\hline
\end{tabular}


Meteorolonicat، Journal, of thIE "Discoviery."

[1902-August.

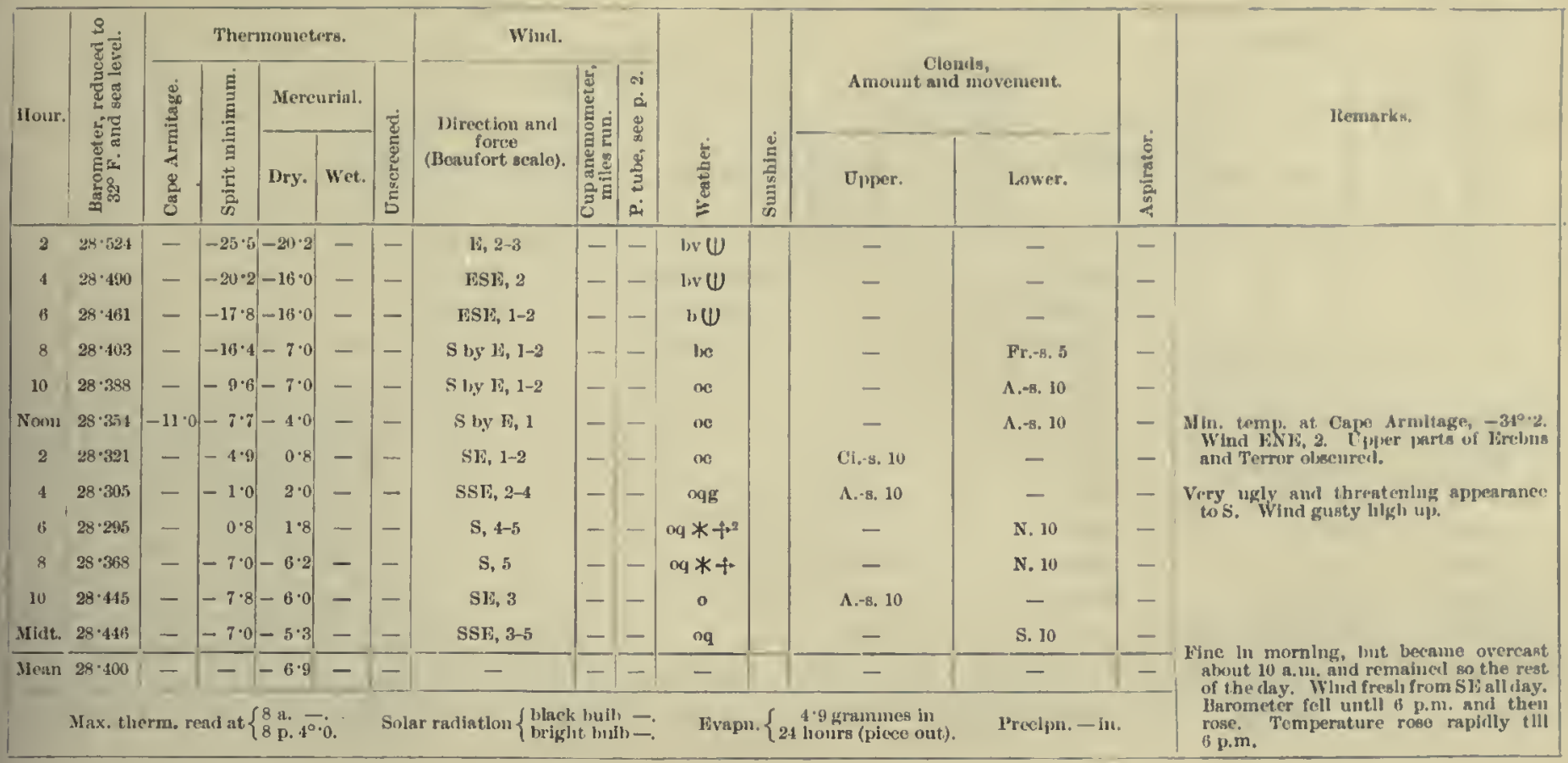

19 th.

\begin{tabular}{|c|c|c|c|c|c|c|c|c|c|c|c|c|c|c|}
\hline 2 & $29 \cdot 450$ & - & $|-7 \cdot 5|$ & $|-6 \cdot 2|$ & - & -1 & SSI, $3-6$ & - & - & oq & - & S. 10 & - & \\
\hline 4 & $28 \cdot 446$ & - & $-9 \cdot 2$ & $-\pi \cdot 2$ & - & - & SII, 2-4 & - & - & oeq & - & S. 10 & - & \\
\hline 6 & $28 \cdot 4.56$ & - & $-10 \cdot 0$ & $-8 \cdot 6$ & - & - & $\mathbf{F}, 2-3$ & - & - & bq & - & - & - & \\
\hline 8 & $28 \cdot 465$ & - & $-12 \cdot 2$ & $-11 \cdot 2$ & - & - & If by $\mathrm{N}, 1-2$ & - & - & be & - & Fr.-8, 3, S (inst) & - & • \\
\hline 10 & $28 \cdot 426$ & - & $-12 \cdot 0$ & $-8 \cdot 8$ & - & - & $\mathbf{F}, 1-2$ & - & - & be & Ci.-8. 5 & Fr.-8. 3, S (fast) & - & Whislwinds down the Strait. \\
\hline Noon & $28 \cdot 362$ & - & -9.5 & $-5 \cdot 0$ & - & - & $S, 4-5$ & - & - & $\log +* *$ & - & N. 10 & - & $\begin{array}{l}\text { 12.30. Temp. at Cape Armitage, }-5^{\circ} \cdot 2 \text { : } \\
\text { min., }-20^{\circ} .5 \text {. Wind S by W, } 4-6 . \text { for. }\end{array}$ \\
\hline 2 & $28: 357$ & - & $-5 \cdot 5$ & $-3 \cdot 0$ & - & - & SSW, 5 & - & - & $\mathrm{bq}$ & - & - & - & \\
\hline 4 & $28 \cdot 390$ & - & $-3 \cdot 8$ & $-2 \cdot 0$ & - & - & SsW, 5 & - & - & $\operatorname{bq} f^{2}$ & - & - & - & Wvaporation dlsh hlown away. \\
\hline 6 & $28 \cdot 398$ & - & $-2 \cdot 5$ & $-1 \cdot 0$ & - & - & S, $2-3$ & - & - & $\operatorname{lng}$ & - & - & - & $\begin{array}{l}\text { At noou, } 2,4 \text {, and } 6 \text { p.m., the sky } \text { พas } \\
\text { apparently elear, and drlit snow ajp }\end{array}$ \\
\hline 8 & $28 \cdot 3 i 7$ & - & $\mid-1 \cdot 2$ & $1 \cdot 0$ & - & - & SE, 1-2 & - & - & bin & - & - & - & prarently very high. \\
\hline 10 & $28 \cdot 377$ & - & $-0 \cdot 5$ & 0.8 & - & - & $\mathrm{S}, 2$ & - & - & le & A.-s. $5, \mathrm{~S}$ (mod,) & - & - & \\
\hline Midt. & $28 \cdot 35,5$ & - & 0.0 & $1 \cdot 1$ & - & -1 & $\mathrm{~F}, 0-1$ & $1-$ & -1 & lne & Ci. -8.6 & Cu. 6, Sw & - & \multirow{2}{*}{$\begin{array}{l}\text { Blue sky with elouds all day. Wind very } \\
\text { variable and strong. Barometer fairly } \\
\text { steady. Temperature feil early, but } \\
\text { rose again quickly li evening. }\end{array}$} \\
\hline Meau & $28 \cdot 405$ & - & - &,$- 4 \cdot 2$ & - & - & - & - & - & - & - & - & - & \\
\hline
\end{tabular}

20 th.

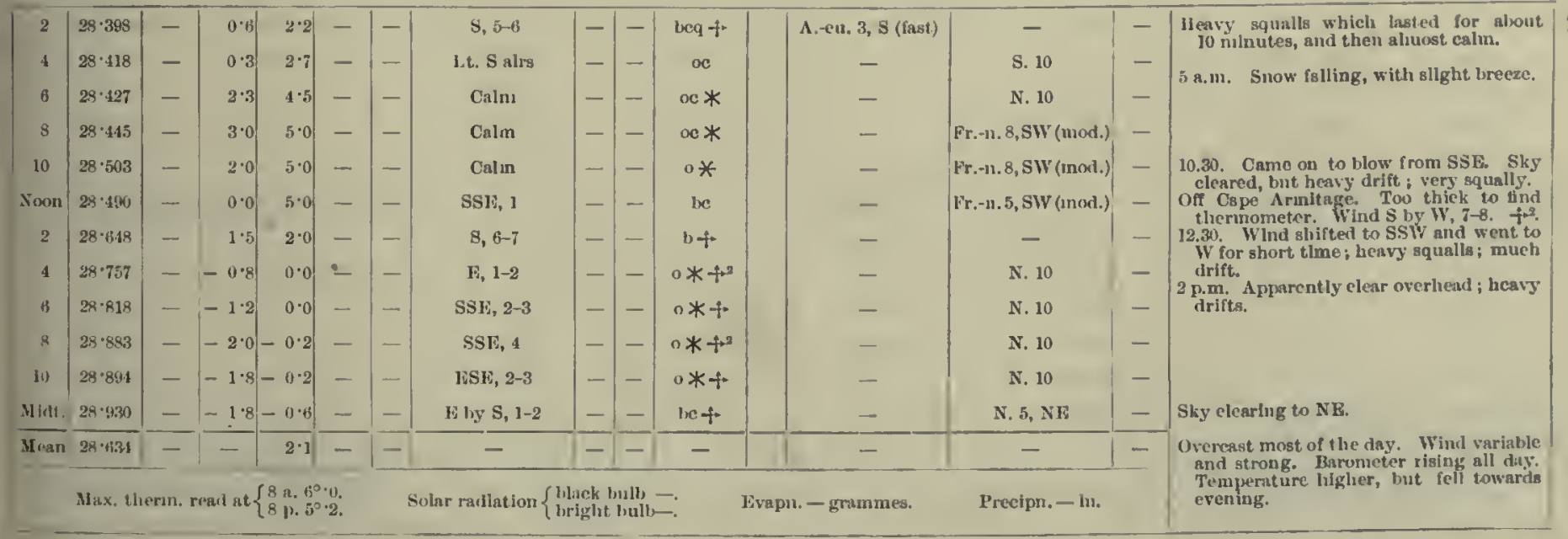


MFteororogical, JoURnal of The "Discovery."

\begin{tabular}{|c|c|c|c|c|c|c|c|c|c|c|c|c|c|c|c|}
\hline \multirow{3}{*}{ Hour. } & \multirow{3}{*}{ 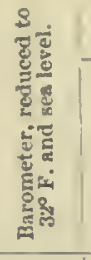 } & \multicolumn{5}{|c|}{ Thermometers. } & \multicolumn{3}{|l|}{ Wind. } & \multirow[b]{3}{*}{ 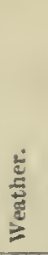 } & \multirow[b]{3}{*}{ 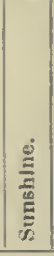 } & \multirow{2}{*}{\multicolumn{2}{|c|}{$\begin{array}{l}\text { Clonds, } \\
\text { Amonnt and movement. }\end{array}$}} & \multirow[b]{3}{*}{ 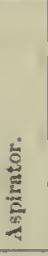 } & \multirow{3}{*}{ Ilemarks. } \\
\hline & & \multirow{2}{*}{ 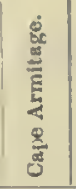 } & \multirow{2}{*}{ 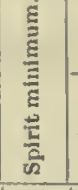 } & \multicolumn{2}{|c|}{ Mercurlal. } & \multirow{2}{*}{ 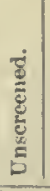 } & \multirow{2}{*}{$\begin{array}{l}\text { Directlon and } \\
\text { foree } \\
\text { (Beanfort scale). }\end{array}$} & \multirow{2}{*}{ 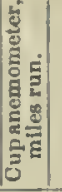 } & \multirow{2}{*}{ 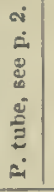 } & & & & & & \\
\hline & & & & Dry. I & Wet. & & & & & & & Upper. & Lower. & & \\
\hline 2 & $28 \cdot 799$ & - & $|-1 \cdot 6|$ & $|-0 \cdot 2|$ & - & - & E by $\mathbf{N}, 2-3$ & -1 & -1 & lec & & - & S.ent 4, 1s & - & \\
\hline 4 & $28^{\bullet} 797$ & - & -0.8 & -0.2 & - & - & Lt. EN II alrs & -1 & - & le & & Th in $A . \cdot 8,6, \mathrm{SW}$ & - & - & \\
\hline 6 & $28 \cdot 867$ & - & $-2 \cdot 0$ & $-1 \cdot 5$ & - & - & NNE, 1-2 & - & -1 & Ix & & $\mathrm{TH} \ln$ A.-S. 2, SE & - & - & \\
\hline 8 & $28 \cdot 831$ & - & $|-4 \cdot 6|$ & $-2 \cdot 4$ & - & - & NNE, I & - & -1 & $\infty$ & & ᄉ.-8. 10 & 一 & - & \\
\hline 10 & $28 \cdot 804$ & - & $-4 \cdot 5$ & $3 \cdot 0$ & - & - & $\mathrm{NE}, 1$ & - & - & b & & - & - & - & Noon, Cape Armitage, $M 1 \mathrm{n}$, temp. \\
\hline Noon & $28 \cdot 805$ & $-i \cdot 1$ & $-1 \cdot 8$ & $0 \cdot 0$ & - & - & $N \mathrm{~N}, 1-2$ & - & - & le & & A.-s. 5 & - & - & 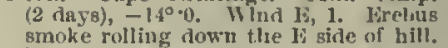 \\
\hline 2 & $28 \cdot 819$ & - & $-7 \cdot 0 \mid$ & $|-5 \cdot 0|$ & - & - & $\mathrm{NE}, 2$ & - & -1 & b & & - & - & - & $\begin{array}{l}\text { Whil st rong on summit and mueh drift } \\
\text { on looth nomntains. Sun observed }\end{array}$ \\
\hline 4 & $28 \cdot 886$ & - & $-9 \cdot 0$ & $-8 \cdot 2$ & - & - & $\mathrm{E}, 3$ & -1 & - & b & & - & - & - & $\begin{array}{l}\text { alove the horlyon for the tirst time. } \\
\text { 12.30. Temp. at high-level statlon, }-10^{\circ} \cdot 0 \text {; }\end{array}$ \\
\hline 6 & $28 \cdot 939$ & - & $-11 \cdot 0$ & $-9 \cdot 0$ & - & - & FNE, 4 & - & - & b & & - & - & - & $\begin{array}{l}\text { min., }-37^{\circ} 0 \text {. } \\
\text { \&.m. Fiaporation dish ern]ty }=45 \cdot 492\end{array}$ \\
\hline 8 & $28 \cdot 998$ & - & $-10 \cdot 2$ & $-9 \cdot 4$ & - & - & FNE, 2 & - & - & b & & - & - & - & $\begin{array}{l}\text { grammes. } \\
\text { 15 p.In. Fvaporation dish full, too heavy }\end{array}$ \\
\hline 10 & $29 \cdot 073$ & - & $-11 \cdot 0$ & $-9 \cdot 2$ & - & - & IHNE, 2 & - & - & b & & - & - & - & $\begin{array}{l}\text { to weigh, being over } 100 \text { grammes. } \\
10 \text { p.m. Slight auroral arcli from is to Sw, }\end{array}$ \\
\hline Minlt. & $29 \cdot 156$ & - & $-12 \cdot 5$ & $-10 \cdot 0$ & - & - & E, 2 & - & -1 & he & & - & Fr.-cir. 2 & - & alt. $14^{\circ}$. 2 streaners in zenith from $\mathrm{Sk}$. \\
\hline \multirow[t]{2}{*}{ Mean } & $28 \cdot 900$ & - & - & $-4 \cdot 3$ & - & - & - & -1 & - & - & & - & . - & $\rightarrow$ & \multirow{2}{*}{$\begin{array}{l}\text { Iine and elear nearly all day. Wind freslt } \\
\text { from the 1. Barouteter rising during } \\
\text { the day. Temperature falling. }\end{array}$} \\
\hline & $\operatorname{Max} . t$ & herm. & ard at. & $\left\{\begin{array}{l}8 \mathrm{a.} .1^{\circ} \\
8 \mathrm{p} .3^{\circ}\end{array}\right.$ & ( ) & & lar radiation $\left\{\begin{array}{l}\mathrm{bl} \\
\mathrm{br}\end{array}\right\}$ & aek lit & $\begin{array}{l}\text { ulb } \\
\text { bulb }\end{array}$ & & Wva & see remarks. & Precipn. - in. & & \\
\hline
\end{tabular}

$23 r d$.

\begin{tabular}{|c|c|c|c|c|c|c|c|c|c|c|c|c|c|c|}
\hline 3 & $29 \cdot 227$ & - & $-12 \cdot 0$ & $|-10.0|$ & - & $1-1$ & E, 2 & $|-|$ & - & the & A.-B. 1 & - & - & \multirow{10}{*}{ 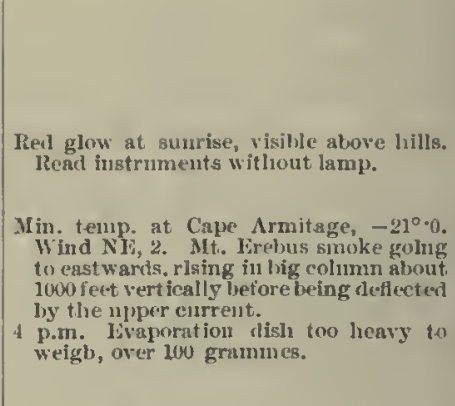 } \\
\hline 4 & $29 \cdot 2 \pi 3$ & - & $-13 \cdot 0$ & $|-8 \cdot 0|$ & - & - & E, I-2 & - & - & be & A.-8. 2 & - & - & \\
\hline 6 & $29 \cdot 291$ & - & $-13 \cdot 0$ & $-6 \cdot 0$ & - & - & E, 2 & - & - & b & - & - & - & \\
\hline 8 & $29 \cdot 325$ & - & $-9 \cdot 3$ & $-7 \cdot 1$ & - & - & Calm & - & - & oc & A.-s. 10 & - & - & \\
\hline 10 & $29-339$ & - & $-11 \cdot 2$ & $-11 \cdot 0$ & - & - & Calm & - & - & b & - & - & - & \\
\hline Nmon & $29 \cdot 319$ & $-12 \cdot 2$ & $-12 \cdot 0$ & $-5 \cdot 0 \mid$ & - & - & SE, 1 & - & - & b & - & - & - & \\
\hline 2 & $29 \cdot 289$ & - & $-10 \cdot 0$ & $|-8 \cdot 0|$ & - & - & Lt. E airs & - & - & b & - & - & - & \\
\hline 4 & $29 \cdot 235$ & - & $-15 \cdot 0$ & $-13 \cdot 0$ & - & - & ENE, 2 & - & - & b & - & - & - & \\
\hline 6 & $29 \cdot 186$ & - & $|-17 \cdot 5|$ & $-16 \cdot 8$ & - & - & ENE, 3 & - & - & b & - & - & - & \\
\hline 8 & $29 \cdot 152$ & - & $-17 \cdot 8$ & $-10 \cdot 2$ & - & - & IFNE, 1 & - & - & b & - & - & - & \\
\hline 10 & $29 \cdot 098$ & - & $-13 \cdot 0$ & $-10 \cdot 0$ & - & - & ENE, 1 & - & - & " & - & - & - & \multirow{2}{*}{$\begin{array}{l}\text { Milt. Anroral arch Nh to SW throngh } \\
\text { zenith, alt. } 70^{\circ} \text {, apparently wint driven. }\end{array}$} \\
\hline MInt. & $29 \cdot 074$ & - & $-18 \cdot 0$ & $-16 \cdot 0$ & - & - & FNE, 4 & -1 & - & $w$ & - & - & - & \\
\hline Mean & $29 \cdot 234$ & - & - & $-10 \cdot 1$ & - & - & - & -1 & -1 & - & - & - & - & \multirow{2}{*}{ 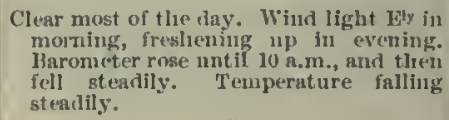 } \\
\hline & M & 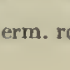 & , & & 1. & & Solar radiation & & & & see remarks. & IPrecipn & & \\
\hline
\end{tabular}

24 th.

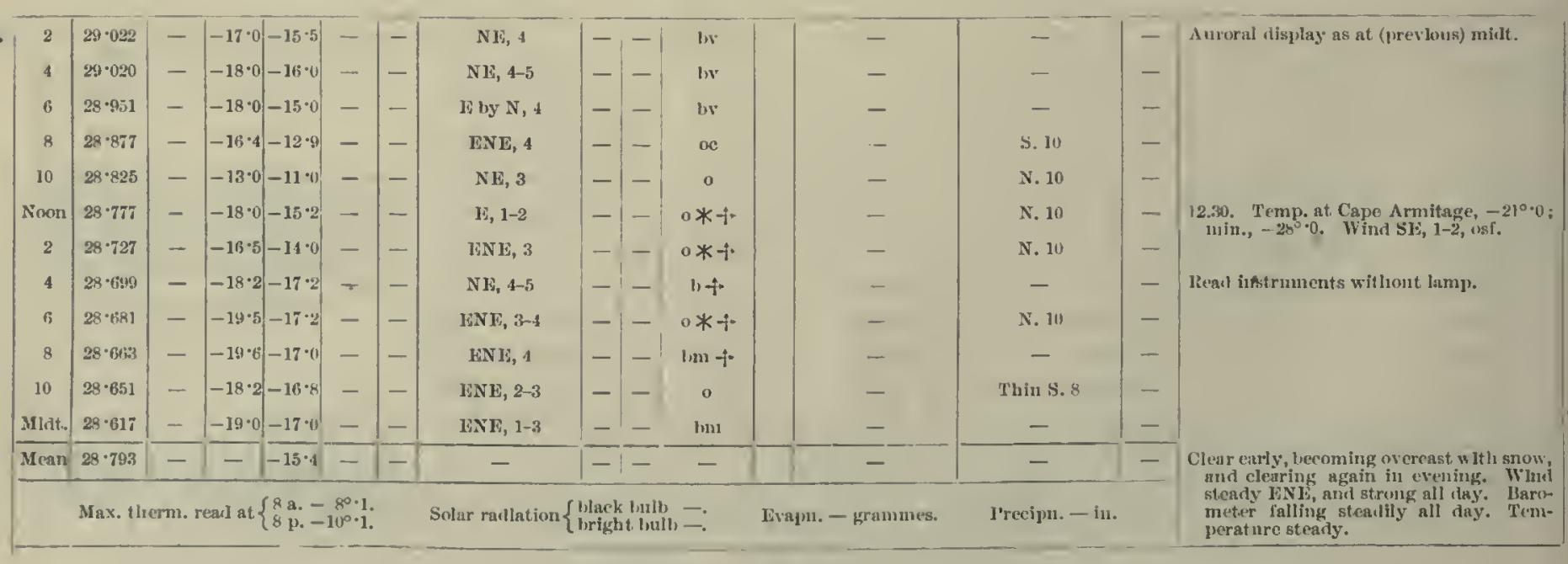


Meteorologicat Joumal of the "DiscoveliY."

[1902-August.

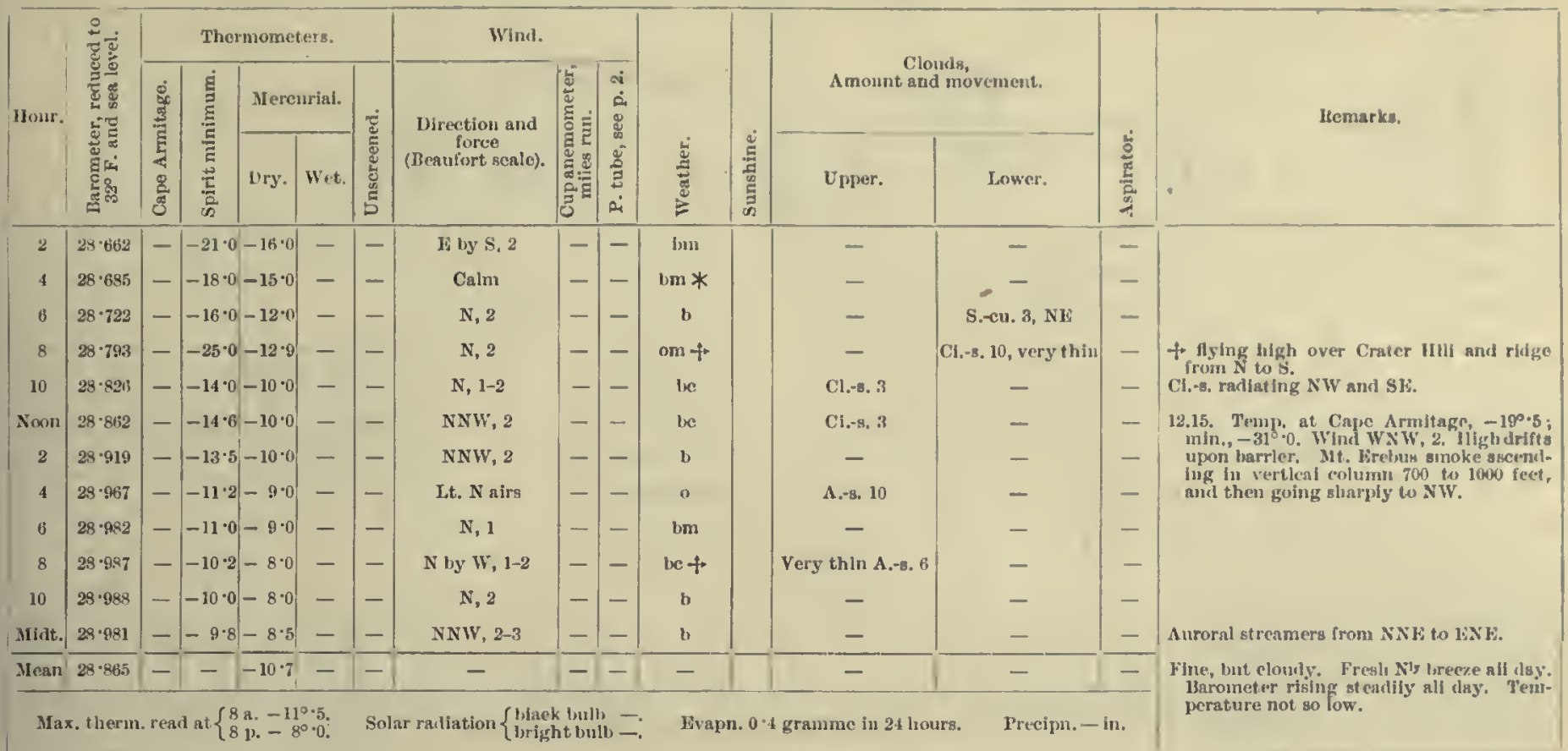

25 th.

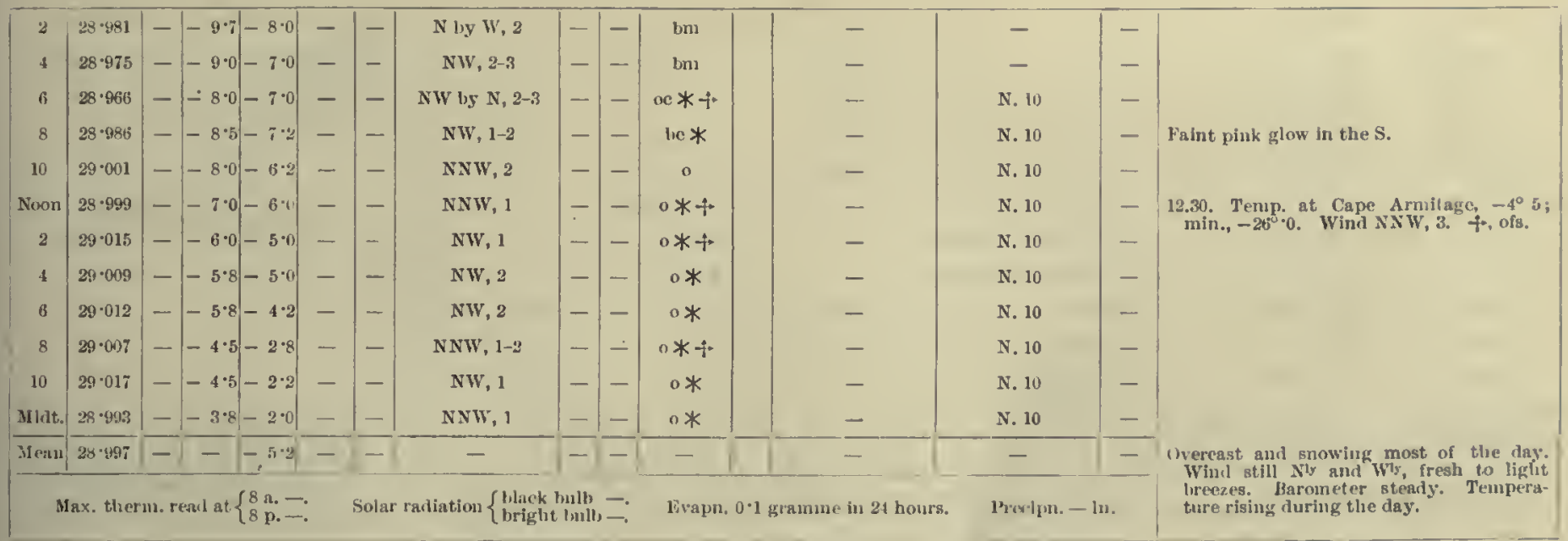

\begin{tabular}{|c|c|c|c|c|c|c|c|c|c|c|c|c|c|}
\hline 2 & $28 \cdot 991$ & - & $-8 \cdot 0-7 \cdot 0$ & - & $1-$ & Calm & - & - & the $\rightarrow$ & - & N. 3 & - & \\
\hline 4 & $29 \cdot 9.90$ & - & $-8 \cdot 3-6 \cdot 5$ & - & - & Calm & - & - & $0 *$ & - & N. 10 & - & \\
\hline is & $2 x \cdot 978$ & - & $|-6.9|-4.5$ & - & - & $\mathrm{SE}, 1$ & - & - & $n *$ & - & N. 10 & - & $*$ \\
\hline 8 & $28 \cdot 970$ & - & $|-5 \cdot 7|-4 \cdot 1$ & - & - & Calm & - & - & om & - & S. 10 & - & \\
\hline ximan & $2 \times \cdot 957$ & - & $-3 \cdot 0 \quad 0 \cdot \pi$ & - & - & W, 1 & - & - & o & Fr.-n.-8. 10 & $'-$ & - & \multirow{7}{*}{ 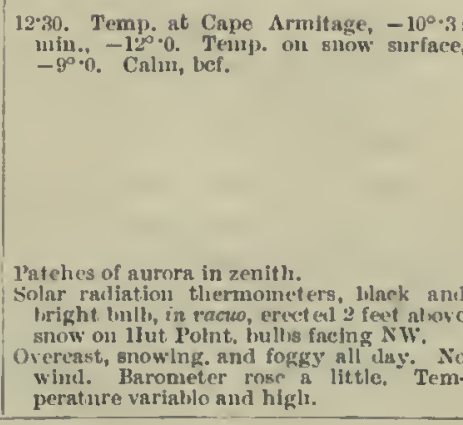 } \\
\hline 2 & $28 \cdot 979$ & - & $-8 \cdot 1-6 \cdot 1)$ & - & - & Calm & - & - & om & - & Thin S. 10 & - & \\
\hline 8 & $29 \cdot 996$ & - & $-4 \cdot 8-4 \cdot 1)$ & - & - & Calm & - & - & of $*$ & - & N. 10 & - & \\
\hline 10 & $2 x \cdot 972$ & - & $-5 \cdot 3-4 \cdot 8 \mid$ & - & - & WSE, 1 & - & - & $\operatorname{limf}$ & - & - & - & \\
\hline Mills. & $2 x \cdot 9: 9$ & - & $-5 \cdot 7-5 \cdot 0$ & - & - & $\mathrm{Ca} / \mathrm{m}$ & -1 & - & 118 & - & - & - & \\
\hline Yean: & $28 \cdot 980$ & - & $-3 \cdot 8$ & - & $1-1$ & - & -1 & - & - & - & - & & \\
\hline & & & & & & & & & Fvg & me in 241 & "receipn & & \\
\hline
\end{tabular}




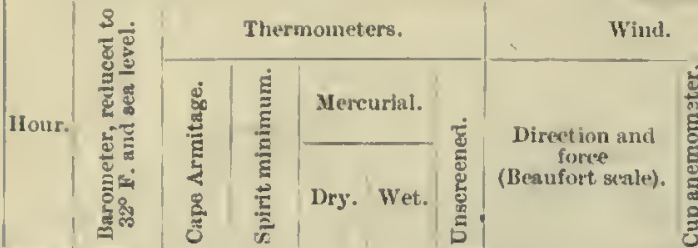

28 th.

\begin{tabular}{|c|c|c|c|c|c|c}
2 & $29 \cdot 000$ & - & $-7 \cdot 0$ & $-5 \cdot 2$ & - & - \\
4 & $28 \cdot 980$ & - & $-6 \cdot 0-4 \cdot 0$ & - & - \\
6 & $29 \cdot 005$ & - & $-7 \cdot 3-6 \cdot 8$ & - & - \\
8 & $29 \cdot 030$ & - & $-8 \cdot 9-7 \cdot 0$ & - & - \\
10 & $29 \cdot 060$ & - & $-10 \cdot 5$ & $-9 \cdot 0$ & - & - \\
Noon & $29 \cdot 078$ & $-16 \cdot 8$ & $-10 \cdot 5-5 \cdot 8$ & - & - \\
2 & $29 \cdot 098$ & - & $-6 \cdot 5-4 \cdot 0$ & - & - \\
4 & $29 \cdot 130$ & - & $-11 \cdot 7-11 \cdot 6$ & - & - \\
6 & $29 \cdot 144$ & - & $-11 \cdot 7-7 \cdot 0$ & - & - \\
8 & $29 \cdot 149$ & - & $-14 \cdot 5-8 \cdot 2$ & - & - \\
10 & $29 \cdot 155$ & - & $-14 \cdot 5-12 \cdot 0$ & - & -
\end{tabular}

BNE, 3

1:NL, 4-5

1) $\lg \mathrm{N}, 4-5$

NT, 3-4

N1, 2-3

$\mathbf{E}, 1$

FSH, 2

SSI:, 1-2

$\mathrm{SF}, 2-3$

E, 1

Midt. $29 \cdot 157-13 \cdot 5-9 \cdot 0-1-$

MPan $\overline{29 \cdot 082}, \overline{-}-\overline{-7 \cdot 5}-\overline{-}$
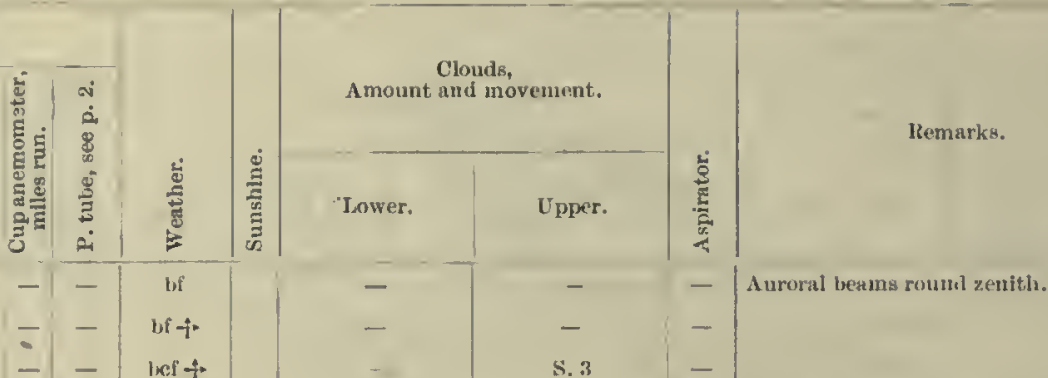

A.-s. 10

-

C1. +8.3

Ci.-cu. 6

Thin A.-B. 10

$15 \quad 12-15$

4 20-25 b

$13 \quad 12-15 \quad$ b

$1328 \quad b+$

$17 \mid \begin{array}{cc}23-25 & \text { b }\end{array}$
Remarks.

Placed sereens on new frame. Freete the Rohinson and smail lynes anemometers.

Min. temp. at Cape Armitage, $-20^{\circ} \cdot 0$ Temp. on stow starface, 1 . 1, $0-17^{\circ} .0$. Temp. at high-lesel station, scures.

Anroral arch extending from $12^{\circ}$ to zenith, N1. Patclies in zenith. All very faint.

Fine overhead, lunt foggy early, clearing after 4 p.m. Wini NE to $\$ 1$, strong in morming, and again hreshetuing a day. Barouter iucline to again.

$29 t h$.

\begin{tabular}{|c|c|c|c|c|c|c|c|c|c|c|c|c|c|}
\hline 2 & $29 \cdot 150$ & - & $-10 \cdot 5-5 \cdot 0$ & - & - & $\mathbf{N}, \mathbf{1}$ & 7 & 12 & $10 \mathrm{E}$ & - & - & - & \multirow{5}{*}{$\begin{array}{l}\text { Donble arch in } \mathrm{SE} \text {, alt. } 12^{\circ} \text { and } 45^{\circ} \text {, } \\
\text { faint. } \\
\text { Auroral display in } \mathrm{N} \text {, streamers; tright } \\
\text { glare in SH. } \\
\text { Twilight platinly visible in } 1 \mathrm{~s} \text { and SE. } \\
\text { Preeipitation in form of minutc } \\
\text { crystals at } 4 \text { and } 6 \text { a.m. }\end{array}$} \\
\hline 4 & $29 \cdot 156$ & - & $-20 \cdot 0-18 \cdot 8$ & - & - & Calm & 4 & 0 & $b \rightarrow$ & - & - & - & \\
\hline 6 & $29 \cdot 160$ & - & $-25 \cdot 0-18 \cdot 8$ & - & - & Calm & 0 & 0 & $b \rightarrow$ & - & - & - & \\
\hline 8 & $29 \cdot 157$ & - & $-19 \cdot 8-11 \cdot 3$ & - & - & 1is by S, 1-2 & 6 & $15-20$ & om $*$ & - & N. 10 & - & \\
\hline 10 & $29 \cdot 131$ & - & $-14 \cdot 8-14 \cdot 0$ & - & -1 & ENE, 2-3 & 13 & $15-25$ & on $*$ & - & N. 10 & - & \\
\hline Noon & $29 \cdot 139$ & $-20 \cdot 4$ & $-16 \cdot 7-16 \cdot 2$ & - & - & ENT, 3 & 18 & $25-29$ & $b c+$ & Fr.-ci.-8. 5, SW & - & - & \multirow{6}{*}{$\begin{array}{l}\text { Min. tenup. at Cape Armitage, }-30^{\circ} \cdot 5 \text {. } \\
\text { Temp. on snow surface, }-19^{\circ} \cdot 2 \text {; min. } \\
\text { on snow, }-27^{\circ} \cdot 0 \text {. Whid } \mathbf{E}, 2-3 . \text { †. }\end{array}$} \\
\hline 2 & $29 \cdot 113$ & - & $-18 \cdot 2-15 \cdot 2$ & - & - & NE by E, 2-3 & 20 & $20-25$ & be & Ci.-s. 3 & - & - & \\
\hline 4 & $29 \cdot 131$ & - & $-16 \cdot 5-12 \cdot 8$ & - & - & NE, 2-3 & 20 & $25-26$ & 0 & A. -5.10 & - & - & \\
\hline 6 & $29 \cdot 127$ & - & $-13 \cdot 3-4 \cdot 8$ & - & - & NE, 0-1 & 2 & 12 & $\circ * 4$ & - & N. 10 & - & \\
\hline 8 & $29 \cdot 128$ & - & $-11 \cdot 3-10 \cdot 2$ & - & - & Calm & 2 & 0 & o & - & S. 10 & - & \\
\hline 10 & $29 \cdot 124$ & - & $-15 \cdot 0-15 \cdot 0$ & - & - & Lt. W airs & 4 & 10 & of & - & S. 10 & - & \\
\hline Midt. & $29 \cdot 110$ & - & $-18 \cdot 4-5 \cdot 8$ & - & - & 1.t. NW airs & 9 & 10 & of & - & S. 10 & - & \multirow{3}{*}{$\begin{array}{l}\text { Fine early, clouding over with snow } \\
\text { and fog later. Wind variable } \mathrm{E} \text {, and } \\
\text { fresh, inax. run of cups for } 2 \text { hours, } \\
40 \text { miles; max. rate olsserved ly pres- } \\
\text { snre tulye, } 29 \text { miles per hour. liaro- } \\
\text { meter fairly steady, but, fell slightly. } \\
\text { Temperature rose quickly in evening. }\end{array}$} \\
\hline MeมีII & $29 \cdot 136$ & - & $-\quad-12 \cdot 3$ & - & -1 & - & - & - & - & - & - & - & \\
\hline & & 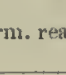 & $\{8$ & & 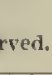 & $\underset{\text { radiation }}{\text { Solar }}\left\{\begin{array}{l}\text { b } \\
\text {, }\end{array}\right.$ & & . & Eva & gramme in $24 \mathrm{~h}$ & 1'recipn & & \\
\hline
\end{tabular}

30 th.

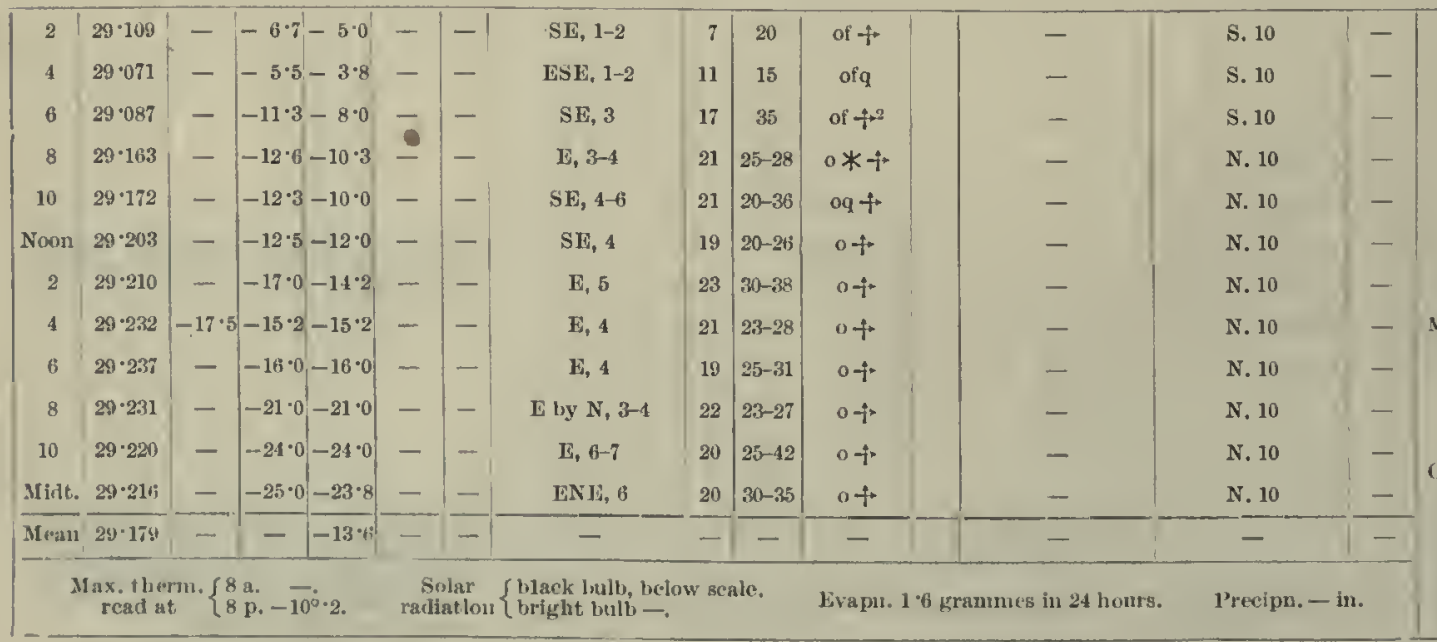

lin. temp. at Cape Armitage, $-23^{\circ} \cdot 8$. Telup. on snow surface, $-13^{\circ} 0$; min.
on strow, $-21^{\circ} 0$. Wind 14,3 , oq $f^{\circ}$.

Orercast, snowing, foggs, and squally all day, Wiud strong to a fresli gale frnm the 19, with nulch drift; max. run of cups for 2 hours, 16 miles; max. rate ohserved by pressure tuhe,
42 miles per hour. J3arometer rose until $f$ p.n., and then fell. Tenperature falling rapilly. 
Meteorological Jouknal, of TiLe " Discovktry."

[1902-August.

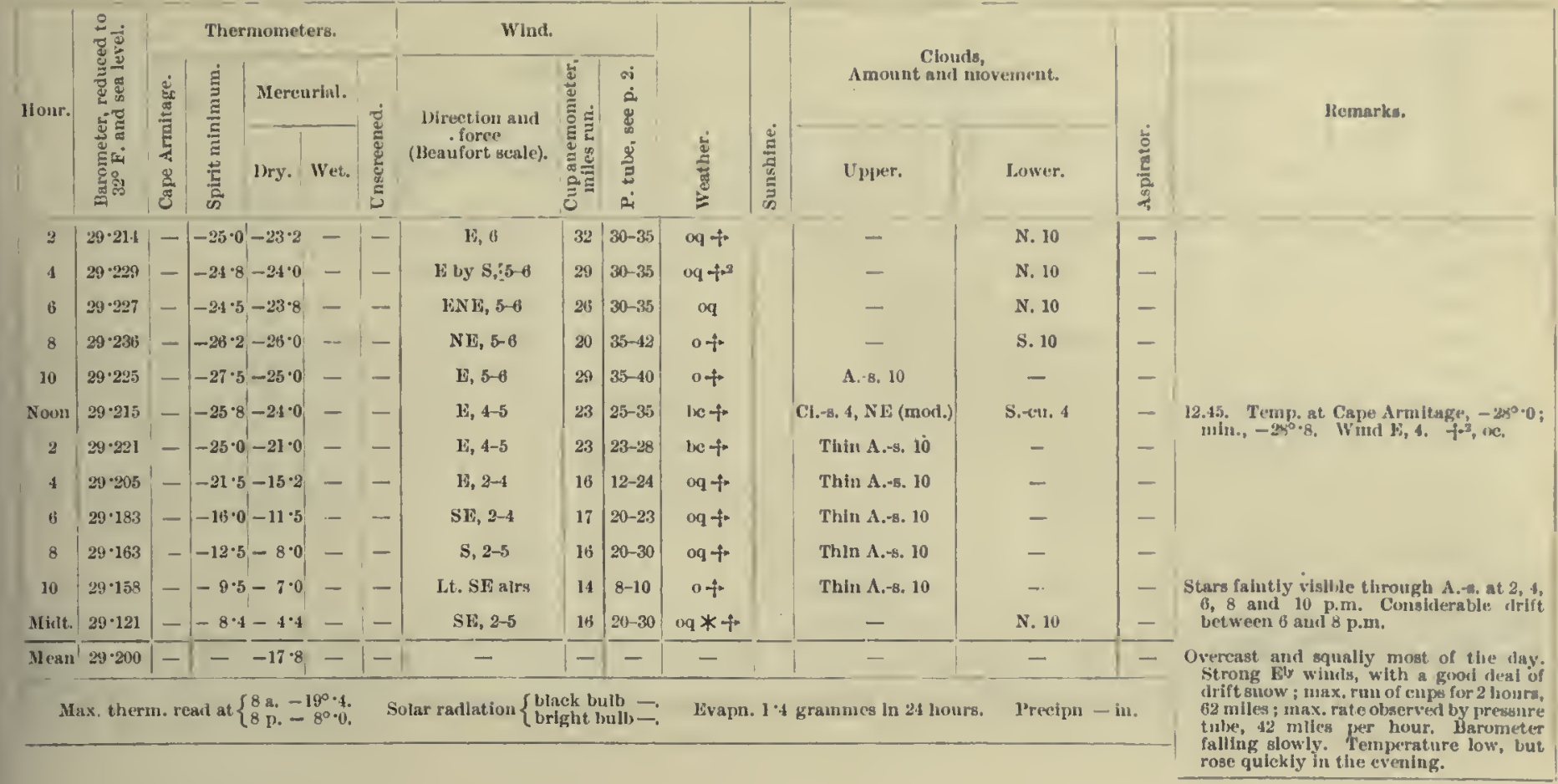


1902 -September.]

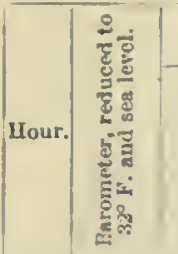

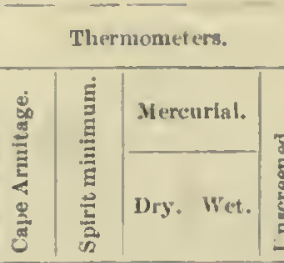

1 st.

\begin{tabular}{|c|c|c|c|c|c|}
\hline 2 & $20 \cdot 121$ & - & $|-6 \cdot 1|$ & $-3 \cdot 2$ & - \\
\hline 4 & $29 \cdot 125$ & - & $-4 \cdot 1$ & -1.0 & - \\
\hline 6 & $29 \cdot 114$ & - & $-1 \cdot 4$ & $1 \cdot 8$ & - \\
\hline 8 & $29 \cdot 120$ & - & $-1 \cdot 5$ & 0.8 & - \\
\hline 10 & $29 \cdot 109$ & - & $-1 \cdot 0$ & $4 \cdot 2$ & - \\
\hline Noons & $29 \cdot 120$ & - & $1 \cdot 0$ & $3 \cdot 8$ & - \\
\hline 2 & $2 y \cdot 143$ & - & 0.8 & $2 \cdot 5$ & - \\
\hline 4 & $29 \cdot 197$ & - & $1 \cdot 1$ & $2 \cdot 8$ & - \\
\hline 6 & $29 \cdot 259$ & - & $-1 \cdot 0$ & $1 \cdot 6$ & - \\
\hline 8 & $29 \cdot 319$ & - & $-2 \cdot 8$ & -0.2 & - \\
\hline 10 & $29 \cdot 329$ & - & $-1 \cdot 0$ & 2.0 & - \\
\hline Midt. & $29 \cdot 355$ & - & -0.5 & 1.5 & - \\
\hline$\overline{\text { II ean }}$ & $29 \cdot 193$ & - & - & $1 \cdot 4$ & - \\
\hline
\end{tabular}

Mkteorological, Journa, of the "Discovery."

Max, therm, read at $\begin{cases}8 & \text { a. } 2^{\circ} \cdot 0 . \\ 8 & p .\end{cases}$

Solar radiation $\left\{\begin{array}{l}\text { black bulb }- \text {. } \\ \text { light bult }- \text {. }\end{array}\right.$

$\left.\begin{array}{c|c}\text { Direction and } \\ \text { foree }\end{array}\right)$
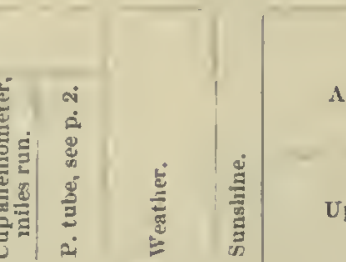

Clouds,
Amount and movement.

Unper.

lienarks.

\begin{tabular}{|c|c|c|c|c|c|c|c|}
\hline$S$ thy $k, 2-5$ & 13 & $|20-25|$ & or $*+t^{3}$ & - & N. 10 & - & \multirow{7}{*}{ 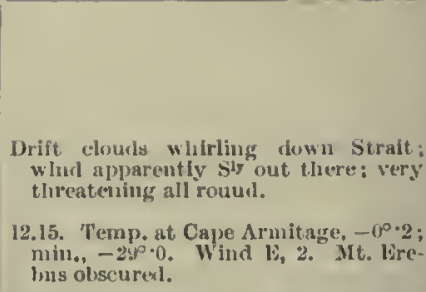 } \\
\hline SW. I & 13 & $15-20$ & oq & - & $\$ .10$ & - & \\
\hline $15,1-2$ & 10 & 20 & $\infty q$ & - & S. 10 & - & \\
\hline $\mathrm{ESE}, 2$ & 13 & 20 & o & - & S. 10 & - & \\
\hline FS1i, 2 & 13 & $18-20$ & o & - & S. 10 & & \\
\hline $1 B, 2$ & 13 & $20-23$ & o & Thin $\Lambda-8.10$ & - & - & \\
\hline E, 1-2 & 13 & 18 & o & Thin A.-8. 10 & - & - & \\
\hline $\mathrm{SE}, 2$ & 13 & $18-23$ & - & A. -8.10 & - & - & \multirow{7}{*}{$\begin{array}{l}\text { sinow drifting down Strait. Very } \\
\text { ihreatening look over W and SW } \\
\text { hills, and to the S. } \\
\text { Overcast and squally most of the day. } \\
\text { Strong Slity wind max, run of cups } \\
\text { for } 2 \text { hours, } 40 \text { miles max. rate } \\
\text { observed by pressure tulse, } 42 \text { miles } \\
\text { per lionr. Barometer rose stemllily } \\
\text { all day. Temperature high. }\end{array}$} \\
\hline ESIE, 2-1 & 16 & $20-30$ & oq & - & S. 10 & - & \\
\hline $\mathrm{E}, 2-6$ & 20 & $15-42$ & oq & - & S. 10 & - & \\
\hline SST, 1-3 & 18 & $10-18$ & oq $*+$ & - & N. 10 & - & \\
\hline $5,5-6$ & 18 & $\mid 18-31$ & nq $*+$ & - & N. 10 & - & \\
\hline- & - & - & - & - & - & - & \\
\hline ion \{ & & -. & & mes in & \multicolumn{2}{|c|}{ P'recipu. - lin. } & \\
\hline
\end{tabular}

2nd.

\begin{tabular}{|c|c|c|c|c|c|c|c|c|c|c|c|c|c|c|}
\hline 2 & $29 \cdot 412$ & - & $|-1 \cdot 0|$ & 00 & - & -1 & $S$ by $E_{0}, 3-6$ & 28 & $15-26$ & oq $*+$ & - & N. 10 & - & \multirow{14}{*}{ 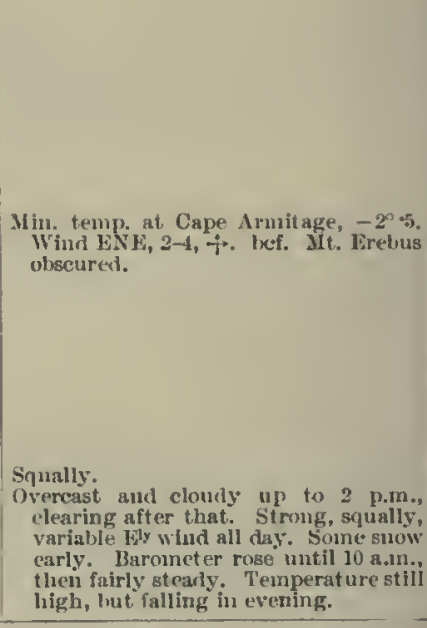 } \\
\hline 4 & $29 \cdot 470$ & - & -1.0 & 10 & - & - & SE, 1-4 & 13 & $10-22$ & $\operatorname{og} *+$ & - & N. 10 & - & \\
\hline 6 & $29 \cdot 504$ & - & -0.8 & $1 \cdot 2$ & - & - & ESE, 2-4 & 18 & $14-22$ & oq $*+$ & - & N. 10 & - & \\
\hline 8 & $29 \cdot 512$ & - & 0.0 & $2 \cdot 2$ & - & - & $\mathrm{SE}, 5-6$ & 21 & $25-38$ & oq $*+$ & - & N. 10 & - & \\
\hline 10 & $29 \cdot 524$ & - & 0.5 & 1.2 & - & - & ESI", 1-2 & 15 & $15-20$ & $0 *+$ & - & N. 10 & - & \\
\hline Noou & $29 \cdot 30,3$ & $-1 \cdot 0$ & $-2 \cdot 0$ & 0.5 & - & - & ENE, 4-5 & 16 & 25 & $x+4$ & Ci.ecu. 8 & - & - & \\
\hline 2 & $29 \cdot 484$ & - & $|-3 \cdot 5|$ & $-2 \cdot 8$ & - & -1 & E, 4-5 & 23 & $25-32 \mid$ & let + & Ci.-8. 6 & - & - & \\
\hline 4 & $29 \cdot 476$ & -1 & $|-7 \cdot 5|$ & $-4 \cdot 8$ & - & - & $E, 5-6$ & 26 & $28-34$ & $1+4$ & - & - & - & \\
\hline 6 & $29 \cdot 455$ & - & $-8 \cdot 0$ & -6.0 & - & -1 & E by N, 5-6 & 26 & $26-39 \mid$ & $b+4$ & - & - & - & \\
\hline 8 & $29 \cdot 474$ & - & $|-7 \cdot 1|$ & -4.5 & - & -1 & ENE, 4-5 & 25 & $24-32$ & 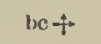 & C.s. 4 & - & - & \\
\hline 10 & $29 \cdot 492$ & - & $|-7 \cdot 5|$ & -40 & - & - & $N E, 4-5$ & 26 & $28-33$ & b & - & - & - & \\
\hline Mirit. & $29 \cdot 482$ & - & -6.4 & -5.0 & - & - & E by S. 2-4 & 19 & 25 & bq & - & - & - & \\
\hline Mean & $29 \cdot 4 \times 2$ & - & -1 & -1.8 & -1 & -1 & - & - & - & - & - & - & - & \\
\hline & Max, the & $\mathrm{mm}, \mathrm{re}$ & ad at \{ & 8 p. 2 & & Solar & tion 1 & bull & & Lvapu. & nes in 2. livurs. & 1'recipm & & \\
\hline
\end{tabular}

3rd.

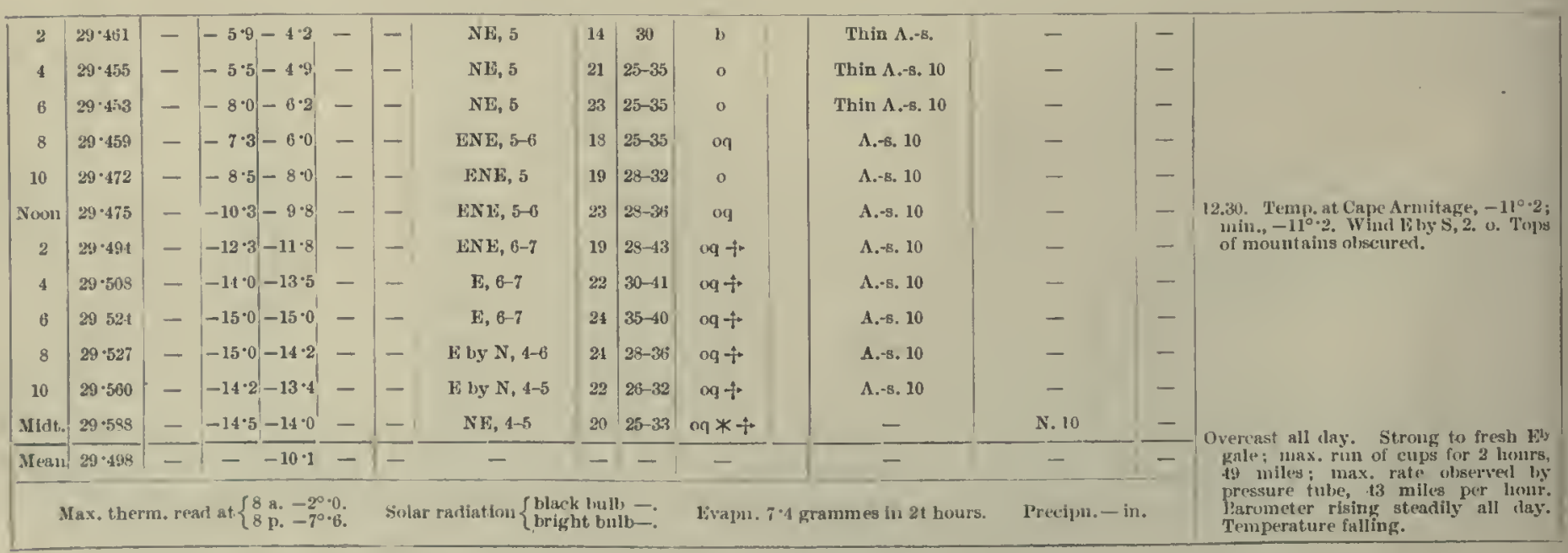


Meteolological Jouinat, or the "Ijiscovimix."

[1902-September.

\begin{tabular}{|c|c|c|c|c|c|c|c|c|c|c|c|c|c|c|c|}
\hline \multirow{3}{*}{ liour. } & \multirow{3}{*}{ 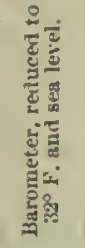 } & \multicolumn{5}{|c|}{ Thernometers. } & \multicolumn{3}{|l|}{ Wind. } & \multirow[b]{3}{*}{ 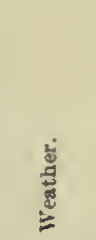 } & \multirow[b]{3}{*}{ 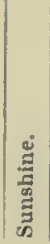 } & \multirow{2}{*}{\multicolumn{2}{|c|}{$\begin{array}{l}\text { Clourls, } \\
\text { Amount and movement. }\end{array}$}} & \multirow{3}{*}{$\begin{array}{l}\frac{3}{3} \\
\frac{2}{2} \\
\frac{2}{2}\end{array}$} & \multirow{3}{*}{ Kemarks. } \\
\hline & & \multirow{2}{*}{ 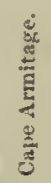 } & \multirow{2}{*}{ 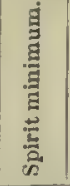 } & \multicolumn{2}{|c|}{ Mereurial. } & \multirow{2}{*}{ 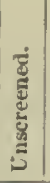 } & \multirow{2}{*}{$\begin{array}{c}\text { Direetion and } \\
\text { force } \\
\text { (Beaufort Bcale). }\end{array}$} & \multirow{2}{*}{ 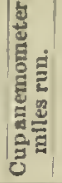 } & \multirow{2}{*}{ 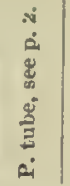 } & & & & & & \\
\hline & & & & $1 \mathrm{ry}$. & Wet. & & & & & & & Upper. & Lower. & & \\
\hline 2 & $29 \cdot 603$ & - & $|-14 \cdot 5|$ & $-14 \cdot 0$ & - & - & WNE, B-4 & 18 & $25-33$ & $0+* *$ & & - & N. 10 & - & \\
\hline 4 & 29.604 & - & $-14 \cdot 5$ & $-8 \cdot 0$ & - & - & $1,1-2$ & 10 & $10-15$ & 0 & & $A-0.10$ & - & - & \\
\hline B & $29 \cdot 610$ & - & $-10^{\circ} 0$ & $-6 \cdot 5$ & - & - & x, 1-2 & 10 & $10-15$ & 0 & & A.-8. 10 & - & - & \\
\hline 8 & $29 \cdot 612$ & - & $-13 \cdot 2$ & $-12 \cdot 8$ & - & - & SH, 1-3 & 5 & $15-20$ & 0 & & A.-s. 10 & - & - & \\
\hline 10 & $29 \cdot 632$ & - & $-14 \cdot 5$ & $-8 \cdot 8$ & - & - & Lt. Kairs & 7 & $8-10$ & 0 & & A.-8. 10 & - & - & \\
\hline Nююи & $29 \cdot 617$ & - & $-11 \cdot 5$ & $-10 \cdot 0$ & - & - & ESH, 2 & 8 & $15-18$ & 0 & & A.-1̀. 10 & - & - & 12.30. Temp. at Cape Armitage, $-13^{\circ}$ \\
\hline 2 & $29 \cdot 650$ & - & $-13 \cdot 7$ & $-13 \cdot 0$ & - & - & E, 1-2 & 10 & $10-15$ & o & & - & S. 10 & - & Mountalns obscured. \\
\hline 4 & $29 \cdot 654$ & - & $-15 \cdot 0$ & $-15 \cdot 0$ & - & - & E, 4-6 & 19 & $25-35$ & $o q+*$ & & - & N. 10 & - & \\
\hline 6 & $29 \cdot 651$ & - & $-17 \cdot 0$ & $-16^{\circ} 0$ & - & - & b., 2-3 & 18 & $\mid 20-22$ & b & & - & - & - & \\
\hline 8 & $29 \cdot 6 \cdot 4$ & - & $-18 \cdot 0$ & $-17 \cdot 0$ &.- & - & $\mathrm{NE}, 3$ & 16 & 23 & b & & - & - & - & \\
\hline 10 & $29 \cdot 640$ & - & $-18 \cdot 0$ & $-13 \cdot 8$ & - & - & Calm & 10 & 0 & b) & & - & - & - & \\
\hline Milt. & $29 \cdot 641$ & - & $-15 \cdot 8$ & $-13 \cdot 0$ & - & - & Lt. E airs & 2 & 0 & $\mathrm{~b}$ & & - & - & - & \multirow{3}{*}{ 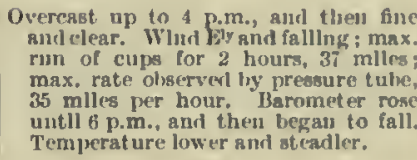 } \\
\hline Mfean & $29 \cdot 633$ & - & - & $-12 \cdot 3 \mid$ & - & - & - & -1 & - & - & & - & - & - & \\
\hline \multicolumn{6}{|c|}{ Max. therm. reari at $\left\{\begin{array}{l}8 \text { 8. } \\
81,-6^{\circ} \cdot 5 .\end{array}\right.$} & \multicolumn{4}{|c|}{ Solar radiation $\left\{\begin{array}{l}\text { blaek huih } \\
\text { briglit luulb }=\text { : }\end{array}\right.$} & Evapn & & imes in 24 & Precll & & \\
\hline
\end{tabular}

4 th.

\begin{tabular}{|c|c|c|c|c|c|c|c|c|c|c|c|c|c|}
\hline 2 & $29 \cdot 630$ & - & $-14 \cdot 5-11 \cdot 4$ & - & - & Nk alrs & 7 & 0 & b & - & - & - & \multirow{9}{*}{ 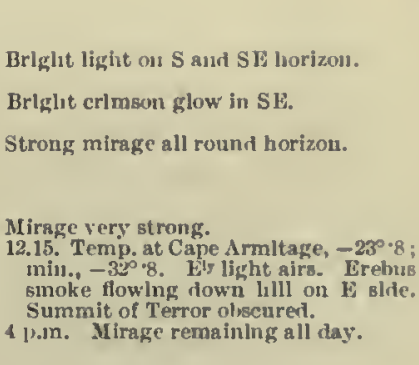 } \\
\hline 4 & $29 \cdot 630$ & - & $-16 \cdot 0-16 \cdot 0$ & - & - & Lt. NE airs & 2 & 0 & b & - & - & - & \\
\hline 6 & $29 \cdot 628$ & - & $-16 \cdot 8-16 \cdot 5$ & - & - & Lt. SE airs & 0 & 0 & o & A. -8.10 & - & - & \\
\hline 8 & $29 \cdot 623$ & - & $-16 \cdot 0-14 \cdot 2$ & - & - & Calm & 0 & 0 & o & A. -8.10 & - & - & \\
\hline 10 & $29 \cdot 606$ & - & $-21 \cdot 0-19 \cdot 0$ & - & - & Calm & 0 & 0 & o & A.-5. 10 & - & - & \\
\hline Noon & $29 \cdot 599$ & - & $-19 \cdot 2-17 \cdot 0$ & - & - & Calm & 0 & 0 & 0 & A.-s. 10 & - & - & \\
\hline 2 & 29.574 & - & $-17 \cdot 6-15 \cdot 8$ & - & - & Calm & 0 & 0 & o & A.-s. 10 & - & - & \\
\hline 4 & $29.5+54$ & - & $-16 \cdot 2-14 \cdot 5$ & - & - & Calm & 0 & 0 & 0 & A. -5.10 & - & - & \\
\hline${ }^{6}$ & $29 \cdot 550$ & - & $-14.7-6.0$ & - & - & Lt. $\mathrm{N}$ alrs & 6 & 5 & ? & A.-8. 10 & - & - & \\
\hline 8 & $29 \cdot 533$ & - & $-11 \cdot 5-10 \cdot 2$ & - & - & Calm & 0 & 0 & 0 & Thin A.-8. 10 & - & - & \multirow{5}{*}{$\begin{array}{l}\text { Overeast nearly all ray, and ealm. } \\
\text { Barometer falling slowly all ray. } \\
\text { Temperature rlsling towards the eveu- } \\
\text { ing. }\end{array}$} \\
\hline 10 & $29 \cdot 5.30$ & - & $-10 \cdot 7-5 \cdot 0$ & - & - & $N, 0-2$ & 2 & $0-20$ & og & A.-8. 10 & - & - & \\
\hline Mint. & $29 \cdot 528$ & - & $-7 \cdot 8-7 \cdot 8$ & - & $=$ & Lt $\mathrm{S}$ airs & 0 & 0 & 0 & A.-8. 10 & - & - & \\
\hline Mran & $29 \cdot 58.3$ & - & $-\quad-12 \cdot 8$ & - & -1 & - & - & - & - & - & - & - & \\
\hline & x. thern & & $t\left\{\begin{array}{l}8 \mathrm{a}_{0}-11^{\circ} \cdot \\
8 \mathrm{p}_{0}-6^{\circ}\end{array}\right.$ & & & ation \{ & & & Eval & ramme in 24 iton & \multicolumn{2}{|c|}{ Precl } & \\
\hline
\end{tabular}

5th.

\begin{tabular}{|c|c|c|c|c|c|c|c|c|c|c|c|c|c|}
\hline 2 & $29 \cdot 521$ & - & $-9 \cdot 5-70$ & - & -1 & Calm & 0 & 0 & 0 & A. -8.10 & - & - & \multirow{12}{*}{$\begin{array}{l}\text { Nin. temp. at. Cape Armltage, }-27^{\circ} \cdot 0 \text {. } \\
\text { WWind Sk, } 1 \text {. os. } \\
\text { Snow very damp, and lallugg lu large } \\
\text { flakes, at } S \text { and } 10 \mathrm{p} \text {. m. and midt. }\end{array}$} \\
\hline 4 & $29 \cdot 518$ & - & $-8 \cdot 0-2 \cdot 0$ & - & - & $N W, 2-4$ & 4 & $15-22$ & $\circ q *$ & - & N. 10 & - & \\
\hline 6 & $29 \cdot 516$ & - & $-3 \cdot 0-2 \cdot 0$ & - & - & $\mathbf{N}, 1-3$ & 9 & $10-20$ & $\infty q *$ & - & N. 10 & - & \\
\hline 8 & $29 \cdot 533$ & - & $-3 \cdot 4-2 \cdot 0$ & - & - & N hy E, 2 & 8 & $15-20$ & $o *$ & - & N. 10 & - & \\
\hline 10 & $294 \cdot 539$ & - & $-3 \cdot 2-2 \cdot 4$ & - & - & N1\%, 1 & 7 & $10-12$ & $o *$ & - & N. 10 & - & \\
\hline Nions & $24: 542$ & $-6 \cdot 2$ & $2-4 \cdot 2-2 \cdot 2$ & - & -1 & BNE, 1 & 4 & 8-10 & $0 *$ & - & N. 10 & - & \\
\hline 2 & $29 \cdot 551$ & - & $|-5 \cdot 7|-5 \cdot 2$ & - & - & Nsis, 1-2 & 8 & 10-15 & $n *$ & - & N. 10 & - & \\
\hline 4 & $2: 1 \cdot \sin 3$ & - & $-13 \cdot 3-4 \cdot 8$ & - & - & Sk, 2 & 7 & $10-20$ & $o *$ & - & N. 10 & - & \\
\hline b & $29 \cdot 575$ & - & $-7 \cdot 0-4 \cdot 0$ & - & - & Cralm & 7 & 0 & $\mathrm{~cm} *$ & - & N. 10 & - & \\
\hline 8 & $29 \cdot 601$ & - & $-8.4-7 \cdot 2$ & - & - & E hy $N, 3-5$ & 7 & 21-29: & $0 *+$ & - & N. 10 & - & \\
\hline 10 & $29 \cdot 626$ & - & $-6 \cdot 7-5 \cdot 2$ & - & - & E. 3 & 13 & $15-18$ & $\circ *$ & - & N. 10 & - & \\
\hline Mint. & $29 \cdot 654$ & - & $-\Omega \cdot 5-8 \cdot 0$ & - & - & 1s by $\mathrm{N}, 3-4$ & 15 & $20-25$ & $\omega *$ & - & N. 10 & - & \\
\hline Mเหมเ & $29 \cdot 5+5$ & - & --43 & - & - & - & - & - & - & - & - & - & $\begin{array}{l}\text { Orerrast and annwing nearly all day. } \\
\text { Wind light and rarialle. Barometer }\end{array}$ \\
\hline
\end{tabular}


1902-September.]

Meteorological Jovinal of the "Discovery."

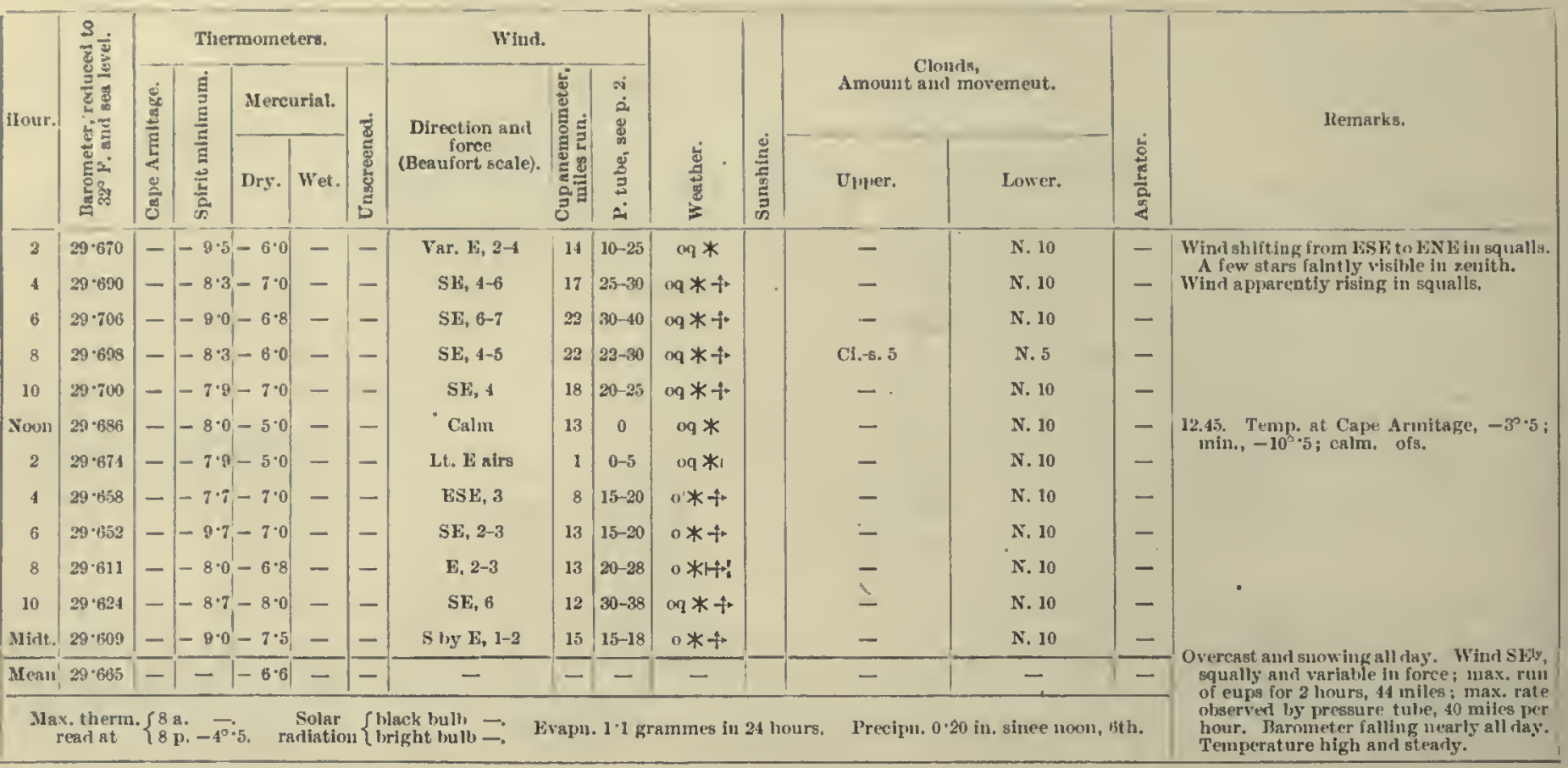

8th.

\begin{tabular}{|c|c|c|c|c|c|c|c|c|c|c|c|c|c|}
\hline 2 & $29 \cdot 575$ & -1 & $|-7 \cdot 9-5 \cdot 2|$ & - & - & S by $16,2-3$ & 18 & $|25-32|$ & $0 *+$ & - & N. 10 & - & \multirow{11}{*}{ 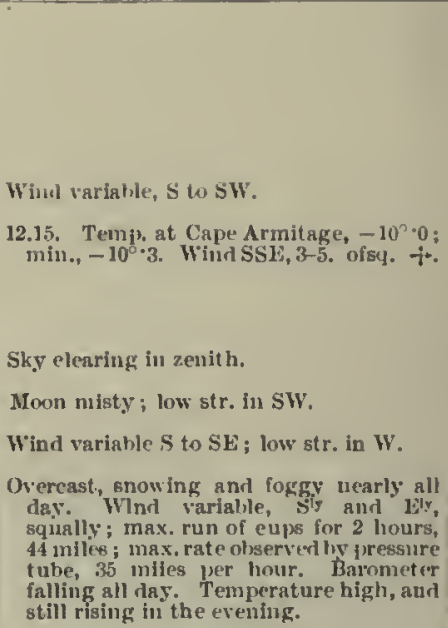 } \\
\hline 4 & $29 \cdot 577$ & - & $-8.5-6.0$ & - & - & S by $E, 1-3$ & 13 & $18-26$ & oq $*+$ & - & N. 10 & - & \\
\hline 6 & $29 \cdot 567$ & - & $-7 \cdot 5-6.8$ & - & - & SSE, 2-3 & 18 & $20-30$ & $0 * i$ & - & N. 10 & - & \\
\hline 8 & $29 \cdot 549$ & - & $-7 \cdot 3-6 \cdot 0$ & - & - & $S E, 1-4$ & 18 & $15-20$ & oq $*+$ & - & N. 10 & - & \\
\hline Noon & $29 \cdot 559$ & -1 & $-7 \cdot 0-5 \cdot 8 \mid$ & - & - & SE, 1-2 & 13 & $15-20$ & of $*+$ & - & N. 10 & - & \\
\hline 2 & $29 \cdot 548$ & - & $-6 \cdot 3-5 \cdot 2$ & - & - & SE, 4-5 & 13 & $25-30$ & of $* 4$ & - & N. 10 & - & \\
\hline 8 & $29 \cdot 536$ & - & $-7 \cdot 7-i \cdot 0$ & - & - & SE, $3-6$ & 20 & $20-35$ & bq q. & - & - & - & \\
\hline 10 & $29 \cdot 515$ & - & $-8 \cdot 3-5 \cdot 8$ & - & - & Var. S, 2-4 & 15 & $15-25$ & hq f- & - & - & - & \\
\hline Mintt. & $29 \cdot 515$ & - & $-11 \cdot 0-2 \cdot 8$ & - & - & E by S, 2-3 & 13 & $20-22$ & be & Ci.-s. 2 & - & - & \\
\hline$\overline{\text { IIean }}$ & $29 \cdot 550$ & -1 & --5.8 & - & - & - & $\overline{-}$ & - & - & - & - & - & \\
\hline & er: & & & & & 二. & & 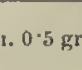 & nme i & Precil & sinet 11 & & \\
\hline
\end{tabular}

9th.

\begin{tabular}{|c|c|c|c|c|c|c|c|c|c|c|c|c|c|}
\hline 2 & $29 \cdot 527$ & -1 & $-5 \cdot 0-4 \cdot 0^{1}$ & - & -1 & $E, 1-2$ & 9 & $10-15$ & $\operatorname{bem} U$ & Ci.-s. 2 & - & - & Ci.-s, radiating NW to SE. \\
\hline 4 & $29 \cdot 529$ & - & $-6 \cdot 0-5 \cdot 4$ & - & - & Lt. SE airs & 6 & 10 & $\mathrm{~cm} U$ & Ci.-s. 7 & - & - & Some low dark str. over $S$ lhorizon. Glow \\
\hline 6 & $20 \cdot 492$ & - & $-8 \cdot 0-8 \cdot 0$ & - & - & Lt. SE airs & 0 & 10 & c & Ci.-s. 6 & A. . $.3 .3, \mathrm{~S}$ & - & Ci.-s. radiating NW to Sli. Otservations \\
\hline 8 & $28 \cdot 477$ & - & $-5 \cdot 3-5 \cdot 0$ & - & - & Lt. NF airs & 2 & 0 & 0 & A. -8.10 & - & - & \multirow{4}{*}{ 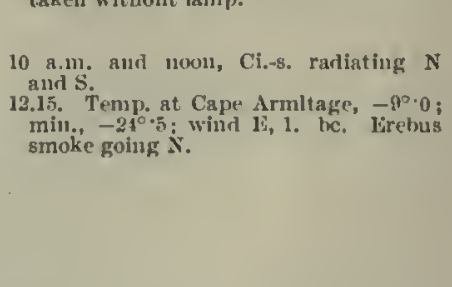 } \\
\hline Noon & $29 \cdot 429$ & - & $\begin{array}{ll}-5 \cdot 3 & 0 \cdot 2\end{array}$ & - & - & Calm & 0 & 0 & $n c \oplus$ & Ci.s. 5 & - & - & \\
\hline 2 & $29 \cdot 423$ & - & $-4 \cdot 2 \quad 2 \cdot 0$ & - & - & ESE, 1-2 & 7 & $10-15$ & ic & Ci.-s. 8 & - & - & \\
\hline 8 & $29 \cdot 325$ & - & $-6.5-6.0$ & - & - & $\mathrm{N}$ by $1 \mathrm{k}, 3$ & i & $15-20$ & hem & Ci.-s. 5 & - & - & \\
\hline 10 & $29 \cdot 28 \pi$ & - & $-11 \cdot 3-1 \cdot 2$ & - & - & Calın & 3 & 0 & b) & - & - & - & \multirow{4}{*}{ 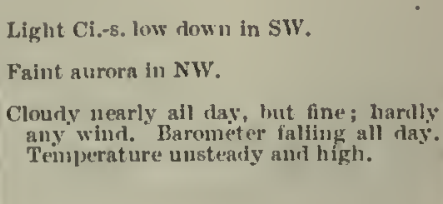 } \\
\hline slint. & $29 \cdot 212$ & - & $-4.8 \quad 0.0$ & - & - & NNR, 1-2 & 4 & $10-15$ & b) & - & - & - & \\
\hline Mean & $\longdiv { 2 9 \cdot 4 0 9 }$ & $\overline{-1}$ & $\mid--2.8$ & - & - & - & - & - & - & - . & - & - & \\
\hline & & & & & & aght bulb & & & & . & l'rectpu & & \\
\hline
\end{tabular}


Mnthorological, Journat, of till, "Jiscovery."

[1902-September.

\begin{tabular}{|c|c|c|c|c|c|c|c|c|c|c|c|c|c|c|c|}
\hline \multirow{3}{*}{ ilowr. } & \multirow{3}{*}{ 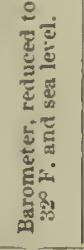 } & \multicolumn{5}{|c|}{ Thermometers. } & \multicolumn{3}{|l|}{ Wind. } & \multirow[b]{3}{*}{ 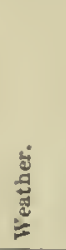 } & \multirow[b]{3}{*}{ 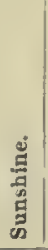 } & \multirow{2}{*}{\multicolumn{2}{|c|}{$\begin{array}{l}\text { Ciousls, } \\
\text { Anumut and thovement. }\end{array}$}} & \multirow[b]{3}{*}{$\frac{\stackrel{5}{5}}{\frac{5}{3}}$} & \multirow{3}{*}{ liemarks. } \\
\hline & & \multirow{2}{*}{ 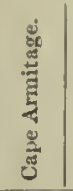 } & \multirow{2}{*}{ 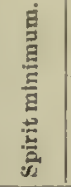 } & \multicolumn{2}{|c|}{ Mercurial. } & \multirow{2}{*}{ 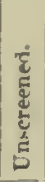 } & \multirow{2}{*}{$\begin{array}{c}\text { Direction ami } \\
\text { forces } \\
\text { (1Beaufort scaipe). }\end{array}$} & \multirow{2}{*}{ 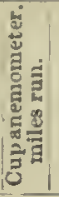 } & \multirow{2}{*}{ 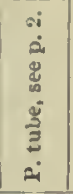 } & & & & & & \\
\hline & & & & Dry. & Wet. & & & & & & & Upper. & Lower. & & \\
\hline 3 & $29 \cdot 182$ & - & $-13 \cdot 3$ & $-11 \cdot 5$ & - & -1 & Calm & 1 & 0 & b) & & - & - & - & \multirow{7}{*}{$\begin{array}{l}\text { If eavy drift driving north wards up the } \\
\text { strait: twoue on board the ahips. }\end{array}$} \\
\hline 4 & $29 \cdot 0.98$ & - & $-12 \cdot 7$ & $-2 \cdot 3$ & - & - & s, 0-1 & 3 & 0 & $b$ & & - & - & - & \\
\hline B & $28 \cdot 9: 0$ & - & $-10 \cdot 3$ & $-4 \cdot 8$ & - & - & liNT, 1-2 & 8 & 20 & b & & - & - & - & \\
\hline 8 & $28 \cdot 977$ & - & $-9 \cdot 0$ & $-2 \cdot 0$ & - & - & $\mathrm{SI}_{2}, 4$ & 14 & $25-28$ & b & & - & - & - & \\
\hline 10 & $28 \cdot 919$ & - & $-13 \cdot 0$ & $-13 \cdot 0$ & - & - & lis by $S, 3-4$ & 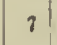 & $20-25$ & b & & - & - & - & \\
\hline Nom & $28 \cdot 890$ & - & $-26 \cdot 2$ & $-5 \cdot 0$ & - & - & SF, 1-2 & 11 & $10-15$ & b $\oplus$ & & - & - & - & \\
\hline 2 & $28 \cdot 841$ & - & $-20 \cdot 8$ & $-7 \cdot 8$ & - & - & S1: 1-2 & 4 & $10-12$ & b) & & - & - & - & \\
\hline 4 & $28 \cdot 791$ & $-34 \cdot 2$ & $-11 \cdot 8$ & $-4 \cdot 8$ & - & - & Calm & 5 & 0 & be & & Fr.-ei.-8. 3 & - & - & \multirow{7}{*}{ 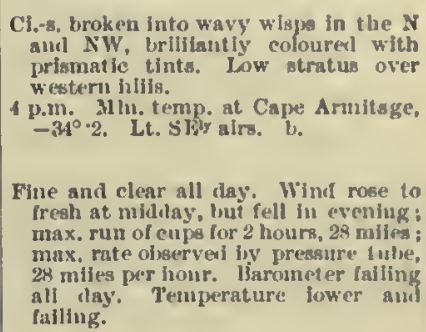 } \\
\hline 6 & $28 \cdot 779$ & - & $-11 \cdot 8$ & -6.8 & - & - & $\mathrm{N} 13,2-3$ & 2 & $15-20$ & b & & - & - & - & \\
\hline 8 & $23 \cdot 748$ & - & $-15 \cdot 8$ & $-10^{\circ} 0$ & - & - & Caim & 2 & 0 & b & & Fr.-ci.-eu. 1 & - & - & \\
\hline 10 & $28 \cdot 758$ & - & $-26 \cdot 0$ & $-10 \cdot 0$ & - & - & SE, 0-1 & 2 & 0 & () & & - & - & - & \\
\hline Mint. & $23 \cdot 684$ & - & -10.5 & $-4 \cdot 0$ & - & - & Lt. Jy airs & 4 & 0 & b) & & - & - & - & \\
\hline $\overrightarrow{\text { Veas }}$ & $28 \cdot 888$ & - & - & $-7 \cdot 3$ & - & -1 & - & $1-$ & - & - & & - & - & - & \\
\hline & & & $\left\{\begin{array}{l}8 \\
8\end{array}\right.$ & a. $0^{\circ}$ & & Sola & aliation $\left\{\begin{array}{l}\text { biack } \\
\text { brigh }\end{array}\right.$ & buil & $46^{\circ} \cdot 4$ & Eva & & вแнนеะ In $21 \mathrm{~h}$ & Precipn & & \\
\hline
\end{tabular}

10 th

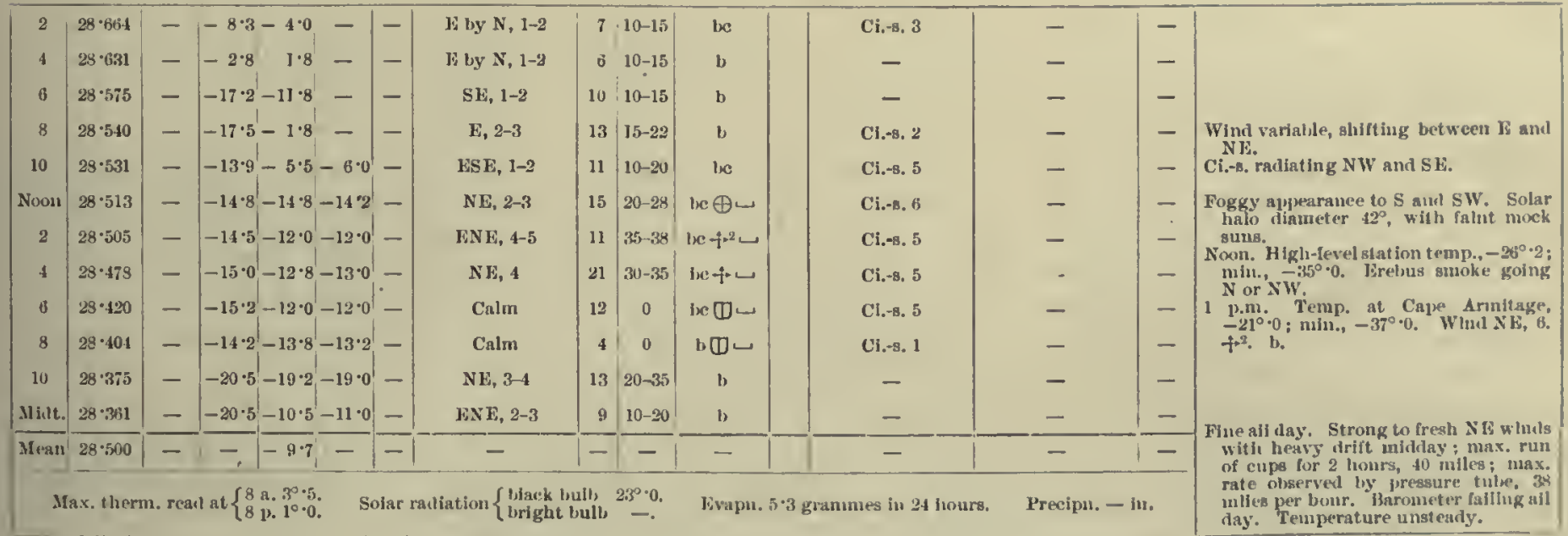

11 th.

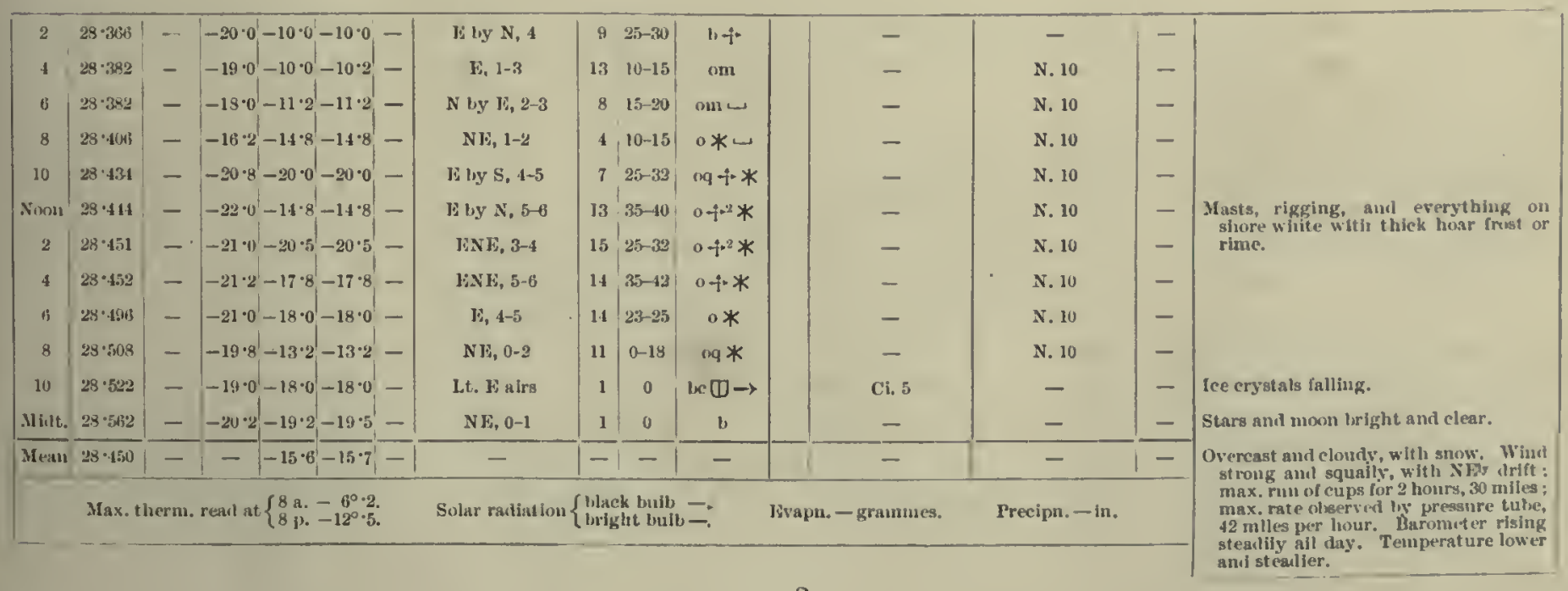

12 th. 
1902-September.]

Metworological Jourina, of the "Discovery."

\begin{tabular}{|c|c|c|c|c|c|c|c|c|c|c|c|c|c|c|c|}
\hline \multirow{3}{*}{, lour. } & \multirow{3}{*}{ 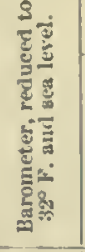 } & \multicolumn{5}{|c|}{ Thernumeters. } & \multicolumn{3}{|l|}{ IWind. } & \multirow[b]{3}{*}{ 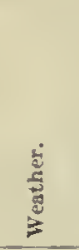 } & \multirow[b]{3}{*}{ 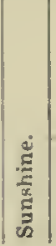 } & \multirow{2}{*}{\multicolumn{2}{|c|}{$\begin{array}{l}\text { Clouds, } \\
\text { Amount and movernent. }\end{array}$}} & \multirow[b]{3}{*}{ 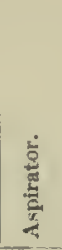 } & \multirow{3}{*}{ Hemarks. } \\
\hline & & \multirow{2}{*}{ 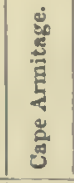 } & \multirow{2}{*}{ 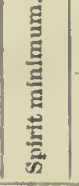 } & \multicolumn{2}{|c|}{ Mereurial. } & \multirow{2}{*}{ 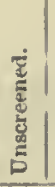 } & \multirow{2}{*}{$\begin{array}{c}\text { Direction and } \\
\text { force } \\
\text { (Beanfort scale). }\end{array}$} & \multirow{2}{*}{ 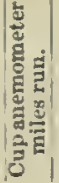 } & \multirow{2}{*}{ 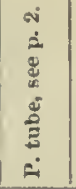 } & & & & & & \\
\hline & & & & Dry. & Wet. & & & & & & & Upper. & Lower. & & \\
\hline 2 & $28 \cdot 504$ & - & $-24 \cdot 0$ & -20.0 & $-19 \cdot 8$ & -1 & Calm & 2 & 0 & b & & $\mathrm{Cl} .-\mathrm{B} .1$ & - & - & \multirow{2}{*}{$\begin{array}{l}2 \text { and } 8 \text { a.m. Fog bank extending irom } \\
\mathrm{S} \text { to W. }\end{array}$} \\
\hline 8 & $28-530$ & - & $-19 \cdot 0$ & $-14 \cdot 0$ & $-14 \cdot 0$ & - & $\mathrm{NE}, 2$ & 6 & $10-15$ & bes & & $\mathrm{Ci},-\mathrm{s} . \mathrm{B}$ & S. 2 & - & \\
\hline 10 & $28 \cdot 5 i 1$ & - & $-20 \cdot 8$ & $-17 \cdot 0$ & $-17 \cdot 0$ & - & Calm & 7 & 0 & be & & Ci.-s. 5 & - & - & \multirow{4}{*}{ 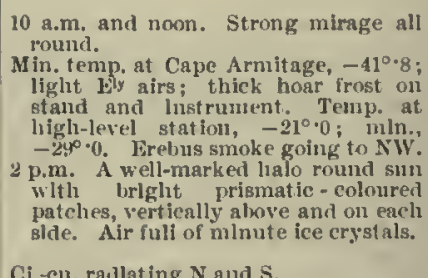 } \\
\hline Noon & $28 \cdot 584$ & $-35 \cdot 0$ & $-25 \cdot 5$ & $-25 \cdot 2$ & $-25 \cdot 2$ & - & Calın & 0 & 0 & lic & & Ci.-s. 6 & Cl. 2 & - & \\
\hline 2 & $28 \cdot 582$ & - & $-35 \cdot 2$ & $-20 \cdot 0$ & $-20 \cdot 0$ & - & SE, 2 & 2 & $15-20$ & $b c \oplus$ & & Ci. 7 & S. 1 & - & \\
\hline 4 & $28 \cdot 665$ & - & -20.8 & $-18 \cdot 8$ & $-18 \cdot 0$ & - & NE, 3-4 & 6 & $20-25$ & $b+$ & & - & - & - & \\
\hline Mean & $28 \cdot 620$ & - & - & $-18 \cdot 5$ & $-18 \cdot 4$ & -1 & - & - & - & - & & - & - & - & \multirow{2}{*}{$\begin{array}{l}\text { Thick lioar frost on everytling all day. } \\
\text { Cloudy, but fine generally. W'Ind } \\
\text { varlable and lighlt. Barouneter rising } \\
\text { steadily all day. Temperature lower } \\
\text { and steady. }\end{array}$} \\
\hline & \multicolumn{6}{|c|}{ Max. therm. read at $\left\{\begin{array}{l}8 \text { a. }-12^{a} \cdot 8 . \\
8 \mathrm{p},-15^{\circ} \cdot 0 .\end{array}\right.$} & \multicolumn{4}{|c|}{ Solar radiation $\left\{\begin{array}{l}\text { black bulb } 17^{\circ} \circ \\
\text { brighlt bulb }\end{array}\right.$} & & grammes. & \multicolumn{2}{|c|}{ Precipn. - In. } & \\
\hline
\end{tabular}

14th.

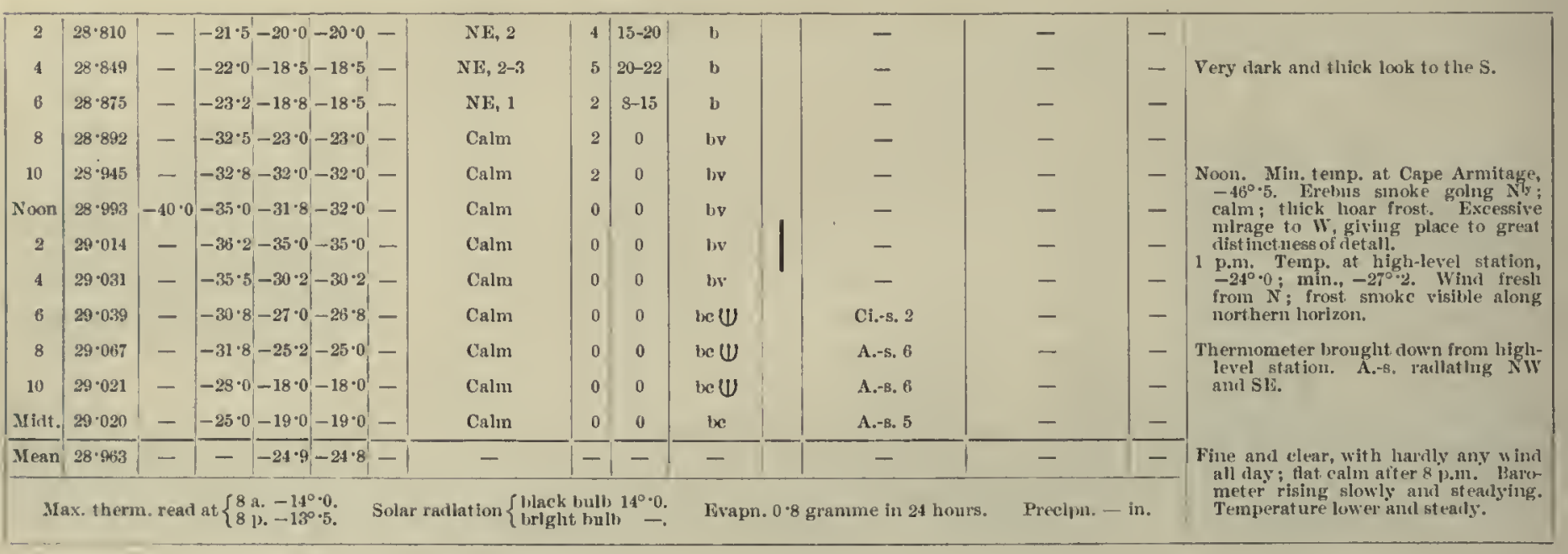

15th.

\begin{tabular}{|c|c|c|c|c|c|c|c|c|c|c|c|}
\hline 2 & $28 \cdot 9.937$ & - & $|-21 \cdot 8-18 \cdot 0 \cdot-18 \cdot 0|-$ & 1.t. NE airs & 0 & 0 & $\mathrm{bm}$ & - & - & - & Some snow fallen since mlduight; \\
\hline 4 & $28 \cdot 929$ & - & $-19 \cdot 2-14 \cdot 8-14 \cdot 8$ & E, 1 & 2 & $12-15$ & o & A.-8. 10 & - & - & lladlant sky in the S. \\
\hline 8 & $28: 895$ & - & $|-14 \cdot 2-12 \cdot 8-12 \cdot 2|-$ & E, $1-2$ & 7 & $10-15$ & om & - & s. 10 & - & \multirow{12}{*}{ 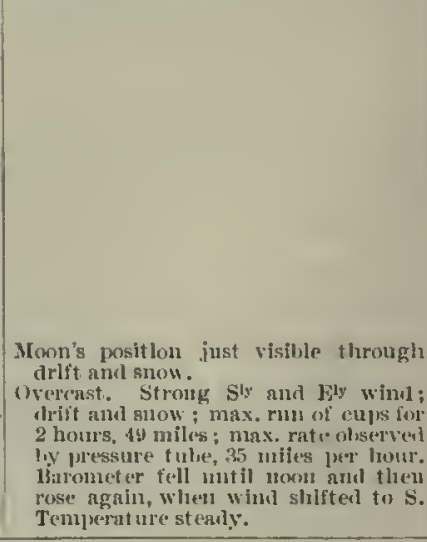 } \\
\hline 8 & $28 \cdot 890$ & - & $|-16 \cdot 8-16 \cdot 8-16 \cdot 2|-$ & SE, $1-2$ & 10 & $10-15$ & om $+2 *$ & - & N. 10 & - & \\
\hline 10 & $28 \cdot 877$ & - & $|-19 \cdot 4-18 \cdot 2-18 \cdot 2|-$ & $\mathrm{NF}, 3-5$ & 15 & $25-35$ & $0+*$ & - & N. 10 & - & \\
\hline Nonn & 28.868 & - & $\left|-18 \cdot 9^{\prime}-16 \cdot 2-16 \cdot 2\right|-$ & E l l,y $N, 4-5$ & 18 & $25-28$ & $0 \uparrow * *$ & - & N. 10 & - & \\
\hline 2 & 28.875 & - & $-16 \cdot 5-15 \cdot 0-15 \cdot 0$ & 1,4 & 16 & $25-28$ & $0+2 *$ & - & N. 10 & - & \\
\hline 4 & 28.893 & - & $-18 \cdot 5-12 \cdot 0-12 \cdot 0 \mid-$ & $\mathrm{s}, 4-5$ & 20 & $25-30$ & $0 \uparrow^{2} *$ & - & N. 10 & - & \\
\hline 6 & $28 \cdot 948$ & - & $-18 \cdot 8-12 \cdot 2-12 \cdot 2-$ & $\mathrm{s}, 2-5$ & 25 & $20-35$ & oq $t^{2} *$ & - & N. 10 & - & \\
\hline 8 & 28.973 & - & $-18 \cdot 8-18 \cdot 0-17 \cdot 8-$ & $\operatorname{SSE}, 2-4$ & 21 & $25-30$ & $04^{2} *$ & - & N. 10 & - & \\
\hline 1) & $29 \cdot 017$ & - & $-18 \cdot 8-18 \cdot 8-18 \cdot 5-$ & $\operatorname{ssc1}, 3-4$ & 21 & $25-3 v$ & oq $+*$ & - & N. 10 & - & \\
\hline Midt. & $29 \cdot 0.31$ & - & $-17 \cdot 5-10 \cdot 0-16 \cdot 0-$ & E, 3 & 13 & $15-20$ & ong $*$ & $=$ & N. 10 & - & \\
\hline Mean & $28 \cdot 927$ & - & $\left|-1^{-15 \cdot 7},-15 \cdot 6,-\right|$ & - & - & - & - & - & - & - & \\
\hline \multicolumn{4}{|c|}{ Max. therm. real at $\left\{\begin{array}{l}8 \mathrm{a} . \\
8 \mathrm{p} .\end{array}-12^{\circ} .5\right.}$. & Solar radinati & & inin & & grannes & \multicolumn{2}{|c|}{ гри. - } & \\
\hline
\end{tabular}


Mithololonicat, Journal, of THl: "1)iscovkity."

[1902-September.

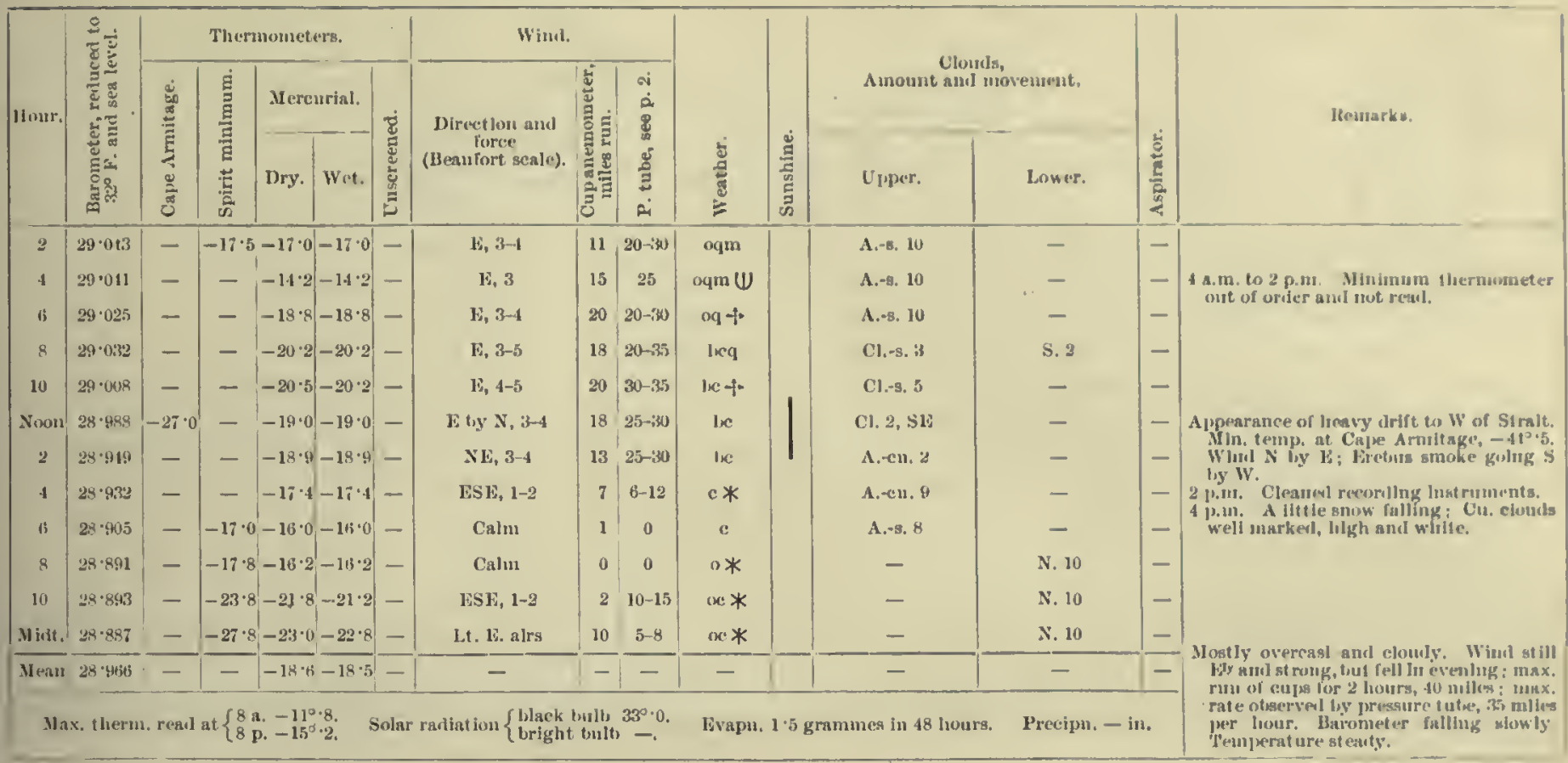

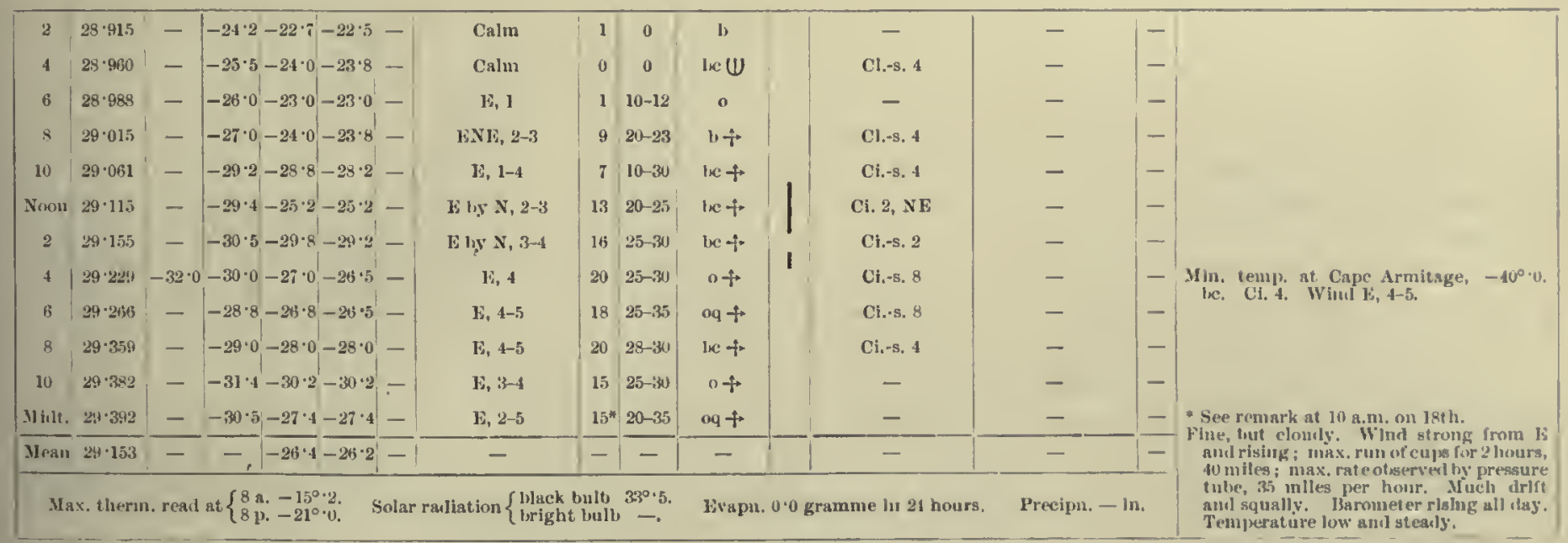

\begin{tabular}{|c|c|c|c|c|c|c|c|c|c|c|c|}
\hline 2 & $29 \cdot 391$ & - & $-28 \cdot 2-25 \cdot 2-25 \cdot 0-$ & 1, 3-6 & 18 & $25-39$ & oq + & - & - & - & \multirow{4}{*}{ Very heavy arlift all uight. } \\
\hline 4 & $29 \cdot 453$ & - & $-27 \cdot 8-24 \cdot 2-24 \cdot 0-$ & $\mathrm{SF}_{2,4-7}$ & 17 & $30-45$ & $q+$ & - & X. 10 & - & \\
\hline 6 & $29 \cdot 178$ & - & $-26 \cdot 4-25 \cdot 2-25 \cdot 0-$ & S1, 5-7 & $2 \pi$ & $35-55$ & $o q f^{2}$ & - & N. 10 & - & \\
\hline 8 & $29 \cdot 483$ & - & $-29 \cdot 8-28 \cdot 5-28 \cdot 0-$ & $1 \mathrm{E}, 4-6$ & 23 & $35-45$ & oq $f^{2}$ & - & x. 10 & - & \\
\hline 10 & $29-560$ & - & $-30 \cdot 2-22 \cdot 0-22 \cdot 0-$ & S, 5-6 & 26 & $35-40$ & oq $*+w^{2}$ & - & N. 10 & - & \multirow{4}{*}{ 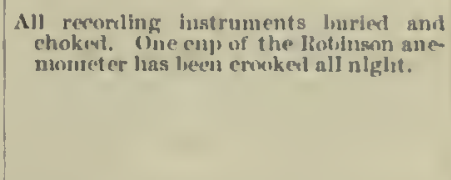 } \\
\hline Nom & $29 \cdot 5 ; 6$ & - & $-21 \cdot 0-21 \cdot 0-21 \cdot 0-$ & $s, 5-6$ & 24 & $40-50$ & $q * *+^{2}$ & - & x. 10 & - & \\
\hline 2 & $29 \cdot 645$ & - & $-25 \cdot 4-21 \cdot 2-21 \cdot 2-$ & Var. SE, 3-6 & 19 & $25-39$ & oq $* f^{2}$ & - & N. 10 & - & \\
\hline 4 & $29 \cdot 693$ & - & $-27 \cdot 2-25 \cdot 5-25 \cdot 5-$ & Var. SE, 2-5 & 21 & $20-35$ & $\operatorname{og} *+2$ & - & N. 10 & - & \\
\hline 6 & $23 \cdot 750$ & - & $-30 \cdot 5-26 \cdot 0-25 \cdot 8-$ & Far. 1, 4-5 & 2.7 & $25-30$ & $\operatorname{og} *+t^{2}$ & - & N. 10 & -1 & \multirow{6}{*}{ 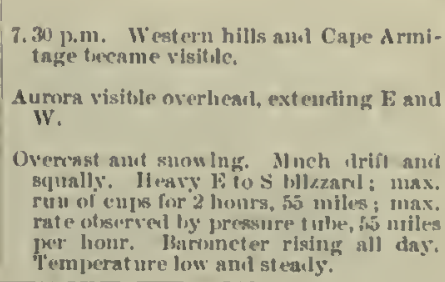 } \\
\hline 8 & $2 y$ & - & $-29 \cdot 9-23 \cdot 0-23 \cdot 0-$ & bSW, 2-3 & $1 / \mathrm{s}$ & $10-20$ & loqe +f & A.-s. 3 & - & - & \\
\hline 10 & $29 \cdot 7+10$ & - & $-28 \cdot 0-25 \cdot 0-25 \cdot 0-$ & 1., $2-3$ & 7 & $10-15$ & lice & A.-s. 1 & - & - & \\
\hline Nhist. & $29 \cdot 752$ & - & $-26 \cdot 0-20 \cdot 0-20 \cdot 0-$ & $1 \mathrm{by} s, 1-2$ & 11 & 10-1:3 & t" & - & - & - & \\
\hline $\mathrm{N} \cdots \mathrm{m}$ & $29 \cdot 6111$ & - & $-\quad-23 \cdot 9-23 \cdot 8-$ & - & - & - & - & - & - & - & \\
\hline & Max the & $\mathrm{rm}, \mathrm{ret}$ & 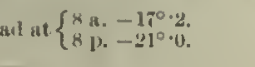 & Solar rautiation & & It lin & & Livajn. - grammes. & Preeipm. - In. & & \\
\hline
\end{tabular}


1902 - Septem ber.]

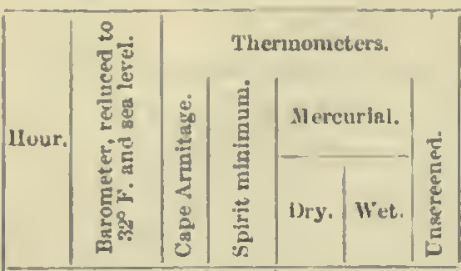

19 th.

\begin{tabular}{|c|c|c|c|c|c|}
\hline 2 & $29 \cdot 7 i 8$ & - & $-23 \cdot 5$ & $-18 \cdot 8-18 \cdot 8$ & - \\
\hline 4 & $2 x \cdot 7+12$ & - & -27 & $27 \cdot 5-27 \cdot 5$ & - \\
\hline 0 & $29 \cdot 710$ & - & -29 & $20 \cdot 0-20 \cdot 0$ & - \\
\hline 8 & $29 \cdot 656$ & - & -27 & $-24 \cdot 0$ & 一 \\
\hline 10 & $29 \cdot 570$ & - & - & $23 \cdot 4$ & 一 \\
\hline Noon & $29-520$ & - & & $-19 \cdot 8$ & - \\
\hline 2 & $29 \cdot 516$ & - & -20 & $-16 \cdot 0-16 \cdot 0$ & 一 \\
\hline 4 & $29 \cdot 468$ & - & $-21 \cdot 0$ & $-10 \cdot 0$ & - \\
\hline 6 & $29 \cdot 452$ & - & & $-18 \cdot 2$ & - \\
\hline 8 & $29 \cdot 422$ & - & -2 & $-22 \cdot 5-22 \cdot 0$ & 一 \\
\hline 10 & $29 \cdot 431$ & - & $-33 \cdot 5$ & $-17 \cdot 0-17 \cdot 0$ & 一 \\
\hline Mhitt. & $29 \cdot 132$ & - & 10 & $-19 \cdot 2$ & - \\
\hline I & $29-5630$ & - & - & $3-20 \cdot 7$ & $1-$ \\
\hline
\end{tabular}

Max, therm. read at $\begin{cases}8 & \text { a. } \\ 8 & p_{0}-\text {. }\end{cases}$

Metrorological, Journal of the "Discovery."

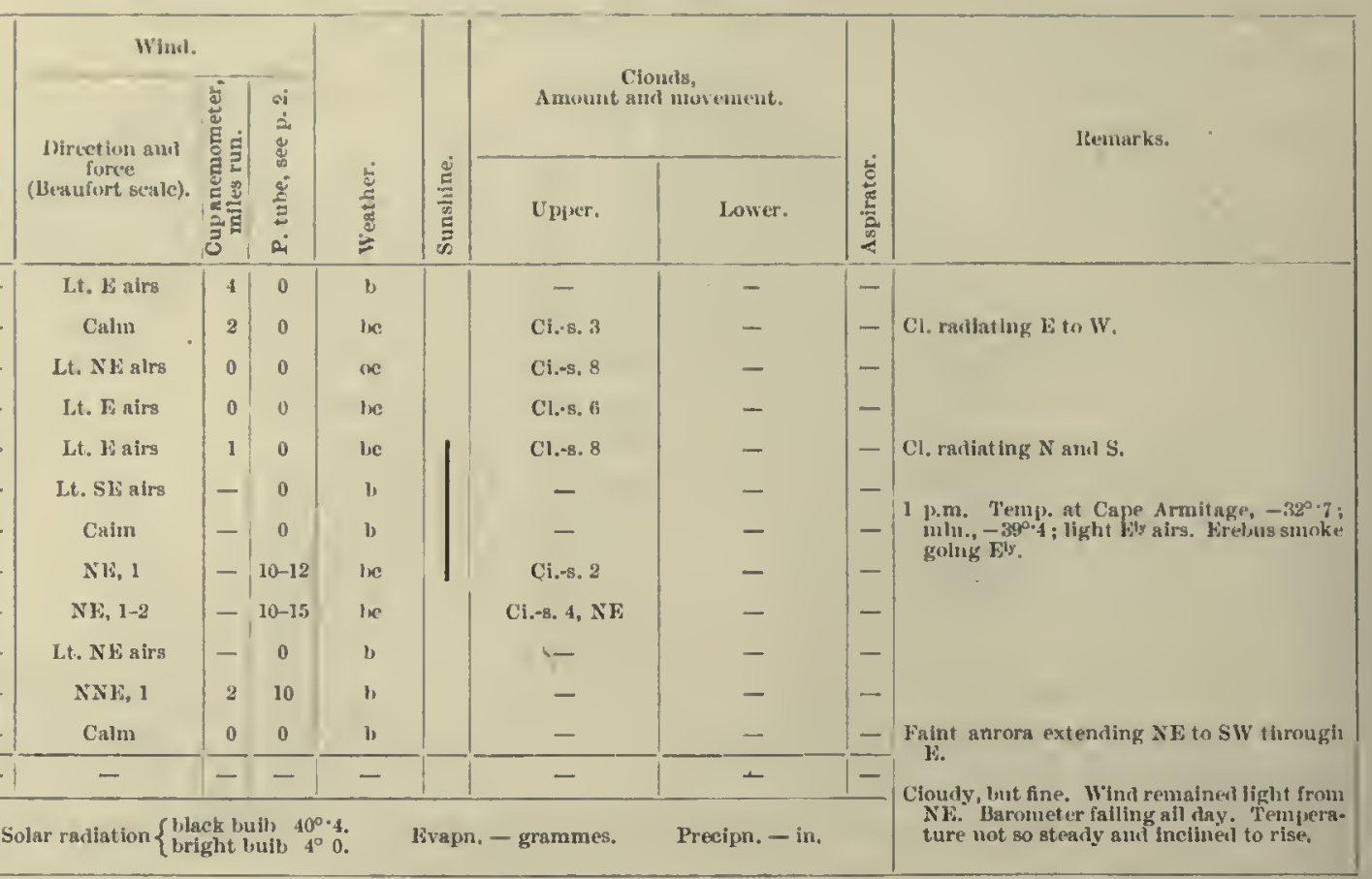

20 th.

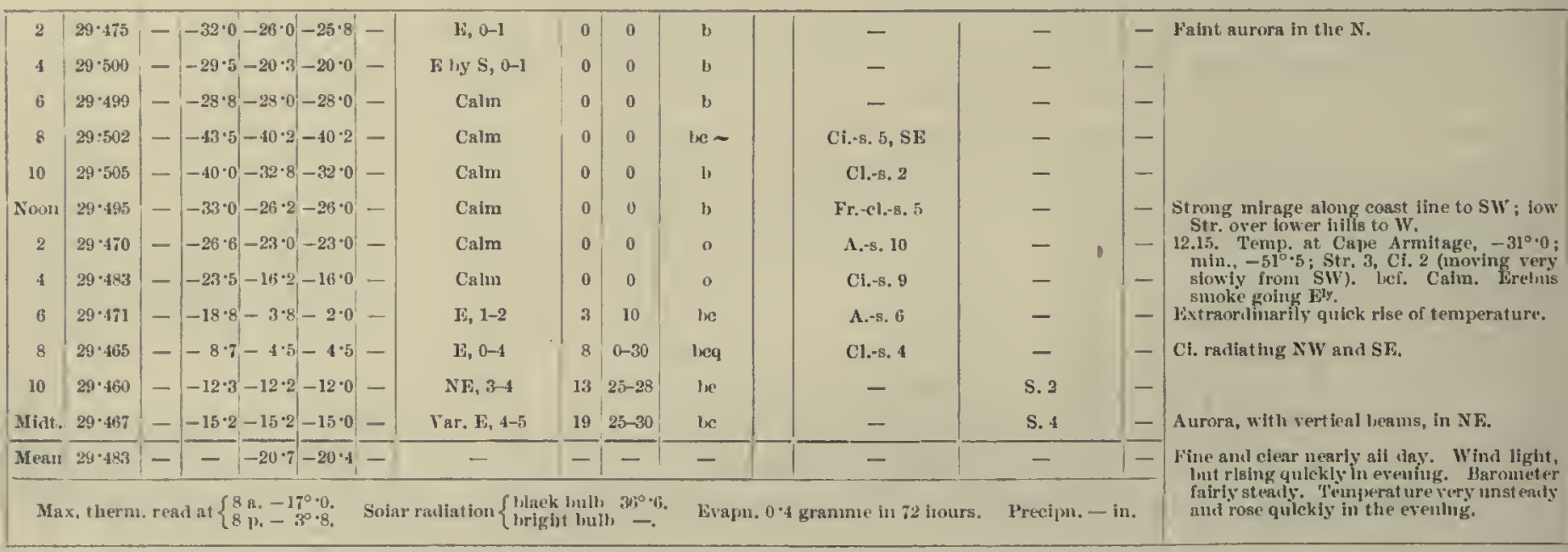

21st.

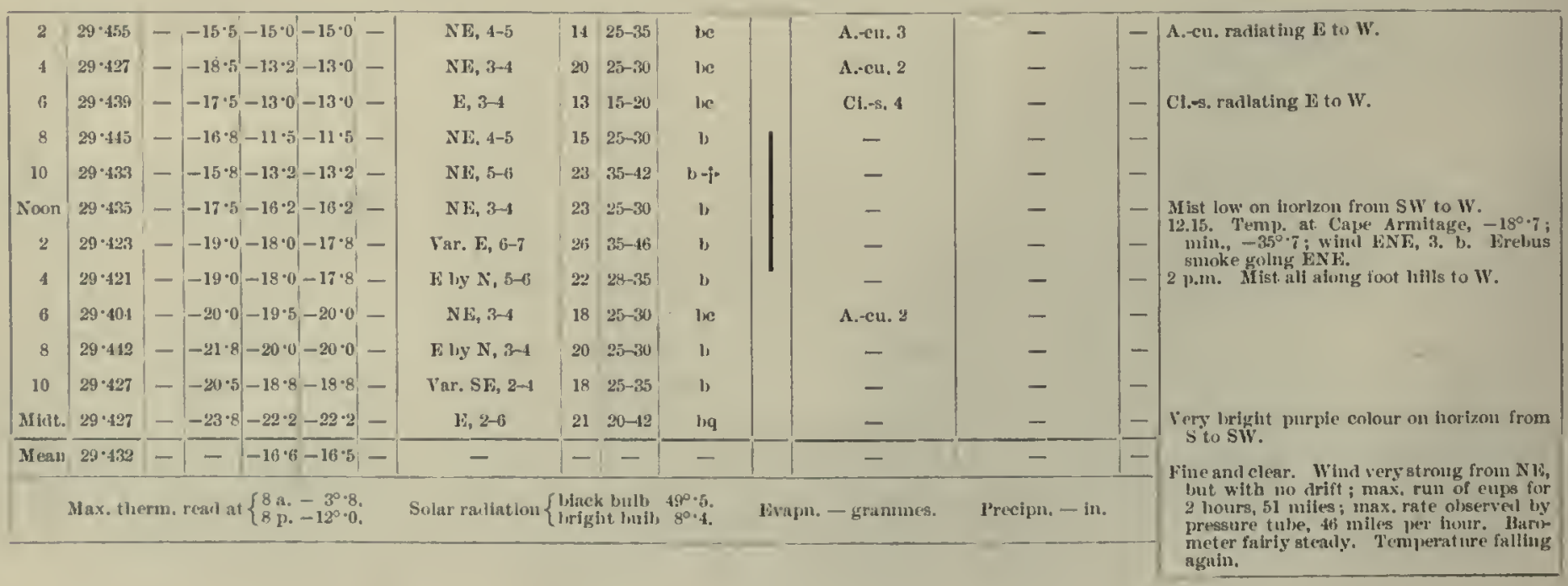


Mhitenotogical, Jourat, of THE" "Discovery."

[1902-September.

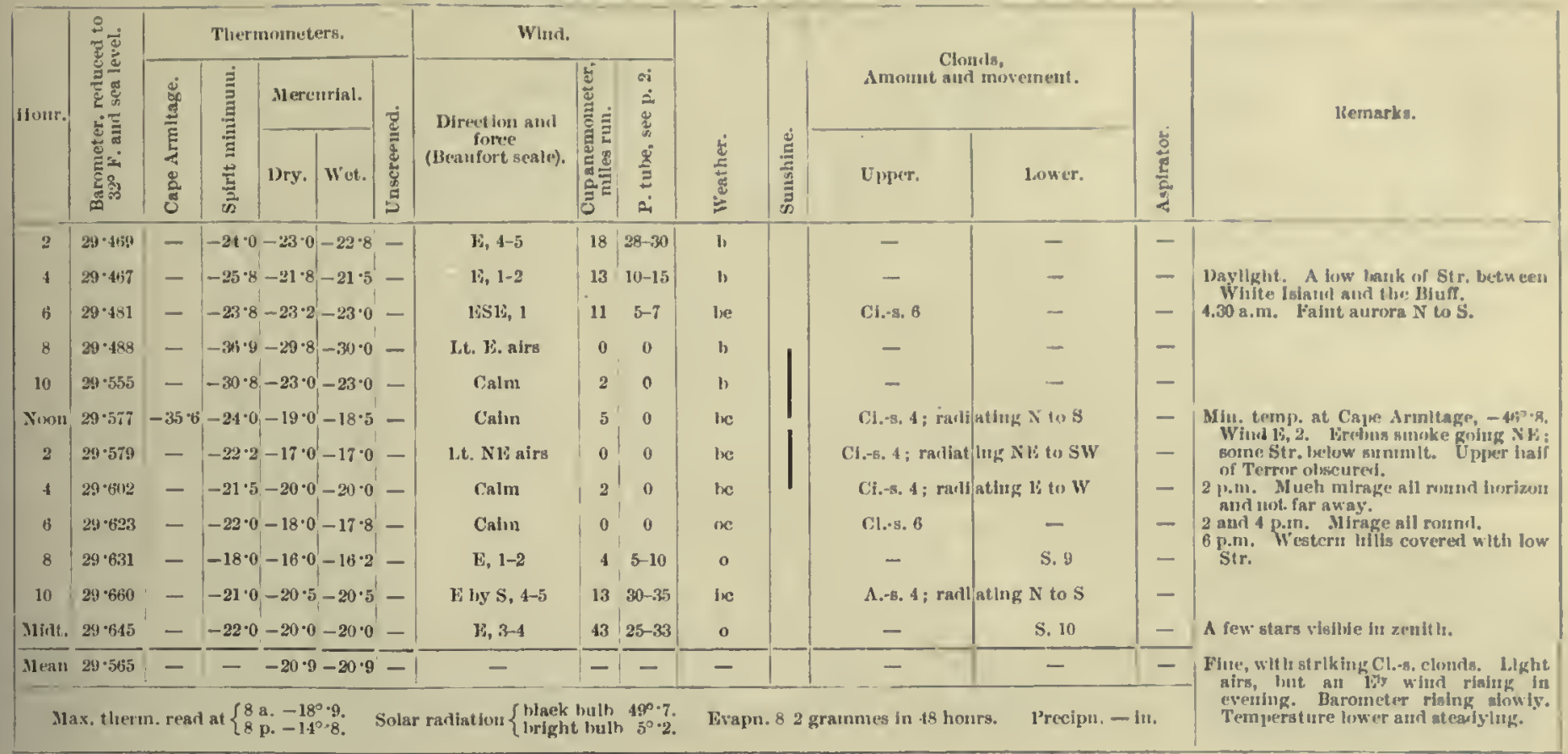

22nd.

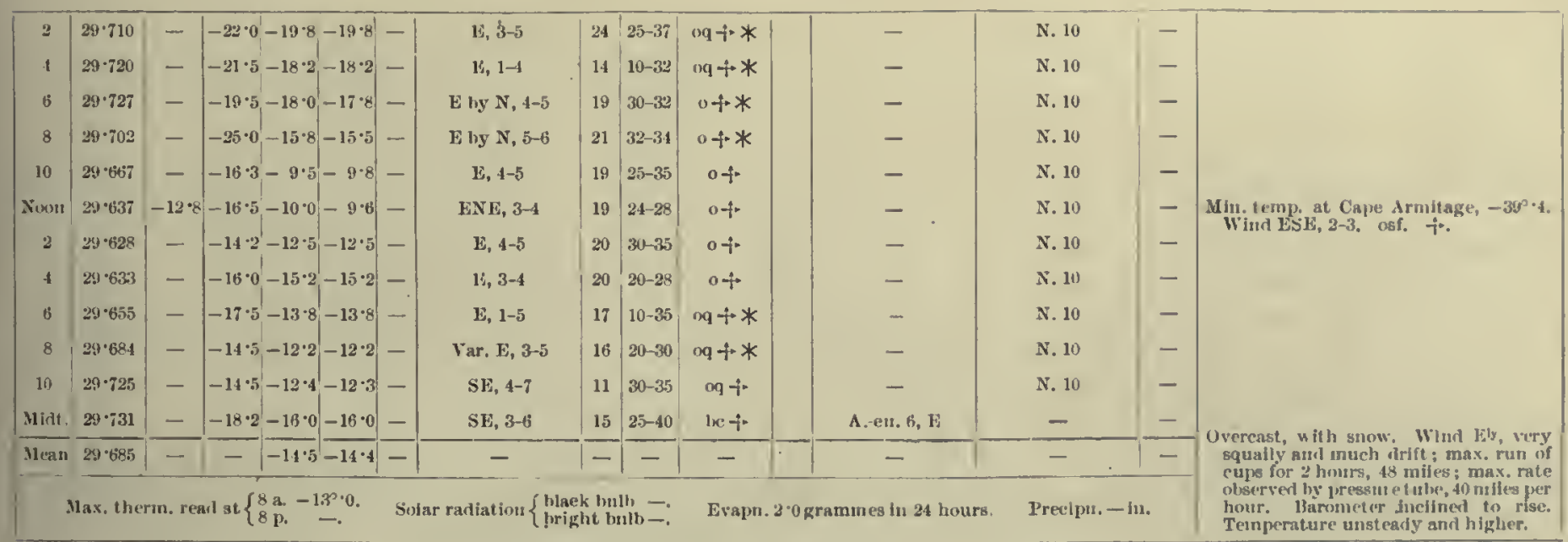

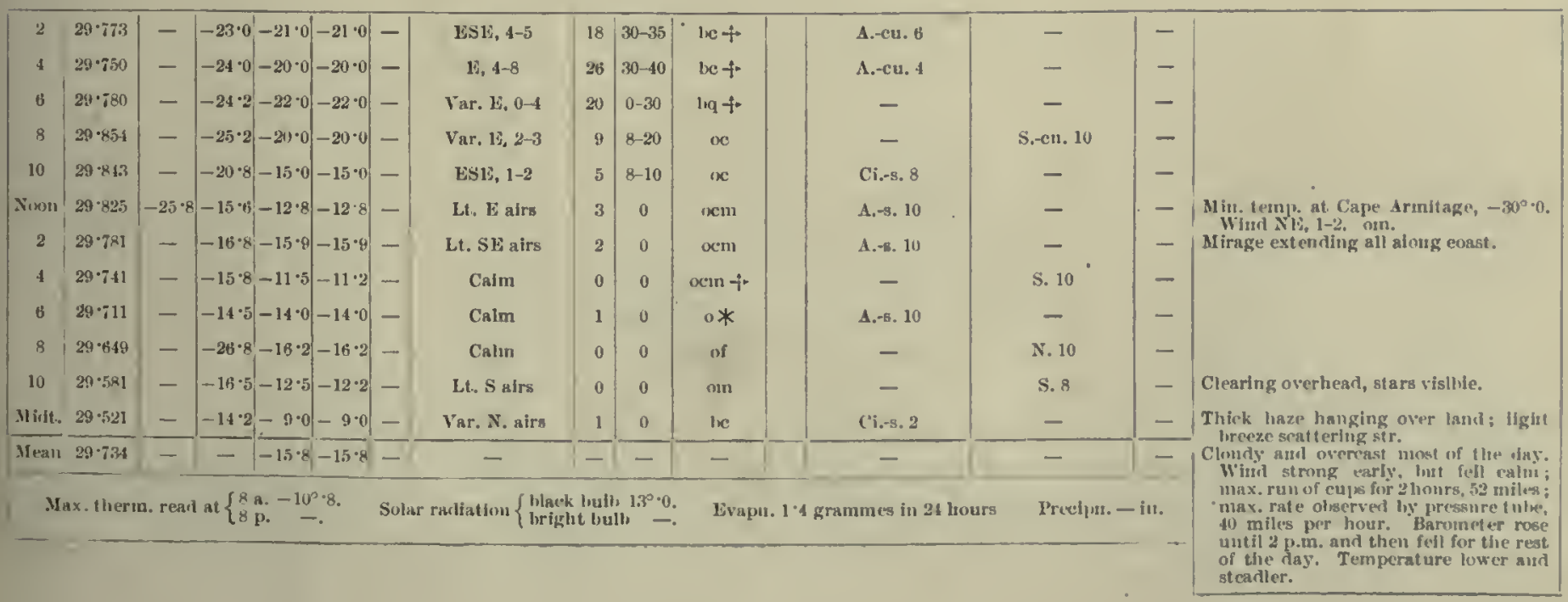


1902-September.]

Meteorological Jouksal of the "I) Iscovery."

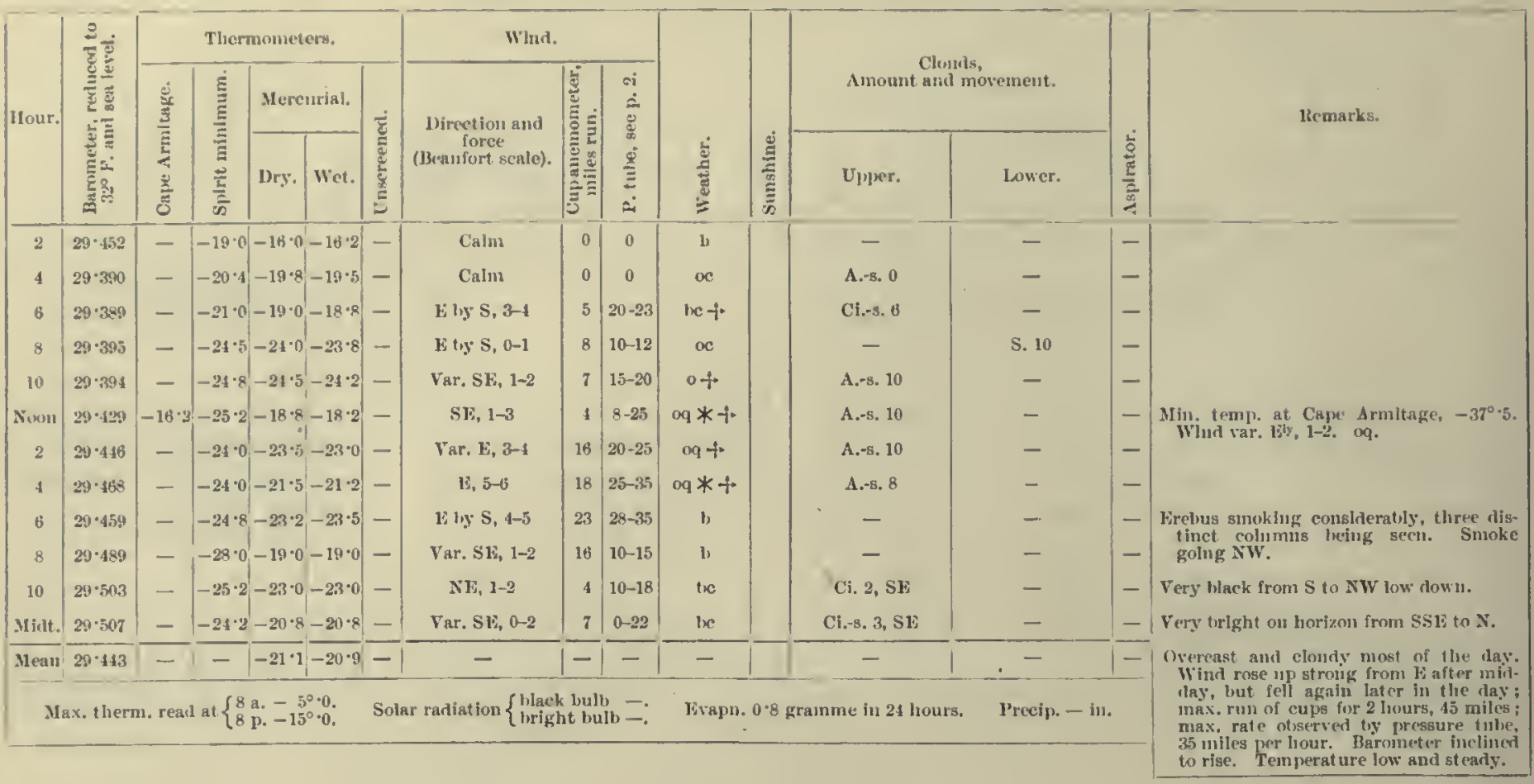

26 th

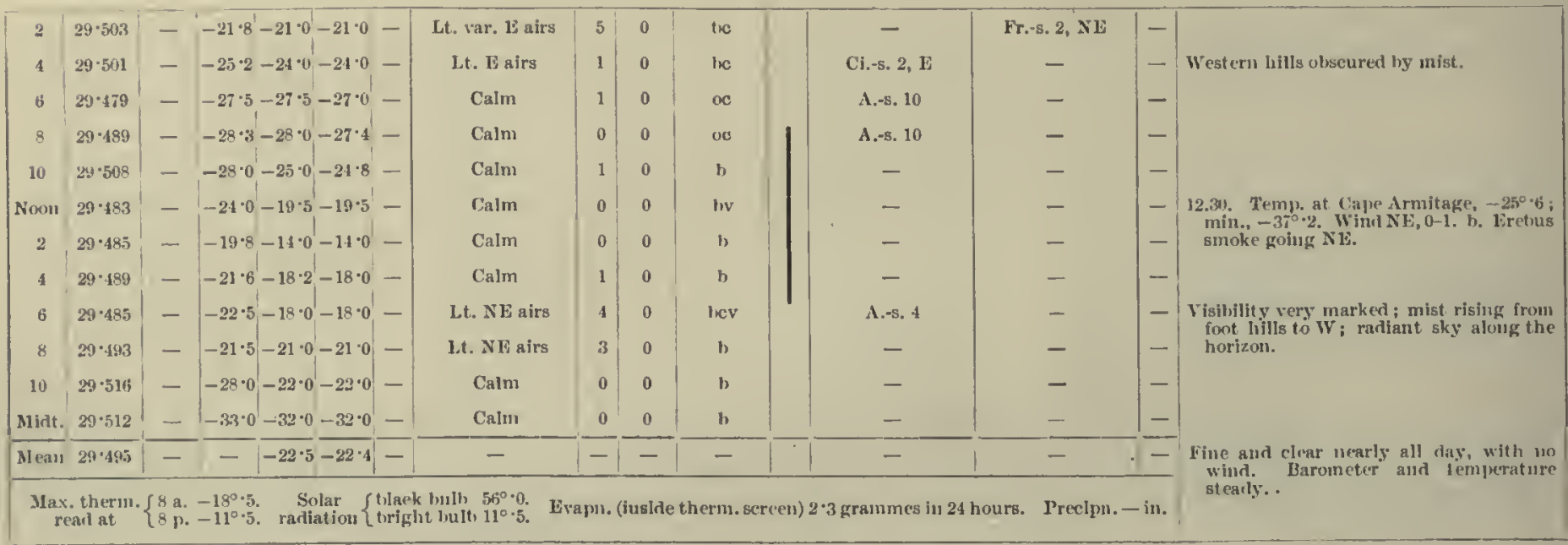

27th,

\begin{tabular}{|c|c|c|c|c|c|c|c|c|c|c|c|c|c|c|}
\hline 2 & 29.517 & - & $-34 \cdot 0-27 \cdot 2$ & $-27 \cdot 0$ & -1 & 1.t. NE aIrs & 0 & 0 & hic & & Ci.-s. $3, \mathrm{NW}$ & - & - & \multirow{5}{*}{ Mlrage all along horizon. } \\
\hline 4 & $29-522$ & - & $-35 \cdot 5-28 \cdot 5$ & $-26 \cdot 0$ & - & Calm & 0 & 0 & b & & - & - & - & \\
\hline 6 & $23 \cdot 547$ & - & $-30 \cdot 5-30 \cdot 0$ & - & - & Calm & 1 & 0 & b & & - & - & - & \\
\hline 8 & $29 \cdot 598$ & - & $-29 \cdot 0-10 \cdot 0$ & $-10 \cdot 0$ & - & $\mathrm{x}, 1-2$ & 2 & $10-15$ & b & & - & - & - & \\
\hline 10 & $29 \cdot 4.41$ & - & $-10 \cdot 4-70-$ & $-7 \cdot 0$ & - & $\mathrm{N}, 1$ & i) & $5-10$ & b & 1 & - & - & - & \\
\hline Noon & $29 \cdot 497$ & $-22 \cdot 8$ & $-17 \cdot 2-13 \cdot 8$ & $-16 \cdot 5$ & - & Calm & 3. & 0 & twe & 1 & A.-s. 4 & - & - & \multirow{7}{*}{$\begin{array}{l}\text { Min. temp. at Cape Armitage, }-45^{\circ} 0 \\
\text { calin. Ve. Ci.-s. } \\
\text {. }\end{array}$} \\
\hline 2 & $29 \cdot 49$ & - & $-17 \cdot 2-5 \cdot 0$ & $-5 \cdot 0$ & - & $N, 1-3$ & 4 & $10-20$ & $n$ & 1 & I. $-\mathrm{s}, 8, \mathrm{~N}$ & - & - & \\
\hline 4 & $29 \cdot 513$ & - & $-13 \cdot 5-7 \cdot 0$ & $-7 \cdot 0$ & - & Calm & 2 & 0 & $x$ & & Ci.-cul. \&, SW & - & -1 & \\
\hline G & $29 \cdot 452$ & - & $-11 \cdot 7-6 \cdot 8$ & -6.8 & - & It. NE airs & 1 & 0 & $\propto$ & & $\begin{array}{l}\text { S.cll. 8, SW } \\
\text { (rapid) }\end{array}$ & - & - & \\
\hline 8 & $28 \cdot 418$ & - & $-7 \cdot 0 \quad 7 \cdot 0$ & $7 \cdot 2$ & - & le, $2-3$ & 4 & $15-20$ & ce & & A.-8. 5, NNW & - & - & \\
\hline 10 & $29 \cdot 378$ & - & $4 \cdot 5 \quad 6 \cdot 5$ & $6 \cdot 2$ & - & Sk, 1 & $i$ & $5-10$ & $x$ & & - & Cu. 10 & - & \\
\hline Midt. & $29 \cdot 3+2$ & - & $3.4 \quad 5.8$ & $5 \cdot 2$ & - & 1.t. s1 airs & 7 & o & $\kappa$ & & - & Cu. 10 & -1 & \\
\hline$M$ +anu & $29 \cdot 476$ & -1 & $1--9 \cdot 8$ & - & -1 & - & -1 & - & - & & - & - & 二 & \multirow{2}{*}{ 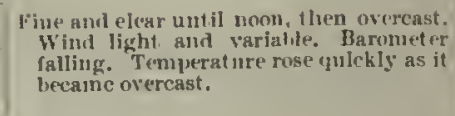 } \\
\hline & ax. ther & rrad & at $\begin{cases}8 \mathrm{a} . & -10^{\circ} \cdot \mathrm{c} \\
8 \mathrm{~m} & \mathrm{p}\end{cases}$ & & Sole & ation $\left\{\begin{array}{l}t, 1 \\
b r\end{array}\right.$ & oult & $\begin{array}{l}84^{\circ} \cdot 0 . \\
18^{\circ} \cdot 0 .\end{array}$ & liva & & anme in 24 hours. & Precipn. - in & & \\
\hline
\end{tabular}


MFtzorologion, JOURNAI, OF THE "DISCOVEIr."

[1902-September.

\begin{tabular}{|c|c|c|c|c|c|c|c|c|c|c|c|c|c|c|c|}
\hline \multirow{3}{*}{ Ilour. } & \multirow{3}{*}{ 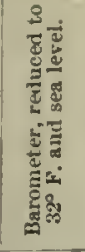 } & \multicolumn{5}{|c|}{ Tilermorneters. } & \multicolumn{3}{|l|}{ Whıd. } & \multirow[b]{3}{*}{ 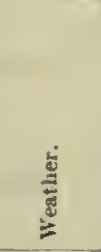 } & \multirow[b]{3}{*}{ 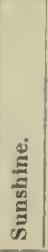 } & \multirow{2}{*}{\multicolumn{2}{|c|}{$\begin{array}{l}\text { Clonds, } \\
\text { Amount all inovement. }\end{array}$}} & \multirow[b]{3}{*}{ 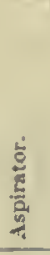 } & \multirow{3}{*}{ liemarks. } \\
\hline & & \multirow{2}{*}{ 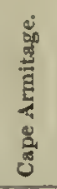 } & \multirow{2}{*}{ 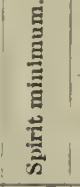 } & \multicolumn{2}{|c|}{ Merenrial. } & \multirow{2}{*}{ 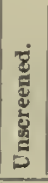 } & \multirow{2}{*}{$\begin{array}{c}\text { Direction ani } \\
\text { force } \\
\text { (Benufort scale). }\end{array}$} & \multirow{2}{*}{ 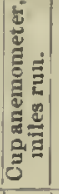 } & \multirow{2}{*}{ 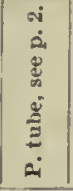 } & & & & & & \\
\hline & & & & Dry. & Wet. & & & & & & & Upper. & Lower. & & \\
\hline 2 & $29 \cdot 331$ & - & $|-1 \cdot 0|$ & $6 \cdot 0$ & - & - & $S, 3-4$ & 7 & $|20-25|$ & $\propto$ & & - & Cu. 10 & - & \\
\hline 4 & $29 \cdot 308$ & $\rightarrow$ & $2 \cdot 0$ & $5 \cdot 0$ & - & - & E, 4-5 & 10 & $25-30 \mid$ & o广* & & - & Cu. 10 & $\rightarrow$ & \\
\hline 6 & $29 \cdot 291$ & - & $3 \cdot 2$ & $7 \cdot 0$ & $8 \cdot 0$ & - & Var. Sle, 1-5 & 12 & $10-32$ & $\operatorname{og}+* *$ & & - & S. 10 & - & \\
\hline 8 & $29 \cdot 25,3$ & - & $4 \cdot 2$ & $7 \cdot 0$ & $8 \cdot 0$ & - & Var. N15, 2-5 & 14 & $15-30$ & og $\mathrm{i}^{-}$ & & ล.ซซ. 10 & - & - & \\
\hline 10 & $29 \cdot 203$ & $\rightarrow$ & $6 \cdot 0$ & $11 \cdot 0$ & $10 \cdot 3$ & $\rightarrow$ & V8r. S1i, 2-4 & 15 & $15-25$ & oc & & A.-cu, 10, S1s & 一 & - & \\
\hline N(x)! & $29 \cdot 175$ & - & $10 \cdot 0$ & $13 \cdot 0$ & $12 \cdot 0$ & - & $\mathrm{SE}, 2-3$ & 4 & $15-20$ & $\infty$ & & A.-eu. 10, SSE & - & - & 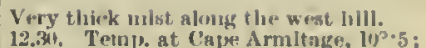 \\
\hline 2 & $29 \cdot 175$ & - & $9 \cdot 5$ & $13 \cdot 8$ & $12 \cdot 0$ & - & SSF, 3-4 & 4 & $20-24$ & 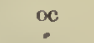 & & A.-8. 9 & - & - & min., $-25^{\circ} \cdot 3$. Wilncl s. of. + . \\
\hline 4 & $29 \cdot 123$ & - & $8 \cdot 8$ & $10^{\circ} 8$ & $10 \cdot 8$ & - & SE, 1-2 & 10 & $10-20$ & $\alpha+$ & & A.en. 9 & - & - & \\
\hline 6 & $29 \cdot 130$ & - & $9 \cdot 0$ & $11 \cdot 0$ & $11 \cdot 0$ & - & S, 4-5 & 15 & $25-30$ & $o c+$ & & - & N. 10 & - & \\
\hline 8 & $29 \cdot 113$ & - & $8 \cdot 8$ & $13 \cdot 0$ & $12 \cdot 2$ & - & E by S, 2-3 & 12 & $10-22$ & o & & $A,-8,10$ & - & - & Vlalbllity very markerl, S to SW. \\
\hline 10 & $29 \cdot 089$ & - & $11 \cdot 5$ & $14 \cdot 0$ & $13 \cdot 8$ & - & Var. S1i, 1-2 & 7 & $10-12$ & $\infty$ & & A.ecu. $10, \mathrm{SSE}$ & - & - & \\
\hline Midt. & $29 \cdot 066$ & - & $12 \cdot 2$ & $14 \cdot 0$ & $13 \cdot 8$ & - & Var. F, 0-1 & 4 & $8-10$ & $\infty$ & & Cl. cu. 10, S1. & - & - & Stars vlslble throngh Cl.ceu. \\
\hline Mean & $29 \cdot 183$ & - & - & $10 \div$ & $1-$ & $1-$ & - & - & - & - & & - & - & $一$ & $\begin{array}{l}\text { Overrust, with strong Sty and varlable } \\
\text { wiluds, with llrift, falllug in evening. }\end{array}$ \\
\hline
\end{tabular}

28 th.

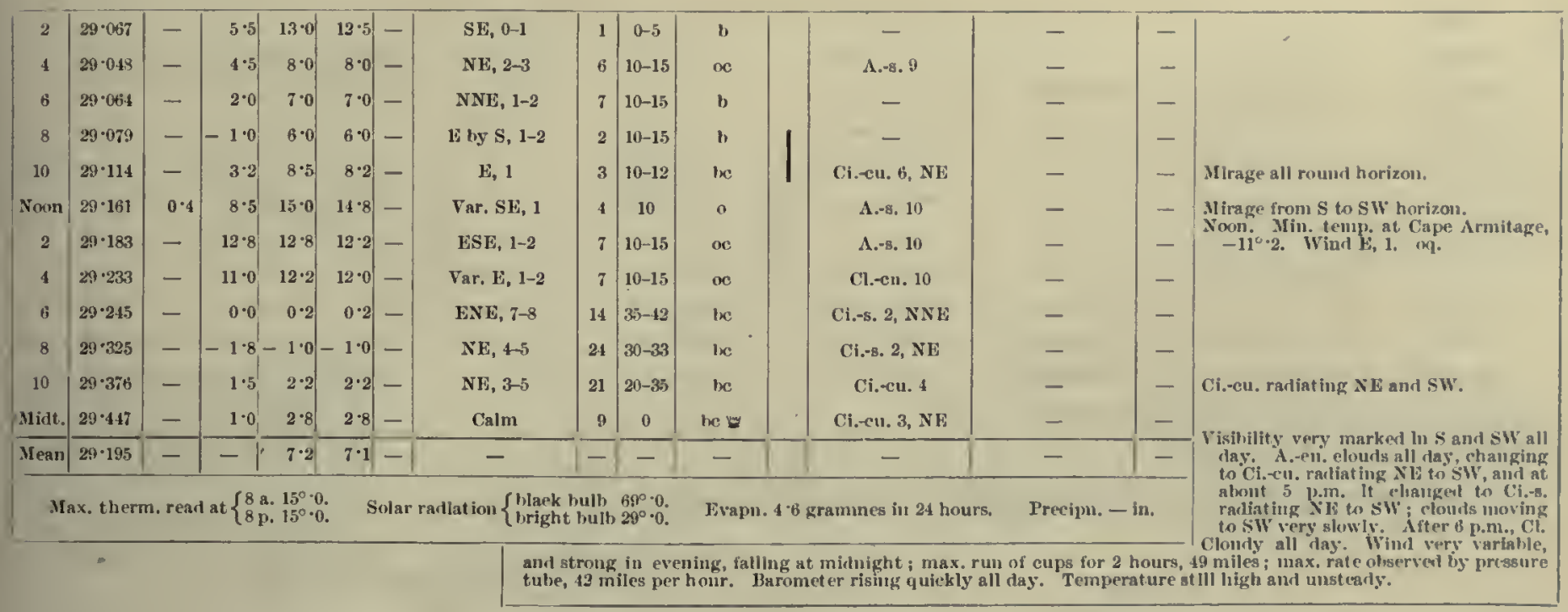

29 th.

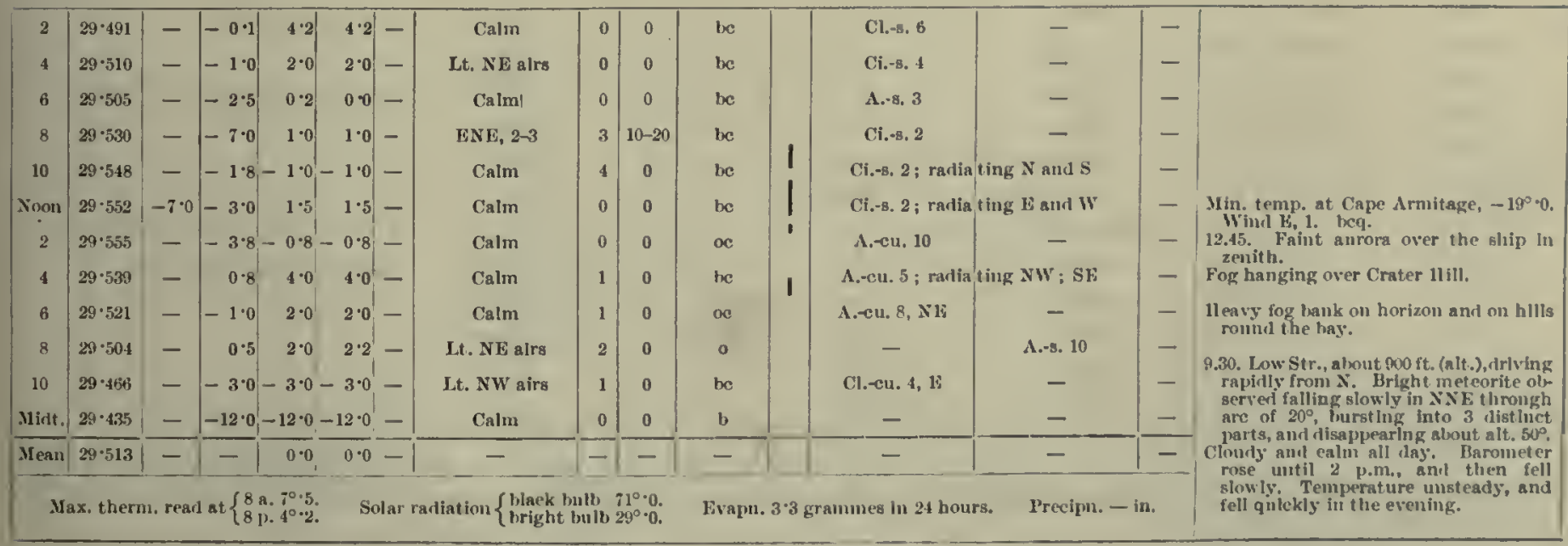


1902 - October.]

Methorological Journal, of THE "Discovery."

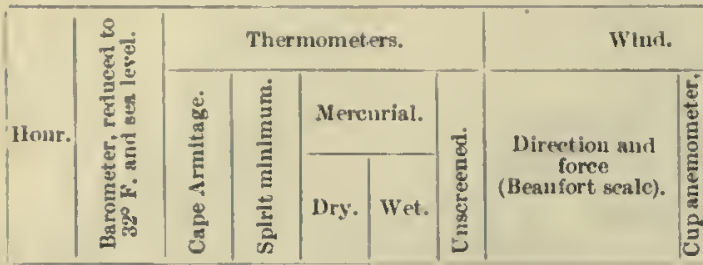

1st.

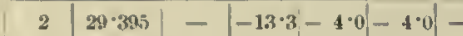

$429 \cdot 2559--5 \cdot 0-1 \cdot 0-1 \cdot 0-$

6 $29 \cdot 333--6 \cdot 5-5 \cdot 0-5 \cdot 0-$

$829 \cdot 314--10 \cdot 0-10 \cdot 0-9 \cdot 5-$

$1029 \cdot 2 \times 2--11 \cdot 0-8 \cdot 0-8 \cdot 0-$

Nю⿻1 $29 \cdot 231-11 \cdot 0-10 \cdot 3-5 \cdot 0-5 \cdot 0-$

$239 \cdot 249--8 \cdot 5-6 \cdot 0-5 \cdot 5-$

$429 \cdot 251--8 \cdot 0-3 \cdot 2-3 \cdot 2-$

6 $29 \cdot 243--7 \cdot 7-5 \cdot 0-5 \cdot 0-$

$8|29 \cdot 249|--6.3-1.0-0.8-$

$10|29 \cdot 244|-3 \cdot 0-1 \cdot 2-1 \cdot 3-$

Mlat. $29.232--3.2-1.5-1.5-$
Calm

Calm

Calm

ESE, 1-2

ESF, 1

FSE, 2-4

Var. 1, 2-3

$\mathrm{SE}, 3-4$

Var. $\mathbf{E}, 2-3$

H, 1-2

H, 3-4
E by S, $3-4$

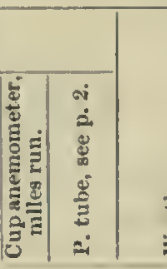

0 010

\begin{tabular}{l|l|l}
0 & 0 & икњ
\end{tabular}

0 of 0

1 10-15 b

7 5-10 W

10 2-4 $\alpha+$

12 15-20 क⿻

6 $10-20$ oc

18 20-25 oc +

$13 \quad 15-20$ oc

6 5-10 oc

$10 \quad 20-25$

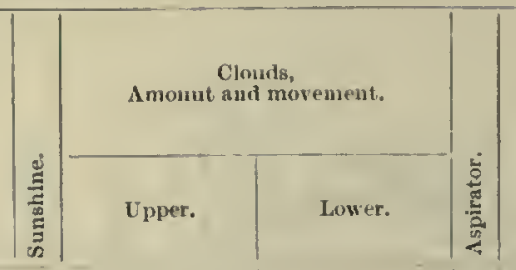

Ci.-8. 10

Ci.-5. 3, SW

Ci.-8. $8, \mathrm{~S}$

C1.-8. 4, SW

Ci.-5. 6

Ci.-8. 9

Ci.-s. 8

"Cl.-cu. 8

Ci.-cu. 10

Ci.cu. 10, sw

Ci.-cu. 9

Ci.-cu, 3
Remarks.

- $\quad$ - 2, 4, and $6 \mathrm{a} . \mathrm{m}$. Heravy rime ou masts - olservations taken wlthout lamp.

Iregvy fog hank atong horizon ; hills clear in the 13 ; tops of high mountains in - Wrisille.

10 a.m. Iow fng hank all ronnd. Bright bulls in rocuo found lirokell.

Non. Miu. temp. at Cape Armitage,

$-19^{\circ} 0$. Wisd $\mathrm{k}, 1-2$. kxq. + . $-$

- Wind shifty, E to NE.

- Overeast and elondy all day. Wind rose strong from $\mathrm{SF}$ in aftericion; $\mathrm{max}$. run of cups for 2 hours, $3 t^{\circ}$ miles; max. rate observer liy pressure tule, 28 miles per hour. Baroneter fell steadily till noon. Temperature higb.

2nd.

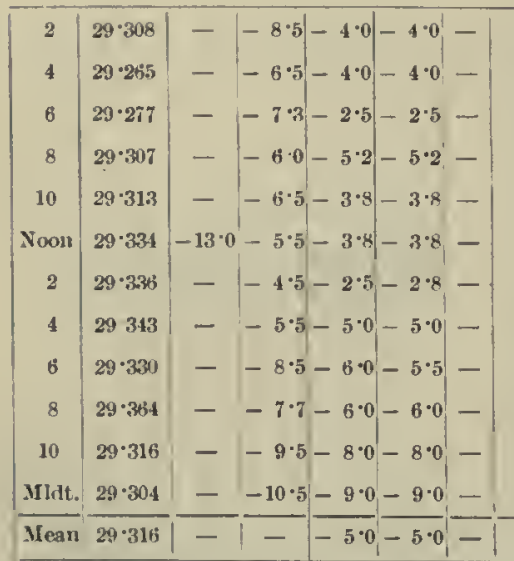

\begin{tabular}{|c|c|c|c|}
\hline E, 1-2 & 10 & $|10-20|$ & we + \\
\hline$E, 1-2$ & 5 & $10-15$ & $\alpha+$ \\
\hline Var. SE, 3-4 & 13 & $25-28$ & $o+$ \\
\hline Var. SE, 2-3 & 15 & $15-25$ & $o c+$ \\
\hline Var. 1, 3-4 & 10 & $20-25$ & $b c+$ \\
\hline Var. $\mathrm{F}^{2}, 2-3$ & 8 & $15-20$ & be \\
\hline E, 2-3 & 15 & $10-20$ & be \\
\hline ENE, 2-3 & 9 & $10-20$ & bc \\
\hline E, 4-5 & 13 & 20-25 & be \\
\hline Lt. E alrs & 6 & 0 & bc \\
\hline Var. NE, 3-4 & 10 & $20-25$ & $b f+$ \\
\hline Lt. var. $\mathrm{E}$ airs & 5 & 0 & bu \\
\hline & - & - & \\
\hline
\end{tabular}

\begin{tabular}{|c|c|}
- & S.4 \\
- & Cu. 10 \\
- & S.cu. 10 \\
Ci. 8 & - \\
Ci.-8. 6, SW & - \\
Ci.-s. 5, w & - \\
Ci. 3 & - \\
Ci. 2 & - \\
Ci.-8. 4, w & - \\
Ci.-s. 5, w & - \\
Ci.-s. 2, w & - \\
- & - \\
\hline- & -
\end{tabular}

Max. therm. read at $\left\{\begin{array}{ll}8 \mathrm{a}, & 0^{\circ} \cdot 2 . \\ 8 \mathrm{p}, & -2^{\circ} \cdot 0 .\end{array}\right.$ Solar radiation $\left\{\begin{array}{l}\text { black bulb } 68^{\circ} \cdot 0 . \\ \text { hright lualb }-.\end{array}\right.$

Clondy most of the day : very striking Cl.s. clonds, Strong Ely wind; max. rate observed hy pressure tube, 28 miles per hour. Barometer fairly steady. Temperature ligh, but falling again at night.

\begin{tabular}{|c|c|c|c|c|c|c|c|c|c|c|c|}
\hline 2 & $29 \cdot 259$ & - & $|-11 \cdot 4|-8 \cdot 0 \mid-8 \cdot 0-1$ & Calm & 9 & 0 & b & - & - & $1-$ & Heavy fog bank to $\mathrm{S}$. \\
\hline 4 & $29 \cdot 281$ & - & $-14 \cdot 5 \mid-11 \cdot 0-11 \cdot 0-$ & Lt. NE airs & 0 & 0 & oc & A.-8. 9 & - & - & \multirow{4}{*}{$\begin{array}{l}\text { Tops of near hills obscured hy fog. Thick } \\
\text { rime on everything. }\end{array}$} \\
\hline b & 29254 & - & $-8 \cdot 0-7 \cdot 5-7 \cdot 0-$ & Calm & 0 & $\mathbf{0}$ & $\propto \sqcup$ & - & S. 10 & - & \\
\hline 8 & $29 \cdot 234$ & - & $|-12 \cdot 5|-6 \cdot 2-6 \cdot 0-$ & Calm & 0 & 0 & c⿻ & - & S. 10 & - & \\
\hline 10 & $29 \cdot 229$ & - & $-12 \cdot 3-5 \cdot 5-5 \cdot 5-$ & Calm & 2 & 0 & of $\smile$ & - & s. 10 & $1-$ & \\
\hline Noon & $29 \cdot 223$ & - & $|-8 \cdot 0-7 \cdot 5|-7 \cdot 5 \mid-$ & Calm & 0 & 0 & of $\sqcup$ & - & S. 10 & - & \multirow{2}{*}{$\begin{array}{l}1 \text { p.m. Temp. at Cape Armitage, }-8^{\circ} 5 \\
\text { min. }-18^{\circ} \cdot 0 \text {, Calm; low dense fog. }\end{array}$} \\
\hline 2 & $29 \cdot 213$ & - & $|-13 \cdot 3|-13 \cdot 0 \mid-12 \cdot 8-$ & Var. SE, 1-2 & 0 & $8-10$ & กई & - & S. 10 & ; & \\
\hline 4 & $29 \cdot 195$ & - & $-12 \cdot 7-11 \cdot 5-11 \cdot 2-$ & Calm & 2 & 0 & bc & - & Fr.-8. 6, NW & - & \multirow[t]{4}{*}{ Heary fog lanks along horizon. } \\
\hline 6 & $29 \cdot 167$ & - & $-13 \cdot 7-12 \cdot 3-12 \cdot 5-$ & Lt. NW alrs & 2 & 0 & $b$ & - & Fr.-8. 3, XW & - & \\
\hline 8 & $29 \cdot 161$ & - & $|-17 \cdot 6|-7 \cdot 9-7 \cdot 2 \mid-$ & Calm & 0 & 0 & om & - & Fr.-в. 10, NW & - & \\
\hline 10 & $29 \cdot 163$ & - & $-21 \cdot 2-20 \cdot 0-20 \cdot 0-$ & Calm & 0 & 0 & bf $\backsim$ & - & - & - & \\
\hline Midt. & $29 \cdot 152$ & - & $-23 \cdot 8-23 \cdot 8-23 \cdot 0-$ & Calm & 0 & 0 & bu & - & - & - & \multirow{3}{*}{ 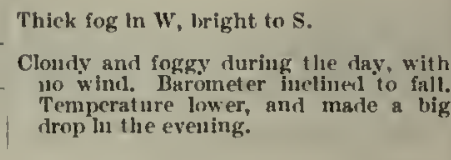 } \\
\hline Mran & $20 \cdot 20191$ & - & $--11 \cdot 2-11 \cdot 0-1$ & - & $1-$ & 一 & - & - & - & $1-$ & \\
\hline & tons & & Sols & ation $\left\{\begin{array}{l}\text { b } \\
\text { b }\end{array}\right.$ & & 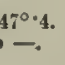 & Kvapm & me in 24 hours. & Preclpn. - in & & \\
\hline
\end{tabular}


Meteorol,ogical Journat, of the "Discovery."

[1902-October.

\begin{tabular}{|c|c|c|c|c|c|c|c|c|c|c|c|c|c|c|c|}
\hline \multirow{3}{*}{ Ifour. } & \multirow{3}{*}{ 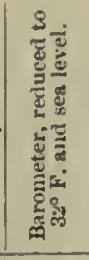 } & \multicolumn{5}{|c|}{ Thermometers. } & \multicolumn{3}{|l|}{ Wind. } & \multirow[b]{3}{*}{ 这 } & \multirow[b]{3}{*}{$\begin{array}{l}\text { 总 } \\
\text { 兽 } \\
\text { 量 }\end{array}$} & \multirow{2}{*}{\multicolumn{2}{|c|}{$\begin{array}{l}\text { Cloudls, } \\
\text { Amnount and mosement. }\end{array}$}} & \multirow[b]{3}{*}{$\mid$} & \multirow{3}{*}{ enurk:. } \\
\hline & & \multirow{2}{*}{ 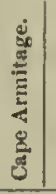 } & \multirow{2}{*}{ 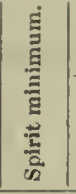 } & \multicolumn{2}{|c|}{ Uercurial. } & \multirow[b]{2}{*}{ 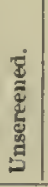 } & \multirow{2}{*}{$\begin{array}{c}\text { Divectlon and } \\
\text { forre } \\
\text { (Beanfort scale). }\end{array}$} & \multirow{2}{*}{ 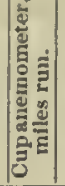 } & \multirow{2}{*}{ 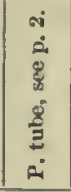 } & & & & & & \\
\hline & & & & Dry. & Wet. & & & & & & & Upper. & Lower. & & \\
\hline 2 & $29 \cdot 129$ & - & $-26 \cdot 0$ & $-23 \cdot 0$ & $-23 \cdot 0$ & - & Catm & 0 & 0 & be & & - & S.-cu. $2, \mathbf{E}$ & $1-$ & Hrillant sky, very rexl in $\mathbf{E}$. Silkgit fog \\
\hline 4 & $29 \cdot 12 \theta$ & - & $-25 \cdot 0$ & $-15 \cdot 5$ & $-15 \cdot 2$ & - & $\mathrm{NE}, 1-2$ & 4 & $10-15$ & te & & - & S.eu. $4, \mathrm{NE}$ & - & \\
\hline 6 & $29 \cdot 098$ & - & $-16 \cdot 8$ & $3-12 \cdot 5$ & $-12 \cdot 5$ & - & $\mathrm{NE}, 2-3$ & 16 & $20-25$ & $\propto$ & & - & A.cu. 10 & - & 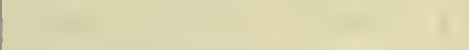 \\
\hline 8 & $29 \cdot 100$ & - & $-16 \cdot 0$ & $-14 \cdot 2$ & $-14 \cdot 2$ & - & Calm & 0 & 0 & œm & & - & S. 10 & - & Heavy fog over land to $\mathrm{W}$. \\
\hline 10 & $29 \cdot 087$ & - & $-15 \cdot 0$ & $-11 \cdot 4$ & $-11 \cdot 8$ & - & Lt. LS1i alrs & 0 & 0 & wem & & - & S. 10 & - & Set up new uright-bulb radiatlou thermo \\
\hline Noon & $29 \cdot 075$ & - & $-12 \cdot 7$ & $-9 \cdot 0$ & $-8 \cdot 5$ & - & Calm & 2 & 0 & $\propto \mathrm{m}$ & & - & S. 10 & $1-$ & \\
\hline 2 & $29 \cdot 069$ & - & $-9 \cdot 7$ & $-8 \cdot 8$ & $-8 \cdot 8$ & - & Calm & 0 & 0 & om & & - & S. 10 & - & Very foggy in $W$ and over ufar lulla. \\
\hline 4 & $29 \cdot 067$ & - & $-11 \cdot 3$ & $-10 \cdot 5$ & $-10 \cdot 5$ & - & Calm & 0 & 0 & o & & - & s. 10 & - & Fog to SW. \\
\hline 6 & $29 \cdot 0 \pi 1$ & - & $-12 \cdot 5$ & $-12 \cdot 0$ & $-12 \cdot 0$ & - & Lt. var. S alrs & 0 & 0 & on & & - & S. 10 & - & \\
\hline 8 & $29 \cdot 049$ & - & $-14 \cdot 3$ & $3-12 \cdot 0$ & $-12 \cdot 0$ & -1 & Lt. SE airs & 3 & 0 & ocm & & - & S. 10 & - & \\
\hline 10 & $29 \cdot 061$ & - & $-13 \cdot 5$ & $5-12 \cdot 0$ & $-12 \cdot 0$ & - & Lt. SE airs & 1 & 0 & ocm $\omega$ & & - & S. 10 & - & \\
\hline Midt. & $29 \cdot 071$ & - & $-12 \cdot 5$ & $-10 \cdot 0$ & $-10 \cdot 0$ & -1 & Calm & 0 & 0 & œin & & - & S. 10 & - & Heavy rhue on everyth thg. \\
\hline$\overline{\text { Iean }}$ & $29 \cdot 084$ & $\overline{-}$ & - & $\overline{-12 \cdot 6}$ & $\overline{-12 \cdot 5}$ & $\overline{\bar{I}_{1}}$ & - & 二 & - & - & & - & - & - & $\begin{array}{l}\text { Overeast and misty all day, Light variable } \\
\text { airs. Barometer fallhg intil } 8 \text { p.m., and }\end{array}$ \\
\hline & Iax, ther & m. r & ead at & $\left\{\begin{array}{l}8 \mathrm{a} \\
8 \mathrm{p}\end{array}\right.$ & $\begin{array}{ll}-12^{\circ} \cdot 2 . \\
-77^{\circ} \cdot 0 .\end{array}$ & & Solar radiation \{ & $\begin{array}{l}\text { black } \\
\text { brlgh }\end{array}$ & hanl & . & & anmes. & Precipn. - & & $\begin{array}{l}\text { then a slow rise. Temperature fower andl } \\
\text { unsteady. }\end{array}$ \\
\hline
\end{tabular}

4 th.

\begin{tabular}{|c|c|c|c|c|c|c|c|c|c|c|c|c|}
\hline 2 & $29 \cdot 093$ & -1 & $-13 \cdot 0 \mid$ & $|-11 \cdot 8-11 \cdot 8|-1$ & Lt. E airs & 0 & 0 & $\propto f \cup$ & - & S.cu. 10 & $1-$ & \multirow{8}{*}{ 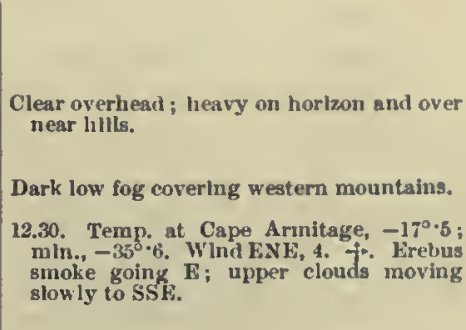 } \\
\hline 4 & $29 \cdot 125$ & - & $-14 \cdot 0$ & $-13 \cdot 5-13 \cdot 0-$ & Calm & 0 & 0 & ocf $\backsim$ & - & S..cu. 10 & - & \\
\hline 6 & $29 \cdot 131$ & - & $-15 \cdot 0$ & $-14 \cdot 0-14 \cdot 0-$ & Calm & 1 & 0 & hef - & Cl. 5 & - & - & \\
\hline 8 & $29 \cdot 159$ & - & $-16 \cdot 0$ & $-16 \cdot 0-16 \cdot 0-$ & NE, 3-4 & 2 & $24-28$ & them $\sqcup$ & Cl.os. 5 & - & - & \\
\hline 10 & $29 \cdot 185$ & - & $-19 \cdot 9$ & $-11 \cdot 0-11 \cdot 0-$ & ENE, 3-4 & 16 & $20-25$ & $b c+$ & Cl. 6 & - & - & \\
\hline Nom! & $29 \cdot 187$ & - & $-17 \cdot 2$ & $-15 \cdot 5-15 \cdot 5-$ & $\mathrm{NE}, 4-5$ & 16 & 28-32 & $b c+$ & Cl. 5 & - & - & \\
\hline 2 & $29 \cdot 241$ & - & $-18 \cdot 0$ & $-11 \cdot 0-11 \cdot 0-$ & E by N, 6 & 18 & $25-38$ & $1 x+7$ & Ci.-8. 6 & - & - & \\
\hline 4 & $29 \cdot 259$ & - & $-19 \cdot 0$ & $-18 \cdot 0-18 \cdot 0-$ & E by $N, G-7$ & 24 & $35-40$ & $\propto+$ & $\mathrm{Ci}_{.}-8.8$ & - & - & \\
\hline$b$ & $29 \cdot 285$ & - & $-19 \cdot 5$ & $-17 \cdot 8-17 \cdot 8-$ & E, 2-6 & 23 & $15-35$ & ocq $\div$ & Ci.-8, $3, \mathrm{NW}$ & S.cu. 5 & - & \multirow{6}{*}{$\begin{array}{l}\text { Red glow th the sky to the SW. } \\
\text { Cloudy, with fog in morning. High Ely } \\
\text { and Ny winds with drift; i max. run of } \\
\text { cups for } 2 \text { hours, } 48 \text { miles ; max, rate } \\
\text { observed by pressure tube, } 40 \text { miles per } \\
\text { hour. Barouneter rising. Temperature } \\
\text { low and steady. }\end{array}$} \\
\hline 8 & $29 \cdot 306$ & - & $-18 \cdot 2$ & $-16 \cdot 0-15 \cdot 8-$ & ENE, 3-5 & 22 & $25-32$ & $\infty f$ & - & S.cu. 10 & - & \\
\hline 10 & $29 \cdot 307$ & - & $-18 \cdot 8$ & $-17 \cdot 0-17 \cdot 2-$ & NE, 4-5 & 23 & $28-33$ & $\infty \mathrm{f}$ & - & A.cu. 10 & - & \\
\hline Midt. & $29 \cdot 318$ & - & $-19 \cdot 2$ & $-18.5-18 \cdot 5-$ & $\mathrm{NE}, 3-4$ & 18 & $25-30$ & $x+$ & - & A.cu. 10 & - & \\
\hline Mean & $29 \cdot 216$ & - & - & $-15 \cdot 0-15 \cdot 0-$ & - & - & - & - & - & - & - & \\
\hline \multicolumn{5}{|c|}{ Max. therm. read at $\left\{\begin{array}{l}8 \mathrm{a} .0 \\
8 \mathrm{p} .\end{array}-10^{\circ} 0\right.}$. & Solar radiatio & $\begin{array}{l}\text { black } \\
\text { brigh }\end{array}$ & $\begin{array}{l}\text { bulb } \\
\text { ht bul }\end{array}$ & 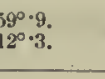 & Evapu. - grammes. & \multicolumn{2}{|c|}{ Precipn. - in. } & \\
\hline
\end{tabular}

5th.

\begin{tabular}{|c|c|c|c|c|c|c|c|c|c|c|c|}
\hline 2 & $29: 357$ & - & $1-\ddot{20} 0^{\circ} 0-18 \cdot 0-18 \cdot 0-$ & WNE, 4-5 & 19 & $30-35$ & $\infty$ & $\mathrm{Cl}_{.}-8.8$ & - & $|-|$ & \multirow{11}{*}{ \}Strong mirage to $\mathrm{NW}$. } \\
\hline 4 & $29 \cdot 361$ & - & $-19 \cdot 0-18 \cdot 0-18 \cdot 0-$ & E by $N, 4-5$ & 20 & $30-35$ & $\boldsymbol{\alpha}$ & - & S. 10 & - & \\
\hline 6 & $29 \cdot 372$ & - & $-20 \cdot 5-18 \cdot 5-13 \cdot 5-$ & $N E, 3-4$ & 18 & $20-25$ & $\infty$ & - & S.cu. 10 & - & \\
\hline 8 & $29 \cdot 371$ & - & $-20 \cdot 8-19 \cdot 0-19 \cdot 0-$ & N1, 4-5 & 21 & $28-35$ & $\propto$ & - & S.eu. 10 & - & \\
\hline 10 & $29 \cdot 369$ & - & $-19 \cdot 8-17 \cdot 0-17 \cdot 0-$ & Var. NE, 3-4 & 18 & $25-30$ & $0+$ & - & S.cu. 10 & - & \\
\hline Noon & $29 \cdot 369$ & - & $-19 \cdot 0-18 \cdot 0-18 \cdot 0-$ & $N x, 3-4$ & 15 & $20-25$ & o & - & S.cu. 10 & - & \\
\hline 2 & $29 \cdot 353$ & - & $-19 \cdot 2-19 \cdot 0-18 \cdot 8-$ & $\mathrm{NB}, 3-4$ & 13 & $25-30$ & 0 & - & S. 10 & - & \\
\hline 4 & $29 \cdot 329$ & - & $-30 \cdot 5-20 \cdot 0-20 \cdot 0-$ & ENE, 2-3 & 17 & $20-28$ & $\propto$ & - & s. 10 & - & \\
\hline 6 & $29 \cdot 234$ & - & $-21 \cdot 0-18 \cdot 0-17 \cdot 5-$ & E by $N, 3-4$ & 10 & $20-28$ & $\propto$ & - & S.cu. 10 & - & \\
\hline 8 & $29 \cdot 315$ & - & $-18 \cdot 8-15 \cdot 5-15 \cdot 5-$ & L, 1-2 & $14_{1}$ & $10-15$ & $\infty$ & - & S.cu. 10 & - & \\
\hline 10 & $29 \cdot 287$ & - & $-16 \cdot 0-15 \cdot 0-15 \cdot 0-$ & Lt. $\mathbf{N}$ alrs & 5 & 0 & $\propto$ & - & S.-cu. 10 & - & \\
\hline Midt. & $29 \cdot 266$ & - & $-19 \cdot 0-16 \cdot 0-16 \cdot 0-$ & Calm & 1 & 0 & $\propto$ & - & S.cu. 10 & - & \multirow{3}{*}{ 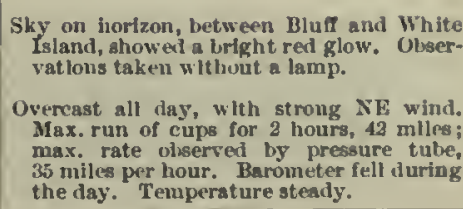 } \\
\hline Mean & $29 \cdot 340$ & - & $-\overline{-17 \cdot 7} \overline{-17 \cdot 8}-$ & - & $=$ & - & - & - & - & - & \\
\hline & x. ther & & read at $\left\{\begin{array}{l}8 \mathrm{a} .-16^{\circ} \cdot 8 . \\
8 \mathrm{p},-15^{\circ} \cdot 0 .\end{array}\right.$ & Soląr radistlon & $\begin{array}{l}\text { black } \\
\text { brigh }\end{array}$ & S Inlb & - & Evapn. - grammes. & Preelpn. - & & \\
\hline
\end{tabular}


1902-October.]

Mfteorologica, Jourat. of the "Discovery."

\begin{tabular}{|c|c|c|c|c|c|c|c|c|c|c|c|c|c|c|c|}
\hline \multirow{3}{*}{ llour. } & \multirow{3}{*}{ 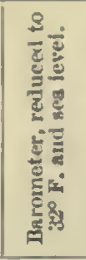 } & \multicolumn{5}{|c|}{ - Thormometers. } & \multicolumn{3}{|l|}{ Winel. } & \multirow[b]{3}{*}{ 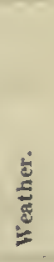 } & \multirow[b]{3}{*}{ 递 } & \multirow{2}{*}{\multicolumn{2}{|c|}{$\begin{array}{l}\text { Clouds, } \\
\text { Anount and movement. }\end{array}$}} & \multirow[b]{3}{*}{ 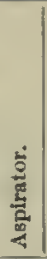 } & \multirow{3}{*}{ Re111ark8. } \\
\hline & & \multirow{2}{*}{$\frac{8}{\substack{g\\
}}$} & \multirow{2}{*}{ 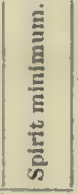 } & \multicolumn{2}{|c|}{ Mencurial. } & \multirow{2}{*}{$\frac{\text { हैं }}{5}$} & \multirow{2}{*}{$\begin{array}{l}\text { Direetion and } \\
\quad \text { foree } \\
\text { (Beaufort scale). }\end{array}$} & \multirow{2}{*}{ 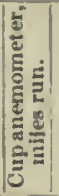 } & \multirow{2}{*}{ 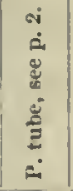 } & & & & & & \\
\hline & & & & Dry. & Wet. & & & & & & & Upper. & Lower. & & \\
\hline 2 & $29 \cdot 214$ & - & $-19 \cdot 0$ & $-15 \cdot 4$ & $-15 \%$ & - & Lt. NIS airs & 2 & 0 & $\infty$ & & - & S. 10 & - & \multirow{5}{*}{$\begin{array}{l}\text { Sum shining on foot of western hills } \\
\text { beinind the fog. }\end{array}$} \\
\hline 4 & $29 \cdot 199$ & - & $-17 \cdot 2$ & $-16 \cdot 8$ & $-17 \cdot 0$ & - & Calın & 0 & 0 & $o *$ & & - & S. 10 & - & \\
\hline 6 & $29 \cdot 179$ & - & $-17 \cdot 0$ & $-15 \cdot 0$ & $-15 \cdot 0$ & - & Calm & 0 & 0 & ne & & - & S. 10 & - & \\
\hline 8 & $29 \cdot 144$ & - & $-15 \cdot 0$ & $-15 \cdot 0$ & $-15 \cdot 0$ & - & Calın & 0 & 0 & $a c$ & & - & S. 10 & - & \\
\hline 10 & $29 \cdot 146$ & - & $-18 \cdot 6$ & $-12 \cdot 8$ & $-12 \cdot 8$ & - & Cain & 0 & 0 & $o c$ & & - & S. 10 & - & \\
\hline Noost & $29 \cdot 118$ & - & $-14 \cdot 5$ & $-10 \cdot 2$ & $-10 \cdot 2$ & - & NE, 1-2 & 2 & $10-18$ & $\infty$ & & - & S. 10 & - & \multirow{4}{*}{$\begin{array}{l}\text { Mirage to tise south horizon. } \\
12.30 . \text { Temp. at Cape Arnitage, }-17^{\circ} \cdot 2 \text {; } \\
\text { min., }-20^{\circ} \cdot 4 \text {; wind BSE, 3. lirelus } \\
\text { obscured. } \\
\text { livaporation dish replacel outslie, having } \\
\text { been mlslail sinee 3rd Inst. }\end{array}$} \\
\hline 2 & $29 \cdot 118$ & - & $-10 \cdot 5$ & $-10 \cdot 0$ & $-10 \cdot 0$ & - & $\mathrm{NF}, 0-1$ & 3 & $0-5$ & o & & - & S. cu. 10 & - & \\
\hline 4 & $29 \cdot 115$ & - & $-11 \cdot 0$ & $-10 \cdot 5$ & $-10 \cdot 5$ & - & N1i, 1-2 & 7 & $12-18$ & o & & - & S.-eu. 10 & - & \\
\hline 6 & $29 \cdot 095$ & - & $-15 \cdot 6$ & -10.0 & $-10 \cdot 0$ & - & Var. $\mathrm{E}, 2-3$ & 11 & $10-20$ & o & & - & S. 10 & - & \\
\hline 8 & $29 \cdot 113$ & - & $-18 \cdot 5$ & $-17 \cdot 0$ & $-17 \cdot 0$ & - & JiNE, 3-4 & 11 & $22-26$ & o & & - & S. 10 & - & \multirow{5}{*}{$\begin{array}{l}\text { Very red in southern sky. } \\
\text { Overcast and elondy all day. Winil } \\
\text { sprang up strong alnout } 5 \text { p.m. Buro- } \\
\text { meter (showeel) a slight fali during the } \\
\text { day. Temperature steady. }\end{array}$} \\
\hline 10 & $29 \cdot 113$ & - & $-18 \cdot 5$ & $-16 \cdot 8$ & $-16 \cdot 8$ & - & N1, 3-4 & 14 & $20-28$ & $o c$ & & - & S. $-\mathrm{cu}, 8$ & - & \\
\hline Mnat. & $29 \cdot 106$ & - & $-21 \cdot 5$ & $-20 \cdot 8$ & $-20 \cdot 8$ & - & NF, $2-5$ & 14 & $12-25$ & ocq & & - & S. 10 & - & \\
\hline Mesn & $29 \cdot 134$ & - & - & $-14 \cdot 2$ & $-14 \cdot 2$ & - & - & -1 & - & - & & - & $\therefore$ & - & \\
\hline \multicolumn{7}{|c|}{ 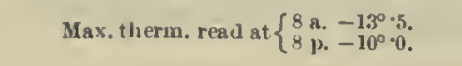 } & \multicolumn{4}{|c|}{ Soiar rariation $\left\{\begin{array}{l}\text { black bull, } 29^{\circ} \cdot 5 \\
\text { bright bulb }\end{array}\right.$} & & rammes. & \multicolumn{2}{|l|}{ Preclpn. - in. } & \\
\hline
\end{tabular}

8 th.

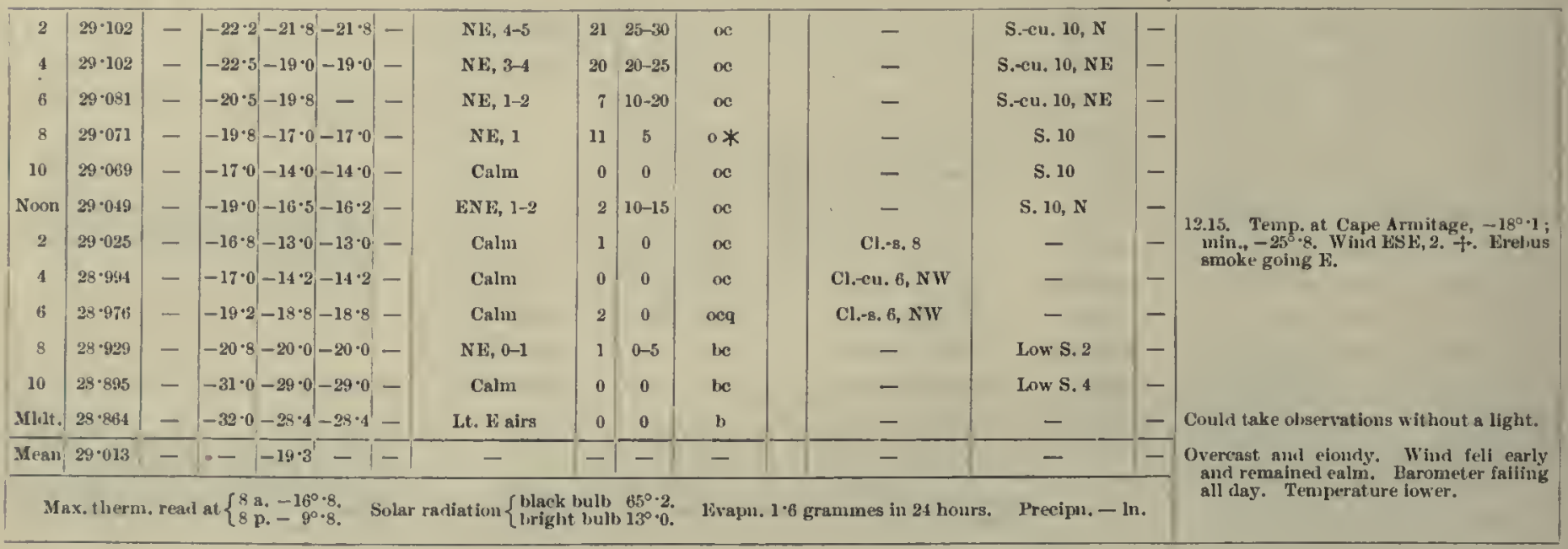

gth.

\begin{tabular}{|c|c|c|c|c|c|c|c|c|c|c|c|}
\hline 2 & $28 \cdot 831$ & - & $-29 \cdot 0^{\prime}-21 \cdot 2^{\prime}-21 \cdot 2^{\prime}-$ & Lt. $N$ E alrs & 1 & 0 & be & Cl. $\bullet .8,2, \mathrm{SW}$ & - & - & \multirow{5}{*}{ Mirage along the western hills. } \\
\hline 4 & $28 \cdot 785$ & - & $-24 \cdot 2-21 \cdot 0-21 \cdot 0-$ & $\mathrm{NNE}, 1-2$ & 7 & $10-15$ & 1) & - & - & - & \\
\hline 6 & $28 \cdot 774$ & - & $-28 \cdot 2-27 \cdot 0-27 \cdot 0-$ & Calm & 4 & 0 & be & $\mathrm{Cl}_{\circ}-\mathrm{s}_{0} 2, \mathrm{~S}^{1 y}$ & - & - & \\
\hline 8 & $28 \cdot 758$ & - & $-32 \cdot 2-20 \cdot 2-20 \cdot 2-$ & Calm & 0 & 0 & $\alpha$ & - & S.-cu. 9, INNE & - & \\
\hline 10 & $28 \cdot 718$ & - & $|-22 \cdot 0|-14 \cdot 9 \mid-15 \cdot 0-$ & Calm & 2 & 0 & $\propto *$ & - & A.-8. 9 & - & \\
\hline Noon & $28 \cdot 701$ & $-27 \cdot 5$ & $-17 \cdot 9-17 \cdot 0-17 \cdot 0-$ & Lt. F airs & 0 & 0 & se* & - & S. 10 & - & \multirow{5}{*}{$\begin{array}{l}\text { Strong mirage alí along horizon. Min. } \\
\text { temp, at caife Armitage, }-43^{\circ} \cdot 0 \text {. Wind } \\
\text { E by S, 2. o. }\end{array}$} \\
\hline 2 & $28 \cdot 641$ & - & $-19 \cdot 0-14 \cdot 0)-14 \cdot 0=$ & Calm & 0 & 0 & $\infty$ & - & S. 10 & - & \\
\hline 8 & $28 \cdot 653$ & - & $-21 \cdot 0-18 \cdot 8-18 \cdot 2-$ & Lt. 1i airs & 0 & 0 & œ & - & S. 10 & - & \\
\hline 10 & $28 \cdot 601$ & - & $-20 \cdot 5-18 \cdot 0-18 \cdot 0-$ & Lt. NR airs & 3 & 0 & $\propto$ & - & S.-en. 9 & - & \\
\hline Mint. & $28 \cdot 5 \times 3$ & - & $-22 \cdot 6-20 \cdot 0-20 \cdot 2-$ & LA. N1E airs & 1 & 0 & ne & - & S.-cu. 9 & -1 & \\
\hline Hean & $28 \cdot 649$ & - & $--18 \cdot 7-18 \cdot 7$ & - & $\overline{-}$ & - & - & - & - & $=$ & \multirow{2}{*}{$\begin{array}{l}\text { Overcast and elondy all day. Slight } \\
\text { snowfall and light airs. Barometer fell } \\
\text { steanlily all day. Temperature lower } \\
\text { and steary. }\end{array}$} \\
\hline & 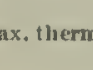 & & $\left\{\begin{array}{l}8 \mathrm{a} .-18^{\circ} \cdot 2 \\
8 \mathrm{p} .-13^{\circ} \cdot \mathrm{s}\end{array}\right.$ & on $\left\{\begin{array}{l}b, 18 \\
1, r i\end{array}\right.$ & & $30^{\circ} \cdot 0$ & Eva & amme In 24 hou & - Precipn. $-i$ & & \\
\hline
\end{tabular}


Methorolonical, Journal, of the "Discovery."

[1902-0ctober.

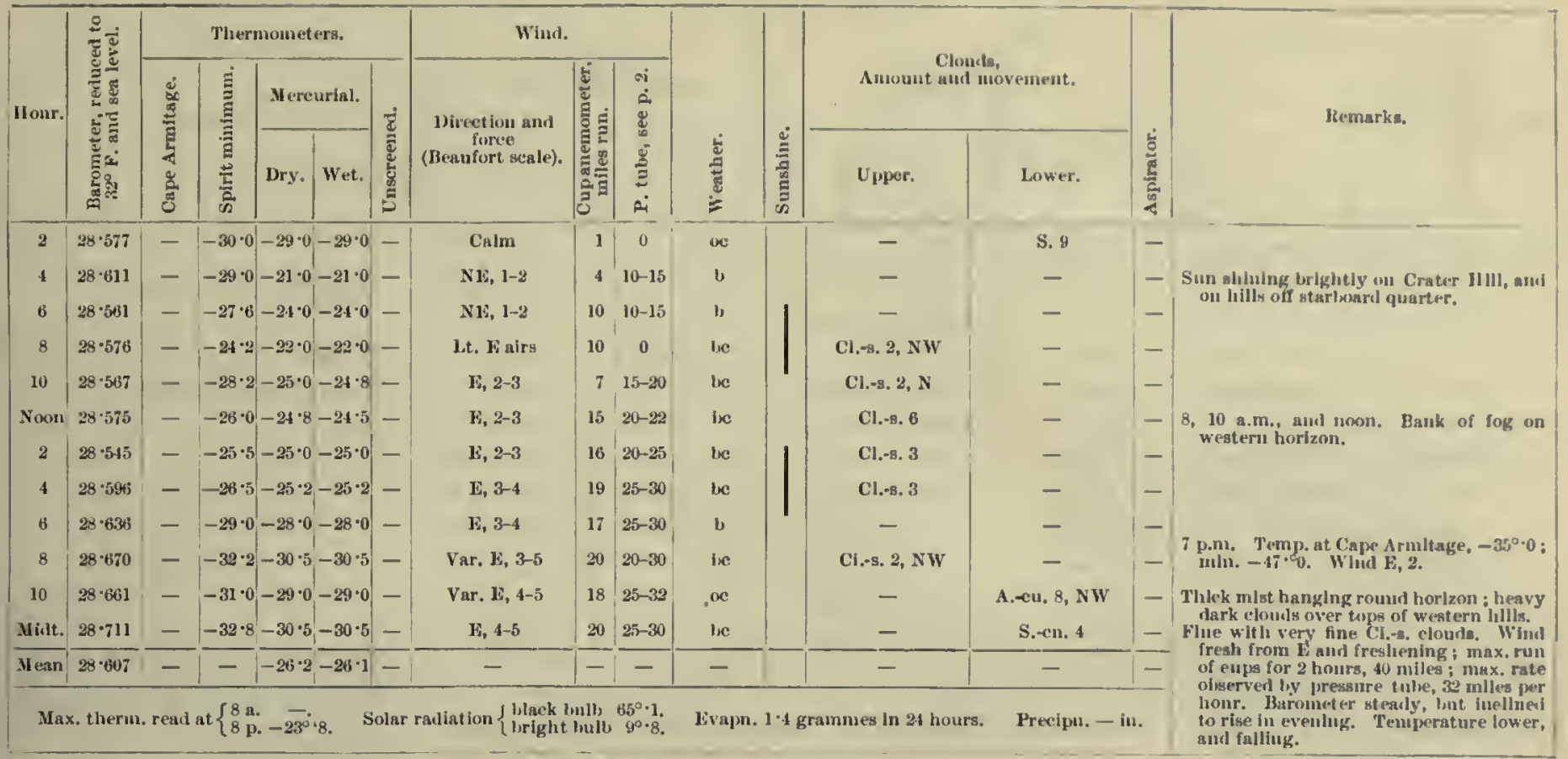

10 th.

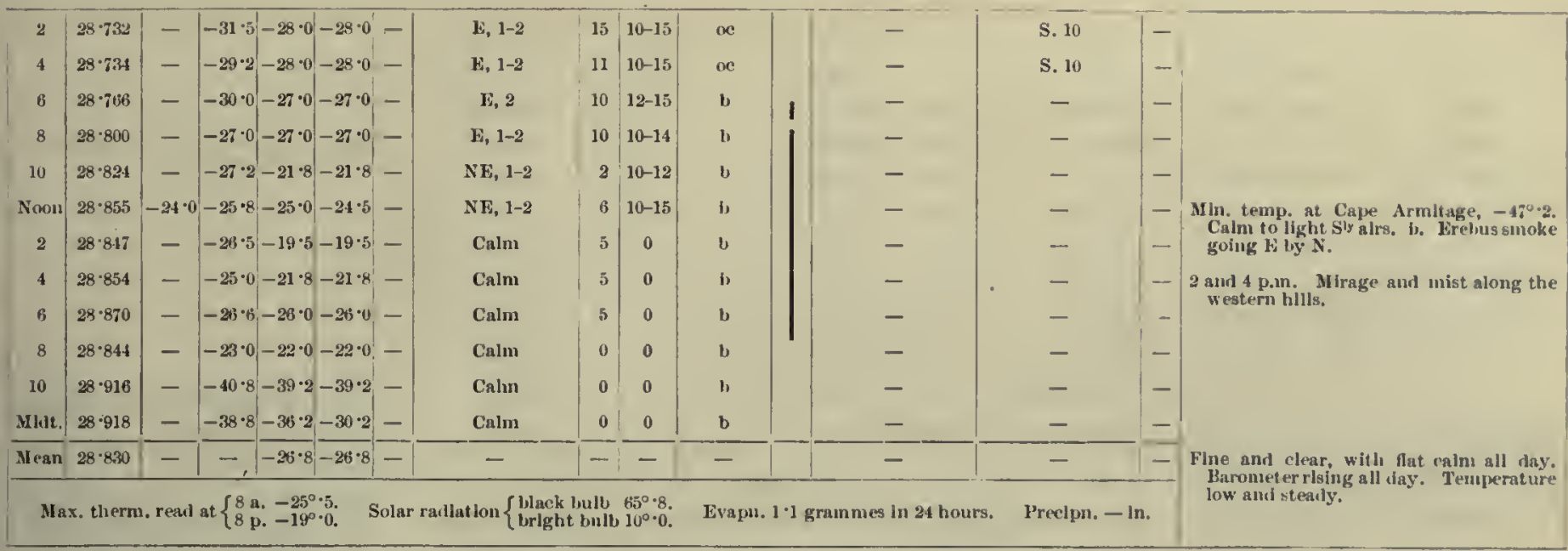

\begin{tabular}{|c|c|c|c|c|c|c|c|c|c|c|c|c|}
\hline 2 & $28 \cdot 920$ & - & $-37 \cdot 2-32 \cdot 0-32 \cdot 0$ & - & Calm & 0 & 0 & oc & - & S. 8 & $1-$ & lfeavy fog bank lu Sls. \\
\hline 4 & $28 \cdot 922$ & - & $-34 \cdot 2-32 \cdot 0-32 \cdot 0$ & - & SE, 2-3 & 5 & $15-20$ & b & - & - & - & \multirow{8}{*}{ 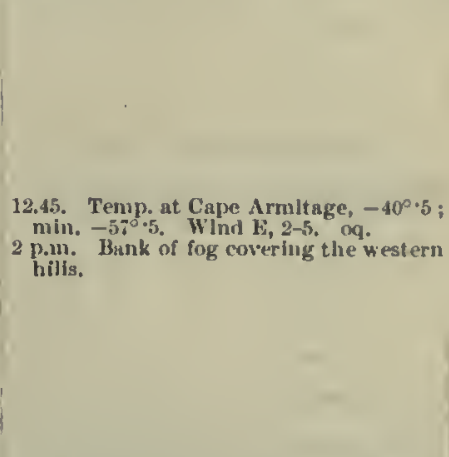 } \\
\hline 6 & $28 \cdot 888$ & - & $-35 \cdot 0-32 \cdot 4-32 \cdot 4$ & - & SE, 2-3 & 5 & $15-25$ & $\infty$ & - & S. 10 & - & \\
\hline 8 & $28 \cdot 873$ & - & $-33 \cdot 2-30 \cdot 0-30 \cdot 0$ & - & SE, 1-4 & 12 & $10-32$ & beq & C1.-8. 4 & - & - & \\
\hline Noon & $28 \cdot 860$ & - & $-25 \cdot 0-23 \cdot 8-23 \cdot 8$ & - & Var. E, 0-1 & 4 & $5-10$ & oc & Cl.-s. 8 & - & - & \\
\hline 2 & $28 \cdot 853$ & - & $-27 \cdot 2-26 \cdot 0-26 \cdot 0$ & - & $\mathrm{NI}, 1-2$ & 8 & $10-15$ & oe & - & S.-сu. 10 & - & \\
\hline 8 & $28 \cdot 827$ & - & $-31 \cdot 8-27 \cdot 0-26 \cdot 8$ & - & ISE, 1-2 & 10 & $5-15$ & $n *$ & - & N. 10 & - & \\
\hline 10 & $29 \cdot 827$ & - & $-28 \cdot 0-28 \cdot 0-26 \cdot 0$ & - & If by $S, 2-3$ & 6 & $10-15$ & $\alpha+$ & - & N. 10 & - & \\
\hline Mldt. & $28 \cdot 828$ & - & $-23 \cdot 2-26 \cdot 0-26 \cdot 0$ & -1 & E by $\mathbf{N}, 3-4$ & 17 & $15-20$ & $o c+$ & $=$ & N. 10 & - & \\
\hline MI $(-\mathrm{an}$ & $23 \cdot 861$ & - & $--27 \cdot 6-27 \cdot 8$ & -1 & - & $1-$ & - & - & - & - & 1 & \multirow{2}{*}{$\begin{array}{l}\text { Overcast and cloudy. Whind varlahle and } \\
\text { gquaslly, freshenlyg In eventrig. Baro } \\
\text { meter falling all day, but very steadily. } \\
\text { Temperature steady and low. }\end{array}$} \\
\hline & 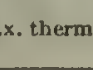 & teat & $\left\{\begin{array}{l}8 \text { a. }-210^{\circ} \cdot 0 \\
8 \text { p. }-20^{\circ} \cdot 0\end{array}\right.$ & ols & ation $\left\{\begin{array}{l}\text { bl } \\
\text { hr }\end{array}\right.$ & ull, & $\begin{array}{l}311^{0 \cdot 2 \cdot 2} \\
8^{\circ} \cdot 2 .\end{array}$ & Evapr & mine in $2 \mathrm{~s}$ hours. & Precipn. - & & \\
\hline
\end{tabular}


1902 -October.]

Meteorological Journal of the "Discovery."

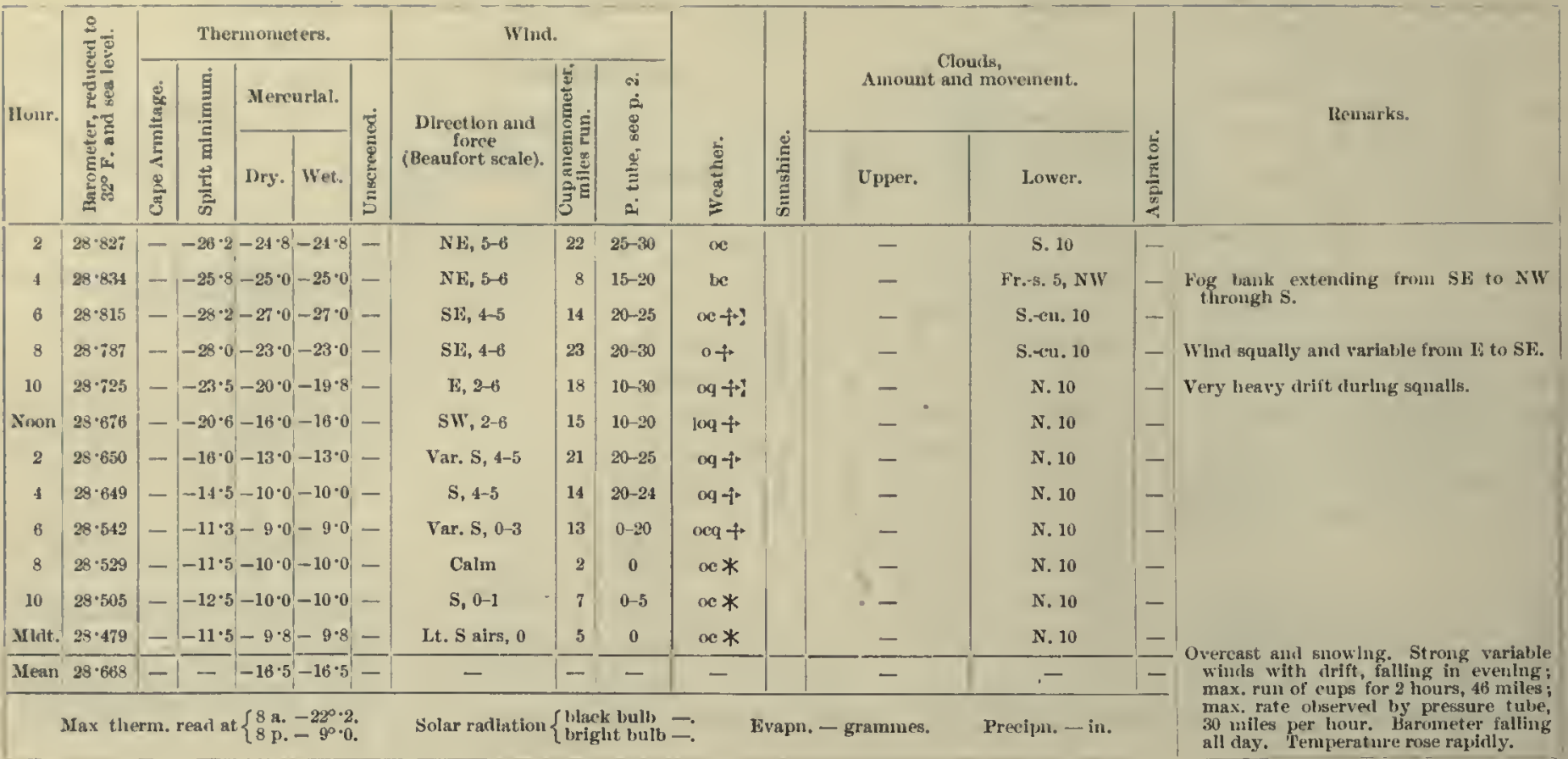

14th.

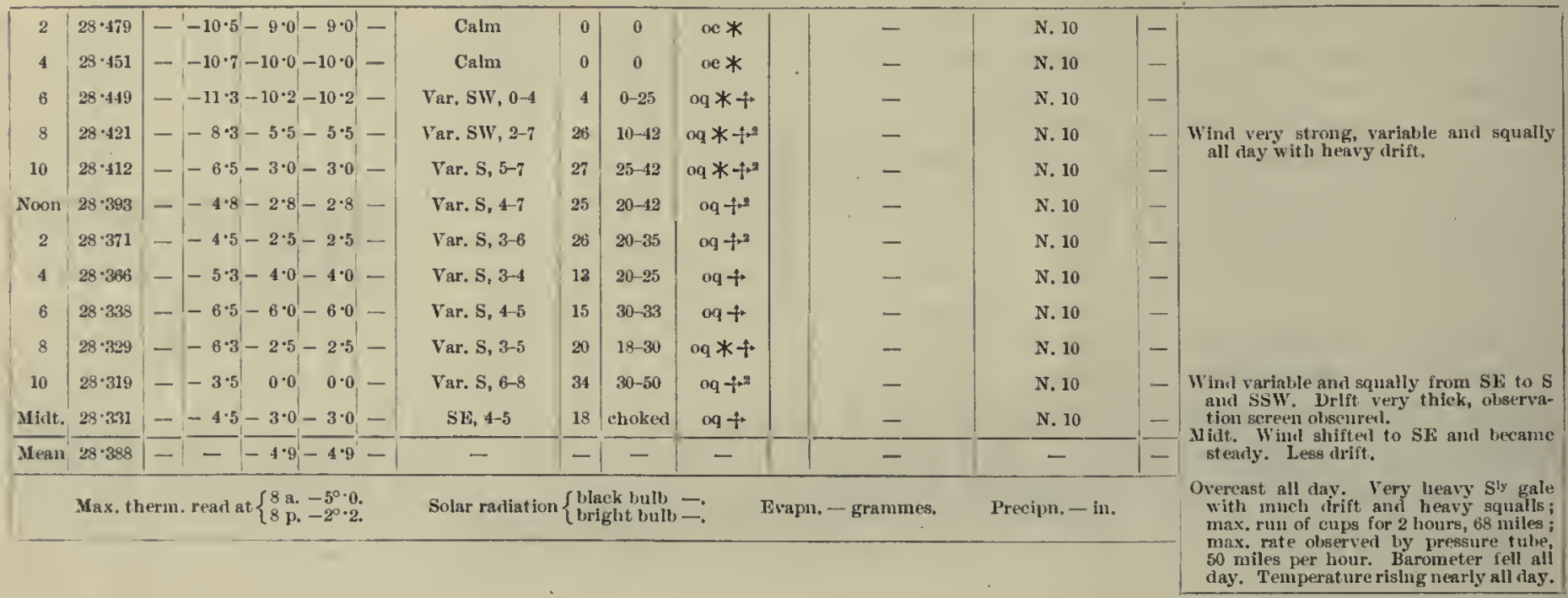

15th.

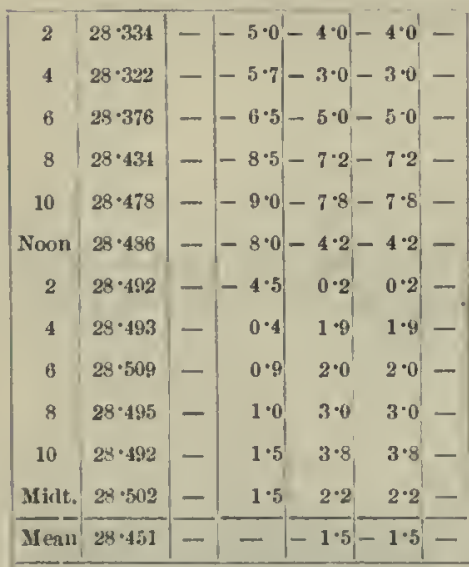

Max. therm. read at $\left\{\begin{array}{l}8 \text { a. } 0^{\circ} \cdot 2 . \\ 8 \text { p. } 3^{\circ} \cdot 0\end{array}\right.$

\begin{tabular}{|c|c|c|c|}
\hline SE, 4-5 & 19 & choked & $n q+$ \\
\hline Var. S, 4-6 & 23 & choked & $\operatorname{cog}+t^{-}$ \\
\hline $\mathrm{F}, 4-6$ & 29 & choked & $0 q-i^{-}$ \\
\hline $\mathrm{I}, 6$ & 21 & ehoked & $o g-t^{*}$ \\
\hline$E, 4-6$ & 23 & $25-32$ & $\infty \mathrm{it}$ \\
\hline $\mathrm{IN}, 3-7$ & 24 & $15-35$ & $\operatorname{og} * f$ \\
\hline ISSE, 2-4 & 21 & $15-20$ & $\alpha *-i$ \\
\hline Var. SE, 2-6 & 13 & $10-25$ & oq $*+$ \\
\hline Var. SE; 2-4 & 18 & $5-15$ & or $*-i$. \\
\hline Var. SE, 3-4 & 15 & $15-20$ & oq + i \\
\hline Var. Sls, 0-5 & 11 & $0-25$ & $\mathrm{oq}+$ \\
\hline Var. N1, 1-4 & 18 & $8-20$ & $o q$ \\
\hline- & - & - & - \\
\hline
\end{tabular}

Solar radiation $\left\{\begin{array}{l}\text { blaek bulb } \\ \text { bright hulb }- \text {. }\end{array}\right.$
Evapn.-grammes.

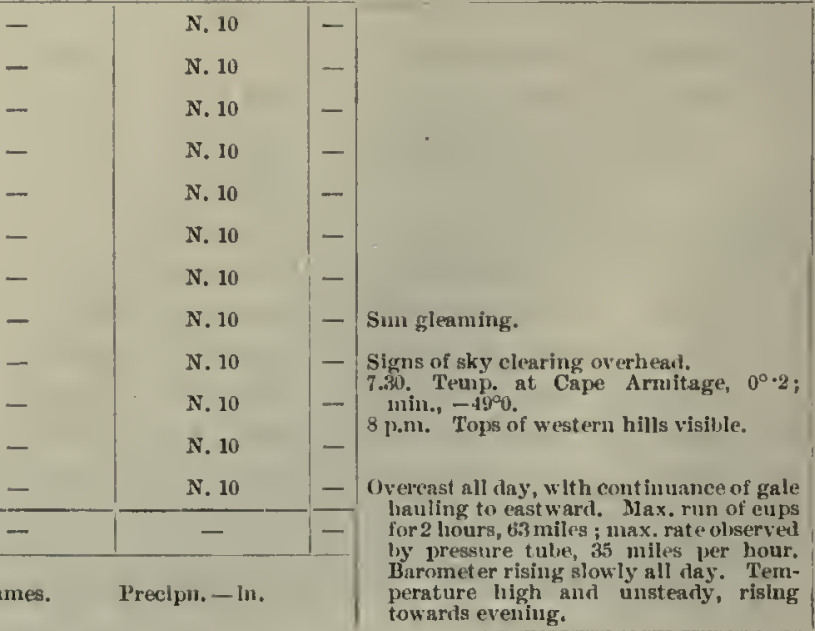




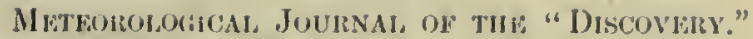

[1902-0ctober.

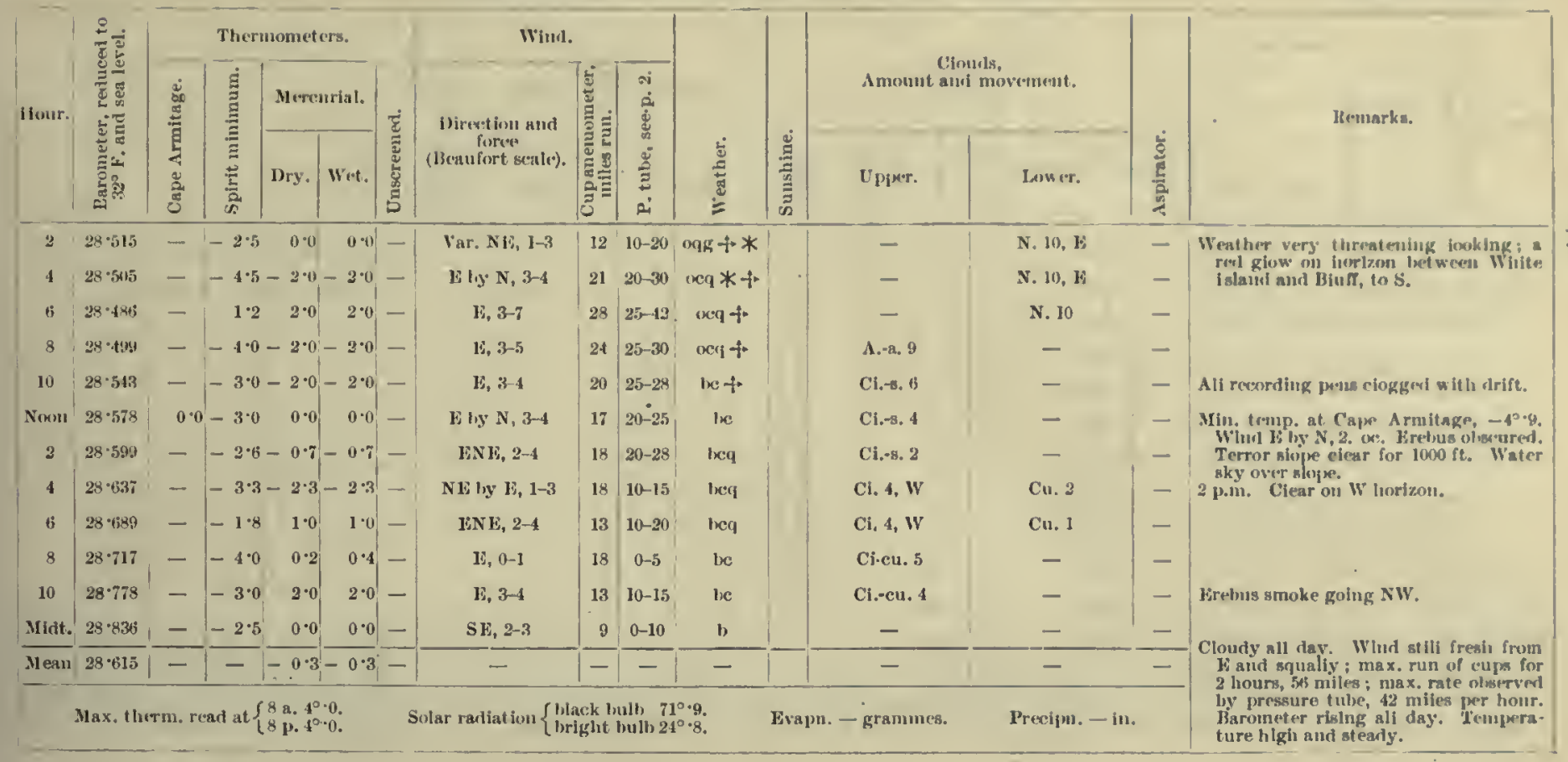

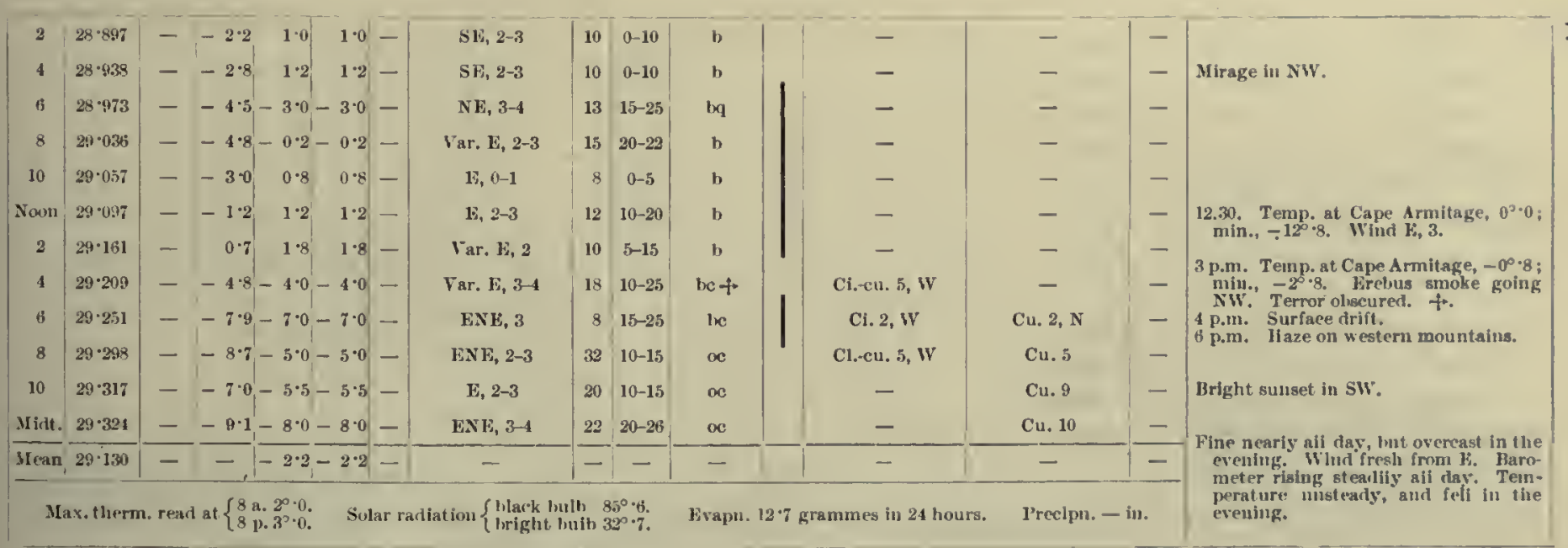

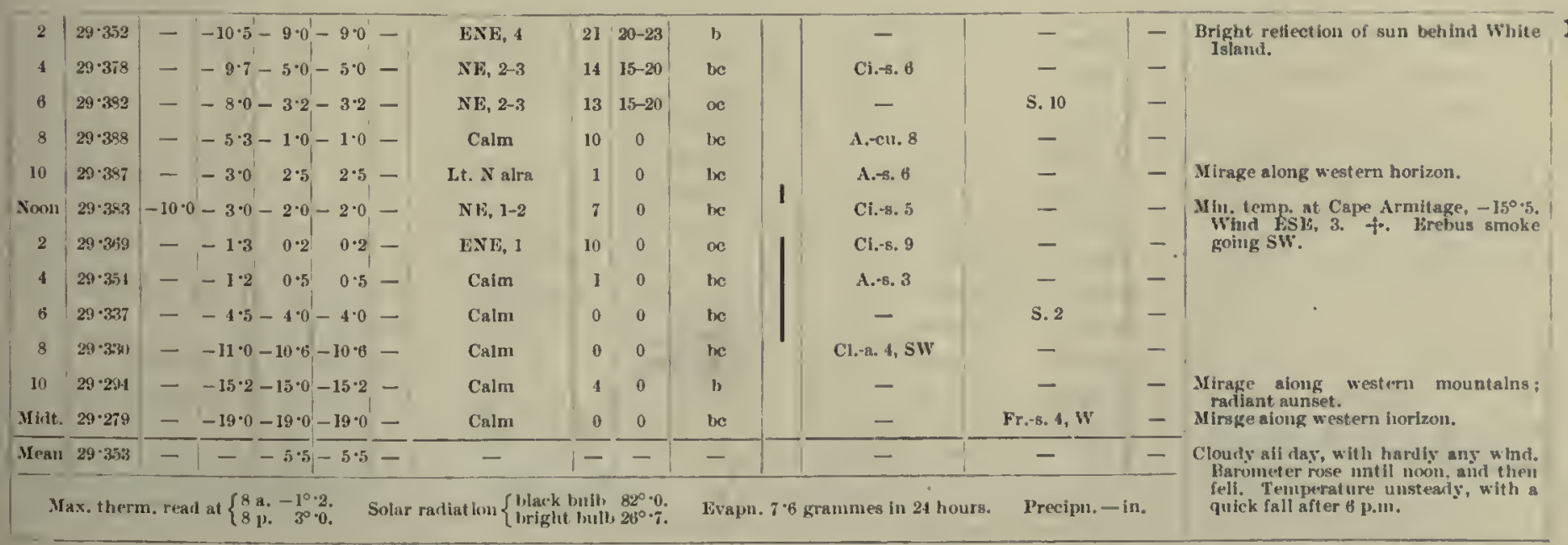


1902 - October.]

M gteonological JourNat, of TIIE "Discovery."

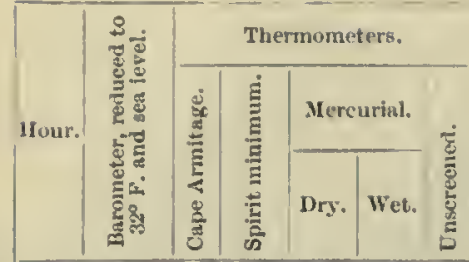

19th.

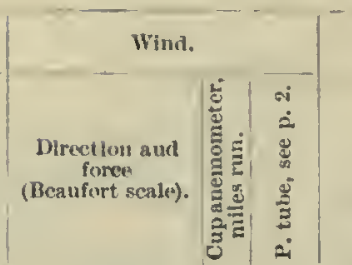

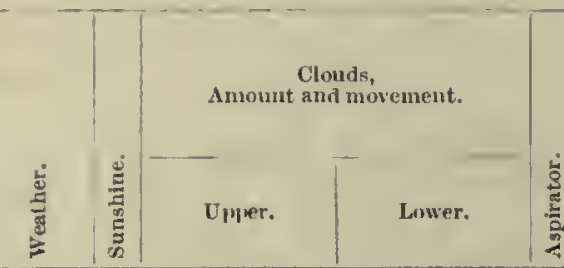

菑

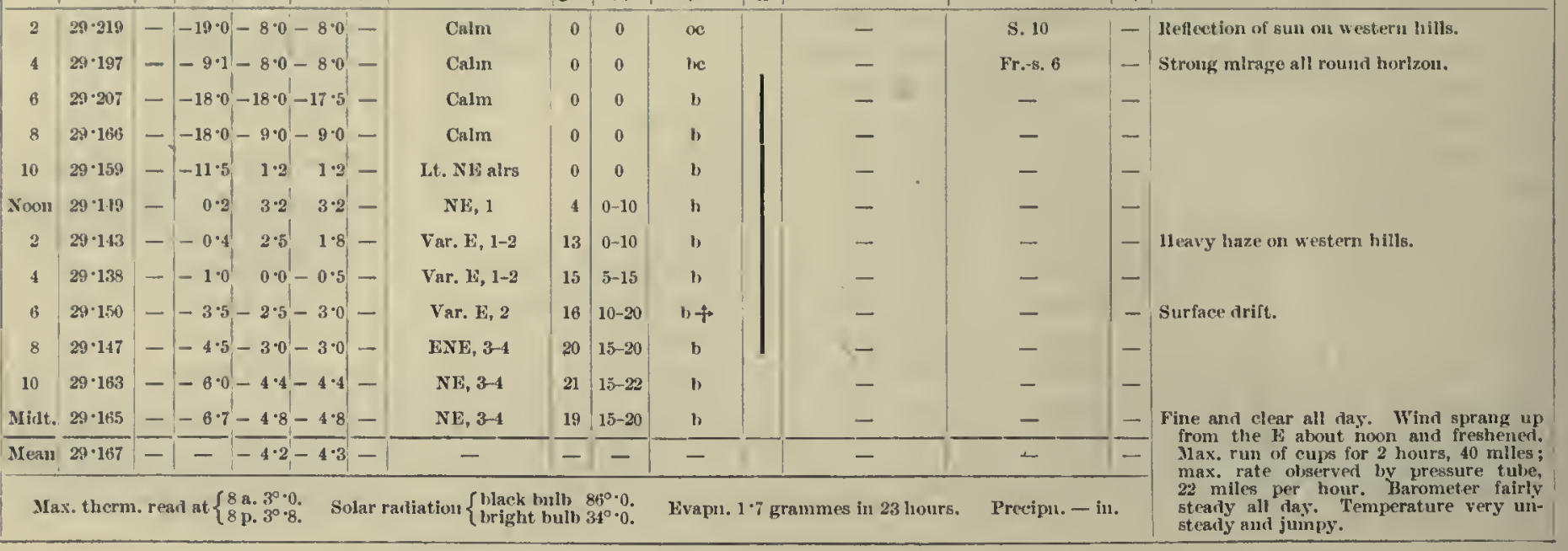

20 th.

\begin{tabular}{|c|c|c|c|c|c|c|c|c|c|c|c|c|}
\hline 2 & $29 \cdot 173$ & -1 & $-5 \cdot 7-4 \cdot 2-4 \cdot 5 \mid-$ & NE, 3-4 & 18 & $10-15$ & b & & - & - & -1 & \multirow{12}{*}{ Il eavy haze all along western horizon. } \\
\hline 4 & $29 \cdot 195$ & - & $-5 \cdot 7-5 \cdot 0-5 \cdot 0-$ & $\mathrm{NE}_{1}, 1-2$ & 15 & 0 & h & & - & - & - & \\
\hline 6 & $29 \cdot 203$ & - & $-6 \cdot 3-3 \cdot 4-3 \cdot 4-$ & Lt. NE airs & 10 & 0 & b & & - & - & - & \\
\hline 8 & $29 \cdot 209$ & - & $|-7 \cdot 7|-2 \cdot 0-2 \cdot 0-\mid$ & Lt. NE alrs & 10 & 0 & b & & - & - & - & \\
\hline 10 & $29 \cdot 243$ & - & $-7 \cdot 0-2 \cdot 0-2 \cdot 0-$ & WNE, 3-4 & 8 & 0 & b & & - & - & - & \\
\hline Noon & $29 \cdot 245$ & - & $-10 \cdot 5-10 \cdot 0-10 \cdot 0-$ & E by $N, 4-5$ & 21 & $25-30$ & $b$ & & - & - & - & \\
\hline 2 & $29 \cdot 253$ & - & $-10 \cdot 5-9 \cdot 0-9 \cdot 2-$ & ENE, 4-5 & 28 & $20-30$ & be & & - & S. 2 & - & \\
\hline 4 & $29 \cdot 263$ & - & $-6 \cdot 7-6 \cdot 0-6 \cdot 4-$ & E, 3-4 & 23 & $10-20$ & oc & & - & Cu. 8 & - & \\
\hline${ }^{\circ} 6$ & $29 \cdot 275$ & - & $-9 \cdot 2-6 \cdot 0-6 \cdot 4-$ & E by $\mathrm{S}, 1-2$ & 17 & 0 & $\propto c$ & & - & Cu. 10 & - & \\
\hline 8 & $29 \cdot 275$ & - & $-6 \cdot 5-4 \cdot 0-4 \cdot 0-$ & Lt. NE airs & 12 & 0 & oc & & - & Cu. 10 & - & \\
\hline 10 & $29 \cdot 307$ & - & $-8.5-6.0-6 \cdot 0-$ & Lt NE airs & 2 & 0 & oc & & - & Cu. 10 & - & \\
\hline Mint. & $29 \cdot 297$ & - & $-11 \cdot 5-11 \cdot 5-11 \cdot 8-$ & Calm & 0 & 0 & $o c$ & & - & Cu. 10 & -1 & \\
\hline Mean & $29 \cdot 245$ & -1 & $--5 \cdot 8-5 \cdot 9,-$ & - & 1 & - & - & & - & - & $1-$ & \multirow{2}{*}{$\begin{array}{l}\text { Fine and clear until noon, when it became } \\
\text { overcast. Wint fresh from ENF, dying } \\
\text { down towards the evening. Baroneter } \\
\text { rising steadily all day, Temperat ure } \\
\text { steadier and falling. }\end{array}$} \\
\hline $\mathrm{Ma}$ & therm & & 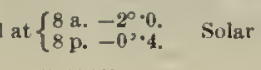 & iation $\left\{\begin{array}{l}\text { blat } \\
\text { brio }\end{array}\right.$ & bul & $\begin{array}{l}80^{\circ} \cdot 7 . \\
25^{\circ} \cdot 0 .\end{array}$ & Bva & $\cdot 0$ & ees in 24 hours. & Precipn & & \\
\hline
\end{tabular}

21st.

\begin{tabular}{|c|c|c|c|c|c|c|c|c|c|c|c|}
\hline 2 & $29 \cdot 291$ & -1 & $-14 \cdot 5-10 \cdot 0-9 \cdot 8-$ & Calm & 0 & 0 & oc & - & Cu. 10 & - & \multirow{14}{*}{$\begin{array}{l}\text { Haze and small detached Cu. to westward. } \\
\text { Mirage to S and W. } \\
\text { Mlirage in front of westem hills. } \\
9 \text { p.m. Temp. at Cape Armitage, }-27^{\circ} \cdot 5 \text {. } \\
\text { Overcast and cloudy all day. Light to } \\
\text { fresh variable wint. Darometer falling } \\
\text { generally all day. Temperature steady. }\end{array}$} \\
\hline 4 & $29 \cdot 290$ & - & $-12 \cdot 0-9 \cdot 8-9 \cdot 8-$ & Calm & 1 & 0 & $\infty$ & - & Cu. 10 & - & \\
\hline 6 & $29 \cdot 285$ & - & $-11 \cdot 5-8 \cdot 0-8 \cdot 0-$ & Calm & 1 & 0 & oc & A.-s. 6 & - & - & \\
\hline 8 & $29 \cdot 277$ & - & $-9 \cdot 5-2 \cdot 8-2 \cdot 8-$ & L.t. S. airs & 5 & 0 & $\alpha$ & A.-5. 8 & - & - & \\
\hline 10 & $29 \cdot 257$ & - & $-8 \cdot 0-4 \cdot 0-4 \cdot 0-$ & Calm & 0 & 0 & oc & A.-8. 9 & - & - & \\
\hline Noon & $29 \cdot 243$ & - & $-5 \cdot 0-5 \cdot 0-5 \cdot 0-$ & $N E, 1-2$ & 2 & 0 & $\infty$ & A.-8. 8 & - & - & \\
\hline 2 & $29 \cdot 240$ & - & $-8 \cdot 2-6 \cdot 0-6 \cdot 4-$ & ENE, 2-3 & 17 & $10-15$ & oc & - & S.-cu. $6, \mathrm{~W}$ & - & \\
\hline 4 & $29 \cdot 241$ & - & $-9 \cdot 2-7 \cdot 0-7 \cdot 3-$ & E by $S, 2-3$ & 21 & $10-15$ & c & - & S.cu. $6, \mathrm{~W}$ & - & \\
\hline 6 & $29 \cdot 233$ & - & $-7 \cdot 7-7 \cdot 0-7 \cdot 2-$ & Var, $\mathrm{E}, 1-2$ & 18 & $0-10$ & oc & A.-5. 6 & - & - & \\
\hline 8 & $29 \cdot 231$ & - & $-5.7-5.0-5.0-$ & Nle, 1-2 & 11 & 10-15 & oc & A.-5. 8, NW & - & - & \\
\hline 10 & $29 \cdot 217$ & - & $-7 \cdot 7-4 \cdot 8-4 \cdot 8-$ & Lt. NE airs & 7 & $0-5$ & oc & - & S.cu. 9 & - & \\
\hline Mllat. & $29 \cdot 211$ & - & $-12 \cdot 0-5 \cdot 0-5 \cdot 0=$ & Lt. NE alrs & 0 & 0 & oc & - & Cu. 10 & $=$ & \\
\hline$\overline{\text { Mean }}$ & $21 \cdot 251$ & $1-1$ & $1-1-6 \cdot 2-6 \cdot 3-1$ & - & $\overline{-}$ & - & - & - & - & - & \\
\hline \multicolumn{4}{|c|}{ Max. therm. read at $\left\{\begin{array}{l}8 \mathrm{a},-2^{\circ} \cdot 5, \\
8 \mathrm{p},-2^{\circ} \cdot 5 .\end{array}\right.$ Sol } & Hation $\{1$ & & ili $24^{\circ} \cdot 7$ & & anmes in $24 \mathrm{ho}$ & \multicolumn{2}{|c|}{ Precipn. - in. } & \\
\hline
\end{tabular}


Methorological Journal of the "Discoviry,"

[1902-October.

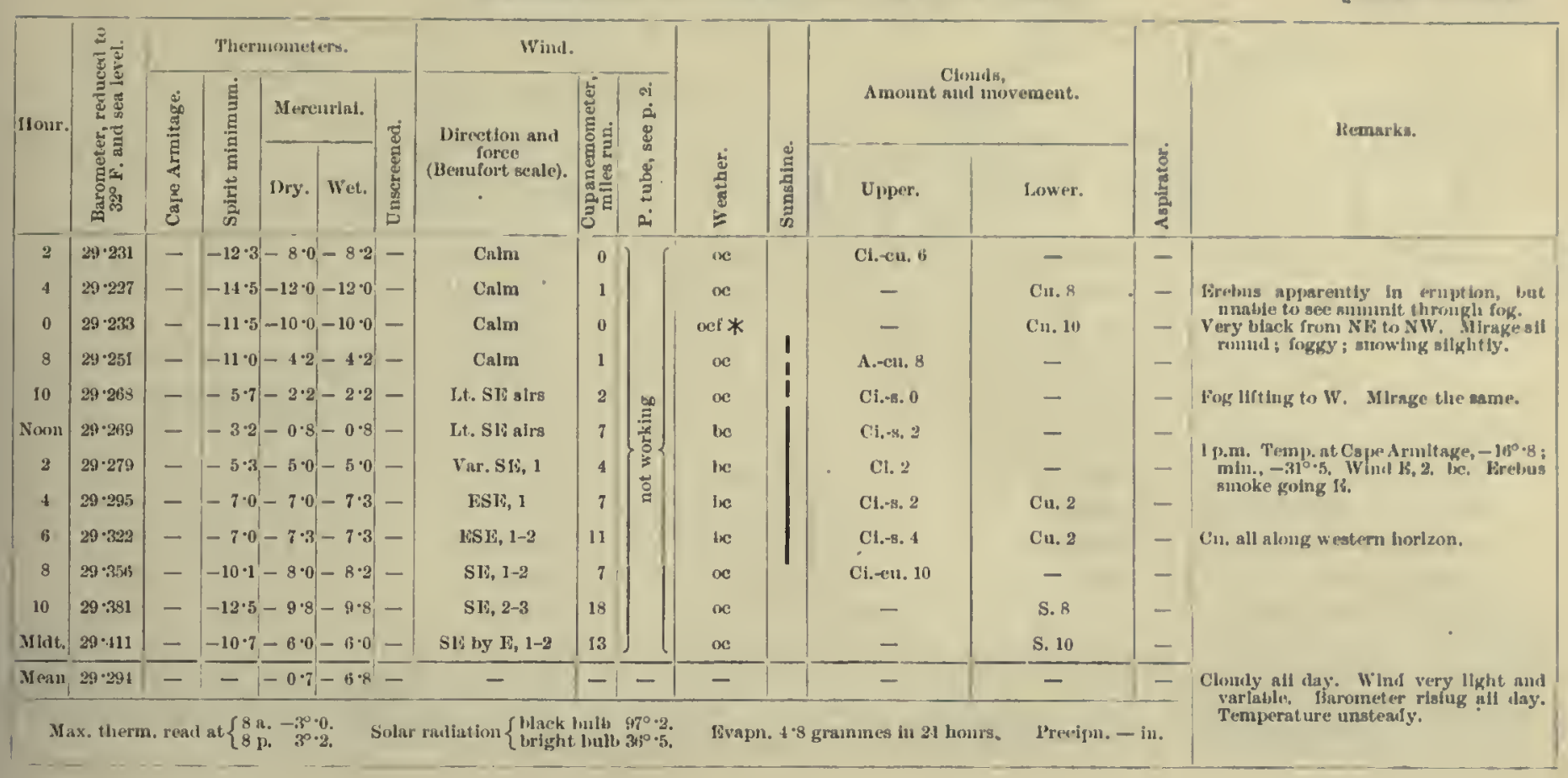

22nd.

\begin{tabular}{|c|c|c|c|c|c|c|c|c|c|c|c|}
\hline 2 & $29 \cdot 11.4$ & - & $-11 \cdot 2-12 \cdot 0-12 \cdot 0$ & SE, 3 & 13 & & oc & - & Cu. 10 & - & \multirow{14}{*}{ 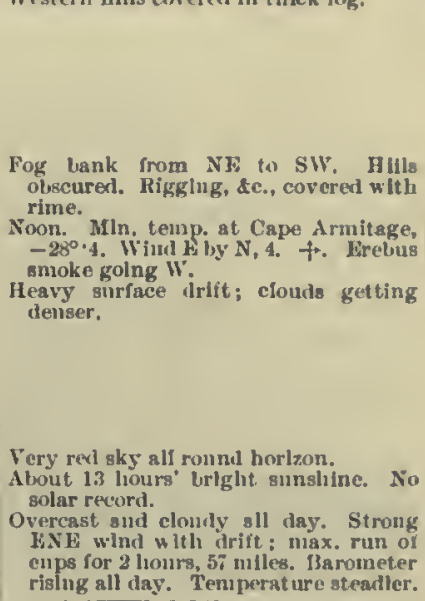 } \\
\hline 4 & $29 \cdot 442$ & - & $-11 \cdot 5-7 \cdot 5-7.5-$ & E, 3-4 & 18 & & $\infty$ & - & Cu. 10 & - & \\
\hline 6 & $29 \cdot 500$ & - & $-11 \cdot 5-8 \cdot 0-8 \cdot 0-$ & $E, 4$ & 22 & & $x+$ & Ci.-s. 8 & - & - & \\
\hline 8 & $29 \cdot 521$ & - & $-11 \cdot 3-8 \cdot 2-8 \cdot 2-$ & $E, 4-5$ & 15 & & $b e+$ & $\mathrm{Ci}_{0.8 .4}$ & - & - & \\
\hline 10 & $29 \cdot 590$ & - & $-10 \cdot 2-9 \cdot 8-9 \cdot 8-$ & E, 4-5 & 18 & 60 & 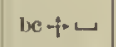 & Ci.cu 5 & - & - & \\
\hline Noon & $29 \cdot 604$ & $-10 \cdot 2$ & $-12 \cdot 7-10 \cdot 0-10 \cdot 0-$ & Var. SE, $4-6$ & 19 & 竞 & $\alpha+w$ & $\mathrm{Ci}_{.88 .8}$ & - & - & \\
\hline 2 & $29 \cdot 613$ & - & $-9 \cdot 5-7 \cdot 5-7 \cdot 5-$ & Far. E, 4-6 & 28 & 5 & ocq + & Ci.-s. 7, SW & - & - & \\
\hline 4 & $29 \cdot 636$ & - & $-9 \cdot 5-7 \cdot 3-7 \cdot 3-$ & ENE, 6-7 & 26 & $\cong$ & $\sec +$ & Cl.-8. $8, \mathrm{SW}$ & - & - & \\
\hline 6 & $29 \cdot 654$ & - & $-9 \cdot 7-9 \cdot 0-8 \cdot 5-$ & if by $N, 4-5$ & 26 & & $n+$ & $\mathrm{Cl} .-5,8, \mathrm{sW}$ & - & - & \\
\hline 8 & $29 \cdot 663$ & - & $-9 \cdot 7-8 \cdot 8-8 \cdot 8-$ & $5,4-5$ & 23 & & $x+$ & Cl.-s. 8 & - & - & \\
\hline 10 & $29 \cdot 669$ & - & $-9.5-9 \cdot 0-9 \cdot 0-$ & 5. $4-5$ & 22 & & $\alpha+$ & Ci.-8. 8 & - & - & \\
\hline Milit. & $29 \cdot 653$ & - & $-11 \cdot 0-8 \cdot 0-8 \cdot 0-$ & Var. E, $\mathbf{4 - 5}$ & 21 & & $\infty+$ & Ci.-8. $n$ & - & - & \\
\hline Mean & $29 \cdot 580$ & - & $-1^{-8 \cdot 8-8.7}-$ & - & - & - & - & - & - & - & \\
\hline & lnt & rea & -8. & liation \{ & & $88^{\circ}$ & Evapm & ammes in 24 & \multicolumn{2}{|c|}{ Preclpn. - in. } & \\
\hline
\end{tabular}

\begin{tabular}{|c|c|c|c|c|c|c|c|c|c|c|c|}
\hline 2 & $29 \cdot 639$ & - & $-11 \cdot 7-9 \cdot 0-9 \cdot 0^{\prime}-$ & Var. $E_{\text {, }}$ 4-5 & 17 & \multirow{12}{*}{ 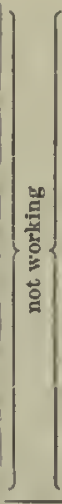 } & oeqto & A.-8. 10 & - & - & \multirow{5}{*}{$\begin{array}{l}\text { Driring lanks of fong and drift, with } \\
\text { necasional visllility. }\end{array}$} \\
\hline 4 & $29 \cdot 630$ & - & $-12 \cdot 5-9 \cdot 8-9 \cdot 8-$ & $\operatorname{Par} .18,4-5$ & 19 & & $o c+$ & A.-8. 10 & 一 & - & \\
\hline 6. & $29 \cdot 600$ & - & $-15 \cdot 8-10 \cdot 0-10 \cdot 0-$ & E, $5-7$ & 23 & & $a+$ & A.-s. 10 & - & - & \\
\hline 8 & $29 \cdot 660$ & - & $-15 \cdot 0-6 \cdot 0-6 \cdot 0-$ & $\mathrm{F}, 5-6$ & 21 & & $\infty+$ & A.-8. 9 & - & - & \\
\hline 10 & $29 \cdot 548$ & - & $-7 \cdot 5-2 \cdot 0-2 \cdot 8-$ & Var. E, 5-b & 21 & & $\alpha+$ & - & s. 10 & - & \\
\hline Noon & $29 \cdot 485$ & $-13 \cdot 8$ & $-12 \cdot 5-12 \cdot 0-12 \cdot 0-$ & E hy S, 6-7 & 26 & & $\infty+4$ & - & S. 10 & - & \multirow{9}{*}{ 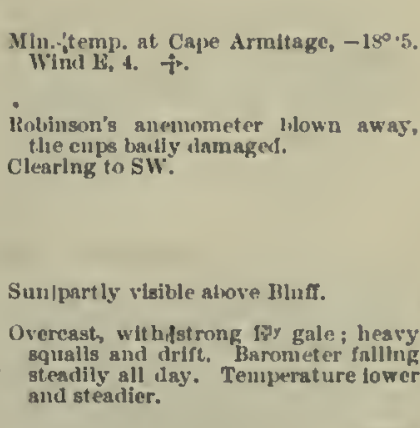 } \\
\hline 2 & $29 \cdot 437$ & - & $-14 \cdot 2-13 \cdot 4-13 \cdot 4-$ & Var. $15,7-8$ & 35 & & $o c f^{3}$ & - & S.cu. 10 & - & \\
\hline 4 & $29 \cdot 351$ & - & $-13 \cdot 0-12 \cdot 2-12 \cdot 2-$ & Var. E, 7-10 & - & & acq to? & - & S.en11. 10 & - & \\
\hline 6 & $29 \cdot 329$ & - & $-12 \cdot 2-10 \cdot 0-10 \cdot 0$ & Var. E, $9-10$ & - & & ocq $++^{2}$ & - & S.ecu. 10 & - & \\
\hline 8 & $29 \cdot 335$ & - & $-13 \cdot 3-11 \cdot 5-10 \cdot 5-$ & Var, $\mathbf{E ,}, 0-10$ & - & & ocuti & $\mathrm{Ci}_{.}-8 . \mathrm{fi}$ & - & - & \\
\hline 10 & $29 \cdot 339$ & - & $-14 \cdot 3-13 \cdot 0-13 \cdot 0-$ & N ty N, s-6 & - & & $x+$ & - & S. 6 & - & \\
\hline Mlat. & $29 \cdot 265$ & - & $-15 \cdot 2-12 \cdot 0-12 \cdot 0-$ & $1: \mathrm{NE}, 5-6$ & - & & $x+$ & C1.-c11. 10 & - & - & \\
\hline Mes:1t & $29 \cdot 4+13$ & - & $=-10 \cdot 1,-10 \cdot 1-$ & - & -1 & - & - & - & - & - & \\
\hline \multicolumn{4}{|c|}{ Max, therm, reat at $\begin{cases}4 \text { a. } & -3^{\circ} 0 . \\
8 \text { p. } & 2^{\circ} \cdot 5 .\end{cases}$} & $(1)$ & (t) & 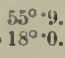 & & ammes in 24 & \multicolumn{2}{|c|}{ Presipm. - in. } & \\
\hline
\end{tabular}


1902-October.]

Mhtzorological Journat, of the "Discoveliy."

\begin{tabular}{|c|c|c|c|c|c|c|c|c|c|}
\hline \multirow{3}{*}{ Hour. } & \multirow{3}{*}{ 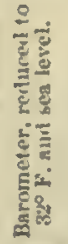 } & \multicolumn{5}{|c|}{ Thernonielers, } & \multicolumn{3}{|l|}{ WInd. } \\
\hline & & is & $E$ & Ner & urlal, & & & & 1 \\
\hline & & $\mid \frac{\pi}{\frac{c}{c}}$ & & Iory. & Wes. & 旁 & & p & 12 \\
\hline
\end{tabular}

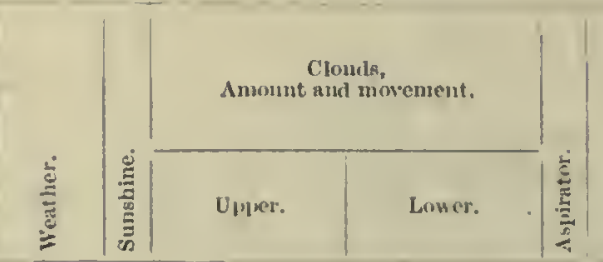

25 th.

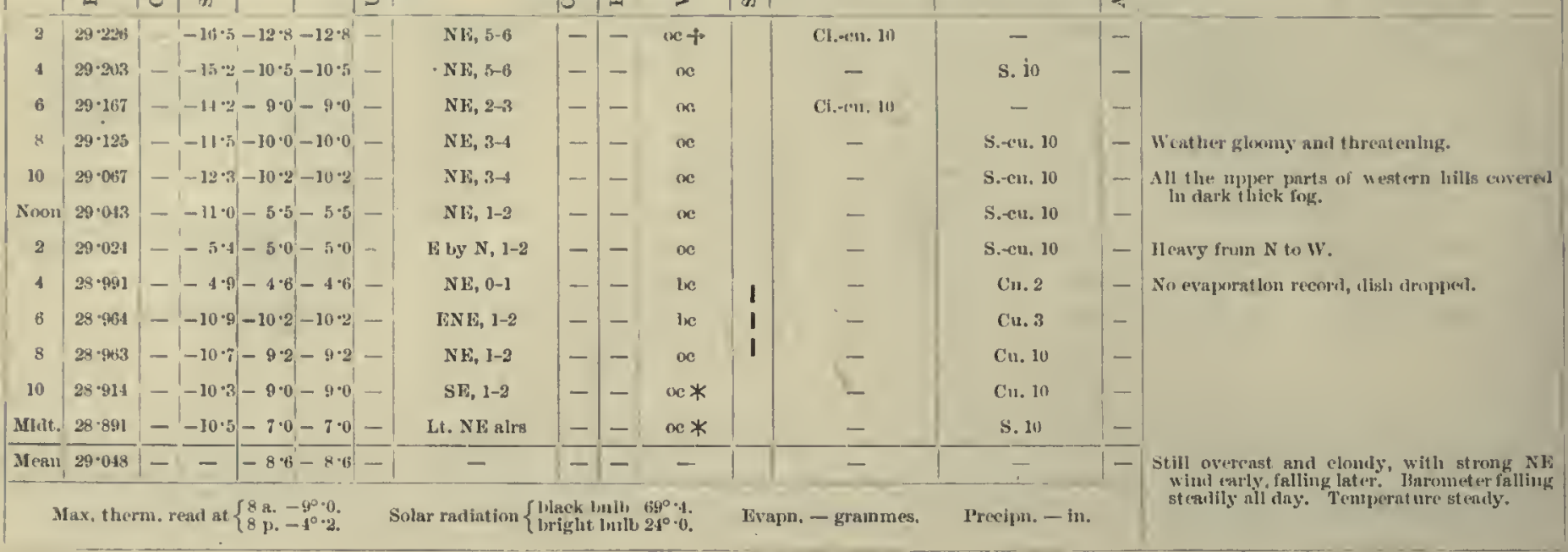

26 th.

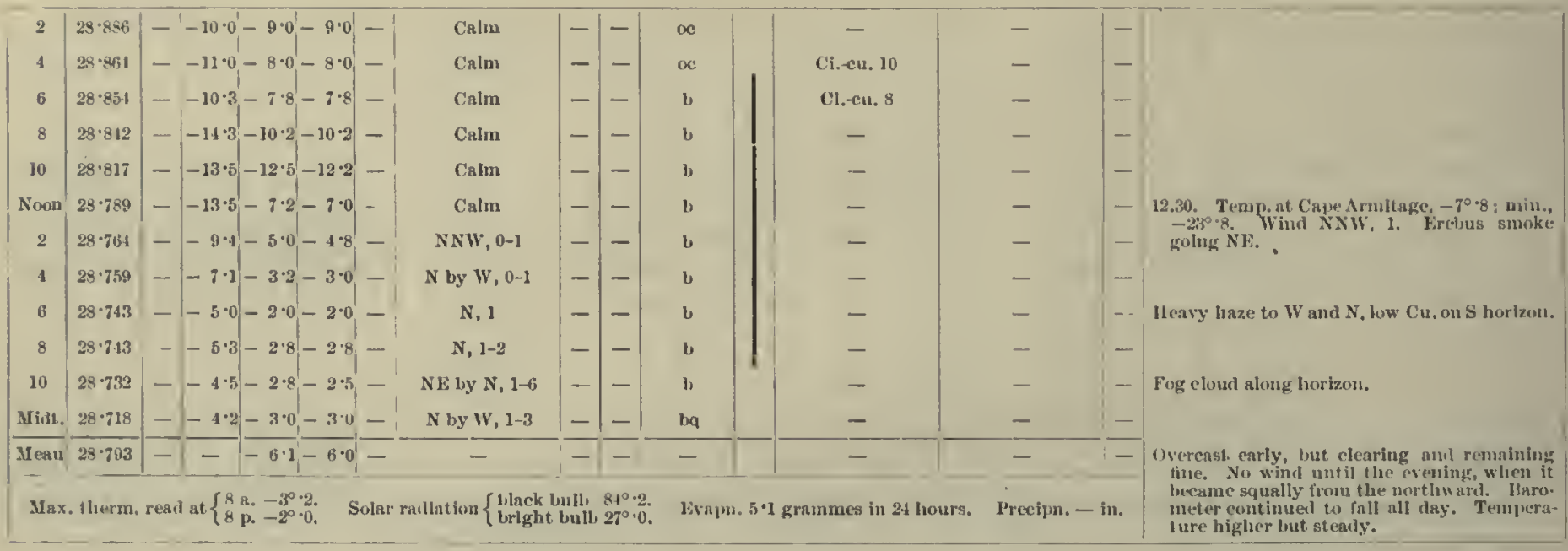

27 th.

\begin{tabular}{|c|c|c|c|c|c|c|c|c|c|c|c|}
\hline 2 & $2 s \cdot 709$ & -1 & $-5 \cdot 5-4 \cdot 8^{\prime}-4 \cdot 8^{\prime}-$ & $\mathrm{NE}, 1$ & -1 & - & $\infty$ & - & S.-en. 8 & $|-|$ & \\
\hline 4 & $28 \cdot 689$ & - & $-12 \cdot 5-6 \cdot 0-6 \cdot 0-$ & $N, 1-2$ & - & - & $\propto$ & - & S.-cu. 8 & - & \\
\hline 6 & $28 \cdot 687$ & - & $-9 \cdot 0-3 \cdot 0-3 \cdot 0-$ & $N, 2-3$ & $\begin{array}{ll}-1 & 1 \\
1 & 1\end{array}$ & - & $n$ & - & S.-cu. 8 & - & \\
\hline 8 & $28 \cdot 6 \overline{3}$ & - & $-3 \cdot 0-1 \cdot 0-1 \cdot 0-$ & $N, 2-3$ & - & - & oc & - & Cu. 10 & - & \\
\hline 10 & $28 \cdot 6 \times 2$ & - & $\begin{array}{lll}-2 \cdot 5 & 3 \cdot 8 & 3.8\end{array}-$ & $\mathbf{N}, 2-3$ & - & - & $\infty$ & - & Cu. 11 & - & \\
\hline Noon & $28 \cdot 691$ & $4 \cdot 5$ & $\begin{array}{llll}2 \cdot 0 & 1 \cdot 2 & 1 \cdot 3 & -\end{array}$ & $N$ by $\mathbf{E}, 2-3$ & - & - & $\infty$ & - & ('ir. 10 & $1-$ & 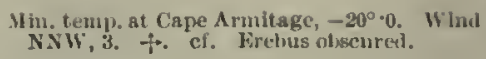 \\
\hline 2 & $28 \cdot 091$ & -1 & $3 \cdot 3 \quad 5 \cdot 0 \quad 5 \cdot 0-$ & N liy w, 0-1 & - & - & $\infty$ & -. & C'11.9 & - & 3 p.111. Temp. at Cape Armitnge, $6^{\circ} .5 ;$ min.. \\
\hline 4 & $28 \cdot 695$ & -1 & $5 \cdot 3 \quad 7 \cdot 4 \quad 6 \cdot 6-1$ & Var. $N, 1-2$ & - & - & 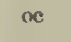 & - & S.ren. 10 & & $4 \%$ \\
\hline 6 & $28 \cdot 701$ & - & $5 \cdot 5) \quad 8 \cdot 0 \quad 3 \cdot 8-$ & N, 1-2 & - & - & $\infty *$ & - & x. 10 & - & Dull to S, clearing ln W. \\
\hline 8 & $28 \cdot 708$ & - & $5.5, \quad 8.0 \quad 600-$ & $N, 2$ & - & - & ae $*$ & - & s. 10 & & \\
\hline 10 & $28 \cdot 729$ & - & $6 \cdot 2 \quad 8 \cdot 0 \quad 7 \cdot 0-$ & $N, 1$ & - & - & or $*$ & - & x. 10 & & Snow falling lightly. \\
\hline Mints. & $2 \times \cdot 737$ & $=$ & $8 \cdot 1) \quad 8 \cdot 8 \quad 8 \cdot 2-1$ & I.t. $\mathbf{N}$ nirs & -1 & - & w* & - & N. 111 & & \\
\hline Mesu, & $\begin{array}{l}28 \cdot 700 \\
\text { ax, thert }\end{array}$ & -1 & 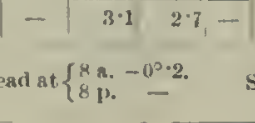 & lar rallations & 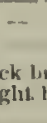 & - & - & Livapn. 9 'f grammes. & l'restimu, - i & I & 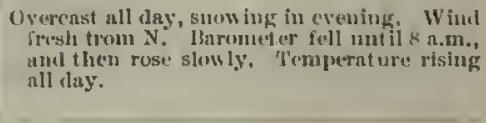 \\
\hline
\end{tabular}


Mhteolological, JoukNal, of TH\% "Discoviky."

[1902-October.

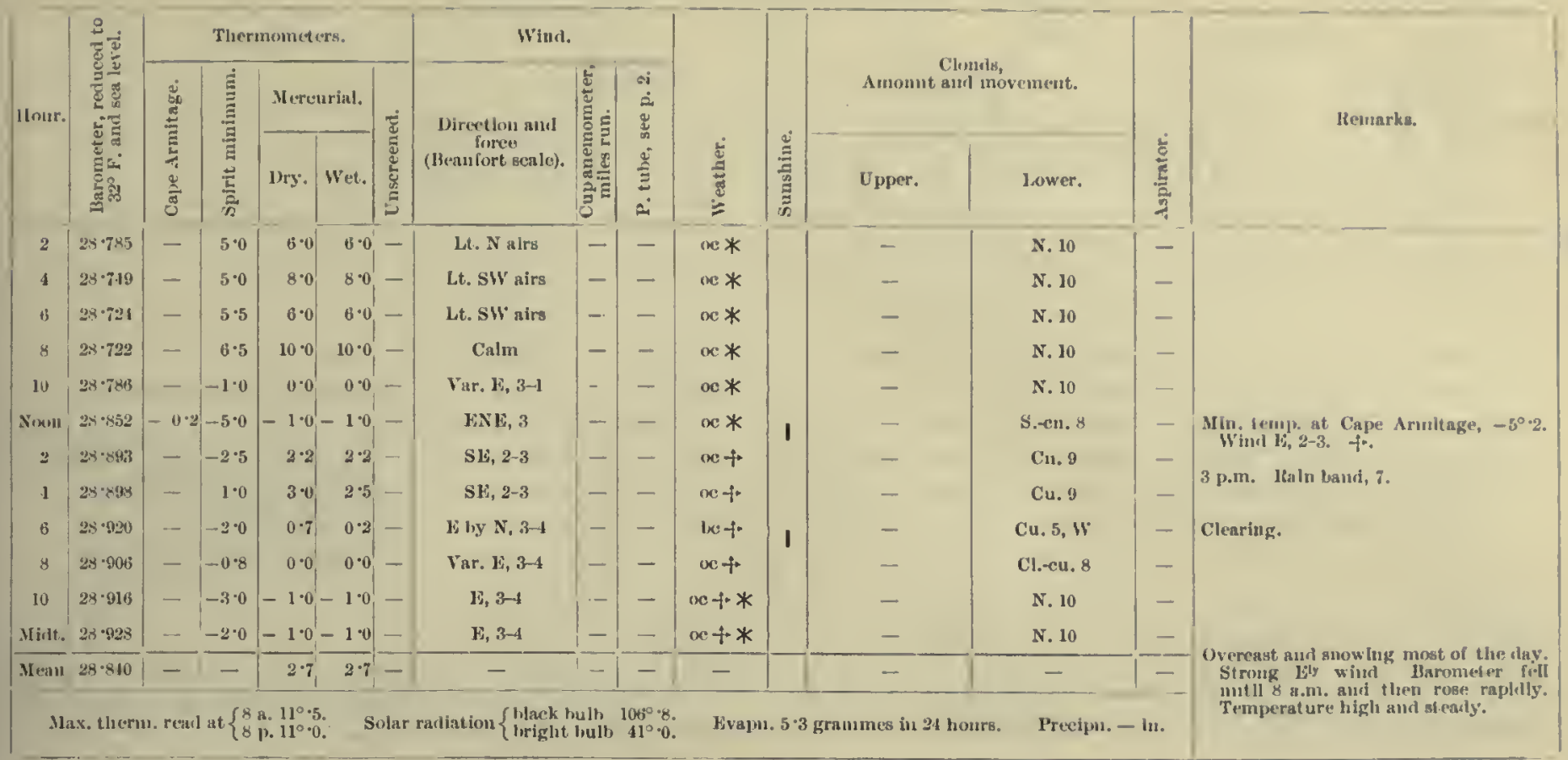

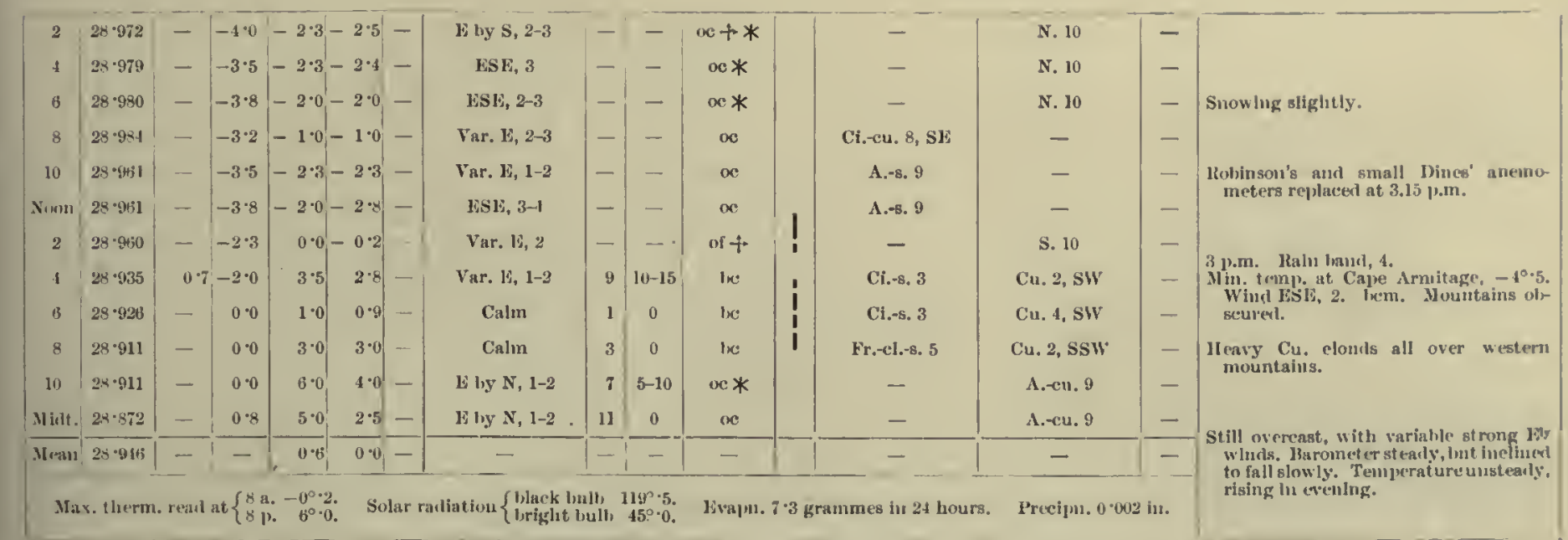

\begin{tabular}{|c|c|c|c|c|c|c|c|c|c|c|c|c|c|c|}
\hline 2 & $24 \cdot 66 \pi$ & - & $2 \cdot 8$ & $3 \cdot 0$ & - & - & lis ly N, 1-2 & 18 & - & $\alpha+*$ & - & N. 10 & - & \multirow{14}{*}{ 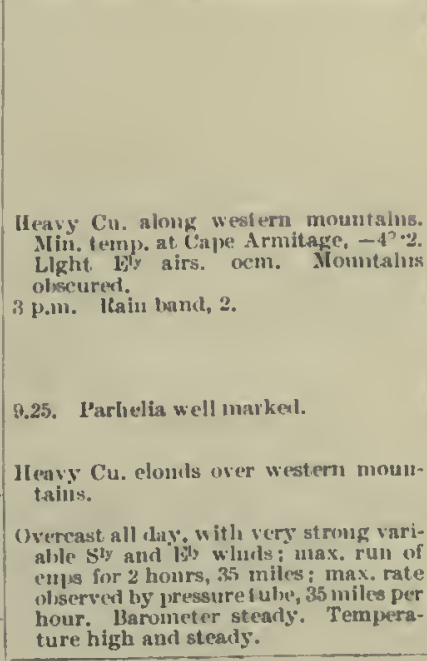 } \\
\hline 4 & $28 \cdot 87 x$ & $\rightarrow$ & $3 \cdot 2$ & $3 \cdot 9$ & - & - & lis E, 1-2 & 0 & - & $\alpha+*$ & - & N. 10 & - & \\
\hline 6 & $2 s \cdot 8 \times 4$ & $=$ & $6 \cdot 5$ & $8 \cdot 3$ & $8 \cdot 1$ & - & si hy $\mathrm{W}, 5-6$ & $160^{\circ}$ & $20-35$ & $v+*$ & - & N. 10 & - & \\
\hline 8 & $25 \cdot x+41$ & - & $5 \cdot 0$ & $6 \cdot 0$ & $5 \cdot 8$ & - & $S$ by $12,2-3$ & 18 & $15-18$ & $\infty+$ & - & S. 10 & - & \\
\hline 10 & $2 s \cdot 870$ & + & $5 \cdot 0$ & $9 \cdot 0$ & $8 \cdot x$ & - & $1 \%, 2-3$ & 11 & $15-20$ & $\propto$ & - & S. 10 & - & \\
\hline Noull & $28 \cdot 480$ & $13 \cdot 0$ & $7 \cdot 2$ & $10 \cdot 8$ & $9 \cdot 8$ & - & 1.t. var. SH airs & b & $0-10$ & orel & - & S. 10 & - & \\
\hline 2 & $2 x \cdot 847$ & - & $9 \cdot i$ & $10 \cdot 4$ & $9 \cdot x$ & - & WSl, 1-2 & 5 & $1 u-15$ & oc & - & S. cu. 10 & - & \\
\hline 4 & $2 x+x+11$ & - & $9 \cdot 3$ & $10 \cdot 0$ & $9 \cdot 5$ & & SE, 1-3 & 9 & $10-20$ & oert & - & S. $-\mathrm{cn} .10$ & a & \\
\hline 6 & $2 x-851$ & - & $9 \cdot 5$ & $11 \cdot 0$ & $10-7$ & - & Sli, 1-3 & 11 & $10-20$ & orr & - & N. 9 & - & \\
\hline s & $2 \cdot 5 \cdot 8.43$ & - & $6 \cdot 3$ & 6.8 & (i) $4 i$ & - & SE, 3-1 & 10 & $20-2 x$ & $0+$ & - & S. 9 & - & \\
\hline 11) & $2 \times-8 \times 3$ & - & $2 \cdot 8$ & $3 \cdot 0$ & $3 \cdot 0$ & - & SF, 3-4 & 11 & $20-28$ & ocq $\div$ & - & S.-c11. \&, SSle & - & \\
\hline Mislt. & $2 \times \cdot 493$ & - & $11 \cdot 8$ & $4 \cdot 0$ & $3 \cdot x$ & - & Var. $1,3-1$ & $1 i$ & $20-25$ & (x) & - & s. $10,5 \mathrm{E}$ & - & \\
\hline Mran & $2 x \cdot \times i t i$ & - & - & $7 \div 2$ & - & - & - & - & - & - & - & - & - & \\
\hline s & $\begin{array}{l}\text { theru } \\
\text { anl at }\end{array}$ & $8 \mathrm{a}$. & . & sol & \{ & rit & ilit $82^{\circ} \cdot 7$. & BII & $\mathrm{gt}$ & nines in 21 lours. & \multicolumn{3}{|c|}{ l'reclpn. $0.003 \mathrm{in}$. sinee 1 p.m. $39 \mathrm{~h}$. } & \\
\hline
\end{tabular}


Meteorological Journal of the "Discovery."

31st.

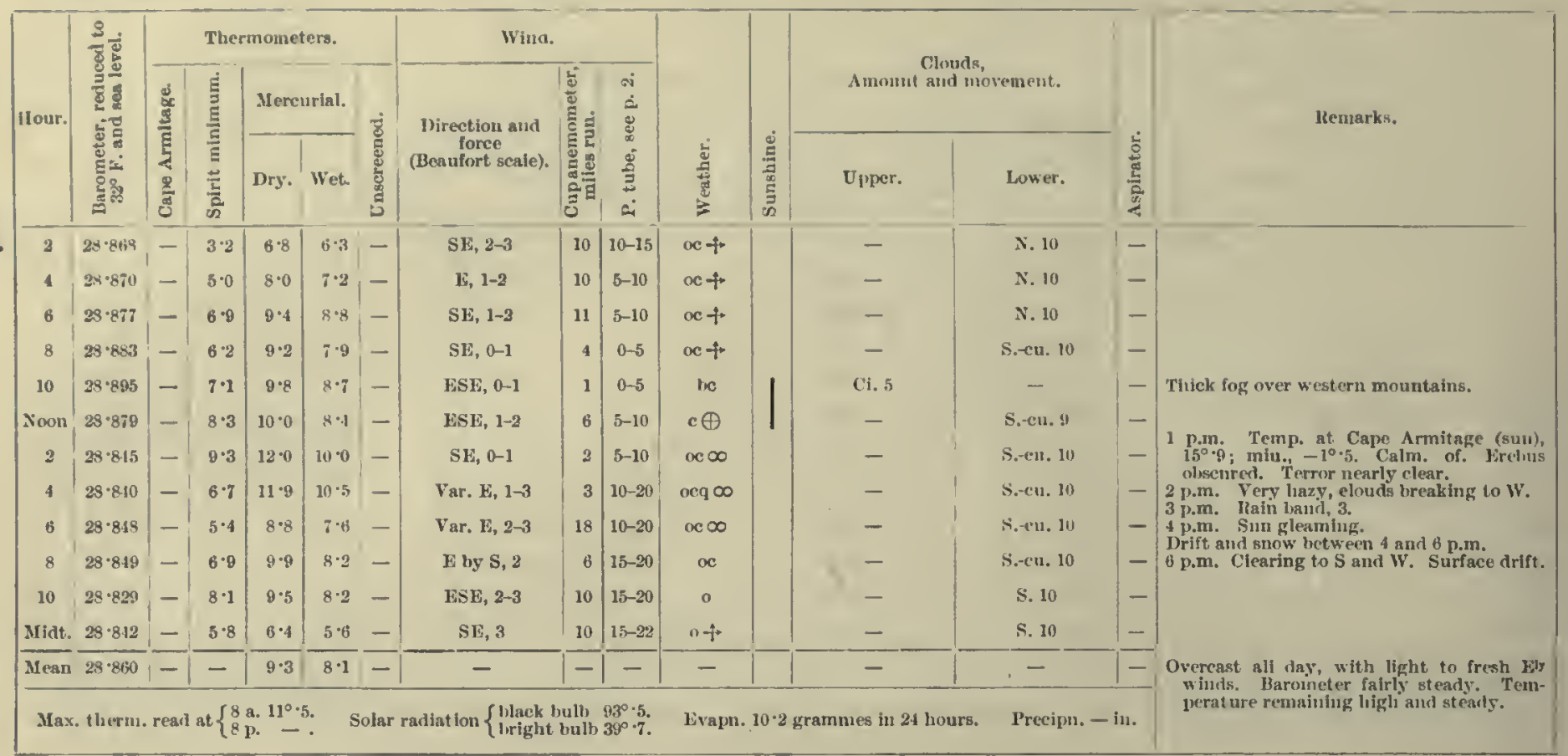


Methonologicat, JoURNa, OF THE: "Discoveley."

[1302-November.

\begin{tabular}{|c|c|c|c|c|c|c|c|c|c|c|c|c|c|c|c|}
\hline \multirow{3}{*}{ Hour. } & \multirow{3}{*}{ 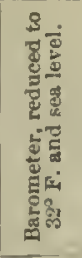 } & \multicolumn{5}{|c|}{ Thermometers. } & \multicolumn{3}{|l|}{ Whad. } & \multirow[b]{3}{*}{ } & \multirow[b]{3}{*}{ 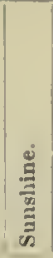 } & \multirow{2}{*}{\multicolumn{2}{|c|}{$\begin{array}{l}\text { Clonds, } \\
\text { Amount and novement. }\end{array}$}} & \multirow[b]{3}{*}{ 竞 } & \multirow{3}{*}{ lkeunarkn. } \\
\hline & & \multirow{2}{*}{ 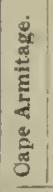 } & \multirow{2}{*}{ 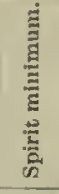 } & \multicolumn{2}{|c|}{ Mercurlal. } & \multirow{2}{*}{ 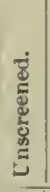 } & \multirow{2}{*}{$\begin{array}{l}\text { Direet.lou and } \\
\text { foree } \\
\text { (Beanfort scale). }\end{array}$} & \multirow{2}{*}{ 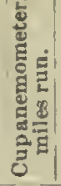 } & \multirow{2}{*}{ 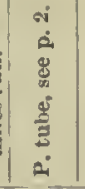 } & & & & & & \\
\hline & & & & Dry. & Wet. & & & & & & & Upuer. & Lower. & & \\
\hline 2 & $28 \cdot 849$ & -1 & $6 \cdot 0$ & $9 \cdot 2$ & $8 \cdot 8$ & - & SSII, 1-2 & 12 & $10-15$ & $\alpha$ & & - & s. $10, \$ 1 \%$ & $\mid-$ & \\
\hline 4 & $28 \cdot 8.17$ & - & $7 \cdot 0$ & $9 \cdot 0$ & 8.8 & - & SSE, 2-3 & 10 & $15-22$ & ocq & & - & S. 10, Sl: & $\rightarrow$ & \\
\hline 6 & $28 \cdot 829$ & - & $8 \cdot 0$ & $9 \cdot 4$ & 9.2 & - & $\mathrm{SI}, 2-1$ & 10 & $20-25$ & $\operatorname{arcto}$ & & - & N. 10 & - & \\
\hline 8 & 28.803 & - & $6 \cdot 8$ & $8 \cdot 8$ & $8 \cdot 6$ & - & sw, 3-5 & 19 & $20-37$ & oegt. & & - & N. 10 & - & \\
\hline 10 & $28 \cdot 820$ & - & $6 \cdot 4$ & $7 \cdot 1$ & 0.5 & - & Var. $S, 4-b$ & 22 & $20-30$ & $\infty \mathrm{q}+$ & & - & N. 10 & - & \\
\hline Noon & $28 \times 867$ & - & $5 \cdot 8$ & $9 \cdot 5$ & $7 \cdot 1$ & - & $1,1-3$ & 18 & $15-20 !$ & neq $f$ & & - & S.e.11. 10 & - & 1 p.m. Sky l,reaklug up overlurad. Small \\
\hline 2 & $28 \cdot 855$ & - & $7 \cdot 7$ & $10 \cdot 0$ & $10 \cdot 5$ & - & Var. SE, 1-3 & 13 & 10-20 & $o c q+$ & & - & S.-'บ1. II & - & $\begin{array}{l}\text { Nin. over Whate. Istani. } \\
2 \text { p.m. Jaln Inaul } 1 \text {. }\end{array}$ \\
\hline 4 & $28 \cdot 869$ & - & 6.6 & $8 \cdot 3$ & $8 \cdot 2$ & - & Var. SE, 3-6 & 18 & $20-35$ & ме1 & & - & S.-c11. 10 & - & 3 p.m. Ileavy squalis wlth drlct. \\
\hline 6 & $28 \cdot 891$ & - & $7 \cdot 4$ & $8 \cdot 8$ & $8 \cdot 8$ & - & Var, S, 4-7 & 27 & $20-40$ & seqtr & & - & N. 10 & - & Foree of wind on llut Polnt 9 to 10 . Sun \\
\hline 8 & $28 \cdot 939$ & - & $7 \cdot 5$ & $8 \cdot 0$ & $7 \cdot 8$ & - & S1E, 3-5 & 21 & $20-30$ & ocq t. & & - & N. 10 & - & \\
\hline 10 & $28 \cdot 915$ & - & $8 \cdot 0$ & $8 \cdot 0$ & $7 \cdot 9$ & - & 1E, 3-5 & 15 & $15-25$ & $o c q+$ & & - & N. 10 & - & \\
\hline Mint. & $28 \cdot 9: 90$ & - & $8 \cdot 8$ & $11 \cdot 0$ & $10 \cdot 2$ & - & $s, 4-7$ & 19 & $20-35$ & ocqt & & - & N. 10 & $1-$ & \\
\hline Mean & $28 \cdot 876$ & - & - & $8 \cdot 9$ & $8 \cdot 5$ & $\overline{-1}$ & - & $1-$ & - & - & & - & - & $1-$ & 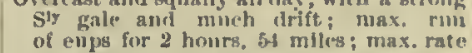 \\
\hline & & & & & & & 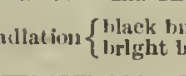 & 11, & 0.0 & 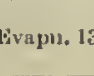 & & & ecilin. & & 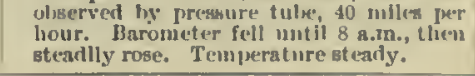 \\
\hline
\end{tabular}

1st.

\begin{tabular}{|c|c|c|c|c|c|c|c|c|c|c|c|c|c|c|}
\hline 2 & $29 \cdot 013$ & - & $8 \cdot 8$ & $9 \cdot 4$ & $9-4$ & - & S, 4-7 & 23 & $25-38$ & ocq & - & N. 10 & $1-$ & Sun gleamling. \\
\hline 4 & $29 \cdot 025$ & $\cdots$ & $8 \cdot 8$ & $9 \cdot 6$ & $9 \cdot 5$ & - & S by $1,5-7$ & 26 & $30-45$ & $\operatorname{org} * \leftarrow$ & - & N. 10 & - & \\
\hline 6 & $29 \cdot 0655^{\circ}$ & - & - & $7 \cdot 0$ & $7 \cdot 0$ & - & Var. S, 4-5 & 18 & $20-30$ & $v q+$ & - & N. 10 & - & \\
\hline 8 & $29 \cdot 101$ & - & - & 10.0 & $10 \cdot 0$ & - & Var. E, 2-5 & 17 & $20-35$ & $\operatorname{ocg}+$ & - & $\mathrm{Cu}, 10, \mathrm{sla}$ & - & $\begin{array}{l}\text { Weather very squally. Wind suclilenly } \\
\text { shlfited from s to Ni. Sky clearlug to }\end{array}$ \\
\hline 10 & $29 \cdot 141$ & - & - & $10 \cdot 8$ & $10 \cdot 0$ & - & Var. $\mathbf{E}, 1-3$ & 17 & $5-25$ & ocq + & - & Cu. 8 & - & southwaris. \\
\hline Noon & $29 \cdot 277$ & - & - & $12 \cdot 2$ & $11 \cdot 2$ & - & s1v, 1-2 & 10 & $10-15$ & oeq + & - & A.-cu. 8 & 1- & \\
\hline 2 & $29 \cdot 3: 32$ & - & - & $12 \cdot 8$ & $12 \cdot 0$ & - & SsN, 2-3 & 12 & $15-20$ & $\alpha+$ & - & S.eu. 10 & - & Sin gleamlug ; clear $\mathrm{S}$ to $\mathrm{W}$. \\
\hline 4 & $29 \cdot 274$ & - & $y \cdot 8$ & $10 \cdot 4$ & $9 \cdot 8$ & - & E, 2-3 & 13 & $15-20$ & $\infty$ & - & Cu. 8 & - & \\
\hline 6 & $29 \cdot 278$ & - & $9 \cdot 7$ & $11 \cdot 0$ & $10 \cdot 0$ & - & Var. SE, 2-4 & 10 & $10-25$ & eq + & $\mathrm{Ci}_{2} 2, \mathrm{NW}$ & Cu. $7, \mathrm{~N}$ & - & Clouds breaklng up and drlvlug Sty. \\
\hline 8 & $29 \cdot 331$ & - & - & $9 \cdot 2$ & $9 \cdot 0$ & - & S1:, $2-4$ & 27 & $20-30$ & ocq & Cl.-8. 2, NW & S.-e11. 6, NW & - & \\
\hline 10 & $29 \cdot 333$ & - & - & $9 \cdot 0$ & $8 \cdot 5$ & - & E, 2-1 & 21 & $20-30$ & oœ1 & - & S.en. 9 & - & \\
\hline Mist. & $29 \cdot 352$ & - & $4 \cdot 2$ & $10 \cdot 0$ & $4 \cdot 8$ & - & Var. 1:, 1-3 & 13 & $5-20$ & $\operatorname{seq} q$ & - & A.evu. 8 & - & Spirit minimum out of order. Overenst, \\
\hline Mean & $29 \cdot 211$ & - & - & $10 \cdot 1$ & $9 \cdot 7$ & -1 & - & - & - & - & - & - & $1-$ & $\begin{array}{l}\text { drifi max. rum of cups for } 2 \text { hours, } \\
53 \text { miles; max. rate olserved hy pressure }\end{array}$ \\
\hline
\end{tabular}

2nd.

\begin{tabular}{|c|c|c|c|c|c|c|c|c|c|c|c|c|c|c|c|}
\hline 2 & $29 \cdot 316$ & -1 & $9 \cdot 8$ & $10 \cdot 8$ & $10 \cdot 1$ & $1-$ & Var. $14,1-3$ & 18 & $10-25$ & $x+1$ & & - & A.-с'u. 9 & $1-$ & \\
\hline 4 & $29 \cdot 365$ & $-\ldots$ & $10 \cdot 7$ & $11 \cdot 0$ & $11 \cdot 0$ & - & E hy S, 1-2 & 10 & $\mid 0-20$ & oeq & & - & A.-cin. . & - & \\
\hline 6 & $29 \cdot 112$ & -1 & $8 \cdot 0$ & $10 \cdot 3$ & $10 \cdot 2$ & - & ES1:, 1-2 & 12 & $5-18$ & be & & - & Cu. 5, SH & - & \\
\hline 8 & $29 \cdot 478$ & - & $8 \cdot 9$ & $10 \cdot 5$ & 10.5 & -1 & ISEE, 1-2 & 11 & $5-10$ & be & & - & S.-e+t. 7, SSE & - & \\
\hline 10 & $29 \cdot 462$ & $=$ & $8 \cdot 0$ & $12 \cdot 0$ & $11 \cdot 6$ & - & Var, $1,1-2$ & 12 & $10-15$ & ix & & C1. 3 & - & - & \\
\hline Noon & $29 \cdot 465$ & - & $11 \cdot 2$ & $14 \cdot 5$ & $14 \cdot 0$ & - & Var. 1, 1-2 & 10 & $10-15$ & $\infty$ & 1 & (?. 8 & - & $1-$ & \\
\hline 2 & $29 \cdot 45 v$ & - & 10.0 & 12.4 & $12 \cdot 1$ & - & E by $N, 1-3$ & 11 & $5-15$ & Ixeq & 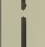 & (ii. s. i, NW & $(11,1, s$ & - & $\begin{array}{l}\text { Low detached Cu. to } \mathrm{S} \text { and } 11 \text {. Very hazy } \\
\text { to k and } \mathrm{S} \text {. Vo }\end{array}$ \\
\hline 4 & $29 \cdot 410$ & - & $10 \cdot 2$ & $12 \cdot 5$ & $12 \cdot 0$ & - & Var. SE, 1-3 & 10 & $0-15$ & $I x: q$ & & Ci. 3, NW & ('u, I, s & . & 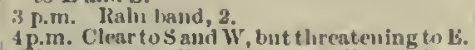 \\
\hline 6 & $29 \cdot 41 m$ & - & $9 \cdot 0$ & $10^{\circ} 0$ & $9 \cdot 0$ & - & Var, L, 1-3 & 13 & $0-15$ & leq & & $\mathrm{Ci}_{2} \mathrm{~s}, \mathrm{NW}$ & - & - & $\begin{array}{l}\text { Whind rery squally. Clearing all round on } \\
\text { loorlzon. }\end{array}$ \\
\hline 8 & $29 \cdot 456$ & - & $11 \cdot 9$ & $12 \cdot 0$ & $11 \cdot 0$ & $1-$ & E by $N, 1$ & 11 & $0-5$ & $1 x$ & ] & Ci. $4, \mathrm{NW}$ & - & - & \\
\hline 10 & $29 \cdot 4: 27$ & - & $10 \cdot 3$ & $12 \cdot 4$ & $11 \cdot 0$ & - & lisli, $0-1$ & 1 & 0 & Ix & & Cl. 4 & - & - & Low Cu. maving from S to $\mathrm{V}$. \\
\hline Mldt. & $29 \cdot 401$ & $=$ & $9 \cdot 0$ & $10 \cdot 1$ & $8 \cdot 4$ & -1 & Lt. WSL airs & 2 & 0 & ise & & Ci. 2 & - & - & \\
\hline Nean! & $29 \cdot 435$ & - & - & $11 \cdot 5$ & $11 \cdot 0$ & $1-1$ & - & -1 & - & - & & - & - & - & 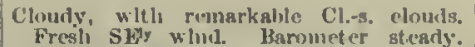 \\
\hline
\end{tabular}

3 rd. 


\begin{tabular}{|c|c|c|c|c|c|c|c|c|}
\hline \multirow{3}{*}{ Hour. } & \multirow{3}{*}{ 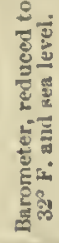 } & \multicolumn{4}{|c|}{ Thermometers. } & \multicolumn{3}{|l|}{ Wind. } \\
\hline & & 赵 & है & Mereurial. & . & Directiou & 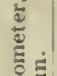 & $\stackrel{\dot{\Xi}}{\vdots}$ \\
\hline & & $\frac{\bar{z}}{\frac{y}{g}}$ & 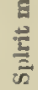 & Dry. Wet. & 递 & (Beaufort seale). & | & $\begin{array}{l}\frac{8}{3} \\
\text { L }\end{array}$ \\
\hline
\end{tabular}

4th.

\begin{tabular}{|c|c|c|c|c|c|c|c|c|c|c|c|c|c|c|}
\hline 3 & $29 \cdot 311$ & - & $8 \cdot 6$ & $13 \cdot 0$ & $11 \cdot 4$ & - & 1.t. ISE airs & 7 & 0 & les & $\mathrm{Ci}, \cdot 3, \mathrm{SW}$ & - & - & Jow Cn, moving from $\mathrm{S}$ to $\mathrm{J}$. \\
\hline 4 & $29 \cdot 31: 1$ & - & 6.5 & $12^{\circ} 0$ & $11 \cdot 2$ & - & Var. 10,0-1 & 6 & $0-5$ & the & C1. $2, \mathrm{SW}$ & Cu. 4, s & - & \\
\hline 6 & $29 \cdot 311$ & - & $6 \cdot 0$ & $8 \cdot 9$ & $9 \cdot 2$ & - & Var. E, 0-1 & 10 & $0-5$ & be & $\mathrm{Cl}, 2, \mathrm{SW}$ & Cu, 3, S & - & \\
\hline 8 & $29 \cdot 393$ & - & $8 \cdot 9$ & $11 \cdot 3$ & $10 \cdot 4$ & - & w ly $\mathrm{N}, 1-2$ & 5 & $5-10$ & the & Ci. 1 & - & - & - \\
\hline 10 & $29 \cdot 399$ & - & $10 \cdot 9$ & $14 \cdot 6$ & $13 \cdot 7$ & - & Calm & 7 & 0 & lic & Ci. 1 & - & - & \\
\hline Noon & $29 \cdot 3 \times 1$ & - & $10 \cdot 2$ & $14 \cdot 5$ & $13 \cdot 0$ & - & Lt. Slis airs & 1 & 0 & ine & Ci.-s. 2 & - & $\rightarrow$ & \\
\hline 2 & $29 \cdot 3 t^{2}$ & - & $12 \cdot 7$ & $17 \cdot 6$ & $15 \cdot 8$ & - & SE, $0-1$ & 1 & 0 & be & Ci.-s. 5, I & - & - & $\begin{array}{l}\text { Low detheher elouils on S horizon. } \\
\text { : p.m. ltain hant, I. }\end{array}$ \\
\hline 4 & $29 \cdot 352$ & - & $10 \cdot 0$ & $11 \cdot 6$ & $10^{\circ} 0$ & - & SH, 1-2 & 8 & $5-20$ & be & Ci.-s. $6, W$ & - & - & Very hazy. \\
\hline 6 & $29 \cdot 351$ & - & $8 \cdot 4$ & 9.4 & $8 \cdot 6$ & - & E, 1-3 & 11 & $5-20$ & be & Ci.-s. b, SW & - & - & 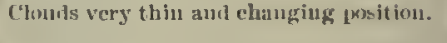 \\
\hline 8 & $29 \cdot 34$ & - & $8 \cdot 3$ & $12 \cdot 4$ & $10 \cdot 8$ & - & L by $\mathbf{N}, 1-3$ & 13 & $5-20$ & be & Ci. 3 & $\mathrm{Cu}, 4, \mathrm{SL}$ & - & $\begin{array}{l}\text { Cu. driving up from SE in detaclied } \\
\text { portions. }\end{array}$ \\
\hline 10 & $29 \cdot 311$ & - & $10 \cdot 0$ & $11 \cdot 5$ & $10 \cdot 2$ & - & ESE, 1-3 & 15 & $5-20$ & be & - & S.-cu. 5 & - & \\
\hline Mint. & $29 \cdot 366$ & - & $7 \cdot 8$ & $10 \cdot 0$ & $8 \cdot 9$ & - & E by N, 1-3 & 10 & $5-25$ & lic & Ci. 2 & $\mathrm{Cn} .1 . \mathrm{SE}$ & 二 & \\
\hline Nlean, & $29 \cdot 340$ & - & - & $12 \cdot 2$ & $11 \cdot 1$ & -1 & - & -1 & - & - & - & - & - & $\begin{array}{l}\text { Fine, lout cloudy. light to frosh lity } \\
\text { winds. Barometer st eary. Tempera- }\end{array}$ \\
\hline
\end{tabular}

5 th.

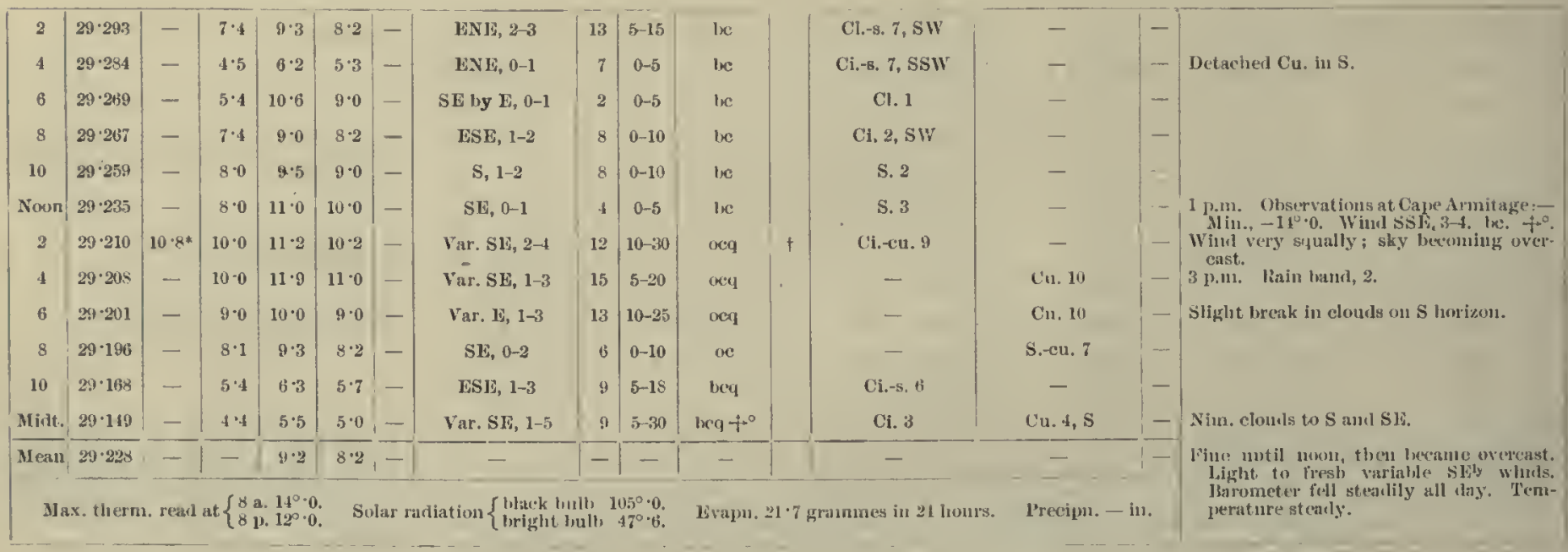

* liead at 1 p.m. † Card blown out of Sunshine Recorder and lost, about I2 lours of bright sunshine estimated.

6 th.

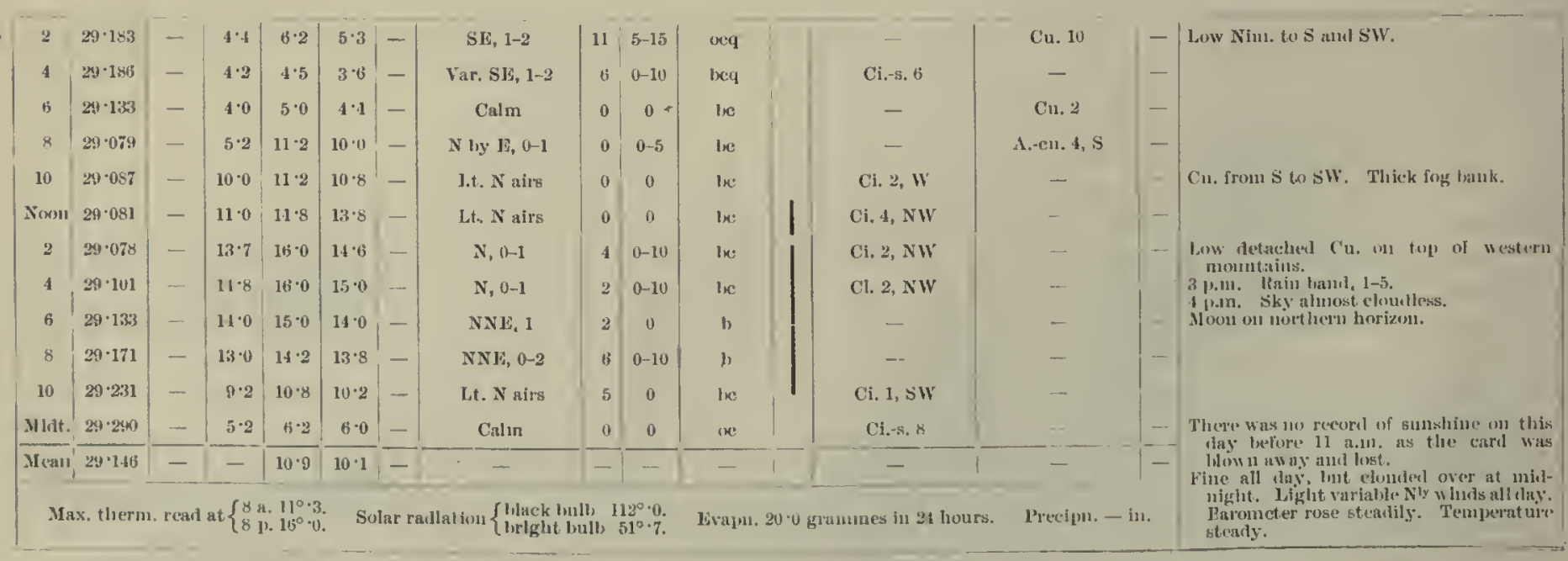




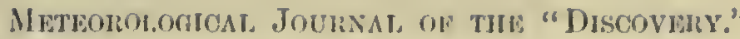

[1302-November.

\begin{tabular}{|c|c|c|c|c|c|c|c|c|c|c|c|c|c|c|c|}
\hline \multirow{2}{*}{11 aur. } & \multirow{2}{*}{ 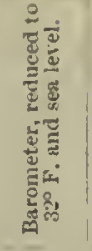 } & \multirow{2}{*}{$\mid$} & \multicolumn{3}{|c|}{ Thermoncters. } & \multirow{2}{*}{ | } & Whin. & & $\stackrel{\oplus i}{\longleftarrow}$ & \multirow[b]{2}{*}{ 就 } & \multirow{2}{*}{ 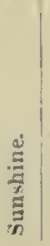 } & \multicolumn{2}{|c|}{$\begin{array}{l}\text { Clousls, } \\
\text { Amount and uovement. }\end{array}$} & \multirow{2}{*}{$\mid$} & \multirow{2}{*}{ Henuarks. } \\
\hline & & & 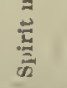 & Dry. & Wet. & & (Beaufort scale). & 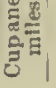 & 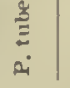 & & & Upper. & Lawer. & & \\
\hline 2 & $37 \cdot 312$ & -1 & $5 \cdot 0$ & $10 \cdot 2$ & $10 \cdot 0$ & -1 & L.t. N birs alrs & $3 \mid$ & 0 & le & 1 & Ci.-8. 6 & - & -1 & \\
\hline l & $29 \cdot 3213$ & - & $13 \cdot 8$ & $11 \cdot 0$ & $10 \cdot s$ & -1 & Calm & 0 & 0 & he & 1 & Cl. 4 & - & 1 & \\
\hline 6 & $29 \cdot 3 n$ & - & $8 \cdot 0$ & 30.5 & $9 \cdot 8$ & -1 & Cylm & 0 & 0 & Ine & & Ci, $6, \mathrm{sw}$ & - & - & \\
\hline 8 & $29 \cdot 311$ & - & $8 \cdot 5$ & $12 \cdot 5$ & $12 \cdot 3$ & -1 & Calm & 0 & 0 & $\boldsymbol{x}$ & & Cl. $-\mathrm{s}, 9$ & - & -1 & 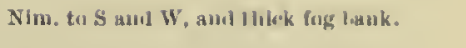 \\
\hline 10 & $28 \cdot 128$ & - & $11 \cdot 3$ & $12 \cdot 8$ & $12 \cdot 8$ & - & WNI, 1-2 & 4 & $5-10$ & o* & & - & N. 111 & -1 & \\
\hline Noon & $29 \cdot 43$ & - & $11 \cdot 2$ & $12 \cdot 5$ & $12 \cdot 5$ & -1 & S $1, y, w, 1-3$ & 4 & $6-15$ & $a *+$ & & - & s. 10 & - & \\
\hline 2 & $29 \cdot 410$ & - & $11 \cdot 4$ & $13 \cdot 0$ & $12 \cdot 5$ & - . & SSIE, 1-2 & 10 & $0-10$ & are $*$ & & - & N. 11 & - & 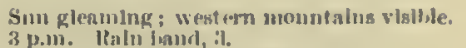 \\
\hline 4 & $29 \cdot 427$ & - & 11.5 & $12 \cdot 5$ & $11 \cdot 0$ & - & 1: $\ln S, 1-2$ & (3) & $0-10$ & c & & Ci. 3, S l: & Cu. if & - & Cfrar to $S$ anel li: sin gleanilug. \\
\hline B & $29 \cdot 401$ & - & $11 \cdot 4$ & $13 \cdot 7$ & $12: 5$ & - & EN1:, 1-2 & 7 & $0-10$ & be & 1 & - & Fr.-ch. 2 & - & Sky almost clondliass. VImblilty sumll. \\
\hline 8 & $29 \cdot 339$ & - & $12 \cdot 0$ & $13 \cdot 0$ & $12 \cdot 0$ & - & 1HN 1, 1-2 & 7 & $5-15$ & be & i & - & Fr.cu. 3 & - & \\
\hline 10 & $29 \cdot 359$ & - & $7 \cdot 4$ & $9 \cdot 0$ & $8 \cdot 0$ & - & Cahn & 2 & 0 & $1, \infty$ & & - & - & - & \\
\hline Mitat. & $29 \cdot 329$ & - & $6 \cdot 0$ & $6 \cdot 5$ & $6 \cdot 5$ & - & Cxhm & 0 & 0 & $1, \infty$ & & - & - & - & \\
\hline Mtan & $29 \cdot 375$ & - & - & $11 \cdot 5$ & $10 \cdot 9$ । & -1 & - & -- & - & - & & - & - & $1=$ & 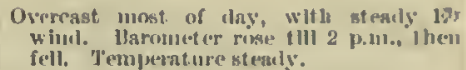 \\
\hline
\end{tabular}

7 th.

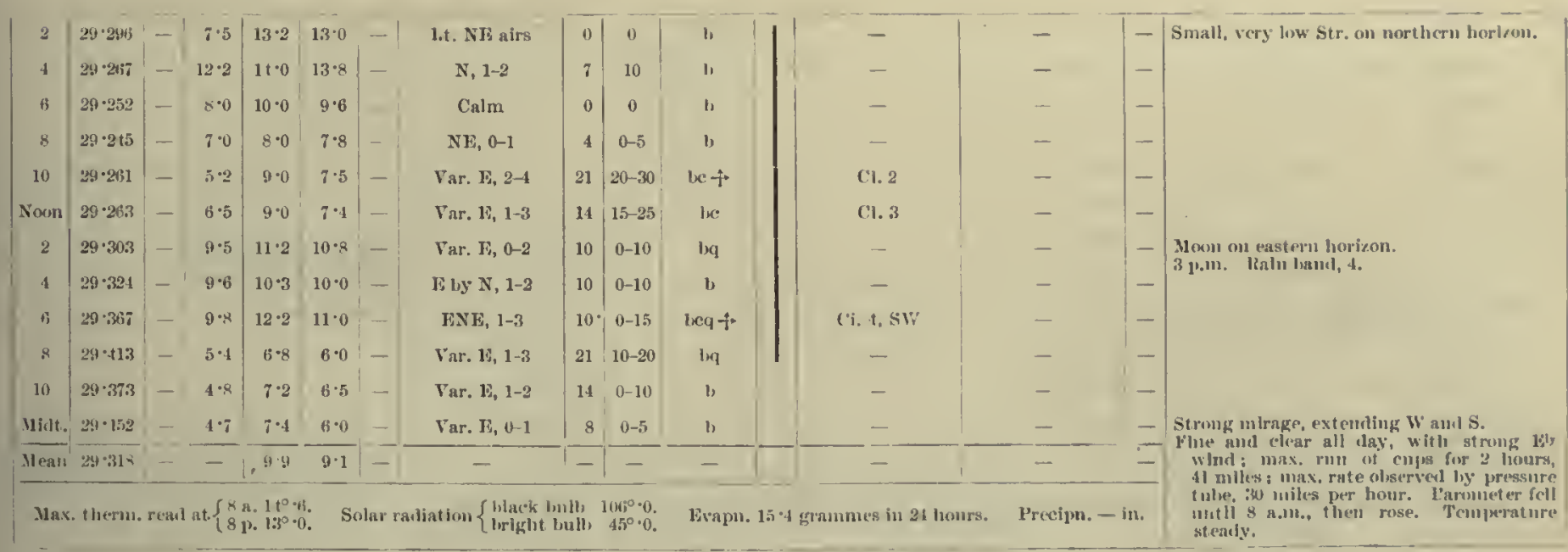

8 th

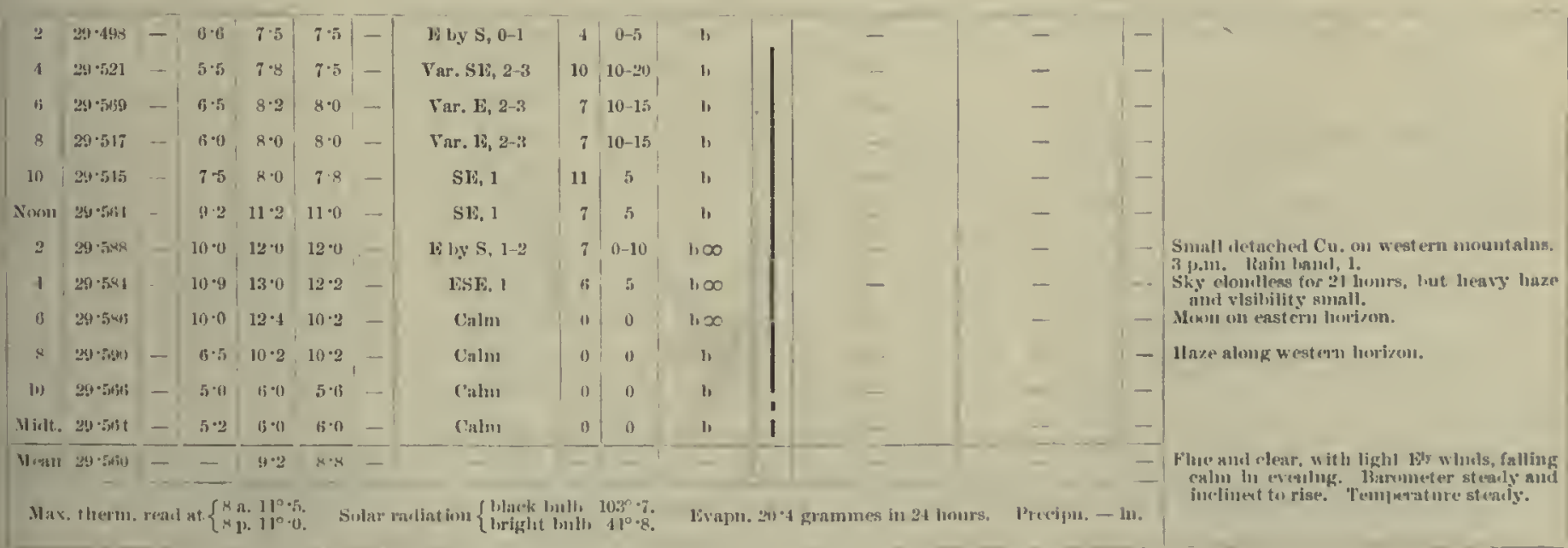




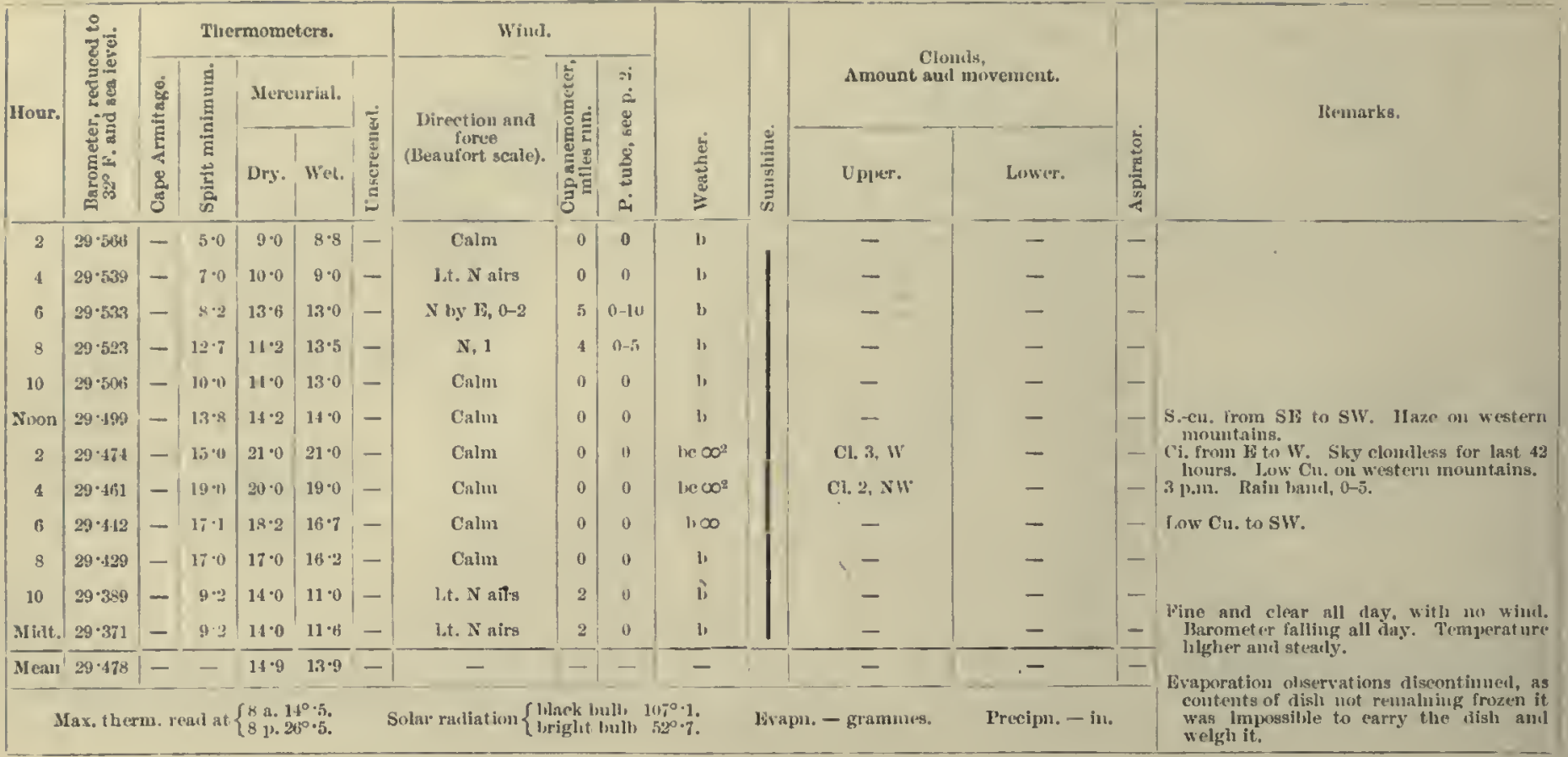

11 th.

\begin{tabular}{|r|r|r|r|r|r|r}
\hline 2 & $29 \cdot 37$ & - & $12 \cdot 6$ & $15 \cdot 6$ & $14 \cdot 9$ & - \\
4 & $29 \cdot 365$ & - & $14 \cdot 0$ & $16 \cdot 0$ & $14 \cdot 8$ & - \\
6 & $29 \cdot 365$ & - & $13 \cdot 5$ & $15 \cdot 2$ & $15 \cdot 0$ & - \\
8 & $29 \cdot 365$ & - & $11 \cdot 8$ & $15 \cdot 2$ & $13 \cdot 8$ & - \\
10 & $29 \cdot 336$ & - & $13 \cdot 2$ & $15 \cdot 0$ & $1.1 \cdot 0$ & - \\
Noon & $29 \cdot 355$ & - & $9 \cdot 0$ & $10 \cdot 2$ & $9 \cdot 2$ & - \\
2 & $29 \cdot 397$ & - & $6 \cdot 3$ & $7 \cdot 4$ & $7 \cdot 0$ & - \\
4 & $29 \cdot 413$ & - & $15 \cdot 5$ & $9 \cdot 0$ & $8 \cdot 2$ & - \\
6 & $29 \cdot 415$ & - & $4 \cdot 9$ & $8 \cdot 3$ & $8 \cdot 0$ & - \\
8 & $29 \cdot 131$ & - & $4 \cdot 0$ & $5 \cdot 0$ & $5 \cdot 0$ & - \\
10 & $29 \cdot 416$ & - & $1 \cdot 2$ & $4 \cdot 0$ & $4 \cdot 0$ & - \\
Midt. & $29 \cdot 423$ & - & $0 \cdot 0$ & $3 \cdot 0$ & $3 \cdot 0$ & - \\
\hline Mean & $29 \cdot 3.39$ & - & - & $10 \cdot 3$ & $9 \cdot 7$ & -
\end{tabular}

Max. theru, reail at $\left\{\begin{array}{l}8 \text { a. } 18^{\circ} \cdot 5 . \\ 8 \text { p. } 16^{\circ} \cdot 2 .\end{array}\right.$

\begin{tabular}{|c|c|c|c|c|}
\hline Calm & 0 & 0 & c & - \\
\hline Lt. NE alrs & 0 & 0 & ine & Ci, s, $5, \mathrm{~S}$ \\
\hline Calm & 0 & 0 & $x$ & Ci. 10 \\
\hline Calm & 2 & $"$ & $\boldsymbol{c c}$ & A.-8. 10 \\
\hline SE, 1 & 7 & 5 & $\alpha$ & A.-8. 10 \\
\hline SE, 1-2 & 10 & - & oc & - \\
\hline SE, 2-4 & 15 & - & oeq & - \\
\hline SE, 2-4 & 14 & - & seq & - \\
\hline Var. E, 1-3 & 14 & - & $e q$ & - \\
\hline Var. E, 2-3 & 11 & - & (x) & - \\
\hline Var. 1), 2-4 & 15 & - & $\propto$ & - \\
\hline Var. $5,4-5$ & 23 & - & $\infty+$ & - \\
\hline$\cdot \quad-$ & - & - & - & - \\
\hline
\end{tabular}

Solar radiation $\left\{\begin{array}{l}\text { llack bult, } \\ \text { lirlgh } 9.9 .\end{array}\right.$
Evapu. - grammes.

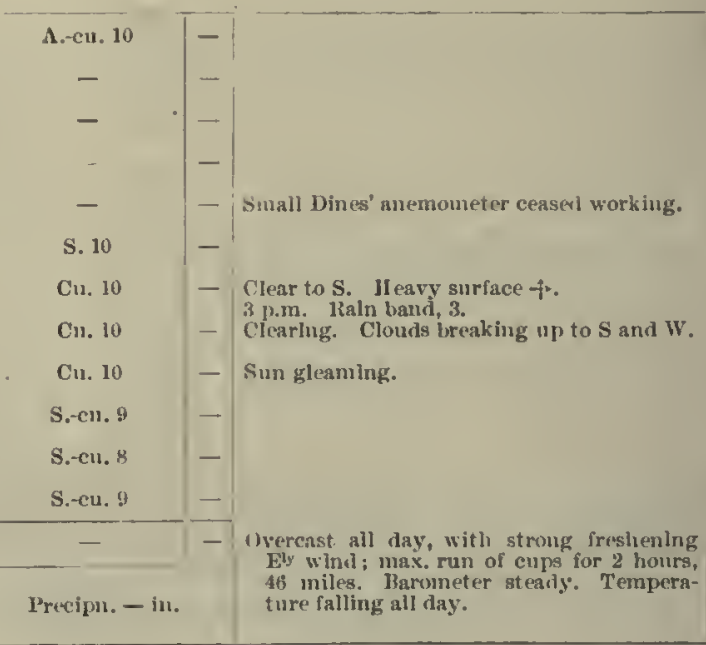

12 th.

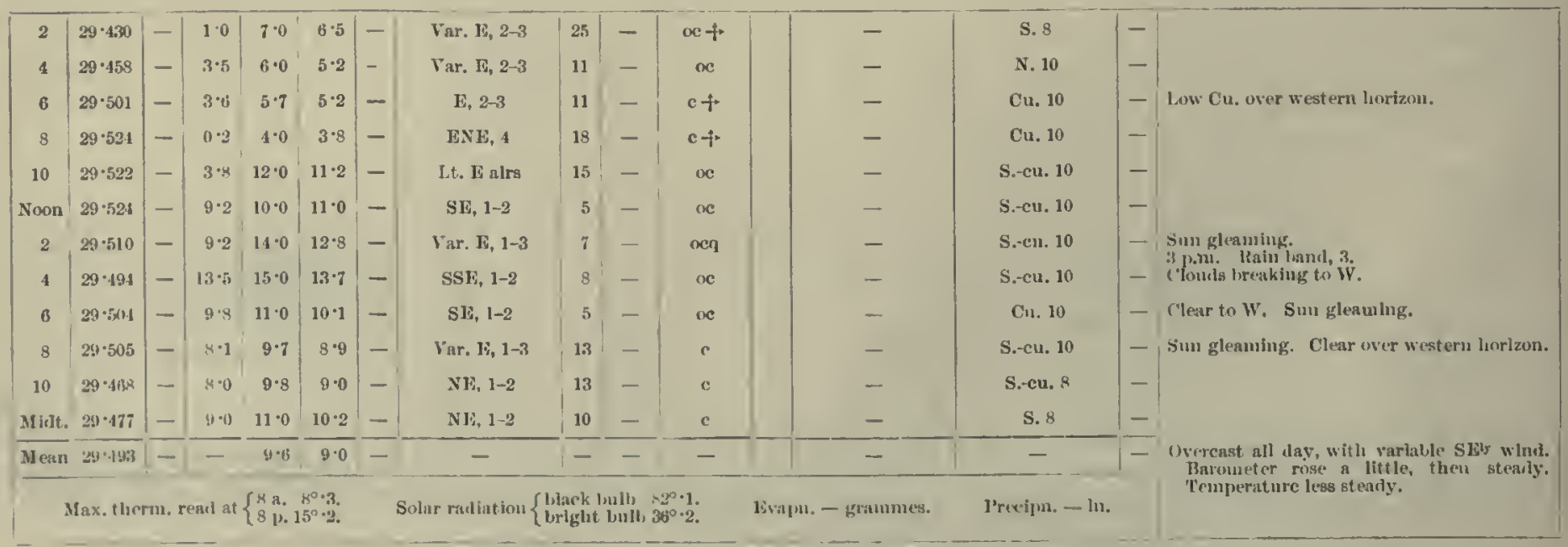


Meteoliological JoukNal, of the "Discuveny."

[1902-November.

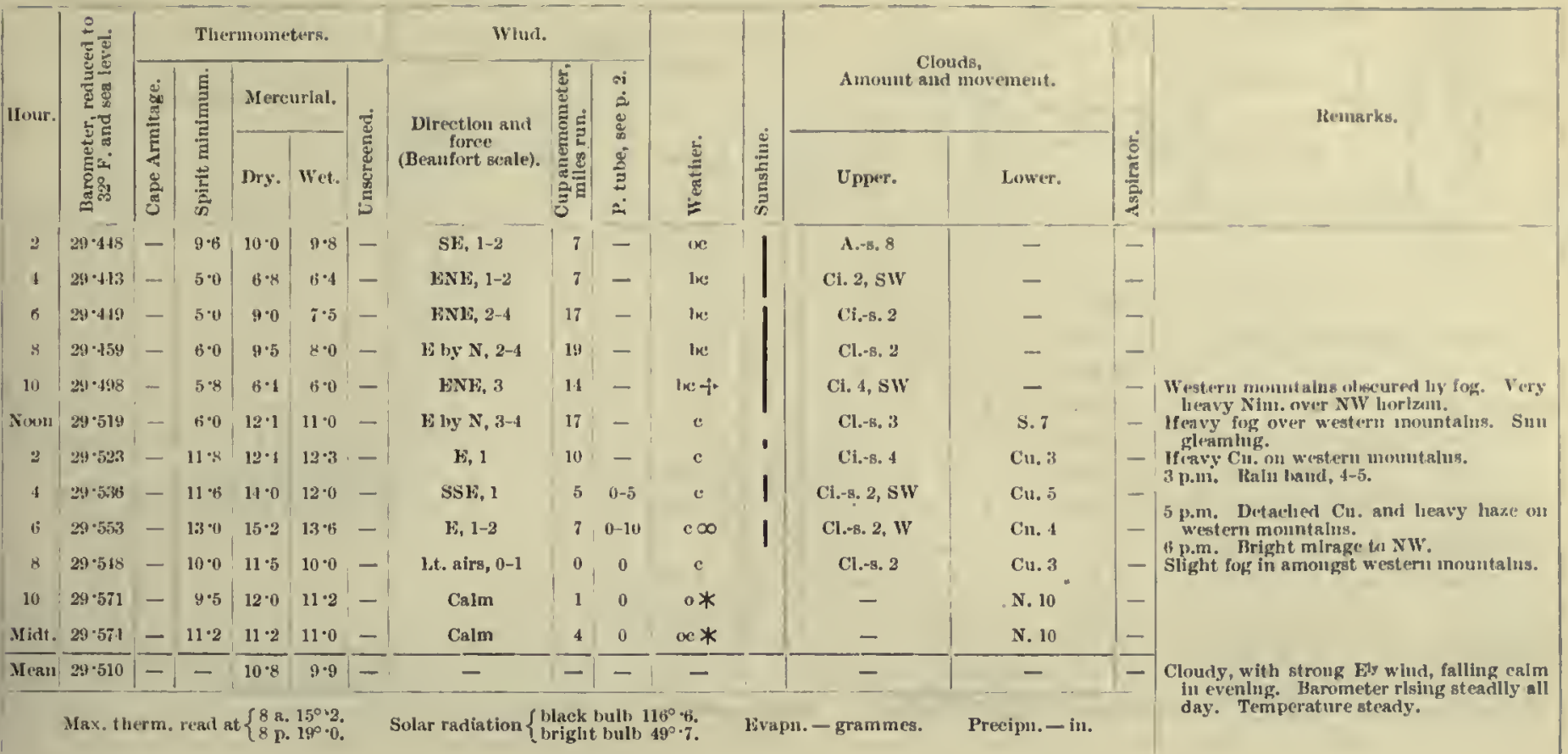

13 th.

\begin{tabular}{|c|c|c|c|c|c|c|c|c|c|c|c|c|c|c|c|}
\hline 2 & $29 \cdot 5 \pi 6$ & $1-1$ & $11 \cdot 0$ & $|13 \cdot 0|$ & $12 \cdot 8$ & - & $\mathrm{Calm}$ & 0 & 0 & $\operatorname{ocm} *$ & & - & N. 10 & $1-$ & \multirow{4}{*}{ Thick mist all round aud very dull. } \\
\hline 4 & $29 \cdot 576$ & - & $12 \cdot 0$ & $13 \cdot 0$ & $12 \cdot 8$ & - & Calm & 0 & 0 & ocm $*$ & & - & N. 10 & - & \\
\hline 6 & $29 \cdot 584$ & - & $13 \cdot 0$ & $14 \cdot 0$ & $13 \cdot 2$ & - & Calm & 0 & 0 & $\operatorname{ocm} *$ & & - & N. 10 & - & \\
\hline 8 & $24 \cdot 551$ & - & $11 \cdot 8$ & $15 \cdot 0$ & $11 \cdot 6$ & - & Calm & 0 & 0 & $\operatorname{acm} *$ & & - & N. 10 & - & \\
\hline 10 & $29 \cdot 511$ & - & $12 \cdot 0$ & $15 \cdot 0$ & $14 \cdot 2$ & - & Lt. airs, $0-1$ & 9 & 0 & $\cos *$ & & - & N. 10 & '- & \multirow[t]{2}{*}{ Sun gleamiug. } \\
\hline Хюю1 & $29 \cdot 523$ & - & $9 \cdot 0$ & $12 \cdot 4$ & $11 \cdot 2$ & - & NNE, $0-1$ & 4 & 0 & ac* & & - & N. 10 & - & \\
\hline 2 & 29.5065 & - & $16 \cdot 1$ & $18 \cdot 0$ & $17 \cdot 8$ & - & $\mathbf{x}, \mathbf{I}$ & 1 & $0-10$ & $\mathrm{c}$ & & - & $\mathrm{Cu} .7$ & - & \multirow{6}{*}{$\begin{array}{l}3 \text { p..n. Raln band, } 1 . \\
5 \text { p.m. Clear overlear. Hanks of Cu. almm } \\
\text { fout of western mountains. } \\
6 \text { p.m. Heavy Nim. on NW horizon. }\end{array}$} \\
\hline 4 & $29 \cdot 4 \times 3$ & - & $17 \cdot 0$ & $18 \cdot 0$ & $17 \cdot 0$ & - & NNE, $1-3$ & 4 & $5-15$ & be & 1 & Ci. 2, SE; & Cu. 1 & - & \\
\hline$b^{\circ}$ & $29 \cdot 460$ & - & $17 \cdot 0$ & $17 \cdot 2$ & $17 \cdot 0$ & - & $N, 1-2$ & s & $5-15$ & $1 x$ & i & Ci. $2, \mathrm{SE}$ & $\mathrm{Cu}_{1} .3$ & - & \\
\hline 8 & $29 \cdot 441$ & - & $11 \cdot 0$ & $12 \cdot 0$ & $11 \cdot 4$ & - & Lt. $\mathbf{N}$ airs & 5 & 0 & oe & & A.-5. 9 & - & - & \\
\hline 10 & $29 \cdot 451$ & - & $1.1 \cdot 0$ & $15 \cdot 2$ & $14 \cdot 3$ & - & Var. N, 1-2 & 5 & $5-10$ & $\mathrm{c}$ & & Cl. 8 & Cu. 2 & - & \\
\hline Midt. & $29 \cdot 461$ & -1 & $9 \cdot 8$ & $11 \cdot 0$ & -10.0 & - & $N, 1-3$ & 6 & $5-10$ & $\mathrm{c}$ & & Ci. 6 & - & - & \\
\hline Mean & $29 \cdot 513$ & $1-1$ & - & $14 \cdot 6$ & $13 \cdot 9$ & 1 & - & $1-1$ & $1-1$ & - & 1 & - & - & - & 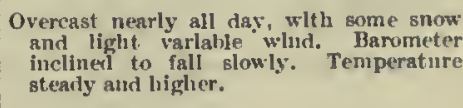 \\
\hline
\end{tabular}

14 th.

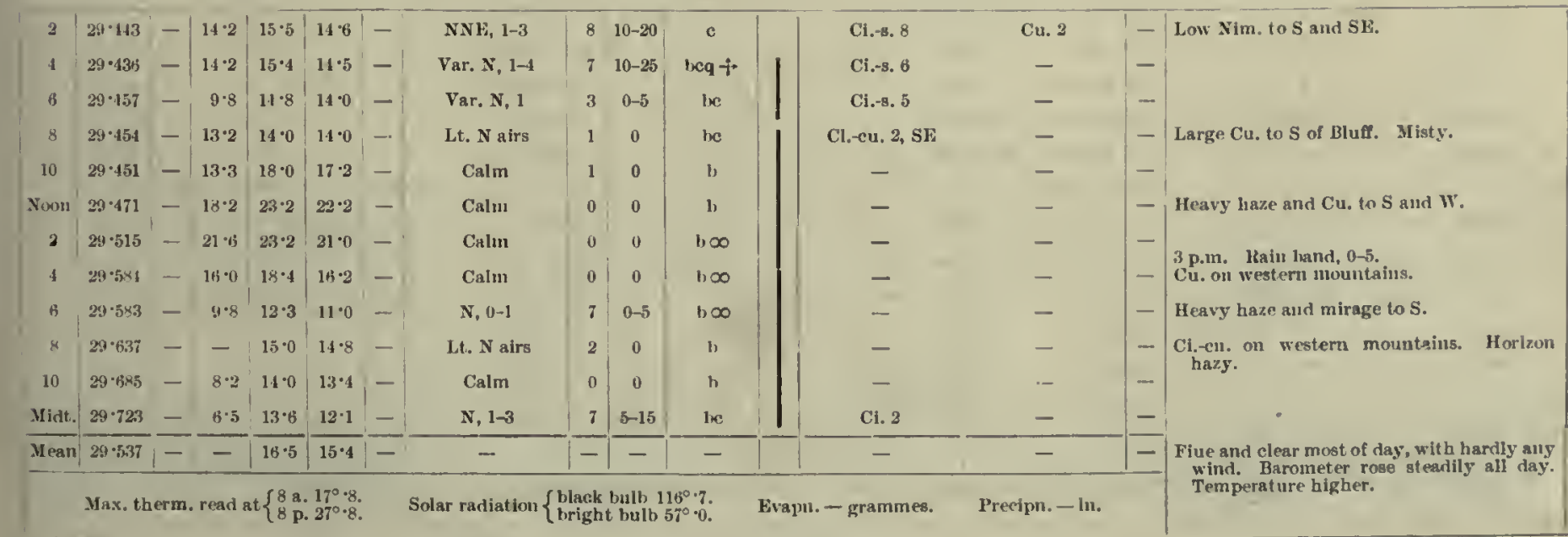


1902-November.]

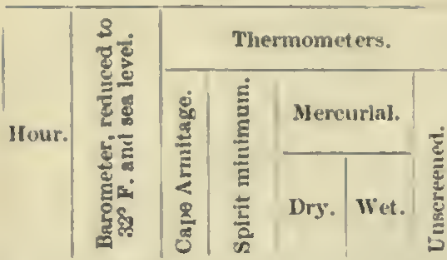

16 th.

\begin{tabular}{|r|r|r|r|r|r|r|}
\hline 4 & $29 \cdot 751$ & - & $5 \cdot 8$ & $10 \cdot 7$ & $10 \cdot 0$ & - \\
\hline 4 & $29 \cdot 797$ & - & $6 \cdot 0$ & $11 \cdot 0$ & $10 \cdot 0$ & - \\
8 & $29 \cdot 858$ & - & $10 \cdot 0$ & $12 \cdot 2$ & $12 \cdot 0$ & - \\
10 & $29 \cdot 927$ & - & $12 \cdot 0$ & $13 \cdot 3$ & $13 \cdot 0$ & - \\
Nuoun & $29 \cdot 968$ & - & $11 \cdot 5$ & $18 \cdot 2$ & $17 \cdot 4$ & - \\
2 & $29 \cdot 970$ & - & $17 \cdot 4$ & $20 \cdot 0$ & $19 \cdot 0$ & - \\
4 & $29 \cdot 966$ & - & $18 \cdot 0$ & $20 \cdot 6$ & $18 \cdot 8$ & - \\
8 & $29 \cdot 961$ & - & $15 \cdot 9$ & $15 \cdot 2$ & $16 \cdot 4$ & - \\
8 & $29 \cdot 943$ & - & $16 \cdot 8$ & $17 \cdot 4$ & $16 \cdot 2$ & - \\
10 & $29 \cdot 927$ & - & $16 \cdot 0$ & $16 \cdot 0$ & $15 \cdot 2$ & - \\
Mint. & $29 \cdot 905$ & - & $12 \cdot 0$ & $15 \cdot 2$ & $15 \cdot 0$ & - \\
\hline Mean & $29 \cdot 912$ & - & - & $15 \cdot 9$ & $15 \cdot 1$ & - \\
\hline
\end{tabular}

Max. therm. read at $\left\{\begin{array}{l}8 \text { a. } 15^{\circ} \cdot 0 . \\ 8 \mathrm{p},\end{array}\right.$
Metrolological Joursa, of The "Discoveriy."

\begin{tabular}{|c|c|c|c|c|c|c|c|c|}
\hline \multicolumn{3}{|l|}{ Wind. } & \multirow[b]{3}{*}{ 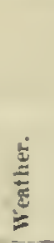 } & \multirow[b]{3}{*}{ 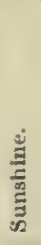 } & \multirow{2}{*}{\multicolumn{2}{|c|}{$\begin{array}{l}\text { Cibuds, } \\
\text { Amonit anl movement. }\end{array}$}} & \multirow[b]{3}{*}{ 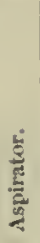 } & \multirow{3}{*}{ liemarks. } \\
\hline \multirow{2}{*}{$\begin{array}{l}\text { Direction and } \\
\text { fore' } \\
\text { (Beaufort seale). }\end{array}$} & \multirow{2}{*}{ 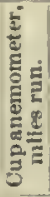 } & \multirow{2}{*}{ 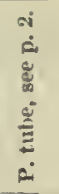 } & & & & & & \\
\hline & & & & & Upper. & Lower. & & \\
\hline$N, 2-4$ & 10 & $5-15$ & lie & 1 & Ci.-s. 4 & - & - & \\
\hline$x, 1-2$ & $i$ & $3-10$ & be & & Ci. 3 & - & - & \\
\hline NXI, 1-2 & 8 & $0-15$ & he & & Ci. 2 & - & - & \\
\hline Caln & 7 & 0 & b & & - & - & - & \\
\hline Caln & 0 & 0 & is & & - & - & - & \\
\hline Caluı & 2 & 0 & b & & - & - & - & \\
\hline Calm & 1 & 0 & $1, \infty$ & & - & - & - & $\begin{array}{l}\text { Large ('u, on White Island, aut Xim. to } \\
\text { F gud W. }\end{array}$ \\
\hline Caln & 2 & 0 & $b \infty$ & & - & - & - & 3 1.m. laiu band, 4 . \\
\hline $\mathrm{N}, 0-1$ & 4 & $5-10$ & le & & Ci.-s. 1. SW & - & - & Long Ci.-5. on NW horizon. \\
\hline $\mathrm{N}, 0-1$ & $b^{3}$ & $0-10$ & b & & - & - & - & \\
\hline Lt. N airs & 7 & $5-10$ & he & I & ci. 2, NW & - & - & \\
\hline Calm & 4 & $0-5$ & be & i & Ci.-eu. $4, \mathrm{NW}$ & - & - & \\
\hline- & - & - & - & & - & - & - & $\begin{array}{l}\text { Fine all day, with hardly auy wind. Barn- } \\
\text { meter rose until } 2 \text { jom., theu foil siowis. }\end{array}$ \\
\hline ar radiatiou $\left\{\begin{array}{l}b \\
b\end{array}\right.$ & k & Ih & & & - grammes. & recipun. & & Tomperature stea \\
\hline
\end{tabular}

17 th.

\begin{tabular}{|c|c|c|c|c|c|c|c|c|c|c|c|c|c|c|c|}
\hline 2 & $29 \cdot 868$ & - & $14 \cdot 0$ & $16^{\circ} \cdot 0$ & $15 \cdot 8$ & - & NH, 1 & 4 & 5 & oc & I & - & A.-cu. 9 & - & \\
\hline 4 & $29 \cdot 848$ & - & $15 \cdot 0$ & $17^{\circ} 0$ & $16 \cdot 0$ & - & Lt. $\mathcal{E}$ airs & 0 & 0 & oc & & - & A.-૯u. 9 & - & \multirow{8}{*}{$\begin{array}{l}\text { Very bright to } \mathrm{S} \text { and } \mathrm{W} \text {. } \\
\text { Suowing lightly. } \\
\text { Hearg Nim. ou western horizon. } \\
3 \text { pom. liain baud, } 5 \text {. } \\
\text { Very lull and threateniug, with beavy } \\
\text { sutface drift. }\end{array}$} \\
\hline 8 & $29 \cdot 832$ & - & $14 \cdot 8$ & 170 & $16 \cdot 2$ & - & It. $X$ airs & 11 & 0 & oc $*$ & & - & న. 10 & - & \\
\hline 8 & $29 \cdot 828$ & - & $15 \cdot 0$ & $17 \cdot 3$ & 16.8 & - & Lt. $N$ airs & 2 & 0 & oc $*$ & & - & N. 10 & - & \\
\hline 10 & $29 \cdot 832$ & - & $17 \cdot 1$ & $18 \cdot 3$ & $18 \cdot 0$ & - & Calm & 0 & 0 & o & & - & S. 10 & - & \\
\hline Noon & $29 \cdot 818$ & - & $18 \cdot 0$ & $18 \cdot 1$ & $16 \cdot 1$ & - & NE, 0-1 & 0 & $0-5$ & o & & - & S. 10 & - & \\
\hline 2 & $29 \cdot 907$ & - & $16 \cdot 0$ & $17 \cdot 2$ & $16 \cdot 3$ & - & ENE, 1-2 & 6 & $5-10$ & ue & & - & N. 10 & - & \\
\hline 4 & $29 \cdot 810$ & - & $10 \cdot 0$ & $11 \cdot 3$ & $10^{\circ} 0$ & - & Var. E, 2-3 & 12 & $20-25$ & $o c t$ & & - & S.-cu. 10 & - & \\
\hline 6 & $-29 \cdot 409$ & - & $8 \cdot 0$ & $9 \cdot 0$ & $8 \cdot 2$ & - & KSF, 2-3 & 17 & $20-25$ & $\alpha$ & & - & S.-cul. 10 & - & \\
\hline 8 & $29 \cdot 799$ & - & 6.0 & $12 \cdot 0$ & $11 \cdot 2$ & - & Var. E, 2-4 & 24 & $20-30$ & $\alpha+$ & & - & S.-eut. 10 & - & \\
\hline 10 & $29 \cdot 818$ & - & $5 \cdot 7$ & $6 \cdot 6$ & $6 \cdot 5$ & - & $\mathrm{ENE}_{2}, 2-4$ & 16 & $25-30$ & $0+$ & & - & S. 10 & - & \\
\hline Midt. & $29 \cdot 813$ & -1 & $5 \cdot 5$ & $6 \cdot 2$ & $6 \cdot 1$ & - & Var. $\mathbf{F}, 3-5$ & 21 & $25-35$ & $0+$ & & - & S.-cu. 10 & - & \\
\hline Mean & $29 \cdot 824$ & - & - & $13 \cdot 8$ & $13 \cdot 1$ & - & - & - & - & - & & - & - & - & 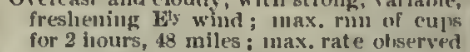 \\
\hline & ex. th & & . & $8 a$ & 8. & & lar radintion \{ & 18 & 11, & & Eve & mmes. & 1'recipn.- - in. & & $\begin{array}{l}\text { hy pressure tube, } 35 \text { milus per lour. } \\
\text { Barometer fell slow ly during day. Ten- } \\
\text { perature fell towards evening. }\end{array}$ \\
\hline
\end{tabular}

18 th.

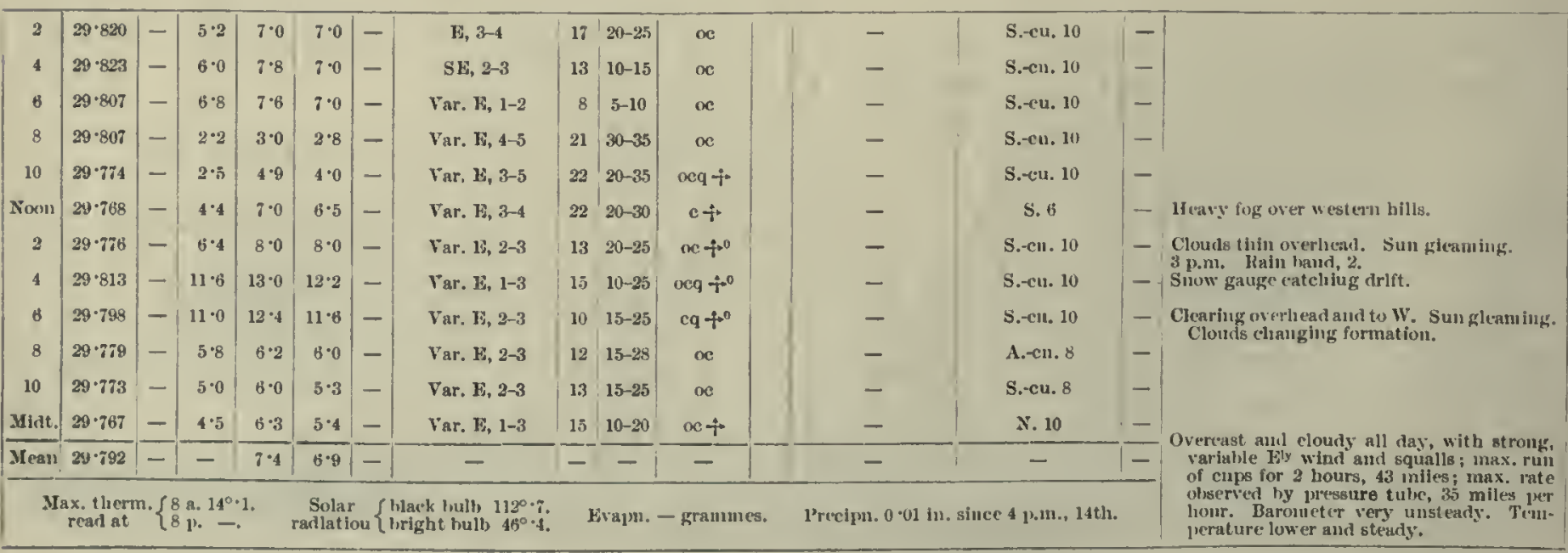




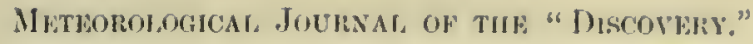

[1902-November.

\begin{tabular}{|c|c|c|c|c|c|c|c|c|c|c|c|c|c|c|c|}
\hline \multirow{3}{*}{ Hlour. } & \multirow{3}{*}{ 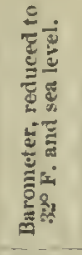 } & \multicolumn{5}{|c|}{ Thermonet ers. } & \multicolumn{3}{|l|}{ Wint. } & \multirow[b]{3}{*}{ 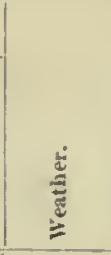 } & \multirow[b]{3}{*}{ 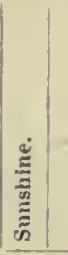 } & \multirow{2}{*}{\multicolumn{2}{|c|}{$\begin{array}{l}\text { Clouds, } \\
\text { Amonut and mavement. }\end{array}$}} & \multirow[b]{3}{*}{$\frac{5}{\frac{5}{2}}$} & \multirow{3}{*}{ Memarks. } \\
\hline & & \multirow{2}{*}{ 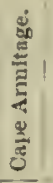 } & \multirow{2}{*}{ 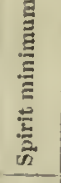 } & \multicolumn{2}{|c|}{ Slereullal. } & \multirow{2}{*}{ 竞 } & \multirow{2}{*}{$\begin{array}{l}\text { Directlou aud } \\
\text { foree } \\
\text { (Beanfort seale). }\end{array}$} & \multirow{2}{*}{ 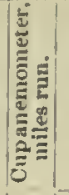 } & \multirow{2}{*}{ 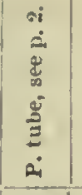 } & & & & & & \\
\hline & & & & Dry. & Wet. & & & & & & & Upper. & Lower. & & \\
\hline 2 & $29 \cdot 772$ & -1 & $3 \cdot 8$ & 18 & 4.7 & - & EN1;, 2-3 & $9 !$ & $10-20$ & $n+*$ & & - & N. 10 & - & \multirow[t]{2}{*}{ Sllght snow. Suu gleaming. } \\
\hline 4 & $2 n-i b i$ & -1 & $3 \cdot 9$ & $5 \cdot 4$ & $5 \cdot 2$ & - & HNE, 3-4 & 13 & $25-30$ & $x: f * *$ & & - & N. 10 & - & \\
\hline ii & 2458 & - & 4.8 & $i \cdot 0$ & 6.8 & - & NW, 2-3 & 13 & $15-20$ & $\propto *$ & & - & N. 8 & - & Snowlug sllghtly, ans hazy. \\
\hline s & $24 \cdot 775$ & - & $0 \cdot 0$ & $7 \cdot 0$ & 6.8 & - & Var, E, 1-2 & 10 & $10-15$ & bc $*$ & & A.-8. $3, \mathbf{E}$ & $\mathrm{Cu}, 4, \mathrm{SE}$ & - & \multirow{3}{*}{$\begin{array}{l}\text { Weatern mountalus oluscured loy thlek } \\
\text { haze. Snowing lightly at tlines. }\end{array}$} \\
\hline 10 & $29 \cdot 752$ & - & $6 \cdot 2$ & $8 \cdot 0$ & $7 \cdot 8$ & - & $\mathrm{N}, 1-2$ & 11 & $10-15$ & lie & & Cl. 2 & - & - & \\
\hline $\mathrm{N} n \mathrm{non}$ & $29 \cdot 764$ & -1 & $5 \cdot 0$ & $8 \cdot 0$ & 78 & - & $E, 1-2$ & 11 & $10-15$ & be & & Ci.-s. 4 & - & - & \\
\hline 2 & $29 \cdot 799$ & - & $5 \cdot 7$ & $6 \cdot 4$ & $6 \cdot 4$ & - & $\operatorname{Var} . \mathbf{E}, 1-3$ & 14 & $10-25$ & beg + & & - & Cu. B, NF & - & \multirow{3}{*}{ 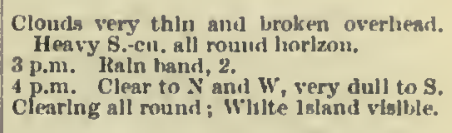 } \\
\hline 4 & $29 \cdot 806$ & - & $5 \cdot 2$ & $6 \cdot 0$ & $5 \cdot 5$ & - & ENE, 2-4 & 15 & $15-30$ & heq $+\infty$ & & - & Cu. $t, \mathrm{NE}$ & - & \\
\hline a & $29 \cdot 810$ & -1 & $4 \cdot 5$ & $5 \cdot 4$ & $5 \cdot 0$ & $8 \cdot 2$ & F by $N, 1-3$ & 17 & $10-25$ & $\operatorname{leq} \infty$ & 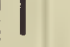 & Ci..8. 2 & S.-cu, ${ }_{2}, \mathrm{NH}$ & - & \\
\hline 8 & $20 \cdot 814$ & - & $4 \cdot 2$ & $5 \cdot 2$ & $5 \cdot 0$ & $8 \cdot 5$ & Var. E, 2-3 & 11 & $15-20$ & be & & Cl.-8. 4, W & - & - & \multirow{3}{*}{$\begin{array}{l}\text { Clearing all round, except to } S \text { of white } \\
\text { lsland, whlch is wearly otseurell lil } \\
\text { milst. }\end{array}$} \\
\hline 10 & $29 \cdot 808$ & - & $4 \cdot 5$ & $5 \cdot 8$ & $5 \cdot 0$ & $5 \cdot 8$ & Var. E, 3-4 & 17 & $20-30$ & be & & $\mathrm{Cl}_{.-8 .} 4$ & - & - & \\
\hline Mrialt. & $29 \cdot 786$ & -1 & $+\cdot 2$ & $5 \cdot 2$ & $5 \cdot 2$ & $5 \cdot 3$ & Var. E, 3-4 & 11 & $20-28$ & bc & & $\mathrm{C}_{i} .8 .6$ & - & - & \\
\hline Mean & $29 \cdot 785$ & - & - & $6 \cdot 2$ & $5 \cdot 9$ & - & - & - & - & - & & - & - & - & \multirow{2}{*}{$\begin{array}{l}\text { Clously all day, with allght snow fall. } \\
\text { Whit stlll strong and gqually from } \mathrm{E} \\
\text { Barometer steady. Temnetratire stendy } \\
\text { and low. }\end{array}$} \\
\hline & ern & & & & & & $\left\{\begin{array}{l}\text { lilack bull, } \\
\text { bright bult }\end{array}\right.$ & & & & & \multicolumn{3}{|c|}{ Preejpn. 0.01 in. slnce 4 p.m. 18 th. } & \\
\hline
\end{tabular}

19 th.

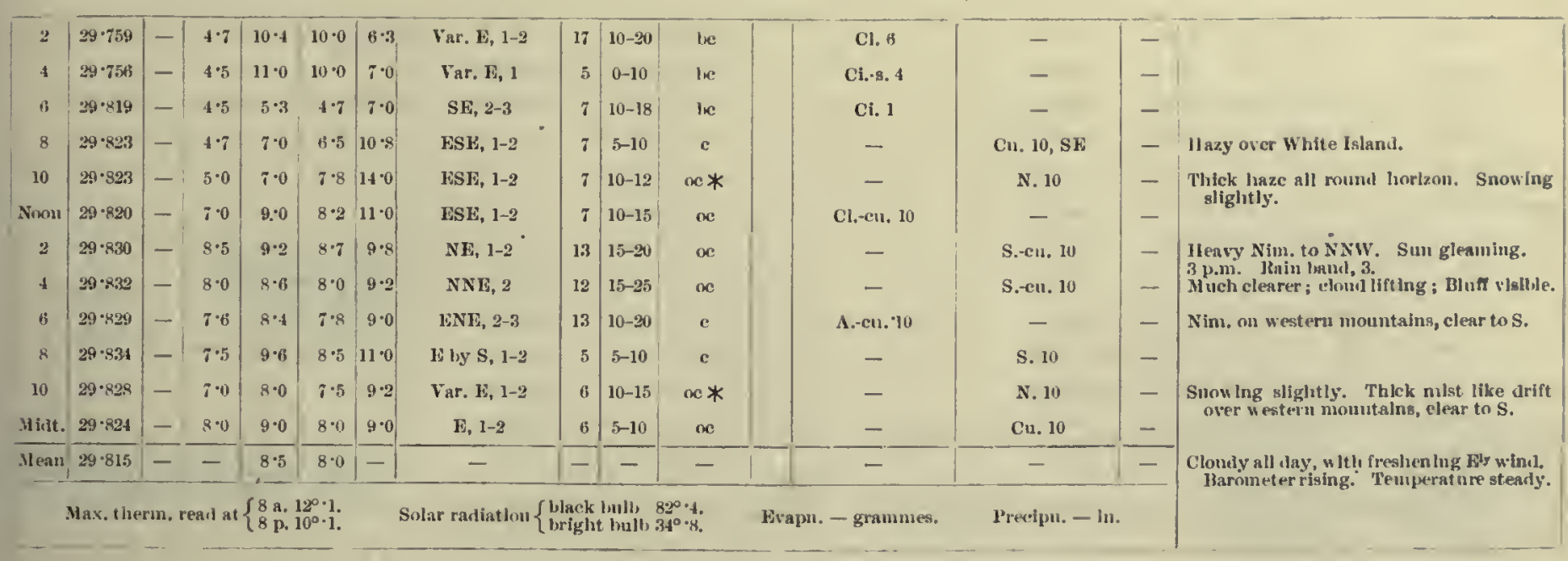

20 th.

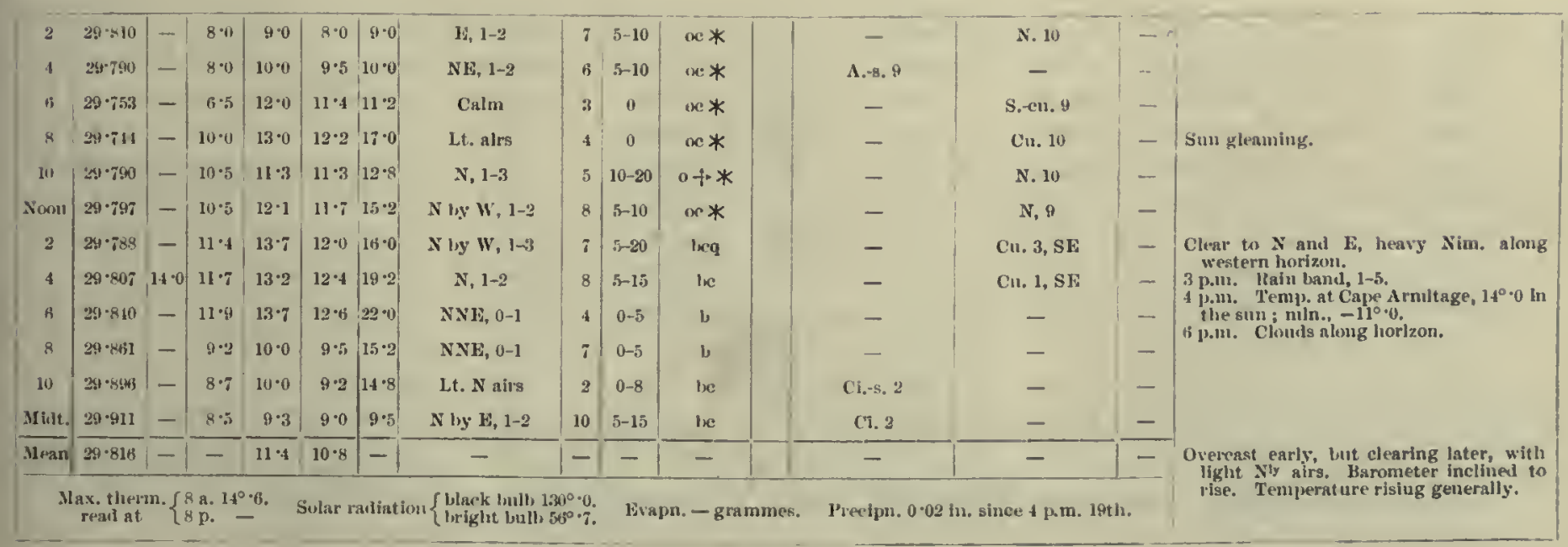


1902-November.]

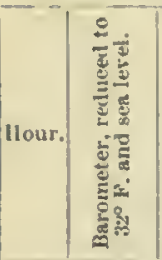

22nd.
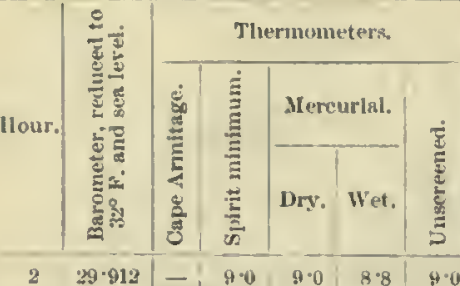

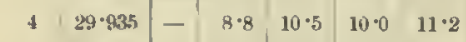

$\begin{array}{llllllll}6 & 29 \cdot 913 & - & 9 \cdot 0 & 11 \cdot 0 & 10 \cdot 0 & 11 \cdot 3\end{array}$

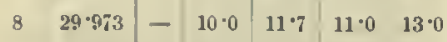

$\begin{array}{ll}10 & 29 \cdot 918\end{array}$

Xoon $30 \cdot 018-11 \cdot 8 \quad 13 \cdot 2 \quad 12 \cdot 5 \quad 16 \cdot 2$

$\begin{array}{llllllll}2 & 30.052 & - & 12.4 & 14.6 & 13.7 & 20.3\end{array}$

$+30 \cdot 057-13 \cdot 214 \cdot 413.8 \quad 17 \cdot 6$

i) $30.061-13 \cdot 7,15 \cdot 0,14 \cdot 0 \quad 16 \cdot 0$

\begin{tabular}{llll|ll}
8 & 30.071 & $-13 \cdot 6$ & 14.5 & $14 \cdot 0$ & $15 \cdot 4$
\end{tabular}

$10|30 \cdot 108-8.5 \quad 14 \cdot 5| 14 \cdot 0 \quad 15 \cdot 0$

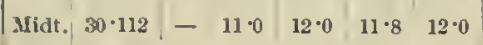

3lean $30 \cdot 016--\frac{12 \cdot 7}{12 \cdot 0}-$

Max. therm. read at $\left\{\begin{array}{l}8 \text { a. } 14^{\circ} \cdot 0 . \\ 8 \text { p. } 15^{\circ} \cdot 6 \text {. }\end{array}\right.$
Metrorological, Jouksal of the "Discovery."

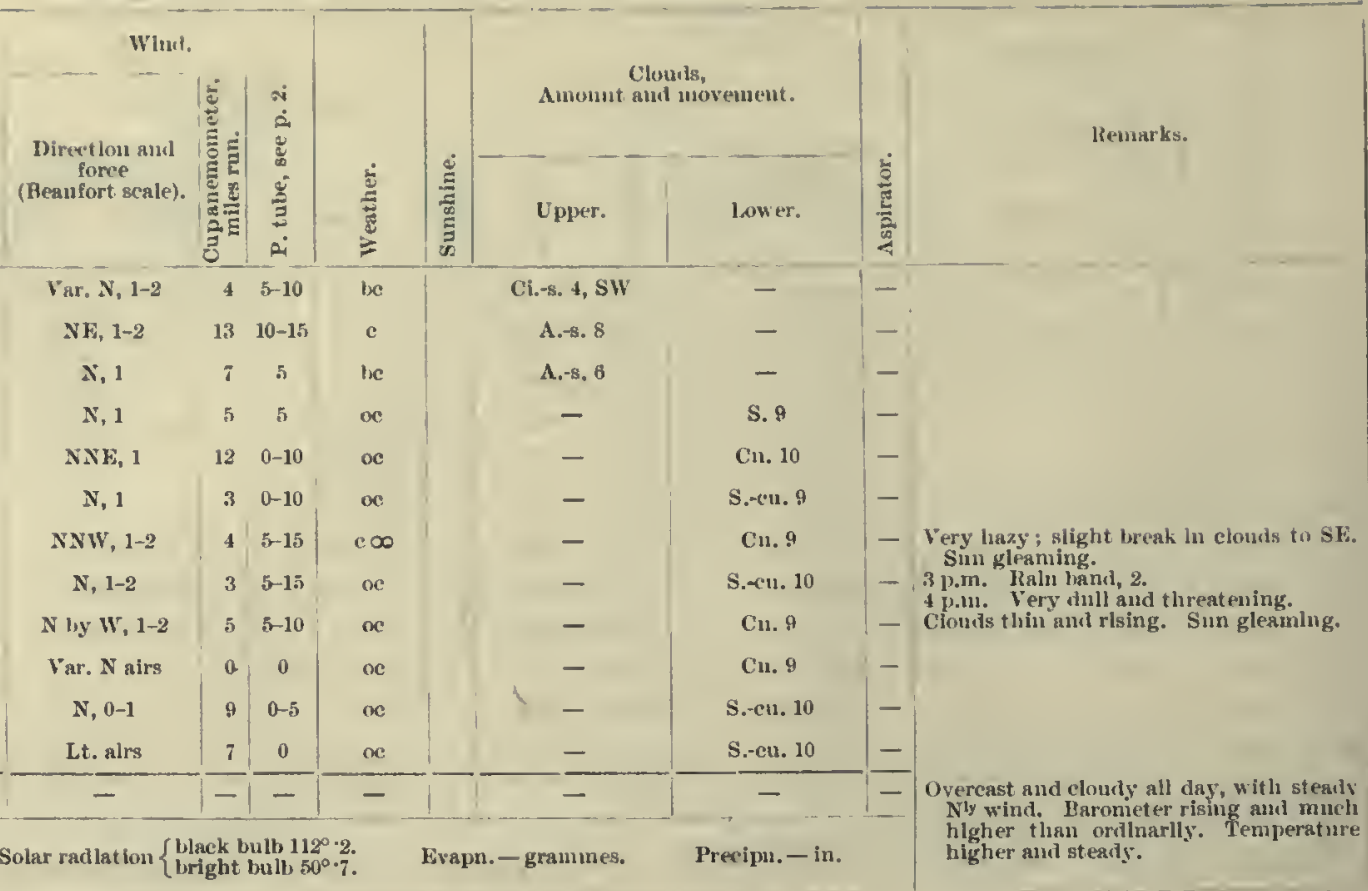

23rd.

\begin{tabular}{|r|r|r|r|r|r|r|}
\hline 2 & $30 \cdot 103$ & - & $11 \cdot 3$ & $14 \cdot 0$ & $14 \cdot 0$ & $14 \cdot 2$ \\
4 & $30 \cdot 099$ & - & $8 \cdot 8$ & $10 \cdot 1$ & $10 \cdot 0$ & $18 \cdot 4$ \\
6 & $30 \cdot 097$ & - & $9 \cdot 0$ & $15 \cdot 0$ & $15 \cdot 0$ & $11 \cdot 2$ \\
8 & $30 \cdot 084$ & - & $13 \cdot 0$ & $13 \cdot 2$ & $13 \cdot 0$ & $27 \cdot 0$ \\
10 & $30 \cdot 057$ & - & $13 \cdot 8$ & $16 \cdot 0$ & $15 \cdot 8$ & $26 \cdot 5$ \\
Nnon & $30 \cdot 045$ & - & $16 \cdot 8$ & $20 \cdot 0$ & $19 \cdot 2$ & $28 \cdot 0$ \\
2 & $30 \cdot 029$ & - & $16 \cdot 4$ & $20 \cdot 0$ & $20 \cdot 0$ & $24 \cdot 0$ \\
4 & $29 \cdot 995$ & - & $18 \cdot 6$ & $20 \cdot 4$ & $18 \cdot 8$ & $23 \cdot 2$ \\
6 & $29 \cdot 971$ & - & $19 \cdot 0$ & $20 \cdot 0$ & $19 \cdot 4$ & $21 \cdot 7$ \\
8 & $29 \cdot 940$ & - & $19 \cdot 0$ & $19 \cdot 8$ & $19 \cdot 8$ & $20 \cdot 2$ \\
10 & $29 \cdot 905$ & - & $17 \cdot 3$ & $18 \cdot 2$ & $18 \cdot 0$ & $18 \cdot 8$ \\
Midt. & $29 \cdot 866$ & - & $17 \cdot 8$ & $19 \cdot 0$ & $18 \cdot 5$ & $19 \cdot 0$ \\
\hline Mleanl & $30 \cdot 016$ & - & - & $17 \cdot 1$ & $16 \cdot 8$ & - \\
\hline
\end{tabular}

Mlax. therm. read at $\left\{\begin{array}{l}8 \text { a. } 17^{\circ} \cdot 5 . \\ 8 \text { p. } 21^{\circ} \cdot 8 .\end{array}\right.$

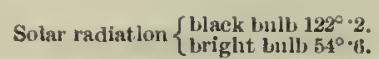

\begin{tabular}{l|c|c|c} 
N, 0-1 & 1 & $0-5$ & be \\
Calm & 2 & 0 & be \\
Calu & 0 & 0 & be \\
Caln & 0 & 0 & be \\
Lt. N airs & 0 & 0 & oc \\
Calm & 0 & 0 & oc \\
NNW, 0-1 & 4 & $0-10$ & e \\
N, 1 & 2 & $5-10$ & $c$ \\
N, 1 & 7 & $5-10$ & c \\
Lt. N airs & 4 & 5 & c \\
Lt. NW airs & 4 & 0 & c \\
N, 1 & 7 & 5 & c \\
\hline - & - & - & - \\
\hline
\end{tabular}

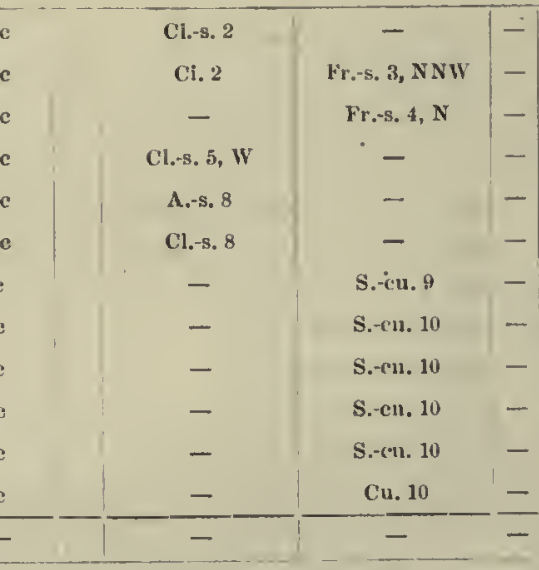

Clondy most of day, with hardly any wind. Barometer filling rather qulcki

Erapn. - grammes.

Precipn. - in Suu gleaming. Nim. to SF. Fog liwuz ing on Crater Ilill.

3-p.m. Rain band, 3 .
4 p.m. Low lank of cumulus on west eru

6 p.m. Clearing to W. Sum gleaning. all day. Temperature ligher.
24 th

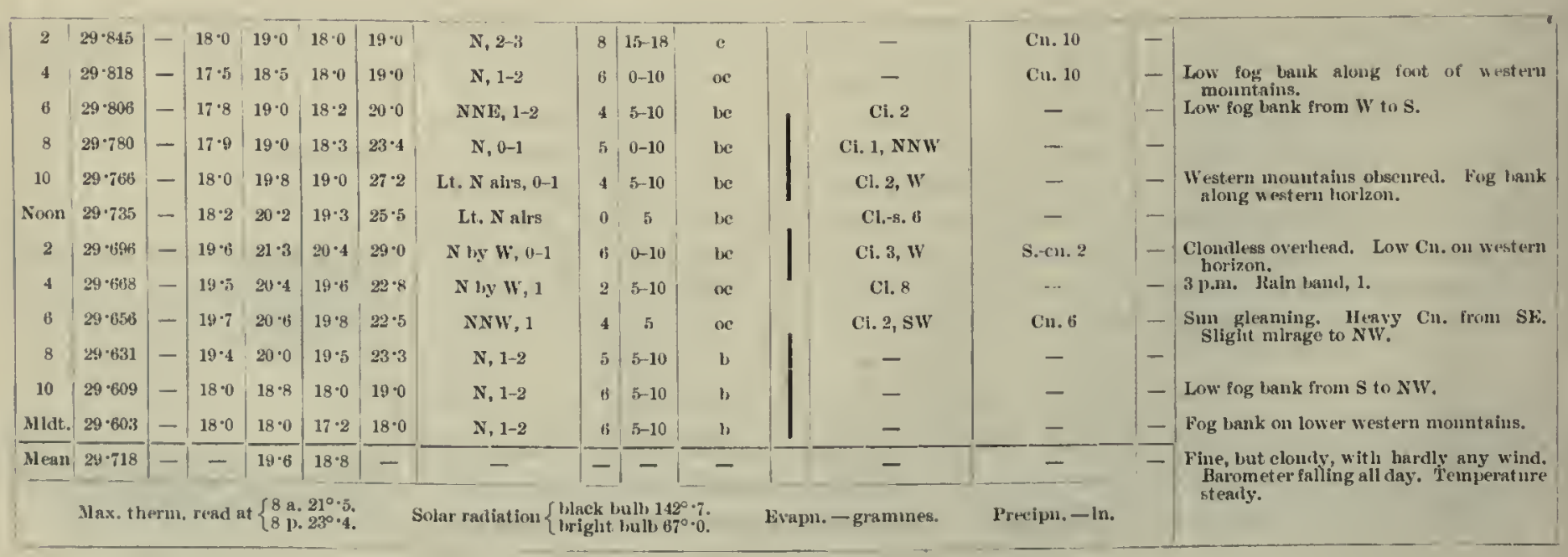




\begin{tabular}{|c|c|c|c|c|c|c|c|c|c|c|c|c|c|c|c|}
\hline \multirow[b]{2}{*}{ Hour. } & \multirow{2}{*}{ 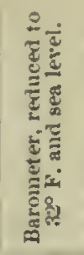 } & \multicolumn{5}{|c|}{ Thermoneters. } & \multicolumn{3}{|l|}{ Whud. } & \multirow[b]{2}{*}{ 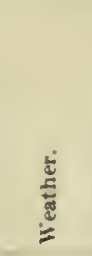 } & \multirow[b]{2}{*}{ 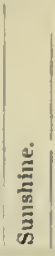 } & \multicolumn{2}{|c|}{$\begin{array}{l}\text { Clomin, } \\
\text { Amout and unoverneut. }\end{array}$} & \multirow[b]{2}{*}{ 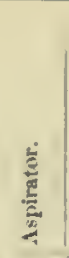 } & \multirow[b]{2}{*}{ Itr-mark\%. } \\
\hline & & 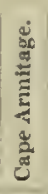 & 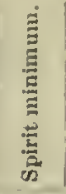 & $\begin{array}{l}\text { Mere } \\
\text { Dry. }\end{array}$ & Wet. & 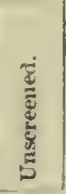 & $\begin{array}{l}\text { Direction and } \\
\text { force } \\
\text { (Beanfort seale). }\end{array}$ & 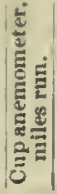 & 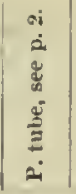 & & & Amosunt a & il moverneut. & & \\
\hline 2 & $29 \cdot 5 \cdot 19$ & - & $15 \cdot 3$ & $17 \cdot 0$ & $16 \cdot 2$ & $17 \cdot 0$ & $\mathrm{~N}, 1-2$ & 6 & $5-10$ & b & & - & - & - & \multirow{6}{*}{ 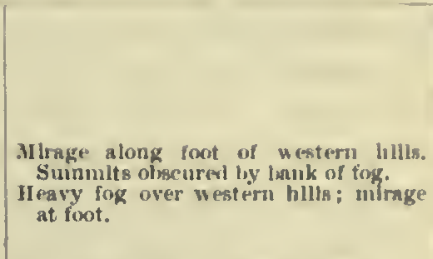 } \\
\hline 1 & $29 \cdot 541$ & - & $15 \cdot 3$ & $16 \cdot 8$ & $16 \cdot 0$ & $17 \cdot 4$ & $\mathrm{~N}, 1-2$ & 6 & $5-10$ & $b$ & & - & - & - & \\
\hline b & $29 \cdot 555$ & - & $14 \cdot 8$ & $16 \cdot 8$ & $16 \cdot 5$ & $18 \cdot 8$ & Lt. $N$ alrs & 4 & 0 & b & & - & - & - & \\
\hline 8 & $29 \cdot 563$ & - & $15 \cdot 0$ & $16 \cdot 8$ & $16 \cdot 0$ & $18 \cdot 5$ & Cahn & 2 & 0 & les & & - & S. 4 & - & \\
\hline 10 & $29 \cdot 563$ & - & $16 \cdot 0$ & $18 \cdot 5$ & $18 \cdot 2$ & $29 \cdot 0$ & Caim & 0 & 0 & be & 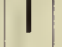 & C1.-8. 2 & A.-en. $3, x$ l, W W & - & \\
\hline Nion & $29 \cdot 552$ & - & $11 \cdot 5$ & $12 \cdot 0$ & $11 \cdot 7$ & $13 \cdot 0$ & NE, 2-3 & 9 & $10-20$ & $\infty$ & & - & C'u. 10, NNE & - & \\
\hline 2 & $29 \div 551$ & - & $9 \cdot 2$ & $10 \cdot 0$ & $9 \cdot 8$ & $13 \cdot 0$ & FNE, 2-3 & 14 & $10-20$ & $\infty *$ & & - & N. 10 & - & \multirow{5}{*}{ 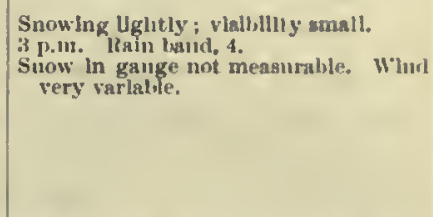 } \\
\hline+ & $29 \cdot 558$ & - & 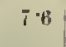 & $8 \% 0$ & $7 \cdot 8$ & $10 \cdot 0$ & Var. 13, 3-4 & 13 & $15-20$ & $\propto *$ & & - & N. 10 & 一 & \\
\hline is & $29 \cdot 559$ & - & $6 \cdot 0$ & $7 \cdot 2$ & $7 \cdot 0$ & $7 \cdot 8$ & Var. F, 4-5 & 20 & $25-35$ & $\alpha e+$ & & - & N. 10 & - & \\
\hline 8 & $29 \cdot 356$ & - & 4.5 & $5 \cdot 0$ & $5 \cdot 0$ & $5 \cdot 0$ & E by N, 4-5 & 19 & $25-30$ & nef & & - & N. 10 & - & \\
\hline 10 & $29 \cdot 5 \pi 9$ & - & $3 \cdot 5$ & $4 \cdot 3$ & $4 \cdot 2$ & $4 \cdot 5$ & Var. IV, $3-5$ & 21 & $20-30$ & oeq + & & - & N. 10 & - & \\
\hline Mint. & $29 \cdot 589$ & - & $2 \cdot 6$ & $3 \cdot 2$ & $3 \cdot 0$ & $3 \cdot 0$ & F, 4-5 & 18 & $25-35$ & $n+4$ & & - & N. 10 & - & \multirow{3}{*}{$\begin{array}{l}\text { West ern hlll obscurenl hy fog. Clear } \\
\text { to S. } \\
\text { Overeast and cloudy nearly all day. } \\
\text { Llght wind in forenom, fresisenlng to } \\
\text { strong from fo for the rest of the day } \\
\text { max. run of cups for } 8 \text { hours, } 48 \text { mlles } \\
\text { max. rate observer liy pressure tube, } \\
35 \text { miles per hour. Barometer st ealy, } \\
\text { incllned to rlse. Tempernt ure falling } \\
\text { all day. }\end{array}$} \\
\hline Mean & $29 \cdot 560$ & - & - & $11 \cdot 3$ & $11 \cdot 0$ & - & - & $\overline{-}$ & - & - & & - & - & - & \\
\hline & Max. th & n. & regul & t $\{8 \mathrm{~B}$ & $20^{\circ} \cdot 5$. & & Solar ralliatlon \{ & ck & ib & - 0. & 8 & - grammues. & Precipn, - In. & & \\
\hline
\end{tabular}

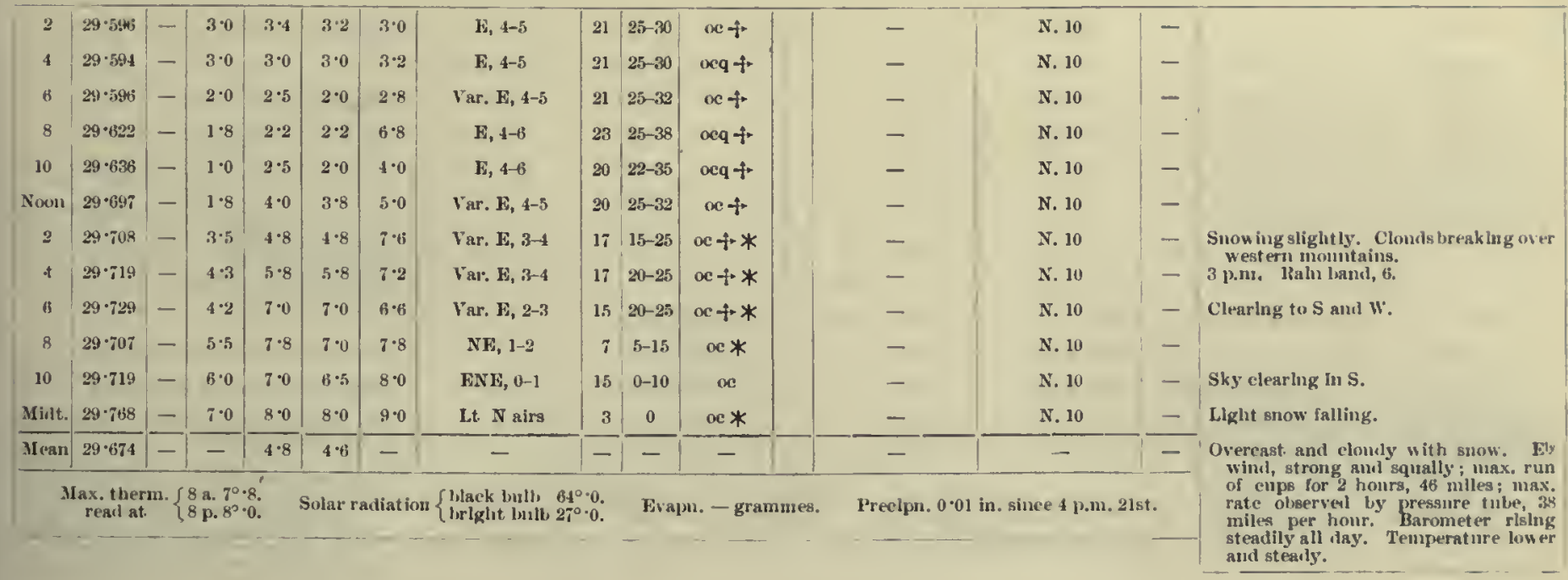

26 th.

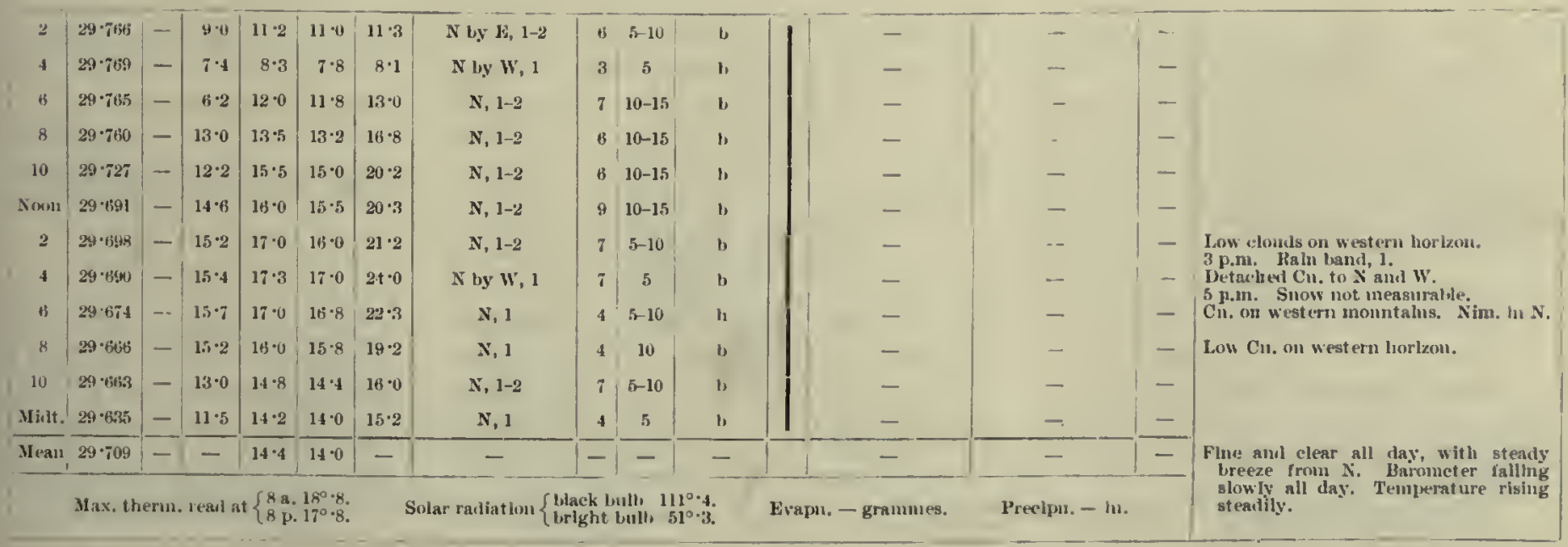


1902-November.

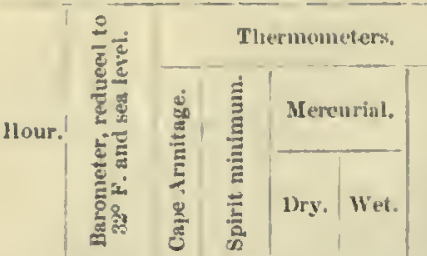

28 th.

$3 2 9 \cdot 6 0 9 - 1 2 \cdot 2 \longdiv { 1 1 0 0 }$

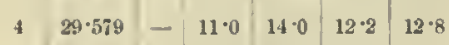

\begin{tabular}{ll|l|l|l|l|}
6 & $29 \cdot 571$ & $-11 \cdot 5$ & $16 \cdot 2$ & $16 \cdot 2$ & $16 \cdot 5$
\end{tabular}

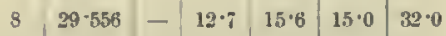

$10 \mid 29 \cdot 523-12 \cdot 5 \quad 10 \cdot 0 \quad 15 \cdot 8 \quad 22 \cdot 3$

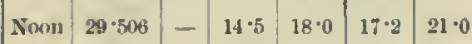

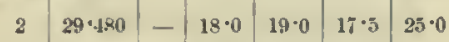

$4 \quad 29 \cdot 459-16 \cdot 5 \mid 17 \cdot 3 \quad 15 \cdot 8 \quad 23 \cdot 0$

b $29 \cdot 451-16 \cdot 0 \quad 20 \cdot 0 \quad 18 \cdot 4 \quad 22 \cdot 3$

$8 \quad 29 \cdot 451-19 \cdot 019 \cdot 017 \cdot 021 \cdot 8$

$10.29 \cdot 443-15 \cdot 4 \quad 15 \cdot 8 \quad 14 \cdot 2 \quad 14 \cdot 5$

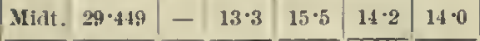

Yean $\overline{29 \cdot 506}-\overline{16 \cdot 7} \overline{15 \cdot 6}-$

Max. therm. reat at $\left\{\begin{array}{l}8 \text { a. } 18^{\circ} \cdot 2 . \\ 8 \text { p. } 23^{\circ} \cdot 0 .\end{array}\right.$
Meteokolofical Jotrnal, of the "Discoveliy."

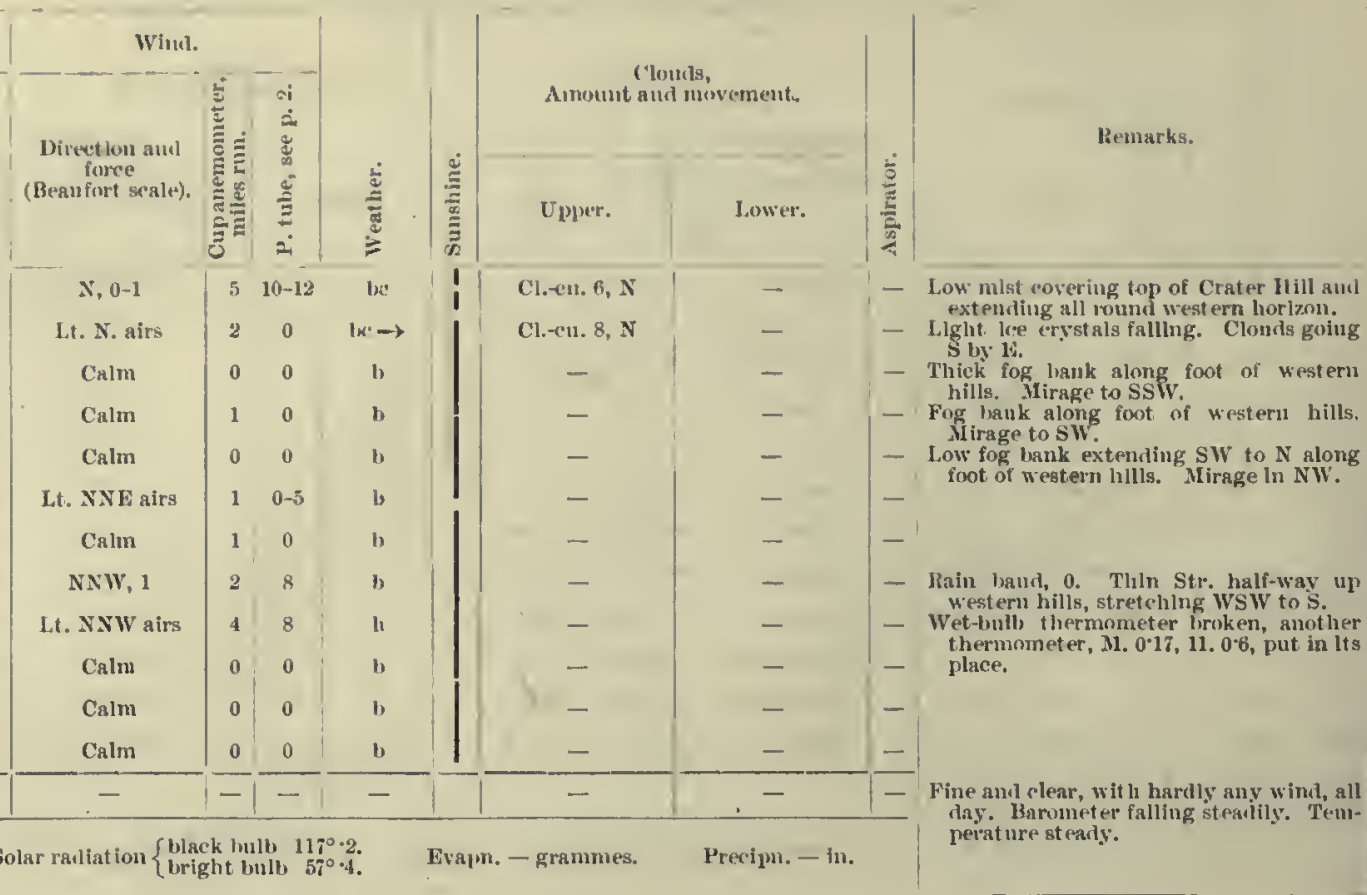

29th.

\begin{tabular}{|c|c|c|c|c|c|c|c|c|c|c|}
\hline 2 & $29 \cdot 411$ & - & $9 \cdot 8$ & $10^{\circ} 2$ & $10 \cdot 2$ & $11 \cdot 5$ & Calm & 0 & 0 & b \\
\hline 4 & $29 \cdot 458$ & - & $5 \cdot 0$ & $14 \cdot 0$ & $14 \cdot 0$ & $15 \cdot 0$ & SE, 1-2 & 5 & $10-15$ & b \\
\hline if & $29 \cdot 485$ & - & $11 \cdot 8$ & $16 \cdot 0$ & $15 \cdot 2$ & $15 \cdot 8$ & SF, 1-2 & 3 & $10-15$ & b) \\
\hline 8 & $29 \cdot 517$ & - & $12 \cdot 2$ & $14 \cdot 2$ & $12 \cdot 8$ & $16 \cdot 2$ & NF, 1-2 & 10 & $10-15$ & b \\
\hline 10 & $29 \cdot 536$ & - & $12 \cdot 0$ & $13 \cdot 2$ & $12 \cdot 0$ & $17 \cdot 2$ & $\mathbf{E}, 3-4$ & 13 & $20-25$ & b \\
\hline Somn & $29 \cdot 545$ & - & $11 \cdot 5$ & $11 \cdot 8$ & $11 \cdot 0$ & $18 \cdot 0$ & $F, 4-5$ & 15 & $23-28$ & b) \\
\hline 2 & $29 \cdot 55 \times 6$ & - & $10 \cdot 0$ & $11 \cdot 2$ & $10 \cdot 0$ & $16 \cdot 0$ & N by N, 4-5 & 20 & $20-26$ & $11+4$ \\
\hline 4 & $29 \cdot 577$ & - & $10 \cdot 0$ & $10 \cdot 8$ & $10 \cdot 0$ & $13 \cdot 8$ & E hy X, 5-6 & 21 & $25-35$ & $b-4$ \\
\hline 6 & $29 \cdot 594$ & - & $10 \cdot 0$ & $11 \cdot 4$ & $10 \cdot 2$ & $13 \cdot 0$ & E by $\mathbf{N}, 5-6$ & 23 & $28-31$ & lict- \\
\hline 8 & $29 \cdot 629$ & - & $10^{\circ} 0$ & $10 \cdot 5$ & $9 \cdot 6$ & $11 \cdot 0$ & F ly $\mathrm{N}, 4-6$ & 18 & $25-30$ & be \\
\hline 10 & $29 \cdot 13 \dot{3} 1$ & - & $12 \cdot 2$ & $13 \cdot 8$ & $12 \cdot 8$ & $12 \cdot 8$ & F by N, $3-4$ & 12 & $20-25$ & le \\
\hline Mllit. & $29 \cdot 685$ & - & $11 \cdot 2$ & $13 \cdot 6$ & $12 \cdot 0$ & $12 \cdot 0$ & E lo N, 1-2 & 7 & $10-15$ & lic \\
\hline Mеаи & $29 \cdot 555$ & -1 & - & $12 \cdot 6$ & $11 \cdot 7$ & - & - & - & - & 一 \\
\hline
\end{tabular}

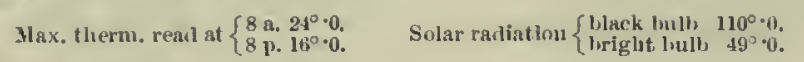

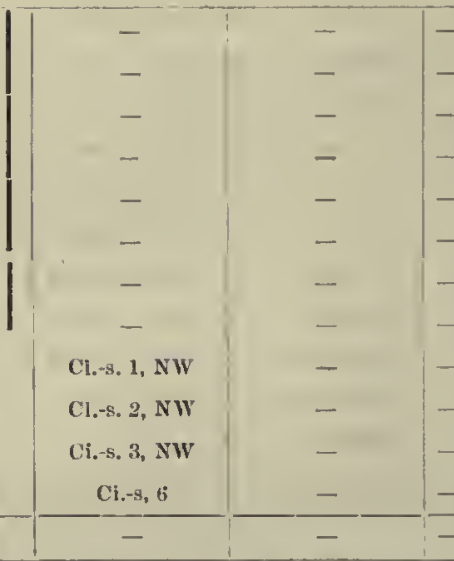

Evapm. - grammes.
Precipn. - іи.

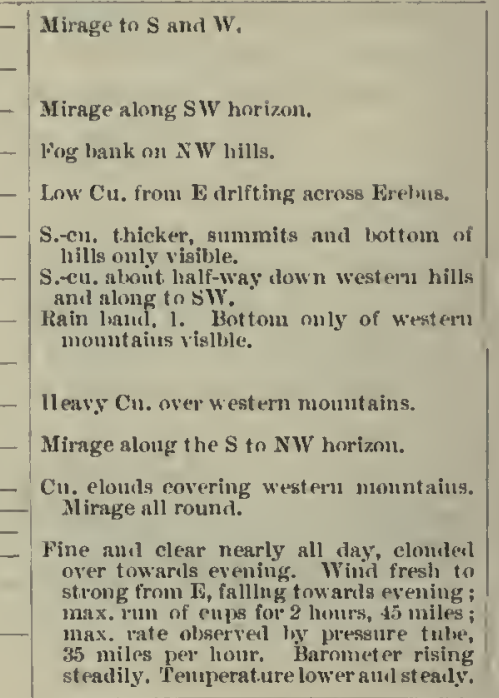

30 th.

\begin{tabular}{|c|c|c|c|c|c|c|}
\hline 2 & $28 \cdot 704 i$ & $-i$ & $10 \cdot 5$ & $11 \cdot 8$ & $11 \cdot 0$ & $14 \cdot 8$ \\
\hline 4 & $29 \cdot 730$ & -1 & $6 \cdot 2$ & $14 \cdot 2$ & $14 \cdot 2$ & $16 \%$ \\
\hline B & $29 \cdot 751$ & $-i$ & $12^{\circ} 0$ & $13 \cdot 5$ & $13 \cdot 0$ & $15 \cdot 0$ \\
\hline 8 & $29 \cdot 781$ & -1 & $11 \cdot 5$ & $13 \cdot 0$ & $12 \cdot 5$ & $22 \cdot 0$ \\
\hline 10 & $29 \cdot 814$ & -1 & $11 \cdot 5$ & $19 \cdot 0$ & $18 \cdot 0$ & $3 \cdot 0$ \\
\hline Xinou & $29 \cdot 822$ & - & $17 \cdot 0$ & $18 \cdot 0$ & $16 \cdot 2$ & $29 \cdot 0$ \\
\hline 2 & $29 \cdot 817$ & - & $18 \cdot 4$ & $20^{\circ} 0$ & $17 \cdot 0$ & $38 \cdot 5$ \\
\hline 4 & $29 \cdot 815$ & - & $18 \cdot 0$ & $18 \cdot 2$ & $16 \cdot 8$ & $28 \cdot 0$ \\
\hline (6) & $29 \cdot 813$ & - & $17 \cdot 5$ & $18 \cdot 6$ & $16 \cdot 5$ & $24 \cdot 0$ \\
\hline 8 & $29 \cdot \sin$ & - & $16 \cdot 6$ & $17 \cdot 5$ & $16 \cdot 2$ & $17 \cdot 0$ \\
\hline 10 & $29 \cdot 797$ & - & $16 \cdot 0$ & $17 \cdot 8$ & $10^{\circ} 0$ & $18 \cdot 0$ \\
\hline Mlist. & $29-727$ & -1 & $15 \cdot 0$ & $17 \cdot 0$ & $15 \cdot 3$ & $17 \cdot 0$ \\
\hline M cens! & $29 \cdot \div 82$ & - & - & $16 \cdot 6$ & $15 \cdot 2$ & - \\
\hline
\end{tabular}

lì $\tan \mathrm{N}, 2-3$

Calm

FS1, 1-2

Lt. $N$ alrs

S1: 1

1.t. SE airs

Lt. SI airs

Lt. $\mathbf{N}$ airs

$\mathrm{s}, 1$

$\mathrm{N}, 1$

$\mathrm{N}, 1$

N, 1-2 i 12-20 te $\rightarrow$

$1201 x \rightarrow$

5 10-15 oc

70 or

$3|8-10|$ oe

$+0-8$ oc

3) 0 be $\rightarrow$

1 i $0-5$ ise $\rightarrow$

410 le

5 7-10 is

| 5 | 10 化

$4+10-15$

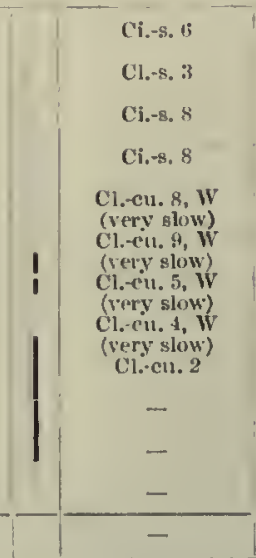

Mirage all romul lorlzon; Nin. clouds hauglug over' western moutut alus.

- Mirnge extending lis to $S$ to W.

Prismatle enlouring in clomis. Summit. of western uounfulng ohsemed all day.

Fr:eu, 2, N - Very henvy Cu, over western hills; slight Cu. 6, $N$ (fast) - wiitage ln S. laaiu bant, 2.

- Weary Cu. dark to $\mathbf{N}$, passing neer from $\mathbf{x}$, Very Sum slulning lu S, lleary Nius, in

Clourly all day, with light variable airs. Barometer rose unt il nom, then fell
slowly. Temperature stealy and ligher. 


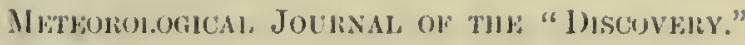

[1902-December.

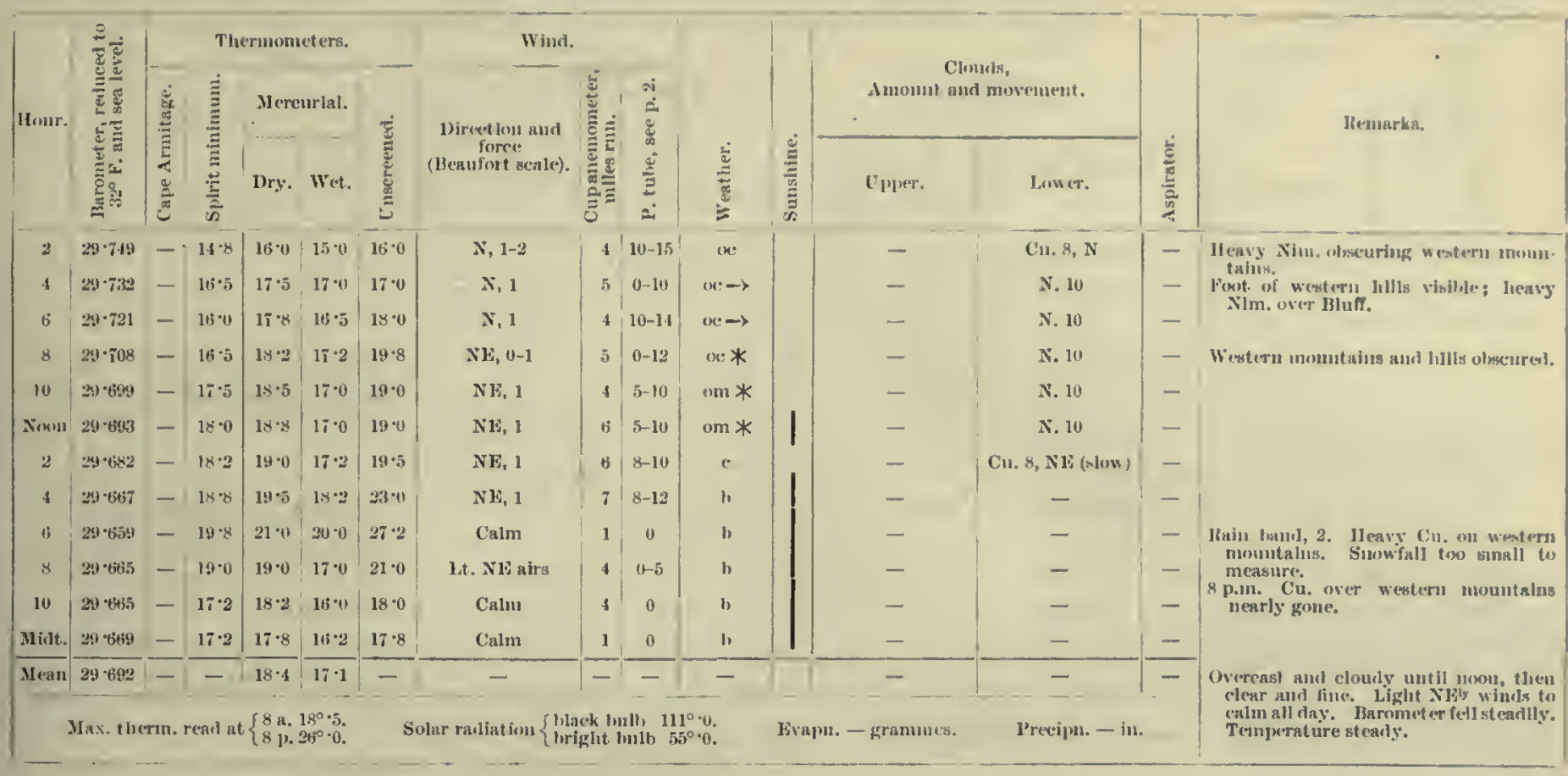

1 st.

\begin{tabular}{|c|c|c|c|c|c|c|c|c|c|c|c|c|c|c|}
\hline 2 & 24 6ั2 & -1 & $9 \cdot 5$ & 110 & $10 \cdot 2$ & $12 \cdot 2$ & Var. $\mathbf{E}, 2-3$ & 10 & $15-22$ & the & - & Cu. $8, \mathrm{NW}$ & - & \\
\hline 4 & $29 \cdot 678$ & - & $7 \cdot 0$ & $7 \cdot 2$ & $7 \cdot 0$ & $7 \cdot 0$ & Var. E, 3-4 & 10 & $20-30$ & $1 x+$ & - & Cu. $5, \mathrm{NW}$ & - & NW hills obseurest; low snowdrift. \\
\hline 6 & 29.896 & - & $9 \cdot 8$ & $10 \cdot 0$ & $10 \cdot 0$ & $11 \cdot 8$ & NE, 4-5 & 15 & $30-35$ & $10+$ & $\mathrm{Cl},-\mathrm{s}, 3, \mathrm{SE}$ & - & - & 11igh drift off hills to $\mathrm{k}$. \\
\hline 8 & $29 \cdot 737$ & - & $5 \cdot 0$ & $6 \cdot 0$ & $6 \cdot 0$ & 10.2 & ENF, 5-6 & 34 & $28-3 i$ & the fo & - & Fr-11. 2, E (fast) & - & Drlit from hill \\
\hline 10 & $29 \cdot 7 \overline{3} 3$ & -1 & 5.2 & 6.2 & 5.8 & $9 \cdot 8$ & 1:NE, 4-5 & 18 & $25-30$ & $1 x+$ & - & Fr--11. 3, E (fast) & - & $\begin{array}{l}\text { Tet wreen } \\
\text { leary d }\end{array}$ \\
\hline Xow & $29 \cdot 759$ & - & $5 \cdot 8$ & 6.8 & 6.2 & 9.8 & FiNE, 4-5 & 21 & $20-28$ & het. & - & Fr.-cu. 1, E & - & 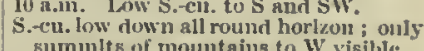 \\
\hline 2 & $29 \cdot 768$ & - & 8.5 & $7 \cdot 4$ & 6.8 & 10.0 & E hy $\mathbb{N}, 4-5$ & 20 & $20-29$ & tee & Cì.cu.s, sw & - & - & \\
\hline 4 & $29 \cdot 7 \pi$ & - & 10.8 & $7 \cdot 9$ & $7 \cdot 2$ & $8 \cdot 0$ & El, $\mathbf{N}, 3-4$ & 1.5 & $18-2 t$ & $x *$ & - & x. 10 & - & \\
\hline 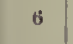 & $29 \cdot 775$ & - & $7 \cdot 5$ & 8.0 & $i \cdot 5$ & $8 \cdot 5$ & 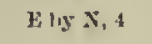 & 18 & $22-2 i$ & $\infty+*$ & - & Cu. 10 & - & 1hain band, 3. \\
\hline 8 & $29 \cdot 769$ & - & $i \cdot 0$ & $8 \cdot 0$ & $7 \cdot 5$ & 8.0 & ENH, 3-4 & 18 & $20-25$ & $\infty$ & - & Cu. 10 & - & \\
\hline 10 & $29 \cdot 760$ & - & 6.0 & $7 \cdot 2$ & $7 \cdot 0$ & $7 \cdot 0$ & ENE, 4 & 13 & $20-25^{\circ}$ & $a \leftrightarrow$ & - & Cu. 10 & - & Sky clearing in $\mathrm{S}$. \\
\hline sidt. & $29 \cdot 758$ & $=1$ & $6 \cdot 0$ & $7 \cdot 2$ & 6.2 & $7 \cdot 0$ & IENE, 4 & 17 & $20-25$ & $x+* *$ & - & Cu. 10 & - & 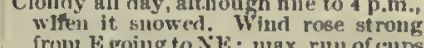 \\
\hline ylean & $29 \cdot 742$ & - & - & $i \cdot 7$ & $5 \cdot 3$ & - & - & - & - & - & - & - & - & 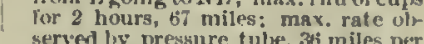 \\
\hline & $M_{\mathrm{s}}$ & & & $\begin{array}{l}8 \mathrm{ag} . \\
8 \mathrm{n} .\end{array}$ & & & thiou & & & & & p'recipu. - is & & 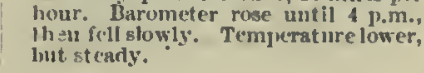 \\
\hline
\end{tabular}

2nd.

\begin{tabular}{|c|c|c|c|c|c|c|c|c|c|c|c|c|c|c|}
\hline 2 & $29 \cdot 743$ & - & $5 \cdot 0$ & 150 & 5.8 & $6 \%$ & ENE, $4-5$ & $15:$ & $24-28$ & ๓* & - & Cu. 10 & - & \multirow{4}{*}{ 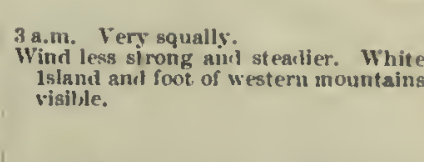 } \\
\hline 4 & $29 \cdot 721$ & - & $4 \cdot 8$ & 52. & 30 & $5 \cdot 0$ & Var. E, 4-5 & $11=$ & $20-25$ & ort** & - & Cu. 10 & - & \\
\hline 6 & $29 \cdot 713$ & - & 4.5 & $5 \cdot 2$ & $5 \cdot 2$ & 6.0 & Var. IE, 3-4 & 13 & $15-20$. & $\propto *$ & - & Cu. 10 & - & \\
\hline 8 & $29 \cdot 702$ & - & $4 \cdot 2$ & $7 \cdot 8$ & 8.0 & $9 \cdot 0$ & $\mathrm{NB}, 0-1$ & 12 & $0-12$ & $\infty$ & - & Cu. 10 & - & \\
\hline 10 & $29 \cdot 710$ & - & in & 8.5 & $8 \cdot 2$ & $12 \cdot 0$ & $\mathrm{NE}, 1$ & 6 & 10 & the & - & Fr.eru. 1 & - & \multirow{3}{*}{ 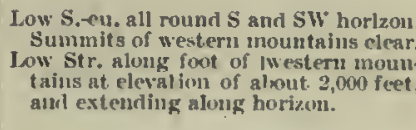 } \\
\hline Noon & 1 $23 \cdot 703$ & - & 8.8 & $13 \cdot 2$ & $12 \cdot 2$ & 20.5 & Calm & 4 & 0 & " & - & - & - & \\
\hline 2 & $29 \cdot 691$ & $1-$ & 10.8 & $14 \cdot 0$ & 13.0 & $20 \cdot 0$ & Lt. NE airs & 2 & $0-5$ & $"$ & - & - & - & \\
\hline 1 & $29 \cdot 695$ & - & $11 \cdot 0$ & $12 \cdot 6$ & $12 \cdot 0$ & $19 \cdot 2$ & Lt. N alrs & 4 & $j-\bar{i}$ & " & - & - & - & \multirow{3}{*}{ 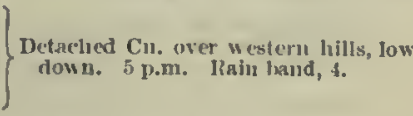 } \\
\hline 6 & $24 \cdot 679$ & - & $11 \cdot 5$ & 13.5 & $12 \cdot 1$ & $19 \cdot 2$ & $\mathrm{~N}, 1$ & 4 & $8-10$ & h & - & - & - & \\
\hline 8 & $29 \cdot 670$ & $1-$ & $12 \cdot 0$ & 140 & $13 \cdot 8$ & $17 \cdot 3$ & $\mathrm{~N}, 1$ & 4 & $8-10$ & 1. & - & - & - & \\
\hline 10 & $29 \cdot 665$ & -1 & $12 \cdot 2$ & $13 \cdot 0$ & $12 \cdot 2$ & $13 \cdot 0$ & $x \log 11,1$ & 5 & 10 & b) & - & -- & - & \multirow[t]{2}{*}{ Detachel Cu. to S and SW. } \\
\hline MIrtt. & $29-649$ & $=$ & $12 \cdot 0$ & $12 \cdot 0$ & $13 \cdot 0$ & $13 \cdot 0$ & $\mathrm{~N}$ l,y E, 1-2 & 6 & $10-15$ & " & - & $=$ & - & \\
\hline Mean & $29 \cdot 694$ & -1 & - & $10 \cdot 4$ & $9 \cdot 5$ & - & - & -1 & - & - & - & - & - & \multirow{2}{*}{ 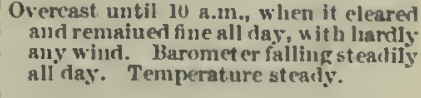 } \\
\hline & sit & & read at & $\left\{\begin{array}{l}8 \mathrm{a} . \\
8 \mathrm{p} .1\end{array}\right.$ & & & diatlon & & 1016 & & ramn & Preceipm. & & \\
\hline
\end{tabular}


1902-December.]

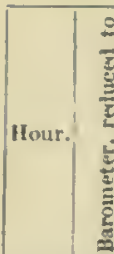

4th.

\begin{tabular}{|c|c|c|c|c|c|c|c|c|c|c|}
\hline & & & & & & & & & & \\
\hline 2 & $29 \cdot 636$ & - & $118^{\prime}$ & $13 \cdot 0$ & $12 \cdot 0$ & $13 \cdot 3$ & $x^{3}, y, 1-2$ & 9 & $10-12$ & b" \\
\hline 4 & $29 \cdot 634$ & - & $12 \cdot 0$ & 13.0 & $12 \cdot 0$ & $13 \cdot 0$ & $x$ i, $\mathbf{k}, 1-2$ & 7 & $10-12$ & " \\
\hline is & $29 \cdot 626$ & - & $11 \cdot 2$ & $12 \cdot 4$ & $12 \cdot 2$ & $13 \cdot 0$ & $N, 1$ & 5 & 10 & " \\
\hline 8 & $24 \cdot 6003$ & - & $10 \cdot 8$ & $13 \cdot 2$ & $12 \cdot 8$ & $16 \cdot 8$ & $x$ ln: $1,1-2$ & 11 & $10-15$ & 1 \\
\hline 10 & 29).567s & - & $12 \cdot 2$ & 14.5 & $13 \cdot 8$ & $20 \cdot 0$ & $x, 1-2$ & 6 & $10-15$ & 4 \\
\hline$X(x)=11$ & $29 \cdot 554$ & - & $13 \cdot 0$ & $15 \cdot 0$ & $14 \cdot 5$ & $18 \cdot 5$ & S hy W, 1-2 & 7 & $10-1.5$ & Ix: \\
\hline 2 & $29: 5613$ & - & $g \cdot 0$ & $16 \cdot 0$ & 1.5 .5 & $21 \cdot 0$ & N hy W, 1-2 & $x$ & $9-13$ & in: \\
\hline 4 & 29! $-4 k 3$ & - & $15 \cdot 0$ & $16 \cdot 5$ & $15 \cdot 5$ & $20 \cdot 2$ & $N \ln w, 1-2$ & 8 & $10-15$ & b \\
\hline 6 & $29 \cdot 449$ & - & $15^{\circ} 6$ & $17 \cdot 0$ & $16 \cdot 6$ & $21 \cdot 5$ & $\mathrm{~N}$ ly $\mathrm{W}, 1$ & 7 & $8-10$ & h \\
\hline 8 & $29 \cdot 42 \pi$ & - & $15 \cdot 2$ & 16.0 & $15 \cdot 2$ & $19 \cdot 2$ & N ly w, l & 5 & $8-8$ & b \\
\hline 10 & $29 \cdot 407$ & - & 11.5 & $12 \cdot 2$ & $12 \cdot 0$ & $12 \cdot 0$ & Lt. $\mathbf{N}$ airs & 4 & 6.8 & b \\
\hline Midt. & $29 \cdot 386$ & - & $11 \cdot 5$ & $13 \cdot 0$ & $12 \cdot 2$ & $14 \cdot 0$ & Lt. var, airs & 4 & 0 & $b$ \\
\hline IIcau & $29 \cdot 521$ & $\overline{-}$ & - & $\overline{14 \cdot 4}$ & $\overline{13 \cdot 7}$ & - & - & $\overline{-1}$ & - & - \\
\hline
\end{tabular}

Jax. therm. read at $\left\{\begin{array}{lll}8 \text { a. } & 14^{\circ} \cdot 0 . \\ 8 & 1 & 17^{\circ} \cdot 0^{\circ}\end{array}\right.$

Solar radlation $\left\{\begin{array}{l}\text { black bulth } 120^{\circ} 0 . \\ \text { liright bu1h } \\ 54^{\circ} \circ\end{array}\right.$.

\section{Meteorological Journal of the "Discovery."}

\begin{tabular}{|c|c|c|c|c|c|c|c|c|}
\hline \multicolumn{3}{|l|}{ Whind. } & \multirow[b]{3}{*}{ 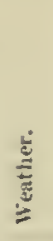 } & \multirow[b]{3}{*}{ 总 } & \multirow{2}{*}{\multicolumn{2}{|c|}{$\begin{array}{l}\text { Clonds, } \\
\text { Amount and movement. }\end{array}$}} & & \multirow{3}{*}{ Newnarks. } \\
\hline \multirow{2}{*}{$\begin{array}{c}\text { Directlou and } \\
\text { forer } \\
\text { (Benufort scalc). }\end{array}$} & \multirow{2}{*}{ 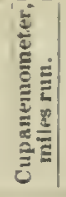 } & \multirow{2}{*}{ 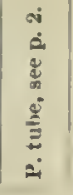 } & & & & & & \\
\hline & & & & & Upper. & Lower. & $\frac{\frac{\pi}{2}}{\frac{\pi}{2}}$ & \\
\hline$X$ ly $1,1-2$ & 9 & 10-12 & 1" & 1 & - & - & - & \\
\hline $\mathrm{X}, \mathrm{y} \mathbf{E}, 1-2$ & 7 & $10-12$ & " & & - & - & - & \\
\hline$N, 1$ & 5 & 10 & 3 & & - & - & - & \\
\hline$x \ln 1,1-2$ & 11 & $10-15$ & 1 & & - & - & - & 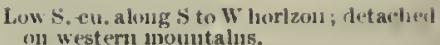 \\
\hline$x, 1-2$ & 6 & $10-15$ & 3 & & - & - & - & 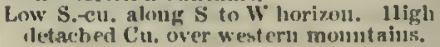 \\
\hline N hy W, 1-2 & 7 & $10-1.5$ & ine & & - & Fr.en, $2, N$ (fast) & - & \\
\hline N hy W, 1-2 & x & 91.3 & lx: & & - & Fr.ru, 1, XNW & - & \\
\hline$N \operatorname{lng} W, 1-2$ & 8 & $10-15$ & b) & & - & - & - & 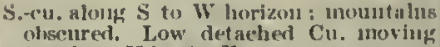 \\
\hline N ly $w, 1$ & 7 & $8-10$ & '1 & & - & - & - & 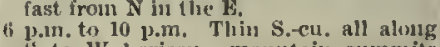 \\
\hline N ly w, 1 & 5 & $8-8$ & b & & - & - & - & $\begin{array}{l}\text { st to W borizon ; monntain summits } \\
\text { clear. }\end{array}$ \\
\hline Lt, $\mathbf{N}$ airs & 4 & $6 \cdot 8$ & $\mathbf{b}$ & & - & - & - & lain hand, 4. \\
\hline L.t. var, airs & 4 & 0 & $\mathrm{~b}$ & 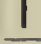 & - & - & - & $\begin{array}{l}\text { Summits of mountans obseured. Low } \\
\mathrm{Cu} \text {. along } \mathrm{S} \text { to } W \text { horizons. }\end{array}$ \\
\hline- & -1 & - & - & & - & - & - & $\begin{array}{l}\text { Flne all day, wilth a steady Ny breeze. } \\
\text { Rarometer falling. Temperature steady. }\end{array}$ \\
\hline
\end{tabular}

Evapu. - grammes. P'recipn.-lu. Rarometer falling. Temperat ure steady.
5 th

\begin{tabular}{|c|c|c|c|c|c|c|c|c|c|c|c|}
\hline 2 & $\mid 29 \cdot 376$ & - & $8 \cdot 0$ & $11 \cdot 0$ & $10 \cdot 5$ & $15 \cdot 0$ & Calm & 1 & 0 & $\alpha *$ & \\
\hline 4 & $29 \cdot 311$ & - & $11 \cdot 0$ & $12 \cdot 0$ & 11.5 & $13 \cdot 2$ & Var. S, 2-3 & 2 & $10-15$ & bc & \\
\hline 6 & $29 \cdot 367$ & - & $12 \cdot 0$ & 160 & 14.0 & $17 \cdot 0$ & ENE, 0-1 & 3 & 6.8 & b & \\
\hline 8 & $29 \cdot 367$ & -1 & $12 \cdot 8$ & $16 \cdot 2$ & 14.0 & $20 \%$ & Lt. $S$ alrs & 1 & 0 & b & \\
\hline 10 & $29 \cdot 376$ & - & $15 \cdot 0$ & $18 \cdot 8$ & $16 \cdot 0$ & $29 \cdot 2$ & Var. $\mathbf{F}, 1$ & 4 & 10 & b & \\
\hline Noon & $29 \cdot 363$ & -1 & 160 & $16 \cdot 8$ & 15.0 & $23 \cdot 8$ & $\mathrm{E}, 2-3$ & 8 & $20-22$ & b & \\
\hline 2 & $29-36 i 1$ & - & $13 \cdot 2$ & $14 \cdot 2$ & $13 \cdot 0$ & $19 \cdot 0$ & E, 2-3 & 15 & $15-25$ & b & \\
\hline 4 & $29 \cdot 353$ & - & $14 \cdot 0$ & $14 \cdot 8$ & $13 \cdot 2$ & $20 \cdot 2$ & E by $N, 3$ & 16 & 22-25 & $b$ & \\
\hline 6 & $29 \cdot 335$ & - & $14 \cdot 0$ & $15 \cdot 0$ & $12 \cdot 2$ & $19 \cdot 2$ & E by $\mathrm{N}, 2-3$ & 14 & $15-22$ & b & \\
\hline 8 & $29 \cdot 312$ & - & $13 \cdot 0$ & $13 \cdot 0$ & $11 \cdot 2$ & $16 \cdot 0$ & E ly $N, 1-2$ & 14 & $12-18$ & b & \\
\hline 10 & $29 \cdot 281$ & - & $12 \cdot 2$ & $13 \cdot 0$ & $11 \cdot 0$ & $13 \cdot 0$ & NE, 1-3 & 15 & $12-22$ & b & \\
\hline Midt. & $29 \cdot 273$ & -1 & $11 \cdot 0$ & $13 \cdot 2$ & $12 \div 5$ & $13 \cdot 2$ & Lt. NE alrs & 2 & 0 & b & I \\
\hline Mean' & $29 \cdot 340$ & -1 & - & $14 \cdot 5$ & $12 \cdot 8$ & - & - & - & - & - & \\
\hline
\end{tabular}

Jax, therm. read at $\left\{\begin{array}{l}8 \mathrm{a} .18^{\circ} \cdot 8 . \\ 8 \mathrm{p.} 20^{\circ} \cdot 1 .\end{array}\right.$ 


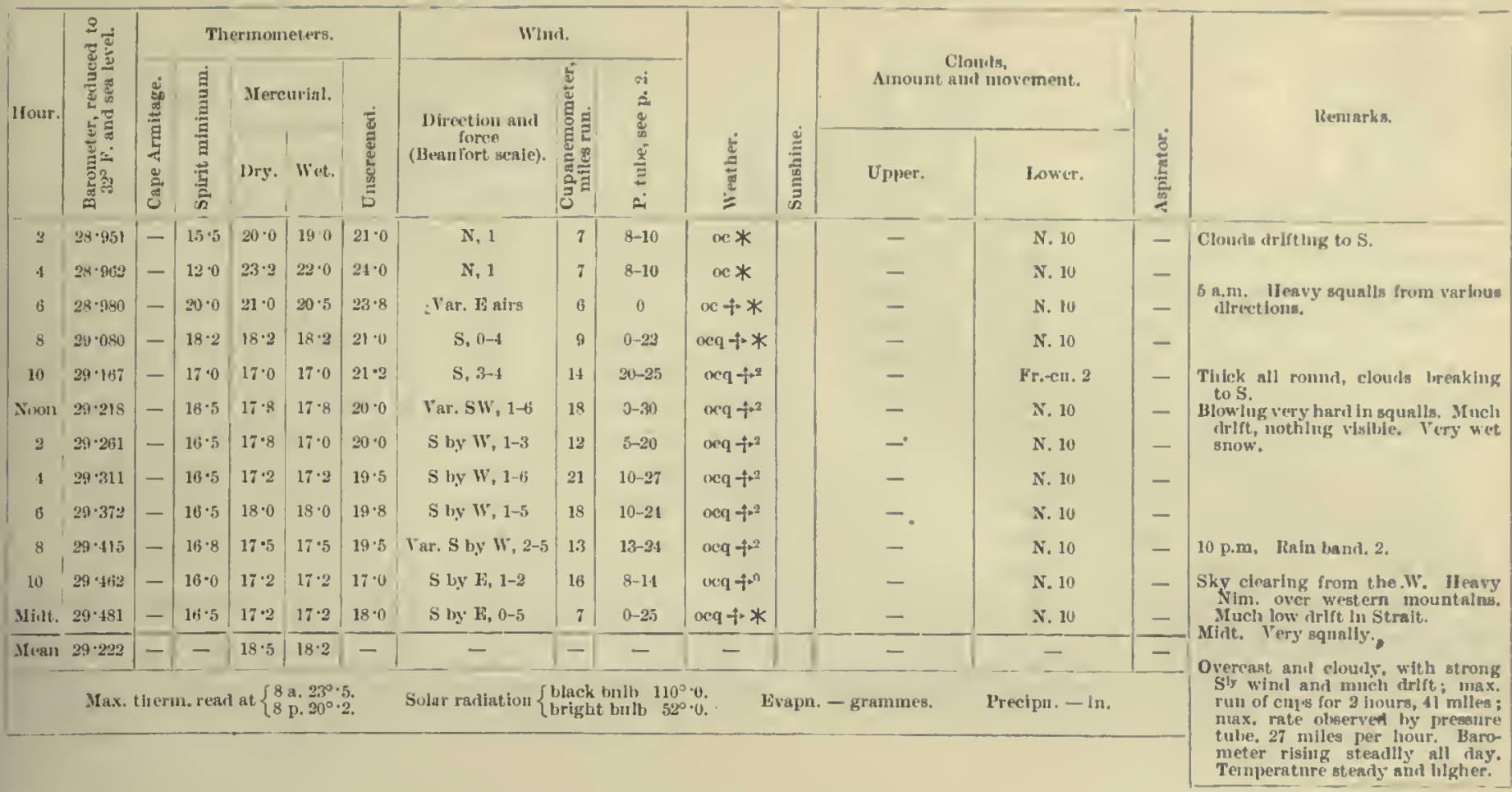

7 th.

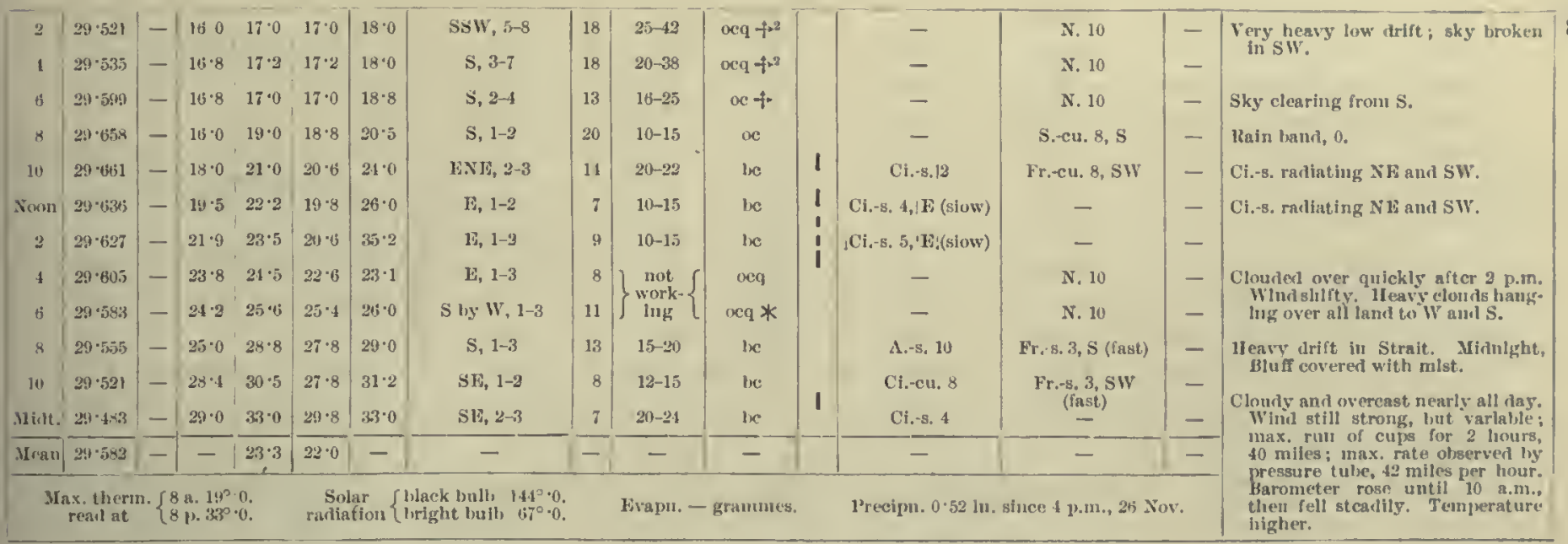

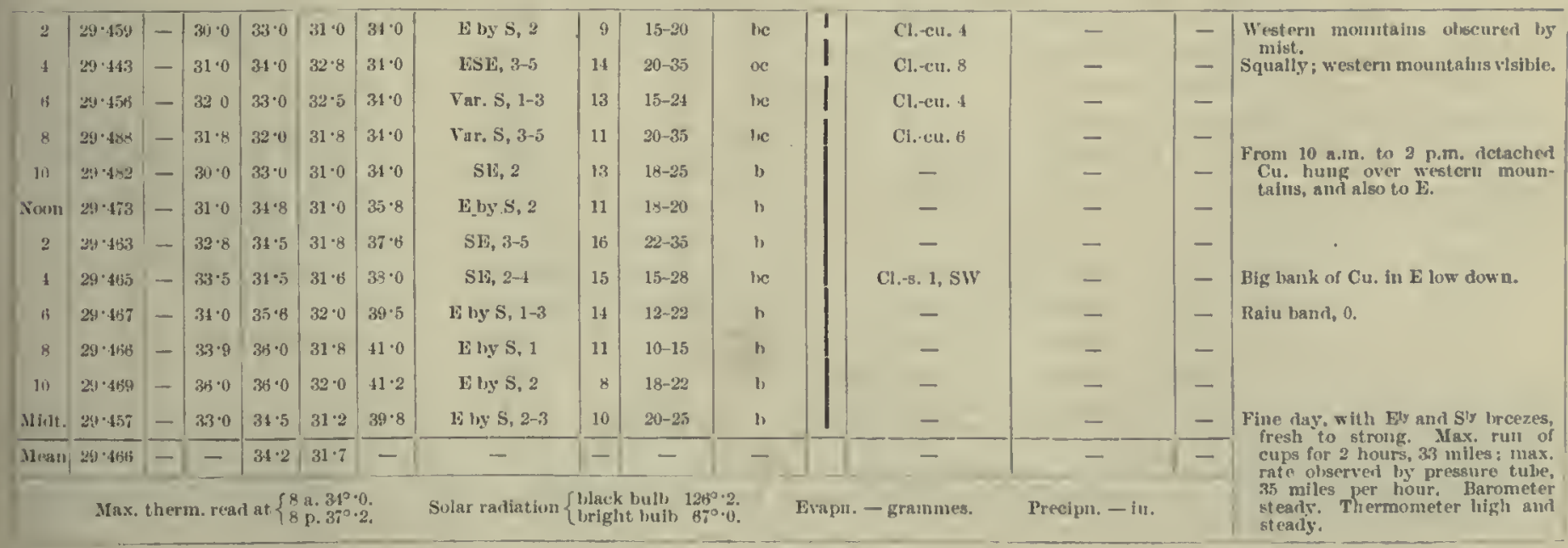


1902 -December.]

Meteolological Jourinal of the "Discovery."

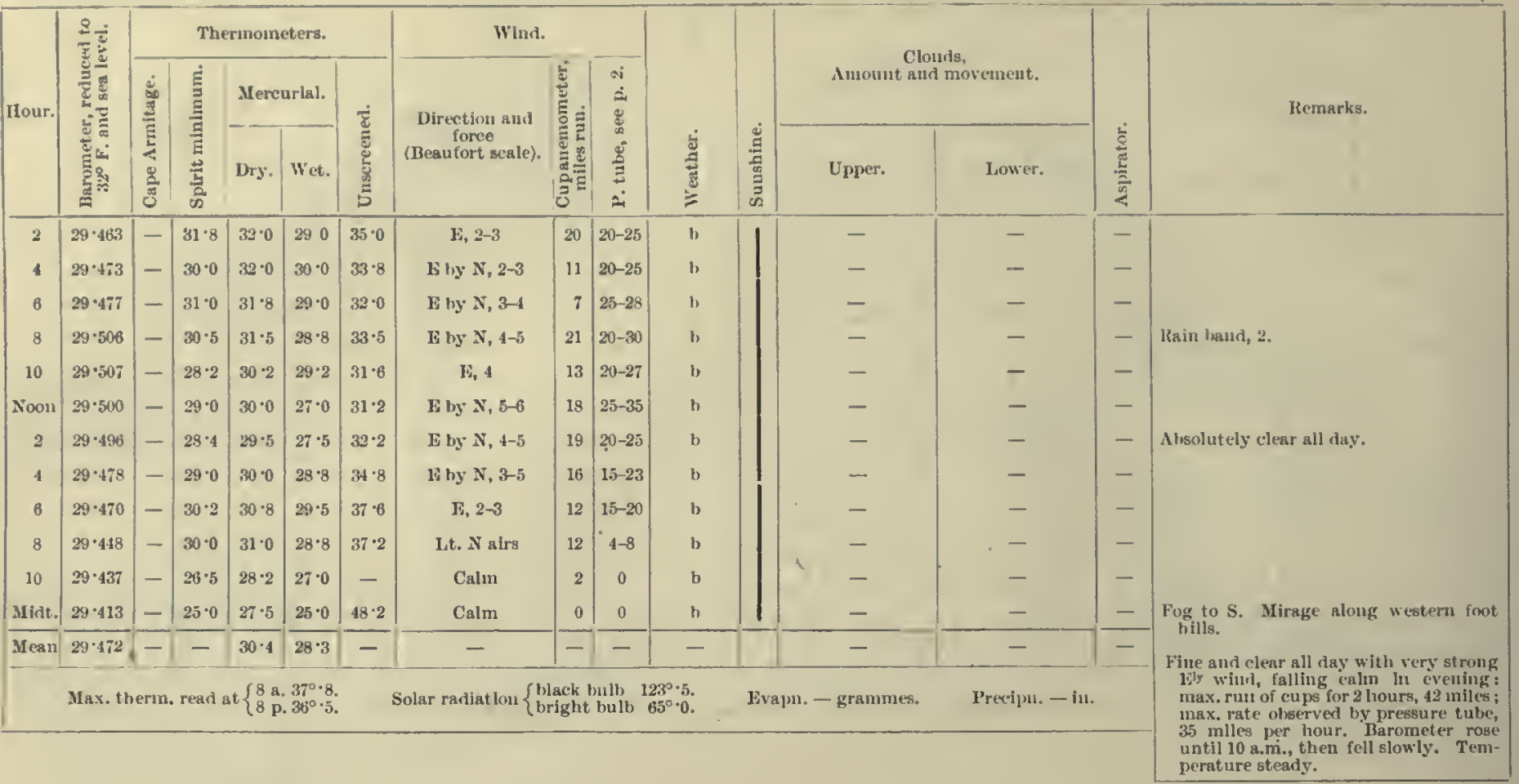

11th,

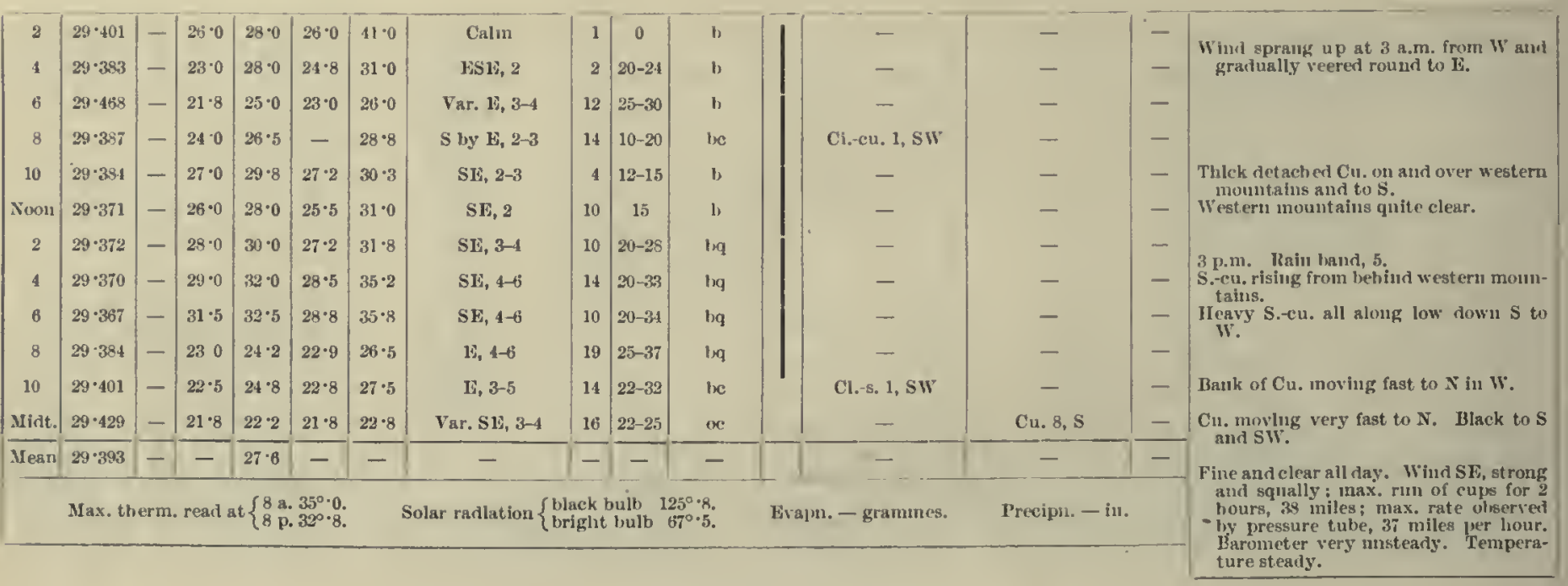

12 th.

\begin{tabular}{|c|c|c|c|c|c|c|c|c|c|c|c|c|c|c|}
\hline 2 & $29 \cdot 434$ & -1 & $21 \cdot 8$ & $24 \cdot 0$ & $23 \cdot 0$ & $24 \cdot 0$ & Var. $\mathrm{SE}_{1}, 3-4$ & 15 & $|20-25|$ & $\infty$ & - & N. 10 & - & \multirow{2}{*}{$\begin{array}{l}\text { SW hills and White Island obscured. } \\
2.30 \text { a.m. Snowing and squally. }\end{array}$} \\
\hline 4 & $29 \cdot 470$ & - & $22 \cdot 0$ & $24 \cdot 8$ & $23 \cdot 8$ & $24 \cdot 8$ & Var. SE, 2-4 & 14 & $15-25$ & $\alpha+* *$ & - & N. 10 & - & \\
\hline 6 & $29 \cdot 516^{\circ}$ & - & $23 \cdot 2$ & $25 \cdot 0$ & $24 \cdot 0$ & $25 \cdot 0$ & Var. SE, 0-b & 18 & $0-35$ & $\operatorname{seq}+*$ & - & N. 10 & - & \multirow{5}{*}{ 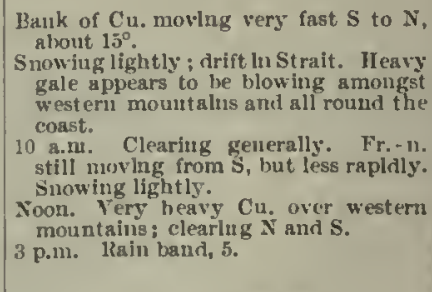 } \\
\hline 8 & $29 \cdot 553$ & - & $24 \cdot 2$ & $26 \cdot 8$ & $25 \cdot 0$ & $28 \cdot 8$ & Var. SE, 4-6 & 19 & $25-30$ & ocg $*$ & - & N. $10, \underset{\text { (fast) }}{\mathrm{Fr}-11.3, \mathrm{~S}}$ & - & \\
\hline 10 & $29 \cdot 587$ & -1 & $25 \cdot 2$ & $25 \cdot 2$ & $24 \cdot 0$ & $27 \cdot 6$ & ESE, $2-3$ & 10 & $15-20$ & $\infty *$ & - & N. 10 & - & \\
\hline Noon & $29 \cdot 601$ & - & $25 \cdot 0$ & $26 \cdot 0$ & $25 \cdot 2$ & $29 \cdot 0$ & ENE, 3-4 & 12 & $20-25$ & $o$ & - & N. 10 & - & \\
\hline 2 & $29 \cdot 810$ & - & $25 \cdot 4$ & $28 \cdot 2$ & $26 \cdot 2$ & 30.0 & E, 1-2 & 14 & $10-18$ & o & - & N. 10 & - & \\
\hline 8 & $29 \cdot 645$ & -1 & $24 \cdot 5$ & $25 \cdot 2$ & $25 \cdot 0$ & $25 \cdot 5$ & SE, 3-4 & 16 & $20-35$ & $o+$ & - & N. 8, SW & - & \multirow{5}{*}{ 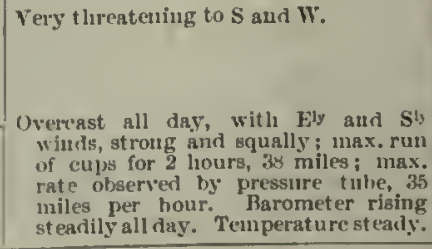 } \\
\hline 10 & $29 \cdot 6.53$ & - & $24 \cdot 8$ & $25 \cdot 5$ & $24 \cdot 8$ & $25 \cdot 5$ & SE, 1-4 & 14 & $|10-30|$ & $\infty+*$ & - & N. 10 & - & \\
\hline MIdt. & $29 \cdot 643$ & -1 & $24 \cdot 0$ & $26 \cdot 0$ & $25 \cdot 5$ & $26 \cdot 5$ & S1:, 0-1 & 11 & $10-12$ & o* & - & N. 10 & - & \\
\hline Mean & $29 \cdot 580$ & -1 & - & 202 & $25 \cdot 1$ & - & - & - & - & - & - & - & - & \\
\hline & & & & & & si & & & $148^{\circ} \cdot 5$ & & imnes. & 1. not measurable. & & \\
\hline
\end{tabular}


Mrteorological Jourvat, of tJi: "Discovery."

[1902-December.

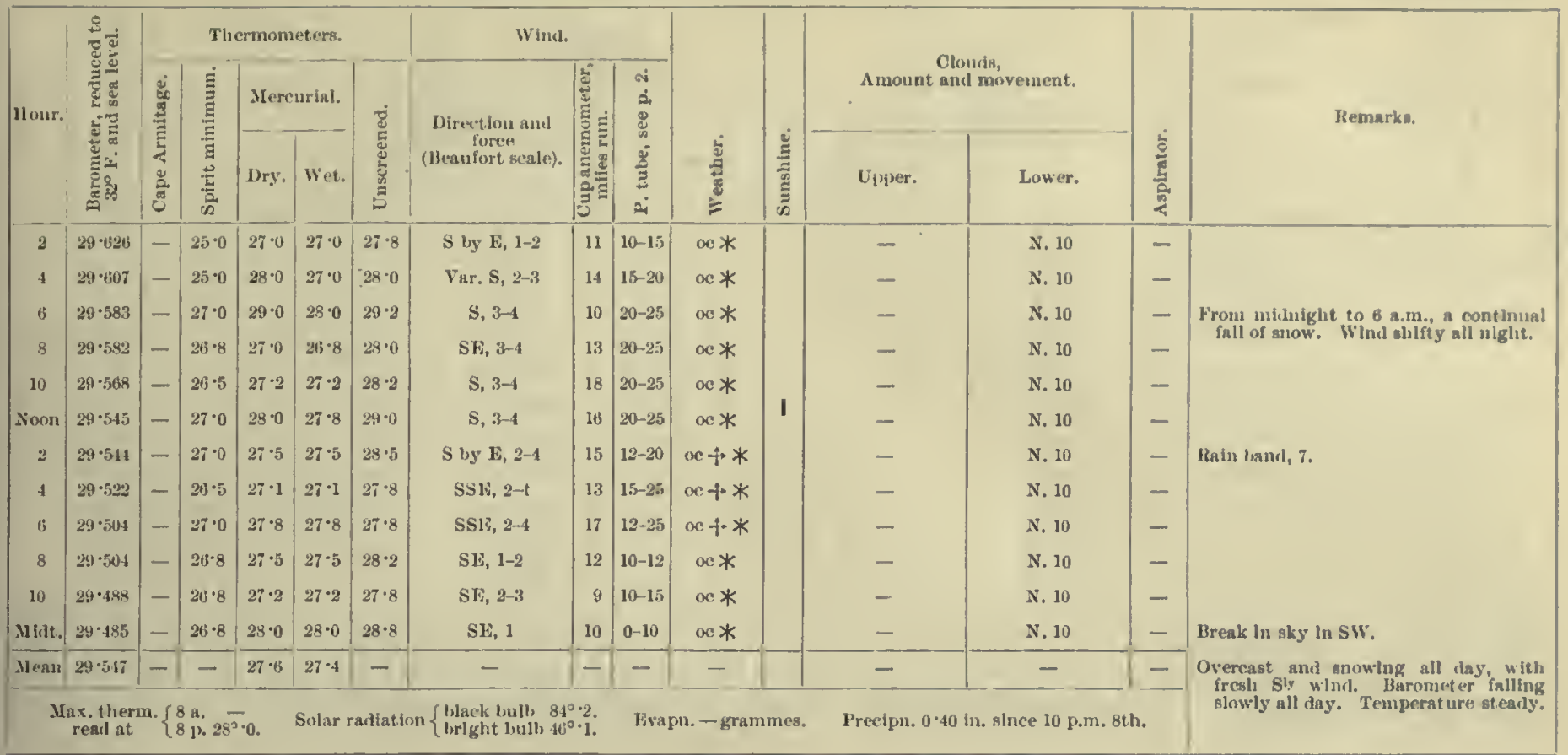

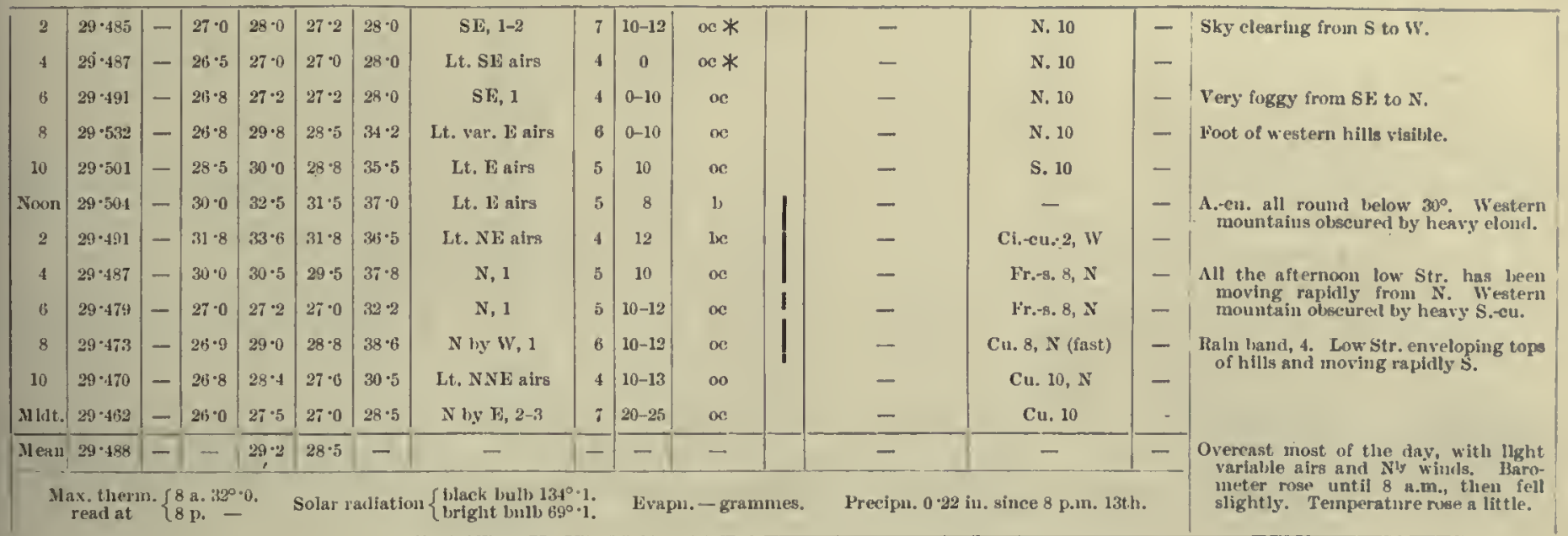

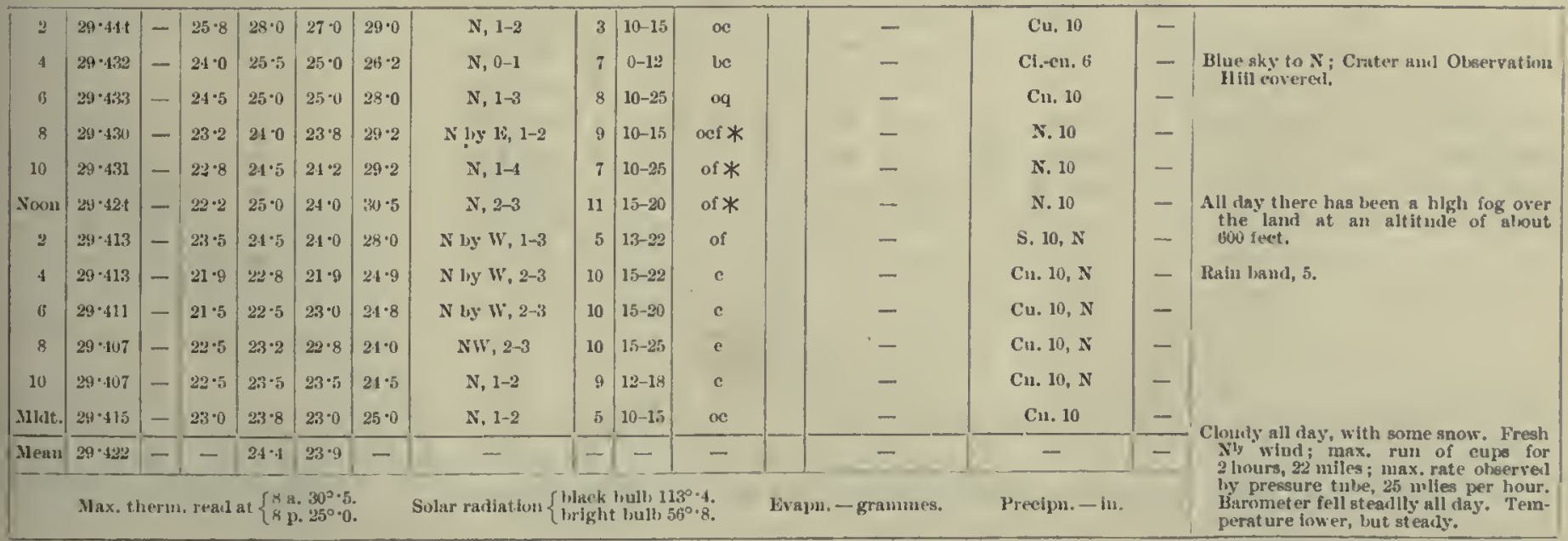




\begin{tabular}{|c|c|c|c|c|c|c|c|c|c|c|c|c|c|c|c|}
\hline \multirow{3}{*}{ Hour. } & \multirow{3}{*}{ 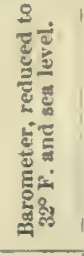 } & \multicolumn{5}{|c|}{ Thermometers. } & \multicolumn{3}{|l|}{ Winti. } & \multirow[b]{3}{*}{ 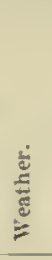 } & \multirow[b]{3}{*}{ 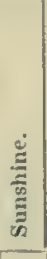 } & & & \multirow[b]{3}{*}{ 咅 } & \multirow{3}{*}{ liemarks. } \\
\hline & & \multirow{2}{*}{ 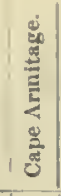 } & \multirow{2}{*}{ 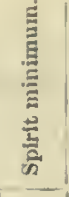 } & \multicolumn{2}{|c|}{ Mereurial. } & \multirow{2}{*}{ 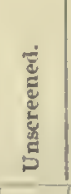 } & \multirow{2}{*}{$\begin{array}{c}\text { Directiou and } \\
\text { force } \\
\text { (Benufort scale). }\end{array}$} & \multirow{2}{*}{ 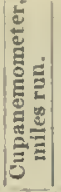 } & \multirow{2}{*}{ 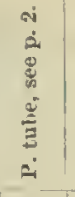 } & & & \multicolumn{2}{|c|}{ Amount and movernent. } & & \\
\hline & & & & Dry. & Wet. & & & & & & & Uplyer. & lower. & & \\
\hline 3 & $29 \cdot 412$ & - & $21 \cdot 0$ & $22 \cdot 2$ & $22 \cdot 0$ & $23 \cdot 8$ & N. $1-2$ & 8 & $10-15$ & oe & & - & Cu. 10 & - & Observation Hill obseured lyy fog. \\
\hline 4 & $29 \cdot 429$ & - & $21 \cdot 0$ & $22 \cdot 0$ & $22 \cdot 0$ & $23 \cdot 8$ & Lt. $N$ alrs & 4 & 0 & $o c$ & & - & $\mathrm{Cu} .10$ & - & \\
\hline 6 & $29 \cdot 418$ & - & $20 \cdot 2$ & $22 \cdot 0$ & $21 \cdot 8$ & $23 \cdot 8$ & Lt. W all's & 4 & 0 & $\infty$ & & - & C11. 10 & - & \\
\hline 8 & $29 \cdot 475$ & - & $21 \cdot 0$ & $22 \cdot 2$ & $22 \cdot 0$ & $25 \cdot 5$ & Lt. $\mathbf{N}$ airs & 4 & $0-8$ & o & & - & Inw S. 10 & - & $\begin{array}{l}\text { All land covered by a hlgh fog at fow feet } \\
\text { altitule. }\end{array}$ \\
\hline 10 & $29 \cdot 481$ & -1 & $21 \cdot 8$ & 23.5 & 23.0 & $31 \cdot 5$ & Lt. $N$ airs & 3 & $0-5$ & 0 & & - & Low S. 11$)$ & - & \\
\hline Xoon & $29 \cdot 493$ & - & 22.0 & $24 \cdot 0$ & $23 \cdot 2$ & $32 \cdot 8$ & Calm & 2 & 0 & 0 & 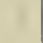 & - & Low S. 10 & - & \\
\hline 2 & $29 \cdot 493$ & - & $22 \cdot 8$ & $25 \cdot 0$ & 21.0 & $3 i \cdot 0$ & Lt. $N$ alrs & 3 & 0 & 0 & & - & Low S. 10 & - & \\
\hline 4 & $29 \cdot 485$ & - & $22 \cdot 8$ & $24 \cdot 2$ & $23 \cdot 5$ & $32 \cdot 8$ & Calm & 2 & 0 & 0 & & - & Low S. 10 & - & $\begin{array}{l}\text { Fing liftel to just below } 1000 \text { feet ; clearing } \\
\text { to } \mathrm{S} \text {. }\end{array}$ \\
\hline 6 & $29 \cdot 502$ & - & $22 \cdot 8$ & $23 \cdot 5$ & $23 \cdot 0$ & $2 n \cdot 0$ & Lt. $\mathbf{N}$ airs & 3 & 0 & o & & - & Jow S. 10 & - & lain bant, 3. \\
\hline 8 & $29 \cdot 500$ & - & $22 \cdot 5$ & $23 \cdot 2$ & $22 \cdot 5$ & $25^{\circ} 6$ & Lt. NW airs & 3 & $0-12$ & 0 & & - & Low S. 10 & - & \\
\hline 10 & $29 \cdot 501$ & - & $22 \cdot 6$ & $23 \cdot 2$ & $22 \cdot 6$ & $23 \cdot 8$ & Lt. $N W$ airs & 4 & $0-6$ & 0 & & - & Low S. 10 & - & \\
\hline Mldt. & $29 \cdot 495$ & -1 & $22 \cdot 0$ & $23 \cdot 0$ & $22 \cdot 5$ & $25 \cdot 0$ & Lt. NW airs & 3 & $0-10$ & $\infty$ & & - & $\mathrm{Cu}, 10$ & - & \\
\hline Mean & $29 \cdot 476$ & - & - & $23 \cdot 2$ & $22 \cdot 7$ & - & - & - & - & - & & - & - & - & $\begin{array}{l}\text { Overcast all day, with liglit Nly and NWly } \\
\text { airs. Barometer rose quickly and }\end{array}$ \\
\hline & & & & $\mathrm{g}$ & & & $\{b]$ & . & $\begin{array}{ll}\text { lib } 88 \\
\text { ulb } 47\end{array}$ & & & mes. & Precipn, - & & remalned stearly. 'Temperature stearly. \\
\hline
\end{tabular}

17 th.

\begin{tabular}{|c|c|c|c|c|c|c|c|c|c|c|c|c|c|c|}
\hline 2 & $29 \cdot 492$ & - & $21 \cdot 0$ & $22 \cdot 0$ & $21 \cdot 8$ & $23 \cdot 0 \mid$ & Lt. NW:airs & 4 & 0 & ne & - & $\mathrm{Cu}, 10$ & $-\mid$ & \multirow{3}{*}{ High wind blowing overhead. } \\
\hline 4 & $29 \cdot 481$ & - & $21 \cdot 0$ & $21 \cdot 8$ & $21 \cdot 5$ & $23 \cdot 5$ & $\mathrm{Nw}, 0-1$ & 6 & $6-12$ & $o c$ & - & $\mathrm{Cn}, 10$ & - & \\
\hline 6 & $29 \cdot 482$ & -1 & $20^{\circ} 0$ & $21 \cdot 8$ & $21 \cdot 0$ & $23 \cdot 2$ & $N W, 1$ & 3 & $0-10$ & oc & - & $\mathrm{Cu}, 10$ & - & \\
\hline 8 & $29 \cdot 467$ & - & $20 \cdot 2$ & $21 \cdot 2$ & $21 \cdot 0$ & $23 \cdot 2$ & $\mathrm{NW}, 1$ & 5 & $0-10$ & o & - & Cus. 10 & - & \multirow[t]{2}{*}{ Fog from NW to SW. } \\
\hline 10 & $29 \cdot 464$ & - & $20 \cdot 0$ & $23 \cdot 0$ & $22 \cdot 2$ & $27 \cdot 0$ & x. 1-2 & 5 & $5-15$ & o & - & S. 10 & - & \\
\hline Nuon & $29 \cdot 447$ & - & $22 \cdot 0$ & $23 \cdot 5$ & $22 \cdot 8$ & $29 \cdot 5$ & $x, 1-2$ & 4 & $\mid 10-15$ & o & - & S. 10 & -1 & \multirow{2}{*}{$\begin{array}{l}\text { Low Str. hanging over all hills the entire } \\
\text { day, at altitude of athout } 1000 \text { feet. } \\
\text { 3p.m. laain hancl, } 2 .\end{array}$} \\
\hline 2 & $29 \cdot 431$ & - & $22 \cdot 9$ & $24 \cdot 0$ & $23 \cdot 2$ & $28 \cdot 0$ & NNW, 1-2 & a) & $10-17\}$ & o & - & S. 10 & - & \\
\hline 8 & $29^{\circ}+05$ & - & $25 \cdot 4$ & $26 \cdot 8$ & .25 .2 & $27 \cdot 6$ & $\mathrm{~N}$ by w, 1-2 & 8 & $10-16$ & 0 & - & S. 10 & - & \multirow{3}{*}{ 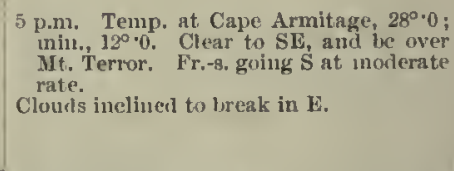 } \\
\hline 10 & $20 \cdot 405$ & - & $25 \cdot 0$ & $26 \cdot 1$ & $25 \cdot 1$ & $27 \cdot 0$ & Lt. NW airs & 7 & $0-8$ & $n$ & - & s. $10, x$ & - & \\
\hline Midt. & $29 \cdot 103$ & - & $24 \cdot 0$ & $25 \cdot 2$ & $24 \cdot 8$ & $28 \cdot 2$ & Calm & 3 & 0 & 0 & - & S. 10 & - & \\
\hline Mean & $29 \cdot 44$ & - & - & $24 \cdot 0$ & $23 \cdot 3$ & - & - & -1 & - & - & - & - & $1-$ & \multirow{2}{*}{$\begin{array}{l}\text { Overeast all day, with steady Nly wincl. } \\
\text { Barometer falling steadily all day. } \\
\text { Temperature steady. }\end{array}$} \\
\hline & ther & & 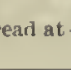 & $8 \mathrm{a}$ & & & uliation & & & & Evapu. - grammes. & Precipn. - int. & & \\
\hline
\end{tabular}

18th.

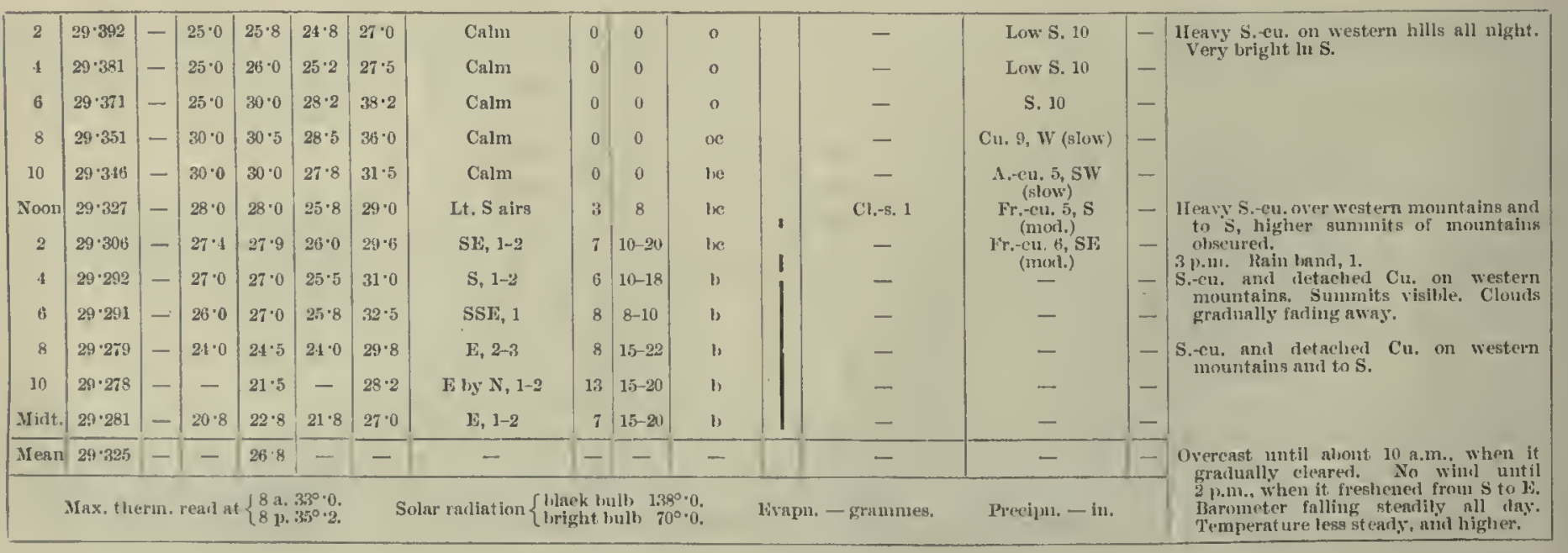


Mhtionological Journat, of tule "Discovehy."

[1902-December.

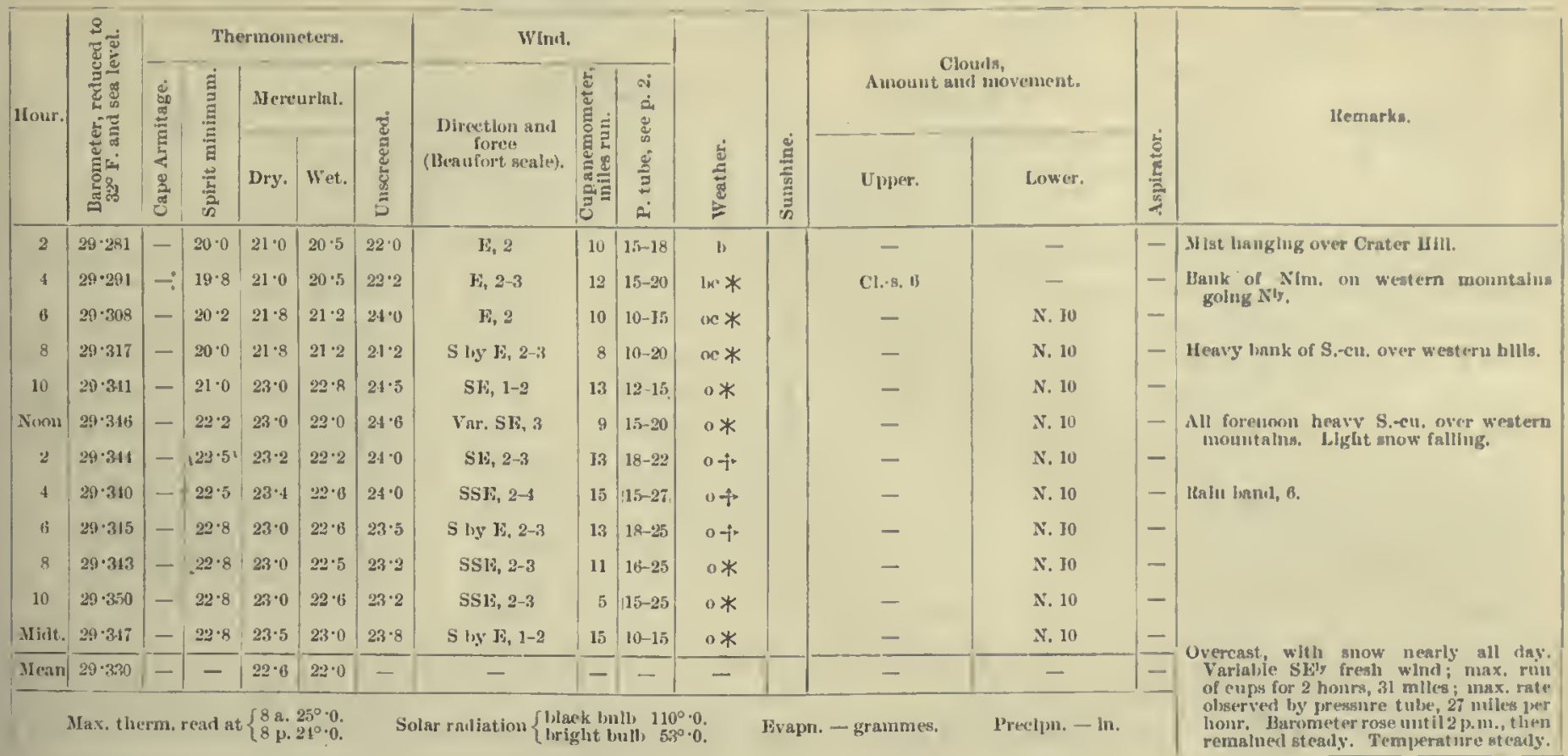

\begin{tabular}{|c|c|c|c|c|c|c|c|c|c|c|c|c|c|c|}
\hline 2 & 24: 339 & -1 & 23.8 & $24 \div 2$ & $24 \cdot 0$ & $21 \cdot 2$ & $\overline{S, 2-3}$ & 8 & $\mid 15-25$ & o* & - & N. 10 & $1-$ & \multirow{6}{*}{$\begin{array}{l}\text { Overeast and snowlug all das. Very } \\
\text { thick all round Top of Oliservatloi } \\
\text { lill and near hills covered. }\end{array}$} \\
\hline 4 & $29 \cdot 332$ & - & $23 \cdot 5$ & $2 t \cdot 0$ & $24 \cdot 0$ & $24 \cdot 2$ & S, I-2 & $\pi$ & $10-15$ & $o *$ & - & N. 10 & - & \\
\hline 6 & $29 \cdot 327$ & - & $23 \cdot 8$ & $21 \cdot 2$ & $23 \cdot 8$ & $25 \cdot 2$ & S by E, 1-2 & 10 & $\mid 10-15$ & $o *$ & - & N. 10 & - & \\
\hline 8 & $29 \cdot 316$ & - & $21 \cdot 0$ & $25 \cdot 0$ & $21 \cdot 8$ & $215 \cdot 8$ & $\mathrm{~S}$ lỵ $\mathrm{F}, 1-2$ & 7 & $10-15$ & $\circ *$ & - & N. 10 & - & \\
\hline Noom & 291311 & - & $23 \cdot 5$ & $21 \cdot 0$ & $23 \cdot 5$ & $28 \cdot 2$ & $\mathrm{E}, \mathrm{I}-2$ & 5 & $12-15$ & $\circ *$ & - & N. 10 & - & \\
\hline 2 & $24 \cdot 298$ & - & 23.6 & $24 \cdot 2$ & $23 \cdot 5$ & $26 \cdot 8$ & $\mathrm{E} ; \mathrm{H}, 2$ & 10 & 18 & $\circ *$ & - & N. 10 & - & \\
\hline 8 & $29 \cdot 30.4$ & - & 120.0 & $20 \cdot 0$ & $19 \cdot 6$ & $21 \cdot 0$ & SE, 1-2 & 7 & $10-12$ & $\circ *$ & - & N. 10 & - & \multirow{2}{*}{ train baut, $\theta$. } \\
\hline 10 & $29 \cdot 317$ & - & $20 \cdot 0$ & $20 \cdot 0$ & $19 \cdot 5$ & $20 \cdot 8$ & BSE, I-2 & 10 & $10-12$ & $o *$ & - & x. 10 & - & \\
\hline Nirit. & $29 \cdot 319$ & $1-$ & 18.0 & $19 \cdot 0$ & $18 \cdot 8$ & 20.0 & 18, $1-2$ & 10 & $10-15$ & $\alpha *$ & - & N. 10 & $1=$ & \multirow{2}{*}{ 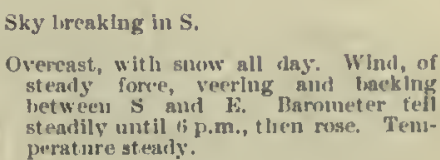 } \\
\hline Nent & $20: 314$ & -1 & - & $22 \cdot 9$ & $22 \cdot 5$ & - & - & - & - & - & - & - & - & \\
\hline
\end{tabular}

20 th.

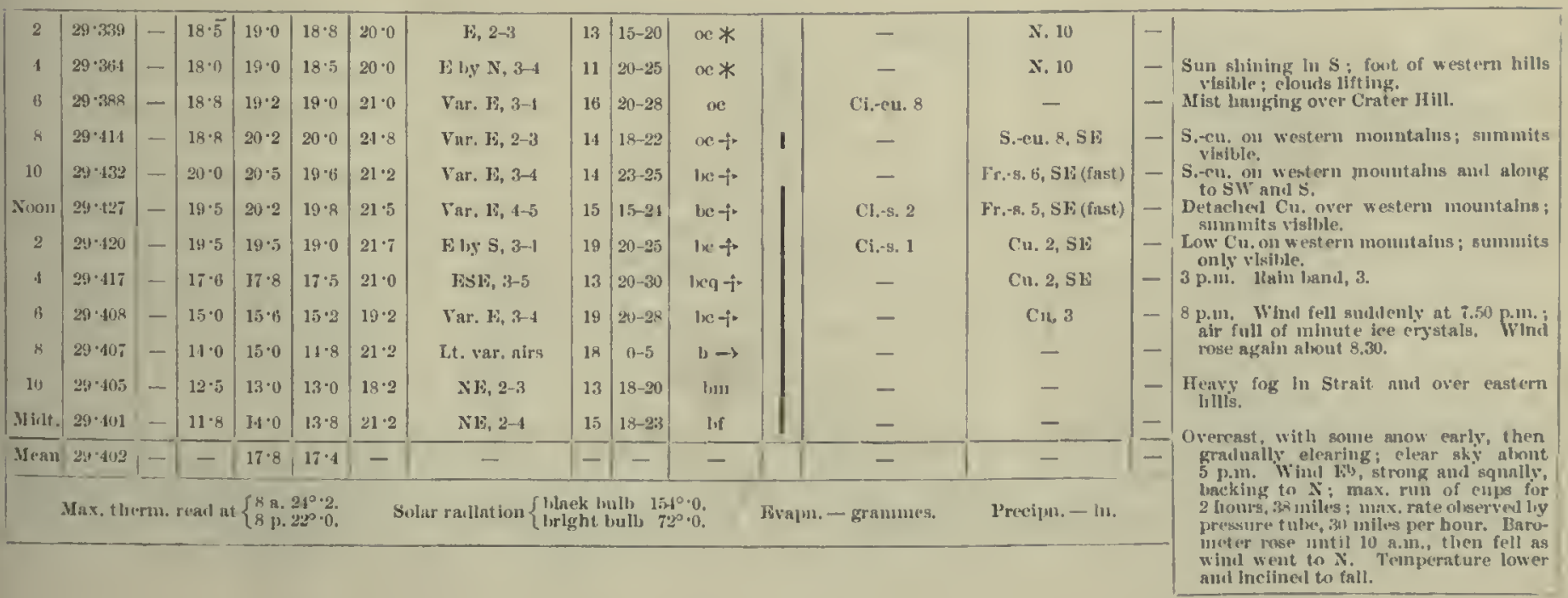


1902-December.]

Mfteorotogical Jourat of thl: "Discovery."

\begin{tabular}{|c|c|c|c|c|c|c|c|c|c|c|c|c|c|c|c|c|}
\hline \multirow{17}{*}{ 22nd. } & \multirow{3}{*}{ IIour. } & \multirow{3}{*}{ 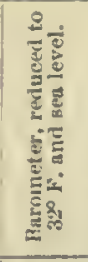 } & \multicolumn{5}{|c|}{ Theruometers. } & \multicolumn{3}{|c|}{ Wincl. } & \multirow[b]{3}{*}{ 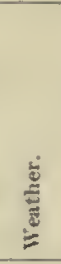 } & \multirow[b]{3}{*}{ 竎 } & \multirow{2}{*}{\multicolumn{2}{|c|}{$\begin{array}{l}\text { Clouds, } \\
\text { Amount and movement. }\end{array}$}} & \multirow[b]{3}{*}{ 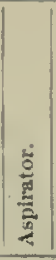 } & \multirow{3}{*}{ Remarks. } \\
\hline & & & \multirow{2}{*}{ 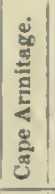 } & \multirow{2}{*}{ 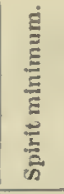 } & \multicolumn{2}{|c|}{ Mercurial. } & \multirow{2}{*}{ 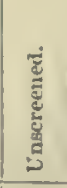 } & \multirow{2}{*}{$\begin{array}{l}\text { Directlon anul } \\
\quad \text { foree } \\
\text { (Bealifort scalc). }\end{array}$} & \multirow{2}{*}{ 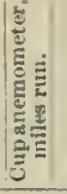 } & \multirow{2}{*}{ 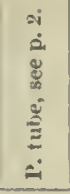 } & & & & & & \\
\hline & & & & & Dry. & Wet. & & & & & & & $v_{\text {pper. }}$ & Lower. & & \\
\hline & 2 & $29 \cdot 399$ & -1 & $12 \cdot 0$ & $11 \cdot 0$ & $13 \cdot 8$ & $20 \cdot 8$ & Le. var. alrs & $i$ & $0-6$ & bf & & - & - & -1 & Thick fog outslde lany, and ou top of near \\
\hline & 4 & $29 \cdot 3: 96$ & - & $11 \cdot 5$ & $12 \cdot 8$ & $12 \cdot 5$ & $16 \cdot 5$ & $\mathrm{~N}] \%, 2-3$ & 8 & $15-20$ & lif & & - & - & - & $\begin{array}{l}\text { hillis. } \\
\text { Thick fog in bay. }\end{array}$ \\
\hline & 6 & $29 \cdot 394$ & - & $10 \cdot 5$ & $14 \cdot 0$ & $13 \cdot 8$ & $16 \cdot 8$ & Sk, 1-2 & 9 & $8-12$ & bf & & - & - & - & Dark blne sky to NW, tlulck fog In bay \\
\hline & 8 & $29 \cdot 388$ & - & $12 \cdot 2$ & $13 \cdot 0$ & $13 \cdot 0$ & $16 \cdot 5$ & Calun & 4 & 0 & b, & & - & - & - & $\begin{array}{l}\text { and on top of uear hills. } \\
\text { Thlek fog all aloug Stralt, Otscrvation }\end{array}$ \\
\hline & 10 & $29 \cdot 393$ & - & $13 \cdot 0$ & $20 \cdot 2$ & $19 \cdot 5$ & $27 \cdot 2$ & Calm & 2 & 0 & b & & - & - & - & Thick fog fog all rouud, Observation vill \\
\hline & Noon & $29 \cdot 374$ & - & $17 \cdot 0$ & 18.0 & $17 \cdot 0$ & $21 \cdot 0$ & Calm & 4 & 0 & ", & & - & - & - & Thick fog all round, smminits of western \\
\hline & 2 & $29 \cdot 251$ & - & $17 \cdot 3$ & $20 \cdot 2$ & $19 \cdot 0$ & $20 \cdot 2$ & NNI, 0-1 & 3 & 0 & жe & i & - & S.-cur. 7 & - & 1)ark fog lank from $N W^{\prime}$ to $S$. \\
\hline & 4 & $29 \cdot 331$ & - & $17 \cdot 7$ & $18 \cdot 8$ & $17 \cdot 6$ & $25 \cdot 5$ & $N, 1$ & 4 & $5-10$ & c & & - & S.-cu. 9 & - & 3 pittle of it. lasln land, 2 . \\
\hline & 6 & $29 \cdot 312$ & - & $17 \cdot 0$ & $19 \cdot 0$ & $18 \cdot 2$ & $24 \cdot 3$ & $N w, 1$ & 4 & $0-10$ & c & & - & S.cu. 10 & - & Ap.m. Mirage to 1 , sun gleaming \\
\hline & 3 & $29 \cdot 280$ & - & $18 \cdot 9$ & $18 \cdot 2$ & $18 \cdot 0$ & $20 \cdot 1$ & NW, 1 & 6 & $0-10$ & ocf & & - & S. 10 & - & $\begin{array}{l}\text { llorlzoul vlsllble all round. Fog alout } \\
\text { foo ft }\end{array}$ \\
\hline & 10 & $20 \cdot 283$ & - & $17 \cdot 0$ & $19 \cdot 0$ & $18 \cdot 8$ & $20 \cdot 0$ & NW, 1 & 5 & $0-10$ & nef & & - & S. 10 & - & \\
\hline & Mintt. & $29 \cdot 194$ & -1 & $17 \cdot 8$ & $20 \cdot 0$ & $19 \cdot 2$ & $20 \cdot 0$ & $\mathrm{NW}, 1$ & 4 & $0-10$ & ocf & & - & S. 10 & - & \\
\hline & Mean & $29 \cdot 337$ & - & - & $17 \cdot 3$ & $16 \cdot 7$ & - & - & - & - & - & & - & - & - & $\begin{array}{l}\text { Clear until nom, beoming quickly over- } \\
\text { east and foggy. Wind very light all }\end{array}$ \\
\hline & & Max. the & man & 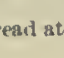 & & & & raliation \{ & b & $\begin{array}{l}\text { thb } 1 \\
\text { ulb }\end{array}$ & & & imes. & l'recipn. $0.05 \mathrm{in.}$ & & $\begin{array}{l}\text { day from Yam Nit. larometer falling } \\
\text { slowly. Temperature low er, lut rlslng. }\end{array}$ \\
\hline
\end{tabular}

$23 \mathrm{rd}$

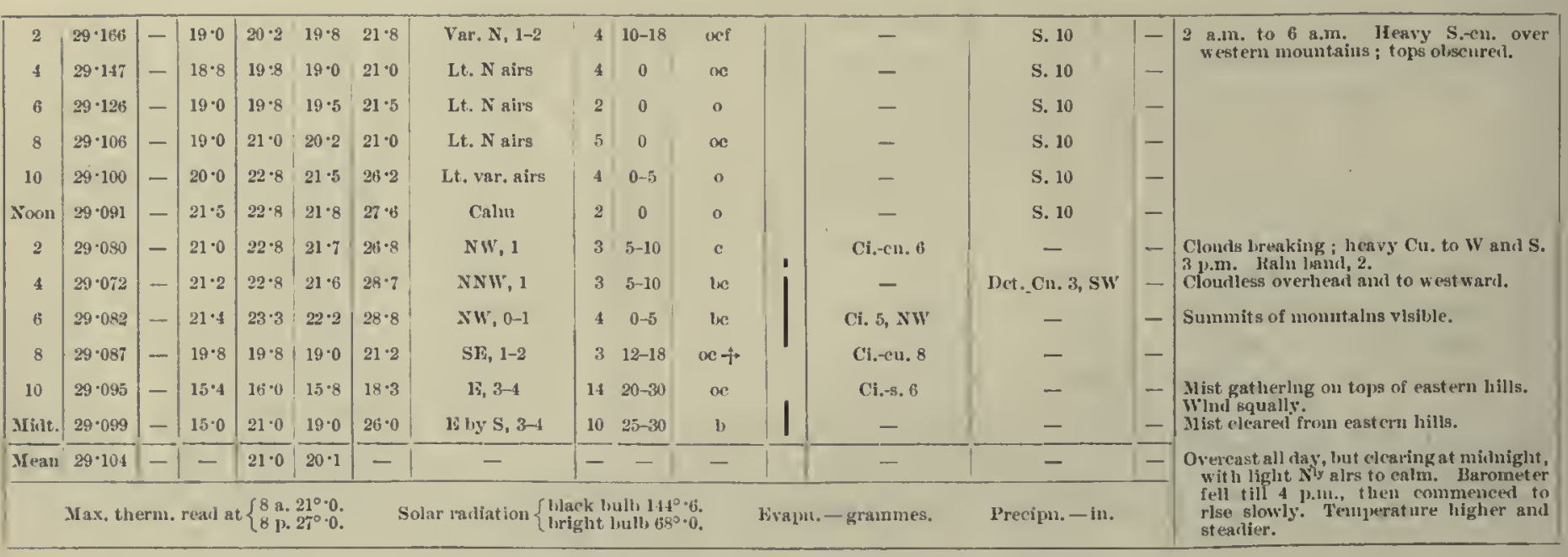

24th,

\begin{tabular}{|c|c|c|c|c|c|c|c|c|c|c|c|c|c|c|c|}
\hline 2 & $29 \cdot 102$ & - & $19 \cdot 8$ & $21 \cdot 8$ & $20 \cdot 0$ & $22 \cdot 0$ & $\mathbf{E}, 2$ & 14 & $10-15$ & 6 & & - & - & - & \multirow{4}{*}{$\begin{array}{l}\text { Strong SWly w lud in Seal 13ay, with drift. } \\
\text { strong mirage all rouml lorizon. }\end{array}$} \\
\hline 4 & $29 \cdot 110$ & - & $20 \cdot 8$ & $21 \cdot 0$ & $19 \cdot 8$ & $25 \cdot 0$ & Lt. S airs & 7 & 0 & b & & - & - & - & \\
\hline 6 & $29 \cdot 135$ & - & $13 \cdot 8$ & $15^{\circ} 0$ & $14 \cdot 5$ & $22 \cdot 0$ & Lt. airs & 2 & 0 & 1, & & - & - & - & \\
\hline 8 & $29 \cdot 142$ & - & $13 \cdot 0$ & $17 \cdot 2$ & $16 \cdot 8$ & $19 \cdot 8$ & Caln & 1 & 0 & b & & - & - & - & \\
\hline 10 & $29 \cdot 142$ & - & $17 \%$ & $20 \cdot 2$ & $19 \cdot 0$ & $25 \cdot 8$ & Calm & 1 & 0 & oc & & Cl.-en. 8 & - & - & \multirow{8}{*}{ 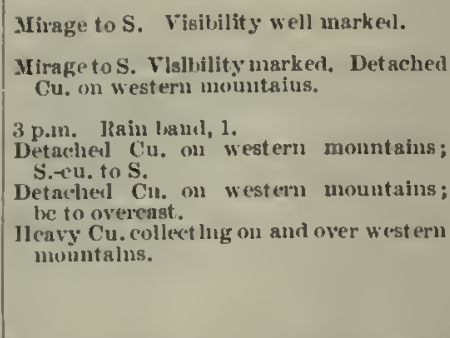 } \\
\hline Noon & $29 \cdot 139$ & - & $20 \cdot 0$ & $25 \cdot 0$ & $23 \cdot 0$ & $36 \cdot 0$ & Calun & 0 & 0 & oc & I & Ci.-cn. 7 & - & - & \\
\hline 2 & $29 \cdot 1.10$ & - & $23 \cdot 0$ & $27 \cdot 0$ & $21 \cdot 6$ & $36-2$ & Calm & 1 & 0 & c & & Ci.-cru. 7, NE & - & - & \\
\hline 4 & $29 \cdot 134$ & -1 & $26 \cdot 4$ & $27 \cdot 9$ & $25 \cdot 3$ & $3 \cdot 2$ & ENI: 1-2 & 4 & $10-15$ & $1 x^{2}$ & 1 & - & S. $2, \mathrm{~S}$ & - & \\
\hline 6 & $29 \cdot 139$ & - & $21 \cdot 7$ & $24 \cdot 7$ & $23 \cdot 1$ & $27 \cdot 3$ & Var. $J, 1-3$ & 7 & $5-20$ & $e q$ & & - & S.-cu. 10 & - & \\
\hline 8 & $29 \cdot 139$ & -1 & $21 \cdot 2$ & $27 \cdot 8$ & $2 t i \cdot 0$ & $33 \cdot 0$ & Calm & 3 & 0 & oc & 1 & Cl.-сu. 9 & - & - & \\
\hline 10 & $29 \cdot 139$ & - & $22 \cdot 0$ & $23 \cdot 0$ & $22 \cdot 0$ & $26 \cdot 0$ & Lt. NF airs & 2 & 0 & $\alpha$ & & - & S.-cu. 5 & - & \\
\hline Mint. & $29 \cdot 080$ & -1 & $23 \cdot 0$ & $30 \cdot 8$ & $20 \cdot 2$ & $32-0$ & Lt. NE airs & 4 & 0 & ine & 1 & Cl.-8. 4 & - & - & \\
\hline Mean & $29 \cdot 129$ & - & - & $23 \cdot 5$ & $21 \cdot 9$ & - & - & - & - & - & & - & - & - & \multirow{2}{*}{ 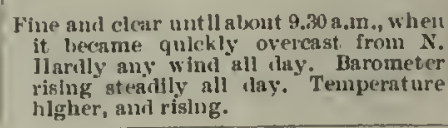 } \\
\hline & 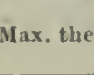 & & 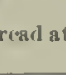 & $\int 8:$ & & & , & & 1 & & & graunues. & 1'recipu. - ln. & & \\
\hline
\end{tabular}


MEt EOROLogica, JoURNat, OF THF "Discovery."

[1902-December.

\begin{tabular}{|c|c|c|c|c|c|c|c|c|c|c|c|c|c|c|c|}
\hline \multirow{3}{*}{ llour. } & \multirow{3}{*}{ 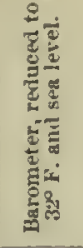 } & \multicolumn{5}{|c|}{ Thermometers. } & \multicolumn{3}{|l|}{ Whlud, } & \multirow[b]{3}{*}{ : } & \multirow[b]{3}{*}{ 竎 } & \multirow{2}{*}{\multicolumn{2}{|c|}{$\begin{array}{l}\text { Clouds, } \\
\text { Amount mul movement. }\end{array}$}} & \multirow[b]{3}{*}{ 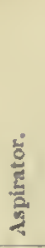 } & \multirow{3}{*}{ Kemarks. } \\
\hline & & \multirow{2}{*}{ 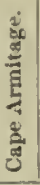 } & \multirow{2}{*}{ 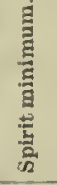 } & \multicolumn{2}{|c|}{ Mereurlal. } & \multirow{2}{*}{ 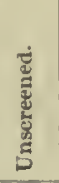 } & \multirow{2}{*}{$\begin{array}{c}\text { Dircetlou and } \\
\text { force } \\
\text { (Bexufort scale). }\end{array}$} & \multirow{2}{*}{ 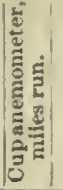 } & \multirow{2}{*}{ 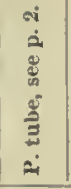 } & & & & & & \\
\hline & & & & Bry. & Wet. & & & & & & & Vpper. & Jow er. & & \\
\hline 2 & $29 \cdot 128$ & - & $25 \cdot 0$ & $31 \cdot 0$ & 29.0 & $3 t \cdot 0$ & N 1 & 2 & 10 & be & I & - & C11. 6, SW (mod.) & - & $\begin{array}{l}2,4 \text {, and is a.m. Very stormy appear- } \\
\text { ance on westeril unountainn; heav'y }\end{array}$ \\
\hline 4 & $29 \cdot 135$ & - & $28^{\circ} 0$ & $32 \cdot 2$ & $29 \cdot 5$ & $40-5$ & Sth, 0-1 & 4 & $0-10$ & le & & $\begin{array}{l}\text { C1.-cn. } 4_{1} \text { SW } \\
\text { (slow) }\end{array}$ & - & - & $\begin{array}{l}\text { Cin. and apparently mueh irlft. Very } \\
\text { striklng Cl.-en. clonds. }\end{array}$ \\
\hline 6 & $29 \cdot 160$ & - & $29 \cdot 5$ & $29 \cdot 5$ & $25 \cdot 8$ & $31 \cdot 0$ & SIi, 0-1 & 6 & $0-8$ & 4 & & - & - & - & $\begin{array}{l}\text { Cl.eri. low down on horfzon, passing to } \\
\text { Nli slowly. }\end{array}$ \\
\hline 8 & $29 \cdot 219$ & - & $24^{\circ} 0$ & $21 \cdot 0$ & $22 \cdot 0$ & $20^{\circ} 0$ & SW, 1-2 & 4 & $10-15$ & us & & $\begin{array}{l}\text { Cl. eu, } 4,5 W \\
\text { (slow) }\end{array}$ & 一 & - & 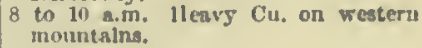 \\
\hline 10 & $29 \cdot 257$ & 一 & $22 \cdot 0$ & $2 t \cdot 2$ & $22 \cdot 6$ & $25 \cdot 2$ & Sk, 2-3 & 13 & $12-25$ & be & & Cl. $-8.2,5 \mathrm{~W}$ & - & - & \\
\hline Noon & $29 \cdot 275$ & - & $22 \cdot 2$ & $26 \cdot 0$ & $24 \cdot 0$ & $27 \cdot 8$ & $\mathrm{H}, 2-3$ & 10 & $10-15$ & b & & - & 一 & - & \\
\hline 2 & $29 \cdot 293$ & - & $25 \cdot 3$ & $30 \cdot 6$ & $28 \cdot 2$ & $32 \cdot 0$ & ESK, 2-4 & 10 & $15-30$ & $\operatorname{lig}+$ & & 一 & 一 & - & $\begin{array}{l}\text { Ifenyy detachwl Cu. ou weatern moun- } \\
\text { tains. Vlsll,ility small. }\end{array}$ \\
\hline 4 & $29 \cdot 300$ & - & $20 \cdot 6$ & $30 \cdot 8$ & $29 \cdot 2$ & $37 \cdot 7$ & $S F, 2-3$ & 10 & $10-20$ & $b f$ & & - & - & - & $\begin{array}{l}3 \text { p.ni. liah laud, } 1 . \\
4 \text { p.un. Str. on west ern mountains and }\end{array}$ \\
\hline 6 & $29 \cdot 347$ & - & $29 \cdot 7$ & $32 \cdot 2$ & $30 \cdot 0$ & $t t \cdot 0$ & Calm & 9 & 0 & $b \infty$ & I & - & - & - & 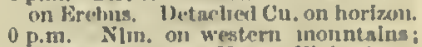 \\
\hline 8 & $29 \cdot 374$ & - & $29 \cdot 0$ & $30 \cdot 2$ & 28.5 & $45 \cdot 0$ & Calm & 1 & 0 ! & b & & - & - & - & $\begin{array}{l}\text { detachel Cu. oi } \mathrm{N} \text { aud } \mathrm{W} \text { Lorizou: } \\
\text { extremely liazy. }\end{array}$ \\
\hline 10 & $29 \cdot 384$ & - & $26 \cdot 0$ & $26 \cdot 8$ & $25^{\circ} 0$ & $43 \cdot 8$ & Calu & 0 & 0 & b & & - & - & - & \\
\hline M idt. & $29 \cdot 398$ & - & $25 \cdot 7$ & $25 \cdot 7$ & $23 \cdot 0$ & $43 \cdot 0$ & Calin & 1 & 0 & b) & & - & - & - & \\
\hline M ean, & $29 \cdot 273$ & - & - & $28 \cdot 6$ & $26 \cdot 4$ & 一 & - & - & - & 一 & & - & - & - & 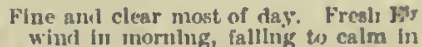 \\
\hline & Max. & & & $\int 8$ & $a$. & & adiatl & $\begin{array}{l}\text { ack } \\
\text { lghh }\end{array}$ & $\begin{array}{l}\text { oulh } \\
\text { bulli) }\end{array}$ & & & grainmes. & reeipm. & & 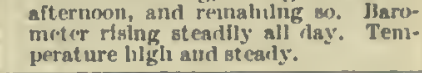 \\
\hline
\end{tabular}

\begin{tabular}{|c|c|c|c|c|c|c|c|c|c|c|c|c|c|}
\hline 2 & $23 \cdot 422$ & -1 & $21 \cdot 0$ & $25 \cdot 0$ & $22 \cdot 0$ & $41 \cdot 0$ & Calm & 1 & 0 & b & - & - & - \\
\hline 4 & $29 \cdot 468$ & - & $24 \cdot 2$ & $27 \cdot 2$ & $2 t \cdot 0$ & $35 \cdot 2$ & Calm & 1 & 0 & In & - & Det. Cu. $2.5 W$ & - \\
\hline 6 & $29 \cdot 48 t$ & - & $25 \cdot 0$ & $29 \cdot 0$ & $28 \cdot 0$ & $35 \cdot 2$ & Calm & 3 & 0 & $\mathbb{l}$ & Ci. $-\mathrm{cu}, 2, \mathrm{SE}$ & Det. Cu. 2, sw & - \\
\hline 8 & $29 \cdot 510$ & - & $28 \cdot 5$ & $36 \cdot 0$ & $35 \cdot 0$ & $42 \cdot 0$ & Calın & 1 & 0 & 1, & - & - & - \\
\hline 10 & $27) \cdot 538$ & - & $35 \cdot 0$ & $37 \cdot 0$ & $35 \cdot 5$ & $39 \cdot 0$ & Lt. SF alrs & 1 & $5-8$ & h & - & 一 & - \\
\hline Noon & $29 \cdot 548$ & - & $33 \cdot 8$ & $35 \cdot 0$ & $32 \cdot 2$ & $37 \cdot 0$ & $N E, 1$ & 10 & $10-12$ & 1) & - & - & - \\
\hline 2 & $29 \cdot 574$ & - & $30 \cdot 8$ & $33 \cdot 2$ & $29 \cdot 7$ & $39 \cdot 0$ & NE, $0-1$ & 3 & 5-15 & $b \infty$ & - & - & - \\
\hline 4 & $20 \cdot 580$ & - & $31 \cdot 0$ & $33 \cdot 3$ & $29 \cdot 3$ & $38 \cdot 2$ & Calun & 8 & 0 & $b, \infty$ & - & - & - \\
\hline 6 & $29 \cdot 594$ & - & $29 \cdot 6$ & $30 \cdot 0$ & $28 \cdot 5$ & $34 \div 3$ & Calm & 4 & 0 & $b \infty$ & - & - & - \\
\hline 8 & $29 \cdot 598$ & - & $28 \cdot 8$ & $30 \cdot 2$ & $28 \cdot 0$ & $41 \cdot 5$ & NNW, 1 & 7 & 8 & b & - & - & - \\
\hline 10 & $29 \cdot 599$ & - & $28 \cdot 0$ & $29 \cdot 0$ & $27 \cdot 5$ & $37 \cdot 1$ & NNW, 0-1 & 4 & $0-8$ & b & - & - & 一 \\
\hline Midt. & $29 \cdot 602$ & - & $22 \cdot 0$ & 28.5 & $25 \cdot 0$ & $33 \cdot 0$ & Lt. NNW airs & 4 & 0 & b & - & - & - \\
\hline Yean & $29 \cdot 5+3$ & - & - & $31 \cdot 1$ & $28 \cdot 9$ & - & - & -1 & - & - & - & - & - \\
\hline
\end{tabular}

10 a.m. to noon. Mirsge all round liorlzon.

Str. on \%estern mountains. 3 j.m. hain band, 0 .

Wached $\mathrm{Cu}$, on westeru mountalns. 列 blowing 1-2 hetween hours.

Visihility well markedl.

1) ist rising all down Strait.

Low fog bank all down Strait.

1 p.m. Mean of 8 olservations : with asplrator, dry lumlb $33^{\circ} 6$, wet hulb $27^{\circ .5}$ rom screen, dry bulh $33^{\circ} .99$, wet bulb $28^{\circ} \cdot 15$. Hygrogra ph show eyl 20 per cent. Temperate Tanter Temperature higher.

6 th.

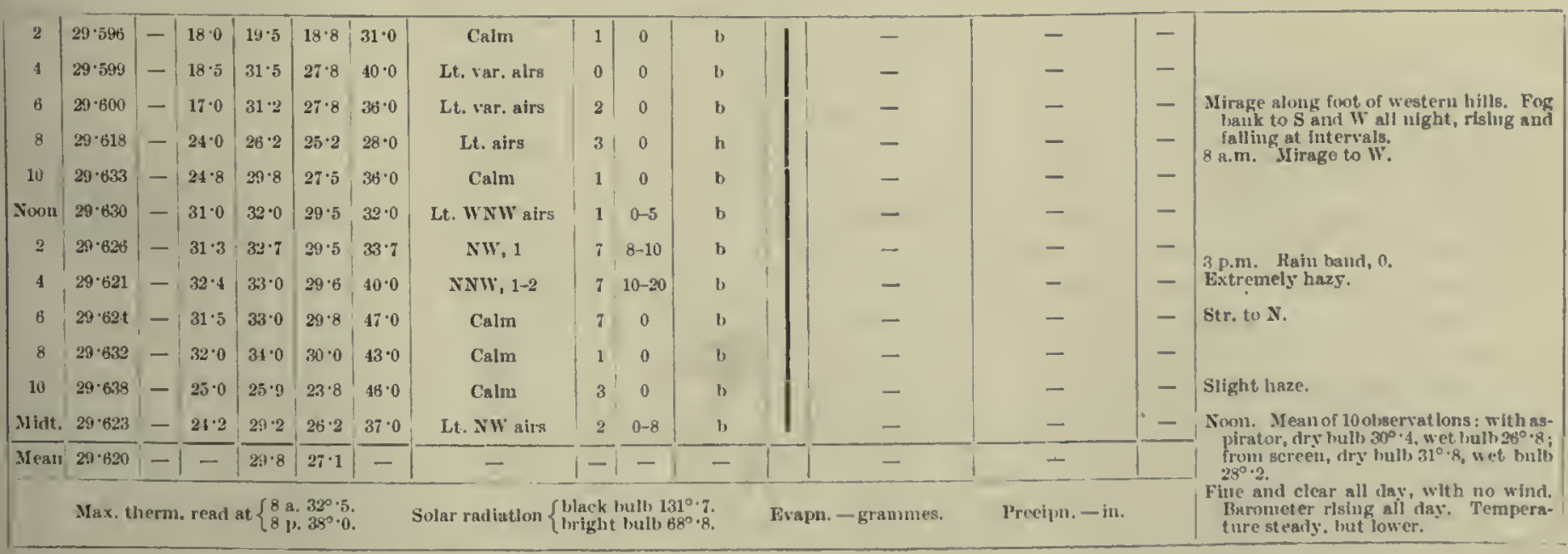

27 th. 
1902 -December.]

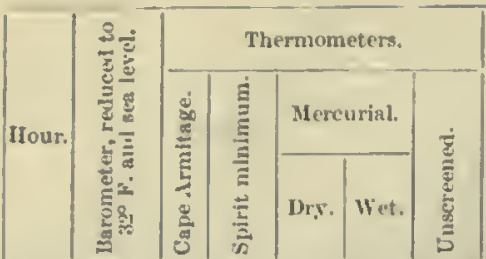

28 th.

\begin{tabular}{|c|c|c|c|c|c|c|}
\hline 2 & $24) \cdot 620$ & - & $23 \%$ & $2+0$ & $2 \% \cdot 0$ & 380 \\
\hline 4 & $29 \cdot 611$ & - & $28 \cdot 0$ & $32 \cdot 0$ & $28 \cdot 2$ & $38 \cdot 0$ \\
\hline 6 & $29+60 \mathrm{~s}$ & - & $31 \cdot 2$ & $32 \cdot 2$ & $28 \cdot 2$ & $3 * 2$ \\
\hline 8 & $29 \cdot 594$ & - & 320 & $333 \cdot 9$ & $28 \cdot 2$ & $3 b \cdot 2$ \\
\hline 10 & 29 'tate & - & $32 \cdot 8$ & $34-8$ & $32 \cdot 3$ & 38.0 \\
\hline Noon & $29 \cdot(6) 1$ & - & $31 \cdot 0$ & $34 \cdot " 3$ & $31 \cdot 8$ & $37 \cdot 0$ \\
\hline 3 & $29 \cdot 5,8$ & - & i & $33 \cdot 3$ & $29 \cdot 4$ & $3 ; \cdot 2$ \\
\hline 4 & $29 \cdot 594$ & - & 300 & 307 & $28 \cdot 6$ & 25.0 \\
\hline 6 & $29 \cdot 587$ & - & $29-6$ & $30 \cdot 0$ & $27 \cdot 8$ & $31 \cdot 0$ \\
\hline 8 & $29 \cdot 5 i 4$ & - & $28 \cdot 0$ & $2 s \cdot 2$ & $27 \cdot 8$ & $33 \cdot 0$ \\
\hline 10 & $29 \cdot 5$ คื & - & 240 & $25 \cdot 0$ & $24 \cdot 8$ & $28 \cdot 0$ \\
\hline
\end{tabular}

Meteorological JuUlinal of the "Discovery."

\begin{tabular}{|l|l|l|l|l|} 
Midt. $24 \cdot 557$ & $-24 \cdot 8$ & $25 \cdot 0$ & $24 \cdot 5$ & $26 \cdot 0$ \\
\hline
\end{tabular}

Mean $\overline{20 \cdot 591}-\overline{30 \cdot 5} \overline{28 \cdot 2}-$

\begin{tabular}{|c|c|c|c|c|c|c|c|}
\hline \multicolumn{3}{|c|}{ Winel. } & \multirow[b]{3}{*}{ 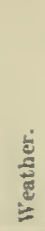 } & \multirow{3}{*}{ 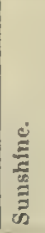 } & \multirow{2}{*}{\multicolumn{2}{|c|}{$\begin{array}{l}\text { Clouds, } \\
\text { Amout and movenent. }\end{array}$}} & \\
\hline \multirow{2}{*}{$\begin{array}{c}\text { Direetion aut } \\
\text { forre } \\
\text { (Heaufort scale). }\end{array}$} & \multirow{2}{*}{ 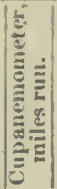 } & \multirow{2}{*}{ 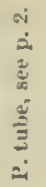 } & & & & & \\
\hline & & & & & Upper. & l.ower. & \\
\hline
\end{tabular}

Jeennarks,

\begin{tabular}{l|l|l}
$\mathrm{NW}, 1$ & 4 & $8-10$
\end{tabular}

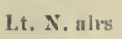

Lt. $x$, airs

Lt. x, airs

L1. N. airs

N. $2-3$

I., $w, 1$

$\mathbf{X} w, 1$

$x \log W, 1-2$

N 1,y W, 2-4

N l,y $:$ W, 1-2

ii $0-8$

b)

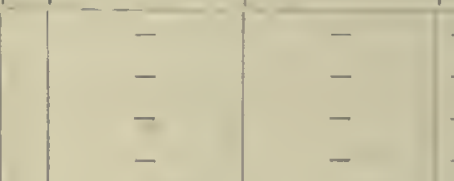

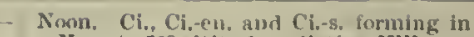
$X$ up to $70^{\circ}$ altitude ralliat ing Nit. 1 p.m. Cl.-s. forming quickly and radiat$\mathrm{ing}$ from NW to Sib. A.-8. forming in
$\mathrm{N}$ and N S. Western momtalus still

2 put clear. zontal hands radiat luy $x W^{\circ}$ to s: p.,n, Lahn band, 3 .

p.n. Ci.eru. clunging lornation,

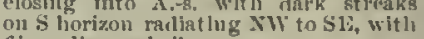
filmy diagonal rilis.

b.m. Ciouds mueh lower than previons fil. S.-ci. to N, aul on westery mountains. 8 p.m. l"leecy streaks radiating NW to Sli. liank of lills. froun $\mathrm{N}$. Pr.-8. passlug over rapially 10 p.m. Thick low Str. passing over from $N$. No land visible to $W$ or $S$ : 1 ops of liils eoveret. Alt it urle of Str., bion leet. Miclt. Very heary Cu. to N. Snowing slightly", and squally, and lhen ralm. eastem hills in fog. A break to $\mathrm{D}$, and Hhe sky visible, with rery streaky anil
heth, $28^{\circ}-4$; from screen, diy lulb $31^{\circ} .83$,

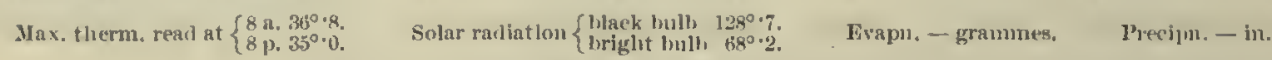

S. $8, X$ (fast) S.-cu. \$, X (rapill)

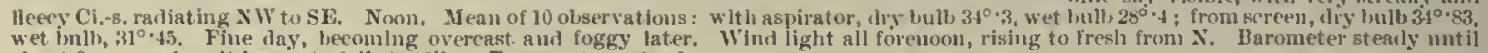
ahout 2 p.m., when it hegan to fnll steadily. Temperature steady.

29th.

\begin{tabular}{|c|c|c|c|c|c|c|c|c|c|c|}
\hline 2 & $29 \cdot 529$ & - & $22 \cdot 0$ & 23.0 & $22 \cdot 8$ & $24 \cdot 0$ & $\mathrm{~N}, 0-2$ & 8 & $0-18$ & ofq $*$ \\
\hline 4 & $29 \cdot 518$ & - & $22 \cdot 2$ & $23 \cdot 5$ & $23 \cdot 0$ & 2.0 & N, 1-2 & 4 & $5-12$ & o:c \\
\hline 6 & 29.495 & -1 & $22 \cdot 0$ & $23 \cdot 0$ & $22 \cdot 8$ & $25 \cdot 2$ & NW, 1-2 & 7 & $8-10$ & oc \\
\hline 8 & $29 \cdot 477$ & - & $22 \cdot 2$ & $21 \cdot 8$ & $24^{\circ} 0$ & $27 \cdot 0$ & Lt. NW airs & 4 & 0 & $\infty$ \\
\hline 10 & $29 \cdot 477$ & - & $2.1 \cdot 0$ & $27 \cdot 1$ & $26^{\circ} 0$ & $30 \cdot 0$ & Lt. $\mathbf{X}$ alrs & 1 & $0-8$ & 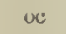 \\
\hline Noou & $29 \cdot 467$ & - & $21 \cdot 5$ & $27 \cdot 0$ & $25 \cdot 5$ & $28 \cdot 0$ & Calm & 2 & 0 & 1. \\
\hline 2 & $29 \cdot 454$ & -1 & $25 \cdot 7$ & $30 \cdot 0$ & $27 \cdot 6$ & $31 \cdot 0$ & Caim & 3 & 0 & 1" \\
\hline 4 & $29 \cdot 438$ & - & $29^{\circ} 6$ & $32 \cdot 0$ & $27 \cdot 6$ & $37 \cdot 8$ & $\mathrm{SH}, 1$ & 4 & $5-12$ & 1) \\
\hline 6 & 29.431 & - & $26 \cdot 5$ & $30 \cdot 2$ & $2 ; ; 6$ & $35 \cdot 2$ & SL, 1-2 & 5 & 10-15 & b) \\
\hline 8 & $29 \cdot 430$ & - & $29 \cdot 5$ & $32 \cdot 0$ & 28.5 & $3 \pi^{\circ} 0$ & $\mathrm{E}, 2$ & 10 & $12-18$ & 1) \\
\hline 10 & $29 \cdot 426$ & - & $30 \cdot 0$ & $31 \cdot 0$ & $28 \cdot 0$ & $35 \cdot 0$ & $1: 2$ & 10 & $10-20$ & b) \\
\hline Miat. & $39 \cdot 419$ & - & $27 \cdot 8$ & 31.5 & $31 \cdot 0$ & $40^{\circ} 0$ & $1 ; 2$ & 10 & $10-15$ & b \\
\hline Meal & $29 \cdot 4 k 4$ & - & - & $28 \cdot 2$ & $24 \cdot 4$ & 一 & - & - & - & - \\
\hline
\end{tabular}

Uax, therm. read at $\left\{\begin{array}{l}8 \mathrm{a}, 28^{\circ} 8 . \\ 8 \mathrm{p}, 36^{\circ} 0 .\end{array} \quad\right.$ Solar radint.jun $\left\{\begin{array}{l}\text { black bulb } 127^{\circ} \cdot 6 . \\ \text { bright hulb } 65^{\circ} \cdot 2 .\end{array}\right.$

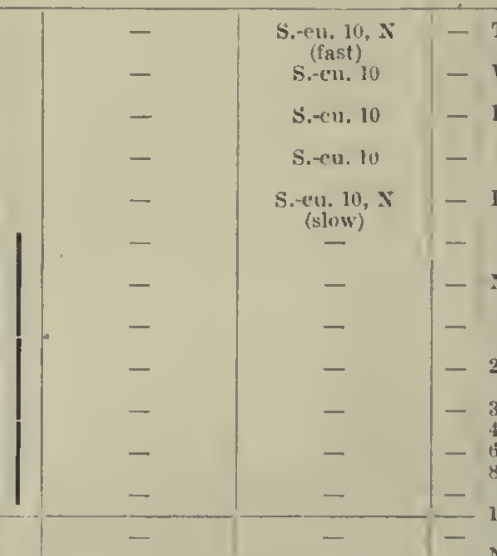

Erapn. - grammes.

Preeipu, - in.

Thick fog over Ohservat ion 11ill; snowlug and squally.
West ern montains visille. Ileary bank or S.-cu. NW tos.
From s to sw very elear.

Low S.-cu., ahout 600 feet in altitude, cutting of all land abose that height. sun gleaming: clonds lireaking overhear com. Thick fog lank langing over al mountains visible. Fog bank of wester mountains visible, Fog hank disappetur ing very fast.

p.th. Dark bank of fog on foot of corn mountains and to $\mathrm{s}$.

p.m. lain hank, 1 iw disappeariug.

6 p.un, Low fog comliug up, bous Saun w. p.m. Low fog alt down Strait from Sl\% to $W$, gradually Iadling away iu W. 0 1).m. Thin fog all dow Strait at altiturle of 200 fect. Quite cluar overlieas. coon. Mean of 10 olservations : with aspirator, dry bull $26^{\circ} \cdot 3$, wet bulh $25^{\circ} \cdot 2$; from sereen, dry lublt $31^{\circ} \cdot 0$, wet bull p.m. If (an of 10 ohservat lons: with as.

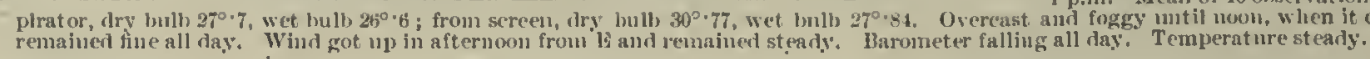

30 th.

\begin{tabular}{|c|c|c|c|c|c|c|c|c|c|c|c|c|c|c|}
\hline 2 & $29 \cdot 414$ & - & $22 \cdot 0$ & 248 & $23 \cdot 0$ & $2 x^{\circ} 0$ & Xw, 3-4 & 8 & $20-25$ & 1, & - & - & $1-$ & Bank of mist all round horizon. \\
\hline 4 & $29 \cdot 417$ & - & $18 \cdot 0$ & $21 \cdot 0$ & $21 \cdot 0$ & $24 \cdot 0$ & N by $W, 2-3$ & 10 & $15-20$ & he & - & Civ. 6 & - & Thick fog. \\
\hline 6 & $211 \cdot 411$ & - & $19 \cdot 8$ & $20 \cdot 8$ & $21 \cdot 0$ & $23 \cdot 0$ & Lt. $N$ airs & 7 & 0 & be - & - & Cu, 4 & - & Fog clearing overluead. \\
\hline 8 & $29 \cdot 409$ & - & $19 \cdot 8$ & 200 & $20 \cdot 0$ & $23 \cdot 0$ & Lt. $N$ airs & 1 & 0 & keu & - & S.-(2)1. 3 & - & \\
\hline 10 & $21 \cdot 901$ & - & $19 \cdot 0$ & $23 \cdot 0$ & $22 \cdot 8$ & $30 \cdot 0$ & Calu & 2 & 0 & औU & - & - & - & Thin mist ium ging in St rait, lut gradually \\
\hline Noon & $29 \cdot 389$ & - & $21 \cdot 2$ & $24 \cdot 0$ & $23 \cdot 8$ & $26 \cdot 0$ & NXW, I & 4 & $8-12$ & $\lim \omega$ & - & - & - & $\begin{array}{l}\text { Mist passlug overhead rapldly from } N \text {. } \\
\text { Nooll }\end{array}$ \\
\hline 2 & $211 \cdot 390$ & - & $22 \cdot 7$ & $24 \cdot 2$ & $24 \cdot 0$ & $31 \cdot 4$ & NNw, 0-1 & 4 & $0-8$ & $o c \sqcup$ & - & Fog 10 & - & $\begin{array}{l}\text { N. Tog all round in Strait. } \\
2 \text { p.n. No visibility. }\end{array}$ \\
\hline 4 & $29 \cdot 374$ & - & $23 \cdot 2$ & $24 \cdot 6$ & $24 \cdot 4$ & $29 \cdot 4$ & $\mathrm{~N}$ by $\mathrm{W}, 1$ & 4 & $5-12$ & $\infty w$ & - & Fog 10 & - & 3 p.m, Rain bank, 3 . \\
\hline 6 & $24 \cdot 364$ & - & $24 \cdot 3$ & $25 \cdot 0$ & $24 \cdot b$ & $27 \cdot 6$ & $\mathbf{x}, 1-3$ & $i$ & $8-25$ & nequ & - & $\begin{array}{l}\text { Seudil } 10, N \\
\text { (fast) }\end{array}$ & - & $\begin{array}{l}\text { shajed fog erystals on surface, } \\
\text { b p.un. Fog erystals oul weather side of }\end{array}$ \\
\hline 8 & $24 \cdot 334$ & - & $23 \cdot 8$ & $24 \cdot 2$ & $24 \cdot 0$ & $25 \cdot 8$ & $\operatorname{Var}, \mathbf{N}, 1$ & 7 & $8-12$ & 0 & - & $\begin{array}{c}F r \cdot-l l .10, \mathrm{I} \\
\text { (fast) }\end{array}$ & - & $\begin{array}{l}\text { all projecting objects. } \\
\text { 8 p.m. Wind slifting from NW in XW }\end{array}$ \\
\hline 10 & $24 \cdot 375$ & - & $23 \cdot 6$ & $21 \cdot 0$ & $2 n \cdot 7$ & $25 \cdot 3$ & Lt. $\mathbf{N}$, airs & 5 & $0-5$ & 0 & - & Fog 10 & - & $\begin{array}{l}\text { and back. Visibillty, } 0 \text {, Altiture of } \\
\text { fog, ton feet. }\end{array}$ \\
\hline Minlt. & 291.388 & - & $23 \cdot 2$ & 2.4 & $24 \cdot 0$ & $26 \cdot 2$ & Lt. N. alrs & 4 & $0-5$ & 0 & - & Fogr 10 & - & 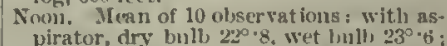 \\
\hline II (21) & $29 \cdot 3 \sin 0$ & - & - & $23 \cdot 3$ & $23 \cdot 0$ & - & 一 & - & - & - & - & - & - & $\begin{array}{l}\text { from screcell, dry lulh } 23^{\circ} \cdot 81 \text {, wet hulb } \\
23^{\circ} \cdot 53 \text {. }\end{array}$ \\
\hline \multicolumn{7}{|c|}{ 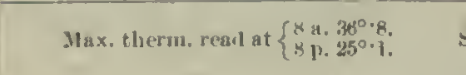 } & \multicolumn{4}{|c|}{ Solar radiatlon $\left\{\begin{array}{l}\text { l)lack bulb, } 111^{\circ} \% \text {, } \\
\text { Wriglit bulb } 57^{\circ} \%\end{array}\right.$} & Bvałn. - grammes. & \multicolumn{2}{|l|}{ Previpu. - in. } & $\begin{array}{l}\text { 1. } 30 \text { p.an. Mern of } 10 \text { olservations : witl } \\
\text { Aspirator, dry lull, } 22^{\circ} \cdot 3 \text {, wet bull, } 23^{\circ} \cdot 0 \text {; } \\
\text { from sereen, dry bulh } 23^{\circ} \cdot 83 \text {, wet bull }\end{array}$ \\
\hline
\end{tabular}


Mntrorotogical, JouRA, OH THE "Discovitiy,"

[1902-Deccmber.

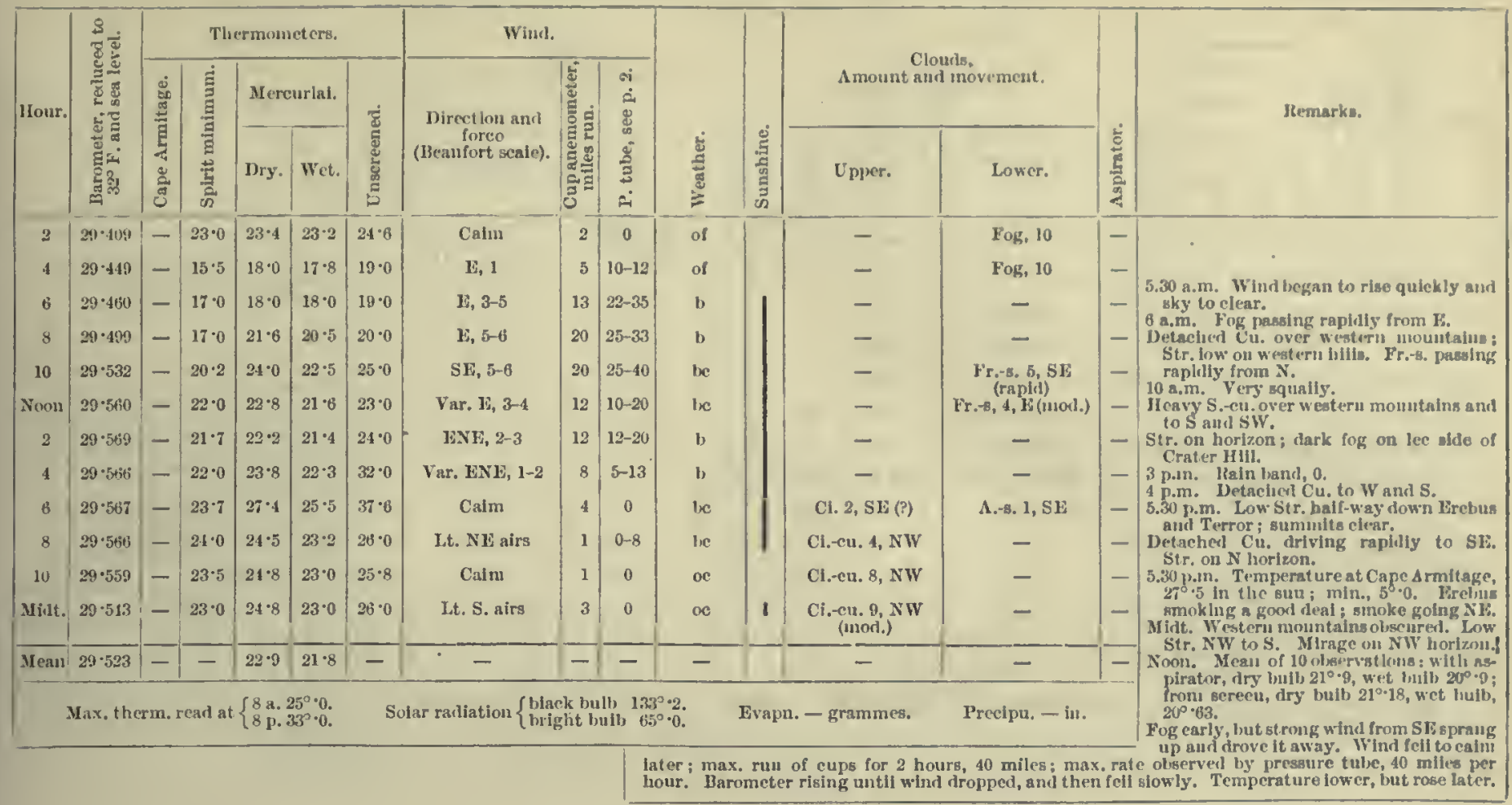

31 st. 
1903-January.]

Mrthorological Journay, of THE "Discovery."

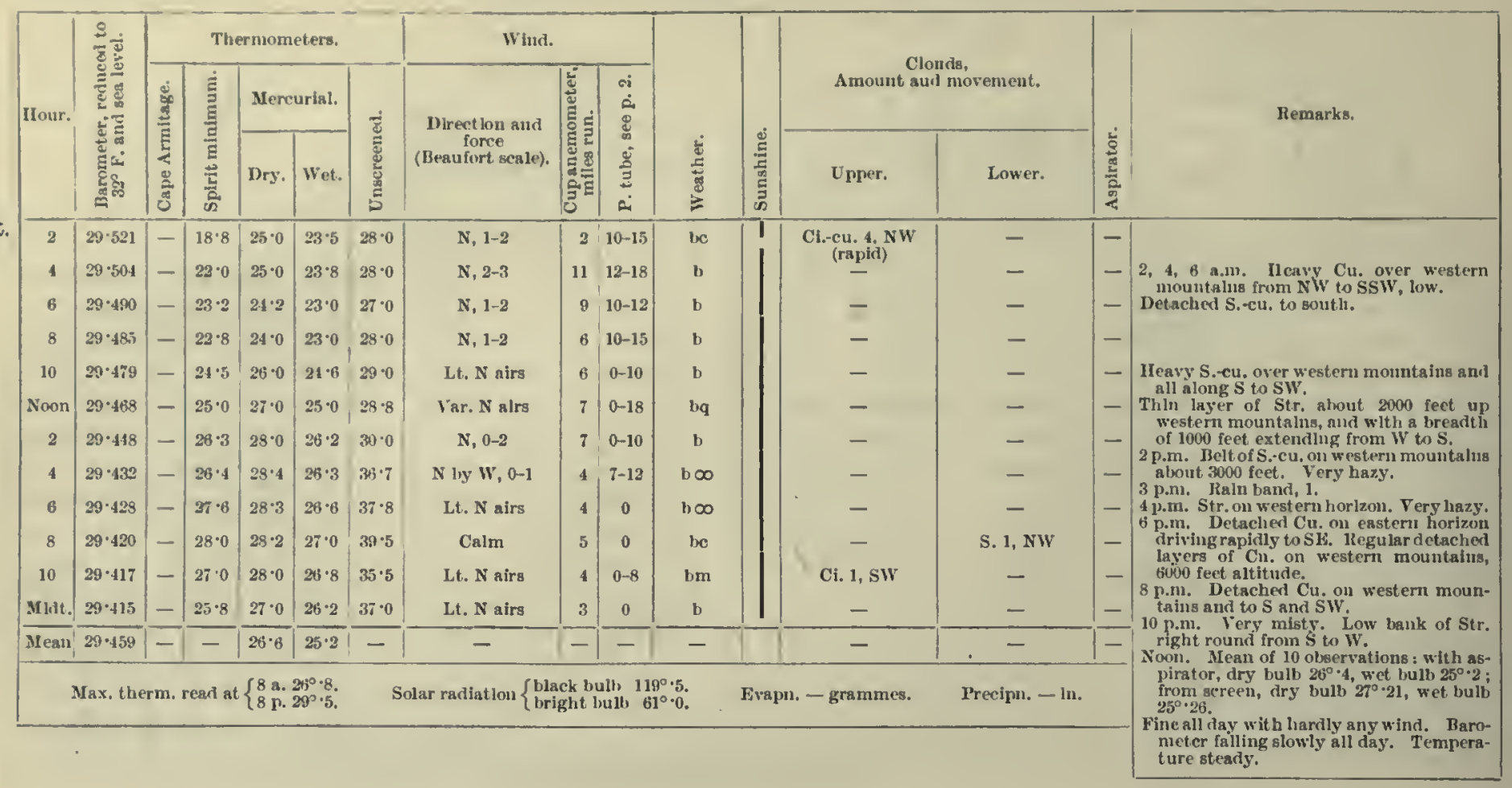

2nd.

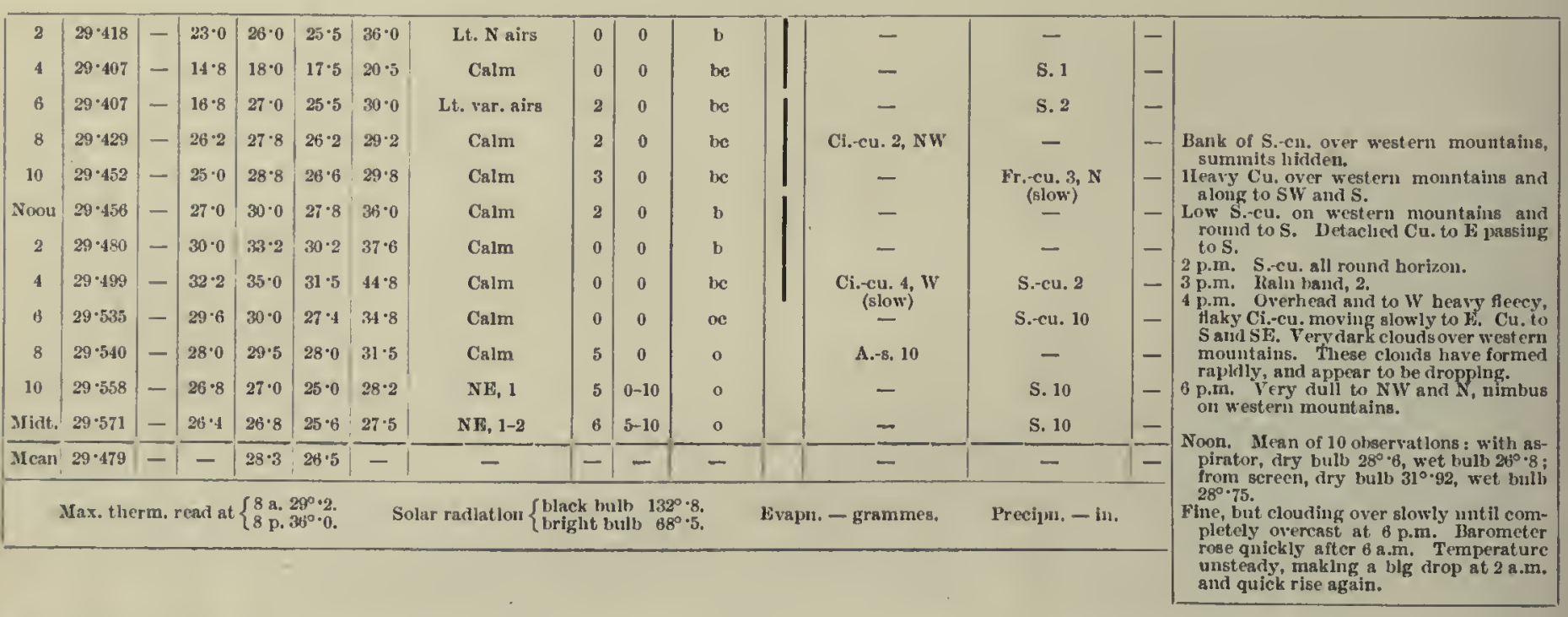

$3 r d$.

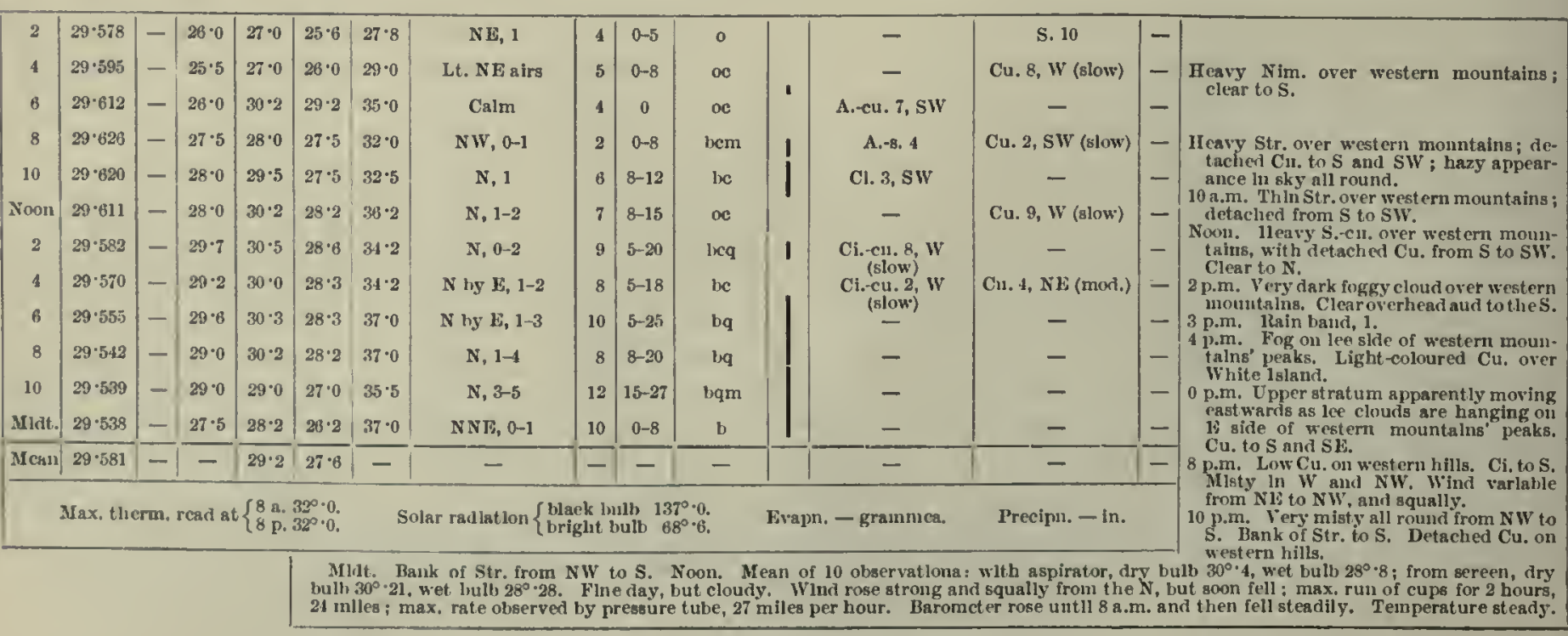


Meteorologicat, Journal of tur "Discovery."

[1903.-January.

\begin{tabular}{|c|c|c|c|c|c|c|c|c|c|c|c|c|c|c|c|}
\hline \multirow{3}{*}{ Ilour. } & \multirow{3}{*}{ 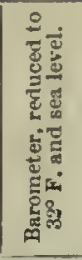 } & \multicolumn{5}{|c|}{ Thermoneters. } & \multicolumn{3}{|l|}{ WInd. } & \multirow[b]{3}{*}{ 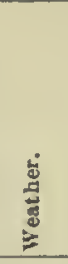 } & \multirow{3}{*}{ 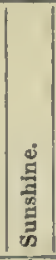 } & \multirow{2}{*}{\multicolumn{2}{|c|}{$\begin{array}{l}\text { Clonds, } \\
\text { Amount and movement. }\end{array}$}} & \multirow[b]{3}{*}{ 謽 } & \multirow{3}{*}{ llemarks. } \\
\hline & & \multirow{2}{*}{ 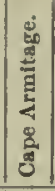 } & \multirow{2}{*}{ 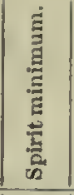 } & \multicolumn{2}{|c|}{ Mercurlat. } & \multirow{2}{*}{ 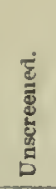 } & \multirow{2}{*}{$\begin{array}{c}\text { Direction and } \\
\text { force } \\
\text { (Reaufort acaie). }\end{array}$} & \multirow{2}{*}{ 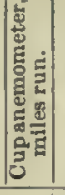 } & \multirow{2}{*}{ 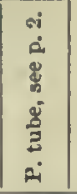 } & & & & & & \\
\hline & & & & Dry. & Wet. & & & & & & & vpper. & Lower. & & \\
\hline 2 & $29 \cdot 540$ & - & $25 \cdot 0$ & $28 \cdot 0$ & $25 \cdot 2$ & $31 \cdot 0$ & Var. N to $W, 0-2$ & 4 & $0-18$ & $b$ & I & - & - & $1-1$ & \multirow{14}{*}{ 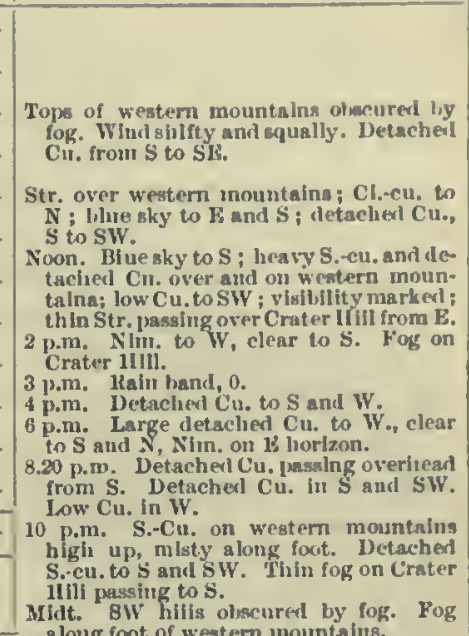 } \\
\hline 4 & $29 \cdot 568$ & - & $24 \cdot 0$ & $25 \cdot 2$ & 24.0 & $29 \cdot 2$ & Var. $N$ to $W, 0-3$ & 5 & $0-20$ & beq & & - & S.-cu. 4, S1 (fast) & - & \\
\hline 6 & $29 \cdot 591$ & - & $23 \cdot 0$ & $24 \cdot 0$ & $23 \cdot 2$ & $27 \cdot 0$ & Var. $S$ to $\mathrm{N}, \mathrm{O}^{-3}$ & 7 & $0-22$ & ber & 1 & - & 8.-etı. 8 & - & \\
\hline 8 & $29 \cdot 615$ & - & $23 \cdot 0$ & $24 \cdot 5$ & $23 \cdot 8$ & $27 \cdot 0$ & E, 4-5 & 14 & $20-28$ & be & & Cl. $\mathrm{cu} .6$ & - & - & \\
\hline 10 & $29 \cdot 629$ & - & $24 \cdot 5$ & $27 \cdot 0$ & $25 \cdot 0$ & $29 \cdot 5$ & Var. E, 5 & 15 & $20-25$ & $\boldsymbol{\alpha c}$ & & - & Cr. 8, N (slow) & - & \\
\hline Noon & $29 \cdot 617$ & - & $23 \cdot 0$ & $28 \cdot 2$ & $26 \cdot 0$ & 310 & Var. E. $2-4$ & 13 & $15-20$ & $o c$ & & - & Cu $9, N$ (slow) & - & \\
\hline 2 & $29 \cdot 673$ & - & $25 \cdot 4$ & $26 \cdot 2$ & $24 \cdot 6$ & $30 \cdot 6$ & Var. $\mathbf{E}, 1-2$ & 13 & $3-18$ & $o c$ & & - & Cu. 9, N (slow) & - & \\
\hline 4 & $29 \cdot 681$ & - & $26 \cdot 3$ & $29 \cdot 6$ & $25 \cdot 6$ & $30 \cdot 8$ & ESE, 0-1 & 8 & $5-8$ & be & 1 & Ci.-cu. 4, W (siow) & Cu. 1, N (slow) & - & \\
\hline 6 . & $29 \cdot 689$ & - & $29 \cdot 0$ & $31 \cdot 2$ & $29 \cdot 0$ & $38 \cdot 4$ & Calm & 3 & 0 & be & & Cl.-8. 2, W (slow) & - & - & \\
\hline 8 & $29 \cdot 689$ & - & $29 \cdot 0$ & $29^{\circ} 0$ & $26 \cdot 2$ & $36 \cdot 2$ & $N, 1-2$ & 1 & $10-15$ & $b$ & & - & - & - & \\
\hline 10 & $29 \cdot 689$ & - & 205 & $28 \cdot 0$ & $27 \cdot 0$ & $36 \cdot 8$ & $\mathrm{~N}, 1$ & 3 & $8-10$ & b & & - & - & - & \\
\hline Mint. & $29 \cdot 672$ & - & $27 \cdot 0$ & $28 \cdot 0$ & $27 \cdot 0$ & $36 \cdot 2$ & $\mathrm{~N}, 1-2$ & 9 & 10-15 & b & 1 & - & - & $1-$ & \\
\hline Mean & $29 \cdot 640$ & - & - & $27 \cdot 2$ & $25 \cdot 6$ & - & - & - & - & - & & - & - & $1-$ & \\
\hline \multicolumn{7}{|c|}{ Max. therm. read at $\left\{\begin{array}{l}8 \text { a. } 30^{\circ} 0 \\
8 \text { p. } 36^{\circ} \cdot 8\end{array}\right.$} & \multicolumn{4}{|c|}{ Solar radiation $\left\{\begin{array}{l}\text { biack buib } 117^{\circ} \cdot 8 . \\
\text { bright buit } 62^{\circ} \cdot 0 .\end{array}\right.$} & \multicolumn{2}{|c|}{ Evapn. - grammea. } & Precipn. - ln. & & \\
\hline & & & & & & & & & & & & & & & $\begin{array}{l}29 \mathrm{mil} \\
\text { ture sten }\end{array}$ \\
\hline
\end{tabular}

\begin{tabular}{|c|c|c|c|c|c|c|c|c|c|c|c|c|c|c|}
\hline 2 & $29 \cdot 664$ & -1 & $26 \cdot 5$ & $28 \cdot 0$ & $27 \cdot 0$ & 350 & $N, 1-3$ & 7 & $10-15$ & b & & - & - & - \\
\hline 4 & $29 \cdot 647$ & - & $25 \cdot 0$ & $28 \cdot 2$ & $27 \cdot 5$ & $32 \cdot 0$ & N, 1-2 & 8 & $10-18$ & b & & - & - & - \\
\hline 6 & $29 \cdot 641$ & - & $27^{\circ} 0$ & $28 \cdot 0$ & $27 \cdot 0$ & $31 \cdot 0$ & $N, 1-2$ & 8 & $8-15$ & b & & - & - & - \\
\hline 8 & $29 \cdot 641$ & - & $25 \cdot 8$ & $27 \cdot 6$ & $26 \cdot 2$ & $29 \cdot 8$ & Calm & 2 & 0 & lic & i & - & Cu. 7, N (fast) & - \\
\hline 10 & $29 \cdot 633$ & - & $27 \cdot 5$ & $29 \cdot 0$ & $28 \cdot 0$ & $30 \cdot 5$ & Calm & 2 & o & $\propto$ & & - & Cu. 9, N (mod.) & - \\
\hline Nonn & $29 \cdot 622$ & - & $28 \cdot 0$ & $28 \cdot 2$ & $27 \cdot 5$ & $30 \cdot 8$ & Cnlm & 5 & 0 & 0 & I & - & S. 10, N (slow) & - \\
\hline 2 & $29 \cdot 616$ & - & $28 \cdot 0$ & $30 \cdot 3$ & $29 \cdot 0$ & $32 \cdot 4$ & Calm & 3 & 0 & $b c$ & 急 & - & Cu. 6, NW (siow) & - \\
\hline 4 & $29 \cdot 601$ & - & $29 \cdot 4$ & $31 \cdot 6$ & $29 \cdot 6$ & $36 \cdot 8$ & Calm & 0 & 0 & te & 然 & Ci.eu. 2, W (slow) & - & - \\
\hline 6 & $29 \cdot 593$ & - & $23 \cdot 3$ & $30 \cdot 0$ & $28 \cdot 2$ & $38 \cdot 3$ & Caim & 0 & 0 & be & 2 & Ci.-cu, 5, W (slow) & - & - \\
\hline 8 & $29 \cdot 585$ & - & $29 \cdot 0$ & $30 \cdot 0$ & $28 \cdot 0$ & $31 \cdot 0$ & Calm & 3 & 0 & $\propto$ & 㟧 & $\begin{array}{l}\text { Cl.-cu. 7, NW } \\
\text { (siow) }\end{array}$ & - & - \\
\hline 10 & $29 \cdot 580$ & - & $28 \cdot 5$ & $29 \cdot 5$ & $27 \cdot 8$ & $30 \cdot 0$ & Lt. $\mathbf{N}$ airs & 2 & 0 & $b$ & $\frac{9}{5}$ & - & - & - \\
\hline Midt. & $29 \cdot 571$ & -1 & $26 \cdot 5$ & $27 \cdot 0$ & $26 \cdot 0$ & 40.0 & $\mathrm{~N}, 1-2$ & 3 & 8-15 & b & है & $=$ & - & $=$ \\
\hline Mean & 23617 & - & - & $29 \cdot 0$ & $27 \cdot 7$ & - & - & - & - & - & & - & - & $1-$ \\
\hline
\end{tabular}

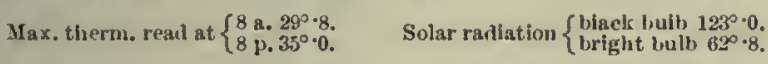

Evapu. - granurues. Precipn. - in. 4th.

5 th.

Pog over Observalion and Crater Hills. Low bank of S.eu. half - way down Western monntains, summits clear. westem mountalns and along to $\mathrm{S}$. Noon. 1 ligin fog over ali lanil a tait. of 600 ft. 2 p.m. Fog on Crater 11 ill and western mountalns up to alout $6000 \mathrm{ft}$. Clear to S. 3 p.in. Lain band, 0.

p.m. S.-cu. from NW to S. Upper cloud formed on NW horizon and are movlng siowiy to SE.

p.m. S.eu. at base and summits of vestcrimountaing mo D.m. Low fog from $W$ to $S$. Detached p.m. on western mourtains. Typlcal Cl.-cu. Cl s, to S. $W$; detarhed Cu. to l\%. Midt. Low fog from S to W. Cloudless sk y. Noon. Blen of 10 observat lona: with aspirator, ary huil, $27^{\circ} \cdot 5$, wet bivils $27^{\circ} \cdot 4$; inom
gereen, dry buib $28^{\circ} \cdot 5$, wet hulb $27^{\circ} \cdot 5$. Fine, with some typical exampies of Cl.-cu. clouds. 1 lardy any wind ali day later. Teruperature steady.

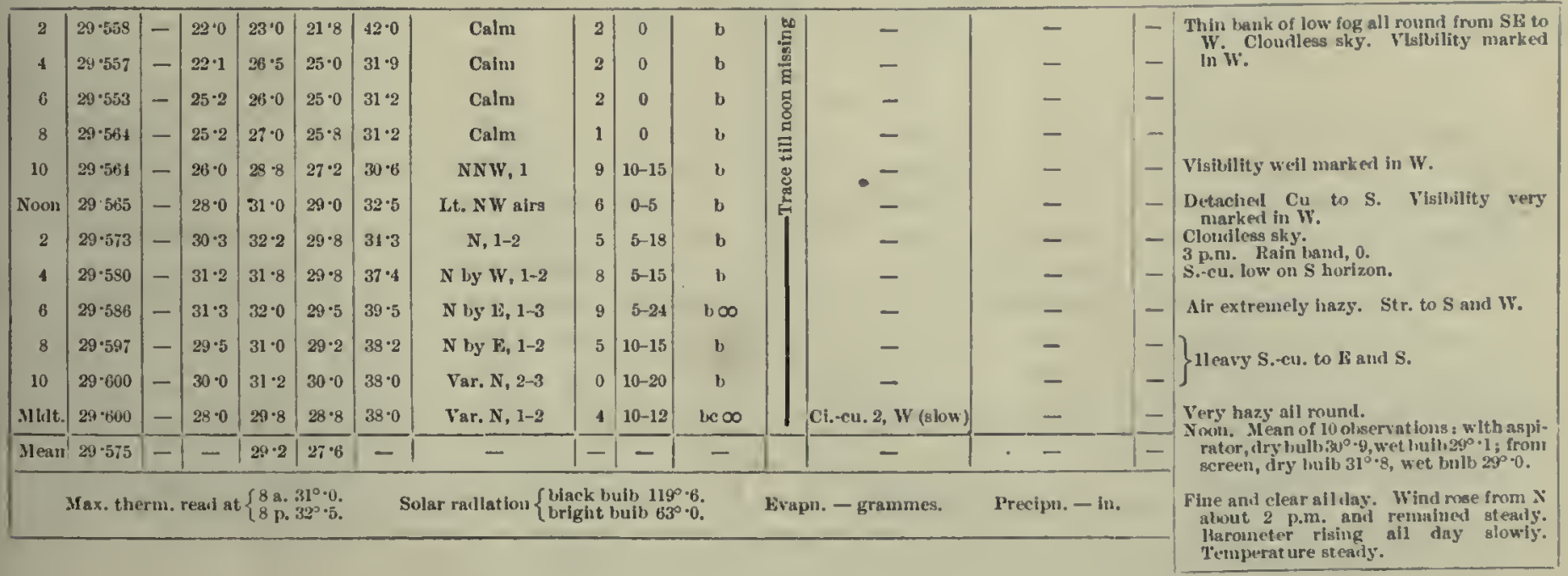


1903 - Jannary.]

Meteorological Journal of tif "Discovery."

\begin{tabular}{|c|c|c|c|c|c|c|c|c|c|c|c|c|c|}
\hline \multirow{3}{*}{ If sour. } & \multirow{3}{*}{ 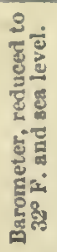 } & \multicolumn{5}{|c|}{ Thermometcrs. } & \multicolumn{3}{|l|}{ Wind. } & \multirow[b]{3}{*}{ 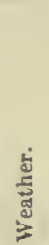 } & \multirow[b]{3}{*}{ 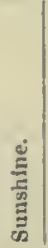 } & \multirow{2}{*}{\multicolumn{2}{|c|}{$\begin{array}{l}\text { Clouls, } \\
\text { Amount and novemeit. }\end{array}$}} \\
\hline & & \multirow{2}{*}{ 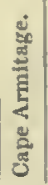 } & \multirow{2}{*}{ 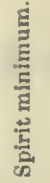 } & \multicolumn{2}{|c|}{ Mercurial. } & \multirow{2}{*}{ 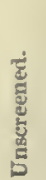 } & \multirow{2}{*}{$\begin{array}{l}\text { Directlou and } \\
\text { force } \\
\text { (Beaufort scale). }\end{array}$} & \multirow{2}{*}{ 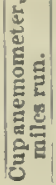 } & \multirow{2}{*}{ 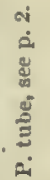 } & & & & \\
\hline & & & & Dry. & Wet. & & & & & & & Upper. & Lower. \\
\hline
\end{tabular}

7th.

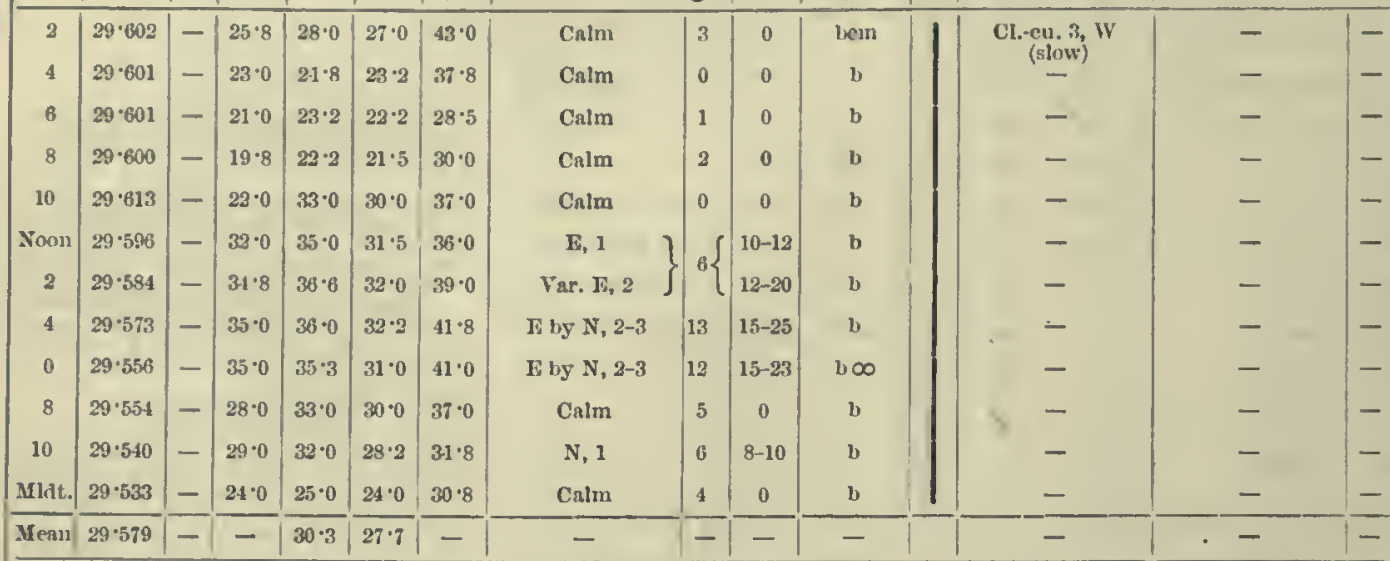

Max. therm, read at $\left\{\begin{array}{l}8 \text { a. } 32^{\circ} 0 . \\ 8 \text { p. } 33^{\circ} \cdot 0 .\end{array}\right.$ Solar radiation $\left\{\begin{array}{l}\text { black bulb } 126^{\circ} \cdot 7 . \\ \text { briglit bulb } 68^{\circ} \cdot 8 .\end{array}\right.$

Evapn.-grammes.

Precipn. - in

- Very heavy bank of S.-cu, all romil horlpassing S over Crater Hill.

taing tains, 600 fect. Tops vislble extending to $\mathrm{S}$

Low fog bank all round from $W$ to $S$. Nilsty over lanid.

Low bank of Str, to $S$.

Extremely hazy, visibility small.

3 p.m. Kain hand, 2. .cu. on S horlzon; detached cn. over Wow

Cu, to 8 str. to $\mathrm{N}$ and $\mathrm{NWV}$; detaclsel Wu. to S and Hoge at foot 8 p.m. Thick fog in Strait and driving in from $N$.

p.m. Low fog along foot of westem hills from $\mathrm{NW}$ to $\mathrm{S}$, detacherl $\mathrm{Cu}$, in Midt. low dark Str. in S. fow fog l,ank all round horizon. Yoon. Aran of 10 olservations : with as pirator, dry bulb $37^{\circ} 8$, wet bulh $30^{\circ} \cdot 6$ from sereen, itry bulb $37^{\circ} 0$, wet bulk

Fine and clear all day. Wind from calm sprang to fresh in aftertioon, and thel fell again to calm. Baroneter fell higher generally.

8 th.

\begin{tabular}{|c|c|c|c|c|c|c|c|c|c|c|c|c|c|}
\hline 3 & $29 \cdot 502$ & -1 & $19 \cdot 8$ & $21 \cdot 0$ & $23 \cdot 0$ & $30 \cdot 0$ & Calm & 0 & 0 & $\mathrm{~b}$ & - & - & $\mid-$ \\
\hline 4 & $29 \cdot 483$ & - & $21 \cdot 8$ & $25 \cdot 0$ & $23 \cdot 8$ & 31.0 & Calm & 0 & 0 & $b$ & - & - & - \\
\hline 6 & $29 \cdot 472$ & - & $21 \cdot 0$ & $24 \cdot 2$ & $23 \cdot 0$ & $27 \cdot 0$ & Calm & 0 & 0 & b & - & - & - \\
\hline 8 & $29 \cdot 473$ & - & $25 \cdot 0$ & $33 \cdot 0$ & 28.8 & $32 \cdot 0$ & Lt. SE airb & 1 & 0 & b & - & - & - \\
\hline 10 & $29 \cdot 454$ & - & $31 \cdot 0$ & $31 \cdot 5$ & 28.8 & $36 \cdot 0$ & SE, 1-2 & 8 & $10-15$ & b & - & - & - \\
\hline Noon & $29 \cdot 430$ & .. & $29 \cdot 0$ & $29 \cdot 6$ & $28 \cdot 0$ & $30 \cdot 0$ & ESE, 2-4 & 10 & $15-23$ & b & - & - & - \\
\hline 2 & $29 \cdot 412$ & - & $29 \cdot 0$ & $31 \cdot 6$ & $29 \cdot 6$ & $32 \cdot 3$ & Yar. F, 2-3 & 14 & $13-20$ & be & - & Det. Cu. 2, SE & - \\
\hline 4 & $29 \cdot 401$ & - & $30^{\circ} 0$ & $30 \cdot 7$ & $28 \cdot 8$ & $33 \cdot 4$ & ENE, 1-2 & 10 & $5-12$ & b & - & - & - \\
\hline 6 & $29 \cdot 389$ & - & $30 \cdot 2$ & $32 \cdot 0$ & $30 \cdot 2$ & $34 \cdot 5$ & Calm & 4 & 0 & b & - & - & - \\
\hline 8 & $29 \cdot 370$ & - & $29 \cdot 5$ & $30 \cdot 0$ & $28 \cdot 2$ & $32 \cdot 5$ & N, 0-1 & 4 & $0-10$ & be & - & S. 1 & - \\
\hline 10 & $29 \cdot 364$ & - & $28 \cdot 2$ & $28 \cdot 9$ & $27 \cdot 8$ & $31 \cdot 5$ & $N, 1$ & 8 & $0-10$ & b & - & - & - \\
\hline Miat. & $29 \cdot 351$ & - & $26 \cdot 9$ & $27 \cdot 1$ & 26.7 & $29 \cdot 0$ & $\mathrm{~N}, 1$ & 6 & 10 & b & - & - & $1-$ \\
\hline Mean & $29 \cdot 425$ & -1 & - & $29 \cdot 0$ & $27 \cdot 2$ & - & - & $1-$ & - & - & - & - & - \\
\hline
\end{tabular}

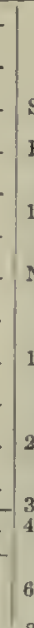

Max. therm. read at $\left\{\begin{array}{l}8 \text { a. } 35^{\circ} \cdot 2 . \\ 8 \mathrm{p}, 39^{\circ} \cdot 0 .\end{array} \quad\right.$ Solar radintion $\left\{\begin{array}{l}\text { black bulh } 119^{\circ} \cdot 0 . \\ \text { bright bulb } 63^{\circ} \cdot 0 .\end{array}\right.$

Evapn. - grammes.

Preclpn. - in. p.m. Rain band, 1.
p.m. Detached Cu, passing. Fog on
Crater Il ill ; S.-cu, all roumi horizonl. Suminits of western monntains visible. p.m. Layers of $\mathrm{Cu}$. on western mon trong mirage all round horizon.

Heavy S.-cu, in S and SE. Bank of fog on western mountains, tops a 0 a.m, Dark S.-cur. on western mountains, simmits and bases

oon. S.cu. over anil on western mountains increasiug. Cu. and low Str. to Sl and S. increasing and rising. Land getting quickly obscured.

.45 p.m. Fog passing over eastern hills from $\mathrm{E}$ rapidly; western mountain obscuren.

p.m. IV steru monntains alunost obp.m. For on Crater and Obsem. on suranut.

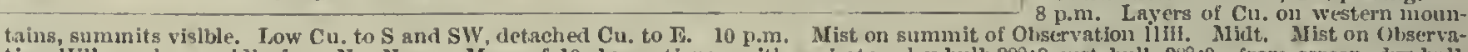
tion Hill unovlng rapidly from $\mathrm{N}$. Noon. Mean of 10 observations : with aspirator, dry bulb $29^{\circ} \cdot 8$, wet bull, $25^{\circ} \cdot 0$; from sereen, dry bulb $29^{\circ} 82$, wet hull, $28^{\circ} \cdot 23$. Fine and clear all day, wlth a repetition of calm and whil as yesterulay, rising in the milllle of the day and going down hithe evening. Barometer continued to fall steadily all day. Temperature sterily.

9th.

\begin{tabular}{|c|c|c|c|c|c|c|c|c|c|c|c|c|c|c|c|}
\hline 2 & $29 \cdot 236$ & -1 & $26 \cdot 0$ & $27 \cdot 6$ & $26 \cdot 6$ & $26 \cdot 0$ & N, 1-2 & 5 & $0-10$ & b & 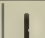 & - & - & -1 & Mist almost gone. \\
\hline 4 & $20 \cdot 327$ & - & $20 \cdot 0$ & $26 \cdot 2$ & $25 \cdot 5$ & $29 \cdot 0$ & $\mathrm{~N}, \mathbf{1 - 2}$ & 12 & $0-15$ & $\mathrm{~b}$ & & - & - & - & 5 a.m. Low fog from SE to SW. Clear \\
\hline 6 & $29 \cdot 327$ & - & $25 \cdot 5$ & $26 \cdot 5$ & $25 \cdot 2$ & $29 \cdot 2$ & $N, 1-2$ & 4 & $8-15$ & b & & - & - & - & Low fog from SE to NIs. Fog passing \\
\hline 8 & $29 \cdot 318$ & - & $25 \cdot 5$ & $26 \cdot 5$ & $25 \cdot 8$ & $29 \cdot 0$ & $\mathbf{N}, \mathbf{1}$ & 5 & $6-12$ & o & & - & S, $10, \mathrm{~N}$ & - & $\begin{array}{l}\text { Lover fog over all linls above } 600 \text { fert, } \\
\text { divilug over from } \mathrm{N} \text {. }\end{array}$ \\
\hline 10 & $29 \cdot 315$ & - & $25 \cdot 0$ & $27 \cdot 0$ & $26 \cdot 0$ & $31 \cdot 0$ & Lt. $\mathbf{N}$ airs & 7 & $5-10$ & 0 & : & - & Thin S. 10, N & - & 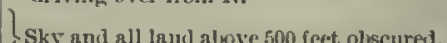 \\
\hline Noon & $29 \cdot 315$ & - & $26 \cdot 0$ & $27 \cdot 5$ & $26 \cdot 8$ & $33 \cdot 0$ & $\mathrm{~N}, 1$ & 7 & $5-12$ & o & & - & Thins. 10, N (fast) & - & 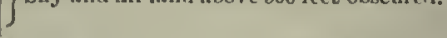 \\
\hline 2 & $29 \cdot 307$ & - & $26 \cdot 0$ & $27 \cdot 4$ & $26 \cdot 6$ & $30 \cdot 0$ & NNW, 1-2 & 7 & $0-10$ & oc & & - & S.-cu. 10, $N$ (fast) & - & Sheet of S.-cu. olscuring all land orer \\
\hline 4 & $29 \cdot 307$ & - & $26 \cdot 7$ & $27 \cdot 3$ & $26 \cdot 5$ & $29 \cdot 7$ & Far, N, 1-2 & 8 & $0-10$ & oc & & - & S. - cla. 10, N (fast) & - & 3 p.m. lain band, 3 . \\
\hline e & $20 \cdot 317$ & - & $25 \cdot 8$ & $26 \cdot 2$ & $25 \cdot 4$ & $28 \cdot 3$ & Far. $\mathrm{N}, 1-2$ & 8 & $0-10$ & $\propto$ & & - & S.-cu, 10, $\mathbb{N}$ (fast) & - & visible, also NIV of White Islanul. \\
\hline 8 & $29 \div 327$ & - & $25 \cdot 0$ & $25 \cdot 5$ & $25 \cdot 0$ & $25 \cdot 8$ & Far. N, 1-2 & 5 & $8-12$ & oc & & - & Fr.-s, 10, $\mathrm{N}$ (fast) & - & Obscrvation linils. \\
\hline 10 & $29 \cdot 332$ & - & $24 \cdot 5$ & $25 \cdot 0$ & $24 \cdot 3$ & $25 \cdot 0$ & Lt. $\mathbf{N}$ airs & 6 & $0-8$ & $o c$ & & 一 & $\mathrm{Fr}_{\mathrm{r}}-\mathrm{s}, 10, \mathrm{~N}$ (fnst) & - & 8 p.m. Fro-8. passing over tops of east ern \\
\hline Mlilt. & $29 \cdot 341$ & -1 & $23 \cdot 2$ & $24 \cdot 0$ & $24 \cdot 0$ & $25 \cdot 0$ & Calm & 2 & 0 & oef & & - & S. $10, \mathrm{~N}$ (fast) & -1 & $\begin{array}{l}10 \text { p.m. For on summits of cast erm hills, } \\
\text { at. about } 500 \text { feet. }\end{array}$ \\
\hline M can & $29 \cdot 322$ & - & - & $26 \cdot 4$ & $25 \cdot 0$ & - & - & - & - & - & & - & - & - & to $\mathrm{S}$, alt. about 150 feet. \\
\hline & 1 & & : & & & & iot & & 1) 1 & & \multicolumn{2}{|c|}{ Eva pn. - grammes. } & 年 & & $\begin{array}{l}\text { Overeast most of the lay, with light to } \\
\text { fresh variable N'y whids. Barometer } \\
\text { steady. T'emperature steary. }\end{array}$ \\
\hline
\end{tabular}


Metrorologicat, Journal of the "Discoveiry."

[1903-January.

\begin{tabular}{|c|c|c|c|c|c|c|c|c|c|c|c|c|c|c|}
\hline \multirow{3}{*}{ Hlour. } & \multirow{3}{*}{ 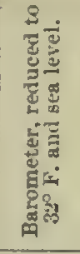 } & \multicolumn{5}{|c|}{ Therunoueters. } & \multicolumn{3}{|l|}{ Whar. } & \multirow[b]{3}{*}{ 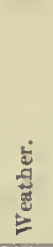 } & \multirow[b]{3}{*}{ 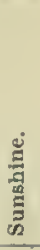 } & \multirow{2}{*}{\multicolumn{2}{|c|}{$\begin{array}{l}\text { Clouds, } \\
\text { Autount and unovenent. }\end{array}$}} & \multirow[b]{3}{*}{$\frac{\stackrel{0}{8}}{\frac{2}{2}}$} \\
\hline & & \multirow{2}{*}{ 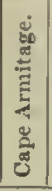 } & \multirow{2}{*}{ 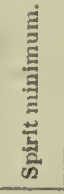 } & \multicolumn{2}{|c|}{ Mercurlai. } & \multirow[b]{2}{*}{ 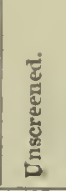 } & \multirow{2}{*}{$\begin{array}{c}\text { Dircetion and } \\
\text { force } \\
\text { (Beanfort scale). }\end{array}$} & \multirow{2}{*}{ 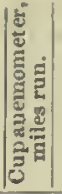 } & \multirow{2}{*}{ 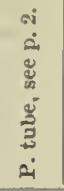 } & & & & & \\
\hline & & & & Dry. & Wet. & & & & & & & Upier. & Lower. & \\
\hline 2 & $29 \cdot 331$ & - & $22 \cdot 2$ & $23 \cdot 2$ & $23 \cdot 2$ & - & - Var. N, 1-3 & $\tau$ & $5-15$ & of & & - & Fr.-8, 10, N (fast) & 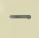 \\
\hline 4 & $29: 329$ & - & $23 \cdot 0$ & $24 \cdot 2$ & $24 \cdot 2$ & - & $\mathrm{N}, 1$ & 4 & $5-10$ & of & & - & Fr.-8, 10, $\mathbf{N}$ (fast) & - \\
\hline 6 & $29 \cdot 333$ & - & $22 \cdot 0$ & $24 \cdot 0$ & $24 \cdot 0$ & - & NW, I & 7 & $6-10$ & be & & - & S. cu, 8 & - \\
\hline 8 & $29 \cdot 351$ & - & $23 \cdot 0$ & $27 \cdot 0$ & $26 \cdot 2$ & $27 \cdot 0$ & Caim & 0 & 0 & be & 1 & - & S.cu. $8, \mathrm{~N}$ & - \\
\hline 10 & $29 \cdot 358$ & - & $24 \cdot 2$ & $26 \cdot 0$ & $25 \cdot 2$ & $23 \cdot 0$ & NNW, 1-2 & 3 & $8-15$ & bc & I & Ci.-cu, 2 (no & S.-eu. 1, N & - \\
\hline Nonil & $29 \cdot 359$ & - & 23.8 & $25 \cdot 6$ & $25 \cdot 0$ & $28 \cdot 0$ & NNW, 1-2 & 11 & $5-15$ & be & 1 & Ci.-cu. 3 & S.cu. $4, \mathrm{~N}$ & - \\
\hline 2 & $29 \cdot 36 i$ & - & $21 \cdot 8$ & $26 \cdot 2$ & $25 \cdot 8$ & $31 \cdot 0$ & $N$ by $W, 1-3$ & 11 & 5-22 & bea & & $\mathrm{Ci}_{*}-\mathrm{cu}, 2$ & S. cu, $6, N$ (fnst) & - \\
\hline 4 & $29 \cdot 382$ & - & $25 \cdot 0$ & $26 \cdot 7$ & $25 \cdot 8$ & $29 \cdot 3$ & N by $W, 0-2$ & 7 & $0-15$ & cq & & - & S.-cu. $10, \mathbf{N}$ (fast) & - \\
\hline 6 & $29 \cdot 405$ & - & $25 \cdot 8$ & $26 \cdot 5$ & $25 \cdot 5$ & $30 \cdot 0$ & $N, 2-4$ & 10 & $0-22$ & eq & & - & S.-cu. 8, Nis (slow) & - \\
\hline 8 & $29 \cdot 399$ & - & $25 \cdot 5$ & $27 \cdot 2$ & $25 \cdot 8$ & $28 \cdot 0$ & $\mathrm{~N}, 2$ & 11 & $8-12$ & oc & 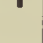 & - & S.-cn. 9, N B (slow) & - \\
\hline 10 & $29 \cdot 417$ & - & $26 \cdot 5$ & $28 \cdot 0$ & $26 \cdot 2$ & $28 \cdot 5$ & Lt. $N$ airs & 7 & $0-5$ & o & & - & S.-cu. 9 & - \\
\hline Midt. & $29 \cdot 417$ & - & $25 \cdot 0$ & $27 \cdot 8$ & $25 \cdot 0$ & - & $N, 1-2$ & 3 & $8-10$ & $\infty$ & & - & S.-eu. 9 & - \\
\hline Mean & $29 \cdot 371$ & -1 & - & $26 \cdot 0$ & $25 \cdot 1$ & - & 一 & - & - & - & & - & - & - \\
\hline
\end{tabular}

Max, therm, read at $\left\{\begin{array}{l}8 \text { a. } 28^{\circ} \cdot 5 \\ 8 \text { p. } 29^{\circ} \cdot 5 .\end{array}\right.$

Solar radiation $\left\{\begin{array}{l}\text { black bull, } \\ \text { Irlglit bulb } \\ 1.37^{\circ} \cdot 2\end{array}\right.$

Fvapu. - grammes.

Precipn, - in. 

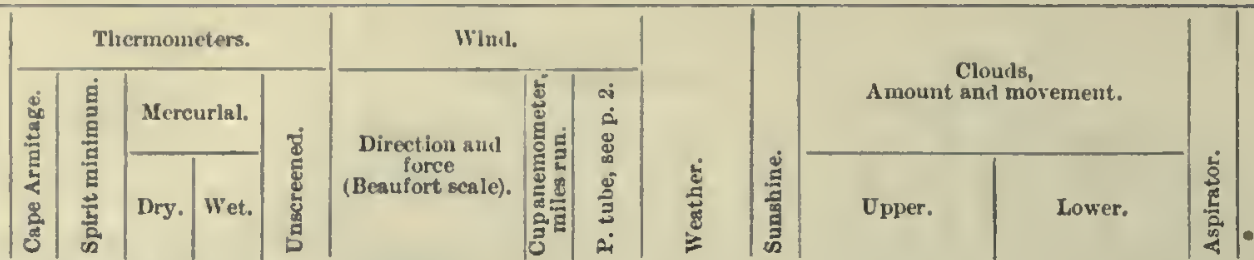

13th.

\begin{tabular}{|c|c|c|c|c|c|c|}
\hline 2 & $29 \cdot 539$ & - & $25 \cdot 0$ & $28 \cdot 0$ & $27 \cdot 5$ & $32 \cdot 0$ \\
4 & $29 \cdot 544$ & - & $27 \cdot 0$ & $28 \cdot 0$ & $27 \cdot 2$ & $32 \cdot 0$ \\
6 & $29 \cdot 553$ & - & $28 \cdot 8$ & $27 \cdot 2$ & $26 \cdot 5$ & $32 \cdot 0$ \\
8 & $29 \cdot 577$ & - & $24 \cdot 0$ & $26 \cdot 0$ & $25 \cdot 0$ & $32 \cdot 0$ \\
10 & $29 \cdot 601$ & - & $24 \cdot 8$ & $26 \cdot 0$ & $25 \cdot 1$ & $31 \cdot 2$ \\
Noon & $29 \cdot 601$ & - & $25 \cdot 0$ & $26 \cdot 8$ & $25 \cdot 8$ & $30 \cdot 8$ \\
2 & $29 \cdot 603$ & - & $25 \cdot 3$ & $27 \cdot 0$ & $26 \cdot 0$ & $31 \cdot 8$ \\
4 & $29 \cdot 605$ & - & $26 \cdot 0$ & $27 \cdot 8$ & $26 \cdot 4$ & $32 \cdot 0$ \\
6 & $29 \cdot 614$ & - & $27 \cdot 0$ & $30 \cdot 5$ & $28 \cdot 6$ & $34 \cdot 3$ \\
8 & $29 \cdot 614$ & - & $28 \cdot 0$ & $29 \cdot 2$ & $27 \cdot 8$ & $31 \cdot 0$ \\
10 & $29 \cdot 624$ & - & $27 \cdot 0$ & $27 \cdot 5$ & $26 \cdot 2$ & $28 \cdot 8$ \\
Midt. & $29 \cdot 616$ & - & $25 \cdot 0$ & $26 \cdot 8$ & $25 \cdot 8$ & $28 \cdot 0$ \\
\hline Mean & $29 \cdot 591$ & - & - & $27 \cdot 6$ & $26 \cdot 5$ & - \\
\hline
\end{tabular}

N, 1-2

Var. $\mathrm{E}, 1$

E by $\mathbf{S}, 1$

E, 1

E, 1

ENE, 1-3

var. E, 2-3

ENE, 1-2

S by E, 0-1

Lt. Is alrs

Calm

E, 0-1 \begin{tabular}{|c|c|c|}
2 & $10-15$ & $0 *$ \\
6 & $5-10$ & of $*$
\end{tabular}

5 5-10 of $*$

13 0-8 oc*

11 5-12 $\circ *$

$10 \quad 12-23 \quad \circ *$

$13 \quad 10-20$ oc $*+$

11 5-10 oc ने

\begin{tabular}{c|c|c}
11 & $5-10$ & oc $-f$ \\
4 & 0 & be \\
3 & 0 & oc
\end{tabular}
$-$

\begin{tabular}{|c|c}
3 & 0 \\
4 & 0 \\
0 & $8-10$
\end{tabular} \mid

Max. therm. $\left\{8\right.$ a. $29^{\circ} 0, \quad$ Solar fblack bulb $119^{\circ}-8$.

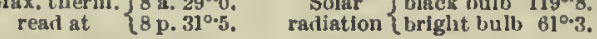

Evapn. - grammes.

\begin{tabular}{|c|c|c|c|}
\hline & - & N. 10 & - \\
\hline & - & N. 10 & - \\
\hline & - & N. 10 & - \\
\hline & 一 & N. 10 & - \\
\hline & - & N. 10 & - \\
\hline & - & N. 10 & - \\
\hline & - & N. 10 & - \\
\hline 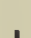 & - & S.-cu. 9 & - \\
\hline 1 & Cl.-cu. 4, NW & S.-cu. 4 & - \\
\hline & - & S.-cu. 10 & - \\
\hline & - & N. 10 & - \\
\hline I & - & Cu. 8 & - \\
\hline & - & , - & \\
\hline
\end{tabular}

Precipn. 0.08 in. since 4 p.m. 12th.

$-$
Remarks.

Snow covering the thermometer (ex pose(i) fin sunshine.

Clouds inclined to loreak overhead and to S1. Very thick all round.

Sun gleaning: White Island visible 3 p.m. Kain band, 2.

ppearance of clianging in $\mathbf{N}$ and $\mathrm{F}$; oun gleaming: tase of Mt. Discovery visible.

p.m. Clear with Cl. to $\mathbf{N}$ and $\mathrm{E}$, very duli to $\mathrm{S}$; mirage to nowing slightly ; very dark to $S$; land visible. ky clearlng; White Island visible; sun ahinlng.

Noon. Mean of 10 observatlons : with aspirator, rry bulb $26^{\circ} 1$, wet bulb $25^{\circ} 1$; from screen, dry bulb $26^{\circ} \cdot 0$, wet bulb $25^{\circ} 0$.

Overeast all day with heavy snowfall. Wind varialle Fly, light. Barometer riaing steadily most of the day. Temrature steady.

14th.

\begin{tabular}{|c|c|c|c|c|c|c|c|c|c|c|c|c|c|c|c|}
\hline 2 & $29 \cdot 617$ & - & $20 \cdot 0 \mid$ & $24 \cdot 0$ & $23 \cdot 0$ & $|28 \cdot 5|$ & Var. E, 1 & 0 & $|10-12|$ & be & & - & $\mathrm{Cu}, 6$ & |- & \multirow{12}{*}{ 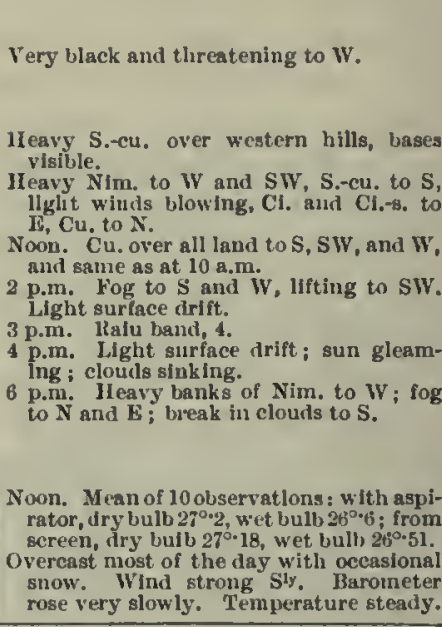 } \\
\hline 4 & $29 \cdot 619$ & - & $22 \cdot 5$ & $26 \cdot 0$ & $24 \cdot 0$ & 30.0 & Var. F, 1 & 8 & $8-10$ & be & & - & S.ent. 4 & - & \\
\hline 0 & $29 \cdot 617$ & - & $23 \cdot 0$ & $25 \cdot 8$ & $25 \cdot 0$ & $28 \cdot 0$ & Lt. var. alrs & 3 & 0 & $\alpha$ & & - & S.-cu. 8 & - & \\
\hline 8 & $29 \cdot 623$ & - & $25 \cdot 0$ & $27 \cdot 0$ & $25 \cdot 0$ & $29 \cdot 5$ & S by E, 2-3 & 3 & $15-20$ & $\infty$ & & - & S.-cu. 8 & - & \\
\hline 10 & $29^{\circ} 633$ & - & $24 \cdot 8$ & $20 \cdot 2$ & $25 \cdot 0$ & $30 \cdot 1$ & $\mathrm{SSE}_{0} 2$ & 9 & $10-15$ & be & $\mid$ & - & S.-cu, 4, S (slow) & - & \\
\hline Noon & $29 \cdot 630$ & - & $25 \cdot 2$ & $26 \cdot 8$ & $25 \cdot 2$ & $27 \cdot 8$ & $S$ by E, 2-3 & 10 & $15-22$ & $b e+$ & i & Ci.-8. 2 & Cu. 4, SE (slow) & - & \\
\hline 2 & $29 \cdot 632$ & - & $25 \cdot 5$ & $28 \cdot 0$ & $26 \cdot 6$ & $30 \cdot 0$ & SSE, 2-4 & 13 & $15-25$ & beq + & & $\begin{array}{l}\mathrm{Ci},-\mathrm{s}, 2, \mathrm{NW} \\
\text { (slow) }\end{array}$ & Cu. 4, SE (slow) & - & \\
\hline 8 & $29^{\circ} 029$ & - & $25 \cdot 0$ & $26 \cdot 0$ & $25 \cdot 0$ & $27 \cdot 5$ & S, 2 & 9 & $10-18$ & o* & & - & N. 10 & - & \\
\hline 10 & $29 \cdot 635$ & - & $21 \cdot 5$ & $25 \cdot 0$ & $24 \cdot 0$ & $25 \cdot 0$ & SSE, 1-2 & 10 & $0-10$ & o* & & - & N. 10 & - & \\
\hline Midt. & $29 \cdot 634$ & - & $23 \cdot 0$ & $24 \cdot 9$ & $24 \cdot 2$ & $25 \cdot 1$ & $\mathrm{SE}, 1-2$ & 8 & $0-10$ & o* & & - & N. 10 & - & \\
\hline Mean & $29 \cdot 628$ & - & - & $28 \cdot 1$ & $24 \cdot 9$ & -1 & - & -1 & - & 一 & & - & - & & \\
\hline \multicolumn{5}{|c|}{$\begin{array}{l}\text { Max. therm. } \\
\text { read at }\end{array}$} & \multicolumn{3}{|c|}{$\begin{array}{l}\text { Solar } \\
\text { radiation }\left\{\begin{array}{l}\text { black bulb } \\
\text { bright bulb }\end{array}{ }^{142^{\circ} \cdot 8} \text {. }\right. \\
72^{\circ} \times 8 .\end{array}$} & \multicolumn{7}{|c|}{ Precipn. $0^{\circ} 01$ in. since 6 p.m. 13 th. } & \\
\hline
\end{tabular}

15th.

\begin{tabular}{|c|c|c|c|c|c|c|c|c|c|c|c|c|c|c|c|}
\hline 2 & $29 \cdot 635$ & - & $24 \cdot 5$ & $|25 \cdot 1|$ & $24 \cdot 5$ & $25 \cdot 4$ & SE, 1-2 & 7 & $5-10$ & o & \multirow{12}{*}{ I } & - & S. 10 & - & \multirow{14}{*}{ 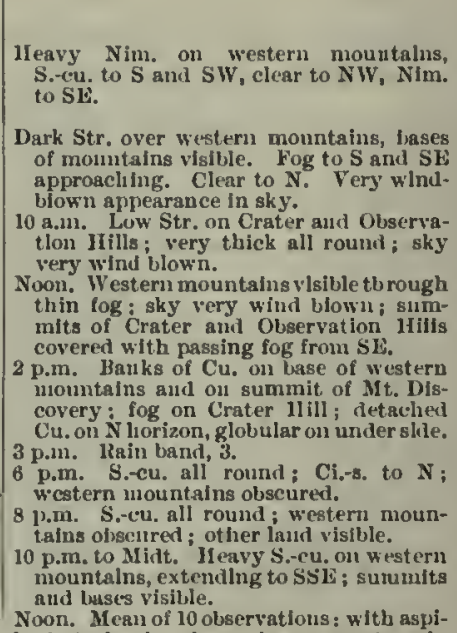 } \\
\hline 4 & $29 \cdot 631$ & - & $24 \cdot 5$ & $28 \cdot 0$ & $24 \cdot 8$ & $28 \cdot 5$ & Var. SE, 1-2 & 9 & $8-12$ & o & & - & Cu. 8, S (slow) & - & \\
\hline 6 & $29 \cdot 627$ & - & $25 \cdot 0$ & $26 \cdot 5$ & $25 \cdot 1$ & $28 \cdot 8$ & Lt. S E airs & 7 & $0-8$ & o & & - & S.-c11. 10 & - & \\
\hline 8 & $29 \cdot 619$ & - & $25 \cdot 5$ & $27 \cdot 1$ & $25 \cdot 5$ & $30 \cdot 5$ & Lt. SE airs & 4 & 5-12 & be & & Cl. 3 & A.-s. 5, SE (slow) & - & \\
\hline 10 & $29 \cdot 615$ & - & $26 \cdot 0$ & $27 \cdot 0$ & $26 \cdot 0$ & $32 \cdot 0$ & E, 1-2 & 11 & $5-15$ & be $*$ & & Ci.-s. 4, S (slow) & Fr.-n. 2, SE & - & \\
\hline Noon & $29 \cdot 596$ & - & $26 \cdot 0$ & $27 \cdot 0$ & $26 \cdot 0$ & $29 \cdot 2$ & E, 1-2 & 8 & $10-20$ & be & & Cl.-s. 5, S (slow) & $\mathrm{Fr}_{0}^{-8.2 .2, \mathrm{SWF}}$ & - & \\
\hline 2 & $29 \cdot 569$ & - & $26 \cdot 6$ & $28 \cdot 0$ & $26 \cdot 2$ & $33 \cdot 0$ & Var. SE, 2-3 & 13 & $10-22$ & be & & $\mathrm{Cl}_{0}-\mathrm{a}, 6, \mathrm{SW}$ & Cu. 2, SE (mol) & - & \\
\hline 4 & $29 \cdot 554$ & - & 287 & $27 \cdot 3$ & $25 \cdot 5$ & $31 \cdot 2$ & Var. E, 2-3 & 9 & $10-20$ & be & & Ct.-8, $6, s W$ & 一 & $1-$ & \\
\hline 0 & $29 \cdot 548$ & - & $26 \cdot 5$ & $26 \cdot 5$ & $25 \cdot 0$ & $28 \cdot 0$ & Var. E, 2-3 & 11 & $5-10$ & bc & & Cl.cu, 4, $\mathrm{w}$ & 一 & - & \\
\hline 8 & $29 \cdot 540$ & - & $25 \cdot 0$ & $26^{\circ} 0$ & $24 \cdot 2$ & $28 \cdot 0$ & Var. E, 1-2 & 9 & $5-12$ & be & & Ci.-cut. 2, w & 一 & - & \\
\hline 10 & $29 \cdot 533$ & - & $22 \cdot 0$ & $25 \cdot 0$ & $23 \cdot 2$ & $25 \cdot 0$ & $\mathrm{ESE}, 1$ & 9 & $5-10$ & $\infty$ & & - & A.cut. 8 & - & \\
\hline Midt. & $29 \cdot 526$ & - & $22 \cdot 0$ & $2: 0$ & $23 \cdot 0$ & $2 \cdot 4 \cdot 0$ & Sw, 1 & 4 & $5-10$ & oc & & - & A.ccu. 8 & - & \\
\hline Nean & $29 \cdot 583$ & - & - & $26 \cdot 31$ & $24 \cdot 9$ & - & - & -1 & - & - & & - & - & & \\
\hline \multicolumn{5}{|c|}{$\begin{array}{l}\text { Max. therm. } \\
\text { read at }\end{array}$} & \multicolumn{3}{|c|}{ 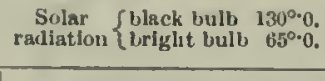 } & & 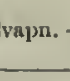 & & \multicolumn{4}{|c|}{ Precipn. 0.004 in. since 4 p.m. 14tli. } & \\
\hline
\end{tabular}

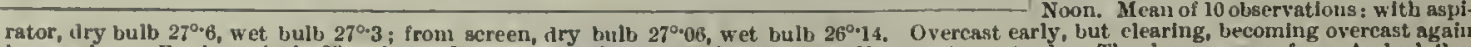
in evening. Fresh variable wily wind Barometer falling steadily all day. Temperature steady. The lce as scen frou Arrival Bay lielghts appeared to have gone out as far as tho lslands; b eavy pack onl horlzon. 
Miteorological, Journal, of the "Discovery."

[1903-January.

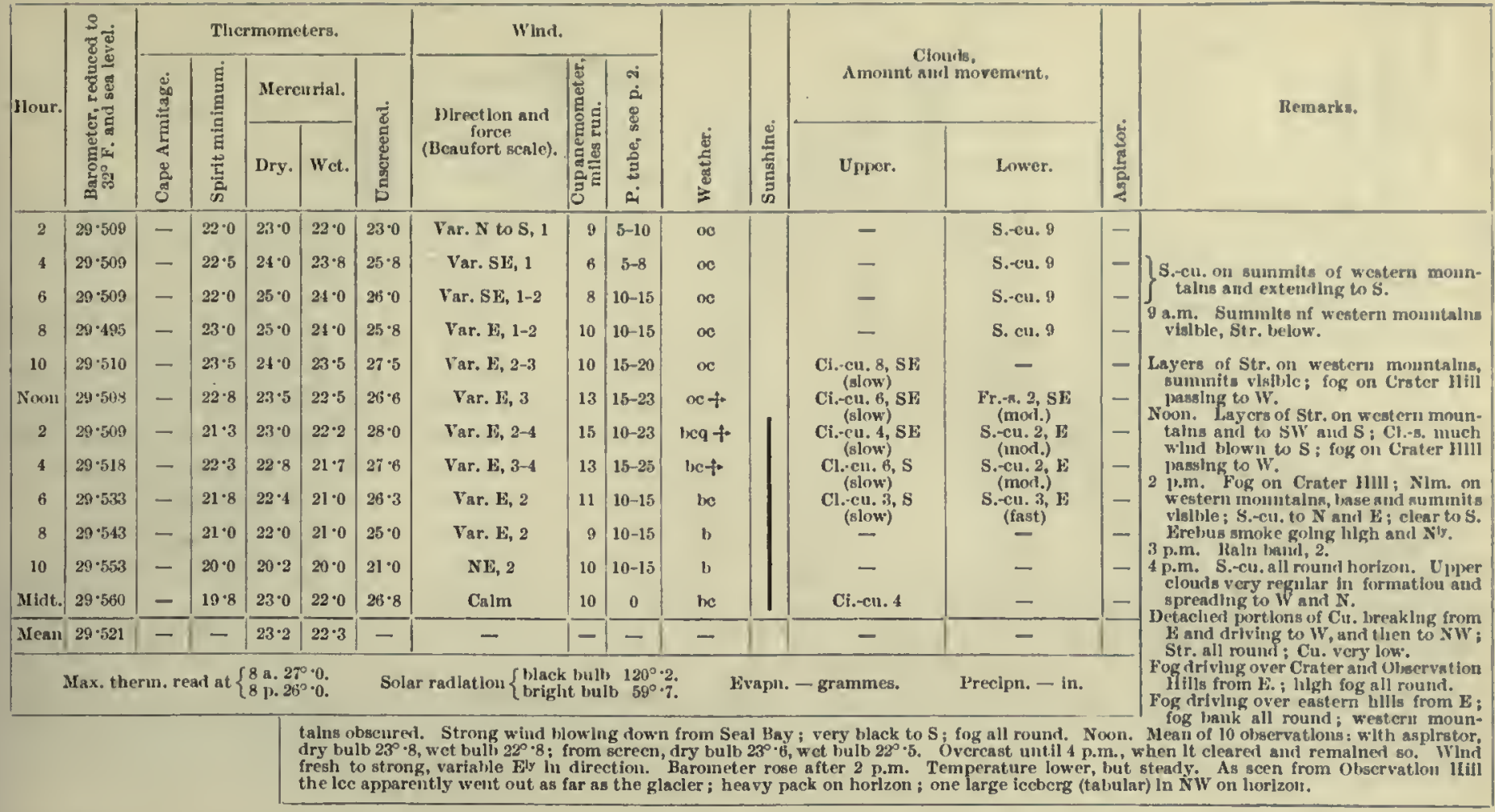

\begin{tabular}{|c|c|c|c|c|c|c|c|c|c|c|c|c|c|c|c|}
\hline 2 & $29 \cdot 575$ & - & 20.0 & $22 \cdot 0 \mid$ & $21 \cdot 0$ & 240 & Calm & 1 & 0 & be & 1 & - & Cu. 6 & -1 & \multirow{6}{*}{$\begin{array}{l}\text { Tops of Crater and Otservation H11ls } \\
\text { ohscured ly mist. } \\
\text { No land visible to W. Fog passing } \\
\text { Observation Jlill from N. } \\
\text { Low S.-cu. all round. }\end{array}$} \\
\hline 4 & $29 \cdot 592$ & - & $21^{\circ} 0$ & $222^{\circ}$ & $21 \cdot 5$ & $26 \cdot 0$ & Calm & 0 & 0 & be & ' & - & Cu. 8 & - & \\
\hline B & $29^{\circ} 603$ & - & $19^{\circ} 0$ & $23 \cdot 0$ & $23 \cdot 0$ & $26 \cdot 8$ & NE, 1 & 0 & $8-10$ & be & \multirow{8}{*}{ 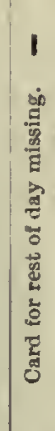 } & - & Cu. 9 & - & \\
\hline 8 & $29 \cdot 626$ & - & $18 \cdot 0$ & $19 \cdot 2$ & $18 \cdot 0$ & $23 \cdot 0$ & NE, 1-2 & 8 & $8-15$ & be & & - & Cu. 9 & - & \\
\hline 10 & $29 \cdot 644$ & - & $19 \cdot 1$ & $23 \cdot 2$ & $22 \cdot 1$ & $29 \cdot 0$ & $N E, 1$ & $i$ & $0-8$ & $\infty$ & & - & Cu. $10, \mathrm{NW}$ & - & \\
\hline Noon & $29 \cdot 646$ & - & $23 \cdot 8$ & 245 & $23 \cdot 1$ & $30 \cdot 8$ & Lt. NE airs & 2 & 0 & o & & - & S. cu. 10 & - & \\
\hline 2 & $29 \cdot 6.15$ & - & $24 \cdot 5$ & 26.8 & $25 \cdot 3$ & 31.0 & Calm & 5 & 0 & $\infty$ & & - & S. cu. 10 & - & \multirow{5}{*}{ 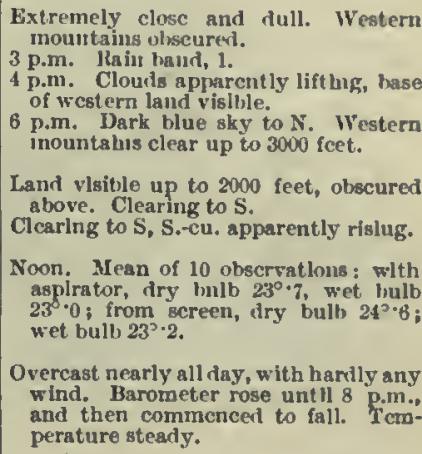 } \\
\hline 8 & $29 \cdot 659$ & - & $23 \cdot 5$ & $24 \cdot 1$ & $23 \cdot 2$ & $26 \cdot 0$ & Calm & 2 & 0 & $\infty$ & & - & S.-cu. 10 & $\begin{array}{l}25 \cdot 5 \\
24: 8\end{array}$ & \\
\hline 10 & $29 \cdot 643$ & - & $23 \cdot 5$ & $21 \cdot 0$ & $23 \cdot 0$ & $25 \cdot 5$ & Calm & 3 & 0 & $\infty$ & & - & S.-cu. 10 & $\left|\begin{array}{r}-4.8 \\
23.8 \\
23.0\end{array}\right|$ & \\
\hline Mldt. & $29 \cdot 635$ & - & $23 \cdot 5$ & $24 \cdot 0$ & $23 \cdot 0$ & $24 \cdot 5$ & Calm & 3 & 0 & $\infty$ & & - & S.cu. 10 & $\begin{array}{l}28 \cdot 8 \\
23 \cdot 0 \\
23.0\end{array}$ & \\
\hline Mean & $29 \cdot 631$ & - & - & $23 \cdot 7$ & $22 \cdot 5$ & - & - & -1 & - & - & & - & - & & \\
\hline
\end{tabular}

17 th.

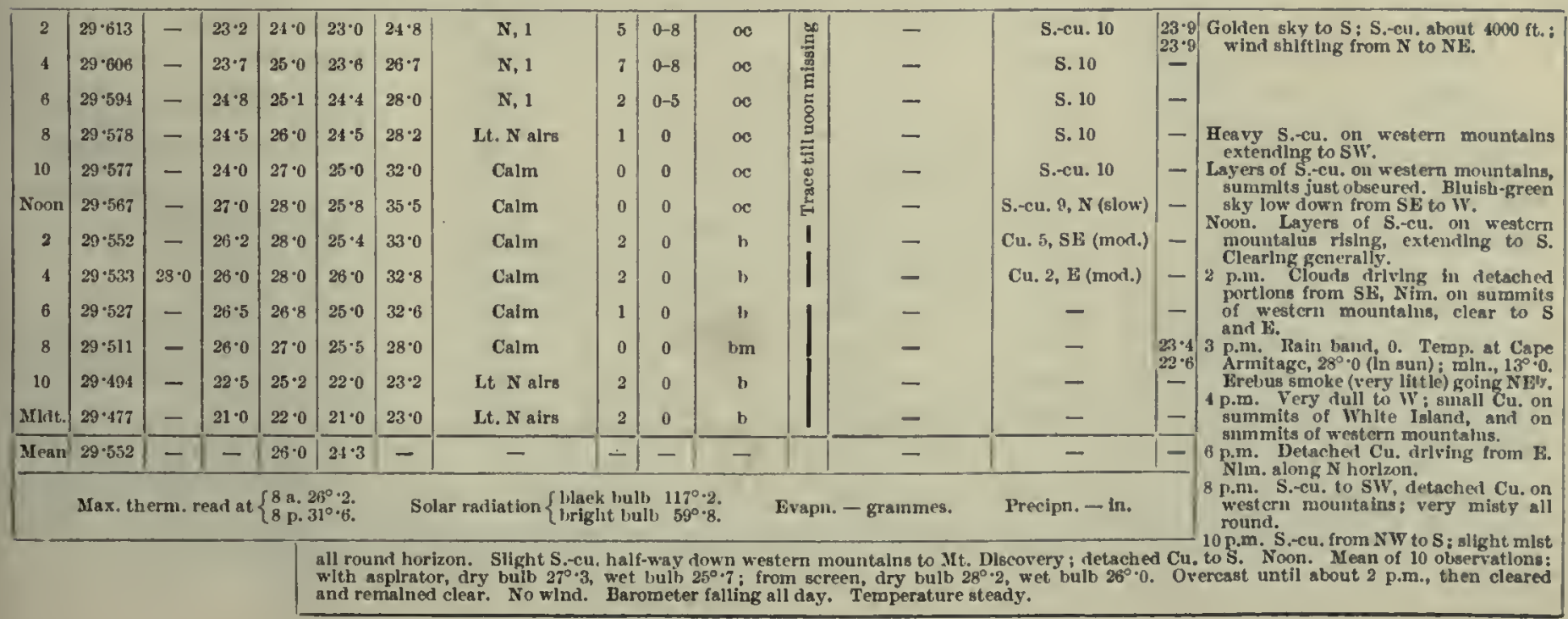


1903 -January.]

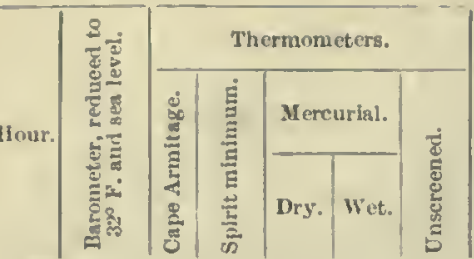

19 th

\begin{tabular}{|c|c|c|c|c|c|c|c|c|c|c|}
\hline 2 & $29 \cdot 451$ & - & 11.8 & 21.5 & 200 & $23 \cdot 0$ & Lt. $\times$ airs & 3 & 0 & $\mathrm{~mm}$ \\
\hline 4 & $29 \cdot 422$ & - & $20 \cdot 0$ & 20.0 & $18 \cdot 0$ & $23 \cdot 0$ & $x, 1$ & 4 & $0-8$ & b \\
\hline 6 & $29 \cdot 400$ & - & $18 \cdot 0$ & $18^{\circ} 0$ & $17 \cdot 2$ & $18 \cdot 0$ & $x, 1$ & 3 & $0-8$ & b \\
\hline 8 & $29 \cdot 393$ & - & $13 \cdot 2$ & $23 \cdot 0$ & 21.8 & $27 \cdot 5$ & Calm & 1 & 0 & b \\
\hline 10 & $29 \cdot 377$ & - & $23 \cdot 8$ & $24 \cdot 8$ & $23 \cdot 1$ & $29 \cdot 2$ & Calın & 1 & 0 & ine \\
\hline Noon & $29 \cdot 350$ & - & $21 \cdot 0$ & $23^{\circ} 0$ & $22 \cdot 0$ & $29 \cdot 5$ & xE, 1 & 5 & $0-8$ & $\propto c$ \\
\hline 2 & $29 \cdot 318$ & - & $21 \cdot 7$ & $22 \cdot 3$ & $21 \cdot 6$ & $28 \cdot 2$ & ENF, 2 & 5 & $10-15$ & $\propto$ \\
\hline 4 & $29 \cdot 292$ & - & $21 \cdot 6$ & $22 \cdot 2$ & $21 \cdot 0$ & $27 \cdot 3$ & Var. SE, 0-2 & 3 & $0-12$ & œе \\
\hline 6 & $29 \cdot 266$ & - & $20 \cdot 8$ & $21 \cdot 4$ & $20 \cdot 2$ & 24.0 & ESE, 1-2 & 3 & $5-10$ & oc \\
\hline 8 & $29 \cdot 222$ & - & $21 \cdot 0$ & $22 \cdot 5$ & $21 \cdot 2$ & $29 \cdot 0$ & Calın & 4 & 0 & oc \\
\hline 10 & $29 \cdot 190$ & - & $21 \cdot 0$ & $22 \cdot 5$ & $21 \cdot 0$ & $230^{\circ}$ & Calm & 1 & 0 & $\propto$ \\
\hline Mint. & $29 \cdot 156$ & - & $18 \cdot 0$ & $20^{\circ} 0$ & $19 \cdot 5$ & $21 \cdot 0$ & Lt. Var. airs & 3 & 0 & $o c$ \\
\hline Nleau & $29 \cdot 330$ & - & - & $21 \cdot 8$ & $20 \cdot 6$ & - & - & -1 & - & - \\
\hline
\end{tabular}

Max, therm. read at $\left\{\begin{array}{l}8 \text { a. } 29^{\circ} \cdot 0 . \\ 8 \text { p. } 27^{\circ} \cdot 0\end{array}\right.$

Solar radlation $\left\{\begin{array}{l}\text { black bulh } 125^{\circ} \cdot 2 \text {. } \\ \text { bright bulb } 59^{\circ} \cdot 8\end{array}\right.$

Mfthorological Jouknal of thr "Discovery."

\begin{tabular}{|c|c|c|c|c|c|c|c|c|}
\hline \multicolumn{3}{|l|}{ Wind. } & \multirow[b]{3}{*}{ 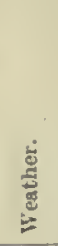 } & \multirow[b]{3}{*}{$\mid$} & \multirow{2}{*}{\multicolumn{2}{|c|}{$\begin{array}{l}\text { Cioudis, } \\
\text { Amount and movement. }\end{array}$}} & \multirow[b]{3}{*}{ 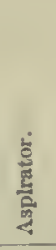 } & \multirow{3}{*}{ Remarks. } \\
\hline \multirow{2}{*}{$\begin{array}{c}\text { Direetim and } \\
\text { foree } \\
\text { (Benufort scalc). }\end{array}$} & \multirow{2}{*}{ 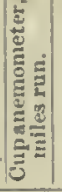 } & \multirow{2}{*}{ 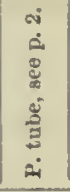 } & & & & & & \\
\hline & & & & & Upper. & Lower. & & \\
\hline Lt. $x$ airs & 3 & 0 & $\mathrm{~lm}$ & & - & - & - & Mist all round horlyon and rery low. \\
\hline$x, 1$ & 1 & $0-8$ & b & & - & - & - & land around. \\
\hline $\mathbf{N}, 1$ & 3 & $0-8$ & b & & - & - & - & Bank of Str. from NW very low cx- \\
\hline Calm & 1 & 0 & b & 1 & - & - & - & $\begin{array}{l}\text { White lsland. Detncher S.-cu. on } \\
\text { western mountalns to } S \text {. }\end{array}$ \\
\hline Calın & 1 & 0 & ine & 1 & - & Cu. $4, x$ & - & 8 a.m. Inigh fog orer western mountalns \\
\hline NE, 1 & 5 & $0-8$ & $\propto$ & & - & S.-eu. $10, \mathrm{~N}$ & - & 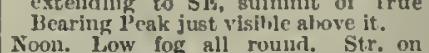 \\
\hline ENE, 2 & 5 & $10-15$ & $\propto$ & & - & S. cu. $10, \mathrm{NE}$ & - & $\begin{array}{l}\text { summits of Crater and Oiservation } \\
\text { 1lills passing from } E \text {. }\end{array}$ \\
\hline Var. SE, 0-2 & 3 & $0-12$ & $\propto ⿻$ & & - & S.cu. 10 & - & 2 p.m. For oun Crater and Olservations \\
\hline ESE, $1-2$ & 3 & $5-10$ & oc & & - & S.cu. 10 & - & $\begin{array}{l}3 \text { p.n. Iain band, } 2 \text {. } \\
1 \mathrm{~nm} \text {. Clouds very low, hase of white }\end{array}$ \\
\hline Calin & 4 & 0 & oc & & - & S.-en. 10 & - & $\begin{array}{l}\text { Islankl to } S \text {, and western mountains } \\
\text { just visible. }\end{array}$ \\
\hline Calm & 1 & 0 & $\alpha$ & & - & S.eu. 10 & - & $\begin{array}{l}6 \text { p.m. Sun gleaming at times, blue sky } \\
\text { on } \mathrm{X} \text { horizon. }\end{array}$ \\
\hline Lt. Var. airs & 3 & 0 & oc & & - & S.-eu. 10 & - & \\
\hline- & - & - & - & & - & - & - & $\begin{array}{l}\text { pirator, dry bulb } 21^{\circ} 2,2 \text {, wet bulth } 20^{\circ} 5 \\
\text { from sereen, ary bulb } 22^{\circ} \cdot 1 \text {, wet bulb }\end{array}$ \\
\hline radiation \{ & & & & & inines. & Precipı. - & & $\begin{array}{l}\text { Fine till after } 10 \text { a.m., whien it bceame } \\
\text { overcast and remained so for the rest } \\
\text { of the day llardly aly winl. Baro } \\
\text { meter falling rapintly all day. Tem- } \\
\text { perature fower aud falling. }\end{array}$ \\
\hline
\end{tabular}

20 th.

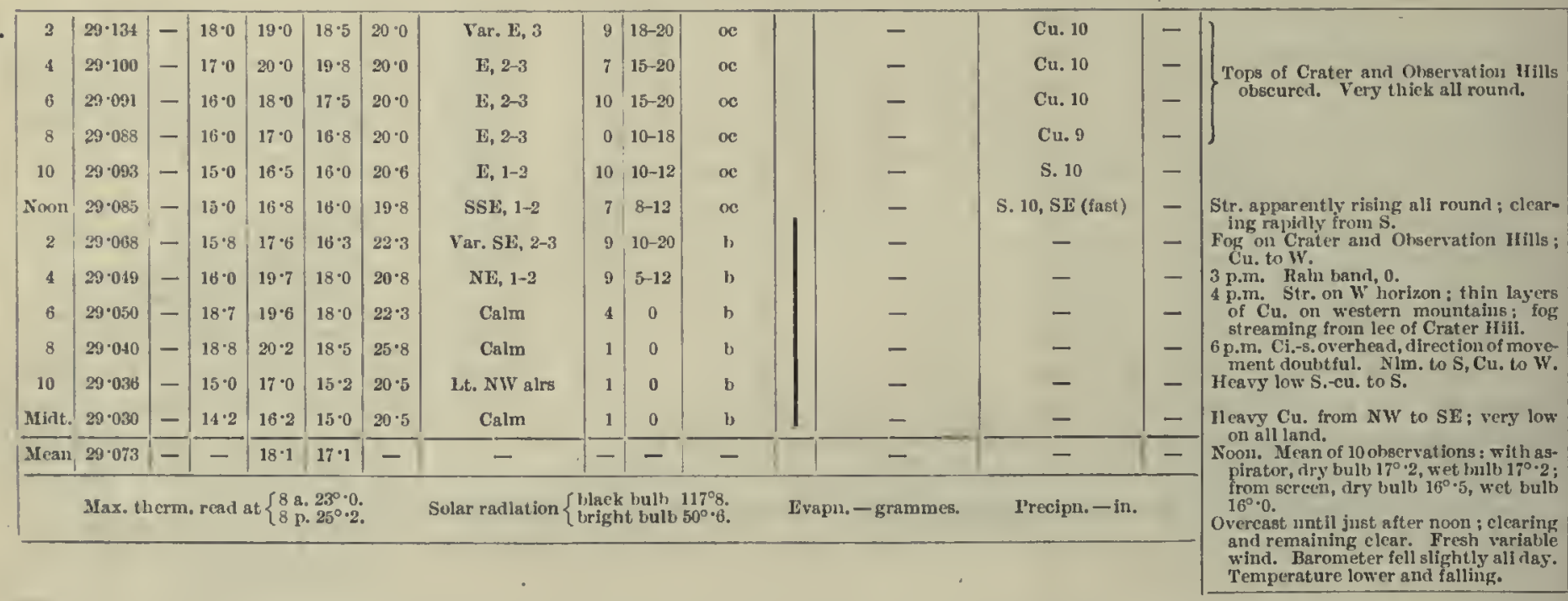

21st.

\begin{tabular}{|c|c|c|c|c|c|c|c|c|c|c|c|c|}
\hline 2 & $29 \cdot 011$ & - & $14^{\circ} 0$ & 20.5 & $18 \cdot 0$ & $23 \cdot 0$ & Var. NE, 1 & 3 & $0-8$ & $\infty$ & - & $\mathrm{Cu} .9, \mathrm{NW}$ (fast) \\
\hline 4 & $28 \cdot 985$ & - & $18 \cdot 0$ & $24 \cdot 2$ & $23 \cdot 0$ & $28 \cdot 0$ & Lt. NE airs & 2 & $0-5$ & oc & - & N. 10 \\
\hline 6 & $28 \cdot 160$ & - & $20 \cdot 2$ & $29 \cdot 0$ & $26 \cdot 5$ & $30 \cdot 0$ & $W, 1-3$ & 0 & $8-20$ & $\infty$ & - & N. 10 \\
\hline 8 & $28 \cdot 964$ & - & $25 \circ$ & $25 \cdot 2$ & $25 \cdot 0$ & $26 \cdot 0$ & $\mathrm{~S}, 3$ & 0 & $15-20$ & $o c *$ & - & N. 10 \\
\hline 10 & $28 \cdot 958$ & - & $14 \cdot 0$ & 16.5 & 16.5 & $19 \cdot 0$ & $\mathrm{NE}, 4-5$ & 24 & $20-27$ & $0+$ & - & N. 10 \\
\hline Noon & $28 \cdot 954$ & - & $15 \cdot 5$ & $22 \cdot 2$ & $21 \cdot 6$ & $27 \cdot 2$ & Var. E, 2-3 & 13 & $10-20$ & $0 f^{\circ}$ & - & N. 10 \\
\hline 2 & $28^{-955}$ & - & $21 \cdot 0$ & $25 \cdot 7$ & $24 \cdot 2$ & $28 \cdot 0$ & s, 2-4 & 9 & $10-25$ & $\operatorname{coq}+$ & - & Cu. $10 \mathrm{~s}$ (mod.) \\
\hline 4 & $28 \cdot 940$ & - & $24 \cdot 2$ & $26 \cdot 2$ & 250 & $27 \cdot 2$ & S, 2-4 & 13 & $10-27$ & ocq 4 & - & Ca. 10, S \\
\hline 0 & $28 \cdot 887$ & - & $24 \cdot 8$ & $20 \cdot 6$ & $25 \cdot 0$ & $28 \cdot 0$ & S, 1-3 & 12 & $5-20$ & ocq & - & Cu. 9, S (morl.) \\
\hline 9 & $28 \cdot 850$ & - & $25 \cdot 0$ & $28 \cdot 5$ & $28 \cdot 2$ & $29 \cdot 0$ & Lt. $S$ alrs & $\theta$ & $0-8$ & $\infty$ & - & Cu. 10 \\
\hline 10 & $28 \cdot 842$ & - & $28 \cdot 0$ & $28 \cdot 0$ & $28 \cdot 2$ & $28 \cdot 8$ & Lt. $\mathbf{S}$ airs & o & 0 & $\infty$ & - & Cu. 9 \\
\hline Midt. & 28.838 & -1 & $24 \cdot 0$ & $27 \cdot 0$ & 25.8 & $28 \cdot 0$ & Lt. S airs & 4 & $5-10$ & $x$ & - & Cu. 8 \\
\hline Mean & $28 \cdot 929$ & -- & - & $25 \cdot 0$ & $28 \cdot 6$ & - & - & - & - & - & - & - \\
\hline
\end{tabular}

Max. therm. $\left\{8 \mathrm{a} .28^{\circ} .8\right.$. Solar \{black bult $110^{\circ} 0$.

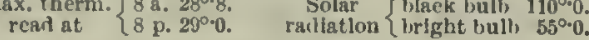

Evapn. - grammes.

Preelpn. $0^{\circ} 003$ in. since 4 p.m. 15 th.
Misty all round horizon; blue sky in $\mathrm{N}$. Very bright to $S$ and $W$.

Very heavy Nim. from $N$ to $W$ over hills, tops obscured; very black and 6.35. Snowing slightly and squally.

Fery thick all round; wind gusty; sun Very dark Nim. to $S$ and $S W$; a break in sky overliead and to $S$; heavy surface drift.

3 p.m. 1hain band, 2. 4 p.m. Western mountains vlsible; clearing on SW horizon.

p.111. Barometer pumping ; rellection of sun on westeru mountains ; wind very squally ; sun
Nim. to $S$ and $W$.

Nim. to S and W $W_{\text {Nim. orer wrestern }}$ p.m. ll eavy Nim. orer western windy appearance in $S$, rark, water sky and $\mathrm{Nim}$. in $\mathrm{N} W$ and $\mathrm{N}$.

Overcast all day, with light snow fall. Wind very squally and strong from $S$, falling to light alrs in the evening. Jarometer falling all day. Temperature higher and unsteary. 
Mhthorological JoURnal, of THE "Discovkiry."

\begin{tabular}{|c|c|c|c|c|c|c|}
\hline \multirow{3}{*}{ Hfour. } & \multirow{3}{*}{ 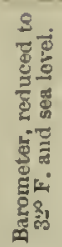 } & \multicolumn{5}{|c|}{ Thermometers. } \\
\hline & & $\stackrel{8}{\circ}$ & छี & Mer & urlat. & \\
\hline & & d: & $\frac{\vec{z}}{\frac{2}{2}}$ & Dry. & Wet. & 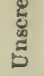 \\
\hline
\end{tabular}

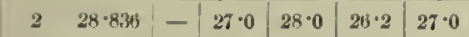

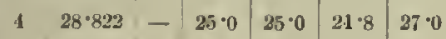

(3) $28 \cdot 82.1-25 \cdot 0 \quad 28 \cdot 0 \quad 27 \cdot 0 \quad 30 \cdot 2$

\begin{tabular}{ll|l|l|l|l}
8 & $28 \cdot 811$ & $-27 \cdot 0$ & $29 \cdot 0$ & $27 \cdot 8$ & $30 \cdot 8$
\end{tabular}

\begin{tabular}{ll|l|l|l|l}
10 & $28 \cdot 80.1$ & $-27 \cdot 0$ & $28 \cdot 2$ & $27 \cdot 8$ & $30 \cdot 0$
\end{tabular}

\begin{tabular}{l|l|l|l|l|l|} 
Noo11 $28 \cdot 8.15$ & $-25 \cdot 9$ & $27 \cdot 2$ & $26 \cdot 0$ & $28 \cdot 7$
\end{tabular}

\begin{tabular}{l|ll|l|l|l|l|}
2 & $28 \cdot 869$ & $-28 \cdot 5$ & $27 \cdot 0$ & $26 \cdot 1$ & $28 \cdot 2$
\end{tabular}

\begin{tabular}{ll|l|l|l|}
$1 \quad 28 \cdot 9113$ & $-26 \cdot 0$ & $26 \cdot 7$ & $25 \cdot 6$ & $29 \% 3$
\end{tabular}

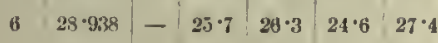

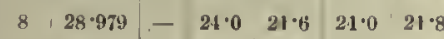

$1029 \cdot 050-20.0 / 21.020^{\circ} 0 \quad 21{ }^{\circ} 0$

Ifirt. $29 \cdot 122-18 \cdot 0 \quad 18 \cdot 0.17 \cdot 8 \quad 18 \cdot 2$

\section{Wind.
Direction and
force
(Beaufort seale).}

Var, S, 1-2

Var. S, 2-3

Var. S1: 2-3

$\mathrm{S}$ by $\mathbf{E}, 1-4$

S, 3-4

S hy 1, 3-1

Var. SE, 2-4

Var. S1, 1-3

SSF, 1-3

SSE, 3-4

Var. 1, 3

E, 3-4
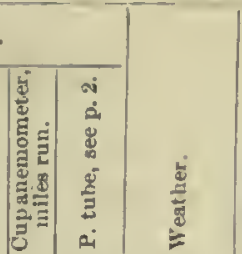

$5|5-10| x$

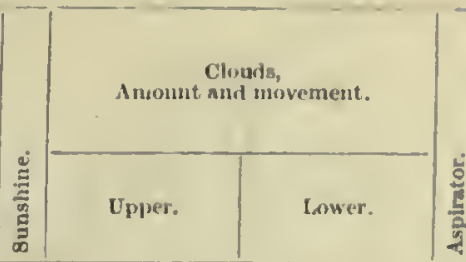

Cu. $9, \mathrm{~N}$

S.-cu. 9, NE

Cu. 8

Ci1. 10

S. c11. $10,8 \mathrm{~W}$
(rapid) - Very thiek all round; wind equally

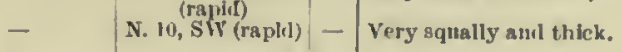

$17 \quad 15-25$ « $\div * *$

$14 \quad 10-30 \quad 0 \mathrm{cq} \div$

8 8-22: neq $\div$

11 10-22 ocq

10 18-26' ocq $f$

13 15-22 ocq $\dot{i}$

13 15-25 bemq + *

$\mid$

Kemarkw - Weather threatening: wind shifty, varyand on w'astern monntaints.

Ai a.m. Very litiek to $\mathrm{S}$, land oincurevi.

- S.en. 10, S (fast) -

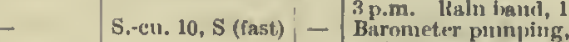

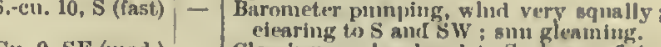
Cu. s, SF (mod.) - Clearing overhearl snd to S ; leavy flaky
Nim. 1o W: sun glea nulig.

Cu. 4, S1 (mor.) - Heary Nim. over Westerit monntalns:

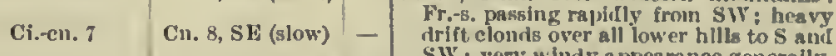

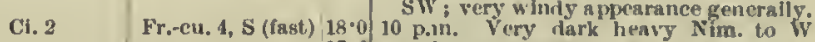

22nd.

$\overline{\text { Mean }} \overline{28 \cdot 901} \overline{-} \overline{25 \cdot 8} \overline{21 \cdot 8} \overline{-}$

Max, therm. rear at $\left\{\begin{array}{ll}8 \text { a. } 31^{\circ} \cdot 2 . \\ 8 \text { p. } 30^{\circ} \cdot 8 .\end{array}\right.$ Solar radiation $\begin{cases}\text { black mulb } & 121^{\circ} \cdot 2 . \\ \text { hright bnlb } & 62^{\circ} \cdot 8 .\end{cases}$

kvapn. - grammes.

Preclpn. - ln. $17 \cdot 6$ aud over westeru mountains; very winiy look all rouni; Clains; very

throngh patches of $\mathrm{Cu}$,
Midt. Very isleak to $\mathrm{W}$; no land in sight froin W lo SW: mlsty look in sky overliead; Ci. very wind-blown; heavy drift in Strait.

Overcast all day, with llgist sunwall.

Wind strong, variable Wty and Sly, and very gusty. Much surface rifift, and at times high drift. Baromater fell until 10 a.m., and then rose

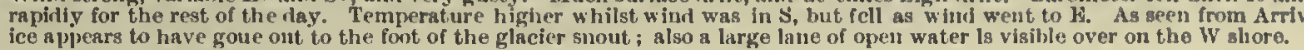

\begin{tabular}{|c|c|c|c|c|c|c|c|c|c|c|c|c|}
\hline 2 & $29 \cdot 179$ & - & $15 \cdot 2$ & $16 \cdot 0$ & $15 \cdot 5$ & $16 \cdot 8$ & SE, 3-4 & 18 & $15-25$ & oeq $f$. & & - \\
\hline 4 & $29 \cdot 194$ & - & $1.1 \cdot 5$ & $15 \cdot 5$ & $15 \cdot 0$ & $16 \cdot 2$ & Var. E, 3-6 & 15 & $20-35$ & ocq $\div$ & 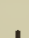 & - \\
\hline 6 & $29 \cdot 213$ & - & $15 \cdot 0$ & $16 \cdot 0$ & $15 \cdot 5$ & $17 \cdot 2$ & $\mathrm{NE}, 5-6$ & 16 & $25-35$ & beq + & I & $\mathrm{Ci}, 2, \mathrm{E}$ \\
\hline 8 & $29 \cdot 259$ & - & $15^{\circ} 0$ & $21 \cdot 0$ & $22 \cdot 0$ & 26.0 & Var. E, 0-1 & 11 & $0-8$ & be & & Ci. 4 \\
\hline 10 & $29 \cdot 248$ & - & $23 \cdot 8$ & $21 \cdot 7$ & $23 \cdot z$ & $30 \cdot 2$ & Calm & 3 & 0 & b & & - \\
\hline Noon & $29 \cdot 288$ & - & 2.10 & $27 \cdot 6$ & $25 \cdot 6$ & $31 \cdot 8$ & Calm & 5 & 0 & $o c$ & & - \\
\hline 2 & $29 \cdot 293$ & - & $27 \cdot 0$ & $29 \cdot 6$ & $27 \cdot 4$ & $37 \cdot 4$ & Calın & 1 & 0 & c & 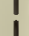 & - \\
\hline 4 & $29 \cdot 3400$ & - & $29 \cdot 2$ & $31 \cdot 0$ & $29 \cdot 0$ & $39^{\circ} 0$ & Calın & 1 & 0 & $\propto c$ & I & - \\
\hline 6 & $29 \cdot 321$ & - & $27 \cdot 0$ & $28 \cdot 2$ & $27 \cdot 2$ & $30^{\circ} 0$ & $\mathrm{NW}, \mathrm{I}$ & 3 & $8-10$ & oc & : & - \\
\hline 8 & $29 \cdot 329$ & - & $27 \cdot 0$ & $27 \cdot 8$ & $26 \cdot 2$ & $29 \cdot 5$ & Calm & 5 & 0 & $o c$ & 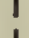 & - \\
\hline 14) & $29 \cdot 321$ & - & $27 \cdot 0$ & $28 \cdot 5$ & $27 \cdot 0$ & $29 \cdot 0$ & $N, 3-4$ & 5 & $20-25$ & bc & & 一 \\
\hline illt. & $20 \cdot 335$ & - & $27 \cdot 2$ & $28 \cdot 5$ & $27 \cdot 0$ & $2: 0$ & $\mathbf{N}, 3$ & 12 & $10-18$ & $o c$ & & - \\
\hline Iean. & $29 \cdot 275$ & - & - & 21.8 & $23 \cdot 4$ & - & - & - & - & - & & - \\
\hline
\end{tabular}

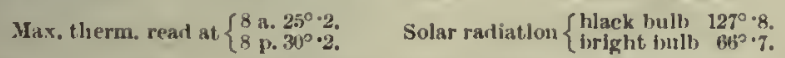

Evapn. - grammes.

A.-cn. $9, \mathrm{~S}$ (slow)

A.-cu. $8, \mathrm{~S}$ (slow)

Very squally ; sky clearing to $\mathrm{S}$; hlue sky to $\mathrm{N}$ : detacher $\mathrm{Cu}$. and very wind hlowi Str. over SW land; If land

$\begin{array}{ll}17 \cdot 8 & \text { obo a.m. Very heavy aqualla; much } \\ 16.9 & \text { drift ln Strait. }\end{array}$

16.9 4 a.1m. Clear and brlght to S : S.-cu. from SW to $W$; foot hills visible; light de-

- $\quad-\quad$ tached Cu. to NII with he; very dark

8.-cu. 9, S (fast) $27 \cdot 2,6$ a.m. Light delached S.-cu. to s ; clear

\begin{tabular}{l|l} 
S.-cu. 9, SW & - Cl.cul extenting NW to W werer western
\end{tabular} (slow)
$\mathrm{Cn} .8$, $\mathrm{SW}$ (slow) C11. 8, W (slow) - Nonn. Mercury in int he thermometers C1. 7, S (slow) jumping rapirily and falling S.-cu. 4, s - 3 gleaming at times. \begin{tabular}{l|l} 
Cu. 9 & - p.un. Nim. on nestem mountains: \\
detacherf clourls in places; sun gleam-
\end{tabular} ing.

p.m. Nim. on western mountains ; Sinn $\mathrm{E}$; blue liazy sky to $\mathrm{N}$. Bu. in large detsched form inonntains: sky : lieavy bauk of roll $\mathrm{Cu}$. on $\mathrm{S}$

Precipn. - in. $\quad \begin{gathered}8 \mathrm{gu}, \text { in la } \\ \text { sky; h } \\ \text { horizmi. }\end{gathered}$ Cloury all day, with very strong variable by wlud early, and heavy squalls, falling calm during afternoon, but rising agaln from $N$ in
evening. Barometer rising quickly all day. Teinperature rose quickly abont 8 p.m., and remainer higher.

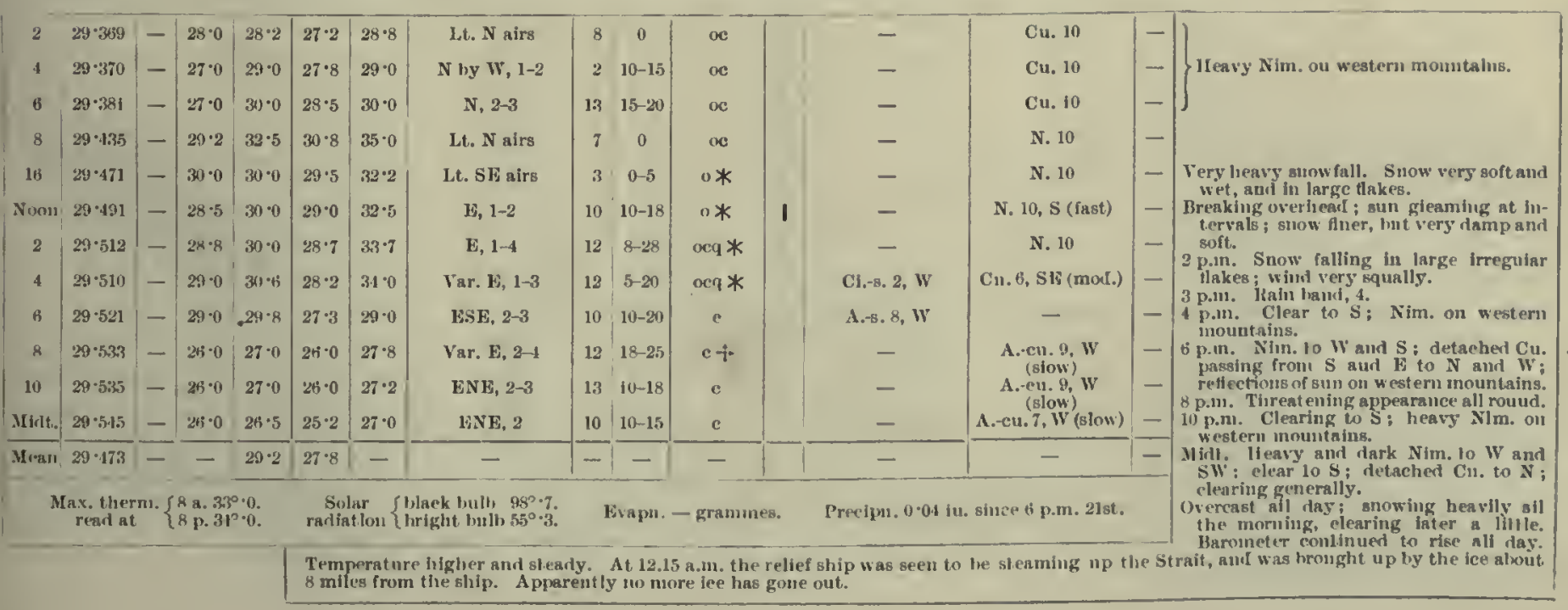

24 th. 
1903-January.]

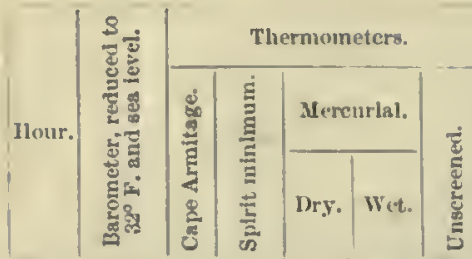

25th.

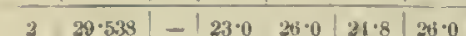

\begin{tabular}{lllll|l}
\hline$\quad 35 \cdot 534$ & $-25 \cdot 0$ & $28 \cdot 0$ & $28 \cdot 2$ & $29 \cdot 0$
\end{tabular}

\begin{tabular}{ll|l|l|l|l|}
3 & $29 \cdot 528$ & $-27 \cdot 2$ & $29 \cdot 2$ & $27 \cdot 8$ & $32 \cdot 0$
\end{tabular}

\begin{tabular}{l|l|l|l|l|l}
$8,29 \cdot 501$ & $-128 \cdot 8$ & 30.2 & 28.8 & 33.0
\end{tabular}

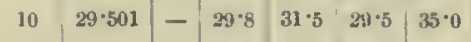

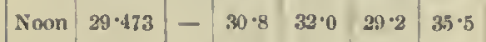

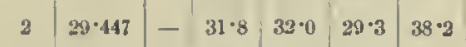

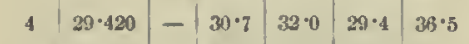

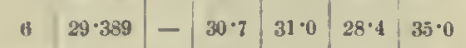

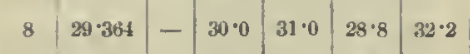

\begin{tabular}{ll|l|l|l|l|}
10 & $29 \cdot 338$ & $-36 \cdot 0$ & $27 \cdot 2$ & $26 \cdot 5$ & $28 \cdot 0$
\end{tabular}

Mlitt. $29 \cdot 319-26 \cdot 5 \quad 27 \cdot 0 \quad 26 \cdot 0 \quad 27 \cdot 0$

Мев11 $29 \cdot 416-\overline{-} \overline{29 \cdot 8} \overline{27 \cdot 9}-$

Max, therm, read at $\left\{\begin{array}{l}8 \text { a. } 31^{\circ} \cdot 2 . \\ 8 \text { p. } 31^{\circ} \cdot 0 .\end{array}\right.$

Solar radiation $\left\{\begin{array}{l}\text { black hulb } 1200^{\circ} \cdot 2 . \\ \text { lright bulb } 63^{\circ} \cdot 0 .\end{array}\right.$

Calm

NF, 1

4

0. 5-8

Calm

NNW, 0-1

Calm

ENE, 1

L.t. $\mathbf{N}$ airs

NF, 1-2

0

0

3 7-10
Meteorological Joutenal of the" "Discovery."

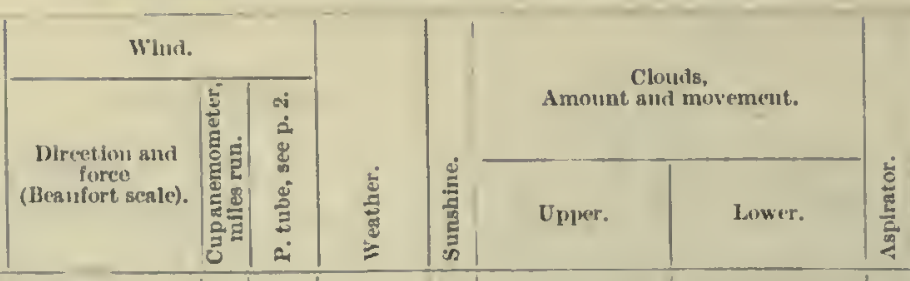

Remarks.

害

Cl. 2, W (slow) S 8, N (mol.) Lo'-1 Layers of Str, mi weshorn monntains ex$24 \cdot 8$ toruling to Sil: iright to $\mathrm{S}$.

NE, $1 \quad|10| 10-12 \mid$ oc

$-$

\begin{tabular}{l|l|l}
2 & 0 & oc
\end{tabular}

3 a-8 h

\begin{tabular}{l|l|l|l|l} 
& 3 & $5-10$ & oeq
\end{tabular}

$10 \mid 10-15$ ac

-

S 10

27.5 Str. all roum at aif. of alwint $2001 \mathrm{ft}$.; 26 -4 dark lin SW.

-1 Mirage to sw, Str. apparently descending.

Ci.-8, $10 \quad-\quad 1-$

Ci.-8. 8 - $\quad-$ Cr. to NE; rery thick to $\mathrm{WV}$; sun gleam-

Ci. 5 - $\quad-\quad \begin{gathered}\text { Western montains olseured. } \\ \text { Noon. Sky clear to N and W; foot hills }\end{gathered}$ ing through patches of blue sky to k;

Ci. 5 - $\quad-\quad \begin{gathered}\text { western mountains olscured. } \\ \text { Noon. Sky clear to } \mathrm{N} \text { and } \mathrm{W}_{\text {; }} \text { foot hills }\end{gathered}$

- $\quad-\quad$ elcas. Detached Cu. passing ; S.cu. W

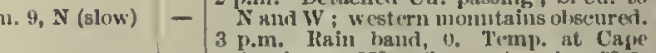

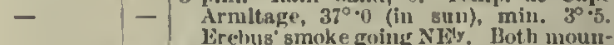
- lirehus smoke going NWly. Hoth moun-
$-\quad 30.4$ tains nearly obseureti, Terror contCu, 10, N $\quad$ - $\begin{aligned} & \text { p.m. Dark flaky Cn. all round horizon. } \\ & \text { 6 p.m. Sky elontless ; Str, to W, SW, }\end{aligned}$ - and $\mathrm{S}$.

- 8 west very misty to W and S ; Str. over $10 \mathrm{pem}$. Very thick mist covering Olıser10 p.m. Very thick mist covering Oliser-
vation Hlill, Crater Ilill, and all western vation Hill,
momtains.

Midt. Ilist cleared from hills in $\boldsymbol{V}$; heavy $\mathrm{Cu}$. onl westers mountains.

Precipu, - iu.

Overcast and cloudy most of the day, with light NF breezes, fresheuing in evening and going to N. Barometer falling steadily all das: Temperature steary. No ice apparcitiy gone out. Heavy pack outside fast iee with some hig hergs.

26th.

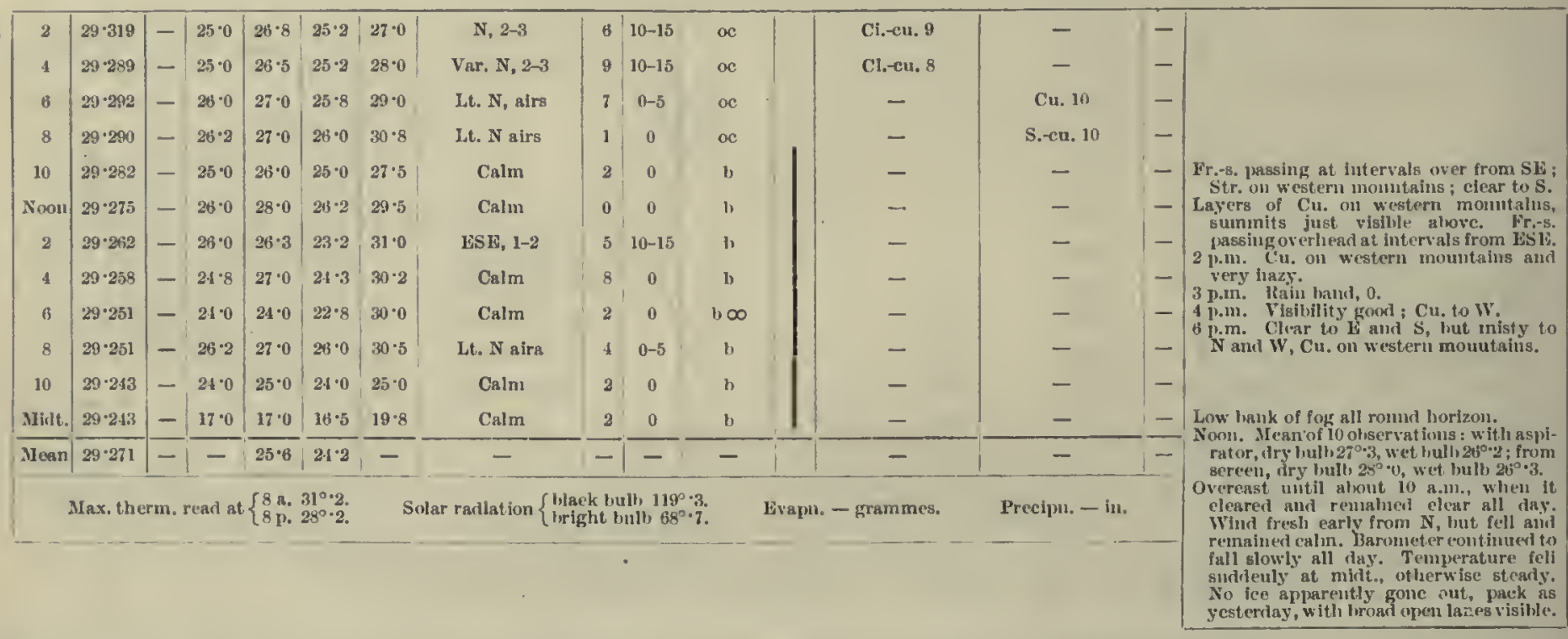

27 th.

\begin{tabular}{|c|c|c|c|c|c|c|c|c|c|c|c|c|c|}
\hline 2 & $29 \cdot 236$ & - & $16 \cdot 0$ & $16 \cdot 5$ & $16 \cdot 2$ & $18 \cdot 5$ & Calm & 0 & 0 & $h$ & - & - & - \\
\hline 4 & $29 \cdot 238$ & - & $12 \cdot 0$ & $14 \cdot 5$, & $13 \cdot 2$ & 24.0 & Calın & 0 & 0 & b) & - & - & - \\
\hline 6 & $29 \cdot 228$ & -1 & $16 \cdot 0$ & $17 \cdot 8$ & $16 \cdot 0$ & $31 \cdot 0$ & Calm & 0 & 0 & b) & - & - & - \\
\hline 8 & $29 \cdot 231$ & - & $15 \cdot 2$ & $22 \cdot 5$ & $21 \cdot 2$ & $32 \cdot 5$ & Calun & 0 & 0 & b & - & - & - \\
\hline 10 & $29 \cdot 226^{\circ}$ & - & $23 \cdot 5$ & $27 \cdot 0$ & $24 \cdot 5$ & $480^{\circ}$ & Calm & 0 & 0 & b & - & - & - \\
\hline Noom & $29 \cdot 228$ & - & $23 \cdot 0$ & $20 \cdot 2$ & $24 \cdot 2$ & $39 \cdot 0$ & Calm & 1 & 0 & b & - & - & - \\
\hline 2 & $29 \cdot 227$ & - & $2 t \cdot 2$ & $30^{\circ} 4$ & 27.5 & $40^{\circ} 0$ & Calın & 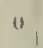 & 0 & b & - & - & - \\
\hline 4 & $29 \cdot 227$ & - & $28 \cdot 3$ & $30 \cdot 0$ & $27 \cdot 6$ & $41 \cdot 8$ & Calm & 1 & $u$ & $n \infty$ & - & - & - \\
\hline 6 & $2 y \cdot 257$ & - & $29 \cdot 2$ & $32 \cdot 2$ & $29 \cdot 0$ & $45 \cdot 2$ & Calm & 3 & 0 & b $\infty$ & - & - & - \\
\hline 8 & $23 \cdot 270$ & - & $25 \cdot 2$ & $25 \cdot 2$ & $24 \cdot 2$ & $32 \cdot 2$ & Calm & 0 & 0 & h & - & - & - \\
\hline 10 & $29 \cdot 290$ & - & $22 \cdot 0$ & $22 \cdot 0$ & $20 \cdot 0$ & $21 \cdot 8$ & Calm & 0 & 0 & b) & - & - & - \\
\hline Mint. & $29 \cdot 293$ & - & $16^{\circ} 0$ & $17 \cdot 0$ & $160^{\circ}$ & $19 \cdot 8$ & Calm & $n$ & 0 & b & - & - & - \\
\hline Mean & $29 \cdot 24 h$ & - & - & $23 \cdot 4$ & $21 \cdot 63$ & - & - & - & - & - & - & - & - \\
\hline
\end{tabular}

Nirage extending SE to SW.

Mirage along western horlzon.

Mirage to $S$, mucl looning in W aud SW.

Low lrank of Cn. on western mountains alout laalf-way down, extending to siv. Mluch mirage in SSw aur Wr.

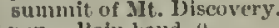

p.m. lian balld, (3. SW, $W$, and $\mathrm{N}$, with unirage.

to p.m. Hxtremely hazy; detached S.epu.

oul horizon to $\mathrm{WV}$ and s.

8 p.m. Very misty all romnd; detacherl

Cu. to SW ; clear to S.

$10 \mathrm{p} . \mathrm{m}$. to midt. Ileavy $\mathrm{S}$-ccu. to the $\mathrm{S}$; detacherl Cu. from s to $\mathrm{N}$.

ont. Nean of 10 ohservat ions: with aspi. rator. Ary bulb $25^{\circ} \cdot 9$, wet bull $24^{\circ} \cdot 4$; from

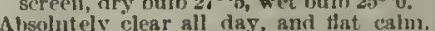
Barometer very st mady, inclined to rise. Tarometer very st early, inclinexi to rise. again in evening. 
Mateorological ,Joutena, of TIE "Discovery."

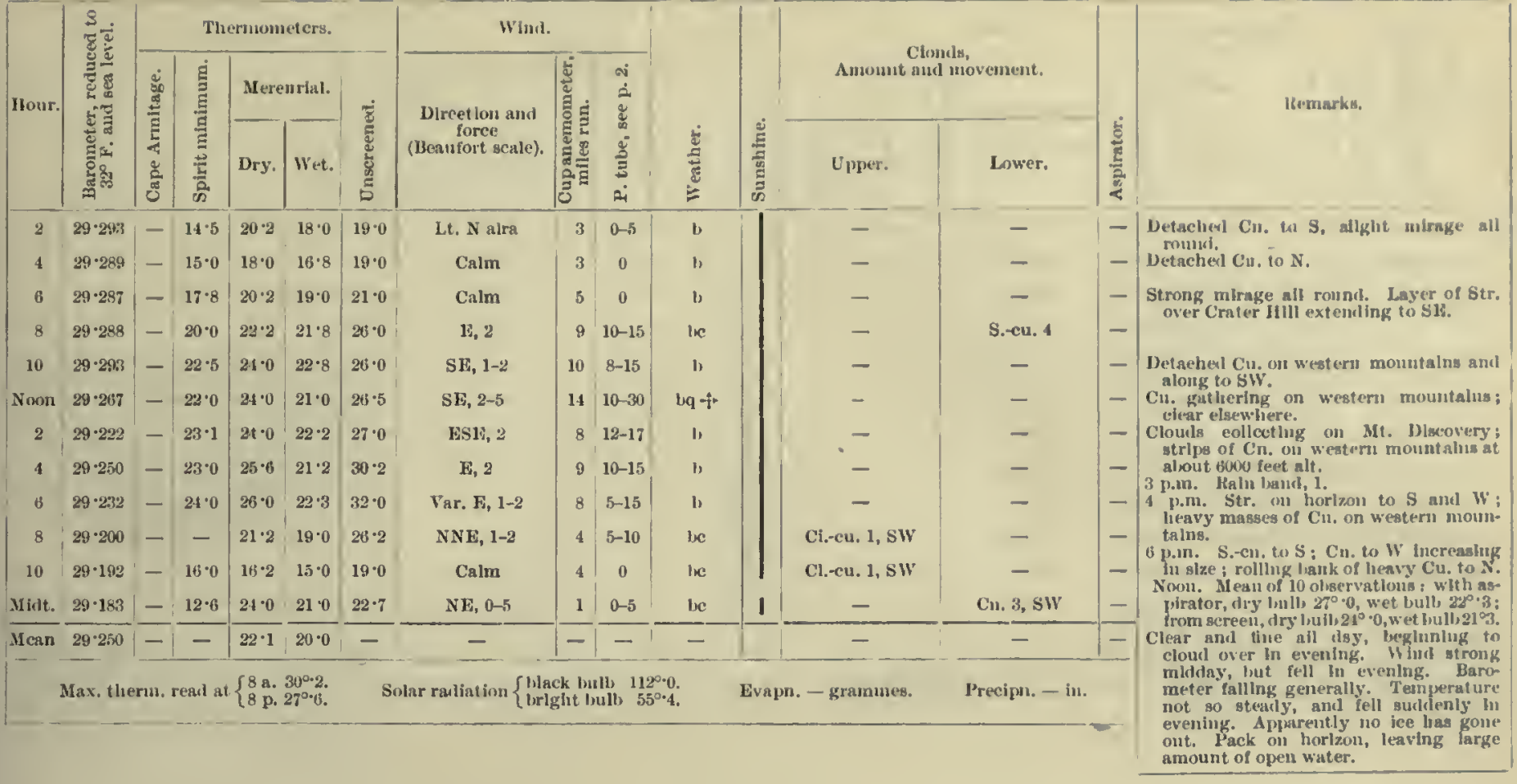

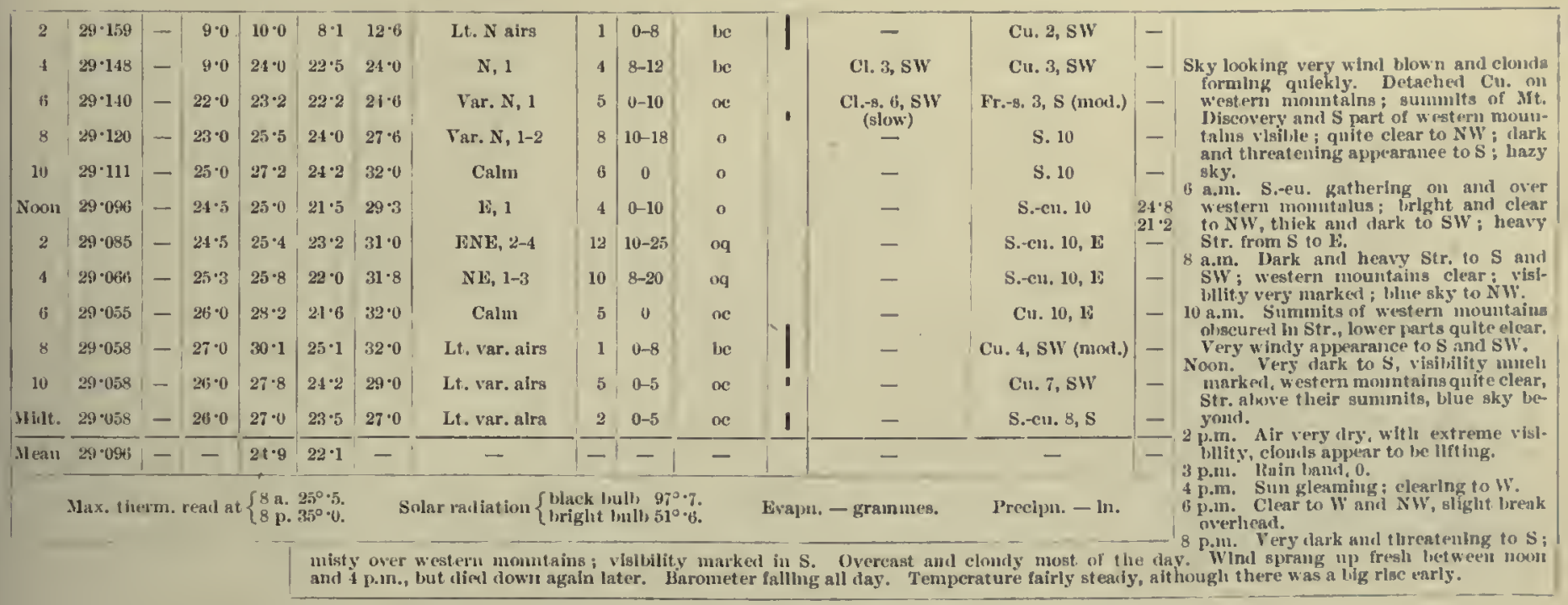

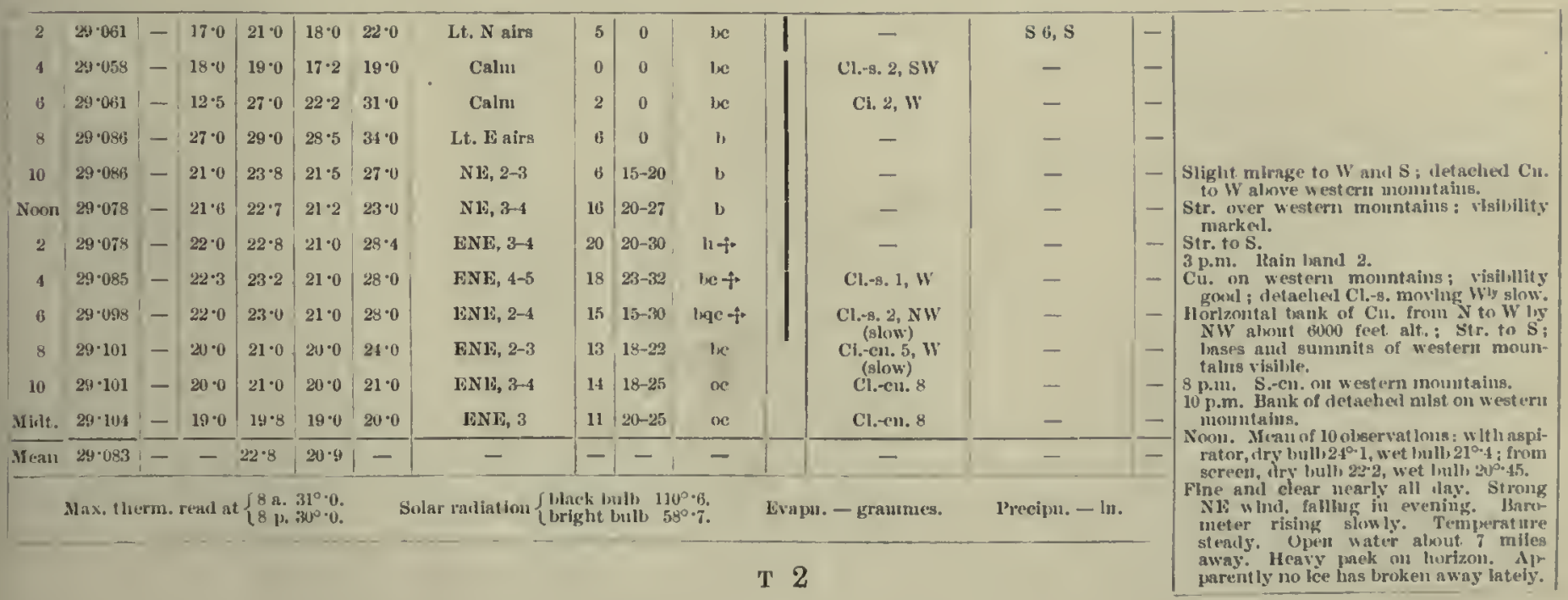


1903 -January.]
Meteorological Journat, of tite "Discovery."

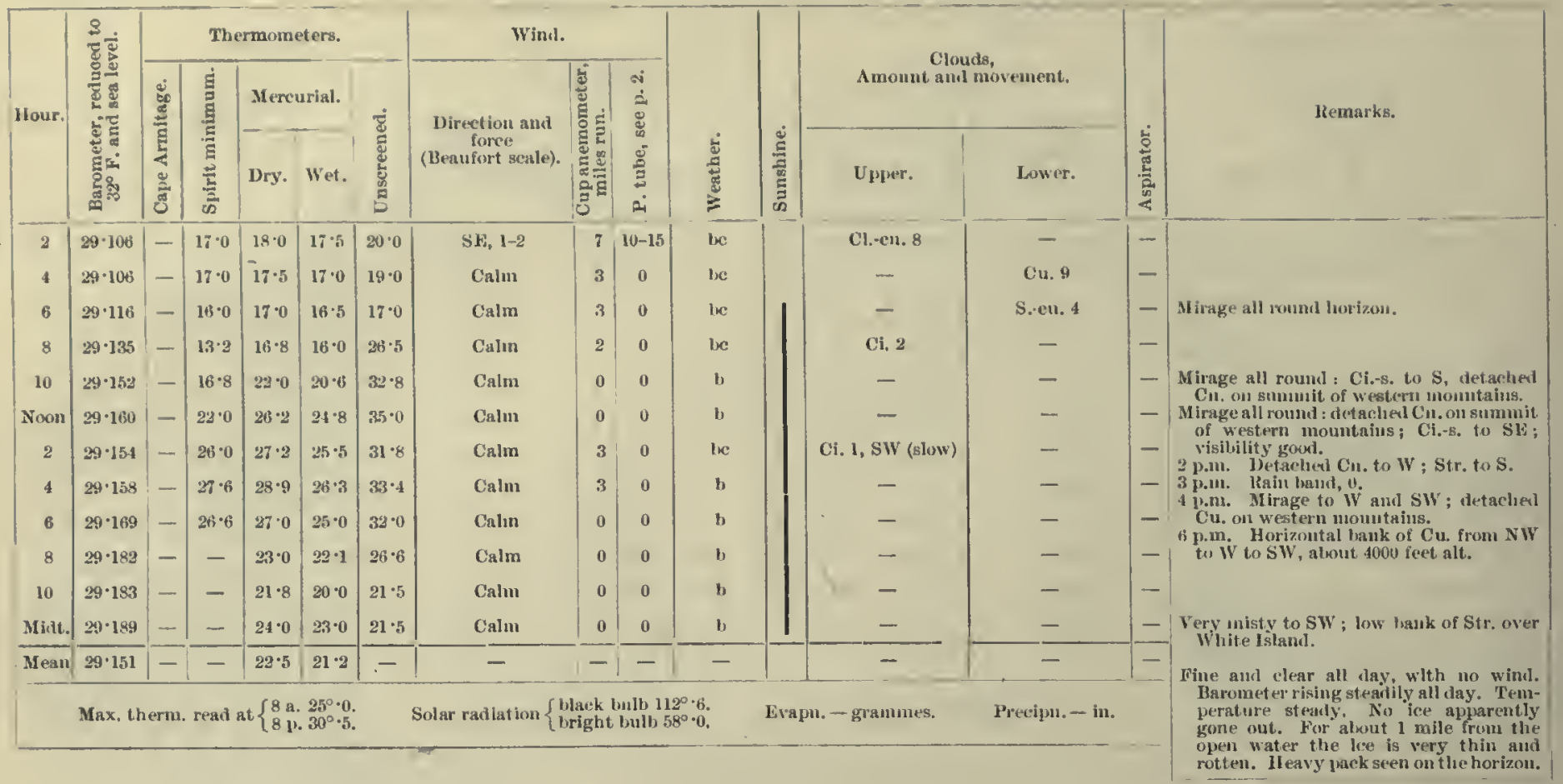


Metrolologicat, Jouknat, of thl, "Discovery."

\begin{tabular}{|c|c|c|c|c|c|c|c|c|c|c|c|c|c|c|c|}
\hline Ilour. & 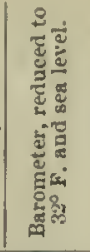 & 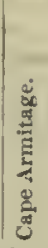 & 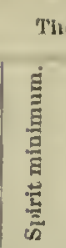 & $\begin{array}{l}\text { Merc } \\
\text { Dry. }\end{array}$ & uriul. & 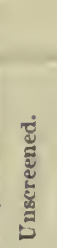 & $\begin{array}{l}\text { Directlou aud } \\
\text { force } \\
\text { (Herulort. neale). }\end{array}$ & 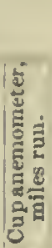 & 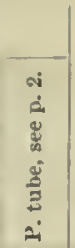 & 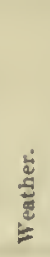 & 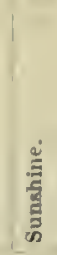 & \multicolumn{2}{|c|}{$\begin{array}{l}\text { Clousls, } \\
\text { Auoust and uovenent. }\end{array}$} & $\begin{array}{l}\frac{\dot{4}}{5} \\
\frac{5}{2} \\
\frac{5}{8}\end{array}$ & Kemarks. \\
\hline 3 & $29 \cdot 193$ & - & - & $13 \cdot 0$ & $12 \cdot 0$ & $24 \cdot 2$ & Caluı & 0 & 0 & 10 & & - & - & $1-$ & \\
\hline 1 & $29 \cdot 211$ & - & $17 \cdot 8$ & $17 \cdot 8$ & $163^{\circ} \cdot 0$ & $27 \cdot 5$ & Cuin & 0 & 0 & b & & - & - & - & Ileavy S.eus. Iaslf- way down weatern \\
\hline 6 & $29 \cdot 211$ & - & $17 \cdot 5$ & $21 \cdot 0$ & $19 \cdot 8$ & $x \cdot 0$ & Calm & 0 & 0 & be & 1 & - & Cu. : 3, N & - & 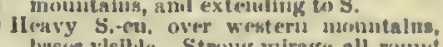 \\
\hline 8 & $29 \cdot 211$ & - & $20 \cdot 0$ & $32 \cdot 0$ & 210 & $27 \cdot 0$ & $51 * 1$ & 5 & $5-8$ & we & ' & - & S.-cur, 7, N & - & Luscen vislbl." Strong mirage all rount \\
\hline 10 & $29 \cdot 219$ & - & $211^{\circ}$ & $23 \cdot 5$ & $29 \cdot 3$ & $28 \cdot 5$ & F, 1 & 5 & $8-10$ & oc & & - & S.ศ*u, 8, N & - & 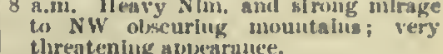 \\
\hline Noon & $29 \cdot 229$ & - & $19 \cdot 8$ & $25 \cdot 0$ & $23 \cdot 0$ & $30 \cdot 0$ & WNI $1-2$ & 8 & $10-15$ & oe & & - & Cu, 10, NE & - & Mirage on $\mathrm{S}$ fortzou. \\
\hline 2 & $29 \cdot 2 \mathrm{mi}$ & - & $21 \cdot 6$ & $2 * 0$ & $21 \cdot 8$ & $34 \cdot 0$ & ENI, 0-1 & 3 & $5-8$ & oc & & - & Cu. 10, H & - & Sllglit lreaks overitesal, mirage to W. \\
\hline 4 & $.29 \cdot 228$ & - & $26 \cdot 2$ & $28 \cdot 0$ & $25 \cdot 8$ & $82 \cdot 3$ & Cuim & 0 & 0 & $\alpha$ & 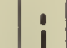 & - & Cu. 8, li & $\begin{array}{l}16 \cdot 7 \\
15 \cdot 4\end{array}$ & Niun. on west teru inountalus ; clear to s : \\
\hline 6 & $29 \cdot 226$ & - & $2 t \cdot b$ & $25 \cdot 0$ & $22 \cdot 4$ & $29 \cdot 0$ & ENE, 1 & 4 & $5-10$ & $b$ & & - & - & -1 & \\
\hline 8 & $29 \cdot 233$ & - & $20 \cdot 0$ & $20 \cdot 2$ & $18 \cdot 6$ & $22 \cdot 3$ & Var. I., 2 & 11 & $10-15$ & $b$ & & - & 一 & - & Cn. approacling from $W$. \\
\hline 10 & $29 \cdot 3: 35$ & - & $19 \cdot 0$ & $22 \cdot 0$ & $20^{-10}$ & $33 \cdot 0$ & $\mathbf{E}, 0-1$ & 8 & 0 & b & & - & - & - & Mirage at foot of western uount alni. \\
\hline Mint. & $29 \cdot 213$ & - & $15 \cdot 0$ & $16 \cdot 0$ & $13 \cdot 5$ & $16 \cdot 0$ & Calm & 4 & 0 & 13. & & 一 & - & - & Mirage on W horlzon; Invavy Cu, over \\
\hline Mean & $20 \cdot 223$ & - & - & $21 \cdot 7$ & $19 \cdot 9$ & - & - & - & - & - & & $一$ & - & 一 & \\
\hline & Yas & 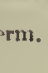 & 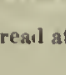 & $\int 8 \mathrm{a}$. & $\mathrm{i}^{\circ} \cdot 2$ & & r rallintiou $\{\mathrm{bl}$ & & all) 10 & & Eva! & ranmes. & Precip.. - in. & & 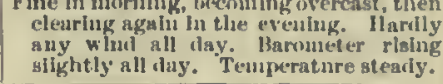 \\
\hline
\end{tabular}

1 st.

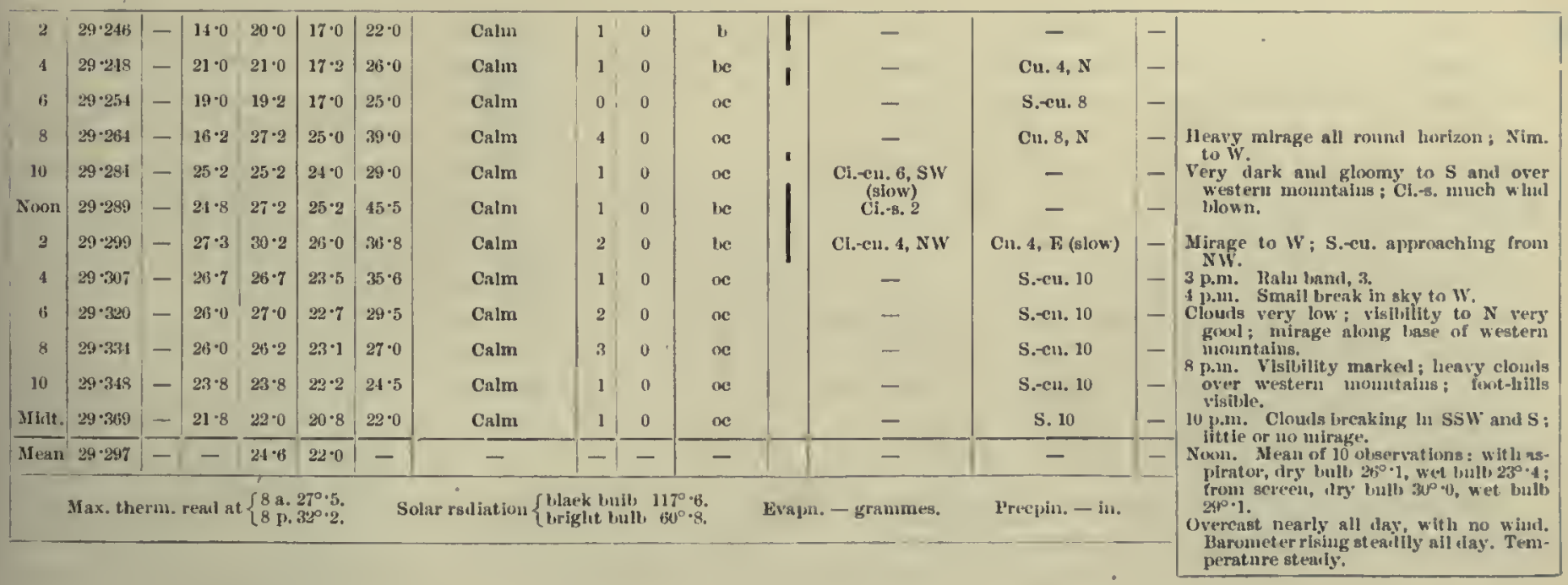

\begin{tabular}{|c|c|c|c|c|c|c|c|c|c|c|c|c|c|c|}
\hline 2 & $|29: 373|$ & -1 & $21 \cdot 5$ & $21 \cdot 0$ & $21 \cdot 5$ & $2 t \cdot 5$ & Calm & 2 & 0 & ne & - & S.eu. 10 & 1- & Cloudis breaklug in SW and S. \\
\hline 4 & $29 \cdot 377$ & - & $22 \cdot 0$ & $26 \cdot 0$ & $21 \cdot 0$ & $26-2$ & Lt. $\mathbf{N}$ airs & 2 & $0-8$ & ne & - & S.-en. 10 & - & $\begin{array}{l}\text { Clouls apuarently rising; vlsibility } \\
\text { markiel to s. }\end{array}$ \\
\hline B & $29 \cdot 381$ & - & $25 \cdot 2$ & $26 \cdot 2$ & $24 \cdot 0$ & $27 \cdot 6$ & Calm & 5 & 0 & ac & - & S.-en. 10 & - & $\begin{array}{l}\text { Heary hank of roll Cu. in } \mathrm{S} \text {; clear to } \\
\text { SIV; clearing gencralls. }\end{array}$ \\
\hline 8 & $29 \cdot 401$ & - & $25 \cdot 0$ & $26 \cdot 6$ & $24 \cdot 0$ & $28 \cdot 5$ & Calm & 1 & 0 & ac & - & S. $-\mathrm{en} .10$ & - & \\
\hline Nom & $29 \cdot 409$ & - & $20 \cdot 0$ & $27 \cdot 5$ & $25 \cdot 2$ & $31 \cdot 6$ & $\mathbf{N}, 1$ & 4 & 10 & be & $\begin{array}{l}\text { Cl.-s, 3, SWY } \\
\text { (slow) }\end{array}$ & - & - & $\begin{array}{l}\text { generally, sun gleamlug. } \\
\text { Nous. lloll Cn. gut westeru mountains, }\end{array}$ \\
\hline 2 & $29 \cdot 401$ & - & $26 \cdot 4$ & $27 \cdot 6$ & $25 \cdot 8$ & $32 \cdot 0$ & $N, 0-1$ & 4 & $0-8$ & b & - & - & - & $\begin{array}{l}\text { summuits risibic: visillility gookl ; sky } \\
\text { clearlng raphilly. }\end{array}$ \\
\hline 8 & $29 \cdot 417$ & - & $27 \cdot 0$ & $29 \cdot 2$ & $24 \cdot 8$ & $30 \cdot 0$ & Calm & 2 & 0 & b & - & - & - & 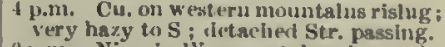 \\
\hline 10 & $29 \cdot 379$ & - & $21 \cdot 0$ & $26 \cdot 0$ & $23 \cdot 0$ & $29 \cdot 0$ & Cslm & 0 & 0 & $b$ & - & - & - & $\begin{array}{l}0 \text { p.m. Nim. in } 16 \text {, monntains onscuret. } \\
\text { 10 p.m. Low bank of mist all rount }\end{array}$ \\
\hline Midt. & $29 \cdot 381$ & - & $17 \cdot 2$ & $23 \cdot 0$ & $20 \cdot 8$ & $21 \cdot 0$ & Calm & 0 & 0 & b & - & - & - & $\begin{array}{l}\text { horizon. } \\
\text { Midt. Cu. to Sw. }\end{array}$ \\
\hline Мея⿰㇇ & $29-393$ & - & - & $246 \cdot 6$ & $24 \cdot 1$ & - & - & - & - & - & - & $\div$ & $1-$ & Overeast mutil 10 a.m., then clearing and \\
\hline & - & & 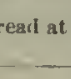 & & & & & & & & 1 & cipn. $-\mathrm{ln}$. & & 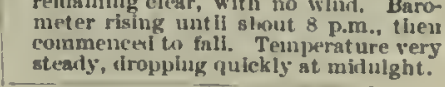 \\
\hline
\end{tabular}




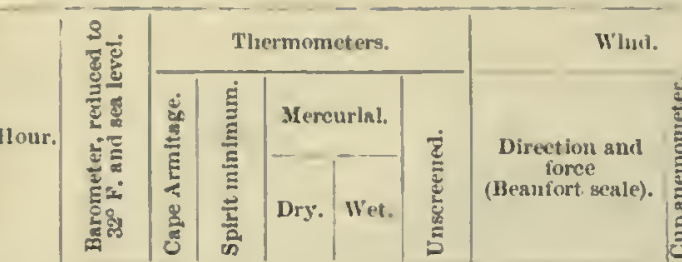

4 th.

\begin{tabular}{|c|c|c|c|c|c|c|}
\hline 2 & $29 \cdot 382$ & - & $16 \cdot 0$ & $16 \cdot 0$ & $15 \cdot 0$ & $24 \cdot 0$ \\
4 & $29 \cdot 360$ & - & $12 \cdot 0$ & $14 \cdot 0$ & $12 \cdot 8$ & $26 \cdot 2$ \\
8 & $29 \cdot 352$ & - & $13 \cdot 0$ & $18 \cdot 0$ & $16 \cdot 8$ & $28 \cdot 0$ \\
8 & $29 \cdot 350$ & - & $14 \cdot 0$ & $20 \cdot 2$ & $19 \cdot 0$ & $29 \cdot 0$ \\
10 & $29 \cdot 34$ & - & $20 \cdot 0$ & $24 \cdot 8$ & $22 \cdot 8$ & $29 \cdot 0$ \\
Noon & $29 \cdot 332$ & - & $25 \cdot 0$ & $27 \cdot 5$ & $25 \cdot 5$ & $38 \cdot 8$ \\
2 & $29 \cdot 306$ & - & $26 \cdot 2$ & $27 \cdot 0$ & $24 \cdot 8$ & $34 \cdot 2$ \\
4 & $29 \cdot 283$ & - & $26 \cdot 8$ & $27 \cdot 8$ & $25 \cdot 0$ & $40 \cdot 0$ \\
6 & $29 \cdot 267$ & - & $27 \cdot 0$ & $27 \cdot 5$ & $25 \cdot 2$ & $34 \cdot 0$ \\
8 & $29 \cdot 2.9$ & - & $25 \cdot 8$ & $26 \cdot 0$ & $25 \cdot 0$ & $26 \cdot 0$ \\
10 & $29 \cdot 251$ & - & $22 \cdot 2$ & $22 \cdot 2$ & $21 \cdot 8$ & $23 \cdot 0$ \\
Mldt. & $29 \cdot 244$ & - & $22 \cdot 0$ & $23 \cdot 0$ & $22 \cdot 0$ & $23 \cdot 0$ \\
\hline
\end{tabular}

Max, therm, real at $\left\{\begin{array}{l}8 \mathrm{a}, 30^{\circ} 0, \\ 8 \mathrm{p}, 32^{\circ} \cdot 0,\end{array}\right.$
Solar ralliation $\left\{\begin{array}{l}\text { black bull, } 115^{\circ} \cdot 6 . \\ \text { bright bulb } 59^{\circ} \cdot 8 .\end{array}\right.$

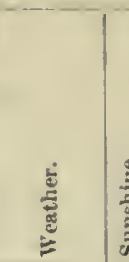

$\mid$\begin{tabular}{c|c|} 
Clouda, \\
Amount and movement.
\end{tabular}

liemarks.

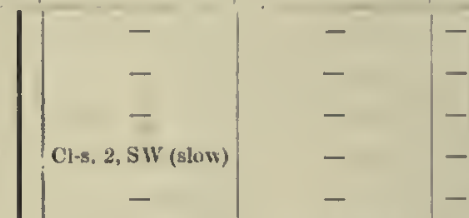
S. $10, \mathbf{N}$ (fast)

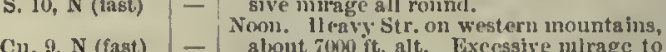
S and SW.

- 2 p.m. Lanil to $W$ and $S$ olsecuresl execpt along horizon. Bank of $\mathrm{Cu}$, extending from $\mathbf{W}$ to $\mathbf{N}$.

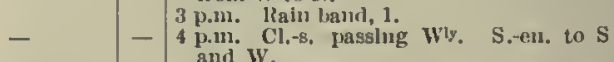
- $\quad-6$ p.m. jetaeled Cu.

C11. 8, NE (slow) - 8 p.m. Very mlsty over western momp.m. Very mlsty over western momm-
tains; detached $\mathrm{Cu}$. to $\mathrm{S}$ ami SW; Ci.-s. to Niv.

10 p.m. Dark Nim, frou SW extending srounat horizon to SE, obscuring tope ot mountalns. Midt. Ileavy lank of Nlm. over the Bluft Fine and clear nearly all day, with no wind. Barometer falling steadily all day. Temperature low early, rlsing by miliday.
5 th,

\begin{tabular}{|c|c|c|c|c|c|c|c|c|c|c|c|c|c|c|}
\hline 2 & $29 \cdot 240$ & - & $16 \cdot 8$ & $19 \cdot 8$ & $18 \cdot 0$ & $19 \cdot 8$ & $\mathrm{NE}, 2-3$ & 5 & $10-15$ & oc & & - & S. cu. $9, N E$ & - \\
\hline 4 & $29 \cdot 240$ & - & $16 \cdot 2$ & $19 \cdot 0$ & $17 \cdot 8$ & $19 \cdot 8$ & Var, E, 3-4 & 17 & $15-22$ & be & & - & NE & - \\
\hline 6 & $29 \cdot 2.42$ & - & $16 \cdot 0$ & $18 \cdot 0$ & $16 \cdot 2$ & $18 \cdot 0$ & Var. 18, 3-4 & 19 & $15-25$ & be & & - & S.-cu. 5,1 & - \\
\hline 8 & $29 \cdot 233$ & - & $17 \cdot 0$ & $18 \cdot 0$ & $16^{\circ} 0$ & $20 \cdot 2$ & $14,2-3$ & 11 & $15-20$ & oc & & - & S.-cu. 8, SE & - \\
\hline 10 & $29 \cdot 237$ & - & $17 \cdot 0$ & 18.5 & 17.0 & $21 \cdot 5$ & $13,3-4$ & 16 & $20-28$ & $\infty$ & & - & Cn. $9, \mathrm{~S}$ (slow) & - \\
\hline Noon & $29 \cdot 231$ & - & $18 \cdot 0$ & $20 \cdot 0$ & $17^{\circ} 0$ & $22 \cdot 3$ & E, $2-8$ & 15 & $|10-22|$ & oc & & - & A.-cu. 8, S (slow) & - \\
\hline 2 & $29 \cdot 225$ & - & $18 \cdot 7$ & $20 \cdot 0$ & $17 \cdot 6$ & $22 \cdot 6$ & ENE, 2-3 & 16 & $15-23$ & oc & & - & A. $\mathrm{cu}, 8, \mathrm{SE}$ & - \\
\hline 4 & $29 \cdot 223$ & - & $19 \cdot 0$ & $20 \cdot 0$ & $17 \cdot 8$ & $21 \cdot 3$ & Var. E, $2-3$ & 11 & $12-20$ & be & ? & - & Cu. 2, E (mod.) & - \\
\hline 6 & $24 \cdot 215$ & - & $18 \cdot 6$ & $20 \cdot 8$ & $18 \cdot 0 !$ & $23 \cdot 4$ & E by $N, 2-3$ & 10 & $15-23$ & $o c$ & ! & - & Cu. 7 , NF (mor.) & - \\
\hline 8 & $29 \cdot 213$ & - & $19 \cdot 5$ & 200 & $17 \cdot 0$ & $20 \cdot 8$ & $\mathrm{NE}, 2$ & 14 & $8-12$ & bc & i & - & Cu. 5, N (mсні.) & - \\
\hline 10 & $\mid 29 \cdot 213$ & - & $19 \cdot 0$ & $19 \cdot 5$ & $16 \cdot 8$ & $20 \cdot 5$ & NL, 1-2 & 8 & $5-10$ & oc & & - & Cn. $\tilde{\text {, N NE }}$ & - \\
\hline Midt. & $29 \cdot 213$ & - & $19 \cdot 8$ & 21.5 & $19 \cdot 2$ & $21 \cdot 0$ & Lt. NE airs & 7 & $5-10$ & oc & & - & Cul. $9, \mathrm{NE}$ & $1-$ \\
\hline Mean? & 1) $24 \cdot 227$ & $1-$ & - & $19 \cdot 6$ & $17 \cdot 4$ & - & - & - & - & - & & - & - & - \\
\hline
\end{tabular}

Vime on western mountains, sumunits $\int$ olscured ; slight mirage in S1.

Very heary Nim. on western mountains cxt eniling from NW to SII. Very thaek and tlurcatening; land otscured in NW.

11eavy S.-cu. on west ern mountains ; dark and tlireatening to SW dark and threatening to SW ; b ; very lin $\mathrm{S}$; visibllity good?

2 p.m. il eavy layers of $\mathrm{Cu}$. on western inomutains $4000 \mathrm{it}$, $\mathrm{np}$. 3 p.m. Rain tasul, 2 . 1 p.m. Cu. on western mountains, but summits and bases visilde.

6 p.m. Cll. to W, Niml. to N, with

8 sp.ight mirage. Clearto $\mathrm{S}$ and SW ; tetached Cu. to $\mathrm{E}$; kow S.-ent, on western monnt ains, sumnits only just visible alover, visibility markerl.

$10 \mathrm{p.m}$. NIm. clouds from $\mathrm{W}$ to $\mathrm{SW}$.

Midit. West.ern monntains olscured; Nim. Un Mit. Discovery.

Noon. Mean of 10 olservations : with aspirator, dry bulb $20^{\circ} 3$, wet binll $15^{\circ}$, $17^{\circ} \cdot 0$.

Cloudy all day, with fresh Fiy $^{1 / 5}$ ind which fell at niglit. Barometer fell all day. Temperat ure stegdy.

\begin{tabular}{|c|c|c|c|c|c|c|c|c|c|c|c|c|c|c|}
\hline 6 th. & $29 \cdot 216$ & - & $17 \cdot 0$ & $17 \cdot 0$ & $15 \cdot 8$ & 13.5 & Calm & 1 & 0 & ne & - & S.eu. 5 & - & \\
\hline 4 & $29 \cdot 206$ & - & $16 \cdot 0$ & $17 \cdot 5$ & $16 \cdot 0$ & $14 \cdot 0$ & Calm & 0 & 0 & oc & - & $\mathrm{Cu} .8$ & - & Mirage in $\mathrm{W}$. \\
\hline 6 & $29 \cdot 222$ & - & $15 \cdot 5$ & $20 \cdot 5$ & $18 \cdot 8$ & $22 \cdot 0$ & Calm & 6 & 0 & oc & - & Cu. 9 & - & Very thick and dirty looking towards \\
\hline 8 & $29 \cdot 222$ & - & $19 \cdot 8$ & $22 \cdot 0$ & $20 \cdot 0$ & $26 \cdot 0$ & Calm & 3 & 0 & ac $*$ & - & C11. 10 & - & $\begin{array}{l}\text { western mountains. } \\
\text { lleavy Nim. to W; suowing slightily. }\end{array}$ \\
\hline 10 & $29 \cdot 246$ & - & 10.5 & 11.8 & $11 \cdot 0$ & $14 \cdot 0$ & SE, 2-3 & 8 & $15-20$ & oc $\div$ & - & S.-cu. 10 & - & Very thlek all round. \\
\hline Noon & $29 \cdot 265$ & -1 & $11 \cdot 0$ & $16 \cdot 0$ & $14 \cdot 2$ & $18 \cdot 0$ & $\mathrm{E}, 3$ & 14 & $12-18$ & $o c+$ & - & s.-en. 10 & - & $\begin{array}{l}\text { Clearing to s ; much drift in Strait ; very } \\
\text { thick to } W \text {. dark water sky to rit and N }\end{array}$ \\
\hline 2 & $29 \cdot 2 \uparrow 2$ & - & $11 \cdot \pi$ & $15 \cdot 2$ & $13 \cdot 6$ & $17 \cdot 4$ & Var. 1s, 2-4 & 17 & $15-39$ & oeq + & - & S.cu. 10 & - & $\begin{array}{l}\text { Dark allatlow over western monntains; } \\
\text { winct terv variable and squally. }\end{array}$ \\
\hline 4 & $29 \cdot 275$ & - & $14 \cdot 0$ & $14 \cdot 7$ & $1.3 \cdot 2$ & $17 \cdot 0$ & Var. E, 2-4 & 16 & $\mid 15-28$ & ocq $f$ & - & S. cu. 10 & - & 3 p.m. llain band, 4 . \\
\hline 6 & $29 \cdot 279$ & - & $12 \cdot 8$ & $14 \cdot 0$ & $12 \cdot 7$ & $16 \cdot 8$ & Var. F, 2-4 & 18 & $16-27$ & $\infty$ & - & N. 10 & - & 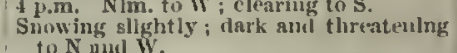 \\
\hline 8 & $29 \cdot 29.3$ & - & $12: 3$ & $14 \cdot 2$ & $12 \cdot 2$ & $15 \cdot 0$ & Var. 1:, 2-3 & 12 & $15-18$ & $o *$ & - & N. 10 & - & $\begin{array}{l}\text { to } \\
\text { Dark and threatening to } \mathrm{W} \text { and } \mathrm{SW} \text {; low } \\
\text { parts of laud visille to } \mathrm{S} \text {. }\end{array}$ \\
\hline 10 & $29 \cdot 3413$ & -1 & $13 \cdot 0$ & $14 \cdot 0$ & $13 \cdot 5$ & $14 \cdot 4$ & F l,y $N, 2-3$ & 14 & $16-20$ & $o *$ & - & N. 10 & - & \\
\hline vilut. & $29 \cdot 307$ & $=$ & $12 \cdot 0$ & $14 \cdot 0$ & $12 \cdot 5$ & $13 \cdot 2$ & E by $N, 1-2$ & 10 & $8-15$ & $o *$ & - & N. 10 & - & \multirow{3}{*}{ 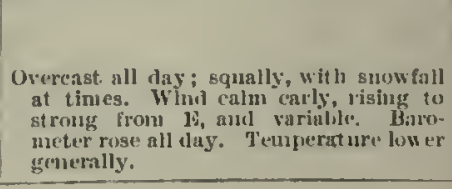 } \\
\hline$\overline{\text { Mean }}$ & $29 \cdot 259$ & - & $\cdot-$ & $\longdiv { 1 5 \cdot 9 }$ & $14: 5$ & - & - & $\overline{-}$ & - & - & - & - & I & \\
\hline & \multicolumn{4}{|c|}{$\begin{array}{l}\text { Max. therm. } \\
\text { real at }\end{array}$} & \multicolumn{3}{|c|}{ 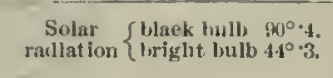 } & \multicolumn{3}{|c|}{ lsoapn. - grammes. } & •. & p.ur. 24th & & \\
\hline
\end{tabular}


Metemolocical, Journat, of tiik "Discovkry."

[1903-February.

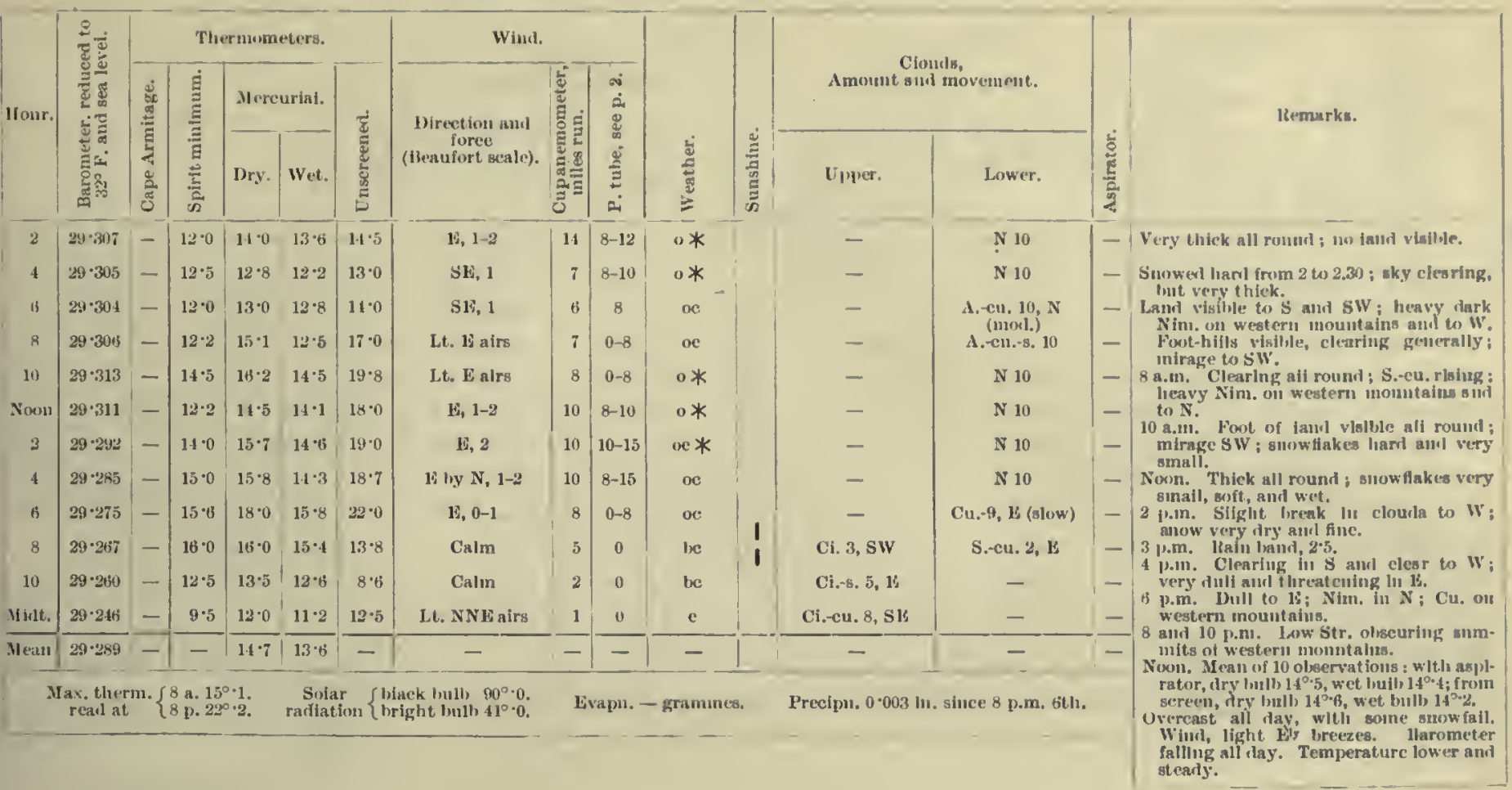

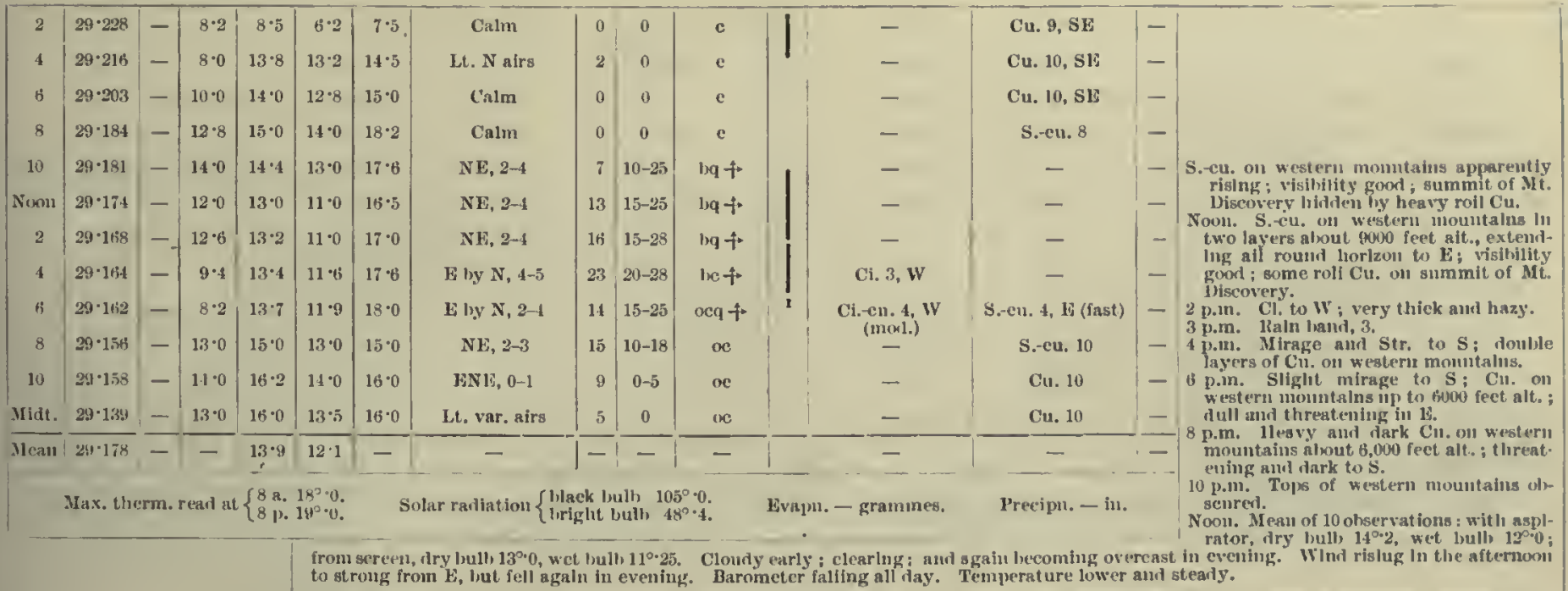

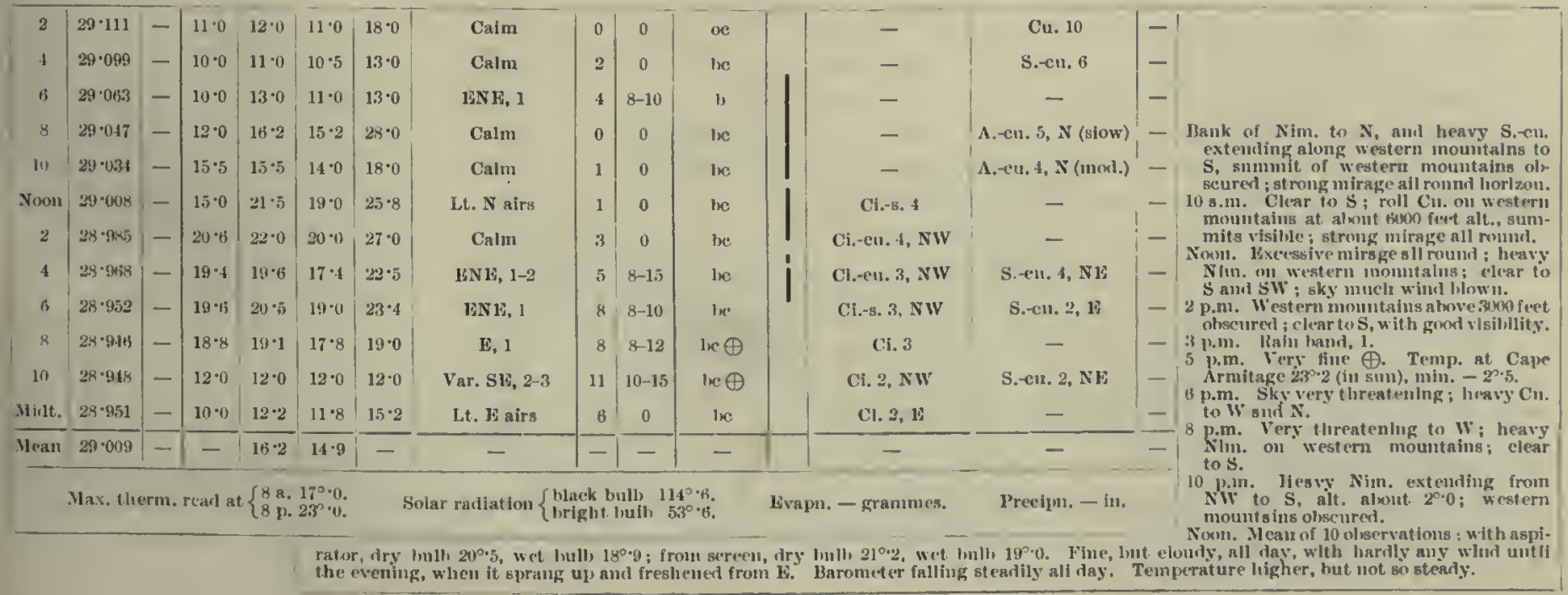


Meteololocical, Jouinal of th: "Discoviry."

[1903-February.

\begin{tabular}{|c|c|c|c|c|c|c|c|c|c|c|c|c|c|c|c|}
\hline \multirow{3}{*}{ Ilour. } & \multirow{3}{*}{ 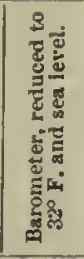 } & \multicolumn{5}{|c|}{ Thermometers. } & \multicolumn{3}{|l|}{ Wind. } & \multirow[b]{3}{*}{ है } & \multirow[b]{3}{*}{ 至 } & \multirow{2}{*}{\multicolumn{2}{|c|}{$\begin{array}{l}\text { Ciouds, } \\
\text { Amount and movennent. }\end{array}$}} & \multirow[b]{3}{*}{$\frac{\overbrace{}^{2}}{2}$} & \multirow{3}{*}{ IRemarks. } \\
\hline & & \multirow{2}{*}{$\mid$} & 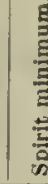 & \multicolumn{2}{|c|}{ Mercurlal. } & \multirow{2}{*}{ 要 } & \multirow{2}{*}{$\begin{array}{l}\text { Direction and } \\
\text { (Borce } \\
\text { (Bcaufort scale). }\end{array}$} & \multirow{2}{*}{ 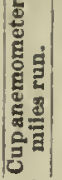 } & \multirow{2}{*}{ 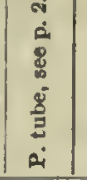 } & & & & & & \\
\hline & & & 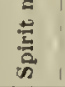 & Dry. & Wet. & & & & & & & Upyer. & Low & & \\
\hline 2 & $29 \cdot 330$ & - & 4.0 & $7 \cdot 0$ & 8.4 & 6.5 & E, $2-4$ & 17 & $|15-36|$ & c & & Cl. $-c u .8, \mathbf{N}$ & - & - & \multirow{4}{*}{ 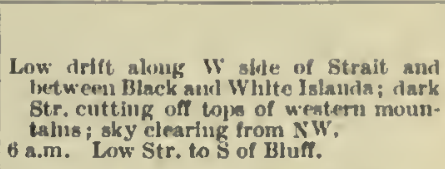 } \\
\hline 4 & $29 \cdot 324$ & - & $4 \cdot 8$ & $3 \cdot 2$ & 6.0 & $6 \cdot 0$ & K by S, 3-5 & 15. & $12-30$ & be & & C1.8. 4, NW & - & - & \\
\hline 6 & $29 \cdot 330$ & -1 & $4 \cdot 0$ & $5 \cdot 8$ & $5 \cdot 2$ & $8 \cdot 0$ & E by $S, 3-5$ & 13 & $12-22$ & b & & - & - & - & \\
\hline 8 & $29 \cdot 330 \mid$ & - & $4 \cdot 0$ & 8.8 & 8.0 & $9 \cdot 0$ & E by S, 4-5 & 17 & $20-25$ & $\mathrm{~h}$ & & - & - & - & \\
\hline 10 & $29 \cdot 334$ & - & 6.5 & 8.8 & 8.0 & $10 \cdot 2$ & Var. E, 3 & 19 & $18-22$ & h & & - & - & - & \multirow{7}{*}{ 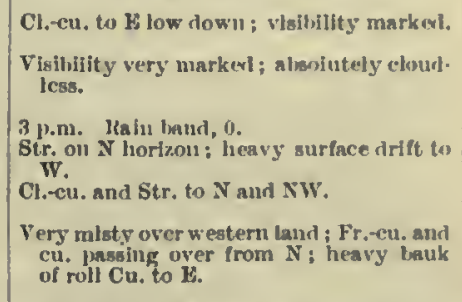 } \\
\hline Nnow & $29 \cdot 324$ & - & $8 \cdot 0$ & $8 \cdot 0$ & 7.5 & $9 \cdot 0$ & Var. $\mathbf{E}, 2-3$ & 15 & $20-22$ & $b$ & & - & - & -. & \\
\hline 3 & $23 \cdot 312$ & -1 & 7.5 & 7.8 & $7 \cdot 0$ & $8 \cdot 0$ & Var. $E_{n} 4$ & 21 & $20-25 \mid$ & $1+\mathrm{f}^{*}$ & & - & - & - & \\
\hline 4 & $29 \cdot 293$ & - & $5 \cdot 0$ & $7 \cdot 8$ & 6.8 & $11 \cdot 0$ & Var. k, 3-5 & 19 & $20-35$ & $b_{1}+$ & & - & - & - & \\
\hline in & $29 \cdot 299$ & - & $6 \cdot 3$ & $8 \cdot 0$ & $7 \cdot 8$ & $11 \cdot \mathrm{b}$ & Es by $8,2-3$ & 18 & $15-25$ & b & & - & - & - & \\
\hline 8 & $|29 \cdot 321|$ & - & $7 \cdot 0$ & $10 \cdot 0$ & $8 \cdot 5$ & $10 \cdot 0$ & $\mathbf{k}, 2-3$ & 17 & 15-22 & we & & - & Cu. 4, N (mox.) $)$ & - & \\
\hline 10 & $29 \cdot 281$ & - & $8 \cdot 0$ & $9 \cdot 0$ & 8.0 & $9 \cdot 0$ & Var. $\mathbf{E}, 4-5$ & 15 & 25-28 & we & & - & Cu. 9 & - & \\
\hline Mint. & $29 \cdot 281$ & - & $4 \cdot 0$ & 8.0 & $4 \cdot 8$ & $8 \cdot 0$ & Var. E, 4 & 18 & $20-23$ & te* $*$ & & - & Cu. 9 & - & \multirow{3}{*}{ 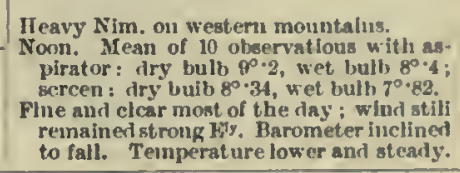 } \\
\hline Меви & $29 \cdot 313$ & -1 & - & $7 \cdot 9$ & $7 \cdot 0$ & - & - & -1 & - & - & & - & - & - & \\
\hline \multicolumn{7}{|c|}{ 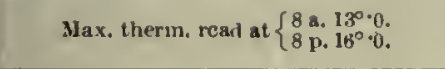 } & \multicolumn{4}{|c|}{ Solar radiat ion $\left\{\begin{array}{l}\text { black luil } 92^{\circ}, 8_{0} \\
\text { bright bulb } 41^{\circ} 0\end{array}\right.$} & & grammes. & \multicolumn{2}{|c|}{ Precipm. - in. } & \\
\hline
\end{tabular}

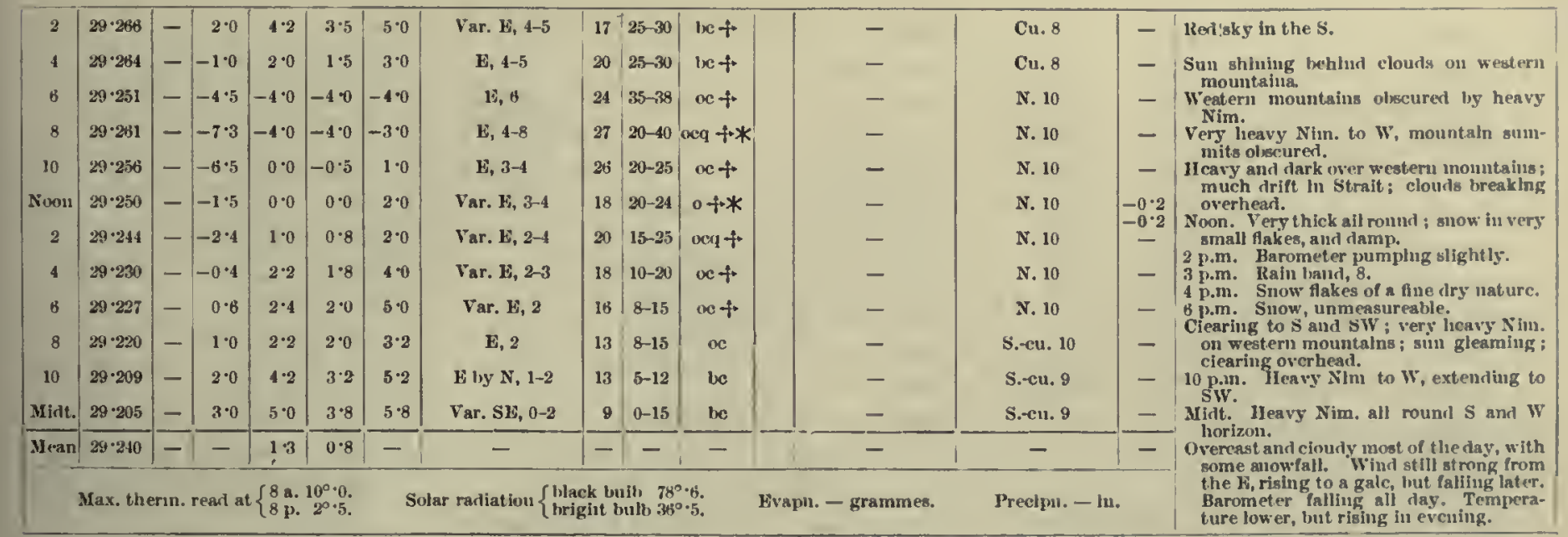

\begin{tabular}{|c|c|c|c|c|c|c|c|c|c|c|c|c|c|c|c|}
\hline 2 & $29 \cdot 197$ & - & 0.0 & 1.0 & 0.0 & $2 \cdot 5$ & $S \operatorname{lng} E_{1}, 1-2$ & 7 & $8-12$ & het thigh $^{\text {hath }}$ & & - & S.ent. $, 0, x$ & - & Heavy Nim. to SW. \\
\hline 4 & $29 \cdot 165$ & - & $1 \cdot 0$ & 1.0 & $1 \cdot 0$ & $2 \cdot 0$ & S hy E, 3-4 & 13 & $15-25$ & $\operatorname{lot}_{-1}^{\text {hlgh }}$ & & - & S.cu. $7, \mathrm{x}$ & - & Sky clearing all round except to $\mathrm{S}$. \\
\hline 6 & $29 \cdot 153$ & - & $-1 \cdot 0$ & 0.2 & 0.0 & 0.5 & Yar. SE airs & 7 & $0-5$ & be & 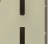 & - & A.cu. 2, N (fast) & - & 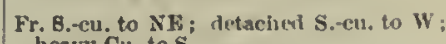 \\
\hline 8 & $28 \cdot 137$ & - & $-1 \cdot 0$ & $4 \cdot 2$ & $2 \cdot 0$ & $5 \cdot 0$ & Lt. var. airs & 3 & 0 & in & i & - & s.eu. 6 & - & \\
\hline 10 & $29 \cdot 100$ & - & $3 \cdot 0$ & 8.0 & 48 & $9 \cdot 0$ & NE, 1 & 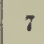 & $0-8$ & be & 1 & - & Fr, cu. 5, $\mathrm{N}$ & - & Clear all rouncl low down; visitility \\
\hline Noon & $29 \cdot 065$ & - & $5 \cdot 0$ & 6.8 & $5 \cdot 6$ & $12 \cdot 0$ & $N E, 1$ & 7 & $0-8$ & $\infty$ & & - & Cu. $8, \mathrm{~N}$ (nod.) & - & mount \\
\hline 2 & 20.034 & - & $5 \cdot 0$ & $5 \% 6$ & 4.0 & $8 \cdot 6$ & E by $N, 1-2$ & 10 & $10-18$ & $\infty$ & & - & Cu, $10, \mathrm{NE}$ & - & Cu. very di \\
\hline 4 & $28 \cdot 014$ & $1-$ & $4 \cdot 8$ & $6 \cdot 2$ & $5 \cdot 0$ & $9 \cdot 8$ & Ex ly $N, 2-3$ & 14 & $15-25$ & x & 1 & - & Cu. 7 & - & 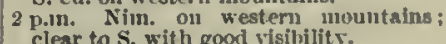 \\
\hline o & $28 \cdot 9865$ & - & $3 \cdot 8$ & $3 \cdot 0$ & $3 \cdot 2$ & $8 \cdot 2$ & Var. E, 2-3 & 17 & $15-25$ & $\propto$ & & - & A.cu. 5 & - & $\Omega_{0}$ \\
\hline 8 & $28 \cdot 974$ & - & $3 \cdot 2$ & 40 & $2 \cdot 9$ & $6 \cdot 0$ & Ex by N, 2-3 & 15 & $12-30$ & be & & - & A.-cu, 3, NNW & - & 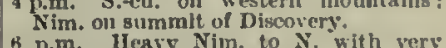 \\
\hline 10 & $28 \cdot 90^{\circ} 0$ & - & 1.8 & $3 \cdot 0$ & 1.5 & $2 \cdot 0$ & Var. E, 2-3 & 13 & $10-15$ & ineq & & - & A.cu. $4, \mathrm{NW}$ & - & 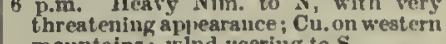 \\
\hline Midt. & $28 \cdot 931$ & $1-$ & $2 \cdot 0$ & $3 \cdot 0$ & $2 \cdot 0$ & $3 \cdot 5$ & N, 2-3 & 13 & $5-10$ & b & & - & A.cu, $2, \mathrm{sW}$ & - & varying $\mathrm{F}$ to \\
\hline M1ean" & $29 \cdot 060$ & $1-$ & - & $3 \cdot 8$ & $2 \cdot 7$ & - & - & - & - & - & & - & - & - & Noni. Mean of 10 ntservatlous with na- \\
\hline \multicolumn{6}{|c|}{ Max, thermil, read at $\left\{\begin{array}{l}8 \mathrm{a}, 5^{0} \\
8 \mathrm{p}, 7^{\circ} \cdot 2 .\end{array}\right.$} & \multicolumn{5}{|c|}{ 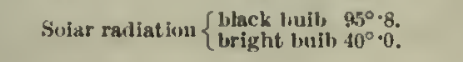 } & \multicolumn{2}{|c|}{ Kvapn. - urammes. } & Prectipn. -1 & & riable \\
\hline
\end{tabular}


Meteorological, JoUkxal of THE " Discovery."

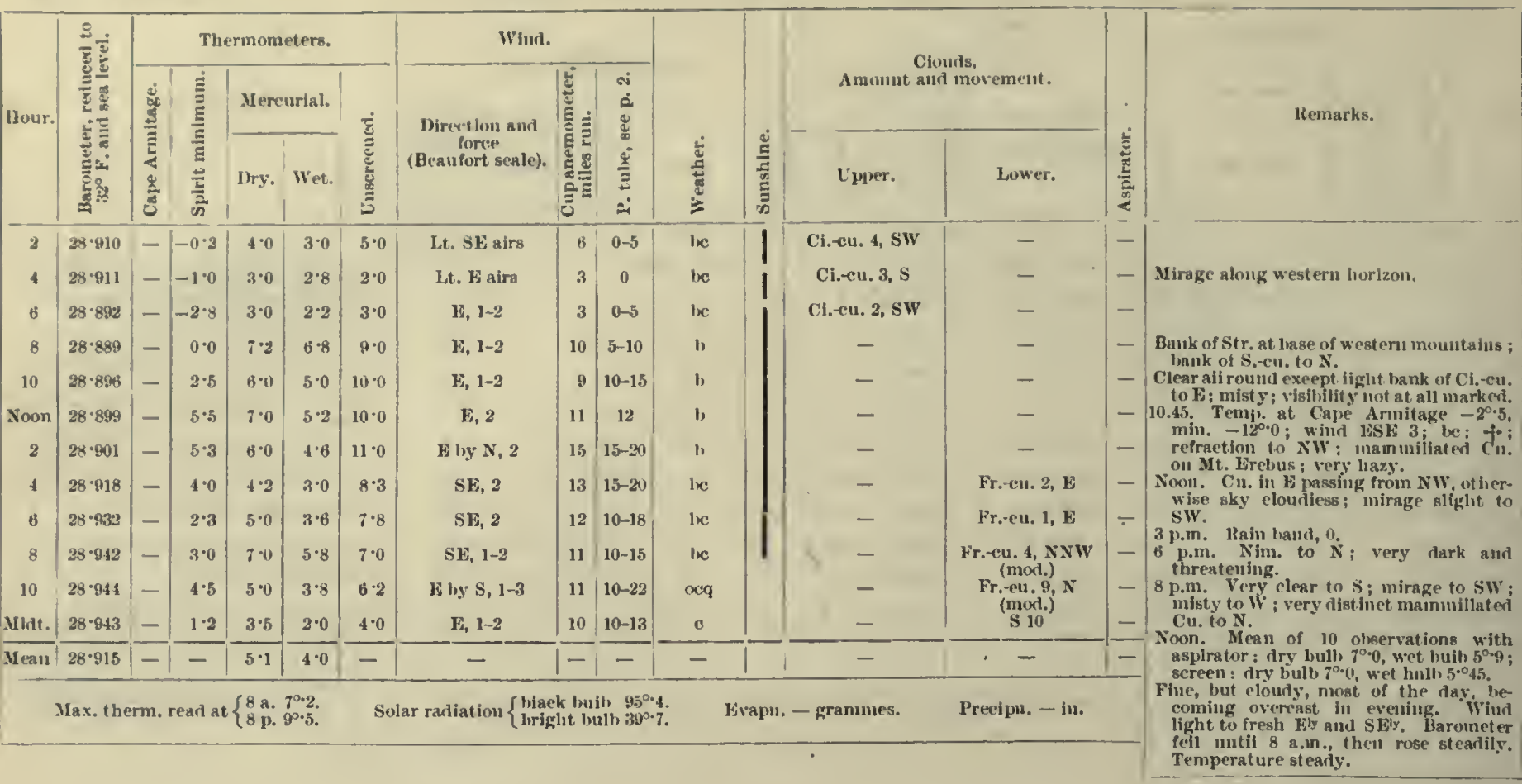

17th.

\begin{tabular}{|c|c|c|c|c|c|c|}
\hline 2 & $28 \cdot 939$ & - & $2 \cdot 0$ & 3.0 & $2 \cdot 8$ & $3 \cdot 2$ \\
\hline 4 & $28 \cdot 939$ & - & 0.0 & 1.8 & 1.5 & 1.8 \\
\hline b & $28 \cdot \Omega 32$ & - & $-4 \cdot 0$ & $2 \cdot 2$ & $2 \cdot 0$ & 30 \\
\hline 8 & 28.992 & - & 0.2 & 1.8 & $1 \cdot 5$ & $3 \cdot 8$ \\
\hline 10 & $29 \cdot 007$ & - & $-0 \cdot 2$ & 1.8 & 1.0 & $2 \cdot 8$ \\
\hline Nooli & $28 \cdot 020$ & - & 0.0 & $2 \cdot 0$ & 1.5 & $4 \cdot 5$ \\
\hline 2 & $233^{\circ} 024^{\circ}$ & - & 1.0 & $2 \cdot 5$ & $2 \cdot 0$ & $5 \cdot 0$ \\
\hline 4 & $29 \cdot 031$ & - & $2 \cdot 0$ & $5 \cdot 0$ & $4^{\circ} 0$ & $8 \cdot 2$ \\
\hline 6 & $29 \cdot 052$ & - & 4.0 & $6 \cdot 2$ & $4 \cdot 8$ & $8 \cdot 5$ \\
\hline 8 & $29 \cdot 070$ & - & $5 \cdot 0$ & $5 \cdot 5$ & $5 \cdot 0$ & 6.0 \\
\hline 10 & $29 \cdot 088$ & - & $3 \cdot 8$ & $3 \cdot 0$ & $3 \cdot 8$ & $3 \cdot 1$ \\
\hline
\end{tabular}

\begin{tabular}{c|c|c|c|} 
E, 3-3 & 15 & $15-20$ & 0 \\
E, 4-6 & 23 & $20-32$ & $0 q+$ \\
E, 2-5 & 21 & $10-25$ & $0 q+$ \\
E, 3-5 & 20 & $20-28$ & $0 q+$ \\
ESE, 3-4 & 16 & $15-22$ & 1 wenq + \\
E, 2-3 & 14 & $10-22$ & heniq \\
Var, E, 1-2 & 15 & $8-18$ & hc \\
E, 1-2 & 12 & $5-12$ & hc \\
E, 0-1 & 10 & $0-10$ & hc \\
E, 1 & 10 & $8-10$ & bc \\
SE, 1-2 & 8 & $8-10$ & b \\
E, 1 & 8 & $8-10$ & hc \\
\hline
\end{tabular}

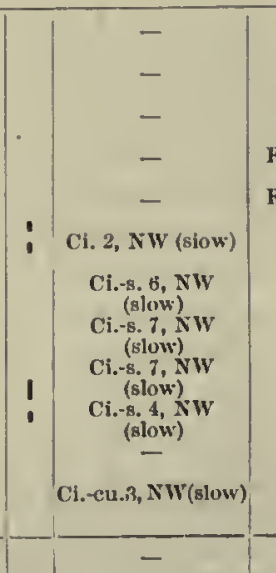

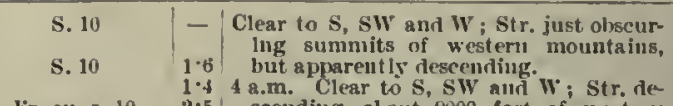

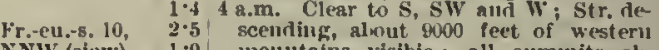

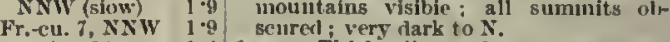
(mor.) 1.6 a.m. Thiek aii rontur; sun gleaming;

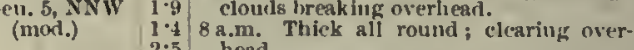
(mod.)

$2 \cdot 3$ hear. Clearing cenerally ; iand visible - to S and SW, thiek to W.

Noon. Very hazy to $\mathrm{W}$, but cloaring: inisty apjearance in sky; wiudy ap-

2 p.m. Thin film of Cu. on west (ron inountans ; very lright to $\mathrm{s}$.

3 p.m. laaiu bauri, 2.

6 p.m. Ci,-s, of a shreddy, duli and $4 \cdot 3$ threatening $k$ ind.

$2 \cdot 78$ p.m. Very misty to W; S.-cu. ou $2 \cdot 5$ in sky". monntahs; wild appearunce 10 p.m. Very misty in sky nveriear, no definlte clouds; Cir. to Fi Fr, Fo, to SW : elear to $S_{\text {; }}$ dark S.-en. over
uesteru mountains, sunmits just visible.

Mist. Dark and threatening to sw: S.-cu. coliecting on western mount ains

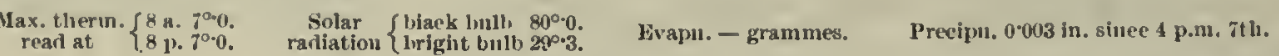
very red $t o s ;$ sum obseured belnind very dark $S$. cu. ; moou visible in $\mathbf{B}$; windy appearance in sky; Str. on land fo s. Ciourly day, with
strong lily wind early, kith drift, falling to fresh lreeze. Barometer rising all day. Temperature fow and stearly.

18 th.

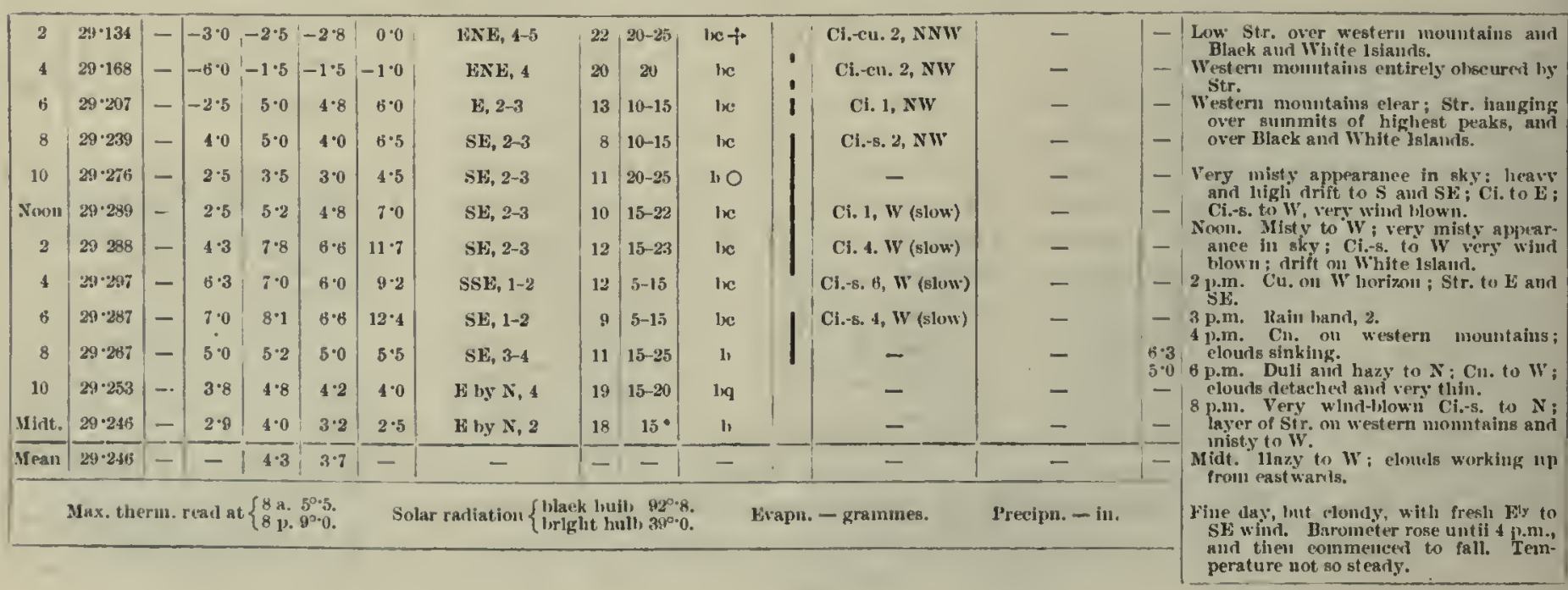


Mfthorological, Journal of thl "Discovfry."

[1903-February.

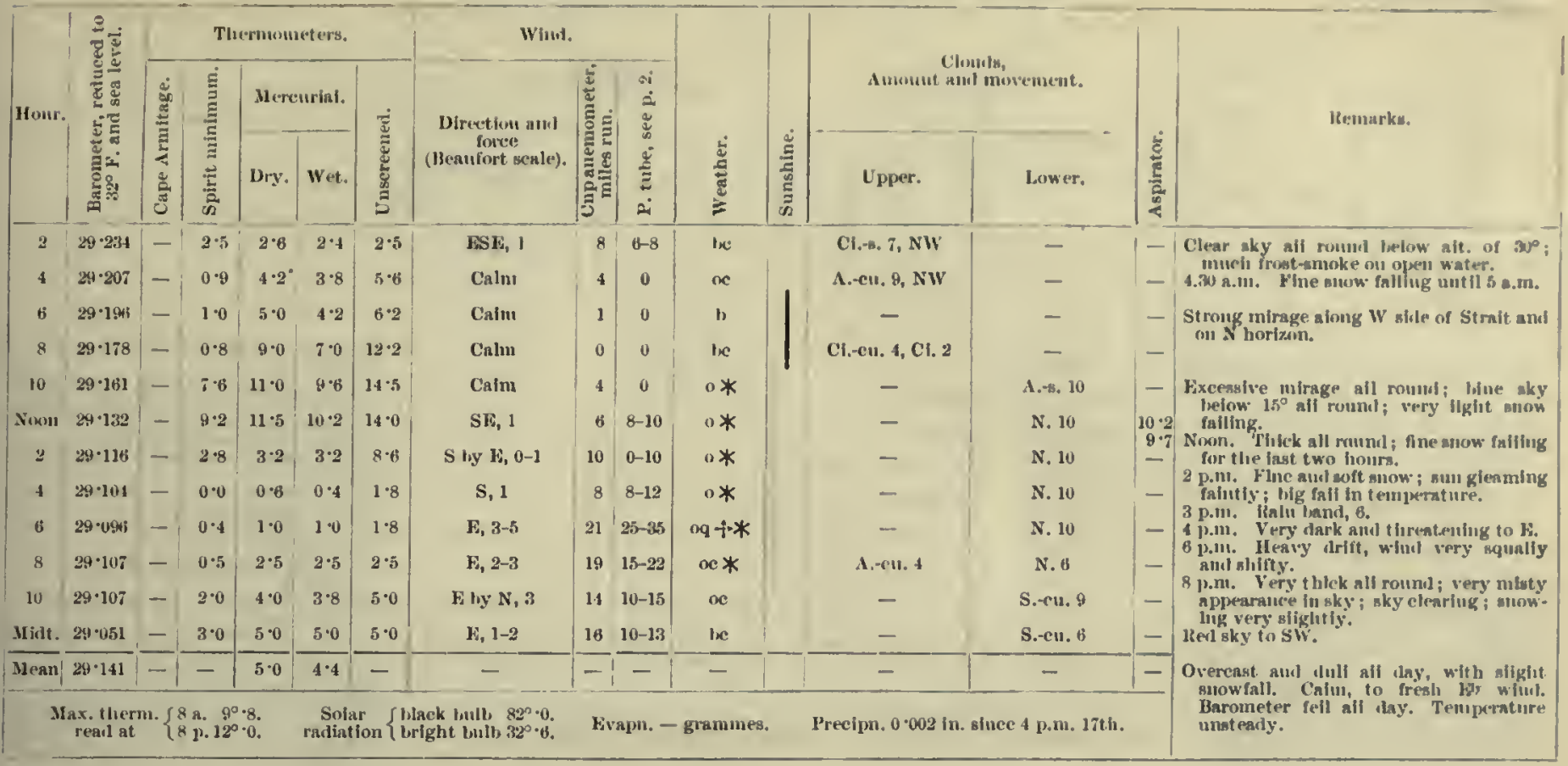

\begin{tabular}{|c|c|c|c|c|c|c|c|c|c|c|c|c|c|c|}
\hline 2 & $|28 \cdot 053|$ & $|-\quad 0.0|$ & $2 \cdot 0$ & $2 \cdot 0$ & $5 \cdot 0$ & Var. E, 1-2 & $\theta$ & $0-8$ & ine & & $C i_{0}-B_{0}-A$ & - & $1-1$ & \multirow{12}{*}{ 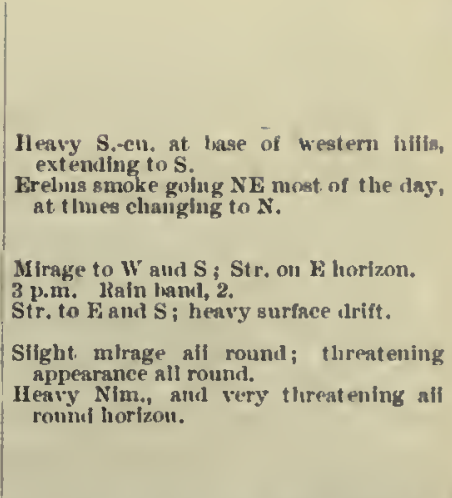 } \\
\hline 4 & $29 \cdot 0.52$ & $|--1 \cdot 0|$ & 1.0 & 1.0 & 40 & Lt. $E$ airs & 9 & 0 & 1. & & - & - & - & \\
\hline 6 & $29 \cdot 091$ & $-1-1 \cdot 0$ & $4 \cdot 0$ & $4 \cdot 0$ & $5 \cdot 0$ & Calm & 2 & 0 & ine & & - & Cur. 4 & - & \\
\hline 8 & $29 \cdot 087$ & -0.0 & $1 \cdot 0$ & $1 \cdot 0$ & $10 \cdot 0$ & Cailı & 1 & 0 & Ine & & - & S.en. 8 & - & \\
\hline 10 & $29 \cdot 079$ & -10.9 & $9 \cdot 0$ & $8 \cdot 0$ & $9 \cdot 0$ & Calm & 2 & 0 & be & & - & Cu. 3 & - & \\
\hline Noon & $29 \cdot \mathrm{w} 39$ & $-9 \cdot 0$ & $9 \cdot 8$ & $8 \cdot 8$ & $11 \cdot 0$ & E, 1-2 & 5 & $3-12$ & ke & I & 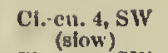 & - & - & \\
\hline 2 & $29 \cdot 0 ; 1$ & $7 \cdot 6$ & $11 \cdot 3$ & 10.0 & 16.0 & LSE, 2-3 & 10 & $15-25$ & thet & 1 & $\begin{array}{l}\text { Cl.-ent, } \\
\text { (mori.) } \\
\text { (nWV }\end{array}$ & - & - & \\
\hline 4 & $29 \cdot 091$ & $-8 \cdot 4$ & $10 \cdot 2$ & $8 \cdot 4$ & $12 \cdot 6$ & E, 3-4 & 18 & $20-30$ & $1 x+$ & I & Cl.-c11. 8 & - & - & \\
\hline " & $29 \cdot 131$ & $8 \cdot 0$ & $8 \cdot 8$ & $7 \cdot 0$ & $15 \cdot 0$ & E hy $x, 3-4$ & 18 & $20-30$ & tec + & & $C i_{0}-\beta_{0}, 2, w$ & S. en. 2, F (fast) & - & \\
\hline 8 & $29 \cdot 146$ & -6.5 & $7 \cdot 5$ & $6 \cdot 2$ & $8 \cdot 1$ & F by $N, 3-4$ & 18 & $20-25$ & $b$ & I & - & - & - & \\
\hline 10 & $29 \cdot 171$ & $-7 \cdot 0$ & $8 \cdot 0$ & $6 \cdot 8$ & $8 \cdot 0$ & Var. NE, 3-4 & 19 & $20-25$ & be & & - & S.-c11. 3, E & - & \\
\hline Midt. & $29 \cdot 183$ & - 6.8 & $7 \cdot 0$ & $6 \cdot 2$ & $7 \cdot 0$ & NE, 3-4 & 17 & $20-25$ & oe & & - & Cu. 10 & $=$ & \\
\hline Mean & $29 \cdot 101$ & - & 6.6 & $5 \cdot 81$ & - & - & - & - & - & & -- & - & $1-$ & $\begin{array}{l}\text { Finc diny, but cioudy. From caim the } \\
\text { w lnd freshented to sifong from } \mathbf{E} \text {. Bame }\end{array}$ \\
\hline
\end{tabular}

20 th.

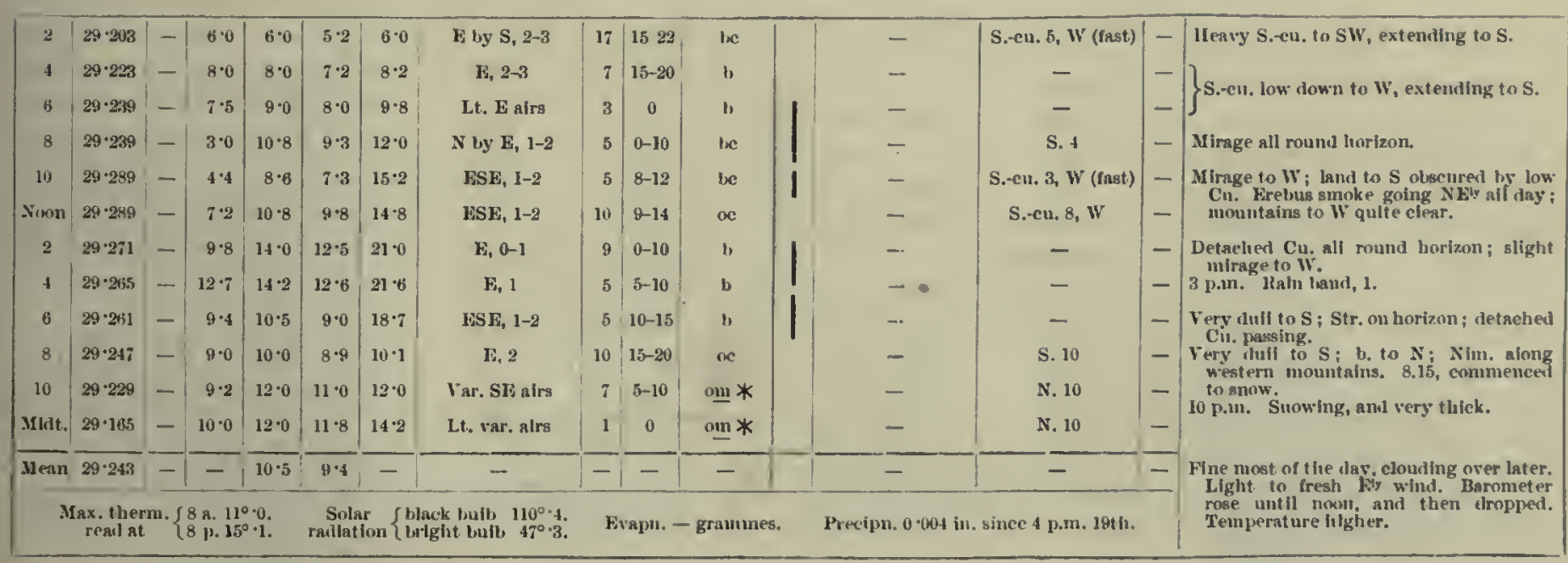




\begin{tabular}{|c|c|c|c|c|c|c|c|c|c|c|c|c|c|c|c|}
\hline \multirow{3}{*}{ INour. } & \multirow{3}{*}{ 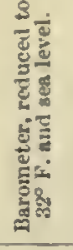 } & \multicolumn{5}{|c|}{ Thermometers. } & \multirow{3}{*}{$\begin{array}{l}\text { Directiou aud } \\
\text { force } \\
\text { (Beau fort seate). }\end{array}$} & \multirow{3}{*}{ 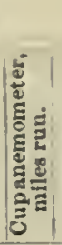 } & \multirow{3}{*}{ 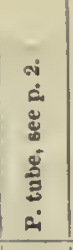 } & \multirow[b]{3}{*}{ 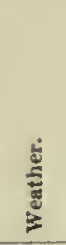 } & \multirow[b]{3}{*}{ 起 } & \multirow{2}{*}{\multicolumn{2}{|c|}{$\begin{array}{l}\text { Ciouds, } \\
\text { Amount sud movement. }\end{array}$}} & \multirow[b]{3}{*}{ 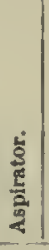 } & \multirow{3}{*}{ Remarks. } \\
\hline & & \multirow{2}{*}{ 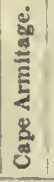 } & \multirow{2}{*}{ 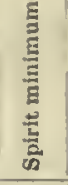 } & \multicolumn{2}{|c|}{ Mencurial. } & \multirow{2}{*}{ 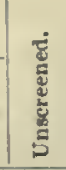 } & & & & & & & & & \\
\hline & & & & Dry. & Wet. & & & & & & & Upper. & Lower. & & \\
\hline 2 & $29 \cdot 161$ & - & $3 \cdot 0$ & $5 \cdot 0$ & $4 \cdot 0$ & $9 \cdot 0$ & Var, $\mathbf{N}$ alra & 3 & $5-10$ & om & & - & $\mathrm{C}$ 11. 10, E & - & \\
\hline 4 & $29 \cdot 145$ & - & $4 \cdot 0$ & $4 \cdot 2$ & $3 \cdot 0$ & $8 \cdot 0$ & $N E_{1}, 1-2$ & 4 & $5-10$ & $\infty$ & & Ci.en. 8 & - & - & • \\
\hline 6 & $29 \cdot 117$ & - & $2 \cdot 0$ & $9 \cdot 2$ & $8 \cdot 0$ & $11 \cdot 0$ & $N, 1-2$ & 4 & $5-10$ & be & 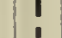 & Ci. 4,5 & - & - & \\
\hline 8 & $29 \cdot 095$ & - & $2 \cdot 2$ & $8 \cdot 8$ & $7 \cdot 8$ & $12 \cdot 0$ & Calu & 3 & 0 & te & I & $\mathrm{Cl}_{0}-\mathrm{s}, 2, \mathrm{~W}$ & - & - & Bank of Str. to W, detacher Cu, to S. \\
\hline 10 & $2 n \cdot 055$ & - & $7 \cdot 5$ & $11 \cdot 8$ & $10^{\circ} 2$ & $13 \cdot 1$ & NNE, 1-2 & 6 & $10-15$ & ise & & Ci. 2 & 一 & - & Erebus coverei with thin mist, sinoke \\
\hline Noon & $29 \cdot 011$ & - & $3 \cdot 9$ & $6 \cdot 0$ & $5 \cdot 5$ & $8 \cdot 2$ & FNE, 3-4 & 20 & $25-33$ & b & & - & - & - & $\begin{array}{l}\text { noon the sunoke rose verticaily and then } \\
\text { broke to } \mathrm{NE} \text {; nountain clear. }\end{array}$ \\
\hline 2 & $29 \cdot 000$ & - & $0 \cdot 0$ & $4 \cdot 0$ & $3 \cdot 0$ & $7 \cdot 2$ & NF, 3-4 & 21 & $25-32$ & $b+f^{2}$ & & - & - & - & 1 p.m. Smoke was going in a big colunin \\
\hline 4 & $28 \cdot 982$ & - & 0.0 & $3 \cdot 5$ & $2 \cdot 7$ & $7 \cdot 0$ & $N F, 3-1$ & 21 & $25-33$ & $1+t^{2}$ & & 一 & - & - & $\begin{array}{l}2 \text { p.un. IIeary surface drlft; sky cloud- } \\
\text { less; vislbifity snali. }\end{array}$ \\
\hline 6 & $28 \cdot 9: 2$ & - & $-4 \cdot 2$ & $4 \cdot 2$ & $3 \cdot 0$ & $9 \cdot 6$ & ENE, 3-5 & 28 & $25-35$ & $b+$ & & - & - & - & $\begin{array}{l}4 \text { p.in. leavy drift on surface; slight } \\
\text { mirage to } S \text {. }\end{array}$ \\
\hline 8 & $28 \cdot 922$ & - & $0 \cdot 0$ & $4 \cdot 8$ & $3 \cdot 7$ & $4 \cdot 1$ & E by $N, 3-5$ & 21 & $30-35$ & $b \div$ & I & - & - & - & $\begin{array}{l}6 \mathrm{p} . \mathrm{In} \text {. Detrehed Nim. to } \mathrm{N} \text {; mirage to } \\
\mathrm{S} \text { ald }\end{array}$ \\
\hline 10 & $28 \cdot 904$ & - & $-0 \cdot 8$ & $2 \cdot 0$ & $1 \cdot 6$ & $1 \cdot 8$ & WNE, 5 & 22 & 32 & he + & & Ci.-s. 7, N & - & - & $\begin{array}{l}8 \text { p.m. Clear bilue sky, oniy ciond a } \\
\text { Ci.-cu. to NF. }\end{array}$ \\
\hline Minit. & $28 \cdot 896$ & - & $-3 \cdot 0$ & $7 \cdot 0$ & $4 \cdot 9$ & 6.8 & ENE, 3-4 & 21 & $25-30$ & be + & & C1.-cu. 4, NF & 一 & - & $\begin{array}{l}10 \text { p.m. Thin band of Str. } 4000 \text { feet up, } \\
\text { along Discovery and western mountnins; }\end{array}$ \\
\hline Mean & $29 \cdot 018$ & - & - & $5 \cdot 9$ & $4 \cdot 8$ & - & - & -1 & - & - & & - & - & - & $\begin{array}{l}\text { clouds moving to } \mathrm{S} \text { fast. } \\
\text { Midt. Bright aloug } \mathrm{S} \text { and } \mathrm{W} \text { horlzou. }\end{array}$ \\
\hline & ax, $t$ & 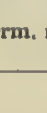 & read & $8 \mathrm{a}$. & 0.0 & & ar radlation $\left\{\begin{array}{l}\text { bi } \\
b r\end{array}\right.$ & bu & ilb 8 & & Eval & grammes. & Precipn، - in. & & $\begin{array}{l}\text { Fine, but cloudy. Wind rose up strong } \\
\text { from } \mathbf{E} \text { and remained strong all day, } \\
\text { with heavy surface drift. Baroineter } \\
\text { failing ail day. Temperature feli lower } \\
\text { again. }\end{array}$ \\
\hline
\end{tabular}

23rat

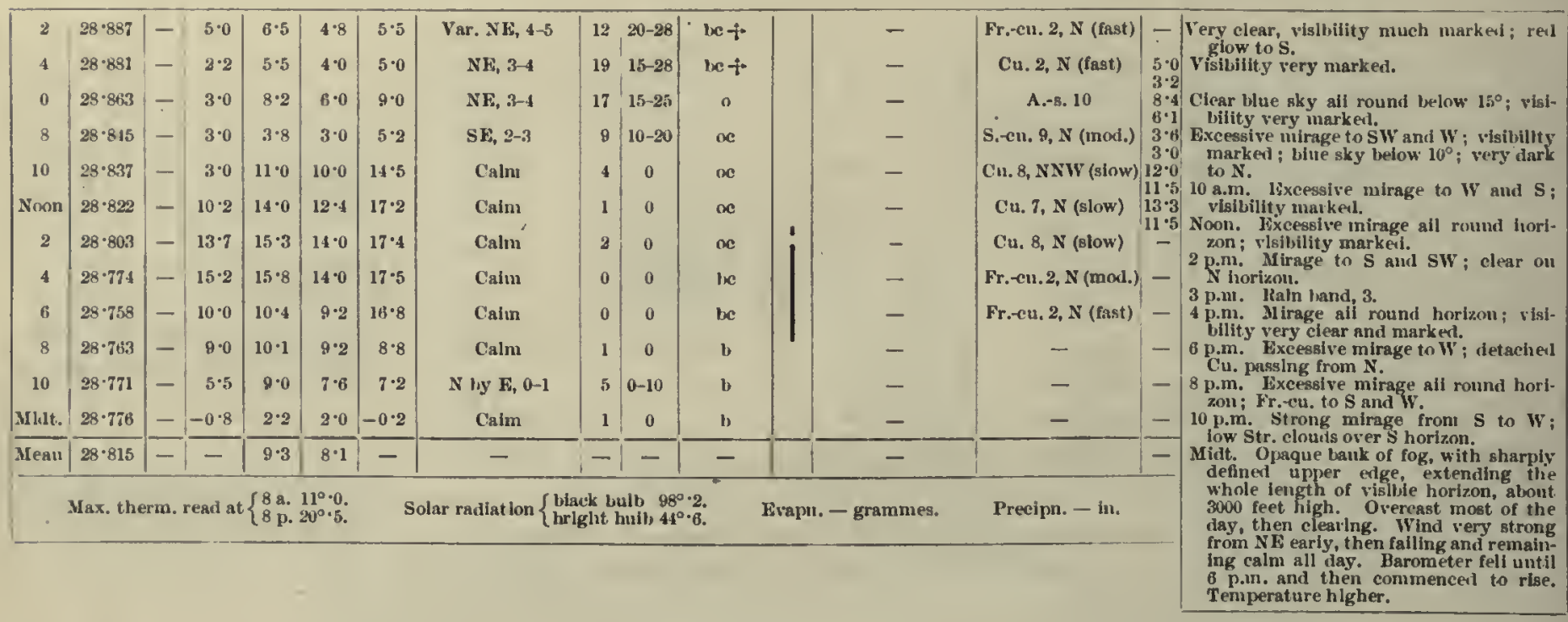

24 th.

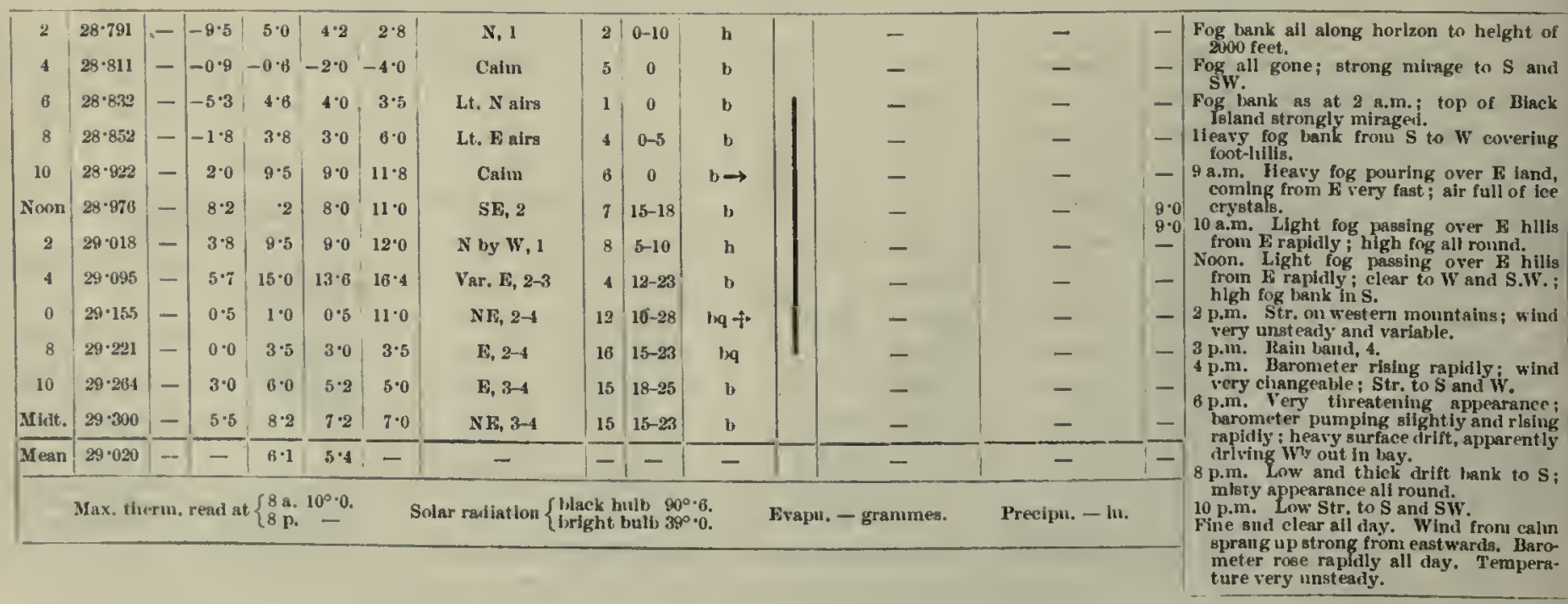


Metborologicat Joursat of the "Discovery."

[1903-February.

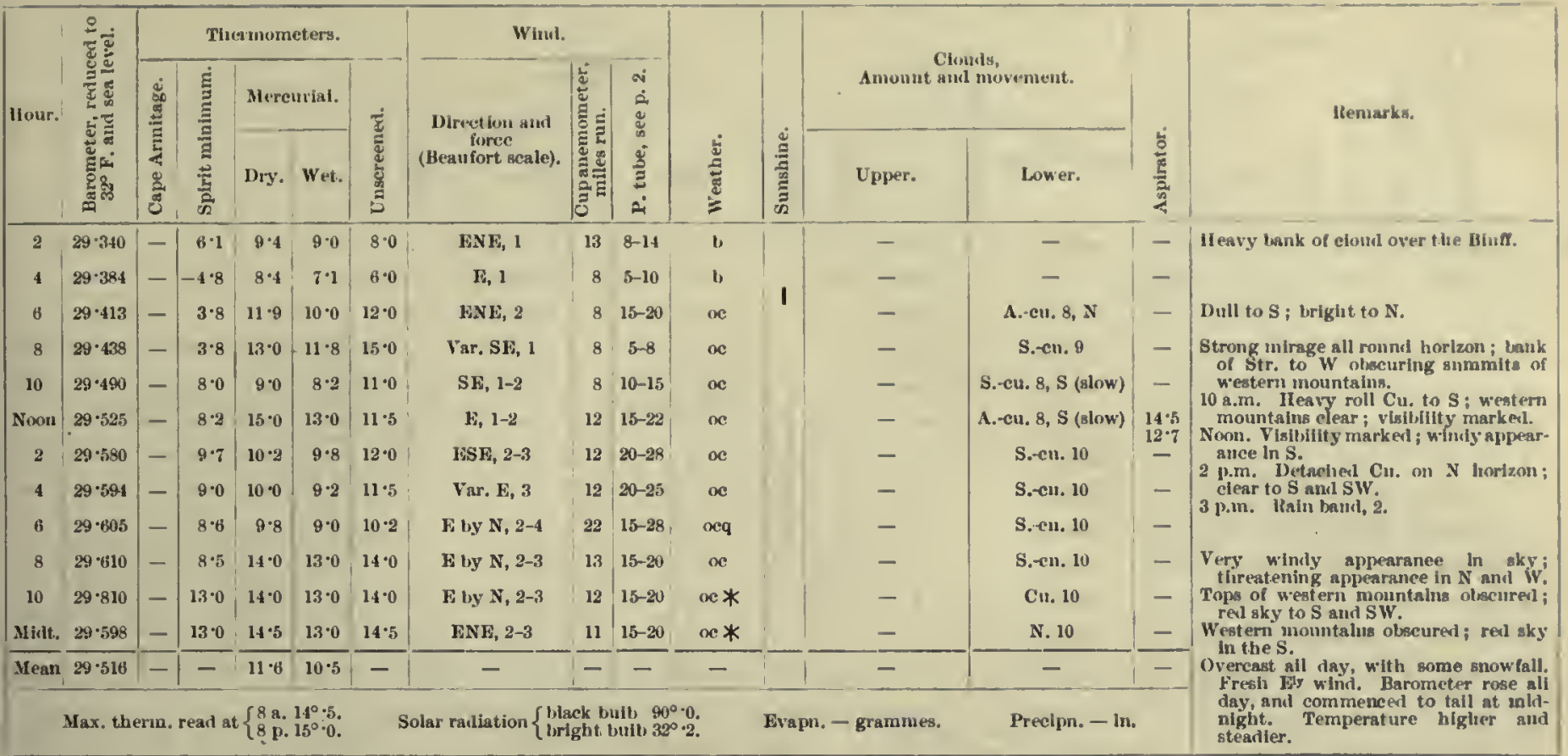

25th

\begin{tabular}{|c|c|c|c|c|c|c|c|c|c|c|c|c|c|c|}
\hline 2 & $29 \cdot 584$ & -1 & $12 \cdot 0$ & $|12 \cdot 0|$ & $12 \cdot 0$ & $12 \cdot 2$ & Lt. $\mathrm{N}$ airs & 11 & 0 & $\infty$ & - & S. en. 8,5 & - & Western mountains vialble; Mt. Dis- \\
\hline+ & $29 \cdot 558$ & - & $8 \cdot 0$ & $11 \cdot 5$ & $11 \cdot 0$ & $12 \cdot 0$ & Caim & 2 & 0 & be & Cil-cul, 8, S & - & - & 1.westerin mosntans \\
\hline B & $29 \cdot 530$ & - & $11 \cdot 0$ & 16.0 & $15 \cdot 0$ & $16 \cdot 0$ & Lt. $W$ alrs & 2 & 0 & m & - & N. 10 & - & \\
\hline 8 & $29 \cdot 498$ & - & $11 \cdot 8$ & $13 \cdot 5$ & $13 \cdot 0$ & $16 \cdot 0$ & SE, 1 & 2 & $8-10$ & $\propto *$ & - & N. 10 & - & Lenry Nim. to $S$; fand to $S$ obscured. \\
\hline 10 & $29 \cdot 404$ & - & $13 \cdot 0$ & $17 \cdot 2$ & $18 \cdot 8$ & $20 \cdot 0$ & $\mathrm{E}, 1$ & 7 & $8-10$ & o* & - & N. 10 & - & Thick all round; snowing slightly. \\
\hline Nom! & $29 \cdot 439$ & - & 14.5 & $17 \cdot 2$ & $18 \cdot 2$ & $19 \cdot 8$ & E., 1 & 7 & $8-10$ & ** & -- & N. 10 & -0.8 & irk Nlm. to W aur SW ; snowing \\
\hline 2 & $29 \cdot 431$ & - & $13 \cdot 2$ & $13 \cdot 4$ & $13 \cdot 0$ & $20 \cdot 7$ & s, 1 & 8 & $5-10$ & o* & - & N. 10 & $-1-8$ & \\
\hline 4 & $29 \cdot 416$ & - & $13 \cdot 8$ & $13 \cdot 6$ & $13 \cdot 0$ & $21 \cdot 0$ & SF, 1-2 & 8 & $5-12$ & o* & - & N. 10 & - & 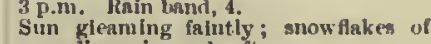 \\
\hline B & $29 \cdot 114$ & - & 8.5 & $9 \cdot 3$ & $8 \cdot 0$ & $15^{\circ} 6$ & $\mathrm{~N} 18,2-4$ & B & $10-25$ & $0 \% *$ & - & N. 10 & - & \\
\hline 8 & $29 \cdot 344$ & - & $3 \cdot 2$ & $7 \cdot 2$ & $7 \cdot 0$ & $7 \cdot 2$ & $\mathrm{NE}, 4-6$ & 15 & $20-30$ & ос $+* *$ & - & N. 10 & - & Sky clearing overhead and to SW; \\
\hline 10 & $29 \cdot 449$ & - & $8 \cdot 0$ & $8 \cdot 0$ & $7 \cdot 0$ & 8.0 & NE, 1-4 & 13 & $8-25$ & oq $+t^{100} *$ & - & s. 10 & - & $\begin{array}{l}\text { squaliy and snowi } \\
\text { Sky clearing to } w \text {. }\end{array}$ \\
\hline silut. & $29 \cdot 466$ & - & $8 \cdot 0$ & $8 \cdot 2$ & 7.8 & $8 \cdot 0$ & Var. NE, 2-4 & 13 & $15-25$ & tet & - & Cu. $8, \mathrm{~S}$ (inst) & - & Very heavy Nim. froun $N$ to S throngh \\
\hline Meani & $29 \cdot 457$ & - & - & $12 \cdot 3$ & $11 \cdot 7$ & - & - & - & - & - & - & - & - & 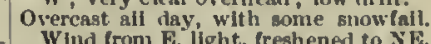 \\
\hline & 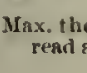 & & & & & & buit & & & & Precipn. & $4 \mathrm{p} \cdot \mathrm{m}$ & & 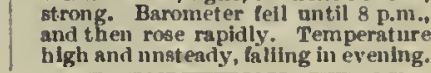 \\
\hline
\end{tabular}

26th.

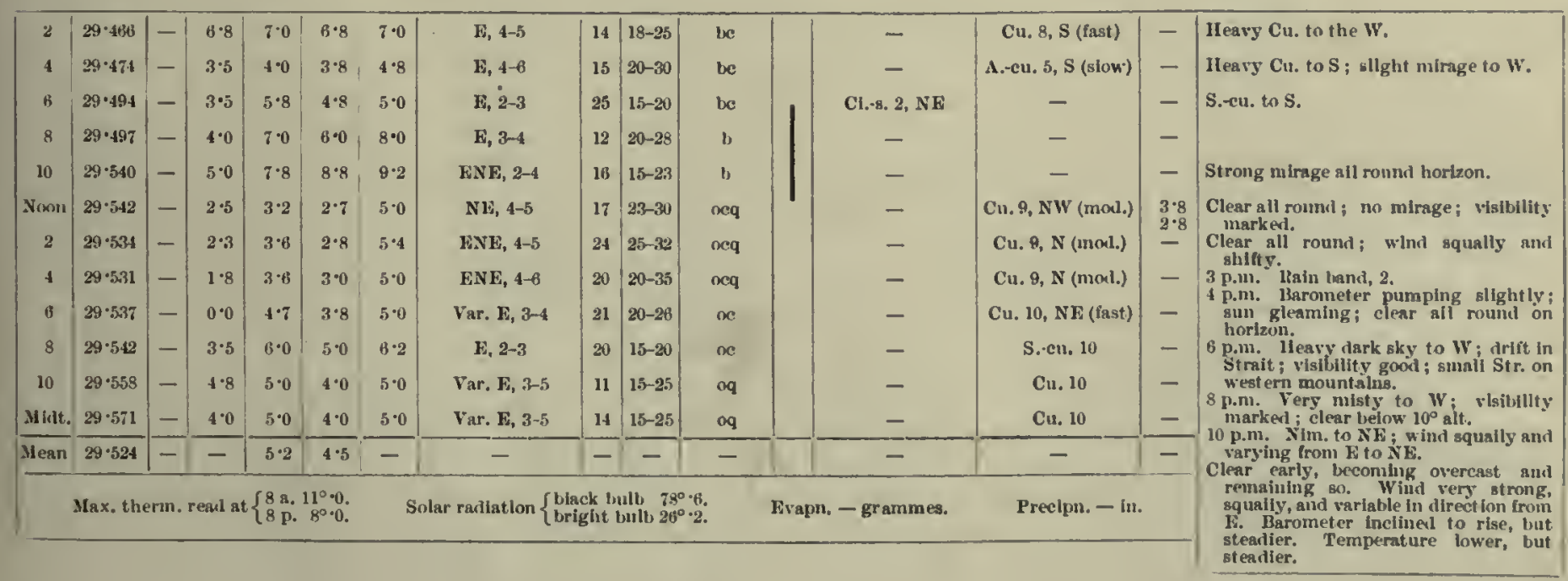


1903-February.]

Meteorolocical Journat, of The "Discovery."

28th.

\begin{tabular}{|c|c|c|c|c|c|}
\hline \multirow{3}{*}{ Hour. } & \multirow{3}{*}{ 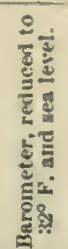 } & \multicolumn{4}{|c|}{ Therinometers. } \\
\hline & & $\dot{\infty}$ & 芜 & Men & urlal. \\
\hline & & है & 䓂 & Dry. & Wet. \\
\hline
\end{tabular}

\begin{tabular}{c|c} 
Winul. \\
Direction nuil \\
fonce \\
(Henfort scale).
\end{tabular}

\begin{tabular}{|c|c|c|c|c|c|c|c|}
\hline 2 & $29 \cdot 591$ & - & $3 \cdot 0$ & $6 \cdot 0$ & $5 \cdot 0$ & $5 \cdot 0$ & NSE, $3-4$ \\
\hline 4 & $29 \cdot 643$ & - & $5 \cdot 0$ & $7 * 0$ & 6.0 & $7 \cdot 2$ & Var. E, 4-5 \\
\hline 6 & $29 \cdot 6 \pi 1$ & - & $4 \cdot 0$ & $8 \cdot 0$ & $7 \cdot 0$ & $8 \cdot 8$ & ESF, 2-3 \\
\hline 8 & $29 \cdot 645$ & - & $5 \cdot 0$ & $6 \cdot 0$ & 4.5 & 6.0 & $S E, 3-4$ \\
\hline 10 & $29 \cdot 681$ & - & $1 \cdot 0$ & $3 \cdot 2$ & $2 \cdot 8$ & $4 \cdot 6$ & N E, $5-6$ \\
\hline Noon & $29 \cdot 697$ & - & $-4 \cdot 0$ & $2 \cdot 0$ & $1 \cdot 2$ & 3.0 & ENE, 6-7 \\
\hline 3 & $29 \cdot 705$ & - & $-5 \cdot 1$ & $2 \cdot 0$ & $1 \cdot 0$ & $5 \cdot 0$ & HNE, 5-6 \\
\hline 4 & $29 \cdot 721$ & - & $-2 \cdot 8$ & $1 \cdot 8$ & 0.6 & $4 \cdot 8$ & $N F, 4-5$ \\
\hline 6 & $29 \cdot 725$ & - & $1 \cdot 0$ & $2 \cdot 3$ & $1 \cdot 5$ & $5 \cdot 7$ & NE, 1-2 \\
\hline 8 & $29 \cdot 739$ & - & $1 \cdot 8$ & $4 \cdot 0$ & $3 \cdot 5$ & $2 \cdot 0$ & Cal in \\
\hline 10 & $29 \cdot 651$ & - & $-2 \cdot 2$ & $-1 \cdot 9$ & $-2 \cdot 0$ & $-7 \cdot 0$ & Calm \\
\hline Mlat. & $29 \cdot \pi 31$ & - & $-1 \cdot 0$ & $-2 \cdot 8$ & $-3 \cdot 0$ & $|-i \cdot 5|$ & Calm \\
\hline ean & $29 \cdot 692$ & - & - & $3 \cdot 1$ & $2 \cdot 3$ & - & - \\
\hline
\end{tabular}

$11|20-25|$

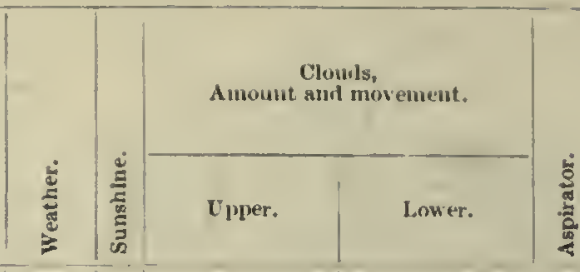

Max, therm, rear at $\left\{\begin{array}{l}8 \text { a. } \\ 8 \text { p. } 7^{\circ} \cdot 2 . \\ 8 \text {. }\end{array}\right.$

Solar radiation $\left\{\begin{array}{l}\text { black bulb } 81^{\circ} 0 . \\ \text { bright bulb } 31^{\circ} 0 .\end{array}\right.$

Evapn. - gramues.

- $\quad$ Ci1. 10

Cu. 10

Cir. 10

IReunarky.

造

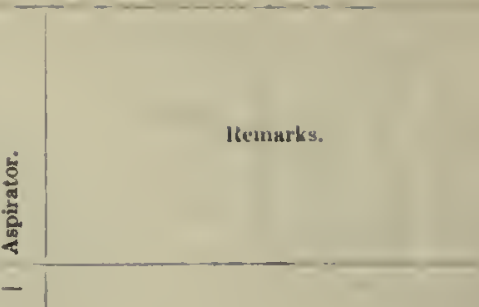

\begin{tabular}{|c|c|c|c|} 
oeq & - & Cu. 10 & - \\
oc & - & C1. 10 & -
\end{tabular}

A.cu.6 - Slight mirage round horizon; lreavy

- $\quad$ - Visibility marked; heavy moll Cu. to $\mathbf{E}$;

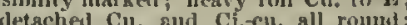
thin layer of Str. on western monstalns,

beq + ! Cl.eu. 3, w

- $\quad \begin{aligned} & 3.0 \\ & \text { - alout 12,000 feet alt. }\end{aligned}$

- - Ne

- $\quad-2$ p.m. Mirage to Wi visilillty very

- $\quad-\quad 3$ p.m. Kaln kand, 0. 3 p.m. Raln tand, 0.
6 p.m. Lixcept lonally elear, witl markeyl

- $\quad$ visibllity i wini W's out in the laty.
8 p.m. Slight mlrage all round; visi-

- $\quad$ - bility markerl.

10.25 p.uns. Wispos of Fr.en. to SW and $-2 \cdot 6$ W, 17,000 feet alt.

Thill layers of Str, to $S:$ frost ferns on glass of anemometer; very red

Overcast early then cleariur aul remaining fine and clear. Wind very strong from ENE to $\mathrm{NH}$, falling to calm alout 8 p.m. Barometer incliner to rise st.ealily all day. Tomperature lower, falling and unstearly. 
Methorological, Jouinal of the " Discovery."

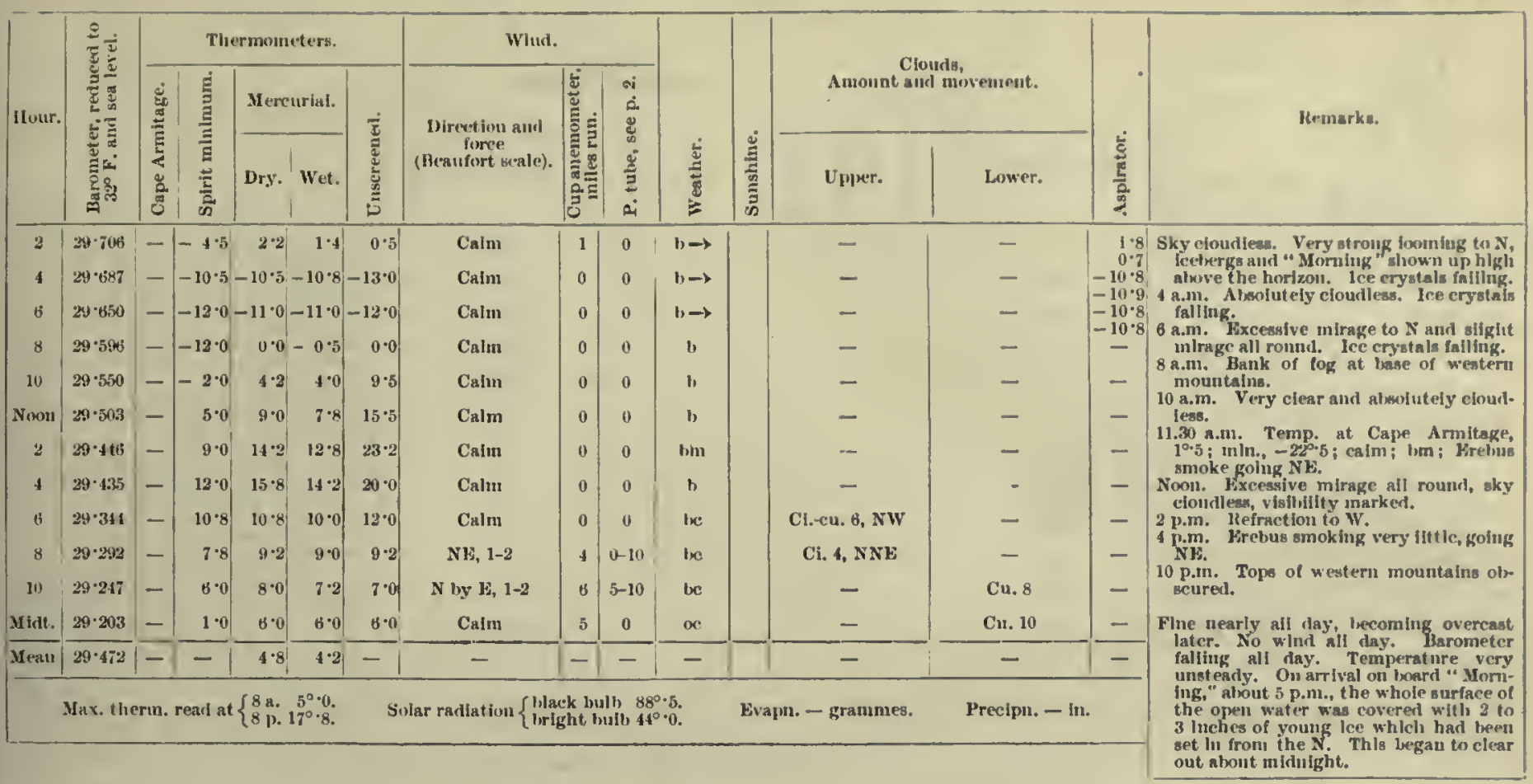

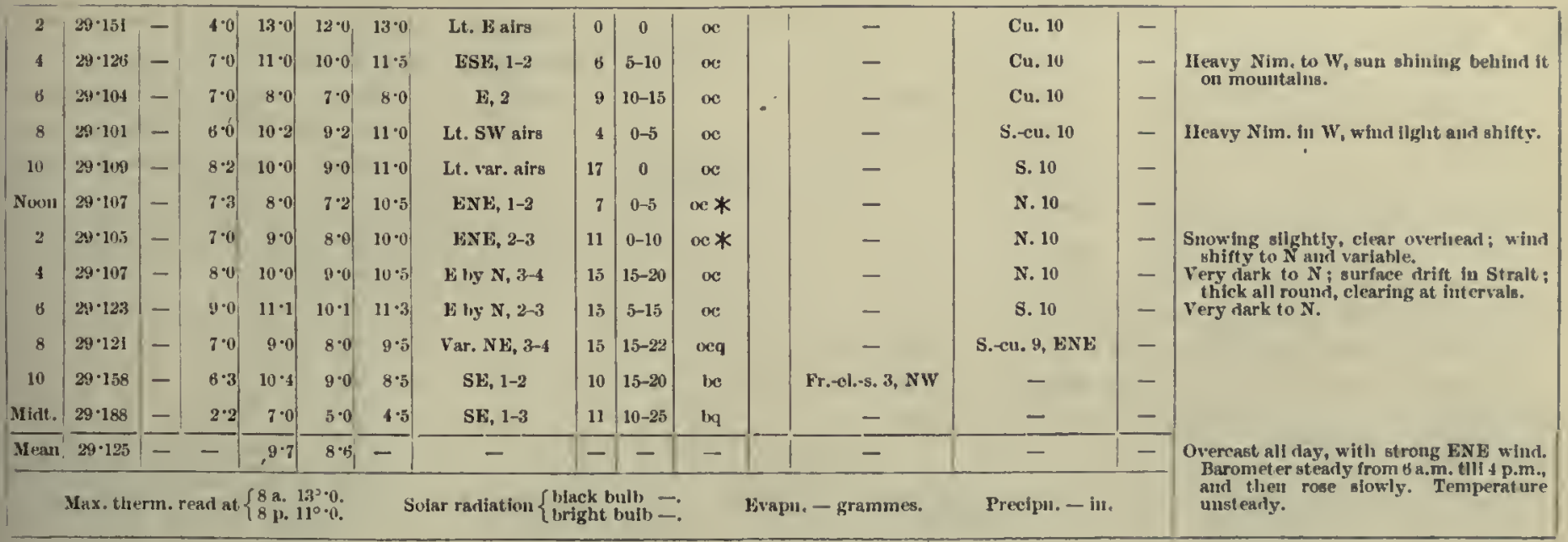

\begin{tabular}{|c|c|c|c|c|c|c|c|c|c|c|c|c|c|c|}
\hline 2 & $29 \cdot 2066^{\circ}$ & -1 & & $2 \cdot 2$ & $1 \cdot 8$ & 0.8 & Var. SSE, 1-2 & 17 & $10-17$ & b & - & - & - & Dark hank of cloud up to 20 \\
\hline 4 & $29 \cdot 2+3$ & - & $0 \%$ & $4 \%$ & 3.5 & $4 \cdot 0$ & SE, 1-2 & 13 & $10-15$ & . & - & s. 10 & - & Low drift ln \\
\hline 8 & $29 \cdot 288$ & - & 1.8 & 3.5 & $2 \cdot 8$ & $3 \cdot 5$ & SE, 2-4 & 9 & $12-32$ & oq & - & ง. 10 & - & \\
\hline 8 & $29 \cdot 290$ & - & $1 \cdot 0$ & $5 \cdot 0$ & $5 \cdot 0$ & $6 \cdot 0$ & s, 3-4 & 11 & $20-25 \mid$ & x & - & s. 10 & - & \\
\hline 10 & $29: 332$ & - & $4 \cdot 6$ & $7 \cdot 8$ & $6 \cdot 9$ & $9 \cdot 2$ & Lt. Sll airs & 15 & 0 & o & - & s. 10 & - & Str. un summit of westcra range; rlgl- \\
\hline Neno & $29: 372$ & - & $5 \cdot 0$ & $5 \cdot 5$ & $5 \cdot 0$ & 6.8 & $s E, 1-2$ & 11 & $5-10$ & $\infty$ & - & S. 10 & & to $\mathrm{S}$. \\
\hline 2 & $29 \cdot 3 ; \mathrm{it}$ & - & $4 \cdot 8$ & $6 \cdot 5$ & $5 \cdot 7$ & $8 \cdot 2$ & SSE, $2-4$ & 15 & $18-28$ & $\propto ⿻ q$ & - & S.ent. 10 & - & w; thick to S. \\
\hline 4 & $29 \cdot 403$ & - & $5 \cdot 3$ & $7 \cdot 0$ & 6.0 & $8 \cdot 0$ & Vur. St, 1-3 & 13. & $5-20$ & $\infty e q$ & - & S.cu. 10 & - & 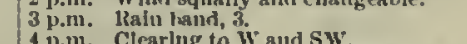 \\
\hline 6 & $29 \cdot 473$ & - & $4 \cdot 8$ & $5 \cdot 2$ & $4 \cdot 3$ & $7 \cdot 8$ & $\operatorname{ssk}$, 1-3 & 13 & 820 & $x: q$ & - & 5.en. 10 & - & suil gleaming faintiy; sky cicaring to $\mathrm{W}$. \\
\hline 8 & $2 y \cdot 524$ & - & $3 \cdot 0$ & $5 \cdot 0$ & $4 \cdot 4$ & $3 \cdot 0$ & SE, 2-4 & 16 & $20-27$ & oq $*$ & - & N. 10 & - & Cleariug to $\mathrm{s}$; elouds covering upper \\
\hline 10 & 24.530 & - & $3 \cdot 5$ & $5 \cdot 0$ & $3 \cdot 9$ & $3 \cdot 8$ & $\mathbf{x}, 2$ & 10 & $15-20$ & $\propto$ & - & S. 10 & - & Citen. to $\mathrm{NW}$; NIm. to $\mathrm{S}$ and SW; ait. \\
\hline Midt. & $29 \cdot 518$ & - & $3 \cdot 5$ & $4 \cdot 2$ & $3 \cdot 2$ & $2 \cdot 4$ & ENE, 1 & 13 & 10 & b & - & - & - & Stars vilsblbie; Xim. to S and SW \\
\hline Nean | & $2 y \cdot 379$ & -1 & -1 & $5 \cdot 1$ & 4.4 & - & - & $1-$ & - & - & - & - & - & 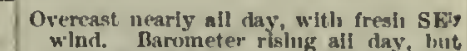 \\
\hline & Max. & & & 80. & & & allation & & itis & & Evapn. - gramines. & Precipn. - & & \\
\hline
\end{tabular}




\begin{tabular}{|c|c|c|c|c|c|c|c|c|c|}
\hline \multirow{3}{*}{ Ionr. } & \multirow{3}{*}{ 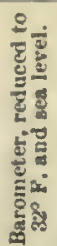 } & \multicolumn{5}{|c|}{ Thermomcters. } & \multicolumn{3}{|c|}{ Wiud. } \\
\hline & & 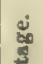 & $\dot{E}$ & Mere & arial. & & & & $\stackrel{1}{\vdots}$ \\
\hline & & $\frac{2}{8}$ & $\begin{array}{l}\text { है } \\
\text { है } \\
\text { है }\end{array}$ & Dry. & Wet. & 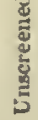 & $\begin{array}{c}\text { Direction antul } \\
\text { fore } \\
\text { (Bcaufort scalc). }\end{array}$ & 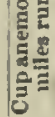 & 雚 \\
\hline
\end{tabular}

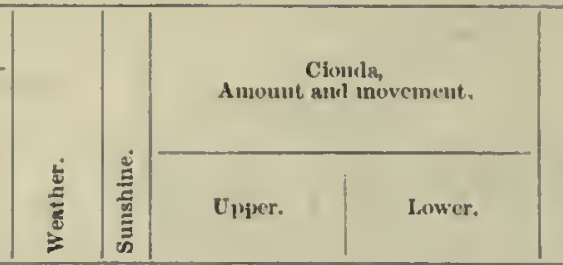

\begin{tabular}{|c|c|c|c|c|c|c|c|c|c|c|c|c|c|c|}
\hline 2 & $29 \cdot 518$ & - & $2 \cdot 1$ & $3 \cdot 0$ & $2 \cdot 0$ & 11 & $\mathbf{F}, 1$ & 6 & $8-10$ & be & - & S.-cul. 2, W & - & $\begin{array}{l}\text { Western mountalus clur; heary Ci.s. } \\
\text { streteluling from Discovery to } \mathrm{NB} \text {; } 4 \text { ilt. }\end{array}$ \\
\hline 4 & $29 \cdot 514$ & - & $-1 \cdot 4$ & $3 \cdot 8$ & $2 \cdot 9$ & $-1 \cdot 5$ & Calm & 0 & 0 & b & - & - & - & 4000 frect. \\
\hline 6 & $29 \cdot 498$ & - & 10 & $9 \cdot 2$ & $7 \cdot 1$ & 9.7 & Calı1 & 2 & 0 & oc & - & 5. 10 & - & 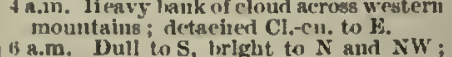 \\
\hline 8 & $29 \cdot 504$ & - & $2 \cdot 0$ & $4 \cdot 5$ & 4.0 & $6 \cdot 2$ & Calm & 1 & o & $\alpha$ & - & S. 10 & - & $\begin{array}{l}\text { Nim. on Mit. Discovery, Str. on } \\
\text { west eru mountahs' summits at glout }\end{array}$ \\
\hline 10 & $29 \cdot 516$ & - & $2 \cdot 0$ & $10 \cdot 0$ & $8 \cdot 5$ & $12 \cdot 0$ & Calm & 1 & 0 & we: & - & Cin, $9, w$ & - & $\begin{array}{l}11,000 \text { feet alt. } \\
8 \text { a.m. Ileavy and dark Nim. to } \mathrm{N} \text {. }\end{array}$ \\
\hline Nikul & $20 \cdot 513$ & - & $10 \cdot 0$ & $12 \cdot 2$ & $10 \cdot 8$ & $15^{\circ} 0$ & Lt. N alrs & 2 & 0 & " & - & - & - & $\begin{array}{l}10 \text { s.m. Clear to S and } N \text {; Str. two-thirds } \\
\text { up westeru montains. }\end{array}$ \\
\hline 2 & $29 \cdot 503$ & - & $11 \cdot 2$ & $18 \cdot 5$ & 16.0 & $24 \cdot 3$ & Caln & 2 & 0 & h & - & - & - & 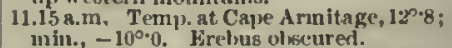 \\
\hline 4 & $29 \cdot 493$ & - & 13.5 & $16 \cdot 7$ & $14^{\circ} 0$ & $20 \cdot 6$ & NNB, 1-2 & 5 & $5-12$ & h & - & - & - & $\begin{array}{l}\text { Noon. Thin mist on sumunit of western } \\
\text { unountalns: detached Cu. to } \mathrm{S} \text {. }\end{array}$ \\
\hline 6 & $29 \cdot 487$ & - & $14 \cdot 2$ & $16 \cdot 0$ & $14 \cdot 0$ & $20 \cdot 2$ & $\mathbf{N}$ ly $\mathrm{k}, 3$ & 7 & $8-15$ & be & Cl. $1, W$ & - & - & $\begin{array}{l}2 \text { p.m. Str. all round horlzon, } \\
6 \text { v.m. Ci.-8, on S trorizon; very dull and }\end{array}$ \\
\hline 8 & $29 \cdot 476$ & - & $14 \cdot 5$ & $17 \cdot 5$ & $16 \cdot 0$ & $16 \cdot 0$ & $x, 1$ & 8 & 8 & be & Ci. $1, w$ & - & - & $\begin{array}{l}\text { threatening to } N \text {. } \\
8 \text { p.m. Very biack and threatening to } S\end{array}$ \\
\hline 10 & $29 \cdot 462$ & - & $15 \cdot 0$ & $18 \cdot 2$ & 16.0 & $18^{\circ} 0$ & $\mathbf{N}, 1$ & 7 & 10 & $\alpha$ & - & Cu. 10 & - & 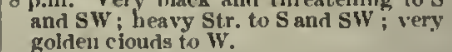 \\
\hline MIAt. & $29 \cdot 470$ & - & $15 \cdot 2$ & $18 \cdot 5$ & $17 \cdot 0$ & 18.0 & Calın & 8 & 0 & $o c$ & - & N. 10 & - & 10 p.m. Tops of western mountalns ol- \\
\hline Mean & $29 \cdot 496$ & - & - & $12 \cdot 3$ & $10^{\circ} 6$ & - & - & - & - & - & - & - & - & $\begin{array}{l}\text { clear to } 5 \text {; necessary to use a light to } \\
\text { read the harometer. }\end{array}$ \\
\hline \multirow{2}{*}{\multicolumn{7}{|c|}{ Max. themn. read at $\left\{\begin{array}{l}8 \text { a, } \\
8 \mathrm{p}, 20^{\circ} \cdot 5\end{array}\right.$}} & \multicolumn{4}{|c|}{ Solur radiatiou $\left\{\begin{array}{l}\text { black bulb } 86^{\circ} \cdot 4 \\
\text { brlght buib } 41^{\circ} .\end{array}\right.$} & Evapu, - grainmes. & \multicolumn{2}{|c|}{ Precipn. - in. } & $\begin{array}{l}\text { Mlit. Very blaek and threatening all } \\
\text { round; tops of western mountains olr } \\
\text { scured by heavy Nim. clouds; nece- } \\
\text { ary to }\end{array}$ \\
\hline & & & & & & & leters & & & & & & & ais. Very little whid all da \\
\hline
\end{tabular}

5 th.

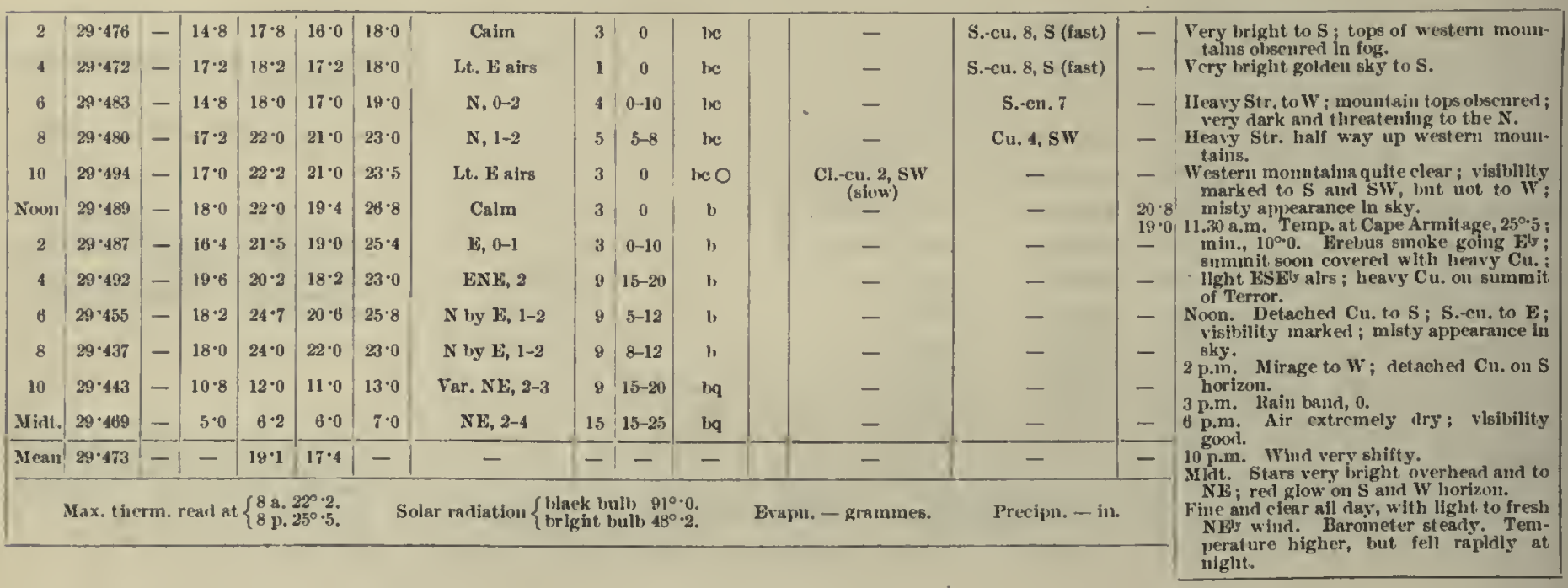

6 th.

\begin{tabular}{|c|c|c|c|c|c|c|c|c|c|c|c|c|c|c|}
\hline 2 & $29 \cdot 469$ & - & $-2 \cdot 5$ & $-1 \cdot 0$ & $-1 \cdot 0$ & 0.0 & Var. $\mathrm{H}, 6-7$ & 24 & inin-40 & $69+$ & - & - & - & $\begin{array}{l}\text { Wind shifted to LXE and freshened; low } \\
\text { drift. }\end{array}$ \\
\hline 4 & $29 \cdot 432$ & - & $-2 \cdot 0$ & $-1 \cdot 0$ & -0.8 & $-2 \cdot 0$ & HNE, 6-8 & 28 & $35-50$ & $\mathrm{bq}+$ & - & - & & \\
\hline 6 & $29 \cdot 427$ & - & $-3 \cdot 2$ & $-1 \cdot 0$ & $-1 \cdot 0$ & $-1 \cdot 0$ & ENE, 5-6 & 24 & $35-40$ & $\operatorname{lng}$ & - & & - & \\
\hline 8 & $29 \cdot 420$ & - & $-7 \cdot 5$ & $-7 \cdot 0$ & $-7 \cdot 0$ & $-6 \cdot 0$ & Var. $\mathbf{E}, 5-6$ & 28 & $35-40$ & be & - & S.-cu. 4, N (fast) & - & \\
\hline 10 & $29 \cdot 408$ & - & $-5 \cdot 0$ & $-1 \cdot 5$ & $-2 \cdot 0$ & $-0 \cdot 6$ & Var. 1, 5-b & 32 & $20-38$ & ocq & - & Cu. $8, \mathrm{~N}$ (fast) & - & Laycrs of S.cu., with blue sky bet ween, ali \\
\hline Nom! & $29 \cdot 401$ & - & $-5 \cdot 0$ & $-4 \cdot 8$ & $-5 \cdot 0$ & $-3 \cdot 0$ & Var. $\mathbf{k}, 6-8$ & 26 & $\mid 28-44$ & neqt & - & S.cu. 10 & $\begin{array}{l}-3 \cdot 9 \\
-4 \cdot 4\end{array}$ & $\begin{array}{l}\text { vlsible; l hases obscured. Low drift in } \\
\text { Strait ; very squally. }\end{array}$ \\
\hline 2 & $29 \cdot 401$ & - & $-6 \cdot 0$ & $-6 \cdot 0$ & $-5 \cdot 6$ & $-3 \cdot 0$ & Var. E, 7-9 & 29 & $28-46$ & ocq + & - & S.-cu. 10 & - & $\begin{array}{l}\text { Nooun. I eavy and dark } N \text { to } 5 w \text {; thick } \\
\text { drift in Strait; western momutains olr }\end{array}$ \\
\hline 8 & $29 \cdot 502$ & - & $-3 \cdot 8$ & 6.0 & $5 \cdot 0$ & $5 \cdot 5$ & SE, 1-2 & 11 & $10-17$ & $1 x$ & Froel.-6, 1 & S. 3 & - & $\begin{array}{l}\text { 11ature } \\
8 \text { p.in. Thiek Str. to } S \text { and } W \text { olscuring }\end{array}$ \\
\hline 10 & $29 \cdot 539$ & -1 & 0.0 & $0 \cdot 1$ & $0 \cdot 1$ & $-2 \cdot 0$ & Calm & 4 & 0 & be & - & Fr.-s. 2, S (mod.) & $\begin{array}{l}-1 \cdot 8 \\
=1 \cdot 8\end{array}$ & $\begin{array}{l}\text { weetern mountains, nleary banks of } \\
\text { low clonds to } \mathrm{f} \text { and } \mathrm{SW} \text {. }\end{array}$ \\
\hline Midt. & 29.560 & - & $-2 \cdot 0$ & $3 \cdot 0$ & $2 \cdot 0$ & $2 \cdot 0$ & Calu & 4 & 0 & be & - & - & $\begin{array}{r}4 \cdot 3 \\
3 \cdot 6 \\
\end{array}$ & 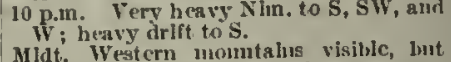 \\
\hline Mean! & $29 \cdot 458$ & -1 & - & $-1 \cdot 3$ & $-1 \cdot 5$ & - & - & $1-$ & - & - & - & - & - & 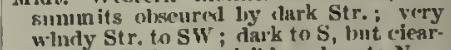 \\
\hline
\end{tabular}


Methorolonical, Journat, of TIIF "Discovery."

[1903-March.

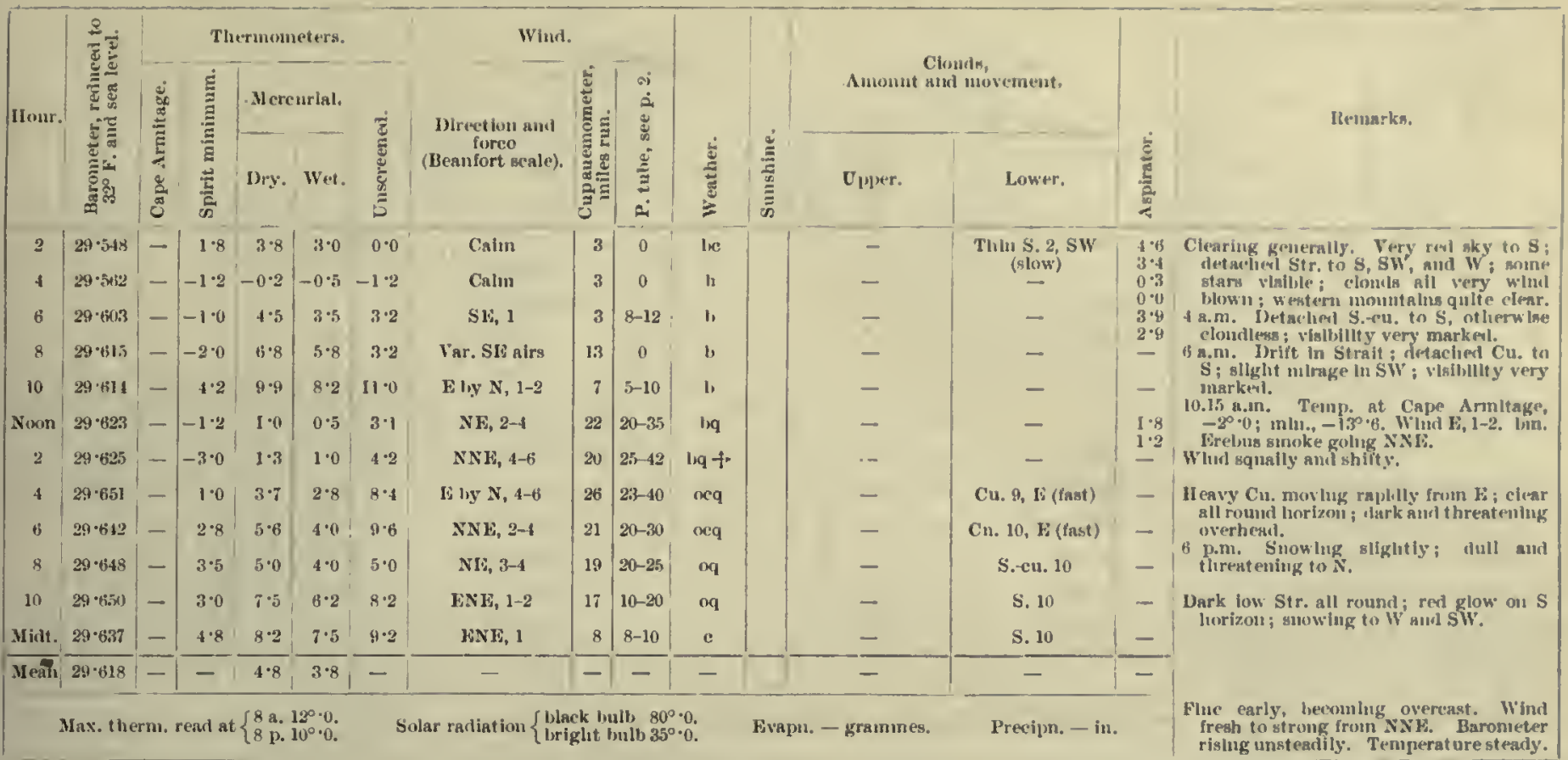

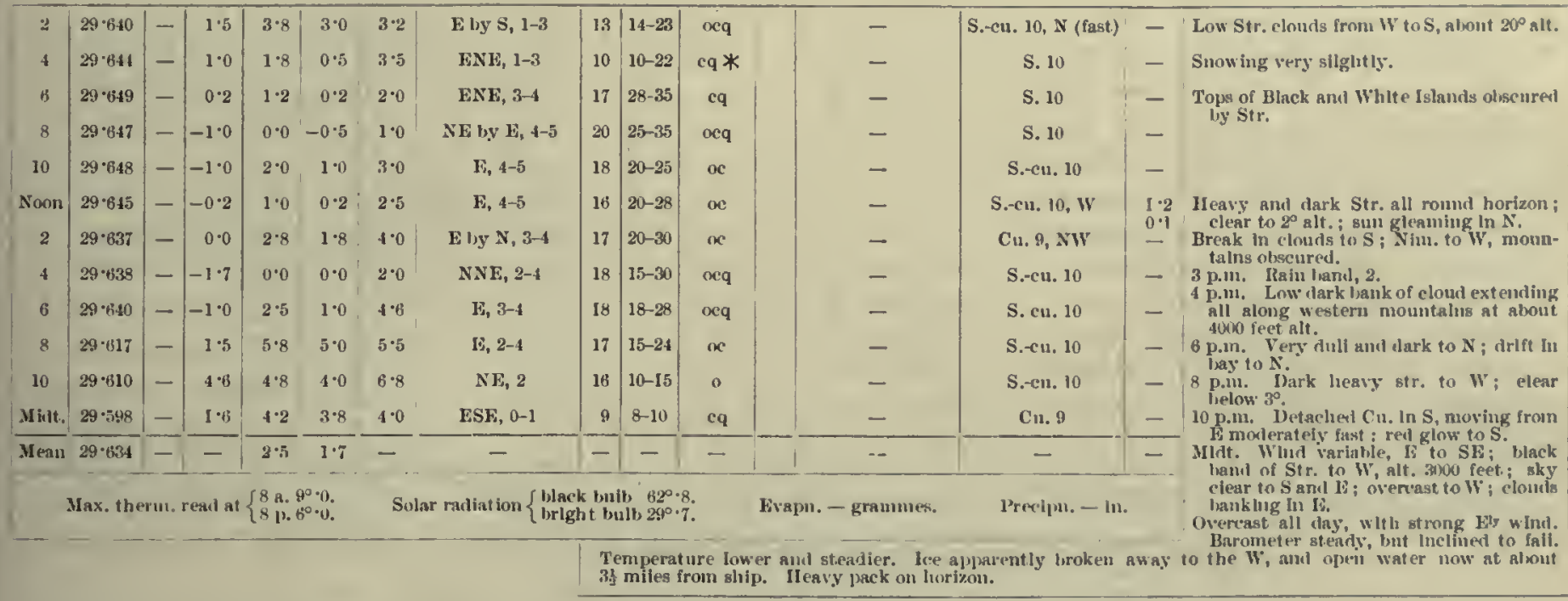

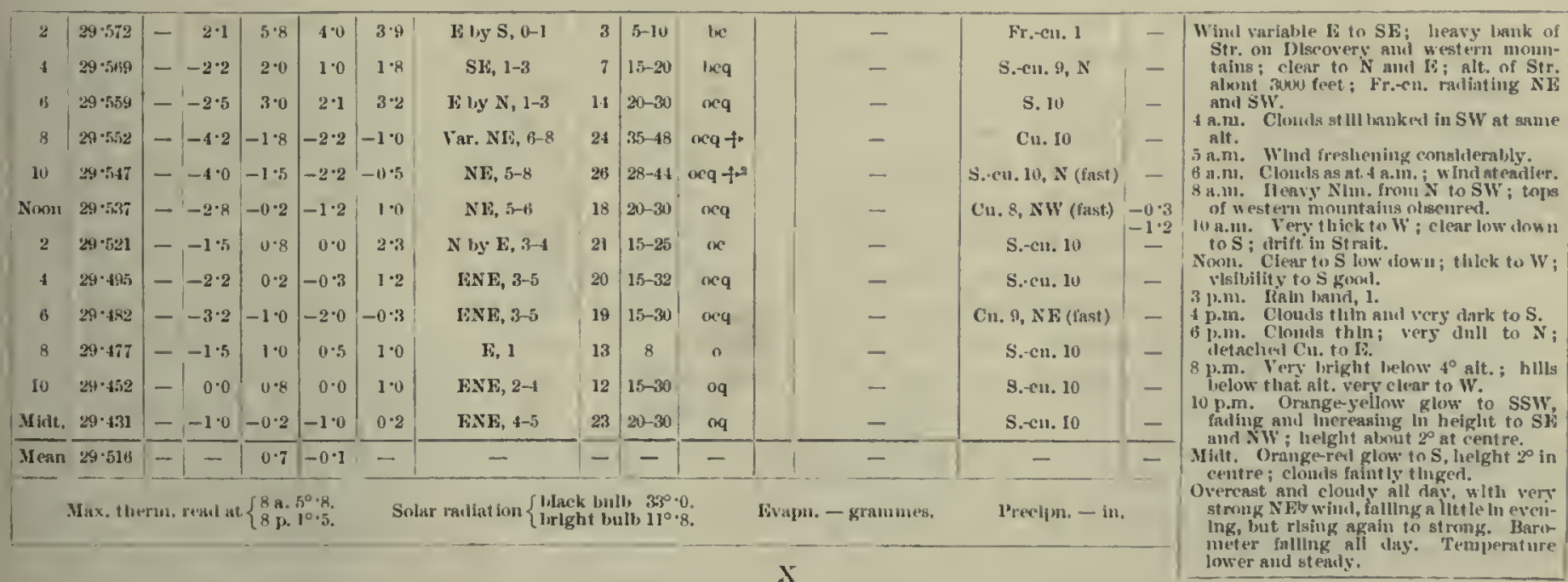


1903-March.]

Meteorological Journat, of tie "Discovery."

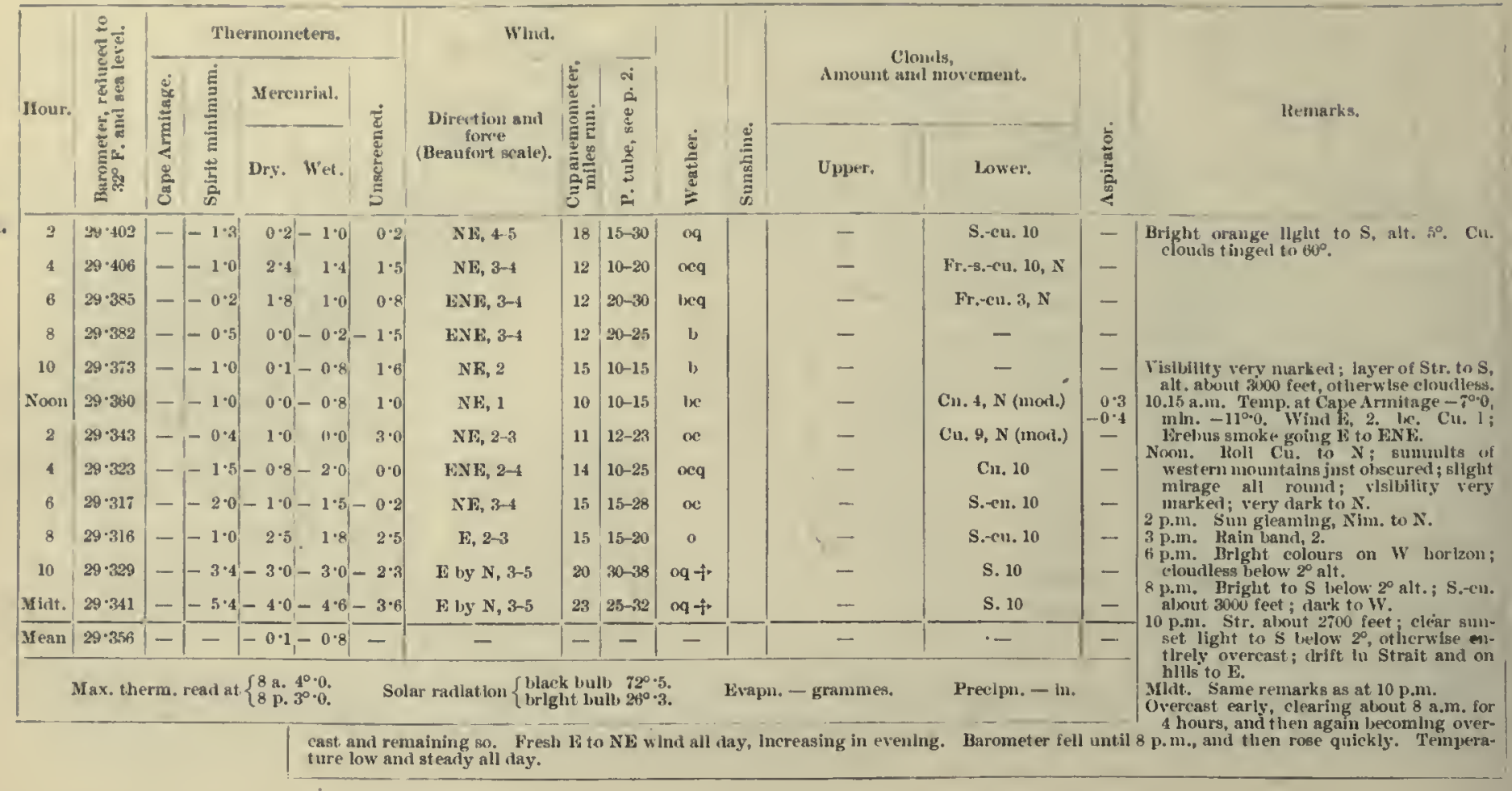

10 th

11 th.

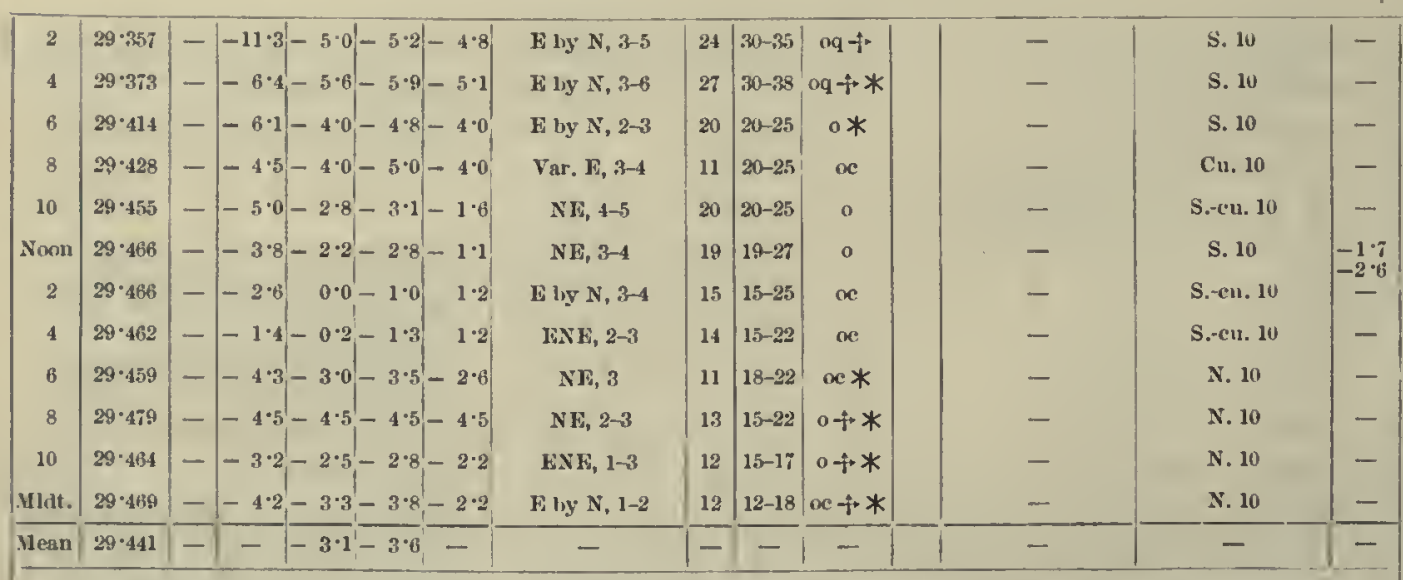

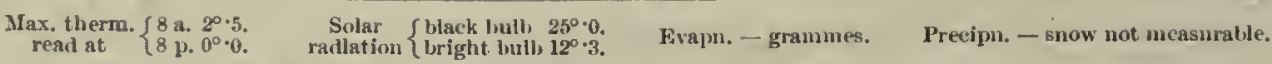

- Str, extending to S, leaving less sunset light clear on the loorizong vess sunset clomis of drift ruming to Niv in Strait. 4 a.m. Western range hidden ly drift; Iluff, otherwise clear to $\mathrm{S}$.

6 a.m. Snowhg very slightly, Bluft clear, foot-hills to $W$ also visible throngh 10 a.m. Clear below $2^{\circ}$ alt.; drlft in Strait; dark and heary Ninn. to w foot-hills only just visitile. 10.15 a.m. Tenip. at Cape Ainnitage $-2^{\circ} 4$,
mln. - 8.0. Wind ESE, 3. oc.

foon. Fintirely overcast except to $S$, alt. $2^{\circ}$; Nim, to W.

p.m. Clear to $S$ and $S W$; snmmits of western mountains olseurei.

3 p.m. Rain band, 4.

p.m. General appearance of snow ; clear 6 p.m. Snowing slightly; drift in Strait to $N$.

1.m. Thiek all round; no land visible. p.m. Very light drift; low parts of $0^{\circ} 30^{\circ}$.

Mlldt. Less drift; clcar on lorizon between SS1s and SW, alt. $1^{\circ} 15^{\prime} ;$ low Bluf sud W end of White 1sland. Overcast all day, witl some slight snow fali. Wind strong bly all day. Barouteter rose slow ly most of the day. Temperature lower, and was below aero all tay.
12 th.

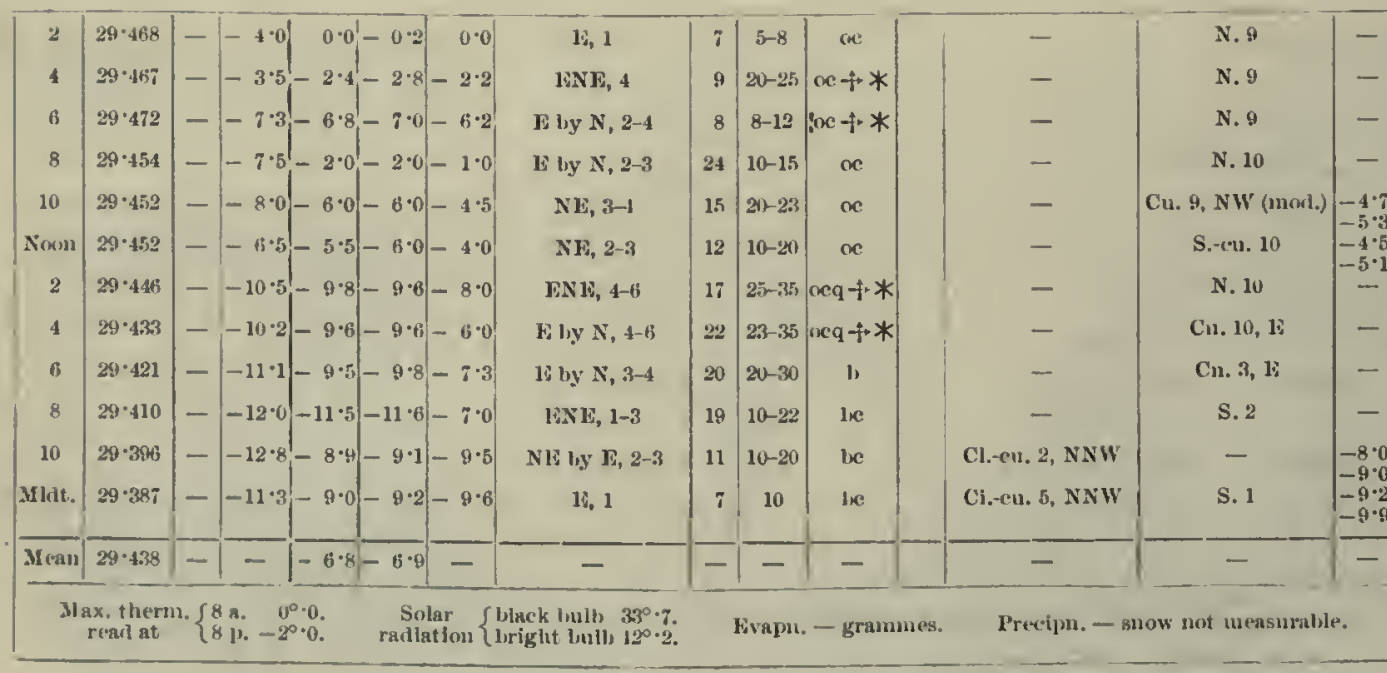

Bank of Str. extending rouml horizon hetwern SSF and SW to an alt of $1^{\circ} 15^{\prime}$. 3.55 a.1m. Wind rising ; drift olsseuring sestern mountains ohscoured; clear sky;

6 a.m. Wind squally; snowing anu ctriftlng; thick all round. a.uu. Dark and threatenlng to $S$; western nountalus vlsible; summits olsseured. Temp. at Cape Armitage
10.15 a,m. Tem.

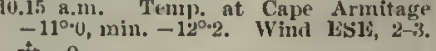
Nơn. Clear to SW low down; visibility very marked; clearlng to $W$, summits of western mountains just olsenrem. 2 p.m. Wind squally and shlifty; snowing 3 p.m. lenin trand, 1 . 4 p.m. Cantonds breaking nu, to $W$ and
l.meconlug detachet lu zentth.

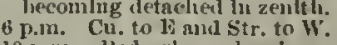
10 p.m. Cu. to bed and str. to $W$.

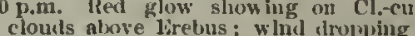
cloukts above lirebus;
bank of Str. over Hluff.

Mint. lleary clowl lanks to $S$ olsscurlng the Bluff.

Overcast most of the day, clearing in evening. Strong lyly wifi, falling in evenlng. Baroneter falling slight ly all clay. Temperature unst cady, and below acro all day. 
Meteorological Journal of THE "Discoveky:"

[1903-March.

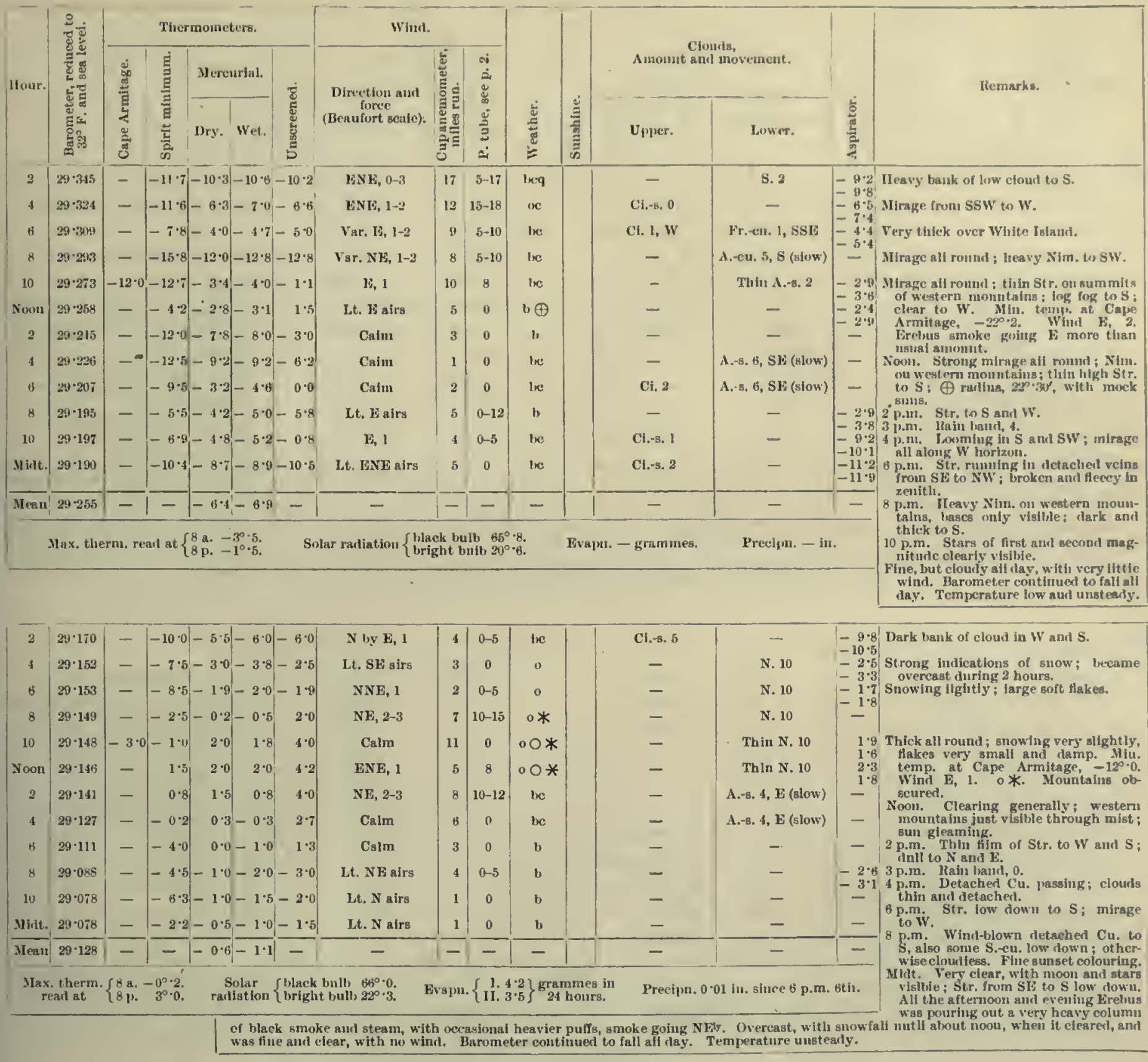

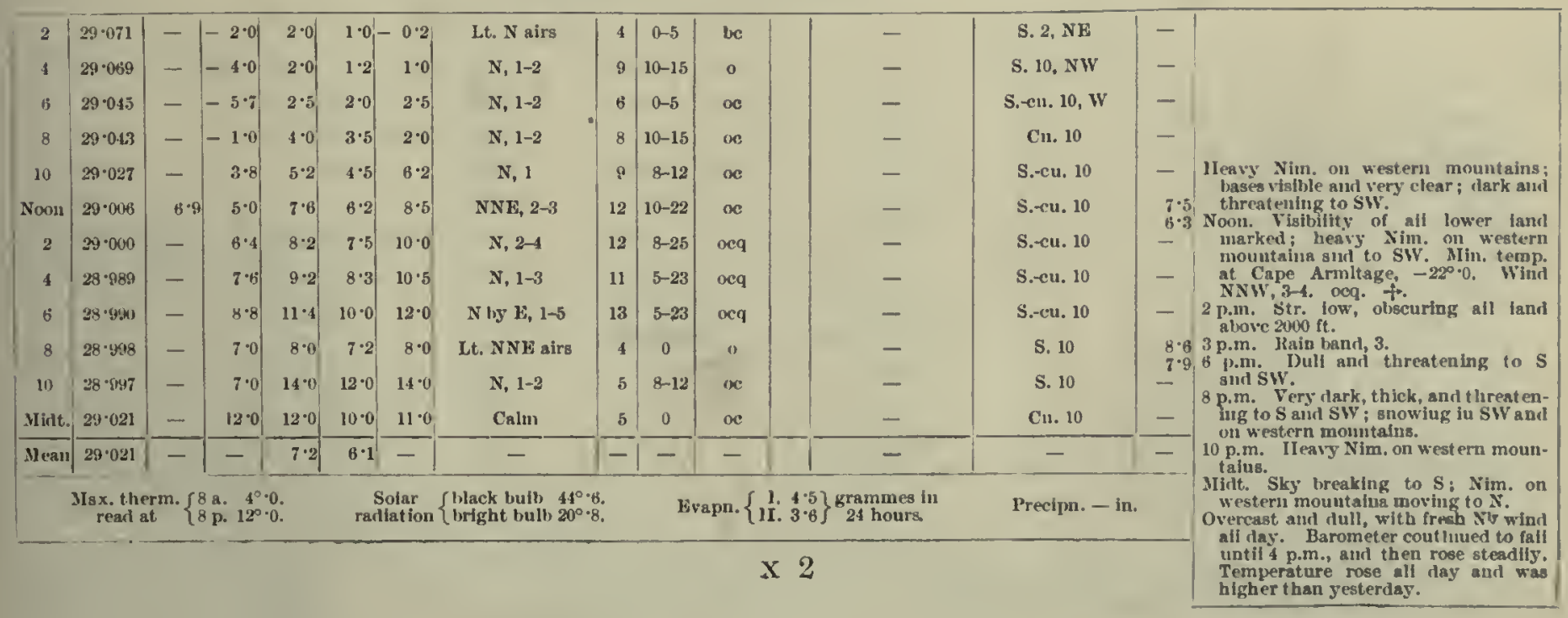




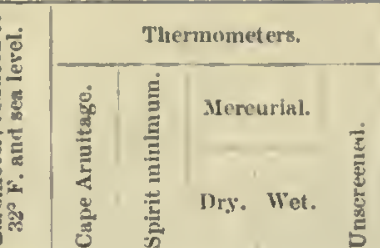

16 th.

\begin{tabular}{|c|c|c|c|c|c|c|c|c|c|c|}
\hline 2 & $29 \cdot 027$ & - & $10 \cdot 0$ & 110 & 10.5 & 11.5 & $x, 0-1$ & 4 & $0-10$ & oc \\
\hline 4 & $29 \cdot 055$ & - & $10 \cdot 0$ & $12 \cdot 0$ & $11 \cdot 0$ & $12 \cdot 0$ & $\mathrm{~N}, \mathrm{I}$ & 6 & $10-12$ & $\omega$ \\
\hline 6 & $29 \cdot 072$ & - & 10.0 & $14 \cdot 0$ & $12 \cdot 0$ & $14 \cdot 5$ & Var. FNE, $2-4$ & 10 & $15-20$ & $\omega * *$ \\
\hline 8 & $29 \cdot 112$ & - & $13 \cdot 8$ & $15 \cdot 0$ & $13 \cdot 8$ & $15 \cdot 2$ & Lt. NE airs & 8 & 0 & oc* $*$ \\
\hline 10 & $29 \cdot 121$ & 14. & $14 \cdot 0$ & $15 \cdot 1$ & $13 \cdot 1$ & $16 \cdot 3$ & E by $N, 1$ & 4 & $8-10$ & be \\
\hline Noon & $28 \cdot 148$ & - & $13 \cdot 8$ & $15 \cdot 0$ & $13 \cdot 5$ & $16 \cdot 8$ & $\mathrm{E}, 1-2$ & 10 & $10-15$ & $\infty 0$ \\
\hline 2 & $29 \cdot 187$ & - & $12 \cdot 0$ & $12 \cdot 3$ & $11 \cdot 3$ & $13 \cdot 7$ & $\mathrm{ESE}_{2}, 1-2$ & 4 & $5-12$ & $\operatorname{ce} \oplus$ \\
\hline 4 & $29 \cdot 201$ & 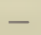 & $2 \cdot 0$ & $2 \cdot 6$ & $2 \cdot 6$ & 2.6 & ENE, 5-T & 18 & $30-42$ & $\operatorname{oeq} i$ \\
\hline 6 & $29 \cdot 227$ & - & $1 \cdot 0$ & 1.0 & 0.4 & 1.0 & NE, 4-6 & 26 & $28-40$ & $\infty q$ i. \\
\hline 8 & $29 \cdot 258$ & - & 0.0 & $2 \cdot 2$ & $2 \cdot 0$ & $2 \cdot 5$ & $\mathbf{E}$ Uy $\mathbf{N}, \mathbf{4 - 5}$ & 24 & $22-33$ & os \\
\hline 10 & $29 \cdot 304$ & - & 0.0 & $1 \cdot 0$ & 0.2 & $1 \cdot 0$ & Var. NE, 4-6 & 21 & $25-35$ & $o c$ \\
\hline idt. & $29 \cdot 335$ & - & $-4 \cdot 2$ & 2.8 & $4 \cdot$ & $3 \cdot 0$ & Var, NE, 5-6 & 22 & $32-37$ & $\cos 4$ \\
\hline & 71 & - & - & $8 \cdot 1$ & $7 \cdot 2$ & - & - & - & - & - \\
\hline
\end{tabular}

\begin{tabular}{c|c} 
Direction and \\
force \\
(Beanfort scalo).
\end{tabular}

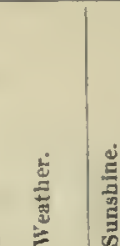

(1)
Clouds,
Amonnt and movement.

| Uner.

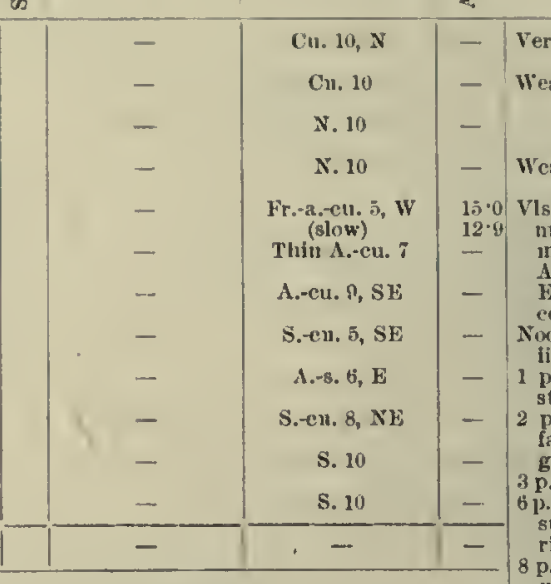

|

Remarks.

ery black to $\mathbf{N}$ and $\mathrm{NW}$.

Heatler very musettled; wint sinisty.

Western mountains obscured.

0 Vlsibllity of lower parts of land very narked; heavy Nhn. on western nountains. Nlin temp at Cape Erebus gnoke going $\mathrm{NE}^{\mathrm{l}} \mathrm{y}$ in a st rong column, fom. Thin Str. on western mount p.m. Frelus smoke going Nivy in strong llue.

p.m. Heavy surface drift; sudden all in temperature. Erehns smoke. going Wiy and a little Sty. p.m. lain band, 3 . p.m. Clouds thin and detaehed; heavs: surface drift in bay. Erelus smoke rising to 3000 feet and going Ny. p.m. Thin layers of S.-cu. on western mountains, otherwise they were clear ; cle

ing to $N$. W light to strong alout $3 \mathrm{p.m}$, and continued to blow hard from $\mathrm{E}$ and $\mathrm{NE}$. Barometer rose steadily all day. Temperat ure rose until wind light to strong alout 3 p.m., and contin
freshened, and then dropped suddenly.

\section{-}

17 th

\begin{tabular}{|c|c|c|c|c|c|c|c|c|c|c|c|c|}
\hline 2 & $29 \cdot 383$ & - & $|-6 \cdot 3|-2 \cdot 2|-2 \cdot 0|$ & $-2 \cdot 0$ & Var. E, 1-6 & 27 & $8-35$ & ocq $\div$ & -. & S. 10 & - & \multirow{10}{*}{ 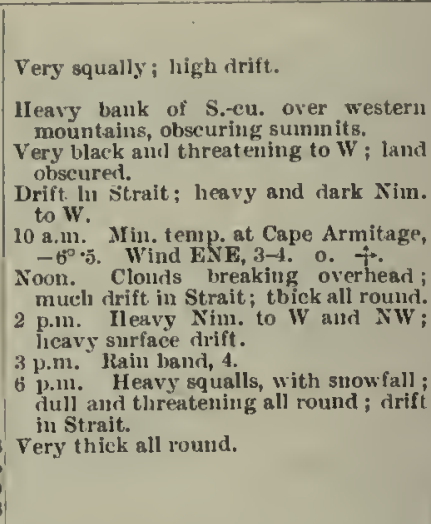 } \\
\hline 4 & $29 \cdot 397$ & - & $\mid-4 \cdot 0-3 \cdot 8-4 \cdot 2$ & $-4 \cdot 0$ & Je, 3-4 & 14 & $20-25$ & oct + & - & S. 10 & - & \\
\hline 6 & $29 \cdot 44$ & - & $|-6 \cdot 0|-4 \cdot 0|-5 \cdot 0|$ & $-5 \cdot 0$ & $\mathrm{E}, 5-6$ & 23 & $30-35$ & oc + & - & S.en. 10 & - & \\
\hline 8 & $29 \cdot 476$ & - & $|-6.7-6.5|-6.8 \mid$ & $-6 \cdot 0$ & Var. E, 5-6 & 22 & $30-40$ & $o q+$ & - & S. 10 & - & \\
\hline 10 & $29 \cdot 513$ & $-6 \cdot 0$ & $|-6 \cdot 5|-5 \cdot 1|-5 \cdot 5|$ & $-4 \cdot 2$ & ENE, 1-6 & 27 & $10-34$ & $\mathrm{oq}+$ & - & S. 10 & - & \\
\hline Noou & $29 \cdot 529$ & - & $|-6.0|-5.5|-6.2|$ & $-4 \cdot 5$ & Var. NE, 4-6 & 23 & $25-33$ & ocq $\div$ & - & S.ent. 8 & - & \\
\hline 2 & $29 \cdot 528$ & - & $|-7 \cdot 8|-2 \cdot 0-2 \cdot 4 \mid$ & $-1 \cdot 7$ & $\operatorname{Var} .12,4-6$ & 23 & $23-36$ & neq $\div$ & - & S.сcu. 10 & - & \\
\hline 8 & $29 \cdot 588$ & - & $|-10 \cdot 0|-10 \cdot 0|-10 \cdot 0|$ & $-9 \cdot 8$ & $E, 5-7$ & 25 & $25-38$ & $0+$ & - & S. 10 & - & \\
\hline 10 & $29 \cdot 564$ & - & $|-11 \cdot 3|-10 \cdot 2|-10 \cdot 2|$ & - & $5,5-6$ & 23 & $25-30$ & o & - & S. 10 & $\begin{array}{r}-9 \cdot 8 \\
-10.5\end{array}$ & \\
\hline Mint. & $29 \cdot 589$ & - & $|-11 \cdot 0|-10 \cdot 2|-10 \cdot 4|$ & - & $E, 4-5$ & 24 & $20-25$ & 0 & - & s. 10 & {$\left[\begin{array}{rl}-9.0 \\
-9.0 \\
-9.8\end{array}\right.$} & \\
\hline slean & $29 \cdot 510$ & - & $--6.4-6.7$ & - & - & $1-1$ & - & - & - & - & - & $\begin{array}{l}\text { Overeast all day, wit ly very strong } \mathrm{v} \\
\text { wind, and squally. Baromet rer reste } \\
\text { all day. Temperature low and steady } \\
\text { all day. }\end{array}$ \\
\hline
\end{tabular}

18 th.

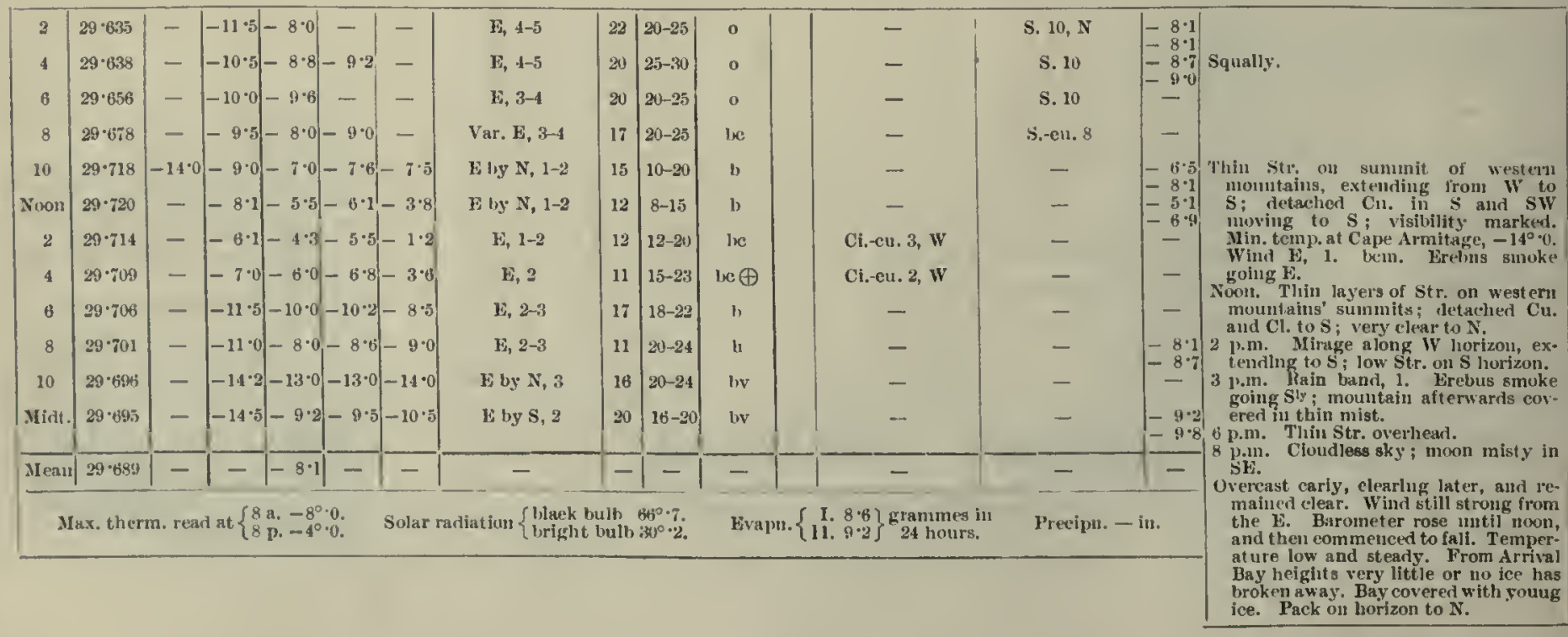




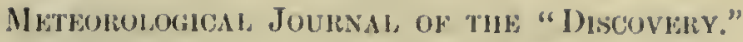

[1903-March.

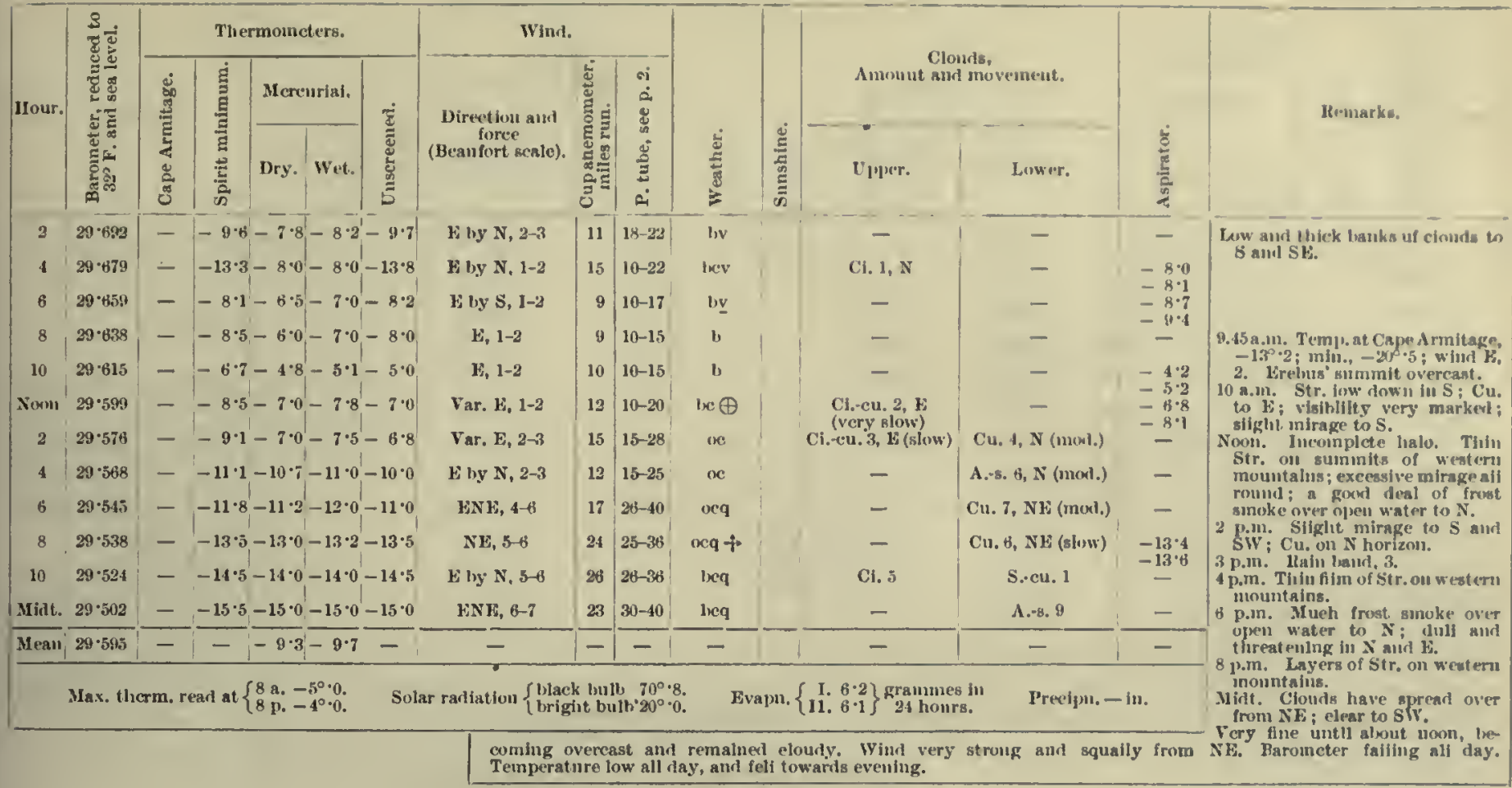

\begin{tabular}{|c|c|c|c|c|c|c|c|c|c|c|}
\hline 2 & $29 \cdot 503$ & - & $-15 \cdot 0^{\prime}-11 \cdot 0-11 \cdot 5-11 \cdot 0^{\prime}$ & Var.|NE, 3-4 & 24 & $15-28$ & beq & - & Cu. 7 & $-11 \cdot 6$ \\
\hline 4 & $29 \cdot 515$ & - & $-11 \cdot 9-9 \cdot 0-9 \cdot 5-9 \cdot 0$ & SE hy $\mathrm{E}, 0-2$ & 8 & $0-10$ & beq & - & Cn. 7 & -119 \\
\hline$\theta$ & $29 \cdot 522$ & - & $-8 \cdot 5-7 \cdot 8-8 \cdot 0-10^{\circ} 0$ & ENE, 0-1 & 5 & 0 & 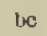 & Ci.-cu. 1 & - & $-8 \cdot 0$ \\
\hline 8 & $29 \cdot 529$ & - & $-12 \cdot 0-10 \cdot 0-10 \cdot 5-11 \cdot 2$ & Lt. NEairs & 1 & 0 & be & Ci.-еu. 1 & - & - \\
\hline 10 & $29 \cdot 534$ & $-21 \cdot 2$ & $-16 \cdot 0-15 \cdot 0-15 \cdot 0-16 \cdot 0$ & Calin & 1 & 0 & bv & - & - & - \\
\hline Noon & $29 \cdot 532$ & - & $-16 \cdot 2-13 \cdot 2-13 \cdot 2-9 \cdot 8$ & Calm & 0 & 0 & bv & - & - & $-14 \cdot 4$ \\
\hline 2 & $29 \cdot 326$ & - & $-13 \cdot 1-8 \cdot 8-9.5-8 \cdot 6$ & Caim & 0 & 0 & h & - & - & - \\
\hline 4 & $29 \cdot 509$ & - & $-11 \cdot 0-9 \cdot 0-9 \cdot 4-8.8$ & Calm & 2 & 0 & b & - & - & - \\
\hline 6 & $29 \cdot 504$ & - & $-14 \cdot 3-13 \cdot 0-13 \cdot 4-12 \cdot 0$ & Calm & 0 & 0 & Ы & - & - & - \\
\hline 8 & $29 \cdot 493$ & - & $-14 \cdot 5-8 \cdot 0-8 \cdot 2-12 \cdot 0$ & Calm & 0 & 0 & b & - & - & -60 \\
\hline 10 & $29 \cdot 477$ & - & $-8.5-1.0-1.8-2 \cdot 2$ & $\mathrm{~N}, 1$ & 9 & $8-12$ & i) & - & - & $\begin{array}{l}-1 \cdot 2 \\
-2 \cdot 0\end{array}$ \\
\hline Midt. & $29 \cdot 469$ & - & $-2 \cdot 2-0.2-i 1 \cdot 2-1.8$ & $\mathrm{~N}, 1$ & 8 & $10-12$ & b & - & - & $\begin{aligned} & 1.0 \\
= & 2.0\end{aligned}$ \\
\hline 31 ean & $29 \cdot 509$ & - & $-\overline{28.8-9.3}-$ & - & - & - & - & - & - & - \\
\hline
\end{tabular}

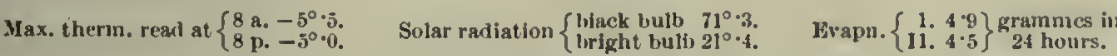

Preclin. - in. 19th.

$\mathrm{V}$ clearing in $\mathrm{SW}$; wind vartable hetween ENE and $\mathbf{E}$ : 110 4 a.m. Sky clearing to $S W$; calun for shout an lourbet ween 2 and 4 a.m. 1ked glow of sunrlse on 10 a.m. Excesslve mirage ali round ; light cir. to $\mathbf{E}$; layers of Str. to SE; otherw ise elourlless sky. Win. temp. at Cape Arritage, $-24^{\circ} \cdot 1$ Caim. Bt romg column. voon. Excessive mirage all rount. pom. Donble mirage $w$ w and 3 p.m. Kain hand, 1 .

6 p.m. Bank of str, to w. moon in Ssw.

p.rn. Thin S.-cun. in IW passing coimuring quickly: fine sunset thermonet er sereens.

10 p.m. A layer of detacherl S.ccu. moving to's at morlerate speed

Mirt." Thin seurl passing fast from N. Fine and clear day, with hardly any wind. Barometer rose until about noon, and then feli steadily. Temperature low all day, rising lin evenlug.

20 th.

\begin{tabular}{|c|c|c|c|c|c|c|}
\hline Calin & 1 & 0 & be & & Thin seud, 2 , & $\begin{array}{l}-9.0 \\
-9.2\end{array}$ \\
\hline Caim & 0 & 0 & the & - & Det. en. 3. & $-10 \cdot 1$ \\
\hline Calm & 1 & 0 & b & - & - & $\begin{array}{r}-6 \cdot 2 \\
-8.5\end{array}$ \\
\hline NE, 2-3 & 7 & $10-15$ & ice & Cl, -cu, $t, \mathrm{NF}$ & - & - \\
\hline E by $S, 2$ & 10) & $10-15$ & be & - & Fr.-s. 4 & $-9 \cdot 8$ \\
\hline S1:, 2-3 & 8 & $15-18$ & i, & - & - & $-5 \cdot 1$ \\
\hline SE, 1-2 & s & $7-\mathrm{i} 2$ & lese & Ci.s. 2, NW & - & - \\
\hline $\mathrm{B}, 2$ & 6 & $12-20$ & we & $\mathrm{Cl}_{0}-8,3, \mathrm{NW}$ & - & - \\
\hline 1,2 & 13 & $12-20$ & the & $\mathrm{Ci}_{0}-5.3, \mathrm{NW}$ & - & - \\
\hline LSE, 1-2 & 10 & $10-15$ & 1. & - & - & - \\
\hline NNE, 0-1 & 7 & $0-\mathrm{i} 2$ & b & - & - & $-9 \cdot 8$ \\
\hline Lt. NE airs & 3 & 0 & b & - & -- & $\begin{array}{r}-9.0 \\
-\quad 9.8 \\
\end{array}$ \\
\hline$=$ & $\overline{-1}$ & - & - & - & - & - \\
\hline
\end{tabular}

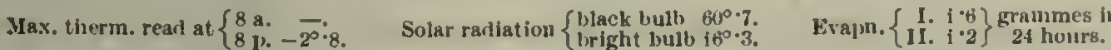
a.m. Bank of Str. to $S$ and over
Precipm. - in.
Layers of Sir. on western mountains ant also to $\mathrm{S}$.

Snk of Cn. $w$ o ; j dark heary monutains "isible: dark over White lelearly detaeherl clouds to SII and ve: a.m. Western mountalus ol senred ly detaelied S.cell, pasaing from $X$ fast : detacheil s.-cn. clourl in $\boldsymbol{E}_{\text {passing from }} \mathrm{x}$ and colfect ing in s.

10 a.m. Str. jist down to top of Black Islanit, aloont 3400) feet. 10.13 a.mo. Temp) at Cape Armiage, $-17^{\circ} 0 ;$ mill., $-25^{\circ} \cdot 8$. roing $\mathrm{Y}_{\mathrm{y}}$

Nixoll. Layers of Str. on western mountains alont linalf-way down; \& thick land of grey fog olsecures the foot of western

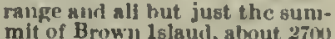
fect; it ext ensis to $\mathrm{S}$, but there is neither fog nur mist. on this side of the Strait.

2 1.111. Hleary lanin of fog in frout of all land to $\mathrm{S}$ and $W$; suinm its of lsiands onjy visible.

p.m. lkin band, 2. के p.m.

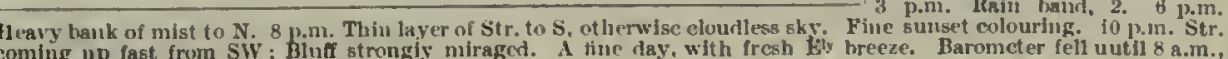

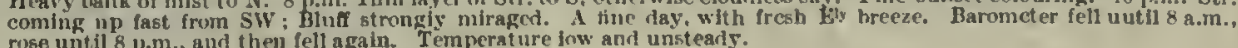

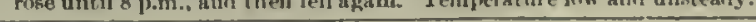




\begin{tabular}{|c|c|c|c|c|c|c|c|c|c|c|c|c|c|c|}
\hline \multirow{3}{*}{ IIour. } & \multirow{3}{*}{ 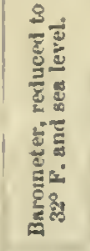 } & \multicolumn{5}{|c|}{ Thernnoineters. } & \multicolumn{3}{|l|}{ Wind. } & \multirow[b]{3}{*}{ 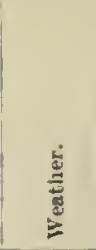 } & \multirow[b]{3}{*}{ 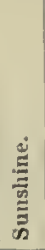 } & \multirow{2}{*}{\multicolumn{2}{|c|}{$\begin{array}{l}\text { Clouds, } \\
\text { Amount aud novemeut. }\end{array}$}} & \multirow[b]{3}{*}{$\frac{\frac{\dot{5}}{\tilde{E}}}{\frac{8}{2}}$} \\
\hline & & \multirow{2}{*}{ 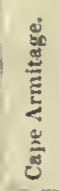 } & \multirow{2}{*}{ 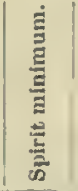 } & \multicolumn{2}{|c|}{ Mercurial. } & \multirow{2}{*}{ 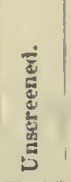 } & \multirow{2}{*}{$\begin{array}{c}\text { Direetion and } \\
\text { force } \\
\text { (Bcanfort scale). }\end{array}$} & \multirow{2}{*}{ 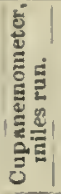 } & \multirow{2}{*}{ 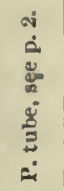 } & & & & & \\
\hline & & & & Dry. & Wet. & & & & & & & Upper. & Lower. & \\
\hline 2 & $29 \cdot 4 \times 2$ & - & $-12 \cdot 5$ & -60 & -6.5 & $-8 \cdot 0$ & Calra & 2 & 0 & oc & & - & S.eu. 10, N & $-6 \cdot 2$ \\
\hline 4 & $29) \cdot 126$ & - & $-12 \cdot 3$ & $-10 \cdot 0$ & $-9 \cdot 8$ & $-10 \cdot 5$ & $\mathbf{S E}, 1$ & 3 & 8-12 & oc & & - & S. 10 & -6 \\
\hline s & $29 \cdot 343$ & - & $-10 \cdot 7$ & $-9 \cdot 2$ & $-9 \cdot 0$ & $-11 \cdot 5$ & ESE, 1 & 3 & 8-14 & $\operatorname{cm}$ & & - & S. 10 & $-10 \cdot 1$ \\
\hline 8 & $29 \cdot 343$ & - & $-13 \cdot 5$ & $-3 \cdot 0$ & -30 & -5.0 & WNE, 2 & 8 & $15-18$ & oc & & $\rightarrow$ & S. 10 & -8.8 \\
\hline 10 & $29 \cdot 324$ & - & $-16 \cdot 5$ & $-12 \cdot 0$ & $-11 \cdot 2$ & $-11 \cdot 5$ & SF, 2-3 & 7 & $18-30$ & $u q$ & & - & S. 10 & $-11 \cdot 6$ \\
\hline Nooli & $29 \cdot 302$ & -15 & $-15 \cdot 5$ & $-11 \cdot 2$ & $-11 \cdot 0$ & $-10 \cdot 2$ & Sls, 3-4 & 17 & $18-24$ & ing & & - & S. 10 & $\begin{array}{l}-11.6 \\
-10.8 \\
-10.8\end{array}$ \\
\hline 2 & $29 \cdot 274$ & - & $-11 \cdot 8$ & $-10 \cdot 6$ & $-10 \cdot 6$ & $-10 \cdot 0$ & $S F, 3-4$ & 13 & $16-25$ & og & & - & S. 10 & - \\
\hline 4 & $29 \cdot 281$ & - & $-14 \cdot 0$ & $-11 \cdot 5$ & $-11 \cdot 2$ & $-11 \cdot 2$ & Var. Es1\%, 3-4 & 16 & $20-30$ & $o c+$ & & - & S.-cu. 10 & - \\
\hline is & $29 \cdot 283$ & - & $-13 \cdot 5$ & $-12 \cdot 0$ & $-12 \cdot 2$ & $-12 \cdot 0$ & Var, Lly S, 4-5 & 22 & $25-35$ & $00-1$ & & - & S.eu. 10 & 一 \\
\hline 8 & $29 \cdot 289$ & - & $-15 \cdot 5$ & $-14 \cdot 8$ & $-14 \cdot 8$ & $-14 \cdot 6$ & var. $\mathbf{N}, 4-5$ & 20 & $24-30$ & oq $+* *$ & & - & N. 10 & - \\
\hline 10 & $29 \cdot 294$ & - & $-17 \cdot 4$ & $-11 \cdot 0$ & $-11 \cdot 0$ & $-9 \cdot 1$ & E by $\mathrm{N}, 2$ & 24 & $18-20$ & he $*$ & & - & S. 5 & - \\
\hline Midt. & $2 y \cdot 305$ & - & $-11 \cdot 7$ & $-8 \cdot 0$ & $-7 \cdot 9$ & $-10^{\circ} 0$ & Var. Ji airs & 9 & $0-5$ & $1 x$ & & - & A.-s. 3 & $\begin{array}{l}-8 \cdot 3 \\
-8 \cdot 7\end{array}$ \\
\hline$\overline{\mathrm{Mean}}$ & $29 \cdot 334$ & - & - & $-9 \cdot 9$ & $-9 \cdot 9$ & - & - & $\overline{-}$ & - & - & & - & - & - \\
\hline
\end{tabular}

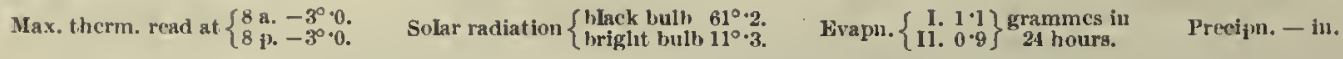

Remarks.

Clear sky from S to NW to ait. $15^{\circ}$; brilliant colouring over sun's posit im.

tr. very low, alt. allout buo feet: inisty to $\mathrm{S}$ and $W$.

Thick ail routud; thin Str. hlowing over ()bservation Hiil from $\$$ rime forming.

thon. lime forming on every thing; thicek mist phuriug over temp. at Cape Armitin. temp. at Cape Armitage, Fogry to $s$ and $W$; iroar frost and rime on instrument.

3 p.m. liain bane?, ?

4 p.m. All laud to in obscuret wind very variable.

p.m. lleavy clouds of drift in inoutli of Strait, direction $\mathrm{S}^{\text {ly }}$; foot of foot-liills visible.

i)m. Very thick ; wind squaily and variable from $\mathrm{N}$ to SSE.

p.m. Stars shining overhead dull fow down; sllowing very

Slight
SSE.

Ciear overhead.

Overeast and dull all day, with strong variable SEly wind. Barometer fell until evening, and then commeuced to rise. Temperature very unsteady and low.

23rd.

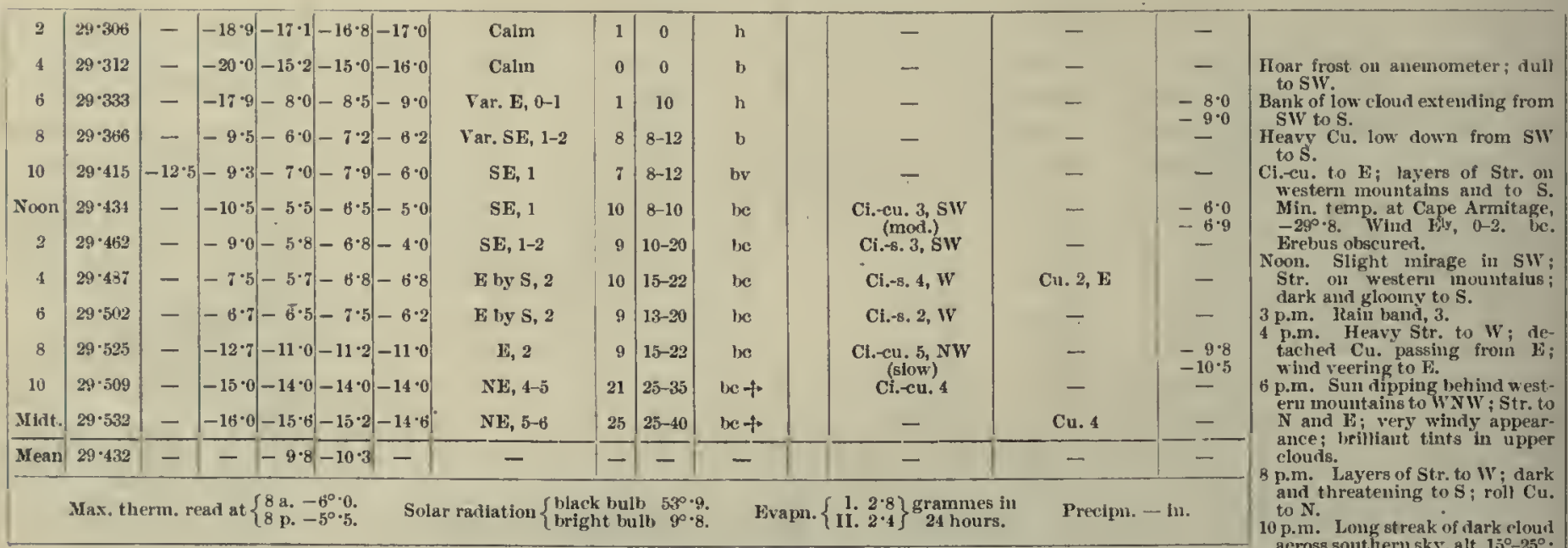

arange-red glow to $\mathrm{S}$. Midt S. across southerusky, alt. $15^{\circ}-25^{\circ}$ orange-red glow to $\mathrm{S}$. Midt. S.-cu. ciouds to S. Fine and clear early, with clouds iater. Wint fresliened from cal
low and unsteady.

24th.

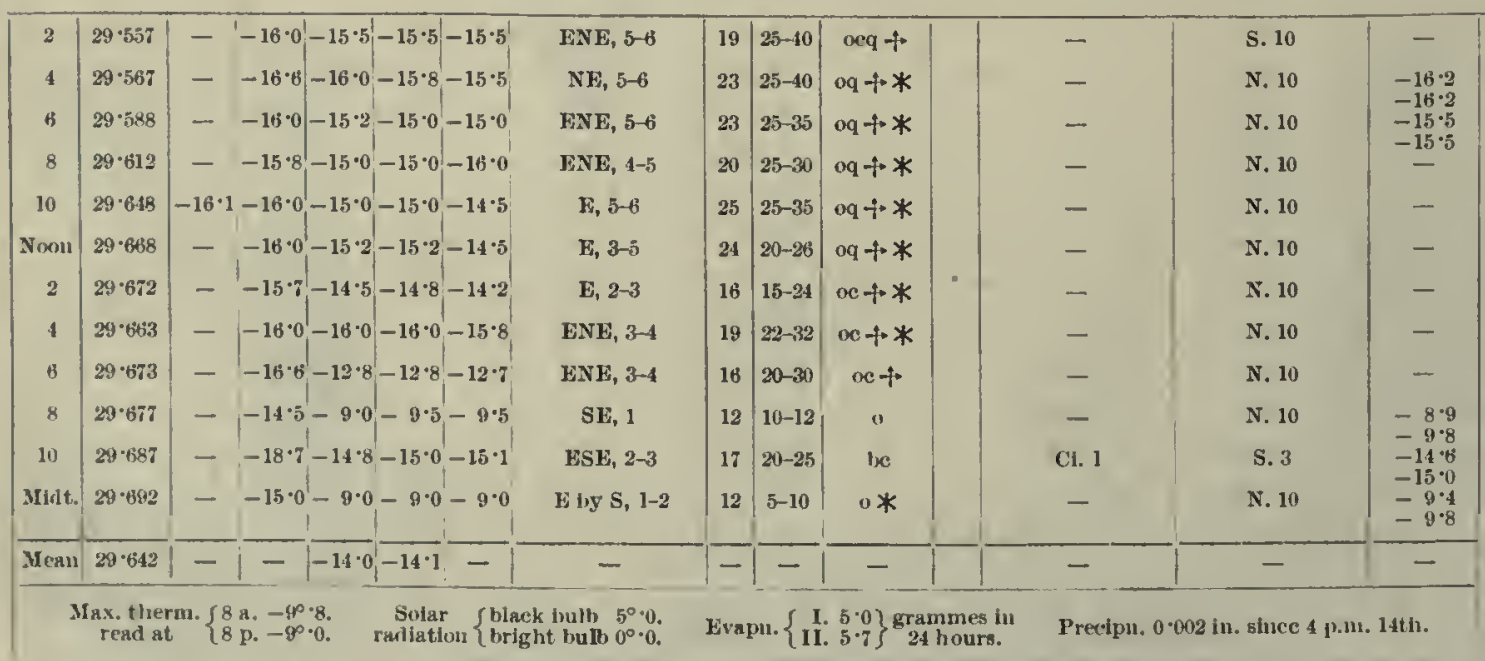

Thick all roumd. Min. temp. at Cape Armitage, $-19^{\circ} \cdot 2$. of., Erelnis olscured.

Light fall of fine powdery snow.

i).m. Rah band, 2 .

Wentlicr showing signs of clcar-

ing. lier glare of sunset seen through seeu from ship, apporarer to be going S'y in long streamers. 10 p.m. Heavy clouds in N, W, and $S$ to the hortzon; many

stars visible.
Midt. These clouds have come up against the wind, aud now completely overcast the sky, except or a thin streak of light on s and Whorizon; uo stars visible; 
Meteorological Journat, of thr" "Discoviry."

[1903-March.

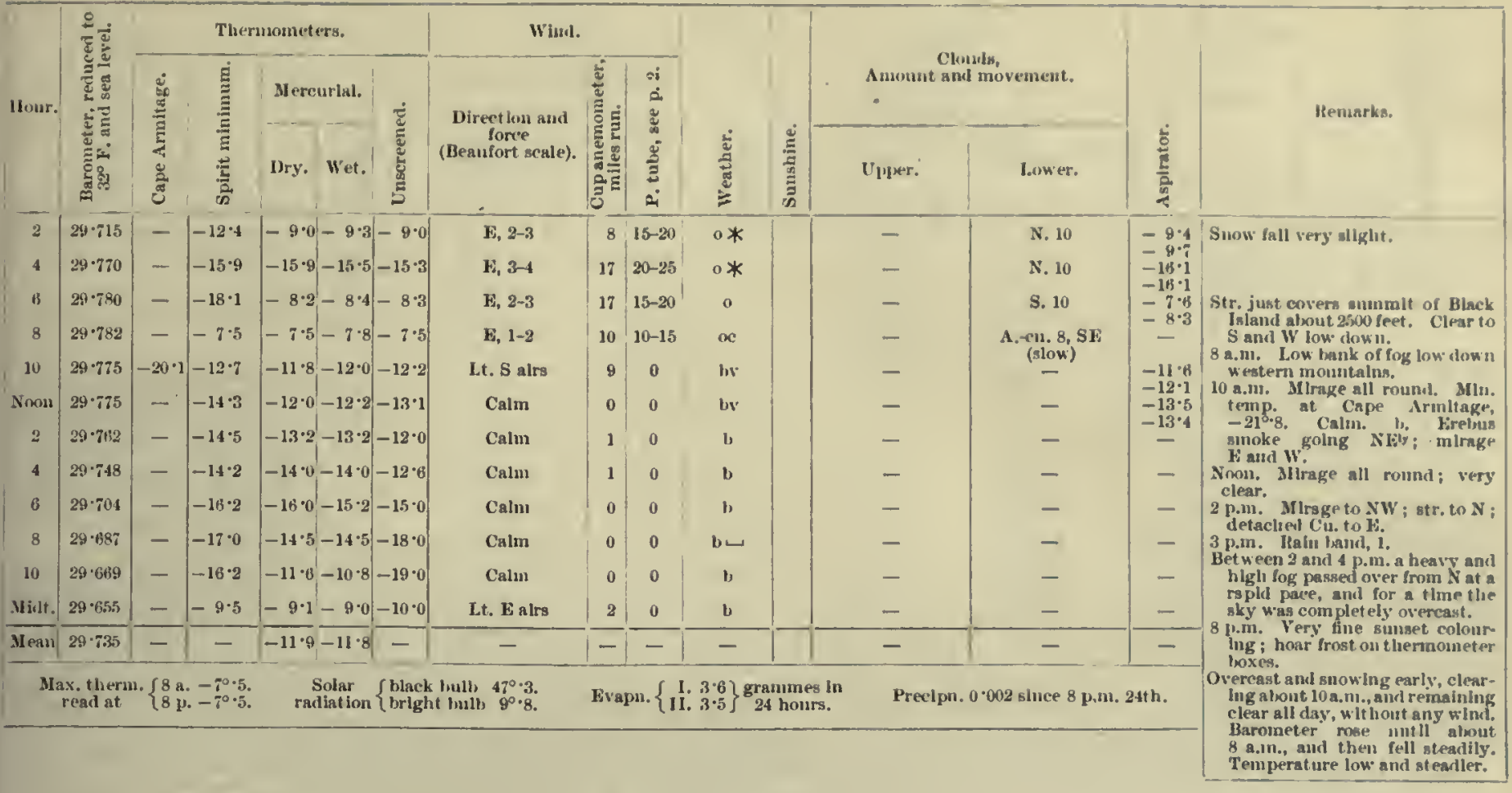

25 th.

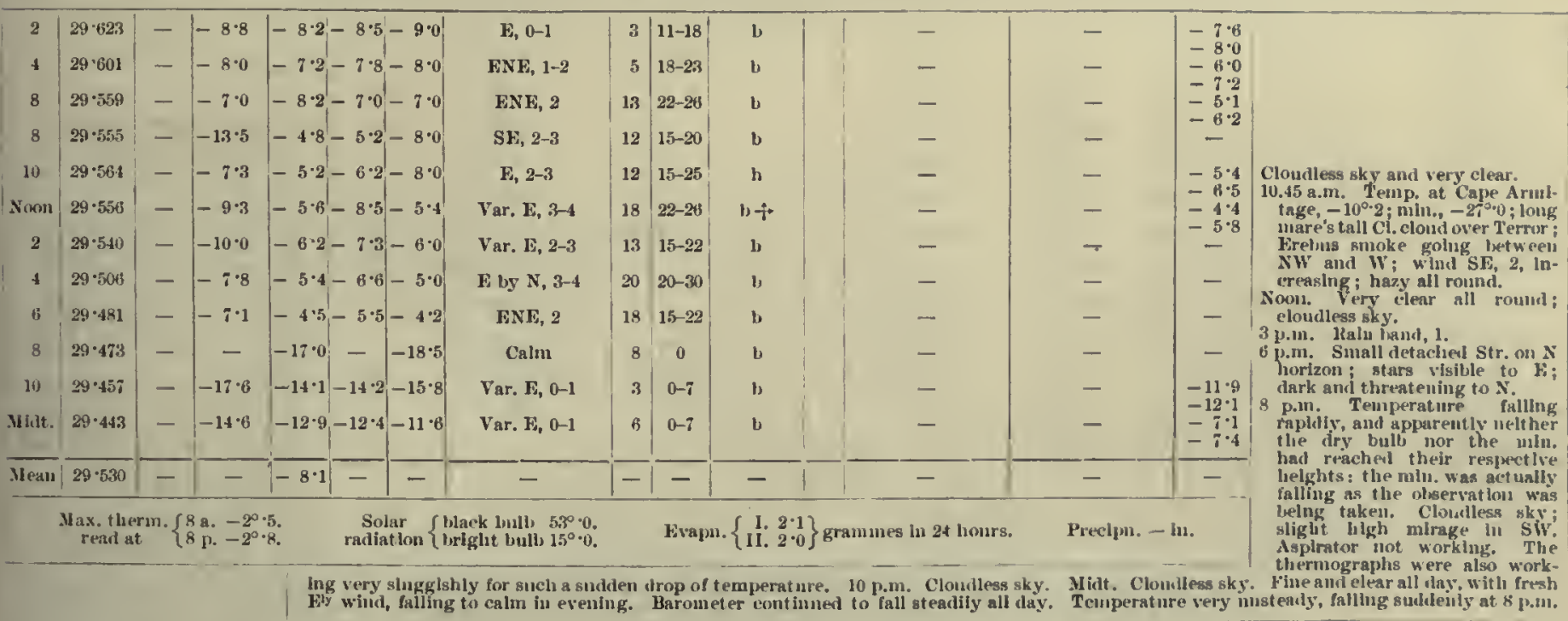

\begin{tabular}{|c|c|c|c|c|c|c|c|}
\hline 2 & $29 \cdot 426$ & - & $-12 \cdot 1$ & $-1 \cdot 7-2 \cdot 8-3 \cdot 8$ & E, 1-2 & 8 & ${ }^{5}-10$ \\
\hline 4 & $29 \cdot 401$ & - & $-4 \cdot 8$ & $-3 \cdot 4-4.3-3.5$ & ISSE, 1-2 & 8 & $8-16$ \\
\hline 6 & $29 \cdot 392$ & - & $-4 \cdot 8$ & $-2 \cdot 0-3 \cdot 1-3 \cdot 2$ & ISSE, 1-2 & 9 & $8-15$ \\
\hline 8 & $29 \cdot 373$ & - & -4.8 & $-3 \cdot 2-4 \cdot 0-4 \cdot 2$ & Var. E, 2-3 & 8 & $15-20$ \\
\hline 10 & $29 \cdot 335$ & $-4 \cdot 5$ & $-4 \cdot 0$ & $-1 \cdot 2-2 \cdot 2-1 \cdot 8$ & $\mathrm{SIE}_{2} 2-3$ & 12 & $12-20$ \\
\hline 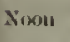 & $29 \cdot 286$ & - & $-2 \cdot 0$ & $1.5 \quad 0.0 \quad 3.0$ & $\mathrm{SE}, 1$ & 9 & $10-12$ \\
\hline 2 & $2 y \cdot 244$ & - & $-7 \cdot 5$ & $-3 \cdot 3-4 \cdot 2-3 \cdot 0$ & Calm & 3 & 0 \\
\hline 1 & $29 \cdot 187$ & - & $-11 \cdot 1$ & $-10 \cdot 0-10 \cdot 5-9 \cdot 3$ & Calm & 0 & 0 \\
\hline ; & $29 \cdot 151$ & - & $-11 \cdot 7$ & $-4.8-5.8-4.0$ & $\mathrm{~N}$ by L, 0-1 & 1 & $30-10$ \\
\hline 8 & $29 \cdot 097$ & - & $-13 \cdot 5$ & $2 \cdot 5$ & N, 1-2 & 4 & $12-15$ \\
\hline 10 & $29 \cdot 068$ & - & $0 \cdot 2$ & $6 \cdot 7 \quad 4 \cdot 2$ & NXE, 1-2 & 7 & $5-10$ \\
\hline Mist. & $29 \cdot 0.53$ & - & $2 \cdot 2$ & $7 \cdot 0 \quad 5 \cdot 5$ & $N E, 1-2$ & 9 & $5-10$ \\
\hline ant & $29 \cdot 251$ & - & - & $-1 \cdot 0-2 \cdot 1-$ & - & -1 & - \\
\hline
\end{tabular}

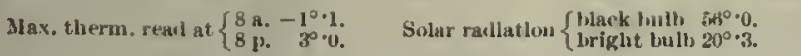
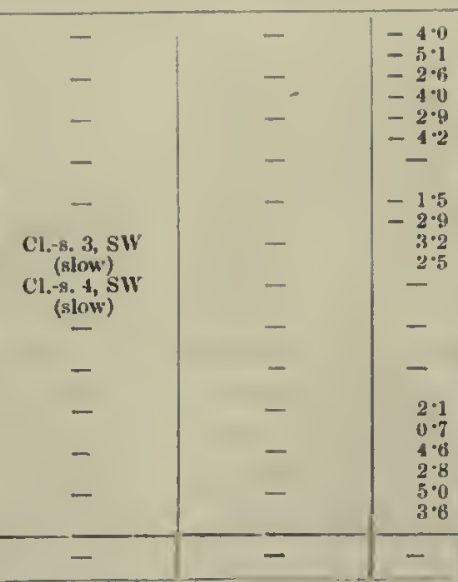

27 th.

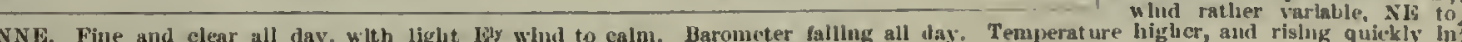
the eveniug. 


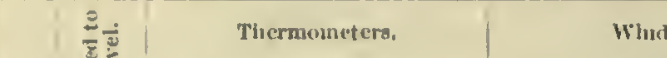

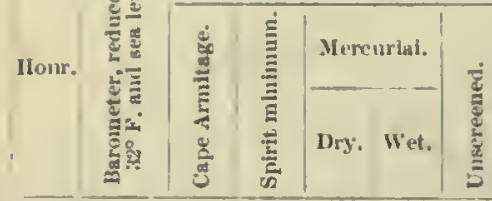

28 th.

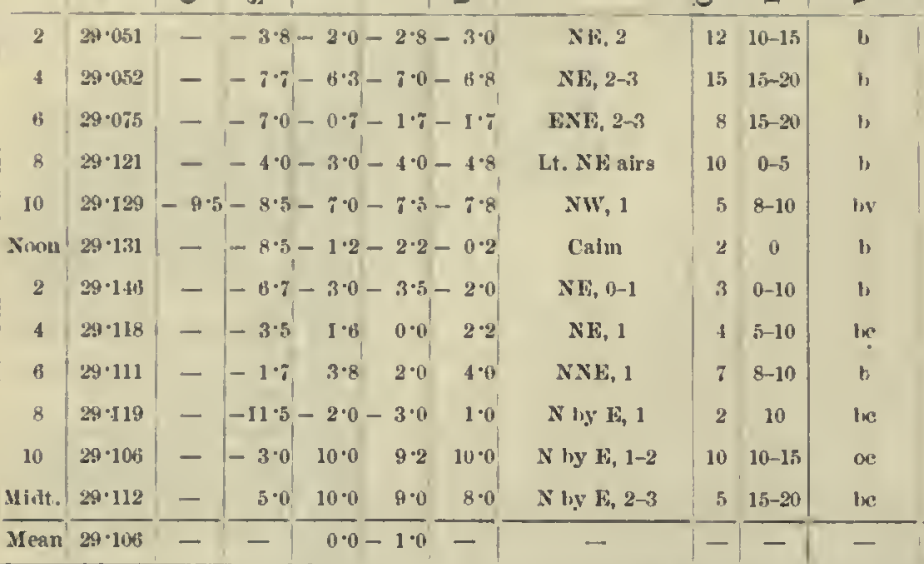
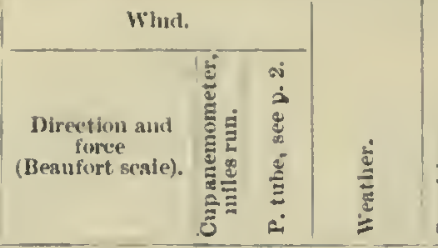

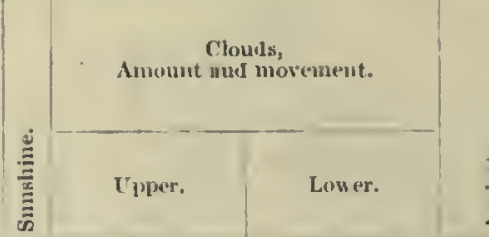

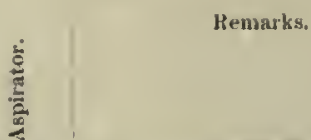

Max. therm. read at $\left\{\begin{array}{l}8 \text { a. } 7^{\circ} \cdot 2 . \\ 8 \text { p. } 4^{\circ} \cdot 5 .\end{array}\right.$ Solar radiation $\left\{\begin{array}{l}\text { black bulb } 57^{\circ} \cdot 3 . \\ \text { bright hulb } 23^{\circ} \cdot 4\end{array}\right.$

Evapu. $\left\{\begin{array}{r}\text { I. } 7 \cdot 0 \\ \text { If. } 7 \cdot 3\end{array}\right\}$ gramnies in

Precipn. - in.

- 2.6 Wiml stesulier and not so gusty $-3 \cdot 3 \quad$ 89 at mininight.

$-6 \cdot 9$
$-\quad 7 \cdot 2$

$0 \cdot 0$
-1.5 Wint variabie, $\mathrm{F}$ to $\mathrm{NE}$, and -15 rather gusty.

- $6 \cdot 3$ Ifeary lank of roll Cu. low down - 7.2 , to S and SW; ligitt and de- 1.8 eru mountains; misty appearance in sky; very clear; visjat Cape Armitage, $-2 f^{\circ} \cdot 0$. Light Nw'y airs; hazy; Hrehus smoking kell, aud going stummit

3*2 Noou, Detacher $\mathrm{Cu}$, forming rapinly in $\mathrm{E}$, and going to $\mathrm{N}$ fast; Cu. low down lit F, moviug i fast ; detached heavy roli $\mathrm{Cn}$. to S; Str. on and over western unountalins summits; windy appearance in SW.

p.m. Str. to $S$ and $W$; detaeher Cu. passing from $N$; Str. on guinmits of moun ta.

4 p.m. Heavy Cu. on Mt. Discovery; Str. on western mountains, sumnits obscured. 6 1).m. Str. on summits of wcstern mountains, and exteuding to S. 8 1.11. Dark and threatening to $S$ and $S W$; windy appearance on western mountains ; much detached Cu. on and over western mountains and to SW, 10 p.m. Henvy Cu. over foot-lílis of westem mountains ; very dark all over. Midt. Cu. coverlng westeru mountains ; clear overhead. Fine and ciear ncarly ail towaris evening. New lee lans formed for about a mile to the $\mathrm{N}$ of the old lce, and is at tached to it. It can be walked upion with safety.

29 th.

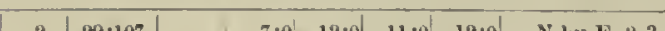

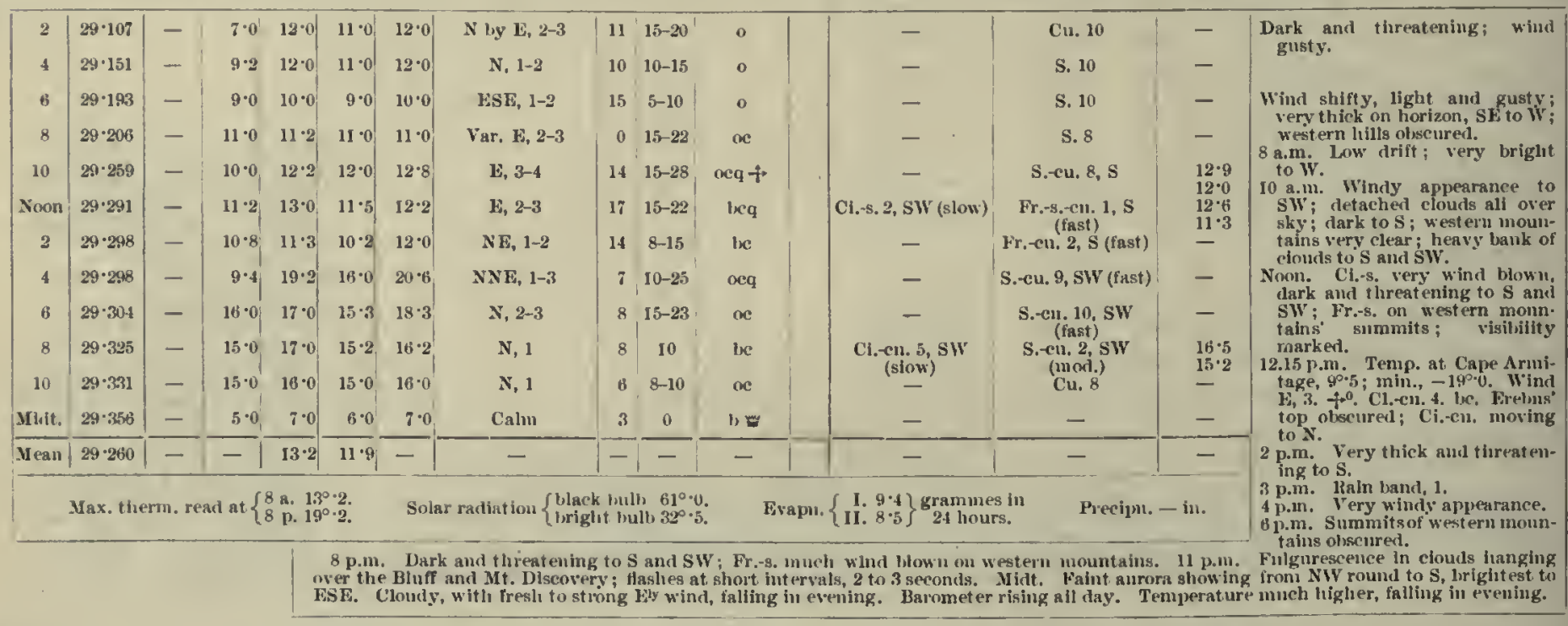

30th.

\begin{tabular}{|c|c|c|c|c|c|c|c|c|c|c|}
\hline 2 & $29 \cdot 35 \times$ & - & $2 \cdot 0$ & $6 \cdot 0$ & $5 \cdot 0$ & $7 \cdot 0$ & Culm & 1 & 0 & b) \\
\hline 4 & $29 \cdot 3943$ & - & $1 \cdot 0$ & $3 \cdot 0$ & $2 \cdot 0$ & $4: 5$ & Calm & 1 & 0 & b \\
\hline bi & $20 \cdot 3994$ & - & $2 \cdot 0$ & $7 \cdot 0$ & $6 \cdot 0$ & $8 \cdot 0$ & $1 \mathrm{SE}, 1$ & 5 & $8-10$ & b \\
\hline 8 & $29 \cdot 450$ & - & $6 \cdot 8$ & $9 \cdot 0$ & $8 \cdot 0$ & $12 \cdot 0$ & NE, $2-3$ & 8 & 10 & $b$ \\
\hline 10 & $29 \cdot 456$ & - & $6 \cdot 8$ & $9 \cdot 0$ & $7 \cdot 4$ & $8 \cdot 0$ & NE, 2-3 & 11 & $15-22$ & 1) \\
\hline Nomu & $29 \cdot 499$ & - & $3 \cdot 8$ & $4 \cdot 2$ & $3 \cdot 5$ & $4 \cdot 1$ & $\mathrm{NIE}, 3-5$ & 17 & $22-30$ & h'q $\oplus$ \\
\hline 2 & $29 \cdot 504$ & - & $2 \cdot 8$ & $3 \cdot 3$ & $2 \cdot 8$ & $3 \cdot 8$ & NIE, 3-5 & 21 & $23-33$ & ireq \\
\hline 4 & $29 \cdot 511$ & - & $1 \cdot 8$ & $2 \cdot 5$ & $2 \cdot 0$ & $2 \cdot 5$ & EN1 4 4-5 & 23 & $30-10$ & orq \\
\hline 6 & $29 \cdot 510$ & - & 0.0 & $1 \cdot 0$ & $0 \cdot 2$ & $1 \cdot 0$ & Is hy N, 3-5 & 22 & $22-33$ & orq \\
\hline 8 & $29 \cdot 517$ & - & -1.2 & $0.0-$ & 0.2 & $0 \cdot 0$ & IS, $3-4$ & 18 & $18-25$ & neq \\
\hline 10 & $29 \cdot 187$ & - & $-1 \cdot 0$ & $0 \cdot 0-$ & $0 \cdot 2$ & $-1 \cdot 0$ & If loy N, 3-4 & 17 & $15-22$ & oe \\
\hline Miste. & $29 \cdot 488$ & - & $-2 \cdot 0$ & $-1 \cdot 0-$ & $1 \%$ & $-1 \cdot 0$ & E, $2-\mathbf{A}$ & 14 & $18-25$ & be \\
\hline IItan & $29 \cdot 453$ & - & - & $3 \cdot 7$ & $2 \cdot 9$ & - & - & - & - & - \\
\hline
\end{tabular}

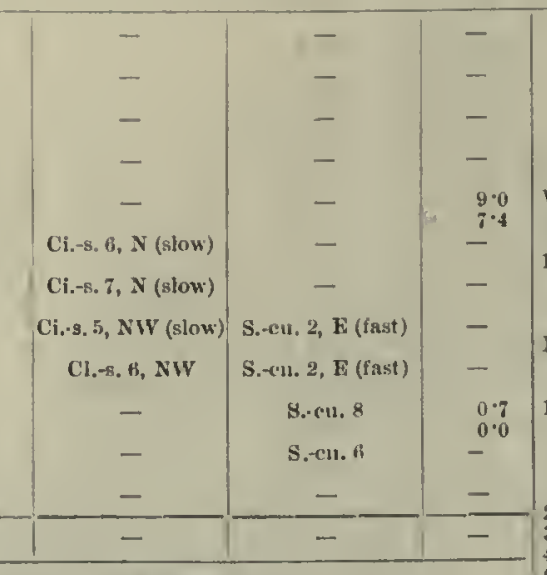

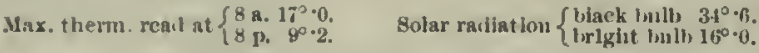

Evapu. $\left\{\begin{array}{r}\text { I. } 8: 3 \\ \text { II. } 9 \cdot 0\end{array}\right\}$ graumues in 24 lionrs.

Precipu, - in,
9.0 Very wiudy Ci. to $\mathrm{N}$; dark and heavy bank of S.-cir. to $S$ and 30 a.m. Tenp. at Cape Armiage, $-7^{\circ} 9 ; \min ,-13^{\circ} 8$. Wind

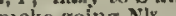
snoke going Nit. on. Ci,-s. very wind blown ciourting up from $W$. p.m. Temp. at Cape Amitage, Frelus smoke going Is; sky round sun. p.m. Very windy a plearance. $3 \mathrm{j} . \mathrm{m}$. lain wand, 4. p.In. Str. on E horizon. p.m. Clonds apparently sinkiing; very feathery Ci.s. streteining in all diret 


\section{1}

Mithorologrcal JoURNAL OF THI: "Discovitiy."

[1903-March.

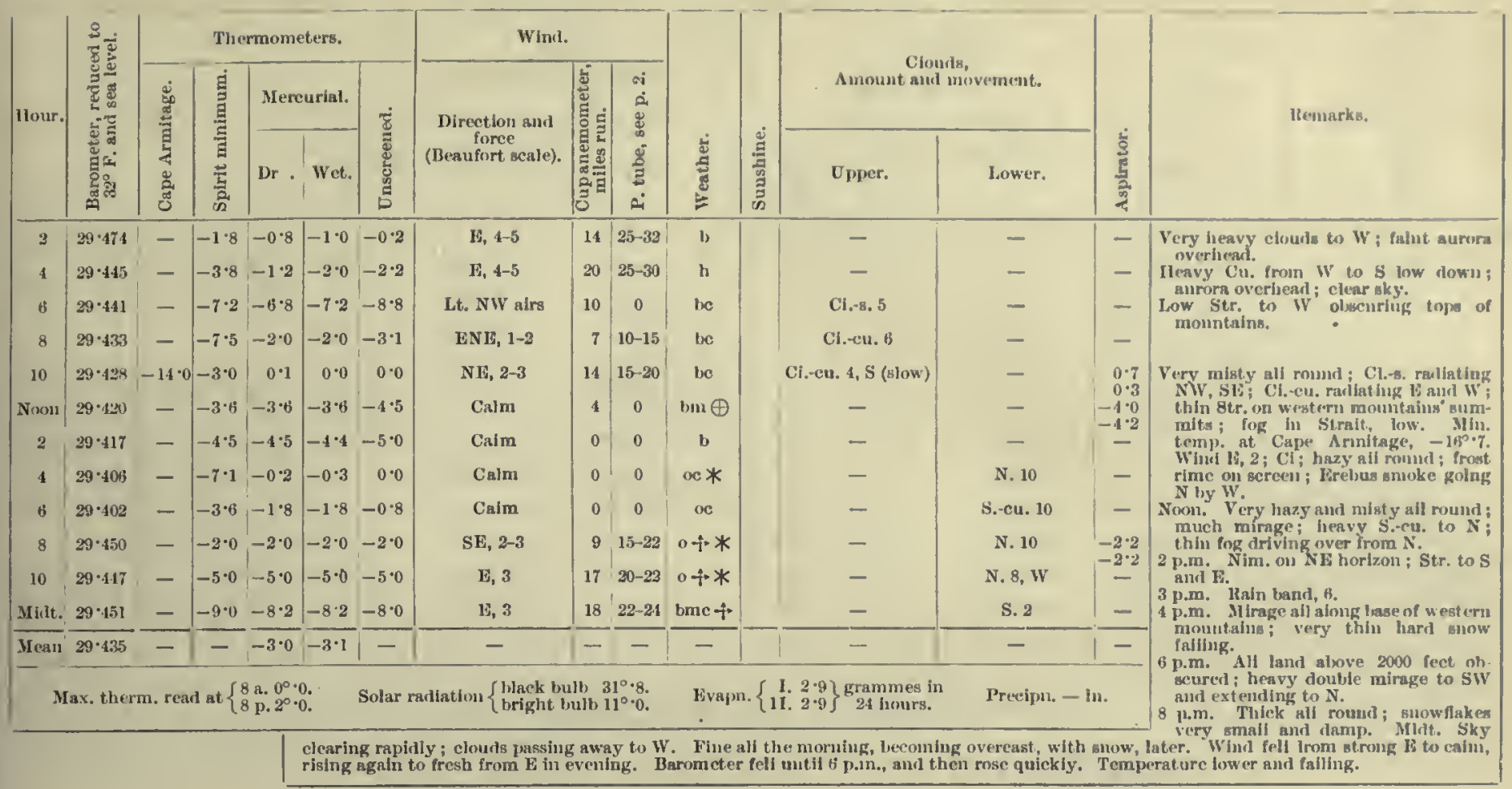


1903-April.]

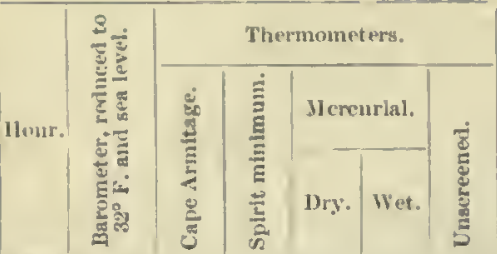

1st.

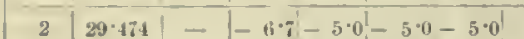

$20.474-6 \cdot 7-500-50-5 \cdot 0$

$4 x \cdot 4 \times 3-4 \cdot 5-2 \cdot 6-3 \cdot 0-3 \cdot 0$

6) $29 \cdot 516--4 \cdot 5-3 \cdot 4-4 \cdot 0-3 \cdot 8$

$8 \quad 29 \cdot 519-9 \cdot 5-6 \cdot 0-6 \cdot 0-8 \cdot 0$

$1029 \cdot 514-12 \cdot 8-10 \cdot 7-8 \cdot 8-8 \cdot 8-8 \cdot 0$

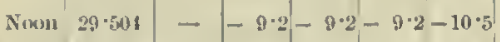

$2 \quad 29 \cdot 4.2--8 \cdot 4-6 \cdot 0-6 \cdot 0-6 \cdot 0$

$429 \cdot 470--7 \cdot 5-6 \cdot 7-6 \cdot 5-5 \cdot 0$

6 $29 \cdot 452--7 \cdot 1-5 \cdot 8-0 \cdot 0-5 \cdot 3$

$8 \quad 29 \cdot 439--7 \cdot 0-6 \cdot 0-6 \cdot 0-6 \cdot 0$

10 (2) $418--7 \cdot 0-3 \cdot 0-3 \cdot 0-3 \cdot 8$

Mislt. $29 \cdot 416-\mid-5 \cdot 5-4 \cdot 2-5 \cdot 0-4 \cdot 0$

$\overline{\text { Mean }} \overline{29 \cdot 475} \overline{-}-\overline{-5 \cdot 6-5 \cdot 7}-$

Max. therm, read at $\left\{\begin{array}{l}8 \mathrm{a},-2^{\circ} \cdot 0 . \\ 8 \mathrm{p},-4^{0}, 0 .\end{array}\right.$

Solar rarlation $\left\{\begin{array}{l}\text { black bulb } 41^{\circ} \cdot 0 . \\ \text { bright lulb } 12^{\circ} \cdot 5 .\end{array}\right.$

Meteorological Journal of THE "Discovkity."
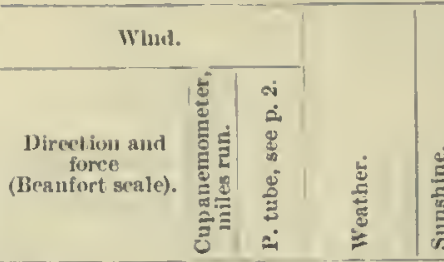

J, 0-1

Calm

Calm

Calm

Calın

Calnı

Is, 1-2

EN1, 3-4

WNE, 2-3

WNF, 1-3
$|22| 20-22 \mid$ bin +

\begin{tabular}{|c|c|c}
11 & 10 & 16 \\
6 & 0
\end{tabular}

\begin{tabular}{l|l|l}
0 & 0 & be \\
0 & inc
\end{tabular}

\begin{tabular}{l|l|l|l}
1 & 0 & b \\
1 & 0 & 1
\end{tabular}

000

4 5-12

$1 1 \longdiv { 2 0 - 2 5 } 0 + *$

\begin{tabular}{ll|l|l|l}
21 & $15-20$ & 0
\end{tabular}

$11 \quad 5-20$ ixeq
Clouts,

Amount and movement.

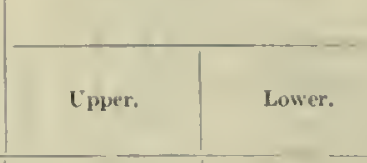

\begin{tabular}{|c|c|}
\hline - & - \\
\hline - & S. 2 \\
\hline- & S. 1 \\
\hline - & S.-en. 2 \\
\hline 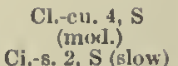 & - \\
\hline Ci.-s. 5, S (slow) & - \\
\hline Ci.-8. 2, S & S.-cu. 5 (t thin) \\
\hline Ci.cu. 2, S & - \\
\hline - & S. 10 \\
\hline- & S. 10 \\
\hline - & Fr.-8. 4 \\
\hline
\end{tabular}

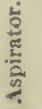

- Alparently a liglit snow fall ratlier $-4 \cdot 7$ than clrift; stars visible, lut a
trifle hazy. $-5 \cdot 1 \quad 4$ a.m. rog aut low Str, rolleyl
$-\quad$ away to $W$.

$-$

- 8.0 Very clear; heavy Cu. SIs to S.

- 10.8 Min. temp. at cape Armitage,

-9.8 In: IIazy; refraetion 15, $S$, and W; lirelus smoke going NIS; lower erater smoke going NW foent. Sliplat mirage all roun.

2 p.m. Fery thin Str, aloug western mountains.

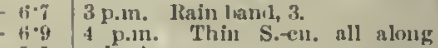

$-5 \cdot 1$
$-5 \cdot 1$

$-5 \cdot 1$
$-2 \cdot 6$

i p.m. ll eavy dirlft all along w p.m. lleary cirlft all along w slite of Strait going from s to in hoar frost.
8 p.m. Iloar frost on thermoMirit. Clours settling down to S.

Fine, but cloudy; becoming over-

fall. Wiud light, rising from $\mathbf{L}$ in afternom, and freshening. Barometer rose mutil 8 a.m. and then fell for the rest of the das. fall. Wind light, ri
Temperature steady.

2nd.

\begin{tabular}{|c|c|c|c|c|c|c|c|c|c|c|c|c|c|}
\hline 2 & $29 \cdot 410$ & - & $|-40|$ & $-2 \cdot 0$ & $|-2 \cdot 0|-3 \cdot 0 \mid$ & EN14, 1-2 & 10 & $5-12$ & 1) & & - & - & $-2 \cdot 2$ \\
\hline 4 & $29 \cdot 381$ & - & $-3 \cdot 0$ & $-2 \cdot 0$ & $-2 \cdot 0-3 \cdot 0$ & WNF, 1 & 5 & 10 & b) & & - & - & $\begin{array}{l}-2.8 \\
-1.8\end{array}$ \\
\hline is & $29 \cdot 368$ & - & $-5 \cdot 5$ & $-5 \cdot 0$ & $\mid-5 \cdot 5-5 \cdot 0$ & Nit by $F, 2-3$ & 18 & $20-25$ & $o g$ & & - & s. 10 & $\begin{array}{l}-2.6 \\
-5.8 \\
-6.2\end{array}$ \\
\hline 8 & $29 \cdot 361$ & - & $-5 \cdot 7$ & $-5 \cdot 0$ & $-5 \cdot 5-5 \cdot 0$ & NI by li, 3-4 & 18 & $20-25$ & $o q *$ & & - & S. 10 & -0 \\
\hline 10 & $29-358$ & $-15 \cdot 2$ & $-6 \cdot 3$ & $-5 \cdot 2$ & $-5 \cdot 3-5 \cdot 1$ & IN $13,3-4$ & 15 & $20-26$ & $o f * *$ & & - & Thln Nim. 10 & $-5 \cdot 4$ \\
\hline Nonn & $29 \cdot 362$ & - & $-5 \cdot 5$ & $-3 \cdot 8$ & $-4 \cdot 0-3 \cdot 2$ & ENE, 2-3 & 14 & $12-20$ & $\circ *$ & & 一 & Thin Nim. 10 & $\begin{array}{l}-30 \\
-\quad 3 \cdot 6 \\
-\quad 4 \cdot 0\end{array}$ \\
\hline 3 & $29 \cdot 3 i 7$ & - & $-7 \cdot 5$ & $-7 \cdot 1$ & $-7 \cdot 6-7 \cdot 0$ & F lyy $\mathrm{S}, 2$ & 11 & $13-20$ & oc & t & Ci.er. 6, S1 & S. 2, Is (mod.) & - \\
\hline 4 & $29-375$ & - & $-9 \cdot 8$ & $-9 \cdot 5$ & $-9 \cdot 3-9 \cdot 4$ & $\mathbf{E}, 3$ & 10 & $22-28$ & b) & 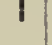 & - & 一 & 一 \\
\hline (i) & $29 \cdot 386$ & - & $-9 \cdot 9$ & $-8 \cdot 7$ & $-9 \cdot 0-8 \cdot 0$ & E\&, 2-3 & 14 & $15-22$ & $\infty$ & & - & S.-e11. J (t hin) & - \\
\hline 8 & $29 \cdot 393$ & - & $-9 \cdot 5$ & $-8 \cdot 8$ & $|-8 \cdot 8|-8 \cdot 5$ & NE, 2-3 & 15 & $15-20$ & b & & - & - & $\begin{array}{l}-8.5 \\
-8.7\end{array}$ \\
\hline 10 & $28 \cdot 398$ & - & $-8 \cdot 7$ & $-8 \cdot 5$ & $-8 \cdot 5-9 \cdot 0$ & LNE, 3 & 13 & $15-23$ & $b+$ & & - & - & $\begin{array}{l}=8.5 \\
=8.5 \\
-8.5\end{array}$ \\
\hline virlt. & $29 \cdot 392$ & - & $-14: 3$ & $-13 \cdot 0$ & $-13 \cdot 0-13 \cdot 5$ & FNF, 3-4 & 18 & $18-25$ & 16 & & 一 & - & - \\
\hline Mean & $29-3382$ & - & - & $-6 \cdot 6$ & $-6 \cdot 7-$ & - & - & - & - & & - & - & - \\
\hline
\end{tabular}

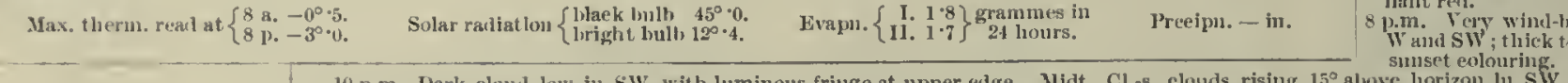

Thick low bank of dark cloud Very gloomy and blaek to $\mathrm{W}$.

Very thick along horizon; snowing slightly. ick all romin; snowflakes hard and small. Mln. teup. at Cape Armitage, $-28^{\circ} \cdot 2$. Whint,

Noon. No land visille. II. In. clear to $\mathrm{S}$; cloudlegs zenith; sumnlts of highest mountalns ulsseured.

3 p.m. Rain bant, 1. from SW ; Str. all round horiroll ; higliest peaks of wertern mountains visible.

p.m. Very thln film of Sir. all round horlzon ; Ci.-eu. passing from SW, eoloured a lorilliant red.

p.m. Very winel-blown Str. to W and SW; thick to S; brilliant

10 p.m. Dark clond low in $s \mathrm{~W}$, with luminous fringe at npper elge. Midt. Cl.-s. clouds rising $15^{\circ}$ above lorizon lin SW. 12.15 a.m. Brilliant met eor descender from zentith to b0 alove horizon, due S. Ove

3rd.

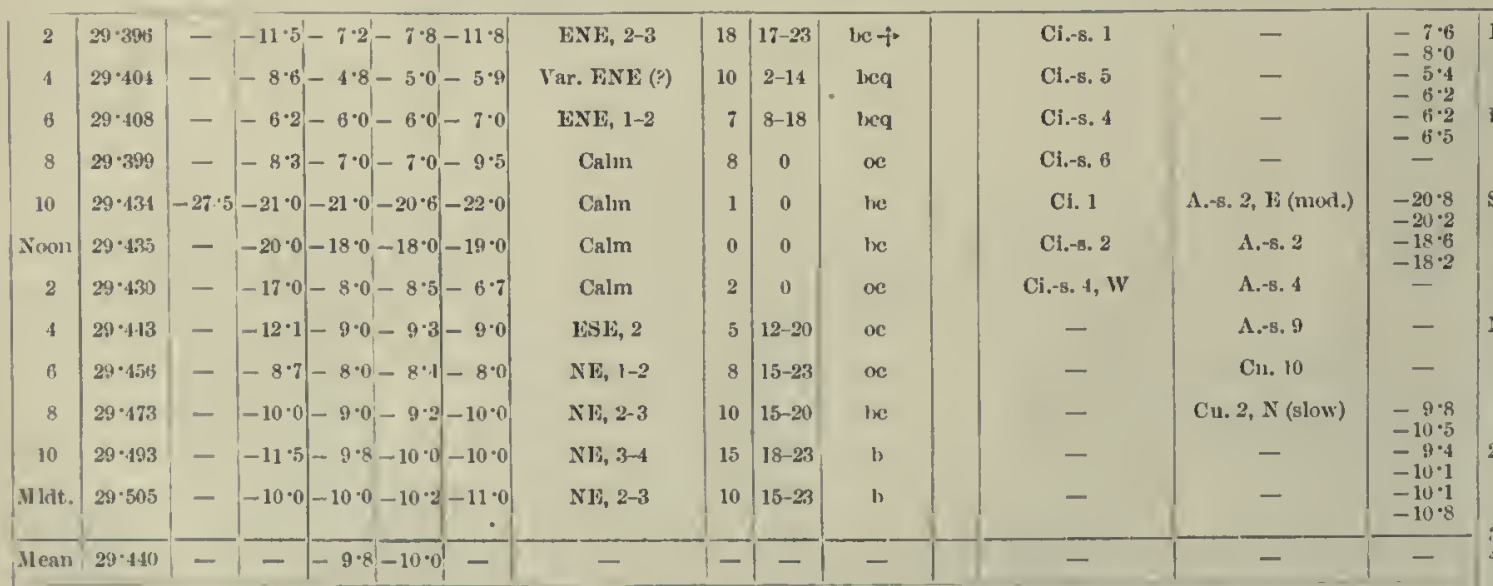

Proad faint auroral streamer, waxing and wanling, 30 alwe SW horizon, extending towards, Refractiou in $\mathrm{SW}$ and 5 .

ight mirage all round; visilility very marked; layers of det. Fir.s. all round; fog at Cape Armitage, $-31^{\circ} \%$; Noou. Strong mirage all round; vou. Strong mirage all round; visibility very marked ; Ci.-s. and wind blown; ligh for coming ln from $\mathrm{N}$; haxy all over sky.

2 p.m. I ipl peaks of westerm mountains ohscured; S1r. all rounl horizon: bigh mlragc tos.

3 1).m. Lain haur, 3.

p.un. Ilorizontal bank of thin Str. With nirage to $\mathrm{W}$; foggs.

p.m. Dark conical masses of
Cu.; clear to $\mathbf{N} W$. Cu.; clear to NW. Max. therm, read at $\left\{\begin{array}{l}8 \mathrm{n},-4^{\circ} \cdot 8 . \\ 8 \mathrm{p},-7^{\circ} \cdot 0 .\end{array} \quad\right.$ Solar radlation $\left\{\begin{array}{l}\text { blaek bulh } 4^{\circ} \\ \text { bright bull, }\end{array}\right.$ 
Meteolological Jounnal of the "Discoveliy."

[1903-April.

\begin{tabular}{|c|c|c|c|c|c|c|c|c|c|c|c|c|c|c|}
\hline \multirow{3}{*}{11 our. } & \multirow{3}{*}{ 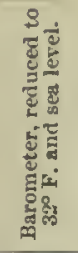 } & \multicolumn{5}{|c|}{ Thermometers. } & \multicolumn{3}{|l|}{ Wind. } & \multirow[b]{3}{*}{ 莣 } & \multirow[b]{3}{*}{ 造 } & \multirow{2}{*}{\multicolumn{2}{|c|}{$\begin{array}{l}\text { Clouls, } \\
\text { Amonnt and movement. }\end{array}$}} & \multirow[b]{3}{*}{$\frac{\frac{5}{8}}{\frac{8}{6}}$} \\
\hline & & \multirow{2}{*}{ 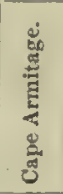 } & \multirow{2}{*}{ 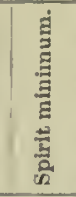 } & \multicolumn{2}{|c|}{ Mlereurial. } & \multirow{2}{*}{ 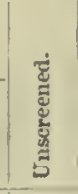 } & \multirow{2}{*}{$\begin{array}{c}\text { Direction and } \\
\text { foree } \\
\text { (Beatifort seale). }\end{array}$} & \multirow{2}{*}{ 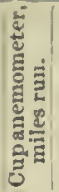 } & \multirow{2}{*}{ 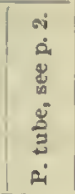 } & & & & & \\
\hline & & & & Dry. & Wet. & & & & & & & Upper. & Lower. & \\
\hline 2 & $29 \cdot 522$ & - & $-10 \cdot 5$ & $-10 \cdot 5$ & $-11 \cdot 0$ & $-12 \cdot 0$ & NE, 2-3 & 13 & $15-23$ & b & & - & & $\begin{array}{l}-10.7 \\
-11.2\end{array}$ \\
\hline 4 & $29 \cdot 536$ & - & $-12 \cdot 0$ & $-12 \cdot 0$ & $-12 \cdot 2$ & $-13 \cdot 0$ & $\mathbf{N E}, 2-3$ & 15 & $15-22$ & b & & - & - & $-12 \cdot 3$ \\
\hline 0 & $29 \cdot 57^{2}$ & - & $-15 \cdot 0$ & $-15 \cdot 0$ & $-15 \cdot 0$ & $-15 \cdot 8$ & Var. NE, 5-6 & 16 & $25-33$ & $11+$ & & - & - & $\begin{array}{l}-13 \cdot 0 \\
-15 \cdot 2\end{array}$ \\
\hline 8 & $29 \cdot 591$ & - & $-16 \cdot 0$ & $-16 \cdot 0$ & $-16 \cdot 0$ & $-15 \cdot 5$ & I) by $\mathbf{N}, 4-5$ & 20 & $20-25$ & $b-t$ & & - & - & $-15 \cdot 5$ \\
\hline 10 & $29 \cdot 624$ & $-24 \cdot 5$ & -15.8 & $-11 \cdot 9$ & $-15 \cdot 1$ & $-15 \cdot 5$ & LNE, 2-4 & $2 . t$ & $20-30$ & le to & & Cl. 2 & - & $-14 \cdot 6$ \\
\hline Noon & $29 \cdot 662$ & - & - & $-18 \cdot 0$ & $-17 \cdot 8$ & $-18 \cdot 0$ & W by $N, 3-4$ & 15 & $25-30$ & $1 x+$ & & Cl. -8.5 & - & $\begin{array}{l}-15 \cdot 2 \\
-17 \cdot 7\end{array}$ \\
\hline 2 & $29 \cdot 685$ & - & $-17 \cdot 6$ & $-16 \cdot 0$ & $-16 \cdot 3$ & $-16 \cdot 0$ & E liy $\mathrm{N}, 3-4$ & 21 & $24-32$ & oe $\rightarrow$ & & Cl. $-8,5, N W$ & S.eu. 3,1 . & -17 \\
\hline 4 & $29 \cdot 700$ & - & $-15 \cdot 7$ & $-15 \cdot 3$ & $-16 \cdot 5$ & $-14 \cdot 8$ & Var. 18, 3-5 & 20 & $25-35$ & xq- & & - & S.cu. 9 & 一 \\
\hline 6 & $29 \cdot 733$ & - & $-17 \cdot 0$ & $-16 \cdot 0$ & $-16 \cdot 0$ & $-16 \cdot 3$ & Var. E, 3-4 & 20 & $22-30$ & $o c$ & & - & A. -8.10 & - \\
\hline 8 & $29 \cdot 763$ & - & $-16 \cdot 5$ & $-16 \cdot 0$ & $-16 \cdot 0$ & $-16 \cdot 0$ & INE, 2-3 & 16 & $10-20$ & $b c$ & & - & A.-ลิ. 2 & $-15 \cdot 0$ \\
\hline 10 & $29 \cdot 762$ & - & $-15 \cdot 0$ & $-14 \cdot 2$ & $-14 \cdot 2$ & $-14 \cdot 5$ & If by $N, 2-4$ & 15 & $19-27$ & b & & - & - & $\begin{array}{l}-10.1 \\
-13.7\end{array}$ \\
\hline Mint. & $29 \cdot 752$ & - & $-15 \cdot 0$ & $-12 \cdot 2$ & $-12 \cdot 6$ & $-13 \cdot 0$ & E by $N, 2-3$ & 16 & $20-25$ & b & & - & - & $\begin{array}{l}-13 \cdot 7 \\
-12 \cdot 1 \\
-13 \cdot 0\end{array}$ \\
\hline Mesn & $29 \cdot 659$ & - & - & $-14 \cdot 7$ & $-14 \cdot 9$ & - & - & - & - & - & & - & - & - \\
\hline
\end{tabular}

Hax. therm. read at $\left\{\begin{array}{l}8 \text { a. }-8^{\circ} \cdot 0 . \\ 8 \text { p. }-14^{\circ} \cdot 5 .\end{array}\right.$ Solar radiatlon $\left\{\begin{array}{l}\text { black luulb } 18^{\circ} \cdot 6 . \\ \text { bright bulb } 8^{\circ} \cdot 0 .\end{array}\right.$

Evapn. $\left\{\begin{array}{l}\text { 1. } 2 \cdot 8 \\ \text { 11. } 2 \cdot 9\end{array}\right\}$ grammes in 2 thours.

Preclpn. - In.

Nlemarks,

Very falint and Illswontuenterl

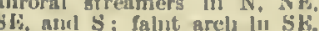
alt. $12^{\circ}$. 4 a.m. is tew sletacherl $\mathrm{Mr},-16$. to 5 , low dowit.

a.m. Low Yr.-s, to $\mathbf{E}$; virry misty on wenteru mountalus vislbility to S. markerl.

0 a.m. A few lands of low Str. half-way mp western inouni talus; visllillty markerl; Cl.-forming ul, from $N$; one or two temp. at Cape Armitage,

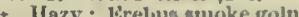
Siv In bir rolume Noon. $\mathrm{Cl}_{1}+\mathrm{B}$. st III thlekest to $\mathrm{N}$ very thlin, thut nore rextenslve overieal; a few streaks of low tr. half-way up western mountalus.

2 p.in. $\mathrm{Cl}_{\text {. }}$-s, overheral; lieavy s.-cu. to 1 .

3 p.m. lain land, 2

p.in. Detached Cu. on wester monntalis, clear to s.

p.m. Clear all roumu; low Nim. p.m.

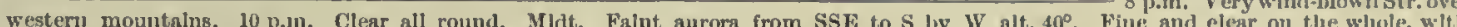
strong to fresh varinble wy wind. Barometer rose until 10 p.m., and then commeneed to fall.' Temperature stendy, but low.

\begin{tabular}{|c|c|c|c|c|c|c|c|c|c|c|c|c|}
\hline 2 & $29 \cdot 731$ & - & $-13 \cdot 0-10^{\prime} 2^{\prime}$ & $-10 \cdot 8^{\prime}-11 \cdot 0$ & F, $2-4$ & 20 & $20-27$ & $b c$ & & - & S. $3, \mathrm{~S}$ & $-10 \cdot 8$ \\
\hline 4 & $29 \cdot 719$ & - & $-12 \cdot 5-11 \cdot 0$ & $-11 \cdot 8-12 \cdot 2$ & E, 1-3 & 15 & $12-20$ & bq & & - & - & $\begin{array}{l}-11 \cdot 6 \\
-11 \cdot 6\end{array}$ \\
\hline 6 & $29 \cdot 701$ & - & $-15 \cdot 5-11 \cdot 8$ & $-12 \cdot 2-12 \cdot 0$ & H, 1-2 & 11 & $10-17$ & $\alpha$ & & - & S. 10 & $\begin{array}{l}-12 \cdot 1 \\
-12 \cdot 6\end{array}$ \\
\hline 8 & $29 \cdot 674$ & - & $-19 \cdot 2-15 \cdot 0$ & $-15 \cdot 5-15 \cdot 5$ & Var. NF, 3-5 & 18 & $25-35$ & oc & & - & S.-eu. 5 & $-13^{\circ}$ \\
\hline 10 & $29 \cdot 677$ & - & $-16 \cdot 0-15 \cdot 5$ & $-16 \cdot 0-16 \cdot 0$ & $\mathrm{E}, 3-5$ & 17 & $20-27$ & beq & & Cl. .8 .3 & - & $-15 \cdot 3$ \\
\hline Noon & $29 \cdot 663$ & - & $-23 \cdot 2-22 \cdot 5$ & $-22 \cdot 5-22 \cdot 6$ & Var. 1., 3-5 & 19 & $20-20$ & 1.eq fo & 1 & $\mathrm{Cl} .-8.4$ & - & $\begin{array}{l}-15 \cdot 9 \\
-22 \cdot 4\end{array}$ \\
\hline 2 & $29 \cdot 635$ & - & $-22 \cdot 5-21 \cdot 0$ & $-21 \cdot 0-21 \cdot 0$ & Var. J, 4-6 & 24 & $28-37$ & exq + & I & $\mathrm{Cl}_{.}-\mathrm{s} .4$ & A.-8. 5 & -227 \\
\hline 4 & $29 \cdot 601$ & - & $-21 \cdot 6-21 \cdot 3$ & $-21 \cdot 2-22 \cdot 5$ & Var. N, $4-t ;$ & 23 & $28-38$ & oeq $\mathrm{ft}$ & I & - & S.ccu. 10 & - \\
\hline 6 & $29 \cdot 578$ & 一 & $-20 \cdot 5-20 \cdot 5$ & $-20 \cdot 5-21 \cdot 0$ & Var. 15, 5-0 & 23 & $28-35$ & $a c t$ & & - & S.-cu. 10 & - \\
\hline 8 & $29 \cdot 560$ & - & $-20 \cdot 0-17 \cdot 2$ & $-17 \cdot 8-18 \cdot 0$ & Tar. E, 4-5 & 22 & $20-27$ & $b$ & & - & - & $\begin{array}{r}-18 \cdot 0 \\
-18 \cdot 4\end{array}$ \\
\hline 10 & $29 \cdot 556$ & - & $-20 \cdot 5-20 \cdot 0$ & $-20 \cdot 2-21 \cdot 0$ & F by $\mathbf{N}, 5$ & 20 & $25-28$ & b) & & - & - & $-20^{\circ} \cdot$ \\
\hline Midt. & $29 \cdot 515$ & - & $-19 \cdot 5-17 \cdot 0$ & $-17 \cdot 2-19 \cdot 0$ & Far. E, 5 & 27 & $25-30$ & $10+$ & & - & - & $\begin{array}{r}-18.0 \\
-18.4\end{array}$ \\
\hline Mean & $29 \cdot 634$ & - & $-\quad-16 \cdot 9$ & $\overline{-17 \cdot 2}-$ & - & - & - & - & & - & - & - \\
\hline
\end{tabular}

Max. therm. read at $\left\{\begin{array}{l}8 \text { a. }-10^{\circ} 0 . \\ 8 \text { p. }-15^{\circ} 0 .\end{array} \quad\right.$ Solar radiation $\left\{\begin{array}{l}\text { black bull, } 20^{\circ} \cdot 4 . \\ \text { bright bulb } 12^{\circ} \cdot 3 .\end{array} \quad\right.$ Kvapn. $\left\{\begin{array}{c}1.5 \cdot 7 \\ 11.5 \cdot 7\end{array}\right\} \begin{array}{c}\text { grammes in } \\ 24 \text { hours. }\end{array}$
Precipn. - In.
One continuous auroral streamer from alt. $30^{\circ}$ SSir throus th to $\mathrm{NNF}$; faint patch in SW. alt. $50^{\circ}$; Str. elouds obseurln tops of Black and White Island and Bluf

4 a.m. $\Delta$ few rapged $S t r$ to $S$ and SE moving to $\mathrm{N}$ slowly.

6 a.m. Overcast all over except in $S$, where there was a cles sky below $20^{\circ}$ alt.; clark to

A.m. Cl,-so, radiating $\mathbf{N}$ and $\mathrm{S}$ sighit mirage all roun ;

voos. Very wilul-hlown Cl-s. clear all round; hazy ln sky.

12.30 y.m. Temp. at Cape Arm trge, $-31^{\circ} \cdot 2 ;$ mill., $-35^{\circ} \cdot 13$ Wlind liNE, 3. $\{$ ". Frebus smoke golung SW.

2 p.m. Clear all round horlzon Str. on summlts of hlghest peaks. IRaln land, 4.

p.m. Very dark and threaten ing to $\mathrm{N}$ and $\mathrm{N} ;$ heavy drlft in

8 p.m. Windy Str. to $S$ and $\mathrm{SW}$. 10 p.m. Fr.-s. tosw . $300^{\circ}$ alt.

Overeast and eloudy most of the day, with strong LNW wind. Barometer falling all day. Temperature lower and fairly steady.

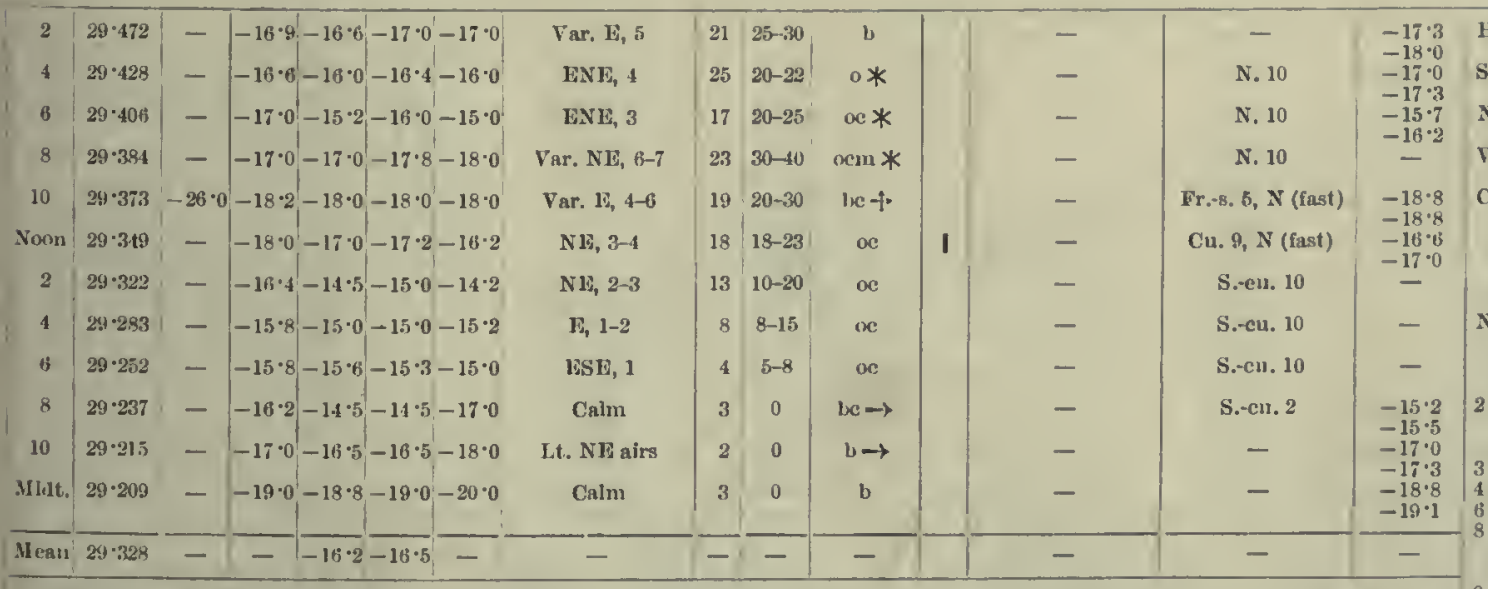

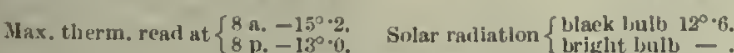

Heavy gusts of wind; sky dull to sky a leaden grey.

No land visible exeept to $\mathrm{E}$.

Fery dirty and thlek all round wind verylug ND to 1 ?

earing rapldty ; very tilek drlf II Stralt; western inountain ing whe lear sky behlne. $-31^{\circ}$. Wind $\mathrm{N}$. W trebus obscured.

Soon. Fery elear below $5^{\circ}$ alt. vlsibility marked; dark nai henry eloud to E; light thl Str. on western mountalis.

p.m. Looming to $W$ and SW inirage to $\mathrm{S}$ sind along foot of western momntalns.

p.m. lRaln harit,

p.m. roggy to $\mathrm{s}$.

p.m. Very heavy Str, to NW, extenling to $S$; lee erystals (alling.

30 p.lin. lee crystals falling perfertly elear sky: stars very birlght.
lidt. Dense rime ou solur ther. Nilt. Dense rime ous solns ther. mometer. Overenst and eloudy most of the dhy
Barometer falling all day. Temperature st.early.
5 th.

th. 


\begin{tabular}{|c|c|c|c|c|c|c|}
\hline \multirow{3}{*}{ Hour. } & \multirow{3}{*}{ 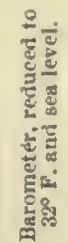 } & \multicolumn{5}{|c|}{ Thermomcters. } \\
\hline & & ذ్ & E & Men & urlal. & \\
\hline & & \&ु. & 竞 & Dry. & Wet. & है \\
\hline
\end{tabular}

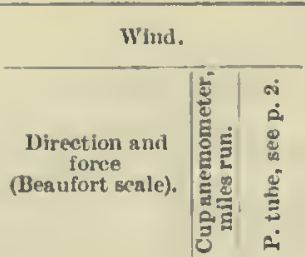
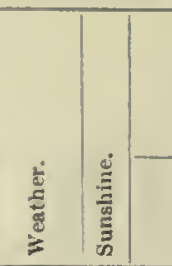

Irean $20 \cdot 26 \cdot$

$229 \cdot 185--25 \cdot 5-20 \cdot 0-20 \cdot 0-24 \cdot 6$

\begin{tabular}{l|l|l|l|} 
SW, 1 & 2 & 10 & $b \rightarrow$
\end{tabular}

$429 \cdot 175--27 \cdot 0-22 \cdot 3-22 \cdot 2-28 \cdot 0$

Calm

$629 \cdot 109--23 \cdot 0-14 \cdot 0-14 \cdot 0-14 \cdot 2$

$829 \cdot 179--15 \cdot 0-15 \cdot 0-15 \cdot 0-15 \cdot 0$

$10 \quad 29 \cdot 177-33 \cdot 1-18 \cdot 8-18 \cdot 0-18 \cdot 0-19 \cdot 0$

Noon $29 \cdot 194--18 \cdot 8-18 \cdot 8-18 \cdot 8-21 \cdot 1$

$229 \cdot 228-\quad-27 \cdot 4-27 \cdot 0-30 \cdot 0$

$429 \cdot 275-28 \cdot 0 \quad-21 \cdot 2-22 \cdot 0-20 \cdot 6$

$6 \quad 29 \cdot 325--33 \cdot 0-31 \cdot 0-31 \cdot 5-39 \cdot 0$

$829 \cdot 376--30 \cdot 0-19 \cdot 8-19 \cdot 8-21 \cdot 0$

$1029 \cdot 427--26 \cdot 0 \quad-25 \cdot 0-25 \cdot 4-27 \cdot 0$

Mldit. $29 \cdot 467--25 \cdot 8 \quad-18 \cdot 7-19 \cdot 0-20 \cdot 0$

Var. $\mathrm{N}, 1$

Lt. Eairs

NE, 1

Calm

Calm

Calm

Calm

ESE, 1

$\overrightarrow{-20 \cdot 9}-21 \cdot 1=$

t. $\mathrm{F}$ by $\mathrm{N}$ alrs

NE, 1-2 \begin{tabular}{l|l|l}
3 & 0 & $0 *$
\end{tabular}

${ }_{4}^{4} c_{8-10}^{0} \quad 0 *$

3. 8 lm

\begin{tabular}{|c|c|c|}
5 & 0 & $b$ \\
2 & 0 & $b$ \\
\hline & 0 & $b$
\end{tabular}

\begin{tabular}{|l|l|l|}
1 & 0 & $b$ \\
0 & 0 & $b$
\end{tabular}

7 $\quad 8-10$

\begin{tabular}{r|r|c|}
5 & $0-10$ & b \\
11 & $15-20$ & b
\end{tabular}

$-$

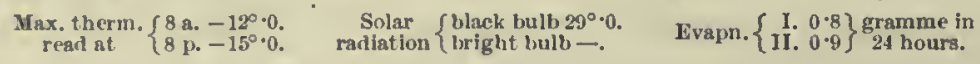

Preclpn, 0.02 in. since 6 p.m., 6 th.
Clouds,

Amount and movement.

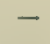

N. 10

N. 10

N. 10

$-13 \cdot 7$
$-13 \cdot 7$

$-\quad-18 \cdot 4$

$-18 \cdot 4$
$-18 \cdot 8$

$-18 \cdot 8$
$-18 \cdot 8$

$-$

$-$

$-$

$-$

$-$

\begin{tabular}{|c|c|c|c|c|c|c|c|c|c|c|c|c|}
\hline 2 & $29 \cdot 476$ & - & $-18 \cdot 1$ & $|-18 \cdot 0|-18 \cdot 1|-18 \cdot 2|$ & $\mathrm{NE}, 1$ & 12 & $10-15$ & $8 w$ & - & - & $\begin{array}{l}-16.6 \\
-17.3\end{array}$ & Briglit aurora with well-marked \\
\hline 4 & $29 \cdot 469$ & - & $-21 \cdot 6$ & $-19 \cdot 2-19 \cdot 2-20 \cdot 6$ & $\mathrm{NE}, 1$ & 3 & $10-15$ & b & Ci.-s. 2 & - & $-18 \cdot 0$ & Bright greenish beams ln $N$, \\
\hline 6 & $29 \cdot 461$ & - & $-18 \cdot 0$ & $-13 \cdot 3-13 \cdot 6-14 \cdot 8$ & NE, 1 & $6^{2}$ & $5-10$ & b & - & - & $\begin{array}{l}-18.6 \\
-14.4\end{array}$ & $\begin{array}{l}\text { arch to fo and s ; some broken } \\
\text { Ci. to } \mathrm{NE} \text {. A urora was plainiy }\end{array}$ \\
\hline 8 & $29 \cdot 4.10$ & 一 & $-19 \cdot 0$ & $-19 \cdot 0-18 \cdot 5-20 \cdot 0$ & Calm & 6 & 0 & $\mathrm{~b}$ & - & - & - & \\
\hline 10 & $29 \cdot 423$ & - & $-23 \cdot 0$ & $-15 \cdot 6-15 \cdot 6-16 \cdot 6$ & Lt. $N$ airs & 3 & $0-5$ & bᄂ & - & - & $\begin{aligned}-15 \cdot 3 \\
-150\end{aligned}$ & Excessive mirage all round : \\
\hline Noon & $29 \cdot 421$ & - & $-30 \cdot 2$ & $-30 \cdot 2-30 \cdot 0-32 \cdot 5$ & Calm & 1 & 0 & b.w & - & - & Mercury & frost rime on thermometer. \\
\hline 2 & $29 \cdot 444$ & - & $-29 \cdot 5$ & $-18 \cdot 0-18 \cdot 3-20 \cdot 0$ & Calm & 3 & $0-5$ & $b$ & - & - & - & tage, $-50^{\circ} 0$ min., $-50^{\circ} 0$ \\
\hline 4 & $29 \cdot 458$ & - & $-18 \cdot 7$ & $-16 \cdot 8-17 \cdot 0-19 \cdot 8$ & $\mathrm{E}, 1$ & 7 & $5-10$ & b & - & - & - & on SE horizon; much mirage \\
\hline 6 & $29 \cdot 486$ & - & $-17 \cdot 5$ & $-16 \cdot 3-16 \cdot 8-18 \cdot 0$ & E, 1 & 6 & $0-8$ & b & - & - & - & NNE or $\mathrm{NI}^{1 /}$ (mostly lifddcn \\
\hline 8 & $29 \cdot 536$ & - & $-25 \cdot 0$ & $-25 \cdot 0 \mid-25 \cdot 0-28 \cdot 0$ & Calm & 5 & 0 & $b$ & - & - & 8 p.m. to & thermometer. \\
\hline 10 & $29 \cdot 55 \mathrm{~A}$ & - & $-28 \cdot 2$ & $|-28 \cdot 2|-28 \cdot 1-$ & Calm & 2 & 0 & $b=$ & - & - & Mercury & ronind; frost rime on therno- \\
\hline Midt. & $29 \cdot 586$ & - & $-33 \cdot 2$ & $-27 \cdot 0-24 \cdot 9-27 \cdot 0$ & $N$ by $\mathbf{E}, 0-1$ & 0 & $10-14$ & $b=$ & - & - & scale & down; Str. to $\mathrm{I}$ and $\mathrm{N}$ ln \\
\hline diean & $29 \cdot 480$ & - & - & $-20 \cdot 6-20 \cdot 6-$ & - & - & -1 & - & - & - & - & 2 p.n. Mirage ail round, \\
\hline \multicolumn{8}{|c|}{ Max, therur, read at $\left\{\begin{array}{l}8 \mathrm{a} .-11^{\circ} \circ \\
8 \mathrm{p} .-15^{\circ} \circ\end{array}\right.$} & . & $a m m$ & \multicolumn{2}{|c|}{ Precipn. - in. } & $\begin{array}{l}4 \text { p.m. Small detached specks } \\
\text { of cloud on summilts of } \\
\text { western mountains. }\end{array}$ \\
\hline
\end{tabular}

\begin{tabular}{|c|c|c|c|c|c|c|c|c|c|c|c|c|}
\hline 2 & $29 \cdot 620$ & - & $-31 \cdot 0$ & $-26 \cdot 5-20 \cdot 3-26 \cdot 2$ & Calm & 0 & 0 & $\mathrm{bm}$ & - & - & Mercury & Faint auror \\
\hline 4 & $29 \cdot 651$ & - & $-29 \cdot 0$ & $-18 \cdot 0-17 \cdot 9-25 \cdot 0$ & Caim & 0 & 0 & b & - & - & & round horizon from $S$ to $W$ \\
\hline 6 & $29 \cdot 681$ & - & $-29 \cdot 2$ & $-22 \cdot 5-22 \cdot 2-22 \cdot 2$ & NNE, $0-1$ & 2 & 0 & $1 x$ & $\mathrm{Ci}_{-}-\mathrm{B}, \mathrm{I}$ & 一 & $-15 \cdot 5$ & alt. $0^{\circ}$; cioudless sky; mlst \\
\hline 8 & $29 \cdot 719$ & - & $-25 \cdot 2$ & $-15 \cdot 8-15 \cdot 2-15 \cdot 8$ & Lt. E airs & 5 & 0 & b & - & - & $-16^{-2}$ & 6 a.m. Light $\mathrm{C}$ i.-s. clouds low \\
\hline 10 & $29 \cdot 763$ & - & $-19 \cdot 5$ & $-15 \cdot 5-15 \cdot 5-15 \cdot 5$ & $\mathrm{NE}, 0-2$ & ? & $0-15$ & be & $\mathrm{Cl} .-8.4$ & - & $\begin{array}{l}-15 \cdot 3 \\
-15 \cdot 5\end{array}$ & $\begin{array}{l}8 \text { a.m. Il Ieavy S.-cn. to W. } \\
10 \text { g.m. Ci.-8. radlating NIF, }\end{array}$ \\
\hline Noon & $29 \cdot 750$ & 一 & $-18 \cdot 0$ & $-14 \cdot 0-14 \cdot 2-15 \cdot 2$ & NE, 2-3 & 10 & $15-20$ & lec + & Ci. $1, \underset{\text { (fast) }}{\mathrm{Cl}-8,2}, \mathrm{SE}$ & - & $\begin{array}{l}-14 \cdot 1 \\
-14 \cdot 4\end{array}$ & $\begin{array}{l}\text { Sti; low lank of fog all rouma } \\
\text { horizon from S to iv : mucl }\end{array}$ \\
\hline 2 & $29 \cdot 772$ & - & $-20 \cdot 8$ & $-20 \cdot 0-20 \cdot 0-22 \cdot 0$ & IESE, 1-2 & 5 & $10-20$ & oc & $\stackrel{-1}{-}$ & A.-cu. $9, \mathbf{E}$ & - & frost rime on thernometers. \\
\hline 8 & $29 \cdot 702$ & - & $-17 \cdot 5$ & $-17 \cdot 0-17 \cdot 0-16 \cdot 2$ & Calm & 0 & 0 & o & - & S. 10 & - & $\begin{array}{c}\text { on guys. } \\
\text { Noon. Ci.-8. radiating NW, SE; }\end{array}$ \\
\hline 10 & $29 \cdot 655$ & - & $-17 \cdot 0$ & $-13 \cdot 0-13 \cdot 1-12 \cdot 8$ & Caim & 2 & 0 & om & - & S. 10 & - & $\begin{array}{l}\text { Ci,-s, to } N \text { and } W \text {; loggy to } N \\
\text { low down; very fleeey and }\end{array}$ \\
\hline Minlt. & $29 \cdot 602$ & $\rightarrow$ & $-15 \cdot 0$ & $-8 \cdot 6-9 \cdot 0-9 \cdot 0$ & Calm & 2 & 0 & om $*$ & - & N. 10 & - & wind-blowin Ci.s, and $\mathrm{Cl}$. \\
\hline \multirow[t]{2}{*}{ Mean } & $29 \cdot 697$ & - & - & $-17 \cdot 0-17 \cdot 0-$ & - & - & - & $\overline{-}$ & - & - & - & 2 p.In. Bxcessive mirage to $W$ \\
\hline & \multicolumn{4}{|c|}{ Max. therin. reat at $\left\{\begin{array}{l}8 \mathrm{a},-14^{\circ} \cdot 8 . \\
8 \mathrm{p} .11^{\circ} \cdot 0 .\end{array}\right.$} & \multicolumn{4}{|c|}{ Solar radiation $\left\{\begin{array}{l}\text { black bulb } 15^{\circ} \cdot 5 . \\
\text { bright buib }\end{array}\right.$} & $-g$ & \multicolumn{2}{|c|}{ Precipn. - in. } & $\begin{array}{l}3 \text { p.m. Rain irand, } 3 \text {. } \\
4 \text { p.m. Fxcessive mirage along } \\
\text { foot of western mountains } \\
\text { very windy appearance. }\end{array}$ \\
\hline
\end{tabular}


Meteonological Journat, of the "Discovery."

[1903-April.

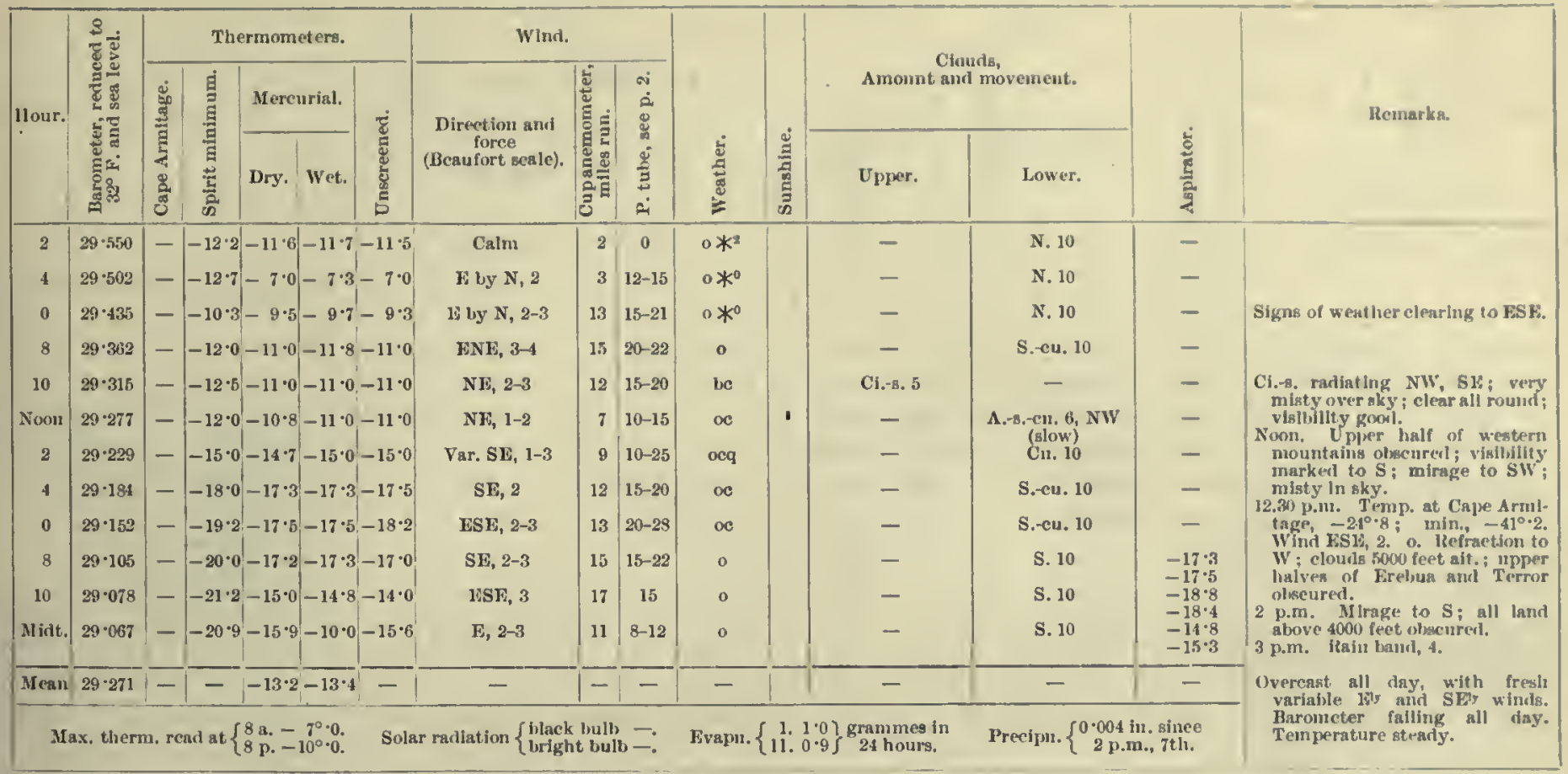

10 th.

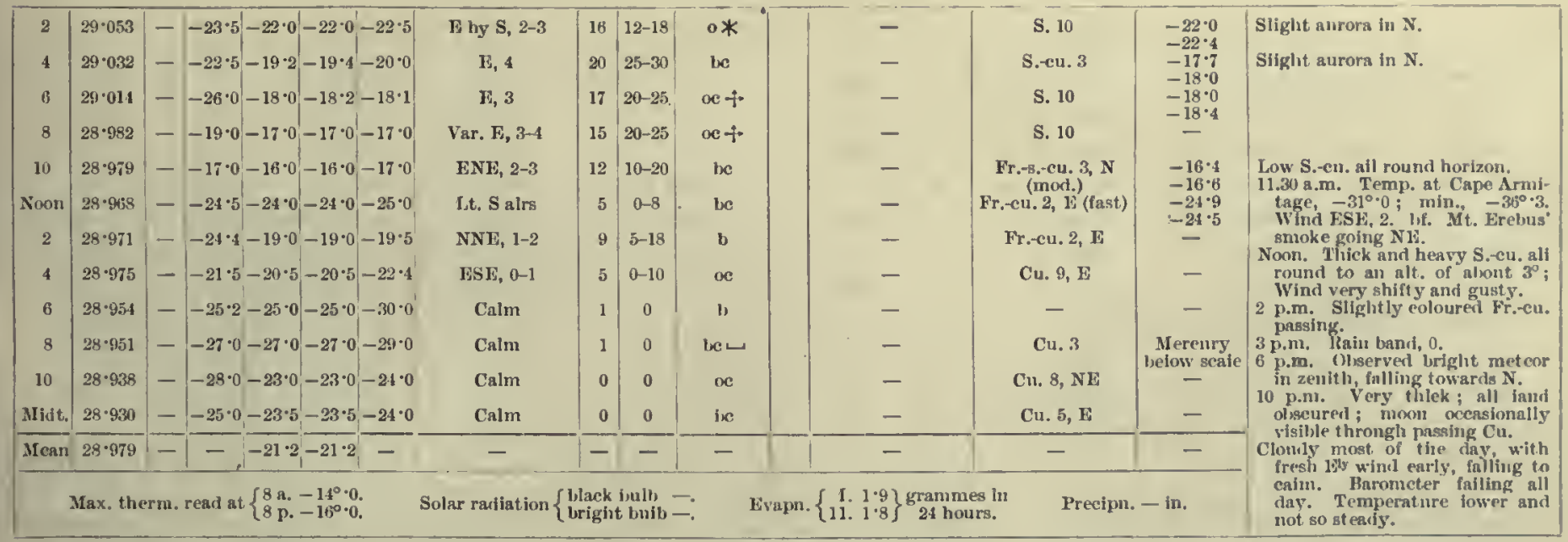

11 th.

\begin{tabular}{|c|c|c|c|c|c|c|c|c|c|c|c|}
\hline 2 & $28 \cdot 908$ & - & $-24 \cdot 0^{\prime}-24 \cdot 0-24 \cdot 0-24 \cdot 0$ & Calm & 0 & 0 & tec & - & Cu. 4, NE & - & \multirow{14}{*}{ 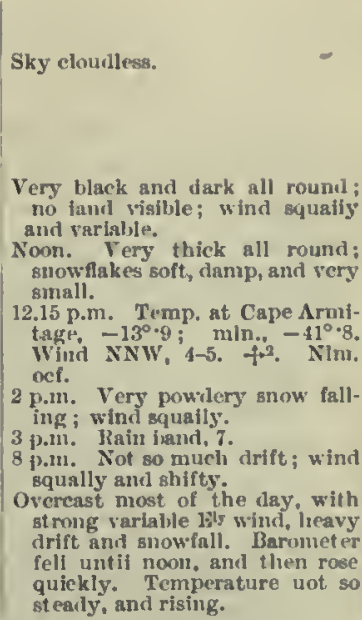 } \\
\hline 4 & $28 \cdot 870$ & - & $-30 \cdot 0-23 \cdot 0-23 \cdot 0-25 \cdot 0$ & L.t. $\mathbf{N}$ alra & 0 & $\mathbf{0}$ & b & - & - & - & \\
\hline 6 & $28 \cdot 854$ & - & $-28 \cdot 0-19 \cdot 0-19 \cdot 0-18 \cdot 0$ & Lt. $\mathbf{N}$ airs & 0 & 0 & b & - & - & - & \\
\hline 8 & $28 \cdot 835$ & - & $-23 \cdot 0-19 \cdot 0-19 \cdot 0-18 \cdot 0$ & Lt. $\mathbf{N}$ airs & 4 & 0 & oc & - & S.cu. 8 & - & \\
\hline 10 & $28 \cdot 803$ & - & $-20 \cdot 5-9 \cdot 0-10 \cdot 0-9 \cdot 5$ & NF, 1-3 & 12 & $8-20$ & $o q$ & - & S. 10 & $-9 \cdot 4$ & \\
\hline Noon & $28 \cdot 795$ & - & $-9 \cdot 7-7 \cdot 2-7 \cdot 6-7 \cdot 0$ & N. 3-4 & 15 & $15-27$ & $\infty+*$ & - & N. 10 & $\begin{array}{l}-10.1 \\
-7.4\end{array}$ & \\
\hline 2 & $28 \cdot 800$ & - & $-7 \cdot 7-5 \cdot 8-6 \cdot 0-5 \cdot 0$ & $N, 2-4$ & 17 & $15-30$ & oq $+* *$ & - & N. 10 & $-7 \cdot 8$ & \\
\hline 4 & $28 \cdot 921$ & - & $-10 \cdot 5-10 \cdot 2-10 \cdot 0-12 \cdot 0 \mid$ & E iny $\mathbf{S}, 2-\mathbf{A}$ & 11 & $18-32$ & og $+^{2} *$ & - & N. 10 & - & \\
\hline 0 & $28 \cdot 957$ & - & $|-19 \cdot 0-17 \cdot 3|-17 \cdot 0-19 \cdot 5)$ & Var. E, 4-6 & 18 & $28-40$ & $o q++^{2} *$ & - & N. 10 & - & \\
\hline 8 & $29 \cdot 009$ & - & $-19 \cdot 0-15 \cdot 0-15 \cdot 0-14 \cdot 5$ & Var. $\mathbf{E}, 4-6$ & 28 & $20-30$ & $o q+* *$ & - & N. 10 & - & \\
\hline 10 & $29 \cdot 0.13$ & - & $-15 \cdot 0-10 \cdot 0-10 \cdot 0-10 \cdot 0$ & E lyy $S, 2-4$ & 25 & $10-20$ & $o+f$ & - & N. 10 & - & \\
\hline Mlat. & $29 \cdot 049$ & - & $-13 \cdot 5-8 \cdot 0-8 \cdot 0-8 \cdot 0$ & ENE, 2-3 & 9 & $10-15$ & $\infty$ & - & Ci1. 10 & - & \\
\hline Mean & $28 \cdot 904$ & - & $--14 \cdot 0-1.1 \cdot 1-$ & - & - & - & - & - & - & - & \\
\hline & th & & $\left\{8 \mathrm{a},-13^{\circ} \cdot 8\right.$ & Solar rallation & lack & oull, & & $\left\{\begin{array}{cc}1 . & 0 \cdot 3 \\
\text { II. } & 0 \cdot 3\end{array}\right\}$ & \multicolumn{2}{|c|}{ Precipn., - in. } & \\
\hline
\end{tabular}




\begin{tabular}{|c|c|c|c|c|c|c|c|c|c|c|c|c|c|c|}
\hline \multirow{3}{*}{ Ilonr. } & \multirow{3}{*}{ 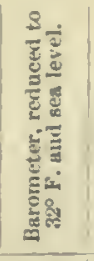 } & \multicolumn{4}{|c|}{ Thormoneters. } & \multicolumn{3}{|l|}{ Whus. } & \multirow[b]{3}{*}{ 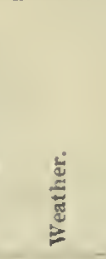 } & \multirow[b]{3}{*}{ 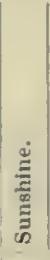 } & \multirow{2}{*}{\multicolumn{2}{|c|}{$\begin{array}{l}\text { Chourds, } \\
\text { Amount and movement. }\end{array}$}} & \multirow[b]{3}{*}{$\frac{\frac{5}{5}}{\frac{d}{2}}$} & \multirow{3}{*}{ Remarks. } \\
\hline & & \multirow{2}{*}{ 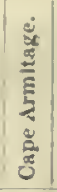 } & \multirow{2}{*}{ 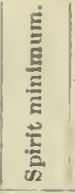 } & \multirow{2}{*}{ Dry. Wet. } & \multirow{2}{*}{ 递 } & \multirow[b]{2}{*}{$\begin{array}{c}\text { Direction and } \\
\text { force } \\
\text { (Beanfort scalc). }\end{array}$} & \multirow{2}{*}{ 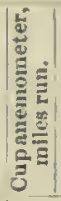 } & \multirow{2}{*}{ 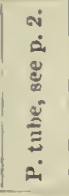 } & & & & & & \\
\hline & & & & & & & & & & & Upper. & Lower. & & \\
\hline 2 & $29 \cdot 0 ; 8$ & - & $-10 \cdot 5$ & $-8 \cdot 0-8 \cdot 0$ & -0.5 & Caln & 6 & 0 & inc & & - & $\mathrm{Cu}, 9, \mathrm{NF}$ & - & \\
\hline 4 & $29 \cdot 095$ & - & $-9 \cdot 5$ & $-6 \cdot 0-6 \cdot 0$ & $-5 \cdot 0$ & CaIm & 4 & 0 & be & & 一 & Cu. 8, NF & - & Sky clearing in SW; waek and \\
\hline 6 & $29 \cdot 125$ & - & -9.5 & $-8 \cdot 0-8 \cdot 0$ & $-7 \cdot 0$ & Culn & 0 & 0 & oc & & - & N. 10 & - & threatening in $\mathrm{N}$. \\
\hline 8 & $29 \cdot 197$ & - & $-16 \cdot 0$ & $-i \cdot 0-8 \cdot 0$ & $-6 \cdot 0$ & ENL, 1-2 & 0 & $8-12$ & oc $f$ & & - & N. 10 & - & Low rrift. \\
\hline 10 & $29 \cdot 237$ & - & $-18 \cdot 8$ & $-16 \cdot 8-16 \cdot 8$ & $-16 \cdot 2$ & Var. SW, 4-6 & 14 & $20-33$ & $o q+* *$ & & - & N. 10 & - & Iteavy drift; no land visithe. \\
\hline Xoon & $29 \cdot 297$ & - & $-21 \cdot 0$ & $-19 \cdot 0-19 \cdot 0$ & $-18 \cdot 8$ & Var. $\mathrm{N}, 4-6$ & 22 & $20-30$ & oq $+* *$ & & - & N. 10 & - & 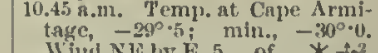 \\
\hline 2 & $29 \cdot 328$ & - & $-25 \cdot 0$ & $-24 \cdot 0-2 \cdot \cdot 0$ & $-24 \cdot 5$ & Var, 1, 5-7 & 31 & $30-40$ & oq $+* *$ & & - & N. 10 & - & 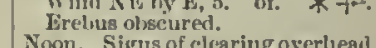 \\
\hline 4 & $29 \cdot 352$ & - & $-25 \cdot 6$ & $-24 \cdot 6-24 \cdot 3$ & $-28 \cdot 0$ & ENW, 5-7 & 26 & $30-42$ & heq $f$ & & Ci.-cu. 4, NI & - & - & $\begin{array}{l}\text { Noon. Signs of chraring overhesul } \\
\text { and to } \mathrm{SH} \text {; wind still varfahle }\end{array}$ \\
\hline 6 & $29 \cdot 3 \%$ & - & $-21 \cdot 4$ & $-21 \cdot 0-21 \cdot 0$ & $-21 \cdot 0$ & Fis by N, 4-6 & 31 & $25-35$ & ocq + & & - & Fr.-n. 8 & - & 2 p.m. Clouds very thin in \\
\hline 8 & $29 \cdot 367$ & - & $-26 \cdot 0$ & $-17 \cdot 0-17 \cdot 0$ & $-16 \cdot 2$ & Var. 2-6 & 24 & $15-28$ & hqm + & & 1) - & - & - & geufth ; gental appearance of \\
\hline 10 & $29 \cdot 359$ & - & $-16 \cdot 8$ & $-7 \cdot 5-7 \cdot 8$ & $-7 \cdot 8$ & Lt. $\mathrm{NE}$ airs & 10 & 0 & oc & & - & Cu. $8, \mathrm{NE}$ (fast) & - & $\begin{array}{l}3 \text { p.m. loain hand, } 2 \text {. } \\
6 \text { p.m. Joon gleaming and show. }\end{array}$ \\
\hline Mirlt. & $29 \cdot 344$ & - & $-13 \cdot 0$ & $-10 \cdot 0-10 \cdot 0$ & $-10 \cdot 8$ & Lt. $N$ airs & $4 !$ & 0 & $\alpha$ & & - & Cu. 8 , NE (not.) & - & $\begin{array}{l}\text { ing hetween squals. } \\
8 \text { p.m. Clear overhear ; very }\end{array}$ \\
\hline Mean & $29 \cdot 262$ & - & 一 & $-14 \cdot 1-14 \cdot 2$ & - & - & -1 & - & - & & - & $\cdot-$ & - & Overe'sist and eloury all day, witl \\
\hline 3 & the & r & at & 8 a. $-6^{\circ} \cdot 0$ & Sol & diation \{llae & bulth & -. & Evapu & & rramo in & Precipn & in. & $\begin{array}{l}\text { and rrifting. Barometer rose } \\
\text { mntil } 8 \text { p.m., and then rom- } \\
\text { menced to fall. Temperature } \\
\text { very unsteady, with big drop in } \\
\text { aflernoon. }\end{array}$ \\
\hline
\end{tabular}

14th.

\begin{tabular}{|c|c|c|c|c|c|c|c|c|c|c|c|c|}
\hline 2 & $29 \cdot 330$ & - & $-15 \cdot 8$ & $-14 \cdot 2-14 \cdot 2-14 \cdot 0$ & Calm & 2 & 0 & o* & & - & N. 10 & - \\
\hline 4 & $29 \cdot 308$ & - & $-14 \cdot 8$ & $-13 \cdot 8-13 \cdot 8-13 \cdot 8$ & Calm & 2 & 0 & o* & & - & N. 10 & - \\
\hline 6 & $29 \cdot 279$ & - & $-15 \cdot 5$ & $-13 \cdot 0-13 \cdot 0-13 \cdot 0$ & Calm & 1 & 0 & $o *$ & & - & N. 10 & - \\
\hline 8 & $29 \cdot 255$ & - & $-14 \cdot 0$ & $-10 \cdot 0-10 \cdot 5-10 \cdot 0$ & Calm & 0 & 0 & om * & & - & N. 10 & - \\
\hline 10 & $29 \cdot 228$ & - & $-14 \cdot 0$ & $-8 \cdot 8-8 \cdot 8-13 \cdot 0$ & Calm & 1 & 0 & he & & Ci. $1, \mathrm{~S}$ & Fr,-a.-cu, 3, SW & - \\
\hline $\mathbf{N}_{(\leftarrow m)}$ & $29 \cdot 207$ & - & $-19 \cdot 2$ & $-13 \cdot 2-13 \cdot 2-14 \cdot 0$ & Calm & - & 0 & lie & 1 & Ci. $4, \mathrm{~s}$ & Fr.-8.-s. 2, SW & - \\
\hline 2 & $29 \cdot 179$ & - & $-17 \cdot 7$ & $-14 \cdot 6-14 \cdot 8-18 \cdot 8$ & Calm & - & 0 & ixc & 1 & Ci.-eu. $2, \mathrm{~s}$ & Fr.-cul. 2, w W & - \\
\hline 4 & $29 \cdot 160$ & - & $-14 \cdot 1$ & $-12 \cdot 8-12 \cdot 8-15 \cdot 0$ & Calm & - & 0 & be & & - & A.-en. $8, W$ & - \\
\hline 6 & $29 \cdot 142$ & - & $-13 \cdot 8$ & $-10 \cdot 0-10 \cdot 0-9 \cdot 0$ & $N \lg W, 0-1$ & - & $0-8$ & oc & & - & N. 10 & - \\
\hline 8 & $29 \cdot 111$ & - & $-17 \cdot 0$ & $\begin{array}{llll}0.5 & 0.0 & 1.2\end{array}$ & $\mathrm{~N}, 1$ & - & $5-10$ & 0 & & - & S. 10 & $1 \cdot 0$ \\
\hline 10 & $29 \cdot 076$ & - & $-2 \cdot 5$ & \begin{tabular}{|l|l|l|}
1.0 & 0.4 & 1.0 \\
\end{tabular} & ENE, 1-2 & - & $8-10$ & $\circ *$ & & - & N. 10 & 1.8 \\
\hline Mintt. & $29 \cdot 0.038$ & - & $-i \cdot i$ & $-4 \cdot 5-4 \cdot 5-4 \cdot 2$ & FSE, 2-3 & - & $10-15$ & $\circ *$ & & - & N. 10 & - \\
\hline Meran & $29 \cdot 1993$ & - & - & $-9 \cdot 5-4 \cdot 6-$ & - & - & - & - & & - & - & - \\
\hline
\end{tabular}

11.15 a.m. Temp, at Cape Armitage, $-22^{\circ} \cdot 5 ;$ min., $-32^{\circ} \cdot \overline{7}$. Canne bee. $\mathrm{Ci}_{-}{ }^{2}$ Str. all scured; hoar frost on thermo-

Noon. Thick to $\mathrm{S}$; clearing to W; sumimits of westernl mountalns olseured in thin Str.; $\mathrm{Ci}$. 2 p.ur. Nleavy Nim. to sW ; de2 p.ni. 3 p.m. Kain land, 3 .

$4 \mathrm{p} . \mathrm{m}$. Nim. to $\mathrm{W}$. of hut, aceompanierl by lissing noise

8 p.m. Very misty to $\mathrm{F}$.

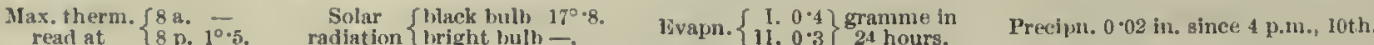
Milit. Str. very thin; noon elearer.

coutly and orercast all day, wit la harrily any wind. Barometer falling an day. Temperatur rise in the evening.

15 th.

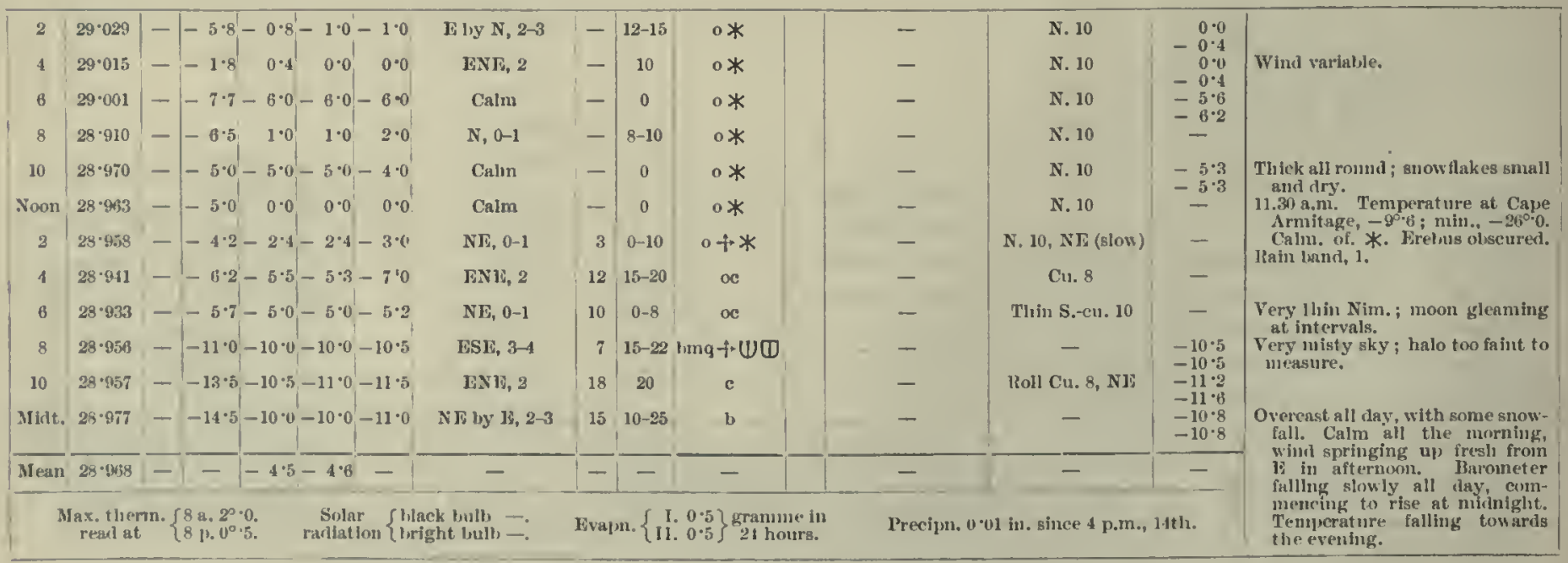


Mathokological, Journal, of the "Discoveny."

\begin{tabular}{|c|c|c|c|c|c|c|}
\hline \multirow{3}{*}{11 our. } & \multirow{3}{*}{ 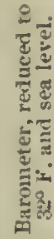 } & \multicolumn{5}{|c|}{ Thermometers. } \\
\hline & & غ્غ & 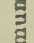 & Sert & urial. & \\
\hline & & ¿ू & है & Diy. & Wet. & 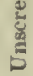 \\
\hline
\end{tabular}

\begin{tabular}{|c|c|c|}
\hline whis. & & \\
\hline $\begin{array}{c}\text { Direction and } \\
\text { forces } \\
\text { (Beanfort scale). }\end{array}$ & 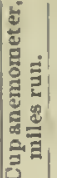 & 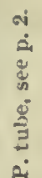 \\
\hline
\end{tabular}
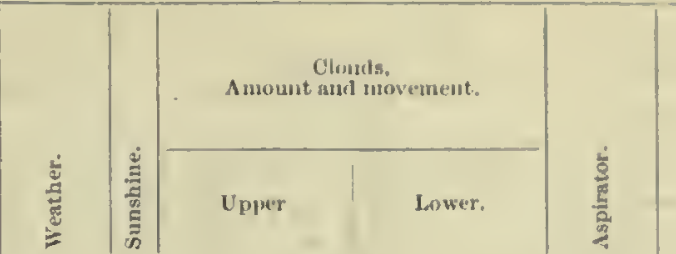

$3|2 k \cdot 971|-|-1.4 \cdot 5|-12 \cdot 8^{\prime}-12 \cdot 8^{\prime}-13 \cdot 0$

$428 \cdot 9 \cdot 1)--14 \cdot 5-13 \cdot 0-13 \cdot 0-13 \cdot 5$

Liv1, 3-4

19. $\quad 22-28$

LNI, 2-3

17 15-29

WNE, 3-1

$20 \quad 22-28 \quad b$

$829 \cdot 056|-|-17 \cdot 0-16 \cdot 0-16 \cdot 0-16 \cdot 0$

$1029 \cdot 091--16 \cdot 5-15 \cdot 5-15 \cdot 0-16 \cdot 0$

22 25-28, bf

Ni, 3-4

17 18-26 icet

NE, 2-3

$15 \quad 15-23 \quad 1 x f$

Calı

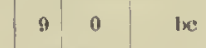

NNE, 0-1

\begin{tabular}{l|l|l|l|l|l|}
7 & $0-10$ & In
\end{tabular}

Calm

Calm

\begin{tabular}{l|l|l|l}
3 & 0 & luc
\end{tabular}

$20 \cdot$

11) $29 \cdot 210--26 \cdot 6-23 \cdot 5-23 \cdot 8-25 \cdot 2$

Calı

\begin{tabular}{c|c|c}
0 & 0 & $b y-$ \\
0 & 0 & $b c \emptyset$ \\
0 & 0 & ve 10
\end{tabular}

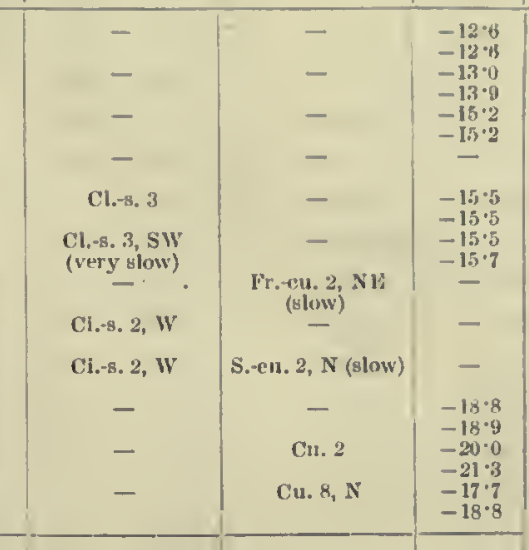

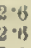

16 th.

$\overline{\text { Mean }} \overline{29 \cdot 120}=-\overline{-16 \cdot 9-17 \cdot 0-}$

Max. therm, read at $\left\{\begin{array}{l}8 \text { a. } \\ 8 \mathrm{p},-\overline{13} 3^{\circ} \cdot 0 .\end{array}\right.$

Evapn. $\left\{\begin{array}{r}1.0 \cdot 4 \\ 1 \mathrm{I} .0 \cdot 2\end{array}\right\} \begin{aligned} & \text { gramme in } \\ & 24 \text { hours. }\end{aligned}$

P'reclpn, - In $10 \mathrm{p}$. ru. Frost riue on outslide

thermometer.
Midt. Frost rime on ontshe thermumeter.

Fine, int clonrly all day, with freah

ery faint anrorai strominurs to $\mathrm{N}$ erystal falling. 10 a.m. No buri vlslhle; inlsey lu 11 a.ni, Tenp. at Cape Armllage, $-24^{\circ} \cdot 8 ;$ mlu., $-27^{\circ} \cdot 0$. Whin!

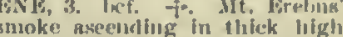
column, and then lireakling away to livu. Clearlug generaliy; miaty

1.711. Very wilud-Hlown $\mathrm{Cl}_{0}=\mathrm{m}$

ealm and remaining so. Barometer rising steadily all day. Temperature falling, and steauller all day.

\begin{tabular}{|c|c|c|c|c|c|c|c|c|c|c|}
\hline 2 & $29 \cdot 273$ & - & $-27 \cdot 9-22 \cdot 5-22 \cdot 8-23 \cdot 0$ & Calım & 0 & 0 & be $\sqcup$ & - & Cu. $6, \mathrm{~N}$ & $-19 \cdot 8$ \\
\hline 4 & $29 \cdot 292$ & - & $-25 \cdot y-14 \cdot 8-15 \cdot 0-14 \cdot 0$ & E, 0-1 & I & 0 & be - & - & Cu. $7, \mathrm{NNW}$ & $-13 \cdot 5$ \\
\hline 6 & $29 \cdot 2965$ & - & $-14 \cdot 5-11 \cdot 2 \mid-11 \cdot 6-11 \cdot 2$ & $1 ;, 0-1$ & 2 & 0 & $\infty *$ & - & N. 10 & $\begin{array}{l}-11 \cdot 3 \\
-11 \cdot 4\end{array}$ \\
\hline 8 & $29 \cdot 313$ & - & $-12 \cdot 5-10^{\circ} 0-10^{\circ} 0-10^{\circ} 0$ & Lt. $\mathbf{N}$ airs & 7 & 0 & oc & - & N. 10 & -114 \\
\hline 10 & $29 \cdot 321$ & - & $-12 \cdot 3-11 \cdot 0-11 \cdot 0-12 \cdot 0$ & Calm & 1 & 0 & $\circ *$ & - & N. 10 & $\begin{array}{l}-9 \cdot 0 \\
-10 \cdot 1\end{array}$ \\
\hline Sivon & $29 \cdot 321$ & - & $-18 \cdot 0-13 \cdot 0-13 \cdot 0-12 \cdot 0$ & Calm & 4 & 0 & $a *$ & - & N. 10 & $\begin{array}{l}13 \cdot 0 \\
-13 \cdot 0\end{array}$ \\
\hline 2 & $29-312$ & - & $-17 \cdot 3-14 \cdot 2-14 \cdot 2-15 \cdot 0$ & Calm & 3 & 0 & $o *$ & - & N. 10 & - \\
\hline 4 & $29-311 i$ & - & $-17 \cdot 0-14 \cdot 5-14 \cdot 5-16 \cdot 5$ & N by E, 1 & 5 & $0-10$ & $o *$ & - & N. 10 & - \\
\hline 6 & $29 \cdot 304$ & - & $-18 \cdot 0-18 \cdot 0-17 \cdot 8-18 \cdot 6$ & N, 1-2 & 9 & $5-12$ & b & - & - & - \\
\hline 8 & $29 \cdot 31)^{2}$ & - & $-19 \cdot 5-19 \cdot 5-19 \cdot 0-20 \cdot 0$ & NE, $3-4$ & 9 & $15-22$ & $1 x-4 \cdot(1)$ & - & Fr.cu. $2, \mathrm{NE}_{\text {(slow) }}$ & $\begin{array}{l}-20 \cdot 2 \\
-20 \cdot 2\end{array}$ \\
\hline 10 & $29 \cdot 317$ & - & $-21 \cdot 0-20^{\circ} 0-19 \cdot 5-20 \cdot 0$ & $\mathrm{NH}, 3-4$ & 20) & 18-25 & $1 x+\div$ & - & A.-eu, $2, \mathrm{~N}$ (slow) & - \\
\hline Misl & $29-316$ & - & $-20 \cdot 5-20 \cdot 0-19 \cdot 8-20 \cdot 6$ & $N E, 2-3$ & 16 & $15-20$ & $b-f \cdot \mathbb{C}$ & 一 & - & $\begin{array}{l}-19 \cdot 5 \\
-19 \cdot 8\end{array}$ \\
\hline can & $29 \cdot 3 \mathrm{3} 7$ & - & $--15 \cdot \hat{\imath}-15 \cdot 7-$ & - & $\overline{-}$ & - & - & - & - & - \\
\hline
\end{tabular}

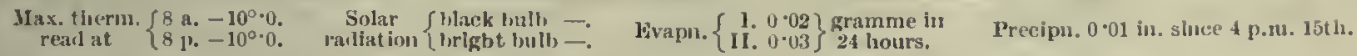
2 a.m. and 1 a.m. Frost rime ou

Snowflakes very small, bard, and iry ; thick all round.

11 a.m. Temp. at Cape Armitage, $-31^{\circ} \cdot 2$ min., $-47^{\circ .5}$. Wind Som. Snowing very lightly; tluick all ronutl.

4 p.m. Thick dry suow mixed with ice erystals.

p.m. lain hand, 1

Visty in $5 \mathrm{ky}$; wind lucreasing.

Ciouds round noon of prismatle

Misty lu sky to Is.

Overeast and snowing most of the clay, clesrlug in evening. Wiud light all the mornlug, rising np from NH, stromg in evening.
Barometer rose until nonn, then fell sliglit ly unt il 8 p.m., and i heu rose again. Temperature fallug ail day.

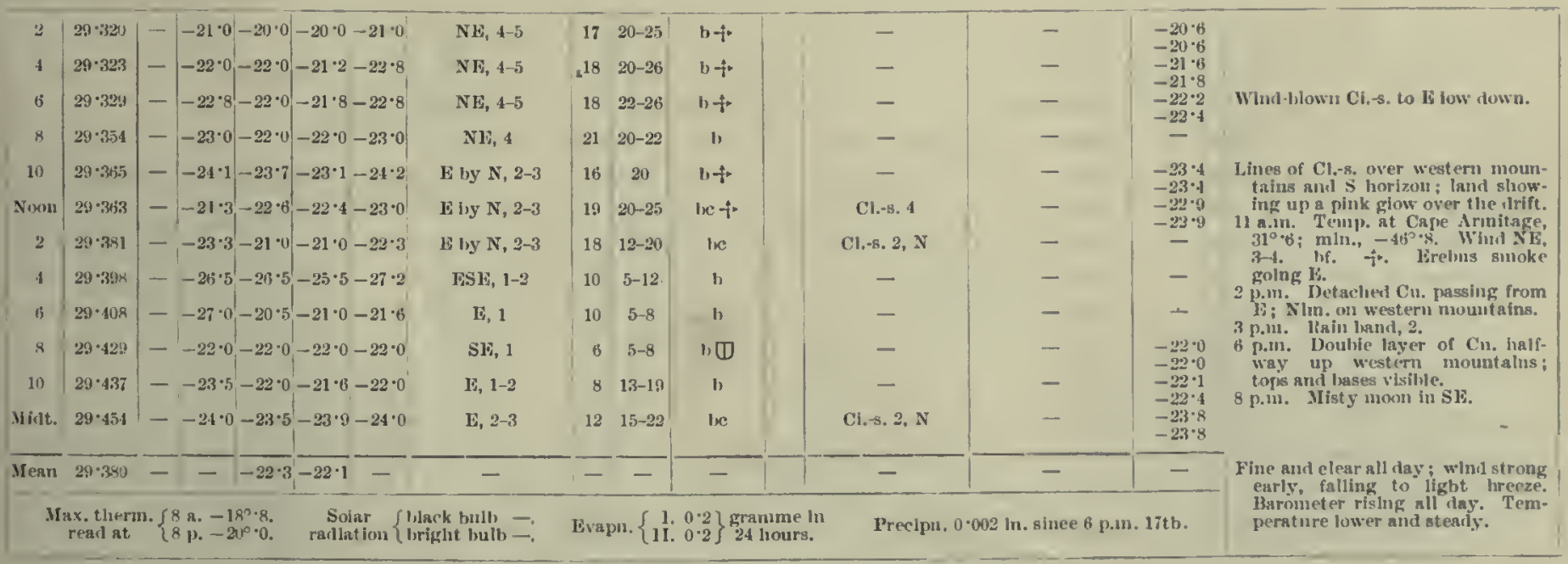




\begin{tabular}{|c|c|c|c|c|c|c|c|c|c|c|c|c|c|c|c|}
\hline \multirow{3}{*}{ Hlour. } & \multirow{3}{*}{ 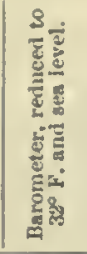 } & \multicolumn{5}{|c|}{ Thermometers. } & \multicolumn{3}{|l|}{ Whind. } & \multirow[b]{3}{*}{ 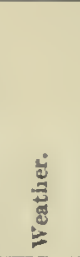 } & \multirow[b]{3}{*}{ 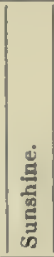 } & \multirow{2}{*}{\multicolumn{2}{|c|}{$\begin{array}{l}\text { Clouds, } \\
\text { Amount and nsovenicut. }\end{array}$}} & \multirow[b]{3}{*}{ 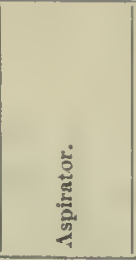 } & \multirow{3}{*}{ lemarks. } \\
\hline & & \multirow{2}{*}{ 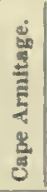 } & \multirow{2}{*}{ 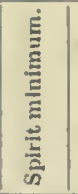 } & \multicolumn{2}{|c|}{ Mercurial. } & \multirow[b]{2}{*}{ 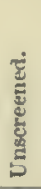 } & \multirow[b]{2}{*}{$\begin{array}{l}\text { Direetion and } \\
\text { force } \\
\text { (Beaufort seale). }\end{array}$} & \multirow{2}{*}{ 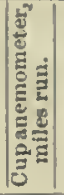 } & \multirow{2}{*}{ 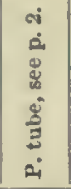 } & & & & & & \\
\hline & & & & Dry. & Wet. & & & & & & & Upper. & Lower. & & \\
\hline 2 & $29 \cdot 462$ & - & $-24 \cdot 2$ & $-21 \cdot 5$ & $-21 \cdot 2$ & - & b, $1-3$ & 15 & $13-23$ & ix & & Cl.-8. 4, N & - & $-22^{\circ} 0$ & \multirow{4}{*}{$\begin{array}{l}\text { No land visible through drlft ln } \\
S \text { and } W \text {. }\end{array}$} \\
\hline 4 & $29 \cdot 470$ & -1 & $-30 \cdot 5$ & $-30 \cdot 0$ & $-2 n \cdot 2$ & - & F by N, 3-4 & 19 & $25-33$ & $10+$ & & Cl.-s. $5, \mathrm{~N}$ & - & Mereury & \\
\hline 6 & $29 \cdot 478$ & - & $-31 \cdot 8$ & $-30 \cdot 2$ & $-29 \cdot 8$ & - & ENE, 2-4 & 21 & $20-35$ & $\log +$ & & $\mathrm{Cl}_{0}-5,2, \mathrm{~N}$ & - & - & \\
\hline 8 & $29 \cdot 472$ & - & $-32 \cdot 0$ & $-27 \cdot 0$ & $-26 \cdot 5$ & - & E by $N, 4-5$ & 21 & $25-30$ & $b+$ & & - & - & - & \\
\hline 10 & $29 \cdot 473$ & - & $-27 \cdot 0$ & $-24 \cdot 0$ & $-24 \cdot 0$ & - & NE, 3-4 & 11 & $15-20$ & b & & - & - & Nercury & \multirow{10}{*}{ 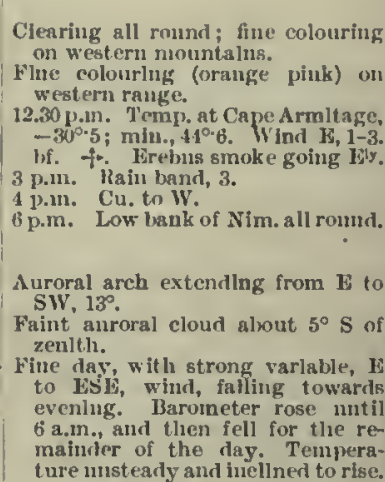 } \\
\hline Nown & $29 \cdot 457$ & - & $-24 \cdot 9$ & $-10 \cdot 4$ & $|-19 \cdot 5|$ & - & Es ly S, I-2 & 12 & $0-5$ & b) & & - & - & $\begin{array}{c}\text { below scale } \\
-19 \cdot 7 \\
-20 \cdot 2\end{array}$ & \\
\hline 2 & $29 \cdot 437$ & - & $-19 \cdot 8$ & $-19 \cdot 0$ & $-19 \cdot 3$ & - & ESE, 2-3 & 11 & $1 \theta-18$ & " & & - & - & - & \\
\hline 4 & $29 \cdot 424$ & - & $-19 \cdot 3$ & $-18 \cdot 0$ & $-18 \cdot 4$ & - & SL, 2-3 & 11 & $10-18$ & b & & $:-$ & - & - & \\
\hline 6 & $29 \cdot 398$ & - & $-18 \cdot 5$ & $-16 \cdot 8$ & $-17 \cdot 0$ & - & ESE, 2-5 & 14 & $10-25$ & ing & & - & - & - & \\
\hline 8 & $29 \cdot 388$ & - & $-19 \cdot 0$ & $-16 \cdot 0$ & $-16 \cdot 0$ & - & $\mathbf{E}, 2-3$ & 13 & $12-24$ & b & & $\checkmark$ & - & $\begin{array}{l}-16.2 \\
-16.8\end{array}$ & \\
\hline 10 & $29 \cdot 330$ & - & $-16 \cdot 9$ & $-15 \cdot 3$ & $-15 \cdot 6$ & - & ESE, 2-3 & 14 & $10-20$ & b & & - & - & $\begin{array}{l}-10.8 \\
-15.2 \\
-15.8\end{array}$ & \\
\hline Midt. & $29 \cdot 292$ & - & $-15 \cdot 8$ & $-12 \cdot 8$ & $-13 \cdot 3$ & - & E loy S, 1-2 & 9 & $10-15$ & b & & - & - & $\begin{array}{l}-110 \cdot \\
-11 \cdot 2 \\
-12 \cdot 5\end{array}$ & \\
\hline Mean & $29 \cdot 423$ & - & - & $-20 \cdot 8$ & $-20 \cdot 8$ & -1 & - & 1 & - & - & & - & - & - & \\
\hline \multicolumn{7}{|c|}{ Max. therm, read at $\left\{\begin{array}{l}8 \mathrm{a},-20^{\circ} 0 \\
8 \mathrm{p},-16^{\circ} 0\end{array}\right.$} & Solar radiation \{ & bla & ba & - . & & $\left\{\begin{array}{l}1.0 \cdot 5 \\
11,0 \cdot 6\end{array}\right\}$ & \multicolumn{2}{|c|}{ Preclpn. - in. } & \\
\hline
\end{tabular}

20 th

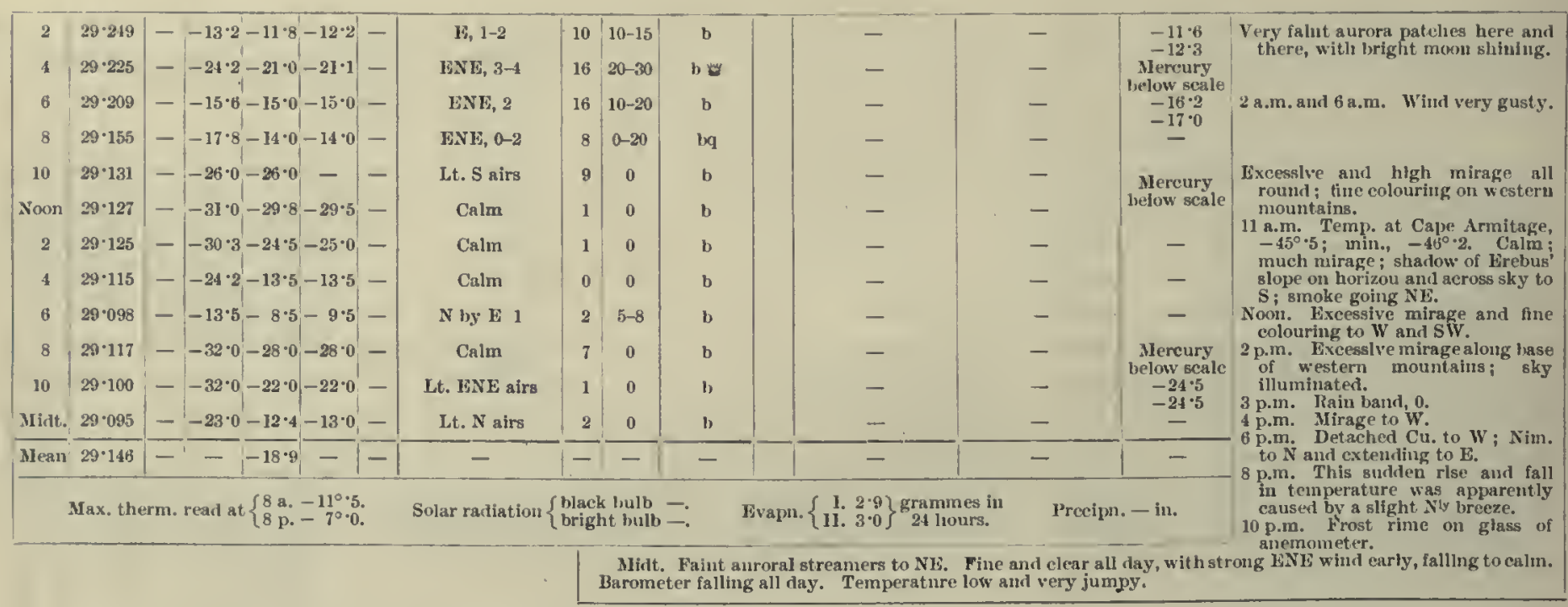

21 st.

\begin{tabular}{|c|c|c|c|c|c|c|c|c|c|c|c|c|}
\hline 2 & $29 \cdot 100$ & - & $-19 \cdot 0$ & $-18 \cdot 5-18 \cdot 0$ & - & NNE, 1 & 4 & 0 & b & - & - & $\begin{array}{l}-20 \cdot 9 \\
-20 \cdot 9\end{array}$ \\
\hline 4 & $29 \cdot 117$ & - & $-20 \cdot 5$ & $-14 \cdot 0-14 \cdot 0$ & - & NNW, 1 & 4 & 0 & bu & - & - & $\begin{array}{l}-2079 \\
-13.7\end{array}$ \\
\hline 6 & $29 \cdot 163$ & - & $-25 \cdot 5$ & $-25 \cdot 5-24 \cdot 5$ & - & Lt. NNW airs & 6 & 0 & $b$ & - & - & Nercury \\
\hline 8 & $29 \cdot 210$ & - & $-28 \cdot 0$ & $-25 \cdot 0-24 \cdot 8$ & - & $\mathrm{SE}, 1$ & 3 & $5-10$ & 1) & - & - & \\
\hline 10 & $29 \cdot 241$ & - & $-25 \cdot 0$ & $-24 \cdot 0-23 \cdot 8$ & - & Calm & 4 & 0 & Иப & - & - & Mlercury \\
\hline Noon & $29 \cdot 265$ & - & $-31 \cdot 0$ & $-29 \cdot 5-29 \cdot 0$ & - & Calm & 0 & 0 & bu & - & - & below scale \\
\hline 2 & $29 \cdot 283$ & - & $-30 \cdot 8$ & $-27 \cdot 8-27 \cdot 8$ & - & Calm & 1 & 0 & ウே & - & - & - \\
\hline 4 & 29.313 & - & $-35 \cdot 2$ & $-35 \cdot 2-$ & - & Calm & 2 & 0 & bบ & - & - & - \\
\hline 6 & $29 \cdot 355$ & - & -40.0 & $-40 \cdot 0-39 \cdot 5$ & - & Calm & 0 & 0 & ดบ & - & - & - \\
\hline 8 & $29: 371$ & - & $-39 \cdot 5$ & $-20 \cdot 0-20.5$ & - & Calm & 1 & 0 & bப. & - & - & $\begin{array}{l}-21 \cdot 6 \\
-22 \cdot 0\end{array}$ \\
\hline 10 & $29 \cdot 390$ & - & $-35 \cdot 7$ & $-23 \cdot 0-24 \cdot 0$ & - & Lt. NE airs & 2 & 0 & $b \sqcup$ & - & - & $\begin{array}{l}-20.4 \\
-20.7\end{array}$ \\
\hline Mildt. & $29 \cdot 412$ & -1 & $-39 \cdot 9$ & $-39 \cdot 0-38 \cdot 9$ & - & Calm & 2 & 0 & b & - & - & $\begin{array}{c}\text { Mercury } \\
\text { below scale }\end{array}$ \\
\hline Mean & $29 \cdot 268$ & - & - & $-26 \cdot 8-$ & - & - & $=$ & - & - & - & - & - \\
\hline
\end{tabular}

Hoar frost on auemometer glass.

Slight mlrage all round; very fine colouring to $W_{;}$frost rime on 11 a.m. Temp. at Cape Armitage, $-48^{\circ} \cdot 8$; min., $-49^{\circ} \cdot 9$. Wind WNE Refractlon great to $\mathrm{E}$ and $\mathbf{N}$. Nit. Erebus' smoke going NW
frost rime on thermometer. $1.40 \mathrm{a.m}$. Frelus smoke goin and pouring down slope. hility mart rim

3 p.m. Rain band, 0 .

p.m. Solar thermometer coated with frost; detached clouds on $\mathrm{N}$ horlzon. coated with rlme. 0 p.m. Anemometer glass again

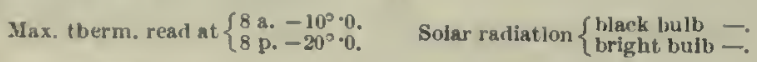
Evapn. $\left\{\begin{array}{l}1.0^{\circ} \cdot 0 \\ 11.0^{\circ} 0^{2}\end{array}\right\} \begin{aligned} & \text { grannine In } \\ & 24 \text { hours. }\end{aligned}$ Precipn. - n. requlred scraping.
vidt. No more preclpitation on glass. Aurora, an extended and rery perfeet areh of vertical rays 
Meteorologicat, Journat, of rhe " Discoverey,"

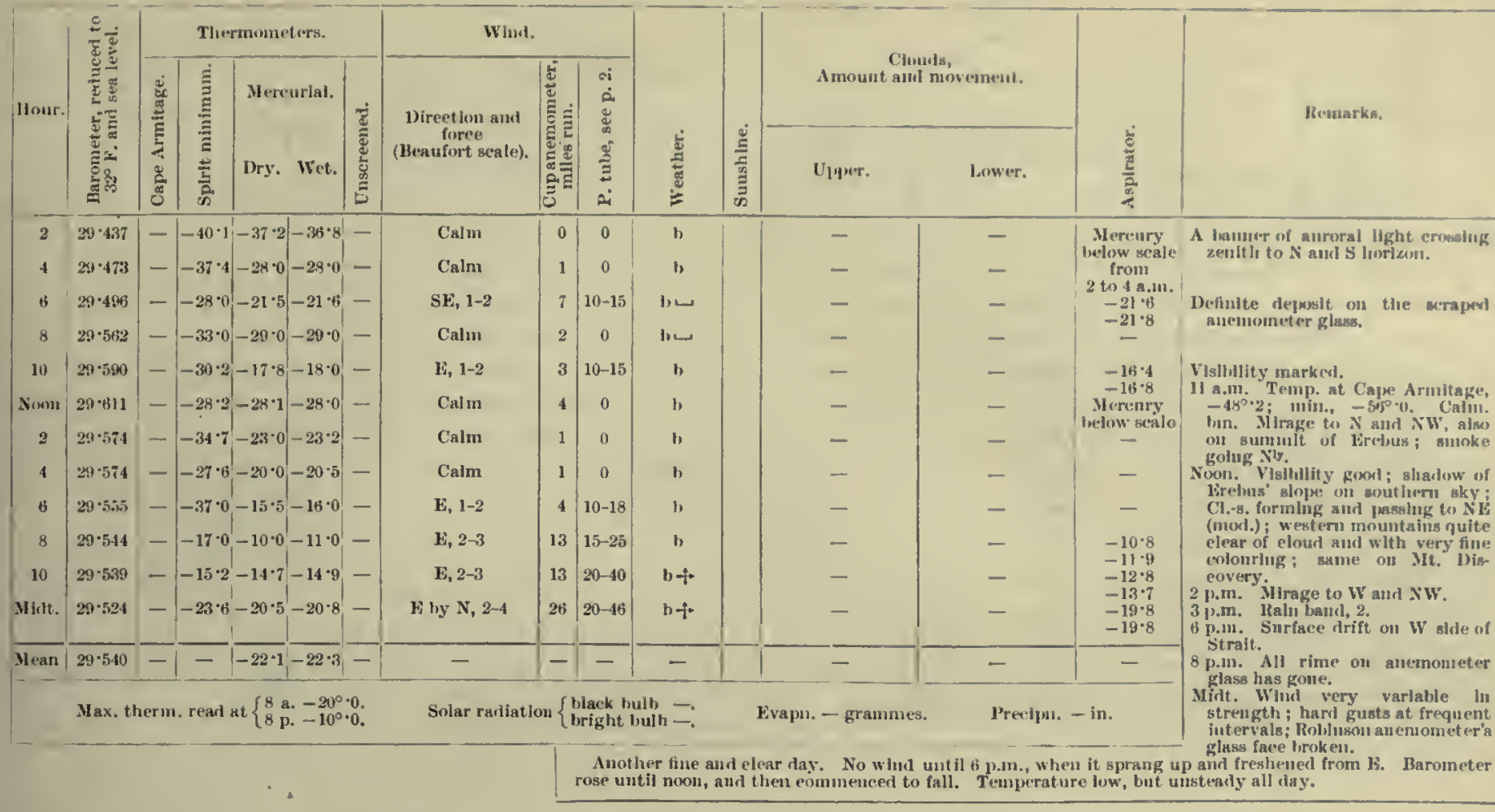

\begin{tabular}{|c|c|c|c|c|c|c|c|c|c|c|c|}
\hline 2 & $29 \cdot 449$ & - & $-25 \cdot 9-15 \cdot 0-15 \cdot 2-$ & E by $N, 2-3$ & -1 & $22-24$ & $b+$ & - & - & $-14 \cdot 4$ & \multirow{4}{*}{ 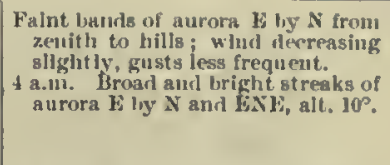 } \\
\hline 4 & $29 \cdot 170$ & - & $-15 \cdot 2-13 \cdot 4-13 \cdot 8-$ & E ly $\mathbf{N}, 2-3$ & 16 & $24-28$ & $b+$ & - & - & -12.4 & \\
\hline i & $29 \cdot+15$ & - & $-16 \cdot 2-16 \cdot 0-16 \cdot 2-$ & E by $\mathbf{N}, 2-3$ & 15 & $15-25$ & $b+$ & - & - & $\begin{array}{l}-13.7 \\
-15 \cdot 2\end{array}$ & \\
\hline 8 & $29 \cdot 417$ & - & $-18 \cdot 0-18 \cdot 0-18 \cdot 0-$ & ESE, $2-3$ & 21 & $15-20$ & bf. & - & - & $-16 \cdot 6$ & \\
\hline 11) & $29 \cdot 385$ & - & $-19 \cdot 2-15 \cdot 0-15 \cdot 5-$ & ENE, 2-3 & 15 & 10-18 & 1. & - & - & $-11 \cdot 7$ & \multirow{9}{*}{ 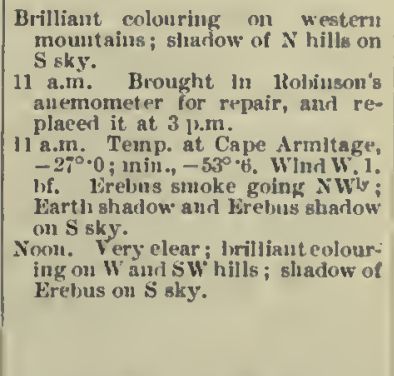 } \\
\hline Xoon & $29 \cdot 327$ & - & $-19 \cdot 2-16 \cdot 2-16 \cdot 8-$ & $\mathbf{E}, 1$ & -1 & $8-10$ & b) & - & - & $\begin{array}{l}-128 \\
-16 \cdot 8 \\
-110\end{array}$ & \\
\hline 2 & $29 \cdot 275$ & - & $-16 \cdot 3-16 \cdot 0-16 \cdot 0-$ & S18, 1 & - & $5-8$ & h & - & - & -10 & \\
\hline 4 & $29 \cdot 234$ & - & $-19 \cdot 0-18 \cdot 3-18 \cdot 0-$ & ESE, 1-2 & - & 5-12 & b & - & - & - & \\
\hline 6 & $29 \cdot 212$ & - & $-20 \cdot 8-18 \cdot 0-19 \cdot 0-$ & E, 2 & 8 & $18-23$ & b & - & .- & - & \\
\hline 8 & $29 \cdot 171$ & - & $-22 \cdot 0-20 \cdot 0-20 \cdot 0-$ & NE, 5-6 & 17 & $25-33$ & b & - & - & $-20 \cdot 6$ & \\
\hline 10 & $29 \cdot 135$ & - & $-22 \cdot 2-19 \cdot 5-19 \cdot 7-$ & E hy N, 3-4 & 23 & $22-27$ & b & - & - & $-19 \cdot 8$ & \\
\hline Millt. & $29 \cdot 082$ & - & $-23 \cdot 8-23 \cdot 6-23 \cdot 5-$ & E, 4-5 & 20 & 28-34 & b & - & - & $\begin{array}{l}-24.2 \\
-24 \cdot 2 \\
-24 \cdot 2\end{array}$ & \\
\hline Nean & $29 \cdot 304$ & - & $-\mid-17 \cdot 5-17 \cdot 6-$ & - & - & - & - & - & - & - & \\
\hline & & & read at $\left\{\begin{array}{l}8 \mathrm{a} .-8^{\circ} \cdot 0 \\
8 \mathrm{p} .-13^{\circ} \cdot 0\end{array}\right.$ & Solar radiatio & & & & & \multicolumn{2}{|c|}{ Precipu. $-\ln$. } & 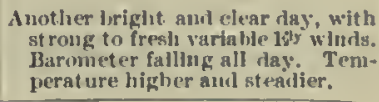 \\
\hline
\end{tabular}

\begin{tabular}{|c|c|c|c|c|c|c|c|c|c|c|c|}
\hline 2 & $29 \cdot 052$ & - & $-24 \cdot 0-19 \cdot 4-19 \cdot 7-$ & E by N, 3-4 & 23 & $22-28$ & b & - & - & $-19 \cdot 1$ & \multirow{4}{*}{$\begin{array}{l}\text { Auroral streamers of very slight } \\
\text { intensity to EN and } \mathrm{SE} \text {, alt. up } \\
\text { to } 30^{\circ} \text {. } \\
\text { Completely overcast, except to } \mathrm{W} \\
\text { and } \mathrm{S} \text {. }\end{array}$} \\
\hline 4 & $29 \cdot 037$ & - & $-21 \cdot 2-16 \cdot 8-17 \cdot 1-$ & $\mathrm{NE}, 3$ & 13 & $21-2-1$ & bc & - & S.-eu. 2 & & \\
\hline B & $29 \cdot 016$ & - & $-18 \cdot 2-11 \cdot 3-12 \cdot 1-$ & ENE, 1-2 & 18 & $12-17$ & oc & - & S.-cu. 9 & -11.7 & \\
\hline 8 & $28 \cdot 998$ & - & $-20 \cdot 0-19 \cdot 0-19 \cdot 2-$ & NE, 3-4 & 22 & $20-25$ & $b$ & - & -. & -126 & \\
\hline 10 & $29 \cdot 997$ & - & $-21 \cdot 0-21 \cdot 0-21 \cdot 0-$ & S, 1 & 7 & $10-15$ & b & - & - & $-22 \cdot 7$ & \multirow{10}{*}{ 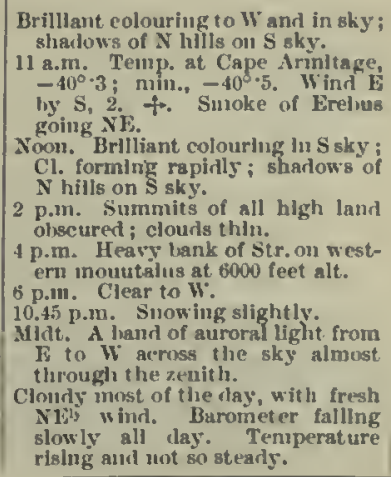 } \\
\hline Noon & $28 \cdot 93 \mathrm{t}$ & - & $-2 t \cdot 0-19 \cdot 0-19 \cdot 0-$ & $\mathrm{NE}_{1}, 4-5$ & 13 & $23-28$ & be & C1. $3, \mathrm{~W}$ (slow) & - & $\begin{array}{l}-19 \cdot 1 \\
-19 \cdot 5\end{array}$ & \\
\hline 2 & $28 \cdot 9+54$ & - & $-19 \cdot 5-17 \cdot 0-17 \cdot 4$ & ENE, 3-4 & 19 & $22-30$ & $\infty$ & - & S.cu. 10 & - & \\
\hline 4 & $28 \cdot 962$ & - & $-19 \cdot 0-17 \cdot 0-17 \cdot 2-$ & ENE, 3-4 & 20 & $20-28$ & the & - & Fr.-cu. 5, N E (fast) & 一 & \\
\hline B & $28 \cdot 9,960$ & - & $-16 \cdot 3-15 \cdot 5-15 \cdot 5-$ & ENE, 3-4 & 21 & $20-28$ & $\infty$ & - & S.cu. 10 & 一 & \\
\hline 8 & $28 \cdot 9 ; 2$ & - & $-17 \cdot 0-15 \cdot 0-15 \cdot 2-$ & NE, 2-3 & 13 & $15-20$ & 0 & - & S. 10 & $\begin{array}{l}-15 \cdot 5 \\
-160.1\end{array}$ & \\
\hline 10 & $28 \cdot 95 \%$ & - & $-16 \cdot 4-11 \cdot 0-11 \cdot 8-$ & NE, 0-1 & 13 & $0-5$ & o & - & S. 10 & $\begin{array}{l}-16.1 \\
-11: 2 \\
-11 .-7\end{array}$ & \\
\hline Nlidt. & $28 \cdot 9 t 50$ & - & $-21 \cdot 0-16 \cdot 6-16 \cdot 4-$ & E, 0-1 & 7 & $0-5$ & $b$ & - & - & $\begin{array}{r}-18 \cdot 9 \\
-19 \cdot 1 \\
\end{array}$ & \\
\hline \multirow[t]{2}{*}{ Means } & $28 \cdot 985$ & - & $--16 \cdot 6-16 \cdot 8-$ & - & - & - & - & - & - & - & \\
\hline & Max. & & read at $\left\{\begin{array}{l}8 \mathrm{a} \\
8 \mathrm{p}--\overline{15^{\circ}} \cdot 0\end{array}\right.$ & (1) & & & & & & & \\
\hline
\end{tabular}


1903-April.]

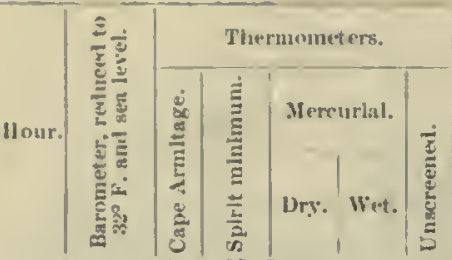

25 th.

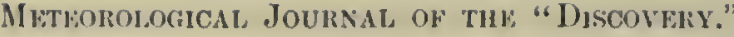

\begin{tabular}{|c|c|c|c|c|c|c|c|c|}
\hline \multicolumn{3}{|l|}{ Wind. } & \multirow[b]{3}{*}{ 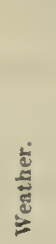 } & \multirow{3}{*}{ 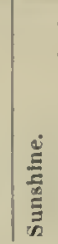 } & \multirow{2}{*}{\multicolumn{2}{|c|}{$\begin{array}{l}\text { Clourls, } \\
\text { Amonit and movement. }\end{array}$}} & \multirow[b]{3}{*}{ 苟 } & \multirow{3}{*}{ Kemarks. } \\
\hline \multirow{2}{*}{$\begin{array}{l}\text { Directlon anul } \\
\text { forre } \\
\text { (Beaufort scale). }\end{array}$} & \multirow{2}{*}{ 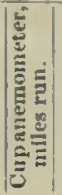 } & \multirow{2}{*}{ 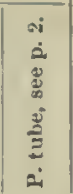 } & & & & & & \\
\hline & & & & & Epiper. & Jow cr. & & \\
\hline bigy $x, 3$ & $|17|$ & $\mid 20-23$ & b & & - & - & $-17 \cdot 7$ & \\
\hline $\mathrm{N} \&, 1$ & 15 & $5-15$ & Ix: & & - & s. 2 & $\begin{array}{l}-18 \cdot 0 \\
-15 \cdot 5 \\
-150\end{array}$ & 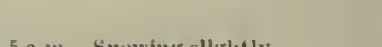 \\
\hline E by S, 0-1 & 7 & $0-10$ & lic & & - & Fr.-eu. 1, 1s & $\begin{array}{l}-159 \\
-21.6\end{array}$ & s a.m. Snowing sllghitly. \\
\hline Lt. Fin irs & 8 & $0-5$ & $\propto$ & & - & S.eu. 8, SE & $-21 \cdot 8$ & \\
\hline Calm & 7 & 0 & $\mathbf{c}$ & & - & Cu. 8 & $-17 \cdot 0$ & Very clear all round; hrillant pink \\
\hline Calm & 0 & 0 & $\infty$ & & - & S.-e11. 8, F (slow) & 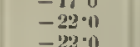 & 11 n.m. Temp. at Cape Armitage, \\
\hline Calm & 0 & 0 & Ix: & & Ci.-cu. 2, W & S.en. $2, \mathrm{E}$ & -220 & 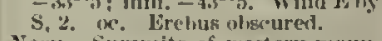 \\
\hline$N E, 0-1$ & 2 & $0-8$ & b & & Cl.-cr. $2, W$ & - & - & 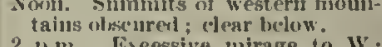 \\
\hline KNE, 1 & 3 & $5-8$ & b & & - & - & - & ${ }^{2}$ pim inf lomeessive mirage to $\mathrm{W}$; \\
\hline Calm & 5 & 0 & b & & - & - & $-22 \cdot 0$ & $\begin{array}{l}3 \text { p.um. lain land, } 1 \text {; } \\
\text { 4 p.m. Mirage to } W_{\text {; }} \text {; summits of }\end{array}$ \\
\hline F, 0-1 & 1 & $0-5$ & b & & - & - & $\begin{array}{c}-22 \cdot 0 \\
\text { Mercury }\end{array}$ & $\begin{array}{l}\text { Westenis mount ains ohscerred. } \\
\text { Neressary to use light to take } 8 \text { a.m. }\end{array}$ \\
\hline E, 0-1 & 8 & $0-5$ & $1, \pm$ & & - & - & helow & \\
\hline- & - & - & - & & - & - & 一 & Cloudy carly, lmit clearing later, \\
\hline Solar radiation \{ & $\begin{array}{l}\text { hlark } \\
\text { hrigh }\end{array}$ & $\begin{array}{l}k \text { bulb } \\
\text { lit bulh }\end{array}$ & & & $\left\{\begin{array}{r}\text { I. } 2 \cdot 0 \\
\text { II. } 2 \cdot 1\end{array}\right\}^{\mathrm{gran}} 24$ & in. & - in & $\begin{array}{l}\text { steady: T'emperature } \\
\text { stearly: }\end{array}$ \\
\hline
\end{tabular}

26th.

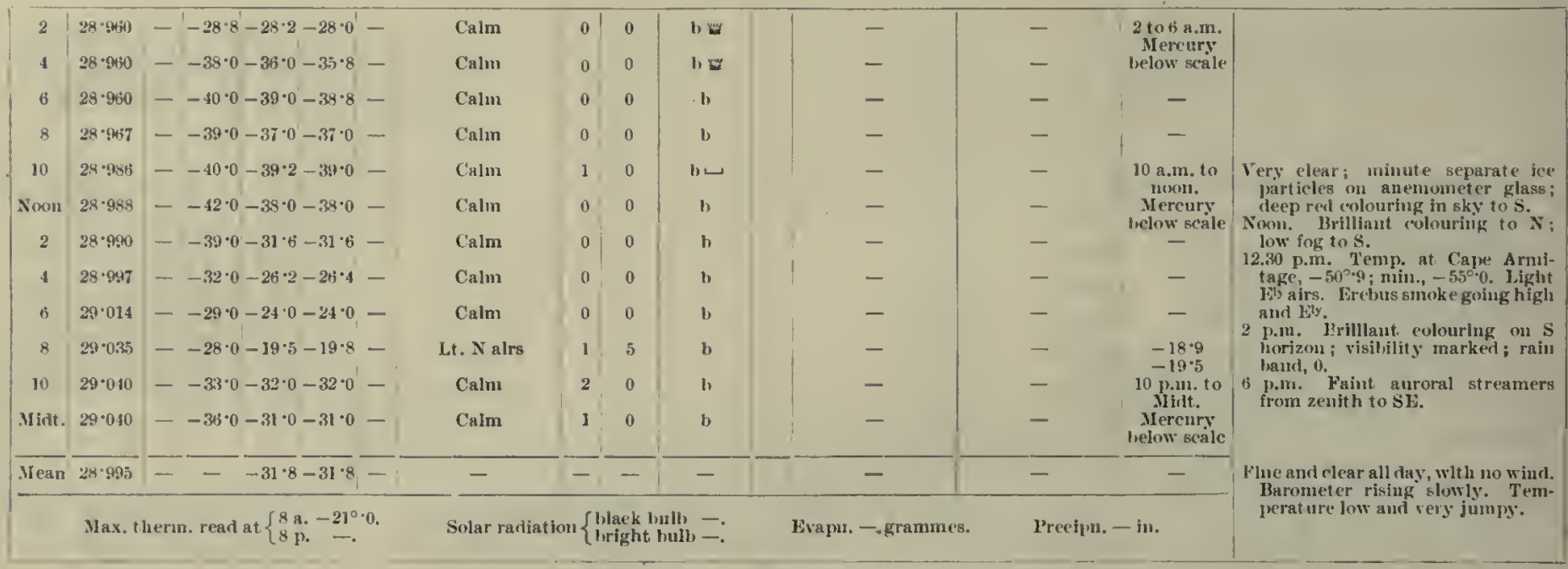

27 th.

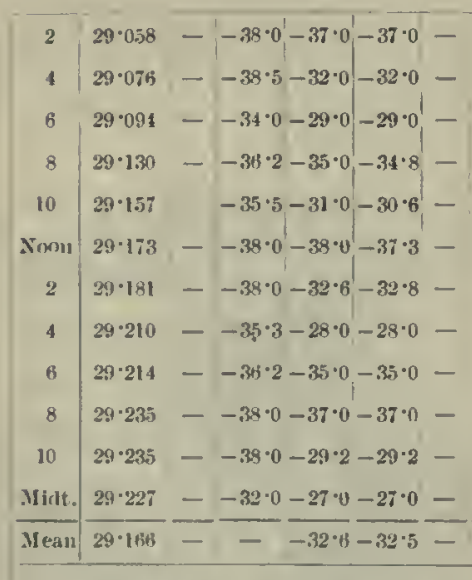

Max, therm. reat at $\left\{\begin{array}{l}8 \text { a. }-11^{\circ} \cdot 8 . \\ 8 \text { p. }-21^{\circ} 0 .\end{array}\right.$
Max, therm. read at $\left\{\begin{array}{l}8 \text { a. }-10^{\circ} 0 \\ 8 \mathrm{p},-16^{\circ} 0\end{array}\right.$

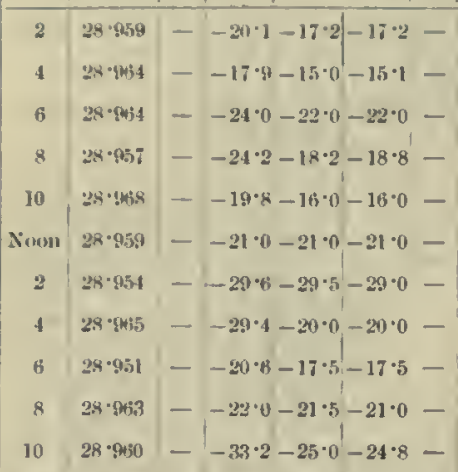

Midt. $28 \cdot \min 0--30 \cdot 5-25 \cdot 2-24 \cdot 8-$ Mean $28 \cdot(k+0)--20 \cdot 7-20 \cdot 6-$

\begin{tabular}{|c|c|c|c|c|c|c|}
\hline Calm & 0 & 0 & b & - & - & 2 to b a.m. \\
\hline Calm. & 0 & 0 & $b=$ & - & - & betow scale \\
\hline Calm & 0 & 0 & b) & - & - & - \\
\hline Calm. & 0 & 0 & b & - & - & - \\
\hline Calm & 0 & 0 & b & - & - & Mercury \\
\hline Calm & 0 & 0 & $b$ & - & - & from $110 \mathrm{am}$. \\
\hline Lt. $S W$ airs & 1 & 0 & b & - & - & - \\
\hline Calm & 2 & 0 & $b=$ & - & - & - \\
\hline Calin & 0 & 0 & b & - & - & - \\
\hline Calın & 0 & 0 & b & - & - & Mercury \\
\hline Calm & 0 & 0 & b & - & - & - \\
\hline Calm & 0 & 0 & b & - & - & - \\
\hline - & - & - & - & - & - & - \\
\hline
\end{tabular}

Evะpu. - grammes.
Slight uirage to $\mathbf{S}$; very elear all round. Temp. at Cape Armitage, thermoneter. lirebus sinok" rlsing $15(10)$ feet, and then break.

Ing to N $\mathrm{h}$. horizon. Temp. outside screcu y standard spirit thermometer, $-425$

2 p.m. Rain band, 0 .
b p.n. Faint auroral streamers from zeuitll to $\mathrm{S}$ aud $\mathrm{SE} ;$; ohserved met eor from zenlt li to $S$, very fast. p.un. Very faint auroral arels of sw, alt. in centre alout. $7^{\circ}$. streamers novlug slowly from $w$ to $\mathbf{E}$.

I idt. A uroral st reaners from ENE 
Meteorological, JoUkNal, OF THE "Discovilty."

[1903-April.

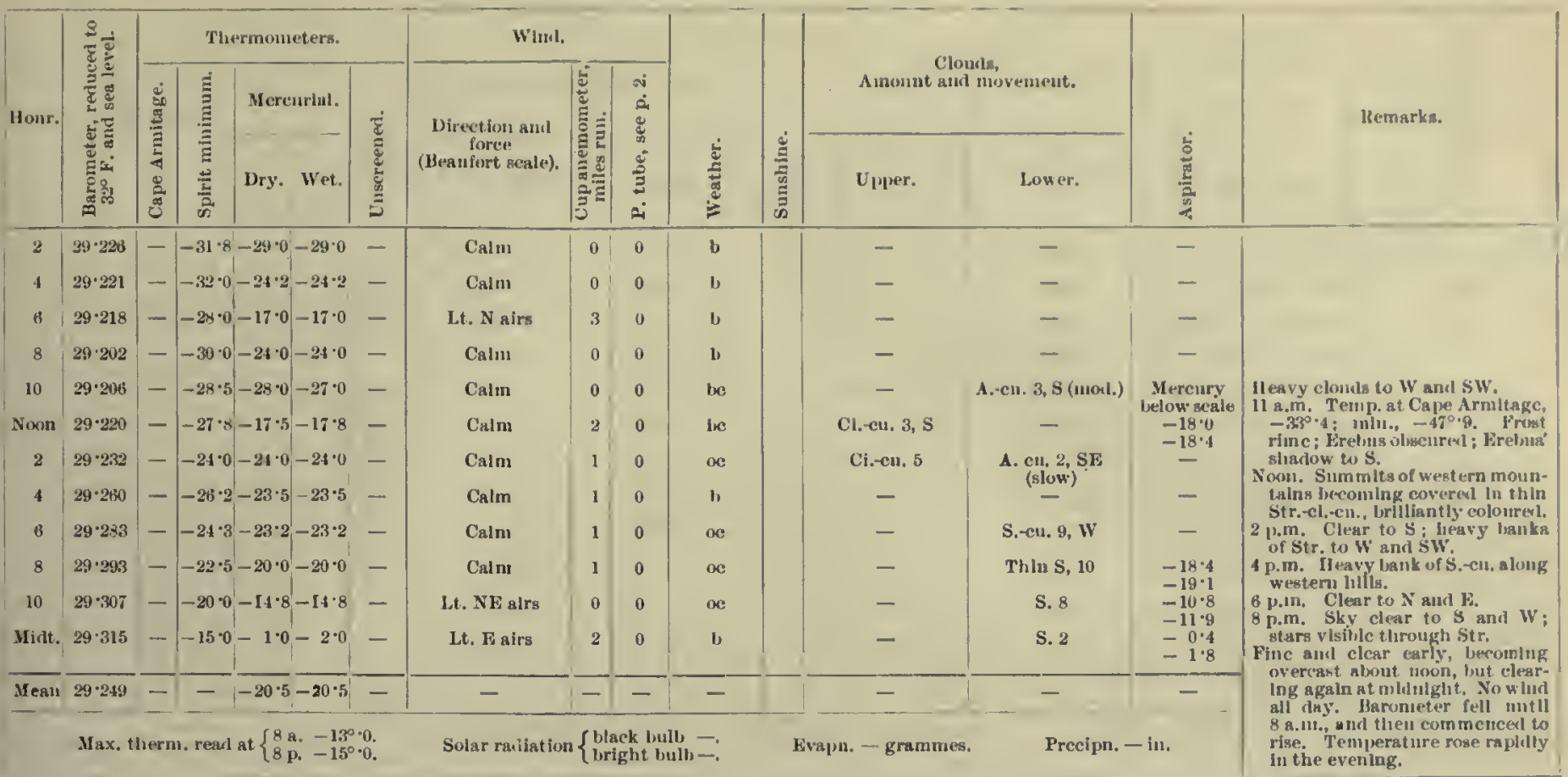

\begin{tabular}{|c|c|c|c|c|c|c|c|c|c|c|c|c|}
\hline 2 & $29 \cdot 338$ & - & $-11 \cdot 3-10^{\circ} 0-10 \cdot 0$ & - & Lt. NNE alrs & 2 & 0 & b & - & - & $-10 \cdot 8$ & \multirow{11}{*}{ 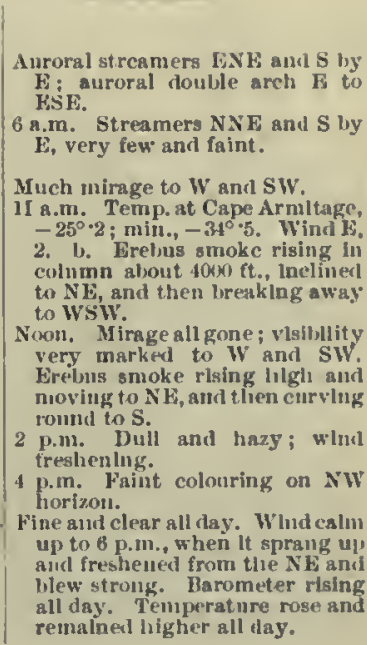 } \\
\hline 4 & $29 \cdot 353$ & - & $-15 \cdot 8-9 \cdot 8-10 \cdot 0$ & - & Culm & 2 & 0 & b & - & - & $\begin{array}{l}-11.6 \\
-3.3\end{array}$ & \\
\hline 6 & $29 \cdot 358$ & - & $-16 \cdot 2-14 \cdot 0-14 \cdot 0$ & - & Caltu & 2 & 0 & b & - & - & -17.0 & \\
\hline 8 & $29 \cdot 417$ & - & $-14 \cdot 2-3 \cdot 8-3 \cdot 8$ & - & Calm & 0 & 0 & b & - & - & -18 & \\
\hline Noon & $29 \cdot 455$ & - & $-21 \cdot 0-15 \cdot 0-15 \cdot 0$ & - & Calın & 0 & 0 & b & - & - & $\begin{array}{l}-19 \cdot 5 \\
-15 \cdot 3 \\
-15 \cdot 7\end{array}$ & \\
\hline 2 & $29 \cdot 459$ & - & $-15 \cdot 0-3 \cdot 0-4 \cdot 0$ & - & N by E, 1-2 & 1 & $10-20$ & b & - & - & - & \\
\hline 8 & $29 \cdot 459$ & - & $-5 \cdot 5-4 \cdot 2-5 \cdot 0$ & - & NF, 4-6 & 16 & $24-34$ & $b q+$ & - & - & $\begin{array}{l}-4.7 \\
-5.4\end{array}$ & \\
\hline 10 & $29 \cdot 505$ & - & $-5 \cdot 0-2 \cdot 0-3 \cdot 0$ & - & NF, 3-7 & 17 & $22-41$ & $\operatorname{lng}$ & - & - & $\begin{array}{l}=3 \cdot 3 \\
=3 \cdot 3\end{array}$ & \\
\hline Midt. & . $29 \cdot 503$ & - & $-4 \cdot 0-3 \cdot 0-4 \cdot 0$ & - & EN1:, 4-6 & 16 & $30-33$ & bq & - & - & $\begin{aligned} & 38 \\
- & 2 \cdot 9 \\
- & 3 \cdot 3\end{aligned}$ & \\
\hline$\overline{M e a n}$ & $29 \cdot 441$ & - & $-\mid \overline{-7 \cdot 9}-8 \cdot 3$ & - & - & - & - & - & - & - & - & \\
\hline & $M_{8}$ & & $\left\{\begin{array}{lr}8 \mathrm{a}, & 5^{\circ} \cdot 2 . \\
8 \mathrm{p}, & -1^{\circ} \cdot 0 .\end{array}\right.$ & & tadiats & & $\begin{array}{l}\operatorname{lomib} \\
\operatorname{lngl}\end{array}$ & & $\left.\cdot 8\}^{8}\right\}$ & \multicolumn{2}{|c|}{ Preclpn, - In. } & \\
\hline
\end{tabular}

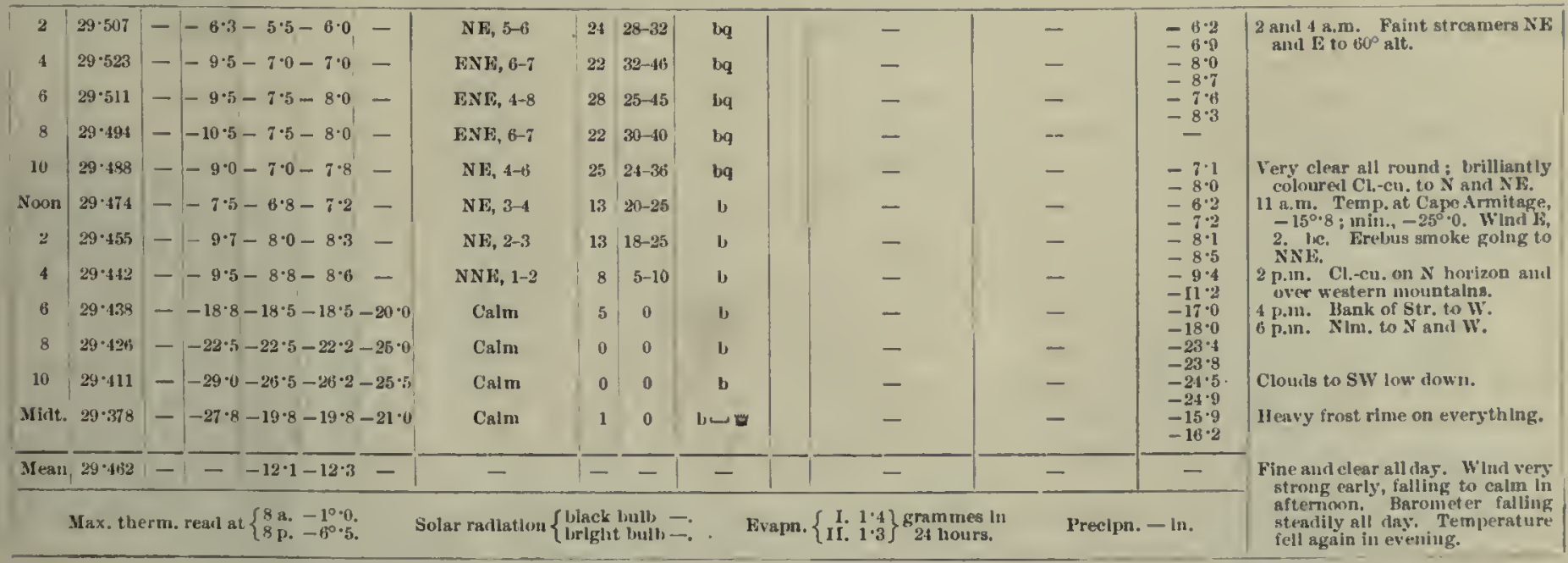


Mhtborological, JoUnNal, of THE "Discovery."

1903 - May.]

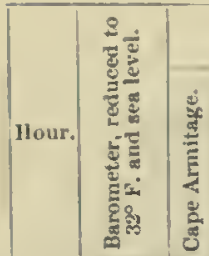

1 st.

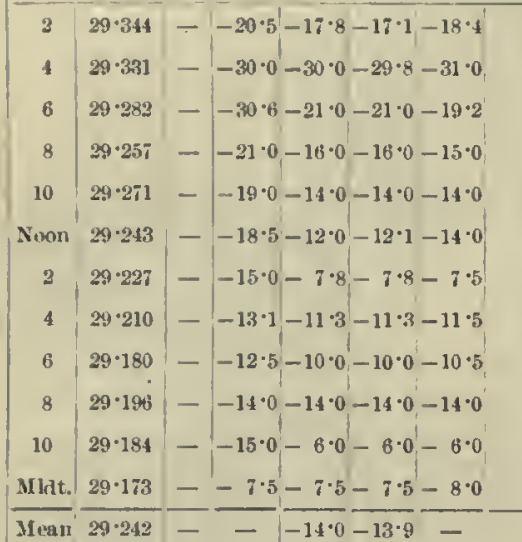

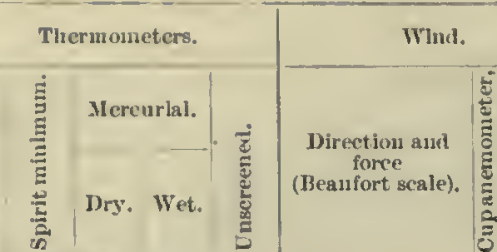

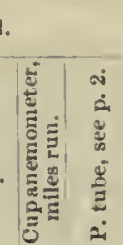

\begin{tabular}{|c|c|c|c|c|c|c|c|}
\hline Calm & 1 & 0 & bus & & - & - & $-17 \cdot 3$ \\
\hline Calm & 0 & 0 & 1) & & - & - & Mercury \\
\hline Calm & 0 & 0 & he & & - & S. $2, \mathrm{~S}$ & -18.8 \\
\hline Calm & 4 & 0 & bu & & - & - & - \\
\hline Lt. SE airs & 0 & $0-5$ & bme & & - & - & $-14 \cdot 1$ \\
\hline $\mathrm{SE}, 1$ & 5 & 10 & $\ln \omega$ & & - & - & $\begin{array}{l}-14.6 \\
-11.6\end{array}$ \\
\hline Var. E, 1-2 & 7 & $10-15$ & thm & & - & - & $\begin{array}{l}-18.0 \\
-8.0\end{array}$ \\
\hline ESE, 3-5 & 12 & $25-35$ & ocq +qu & . & - & Fog, 10 & $-11 \cdot 2$ \\
\hline SE, 2-5 & 11 & $18-35$ & oeq キேப & & - & Fog, 10 & $\begin{array}{l}10.8 \\
-10 \%\end{array}$ \\
\hline E, 1-4 & 8 & $10-26$ & $o q \backsim$ & 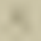 & - & Fog, 10 & -14.8 \\
\hline E, 2-4 & 11 & $18-25$ & $q+* *$ & & - & Fog, 10 & - \\
\hline F, 2-4 & 11 & $15-22$ & $0+* *$ & & - & N. 10 & - \\
\hline- & - & - & - & & - & - & - \\
\hline
\end{tabular}

Max. therm. $\left\{8\right.$ a. $-13^{\circ} \cdot 0$.

Solar \{black bulh - .

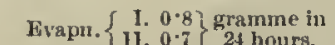

Precipn. 0.004 ln. siuce 6 p.m. 18tlı ult.

Clonds,

Amount and movement.
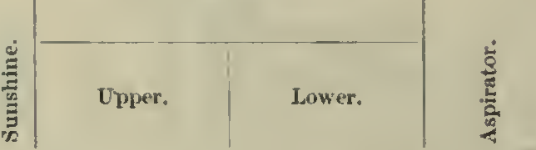

Remarks.

Auroral streainers to $\mathrm{S}$ awd $\mathrm{SE}$ faint ; frost rime ous therwometers. sc. a.n. Aurora over land to $E$ and thlug.

6 a.m. Fog commenelug to come up from $S$ and pouring down

11 a.m. Temp. at Cape Armltage.

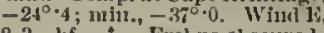
2-3. 1,f. +. Ereluns olsceured hoar frost on tlermometer and

a.m. Low fog all round horizon; inisty appearanee in sky

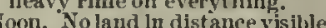

fog. On all engtern hills; fros rime on everything.

2 p.m. Everything thickly coater with fog erystals.

4 p.un. Everything thickly coated with fog erystals.

p.m. Fog crystals on everyt ling very thiek and inclineid totloek anemometer arms arri eups heavily coater.

10 p.11. Brouglit aspirator on hoard thickly coaterd with

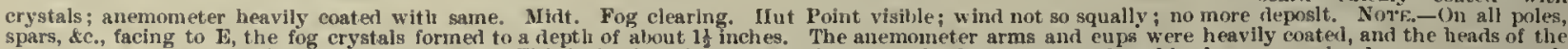
spars, \&c., facing to $\mathrm{E}$, the fog crystals formed to a depth of alout $1 \frac{1}{2}$ inches. The anemoneter arms and eups were heavily costed, and the headis of the pressurc-tubes were partially choked by the same. This is the first time since we have been in these quarters that this phenomenonl has heen so pronouneed,

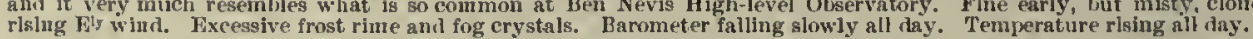

2nd.

\begin{tabular}{|c|c|c|c|c|c|c|c|c|c|}
\hline 2 & $29 \cdot 173$ & $-|-8 \cdot 5-8 \cdot 0-8 \cdot 0-8 \cdot 0|$ & 1., 1-3 & 13 & $|12-22|$ & $0+*$ & - & N. 10 & - \\
\hline 4 & $29 \cdot 171$ & $--9 \cdot 5-5 \cdot 0-5 \cdot 0-5 \cdot 0$ & E, 3-4 & 16 & $18-25$ & $0+*$ & 一 & N. 10 & - \\
\hline 6 & $29 \cdot 155$ & $--5 \cdot 5-2 \cdot 3-2 \cdot 3-2 \cdot 3$ & E, 2-3 & 19 & $15-20$ & $0+*$ & - & N. 10 & - \\
\hline 8 & $29 \cdot 150$ & $--3 \cdot 0-1 \cdot 0-1 \cdot 0-1 \cdot 0$ & S hy E, 4-5 & 25 & $30-35$ & $0-4 *$ & - & N. 10 & - \\
\hline 10 & $29 \cdot 174$ & $--2 \cdot 1-1 \cdot 4-1 \cdot 4-1 \cdot 2$ & $\mathrm{sE}, 3-6$ & 16 & $20-50$ & oq $+* \dot{*}$ & - & N. 10 & - \\
\hline Noon & $29 \cdot 182$ & $-3 \cdot 7-3 \cdot 0-3 \cdot 0-3 \cdot 1$ & E by $S, 2-4$ & 15 & $20-30$ & oq $-\neq * *$ & - & N. 10 & - \\
\hline 2 & $29 \cdot 187$ & $--3 \cdot 5-3 \cdot 0-3 \cdot 0-2 \cdot 3$ & E, $1-2$ & 13 & $5-15$ & oc $*$ & - & N. 10 & 一 \\
\hline 4 & $29 \cdot 172$ & $--5 \cdot 7-5 \cdot 0-5 \cdot 0-5 \cdot 2$ & E by N, 2-4 & 14 & $15-27$ & ocq $+*$ & - & N. 10 & - \\
\hline b & $29 \cdot 146$ & $-6 \cdot 8-4 \cdot 2-4 \cdot 2-4 \cdot 2$ & Var. E, 3-5 & 26 & $25-35$ & ocq $\tau^{2}=2 *$ & - & N. 10 & - \\
\hline 8 & $29 \cdot 163$ & $--47-3 \cdot 0-3 \cdot 0-3 \cdot 0$ & Var. SE, 36 & 18 & $20-35$ & $o c+$ & - & S. 9 & - \\
\hline 10 & $29 \cdot 154$ & $--5 \cdot 7-5 \cdot 5-5 \cdot 5-5 \cdot 5$ & SE, 4-6 & 22 & $25-33$ & $o q+$ & - & S. 10 & - \\
\hline Midt. & $29 \cdot 152$ & $--6.7-5.2-5.8-5.5$ & SSE, 2-4 & 17 & $20-27$ & oq + & - & S. 10 & - \\
\hline Mean & $29 \cdot 165$ & $-\quad--3 \cdot 9-3 \cdot 9-$ & - & $1-$ & - & - & - & - & - \\
\hline
\end{tabular}

2, 4, 6 a.m. Otservation IIjill visible, fog clearing; hoar damp, and very sulall.

Shore only visible at times. 11 a.m. Temp. at Cape Armitage, $-3^{\circ} 6$; min., $-27^{\circ} 0$. Wind E Signs of clearing to Sw. Signs of clearing to $\mathrm{SW}$.

Very thick; instruments coatel in drift.

Wind very squally and varialle, St to $S$ stars dilmly vlsible Dines.

$10 \mathrm{pm}$. Il eavy lank of cloud to SW and NW : 110 drift alove 20 feet; thin Str.; stars dinly vislble through it. Nidt. Same as at 10 p.m., but with more drift. vercast and snowing most of the day, with strong varialie $E^{\text {ly }}$ and SWly winds. Barometer Temperature higher and steady.

$3 r d$

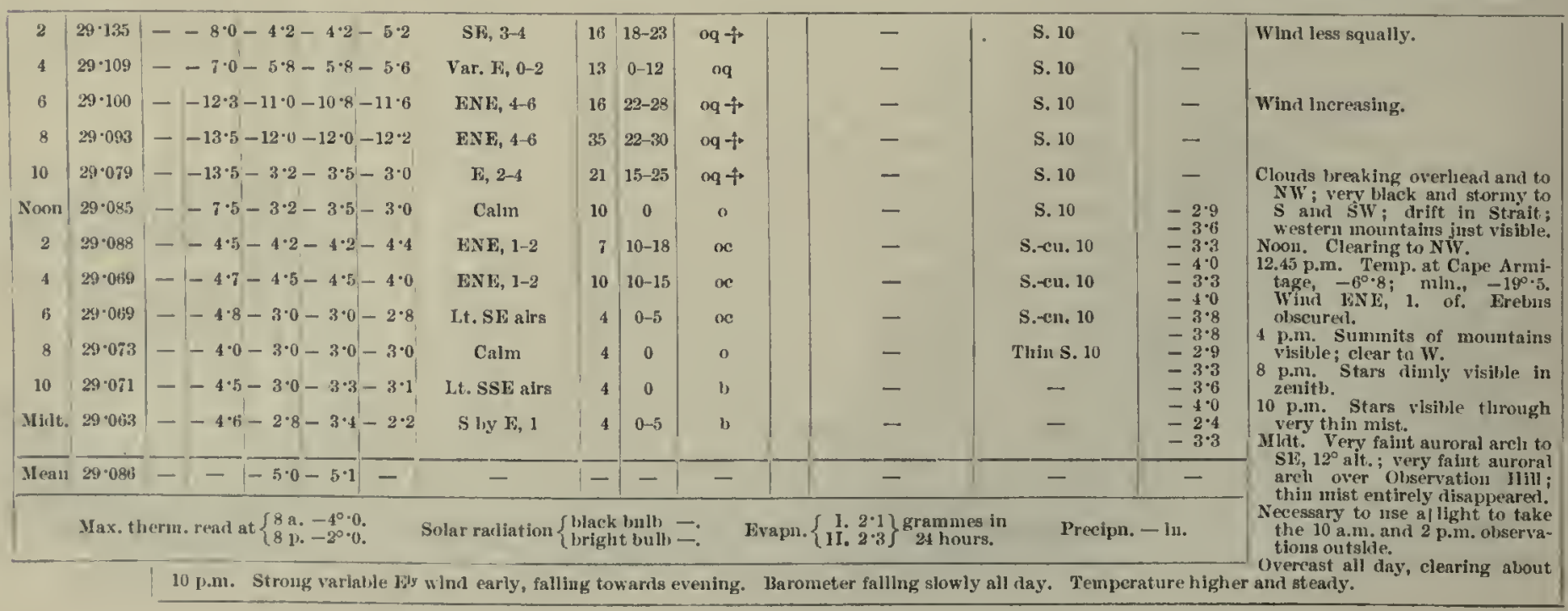


Methorological, Journal of tile "Discoveity."

\begin{tabular}{|c|c|c|c|c|c|c|c|c|c|c|c|c|c|c|c|}
\hline \multirow{3}{*}{ lionr. } & \multirow{3}{*}{ 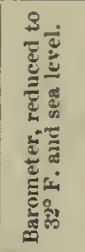 } & \multicolumn{5}{|c|}{ Tirermoneters. } & \multicolumn{3}{|l|}{ Winui. } & \multirow[b]{3}{*}{ 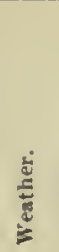 } & \multirow[b]{3}{*}{ 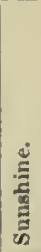 } & \multirow{2}{*}{\multicolumn{2}{|c|}{$\begin{array}{l}\text { Clomis, } \\
\text { Amoun ani novensent. }\end{array}$}} & \multirow[b]{3}{*}{$\frac{\frac{5}{\frac{5}{2}}}{\frac{2}{2}}$} & \multirow{3}{*}{ It-numak. } \\
\hline & & \multirow{2}{*}{ 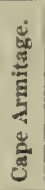 } & \multirow{2}{*}{ 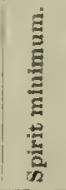 } & \multicolumn{2}{|c|}{ Jlercurial. } & \multirow{2}{*}{ 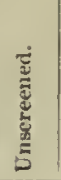 } & \multirow{2}{*}{$\begin{array}{c}\text { Direction and } \\
\text { force } \\
\text { (Beraufort seale). }\end{array}$} & \multirow{2}{*}{ 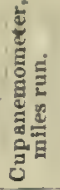 } & \multirow{2}{*}{ 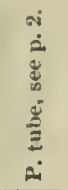 } & & & & & & \\
\hline & & & & Dry. & Wet. & & & & & & & Upper. & Lower. & & \\
\hline 2 & $29 \cdot 0+7$ & - & $-5 \cdot$ & $-3 \cdot 3$ & $-3 \cdot 7$ & $-3 \cdot 6$ & Caim & 2 & 0 & 1) & & - & - & $-3 \cdot 5$ & Very thin miat over aky, lut atark \\
\hline 4 & $29 \cdot 028$ & - & $-8 \cdot$ & $-7 \cdot 0$ & $-7 \cdot 0$ & $-8 \cdot 3$ & IS 1\%, 1-2 & 2 & $5-10$ & i) & & - & - & $-7 \cdot 6$ & al ist difinerseri. \\
\hline 6 & $29 \cdot 027$ & - & $-9 \cdot$ & $-7 \cdot 0$ & $-7 \cdot 5$ & $-10 \cdot 5$ & Lt. NE airs & 7 & 0 & b & & - & - & $-10 \cdot 1$ & \\
\hline 8 & $29 \cdot 020$ & - & $-14 \cdot$ & $-12 \cdot 0$ & $-12 \cdot 0$ & $-12 \cdot 0$ & Lt. NE airs & 1 & 0 & be & & - & Fr. $-8.4, \mathrm{Nl}$ & - & \\
\hline 10 & $29 \cdot 007$ & - & -13 & $-10 \cdot 2$ & $-10 \cdot 2$ & $-12 \cdot 0$ & Caim & 2 & 0 & be & & - & S.-cu. 4, F & $-10 \cdot 8$ & Threntening look in s and SW. \\
\hline Noon & $29 \cdot 00 \cdot t$ & - & $-15 \cdot$ & $-15 \cdot 0$ & $-15 \cdot 0$ & $-15 \cdot 0$ & Calm & 1 & 0 & oc & & - & S.en, b, w & $\begin{array}{l}-11.2 \\
-14.4 \\
-11.8\end{array}$ & 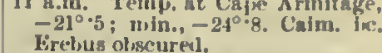 \\
\hline 2 & $29 \cdot 003$ & - & $-15 \cdot$ & $-4 \cdot 0$ & $-4 \cdot 5$ & $-3 \cdot 8$ & FSt, 0-1 & 2 & $0-8$ & oe & & - & A.-en. 9, Is & $\begin{array}{l}-3 \cdot 3 \\
-\quad 4 \cdot 4\end{array}$ & Nonir. Sight refraction all rumbl \\
\hline 4 & $29 \cdot 005$ & - & $-9 \cdot$ & $-6 \cdot 0$ & $-6 \cdot 0$ & $-6 \cdot 0$ & F by $\mathrm{N}, 1-2$ & 4 & $10-18$ & be & & - & Cu. 5, F & $\begin{array}{l}-5 \cdot 8 \\
-0.5\end{array}$ & $\begin{array}{l}\text { SW on wistern monntains. } \\
\text { p.m. }\end{array}$ \\
\hline$b$ & $29 \cdot 009$ & - & $-7 \cdot$ & $-4 \cdot 2$ & $-5 \cdot 0$ & $-4 \cdot 6$ & ENE, $2-3$ & 17 & $20-28$ & oe & & - & S.en. 10 & $\begin{array}{l}=4.5 \\
=4.8\end{array}$ & 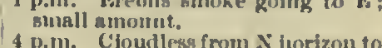 \\
\hline 8 & $29 \cdot 017$ & - & $-4 \cdot$ & $-4 \cdot 2$ & $-5 \cdot 0$ & $-5 \cdot 0$ & NF, $2-4$ & 16 & 12-24 & o & & - & S. 10 & $\begin{array}{l}-4 \cdot 4 \\
=5 \cdot 4\end{array}$ & zenilth. \\
\hline 10 & $29 \cdot 038$ & - & $-7 \cdot$ & $-6 \cdot 4$ & $-7 \cdot 0$ & $-6 \cdot 6$ & ENE, 3 & 17 & $20-30$ & o & & - & S. 10 & $=8 \cdot 7$ & \\
\hline Mirlt. & $29 \cdot 042$ & - & $-8 \cdot$ & -6.5 & $-6 \cdot 9$ & $-6 \cdot 0$ & $\mathrm{NE}, 3$ & 16 & $25-35$ & $0+$ & & - & S. 10 & $\begin{array}{l}=8 \cdot 3 \\
-8 \cdot 7 \\
\end{array}$ & Citar early, jueroming clouly and \\
\hline Mean & $29 \cdot 021$ & - & - & $-7 \cdot 2$ & $\overline{-7 \cdot 5}$ & - & - & - & - & - & & - & - & - & from Sly, variable and fresilienlng. \\
\hline
\end{tabular}

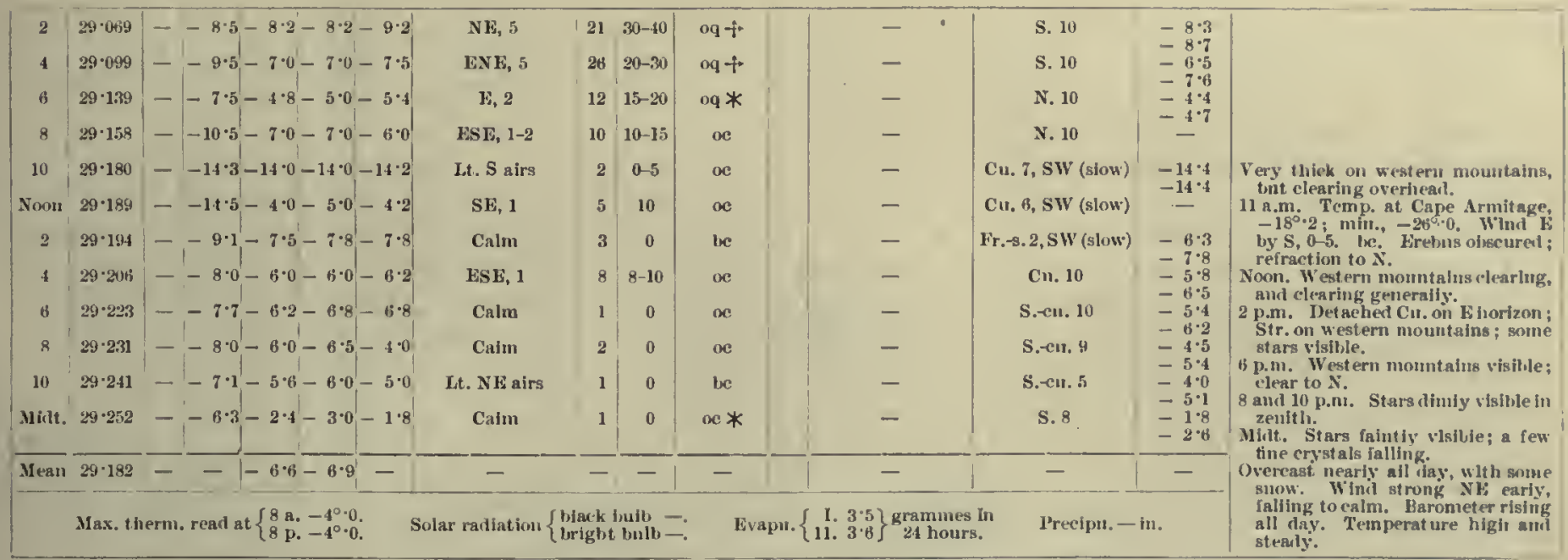

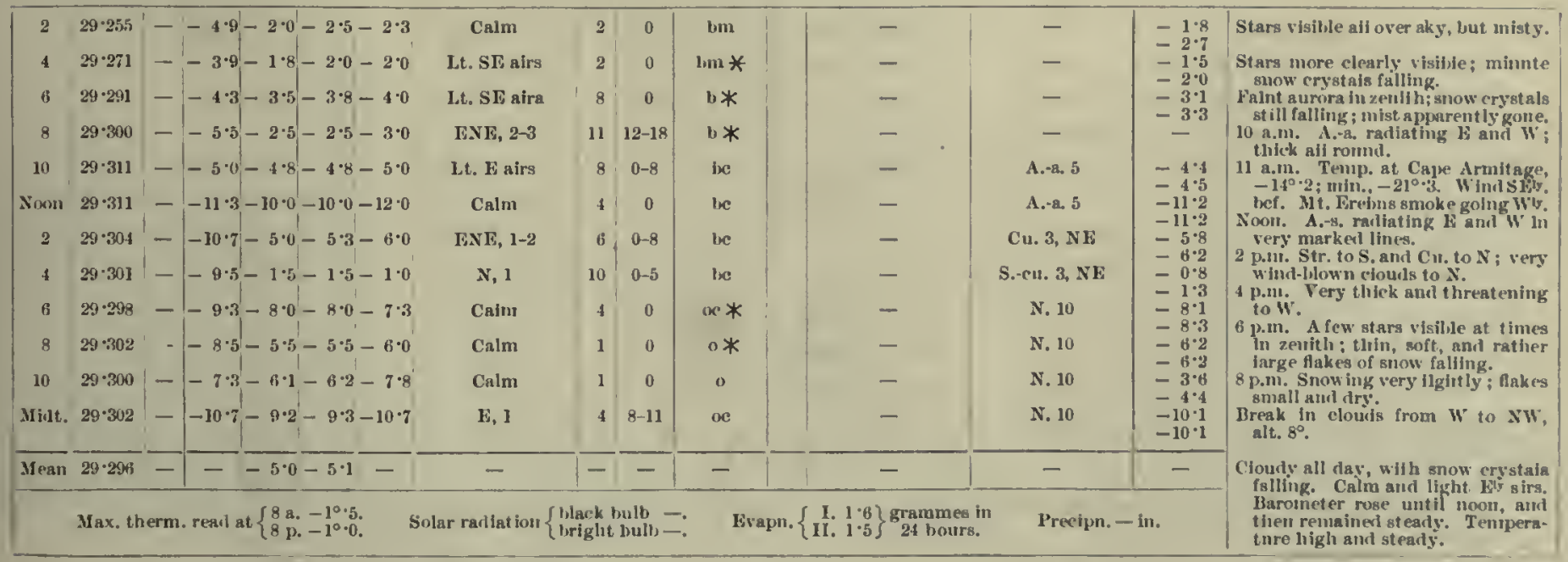


1903 -May.]

MetForological JoURsal, of THE " MIscovery."

\begin{tabular}{|c|c|c|c|c|c|c|c|c|}
\hline$\stackrel{5}{\square}$ & \multicolumn{5}{|c|}{ Thicrmomet ers. } & \multicolumn{3}{|c|}{ Wiml. } \\
\hline 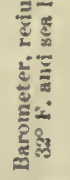 & 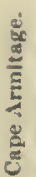 & 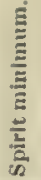 & $\mathrm{Irer}$ & Irial. & 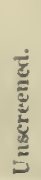 & $\begin{array}{c}\text { Direction am } \\
\text { fore } \\
\text { (Beaufort seale). }\end{array}$ & 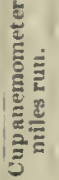 & 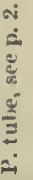 \\
\hline
\end{tabular}

7 th.

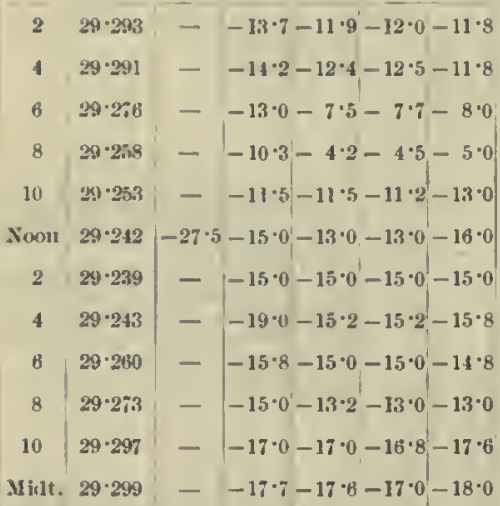

lis by $\mathrm{S}, \mathrm{I}$

Fin $S, 0-1$

Caln

$X$ ing $E, 1-2$

Calu

SE, 1

Calm

E. by $N, 2-4$

Var, $\mathbf{L}, 2-4$

var. $\mathrm{F}, 1-2$

ESE, 0-2

ESE, 1-2
(9 8-12 om

4) 8-11 bem

3 0 k.m *

2 5-10 oc

50 lice

5. 8-12 lveto

30 is

12 15-25 ocq +

14 12-25 ocq f-

14 8-15 oc

$7 \quad 0-14$

$10 \quad 7-15$

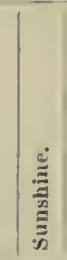

Clouris,

A mount unl movenent.

Upper.

N. 10

Cu. 1, N. 8

N. 4

N. 8

A.- -4

Ci. $-8,1$

-

Cl.-8. 3, W

$-$

S.-cu. 10 (thlin)

S.-c11. 4

S.-cu. 6

S.-cั11. 9

S.- $\mathrm{cll} .8$
IRemarks.

焉

\section{$-11 \cdot 2$}

$-10 \cdot 4$

$-10 \cdot 4$
$-7 \cdot 6$

$-8.0$

Sturs just visible in zenlt lu.

A uroral arch between 1 and $\mathrm{S}$ alt. $12^{\circ}$; hand of auroral ligh

-11.9 A.-8, rullathy $\mathrm{K}$ and $\mathrm{W}$ aлut

$-11 \cdot 9$

$-15 \cdot 5$

$-16 \cdot 2$

$-16 \cdot 4$

rely istinct, witlt hly clust ers of ractiating cloucls at the

Noon. Ilisty to $\mathrm{S}$ and $\mathrm{W}$, the radiating hands lave apparently (iis)kersen, nlthough thery distuctly markel; low ery istincty shiilue in $\mathrm{X} \times \mathrm{W}$ about $30^{3}$ alt Noon, Nin, temul at cape mit ape $-28^{\circ} \cdot 5$. Whe Wu $A r-$ bef. Hrebus smoke going $\mathbf{B}$

\begin{tabular}{|c|c|c|c|}
\hline LSE, 1 & 9 & 7 & $i k=-$ \\
\hline Calm & 6 & 0 & hem \\
\hline Culm & 2 & 0 & $\infty *$ \\
\hline Calm & 1 & 0 & bu \\
\hline Calin & 1 & 0 & b \\
\hline Calm & 0 & 0 & bw \\
\hline Calm & 3 & 0 & b \\
\hline SE, 2-4 & 5 & $10-20$ & leqm $f$ \\
\hline F ly $5,2-4$ & 15 & $15-22$ & $\operatorname{irgmu}(U$ \\
\hline $1,3-4$ & 14 & $18-24$ & к'qu U \\
\hline E, 3 & 20 & $22-28$ & he $\bar{U}+$ \\
\hline E, 4 & 21 & $25-35$ & bf. \\
\hline
\end{tabular}
lis gool big column; Terror oiscertert.

8 p.m, Moon sltining on clourls to Niv.

0 p.m. Afoon shining belint

clomis to N.
Mlit. Clear sky to N.NW and Nit and round the mont. but cloucly, with fresli

breeze in aftemoon, falling in evening. Burometer fell until 2 p.m., and tlen rose slowly. Tenpcrature falling steadily.

8th.

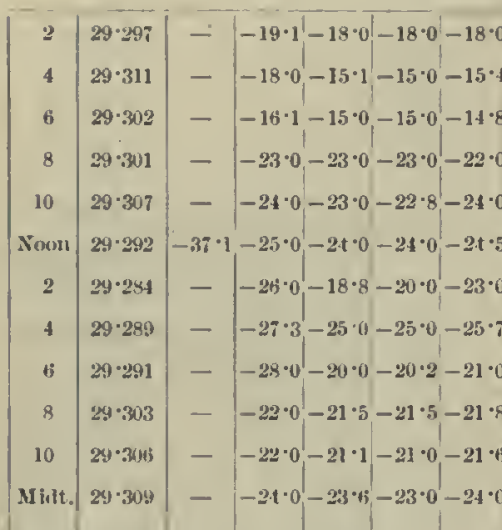

\section{S.eu. 2 \\ A.-s, 2}

A.-s. 2, N. 6

$-$

$-$

$-$

S. $2, \mathrm{~N}$

Cl.-8, 2

Ci.cus 1
S.-e1. 3, E

S.-cu. 6, SE

$\overline{\text { Mean }} \overline{29 \cdot 294}-\overline{-} \overline{-20 \cdot 7}-20 \cdot 7-$

Max. therm. reall at $\left\{\begin{array}{l}8 \mathrm{a},-12^{\circ} \circ \\ 8 \mathrm{po},-16^{\circ} \cdot 5 .\end{array}\right.$

Evapn. $\left\{\begin{array}{l}\text { I. } \\ 11.004 \\ 0 \cdot 01\end{array}\right\}$ gramuc in

Precipn. - in.

-18.0 Frost rime on instruments; -18.0
$-18 \cdot 0$
$-16 \cdot 3$ -16.24 NW. Frost rime on instru-15.2 ments; crystuls of large size $-15 \cdot 3$ falling thickly.

$-22 \cdot 7$ Low Str. to S and SW,

Str. to S and SW; refraction anl round, low down.

Nom, Mlin. temp. at Cape 1) Frebur smoke in some. colume, rislug 2000 feet and theel going to WNW.

1 p.ru. Firelous smoke rising in pist columu alout 1000 feet. anil then breaking away tu a NWh slirectlon.

4 1.1n. Whind-hlown Ci.-cus. to $\mathrm{N}$ ant $\mathrm{NW}$; leevy hank of

\begin{tabular}{|c|c|c|}
\hline Ci. 1 & - & $-22 \cdot 2$ \\
\hline - & - & $-22 \cdot 1$ \\
\hline- & - & $-22 \cdot 1$ \\
\hline - & Cu. 10 & - \\
\hline- & S.-cur. 8, K (slow) & $-20 \cdot 9$ \\
\hline- & S.-ent. 9 & $\begin{array}{l}-18 \cdot 0 \\
-18.4\end{array}$ \\
\hline - & Tliu S. 9 & $\begin{array}{l}-18 \cdot 0 \\
-15 \cdot 4\end{array}$ \\
\hline - & S. 4 & $\begin{array}{l}-18 \cdot 2 \\
-18 \cdot 6\end{array}$ \\
\hline- & s.s & $\begin{array}{l}-18 \cdot 8 \\
-18 \cdot 4 \\
-18.4\end{array}$ \\
\hline - & A..s. 5 & $\begin{array}{l}-17 \cdot 3 \\
-17.7\end{array}$ \\
\hline - & A. $-5,6$ & - \\
\hline- & A.-8. 3 & $=$ \\
\hline - & - & - \\
\hline
\end{tabular}
Str. to $s$.

1,all. Dianeter of lialo $45^{\circ}$; this appeared a pparently elese. to slip, as lt was betweenl us
and the lills to $1 \mathrm{ki}$; rime our all instruments. p.mi. leime or

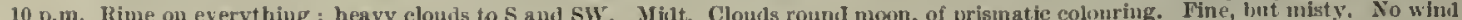
until 4 p.m., when it sprang up strong from SE. Barometer fell until 2 p.m., and then rose steally. Temperature lower anil steady.

9th.

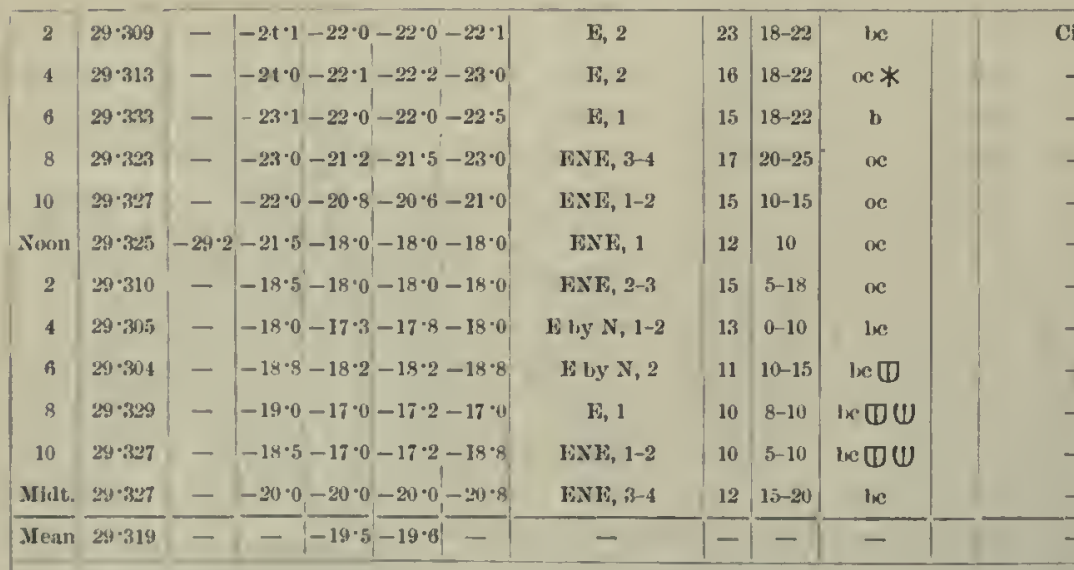

Wax, therm. reall at $\left\{\begin{array}{l}8 \text { a. }-17^{\circ} \cdot 0 . \\ 8 \text { p. }-17^{\circ} \cdot 0 .\end{array}\right.$
I'recipn. - in.
II cavy clouls to NW.

Aurora to H.

Thick all roumi.

Nin. tenip. at Cape Armitare, $-48^{\circ} \cdot 2$. 1\% hy is, 1 . Hrelus ohseured; fog all romal hor

Non. Thick sll roumul : necessary to use a light for olsser

13.n. Clomls thlu in zenitlr, and loroken in.

p.m. Very faine lase; clomu-

8 1.11.. Ilalo faint, dlameter

lonily and misty, witl frosl W'y wind all day, ant freshening more towaris the evenang. Buclinet to rise. 'Temperature st ealy. 
Mrthololocical, JuURAal, of TH: "Discoveliy."

$[1903$ - May.

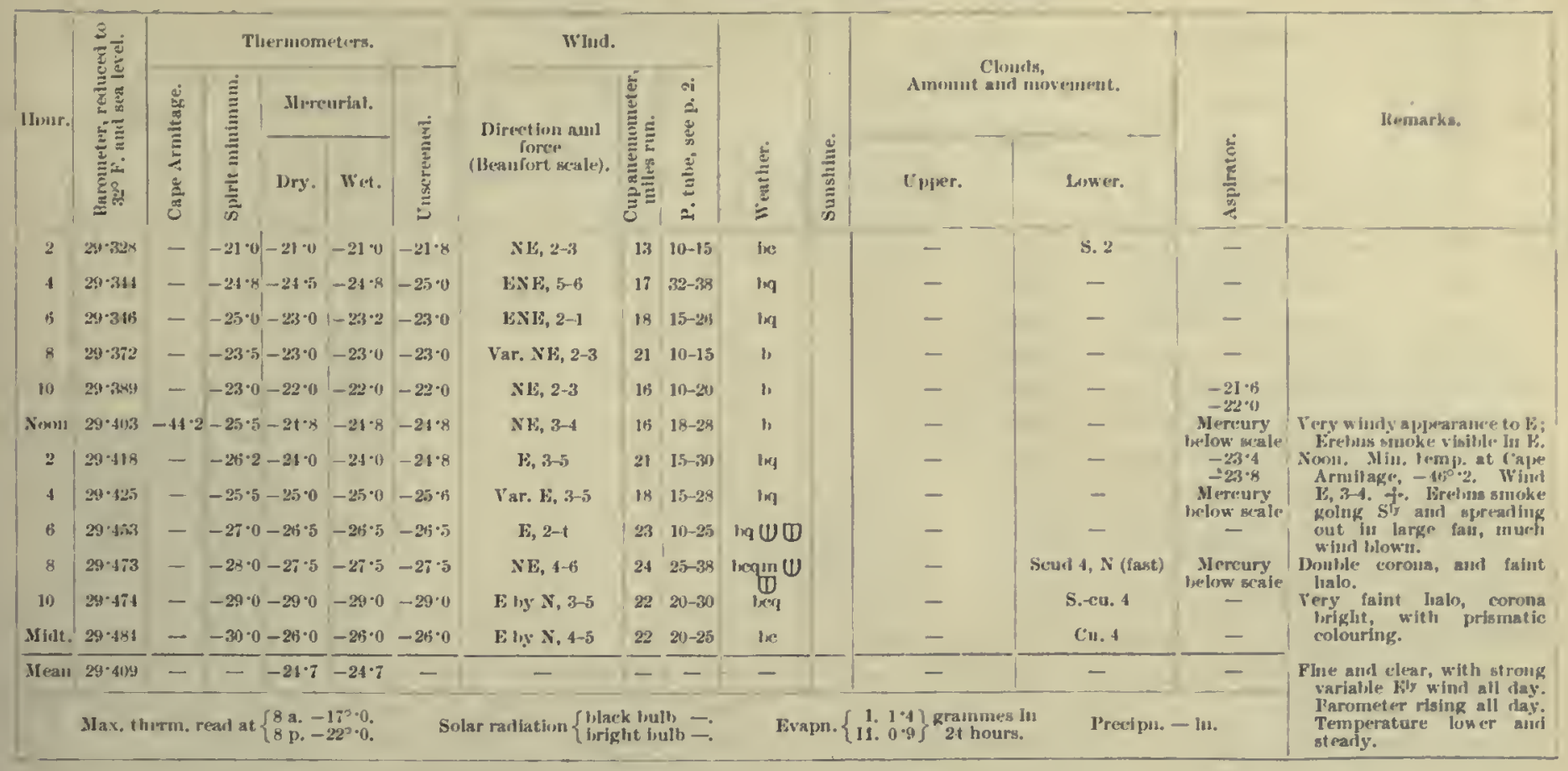

10 th.

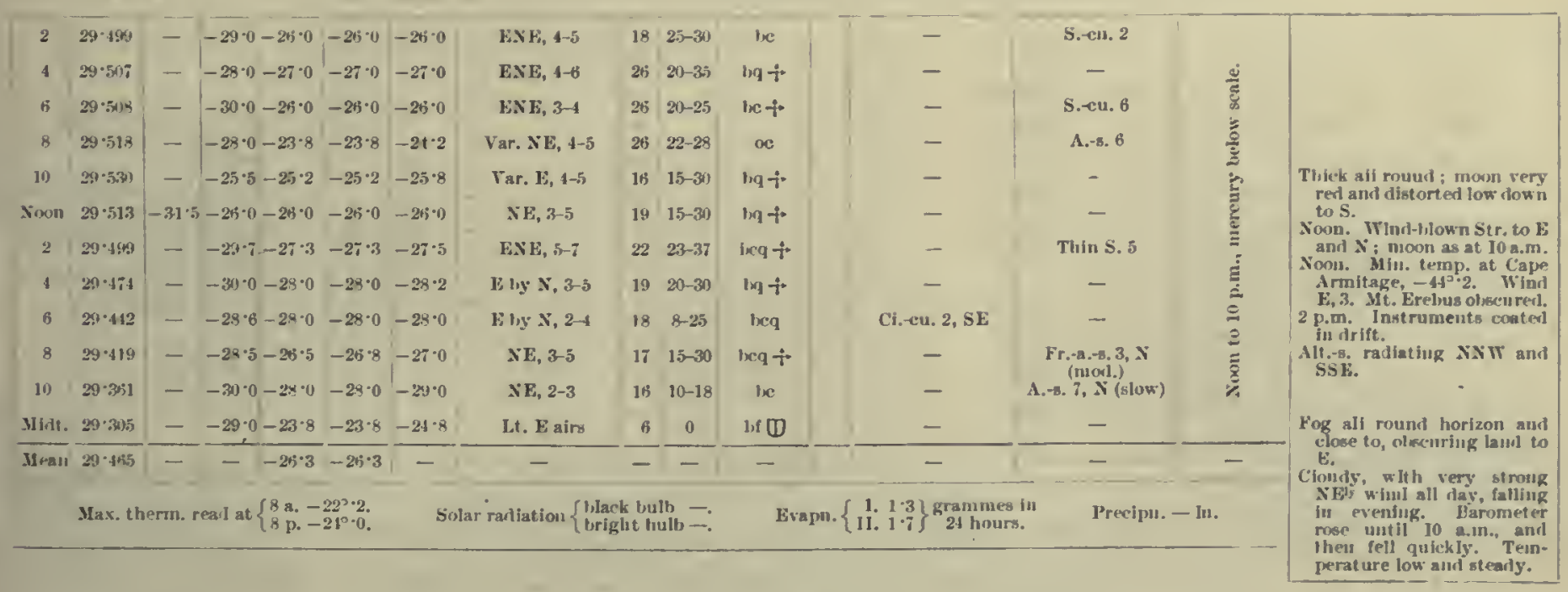

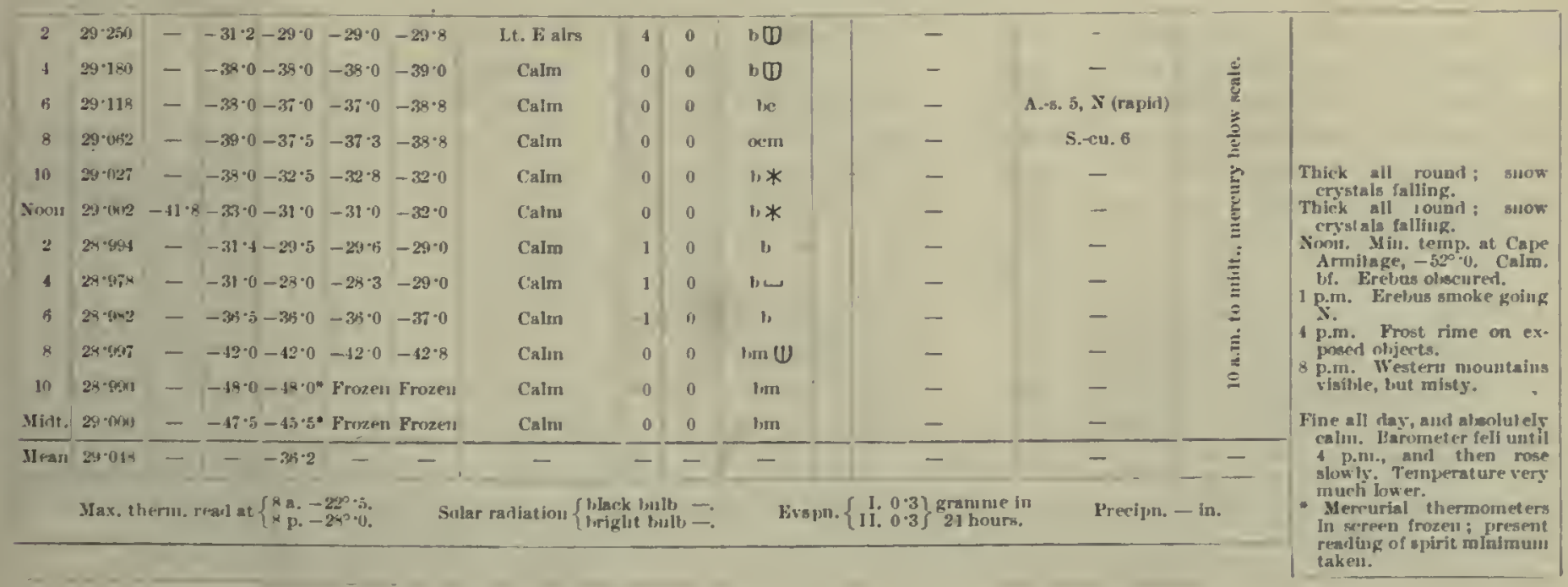

12 th. 
1903 - May.]
Methorological, Journal, of tile "Discovery."

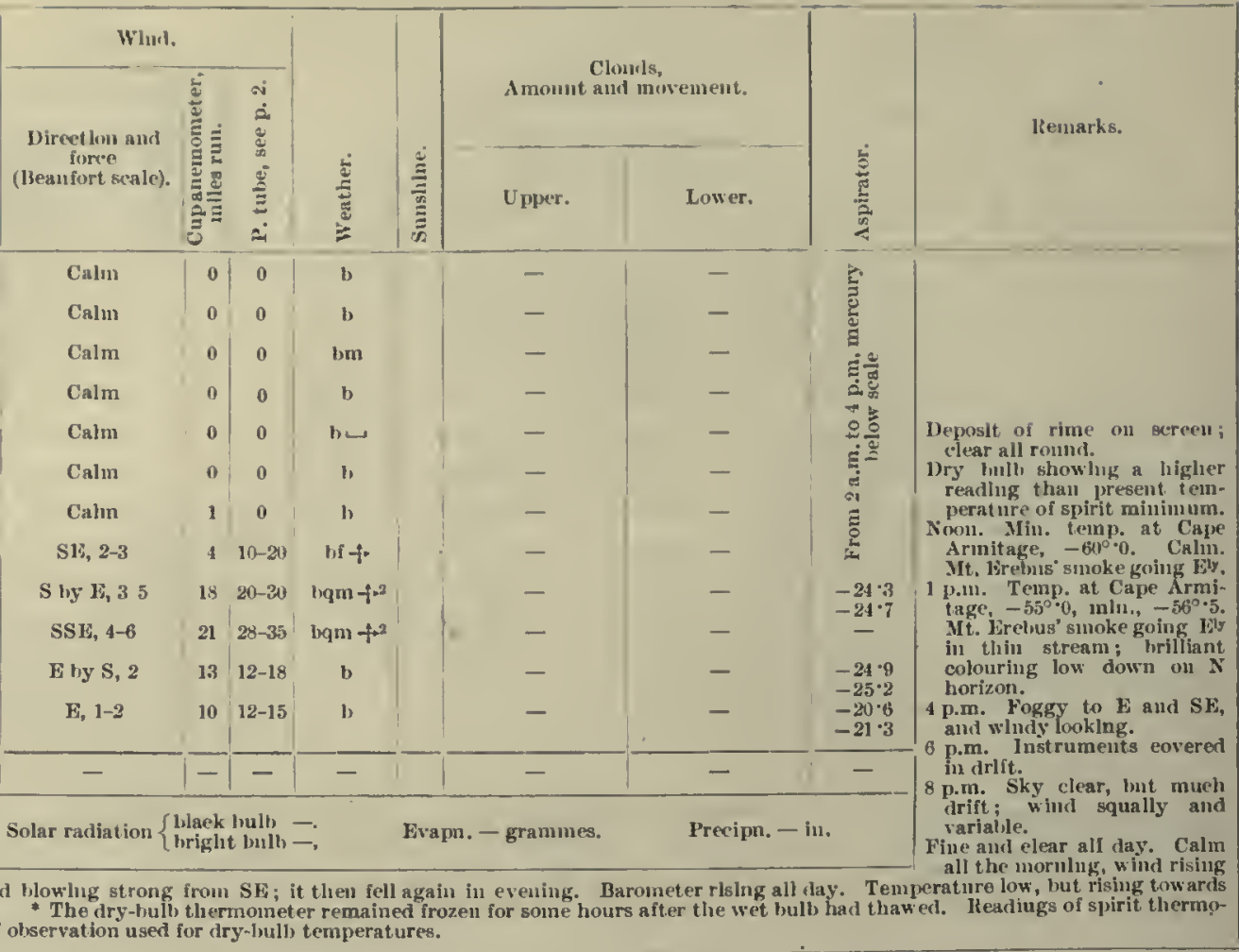

about 4 p.m. and blowing strong from SE; it then fell again in evening. Barometer rlsing all day. Temperature low, but rising towards

13th.

\begin{tabular}{|c|c|c|c|c|c|c|}
\hline \multirow{3}{*}{ llour. } & \multirow{3}{*}{ 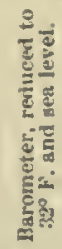 } & \multicolumn{5}{|c|}{ Thermometers. } \\
\hline & & 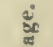 & $\underline{E}$ & Mere & Irlal. & \\
\hline & & हैं & $\frac{\vec{z}}{\hat{*}}$ & Dry. & Wet. & 窇 \\
\hline
\end{tabular}

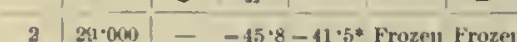

$429 \cdot 011-48 \cdot 2-47 \cdot 3^{*}$ Frozen Frozen

$629 \cdot 028--47 \cdot 5-42 \cdot 5 n$. Fruzent $-43 \cdot 0$

$8 \quad 29 \cdot 100--46 \cdot 0-43 \cdot 0^{\circ}-40 \cdot 0-40 \cdot 0$

$10 \quad 29 \cdot 147--41 \cdot 5-32 \cdot 0 *-33 \cdot 0-33 \cdot 0$

Xcon $29 \cdot 1 \mathrm{~s},-55 \cdot \times-39 \cdot 0-35 \cdot 8-34 \cdot 0-35 \cdot 0$

$2 \quad 20 \cdot 2.11--40 \cdot 0-31 \cdot 0-31 \cdot 0-30 \cdot 0$

$423 \cdot 326--34 \cdot 0-27 \cdot 5 \cdot-27 \cdot 5-26 \cdot 0$

$\begin{array}{lllll}629 \cdot 365 & - & -29 \cdot 2-24 \cdot 0 & -24 \cdot 5 & -23 \cdot 8\end{array}$

$829 \cdot 4.22--29 \cdot 5-23 \cdot 0,-23 \cdot 0-24 \cdot 0$

$1028 \cdot 456--25 \cdot 0-25 \cdot 0-25 \cdot 0-26 \cdot 0$

Midt, $29 \cdot 497-\quad-25 \cdot 0-21 \cdot 5-21 \cdot 5-22 \cdot 0$

$\overline{\text { Mean }} \overline{29 \cdot 234} \mid \overline{-}-\overline{-32 \cdot 0}-\overline{-}$

Max. therm. read at $\left\{\begin{array}{l}8 \text { a. }-42^{\circ} \circ, \\ 8 \text { p. }-22^{\circ} \cdot 0^{\prime}\end{array}\right.$ evening,
meter at time of olservation used for dry-ludl) temperatures.

14th.

\begin{tabular}{|c|c|c|c|c|c|c|c|c|}
\hline 2 & $29 \cdot 513$ & - & $-24 \cdot 0-23 \cdot 8$ & $-24 \cdot 0$ & $-24 \cdot 0$ & FSE, 1-2 & 8 & 12-15 \\
\hline 4 & $29 \cdot 533$ & - & $-24 \cdot 0-23 \cdot 0$ & $-23 \cdot 0$ & $-24^{\prime} 0$ & Lt. SF airs & 1 & $0-8$ \\
\hline 6 & $29: 514$ & - & $-26 \cdot 0-24 \cdot 8$ & $-24 \cdot 8$ & $-24 \cdot 0$ & $\mathrm{SE}, 1-2$ & 18 & $12-15$ \\
\hline 8 & $29 \cdot 490$ & - & $-27 \cdot 0-23 \cdot 0$ & $-23 \cdot 0$ & $-23 \cdot 2$ & $\mathrm{NE}, 1-2$ & 10 & $10-15$ \\
\hline 10 & $29 \cdot 483$ & - & $-33 \cdot 0-33 \cdot 0$ & $-32 \cdot 0$ & $-35 \cdot 0$ & Calm & 4 & 0 \\
\hline Xioon & $29 \cdot 460$ & $-5 \overline{5} \cdot 0$ & $-41 \cdot 5-40 \cdot 0$ & $-40 \cdot 0$ & $-41 \cdot 5$ & Calm & o & 0 \\
\hline 2 & $29 \cdot 408$ & - & $-41 \cdot 8-38 \cdot 0$ & $-38 \cdot 0$ & $-37 \cdot 7$ & Calm & 0 & 0 \\
\hline 4 & $29 \cdot 3.88$ & - & $-40 \cdot 0-40 \cdot 0$ & $-40 \cdot 0$ & $-41 \cdot 0$ & Calm & 0 & 0 \\
\hline 6 & $29 \cdot 330$ & - & $-41 \cdot 2-40 \cdot 0$ & $-40 \cdot 0$ & $-41 \cdot 3$ & Calm & 0 & 0 \\
\hline 8 & $29 \cdot 313$ & - & $-45 \cdot 5 \mid-45 \cdot 0$ & $-44 \cdot 8$ & $-46 \cdot 8$ & Calm & 0 & 0 \\
\hline 10 & $29 \cdot 284$ & - & $-47^{\circ} 0-43^{\circ} 0^{*}$ & $-42 \cdot 0$ & $-43 \cdot 0$ & Calm & 0 & 0 \\
\hline Midt. & $29 \cdot 257$ & - & $-45 \cdot 2-41 \cdot 0^{*}$ & $-42 \cdot 8$ & $-44 \cdot 0$ & Calm & 0 & 0 \\
\hline Mean & $29 \cdot 417$ & - & $-\quad-34 \cdot 8$ & - & - & - & - & - \\
\hline
\end{tabular}

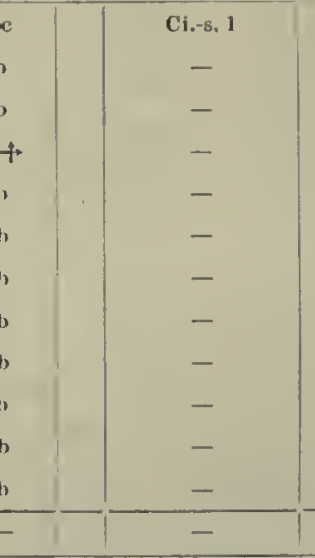

Evapu. $\left\{\begin{array}{l}\text { I. } 0.2 \\ \text { Ii. } 0.8\end{array}\right\}$ gramme in 24 hours.

\begin{tabular}{|c|c|}
\hline S.cu. 3 & -238 \\
\hline- & $\begin{array}{r}-23.8 \\
-23 \cdot 4 \\
-230.4\end{array}$ \\
\hline- & $\begin{array}{l}-20.7 \\
-24 \cdot 9\end{array}$ \\
\hline - & \\
\hline- & \\
\hline -. & $\Xi$ \\
\hline- & \\
\hline- & 官总 \\
\hline - & Eे \\
\hline- & 5 \\
\hline- & 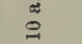 \\
\hline- & \\
\hline- & - \\
\hline
\end{tabular}

Precipn. - ill.
Could hear wind blowing in int ermittent gusts leet ween a.m. and 6 a.m.

Clear all round. Cleared heads of Dines' anemometers. Min, temp. at Cape Armitage, $-5 \tilde{r}^{\circ} \cdot 0$. Calm. bm. Erebus

smoke going jilv,
2 p.m. st. Hilmo's fire olserverl

4 p.m. Colouring on NW

6 p.m. Foggy over western

p.m. Clear aud bright moonlight.

During these low temperatures the elocks of only two of the revorling instruments contiune to work-one hygroThaph and oue thermograph. The Dines ".lock is inclined
Therature low and falling in to stop on belug handled; it stopped last night. Fine and ealm all day.
"leadings of spirit thermometer at times of observation. 15th.

\begin{tabular}{|c|c|c|c|c|c|c|c|c|c|c|c|c|c|}
\hline 2 & $29 \cdot 241$ & - & $-46 \cdot 2-4 \mathrm{t} \cdot 0^{*}$ & $-42 \cdot 0$ & $-40 \cdot 6$ & Calm & 0 & 0 & bi & - & - & \multirow{10}{*}{ 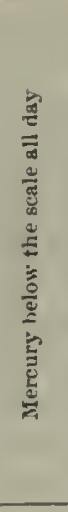 } & \multirow{12}{*}{ 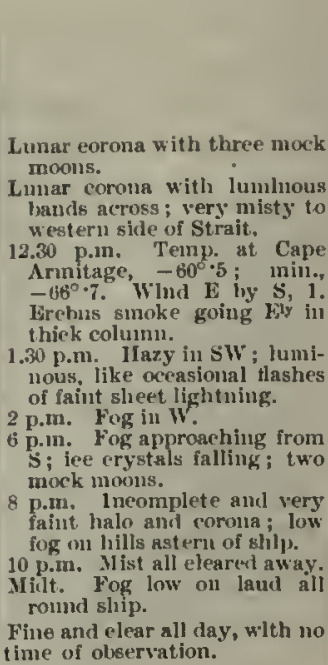 } \\
\hline 4 & $29 \cdot 2: 11$ & - & $-43 \cdot 8-41 \cdot 0^{*}$ & $-39 \cdot 8$ & $-38 \cdot 8$ & Calm & o & 0 & if & - & - & & \\
\hline 6 & $29 \cdot 214$ & - & $-44 \cdot 8-43 \cdot 6^{*}$ & $*-42 \cdot 0$ & $-41 \cdot 5$ & Calm & 0 & 0 & hi $U \approx$ & - & - & & \\
\hline 8 & $29 \cdot 200$ & - & $-43 \cdot 0-28 \cdot 0$ & $-28 \cdot 0$ & $-29 \cdot 0$ & Lt. E airs & 3 & $10-12$ & $b \omega$ & - & - & & \\
\hline 10 & $29 \cdot 202$ & - & $-29 \cdot 5-29 \cdot 0$ & $-28 \cdot 0$ & $-23 \cdot 5$ & $S E, 1$ & 2 & 0 & b $U$ & - & - & & \\
\hline Noon & $29 \cdot 193$ & - & $-34 \cdot 0-34 \cdot 0$ & $-33 \cdot 8$ & $-35 \cdot 2$ & Calm & 6 & 0 & b) & - & - & & \\
\hline 2 & $29 \cdot 184$ & - & $-40 \cdot 5-40 \cdot 0$ & $-39 \cdot 7$ & $-41 \cdot 3$ & Calm & 1 & 0 & " & - & - & & \\
\hline 8 & $29 \cdot 165$ & - & $-50 \cdot 0-50 \cdot 0^{*}$ & $-49 \cdot 2$ & Frozen & Calm & 0 & 0 & $\ln \mathbb{U}$ & - & - & & \\
\hline 10 & $29 \cdot 130$ & - & $-51 \cdot 2-46 \cdot 2^{*}$ & $-45^{*} 0$ & Frozen & Calm & 0 & 0 & bu & - & - & & \\
\hline Mlatt. & $29 \cdot 121$ & - & $-49 \cdot 0-47 \cdot 2^{*}$ & $-47 \cdot 0$ & Frozen & Calın & o & 0 & b & - & - & & \\
\hline Mean & $29 \cdot 187$ & - & --40.8 & - & - & - & - & - & - & - & - & - & \\
\hline \multicolumn{6}{|c|}{ Max, therm. read at $\left\{\begin{array}{l}8 \mathrm{a},-27^{\circ} \cdot 0 \\
81,-28^{\circ} \cdot 0 .\end{array}\right.$} & radiation & igl & $\begin{array}{l}\text { loulb } \\
\text { linults }\end{array}$ & & Evapu. - graumes. & \multicolumn{2}{|l|}{ Preeipn1. - ln. } & \\
\hline
\end{tabular}


Metronological Journal of the "Discovery."

[1903-May.

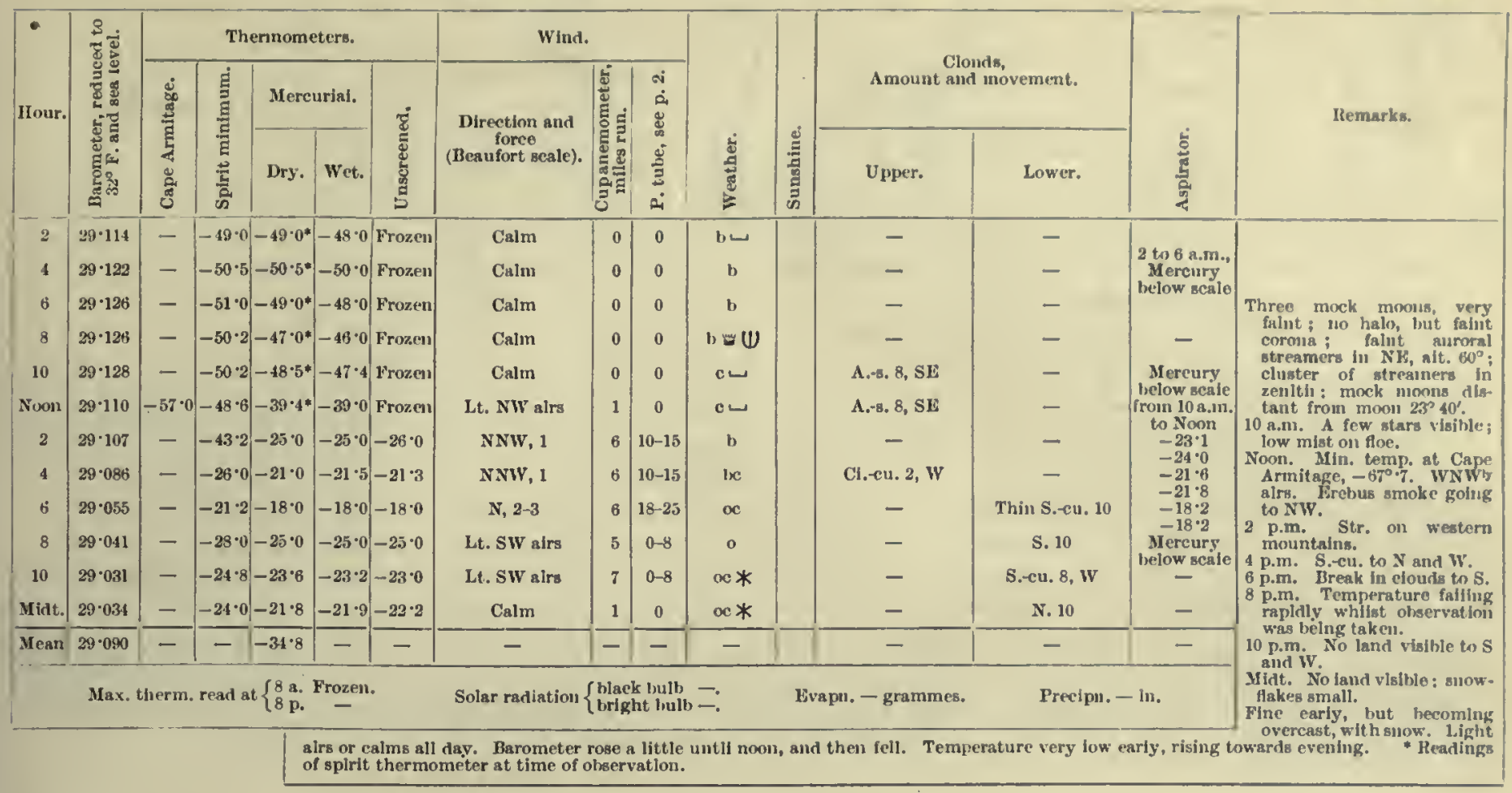

16 th.

\begin{tabular}{|c|c|c|c|c|c|c|c|c|c|c|c|c|}
\hline 2 & $29 \cdot 035$ & - & $|-22 \cdot 2|-21 \cdot 5$ & $-21 \cdot 5-20 \cdot 5$ & ENE, 2-4 & 7 & $18-27$ & og $4+*$ & - & N. 10 & - & \multirow{14}{*}{ 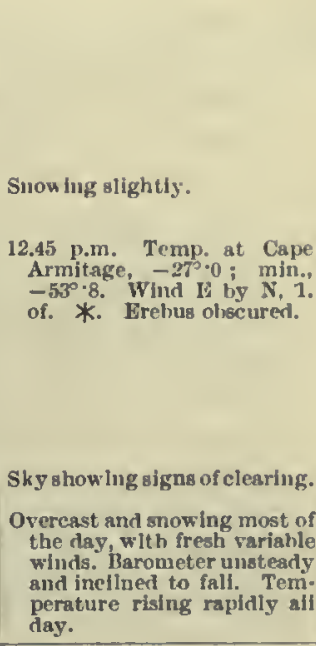 } \\
\hline 4 & $29 \cdot 016$ & - & $-22 \cdot 6-16 \cdot 8$ & $-16 \cdot 8-16 \cdot 0$ & Var. E, 3-4 & 17 & 20-34 & $0+$ & - & N. 10 & - & \\
\hline 6 & $29 \cdot 034$ & - & $-18 \cdot 0-13.5$ & $-13 \cdot 5-13 \cdot 2$ & ENE, 2-4 & 17 & $18-35$. & oq $+* *$ & - & N. 10 & - & \\
\hline 8 & $28 \cdot 993$ & - & $-19 \cdot 0-11^{\circ} 0$ & $-11 \cdot 0-11 \cdot 0$ & E by $S, 3-4$ & 18 & $20-25$ & $\infty+$ & - & N. 10 & - & \\
\hline 10 & $29 \cdot 002$ & - & $-15 \cdot 0-8 \cdot 0$ & $-8.0-6.0$ & $\mathrm{E}, 1$ & 13 & 10 & o* & - & N. 10 & $-5 \cdot 1$ & \\
\hline Noon: & 28.995 & - & $-17 \cdot 0-15 \cdot 5$ & $-15 \cdot 5-15^{\circ} 0$ & Calm. & 4 & 0 & o* & - & N. 10 & -15.2 & \\
\hline 2 & $28: 987$ & - & $-15 \cdot 4-12 \cdot 0$ & $-12 \cdot 0-12 \cdot 8$ & N, 1-2 & 4 & $10-20$ & oq $+*$ & - & N. 10 & $\begin{array}{l}-13.0 \\
-13.0 \\
-13.3\end{array}$ & \\
\hline 4 & 28.978 & - & $-14 \cdot 5-11 \cdot 8$ & $-11 \cdot 8-11 \cdot 8$ & N hy W, 1 & 8 & 8-12 & $0+*$ & - & N. 10 & $\begin{array}{l}-11.9 \\
-11.9\end{array}$ & \\
\hline 6 & $28 \cdot 973$ & - & $-12 \cdot 1-8 \cdot 5$ & $-8.8-8.5$ & NNW, 1-2 & 10 & $10-15$ & $\circ *$ & - & N. 10 & $\begin{array}{l}-6 \cdot 2 \\
=-6.3\end{array}$ & \\
\hline 8 & $28 \cdot 917$ & - & \begin{tabular}{|l|l|}
$-10 \cdot 3$ & $1 \cdot 5$
\end{tabular} & \begin{tabular}{l|l}
$1 \cdot 2$ & $2 \cdot 0$
\end{tabular} & NE, 1 & 7 & $10-12$ & $\circ *$ & - & N. 10 & $\begin{array}{l}1.4 \\
1.0\end{array}$ & \\
\hline 10 & $28: 910$ & - & $\begin{array}{lll}0.0 & 2 \cdot 0\end{array}$ & $1 \cdot 8: 2.0$ & ESE, 1 & $\theta$ & $0-5$ & $0 *$ & - & N. 10 & $\begin{array}{l}0.7 \\
0.5\end{array}$ & \\
\hline Miat. & 28.905 & - & $-57-2 \cdot 0$ & $-2 \cdot 4-1 \cdot 2$ & $\mathrm{E}, 1$ & 4 & $0-5$ & $o *$ & - & N. 10 & $\begin{array}{r}0 \% \\
-0.8\end{array}$ & \\
\hline Mean & $28 \cdot 931$ & - & $|-|-8.8 \mid$ & $|-9 \cdot 9|-$ & - & - & - & - & - & - & - & \\
\hline \multicolumn{4}{|c|}{$\begin{array}{l}\text { Max. therm. } \\
\text { read at }\end{array}\left\{\begin{array}{l}8 \mathrm{a},-11^{\circ} \circ \\
8 \mathrm{p} .\end{array}\right.$} & $\begin{array}{l}\text { Sole } \\
\text { radiat }\end{array}$ & & & & & & & & \\
\hline
\end{tabular}

17 th.

\begin{tabular}{|c|c|c|c|c|c|c|c|c|c|c|c|c|c|}
\hline 2 & 28.883 & - & $-3 \cdot 8$ & $4 \cdot 2$ & \begin{tabular}{l|l|}
$3 \cdot 8$ & $6 \cdot 2$ \\
\end{tabular} & E, $0-1$ & 3 & 0 & 0 & - & N. 10 & $5 \cdot 7$ & \multirow{14}{*}{ 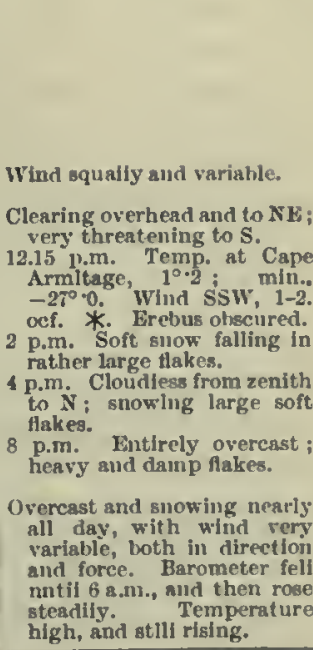 } \\
\hline 4 & 28.8615 & - & $-5 \cdot 5-$ & $2 \cdot 0$ & $-1 \cdot 3-1 \cdot 6$ & NW by $\mathbf{N}, 1$ & 5 & $5-10$ & o & - & N. 10 & -0. & \\
\hline 6 & $28 \cdot 819$ & - & $-3 \cdot 0$ & $2 \cdot 7$ & \begin{tabular}{l|l|}
$2 \cdot 3$ & $2 \cdot 3$ \\
\end{tabular} & $N, 2-4$ & 10 & $15-25$ & c & - & N. 7 & $\begin{array}{l}2.5 \\
2.5 \\
1.8\end{array}$ & \\
\hline 8 & $28 \cdot 854$ & - & $1 \cdot 0$ & 4.0 & \begin{tabular}{ll|}
$3 \cdot 8$ & $4 \cdot 2$
\end{tabular} & Calm & 7 & 0 & $\propto$ & - & S. 10 & - & \\
\hline 10 & $28 \cdot 890$ & - & $3 \cdot 0$ & 6.0 & \begin{tabular}{l|l|}
$6 \cdot 0$ & $6 \cdot 2$ \\
\end{tabular} & Var. S, 1-5 & 11 & $12-33$ & og + & - & S. 10 & - & \\
\hline Nnon & 28.901 & - & $5 \cdot 0$ & 6.8 & $6 \cdot 2 \quad 7 \cdot 5$ & Calm & 7 & 0 & o* & - & N. 10 & $7: 8$ & \\
\hline 2 & $28 \cdot 894$ & - & $6 \cdot 6$ & $7 \cdot 5$ & \begin{tabular}{rl|}
$7 \cdot 0$ & $8 \cdot 0$
\end{tabular} & ENE, 1-2 & 8 & $5-15$ & og $*$ & - & N. 10 & $8 \cdot 2$ & \\
\hline 4 & $28 \cdot 912$ & - & 6.7 & $7 \cdot 8$ & $7 \cdot 8 \quad 8 \cdot 0$ & ENE, 1-3 & 5 & $10-23$ & 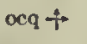 & - & N. $7, \mathrm{NE}$ & $\begin{array}{l}7.0 \\
7 \cdot 6 \\
8.6\end{array}$ & \\
\hline 6 & $28 \cdot 938$ & - & $7 \cdot 5$ & $8 \cdot 6$ & $\begin{array}{ll}8 \cdot 6 & 10.0\end{array}$ & $N E, 1-2$ & 13 & 5-12 & oc $*$ & - & N. 10 & $8 \cdot 7$ & \\
\hline 8 & $28 \cdot 985$ & - & $8 \cdot 0$ & $8 \cdot 2$ & $8.0 \quad 8.0$ & $N, 1$ & 6 & $a-8$ & o* & - & N. 10 & - & \\
\hline 10 & $29 \cdot 028$ & - & $8 \cdot 0$ & $8 \cdot 2$ & $8 \cdot 2 \quad 9 \cdot 0$ & NNE, 1-2 & 13 & 10 & om & - & N. 10 & - & \\
\hline Midt. & $29 \cdot 074$ & - & $7 \cdot 0$ & $8 \cdot 2$ & $7.0 \quad 7 \cdot 5$ & Lt. NE alrs & 7 & 0 & $o *$ & - & N. 10 & - & \\
\hline Mean & 28.920 & - & - & $5 \cdot 9$ & $5 \cdot 6 \cdot-$ & $=$ & -1 & - & - & - & - & - & \\
\hline \multicolumn{5}{|c|}{ 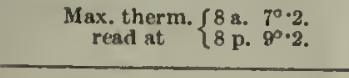 } & \multicolumn{2}{|c|}{ 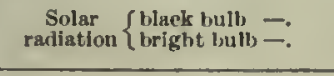 } & \multicolumn{6}{|c|}{ 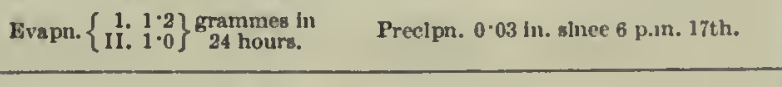 } & \\
\hline
\end{tabular}

18 th. 
1903-May.]

Mktzonologicai, Jouknal of the "Discovfizy."

\begin{tabular}{|c|c|c|c|c|c|c|c|c|c|c|c|c|c|c|c|}
\hline \multirow{3}{*}{ Ilour. } & \multirow{3}{*}{ 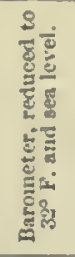 } & \multicolumn{5}{|c|}{ Thermoneters. } & \multicolumn{3}{|l|}{ Wiud. } & \multirow[b]{3}{*}{ 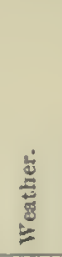 } & \multirow[b]{3}{*}{ 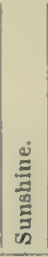 } & \multirow{2}{*}{\multicolumn{2}{|c|}{ Amount and movement. }} & \multirow[b]{3}{*}{$\frac{b}{\frac{3}{3}}$} & \multirow{3}{*}{ Remarks. } \\
\hline & & 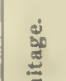 & छٌ & \multicolumn{2}{|c|}{ Mencuriai. } & \multirow{2}{*}{ | } & \multirow{2}{*}{$\begin{array}{c}\text { Direetious aud } \\
\text { forco } \\
\text { (Beaufort scale). }\end{array}$} & \multirow{2}{*}{ 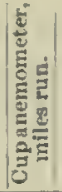 } & \multirow{2}{*}{ 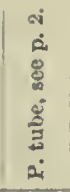 } & & & & & & \\
\hline & & 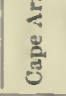 & 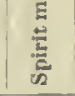 & Dry. & Wet. & & & & & & & Upper. & Lower. & & \\
\hline 2 & $29 \cdot 125$ & - & $\overline{5 \cdot 5}$ & 70 & 6.8 & $6 \cdot 4$ & Lt. Sti airs & 4 & 0 & $o *$ & & - & N. 10 & - & \\
\hline 4 & $29 \cdot 213$ & - & $6 \cdot 5$ & $8 \cdot 0$ & $7 \cdot 8$ & $7 \cdot 2$ & $1 ;, 2$ & 10 & $10-15$ & $0 *$ & & - & N. 10 & - & \\
\hline 6 & $29 \cdot 215$ & - & $5 \cdot 5$ & $6 \cdot 5$ & $6 \cdot 2$ & $3 \cdot 0$ & Fi by $N, 1-2$ & 8 & 10 & ine & & - & S.cu. 2 & - & \\
\hline 8 & $29 \cdot 266$ & - & $-3 \cdot 0$ & 0.0 & $0 \cdot 0 !$ & 0.0 & $N, 0-1$ & 11 & $8-10$ & b & & - & - & - & \\
\hline 10 & $29 \cdot 319$ & - & $-10 \cdot 5$ & $-4 \cdot 8$ & $-4 \cdot 8$ & $-5 \cdot 0$ & Culm & 1 & 0 & $b$ & & - & - & $-6 \cdot 5$ & \\
\hline Noon & $29 \cdot 316$ & $-28 \cdot 9$ & $-6 \cdot 5$ & $3 \cdot 2$ & $2 \cdot 8$ & $3 \cdot 0$ & $\mathrm{~N}, 1-2$ & 8 & 10 & b & & - & - & & Min. temp. at Cape Armitage, \\
\hline 2 & $29 \cdot 351$ & - & $-7 \cdot 5$ & $1 \cdot 2$ & $1 \cdot 0$ & $0 \cdot 8$ & $N, 0-1$ & 7 & 0 & $\mathrm{~b}$ & & - & - & $1 \cdot 4$ & Erebus smoke going NNE. \\
\hline 4 & $29 \cdot 359$ & - & -3.8 & $0 \cdot 0$ & $0 \cdot 0$ & 0.0 & $\mathbf{N}, 1$ & 4 & 10 & b & & - & - & $\begin{array}{r}1.0 \\
-0.8 \\
\end{array}$ & Foggy to S aud $\mathrm{E}$. \\
\hline 6 & $29 \cdot 3 x \cdot 3$ & - & $-2 \cdot 2$ & 0.0 & 0.0 & $-1 \cdot 0$ & N by $w, 1$ & $B$ & 10 & b & & - & 一 & $\begin{array}{l}-0.8 \\
-0.4 \\
-0.8\end{array}$ & Meteor from zenitli enstwards. \\
\hline 8 & $29 \cdot 367$ & - & $-4 \cdot 0$ & $0 \cdot 0$ & -0.2 & $-0 \cdot 2$ & Lt. $\mathbf{N}$ airs & 7 & 0 & b & & 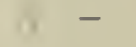 & - & $\begin{array}{r}0.0 \\
-0.4\end{array}$ & \\
\hline 10 & $29 \cdot 368$ & - & $-18 \cdot 2$ & $-15 \cdot 0$ & - & $-17 \cdot 7$ & Calm & 1 & 0 & bu & & - & - & $\begin{array}{l}-13 \cdot 4 \\
-15 \cdot 5\end{array}$ & $\begin{array}{l}\text { i)eposition of fairiy large sparkling } \\
\text { flate-like iee erystals, }\end{array}$ \\
\hline Mintt. & $29 \cdot 36.4$ & - & $-15 \cdot 2$ & -6.0 & -6.5 & $-5 \cdot 0$ & Lt. $X E$ airs & 2 & 0 & bே & & - & - & $\begin{aligned} & 5.4 \\
&-5.4 \\
&-5.4 \\
&\end{aligned}$ & Further deposit. \\
\hline Mean & $29 \cdot 304$ & 一 & - & $0^{\circ} 0$ & - & - & - & - & - & - & & - & - & 一 & fing eiear all day, with light to \\
\hline & $\begin{array}{l}\text { lax. th } \\
\text { read a }\end{array}$ & $\{8$ & $\begin{array}{l}9^{\circ} \cdot 0 . \\
5^{\circ} \cdot 0 \text {. }\end{array}$ & & $\begin{array}{c}\text { Solar } \\
\text { adiatio }\end{array}$ & $\left\{\begin{array}{l}b l \\
b r\end{array}\right.$ & h bulb - & R & $\left\{\begin{array}{l}\text { II. } \\
\text { II. }\end{array}\right.$ & \}$_{2}^{g r}$ & & Prec & ince & 8 th. & $\begin{array}{l}\text { steadily all day. Temperature } \\
\text { very nusteady and inclined to } \\
\text { fall. }\end{array}$ \\
\hline
\end{tabular}

20th.

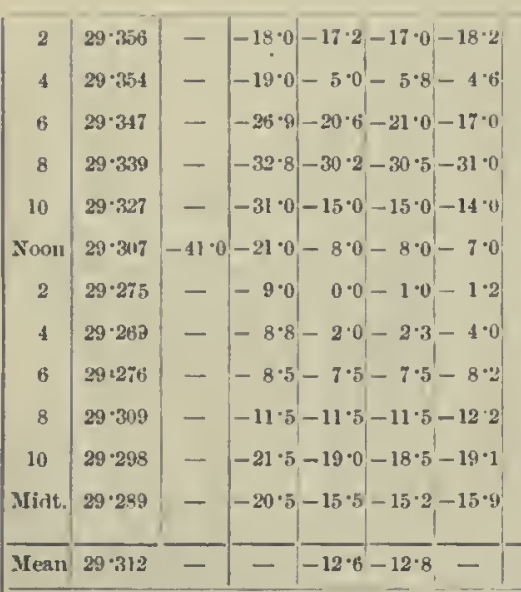

\begin{tabular}{|c|c|c|c|}
\hline Calm & 4 & 0 & b \\
\hline Lt. $\mathbf{N}$ airs & 2 & 0 & b \\
\hline Caln & 3 & 0 & b๘ \\
\hline Calm & 0 & 0 & bu \\
\hline Calın & 0 & 0 & b๘ \\
\hline ISS1, 1 & 3 & $10-12$ & b \\
\hline Var., 0-2 & 10 & $0-20$ & bq t. \\
\hline SE, 3-6 & 18 & $23-25$ & $\operatorname{loq}+$ \\
\hline Var. $18,5-7$ & 21 & $28-40$ & $\log +5$ \\
\hline Calm & 7 & 0 & b \\
\hline Lt. $\mathbf{N W}$ airs & 6 & $5-10$ & $\mathrm{bm} \sqcup$ \\
\hline Caim & 7 & 0 & $\mathrm{bm}$ \\
\hline- & - & - & - \\
\hline
\end{tabular}

Max, therm, rear at $\left\{\begin{array}{l}8 \mathrm{a}, \\ 8 \mathrm{p}, 0^{\mathrm{c}} \cdot 5\end{array}\right.$

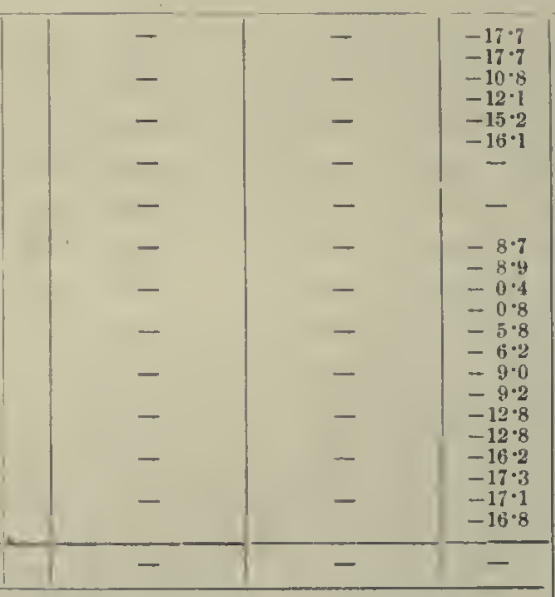

Frapn. $\left\{\begin{array}{c}\text { I. } 0.0 \\ \text { I1, } 0.0\end{array}\right\} \begin{aligned} & \text { gramme in } \\ & 24 \text { hours. }\end{aligned}$
Preeipn. - in. llime all disappeared from glass since last observation.

Some more rime deposited.

Slight auroral streamers $\mathrm{SE}$ to $\mathrm{W}$, $25^{\circ}$ to $45^{\circ}$.
Asplinator falling too raplaly for an observation.

Whind very puffy, and from various directions ; temjerat ure varring according to direction of wins. Noou. Min. Temp. at Cape Armitage, $-43^{\circ} \cdot 6$. Wind $\mathrm{E}$ by $\mathrm{S}, 1-2$. bf. Foggy to W'; Erebus smoke going NNL.

2 p.m. Calm on going out, and then a squail from $S$, and so it from $\mathrm{y}$ and carious ant squals p.m. Fogry all round.

10 p.m. Low mist olscuring land to $\mathrm{S}$ and $\mathrm{W}$; rime on rnemo-

meter glass.
Mlidt. No inore rime fallen.

Fine and clear all day, with little wind except at Noon, but in afternoon it biew up pretty strong alu variabie from $\mathrm{E}$. Nol'E.-The temperature varied considerably all day, aecordingly as the wind blew from $\mathrm{N}$ or $S$.

21 st.

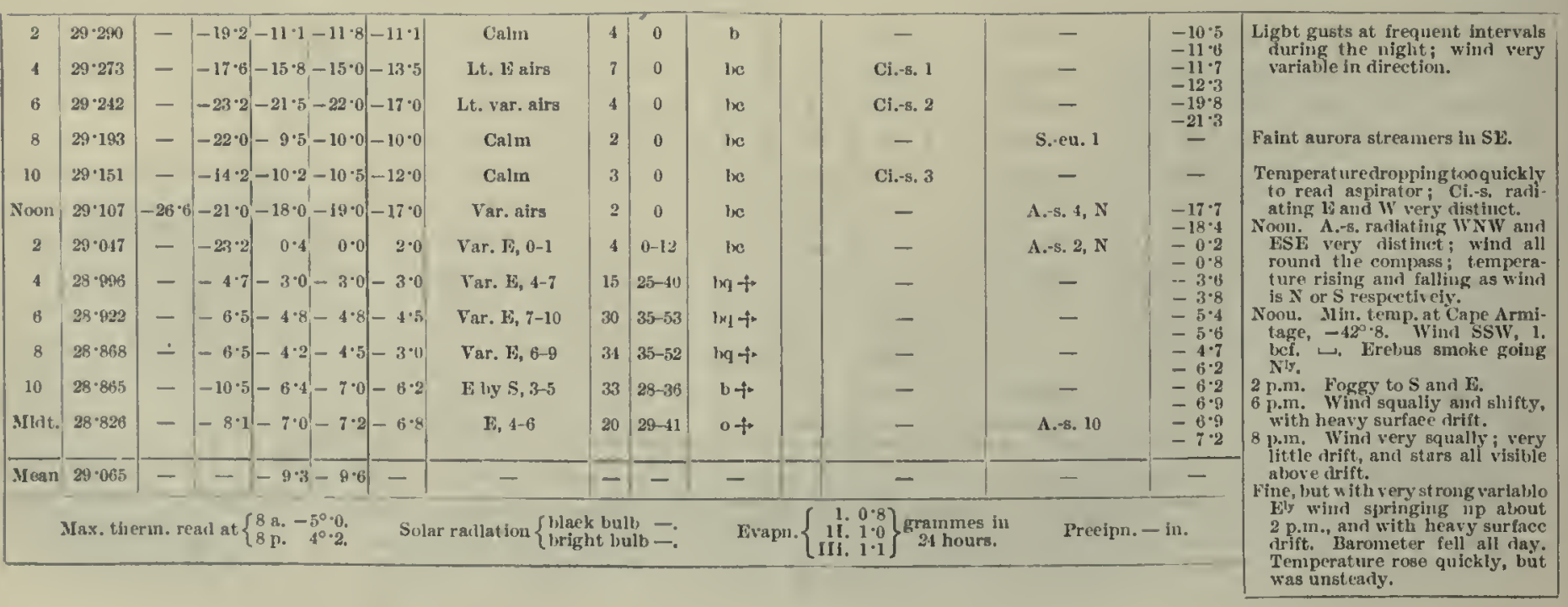


Meteorological, Journa of the "Discoviry."

[1903-May.

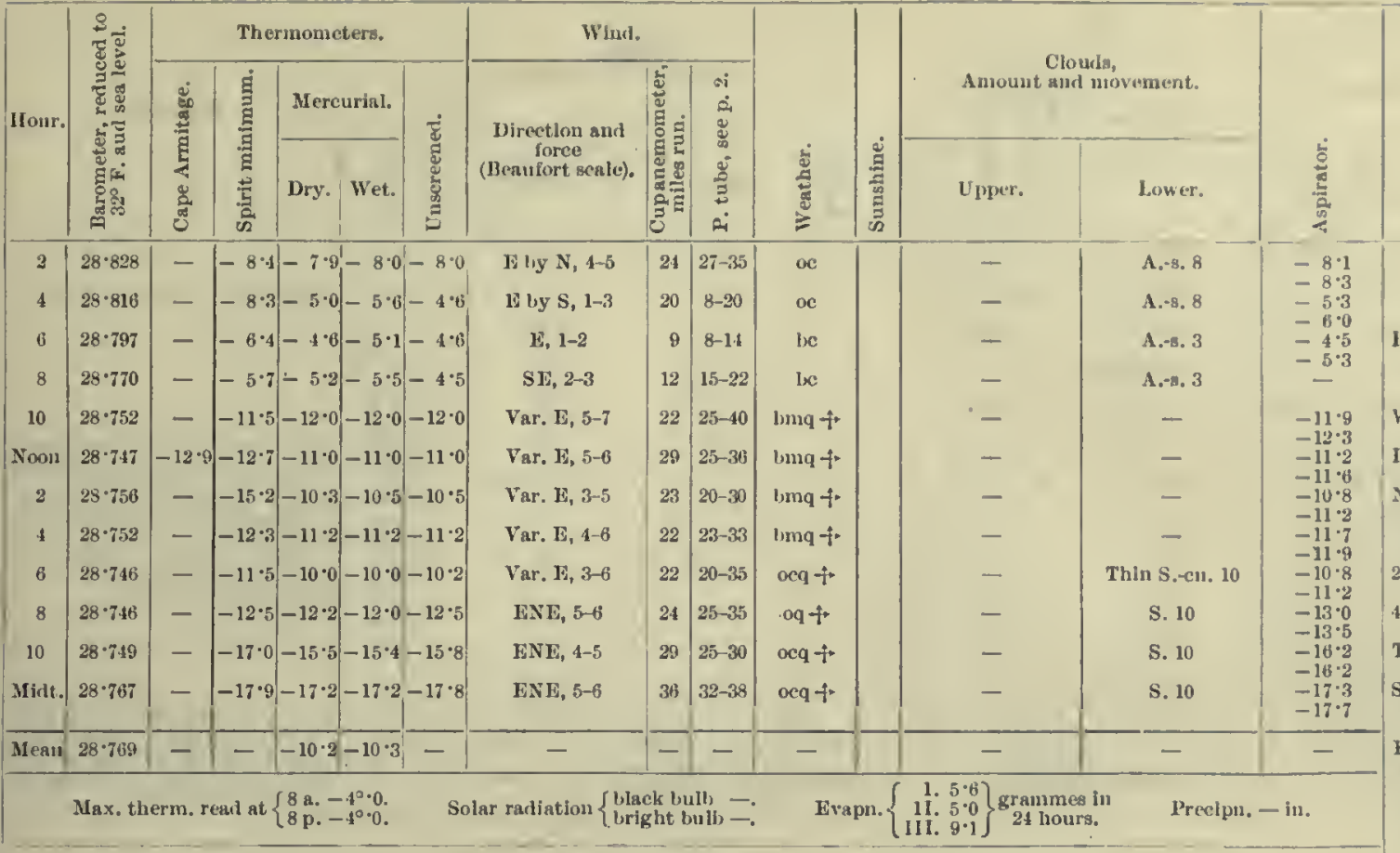

leemarks.

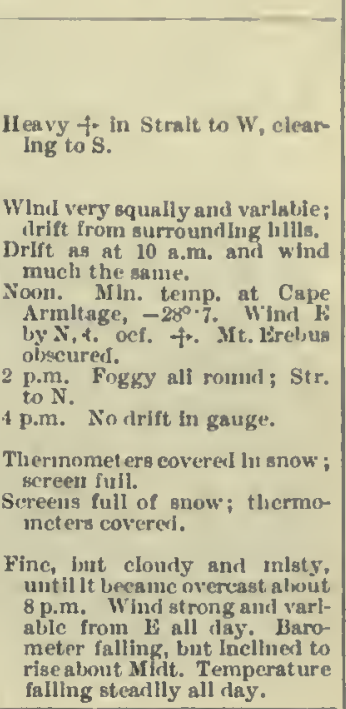

22nd.

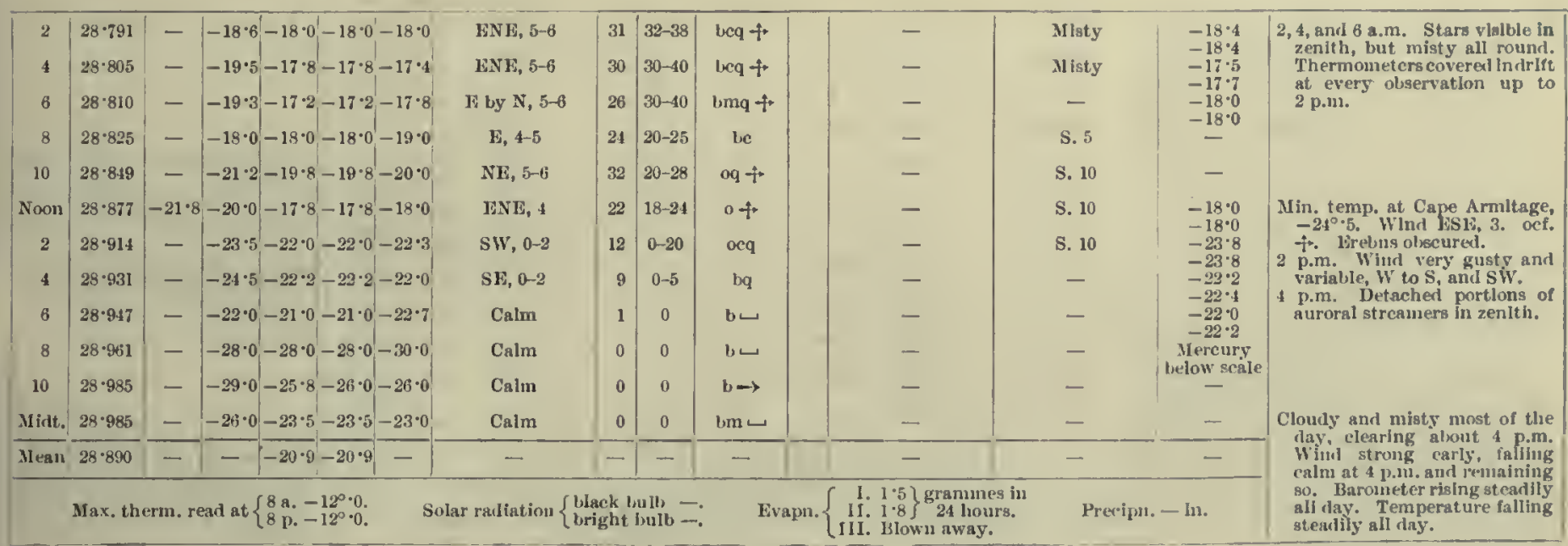

23rd. il. Thermometers coverel indrift at every observation up to . 

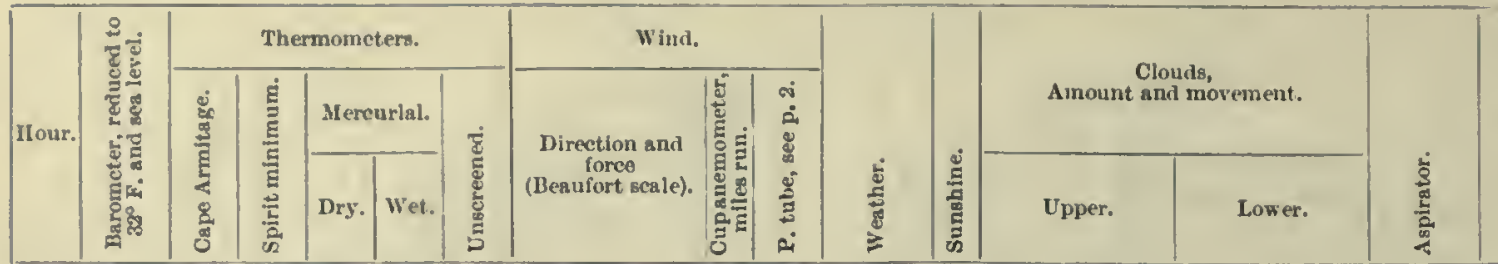

25th.

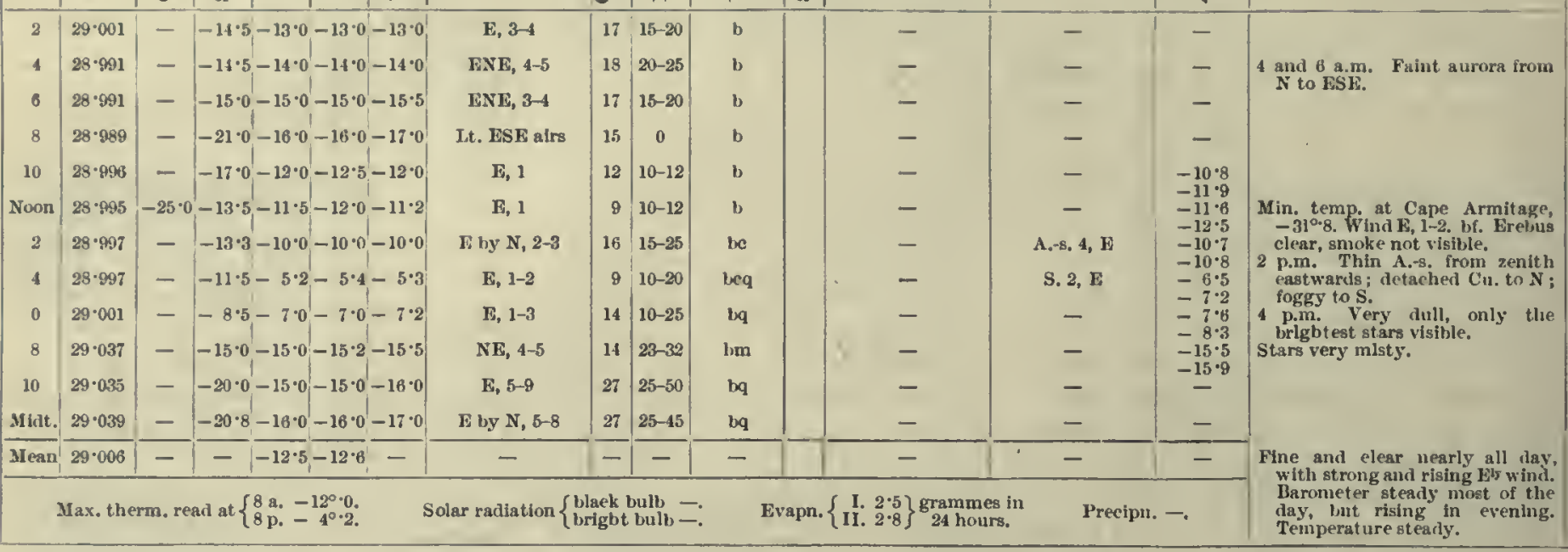

26th.

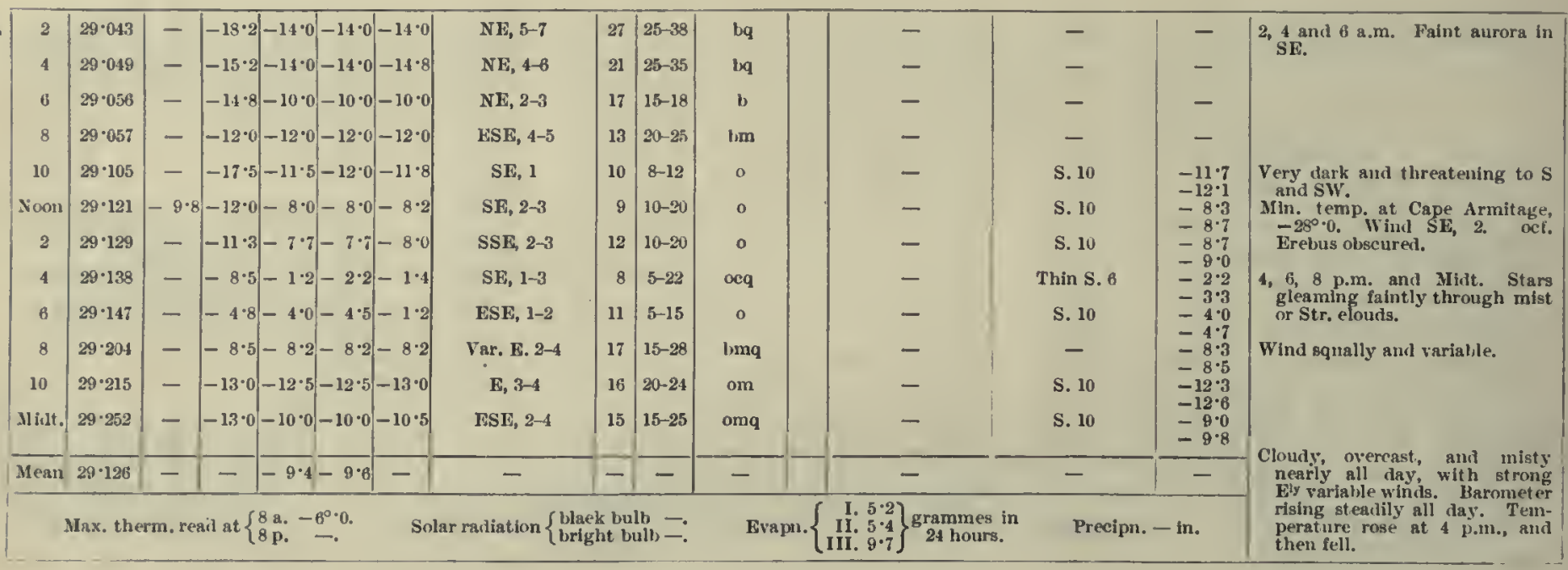

\begin{tabular}{|c|c|c|c|c|c|c|c|c|c|c|c|c|}
\hline 27th. & 2 & $29 \cdot 253$ & - & $-11 \cdot 0-8 \cdot 0-8 \cdot 2-8 \cdot 5$ & E by $S, 2-3$ & 14 & $\mid 10-16$ & $\mathrm{bm}$ & - & - & $\begin{array}{l}-8 \cdot 3 \\
=8 \cdot 3\end{array}$ & \multirow{5}{*}{$\begin{array}{l}\text { Falnt auroral streamers due } \mathbf{E} \\
\text { Rlt. } 25^{\circ} \text {. }\end{array}$} \\
\hline & 4 & $29 \cdot 261$ & - & $-10 \cdot 0-8 \cdot 0-8 \cdot 2-8 \cdot 0$ & E by $N, 2-3$ & 9 & $10-15$ & $\mathrm{bm}$ & - & - & $\begin{array}{l}-8 \cdot 3 \\
-8 \cdot 3\end{array}$ & \\
\hline & 0 & $29 \cdot 259$ & - & $-9 \cdot 5-8 \cdot 0-8 \cdot 0-8 \cdot 2$ & E, 1-2 & 9 & $8-10$ & bm & - & - & $\begin{array}{l}=8.0 \\
=8.0\end{array}$ & \\
\hline & 8 & $29 \cdot 292$ & - & $-10 \cdot 7-9 \cdot 8-9 \cdot 8-9 \cdot 8$ & Calm & 7 & 0 & b & - & - & $-8 \cdot 3$ & \\
\hline & 10 & $29 \cdot 311$ & - & $-14 \cdot 5-13 \cdot 8-13 \cdot 8-13 \cdot 2$ & Calm & 1 & 0 & b & - & - & $\begin{array}{l}-13 \cdot 0 \\
-13 \cdot 4\end{array}$ & \\
\hline & Noon & $29 \cdot 309$ & $-18 \cdot 6$ & $-14 \cdot 5-13 \cdot 0-13 \cdot 0-13 \cdot 2$ & Lt. $\mathrm{E}$ airs & 3 & 0 & b & - & - & $\begin{array}{l}-1.3 \cdot \\
-13 \cdot 4 \\
-13 \cdot 5\end{array}$ & \multirow{9}{*}{ 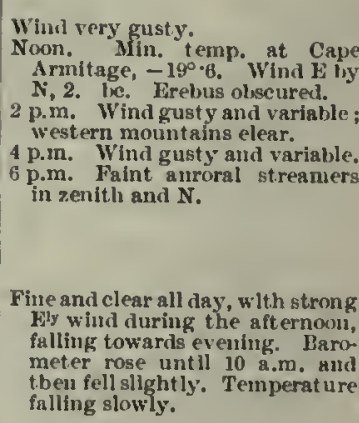 } \\
\hline & 2 & $29 \cdot 283$ & - & $-15 \cdot 0-12 \cdot 5-12 \cdot 5-12 \cdot 7$ & INNE, 2-3 & 12 & $15-25$ & b & - & - & $-12 \cdot 6$ & \\
\hline & 4 & $28 \cdot 279$ & - & $-20 \cdot 0-20 \cdot 0-20.0-20 \cdot 0$ & E, 5-8 & 26 & $30-48$ & $\mathrm{bq}=$ & - & - & $-20 \cdot 2$ & \\
\hline & 0 & $29 \cdot 2 \pi 6$ & - & $-20 \cdot 0-16 \cdot 8-16 \cdot 8-16 \cdot 5$ & NE, 3-6 & 28 & $25-37$ & $\operatorname{lng} \backsim$ & - & - & $\begin{array}{l}-20.6 \\
-17.3 \\
-17.3\end{array}$ & \\
\hline & 8 & $29 \cdot 281$ & - & $-17 \cdot 5-14 \cdot 0-15 \cdot 0-14 \cdot 0$ & NE, 2-3 & 19 & $10-18$ & b & - & - & $\begin{array}{l}-17.8 \\
-13 \cdot 5\end{array}$ & \\
\hline & 10 & $29 \cdot 284$ & - & $-21 \cdot 0-21 \cdot 0-20 \cdot 5-23 \cdot 0$ & Calm & 6 & 0 & b & - & - & -14 & \\
\hline & Midt. & $29 \cdot 2.33$ & - & $-30 \cdot 0-21 \cdot 0-21 \cdot 2-21 \cdot 0$ & NNE, 0-1 & 1 & 0 & b & - & - & - & \\
\hline & Mean & $29 \cdot 281$ & - & $-\mid-13 \cdot 8-13 \cdot 9-$ & - & - & - & - & - & 一 & - & \\
\hline & & & & & diation \{ & & - & & 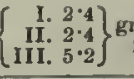 & & in. & \\
\hline
\end{tabular}


Meteorolonital Journal of tife "Discovery."

[1903-May.

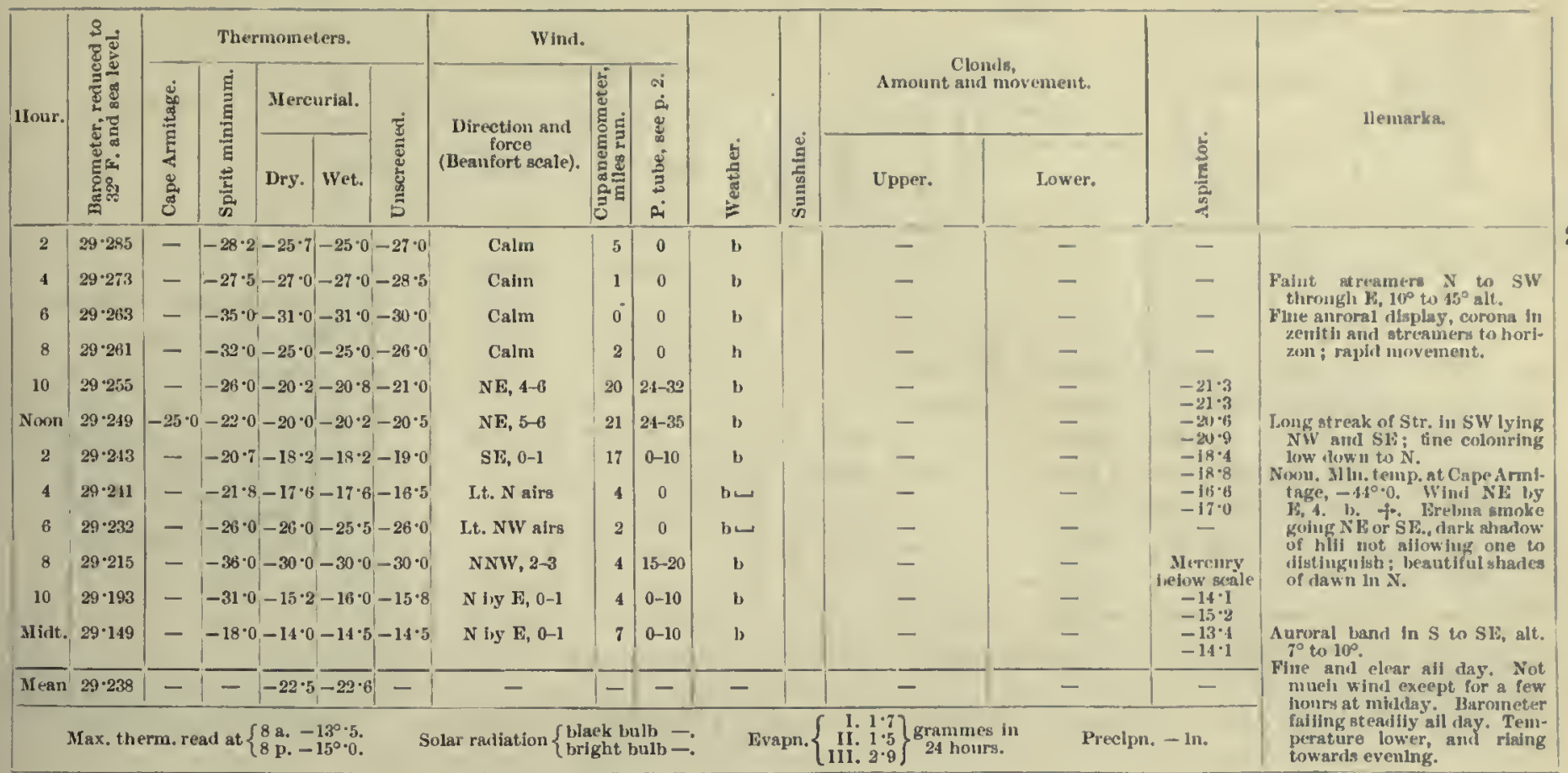

28th.

\begin{tabular}{|c|c|c|c|c|c|c|c|c|c|c|}
\hline 2 & $29 \cdot 124$ & $\dot{-}$ & $|-29 \cdot 6|-29 \cdot 5-29 \cdot 0|-30 \cdot 0|$ & Caim & 0 & 0 & b & - & - & Mercury \\
\hline 4 & $29 \cdot 110$ & - & $-30 \cdot 2-23 \cdot 2-23 \cdot 4-22 \cdot 1$ & Calm & 0 & 0 & b & - & - & $\begin{array}{c}\text { helow scaie } \\
-20 \cdot 6\end{array}$ \\
\hline 6 & $29 \cdot 104$ & - & $\mid-24 \cdot 8-18 \cdot 8-19 \cdot 0-18 \cdot 8$ & ENE, 2-3 & 8 & $15-20$ & $\mathrm{liq}$ & - & - & -26 \\
\hline 8 & $29 \cdot 094$ & - & $-18 \cdot 0-18 \cdot 0-18 \cdot 0-18 \cdot 0$ & ENE, 2-3 & 21 & $15-20$ & b & - & - & - \\
\hline 10 & $29 \cdot 098$ & - & $-19 \cdot 5 \mid-19 \cdot 5,-19 \cdot 8-20 \cdot 0$ & Var. F, 2-4 & 7 & $15-25$ & $b$ & - & - & $-19 \cdot 8$ \\
\hline Noon & $29^{\circ} 0993$ & $-33 \cdot 8$ & $|-23 \cdot 5|-23 \cdot 0-23 \cdot 0 \mid-23 \cdot 0$ & ENE, 4-6 & 18 & $20-30$ & $b+$ & - & - & $\begin{array}{l}-20.6 \\
-23.6\end{array}$ \\
\hline 2 & $29 \cdot 092$ & - & $-27 \cdot 0|-22 \cdot 6-22 \cdot 0-22 \cdot 0|$ & FNE, 2-3 & 18 & $15-23$ & b & - & - & $-22 \cdot 0$ \\
\hline 4 & $29 \cdot 098$ & - & $-25 \cdot 0-24 \cdot 0-24 \cdot 0-24 \cdot 0$ & E, 0-1 & 8 & $0-5$ & b & - & - & \\
\hline 6 & $29 \cdot 10 \tau$ & - & $-34 \cdot 0 \mid-32 \cdot 5-32 \cdot 5-37 \cdot 4$ & Calm & 2 & 0 & b & - & - & \\
\hline 8 & $29 \cdot 099$ & - & $-32 \cdot 8-32 \cdot 0-31 \cdot 0-33 \cdot 8$ & Calm & 2 & 0 & bu & - & - & $\begin{array}{l}6 \mathrm{p} . \mathrm{m} \text {. to } \\
\text { Miltt. }\end{array}$ \\
\hline 10 & $29 \cdot 101$ & - & $-37 \cdot 0-32 \cdot 2-32 \cdot 0-33 \cdot 0$ & Calm & 0 & 0 & bu & - & - & $\begin{array}{l}\text { Merenry } \\
\text { bejow scife }\end{array}$ \\
\hline s1ut. & $29 \cdot 085$ & - & $|-34 \cdot 5|-28 \cdot 5-29 \cdot 0-30 \cdot 0$ & Calm & 0 & 0 & bص & - & - & \\
\hline Ml ean & $\longdiv { 2 9 \cdot 1 0 1 }$ & - & $-^{\prime}-25 \cdot 3-25 \cdot 3-$ & - & - & - & - & - & - & - \\
\hline
\end{tabular}

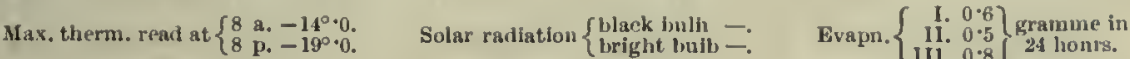

Precipn, - in.
Auroral streamess to Nes and $N$; giow to $s$.

Ners in $\mathrm{N}$ and $\mathrm{S}$.

Pattelieg of faint aurora in $\mathrm{N}$ Fr.-B. to $N$ and I: low down.

Low bank of Str. W $\mathrm{X}$ and extening to $W$.

Noon. Min. temp. at Cape Armitage, $-41^{\circ} 4$. Wint 1.Ne, 1-2. b. Large eoiumn of lirebus smoke forming mushroon (low) and theis going $N$; low bank of Str.

p.m. Fine colouring on NW horizonn.

and 6 p.m. Brigit aumora low filt. Alrorai arch extending from NE to SW througit in alt.. $z^{\circ}$ to $20^{\circ}$, mov ement to $W$ Fine and cear ail day. Wiul strong during the forenmon, bast. raim for the remsinder of the day. Barometer falling silghtity uil day. Tempera ture low and stendy

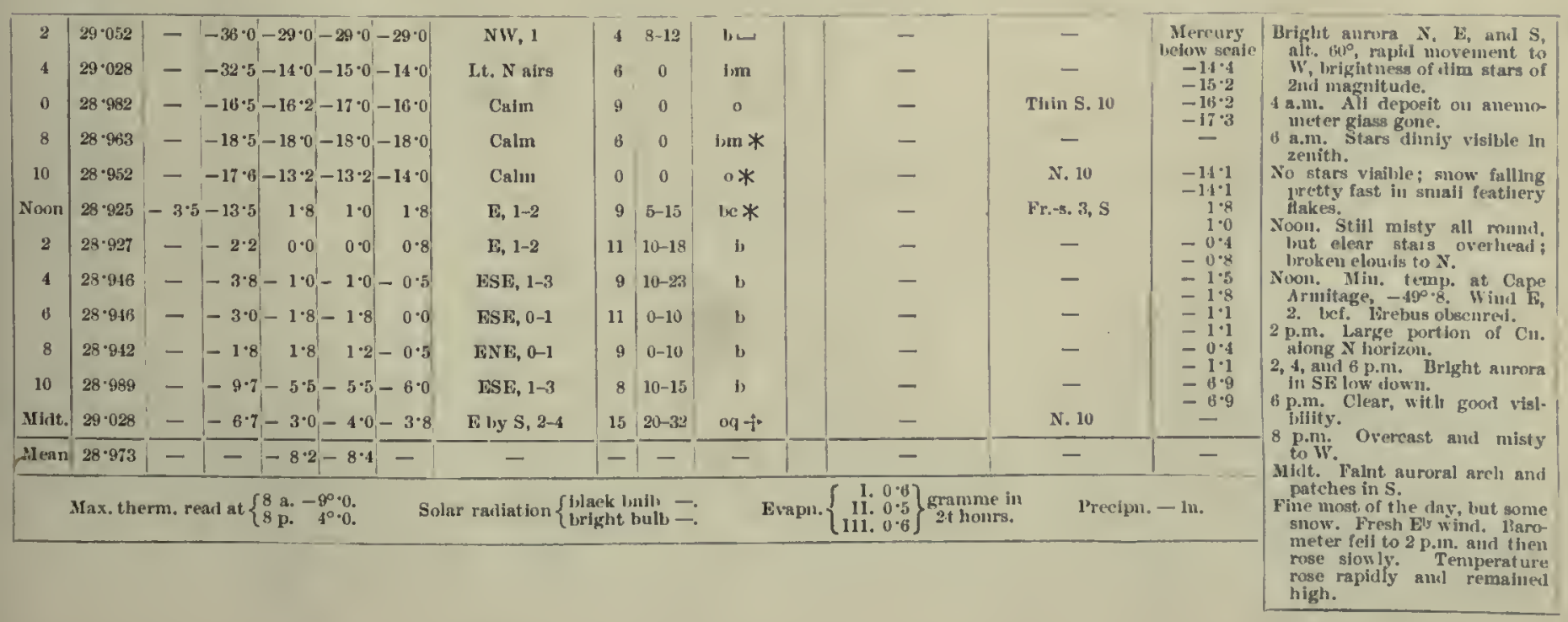

29th.

30 th. 
182

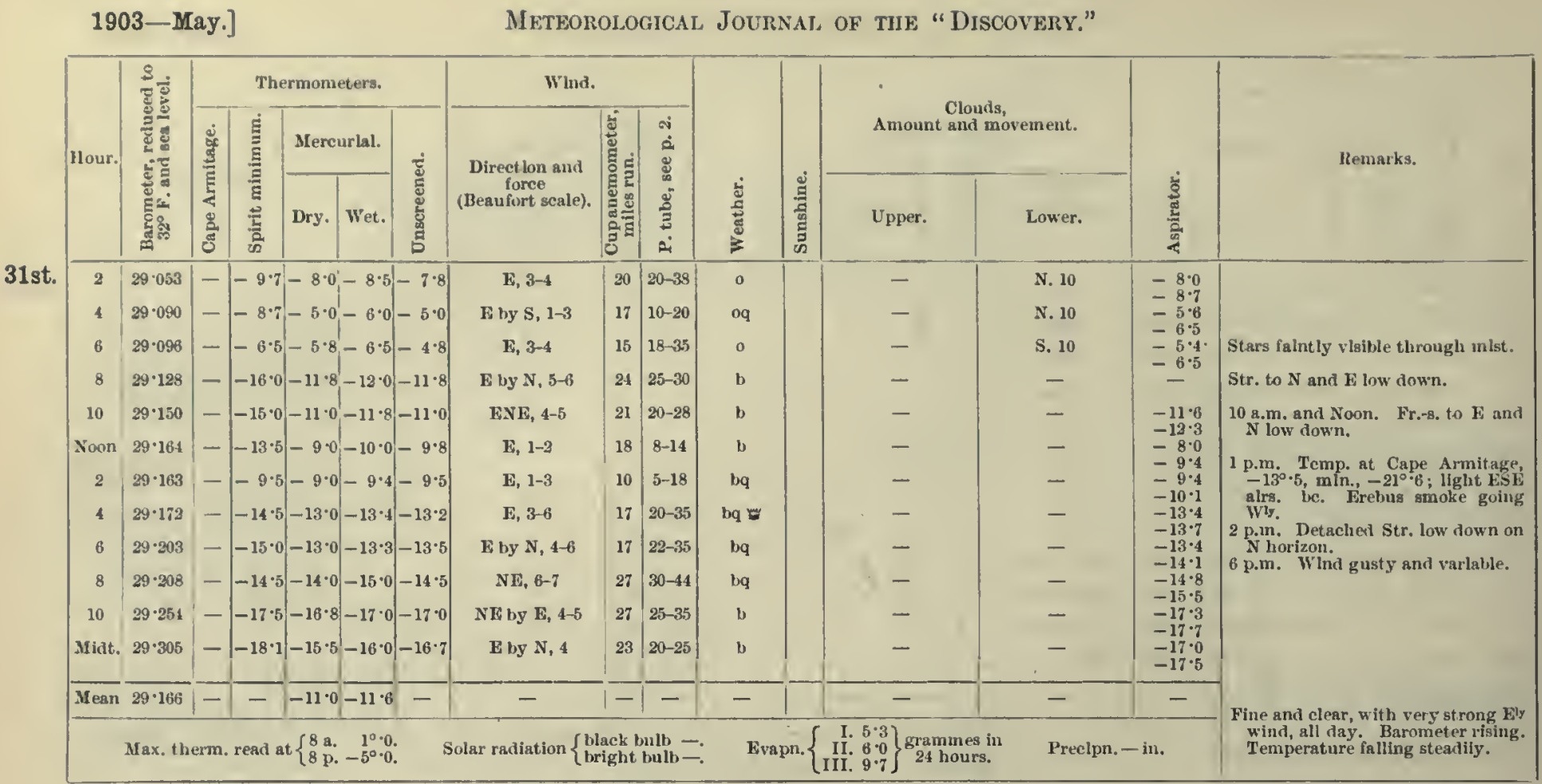


Meteorological Journat of THE "Discovery."

[1903-June,

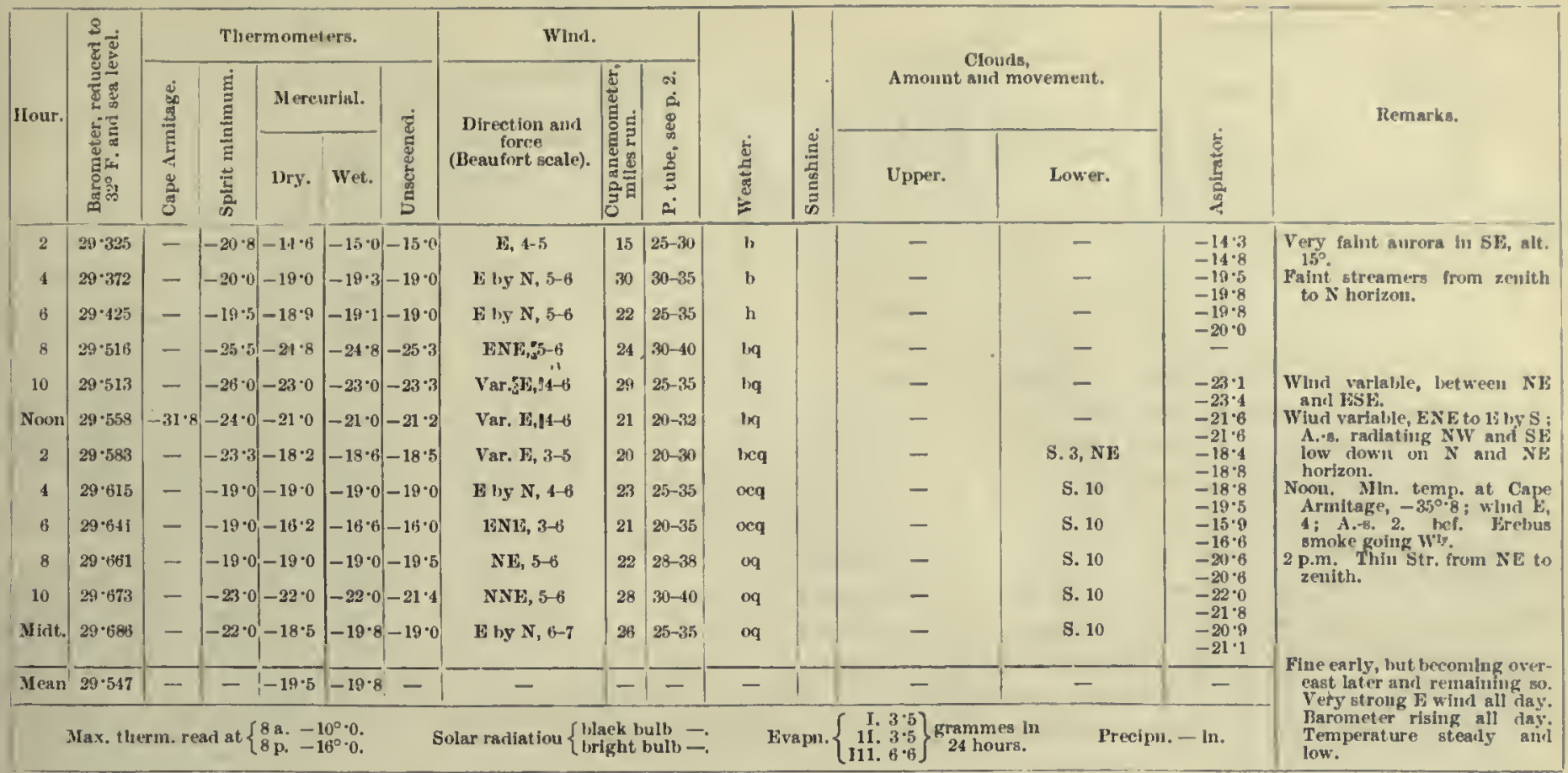

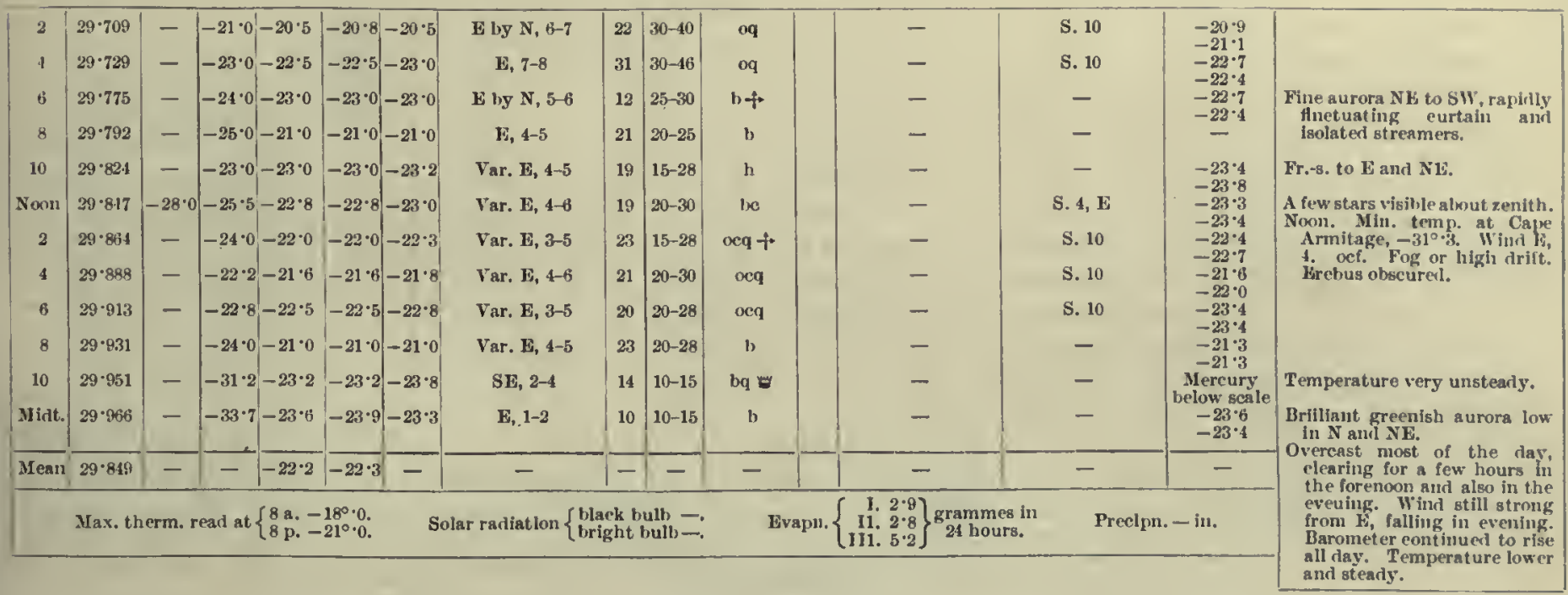

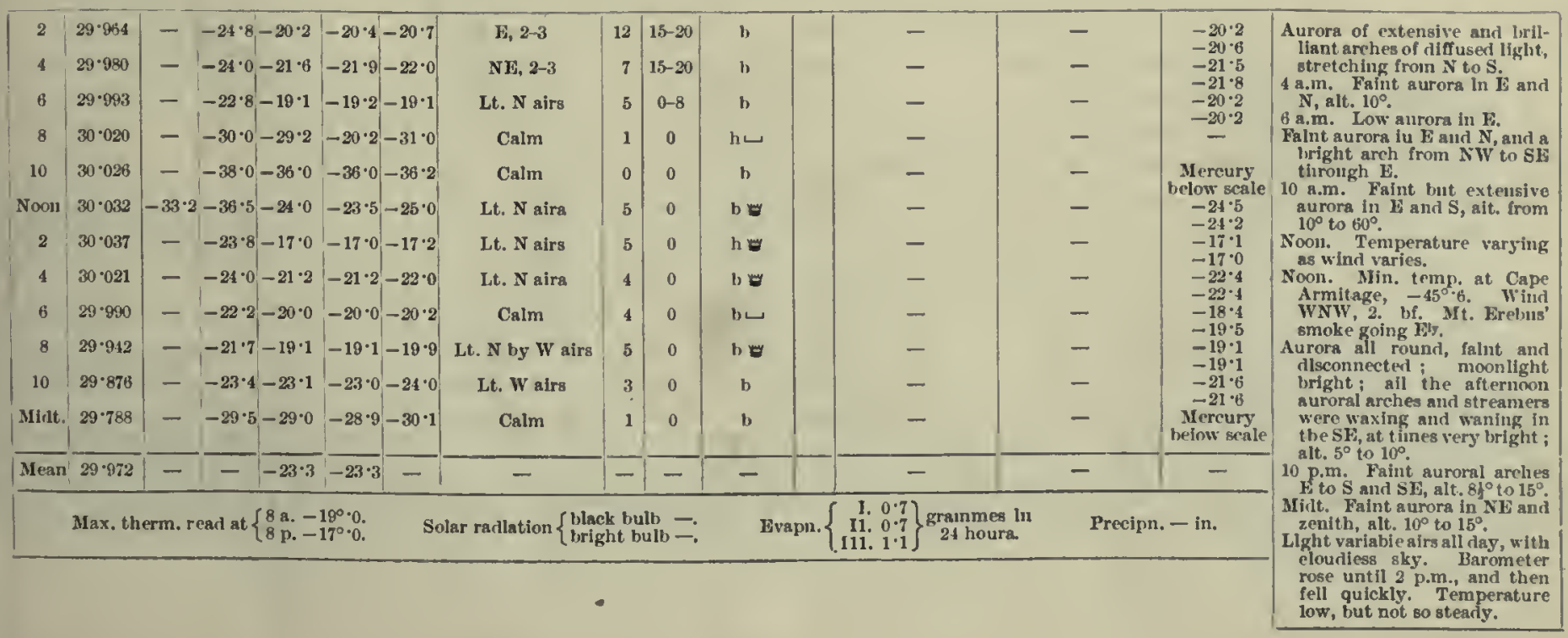


1903 - June.]

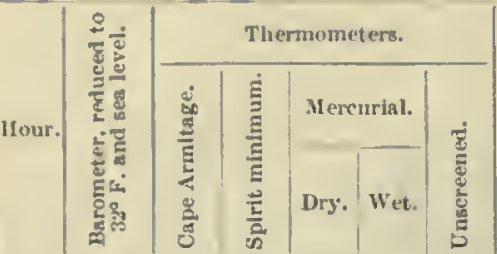

4 th.

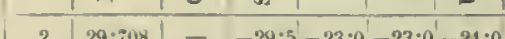

$429 \cdot(12--28 \cdot 9-22 \cdot 1-22 \cdot 1-22 \cdot 5$

$629 \cdot 501-23 \cdot 5-19 \cdot 0-19 \cdot 1-19 \cdot 1$

$829 \cdot 406--18 \cdot 0-17 \cdot 0-17 \cdot 0-17 \cdot 0$

$1029 \cdot 372--24 \cdot 0-23 \cdot 8-23 \cdot 2-23 \cdot 3$

Nooll $29 \cdot 356-28 \cdot 0-30 \cdot 0-25 \cdot 0-25 \cdot 0-25 \cdot 5$

$2 \quad 29 \cdot 320-32 \cdot 0-16 \cdot 0-16 \cdot 0-15 \cdot 8$

$429 \cdot 350-\quad-208-15 \cdot 7-15 \cdot 7-15 \cdot 7$

$629 \cdot 377--23 \cdot 8-17 \cdot 0-17 \cdot 0-17 \cdot 3$

$8 \mid 29 \cdot 381--19 \cdot 0-19 \cdot 0-19 \cdot 0-19 \cdot 5$

$10 \mid 29 \cdot 391-22 \cdot 1-21 \cdot 3-21 \cdot 3-21 \cdot 5$

Nidt. $29 \cdot 423--25 \cdot 4-25 \cdot 3-25 \cdot 3-25 \cdot 7$

Mean $\overline{29 \cdot 131}-\overline{-} \overline{-20 \cdot 4}-20 \cdot 3=$

Max. therm, read at $\left\{\begin{array}{l}8 \mathrm{a},-17^{\circ} \cdot 0 . \\ 81,\end{array}\right.$

Mrteorological Journal of the "Discoviry."

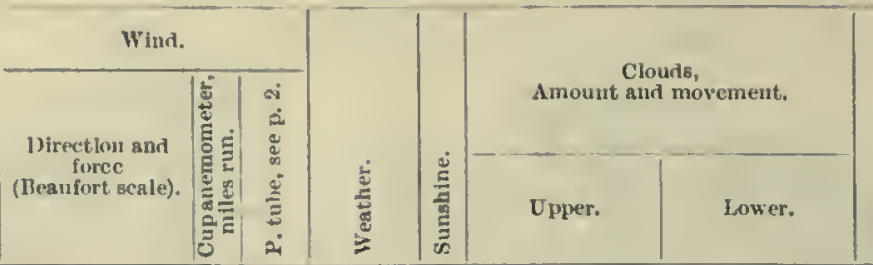

\section{Calm}

\section{Calm}

$\mathrm{E}, 0-1$

L, 2-3

S by $\mathbf{b}, 1$

$\mathrm{S}$ by $1,1-4$

ISE, 1-3

E loy S, 1-5

B, $1-5$

$\mathrm{SF}, 1-6$

SE, 3-9

S1, 2-5

10

bu

|

\begin{tabular}{l|l}
- & - \\
- & - \\
- & - \\
- & -
\end{tabular}
Solar radiation $\left\{\begin{array}{l}\text { black bulb } \\ \text { lright bulb }=\text { - }\end{array}\right.$

Evapn. - grammes.

Precipn. - ln.

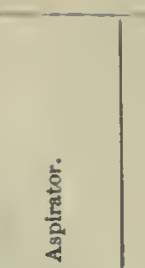

Remarks.

and variable. Nidt. Wind squally and varialle; weather clearing. Clear all the forenoon, becoming overcast later, and remaining so.

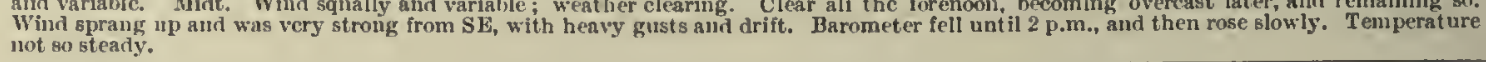

5th.

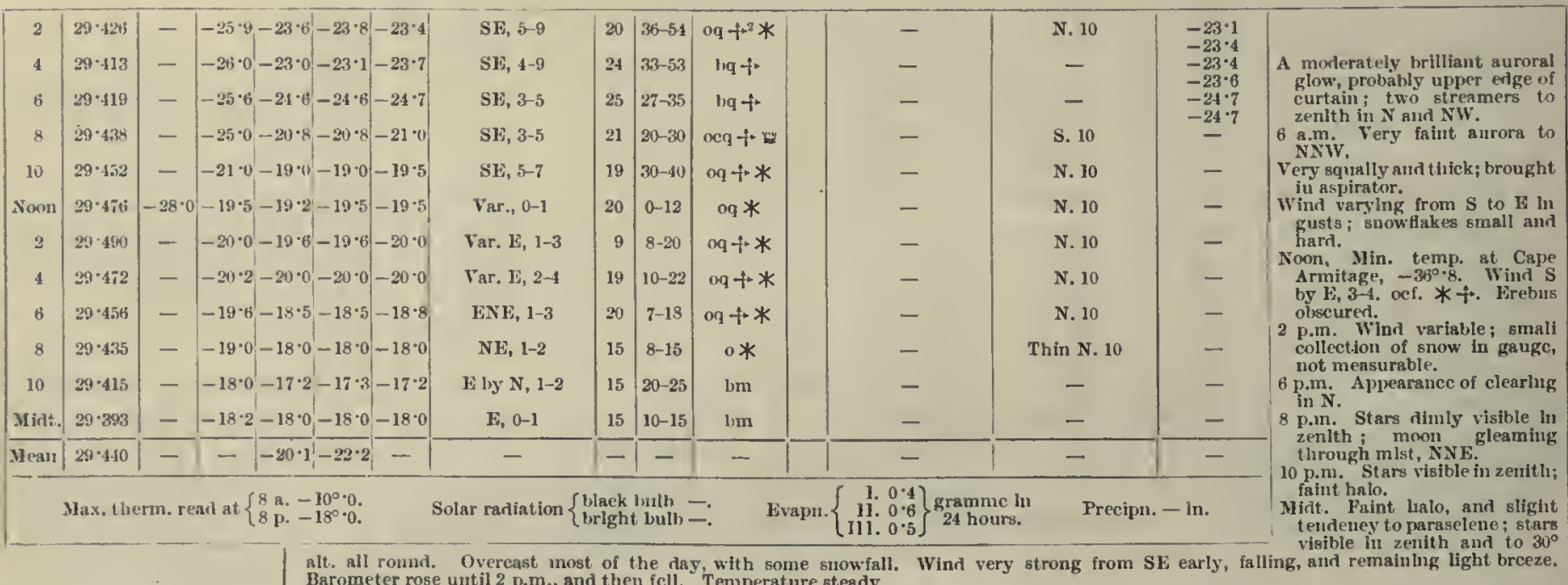

alt. all ronnd. Overeast most of the day, with some snowfall.
Barometer rose until 2 p.m., and then fcll. Temperature steady.

6 th

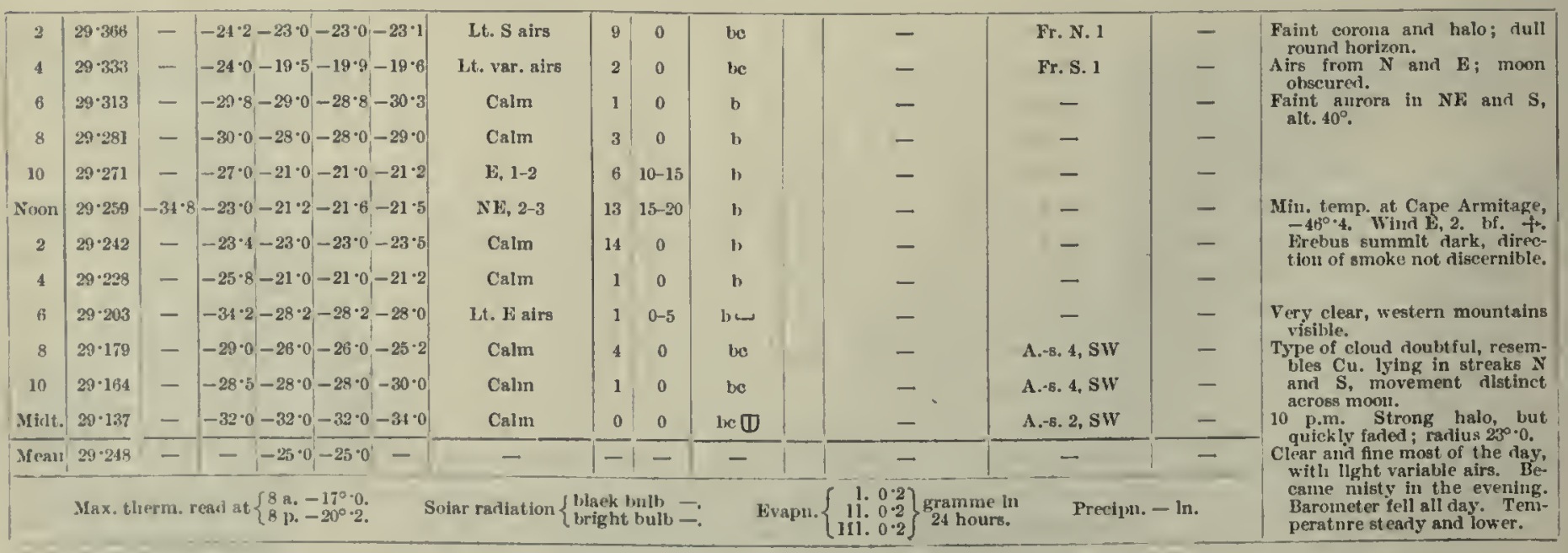




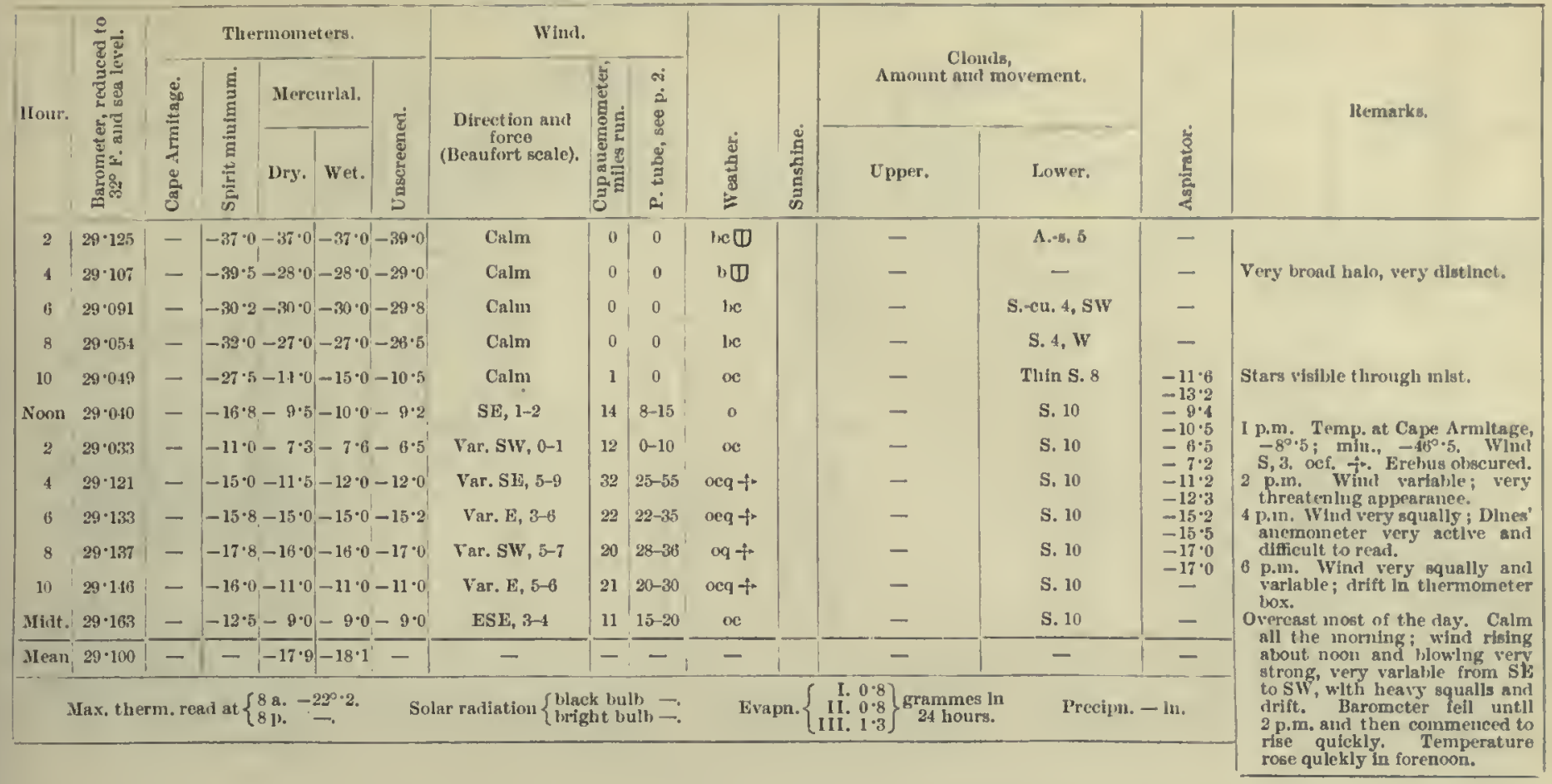

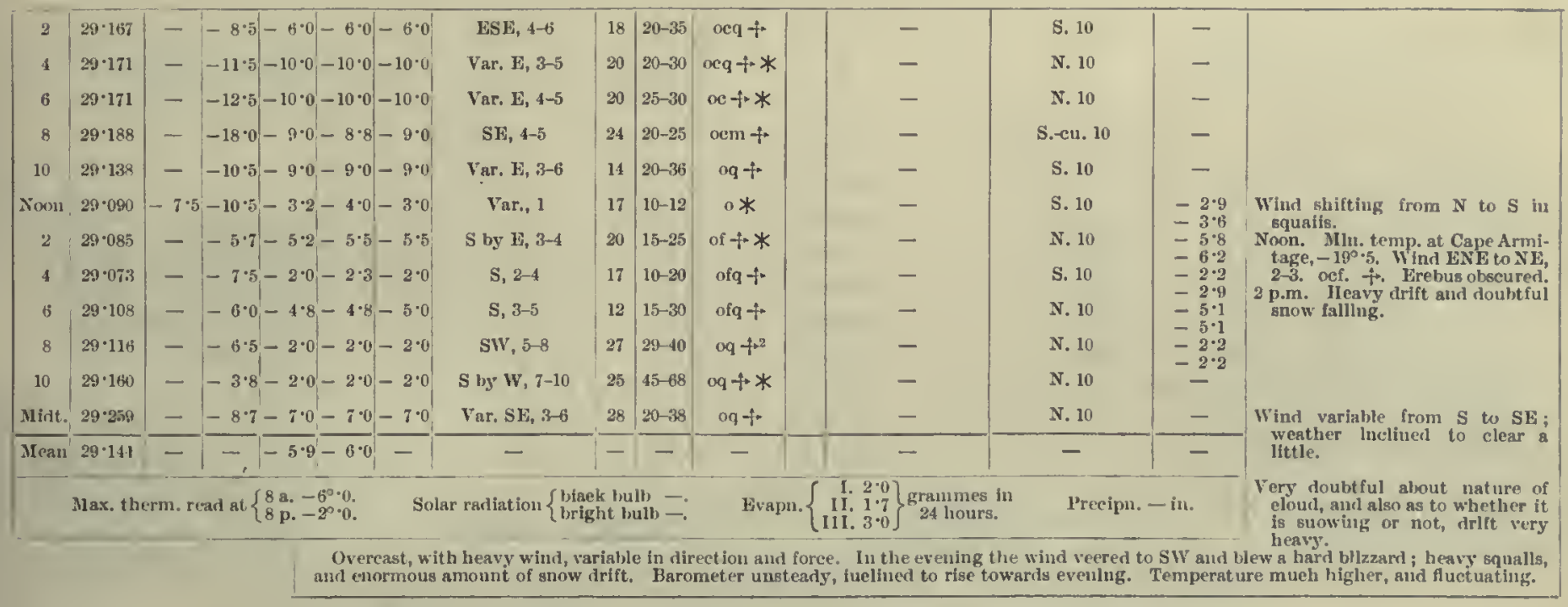

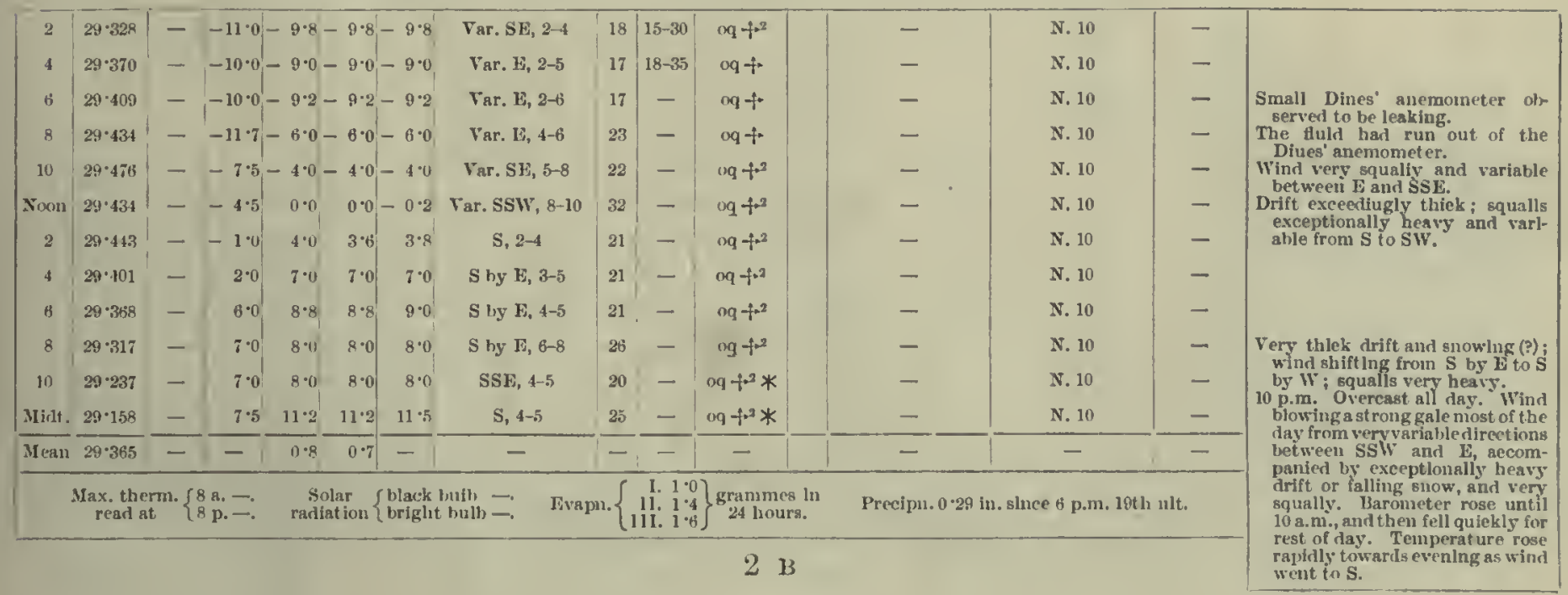




\begin{tabular}{|c|c|c|c|c|c|c|c|c|c|}
\hline \multirow{2}{*}{ Hour. } & \multirow{2}{*}{ 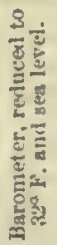 } & \multicolumn{5}{|c|}{ Thermometers, } & \multicolumn{3}{|l|}{ wind. } \\
\hline & & 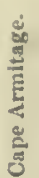 & 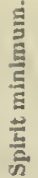 & Mere & Wet. & 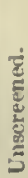 & $\begin{array}{c}\text { Directlon and } \\
\text { force } \\
\text { (Beaufort scale). }\end{array}$ & 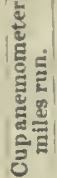 & 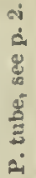 \\
\hline
\end{tabular}

10 th.

\begin{tabular}{|c|c|c|c|c|c|c|c|c|c|c|c|c|c|}
\hline 2 & $29 \cdot 093$ & - & $11 \cdot 0 !$ & $12 \cdot 0$ & $12 \cdot 0$ & $12 \cdot 0$ & SSW, 7-10 & 33 & - & $\operatorname{og}+x^{2} *$ & - & N. 10 & - \\
\hline 4 & $29 \cdot 045$ & - & $11 \cdot 5$ & $12 \cdot 0$ & $12 \cdot 0$ & $12 \cdot u$ & S by W, 7-10 & 40 & - & $\operatorname{og}-j^{2} *$ & - & N. 10 & - \\
\hline 6 & $29 \cdot 042$ & 一 & - & - & - & - & S, 8-11 & 48 & - & $\infty+^{2} *$ & - & N. 10 & - \\
\hline 8 & $29 \cdot 112$ & - & $7 \cdot 0$ & $0 \cdot 0$ & $9 \cdot 0$ & $9 \cdot 0$ & $5,6-7$ & 40 & - & $0-i^{2} *$ & - & N. 10 & 一 \\
\hline 10 & $29 \cdot 239$ & - & $4 \cdot 0$ & $4 \cdot 6$ & $4 \cdot 4^{\prime}$ & $4 \cdot 8$ & $S, 3-5$ & 21 & - & $\propto q$ & - & N. 10 & - \\
\hline Xool1 & $29 \cdot 301$ & $0 \cdot 2$ & 0.0 & $1 \cdot 0$ & $1 \cdot 0$ & $0 \cdot 8$ & SE, 3-5 & 12 & - & leeq 4 . & - & Fr. -8.5, Sli (fast) & - \\
\hline 2 & $29 \cdot 392$ & - & $-1 \cdot 3$ & $.0 \%$ & 0.0 & 0.0 & $\mathbf{E}, 2-3$ & 13 & - & of & - & S. 10 & - \\
\hline 4 & $39 \cdot 422$ & $-0 \cdot 6-$ & $-3 \cdot 6$ & $2 \cdot 0$ & $2 \cdot 0$ & $2 \cdot 3$ & Var. $12,2-4$ & 13 & - & $o q$ & - & N. 10 & - \\
\hline 6 & $29 \cdot 420$ & - & 0.8 & $2 \cdot 8$ & $2 \cdot 8$ & $3 \cdot 0$ & If hy $\mathrm{N}, 3-5$ & 17 & - & oq $*$ & - & X. 10 & 一 \\
\hline 8 & $29 \cdot 4.53$ & - & $1 \cdot 0$ & $2 \cdot 0$ & $2 \cdot 0$ & $2 \cdot 0$ & E, 2-3 & 14 & - & $\circ *$ & - & N. 10 & - \\
\hline 10 & $29 \cdot 459$ & - & $1 \cdot 0$ & $4 \cdot 0$ & $4 \cdot 0$ & $4 \cdot 0$ & ExE, 3 & 14 & - & og & - & S.-๖u. 10 & - \\
\hline Midt. & $29 \cdot 445$ & - & $3 \cdot 0$ & $8 \cdot 0$ & $7 \cdot 0$ & $8^{\circ} 0$ & ENE, 3 & 12 & - & og & - & S.-cu. 10 & - \\
\hline Меan & $29 \cdot 288$ & - & -1 & $5 \cdot 2$ & $5 \cdot 1$ & - & - & 1 & - & 一 & - & - & - \\
\hline
\end{tabular}

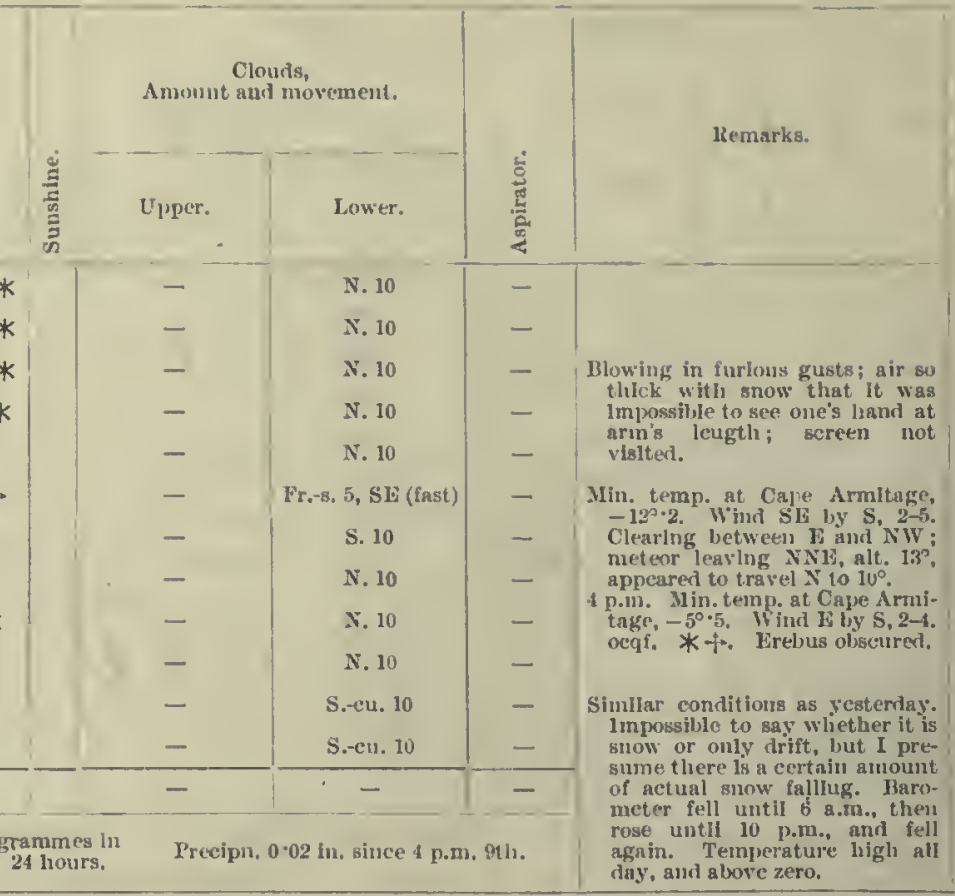

11th.

\begin{tabular}{|c|c|c|c|c|c|c|c|c|c|c|c|c|c|c|}
\hline 2 & $29 \cdot 428$ & - & 6.5 & $10 \cdot 8^{1}$ & $10 \cdot 5^{\prime}$ & 10.8 & E by $\mathrm{N}, 2$ & 10 & - & og & - & S.cu. 10 & - & \multirow{5}{*}{$\begin{array}{l}\text { Mint. to } 6 \text { a.m. Very gloomy and } \\
\text { threatening, but clear, western } \\
\text { range being vislble. } \\
\text { Fine hard snow fallng. }\end{array}$} \\
\hline 4 & $29 \cdot 383$ & - & 10.5 & $15 \cdot 0$ & $14 \cdot 0^{\prime}$ & $15 \cdot 0$ & E, 3 & 4 & - & $\operatorname{og}$ & - & S.-cu. 10 & - & \\
\hline 6 & $29 \cdot 355$ & - & $13 \% 5$ & $17 \cdot 0$ & 16.0 & $17 \cdot 0$ & E, 1-2 & 14 & - & $\operatorname{og} *$ & - & N. 11) & - & \\
\hline 8 & $29 \cdot 390$ & - & $5 \cdot 0$ & $9 \cdot 0$ & $8 \cdot 8$ & $9 \cdot 0^{\prime}$ & Lit by $\mathrm{N}, 2-4$ & 19 & - & $0+$ & - & N. 10 & - & \\
\hline 10 & $29 \cdot+34$ & - & $5 \cdot 0$ & $5 \cdot 8$ & $5 \cdot 2$ & $5 \cdot 5$ & $1,2-3$ & 9 & - & $\mathrm{nm}+\mathrm{fr} *$ & - & N. 10 & - & \\
\hline Xoon & $29 \cdot 486$ & $8 \cdot 5$ & $4 \cdot 0^{\circ}$ & $8 \cdot 0^{\prime}$ & $8 \cdot 0$ & $8 \cdot 0$ & 1s, I-2 & 18 & - & om $+*$ & - & N. 10 & - & \multirow{7}{*}{ 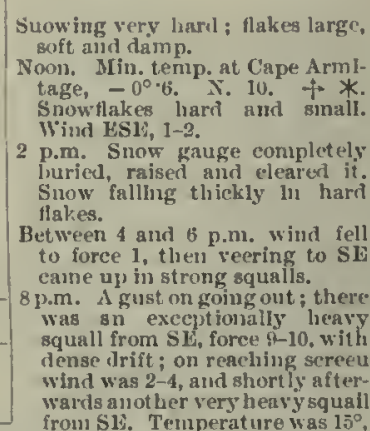 } \\
\hline 2 & 29.542 & -1 & $4 \cdot 8$ & $9 \cdot 0$ & $9 \cdot 0$ & $9 \cdot 3$ & L by $N, 2-3$ & 18 & - & of $+* *$ & - & N. 10 & - & \\
\hline 8 & $29 \cdot 613$ & - & $10^{\circ} 0$ & $15 \cdot 0$ & $14 \cdot 0$ & $14 \cdot 2$ & Var. 18,4-9 & 17 & - & oq $+2^{2} *$ & - & x. 10 & - & \\
\hline 10 & $29 \cdot 620$ & - & $11 \cdot 0$ & $13 \cdot 0$ & $12 \cdot \theta$ & $13 \cdot 0$ & S, 5-7 & 25 & - & $\operatorname{og}[+2 *$ & 一 & N. 10 & - & \\
\hline Midt. & $29 \cdot 713$ & - & $8 \cdot 5$ & $9 \cdot 1$ & 9.0 & $8 \cdot 0$ & Var. 1), 3-5 & 20 & - & ocqf $+* *$ & - & S. 10 & 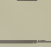 & \\
\hline \multirow[t]{2}{*}{$\overline{\text { Mean }}$} & $29 \cdot 516$ & - & -1 & $11 \cdot 1$ & $10 \cdot 7$ & - & - & -1 & - & - & $=$ & - & - & \\
\hline & Max, th & $\mathrm{m}$ & 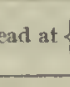 & & & & adlation $\left\{\begin{array}{l}b \\
1\end{array}\right.$ & & -. & Erap & $\left\{\begin{array}{r}1.2 \cdot 1 \\
11.3 \cdot 4 \\
\text { I1I. } 5 \cdot 8\end{array}\right\} \mathrm{gr}$ & \multicolumn{2}{|c|}{ Precipn. - In. } & \\
\hline
\end{tabular}

12 th.

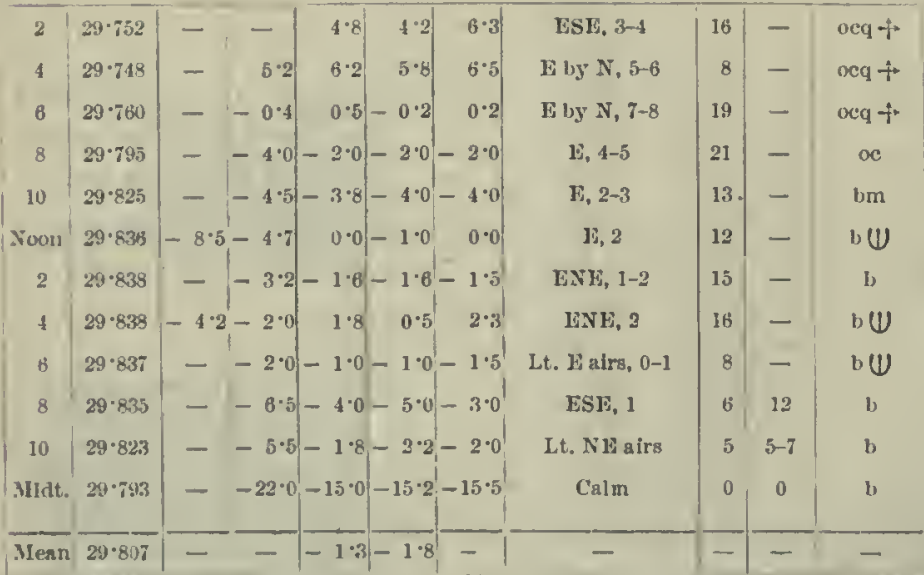

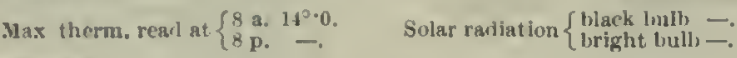

\begin{tabular}{|c|c|}
\hline S. 10 & - \\
\hline S. 10 & - \\
\hline S. 10 & - \\
\hline S. 10 & - \\
\hline- & - \\
\hline- & $\begin{array}{r}0.0 \\
-1.1\end{array}$ \\
\hline - & $\begin{array}{r}-1 \cdot 8 \\
=2 \cdot 6\end{array}$ \\
\hline- & $\begin{array}{l}1.0 \\
0.7\end{array}$ \\
\hline- & $\begin{array}{l}-2 \cdot 2 \cdot 8 \\
=2 \cdot 9\end{array}$ \\
\hline- & $\begin{array}{l}-4 \cdot 2 \cdot 7 \\
=4 \cdot 7\end{array}$ \\
\hline - & $\begin{array}{l}=1.8 \\
=2.6\end{array}$ \\
\hline- & $\begin{array}{l}-18 \cdot 4 \\
-18 \cdot 2\end{array}$ \\
\hline
\end{tabular}

Clkaring generally.

Clearing rapldly; heavy Str. to IV; moon misty. Str. to " and sti moving iu a Why sirection.

conl. Min temp. at Cape Armntage, $-9^{\circ} \cdot 7$. Whid $E$ by $S$ lirebiss summit obseured. 2 p.u1. Str. on

gauge ehoked,
p.m. Faint aurora, only visible

p.m. Mn. temp. at Cape Armitage, $-14^{0} \cdot 2$. What by $\mathrm{N}$ dark water sky clous to $\mathrm{si}$. $f$. Sunall corona.

p.m. - Ohserverl hright and larg." flame rise up higls from lirelus, followed lyy a glow, and then a thick enlumus of smoht; smoke golug X13.

wind remalued strong untilatout 10 a.m. then it gradually fell and sky eleared. Baroineter rose until 4 p.m., and then fel slowly. Temperature fell gradually as wind drouperi. 
Mutforolonical, Journat, of the "Discovery."

[1903-June.

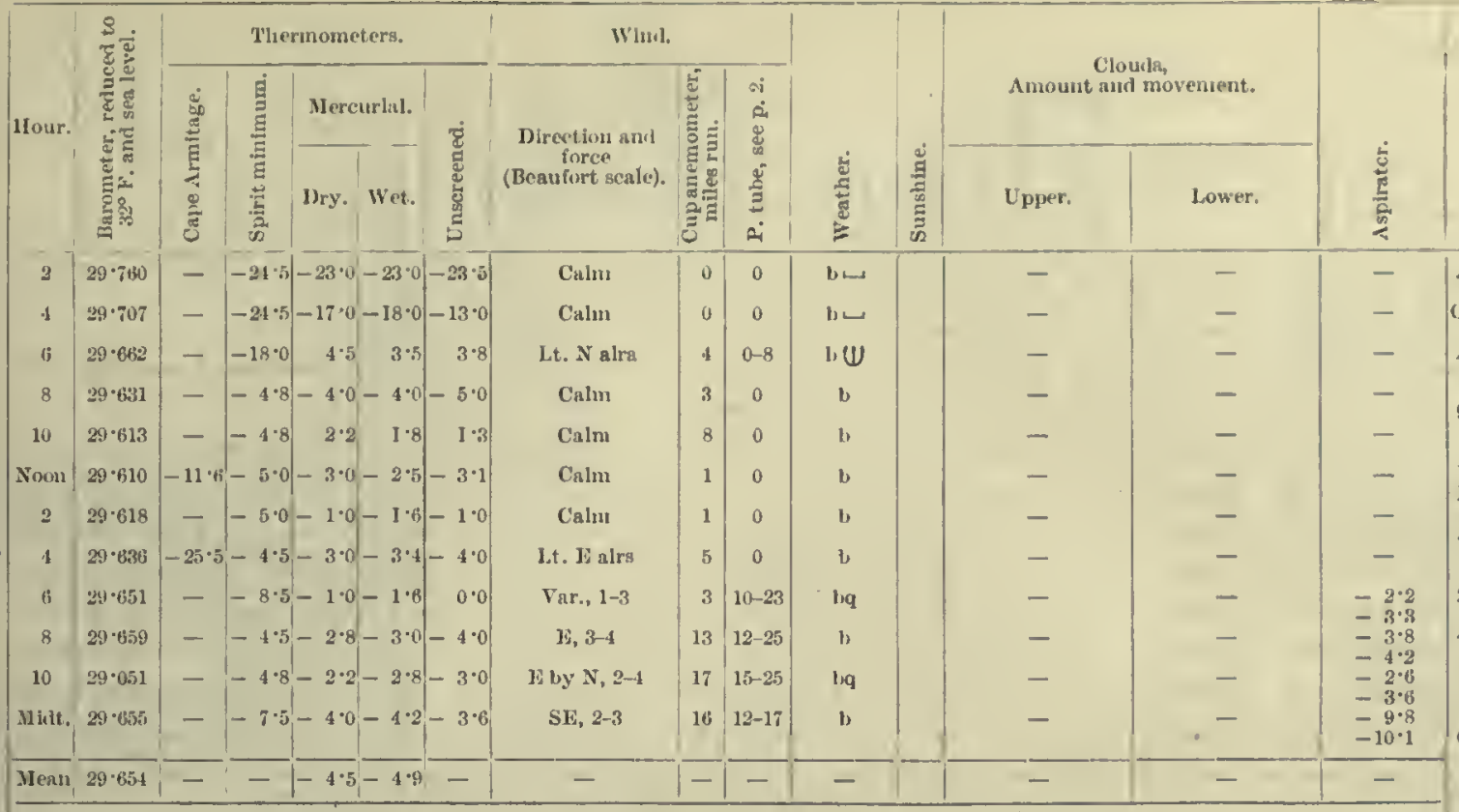

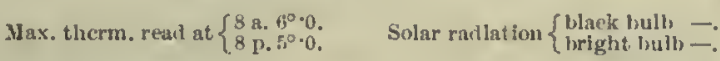

Evapn. $\left\{\begin{array}{rr}1 . & 2 \cdot 4 \\ 11 . & 3 \cdot 4 \\ \text { III. } 5 \cdot 2\end{array}\right\} \begin{aligned} & \text { grammes in } \\ & 24 \text { lours. }\end{aligned}$

Precipn. - ln.

A covererl it fern-like structuren.
Jutshle thermonicter coaterl with rime. corona.

9 a.m. Nig hrecee blowlng, force 1-2.

Vlslthllty markedly good.

Nonn. Mln. temp. at Cape Armitage, $-320 \cdot 5$. Calm. ol low fog between If and s close to lant; rine out ther

2 p.m. Bank of Str. on S anil

4 p.m. Mln. temp. at. Cape 1. bme haze; frost rlme on ther

p.m. Heary surface drlft rumulng front Cape Amoitane towards $W$

p.m. Western nountalus falntly vislble; low virlt lu Strait; small detached clonds to $S$.
vidt.

idt. All distant land ver dlstinct; no drift. Calm most of the day and clear. Wind sprang up in the evening from an casterly
direction and blew very fresh. Barometer rising and falling in regular waves. Temperature very unstealy.

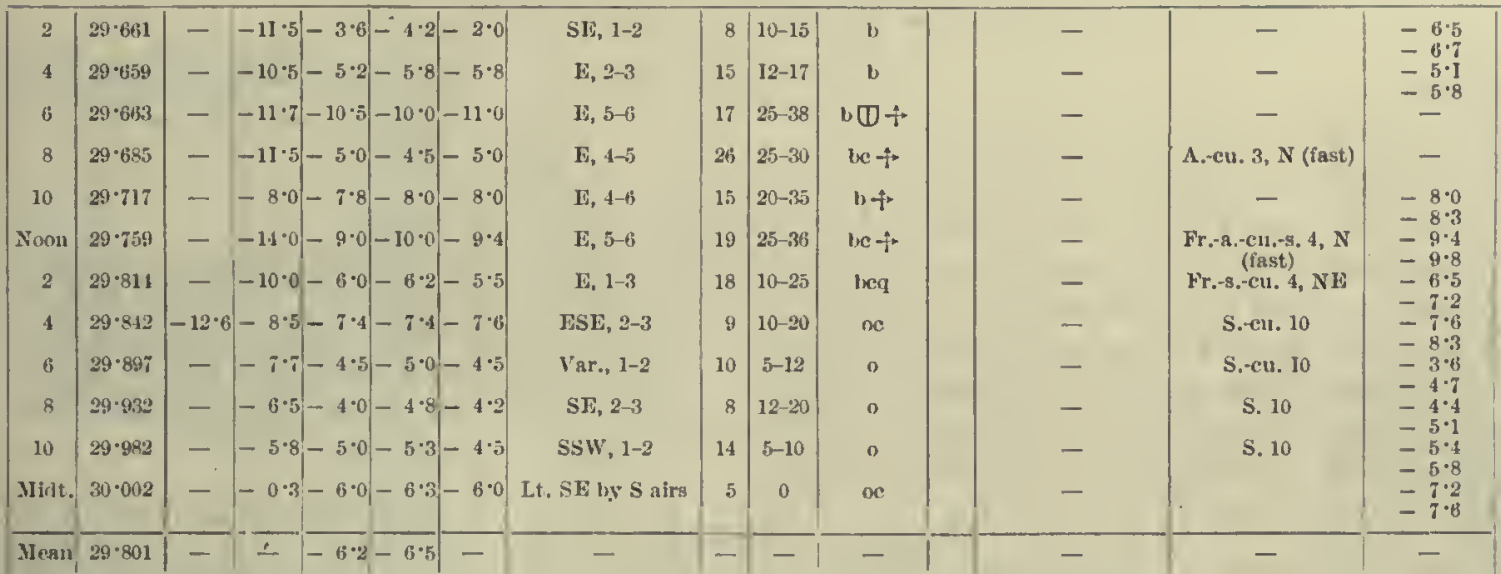

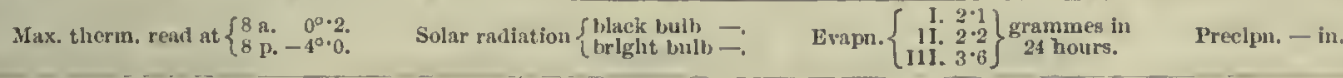

One bright aurora streasner passing throu

Driftlng heavily; land olsscurer except close to shlp.

Jieavy $\mathrm{Cu}$, to $\mathrm{N}$.

Detached Cu. to $\mathrm{W}$ passing to $\mathrm{S}$ fast ; drift from hlll-tops.

1.20 p.m. Teup. at Cape Armi-

tage, $-15^{\circ} .5$; mln., $-33^{\circ} \cdot 0$.

Tloud 0000 it olrecurlug oc. Cln onomito thermometer.

p.m. Jleavy hank of S.-cu. over western mountalns.

4 p.ni. Min. temp. at Cape Armitsuge, $-19^{\circ} \cdot 2$. Vilnd lyy S, 1 nc. Erebus obscurevt. 6 p.in, Wind varying froil vitit. Slyyclearlng to $\mathrm{S}$ and $\mathrm{SW}$

Fresh Fly wind all day, falling in evening. Clear until 2 p.m. and then overcast. Maronieter rising all day, Tetuperature steadier, but still remaining high.

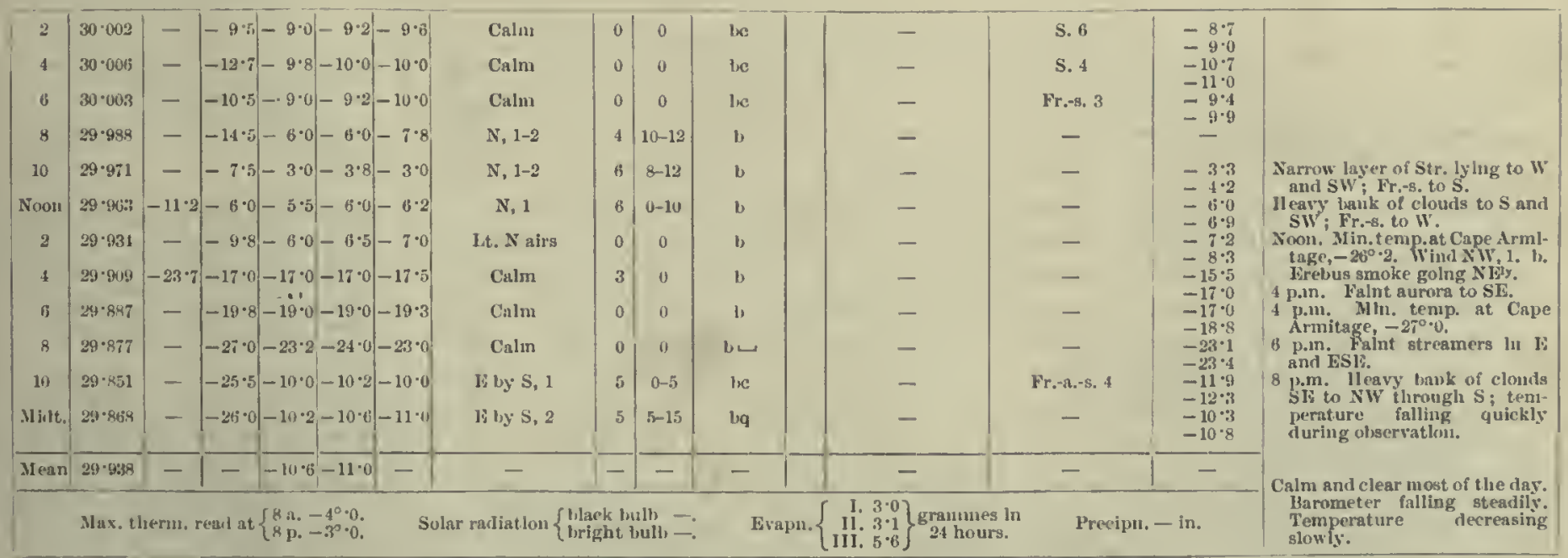




\begin{tabular}{|c|c|c|c|c|c|c|c|c|c|}
\hline \multirow{3}{*}{ Ilour. } & \multirow{3}{*}{ 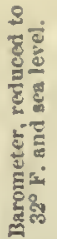 } & \multicolumn{5}{|c|}{ Thermometers. } & \multicolumn{3}{|l|}{ Wint. } \\
\hline & & 这 & $\stackrel{\Xi}{E}$ & Merc & urial. & & & 㐫 & $\dot{\sim}$ \\
\hline & & 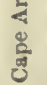 & 点 & Dry. & Wet. & : & (Beaufort scaie). & 莺 & : \\
\hline
\end{tabular}

16th.

\begin{tabular}{|c|c|c|c|c|c|c|c|c|c|c|}
\hline 2 & $29 \cdot 868$ & - & $-23 \cdot 0-11 \cdot 0-11 \cdot 0-12 \cdot 0$ & Nit by $\mathrm{X}, 4-5$ & 17 & $10-25$ & b & - & - & $-11^{\circ} 6$ \\
\hline 4 & $29 \cdot 854$ & - & $-22 \cdot 0-11 \cdot 0-11 \cdot 0-12 \cdot 0$ & E by $N, 6-\bar{\tau}$ & 18 & $20-40$ & $b+$ & - & - & $-11 \cdot 6$ \\
\hline b & $29 \cdot 873$ & - & $-22 \cdot 0-10 \cdot 8-10 \cdot 8-11 \cdot 0$ & E, 6-7 & 23 & $30-50$ & $b+$ & - & - & $\begin{array}{l}-11 \cdot 9 \\
-11 \cdot 2\end{array}$ \\
\hline 8 & $29 \cdot 881$ & - & $-8 \cdot 0-6 \cdot 0-6 \cdot 2-7 \cdot 0$ & SE, $2-3$ & 16 & $10-15$ & b & - & - & -110 \\
\hline 10 & $29 \cdot 955$ & - & $-10 \cdot 5-7 \cdot 0-8 \cdot 0-7 \cdot 0$ & E, $2-3$ & 12 & $10-20$ & $b e+$ & - & Det. Fr.-s. $2, \mathrm{~S}$ & $\begin{array}{l}=7 \cdot 6 \\
=8 \cdot 5\end{array}$ \\
\hline Noon & $29 \cdot 967$ & -13 & $3-8 \cdot 7-7 \cdot 5-8 \cdot 0-7 \cdot 8$ & ESE, 1-2 & 9 & $8-14$ & b & - & - & $\begin{array}{l}=8.0 \\
=8.7 \\
-8.7\end{array}$ \\
\hline 2 & $29 \cdot 983$ & - & $-8 \cdot 5-.8 \cdot 2-9 \cdot 0-10 \cdot 5$ & Calm & 7 & 0 & $b$ & - & - & $-10 \cdot 5$ \\
\hline 4 & $29 \cdot 989$ & $-25^{\circ}$ & $-11 \cdot 7-8 \cdot 5-9 \cdot 0-8 \cdot 5$ & $\mathrm{E}, 0-1$ & 5 & $0-10$ & bu & - & - & $\begin{array}{l}-116 \\
-10 \cdot 8\end{array}$ \\
\hline 6 & $29 \cdot 990$ & - & $-13 \cdot 3-8 \cdot 0-9 \cdot 0-8 \cdot 6$ & ESE, 1 & 9 & $5-10$ & เே & - & - & $\begin{array}{l}-9.0 \\
-10.5\end{array}$ \\
\hline 8 & $3 w \cdot 007$ & - & $-11 \cdot 5-10 \cdot 2-10 \cdot 8-10 \cdot 8$ & $N E, 1-2$ & 5 & $10-15$ & b & - & - & $\begin{array}{l}-10 \cdot 8 \\
-10 \cdot 4\end{array}$ \\
\hline 10 & $30 \cdot 007$ & - & $-12 \cdot 9-11 \cdot 2-11 \cdot 7-11 \cdot 6$ & NH, 3 & 16 & $10-20$ & be & Fr.-ci.-s. 4 & - & $\begin{array}{l}-11 \cdot 4 \\
-12 \cdot 3\end{array}$ \\
\hline Mlidt. & $30^{\circ} 006$ & - & $-19 \cdot 2-11 \cdot 0-11 \cdot 5-11 \cdot 5$ & Lt. NE airs & 5 & 0 & be & Fr.ci. s. 5 & - & $\begin{array}{l}-12 \cdot 6 \\
-11 \cdot 6 \\
-12 \cdot 3 \\
\end{array}$ \\
\hline ILean & $29 \cdot 949$ & - & $-|-9 \cdot 3-9 \cdot 7|-$ & - & $1-$ & - & - & - & - & - \\
\hline & & & 0.0. & iat & & ulb $=$ : & & $\left\{\begin{array}{r}1.2 \cdot 2 \\
\text { II. } 2 \cdot 3 \\
\text { III. } 3 \cdot 7\end{array}\right\}^{g r}$ & \multicolumn{2}{|c|}{ Precipn. $-\ln$. } \\
\hline
\end{tabular}

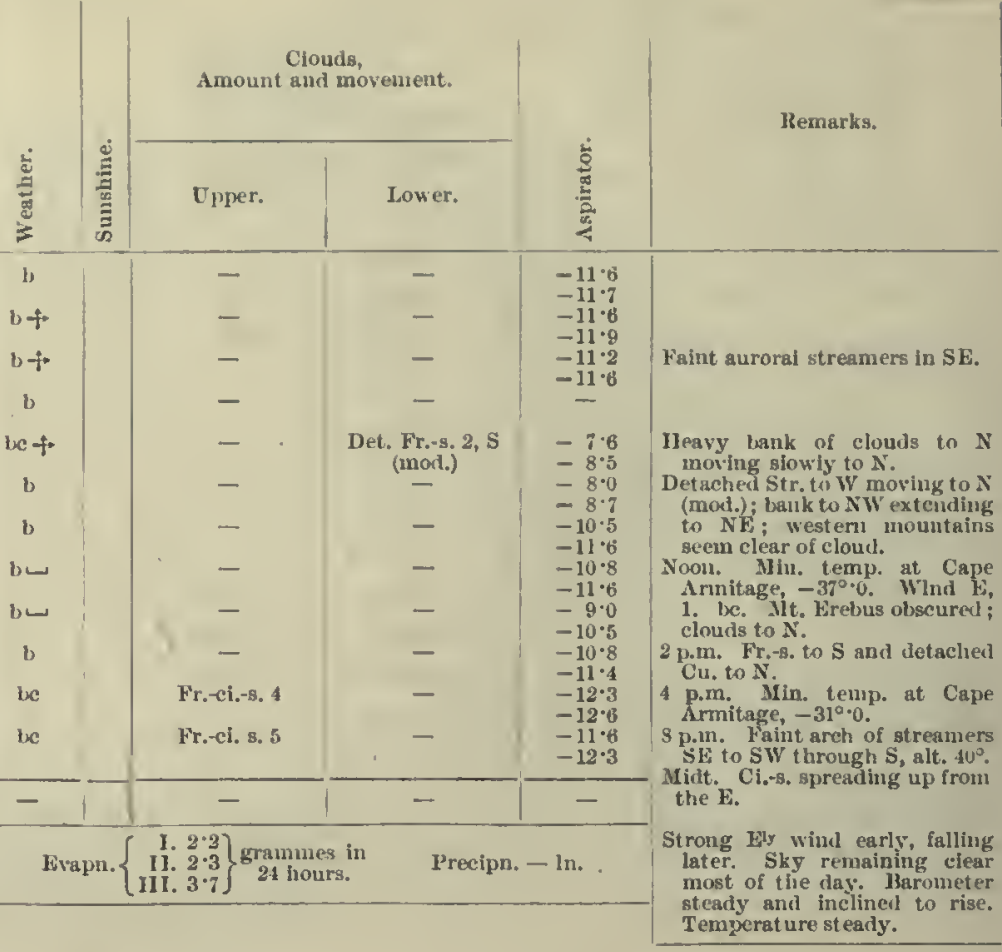

17 th

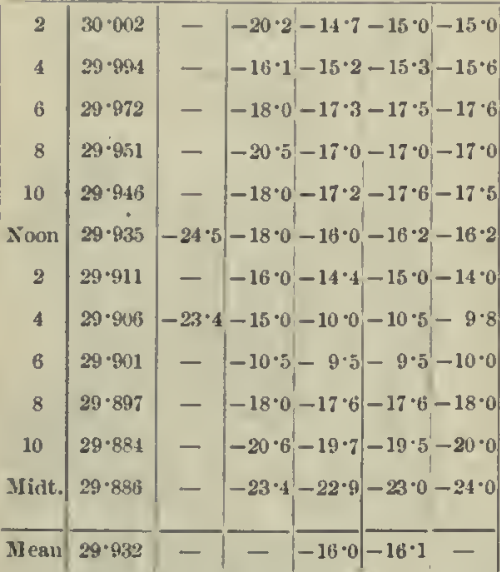

\begin{tabular}{|c|c|c|c|}
\hline NE, 2-3 & 5 & 15 & b \\
\hline NE, 3-4 & 18 & $20-25$ & b \\
\hline $\mathrm{NF}_{0} 4-5$ & 21 & $30-33$ & $b+$ \\
\hline NE, $4-\bar{t}$ & 29 & $25-42$ & ocq \\
\hline NE, 5-7 & 21 & $25-40$ & $o q f^{\circ}$ \\
\hline NE, $5-6$ & 21 & $25-35$ & eq \\
\hline E ly $N, 3-4$ & 19 & $20-30$ & o \\
\hline E by $N, 2-3$ & 18 & $12-20$ & 0 \\
\hline Lt. NE airs & 10 & 0 & oc $*$ \\
\hline Calm & 1 & 0 & $\operatorname{lm} *$ \\
\hline Lt. NW airs & 1 & 0 & bm $*$ \\
\hline Calm & 2 & 0 & $\propto * *$ \\
\hline- & & - & - \\
\hline
\end{tabular}

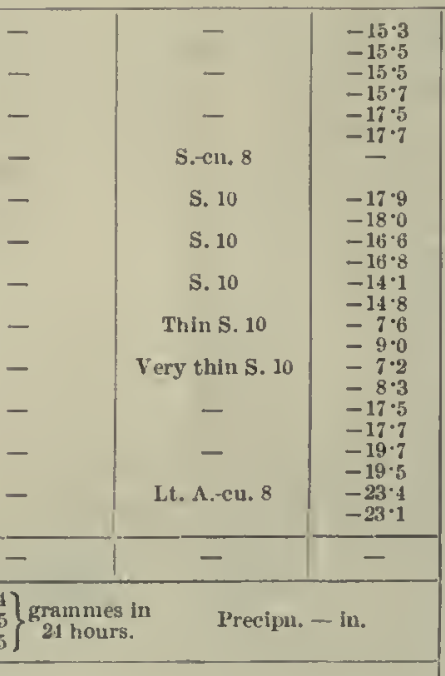

Bxtensive arch from $\mathrm{N}$ to $\mathrm{S}$ across zenith plainly visible about 3 uninut es.

Min. temp. at Cape Armitage, $-30^{\circ} \cdot 2$. Wind b by $N, 3-4$ oq. -7. Karebus olsccured. Min. tem p. at Cape Armitage, Str. 10. Fine cristals falling. 4, 6,8 p.m. and Midt. Suow: crystals falling.

Faint aurora ESE, alt. $6^{\circ}$ to $10^{\circ}$. Very strong Nkit whind up to noon, when it fell and graduany bertecalm. Orerearly and clearing bit clear arly and clearing againl later. Barometer falling steadlly all inclined to fall.
$18 \mathrm{th}$.

\begin{tabular}{|c|c|c|c|c|c|c|c|c|c|c|}
\hline 2 & $29 \cdot 885$ & - & $-23 \cdot 5-18 \cdot 8-18 \cdot 9-18 \cdot 8$ & Lt. NE airs & 2 & 0 & $\infty$ & - & Lt. Cu. 10 & $-18 \cdot 0$ \\
\hline 4 & $29 \cdot 877$ & - & $-19 \cdot 1-13 \cdot 9-14 \cdot 0-14 \cdot 1$ & Lt. NE airs & 4 & 0 & $o c$ & - & l.t. Cn. 10 & $-\underline{-17 \cdot 9}$ \\
\hline 6 & $29 \cdot 871$ & - & $-15 \cdot 8-15 \cdot 2-15 \cdot 3-15 \cdot \gamma$ & $\mathbf{E}, 1$ & 6 & $8-10$ & oc $*$ & - & Det. Cu. 7 & $-15 \cdot 5$ \\
\hline 8 & $29 \cdot 870$ & - & $-19 \cdot 0-19 \cdot 0-19 \cdot 0-19 \cdot 0$ & ENL, 1 & 3 & $8-10$ & Ixe & - & Cu. 2 & \\
\hline 10 & $29 \cdot 8 i 0$ & - & $-21 \cdot 0-20 \cdot 0-20 \cdot 0-21 \cdot 0$ & Calm & 3 & 0 & bu & - & - & $-20 \cdot 9$ \\
\hline Noon & $29 \cdot 873$ & $-41 \cdot 5$ & $-29 \cdot 5-18 \cdot 1-18 \cdot 2-19 \cdot 0$ & Lt. E airs & 4 & 0 & bし & - & - & $\begin{array}{l}-20.9 \\
-18.9 \\
-10.9\end{array}$ \\
\hline 2 & $29 \cdot 883$ & - & $-21 \cdot 0-18 \cdot 0-18 \cdot 0-17 \cdot 5 \mid$ & ESE, $0-1$ & 7 & 0 & $b$ & - & - & $\begin{array}{l}-19.1 \\
-19 \cdot 8\end{array}$ \\
\hline 4 & $29 \cdot 8 \times 5$ & $-46^{\circ} \cdot 4$ & $-33 \cdot 0-21 \cdot 4-21 \cdot 2-22 \cdot 0$ & Lt. Wairs & 4 & 0 & $b=\sqcup$ & - & - & $\begin{array}{l}-19 \cdot 8 \\
-22 \cdot 0\end{array}$ \\
\hline 6 & $29 \cdot 901$ & - & $-30 \cdot 8-21 \cdot 0-21 \cdot 0-22 \cdot 5$ & s, $0-1$ & 4 & $5-10$ & b & - & - & $\begin{array}{l}-23 \cdot 4 \\
-23 \cdot 8\end{array}$ \\
\hline 8 & $20 \cdot 917$ & - & $-28 \cdot 0-21 \cdot 2-25 \cdot 0-21 \cdot 8$ & Caim & 1 & 0 & $b$ & - & - & $\begin{array}{l}-23 \cdot 8 \\
-22 \cdot 0\end{array}$ \\
\hline 10 & $29 \cdot 958$ & - & $-35 \cdot 0-31 \cdot 8-31 \cdot \tau-35 \cdot 0$ & Calm & 1 & 0 & b & - & - & Mercury \\
\hline Mint. & $2 y-9.5 \%$ & - & $-42 \cdot 0-36 \cdot 2-36 \cdot 2-3 i \cdot 0$ & Calm & 1 & 0 & b & - & - & $\begin{array}{l}\text { lelow seale } \\
\text { from } 10 \text { p.t1 } \\
\text { to midt. }\end{array}$ \\
\hline $\overrightarrow{\text { I ean }}$ & $29 \cdot 896$ & - & $--21 \cdot \pi-21 \cdot 8-$ & - & - & - & - & - & - & - \\
\hline & $y$ & 然然 & $-13^{\circ} \cdot 0$ & 皮 & & & & & & -in. \\
\hline
\end{tabular}

Paint arch from $\mathrm{E}_{\text {, alt. }} 3^{\circ}$, to $\mathrm{S}$, alt. $6^{\circ}$; apex $\mathrm{KSL}^{\circ}, 11^{\circ}$. Faint patelies Very fiue suow crystals falling.:

Briglit aurora streamers in SE, $N E$, suld $N_{i}$ part of coroua

near zenlth.
Niton. Min. temp. at Cape Armitage, $-13^{\circ} 0$. Wind going NE.y, small quautity. 4 porm. Hin. temp. at Cape Arnitage, $-47^{\circ} \cdot 3$.

Temperature was very." "junupy" during the olsservition, snd few minutes afterwards the mercury in the aepirator was below the scale. Practlcally during all the after uoon there was a bright and extensive aurora low ulcun extending froml helintil Mt. T trror to the tend of White lsland; at limes streaners roso to $5^{\circ}$ alt. and were very lright; movement laterally, very rapid is

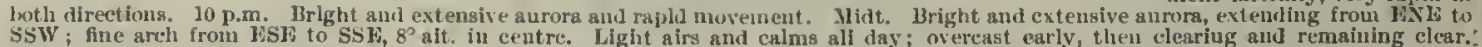
Barometer fell a little, and, after being steady for 8 loours, rose quickly. Tempernt ure lower, and falling unsteadily... 


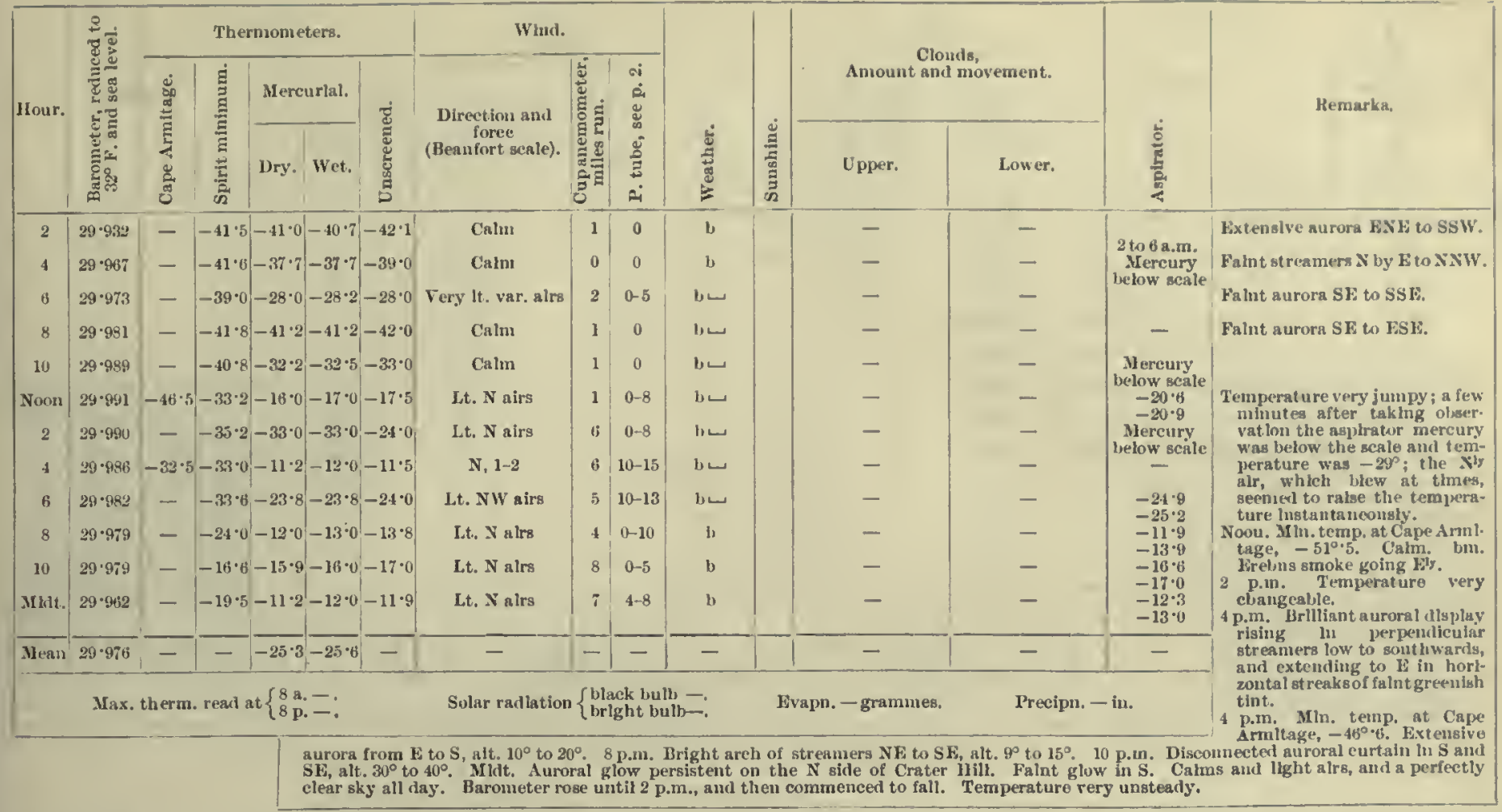

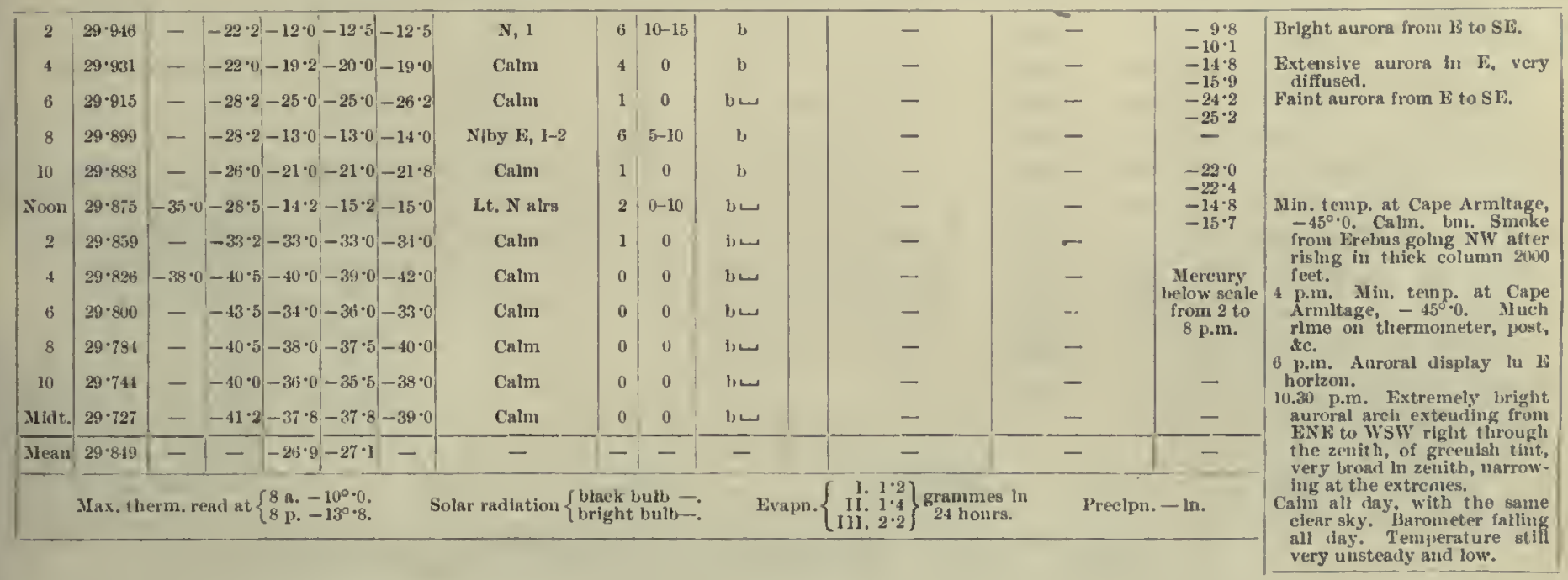

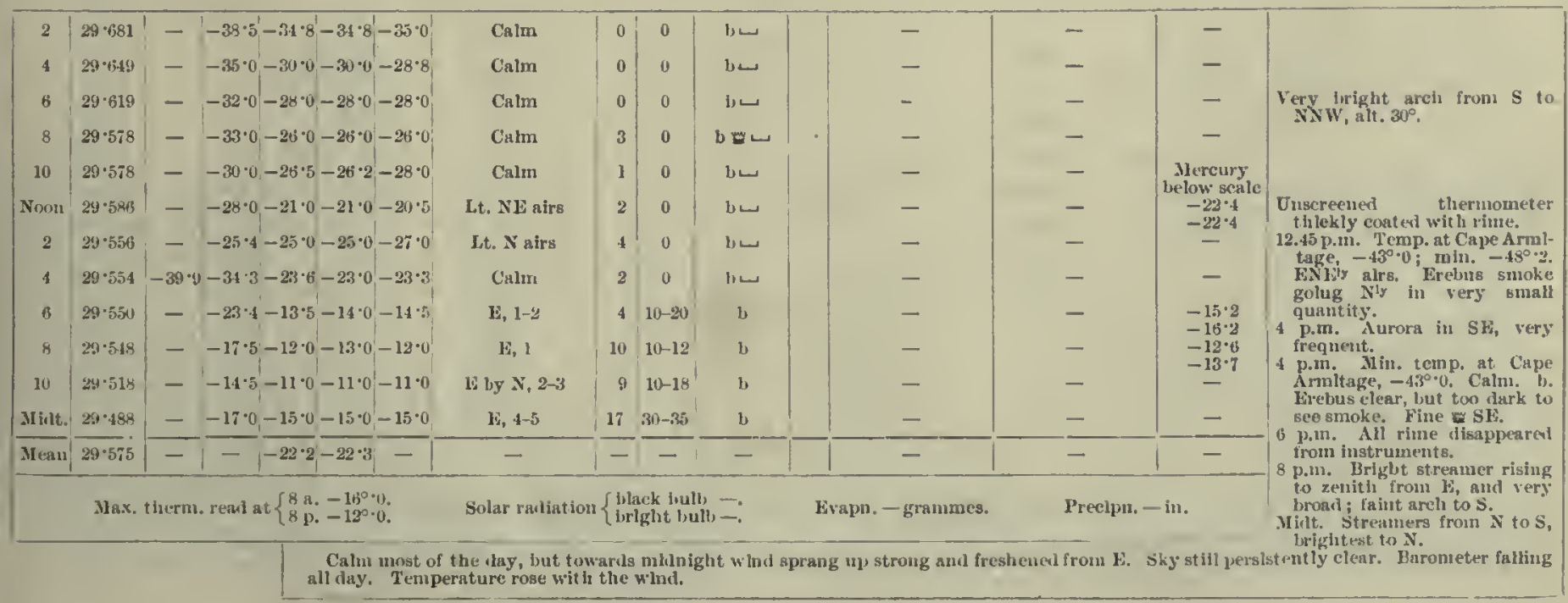




\begin{tabular}{|c|c|c|c|}
\hline \multicolumn{4}{|c|}{ Thermometers. } \\
\hline$\dot{\mathrm{s}}$ & 总 & Mere & irlal. \\
\hline है & 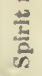 & Dry. & wet. \\
\hline
\end{tabular}

Wind.
Direction and
forec
(1Beaufort scale).

22nd.
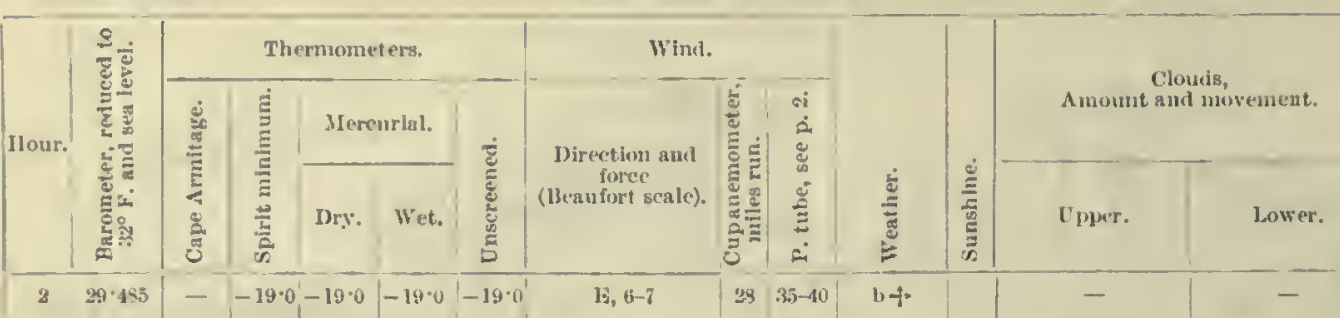

$+29 \cdot 4 \times 3--20 \cdot 0-17 \cdot 0-17 \cdot 0-17 \cdot 0$

E, 6-7

-

b-

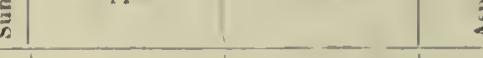

$6 \quad 29 \cdot 447-\quad-17 \cdot 0-15 \cdot 0-15 \cdot 0-15 \cdot 0$

$8 \quad 29 \cdot 445--23 \cdot 0-22 \cdot 0-21 \cdot 8-24 \cdot 0$

$\begin{array}{lllllll}10 & 29 \cdot 402 & - & -28 \cdot 0 & -27 \cdot 5 & -27 \cdot 5 & -28 \cdot 0\end{array}$

Noo1 $29 \cdot 381-41 \cdot 4-41 \cdot 0-40 \cdot 5-41 \cdot 5-42 \cdot 0$

$229 \cdot 320--41 \cdot 0-3 k \cdot 0-36 \cdot 0-3 i \cdot 0$

$4 \quad 39 \cdot 312-18 \cdot 2-41 \cdot 8-34 \cdot 5-34 \cdot 5-35 \cdot 0$

6 29.267 - $-43 \cdot 5-43 \cdot 0-43 \cdot 0-44 \cdot 0$

$829 \cdot 24 ;-13 \cdot 8-35 \cdot 0-35 \cdot 0-37 \cdot 3$

$10 \quad 29 \cdot 228 !-\quad-47 \cdot 0-46 \cdot 8 *$ Froz $\cdot 1 \mathrm{x}-46 \cdot 0$

31int. $29 \cdot 210-43 \cdot 0-38 \cdot 0-39 \cdot 0-38 \cdot 0$

I., 3-4 $13 \quad 15-20 \mid 1,-4$

1., 0-1 11 o-5 1,

Lt. SF airs

Caim

Calm

Calm

Calm

Calm

1.t. SE airs

Calm

Calm

Mean $29 \cdot 354$

Max, them. reat at $\left\{\begin{array}{l}8 \text { a. } \\ 8 \text { p. }-18^{\circ} \cdot 8^{\circ}\end{array}\right.$

Solar radiation $\left\{\begin{array}{l}\text { black lwull, } \\ \text { lrigbt lualb }- \text {. }\end{array}\right.$

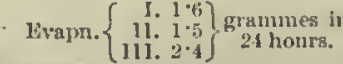

Precipu. - in.

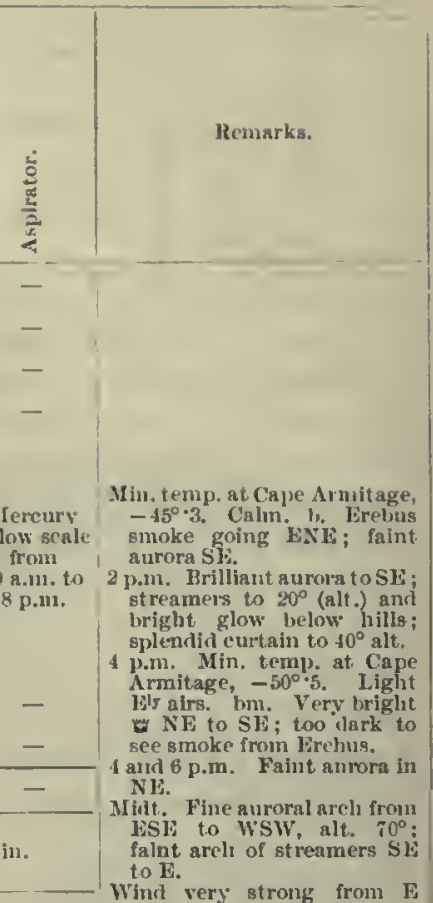
early, falling quickly and remaining calm, with bright clear sky all day. Barometer cont in ued to fall all day. Temperature much lower and steartier. "Nercury frozen, reading is from spirit mininum themometer.

$23 \mathrm{rd}$

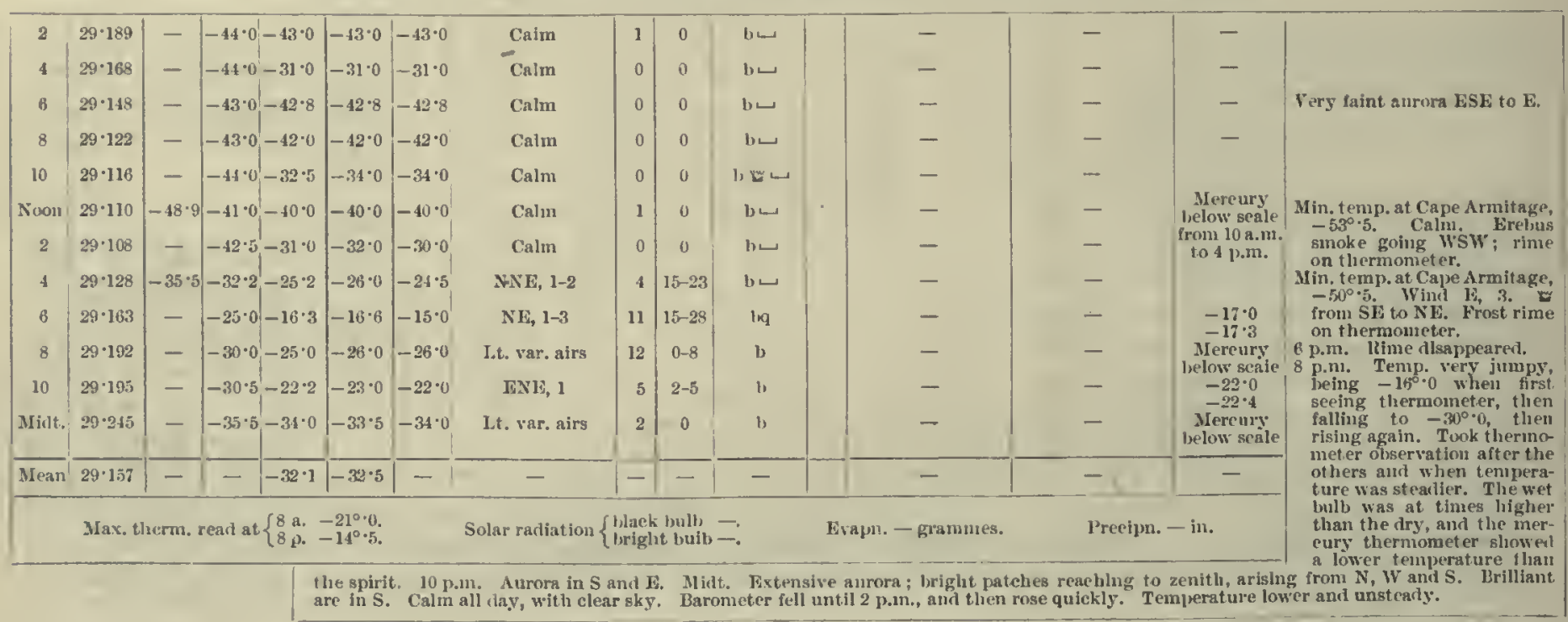

24th.

\begin{tabular}{|c|c|c|c|c|c|c|c|c|c|c|c|c|}
\hline 2 & $29 \cdot 288$ & - & $-40 \cdot 5-18 \cdot 2$ & $-17 \cdot 8$ & $-18 \cdot 2$ & Calm & 2 & 0 & b) & - & - & $\begin{array}{l}-18.4 \\
-19.5\end{array}$ \\
\hline 4 & $29 \cdot 308$ & - & $-41 \cdot 0-28 \cdot 2$ & $-28 \cdot 2$ & $-29 \cdot 0$ & Calm & 2 & 0 & 1) & - & - & $\begin{array}{l}\text { Merenry } \\
\text { Lelow serale }\end{array}$ \\
\hline B & $29 \cdot 297$ & - & $-38 \cdot 0-17 \cdot 2$ & $-18 \cdot 0$ & $-19 \cdot 0$ & E, 2 & 3 & $10-12$ & b & - & - & $\begin{array}{l}-19 \cdot 4 \\
-20 \cdot 6\end{array}$ \\
\hline 8 & $29 \cdot 325$ & - & $-33 \cdot 0-23 \cdot 2$ & $-24 \cdot 0$ & -23.8 & Caim & 4 & 0 & b. & - & - & - \\
\hline 10 & $29 \cdot 325$ & - & $-29 \cdot 0-27 \cdot 0$ & $-27 \cdot 0$ & $-28 \cdot 0$ & Calm & 0 & 0 & b & - & - & $\begin{array}{l}\text { Mercury } \\
\text { below serile }\end{array}$ \\
\hline Noon & $29 \cdot 311$ & $-28 \cdot 8$ & $-33 \cdot 5-21 \cdot 0$ & $-24 \cdot 2$ & $-25 \cdot 0$ & Lt, WNE airs & 2 & $5-12$ & 1, & - & - & $\begin{array}{l}\text { from } 10 \text { i.m. } \\
\text { to nooll }\end{array}$ \\
\hline 2 & $29 \cdot 308$ & - & $-29 \cdot 0-14 \cdot 8$ & $-15 \cdot 0$ & $-15 \cdot 0$ & It. NE airs & 2 & $0-5$ & $x * *$ & - & S. $B$, F. & $\begin{array}{l}-18.0 \\
-18 \cdot 4\end{array}$ \\
\hline 4 & $29 \cdot 291$ & $-2 \cdot 8$ & $-15 \cdot 4-2 \cdot 0$ & $-2 \cdot 5$ & $-2 \cdot 0$ & $\mathrm{Sk}_{3}, 1-2$ & 10 & $10-18$ & of $-i$ & - & N. 10 & $\begin{array}{l}-1 \cdot 8 \\
-2 \cdot 6\end{array}$ \\
\hline 6 & $29 \cdot 282$ & 一 & $-4 \cdot 0-1 \cdot 0$ & $-1 \cdot 0$ & $\quad 0.0$ & $\mathrm{SF}_{2}, 2-1$ & 17 & $10-25$ & ofq $\neq * *$ & - & N. 10 & $\begin{array}{l}=0.8 \\
=1.5\end{array}$ \\
\hline 8 & $29 \cdot 310$ & - & $-3.0 \quad 1.0$ & $1 \cdot 0$ & $1 \cdot 6$ & S, 1-4 & 20 & $10-22$ & $\operatorname{mgf}+* x$ & - & N. 10 & $\begin{array}{l}0 \cdot \overline{7} \\
0 \cdot 7\end{array}$ \\
\hline 10 & $29 \cdot 3014$ & - & $-2 \cdot 0-0 \cdot 5$ & $-1 \cdot 0$ & $-1 \cdot 0$ & ISE, 3-4 & 12 & $10-22$ & $m$ & - & S. 10 & $\begin{array}{l}-0.8 \\
-1 \cdot 1\end{array}$ \\
\hline Mlat. & $x \cdot 205$ & - & $-3 \cdot 0-2 \cdot 0$ & $-2 \cdot 2$ & $-2 \cdot 0$ & SE, 4 & 14 & $10-30$ & oq - $f^{\prime}$ & - & S. 10 & $\begin{array}{r}-1 \cdot 7 \\
-4.7 \\
\end{array}$ \\
\hline Mesn & $20 \cdot 304$ & - & $-\left.\right|^{-13 \cdot 1}$ & $-13: 3$ & - & - & - & - & - & - & - & - \\
\hline
\end{tabular}

r.-s. to E, aptarently approaching. Armitage, $-0^{\circ} 0^{\circ}$. Wint NNW, 1. NE; clourded to $\mathrm{S}$

p.n. Fine jowlery snow giling; thin haze: stars visible in zentll.

p.m. lintirely wercast ; sery threatening apperr-

ance. Yin. temp. at Cape rmitage, $-33^{\circ} \cdot$ Wind SF, $f-5$. oqf. $*-f^{2}$, p.m. Snow falling in smal p.m. Wind squally inowflakes harl and small.

alm nearly all day. Sky

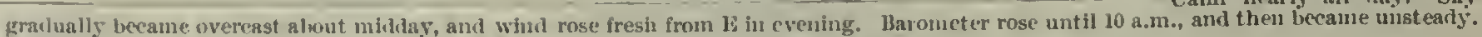
Temperature rising rujidly tow ards the eveuing. 
Metborological, Joulinal of Thl "Discoviliy."

[1903-June.

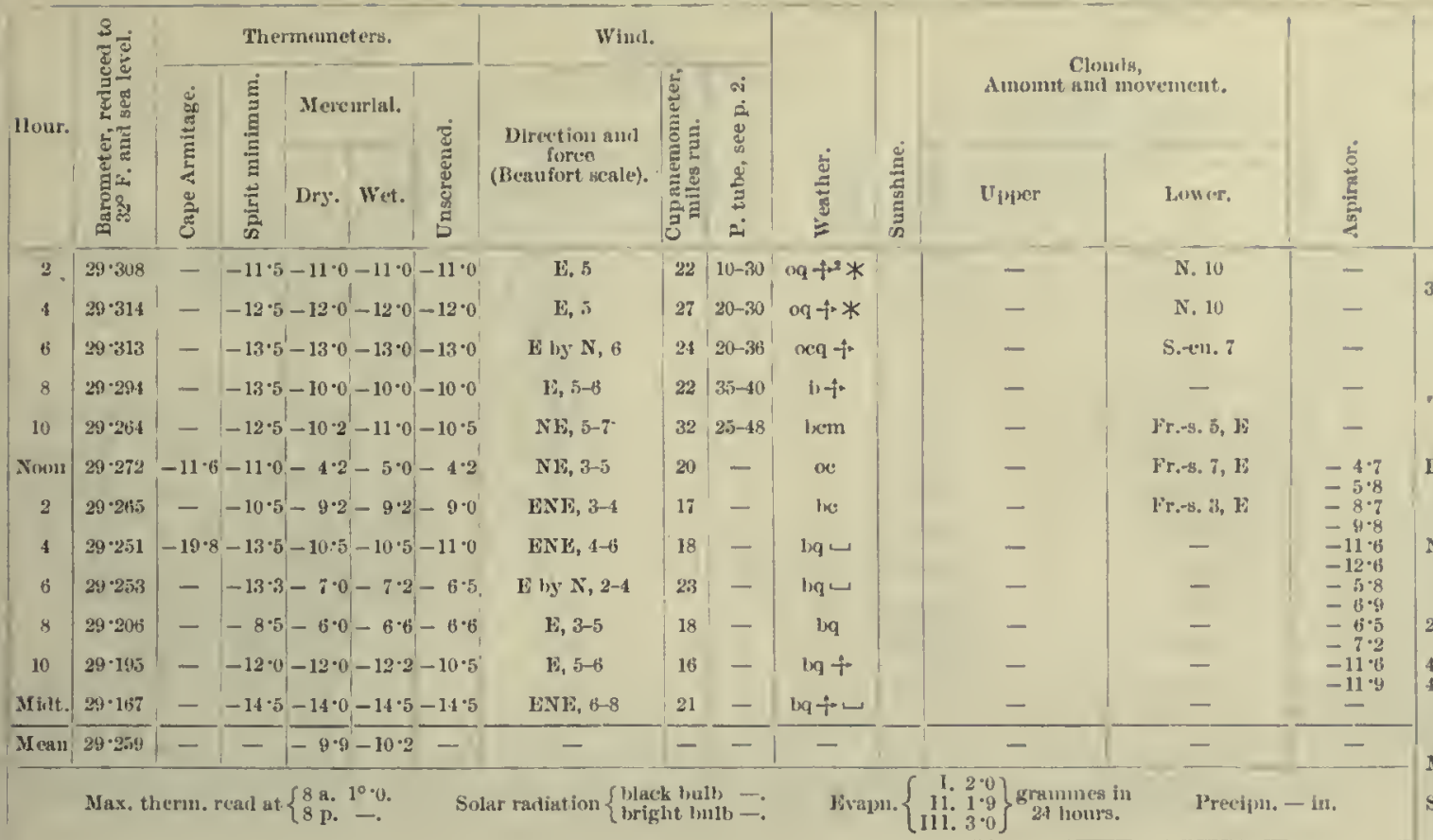

Nemarks.

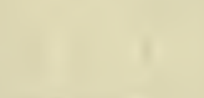

3 a.ın. Furlous squall, force $8-9$

25 th

Break ln clouds in $\mathrm{S}$ and us $\mathrm{X}$. Dinea small anemometer was not working properly, so was Nown

Arunita S, 13 , 8 . Wh rebus otascured.

2 p.ur. Dietacherl Str. on $\mathrm{X}$ and $\boldsymbol{H}$ horlkon.

4 p.tn. Foggy to 5 .

p.m. Mir. temp. at Capre Armitage, $-21^{\circ} \cdot 2$. Whul and SST.

Nirlt. l'atches of streauers to $\mathrm{K}$, alt $10^{\circ}$.

trong by wind all day. Overcast early, hut cleariug and remaining clear. Barometer steadier and higher.

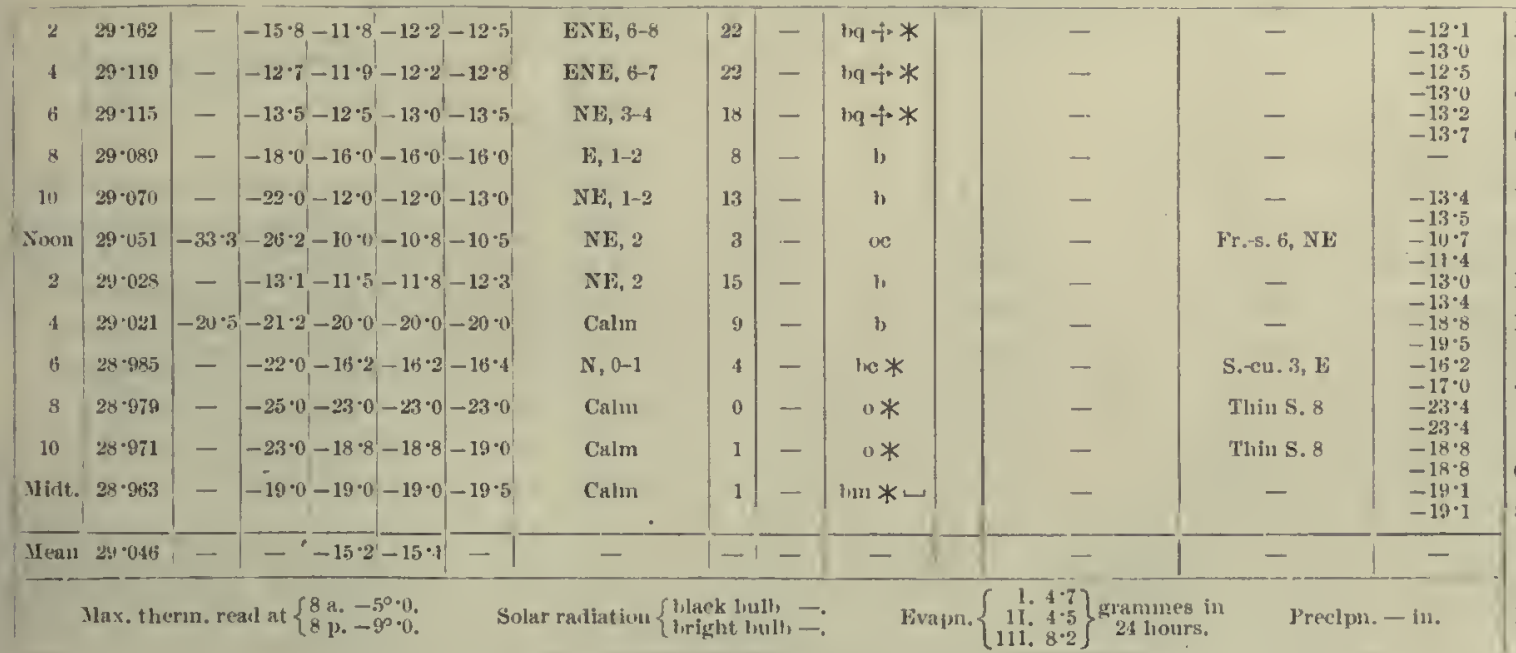

Bind of aurora running across Bky a little li of zenith from $\mathrm{N}$ to $\mathrm{S}$; odd jatches fir $\mathrm{E}$. 4 A.lu. Aurora streamers from NE to $S$. a.m. Irregular streamers from NE to $S$, anu iu SW.

Viry falut aurora areh from $\mathrm{F}$ to $\mathrm{SE}$. Erebus smoke visible from ship going SW, Bank of Fr.-8. to $x$.

Noun. Hovement of elouds very loubtiul.

Arm. Mln. temp, at Cape Armitage, $-40^{\circ} \cdot 1$. Wiun Ask, 1. Krebus ouscurerd. p.m, Mint. temis. at Cape ExE, 1-2. 3. Erebus smoke not visihle, $t$ on dark.

6 p.m. Vory tine snow falling ; Str. to E.

p.m. Very fine snow falling; stars dimly visille in kenith. 10 p.m. Silow erystals falling. very small ; stars dimly Hsiblo in zenith.

Milt. Very misty all romm ; suow erystals falling; rlme

Very strong lily wind carly, gralually falling ealın. Sky elear generally. Barometer continued to fall all day. Temperature stealy and
lower. lower.

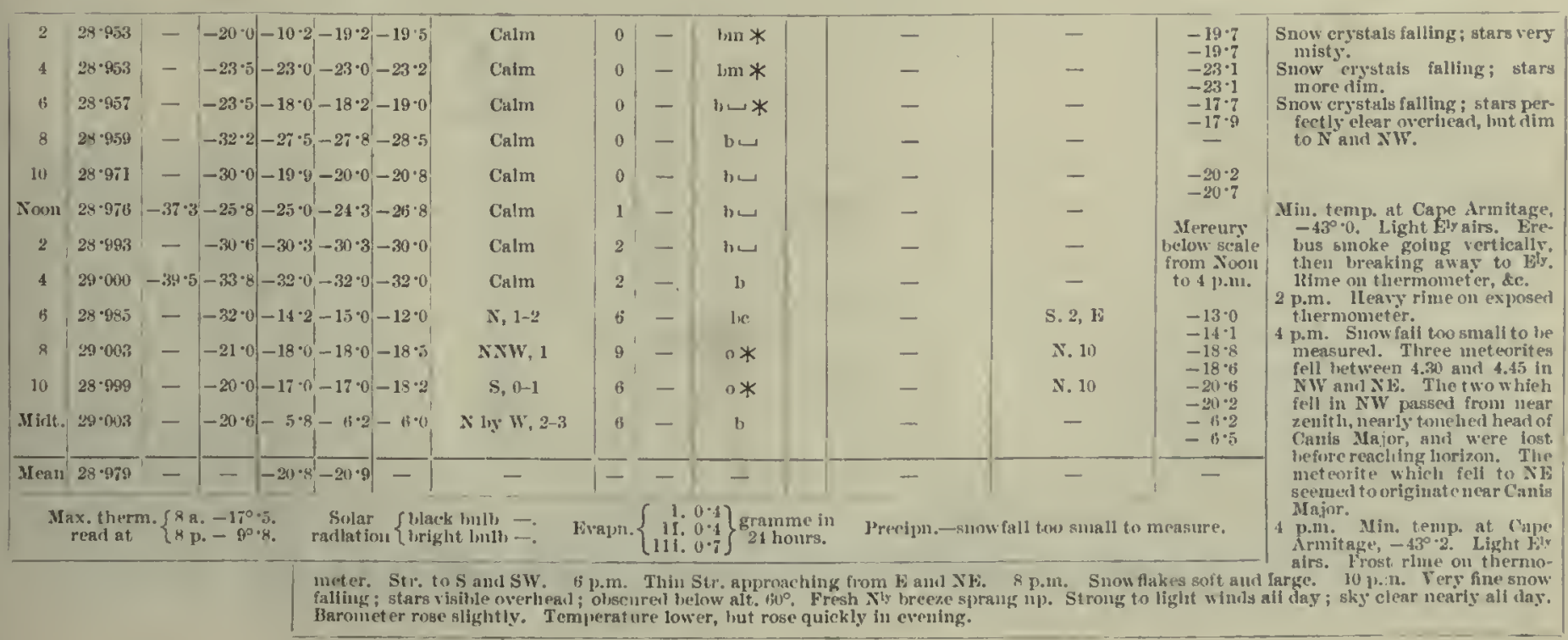




\begin{tabular}{|c|c|c|c|c|c|c|c|}
\hline \multirow{3}{*}{1.} & \multirow{3}{*}{ 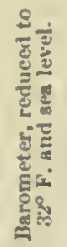 } & \multicolumn{5}{|c|}{ Thermoneters. } & \multirow[t]{3}{*}{ Wland. } \\
\hline & & \multirow{2}{*}{ 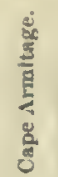 } & \multirow{2}{*}{ 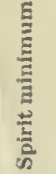 } & \multicolumn{2}{|c|}{ Mercurial. } & \multirow{2}{*}{ - } & \\
\hline & & & & Dry. & wet. & & \\
\hline
\end{tabular}

28th.

\begin{tabular}{|c|c|c|c|c|c|c|c|c|c|c|c|}
\hline & & & & & & & & & & & \\
\hline 2 & $29 \cdot 011$ & & $-7 \cdot 3-6 \cdot 0-600-6 \cdot 8$ & Lt. $\mathbf{N}$ alrs & 8 & - & $\circ *$ & - & N. 10 & $\begin{array}{l}-6 \cdot 2 \\
-6.5\end{array}$ & $\begin{array}{l}\text { Fine auow falllig; stars falutly } \\
\text { visihle overbead, }\end{array}$ \\
\hline 4 & $29 \cdot 032$ & - & $|-10.0|-3.5-3.8-3.8 \mid$ & $1,0-2$ & 4 & - & oq $*$ & - & N. 10 & $\begin{array}{l}-0.3 \\
-3 \cdot 3\end{array}$ & Snowing liearlly; small Hakes; \\
\hline 6 & $29 \cdot 024$ & - & $|-4 \cdot 7|-2 \cdot 4-3 \cdot 0|-2 \cdot 5|$ & 19,1 & 3 & - & o* & - & N. 10 & $\begin{array}{l}-4.0 \\
-2 \cdot 9\end{array}$ & \\
\hline 8 & $29 \cdot 043$ & - & $-4 \cdot 0|-2 \cdot 0-2 \cdot 0-2 \cdot 0|$ & F ty $x, 1-2$ & 10 & - & $\circ *$ & - & N. 10 & -36 & \\
\hline 10 & $29 \cdot 0.46$ & - & $\begin{array}{lllll}-3.0 & 0.0 & 0.0 & 0.0\end{array}$ & E, 2-8 & 14 & - & $\circ *$ & - & Thin N. 10 & - & \multirow{10}{*}{ 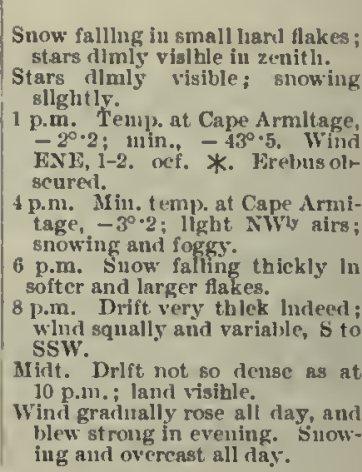 } \\
\hline Nomu & $29 \cdot 037$ & - & \begin{tabular}{lll|l|}
-0.8 & 1.8 & 1.0 & 1.0
\end{tabular} & N1. 1-3 & 6 & - & $\circ *$ & - & Thln N. 10 & 0.7 & \\
\hline 3 & $29 \cdot 033$ & - & $\begin{array}{llll}-0.2 & 3.2 & 3.0 & 4.0\end{array}$ & ENE, 1 & 15 & - & $o * 0$ & - & x. 10 & $\begin{array}{l}0.3 \\
3: 2 \\
0.2\end{array}$ & \\
\hline 4 & $29 \cdot 017$ & $-2 \cdot 8$ & $-2 \cdot 5-2 \cdot 0-2 \cdot 0-2 \cdot 0$ & $N W, 1-2$ & $i$ & - & )* & - & N. 10 & $\begin{aligned} 2.8 \\
-1.5\end{aligned}$ & \\
\hline 6 & $28 \cdot 987$ & - & $\left|\begin{array}{lll}-2 \cdot 8 & 2 \cdot 0 & 1 \cdot 5\end{array}\right| 2 \cdot 6$ & Calm & 6 & - & $0 *^{3}$ & - & N. 10 & $\begin{array}{rl}-1 & 1 \\
2.5 \\
1.8\end{array}$ & \\
\hline 8 & $28 \cdot 986$ & - & $\begin{array}{llll}-1.0 & 5.5 & 5.5 & 5.5\end{array}$ & S, 5-7 & 9 & - & $\infty t^{2} *^{2}$ & - & N, 10 & $1 \cdot$ & \\
\hline 10 & $29 \cdot 007$ & - & $-5 \cdot 0-5 \cdot 0-5 \cdot 0-5 \cdot 0$ & $\mathrm{E}, 7$ & 23 & - & $n q 4^{2} *^{a}$ & - & N. 10 & $\begin{aligned}-5 \cdot 1 \\
-5.1\end{aligned}$ & \\
\hline Mint. & $39 \cdot 055$ & - & $-8 \cdot 5-6.5-6.5-6.8$ & F, 5-7 & 23 & - & oq $+* *$ & - & N. 10 & $-\underline{a}$ & \\
\hline Xean & $28 \cdot 023$ & - & $1-1-1 \cdot 2-1 \cdot 4-$ & - & 1 & - & -- & - & - & - & \\
\hline & & & & & & & & & $2 \mathrm{ins}$ sluc & m. 10th. & \\
\hline
\end{tabular}

29th.

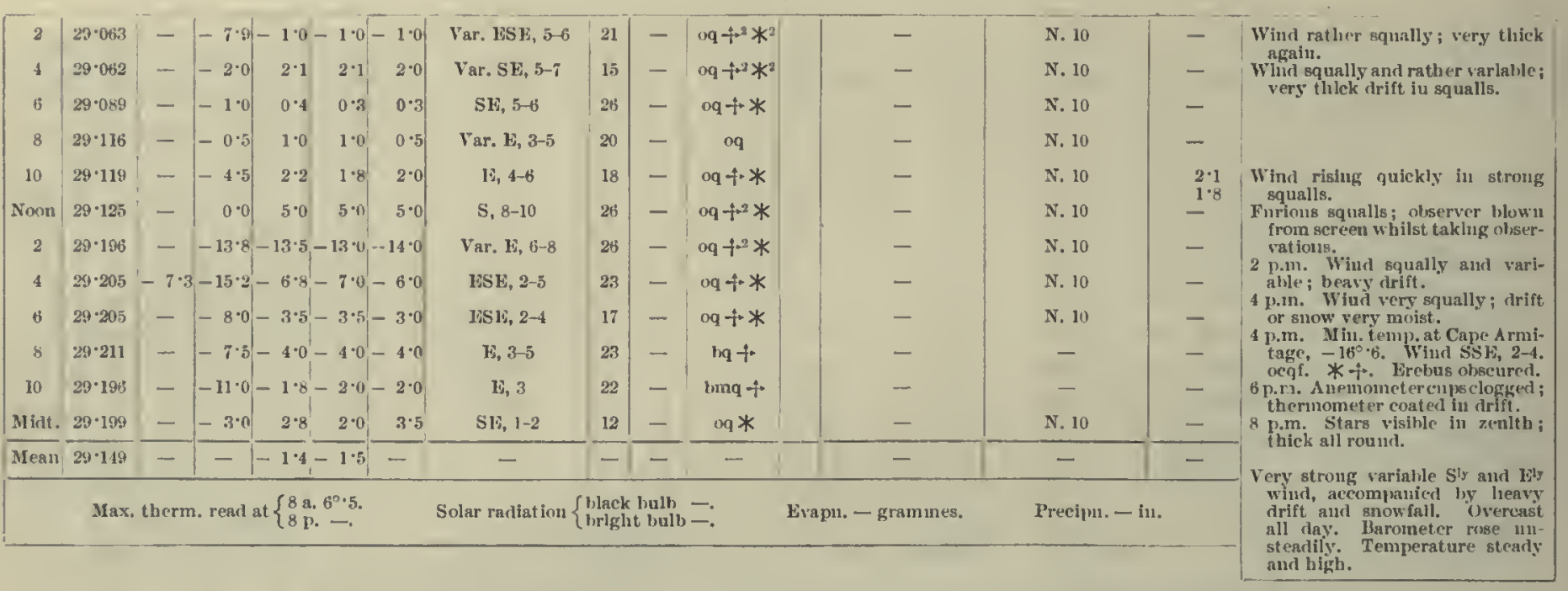

30 th.

\begin{tabular}{|c|c|c|c|c|c|c|c|c|c|c|}
\hline 2 & $29 \cdot 185$ & - & $|-5 \cdot 0|-5 \cdot 0-5 \cdot 0|-5 \cdot 0|$ & Calm & 3 & - & b & - & - & - \\
\hline 4 & $29 \cdot 208$ & - & $|-6 \cdot 5|-2 \cdot 4-2 \cdot 4-7 \cdot 0 \mid$ & $N E, 1-2$ & 7 & - & $o q$ & - & N. 10 & - \\
\hline 6 & $29 \cdot 190$ & - & $-11 \cdot 5|-10 \cdot 0-10 \cdot 0-10 \cdot 0|$ & F, 3-5 & 21) & - & oq & - & N. 10 & - \\
\hline 8 & $29 \cdot 193$ & - & $-10 \cdot 5|-4 \cdot 0-4 \cdot 0-4 \cdot 0|$ & E, 1-3 & 18 & - & loqu & - & - & - \\
\hline 10 & $29 \cdot 212$ & - & $-11 \cdot 5-5 \cdot 2-5 \cdot 8-5 \cdot 5$ & NE, 2-3 & 13 & - & $\mathrm{bm}$ & - & - & - \\
\hline Nioon & $29 \cdot 215$ & $-12 \cdot 2$ & $-7 \cdot 5|-2 \cdot 0-2 \cdot 0|-3 \cdot 0$ & Calm & 10 & - & $o *$ & - & x. 10 & - \\
\hline 2 & $29 \cdot 217$ & - & $|-6 \cdot 0|-5 \cdot 3-5 \cdot 3 \mid-6 \cdot 0$ & NE, 1-2 & 4 & - & oc & - & S. 10 & $\begin{array}{l}-4 \cdot 0 \\
-4 \cdot 7\end{array}$ \\
\hline 4 & $23 \cdot 215$ & $-14 \cdot 4$ & $-7.0-6.5-6.5-5.5$ & NF, 0-1 & 7 & - & b & - & - & $\begin{array}{l}-4 \cdot 8 \\
-5 \cdot 1 \\
-5.8\end{array}$ \\
\hline B & $29 \cdot 216$ & - & $-14 \cdot 3-7 \cdot 0-7 \cdot 0-7 \cdot 5$ & Lt. SE airs & 5 & $0-8$ & $b \sqcup$ & - & - & $\begin{array}{l}-5.9 \\
=5.8\end{array}$ \\
\hline 8 & $29 \cdot 240$ & - & $-20 \cdot 0 \mid-17 \cdot 0-17 \cdot 0-17 \cdot 5$ & Calm & 2 & 0 & b & - & - & $\begin{array}{l}-17 \cdot 3 \\
-17 \cdot 3\end{array}$ \\
\hline 10 & $29 \cdot 248$ & - & $-16 \cdot 4 \mid-16 \cdot 0-15 \cdot 4)-16 \cdot 5 \mid$ & Lt. SF airs & 5 & 0 & $\lim \rightarrow$ & - & - & $\begin{array}{l}-11 \cdot 3 \\
-17 \cdot 1 \\
-17 \cdot 0\end{array}$ \\
\hline Mlat. & $29 \cdot 2455$ & - & $-20 \cdot 7 \mid-13 \cdot 2-12 \cdot 5-12 \cdot 5$ & Caluu & 0 & 0 & $\mathrm{~m}$ & - & Fogger, 10 & $\begin{array}{l}-10 \cdot 8 \\
-11.6\end{array}$ \\
\hline Nean & $29 \cdot 217$ & - & $-\mid-7 \cdot 8-i \cdot 8-$ & - & $\overline{-}$ & - & - & - & 二 & - \\
\hline
\end{tabular}

Max. thern. read ut $\left\{\begin{array}{l}8 \mathrm{~A}_{0} \\ 8 \mathrm{p},-3^{\circ} \%\end{array}\right.$

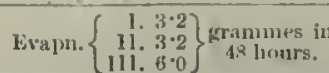

Precipn. - in.

Remarks. lug and overcast all day. 
Meteorological Journal of the "Discoviry."

[1903-July.

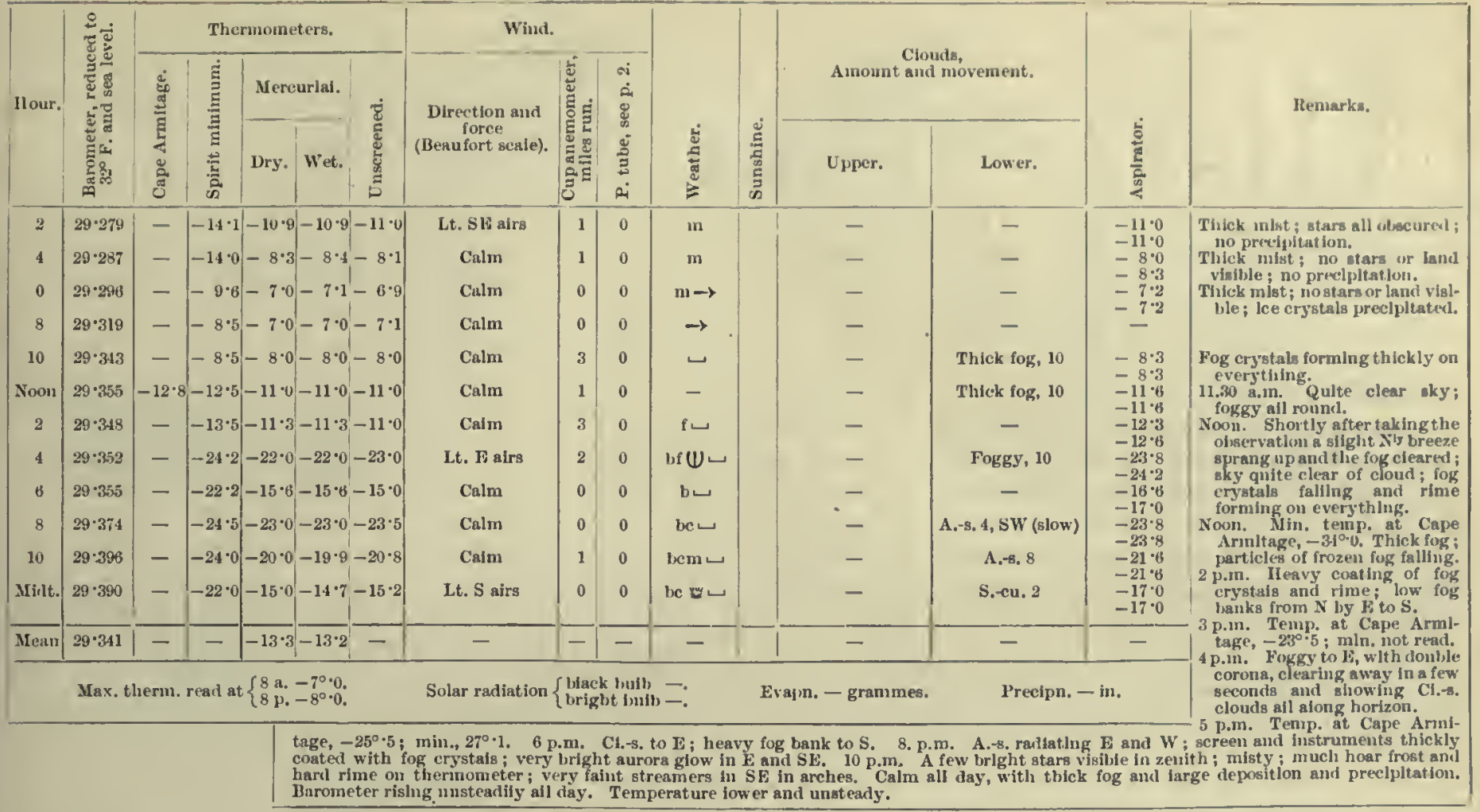

\begin{tabular}{|c|c|c|c|c|c|c|c|c|c|c|c|}
\hline 2 & $29 \cdot 394$ & - & $-24 \cdot 2$ & $-21 \cdot 0|-21 \cdot 0-22 \cdot 3|$ & Lt. $S$ airs & 1 & 0 & be & - & S.en. 2 & $-21 \cdot 1$ \\
\hline 4 & $29 \cdot 390$ & 一 & $-24 \cdot 1$ & $-12 \cdot 6-12 \cdot 4-12 \cdot 1$ & 1.t. È airs & 2 & 0 & be w⿰ & - & Cu. 3 & $-14 \cdot 4$ \\
\hline 6 & $29-386$ & - & $-18 \cdot 8$ & $-18 \cdot 0-17 \cdot 7-18 \cdot 7$ & Lt. var. airs & 3 & 0 & bem乚 & - & Cu. 4 & $\begin{array}{l}-14.8 \\
-16^{\circ} 6\end{array}$ \\
\hline 8 & $29 \cdot 375$ & - & $-21 \cdot 0$ & $-18 \cdot 0-180^{\circ}-18^{\circ} 0$ & ESE, 1-2 & 3 & $5-10$ & b & - & - & $-16 \cdot 2$ \\
\hline 10 & $29 \cdot 385$ & - & $-19 \cdot 0$ & $-7 \cdot 5-7 \cdot 8-7 \cdot 8$ & E, 2-3 & 9 & $12-18$ & b & - & - & $-8 \cdot 0$ \\
\hline Noon & $29 \cdot 372$ & $-26 \cdot 3$ & $-10 \cdot 5$ & $-8 \cdot 5-8 \cdot 5-8 \cdot 8$ & E, $3-4$ & 13 & $18-25$ & $b+$ & - & - & $\begin{array}{l}=8.0 \\
=9 \cdot 0\end{array}$ \\
\hline 2 & $29 \cdot 363$ & - & $-15 \cdot 2$ & $-10 \cdot 0-11 \cdot 0-10 \cdot 5$ & Var. E, 2-4 & 14 & $15-25$ & beq $f$ & Cl.cu. 1, E & - & $-10 \cdot 1$ \\
\hline 4 & $29 \cdot 3 \hbar 3$ & $-27 \cdot 8$ & -1 & $-15 \cdot 0-15 \cdot 0-16 \cdot 0$ & Var. IE, 3-6 & 18 & $20-30$ & beq $t$ & Ci.-cu. 2, E & - & $\begin{array}{l}-10 \% 0 \\
-17 \cdot 0\end{array}$ \\
\hline 6 & $20 \cdot 369$ & - & & $-20 \cdot 2-20 \cdot 2-21 \cdot 5$ & Var. E, 3-6 & 18 & $22-30$ & bq $+\bullet \bullet$ & - & - & $\begin{array}{l}-180 \\
-32 \cdot 0 \\
-22 \cdot 4\end{array}$ \\
\hline 8 & $29 \cdot 388$ & - & & $-19 \cdot 5-19 \cdot 5-19 \cdot 8$ & NE, 4-6 & 29 & $25-30$ & beq $+U$ & - & A. - s. 3 & $-19 \cdot 5$ \\
\hline 10 & $29 \cdot 386$ & - & $-21 \cdot 1$ & $-20 \cdot 0-20 \cdot 0-20 \cdot 0$ & ENE, 5-6 & 24 & $30-37$ & $b c+U$ & - & A. -8.2 & $\begin{array}{l}-18 \cdot 6 \\
-20 \cdot 6\end{array}$ \\
\hline Midt. & $29 \cdot 396$ & - & $-21 \cdot 9$ & $-21 \cdot 9-22 \cdot 0-21 \cdot 9$ & LNE, 46 & 20 & $29-37$ & $b c+$ & - & A. -6.1 & $\begin{array}{r}-22 \cdot 0 \\
-22.0 \\
\end{array}$ \\
\hline Mean & $29 \cdot 381$ & - & - & $-16 \cdot 0-16 \cdot 1-$ & - & - & - & - & - & - & $\overline{-}$ \\
\hline
\end{tabular}

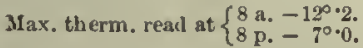

Solar radiation $\left\{\begin{array}{l}\text { biack bulh } \\ \text { bright buib }=\text {. }\end{array}\right.$
Preclpn. - In.

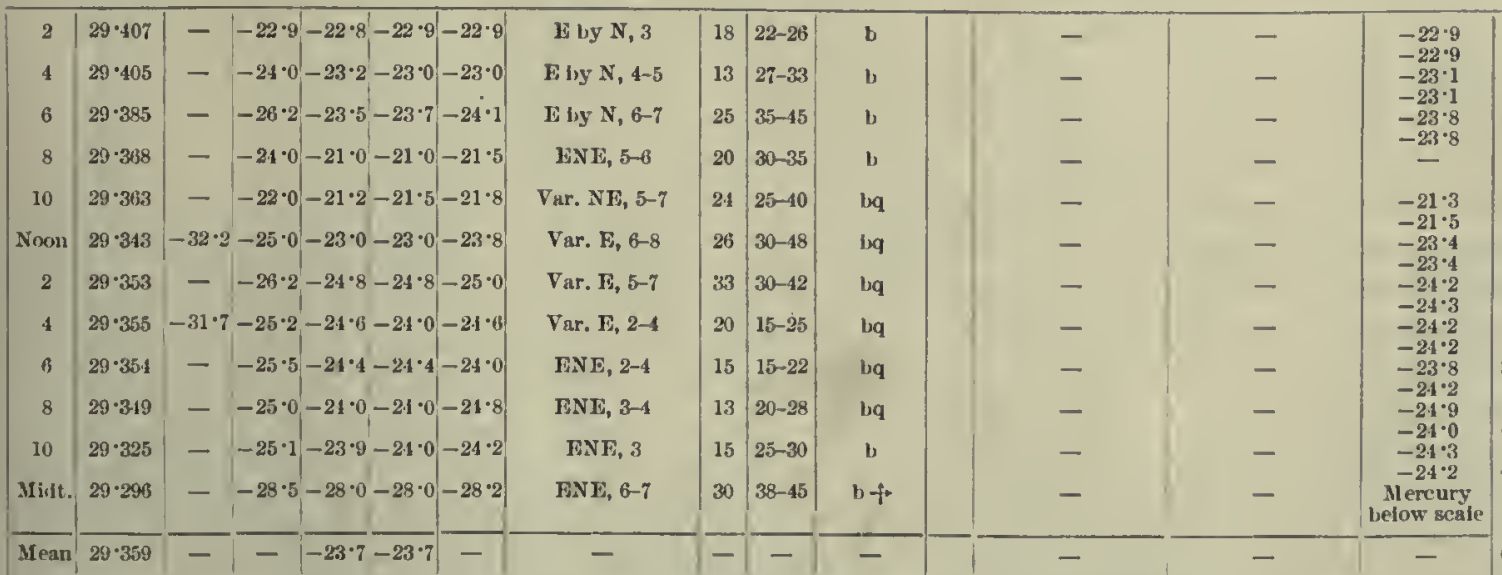

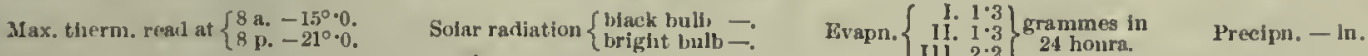

Auroral bands to ENE aud 2nd.

Bright patehes (aurora) between SW and NNE, alt. $10^{\circ}$ to $20^{\circ}$ very misty to s; rine on ther moineters all night.

8 a.m. Arches and streanters S to NW, bright.

10 a.m. Precipitation has ap parentily ccased.

Noon. Some detached Str. to

Nooin. Min.temp, at Cape Arnultage, $-33^{\circ} \cdot 5$. Wind FNE, 4. 11. ${ }^{2}$, on thermometer. Erelns ouscured.

2 p.m. Cl.-cll. along $\mathrm{E}$ horizon

foggy to S. tage, $-30^{\circ} \cdot 2$. Wind $\mathrm{E}$ by $\mathrm{S}$, 2-3. 1. to.

6 p.m. A meteor from zenith nortiwards, very bright. and graduatiy freshened untlil
It blew very strong from $\mathbf{B}$, It blew very strong from $\mathbf{B}$, varlabie. Sky pretty cicar of toun. towang thermening. ind sprang up about 8 a.m.

1 st.

A bank of Str. over western 3 rd nountains.

llapidly changing, but generally faint, anrora, NE to SSE.

Faint areh XXE to SSE, alt. $10^{\circ}$

streamers in N.iling qulckiy
Temperature falling during observation.

Noon. Nin. tempat Cape A rmaltage, $-3.4^{\circ} \cdot 5$. Wild KNE, $4-5$. in Fis direction. 2 p.m. Very clear; western range quite viaibie (moonlighit).

p.on. Dright aurorsl anch froin $\mathrm{S}$ to NE through $\mathrm{E}$.

4 p.m. Nin. temp. at Cape Armitage, $-33^{\circ} \cdot 5$. Wind $E$ by S, 1. b. E to E and SS kirebus smoke golng EN E. ${ }^{6} \mathrm{p}$.13..

Mlict. Snow drifting on surface of hloe.

Very strong to fresh NVI wind ail dlay, wit in perfectiy clear sky. Temperature low and steady. 


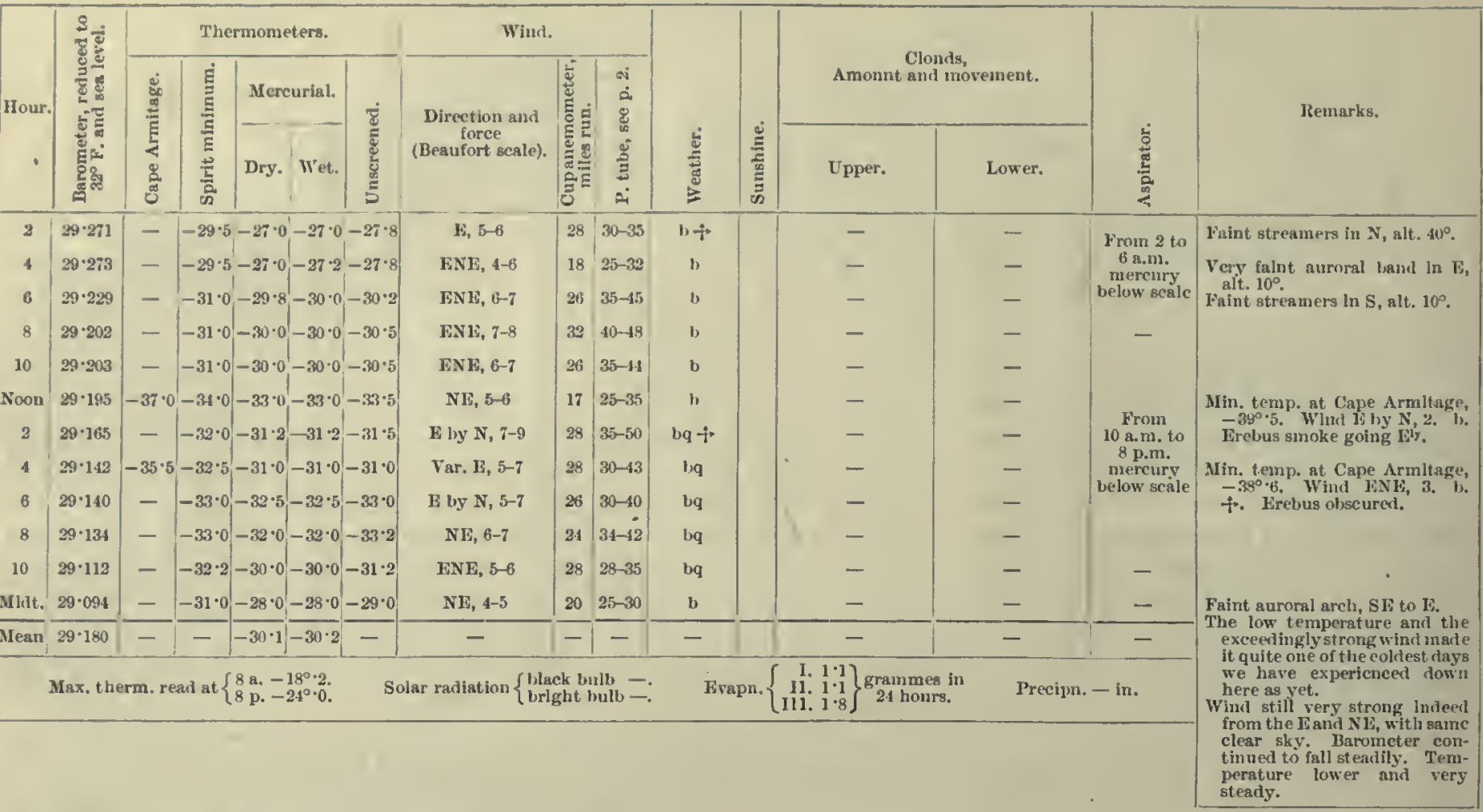

5 th.

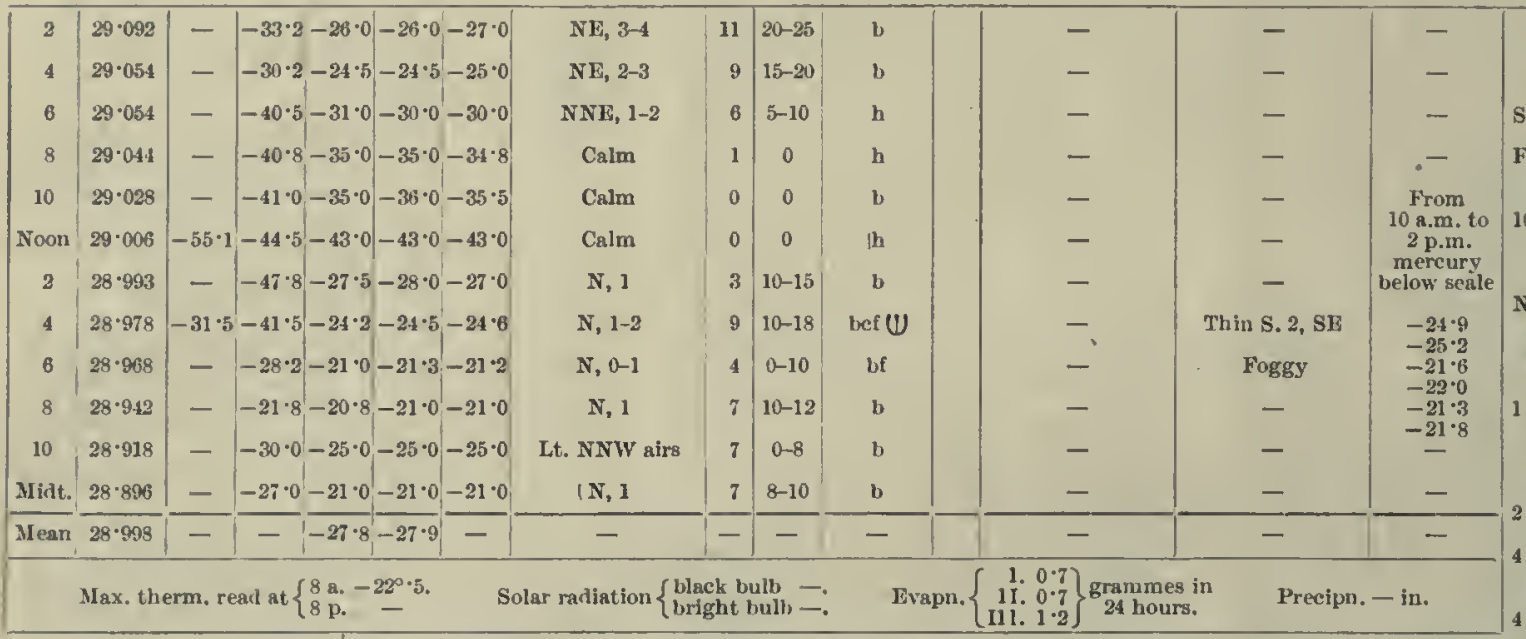

Str. on western mountains.

Sint auroral arch from NE to SW, alt. $10^{\circ}$; a small hank of a.m. Some detaehel Str. low down to $S$; very faint anroral arch extending from Noon. Min. tcml\% at Cape Armitage, $-58^{\circ} \cdot 1$. Calm. b. Hrebus smoke aseending then breaking awry to ty vw.

p.m. Irebus smoke ascend p.m. Trebus smoke ascendbreaking away to Sly $^{\prime}$ direc tion; apparently sevariable winds up there. $\mathrm{E}$ and $\mathrm{SF}$ horizon.

p.m. Lunar lialo, rscius $22^{\circ} 20^{\prime}$; foggy to $\mathrm{S}$ and $\mathrm{SI}$

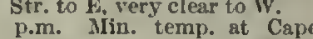

NW, 2. Ci.-8. 5. he. Frebus smoke going SE in large volume. 4.30 p.m. Min. temp. at Cape Armitage, $-33^{\circ} 0$. W U U. 6 p.m. Thin loggy Str. passing across moon; falnt corona and halo, the latter of ordinary radlus. 8 p.m. Thlu Str. to 13 and fogigy to $S$. calm, with the same clear sky, except for about 5 hours in afternoon, when it became foggy. Barometer still continued to fall slowly.
Temperature lower and not so steady.

6th.

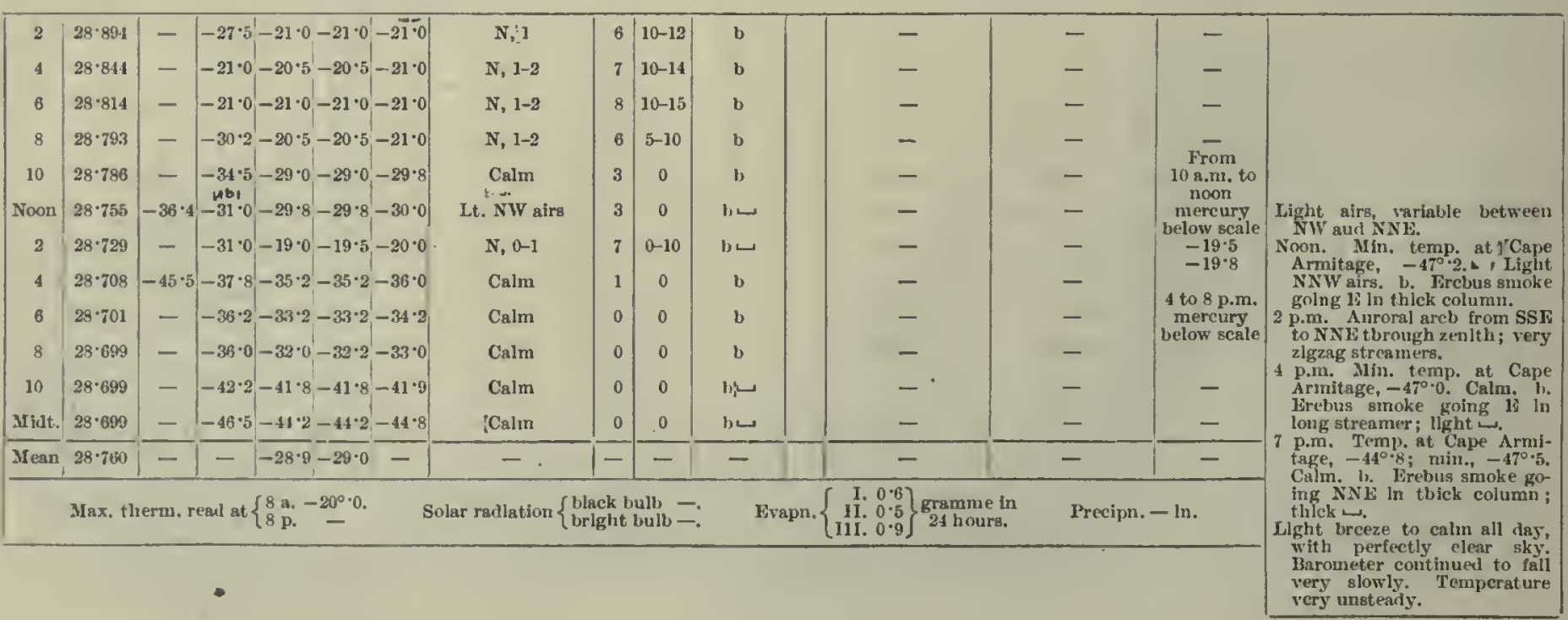


Metrorological Journal, of the "Discovery."

[1903-July.

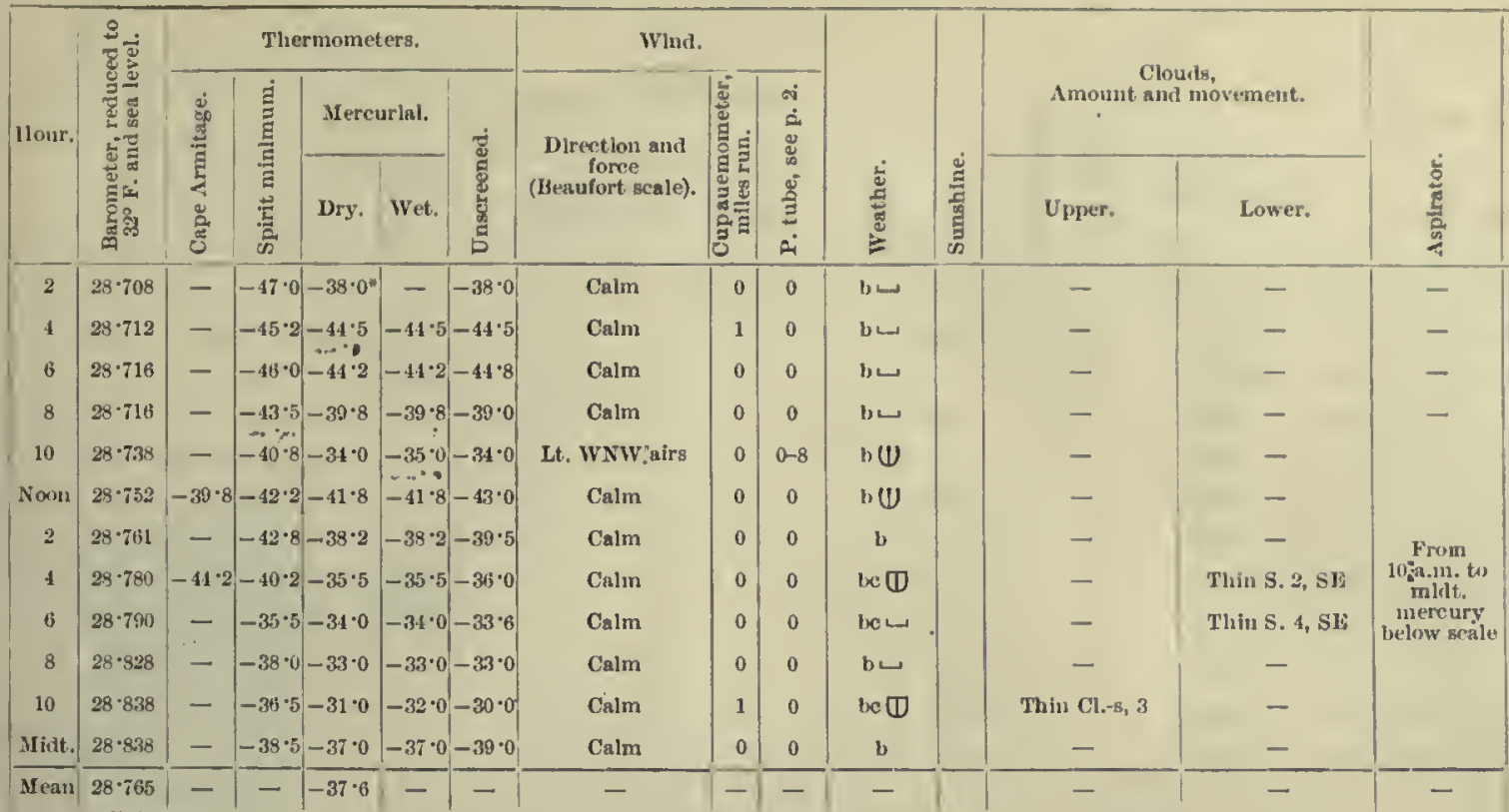

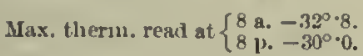

Solar radiatlon $\left\{\begin{array}{l}\text { black bulh }- \text {. } \\ \text { bright bulb- }\end{array}\right.$

Evapn. - grammes.

Precipu, - in.

Reขnarkn.

Dry-bulb mercury down II

Rlme on anemoneter glasu anu thermometer; detaclied Str. hal-way up western mounround.

Ba.m. lieary S, en. on western mountalus, extending to Bluft ; tops vislble.

8 a.m. Heavy liank of fog $u$ shooting from lt.

$10 \mathrm{a} .1 \mathrm{n}$. If Cavy henk of fog or cloud low down to S and SW. conl. Heavy clouds to $\mathrm{S}$, hankerl up; detaclied $\mathbf{F r}$ - $\mathrm{s}$.

toon. Min. temp. at Cape Armitage, $-59{ }^{1}$; llght st aira. b. Erehus smoke going p.m. Str. to $S$ and $S E$. forgy p.m. Str. to $S$ and $S E$; f foggy p.m. Str. approachlug from SE. Mn. temp, at Cape p.m. Mln. temp, at Cape airs. Ci.-s. 5. we. $\mathbb{D} \Psi$. $6 \mathrm{p} . \mathrm{m}$. Thin Str. In detaehed banks aeross zenith. $7 \mathrm{p} . \mathrm{m}$. Temp. at Cape Armitage, $-51^{\circ} \cdot 0 ;$ mln., $-51^{\circ} \cdot 0$. Frebus smoke golng NW in strong column. 8 p.m. Detaehed $S$ tr, to $\mathrm{S}$ and $\mathrm{SF}$, and all along $\mathrm{W}$ horlzon to $\mathrm{N}$. Calm all day, and clear sky except for some thln stratus
in the afternoon. Barometer risling steadily all day. Temperature lower and steady. * leadlng of spirit mlulmum thermometer at time of observation.

\begin{tabular}{|c|c|c|c|c|c|c|}
\hline 2 & $28 \cdot 877$ & - & $-38 \cdot 5$ & $-38 \cdot 0$ & $-38 \cdot 0$ & $-38 \cdot 0$ \\
4 & $28 \cdot 917$ & - & $-45 \cdot 5$ & $-45 \cdot 0$ & $-45 \cdot 0$ & $-46 \cdot 0$ \\
6 & $28 \cdot 941$ & - & $-46 \cdot 0$ & $-43 \cdot 0$ & $-43 \cdot 0$ & $-43 \cdot 0$ \\
8 & $29 \cdot 003$ & - & $-43 \cdot 0$ & $-26 \cdot 0$ & $-26 \cdot 0$ & $-26 \cdot 0$ \\
10 & $29 \cdot 024$ & - & $-33 \cdot 0$ & $-24 \cdot 0$ & $-24 \cdot 0$ & $-23 \cdot 8$ \\
Nonn & $29 \cdot 053$ & $-4.5 \cdot 2$ & $-39 \cdot 0$ & $-38 \cdot 5$ & $-38 \cdot 5$ & $-40 \cdot 0$ \\
2 & $29 \cdot 0 \cdot 0$ & - & $-42 \cdot 5$ & $-37 \cdot 8$ & $-38 \cdot 0$ & $-37 \cdot 5$ \\
4 & $29 \cdot 082$ & $-41 \cdot 0$ & $-38 \cdot 2$ & $-35 \cdot 0$ & $-35 \cdot 2$ & $-35 \cdot 0$ \\
6 & $29 \cdot 076$ & - & $-38 \cdot 0$ & $-30 \cdot 0$ & $-30 \cdot 0$ & $-30 \cdot 5$ \\
8 & $29 \cdot 088$ & - & $-38 \cdot 0$ & $-20 \cdot 8$ & $-21 \cdot 0$ & $-21 \cdot 0$ \\
10 & $29 \cdot 102$ & - & $-39 \cdot 0$ & $-21 \cdot 0$ & $-21 \cdot 2$ & $-21 \cdot 0$ \\
Micit. & $29 \cdot 124$ & - & $-22 \cdot 0$ & $-20 \cdot 8$ & $-21 \cdot 0$ & $-20 \cdot 0$ \\
\hline Mean1 & $29 \cdot 029$ & - & - & $-31 \cdot 7$ & $-31 \cdot 7$ & - \\
\hline
\end{tabular}

Max. therm. read at $\left\{\begin{array}{l}8 \text { a. } \\ 8 \mathrm{p},-24^{\circ} \cdot 0 .\end{array}\right.$

\begin{tabular}{|c|c|c|c|}
\hline Calm & 0 & 0 & beu \\
\hline Calm & 0 & 0 & ис Ф七 \\
\hline Calm & 0 & 0 & be -4 \\
\hline Calm & 2 & 0 & bm \\
\hline Lt. Ji airs & 2 & $0-8$ & bU山 \\
\hline Calm & 2 & 0 & $b e$ \\
\hline Calm & 1 & 0 & $\operatorname{orf} U *$ \\
\hline Calm & 0 & 0 & bf \\
\hline Lt. $S W$ alrs & 2 & $0-5$ & bru \\
\hline $\mathrm{SE}, 1$ & 6 & $10-14$ & b \\
\hline SE, 1-2 & 10 & $15-20$ & bv \\
\hline ESE, 1-2 & 9 & $15-20$ & by \\
\hline
\end{tabular}

Solar radlation $\left\{\begin{array}{l}\text { hlack luull, }- \text {. } \\ \text { lright bull }-\cdots\end{array}\right.$

\begin{tabular}{|c|c|c|}
\hline & & \\
\hline Thin $\mathrm{Ci}_{.}-\mathrm{s}_{0} 3$ & - & From 2 to \\
\hline Thln Ci.-8. 8 & - & $\begin{array}{l}\text { 6 a.m.. } \\
\text { mereury }\end{array}$ \\
\hline Thin Ci.-8. 5 & - & below scale \\
\hline - & - & - \\
\hline - & - & $-21 \cdot 0$ \\
\hline - & Thitu S.-ch. 4, SE? & $-24 \cdot 0$ \\
\hline - & Thhln N. 8, sk & 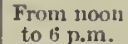 \\
\hline - & Misty & $\begin{array}{l}\text { mercury } \\
\text { below scalc }\end{array}$ \\
\hline - & - & \\
\hline - & - & $-22 \cdot 0$ \\
\hline - & - & -20.9 \\
\hline - & - & $\begin{array}{l}-21.6 \\
-21: 3 \\
-21.6\end{array}$ \\
\hline - & - & - \\
\hline
\end{tabular}

Precip11. - in.
7 th,

8 th.

9th.

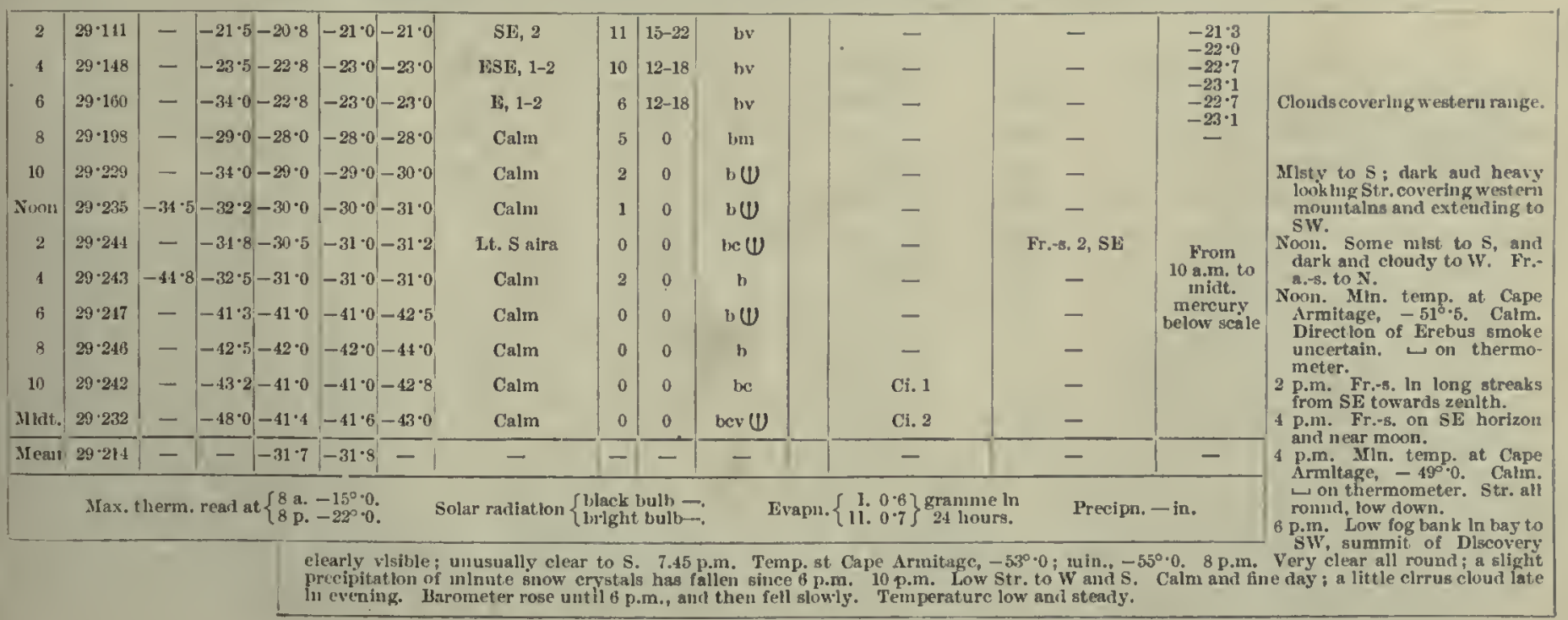


1903 -July.]

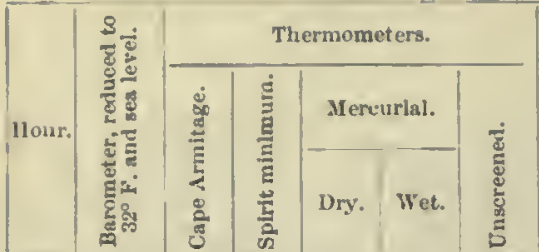

10 th.

$429 \cdot 217--32 \cdot 5-29 \cdot 2-28 \cdot 8-30 \cdot 0$

6 $29 \cdot 211--31 \cdot 5-31 \cdot 2-31 \cdot 5-30 \cdot 5$

$8 \quad 29 \cdot 210--35 \cdot 8-35 \cdot 0-35 \cdot 0-36 \cdot 0$

$1029 \cdot 217--43 \cdot 5-43 \cdot 0-43 \cdot 0-44 \cdot 0$

\begin{tabular}{|l|l|l|l|} 
Noon $29 \cdot z 20$ & $-58 \cdot 5-48 \cdot 0-48 \cdot 0^{*}-47 \cdot 8-49 \cdot 0$
\end{tabular}

$2|20 \cdot 211|-\quad-49 \cdot 0-46^{\circ} 0^{*}-45 \cdot 3-49 \cdot 0$

$429 \cdot 218-56 \cdot 0-51 \cdot 2-51 \cdot 2^{*}-50 \cdot 0$ Frozen

6 $29 \cdot 216-\mid-58 \cdot 4-52 \cdot 2 *$ Frozen' Frozen

$829 \cdot 230--52 \cdot 4-50 \cdot 2 *$ Frozen Frozen

$1029 \cdot 235-\quad-52 \cdot 5-50 \cdot 0^{*}$ Frozen Frozen

slidt. $29 \cdot 237--52 \cdot 5-51 \cdot 2 *$ Frozen Frozen

Mean \begin{tabular}{llll|l|l|}
$29 \cdot 221$ & - & $-43 \cdot 3$ & - & -
\end{tabular}

Max. therm. rean at $\left\{\begin{array}{l}8 \text { a. }-29^{\circ} 0 . \\ 8 \text { p. }-31^{\circ} \cdot 2 .\end{array}\right.$

Solar radiation $\left\{\begin{array}{l}\text { blaek bulb } \\ \text { lrigbt inll }- \text { - }\end{array}\right.$

Evapu. $\left\{\begin{array}{r}\text { I. } 0 \cdot 2 \\ \text { II. } 0 \cdot 2 \\ \text { III. } 0 \cdot 2\end{array}\right\} \begin{gathered}\text { gramme in } \\ 24 \text { hours. }\end{gathered}$

Precipu. - in.

Clouds,

Amount and morement.

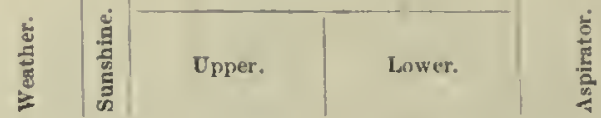

\begin{tabular}{|c|c|c|c|c|c|c|}
\hline NF by $\mathbf{E}, 3-4$ & 15 & $18-22$ & bev $U+$ & Ci. 1, Cl.-8. 2 & - & \\
\hline ENE, 3 & 15 & $18-22$ & br $U$ fr & - & - & 2 to 6 a.m. \\
\hline N Is by $F_{*}, 2-3$ & 15 & $10-21$ & bv $U \div$ & - & 一 & inelow scale \\
\hline Calm & 2 & 0 & $b U$ & - & - & - \\
\hline Calm & 1 & 0 & $b U$ & - & - & \\
\hline Calm & 0 & 0 & $\ln U$ & - & - & \\
\hline Calm & 0 & 0 & $\lim U$ & - & - & From \\
\hline Calm & 0 & 0 & bm $U$ & 一 & - & $\begin{array}{l}10 \text { a.m. to } \\
\text { mlat. }\end{array}$ \\
\hline Calm & 0 & 0 & $b m U$ & - & - & $\begin{array}{l}\text { mereury } \\
\text { inelow scale }\end{array}$ \\
\hline Calm & 0 & 0 & $\operatorname{bm} U$ & - & - & \\
\hline Calm & 0 & 0 & $\operatorname{sm} U$ & - & - & \\
\hline Calm & 0 & 0 & $\operatorname{bin} U \sqcup$ & - & - & \\
\hline- & - & - & - & - & - & - \\
\hline
\end{tabular}

Misty all aloug surface of

Thin mist apparentily rising froin all over toe.

Arm. Min. triup. at Cape smoke going $F$ in heavy column ; misty all round. 1.n1. Sirface drift in bay:

fogky to $1 \mathrm{l}$ and Sli. p.m. Fog running along surface of floe, ani

p.m. Mln. temp. at Cape Armitage, $-60^{\circ} \cdot 5$; light $N^{\prime}$ goting $\mathrm{E}$ in large volume. UU. Alt inercury ther mometers frozen : low for rising from floe and land. rising from floe and land. tage, $-61^{\circ} \cdot 8$, min., $-61^{\circ} .8$. Frebus' sinoke going FSE. 8 p.m. Tery mlsty all aloug surface of floe, and all land astern of ship. 10 p.m. Simllar conditions as at

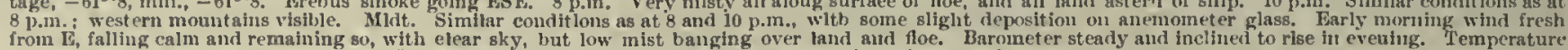
very mieh lower aud steady. * Readings of spirit minimum thermoneter at time of observation.

11th.

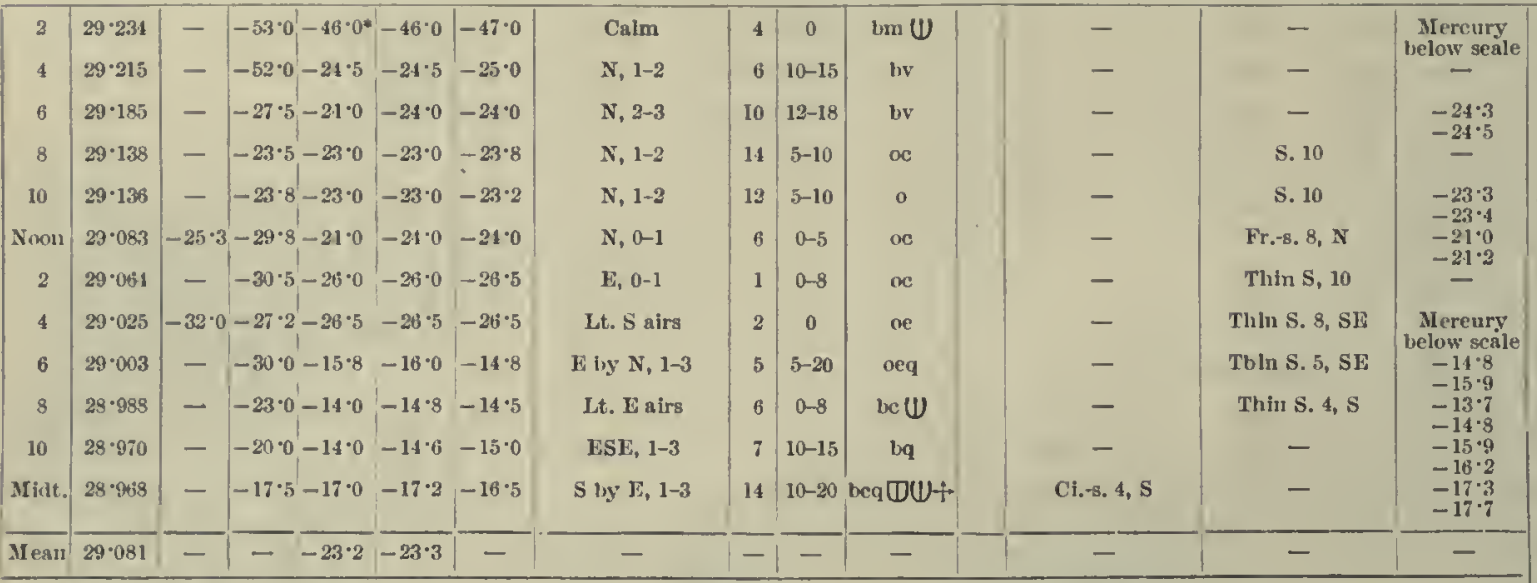
a.m. Sliglit Niy lreeze
sprang up, and temperature rose at onee to $-26^{\circ} \cdot 0$,
faling as scon as wind dier faling as scon as wind dies
away. The dry butb remalied frozen, but the other mereurial t.

meters thawed out. but thick so misty in Bay

3 a.m. Nis breeze, foree 1 ; remarkably elear all all distant land visible, no mist anywhere. Temperature, $-28 \cdot 2$.

a.m. Visibility very marker. Some detached Str. to SW ahove Mit. Diseovery.

a.m. A.-6. to $S$ and extending to NW; nlove wester range detacher Str. to $\mathrm{F}$. 10 a.ru. Streak of light oniy on the Wreaking hrom some stars vistble hearl.

Noon. Min. temp. at Cape Armitage, $-62^{\circ}-2$; NW by Max. therm. read at $\left\{\begin{array}{l}8 \mathrm{a},-22^{\circ} \cdot 5 \\ 8 \mathrm{p} .-12^{\circ} \cdot 0\end{array}\right.$ Solar radiation $\left\{\begin{array}{l}\text { llack bull } \\ \text { bright buli }- \text { - }\end{array}\right.$ Evapn. $\left\{\begin{array}{r}\text { I. } 0 \cdot 3 \\ \text { II. } 0 \cdot 2 \\ \text { III. } 0 \cdot 3\end{array}\right\}$ gramme in

Preeipn. - ln

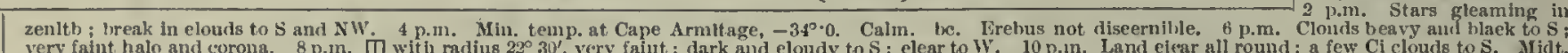
very faint halo and coroma. 8 p.m.

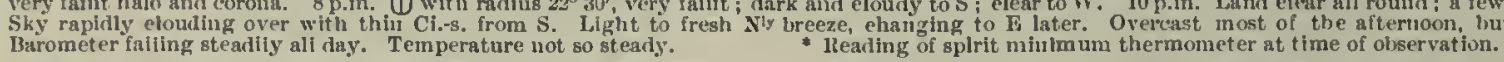

12 th.

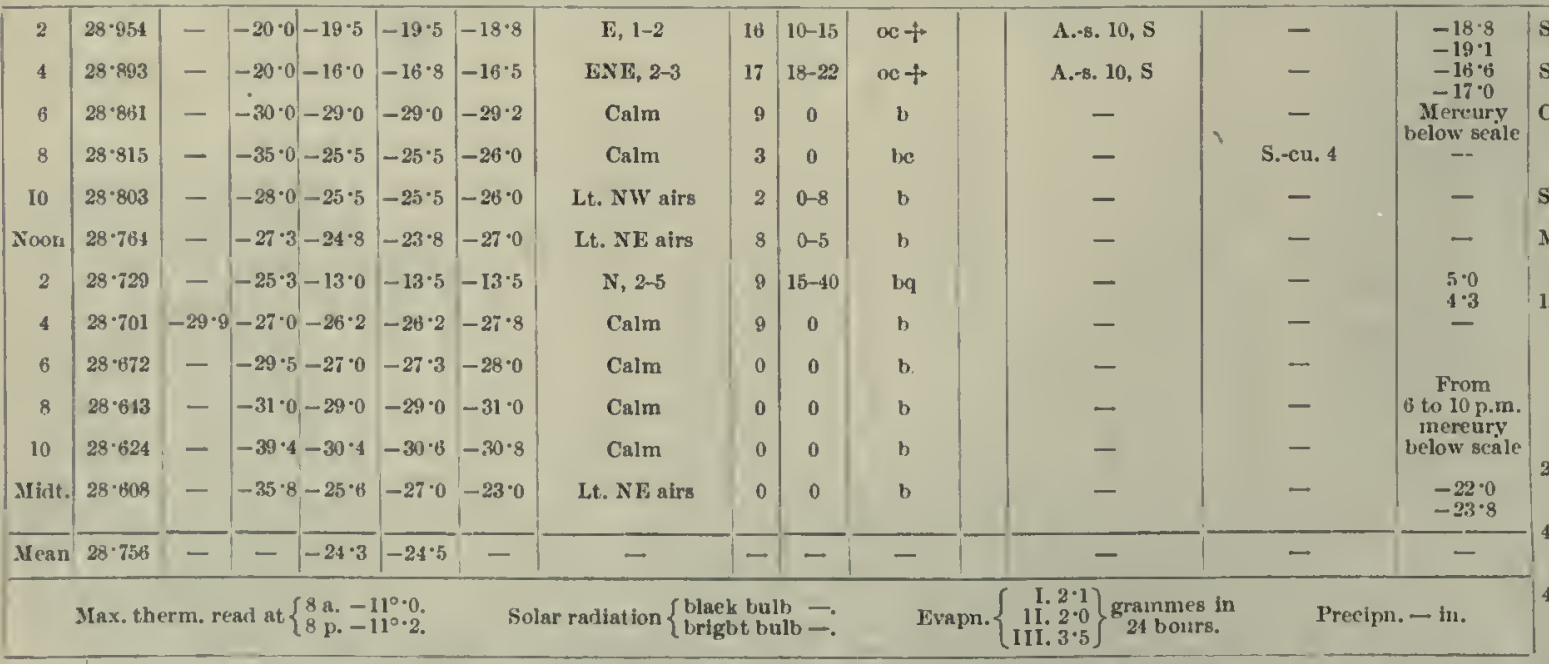

Stars in zenit b faintly visible. Stars in zeuith fatutly visible. Clmulless; dist ant land very

Str. to $W$ and $E$ moving from $\mathrm{S}$

oon on southern lorizon nuch refracterl; some Str. 12.30 p.m. Temp. at Cape Amitage, $-31^{\circ} 0^{\circ}$ min., b. Misty to F. Mt. Erebus' summit obseured; pe euliar eloud been hangin to $\mathbf{N}$ of Mt. Discovery all forenoon.

p.m. Str. on soutbern bortzon ; m
hind Biuff.

p.m. II eavy Cu. to $\mathrm{S}$ and F: : extensive faint auror p.m. Min. tem and p.m. Min. temp. at Cape Armitage, $-36^{\circ} \cdot 1$. Win
Nw, 1. Erehus not disNW 1. Erebus not dis-

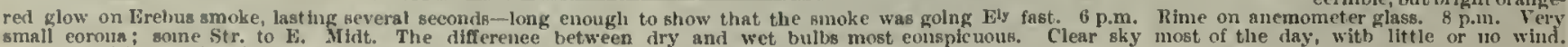
liarometer feli steadily all day. Tempernture low, but not so steady. 
Mrtwolologicat JoURnal of the "Discovery."

$[1903$-July.

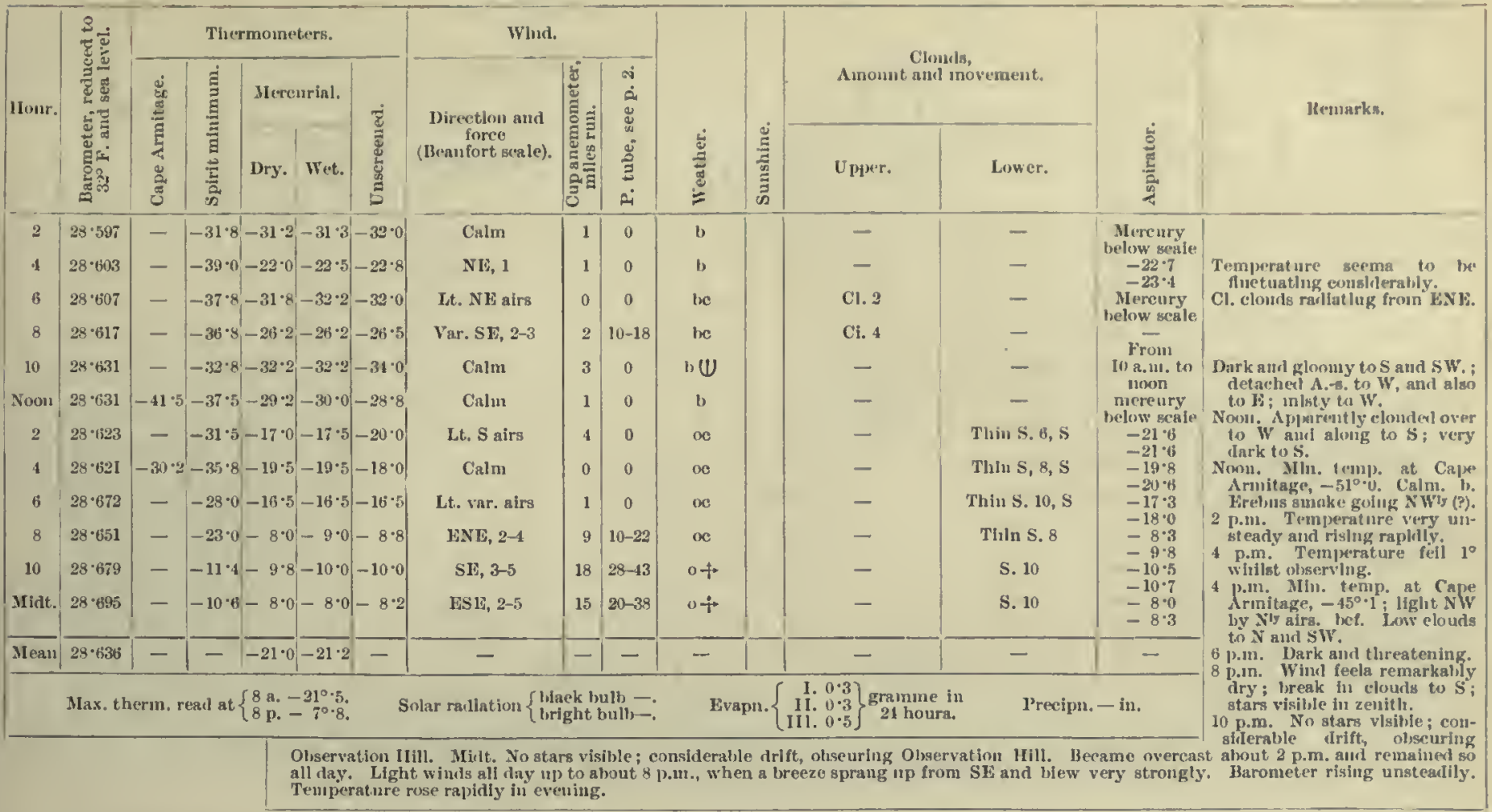

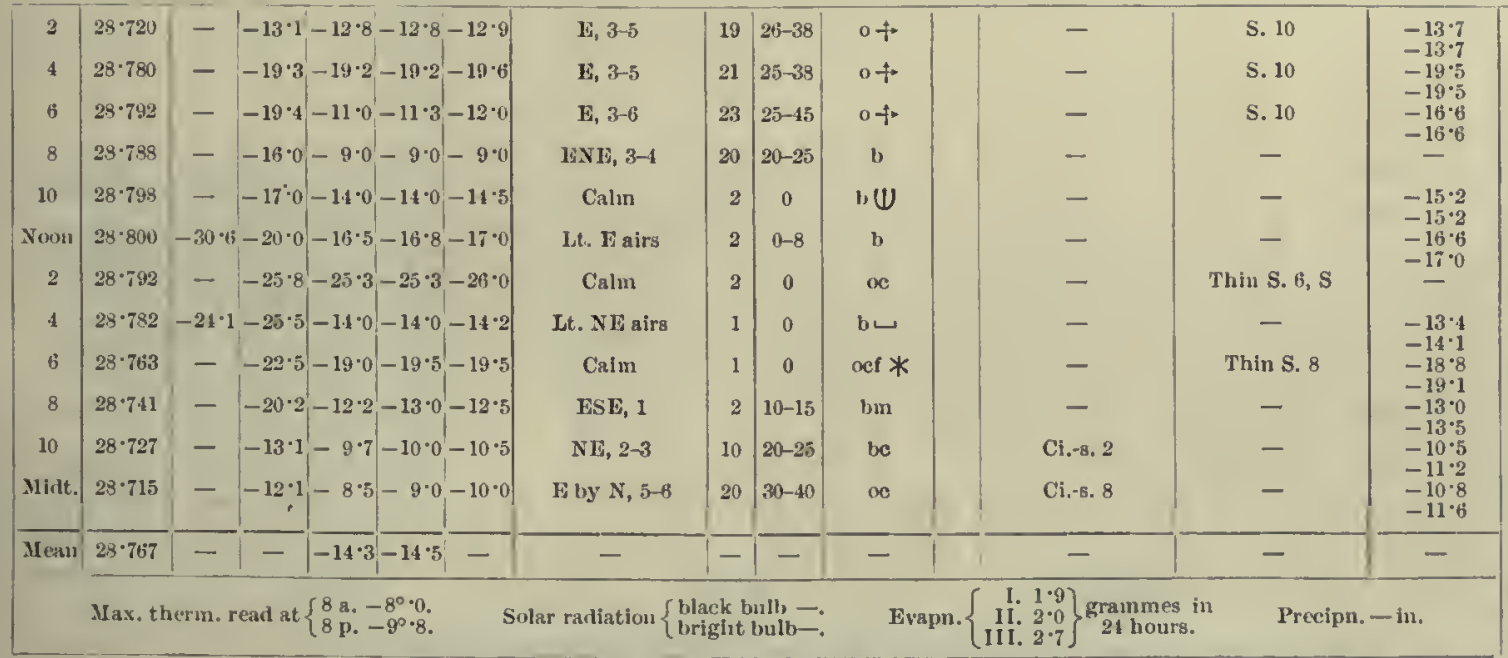
Wind remained stroug all ulgitt, faliug during the day, and agin rising from NB in evening, when it eommenced to blow rery tresh. Barometer rose until noon, mul then fell. Temperature bigher and nustealy.

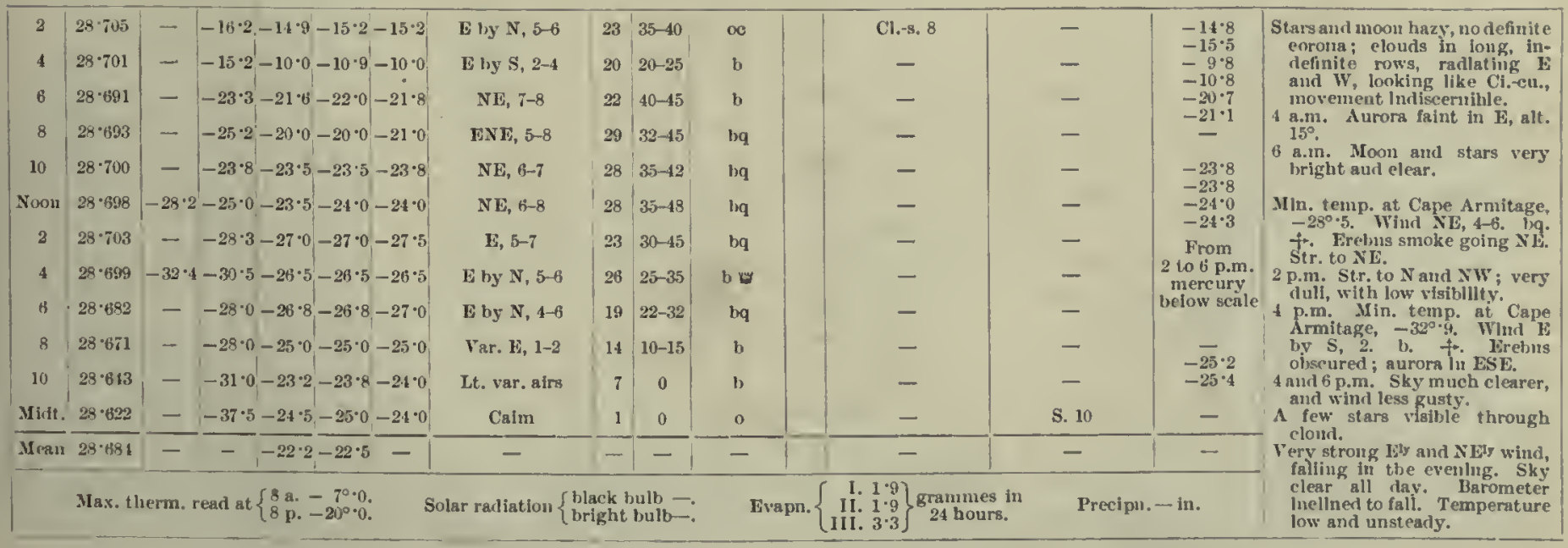




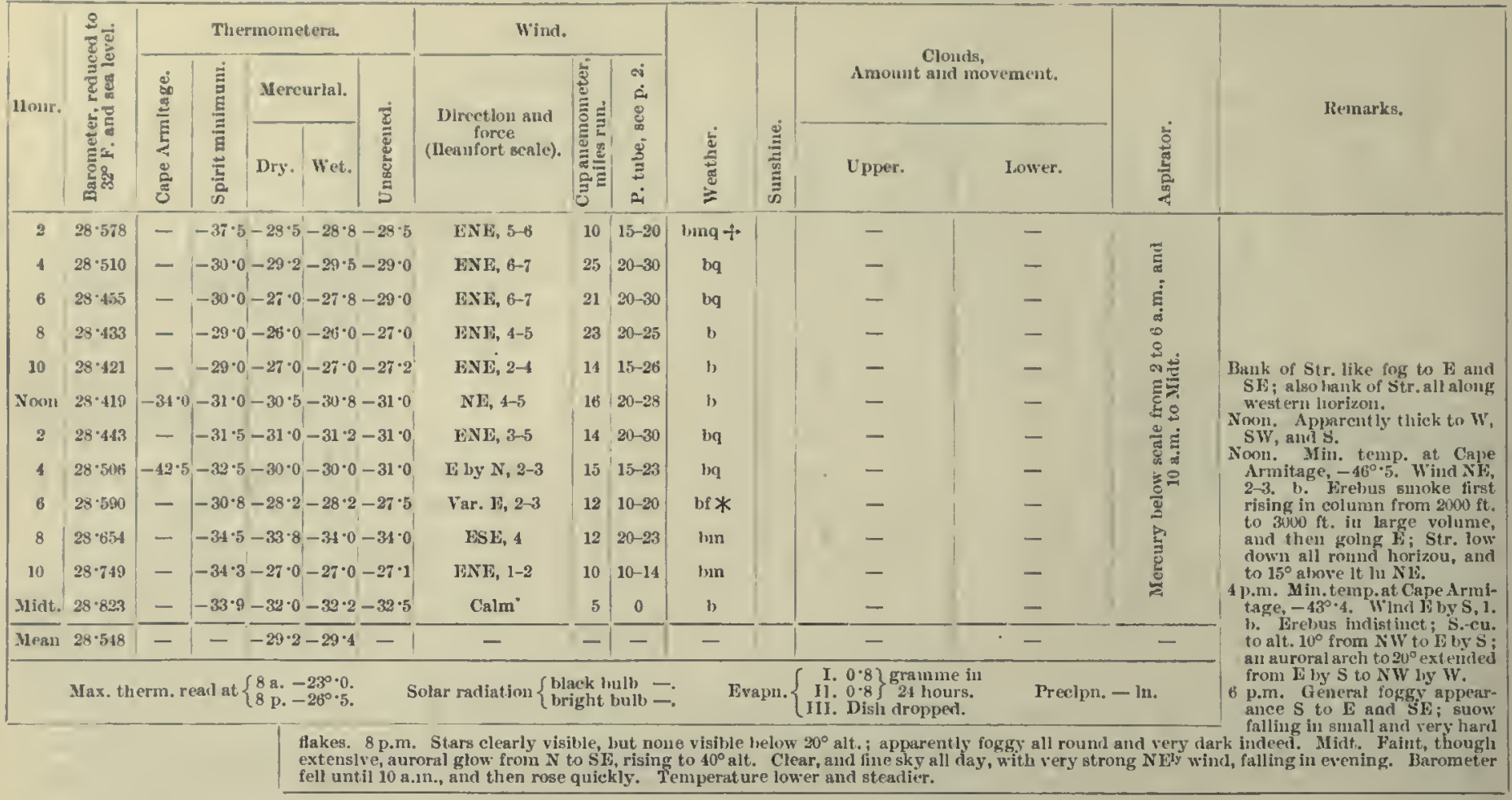

17 th.

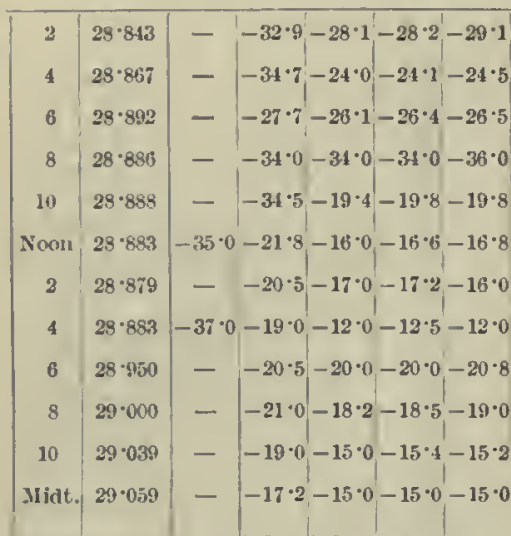

Calm

Lt. E airs

E, 1

Calm

$\mathrm{N}, 2$

$\mathrm{N}, 1-2$

$N, 3-4$

$N, 3-4$

ESE, 0-1

Calm

NE airs

Lt. $\mathbf{S}$ airs

\begin{tabular}{|r|c|c}
\hline 2 & 0 & bc \\
4 & 0 & be \\
3 & 5 & $b$ \\
2 & 0 & $b$ \\
3 & $10-15$ & $b$ \\
10 & $10-12$ & oc \\
14 & $20-28$ & be \\
15 & $20-30$ & bc \\
10 & $0-5$ & bcf \\
1 & 0 & bm \\
1 & 0 & bcm $*$ \\
1 & 0 & hem $*$ \\
\hline
\end{tabular}

S. 2

Thlu S. 3

$$
-
$$$$
-
$$

Thlu S. 8, SW

Thin S. 2, W

Tbin S. 4

Thin S. 6

-

S. 5

S. 6
Mermiry $/$ Thin Str. clouds, dictacbed to below scale NE and I.
$-25 \cdot 2$ Mercury below sealc

-19.8 Wlnd sprang $u p$ from $\mathrm{N}$ just $-20 \cdot 4$ hefore 10 a.m., and tempers $-16 \cdot 8$ ture rose immelist ely.
$-17 \cdot 3 \quad$ Noon. Sky overeast, with stars dimly visible. Shortly aftror olservat ion the sky in zenit was quite clear again; tark

Noon. Min. temp. at Cape
Armitage, $-44^{\circ} \cdot 8^{2}$. Winl S liy Armitage, $-44^{\circ} 8$. Winuls ly
$W, 1$. be. Erehins ofseured, W. 1 . 8 , going to $\mathrm{NE}$.

2 p.m. Dark $S$. cen. to $N$ and $W$ i p.m. Foggy to $\mathrm{s}$. 4 p.m. Min.temp. at Cape Armi-

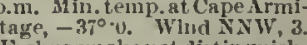
Firelius smoke not distinguislable.

6 p.m. Stars visible only in zonith. Stars all visible, but
p.ul. Stars

dim. stars visible overhesul.

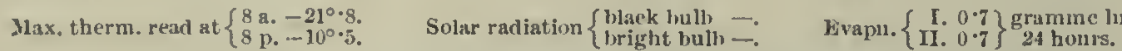

Prechp), - In

slight snow falling. Hidt. Stara visille overhead; snow falling morc thickly. Clondy frequentiy
midday. Barometer rising slowly and rather unsteadily. Temperature rose quickly before 10 a.m.

18 th

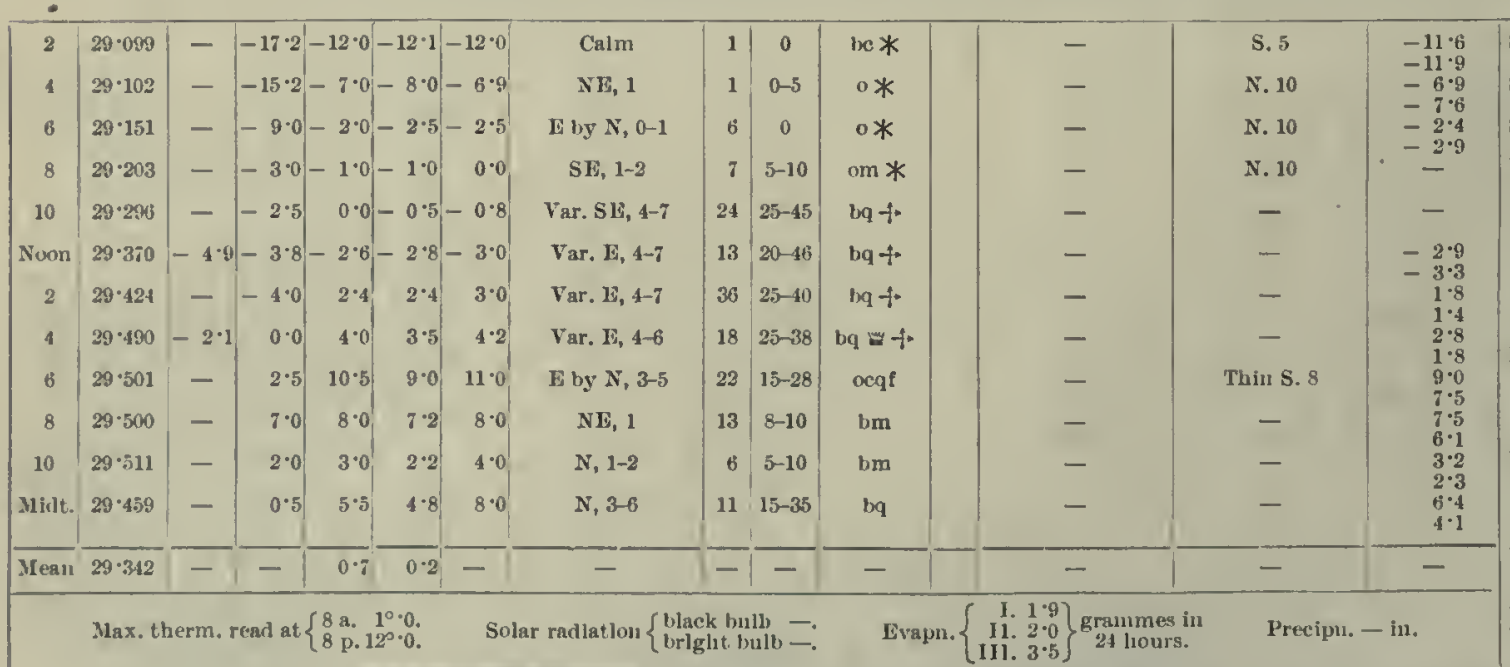

Stars visible from $20^{\circ}$ alt. to zenith. hard, falling thickly. Snowflakes falling thickly.

Very thick all round; sky black aud threatening; every appearance of a blizzard ; wind warrls the $S$.

$10 \mathrm{a.m}$. Str. along to $\mathrm{E}$, passing to $X$ rapidily; drift rery thick wind rariable, ESE to SSL li

squan. liea vy bank of Str. to $\mathrm{N}$, and along $\mathbf{E}$, moving to $\mathrm{N}_{\mathrm{i}}$ round; drift very thick ln squalls. Noon. $\mathrm{XI}$

tage, $-36^{\circ} \cdot 5$. Wind $\mathbf{S E}, 4-6$. + Erebus obscured; storny looking.

p.in. Wind squally and varlable, heavy lyank of Str. on $\mathrm{N}$ horizon. Fogg: all round.

p.m. In temp. at Cape Armitage, $-5^{\circ} 4$. Whin $1,1-3$. 1 . +* Frebus obscured; coufused o SF, alt. $8^{\circ}$. $b$ p.m. Stars dimly vislble, $8 \mathrm{p}$. m. Stars all dimly vislble. Clondy and orercast early; clearing and remaining clear for rist of day. Wind strong all mldday from $\mathbf{E}$ ast 
Metforotogtcat, Journat, of trie "Discovery."

$[1903-J u l y$.

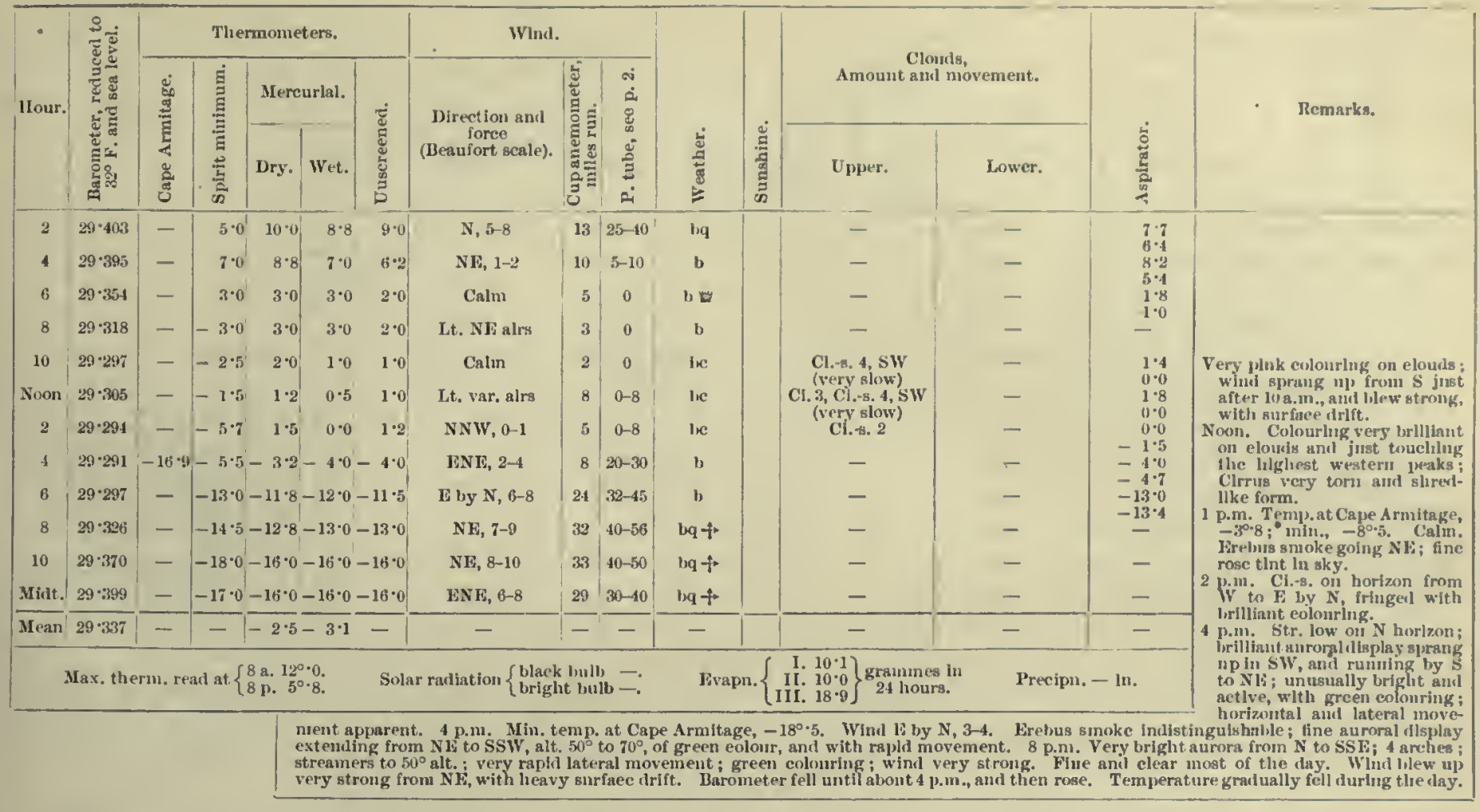

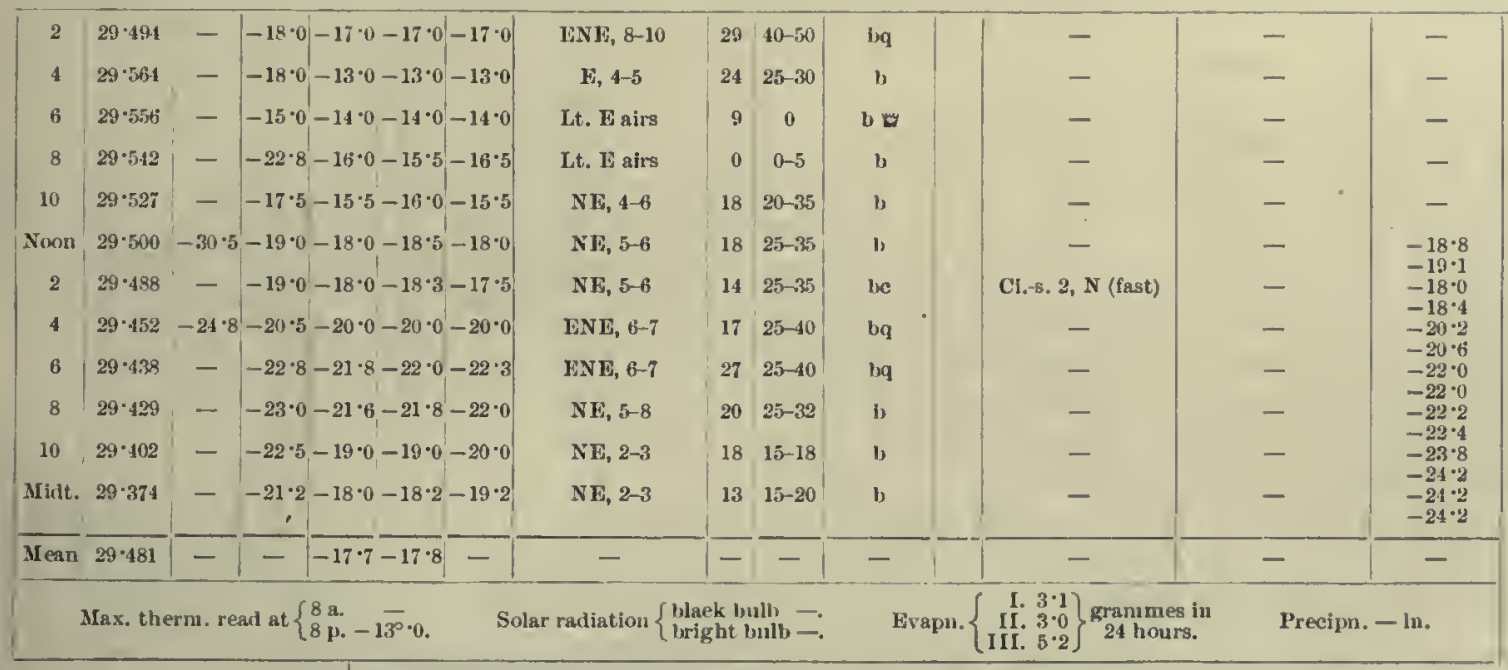

inehind hills to NE. Fine and elear sky all day. Wind very strong NGw, falling towards evening. Barometer palling pretty steadily gince $6 \mathrm{a.m}$. Temperature lower and steady.

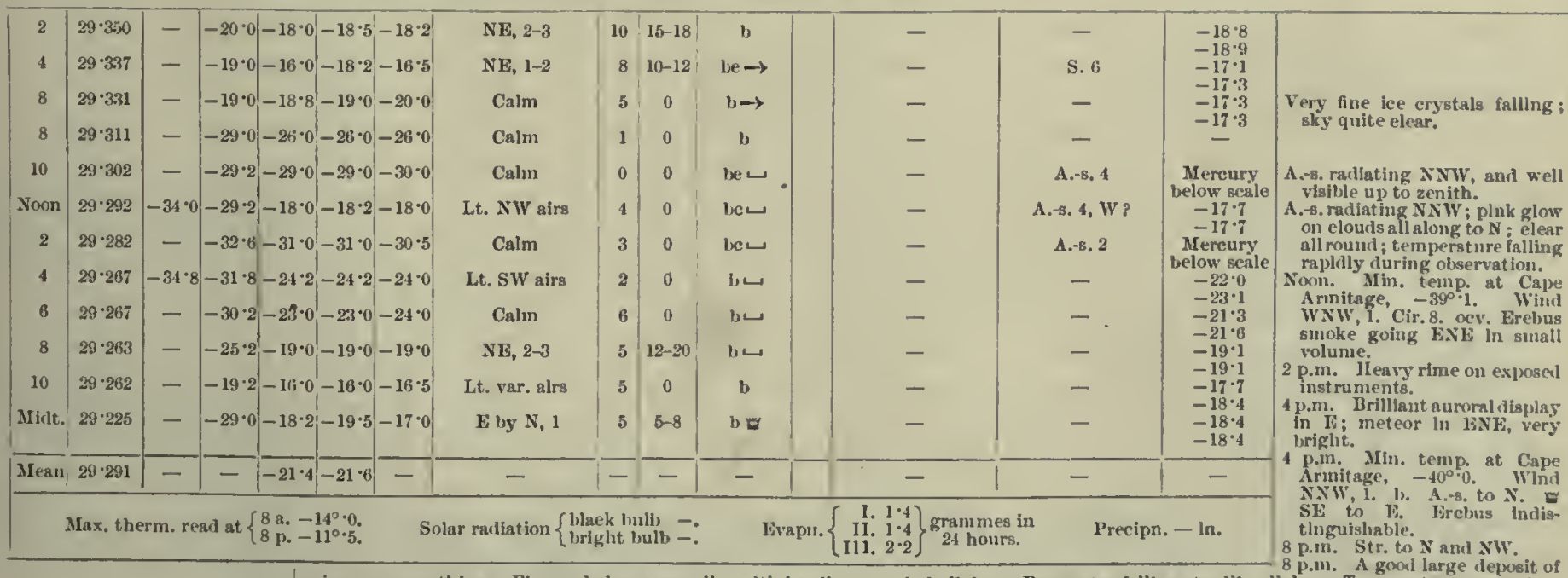


-

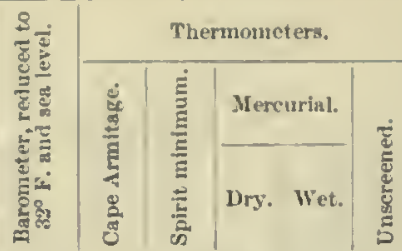

22nd.
Wind.

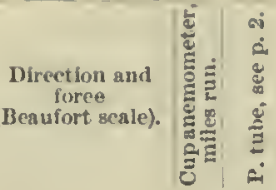

\section{|}

Reuuarks.

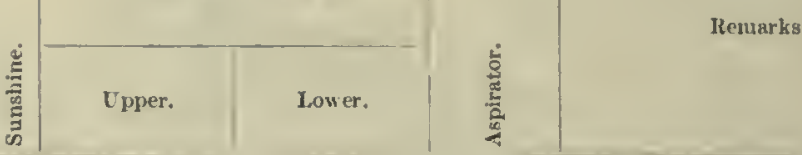

\begin{tabular}{|c|c|c|}
\hline & & \\
\hline - & - & From \\
\hline 一 & - & $\begin{array}{c}2 \text { to } 8 \text { a.m., } \\
\text { nerveury }\end{array}$ \\
\hline - & - & leclow seale \\
\hline- & - & - \\
\hline $\mathrm{Ci}_{0}+\mathrm{B}, 2, \mathrm{SW}$ & S. $1, \mathrm{~S}$ (slow) & -23.8 \\
\hline- & S. 5, S & - \\
\hline - & S. 10 & $-20 \cdot 6$ \\
\hline - & Thin S. 10 & $-13 \cdot 0$ \\
\hline- & - & $-11 \cdot 2$ \\
\hline - & - & $\begin{array}{l}-1106 \\
-16.6\end{array}$ \\
\hline- & - & $\begin{array}{r}-17.0 \\
0.3\end{array}$ \\
\hline - & - & $-17 \cdot 0$ \\
\hline & 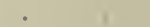 & $-17 \cdot 0$ \\
\hline- & - & - \\
\hline
\end{tabular}

Dark to $\mathrm{S}$.

Dark to $\mathrm{S}$ and $\mathrm{SW}$; Str. all rouncl, low down.

an. Arn. (temp), at Cape Arnitage, $-46^{\circ} \cdot 5$. Calm, Cir. 7 . bis obscured. 2 p.in. Break in clouds to $\mathrm{NW}$ temperat ure falling rapilly. 4 p.m. Stars gleaning iaintly iu zenith. 4 j..n. Min. temp. at Cape Ar Str. 10. Stars dimly visible. ᄂ, Frebus olusetured. 6 p.m. Very dark,
8 p.m. Stars visible, but dim. Overcast during the afternoon other wise fine and clear, witb no wind. Barometer falling all day, hut instralily early. the evening.

$23 r d$

\begin{tabular}{|c|c|c|c|c|c|c|c|c|c|c|c|c|c|}
\hline 2 & $28 \cdot 961$ & - & $-20 \cdot 0$ & $2 \cdot 0$ & 0.5 & $2 \cdot 0$ & E, 1-2 & 6 & 5-12 & b & - & - & 1.8 \\
\hline 4 & $28 \cdot 939$ & - & $-4 \cdot 0$ & 1.5 & 0.0 & 1.5 & F by $S, 2$ & 18 & 10-15 & o & - & S. 10 & 2.8 \\
\hline 6 & $23 \cdot 944$ & - & -0.5 & $3 \cdot 5$ & $2 \cdot 0$ & $3 \cdot 5$ & E, 1-2 & 5 & $5-12$ & $\operatorname{og}$ & - & S. 10 & $\begin{array}{l}1.2 \\
3 \cdot 2\end{array}$ \\
\hline 8 & $28 \cdot 950$ & - & $3 \cdot 4$ & $4 \cdot 2$ & $2 \cdot 4$ & $5 \cdot 5$ & $\mathrm{NL}, 2-3$ & 19 & $10-15$ & o & - & S. 10 & $5 \cdot 0$ \\
\hline 10 & $28 \cdot 963$ & - & $4 \cdot 0$ & $7 \cdot 8$ & $5 \cdot 8$ & $6 \cdot 5$ & $\mathrm{SF}_{2}, 2-4$ & 13 & $1:-22$ & o & - & S. 10 & $\begin{array}{l}8.9 \\
6.4\end{array}$ \\
\hline Noon & $29 \cdot 936$ & $1 \cdot 8$ & 4.0 & $6 \cdot 0$ & 4.5 & $6 \cdot 0$ & NE, 2-3 & 8 & 10-14 & $\infty$ & - & S. $8, \mathrm{~S}$ (slow) & $5 \cdot 0$ \\
\hline 2 & $28 \cdot 908$ & - & $3 \cdot 8$ & $9 \cdot 0$ & $8 \cdot 2$ & $8 \cdot 0$ & $N E, 1-2$ & 12 & $5-15$ & oc & Ci.-cu. 2 & S. $6 . \mathrm{S}$ (slow) & 8.2 \\
\hline 4 & $29 \cdot 008$ & $-3 \cdot 5$ & 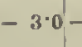 & $2 \cdot 5$ & $2 \cdot 5$ & $3 \cdot 0$ & SSE, 2-4 & 12 & $20-30$ & $\infty q$ & - & S. 10 & $\begin{array}{r}-2 \cdot 6 \\
=2.6\end{array}$ \\
\hline 6 & $29 \cdot 003$ & - & $-4 \cdot 0$ & 0.2 & 0.2 & 0.0 & SI, 2-4 & 13 & $15-20$ & oq + & - & S. 10 & $\begin{array}{r}0.0 \\
-0.8\end{array}$ \\
\hline 8 & $29 \cdot 010$ & - & $-4 \cdot 0$ & 0.0 & 0.2 & 0.0 & $5,4-6$ & 20 & $20-30$ & oq $+* *$ & - & N. 10 & $\begin{aligned} & 0.0 \\
= & 0.4\end{aligned}$ \\
\hline 10 & $29 \cdot 0.49$ & - & $-1 \cdot 5$ & $2 \cdot 0$ & 10 & $1 \cdot 5$ & E by $N, 1-3$ & 10 & $0-10$ & oq $\div *$ & - & N. 10 & $\begin{array}{l}2 \cdot 5 \\
0.5\end{array}$ \\
\hline Midt. & $29 \cdot 0 ; 1$ & - & -0.2 & $3 \cdot 0$ & $2 \cdot 0$ & $3 \cdot 0$ & E hy N, 2-3 & 8 & $5-15$ & beq & - & S. 5 & $\begin{array}{l}2.5 \\
1.4\end{array}$ \\
\hline Iean $_{i}$ & $28 \cdot 979$ & - & - & $3 \cdot 1$ & $2 \cdot 0$ & - & - & - & - & $\dot{-}$ & - & - & - \\
\hline
\end{tabular}

Faint auroral streamers $\mathbf{N}$ to $\mathbf{E}$,

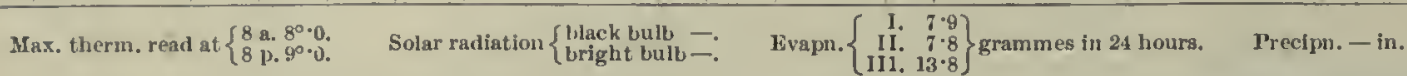
Lrebus obscured. 6 p.m. Stars visible to $S$.

Overcast nearly all day, with fresh variable 18ly wind. Barometer inclined to rise unsteadily. Temperature remained ahove zero nearly all day, with extraordinary differences between the dry and wet bulbs.

24 th.

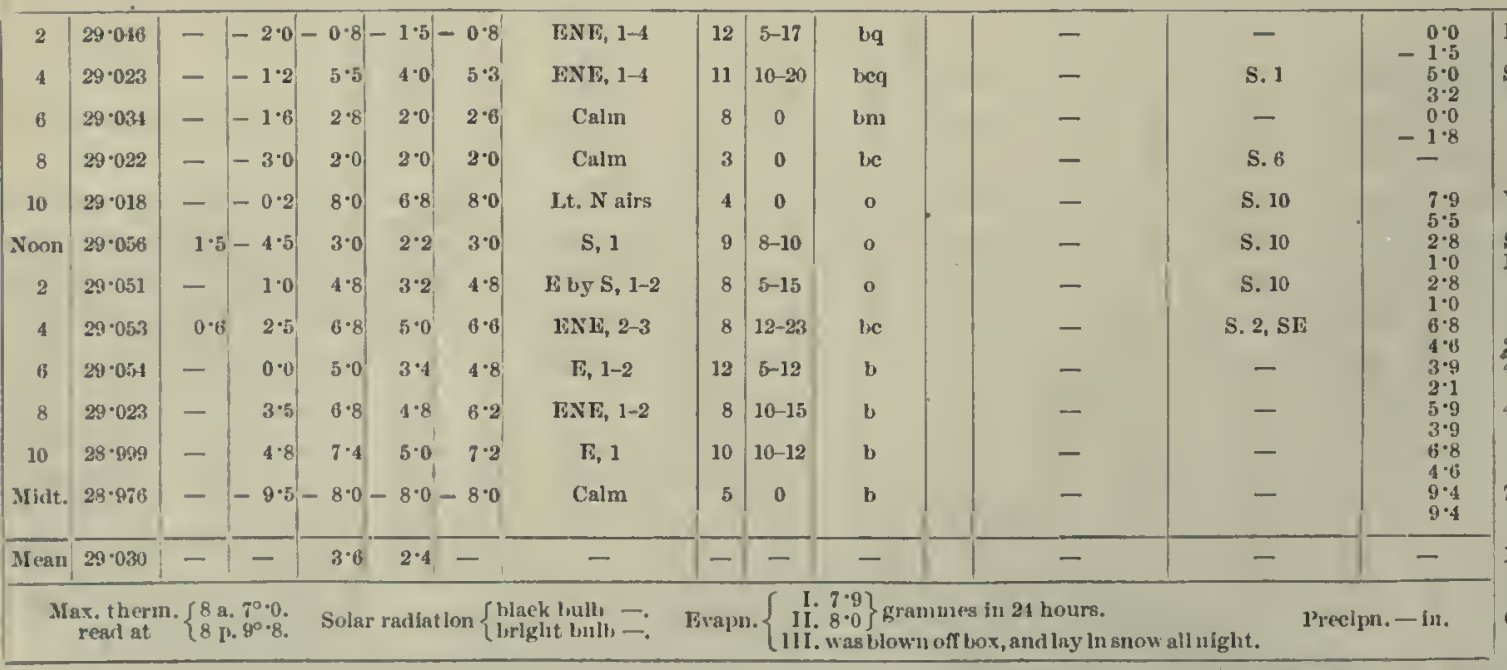

Diffused aurora in SE and S. Slight tracc of anrora in SE.

Very dull and lieavy-lookíg weather. Noon. Min, temp. at Cape Armitage, $-12^{\circ}$. $\%$ Wind ssw, o-1. Str. 9. Brebus

$2 \mathrm{p} . \mathrm{m}$. Brenk in sky to $\mathrm{S}$. 1).m. Clearing raphlly; Str.

to N. Min. temp. at Cape Armitage, $-4^{\circ} 0$. Wind $\mathbf{E}$ by Niw. Sirebus ol sscured $.30 \mathrm{p} . \mathrm{m}$. Flames, followed by a diep red glow, were seen on simmit of lirebus. lidt. Very fuint aurora band from NE to Sw through zenith.

Overenst in the aft ernoon, ot herwise fine, with fresh lyly wind. 
Meteorological, JoURNat, of Tite "DiscovenY."

[1903-July.

\begin{tabular}{|c|c|c|c|c|c|c|c|c|c|c|c|c|c|c|}
\hline \multirow{3}{*}{ Hour. } & \multirow{3}{*}{ 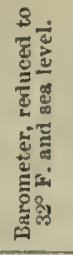 } & \multicolumn{5}{|c|}{ Thermometers. } & \multicolumn{3}{|l|}{ Wind. } & \multirow[b]{3}{*}{ 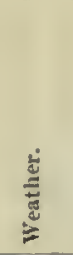 } & \multirow[b]{3}{*}{ 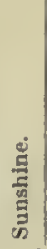 } & \multirow{2}{*}{\multicolumn{2}{|c|}{$\begin{array}{l}\text { Clouds, } \\
\text { A mount aud movement. }\end{array}$}} & \multirow[b]{3}{*}{ 劳 } \\
\hline & & \multirow{2}{*}{ 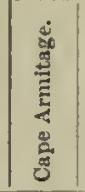 } & \multirow{2}{*}{ 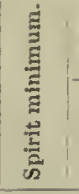 } & \multicolumn{2}{|c|}{ Mercurial. } & \multirow[b]{2}{*}{ 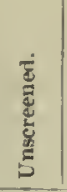 } & \multirow{2}{*}{$\begin{array}{c}\text { Direction and } \\
\text { foree } \\
\text { (Beaufort Bcale). }\end{array}$} & \multirow{2}{*}{ 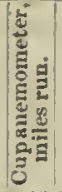 } & \multirow{2}{*}{ 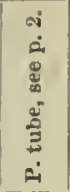 } & & & & & \\
\hline & & & & Dry. & Wet. & & & & & & & Upper. & Lower. & \\
\hline 2 & $28 \cdot 934$ & - & $-9 \cdot 5$ & $-1 \cdot 5$ & -3.0 & $-1 \cdot 5$ & Lt. $N$ alrs & 2 & $0-5$ & b) & & - & - & $\begin{array}{l}-0 \cdot 4 \\
-3 \cdot 4\end{array}$ \\
\hline 4 & $28 \cdot 839$ & - & $-5 \cdot 5$ & $9 \cdot 2$ & $7 \cdot 0$ & $8 \cdot 8$ & $N, 1$ & 4 & 10 & b & & - & - & $\begin{array}{r}-3.6 \\
8 \cdot 6\end{array}$ \\
\hline 6 & $28 \cdot 763$ & - & $-7 \cdot 0$ & $10^{\circ} 0$ & $7 \cdot 0$ & $9 \cdot 2$ & $N, 1-2$ & 8 & $10-18$ & b & & - & - & $\begin{array}{l}16.8 \\
9.3\end{array}$ \\
\hline 8 & $28 \cdot 734$ & - & $-12 \cdot 5$ & $-2 \cdot 0$ & $-3 \cdot 0$ & $-3 \cdot 0$ & $\mathrm{NE}, 2-3$ & 7 & $20-25$ & b & & - & - & $\begin{array}{r}0.8 \\
-2.0\end{array}$ \\
\hline 10 & $28 \cdot 700$ & - & $-11 \cdot 7$ & $-10 \cdot 2$ & $-10 \cdot 0$ & $-10 \cdot 0$ & F by $N, 4-6$ & 12 & $30-38$ & heq & & $\mathrm{Cl}_{0}-\mathrm{s}, 2$ & - & $-11 \cdot 6$ \\
\hline Neon & 25.638 & $-18 \cdot 0$ & $-14 \cdot 3$ & $-12 \cdot 1$ & $-12 \cdot 4$ & $-13 \cdot 3$ & E by N, 6-8 & 32 & $40-49$ & lheg & & Cl.-8. 3 & - & $\begin{array}{l}-10 \cdot 7 \\
-12 \cdot 8\end{array}$ \\
\hline 2 & $28 \cdot 639$ & - & $-14 \cdot 5$ & $-11 \cdot 0$ & $-11 \cdot 5$ & $-12 \cdot 0$ & NE, 5-7 & 24 & $28-40$ & bq & & - & - & $\begin{array}{l}-10.4 \\
-10.8\end{array}$ \\
\hline 4 & $28 \cdot 664$ & $-14 \cdot 6$ & $-14 \cdot 0$ & $-5^{\circ} 0$ & $-6 \cdot 0$ & $-4 \cdot 8$ & NE, 3-4 & 26 & $20-30$ & loq $\sqcup$ & & - & - & -5.8 \\
\hline 6 & $28 \cdot 675$ & - & $-14 \cdot 3$ & $-12 \cdot 2$ & $-12 \cdot 4$ & $-13 \cdot 0$ & ENE, 5-7 & 17 & $20-42$ & bqu & & - & - & $\begin{array}{l}-69 \\
-12.6\end{array}$ \\
\hline 8 & $28 \cdot 714$ & - & $-18 \cdot 0$ & $-14 \cdot 0$ & $-15 \cdot 0$ & $-15 \cdot 0$ & Caln & 9 & 0 & b & & - & - & $\begin{array}{l}-13 \cdot 2 \\
-13 \cdot 7\end{array}$ \\
\hline 10 & $28 \cdot 725$ & - & $-12 \cdot 1$ & $-9 \cdot 2$ & -9.8 & $-9 \cdot 5$ & ENE, 3-4 & 16 & $25-35$ & b) & & - & - & $\begin{array}{l}=-1.0 \\
=8.0 \\
=8.8\end{array}$ \\
\hline Mirlt. & $28 \cdot 751$ & - & $-17 \cdot 0$ & $-14 \cdot 2$ & $-14 \cdot 8$ & $-16 \cdot 0$ & NE, 1-3 & 19 & $10-23$ & b) & & - & - & $\begin{array}{l}-15.2 \\
-15.5\end{array}$ \\
\hline Meau & $28 \cdot 731$ & - & - & -6.0 & $-7 \cdot 0$ & - & - & $1-1$ & - & - & & - & - & - \\
\hline
\end{tabular}

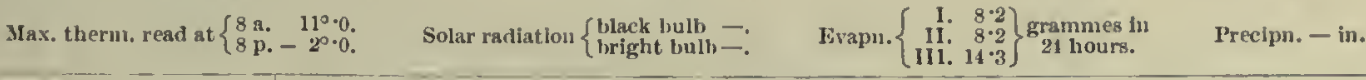

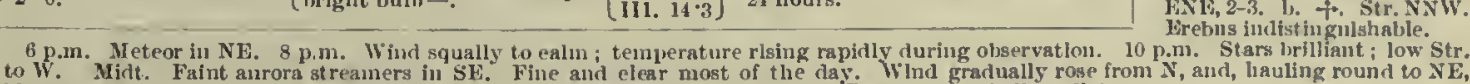
blew up very strong. Barometer fell until noon, and then commencer to rise. Temperature falling all day.

\begin{tabular}{|c|c|c|c|c|c|c|c|c|c|c|}
\hline 2 & $28 \cdot 798$ & - & $-18 \cdot 0-11 \cdot 2-12 \cdot 0-12 \cdot 2$ & NH, 1-3 & 12 & $10-25$ & bq & - & - & $-11 \cdot 2$ \\
\hline 4 & $28 \cdot 816$ & - & $-14 \cdot 3-10 \cdot 0-11 \cdot 0-11 \cdot 0$ & E by $\mathbf{N}, 1-3$ & 15 & $10-22$ & $b=$ & - & - & $\begin{array}{l}-119 \\
-10.5\end{array}$ \\
\hline 6 & $28 \cdot 839$ & - & $-16 \cdot 6-16 \cdot 0-16 \cdot 0-17 \cdot 0$ & ENF, 2-4 & 15 & $15-36$ & b) & - & - & -16.2 \\
\hline 8 & $28 \cdot 933$ & - & $-18 \cdot 0-15 \cdot 0-14 \cdot 8-15 \cdot 0$ & ENE, 5-6 & 16 & $30-35$ & b & - & - & $\begin{array}{l}-10.0 \\
-15 \cdot 2\end{array}$ \\
\hline 10 & $28 \cdot 958$ & - & $-15 \cdot 0-11 \cdot 2-12 \cdot 0-12 \cdot 0$ & NL, 2-4 & 14 & $15-20$ & b & - & - & $\begin{array}{l}-10 \cdot 6 \\
-11.6\end{array}$ \\
\hline Nom & $29 \cdot 994$ & - & $-33 \cdot 0-21 \cdot 0-21 \cdot 2-21 \cdot 0$ & E, 3-4 & 9 & $18-20$ & b & - & - & $\begin{array}{l}-12.0 \\
-21 \cdot 3 \\
-9.8\end{array}$ \\
\hline 2 & 29.037 & - & $-20 \cdot 8-13 \cdot 0-14 \cdot 2-13 \cdot 5$ & $\mathbf{E}, 1$ & 7 & $0-8$ & b & - & - & $\begin{array}{l}-21.8 \\
-14.4\end{array}$ \\
\hline 4 & $29 \cdot 085$ & $-35 \cdot 0$ & $-28.5-20.0-20.0-21 \cdot 0$ & F, 1-2 & 7 & $10-18$ & b & - & - & $\begin{array}{l}-10.9 \\
-20 \cdot 9 \\
-20.9\end{array}$ \\
\hline 6 & $29 \cdot 118$ & - & $-20 \cdot 0-19 \cdot 2-19 \cdot 8-20 \cdot 0$ & $\mathrm{ENE}, 5-6$ & 20 & $22-30$ & b & - & - & $-19 \cdot 1$ \\
\hline 8 & $29 \cdot 160$ & - & $-19 \cdot 5-14 \cdot 0-14 \cdot 8-14 \cdot 4$ & NE, 3-4 & 21 & $15-22$ & b & - & - & -14.4 \\
\hline 10 & $29 \cdot 194$ & - & $-35 \cdot 0-35 \cdot 0-35 \cdot 1-35 \cdot 0$ & Calm & 9 & 0 & $b$ & - & - & Fromlop.m \\
\hline sliat. & $29 \cdot 210$ & - & $-36 \cdot 2-27 \cdot 1-27 \cdot 7,-26 \cdot 2$ & Calm & 0 & 0 & $b$ & - & - & $\begin{array}{c}\text { to Mintit. } \\
\text { mereury scale } \\
\text { below }\end{array}$ \\
\hline Mlean & $29 \cdot 014$ & - & $-17 \cdot 7-18 \cdot 2-$ & - & $\overline{-}$ & - & - & - & - & - \\
\hline
\end{tabular}

Clear all round; falut furora in 26 th.

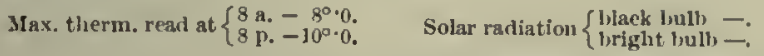
Isvapu. $\left\{\begin{array}{r}\text { I1. } \\ \text { I1. } 4 \cdot 1 \\ \text { III. } 7 \cdot 0\end{array}\right\}$ grammes in 24 hours.

Prrcipu. - in.

Fajit aurora to SF.

Cloudless sky ; fiue colourlug in 2.45 p.m. Temp. at Cape Armitage, $-36^{\circ} \circ 0$; mill., $-40^{\circ} 0$. Calm. b. Krebus smoke in lose plow in sky to 2 as well p.m. Colouring on $\mathrm{NW}$ horizo

p.m. Min. temp. at. Cape Armitage. $-428^{\circ}$. Lt. Fily airs. Frebus sinoke going $\mathrm{B}^{\text {ly }}$; a bright bigh flame, aff erwards driven towards the $\mathrm{E}$, was seen coluing ont of Frelus' erater. p.m. Auroral display In Ean $\mathrm{NE}$; low hanks of Cu. to S. P.In. Diffusive and extenslve
aurora fxtending from $\mathrm{E}$ to SW, very faln

1) p.m. Aurora light just showing above hills, extending from Observation 11 ill to a lit tle $\mathrm{N}$ of $\mathrm{N}$. Mlat. Aurora light just showing above

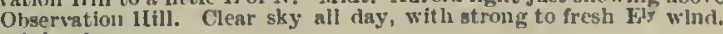
Barometer rising steadily all day. Temperature falling all day and remaining low.

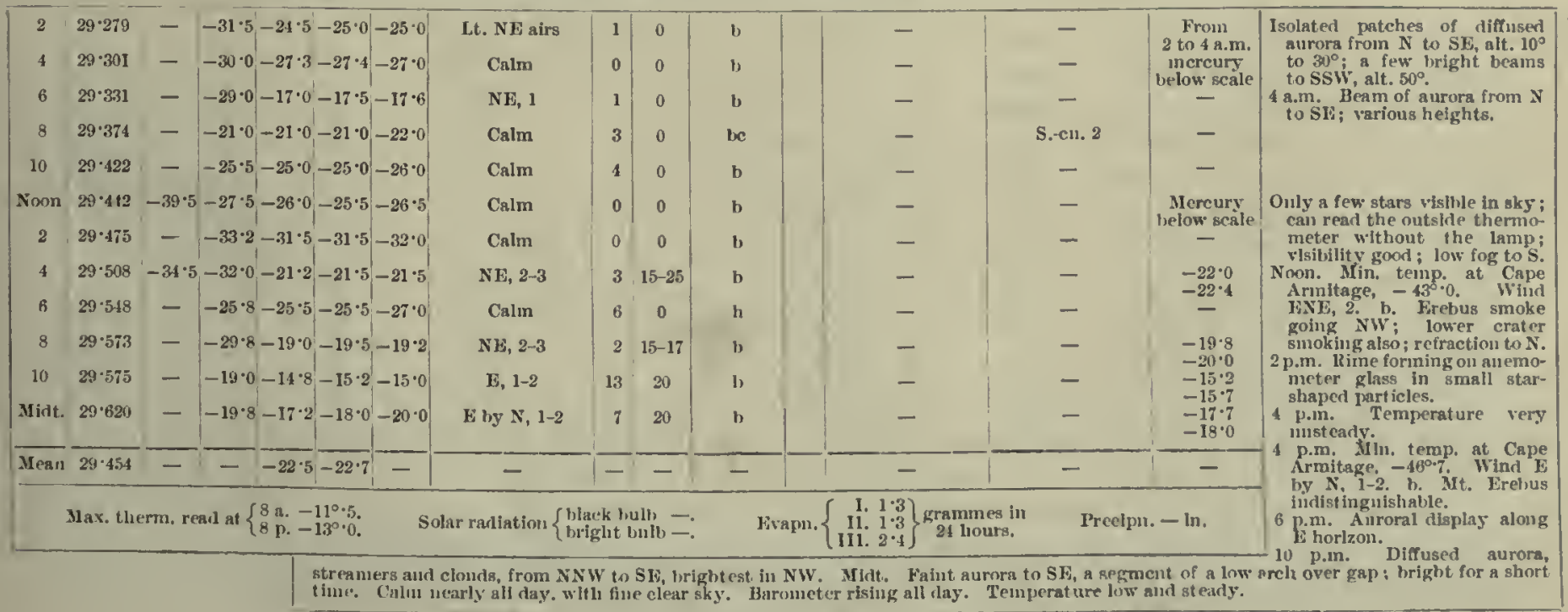


Metroliologicat Jounal of the " Discoviny."
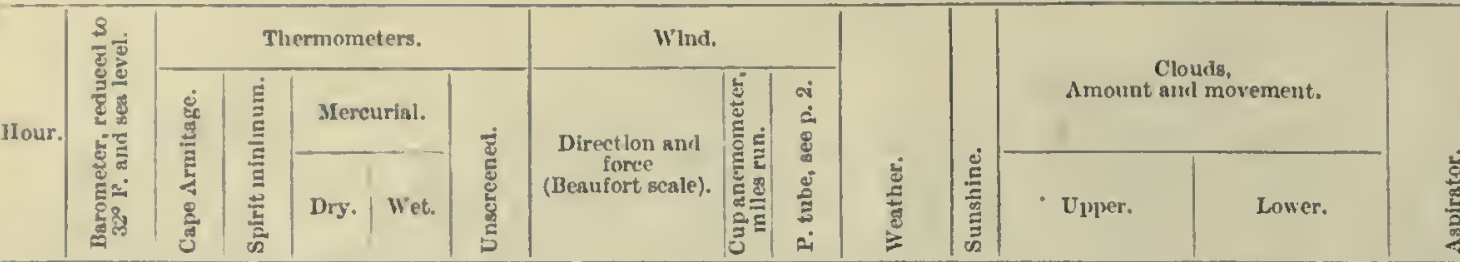

28 th.

\begin{tabular}{|c|c|c|c|c|c|c|c|c|c|c|c|c|}
\hline 2 & $20 \cdot 665$ & - & $-23 \cdot 0 \mid-18 \cdot 0$ & $-18 \cdot 2$ & $-19 \cdot 5$ & $\mathrm{~K}, 2-3$ & 10 & 20 & bq & - & - & $-19 \cdot 5$ \\
\hline 4 & $29 \cdot 695$ & - & $-23 \cdot 0-18 \cdot 0$ & $-18 \cdot 8$ & $-19 \cdot 5$ & ESE, 1-3 & 10 & $15-20$ & $b q$ & - & - & $\begin{array}{l}-202 \\
-23 \cdot 4\end{array}$ \\
\hline 6 & $29 \cdot 702$ & - & $-22 \cdot 0-17 \cdot 0$ & $-17 \cdot 5$ & $-17 \cdot 0$ & E, 1-2 & 10 & $15-25$ & bq & - & - & -17.0 \\
\hline 8 & $29 \cdot 740$ & - & $-20 \cdot 0-18 \cdot 2$ & $-19 \cdot 0$ & $-18 \cdot 0$ & Var. E, 2-3 & 10 & $10-20$ & $b=$ & - & - & $\begin{array}{l}-180 \\
-17 \cdot 0\end{array}$ \\
\hline 10 & $29 \cdot 736$ & - & $-28 \cdot 0-28 \cdot 0$ & $-28^{\circ} 0$ & $-29 \cdot 0$ & Calm & 6 & 0 & b & - & - & From10 R...m. \\
\hline Noon & $29 \cdot 735$ & $-41 \cdot 8$ & $-36 \cdot 0-28 \cdot 5$ & $-28 \cdot 5$ & $-29 \cdot 0$ & Calm & 1 & 0 & b & - & - & $\begin{array}{l}\text { to noon } \\
\text { mereury }\end{array}$ \\
\hline 2 & $29 \cdot 732$ & - & $-29 \cdot 2-23 \cdot 8$ & $-24 \cdot 0$ & $-23 \cdot 8$ & $\mathrm{I}, 1$ & 8 & $7-10$ & b & - & - & $\begin{array}{l}-24 \cdot 5 \\
-25 \cdot 2\end{array}$ \\
\hline 4 & $29 \cdot 729$ & $-35 \cdot 5$ & $-21 \cdot 0-19.0$ & $-20 \cdot 0$ & $-20 \cdot 0$ & b, 1 & 0 & $5-10$ & bo w & - & S. $3, N$ & $\begin{array}{l}-202 \\
-20 \%\end{array}$ \\
\hline 6 & $29 \cdot 685$ & - & $-29 \cdot 2-29 \cdot 2$ & $-29 \cdot 2$ & $-31 \cdot 5$ & Calm & 2 & 0 & b & - & - & Mercury \\
\hline 8 & $29 \cdot 665$ & - & $-32 \cdot 2-25 \cdot 5$ & $-26^{\circ} 0$ & $-26 \cdot 0$ & Calm & 1 & 0 & bm & - & - & - \\
\hline 10 & $29 \cdot 633$ & - & $-2 \pi \cdot 4-23 \cdot 5$ & $-23 \cdot 9$ & $-25 \cdot 0$ & Calın & 1 & 0 & b & - & - & $-22 \cdot u$ \\
\hline Mldt. & $29 \cdot 593$ & - & $-31 \cdot 2-31 \cdot 2$ & $-30 \cdot 6$ & $-33 \cdot 0$ & Calm & 0 & 0 & b & - & - & $\begin{array}{l}\text { 3yereury } \\
\text { below seale }\end{array}$ \\
\hline Jlean & $29 \cdot 693$ & - & $\mid--23 \cdot 3$ & $-23 \cdot 6$ & - & - & - & - & - & - & - & - \\
\hline
\end{tabular}

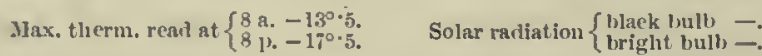

Evapm. $\left\{\begin{aligned} \text { I. } & 1 \cdot 8 \\ \text { II. } & 1 \cdot 7 \\ 11 . & 3 \cdot 1\end{aligned}\right\}$ grammes in hours.

Preclpn. - in.

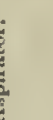

Remarks.

Falnt aurora diffused over $\mathbf{E}$, narrow curlain with sliort a.m. Wind varialle; aurora Wi to S, liright in $N$, var a m displar, remain 6 a.m. Fain

a.m. Faint auroral in SE, and auroral glow over white lsland. a.m. Clear all all round; sky clourless. wester isly on summit of Noon. Min. temp. at. C Arnitage, $-48^{\circ} \cdot 5$. Caln. 2 p.m. Ci.s, to N and NW; thln Str. near moon ; summits of vestern mountalns olseuren. p.m. Clouds diffieult to aetinc : apparently
approaehing from $\mathrm{N}$. p.n. Min. temp. at Cape Arnitage, $-50^{\circ} 4$. Light
BNi a airs. Ci. 3. 1.rebus obscurerl. " falut

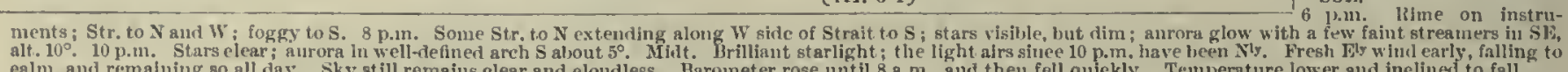
ealm, ani remaining so all day. Sky st ill remains clear and eloudiess. Baroneter rose until 8 a.m., and then fell quiekly. Teniperature lower and inelined to fall.

29th.

\begin{tabular}{|c|c|c|c|c|c|c|c|c|c|c|c|c|}
\hline 2 & $29 \cdot 538$ & - & $-42 \cdot \theta^{\prime}-41 \cdot 5 t$ & $-40 \cdot 5 t$ & $-42 \cdot 6 t^{\prime}$ & Calm & 0 & 0 & b) & - & - & \\
\hline 4 & $29 \cdot 487$ & - & $-43 \cdot 2-38 \cdot 5$ & $-38 \cdot 0$ & $-39 \cdot 5 t$ & Calm & 0 & 0 & b & - & - & 旁 \\
\hline 6 & $29 \cdot 434$ & - & $-42 \cdot 2-37 \cdot 0$ & $-36 \cdot 4$ & $-38 \cdot 0$ & Calm & 1 & 0 & b & - & - & 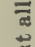 \\
\hline 8 & $29 \cdot 352$ & - & $-43 \cdot 8-35 \cdot 2$ & $-35 \cdot 0 \mid$ & $-36 \cdot 0$ & Calm & 0 & 0 & b & - & - & 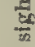 \\
\hline 10 & $29 \cdot 300$ & - & $-36 \cdot 5-28 \cdot 0$ & $-28 \cdot 2$ & $-28 \cdot 5$ & Calm & 0 & 0 & b & - & - & : \\
\hline Sioon & $29 \cdot 230$ & $-46 \cdot 8$ & $-33 \cdot 0-31 \cdot 5$ & $|-31 \cdot 2|$ & $-32 \cdot 0$ & Calm & 0 & 0 & b๘ & - & - & 5 \\
\hline 2 & $29 \cdot 172$ & - & $-31 \cdot 2-33 \cdot 3$ & $-33 \cdot 3$ & $-34 \cdot 0$ & Calin & 0 & 0 & b & - & - & 爮 \\
\hline 4 & $29 \cdot 118$ & $-53 \cdot 0$ & $-42^{\circ} 0-29^{\circ} 5$ & $-29 \cdot 5$ & $-30 \cdot 0$ & Calm & 0 & 0 & b $y$ & - & - & 言 \\
\hline 6 & $29 \cdot 069$ & - & $-43 \cdot 6-43 \cdot 0 t$ & $-43 \cdot 0 t$ & $-43 \cdot 04$ & Lt. $S$ airs & 0 & 0 & bu & - & - & $\Xi$ \\
\hline 8 & $29 \cdot 0.10$ & - & $-43 \cdot 8-40 \cdot 0 t$ & $-40.0 t$ & $-41 \cdot 0 t$ & Calm & 0 & 0 & bu & - & - & 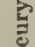 \\
\hline 10 & $29 \cdot 017$ & - & $-48 \cdot 5-45 \cdot 5$ & Frozen & Frozen & Calm & 1 & 0 & $\mathrm{bm}$ & - & - & 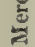 \\
\hline Midt. & $28 \cdot 980$ & - & $-46 \cdot 0-31 \cdot 2$ & $-30 \cdot 9$ & $-33 \cdot 8$ & Calm & 1 & 0 & $b m$ & - & - & \\
\hline Mean & $29 \cdot 228$ & - &.$--36 \cdot 2$ & - & - & - & -1 & - & - & - & - & - \\
\hline & & & & & & & & & & & & \\
\hline
\end{tabular}

Portions of arches at all elevitions up to the zenith,

4 a.m. Wirle curtain from $\mathrm{N}$ to zenith and porlions from $E^{2}$ and $S$.

6 a.m. Faint arches $\mathrm{S}$ and 6 a.m. Faint arches
Eito $10^{\circ}$; stars brilliant all nlght.

Sky clourless; risibility foorl; rime on outside instruments.

Noon. Min. temp. at Cape Armitage, $-51^{\circ} 0$. Wind Wi by $\mathrm{N}, 2$. 1\%. Erebus smoke going towards $\mathrm{k}$.

2 p.m. Clearing ous tops of western mountains; de
taehed $\mathrm{Cu}$. on Mt. Distaeher Cu. on
covery summit. 4 p.m. 11 in. temip. at Cape S'y airs. we to S1B. Firebus smoke indistinguishable. p.n. Heary rime on all exposerl instruments. p.m. Bright auroral arch of streamers, $\mathrm{N}$ to WSF, alt.

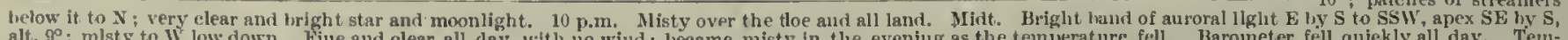

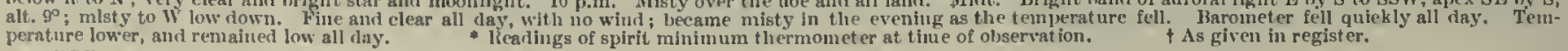

30 th.

\begin{tabular}{|c|c|c|c|c|c|c|c|c|c|c|c|c|}
\hline 2 & $28 \cdot 949$ & - & $-43 \cdot 0-36 \cdot 8$ & $-37 \cdot 0$ & $-38 \cdot 6$ & Calm & 2 & 0 & b & - & - & \\
\hline 4 & $28 \cdot 958$ & - & $-41 \cdot 5-27 \cdot 3$ & $-27 \cdot 0$ & $-28 \cdot 2$ & Calm & 2 & 0 & b & - & - & 至 \\
\hline 6 & $28 \cdot 936$ & - & $-42 \cdot 3^{\prime}-23 \cdot 2$ & $-23 \cdot 6$ & $-23 \cdot 3$ & Lt. ESE airs & 4 & 0 & b & - & - & డే \\
\hline 8 & $29 \cdot 922$ & - & $-46 \cdot 0-41 \cdot 0^{*}$ & $-44 \cdot 0 t$ & Frozen & Calın & 0 & 0 & $\mathrm{lim}$ & - & - & 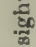 \\
\hline 10 & $28 \cdot 918$ & - & $-44 \cdot 0-41 \cdot 0^{*}$ & $-41 \cdot 0+$ & $-4.1 \cdot 2 \uparrow \mid$ & Calm & 0 & 0 & $b m \sqcup$ & - & - & 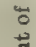 \\
\hline Noon & $28 \cdot 910$ & $-60 \cdot 5$ & $-54 \cdot 0-52 \cdot 5^{*}$ & Frozen & Frozen & Calm & 0 & 0 & $\mathrm{bm} \sqcup U$ & - & - & हี \\
\hline 2 & $28 \cdot 916$ & - & $-52 \cdot 8-49^{\circ} 0^{*}$ & $-48 \cdot 0 \dagger$ & $-51 \cdot 0 \dagger$ & Lt. $\mathbf{N}$ airs & 0 & 0 & $1 m \sqcup$ & - & - & 苕 \\
\hline 4 & $28 \cdot 923$ & $-58 \cdot 0$ & $-54 \cdot 2-51 \cdot 2$ & Frozen & Frozen & Calm & 0 & 0 & bm $\sim \Psi$ & - & - & 吾 \\
\hline 6 & $28 \cdot 955$ & - & $-52 \cdot 3-45 \cdot 5^{*}$ & $-45 \cdot 5 t$ & Frozen & Calm & 0 & 0 & $m \sqcup U$ & - & - & $\Xi$ \\
\hline 8 & $28 \cdot 995$ & - & $-45 \cdot 5-40^{\circ} 0 t$ & $-40.0 t$ & $-41 \cdot 0+$ & Calm & 2 & 0 & $\mathrm{bm} \sqcup U$ & - & - & 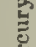 \\
\hline 10 & $29 \cdot 051$ & - & $-41 \cdot 0-36 \cdot 0$ & $-35 \cdot 9$ & $-37 \cdot 0$ & Lt. $N E$ airs & 0 & 0 & b & - & - & 选 \\
\hline Minlt. & $29 \cdot 121$ & - & $-36 \cdot 0-31 \cdot 0$ & $-31 \cdot 0$ & $-31 \cdot 0$ & Calm & 0 & 0 & lec & Cl.-8., W & - & \\
\hline IIean & $28 \cdot 9.93$ & - & $-\overline{-39 \cdot 8}$ & - & - & - & - & - & - & - & - & - \\
\hline
\end{tabular}

Max. therm, reat at $\left\{\begin{array}{l}8 a_{1}-20^{\circ} 0 . \\ 8 p_{1}-22^{\circ} 0 .\end{array}\right.$

Solar radiatlon $\left\{\begin{array}{l}\text { black bulb } \\ \text { lorlght bulb }- \text {. }\end{array}\right.$

Jisapn. - granme.

Preeipn, - In.

Short arches of auroral light, from $8^{\circ}$ to $30^{\circ}$ alt., between in and $\mathrm{SH}_{\text {, very }}$ rariable int streligth and position. a.m. Faint arches of $\mathrm{S}$, alt. $8^{\circ}$ to $60^{\circ}$, very varihble in strength and possidirection of Black all night. a.nu $A$ A bank of fog all 10 a.m. There apparent precipitation an ice crystals, as well as deposition, on anemomet er glass during the night; during the observation a very light $W^{-1 y}$ air sprang up. oon. Heary mist rlsing up from the floe; light air comiug from all quarters. Armitage, $-60^{\circ} .5$. Llght Armitage, $-40^{\circ}$. L Llght
S'y airs, b. Str. low to $\mathrm{N}$. $\checkmark$. lrehus sunoke golug $\mathrm{E}$. 2 p.m. Dry, mlsty vapour rising from the floe; low lank of Cu. on western mom ains; temperature rlsing with Niy airs, and then falling again. 4 p.m. IIin. temp,

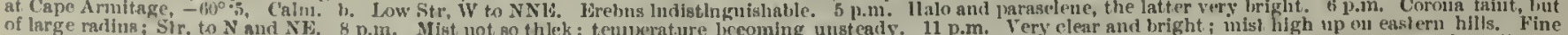

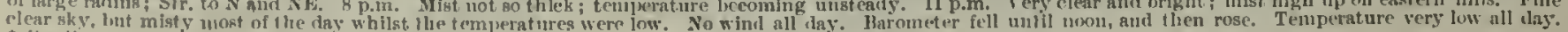

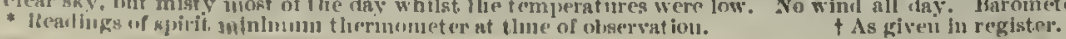




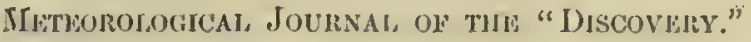

$[1903-J$ uly.

\begin{tabular}{|c|c|c|c|c|c|c|c|c|c|c|c|c|c|c|c|}
\hline \multirow{3}{*}{ Hour. } & \multirow{3}{*}{ 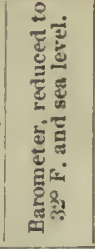 } & \multicolumn{5}{|c|}{ Thermometers. } & \multicolumn{3}{|l|}{ Wint. } & \multirow[b]{3}{*}{ 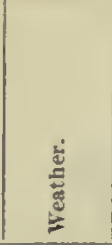 } & \multirow[b]{3}{*}{ 龸 } & \multirow{2}{*}{\multicolumn{2}{|c|}{$\begin{array}{l}\text { Clonds, } \\
\text { Amonnt anl movernent. }\end{array}$}} & \multirow[b]{3}{*}{$\frac{5}{\stackrel{5}{0}}$} & \multirow{3}{*}{ Kemarks. } \\
\hline & & \multirow{2}{*}{ 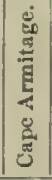 } & \multirow{2}{*}{ 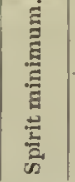 } & \multicolumn{2}{|c|}{ Mercuriai. } & \multirow{2}{*}{ 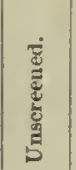 } & \multirow{2}{*}{$\begin{array}{c}\text { Direction and } \\
\text { force } \\
\text { (Bemifort scale). }\end{array}$} & \multirow{2}{*}{ 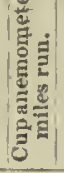 } & \multirow{2}{*}{ 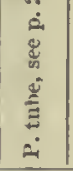 } & & & & & & \\
\hline & & & & Dry. & Wet. & & & & & & & Upper. & Low er. & & \\
\hline 2 & $29 \cdot 209$ & - & $-36 \cdot 1$ & $-30 \cdot 6$ & $-30 \cdot 9$ & $-30 \cdot 5$ & Calur & 0 & 0 & Ine & & $\mathrm{Cl}_{1}-8,3, \mathrm{IT}$ & - & M ireury & Moon getting SW l,y W, very \\
\hline 4 & $24 \cdot 2 s 2$ & - & $-32 \cdot 0$ & $-19 \cdot 1$ & $-19 \cdot 3$ & $-19 \cdot 3$ & $\mathbf{H}, 2$ & 5 & $22-24$ & $\infty$ & & - & s. 7 & $\begin{array}{c}\text { Delow sca } \\
-19 \cdot 7\end{array}$ & $\begin{array}{l}\text { nuch dlstortet } \\
\text { and very resl. }\end{array}$ \\
\hline 6 & $29 \cdot 381$ & - & $-24 \cdot 5$ & $-17 \cdot 9$ & $-18 \cdot 2$ & $-18 \cdot 0$ & k, 1-2 & 10 & $15-20$ & o & & - & S. 10 & $\begin{array}{l}-20 \cdot 2 \\
-18 \cdot 2 \\
-18.8\end{array}$ & [Weatier was too liad to go out to \\
\hline 8 & $29 \cdot 53.4$ & - & $-20 \cdot 0$ & $-19 \cdot 0$ & $-19 \cdot 0$ & $-20 \cdot 0$ & Var. S, 6-7 & 17 & $35-42$ & $\alpha+t^{-}$ & & - & S. 10 & -158 & Cape Armintage.] \\
\hline 10 & $29 \cdot 660$ & - & -20.5 & $-15 \cdot 0$ & $-15 \cdot 0$ & $-15 \cdot 0$ & SSE, 5-6 & 22 & $20-30$ & $0 \div$ & & - & S. 10 & $-15 \cdot 2$ & Thiek ali round. \\
\hline Noon & $29 \cdot 729$ & - & $-17 \cdot 0$ & $-16 \cdot 0$ & $-16 \cdot 0$ & $-16 \cdot 0$ & Var. S, 6-9 & 18 & $30-52$ & $\log f^{2} *$ & & - & N. 10 & -15 & Whind varying between SSF and \\
\hline 2 & $29 \cdot 820$ & - & $-16 \cdot 2$ & $-13 \cdot 0$ & $-13 \cdot 2$ & $-13 \cdot 5$ & $S, 4-6$ & 31 & $15-30$ & ofq $+* *$ & & - & N. 10 & - & ehroked witl dery ditt und falling \\
\hline 4 & $29 \cdot 913$ & - & $-14 \cdot 3$ & $-11 \cdot 3$ & $-11 \cdot 3$ & $-12 \cdot 5$ & $\mathrm{SF}, 3-5$ & 19 & $20-30$ & of $\nrightarrow * *$ & & - & N. 10 & - & 2 p.m. Wind very gusty ; drift \\
\hline 6 & $29 \cdot 997$ & - & $-13 \cdot 5$ & $-12 \cdot 0$ & $-12 \cdot 0$ & $-12 \cdot 5$ & SIE, 3-5 & 14 & $15-28$ & $o q+* *$ & & - & N. 10 & - & not so tinek; sirow in face of \\
\hline 8 & $30 \cdot 033$ & - & $-12 \cdot 5$ & $-11 \cdot 0$ & $-11 \cdot 0$ & $-11 \cdot 2$ & Var. S12, 1-5 & 15 & $12-30$ & begf $f+W$ & & - & S. 4 & $-11 \cdot 7$ & 4 p.m. Squalls at intervais, with \\
\hline 10 & $30 \cdot 061$ & - & $-13 \cdot 1$ & $-12 \cdot 0$ & $-12 \cdot 2$ & $-12 \cdot 8$ & Finy S, 3-6 & 18 & $20-30$ & heqf fr & & - & S. 8 & $-11 \cdot 9$ & 4.30 p.m. Temp. nt Cape Armi- \\
\hline Midt. & $30 \cdot 057$ & - & $-13 \cdot 0$ & $-12 \cdot 0$ & -12.4 & $-12 \cdot 6$ & $1 ;, 3$ & 19 & $25-30$ & let? & & - & S. 2 & - & Wind SSI, 2-4. Nim, 10, 千 $*$. \\
\hline \multirow[t]{3}{*}{$\overline{\text { Mean }}$} & $29 \cdot 723$ & - & - & $-15 \cdot 7$ & $-15 \cdot 9$ & - & - & $=$ & - & - & & - & - & - & parge accumulation of drift in \\
\hline & \multirow{2}{*}{\multicolumn{4}{|c|}{ Max. therm. read at }} & $-16^{\circ} \cdot 2$ & & \multicolumn{5}{|c|}{ Solar radiation $\left\{\begin{array}{l}\text { black buib }- \text {. } \\
\text { luriglit bulb-- }\end{array}\right.$} & 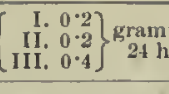 & \multicolumn{2}{|c|}{ I'recipn. - in. } & $\begin{array}{l}8 \text { p.m. Stars visible, but rlim; } \\
\text { moon gitaming lu NWW. Wind } \\
\text { variable between } \mathbf{E} \text { anil SSE, } \\
\text { and squaliy; foggy all round. }\end{array}$ \\
\hline & & & & & so & in & $\begin{array}{l}\text { very unst es } \\
\text { t all day. } \\
\text { eter rose rapi }\end{array}$ & & 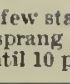 & iibl & & $\begin{array}{l}\text { incon rlim. } \\
\text { Very stror }\end{array}$ & & 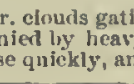 & $\begin{array}{l}\text { ered alout western mountains to } \\
\text { drift or snowfall, and ty heavy } \\
\text { d remained fairly steady. }\end{array}$ \\
\hline
\end{tabular}


1903-August.]

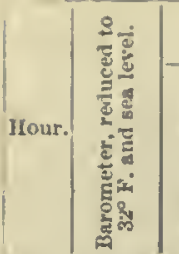

st.

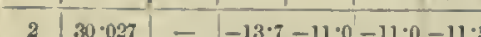

$230 \cdot 027-13 \cdot 7-11 \cdot 0-11 \cdot 0-11 \cdot 5$

$429 \cdot 823--12 \cdot 7-12 \cdot 0-12 \cdot 2-12 \cdot 4$

$6 \quad 29 \cdot 950$

$829 \cdot 898$

$10 \quad 29 \cdot 848$

Noon $29 \cdot 765-18 \cdot 0-17 \cdot 0-17 \cdot 0-17 \cdot 2-17 \cdot 2$

$229 \cdot 612--23 \cdot 5-16 \cdot 8-17 \cdot 0-16 \cdot 5$

$429 \cdot 515 i-11 \cdot 5-20 \cdot 0-8 \cdot 5-9 \cdot 0-8 \cdot 0$

$629 \cdot 354-1-9 \cdot 0-4 \cdot 2-5 \cdot 2-4 \cdot 0$

$829 \cdot 295--17 \cdot 8-15 \cdot 0-15 \cdot 0-15 \cdot 5$

$1029 \cdot 254-1-24 \cdot 0-22 \cdot 0-22 \cdot 0-23 \cdot 0$

Mldit. $29 \cdot 199-26 \cdot 0-25 \cdot 0-25 \cdot 0-24 \cdot 0$

Measi $\overline{29 \cdot 644}-\overline{-13 \cdot 3-13 \cdot 5-}$
Meteorological, Journal, of the "Discovery.'

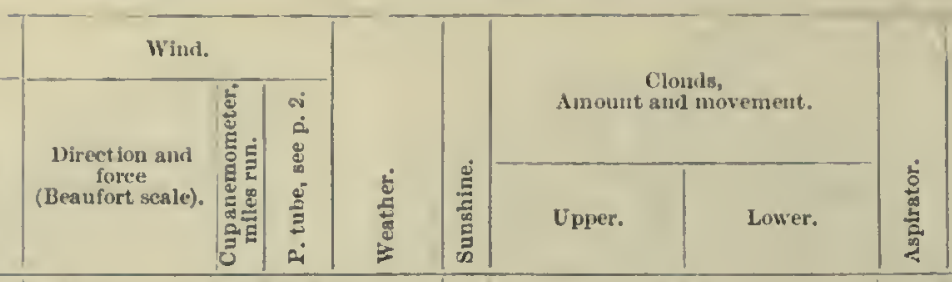

\begin{tabular}{|c|c|c|c|c|c|c|}
\hline F ly $N, 1-3$ & 16 & $22-26$ & $\log 7=$ & - & - & \\
\hline ENE, 2-3 & 15 & 22-26 & b & - & - & \\
\hline Niv, 3-4 & 18 & $25-30$ & $\infty+* *$ & - & N 10 & \\
\hline N1s, 4-5 & 23 & $30-35$ & be & - & S.cu. 4 & \\
\hline Lt. var. airs & 10 & $0-8$ & oc & - & S. 8 & $\stackrel{\infty}{0}$ \\
\hline Lt. var. alrs & 3 & $0-8$ & $x$ & A.-8. 5 & Fr.-8. 2, NW & 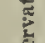 \\
\hline Lt. var. airs & 4 & $0-5$ & $b \in U$ & Ci.-cul. 2 , W & S. $2, N$ & $\frac{5}{0}$ \\
\hline N by $E, 1-3$ & 3 & $5-20$ & bq & - & - & 8 \\
\hline$N$ iby $F, 2-3$ & 10 & $10-25$ & b & - & - & \\
\hline E, 1-2 & 7 & $10-15$ & b & - & - & \\
\hline ESE, 5-7 & 9 & $30-40$ & bq + & - & - & \\
\hline $\operatorname{Var} 1,7-8$ & 20 & $38-45$ & $b+$ & - & - & \\
\hline- & - & - & - & - & - & - \\
\hline
\end{tabular}

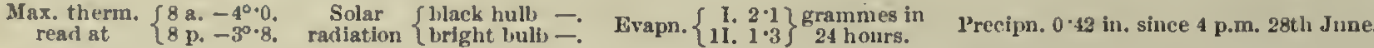

Renuarks

Bank of Str. in W, stars very bright. Dull round the horlzon, and lower rim. liowiug very slightly.

Wind very variable, NW to $\mathbf{E}$ to $S$ anc back to NW again wbilst olserving temperature failing quickly during olservation ; dill and thick looking Str. luing in a Niv and SSE lirec tion; this is below the ligher Str. conn. Western mountains visihle buit misty about summits ; dark, and misty abont summits; dark, ant $\mathrm{S}$ and SW; hank of Str. to $\mathrm{N}$, move ment imperceptible.

oon. Min. temp. at Cape Armitage, $-20^{\circ} \cdot 5$. Wind NW, 1. Le. Ci.-s. 3 Fr.-8. 3, the latter going $\mathrm{F}^{1 y}$; Ere bus s moke going Ely.

p.m. Wind-blown $\mathrm{Cl}$-cu, passing from $\mathbf{E}$; Str. to $\mathrm{N}$ and $\mathrm{NW}$

p.m. Clear whilst taking olservation, bint hin foggy str. passing at

p.m. Min. teinp. at Cape Armitage.

$-24^{\circ} \cdot 6$. Wind NNW, 2, b. - ir. Two small wisps of Str. to $\mathrm{N}$; Erebus indiscernible. 5 p.m. Wind very variahle for about 3 minutes; wind blew in sinal squalls from $\mathrm{X}, \mathrm{NT}, \mathrm{S}, \mathrm{W}$ and $\mathrm{NW}$, and then back to $\mathrm{N}$ by $\mathrm{E}$, backing to $\mathrm{NW}$ every now and thell; drift eame with $\mathrm{F}$ and $\mathrm{NF}$ puffs, and fog witli $\mathrm{N}$ and $\mathrm{XW}$. 6 p.m. Same as at 4 p.m.; foggy and clear at intervals; excessive drop in larometer. 8 p.u. Wind inelined to be varialle. Fine generally, but dull weather,

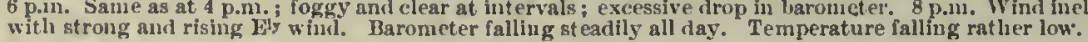

2nd.

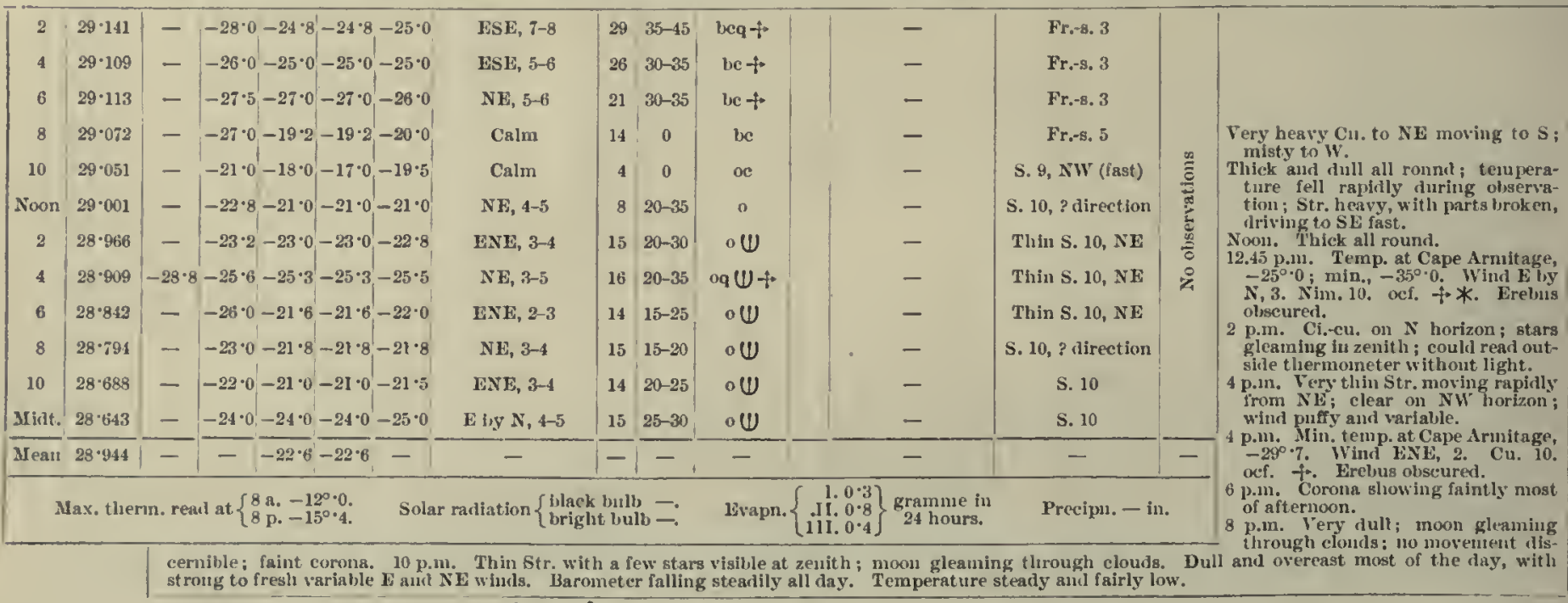

3rd.

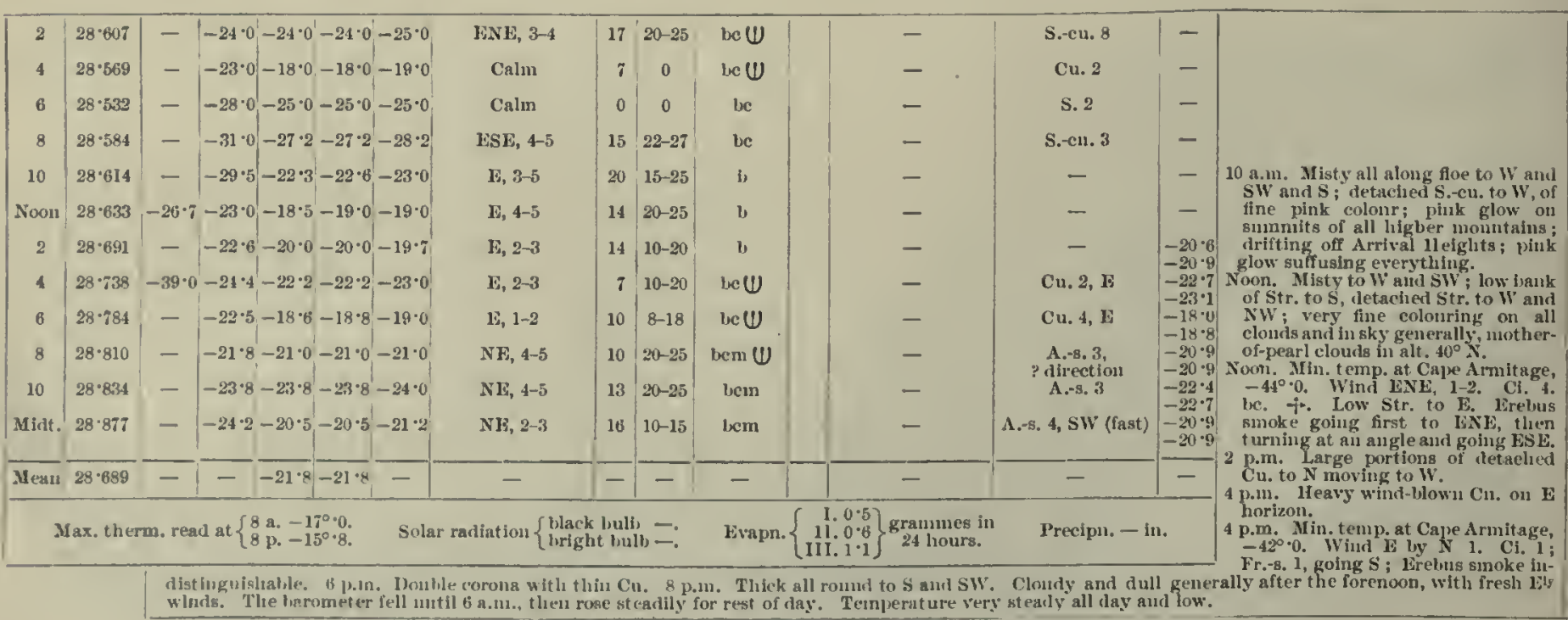


Methorological Journal of the "Discovery."

$[1903-$ A ugust.

\begin{tabular}{|c|c|c|c|c|c|c|c|c|c|c|c|c|c|c|}
\hline \multirow{3}{*}{ Ifour. } & \multirow{3}{*}{ 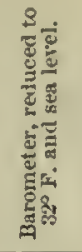 } & \multicolumn{5}{|c|}{ Thermouteters. } & \multicolumn{3}{|l|}{ Wlud. } & \multirow[b]{3}{*}{ 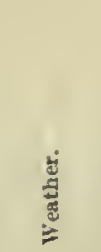 } & \multirow[b]{3}{*}{ 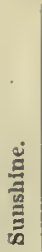 } & \multirow{2}{*}{\multicolumn{2}{|c|}{$\begin{array}{l}\text { Clouds, } \\
\text { Anount and movement. }\end{array}$}} & \multirow[b]{3}{*}{ 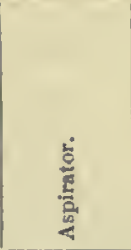 } \\
\hline & & \multirow{2}{*}{ 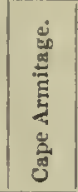 } & \multirow{2}{*}{ 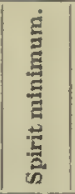 } & \multicolumn{2}{|c|}{ Mereurial. } & \multirow{2}{*}{ 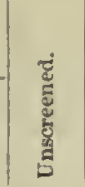 } & \multirow{2}{*}{$\begin{array}{l}\text { Direct lou and } \\
\text { force } \\
\text { (Beau fort scale). }\end{array}$} & \multirow{2}{*}{ 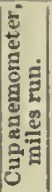 } & \multirow{2}{*}{ 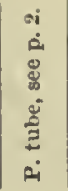 } & & & & & \\
\hline & & & & Dry. & Wet. & & & & & & & Upier. & Lower. & \\
\hline 2 & $28 \cdot 917$ & - & $-29 \cdot 0 \mid$ & $-28 \cdot 0$ & $-27 \cdot 5$ & $-28-2$ & S, I-2 & 8 & $8-12$ & be $U$ & & - & A. -8.3 & Mercury \\
\hline 4 & $28 \cdot 947$ & - & $-29 \cdot 0$ & $-23 \cdot 2$ & $-23 \cdot 2$ & $-24 \cdot 5$ & $\operatorname{Var} . \mathrm{NE}_{2}, 1$ & 3 & $0-5$ & be & & - & $\Lambda,-B, 2$ & $\begin{array}{l}-24 \cdot 2 \\
-24 \cdot 5\end{array}$ \\
\hline 6 & $28 \cdot 973$ & - & $-26 \cdot 0$ & $-23 \cdot 0$ & $-23 \cdot 0$ & $-24 \cdot 0$ & Fily $\mathrm{S}, 1$ & 5 & $5-7$ & be & & 一 & A.-B. 2 & $\begin{array}{l}-22.0 \\
-24 \cdot 2\end{array}$ \\
\hline 8 & $28 \cdot 991$ & - & $-26 \cdot 0$ & $-26^{\circ} 0$ & $-26 \cdot 0$ & $-27 \cdot 0$ & Lt. Wairs & 3 & 5 & be & & - & A. $-\mathrm{s} .2$ & - \\
\hline 10 & $28 \cdot 991$ & - & $-27 \cdot 0$ & $-23 \cdot 2$ & $-23 \cdot 5$ & $-24 \cdot 2$ & NE, 4-63 & 9 & $20-30$ & $b+$ & & - & - & $-24 \cdot 2$ \\
\hline Noon & $28 \cdot \$ 96$ & $-26 \cdot 6$ & $-23 \cdot 8$ & $-21 \cdot 8$ & $-21 \cdot 8$ & $-22 \cdot 2$ & NE, 4-5 & 13 & $20-25$ & $\alpha+U$ & & A. $-8,6, \mathrm{NW}$ & $\mathrm{Fr}_{\mathrm{r}-\mathrm{s.} .4}$ & $\begin{array}{l}-21.6 \\
-21.6\end{array}$ \\
\hline 2 & $28 \cdot 995$ & - & $-23 \cdot 0$ & $-22 \cdot 5$ & $-22 \cdot 5$ & $-22 \cdot 0$ & HNF, 2-3 & 19 & $20-28$ & $b c+U$ & & - & S. $2, \mathbf{E}$ & $\begin{array}{l}-22 \cdot 0 \\
-22 \cdot 7\end{array}$ \\
\hline 4 & $28 \cdot 999$ & $-41 \cdot 8$ & $-24 \cdot 0$ & $-23 \cdot 0$ & $-23 \cdot 0$ & $-23 \cdot 3$ & NI, 1-2 & 10 & $16-18$ & $b e+U$ & & 一 & S. $4, \mathrm{~F}$ & $\begin{array}{l}-23 \cdot 8 \\
-21 \cdot 2\end{array}$ \\
\hline 6 & $28 \cdot 991$ & - & $-25 \cdot 2$ & $-24 \cdot 5$ & $-24 \cdot 5$ & $-25 \cdot 0$ & $\mathrm{NE}, 2-3$ & 14 & $18-25$ & $b c-4$ & & - & S. 4, F & - \\
\hline 8 & $28 \cdot 989$ & - & $-26 \cdot 0$ & $-25 \cdot 0$ & $-25 \cdot 0$ & $-25 \cdot 2$ & NE, 4-6 & 15 & $20-32$ & be + & & 一 & A.-s. 4 & $\overline{-}$ \\
\hline 10 & $28 \cdot 959$ & - & $-28 \cdot 5$ & $-28^{\circ} y$ & $-28 \cdot 0$ & $-29 \cdot 0$ & NE by $E, 4-5$ & 18 & $15-18$ & ocm & & - & & $\begin{array}{l}\text { I0 p.m. to } \\
\text { Midt. }\end{array}$ \\
\hline M int. & $28 \cdot 958$ & - & $-28 \cdot 8$ & $-25 \cdot 0$ & $-25 \cdot 0$ & $-25 \cdot 5$ & ENE, 3 & 11 & 18 & $o c n^{2} \rightarrow$ & & - & S. 10 & $\begin{array}{c}\text { mercury } \\
\text { below scale }\end{array}$ \\
\hline Mean & $28 \cdot 976$ & - & - & $-24 \%$ & $-24 \cdot 4$ & - & - & - & - & - & & - & - & - \\
\hline
\end{tabular}

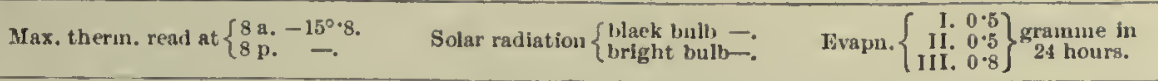

Irecipn. - in.

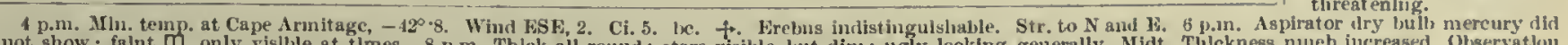

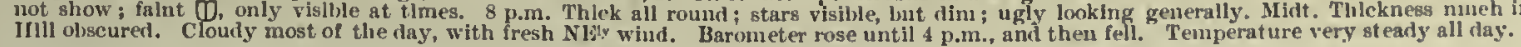

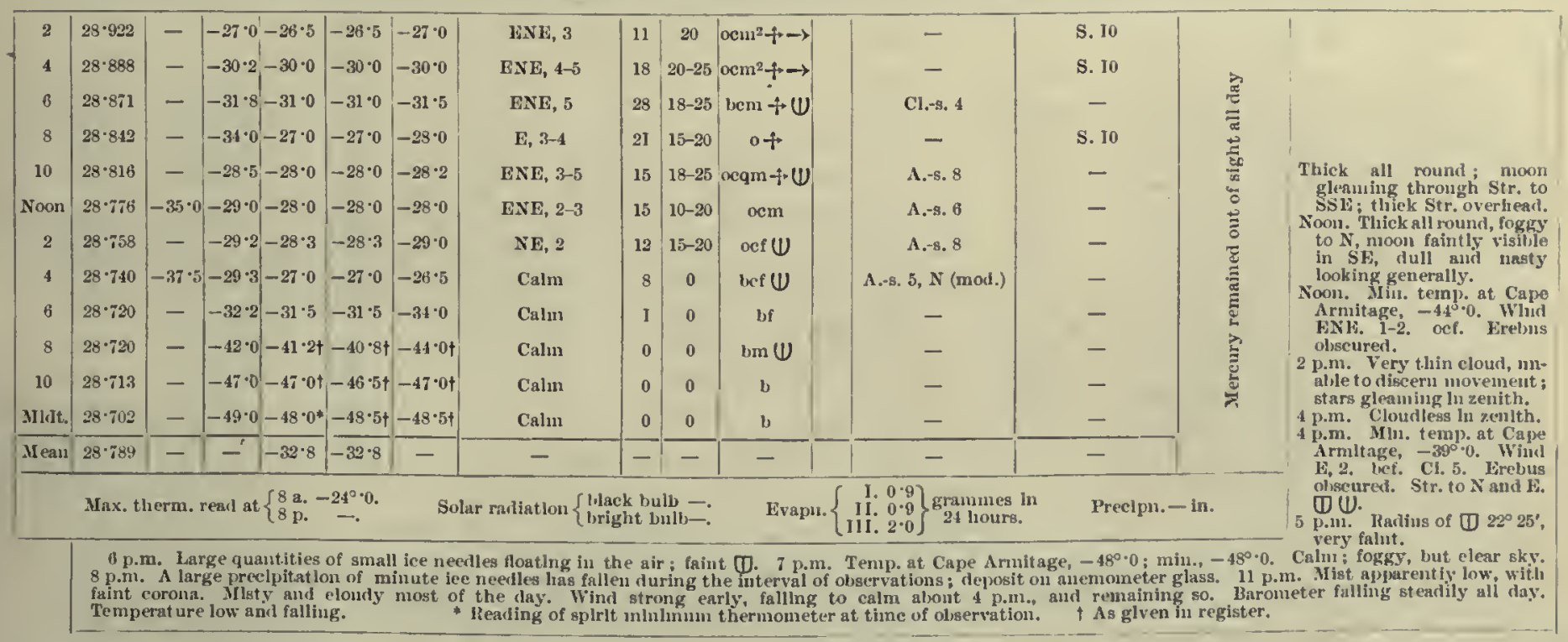

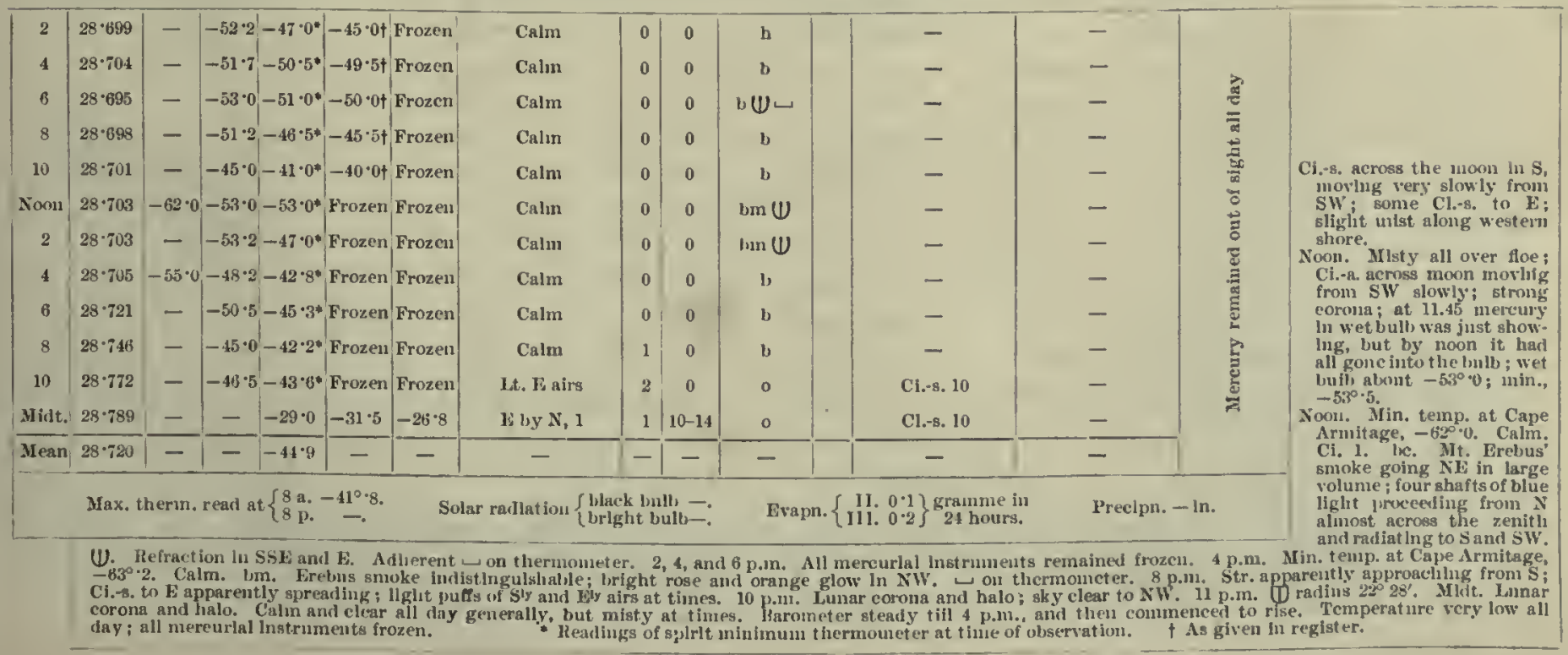


1903-Angust.]

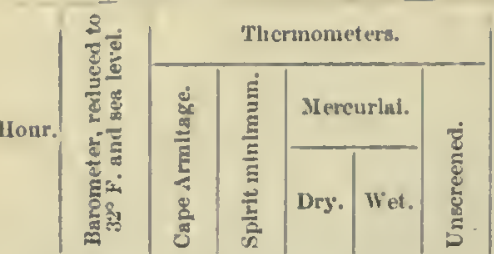

7th.

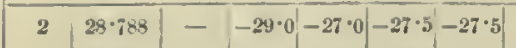

$428 \cdot 800-\quad-29 \cdot 0-27 \cdot 0-27 \cdot 5-27 \cdot 5$

$628 \cdot 854--29 \cdot 9-29 \cdot 1-29 \cdot 5-30 \cdot 0$

$8 \quad 28 \cdot 899--40 \cdot 0-39 \cdot 0-39 \cdot 0-42 \cdot 0$

$1028 \cdot \Omega 39 \rightarrow-44 \cdot 0-34 \cdot 0-34 \cdot 5-35 \cdot 0$

Noon $28 \cdot 94-39 \cdot 5-33 \cdot 0-33 \cdot 5-33 \cdot 0-35 \cdot 0$

$228 \cdot 930--37 \cdot 2-32 \cdot 2-32 \cdot 8-33 \cdot 0$

$428 \cdot 960-50 \cdot 6-35 \cdot 5-31 \cdot 2-31 \cdot 2-28 \cdot 5$

$028 \cdot 964--39 \cdot 5-37 \cdot 0-37 \cdot 0-39 \cdot 5$

$828 \cdot 960--38 \cdot 0-22 \cdot 6-22 \cdot 8-23 \cdot 0$

$1023 \cdot 916--31 \cdot 0-27 \cdot 5-27 \cdot 8-27 \cdot 2$

Midt. $28 \cdot 957-28 \cdot 3-16 \cdot 8-17 \cdot 0-17 \cdot 0$

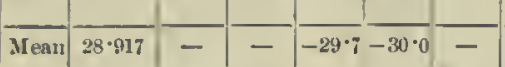

Metrokologicat, Journal of tHe "Discoveliy."

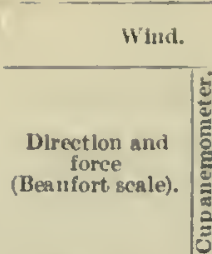

ENl: 3i-4

E. by $\mathbf{N}, 3$

LNE, 0-1

Calu

Calm

L.t. var, alrs

E, 1-2

N, 1-2

NNL, 0-1

NNT, 1

Calm

Lt. SE alrs
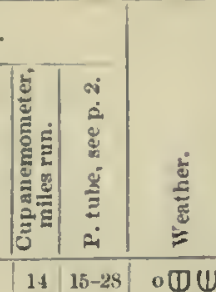

$14 \quad 15-28$ oШU

40 be

\begin{tabular}{l|l|l}
2 & 0 & b
\end{tabular}

\begin{tabular}{c|c|c}
0 & 0 & $b U$ \\
3 & $0-8$ & $b c \Psi$
\end{tabular}

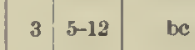

2 $5-15 \quad$ b

\begin{tabular}{l|l|l}
7 & $5-10$ & 0 \\
5 & $5-12$ & 0
\end{tabular}

\begin{tabular}{c|c|c}
2 & 0 & 0 \\
1 & $0-8$ & 0
\end{tabular}

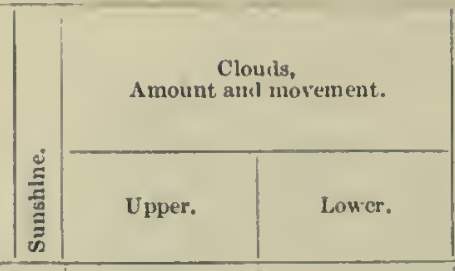

C1. 10

\begin{tabular}{|c|c|c|}
\hline Cl. 10 & - & \\
\hline Cl. 2 & 一 & \\
\hline Ci. 5 & - & 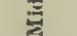 \\
\hline - & - & $\Xi$ \\
\hline - & - & $\Xi$ \\
\hline Ci.-s. 4, & - & $\frac{50}{10}$ \\
\hline Cl. -5.2 , & - & $\ddot{c}$ \\
\hline $\begin{array}{l}\text { ?direction } \\
\text { Ci.-8. 4, W }\end{array}$ & 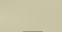 & $\vec{\Xi}$ \\
\hline- & S. 10 & 5 \\
\hline- & S. 10 & 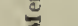 \\
\hline - & S. 10 & \\
\hline - & S. 10 & $\begin{array}{l}-17 \cdot 3 \\
-17 \cdot 7\end{array}$ \\
\hline- & - & - \\
\hline
\end{tabular}

Max. therm. rear at $\left\{\begin{array}{l}8 \text { a. }-25^{\circ} \circ 0 \\ 8 \text { p. }-21^{\circ} 0\end{array}\right.$

Solar radiation $\left\{\begin{array}{l}\text { black bulb } \\ \text { bright bulb }- \text {. }\end{array}\right.$

Evapn. $\left\{\begin{array}{c}\text { II. } 0 \cdot 2 \\ \text { III. } 0 \cdot 3\end{array}\right\} \begin{aligned} & \text { granume in } \\ & 24 \text { hours. }\end{aligned}$

Precipn. - ln.

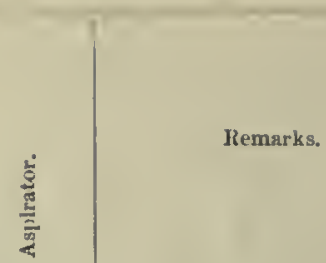

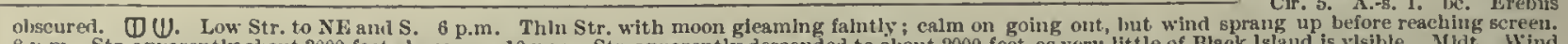
8 1.n. Str. apparently alout 3000 feet above us. 10 p.m. Str. apparently descended to alout 2000 feet, as very little of Black lsland is visible. Midt. Wind

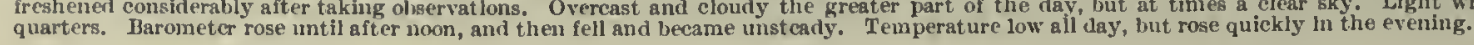

\section{8th.}

\begin{tabular}{|c|c|c|c|}
\hline 2 & $28 \cdot 975$ & - & $-22 \cdot 0-22 \cdot 0-22 \cdot 0^{\prime}-22 \cdot 0$ \\
\hline 4 & $28 \cdot 946$ & - & $9 \cdot 2-19 \cdot 6-19 \cdot 0$ \\
\hline 0 & $28 \cdot 931$ & - & $-20 \cdot 0-19 \cdot 0-19 \cdot 2-19 \cdot 0$ \\
\hline 8 & $28 \cdot 939$ & - & $0.5-20 \cdot 2-21 \cdot 0$ \\
\hline 10 & $28 \cdot 9.40$ & - & $-21 \cdot 8-22 \cdot 6$ \\
\hline Soon & $28 \cdot 881$ & $-22 \cdot 0$ & $19 \cdot 7-19 \cdot 9-20 \cdot 0$ \\
\hline 2 & $28 \cdot 849$ & - & $-22 \cdot 0-22 \cdot 0-23 \cdot 0$ \\
\hline 4 & $28 \cdot 805$ & $-31 \cdot 2$ & $-25 \cdot 0-25 \cdot 2$ \\
\hline 6 & $23 \cdot 747$ & - & $-24 \cdot 3-16 \cdot 3-16 \cdot 5-17 \cdot 0$ \\
\hline 8 & $28 \cdot 651$ & - & $-20 \cdot 2-21 \cdot 0$ \\
\hline 10 & $28 \cdot 663$ & - & $-20 \cdot 5-20 \cdot 5-20 \cdot 5$ \\
\hline IIlut. & $28 \cdot 658$ & - & $-21 \cdot 8-22 \cdot 5$ \\
\hline
\end{tabular}

\section{E, 4}

NF, 4-5

ENE, $1-2$

ESE, 4-5

E, 1

E, 1-3

E, 1-3

ENE, 3-4

Var. E, 1-3

WSW, 1-3

Lt. $\mathbf{E}$ airs

E, 2-3

\begin{tabular}{|c|c|c|c|c|c|}
\hline 10 & $|22-25|$ & o & - & S. 10 & $\begin{array}{l}-22 \cdot 0 \\
-220.2\end{array}$ \\
\hline 14 & $23-27$ & o & - & s. 10 & $-19 \cdot 1$ \\
\hline 10 & $12-15$ & 0 & - & S. 10 & $-19 \cdot 1$ \\
\hline 17 & $20-25$ & om $+*$ & - & N. 10 & $\begin{array}{l}170 . \\
-23.8 \\
-23.4\end{array}$ \\
\hline 12 & $5-10 \mid$ & o* & - & N. 10 & $\begin{array}{l}-22.7 \\
-22.7\end{array}$ \\
\hline ミ14 & $10-23$ & $0+*$ & - & N. 10 & $\begin{array}{l}-19 \cdot 8 \\
-21 \cdot 8\end{array}$ \\
\hline 20 & $10-20$ & $\operatorname{cof}+$ & - & S.cu. 10 & $\begin{array}{l}-22 \cdot 0 \\
-22 \cdot 4\end{array}$ \\
\hline 17 & $15-25$ & of +1 & - & S.cu. 10 & $\begin{array}{l}\text { Mercury } \\
\text { betow segale }\end{array}$ \\
\hline 14 & $8-15$ & $o q f+\cdots$ & - & N. 10 & $\begin{array}{l}-16 \cdot 2 \\
-17 \cdot 0\end{array}$ \\
\hline 16 & $10-15$ & oqf $*$ & - & N. 10 & $\begin{array}{l}-21 \cdot 3 \\
-20 \cdot 9\end{array}$ \\
\hline 1 & 0 & $0 * *$ & - & N. 10 & $\begin{array}{l}-2006 \\
-20.6\end{array}$ \\
\hline 14 & 10-20 & $0 \uparrow *$ & - & N. 10 & $\begin{array}{l}-21 \cdot 3 \\
-21 \cdot 6\end{array}$ \\
\hline- & - & - & - & - & - \\
\hline & & & & \multicolumn{2}{|c|}{ Preclpn. - In. } \\
\hline
\end{tabular}

Wind Inclined to be varlatie and gusty.

Wind lnctined to haul to $S$ of $E$. antl be squally; Str. apparcntly lifting to $\mathrm{S}$ and $\mathrm{SW}$,
Biack Istand visible to ait. of Biack lstand vis
about 2000 feet.

Min. temp. at Cape Armltage, 10. ocf. $t$. Ereisus olscured 2 p.m. Break in clourds to $\mathrm{N}$;
mlsty to $\mathrm{S}$.

misty to $\mathrm{S}$. Conting of drift on ther-
4.m.

4 p.m. Miu. temp. at Cape p.m. Min. temp. at Cape $4-\tilde{v}$. Str. 10. ocfq. †े. Erehus obscured.

6 p.n. Wind very squally and varlable; fine snow falling. $8 \mathrm{p} . \mathrm{m}^{2}$. This is the first o.casion that I remember a WSW wind belng reeorded; light snow falling, sllow hard and small; foggy all round ; just befor

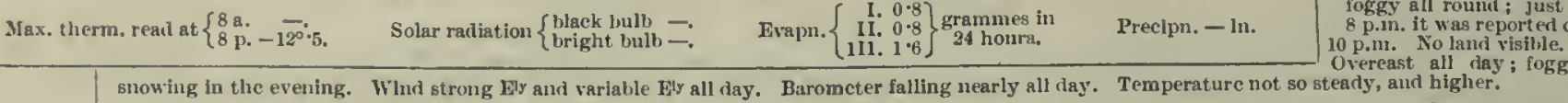

p.m. No land visille.
pvercast all day; foggy an

snowing in the evening. Whid strong Ely and variable Fiy all day. Baromcter falling nearly all day. Temperature not so steady, and higher.

9th.

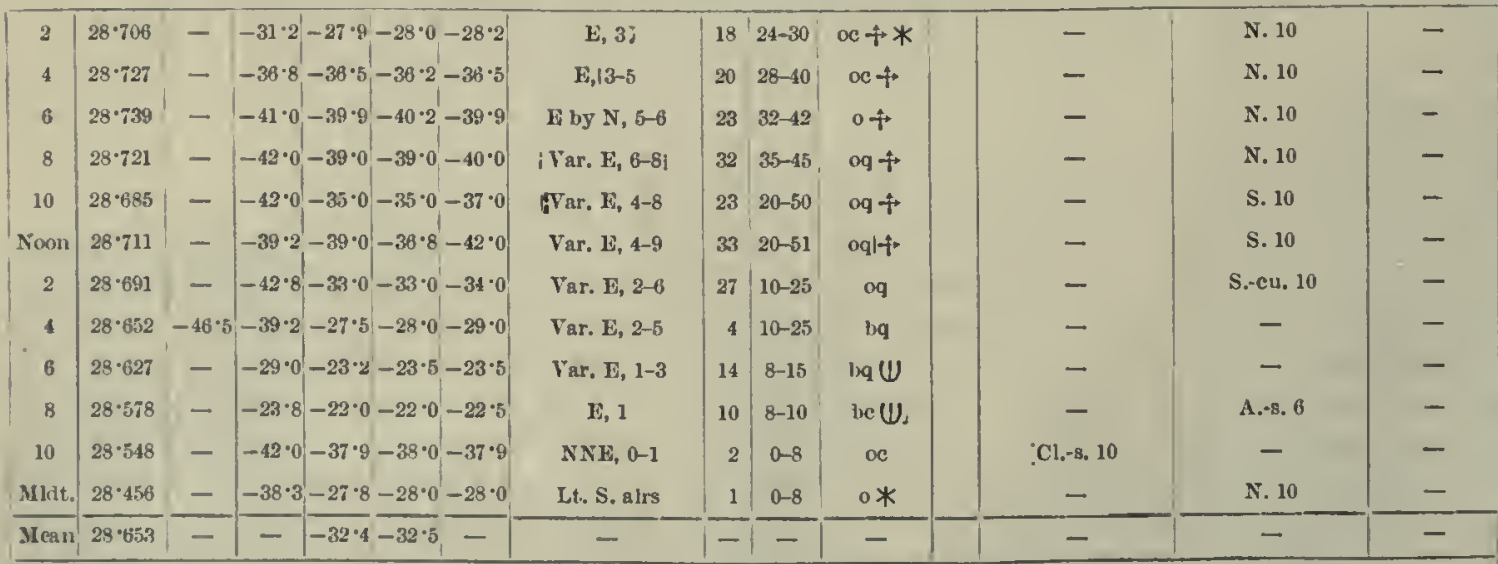

Surface drift and snow; lane Dritiling and snowlng heavily; land obscured.
now in llobinson's anemoneter.

Squalls up to force 9 and 10 at times; wind varlalle from FNli to SE; temperature falls raplaly lin the lulls, generally to $-40^{\circ}$ and rises at once with squall; drift very thick during Nome squals.

$10 \mathrm{am}$ wind weather as at 10 a.m.; wind from NE to temperature as variable.
p.m. Clouds Inelmerl to oreal to $\mathrm{N}$, alt. $30^{\circ}$; Str. very thin overhead.

1 p.m. Temp, at Cape Armitage, $-45^{\circ} \cdot 5 ; \mathrm{min},-46^{\circ} \cdot 5$. Wind $\mathrm{D}, 6-7$. befq. $\rightarrow$. Erelus obscured. Str. to $\mathbf{N}$ and $\mathrm{F}$. 2 p.m. Hreak in clouds to $\mathrm{N}$ ing overhead.

p.su. Str. all round horizon.

Max. therm. read at $\left\{\begin{array}{l}8 \mathrm{a},-16^{\circ} \cdot 0 . \\ 8 \mathrm{p},\end{array}\right.$

Evapn. - gramues.

Prceipn. - In

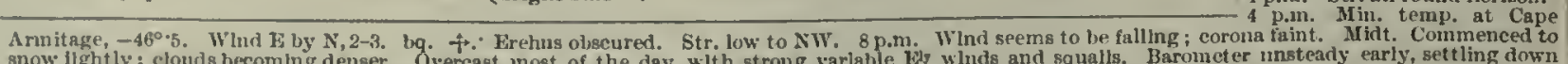

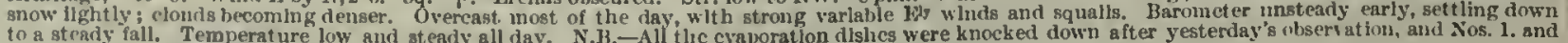
to a strady fall. Temperat ure low 
Metkorological JoUkna. OF THE "Discovery."

[1903-A ugust.

\begin{tabular}{|c|c|c|c|c|c|c|c|c|c|c|c|c|c|c|}
\hline \multirow{3}{*}{ Hour. } & \multirow{3}{*}{ 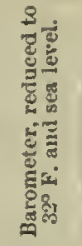 } & \multicolumn{5}{|c|}{ Tuermometers. } & \multicolumn{3}{|l|}{ Whad. } & \multirow[b]{3}{*}{ 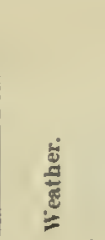 } & \multirow[b]{3}{*}{ 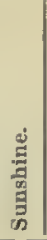 } & \multirow{2}{*}{\multicolumn{2}{|c|}{$\begin{array}{l}\text { Clourds, } \\
\text { Amount and movement. }\end{array}$}} & \multirow[b]{3}{*}{ 客 } \\
\hline & & \multirow{2}{*}{ 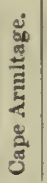 } & \multirow{2}{*}{ 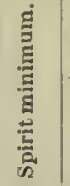 } & \multicolumn{2}{|c|}{ Mercurlas. } & \multirow{2}{*}{ 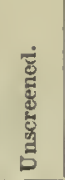 } & \multirow{2}{*}{$\begin{array}{c}\text { Dlreet lou and } \\
\text { force } \\
\text { (Beaufort seale). }\end{array}$} & \multirow{2}{*}{$\mid$} & \multirow{2}{*}{ 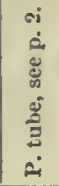 } & & & & & \\
\hline & & & & Dry. & Wet. & & & & & & & Upper. & Lower. & \\
\hline 2 & $28 \cdot 415$ & - & $-28 \cdot 1$ & $-24 \cdot 0$ & $-24 \cdot 0$ & $-24 \cdot 0 \mid$ & E, $0-1$ & 1 & $0-8$ & o* & & - & N. 10 & - \\
\hline 4 & $28 \cdot 368$ & - & $1 \cdot 2$ & $8 \cdot 0$ & $8 \cdot 0$ & $-8 \cdot 0$ & SSE, 2-4 & 13 & $10-25$ & $o *$ & & - & N. 10 & - \\
\hline 6 & $28 \cdot 314$ & - & $-8 \cdot 5$ & 0.0 & 0.0 & 0.0 & $\mathrm{SW}, 4-5$ & 16 & $15-25$ & $0 \rightarrow$ & & - & N. 10 & - \\
\hline 8 & $28 \cdot 301$ & - & $-2 \cdot 0$ & $3 \cdot 0$ & $3 \cdot 0$ & $3 \cdot 0$ & $S$ by $\mathrm{E}, 5-10$ & 30 & $30-58$ & og $t^{2} *$ & & - & N. 10 & - \\
\hline 10 & $28 \cdot 311$ & - & $1 \cdot 8$ & $4 \cdot 0$ & $4 \cdot 0$ & $3 \cdot 6$ & S, 6-8 & 20 & $30-48$ & $\operatorname{og} t^{2} *$ & & - & N. 10 & - \\
\hline Nom & $28 \cdot 303$ & - & $3 \cdot 5$ & $5 \cdot 0$ & $5 \cdot 0$ & $5 \cdot 0$ & Var. S, 6-10 & 26 & $30-61$ & $\operatorname{og} t^{2} *$ & & - & N. 10 & - \\
\hline 2 & $28 \cdot 300$ & - & $4 \cdot 5$ & $6 \cdot 0$ & $5 \cdot 0$ & $6 \cdot 5$ & SSW, 4-9 & 29 & $20-50$ & $\operatorname{og} t^{2} *$ & & - & N. 10 & - \\
\hline 4 & $28 \cdot 325$ & - & $4 \cdot 0$ & 6.5 & $6 \cdot 0$ & $6 \cdot 5$ & Var. S, 6-10 & 43 & $35-55$ & $\operatorname{og} t^{2} *$ & & - & N. 10 & - \\
\hline 6 & $28: 344$ & - & - & - & - & - & Var. SW, 11-12 & 41 & - & $\operatorname{og}+2 *$ & & - & N. 10 & - \\
\hline 8 & $28 \cdot 385$ & - & $5 \cdot 8$ & $7 \cdot 8$ & $7 \cdot 8$ & $7 \cdot 8$ & $S, 6-10$ & 41 & $30-55$ & $\operatorname{og} t^{2} *$ & & - & N. 10 & - \\
\hline 10 & $28 \cdot 411$ & - & $7 \cdot 0$ & $9 \cdot 0$ & 0.0 & $8 \cdot 5$ & S, $7-8$ & 31 & 30 & $\alpha q t^{2} *$ & & - & N. 10 & - \\
\hline Mint. & $28 \cdot 380$ & - & $9 \cdot 0$ & $9 \cdot 5$ & $9 \cdot 5$ & $9 \cdot 5$ & S, $7-9$ & 28 & $30-50$ & $n q+2 *$ & & - & N. 10 & - \\
\hline Mean & $28 \cdot 314$ & - & - & 1.7 & $1 \cdot 6$ & - & - & - & - & - & & - & - & - \\
\hline
\end{tabular}

Max. therm, rearl at $\left\{\begin{array}{l}8 \text { a. } \\ 8 \text { p. } 7^{\circ} \cdot 0.8 .\end{array}\right.$
Solar radiation $\left\{\begin{array}{l}\text { blaek bull, } \\ \text { bright bulb }- \text {. }\end{array}\right.$

Erapn. - grammes.
Preeipn. - ln.

$\frac{\text { Remarkw. }}{-[\text { [No ohservations were talen of Caje }}$

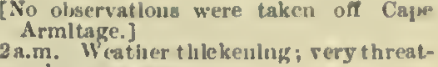
- enlug appenrance. force of winl very varlable. 6 a.m. Wlud varlable ln force and difec. tion.

8 a. in. Very heavy squalls; eatuot see anythlug from shi

- $10 \mathrm{a} . \mathrm{m}$. Drift very thlek; wind stealy in S, int squally.

Noon. Blowing very much larrier in the squalls; wluil shlftug to SSW and back to $S$; drift extremely thilck; baroineter not pumplng in the squalls; wind linereasing lin force and frequeney of squalls

2 p.in. Whi swnig from $W$ to $S$ and the squalls.
thend b bilnding drift witl the squalls.

p.m. No outslile olservatlon taken: The olservat lon for $6 \mathrm{fm}$. was lot taken, hy captalu's oriers: Crankr. the onserver, aflirins that the force 11-12 is not exaggeraterl hi hls oplujon. Several of the oflicers were on deck shortly hefore 6, and they: give 11 as the
force, aud even higlier ln the squalls Whieh followed shortly after 6 p.in. 8 p.m. It appeared to lull, and observations were taken, but shortly afterwards it caine on with inercased violence; squnlls suenn

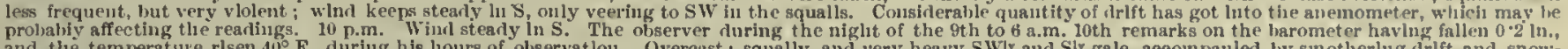

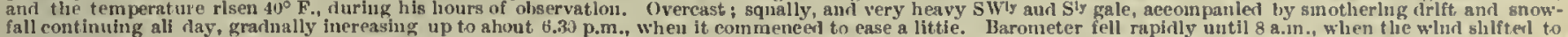

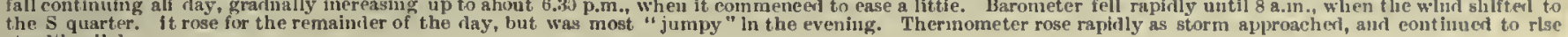
the $s$ quarter.
stearlily ail ray.

\begin{tabular}{|c|c|c|c|c|c|c|c|c|c|c|c|c|c|}
\hline 2 & $28 \cdot 459$ & $1-$ & $8 \cdot 0$ & $9 \cdot 0^{\prime}$ & 0.0 & $9 \cdot 0$ & $S$ by $W, 6-8$ & 31 & $20-50$ & $\log +2 *$ & - & N. 10 & $1-$ \\
\hline 4 & $28 \cdot 490$ & - & $7 \cdot 5$ & $8 \cdot 0^{1}$ & $8 \cdot 0$ & $8 \cdot 0$ & SE, 2-4 & 24 & $12-20$ & oq $-4^{2} *$ & - & N. 10 & - \\
\hline 6 & $28 \cdot 473$ & - & $7 \cdot 0$ & $9 \cdot 2$ & $9 \cdot 0$ & $8 \cdot 5$ & SE, 2-4 & 9 & $14-22$ & oq $+*$ & - & N. 10 & - \\
\hline 8 & $28 \cdot 470$ & - & $8 \cdot 0$ & $10 \cdot 0$ & $10 \cdot 0$ & $9 \cdot 5$ & Var. S, 6-8 & 16 & $25-40$ & $\operatorname{og}+*$ & - & N. 10 & - \\
\hline 10 & 28.412 & - & $8 \cdot 2$ & $10 \cdot 8$ & $10 \cdot 8$ & $10 \cdot 5$ & Var, SW, 7-9 & 35 & $35-48$ & $\operatorname{og}+2 *$ & - & N. 10 & - \\
\hline Noon & $28 \cdot 531$ & - & $10 \cdot 0$ & $11 \cdot 2$ & $11 \cdot 2$ & $11 \cdot 2$ & Var. S, 5-7 & 38 & $25-41$ & $\operatorname{og}-f^{2} *$ & - & N. 10 & - \\
\hline 2 & $28 \cdot 507$ & - & $8 \cdot 0$ & $8 \cdot 5$ & $8 \cdot 5$ & $8 \cdot 5$ & SSW, 8-10 & 22 & $33-55$ & $\operatorname{og} t^{2} *$ & - & x. 10 & - \\
\hline 4 & $28 \cdot 707$ & - & 6.5 & $6 \cdot 5$ & $6 \cdot 5$ & $6 \cdot 0$ & Var. SSE, $6-8$ & 43 & $25-40$ & $\operatorname{og} f^{2} *$ & - & N. 10 & - \\
\hline is & $28 \cdot 919$ & - & $5 \cdot 5$ & $6 \cdot 0$ & $6 \cdot 0$ & $6 \cdot 0$ & Var. S, 8-10 & 38 & $44-56$ & $\log f^{2}+2$ & - & N. 10 & - \\
\hline 8 & $29 \cdot 143$ & - & -13.5 & -13.0 & $-13 \cdot 0$ & $-13 \cdot 0$ & S, 6-9 & 29 & $30-46$ & $\operatorname{og} t^{2} *$ & - & N. 10 & - \\
\hline 10 & $24 \cdot 206$ & - & $-17 \cdot 5$ & $-17 \cdot 0$ & $-17 \cdot 0$ & $-17 \cdot 5$ & SE, 2-4 & 25 & $30-33$ & bem & - & S. 4 & - \\
\hline Mirtt. & $29 \cdot 154$ & - & $-20 \cdot 5$ & $-16 \cdot 0$ & $-16 \cdot 2$ & $-14 \cdot 4$ & E by $N, 4-6$ & 24 & $38-42$ & bc & $\mathrm{Ci},-\mathrm{s} .4$, ?rlirection & - & - \\
\hline Mern & $28 \cdot 7 i 1$ & - & - & $2 \cdot 8$ & $2 \cdot 7$ & - & - & - & - & - & - & - & - \\
\hline
\end{tabular}

Iax, therm. $\left\{\begin{array}{l}8 \text { a. } 10^{\circ} \cdot 0 . \\ 8 \mathrm{p} .11^{\circ} 5\end{array}\right.$
real at $\quad$ Solar radiatlon $\left\{\begin{array}{l}\text { black hulb } \\ \text { bright bulb }- \text {. }\end{array}\right.$

Evapn, 11. 1.2 grammes in 48 hours.

Precipn. - in. Land visible.

- Land plalnly visible, lnt not Ohservatlon Hill; snow harr and failing sharply. Land plalnly visihle; Olservation hil only seen at times.

- A ppears to be cleariug ; very squally ; lanr visible at times; ail thermometer covered with rift.
lo a.m. Wuther sim

10 a.m. Wither simllar to yesterday woon. Weather seemed better

Noon. Wenther seemer better at the afterwards eame on as had as ever; was steadier from 11 a.m. to Noon, hut very gursty, reaching force 9 at times; to SW

2 p.m. Wind stearlier anrl less gusty.

4 p.m. Breaklng to the $F$, with less drift. p.m. lemarkahle rlse in harometer wind veering from $\mathrm{E}$ to $\mathrm{W}$ i,y $\mathrm{S}$. 8 p.m. Thiserl ther mometer box annfluer
2 ft. above suow. 10 p.m. Clearing mpidly; lieavy banka of Str. W and s along the mountalns: stars rapialy clearing overhead and ill-definell halo.

11 p.m. A.-s. eoming frmu S direvtlom

at morlerate speer. Mirt. There may be more Ci.s. or A.-s., iut it must be thlm, as the stars ean be seen elearly through, the moon alone shows lt up. No observations taken of Cape Armitage. Overcast; squally, and heavy' S's gale biowing, accompanied hy the same smothering rrift and suow fall. Barometcr very unst ealy farly, and then rose rapidly to 10 p.m., when it fell again. Temperature ingh all day until hetween 6 and 8 p.11., winen lt dropped quickly.

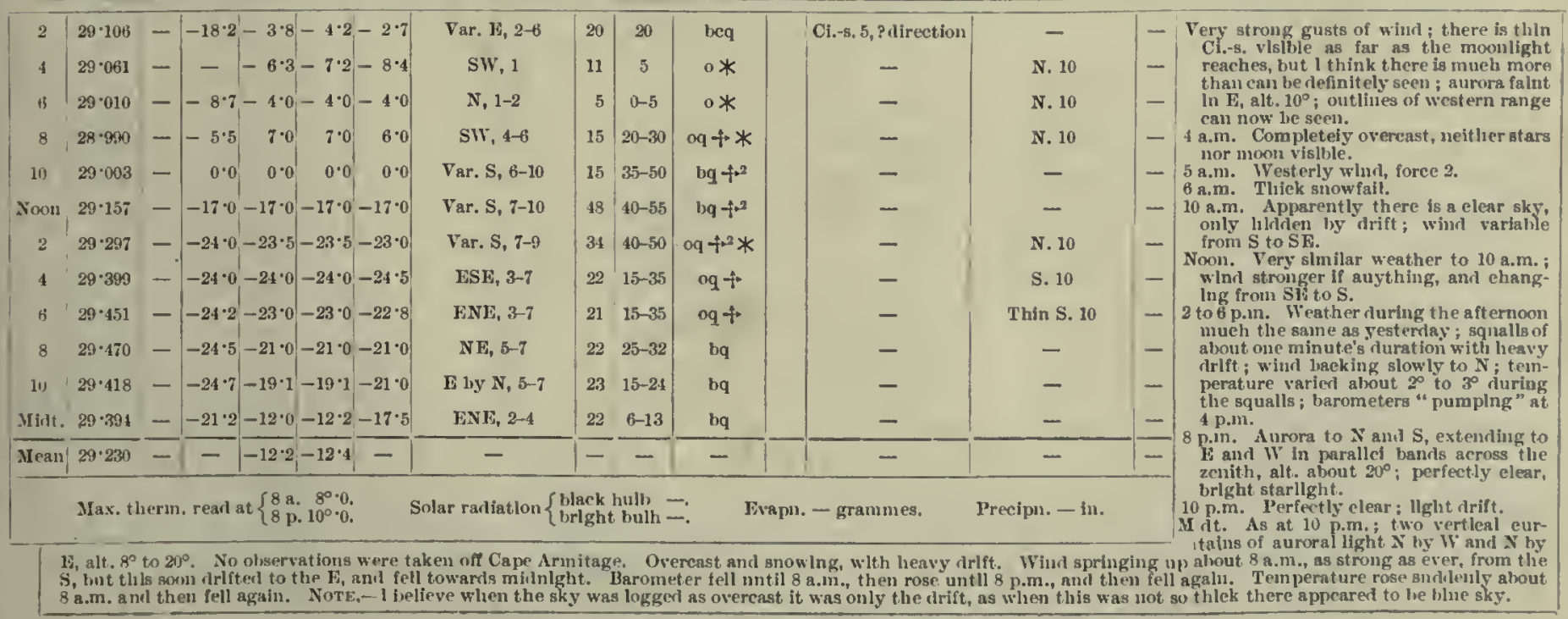


1903-Angust.]

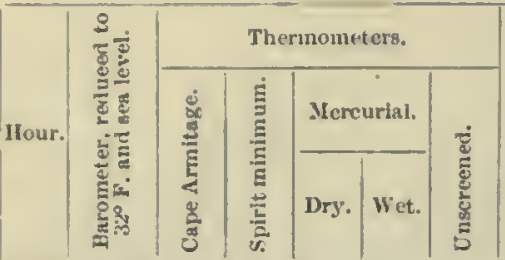

Mrthoriological, JoURxat, of TIIF "DiscovkRY,"

13 th.

\begin{tabular}{|c|c|c|c|c|c|c|c|c|c|c|}
\hline 2 & $29 \cdot 362$ & - & $-\quad-30 \cdot 2-29 \cdot 6-32 \cdot 0$ & L.t. $S$ airs & 9 & 0 & b & - & - & - \\
\hline 4 & $29 \cdot 263$ & - & $-28 \cdot 5-10 \cdot 5-11 \cdot 0-10 \cdot 9$ & NE by $N, 2-4$ & 5 & $15-21$ & $\mathrm{bq}$ & - & - & - \\
\hline 6 & $29 \cdot 155$ & - & $-11 \cdot 1-6 \cdot 2-6 \cdot 4-6 \cdot 6$ & $N$ and $E, 2-3$ & 15 & $i-13$ & $\operatorname{lng}$ & - & - & - \\
\hline 8 & $29 \cdot 006$ & - & $-11 \cdot 5-6 \cdot 0-6 \cdot 2-6 \cdot 4$ & N by 13, 1-6 & 14 & $5-35$ & bq $?$ & - & Fog 10 & - \\
\hline 10 & $28 \cdot 961$ & - & $-7 \cdot 0-7 \cdot 0-7 \cdot 0-7 \cdot 0$ & Var, $\mathbf{N}, 1-4$ & 10 & $5-25$ & bqm+七 & -. & - & - \\
\hline Nion & $28 \cdot 901$ & $-18 \cdot 0$ & $-16 \cdot 0-16 \cdot 0-16 \cdot 0-17 \cdot 0$ & Calm & 3 & 0 & bே & -- & - & - \\
\hline 2 & $28 \cdot 845$ & - & $-19 \cdot 0-12 \cdot 8-13 \cdot 0-13 \cdot 5$ & Lt. F airs & - & 0 & b & - & - & - \\
\hline 4 & $28 \cdot 793$ & $-24 \cdot 2$ & $-13 \cdot 5-8 \cdot 0-9 \cdot 0-7 \cdot 5$ & KNE, $0-1$ & 0 & - & b) & - & - & - \\
\hline 6 & $28 \cdot 769$ & - & $-14 \cdot 5-12 \cdot 3-13 \cdot 0-11 \cdot 3$ & E, 0-1 & 3 & $0-10$ & h & - & - & - \\
\hline 8 & $25 \cdot 757$ & - & $-18 \cdot 0-8 \cdot 8-8 \cdot 8-9 \cdot 0$ & $\mathrm{NE}, 1$ & 6 & 10 & b & - & - & - \\
\hline 10 & $28 \cdot 7 i 3$ & - & $-16 \cdot 0-13 \cdot 2-13 \cdot 0,-13 \cdot 7$ & SE, 2-3 & 13 & $16-26$ & $b-i$ & - & - & $\begin{array}{l}-13 \cdot 2 \\
-12.8(\text { sic })\end{array}$ \\
\hline Mirt. & $28: 813$ & - & $-14 \cdot 4-12 \cdot 0-12 \cdot 2-12 \cdot 0$ & E by S, 3-4 & 18 & $25-35$ & b & - & - & $\begin{array}{l}-11 \cdot 7 \\
-12 \cdot 3 \\
\end{array}$ \\
\hline Mean & $28 \cdot 950$ & - & $-\mid-11 \cdot 9-12 \cdot 1\}-$ & - & $\overline{-1}$ & - & - & - & - & - \\
\hline
\end{tabular}

Faint auroral areh between hy S and SE: alt. $8^{\circ}$ to $12^{\circ}$. Cleared snow off thermoneter linslde screen; the minimum thermometer was thickly coaterl with sulow, as was als the wet bulb thermometer.
th.111. Falnt guroral streamer

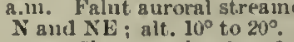
a.m. Clear overliead and anl round, except. low Cn. clonds
to $S$ to an alt. of $3^{\circ}$; wind very variable in direction. 8 a.mil. Very thlek all round, no land visible; very heavy sur-
face drlft, and fog sy. $10 \mathrm{n.m}$ Wind variable between NW and NE, shifting quiekly In gusts; frost. smoke appa-
rently pouring in from iñ: rently pouring in from in: fine colouring in sky. Shifter
sereen to new position ant eleaned loxes and instriments. Snow gauge buried 20 inches under the snow. Noon. Fine eolonrlug lin sky. Noon. Min. temp. at Cape Armitage, $-38^{\circ}$.5. Calm. Ww:
lireluns smoke going N13, frowing elose down $\mathrm{n}$ slope ; 2 inches

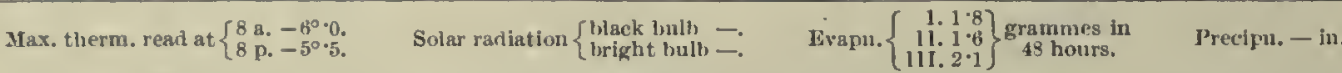

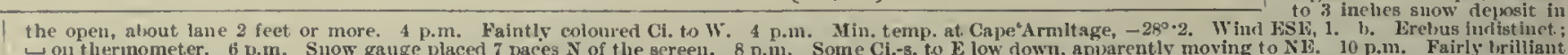

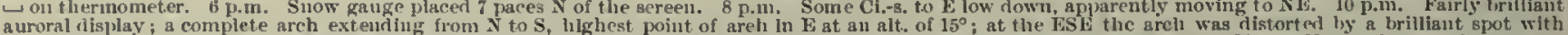

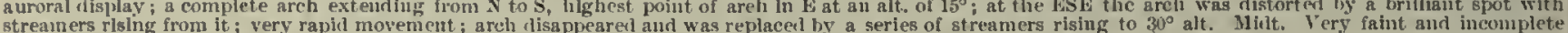

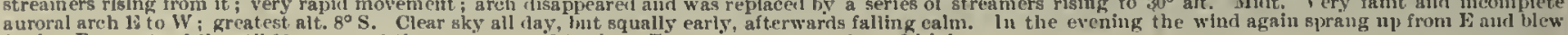

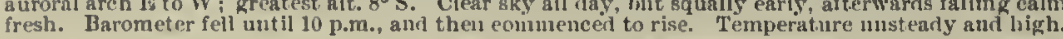

14 th.

\begin{tabular}{|c|c|c|c|c|c|c|c|c|c|c|}
\hline 2 & $28 \cdot 811$ & - & $|-14 \cdot 2-8 \cdot 8-9 \cdot 1|-8 \cdot 6 \mid$ & $\mathrm{SF}, 3-8$ & 19 & $25-54$ & lyq & - & - & -8.7 \\
\hline 4 & $28 \cdot 882$ & - & $-16 \cdot 2-15 \cdot 9-15 \cdot 6-16 \cdot 9$ & S1s, 0-2 & 18 & $0-20$ & b & - & - & -16.4 \\
\hline 6 & $28 \cdot 915$ & - & $-21 \cdot 0-13 \cdot 0-13 \cdot 2-12 \cdot 6$ & NE loy $N, 2-3$ & 13 & $15-24$ & b & - & - & $\begin{array}{l}-16.4 \\
-12 \cdot 6\end{array}$ \\
\hline 8 & $28 \cdot 9.93$ & - & $-13.5-10.0-9.2-9.8$ & $\mathrm{NE}, 2-3$ & 13 & $15-18$ & b & - & - & $\begin{array}{l}-128 \\
-10 \cdot 8 \\
-11 \cdot 2\end{array}$ \\
\hline 10 & $28 \cdot 961$ & - & $--25 \cdot 0--28 \cdot 2$ & $\mathrm{~S}, 1$ & 6 & 10 & bev & Ci.-8. 4, SW (slow) & - & $\begin{array}{l}\text { Mencury } \\
\text { below scale }\end{array}$ \\
\hline Nown & $28 \cdot 993$ & $-27 \cdot 5$ & $-28 \cdot 0-14 \cdot 9-15 \cdot 0-14 \cdot 9$ & $\mathrm{E}, 2$ & 4 & 14 & hev & Cl.-s. $5, \mathrm{SW}$ (slow) & - & $\begin{array}{l}-15 \cdot 5 \\
-16 \cdot 2\end{array}$ \\
\hline 2 & $29 \cdot 015$ & - & $-22 \cdot 5-13 \cdot 0-14 \cdot 0-13 \cdot 5$ & N, 0-1 & 3 & $0-10$ & b & - & - & $-12 \cdot 6$ \\
\hline 4 & $29 \cdot 027$ & $-20 \cdot 4$ & $-18 \cdot 5-13 \cdot 0-14 \cdot 2-15 \cdot 0$ & $\mathrm{Nw}, 1$ & 7 & $8-10$ & oc & - & Cu. 9, $\mathbf{X}$ (iast) & $\begin{array}{l}-18.4 \\
-13 \cdot 4\end{array}$ \\
\hline 6 & $29 \cdot 030$ & - & $-17 \cdot 4-6 \cdot 0-7 \cdot 0-6 \cdot 0$ & $\mathrm{~N}, 2-3$ & 9 & $15-25$ & b & - & - & $\begin{array}{l}-1.5 .4 \\
-5.1 \\
-6.5\end{array}$ \\
\hline 8 & $29 \cdot 035$ & - & $-7 \cdot 0-4 \cdot 2-5 \cdot 0-4 \cdot 2$ & $x, 3-4$ & 9 & $18-24$ & o & - & S. 10 & $\begin{array}{l}-4 \cdot 7 \\
-5.6\end{array}$ \\
\hline 10 & $29 \cdot 045$ & - & $-5.5-3 \cdot 0-3 \cdot 8-3 \cdot 6$ & $N, 0-1$ & 11 & $0-5$ & 0 & - & s. 10 & $\begin{array}{l}=2.9 \\
=3.8\end{array}$ \\
\hline Miat. & $29 \cdot 0 \div 3$ & - & $-4 \cdot 9-3 \cdot 8-4 \cdot 0-4 \cdot 0$ & $N, 1-2$ & 4 & 8-10 & o & - & S. 10 & $\begin{array}{l}=4.0 \\
-\quad 5.3 \\
\end{array}$ \\
\hline$\overline{\text { Iean }}$ & $28 \cdot 981$ & - & $--10 \cdot 9-1-$ & - & $\overline{-}$ & - & - & - & - & 一 \\
\hline
\end{tabular}

Very falut but extensive aurora extending in ond pateles clianging. 4 a.mln. Extremelv faint aurora, $\mathrm{X}$, NNR, and $\mathrm{B}$; Str. cloud a.m. Two very faiut auroral streamers to the 1 , rising to $15^{\circ}$ alt.

10 a.m. Temperature falling raptlly during olservation; aspirator below scale ; extensive refraetion all round; Ci.-s. to $W$, and all along to tine red s glow in clouds : Fr.

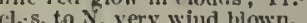
xoon. Refraction all eleared awar visitility stll marked wlut-ilown $C_{1}-8$. to $\mathbf{N}$ and NW : western inountains clear; a thin streak of Str. slout 1200 feet alt t. ; fine recj colouring, violet and reddish glow to $\mathrm{S}$; wluk was calm at.
nown, and then rose from $\mathrm{E}$. Xom. Min. temp. at Cape Armitage, $-38^{\circ} 0:$ liglit
FSlity airs. b. Firetuis smoke FSlily airs. b. Rirethus smoke
gouing $\mathrm{XV}$; some Cl.-8., Fr.-s., and $\mathrm{Str}$. In $\mathrm{X}$ and NL. 2 p.m. Ci. to NW. 4 p.m. Temperature fell a degree whilst reading thermometers. 4 p.m. Min. temp. at Cape Armitage, $-32^{\circ} \cdot 1 ;$ NWly airs; clouds travclling Siy fast; read wlthont a lamp. 8 p.m. Completely overcast. 10 p.m. Completely overcast. Midt. Stars dimly visible in zenith. Fine most of the day,
unstearly and still ligh.

15th.

\begin{tabular}{|c|c|c|c|c|c|c|c|c|c|c|}
\hline 2 & $29 \cdot 111$ & - & $-4 \cdot 6-3 \cdot 2-3 \cdot 6-4 \cdot 3$ & Lt. $S$ alrs & 4 & $0-2$ & $o *$ & - & N. 10 & $-4 \cdot 4$ \\
\hline 4 & $29 \cdot 131$ & - & $-6 \cdot 0-3 \cdot 2-3 \cdot 9-3 \cdot 0$ & Var. E, 1-2 & 5 & $10-15$ & $0 *$ & - & N. 10 & $\begin{array}{l}-4.6 \\
-3.6\end{array}$ \\
\hline 6 & $29 \cdot 137$ & - & $-4 \cdot 5-3 \cdot 2-3 \cdot 8-3 \cdot 5$ & Var. F, 2-3 & 13 & $10-20$ & bm* & - & - & $\begin{array}{l}3.6 \\
=4.0\end{array}$ \\
\hline 8 & $29 \cdot 149$ & - & $-9 \cdot 5-9 \cdot 0-9 \cdot 0-9 \cdot 0$ & ENE, 3-4 & 17 & $15-20$ & be & - & S.-cu. 2 & - \\
\hline 10 & $29 \cdot 197$ & - & $-9 \cdot 5-3 \cdot 6-4 \cdot 1-3 \cdot 8$ & Lt. var. E airs & 13 & $0-8$ & be & Fr.-a.-s. 3, SW & - & $\begin{array}{l}-4.4 \\
-4.7\end{array}$ \\
\hline Non & $29 \cdot 209$ & $-17 \cdot 5$ & $-13.7-6.8-7.0-6.5$ & Very I1.. E airs & 5 & 0 & be & 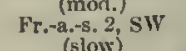 & - & -1 \\
\hline 2 & $29 \cdot 213$ & - & $-10.0-6.0-7.0-6.0$ & Lt. var. E airs & 10 & $0-5$ & b & $\stackrel{\text { (siow) }}{-}$ & - & $\begin{array}{l}-6.9 \\
-8.0\end{array}$ \\
\hline 4 & $29 \cdot 219$ & $-18 \cdot 8$ & $-19 \cdot 0-7 \cdot 2-8 \cdot 0-7 \cdot 2$ & ENE, 2-3 & 3 & $20-25$ & b & - & - & $\begin{aligned} & =7.6 \\
- & 8.5\end{aligned}$ \\
\hline 6 & $29 \cdot 231$ & - & $-15 \cdot 2-12 \cdot 5-13 \cdot 0-13 \cdot 8$ & E, $0-1$ & 8 & $3-12$ & bШ & - & - & $\begin{array}{l}-13 \cdot 7 \\
-14 \cdot 1\end{array}$ \\
\hline 8 & $29 \cdot 228$ & - & $-13 \cdot 5-5 \cdot 0-6 \cdot 2-5 \cdot 0$ & L.t. $\mathbf{N}$ airs & 3 & $5-8$ & b & - & - & $\begin{array}{l}=5.8 \\
-6.9\end{array}$ \\
\hline 10 & $29 \cdot 239$ & - & $-16 \cdot 0-12 \cdot 0-12 \cdot 0-12 \cdot 0$ & Calm & 1 & 0 & b & - & - & $\begin{aligned} & 9 \cdot 8 \\
&-10 \cdot 1\end{aligned}$ \\
\hline MIdt. & $29 \cdot 229$ & - & $-11 \cdot 5-3 \cdot 0-2 \cdot 8-3 \cdot 2$ & $N, 2-3$ & 4 & $12-16$ & b & - & - & $\begin{aligned} & 2 \cdot 7 \\
&- 3 \cdot 7 \\
&\end{aligned}$ \\
\hline Meau & $\overline{29 \cdot 191}$ & - & $-6.3-6.7-$ & - & $\overline{-}$ & - & - & - & $\overline{-}$ & - \\
\hline & Max. t & & 象 $\{8$ & tination & & & & 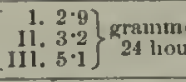 & & \\
\hline
\end{tabular}

Sunw in very small flakes, falling fast. unsteady. Slight isnow; stars rlslble in zenith.

Str. all along, alout $8^{\circ}$ broat, from SE to WSW; some peculiar curved Ci,-a in in various parts of the heavens below $20^{\circ}$ ait. ; fite colouring ln sky to $\mathbf{N}$ and $\mathrm{S}$; read ol olservations wltbont lamp?. Noon. Ci.-s. clouds disappearing; niso Str. lu S appears to be fading out Noonl. Nint. templs at Cape Armitage, $-223^{\circ} 5$. Calm.
bev. Ci.s. 1. Wretius amoke going XNW. NW.

2 p.m. Cil. to NW. liorizon.

p.n. Min. temp. at Cape 1:Nk, 1, b. C1. 1. Trebus smoke indistinct, seems to be going s'y and lity.

B prin. Meteor, from near z.pnith, fell southwards. Light varlable airs to a fresh breeze all day; cloudy carly, but elearing later, Harometer rose most of the 6h prin. Meteor, from near zenith, fell southwards. Light varlalle airs
day, unsteady in evenlug. Temperature highl and stlll very fuctuatlng. 
Meteorologicat. Journat, of th: "Discovery."

[1903-August.

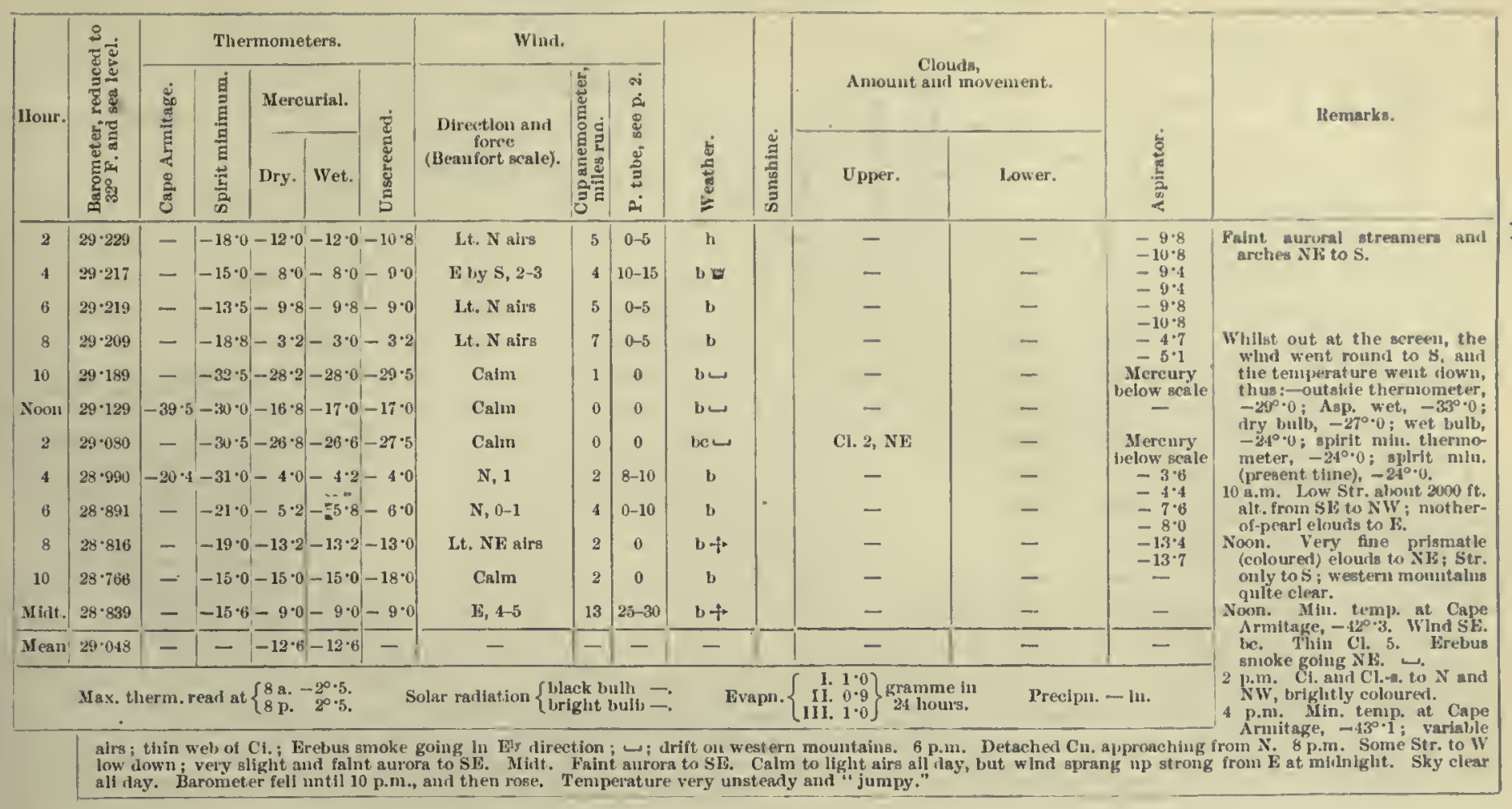

\begin{tabular}{|c|c|c|c|c|c|c|c|c|c|c|}
\hline 2 & $28 \cdot 907$ & - & $-11 \cdot 5-7 \cdot 0-7 \cdot 0-7 \cdot 0$ & SSE, 5- & 18 & $30-40$ & bq + & - & - & - \\
\hline 4 & $28 \cdot 947$ & - & $-11 \cdot 5-9 \cdot 0-9 \cdot 0-9 \cdot 0$ & ESE, 5-6 & 22 & $30-35$ & $b+w$ & - & - & - \\
\hline 6 & $28 \cdot 951$ & - & $-15 \cdot 0-13 \cdot 0-13 \cdot 0-13 \cdot 5$ & ENF, 4-5 & 18 & $25-30$ & $b+f$ & - & - & $\therefore$ \\
\hline 8 & $29 \cdot 015$ & - & $-13 \cdot 5-10 \cdot 0-10 \cdot 0-10 \cdot 0$ & ENE, 4-6 & 23 & $25-35$ & $n+4$ & $\mathrm{Cl}_{.-8 .} 4$ & - & - \\
\hline 10 & $29 \cdot 049$ & - & $-18 \cdot 2-16 \cdot 0-16 \cdot 2-16 \cdot 0$ & E, $4-6$ & 17 & $25-33$ & be & Ci.-s. 3 (? dir.) & - & $\begin{array}{l}-16 \cdot 1 \\
-16 \cdot 6\end{array}$ \\
\hline Noon & $29 \cdot 075$ & $-25 \cdot 8$ & $-20 \cdot 0-19 \cdot 5-19 \cdot 5-19 \cdot 8$ & ENE, G-7 & 10 & $30-42$ & ineq & $\begin{array}{l}\text { Ci-s. } 5, \text { SW } \\
\text { (very slow) }\end{array}$ & - & $\begin{array}{l}-19 \cdot 8 \\
-19.8\end{array}$ \\
\hline 2 & $29 \cdot 107$ & - & $-20 \cdot 2-15 \cdot 3-16 \cdot 0-16 \cdot 0$ & Var. E, 3-6 & 31 & $25-40$ & beq & Ci.-s. 3, WV & - & $-16 \cdot 2$ \\
\hline 4 & $29 \cdot 137$ & $-23 \cdot 5$ & $-16 \cdot 2-14 \cdot 5-15 \cdot 0-13 \cdot 8$ & Var. E, 3-4 & 20 & $20-30$ & be & Ci.-8. 2 (? alir.) & - & $\begin{array}{l}-14.0 \\
-1.4\end{array}$ \\
\hline 6 & $29 \cdot 153$ & - & $-16 \cdot 3-15 \cdot 0-15 \cdot 5-16 \cdot 0$ & Var, E, 4-6 & 23 & $28-12$ & bq & - & - & $\begin{array}{l}-11.4 \\
-15.5 \\
-16.2\end{array}$ \\
\hline 8 & $29 \cdot 220$ & - & $-19 \cdot 5-19 \cdot 0-19 \cdot 0-19 \cdot 6$ & NE, 2-5 & 20 & $10-25$ & bq & - & - & -19.8 \\
\hline 10 & $29 \cdot 220$ & - & $-20 \cdot 0-11 \cdot 8-12 \cdot 0-13 \cdot 2$ & NNE, 4-5 & 15 & $20-25$ & b & - & - & $\begin{array}{l}-10.0 \\
-10.1\end{array}$ \\
\hline Minit. & $29 \cdot 249$ & - & $-21 \cdot 2-15 \cdot 2-16 \cdot 0-16 \cdot 2$ & NE, 4-5 & 13 & $20-25$ & b & - & - & $\begin{array}{l}-10.1 \\
-15.5 \\
-15.5 \\
\end{array}$ \\
\hline$\overline{1 \mathrm{ean}}$ & $29 \cdot 083$ & - & $--13 \cdot 8,-14 \cdot 0-$ & - & - & - & - & - & - & - \\
\hline
\end{tabular}

17 th.

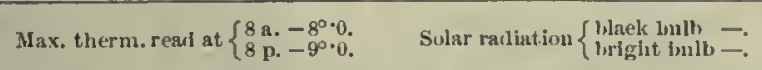

Evagn. $\left\{\begin{array}{r}\text { 1. } 2 \cdot 4 \\ \text { II. } 2 \cdot 5 \\ \text { III. } 3 \cdot 3\end{array}\right\}$ grammes in 24 liours.

Irecipn. - in.

Some fine eolouring on elouds to $W$; they look like S.-cu. 11 a.n. Fine prismatie ing on elouds to NE. Noon. Very fine prismatic
eolourlug in Ci.-s. eloudis heavy surface drift in Stralt western mountains qulte clear; slight $\Lambda_{-s}^{-s}$. to $W_{\text {anil }}$ SW.

Nonil Min. temp. at Cape by 1i, 2-i. Ci.-8. b. 1c. Fine pirismatic colouring. Brebus sic.). Fo to

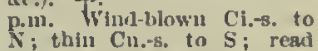
observations wit hout lamp. 4 1.m. Wind less squally; A.-B. to N. Nin. temp. at Cape
p.m. Min.

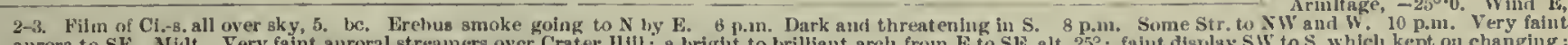

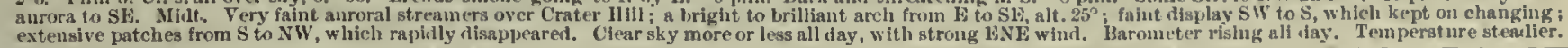

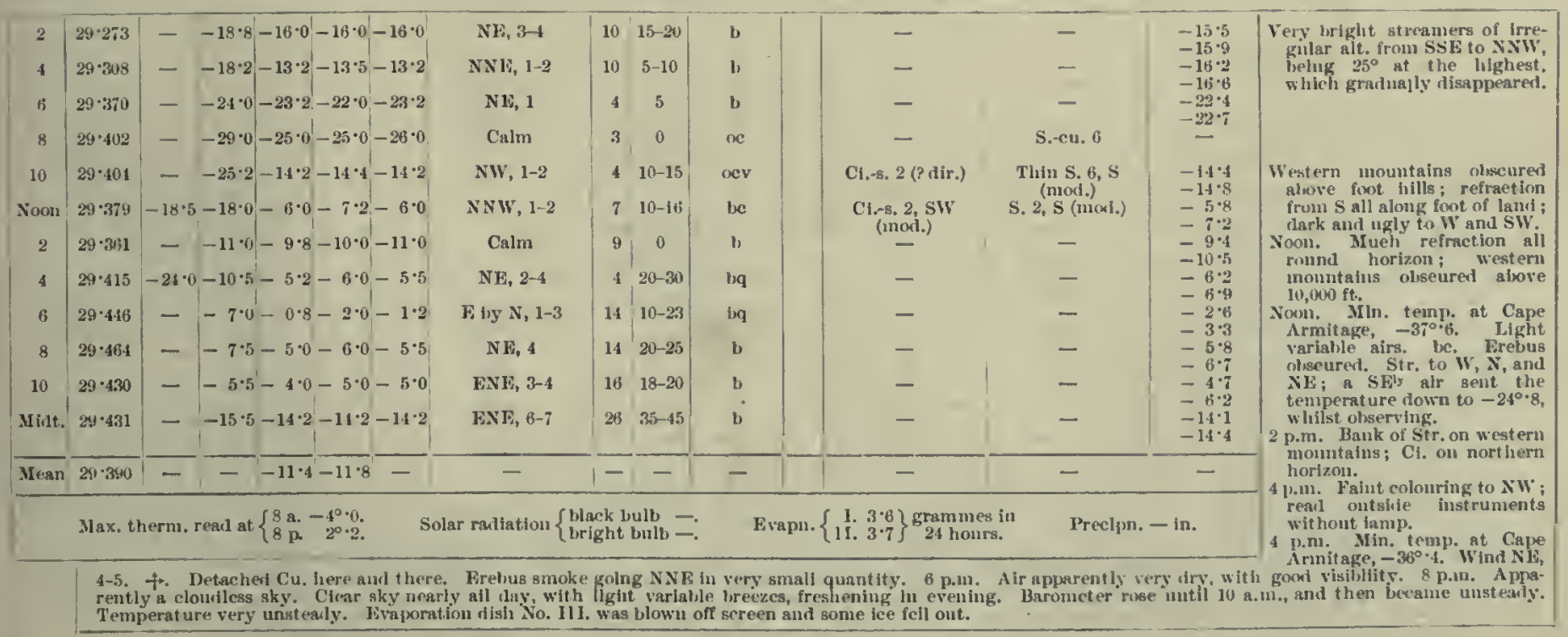

18 th. 

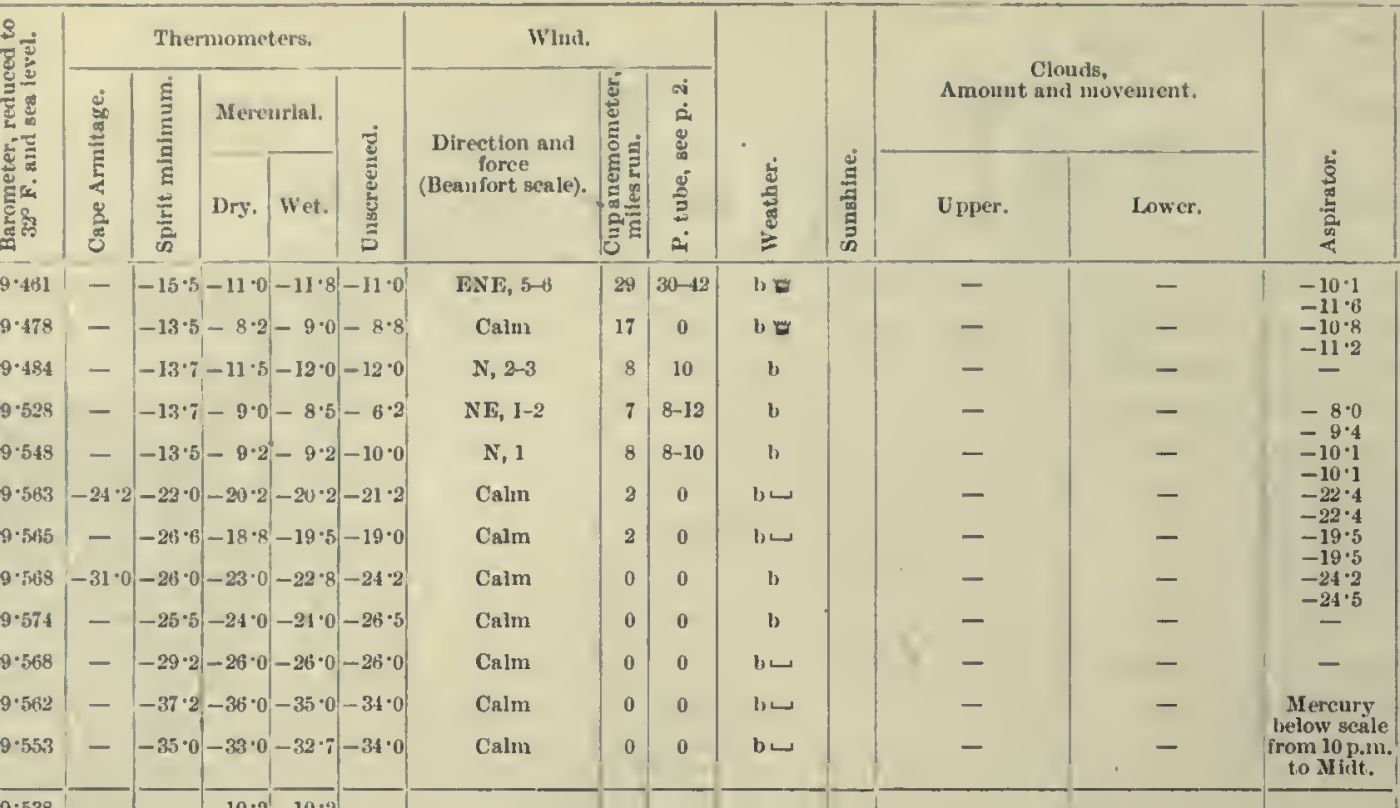

Cloudless sky, with very finc culourlng ; western inon! tains clear: refract Noon. Shadow to S very markm to an angle of $17^{\circ}$

voon. Min, temp, at Cape $A r$ initage, $-32^{\circ} \cdot 4$. Calun. Erelns smoke going to bN 1: or NE ly S, tirst rising lit small column 500 to 1000 teet. (Earth shadow to $S$, also angular shadow rising to $\mathbf{L}$ from at angle of $17^{\circ}$.)

$10|29 \cdot 562|-\quad-37 \cdot 2-36 \cdot 0-35 \cdot 0-34 \cdot 0$

Midt. $29 \cdot 553--35 \cdot 0-33 \cdot 0-32 \cdot 7-34 \cdot 0$

Calm

Calm

Calm

m. lime formin

Mean $\overline{29 \cdot 539}-\overline{-} \overline{-19 \cdot 2}-19 \cdot 2-\overline{-}-\overline{-}$

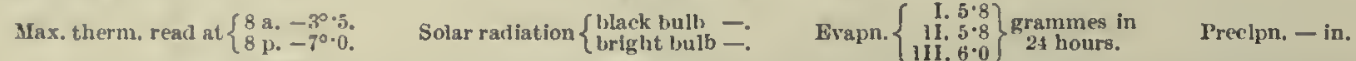
p.m. Outside thernorncter puch affected by aolar raliation. Could read outside therinometer and anemonicter without lamp. W.om. Min. teinp. at Cape Armitage, $-37^{\circ} 0$. Wlud bise, 1-2. b. Frehns smoke going NE; refractlon to NW. 6 p.m. Colouring in western sky ; temperature fell $2^{\circ}$ whilst oluserving. 8 p.in. lleavy deposit of rime on then fell. Temperatire fell quiekly towards the cvening.

20 th

\begin{tabular}{|c|c|c|c|c|c|c|c|c|c|c|c|}
\hline 2 & $29 \cdot 534$ & - & $-38 \cdot 0-3 \cdot 4^{\cdot} 0$ & $-34 \cdot 0-34 \cdot 0$ & Calm & 0 & 0 & $b \sqcup$ & - & - & \\
\hline 4 & $29 \cdot 530$ & - & $-35 \cdot 0-34 \cdot 0$ & $-34 \cdot 5-34 \cdot 0$ & Calm & 0 & 0 & bし & - & - & ⿷匚 \\
\hline 6 & $29 \cdot 499$ & - & $-37 \cdot 5-30 \cdot 0$ & $-30 \cdot 0-20 \cdot 5$ & Calm & 0 & 0 & bu & - & - & $\begin{array}{l}\infty \\
\S\end{array}$ \\
\hline 10 & $29 \cdot 143$ & - & $-39 \cdot 0-33 \cdot 0$ & $-33 \cdot 0-35 \cdot 0$ & Calm & 0 & 0 & bu & - & - & 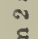 \\
\hline Noon & $29 \cdot 398$ & $-45 \cdot 6$ & $-39 \cdot 2-37 \cdot 8$ & $-37 \cdot 0-39 \cdot 0$ & Calm & 0 & 0 & bu & - & - & है \\
\hline 4 & $29 \cdot 352$ & $-49 \cdot 8$ & $-46 \cdot 5-46 \cdot 0$ & $-45 \cdot 0-47 \cdot 0$ & Calm & 0 & 0 & bm七 & - & - & 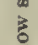 \\
\hline 6 & $29 \cdot 315$ & - & $-46 \cdot 6-39 \cdot 0$ & $-38 \cdot 2-42 \cdot 0$ & Calm & 0 & 0 & bmu & - & - & ప \\
\hline 8 & $29 \cdot 298$ & - & $-42 \cdot 5-39 \cdot 0$ & $-38 \cdot 0-41 \cdot 0$ & Calm & 3 & 0 & bn $\iota$ & - & - & ? \\
\hline 10 & $29 \cdot 291$ & - & $-42 \cdot 5-21 \cdot 2$ & $-\quad-21 \cdot 8$ & Very lt. $\mathbf{N}$ airs & 0 & 0 & bmu & - & - & $\frac{\mathrm{L}}{\mathrm{V}}$ \\
\hline Midt. & $29 \cdot 290$ & - & $-30 \cdot 5-20 \cdot 2$ & $-20 \cdot 4-21 \cdot 8$ & Calm & 2 & 0 & hus & - & - & \\
\hline
\end{tabular}

Sverythling covered with rime took the observat ions wit hout

Str. on westèrn mountains and to $S$ at various altitudes. oon. Min. temp. at Crpe $\Lambda \mathbf{r}$ bus smoke Refraction to $\mathrm{N}$.

2 p.m. Str. on western mountains, ahout 6000 feet up, an all to $\mathrm{S}$.

p.m. Accumnlation of $f \circ g$ crystals, fern shaped, forming on $\mathbf{H}$ and $\mathbf{N}$ slite of thlings very misty to $\mathrm{k}$; large sheet of Str. to W; hase and summits of monntains visible.

p.m. Min, temp. at Cape ArMax. therm. read at $\left\{\begin{array}{l}8 \mathrm{a},-22^{\circ} 0 . \\ 8 \mathrm{~F},-21^{\circ} 0 .\end{array} \quad\right.$ Solar radiation $\left\{\begin{array}{l}\text { black bnlb } \\ \text { bright bulb }- \text {. }\end{array}\right.$

Evapn. - grammes.

Preclpn. - in. Frebus sinoke going $N$; foggy all round.

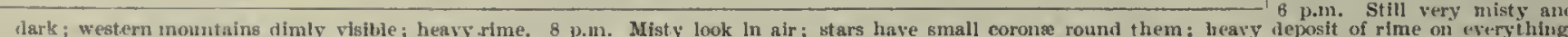

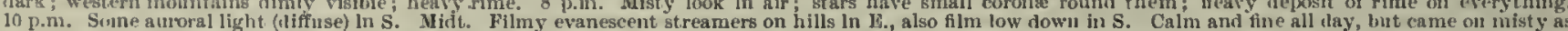
nsual with the colder temperature. Barometer falling all day. Temperature much lower, but rose quickly in the eveulng.

21st.

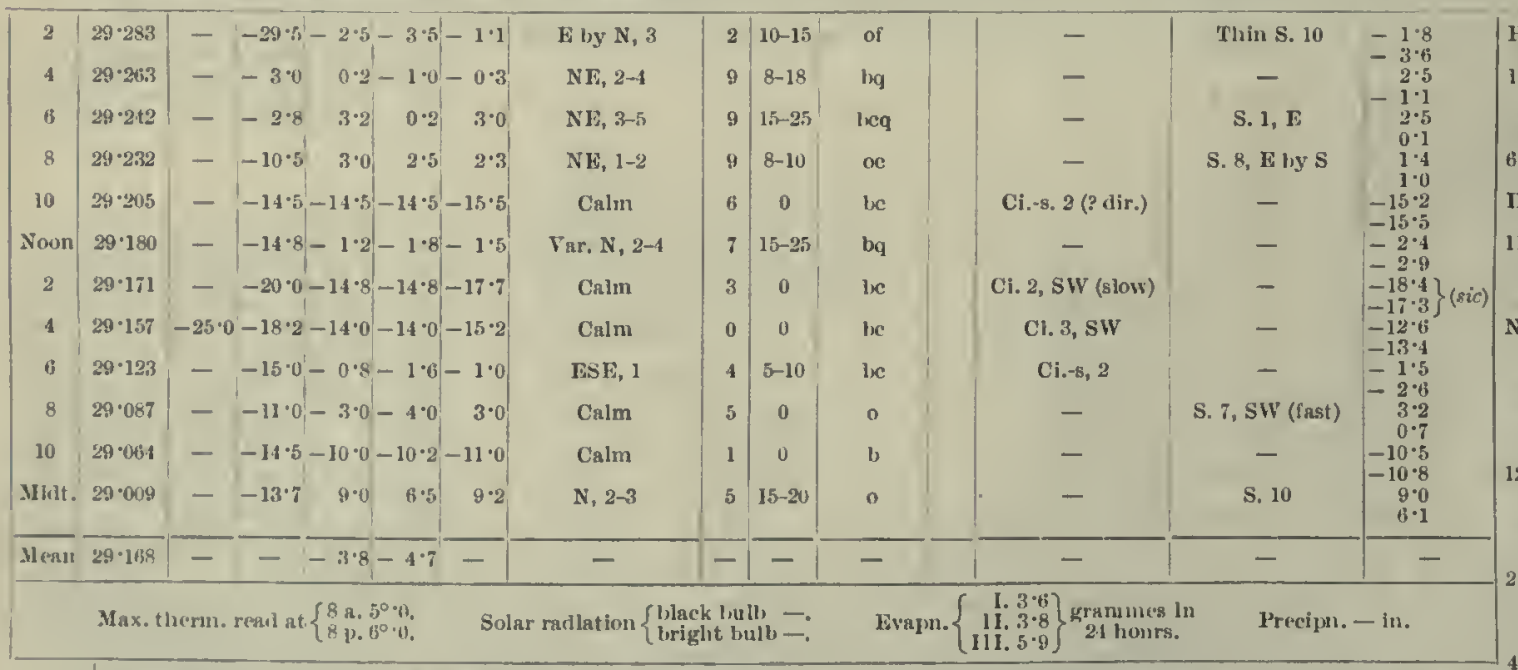

Patch of aurora in S, extemling along horizon and 20 alove it. 1 - defined and evanescent streamers and films of light extenting alove hils a.n. Sone low sir. to

nill look to $S$ and $S W$, and visibility marked.

.45 a.m. Temp. at Cape ArWind NE, 2-3. smoke roing NEly.

Noon. Wind very variable force and direction, at timges falling calm, and then suringing from $\mathrm{E}, \mathrm{S}$ or $\mathrm{N}$, and bloning strongly; temperature fell $4^{\circ}$ whllst olserving; Str. to $S$ and $\mathbf{E}$.

2.50 p.m. Temp. at Cape Armitage, $-18^{\circ} \cdot 2$; nin., $-20^{\circ} \cdot 5$

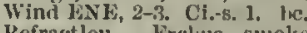
Refractlon, lirelus smok going $\mathbf{N}$ and varialile.

p.m. Faintly eolonred Ci. to S, detaches portlons over whilst olserving.

p.I11. Falnt columring on

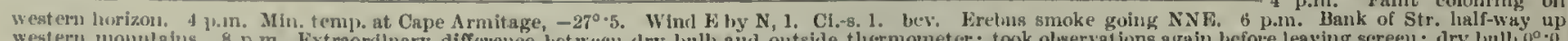

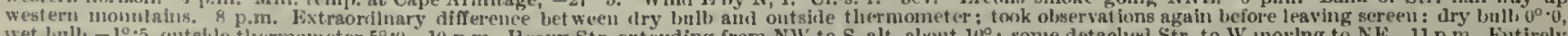

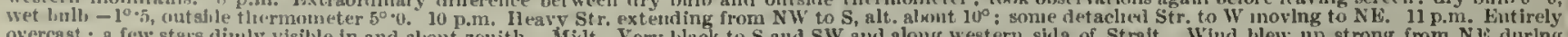

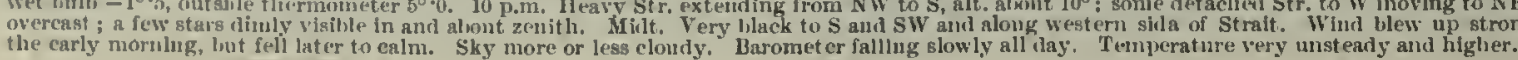


Metborological Journat, OH THE "Discoviry."

[1903-August.

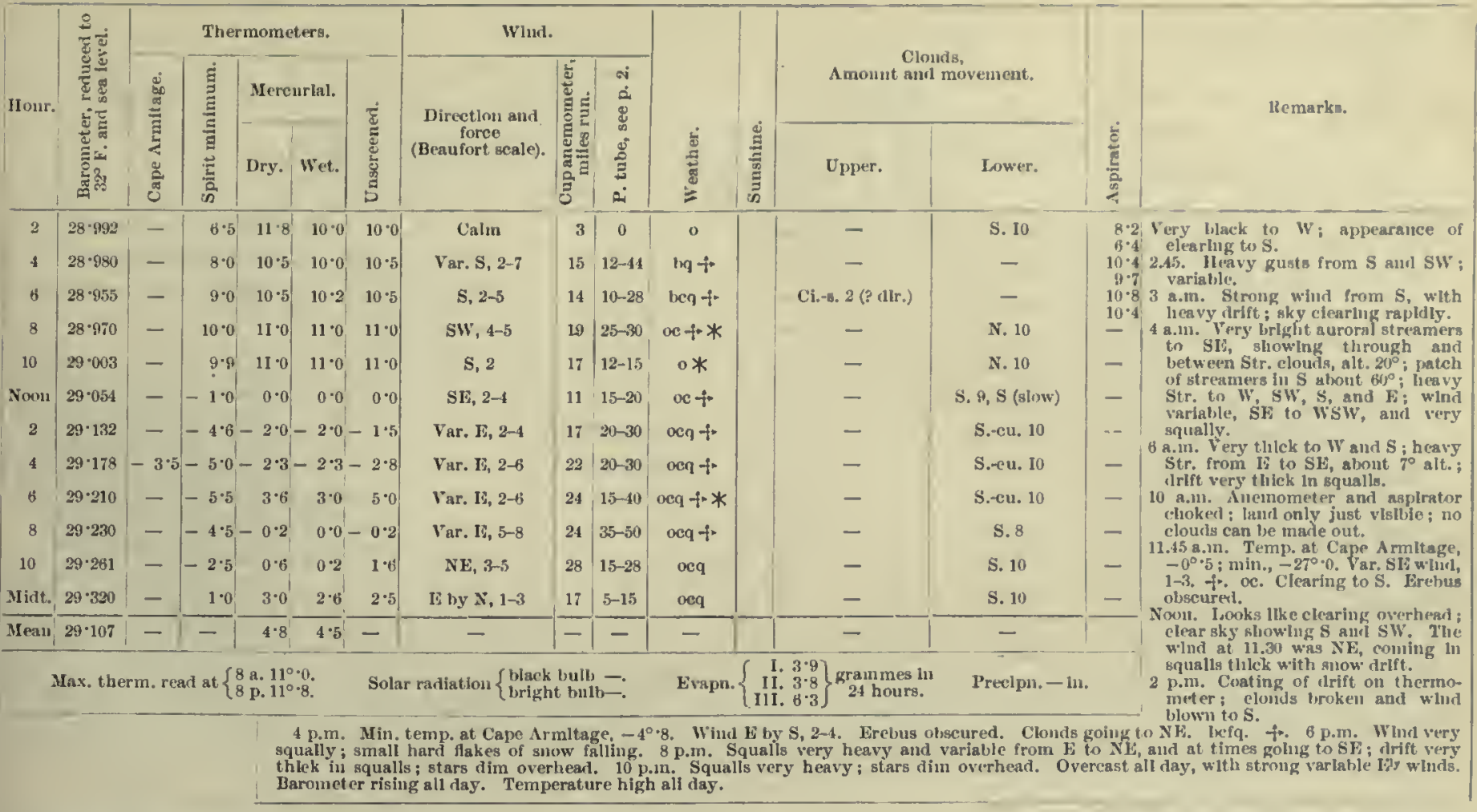

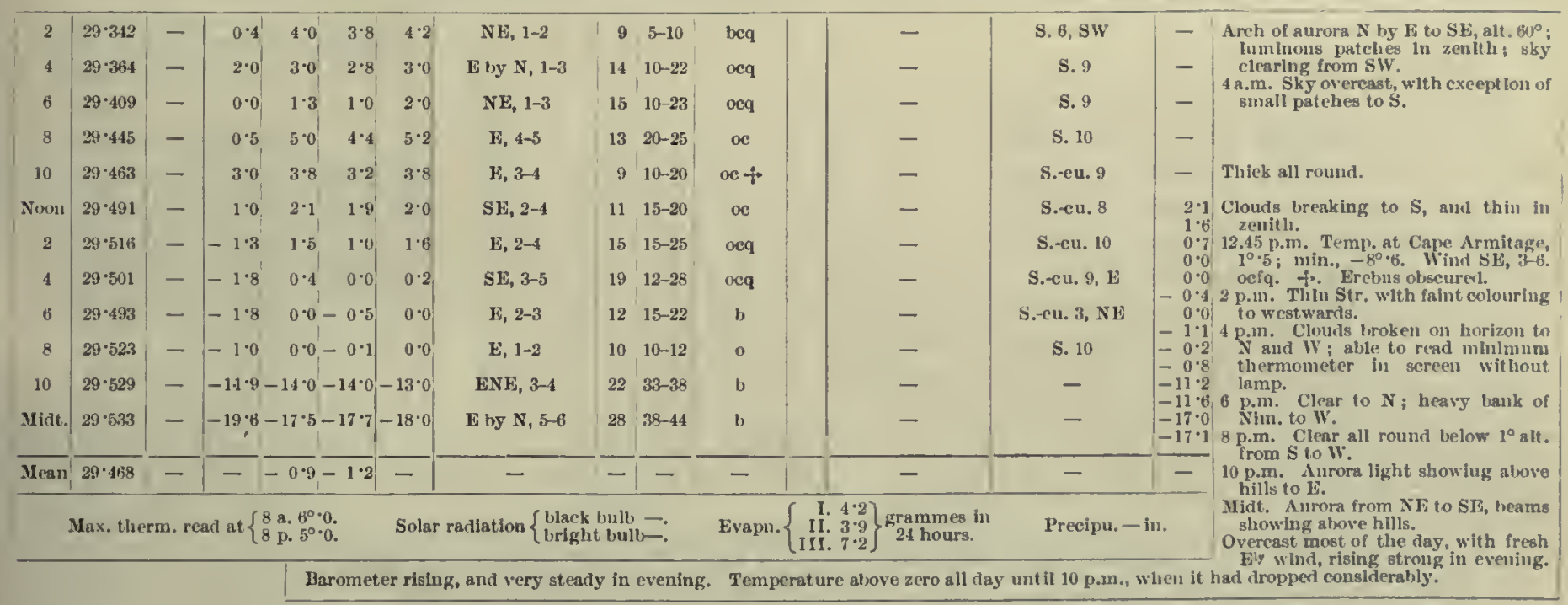

23rd.

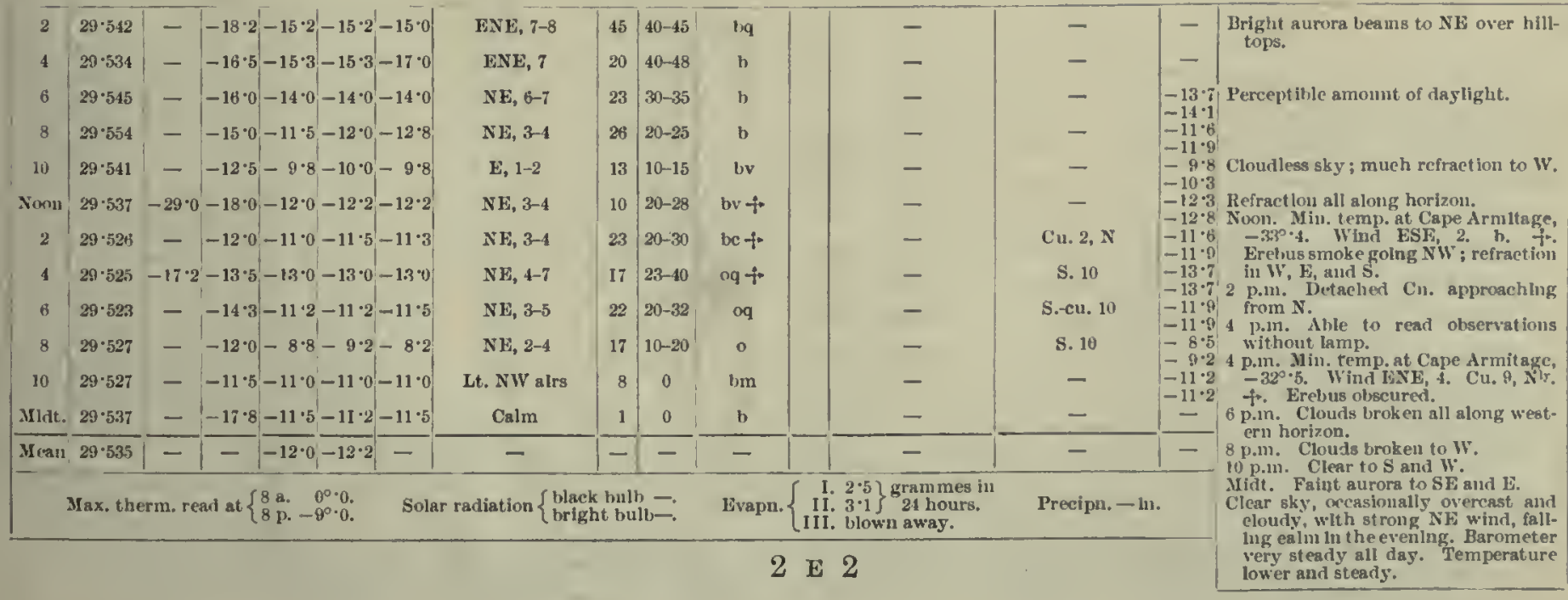



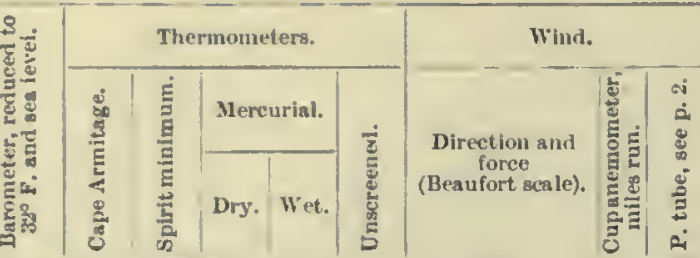

25 th.

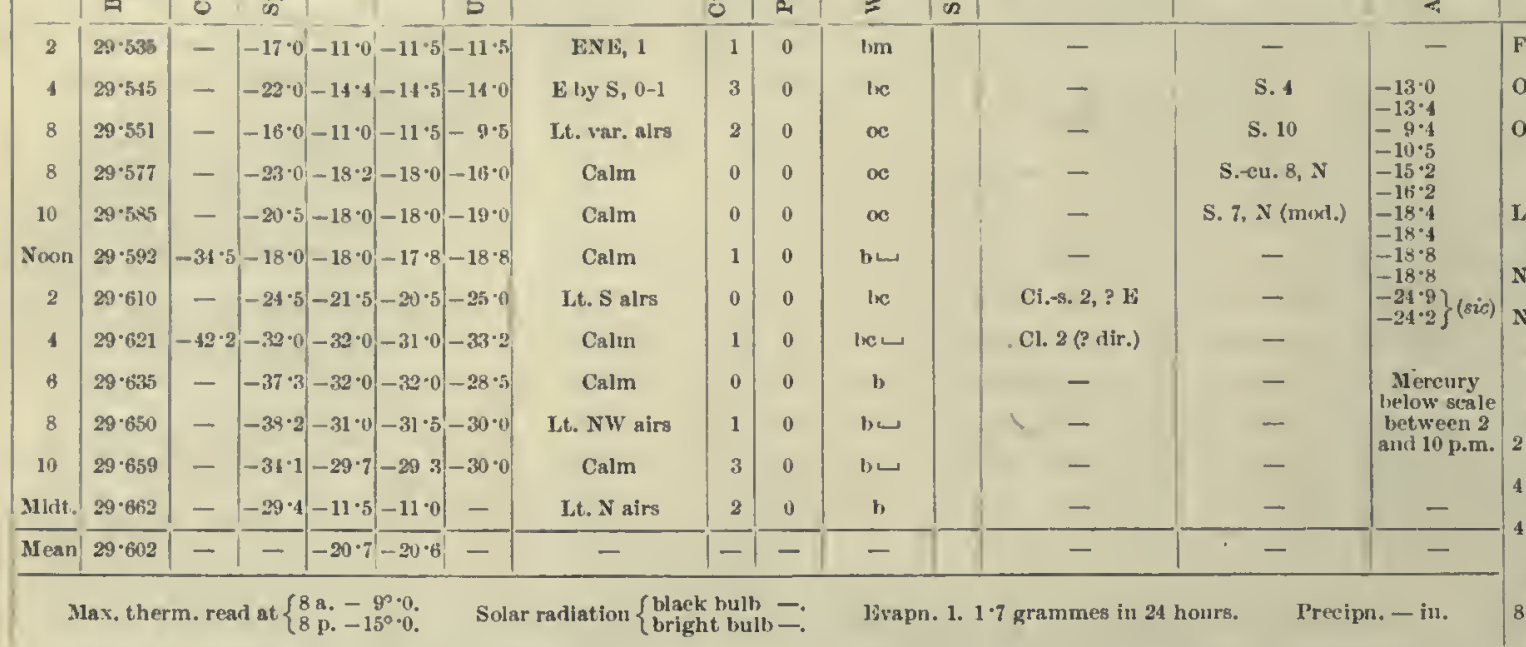

\begin{tabular}{|c|c|c|c|c|c|}
\hline \multirow[b]{2}{*}{ 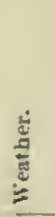 } & \multirow{2}{*}{ 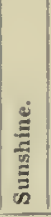 } & \multicolumn{2}{|c|}{$\begin{array}{l}\text { Clouds, } \\
\text { Amount and inovement. }\end{array}$} & \multirow[b]{2}{*}{$\frac{\text { है }}{\frac{5}{2}}$} & \multirow{2}{*}{ liemarks. } \\
\hline & & Upper. & Lower. & & \\
\hline $\mathrm{lm}$ & & - & - & - & Falnt aurora to $\mathrm{S}$, alt. $40^{\circ}$. \\
\hline le & & - & S. 4 & $\begin{array}{l}-13 \cdot 0 \\
-13 \cdot 4\end{array}$ & Only a few stars visible. \\
\hline oc & & - & S. 10 & $\begin{array}{l}-9.4 \\
-9 \cdot 4 \\
-10.5\end{array}$ & $\begin{array}{l}\text { Overcast, except low down to } \\
\text { W and } S \text {. }\end{array}$ \\
\hline oc & & - & S. -cu. $8, \mathrm{~N}$ & $\begin{array}{l}-15 \cdot 2 \\
-16 \cdot 2\end{array}$ & \\
\hline$\infty$ & & - & $\begin{array}{c}\text { S. } 7, \mathrm{~N} \text { (mod.) } \\
-\end{array}$ & $\begin{array}{l}-18 \cdot 4 \\
-18 \cdot 4 \\
-15 \cdot 8\end{array}$ & 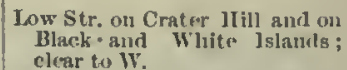 \\
\hline lic & & Ci.-s. 2, ? E & - & $\left\{\begin{array}{l}-18 \cdot 8 \\
-24.9 \\
-24.2\end{array}\right\}$ (sic) & 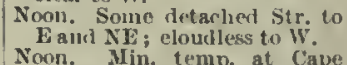 \\
\hline はே匕 & & Cl. 2 (? dir.) & - & & 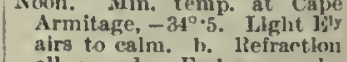 \\
\hline b & & - & - & $\begin{array}{l}\text { Mereury } \\
\text { below seale } \\
\text { between }\end{array}$ & all round. Frebus smoke \\
\hline bப & & - & - & and 10 p.m. & 2 p.m. Temperature falling \\
\hline b & & - & - & - & 4 pime on ont to to liste and is \\
\hline- & & - & - & - & Armitage, - $42^{\circ}$. Calm. \\
\hline liva & & ammes in & Pre & . - in. & 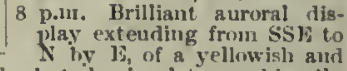 \\
\hline
\end{tabular}

greenish colour. 10 p.m. Brilliant starlight; no elouds on the horizon; light westerly airs sprang up as I eame ln. Overcast early, but clearing later, aud lani ally wind all day. Barometer rising slowly all day. Tenperature lower, but not at all steady.

26th.

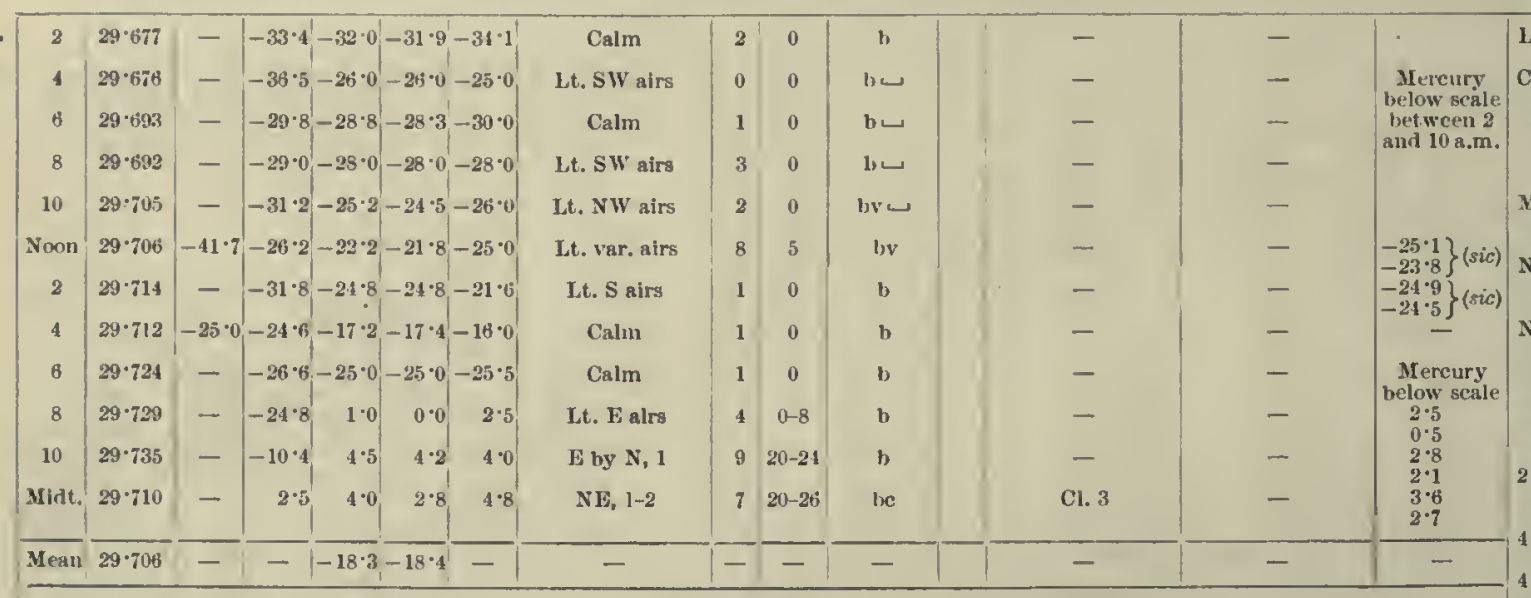

Low arches to Eaud S.

Max. tlierm. rear at $\left\{\begin{array}{lr}8 \mathrm{a} . & -9^{\circ} \cdot 0 . \\ 8 \mathrm{p} . & 4^{\circ} \cdot 5 .\end{array}\right.$ Solar radiation $\left\{\begin{array}{l}\text { black bulb } 9^{\circ} \cdot 5 . \\ \text { hright bulb } 5^{\circ} \cdot 0\end{array}\right.$

Evapn. 11. 0.2 gramme in 24 hours.

Precipı. - in.

Curtain rising tozenith from $\mathrm{X}$. arches in all parts of $E$, arch, and $N$ ls, at all altituctes,

Much refraction all round horizon to W and S; visibility

very marked.
Toon. Tenperat ure falling rapilly; mucl refraction; ; visilility very marken. oonl. Min. temp. at Cape Armilage, $-45^{\circ} \cdot 2$. light jis alra, b. Refraetion all round.
lirebus smoke, ill large volume, rising some 1000 to 2000 feet, and theu going in pur direction.

western and soutbring on zous and in sky.

p.sn. Solar therinometer read for tirst time since placeri. p.m. Min. temp. at Cape Armitage, $-43^{\circ} \cdot 0$. Calm. 1 .
lrebus smoke ln large volime rising some 1000 feet, and tlienl going Nibly very grest

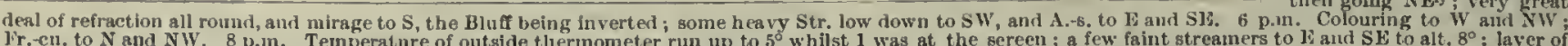

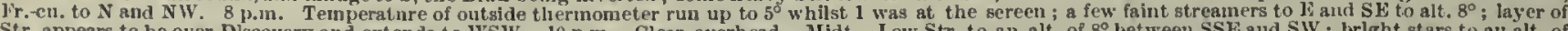
Str. appears to be over Dlscovery and extends to WSW. 10 p.m. Clear overhead. Mitdt. Low Str. to an alt. of $8^{\circ}$ between SSE and SW, brlght stars to ant alt. of
$60^{\circ}$ between NE and $W$ appeared to le shining through a mist - ? Ci. 3. Clear sky all day, wlth no wlud. Barometer rising slowly nearly all day. Temperature $61^{\circ}$ between $\mathrm{NE}$ and $W$ appeared to he shining th
lower early, rising up sudderly iu the evening.

27th.

\begin{tabular}{|c|c|c|c|c|c|c|c|c|c|c|c|}
\hline 2 & $29 \cdot 683$ & - & $-2 \cdot 0-0.3-1 \cdot 0$ & 0.5 & NE, 3 & 21 & $25-40$ & be $=$ & Ci.-s, 3, Ci. 4 & - & $\begin{array}{r}0.0 \\
-0.0\end{array}$ \\
\hline 4 & $29 \cdot 681$ & - & \begin{tabular}{l|l|l|}
-2.7 & 1.1 & 0.1
\end{tabular} & $1 \cdot 5$ & $\mathrm{NE}, 2-3$ & 24 & $25-28$ & $b e+$ & Cl.-в, 6, Cl. 2 & - & $\begin{array}{l}-0.8 \\
1.8\end{array}$ \\
\hline 6 & $29 \cdot 673$ & - & $\begin{array}{lll}-0.2 & 0.2 & -0.1\end{array}$ & 0.0 & $N F, 3-4$ & 15 & $25-30$ & $\boldsymbol{\alpha c}$ & - & Cu. 9 & -0.4 \\
\hline 8 & $29 \cdot 664$ & - & $-6 \cdot 5-8 \cdot 0-6 \cdot 0$ & $-8 \cdot 0$ & Var. 2-5 & 12 & $10-35$ & oeq & - & A.-cu. 8 & $\begin{aligned} & =7.8 \\
- & -8.0\end{aligned}$ \\
\hline 10 & $29-844$ & - & $-9 \cdot 5-7 \cdot 8-8 \cdot 0$ & $-7 \cdot 8$ & NE, $5-6$ & 21 & $28-40$ & $\infty q$ & - & A.eu. 8 & $\begin{array}{r}-8.0 \\
-8.3\end{array}$ \\
\hline Noon & $29 \cdot 844$ & $-11 \cdot 5$ & $-8 \cdot 5-2 \cdot 5-3 \cdot 0$ & $-2 \cdot 0$ & NE, 3-4 & 13 & $15-20$ & be & Ci. $\mathrm{cu}, 3, \mathrm{SW}$ & - & $\begin{array}{l}-8 \cdot 3 \\
-2 \cdot 2 \cdot 9\end{array}$ \\
\hline 2 & $29 \cdot 686$ & - & $-10 \cdot 0-8 \cdot 8-9 \cdot 0$ & $-8 \cdot 0$ & $\mathrm{~F}, 2$ & 4 & $12-15$ & be & Ci.eu. 1, sw & - & $\begin{aligned} & 8.7 \\
- & 9.0\end{aligned}$ \\
\hline 4 & $29 \cdot 619$ & $-21 \cdot 0$ & $-9 \cdot 7-5 \cdot 0-5 \cdot 2$ & -5.8 & $\mathrm{NK}, 2-3$ & 8 & $18-28$ & lic & Ci.-s. 2, W W & - & $\begin{array}{l}-5.1 \\
-5.8\end{array}$ \\
\hline B & $29 \cdot \overline{6} 82$ & - & $7-12 \cdot 0$ & $-12 \cdot 7$ & $N E, 3-6$ & 10 & $30-40$ & bq & - & - & $\begin{array}{l}-12 \cdot 8 \\
-13 \cdot 0\end{array}$ \\
\hline 8 & $29 \cdot 580$ & - & $-14 \cdot 8-10 \cdot 8-12 \cdot 0$ & -8.0 & NE, 1-4 & 17 & $10-20$ & $\mathrm{bq}$ & - & - & $\begin{array}{l}-9.4 \\
-10.5\end{array}$ \\
\hline 10 & 29.579 & - & $-15 \cdot 1-12 \cdot 0-12 \cdot 2$ & $-9 \cdot 8$ & Lt. $\mathbf{N}$ alrs & 2 & $0-10$ & b & - & - & $\begin{array}{l}-8.0 \\
-8.7\end{array}$ \\
\hline Midt. & $29 \cdot 548$ & - & $-19 \cdot 2-18 \cdot 0-17 \cdot 8$ & $-18 \cdot 0$ & Calm & 1 & 0 & b & - & - & $\begin{array}{l}-18 \cdot 4 \\
-18 \cdot 4 \\
\end{array}$ \\
\hline $\overrightarrow{\text { Mean }}$ & $29 \cdot 629$ & - & $--6 \cdot 8-7 \cdot 2$ & -1 & - & $1-$ & - & - & - & - & - \\
\hline
\end{tabular}

Str. elouds to an alt of $15^{\circ}$ all round; light elouds overliead as at nidnight.

$4 \mathrm{am}$. No stara visible bet ween SSE and NW to an alt. of $60^{\circ}$. light elourds in zenitl ; faint patches of auroral light leetween Nls and SSE during appeared to be dulled by

8 a.m. Wind very variable going all round the conipass. $\mathrm{s} . \mathrm{m}$. Cloudless low down to
$\mathrm{S}$ and on western mountains : valsibility marked.

Youn shy clearing oon. Sky elearing rapiclly marked. Noou. Min. temp. at Cape Armitage, $-33^{\circ}-5$. Wind $\mathrm{l}$ by
$\mathbf{N}, 3$. Cl. cu. 4. bc. Erebus smoke rising 3000 to 4000 feet, and then going to WNW.

2 p.m. Pr.ecu. to S.

4 p.m. Net cor ou eastern lworzoll.

11.n1. Min, temp. at Cape

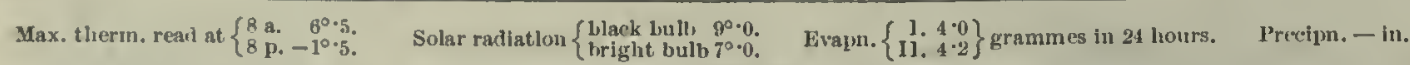
3-4. Cita, $-26^{\circ} \cdot 5$. Wind $\mathrm{Nl} \%$, $3-4.2$ Ci.s. 2. Erehus smoke
rising for 1000 feet, and then

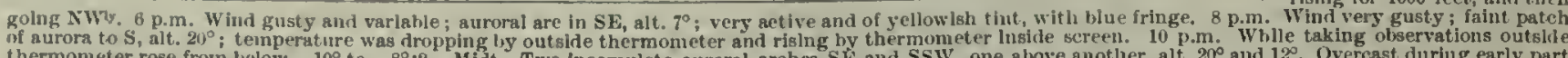
thermometer rose from lelow $-10^{\circ}$ to $-8^{\circ} \cdot 2$. Mint. Two tncomplete auroral arches SI and SSW, one above another, alt. $20^{\circ}$ and $12^{\circ}$. Overcast duriug
of day, but clearing later; wind strolig from Nli, but fell towards the evening. Barometer fell throughout the day. Temperature fell towards evening. 


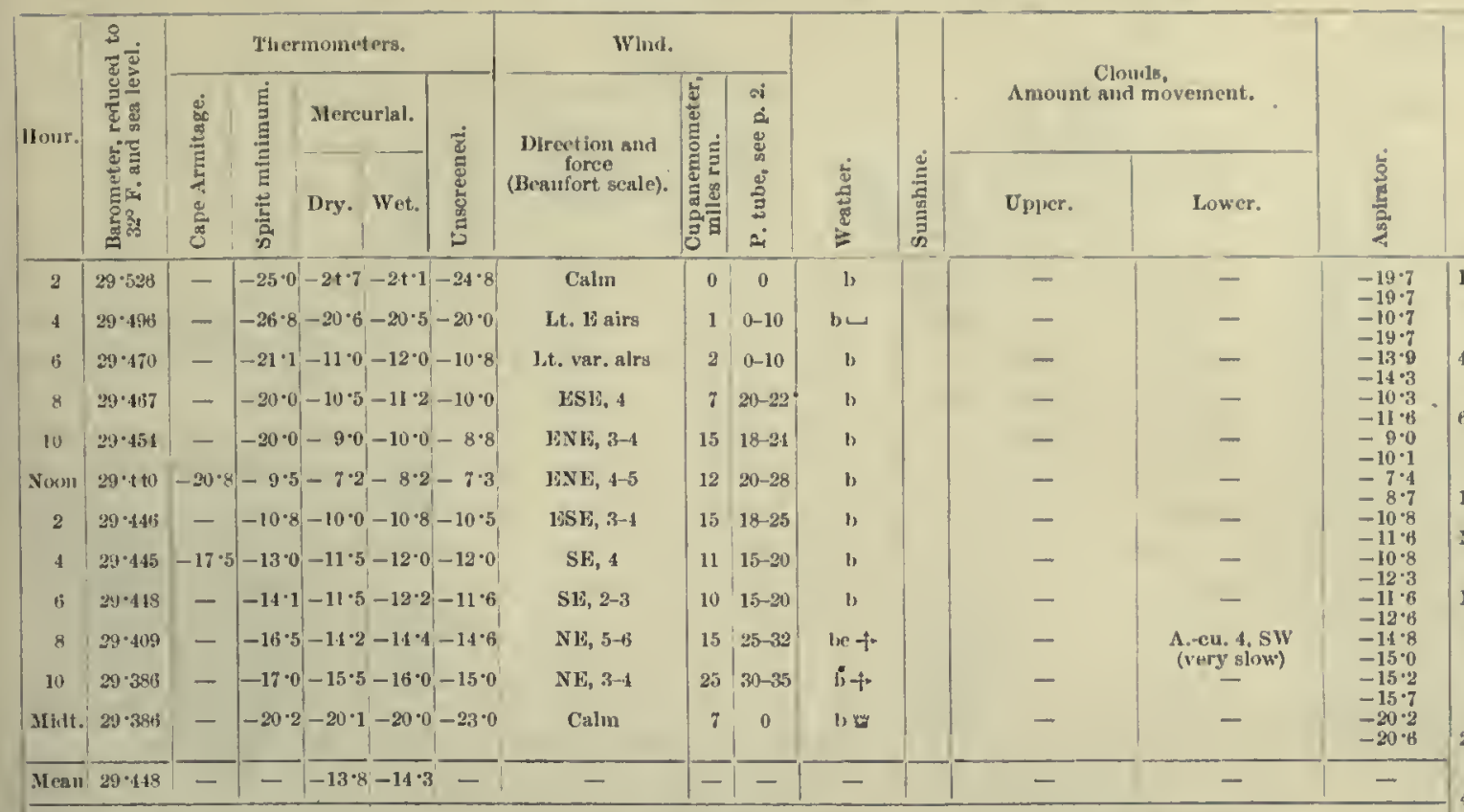

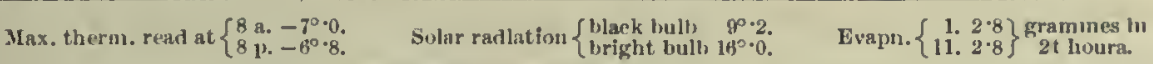

Ireclm. $-1 \mathbf{u}$.

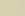

$8 \mathrm{p} . \mathrm{m}$. S.en. approachlug from S and W. $8 \mathrm{pm}$. Clonds moving very slowly indeed, and they are thln. 10 p.m. Small wisps of eloud liemarks.

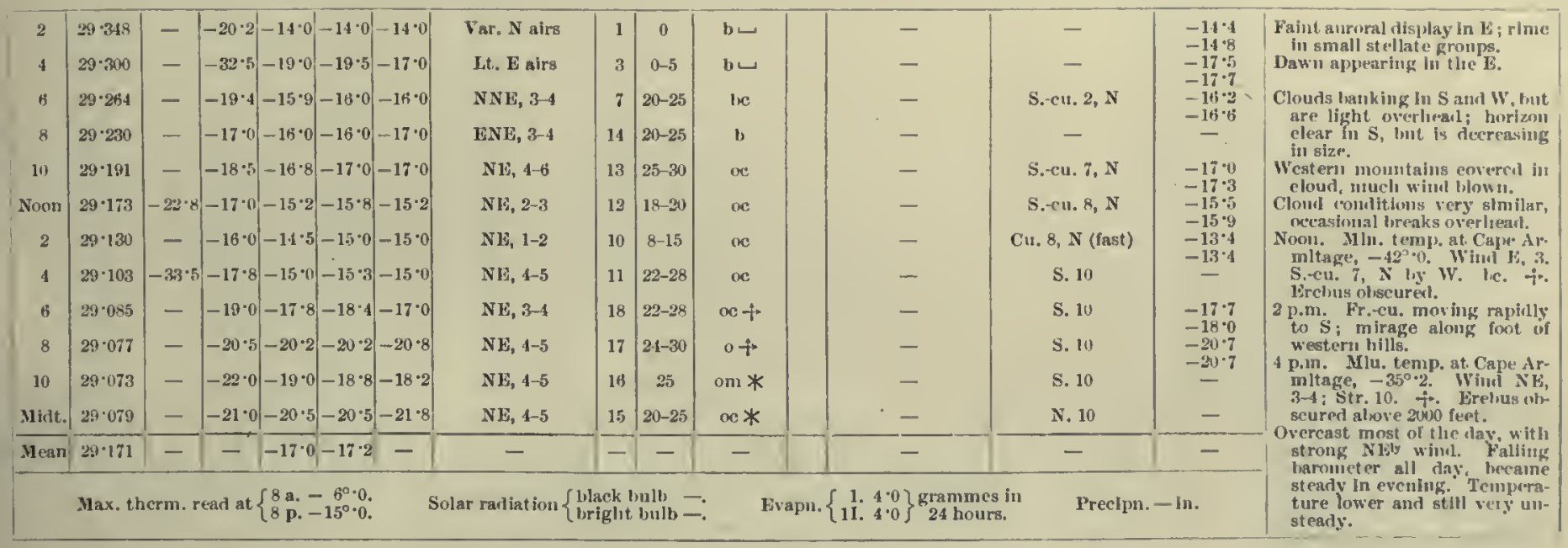

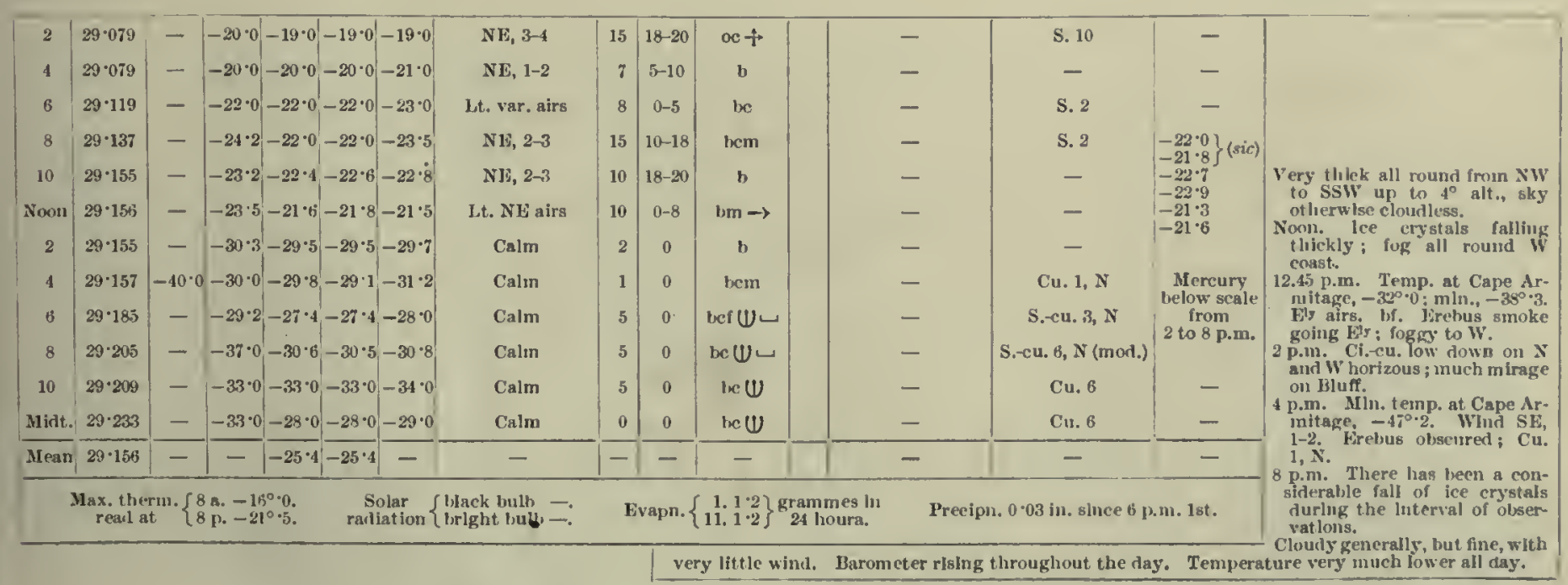


1903 - Angust.]

Meteorological, Jounat of TIF, "Discovery."

31st.

\begin{tabular}{|c|c|c|c|c|c|c|c|c|c|c|c|c|c|c|c|}
\hline \multirow{3}{*}{ llour. } & \multirow{3}{*}{ 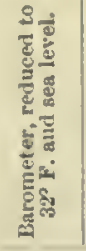 } & \multicolumn{5}{|c|}{ Thermometers. } & \multicolumn{3}{|l|}{ Wind. } & \multirow[b]{3}{*}{ 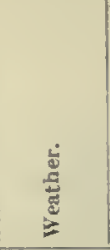 } & \multirow[b]{3}{*}{ 总 } & \multirow{2}{*}{\multicolumn{2}{|c|}{$\begin{array}{l}\text { Clouds, } \\
\text { Anount and movement. }\end{array}$}} & \multirow[b]{3}{*}{ 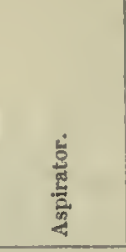 } & \multirow{3}{*}{ Memarks. } \\
\hline & & \multirow{2}{*}{ 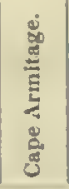 } & \multirow{2}{*}{ 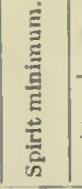 } & \multicolumn{2}{|c|}{ Mercurial. } & \multirow{2}{*}{ 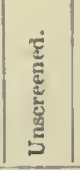 } & \multirow{2}{*}{$\begin{array}{l}\text { Directlon and } \\
\text { force } \\
\text { (Beaufort scalc). }\end{array}$} & \multirow{2}{*}{ 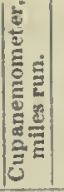 } & \multirow{2}{*}{ 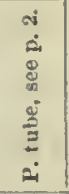 } & & & & & & \\
\hline & & & & Dry. & Wet. & & & & & & & Upper. & Lower. & & \\
\hline 2 & $99 \cdot 257$ & - & $-27 \cdot 0$ & $-26 \cdot 0 !$ & $-26 \cdot 0$ & $-27 \cdot 0 \mid$ & LSE, $2-3$ & 5 & $15-18$ & he $U$ & & - & S.cu. 4 & - & \\
\hline 4 & $29 \cdot 297$ & - & $-25 \cdot 0$ & $-23 \cdot 0$ & $-23 \cdot 0$ & $-23 \cdot 0$ & Calm & 4 & 0 & be $U$ & & - & S. 8 & - & Westem monntains covered \\
\hline 6 & $29 \cdot 349$ & - & $-35 \cdot 0$ & $-28 \cdot 0$ & $-28 \cdot 0$ & $-27 \cdot 0$ & Var. NSF, 3-4 & 9 & $20-25$ & $b+$ & & - & 一 & - & lleavy drlft blowing off Crater \\
\hline 8 & $29 \cdot 400^{\circ}$ & - & $-32 \cdot 0$ & $-2.1 \cdot 0$ & $-24 \cdot 0$ & $-24 \cdot 2$ & $\mathrm{NE}, 2-3$ & 15 & $10-15$ & $b x+$ & & - & S.-cu. 2 & - & \\
\hline 10 & $29 \cdot 152$ & - & $-25 \cdot 8$ & $-21 \cdot 2$ & $-21 \cdot 2$ & $-24 \cdot 2$ & $\mathrm{SE}_{3}, 3-4$ & 14 & $15-20$ & b & & - & - & $\begin{array}{l}-21 \cdot 3 \\
-24 \cdot 3\end{array}$ & Cloudless sky, some fog at base \\
\hline Noon & $29 \cdot 403$ & $-52 \cdot 8$ & $-21 \cdot 8$ & $-22 \cdot 0$ & $-22 \cdot 0$ & -220 & $\mathrm{E}, 2-3$ & 9 & $15-18$ & b & & - & - & $\begin{array}{l}-22 \cdot 4 \\
-22 \cdot 5\end{array}$ & Conditions similar to those at \\
\hline 2 & $29 \cdot 541$ & - & $-28 \cdot 8$ & $-22 \cdot 0$ & $-22 \cdot 2$ & $-21 \cdot 2$ & Lt. $\mathbf{X}$ airs & 5 & $0-8$ & b & & 一 & 一 & $\begin{array}{l}-21 \cdot 3 \\
-21 \cdot 6\end{array}$ & Noon, Iin, temp. at Cape Ar- \\
\hline 4 & $29 \cdot 576$ & $-55 \cdot 0$ & $-39 \cdot 8$ & $-38 \cdot 9$ & $-3 ; \cdot 9$ & $-39 \cdot 9$ & Calm to lt. $\mathrm{S}$ airs & 1 & 0 & $b$ & & - & - & & $\begin{array}{l}\text { N, } 2-3 \text {, bm. Foggy in Ere } \\
\text { bus Bight and over Barrier li }\end{array}$ \\
\hline 6 & 2) $\cdot 502$ & - & $-41 \cdot 0$ & $-26 \cdot 5$ & $-26 \cdot 5$ & $-24 \cdot 5$ & N ly W, 0-1 & 3 & $0-10$ & bし & & - & - & $\begin{array}{c}\text { Mercury } \\
\text { below scale }\end{array}$ & and SF; icy spicules falling. \\
\hline 8 & $29 \cdot 627$ & - & $-42 \cdot 0$ & $-41 \cdot 0$ & $-40 \cdot 0$ & $-42 \cdot 2$ & Calm & 2 & 0 & $\operatorname{bin} U \smile$ & & - & - & $\begin{array}{l}\text { from } \\
4 \text { p.m. to }\end{array}$ & $\begin{array}{l}\text { of Ci. to } N \text {. Erebus smoke } \\
\text { rising } 3000 \text { to } 4000 \text { fect in }\end{array}$ \\
\hline 10 & $29 \cdot 637$ & - & $-42 \cdot 0$ & $-40 \cdot 0$ & $-40 \cdot 0$ & $-42 \cdot 5$ & Calm & 1 & 0 & $\mathrm{bm} \uplus \sqcup$ & & - & - & Mít. & $\begin{array}{l}\text { height, forming a torn musl- } \\
\text { room, and then going to } \mathrm{E} \text {. }\end{array}$ \\
\hline Midt. & $29 \cdot 618$ & - & $-41 \cdot 0$ & $-30 \cdot 0$ & $-30 \cdot 0$ & $-31 \cdot 2$ & Calin & 1 & 0 & bU二 & & - & - & & $\begin{array}{l}2 \text { p.m. Small detached portions } \\
\text { of } \mathrm{Ci} \text {. On N horizon. }\end{array}$ \\
\hline \multirow[t]{3}{*}{ Mpar } & $29 \cdot 487$ & - & - & $-28 \cdot 8$ & $|-28 \cdot 7|$ & - & - & - & - & - & & - & - & - & $\begin{array}{l}4 \mathrm{p} . \mathrm{m} \text {. Mother-of-pearl wisps } \\
\text { of Ci. to NW; Ci. low in WV }\end{array}$ \\
\hline & \multicolumn{6}{|c|}{ Max. thermn. read at $\left\{\begin{array}{l}8 \text { a. }-18^{\circ} \cdot 0 \\
8 \text { p. }-17^{\circ} \cdot 0\end{array}\right.$} & \multicolumn{4}{|c|}{ Solar radiation $\left\{\begin{array}{l}\text { black bulb }- \text {. } \\
\text { bright bulb }- \text {. }\end{array}\right.$} & & Evapn. - grammes. & \multicolumn{2}{|c|}{ Precipn. - in. } & $\begin{array}{l}\text { and S ; outside thermometer } \\
\text { fell during time I was at } \\
\text { serecu to }-41^{\circ} \cdot 5 \text {. } \\
4 \text { p.m. Min. temp. at Cape }\end{array}$ \\
\hline & & & & & hrm & & $\begin{array}{l}0 . \text { Wind } \mathrm{W}, \\
\text { n rime. } 8 \mathrm{p} \cdot \mathrm{m} \\
\text { naining so. } \mathrm{H}\end{array}$ & & & & & 4. Simall & wi & riabl & $\begin{array}{l}\text { to } \mathrm{N} .6 \mathrm{p} . \mathrm{m} \text {. Thermoneter } \\
\text { winds early, falling calm about } \\
\text { and very finctuating. }\end{array}$ \\
\hline
\end{tabular}


Meteorologicai, Journal of tile "Discovery,"

[1903-September.

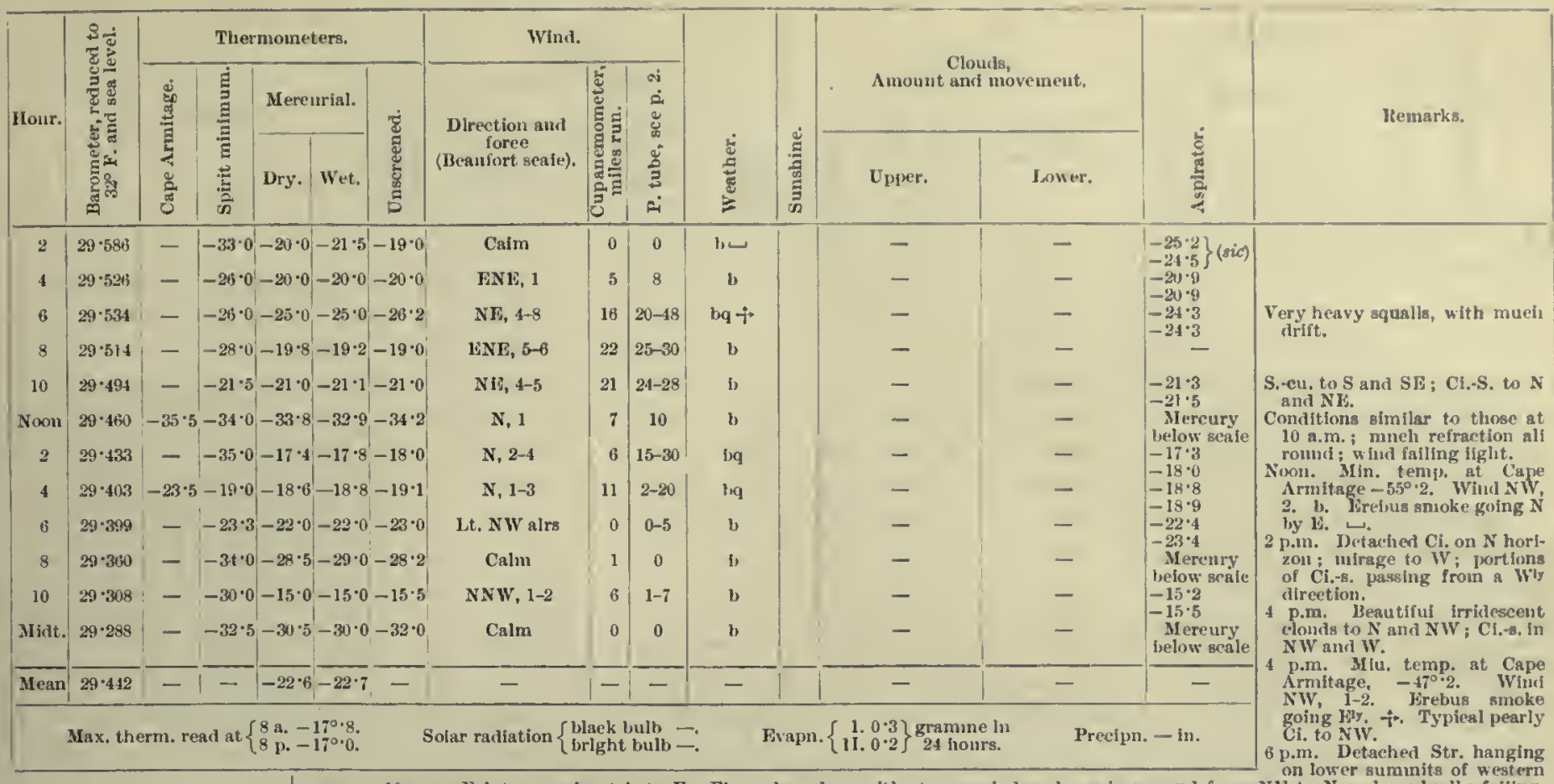
rauge. 10 p.m. Faint anrorai pateh to $\mathbf{E}$. Fine, elear day, with strong wind early, going round from $\mathrm{N} 1 \mathrm{j}$ to $\mathrm{N}$ and gradually faliing.
Barometer fafíng afl day. Temperature very unsteady.

\begin{tabular}{|c|c|c|c|c|c|c|c|c|c|c|c|c|}
\hline 2 & $29 \cdot 275$ & - & $-34 \cdot 0$ & $-32 \cdot 5$ & $-32 \cdot 0-33 \cdot 0$ & Calm & 0 & 0 & b & 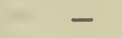 & - & Mfereury \\
\hline 4 & $29 \cdot 214$ & - & $-34 \cdot 0$ & $-25 \cdot 0$ & $-25 \cdot 0-25 \cdot 0$ & Lt. S airs & 3 & 0 & b & - & - & from \\
\hline 6 & $29 \cdot 209$ & - & $-23 \cdot 0$ & $-22 \cdot 0$ & $-22 \cdot 0-23 \cdot 2$ & SE, 0-1 & 4 & 0 & be & Cl. 2 & - & $\begin{array}{c}2 \text { fo } 4 \mathrm{am} . \mathrm{m} \\
-19 \cdot 8\end{array}$ \\
\hline 8 & $29 \cdot 193$ & - & $-22 \cdot 0$ & $-17 \cdot 8$ & $-18 \cdot 0-18 \cdot 5$ & ESF, $5-6$ & 16 & $30-35$ & b & - & - & $-18 \cdot 0$ \\
\hline 10 & $29 \cdot 177$ & - & $-18 \cdot 8$ & $-18 \cdot 5$ & $-18 \cdot 8-19 \cdot 0$ & $E, 4-6$ & 18 & $22-32$ & $b y+$ & - & - & $-19 \cdot 1$ \\
\hline Noon & $29 \cdot 169$ & $-35 \cdot 8$ & $-32 \cdot 0$ & $-27 \cdot 0$ & $-26 \cdot 8-28 \cdot 0$ & SE, 3-4 & 15 & $18-24$ & $b v f$ & - & - & Mercury \\
\hline 2 & $29 \cdot 145$ & - & $-27 \cdot 6$ & $-19^{\circ} 0$ & $-19 \cdot 3-19 \cdot 7$ & ENE, 4-6 & 19 & $25-38$ & $\operatorname{loq}+$ & - & - & $\begin{array}{c}\text { betow seate } \\
-19 \cdot 5\end{array}$ \\
\hline 4 & $29 \cdot 139$ & $-31 \cdot 2$ & $-19 \cdot 3$ & $-18 \cdot 0$ & $-18 \cdot 8-18 \cdot 0$ & $\mathrm{NE}, 3-5$ & 18 & $23-32$ & $\log 7$ & - & - & $\begin{array}{l}-18.0 \\
-18.0 \\
-19.1\end{array}$ \\
\hline 6 & $29 \cdot 131$ & - & $-18 \cdot 5$ & $-15 \cdot 0$ & $-16 \cdot 0-15 \cdot 7$ & NE, 2-3 & 13 & $12-22$ & b & - & - & $\begin{array}{l}-14.8 \\
-15.9\end{array}$ \\
\hline 8 & $29 \cdot 127$ & - & $-3 \cdot t^{\prime} 6$ & $-22 \cdot 0$ & $-22 \cdot 0-22 \cdot 2$ & $N, 1$ & 3 & $8-10$ & b & - & - & $\begin{array}{l}-22 \cdot 0 \\
-22 \cdot 4\end{array}$ \\
\hline 10 & $29 \cdot 111$ & - & $-25 \cdot 0$ & $-24 \cdot 2$ & $-24 \cdot 5-24 \cdot 5$ & NE, 4 & 6 & $28-35$ & b & - & - & $\begin{array}{l}-24 \cdot 2 \\
-2.99\end{array}$ \\
\hline slidt. & $29^{\circ} 089$ & - & $-25 \cdot 0$ & $-23 \cdot 2$ & $-23 \cdot 8-24 \cdot 0$ & NE, 4-5 & 33 & $30-37$ & b & - & - & $\begin{array}{l}-24 \cdot 2 \\
-24 \cdot 5\end{array}$ \\
\hline Меяп & $29 \cdot 165$ & - & - & $|-22 \cdot 0|$ & $-22 \cdot 3-$ & - & - & - & - & - & - & - \\
\hline
\end{tabular}

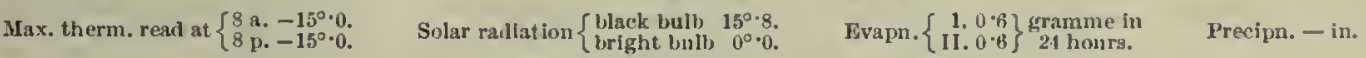




\begin{tabular}{|c|c|c|c|c|c|c|c|c|c|c|c|c|c|c|}
\hline \multirow{3}{*}{ Ilour. } & \multirow{3}{*}{ 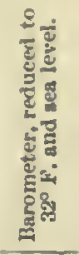 } & \multicolumn{5}{|c|}{ Thermometers. } & \multicolumn{3}{|l|}{ Wind. } & \multirow[b]{3}{*}{ 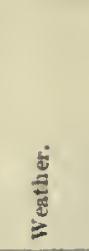 } & \multirow[b]{3}{*}{ 萢 } & \multirow{2}{*}{\multicolumn{2}{|c|}{$\begin{array}{l}\text { Clonis, } \\
\text { Ainomt and morement. }\end{array}$}} & \multirow[b]{3}{*}{ 产 } \\
\hline & & \multirow{2}{*}{ 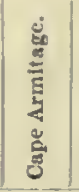 } & \multirow{2}{*}{ 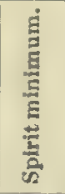 } & \multicolumn{2}{|c|}{ Mercurial. } & \multirow{2}{*}{ 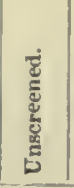 } & \multirow{2}{*}{$\begin{array}{c}\text { Direction and } \\
\text { foree } \\
\text { (Beanfort scale). }\end{array}$} & \multirow{2}{*}{ 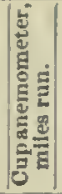 } & \multirow{2}{*}{ 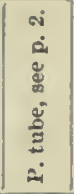 } & & & & & \\
\hline & & & & Dry. & Wet. & & & & & & & Upper. & Lower. & \\
\hline 2 & $29 \cdot 239$ & - & $-21 \cdot 0$ & $-18 \cdot 6$ & $-18 \cdot 8$ & $-19 \cdot 0$ & E, 3-4 & 11 & $18-28$ & be $U$ & & Ci. 2 & - & 1- \\
\hline 4 & $29 \cdot 211$ & - & $-19 \cdot 0$ & $-14 \cdot 5$ & $-15 \cdot 0$ & $-14 \cdot 3$ & E, 0-1 & 11 & $0-5$ & $\operatorname{bm} \omega$ & & - & - & - \\
\hline 6 & $29 \cdot 178$ & - & $-17 \cdot 8$ & $-17 \cdot 8$ & $-18 \cdot 2$ & $-18 \cdot 4$ & Lt. S by $\mathrm{N}$ alrs & 8 & 0 & b & & - & - & - \\
\hline 8 & $29 \cdot 112$ & - & $-32 \cdot 0$ & $-26 \cdot 0$ & $-28 \cdot 0$ & $-26 \cdot 8$ & Calm & 0 & 0 & be & & - & S. 4 & - \\
\hline 10 & $29 \cdot 065$ & - & $-26 \cdot 8$ & $-21 \cdot 2$ & $-21 \cdot 5$ & $-21 \cdot 2$ & Lt. NE airs & 1 & 5-8 & bx & & - & - & - \\
\hline Noon & $29 \cdot 023$ & $-35 \cdot 3$ & $-2 b \cdot 0$ & $-21 \cdot 0$ & $-24 \cdot 0$ & $-24 \cdot 2$ & $N F, 4$ & 10 & $20-23$ & $b v+$ & 1 & - & - & - \\
\hline 2 & $28 \cdot 950$ & - & $-27 \cdot 6$ & $-27 \cdot 0$ & $-27 \cdot 0$ & $-27 \cdot 5$ & Nls, 5-6 & 22 & $28-35$ & $b+$ & 1 & - & - & $1-$ \\
\hline 4 & $23 \cdot 927$ & $-36 \cdot 0$ & $-2 y \cdot 2$ & $-28 \cdot 2$ & $-28 \cdot 2$ & $-29 \cdot 2$ & $\mathrm{NE}, 5-6$ & 19 & $25-33$ & $b \div$ & & - & - & - \\
\hline 6 & $28 \cdot 875$ & - & $-29 \cdot 2$ & $-28 \cdot 5$ & $-26 \cdot 5$ & $-27 \cdot 0$ & NE, 3-5 & 18 & $20-30$ & $\ln +6$ & & - & - & - \\
\hline 8 & 28 '836 & - & $-28 \cdot 5$ & $-28 \cdot 2$ & $-23 \cdot 0$ & $-29 \cdot 0$ & $N E, 1-2$ & 14 & $10-18$ & ᄂ & & - & - & $1-$ \\
\hline I0 & $28 \cdot 809$ & - & $-33 \cdot 2$ & $-32 \cdot 2$ & $-32 \cdot 0$ & $-33 \cdot 0$ & SSE, 1 & 9 & $8-10$ & is & & - & - & - \\
\hline Mlat. & $28 \cdot 777$ & - & $-31 \cdot 8$ & $-32 \cdot 0$ & $-32 \cdot 0$ & $-32 \cdot 6$ & $\mathrm{SE}, 1$ & 7 & 10 & b & & - & - & $=$ \\
\hline an & & - & - & $-24 \cdot 7$ & $-24 \cdot 8$ & - & - & - & - & - & & - & - & - \\
\hline
\end{tabular}

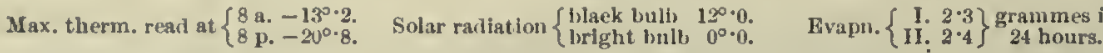

Precipu. - in.
Reunarks,

Some Str. to 8 and $\mathrm{NW}$, also a bank of Str. to $S$ and $S E$.
Str, to $S$ alui $S E$, Cl.s. to W.

Clourlless except for bank of Str. to s atul SE; whul sprang up fresh fron Non and temperature rose quirkly. Irift in Strait.

Noon. Min, temp. at Cape Aruitage $-50^{\circ} \cdot 0$. Whid N1, 3. b. Lines of Str. low F; Frebus smoke uing tirst downwards and then Ity in a long pendant as far as Mt. Terror. U on therino

2 p.in. Str. on lower peaks of wester mountains, and Str, on S horizon. p.m. liain band, 3.

p.un. S.-cu. passing over, and on, western monntains, and passing at rapid pace in $S^{l y}$ direction

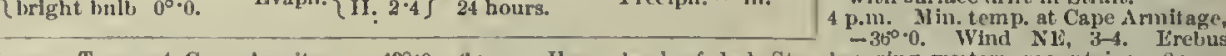

smoke going Nk. Fou western side of Stralt. 5 p.m. Temp. at Cape Armitage, $-43^{\circ} \cdot 0.6$ p.m. Ileavy bank of dark Str. olscuring westerni mountains. 8 p.m.

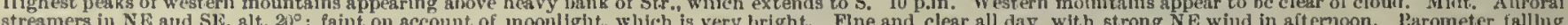
all day. Temperature falling cont inuously.

5 th.

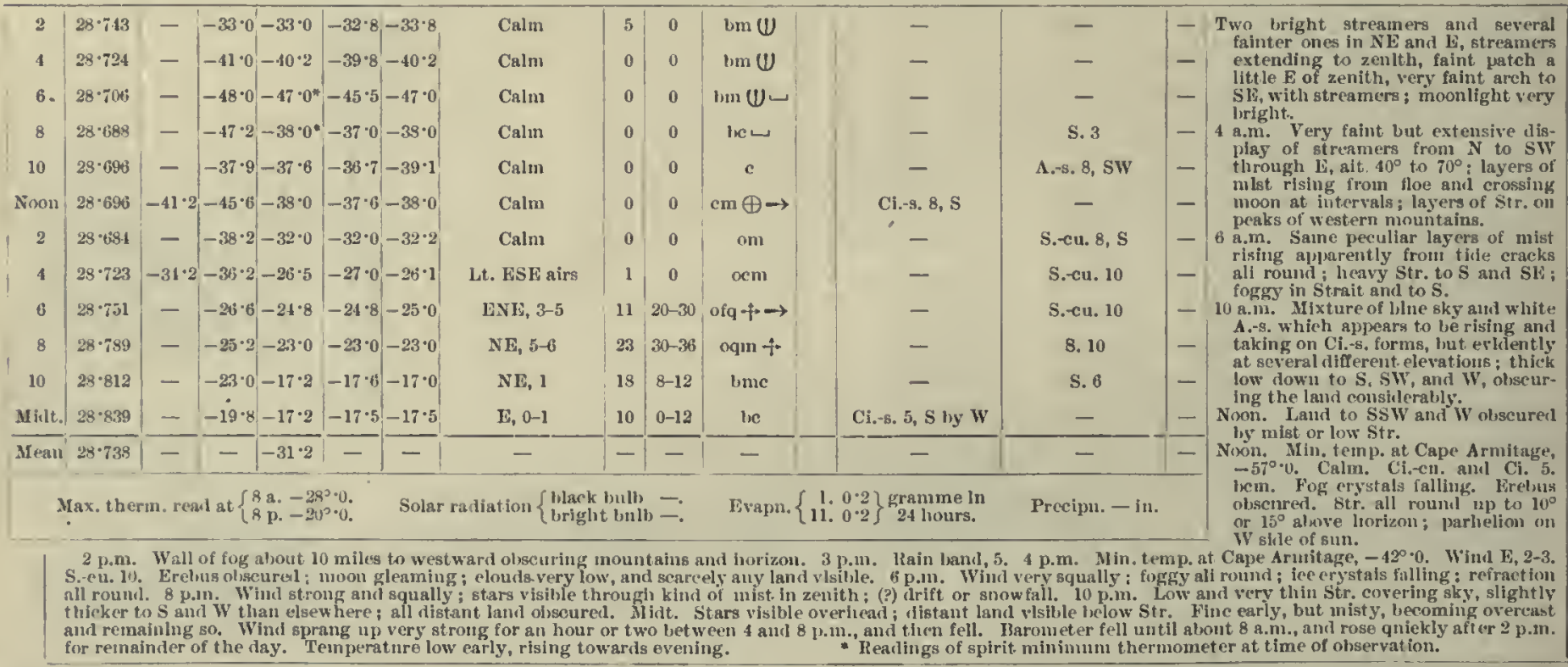

6th.

\begin{tabular}{|c|c|c|c|c|c|c|c|c|c|c|c|}
\hline 2 & $28 \cdot 859$ & - & $|-18 \cdot 0|-14 \cdot 2$ & $-14 \cdot 8-14 \cdot 0$ & Lt. $S$ by $W$ airs & 9 & $0-10$ & oc & - & S. 10 & L \\
\hline 4 & $28 \cdot 845$ & - & $-16 \cdot 6-13 \cdot 8$ & $-14 \cdot 2-14 \cdot 0$ & E, 2-3 & 7 & $15-23$ & $\alpha+$ & - & S. 10 & - \\
\hline 3 & $28 \cdot 898$ & - & $-15 \cdot 0-14 \cdot 0$ & $-14 \cdot 2-12 \cdot 2$ & E by $N, 3-4$ & 10 & $22-35$ & ocq t* $^{+}$ & - & S. 10 & - \\
\hline 8 & $28 \cdot 883$ & - & $-13.5-11 \cdot 0$ & $-11 \cdot 0-11 \cdot 0$ & Var. ESE, 5-7 & 15 & $20-35$ & $\operatorname{ocg} t$ & - & S. 10 & - \\
\hline 10 & $23 \cdot 891$ & - & $-12 \cdot \frac{1}{10}-10 \cdot 8$ & $-11 \cdot 3-10 \cdot 8$ & SE, 2-4 & 13 & $10-20$ & beq & $\mathrm{Ci}_{-}-\overline{\mathrm{s}}, 3, \mathrm{sw}$ & $\mathrm{Fr}_{0}-\mathrm{s}, 4, \mathrm{~S}$ & - \\
\hline Noon & $28 \cdot 88.3$ & $-17 \cdot 2$ & $-17 \cdot 0-15 \cdot 6$ & $-15 \cdot 9-16 \cdot 0$ & Var. $\mathrm{X}, 4-5$ & 1.4 & $18-21$ & oeq $+\frac{t}{t}$ & Ci.-s. 4, sw & S. $5, \mathrm{~S}$ & $1-$ \\
\hline 2 & $28 \cdot 8.55$ & - & $-16 \cdot 3-11 \cdot 3$ & $-12 \cdot 0-11 \cdot 2$ & F, 2-3 & 15 & $12-25$ & be & $\mathrm{Ci}_{0}-\mathrm{s}, 2, \mathrm{SW}$ & Cu. 2, s & - \\
\hline 4 & $28 \cdot 855$ & $-11 \cdot 1$ & $-12 \cdot 6-8 \cdot 8$ & $-9.5-8.8$ & ESE, $2-5$ & 11 & $20-30$ & ocq & - & S. 10 & - \\
\hline 6 & $28 \cdot 839$ & - & $-10 \cdot 1-8 \cdot 0$ & $-9 \cdot 5-8 \cdot 8$ & SE, 1-2 & 10 & $8-15$ & - & - & S. 10 & - \\
\hline 8 & $28 \cdot 802$ & - & $-10 \cdot 0-7 \cdot 0$ & $-8.0-6.8$ & Lt. E airs & 5 & 0 & $x$ & - & S.en, 10, S (slow) & - \\
\hline 10 & $28 \cdot 798$ & - & $-14 \cdot 3-14 \cdot 1$ & $-14 \cdot 2-15 \cdot 2$ & Lt. F airs & 6 & 0 & be $\mathbb{U}$ & $C i_{0}-8.4$ & - & - \\
\hline Mlielt. & $28 \cdot 815$ & 一 & $-15 \cdot 0-12 \cdot 8$ & $-13 \cdot 0-13 \cdot 2$ & Lt, var, airs & 3 & 0 & $c \mathbb{O}$ & Ci. 8 & - & - \\
\hline ean & $29 \cdot 8.53$ & - & $-\quad-11 \cdot 9$ & $-12 \cdot 3-$ & - & - & - & - & - & - & - \\
\hline
\end{tabular}

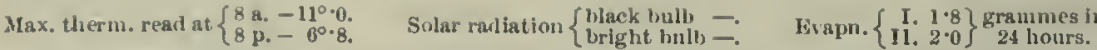

Precipm. - in.

Moon dimly visible; stars obsenred.

Dark sky to $\mathrm{S}$ and $\mathrm{SW}$; no land visible

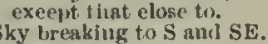

Very windy looking all round; western 政,

hon. Conlitions very slmilar ; muvements of clouds are fast and direet : land. Strall, and to $\mathrm{S}$ and st. Noon. Min. temp, at Cape Arnitage, Erebus olscured. "Str. ail rouni loorizon.

2 p.m. lieavy Str. sloug S liorizon; small detay
from SE. 3 p.m. lain band, 3 p.m. Some lreak in elouds to $W$ and $\mathrm{X}$ W.

$1, m$. Min, temp), at Cape Armitage $-17^{\circ} \cdot{ }^{\circ}$. Wind Sli, $1-2$. Clmuds of irifít to Sls all going to Nkifoc.
Str. 10. Tons of Binek mul Witite Str. 10. This of Biack minl Wirte

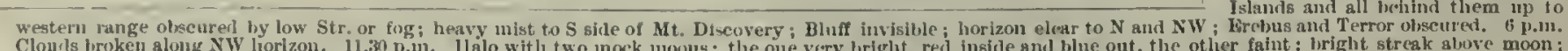

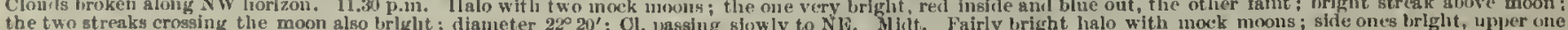

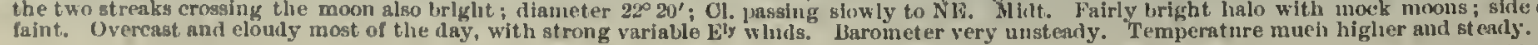


Metlonotogicat, Joulinal, of thi: "Discovetiy."

$[1903$ - September.

\begin{tabular}{|c|c|c|c|c|c|c|c|c|c|c|c|c|c|}
\hline \multirow{3}{*}{ Ifour. } & \multirow{3}{*}{ 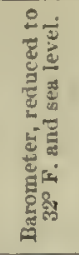 } & \multicolumn{4}{|c|}{ Thermometers. } & \multicolumn{3}{|l|}{ Wind. } & \multirow[b]{3}{*}{ 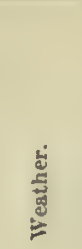 } & \multirow[b]{3}{*}{ 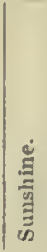 } & \multirow{2}{*}{\multicolumn{2}{|c|}{$\begin{array}{l}\text { Cloutls, } \\
\text { Aurout unul movenent. }\end{array}$}} & \\
\hline & & \multirow{2}{*}{ 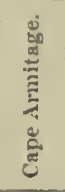 } & \multirow{2}{*}{ 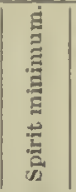 } & \multirow{2}{*}{$\frac{\text { Mereurial. }}{\text { Diy. Wet. }}$} & \multirow[b]{2}{*}{ 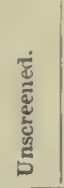 } & \multirow[b]{2}{*}{$\begin{array}{c}\text { Directiont aud } \\
\text { force } \\
\text { (Beaufort seale). }\end{array}$} & \multirow{2}{*}{ 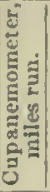 } & \multirow{2}{*}{ 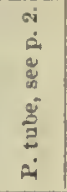 } & & & & & \\
\hline & & & & & & & & & & & Upper. & Lower. & \\
\hline 2 & 28.807 & - & -14.5 & $\mid-4 \cdot 0-5 \cdot 5$ & -4.4 & $N, 1-2$ & 4 & $7-15$ & Ine & & CI. 3 & $\overline{-}$ & - \\
\hline 4 & $29 \cdot 806$ & - & $-18 \cdot 7$ & $-4 \cdot 8-5$. & $-1 \cdot 8$ & N, 2-3 & 5 & $15-213$ & le & & Cl. 4 & - & - \\
\hline 6 & $28 \cdot 820$ & - & $-5 \cdot 1$ & $-2 \cdot 1-3 \cdot 0$ & $-2 \cdot 0$ & N, 1-2 & 10 & $7-16$ & oc & & - & S. 11) & - \\
\hline 8 & $28 \cdot 885$ & - & $-7 \cdot 5$ & $-2 \cdot 8-2 \cdot 8$ & $-2 \cdot 2$ & SE, 2-3 & 4 & $10-15$ & $\infty$ & & - & S. 10 & 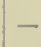 \\
\hline 10 & $2 R \cdot 929$ & - & $-4 \cdot 2$ & $-1 \cdot 0-1 \cdot 2$ & $-1 \cdot 0$ & SW, 3-4 & 9 & $25-30$ & orq & & 一 & S. 10 & - \\
\hline $\mathrm{N}(m)$ & $28 \cdot 988$ & $-3 \cdot 6$ & $-7 \cdot 3$ & $\mid-4 \cdot 0-4 \cdot 3$ & $-4 \cdot 0$ & SH, 3 & 11 & $15-18$ & orq 7 - & & - & S. 8 & - \\
\hline 2 & $29 \cdot 055$ & - & -5.5 & $-5 \cdot 0-5 \cdot 0$ & $-5 \cdot 0$ & SE, 3-4 & 17 & $20-28$ & of 7 . & & - & S. 10 & - \\
\hline 4 & $29 \cdot 130$ & - & $-7 \cdot 5$ & $\mid-7 \cdot 2-7 \cdot 2$ & $-6 \cdot 8$ & Sin, 3-5 & 15 & $20-30$ & ofg $\rightarrow^{\circ}$ & & - & S.-C11. 10 & - \\
\hline 6 & $29 \cdot 142$ & - & $-8 \cdot 9$ & $-7 \cdot 0-7 \cdot 2$ & $-7 \cdot 0$ & $\mathrm{ESE}, 2-3$ & 12 & $12-18$ & $0 \div$ & & - & S. 10 & - \\
\hline 8 & $29 \cdot 148$ & - & $-7 \cdot 5$ & $-4 \cdot 9-5 \cdot 0$ & $-4 \cdot 9$ & E ly N, 0-1 & 10 & 0 & bme & & - & S. 2 & - \\
\hline 10 & $29 \cdot 142$ & - & $-7 \cdot 8$ & $-3.5-4.5$ & $-3 \cdot 0$ & NNE, 0-1 & 5 & $5-6$ & inc & & Cl. $\approx \mathrm{Z}_{2} 8$ & - & - \\
\hline Mislt. & $29 \cdot 141$ & - & $-10 \cdot 7$ & $-5.0-5.4$ & $-5 \cdot 0$ & NNE, 2-3 & 7 & $15-21$ & le & & Ci,-s, 6 & - & - \\
\hline$M$ can' & $\overline{28 \cdot 999}$ & - & - & $|-4 \cdot 3-4 \cdot 7|$ & - & - & $1-$ & - & - & & - & - & \\
\hline
\end{tabular}

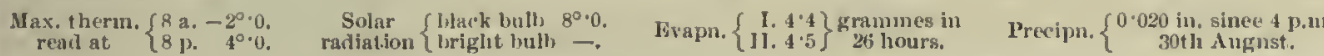
all rominl.

Wind very gust y ; suddenly diropped at 10.30, and sky began clearlug from Niv, whlch quickly extenuthl roum tus SW ; all westerin mountalis clearim $-27^{\circ} \cdot 2$. Whul $s, 3-5$. Sir. $y_{\text {. ocín }}$

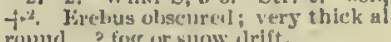

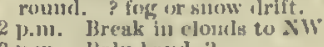

. Thlo hand,

Tiv, Hroken and detached Cu. slong 4.45 pall. Ternp, at Cape Irmltage $-9^{3} 9:$ min., $-10^{\circ} 1$. Wiml s las

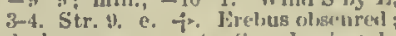
dark appearance to $S$; elearing ln Nw.

6 p.m. Clear to NW and W, with colour on horlan.

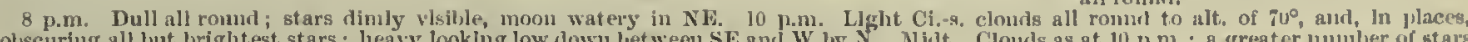

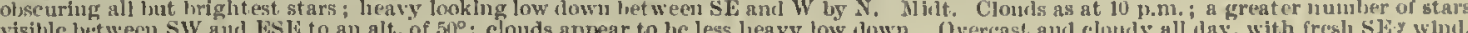
bamnuter rose until 8 p.m., and then liegan to fall. Tenperature high, but st ealy.

\begin{tabular}{|c|c|c|c|c|c|c|c|c|c|c|c|}
\hline 2 & $29 \cdot 139$ & - & $-5 \cdot 9^{\prime}-2 \cdot 0-2 \cdot 1-2 \cdot 2$ & $\mathrm{~N}, 2-3$ & 16 & $|24-28|$ & lie & - & S. $1, \mathrm{~W}$ & - & Faint auroral streamer $\mathbf{S} \mathbf{F}, 10^{\circ}$ to $30^{\circ}$; \\
\hline 4 & $29 \cdot 133$ & - & $-6.9-0.6-1.2-1.0$ & $\mathbf{N}, 2-3$ & 18 & $22-32$ & le & - & S. 2 & - & 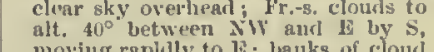 \\
\hline 6 & $29 \cdot 157$ & - & $\begin{array}{lll}-2.0 & 0.1-0.4 \quad 0.0\end{array}$ & $\mathrm{~N}, 1-2$ & 9 & $20-25$ & lw & - & S. 2 & - & $\begin{array}{l}\text { moving raphily to } 15 \text {; loguks of cloud } \\
\text { over western hills and round to } S \text { to }\end{array}$ \\
\hline 8 & 2): $\cdot 195$ & - & $-6 \cdot 5-6 \cdot 2-6 \cdot 2-8 \cdot 0$ & $\mathbf{N}, 0-\mathbf{I}$ & 8 & $0-5$ & lse & - & S. 1 & - & 4 a.m. Clonls simllar to those at $2 \mathrm{am}$. \\
\hline 10 & $29 \cdot 201$ & - & $-7 \cdot 4-2 \cdot 0-2 \cdot 8-2 \cdot 3$ & $\mathrm{~N}, \mathbf{1 - 2}$ & 8 & $15-20$ & le & Ci.-s, I, NW & - & - & 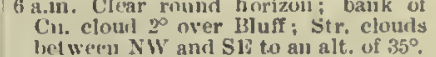 \\
\hline Noon & $29 \cdot 217$ & $-7 \cdot 4$ & $-9.5-2.5-3.0-2 \cdot 4$ & N by W, 2-3 & 9 & $20-25$ & be & Ci. 2 & - & - & $10 \mathrm{a}$. II. Streamers of clouds extendlag \\
\hline 2 & $29 \cdot 244$ & - & $-10 \cdot 3-10 \cdot 0-10 \cdot 2-11 \cdot 0$ & Calm & 8 & 0 & be & $C i,-8,6, N I V$ & - & -1 & Nonn. Ci. clouds radiating from $N 1 W$. \\
\hline 4 & $29 \cdot 273$ & $-20 \cdot 0$ & $-12 \cdot 4-10 \cdot 1-10 \cdot 5-10 \cdot 2$ & Calm & 0 & 0 & lic & $\mathrm{Ci}_{*}-\mathrm{s}, 6$ & 一 & - & 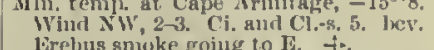 \\
\hline 6 & $29 \cdot 290$ & - & $-13 \cdot 8-10.5-11.0-10.0$ & Calm & 0 & 0 & oe & - & S.-en. 10 & - & 2 p.m. Ci. ruming in veins from NW \\
\hline 8 & $29 \cdot 313$ & - & $-13 \cdot 5-13 \cdot 1-13 \cdot 0-15 \cdot 0$ & Culm & 2 & 0 & $\infty$ & - & S. 10 & - & lami, 1 . \\
\hline 10 & $29 \cdot 3326$ & - & $-14 \cdot 9-4 \cdot 2-5 \cdot 0-5 \cdot 0$ & NE, 2-3 & 6 & $15-20$ & o & - & S. 10 & - & 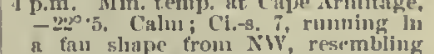 \\
\hline Milt. & $29 \cdot 372$ & - & $-5.4-1 \cdot 7-2 \cdot 8-2 \cdot 2$ & $\mathrm{NE}, 2-3$ & 11 & $15-20$ & 0 & $\overline{-}$ & S. 10 & - & Ci.-ru. in zenitli ; no smoke fron \\
\hline Neaur & $29 \cdot 298$ & - & $|-|-.5 \cdot 2|-5 \cdot 7|-$ & - & - & - & - & - & - & \multirow{2}{*}{\multicolumn{2}{|c|}{ 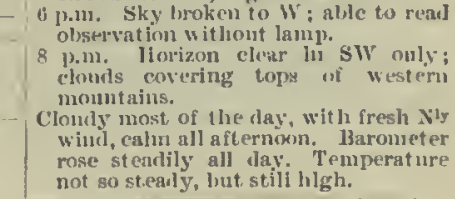 }} \\
\hline \multicolumn{11}{|c|}{ 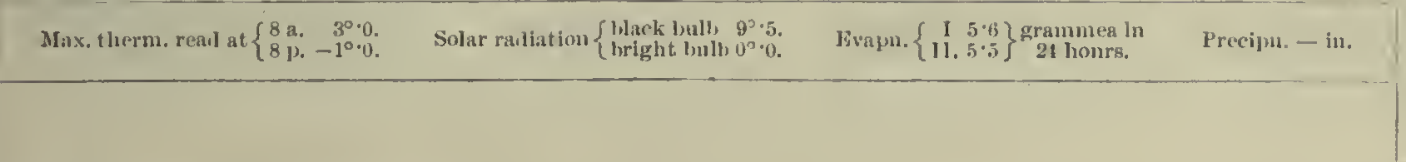 } & \\
\hline
\end{tabular}

8th.

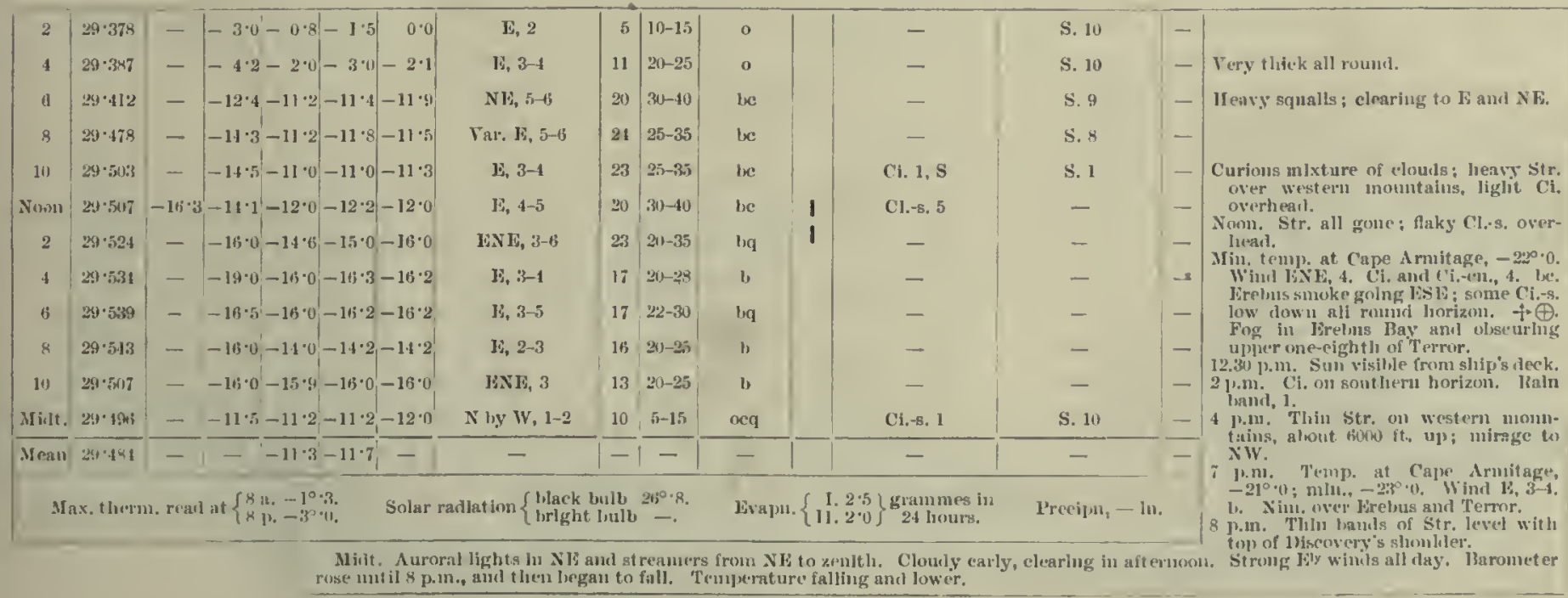


1903-September:.]

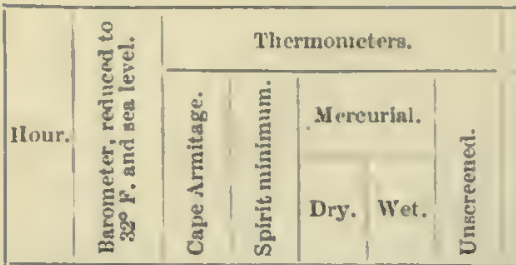

10 th.

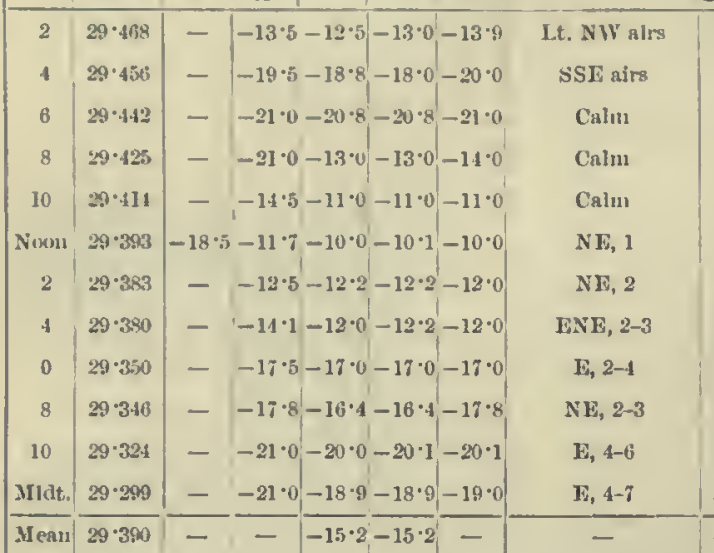
Max. therm.
read at $\left\{\begin{array}{l}8 \mathrm{a} . \mathbf{g}^{\circ} \cdot 0 . \\ 8 \mathrm{p} .-8^{\circ} \cdot 5 .\end{array}\right.$

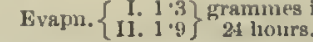

Metrolotofical Journat, of the "Discovery."

0 p

10 p.m. Stars dim oyerhcad; snow and irift; fog seems to be lifting. Milt. No stars; ? drift or snow ; fresh deposit of rime; rer squaly. Orercast, dull, and foggy day. Calm all the forencon,

11 th.

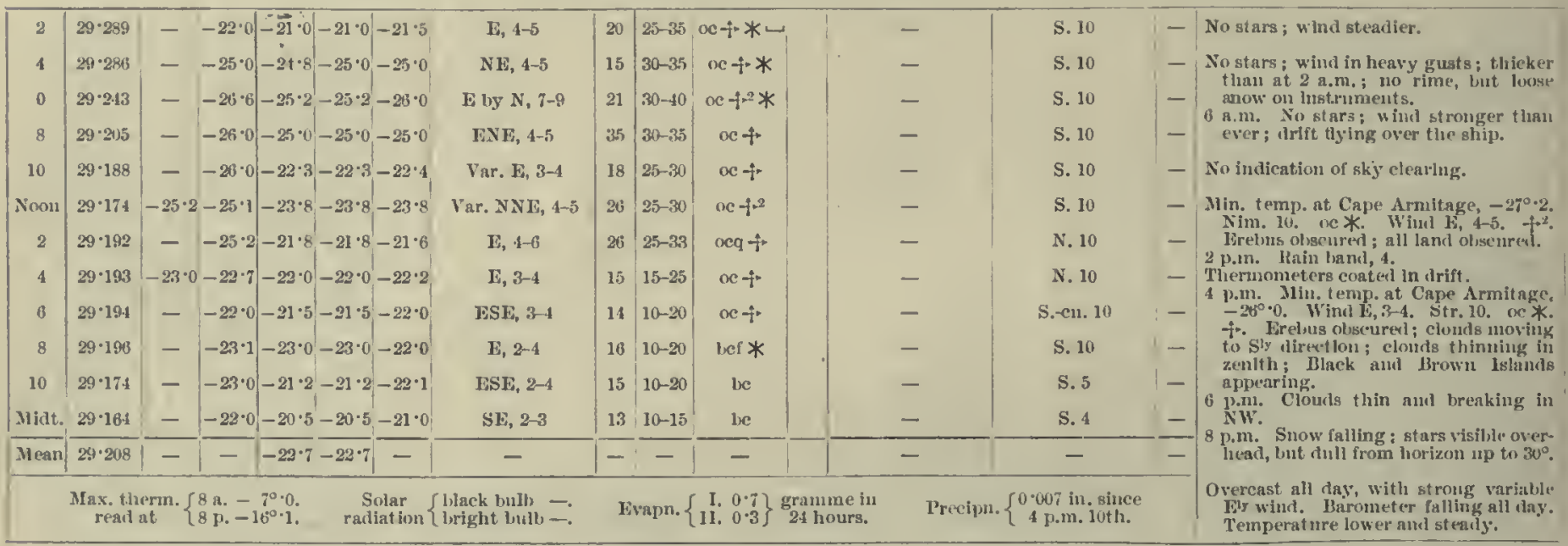

12 th

\begin{tabular}{|c|c|c|c|}
\hline 2 & $29 \cdot 117$ & - & $-22 \cdot 0-21 \cdot 5-21 \cdot 5-23 \cdot 0$ \\
\hline 4 & $29 \cdot 115$ & - & $-21 \cdot 0-20^{\circ} 0^{\prime}-20^{\circ} 0-22 \cdot 0$ \\
\hline b & $29 \cdot 105$ & - & $-25 \cdot 0-24 \cdot 0-24 \cdot 0-24 \cdot 0$ \\
\hline 8 & $29 \cdot 080$ & - & $-29 \cdot 3-26 \cdot 7-27 \cdot 0-25 \cdot 0$ \\
\hline 10 & $29 \cdot 0.47$ & - & $5 \cdot 5-25 \cdot 5-25 \cdot 5$ \\
\hline Nom & $29 \cdot 014$ & $-31 \cdot$ & $3-21 \cdot 8-21 \cdot 5$ \\
\hline 2 & $29 \cdot 0.31$ & - & $-22 \cdot 2-21 \cdot 2,-21 \cdot 4-22 \cdot 0$ \\
\hline 4 & $29 \cdot 021$ & $-3 B$. & $-22 \cdot 8-21 \cdot 0-21 \cdot 3-1 \cdot 0$ \\
\hline 6 & $29 \cdot 011$ & - & $-34 \cdot 0-32 \cdot 6-32 \cdot 6-31 \cdot 3$ \\
\hline 8 & $29^{\circ} 010$ & - & $-333 \cdot 5-25 \cdot 0-25 \cdot 0-25 \cdot 5$ \\
\hline 10 & $28 \cdot 9 \$ 1$ & - & $-25 \cdot 0-24 \cdot 0-24 \cdot 0-24 \cdot 0$ \\
\hline Mlit. & $28 \cdot 980$ & - & $\mid-31 \cdot 0-30 \cdot 0-30 \cdot 0-30 \cdot 0$ \\
\hline Iean & $23 \cdot 045$ & - & $-\quad-21 \cdot 4|-24 \cdot 5|-$ \\
\hline
\end{tabular}

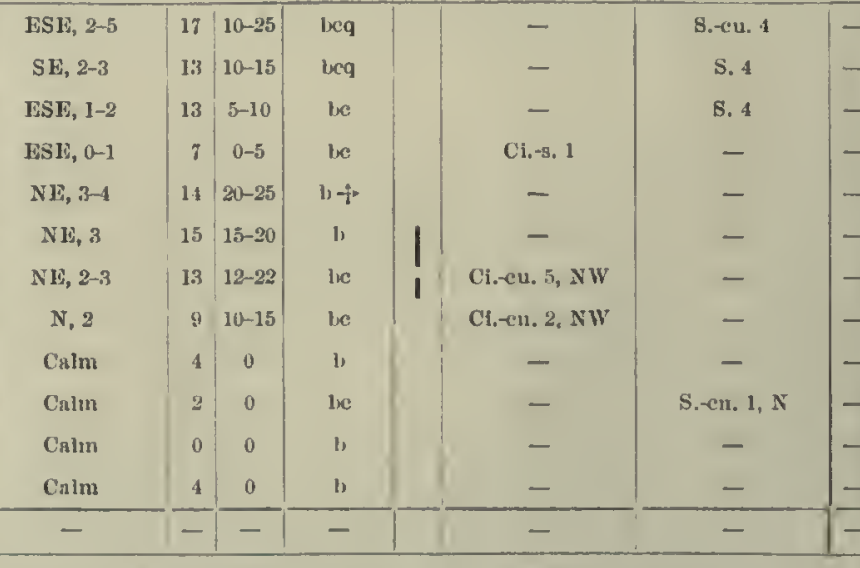

Max. therm. read at $\left\{\begin{array}{l}8 \mathrm{a}, 0 \\ 3 \mathrm{p},-10^{\circ} \cdot 2 .\end{array}\right.$
Solur radiatlon $\left\{\begin{array}{l}\text { black bull, } 32^{\circ} \cdot \% \text {. } \\ \text { briglit bulb, }\end{array} 0^{\circ} 0_{0}\right.$.

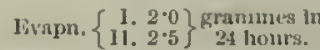

Precipm. - in.
Remarks.

- Falnt anroral arch SK to SW, but vely

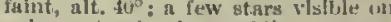
going out: clearing rathltly. with henes clonds all round horiyon, b a.min. Clonds only forming a very thlu lim, cepperedinly nenr zenith

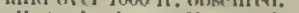
silghtly; 310 lam to $W^{\prime}$ visilile.

lint temp. at Cape Armitage, $-27^{\circ} 5$. tion to W. Ninst dlatant land of

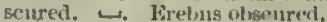

1 p.n. Brethis' summit eleared, smoke polng SSE. hanks of semi passing orer enst ern liflls; surumits of Ohservat lou alul Crater nills obsenrerl. Rain band, 3.

on $\mathrm{N}$, NE, and $\mathrm{Fing}$ sides of exinstal ohjects.

p.m. Wixcessive coatlung of rime $m$ anemometer glass, also on thernometers inslle sereen; clonds troken and thlin a long N horizont.

p.m. lime on everything; smo ju 
Methonological Journa, of the "Dliscovery."

[1903 - September.

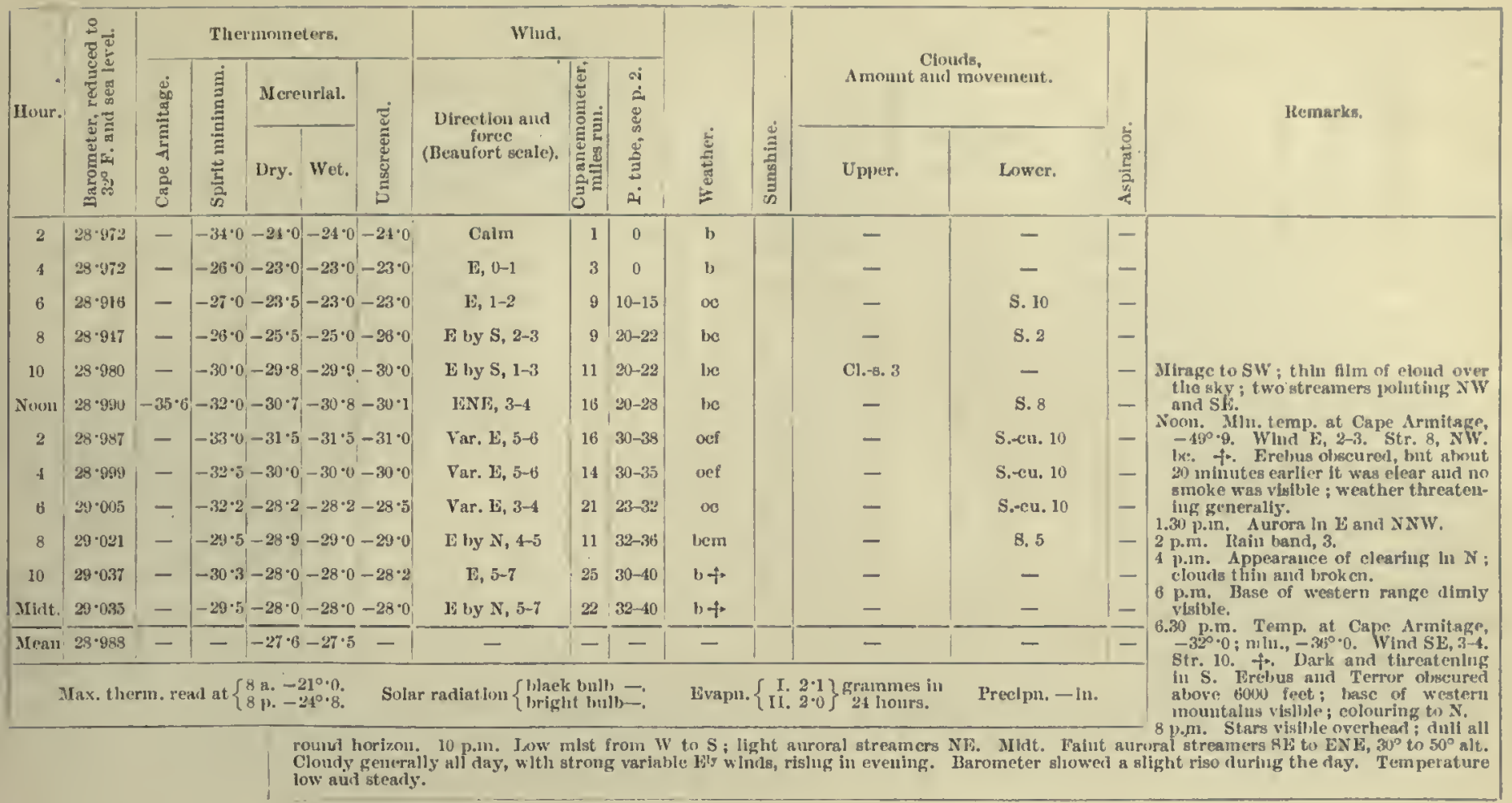

13th.

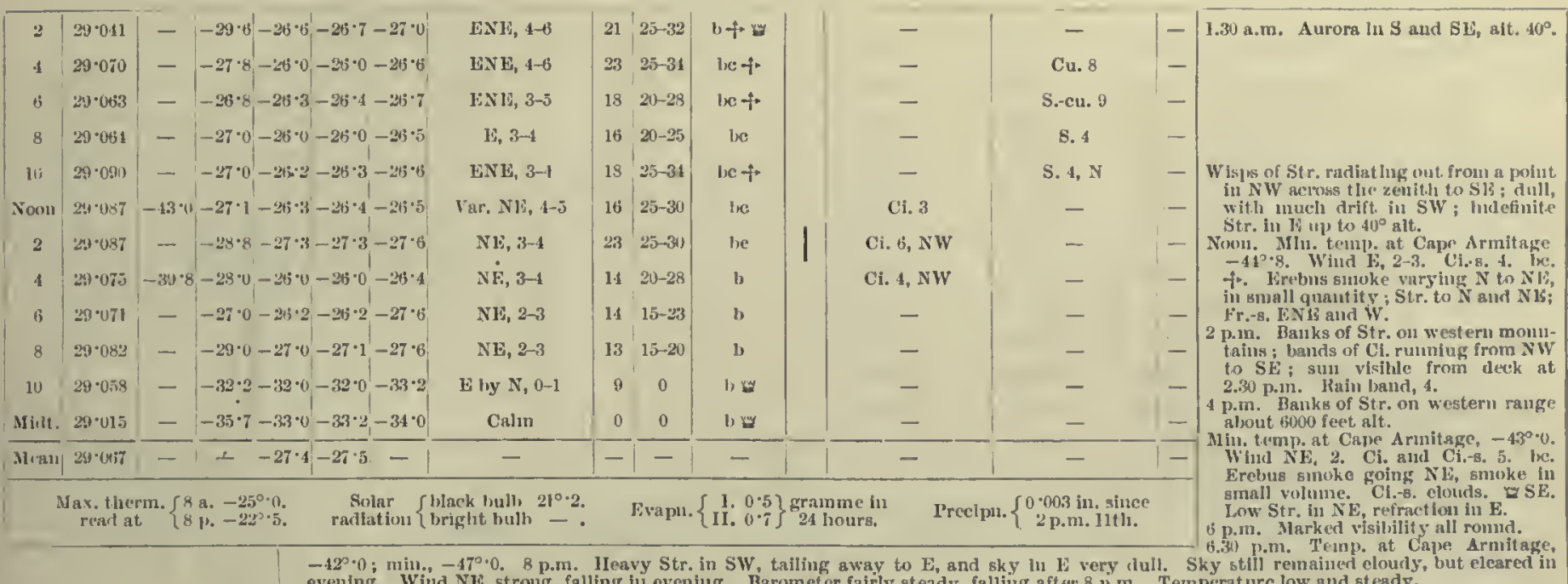

14 th. $-42^{\circ} 0$; min.,
evening. Wind NE, strong, falling in eveniug. Barometer fairly steady, falling after 8 p.m. Temperat urc low and steady.

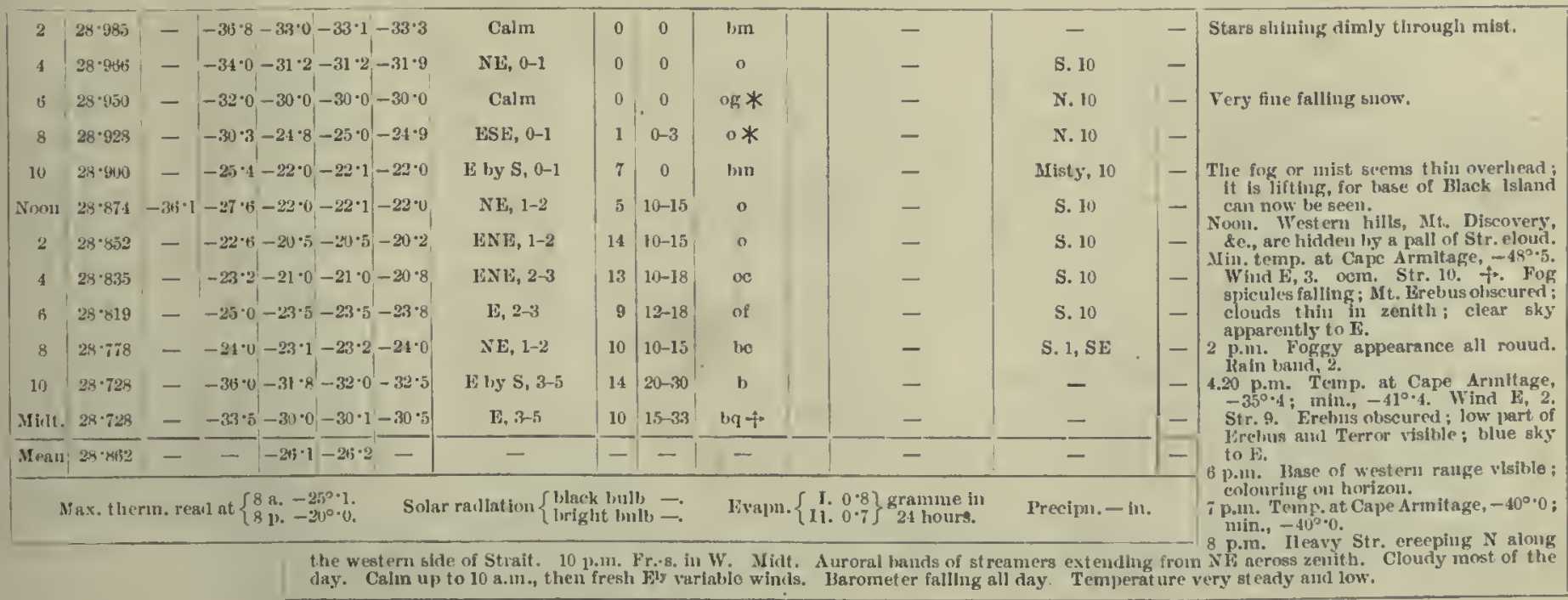


1903 -Septenber.]

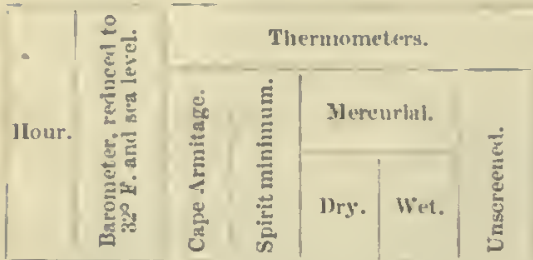

16 th.

\begin{tabular}{|c|c|c|c|c|c|}
\hline 2 & $2 s+675$ & - & $-30 \cdot 5-26 \cdot 2$ & $-2 t i \cdot 8$ & $-27 \cdot 0$ \\
\hline 4 & $2 x-633$ & - & $-38 \cdot 5-28 \cdot 2$ & $-2 \times \cdot 2$ & $-28 \cdot 2$ \\
\hline 6 & $28 \cdot 671$ & - & $-28 \cdot 5-28 \cdot 1$ & $-28 \cdot 1$ & $-28 \cdot 9$ \\
\hline 8 & $28 \cdot 693$ & - & $-31 \cdot 4-30 \cdot 5$ & $-30 \cdot 4$ & $-30 \cdot 2$ \\
\hline 10 & $28 \cdot 70.1$ & - & $-32 \cdot 4-31 \cdot 1$ & $-31 \cdot 4$ & $-31 \cdot 9$ \\
\hline Nimon & $28 \cdot 710$ & - & $-35 \cdot 0-31 \cdot 0$ & $-31 \cdot 0$ & $-81 \cdot 0$ \\
\hline 2 & $28 \cdot 782$ & - & $-35 \cdot 2-34 \cdot 0$ & $-31 \cdot 0$ & $-31 \cdot 2$ \\
\hline 4 & $28 \cdot 819$ & - & $-37 \cdot 2-31 \cdot 5$ & $-31 \cdot 5$ & $-31 \cdot 0$ \\
\hline 6 & $28 \cdot 898$ & - & $-37 \cdot 0-31 \cdot 2$ & $-31 \cdot 2$ & $-31 \cdot 2$ \\
\hline 8 & $28 \cdot 950$ & - & $-32 \cdot 6-30 \cdot 9$ & $-30 \cdot 9$ & $-31 \cdot 0$ \\
\hline 10) & $2 s \cdot 9 \pi 3$ & - & $-31 \cdot 0-32 \cdot 1$ & $-32 \cdot 1$ & $-32 \cdot 8$ \\
\hline MInilt. & $29 \cdot 022$ & - & $-35 \cdot 1-34 \cdot 8$ & $-31 \cdot 8$ & $-33 \cdot \tau$ \\
\hline
\end{tabular}

Mhtrolological Jourial of the "Discoviky."

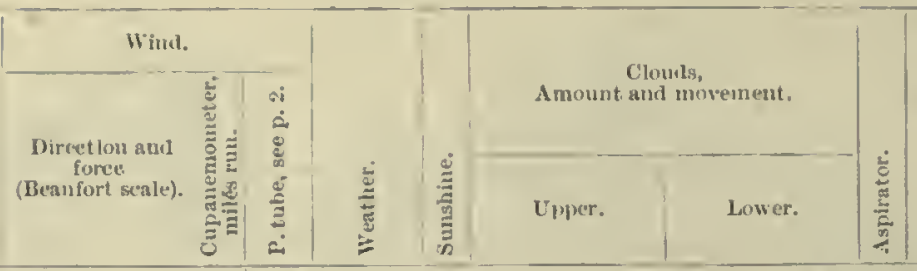

lemarks.

Var. E, 4-7 24 $25-40$ beq के

1i, 3-4 $15 \quad 15-30$ beq -

Var. 15, 4-7 26 25-42 beq + .

1s, 4-4

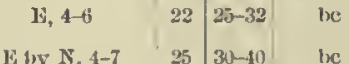

N15, 4-5 $23 \mid 30-35$ 1,

FNE, 6-8 29 $30-40$ liq

ENE, $0-7$

NE, 6-7

พา1, 7-8

N1\%, 7-8

N1: 6-7

26 25-35 b-

28 25-3is b-

$32 \quad 40-45 \quad b-i^{2}$

$3340-45^{\circ}$ b)

$24 \quad 35-42$ be

\begin{tabular}{|c|c|}
\hline - & Det. S. 1, ? Sl: \\
\hline- & S. 3 \\
\hline- & Fr.-8. 2 \\
\hline - & Fr.-s. 3,1 \\
\hline- & S.-cu. 1, Sl: \\
\hline- & s.-eu. 2, sli \\
\hline- & S. $2, \mathrm{SL}$ \\
\hline- & - \\
\hline - & - \\
\hline - & - \\
\hline- & - \\
\hline- & s. 2 \\
\hline- & - \\
\hline
\end{tabular}
round horizon.

Iеan $28 \cdot \sin -1-\overline{-31 \cdot 1}-31 \cdot 1-$

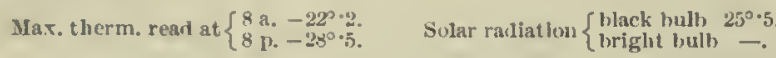

Evapu. $\left\{\begin{array}{cc}1 . & 0 \cdot 7 \\ 11 & 0.5\end{array}\right\}$ gramme in

2,4 , anแ 6 a.m. Detached Str, all

Preelyn. - in. 
Methorological, Joviral, of the "DiscoviliY."

[1903-September.

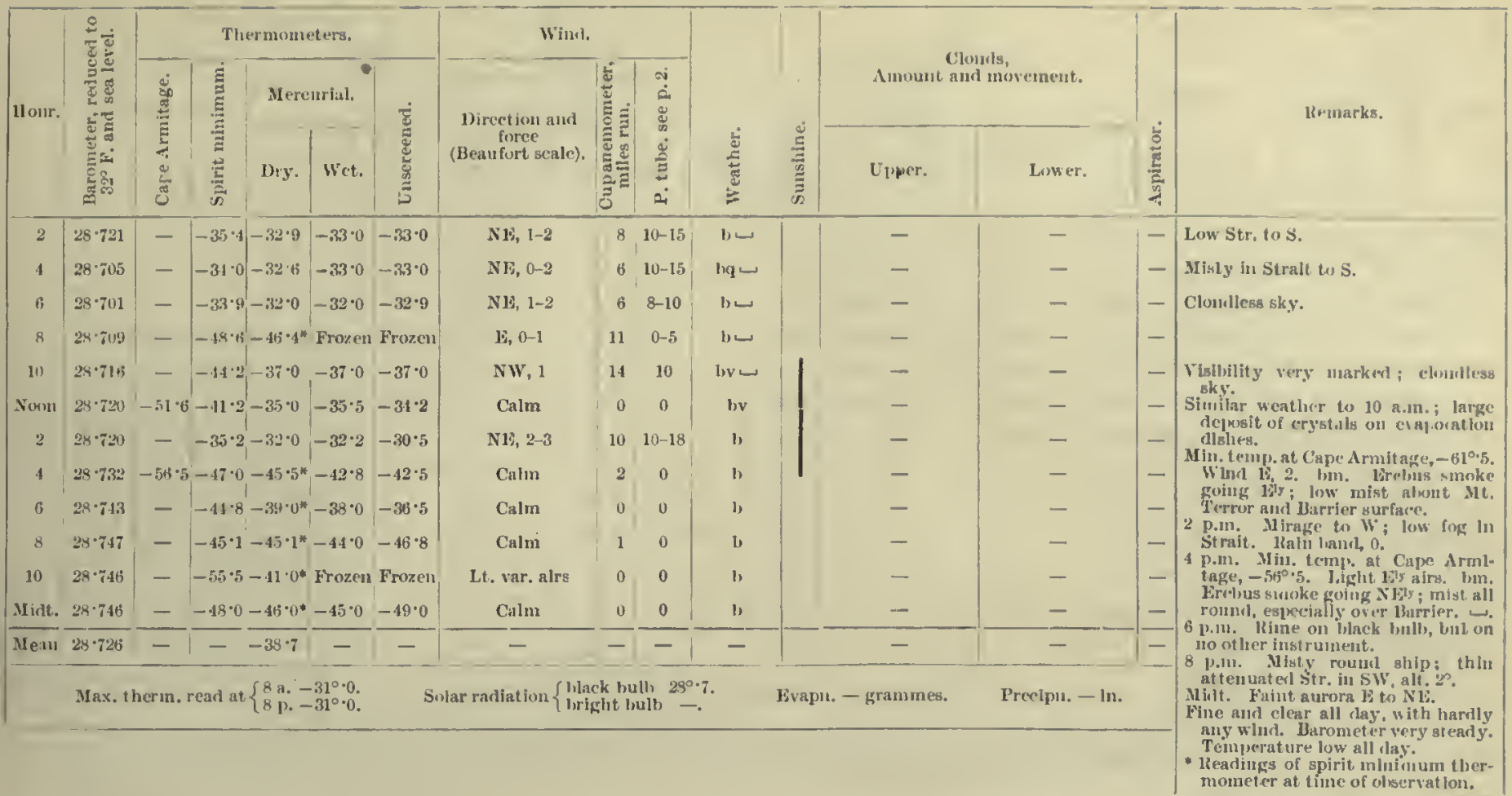

19 th.

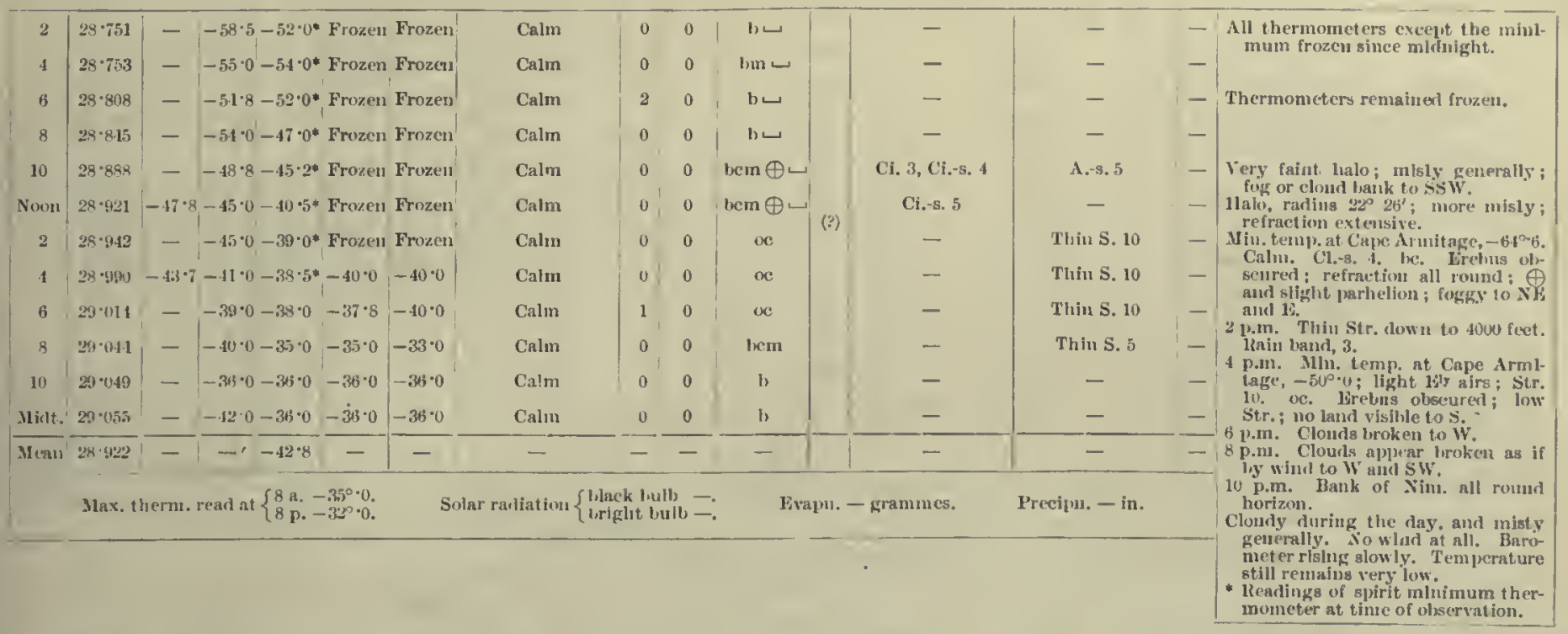

\begin{tabular}{|c|c|c|c|c|c|c|c|c|c|c|c|c|c|}
\hline 2 & $2 y \cdot 05 j$ & - & $-39 \cdot 0-31 \cdot 0$ & $-31 \cdot 0 \mid$ & $-33 \cdot 0 \mid$ & Colm & 0 & 0 & b) & - & - & - & Day lireak to S and SW. \\
\hline 4 & $29 \cdot 0 \overline{5} 6$ & - & $-39 \cdot 5-39 \cdot 0$ & $-39 \cdot 0$ & $-40 \cdot 0$ & Lt. $S$ airs & 0 & 0 & b & - & - & - & \\
\hline "3 & $20 \cdot 0.15$ & - & $-43 \cdot 0-41 \cdot 0$ & $-41 \cdot 0$ & $-43^{\circ} 0$ & Crim & 0 & 0 & lic & Cl.-8. 7 & - & - & Low lank of fog all rounl horizon. \\
\hline 8 & $29 \cdot 052$ & - & $-50 \cdot 5-46 \cdot 2 *$ & $-4.5 \cdot 0$ & Frozen & Calm & 0 & 0 & bem $\sqcup$ & Ci.-s. ${ }^{4}$ & S. 3 & - & Mist $y$ gencrally : Ci - -s. radiathng $\mathrm{N}$; \\
\hline 10 & $2 y \cdot 044$ & - & $-48 \cdot 0-41 \cdot 0^{*}$ & $-43 \cdot 0$ & Frozen' & Calm & 0 & 0 & $\operatorname{lem} \oplus \sqcup$ & Ci.-s. 5 & - & - & 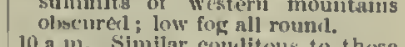 \\
\hline Noon & $29 \cdot 021$ & $-47 \cdot 0$ & $-44 \cdot 0-28 \cdot 8$ & $-2 \% \cdot 0$ & $-28 \cdot 8$ & $12,1-2$ & 2 & $111-18$ & $\operatorname{bem} \oplus$ & Ci.-8. 45 & - & - & $\begin{array}{l}10 \text { a.m. Siminar confitons to thrse } \\
\text { at on.m., hat molst thinner; Clo-s. }\end{array}$ \\
\hline 2 & $29 \cdot 604$ & - & $-31 \cdot 2-27 \cdot 0$ & $-27 \cdot 2$ & $-27 \cdot 3$ & SS1s, 1-2 & 8 & $8-12$ & $\boldsymbol{x}$ & - & Thin S. 10 & - & low-lying fog all romd; visibillty \\
\hline 8 & $28 \cdot 912$ & - & $-26 \cdot 8-22 \cdot 0$ & $-22 \cdot 0$ & $-22 \cdot 0$ & $13,3-4$ & 13 & $20-25$ & o & - & S. 10 & - & 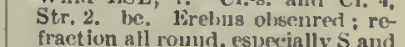 \\
\hline 19 & $28 \cdot 920$ & - & $-22 \cdot 3-21 \cdot 0$ & $-21 \cdot 0$ & $-21 \cdot 2$ & 1SNE, 2-3 & 11 & $\mid 2-17$ & be & - & Fr.-s. 8 & - & W : str, moving from $\mathrm{NE}$. \\
\hline Mint. & $28 \cdot \cong 1$ & - & $-27 \cdot 3-22 \cdot 0$ & $-22 \cdot 7$ & $-20 \cdot 6$ & 18. $2-4$ & 8 & $15-24$ & Ise & - & Fr.-8. 8 & - & $\begin{array}{l}\text { mirage to W. lain baud, } 5 \\
4 \text { p.m. }\end{array}$ \\
\hline Mcaul & 2,0002 & - & $1--31 \cdot 3$ & - & - & - & - & -1 & - & - & - & - & $\begin{array}{l}\text { tage, }-47^{\circ} \text {. o. Wind S1, } 1-2 . \text { Str. } \\
\text { 10. }\end{array}$ \\
\hline \multicolumn{5}{|c|}{ 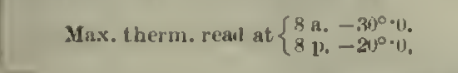 } & \multicolumn{3}{|c|}{ Solar raslat tom $\left\{\begin{array}{l}\text { blark bull, } \\
\text { Hrright luull, }\end{array}\right.$} & & \multicolumn{2}{|c|}{ Fivapu. $\left\{\begin{array}{l}1.0 .1 \\
11,0 \cdot 1\end{array}\right\}$ graume in hours. } & \multicolumn{2}{|l|}{ l'recipn. - int. } & $\begin{array}{l}\text { fraet lon excesslve ; } \smile \text { on thermo- } \\
\text { mieter. Minge to } W \text { all aftermoon. } \\
6 \text { p.m. Mina. }\end{array}$ \\
\hline
\end{tabular}


1903-September.]
Mrthorolonical Journal, of the "Discovery."

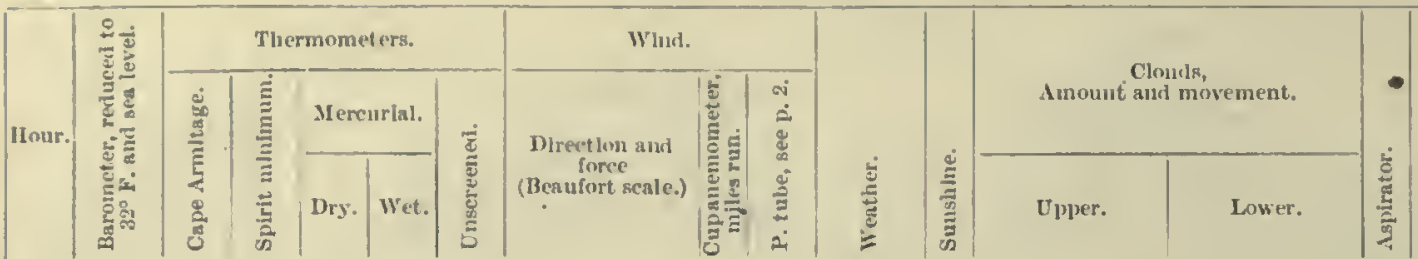

22nd.

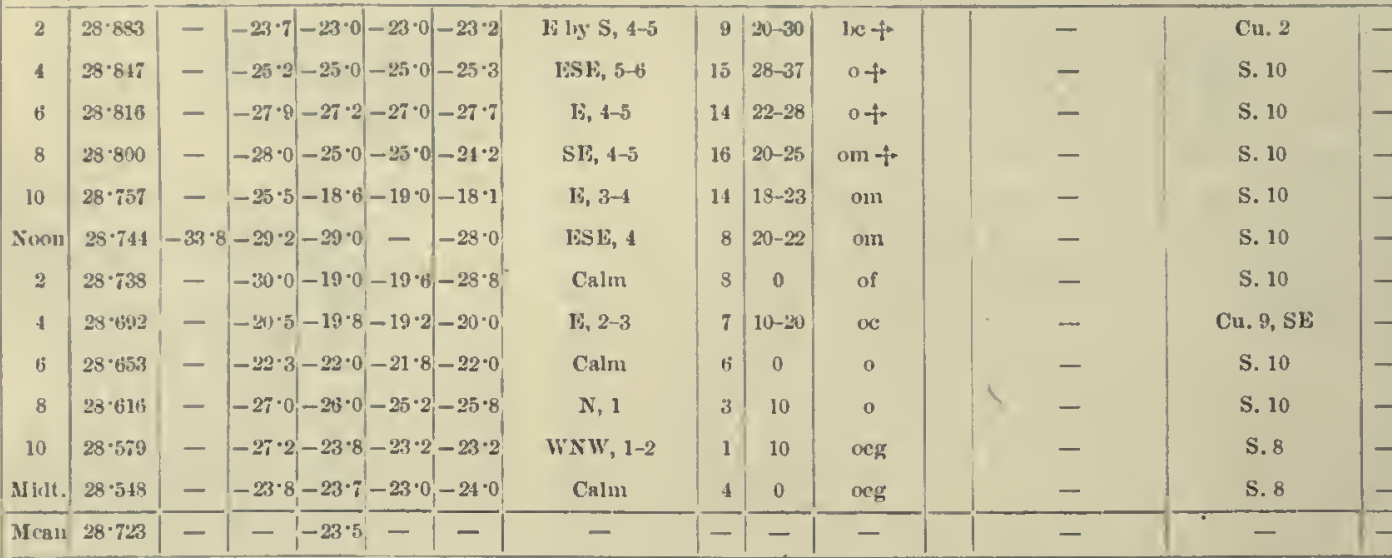

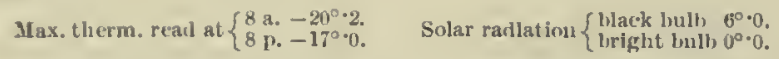

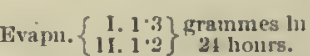

Precipu. - lı.

liemarks.

No land visible from SE to $W$.

Clearlug; Str. thin overhead; Brown Islant and western foot hllls visille up to $6000 \mathrm{ft}$.; very thick still to $\mathrm{S}$; visibility very nnarked; smin gleaming. Noon. Brown Island agaln olscurerd, and thick to s; Str. more dense; westeru monntains elear 113 to $12,000 \mathrm{ft}$. from thelr northern end to half-wny along range.

Min. tenul, at Cape Armitrge, $-41^{\circ} \cdot 8$.

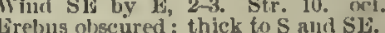
3 p.m. lRaln band, 3 . rears hlgher than iry; clouds broken. perature falliug at timeof high, temperature IIeavy clouds to $\mathrm{S}$ and $\mathrm{SW}$; l,right and angry looking cloulds to SW; thlek to $S$, and dark and thiek to $\mathrm{E}$.

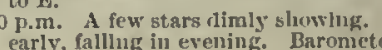
Midt. A few stars dimly showling; wind wane po
falling all day. Temperature very steady all day. 23rd.

\begin{tabular}{|c|c|c|c|}
\hline 2 & $28 \cdot 475$ & - & $-25 \cdot 0^{\prime}-20 \cdot 0|-20 \cdot 0|-20 \cdot 0$ \\
\hline 4 & $28 \cdot 430$ & - & $-18 \cdot 3-19 \cdot 1$ \\
\hline 6 & $28 \cdot 359$ & - & $-23 \cdot 0-23 \cdot 0-22 \cdot 8-23 \cdot 0$ \\
\hline 8 & $28 \cdot 351$ & - & $-23 \cdot 2-18 \cdot 0-17 \cdot 8-17 \cdot 5$ \\
\hline 10 & $28 \cdot 322$ & - & $-5 \cdot 5$ \\
\hline Noon & $28 \cdot 30.3$ & $-4 \cdot 8$ & $4 \cdot 0-3 \cdot 5$ \\
\hline 2 & $28 \cdot 3 k 3$ & - & $-3 \cdot 2$ \\
\hline 4 & $28 \cdot 371$ & $-2 \cdot 2$ & -1.5 \\
\hline ti & $28 \cdot 404$ & - & -0.7 \\
\hline 8 & $28 \cdot 490$ & - & $-8 \cdot 5$ \\
\hline 10 & $28 \cdot 534$ & - & $-12 \cdot 6-11 \cdot 5-12 \cdot 0-12 \cdot 0$ \\
\hline Vlidt. & $28 \cdot 600$ & - & $-13 \cdot 0-10$ \\
\hline
\end{tabular}

S. 10

S. 10

N. 10

N. 10

N. 10

N. 10

N. 10

S. 10

న. 10

S. 10

S. 8

N. 10

М ея11 28.418

ISW, 3-8

$20 \mid 15-58$ oeg $+2 *$

Max, therm. $\left\{8\right.$ a. $-16^{\circ} \cdot 0$. Solar
railiation black bulb $13^{\circ} \cdot 8$.
bright bulb

Wvapm. $\left\{\begin{array}{l}1,0 \cdot 7 \\ \text { II. } 0.6\end{array}\right\}$ gramme in

Procipu. $\{0 \cdot 056$ in. slue 0 real at $\left\{\begin{array}{l}8 \text { a. } \\ 8 \mathrm{p}\end{array}\right.$

Overenst all day, with suowfall and drift; wind very strong and

24 tll.

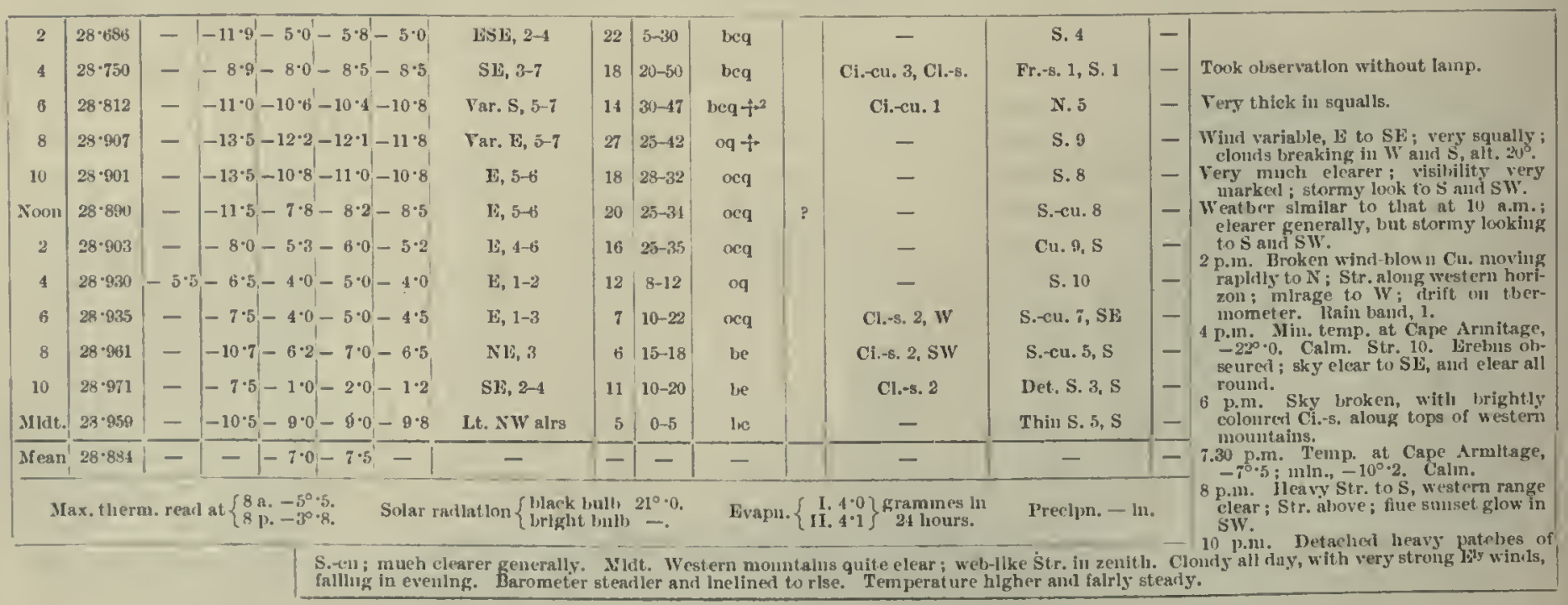


Metgohologicat, Journat, of tue "Discovery."

[1903-September.

\begin{tabular}{|c|c|c|c|c|c|c|c|c|c|c|c|c|c|c|}
\hline \multirow{3}{*}{ llour. } & \multirow{3}{*}{ 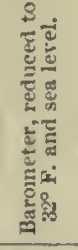 } & \multicolumn{5}{|c|}{ Theruomet ers. } & \multicolumn{3}{|l|}{ Wind. } & \multirow[b]{3}{*}{ 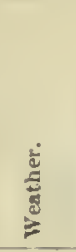 } & \multirow[b]{3}{*}{ 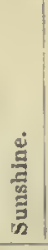 } & \multirow{2}{*}{\multicolumn{2}{|c|}{$\begin{array}{l}\text { Clouds, } \\
\text { Amount anil movenent. }\end{array}$}} & \multirow[b]{3}{*}{ 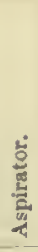 } \\
\hline & & \multirow{2}{*}{ 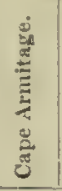 } & \multirow{2}{*}{ 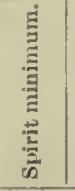 } & \multicolumn{2}{|c|}{ Mereurial. } & \multirow[b]{2}{*}{ 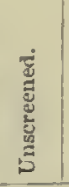 } & \multirow{2}{*}{$\begin{array}{l}\text { Direction and } \\
\text { force } \\
\text { (Beaufort seale) }\end{array}$} & \multirow{2}{*}{ 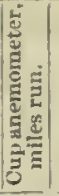 } & \multirow{2}{*}{ 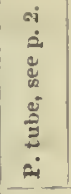 } & & & & & \\
\hline & & & & Dry. & Wet. & & & & & & & Upper: & Lower. & \\
\hline 2 & $28 \cdot 949$ & - & -10.5 & $-4 \cdot 0$ & $-4 \cdot 5$ & $-4 \cdot 5$ & $N, 2-3$ & 61 & $12-18$ & be & & - & S. 4 & - \\
\hline 4 & $28 \cdot 961$ & - & $-14 \cdot 0$ & $-11 \cdot 0$ & $-11 \cdot 9$ & $-14 \cdot 0$ & Calm & 4 & 0 & b & & - & - & - \\
\hline 6 & $28 \cdot 917$ & - & $-13 \cdot 4$ & $-6 \cdot 8$ & $-7 \cdot 5$ & $-7 \cdot 0^{\prime}$ & NNW, 1 & 5 & $8-10$ & bev & & Cl.-s, 3, SW (fast) & S. $2, \mathrm{~S}$ & - \\
\hline 8 & $23 \cdot 890$ & - & $-10 \cdot 5$ & $-5 \cdot 0$ & $-5 \cdot 8$ & $-5 \cdot 5$ & Var. N, 4-5 & 10 & $25-32$ & b & & - & - & - \\
\hline 10 & $23 \cdot 8,8 ;$ & - & $-6 \cdot 4$ & $1 \cdot 7$ & 0.3 & $3 \cdot 4$ & $\mathbf{N}, 1-2$ & 12 & $0-5$ & b) & & - & - & - \\
\hline Noon & $28 \cdot 87 i$ & 0.9 & $-2 \cdot 2$ & 3.5 & $2 \cdot 1$ & $3 \cdot 9$ & Var. N, 1-3 & 8 & $15-20$ & lwe & & Ci.-s. 6, SSW (fast) & - & - \\
\hline 2 & $28 \cdot 818$ & - & $0 \cdot 3$ & $4 \cdot 0$ & $3 \cdot 0$ & $4 \cdot 5$ & $N, 4-6$ & 14 & $23-35$ & by & & 一 & - & 一 \\
\hline 4 & $28 \cdot \pi 50$ & $9 \cdot 0$ & $2 \cdot 3$ & $6 \cdot 4$ & $5 \cdot 8$ & $6 \cdot 0$ & $N, 3-4$ & 18 & $18-25$ & b & | & - & - & - \\
\hline ; & $23 \cdot 771$ & - & $-11 \cdot 1$ & $-7 \cdot 0$ & $-7 \cdot 8$ & $-8 \cdot 0$ & NE, 3-4 & 19 & $20-28$ & b & & 一 & - & 一 \\
\hline 8 & $28 \cdot 733$ & - & $-11 \cdot 0$ & $-9 \cdot 0$ & $-9 \cdot 2$ & $-3 \cdot 8$ & INE, 7-8 & 20 & $35-48$ & $b q+$ & & - & - & - \\
\hline 10 & $28 \cdot 820$ & - & $-11 \cdot 5$ & -0.1 & -1.8 & $-1 \cdot 2$ & ESE, 2-4 & 21 & $15-25$ & heq & & - & S. 7 & - \\
\hline Mirlt. & $28 \cdot 814$ & - & -6.0 & $-4 \cdot 2$ & $-5 \cdot 0$ & $-4 \cdot 1$ & Lt. $\mathbf{N}$ airs & 7 & 0 & he & & - & S. 4 & - \\
\hline Meran & $28 \cdot 8513$ & - & - & $-2 \cdot 6$ & $-3 \cdot 5$ & - & - & -1 & - & - & & - & - & - \\
\hline
\end{tabular}

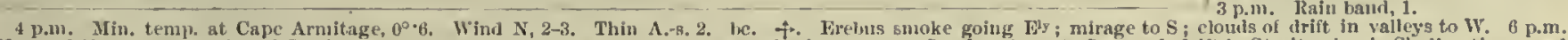

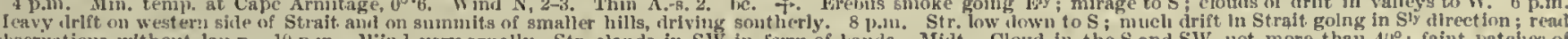
olservations withont laup. 10 p.1n. W'ind very squally, Str. clonds in SW in form of lands. Midt. Cloud in the $S$ and SW, not more than $40^{\circ}$; faint pateiles of aurora in $\mathrm{F}$, alt. $15^{\circ}$, and also a lltitle to $\mathrm{W}$ of
to fall. Temperaturo unst endy anil higher.

\begin{tabular}{|c|c|c|c|c|c|c|c|c|c|c|c|c|c|}
\hline $29 \cdot 7 \pi 3$ & -- & $-6 \cdot 5$ & 0.2 & $4^{\circ} 0$ & $8 \cdot 0$ & Lt. NW airs & 7 & 0 & be & & - & S. 9 & - \\
\hline $28 \cdot 771$ & - & $-0 \cdot 2$ & $2 \cdot 1$ & 0.8 & $2 \cdot 5$ & Very var. gusts & 5 & $0-5$ & ine & & - & S. 9 & 一 \\
\hline $28 \cdot 920$ & - & -0.1 & $5 \cdot 2$ & $5 \cdot 0$ & $5 \cdot 2$ & Var. SSW, 3-5 & 15 & $20-30$ & beq ${ }^{-5}$ & & - & S. 9 & - \\
\hline $28 \cdot 808$ & - & $5 \cdot 0$ & $\pi \cdot 8$ & $7 \cdot 2$ & $8 \cdot 0$ & S, 4-7 & 14 & $20-35$ & neq $i^{*}$ & & - & S. $8, \mathrm{~S}$ & - \\
\hline $28 \cdot 831$ & - & $7 \cdot 0$ & $i \cdot \pi$ & $6 \cdot 9$ & $7 \cdot 8$ & Far. SI, 1-5 & 17 & $10-25$ & ocq + & & - & S. 8 & - \\
\hline Nix) $28 \cdot 831$ & $6 \cdot 1$ & $6 \cdot(3)$ & $7 \cdot 1$ & $6 \cdot 1$ & $7 \cdot 0$ & SSF, 2-1 & 10 & $15-20$ & leq - & & - & S.-cu. $2, \mathrm{SW}$ & - \\
\hline $28 \cdot 818$ & - & $4 \cdot 0$ & $7 \cdot 2$ & $8 \cdot 0$ & $8 \cdot 2$ & S $1, y k, 2-4$ & 11 & $18-25$ & leq $t^{2}$ & ' & - & Cu. 2, Sw & - \\
\hline $29 \cdot 789$ & $6 \cdot 5$ & $4 \cdot 0$ & $7 \cdot 5$ & $8 \cdot 2$ & $7 \cdot 8$ & Lt. SW airs & 9 & 0 & lice & & $\mathrm{Cl}_{0}-8.5, \mathrm{SW}$ & - & 一 \\
\hline $29 \cdot 7.55$ & - & $4 \cdot 5$ & $11 \cdot 0$ & $8 \cdot 3$ & $10 \cdot 3$ & $\mathrm{~N}, 1$ & 5 & $0-8$ & b & 1 & - & - & - \\
\hline $28 \cdot i 38$ & - & $5 \cdot 8$ & $10 \cdot 0$ & $8 \cdot 0$ & $9 \cdot 5$ & Lt. $N$ airs & 4 & (1-8 & b & & - & - & - \\
\hline $28 \cdot 709$ & - & $3 \cdot 3$ & $3 \cdot 7$ & $2 \cdot 5$ & $3 \cdot 0$ & Calm & 4 & 0 & b & & - & - & $\begin{array}{l}-1.8 \\
-1.8\end{array}$ \\
\hline Mhilt. $28 \cdot 669$ & - & $-3 \cdot \pi \mid$ & $2 \cdot 0$ & 0.9 & $2 \cdot 1$ & $\mathrm{NE}, 1-3$ & 9 & $5-15$ & b & & - & - & $\begin{array}{r}1 \cdot 8 \\
3 \cdot 4 \\
1.6\end{array}$ \\
\hline an $28 \cdot 778$ & - & - & $6 \cdot 4$ & $5 \cdot 2$ & - & - & - & - & - & & $\longrightarrow$ & - & - \\
\hline
\end{tabular}

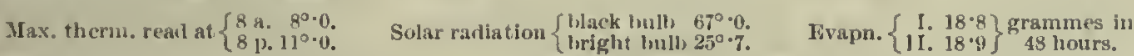

Precipn. - in.

to W ; leavy drift on surface.

3 p.m. Kain banl, o.

1.m. Min. temp. at Cape Amitage, Colut Niy. Ci.s,

8 p.m. Soune Ci.s. to S aml over western mountains; very gorgeous sunset colouring in SW. 10 p.m. Some Str. on the S, SW, and W horizon, below $30^{\circ} 1 \mathrm{n}$ alt. Micit. Some Str. still on $S$ horizon; clear to W and
inclinerl to fall. Tempcrnture high anil fairly steatly.

\begin{tabular}{|c|c|c|c|c|c|c|c|c|}
\hline 2 & $28 \cdot 625$ & - & $-7 \cdot 7-6 \cdot 2-6 \cdot 3-6 \cdot 7$ & $\mathrm{NE}, 5-6$ & 15 & $25-40$ & b & - \\
\hline 4 & $28 \cdot 589$ & - & $-10 \cdot 6-9.5-9.5-9 \cdot 4$ & Niv, 7-8 & 30 & $30-47$ & be & - \\
\hline 6 & 28.582 & 一 & $-11 \cdot 1-9 \cdot 9-10 \cdot 0-9 \cdot 9$ & $\mathrm{NE}, \mathrm{B}-7$ & 31 & $35-43$ & be & $\mathrm{Ci}_{.-s .} 6, \mathrm{~W}^{\circ}$ \\
\hline 8 & $28 \cdot 5.81$ & - & $-11 \cdot 5-5 \cdot 2-6 \cdot 0-5 \cdot 0$ & NE, 6-7 & 32 & $30-42$ & hev & $\mathrm{Ci}_{.}-\mathbf{s .} 5$ \\
\hline 10 & $29 \cdot 6 u 2$ & - & $-8 \cdot 3-5 \cdot 2-6 \cdot 0-4 \cdot 8$ & $N E, 4-5$ & 21 & $18-25$ & her & $\mathrm{Ci}_{.-3.6} 6$ \\
\hline Xinon & $23-723$ & $-12 \cdot 6$ & $-13 \cdot 3-2 \cdot 0-3 \cdot 2-1 \cdot 5$ & $N F, 3-5$ & 20 & $15-25$ & erq & $\mathrm{Ci}_{-}-\mathrm{s}, 4$ \\
\hline 2 & $28 \cdot 6.55$ & - & $-3 \cdot 2-1 \cdot 0-2 \cdot 0-1 \cdot 0$ & Lt. $\mathbf{N}$ airs & 7 & $0-i$ & $(x$ & Ci.cu. 3, SW \\
\hline 4 & $28 \cdot 6163$ & $-8 \cdot 0$ & $-7 \cdot 7-4 \cdot 5-5 \cdot 3-5 \cdot 2$ & Lt. SW airs & 3 & 0 & ac & Ci.cu. $6, \mathrm{SW}$ \\
\hline 15 & $28 \cdot 6 \cdot 83$ & - & $-12 \cdot 1-9 \cdot 0-9 \cdot 4-10 \cdot 0$ & Calm & 1 & 0 & be & Ci.-cu. 4, SW \\
\hline 8 & $28 \cdot 6.99$ & - & $-11 \cdot 0-10 \cdot 5-10 \cdot 8-10 \cdot 0$ & Calm & 0 & 0 & nc & - \\
\hline (1) & $2 * \cdot 689$ & $\dot{-}$ & $-10 \cdot 5-2 \cdot 2-2 \cdot 8-3 \cdot 0$ & Calın & 2 & 0 & o & - \\
\hline int. & $28 \cdot x+8$ & - & $\begin{array}{llll}-5.5 & 9 \cdot 5 & 7.5 & 9 \cdot 0\end{array}$ & SSE, 1-3 & 2 & $5-20$ & oq & - \\
\hline an & 28 & - & $--4 \cdot 6-5 \cdot 3$ & - & - & - & - & - \\
\hline
\end{tabular}

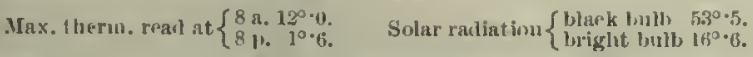

livapn. $\left\{\begin{array}{r}1.8 \cdot 5 \\ 11.8 \cdot 0\end{array}\right\}$ grammes in
- $\quad-5 \cdot 8$ The same streaks of Str. in $\mathrm{S}$ and - 6.5 sone In SW low dorrin on horlzen; - 9.8 .4 9.13 Similar coullitions to

s. to those at -10.1 6 2 a.m. Ci.n. -4.9 A.-s.ehiffly W over the mountalus; $-5 \cdot 8$ no drift. all night.

- 4.98 a.m. Visibllity marked; S.cu. in $-5 \cdot 6$ no general formation.

\begin{tabular}{l|l|l|l} 
A.-8. 5 & $-1 \cdot 7$ & 10 a.m. Much refraction low down
\end{tabular} S.-cu. $6 \quad-2$ all roumu, except in $\mathrm{S}$.

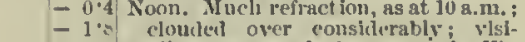
- $\quad-4.4$ billty very markers over to the W; - 5.4 Cnl. to N low down.

- $\quad-8 \cdot 3$ Min. temp. at Cape Armitage, HIgh S.-cn. $7, \mathrm{~S}-9.4$ MIt. Hrelus smoke going in $\mathrm{X}^{\prime}$ S. $10 \quad-40 \cdot 7 \cdot 2$ p.m. Dlstant movement of clouds

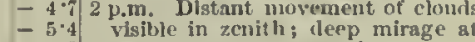
\begin{tabular}{l|l|l|l} 
S. 10 & $9 \cdot 5$ & base of west.erll mount ains.
\end{tabular} 8.1 3 p.m. Rain luand, 1 . 4 p.m. Ci.cu. In detached masses ruming from SW to NE. MIn. temp at Cape Arultage, $-14^{\circ} \cdot 3$. 1, ight NE airs. Mit. Nirthus smoke ascentiling vertically in thin columm only.

Precipu. - In.

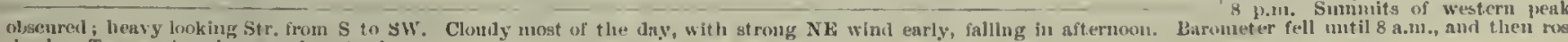
slowly. Temperature lower int unst ealy. 
1903 -September.]

Meteorotogicat, Jounat. of the "Discovery."

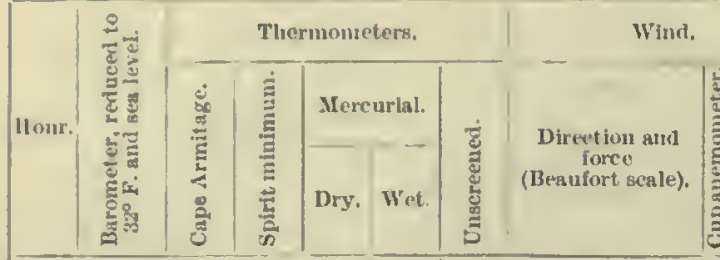

28th.

\begin{tabular}{|c|c|c|c|c|c|c|c|c|c|c|}
\hline 2 & $28 \cdot 708$ & - & $5 \cdot 8$ & $8 \cdot 0$ & $6 \cdot 8^{\prime}$ & $7 \cdot 9$ & S1: 0-1 & 11 & $0-5$ & 0 \\
\hline 4 & $28 \cdot 714$ & - & $6 \cdot 5$ & $11 \cdot 2$ & $11 \cdot 0$ & $11 \cdot 0$ & S l, $1: 4-6$ & 9 & $20-40$ & beq f- \\
\hline 6 & $28 \cdot 746$ & - & $6 \cdot 8$ & $12 \cdot 0$ & $10 \cdot \pi$ & $12 \cdot 0$ & $\mathrm{SF}_{2}, 2-6$ & 10 & $5-10$ & heq \\
\hline 8 & $28 \cdot 746$ & - & $8 \cdot 0$ & 9.8 & $9 \cdot 5$ & $9 \cdot 8$ & $S, 4-7$ & 17 & $20-38$ & bea $4+2$ \\
\hline I0 & $23 \cdot 766$ & - & $8 \cdot 5$ & $12 \cdot 0$ & $11 \cdot 8$ & $13 \cdot 8$ & Var. S, 5-9 & 21 & $25-56$ & oeq $+^{2}$ \\
\hline Nown & $23 \cdot 785$ & - & $11 \cdot 0$ & $12 \cdot 8$ & $12 \cdot 5$ & $12 \cdot 8$ & $S, 6-n$ & 26 & $35-58$ & oq +2 \\
\hline 2 & $28 \cdot 8.44$ & - & $10 \cdot 2$ & $12 \cdot 0$ & $11 \cdot 5$ & $12 \cdot 2$ & $\mathrm{SSE}, 1-1$ & 20 & $20-35$ & oq $\div+2$ \\
\hline 4 & $23 \cdot 879$ & $8 \cdot 0$ & $9 \cdot 0$ & $10 \cdot 2$ & $10 \cdot 0$ & $10 \cdot 4$ & $\mathrm{SE}, 4-6$ & 18 & $20-33$ & oq $\div$ \\
\hline 6 & $28 \cdot 9.21$ & - & $7 \cdot 3$ & $8 \cdot 0$ & $8 \cdot 0$ & $7 \cdot 3$ & $\mathrm{~N}, 4-6$ & 28 & $20-32$ & $o q+$ \\
\hline 8 & $28 \cdot 993$ & - & $-9 \cdot$ & $-9 \cdot 5$ & $-9 \cdot$ & $-9 \cdot 8$ & $\mathrm{~F}, 6$ & 22 & $28-36$ & beq \\
\hline 10 & $29 \cdot 083$ & - & $-11 \cdot 5$ & $9 \cdot 8$ & $\theta$ & $-10 \cdot 2$ & $\mathrm{E}$ by $\mathrm{N}, 4-6$ & 24 & $31-45$ & beq + \\
\hline Mirt. & $29 \cdot 229$ & - & $-10^{\circ} 0$ & $-10 \cdot 0$ & -10.0 & $-10 \cdot 6$ & E, $3-5$ & 21 & $23-36$ & beq + \\
\hline Mean & $28 \cdot 865$ & - & - & $5 \cdot 6$ & $5 \cdot 3$ & - & - & - & - & - \\
\hline
\end{tabular}

Clourts,

Amount and movement.

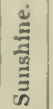

Epper.

\begin{tabular}{|c|c|}
\hline - & S. $10, \mathrm{~s}$ \\
\hline Cl. -8.5 & S. 7 \\
\hline Ci.-s. & $\mathrm{Cu} ., \mathrm{S}$ \\
\hline$C i,-8,2$ & S.-cu. 1 \\
\hline - & $\begin{array}{l}\text { Iligh S. 9, s } \\
\text { (slow) } \\
\text { S. } 10\end{array}$ \\
\hline 一 & S. 10 \\
\hline - & S. 10 \\
\hline - & N. 10 \\
\hline A. -8.4 , SSW & Fr.-n, 3, S (rapld) \\
\hline - & S. cu. 6, $\mathrm{S}$ (fast) \\
\hline - & S. 5, SSW \\
\hline
\end{tabular}

$\cdot 2$ Clours dark over west ern mountains. 11.3 Thick to $\mathbf{N}$ and $W$; lurighter to $\mathrm{S}$ 12.2 Dense clonds to $\mathrm{N}$; Cu. travelling 10.8 very mpidly; curlons Str. clond. 10.0 orer western mountalns. $7 \cdot 58$ a.m. 1) 1rift too thlek to see clouds 12.9 aromil ; blue sky overliead. $12 \cdot 010$ a.m. Winl variahle het ween SSF 1.1. and SW; very gusty ; no land $12 \cdot 2$ visithe througli arit. $\cdot 2$ Noon. Very squally and heavy drift. 11.5 no land visilile; evaporation olser10.0 vation not taken.

$\cdot 02$ p.m. Wind squally and variable lieary nirit on surface.

S. 5, SSW

$0 \cdot 4 \quad-18^{\circ} \cdot 5$. Wind SSl, 3-5, Amitage

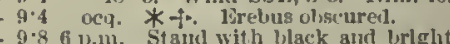
-10.1 bullhs blown over, bulls hroker.

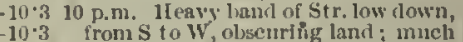
lrift during squalls. Overeast and cloudy most of the day with very strong varial,le $\mathbf{E}$ and whut. Barometer risling all oluy Temperature high, but fell quickly in evening.

29 th.

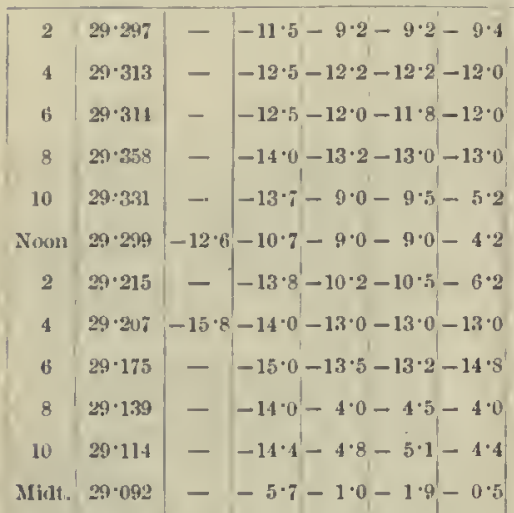

\begin{tabular}{|c|c|c|c|c|c|}
\hline FSE, 2-4 & 20 & $19-35$ & oc $f$ & - & S. 10 \\
\hline E by N, 2-4 & 14 & $16-32$ & $b c+$ & - & S. $2, \mathbf{E}$ \\
\hline ENE, :3-4 & 24 & $22-36$ & be & - & S. 1, S1: \\
\hline ENE, 4-5 & 22 & $28-30$ & be & $\mathrm{Cl},-8,5, \mathrm{SE}$ (mot.) & S. 2 \\
\hline Lt. E airs & 9 & 0 & lie & Ci.-s. 2, E & - \\
\hline Calm & 2 & 0 & bem & Ci.-s. 2, E & - \\
\hline Lt., $N$ alrs & 3 & 0 & $b$ & - & - \\
\hline $\mathrm{NW}, 1-2$ & 6 & $8-12$ & b & - & - \\
\hline Caln & 4 & 0 & lwe & $\mathrm{Ci} . .8 .2$ & - \\
\hline Lt. $\mathbf{N}$ airs & 2 & 0 & be $(U$ & $\mathrm{Ci}_{.-8 .} 3$ & - \\
\hline NNE, 2-3 & 2 & $15-20$ & $b f=L$ & - & - \\
\hline NE, 4-5 & 18 & $20-30$ & b $+\frac{2}{2}$ & - & - \\
\hline- & - & - & - & - & - \\
\hline
\end{tabular}

- 8.7 Drifting heavily at times, land only - $9 \cdot 0$ visible close to. $-12 \cdot 1$ Very low Str. having apprarance of $-12 \cdot 6$ fog pouring over hit -12.1 gap; surface drift. -13.0 detached Str. elomis laving peculiar -13.4 wavy shape orer western mour-

- 7.2 tains. Clearing generally; inlsty $-10 \cdot 3$ over land tos, sw, and $w$. $-10 \cdot 3$
$-10 \cdot 3$
-100 Non. Misty all round.

$10 \cdot 3$ II in. temp, at Cape Armitrge, $-18^{\circ} \cdot 9$ 13.0 Calm. Fr.ci.-s. 3, lirehus smoke $-13 \cdot 4$ going to $\mathrm{NW}$.

\begin{tabular}{l|l}
$-13 \cdot 0$ & $3 \mathrm{p} . \mathrm{m}$. \\
$-13 \cdot 7$ & $4 \mathrm{p} . \mathrm{m}$. Cin band, $\mathrm{N}$. $\mathrm{N}$. \\
- &
\end{tabular}

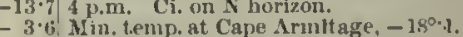
$-4 \cdot 2$ Min. lemp. at Cape Armit age, -180.1,
-4.2 $-3 \cdot 3$
to $\mathrm{N} W$ $4 \cdot 7$ to p.n. Colonred Str. in $W$. - 0.48 p.m. Ci.s. over west erru mountains -1.8 in long lines lying $\mathrm{N} 1 \mathrm{w}$ and $\mathrm{SW}$.

$\overline{11+111} \overline{29 \cdot 211}-\overline{-}-\overline{-9.3-9.4}-$

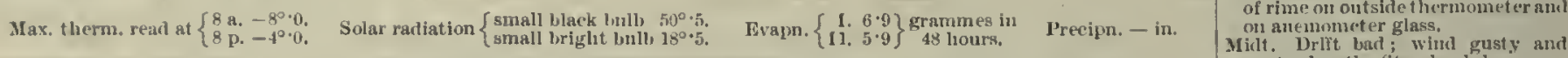
p.m. Ci, -s. over western mounSW; thin Str. tos; stellateerystals of rimeon ontside thermomet erant

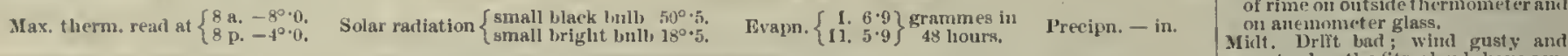
centrated in the SW and SSW, and appear to lle $\mathrm{E}$ and W. Fine generally mul with less wind. $\Lambda$ NE wint ireshencel up in evening. Barometer rose until 8 a.m., and then fell for rest of day. Temperature very unsteady.

30 th

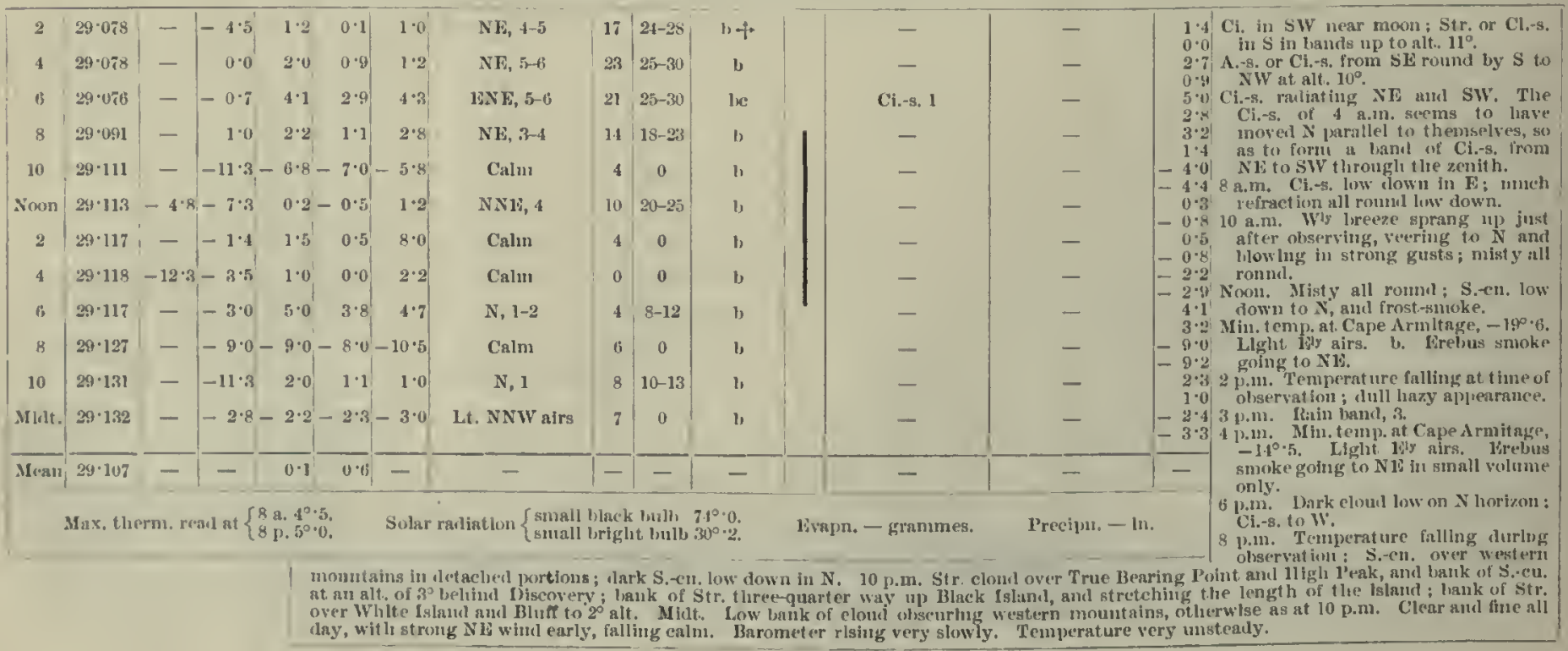


Meteorologicat, Jouinal of tire "Discovery."

[1903-October.

\begin{tabular}{|c|c|c|c|c|c|c|c|c|c|c|c|c|c|c|}
\hline \multirow{3}{*}{ liour. } & \multirow{3}{*}{ 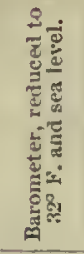 } & \multicolumn{5}{|c|}{ Thirmonicters. } & \multicolumn{3}{|l|}{ Wiul. } & \multirow[b]{3}{*}{$\stackrel{\dot{I}}{=}$} & \multirow{3}{*}{ 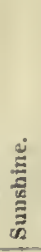 } & \multirow{2}{*}{\multicolumn{2}{|c|}{$\begin{array}{l}\text { Clourls, } \\
\text { Ainount aurl niovemuent. }\end{array}$}} & \multirow{3}{*}{$\frac{\grave{5}}{\frac{5}{2}}$} \\
\hline & & \multirow{2}{*}{ 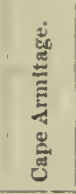 } & \multirow{2}{*}{ 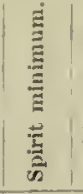 } & \multicolumn{2}{|c|}{ Mercurial. } & \multirow{2}{*}{ | } & \multirow{2}{*}{$\begin{array}{c}\text { Direction andi } \\
\text { force } \\
\text { (Benufort sule). }\end{array}$} & \multirow{2}{*}{ 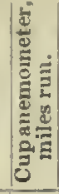 } & \multirow{2}{*}{ 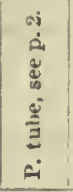 } & & & & & \\
\hline & & & & Dry. & Wet. & & & & & & & Upier. & Lower. & \\
\hline 2 & $29 \cdot 129$ & - & $-5 \cdot 0$ & -4.9 & $-5 \cdot 0$ & $-5 \cdot 4$ & NNF, 1 & 3 & $8-12$ & b & & - & - & -4.4 \\
\hline 4 & $29 \cdot 124$ & $\rightarrow$ & $-11 \cdot 0$ & $-10 \cdot 1$ & $-10 \cdot 0$ & $-10 \cdot 5$ & NE ly F, $5-6$ & 15 & $25-31$ & $b+$ & & - & - & $\begin{array}{l}-5.4 \\
=9.4\end{array}$ \\
\hline B & $29 \cdot 130$ & - & $-13 \cdot 5$ & $-11 \cdot 0$ & $-11 \cdot 0$ & $-11 \cdot 1$ & NE, 5-6! & 22 & $28-33$ & $b+$ & & - & - & $=10.8$ \\
\hline 8 & $29 \cdot 133$ & - & $-13 \cdot 0$ & $-10 \cdot 2$ & $-10 \cdot 5$ & $-10 \cdot 2$ & $\mathrm{NE}, \mathrm{E}-1)$ & 23 & $30-35$ & ux & ' & - & $\begin{array}{l}\text { Det. Fr, }-5,5,5 \\
\text { NNW (fast) }\end{array}$ & $\begin{array}{l}-10.3 \\
-10.8 \\
-10.8\end{array}$ \\
\hline 11) & $29 \cdot 139$ & - & $-11 \cdot 5$ & $-8 \cdot 8$ & $-9 \cdot 0$ & $-8 \cdot 5$ & NE, 4-5 & 21 & $20-30$ & oe & & - & S.-cu, $9, N$ & $\begin{array}{r}-10.8 \\
-8.7\end{array}$ \\
\hline Noun & $29 \cdot 121$ & $-12 \cdot 0$ & $-11 \cdot 0$ & $-9 \cdot 5$ & $-9 \cdot 8$ & $-9 \cdot 2$ & N $\mathbf{E}, 4-5$ & 19 & $20-30$ & $\infty$ & & - & S. 10 & $=8.4$ \\
\hline 2 & $29 \cdot 0007$ & - & $-13 \cdot 0$ & $-12 \cdot 0$ & $-12 \cdot 2$ & $-11 \cdot 8$ & NE, 4-6 & 23 & $2 i n-35$ & $\mathrm{oq}$ & & - & S. 10 & $-12 \cdot 3$ \\
\hline 4 & $29 \cdot 093$ & $-14 \cdot 0$ & $-13 \cdot 3$ & $-12 \cdot 0$ & $-12 \cdot 0$ & $-12 \cdot 0$ & HNH, 5-6 & 22 & $28-33$ & o & & - & S. 10 & $-11 \cdot 7$ \\
\hline$B$ & $29 \cdot 078$ & - & $-13 \cdot 5$ & $-12 \cdot 0$ & $-12 \cdot 4$ & $-12 \cdot 3$ & $\mathrm{NF}, 5-0$ & 22 & $25-32$ & o & & - & S. 10 & $-12 \cdot 1$ (sic) \\
\hline 8 & $29 \cdot 065$ & - & $-14 \cdot 2$ & $-14^{\circ} 0$ & $-14 \cdot 0$ & $-14 \cdot 0$ & Var. $5,5-7$ & 22 & $20-40$ & $o q$ & & - & S. 10 & $\begin{array}{l}-14.1 \\
-14.1\end{array}$ \\
\hline 10 & $29 \cdot 051$ & - & $-14 \cdot 2$ & $-14 \cdot 0$ & $-14 \cdot 0$ & $-14 \cdot 0$ & $\mathrm{NE}, 5-6$ & 19 & $25-30$ & 0 & & - & S. 10 & $-14 \cdot 4$ \\
\hline Midt. & $29 \cdot 015$ & - & $-\overline{14} \cdot 5$ & $-13 \cdot 8$ & $-14 \cdot 0$ & $-13 \cdot 8$ & NE, 4-5 & 21 & $20-25$ & 0 & & - & S. 10 & $\begin{array}{l}-14 \cdot 4 \\
-14 \cdot 8\end{array}$ \\
\hline Mean & $29 \cdot 098$ & - & - & $\overline{-11 \cdot 0}$ & $-11 \cdot 2$ & - & - & - & - & - & & - & - & - \\
\hline
\end{tabular}

Max. therm, rearl at $\left\{\begin{array}{l}8 \text { a. } 3^{\circ} \cdot 5 . \\ 8 \text { p. }-.\end{array} \quad\right.$ Solar radlation $\left\{\begin{array}{l}\text { small hiack bult } 23^{\circ} \cdot 5 . \\ \text { small bright bulb } 5^{\circ} \cdot 5 .\end{array}\right.$

Evapn. - grammes.

Precipn. - In

Western unountalua clear; complete bank of Str. from SSE to SW at $2^{\circ}$ alt. Western mountains obsenred iy drift; conulderable surface and a.m. Ali higin land between $W$ and SSly ohacured by rlouds; only lower parts of liack,

8 a.m. Temperature failing during olsservation; Str, olscuring aif isur alove 2000 feet.
10 a.m. All fand obscuren above foon. Ent ireiy overeast; laul below 1500 feet sliowing.

Min, temp. at Cape Armitage, $-24^{\circ} \cdot 1$. W'ind NE, 2. Str. 10, Frebus obscurerl; clowis down to 2000 feet. p.m. Appearance of irift In Strait. p.m. Ram band, 2.

p.m. Mln. temp. at Cape Armitage, scured. Str, 1500 feet in alt.

p.m. Durk lilue streak (uroter sky) at entrance to Bay; lreak lu cloud to $W$. 8 p.m. Land oniy visibie below variable, NE to $\mathrm{L}$ by $\mathrm{S}$.

Clear early, hut becming overeast about $10 \mathrm{am}$., and remaining $\mathrm{so}$. Wind very stroug from N E all day. Barometer failing all day. Temperature very steady.

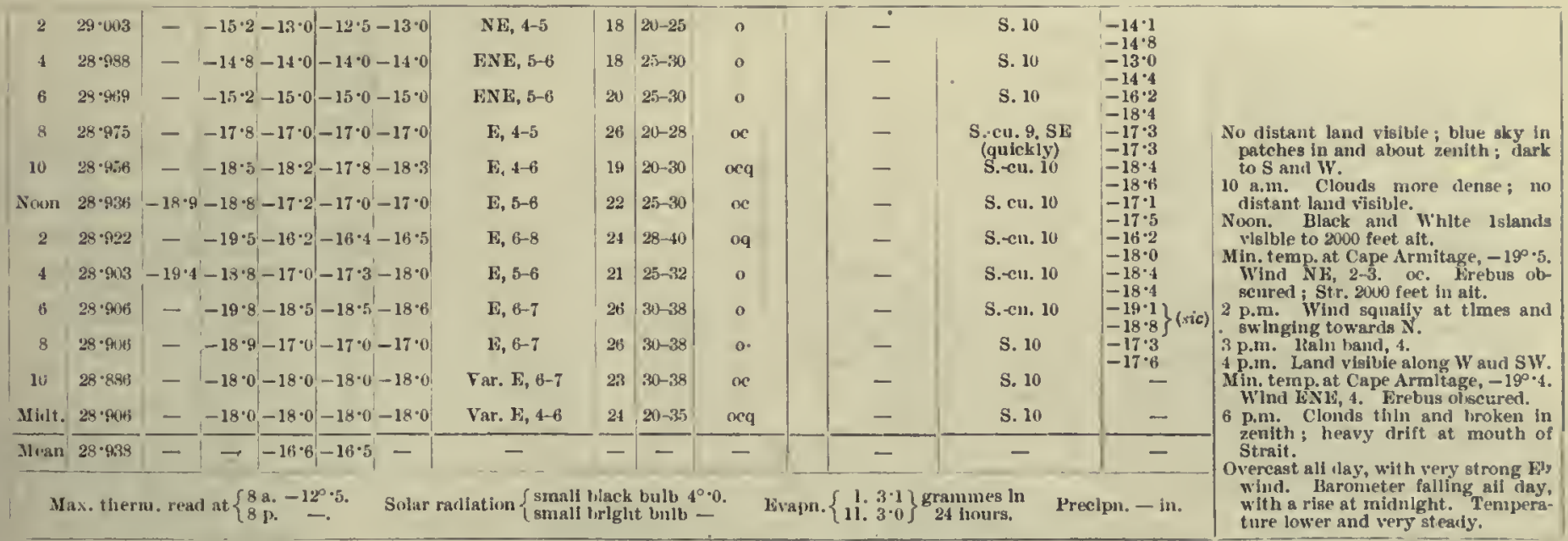

2nd.

\begin{tabular}{|c|c|c|c|c|c|c|c|c|c|c|c|c|}
\hline 2 & $23 \cdot 908$ & - & $|-21 \cdot 0-21 \cdot 0-21 \cdot 0|$ & $-21 \cdot 0$ & Var. E, 7-9 & 28 & $|30-45|$ & $o c q$ & & $-\cdot$ & S. 10 & - \\
\hline 4 & $28 \cdot 911$ & - & $-21 \cdot 0-21 \cdot 0-21 \cdot 0$ & $-21 \cdot 0$ & Var. W, 6-8 & 28 & $|30-41|$ & seq & & - & S. 10 & - \\
\hline if & $28 \cdot 912$ & - & $-22 \cdot 0-22 \cdot 0-22 \cdot 0$ & $-22 \cdot 0$ & 1., 6-8 & 28 & $30-40$ & $\operatorname{seq} *$ & & - & S. 10 & - \\
\hline 8 & $28 \cdot 931$ & - & $-23 \cdot 2-22 \cdot 2-22 \cdot u$ & $-22 \cdot 0$ & $E, B-7$ & 29 & $30-40$ & $\operatorname{ogf} \neq$ & & - & S. 10 & $\begin{array}{l}-22 \cdot 0 \\
-22 \cdot 2\end{array}$ \\
\hline 10 & $28 \cdot 9.9$ & - & $-23 \cdot 6-22 \cdot 0-22 \cdot 0$ & $-21 \cdot 0$ & $\mathrm{~N}, 5-7$ & 27 & $28-38$ & ireq & $i$ & - & Fr.-8. 1, Str. 8, NW & $\begin{array}{l}-22.2 \\
-21 \cdot 1 \\
-21 \cdot 6\end{array}$ \\
\hline Xom & $28 \cdot 948$ & $-25 \cdot 9$ & $-24 \cdot 8-22 \cdot 9-22 \cdot 6$ & $-22 \cdot 0$ & $18,6-7$ & $2 t$ & $30-40$ & be & i & - & 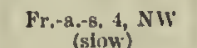 & $\begin{array}{l}-210 \\
-22 \cdot 0 \\
-22 \cdot 7\end{array}$ \\
\hline 2 & $28 \cdot 948$ & - & $-23 \cdot 6-22 \cdot 0-22 \cdot 0$ & $-20 \cdot 2$ & EN1, ti-7 & $2 i j$ & $28-35$ & lic & 1 & Cl.-8. 4 & (silow) & $\begin{array}{l}-22 \cdot 7 \\
-21 \cdot 1\end{array}$ \\
\hline 4 & $28 \cdot 915$ & $-27 \cdot 6$ & $-25 \cdot 5-22 \cdot 8-23 \cdot 8$ & $-23 \cdot 0$ & $15,5-6$ & 24 & $20-32$ & be & | & C1.-8. 4 & - & $\begin{array}{l}-23 \cdot 8 \\
-23 \cdot 8\end{array}$ \\
\hline B & $28 \cdot 9 \cdot 1.5$ & - & $-2 k \cdot 6-25 \cdot v-25 \cdot 0$ & $-25 \cdot 0$ & $1 \mathrm{n}, 5-6$ & 21 & $25-32$ & In & & Ci.-s. 3 & - & $-23 \cdot 8$ \\
\hline 8 & $28 \cdot 918$ & - & $-26 \cdot 8-25 \cdot 5-25 \cdot 5$ & $-2 s \cdot 0$ & N11, 5-6 & 23 & $28-34$ & he & & Ci.-s. 2 & S. 2 & $\begin{array}{l}-32.6 \\
-25 \cdot 6 \\
-20,0.0\end{array}$ \\
\hline 10) & $22 \times(M+M i)$ & - & $-2 \pi \cdot 0-25 \cdot 2-25 \cdot 2$ & -240 & NL, $5-16$ & 26 & 2835 & lice & & C1.-8. 2 & S. 3 & $-2 x \cdot 0$ \\
\hline M[iult. & $28 \cdot 674$ & - & $-2 \pi \cdot 0-25 \cdot 8-25 \cdot 8$ & $-26 \cdot 0$ & $N 14,5-6$ & 20) & $25-32$ & is & & $\mathrm{Ci}_{.-8 .} 2$ & S. 3 & $\begin{array}{r}-250 \\
-250 . \\
-20.0 \\
\end{array}$ \\
\hline$M \cos 1$ & $28 \cdot 910$ & $\rightarrow$ & $-\quad-23 \cdot 2-23 \cdot 2$ & - & - & - & - & - & -1 & - & - & - \\
\hline
\end{tabular}

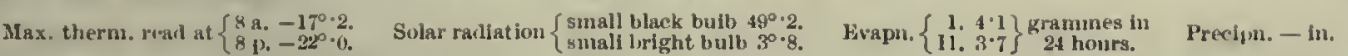


1903-October.]

4 th.

Methorolonical. Journal, of ThF "Discovery."

(s)

\begin{tabular}{|c|c|c|c|c|c|}
\hline \multirow{3}{*}{ Hour. } & \multirow{3}{*}{ 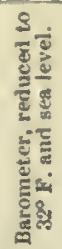 } & \multicolumn{4}{|c|}{ Thermometers, } \\
\hline & & \&્ष & $\begin{array}{l}\text { घี } \\
\underline{\Xi}\end{array}$ & Mereurial. & 8 \\
\hline & & $\frac{1}{8}$ & $\frac{5}{\frac{2}{2}}$ & Dry. Wet. & $\begin{array}{l}\text { 逽 } \\
5\end{array}$ \\
\hline
\end{tabular}

\begin{tabular}{|c|c|c|c|c|c|c|c|c|c|c|c|c|c|c|}
\hline \multirow{3}{*}{ liour. } & \multirow{3}{*}{ 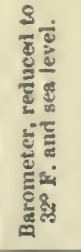 } & \multicolumn{5}{|c|}{ Thermometers, } & \multicolumn{3}{|l|}{ Wind. } & \multirow[b]{3}{*}{ 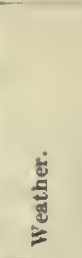 } & \multirow[b]{3}{*}{ 营 } & \multirow{2}{*}{\multicolumn{2}{|c|}{$\begin{array}{l}\text { Clouds, } \\
\text { Amount and inovement. }\end{array}$}} & \multirow[b]{3}{*}{ 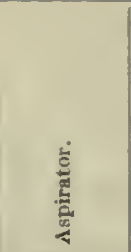 } \\
\hline & & \multirow{2}{*}{ 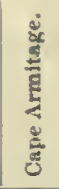 } & \multirow{2}{*}{ 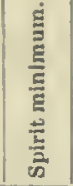 } & \multicolumn{2}{|c|}{ Mereurlal. } & \multirow{2}{*}{ 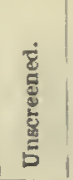 } & \multirow{2}{*}{$\begin{array}{c}\text { Direction and } \\
\text { force } \\
\text { (Beaufort seale). }\end{array}$} & \multirow{2}{*}{ 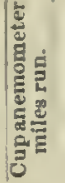 } & \multirow{2}{*}{ 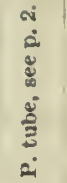 } & & & & & \\
\hline & & & & Dry. & wet. & & & & & & & Upper. & Lower. & \\
\hline 2 & $28 \cdot 983$ & - & $-30 \cdot 8$ & $-29 \cdot 8$ & $-29 \cdot 8$ & $-30 \cdot 2$ & NIs, 5-6 & 23 & $|25-30|$ & be & & C1.-8. 2 & Det. Fr. -s. 4, NIV & \\
\hline 4 & $28 \cdot 987$ & - & $-30 \cdot 8$ & $-30 \cdot 0$ & $-30 \cdot 0$ & $-30 \cdot 8$ & NE, E- B & 24 & $25-34$ & be & & $\mathrm{Ci}_{.-8,2} 2$ & Det. Fr.-8. $5, \mathrm{NW}$ & $\begin{array}{l}2 \text { to } 8 \text { a a.m. } \\
\text { mereury }\end{array}$ \\
\hline 6 & $28 \cdot$ : pou & - & $-31 \cdot 0$ & $-28 \cdot 0$ & $-28 \cdot 0$ & $-28 \cdot 8$ & NE, 5-6 & 22 & $25-32$ & be & & - & Det. Fr.-8. 5 & below scale \\
\hline 3 & $28 \cdot$ mon & - & $-29 \cdot 0$ & $-26 \cdot 0$ & $-26 \cdot 0$ & $-25 \cdot 9$ & N1E, 4-6 & 20 & $20-30 \mid$ & neq & & $\mathrm{Cl}_{.}-\mathrm{B} .2$ & S. 6 & $-25 \cdot 6$ \\
\hline 10 & $20 \cdot 002$ & - & $-27 \cdot 2$ & $-26 \cdot 3$ & $-26 \cdot 3$ & $-2 B \cdot 0$ & N1, 5-6 & 29 & $25-30$ & ne & & Ci.s. $3, \mathrm{NW}$ & A."- 7 & $\begin{array}{l}-260 \\
-2600\end{array}$ \\
\hline Nonn & $29 \cdot 018$ & - & $-29 \cdot 0$ & $-26 \cdot 0$ & $-23 \cdot 0$ & $-26 \cdot 0$ & NE, 4-8 & $2 \mathbf{i}$ & $20-34$ & $\operatorname{seq} \oplus$ & & $\mathrm{C1}_{0}-\mathrm{s}, \mathrm{t}, \mathrm{W}$ & A. -8.3 & $\begin{array}{l}-250 \\
-2500\end{array}$ \\
\hline 2 & $29 \cdot 023$ & - & $-28 \cdot 0$ & $-26 \cdot 0$ & $-26 \cdot 0$ & $-23 \cdot 0$ & NE, 3-4 & 27 & $15-23$ & the & & $\mathrm{Ci},-s, 4$, W & - & $\begin{array}{l}-21.7 \\
-23 \cdot 3\end{array}$ \\
\hline 4 & $29 \cdot 017$ & $-32 \cdot 8$ & $-27 \cdot 6$ & $-26 \cdot 8$ & $-2 t \cdot 8$ & $-25 \cdot 2$ & $\mathrm{NE}, 3-4$ & 20 & $13-20$ & toe & & Cl.-B, 4, W & - & -263 \\
\hline 6 & $29 \cdot 022$ & - & $-27 \cdot 5$ & $-28 \cdot 2$ & $-26 \cdot 3$ & $-26 \cdot 5$ & NE, 3-4 & 18 & $12-18$ & ine & & Cl.*s. 3. W & - & $\begin{array}{l}-24 \cdot 3 \\
-24 \cdot 9\end{array}$ \\
\hline 8 & $29 \cdot 012$ & - & $-26 \cdot 8$ & $-23 \cdot 0$ & $-23 \cdot 2$ & $-23 \cdot 0$ & $\mathrm{NE}, 2-3$ & 16 & $12-18$ & ise & & - & A.-s. 2. NNW & $\begin{array}{l}-2 \cdot .9 \\
-23 \cdot 4\end{array}$ \\
\hline 10 & $29 \cdot 017$ & - & $-24 \cdot 9$ & $-22 \cdot 0$ & $-22 \cdot 3$ & $-21 \cdot 8$ & $N, 0-1$ & 9 & 0 & 1) & & - & 一 & $\begin{array}{r}-24.3 \\
-224.9\end{array}$ \\
\hline Midt. & $29 \cdot 015$ & - & $-40 \cdot 6$ & $-40 \cdot 0$ & $-39 \cdot 2$ & $-41 \cdot 5$ & Calm & 1 & 0 & b & & - & - & $\begin{array}{l}\text { Nercury } \\
\text { below scale }\end{array}$ \\
\hline Ilean & $29 \cdot 006$ & - & - & $-27 \cdot 5$ & $-27 \cdot 5$ & - & - & $1-1$ & - & - & & - & - & - \\
\hline
\end{tabular}

Max. therm. read at $\left\{\begin{array}{l}8 \mathrm{a} . \\ 8 \mathrm{p.}\end{array}-24^{\circ} \cdot 2 . \quad\right.$ Solar radiation $\left\{\begin{array}{l}\text { small black bulb } 45^{\circ} \cdot 0 . \\ \text { small bright bulb } 0^{\circ} \cdot 0 .\end{array}\right.$

Evapn. $\left\{\begin{array}{l}\text { I. } 2 \cdot 9 \\ \text { 1I. } 2 \cdot 6\end{array}\right\} \underset{24 \text { hours. }}{\text { grammes in }}$

Precipn. - ln.

dimly visible and apparently very high. 8 p.m. Western mountaius quite clear; some Str. to SW hehind Mt. Discovery, aud also to $\mathrm{N}$ and $\mathrm{k}$.

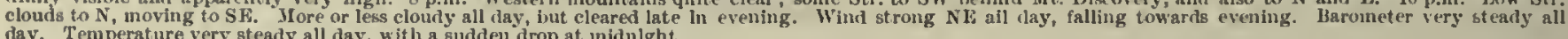
day. Temperature very steady all day, with a sudden drop at midnlght.
Remarks.

Western mountains visible below 5000 feet; thin Str. obscurlng all land at
visibility very markerl. of halo $22^{\circ} 33^{\circ}$. tage, $-29^{\circ} \cdot 6 ;$ min. $-33^{\circ} \cdot 5$. Wind WNE, 5. Thin Fr.-8.3, ait. 3000 to 4000 fect. Ci. 2 . T.
kirebus obscured; ialo with prismatic colours.

p.m. Str. all round northern horizon in loug, northern streaks, 1 in band 4

p.m. Deep mirage to west ward; irfit out in Strait.

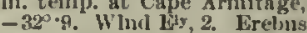
amoke going li. ixe. Mirage in $N$ and $W$ non. Similar weather; radins

.30 p.nı. Temp. at Cape Arml- 5th.

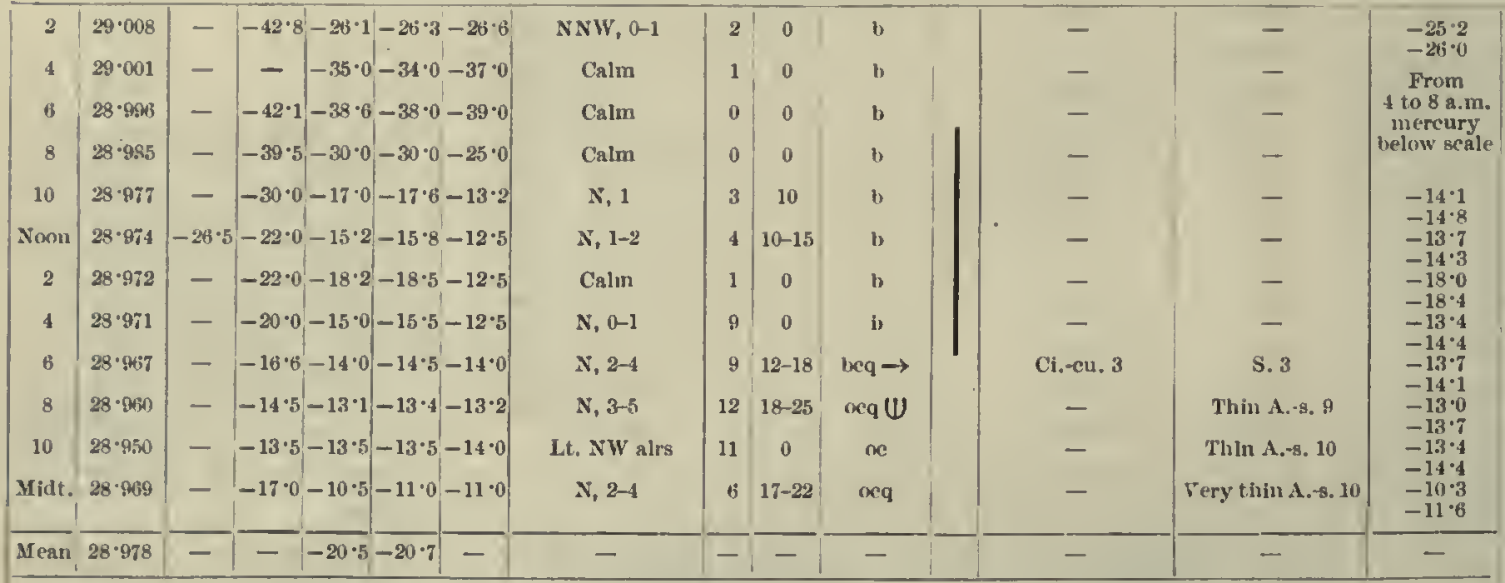

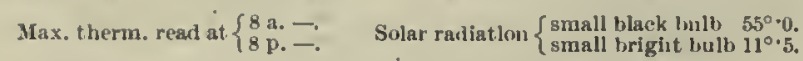

Fvapn. $\left\{\begin{array}{cc}1 . & 1 \cdot 7 \\ \text { II. } 1 \cdot 9 & \cdot 9\end{array}\right\} \begin{aligned} & \text { grammes In } \\ & 24 \text { hours. }\end{aligned}$

Precipn. - in.
Cloudiess exeept for line of $\mathrm{S} t \mathrm{r}$ from $\mathrm{S}$ to $\mathrm{SW}$ in detached $10 \mathrm{portions}$. Clourless except for some Str. low down to $\mathrm{S}$. Noon. Cloudless; Erebus si Noon, Cloudless : kreburs
visible going to WSW. Min. temp. at Cape Arinitage, $-43^{\circ} \cdot 5$. Erelvus smoke going to WsW lin large volume and from many orifices onn northern side; suloke looked like Ci.-8. as it strenmed a way tow. p.m. Air appents

3 p.m. Kain band, 5 .

4 p.m. Ci. in $\mathrm{N}$ and $\mathrm{n}$; str. to $S$ and $W_{;}$Mt. Discovery olus ures at sumit.

Sun was on thermometer at Cape Armitage, so ohsersa6 p.m. Templerature falling at time of observation, ; thipeatenlng apperance to $w$; ice crystals falling. 8 p.m. Very faint corona ; dark and tbreatening all round. 10 p.m. All dlstant land obscured. Midt. All distant land olscuret. Fine and ciea very steady. Temperature rising all day.

6 th

\begin{tabular}{|c|c|c|c|c|c|c|c|c|c|c|}
\hline 2 & $28 \cdot 990$ & - & $|-13 \cdot 5-8 \cdot 0-9 \cdot 0-11 \cdot 0|$ & $N, 1$ & 5 & 0 & e & A. $.8 .3, \mathrm{~S}$ (rapid) & S. 2 & $1-8.1$ \\
\hline 4 & $28 \cdot 969$ & - & $-14 \cdot 5-11 \cdot 8-12 \cdot 0-8 \cdot 0$ & Lt. var, alrs & 4 & 0 & e & A.-s. 3, S (rapid) & S. 3 & $-10 \cdot 1$ \\
\hline 6 & 28.963 & - & $-14 \cdot 5-13 \cdot 5-13 \cdot 2-11 \cdot 5$ & Caim & 2 & 0 & c & A.-8. $6, \mathrm{~S}$ & S. 4 & $\mid-12 \cdot 3$ \\
\hline 8 & $28 \cdot 897$ & - & $-14 \cdot 0-6 \cdot 4-6.8-6.5$ & NE, 3-4 & 2 & $18-23$ & $0+*$ & - & S. 10 & $\begin{array}{l}-13.2 \\
-6.3 \\
-6.9\end{array}$ \\
\hline 10 & $29 \cdot 005$ & - & $-10 \cdot 0-5 \cdot 2-5 \cdot 4-5 \cdot 0$ & NIS, 1-2 & 13 & $10-15$ & $0 \uparrow * *$ & - & N. 10 & $\begin{array}{l}-6 \cdot 1 \\
-5 \cdot 1\end{array}$ \\
\hline Noon & $28 \cdot 999$ & $-8 \cdot$ & $-5.5-0.2-0.60 .8$ & $\mathbf{N E}, 1$ & 7 & $8-10$ & of $*$ & - & N. 10 & $\begin{array}{r}-0.0 \\
-0.0 \\
-0.9\end{array}$ \\
\hline 2 & $28 \cdot 997$ & - & $-7 \cdot 5-7 \cdot 0-7 \cdot 0-5 \cdot 5$ & Lt. S alrs & 5 & $0-5$ & of $*$ & - & N. 10 & 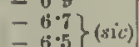 \\
\hline 4 & $29 \cdot 006$ & $-6^{\circ}$ & $\cdot 2-6 \cdot 2-6 \cdot 0$ & Calm & 1 & 0 & of $*$ & - & N. 10 & $\begin{array}{l}-8.5 \\
=6.5\end{array}$ \\
\hline 6 & $29 \cdot 005$ & - & \begin{tabular}{|lll}
$-7 \cdot 5$ & $4 \cdot 0$ & $3 \cdot 6$
\end{tabular} & NE, 1 & 3 & $8-10$ & of $*$ & - & N. 10 & $\begin{array}{r}-8.5 \\
4 \cdot 5 \\
3.9\end{array}$ \\
\hline 8 & $29 \cdot 014$ & - & $-5 \cdot 5-5 \cdot 2-5 \cdot 0-6 \cdot 8$ & Calm & 4 & 0 & of $*$ & 一 & Very thin $\mathbf{N}$. 10, & \\
\hline 10 & $29 \cdot 0 \mathrm{BB}$ & - & $-7 \cdot 7-5 \cdot 0-5 \cdot 0-5 \cdot 0$ & $\mathrm{x}, 0-1$ & 1 & 0 & b & - & seld, 1, SSW (tast) & $=-4.0$ \\
\hline Mlit. & $29 \cdot 023$ & - & $-5.7-1.0-1.0-0.8$ & Calm & 4 & 0 & ine & $\mathrm{Ci}_{\mathrm{i}}-\mathrm{s}, 3$ & - & $\begin{array}{l}=0.4 \\
=1.8\end{array}$ \\
\hline Mean & $28 \cdot 999$ & - & $-\quad-5 \cdot 5-5 \cdot 6-1$ & - & $\overline{-}$ & - & - & - & - & - \\
\hline & & & [smant & $\begin{array}{l}\text { ull, } 21^{\circ} \cdot 2.2 . \\
\text { bulb } 10^{\circ} \cdot 5 .\end{array}$ & & . & & $\begin{array}{l}\text { in } \\
\text { is. }\end{array}$ & $75 \mathrm{in}$. since $60 \mathrm{p}, \mathrm{m}$ & 11. 23 Sept. \\
\hline
\end{tabular}

Islands and west tern land elear: fragunents of Str. to SH, inuo teet alt., travelling rapidly to uorthwarts; clear overheas. 4a.1n. Islandis nearly olssenred; Str. descending on western hillis. Str. very low on western momintains, and land obseured. a.m. Onily land close to Mingle : there has been a
Blight deposit of snow erystals Blight deposit of snow erystals
betwecen 6 and 8 a.m. 10 a.me. Flakes small and hard, and not falling thickly; Nom, snowtlakes small ain bari, anu very thin ; fog and Min. Iemp. at Cape Aruitage, $-21^{\circ} \cdot 1$. Wind SK, 1. lirelns ohscured. Nim. 10, ov, $* 4$. 3 1.m. Llain hand, 7 . 4 p.m. Mlin. temp. at Cupe Arrmltage, $-10^{\circ} \cdot 6$. Calne. lirelus olsseured. Nim 10.

p.m. Snow fell continuonsly durimg tlue afternton, and reels hard and erisp when
troxlden on ; clomsls tinin to $\mathrm{B}$. W wth every appearance of lreaking ; temperature very unsteady, at 3.40 it was $-2^{\circ} \cdot 0$, and at 3.50 it was $-4^{\circ} 0$. 8 p.m. Nim. very thin overh ead, and scud passlug over

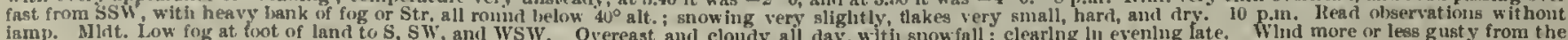
In. Baroneter lneifned to rise slowiy. Temperat ure made a qulek rise, and was then falrly steady. 
Meteorological Journal of the "Discovery."

[1903-0ctober.

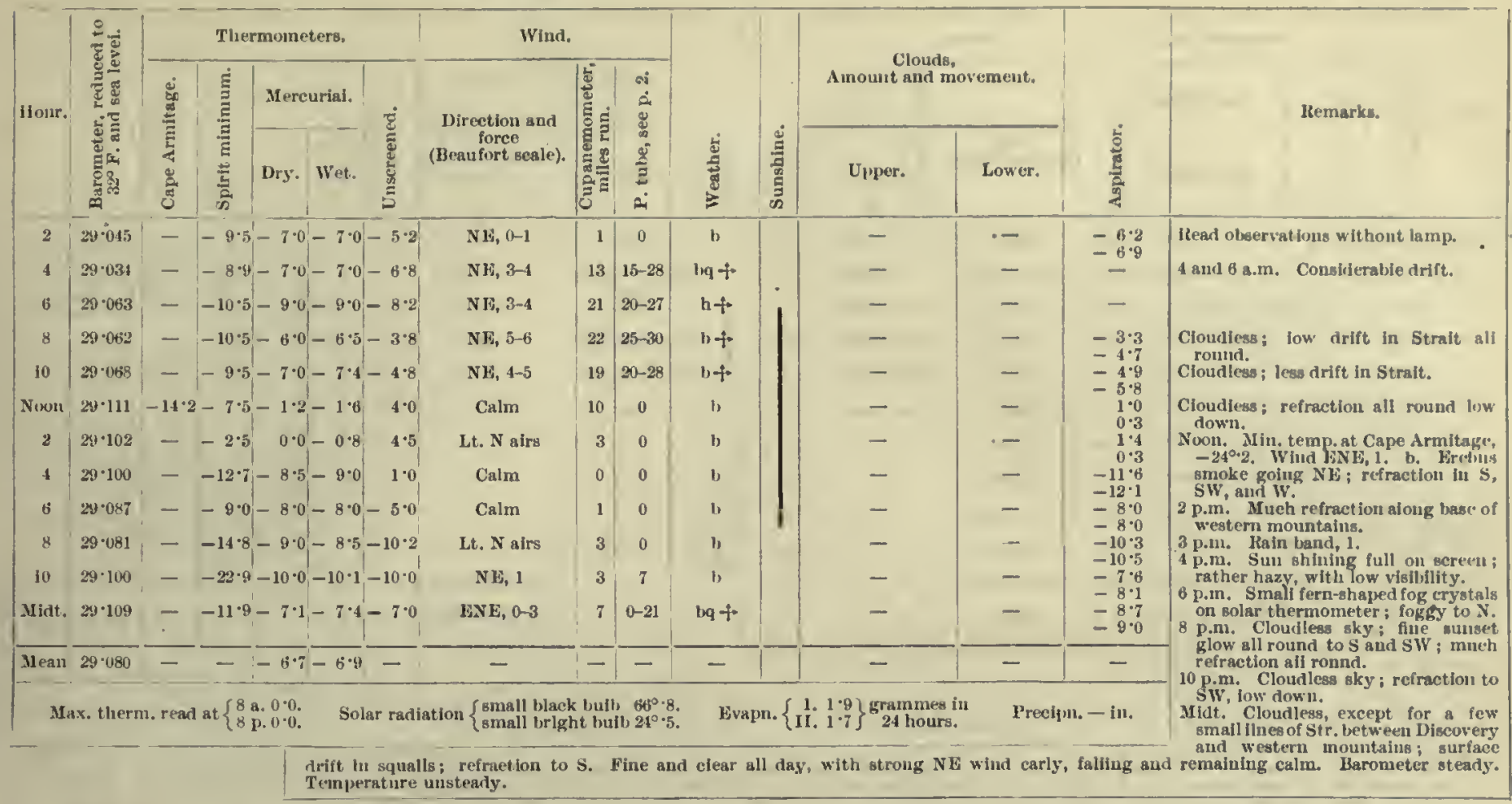

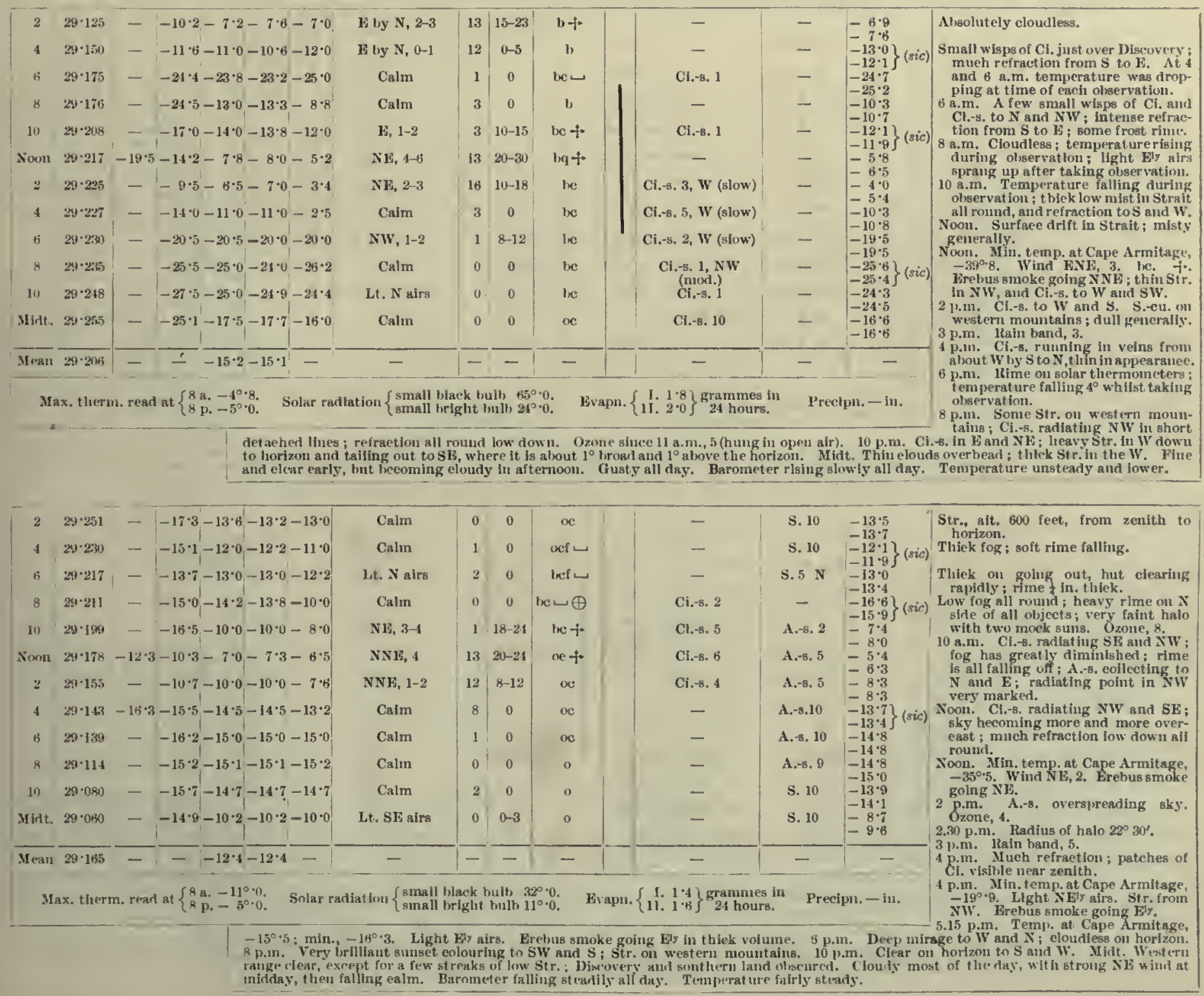


1903 -October.]

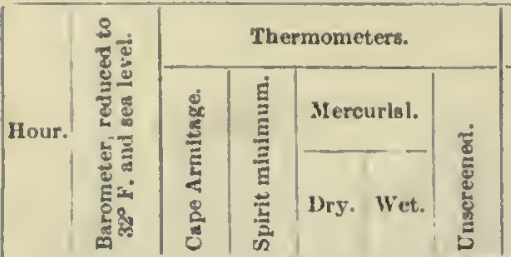

10 th.

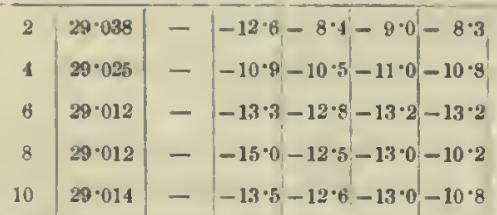

Noon $29 \cdot 018-14 \cdot 6-13 \cdot 5-9 \cdot 2-10 \cdot 2-6 \cdot 5$

$229 \cdot 012-\mid-11 \cdot 9-11 \cdot 5-11 \cdot 5-4 \cdot 8$

$429 \cdot 013--12 \cdot 5-9 \cdot 0-9 \cdot 2-5 \cdot 5$

6 29.018 - $-17 \cdot 3-16 \cdot 0-16 \cdot 0-13 \cdot 5$

$820 \cdot 014--19 \cdot 1-17 \cdot 9-17 \cdot 9-18 \cdot 8$

10 $29 \cdot 008--19 \cdot 2-14 \cdot 0-13 \cdot 8-13 \cdot 0$

Mint. $26 \cdot 988-(3 \cdot 3-11 \cdot 0-11 \cdot 0-11 \cdot 0$

Mfteorological JoukNal of the "Discovery."

\begin{tabular}{|c|c|c|c|c|c|c|}
\hline \multicolumn{3}{|l|}{ Wind. } & \multirow[b]{3}{*}{ 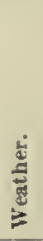 } & \multirow{3}{*}{ 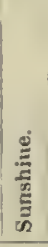 } & \multirow{2}{*}{\multicolumn{2}{|c|}{$\begin{array}{l}\text { Clouds, } \\
\text { Ainount and movement. }\end{array}$}} \\
\hline \multirow{2}{*}{$\begin{array}{c}\text { Directlou and } \\
\text { foreo } \\
\text { (Beaufort seale). }\end{array}$} & \multirow{2}{*}{ 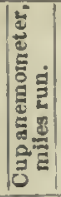 } & \multirow{2}{*}{ 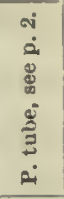 } & & & & \\
\hline & & & & & Upuer. & Lower. \\
\hline NE, 2 & 11 & $15-20$ & $\propto$ & & A.-8. 10 & - \\
\hline E) by $x, 3-4$ & 11 & $18-23$ & c & & Fr.-cl.-5. 8, NW & - \\
\hline$N E, 3-4$ & 21 & $21)-28$ & be & I & Ci. 5 & - \\
\hline NE, 4-5 & 19 & 20-28 & lbc & & Cl.-s. 4, $\mathrm{N}$ (slow) & - \\
\hline$N E, 3-4$ & 20 & $18-24$ & be & & Ci.-8, $\mathbf{1}, \mathbf{N}$ (slow) & - \\
\hline NE, 2-3 & 16 & $18-22$ & b & 1 & 一 & - \\
\hline Culm & 4 & 0 & b & 氙 & - & - \\
\hline Calm & 1 & 0 & b & ــ & - & - \\
\hline Lt. $\mathbf{N}$ airs & 2 & 0 & b & 동 & - & - \\
\hline Calm & 1 & 0 & be & जี & Ci.-cu. $3, \mathrm{~S}$ (slow) & - \\
\hline Lt. NNW alrs & 1 & 0 & oc & 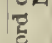 & - & S. $10, \mathrm{SW}$ (slow) \\
\hline Calm & 2 & 0 & bc & $\cong$ & - & S. $8, \mathrm{SW}$ (slow) \\
\hline
\end{tabular}

Mean $29 \cdot 014-1-12 \cdot 1-12 \cdot 4-$

-

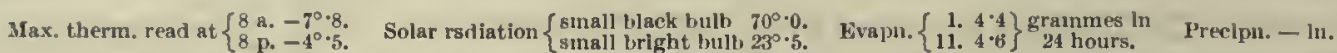

Remarks.

- 8.5, West eru range and upper thiri of Blaek - 9.4. Island clear ; mldale third of Black -10.5 1sland ant correspondlng helghts

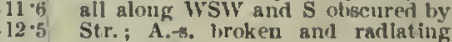

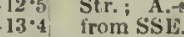

-13.5 from SSl.
a.m. Looks very stormy at the 11.6 Blufi, clearing clsewhere; clond 10.3 all tenritin

6.56 8.m. Cl. radlating $\mathbf{N}$ and $\mathrm{S}$; lone 7.4 hand of low Str $\mathbf{N}$ and $\mathrm{S}$; long Q.1 hand of low Str. alnng uestern Q.8 Discovery; clearing at Bluff. -6.78 a.m. 11eavy bank of Str. ext enting -6.9 from $W$ to Ssiv, alt. $1^{\circ}$.

$-15 \cdot 10 \mathrm{a} . \mathrm{m}$. Str. to $\mathrm{W}^{\circ}$ and SSW, rising

$-15 \cdot 7$ and dlscolving; detacher patches $-19 \cdot 7$ of clourd in Fand SK.

$-19 \cdot 7$ Noon. Cloudless in zenlth; some patches of clour to $\mathrm{S}$ and $\mathrm{Sls}$. Ozone, 6.

$10 \cdot 8$ Noon. Min. temp, at Caple Armitage, $-22^{\circ} \cdot 9$. Wind $\mathbf{E}$
smoke golng $\mathbf{X} \mathrm{E}$. smoke golng $\mathrm{NE}_{\text {, }}$ proachlng. Ozone, 4.

3 p.m. Kain band, 3.

p.m. Temperature fell rapldty. 23 p.m. Temp. at Cape Armitage

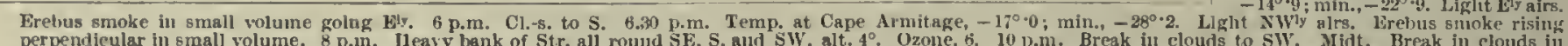

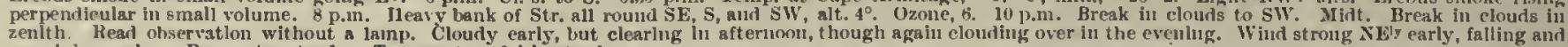
remainlng calm. Barometer steady. Tenperature fairly stearly.

11th.

\begin{tabular}{|c|c|c|c|c|c|c|c|c|c|c|c|c|}
\hline 2 & $28 \cdot 955$ & - & $-11 \cdot 5-$ & $7 \cdot 0-$ & $7 \cdot 8-$ & $-7 \cdot 0$ & Lt. SW alrs & 1 & 0 & oc & - & S. 10 \\
\hline 4 & $28 \cdot 935$ & - & $-9 \cdot 3$ & $7 \cdot 0$ & $6 \cdot 0$ & $7 \cdot 1$ & SE, 0-3 & 4 & $0-15$ & oeqfg & - & S. 10 \\
\hline 6 & $28: 910$ & - & $5 \cdot 0$ & $7 \cdot 0$ & $6 \cdot 4$ & $7 \cdot 0$ & $S$ by $W, 1-4$ & 8 & $5-29$ & $\operatorname{ocq} *$ & - & N. 10 \\
\hline 8 & $28 \cdot 910$ & - & $5 \cdot 5$ & $7 \cdot 8$ & $7 \cdot 5$ & $8 \cdot 0$ & Var. S, 7-9 & 31 & $40-56$ & ocq $+8 *$ & - & N. 10 \\
\hline 10 & $28 \cdot 9544^{\circ}$ & - & $6 \cdot 8$ & $7 \cdot 2$ & $\pi \cdot 2$ & $7 \cdot 8$ & $S, 8-9$ & 35 & $48-60$ & ocq $-i^{2} *$ & - & N. 10 \\
\hline Noon & $28 \cdot 993$ & - & $5 \cdot 8$ & $7 \cdot 0$ & $6 \cdot 2$ & $7 \cdot 5$ & Far. SE, 7-8 & 27 & $36-55$ & ocg & - & S. 10 \\
\hline 2 & $29 \cdot 032$ & - & $4 \cdot 6$ & $7 \cdot 0$ & $6 \cdot 5$ & $8 \cdot 0$ & S1, 4-8 & 28 & $25-55$ & ocq 4 & Ci.eu. 3, W & S. $6, \mathrm{~S}$ \\
\hline 4 & $28 \cdot 051$ & $5 \cdot 2$ & $4 \cdot 6$ & $7 \cdot 0$ & $6: 3$ & $7 \cdot 5$ & WSE, 4-9 & 22 & $25-28$ & ocq + & Ci.cu. 3, w & Cu. $5, \mathrm{~s}$ \\
\hline 6 & $29 \cdot 068$ & - & $2 \cdot 8$ & $7 \cdot 0$ & 6.0 & $7 \cdot 6$ & Var. SE, 4-7 & 24 & $20-40$ & ocq & Ci.-cu. b, W & Cu. $2, \mathrm{~s}$ \\
\hline 8 & $29 \cdot 081$ & - & $5 \cdot 8$ & $7 \cdot 0$ & $6 \cdot 0$ & $7 \cdot 1$ & Var. E, 4-6 & 17 & $20-36$ & beq & A.-s. $5, W$ & S. eur. $2, \mathrm{~S}$ (fast) \\
\hline 10 & $29 \cdot 080$ & - & - & $5 \cdot 8$ & $4 \cdot 5$ & $6 \cdot 0$ & E, 4-6 & 23 & $25-35$ & beq & A. -8.5 & Cu. 4 \\
\hline Mint. & 29.089 & - & $3 \cdot 0$ & 4.4 & $3 \cdot 2$ & $4 \cdot 0$ & E, 3-5 & 19 & $15-30$ & beq & Ci..s. 2 & S.-cu. 2 \\
\hline Nean! & $29 \cdot 010$ & - & - & $5 \% 6$ & $4 \cdot 8$ & - & - & - & - & - & - & - \\
\hline
\end{tabular}

6.7 Break ln elouds in SW and $W$; Blaek 1sland and White lslaul partially 7.2
$5 \cdot 4$ obscured by fog or cloud.
a.m. Dark in 1 and glomy look0 ing: Black and White Island 4 totally obscured; western foot hills and Brown lsland visible.

a.m. All distant land obseured ; tinetly vestern tinctly ylsible a.m. No land risible; wind varlable, S to SSW. Ozone, 10. and wet bulb broken; ; whid steady. xoon. Clearing to $\mathrm{S}$; sun gleaming through Str., which appears to be going $N W$; heavy dritt ln Strait; sunsline card blown away. Ozone, 7 . p.m. Extremely squally; clearing in $\mathbf{B}$ and $S$; very thlck in $W$; baro meter pumpling. 3 p.m. Raln laand, 4 . p.m. 11 eavy drift in Strait obscuring
hase of westeru mountalns; detacher Cu. driving rapldly uorthwards.

Max. therm. read at $\left\{\begin{array}{c}8 \mathrm{a} .8^{\circ} \cdot 2 . \\ 8 \mathrm{p} \cdot 8^{\circ} \cdot 0 .\end{array} \quad\right.$ Solar radiation $\left\{\begin{array}{l}\text { small black bull, } 38^{\circ} \cdot 6 . \\ \text { small brlglt bulb } 19^{\circ} \cdot 0 .\end{array} \quad\right.$ Evapn. $\left\{\begin{array}{c}\text { I. } 2 \cdot 8 \\ 11.2 \cdot 8\end{array}\right\} \begin{array}{c}\text { grammes in } \\ 24 \text { lours. }\end{array} \quad$ Precipn. - in. 4 p.m. Min.temp. at Cape Arnitage, seurer.

scured. Fo. frot smoke rising in clouds

at entrance to Strait; $\mathrm{Cl}_{\text {-s. }}$. on horizon to $\mathrm{N}$ and F. 8 p.m. Send passing over rapldly to $\mathrm{N}$; bases of western mountalus vlsible; all eloutls heavy and stormy leokiug. Ozone, 10. 10 p.m. and Midt. Gloony to W. 11 p.m. Ozone, 6. Overcast and cloudy all day, with very lieavy blizzard early in forenoon, wiud slift ting to SE just before noon and remalning very strong all day. Barometer rising steadily slnce $6 \mathrm{a} . \mathrm{m}$. Temperat ure rose to alove zero and revinained very stead!:

12 th

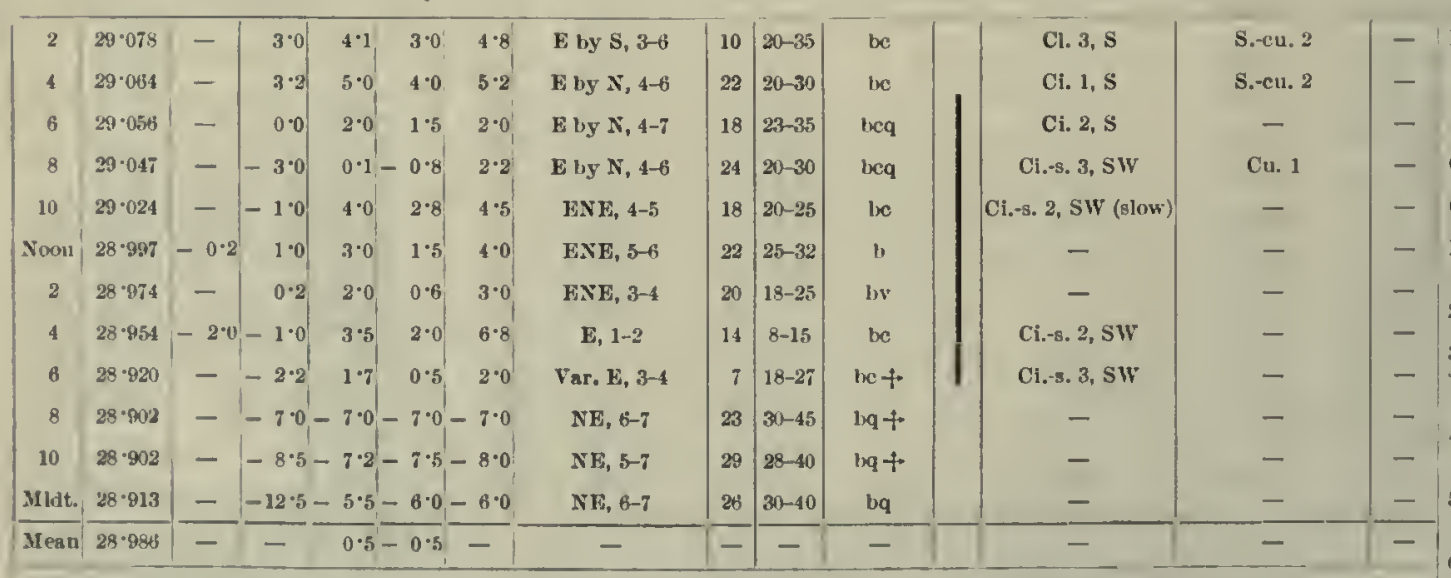

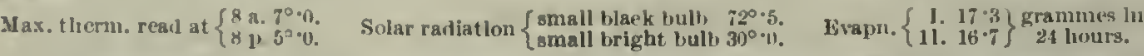

Prorelpn. - lin.
Dark elouds in W; bruad bands of clour, more or
teuding to SE.

Ozone, $\overline{7}$.

Clouds elearing, and weather finer generally.

i11. temp. at Cape Armitage, $-50 \cdot 1$. Wind LNE, 3. Erebus smoke going Wy; irift on Erebus' slopes. mountalns.

4 p.m. $\mathrm{Ci}_{\circ} \mathrm{s}$. on western horizon Str. on westeru mouutalns; marked vislbility.

p.m. Mini. temp. at Cape Armitage, $-4^{\circ} 0$. Wind N1s, 1-2. Erebus smoke invislble.

p.m. Cl.s. running $S W$ ant XE, very thln and indistinct; refraetion slight to W. in NW ; wind very varlal,le. (jility low: surface drfft lu strait.

$10 \mathrm{a.m}$. Fine sunset glnw all roum S horlzon; detached elonds, Inoking like Ci.-B., low down S and SW. Midt. More detachel clouds in S; thlı layer of Str. ou western mountalus, $8000 \mathrm{ft}$. alt.; no surface drlfi, although wind ls just is strong. More or less eloudy, with 11ght upper clouds, and with very strong NE's wlud all day. Barometer fell until io $\mathrm{p}$ m., and then connenced to rlse. Temperature falling towaris the evening. 
Metrorological Journal of the "Discovetr."

[1903-October.

\begin{tabular}{|c|c|c|c|c|c|c|c|c|c|c|c|c|c|c|}
\hline \multirow{3}{*}{ Honr. } & \multirow{3}{*}{ 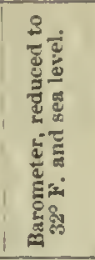 } & \multicolumn{5}{|c|}{ Thermoneters. } & \multicolumn{3}{|l|}{ Whind. } & \multirow[b]{3}{*}{ 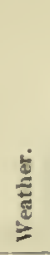 } & \multirow[b]{3}{*}{ 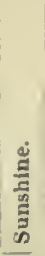 } & \multirow{2}{*}{\multicolumn{2}{|c|}{$\begin{array}{l}\text { Cloudis, } \\
\text { A mount and novement. }\end{array}$}} & \\
\hline & & \multirow{2}{*}{ 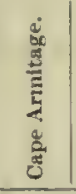 } & \multirow{2}{*}{ 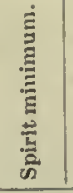 } & \multicolumn{2}{|c|}{ Mercurlal. } & \multirow{2}{*}{ 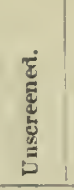 } & \multirow{2}{*}{$\begin{array}{l}\text { Direetlou and } \\
\text { force } \\
\text { (Beaufort scale). }\end{array}$} & \multirow{2}{*}{ 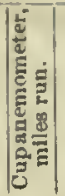 } & \multirow{2}{*}{ 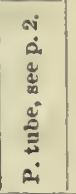 } & & & & & \\
\hline & & & & Dry. & Wet. & & & & & & & Upper. & r. & \\
\hline 2 & $28 \cdot 911$ & - & $-8 \cdot 0$ & $-6 \cdot 5$ & $-7 \cdot 0$ & -6.3 & $\mathrm{NL}, 5-6$ & 27 & $28-36$ & $\operatorname{lng}$ & & - & - & - \\
\hline 4 & $28 \cdot 918$ & - & $-10 \cdot 5$ & $-8 \cdot 0$ & -8.0 & $-9 \cdot 0$ & N $B, 3-5$ & 19 & $18-28$ & theq & & $\mathrm{Cl}_{.-8 .} 2$ & - & - \\
\hline 6 & $28 \cdot 092$ & - & $-8 \cdot 5$ & $-2 \cdot 0$ & $-3 \cdot 2$ & 0.0 & Es $1: 2-4$ & 17 & $12-22$ & be & & $\mathrm{Cl}_{.-8.2} 2$ & & \\
\hline 8 & $29 \cdot 034$ & - & $-5 \cdot 0$ & $-4 \cdot 2$ & $-5 \cdot 0$ & $-2 \cdot 0$ & HSE, 2-4 & 11 & $12-20$ & be & & Ci..2; Cl.-8. 2, SW & & - \\
\hline 10 & $29 \cdot 070$ & - & $-5 \cdot 0$ & -1.0 & $-2 \cdot 3$ & $2 \cdot 0$ & Fi, 1-2 & 9 & $10-18$ & le & & Cl.-n, 1, SW (slow) & - & - \\
\hline Noon & $29 \cdot 096$ & $-5 \cdot 1$ & $-4 \cdot 5$ & $-2 \cdot 0$ & $-3 \cdot 0$ & 0.2 & SE, 1 & 12 & $5-12$ & be & & $\mathrm{Cj},-$ s. 1 & A. $-8.3, \mathrm{SW}$ (morl.) & - \\
\hline 2 & $29 \cdot 113$ & - & $-5 \cdot 5$ & -0.3 & $-1 \cdot 5$ & $2 \cdot 5$ & SE, 2-3 & 13 & $15-22$ & be & & - & Cu. $8, \sin$ (morl.) & - \\
\hline 4 & $29 \cdot 125$ & $-1 \cdot 6$ & $-2 \cdot 5$ & $0 \cdot 0$ & $-1 \cdot 0$ & $2 \cdot 5$ & $\varepsilon, 0-1$ & 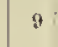 & 0 & $o c$ & & - & Cu. 10, SE (moxl.) & - \\
\hline 6 & $29 \cdot 156$ & - & $-4 \cdot 0$ & 1.0 & $-0 \cdot 3$ & $2 \cdot 3$ & Calm & 5 & 0 & be & ' & Ci.-c11. 5 & - & - \\
\hline 8 & $29 \cdot 164$ & - & $-4 \cdot 5$ & 10 & -0.8 & 0.8 & Lt. $\mathrm{S}$ airs & 5 & $0-8$ & oc & & - & Fr.-A,-s. 7, S (slow) & - \\
\hline 10 & $29 \cdot 180$ & - & -0.5 & $2 \cdot 5$ & $1 \cdot 0$ & 0.5 & Var. SE, 2-3 & 9 & $15-20$ & be & & - & A.-8. 5 & - \\
\hline Midt. & $29 \cdot 200$ & - & $\rightarrow 1 \cdot 0$ & $4 \cdot 0$ & $2 \cdot 0$ & $3 \cdot 3$ & Var. SSE, 1-2 & 9 & 10-15 & bx: & & - & A.-B. 8 & 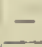 \\
\hline$\overline{\text { Meau }}$ & $29 \cdot 083$ & - & -1 & -13 & $3-2 \cdot 4$ & - & - & - & -1 & - & & - & - & \\
\hline
\end{tabular}

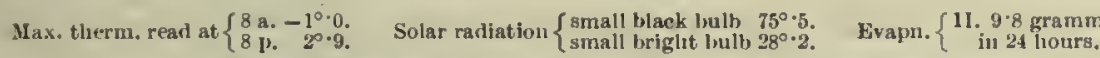

Precipm. - in.

Ilemarks.

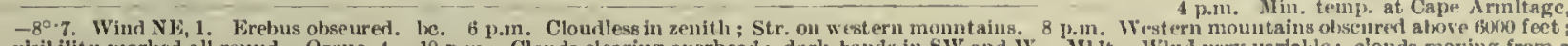

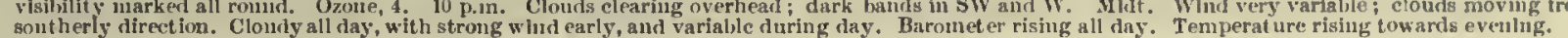

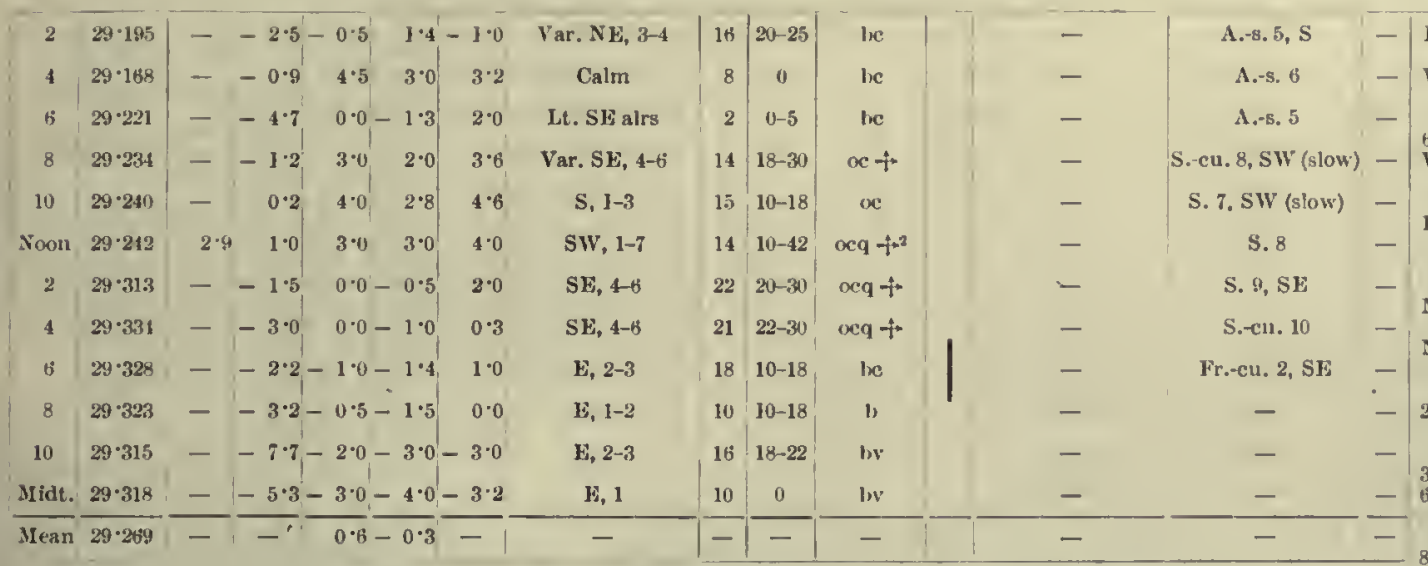

Mlax. therm. read at $\left\{\begin{array}{l}8 \text { u. } 5^{\circ} \cdot 4 . \\ 8 \mathrm{p} .4^{\circ} \cdot 2 .\end{array} \quad\right.$ Solar radiation $\left\{\begin{array}{l}\text { small liaek bulh } 47^{\circ} \cdot 3 . \\ \text { small bright hulb } 18^{\circ} \cdot 5 .\end{array}\right.$ Wivapn. $\left\{\begin{array}{l}1,13 \cdot 1 \text { grammes } \\ \text { in } 24 \text { bours. }\end{array} \quad\right.$ Precipm. - in.
Str. low down to s and Sl: Str.
western monntalis has thlckevied.

II mavy Inutk of S. -cu. In N, alt. $8^{\circ}$; Str.

in same place in 8 and ont western

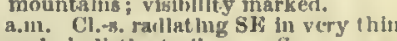

anl Indistlinct llnes: S.cul. very
slowly comlug over from $\mathbf{V}$ : very slowly comling over from 1 ; very
clear to $\mathrm{S}$; vlsitility marked all round, a.111. S.ceu. has gone back towarda the $X$, and is ntow alout $2^{\circ}$ alove $l$ tre bank of Str. to $S$. Wzolle, 7 . $10 \mathrm{a} . \mathrm{m}$. Bank of Str. to $\mathrm{S}$ is dilsappearling; detached port lons of Cl.-s. in S and at various [oluts ; thin strly,
Str. across centro of wetest ern ranuse Str. across centro of wrest ern range olsecured dow to to 8000 feet by thlu layers of detached Str. Poon. Nin. tenp. at Capu Armltage. $-19^{\circ}$. Wind $S$ ly 1,3 . Hirelus smoke going $\mathbf{N W}^{\mathrm{t} y}$.

p.m. Snow crystals fallug ; clouda noving moderately; Discovery summit olsscures.

3 p.t11. lain land, 3.

p.m. Completely overeast; alt. of chonds alout sero feet.

13th.

14th.

Hervy squalls of wind alout 1 a.m force 8. : ; why very variable.

Wind diefl away shortly leefore 4 a.m. range.

65 a.m. Clcaring over western range. Western rango nearly obseured; ; whin squally and variable, $\mathrm{S}$ to SF.

10 a.m. Western mountalıs olsscured above 8000 feet; heary drift in Strait wind variable, blowing from $1,3-4$, at
10.15 A.nn. Noon, Q.m.

Noon. Very squally, with heary drift, lut looks like clearing. Ozone, 7 . Noon. Min. temp, at Cape Armitage, -8 . Wind 5,3

- 2 p.m. Sun gleamlng ; clouds breaking In W, only summits of western moun talns obscured.

3 p.m. Main lant, p.un. Wind-blowin detaelied $\mathrm{Cu}$. in $\mathrm{S}$ and approaching; Str. on slummits of western mountains; ilark clourl in $\mathrm{N}$. 8 p.m. Ozone, 9.

Orarcast, more or less, early anil during forenoon, with a sliort attempt at a Sly blizzard, but later if cleared wind remaining still strong from $\mathrm{E}$. Barometer rose during the day, with necasionin
steady.

\begin{tabular}{|c|c|c|c|c|c|c|c|c|c|c|c|}
\hline 2 & $24 \cdot 2966$ & - & $|-4 \cdot 5-3 \cdot 0-4 \cdot 0|-3 \cdot 7 \mid$ & Calm & 7 & 0 & iv & & - & - & - \\
\hline 4 & $29 \cdot 283$ & - & $-19 \cdot 8-11 \cdot 3-11 \cdot 8-11 \cdot 2$ & ENE, 3-4 & 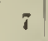 & $25-30$ & Iiv & & - & - & - \\
\hline 6 & $24 \cdot 280$ & - & $-14 \cdot 0-10 \cdot 0-11 \cdot v-10 \cdot 0$ & ENR, 4 & 12 & $28-33$ & w & 1 & - & - & - \\
\hline 8 & $2 y \cdot 235$ & - & $-10.5-6.5-7.2-4.0$ & $\mathrm{NI}, 3-5$ & 35 & $15-28$ & loev & & Ci.-8. 1 & - & - \\
\hline 10 & $29 \cdot 225$ & - & $-7 \cdot 5-6 \cdot 8-7 \cdot 2-4 \cdot 2$ & GNE, 4-6 & 17 & $20-30$ & levev & & C1.-s. 2 & - & - \\
\hline Noon & $29 \cdot 197$ & $-16 \cdot 0$ & $-10 \cdot 5-10 \cdot 3-10 \cdot 5-10^{\circ} 0$ & $N E, 4-6$ & 16 & $18-30$ & ine & & C1.-\$. 2 & Cu. $2, \mathbf{X}$ (fast) & - \\
\hline 2 & $29 \cdot 186$ & - & $-13 \cdot 0-5 \cdot 0-6 \cdot 0-4 \cdot 2$ & NE, 4-5 & 18 & $18-28$ & $\boldsymbol{c o s}$ & 1 & - & C11. $8, x$ (fast) & - \\
\hline 4 & $2 y \cdot 153$ & - & $-7 \cdot 7-3 \cdot 8-4 \cdot 2-3.5$ & $\mathrm{NE}, 2-3$ & 15 & $0-5$ & oc & & - & S. 10 & - \\
\hline$b^{5}$ & $29 \cdot 141$ & - & $-15 \cdot 3-11 \cdot 0-11 \cdot 0-11 \cdot 0$ & E, 2-3 & 10 & $0-10$ & $\infty *$ & & - & ง. 10 & - \\
\hline 8 & $29 \cdot 124$ & - & $-12 \cdot v-2 \cdot 2-3 \cdot v-1 \cdot 8$ & NE, 1-3 & 7 & $0-12$ & o & & - & S. 10 & - \\
\hline 11) & $29 \cdot 128$ & - & $-14 \cdot 2-13 \cdot 5-13 \cdot 2-13 \cdot 2$ & $S E, 3-6$ & 13 & $20-35$ & $\propto ⿻ q$ & & - & S. 10 & - \\
\hline Mllat. & $29 \cdot 127$ & - & $-15 \cdot 0-10 \cdot 2-10 \cdot 2-10 \cdot 2$ & SE, a-5 & 15 & $0-28$ & $\alpha \cdot q$ & & - & S. 10 & - \\
\hline ean & $29 \cdot 107$ & - & $-|-7 \cdot 8-8 \cdot 3|-$ & - & - & - & - & & - & - & - \\
\hline
\end{tabular}

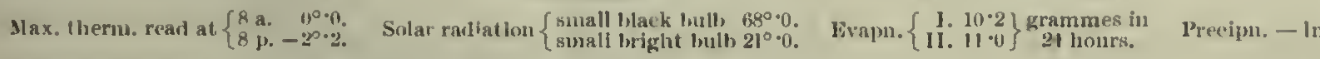

15 th.

Clondless ail roumd, except for layers of Ci,-8, in zenith, lying $\mathbf{E}$ asid $W$. 10 a.m. Hore Ci.-s. bas collected, and points of radiation are very marked

Nooll. Cu. pouring over from $\mathbf{N}$ very radiatling $\mathrm{E}$ and $\mathrm{W}$.

voon. Min. tennp at Cape

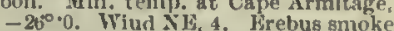
going 1 1'y, but soon obscured. 3 poing. Deep mirage at base of west crn inountains : sun glesming at thmes. 31 .m. Rain band, 5 .

4 p.m. Thin Str.; drift in Stralt. p.m. Break in clouds in $\mathrm{S}$ and $\mathrm{SW}$; Nim. on western nountains and in $\mathrm{X}$; Ozonle, b.

1.m. Appearauce of snowing all lounid : resl glow visible alout su" alt.

101) p.m. ant Mirlt. Mreak in clouls low down ln sky near horizon ln SSW and

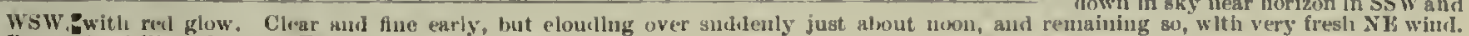
Haronicter falling all day. Temperature not so st eady. 
1903-October.]

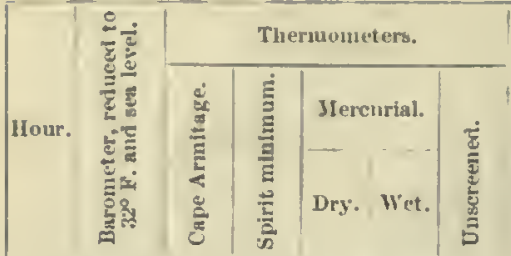

16 th.

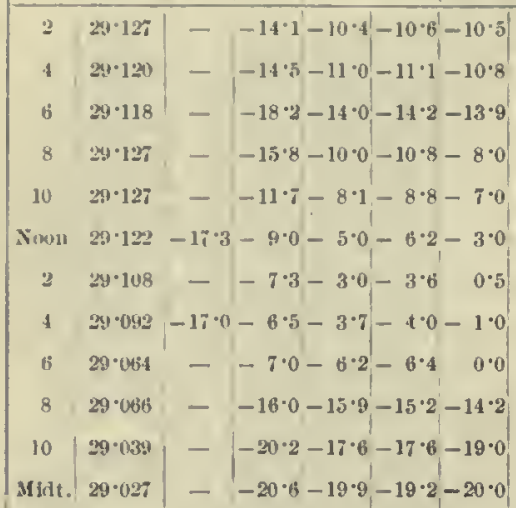

Meteorological JuUral of the "Discovery."

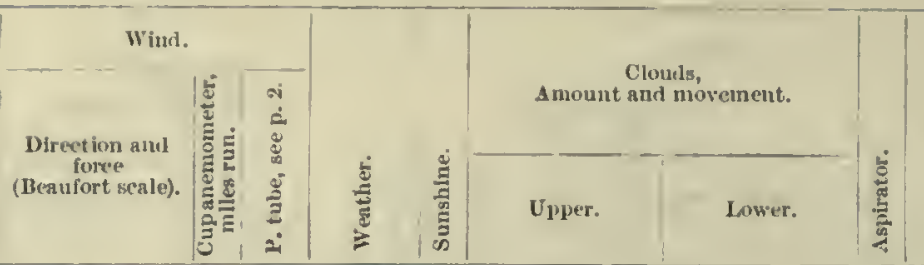

Hemarks.

\begin{tabular}{|c|c|c|c|c|c|c|}
\hline $\mathrm{H}_{6}, \mathrm{O}-\mathrm{H}$ & 15 & $0-20$ & ocq $+*$ & - & S. 10 & - \\
\hline EN12, 3-5 & 16 & $10-36$ & beq & - & Yr.-8.-cu, 2, WNW & - \\
\hline ENE, 4-7 & 22 & $20-30$ & lseg & A. .8 .5 & S. 2 & - \\
\hline $12,4-5$ & 21 & $20-28$ & be & Ci.-s. 3 & Det. S. 2, SW . & - \\
\hline 1XNY, $2-4$ & 13 & $10-24$ & $1 x$ & Ci..8. 2 & Det. A.-s. 2, W & - \\
\hline EXE, 1-2 & 15 & $10-14$ & Ine & - & A.-cu. 4, NW & 一 \\
\hline $1,, 1-2$ & 13 & $0-8$ & lie & Ci.-8. $2, \mathrm{sW}$ & - & 一 \\
\hline $\mathrm{NE}, 2-3$ & 8 & $v-10$ & be & - & A.-cu. $4, \mathbf{N}$ & - \\
\hline Calm & ö & 0 & oe & - & A.-cu. $8, \mathrm{NW}$ & - \\
\hline Calm & 0 & 0 & b & - & - & - \\
\hline Calın & 0 & 0 & $h \sqcup$ & - & - & - \\
\hline Calm & 0 & 0 & oe & - & Cu. 7, NW (morl.) & - \\
\hline- & -1 & - & - & . - & - & - \\
\hline
\end{tabular}

The break in clonds low down near horlclonds are hreaking and sky apparently clearing. Distant la

Distant land to $\mathrm{S}$ vlslble, but misty western mountalns quite obscuren.

Ozone, 4 .
10a.m. J1 st clearlng trom land; west mount ains visible, but mist $y$; 1 arhelia visible to W of sun, very brightly colouret.

Noml. Yery dark sky to $\mathrm{N}$, and bank of to cull all round from NNL to $\mathrm{J}$, in Noou, Mill. temp. at Cape Amltage, $-19^{\circ} \cdot 1$. Wind SE, 2. lirebus obsetred. A.-cu. 3 . 2 A. -cn. Detachel portions of $\mathrm{Ci}_{0}-3$, on horlzou all routur; fogigy to $\mathrm{W}$. p.m. lain baud, 2. p.m. A.-cu. oyerspreading sky from Nw. p.m. Min. temp. at Cape Armitage, $-19^{\circ} \cdot 0$. Wind $\mathrm{Ni}, 1-2$. Wrebus smok

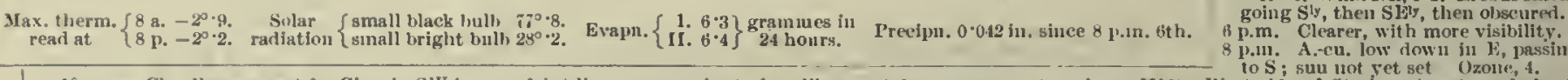

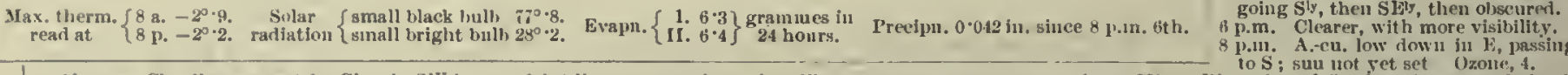

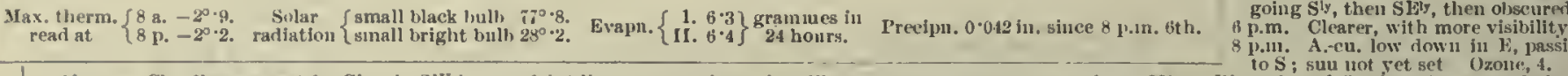
10 p.m. Clourless, except for Ci.s. in SW in very faint lines; very minute fern-like crystals on anemometer glass. Mint. West side of Strait. quite elear of cloncl,

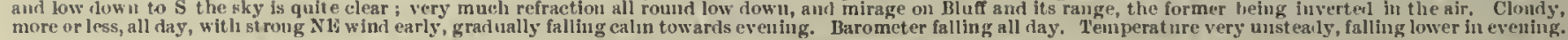

17 th.

\begin{tabular}{|c|c|c|c|c|c|c|c|c|c|c|c|}
\hline 2 & $29 \cdot 004$ & - & $|-20 \cdot 0-19 \cdot 3-19 \cdot 0|-19 \cdot 5 \mid$ & Culm & 0 & 0 & $o c \rightarrow$ & & - & $\mid$ Cu. 8, NW (mod.) $\mid$ & - \\
\hline 4 & $28 \cdot 979$ & - & $-19 \cdot 2-10 \cdot 0-10 \cdot 0-9 \cdot 4$ & Lt. NW alrs & 1 & $0-5$ & $\infty *$ & & - & Cu. 8, NW (nnorl.) & - \\
\hline 6 & $28 \cdot 9 n i$ & - & $-18 \cdot 5-16 \cdot 0-16 \cdot 0-14 \cdot 5$ & Calm & 0 & 0 & $\infty *$ & & - & C1. 8, NW (mori.) & - \\
\hline 8 & $28 \cdot 9 \cdot 9$ & - & $-16 \cdot 0-7 \cdot 2-7 \cdot 6-6.5$ & Calun & 0 & 0 & oc & & - & Cu. 9, SW (slow) & - \\
\hline 10) & $28 \cdot 934$ & - & $-10 \cdot 0-3 \cdot 2-4 \cdot 0 \quad 0.0$ & Calın & 0 & 0 & ee & & -- & Cu. B, NW (slow) & - \\
\hline Nivon & $28 \cdot 923$ & $-14 \cdot 4$ & $-11 \cdot 3-6 \cdot 0-6 \cdot 5-5^{\circ} 0$ & $12,2-3$ & 8 & $10-18$ & oc & & - & S. -cu. $\$$, W (slow) & - \\
\hline 2 & $28 \cdot 919$ & - & $-12 \cdot 2-5 \cdot 0-6 \cdot 0-2 \cdot 5$ & $11,2-3$ & 12 & $12-18$ & oe & & - & Cu. 9, NW (slow) & $1-$ \\
\hline 4 & $28 \cdot 911$ & $-12 \cdot 2$ & $-10 \cdot 7-8 \cdot 0-8 \cdot 3-7 \cdot 5$ & E, 3-4 & 11 & $22-28$ & $\propto$ & & - & Cu. $4, \mathbf{N}$ (slew) & - \\
\hline 6 & $28 \cdot 165$ & - & $-13 \cdot 1-11 \cdot 4-11 \cdot 6-12 \cdot 0$ & 19N1: $8-4$ & 15 & $18-25$ & lnc & & - & Det. Cu. $5, N$ (fast) & - \\
\hline 8 & $24 \cdot 499$ & - & $-12 \cdot 5-11 \cdot 0-11 \cdot 2-11 \cdot 2$ & $x]: 3-4$ & 10 & $18-22$ & be & 1 & - & A.-cu. 3, var. & - \\
\hline 1) & $28 \cdot 675$ & - & $-12 \cdot 5-12 \cdot 0-12 \cdot 0-12 \cdot 0$ & $N 15,2-3$ & 14 & $15-20$ & b & & - & - & - \\
\hline slidt. & $29 \cdot 875$ & - & $-14 \cdot 5-12 \cdot 2-12 \cdot 5-12 \cdot 4$ & $N E, 2-3$ & 13 & $18-22$ & 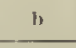 & & - & - & - \\
\hline Ilean & $28 \cdot 928$ & - & $-\mid-10 \cdot 1-10 \cdot 4-$ & - & -1 & - & - & & - & - & - \\
\hline
\end{tabular}

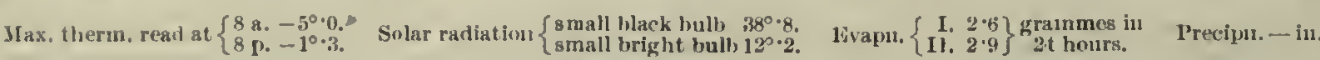

- A slight deposit of ice crystals between observations; clouds thinsing; ex4 r.m. Very miunte

a.m. Very thinute ice crystals falling : a.m. Conditions sim misty appearaluce over Wilar to 4 a.m. very excesslve refraction. 8 a.ro. 0\%olle, 3 .

8 to 10 a.m. Extensive refraction clouds and conditions similar. Noon. Clonds now tonehing simmints of $\mathrm{n}$ estern umutains.

Noon. Slin. temp, at Cape Armitage. $-35^{\circ} 0$. Wind $\mathrm{NL}, 2$. Frebus senree ; reiraction to $\mathrm{W}$. p.m. largo masses of detaeherl Cu. apparently low and very whit

1.11n. Rain hand, 1.

p.m. Sun gleauning fautly ; unirage all roming to westward. b.in. Min, temp, at Cape Arunitage
$-15^{\circ} 0$. Wind $\mathrm{Ni}, 2$. Lirchus ol seured

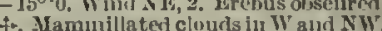
p.m. Cloudless in zenith and in $\mathrm{N}$ simin olsceurerl.

p.m. Clouls moving in various direotions. Orone, 7.

10 1.un. Baun of grey Str. along westeru

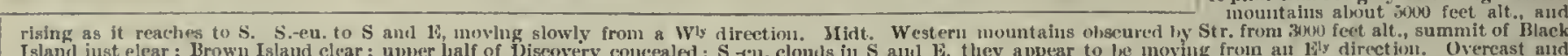

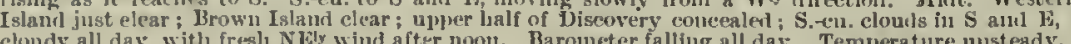
clondy all day, with fresl $\mathrm{N}$ ily wind after noon. Baroncter falling all day. Temperature unsteady.
18 th.

\begin{tabular}{|c|c|c|c|c|c|c|c|c|c|}
\hline 2 & 28.874 & - & $-14 \cdot 0 \mid$ & $-12 \cdot 2-12 \cdot 8-12 \cdot 8$ & NN17, 2-3 & 14 & $15-22$ & b & \\
\hline 4 & $28 \cdot 873$ & - & $-13 \cdot 5$ & $-10 \cdot 9-11 \cdot 0-11 \cdot 0$ & ENE, 1-3 & 11 & $5-15$ & b & \\
\hline b & 28.879 & - & $-16 \cdot 0$ & $-11 \cdot 0-11 \cdot 5-11 \cdot 0$ & 1), 1-2 & 7 & $5-10$ & inc & \\
\hline 8 & $28 \cdot 4,85$ & - & $-11 \cdot 5$ & $-8 \cdot 5-10 \cdot 0-8 \cdot 8$ & $N E, 3-4$ & 8 & $18-21$ & ine & \\
\hline 10) & $28 \cdot 893$ & - & $-15 \cdot 0$ & $-13 \cdot 8-13 \cdot 8-13 \cdot 0$ & NH, 4-5 & 16 & $2010-28$ & $\infty+*$ & \\
\hline X̌on & $38 \cdot 804$ & - & $-15 \cdot 7$ & $-14 \cdot 0-1 t \cdot 0-13 \cdot 1$ & N15, 4-5 & 201 & $25-32$ & $0+* *$ & \\
\hline 2 & $28 \cdot 912$ & - & $-15 \cdot 3$ & $-11 \cdot 5-11 \cdot 8-10 \cdot 0$ & $\mathbf{N E}, 3-4$ & 18 & $25-30$ & o & \\
\hline 4 & $28-919$ & $-2 x \cdot \cdot 6$ & $-16^{\circ}$ & $-11 \cdot 0-11 \cdot 5-8 \cdot 8$ & $N E, 3-4$ & 14 & $20-28$ & b & \\
\hline 6 & $28 \cdot 022$ & - & $-16 \cdot 3$ & $-9 \cdot 0-9 \cdot 6-8 \cdot 0$ & $\mathbf{N E}, 1-3$ & 14 & $10-16^{\circ}$ & 0 & \\
\hline 8 & $28 \cdot 928$ & - & $-11 \%$ & $-9 \cdot 0-9 \cdot 5-8 \cdot 2$ & $\mathbf{N}, \mathbf{l}$ & 4 & $8-10$ & b & \\
\hline 10 & $28 \cdot 932$ & - & $-15 \cdot 8$ & $-15 \cdot 2-15 \cdot 2-16 \cdot 2$ & Calm & 5 & 0 & h & \\
\hline Mirlt. & $28 \cdot 944$ & - & $-21 \cdot 4$ & $-15 \cdot 4-16 \cdot 2-17 \cdot 8$ & Calm & 0 & 0 & b & \\
\hline Hean & $28 \cdot 905$ & - & - & $-11 \cdot 9-12 \cdot 2-$ & - & - & - & $\rightarrow$ & \\
\hline
\end{tabular}

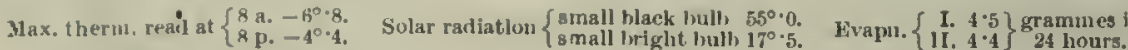

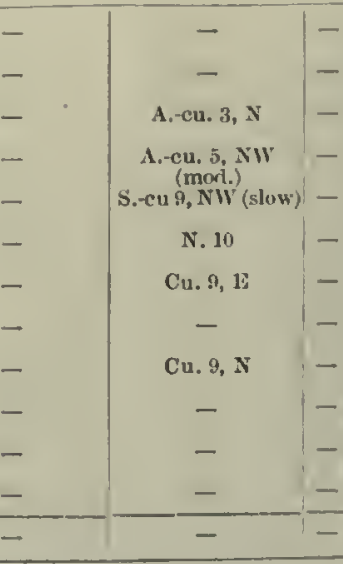

Precipn. - ill.
Western mountains obseured by Str. from 3000 fcet alt. ; a thin band of S.-cu. extending io Bluff, and seme

a.m. Foot hills visible: summits of western montains hidien by S.-cu. a hand of which extents acrome chlon in NW.

a.m. S.-c11. obscuring sumnits of wand inountains, and a tuarrow S.cu. and A.s. Ji NW a.m. Appes rance of snowing in W and Sw. Ozonc, 6.

10 a.m. S1rowing very sllghtly; no land visible except close to ship. p.m. Detached Cu. passing from alyut

p.m. Itain band, 4. p.m. Ci. on easteru lorizon ; Cu. to $\mathrm{N}$ and $\mathrm{W}$.

p.m. Mir. temp. at Cape Armitage 4. Wind Nis by $\mathbf{F}, 2-3$. Frelou silloke going $\mathrm{NE}_{\text {. }}+$ p.m. Summits of western range 8 p.m. Detached Str. to SH, S, sud SW: p.m. Det

Fine day, w th ha little snow iu forenom and a fiesh NE wind, which fell to calin in eventhig. Barometer risiug al day. Temperature lower and steary: 
Meteorological. Journat, of THE "Discoveriy."

[1903-October.

\begin{tabular}{|c|c|c|c|c|c|c|c|c|c|c|c|c|c|c|}
\hline \multirow{3}{*}{ Ionr. } & \multirow{3}{*}{ 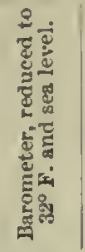 } & \multicolumn{5}{|c|}{ Thermoneters. } & \multicolumn{3}{|l|}{ Wind. } & \multirow[b]{3}{*}{ 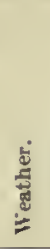 } & \multirow[b]{3}{*}{ 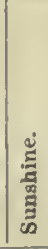 } & \multirow{2}{*}{\multicolumn{2}{|c|}{$\begin{array}{l}\text { Clousts, } \\
\text { Amount and novement. }\end{array}$}} & \multirow[b]{3}{*}{$\frac{5}{\frac{5}{5}}$} \\
\hline & & \multirow{2}{*}{ 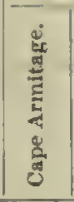 } & \multirow{2}{*}{ 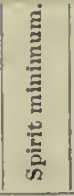 } & \multicolumn{2}{|c|}{ Mercurisl. } & \multirow[b]{2}{*}{$\begin{array}{l}\tilde{z} \\
\tilde{d} \\
\tilde{d}\end{array}$} & \multirow[b]{2}{*}{$\begin{array}{c}\text { Direction aud } \\
\text { force } \\
\text { (Beanfort sealc.) }\end{array}$} & \multirow{2}{*}{ 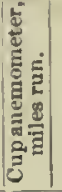 } & \multirow{2}{*}{ 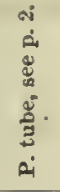 } & & & & & \\
\hline & & & & Dry. & Wet. & & & & & & & Viper. & Lower. & \\
\hline 2 & $28 \cdot 957$ & - & $-23 \cdot 2$ & $-19 \cdot 0$ & $-19 \cdot 0$ & $-19 \cdot 1$ & Calm & 0 & 0 & b & & - & - & - \\
\hline 4 & $28 \cdot 9 \times 3$ & - & $-27 \cdot 2$ & $-22 \cdot 5$ & $-22 \cdot 6$ & $-23 \cdot 0$ & Calm & 0 & 0 & ', & & - & - & - \\
\hline 13 & $23 \cdot 909$ & - & $-23 \cdot 4$ & $-16 \cdot 2$ & $-16 \cdot 6$ & $-11 \cdot 3$ & Calm & 1. & 0 & b & & - & - & - \\
\hline 8 & 29098 & - & $-25 \cdot 2$ & $21 \cdot 8$ & $-21 \cdot 5$ & $-9 \cdot 0$ & Calm & 0 & v & $b$ & & - & - & - \\
\hline in & $29 \cdot 0692$ & - & $-22 \cdot 8$ & $8-20 \cdot 0$ & $-20 \cdot 0$ & $-0 \cdot 0$ & Calun & 1 & 0 & b & & - & - & - \\
\hline Noon & $29 \cdot 684$ & $-22 \cdot 0$ & $-19 \cdot 6$ & $-15 \cdot 0$ & $-15 \cdot 0$ & $-8 \cdot 0$ & Caluu & 0 & 0 & b. & & - & - & - \\
\hline 2 & $29 \cdot 102$ & - & $-23 \cdot 2$ & $2-18 \cdot 8$ & $8-18 \cdot 8$ & $-5,0$ & Calm & o & 0 & b & & - & - & - \\
\hline 4 & $29 \cdot 12 t$ & - & $-18 \cdot 0$ & $-16 \cdot 0$ & $-16 \cdot 6$ & $-3 \cdot 2$ & Calm & 0 & 0 & b) & & - & - & - \\
\hline : & $29 \cdot 129$ & - & $-17 \cdot 2$ & $2-15 \cdot$ & $-15 \cdot 5$ & $-8 \cdot 0$ & Calm & 0 & 0 & b & & - & - & - \\
\hline 8 & $29 \cdot 142$ & - & -18.0 & $0-14 \cdot 0$ & $0-14^{\circ} 0$ & $-8 \cdot 0$ & Calun & 0 & 0 & b & 1 & - & - & - \\
\hline 10 & $29 \cdot 164$ & - & $-18 \cdot 2$ & $-13=$ & $2-13 \cdot 4$ & $-14 \cdot 0$ & Calm & 0 & 0 & Iw & & - & - & $1-$ \\
\hline MIrat. & $29 \cdot 191$ & - & $-23 \cdot 0$ & $0-16 \cdot 8$ & $-17 \cdot 8$ & $-16 \cdot 0$ & Calm & 0 & 0 & bv & & - & - & - \\
\hline Nean & $29-0181$ & - & - & -17 & $\mid-17 \cdot 5$ & - & - & $1-1$ & - & - & & - & - & - \\
\hline
\end{tabular}

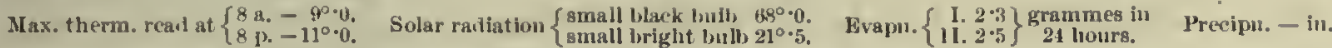

Marnlficent day; clomulless mul malu. laronicter rising all day. Tenuperature lower mul stealy:

Remarks.

oggy appearance; mirage to W. cloudless; excestulve refraction all romul low tho ho. clondless; excesslve refrnction all Non. Min. tenup, at. Cape Armituge, golng to $\mathrm{Nb}$. Much refration :

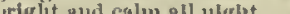
ing (ancept some VW; lazy, with mucli refraction. Ci.-s to NiW and 19 th

\begin{tabular}{|c|c|c|c|c|c|c|c|c|c|}
\hline 2 & $29 \cdot 200$ & - & $-18 \cdot 0-3 \cdot 8-4 \cdot 2-4 \cdot 0$ & $N_{0} 1-2$ & 6 & 10 & bv & - & - \\
\hline 4 & $29 \cdot 222$ & - & $-4 \cdot 5-2 \cdot 2-3 \cdot 0-2 \cdot 0$ & NNW, o-1 & 5 & 0 & bv & - & - \\
\hline 6 & $29 \cdot 232$ & - & $-25 \cdot 2-18 \cdot 2-18 \cdot 0-18 \cdot 0$ & $N W, 0-1$ & 2 & 0 & iw & - & - \\
\hline 8 & $29 \cdot 286$ & - & $-22 \cdot 8-22 \cdot 5-21 \cdot 5-11 \cdot 2$ & Lt. SW airs & 4 & 0 & w & - & - \\
\hline 10 & $29 \cdot 292$ & - & \begin{tabular}{l|l|l}
-22.5 & $0.8-1.8$ & 75
\end{tabular} & Var. lt. $\mathbf{X}$ airs & 1 & 0 & by & - & - \\
\hline Nom & $29 \cdot 3215$ & $-3 \cdot 4$ & \begin{tabular}{l|ll}
-8.5 & $0.0-1 \cdot 0$ & 3.8
\end{tabular} & Calun & 2 & 0 & in & - & - \\
\hline 2 & $29 \cdot 33 x$ & - & $\begin{array}{llll}-2.8 & 50 & 4 \cdot 0 & 7.5\end{array}$ & $N W, 1-2$ & 8 & 5-12 & b) & - & - \\
\hline 4 & $29 \cdot 32 v$ & - & $\begin{array}{llll}2 \cdot 8 & 5 \cdot 0 & 4 \cdot 0 & 9 \cdot 3\end{array}$ & NNW, 2-3 & 8 & $10-15$ & b & - & - \\
\hline 6 & $29 \cdot 311$ & - & $-1 \cdot 0-0 \cdot 2-1 \cdot 0 \quad 5 \cdot 0$ & Calu & 4 & .0 & ine & Ci. 2 & - \\
\hline 8 & $29 \cdot 313$ & - & $-11 \cdot \hat{\imath}-10 \cdot 2-10 \cdot 0-9 \cdot 2$ & Calm & 1 & 0 & Ixenv & Ci. $4, \mathrm{Cl}_{-}-\mathrm{s}, 2, \mathrm{~W}$ & - \\
\hline 10 & $29 \cdot 315$ & - & $-9 \cdot 7-4 \cdot 8-5 \cdot 0-5 \cdot 6$ & Lt. F airs & 0 & 0 & lic & $\left(\mathrm{Ci}_{.-\mathrm{s} .1}\right)$ & - \\
\hline M Iat. & $29 \cdot 314$ & - & $-7 \cdot 3-3 \cdot 8-4 \cdot 5-4 \cdot 2$ & Calm & 3 & 0 & be & Ci.-s. 5 & - \\
\hline IIean? & $29 \cdot 2 \times 8$ & - & $-|-4 \cdot 6|-5 \cdot 2-$ & - & - & - & - & - & - \\
\hline
\end{tabular}

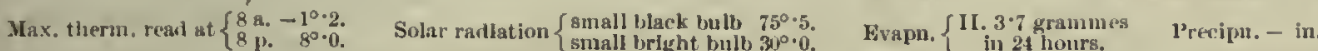

- 2 to 6 a.m. Nuch refraction in W.

20th,

Ozone, 6.

- Some CI,-8. to NW, otherwise clourless Vind sprang up fresli from $N$ at 12.15 p.m.; Cu. to S; Cl,-8. to $\mathrm{N}$ anil Toon. Min. temp. at Cape Armitage, $-34^{\circ} \cdot 2$. Light Why airs. Wirebus smoke golng N.Xl; sllght refrartion. 2 p.un. IIazy, with little visibllity. (3) p.m. Thin Ci.-8, running Fond $W$; Str. to N.

very wind blormy look in $\mathrm{XV}$; Cl.-8, blown, radiat lng $\mathrm{k}$ and $\mathrm{W}$. 10 p.m. Ci.-s, and A.-s, thick behind

west ern range,
Mint. Simllar conditions; elourls are beantifully rose tiut erl.

Another very tine day, nearly clomiless and calm, but clonierl a bit in evening. Barometer rishng until 2 p.m., then stealy. Temperature very nnstearly:

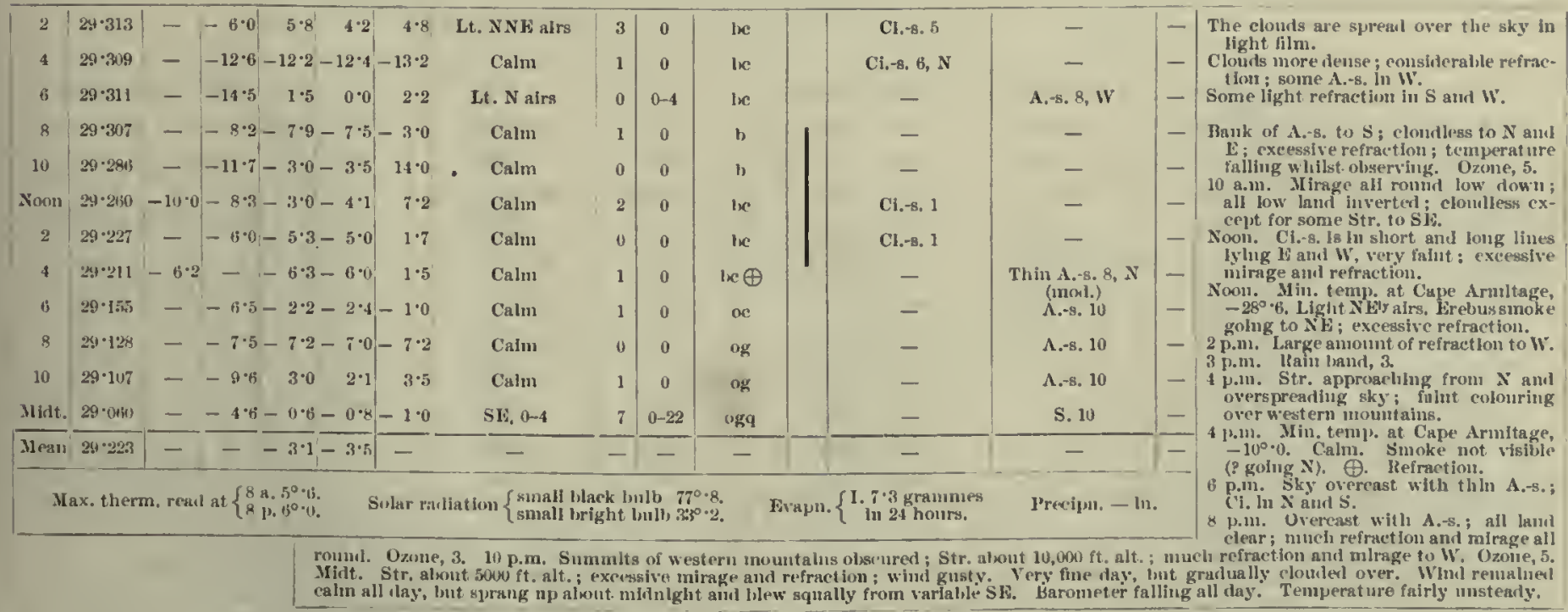



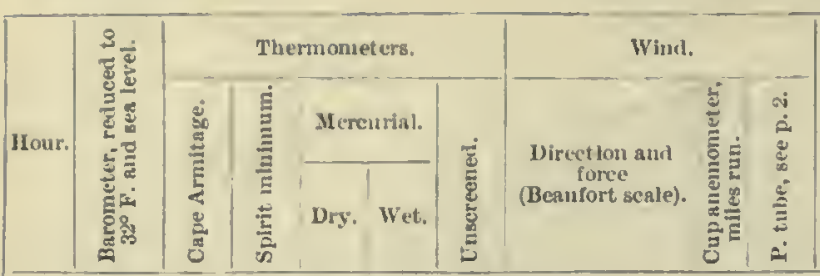

22nd.

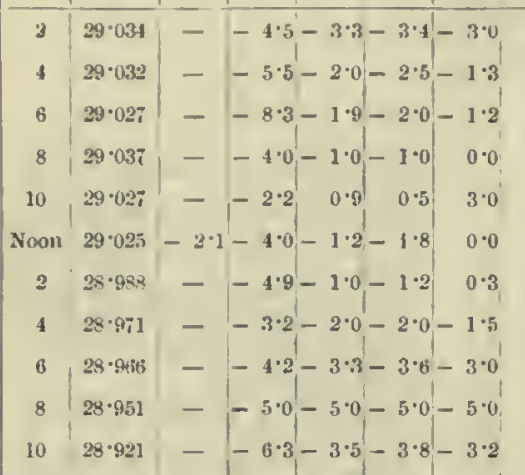

\section{$\mathbf{H}, 5-i$}

Var, $\mathrm{F}, 1-5$

F.XF, 1-3

ENF, 3-5

NF, 4-5

NF, 5-i

Var. $F_{4,4-5}$

ar. F, 3-4 $18 \mid 20-28$ oc

Var, \&, 4-5 $14 \quad 20-28$ oc

WNi, 4-5 $|21| 20-33 \mid$ of -

E, 5-6
Var. F, I-5

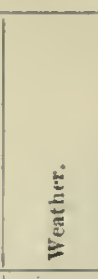

$\mid$\begin{tabular}{c|c|} 
Clouds, \\
Amount and movement.
\end{tabular}

S. 10 - Str, down to 1,500 feet. Ozone, 8 .

x. 10

S. 10

N. 10

C1.-8, 3, NE A, -cu, 3, S (niol.)

Cn. 8, SE (fast) - 10 a.m. Thick ail roune; ; ieary bank

Cn. 8, SE (fast) - 10 a.m. Thick ail round

Noon. Ozone, 8 . -130.9. Whin ENE, 3. †. Frebus obseurerl. summits of western monntuins obseirert. 3 p.ur. Ram bant, 2.

Millt. $25 \cdot 899--6 \cdot 3-2 \cdot 2-2 \cdot 8-3 \cdot 0$

$\overline{\text { IIean }} \overline{28 . \mathrm{ggN}} \overline{-} \overline{-2.1}-\overline{-2.4} \overline{-}$

Max. therm, real at $\left\{\begin{array}{l}8 \text { a. } 5^{\circ} \cdot 0 . \\ 8 \text { p. } 1^{\circ} \cdot 0 .\end{array}\right.$ Solar radiat ion $\left\{\begin{array}{l}\text { small black lulb, } 92^{\circ} \cdot 0 \\ \text { small bright bulin } 38^{\circ} \cdot 2\end{array}\right.$ $\frac{21}{-} \frac{25-37}{-} \mid$ r.-eir, $3, \mathrm{SF}$ (fast)

- $\quad$ Fr.eu. 7, F (fast)

Ci.-8. 5

A. -8.10
S. 5

S. - en. $b^{2}$

S.-eu. 2, S. 9 and curluds of drift rising lin patches and curlung for some distance northwards. p.m. Qulte clear in S aurd SW; dlark and threatening to $\mathrm{N}$.

p.m. Wind variable from ENE to LSE; visilility very markes ; threatening look to S ani SW. Ozone, 10. mountalns and also summit of Dis-

covery hidden by Str.; islauds are clear of cloul. Overcast and eloudy most. of the day, with snow early, and drifting. Very strong

Fovery hidrien by Str.; islauds are clear of clond. Overcast and eloury most. of the day, with
F and wind all day. Barometer faling thronginout the day. Temperature fairly steally all day.

23rd,

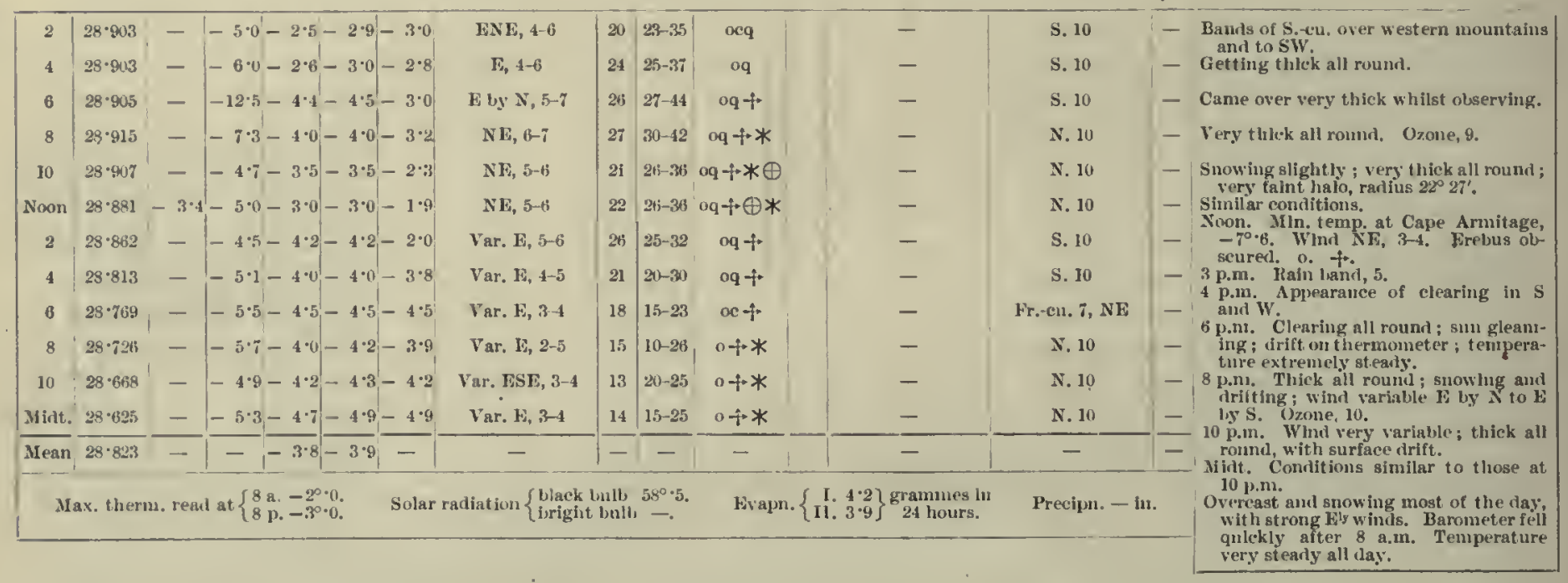

24th.

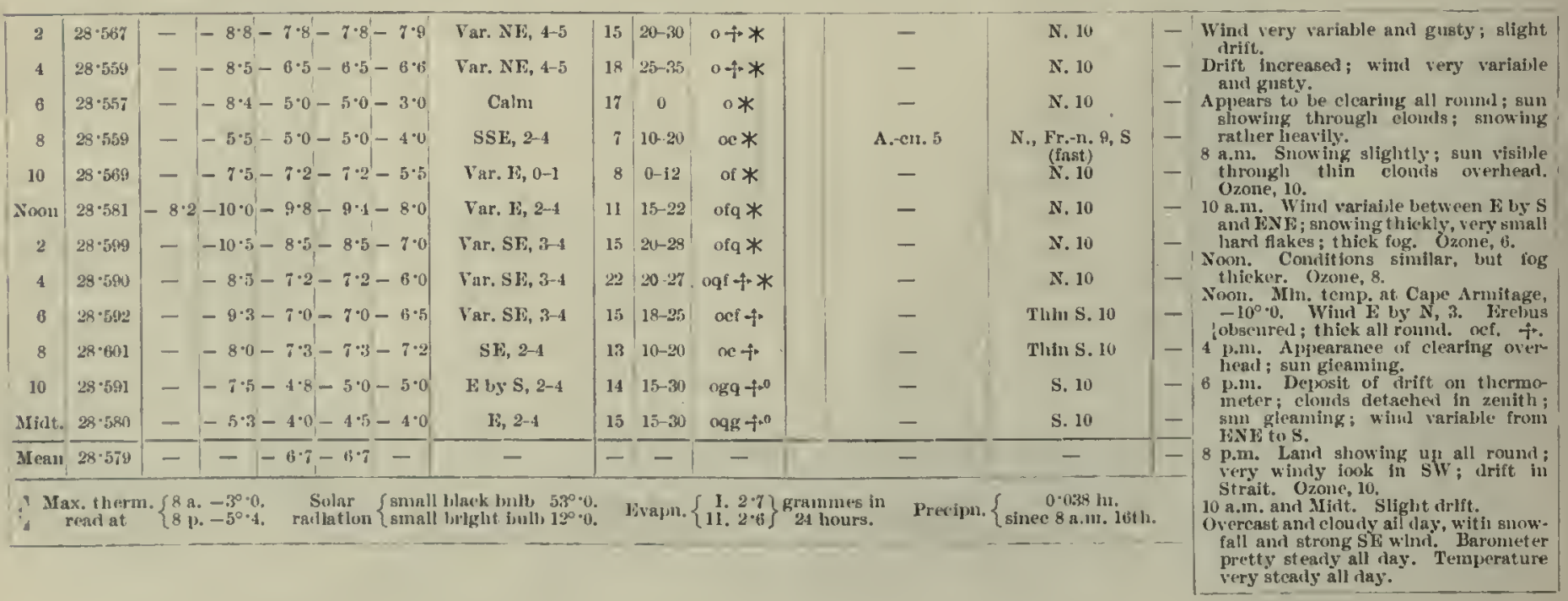


Meteorolonical, Journat of The "Discovery."

[1903-October.

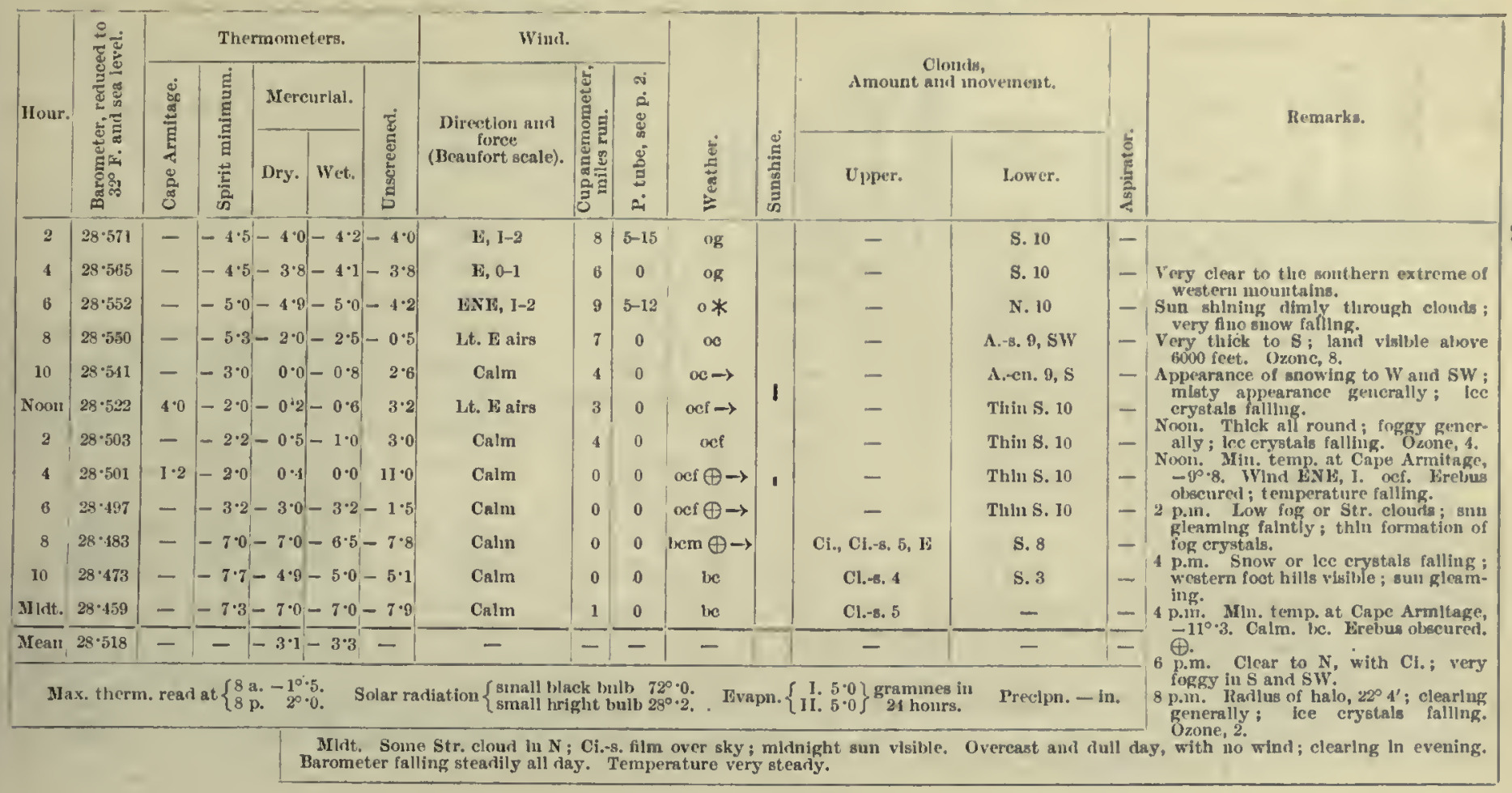

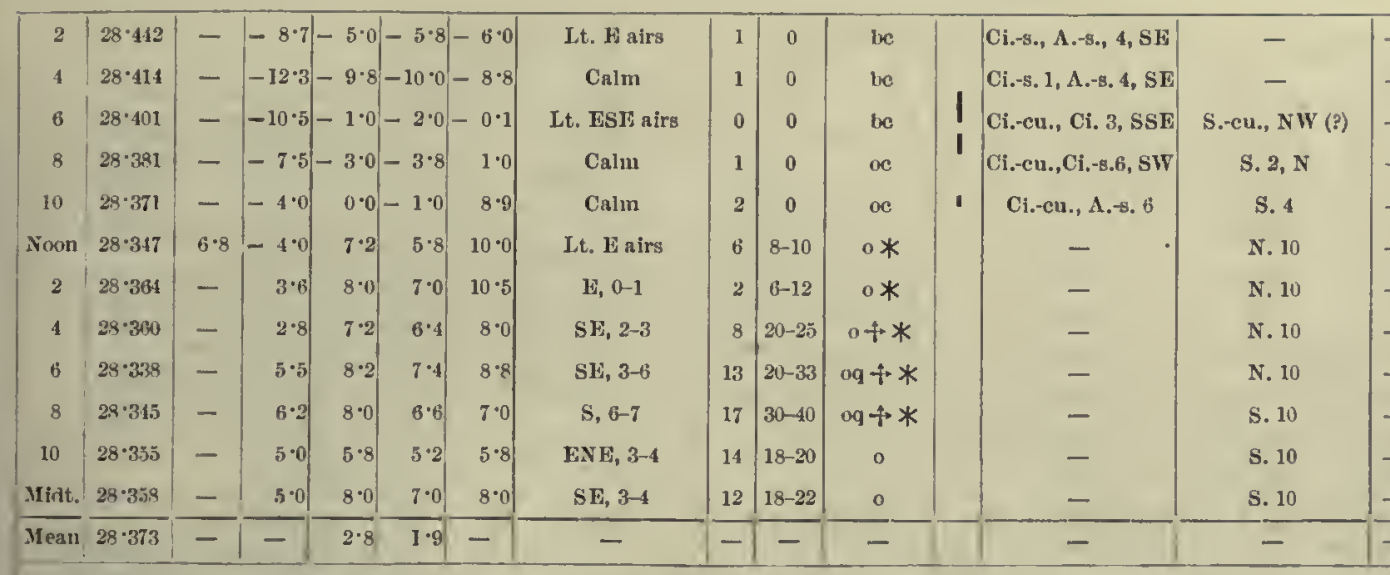

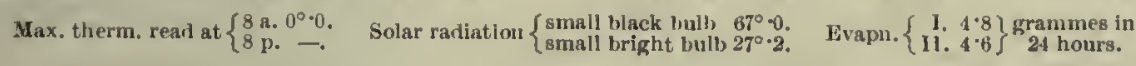

Precipn. - in.

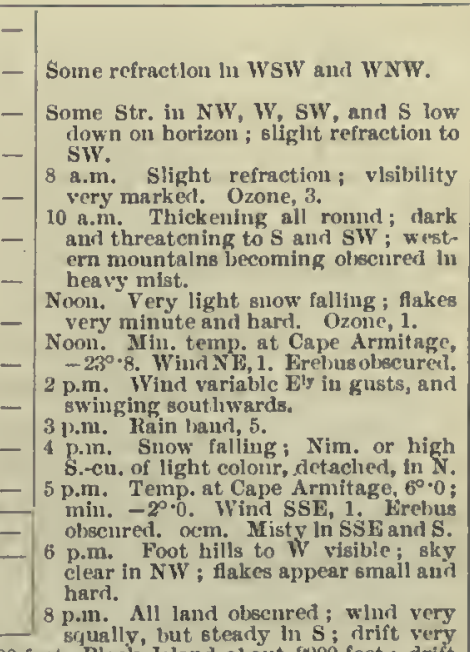

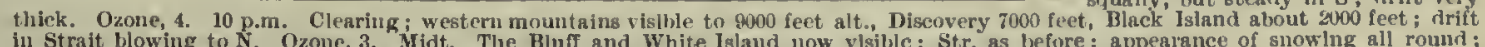
in Strait blowing to N. Ozone, 3. Midt. The Blunt and White Island now vlsible; Str. as hefore; a ppearance of snowing all round;

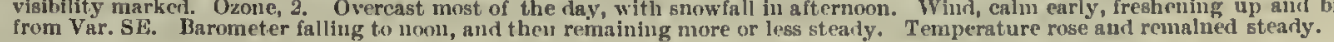

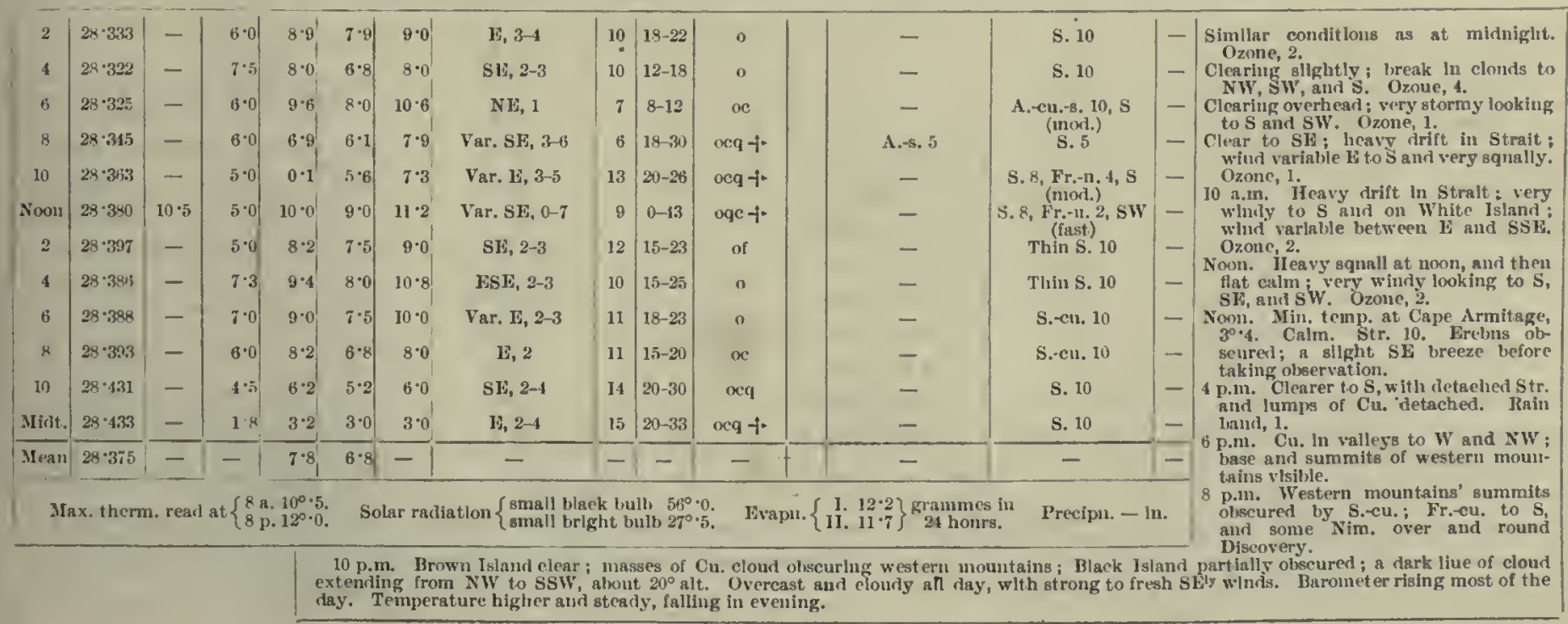


1903-0ctober.]

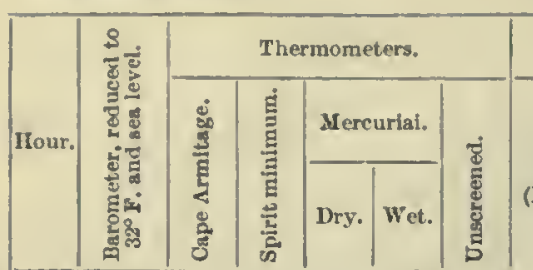

Meteonological Jourial of the "Discovery."

28th.

\begin{tabular}{|c|c|c|c|c|c|c|c|c|c|c|c|c|c|}
\hline 2 & $|28 \cdot 446|$ & - & $2 \cdot 0$ & $5 \cdot 0$ & $4 \cdot 2$ & $4 \cdot 5$ & $E, 1-3$ & $\theta$ & $|10-25|$ & oeq $+*$ & - & N. 10 & - \\
\hline 4 & $28 \cdot 477$ & - & 0.0 & $2 \cdot 0$ & 1.5 & $2 \cdot 0$ & E, 2-4 & 17 & $15-30$ & $o c q+*$ & - & N. 10 & - \\
\hline 6 & $28 \cdot 510$ & - & -1.5 & 0.0 & -0.5 & 0.0 & Far. SE, 3-7 & 18 & $15-45$ & $\operatorname{seq}+$ & - & S. 10 & - \\
\hline 8 & $28 \cdot 528$ & - & $-1 \cdot 8$ & 1.6 & $1 \cdot 6$ & $2 \cdot 0$ & Var. SE, 5-7 & 10 & $28-42$ & ogf - $^{-}$ & - & S. 10 & - \\
\hline 10 & 28.552 & - & $-3 \cdot 0$ & 0.0 & 0.0 & $1 \cdot 1$ & Var. ESE, 4-7 & 19 & $23-45$ & ocqf $f$ & Ci.s. 5 & S.cu. $4, \mathrm{SIV}$ & - \\
\hline Noon & 28.582 & - & $-5 \cdot 5$ & $-3 \cdot 1$ & $-3 \cdot 6$ & $-2 \cdot 0$ & NE, 4-5 & 19 & $20-27$ & ocq & Ci.-cu. 6. SW & Cu. 4, det. Cu. 2, & - \\
\hline 2 & $28 \cdot 5 i 7$ & - & $-5 \cdot 5$ & $-1 \cdot 6$ & $-1 \cdot 6$ & $1 \cdot 0$ & ENE, 4-5 & 18 & $20-28$ & $o q$ & - & A.-cu. 10 & - \\
\hline 4 & $28 \cdot 501$ & $-0 \cdot 1$ & $-4 \cdot 9$ & $1 \cdot 3$ & 0.8 & $4 \cdot 5$ & Var. Ea, 4-6 & 21 & $25-33$ & oq & - & A.cu. 10 & - \\
\hline 6 & $28 \cdot 517$ & - & -0.2 & $5 \cdot 0$ & $4 \cdot 0$ & $5 \cdot 2$ & Var. E, 4-5 & 20 & $20-25$ & o & - & A. cu. 10 & - \\
\hline 8 & 28.511 & - & $2 \cdot 2$ & 6.2 & $5 \cdot 6$ & 6.5 & Lt. E airs & 9 & $5-8$ & $x$ & - & S. 8, Fr.-8. $_{\text {(mod.) }} 4, \mathrm{~S}$ & - \\
\hline 10 & $28 \cdot 511$ & - & 0.9 & $2 \cdot 1$ & $1 \cdot 2$ & $2 \cdot 0$ & SE, 4-5 & 8 & $20-30$ & oc & Ci., Ci.-cu. 1 & S. 7 & - \\
\hline Midt. & $28 \cdot 492$ & - & -0.2 & $2 \cdot 0$ & $1 \cdot 0$ & $1 \cdot 7$ & Var. ESE, 4-5 & 11 & $20-25$ & $\infty$ & - & S. 9 & $1=$ \\
\hline Nean & 28.522 & - & - & $1 \cdot 7$ & $1 \cdot 2$ & - & 一 & - & - & - & - & - & - \\
\hline
\end{tabular}

Max, therm. read at $\left\{\begin{array}{l}8 \text { a. } 8^{\circ} \circ . \\ 8 \text { p. } 8^{\circ} 0 .\end{array}\right.$ Solar radiation $\left\{\begin{array}{l}\text { small black bulb } 50^{\circ}, 0 . \\ \text { small bright bulb } 20^{\circ} .\end{array}\right.$

Evapn. $\left\{\begin{array}{l}\text { I. } 10^{\circ} 9 \\ 1 \mathrm{I}, 10^{\circ}\end{array}\right\}$ grammes in 24 hours.

Clouds,

Amount and moverneut.

Direction and force
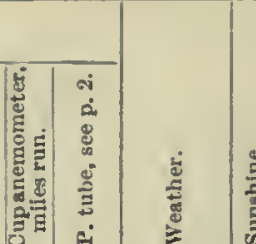

Amount and movement.

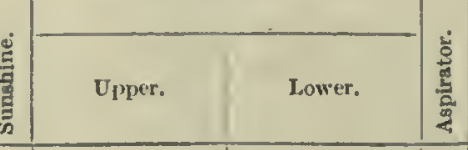

Remarks.

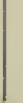

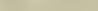

Thiek alt round; whd wariable from $S E$

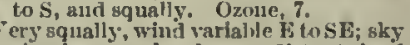
clearing overhead; 10 distant iani voon. Clearing to $8 \mathrm{~F}$ and generally: drift in Strait; western mountalus abscured. Ozolle, 4 rapidly iain band, 1.

p.m. Alt. of clouds about movo feet : all highest summits to $\mathrm{W}$ olsscured. 4 p.m. Min. temp. at Cape Armitage, $7 \%$. Str. 10 . p.m. Drift on surface of western side of Strait, obscuring bases of western mountalns.

p.1n. Clearing to SE ; elouds torn and heavy looking generally. Ozone, 2. p.m. Very ragged Str. over western rallge; patches of $\mathrm{Ci}_{\text {. }}$ and $\mathrm{Ci}$-cu Overcast ail day, with strong to fresi winds. Barometer rose until noon and then fell sgain for remainder of day. Temperature fairiy steady ali day.

29th.

\begin{tabular}{|c|c|c|c|c|c|c|c|c|c|c|c|c|c|c|}
\hline 2 & $28 \cdot 493$ & - & 0.0 & $1 \cdot 6$ & 0.9 & 1.8 & Var. $E, 3-4$ & 8 & $15-20$ & he & & Ci. 1 & S. 8 & - \\
\hline 4 & $28 \cdot 453$ & - & 1.0 & $3 \cdot 8$ & $2 \cdot 7$ & $4 \cdot 6$ & Var. SE, 4-5 & 15 & $20-30$ & $b c+$ & 1 & - & S., S.-cu. 8 & - \\
\hline 6 & $28 \cdot 450$ & - & $-2 \cdot 2$ & $-1 \cdot 2$ & $-1 \cdot 8$ & $-1 \cdot 1$ & ENE, 3-4 & 16 & $20-22$ & be & 1 & Ci., Ci.-eu. 3 & S., S.-cu. 4 & - \\
\hline 8 & $28 \cdot 460$ & - & $-6 \cdot 3$ & $-4 \cdot 8$ & $-5 \cdot 0$ & $-4 \cdot 0$ & ENE, 4-5 & 15 & $20-26$ & the & & Ci.-cu. 5, SW & S. $3, \mathrm{sw}$ & - \\
\hline 10 & $28 \cdot 460$ & - & $-8 \cdot 5$ & $-6 \cdot 0$ & $-6 \cdot 2$ & $-5 \cdot 0$ & ENE, 5-6 & 16 & $28-35$ & be & & Ci.-s. $5, \mathbf{S}$ & A.-5. 3,5 & - \\
\hline Noon & $28 \cdot 445$ & $-2 \cdot 6$ & $-7 \cdot 4$ & $-2 \cdot 0$ & $-2 \cdot 8$ & 0.0 & NF, 5-6 & 21 & $24-30$ & be & I & Ci.-s. 6, sw & A.-s. $2, \mathrm{SW}$ & - \\
\hline 2 & $28 \cdot 450$ & - & $-8 \cdot 0$ & -0.5 & $-1 \cdot 2$ & 2.5 & $\mathbf{E}, 15$ & 20 & $23-25$ & oc & ; & Ci.cu. 7, ș & A.-8, 2, SE & - \\
\hline 4 & $28 \cdot 448$ & $-3 \cdot 0$ & $-4 \cdot 0$ & $-1 \cdot 2$ & $-2 \cdot 0$ & $-1 \cdot 0$ & E by $N, 14-5$ & 15 & $20-25$ & $\propto$ & 1 & Ci.-s. 5, SW & A.-s, 4, SE & - \\
\hline 6 & $28 \cdot 416$ & - & $-4 \cdot 5$ & $-3 \cdot 2$ & $-4 \cdot 0$ & $-3 \cdot 0$ & E by $N, 3-4$ & 14 & $18-25$ & $\infty$ & & Ci.-s. $6, \mathrm{sW}$ & A.-s. 3, SE & - \\
\hline 8 & $28 \cdot 446$ & - & $-4 \cdot 6$ & $-1 \cdot 0$ & $-2 \cdot 0$ & -0.8 & NE, 1-2 & 10 & $10-18$ & $\infty$ & & - & A.-s. $8, \mathrm{~S}$ & - \\
\hline 10 & $28 \cdot 453$ & - & $-2 \cdot 0$ & $1 \cdot 0$ & 0.0 & $1 \cdot 0$ & NE, 1-2 & 8 & $5-10$ & 。 & & - & s. 10 & - \\
\hline Midt. & $28 \cdot 453$ & - & 0.0 & $1 \cdot 0$ & 0.5 & $1 \cdot 0$ & E, 2 & 7 & 12 & ise & & $\mathrm{Ci}_{0}-8.3, \mathbf{E}$ & S. $5, \mathbf{E}$ & - \\
\hline $\overrightarrow{\text { Jean }}$ & $28 \cdot 455$ & - & - & $-1 \cdot 0$ & $-1 \cdot 7$ & - & - & $\overline{-}$ & - & $\overline{-}$ & & - & - & - \\
\hline
\end{tabular}

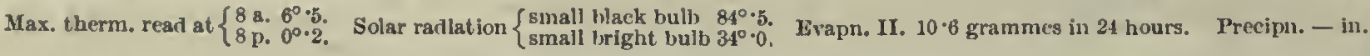

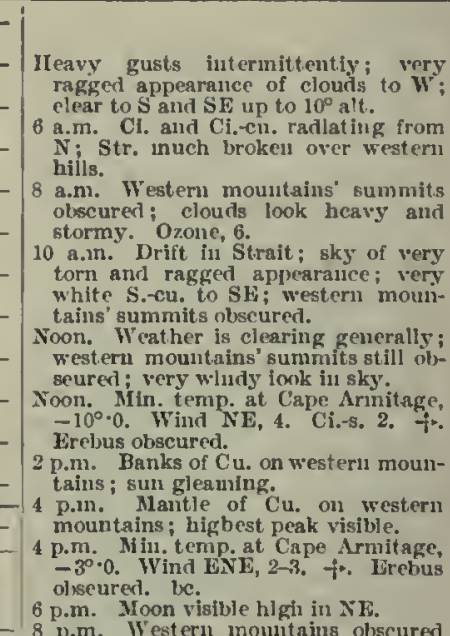

ahove 7000 feet; stormy lonk to SW and S. Ozone, 3. $10 \mathrm{p} . \mathrm{m}$. With exception of upper part of western mountains, land is clear; windy look to $\mathrm{S}$. Midt. Very windy appearance to E. Cloudy and practically overcast ali day, with fresh E's and N Wly whind. Barometer steady. Temperature fairly steady.

30th.

\begin{tabular}{|c|c|c|c|c|c|c|c|c|c|c|c|c|}
\hline 2 & $28 \cdot 459 \mid$ & - & 0.0 & $4 \cdot 0$ & $2 \cdot 2$ & $4 \cdot 0$ & $\mathrm{E}$ by $\mathrm{S}, 1-2$ & 8 & $6-12$ & be & Ci.-8. 3 & S. 6 \\
\hline 4 & $28^{\circ} 468$ & - & $-1 \cdot 4$ & $3 \cdot 0$ & $1 \cdot 4$ & $3 \cdot 0$ & Calm & 4 & 0 & 0 & - & s. 10 \\
\hline 6 & $28 \cdot 489$ & - & $-2 \cdot 9$ & -0.5 & $-1 \cdot 4$ & $-1 \cdot 0$ & SSE, 1 & 1 & 0 & og & - & S. 10 \\
\hline 8 & $28 \cdot 549$ & - & $-4 \cdot 5$ & $-3 \cdot 0$ & $-3 \cdot 0$ & $-2 \cdot 2$ & S1, 5-6 & 14 & $20-35$ & ogf $+* *$ & - & N. 10 \\
\hline 10 & $28 \cdot 615$ & - & $-4 \cdot 9$ & $-1 \cdot 0$ & $-1 \cdot 4$ & 0.0 & Var. SE, 2-7 & 19 & $10-40$ & oqm $-f * *$ & - & S. 10 \\
\hline Noon & $28 \cdot 651$ & - & $-5 \cdot 7$ & $-5 \cdot 0$ & $-5 \cdot 0$ & $-3 \cdot 5$ & SE, 3-5 & 17 & $18-28$ & ogf $f * *$ & - & N. 10 \\
\hline 2 & $28 \cdot 693$ & - & $-5 \cdot 5$ & $-2 \cdot 0$ & $-2 \cdot 5$ & $-1 \cdot 0$ & E, 3-4 & 13 & $20-28$ & of $\rightarrow$ & - & Thin S. 10 \\
\hline 4 & $28 \cdot 699$ & $-2 \cdot 8$ & $-4 \cdot 5$ & $-2 \cdot 6$ & -3.0 & $-1 \cdot 8$ & $E, 3-4$ & 14 & $15-23$ & og -4 & - & Thin S. 10 \\
\hline 6 & $28 \cdot 722$ & - & $-4 \cdot 2$ & $-2 \cdot 0$ & $-2 \cdot 3$ & $-1 \cdot 5$ & E, 2-3 & 15 & $12-20$ & $o g$ & - & S. 10 \\
\hline 8 & $28 \cdot 732$ & - & $-4 \cdot 0$ & $-1 \cdot 0$ & $-1 \cdot 8$ & -0.8 & F, 2-3 & 11 & $12-18$ & 0 & - & S. 10 \\
\hline 10 & $28 \cdot 746$ & - & $-2 \cdot 3$ & -0.8 & $-1 \cdot 0$ & -0.9 & Var. E. $2-3$ & 9 & $9-14$ & om $+*$ & - & N. $10, \mathrm{~S}$ \\
\hline Midt. & $28 \cdot 761$ & - & $-1 \cdot 9$ & $-1 \cdot 0$ & -1.0 & $-1 \cdot 0$ & Var. SE, 2-3 & 6 & $8-20$ & omq $+*$ & - & N. 10 \\
\hline Nean & $28 \cdot 632$ & - & -1 & $-1 \cdot 0$ & $-1 \cdot 6$ & - & - & -1 & - & - & - & - \\
\hline
\end{tabular}

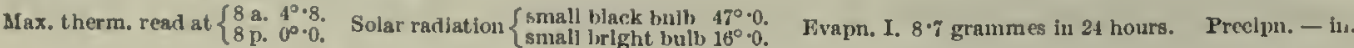

Nuch refraction to $S$.

Whirls of driving snow visible to $\mathrm{S}$ ln western mountains obseuren.

No land visible; drift very thiek in Wind variable, SSE to $E$, and very squally, there being a hearts squall at time of observation;

thick, sun gleaming.
Nooul. Brighter appearanec gencrally.

2 p.m. Dark bank of Cu. in S, SW and : solnc clear patehes to $W$

4 p.in. Heavy banks of whd-blown Cin. all along to west ward; clear patches to SW. 4 p.m. Min, temp. at Cape Armitage, $-g^{\circ} 6$. Wind is by S, 4-5; Frebus olseured. Whirlwind elouds to $W$ on 6 p.n. Clearing on linrlzon all round, fog In vallers and amongst the hills to W and XW.

p.m. Very windy looking in SW and S; sky breaking in s1:. Ozone, 3 . Orereast and dull, with strong winds
all day. Darometer rising all day. Temperat ure steady.
Temp. 
Meteorotogical Journal of THE "Discovery."

[1903-October.

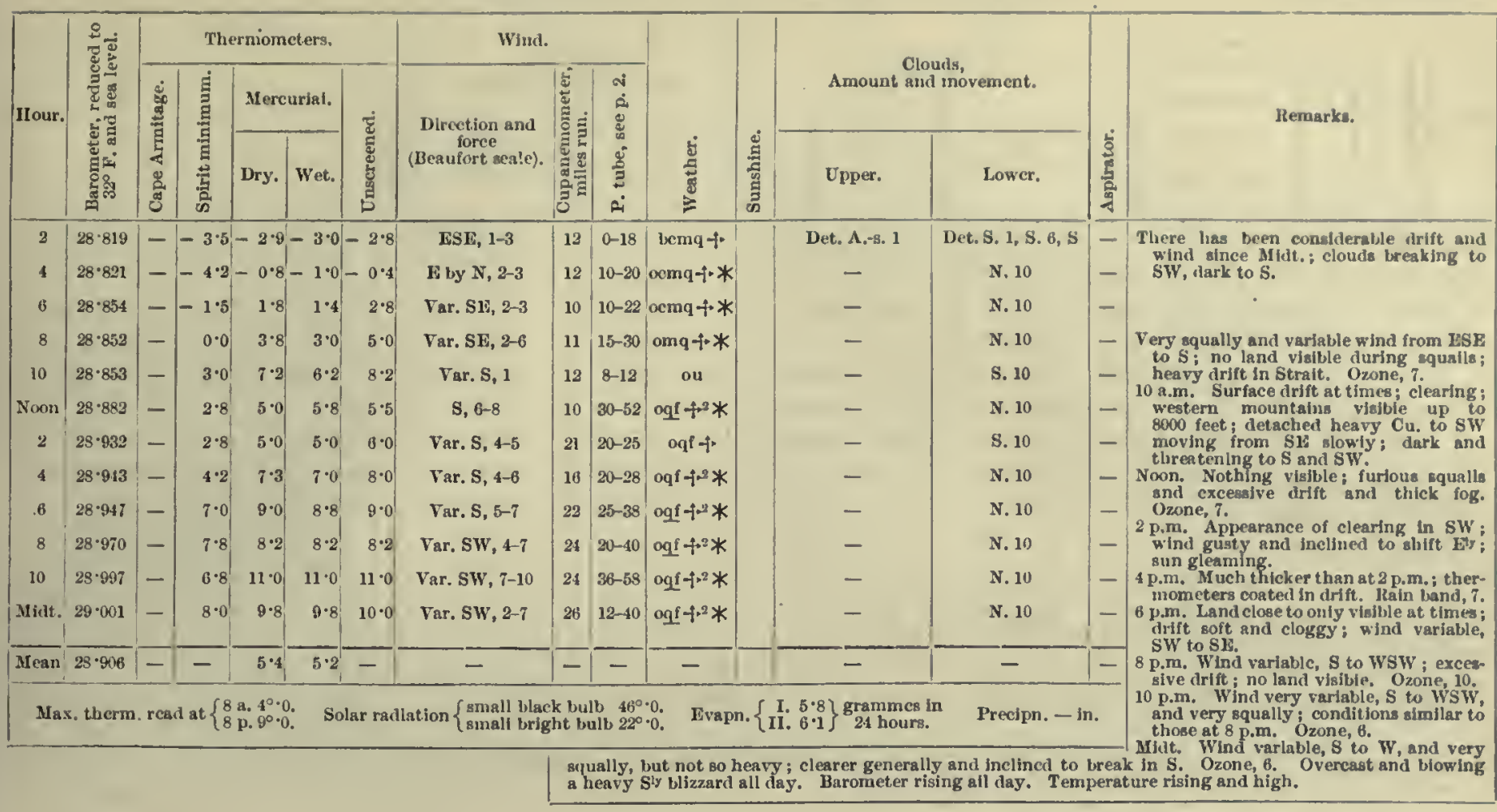


1903-November.]

Mfteorological JoUrnal of the "Discovery."

\begin{tabular}{|c|c|c|c|c|c|c|c|c|c|c|c|c|c|c|}
\hline \multirow{3}{*}{11 our. } & \multirow{3}{*}{ 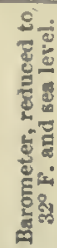 } & \multicolumn{5}{|c|}{ Titermometers. } & \multicolumn{3}{|l|}{ Wind. } & & & \multirow{2}{*}{\multicolumn{2}{|c|}{$\begin{array}{l}\text { Clouds, } \\
\text { Anount and movenent. }\end{array}$}} & \multirow[b]{3}{*}{$\frac{3}{4}$} \\
\hline & & $\stackrel{s}{\circ}$ & $\dot{\theta}$ & Merc & urial. & & & एँ & ฉั & & & & & \\
\hline & & 仓ैं & 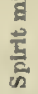 & Dry. & Wet. & 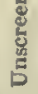 & $\begin{array}{l}\text { fore } \\
\text { (Beaufort seale). }\end{array}$ & 爮 & 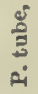 & 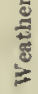 & 兘 & Opjer. & Lower. & \\
\hline
\end{tabular}

1st.

\begin{tabular}{|c|c|c|c|c|c|c|c|c|c|c|c|c|c|}
\hline 2 & $28 \cdot 997$ & - & $9 \cdot 0$ & $11 \cdot 2^{\prime}$ & $11 \cdot 2$ & $11 \cdot 2$ & Var. SW, 7-9 & 28 & $35-50$ & ogf $f+2 *$ & - & N. 10 & - \\
\hline 4 & $29 \cdot 009$ & - & $10 \cdot 2$ & $13 \cdot 0$ & $13 \cdot 0$ & $13 \cdot 2$ & Var. $S, 5-8$ & 25 & $25-50$ & $\operatorname{oqf} f+2$ & - & S. 10 & - \\
\hline 6 & $29 \cdot 036$ & - & $11 \cdot 8$ & $13 \cdot 2$ & $13 \cdot 0$ & $14 \cdot 0$ & SF, 4-7 & 19 & $20-40$ & oqu $\uparrow^{+}$ & - & S. 10 & - \\
\hline 8 & $29 \cdot 052$ & - & $12 \cdot 0$ & $13 \cdot 8$ & $13 \cdot 1$ & $14 \cdot 6$ & SE, 3-5 & 14 & $15-28$ & $\infty q$ & - & S. 10 & - \\
\hline 10 & $29 \cdot 070$ & - & $12 \cdot 5$ & $14 \cdot 0$ & $13 \cdot 8$ & $15 \cdot 0$ & $51,3-4$ & 17 & $15-30$ & ogf + & - & S. 10 & - \\
\hline Noon & $29 \cdot 080$ & $13 \cdot 3$ & $12 \cdot 8$ & $13 \cdot 8$ & $13 \cdot 2$ & $15 \cdot 0$ & SE, 3-4 & 16 & $20-27$ & oqf + & - & S. 10 & - \\
\hline 2 & $29 \cdot 083$ & - & $12 \cdot 3$ & $13 \cdot 5$ & $13 \cdot 0$ & $15 \cdot 0$ & Var. Sls, 3-5 & 14 & $20-30$ & ogf $+*^{\circ}$ & 一 & N. 10 & - \\
\hline 4 & $29 \cdot 083$ & - & $12 \cdot 4$ & $13 \cdot 7$ & $13 \cdot 0$ & $11 \cdot 8$ & Far. S1, 3-4 & 18 & $18-23$ & oqf + & 一 & S. 10 & - \\
\hline 6 & $29 \cdot 075$ & - & $12 \cdot 8$ & $16 \cdot 0$ & $15 \cdot 0$ & $16 \cdot 6$ & Var. SE, 2-3 & 9 & $12-18$ & of $*^{\circ}$ & - & N. 10 & - \\
\hline 8 & $29 \cdot 0+3$ & 一 & $14 \cdot 0$ & $15 \cdot 1$ & $14 \cdot 3$ & $15 \cdot 8$ & Lt. I airs & 4 & $0-5$ & og & - & S. 10 & - \\
\hline 10 & $29 \cdot 044$ & - & $13 \cdot 6$ & $14 \cdot 2$ & $13 \cdot 8$ & $14 \cdot 0$ & $\operatorname{SsI}, 1-3$ & 7 & $10-22$ & $o c q \neq$ & - & S. 10 & - \\
\hline Mlat. & $29 \cdot 047$ & - & $11 \cdot 0$ & $13 \cdot 2$ & $12 \cdot 8$ & $12 \cdot 2$ & SE, 1-3 & 15 & $12-25$ & oeq & Ci.-s.s. 2 & S. 10 & - \\
\hline Mean & $29 \cdot 0.053$ & - & - & $13 \cdot 7$ & $13 \cdot 3$ & - & -1 & - & - & - & - & . - & - \\
\hline
\end{tabular}

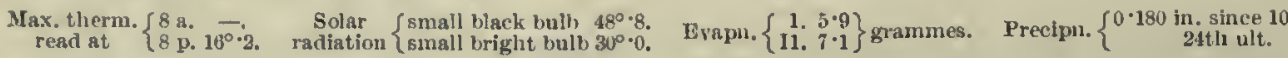

- Wind varlaile bet ween $\mathrm{S}$ and WS W; no

Renarks. and risible; drift excessive; Dines 0 \%one, 7 .

4 a.m. Wind varialse between $\mathrm{S}$ and and no snow fnot so heavy; less 6 a.1n. Wind shifted just after 5 a.m. clearing generally; Brown Island visiile at times; drift in Stralt. 8 a.m. Bri Strait ; Brown generally; drift In Strait; Brown 1sland visible; Hack 10 a.m. Black and Brown Islands elear; sigint drift only.

intervals near land vislble, exeept at Brown lslands showeri up ; considerable $\rightarrow$ (? snow fallugh); sun dimly visible at times. Ozone, 6 . Noon. Min. temp. at Cape Armitage, $-10^{\circ} \cdot 5$. Whind SSE, 5. Nim. 10. Erebus obscured. $t$ 2 p.m. Land to $W$ and $S W$ visible at times. Wind squally and varialule. 3 p.m. Rain bani, 5 .

$6 \mathrm{p.m}$. Appearance of elearing all round ; alt. of clouds, 3000 fcet.

$8 \mathrm{p.m}$. Low land risille all round up to 3000 feet; gloomy and threatening look generally. Ozone, 9 . Midt. Only a very thlu film of cloud in zenitls; hanks of

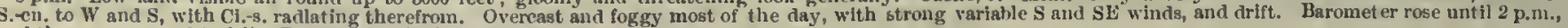
and then commenced to fali. Temperature high and stcady.

2 nd

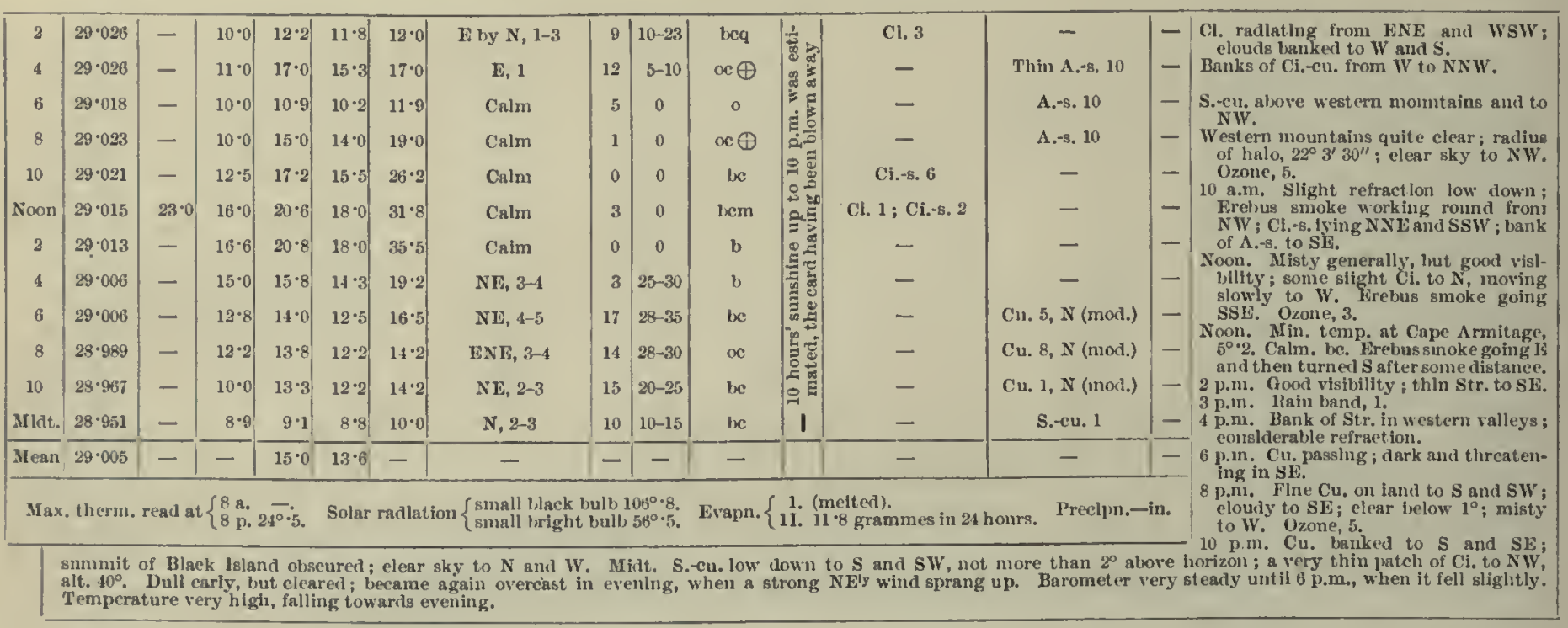

$3 \mathrm{rd}$

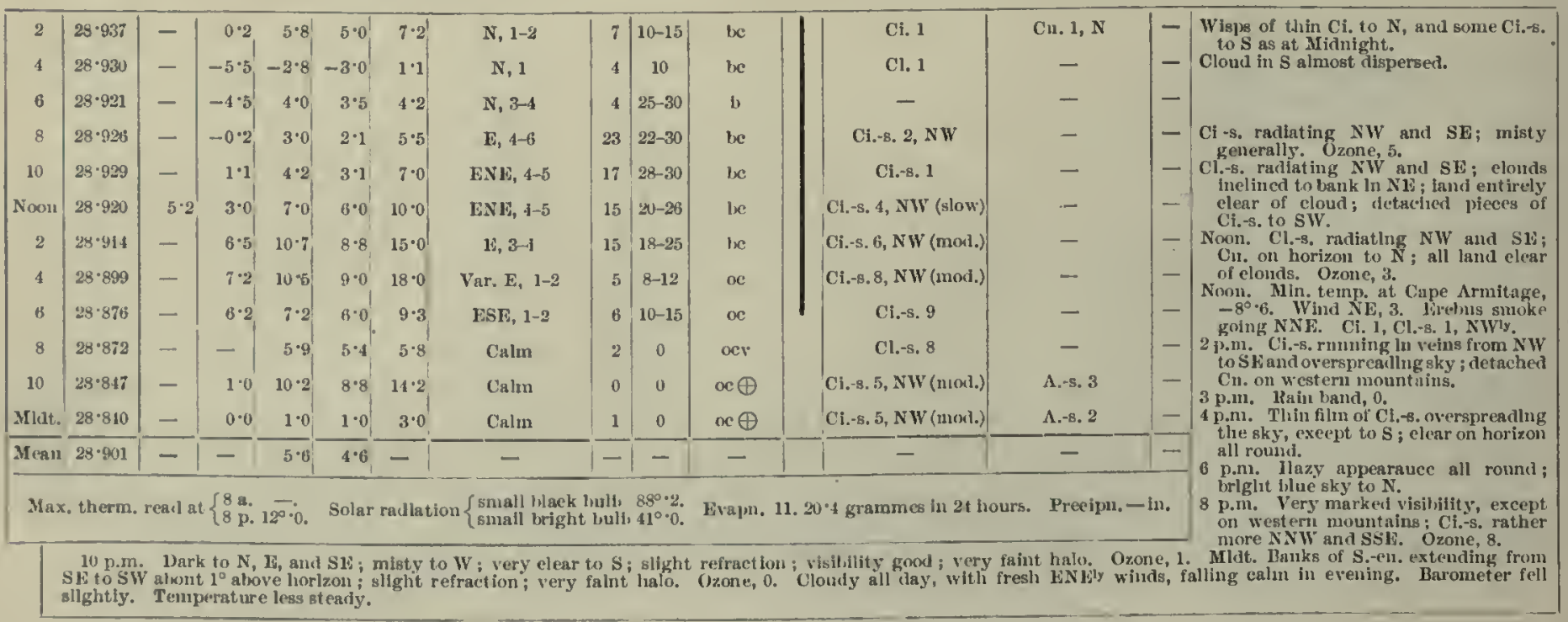


Mfteonologicat, Journal of THE "Discovery."

[1903-November.

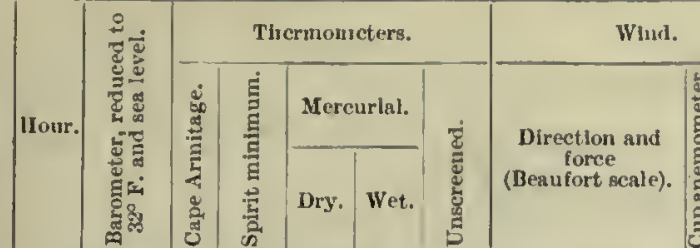

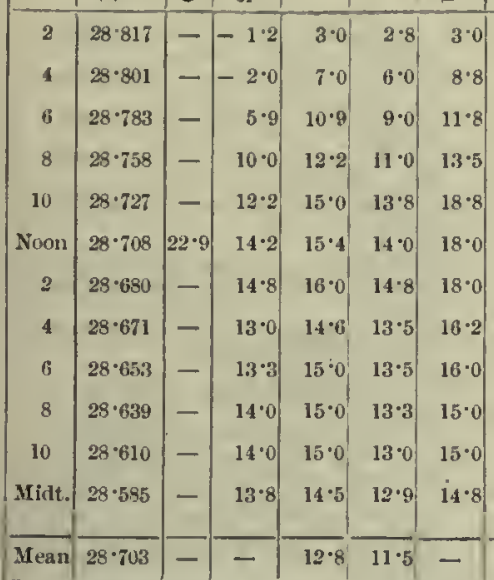

Calın

Lt. $\mathrm{N}$ alrs

N, 1

N, 1

N, 1-2

N, 2-3

N, 1-2

NNW, 1-3

N by $\mathbf{W}, 2-3$

N, 1-2

N, 1-2

$\mathbf{N}, 0-1$

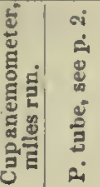

\begin{tabular}{c|c|}
0 & 0 \\
5 & $0-8$ \\
5 & 10 \\
7 & 10
\end{tabular}

$7 \longdiv { 1 0 - 1 5 }$

$8|12-18|$

5 10-15

\begin{tabular}{l|l|}
3 & $12-18$ \\
6 & $13-20$
\end{tabular}

$7 \quad 10-15$

$7 \quad 10-16$ be

Cloudis,

Amount ancí movement.

Hemarks.

\begin{tabular}{|c|c|}
\hline Upper. & Lower. \\
\hline
\end{tabular}

C1.-8. 1, NW $\mid$ Det, A,-cu, 1, SW - Much elearer overiteal; unore S.-cu. to S

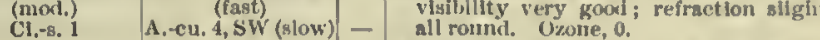

Cu. 9 , SW (slow)

$-$

Ci.-cu. 5, Sw

Ci.-cu. 5, SW

C1.-en. 3, SW

C1.-e1r. 2 Cn. 7, SW (slow)

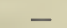

Ct.-cu. 2

ci.-eu. 3
-

A.-s, 2, SW

Cu. 6, S

S.-c11. 8

S.-cu. 10

S. 10

A. -8.3 $\underset{\text { SSW }}{\text { Jagged }}$ A.-g. 2 ,

Max, therm.
reaul at $\left\{\begin{array}{l}8 \mathrm{a}, 12^{\circ} \cdot 9 . \\ 8 \mathrm{p} .16^{\circ} \cdot 7 .\end{array} \quad\right.$ Solar $\left\{\begin{array}{l}\text { small black bulb } 108^{\circ} \cdot 0 . \\ \text { small bright bulb } 49^{\circ} \cdot 8 .\end{array}\right.$

Evapn. $\{$ I. $6 \cdot 10.1\}$ grammes in

4 a.m. Dark ami gloony to S and SW ame bedilnd Whito Islani ; southern end of westeni mountains of ncured ; clear anc fine to NW. Ozone,

6 a.m. Western moun tains obscured down to $5000 \mathrm{ft}$. ; winly looking to $\mathrm{SW}$; dar

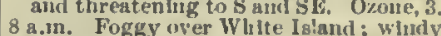
8.in. Foggy over White Island; whidy
looking to $\mathrm{SW}$; westent monntalus acoling the

$10 \mathrm{am}$. Ali clonds apjear to have risen. Noon. Fin

they tass prismatlc colour on clouls as very fraverl triges. $A$. to $S E$ andi $\mathrm{L}$, witi Noon. Min. temp. at Cape Armitage. $-9^{\circ} \cdot 6$, Wrebus smoke golng NI: Wini NW, 1-2. Hc, 2 p.un. Ci.-cu, in zenith; Str, all round. Ozone, 3. p.m. Ci.-cu. in zenIth; westeris mom talns olseured alsove tiv0 $\mathrm{ft}$

6 p.m. Clouds thin, witl sun gleaming. p.ni. All distant land olscured down to alront 2000 feet; appearanee of snowing in SW, S, and W. Ozone, 5 . 10 p.m. Str. in $\mathrm{S}$, sun gleaning.

Very fine clouds all the forenoon; sky gradually cloudel over. Fresh Nly breezes. Barometer continued to fall all day. Temperature higir and st eady.

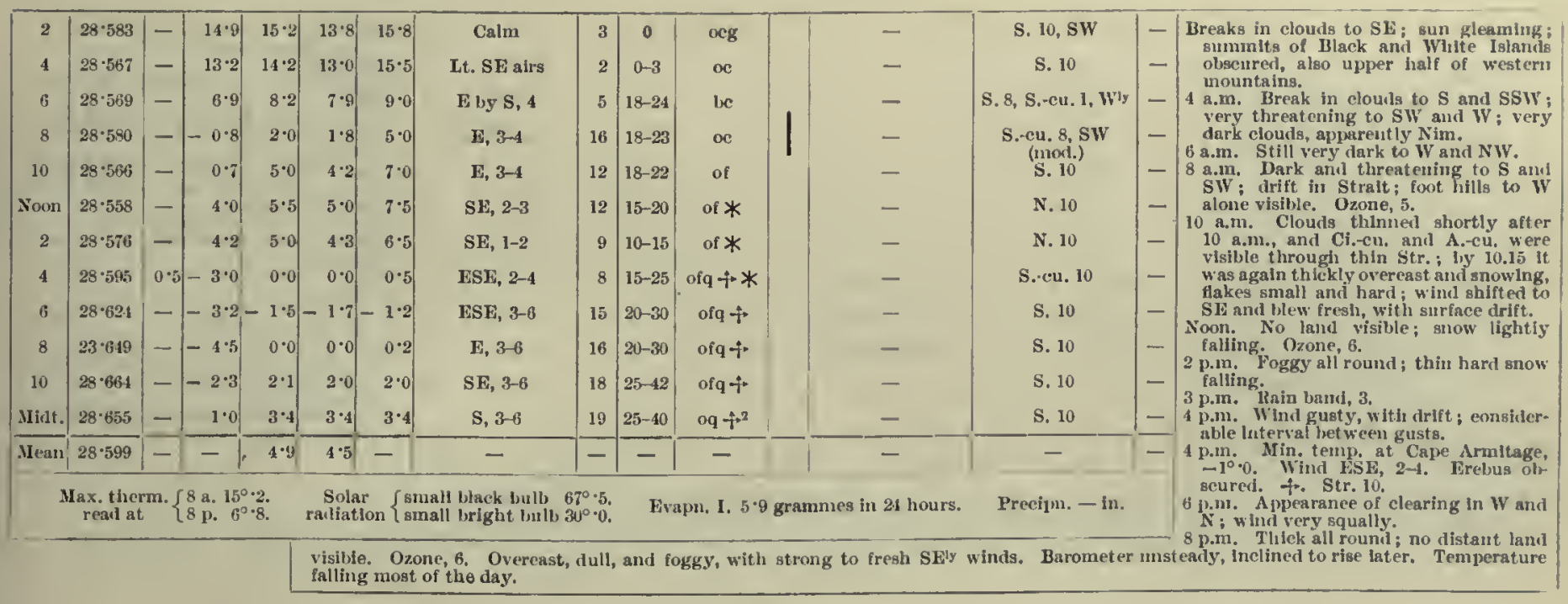

5 th.

th.

\begin{tabular}{|c|c|c|c|c|c|c|c|c|c|c|c|c|c|c|}
\hline 2 & $28 \cdot 660$ & - & $2 \cdot 5$ & $4 \cdot 5$ & $4 \cdot 5$ & $4 \cdot 5$ & $\mathrm{SE}, 3-6$ & 21 & $|15-40|$ & oqf + & 一 & S. 10 & - & \\
\hline 4 & $28-690$ & - & $4 \cdot 0$ & $6 \cdot 1$ & $6 \cdot 0$ & $6 \cdot 5$ & SE, 3-6 & 19 & $15-35$ & ogf $\tau^{-}$ & - & S. 10 & - & $\begin{array}{l}\text { Dark fooking cioudis over western moun- } \\
\text { tains, olsscuring them. }\end{array}$ \\
\hline 6 & $28 \cdot 714$ & - & $5 \cdot 5$ & 6.8 & $6 \cdot 5$ & $7 \cdot 0$ & ST, 3-6 & 17 & $15-35$ & oqf + & 一 & S. 10 & - & \\
\hline 8 & $28 \cdot 739$ & - & $4 \cdot 0$ & $7 \cdot 5$ & $7 \cdot 2$ & $8 \cdot 0$ & $\mathrm{SSE}, 3-5$ & 23 & $25-30$ & oqf + & - & S. 10 & - & $\begin{array}{l}\text { Ileary drift; whilst taking observations } \\
\text { drift stoppeil for } 2 \text { or } 3 \text { luinutes, and a }\end{array}$ \\
\hline 10 & $28 \cdot 748$ & - & $6 \cdot 0$ & $7 \cdot 8$ & $7 \cdot 2$ & $9 \cdot 5$ & Var. SE, 4-5 & 13 & $20-25$ & oing $f$ & - & S. 10 & - & $\begin{array}{l}\text { small portlon of sky was visible in } \mathrm{S} \text { : } \\
\text { Ci.-8., but no moventent seen. }\end{array}$ \\
\hline Soon & $28 \cdot 758$ & $9 \cdot 8$ & $6 \cdot 5$ & $9 \cdot 8$ & $8 \cdot 8$ & $11 \cdot 2$ & E, 2-3 & 14 & $15-23$ & ogs & - & N. 10 & - & 10 a.m. Land beginning to show $11 \mathrm{p}$ all \\
\hline 2 & $28 \cdot 753$ & - & $8 \cdot 2$ & $9 \cdot 5$ & $8 \cdot 0$ & $10 \cdot 8$ & Var, E, 2-3 & 13 & $18-25$ & $0 \div *$ & - & N. 10 & - & $\begin{array}{l}\text { Owone, } 6 . \\
\text { Non. VWhite Island beginning to show }\end{array}$ \\
\hline 4 & $28 \cdot 746$ & - & $4^{\prime} 6$ & $6{ }^{\circ} 6$ & $6 \cdot 0$ & $8 \cdot 2$ & Var. E, 2-4 & 14 & $15-25$ & $o+*$ & - & Thin N. 10 & - & $\begin{array}{l}\text { up; ot lier land as before, and condit ions } \\
\text { sinilar. }\end{array}$ \\
\hline 6 & $28 \cdot 737$ & - & $3 \cdot 7$ & $8 \cdot 2$ & $7 \cdot 5$ & $10 \cdot 0$ & Var. F, 3-6 & 16 & $25-35$ & $0-4$ & - & Thin S.ck. 10 & - & $\begin{array}{l}\text { Nonn. Nin. temp, at Cape Armitage, } \\
-3^{\circ} .6 \text {. Whi LNE, 3. Brebus obscurted. }\end{array}$ \\
\hline 8 & $28 \cdot 723$ & - & $B \cdot 0$ & $9 \cdot 8$ & $9 \cdot 0$ & $10 \cdot 0$ & $\mathbf{E}, 3-4$ & 13 & $15-22$ & of $+* *$ & - & N. 10 & - & $\begin{array}{l}2 \text { p.m. Dark and threatening In } 5 \text {; thin } \\
\text { soft snow falling. }\end{array}$ \\
\hline 10 & $28 \cdot 083$ & - & 10 & $9 \cdot 0$ & $7 \cdot 0$ & $10 \cdot 0$ & Vnr., $0-6$ & 19 & $0-32$ & ofg $+*^{\circ}$ & Ci.-cu. 6, S (mokl.) & N. 10 & - & 3 p.n. Kain land, 2. \\
\hline sinlt. & $28 \cdot 685$ & - & $B \cdot 0$ & $9-5$ & $9 \cdot 4$ & $10 \cdot 0$ & S, 1-6 & 12 & $10-30$ & ofg $+2 *$ & - & N. 10 & - & $\begin{array}{l}\text { o p.m. Sky clondless low dow on on WSW } \\
\text { horizon; detached elonds in S and SW. }\end{array}$ \\
\hline I tean & $28 \cdot 720$ & - & 一 & $7 \cdot 9$ & $7 \cdot 3$ & - & 一 & 一 & - & - & - & - & - & 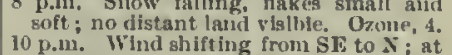 \\
\hline & \multicolumn{4}{|c|}{$\begin{array}{l}\text { Max. therm. } \\
\text { read at }\end{array}\left\{\begin{array}{l}8 \text { a. } \\
8 \mathrm{p}, 10^{\circ}\end{array}\right.$} & $\begin{array}{r}S \\
\text { radi }\end{array}$ & lar & $\begin{array}{l}\text { suall black b } \\
\text { small bright }\end{array}$ & 50 & & \multicolumn{2}{|c|}{ Fvapn. $\left\{\begin{array}{c}\text { I. } 1 \cdot 1 \\
\text { II. } 6.9\end{array}\right\} \begin{array}{c}\text { grammes in } \\
\text { 2: hours. }\end{array}$} & Precilu. 一in. & & $\begin{array}{l}\text { times blowing strong, and then falling } \\
\text { ealm, Ozone, } 4 . \\
\text { Midt. Very gusty, but wind steady } \\
\text { in direction ;now falling thekiy in }\end{array}$ \\
\hline
\end{tabular}


1903-November,]

Meteorologicat, Journat, of the "Discovery."

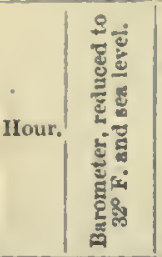

7th.

\begin{tabular}{|c|c|c|c|c|c|c|c|c|c|c|}
\hline 2 & $28 \cdot 673$ & - & 0.0 & 788 & $7 \cdot 8$ & $8 \cdot 0$ & ESE, 4-7 & 15 & $20-45$ & $\log f+$ \\
\hline 4 & $28 \cdot 657$ & - & $2 \cdot 9$ & $3 \cdot 2$ & $3 \cdot 2$ & $3 \cdot 5$ & ENE, 5-7 & 15 & $28-42$ & 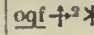 \\
\hline 6 & $23^{\circ} 664$ & - & 1.5 & $2 \cdot 2$ & $2 \cdot 2$ & $2 \cdot 9$ & $\mathrm{E}, 5-6$ & 21 & $25-32$ & \\
\hline 8 & $23 \cdot 689$ & - & 0.5 & $1 \cdot 5$ & 1.5 & $2 \cdot 5$ & E, 4-6 & 17 & $25-30$ & \\
\hline 10 & $23 \cdot 700$ & - & 1.5 & $3 \cdot 5$ & $3 \cdot 5$ & $4 \cdot 5$ & ENE, 4-6 & 21 & $18-25$ & \\
\hline Noon & $28 \cdot 70 \pi$ & $4 \cdot 5$ & $2 \cdot 5$ & $4: 5$ & 4.5 & $5 \cdot 5$ & ENT, 4-5 & 15 & 22-28 & \\
\hline 2 & $28 \cdot 717$ & - & $3 \cdot 2$ & $4 \cdot 5$ & $4 \cdot 2$ & $5 \cdot 0$ & ENE, 4-5 & 18 & $20-27$ & $0+$ \\
\hline 4 & $28 \cdot 714$ & - & $3 \cdot 5$ & $5 \cdot 0$ & $4 \cdot 7$ & $5 \cdot 2$ & ENE, 4-6. & 15 & $20-30$ & \\
\hline 6 & $28 \cdot 701$ & - & $3 \cdot 8$ & $7 \cdot 8$ & $7 \cdot 0$ & $7 \cdot 0$ & $\mathrm{NE}, 4-7$ & 20 & $25-33$ & \\
\hline 8 & $28 \cdot 68 \pi$ & - & $3 \cdot 2$ & $10 \cdot 0$ & $8 \cdot 2$. & $10 \cdot 5$ & E, 3-4 & 12 & $18-24$ & 0 \\
\hline 10 & $29 \cdot 678$ & - & 5.0 & $6 \cdot 2$ & $6 \cdot 1$ & $6 \cdot 2$ & NE, 4-5 & 18 & $25-32$ & $x-$ \\
\hline Midt. & $28 \cdot 677$ & -1 & $5 \cdot 5$ & $9 \cdot 8$ & $9 \cdot 8$ & 9.8 & F, 2-3 & 15 & $20-25$ & be \\
\hline Mean & $28 \cdot 689$ & - & - & $5 \cdot 5$ & $5 \cdot 2$ & - & - & - & - & \\
\hline
\end{tabular}

Winl.

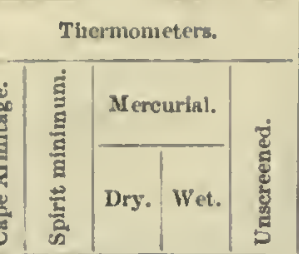

Max. therm. $\left\{8\right.$ a. $g^{\circ \cdot 5}$.

Solar s small black brilb $60^{\circ} \cdot 5$.

Evapn. $\{11.2 \cdot 4 \cdot 5\}$ grammes in

Precipn. $0.045 \mathrm{in}$. since 6 p.m. 1st.

Clonds,

Amount and movement.

\begin{tabular}{|c|c|}
\hline Upper. & Lower. \\
\hline
\end{tabular}

llemarks.

N. $10 \quad-$ No land visible anywhere; drift excessive;

N. 10

N. 10

N. 10

N. 10

N. 10

S. 10

S. 10

N. 10

Cl.-en. 2, SW

N. 9, SW

Ci, 5

S. 4, SW

- Land dimly visible, close to ship; snow

- Woft and very wet. Ozonc, 6

Ozone, 7 .

- No land visible, except close to; much

- drift. Ozone, 5 .

Land cicarer close to slup, no distant ianı visilbie. Ozone, 5.

Ozone, 4.7 . Winul ESE, 1-2. Erebus obscuret.' Fog. $+*$.

2 p.m. Black, Brown, and White 1slands visible betw een squalis.

4 p.n. Dark and foggy in $S$ and $D$.

6 p.m. Soft wet snow falling in large flakes. p.m. Sky breaking in zenith; smin gleaming; brighter generally. Ozonc, 8 .
10 p.m. Hifeavy and dark still to $\mathrm{S}, \mathrm{Sl}, \mathrm{E}, \mathrm{E}$, and $S W$, but White 1 sland can be seen: overhead sky is clearing to blue, flecked
with very tinely divided foathery $\mathrm{Cl}$. Ozone, 5 .

Midt. Clear now on southern horizon, and can see Bluff, White 1sland, and ali the nearer western hilis and islands: blue sky orerhead; Str. still a bit to $N$ :
dark over $S E, E$, and western moundark over $\mathbf{S E}, \mathrm{E}$, and western moun-
tains, but breaking everywhere; drift taims, but breaking every
almost stopped. Ozone, 4.

8th.

\begin{tabular}{|c|c|c|c|c|c|c|c|c|c|c|c|c|c|c|}
\hline 2 & $28 \cdot 677$ & - & $5 \cdot 2$ & $9 \cdot 9$ & $8 \cdot 9$ & $10 \cdot 4$ & E l,y $N, 2-3$ & 19 & $\mid 15-25$ & oc $*$ & Fr.-cl.-s. 4 & N. 6 & - & Stili clear on horizo \\
\hline 4 & $28 \cdot 686$ & - & $7 \cdot 0$ & 11.0 & $10^{\circ} 0$ & $12 \cdot 0$ & E by_S, 1-2 & 13 & $8-20$ & $0 *$ & - & N. 10 & - & 1cent that near \\
\hline 6 & $28 \cdot 695$ & - & $10 \cdot 0$ & $10 \cdot 9$ & $10 \cdot 1$ & $12 \cdot 2$ & E by S, 1-2 & 8 & $5-10$ & 0 & - & S. 10 & - & $\begin{array}{l}4 \text { and, No land visibl } \\
\text { ship. }\end{array}$ \\
\hline 8 & $28 \cdot 692$ & - & $10 \cdot 0$ & $13 \cdot 2$ & $12 \cdot 2$ & $15 \cdot 0$ & Var. E, 0-4 & 2 & $8-20$ & $\propto$ & - & S.-eu. 9, SW (fast) & - & $\begin{array}{l}6 \text { a.m. White Island and Bluff clear } \\
\text { again, the former with the sur on it; }\end{array}$ \\
\hline 10 & $28 \cdot 688$ & - & $11 \cdot 5$ & $13 \cdot 4$ & $13 \cdot 0$ & $15 \cdot 5$ & ENE, 1-2. & 10 & $10-18$ & of $*$ & - & N. 10 & - & 8 a.m. Western nountains foot hills and \\
\hline Noon & $28 \cdot 687$ & - & $11 \cdot 2$ & $16 \cdot 0$ & $15 \cdot 2$ & $17 \cdot 5$ & $E, 1-2$ & 10 & $10-15$ & of $*$ & - & N. 10 & - & $\begin{array}{l}\text { visible. Ozone, } 3 \text {. } \\
\text { Only }\end{array}$ \\
\hline 2 & $28 \cdot 680$ & - & $14 \cdot 4$ & $15 \cdot 8$ & $15 \cdot 2$ & $17 \cdot 5$ & E, 2-3 & 7 & $8-15$ & of $+* *$ & - & N. 10 & - & 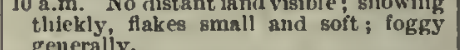 \\
\hline 4 & $28 \cdot 671$ & - & $14 \cdot 5$ & $16 \cdot 8$ & $16^{\circ} 0$ & $180^{\circ}$ & E by $N, 2-3$ & 10 & $12-18$ & of $+* *$ & - & N. 10 & - & $\begin{array}{l}\text { generally. Weather similar; sun shining } \\
\text { throngh. }\end{array}$ \\
\hline 6 & $28 \cdot 668$ & - & $13 \cdot 7$ & $17 \cdot 5$ & $16 \cdot 8$ & $18 \cdot 5$ & Var, 0-2 & 7 & $8-12$ & of $\uparrow^{2} *^{2}$ & - & N. 10 & - & 2 p.um, Snow falling thickly in soft flaker. \\
\hline 8 & $28 \cdot 651$ & - & $16 \cdot 0$ & $17 \cdot 0$ & $17 \cdot 0$ & $17 \cdot 2$ & $S, 5-8$ & 13 & $28-45$ & of $+^{2} *^{2}$ & - & N. 10 . & - & 3p.m. Wind vane was pointing nortinwards \\
\hline 10 & - & - & - & - & - & - & - & - & - & - & - & - & - & southwards and then west wards, finaliy \\
\hline Midt. & - & - & - & - & - & - & - & - & - & - & - & - & - & 8 p.m. Very thick indeed; drift exeessive; \\
\hline Mean & $28 \cdot 630$ & -1 & - & $14 \cdot 2$ & $13 \cdot 4$ & - & - & - & - & - & - & - & - & \\
\hline \multicolumn{5}{|c|}{$\begin{array}{l}\text { Max. therm. } \\
\text { read at }\end{array}\left\{\begin{array}{l}8 \text { a. } 13^{\circ} \cdot 2 \\
8 \mathrm{p} .\end{array}\right.$} & \multicolumn{4}{|c|}{ 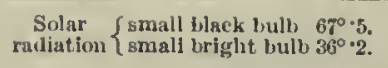 } & \multicolumn{3}{|c|}{ Eva1н. $\left\{\begin{array}{r}1,4 \cdot 1 \\
11,8 \cdot 3\end{array}\right\}{ }_{22}^{\text {grammes in }}$} & $\begin{array}{l}\text { ipn. } 0.024 \\
4 \mathrm{j}, \mathrm{m}, 7\end{array}$ & & \\
\hline
\end{tabular}

9th.

\begin{tabular}{|c|c|c|c|c|c|c|c|c|c|c|c|c|c|c|c|}
\hline 2 & - & -1 & - & - & - & - & - & - & - & - & & - & - & $1-$ & \\
\hline 4 & - & - & - & - & - & - & - & - & - & - & & - & - & - & \\
\hline 6 & - & - & - & - & - & - & - & - & - & - & & - & - & - & \\
\hline 8 & $28 \cdot 693$ & - & $11 \cdot 8$ & $12 \cdot 8$ & $12 \cdot 2$ & $13 \cdot 5$ & SE, 2-7 & 23 & $10-47$ & $1 x+f^{2}$ & & - & S. 9 & - & velocity of wind since 8 /p.m. \\
\hline 10 & $28 \cdot 704$ & - & $11 \cdot 2$ & $13 \cdot 0$ & $12 \cdot 0$ & 16.0 & $\mathrm{SE}, 2-6$ & 23 & $20-25$ & oq + . & & - & S. 9 & - & Very squally, wind scems inclined to slift \\
\hline Noon & $28 \cdot 678$ & - & $9 \cdot 8$ & $15 \cdot 0$ & $14 \cdot 0$ & $17 \cdot 1$ & E, 4-B & 17 & $20-32$ & neq + & & - & S. $6, A_{0}-3,2, S S W$ & - & $\begin{array}{l}\text { and parts of wrestern mountains isible; } \\
\text { heavy hanks of elouds in W, apparently }\end{array}$ \\
\hline 2 & $25 \cdot 685$ & - & $11 \cdot 2$ & $13 \cdot 0$ & $12 \cdot 2$ & $13 \cdot 5$ & ESE, 2-6 & 18 & $15-30$ & oeq $t$ & & - & S. 0 & - & $\begin{array}{l}\text { Nim. Ozone, } \\
\text { Nom. Clearlng generally, blue sky and }\end{array}$ \\
\hline 4 & $28 \cdot 678$ & - & $10 \cdot 8$ & $12 \cdot 2$ & $11 \cdot 5$ & $12 \cdot 5$ & ESE, 5-8 & 19 & $28-45$ & ocq + & & - & S. 9 & - & $\begin{array}{l}\text { troken clouds to S, SE, and SW ; land } \\
\text { becoming visible ; drift in Strait. }\end{array}$ \\
\hline 6 & $23 \cdot 671$ & - & $9 \cdot 6$ & $12 \cdot 2$ & $11 \cdot 5$ & $12 \cdot 2$ & E, 2-4 & 21 & $18-28$ & cq + & & Ci.-cu. 3 & Cu. 5 & - & $\begin{array}{l}2 \text { p.m. Not so mucle drift; White Island } \\
\text { only partly vislble; Brown lsland and }\end{array}$ \\
\hline 8 & $28 \cdot 671$ & - & $11 \cdot 5$ & $14 \cdot 5$ & $14 \cdot 0$ & $10 \cdot 0$ & LN 1, 3-4 & 12 & $18-29$ & the + & & Ci. cu. 2, s & A.-s. 4, S.-cu. 1, S & - & $\begin{array}{l}\text { Mt. Discovery visible, both having } \\
\text { detached Str. hanging over then ; not }\end{array}$ \\
\hline 10 & $28 \cdot 661$ & - & $7 \cdot 5$ & $8 \cdot 0$ & $7 \cdot 5$ & $8 \cdot 5$ & ENE, 3-4 & 14 & $18-21$ & le & & Ci.-cn. 1, s & A.-s. $3, \mathrm{~S}$ & - & so bright; wind linelined to go $\mathrm{s}$. \\
\hline Mlat. & - & - & - & - & - & - & - & - & - & - & 1 & - & - & - & $\begin{array}{l}4 \text { p.m. Very heavy drift, no land visible, } \\
\text { creent that close to. Ozone, } B \text {. }\end{array}$ \\
\hline Ien & $28 \cdot 680$ & - & - & $12 \cdot 6$ & $11 \cdot 0$ & - & - & - & - & 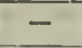 & & - & - & - & $\begin{array}{l}8 \text { p.m. Weather bright ening generally; } \\
\text { all land visible, exeept smali upper por }\end{array}$ \\
\hline \multicolumn{5}{|c|}{ 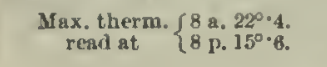 } & \multicolumn{4}{|c|}{ 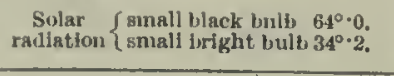 } & \multicolumn{4}{|c|}{ Evapn. $\left\{\begin{array}{l}\text { I. } 4 \cdot 5 \\
\text { I1. } 5 \cdot 4\end{array}\right\} \begin{array}{c}\text { grammes in } \\
24 \text { hours. }\end{array}$} & $\begin{array}{l}\text { Precipn. } 0.03 \text { in. since } \\
4 \text { p.m. } 8 \text { th. }\end{array}$ & & 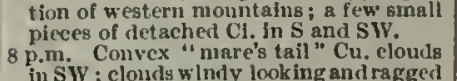 \\
\hline
\end{tabular}


Mhteorological JoURNAI, of TIE "J)ISCOVERY,"

[1903-November.

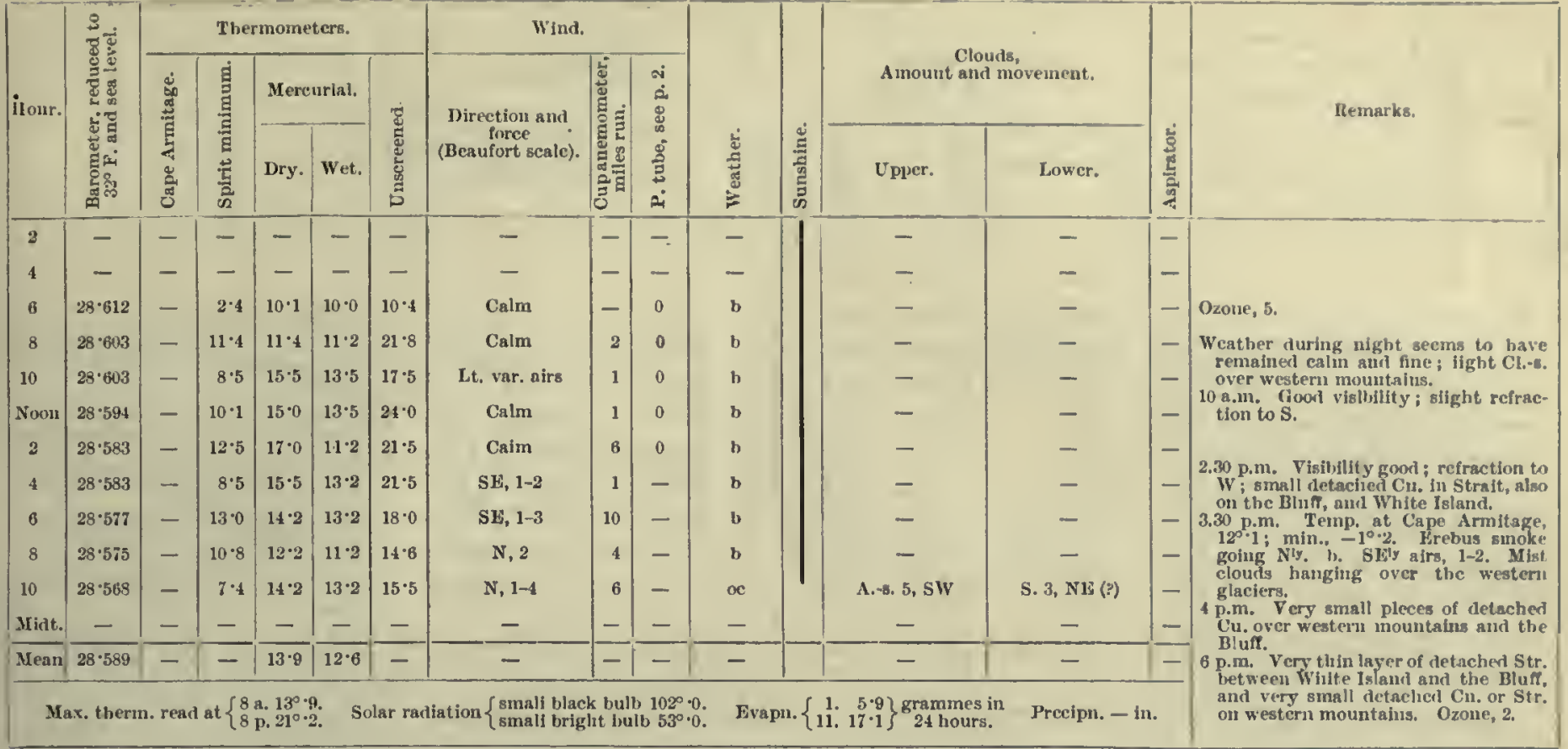

10 th.

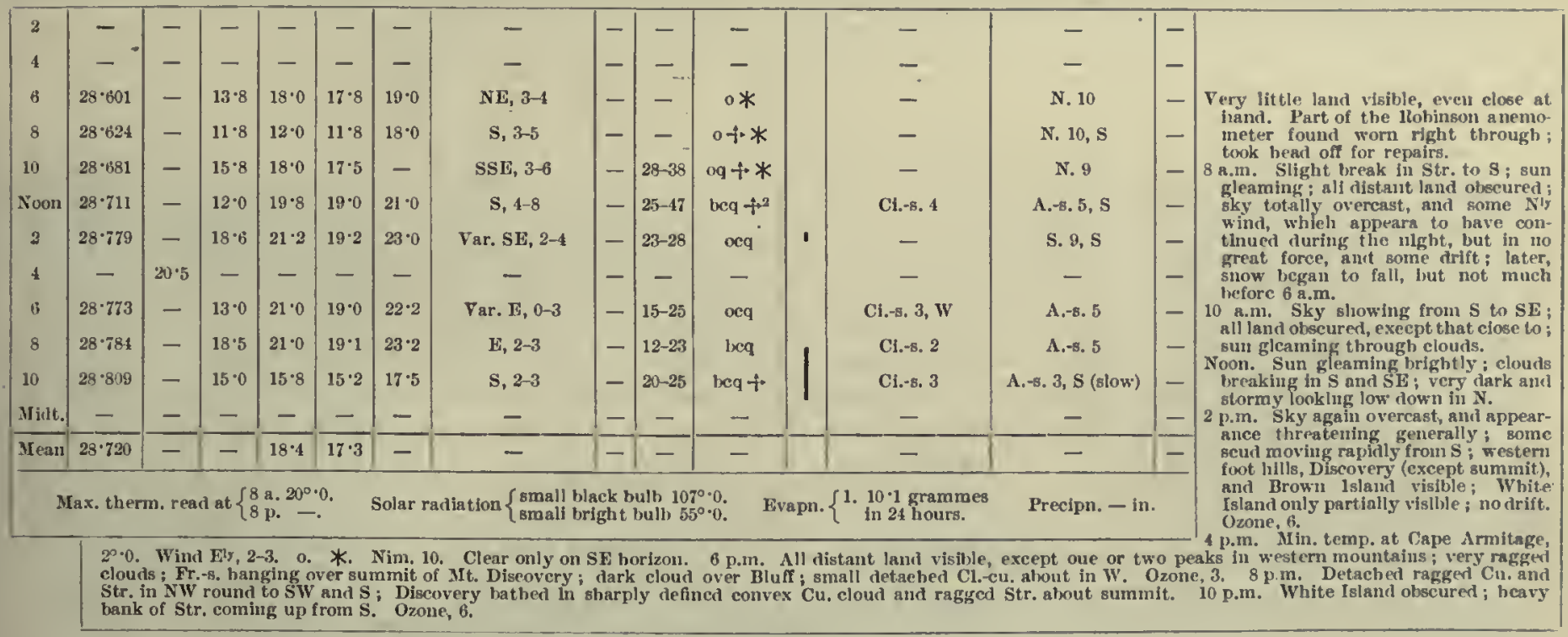

\begin{tabular}{|c|c|c|c|c|c|c|c|c|c|c|c|c|c|c|}
\hline 2 & - & - & - & - & - & - & - & -1 & - & - & - & - & $1-1$ & \\
\hline 4 & - & - & - & - & - & - & - & - & - & - & - & - & - & \\
\hline 6.15 & $28 \cdot 807$ & - & $14 \cdot 5$ & $19 \cdot 9$ & 18.4 & $20 \cdot 5$ & $\mathrm{SE}, \mathbf{2 - 3}$ & 12 & $20-25$ & h & Ci. 1 & - & - & nasses of Str. still irauging on \\
\hline 8 & $28 \cdot 812$ & - & $12 \cdot 0$ & $18 \cdot 0$ & $17 \cdot 3$ & $20 \cdot 5$ & S, $0-1$ & 6 & $0-8$ & be & - & Det. Fr.cu.-s, 3, & - & also in the direetiou of Wrebus; very \\
\hline Noon & $28 \cdot 855$ & - & $19 \cdot 0$ & $24 \cdot 8$ & $22 \cdot 3$ & $24 \cdot 0$ & Var. SE, 2-4 & 12 & $22-32$ & weq - & - & A.-8. 9, SW & - & $\begin{array}{l}8 \text { a.m. Ci.s. and Str. in SE and S-tine } \\
\text { latter covers the upper levels of } W \text { bitc }\end{array}$ \\
\hline 2 & $28 \cdot 872$ & - & $20 \cdot 6$ & $23 \cdot 2$ & $21 \cdot 2$ & $24 \cdot 2$ & Var. SE, 2-4 & 13 & $23-30$ & oeq & - & A.-8. 9, SW & - & $\begin{array}{l}\text { lsland-also in } 5 \text { Wand } W \text {, ohscuriug } \\
\text { upper hilf of } 31 \text {. Discovery and ali but }\end{array}$ \\
\hline 8 & $28 \cdot 853$ & - & $19 \cdot 0$ & $21 \cdot 5$ & $20 \cdot 2$ & $22 \cdot 4$ & $\mathrm{~s}, 3$ & 16 & $20-21$ & be + & - & Det. A.-8. $2 ; A_{-}-5,2 ;$ & - & $\begin{array}{l}\text { roliing nass of Str. Ozone, } 6 \text {. } \\
\text { Noon. }\end{array}$ \\
\hline 10 & 28.814 & - & $18 \cdot 8$ & $22 \cdot 0$ & $20 \cdot 5$ & $21 \cdot 8$ & SE, 1 & 7 & $15-18$ & $\infty$ & - & A.-s. $8 ;$ S.-cu. 1 & - & mpper parts of White Island and Black \\
\hline Miat. & - & - & - & - & - & - & - & - & - & - & - & - & - & seured also; westerm mountains foot \\
\hline $3 \mathrm{Iran}$ & $28 \cdot 839$ & - & - & $\overline{21 \cdot 5}$ & $20 \cdot 0$ & - & - & - & - & - & - & - & $\overline{-}$ & 2 p.nit. Western foot hills, bases of \\
\hline \multicolumn{6}{|c|}{ Max. therm. read at $\left\{\begin{array}{l}8 \text { a. } 21^{\circ} \circ \\
8 \mathrm{p} .\end{array}\right.$} & \multicolumn{5}{|c|}{ Solar raliation $\left\{\begin{array}{l}\text { small black bulb } 97^{\circ} \cdot 1 . \\
\text { small bright bull, } 54^{\circ} \cdot 0 .\end{array}\right.$} & am & \multicolumn{2}{|l|}{ Precipn. - in. } & $\begin{array}{l}\text { part of Discovery visible; Bluff ob- } \\
\text { scurer, lutut outllned by hesty mass of } \\
\text { dark elout ; some Fr.-s. moving SW }\end{array}$ \\
\hline
\end{tabular}

11th.

12 th. 
1903-November.]

Meteorological Journal, of the "Discovery."

\begin{tabular}{|c|c|c|c|c|c|c|c|c|c|c|c|c|c|c|c|}
\hline \multirow{3}{*}{ Hour. } & \multirow{3}{*}{ 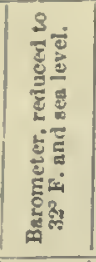 } & \multicolumn{5}{|c|}{ Thermoneters. } & \multicolumn{3}{|l|}{ Wind. } & \multirow[b]{3}{*}{ 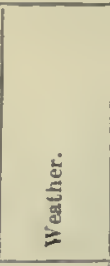 } & \multirow[b]{3}{*}{ 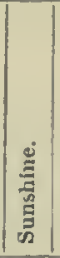 } & \multirow{2}{*}{\multicolumn{2}{|c|}{$\begin{array}{l}\text { Clouds, } \\
\text { Amount and movement. }\end{array}$}} & \multirow{3}{*}{ 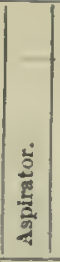 } & \multirow{3}{*}{ Remarks, } \\
\hline & & \multirow{2}{*}{ 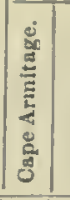 } & \multirow{2}{*}{ 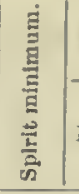 } & \multicolumn{2}{|c|}{ Mercurlal. } & \multirow{2}{*}{ 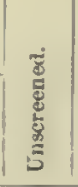 } & \multirow{2}{*}{$\begin{array}{c}\text { Diroct.lon and } \\
\text { force } \\
\text { (Beaufort scale). }\end{array}$} & \multirow{2}{*}{ 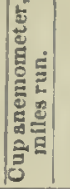 } & \multirow{2}{*}{ 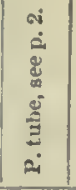 } & & & & & & \\
\hline & & & & Dry. & Wet. & & & & & & & Upper. & Lower. & & \\
\hline 2 & - & -1 & - & - & - & - & - & -1 & - & - & & - & - & \multirow{13}{*}{\multicolumn{2}{|c|}{ 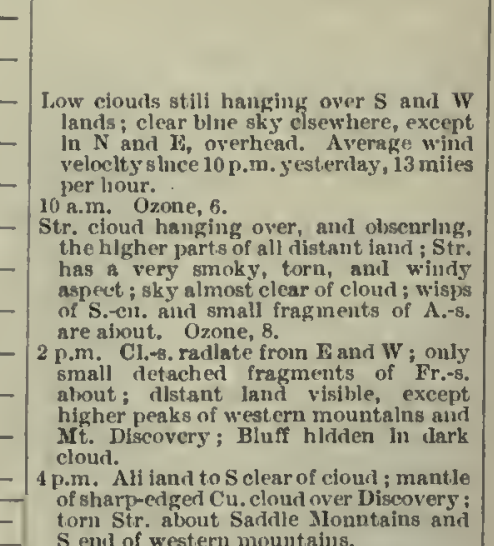 }} \\
\hline 4 & - & - & - & - & - & - & - & - & - & - & ' & - & - & & \\
\hline 6.15 & $28 \cdot 881$ & - & $14 \cdot 5$ & $15 \cdot 2$ & $14 \cdot 8$ & $16 \cdot 3$ & SE, $3-4$ & 13 & $25-28$ & $n c+$ & 1 & - & A.-s.-cu. 4 & & \\
\hline 8 & $28 \cdot 852$ & - & $12 \cdot 5$ & $15 \cdot 0$ & $14 \cdot 0$ & $17 \cdot 5$ & Var. NE, 3-6 & 16 & $20-38$ & $\operatorname{beq}+4$ & & Cl.-s. 2 & 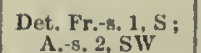 & & \\
\hline 10 & $28 \cdot 876$ & - & $13 \cdot 8$ & $20 \cdot 0$ & $19 \cdot 2$ & - & Var. S, 4-7 & 15 & $33-43$ & iseg $t$ & & Ci.-8. 1 & \begin{tabular}{|l|} 
A. \\
A..s. 2
\end{tabular} & & \\
\hline Noon & $28 \cdot 8 i 6$ & -1 & $18 \cdot 8$ & $21 \cdot 0 \mid$ & $20 \cdot 0$ & $24 \cdot 6$ & $S, 4-7$ & 23 & $25-42$ & $\operatorname{leq} \mathrm{f}^{2}$ & & Cl.-s. 5 & Det. Fr,-a,-s. 5, & & \\
\hline 2 & $28 \cdot 895$ & - & $19 \cdot 6$ & $22 \cdot 2$ & $20 \cdot 5$ & $25 \cdot 0$ & S, 1-4 & 25 & 23 & beq + & & Cl.-s. 3 & Det. Fr,-a.-s. 5, & & \\
\hline 4 & $28 \cdot 893$ & $21 \cdot 5$ & $20 \cdot 0$ & $26 \cdot 0$ & $24 \cdot 2$ & $25 \cdot 0$ & Var. SE, 4-7 & 8 & $25-42$ & beq - + & 1 & Ci..s. 1 & 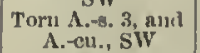 & & \\
\hline 8 & $29 \cdot 899$ & - & $17 \cdot 8$ & $19 \cdot 5$ & $17 \cdot 5$ & $21 \cdot 5$ & $\mathrm{E}, 1-3$ & 19 & $27-30$ & ocq & & Ci.-8. I & A.-s. 5, A.cu. 2 & & \\
\hline 8 & $28 \cdot 922$ & - & $16 \cdot 8$ & $19 \cdot 0$ & $17 \cdot 0$ & $20 \cdot 0$ & Var. SE, 3-5 & 20 & $18-30$ & beq & & - & A.-8. 7, SW & & \\
\hline 10 & $28 \cdot 926$ & - & $9 \cdot 0$ & $9 \cdot 8$ & $9 \cdot 2$ & $9 \cdot 8$ & Var. E, 4-7 & 19 & $33-43$ & ocq + & & Ci.-s. 2 & A. $\rightarrow$. $5, \mathrm{SW}$ & & \\
\hline Midt. & - & -1 & - & - & - & - & - & -1 & - & - & 1 & - & - & & \\
\hline Meanis & $28 \cdot 894$ & $1-1$ & -1 & 18.6 & $17 \cdot 4$ & - & - & -1 & - & - & & - & - & & \\
\hline \multicolumn{6}{|c|}{ Max, therm. read at $\left\{\begin{array}{l}8 \text { a. } 22^{\circ} \cdot 8 . \\
8 \text { p. }-\end{array}\right.$} & \multicolumn{5}{|c|}{ Solar radlation $\left\{\begin{array}{l}\text { small biack bulb } 103^{\circ} \cdot 5 \\
\text { small brlght bult } 54^{\circ} \cdot 8\end{array}\right.$} & \multicolumn{4}{|c|}{ Evapm._grammes. } & 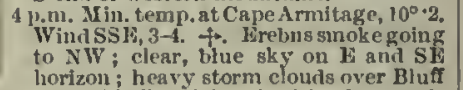 \\
\hline
\end{tabular}

14 th.

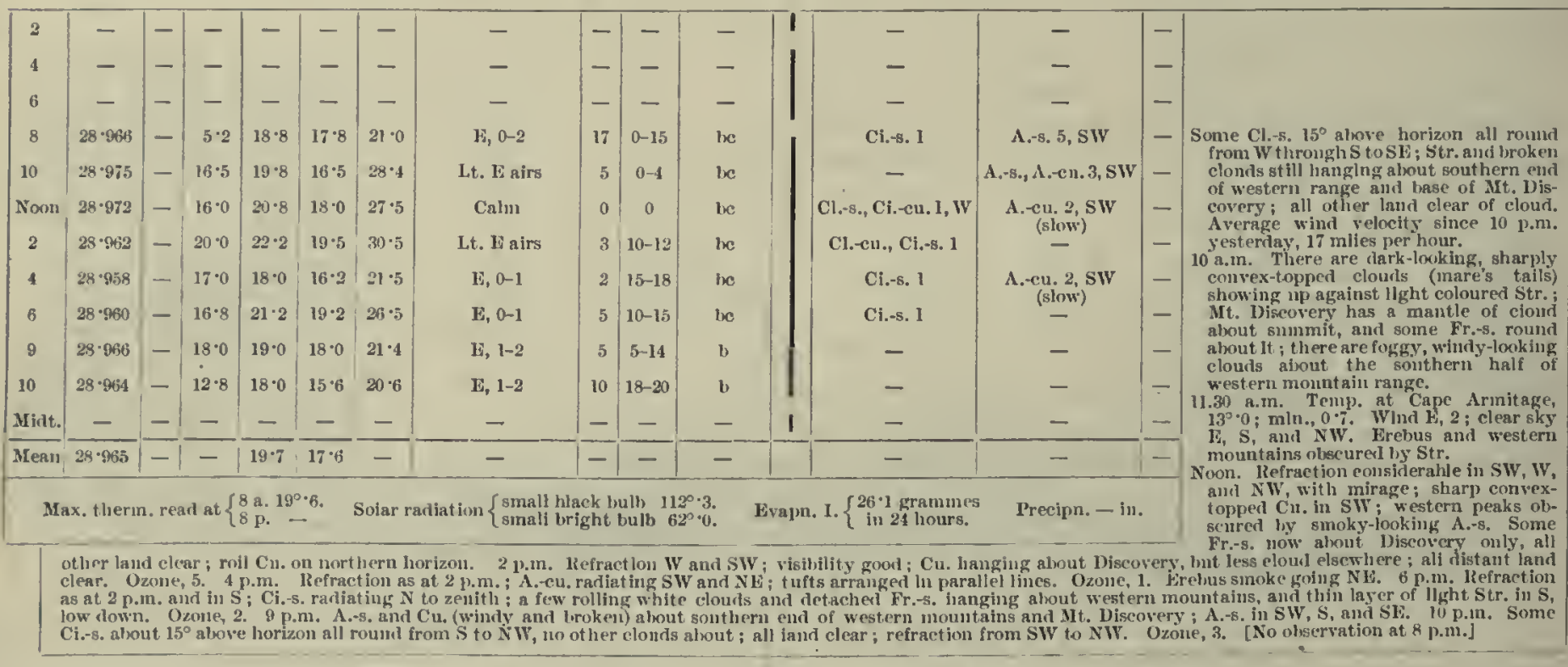

15th.

\begin{tabular}{|c|c|c|c|c|c|c|c|c|c|c|c|c|c|c|}
\hline 2 & - & - & - & - & - & - & - & - & - & - & - & - & -7 & \\
\hline 4 & - & - & - & - & - & - & - & -1 & - & - & - & - & - & \\
\hline 6 & $29 \cdot 021$ & - & $4 \cdot 1$ & $13 \cdot 0$ & $12 \cdot 2$ & $11 \cdot 9$ & Calm & 3 & 0 & inc & lir.ci.-s, 6, Nls & - & -1 & $\begin{array}{l}\text { Mirage. Average wind velority sluce } \\
10 \text { p.u. yestertas, } 3 \text { miles per hour. }\end{array}$ \\
\hline 8 & $29 \cdot 019$ & -1 & $7 \cdot 7$ & $10 \cdot 5$ & $10 \cdot 0$ & $14 \cdot 0$ & Calm & 0 & 0 & be & - & S.-cis. $7, \mathrm{NW}$ & - & 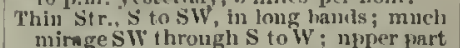 \\
\hline 10 & $29 \cdot 012$ & - & $9 \cdot 9$ & $18 \cdot 0$ & $17 \cdot 0$ & $21 \cdot 0$ & Calm & 0 & 0 & c & - & S.-ell. $9, \mathrm{NW}$ & - & $\begin{array}{l}\text { of Brown istand and liglier portions } \\
\text { of western mountains olseured; sun }\end{array}$ \\
\hline Noon & $29 \cdot 003$ & - & $12 \cdot 0$ & $20 \cdot 8$ & 19.4 & $28 \cdot 6$ & Lt. $\mathbf{N}$ airs & 2 & $0-3$ & he & - & S.ecu. 5, NNW & - & $\begin{array}{l}\text { gleaning } \\
\text { 10 a.m. The thin Str. in } \mathrm{S} \text { has iroadened }\end{array}$ \\
\hline 2 & $28 \cdot 985$ & -1 & $20 \cdot 0$ & 21.5 & $20^{\circ} 0$ & $25 \cdot 0$ & Calm & 2 . & 0 & $\propto$ & - & S.eu. $9, \mathrm{NW}$ & - & $\begin{array}{l}\text { ant extender so that now the uyper } \\
\text { parts of Black ant White Islands }\end{array}$ \\
\hline 4 & $28 \cdot 959$ & - & $18 \cdot 5$ & $10 \cdot 5$ & $19 \cdot 2$ & $22 \cdot 2$ & Lt. Ha airs & 2 & $0-3$ & $\alpha$ & - & S.-cor. s, SW & -1 & $\begin{array}{l}\text { are ohseured as this str. extellds } \\
\text { rouml to } \mathrm{W} \text { it darkens, unt in it has }\end{array}$ \\
\hline 6 & $28 \cdot 052$ & - & $17 \cdot 5$ & $18 \cdot 2$ & $17 \cdot 2$ & $23 \cdot 0$ & Calm & 1 & 0 & c & Ci.-cu. 2 & s.cu. 5, SW & - & $\begin{array}{l}\text { a threatening appearance; the upper } \\
\text { two - thirds of NIt. Diseovery, } 1000 \text {. }\end{array}$ \\
\hline 8 & $28 \cdot 9.4$ & - & $14 \cdot 5$ & $15 \cdot 5$ & $15 \cdot 2$ & $15 \cdot 8$ & L.t. S airs & 1 & $0-2$ & $\operatorname{ocg} *$ & - & N. $10, \mathrm{sw}$ & - & $\begin{array}{l}\text { feet of Brown 1sland, and ail himt } \\
\text { the bases of the western foot hills }\end{array}$ \\
\hline 10 & $28 \cdot 939$ & - & $11 \cdot 0$ & 12.5 & $12 \cdot 0$ & 13.5 & Calm & 0 & 0 & ac $*$ & - & N. 10 & - & $\begin{array}{l}\text { are obsenred; refractiont excessive, } \\
\text { especialiy to } W ; \text { sut gleamluth. }\end{array}$ \\
\hline Midt. & - & - & - & - & - & - & - & - & - & - & $=$ & - & $1-$ & $\begin{array}{l}\text { Mirage. } \\
\text { Noon. Clouds in w heavier; sone mIrage }\end{array}$ \\
\hline$M \operatorname{can} 1$ & $28 \cdot 981$ & - & - & $16 \cdot 6$ & $15 \cdot 7$ & - & - & -1 & - & - & - & - & $1-$ & $\begin{array}{l}\text { stili ; at } 12.15 \text { the out side themometer } \\
\text { showeet } 20^{\circ} 5 \text {. }\end{array}$ \\
\hline \multicolumn{6}{|c|}{ Max. therm. read at $\left\{\begin{array}{l}8 \text { a. } 19^{\circ} \cdot 4 . \\
8 \mathrm{p} .23^{\circ} \cdot 8 .\end{array}\right.$} & \multicolumn{5}{|c|}{ Soiar radiation $\left\{\begin{array}{l}\text { small black lmull, } 100^{\circ} \cdot 2, \\
\text { small bright bali, } \\
53^{\circ} \cdot 2 .\end{array}\right.$} & ainmes. & \multicolumn{2}{|l|}{ Precip. - in. } & 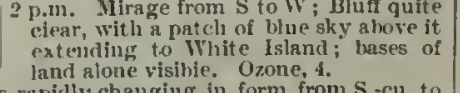 \\
\hline
\end{tabular}


Metiorotogtcal, Journat, of THF "Discovery."

[1903-November.

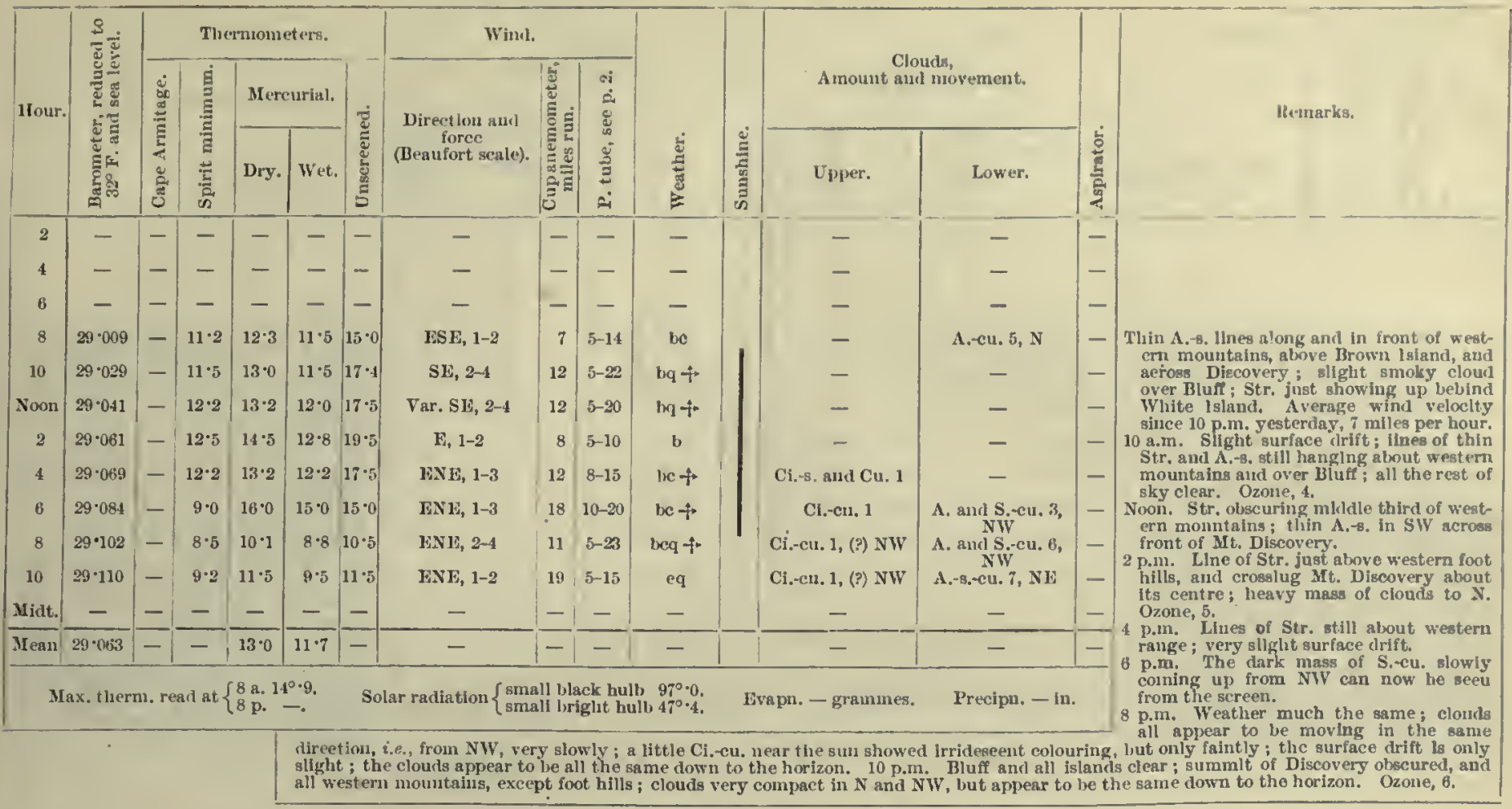

\begin{tabular}{|c|c|c|c|c|c|c|c|c|c|c|c|c|c|c|}
\hline 2 & - & -1 & - & - & - & -1 & - & - & - & - & & - & - & -1 \\
\hline 4 & - & $=$ & - & - & - & - & - & - & - & - & & - & - & - \\
\hline 6 & - & - & - & - & - & - & - & - & - & - & ' & - & - & - \\
\hline 8 & $29 \cdot 188$ & - & -0.7 & 6.5 & $6 \cdot 2$ & $i \cdot 0$ & $N F$ by $F, 3-4$ & 17 & $15-23$ & $\operatorname{ocg}$ & & - & S., A.-8. 10, NNE & - \\
\hline 10 & $29 \cdot 214$ & - & $5 \cdot 1$ & $8 \cdot 2$ & 8.0 & $10 \cdot 0$ & E by $S, 3-4$ & 16 & $15-24$ & oeqg & & - & S. $10, \mathrm{NE}$ & - \\
\hline Noon & $29 \cdot 228$ & - & $7 \cdot 4$ & $9 \cdot 3$ & $9 \cdot 0$ & $10 \cdot 8$ & ENE, 3-4 & 14 & $16-25$ & ocgg $+* *$ & & - & N. 10 & - \\
\hline 2 & $29 \cdot 234$ & - & $7 \cdot 2$ & $8 \cdot 2$ & $8 \cdot 0$ & $9 \cdot 8$ & ENE, 1-3 & 13 & $10-17$ & $\operatorname{oog} \div * *$ & & - & N. 10 & - \\
\hline 4 & $29 \cdot 241$ & - & $7 \cdot 0$ & $9 \cdot 0$ & $8 \cdot 0$ & $10 \cdot 3$ & ENE, 1-3 & 15 & $13-18$ & ocq & & - & S. 10 & - \\
\hline 6 & $29 \cdot 248$ & - & $7 \cdot 2$ & $8 \cdot 5$ & $7 \cdot 8$ & $9 \cdot 5$ & ENE, 1-3 & 18 & $10-20$ & $o c q+$ & & - & S. 10 & - \\
\hline 8 & $29 \cdot 250$ & - & $7 \cdot 0$ & $8 \cdot 0$ & $7 \cdot 0$ & $9 \cdot 0$ & E by N, 3-4 & 17 & $15-24$ & $b c+$ & & Ci.-8. 7 & - & - \\
\hline 10 & $29 \cdot 216$ & - & $6 \cdot 2$ & $7 \cdot 2$ & 6.2 & $7 \cdot 5$ & ENE, 1-3 & 12 & $10-15$ & cq & & Ci.-s. 9 & - & - \\
\hline Midt. & - & - & - & - & - & - & - & - & - & - & & - & - & - \\
\hline Mean & $29 \cdot 231$ & -1 & - & $8 \cdot 1$ & $7 \cdot 5$ & $\overline{-}$ & - & $\overline{-}$ & - & - & & - & - & - \\
\hline
\end{tabular}

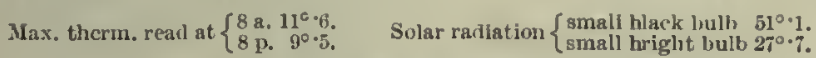

Rvapn. - gramnes. Precipn. - In.

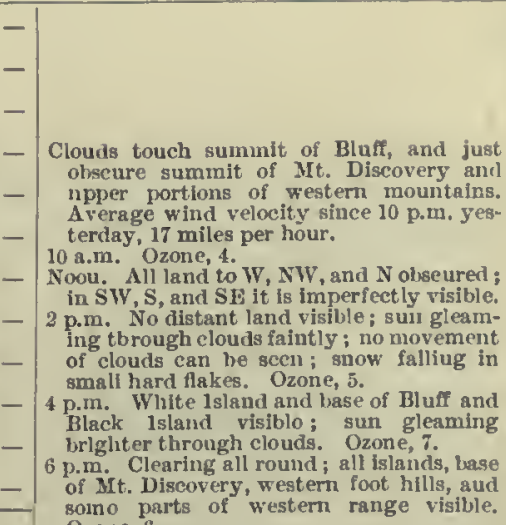
Ozone, 6.

8 p.m. Clouds look stormy and windy about Diseovery; midrle thirl of west en mountains wrapped in thark Str. Fr.-a.-s.
In SW and SE; tbin filn of $\Lambda .-s$. over 16 th. 10 p.m. Weather improving; thin film of Ci.-s. all over sky; all distant land visiblc; lines of Str. still at middle beight of western
mountains; some detached Fr,-8, alout SW and W. Ozone, 4 .

\begin{tabular}{|c|c|c|c|c|c|c|c|c|c|c|c|c|c|}
\hline 2 & - & -1 & - & - & - & -1 & - & - & - & - & - & - & - \\
\hline 4 & - & - & - & - & - & -1 & - & - & - & - & - & - & - \\
\hline 6 & - & - & - & - & - & - & - & - & - & - & - & - & - \\
\hline 8 & $29 \cdot 199$ & - & $5 \cdot 2$ & $15 \cdot \beta$ & $14 \cdot 8$ & $20 \cdot 0$ & Calm & 7 & 0 & be & Cl.-5. 5 & - & - \\
\hline 10 & $29 \cdot 201$ & - & $13 \cdot 0$ & $17 \cdot 0$ & $14 \cdot 8$ & $26 \cdot 6$ & Caln & 0 & 0 & $\mathrm{bc} \oplus$ & Ci.-s. 4 & - & - \\
\hline Noon & $29 \cdot 196$ & - & $17 \cdot 0$ & $17 \cdot 2$ & 14.5 & $27 \cdot 0$ & Lot. E alrs & 0 & 0 & $\mathrm{bc} \oplus 0$ & Ci. -8.3 & - & - \\
\hline 2 & $29 \cdot 192$ & - & $15 \cdot 5$ & $18 \cdot 2$ & $16 \cdot 0$ & $25 \cdot 0$ & Calm & 1 & 0 & $b c$ & Cl.-8. 2 & - & - \\
\hline 4 & $29 \cdot 188$ & - & $15 \cdot 8$ & $18 \cdot 0$ & $15 \cdot 5$ & $25 \cdot 0$ & Calm & 0 & 0 & be & C1.-8. 2 & - & - \\
\hline 6 & $29 \cdot 192$ & - & $17 \cdot 2$ & $19^{\circ} 0$ & $17 \cdot 2$ & $32 \cdot 0$ & Calm & 0 & 0 & be & Ci.-8. 2 & - & - \\
\hline 8 & $29 \cdot 188$ & - & $12 \cdot 8$ & $15 \cdot 3$ & $13 \cdot 0$ & $19 \cdot 4$ & E, 1-2 & 3 & $10-16$ & we $\oplus$ & $\mathrm{Ci}_{.-8 .} 2$ & - & - \\
\hline 10 & $29 \cdot 184$ & - & $12 \cdot 0$ & $14 \cdot 2$ & 115 & $17 \cdot 5$ & L, $0-1$ & 9 & $10-15$ & be & CI.-s. 2 & - & - \\
\hline Midt. & - & - & - & - & - & $=$ & - & - & - & - & - & - & - \\
\hline$\Delta$ eas1 & $29 \cdot 193$ & - & - & 16.8 & $14 \cdot 7$ & -1 & - & $\overline{-}$ & - & - & - & - & - \\
\hline
\end{tabular}

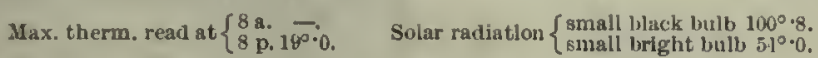

Livapu, - grammes. Precipu, - li. 18th.

The Ci.-s. is spread as an exceedingly thin tilm all over the sky, being thinuer than last night; a thin band of Str. can be seen anong the western mountalus above foot of it in $\mathrm{N}$; considerable refraction $\mathrm{ln} \mathrm{SW}$ aiso in $\mathbf{S}$ and $\mathrm{SE}$; average wind velocity since 10 p.m. yesterday, 7 miles per hour. R.m. The film of $\mathrm{Ci}_{0}-\mathrm{s}$, does not so cover the sky as at 8 a.m. the layers on the western mountains bave hicreased to some extent, and int ersect the range in the midale:

Noon. Solar corona and halo; refraction every where. every where. Gisilility; no refraction. Ozone, 7 .

p.m. Goud visibllity ; no refraction; deover western fuot hilis. Ozone, 0 .

6 p.in. Ozone, 3.

8 p.m. The Ci, $-\mathrm{s}$. most of the day has radiated in lines from the $\mathrm{N}$; some layers of Str. alout western
Fr.-s. in SW; solar halo. 
1903-November.]

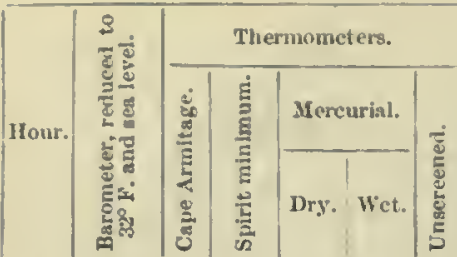

19th,

\begin{tabular}{|c|c|c|c|c|c|c|c|c|c|c|}
\hline 2 & - & - & - & - & - & - & - & - & - & - \\
4 & - & - & - & - & - & - & - & - & - & - \\
6 & - & - & - & - & - & - & - & - & - & - \\
8 & $29 \cdot 167$ & - & $-2 \cdot 2$ & $17 \cdot 8$ & $15 \cdot 8$ & - & SE, 1-2 & 3 & $10-16$ & hev $\oplus$ \\
10 & $29 \cdot 161$ & - & $16 \cdot 8$ & $18 \cdot 0$ & $14 \cdot 6$ & $22 \cdot 2$ & E by S, 2-3 & 7 & $12-20$ & hevq \\
Noon & $29 \cdot 159$ & - & $12 \cdot 1$ & $19 \cdot 0$ & $16 \cdot 2$ & - & E by S, 2-3 & 7 & $15-24$ & bevq \\
2 & $29 \cdot 158$ & - & $17 \cdot 4$ & $20 \cdot 0$ & $17 \cdot 2$ & $23 \cdot 5$ & E by S, 1-3 & 7 & $10-28$ & beq $\Theta$ \\
4 & $29 \cdot 161$ & - & $17 \cdot 8$ & $19 \cdot 0$ & $17 \cdot 4$ & $24 \cdot 0$ & SE, 2-3 & 3 & $10-20$ & beqv \\
6 & $29 \cdot 164$ & - & $15 \cdot 8$ & $18 \cdot 2$ & $15 \cdot 2$ & $22 \cdot 5$ & Var. E, 0-2 & 15 & $10-13$ & beq $\oplus$ \\
8 & $29 \cdot 171$ & - & $16 \cdot 4$ & $17 \cdot 0$ & $14 \cdot 5$ & $19 \cdot 9$ & ENE, 2-3 & 8 & $10-15$ & be $\Theta$ \\
10 & $29 \cdot 169$ & - & $15 \cdot 5$ & $17 \cdot 2$ & $14 \cdot 0$ & $19 \cdot 0$ & E, 0-1 & 5 & $0-5$ & be \\
Midt. & - & - & - & - & - & - & - & - & - & - \\
\hline Mean & $29 \cdot 164$ & - & - & $18 \cdot 3$ & $15 \cdot 6$ & - & - & - & - & - \\
\hline
\end{tabular}

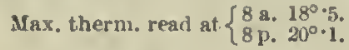

Meteorological Journal, of the "Discovery."

\begin{tabular}{|c|c|c|c|c|c|c|c|c|}
\hline \multicolumn{3}{|l|}{ Wind. } & \multirow[b]{3}{*}{ 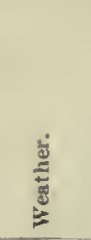 } & \multirow[b]{3}{*}{ 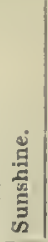 } & \multirow{2}{*}{\multicolumn{2}{|c|}{$\begin{array}{l}\text { Cloulds, } \\
\text { Amount and movement. }\end{array}$}} & \multirow[b]{3}{*}{ 离 } & \multirow{3}{*}{ Remarks. } \\
\hline \multirow{2}{*}{$\begin{array}{l}\text { Directiou and } \\
\text { forro } \\
\text { (Benufort senle). }\end{array}$} & \multirow{2}{*}{ 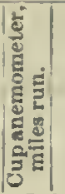 } & \multirow{2}{*}{ 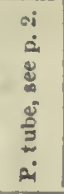 } & & & & & & \\
\hline & & & & & Upler. & Lower. & & \\
\hline - & - & - & - & 1 & - & - & - & \\
\hline - & - & - & - & & - & - & - & \\
\hline - & - & - & - & & - & - & - & \\
\hline SE, 1-2 & 3 & $10-16$ & $\operatorname{hev} \oplus$ & & Cl. $-8 ., 2$ & - & - & There appears to have been no elange in the \\
\hline F by $S, 2-3$ & 7 & $12-20$ & hevq & & $\mathrm{Cl}_{-}-\mathrm{B}, 2, \mathrm{~N}$ & - & - & land clear. Average wind velocity since \\
\hline $\mathrm{F}$ by $\mathrm{s}, 2-3$ & 7 & $15-24$ & berq & & $\mathrm{Cl}_{0}=8.3, \mathrm{~N}$ & - & - & $10 \mathrm{a} . \mathrm{m} . \mathrm{Ci}-\mathrm{s}$, radiating WNW and $\mathrm{FSE}$; \\
\hline E by $\mathrm{S}, 1-3$ & 7 & $10-28$ & heq $\oplus$ & & Ci.-s. 3 & - & - & Noon. Some str. on western mountahs \\
\hline $\mathrm{SE}, 2-3$ & 3 & $10-20$ & beqv & & Ci.-s. 3 & - & - & 2 p.m. Ci.s. radiating WNW and Fsk; \\
\hline Var. $\mathbf{E}, 0-2$ & 15 & $10-13$ & beq $\oplus$ & & Ci.-B. 2 & - & - & mountains. \\
\hline ENE, 2-3 & 8 & $10-15$ & 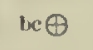 & 1 & Ci.-s. 2 & - & - & $\begin{array}{l}4 \text { p.un. A-s. obscuring suminits of western } \\
\text { mountains: there has been some drift }\end{array}$ \\
\hline E, 0-1 & 5 & $0-5$ & the & & Ci.-s. 4 & - & - & 6 p.lu. Nock sun at $N$ side and upper edige, \\
\hline- & - & - & - & . & - & - & - & $\begin{array}{l}\text { the latter not so elear as the former } \\
\text { upper lialf of western mountains and }\end{array}$ \\
\hline - & - & - & - & & - & - & - & 8 p.m. $A .-$. is spreading over from WNW in \\
\hline adiation \{ & & & & & - grannu & Precipn & & 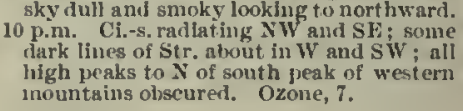 \\
\hline
\end{tabular}

20th.

\begin{tabular}{|c|c|c|c|c|c|c|}
\hline 2 & - & - & - & - & - & - \\
4 & - & - & - & - & - & - \\
6 & - & - & - & - & - & - \\
8 & $29 \cdot 150$ & - & $-0 \cdot 6$ & $14 \cdot 0$ & $13 \cdot 2$ & $17 \cdot 0$ \\
10 & $29 \cdot 145$ & - & $12 \cdot 2$ & $16 \cdot 3$ & $14 \cdot 0$ & $20 \cdot 0$ \\
Noon & $29 \cdot 120$ & - & $13 \cdot 8$ & $17 \cdot 4$ & $14 \cdot 8$ & $21 \cdot 3$ \\
2 & $29 \cdot 098$ & - & $15 \cdot 8$ & $18 \cdot 2$ & $16 \cdot 0$ & $23 \cdot 8$ \\
4 & $29 \cdot 070$ & - & $17 \cdot 0$ & $18 \cdot 2$ & $16 \cdot 2$ & $22 \cdot 4$ \\
6 & $29 \cdot 054$ & - & $17 \cdot 2$ & $18 \cdot 0$ & $16 \cdot 4$ & $21 \cdot 5$ \\
8 & $29 \cdot 045$ & - & $17 \cdot 0$ & $18 \cdot 0$ & $13 \cdot 8$ & $22 \cdot 0$ \\
10 & $29 \cdot 023$ & - & $15 \cdot 0$ & $15 \cdot 2$ & $12 \cdot 5$ & $18 \cdot 0$ \\
\hline
\end{tabular}

\begin{tabular}{c|c|c|c|}
- & - & - & - \\
- & - & - & - \\
- & - & - & - \\
ESE, 3-5 & 0 & $15-28$ & beq - \\
SEiby" E, 2-4 & 15 & $8-20$ & beq \\
E by S, 2-5 & 10 & $12-24$ & beq $\Theta-f$ \\
Var. F, 1-3 & 9 & $12-18$ & beq \\
WNE, 2-4 & 15 & $15-23$ & theq \\
ENE, 2-4 & 10 & $15-23$ & beq \\
ENE, 1-2 & 12 & $2-10$ & bq \\
NE, 0-2 & 6 & $0-10$ & b \\
- & - & - & -
\end{tabular}

- - -

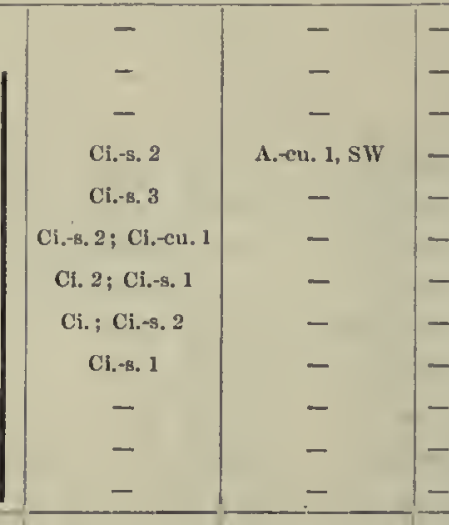

Precipn. - in.

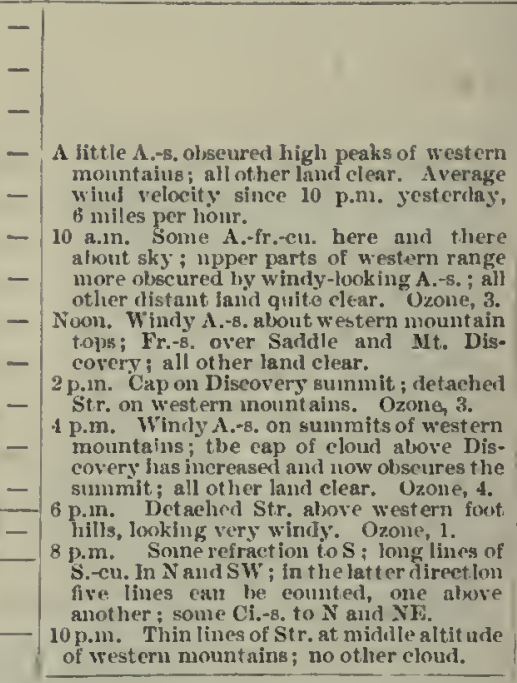

21st.

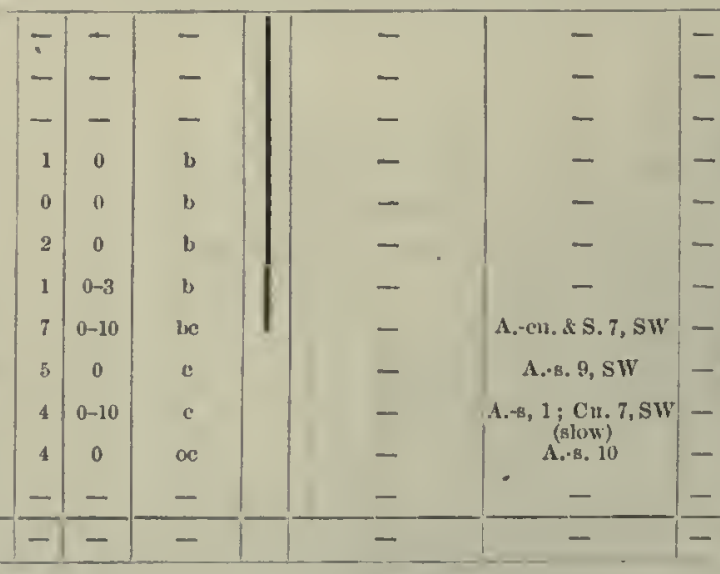

Precipn. - in.

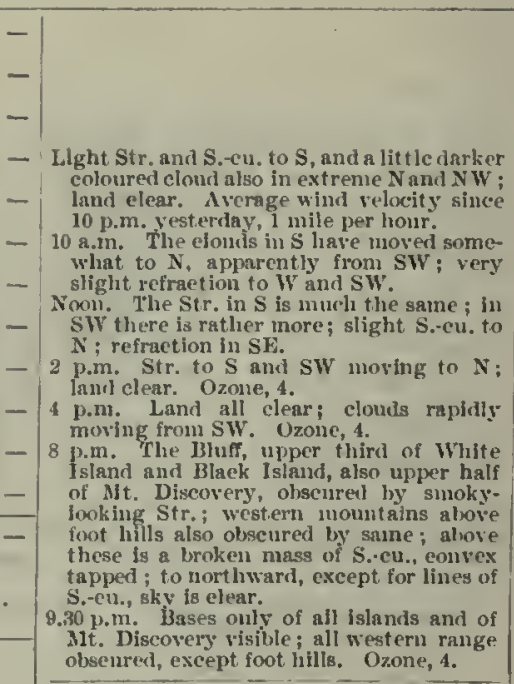


Meteorological Joukna, of the "Discovery."

[1903-November,

\begin{tabular}{|c|c|c|c|c|c|c|c|c|c|c|c|c|c|}
\hline \multirow{3}{*}{ Honr. } & \multirow{3}{*}{ 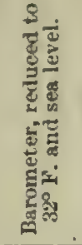 } & \multicolumn{5}{|c|}{ Thermometers. } & \multicolumn{3}{|l|}{ Wlind. } & \multirow[b]{3}{*}{ 岇 } & \multirow[b]{3}{*}{ 总 } & \multirow{2}{*}{\multicolumn{2}{|c|}{$\begin{array}{l}\text { Clouds, } \\
\text { Anomit and movement. }\end{array}$}} \\
\hline & & \multirow{2}{*}{ 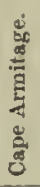 } & \multirow{2}{*}{ 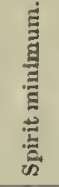 } & \multicolumn{2}{|c|}{ Mercurial. } & \multirow[b]{2}{*}{ 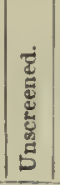 } & \multirow{2}{*}{$\begin{array}{c}\text { Direction aud } \\
\text { foree } \\
\text { (Beanfort seale). }\end{array}$} & \multirow{2}{*}{ 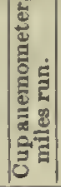 } & \multirow{2}{*}{ 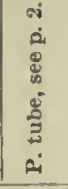 } & & & & \\
\hline & & & & 1)ry. & Wet. & & & & & & & Upper. & Jower. \\
\hline 2 & - & - & - & - & - & - & - & - & - & - & I & - & - \\
\hline 4 & - & - & - & 一 & - & - & 一 & - & - & - & I & - & - \\
\hline 6 & - & - & - & - & - & - & - & - & - & - & & - & - \\
\hline 8 & $29 \cdot 013$ & - & $18 \cdot 6$ & $25 \cdot 4$ & $23 \cdot 6$ & $21 \cdot 6$ & HSE, 1-4 & 7 & $10-20$ & beq & 1 & Cí.-en. 1 & $\begin{array}{c}\text { A. cu, and S. } 5 \text {, } \\
\text { SW }\end{array}$ \\
\hline 10 & $29 \cdot 039$ & - & $20 \cdot 2$ & $25 \cdot 0$ & $22 \cdot 2$ & $33 \cdot 0$ & J.t. W airs & 5 & 0 & be $\oplus$ & 1 & C1.-8. 4 & A. $-\mathrm{s}, 1, \mathrm{sW}$ \\
\hline Noon & $29 \cdot 0.52$ & - & $24 \cdot 5$ & $31 \cdot 1$ & $27 \cdot 4$ & $32 \cdot 2$ & Calm & 0 & 0 & $\operatorname{te} \Theta$ & 1 & - & A. -8.5 \\
\hline 2 & $29 \cdot 075$ & - & $25 \cdot 2$ & $26 \cdot 0$ & $22 \cdot 0$ & $20 \cdot 2$ & Lt. W airs & 4 & $0-3$ & e & 1 & - & A. ${ }^{8 .} 10$ \\
\hline 4 & $29 \cdot 090$ & 一 & $24 \cdot 0$ & $25 \cdot 0$ & $22 \cdot 2$ & $29 \cdot 0$ & Var. 1, 1-2 & 5 & $10-20$ & $\mathrm{c} \oplus$ & I & C1.-8. 4 & A.-8. 6 \\
\hline 6 & $29 \cdot 122$ & - & $23 \cdot 0$ & $24 \cdot 0$ & $22 \cdot 0$ & $25 \cdot 0$ & E, 1-2 & 12 & 10-15 & c & & - & A.-8. 9 \\
\hline 8 & $29 \cdot 151$ & - & $23 \cdot 0$ & $24 \cdot 2$ & $22 \cdot 5$ & $25^{\circ} 0$ & ESE, $1-3$ & 7 & $0-17$ & be & & - & $\begin{array}{l}\text { A.-cu. and } S . b \text {, } \\
\text { N by } \mathrm{W}\end{array}$ \\
\hline 10 & $29 \cdot 173$ & - & $20^{\circ} 0$ & $20 \cdot 1$ & $18 \cdot 3$ & $20 \cdot 5$ & Lt. $\mathbf{N}$ alrs & 6 & 0 & oc & & Fr. c1., s, 7, SW & S 3 \\
\hline Mint. & - & - & - & - & - & - & - & $=$ & - & - & 1 & - & - \\
\hline M ean & $29 \cdot 089$ & - & - & $25 \cdot 1$ & $22 \cdot 5$ & - & - & t- & - & - & - & - & - \\
\hline
\end{tabular}

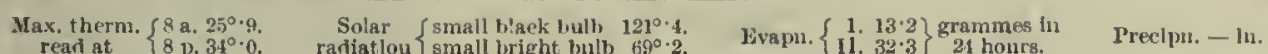

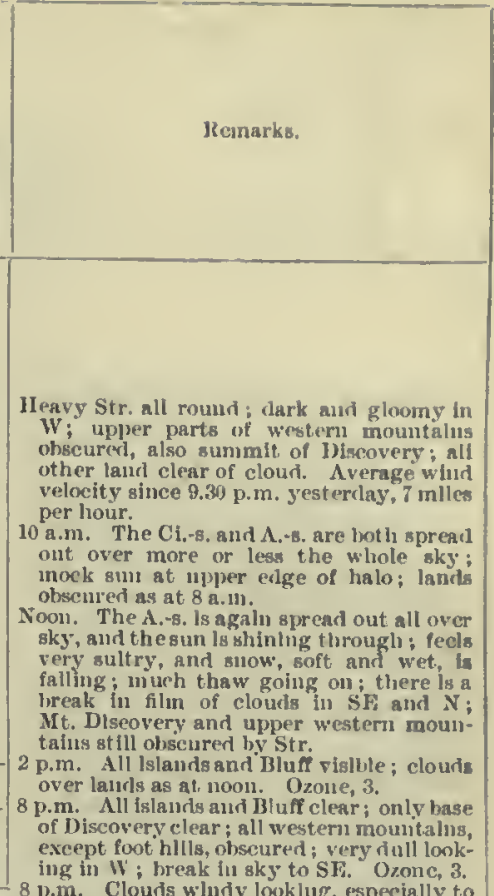

22nd.

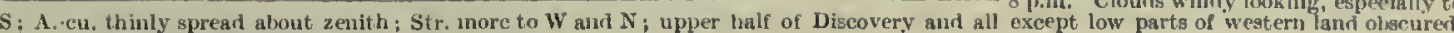
S: A.cu, thinly spread about zenith; Str. nore to W
10 p.m. Ileavy low Str. in W and Sw. Ozonc, 2 to 3 .

\begin{tabular}{|c|c|c|c|c|c|c|c|c|c|c|c|c|c|c|c|}
\hline 2 & - & -1 & - & - & - & -1 & - & $1-$ & - & - & ' & - & - & - & \\
\hline 4 & - & - & - & - & - & - & - & - & - & - & & - & - & - & \\
\hline 6 & - & - & - & - & - & - & - & - & - & - & & - & - & - & \\
\hline 8 & $29 \cdot 290$ & - & $14 \cdot 6$ & $18 \cdot 2$ & $17 \cdot 0$ & $19 \cdot 5$ & SE, 4-7 & 5 & $20-36$ & beq $\neq$ & 1 & - & 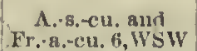 & - & $\begin{array}{l}\text { Bluff almost obse } \\
\text { all hlgher west }\end{array}$ \\
\hline 10 & $29 \cdot 322$ & - & $13 \cdot 4$ & $15 \cdot 0$ & $13 \cdot 4$ & $16 \cdot 3$ & E, 3-5 & 18 & $20-27$ & beq $t^{*}$ & & - & A.-cu. 5 & - & seenis to have \\
\hline Noon & $29 \cdot 332$ & - & $12 \cdot 0$ & $13 \cdot 0$ & $12 \cdot 0$ & $14 \cdot 2$ & Var. 3-8 & 21 & $22-52$ & $\mathrm{cq}+$ & & - & $\begin{aligned} F r,-a,-s, 1 \\
A,-c u\end{aligned}$ & - & $\begin{array}{l}\text { recently : elouds have a torn and windy } \\
\text { look. Average wiud velocity since } 10 \text { p.m. }\end{array}$ \\
\hline 2 & $29 \cdot 337$ & - & $11 \cdot 0$ & $12 \cdot 0$ & $11 \cdot 2$ & $13 \cdot 0$ & Var. $\mathbf{E}, 4-7$ & 16 & 20-38 & $\mathrm{cq}+$ & I & Fr.-ci,-s. 3 & 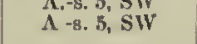 & - & 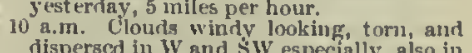 \\
\hline 4 & $29 \cdot 345$ & - & $10 \cdot 0$ & $12 \cdot 0$ & $11 \cdot 2$ & $13 \cdot 0$ & $\mathrm{E}, 4-7$ & 19 & $20-30$ & $c q \rightarrow$ & I & Ci.cu. 3 & A.-c1., $\Lambda$.-s. 5 & - & $\begin{array}{l}\text { dispersed in W and sw esy } \\
\text { S; confitions generally t }\end{array}$ \\
\hline 8 & $29 \cdot 331$ & - & $9 \cdot 0$ & $11 \cdot 0$ & 10.0 & 12.0 & SE, $4-6$ & 17 & 23-33 & $e q+$ & & Ci - cu. 1 & A.-ch. 5, A..s. 2 & - & $\begin{array}{l}8 \text { a.1. } \\
\text { bitc } \\
\text { bat }\end{array}$ \\
\hline 8 & $29 \cdot 324$ & - & $9 \cdot 5$ & $11 \cdot 6$ & $11 \cdot 0$ & $12 \cdot 0$ & Var. SE, 2-7 & 18 & $10-48$ & leq $\rightarrow$ & ' & - & A.-cu. 4, 8.2, S & - & 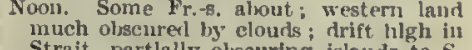 \\
\hline 10 & $29 \cdot 297$ & - & $10 \cdot 6$ & $13 \cdot 2$ & $12 \cdot 2$ & $14 \cdot 8$ & SF, 3-6 & 16 & $15-37$ & ocq + & & - & A.-s.-eu, $9,8 W$ & - & ant totally \\
\hline Mint. & - & -1 & - & - & - & - & - & - & - & - & . & - & - & - & $\begin{array}{l}\text { Ig very rapldy east; } \\
\text { stern range obseuredl }\end{array}$ \\
\hline Heau & $29 \cdot 322$ & $\overline{-1}$ & - & $13 \cdot 3$ & $\overline{12 \cdot 3}$ & $\overline{-}$ & - & - & - & - & - & - & - & - & $\begin{array}{l}\text { 1standis rimy vis } \\
4 \text { p.m. Ozone, } 2 \text {. }\end{array}$ \\
\hline \multicolumn{6}{|c|}{ IIax, therm. read at $\left\{\begin{array}{l}8 \text { a. } 24^{\circ} \cdot 5 \\
8 \mathrm{p}, 17^{\circ} \cdot 8 .\end{array}\right.$} & \multicolumn{5}{|c|}{ Solar radiation $\left\{\begin{array}{l}\text { small black bulb } 108^{\circ} \cdot 8 . \\
\text { small bright tutb } 55^{\circ} \cdot 3 .\end{array}\right.$} & & ta & \multicolumn{2}{|l|}{ I'recipu. - in. } & $\begin{array}{l}\text { dritt ; parts of islands show wecaslonally. } \\
8 \text { p.m. Very dark, wind, ragred Str. cloud } \\
\text { coming over Sadtle, behind It. Discovery, } \\
\text { enveloping western side of Mt. Discovery }\end{array}$ \\
\hline & & & & & & & & & & & & & & & foot \\
\hline
\end{tabular}

\begin{tabular}{|c|c|c|c|c|c|c|c|c|c|c|c|c|c|c|c|}
\hline 2 & - & - & - & - & - & - & - & - & - & - & I & - & - & - & \multirow{11}{*}{ 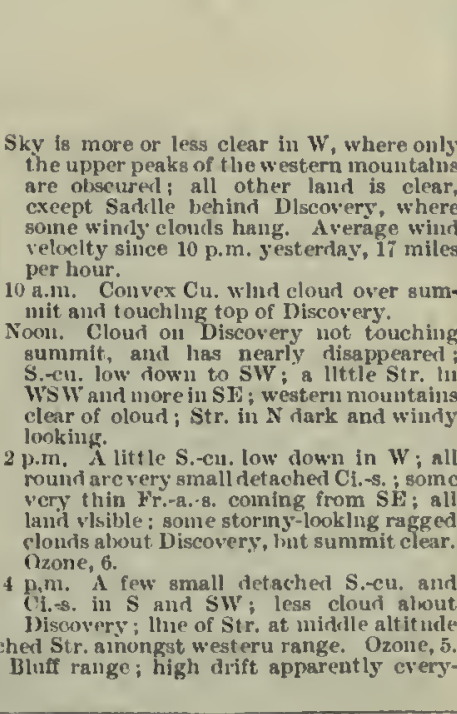 } \\
\hline 4 & - & - & - & - & - & - & - & - & - & - & & - & - & - & \\
\hline 6 & - & - & - & - & - & - & - & - & - & - & & - & - & - & \\
\hline 8 & $29 \cdot 103$ & - & $8 \cdot 2$ & $13 \cdot 0$ & $12 \cdot 3$ & $19 \cdot 5$ & SSE, 2-6 & 17 & $10-34$ & $\mathrm{eq}$ & & - & S. 7, Cu. $2, \mathrm{SW}$ & - & \\
\hline Nowon & $28 \cdot 979$ & -1 & $13 \cdot 2$ & $16^{\circ} 0$ & $14 \cdot 2$ & $20 \cdot 0$ & E by $N, 3-8$ & 11 & $15-28$ & be & 1 & - & A. and Fr.-a.cu, 1 , & - & \\
\hline 2 & $28 \cdot 932$ & - & $12 \cdot 6$ & $13 \cdot 5$ & $12 \cdot 8$ & $18 \cdot 0$ & Var. E, 4-8 & 14 & $20-30$ & loqc & & - & Fr.-a, $-8,1, \mathrm{SE}$ & - & \\
\hline 8 & $28 \cdot 814$ & - & $11 \cdot 2$ & $12 \cdot 0$ & $10 \cdot 8$ & $15 \cdot 0$ & Var. 1, 3-6 & 16 & $12-26$ & $\log$. & & Det. S.-cu. 1 & - & - & \\
\hline 10 & $28 \cdot 781$ & - & $10 \cdot 0$ & $10 \cdot 8$ & $8 \cdot 2$ & $11 \cdot 2$ & NF, 2-3 & 13 & $20-23$ & b & & - & - & - & \\
\hline Mint. & - & - & - & $=$ & - & - & - & - & - & - & 1 & - & - & - & \\
\hline Mean & $28 \cdot 926$ & - & - & $13 \cdot 2$ & $12 \cdot 1$ & - & - & $1-$ & - & - & & - & - & $\rightarrow$ & \\
\hline \multicolumn{6}{|c|}{ Max. therm, reall at $\left\{\begin{array}{l}8 \text { a. } 15^{\circ} \cdot 8 . \\
8 \text { p. } 17^{\circ} \cdot 0 .\end{array}\right.$} & \multicolumn{5}{|c|}{ Solar rodlatlon $\left\{\begin{array}{l}\text { small black bulb } 100^{\circ} \cdot 2 \\
\text { smatl brlght bulb } 52^{\circ} \cdot 0 .\end{array}\right.$} & \multicolumn{4}{|c|}{ Preclpus. - in. } & \\
\hline
\end{tabular}

24th. 
1903-November.]

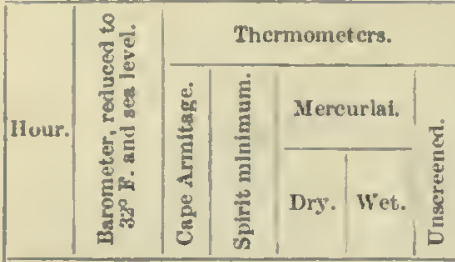

25th.

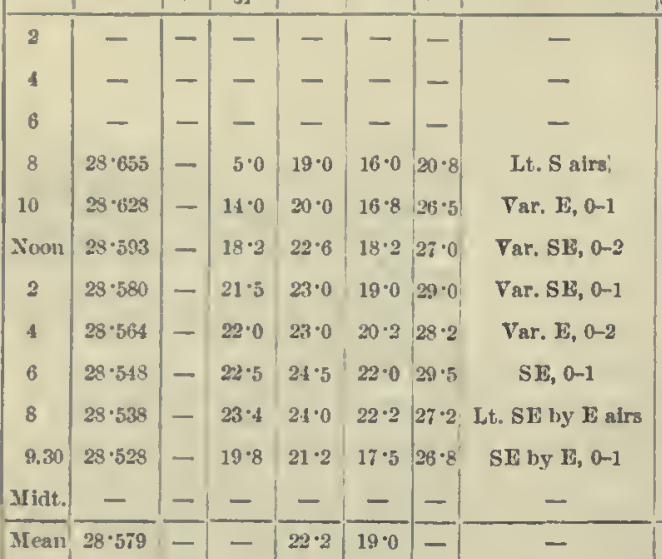

Meteorological Journal of the "Discovery,"

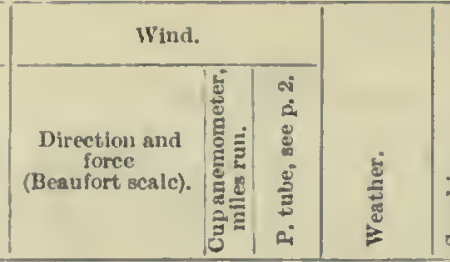

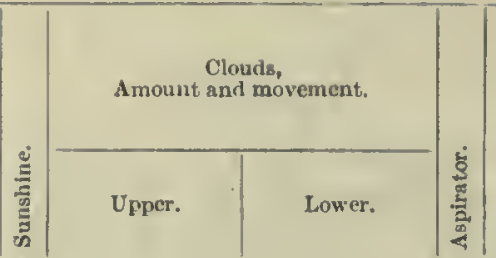

Rewarks.

\begin{tabular}{l|l|l|}
- & - & - \\
- & - & - \\
- & - & - \\
- & - & - \\
- & - & - \\
- & - & - \\
- & - & - \\
- & - & - \\
- & - & - \\
- & - & - \\
- & - & -
\end{tabular}

\section{$-$} 12 miles per hour. western range. Mirage. and SE. Ozone, 3.

Max. therm. read at $\left\{\begin{array}{l}8 \text { a. } 19^{\circ} \cdot 2 . \\ 8 \text { p. } 25^{\circ} \cdot 6^{\circ}\end{array}\right.$. Solar radiation $\left\{\begin{array}{l}\text { small blaek bulh } 106^{\circ} \cdot 5 . \\ \text { small brlght bulb } 57^{\circ} \cdot 5 .\end{array}\right.$ Bvapn. $\left\{\begin{array}{l}1.18 \cdot 1 \text { granmes } \\ \text { in } 24 \text { hours. }\end{array} \quad\right.$ Precipn. - in.

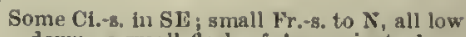
down; a gmall fleck of $\mathrm{A}$. cur. just ahove suminit of inlgh western peak ; refractlon conslderable in Wanl1 SWand thins duwn'1 remained settled; wind secms to have decreased gradually, and there has been continuous sunshine. Mirage. Average wind velocity slnce 10 p.m. yesterday,

a.m. Refraction the same as at 8 a.m.; warit the only other cloud is a to northalout the southem

Nome Refraetion has almost disn everywhere there is a ittle in $S$ apeared loses itself $W$ and NW. The Ci.-s, in SE is alout the sane, and the small cloud remains stationary upon the ieak. Mirage. 2 p.m. Very little thin $\mathrm{Cl}$.-8. low dowin in $\mathrm{S}$

30 p.m. Tein p. at Cape Armitage, $25^{\circ} \cdot 8$;

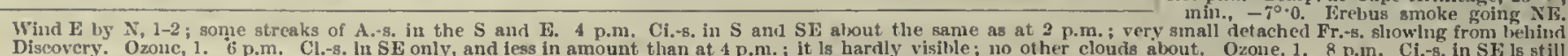

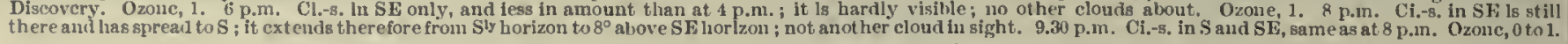

26th.

\begin{tabular}{|c|c|c|c|c|c|c|c|c|c|c|c|c|c|}
\hline 2 & - & -1 & - & - & - & - & - & - & - & - & & - & - \\
\hline 4 & - & - & - & - & - & - & - & - & - & - & & - & - \\
\hline 6 & - & - & - & - & - & - & - & - & - & - & & - & - \\
\hline 8 & $28 \cdot 512$ & - & $7 \cdot 9$ & $23 \cdot 4$ & $21 \cdot 6$ & $28 \cdot 5$ & Lt. NE airs & 1 & 0 & bev & & - & A.-cu., S \\
\hline 10 & $28 \cdot 508$ & - & $20 \cdot 6$ & $25 \cdot 0$ & $20 \cdot 2$ & $27 \cdot 8$ & Lt. S airs, 0-2 & 2 & $0-20$ & bevq fo & 1 & - & A. and Fr.-a.cu. \\
\hline Noon & 28.538 & -1 & $22 \cdot 1$ & $23 \cdot 2$ & $22 \cdot 0$ & $24 \cdot 8$ & S by W, 1-4 & 12 & $5-25$ & beq + & & - & $\begin{array}{l}\text { A.-s, cu. } 2 \text {, and } \\
\text { A. }=8,5, S\end{array}$ \\
\hline 2 & $28 \cdot 528$ & -1 & $21 \cdot 4$ & $22 \cdot 5$ & $21 \cdot 5$ & $23 \cdot 0$ & Var. S, 2-4 & 14 & $20-32$ & cq $f$ & & Ci.-s. 1 & A and $\mathrm{Fr}_{\mathrm{s}}-\mathrm{a}, \mathrm{cu}$ \\
\hline 4 & $28 \cdot 520$ & - & $21 \cdot 4$ & $22 \cdot 2$ & $21 \cdot 8$ & $23 \cdot 0$ & Var. S, 2-6 & 22 & $15 m-4$ & cq $\div$ & & - & A.-8. 9 \\
\hline 6 & $28 \cdot 526$ & - & $21 \cdot 0$ & $22 \cdot 2$ & $21 \cdot 8$ & $22 \cdot 5$ & Var. S, 2-6 & 31 & $15-40$ & $c q \div$ & & - & S. 10 \\
\hline 8 & $28 \cdot 547$ & -1 & $210 \cdot 2$ & $21 \cdot 0$ & $21 \cdot 0$ & $21 \cdot 5$ & $\mathrm{~S}$ by $\mathrm{W}, 4-8$ & 23 & $25-52$ & ocq $f t^{2} *$ & & - & N. 10 \\
\hline 10 & $28 \cdot 574$ & - & $18 \cdot 5$ & $19 \cdot 7$ & $19 \cdot 2$ & $20 \cdot 2$ & S, 3-5 & 19 & $25-33$ & $0 \operatorname{tin}^{2} *$ & & - & N. 10 \\
\hline Mrlit. & - & - & - & - & - & - & - & - & - & - & & - & - \\
\hline Mean & $28 \cdot 532$ & - & - & $22 \cdot 1$ & $21 \cdot 1$ & -1 & - & - & - & - & & - & - \\
\hline
\end{tabular}

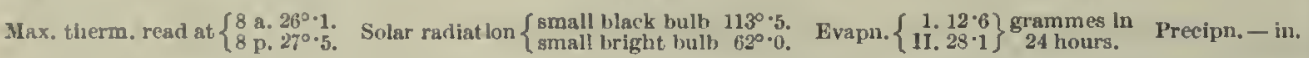

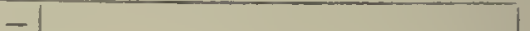

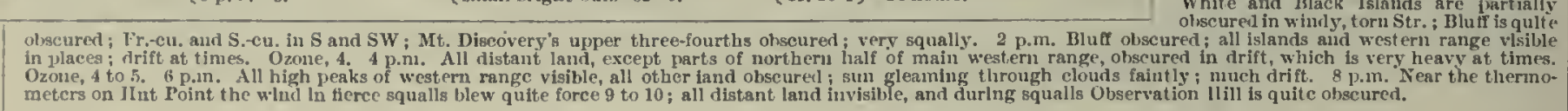

27 th.

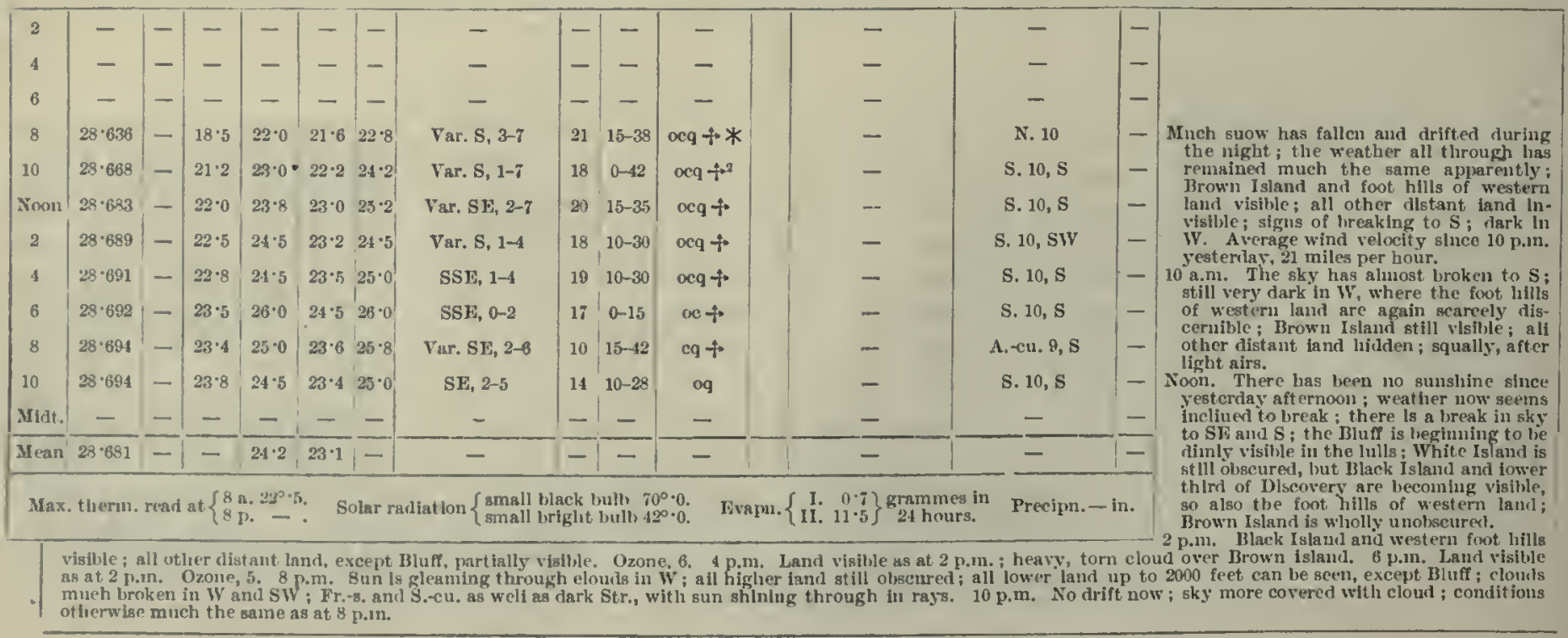


Meteorological Journal of tile "Discovery,"

[1903-November.

\begin{tabular}{|c|c|c|c|c|c|c|c|c|c|c|c|c|c|c|}
\hline \multirow{3}{*}{ IItour. } & \multirow{3}{*}{ 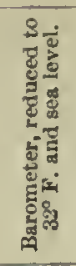 } & \multicolumn{5}{|c|}{ Tliermometers. } & \multicolumn{3}{|c|}{ Wind. } & \multirow[b]{3}{*}{ 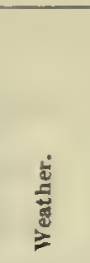 } & \multirow[b]{3}{*}{ 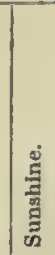 } & \multirow{2}{*}{\multicolumn{2}{|c|}{$\begin{array}{l}\text { Clouds, } \\
\text { Amount and movement. }\end{array}$}} & \\
\hline & & \multirow{2}{*}{ 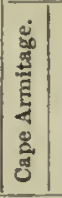 } & \multirow{2}{*}{ 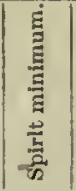 } & \multicolumn{2}{|c|}{ Mercurial. } & \multirow{2}{*}{ 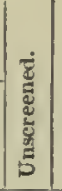 } & \multirow{2}{*}{$\begin{array}{c}\text { Direction and } \\
\quad \text { force } \\
\text { (Beaufort scale). }\end{array}$} & \multirow{2}{*}{ 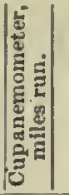 } & \multirow{2}{*}{ 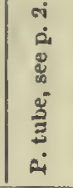 } & & & & & \\
\hline & & & & Dry. & Wet. & & & & & & & Upper, & Lower. & 要 \\
\hline 2 & - & -1 & - & - & - & - & - & - & - & - & & - & - & - \\
\hline 4 & - & - & - & - & - & - & - & - & - & - & & - & - & - \\
\hline$\theta$ & - & - & - & - & - & - & - & - & - & - & 1 & - & - & - \\
\hline 8 & $28 \cdot 675$ & - & $22 \cdot 6$ & 25.0 & $24 \cdot 0$ & $26 \cdot 4$ & JSE, $1-5$ & 14 & $5-25$ & $\infty q$ & & - & A.cu, 8, SSE (slow); & - \\
\hline 10 & 28.854 & -1 & $24 \cdot 8$ & $28 \cdot 2$ & 24.7 & $28 \cdot 0$ & Var. SE, 1-5 & 13 & $5-28$ & ocq & & - & 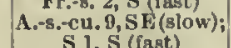 & :- \\
\hline Noon & $28 \cdot 639$ & - & 25.8 & $28 \cdot 2$ & $26 \cdot 6$ & $30 \cdot 5$ & Vur. $\mathrm{S}, 1-6 \mathrm{t}$ & 11 & $5-27$ & $c q+$ & . & - & 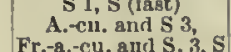 & - \\
\hline 2 & $28 \cdot 820$ & - & $27 \cdot 5$ & $28 \cdot 2$ & $26 \cdot 2$ & $29 \cdot 0$ & Var. $\mathrm{S}, 1-3$ & 10 & $10-28$ & eqt & & - & $\begin{array}{l}\text { cus and } \mathrm{S} \\
\mathrm{S} .10\end{array}$ & - \\
\hline 4 & $28 \cdot 614$ & - & $24 \cdot 8$ & $25 \cdot 2$ & $25 \cdot 0$ & $27^{\circ} 0$ & Var. $5,2-5$ & 8 & $10-38$ & $\mathrm{cq}+$ & & - & S. 10 & - \\
\hline 6 & $28 \cdot 620$ & - & $23 \cdot 2$ & 25.0 & $24 \cdot 0$ & $25 \cdot 0$ & Var. $S, 2-6$ & 17 & $10-40$ & $\operatorname{cq} \uparrow$ & & - & S. 10 & - \\
\hline 8 & $28 * 621$ & - & 23.5 & $25 \cdot 4$ & $24 \cdot 0$ & $25 \cdot 5$ & Var. S, 2-8 & 19 & $10-35$ & $\operatorname{ocg} q+$ & & - & A.-B. $10, S$ & - \\
\hline 10 & $28 \cdot 623$ & - & $24 \cdot 6$ & $26 \cdot 0$ & $24 \cdot 8$ & $26 \cdot 0$ & S, $2-8$ & 15 & $10-48$ & $o q+t^{2}$ & & - & s. 10 & - \\
\hline Midt. & - & -1 & - & - & - & -1 & - & - & - & - & & - & - & $1-$ \\
\hline Mean & $28 \cdot 634$ & $=$ & - & $26 \cdot 2$ & $24 \cdot 9$ & $=$ & - & $=1$ & - & - & & - & - & $1-$ \\
\hline
\end{tabular}

Max, therm. read at $\left\{\begin{array}{l}8 \text { \%. } 25^{\circ} .2 . \\ 8 \mathrm{p} .31^{\circ} 0 .\end{array} \quad\right.$ Solar radiation $\left\{\begin{array}{l}\text { small black hulb } 111^{\circ} \cdot 0 . \\ \text { small bright bulb } 62^{\circ} 0 .\end{array} \quad\right.$ Exapn. $\left\{\begin{array}{l}\text { I. } 6.9 \text { grammea } \\ \text { in } 24 \text { hours. }\end{array} \quad\right.$ Precipn. - in。

Hemarks.

Windy-lonking A.cu., with sharp ugper eiges, ofteit conver, in $W$ and $S W$.

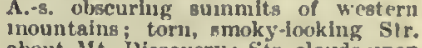

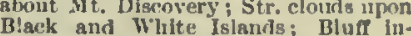
vischle. Averaze wind velocity since 10 p.m. yesterday, 14 iniles per hour. 10 a.m. There are more clouds, windylooking Strr., atrout all the higher laind

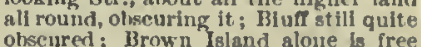

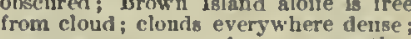
every appearance of worse weather

Noon. Sun gleaming urightly at inter. vals; gloony looklig in $W$ and $S W$; Str. cloud eovers summit of Brown
Island; the clonds alout the zenith are of a wind y kind, as at 10 a.m.

2 p.m. Islaurs and western foot hills visible; surface drift. Ozone, 3

p.m. Blaek and Brown Islands and

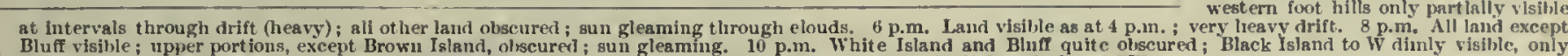
lower fourth of Mt. Discovery not olseured; Brown Island visible, also foot hills of western land; ;ky lighter in S and SW.

\begin{tabular}{|c|c|c|c|c|c|c|c|c|c|c|c|c|c|c|}
\hline 2 & - & - & - & - & - & - & - & - & - & - & - & - & - & \\
\hline 4 & - & - & - & - & - & - & - & - & - & - & - & - & - & \\
\hline 6 & - & - & - & - & - & - & - & - & - & - & - & - & - & \\
\hline 8 & $28 \cdot 750$ & - & 20.5 & $2: \cdot 5$ & $24 \cdot 0$ & $26 \cdot 0$ & SSE, 2-6 & 18 & $10-30$ & $o c q+$ & - & S. $10, \mathrm{~S}$ & - & own Island wholly visible, White and \\
\hline 10 & $28 \cdot 770$ & - & $24 \cdot 0$ & $26 \cdot 2$ & $25 \cdot 0$ & $28 \cdot 2$ & Var. SE, 2-5 & 15 & $5-20$ & $o q+$ & - & S 10 & - & Diseovery and lower foot hills of western \\
\hline Noon & $28 \cdot 788$ & - & $25 \cdot 2$ & $26 \cdot 0$ & $25 \cdot 0$ & $28 \cdot 2$ & $\mathrm{SF}, 2-6$ & 12 & $10-35$ & oq $+* *$ & - & N. $10, \mathrm{~s}$ & - & $\begin{array}{l}\text { land only dimly rishle; a lower scud } \\
\text { Str. cloud is moving rapidly, about }\end{array}$ \\
\hline 2 & $28 \cdot 802$ & - & $24 \cdot 0$ & $25 \cdot 0$ & $24 \cdot 0$ & $25 \cdot 8$ & Var. SE, 1-4 & 14 & $8-30$ & oeq + & - & S. 10 & - & $\begin{array}{l}900 \text { feet in alt., from the S19; the weather } \\
\text { during the night appars to have re }\end{array}$ \\
\hline 4 & $28 \cdot 820$ & - & $24 \cdot 0$ & $25 \cdot 0$ & $24 \cdot 0$ & $26 \cdot 0$ & Var. SE, 1-3 & 15 & $0-20$ & ocq & - & A.-cu, and S. 10 & - & to 5 . Average wlur relocity since \\
\hline 6 & $28 \cdot 840$ & - & $24 \cdot 0$ & $25 \cdot 0$ & $24 \cdot 0$ & $25 \cdot 2$ & Var. SE, 1-2 & 16 & $0-15$ & oc & - & A.-s. 8 & - & $\begin{array}{l}10 \text { p.m. yesterday, } 16 \text { miles per hour. } \\
10 \text { a.m. Very dark and tlreatening to }\end{array}$ \\
\hline 8 & $28 \cdot 846$ & - & $23 \cdot 6$ & $24 \cdot 1$ & $23 \cdot 2$ & $24 \cdot 6$ & $\mathrm{SE}, 2-6$ & 13 & $10-30$ & ocq + & - & s. $10, \mathrm{~s}$ & - & $\begin{array}{l}\text { and } \\
\text { dark elouds; foot hills to } W \\
\text { yust visible. }\end{array}$ \\
\hline 10 & $28 \cdot 865$ & - & $22 \cdot 9$ & $25 \cdot 0$ & $24 \cdot 0$ & $25 \cdot 0$ & Var. SE, $2-7$ & 15 & $10-40$ & oq + & - & 5. $10, \mathrm{~s}$ & - & ginn ing to show up; the Bluff seems to \\
\hline Midt. & - & -1 & - & - & - & - & - & - & - & - & - & - & - & in SW, and the blackness extends to \\
\hline$\overline{M e a n}$ & $28 \cdot 810$ & - & - & $\overline{25 \cdot 1}$ & $24 \cdot 2$ & $1-$ & - & - & - & - & - & - & $\overline{-}$ & 2 p.n. Bases of White and Brown Islands \\
\hline \multicolumn{6}{|c|}{ Max. therm, read at $\left\{\begin{array}{l}8 \text { a. } 26^{\circ} \cdot 8 \\
8 \text { p. } 26^{\circ} \cdot 8 \text {. }\end{array}\right.$} & \multicolumn{5}{|c|}{ Solar radiation $\left\{\begin{array}{l}\text { small blaek bulb } 80^{\circ} \cdot 5 . \\
\text { small bright bulb } 42^{\circ} \cdot 6^{\circ}\end{array}\right.$} & Evapn. $\left\{\begin{array}{l}1.7 \\
\text { in }\end{array}\right.$ & \multicolumn{2}{|c|}{ Precipn. - in. } & $\begin{array}{l}\text { cloud, olscuring upper part; Black } \\
\text { Island, western foot lills, and base of } \\
\text { Discovery visihle, Ititlle sky slowing in }\end{array}$ \\
\hline
\end{tabular}

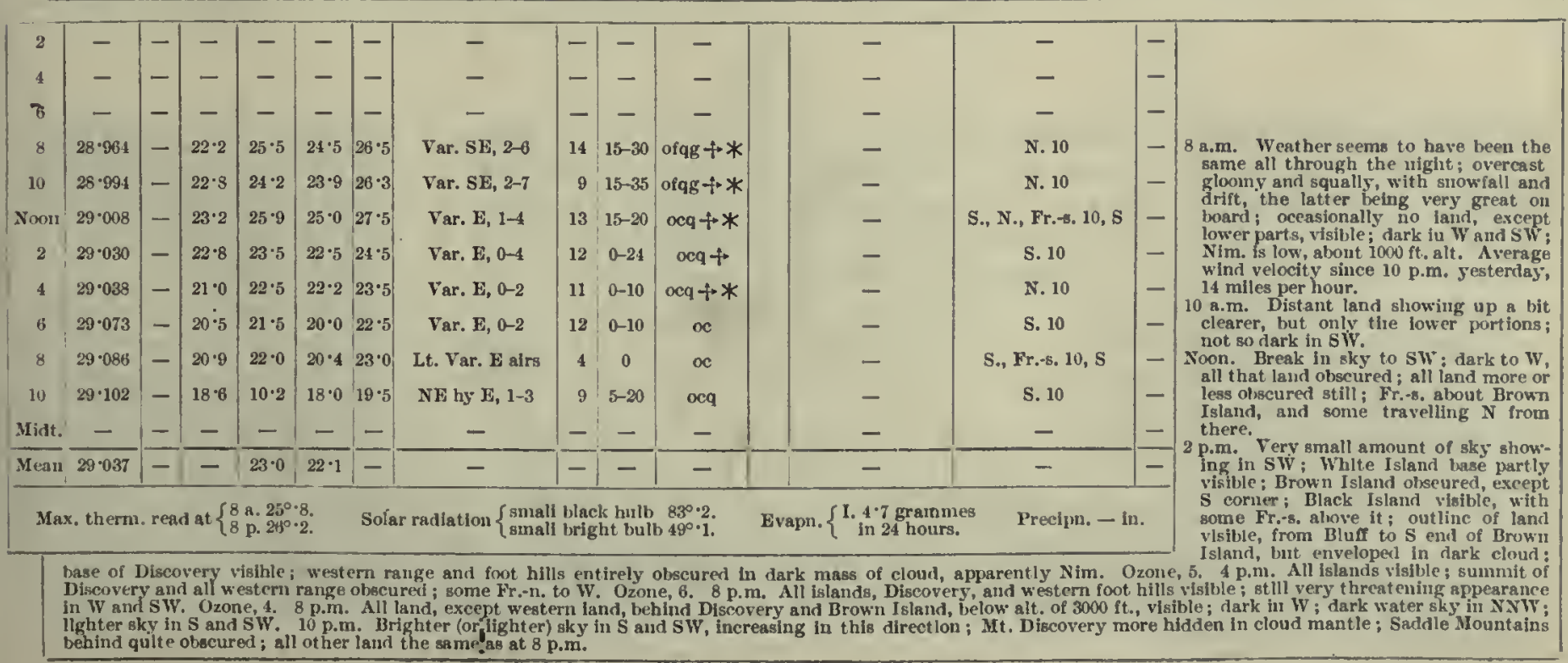

29th. 


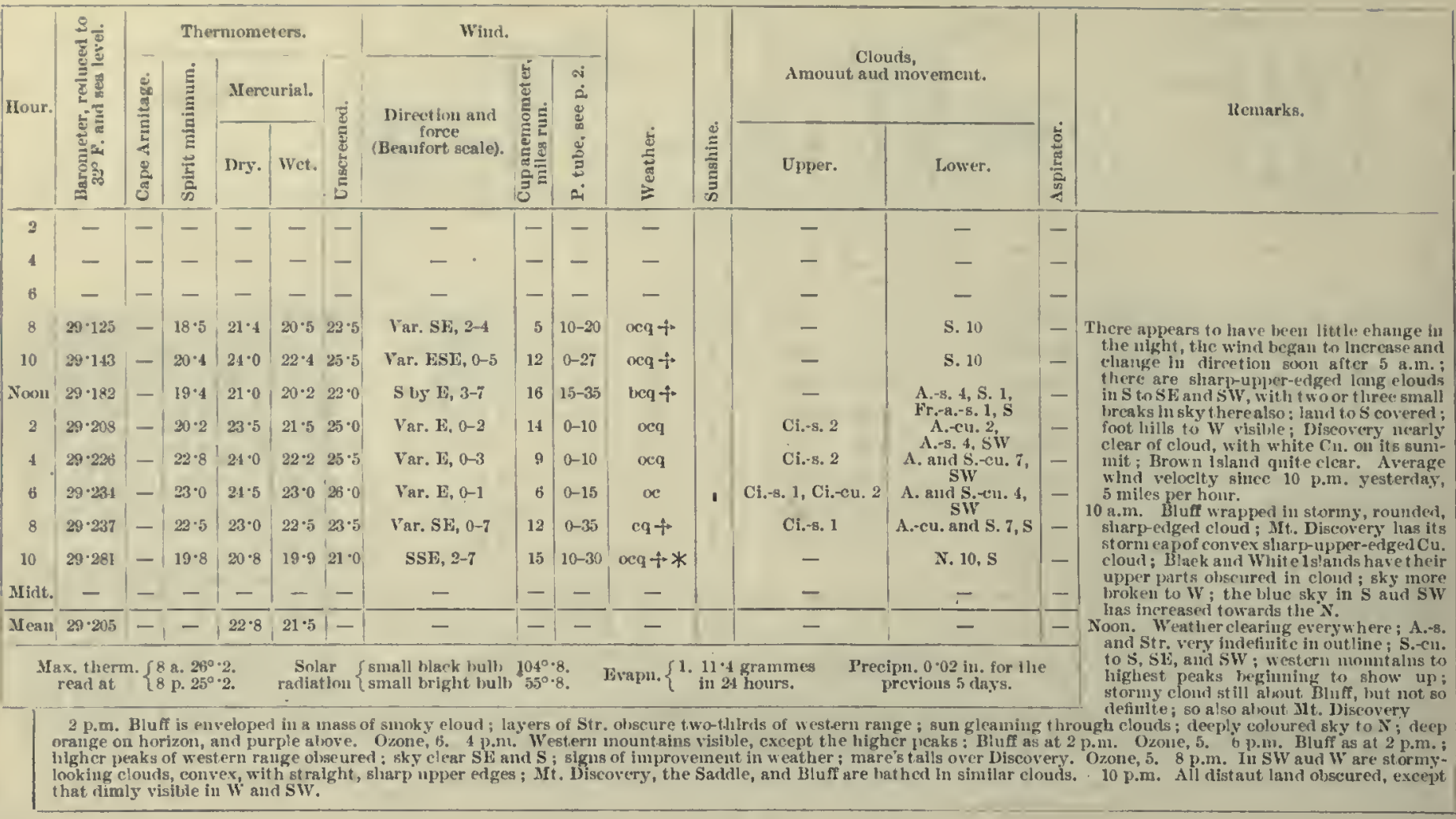

2ud.

\begin{tabular}{|c|c|c|c|c|c|c|c|c|c|c|}
\hline 2 & - & - & - & - & - & - & - & -1 & - & - \\
\hline 4 & - & - & - & - & - & - & - & - & - & - \\
\hline 6 & - & - & - & - & - & - & - & - & - & - \\
\hline 8 & $2: 404$ & - & $13 \cdot 5$ & $18 \cdot 1$ & $17 \cdot 8$ & $20 \cdot 0$ & Var. S1, 2-7 & 18 & $10-30$ & $c q+$ \\
\hline 10 & $29 \cdot 452$ & - & $16 \cdot 8$ & $19 \cdot 4$ & $18 \cdot 4$ & $22 \cdot 0$ & Var. ST, 1-7: & 17 & $5-30$ & $\operatorname{ocg} f+*$ \\
\hline Noon & $29 \cdot 46 \pi$ & - & $18 \cdot 1$ & $20 \cdot 4$ & $20 \cdot 0$ & $22 \cdot 5$ & $\operatorname{SSE}, 1-5$ & 15 & $5-20$ & oqf $+* *$ \\
\hline 2 & $29 \cdot 479$ & - & $20^{\circ} 0$ & $21 \cdot 5$ & $20 \cdot 5$ & $23 \cdot 2$ & Var. S, 0-2 & 12 & $0-10$ & $x+*$ \\
\hline 4 & $29 \cdot 477$ & - & $20 \cdot 4$ & $22 \cdot 0$ & $21 \cdot 2$ & $23 \cdot 5$ & Var. SE, 0-3 & 10 & $0-20$ & $\operatorname{ocq}+$ \\
\hline 6 & $29 \cdot 480$ & - & $20 \cdot 8$ & $23 \cdot 0$ & $21 \cdot 8$ & $3 \cdot 8$ & Var. S1, 0-4 & 12 & $0-25$ & cq \\
\hline 8 & $29 \cdot 470$ & - & $21 \cdot 4$ & $23 \cdot 4$ & $21 \cdot 4$ & 24.0 & E by N, 2-4 & 16 & $5-15$ & beat $f^{-}$ \\
\hline 10 & $29 \cdot 451$ & - & $21 \cdot 9$ & $23 \cdot 0$ & $21 \cdot 2$ & $25 \cdot 2$ & E by $\mathbf{N}, 2-5$ & 11 & $10-24$ & $b x+$ \\
\hline Fidt & - & - & - & - & - & - & - & - & - & - \\
\hline
\end{tabular}

Mean $\overline{29 \cdot 480}=\overline{-1} \overline{21 \cdot 4} \overline{20 \cdot 3}=$

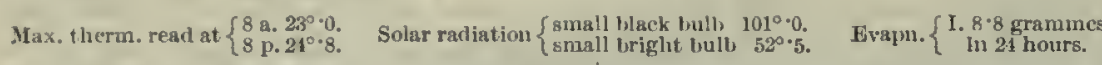

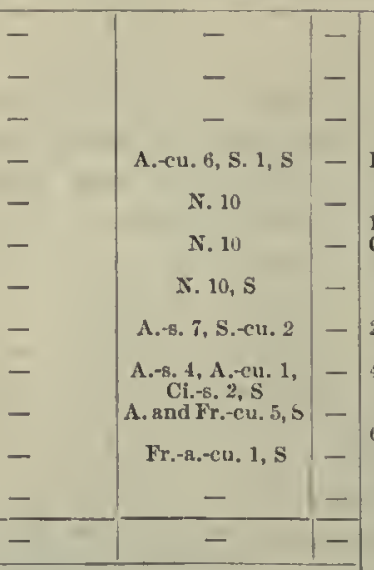

Precipu, - in.
Distant land nearly all obsenred. Average wind velocity sinee 10 [1.1n. yesterdsy, 18 miles per tiour. 10 a.m. All land hut Brown Yslaud ohseured. Island becoming visible; all other land obscured; some scud is passing from and toueling summit of Crater IIill. p.m. Land beconing more visible: heary storm clouds hanghig over it. Ozonc, 9. .m. Conditions slmilar; Bluff obseured hy blanket of eloud, taking same shape as
tänd. Ozone, 5 . " p.m. All land visihle; Bluff still enveloped in eloud, bint it is not so torn and ragged;
gencrally the clouds in $S$, sW, and $W$ gencrally the clouds in $S, S W$, and $W$ look less windy than they have for the
past few days; Bome lines of Stro glout past few days; some lines of Str. Buout
western range, and sumumit of Discovery is covered witl a mass of cloud.

8 p.m. All land, exeept suun mit.of Discovery, Saddle, and western mount aius now clear S.-cil. cover tops.

10 p.m. Muel thuer; brilliant sunshine,

with very little eloud, cxeept about $\mathrm{SW}$ and $\mathrm{W}$; western momtains now nearly clear of clouds, thin lines of A.-s. over them; odd Sir. like Ci.-s. in $W$, and SW.

$3 r d$.
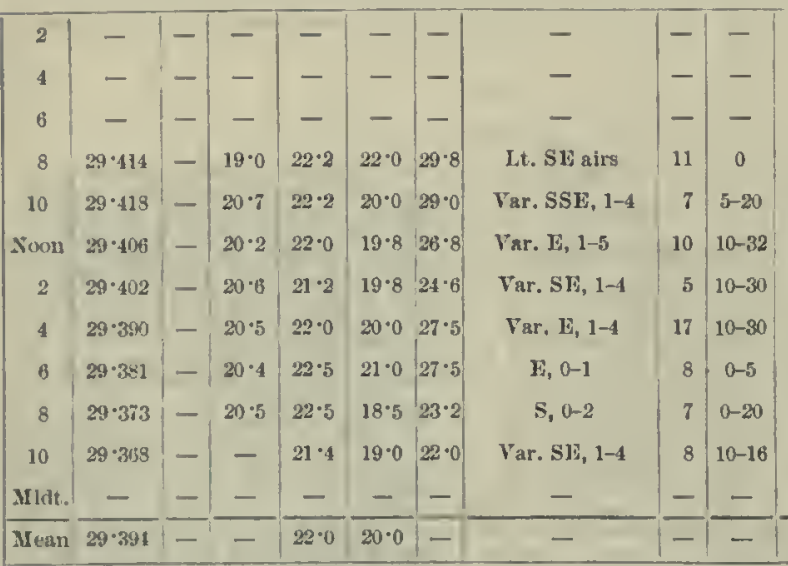

Mlax, therm, real at $\left\{\begin{array}{l}8 \text { a. } 24^{\circ} \circ 0 . \\ 8 \text { p. } 25^{\circ} \cdot 5 \text {. }\end{array}\right.$

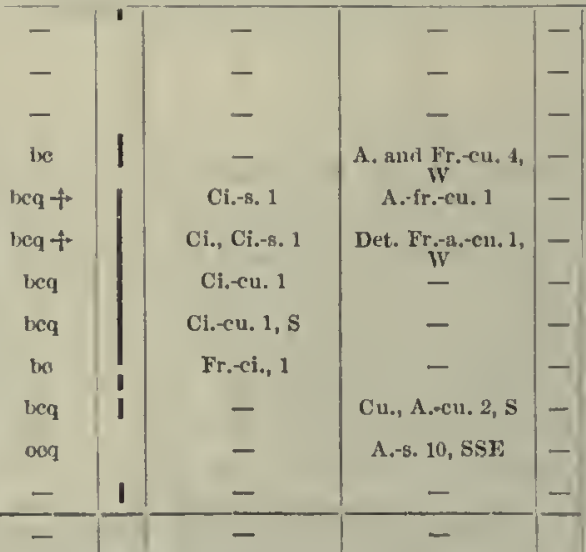

Precipn. - In

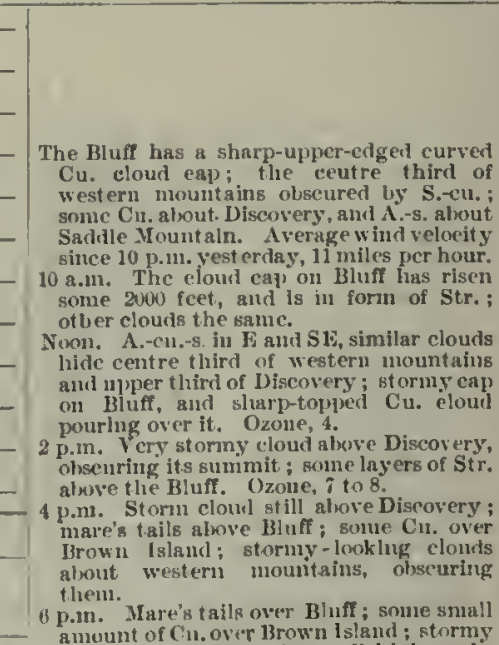

p.m. Mare's tails over Bluff; some smal

clonds In lines; S.-cu. about western mountains aus over Discovery; bank of A.-cu. betwcen Discoverv and sonthern peak of western unountains; all highl peaks

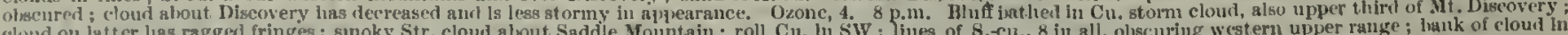

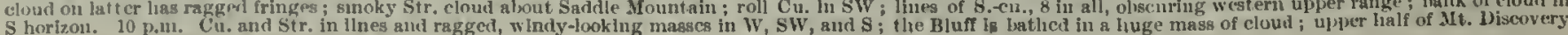
is also batived in storny-lonking cloud. 
Meteorotogical, Journat of the "Discovery."

[1903-December.

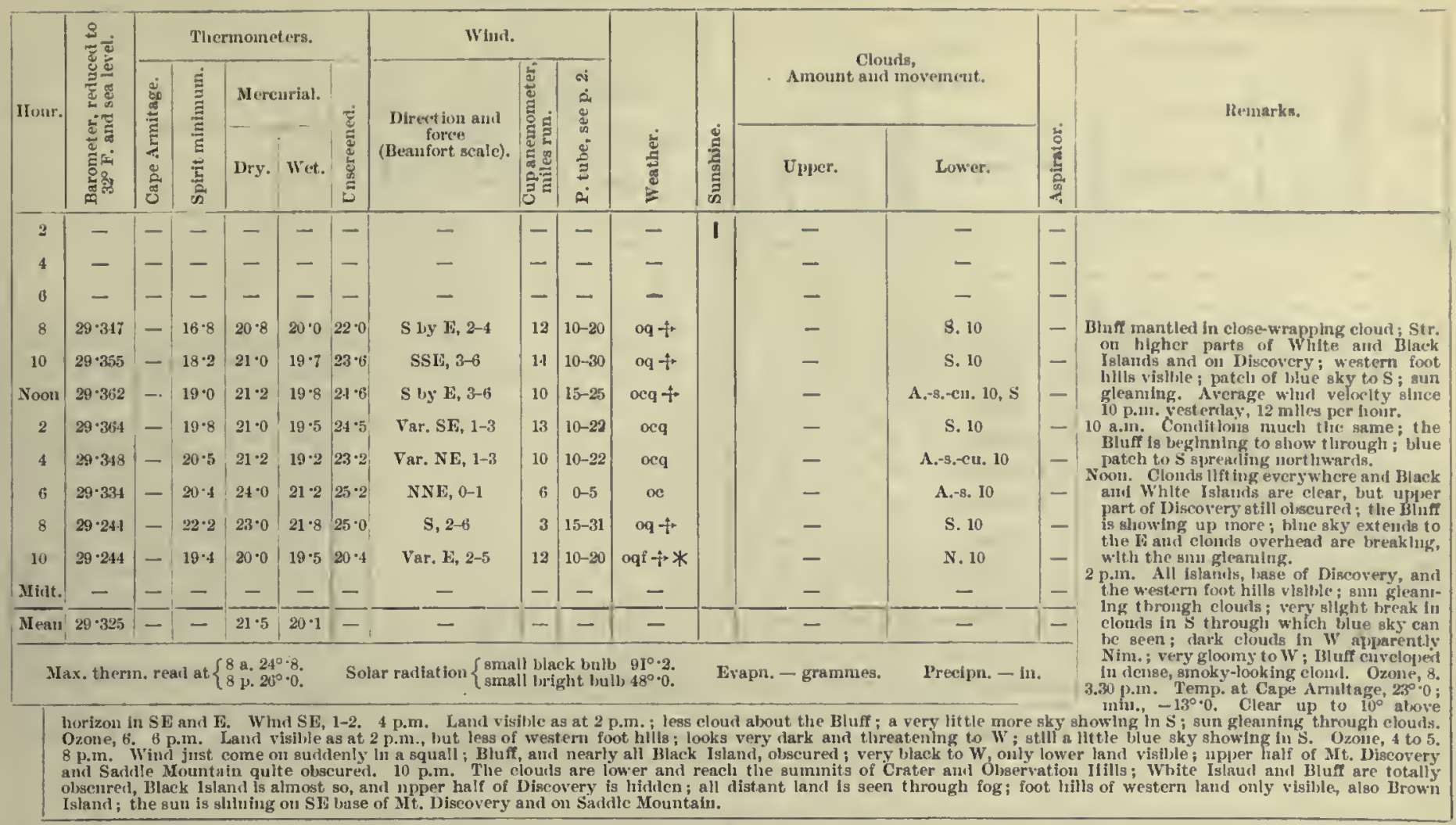

\begin{tabular}{|c|c|c|c|c|c|c|c|c|c|c|c|c|c|c|}
\hline 2 & - & -1 & - & - & - & L- & - & - & - & - & & - & - & - \\
\hline 4 & - & - & - & - & - & -1 & - & - & - & - & & - & - & - \\
\hline 6 & - & - & - & - & - & - & - & - & - & - & & - & - & - \\
\hline 8 & $29 \cdot 371$ & - & $18 \cdot 4$ & $19 \cdot 2$ & $19 \cdot 0$ & $20 \cdot 6$ & SSE, $20-5$ & 12 & $20-28$ & $\operatorname{og} f+*^{2}$ & & - & N. 10 & - \\
\hline 10 & $29 \cdot 393$ & - & $18 \cdot 2$ & $19 \cdot 2$ & $19 \cdot 0$ & $21 \cdot 2$ & SSE, $2-5$ & 14 & $10-32$ & oqf $-f *^{2}$ & & - & N. 10 & - \\
\hline Noon & $29 \cdot 415$ & - & $17 \cdot 9$ & $21 \cdot 2$ & $19 \cdot 0$ & $21 \cdot 8$ & Var. SSE, 3-6 & 20 & $15-35$ & ocq + & & - & S. 10, S (rapid) & - \\
\hline 2 & $29 \cdot 396$ & - & $18 \cdot 8$ & $21 \cdot 4$ & $19 \cdot 8$ & $23 \cdot 0$ & E, 2-5 & 12 & $15-32$ & $\operatorname{ceq}+$ & & - & S. 10, S (rapid) & - \\
\hline 4 & $29 \cdot 412$ & - & $17 \cdot 6$ & $19 \cdot 0$ & $19 \cdot 0$ & $25 \cdot 5$ & SSE, 4-8 & 26 & $25-50$ & oq $+* *$ & & - & N. 10 & - \\
\hline 6 & $29 \cdot 433$ & - & $16 \cdot 0$ & $19 \cdot 0$ & $18 \cdot 2$ & $19 \cdot 2$ & Var. E, 2-i & 14 & $15-42$ & cq + & $i$ & $\mathrm{Cl}_{.-8.1} 1$ & 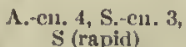 & - \\
\hline 8 & $29 \cdot 453$ & - & $15 \cdot 1$ & $16 \cdot 2$ & $15 \cdot 6$ & $170^{\circ}$ & Var. F, 3-6 & 18 & $15-31$ & eqf $+* *$ & & - & N. T, SSW & 一 \\
\hline 10 & $29 \cdot 478$ & - & $10 \cdot 9$ & $12 \cdot 0$ & $11 \cdot 7$ & $12 \cdot 2$ & E by N, 5-7 & 20 & $25-45$ & bef $+*$ & & Cl. 1 & N. $2, \mathrm{~S}$ & - \\
\hline Mlatt. & - & - & - & - & - & - & - & 二 & - & 二 & & - & - & - \\
\hline I ean & $249 \cdot 419$ & - & - & $18 \cdot 4$ & $17 \%$ & - & - & - & - & - & & - & - & - \\
\hline
\end{tabular}

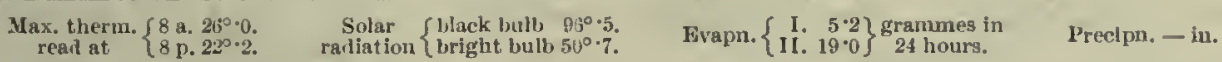
sight; furlous squalis. 10 p.m. Furious squalls; snow is falling in rather large flakes; the Nim. is all to the $\mathrm{S}$ and $\mathrm{E}$, while blne sky is to N, and to well over the
zeitith.

\begin{tabular}{|c|c|c|c|c|c|c|c|c|c|c|c|c|c|c|}
\hline 2 & - & - & - & - & - & - & - & - & - & - & - & - & -1 & . \\
\hline 4 & - & - & - & - & - & - & - & - & - & - & - & - & - & \\
\hline 6 & - & - & - & - & - & - & - & - & - & - & - & - & - & \\
\hline 8 & $29 \cdot 533$ & - & $7 \cdot 8$ & $14 \cdot 0$ & $13 \cdot 1$ & $17 \cdot 0$ & Var. E, 3-6 & 19 & $15-30$ & lec $t^{*}$ & Ci.-8, 2, S & Fr.e11. 1, $\mathrm{s}$ & - & \multirow{11}{*}{ 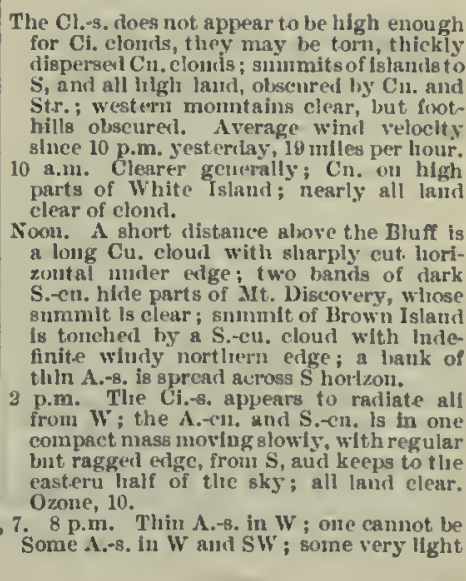 } \\
\hline 10 & $29 \cdot 540$ & - & $12 \cdot 9$ & $15 \cdot 8$ & $14 \cdot 9$ & $17 \cdot 0$ & Var. E, 3-b & 15 & $15-28$ & ineq & Ci., Ci.-s. 3, s & - & - & \\
\hline Noon & $29 \cdot 550$ & - & $11 \cdot 2$ & $17 \cdot 0$ & $15 \cdot 8$ & $20 \cdot 0$ & Var. $15,2-5$ & 15 & $10-23$ & beq + & Ci., Cl.-s. 3, S & - & - & \\
\hline 2 & $29 \cdot 541$ & - & $15 \cdot 5$ & $18 \cdot 2$ & $17^{\circ} 0$ & $20 \cdot 0$ & Var. $\mathbf{E}, 2-5$ & 13 & $15-25$ & eq & Cl., Cl.-8. 2 & A. and S.cu. 7, S & - & \\
\hline 4 & $29 \cdot 531$ & - & $17 \cdot 5$ & 20.0 & $18 \cdot 5$ & $25 \cdot 5$ & Var, $\mathbf{E}, 2-4$ & 13 & $10-20$ & eq & Ci., Ci.-cu. 4 & A.- $-\mathrm{c} 11,2, \mathrm{~S}$ & - & \\
\hline 6 & $29 \cdot 531$ & - & $17 \cdot 5$ & $20^{\circ} 4$ & $19 \cdot 2$ & $25 \cdot 0$ & E, 0-2 & 11 & $0-15$ & licq & Ci.-s. 2 & 一 & - & \\
\hline 8 & $29 \cdot 532$ & - & $19 \cdot 5$ & $21 \cdot 9$ & $18^{\circ} 0$ & $20 \cdot 4$ & E by $\mathbf{N}, 1-2$ & 6 & $0-10$ & leqv & Cl., Cl.-s. 1 & - & - & \\
\hline 10 & $29 \cdot 525$ & - & $21 \cdot 0$ & $23 \cdot 0$ & $19 \cdot 0$ & $27 \cdot 0$ & NE by $\mathbf{E}, 0-1$ & 6 & $0-3$ & bv & - & - & - & \\
\hline Mint. & - & - & - & - & - & - & - & - & - & - & - & - & - & \\
\hline Irean & $\overline{29 \cdot 5366}$ & $\overline{-}$ & - & $18 \cdot 8$ & $16 \cdot 9$ & $\overline{-}$ & - & - & - & - & - & - & - & \\
\hline \multicolumn{5}{|c|}{$\begin{array}{l}\text { Max, therm. } \\
\text { real at. }\end{array}\left\{\begin{array}{l}8 \text { a } 17^{\circ} \cdot 5 . \\
8 \text { p. } 23^{\circ} \cdot 1 .\end{array}\right.$} & \multicolumn{2}{|c|}{$\begin{array}{l}\text { Solar } \\
\text { radiation }\end{array}$} & $\begin{array}{l}\text { mall hlack bnl } \\
\text { mall loriglat be }\end{array}$ & $\begin{array}{r}114 \\
57\end{array}$ & & Tivapn. \{ & $\begin{array}{l}\left.\text { I. } \begin{array}{r}6 \cdot 9 \\
\text { II. } 13 \cdot 3\end{array}\right\} \text { grammes in } \\
\end{array}$ & \multicolumn{2}{|l|}{ Precipur, - in. } & \\
\hline
\end{tabular}


1903 -December.]

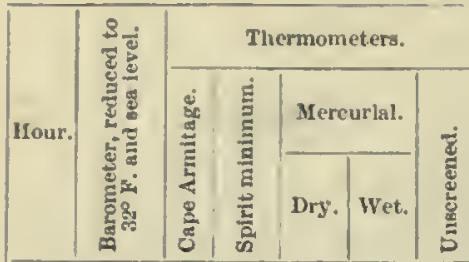

7th.

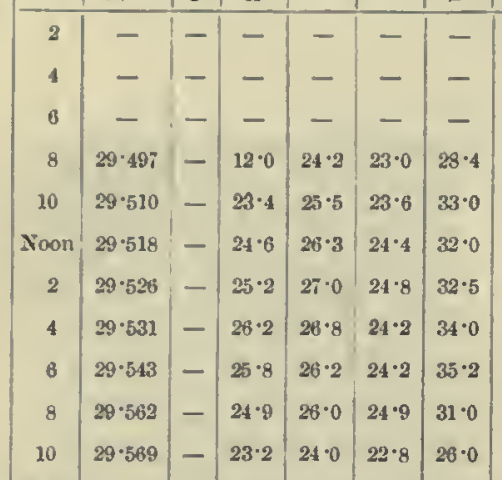

Metrorological Journat. of the "Discovery."

force
(Benufort scale).

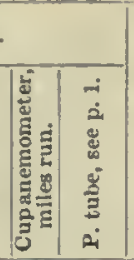

\begin{tabular}{|c|c|c|c|}
\hline- & - & - & - \\
- & - & - & - \\
- & - & - & - \\
N. 0-1 & 4 & $0-10$ & $b v$ \\
N airs & 3 & $0-5$ & bev \\
N_airs & 5 & 0 & $b v$ \\
Lt. W airs & 2 & 0 & bv \\
Lt. NW airs & 2 & 0 & bv \\
Lt. NW airs & 2 & 0 & bv \\
CCalm & 2 & 0 & bv \\
Lt. NW airs & 3 & 0 & bv \\
- & - & - & - \\
\hline- & - & - & - \\
\hline
\end{tabular}

$\mid$

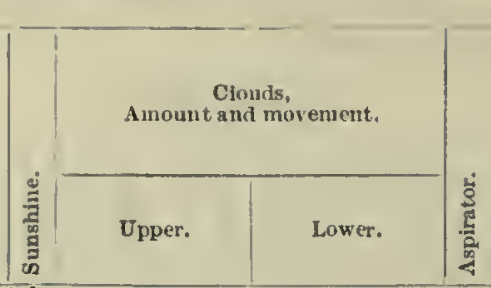

Kemarks. Max. therm. real at $\left\{\begin{array}{l}8 \text { a. } 24^{\circ} \cdot 8 . \\ 8 \text { p. } 27^{\circ} \cdot 6 .\end{array} \quad\right.$ Solar radiation $\left\{\begin{array}{l}\text { blaek bulb } 110^{\circ} \cdot 0 . \\ \text { bright bulb } 59^{\circ} \cdot 8 .\end{array} \quad\right.$ Evapn. $\left\{\begin{array}{l}1.12 \cdot 9 \mathrm{gramm} \\ \text { in } 24 \text { hours. }\end{array}\right.$

Precipn. - in.

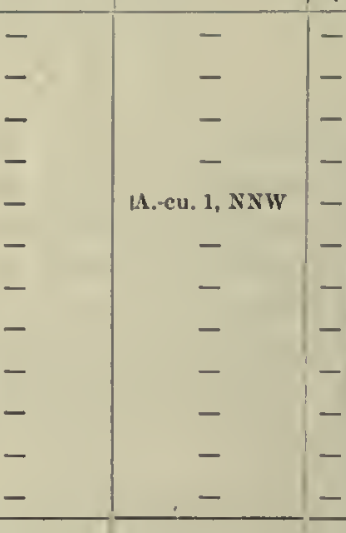

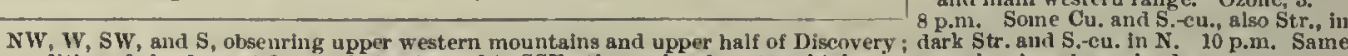
eondition of clouds as at 8 p.m., but extended to SSE; the peaks of western ligh range are showing above eloudis.

8th.

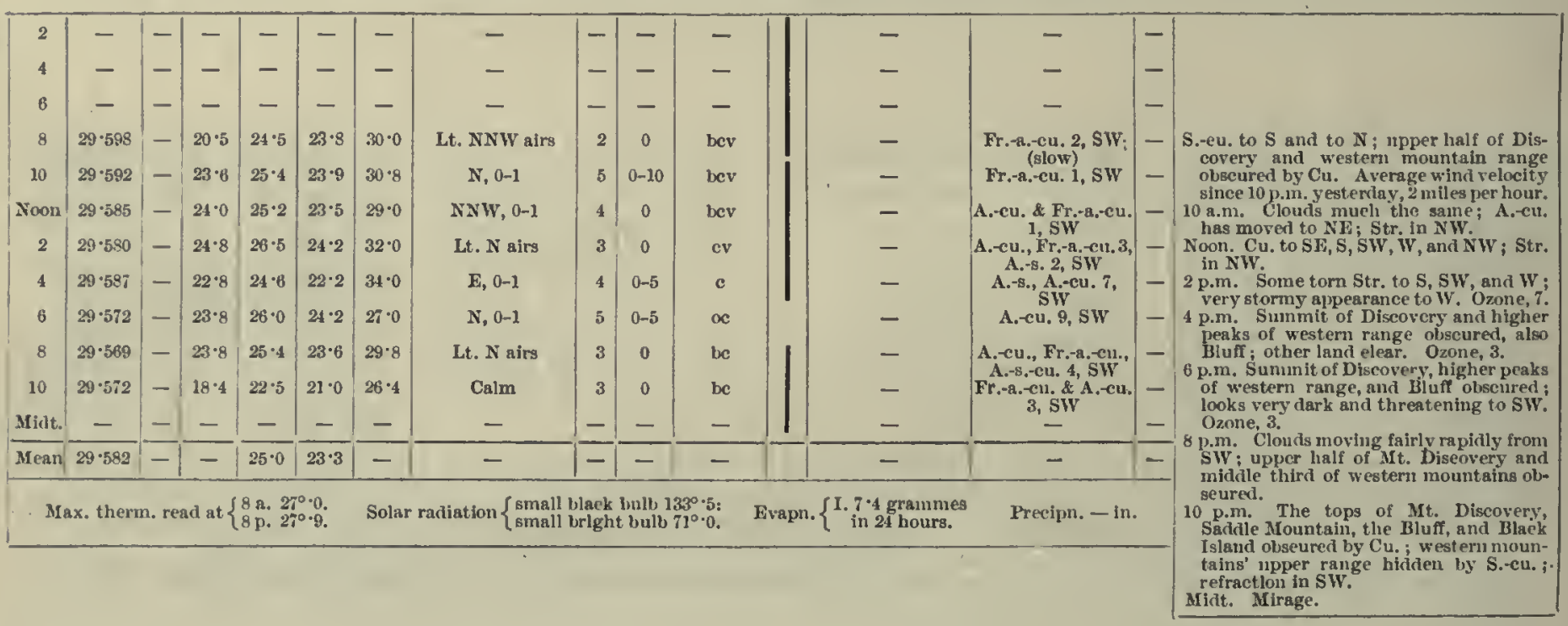

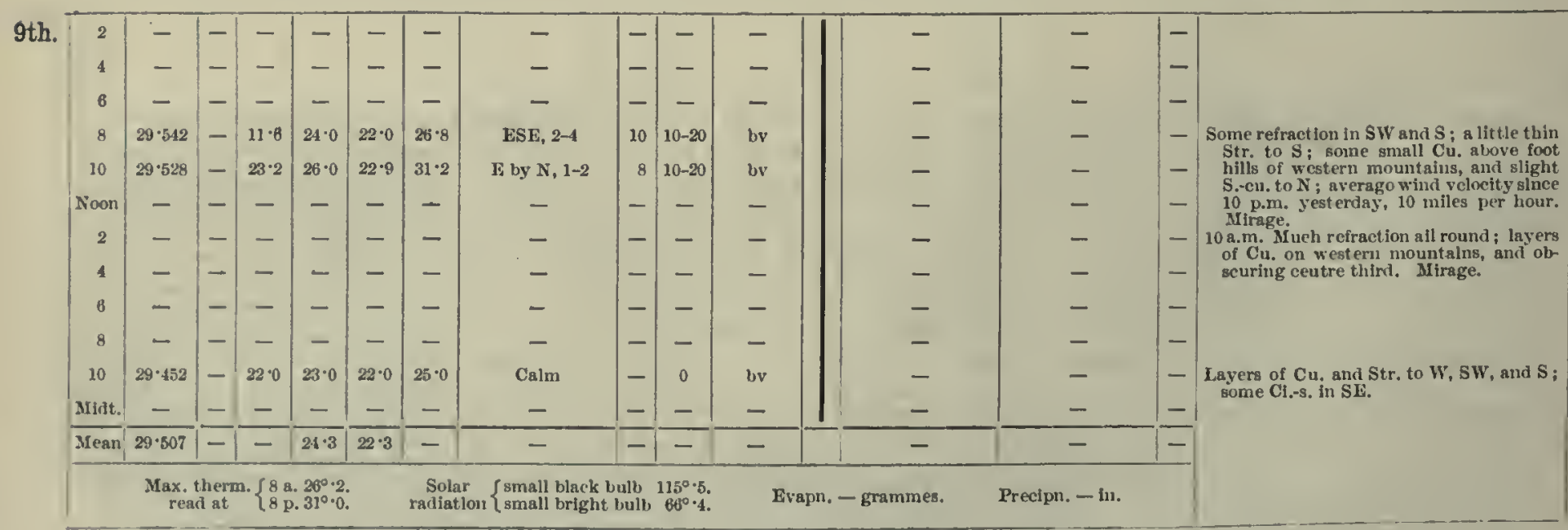


Meteorologicat, Journal, of the: "Discovery."

[1903-December.

\begin{tabular}{|c|c|c|c|c|c|c|c|c|c|c|c|c|c|c|c|}
\hline \multirow{3}{*}{ Hlour. } & \multirow{3}{*}{ 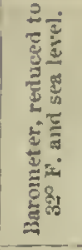 } & \multicolumn{5}{|c|}{ Thermometers. } & \multicolumn{3}{|c|}{ Wiml. } & \multirow[b]{3}{*}{ 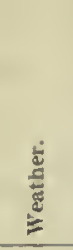 } & \multirow{3}{*}{ 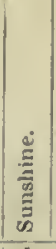 } & \multirow{2}{*}{\multicolumn{2}{|c|}{$\begin{array}{l}\text { Clouds, } \\
\text { A nomut aud incement. }\end{array}$}} & \multirow{3}{*}{$\left|\begin{array}{c}\frac{5}{2} \\
\frac{2}{2} \\
\frac{a}{2}\end{array}\right|$} & \multirow{3}{*}{ letmarks. } \\
\hline & & \multirow{2}{*}{ 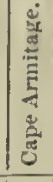 } & \multirow{2}{*}{ 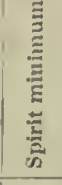 } & \multicolumn{2}{|c|}{ Mlercurial. } & \multirow{2}{*}{ 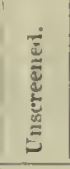 } & \multirow{2}{*}{$\begin{array}{c}\text { Direct ion aurl } \\
\text { furer } \\
\text { (Beanfort scale). }\end{array}$} & \multirow{2}{*}{ 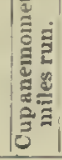 } & \multirow{2}{*}{ 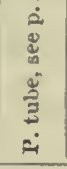 } & & & & & & \\
\hline & & & & $\mathbf{D}_{\mathrm{ry}}$ & Wet. & & & & & & & Upper. & Lower. & & \\
\hline 2 & - & - & - & - & - & - & - & - & - & - & & - & - & $1-$ & \\
\hline 4 & - & - & - & - & - & - & - & - & - & - & & - & - & - & \\
\hline 6 & - & - & - & - & - & - & - & - & - & - & & - & - & - & \\
\hline 8 & $29 \cdot 399$ & - & $15 \cdot 5$ & $23 \cdot 0$ & $22 \cdot 2$ & $28 \cdot 0$ & Calm & 0 & 0 & bv & & - & - & - & $\begin{array}{l}\text { Some refraction ail round horizon to } W \\
\text { and } E_{\text {; }} \text { thin } \operatorname{line} \text { of } C l .-\sin S \text { and } S E \text {; }\end{array}$ \\
\hline 10 & $29 \cdot 393$ & - & $20 \cdot 6$ & $22 \cdot 2$ & $21 \cdot 2$ & $31 \cdot 4$ & Calm & 0 & 0 & bv & & 一 & - & - & $\begin{array}{l}\text { long line of Str. dividing mildile third } \\
\text { of western range horlzontally ; Fr.cu. }\end{array}$ \\
\hline Sinnn & $29 \cdot 342$ & - & $22 \cdot 2$ & $24 \cdot 8$ & $23 \cdot 2$ & $30 \cdot 0$ & NW, $0-2$ & 0 & 0 & hw & & - & - & - & $\begin{array}{l}\text { to SE. Mirage. } \\
10 \text { g.m. Clouds much the same in W and }\end{array}$ \\
\hline 2 & $29 \cdot 333$ & - & $24 \cdot 0$ & $24 \cdot 5$ & $23 \cdot 0$ & $29 \cdot 0$ & Lt. NW airs & 4 & $0-5$ & Is: & & - & - & - & $\begin{array}{l}\text { 10; Cul has formed in horizontal line } \\
\text { SE; Black Island. Mirage. }\end{array}$ \\
\hline 4 & $29 \cdot 3 \pm 8$ & - & $21 \cdot 0$ & $27 \cdot 2$ & $25: 5$ & $34 \cdot 6$ & Calm & 2 & 0 & $w$ & & - & - & - & Noon. Ozone, 8 . Min. Mge. \\
\hline " & 24313 & - & $24 \cdot 4$ & $2 \pi^{\circ} 0$ & $25 \cdot 0$ & $32 \cdot 5$ & 1.1. NW airs & 1 & 0 & lw & & - & - & - & 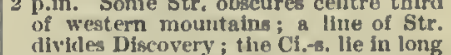 \\
\hline s & $24 \cdot 345$ & - & - & $25 \cdot 4$ & $24 \cdot 4$ & $31 \cdot 0$ & 1t. N1E airs & 1 & 0 & $w$ & & - & - & - & $\begin{array}{l}\text { thin lines from } \mathrm{b} \text { to } \mathrm{W} \text {; no inoventent } \\
\text { dlscernible. Oze, } 3 \text {. }\end{array}$ \\
\hline 10 & $29 \cdot 287$ & - & $23 \cdot 8$ & $26 \cdot 8$ & $25 \cdot 0$ & $30 \cdot 8$ & Calm & 1 & 0 & $\cdot$ tw & & - & - & - & $\begin{array}{l}4 \text { p.m. Str. as at } 2 \text { p.m. in } W . \\
6 \text { p.nu. Some Cu and Str. liv } w \\
\text { appears }\end{array}$ \\
\hline Midt. & - & - & - & - & - & - & - & - & - & - & 1 & - & - & - & 6 p.n. \\
\hline Yenn & $29 \cdot 339$ & - & - & $25 \cdot 1$ & $23 \cdot 7$ & - & - & $1-$ & - & - & & - & - & $1-$ & $\begin{array}{l}8 \text { pome Ci.-s. In } \mathrm{SE}, \mathrm{S} \text {, and SV } \\
\text { Brown Island summit olscured by Cu.; }\end{array}$ \\
\hline & th & & and at & $\left\{\begin{array}{l}8 \mathrm{k} . \\
8 \mathrm{l} .\end{array}\right.$ & 0. & Sr & radiation $\left\{\begin{array}{l}\mathrm{s} \\
\mathrm{s}\end{array}\right.$ & $\begin{array}{l}\text { blat } \\
\text { hirig }\end{array}$ & & & & gxammes. & Precipn. - in. & & 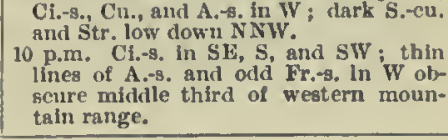 \\
\hline
\end{tabular}

\begin{tabular}{|c|c|c|c|c|c|c|c|c|c|c|c|c|c|}
\hline 2 & - & -1 & - & - & - & - & - & - & - & - & - & - & $1-$ \\
\hline 4 & - & - & - & - & - & - & - & - & - & - & - & - & - \\
\hline 6 & - & - & - & - & - & - & - & - & - & - & - & - & - \\
\hline 8 & $29 \cdot 238$ & - & $13 \cdot 0$ & $25 \cdot 0$ & $24 \cdot 0$ & $31 \cdot 0$ & Calm & 0 & 0 & w & - & - & - \\
\hline 10 & $29 \cdot 2233$ & - & $24 \cdot 2$ & $26^{\circ} 0$ & $24 \cdot 0$ & $33 \cdot 2$ & L.t. NW airs & 1 & 0 & bv & - & - & - \\
\hline Noon & $29 \cdot 225$ & - & $25 \cdot 2$ & $26 \cdot 2$ & $25 \cdot 0$ & $31 \cdot 0$ & Lt. $\mathrm{NW}$ airs & 4 & $0-2$ & by & - & - & - \\
\hline 2 & $29 \cdot 217$ & - & $26^{\circ} 0$ & $27 \cdot 5$ & $21 \cdot 2$ & $.23 \cdot 0$ & Lt. NW sirs & 2 & 0 & w & - & - & - \\
\hline 4 & $29 \cdot 214$ & - & $27 \cdot 0$ & $29 \cdot 0$ & $25 \cdot 5$ & $3 i \cdot 0$ & Calm & 1 & 0 & lwe & cí. & - & - \\
\hline 6 & $29 \cdot 217$ & - & $28 \cdot 5$ & $28 \cdot 6$ & $27 \cdot 0$ & $.25 \cdot 0$ & Calm & 0 & 0 & bre & Cí.-s. & - & - \\
\hline 8 & $29 \cdot 218$ & - & $24^{\circ} 0$ & $2 k \cdot 0$ & $25 \cdot 0$ & $\left.28^{\circ} \cdot\right)$ & Calm & 0 & 0 & lwe & - & A.-ert. 3, SE & - \\
\hline 10 & $29 \cdot 225$ & - & $22 \cdot 8$ & $28 \cdot 0$ & $2 t: 0$ & $2 \pi \cdot 5$ & Calm & 1 & 0 & bre & Ci.eu. 2, SE & - & - \\
\hline Midt. & - & -1 & - & - & - & - & - & - & - & - & - & - & - \\
\hline Mlear. & $29 \cdot 223$ & -1 & - & $27 \cdot 0$ & $25 \cdot 1$ & - & - & - & - & - & - & - & - \\
\hline
\end{tabular}

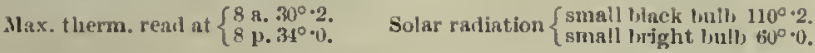

Evapn. - grammes, Preclpn, - in.
10 th.

11 th, 10 p.m. In the $\mathrm{NW}, \mathrm{W}, \mathrm{SW}$, and $\mathrm{S}$ are $\mathrm{A}$-s. fringed with Ci.-cu. ; Cu. cap is sitll upon

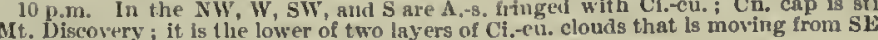

i.-s, to $\mathrm{S} ; \mathrm{Cu}$, to $\mathrm{W}$ olscuring centre third of western mountains ; refraction all round to $W$ and $S$. Mrirage.

C.m. Lines of Cl.-s, in S and SE; tive Cu. to Whas changed into smoky -edget ern mountain range ; mirage in $\mathrm{S}$ and Sw.

Don. Clouds much the same; Cu, on $\mathrm{N}$ $\mathrm{SW}$ low down and rising towards $\mathrm{S}$; no mirage.

p.m. Some detacied $\mathrm{Ci}^{2}-\mathrm{s}$, low down to SW, S, and SW: Str. about centre of p.m. Some Str. shout Discovery and western range. Ozone, 3 p.in. Str. obscures higher peaks of western range. Ozone, $2-3$

p.m. The Cu. clouds are very light and Cirrns-like ; some A.-s, in S and SW and W, olscuring the higher peaks of west-

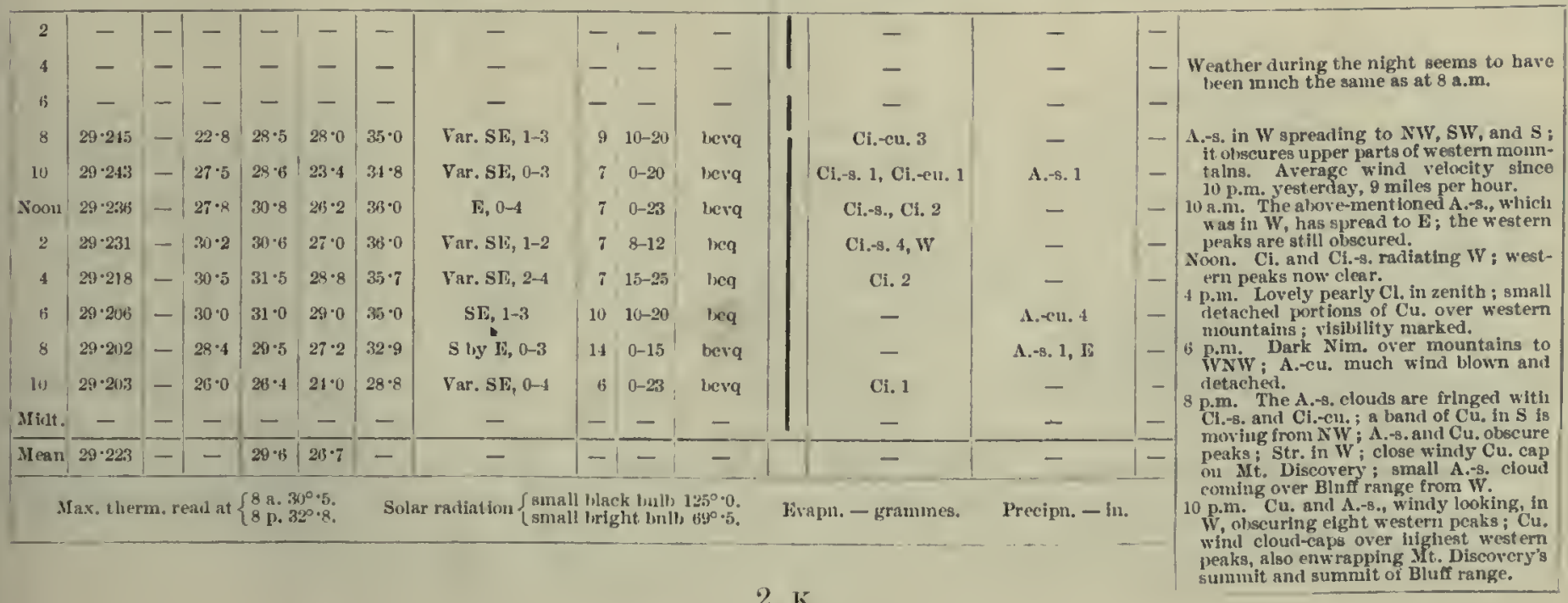




\section{3-December.]}

13th.
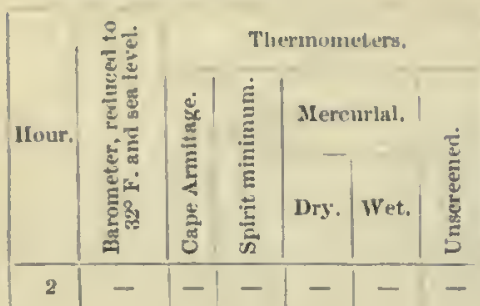

$4-1-1)-$

\begin{tabular}{ccc|c|c|c|c}
0 & - & - & - & - & - & - \\
8 & $\approx \cdot 193$ & - & $19 \cdot 5$ & $25 \cdot 0$ & 24.8 & $28 \cdot 2$
\end{tabular}

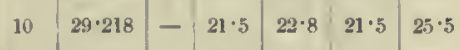

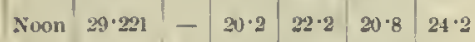

\begin{tabular}{|l|l|l|l|l|l|}
2 & 29.203 & -20.0 & $21 \cdot 3$ & 20.5 & 24.5 \\
\hline
\end{tabular}

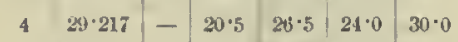

\begin{tabular}{ll|l|l|l|l|}
$6 \quad 29 \cdot 230$ & - & - & $23 \cdot 5$ & $22 \cdot 8$ & $27 \cdot 5$ \\
\hline
\end{tabular}

\begin{tabular}{ll|l|l|l|l|l|}
8 & $29 \cdot 229$ & - & $21 \cdot 6$ & $23 \cdot 8$ & $22 \cdot 0$ & $27 \cdot 0$
\end{tabular}

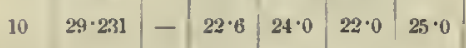

Mean $\frac{-}{29 \cdot 218}-\frac{-}{-} \frac{-}{23 \cdot 0} \frac{-}{22 \cdot 3}-$

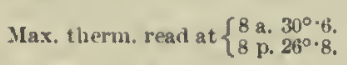

Meteorotogical, Journat, of the "I)Iscovery."

\begin{tabular}{|c|c|c|c|c|c|c|c|c|}
\hline \multicolumn{3}{|l|}{ wind. } & \multirow[b]{3}{*}{ 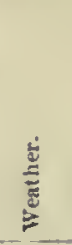 } & \multirow[b]{3}{*}{$\mid$} & \multirow{2}{*}{\multicolumn{2}{|c|}{$\begin{array}{l}\text { Cloonds, } \\
\text { Ament and movement. }\end{array}$}} & \multirow[b]{3}{*}{ 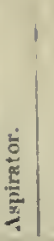 } & \multirow{3}{*}{ Mesharks. } \\
\hline \multirow{2}{*}{$\begin{array}{l}\text { Dircertlon and } \\
\text { forcere } \\
\text { (Beanfort seale). }\end{array}$} & \multirow{2}{*}{ | } & \multirow{2}{*}{ 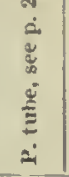 } & & & & & & \\
\hline & & & & & Upper. & Lower. & & \\
\hline - & -1 & - & - & & - & - & - & \\
\hline - & - & - & - & & - & - & - & \\
\hline - & - & - & - & & - & - & - & \\
\hline 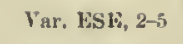 & 11 & $15-27$ & hevq & & Ci.ren. 1 & - & - & $\mathrm{Ci}_{\text {i-s. in }} \mathrm{SW}$; Ci.-en, in $\mathrm{NW}$; all distant \\
\hline Var. $1 \mathrm{ESE}, 4-6$ & 15 & $2 n-34$ & bevq & & ci. & - & - & $\begin{array}{l}\text { lind clear. Averagg wind velocity gince } \\
10 \text { p.m. vesterday, } 11 \text { niles per tour. }\end{array}$ \\
\hline Var. $\mathrm{ENE}_{3}, 3-6$ & 12 & $18-34$ & hevig & & - & A.-fr.-s. 1 & - & 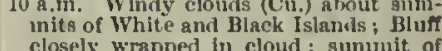 \\
\hline Far. ENk, 4-j; & 23 & $\mid 28-38$ & $\operatorname{lng}$ & & - & - & - & 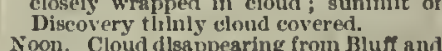 \\
\hline Var. E, 3-1\} & 18 & $20-35$ & $\log$ & & - & - & - & 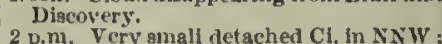 \\
\hline Var. ENk, 3-5 & 13 & $20-30$ & $\operatorname{lng}$ & & - & - & - & $\begin{array}{l}\text { Bluff blanketed. } \\
\text { 4 p.m. Small detached Cl-s, in SW : CI. }\end{array}$ \\
\hline Far. E, 2-6 & 1.3 & $10-30$ & bigr & & 1 - & - & - & 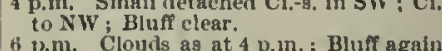 \\
\hline Var. $\mathbf{E}, 1-\overline{5}$ & 11 & $0-28$ & bqv & & - & - & - & 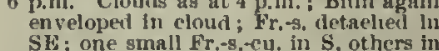 \\
\hline - & - & - & - & 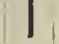 & - & - & - & 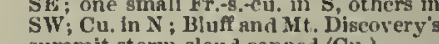 \\
\hline- & $1-$ & - & - & & - & - & - & 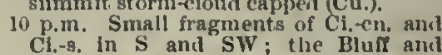 \\
\hline diation \{ & & & & & -gram & Precipn. & & \\
\hline
\end{tabular}

14th.

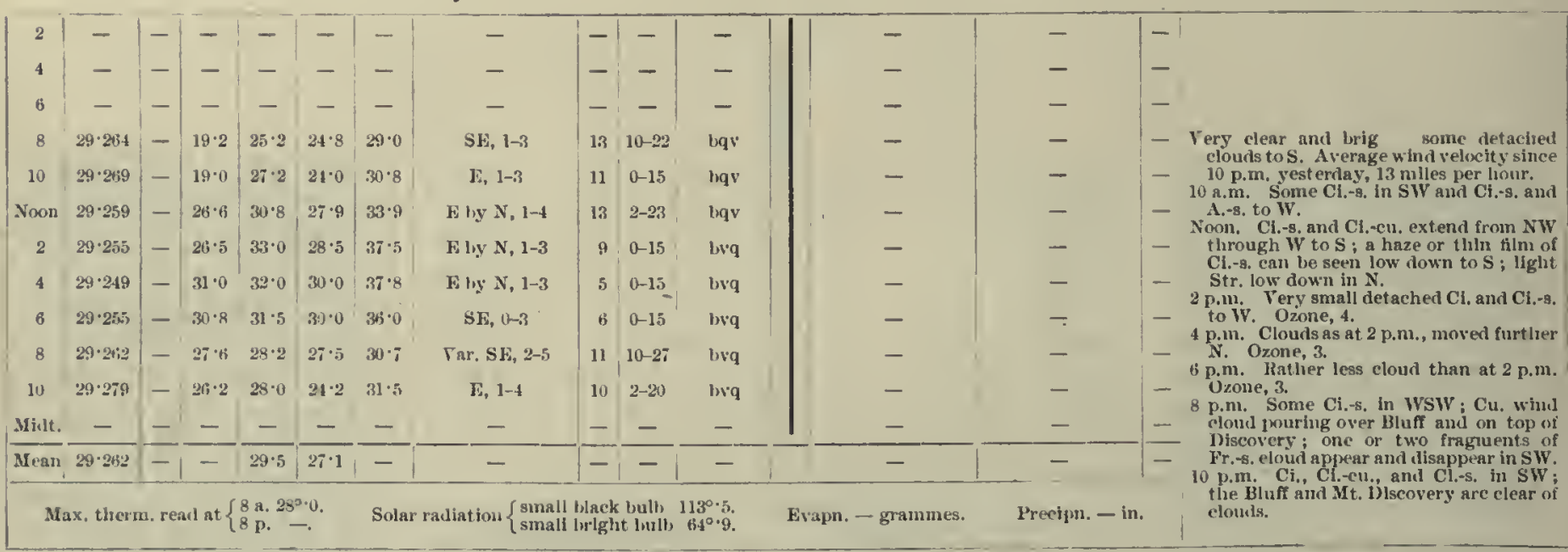

15th.

\begin{tabular}{|c|c|c|c|c|c|c|c|c|c|c|c|c|c|}
\hline 21 & - & -1 & - & - & - & - & - & -1 & - & - & - & - & - \\
\hline 4 & - & - & - & - & - & - & - & - & - & - & - & - & - \\
\hline 6 & - & - & - & - & - & - & - & - & - & - & - & - & - \\
\hline 8 & $29 \cdot 2 \pi 0$ & - & $23 \cdot 4$ & $30 \cdot 2$ & $29 \cdot 0$ & $33 \cdot 2$ & $E, 0-1$ & 7 & $0-10$ & $w$ & - & - & - \\
\hline 10 & $29 \cdot 262$ & - & $29 \cdot 1$ & $30 \cdot 4$ & 2000 & $35 \cdot 2$ & Var. $18,1-3$ & 6 & $2-15$ & wNq & - & - & - \\
\hline Noon & $29 \cdot 241$ & - & $28 \cdot 0$ & $30 \cdot 0$ & $2 \pi \cdot 0$ & $35 \cdot 0$ & E, $1-2$ & 8 & $2-10$ & bwq & - & - & -1 \\
\hline 2 & $29 \cdot 235$ & - & $29 \cdot 2$ & $3 v \cdot 0$ & $26 \cdot 5$ & $35 \cdot 5$ & $N, 0-1$ & 3 & 0 & $\ln w$ & - & - & - \\
\hline 4 & $23 \cdot 231$ & - & $24 \cdot 8$ & 25.5 & $24 \cdot 0$ & $29 \cdot 5$ & $\mathrm{NE}, 1-2$ & 7 & $0-15$ & bre & - & $\mathrm{Fr}_{\mathrm{r}-\mathrm{-a},-\mathrm{s} . \mathrm{s}}$ & - \\
\hline 6 & $29 \cdot 223$ & - & $24 \cdot 0$ & 26.5 & 23.5 & $31 \cdot 0$ & $\mathrm{NE}, 1-2$ & 11 & $0-10$ & wee & - & A.-s, 1 & - \\
\hline 8 & $29 \cdot 216$ & - & $35 \cdot 2$ & 290 & 26.5 & $34 \cdot 8$ & $N, 0-1$ & 4 & 0 & bre & - & A.cu- $1, N N E$ & - \\
\hline 10 & $29 \cdot 210$ & - & - & $2 \pi \cdot 0$ & $24 \cdot 2$ & $37 \cdot 0$ & Ca!n & 1 & 0 & $\mathrm{~mm}$ & - & - & - \\
\hline Mlatt. & $29 \cdot 2017$ & - & - & $23 \cdot 13$ & $22 \cdot 0$ & $27 \cdot 0$ & Calu & a & 0 & $\lim$ & - & - & - \\
\hline Mean & 29.233 & -1 & - & 28.0 & $25 \cdot 4$ & - & - & - & - & - & - & - & - \\
\hline \multicolumn{6}{|c|}{ 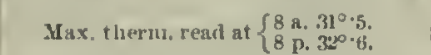 } & \multicolumn{5}{|c|}{ Solar radiatlon $\left\{\begin{array}{l}\text { small black bufl } 111^{\circ} \cdot 1 . \\
\text { small bright bulth } 69^{\circ} \cdot 2 .\end{array}\right.$} & nim & \multicolumn{2}{|l|}{ Precipn. - in. } \\
\hline
\end{tabular}

Some refraction all round. A verage w Ind velocity since 10 p.m. yesterilay, $i$ miles per hour. a.m. Some windy-inoklng Cu. is and western ranges. Fcon. Fr.cu, alout aummit of Diseovery. Cu. lying In anongst soutliern and 2 p.m. Layer of Str. to W. Ozone, 9. p.m. Layer of Str. In $W$ has increased to sw. Ozone 4.

p.m. Layer of Str, in W and SW; Cu. to W obseuring centre thirrl of western range, Ozone, 4.

o.m. Cu., S.-cur, and Str. in $\mathrm{N}$, stretching round to $W$ and $S W$, obscuring of hills.

1. m. Clouds much the same in N to Miclt. Misty

fine roll $C u$ all round on distant land: between western mountains and western foot hilis; thin layer of S. ev. apparently banging over Strait. 
Metgorological Jourxal of the "Discoveliy."

[1903-December.

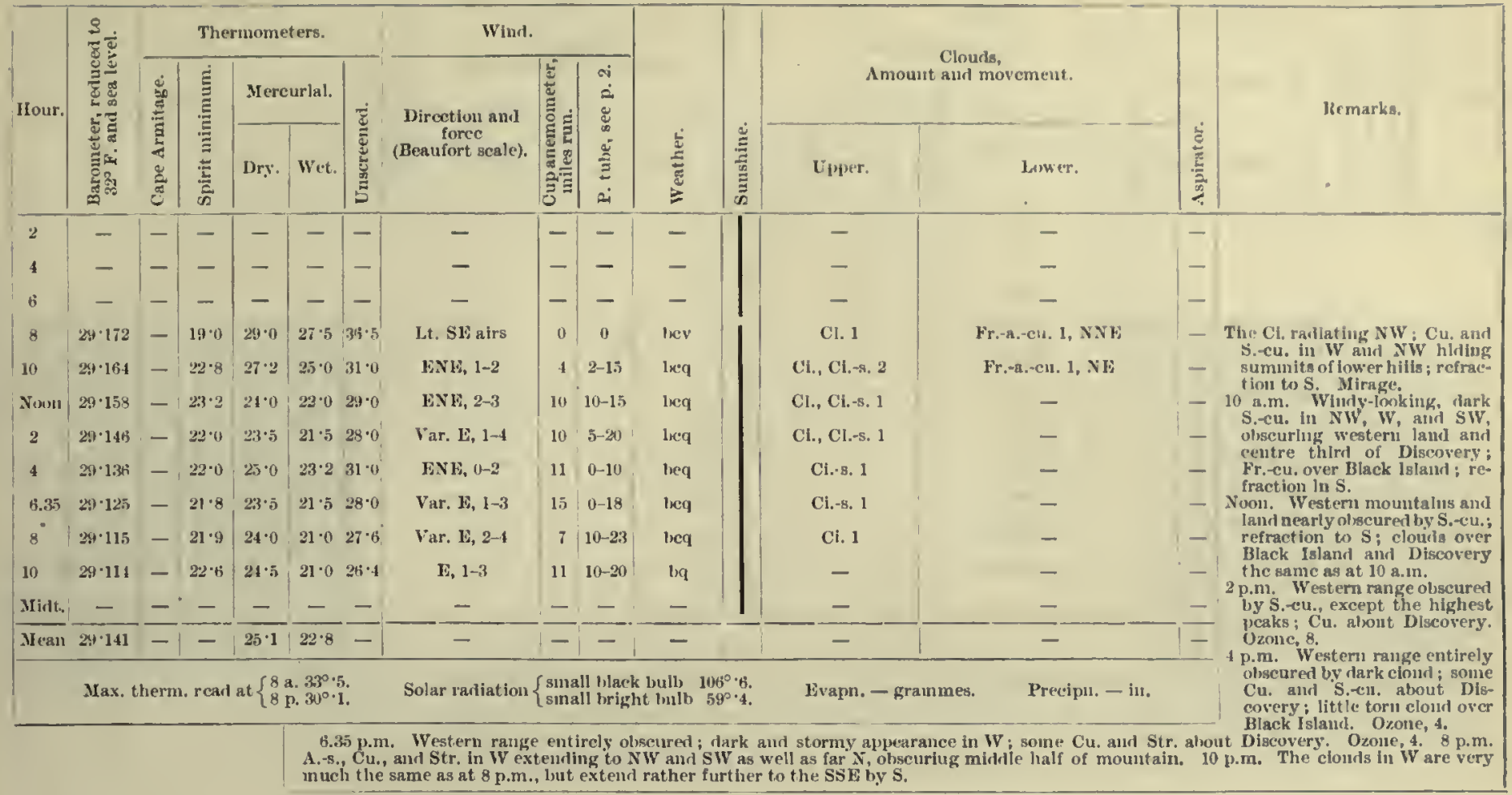

16 th.

\begin{tabular}{|c|c|c|c|c|c|c|c|c|c|c|c|c|c|c|}
\hline 2 & - & - & - & - & - & - & - & -1 & - & - & - & - & - & \\
\hline 4 & - & - & - & - & - & - & - & -1 & - & - & - & - & - & \\
\hline i & - & -1 & - & - & - & - & - & - & - & - & - & - & - & \\
\hline 8 & $24 \cdot 073$ & - & $14 \cdot 5$ & $17 \cdot 6$ & $17 \cdot 2$ & $20 \cdot 5$ & Ely $\mathrm{N}, 3-6$ & 12 & $20-38$ & liveq & Ci., $\mathrm{Ci}_{-}-\mathbf{s} .3$ & - & \multirow{9}{*}{\multicolumn{2}{|c|}{ 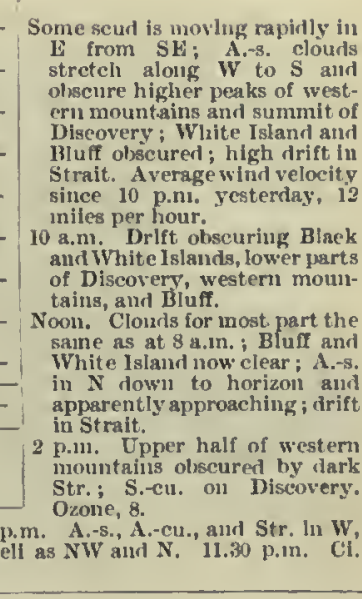 }} \\
\hline 10 & $29 \cdot 06 \dot{b}$ & - & $14 \cdot 8$ & $17 \cdot 4$ & $17 \cdot 2$ & $17 \cdot 6$ & ENE, 3-4 & 16 & $15-24$ & lweq & Cl., Ci.-s. 4 & - & - & \\
\hline Suon & $29 \cdot 061$ & -1 & 15.5 & $16 \cdot 6$ & $16 \cdot 0$ & $20 \cdot 0$ & E, 3-5 & 16 & $15-30$ & lineq & Ci., Ci.-s. 3 & - & & \\
\hline 2 & $29 \cdot 054$ & - & $15 \cdot 4$ & 19.5 & $18 \cdot 5$ & $24 \cdot 5$ & E, 1-3 & 11 & $v-15$ & lieq & Cl. , Cl.-s. 4 & - & & \\
\hline 8 & $2 x \cdot 016$ & - & $21 \cdot 63$ & $23 \cdot 0$ & $21 \cdot 0$ & $25 \cdot 4$ & SSF, $0-2$ & 9 & $0-1 \overline{0}$ & be & Ci.-cu. 2, SE & A.-cu., A.-s., Fr.-a.-cu. 3, SE & & \\
\hline 10 & $28 \cdot 997$ & - & $19 \cdot 8$ & 217 & $19 \cdot 2$ & $26 \cdot 0$ & NE hy E, Q-1 & 9 & $0-8$ & 16 & Ci. 5 & - & $1-$ & \\
\hline Mist. & - & - & - & - & - & -1 & - & - & - & - & - & - & & \\
\hline$\overline{\text { Mean }}$ & $\overline{29 \cdot 046}$ & - & - & $\overline{19 \cdot 9}$ & $\overrightarrow{18 \cdot 8}$ & - & - & -1 & - & - & - & - & $\overline{-}$ & \\
\hline & & & & & $\begin{array}{l}25^{\circ} \cdot 2 \\
24^{\circ} \cdot 7\end{array}$ & & Solar radiation & $\left\{\begin{array}{l}\mathrm{smal} \\
\mathrm{smal}\end{array}\right.$ & $\begin{array}{l}\text { Al blac } \\
\text { Il brig }\end{array}$ & $\begin{array}{ll}a l b \\
\text { anlib }\end{array}$ & \multicolumn{3}{|c|}{ Wvapu. - gramunes. } & \\
\hline
\end{tabular}

17 th.

\begin{tabular}{|c|c|c|c|c|c|c|c|c|c|c|c|c|c|c|}
\hline 2 & - & - & - & - & - & - & - & - & - & - & & - & - & \multirow{14}{*}{ 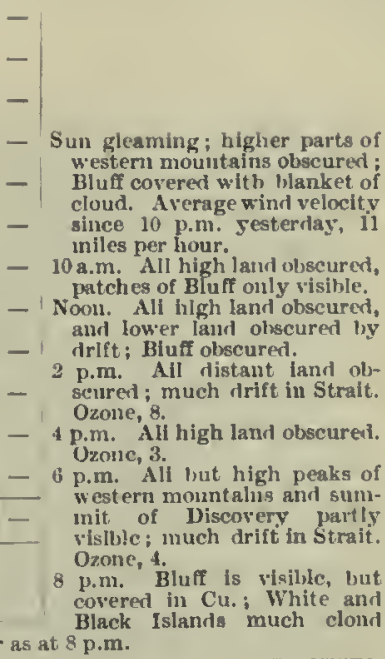 } \\
\hline 4 & - & - & - & - & - & - & - & - & - & - & & - & - & \\
\hline 6 & - & - & - & - & - & - & - & - & - & - & & - & - & \\
\hline 8 & $28 \cdot 910$ & - & $17 \cdot 8$ & $21 \cdot 2$ & $201 \cdot 4$ & $22 \cdot 5$ & E by $\mathrm{S}, 2-5$ & 11 & $15-25$ & $\mathrm{eq} \oplus$ & & - & A.-8., A.cu. 1 & \\
\hline 10 & $28 \cdot 911$ & - & 19.5 & $21 \cdot 5$ & $19 \cdot 6$ & $23 \cdot 2$ & SE by E, 2-4 & 15 & $17-23$ & eq & & Ci. 2 & A.-eu. 6 & \\
\hline Noon & $28 \cdot 914$ & - & $16 \cdot 0$ & $16 \cdot 5$ & $16 \cdot 0$ & $19 \cdot 0$ & Var. SE, 2-7 & 15 & $15-43$ & $\mathrm{eq}$ & & Ci. 1 & A.-cu, 4, S; S 2, SSL & \\
\hline 2 & $28 \cdot 424$ & - & $14 \cdot 8$ & $16 \cdot 0$ & $15 \cdot 2$ & $17 \cdot 6$ & Var. SE, 2-7 & 16 & $0-35$ & $\mathrm{eq} \oplus$ & 1 & Cl., Ci.-s, 3 & A.cu. $3, \Lambda$. s. $4, \mathrm{~S}$ and $\mathrm{SE}$ & \\
\hline 4 & $28 \cdot 927$ & - & $14 \cdot 6$ & $17 \cdot 5$ & $16 \cdot 5$ & $20 \cdot 0$ & SE by E, 2-7 & 15 & $10-40$ & $\mathrm{cq}$ & & Ci., $\mathrm{Cl}_{.}-\mathrm{B} .4$ & A.-cu. 5, S ; A.-s., SE (rap)lily) & \\
\hline B & $28 \cdot 926$ & - & $16 \cdot 5$ & $19 \cdot 0$ & $17 \cdot 5$ & $23 \cdot 0$ & Var, $\mathbf{E}, 1-4$ & 18 & $10-25$ & eq & & \multicolumn{2}{|c|}{ 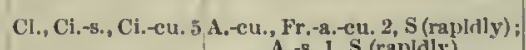 } & \\
\hline 8 & $28 \cdot 930$ & - & $17 \cdot 8$ & $18 \cdot 5$ & $17 \cdot 2$ & $21) \cdot 1$ & ESL, 2-6 & 10 & $10-35$ & Ix:q & & Ci. 2 & $A_{,}-5$, , A.-cll, 2, SE (rapidly); & \\
\hline 10 & $28 \cdot 94$ & - & $16 \cdot 1$ & $17 \cdot 2$ & $15^{\circ} \cdot 3$ & $18 \cdot 0$ & F, $2-4$ & 14 & $15-30$ & leq & & Ci. $4, \mathrm{~W}$ & Light Cn. $2, \mathrm{~S}$ (rapisI & \\
\hline Mlint. & - & - & - & - & - & 二 & - & - & - & - & & - & - & \\
\hline Ifran & $28 \cdot 923$ & -1 & - & 18.4 & $17 \cdot 3$ & $1-1$ & - & - & - & - & & - & - & \\
\hline & & & & & & & very & & bit: & Ib & & \multicolumn{2}{|c|}{ Frapn. - grammes. } & \\
\hline
\end{tabular}

18 th. 
1903 -December.]

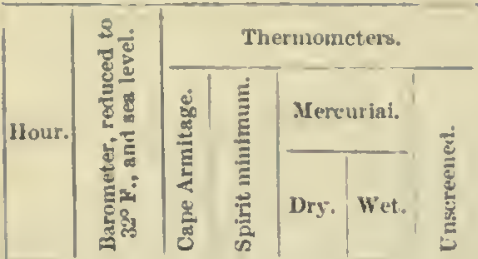

19th.

\begin{tabular}{|c|c|c|c|cc|c|}
\hline 3 & - & - & - & - & - & - \\
4 & - & - & - & - & - & - \\
6 & - & - & - & - & - & - \\
8 & $28 \cdot 415$ & - & $16 \cdot 2$ & $25 \cdot 0$ & $23 \cdot 6$ & $34 \cdot 4$ \\
10 & $23 \cdot 938$ & - & $20 \cdot 5$ & $21 \cdot 4$ & $21 \cdot 5$ & $34 \cdot 0$ \\
N(xon & $23 \cdot 921$ & - & $21 \cdot 0$ & $23 \cdot 8$ & $21 \cdot 1$ & $30 \cdot 0$ \\
2 & $28 \cdot 90.4$ & - & $25 \cdot 8$ & $26 \cdot 5$ & $25 \cdot 0$ & $29 \cdot 0$ \\
4 & $25 \cdot 849$ & - & $25 \cdot 5$ & $26 \cdot 5$ & $23 \cdot 0$ & $28 \cdot 5$ \\
6 & $28 \cdot 893$ & - & $25 \cdot 8$ & $20 \cdot 5$ & $26 \cdot 2$ & $37 \cdot 0$ \\
8 & $28 \cdot 893$ & - & $25 \cdot 6$ & $26 \cdot 0$ & $23 \cdot 3$ & $26 \cdot 9$ \\
10 & $28 \cdot 895$ & - & $23 \cdot 5$ & $24 \cdot 3$ & $22 \cdot 0$ & $39 \cdot 0$
\end{tabular}

Metrorological Jovinal, of the "Discoveik."

\begin{tabular}{c|c|c|c|c|c|c|c|c|}
\hline $\begin{array}{c}\text { Direction and } \\
\text { force }\end{array}$ \\
(BBcalfort seale).
\end{tabular}

20 th

\begin{tabular}{|c|c|c|c|c|c|c|c|c|c|c|c|c|c|c|}
\hline 2 & - & - & - & - & - & - & - & - & - & - & - & - & - & \multirow{12}{*}{ 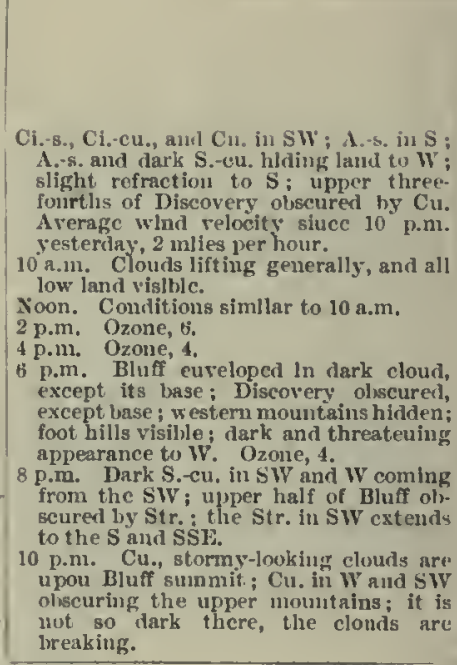 } \\
\hline 4 & - & - & - & - & - & - & - & - & - & - & - & - & - & \\
\hline 6 & - & - & - & - & - & - & - & - & - & - & - & - & - & \\
\hline 8 & $2 y \cdot 014$ & - & $22 \cdot 1$ & $2 i \cdot 0$ & $25 \cdot 0$ & $32 \cdot 0$ & Lt. E airs & 2 & 0 & be & Ci.-cu. 1, s & Fr.cu., $\mathrm{E}$ & - & \\
\hline 10 & $29 \cdot 034$ & - & $25 \cdot 4$ & $27 \cdot 0$ & $24 \cdot 1$ & $35 \cdot 0$ & Lt. SE airs & 2 & $0-8$ & bc & $\begin{array}{l}\text { Ci. 3, Nw (very } \\
\text { slow) }\end{array}$ & Frr.a.t.c1, 1, E & - & \\
\hline Xons & $29 \cdot 065$ & - & $24 \cdot 1$ & $25 \cdot 0$ & $22 \cdot 9$ & $2.9 \cdot 5$ & Var. E, Q-2 & 6 & $0-16$ & beq & Ci. 1 & - & - & \\
\hline 2 & $29 \cdot 125$ & - & $23 \cdot 5$ & $24 \cdot 5$ & $22 \cdot 8$ & $30 \cdot 0$ & Var. E, & 6 & $0-10$ & beq & Ci., Ci.-cu. 2 & - & - & \\
\hline 8 & $29 \cdot 137$ & - & $22 \cdot 5$ & $23 \cdot 8$ & $22 \cdot 0$ & $29 \cdot 1$ & $E$ by $N, 0-1$ & 8 & $0-10$ & be & Ci.-cn. 1 & - & $1-$ & \\
\hline 10 & $20 \cdot 142$ & - & $20 \cdot 6$ & $21 \cdot 1$ & $20 \cdot 0$ & $29 \cdot 1$ & ENE, 1-2" & 8 & $0-10$ & be & - & 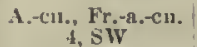 & !- & \\
\hline Midt. & - & - & - & - & - & - & - & - & - & - & - & - & - & \\
\hline Nean & $29 \cdot 096$ & - & - & $21 \cdot 6$ & $22 \cdot 8$ & -1 & - & $1-$ & -1 & - & - & - & $1-$ & \\
\hline \multicolumn{6}{|c|}{ Max. therm. read at $\left\{\begin{array}{l}8 \text { a. } 32^{\circ} \cdot 4 . \\
8 \text { p. } 28^{\circ} \cdot 0 .\end{array}\right.$} & \multicolumn{5}{|c|}{ Solar radiatiou $\left\{\begin{array}{l}\text { small black bulb } 109^{\circ} \cdot 2 . \\
\text { small bright bulb } 61^{\circ} \cdot 5 .\end{array}\right.$} & livajm. - grammes. & \multicolumn{2}{|l|}{ Precipn. - in. } & \\
\hline
\end{tabular}

21st.

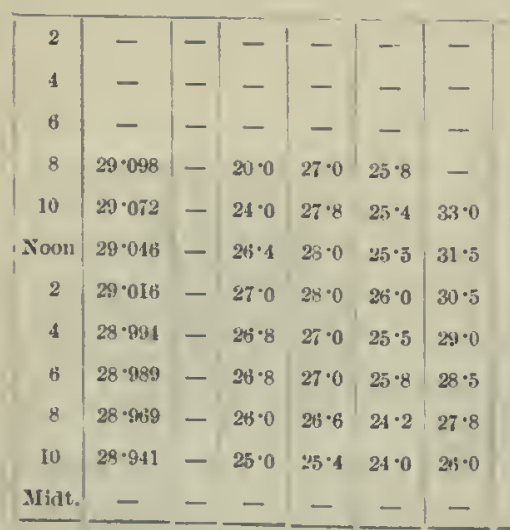

\begin{tabular}{|c|c|c|c|}
\hline- & - & - & - \\
- & - & - & - \\
- & - & - & - \\
Lt. N airs, 0-1 & 5 & 0 & bv \\
Lt. N lị W airs & 1 & $0-10$ & bv \\
N by W, 0-1 & 4 & $5-12$ & bvcq \\
N lyy W, 0-1 & 7 & $5-12$ & cq \\
Niv, 0-1 & 3 & $0-5$ & $o c$ \\
Calm & 0 & 0 & $o c$ \\
Lt. NNW airs & 2 & 0 & $c$ \\
Lt. NW airs & 5 & $2-9$ & $n$ \\
- & - & - & - \\
\hline
\end{tabular}

Max. therm. read at $\left\{\begin{array}{l}8 \mathrm{a}, 27^{2} \cdot 4 . \\ 8 \mathrm{p}, 20^{\circ} \cdot 1 .\end{array} \quad\right.$ Solar rad iat lon $\left\{\begin{array}{l}\text { small black bulb } 122^{\circ} \cdot 8 . \\ \text { sinall hright bull, } 66^{\circ} \cdot 2 .\end{array}\right.$

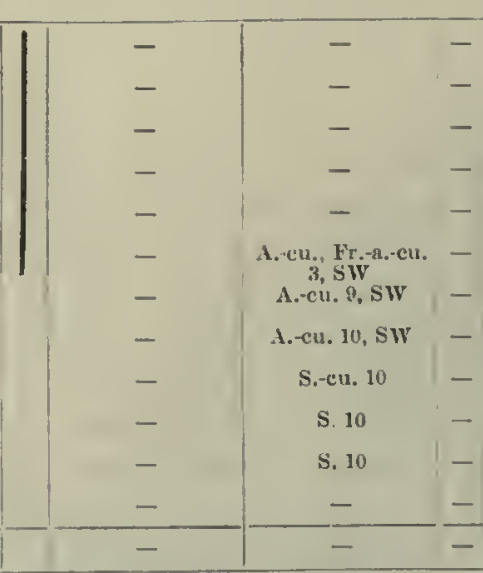

Evapu. - grammes. 1'vecipn. - in. 


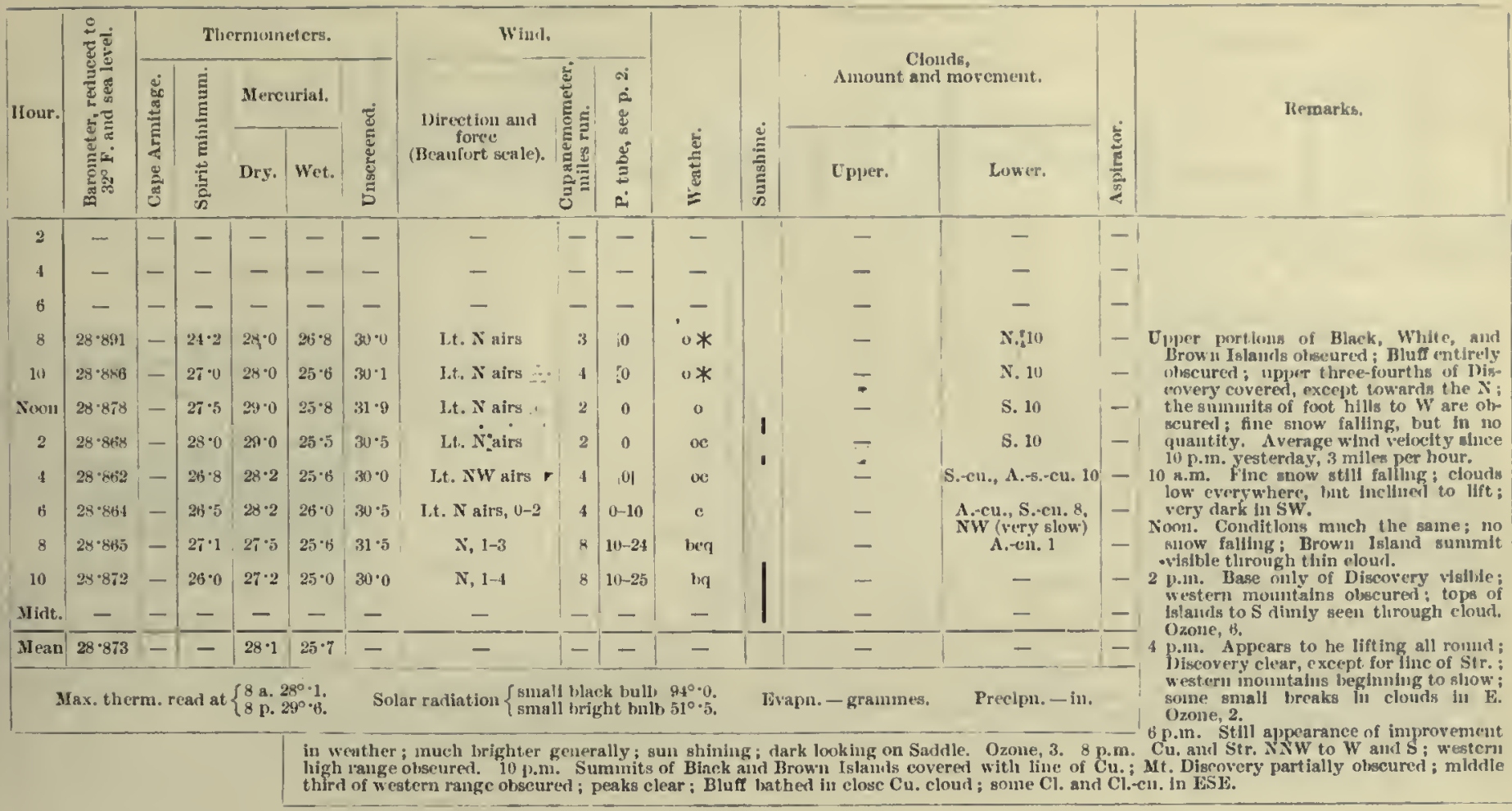

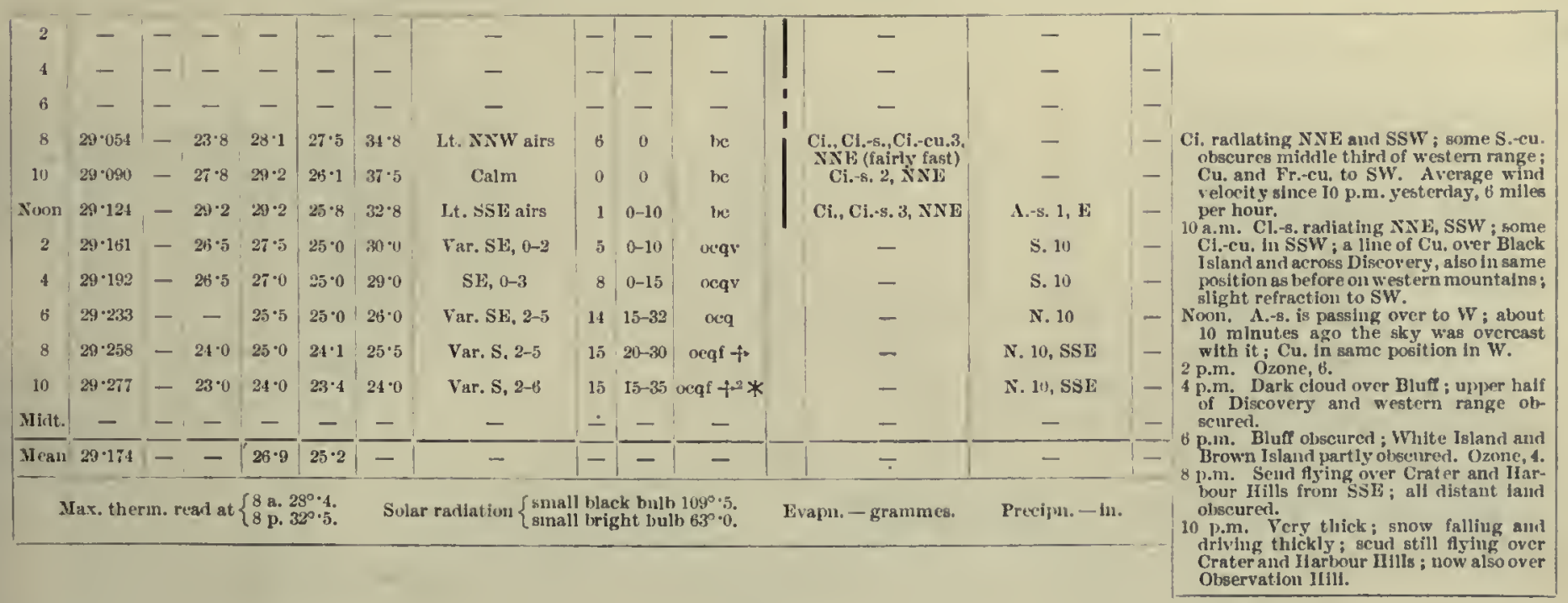

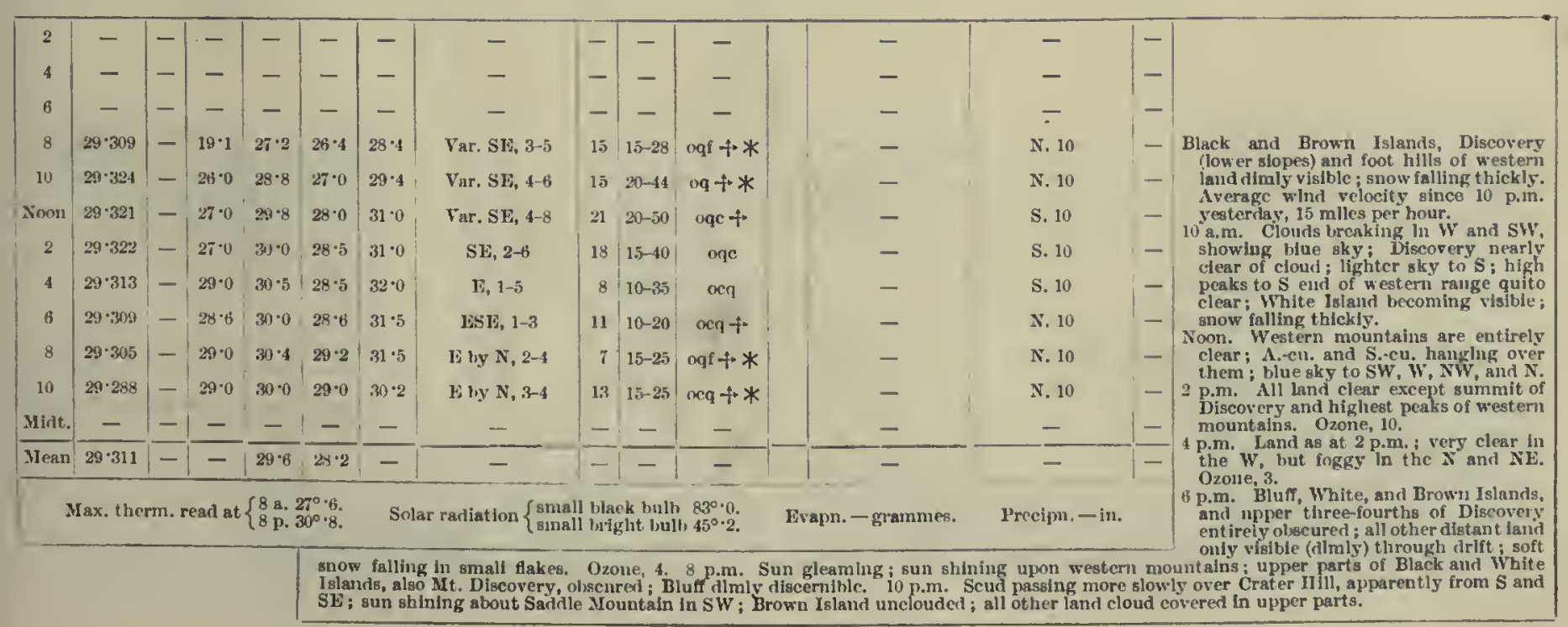


1903 -December.]

\begin{tabular}{|c|c|c|c|c|c|c|c|c|c|}
\hline \multirow{3}{*}{ liour } & \multirow{3}{*}{ 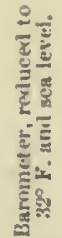 } & \multicolumn{5}{|c|}{ Thermoneters. } & \multicolumn{3}{|l|}{ Wind. } \\
\hline & & 密 & 莽 & 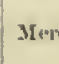 & uriai. & & & & i \\
\hline & & 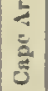 & 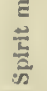 & Dry. & vict. & 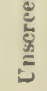 & (Beanfort scale). & & 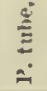 \\
\hline
\end{tabular}

25 th.

\begin{tabular}{|c|c|c|cc|c|c|}
\hline 2 & - & - & - & - & - & - \\
4 & - & - & - & - & - & - \\
6 & - & - & - & - & - & - \\
8 & $29 \cdot 259$ & - & $27 \cdot 0$ & $32 \cdot 0$ & $31 \cdot 0$ & $2.9 \cdot 8$ \\
10 & $29 \cdot 2 \cdot 11$ & - & $26 \cdot 8$ & $33 \cdot 0$ & $34 \cdot 8$ & $20 \cdot 9$ \\
Xoo11 & $29 \cdot 2.23$ & - & $32 \cdot 6$ & $35 \cdot 0$ & $33 \cdot 0$ & $31 \cdot 0$ \\
2 & $29 \cdot 229$ & - & $33 \cdot 5$ & $34 \cdot 0$ & $33 \cdot 2$ & $40 \cdot 0$ \\
4 & $29 \cdot 217$ & - & $35 \cdot 0$ & $34 \cdot 0$ & $34 \cdot 5$ & $40 \cdot 4$ \\
6 & $29 \cdot 207$ & - & $35 \cdot 5$ & $37 \cdot 2$ & $32 \cdot 5$ & $41 \cdot 0$ \\
8 & $29 \cdot 182$ & - & $35 \cdot 6$ & $37 \cdot 0$ & $33 \cdot 0$ & $41 \cdot 0$ \\
10 & $29 \cdot 184$ & - & $34 \cdot 2$ & $34 \cdot 8$ & $32 \cdot 0$ & $37 \cdot 8$
\end{tabular}

Meteorological Jourata of the "Dlscovery."

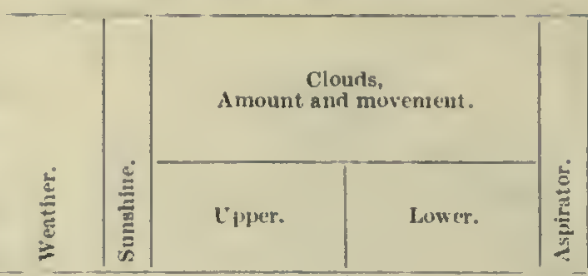

Remarks.

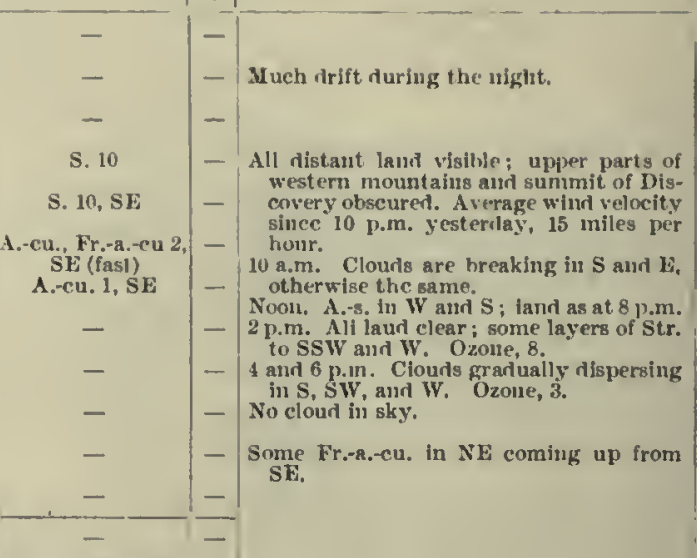

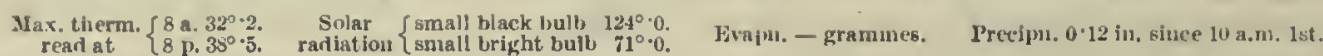

26th.

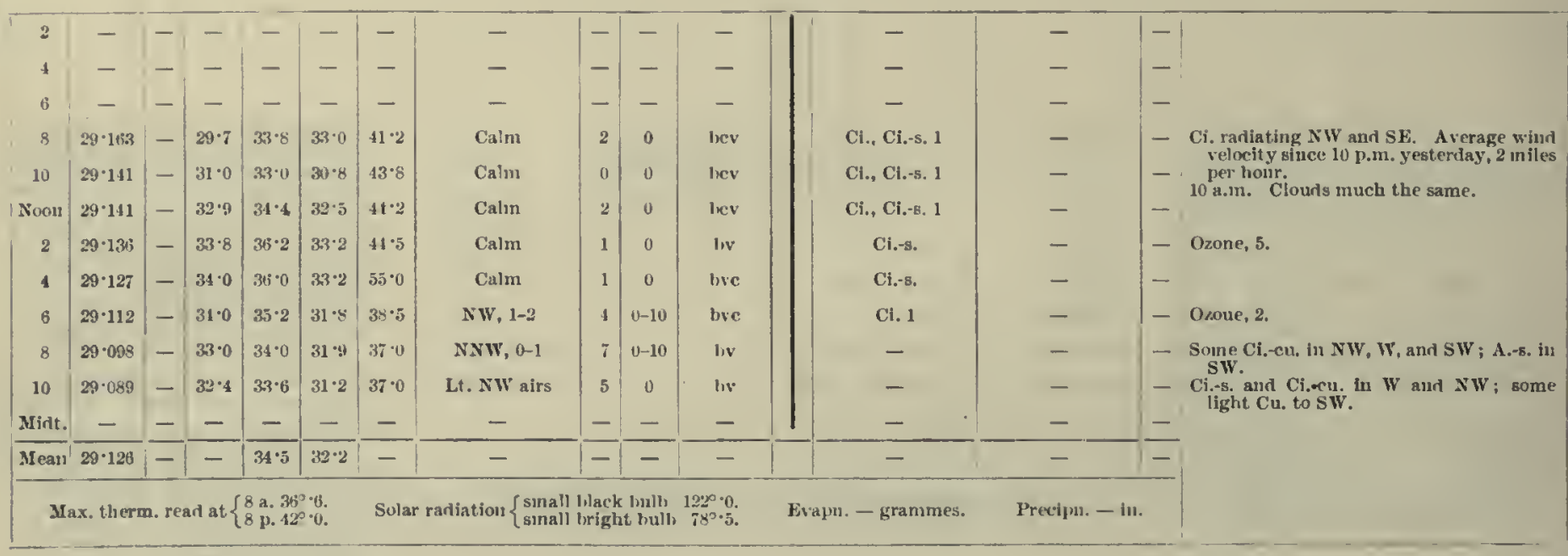

27 th.

\begin{tabular}{|c|c|c|c|c|c|c|c|c|c|c|c|c|c|c|}
\hline 2 & - & -1 & - & - & - & - & - & $1-$ & - & - & - & - & - & \multirow{13}{*}{ 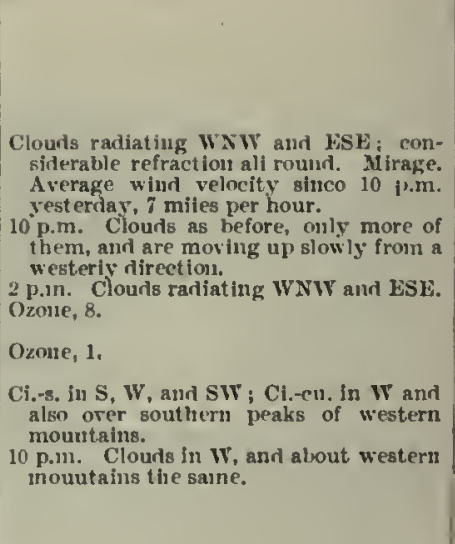 } \\
\hline 4 & - & - & - & - & - & - & - & - & - & - & - & - & - & \\
\hline 6 & - & - & - & - & - & - & - & - & - & - & - & - & - & \\
\hline 8 & $29 \cdot 0 i 4$ & - & $26 \cdot-2$ & $38 \cdot 0$ & $32 \cdot 6$ & $42 \cdot 8$ & ENE, 1-2 & $i$ & $2-15$ & bev & Ci.-8., Ci.-cu. 1, W & - & $1-$ & \\
\hline 10 & $29 \cdot 068$ & - & $28 \cdot 9$ & $33 \cdot 5$ & $31 \cdot 2$ & $37 \cdot 0$ & $N \operatorname{ly} w, 1-3$ & 5 & $12-20$ & leve & Ci.-s., Ci.-cu. 2, W & - & - & \\
\hline Noon & $29 \cdot 078$ & - & $32 \cdot 8$ & $31 \cdot 4$ & $32 \cdot 1$ & $33 \cdot 4$ & NNW, 1-2 & 9 & $2-10$ & Ixviq & $\mathrm{Ci}_{-}-\mathrm{s}, \mathrm{Ci}, \mathrm{cu} .3, \mathrm{w}$ & $A,-5,1, W$ & - & \\
\hline 2 & $29 \cdot 078$ & - & $32 \cdot 5$ & $33 \cdot 5$ & $32 \cdot 0$ & $36 \cdot 0$ & sw, $0-1$ & 7 & 0 & $\mathrm{cv}$ & Cl.-s., Ci.-cu. 4 & A.-eu. 3 & - & \\
\hline 4 & $29 \cdot 074$ & - & $32 \cdot 5$ & $25 \cdot 0$ & $32 \cdot 0$ & $40 \cdot 0$ & Calnn & 2 & 0 & $\mathrm{ev}$ & Ci.-8., Ci.-cu. 8 & - & 一 & \\
\hline 6 & $29 \cdot 079$ & - & $34 \cdot 5$ & $36^{\circ} \cdot 0$ & $34 \cdot 5$ & $46 \cdot 5$ & Calm & 0 & 0 & lev & Ci.-s., Cl.-C11. 3 & - & - & \\
\hline 8 & $29 \cdot 088$ & - & $34 \cdot 3$ & $35 \cdot 2$ & $31 \cdot 0$ & $39 \cdot 0$ & NNE, 1-2 & 3 & $3-15$ & bv & - & - & - & \\
\hline 10 & $29 \cdot 090$ & - & 35.8 & $35 \cdot 8$ & $31 \cdot 9$ & $46 \cdot 0$ & Calm & 2 & 0 & hev & Ci.-s., Ci.-cu. 1 & - & - & \\
\hline Mist. & - & -1 & - & - & - & - & - & $1-$ & - & - & - & - & - & \\
\hline Mean & $29 \cdot 079$ & -1 & - & $35 \cdot 2$ & $32 \cdot 2$ & - & - & $1-$ & - & - & - & - & - & \\
\hline & & & & & & & lation $\int^{8}$ & & & & . & . & & \\
\hline
\end{tabular}




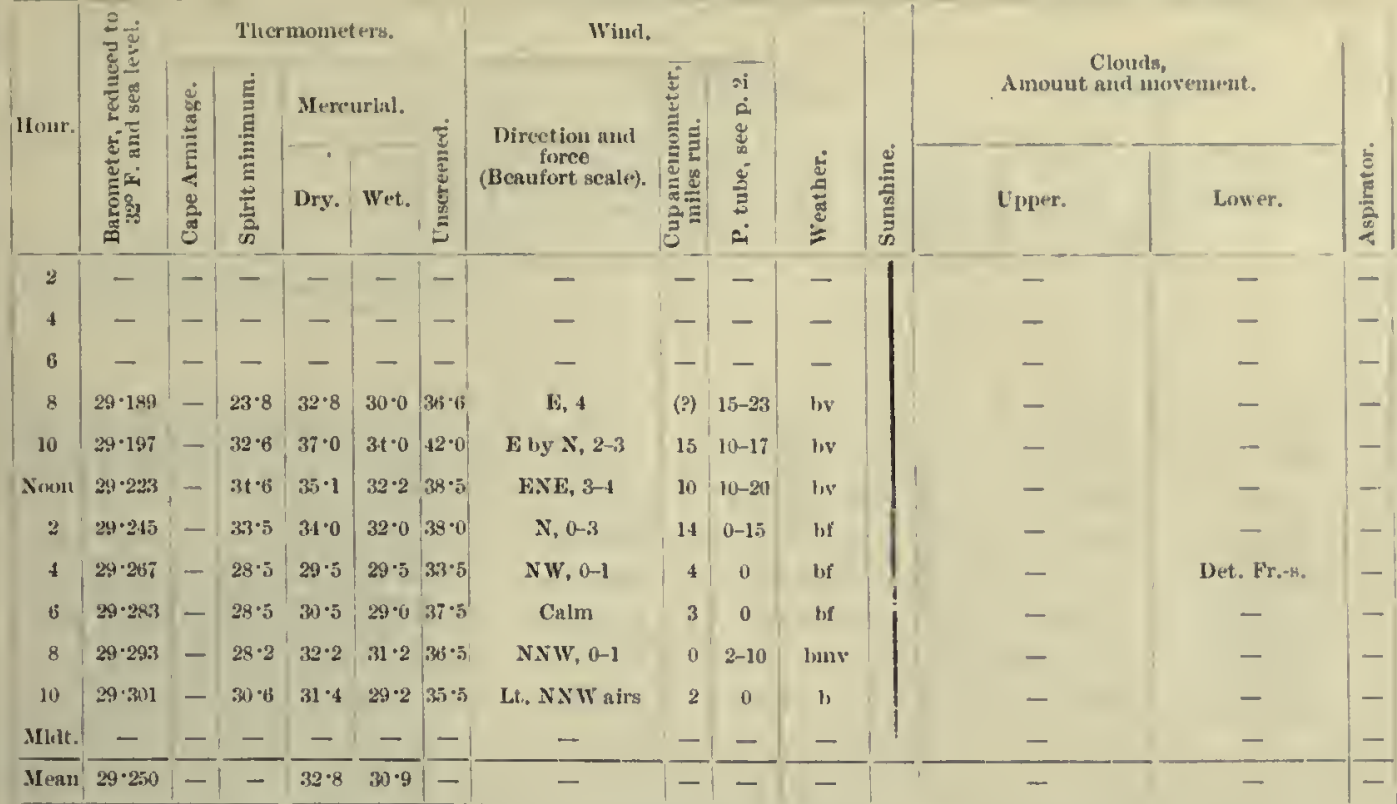

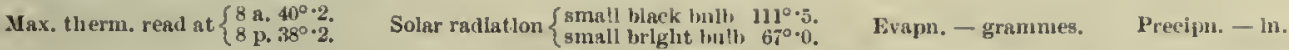

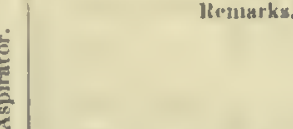

ltentarks.

Weatleer churlug nlght has apuarentl been fine and calm; whid rising abou 6 a.m.

- Slight refraction to $\mathrm{S}$. Mirage. Avernge wind velocity since 10 p.m. yest crray.

a.m. liather inore refraction in $\mathrm{S}$.

foon, Solne Fr.-cu. over Black Island;

p.m. All land clear ; 2.30, a bank of fog lint Point ; by 3.10 it had reached and olsscured Discovery; it then appeared to rlse and form Fr.-s. clouils ; by 3.30 Binf and White Island were obseured. Ozonif, 8.

4 p.m. All distant land, Capc Armitage. and IInt Polnt obscured in dense fog: other land close to dlumly visihic.

o p.m. Land vistble as at 4 p.m. ; air full of minute fog crystals, Ozone, 4.

.30 p.in. All land clear, except loasc of western hills $N$ of Discovery.

p.m. Low mist movlng rapidly in $W$ to $S ; A_{-}-8$. and Fr.cu. in SW; odd Fr.-eu. among hills of west ern range.

i0 p.M. Ci.s. In W and SWF; ordd Cu. well as alout Saddle Mountain.

29 th.

\begin{tabular}{|c|c|c|c|c|c|c|c|c|c|c|c|c|c|c|c|}
\hline 2 & - & - & - & - & - & - & - & - & - & - & & - & - & - & \\
\hline 4 & - & - & - & - & - & - & - & - & - & - & & - & - & - & Yiue, with apparently little wind during \\
\hline 6 & - & - & - & - & - & - & - & - & _ & - & & - & - & - & the unght. \\
\hline 8 & $29 \cdot 344$ & - & $27 \cdot 9$ & $34 \cdot 8$ & 32.0 & $40 \cdot 0$ & E by $x, 1-2$ & 3 & 2-12 & bv & & - & - & - & Cu. and Fr.-cu. In SW over Brown Island. \\
\hline 10 & $29 \cdot 351$ & - & $33 \cdot 8$ & $36 \cdot 8$ & $32 \cdot 0$ & $42 \cdot 0$ & ENF, 0-1 & 7 & $2-10$ & b) & & - & - & - & 10 p.m. yesterday, 3 miles per hour. \\
\hline Noon & $29 \cdot 347$ & - & $36 \cdot 0$ & $39 \cdot 0$ & $33 \cdot 8$ & $45 \%$ & E 1,y N, 0-1 & 2 & 0 & b & & - & - & - & SW has gone. Hirage. \\
\hline 2 & $29 \cdot 329$ & - & $35 \cdot 5$ & $38 \cdot 4$ & $33 \cdot 4$ & $45 \cdot 0$ & bi, 0-1 & 4 & 0 & b & & - & - & - & Nound; the Cu. in SW appears and dis- \\
\hline 8 & $29 \cdot 256$ & - & $27 \cdot 5$ & $29 \cdot 2$ & $28 \cdot 2$ & $37 \cdot 0$ & Calm & 0 & 0 & lice & & Ci., Cl._eu. 3, SW & - & - & diat ely, dry and wet bult showed the \\
\hline 10 & $29 \cdot 243$ & - & $2 \pi \cdot 0$ & $27 \cdot 4$ & $2 i \cdot 4$ & $27 \cdot 8$ & Calm & 1 & 0 & c & & - & A.-ch. 9, SW & - & jumperd to atore luo. Ozone, \\
\hline Midt. & - & 二 & - & - & - & - & - & - & - & - & 1 & - & - & - & 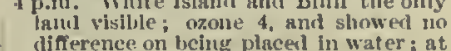 \\
\hline Ierai & $29 \cdot 310$ & $\overline{-}$ & - & $32 \cdot 8$ & $\overline{30 \cdot 3}$ & $1-1$ & - & $\overline{-}$ & - & - & & - & - & - & $\begin{array}{l}4.30 \text { no distant lanil was visible, and } \\
\text { sumuntes of tand close to only dimly }\end{array}$ \\
\hline \multicolumn{6}{|c|}{ Max, therm. read at $\left\{\begin{array}{l}8 \text { a. } 25^{\circ} \cdot 1 . \\
8 \text { p. } 40^{\circ} \cdot 2 .\end{array}\right.$} & \multicolumn{2}{|c|}{ Solar radiat ion \{} & $\begin{array}{l}\text { I bla } \\
\text { I bri }\end{array}$ & $\begin{array}{l}\text { cek bul } \\
\text { ght bu }\end{array}$ & $\begin{array}{l}115^{\circ} 0 \\
71^{\circ} 0\end{array}$ & & Evapn. - grannnes. & \multicolumn{2}{|l|}{ Precijn. -1 in. } & $\begin{array}{l}\text { visible. } \\
\text { p.m. No dlstant land vlsible : Observa- } \\
\text { tlon and Craterll ills and ot her land close }\end{array}$ \\
\hline
\end{tabular}

\begin{tabular}{|c|c|c|c|c|c|c|c|c|c|c|c|c|c|}
\hline 2 & - & - & - & - & - & - & - & - & - & - & & - & - \\
\hline 4 & - & - & - & - & - & - & - & - & - & - & & - & - \\
\hline 6 & - & - & - & - & - & - & - & - & - & - & & - & - \\
\hline 8 & $29 \cdot 9 \pi i$ & - & $24 \cdot 8$ & $32 \cdot 0$ & $30 \cdot 0$ & 360 & Calm & 0 & 0 & inc & & Ci.-cur. $4, \mathrm{sW}$ & - \\
\hline 10 & $28 \cdot 971$ & - & $31 \cdot 5$ & $36^{\circ} \cdot 16$ & $32 \cdot 5$ & $43 \cdot 5$ & Culm & 1 & 0 & tev & & Cl.-s., Ci.-cu. 2, SW & - \\
\hline Noon & $28 \cdot 9+95$ & - & $34 \cdot 1$ & $34 \cdot 9$ & $31 \cdot 8$ & $42 \cdot 2$ & Ca'm & $\theta$ & 0 & ixe & | & $\begin{array}{l}\text { C1.eu. } 4 \text { (higher } \mathrm{NW} \text {, } \\
\text { lower SW }\end{array}$ & - \\
\hline 2 & $28 \cdot 9 \pi 4$ & - & - & $31 \cdot 0$ & $29 \cdot 0$ & $32 \cdot 0$ & $\operatorname{ssl} 1,0-2$ & 3 & $0-15$ & xic & & Ci.-c1l. $3, N w$ & A.-eno, A. s. 7 \\
\hline 4 & $28-982$ & - & $29 \cdot 0$ & $28 \cdot 8$ & $27 \cdot 5$ & $3 w \cdot 8$ & Var. 12, 1-4 & 11 & $0-20$ & seq & & - & S. 10 \\
\hline 6 & $28 \cdot 994$ & - & $2 i \cdot 0$ & $27 \cdot 5$ & $25 \cdot 0$ & $28 \cdot 0$ & ESE, 2- $\tau$ & 12 & $0-35$ & мथा & & - & S., S.-cu. 10 \\
\hline 8 & $29 \cdot(10$ & - & $25 \cdot 2$ & $2: \cdot 0$ & $24 \cdot 6$ & $27 \cdot 5$ & E, 1-4 & 11 & $(0-20$ & nq & & - & S. $10, x$ \\
\hline 10 & $29 \cdot 026$ & - & $24 \cdot 5$ & $25 \cdot 0$ & $21 \cdot 0$ & $25 \cdot 6$ & Sti, 2-4 & 12 & $111-25$ & eq & & Ci.-s. 1, SW & A.-e11. 2, A.-so 1, \\
\hline Midt. & - & $=$ & - & - & - & -1 & - & - & - & - & & - & - \\
\hline M ean & $28-938$ & - & - & $30 \cdot 4$ & $28 \cdot 1$ & -1 & - & $\overline{-}$ & - & - & & - & - \\
\hline
\end{tabular}

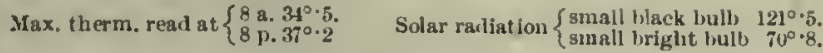

Evapn, - grainmes.

Precipn. - in.

Refraction all round; some A.-S, in S, anc Fr.-cu. to $S W_{\text {; }}$ sorue $C u$. among wester mountaina Mirage.

10 R.m. A.-s. and $\mathrm{Cn}_{1}$ to $\mathrm{W}$ obsectring part of west ern mountains; thin $A_{\text {. }}-\mathrm{s}$. in $\mathrm{S}$. SE, and SW : cloutds passing to X.o. Noon. A.-sis and Cu. extend from Xi through W, S, and SE; Cu. on the
Bluff; sumnit of Discovery olseurest. 30 p.n. Teemp. at Cape Armitage, H $^{\circ} .5$, nuin., $7^{\circ} 0$. Vrebus smoke torn. ing a mushrnom (2uvt) feet). 0, i .-8, 10. Wind $\mathrm{NE}, 3$.

2 p.m. Iligit peaks of western mountains and summit of Discuvery olscuret Bluff has heavy eloud on it. Ozone, 8 . 4 p.m. Clouds as at 2 p.m. Ozone, 4 . p.m. All high land olscured; west en foot hills and all islands visible; Blun clear; heayy dark clouds, apparentiy Nim. " hery dark in iv azd Si

p.m. Very dark in in and SW, obscurIng western land and all but very lowest. slopes of Brown Island and Mt.

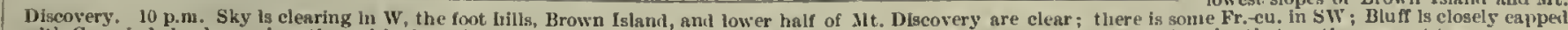
with Cn. wind cloud; ; prismatic zenith clrcle (not parbelion) can be secn, apparently $45^{\circ}$ from sun; a third of circle is visilble ouly, that portion nearest to suni. 
1903-December.]

31 st.

\begin{tabular}{|c|c|c|c|c|c|c|c|c|c|c|c|c|c|c|c|}
\hline \multirow{2}{*}{ Illour. } & \multirow{2}{*}{ 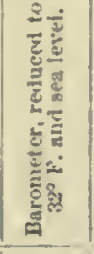 } & \multicolumn{5}{|c|}{ Thermomet ers. } & \multicolumn{3}{|c|}{ W'ind. } & \multirow[b]{2}{*}{ 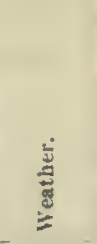 } & \multirow{2}{*}{ 总 } & \multicolumn{2}{|c|}{$\begin{array}{l}\text { Cllouds, } \\
\text { Anount and novenente. }\end{array}$} & \multirow{2}{*}{ 方 } & \multirow{2}{*}{ Remarks. } \\
\hline & & 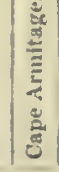 & 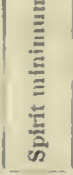 & $10 \%$ & Wet. & 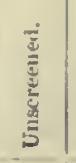 & $\begin{array}{c}\text { Direetion antl } \\
\text { forec } \\
\text { (Beaufort seale). }\end{array}$ & 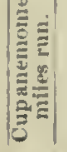 & 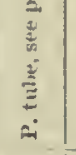 & & & Upper. & Lower. & & \\
\hline 2 & - & - & - & - & - & - & - & - & - & - & & - & - & -1 & Wenther during the night seen \\
\hline 4 & - & - & - & - & - & - & - & - & - & - & & - & - & - & \\
\hline B & - & - & - & - & - & - & - & - & - & - & 1 & - & - & - & \\
\hline 8 & $29 \cdot 0+2$ & - & $22 \cdot 8$ & $2 i \cdot 2$ & $25 \cdot 8$ & $29 \cdot 0$ & Var. E. 1-2 & 12 & $8-15$ & c & & $\mathrm{Cl}_{2} \cdot \mathrm{Cu}, 2$ & A.-8. 5 & - & The sky appenrs to he clearing; still lark \\
\hline 10 & $29 \cdot 075$ & - & $24 \cdot 5$ & $27 \cdot 8$ & $25 \cdot 2$ & $3 \cdot 0$ & SSE, 1-2 & 10 & $8-15$ & ix & 1 & 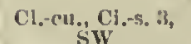 & - & - & and foot of Diseovery are visible; Bluff \\
\hline Noon & $28 \cdot 186$ & - & $2 i \cdot 8$ & $20 \cdot 2$ & $27 \cdot 0$ & $33 \cdot 0$ & s, $0-1$ & ti & $0-15$ & the & & Ci.-ch1, Cl.-\&. 2, & A.-s. $1, \mathrm{sW}$ & - & winl velocitr since 10 p.m, yesterday, \\
\hline 2 & $29 \cdot 084$ & - & $27 \cdot 5$ & $28 \cdot 0$ & $25 \cdot 5$ & $34 \cdot 0$ & $\mathrm{SK}_{4} 1-3$ & 8 & $0-20$ & eq & I & Ci., Ci.-s. 2 & A. $\cdot 8.5$, SW (slow) & -1 & 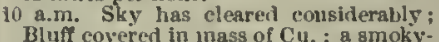 \\
\hline 4 & $29 \cdot 073$ & - & $27 \cdot 5$ & $28 \cdot 0$ & 265 & $31 \cdot 0$ & S1: $0-2$ & 11 & $0-21)$ & $e q$ & & Cl., Ci.-8. 6 & A.-s. 3, SW (stowi) & - & looking, torn Str. llildes most of western \\
\hline 6 & $29 \cdot 068$ & - & $28 \cdot 5$ & $24: 5$ & $24 \cdot 5$ & $32 \cdot 0$ & Var. Six, 0-2 & 11 & $0-10$ & cq & 1 & Ci., Ci.-8. 3 & 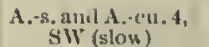 & - & $\begin{array}{l}\text { parts alone showing : a line of heary } \\
\text { Cu. over Brown and Black Islands. }\end{array}$ \\
\hline 8 & $29 \cdot 066$ & - & $26 \cdot 9$ & $2 x \cdot 1$ & $25 \cdot 0$ & $33 \cdot 0$ & Var. $\mathbf{E}, 1-2$ & 8 & $8-12$ & le & & - & A.-e11. $5,5 W$ & -8 & $\begin{array}{l}\text { Nool. Bluf almost complitetely obseurch } \\
\text { by Str., of which there is a good deal in }\end{array}$ \\
\hline 10 & $29 \cdot 049$ & - & $27: 2$ & $28 \cdot 11$ & $25 \cdot 8$ & $34 \cdot 8$ & NE, 2-33 & 7 & $10-18$ & Inc & I & Cl. 1, sw & A.-el1. $2, \mathrm{SW}$ & - & Sald SW, also Cu.; some of the upper \\
\hline Mlat. & $\rightarrow$ & - & - & - & - & - & - & - & - & - & 1 & - & - & - & $\begin{array}{l}\text { becoming visible; intermediste helght } \\
\text { alove foot hills are hidden; IIt. }\end{array}$ \\
\hline Mean & $29 \cdot 0 ; 0$ & - & - & $27 \cdot 7$ & $25 \cdot 7$ & - & - & $1-$ & - & - & & - & - & - & $\begin{array}{l}\text { Discovery almost completely hidden in } \\
\text { torn, snioke-like Str. clout; line of }\end{array}$ \\
\hline $\mathrm{Ma}$ & x. therm & n. $r$ & 1 at & 8 А. 27 & & Sols & diation $\left\{\begin{array}{l}\mathrm{Bn} \\
\mathrm{Bm}\end{array}\right.$ & & ulb & $\begin{array}{l}3^{\circ} \cdot 0 \\
5^{\circ} \cdot 0\end{array}$ & & - gramues. & Preclun. - In. & & $\begin{array}{l}\text { detached Cn. over Brown Island. } \\
2 \text { p.me Bluff and nain range and Dis- } \\
\text { covery olsseured. Ozone, } 7 \text {. } \\
\text { t p.m. Bnse of Biluff visible; Discovery, }\end{array}$ \\
\hline & & & & & $\begin{array}{l}\mathrm{ma} \\
\text { par } \\
\text { clo } \\
10 \\
\text { da }\end{array}$ & & $\begin{array}{l}\text { main wes } \\
\text { r Cun, elou } \\
\text { W; nppe } \\
\text { clear; th }\end{array}$ & & & $\begin{array}{l}18 \mathrm{c} \\
\text { nou }\end{array}$ & & $\begin{array}{l}\text { 3. } 6 \text { p.m. Bas } \\
\text { 4. } 8 \text { p.m. Pea } \\
\text { Discovery is oh: } \\
\text { otning clear ; c }\end{array}$ & $\begin{array}{l}\text { f Buff more vils } \\
\text { of western moun } \\
\text { red by acrles of } \\
\text { li siv all exce }\end{array}$ & $\begin{array}{l}\text { sible t } \\
\text { stalns } \\
\text { f dark, } \\
\text { ppt lov }\end{array}$ & $\begin{array}{l}\text { than at 4p.m.; Brown and Black Islanils } \\
\text { thare hidien behind Cu.; a cap of whind } \\
\text { s, wind-torn Str. clouds; some Str. In SW. } \\
\text { west slope of Mt. Dlscovery obscured by }\end{array}$ \\
\hline
\end{tabular}

Metforolorical JoUhxal of THE "Discovery." 
Miteorolorical, Jourial, of the "Discovery."

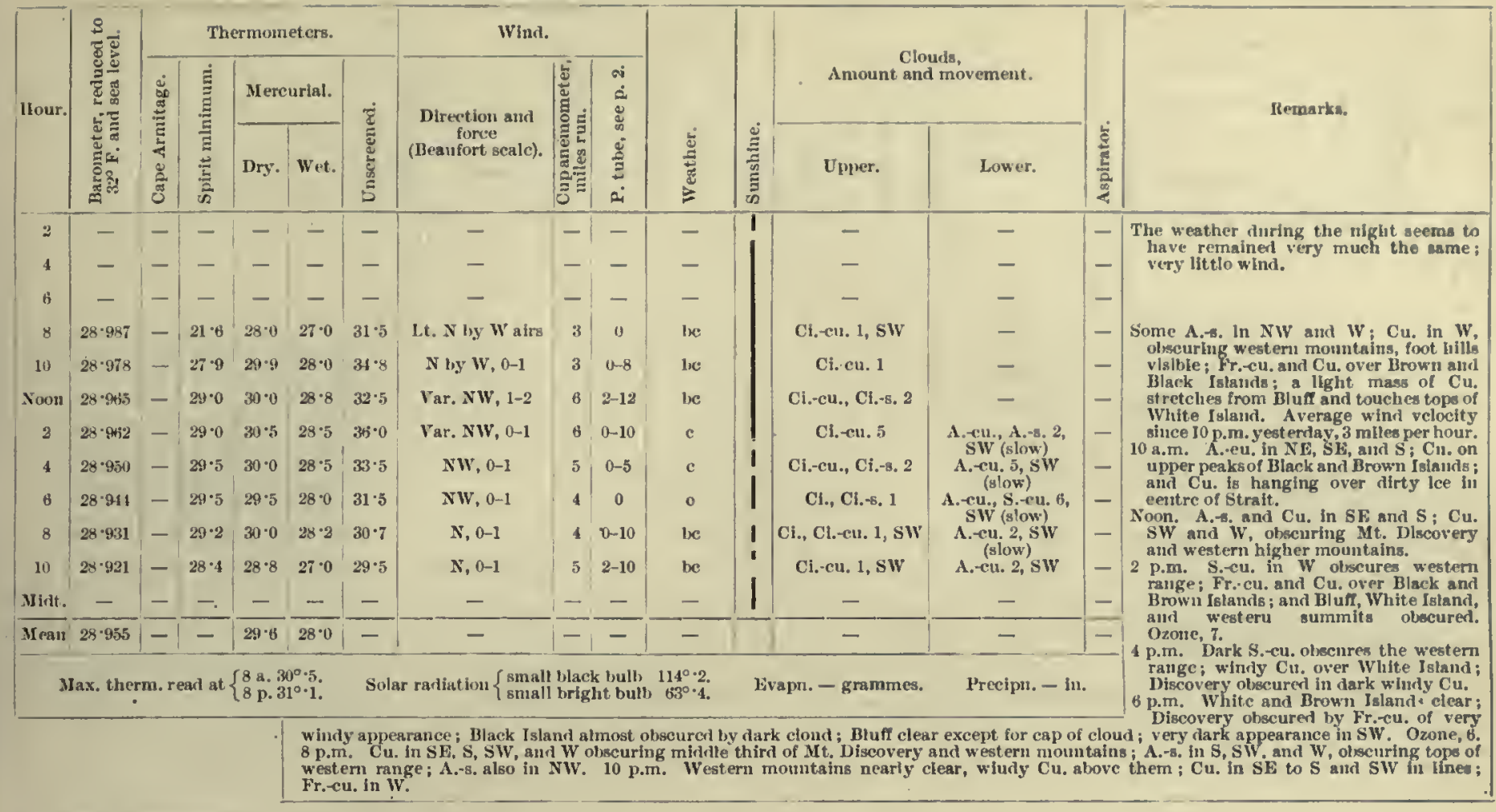

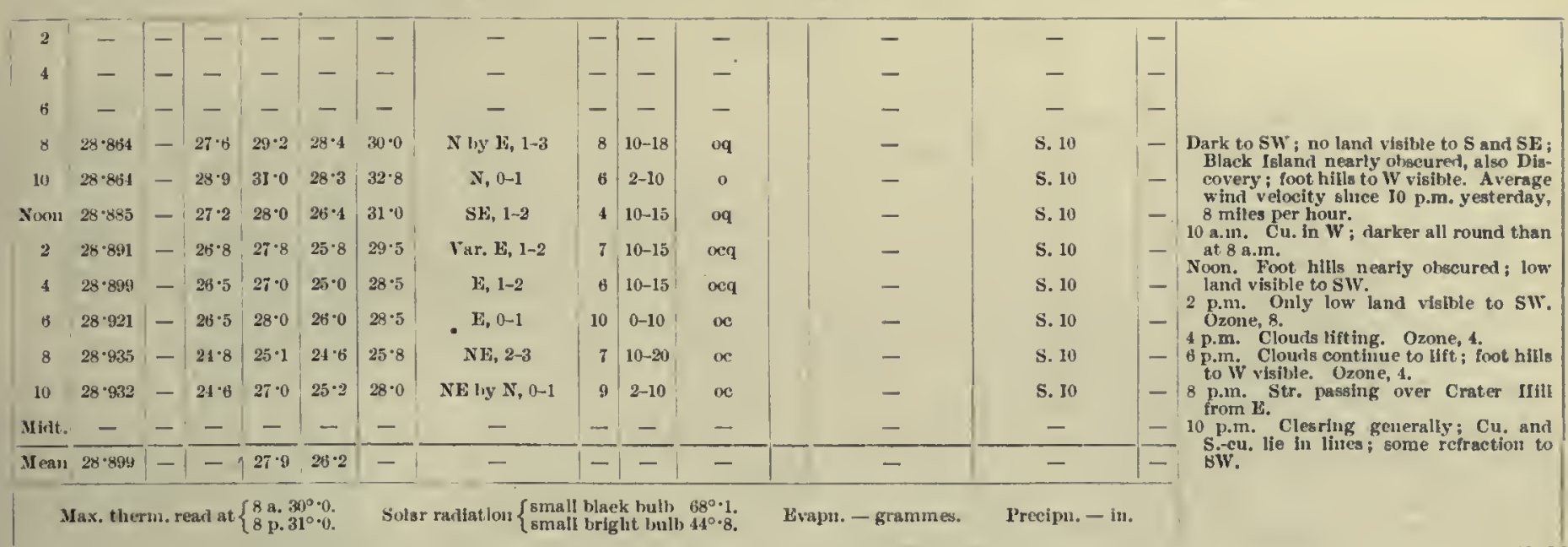

\begin{tabular}{|c|c|c|c|c|c|c|c|c|c|c|c|c|c|c|c|}
\hline 2 & - & - & - & - & - & - & - & - & - & - & & - & - & -1 & \multirow{12}{*}{ 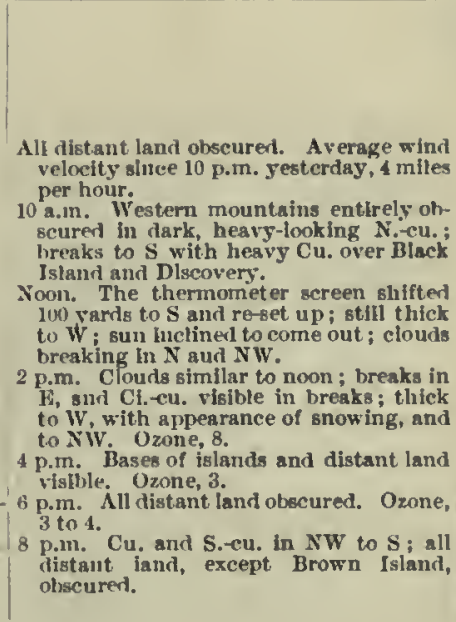 } \\
\hline 4 & - & - & - & - & - & - & - & - & - & - & & - & - & - & \\
\hline 6 & - & - & - & $\cdot-$ & - & - & - & - & - & - & & - & - & - & \\
\hline 8 & $28 \cdot 928$ & - & $26 \cdot 4$ & $28 \cdot 4$ & $27 \cdot 6$ & $31 \cdot 0$ & Calm & 4 & 0 & of $*$ & & - & N. 10 & - & \\
\hline 10 & $28 \cdot 9665$ & - & $25 \cdot 5$ & $26^{\circ} \cdot 1$ & $24 \cdot 8$ & $28 \cdot 0$ & SE, 1-2 & 2 & $10-15$ & o & & - & S. 10 & - & \\
\hline Noron & $28 \cdot 993$ & - & $22 \cdot 5$ & $23 \cdot 8$ & $23 \cdot 0$ & $27 \cdot 0$ & $\mathrm{SE}, 2-3$ & 9 & $12-18$ & œ-i & . & Ci.-5. 3, SW ; & Scurd 1, S (raplet) & - & \\
\hline 2 & $29 \cdot 601$ & - & $23 \cdot 0$ & $26 \cdot 0$ & $24 \cdot 2$ & $28 \cdot 0$ & E, 2-3 & 10 & $12-16$ & oc & ' & $\begin{array}{l}\text { A.-cul. } 4 \text {, SW } \\
\text { A.-cu. SW }\end{array}$ & Cu. 3, SE (fast) & - & \\
\hline 8 & $29 \cdot 034$ & - & $24 \cdot 2$ & $21 \cdot 9$ & $23 \cdot 5$ & $26^{\circ} 0$ & Calm & 7 & 0 & ef $*$ & & - & N. 6, S.-cu. $3, \mathrm{~s}$ & - & \\
\hline 10 & $29 \cdot 050$ & - & $23 \cdot 2$ & $24^{\circ} 0$ & $23 \cdot 4$ & $29 \cdot 2$ & SE, 4 & 11 & $22-28$ & oc $*$ & & - & N. 10 & - & \\
\hline Midt. & - & - & - & - & - & - & - & - & - & $=$ & & - & - & $1-$ & \\
\hline Nean & $28 \cdot 996$ & $1-$ & - & $25 \cdot 6$ & $24 \cdot 2$ & - & - & - & - & - & & - & - & -1 & \\
\hline & & & & & & So & 10 & & & $\begin{array}{l}111^{\circ} \cdot 8 . \\
62^{\circ} \cdot 2 .\end{array}$ & & pn. - grammes & Precipn. - In. & & \\
\hline
\end{tabular}


1904 -January.]

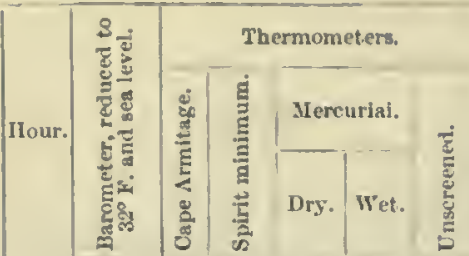

4th.

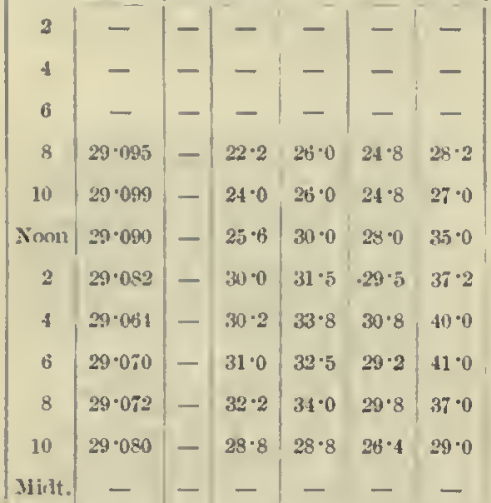

Nean $23 \cdot 082=\overline{30 \cdot 3} \overline{27 \cdot 9}-$

Max. therm. read at $\left\{\begin{array}{l}8 \mathrm{a} .24^{\circ} \cdot 6 . \\ 8 \mathrm{p}, 37^{\circ} \cdot 5 .\end{array}\right.$
MetEOROLOGICAL JoUliNal OF THE "DISCOVERY."

\begin{tabular}{|c|c|c|c|c|c|c|c|c|}
\hline Wind. & & هi & \multirow[b]{2}{*}{ 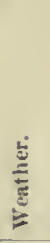 } & \multirow[b]{2}{*}{ 语 } & \multicolumn{2}{|c|}{$\begin{array}{l}\text { Clonds, } \\
\text { Amount and movement. }\end{array}$} & \multirow[b]{2}{*}{ है } & \multirow{2}{*}{ Remarks. } \\
\hline $\begin{array}{c}\text { force } \\
\text { (Beanfort sea!t). }\end{array}$ & 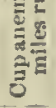 & $\frac{\mathscr{L}^{-}}{3}$ & & & Epper. & Lower. & & \\
\hline- & -1 & - & - & & - & - & -1 & \\
\hline- & -1 & - & - & 1 & - & - & - & \\
\hline- & - & - & - & & - & - & - & \\
\hline Caim & 7 & 0 & 0 & & - & S. $\cdot c u \cdot 10$ & - & Sky clear over western mountains, anit \\
\hline Calm & 2 & 0 & oe & & - & $\begin{array}{l}\text { A.-eu, } x_{,}, \mathrm{SW} \\
\text { (slow) }\end{array}$ & - & $\begin{array}{l}\text { wind velocity sinec } 10 \mathrm{j} . \mathrm{m} \text {. yest eriliy, } \\
7 \text { miles per fiour. }\end{array}$ \\
\hline Calin & 0 & 0 & oc & & - & $\begin{array}{l}\text { A.-cu, } 7, \text { SW } \\
\text { (stow) }\end{array}$ & - & $\begin{array}{l}10 \mathrm{am} \text {. Layers of Str. On westeru moun- } \\
\text { tains clearing geuterally }\end{array}$ \\
\hline Calm & 0 & 0 & b & & - & - & - & $\begin{array}{l}\text { smaller Cu. than proper A.-cn., but not } \\
\text { small enough or high enough for Cl.-cu. }\end{array}$ \\
\hline L.t. NW alrs & 1 & $0-5$ & i, & & - & - & - & $\begin{array}{l}\text { Noon. A.-cu. apparently ascending and } \\
\text { look ing more like Ci.-cu. Some Str. to }\end{array}$ \\
\hline Calm & 2 & 0 & b & & - & - & -1 & W on monntains and on Discovery. \\
\hline Lt. $N$ airs & 1 & 0 & b & & - & - & -1 & ceutre third of Discovery and westeru \\
\hline HXX: 1-2 & 6 & $7-14$ & he & & - & Cu. $2, x$ by & - & $\begin{array}{l}4 \mathrm{pm} \text { Ci. quickly disappcaring ; heavy } \\
\text { roll Cu. on western molntains, olseuring }\end{array}$ \\
\hline - & $\underline{-}$ & - & - & 1 & - & - & 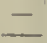 & $\begin{array}{l}\text { ecntre portion. } \\
6 \text { p.m. Bluff obseured in Str. : heave Str. }\end{array}$ \\
\hline- & - & - & - & & - & - & - & $\begin{array}{l}\text { and S.-cu. ohscure Discovery and all } \\
\text { western main range. Ozonc, } 4 \text {. }\end{array}$ \\
\hline radiation $\left\{\begin{array}{l}\text { sma } \\
\text { sma }\end{array}\right.$ & $\begin{array}{l}\text { hlac } \\
\text { hrig }\end{array}$ & $\begin{array}{l}\text { bu!n } \\
\text { thul }\end{array}$ & - l. & & grammes. & Precipu. - in & & $\begin{array}{l}8 \text { p.m. Western monntains and ali ristant } \\
\text { land obsenred by a heavy bank of roll } \\
\text { Cu. and S.-cu., with very massive rolls ; } \\
\text { ot hierwise cloudless. } \\
10 \text { p.m. liefraction to the SW. }\end{array}$ \\
\hline
\end{tabular}

5 th.

\begin{tabular}{|c|c|c|c|c|c|c|c|c|c|}
\hline 2 & - & -1 & - & - & - & - & - & - & - \\
\hline 4 & - & - & - & - & - & - & - & - & - \\
\hline 6 & $2 y \cdot 100$ & - & $21 \cdot 0$ & $23 \cdot 2$ & $22 \cdot 5$ & $23 \cdot 8$ & HXV, 3-4 & 12 & $15-2 n$ \\
\hline 8 & $29 \cdot 126$ & - & $22 \cdot 6$ & $24 \cdot 2$ & 23.0 & $24 \cdot 5$ & E, 3-4 & 13 & $18-25$ \\
\hline 10 & $29 \cdot 142$ & -1 & 23.0 & $25 \cdot 2$ & $26 \cdot 0$ & $26 \cdot 0$ & E, 1-2 & 13 & $10-18$ \\
\hline Ncron & $29 \cdot 152$ & - & $23 \cdot 2$ & $26 \cdot 0$ & $25 \cdot 0$ & $26 \cdot 8$ & E, 2-3 & 10 & $10-18$ \\
\hline 2 & $29 \cdot 168$ & - & $25 \cdot 0$ & $260^{\circ}$ & $25 \cdot 0$ & $27 \cdot 5$ & E, 1-2 & 12 & $10-18$ \\
\hline 4 & $29 \cdot 168$ & -- & $25 \cdot 0$ & $25 \cdot 8$ & $2 \cdot 1 \cdot 9$ & $26 \cdot 0$ & E, 1-2 & 13 & $10-17$ \\
\hline i & $29 \cdot 183$ & - & $25 \cdot 0$ & $25 \cdot 2$ & 24.0 & $25 \cdot 5$ & ENE, 1-4 & 12 & $8-22$ \\
\hline 8 & $29 \cdot 191)$ & - & $25 \cdot 0$ & $27 \cdot 4$ & $25 \cdot 0$ & $28 \cdot 0$ & Calm & 10 & 0 \\
\hline ro & $29 \cdot 202$ & - & $2 \pi \cdot 0$ & $29 \cdot 6$ & $26 \cdot 4$ & $29 \cdot 0$ & $\mathrm{E}$ by $\mathrm{N}, 0-1$ & 3 & 0 \\
\hline Miltt. & - & -1 & - & - & - & - & - & -1 & - \\
\hline $\overrightarrow{\text { ean }}$ & $29 \cdot 159$ & -1 & - & $25 \cdot 8$ & 24.4 & - & - & - & - \\
\hline
\end{tabular}

Max. therm. read at $\left\{\begin{array}{l}8 \text { a. } \\ 8 \text { p. } 28^{\circ \circ} \cdot 0 .\end{array}\right.$

Solar $\left\{\begin{array}{l}\text { small black bulb } 92^{\circ} \cdot 0 . \\ \text { small bright bultb } 40^{\circ} 0\end{array}\right.$

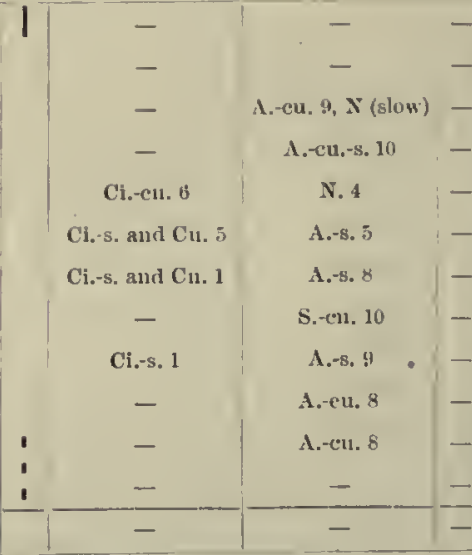

Lvapu. - grammes. Precipu. - in.
- Very black-looking Str. ower to W aul SW, with grey Str. over it; A.-cu. very like look generally. Arerage wind velocity. since 10 p.m. yesterday, 12 miles per

lour. No land in distance visible ; rark grey and biack-looking clouds all round. $10 \mathrm{a.m}$. Sky looks inclined to break ; just commenced to snow, very small dry"
fiakes; White Islaud only dimly visille; drift to $S$ in Strait.

Noon. White Island and westeru momtains stili obscured ; land visible to : ant $\mathrm{SW}$; stormy looking to $\mathrm{SW}$; henvy

Cht. OnI Black Island,
p.11. Ali distant land, cxcept small islet $\mathrm{N}$ of Black Island, obscureed; a small amount of seud in $\mathbf{E}$ moving fast from p.in. Very minutc and hard snow Alakes falling; io dlstant land visible; clouds are nint Nim., but there is some seml passing over at times from se at a falling.

falling.

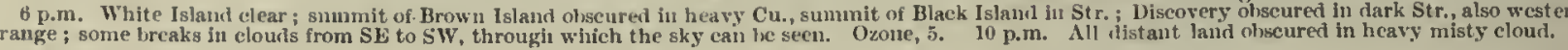

6th.

\begin{tabular}{|c|c|c|c|c|c|c|c|c|c|c|c|c|c|c|c|}
\hline 2 & - & - & - & - & - & - & - & - & - & - & & - & - & -1 & \multirow{11}{*}{ 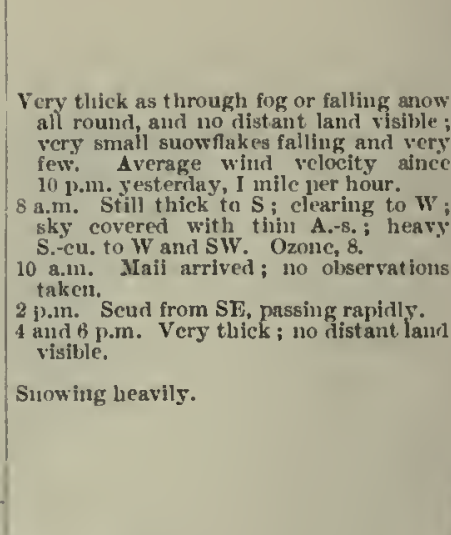 } \\
\hline 4 & - & - & - & - & - & $\dot{-}$ & - & - & - & - & 1 & - & - & - & \\
\hline 6 & $29 \cdot 206$ & - & $21 \cdot 2$ & $25-8$ & 21.8 & $27 \cdot 0$ & L.t. SE airs & 1 & 0 & ine $*$ & 1 & Ci..s. 2 & A.-cu. 5, SW & - & \\
\hline 8 & $29 \cdot 217$ & - & $25 \cdot 0$ & $27 \cdot 0$ & $25 \cdot 6$ & $31 \cdot 0$ & Calm & 0 & 0 & $o c$ & 1 & A. -8.8 & Cu. $2, \mathrm{~N}$ & - & \\
\hline 10 & - & - & - & - & - & - & - & - & - & - & 1 & - & - & - & \\
\hline Noon1 & $29 \cdot 217$ & - & $28 \cdot 8$ & $28 \cdot 0$ & $20 \cdot 6$ & $31 \cdot 2$ & Calm & 2 & 0 & oc $*$ & & - & N. 10 & - & \\
\hline 2 & $29 \cdot 210$ & - & $28 \cdot 2$ & $30 \cdot 4$ & $28 \cdot 5$ & $31 \cdot 5$ & $\mathbf{N W}, 0-1$ & 1 & $v-\tilde{s}$ & $\alpha *$ & & - & s. 10 & - & \\
\hline 8 & $29 \cdot 202$ & - & 250 & $27 \cdot 5$ & $2 i \cdot 2$ & 28.8 & Calm & 7 & 0 & $0 \mathbb{*}^{2}$ & & - & x. 10 & - & \\
\hline 10 & - & - & - & - & - & - & - & - & - & - & & - & - & - & \\
\hline Mluit. & - & - & - & - & - & - & - & - & - & - & & - & - & - & \\
\hline دi exu & $29 \cdot 210$ & - & - & $2 \times 0$ & $23 \div 5$ & - & - & - & - & - & & - & - & $=$ & \\
\hline
\end{tabular}


Mfteorologicai, Journat, of tite "Discovery."

[1904-January.

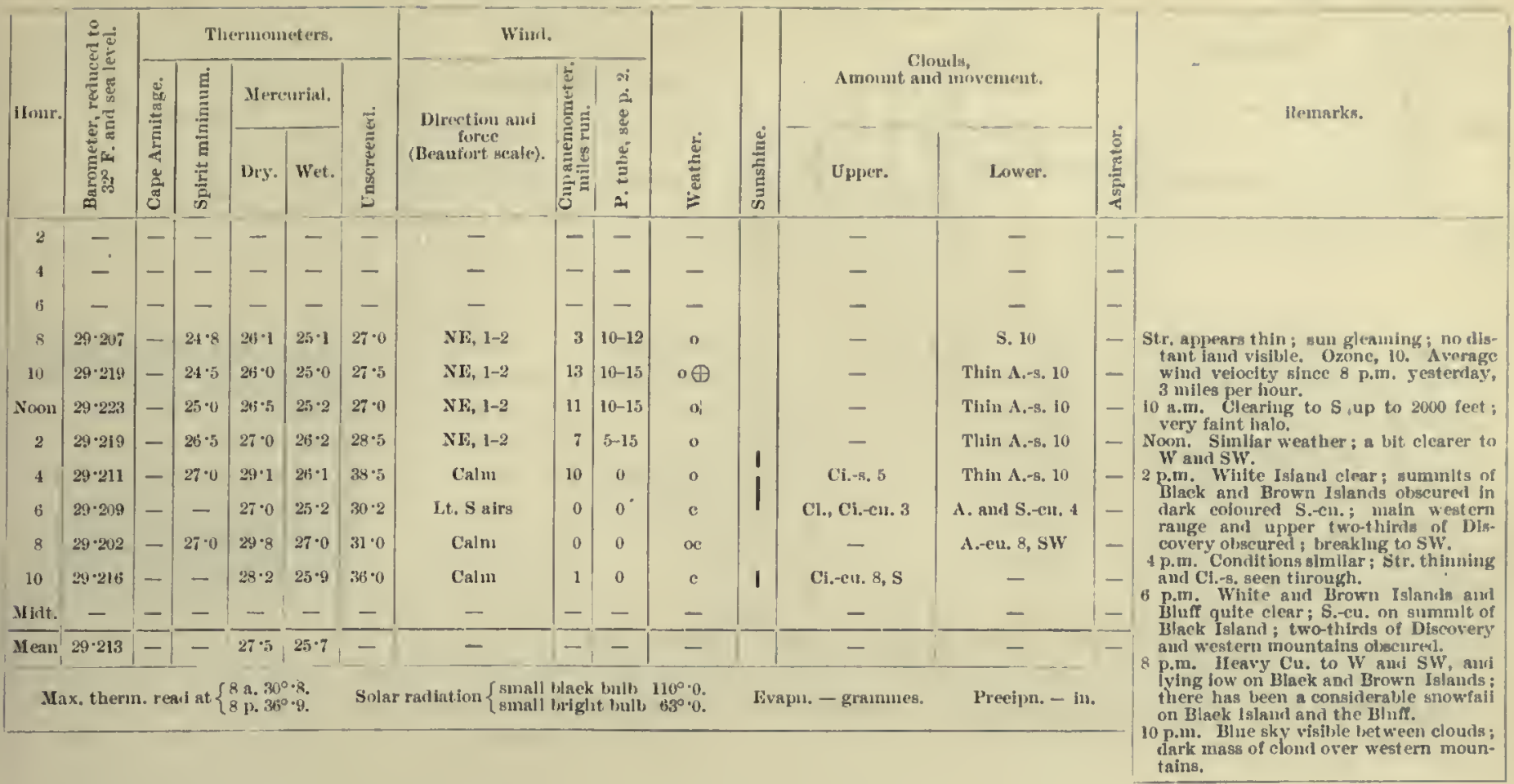

7 th.

\begin{tabular}{|c|c|c|c|c|c|c|c|c|c|c|c|c|c|c|}
\hline 2 & - & - & - & - & - & - & - & - & - & - & - & - & $1-$ & \multirow{14}{*}{ 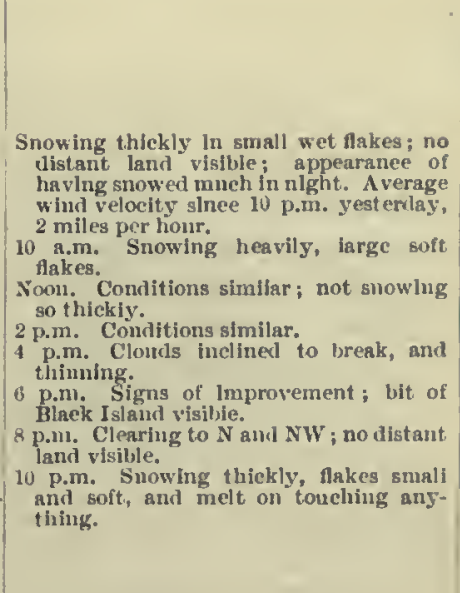 } \\
\hline 4 & - & - & - & - & - & - & - & - & - & - & - & - & - & \\
\hline 6 & - & - & - & - & - & - & - & - & - & - & - & - & - & \\
\hline 8 & $28 \cdot 172$ & - & $22 \cdot 0$ & $30 \cdot 2$ & $28 \cdot 2$ & $31 \cdot 0$ & Catun & 2 & 0 & $n *$ & - & N. 10 & - & \\
\hline I0 & $.29 \cdot 169$ & - & $28 \cdot 0$ & $2 x \cdot 0$ & $28 \cdot 0$ & $32 \cdot 2$ & Calm & 1 & 0 & $o *$ & - & N. 10 & - & \\
\hline Nomn & $29 \cdot 1665$ & - & - & 270 & $26 \cdot 0$ & $32 \cdot 2$ & Lt. $S$ airs & 2 & 0 & $n *$ & - & N. 10 & - & \\
\hline 2 & $29 \cdot 160$ & - & $26 \cdot 5$ & $27 \cdot 0$ & $25 \cdot 5$ & $29 \cdot 5$ & Lt. SE alrs & 4 & 0 & $n *$ & - & N. 10 & - & \\
\hline 4 & $29 \cdot 152$ & - & $26 \cdot 0$ & $27 \cdot 0$ & $26^{\circ} 0$ & $28 \cdot 8$ & Lt. E airs & + & 0 & $\circ *$ & - & N. 10 & - & \\
\hline 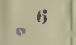 & $29 \cdot 133$ & - & $26 \cdot 2$ & $30 \cdot 0$ & $27 \cdot 5$ & $32 \cdot 5$ & NE, 0-2 & 8 & $0-15$ & осq & Ci.-en. 1 & A.-s. and Cur. ${ }_{\text {SW }}$ & - & \\
\hline 8 & $29 \cdot 1 \mathrm{i} 9$ & - & $28 \cdot 0$ & $28 \cdot 0$ & $26 \cdot 0$ & $28 \cdot 8$ & Lt. SE airs & 5 & 0 & o & - & s. $10, \mathrm{SW}$ & $1-$ & \\
\hline 10 & $29 \cdot 127$ & - & $25 \cdot 5$ & $2 n \cdot 0$ & $25 \cdot 0$ & $27^{\circ} 0$ & 1.t. $S$ airs & 2 & 0 & $0 *$ & - & N. 10 & - & \\
\hline Mislt. & - & - & - & - & - & - & - & $1-$ & - & - & - & - & $1-$ & \\
\hline Mean & $29 \cdot 150$ & - & - & $28 \cdot 0$ & $26 \cdot 5$ & - & - & - & - & - & - & - & - & \\
\hline & & & & & & & 年 & & & & mes. & Preeipn. - & & \\
\hline
\end{tabular}

8 th.

\begin{tabular}{|c|c|c|c|c|c|c|c|c|c|c|c|c|c|}
\hline 2 & - & -1 & -1 & - & - & - & - & - & - & - & - & $1-$ & \\
\hline 4 & - & - & - & - & - & - & - & - & - & - & - & - & \\
\hline 6 & - & - & - & - & - & - & - & - & - & - & - & - & \\
\hline 8 & - & - & - & - & - & - & - & - & - & - & - & - & \\
\hline 10 & $29 \cdot 035$ & - & $25 \cdot 2$ & 280 & $27 \cdot 2$ & $30 \cdot 0$ & Calm & 2 & $\circ *$ & - & N. 10 & - & $\begin{array}{l}\text { Snowing heavily, and there has been a } \\
\text { very heavy fall during, the night. }\end{array}$ \\
\hline NoOn & $29 \cdot 035$ & - & $2 \pi \cdot 2$ & $32 \cdot 2$ & $30 \%$ & $34 \cdot 0$ & Calm & 1 & $a *^{2}$ & - & X. 10 & $1-$ & $\begin{array}{l}\text { Average wind velocity since } 10 \text { p.m. } \\
\text { yesterday, } 2 \text { uiles per hour. }\end{array}$ \\
\hline 2 & $29 \cdot 081$ & - & - & $29 \cdot 0$ & 28.0 & $33 \cdot 5$ & Lt. var, airs & 1 & o* & - & N. 10 & - & $\begin{array}{l}\text { Noon. Snow alling very heavliy; small, } \\
\text { wet. soft tfakes, melt ting almost imme- }\end{array}$ \\
\hline 4 & $29 \cdot 069$ & - & $28 \cdot 5$ & $29-9$ & $28 \cdot 5$ & $32 \cdot 0$ & Lt. SE airs & 4 & $0 *$ & - . & N. 10 & - & $\begin{array}{l}\text { diately. } \\
2 \text { p.m. Conlitions similar. }\end{array}$ \\
\hline 6 & $29 \cdot 069$ & - & $28 \cdot 5$ & $29 \cdot 0$ & $28 \cdot 0$ & $20 \cdot 5$ & Lt. Ev airs & 2 & $n *^{2}$ & - & N. 10 & - & 4 and 6 p.m. Snow falling; almost ralu. \\
\hline 8 & $29 \cdot 075$ & - & $28 \cdot 2$ & $28 \cdot 2$ & $27 \cdot 8$ & $30 \cdot 0$ & Lt. S airs & 4 & $o *^{2}$ & - & N. 10 & - & \\
\hline 10 & - & -1 & - & - & - & - & - & - & - & - & - & - & \\
\hline Minlt. & - & -1 & - & - & - & - & - & $=$ & - & - & $=$ & 1 & \\
\hline$\overline{\text { Ifean }}_{\text {| }}$ & 29.061 & -1 & - & $29 \cdot 4$ & $28 \cdot 4$ & - & - & -1 & - & - & - & - & \\
\hline
\end{tabular}


1904 _January.]

Methorological, Journat, of tule "Discovery."

\begin{tabular}{|c|c|c|c|c|c|c|c|}
\hline \multirow{3}{*}{ Ilour. } & \multirow{3}{*}{ 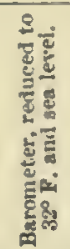 } & \multicolumn{5}{|c|}{ Thermometers. } & \multirow[t]{3}{*}{ Wind. } \\
\hline & & $\dot{0}$ & $\bar{E}$ & \multicolumn{2}{|c|}{ Meneurial. } & & \\
\hline & & 竞 & $\frac{\vec{z}}{\frac{2}{0}}$ & Dry. & Wet. & 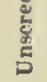 & \\
\hline
\end{tabular}
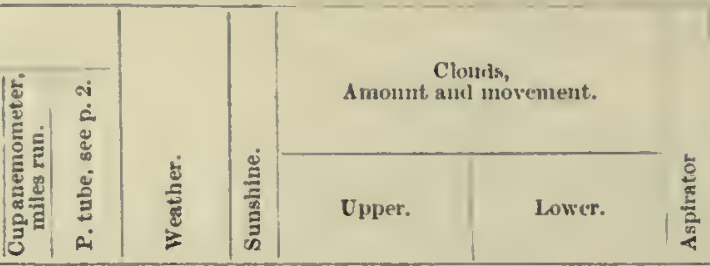

10 th.

\begin{tabular}{|c|c|c|c|c|c|cc|}
\hline 2 & - & - & - & - & - & - & - \\
4 & - & - & - & - & - & - & - \\
6 & - & - & - & - & - & - & - \\
8 & $29 \cdot 102$ & - & $23 \cdot 8$ & $24 \cdot 0$ & $23 \cdot 2$ & $25 \cdot 6$ & SE, 1 \\
10 & $29 \cdot 112$ & - & $23 \cdot 8$ & $29 \cdot 0$ & $27 \cdot 0$ & $30 \cdot 0$ & E, 0-1 \\
Noon & $29 \cdot 125$ & - & $27 \cdot 2$ & $27 \cdot 5$ & $26 \cdot 0$ & $28 \cdot 0$ & Lt. NE airs \\
2 & $29 \cdot 124$ & - & $28 \cdot 0$ & $28 \cdot 5$ & $27 \cdot 5$ & $29 \cdot 5$ & Calm \\
4 & $29 \cdot 127$ & - & $27 \cdot 0$ & $30 \cdot 2$ & $28 \cdot 6$ & $35 \cdot 5$ & Lt. V airs \\
6 & $29 \cdot 127$ & - & - & $30 \cdot 2$ & $28 \cdot 0$ & $29 \cdot 8$ & Lt. NE airs \\
8 & $29 \cdot 131$ & - & $26 \cdot 8$ & $27 \cdot 3$ & $26 \cdot 8$ & $31 \cdot 0$ & Lt. E airs \\
10 & - & - & - & - & - & - & - \\
XIldt. & - & - & - & - & - & - & - \\
\hline
\end{tabular}

$\mid$\begin{tabular}{c|c|c|c|}
- & - & - & \\
- & - & - & \\
- & - & - & \\
2 & $10-12$ & $o c$ & \\
7 & $8-10$ & $o c$ & $:$ \\
5 & 0 & $0 *$ & \\
3 & 0 & $0 *$ & \\
3 & 0 & $c$ & $:$ \\
3 & 0 & $o c$ & \\
1 & 0 & $c$ & \\
- & - & - & $\mid$ \\
- & - & - & $\mid$ \\
\hline
\end{tabular}

$-$

Ci.-8. 4, SW

Cl.-cu, 3, SW

-cu, 4, Sw

$-$

Ci. 8, w ly S

Ci.-s. 4

$-$

$-$ Max. therm.
read at $\left\{\begin{array}{l}8 \text { a. } 29^{\circ} .0 . \\ 8 \text { p. } 34^{\circ} .8 .\end{array} \quad \begin{array}{c}\text { Solar } \\ \text { radiation }\end{array}\left\{\begin{array}{l}\text { sunall black bulb } 122^{\circ} \cdot 5 . \\ \text { small bright bulb } 66^{\circ} \circ 9^{\circ}\end{array}\right.\right.$

Evapn. - gramines. Precipn, 0.41 in, since 10 a.m. 9 th.

11 th.

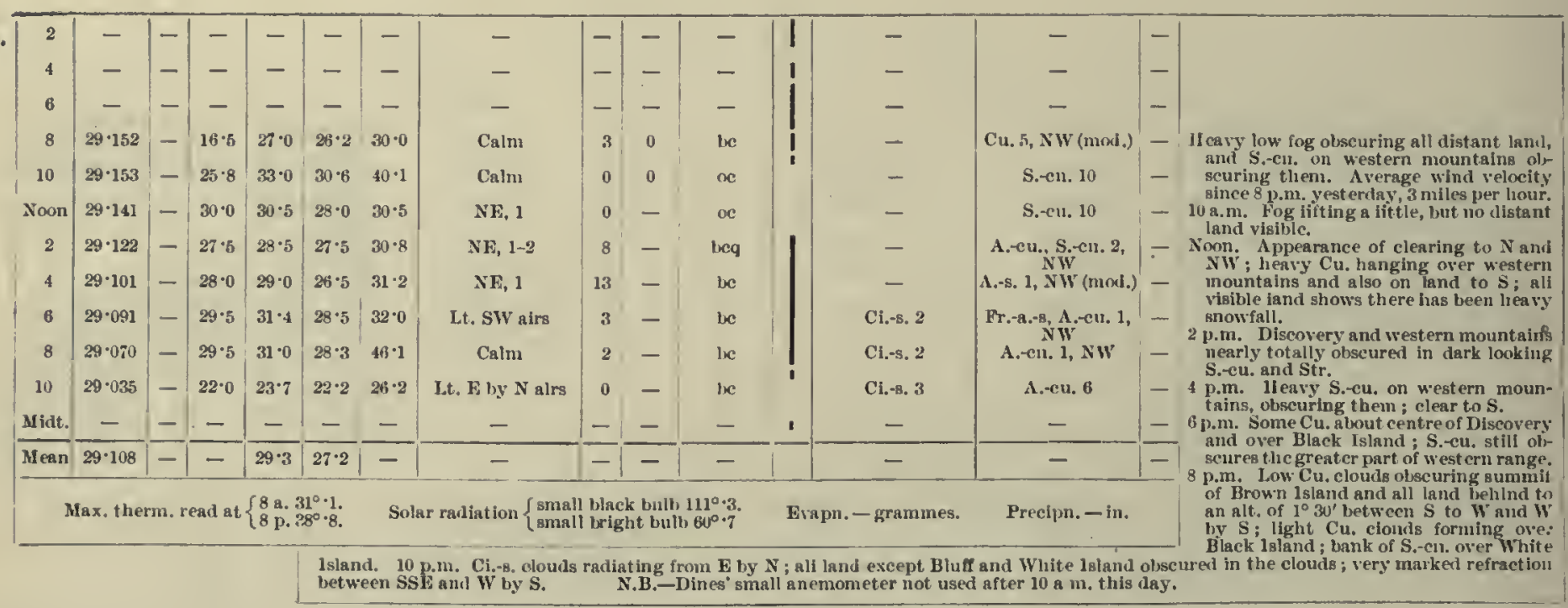

12th.

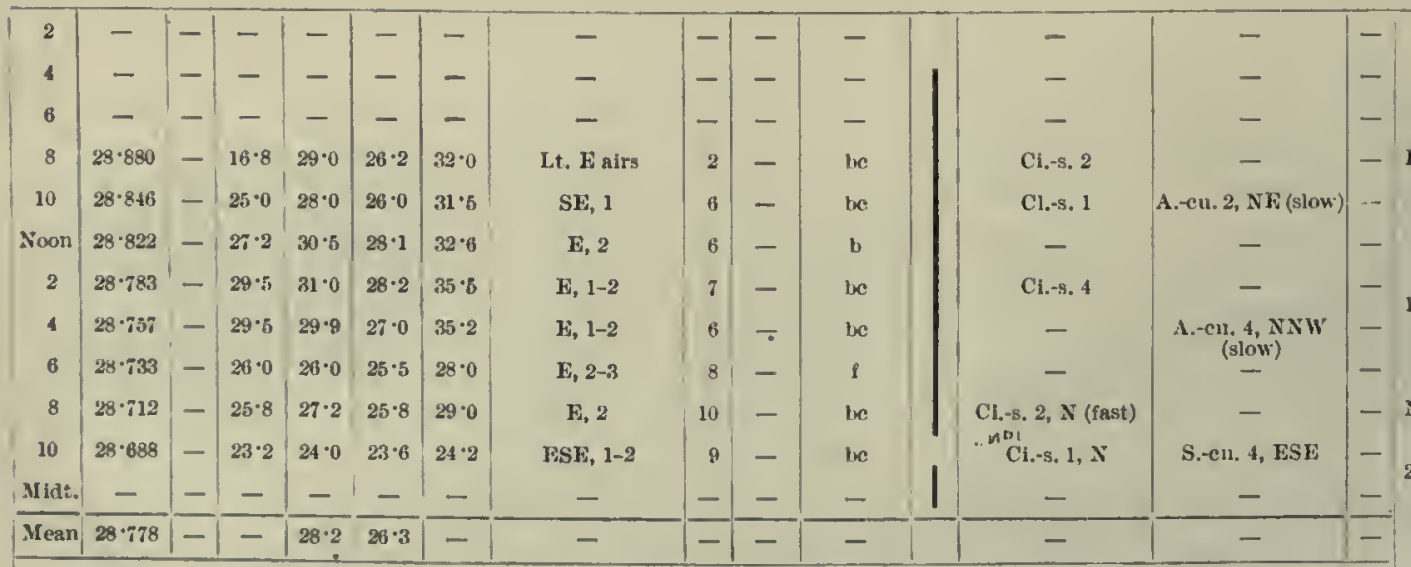

Max. therma. read at $\left\{\begin{array}{l}8 \text { a. } 37^{\circ} \cdot 0 . \\ 8 \text { p. } 31^{\circ} \cdot 8 .\end{array}\right.$ Solar radiation $\left\{\begin{array}{l}\text { small liack bulb } 110^{\circ} \cdot 0 . \\ \text { small loright butb } 64^{\circ} \cdot 0\end{array}\right.$
Evapn.-grammes. Precipn.-in. liemarks.

II eavy fall of snow during night, and all Average uind velocity sluce 8 p.in. Average wimd velocity slite
yesterilay, 2 miles per hour. Sriow falling ; very near to rain.
yesterlay, 2 miles per lions

- Same as at noon.

Bluc sky visible tlirough upper elouds.

Sky risible in SW through breaks in clouds; sun gleaming through A.s. White lsland partly visible; summit of Brown Island olscured by dark Cu. ; base and summit of Discovery visible
western range beginning to sliow up. 
Meteonotogicat. Journal of tire "Discovery.

[1904-January,

\begin{tabular}{|c|c|c|c|c|c|c|c|c|c|c|c|c|c|c|c|c|c|}
\hline \multirow{3}{*}{ ilionr. } & \multirow{3}{*}{ 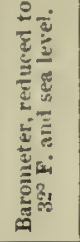 } & \multicolumn{5}{|c|}{ Thermometers. } & \multicolumn{3}{|l|}{ Wind. } & \multirow[b]{3}{*}{ 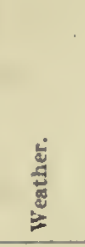 } & \multirow[b]{3}{*}{ 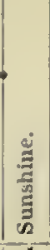 } & \multirow{2}{*}{\multicolumn{4}{|c|}{$\begin{array}{l}\text { Clouds, } \\
\text { Amonut and moveruost. }\end{array}$}} & \multirow[b]{3}{*}{$\frac{5}{\frac{5}{3}}$} & \multirow{3}{*}{ IRemarkn. } \\
\hline & & \multirow{2}{*}{ 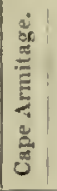 } & \multirow{2}{*}{ 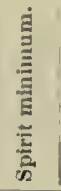 } & \multicolumn{2}{|c|}{ Mercuriul. } & \multirow{2}{*}{ 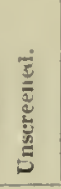 } & \multirow{2}{*}{$\begin{array}{c}\text { Direction and } \\
\text { forces } \\
\text { (lleaturt seale). }\end{array}$} & \multirow{2}{*}{ 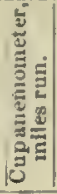 } & \multirow{2}{*}{ 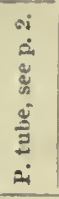 } & & & & & & & & \\
\hline & & & & Dry. & Wet. & & & & & & & - & Lpper. & & Lowwer. & & \\
\hline 2 & - & -1 & - & - & - & - & - & -1 & - & - & & & - & & - & - & \\
\hline 4 & - &. & - & - & - & - & - & - & - & - & & & - & & - & - & \\
\hline 6 & - & - & - & - & - & - & - & - & - & - & & & - & & - & - & \\
\hline 8 & $28 \cdot 680$ & - & $19 \mathrm{~b}$ & $22 \cdot 2$ & $20 \cdot 8$ & $21 \cdot 0$ & Var. F, 1-5 & 2 & - & $\operatorname{lnq}+$ & & & $\div$ & i & - & - & Bluff wered with blanket ; smme Str. on \\
\hline 10 & $28 \cdot 689$ & - & $21 \cdot 5$ & 22.5 & $21 \cdot 0$ & $21 \cdot 0$ & $13,3-5$ & 12 & - & $\operatorname{bq}+$ & & & - & & - & - & $\begin{array}{l}\text { marked; wind aqnaily and sinify is to } \\
\mathrm{SF} \text {. Average wind velocity since }\end{array}$ \\
\hline Xion & $28 \cdot 698$ & - & $19^{\circ} 0$ & $21 \cdot 2$ & $19 \cdot 8$ & $24^{\circ} 0$ & $\mathrm{~F}, 3-5$ & 13 & - & iveq i- & & & - & I & Fr.-n.-cul. 2, Fi & - & 10 p.ru. yesterday, 2 miles per hour. \\
\hline 2 & $28 \cdot 703$ & - & $30 \cdot 5$ & $22 \cdot 5$ & $21 \cdot 0$ & $26 \cdot 0$ & H, 3-5 & 10 & - & luge & & & - & & Fr,-4.,-ru. 1 & - & $\begin{array}{l}\text { apparentiy approaching ; heavy cap on } \\
\text { Bluff and Discovery; Cu, on wetern }\end{array}$ \\
\hline 4 & - & 一 & - & - & - & - & - & - & - & - & & & - & 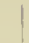 & - & - & $\begin{array}{l}\text { mountains in layers; detached cionda } \\
\text { in SW and W. }\end{array}$ \\
\hline 6 & $28 \cdot 7233$ & - & $18 \cdot 5$ & $19 \cdot 0$ & $18 \cdot 5$ & $24 \cdot 0$ & SI, 3-5 & 13 & - & inge $f$ & & & - & & A. $-(\cdot), 2, \mathrm{SE}$ & - & Noon. Conditions similar. \\
\hline 8 & $28 \cdot 744$ & - & $18 \cdot 5$ & $20 \cdot 1$ & $19 \cdot 1$ & $20 \cdot 5$ & Var. $12,1-5$ & $10^{-}$ & - & oeq + & & & - & & A.-ค11. 7 & - & i p.r. Blut obsenrerl; summits of \\
\hline 111 & - & - & - & - & - & - & - & - & - & - & & - & - & & - & - & $\begin{array}{l}\text { seured; sonre layers of } S \text {.-cu. are about } \\
\text { Discovery and west thn range; the hank }\end{array}$ \\
\hline Midt. & 一 & - & - & - & - & - & - & - & 一 & 一 & & & - & & - & - & of $\mathrm{A}, \mathrm{-g}$. Is moving very slowiy from SE. \\
\hline УЕя & $28 \cdot 708$ & - & - & $21 \cdot 3$ & $20 \cdot 0$ & - & 一 & -1 & - & - & & & - & & - & - & $\begin{array}{l}\text { Arift, in Stralt to } S \text {; seudi flylng raphliy } \\
\text { to } \mathrm{N} \text {; land to } S \text { covereal wilh storin }\end{array}$ \\
\hline & s. thern & 2. res & i at \{ & a. 2 & & Sol & arliation $\left\{\begin{array}{l}\text { sma } \\
\text { sma }\end{array}\right.$ & blac & ondts & $7^{\circ \cdot 0}$ & & gip. & grain & & Precipn. - & & $\begin{array}{l}\text { clourts; heavy masslve roll Cu, to } \mathrm{W} \\
\text { and SW; irlft in squalls. }\end{array}$ \\
\hline
\end{tabular}

13 th.

\begin{tabular}{|c|c|c|c|c|c|c|c|c|c|c|c|c|c|c|}
\hline 2 & - & - & - & - & - & - & - & - & - & - & - & - & - & \multirow{14}{*}{ 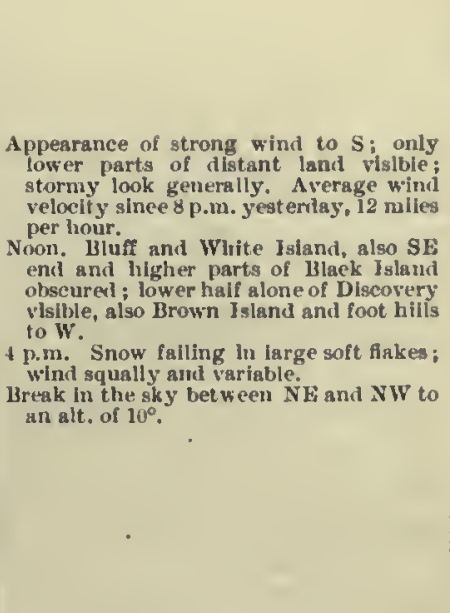 } \\
\hline 4 & - & - & - & - & - & - & - & - & - & - & - & - & - & \\
\hline 6 & - & - & - & - & - & - & - & - & - & - & - & - & - & \\
\hline 8 & $28 \cdot 816$ & - & $19 \cdot 2$ & $24 \cdot 0$ & $23 \cdot 0$ & $25 \cdot 0$ & SSE, 2-4 & 12 & - & ocat & - & S.-cu. 10 & - & \\
\hline 10 & - & - & 一 & - & - & - & - & - & - & - & - & - & - & \\
\hline Nom & $28 \cdot 859$ & - & $21 \cdot 0$ & $23 \cdot 1$ & $21 \cdot 8$ & $2 x \cdot 1$ & Var. SE, $2-\vec{\imath}$ & 15 & - & $o q+$ & - & S. 10 & - & \\
\hline 2 & - & - & - & - & - & - & - & - & - & - & - & - & - & \\
\hline 4 & $2 x \cdot 878$ & - & $21 \cdot 0$ & $23 \cdot 5$ & $22 \cdot 5$ & $24 \cdot 6$ & F, 3-4 & 11 & - & $o q+* *$ & - & N. 10 & - & \\
\hline 6 & - & - & - & - & - & - & - & - & - & - & - & - & - & \\
\hline 8 & $28 \cdot 898$ & - & $20 \cdot 9$ & $21 \cdot 6$ & $21 \cdot 2$ & $22 \cdot 0$ & FNF, 1-2 & 10 & 一 & ocq $+=$ & - & N. 10, SSE & - & \\
\hline 10 & - & - & - & - & - & - & - & - & - & - & - & - & - & \\
\hline Midt. & - & - & - & - & - & - & - & - & - & - & - & - & - & \\
\hline \multirow[t]{2}{*}{ Mean } & $28 \cdot 863$ & - & - & $23 \cdot 1$ & $22 \cdot 1$ & - & - & - & - & - & $\rightarrow$ & - & - & \\
\hline & \multicolumn{6}{|c|}{ Max. titern. rearl at $\left\{\begin{array}{l}8 \text { a. } 24^{\circ} 0 \\
8 \mathrm{p} \cdot 25^{\circ} \cdot 3\end{array}\right.$} & 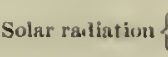 & orrgm & & \multicolumn{2}{|c|}{ Evapn. - graunues. } & \multicolumn{2}{|l|}{ Precipn. - in. } & \\
\hline
\end{tabular}

14 th.

\begin{tabular}{|c|c|c|c|c|c|c|c|c|c|c|c|c|c|c|}
\hline 2 & - & - & - & - & - & - & - & -1 & - & - & - & - & - & \multirow{11}{*}{ 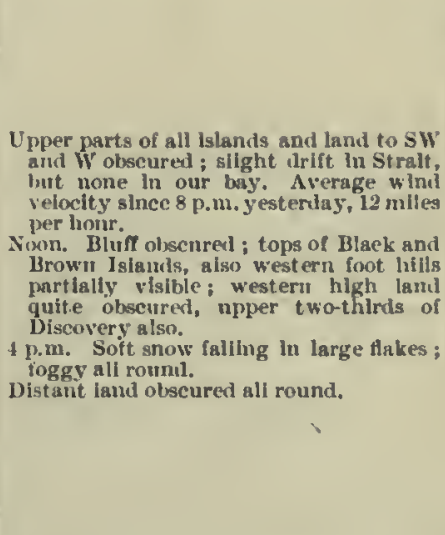 } \\
\hline 4 & - & -1 & - & - & - & - & - & - & - & - & - & - & - & \\
\hline ; & - & - & - & - & - & - & - & - & - & - & - & - & - & \\
\hline 8 & $29 \cdot 912$ & - & $18 \cdot 6$ & $211^{\circ}$ & $20 \cdot 8$ & $21: 5$ & ENE, 2-3 & 12 & - & oc & - & S. 10 & - & \\
\hline 10 & - & - & - & - & - & - & - & $1-$ & - & - & - & - & - & \\
\hline Noon & $28 \cdot 919$ & - & $21) \cdot 2$ & $24: 0$ & $23 \cdot 9$ & $28 \cdot 0$ & EXIs, 2 & 12 & - & $n+* *$ & - & X. 30, ST: & - & \\
\hline 2 & - & - & $\dot{-}$ & - & - & - & - & - & - & - & - & - & - & \\
\hline 9 & $28 \cdot 992$ & - & $24 \cdot 6$ & $25 \cdot 2$ & $25 \cdot 0$ & $28 \cdot 0$ & Lt. S nirs & 3 & - & $\alpha *$ & - & N. 10, S & - & \\
\hline 10 & - & - & $\ddot{-}$ & - & - & - & - & - & - & - & - & - & - & \\
\hline Mirit. & - & $=$ & - & - & - & - & - & - & - & - & - & $=$ & $=$ & \\
\hline Mean & $28-939$ & $1-$ & - & $24 \cdot 2$ & $23 \cdot 8$ & - & - & - & -1 & - & - & - & - & \\
\hline
\end{tabular}


1904-January.]

16th,

\begin{tabular}{|c|c|c|c|c|c|c|c|c|c|}
\hline \multirow{3}{*}{ IIour. } & \multirow{3}{*}{ 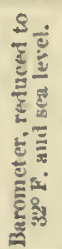 } & \multicolumn{5}{|c|}{ Thorinometerss. } & \multicolumn{3}{|c|}{ Wind. } \\
\hline & & घั & $\underline{E}$ & Mer & urial. & & & & $\stackrel{3}{4}$ \\
\hline & & हैं & 至 & Dry. & Wet. & 竧 & (Beaufort scale) & 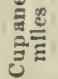 & نे \\
\hline
\end{tabular}

Meteorologicat، Journat, of tile "Discovfry."

\begin{tabular}{|c|c|c|c|c|c|c|c|c|c|c|c|c|c|c|c|}
\hline 2 & - & - & - & - & - & - & - & -1 & - & - & & - & - & - & \\
\hline 4 & - & - & - & - & - & - & - & - & - & - & & - & - & - & \\
\hline 6 & - & - & - & - & - & - & - & - & - & - & & - & - & - & \\
\hline 8 & $29 \cdot 098$ & - & $17 \cdot 8$ & $18 \cdot 8$ & $18 \cdot 5$ & $19 \cdot 5$ & FNE, 3-4 & 7 & - & efr & • & - & Cu. 5, ESE & - & Very sllght surface drift. Average wind \\
\hline 10 & - & - & - & - & - & - & - & - & - & - & 1 & - & - & - & 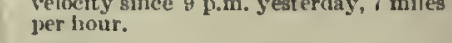 \\
\hline Nuon & $29 \cdot 048$ & - & $17 \cdot 0$ & $19 \cdot 0$ & $19 \cdot 0$ & $22 \cdot 0$ & $\mathbf{F}, 5-7$ & 21 & - & bc + & & $\begin{array}{l}\text { Cl.-er. 1, SW } \\
\text { (slow) }\end{array}$ & S.-cu., EXlin,(fast) & -1 & $\begin{array}{l}\text { Land to W quite olseured by drift, that } \\
\text { In SW and S partially so. }\end{array}$ \\
\hline 2 & - & - & - & - & - & - & - & - & - & - & & - & - & - & \\
\hline 4 & $29 \cdot 078$ & 一 & $18 \cdot 0$ & $20 \cdot 0$ & $19 \cdot 5$ & $24 \cdot 6$ & E, 4-7 & 17 & - & $\operatorname{lnq} \div$ & & - & - & - & $\begin{array}{l}\text { Detrelied C11. passing rapidly from } \mathrm{W} \text {; } \\
\text { heavy drift. }\end{array}$ \\
\hline 6 & - & - & - & - & - & - & - & - & - & - & 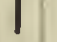 & - & - & - & \\
\hline 8 & $29 \cdot 063$ & - & $17 \cdot 7$ & $20 \cdot 0$ & $19 \cdot 0$ & $26 \cdot 3$ & 15,4 & 19 & - & $h e i^{*}$ & 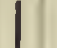 & Cl.-elt, 2 & Cu. 5, NXW & - & $\begin{array}{l}\text { Low bank of Cn. behind Brown Island, and } \\
\text { obscuring westem mountains'and parts }\end{array}$ \\
\hline 10 & - & - & - & - & - & - & - & - & - & - & ? & - & - & - & $\begin{array}{l}\text { of Discovery refraction to } S \text { and SW; } \\
\text { drift in Strait. }\end{array}$ \\
\hline Misit. & - & - & - & - & - & - & - & - & - & - & & - & - & - & \\
\hline Mrean & $29 \cdot 081$ & -1 & - & $19 \cdot 5$ & $19 \cdot 0$ & - & - & - & - & - & & - & - & - & \\
\hline
\end{tabular}

Henurks.

C'louds,
Amount and uovencut.
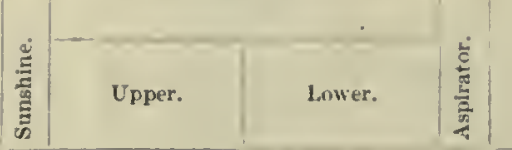

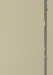


Methorological, JourNal, of th: "Discoveliy."

[1904-January.

\begin{tabular}{|c|c|c|c|c|c|c|c|c|c|c|c|c|c|c|c|}
\hline \multirow{3}{*}{ Hour. } & \multirow{3}{*}{ 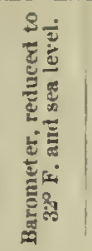 } & \multicolumn{5}{|c|}{ Thermoineters. } & \multicolumn{3}{|l|}{ Wind. } & \multirow[b]{3}{*}{ 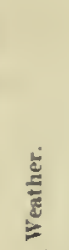 } & \multirow[b]{3}{*}{ 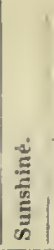 } & \multirow{2}{*}{\multicolumn{2}{|c|}{$\begin{array}{l}\text { Clours, } \\
\text { Anount aud movement. }\end{array}$}} & \multirow[b]{3}{*}{$\frac{\stackrel{5}{5}}{\frac{5}{2}}$} & \multirow{3}{*}{ Remarks. } \\
\hline & & \multirow{2}{*}{ 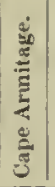 } & \multirow{2}{*}{ 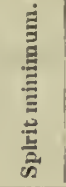 } & \multicolumn{2}{|c|}{ Mercurial. } & \multirow{2}{*}{ 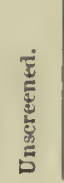 } & \multirow{2}{*}{$\begin{array}{c}\text { Dlrection and } \\
\text { force } \\
\text { (Beaufort scale). }\end{array}$} & \multirow{2}{*}{ 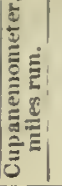 } & \multirow{2}{*}{ 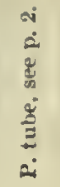 } & & & & & & \\
\hline & & & & Dry. & Wet. & & & & & & & Ipper. & Lower. & & \\
\hline 2 & - & -1 & - & - & - & - & - & -1 & + & - & 1 & - & - & - & \multirow{5}{*}{ 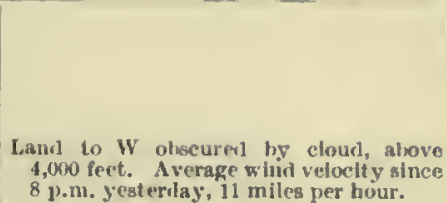 } \\
\hline 4 & - & - & - & - & - & - & - & -1 & - & - & & - & - & - & \\
\hline 6 & - & - & - & - & - & - & - & -1 & - & - & 1 & $\cdot$ & - & - & \\
\hline 8 & 23) 035 & - & $17 \cdot 5$ & $19 \cdot 7$ & $18^{\circ} 0$ & $19 \cdot 5$ & $1 ;, 3-4$ & 11 & - & e & 1 & - & Cu. 6 & - & \\
\hline 10 & - & - & - & - & - & - & - & -1 & - & - & 3 & - & - & - & \\
\hline Noon & $29 \cdot 002$ & - & $17 \cdot 2$ & $20 \cdot 0$ & $18 \cdot 0$ & $2 u \cdot 2$ & $\mathrm{E}$ by $\mathrm{N}, 4$ & 12 & - & $c+4$ & & - & A. $-8 ., A_{.}-11.9$. Xis & - & \multirow{2}{*}{$\begin{array}{l}\text { Sun gleaming ; Str. in SW and w } \\
\text { obscuriug ujper haif of Discovery and } \\
\text { western range. }\end{array}$} \\
\hline 2 & - & - & - & - & - & - & - & - & - & - & & - & - & - & \\
\hline 4 & $29 \cdot 05 \%$ & - & $18 \cdot 0$ & $20 \cdot 0$ & $18 \cdot 2$ & $21 \cdot 0$ & E, 3-4 & 13. & - & $0 \div$ & 1 & - & . S. 11) & - & \multirow[t]{2}{*}{ Low fog banks approaching from $w$. } \\
\hline ii & - & - & - & - & - & - & - & - & - & - & $i$ & - & - & - & \\
\hline 8 & $28 \cdot 965$ & - & - & $19 \cdot 0$ & $17 \cdot 2$ & $19 \cdot 3$ & $\mathrm{E}, 3-4$ & 13 & - & $b e+$ & 1 & Ci. 4 & - & - & \multirow{5}{*}{$\begin{array}{l}\text { Discovery summit ohscured in iight Str. } \\
\text { moklerate drift in Strait. }\end{array}$} \\
\hline 10 & - & - & - & - & - & - & - & - & - & - & & - & - & - & \\
\hline Mint. & - & - & - & - & - & - & - - & - & - & - & & - & - & - & \\
\hline$\overline{\text { Mexu }}$ & $29 \cdot 029$ & -1 & - & $19 \cdot 7$ & $17 \cdot 9$ & - & - & -1 & - & - & & - & - & - & \\
\hline \multicolumn{6}{|c|}{ 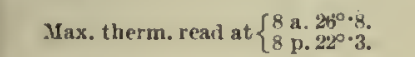 } & Sola & $r$ rarlation $\left\{\begin{array}{l}\text { small } \\
\text { small }\end{array}\right.$ & $\begin{array}{l}\text { black } \\
\text { brigit }\end{array}$ & $\begin{array}{l}\text { bulb } \\
\text { t bull }\end{array}$ & (o. & \multicolumn{2}{|c|}{ Esapu. - grammes. } & l'recipul. - ill. & & \\
\hline
\end{tabular}

19 th.

\begin{tabular}{|c|c|c|c|c|c|c|c|c|c|c|c|c|c|c|}
\hline 2 & - & - & - & - & - & - & - & - & - & - & - & - & - & \multirow{5}{*}{$\begin{array}{l}\text { Very thick snow falling; limit of vision } \\
1 \text { milc. Average wind velocity shice } \\
8 \text { p.m. yest eriay, } 6 \text { miles per hour. }\end{array}$} \\
\hline 4 & - & - & - & - & - & - & - & - & - & - & - & - & - & \\
\hline 6 & - & - & - & - & - & - & - & - & - & - & - & - & - & \\
\hline 8 & 28.991 & - & $13 \cdot 0$ & $17 \cdot 6$ & $17 \cdot 2$ & $18 \cdot 8$ & S by 1:, 2 & G & - & $\operatorname{nef} *^{2}$ & - & N. 10 & - & \\
\hline 10 & - & - & - & - & - & - & - & - & - & - & - & - & - & \\
\hline Xoon & $29 \cdot(001$ & - & $16 \cdot 8$ & $18 \cdot 2$ & $18 \cdot 0$ & $20 \cdot 9$ & Var. SSE, 3-4 & 5 & - & ofq $*^{2}$ & - & X. 10 & - & \multirow{7}{*}{$\begin{array}{l}\text { Very thick, uo distant iand visible, and } \\
\text { near land also obscured; snowing In } \\
\text { iarge flakes and tiiekiy. } \\
\text { Snow falling, soft and large flakes. } \\
\text { Thick suow fallug, obsenring all land but } \\
\text { Ilut Point. }\end{array}$} \\
\hline 2 & - & - & - & - & - & - & - & $1-$ & - & - & - & - & - & \\
\hline 4 & $29 \cdot 012$ & - & $16 \% 5$ & $17 \cdot 0$ & $17 \cdot 0$ & - & SE, 2-3 & 10 & - & of $+*$ & - & N. 10 & - & \\
\hline$B$ & - & - & - & - & - & - & - & - & - & - & - & - & - & \\
\hline 8 & $29^{\circ} 024$ & - & $16 \cdot 5$ & 16.5 & 165 & $17 \cdot 2$ & $\mathrm{E} \log \mathrm{N}, 2-3$ & 10 & - & o* & - & x. 10 & - & \\
\hline 10 & - & - & - & - & - & - & - & - & - & - & - & - & - & \\
\hline Midt. & - & $=$ & - & - & - & - & - & $=$ & - & - & - & - & - & \\
\hline$\overline{\text { Meau }}$ & $29 \cdot 007$ & -1 & - & $77 \cdot 3$ & $17 \cdot 2$ & - & - & 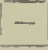 & - & - & - & - & - & \\
\hline & $\begin{array}{l}\text { x. therm } \\
\text { read at }\end{array}$ & & & & & $\mathrm{sm}$ & $\begin{array}{l}\text { ch bull } \\
\text { ght bilh }\end{array}$ & & & nom & recimn & $8 \mathrm{a}$ & & \\
\hline
\end{tabular}

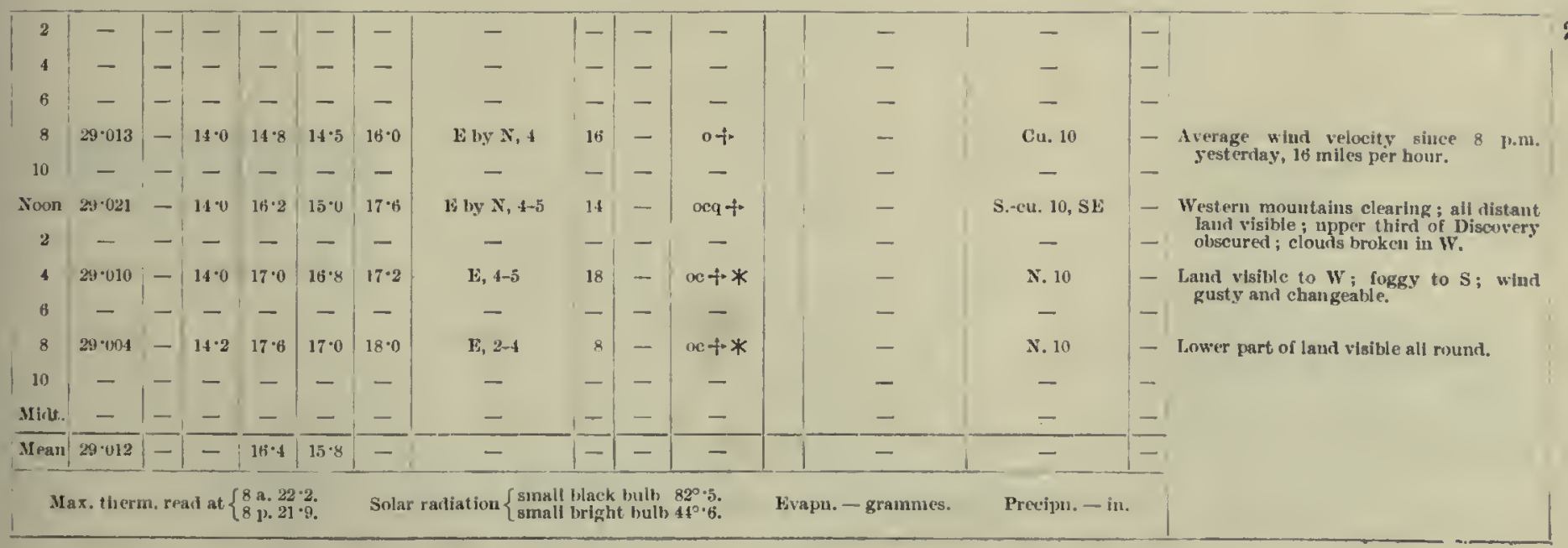


1904 -January, ]

22ud.

\begin{tabular}{|c|c|c|c|c|c|c|c|c|c|}
\hline \multirow{3}{*}{ Hqur } & \multirow{3}{*}{ 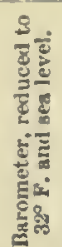 } & \multicolumn{5}{|c|}{ Thermometers. } & \multicolumn{3}{|l|}{ Wind. } \\
\hline & & 咹 & $\vec{z}$ & Men & surlal. & & & & $\stackrel{\text { ه }}{\stackrel{2}{\alpha}}$ \\
\hline & & 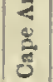 & 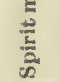 & Dry. & Wet. & 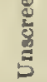 & (Be & 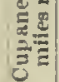 & ڤેّ \\
\hline
\end{tabular}$$
-
$$

Meteolological Jourat of the "Discoveliy."

(2)
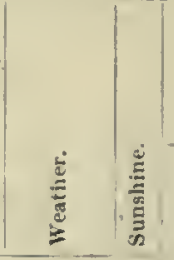

Clouds,
Imount aud novement.

Lower.
Remark8.

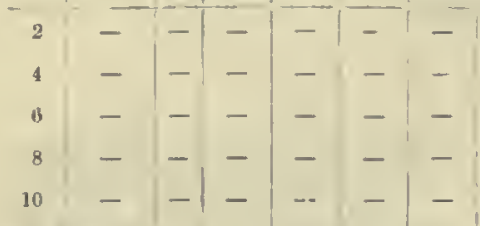

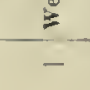

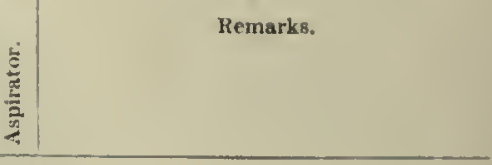

$$
\begin{array}{llll}
- & - & - & - \\
- & - & - & - \\
- & - & - & -
\end{array}
$$

by $x, 4-5) \quad 16-$ ocq $+* *$

Nuon $29 \cdot 0321-\quad 9 \cdot 0 \quad 13 \cdot 0 \quad 13 \cdot 0 \quad 14 \cdot 2$

\begin{tabular}{c|c|cc|cc|c}
2 & - & - & - & - & - & - \\
4 & $29 \cdot 056$ & - & $11 \cdot 0$ & 13.8 & $13 \cdot 4$ & $14 \cdot 2$
\end{tabular}

\begin{tabular}{cc|c|c|cc|c}
6 & - & - & - & - & - & - \\
8. & $29 \cdot 062$ & - & $13 \cdot 3$ & $15 \cdot 9$ & $15 \cdot 6$ & $13 \cdot 3$
\end{tabular}

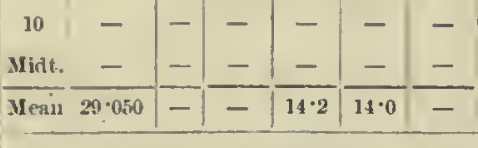

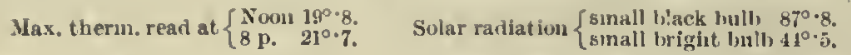

$\mathrm{XE}, 3-4 \cdot 15:-$ of $+*$

VNE, 2

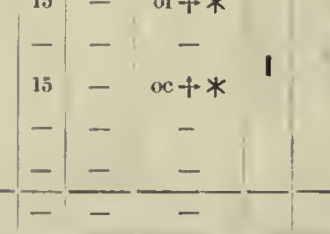

23rd.

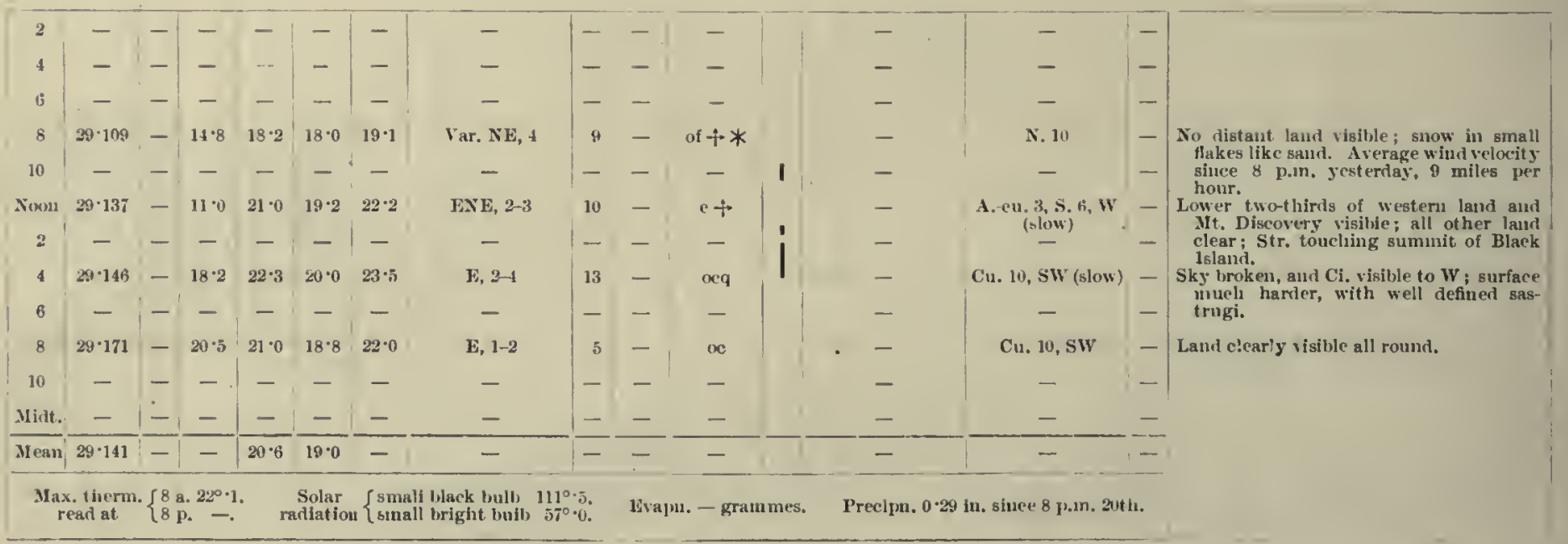

24th.

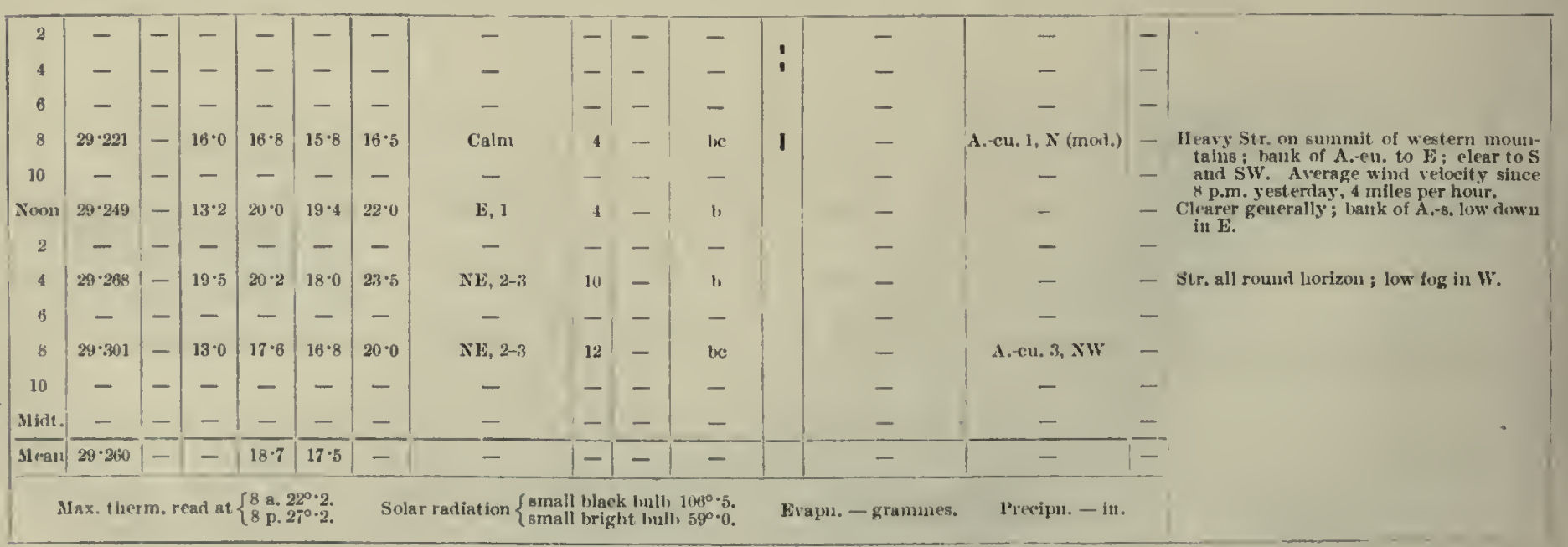


Mrteonological Jounal, of THe: "Discovery."

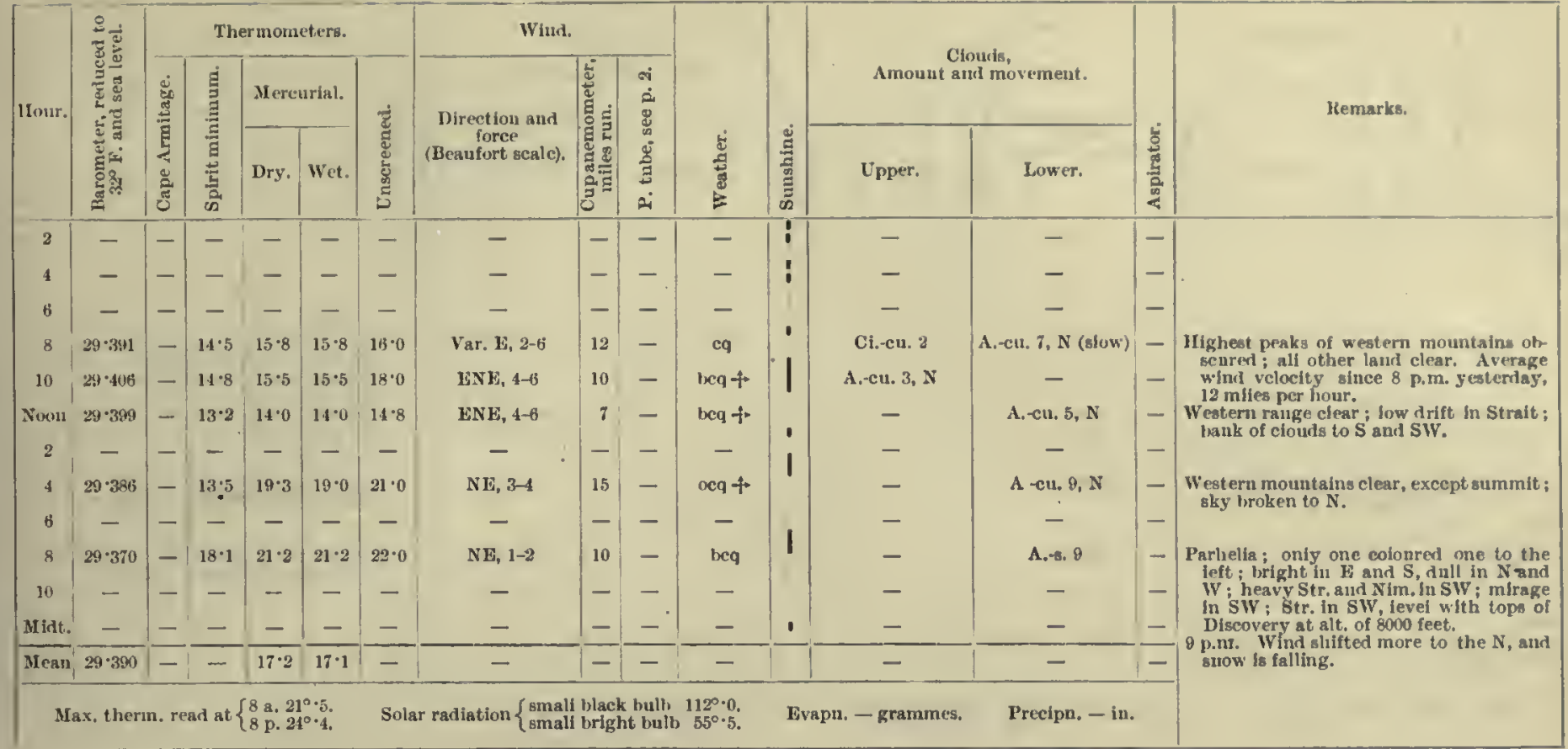

\begin{tabular}{|c|c|c|c|c|c|c|c|c|c|c|c|c|c|c|}
\hline 2 & - & - & - & - & - & 一 & - & - & - & - & $\div$ & - & - & \\
\hline 4 & - & - & - & - & - & - & - & - & - & - & - & - & - & \\
\hline is & - & - & - & - & - & - & - & - & - & - & - & - & - & \\
\hline 8 & $2 y \cdot 310$ & - & $10 \cdot 3$ & $12 \cdot 2$ & $12 \cdot 2$ & $12 \cdot 2$ & Var. E, 4-5 & - & $\rightarrow$ & of $-1+*$ & - & N. 10 & - & No land visible to $W$; hcavy surface drift. \\
\hline 10 & - & - & - & - & - & - & - & - & - & - & - & - & - & \\
\hline Noon & $29 \cdot 271$ & - & $11 \cdot 2$ & $15 \cdot 5$ & $15 \% 5$ & $16 \cdot 5$ & $\mathbf{k}, 3-5$ & 14 & 一 & ocq $\mathrm{i}^{-}$ & - & S.-cu. $\theta$ & - & Cleariug to $\mathrm{S}$ aud round to $\mathrm{w}$; drift fow \\
\hline 2 & - & - & - & - & - & 一 & - & - & - & - & - & - & - & westem range aud on biscovery; de- \\
\hline 4 & $29 \cdot 237$ & - & $11 \cdot 5$ & 18.8 & $18 \cdot 0$ & $20 \cdot 4$ & Eพพพ, 2-3 & 13 & - & $\alpha$ & - & A.-cu. 9, NW & - & $\begin{array}{l}\text { generally. } \\
\text { p.m. Ifeavy detached } N \text { iin. to } W \text { and } N \text {. }\end{array}$ \\
\hline 6 & 一 & - & - & - & - & - & - & - & - & - & - & - & - & \\
\hline 8 & $29 \cdot 206$ & - & $17 \cdot 0$ & $20 \cdot 8$ & $10 \cdot 2$ & $22 \cdot 2$ & NE, $2-3$ & 10 & - & be & - & A.-cu. 5, NW & - & $\begin{array}{l}\text { Clearing generally; heary roll } \mathrm{Cu} \text {. on } \\
\text { western mountains ; bank of cloud to } \mathrm{S} \text {. }\end{array}$ \\
\hline 10 & - & - & - & - & - & - & - & - & - & 一 & - & - & - & \\
\hline Mrist. & - & - & - & - & - & - & - & - & - & - & - & - & - & \\
\hline Mean & $29 \cdot 256$ & $1-1$ & - & $16 \cdot 8$ & $16 \cdot 2$ & - & - & $\overline{-}$ & - & - & - & - & - & \\
\hline
\end{tabular}

26 th.

\begin{tabular}{|c|c|c|c|c|c|c|c|c|c|c|c|c|c|c|}
\hline 2 & - & -1 & - & - & - & - & - & -1 & - & - & - & - & -1 & \multirow{10}{*}{ 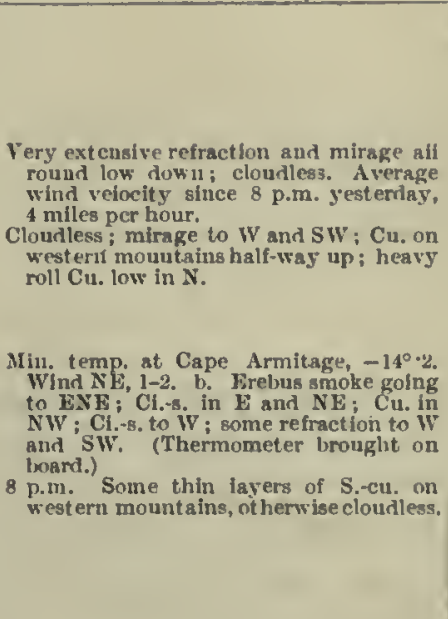 } \\
\hline 4 & - & - & - & - & - & - & - & - & - & - & - & - & - & \\
\hline 6 & - & - & - & - & - & - & - & - & - & - & - & - & - & \\
\hline 8 & $29 \cdot 121$ & - & $4 \cdot 0$ & $11 \cdot 1$ & $9 \cdot 0$ & $23 \cdot 0$ & Calm & 4 & - & b & - & - & - & \\
\hline Noon & $29 \cdot 065$ & 0.0 & $11 \cdot 0$ & $24 \cdot 8$ & $22 \cdot 0$ & $26 \div 5$ & Lt. $N E$ airs & 2 & - & $b$ & - & - & - & \\
\hline 2 & - & $1-$ & - & - & - & - & - & - & - & - & - & - & - & \\
\hline 8 & $29 \cdot(6013$ & - & $14 \cdot 0$ & $15 \cdot 0$ & $14 \cdot 0$ & 160 & EXF, 2-3 & 14 & - & $b$ & - & - & - & \\
\hline 10 & - & - & - & - & - & - & - & - & - & - & - & - & - & \\
\hline Mint. & - & $1-$ & - & - & - & - & - & - & - & - & - & - & -1 & \\
\hline$\overline{\text { мели }}$ & $\overline{439} \cdot 05.1$ & $1-$ & - & $\overline{17 \cdot 1}$ & $\overline{15 \cdot 1}$ & - & - & $\overline{-}$ & - & - & - & - & - & \\
\hline
\end{tabular}


1904-January.]

Meteorological JoUrNal of til: "Discovery."

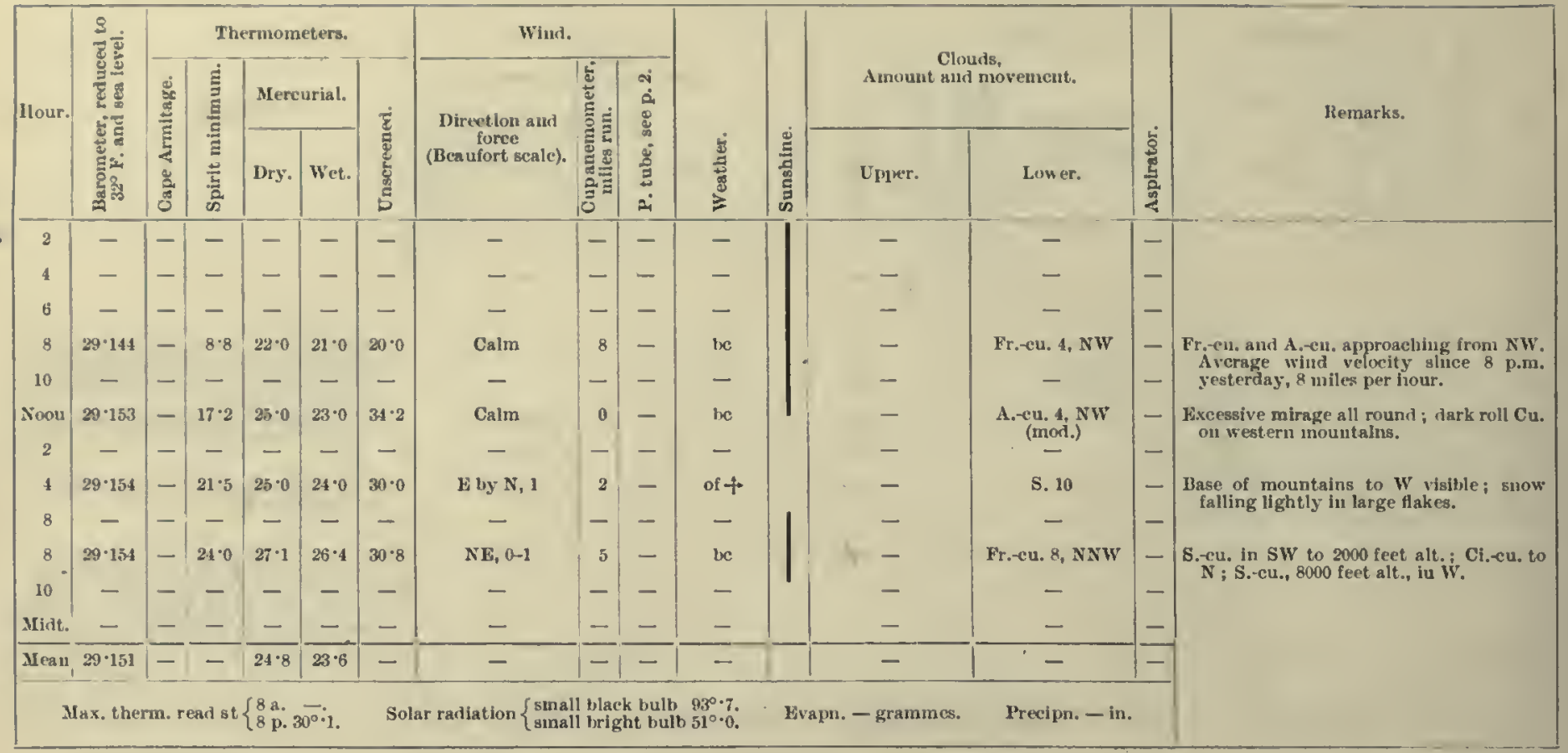

28th.

29 th

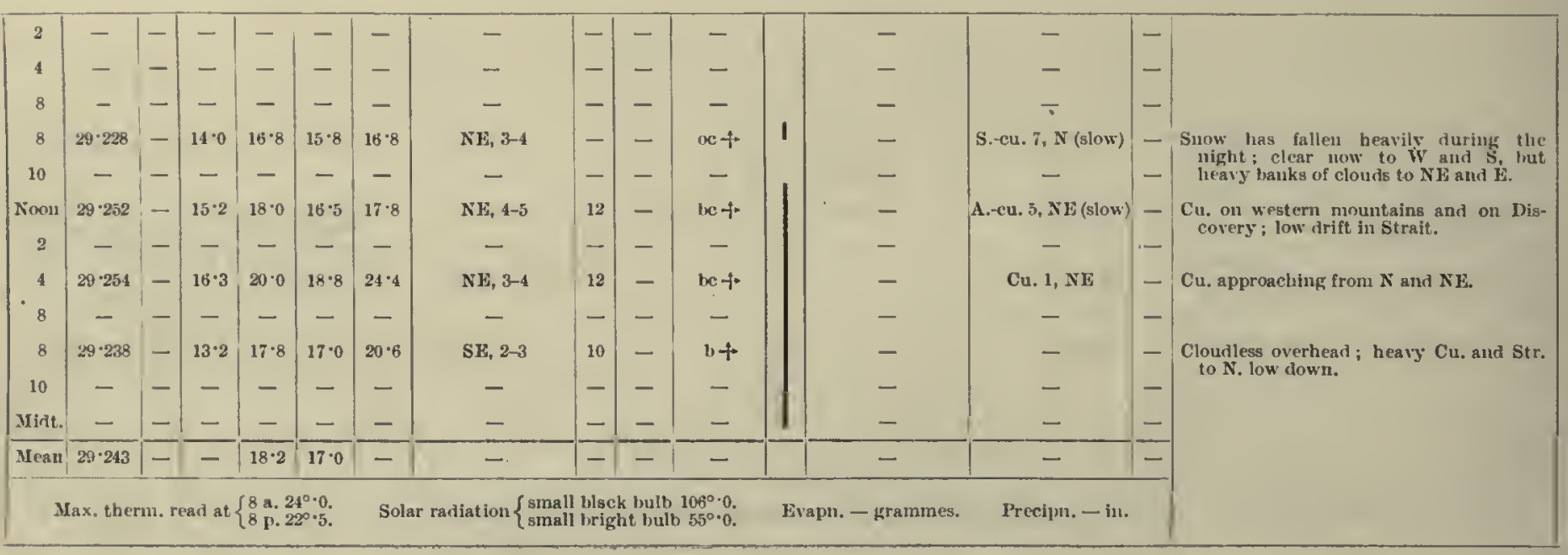

30 th.

\begin{tabular}{|c|c|c|c|c|c|c|c|c|c|c|c|c|c|c|c|}
\hline 2 & - & - & - & - & - & $1-$ & - & $1-$ & - & - & 1 & - & - & - & \multirow{12}{*}{ 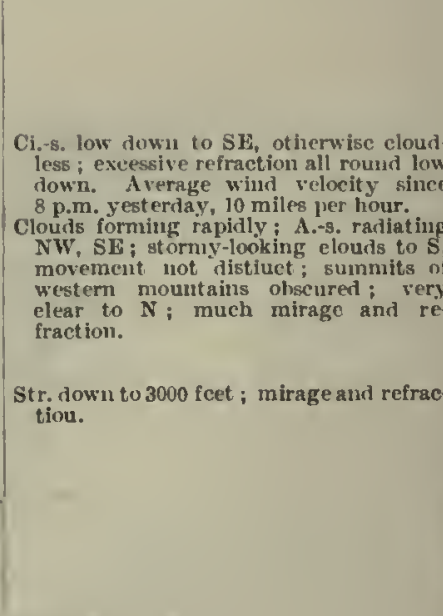 } \\
\hline 4 & - & - & - & - & - & - & - & - & - & - & & - & - & - & \\
\hline 6 & - & - & - & - & - & - & - & - & - & - & & - & - & - & \\
\hline 8 & $29 \cdot 122$ & - & $13 \cdot 2$ & 15.5 & $14: 5$ & $19 \cdot 5$ & $N E_{,}, 2-3$ & 10 & - & bv & & - & - & - & \\
\hline 10 & - & - & - & - & - & - & - & - & - & - & & - & - & - & \\
\hline Nonn & $29 \cdot 080$ & - & 13.0 & $22 \cdot 0$ & $18 \cdot 0$ & $25 \cdot 0$ & NF, $1-2$ & 10 & - & $\operatorname{bev} \oplus$ & 1 & Ci.-s. 3 & A.-5. 4 & - & \\
\hline 2 & - & - & - & - & - & - & - & - & - & - & ! & - & - & - & \\
\hline 8 & $28 \cdot 952$ & - & $22 \cdot 0$ & $23 \cdot 0$ & $21 \cdot 0$ & $23 \cdot 8$ & Calm & 2 & - & $\propto$ & & - & S.eur. 10 & - & \\
\hline 10 & - & - & - & - & - & - & - & - & - & - & & - & - & - & \\
\hline Midt. & - & - & - & - & - & - & - & $1-$ & - & - & & - & - & - & \\
\hline Nean & $29 \cdot 042$ & -1 & - & $22 \cdot 2$ & $19 \cdot 4$ & - & - & $\overline{-}$ & - & - & & - & - & 1 & \\
\hline \multicolumn{6}{|c|}{ Max, therra. rearl at $\left\{\begin{array}{l}8 \text { a. } 24^{\circ} \cdot 0 \\
8 \text { p. } 31^{\circ} \cdot 2 .\end{array}\right.$} & \multicolumn{5}{|c|}{ Solar radlatiou $\left\{\begin{array}{l}\text { small black bulb } 124^{\circ} \cdot 0 . \\
\text { small bright bulth } 64^{\circ} \cdot 2 .\end{array}\right.$} & & yramnes. & \multicolumn{2}{|c|}{ P'rceipu. - in. } & \\
\hline
\end{tabular}


Methorological, Journat, of The "Discoveiry."

[1904-January.

\begin{tabular}{|c|c|c|c|c|c|c|c|c|c|c|c|c|c|c|c|}
\hline \multirow{3}{*}{ IIour. } & \multirow{3}{*}{ 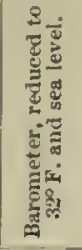 } & \multicolumn{5}{|c|}{ Therinometers. } & \multicolumn{3}{|l|}{ Whu. } & \multirow[b]{3}{*}{ 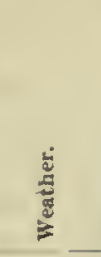 } & \multirow[b]{3}{*}{ 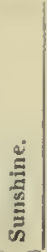 } & \multirow{2}{*}{\multicolumn{2}{|c|}{$\begin{array}{l}\text { Clouris, } \\
\text { Amount and movenent. }\end{array}$}} & \multirow[b]{3}{*}{ 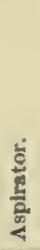 } & \multirow{3}{*}{ Reniarks. } \\
\hline & & \multirow{2}{*}{ 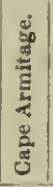 } & \multirow{2}{*}{ 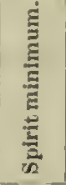 } & \multicolumn{2}{|c|}{ Mereurial. } & \multirow{2}{*}{ 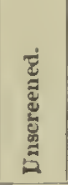 } & \multirow{2}{*}{$\begin{array}{l}\text { Direction auri } \\
\text { force } \\
\text { (Beaufurt scale). }\end{array}$} & \multirow{2}{*}{ 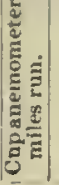 } & \multirow{2}{*}{ 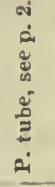 } & & & & & & \\
\hline & & & & Dry: & Wet. & & & & & & & Upper. & Lower. & & \\
\hline 2.30 & - & $1-$ & - & - & - & - & $\mathrm{sW}, 1$ & -1 & - & $o *$ & & - & N. 10 & -1 & Wind sW, 1 ; anowing in heavy thaten, \\
\hline 4 & - & - & - & - & - & - & $\cdots$ & - & - & - & & - & - & - & \\
\hline b & - & - & - & - & - & - & - & - & - & - & & - & - & - & \\
\hline 8 & $28 \cdot 822$ & - & $16 \cdot 8$ & $19 \cdot 0$ & $18 \cdot 0$ & $25 \cdot 5$ & Culns & 2 & - & of $*^{2}$ & & - & N. 10 & - & Snow ing heavily; no diatant faud visible: \\
\hline 10 & - & - & - & - & - & - & - & - & - & - & & - & - & - & velocity since 8 p.m. yest erday, 2 milfes \\
\hline Noou & $28 \cdot 790$ & - & $17 \cdot 8$ & $24 \cdot 0$ & $23 \cdot 2$ & $25 \cdot 8$ & SF, 1-2 & 2 & - & of $*^{2}$ & & - & N. 10 & - & $\begin{array}{l}\text { Snow ing very heavily : flakes sumaller and } \\
\text { yery gott. }\end{array}$ \\
\hline 2 & - & - & - & - & - & - & - & - & - & - & & - & - & - & on loard; no land visibie. \\
\hline 4 & $28 \cdot 767$ & - & $20 \cdot 0$ & $21 \cdot 5$ & $21 \cdot 0$ & $22 \cdot 0$ & HST, 2-3 & 10 & - & of $f * *$ & & - & N. 10 & - & Snowing ; flakes very soft and falling \\
\hline 6 & - & - & - & - & - & - & - & - & - & - & & - & - & - & \\
\hline 8 & $23 \cdot 760$ & - & $20 \cdot 8$ & $21 \cdot 1$ & $20 \cdot 8$ & $22 \cdot 1$ & SF, 1-3 & 12 & - & of $+*$ & & - & N. 10 & - & \\
\hline 10 & - & - & - & - & - & - & - & - & - & - & & - & - & - & \\
\hline Minlt. & - & - & - & - & - & - & - & - & - & - & & - & - & -1 & \\
\hline Mean & $28 \cdot 786$ & - & - & $21 \cdot 4$ & $20 \cdot 8$ & - & - & -1 & - & - & & - & - & - & \\
\hline & the & & at & $\begin{array}{l}\text { a. } 23 \\
\text { p. } 25\end{array}$ & & So & diation $\left\{\begin{array}{l}\text { smal } \\
\text { smal }\end{array}\right.$ & lack & ul! & 0. & & . - grammes. & Precipu. & & \\
\hline
\end{tabular}


1904-Febrnary.]

-

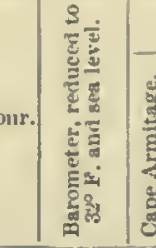

1 st.

\begin{tabular}{|c|c|c|c|c|c|c|c}
\hline 2 & - & - & - & - & - & - & - \\
4 & - & - & - & - & - & - & - \\
8 & - & - & - & - & - & - & - \\
8 & $23 \cdot 750$ & - & $14 \cdot 2$ & $20 \cdot 9$ & $19 \cdot 3$ & $24 \cdot 0$ & Calm \\
10 & $28 \cdot 749$ & - & $20 \cdot 0$ & $22 \cdot 0$ & $20 \cdot 6$ & $25 \cdot 0$ & Calm \\
N.601 & $28 \cdot 734$ & - & $20 \cdot 8$ & $29 \cdot 1$ & $27 \cdot 0$ & $32 \cdot 0$ & Calm \\
2 & - & - & - & - & - & - & - \\
4 & $28 \cdot 721$ & - & $25 \cdot 3$ & $27 \cdot 0$ & $24 \cdot 5$ & $30 \cdot 6$ & NE, 2 \\
6 & - & - & - & - & - & - & - \\
8 & $28 \cdot 728$ & - & $24 \cdot 9$ & $25 \cdot 0$ & $23 \cdot 0$ & $25 \cdot 8$ & Calm \\
10 & - & - & - & - & - & - & - \\
Miidt. & - & - & - & - & - & - & - \\
\hline Mean & $28 \cdot 737$ & - & - & $24 \cdot 8$ & $22 \cdot 9$ & - & - \\
\hline
\end{tabular}

Metroroloficai, Joursai, of the "Discovery."

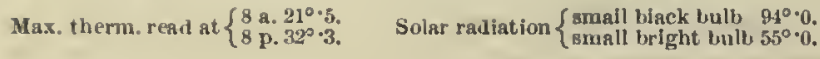

Anumut and noventent.

\begin{tabular}{r|r}
2 & - \\
- & - \\
- & -
\end{tabular}

A.-B. 9
Liเขnarks.

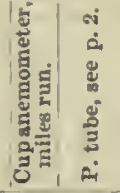

\begin{tabular}{r|r|l}
- & - & - \\
- & - & - \\
- & - & - \\
$z$ & - & $n$
\end{tabular}

$\begin{array}{rrr}0 & - & \text { n } \\ 0 & - & 0 \\ - & - & -\end{array}$

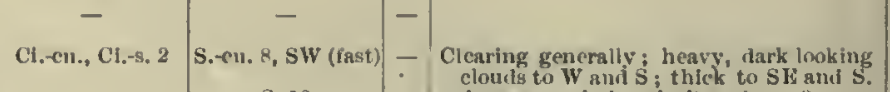

S. 10 vesteriay, 7 miles per liour.

N. 10 but is now elearing quickly. Average whind velneity slnee 8 [p.ul. Snowed hard between! 10 a.m. and noon, Cu. on western luountain leaks.
Cun Water sky to $\mathbf{N}$ and $W$.

\section{2nd.}

\begin{tabular}{|c|c|c|c|c|c|c|c|c|c|c|c|c|c|c|c|}
\hline 2 & - & -1 & - & - & - & - & - & -1 & - & - & & - & - & - & \\
\hline 4 & - & - & - & - & - & - & - & - & - & - & & - & - & - & . \\
\hline 6 & - & - & - & - & - & - & - & - & - & - & & - & - & - & \\
\hline 8 & $28 \cdot 889$ & - & 13.5 & $18 \cdot 0$ & $17 \cdot 2$ & $18 \cdot 6$ & ENE, 3-6 & - & - & $0+$ & & - & S. 10, S (fast) & - & Scud moving up rapidly from $\mathrm{S}$. \\
\hline 10 & - & - & - & - & - & - & - & - & - & - & & - & - & - & \\
\hline Soon & $28 \cdot \sin 0$ & - & $14 \cdot 5$ & $16 \cdot 2$ & $16 \cdot 2$ & $18 \cdot 0$ & Var. $\mathbf{k}, 3-7$ & 17 & - & $0+$ & & - & S. $10, \mathrm{~s}$ & - & Tery heavy gusts all forenoon; no land \\
\hline 2 & - & - & - & - & - & - & - & - & - & - & 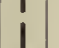 & - & - & - & Sw. \\
\hline 8 & $29 \cdot 015$ & - & $9 \cdot 8$ & $11 \cdot 6$ & $11 \cdot 2$ & $12 \cdot 0$ & NE by $\mathrm{E}, 4-5$ & 23 & - & be +" & i & Cl. 2 & Cu. 3 & - & \\
\hline 10 & $\rightarrow$ & - & - & - & - & - & - & -1 & - & - & & - & - & - & \\
\hline Midt. & - & - & - & - & - & - & - & -1 & - & - & & - & - & - & \\
\hline M can & $28 \cdot 98$ & -1 & - & $14 \cdot 6$ & $14 \cdot 3$ & - & - & -1 & - & - & & - & - & - & \\
\hline & $\begin{array}{l}\text { x. therm } \\
\text { read at }\end{array}$ & & 300 & & $\begin{array}{l}\text { Solar } \\
\text { alliatio }\end{array}$ & $n\left\{\begin{array}{l}\text { sn } \\
\text { sn }\end{array}\right.$ & $\begin{array}{l}\text { ack fiuib } \\
\text { ight ball, }\end{array}$ & & E & $\mathbf{g r}$ & s. & recip & . since 8 a.m. $23 x$ & & \\
\hline
\end{tabular}

$3 r d$.

\begin{tabular}{|c|c|c|c|c|c|c|c|c|c|c|c|c|c|c|c|}
\hline 2 & - & -1 & - & - & $1-$ & - & - & - & - & - & I & - & - & - & \\
\hline 4 & - & - & - & - & - & - & - & - & - & - & $i$ & - & - & - & \\
\hline 6 & - & - & - & - & - & - & - & - & - & - & ' & - & - & - & \\
\hline 8 & $28 \cdot 926$ & - & $7 \cdot 2$ & $17 \cdot 8$ & $16 \cdot 8$ & 22.8 & Calm & s & - & ac & & Ci.-8. 1 & A.-8. 9 & - & $\begin{array}{l}\text { Bluff obseured, also summits of Blaek and } \\
\text { Brown Islands and western range in }\end{array}$ \\
\hline 10 & - & - & - & - & - & - & - & - & - & - & I & - & - & - & $\begin{array}{l}\text { ragged, torn clond; breaks in sky in s } \\
\text { and } \mathrm{s} \text {. Average winl velocity since }\end{array}$ \\
\hline Nonon & $28 \cdot 828$ & - & $16: 5$ & $25 \cdot 0$ & $24 \cdot 0$ & $24 \cdot 0$ & Lt. SW alrs & 4 & - & ac & ' & Ci.s. $4, \mathrm{SW}$ & A.-s. 6, S & - & 8 p.m. yesteriay, 6 miles per hour. \\
\hline 2 & - & - & - & - & - & - & - & - & - & - & . & - & - & - & romid, especlasly to $\mathrm{S}$ and $\mathrm{SW}$; only \\
\hline 8 & $28 \cdot 721$ & - & $200^{\circ}$ & $22 \cdot 0$ & $20 \cdot 8$ & $22 \cdot 0$ & NE, 1-2 & 9 & - & 0 & & - & S. 10 & - & 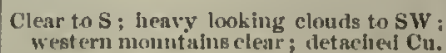 \\
\hline 10 & - & - & - & - & - & - & - & - & - & - & & - & - & - & on Discovery. \\
\hline Miat. & - & -1 & - & - & - & - & 一 & - & - & - & ' & - & - & - & \\
\hline$\overline{\mathrm{Menn}}$ & $\overline{28 \cdot 854}$ & $\overline{-1}$ & - & $\overline{21 \cdot 7}$ & $20 \cdot 7$ & - & - & - & - & - & & - & - & - & \\
\hline
\end{tabular}


Mfteorotogtcat, Journat, of THE "Discovrry."

[1904-February.

\begin{tabular}{|c|c|c|c|c|c|c|c|c|c|c|c|c|c|c|c|}
\hline \multirow{3}{*}{ 1lonr. } & \multirow{3}{*}{ 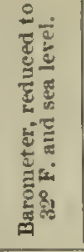 } & \multicolumn{5}{|c|}{ Thermometers, } & \multicolumn{3}{|l|}{ Wind. } & \multirow[b]{3}{*}{$\begin{array}{l}\stackrel{L}{E} \\
\stackrel{E}{E}\end{array}$} & \multirow[b]{3}{*}{ 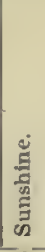 } & \multirow{2}{*}{\multicolumn{2}{|c|}{$\begin{array}{l}\text { Clouds, } \\
\text { Anomit alul unoventent. }\end{array}$}} & \multirow[b]{3}{*}{ 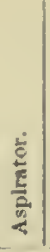 } & \multirow{3}{*}{ IRemarks. } \\
\hline & & \multirow{2}{*}{ 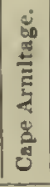 } & \multirow{2}{*}{ 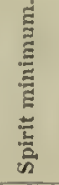 } & \multicolumn{2}{|c|}{ Mereurial. } & \multirow{2}{*}{ 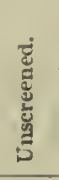 } & \multirow{2}{*}{$\begin{array}{l}\text { Dircetion and } \\
\text { force: } \\
\text { (Branfort seale). }\end{array}$} & \multirow{2}{*}{ 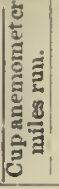 } & \multirow{2}{*}{ 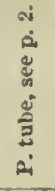 } & & & & & & \\
\hline & & & & Dry. & Wet. & & & & & & & Upler. & Lower. & & \\
\hline 2 & - & - & - & - & - & - & - & -1 & - & - & & - & - & - & \\
\hline 4 & - & - & - & - & - & - & - & - & - & - & 1 & - & - & - & \\
\hline 6 & - & - & - & - & - & - & - & - & - & - & & - & - & - & \\
\hline 8 & $28 \cdot 711$ & - & $7 \cdot 5$ & $17 \cdot 0$ & $16 \cdot 0$ & 30.0 & Calm & - & - & live & & $\begin{array}{c}\text { Cl. } 8.2, \text { SE (very } \\
\text { siow); C1. } 3\end{array}$ & - & - & $\begin{array}{l}\text { Cl.-s. radiating } W \text { and } F \text { : mueh refract lou; } \\
\text { besvy roll Cin. on wistern monntslus, }\end{array}$ \\
\hline lu & - & - & - & - & - & - & - & - & - & - & & - & - & - & 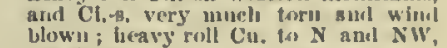 \\
\hline $\mathbf{N}($ (x)แ & $28 \cdot 7: 5$ & - & $16 \cdot 8$ & $3 i) \cdot 5$ & in) 0 & $28 \cdot 0$ & Calm & 2 & - & In & & $\begin{array}{c}\text { Ct.-s, } 5, \text { SF (very } \\
\text { sion) }\end{array}$ & - & - & $\begin{array}{l}\text { low dowid. } \\
\text { Noon. Cl.-s. as at. } 8 \text { oclork ; detached Cu. }\end{array}$ \\
\hline 3 & - & - & - & - & - & - & - & 一 & - & - & & - & - & - & on smmults of westeri inountalus ; \\
\hline 4 & $\begin{array}{c}28 \cdot 765 \\
-\end{array}$ & - & $\begin{array}{c}22 \cdot 5 \\
-\end{array}$ & $\begin{array}{l}25 \cdot 0 \\
-\end{array}$ & $\begin{array}{c}22 \cdot 3 \\
-\end{array}$ & $\begin{array}{c}28 \cdot 5 \\
-\end{array}$ & $\begin{array}{l}\text { Nis, } 1 \\
-\end{array}$ & $\begin{array}{l}2 \\
-\end{array}$ & - & - & 1 & - & $\begin{array}{c}\Lambda_{0}-9.8, N \text { (fust) } \\
-\end{array}$ & - & $\begin{array}{l}\text { to N. } \\
\text { p.nh. Thin A.-8, with Cl. ln Slin and Cu. } \\
\text { to W. }\end{array}$ \\
\hline 8 & - & - & $23 \cdot 0$ & $24 \cdot 2$ & $23 \cdot 0$ & $24 \cdot 8$ & Caim & 2 & - & ov & & - & S. 10 & - & Str, alout 3100 leet alt. \\
\hline 10 & - & - & - & - & - & - & - & - & - & - & & - & - & - & \\
\hline Midt. & - & - & - & - & - & - & - & - & - & - & & - & - & - & \\
\hline Mean & $28 \cdot 738$ & - & - & $24 \cdot 3$ & $\overline{22 \cdot 8}$ & - & - & - & - & - & & - & - & - & \\
\hline
\end{tabular}

4 th.

\begin{tabular}{|c|c|c|c|c|c|c|c|c|c|c|c|c|c|c|c|}
\hline 2 & - & -1 & $1-$ & - & - & - & - & -1 & - & - & & - & - & - & \\
\hline 4 & - & - & - & - & - & - & - & - & - & - & & - & - & - & \\
\hline 6 & - & - & - & - & - & - & - & - & - & - & & - & - & - & \\
\hline 8 & $28 \cdot 898$ & - & $22 \cdot 8$ & $25 \cdot 4$ & $24 \cdot 8$ & $27 \cdot 5$ & Calm & - & - & c & . & $\mathrm{Ci}_{\mathrm{i}}, \mathrm{Cl}_{1-\mathrm{s}_{3} 3}$ & A.-8., A.-cil. 4, SW & - & Sllght snowfall during night. \\
\hline Noon & $28 \cdot 950$ & - & $25 \cdot 0$ & $30 \cdot 0$ & $26 \cdot 5$ & $31 \cdot 5$ & Lt. NE alrs & 2 & - & be & & Ci., Ci.-s. 1 & - & - & Sone torn S.-cu. and Cu, olscure Bluff \\
\hline 2 & - & - & - & - & - & - & - & - & - & - & & - & - & - & over Brown Island. \\
\hline 8 & $29 \cdot 063$ & - & - & $27 \cdot 2$ & $25 \cdot 6$ & $29 \cdot 6$ & $N F, 0-1$ & 1 & - & le & & Ci.-s. 4 & - & - & Ci. clouts radlating NNW. \\
\hline 10 & - & - & - & - & - & - & - & - & - & - & & - & - & - & \\
\hline Milt. & - & - & - & - & - & - & - & - & - & - & 1 & - & - & - & \\
\hline Mean & $28 \cdot 980$ & 1 & -1 & $\overline{28 \cdot 4}$ & $\overline{26 \cdot 0}$ & - & - & $\overline{1}$ & - & - & & - & - & $\overline{-}$ & \\
\hline
\end{tabular}

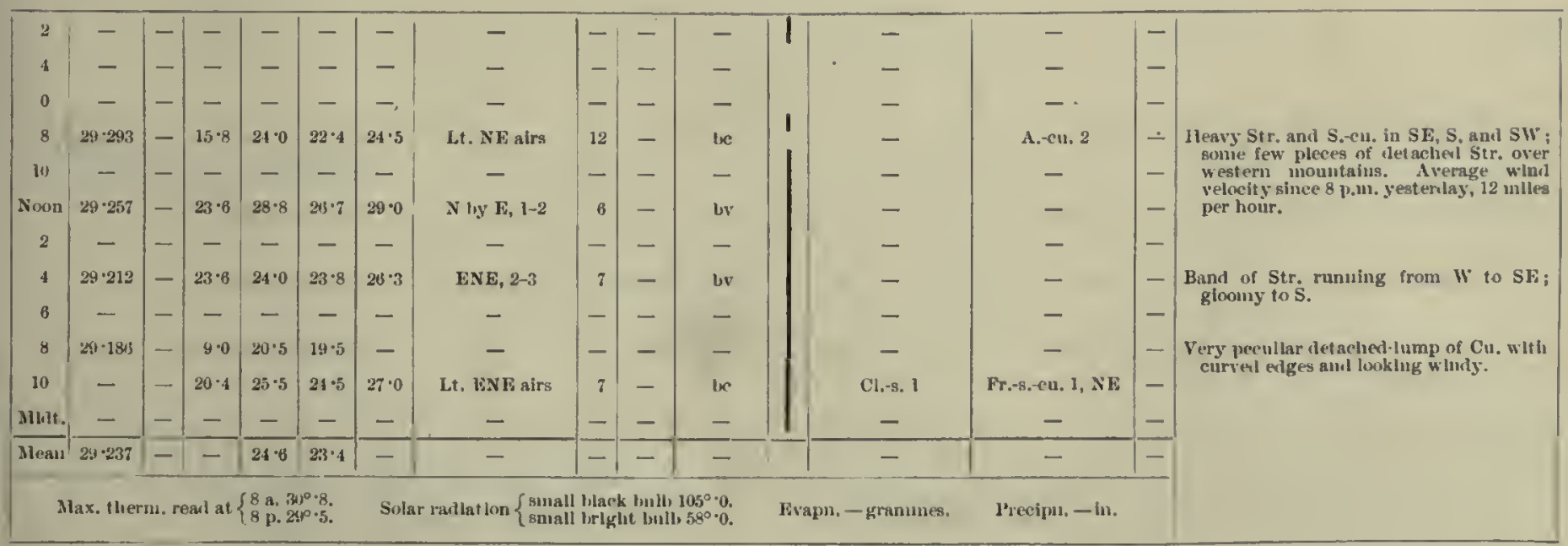


Mrthorolonica, Jour.xal of THE "Discovery."

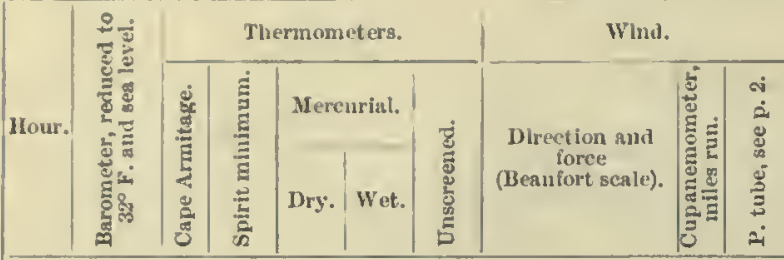

7 th.

\begin{tabular}{|c|c|c|c|c|c|c|c|c|c|c|c|c|c|c|c|}
\hline & ต็ & 8ீ & की & & & 5 & & 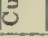 & $2 i$ & $=$ & क & & & $\div$ & \\
\hline 2 & - & -1 & - & - & - & - & - & - & - & - & 1 & - & - & - & \multirow{7}{*}{ 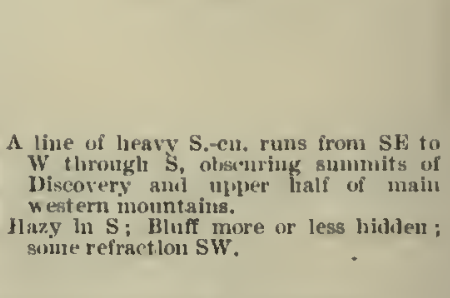 } \\
\hline 4 & - & - & - & - & - & - & - & - & - & - & I & - & - & - & \\
\hline 6 & - & - & - & - & - & - & - & - & - & - & & - & - & - & \\
\hline 8 & $29 \cdot 087$ & - & $n \cdot 4$ & $26 \cdot 8$ & $25 \cdot 8$ & $28 \cdot 5$ & N, 1-3 & - & - & c & & Ci., Ci.-8. 4 & $\mathbf{\Lambda} .-\mathbf{c u}, \mathbf{\Lambda}_{0}-3,3, \mathbf{N}$ & & \\
\hline 10 & - & - & - & - & - & - & - & - & - & - & & - & - & - & \\
\hline Xionu & $29-0.12$ & - & $2 s \cdot 2$ & $28 \cdot 1$ & $27 \cdot 3$ & $29 \cdot 2$ & ENE, 2-8 & - & - & liv & & - & - & - & \\
\hline 3 & - & - & - & - & - & - & - & - & - & - & & - & - & - & \\
\hline 4 & $29 \cdot 016$ & - & $21 \cdot 0$ & $21 \cdot 5$ & $20 \cdot 5$ & $21 \cdot 0$ & F. 3-4 & 12 & - & b & & - & - & - & \multirow{5}{*}{$\begin{array}{l}\text { Fr.s. in lines in SW, S, and SSE. } \\
\text { Cu. on western mountains and to SW; } \\
\text { oflerwise clear. }\end{array}$} \\
\hline 0 & - & - & - & - & - & - & - & - & - & - & & - & - & - & \\
\hline 8 & $29 \cdot 044$ & - & $19 \cdot 6$ & $20 \cdot 2$ & $18 \cdot 9$ & $21 \cdot 2$ & ENE, 5-6 & 15 & - & $b+$ & & - & - & - & \\
\hline 10 & - & - & - & - & - & - & - & - & - & - & & - & - & - & \\
\hline Midt. & - & - & - & - & - & - & - & $1-$ & - & - & 1 & - & - & - & \\
\hline Mesi, & $29-055$ & - & - & $24 \cdot 2$ & $23 \cdot 1$ & - & - & - & - & - & & - & . - & - & \\
\hline & the & & at & a. & & Sol & $\operatorname{tion}\left\{\begin{array}{l}\mathrm{s} \\
\mathrm{s}\end{array}\right.$ & & ulb & & & n. - gramnies. & Preclpn. - In. & & \\
\hline
\end{tabular}

8th.

\begin{tabular}{|c|c|c|c|c|c|c|c|c|c|c|c|c|c|c|c|}
\hline 2 & - & $1-$ & - & - & - & - & - & -1 & - & - & & - & - & $1-$ & \multirow{7}{*}{ 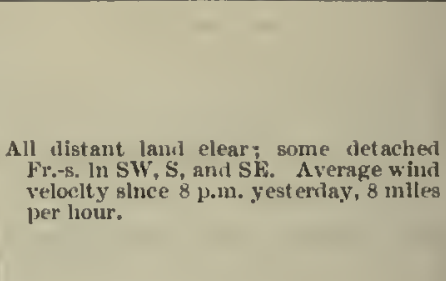 } \\
\hline 4 & - & - & - & - & - & - & - & - & - & - & & - & .- & - & \\
\hline 6 & - & - & - & - & - & - & - & - & - & - & : & - & - & - & \\
\hline 8 & $29 \cdot 142$ & - & $13 \cdot 4$ & $23 \cdot 4$ & $23 \cdot 4$ & $27 \cdot 0^{\circ}$ & E by S, 0-1 & 8 & - & bv & I & - & - & - & \\
\hline 10 & $29 \cdot 168$ & - & $22 \cdot 2$ & $23 \cdot 0$ & $22 \cdot 2$ & $27 \cdot 6$ & ST, 1 & 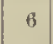 & - & $b$ & & - & - & - & \\
\hline Noon & $29 \cdot 173$ & - & $22 \cdot 5$ & $24 \cdot 0$ & $23 \cdot 5$ & $28 \cdot 5$ & FSE, 1 & 5 & - & b & & - & - & - & \\
\hline 2 & - & - & - & - & - & - & - & - & - & - & & - & - & - & \\
\hline 8 & $29 \cdot 214$ & - & $18 \cdot 6$ & $19 \cdot 2$ & $18 \cdot 2$ & $20 \cdot 0$ & Calun & 1 & - & $\propto c$ & & - & S.-cu. 10, SW & - & \multirow{5}{*}{$\begin{array}{l}\text { Detacherl Cu, in } \mathrm{S} \text { and } \mathrm{E} \text {. } \\
\text { Cloudlng over from } \mathrm{SW} \text {. } \\
\text { Western mountains obscured down to } \\
5000 \text { feet. }\end{array}$} \\
\hline 10 & - & - & - & - & - & - & - & - & - & - & & - & - & - & \\
\hline Miat. & - & - & - & - & - & - & - & - & - & - & & - & - & - & \\
\hline Mean & $29 \cdot 178$ & - & - & $22 \cdot 9$ & $21 \cdot 7$ & - & - & - & - & - & & - & - & - & \\
\hline & ax. ther & & ad at \{ & $\begin{array}{l}8 \text { k. } 2 \\
8 \text { p. } 2\end{array}$ & & Sol & ition $\left\{\begin{array}{l}\mathrm{s} s \\
\mathrm{~s}\end{array}\right.$ & & & & & grammes. & Precipn. -1 & & \\
\hline
\end{tabular}

9th.

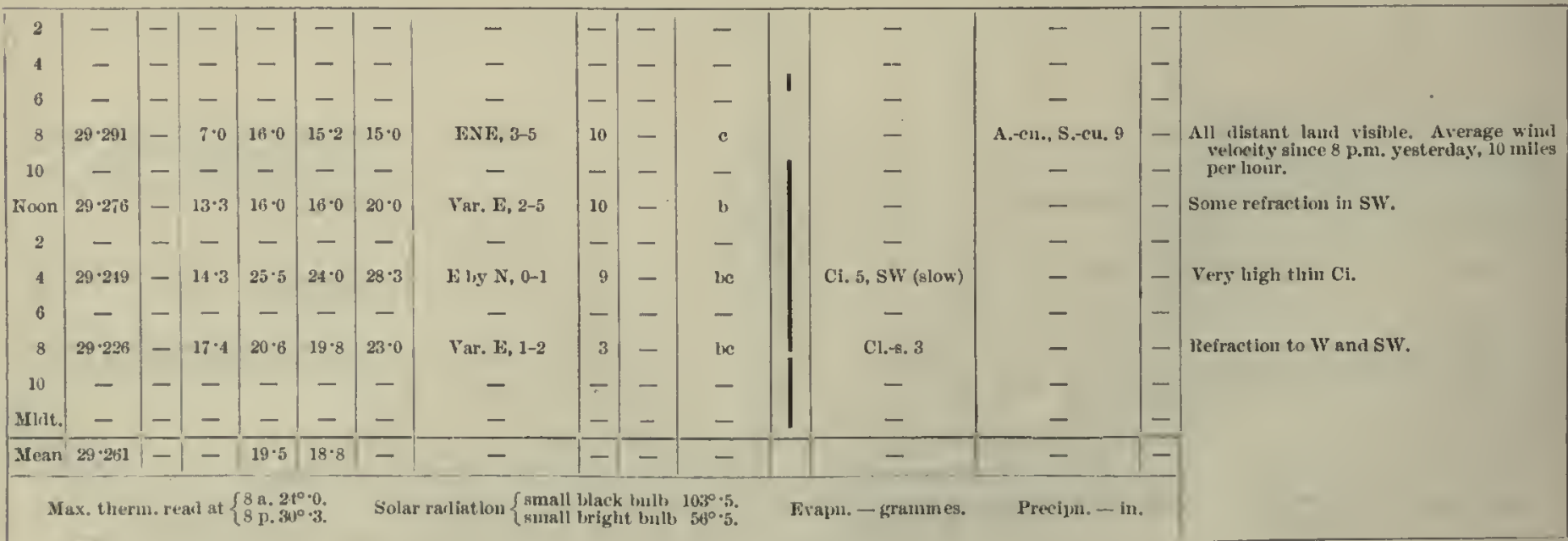


Methorological Joulnal, of the "Discovery."

[1904-February.

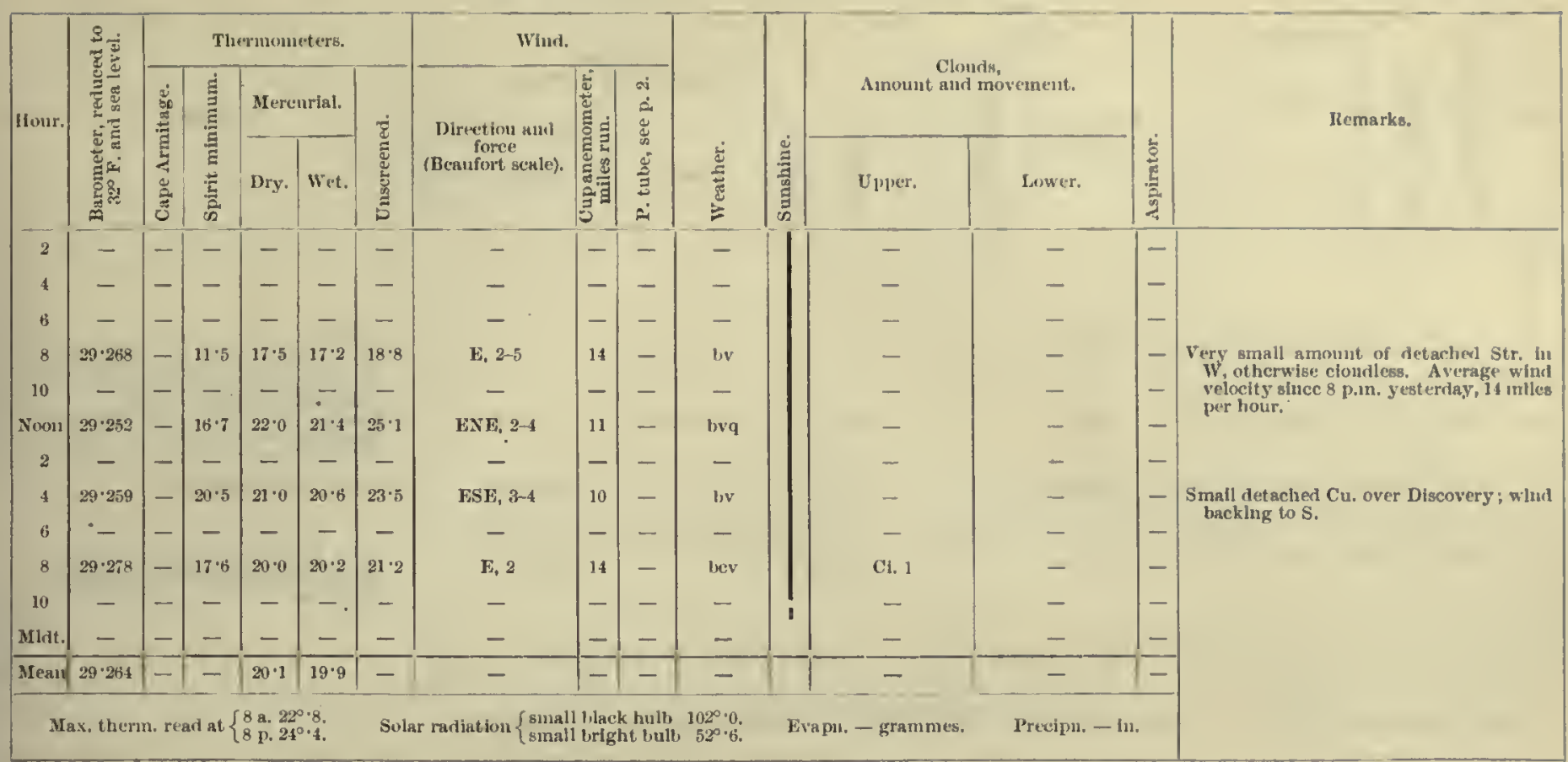

10 th.

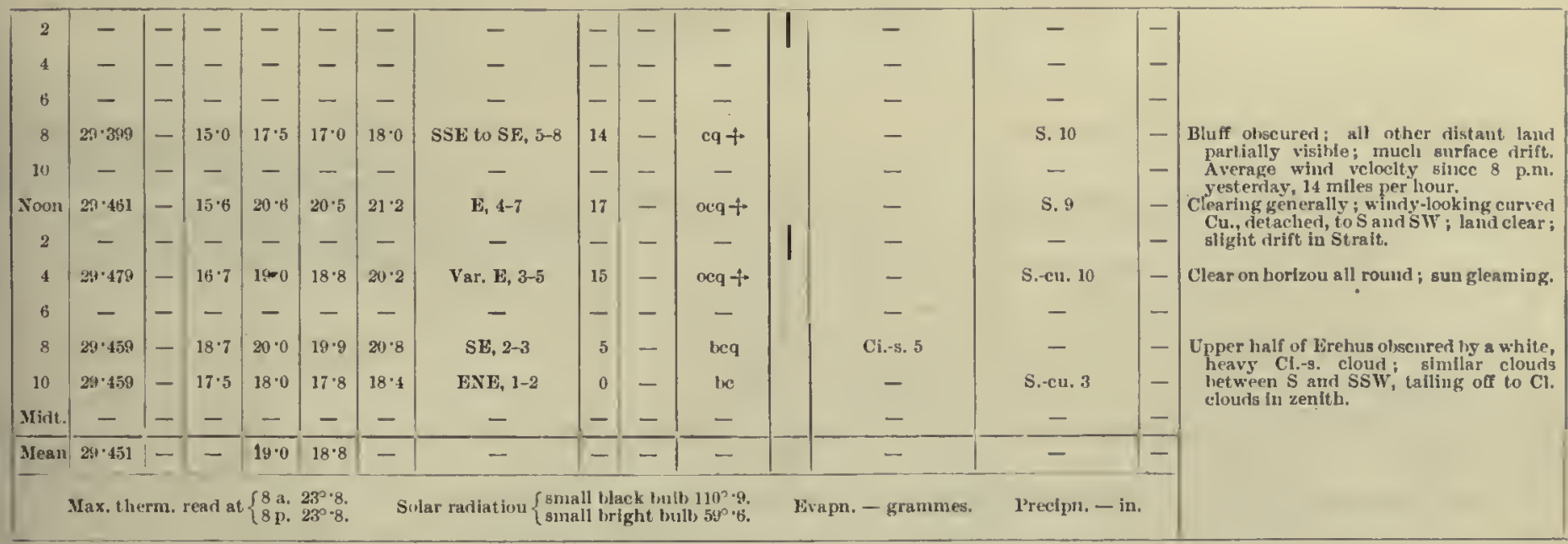

11th.

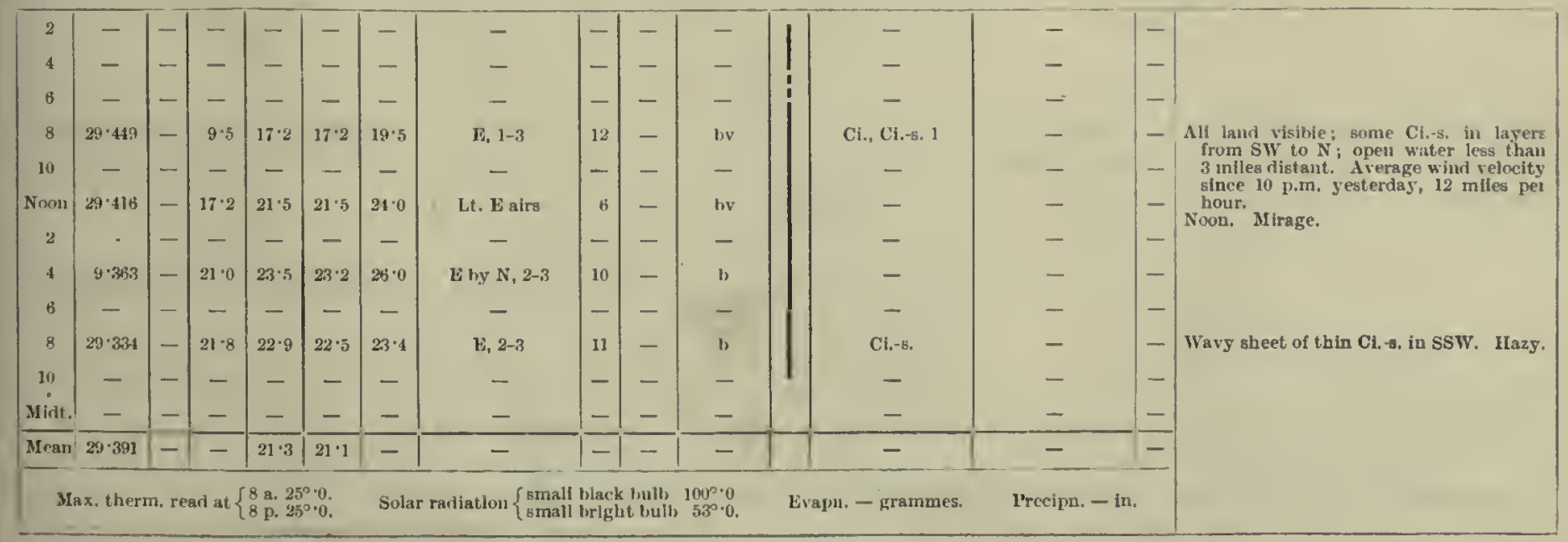


1904 February.]

Meteorological Jounal of the "Discovery."

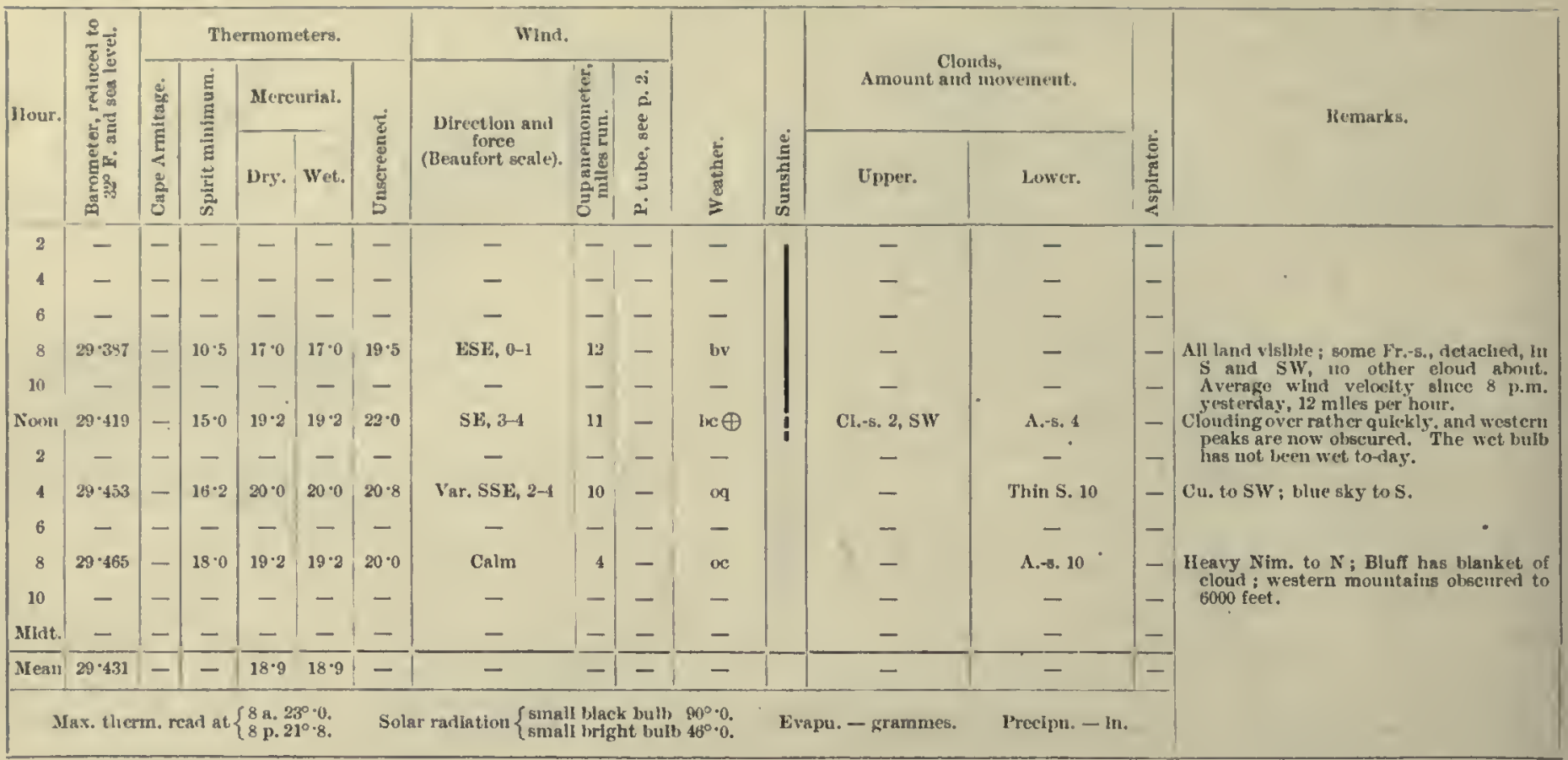

14th.

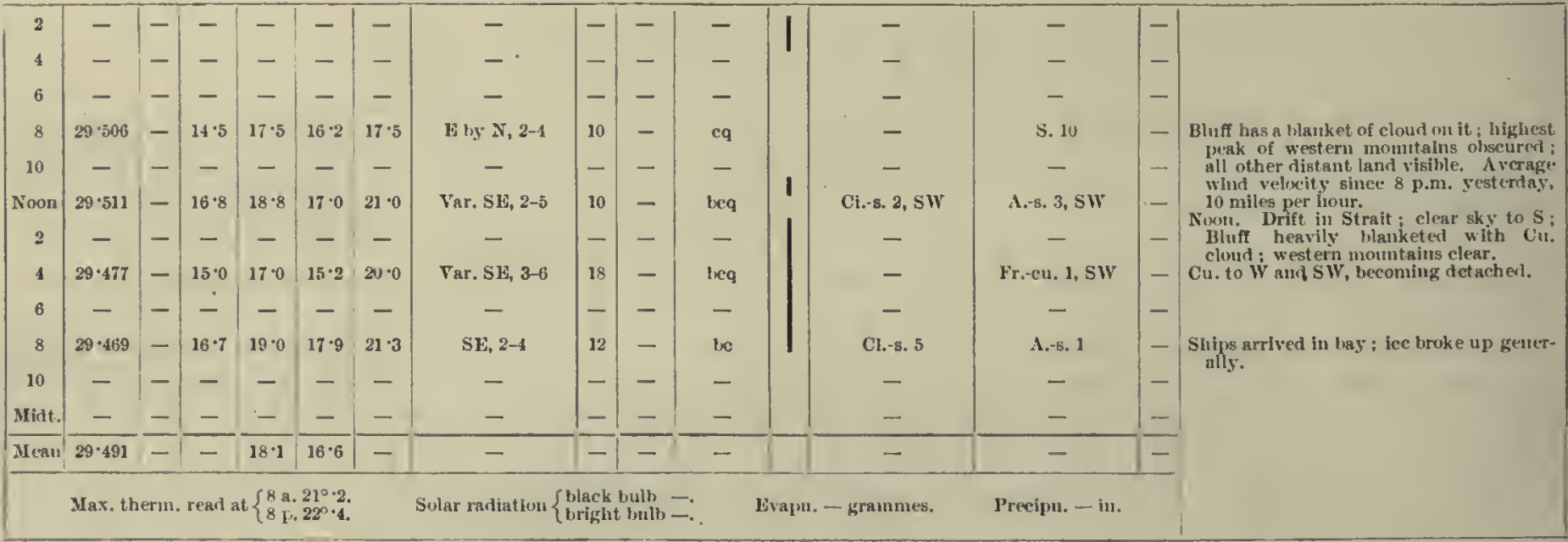

15th.

\begin{tabular}{|c|c|c|c|c|c|c|c|c|c|c|c|c|c|c|c|}
\hline 2 & - & - & - & - & - & - & - & - & - & - & & - & - & $1-$ & \\
\hline 4 & - & - & - & - & - & - & - & - & - & - & & - & - & - & \\
\hline 6 & - & - & - & - & - & - & - & - & - & - & & -- & - & - & \\
\hline 8 & $29 \cdot 408$ & - & $14 \cdot 0$ & $20 \cdot 8$ & $20 \cdot 0$ & $21 \cdot 0$ & v, 1-2 & $\pi$ & - & oc & & - & S. 9 & - & A verage whd velocity since 8 p.m. 5 ester- \\
\hline Nion & $29 \cdot 371$ & - & $22 \cdot 5$ & $24^{\circ} 0$ & $23 \cdot 5$ & $24 \cdot 5$ & $\mathbf{E}, 1-2$ & 5 & - & $\propto$ & ! & - & A.-s. 8 & - & \\
\hline 2 & - & - & - & - & - & - & - & - & - & - & & - & - & - & \\
\hline 8 & - & - & - & - & - & - & - & - & - & - & & - & - & - & \\
\hline 10 & - & - & - & - & - & - & - & - & - & - & & - & - & - & \\
\hline MINt. & - & - & - & - & - & - & - & - & - & - & & - & - & - & \\
\hline Mean & $29 \cdot 359$ & -1 & - & $23 \cdot 3$ & $22 \cdot 3$ & - & - & - & - & - & & - & - & - & \\
\hline
\end{tabular}




\title{
METEOROLOGICAL OBSERVATIONS
}

MADE AT

\author{
EREBUS ISLAND CAMP
}

(LAT. $77^{\circ} 51^{\prime}$ S. ; LONG. $166^{\circ} 45^{\prime}$ W.)

AND ON THE

SLEDGE JOURNEYS FROM WINTER QUARTERS. 
TABLE I.METEOROLOGICAL REGISTER KEP'T $\Lambda$ T THE EREBUS

\begin{tabular}{|c|c|c|c|c|c|c|c|c|c|c|c|c|c|c|c|c|c|c|c|}
\hline \multirow[b]{3}{*}{ Date. } & \multirow{3}{*}{\multicolumn{2}{|c|}{ Time. }} & \multirow[b]{3}{*}{ 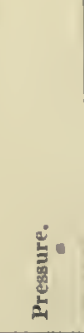 } & \multicolumn{5}{|c|}{ Tenperature. } & \multirow{3}{*}{ 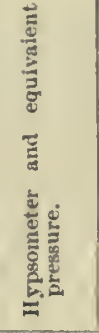 } & \multicolumn{2}{|l|}{ Wind. } & \multirow[b]{3}{*}{ 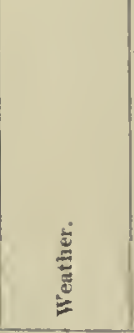 } & \multicolumn{4}{|c|}{ Ciouri. } & \multirow[b]{3}{*}{ Suusinine. } & \multicolumn{2}{|c|}{ Ozone. } \\
\hline & & & & \multirow[b]{2}{*}{$\begin{array}{l}\dot{\bar{\Xi}} \\
\vdots \\
\vdots \\
\vdots\end{array}$} & \multirow[b]{2}{*}{$\begin{array}{l}\stackrel{\tilde{a}}{\Xi} \\
\vdots \\
\Xi\end{array}$} & \multirow[b]{2}{*}{ 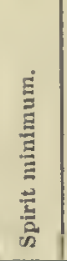 } & \multirow[b]{2}{*}{ 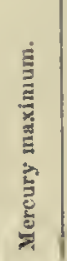 } & \multirow{2}{*}{ 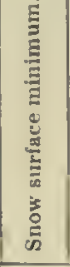 } & & \multirow[b]{2}{*}{ 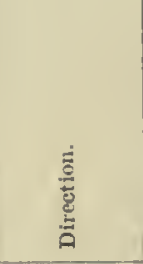 } & \multirow[b]{2}{*}{ 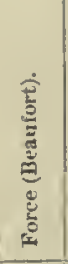 } & & Upper. & & Lov & & & & \\
\hline & & & & & & & & & & & & & 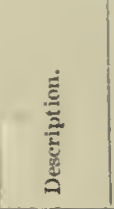 & 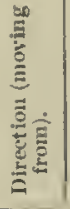 & 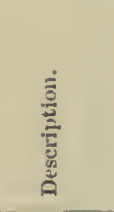 & 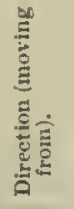 & & 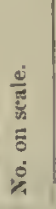 & 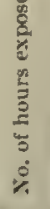 \\
\hline $\begin{array}{l}1903 . \\
\text { Dec. } 16\end{array}$ & 8 & p.m. & $\begin{array}{c}\text { ins.s. } \\
29 \cdot 100\end{array}$ & $\begin{array}{c}0 \\
29 \cdot 5\end{array}$ & 20.8 & $20 \cdot 6$ & $\begin{array}{c}0 \\
30.5\end{array}$ & $\stackrel{\circ}{-}$ & $\stackrel{\circ}{-}$ & Calm & 0 & b & - & - & - & - & Very hrigist & - & - \\
\hline & 10 & " & $29 \cdot 110$ & $26 \cdot 0$ & $23 \cdot 8$ & $26 \cdot 0$ & - & $21 \cdot 0$ & - & Cain & 0 & b) & - & - & - & - & Very lurigit & 5 & 4 \\
\hline Dec. 17 & $2=$ & a.m. & $29 \cdot 065$ & $17 \cdot 2$ & $16 \cdot 8$ & $17 \cdot 0$ & - & - & - & Sl: & $1-2$ & itc & Ci. 2 & - & - & - & Very hright & $i$ & 4 \\
\hline & 6 & $"$ & $29 \cdot 075$ & $18 \cdot 2$ & $17 \cdot 8$ & $16 \cdot 2$ & - & $19 \cdot 5$ & - & F & $2-3$ & be & Ci.-s. 6 & - & - & - & Tery trigit & - & - \\
\hline & 8 & ". & $29 \cdot 060$ & $17 \cdot 0$ & $16 \cdot 8$ & $14 \cdot 8$ & $31 \cdot 2$ & - & - & Fo & 3 & be & $\mathrm{Ci}_{0.8 .4}$ & - & - & - & Bright & 9 & 6 \\
\hline & 10 & $"$ & $29 \cdot 0032$ & $20 \cdot 0$ & $19 \cdot 2$ & $17 \cdot 0$ & - & - & - & F & $2-3$ & be & Ci.-s. 3 & - & - & - & Very trigit & - & - \\
\hline & 21 & p.m. & $29 \cdot 032$ & $19 \cdot 2$ & 18.6 & $17 \cdot 0$ & - & - & $\begin{array}{c}210 \cdot 8 \\
29 \cdot 215\end{array}$ & E & $2-3$ & be & Ci..8. 4 & - & - & - & Bright & 9 & 6 \\
\hline & 4 & $"$ & $29 \cdot 012$ & $21 \cdot 0$ & $19 \cdot 5$ & $18 \cdot 5$ & - & - & - & $1:$ & $3-4$ & be & Ci.-s. 5 & w & - & - & Bright & - & - \\
\hline & 6 & " & $29 \cdot 005$ & $20 \cdot 5$ & $19 \cdot 0$ & $19 \cdot 0$ & - & - & - & 13 & $3-4$ & be & Ci..s. 4 & $w$ & - & - & Bright & - & - \\
\hline & 8 & " & $29 \cdot 000$ & $19 \cdot 1$ & $18 \cdot 1$ & $18 \cdot 2$ & $21 \cdot 0$ & $15 \cdot 2$ & - & $\mathbf{E}$ & 3 & be & $\mathrm{Ci}_{\mathrm{i}-\mathrm{-b} .4}$ & - & - & - & Very bright & 10 & 6 \\
\hline Dec. 18 & 4 & a.m. & $28 \cdot 908$ & $19 \cdot 0$ & $18 \cdot 0$ & $17 \cdot 8$ & - & - & - & $\mathrm{SE}$ & 2 & 0 & - & - & S. 10 & - & None & 8 & 8 \\
\hline & 8 & $"$ & $28 \cdot 900$ & $20 \cdot 0$ & $19 \cdot 0$ & $18 \cdot 2$ & $20 \cdot 8$ & $17 \cdot 5$ & - & SF & $2-4$ & $\circ q O \oplus$ & - & - & S.-cu. 10 & - & Glean & 8 & 4 \\
\hline & 10 & $"$ & $28 \cdot 905$ & $20 \cdot 0$ & $18 \cdot 6$ & $18 \cdot 2$ & - & - & $\begin{array}{l}210 \cdot 4 \\
28 \cdot 98\end{array}$ & E & $2-4$ & oqc $O \oplus+$ & Ci.-s. 2 & - & S.-cu. 5 & $\mathrm{~s}$ & Gleam & $i$ & 2 \\
\hline & Nor & on & $28 \cdot 908$ & 19.8 & $19 \cdot 0$ & $19 \cdot 0$ & - & - & - & $\mathrm{x}$ & $2-3$ & $\alpha q+$ & Ci.-s. 4 & - & S.-сu. 4 & - & Gleam & - & - \\
\hline & 2 & p.m. & $28 \cdot 922$ & $18 \cdot 2$ & $17 \cdot 6$ & $16 \cdot 8$ & - & - & - & E & $3-4$ & $o q+$ & Ci.-8. 6 & - & S.-(11. 3 & - & Gleam & - & - \\
\hline & 4 & $"$ & $28 \cdot 933$ & $18 \cdot 2$ & $17 \cdot 9$ & $16 \cdot 5$ & - & - & - & E & $4-t^{2}$ & ocq $\rightarrow+$ & Ci.-s. 5 & - & A.-cu. 2 & Sli & Gieam & 9 & 6 \\
\hline & 6 & " & $28 \cdot 936$ & $17 \cdot 7$ & $17 \cdot 4$ & $16 \cdot 6$ & - & - & - & ESN & $4-5$ & $1 x-7$ & Ci.-s. 1-2 & - & A.eu. 6 & SIS & Glean & - & - \\
\hline & 8 & $"$ & $28 \cdot 952$ & $18 \cdot 0$ & $17 \cdot 6$ & $16 \cdot 6$ & $21 \cdot 0$ & - & - & $1 s$ & $3-4$ & $1 x$ & C1.-8. 2 & - & A.-«u. 4 & $\mathrm{SE}$ & Bright & $i$ & 4 \\
\hline & 10 & $"$ & $28 \cdot 975$ & $17 \cdot 9$ & $17 \cdot 4$ & $17 \cdot 4$ & - & - & - & $\mathbf{E}$ & $3-4$ & lee & Ci.-s. 2 & - & A.C11. 5 & SE & Gleam & 5 & 2 \\
\hline & $\mathrm{Mis}$ & idt. & - & - & - & - & - & - & - & SE airs & - & be & Ci.-s. 3 & - & $\cdot-$ & - & Very luright & - & - \\
\hline Dec. 19 & 2 & a.m. & - & - & - & - & - & - & - & Lt. E airs & - & be & C1. 2 & - & - & - & Very bright & - & - \\
\hline & 4 & & $28 \cdot 958$ & $22 \cdot 5$ & $21 \cdot 2$ & 16.5 & - & - & - & Culm & 0 & b & - & - & - & - & Very bright & 5 & 6 \\
\hline & 6 & $"$ & 28.955 & $23 \cdot 9$ & $22 \cdot 0$ & $23 \cdot 8$ & - & - & - & Cahm & 0 & b & - & - & - & - & Very lıright & - & - \\
\hline & 10 & " & $28 \cdot 930$ & $24 \cdot 0$ & $23 \cdot 0$ & $21 \cdot 2$ & $21 \cdot 2$ & $15 \cdot 2$ & - & Calm & 0 & 1) & - & - & - & - & Very lright. & 5 & it \\
\hline & No & on & $28 \cdot 912$ & $30 \cdot 0$ & $27 \cdot 6$ & 25.5 & - & - & - & $\mathrm{x}$ & $0-1$ & be & Ci. 1 & - & - & - & Very bright & - & - \\
\hline & 4 & p.m. & $28 \cdot 902$ & $27 \cdot 0$ & $25 \cdot 2$ & $27 \cdot 0$ & - & - & - & N1: & 1 & be & Ci.-s. 6 & - & - & - & Very brigit & - & - \\
\hline & 8 & " & $28 \cdot 905$ & $28 \cdot 2$ & $26 \cdot 2$ & $25 \cdot 8$ & $36 \cdot 2$ & - & - & $\mathrm{x}$ & $1-2$ & lec & Ci.-s. 5 & - & - & - & Brigint & $\tau$ & 10 \\
\hline & 10 & $"$ & $28 \cdot 915$ & - & - & - & - & - & - & Lt. $N$ hreezes & - & be & Ci.-8. 6 & - & - & - & Very hright & - & - \\
\hline Dec. 20 & 5 & a.m. & $28 \cdot 970$ & $25 \cdot 0$ & 23.5 & $21 \cdot 0$ & - & - & - & Calm & $"$ & lec & Ci. 1 & - & - & - & Very lright & 7 & : \\
\hline & 8 & ", & $29 \cdot 020$ & $31 \cdot 0$ & $27 \cdot 3$ & 21.0 & $35 \cdot 2$ & $23 \cdot 0$ & $210 \cdot 8$ & Calu & 0 & inc & Cl.-s. 2 & - & - & - & Very bright & 3 & 4 \\
\hline & 10 & $"$ & $29 \cdot 050$ & $27 \cdot 2$ & $24 \cdot 8$ & $24 \cdot 8$ & - & - & - & It. Ts airs & - & lec & $\mathrm{Ci}_{--8 .} 3$ & - & - & - & Brigint & - & - \\
\hline & & on & $29 \cdot 062$ & 28.5 & $25 \cdot 8$ & $25 \cdot 0$ & - & - & - & $1:$ & $0-1$ & 16 & Ci.-s. 1 & - & A.- -(n). & Sk & Very liright & - . & - \\
\hline & & p.m. & $29 \cdot 115$ & 23.6 & $22 \cdot 0$ & $22 \cdot 8$ & - & - & - & SE & $1-2$ & be & Ci.-s. 3 & $\mathrm{xw}$ & A.-cu. 1 & Sw & Very lriglut & 2 & $8 \cdot 5$ \\
\hline & 6 & " & $29 \cdot 135$ & $23 \cdot 0$ & $22 \cdot 2$ & $22 \cdot 8$ & - & - & - & S1: & $1-2$ & the & - & - & A.r.u. 2 & - & Very bright & - & - \\
\hline & 8 & $"$ & $29-145$ & $21 \cdot 5$ & $20 \cdot 8$ & $21 \cdot 2$ & $34 \cdot 1$ & - & - & $\mathrm{slis}$ & 1 & be & Ci. 1 & - & - & - & Very bright & - & - \\
\hline & 10 & " & $29 \cdot 148$ & 20.8 & $19 \cdot 9$ & $200^{\circ}$ & - & - & - & SE airs & - & be & - & - & S.-c11. 1 & - & Very brigit & - & - \\
\hline & & idt. & $29 \cdot 140$ & $17 \cdot 2$ & $16 \cdot 8$ & $17 \cdot 2$ & - & - & - & Lt. SF airs & - & ix & - & - & A.--cu. 4 & - & Olsscuret & - & - \\
\hline Dec. 21 & & a.nı. & - & - & - & - & - & - & - & Calm & 0 & Iwer & - & - & A.-**1. 2 & sw & Very lright & -- & -- \\
\hline & 4.30 & $"$ & $29 \cdot 118$ & $22 \cdot 8$ & $21 \cdot 2$ & $17 \cdot 1$ & - & - & - & Lt. S airs & - & hir & - & - & - & - & Very brigit & 6 & 12 \\
\hline & 6 & $"$ & $20 \cdot 122$ & $20 \cdot 5$ & $19 \cdot 9$ & $21 \cdot 0$ & - & - & - & Lt. S airs & - & lw & - & - & - & - & Very luright & - & -- \\
\hline & 8 & " & $29 \cdot 070$ & $31 \cdot 0$ & $29 \cdot 1$ & $21 \cdot 0$ & - & - & - & Calın & 0 & b & - & - & - & - & Very bright & 4 & 4 \\
\hline
\end{tabular}


ISLAND CAMP, DECEMBER 16, 1903, TO JANUARY 9, 1904.

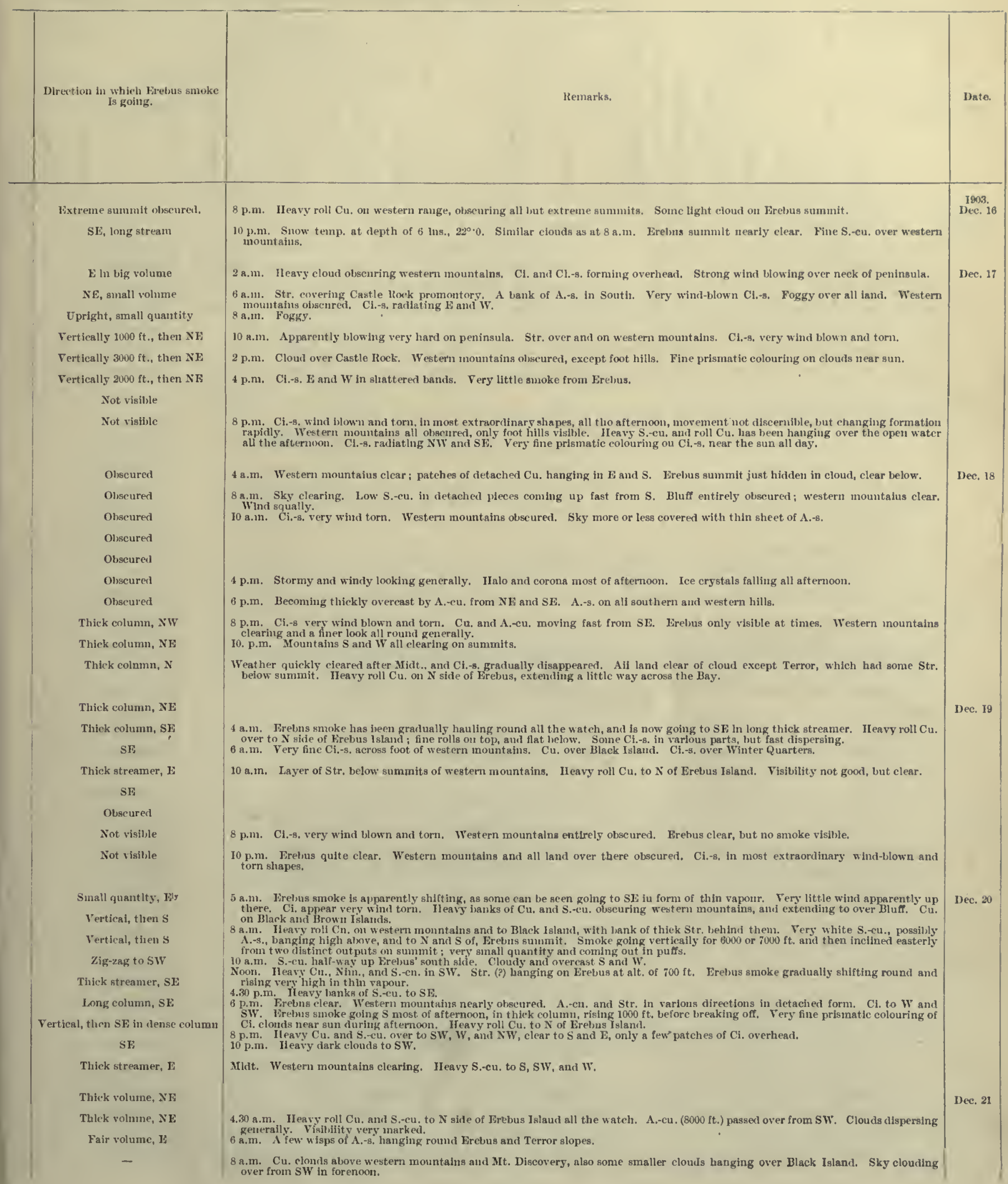


TABLE I.-METEOROLOGICAL REGISTER KEPT AT THE EREBUS

\begin{tabular}{|c|c|c|c|c|c|c|c|c|c|c|c|c|c|c|c|c|c|c|c|}
\hline \multirow[b]{3}{*}{ Date. } & \multirow{3}{*}{\multicolumn{2}{|c|}{ Time. }} & \multirow[b]{3}{*}{ 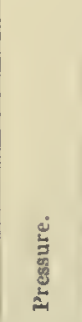 } & \multicolumn{5}{|c|}{ Temperature. } & \multirow{3}{*}{ 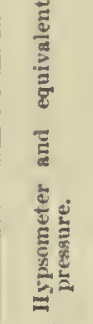 } & \multicolumn{2}{|l|}{ Whac. } & \multirow[b]{3}{*}{ } & \multicolumn{4}{|c|}{ Clouit. } & \multirow[b]{3}{*}{ Sunshine. } & \multicolumn{2}{|c|}{ Ozone. } \\
\hline & & & & \multirow[b]{2}{*}{$\begin{array}{l}\frac{8}{3} \\
\vdots \\
\vdots\end{array}$} & \multirow[b]{2}{*}{$\begin{array}{l}0 \\
\vdots \\
\vdots \\
\vdots\end{array}$} & \multirow[b]{2}{*}{ 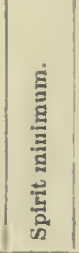 } & \multirow[b]{2}{*}{ 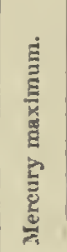 } & \multirow[b]{2}{*}{ 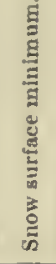 } & & \multirow{2}{*}{\multicolumn{2}{|c|}{ 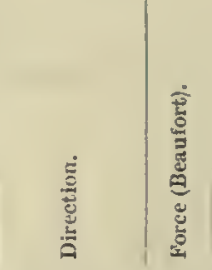 }} & & Uppe & & Lo & & & & \\
\hline & & & & & & & & & & & & & 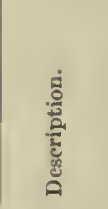 & 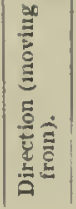 & 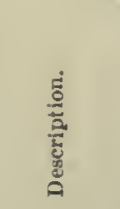 & 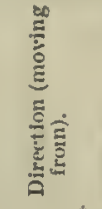 & & 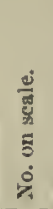 & 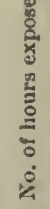 \\
\hline $\begin{array}{l}1900 . \\
\text { Dec. } 21\end{array}$ & 10 & a.m. & $\underset{29 \cdot 065}{\text { ins. }}$ & $\begin{array}{c}0 \\
30 \cdot 7\end{array}$ & $28 \cdot 0$ & $\begin{array}{c}\circ \\
30 \cdot 9\end{array}$ & $\stackrel{0}{-}$ & $\stackrel{\circ}{-}$ & $\stackrel{-}{-}$ & Lt. $S$ airs & - & b & - & - & - & - & Very bright & 2 & $2-3$ \\
\hline & 0.30 & p.n. & $29 \cdot 042$ & $33 \cdot 2$ & $29 \cdot 5$ & $31^{\circ} t$ & - & - & - & Lt. $S$ airs & - & be & - & - & A.๕u. 2 & - & Very briglit & 2 & $1-2$ \\
\hline & & " & $29 \cdot 010$ & $32 \cdot 2$ & 28.8 & $31 \cdot 9$ & - & - & - & Lt. $S$ airs & - & c & - & - & A.-cu. 9 & S by W & Very tright & 2 & 2 \\
\hline & 4 & ", & $28 \cdot 980$ & $30 \cdot 7$ & $27 \cdot 1$ & $31 \cdot 2$ & - & - & - & Lt var, airs & - & $\mathrm{cg}$ & - & - & S.-cu. 10 & SW & Obsenred & 2 & 2 \\
\hline & 6 & . & 28.980 & $30 \cdot 0$ & $26 \cdot 3$ & $29 \cdot 2$ & - & - & - & Caln & 0 & oeg & - & - & $\mathrm{Cu} .10$ & sw & Otscuret & 4 & 2 \\
\hline & 8 & ., & 28.962 & $25 \cdot 0$ & $23 \cdot 9$ & $25 \cdot 2$ & $37 \cdot 0$ & - & - & Lt. $S$ airs & - & $\operatorname{cog}$ & - & - & S. 10 & - & Olscurel & - & - \\
\hline & 10 & & $28 \cdot 935$ & $25 \cdot 8$ & $24 \cdot 4$ & $25 \cdot 0$ & - & - & - & Calm & 0 & $\infty$ & - & - & S. 10 & - & Olseurerl & - & - \\
\hline & 11.30 & " & $28 \cdot 922$ & $26 \cdot 5$ & $24 \cdot 2$ & $25 \cdot 3$ & - & - & - & Lt. $N W$ airs & - & o & - & - & S. 10 & - & Olscured & 4 & 6 \\
\hline Dec. 22 & 3.30 & a.m. & $23 \cdot 900$ & $24 \cdot 5$ & $23 \cdot 7$ & $24 \cdot 2$ & - & - & - & Calm & 0 & $o *$ & - & - & s. 10 & - & Obseured & - & - \\
\hline & 6 & " & $28 \cdot 895$ & $25 \cdot 6$ & $24 \cdot 0$ & $24 \cdot 2$ & - & - & - & Calm & 0 & o & - & - & S. 10 & - & Obseured & 3 & 6 \\
\hline & 8 & ." & 28.885 & $27 \cdot 0$ & $25 \cdot 2$ & $25 \cdot 2$ & $32 \cdot 0$ & - & - & Lt. S airs & - & $o *$ & - & - & s. 10 & - & Olscured & - & - \\
\hline & 10 & $"$ & $28 \cdot 880$ & $30 \cdot 8$ & $28 \% 6$ & $25 \cdot 0$ & - & - & - & Calm & 0 & o & - & - & S. cu. $10^{\circ}$ & - & Obscuret & - & - \\
\hline & No & 2013 & 28.875 & $30 \cdot 9$ & $28 \cdot 2$ & $31 \cdot 2$ & - & - & - & Calm & 0 & 0 & - & - & S.-cu. 10 & - & Ohscurerl & $5-6$ & 6 \\
\hline & 2 & p.m. & 28.832 & $31 \cdot \bar{T}$ & $28 \cdot 2$ & $30 \cdot 8$ & - & - & - & Cahn & 0 & o & - & - & A.-cu. 9 & - & Obscured & - & - \\
\hline & 4 & ", & $28 \cdot 855$ & $30 \cdot 4$ & $2 \pi \cdot 3$ & $30 \cdot 4$ & - & - & - & I.t. airs & 1 & $\infty$ & - & - & A.-cu. 7 & $s$ & Obscured & - & 4 \\
\hline & 6 & " & $28 \cdot 868$ & $30 \cdot 5$ & $27 \cdot 2$ & $28 \cdot 2$ & - & - & - & Lt. $N$ airs & $0-1$ & be & - & - & A.ceu. 4 & $s$ & Very bright & - & - \\
\hline & 8 & " & $28 \cdot 865$ & $29 \cdot 0$ & 26.5 & $29 \cdot 6$ & $35 \cdot 5$ & - & $210^{\circ} \cdot 4$ & $\mathrm{~N}$ & $1-2$ & be & - & - & S.-cu. 1 & $N$ & - & - & - \\
\hline & 11.30 & $"$ & $29 \cdot 890$ & $26 \cdot 6$ & $28 \cdot 5$ & $24 \cdot 5$ & - & - & - & $\mathrm{s}$ & 1 & be & Ci.-s. 1 & - & - & - & Very bright & 5 & 8 \\
\hline Dec. 23 & 4 & a.m. & $28 \cdot 948$ & $25 \cdot 8$ & $23 \cdot 0$ & $23 \cdot 9$ & - & - & - & N ly $\mathbf{E}$ & $1-2$ & lieq & Ci. 5 & NNE & - & - & Gleam & 5 & 4 \\
\hline & 6 & & $23 \cdot 990$ & $28 \cdot 1$ & $24 \cdot 8$ & $25 \cdot 2$ & - & - & - & Lt. $\mathbf{X}$ airs & - & be & Ci.-s. & $\mathrm{NE}$ & - & - & Very bright & - & - \\
\hline & 8.30 & ". & $27 \cdot 072$ & $30 \cdot 6$ & $28 \cdot 3$ & $28 \cdot 2$ & $\begin{array}{l}33 \cdot 8 \\
83.9\end{array}$ & - & - & Lt. S airs & - & be & Ci. 4 & NW & - & - & Very bright & - & - \\
\hline & 10 & & $29 \cdot 098$ & $31 \cdot 2$ & $30 \cdot 6$ & $31 \cdot 0$ & - & - & $\begin{array}{c}210 \cdot 9 \\
27 \cdot 27\end{array}$ & E airs & - & ixc & A.-8. 2 & $N$ & - & - & Very bright & 6 & 6 \\
\hline & Noo & on. & - & - & - & - & - & - & - & - & - & be $0 \oplus$ & A. -8.4 & - & S. 2 & $\mathrm{~s}$ & Glean & - & - \\
\hline & 2 & p.m. & - & - & - & - & - & - & - & $\mathbf{E}$ & 1 & $b e \oplus$ & - & - & S. 6 & $\mathrm{~S}$ (fast) & Gleam & - & - \\
\hline & 4.30 & ", & $29 \cdot 195$ & $25 \cdot 0$ & $25 \cdot 0$ & $24 \cdot 2$ & - & - & - & $E$ & $4-5$ & $\circ *$ & - & - & N. 10 & - & Obscurerl & 7 & 6 \\
\hline & 8 & ". & $29 \cdot 228$ & $24 \cdot 2$ & $24 \cdot 2$ & $24 \cdot 4$ & 38.0 & - & - & $E$ & $3-4$ & $o *$ & - & - & N. 10 & - & Obseured & - & - \\
\hline & 11.30 & " & $29 \cdot 262$ & $23 \cdot 6$ & $23 \cdot 6$ & $23 \cdot 0$ & - & - & - & E & $3-4$ & $o *^{2}+$ & - & - & N. 10 & - & Obscured & 10 & 8 \\
\hline Dec. 24 & 3 & a.m. & $29 \cdot 230$ & 250 & $25 \cdot 0$ & $23 \cdot 0$ & - & - & - & E & $4-5$ & $a *^{2}+$ & - & - & N. 10 & - & Obseured & - & - \\
\hline & 2 & p.m. & $29 \cdot 292$ & $2 s \cdot 0$ & $27 \cdot 8$ & $23 \cdot 8$ & $28 \cdot 2$ & - & - & E & $3-4$ & $o c+$ & - & - & S. 9 & s & Olscured & 10 & 13 \\
\hline & 4 & $"$ & $29 \cdot 292$ & $28 \cdot 0$ & $28 \cdot 0$ & $27 \cdot 0$ & - & - & - & E & 3 & $a *$ & - & - & N. 10 & - & Gleam & - & - \\
\hline & 6 & " & $29 \cdot 295$ & $23 \cdot 5$ & $28 \cdot 9$ & $27 \cdot 6$ & - & - & - & E & $2-3$ & $0 *$ & - & - & N. 10 & - & Ohscured & - & - \\
\hline & 8 & ", & $29 \cdot 282$ & $28 \cdot 0$ & $28 \cdot 0$ & $27 \cdot 2$ & $30 \cdot 2$ & - & - & E & $2-3$ & $o *$ & $\dot{-}$ & - & N. 10 & - & Obscured 1 & 8 & 6 \\
\hline & 10 & $"$ & $29 \cdot 270$ & $30 \cdot 6$ & $29 \cdot 9$ & $28 \cdot 5$ & - & - & - & $\mathbf{E}$ & $2-3$ & $\circ *$ & - & - & N. 10 & - & Ouscured & - & - \\
\hline Dec. 25 & 2 & a.m. & $29 \cdot 2 \pi 0$ & - & - & - & - & - & - & $\mathrm{xE}$ & $2-3$ & $o *$ & - & - & N. 10 & - & Obscured & - & - \\
\hline & 4 & ", & $29 \cdot 2 \pi 0$ & - & - & - & - & - & - & $\mathrm{NE}$ & $2-4$ & oqg & - & - & S. 10 & - & Ohscured & - & - \\
\hline & 6 & $"$ & $29 \cdot 258$ & $30 \cdot 2$ & $2: ? \cdot 3$ & $28 \cdot 3$ & - & - & - & $\mathbf{E}$ & $3-4$ & oc & A.-cu. 3 & - & S. 7 & $\mathrm{SE}$ & Olscured & 6 & 10 \\
\hline & 8 & " & $29 \cdot 248$ & $31 \cdot 3$ & $30^{\circ} 0$ & $28 \cdot 2$ & $32 \cdot 0$ & - & $211 \cdot 2$ & $\mathbf{E}$ & $2-3$ & $\propto c$ & - & - & A.cu. 9 & - & Gleam & - & - \\
\hline & 31 & p.rm. & $29 \cdot 200$ & $33 \cdot 2$ & $31 \cdot 3$ & $31 \cdot 5$ & - & - & - & SE & 1 & bv & - & - & - & - & Very tright & 5 & 8 \\
\hline & 4 & " & $29 \cdot 185$ & $33 \cdot 5$ & $31 \cdot 5$ & $33 \cdot 0$ & - & - & - & $E$ & 1 & bv & - & - & - & - & Very bright & - & - \\
\hline & 6 & " & $29 \cdot 178$ & 33.8 & 31.5 & 33.5 & - & - & - & Lt. E airs & - & bv & - & - & - & - & Very bright & 4 & 4 \\
\hline & 8 & $"$ & $29 \cdot 181$ & $31 \cdot 8$ & $32 \cdot 7$ & $33 \cdot 2$ & $39 \cdot 3$ & - & - & NW by & $1-2$ & bv & - & - & - & - & Very bright & - & - \\
\hline & 10 & " & $29 \cdot 175$ & $35 \cdot 4$ & $33 \cdot 6$ & $34 \cdot 0$ & - & - & - & Calm & 0 & bv & - & - & - & - & Very bright & - & - \\
\hline & 11.30 & ". & $29 \cdot 170$ & $32 \cdot 0$ & $30 \cdot 2$ & $33 \cdot 8$ & - & - & - & Lt. NE airs & - & $\mathrm{bm}$ & - & - & - & - & Very bright & 4 & 6 \\
\hline
\end{tabular}


ISLAND CAMP, DECEMBER 16, 1903, TO JANUARY 9, 1904 (continued).

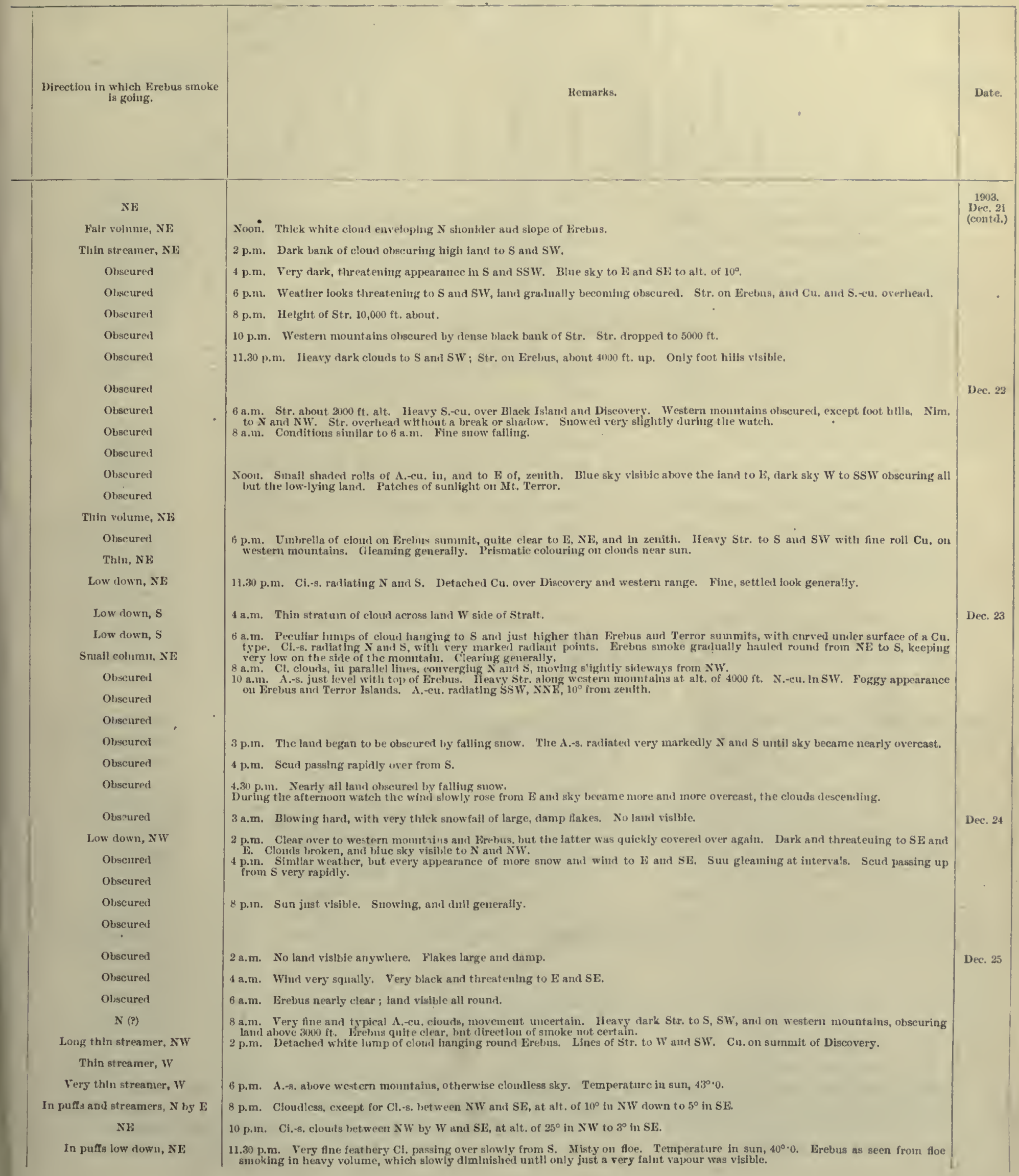


TABLE I-METEOROLOGICAL REGISTER KEPT AT THE EREBUS

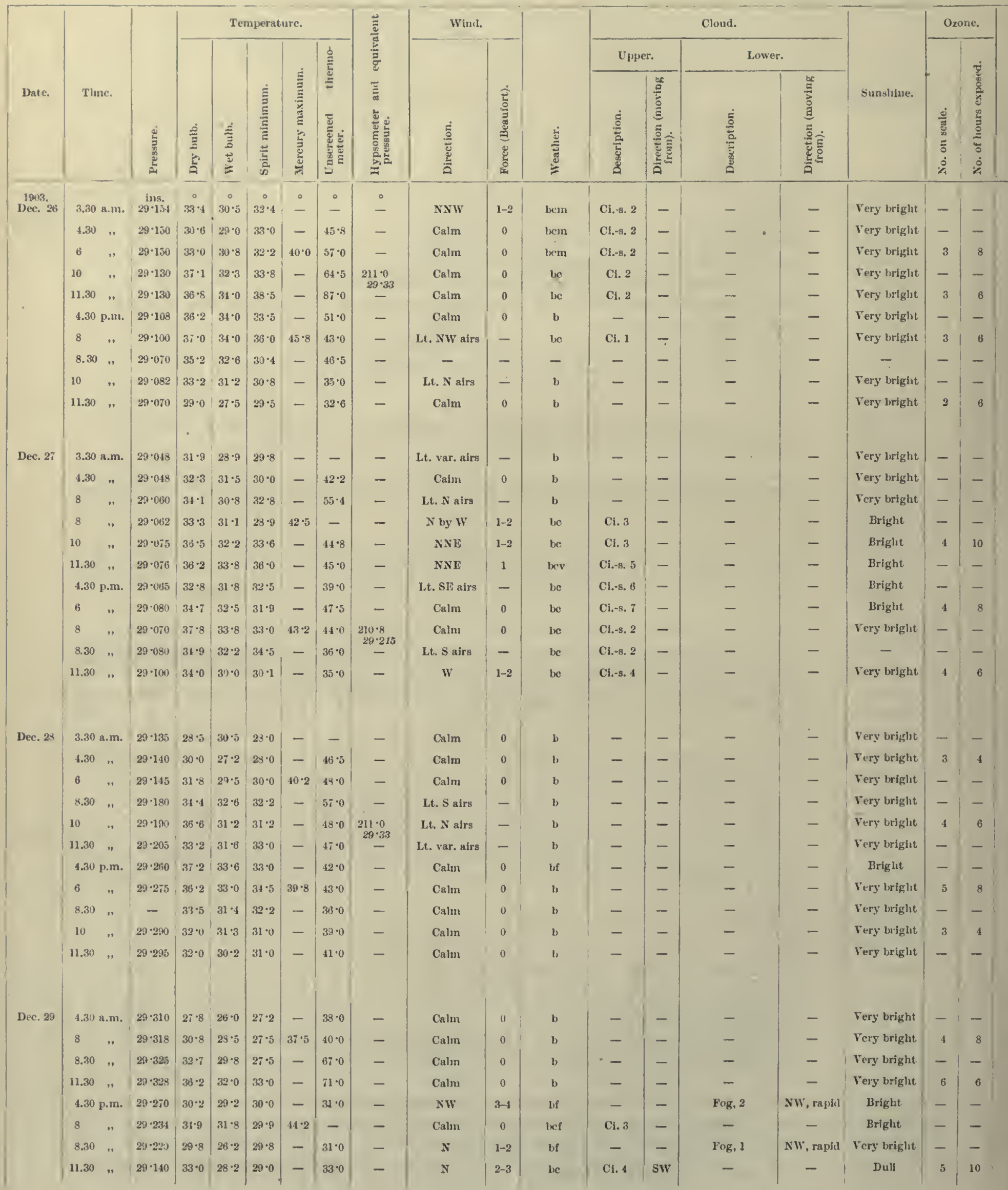


ISLAND CAMP, DECEMBER 16, 1903, TO JANUARY 9, 1904 (continned).

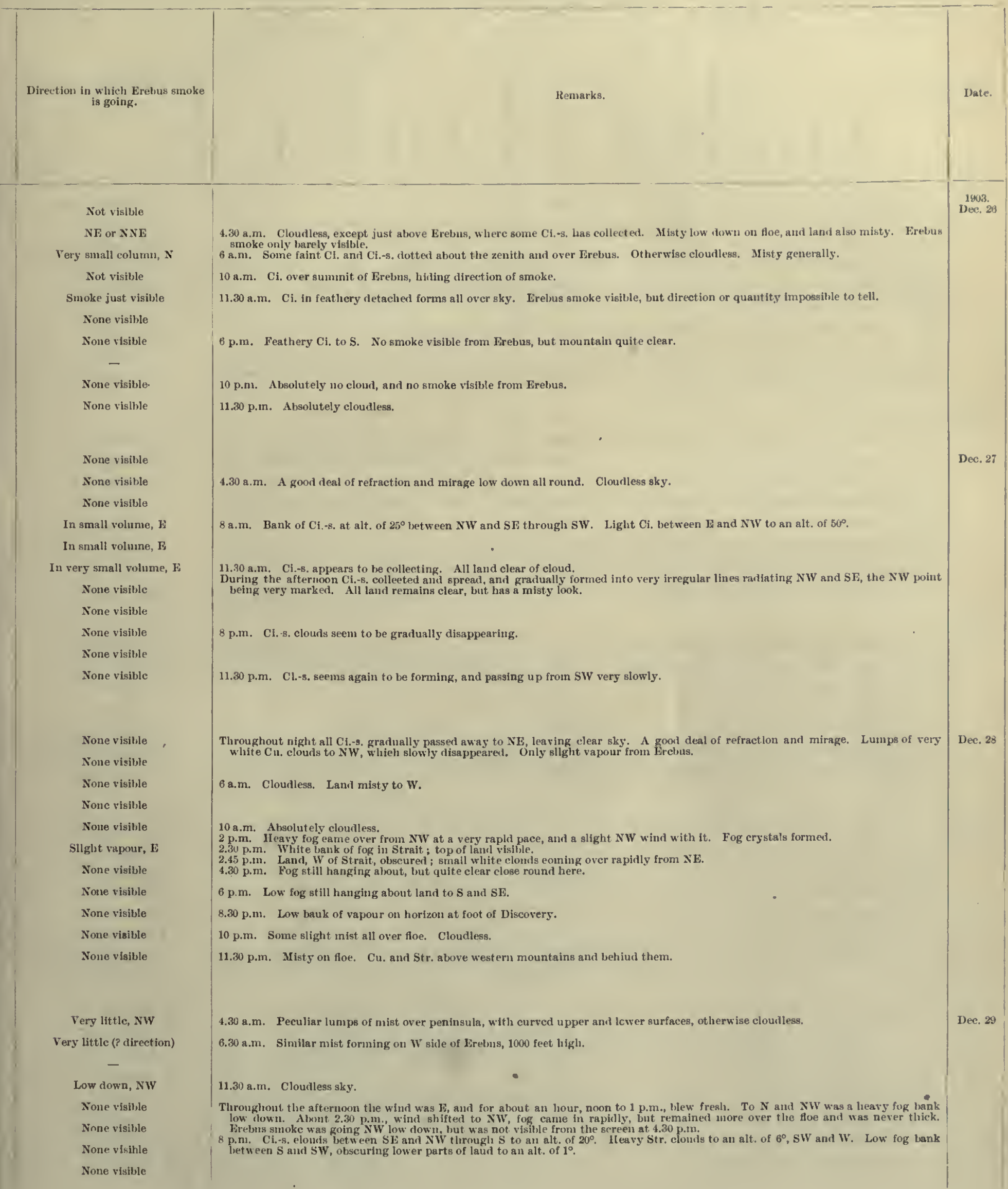


'TABLE I.-METEOROLOGICAL REGISTER KEP'T AT TIE EREBUS

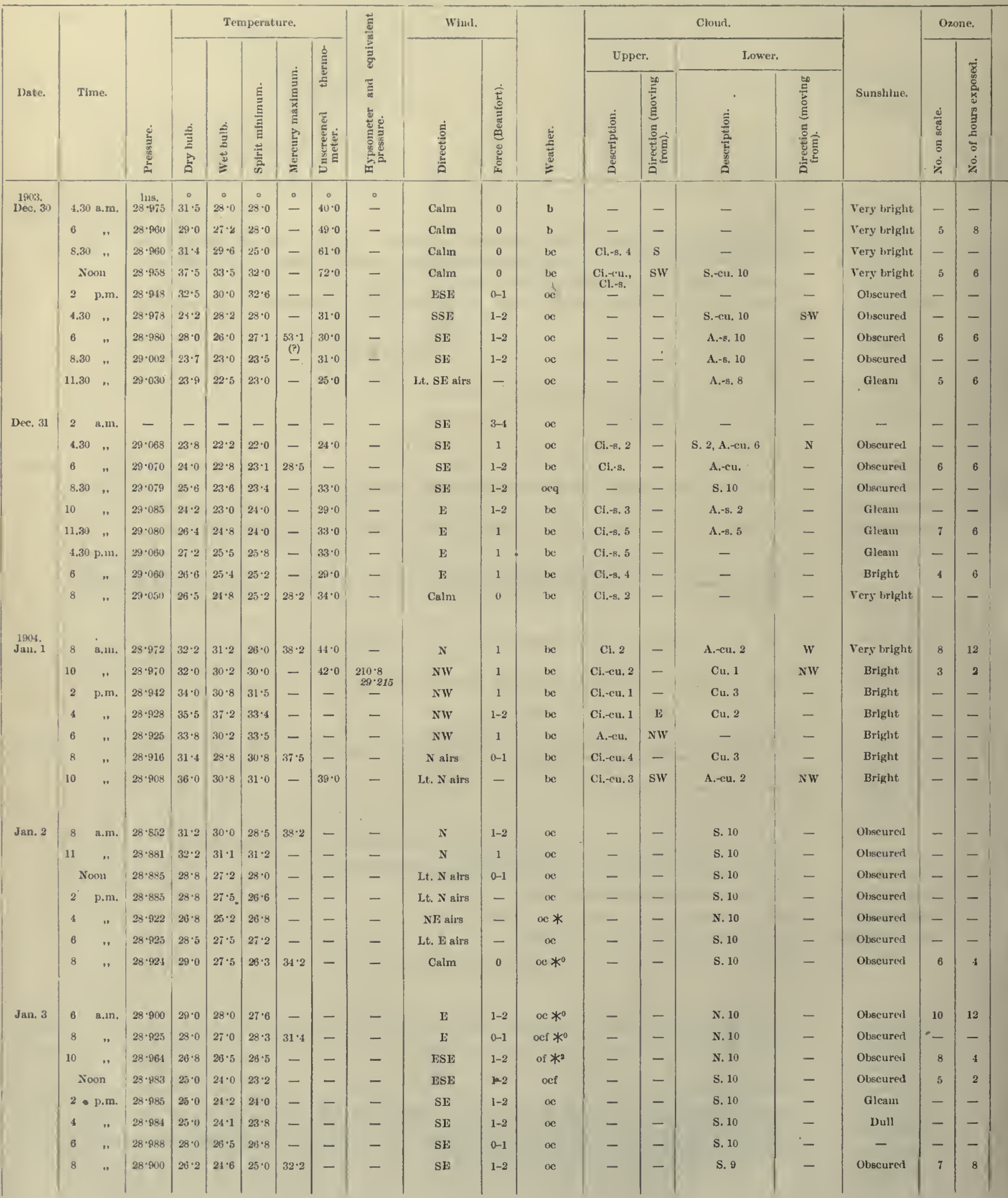


ISLAND CAMP, DECEMBER 16, 1903, TO JANUARY 9, 1904 (coutinued).

\begin{tabular}{|c|c|}
\hline $\begin{array}{l}\text { Direetion in whleh Errbus smoke } \\
\text { is going. }\end{array}$ & Remarks. \\
\hline
\end{tabular}

II eavy eolumn, NE

Large quantity, NH

Largo quantity, N13

Largo quantity, $\mathrm{NF}$

I'artially obseuring summit

Olseured

Obseured

Obseurerl

Obscired

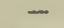

Ohseured

Obseured

Obscured

Ohscured

Obseured

Ohseured

Obseured

Thiek volume, NE

Thick volume, 1

Thiek volume, NI

NE

$\mathrm{NH}$

Ohseurert

Couslderable volume $\mathrm{N} \mathbf{H}$, then $\mathrm{N}$ Itising high, NE

Ohscured

Oisscured

Obscurert

Obscured

olseured

Olscurer

Ohseurert

Ohscured

Olsseured

Olsseureal

Obseured

NE

Ohseuren

Ohseured

Olscured
6 a.m. Dense bank of A.-s. fringed with Ci., Cl.-s., and Ci.-eu., extending from $\mathbf{E}$ to SW. Ci.-s, passed overhead from SW during wat ell at morterate speed. Wind fell about $3 \mathrm{a} . \mathrm{m}$., having been fresl $\mathrm{N}^{\mathrm{l}} \mathrm{g}$ and gusty up to then,.

Nonn. Western mountains almost entirely obseuriel by heavy S.-cu., whieh also obscures summit of Discovery. Fine Ci.cu. overhead, and very fine $C i .-8$, in lines runnlng 1 and $W$.

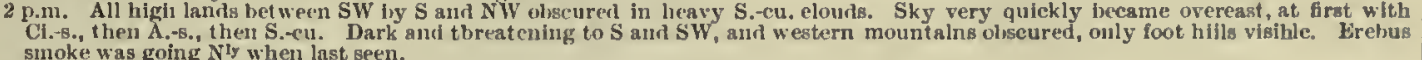

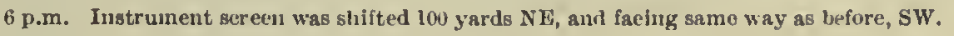

8.30 p.m. Alt. of Str. equals that of top of Erebus.

11.30 p.m. Dark and stormy looking to $\mathrm{S}$, and on western mountains. Ci. and Cl.-s, visible through thin A.-8., very whd hiown aud torn.

4.30 a.m. Heavy and dark Str. to S, SW, and on western mountains. Very fine A.-cu. and Ci.-cu. Nrehus nearly clear; only summit

obseured. Dark to $\mathrm{N}$, with lieavy Cu. and water sky.
6 a.11. Clours similar to those at 4.30 a.n.

10 a.m. Clouds appear to be hreaking up; Ci,-s, very torn and ragged. Western mountains obscured, Summit of Erehus covered witli thin cloud, probably $\mathrm{A} .-\mathrm{s}$.

During the afternoon Ci.s. more or less eovered tile sky, very wind blown and torn, but no direction of movement visible. Very fine prismatic colouring near suu. Frebus smoke going in heavy column all day to $\mathrm{N}$.

8 p.m. A.-s. alhout 1000 feet below summit of Erebus. II eavy bank of eloun from E to S. Very fine roll Cu. on western mountains. Dark and threatening to $\mathrm{N}$ and $\mathrm{NW}$.

8 a.m. Very fine massive banks of roll Cu. to the $\mathbf{N}$, hanging round Frehus slopes. Heavy roll Cu. on western mountains, nearly

$10 \mathrm{a} . \mathrm{m}$. Clouds similar to tlose at 8 a.m., moving fast.

8 p.in. Low Cu. clours to an alt. of $3^{\circ}$ between SSW and SW, clinging to the land, with Str. upper clouds. All but foot hills of westerin range ohscured in heavy Cu, elouds.

Swronghout the day the Cu. clouds have been exceptionally masslve and fine, especially to $\mathbf{N}$ low fown, and over ali land to $\mathrm{SW}$ and in $\mathrm{S}$. The $\mathrm{Ci}$-s. and $\mathrm{Ci}$-cu, have also been very fine, and of most folicate forms. Freins smoke has been pouring out in a very heavy volume, and going NW.

8 p.m. Sky broken on horlzon to $W$ and $N W$. Fog hask on Krebus.

10 p.m. Sky clearing ou horizon all round. Snow falling lightly fil hard tlakes.

6 a.m. Very liard round suow flakes falling.

$10 \mathrm{a} . \mathrm{m}$. Snow falling thlckly ln large soft flakes. Very foggy.

Nonl. Sky hroken to SW on horizon.

2 p.n. Sky lroken in $\mathbf{N}$ and $\mathrm{H}$; appearance of clearing.

4 p.n. Dull ali over. Str. at alt. of 40 no fect probahly.

10 p.m. Sky luroken in the west.

Date. 
TABLE I.-METEOROLOGICAL REGISTER KEPT $\Lambda T$ THE EREBUS

\begin{tabular}{|c|c|c|c|c|c|c|c|c|c|c|c|c|c|c|c|c|c|c|}
\hline \multirow[b]{3}{*}{ Date. } & \multirow[b]{3}{*}{ Time. } & \multirow[b]{3}{*}{ 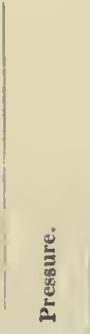 } & \multicolumn{5}{|c|}{ Temperature. } & \multirow{3}{*}{ 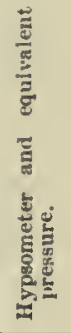 } & \multicolumn{2}{|c|}{ Wind. } & \multirow[b]{3}{*}{ 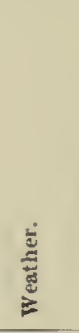 } & \multicolumn{4}{|c|}{ Сіои. } & \multirow[b]{3}{*}{ Sunshine. } & \multicolumn{2}{|c|}{ Ozone. } \\
\hline & & & \multirow[b]{2}{*}{$\frac{\hat{\Xi}}{\tilde{E}}$} & \multirow[b]{2}{*}{$\frac{\partial}{2}$} & \multirow[b]{2}{*}{ 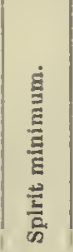 } & \multirow[b]{2}{*}{ 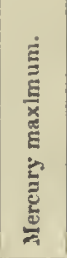 } & \multirow{2}{*}{ 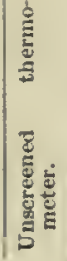 } & & \multirow{2}{*}{\multicolumn{2}{|c|}{ 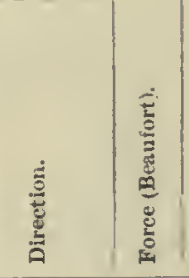 }} & & \multicolumn{2}{|c|}{ Upper. } & \multicolumn{2}{|c|}{ Lower. } & & \multirow[b]{2}{*}{ 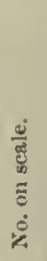 } & \multirow[b]{2}{*}{ 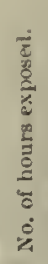 } \\
\hline & & & & & & & & & & & & 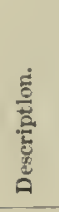 & 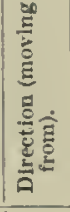 & 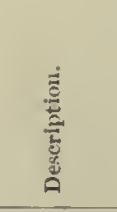 & 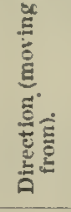 & & & \\
\hline 1904. & & Ins. & 。 & $\circ$ & 。 & $\circ$ & 。 & 。 & & & & & & & & & & \\
\hline \multirow[t]{4}{*}{ Jau. 4} & \& a.m. & $29 \cdot 085$ & $28 \cdot 8$ & $26 \cdot 8$ & 21.5 & $30 \cdot 0$ & - & - & $\mathbf{N E}$ & $0-1$ & $\alpha$ & - & - & S.-eu. 10 & - & Obscured & 7 & 10 \\
\hline & Noon. & $29 \cdot 069$ & $35 \cdot 0$ & $32 \cdot 0$ & $26 \cdot 5$ & - & - & - & Calm & 0 & $w$ & - & - & Cu. 3 & $\mathbf{E}$ & Very luriglat & 4 & 4 \\
\hline & $4 \quad$ p.m. & $28 \cdot 045$ & $35 \cdot 0$ & $33 \cdot 2$ & $35 \cdot 0$ & - & - & - & $\mathrm{NE}$ & $0-1$ & he & - & - & - & - & Very bright & 5 & 4 \\
\hline & $8 \quad$ " & $29 \cdot 063$ & 38.5 & $33 \cdot 6$ & $32 \cdot 5$ & $49 \cdot 3$ & - & - & Calm & 0 & b & - & - & - & - & Very loright & 3 & 4 \\
\hline \multirow[t]{8}{*}{ Jan. 5} & $8 \quad$ a.m. & $29 \cdot 108$ & $26 \cdot 2$ & $25 \cdot 2$ & $18 \cdot 8$ & $41 \cdot 7$ & - & - & SE & $2-3$ & $\omega$ & - & - & S. 10 & - & Gleam & $i$ & - \\
\hline & $10 \quad 1$. & $29 \cdot 121$ & $26 \cdot 5$ & $25 \cdot 0$ & 21.5 & - & - & - & SE & $2-4$ & ine & - & - & S.-cu. & - & Glenm & - & - \\
\hline & Noon. & $29 \cdot 142$ & $28 \cdot 2$ & $2 i \cdot 2$ & $25 \cdot 5$ & - & - & - & SE & $1-2$ & be & - & - & S.cu. & - & Gleain & - & - \\
\hline & $2 \quad$ p.m. & $29 \cdot 160$ & $27 \cdot 0$ & $25 \cdot 2$ & $24 \cdot 5$ & - & - & - & SE & $2-3$ & - & - & - & - & - & Gilean! & - & - \\
\hline & $4 \quad 1$ & $29 \cdot 162$ & - & - & - & - & - & - & - & - & - & - & - & - & - & - & - & - \\
\hline & $6 \quad$. & $29 \cdot 165$ & $25 \cdot 5$ & $21 \cdot 0$ & $24 \cdot 2$ & - & - & - & s & $1-2$ & $x$ & - & - & S. 10 & - & Otsseured? & 8 & 10 \\
\hline & $8 \quad$ " & $29 \cdot 182$ & $25 \cdot 8$ & $24 \cdot 2$ & $24 \cdot 0$ & $31 \cdot 2$ & - & - & Calm & 0 & $\alpha$ & - & - & S. 10 & - & Ohseired & - & - \\
\hline & $10 \quad$ " & $29 \cdot 195$ & $28 \cdot 2$ & $2 b \cdot 3$ & $25 \cdot 0$ & - & - & - & Calm & 0 & $\infty$ & - & - & S. 10 & - & Obscured & 4 & 4 \\
\hline \multirow[t]{6}{*}{ Jan. 6} & Noon. & $29 \cdot 200$ & $29 \cdot 0$ & $30 \cdot 0$ & $24 \cdot 0$ & - & - & - & SE & $1-2$ & - & - & - & N. 10 & - & Dull & - & - \\
\hline & 2 p.m. & $29 \cdot 200$ & $28 \cdot 0$ & $29 \cdot 0$ & $24 \cdot 0$ & - & - & - & SE & $1-2$ & - & - & - & N. 10 & - & - & - & .. \\
\hline & $4 \quad n$ & $29 \cdot 109$ & $28 \cdot 0$ & $29 \cdot 0$ & $24 \cdot 0$ & - & - & - & s & $0-1$ & be & - & - & - & - & - & - & - \\
\hline & $6 \quad$ " & $29 \cdot 200$ & $26 \cdot 0$ & $27 \cdot 0$ & 26.5 & - & - & - & sw & $0-1$ & - & - & - & - & - & - & - & - \\
\hline & $8 \quad$ & $29 \cdot 200$ & $31 \cdot 0$ & $28 \cdot 0$ & $26 \cdot 0$ & - & - & - & Calm & 0 & - & - & - & - & - & - & - & - \\
\hline & $10 \quad$ " & $29 \cdot 109$ & $28 \cdot 0$ & $26 \cdot 5$ & $27 \cdot 0$ & - & - & - & NW & $0-1$ & - & - & - & - & - & - & - & - \\
\hline \multirow[t]{2}{*}{ Jan. 7} & 8 p.m. & $29 \cdot 109$ & $34 \cdot 5$ & 34.0 & $22 \cdot 8$ & $42 \cdot 0$ & - & - & Calm & 0 & ocf & - & - & S. 10 & - & 3 hour & - & - \\
\hline & $10 \quad$ " & $29 \cdot 108$ & $26 \cdot 2$ & $25 \cdot 0$ & $25 \cdot 2$ & - & - & - & SE & - & oef & - & - & S. 10 & - & Olscured & 5 & 10 \\
\hline \multirow[t]{4}{*}{ Jan. 9} & $8 \quad$ a.m. & $29 \cdot 082$ & $35 \cdot 0$ & $33 \cdot 4$ & $25 \cdot 4$ & $38 \cdot 0$ & - & - & Calm & 0 & $\operatorname{cof}$ & - & - & \$. 10 & - & Olsscured & - & - \\
\hline & 6 p.m. & $29 \cdot 072$ & $30 \cdot 2$ & $30 \cdot 2$ & $30 \cdot 0$ & - & - & - & Calm & 0 & of $*$ & - & - & N. 10 & - & Ohscurevi & - & - \\
\hline & . & $29 \cdot 068$ & $28 \cdot 0$ & $28 \cdot 0$ & $27 \cdot 6$ & $36 \cdot 8$ & - & - & lESE & 1 & of $*$ & - & - & N. 10 & - & Obseured & - & - \\
\hline & 10 & $29 \cdot 068$ & $27 \cdot 5$ & $27 \cdot 3$ & $27 \cdot 0$ & - & - & - & SE & v-1 & ocf $*$ & - & - & s. 10 & - & Otsseured & 10 & - \\
\hline
\end{tabular}


ISLAND CAMP, IDECEMBER 16, 1903, TO JANUARY 9, 1904 (continued).

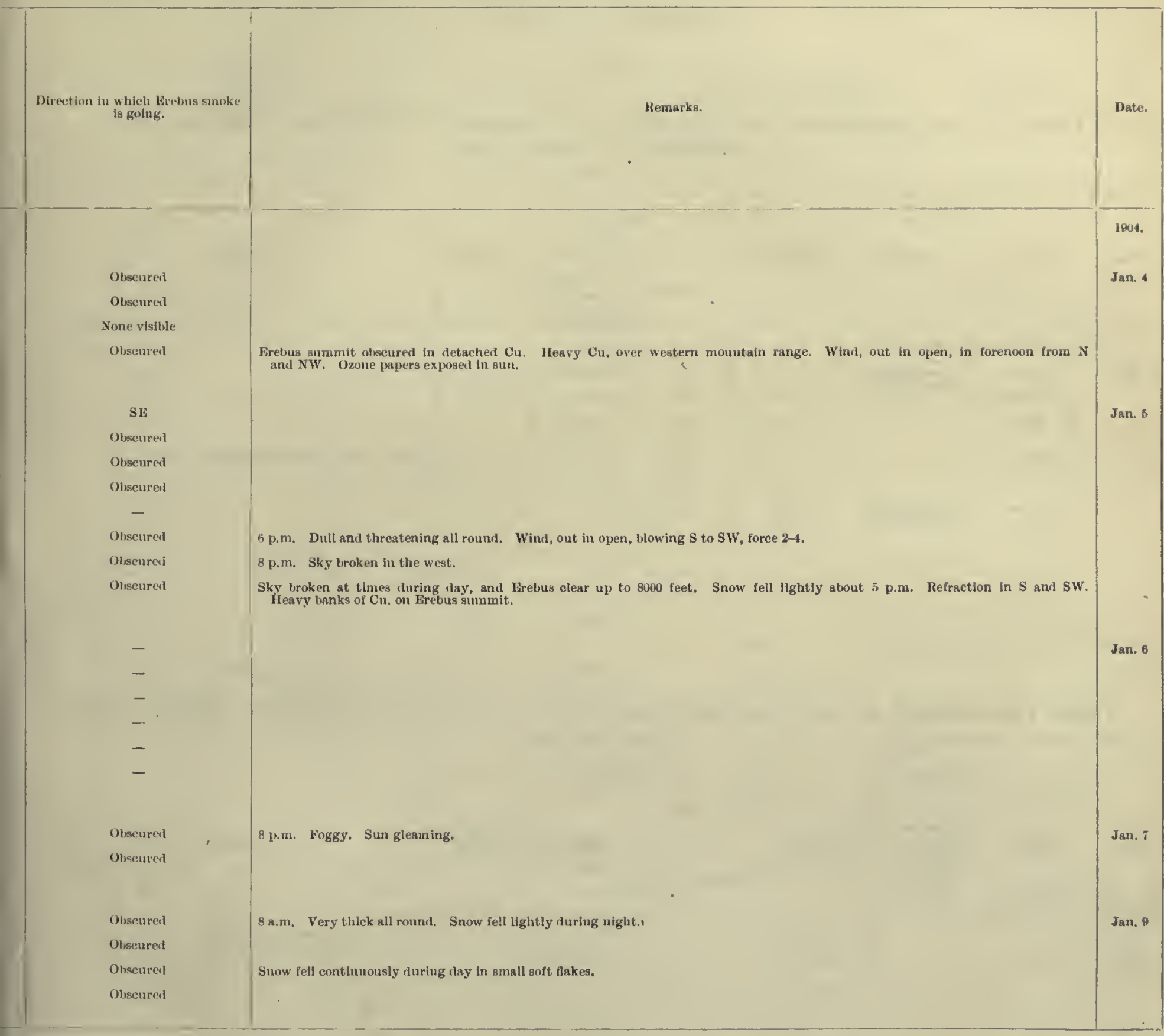




\section{SLEDGE JOURNEYS}

TABLE II.-MR. BERNACCHI'S TRIP ON GREAT ICE BARRIER TO 15 MILES SOUTH OF SHIP, FEBRUARY 3 AND 4, 1902.

\begin{tabular}{|c|c|c|c|c|c|c|c|}
\hline \multirow{2}{*}{ Date. } & \multirow{2}{*}{ Time. } & \multirow{2}{*}{ Position. } & \multirow{2}{*}{ Temperature. } & \multicolumn{2}{|c|}{ Wind. } & \multirow{2}{*}{ Weather. } & \multirow{2}{*}{ Renuarks. } \\
\hline & & & & Direction. & $\begin{array}{c}\text { Force } \\
\text { (Besufort). }\end{array}$ & & \\
\hline \multirow[t]{3}{*}{$\begin{array}{l}1902 . \\
\text { Feb. } 3\end{array}$} & Noon & Lat. $78^{\circ} \cdot 13 \mathrm{~S}$ & - & - & - & - & \\
\hline & 8 p.m. & & 10.5 & Calm & 0 & $\boldsymbol{o c}$ & \\
\hline & Mint. & & $5 \cdot 0$ & NW & $1-2$ & $o c$ & \\
\hline \multirow[t]{3}{*}{ Feb. 4} & $3.30 \mathrm{a} . \mathrm{m}$. & & 0.0 & Calm & 0 & be & 3.30 8.m. Sastrugi lay $\mathrm{SE}$ and $\mathrm{NW}$ on surface. \\
\hline & $"$ & & $2 \cdot 0$ & Calm & 0 & be & \\
\hline & Nom & $\begin{array}{r}\text { Lat. } 78^{\circ} \cdot 298 \\
\text { Long. } 163^{\circ} \cdot 45 \mathrm{~W}\end{array}$ & (in sun) & Calm & 0 & b & \\
\hline
\end{tabular}

TABLE III.-JOURNEY OF CAPTAIN SCOTT, R.N., TO LAY OUT DEPÔT TO SOUTH, MARCH 31 TO APRIL 3, 1902.

\begin{tabular}{|c|c|c|c|c|c|c|c|c|}
\hline \multirow{2}{*}{ Date. } & \multirow{2}{*}{ Time. } & \multirow{2}{*}{$\begin{array}{c}\text { Noon } \\
\text { bearing and } \\
\text { distance } \\
\text { in miles from } \\
\text { Whiter } \\
\text { Quarters. }\end{array}$} & \multirow{2}{*}{$\begin{array}{l}\text { Dry bulb } \\
\text { thermoneter. }\end{array}$} & \multicolumn{2}{|c|}{ Wind. } & \multirow{2}{*}{ Weather. } & \multirow{2}{*}{$\begin{array}{c}\text { Mis- } \\
\text { cellaneous } \\
\text { plienomena. }\end{array}$} & \multirow{2}{*}{ Jemarks. } \\
\hline & & & & Direction. & $\begin{array}{c}\text { Foree } \\
\text { (Beaufort). }\end{array}$ & & & \\
\hline \multirow[t]{2}{*}{$\begin{array}{l}1902 . \\
\text { Jiarob } 31\end{array}$} & $5.30 \mathrm{p} . \mathrm{m}$. & & $\stackrel{\circ \mathrm{F}: \mathrm{F}}{-37_{0}}$ & - & - & - & - & \\
\hline & $9 \quad "$ & & $-42 \cdot 0$ & - & - & - & - & \\
\hline \multirow[t]{3}{*}{ Aprll 1} & 7 a.m. & & $-26 \cdot 0$ & ESE & $4-5$ & oc & + & \\
\hline & 2 p.m. & $\mathrm{S} 47^{\circ} \mathrm{K}, 4 \mathrm{~m}$. & $-30 \cdot 0$ & - & - & - & - & llemaned howing all day. Clearlng later. \\
\hline & $8 \quad "$ & & $-42 \cdot 0$ & - & - & - & - & \\
\hline \multirow[t]{2}{*}{ April 2} & 11 a.m. & $\mathrm{S} 45^{\circ} \mathbf{F}, 9 \mathrm{~m}$. & $-40 \cdot 0$ & - & - & - & - & $\begin{array}{l}\text { Thromghout the day the thermometer did not rise atove } \\
-35^{\circ} \cdot 0 \text {. }\end{array}$ \\
\hline & $9 \quad$ " & & $-47 \cdot 0$ & - & - & - & - & . \\
\hline \multirow[t]{3}{*}{ April 3} & 7.30 R.m. & & $-40 \cdot 0$ & Lt. Fi airs & - & $\operatorname{ocg}$ & - & Very threatening appearance. \\
\hline & $10 \quad$ " & & $-27 \cdot 0$ & - & - & - & - & \\
\hline & 2 p.m. & & $-25 \cdot 0$ & - & - & - & - & \\
\hline
\end{tabular}


TABLE IV.-FIRST JOURNEY OF LIEUTENANT ROYDS, R.N., TO CAPE CROZIFR, MARCH 4 TO 19 , 1902.

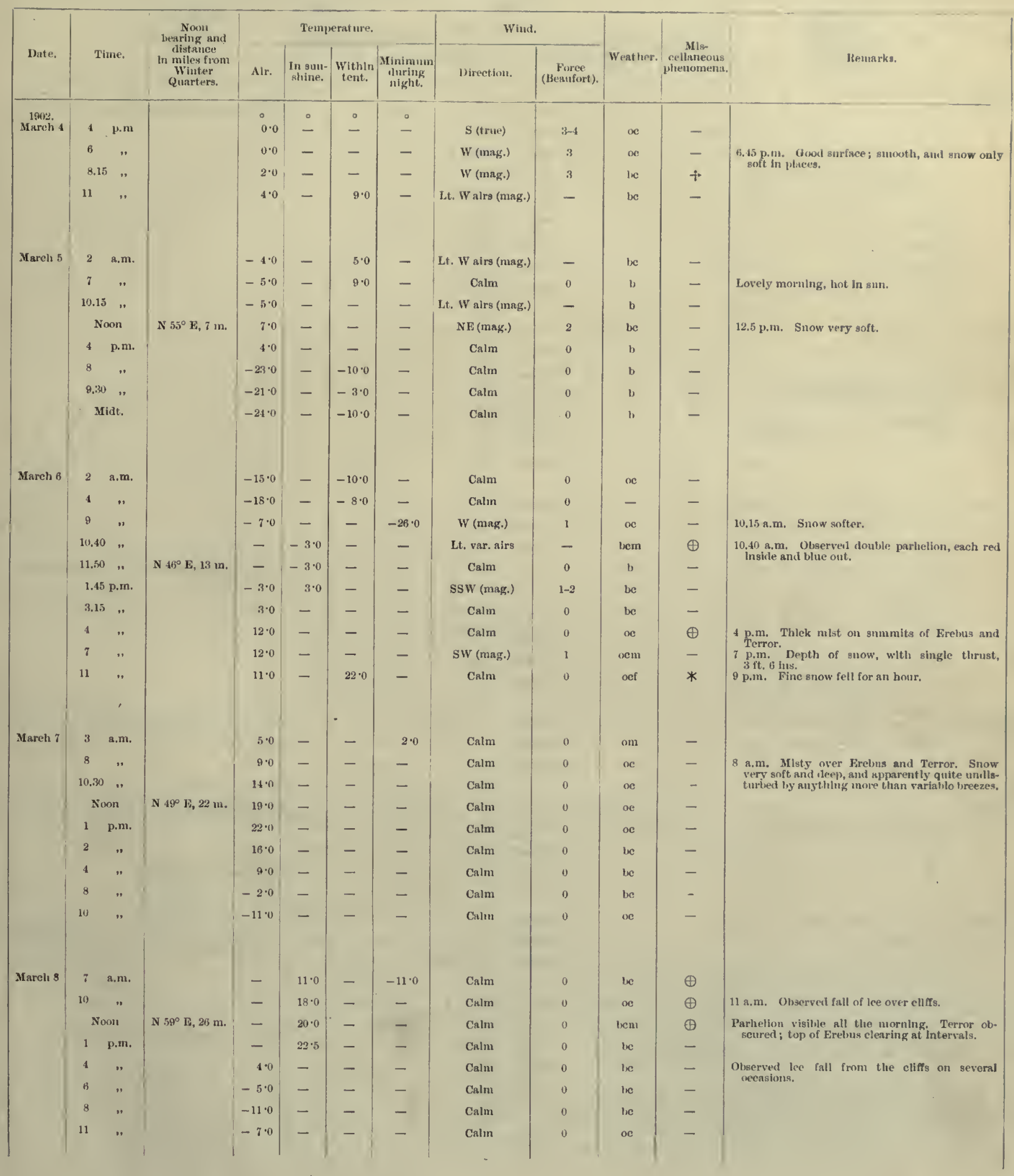


TABLE IV.-FIRST JOURNEY OF IJEUTENANT ROYDS, R.N., TO CAPE CROZIER, MARCH 4 TO 19, 1902 (continued).

\begin{tabular}{|c|c|c|c|c|c|c|c|c|c|c|c|c|}
\hline \multirow[b]{2}{*}{ Date. } & \multirow{2}{*}{\multicolumn{2}{|c|}{ Time. }} & \multirow{2}{*}{$\begin{array}{l}\text { Noou } \\
\text { learing and } \\
\text { ilstance } \\
\text { in mlles from } \\
\text { Whinter } \\
\text { Quniters. }\end{array}$} & \multicolumn{4}{|c|}{ Teuperst ure. } & \multicolumn{2}{|l|}{ Wiuk. } & \multirow[b]{2}{*}{ Weatice. } & \multirow{2}{*}{$\begin{array}{c}\text { Mis- } \\
\text { cellaneots } \\
\text { phenomena. }\end{array}$} & \multirow[b]{2}{*}{ Remarks. } \\
\hline & & & & Air. & $\begin{array}{l}\text { In sun- } \\
\text { shine. }\end{array}$ & $\begin{array}{c}\text { Within } \\
\text { tent. }\end{array}$ & $\begin{array}{l}\text { Mininum, } \\
\text { during } \\
\text { nlght. }\end{array}$ & Direetion. & $\begin{array}{l}\text { Force } \\
\text { (Besulfort). }\end{array}$ & & & \\
\hline \multirow[t]{9}{*}{$\begin{array}{l}19012 . \\
\text {.larcli } 9\end{array}$} & 8 & a.m. & & $i \cdot 0$ & $\stackrel{\circ}{-}$ & $\stackrel{\circ}{-}$ & $\stackrel{\circ}{i \cdot 0}$ & Calm & 0 & on & - & i a.m. Suow suriace getting harder. \\
\hline & 11.30 & . & & - & $20 \cdot 0$ & - & - & Calm & 0 & oe & * & \\
\hline & xi & min & $\mathrm{N} 0 \mathrm{H}^{\circ} \mathrm{E}, \mathrm{BR} \mathrm{m}$ & - & $15 \cdot 0$ & - & - & Calm & 0 & $\alpha$ & - & \\
\hline & 2.30 & p.m. & & 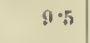 & - & - & - & Calm & 0 & bc & - & \\
\hline & 3 & " & & $8 \cdots$ & - & - & - & Calm & 0 & le & - & \\
\hline & 3.30 & ". & & $0 \cdot 0$ & - & - & - & Calm & 0 & lit & - & \\
\hline & 4.30 & " & . & $5 \cdot 0$ & - & - & - & Calm & 0 & b & - & \\
\hline & 6.25 & " & & $-5 \cdot 0$ & - & $\rightarrow$ & - & Calm & 0 & be & - & \\
\hline & 10 & $"$ & & $4 \cdot 0$ & - & - & - & Calm & 0 & $\infty$ & * & \\
\hline \multirow[t]{7}{*}{ March lo } & 6 & ฉ.m. & & $0 \cdot 0$ & - & - & $-11 \cdot 0$ & Calm & 0 & ост & * & \\
\hline & 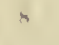 & , & & - & - & - & - & Calm & 0 & b & - & \\
\hline & 10 & " & & - & $10 \cdot 0$ & - & - & NW (mag.) & 1 & b) & 一 & \\
\hline & 11.50 & $"$ & $\times 65^{\circ} \mathrm{E}, 40 \mathrm{~m}$ & 一 & $3 \cdot 0$ & - & - & NW (mag.) & 1 & b) & - & \\
\hline & 4.15 & p.m. & & $-2 \cdot 0$ & - & - & - & SE (true) & 1 & b) & - & \\
\hline & 6 & & & $-15 \cdot 0$ & - & - & - & $\mathrm{SE}$ (true) & 1 & b & - & \\
\hline & 8 & " & & $0 \cdot 0$ & - & - & - & $\mathrm{N}$ (t rue) & 1 & b) & - & \\
\hline \multirow[t]{9}{*}{ March 11} & 6 & a.1n. & & $-5 \cdot 0$ & - & - & $-11 \cdot 0$ & Lt. var. airs & - & be & - & \\
\hline & 9.40 & ". & & $0 \cdot 0$ & - & - & - & Lt. var, alrs & - & $\infty$ & $*$ & \\
\hline & 11 & , & & $0 \cdot 0$ & - & - & - & Calm & 0 & oef & - & \\
\hline & & oni & $\mathrm{N} 55^{\circ} \mathrm{E}, 44 \mathrm{~m}$ & $1 \cdot 0$ & - & - & - & Calm & 0 & ocf & $*$ & $\begin{array}{l}\text { Noon. Fxeellent travelling, suow surfacc hard. } \\
\text { Light very bad. }\end{array}$ \\
\hline & 12.311 & p.m. & & $2 \cdot 0$ & - & - & - & Calm & 0 & nef & * & \\
\hline & 2.15 & " & & $1 \cdot 0$ & - & - & - & Calm & 0 & ocf & * & \\
\hline & 3.10 & " & & 0.0 & - & - & - & NW (mag.) & 2 & ocf & $*$ & \\
\hline & 6 & " & & $-5 \cdot 0$ & - & - & - & NW (mag.) & 4 & ocf & $*+$ & \\
\hline & 8 & . & & $-5 \cdot 0$ & - & - & - & NW (mag.) & 4 & off & $*+$ & \\
\hline \multirow[t]{3}{*}{ March 12} & $i$ & a.m. & & 0.0 & - & - & $0 \cdot 0$ & NF (mag.) & 4 & nef & $*$ & Blew hard all night, with much drift. \\
\hline & No & on & $\mathrm{x} 52 \mathrm{~m}, 12 \mathrm{~m}$ & $-2 \cdot 0$ & - & - & - & NE (mag.) & $3-4$ & $\mathrm{~cm}$ & - & $\begin{array}{l}\text { Noon. Tery soft sticky snow, elogging skis and } \\
\text { ruuners. }\end{array}$ \\
\hline & 9 & p.m. & & $-2 \cdot 0$ & - & - & - & NE (mag.) & 3 & $\mathrm{ocm}$ & - & \\
\hline \multirow[t]{4}{*}{ Marcly 13} & $i$ & a.m. & & $-2 \cdot 0$ & - & - & $-3 \cdot 0$ & $\mathrm{NE}$ (mag.) & 4-5 & lne & - & High wind all night. \\
\hline & $12.31)$ & p.m. & X $6 n^{\circ} \mathrm{E}, 4.5 \mathrm{~m}$ & $-10 \cdot 0$ & - & - & - & NE (mag.) & $6-7$ & loq & $t^{2}$ & \\
\hline & 1 & " & & $-3 \cdot 0$ & - & - & - & NE (mag.) & $4-5$ & b & $++^{3}$ & \\
\hline & 8 & " & & $-5 \cdot 0$ & - & - & - & NE (mag.) & $6-i$ & b & $t^{2}$ & Very heavy drift all day. \\
\hline \multirow[t]{3}{*}{ Marcla 1 i } & i & a.m. & N $67^{\circ} 13,42$ 1n. & $-3 \cdot 0$ & - & - & $-10 \cdot 0$ & NE (mag.) & 2 & b) & + & \\
\hline & & p.m. & & $-10 \cdot 0$ & - & - & - & NE (mag.) & 6 & $\alpha$ & $*-i^{2}$ & $\begin{array}{l}\text { lleavy wint rose alout } 10.0 \text { a.m. (?), acempanicil } \\
\text { by considernlle drift. }\end{array}$ \\
\hline & 8 & " & & $-3 \cdot 0$ & - & - & - & NF (mag.) & 3 & $\propto$ & + & \\
\hline \multirow[t]{7}{*}{ March 1 is } & $s$ & a.ı. & & $-15 \cdot 0$ & - & - & $-16 \cdot 0$ & NF (mag.) & 2 & be & - & \\
\hline & 11.31 & , & $\mathrm{N} 63^{\circ} \mathrm{F}, 32 \mathrm{~m}$. & $0 \cdot 1$ & - & - & - & Calm & 0 & $\boldsymbol{\omega c}$ & - & Noon. Gool t ravelling: snow surface liard. \\
\hline & 1.30 & p.m. & & - & $12 \cdot 0$ & - & - & Calm & 0 & b & - & \\
\hline & 2.20 & .. & & - & $9 \cdot 0$ & - & - & Calm & 0 & $b$ & - & \\
\hline & 4 & ". & & $-8 \cdot 0$ & - & - & - & Calm & 0 & b) & - & \\
\hline & 6 & $"$ & & $-18 \cdot 0$ & - & - & - & Lt. N alrs (mag.) & - & b & - & \\
\hline & 8 & " & & $-15 \cdot 0$ & - & - & 一 & NE (mag.) & $1-2$ & b) & - & \\
\hline
\end{tabular}


TABLE IV.-FIRST JOURNEY OF LIEUTENANT ROYIS, R.N., TO CAPE CROZIER, MARCH 4 TO 19, 1902 (continued).

\begin{tabular}{|c|c|c|c|c|c|c|c|c|c|c|c|}
\hline \multirow{2}{*}{ Date. } & \multirow{2}{*}{ 'Time. } & \multirow{2}{*}{$\begin{array}{c}\text { Noon } \\
\text { bearing and } \\
\text { distanee } \\
\text { in miles from } \\
\text { Winter } \\
\text { Quarters. }\end{array}$} & \multicolumn{4}{|c|}{ Temperature. } & \multicolumn{2}{|c|}{ Wint. } & \multirow{2}{*}{ Weather. } & \multirow{2}{*}{$\begin{array}{c}\text { Mis- } \\
\text { ecliareous } \\
\text { phenomena. }\end{array}$} & \multirow{2}{*}{ llemarks. } \\
\hline & & & Alr. & $\begin{array}{l}\text { In suil- } \\
\text { shine. }\end{array}$ & $\begin{array}{c}\text { Wlthin } \\
\text { tent. }\end{array}$ & $\begin{array}{c}\text { Minimum } \\
\text { during } \\
\text { nightit. }\end{array}$ & Direction. & $\begin{array}{c}\text { Foree } \\
\text { (Beaufort). }\end{array}$ & & & \\
\hline \multirow[t]{10}{*}{$\begin{array}{c}1902 . \\
\text { Marcli } 16\end{array}$} & 2 a.m. & . & $\stackrel{\circ}{-32 \cdot 0}$ & $\stackrel{\circ}{-}$ & $\stackrel{\circ}{-}$ & $\stackrel{\circ}{-42 \cdot 0}$ & Calm & 0 & b & - & \\
\hline & $4 \quad$, & & $-38 \cdot 0$ & - & - & - & Calm & 0 & b & - & \\
\hline & $8 \quad$ " & & $-14 \cdot 0$ & - & - & - & Calm & 0 & 1, & - & Surfaet of snow getting anfter. \\
\hline & $11.25 \quad$ " & & $-19 \cdot 0$ & - & - & - & Calm & 0 & b & - & Strong inlrage in W (true) all day. \\
\hline & Noon & $\mathrm{N} 66^{\circ} \mathbf{H}, 23 \mathrm{~m}$ & $-19 \cdot 0$ & - & - & - & Calm & 0 & b & - & \\
\hline & $2.310 \mathrm{p} . \mathrm{m}$. & & $-19 \cdot 0$ & - & - & - & Calm & 0 & b & - & \\
\hline & $3.25 \quad "$ & & $-16 \cdot 5$ & - & - & - & Calm & 0 & $b$ & - & \\
\hline & $5.15 \quad$, & & $-10 \cdot 0$ & - & - & - & Calm & 0 & $\infty$ & - & 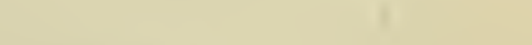 \\
\hline & $6 \quad$ " & & $-5 \cdot 0$ & - & - & - & Calm & 0 & oe & - & 6 p.m. Very suft snow. \\
\hline & $8 \quad$, & & $-5 \cdot v$ & - & - & - & Calm & 0 & of & - & \\
\hline \multirow[t]{8}{*}{ March 17} & $+\quad$ a.m. & & $10 \cdot 0$ & - & - & $-5 \cdot 0$ & Calm & 0 & omf & - & \\
\hline & $8 \quad "$ & & $10 \cdot 0$ & - & - & - & Calm & 0 & $\omega ;$ & $*$ & \\
\hline & $11 \quad$ " & & 110 & - & $=$ & - & Lt. var. airs & - & orf & * & $\begin{array}{l}11 \text { a.m. Very lieavy going ; suow soft and sticky. } \\
\text { Very bad light; to land vislble. }\end{array}$ \\
\hline & Noon & $\mathrm{N} 67^{\circ} \mathrm{F}, 15 \mathrm{~m}$. & $12 \cdot 0$ & - & - & - & Calm & 0 & ocf & $*$ & \\
\hline & 2 p.m. & & $12 \cdot 0$ & - & - & - & Calm & 0 & ocf & * & $\begin{array}{l}\text { Same heavy going all the afternoon; snow } \\
\text { elogglng hadly: }\end{array}$ \\
\hline & $3.30 \quad$, & & $9 \cdot 0$ & - & - & - & Var. & $1-2$ & ocf & 一 & \\
\hline & $6.30 \quad$, & & $8 \cdot 0$ & - & - & - & Var. & $1-3$ & xiq & * & - \\
\hline & $8 \quad$, & & $7 \cdot 0$ & - & - & - & Calm & 0 & ocf & * & \\
\hline \multirow[t]{6}{*}{ March 18} & $7 \quad$ a.m. & & $9 \cdot 0$ & - & - & $7 \cdot 0$ & Lt. var. airs & - & nef & - & Snowed duriug the night. \\
\hline & $10 \quad$, & & $15 \cdot 0$ & - & - & - & Calm & 0 & ocf & - & 10.45 a.m. Surface getting haricr. \\
\hline & Noon & $\mathrm{N} 65^{\circ} \mathrm{E}, 7 \mathrm{~m}$ & $17 \cdot 0$ & 一 & - & 一 & Lt. var. airs & - & $v(m$ & - & \\
\hline & $2 \quad$ p.m. & & $15 \cdot 0$ & - & - & - & Lt. var, airs & - & oem & - & \\
\hline & $3.20 \quad$, & & $12 \cdot 5$ & - & - & - & Var. grusts & $1-3$ & ocmq & - & 3.20 p.m. Very storny aplearauee all roumi. \\
\hline & $6.30 \quad "$ & & $7 \cdot 0$ & - & - & - & W by $N$ (mag.) & 3 & ocm & * & \\
\hline \multirow[t]{3}{*}{ Mareh 19} & s a.m. & & $0 \cdot 0$ & - & - & - & Calm & 0 & ine & - & Blew hard during the night, with heavy gusts \\
\hline & 8 & & $-5 \cdot 0$ & - & - & - & Calm & 0 & be & - & $10 \mathrm{a} . \mathrm{m}$. Excellent travelling. \\
\hline & 2.30 p.m. & & $-3 \cdot 0$ & - & - & - & Lt. var. airs & - & be & - & $\begin{array}{l}\text { Temperature rose as we appronched the fand, and } \\
\text { wind freshened SF; (truc). }\end{array}$ \\
\hline
\end{tabular}

TABLE V.-JOURNEY OF LIEUTENANT ARMITAGE, R.N.R., TO BRING IN DEPÔT. SEPTEMBER 4 AND 5, 1902.

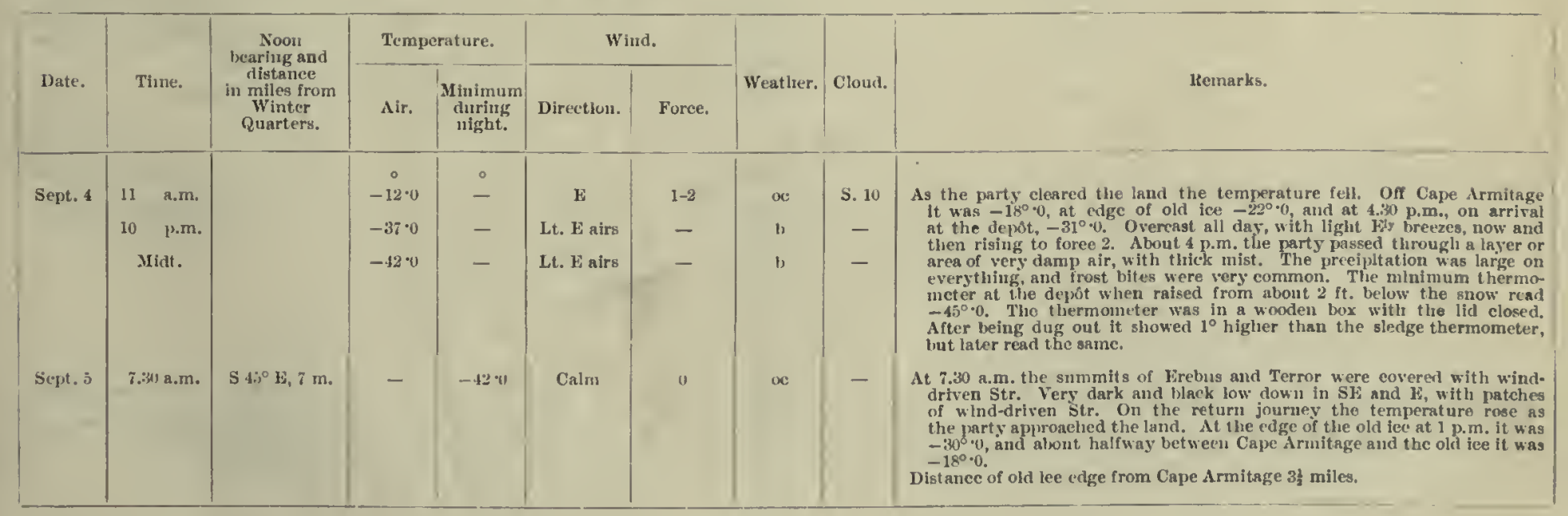


TABLE VI.-JOURNEY OF LIEUTENANT ROYDS, R.N., TO THE SOUTH-WEST, SEPTEMBER 10 TO $19,1902$.

\begin{tabular}{|c|c|c|c|c|c|c|c|c|c|c|c|c|c|}
\hline \multirow{2}{*}{ Date. } & \multirow{2}{*}{ Time. } & \multirow{2}{*}{$\begin{array}{l}\text { Nom } \\
\text { bearing and } \\
\text { distance } \\
\text { wo ninles from } \\
\text { Whuter } \\
\text { Quarters. }\end{array}$} & \multirow{2}{*}{$\begin{array}{l}\text { Pressure: } \\
\text { (Aneroid). }\end{array}$} & \multicolumn{4}{|c|}{ Temperature. } & \multicolumn{2}{|c|}{ Whut. } & \multirow{2}{*}{ Weather. } & \multirow{2}{*}{$\begin{array}{c}\text { MHls- } \\
\text { ccllanerons } \\
\text { phenomena. }\end{array}$} & \multirow{2}{*}{ Clom. } & \multirow{2}{*}{ le'mark8. } \\
\hline & & & & Air. & $\begin{array}{l}\text { Spirit } \\
\text { ninit- } \\
\text { muin. }\end{array}$ & $\begin{array}{c}\text { Within } \\
\text { teit. }\end{array}$ & $\begin{array}{l}\text { Ninimunn } \\
\text { durtng } \\
\text { ulght. }\end{array}$ & Direction. & $\begin{array}{c}\text { Force } \\
\text { (Berzufort). }\end{array}$ & & & & \\
\hline \multirow[t]{9}{*}{$\begin{array}{l}\text { 19we. } \\
\text { Sept. } 10\end{array}$} & 11 A.m. & & ins. & $-28 \cdot 0$ & $\stackrel{-}{-}$ & $\stackrel{\circ}{-}$ & $\stackrel{-}{-}$ & SE & 2 & h & - & - & \multirow{9}{*}{ 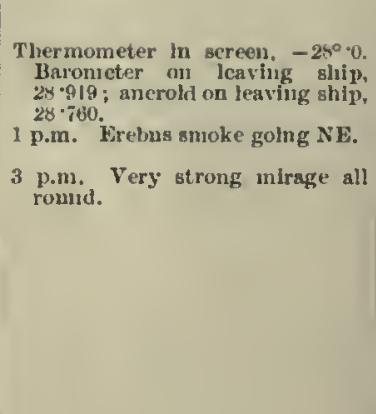 } \\
\hline & 12.30 p.m. & & - & $-28 \cdot 4$ & $-31 \cdot 2$ & - & - & SE & $3-4$ & " & + & - & \\
\hline & $2.30 \quad$, & & - & $-33 \cdot 0$ & $-35 \cdot 5$ & - & - & Calın & 0 & b & - & - & \\
\hline & $3 \quad "$ & & - & $-34 \cdot 0$ & $-37 \cdot 5$ & - & - & Cnlm & 0 & " & - & - & \\
\hline & 3.3n , & & - & $-35 \cdot 0$ & $-37 \cdot 4$ & - & - & - & - & - & - & - & \\
\hline & $4 \quad "$ & & - & $-37 \cdot 2$ & $-38 \cdot 7$ & - & - & Calm & 0 & $\mathrm{bm}$ & - & - & \\
\hline & $4.30 \quad "$. & & - & $-38 \cdot 0$ & $-39 \cdot 2$ & - & - & Calın & 0 & $\lim$ & - & - & \\
\hline & $6 \quad "$ & & - & $-39 \cdot 5$ & - & - & - & Calm. & 0 & om & - & - & \\
\hline & $7 \quad$ & & $28 \cdot 620$ & - & - & $-14 \cdot 0$ & - & - & - & - & - & - & \\
\hline \multirow[t]{4}{*}{ Sept. 11} & 8 a.m. & & - & $-3 ! \cdot 0$ & $-39 \cdot 5$ & $-12 \cdot 0$ & $-41 \cdot 5$ & Sk & 3 & b & - & - & \multirow{2}{*}{$\begin{array}{l}\text { Wind Gusty during the might. } \\
\text { Very little suroke from Eretus. }\end{array}$} \\
\hline & $\begin{array}{l}\text { Noont } \\
3.30 \mathrm{pm} . \mathrm{m} \text {. }\end{array}$ & $\mathrm{s} 36^{\circ} \mathrm{W}, 8 \mathrm{~m}$ & $\begin{array}{c}28 \cdot 300 \\
-\end{array}$ & $\begin{array}{l}-27 \cdot 0 \\
-26 \cdot 0\end{array}$ & $\begin{array}{l}-34 \cdot 0 \\
-30 \cdot 0\end{array}$ & $\begin{array}{l}- \\
-\end{array}$ & $\begin{array}{l}- \\
-\end{array}$ & $\begin{array}{l}\text { Calsm } \\
\text { Calm }\end{array}$ & $\begin{array}{l}0 \\
0\end{array}$ & of & - & $\begin{array}{c}- \\
\text { Fog } 10\end{array}$ & \\
\hline & $5 \quad$. & & - & $-26 \cdot 0$ & $-34 \cdot 0$ & - & - & Calm & 0 & of & - & Fog 10 & $\begin{array}{l}\text { on everything. } \\
5 \text { p.m. Very heavy rime deposit. }\end{array}$ \\
\hline & $"$ & & $28 \cdot 164$ & -260 & $-32 \cdot 0$ & -6.0 & - & Calm & 0 & of & - & Fog 10 & \\
\hline \multirow[t]{6}{*}{ Sept. 12} & 6.31 a.m. & & - & -260 & $-32 \cdot 0$ & - & $-32 \cdot 0$ & Calm & 0 & of & - & Fug 10 & \multirow{6}{*}{ 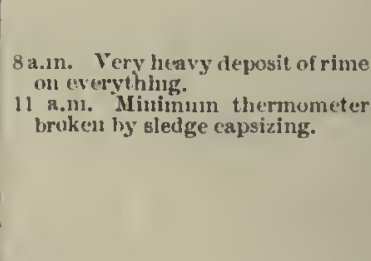 } \\
\hline & 8 & & $28 \cdot 140$ & $-24 \cdot 0$ & $-20 \cdot 0$ & - & - & Calm & 0 & of & - & Fog 10 & \\
\hline & $11 \quad "$ & & - & -220 & $-26 \cdot 0$ & - & - & Caln & 0 & of & $*$ & Fog, $N 10$ & \\
\hline & 0.45 p.m. & $\mathrm{S} 333^{\circ} \mathrm{W}, 14 \mathrm{~m}$. & - & $-24 \cdot 0$ & - & - & - & Calm & 0 & of & - & Fog 10 & \\
\hline & ". & & $2 s \cdot 300$ & $-24 \cdot 0$ & - & - & - & Calm & 0 & of & - & Fog 10 & \\
\hline & $8 \quad "$ & & - & $-28 \cdot 0$ & - & $-7 \cdot 0$ & - & Calm & 0 & of & - & Fog 10 & \\
\hline \multirow[t]{9}{*}{ Sept. 13} & o a.m. & & $28 \cdot 306$ & - & - & - & - & - & - & - & - & - & \multirow{2}{*}{$\begin{array}{l}\text { 6.31) a.m. Tent temperature, } \\
-23^{\circ} \cdot 0 \text {. }\end{array}$} \\
\hline & $8 \quad$ " & & - & $-38 \cdot 5$ & - & - & - & Calm & 0 & $\mathrm{~b}$ & - & - & \\
\hline & $10 \quad "$ & & - & $-39 \cdot 2$ & - & - & - & Calm & 0 & b & - & - & \multirow{3}{*}{$\begin{array}{l}10 \text { r.m. Wrebus smoke gohng NE. } \\
\text { Nom. Mensty on western noun- } \\
\text { tains. }\end{array}$} \\
\hline & Nown & $\mathrm{S} 35^{\circ} \mathrm{W}, 21 \mathrm{~m}$ & - & $-38 \cdot 5$ & - & $\div$ & - & Calm & 0 & $b$ & - & - & \\
\hline & 1 p.m. & & - & $-35 \cdot 0$ & - & - & - & Calm & 0 & b & - & - & \\
\hline & $2 \quad$ " & & - & $-42 \cdot 5$ & - & - & - & Calm & 0 & $\mathrm{bm}$ & - & - & 2 p.m. Very misty on lilll tops. \\
\hline & $4 \quad "$ & & - & $-40 \cdot 0$ & - & - & - & Calm & 0 & lice & - & $\mathrm{Cn} .3$ & $\begin{array}{l}4 \text { p.m. All western hills covered. } \\
\text { Erebus aud Terror clear; smoke }\end{array}$ \\
\hline & $6 \quad$ " & & - & $-41 \cdot 0$ & - & - & - & Calm & 0 & he & - & Cr. 4 & gohng NX. \\
\hline & $8 \quad "$ & & $2 s \cdot 350$ & $-44 \cdot 0$ & - & $-26 \cdot 0$ & - & Calm & 0 & $\ln$ & $\psi$ & Cu. 3 & 8 p.m. Mirage to $\mathrm{E}$. \\
\hline \multirow[t]{5}{*}{ Sept. 14} & $8 \quad$ a.m. & & - & $-49 \cdot 8$ & - & - & - & Calm & 0 & b & - & - & \multirow{5}{*}{$\begin{array}{l}\text { 6.30 a.m. Temperature iu tent. } \\
-27^{\circ} 0^{\circ} \text {. } \\
8 \text { a.m. Oil lamp frozen solld. }\end{array}$} \\
\hline & 10 & & - & $-14 \cdot 0$ & - & - & - & Calm & 0 & b & - & - & \\
\hline & 1.30 p.m. & $\mathrm{S} 35^{\circ} \mathrm{W}, 27 \mathrm{~m}$. & - & $-40 \cdot 0$ & - & - & - & Calin & 0 & b & - & - & \\
\hline & $4.45 \quad$. & & - & $-45 \cdot 0$ & - & - & - & $N$ & $1-2$ & h & + & - & \\
\hline & $8 \quad$, & & $28 \cdot 620$ & $-41 \cdot 0$ & - & $-22 \cdot 0$ & - & s & 1 & b & - & - & \\
\hline \multirow[t]{3}{*}{ Sept. 15} & $8 \quad$ a.m. & & $28 \cdot 500$ & $-28 \cdot 0$ & - & - & - & $s w$ & $2-3$ & om & - & - & 6.30 a.m. Thent temperature, \\
\hline & 12.45 p.1n. & $\mathrm{S}, 37^{\circ} \mathbf{W}, 33 \mathrm{~m}$. & - & $-22 \cdot 0$ & - & - & - & $w$ & 1 & in & - & - & $\begin{array}{l}\text { 1.20 p.m. Blizzard came on on } \\
\text { sucldenly from SW. Very }\end{array}$ \\
\hline & $4 \quad "$ & & - & - & - & $-14 \cdot 0$ & - & sw & $7-8$ & o & $t^{2}$ & - & \\
\hline
\end{tabular}


TABLE VI.-JOURNEY OF LIEUTENANT ROYDS, R.N., TO THE SOUTH-WEST, SEPTEMBER 10 TO 19,1902 (continued).

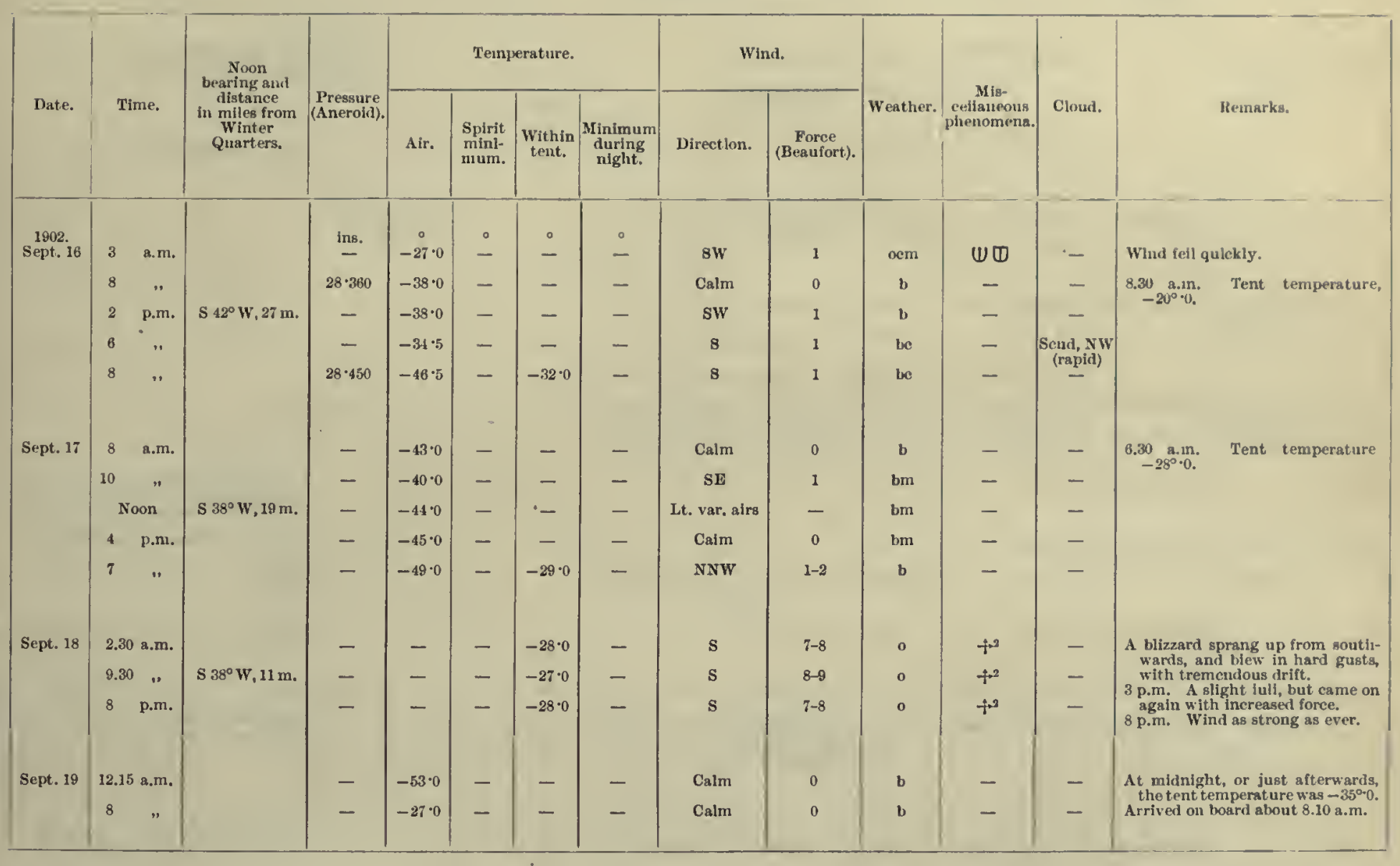


TABLE VII_-JOURNEY OF LIEUTENANT ARMITAGE, R.N.R., TO THE WEST, SEPTEMBER 11 TO $26,1902$.

\begin{tabular}{|c|c|c|c|c|c|c|c|c|c|c|c|}
\hline \multirow{2}{*}{ Date. } & \multirow{2}{*}{ Time. } & \multirow{2}{*}{$\begin{array}{l}\text { Noon } \\
\text { benring and } \\
\text { distance } \\
\text { in miles from } \\
\text { Winter } \\
\text { Quarters. }\end{array}$} & \multirow{2}{*}{$\begin{array}{c}\text { Pressure } \\
\text { (aneroid). }\end{array}$} & \multirow{2}{*}{$\begin{array}{c}\text { Height } \\
\text { above } \\
\text { sea level. }\end{array}$} & \multirow{2}{*}{$\begin{array}{l}\text { Tem- } \\
\text { perature, } \\
\text { air. }\end{array}$} & \multicolumn{2}{|c|}{ Wind. } & \multirow{2}{*}{ Weather } & \multirow{2}{*}{$\cdot \begin{array}{c}\text { Mis- } \\
\text { celianeous } \\
\text { phenomena. }\end{array}$} & \multirow{2}{*}{ Clour. } & \multirow{2}{*}{ Remarks. } \\
\hline & & & & & & Directlon. & $\begin{array}{c}\text { Force } \\
\text { (Beaufort). }\end{array}$ & & & & \\
\hline \multirow[t]{4}{*}{$\begin{array}{l}1903 . \\
\text { sept. } 11\end{array}$} & 10.30 a.m. & & $\begin{array}{l}\text { ins. } \\
29 \cdot 02\end{array}$ & ft. & $\begin{aligned} & 8 \\
- & 8 \cdot 0\end{aligned}$ & L.t. to fresh & - & - & - & - & $\begin{array}{l}\text { Left ship at } 10.30 \text { a.m. Barometer } \\
\text { (corrected), } 28.531 \text { ins. }\end{array}$ \\
\hline & 1.15 p.m. & & - & - & $-20 \cdot 0$ & Var. Fi winds & - & - & - & 一 & \\
\hline & 2.15, & & - & - & - & & - & om & - & - & \\
\hline & $8 \quad$ " & & $28 \cdot 92$ & - & $-23 \cdot 0$ & & - & - & - & - & 8 p.m. Clearing slightly. \\
\hline \multirow[t]{2}{*}{ Sept. 12} & 8 a.m. & $\mathrm{N} 65^{\circ} \mathrm{W}, 6 \mathrm{~m}$. & $28 \cdot 82$ & - & $-15 \cdot 0$ & $\begin{array}{l}\text { Var. W, with } \\
\text { occasional }\end{array}$ & $2-4$ & om & - & $\begin{array}{l}\text { Clouds yassing } \\
\text { overhend from }\end{array}$ & \\
\hline & 8 p.m. & & $28 \cdot 97$ & - & $-19 \cdot 0$ & calins & - & on & - & NE & \\
\hline \multirow[t]{2}{*}{ Sept. 13} & 7.30 a.m. & $\mathrm{N} 65^{\circ} \mathrm{W}, 10 \mathrm{~m}$. & $29 \cdot 02$ & 20 & $-38 \cdot 0$ & Lt. E alra & - & c & - & - & \\
\hline & 7.30 p.m. & & $29 \cdot 17$ & - & $-32 \cdot 0$ & & - & c & - & - & - \\
\hline \multirow[t]{2}{*}{ Sept. 14} & 8 a.m. & $\mathrm{N} 68^{\circ} \mathrm{W}, 16 \mathrm{~m}$. & $29 \cdot 35$ & 20 & -450 & Caim & 0 & b & - & - & \\
\hline & 8 p.m. & & $29 \cdot 40$ & - & -40.0 & NW & $0-1$ & b & - & - & \\
\hline Sept. 15 & i a.m. & $\mathrm{N} 68^{\circ} \mathrm{W}, 21 \mathrm{~m}$. & $29 \cdot 30$ & 20 & $-23 \cdot 0$ & ESE & $2-4$ & o & $*$ & - & $\begin{array}{l}7 \text { a.m. Temperature rose to }-15^{\circ} 0 \\
\text { keeping steady. Weather cleared for } \\
\text { hali-an-hour, but again came on thick. }\end{array}$ \\
\hline \multirow[t]{2}{*}{ Sept. 16} & $9.30 \mathrm{a.m.}$ & $\mathrm{N} 69^{\circ} \mathrm{W}, 25 \mathrm{~m}$ & $29 \cdot 50$ & 20 & $-25 \cdot 0$ & - & - & - & - & - & 9.30 a.m. Fine and clear generally. \\
\hline & 6.15 p.m. & & $29 \cdot 42$ & - & $-36 \cdot 0$ & - & - & - & $\ldots$ & - & $\begin{array}{l}\text { 6.15 p.m. IIeavy cloud over Erebus' } \\
\text { side. }\end{array}$ \\
\hline Sept. 17 & $9 \quad$ a.m. & x $70^{\circ} \mathrm{W}, 32 \mathrm{~m}$. & $29 \cdot 60$ & 10 & $-36 \cdot 0$ & Caims and $E$ & - & $q$ & - & - & $\begin{array}{l}\text { Temperature remained steady all day. } \\
\text { Fine and elear. }\end{array}$ \\
\hline \multirow[t]{3}{*}{ Sept: 18} & $5.30 \mathrm{a} . \mathrm{m}$. & & - & - & $-23 \cdot 0$ & & - & om & - & - & \\
\hline & Sioon & $\mathrm{N} \pi 1^{\circ} \mathrm{W}, 36 \mathrm{~m}$ & $29 \cdot 40$ & 500 & - & $\begin{array}{l}\text { Calms and } \\
\text { L.t. Nk winds } \\
\text { with squalls }\end{array}$ & - & om & - & - & $\begin{array}{l}\text { Cleared for a bit at noon, but again } \\
\text { came on thick until } 5 \text { p.m. }\end{array}$ \\
\hline & $\begin{array}{cc}\text { s } & \text { p.m. } \\
\text { i } & \text {, }\end{array}$ & & $\begin{array}{c}- \\
29 \cdot 54\end{array}$ & - & $\begin{array}{l}-35 \cdot 0 \\
-41 \cdot 0\end{array}$ & $\begin{array}{l}\text { with squalls } \\
\text { of torce 4-5 }\end{array}$ & - & om & - & - & \\
\hline Sept. 19 & - & $\mathrm{N} 70^{\circ} \mathrm{W}, 40 \mathrm{~m}$. & - & - & - & Caim & 0 & - & - & - & $\begin{array}{l}\text { Low temperature all day, belng }-45^{\circ} 0 \\
\text { several tir.es. Fine and clear. }\end{array}$ \\
\hline \multirow[t]{3}{*}{ Sept. 20} & 5 a.m. & & $29 \cdot 30$ & 500 & $-45 \cdot 0$ & - & - & - & - & - & \\
\hline & $10 \quad "$ & N $71^{\circ} \mathrm{W}, 36 \mathrm{~m}$. & - & - & $-25 \cdot 0$ & Cailn & 0 & $\mathrm{~b}$ & - & - & \\
\hline & Mlit. & & - & - & - & $E$ & $1-2$ & - & - & - & \\
\hline Sept. 21 & - & N $69^{\circ} \mathrm{W}, 30 \mathrm{~m}$. & - & - & - & $\begin{array}{l}\text { Lt. NW airs } \\
\text { and caims }\end{array}$ & - & ogu & - & - & $\begin{array}{l}\text { Temperature remalned at about }-35^{\circ}{ }^{\circ} 0 \\
\text { all day. }\end{array}$ \\
\hline \multirow[t]{3}{*}{ Sept. 22} & Noon & N $72^{\circ} \mathrm{W}, 25 \mathrm{~m}$. & $30 \cdot 00$ & - & $-36 \cdot 0$ & - & - & - & - & - & \\
\hline & 4.30 p.m. & & - & - & $-25 \cdot 0$ & - & - & - & - & - & \\
\hline & $9.30 \quad$, & & - & - & $-36 \cdot 0$ & - & - & - & - & - & \\
\hline Sept. 23 & i a.m. & s $68^{\circ} \mathrm{W}, 19 \mathrm{~m}$ & $30 \cdot 30$ & - & $-30 \cdot 0$ & Calm & - & - & - & - & $\begin{array}{l}\text { Barometer steady ail day, } 30 \cdot 12 \text { ins. } \\
\text { Thermometer ranged }-10^{\circ} \cdot 0 \text { to }-26^{\circ} 0 \\
\text { in evening. Lighit NWly winds and } \\
\text { calms all day. Very thick mist all } \\
\text { day. }\end{array}$ \\
\hline Sept. 24 & 6.30 p.m. & & $29 \cdot 98$ & - & $-15 \cdot 0$ & $w$ & 3 & ocm & $*$ & - & \\
\hline \multirow[t]{4}{*}{ Sept. 25} & 10 .m. & $N 66^{\circ} \mathrm{W}, 6 \mathrm{~m}$. & $29 \cdot 98$ & - & $-19 \cdot 0$ & $\mathrm{kSE}$ & 3 & - & - & - & \\
\hline & 2 p.m. & & - & - & $-30 \cdot 0$ & ESE & 4 & - & + & - & \\
\hline & $4 \quad "$ & & - & - & $-32 \cdot 0$ & ESE & 5 & - & + & - & Overeast and thick. \\
\hline & Midt. & & - & - & $-36 \cdot 0$ & Bluzzard & $8-9$ & - & م & - & \\
\hline Sept. 28 & 6 a.m. & & 30.01 & - & $-27 \cdot 0$ & - & - & - & - & - & 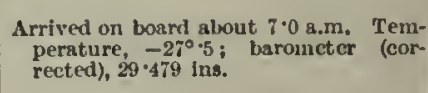 \\
\hline
\end{tabular}


TABLE VIII.-DR. KOETTLITZS TRIP ROUND BLACK ISIAAND, SEPTEMBER 24 TO OCTOBER 2, 1902.

\begin{tabular}{|c|c|c|c|c|c|}
\hline \multirow{2}{*}{ Date. } & \multirow{2}{*}{ Tinnc. } & \multirow{2}{*}{$\begin{array}{l}\text { Noon } \\
\text { bearing and } \\
\text { distance } \\
\text { in miles from } \\
\text { Winter } \\
\text { Quarters. }\end{array}$} & \multicolumn{2}{|c|}{ Temperature. } & \multirow[b]{2}{*}{ JRemarks. } \\
\hline & & & Air. & $\begin{array}{l}\text { Minimurn } \\
\text { during } \\
\text { night. }\end{array}$ & \\
\hline $\begin{array}{l}1902 . \\
\text { Sept. } 24\end{array}$ & $\begin{array}{l}10 \text { a.n. } \\
\text { Noon } \\
6 \text { p.m. }\end{array}$ & & $\begin{array}{l}- \\
-22 \cdot 0\end{array}$ & $\begin{array}{l}- \\
-\end{array}$ & $\begin{array}{l}10 \text { a.m. Fasterly brceze and overeast. } \\
\text { Noon. Threatening sky with fog, very variable winds and squally. Wind from ali round the com jass. } \\
6 \text { p.m. Calm. }\end{array}$ \\
\hline Sept. 25 & $\begin{array}{l}7 \text { a.m. } \\
8 \text { " } \\
10 " \\
8 \text { p.m. }\end{array}$ & $\mathrm{S} 54^{\circ} \mathrm{W}, 15 \mathrm{~m}$ & $\begin{array}{l}- \\
- \\
- \\
-\end{array}$ & $\begin{array}{c}-29 \cdot 0 \\
- \\
- \\
-\end{array}$ & $\begin{array}{l}7 \text { a.m. Thick w cather. } \\
8 \text { a.m. Fasterly wind, } 3 \text {; weather otherwise the same. } \\
10 \text { a.m. Clouds not so dense, and the light became better. Distant land to NF visible. Later in } \\
\text { afternoon, land to } \mathbf{E} \text { visible. } \\
\text { Squalls of force } 5-6 \text { from } S E \text { and Sly directions. Temperature ranged during day from }-21^{\circ} 0 \text { to } \\
-31^{\circ} 0 \text { at } 9 \text { p.m. Variable E'b breezes all day of force } 2-3 \text {. }\end{array}$ \\
\hline Sept. 26 & $\begin{array}{l}4 \text { a.m. } \\
7 . " \\
6 \text { p.m. }\end{array}$ & $\mathrm{S} 27^{\circ} \mathrm{W}, 20 \mathrm{~m}$ & $\begin{array}{l}- \\
-25 \cdot 0 \\
-32 \cdot 0\end{array}$ & $\begin{array}{l}-35 \cdot 0 \\
-\end{array}$ & $\begin{array}{l}\text { Calm and fine. Temperature ranged from }-25^{\circ} \cdot 0 \text { to }-28^{\circ} \cdot 0 \text { during day. Mostly ealm or light Sty airs, } \\
\text { the latter most pronounced towards evening. } \\
6 \text { p.m. Calm and tine. }\end{array}$ \\
\hline Sept. 27 & $\begin{array}{l}7 \text { a.m. } \\
6 \text { p.m. } \\
9 \text {, }\end{array}$ & $\mathrm{S} 27^{\circ} \mathrm{W}, 20 \mathrm{~m}$ & $\begin{array}{l}- \\
-\end{array}$ & $\begin{array}{r}-40 \cdot 0 \\
- \\
-\end{array}$ & $\begin{array}{l}\text { Calm all night; fine all forenoon. Thermometer out of order; no temperatures were taken after this. } \\
6 \text { p.m. Weather threatening. Ci.-cu. clouds rapidly spreading over sky. } \\
9 \text { p.m. Squalls of wind from } \mathrm{S} \text { by } \mathbf{W} \text {. }\end{array}$ \\
\hline Sept. 28 & - & $\mathrm{S} 27^{\circ} \mathrm{W}, 20 \mathrm{~m}$. & - & - & Overcast, with fresh gale and driving snow all day from $\mathrm{S}$ by $\mathbf{W}$. \\
\hline Sept. 29 & $\begin{array}{l}9 \text { p.m. } \\
2 \text { p.m. } \\
4 \quad " \\
8 \quad \text { " }\end{array}$ & $\mathrm{S} 27^{\circ} \mathrm{W}, 20 \mathrm{~m}$ & $\begin{array}{l}- \\
- \\
- \\
-\end{array}$ & $\begin{array}{l}- \\
- \\
-\end{array}$ & $\begin{array}{l}9 \text { a.m. Fine, but nnsettled looking. Cu. } 4, \text { Ci. 1. be. Gusty Sly wind. } \\
2 \text { p.m. Windy sky and threatening appearance. } \\
4 \text { p.m. Sky cleared. } \\
8 \text { p.m. Fine and calm. }\end{array}$ \\
\hline Sept. 30 & $\begin{array}{l}\text { i a.m. } \\
8 \text { p.m. }\end{array}$ & $\mathrm{S} 12^{\circ} \mathrm{W}, 32 \mathrm{~m}$. & - & - & $\begin{array}{l}7 \text { a.m. Fine day, sky more or iess clear of cloud. Detached Ci.cu. to } \mathrm{S} \text {. } \\
8 \text { p.m. Temperature seemed below }-40^{\circ} \cdot 0 \text { (position } \mathrm{S} \text { of Black Island). Fine auil calm. Much } \\
\text { refraction and mirage on barrier about Bluf all afternoou. }\end{array}$ \\
\hline Oct. 1 & $\begin{array}{l}7 \text { a.m. } \\
\text { Noon } \\
2 \text { p.m. } \\
4 \text { ". } \\
8 \text {." }\end{array}$ & $\mathrm{S} 6^{\circ} \mathbf{E}, 18 \mathrm{~m}$ & $\begin{array}{l}- \\
- \\
- \\
-\end{array}$ & $\begin{array}{l}- \\
- \\
- \\
-\end{array}$ & $\begin{array}{l}\text { 7 a.m. Thick fog, calm. Thick frost rime on everything. } \\
\text { Noon. Fog cleared; weathcr threatening; squally. Cu. and Str. clouds. } \\
2 \text { p.m. Weather looking worse. Cu. and Str. clouds coming over rapidly from SW. } \\
4 \text { p.m. Weather improving, but strong S'y brecze, force } 3 \text {, } \\
8 \text { p.m. Sky overcast ; calm and foggy. }\end{array}$ \\
\hline Oet. 2 & $\begin{array}{l}7 \text { a.m. } \\
2 \text { p.m. } \\
5 .\end{array}$ & & $\begin{array}{l}- \\
-\end{array}$ & $\begin{array}{l}- \\
-\end{array}$ & $\begin{array}{l}\text { Fine and calm. Foggy in distance, and remained foggy all the forenoun. } \\
2 \text { p.m. Fog thicker, obscuring all land. } \\
5 \text { p.m. Fog lifted slightly, land to } \mathrm{N} \text { just visible. }\end{array}$ \\
\hline
\end{tabular}


TABLE IX. -SECOND JOURNEY OF LIEUTENANT ROYDS, R.N., TO CAPE CROZIER, OCTOBER 4 TO 24, 1902.

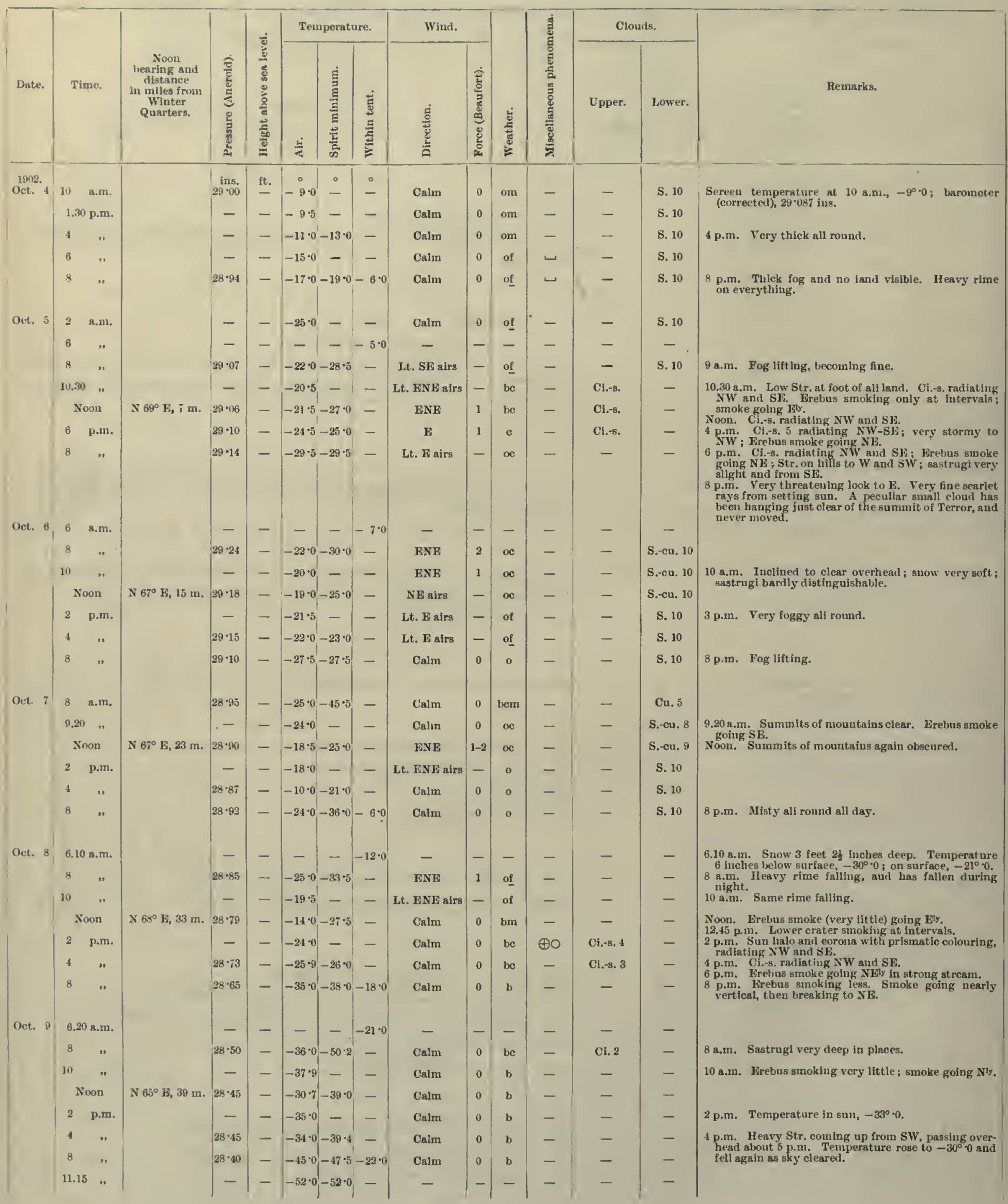


TABLE IX.-SECOND JOURNEY OF LIEUTENANT ROYDS, R.N., TO CAPE CROZIER, OCTOBER 4 TO 24, 1902 (continued).

\begin{tabular}{|c|c|c|c|c|c|c|c|c|c|c|c|c|c|c|}
\hline \multirow[b]{2}{*}{ Date. } & \multirow[b]{2}{*}{ Time. } & \multirow[b]{2}{*}{$\begin{array}{c}\text { Noon } \\
\text { bearing and } \\
\text { distance } \\
\text { ju niles from } \\
\text { Winter } \\
\text { Quarters. }\end{array}$} & \multirow[b]{2}{*}{ 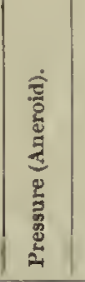 } & \multirow[b]{2}{*}{ 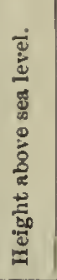 } & \multicolumn{3}{|c|}{ Temperature. } & \multicolumn{2}{|l|}{ Wlnd. } & \multirow[b]{2}{*}{ 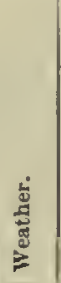 } & \multirow{2}{*}{ 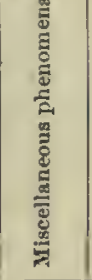 } & \multicolumn{2}{|c|}{ Clouds. } & \multirow[b]{2}{*}{ Remarks. } \\
\hline & & & & & $\stackrel{2}{2}$ & 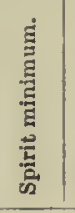 & 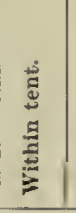 & 苋 & & & & Upper. & Lower. & \\
\hline \multirow[t]{8}{*}{$\begin{array}{l}1902 . \\
\text { Oet. } 10\end{array}$} & 3 a.m. & & ins. & ft. & $\begin{array}{c}0 \\
-57 \cdot 0\end{array}$ & $\begin{array}{c}\circ \\
-58 \cdot 0\end{array}$ & $\begin{array}{c}0 \\
-\end{array}$ & 一 & -1 & $\overline{-}$ & - & - & - & \\
\hline & $6.30 \quad "$ & & - & - & - & - & $-33 \cdot 0$ & - & - & - & - & - & - & \\
\hline & $8 \quad$ " & & $28 \cdot 39$ & - & $-44 \cdot 0$ & $-58 \cdot 5$ & - & Calm & 0 & be & - & $\mathrm{Cl} . .8 .4$ & 一 & 8 a.m. Sunmlt of Terror very misty. \\
\hline & $10 \quad$ " & & - & 一 & $-41 \cdot 0$ & - & 一 & Calm & 0 & be & - & Ci.-8. 5 & - & $\begin{array}{l}10 \text { a.m. Sastrugi very deep, and surface of snow very } \\
\text { hard and sllppery. }\end{array}$ \\
\hline & Noon & N $64^{\circ} \mathrm{E}, 45 \mathrm{~m}$ & $28 \cdot 18$ & - & $-24 \cdot 0$ & $-44 \cdot 0$ & 一 & SW & 2 & oc & - & $\mathrm{Cl} .-8.7$ & - & \\
\hline & $2 \quad$ p.m. & & - & - & $-23 \cdot 0$ & - & - & $8 W$ & 2 & oe & + & - & 一 & \\
\hline & $8 \quad$, & & $27 \cdot 78$ & 940 & $-31 \cdot 0$ & $-31 \cdot 0$ & - & sw & $4-5$ & $\infty$ & + & - & - & 8 p.m. Arrived at furthest camp. \\
\hline & $9 \quad "$ & & - & - & - & - & $-18 \cdot 0$ & - & - & - & - & - & 一 & \\
\hline \multirow[t]{5}{*}{ Oet. 11} & Noon & $\mathrm{N} 64^{\circ} \mathrm{E}, 45 \mathrm{~m}$ & $27 \cdot 815$ & 940 & $-26 \cdot 0$ & - & $-12 \cdot 0$ & sw & $1-2$ & be & $O \oplus$ & Ci.-8. 3 & - & \\
\hline & $2 \quad$ p.m. & & - & - & $-25 \cdot 5$ & - & $-7 \cdot 0$ & Calm & 0 & $b$ & - & - & 一 & \\
\hline & $4 \quad "$ & & $27 \cdot 83$ & - & $-25 \cdot 0$ & $-31 \cdot 0$ & $-11 \cdot 0$ & Lt. NW airs & - & b) & - & - & - & 1 \\
\hline & $6 \quad "$ & & - & - & $-28 \cdot 0$ & - & $-10^{\circ} 0$ & Calm & 0 & b & - & - & - & \\
\hline & $8 \quad " \quad$ & & $27 \cdot 85$ & - & $-35 \cdot 5$ & $-35 \cdot 5$ & $-12 \cdot 0$ & Calm & 0 & b & - & - & - & 8 p.m. llecord was deposited at Cape Crozier. \\
\hline \multirow[t]{7}{*}{ Oct. 12} & $7 \quad \mathrm{a.m}$ & & - & - & - & - & $-13 \cdot 0$ & 一 & - & - & - & - & - & \\
\hline & $8 \quad$ " & & $27 \cdot 82$ & 940 & $-30 \cdot 0-$ & $-40 \cdot 0$ & - & Calm & 0 & ocm & - & A. -8.8 & - & \\
\hline & $10 \quad "$ & & - & - & $-29 \cdot 0$ & 一 & - & sw & $1-2$ & oc & - & A. -8.8 & - & \\
\hline & Noon & $\mathrm{N} 64^{\circ} \mathrm{IG}, 45 \mathrm{~m}$ & $27 \cdot 810$ & - & $-26 \cdot 0$ & $-30 \cdot 0$ & - & SW & 2 & ocm & $\oplus 0+$ & A.-8. 9 & - & $\begin{array}{l}\text { Noon. Very fine solar bals and corona, prismatlc } \\
\text { colouring. }\end{array}$ \\
\hline & 2 p.m. & & - & - & $-27 \cdot 0$ & - & - & SW & 3 & oem & ФO+ & A.-8. 8 & - & \\
\hline & $4 \quad "$ & & - & - & $-27 \cdot 0\}$ & $-29 \cdot 5$ & $-17 \cdot 0$ & SW & $2-3$ & ocin & $\oplus 0 \div$ & A.-8. 10 & - & \\
\hline & $"$ & & $27 \cdot 75$ & - & $-29 \cdot 5 \mid$ & $-30 \cdot 0$ & $-19 \cdot 5$ & sw & $3-1$ & ocm & + & A.-8. 10 & - & $\begin{array}{l}8 \text { p.m. Whind rose gradually all day. Ugly appearance } \\
\text { of weatber to S. }\end{array}$ \\
\hline \multirow[t]{3}{*}{ Oet. 13} & Noon & $\mathrm{N} 50^{\circ} \mathbf{E}, 43 \mathrm{~m}$. & $27 \cdot 60$ & 940 & - & - & - & SW & $7-8$ & o & +2 & - & - & \multirow{3}{*}{$\begin{array}{l}\text { Wind sprang up very strong durlng tbe night } \\
\text { and blew hard all day, with a short lull at } 4 \text { p.m. } \\
\text { Finormous quantlty of drift collecting round teuts. } \\
\text { Overcast all day. }\end{array}$} \\
\hline & 4 p.m. & & - & - & $-18 \cdot 5$ & $-30^{\circ} 0$ & - & SW & 8 & o & -2 & 一 & - & \\
\hline & $8 \quad "$ & - & $27 \cdot 55$ & - & - & - & - & SW & $8-9$ & 0 & $t^{2}$ & - & 一 & \\
\hline Oct. 14 & - & $\mathrm{N} 64^{\circ} \mathrm{E}, 45 \mathrm{~m}$. & - & - & - & - & - & - & - & - & - & - & - & $\begin{array}{l}\text { Exactly sinflar weather to yesterday's. Wind linlied } \\
\text { for about fialf an hour at } 4 \text { a.m., but again came on } \\
\text { witb increasing heavy gusts. Drift very thlek and } \\
\text { tents nearly burled. Barometer fell all day. }\end{array}$ \\
\hline Oet. 15 & - & $\mathrm{N} 62^{\circ} \mathrm{E}, 36 \mathrm{~m}$. & - & - & - & - & - & - & - & - & - & - & 一 & $\begin{array}{l}\text { Wind about the same, blowing a whole gale, accom- } \\
\text { panied by lieavy drift. Barometer started to rise. } \\
\text { Temperature in tent keeplug between } 0^{\circ} 0 \text { aud }-5^{\circ} \cdot 0 \text {. }\end{array}$ \\
\hline Oet. 16 & - & N $62^{\circ} \mathbf{E}, 36 \mathrm{~m}$. & - & - & - & - & 一 & - & - & - & - & 一 & - & $\begin{array}{l}\text { Simllar conditions to yesterday's. Wind stlli a strong } \\
\text { SW gale wlth beavy drift. In the afternoon the sunt } \\
\text { slone out a bit and the wind legan to lull. Baro- } \\
\text { meter rislng steadily, temperature in tent about tho } \\
\text { same. }\end{array}$ \\
\hline Oct. 17 & 8 p.m. & N $62^{\circ} \mathrm{E}, 36 \mathrm{~m}$. & $28 \cdot 115$ & 910 & $-11 \cdot 0$ & $-13 \cdot 0$ & - & sw & $5-6$ & b & - & - & - & $\begin{array}{l}8 \text { p.m. Wind still remained strong, about force 7, but } \\
\text { sky was cloudless and wind gradually fell towards } \\
\text { evenlug. }\end{array}$ \\
\hline \multirow[t]{6}{*}{ Oct. 18} & $1.30 \mathrm{a.m}$. & & - & - & 一 & - & $-9 * 0$ & - & - & - & - & 一 & 一 & \\
\hline & Noon & N $62^{\circ} \mathrm{E}, 36 \mathrm{~m}$ & $29 \cdot 038$ & - & -8.0 & $-11 \cdot 0$ & - & Lt. ENE alrs & - & b & - & - & - & \\
\hline & 2 p.m. & & - & - & $-8 \cdot 0$ & $-10 \cdot 0$ & - & Calm & 0 & b & - & - & - & 2 p.m. Glorious day, sky cloudless. \\
\hline & $4 \quad "$ & & $29 \cdot 00$ & - & $-8 \cdot 0$ & $-9 \cdot 0$ & - & Calm & 0 & b & - & 一 & - & \\
\hline & $"$ & & - & - & $-12 \cdot 0$ & - & - & Calın & 0 & b & - & - & - & \\
\hline & " & & $28 \cdot 94$ & - & $-18 \cdot 0$ & $-18 \cdot 0$ & -1.0 & Caim & 0 & b & - & - & - & \\
\hline Oct. 19 & 10 a.m. & & $28 \cdot 84$ & - & $-4 \cdot 0$ & $-20 \cdot 0$ & - & Caim & 0 & $b$ & 一 & - & - & 10 a.m. Koggy early, but graduslty cleared. \\
\hline & Noon & N $62^{\circ} \mathrm{E}, 36 \mathrm{~m}$ & - & - & $-6 \cdot 0$ & $-10 \cdot 0$ & - & Calm & 0 & b & - & - & - & \\
\hline & 2 p.m. & & 一 & - & $-9 \cdot 0$ & - & 一 & Calm & 0 & b & - & - & - & Sun shining brightly all day. \\
\hline & $8 \quad "$ & & $29 \cdot 00$ & - & $\mid-22 \cdot 0$ & $|-22 \cdot 0|$ & $-10^{\circ} 0$ & Calm & 0 & b & - & 一 & - & \\
\hline
\end{tabular}


TABLE IX.-SECOND JOURNEY OF LIEUTENANT ROYIS, K.N., TO CAPE CROZIER, OCTOBER 4 TO 24, 1902 (continued).

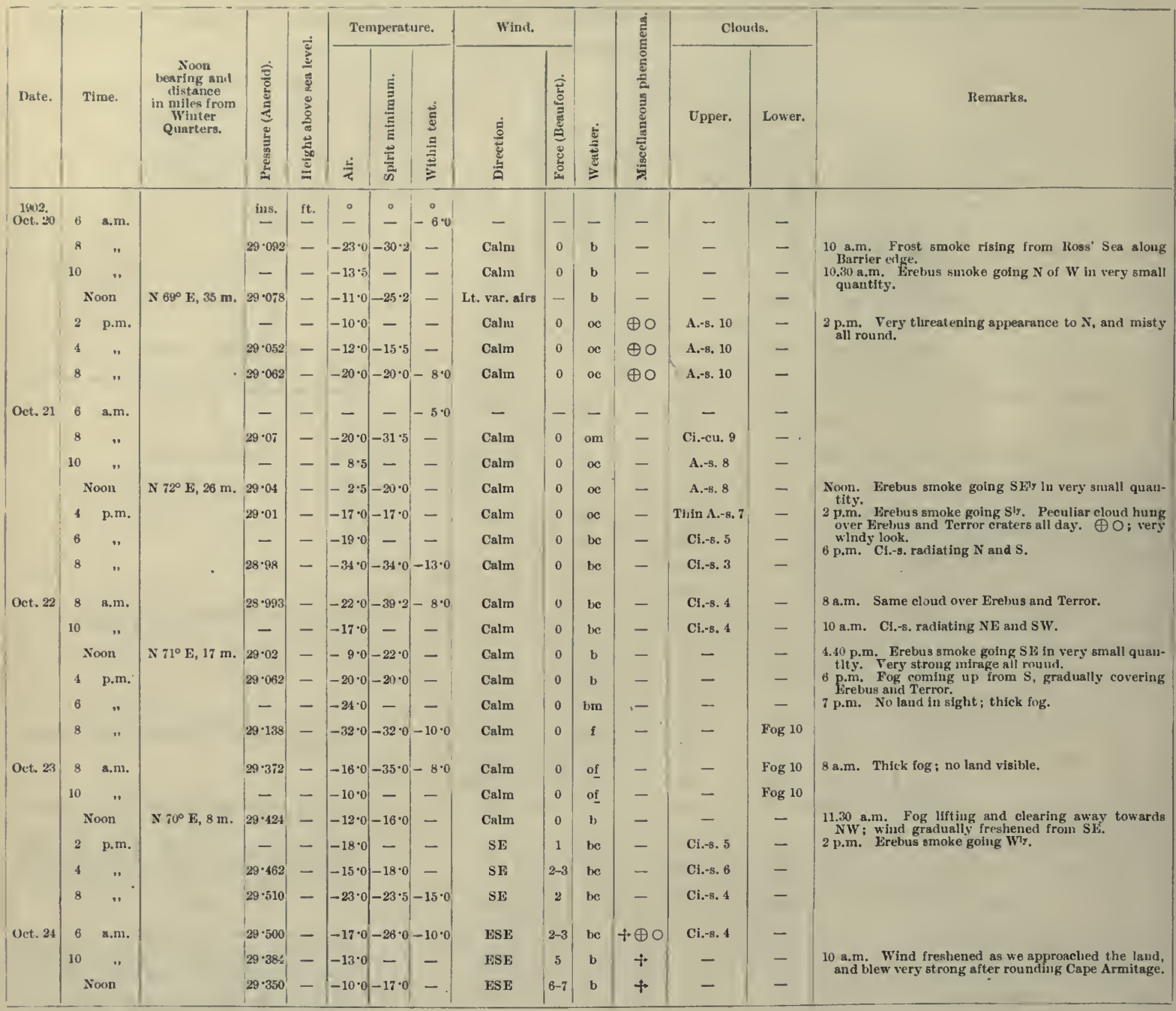

TABLE X.-DR. KOETTLITZ'S TRIP TO EREBUS BAY, NOVEMBER 3 TO 5, 1902.

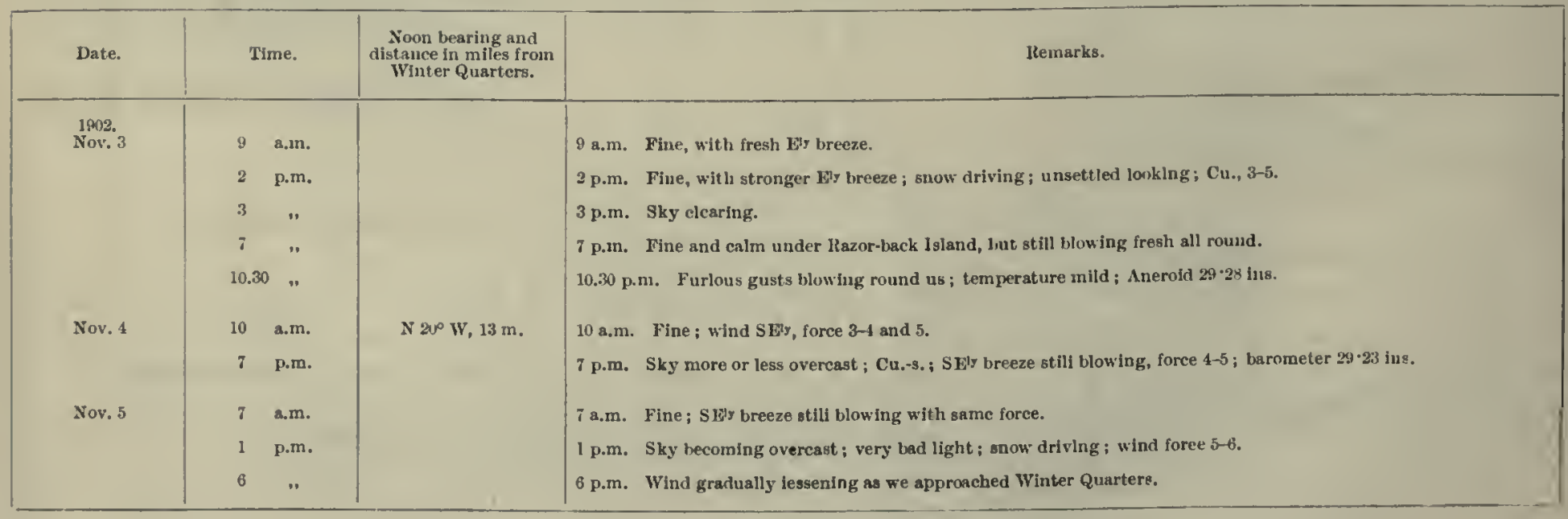


TABLE XI.-THIRD JOURNEY OF LIEUTENANT IOYDS, R.N., TO CAPE CROZIER, NOVEABRR 3 TO $17,1902$.

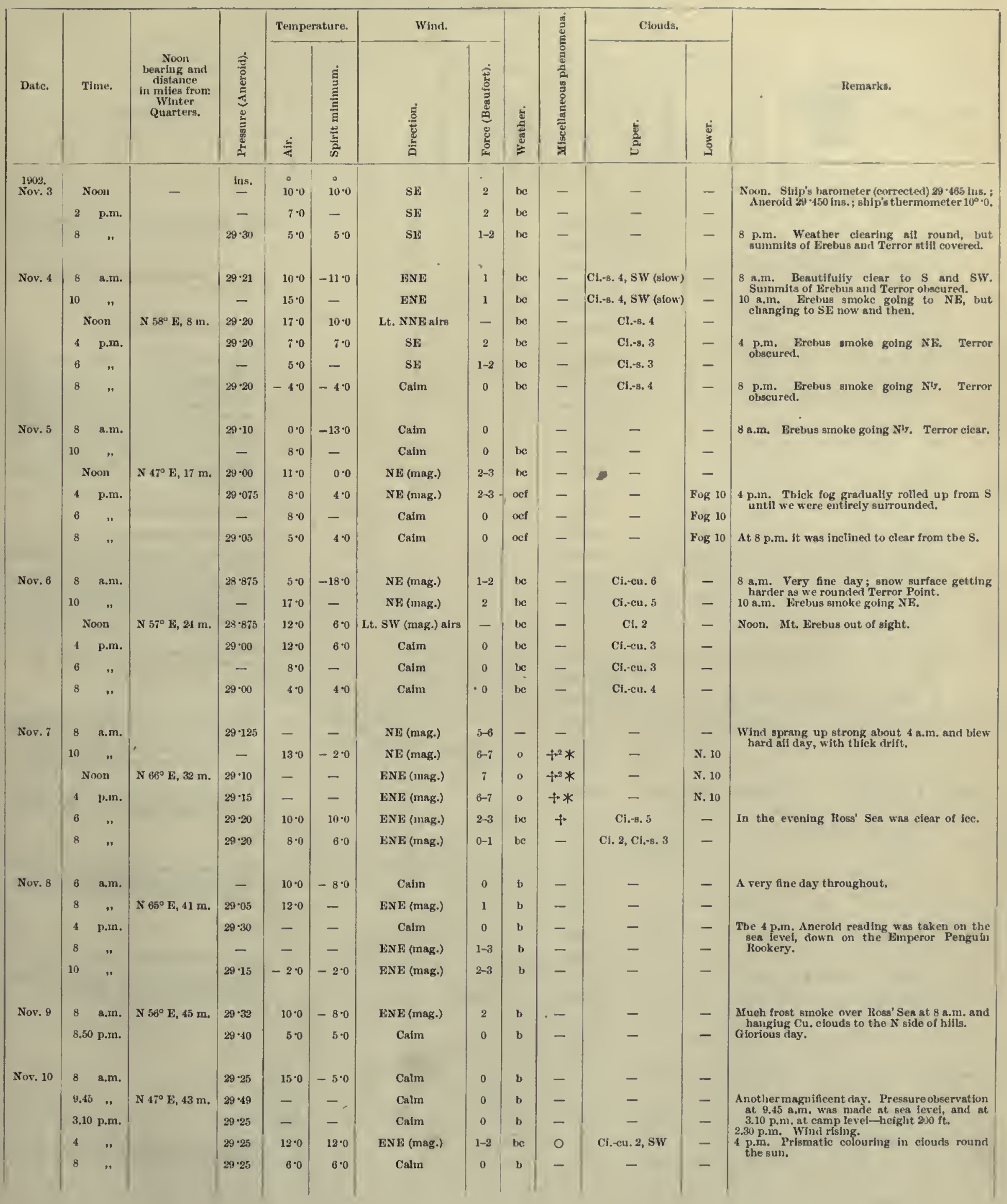


TABLE XI.-THIRD JOURNEY OF LIEUTENANT ROYDS, R.N., TO CAPE CROZIER, NOVENBER 3 TO 17,1902 (continued).

\begin{tabular}{|c|c|c|c|c|c|c|c|c|c|c|c|c|}
\hline \multirow[b]{2}{*}{ Date. } & \multirow[b]{2}{*}{ Time. } & \multirow[b]{2}{*}{$\begin{array}{l}\text { Noon } \\
\text { bearing and } \\
\text { distance } \\
\text { in miltes from } \\
\text { Winter } \\
\text { Quarters. }\end{array}$} & \multirow[b]{2}{*}{ 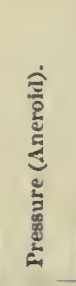 } & \multicolumn{2}{|c|}{ Temperature. } & \multicolumn{2}{|l|}{ Wind. } & \multirow[b]{2}{*}{ 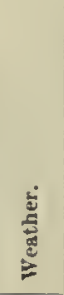 } & \multirow{2}{*}{ 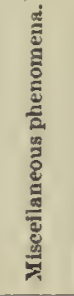 } & \multicolumn{2}{|l|}{ Clouds. } & \multirow[b]{2}{*}{ Remarks. } \\
\hline & & & & 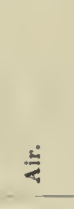 & $\begin{array}{l}\text { 咅 } \\
\text { 总 } \\
\text { 紊 }\end{array}$ & 密 & 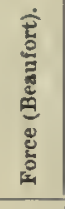 & & & हैँ & 产 & \\
\hline \multirow[t]{5}{*}{$\begin{array}{l}19012 . \\
\text { Nov. j1 }\end{array}$} & \begin{tabular}{cc|}
6 & a.m. \\
8 &
\end{tabular} & & Ins. & 6.0 & -10.0 & ENE (mag.) & 4 & 0 & + & - & A.-8. 10 & $\begin{array}{l}\text { Wind sprang up in heavy gusts at } 3 \text { a.m., and } \\
\text { st } 4 \text { a.m.1. it was blow ing very hard tndeeti. }\end{array}$ \\
\hline & Noon & $\mathrm{X} 59^{\circ} \mathrm{E}, 45 \mathrm{~m}$ & $29 \cdot 10$ & - & - & ENE (mag.) & $a-7$ & q & - & - & - & \\
\hline & 4 p.m. & & $29 \cdot 20$ & - & - & $\operatorname{ENE}$ (mag.) & $6-7$ & q & - & - & - & $\begin{array}{l}3 \text { p.m. Wind lufled, but came on worse shortly } \\
\text { afterwards. }\end{array}$ \\
\hline & $6 \quad 1$ & & $29 \cdot 22$ & - & - & ENE (mag.) & 6 & $q$ & - & - & - & \\
\hline & $8 \quad "$ & & $29 \cdot 25$ & - & - & ENE (mag.) & $6-8$ & $q$ & - & - & - & 8 p.m. Drift excessive all day. \\
\hline \multirow[t]{5}{*}{ Nov. 12} & 8 a.m. & & $29 \cdot 32$ & - & - & FNE (mag.) & $4-5$ & of & - & - & - & $\begin{array}{l}8 \text { a.m. Still blowing and drifting, with dense } \\
\text { fog. }\end{array}$ \\
\hline & $10 \quad "$ & & $29 \cdot 35$ & - & - & ENE (mag.) & $4-5$ & of & - & - & - & $\begin{array}{l}4 \text { p.m. Wind rose in heavy squalls, with short } \\
\text { lulls. Nothing visible. Sky showing signas }\end{array}$ \\
\hline & Nown & $\mathrm{N} 65^{\circ} \mathrm{E}, 41 \mathrm{~m}$ & $29 \cdot 38$ & - & - & ENE (mag.) & $4-5$ & of & - & - & - & $\begin{array}{l}\text { of breaking, and sun gleaming. No temper- } \\
\text { atures taken alf day: thermometer burled. }\end{array}$ \\
\hline & $\begin{array}{cc}4 & \text { p.m. } \\
6 & \end{array}$ & & $29 \cdot 30$ & - & - & $\begin{array}{l}\text { ENE (mag.) } \\
\text { ENE (mag.) }\end{array}$ & $0-7$ & ${ }^{\mathrm{oq}}$ & - & - & - & $\begin{array}{l}6 \text { p.m. Biowing very hard, with heavy squalls. } \\
9 \text { p.1m. Wind lutied considerably, and by }\end{array}$ \\
\hline & $8 \quad$. & & $29 \cdot 25$ & - & - & ENE (mag.) & $2-3$ & $\underline{y}$ & - & - & - & $\begin{array}{l}11 \text { p.m. Wind sprang up in heavy gusts, } \\
\text { lasting for a about balf an hour, and then fell } \\
\text { to dead calm. }\end{array}$ \\
\hline \multirow[t]{6}{*}{ Nov. 13} & 8 a.m. & & $29 \cdot 30$ & $15 \cdot 0$ & $-8 \cdot 0$ & Calm & 0 & be & - & $\mathrm{Cl}_{0}-\mathrm{s}, \mathrm{A}$ & - & 8 a.m. Ci.-s. radiating W. \\
\hline & $10 \quad "$ & & - & $13 \cdot 0$ & - & Calm & 0 & be & - & Cl.-8. 5 & - & 10 a.m. Cl.-s. radiating $W$. \\
\hline & Noon & $\mathrm{N} 66^{\circ} \mathrm{E}, 32 \mathrm{~m}$. & $29 \cdot 30$ & $11 \cdot 0$ & $8 \cdot 0$ & Calm & 0 & bein & - & Ci.:s. 6 & - & $\begin{array}{l}\text { Noon. Ci-s. radiating } W \text {, From } 1.30 \text { p.m. to } \\
3 \text { p.m., } \oplus, O, \text { and parhelia visible. }\end{array}$ \\
\hline & 4 p.m. & & $29 \cdot 40$ & $6 \cdot 0$ & $6 \cdot 0$ & SE (mag.) & $2-3$ & be & - & Ci.-s. 5 & - & $\begin{array}{l}4 \text { p.m. Land to } S W \text { mueh miraged. Summit } \\
\text { of Terror very misty. Ci.-B. radiating } W \text {. }\end{array}$ \\
\hline & $8 \quad "$ & & - & $5 \cdot 0$ & - & SE (mag.) & 3 & be & - & Ci.-8. 6 & - & $\begin{array}{l}8 \mathrm{p} . \mathrm{m} \text {. Very threatening appearance to } \mathrm{S} \text {. } \\
\text { Tops of Erebus aud Terror clearing. Erebu }\end{array}$ \\
\hline & o $"$ & & 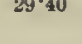 & 40 & 4.0 & Calm & 0 & b & - & - & - & smoke going Niy. \\
\hline \multirow[t]{6}{*}{ Nov. 14} & 8 a.m. & & $29 \cdot 40$ & $12 \cdot 0$ & $-2 \cdot 0$ & SE (mag.) & $3-4$ & oq & 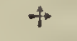 & - & A. -8.10 & $\begin{array}{l}\text { Wind sprang up about } 5 \text { a.m., and blew In } \\
\text { strong gusts, with nuch drift. and }\end{array}$ \\
\hline & $10 \quad "$ & & - & $13 \cdot 0$ & - & ESE (mag.) & 3 & oq & + & - & A.-B. 10 & 10 a.m. Wind very gusty. \\
\hline & Noon & $\mathrm{N} 6 \mathbf{i}^{\circ} \mathrm{E}, 23 \mathrm{~m}$ & $29 \cdot 30$ & - & - & ESE (niag.) & $1-2$ & o & $\oplus 0$ & - & A.-8. 10 & 4 p.m. Prismatic coloured clouds. \\
\hline & 4 p.m. & & $29 \cdot 28$ & $12 \cdot 0$ & $10 \cdot 0$ & Calm & 0 & be & $\oplus 0$ & $\begin{array}{l}\text { Ci.-cu. 5, SW } \\
\text { (slowi) }\end{array}$ & - & $\begin{array}{l}6 \text { p.m. All upper clouds (appeared) mother-of- } \\
\text { pearl near sun. Erebus smoke going NF; ; }\end{array}$ \\
\hline & $6 \quad "$ & & - & $11 \cdot 0$ & - & Calm & 0 & $\propto$ & - & Ci.-cu. 5, Ci.-8. 4 & - & $\begin{array}{l}\text { second crater smoking, smoke going } \mathrm{NE} \text {. } \\
\text { Storny appearance to } \mathrm{S} \text { and SW. }\end{array}$ \\
\hline & $8 \quad "$ & & $29 \cdot 30$ & $9 \cdot 0$ & $9 \cdot 0$ & Calm & 0 & be & - & $\mathrm{Ci}_{.-8.5} 5$ & - & 8 p.m. Irountalns obscured, but clearing. \\
\hline \multirow[t]{6}{*}{ Nov. 15} & $8 \quad \mathrm{~m}$. & & $29 \cdot 30$ & $10 \cdot 0$ & $2 \cdot 0$ & Calm & 0 & b & - & - & - & 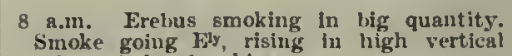 \\
\hline & $10 \quad "$ & & - & $13 \cdot 0$ & - & Calm & 0 & b & - & - & - & columm before breaking away. \\
\hline & Noon & $\mathrm{N} 67^{\circ} \mathrm{K}, 15 \mathrm{~m}$. & $29 \cdot 30$ & $14 \cdot 0$ & $10^{\circ} 0$ & Calm & 0 & b & - & - & - & \\
\hline & 1 p.m. & & $29 \cdot 32$ & $13 \cdot 0$ & $11 \cdot 0$ & Calun & 0 & b & - & - & - & A very glorious day. Frebus smoke was going \\
\hline & $6 \quad "$ & & - & $8 \cdot 0$ & - & Calm & 0 & b & - & - & - & $\begin{array}{l}\text { in any dirertion up to about noon, but then } \\
\text { it setilet, going } \text { sly; }^{\text {; }} \text { apparently hittle wind }\end{array}$ \\
\hline & $8 \quad "$ & & $29 \cdot 48$ & $2 \cdot 0$ & $2 \cdot 0$ & Calm & 0 & b & - & - & - & np there. \\
\hline \multirow[t]{6}{*}{ Xov. 16} & 8 a.m. & & $29 \cdot 90$ & $8 \cdot 0$ & $-6 \cdot 0$ & Calm & 0 & b & - & - & - & \\
\hline & $10 \quad "$ & & - & $14^{\circ} 0$ & - & Calm & 0 & be & - & Cl.eu. 4 & - & 10 a.m. Erebus smoke going $\mathrm{E}^{\text {by. }}$ \\
\hline & Noon & X $68^{\circ} \mathbf{E}, 7 \mathrm{~m}$. & $29 \cdot 95$ & $15 \cdot 0$ & $8 \cdot 0$ & Calm & 0 & b & - & - & - & $\begin{array}{l}\text { Noon. Fery little smoke from Frebus; smoke } \\
\text { going yyy. Much nirage all round. }\end{array}$ \\
\hline & 4 p.m. & & $30 \cdot 00$ & $12 \cdot 0$ & $12 \cdot 0$ & Calm & 0 & $\mathbf{b}$ & - & - & - & 4 p.m. krebus smoke going NE'y. \\
\hline & $6 \quad "$ & & - & $12 \cdot 0$ & - & Calm & 0 & b & - & - & - & \\
\hline & $8 \quad "$ & & $29 \cdot 90$ & $12 \cdot 0$ & 9.0 & Calm & 0 & b & - & - & - & \\
\hline \multirow[t]{5}{*}{ Nov. 17} & 8 s.m. & & $29 \cdot 88$ & $14 \cdot 0$ & $-10 \cdot 0$ & Calm & 0 & oc & + & A.-8. 10 & - & \\
\hline & $10 \quad "$ & & - & - & - & Lt. SE airs & - & o & - & - & S. 10 & \\
\hline & Noon & & $29 \cdot 80$ & $14 \cdot 0$ & $14 \cdot 0$ & SE & 1 & o & - & - & S. 10 & \\
\hline & 2 p.m. & & - & $14 \cdot 0$ & - & SE & $3-4$ & $\circ$ & $t^{2}$ & - & S. 10 & Arrived on board ship at 5.10 p.m. (Ships \\
\hline & $4 \quad$ & & 29.76 & $15 \cdot 0$ & $14 \cdot 0$ & SE & 3 & 0 & + & - & s. 10 & \\
\hline
\end{tabular}


TABLE XIL,-JOURNEY OF DR. KOETTLIT\% TO THE WESTERN MOUNTAINS, NOVEMHER 29 TO DLCEMBER 17, 1902.

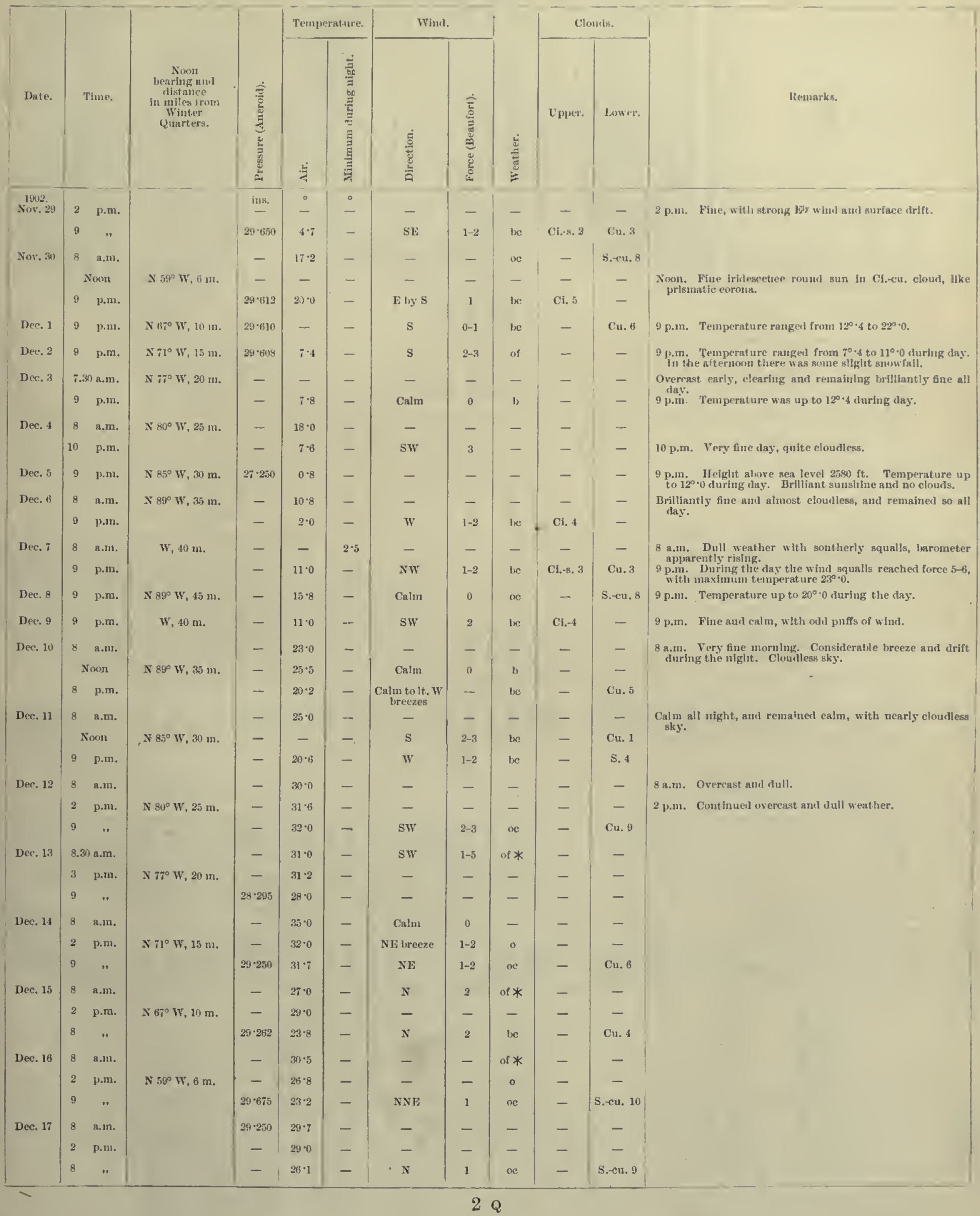


.

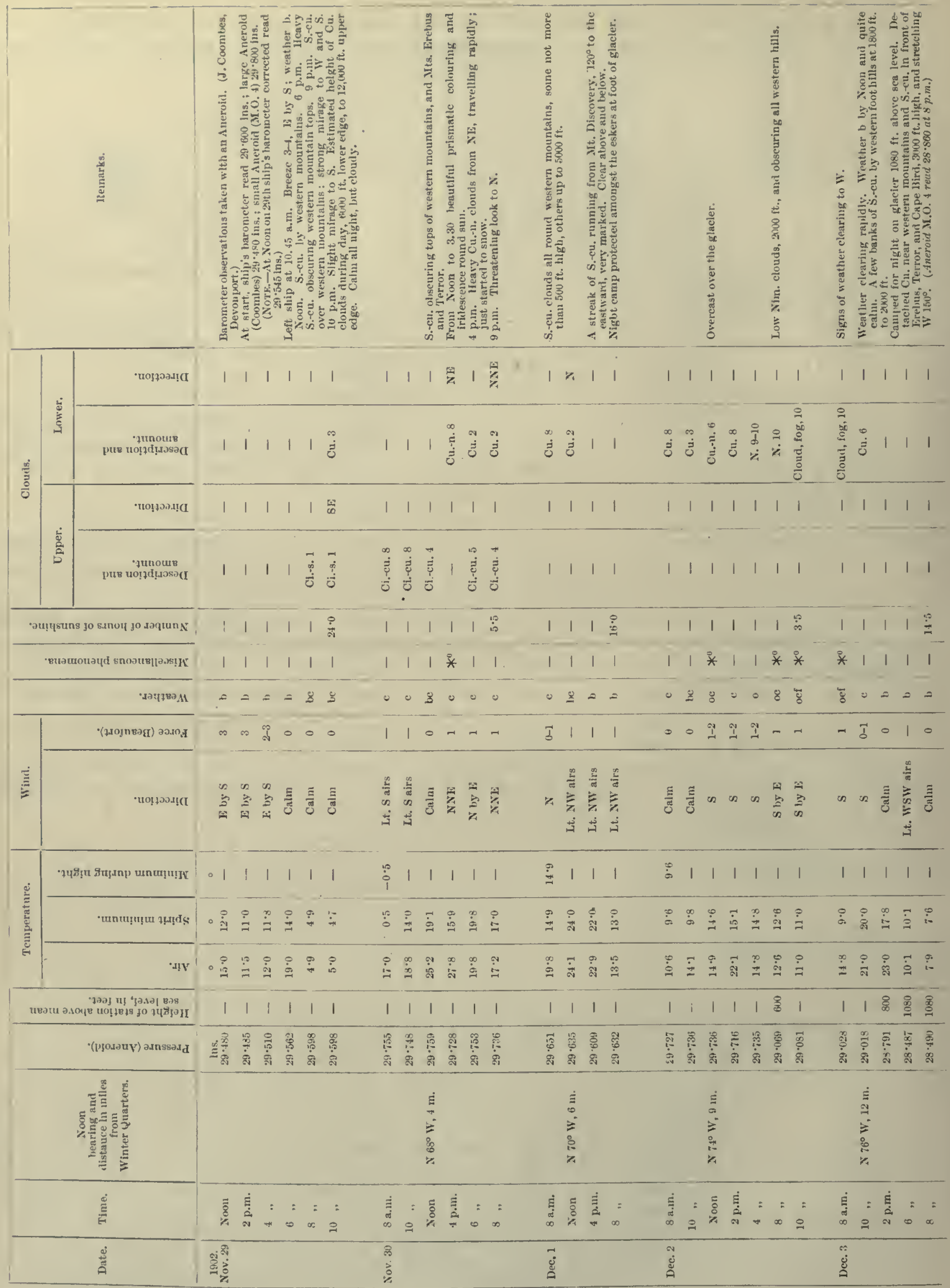



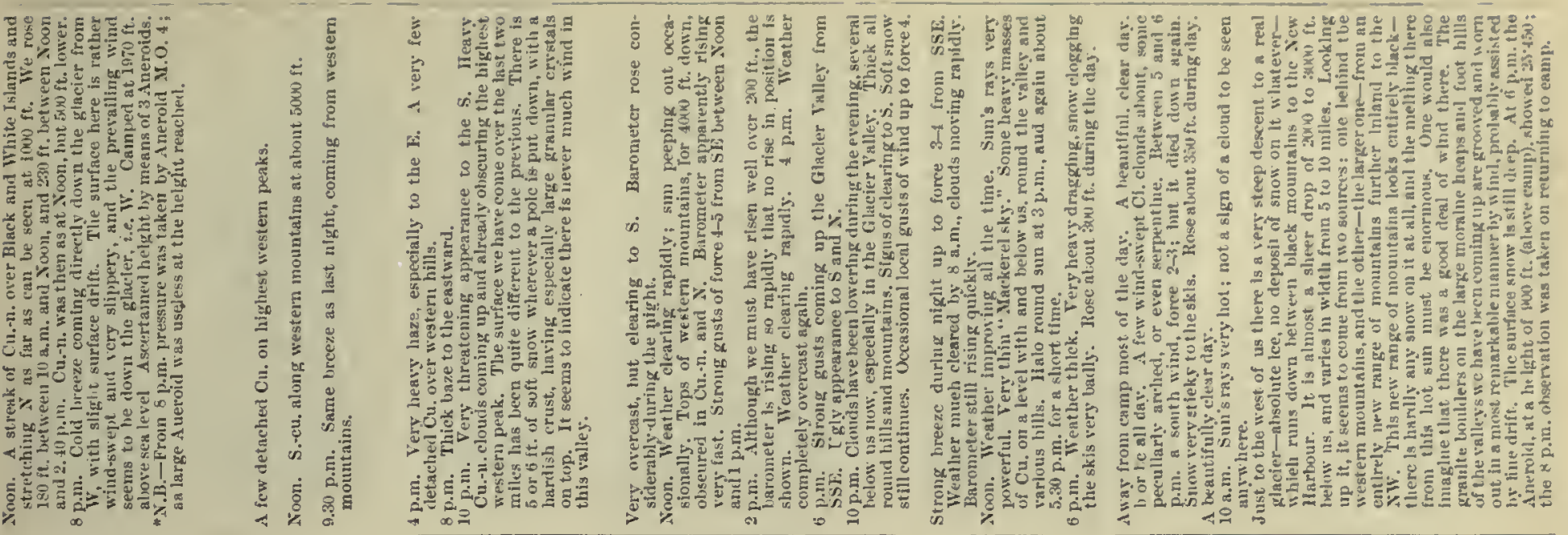

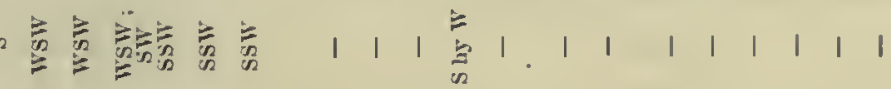

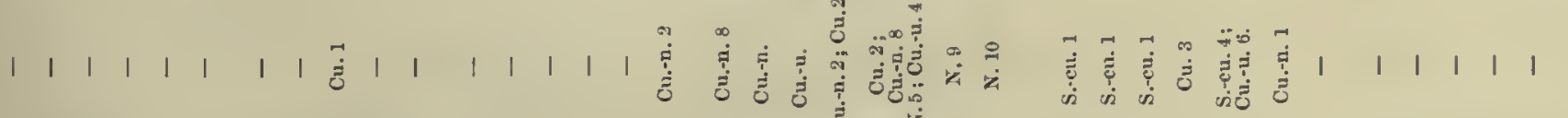

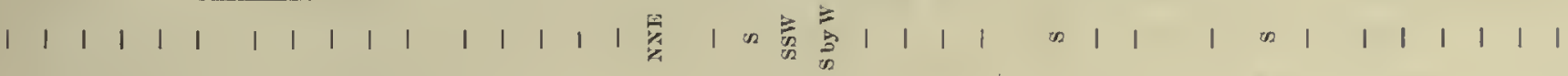

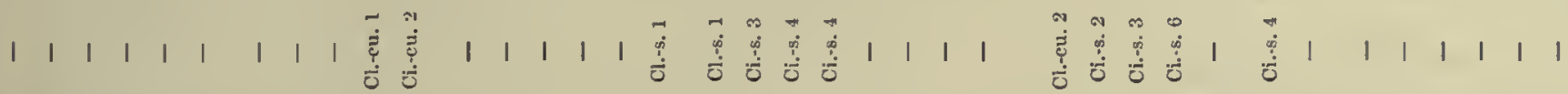

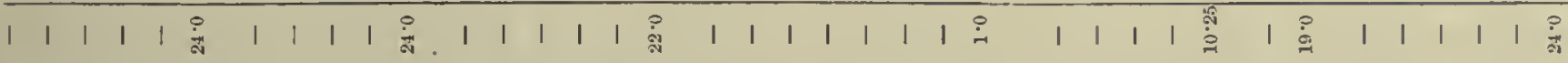

| 1 1 1 1 赵

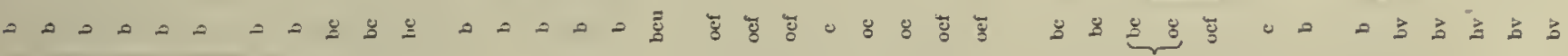

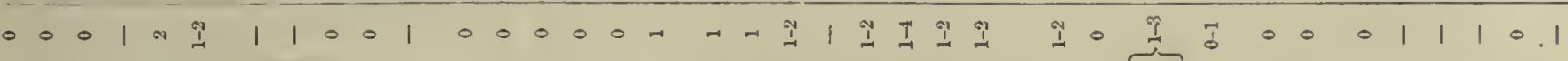

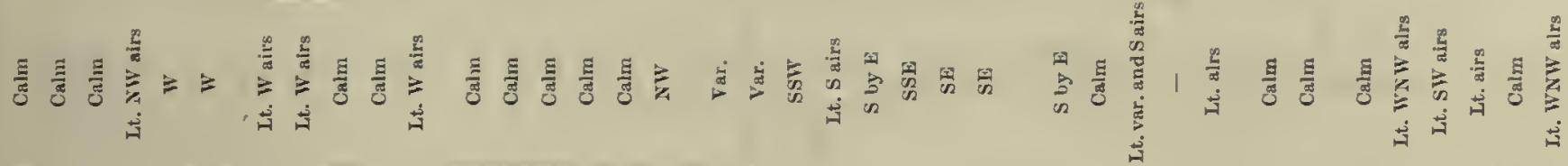

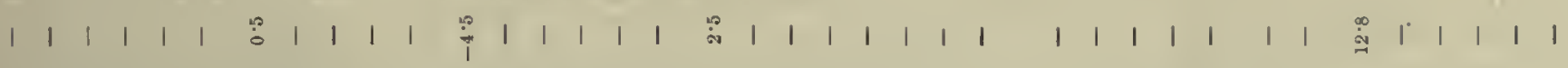

ப

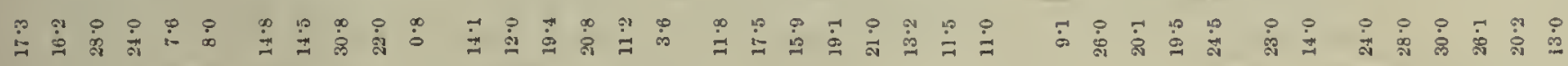

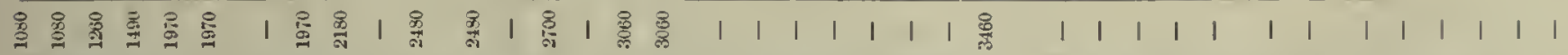

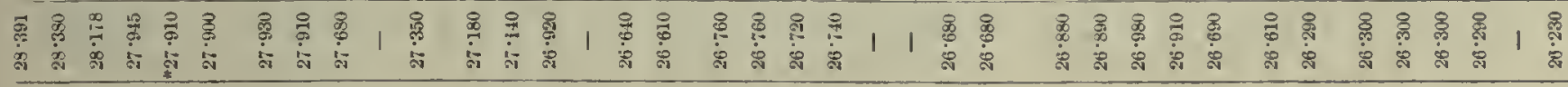

\begin{tabular}{|c|c|c|c|c|c|}
\hline 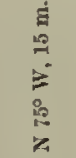 & 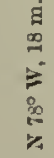 & 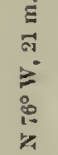 & 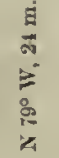 & 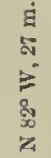 & 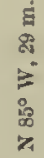 \\
\hline
\end{tabular}

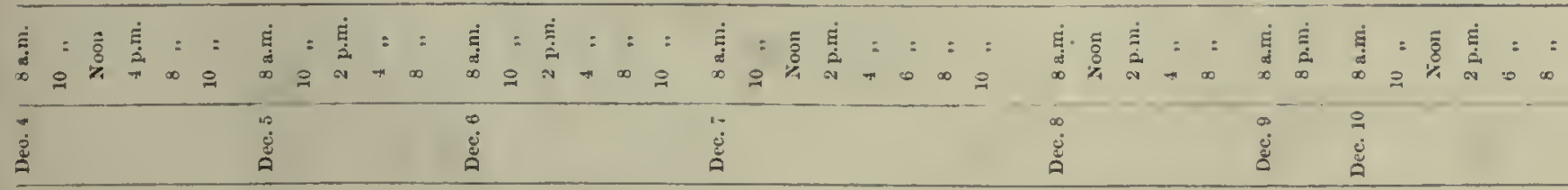


政

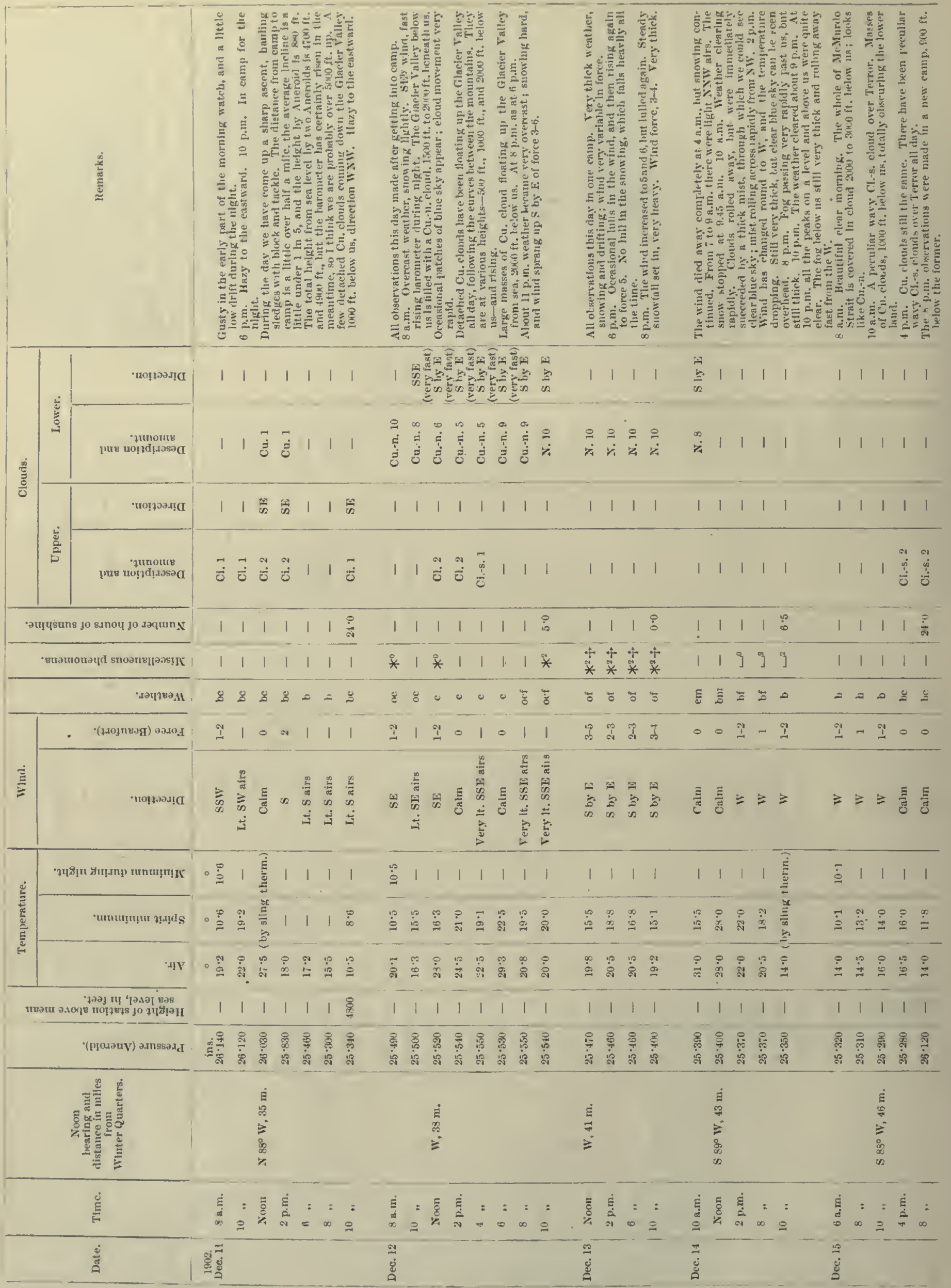




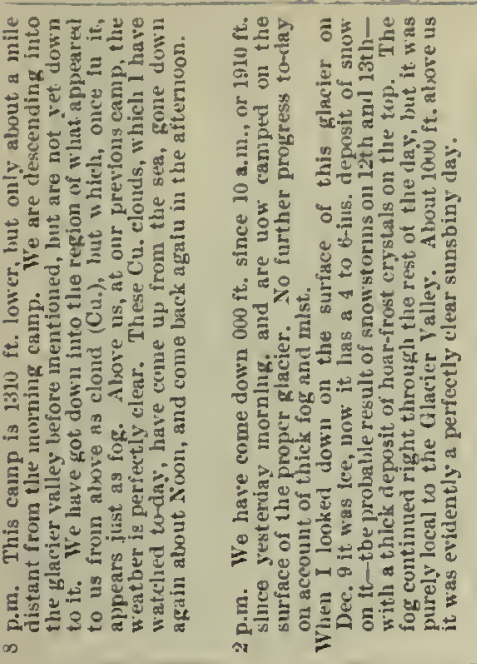

1 11

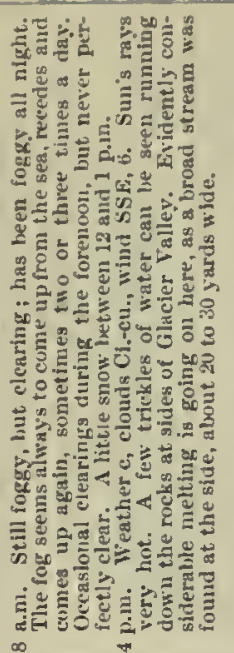

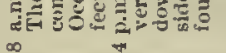

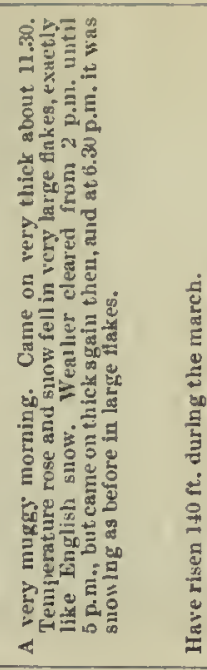

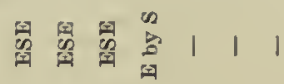
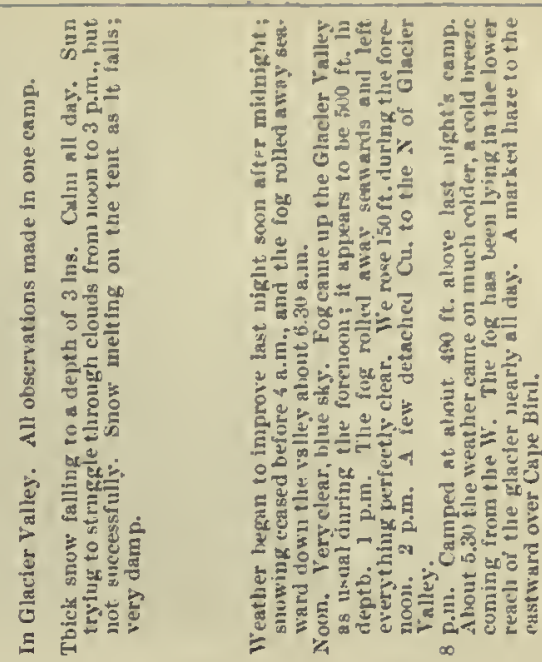

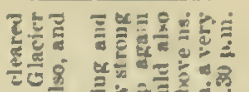

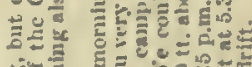

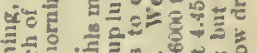

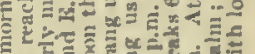

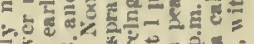
Evon

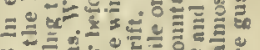
三 오을

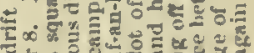

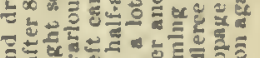
हैं

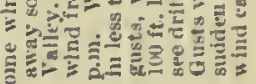

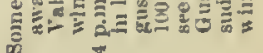

$\begin{array}{lllll}1 & 1 & 1\end{array}$

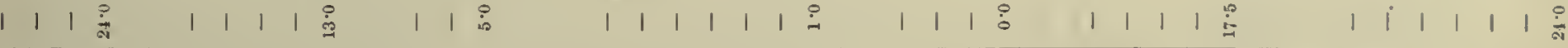

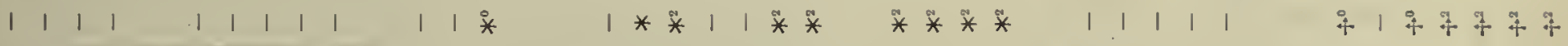

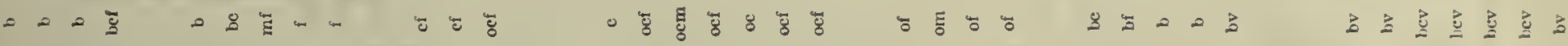

$=0000000110$ 0 0 0 I-0.

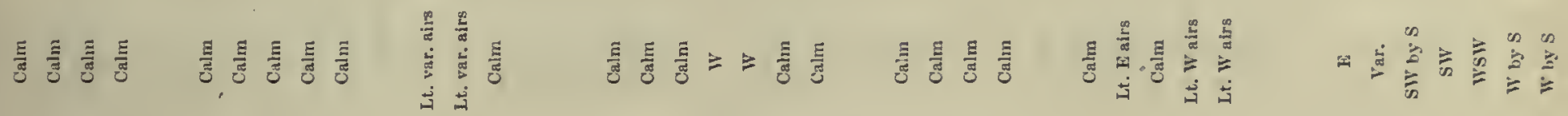

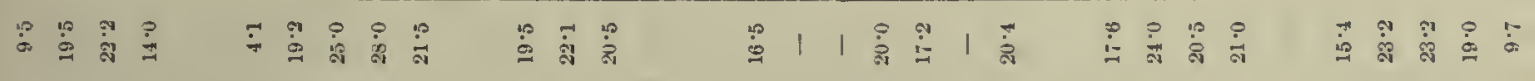

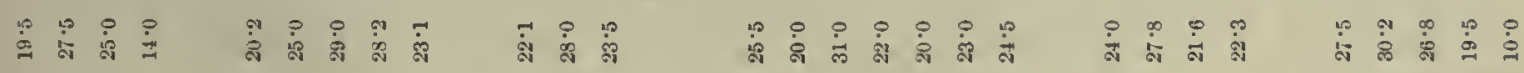

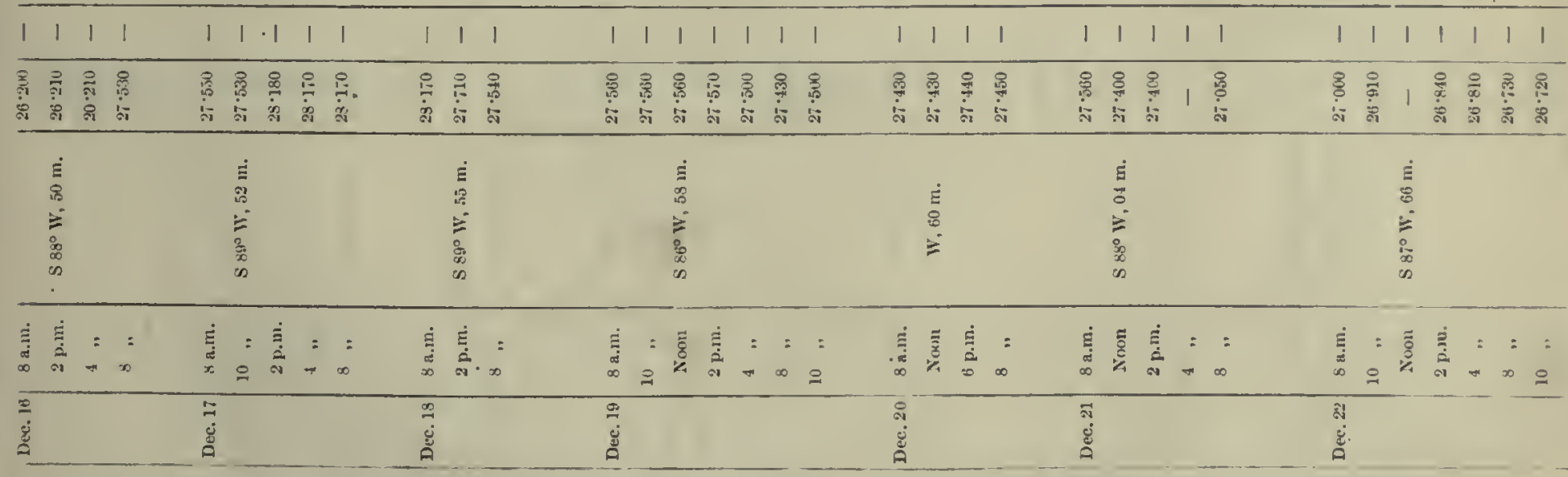




\begin{tabular}{|c|c|c|c|c|c|c|c|c|c|}
\hline & \multirow[t]{2}{*}{ 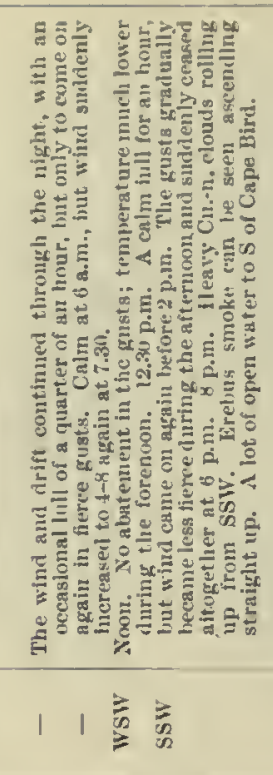 } & \multicolumn{2}{|c|}{ 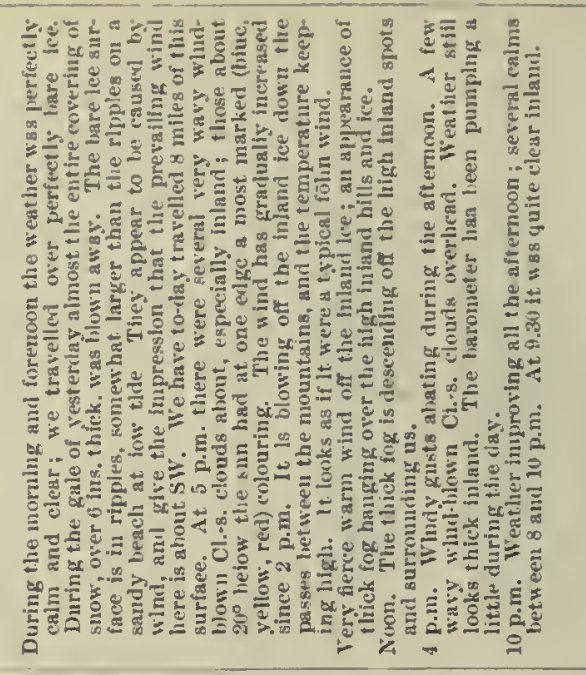 } & 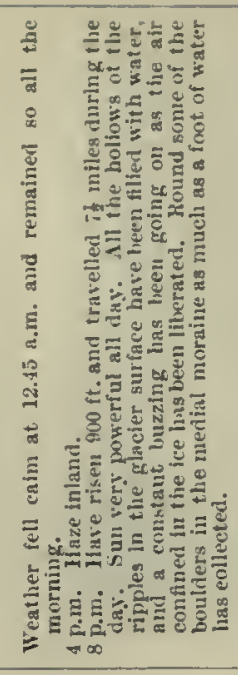 & \multirow{2}{*}{\multicolumn{3}{|c|}{ 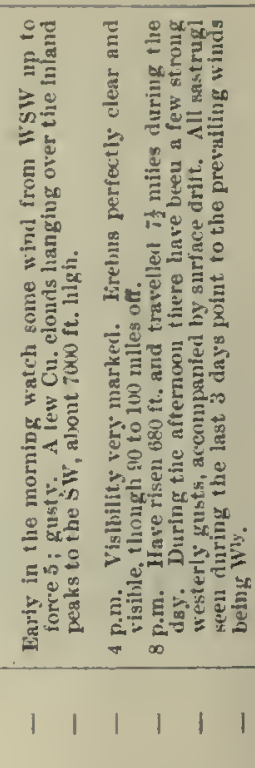 }} \\
\hline \multirow{4}{*}{$\frac{\tau}{\tilde{z}}$} & \multirow[b]{2}{*}{ 言 } & 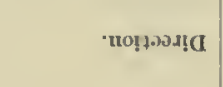 & & 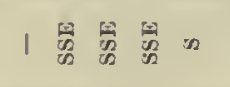 & | $1 \geqslant \bar{m}$ & $\begin{array}{lllll}1 & 1 & 1 & 1 & 1\end{array}$ & & & \\
\hline & & 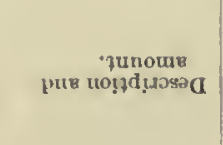 & 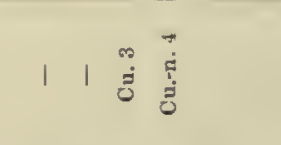 & 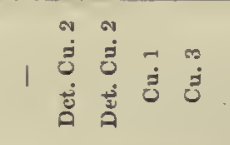 & 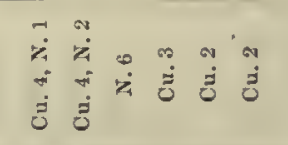 & 11111 & 11 & & 111 \\
\hline & \multirow[b]{2}{*}{ : } & 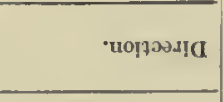 & 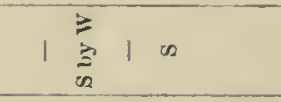 & 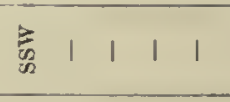 & $\begin{array}{ll}\infty \\
2 \\
E\end{array}$ & | | 言言 | & 11 & & 111 \\
\hline & & 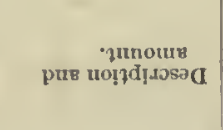 & 1 1 & 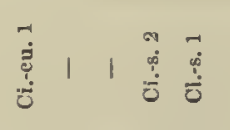 & 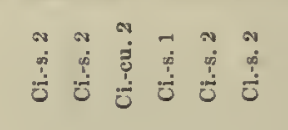 & 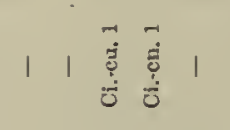 & 11 & & 111 \\
\hline \multicolumn{3}{|c|}{ 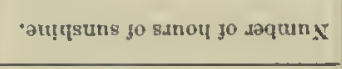 } & $1 \quad 11 \stackrel{\circ}{\dot{2}}$ & 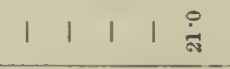 & $1 \quad 1 \quad 1 \quad 1 \quad \vdots$ & $1 \quad 1 \quad 1 \quad 0$ & 11 & 1 & $11 \stackrel{\circ}{\dot{a}}$ \\
\hline \multicolumn{3}{|c|}{ 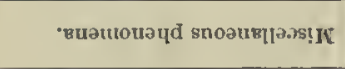 } & $7+\$ 1$ & 11111 & $1 \mid \begin{array}{llll}+ & + & 1 & 1\end{array}$ & $1 \quad 1 \quad 1 \quad 1$ & 11 & 1 & +11 \\
\hline & & 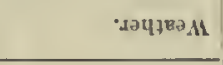 & $\Xi \Xi$ & 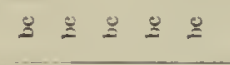 & $\& 8 \overline{8} \cong \cong$ & 茫 & 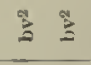 & & $\Xi \Xi$ \\
\hline \multirow[b]{2}{*}{ 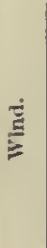 } & & 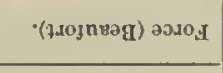 & of & - 0 a $q$ & 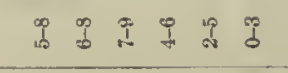 & $0 \quad 1111$ & \% I & & \& \\
\hline & & 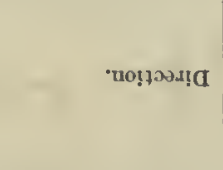 & 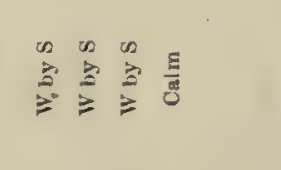 & 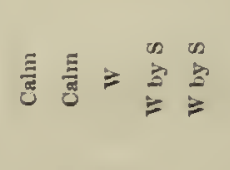 & 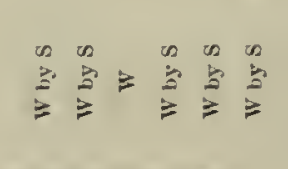 & 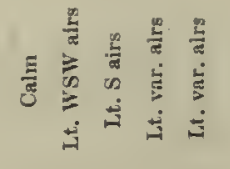 & & & 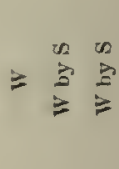 \\
\hline \multirow{3}{*}{ 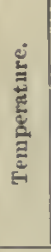 } & & 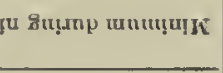 & $0 \begin{array}{llll}1 & 1 & 1 & 1\end{array}$ & $\begin{array}{lllll}1 & 1 & 1 & 1 & 1\end{array}$ & $\begin{array}{llllll}1 & 1 & 1 & 1 & 1 & 1\end{array}$ & $\begin{array}{lllll}1 & 1 & 1 & 1 & 1 \\
\end{array}$ & 11 & & 111 \\
\hline & & 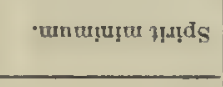 & 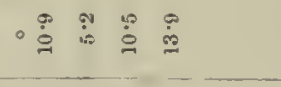 & 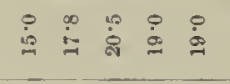 & 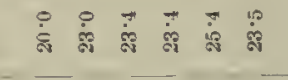 & 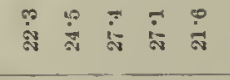 & 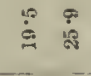 & & $\overrightarrow{\mathrm{\Sigma}} \overrightarrow{\mathrm{s}}$ \\
\hline & & $\cdot \ln$ & 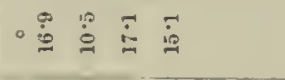 & 品 & 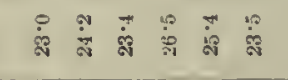 & 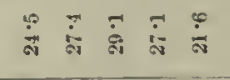 & 离菑 & & $\overrightarrow{\dot{\Delta}}$ \\
\hline \multicolumn{3}{|c|}{ 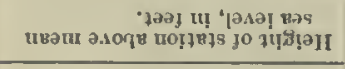 } & 1111 & 11111 & 111111 & 11111 & $1 !$ & & 111 \\
\hline & & 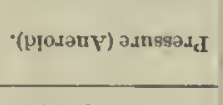 & 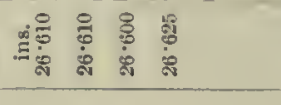 & 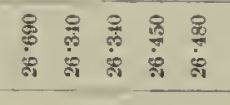 & 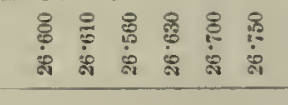 & 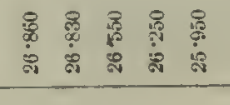 & 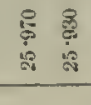 & & 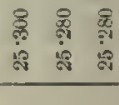 \\
\hline & & 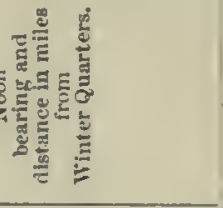 & 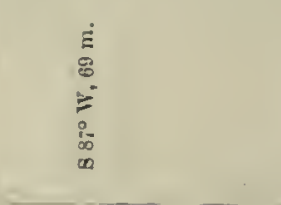 & 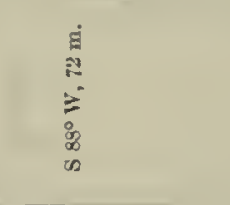 & 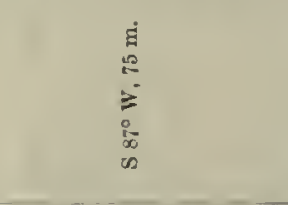 & 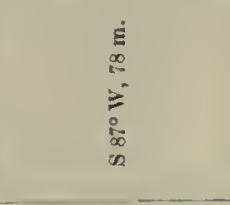 & 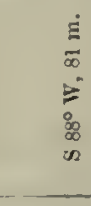 & & \\
\hline & & $\stackrel{\stackrel{\Xi}{E}}{E}$ & 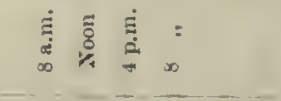 & 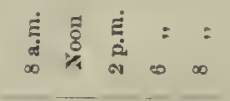 & 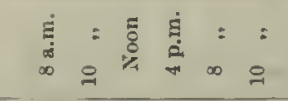 & 章 = & $\begin{array}{l}\dot{1}= \\
\infty \\
\infty\end{array}$ & & $=:=$ \\
\hline & & 亗 & 旅 & $\begin{array}{l}\overline{\text { İ }} \\
\text { ֻั̆ }\end{array}$ & 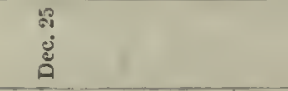 & 离 & 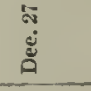 & & \\
\hline
\end{tabular}



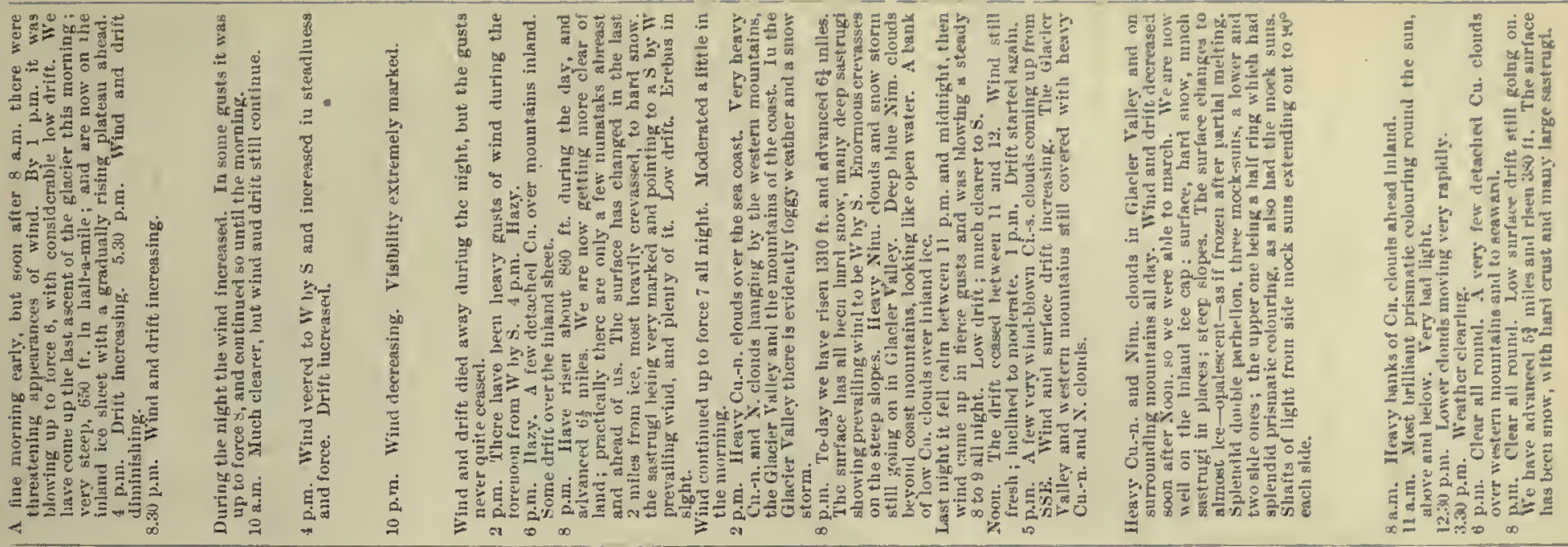

$1 \mid 11$

1 1 | | | $\begin{array}{lllll}1 & 1 & 1 & 1 & 1\end{array}$ $1 \quad 1 \quad 1$ $1 \quad 1 \quad 1$

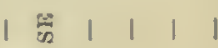

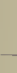

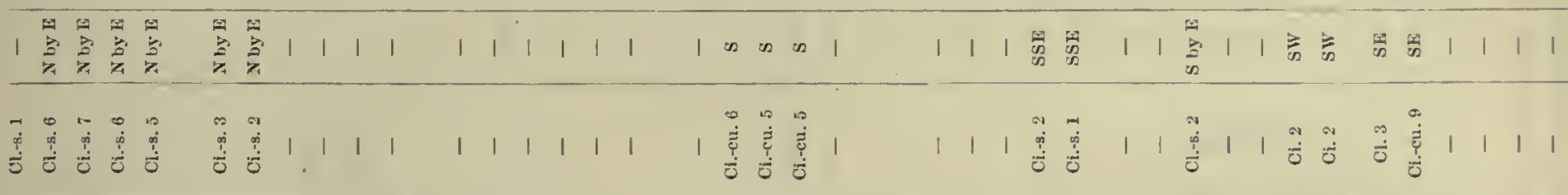

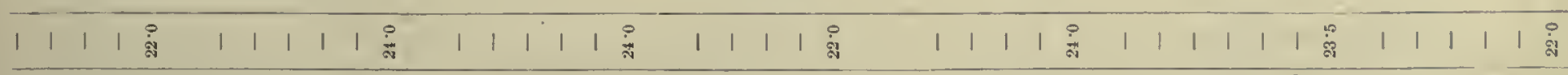

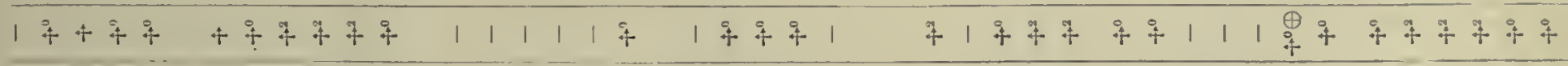

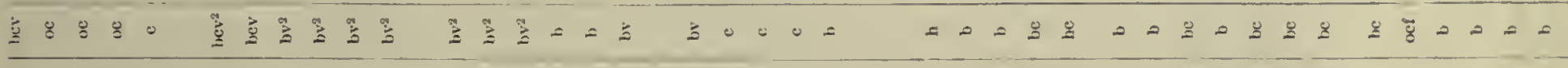

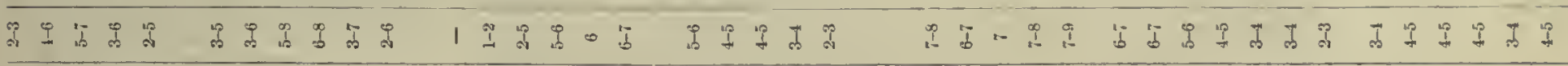

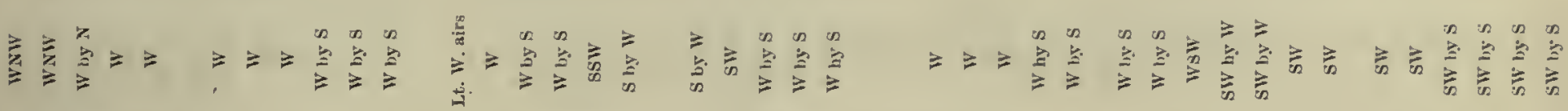

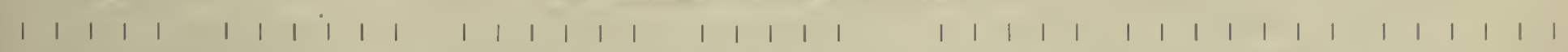

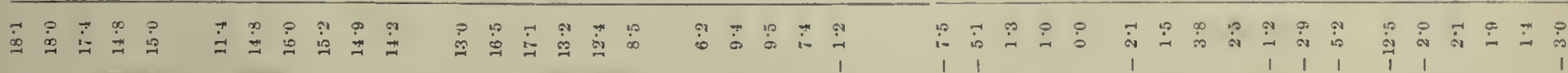

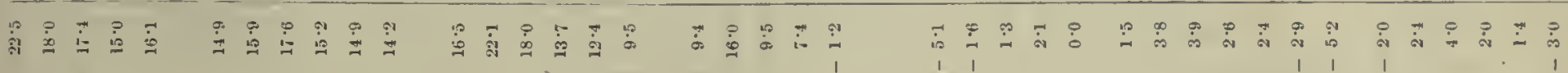

\begin{tabular}{|c|c|c|c|c|c|c|c|c|c|c|c|c|c|c|c|c|c|c|c|c|c|c|c|c|c|c|c|c|c|c|c|c|c|c|c|c|c|c|c|}
\hline 1 & 1 & I & 1 & 1 & 1 & I & 1 & 1 & 1 & 1 & I & I & 1 & I & 1 & 1 & I & 1 & 1 & 1 & 1 & 1 & 1 & 1 & 1 & 1 & 1 & 1 & 1 & I & 1 & I & 1 & 1 & 1 & I & i & 1 & 1 \\
\hline 票 & के & $\begin{array}{l}8 \\
: 3 \\
: 3 \\
\end{array}$ & $\frac{8}{0}$ & $\begin{array}{l}8 \\
\vdots \\
\vdots\end{array}$ & $\begin{array}{l}0 \\
\vdots \\
\text { है }\end{array}$ & 苂 & 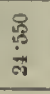 & 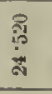 & \begin{tabular}{l}
$\frac{0}{5}$ \\
\hdashline \\
4
\end{tabular} & 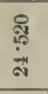 & 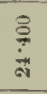 & 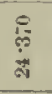 & $\frac{8}{3}$ & 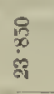 & $\stackrel{8}{8}$ & 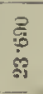 & :- & 苛 & $\begin{array}{l}\overrightarrow{0} \\
\overrightarrow{8} \\
\dot{8}\end{array}$ & 疍 & है & ฟิ & \& & $\begin{array}{l}\text { 옹 } \\
\text { \& } \\
\text { \& }\end{array}$ & 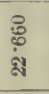 & 离 & 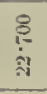 & $\begin{array}{l}8 \\
? \\
\text { औิ }\end{array}$ & 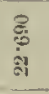 & ஸั & 疍 & สิ & $\begin{array}{l}8 \\
\text { के } \\
\text { สे }\end{array}$ & ลู่ & है & $\frac{8}{0}$ & $\begin{array}{c}0 \\
\frac{8}{2} \\
\dot{a}\end{array}$ & הิ & $\begin{array}{l}\text { ลे } \\
\dot{3}\end{array}$ \\
\hline & 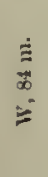 & & & & & 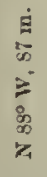 & & & & & & $\begin{array}{l}\dot{E} \\
\dot{a} \\
\dot{2} \\
\dot{0} \\
\dot{8} \\
3\end{array}$ & & & & & & 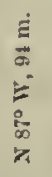 & & & & & 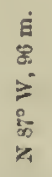 & & & & & $\begin{array}{l}\stackrel{3}{E} \\
\mathbb{E} \\
2 \\
8 \\
8 \\
8\end{array}$ & & & & & & & 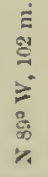 & & & & \\
\hline 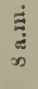 & $\underset{\infty}{\dot{\Xi}}$ & $=$ & $:$ & $=$ & $\begin{array}{l}\dot{E} \\
\stackrel{d}{\alpha} \\
\stackrel{g}{0}\end{array}$ & $\begin{array}{l}5 \\
8 \\
\not 12\end{array}$ & $\underset{\text { से }}{\dot{\Delta}}$ & $=$ & $\begin{array}{l}: \\
\infty\end{array}$ & : & 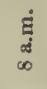 & $:$ & $\underset{\sim}{\stackrel{\Xi}{\Xi}}$ & $=$ & $=$ & $:$ & $\underset{\substack{\dot{d} \\
\infty \\
\infty}}{\infty}$ & $\underset{\Delta}{\dot{\varepsilon}}$ & $\begin{array}{l}= \\
+\end{array}$ & $=$ & $:$ & 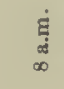 & हี & $\underset{\sigma}{\dot{E}}$ & $=$ & $:$ & $\underset{\substack{\dot{B} \\
\infty}}{\dot{g}}$ & $\begin{array}{l}5 \\
8 \\
3 \\
4\end{array}$ & $\underset{\dot{\Xi}}{\dot{\Xi}}$ & $=$ & $=$ & $=$ & $:$ & $\begin{array}{l}\dot{\Xi} \\
\dot{d} \\
\infty\end{array}$ & $\bar{E}$ & $\underset{\text { ล }}{\text { \& }}$ & $=$ & $=$ & $\begin{array}{l}= \\
\infty\end{array}$ \\
\hline है & & & & & $\begin{array}{l}\text { \& } \\
\dot{8} \\
\stackrel{8}{0}\end{array}$ & & & & & & 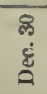 & & & & & & $\begin{array}{l}\vec{m} \\
\text { हूँ }\end{array}$ & & & & & है & & & & & ब & & & & & & & $\stackrel{\vec{E}}{\longrightarrow}$ & & & & & \\
\hline
\end{tabular}




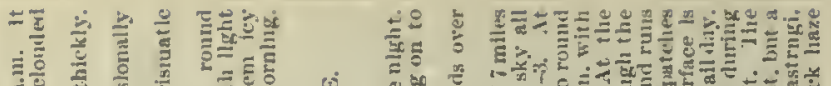
\%

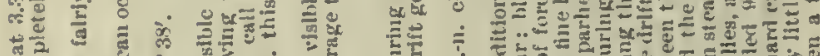
三

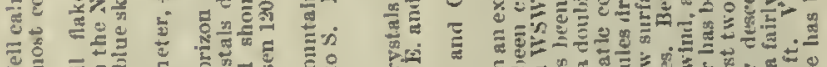

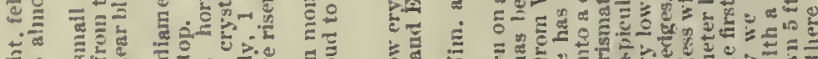

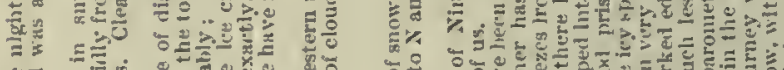

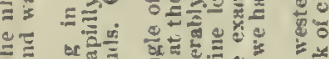

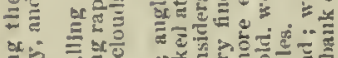

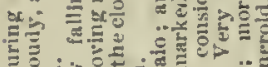

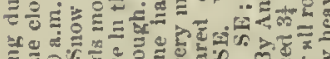

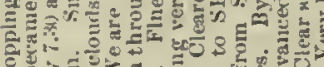

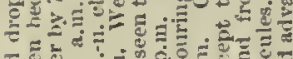
年

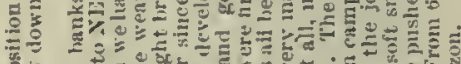
言晒 C to 6rove E.

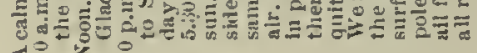

of

2

है

25

盗

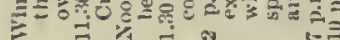

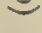

号

i
कृष्ठ

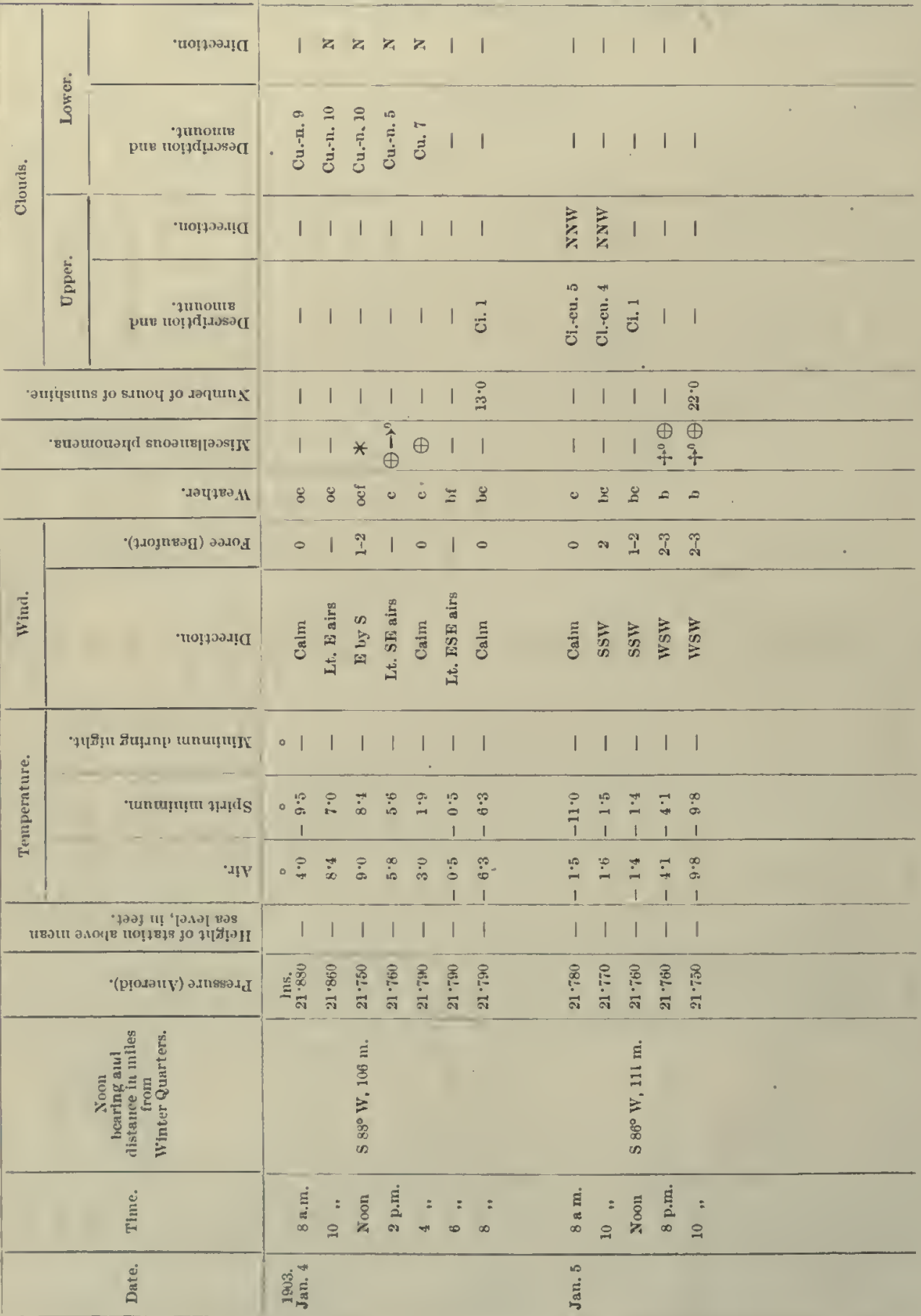

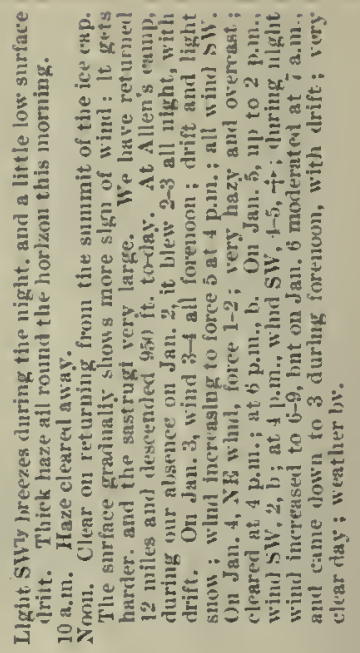

$\begin{array}{lllllll}1 & 1 & 1 & 1 & 1 & 1 & 1\end{array}$

窟

\begin{tabular}{|c|c|}
\hline & $1 \quad 1 \quad 1 \quad 1 \quad 13$ \\
\hline q & 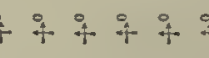 \\
\hline$=$ & $0 \pm Z \pm \geq$ \\
\hline & I \\
\hline
\end{tabular}

$\begin{array}{lllllll}1 & 1 & 1 & 1 & 1 & 1 & 1\end{array}$

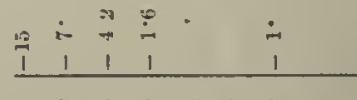

i

1111

$\begin{array}{lllllll}1 & 1 & 1 & 1 & 1 & 1 & 1\end{array}$

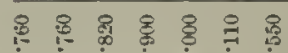

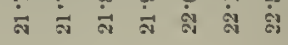

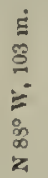

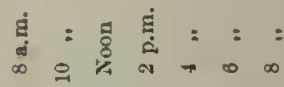




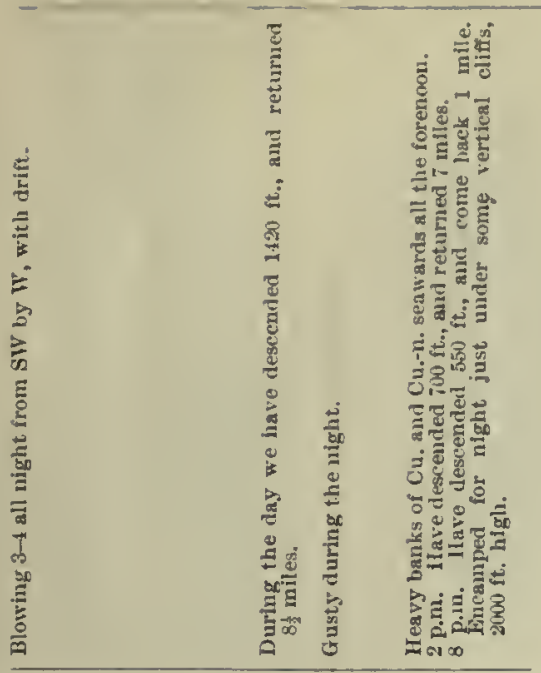

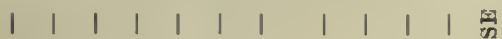

$\begin{array}{llllllllllll}1 & 1 & 1 & 1 & 1 & 1 & 1 & 1 & 1 & 1 & 1 & \bar{j}\end{array}$

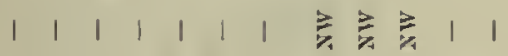

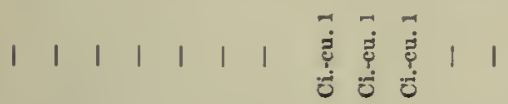

$\begin{array}{llllllllllll}1 & 1 & 1 & 1 & 1 & 1 & \frac{9}{4} & 1 & 1 & 1 & 1 & \frac{9}{4}\end{array}$

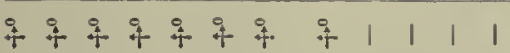

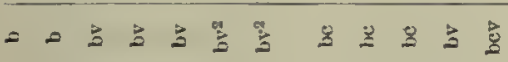

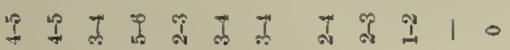

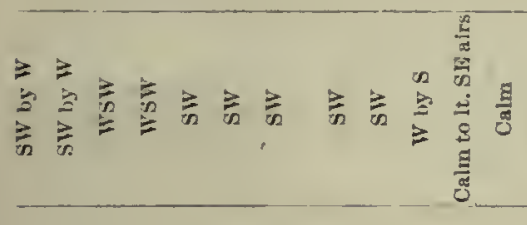

$\begin{array}{lllllllllllllllll}1 & 1 & 1 & 1 & 1 & 1 & 1 & 1 & 1 & 1 & 1 & 1\end{array}$

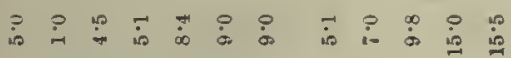

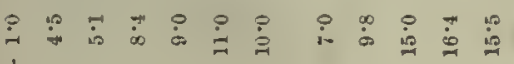

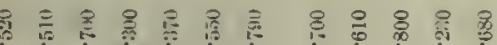

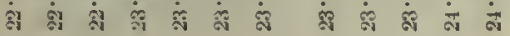

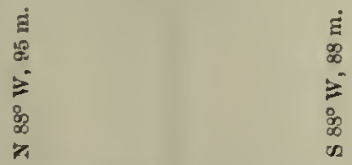

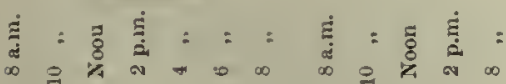

เี่

雍
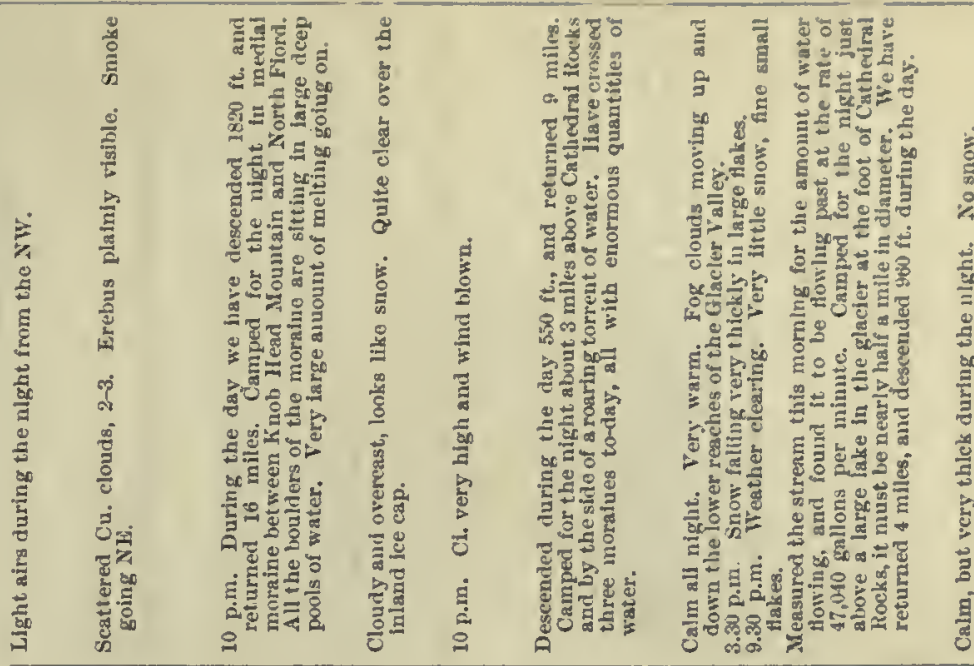

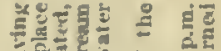

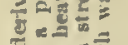

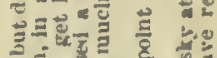
불.

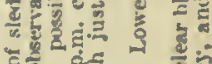

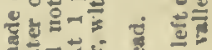

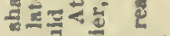
두 $<8$ का एक

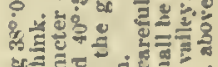

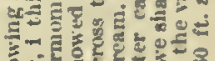

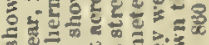
w

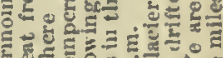

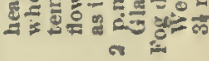

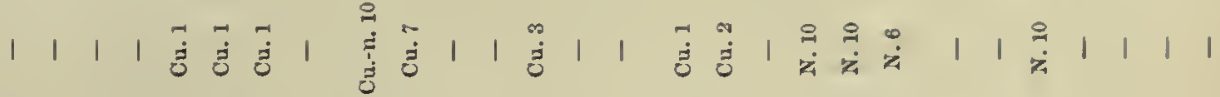

空

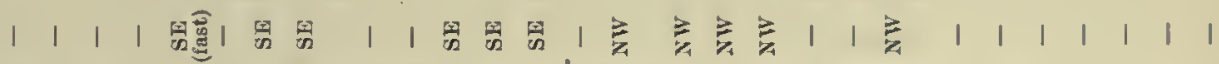

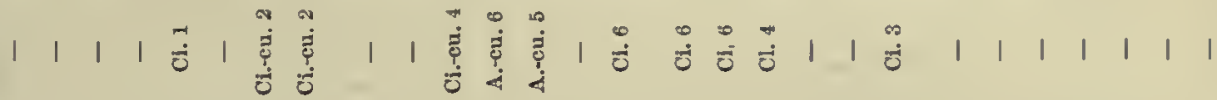

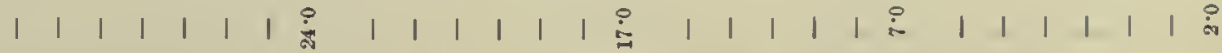

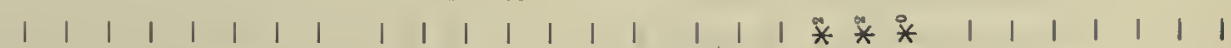
ב

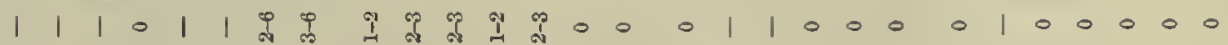

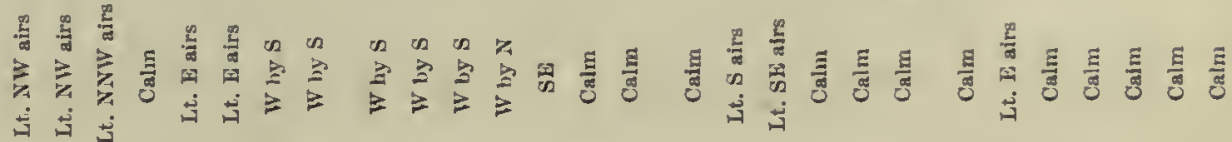
$\begin{array}{lllllllllllllllllllllllllllll}1 & 1 & 1 & 1 & 1 & 1 & 1 & 1 & 1 & 1 & 1 & 1 & 1 & 1 & 1 & 1 & 1 & 1 & 1 & 1 & 1 & 1 & 1 & 1 & 1 & 1 & 1 & 1\end{array}$

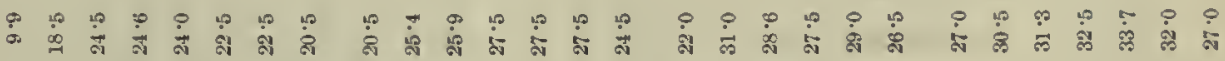

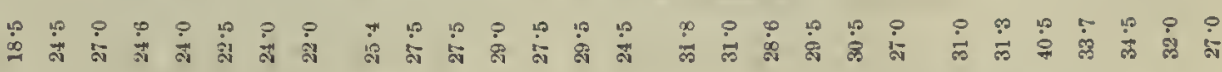

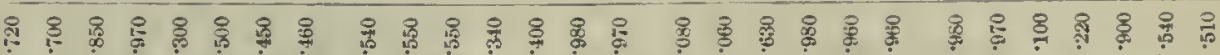

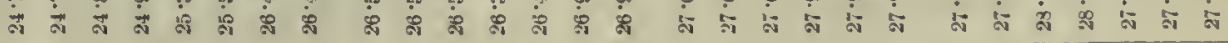

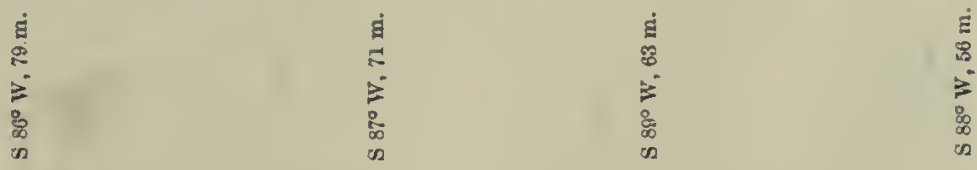

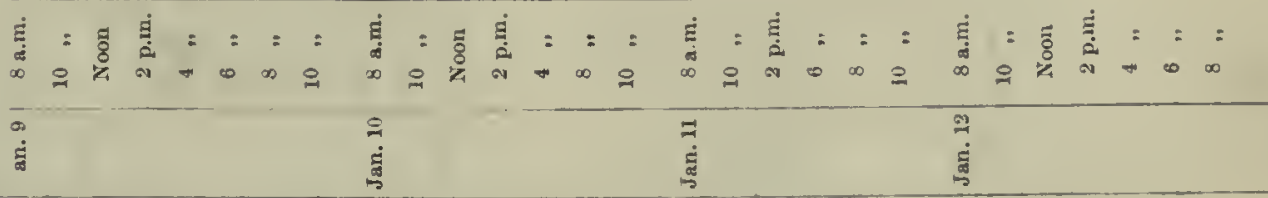




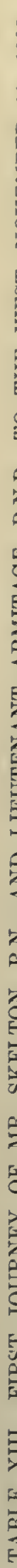

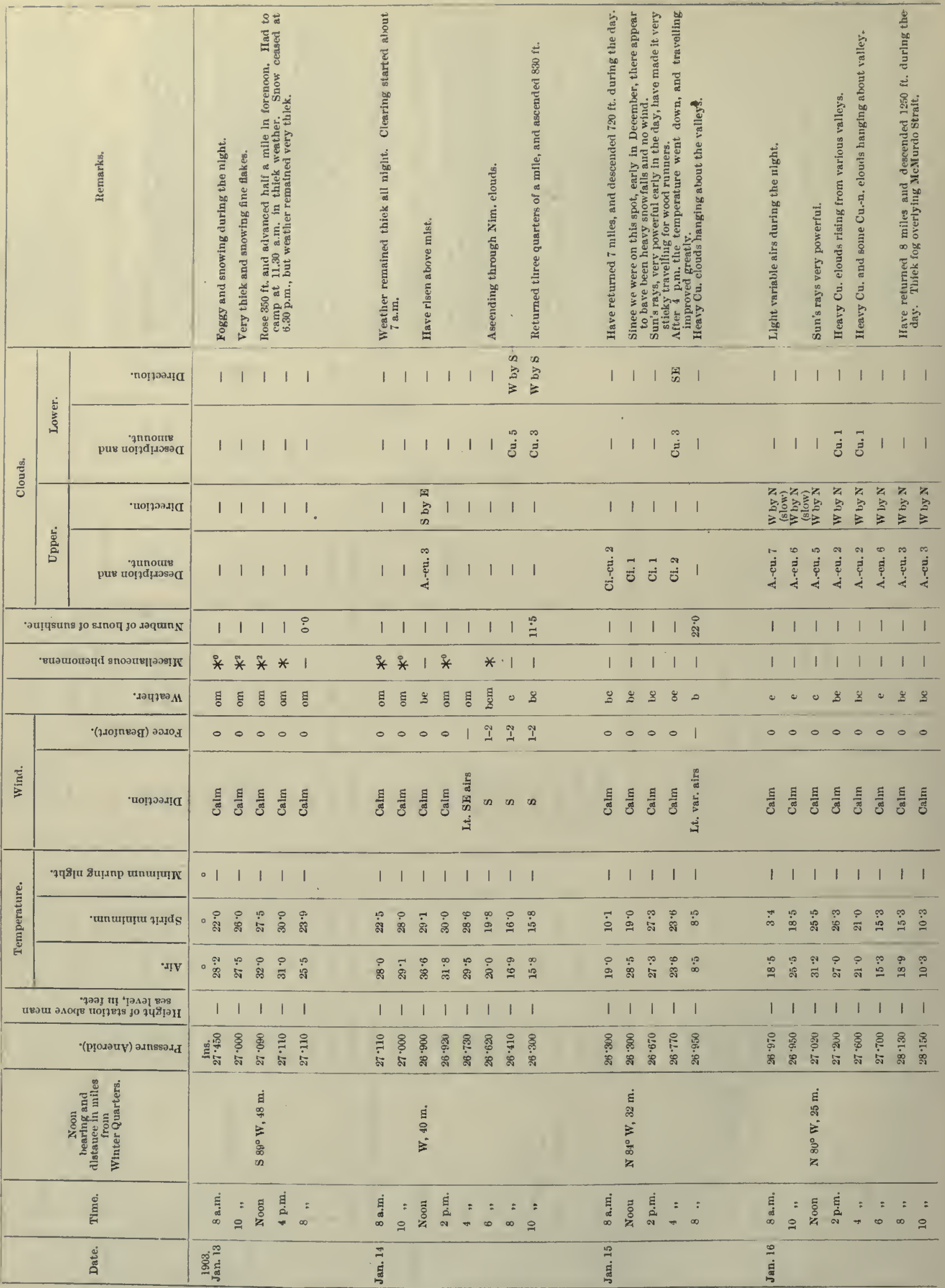




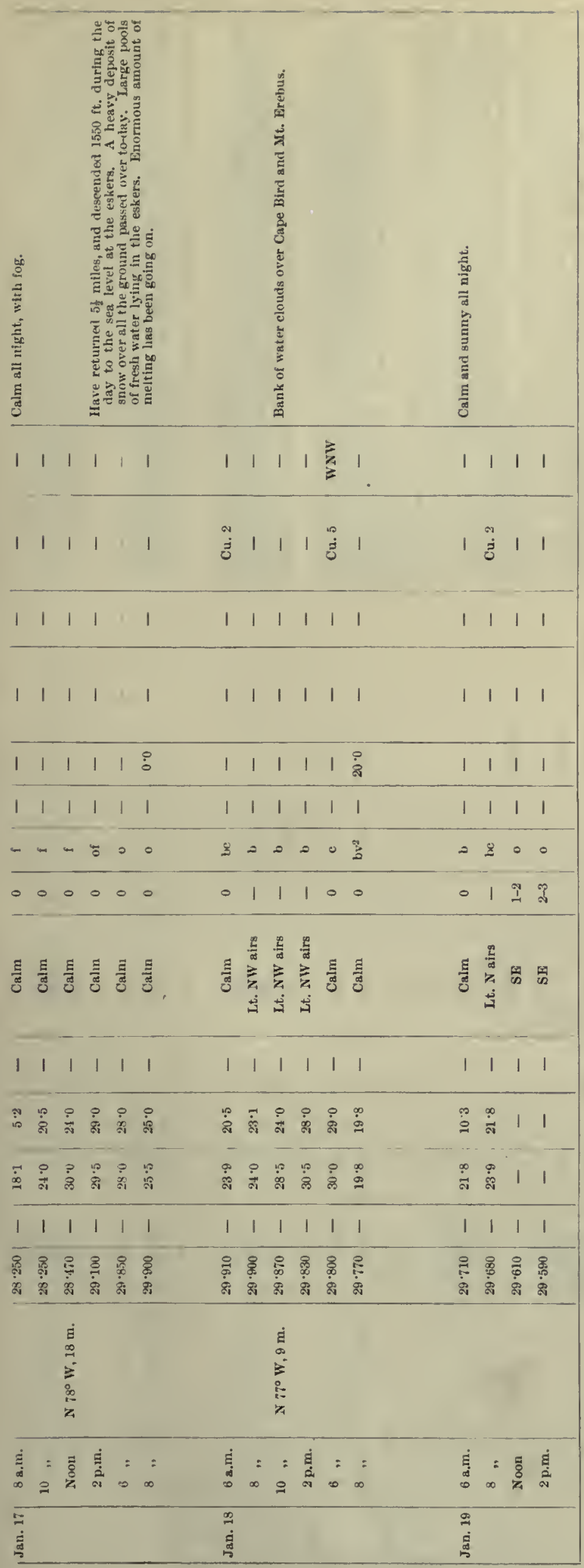


唫

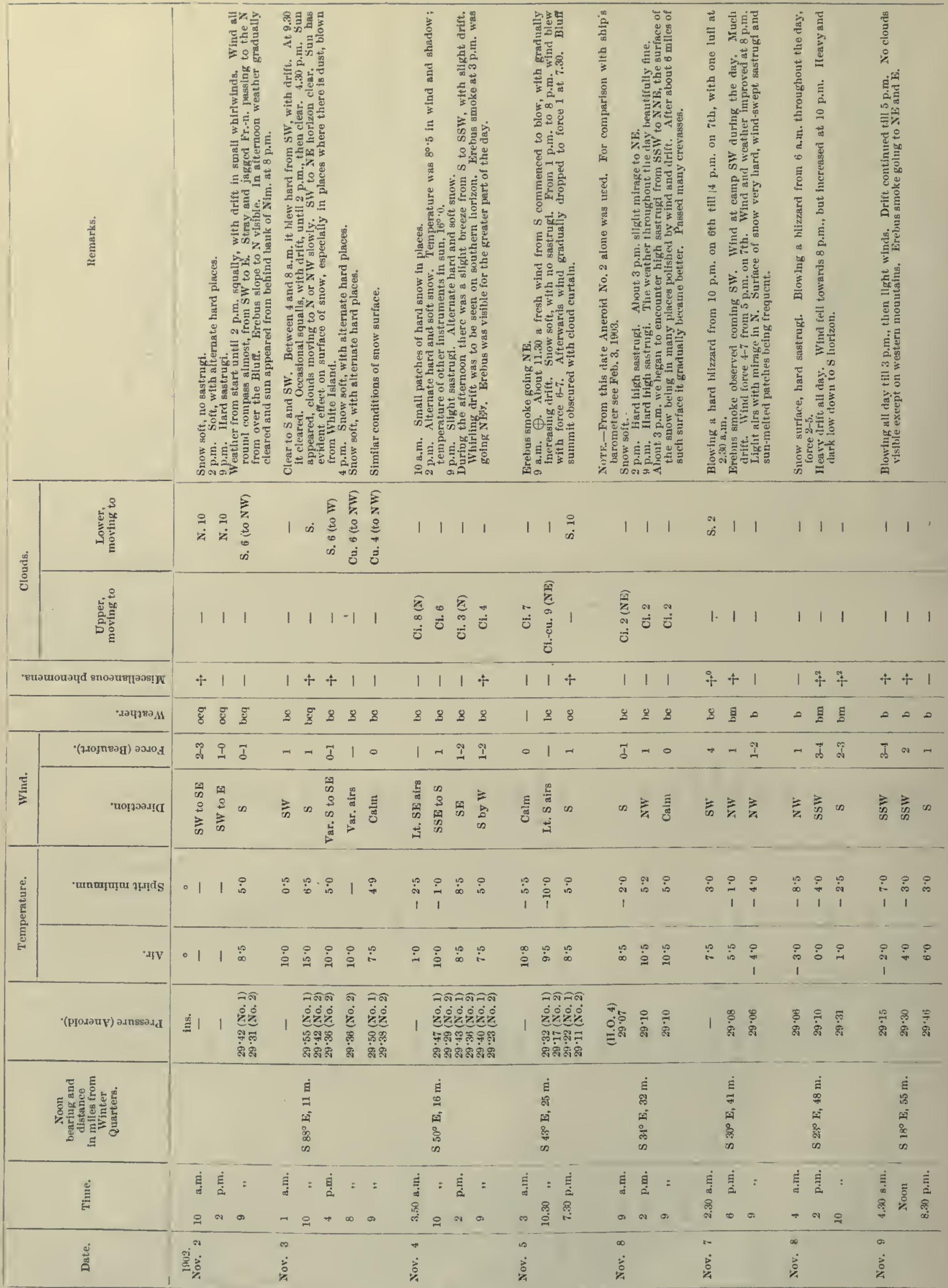



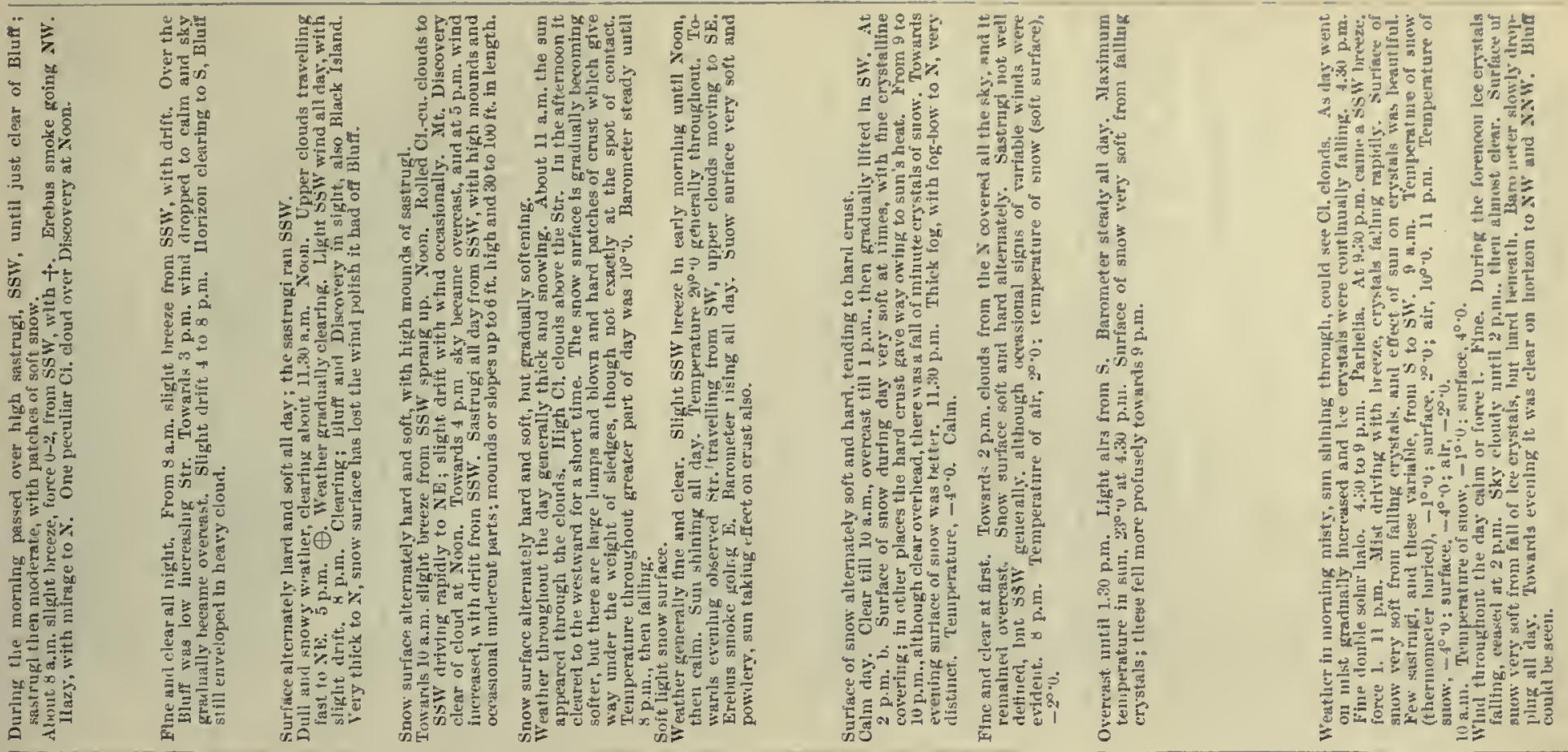

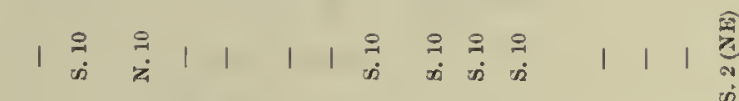

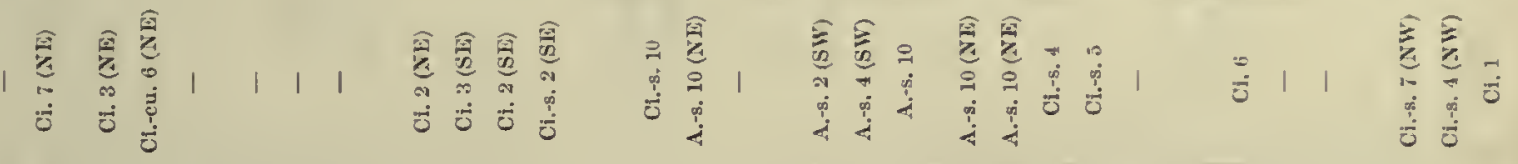

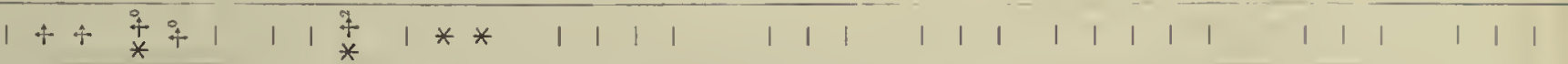

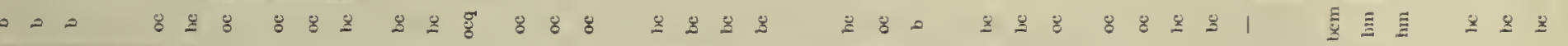

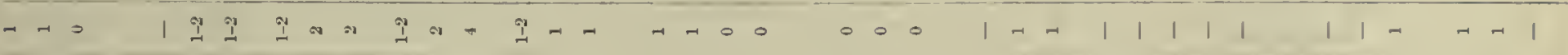

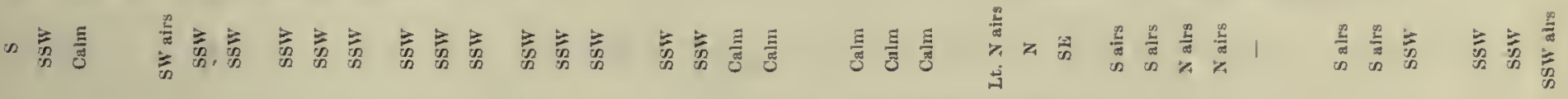

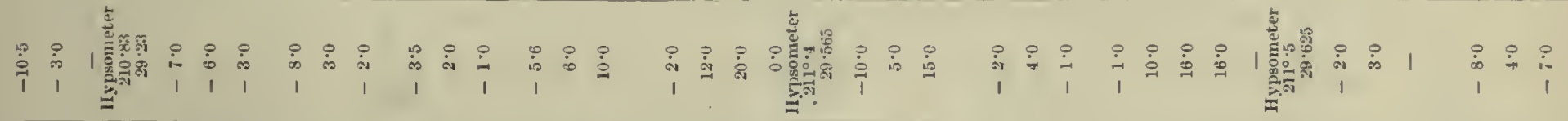

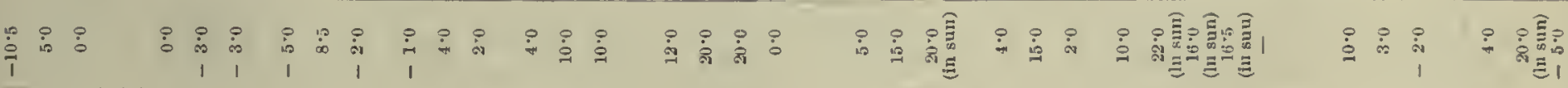

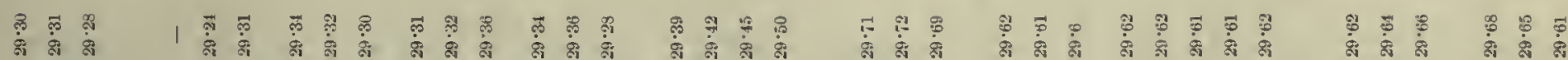

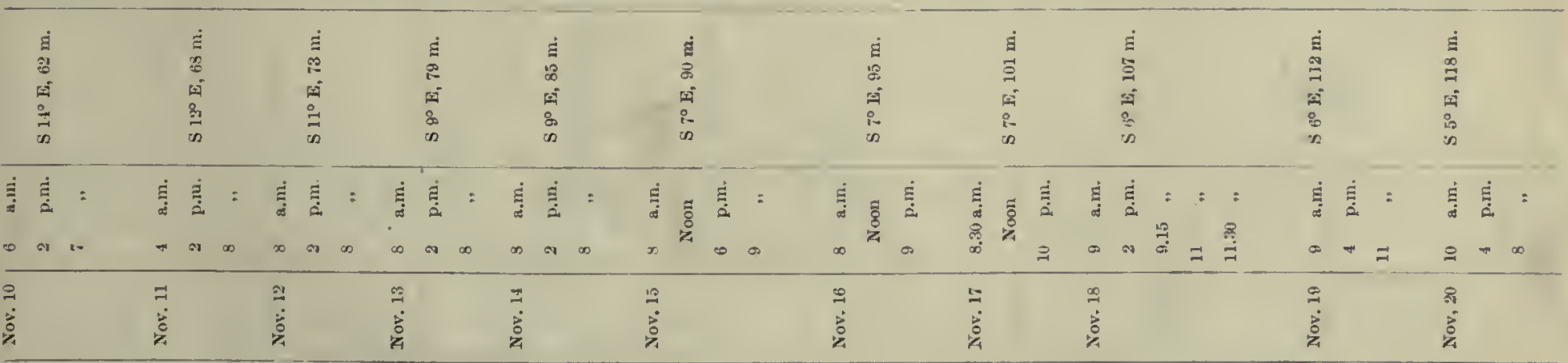




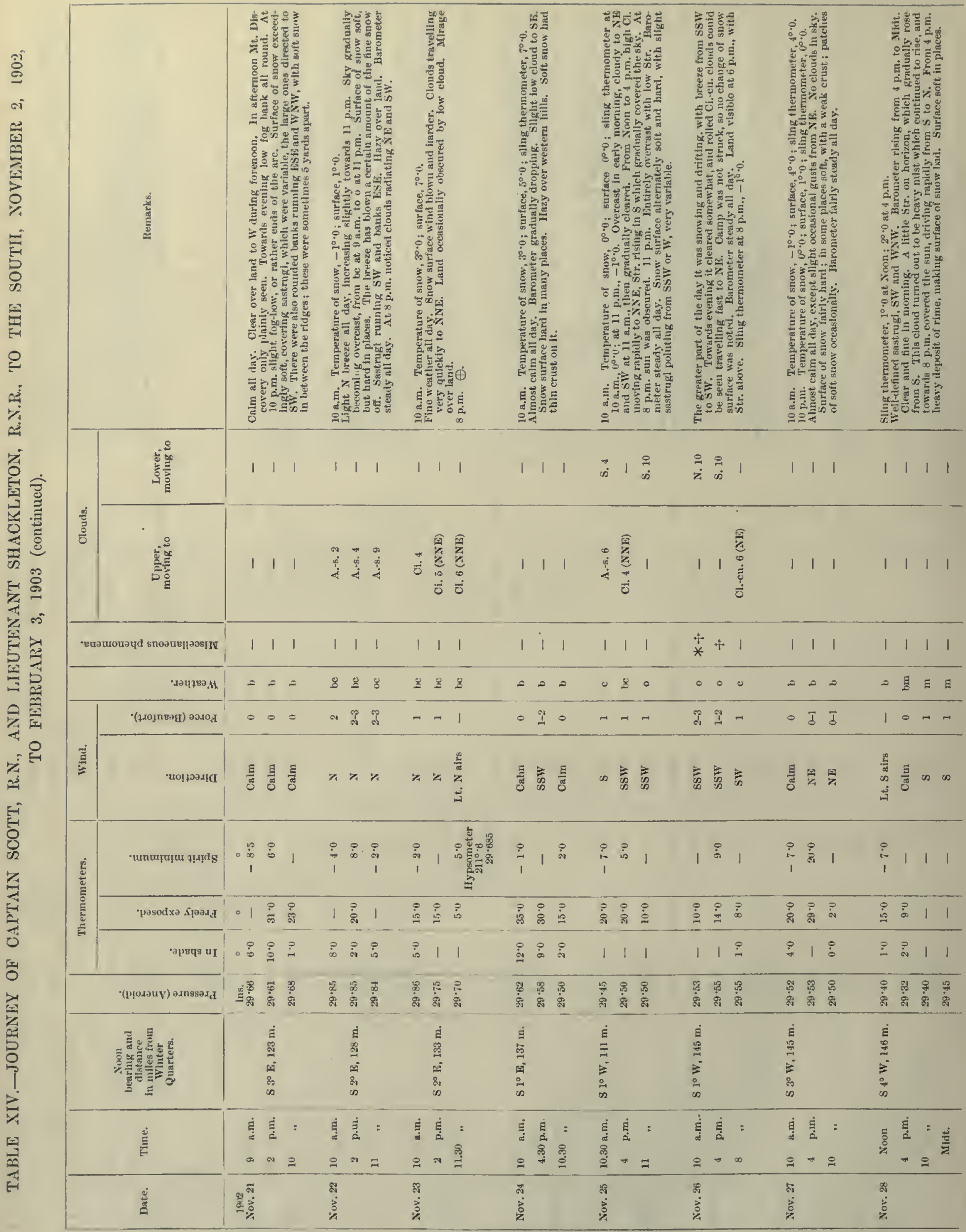




\begin{tabular}{|c|c|c|c|c|c|c|c|c|c|}
\hline 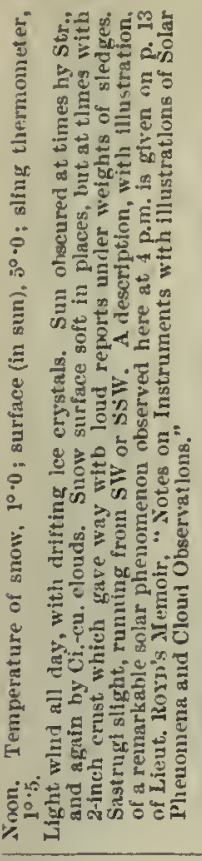 & 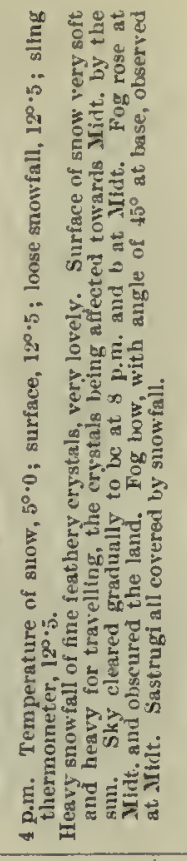 & 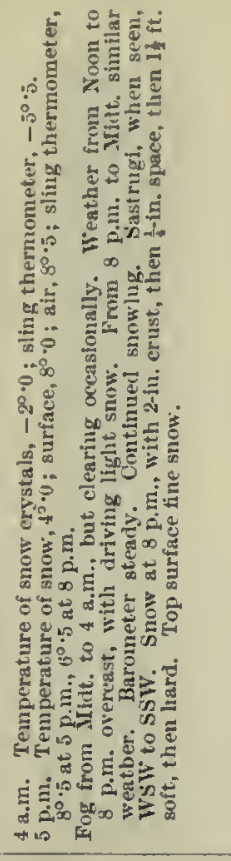 & 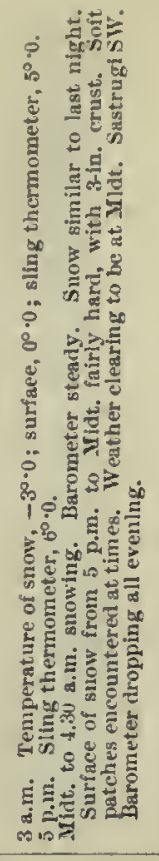 & 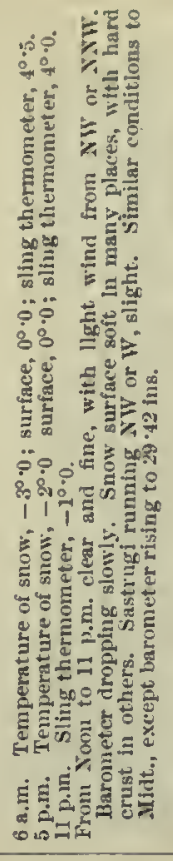 & 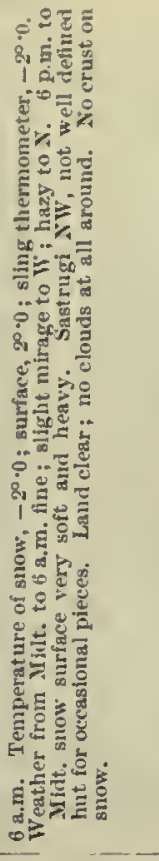 & 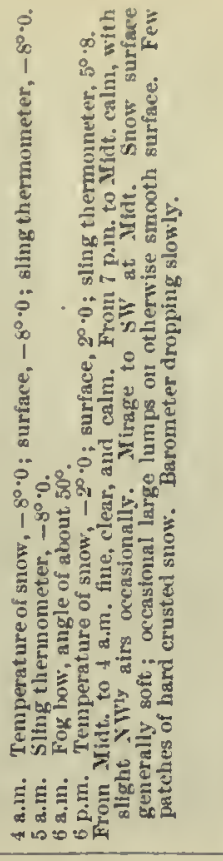 & 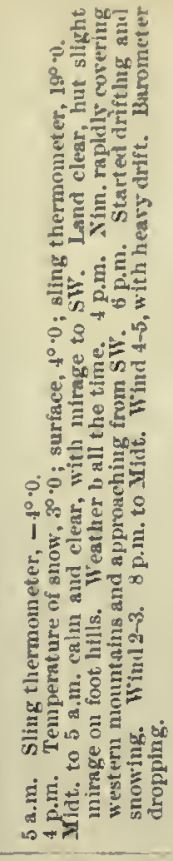 & & 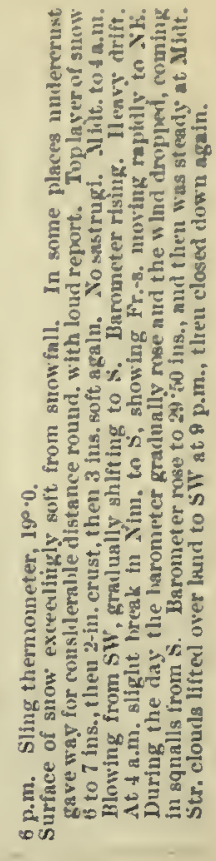 \\
\hline 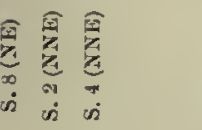 & 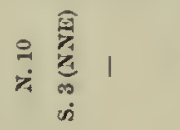 & 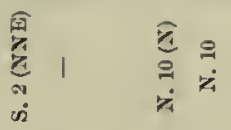 & 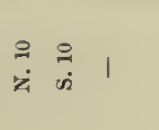 & $\begin{array}{lll}1 & 1 & 1\end{array}$ & $\begin{array}{llll}1 & 1 & 1 & 1\end{array}$ & $\begin{array}{lll}1 & 1 & 1\end{array}$ & 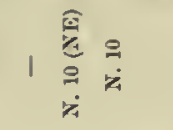 & $\bar{\pi}$ & 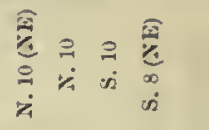 \\
\hline
\end{tabular}

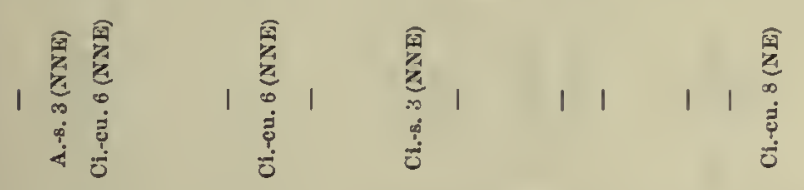

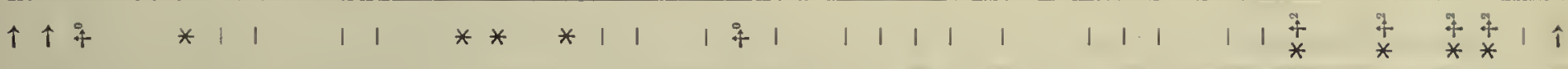

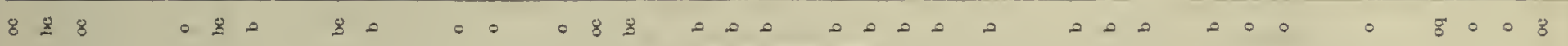
I I I

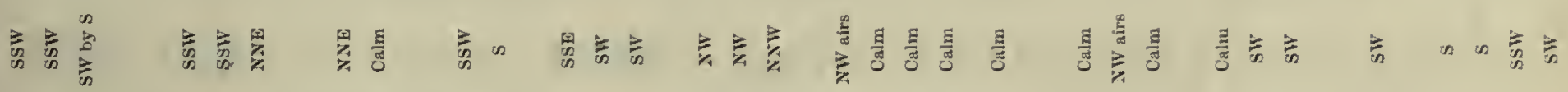

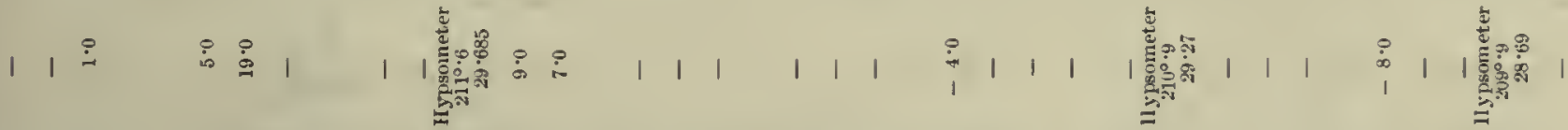

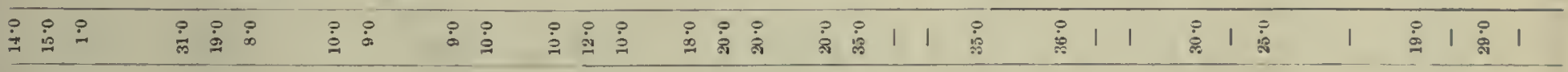

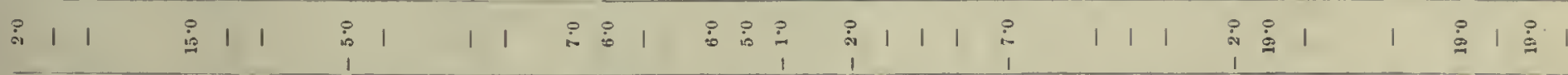

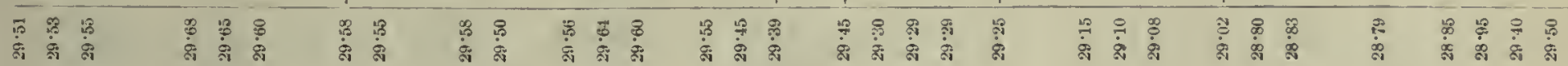

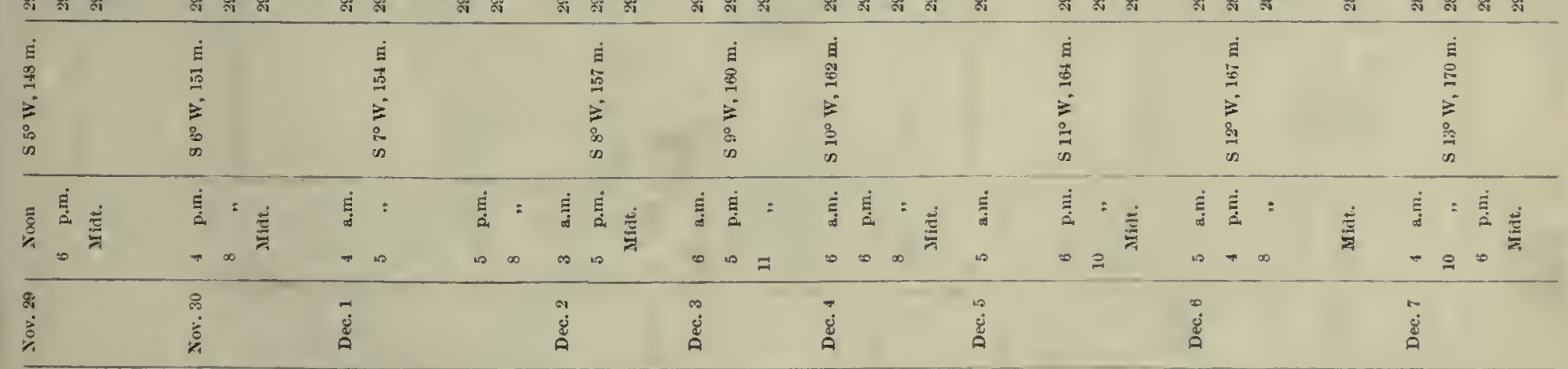




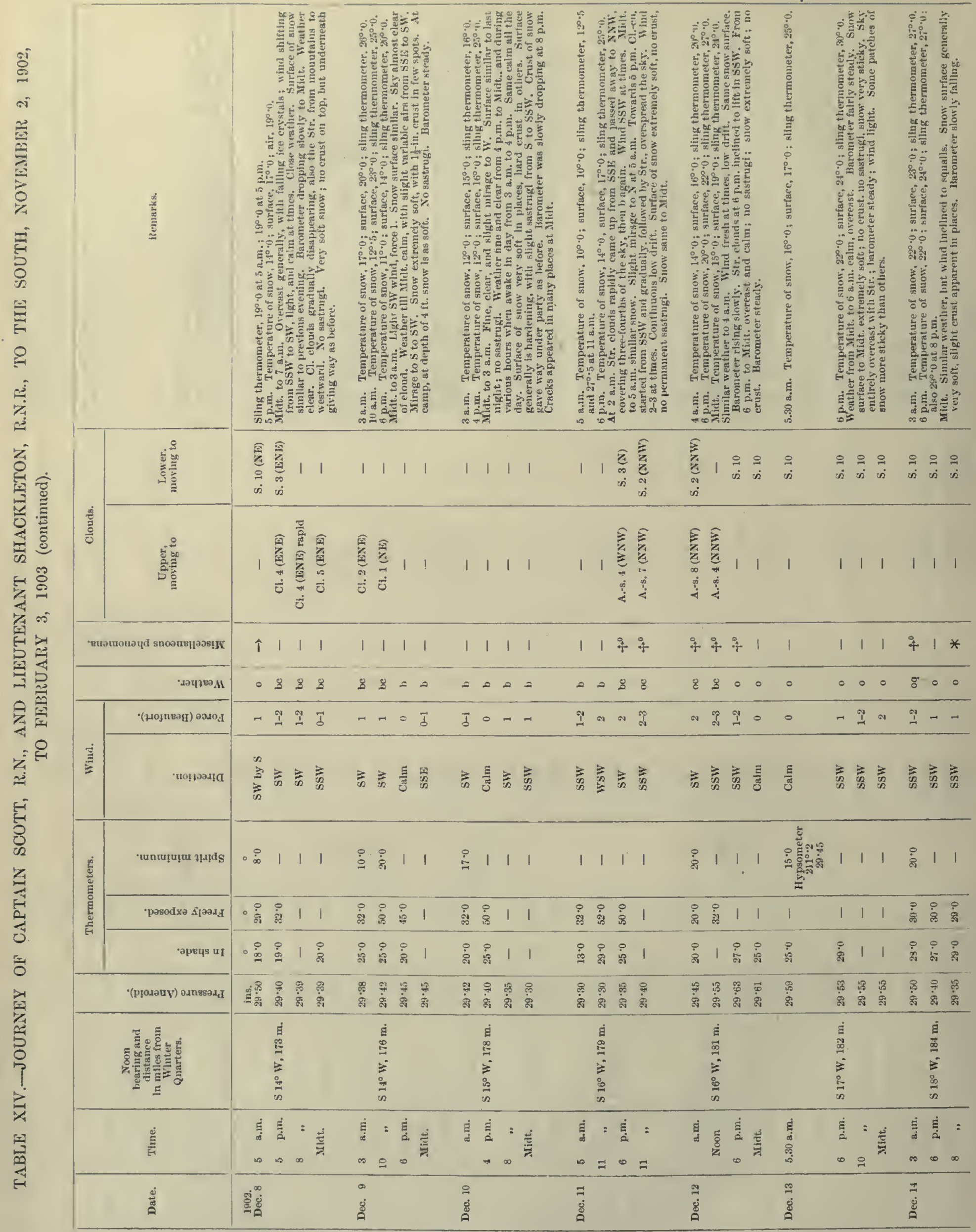




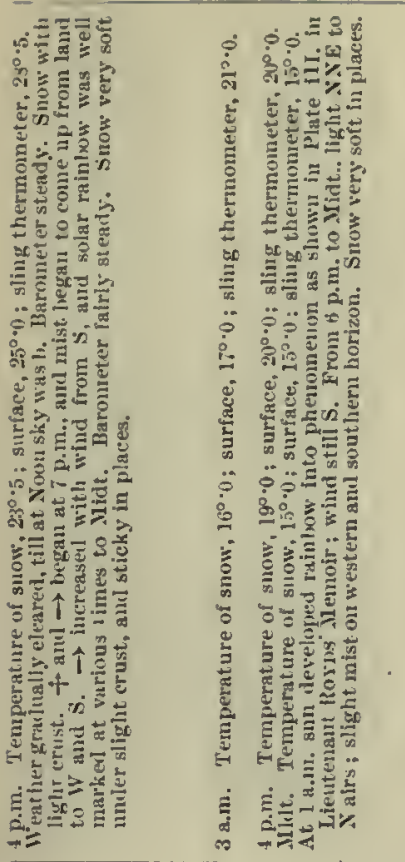

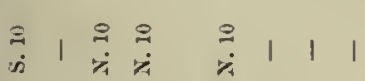

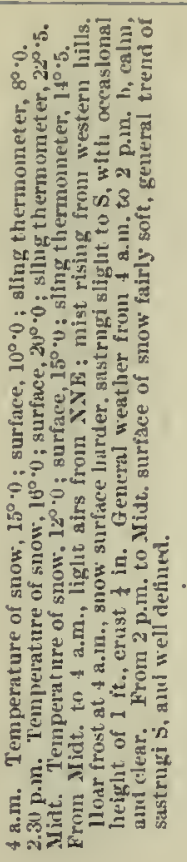

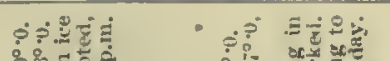

คำำ

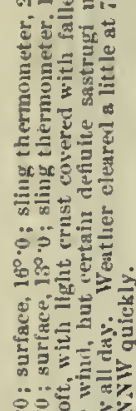

io

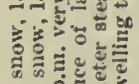

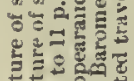

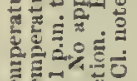

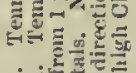

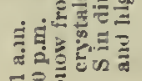

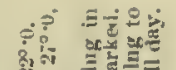

3.ำ

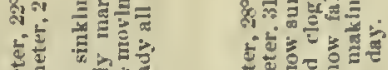

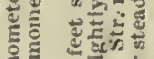

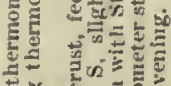

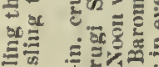

क.

年

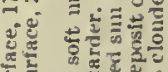

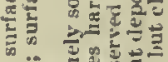

管

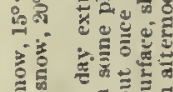

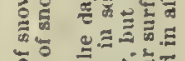

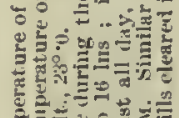

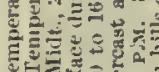

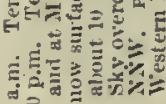

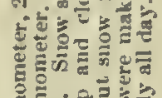
है.

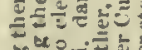

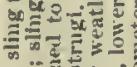

等

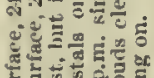

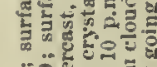

等

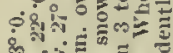

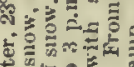

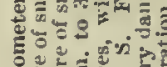

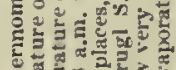

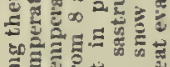

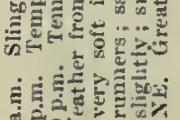

ร.

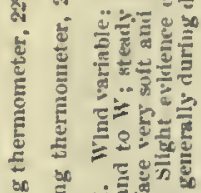

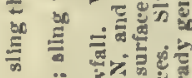

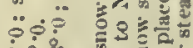

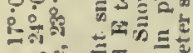

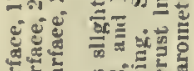

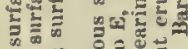

a.

ํ.

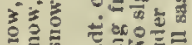

異

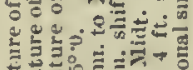

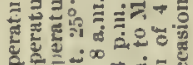

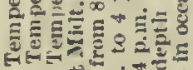

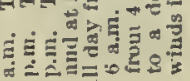

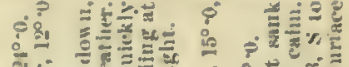

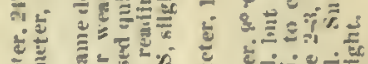

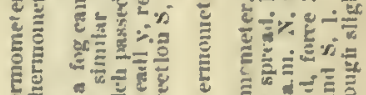

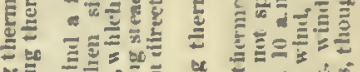

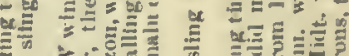

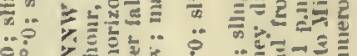

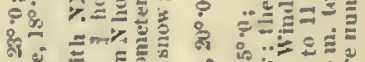

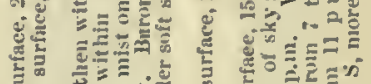
家.. 管

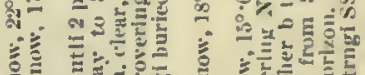

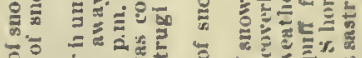

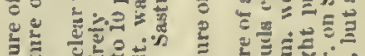

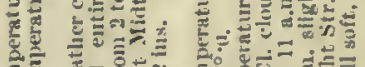
策

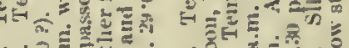

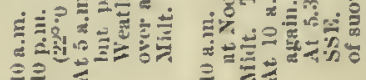

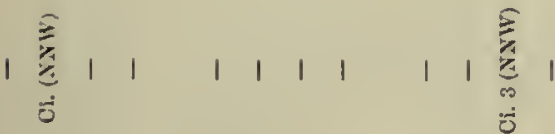

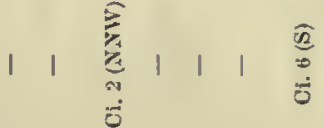

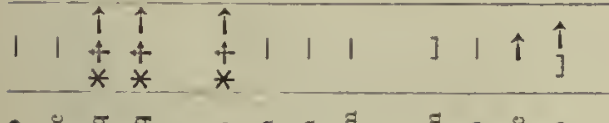

- $\$ 8$ I I $\vec{\delta} \vec{o}$ I จ ล ล

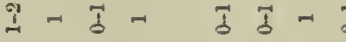
53

$-3$

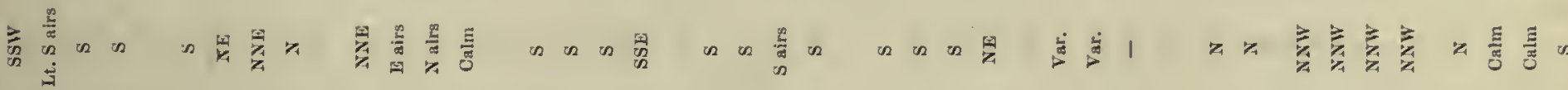

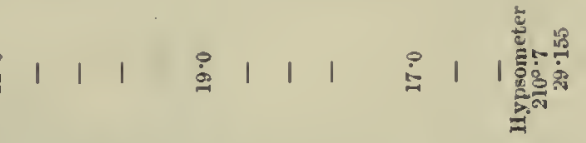

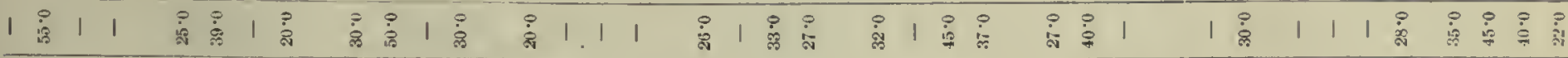

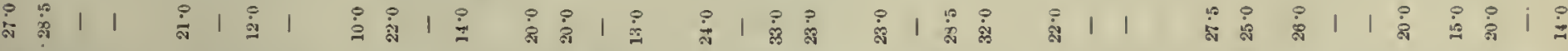

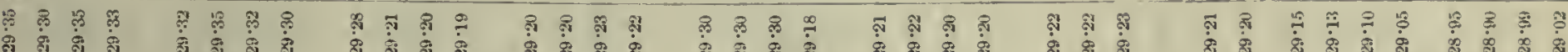

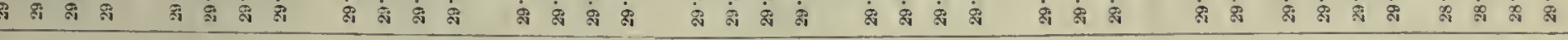

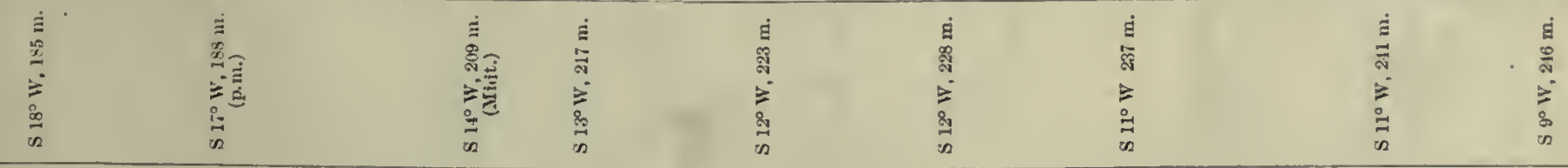

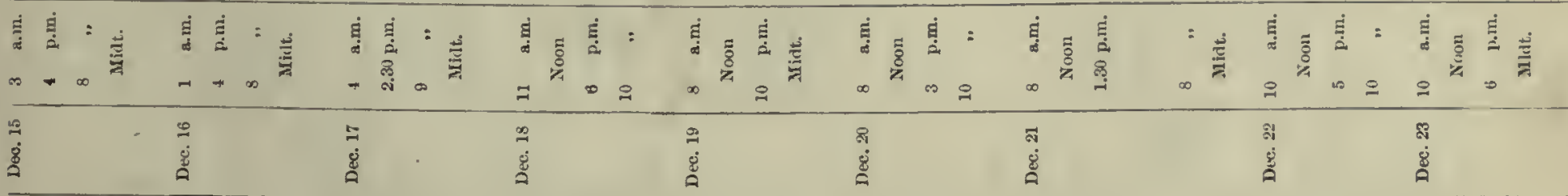




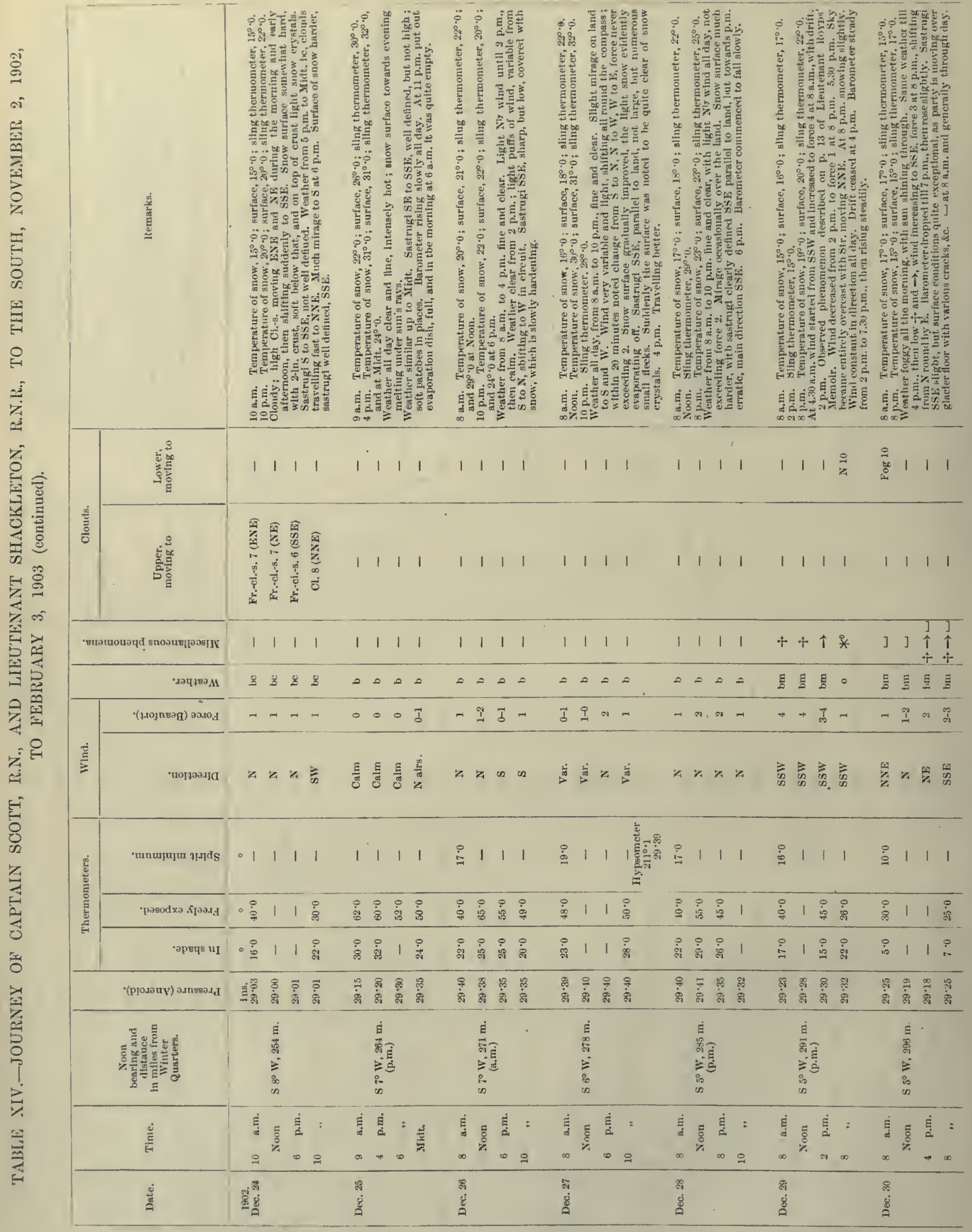




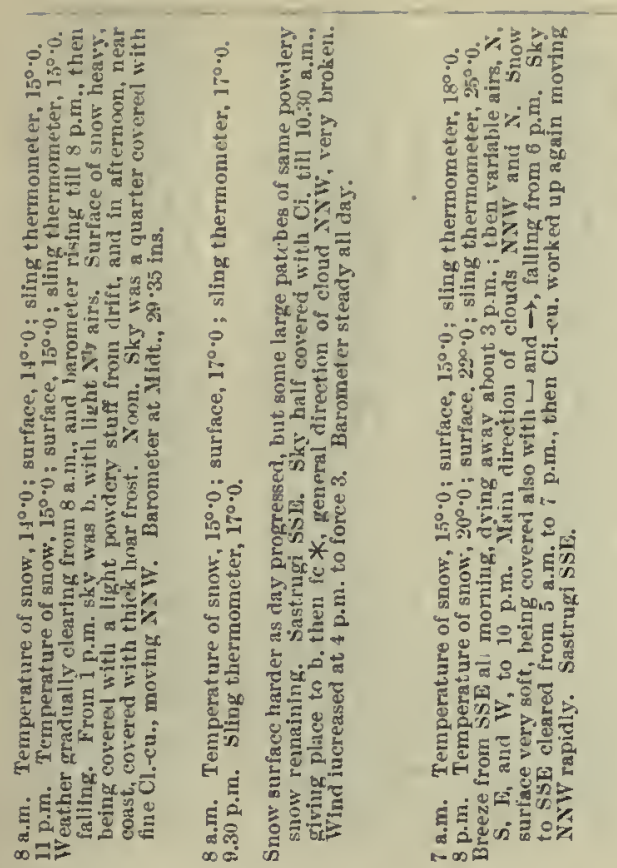

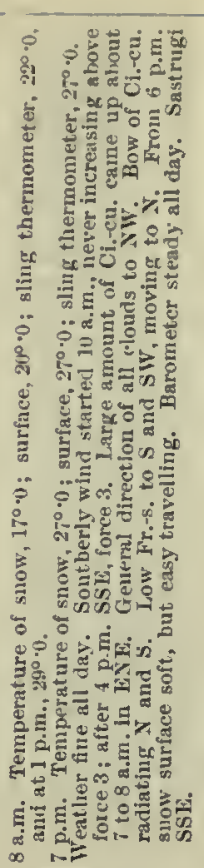

赔

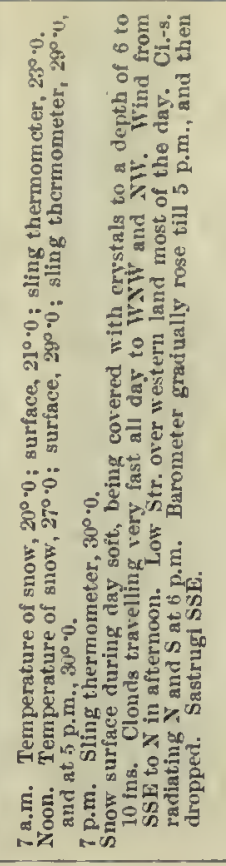

$\sum_{\infty}\left|\sum_{i}\right|$

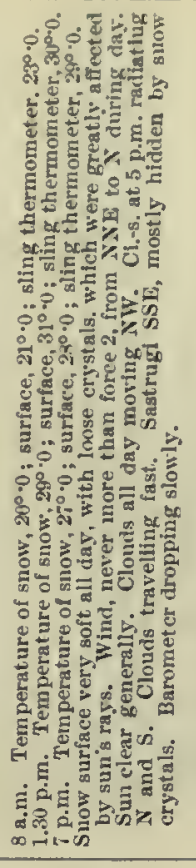

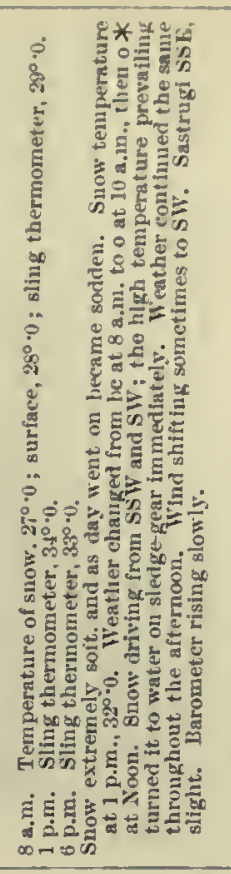

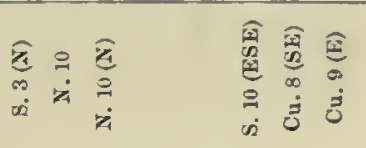

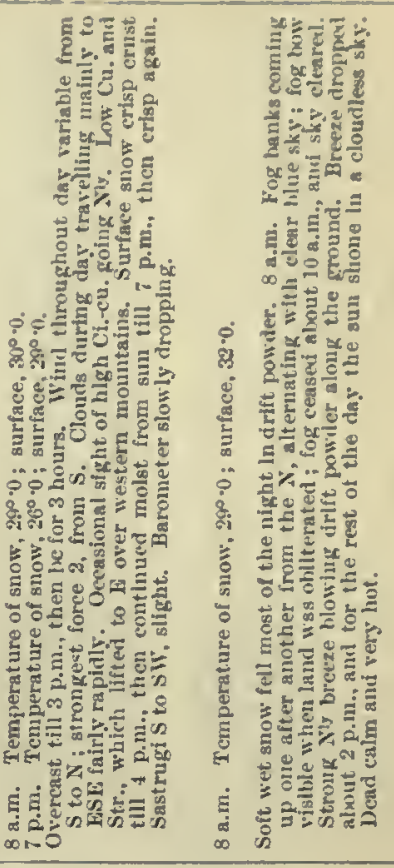

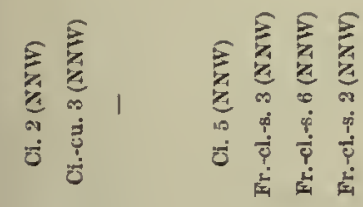

$|1| 1|1| 1 \mid \hat{i}$

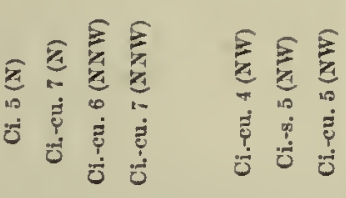

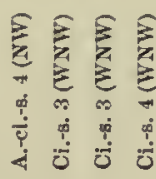

$\begin{array}{lll}\substack{a \\ \infty \\ 0} & 1 & 1 \\ 0 & 1 & 1\end{array}$

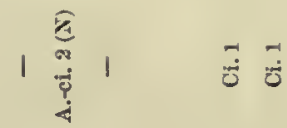

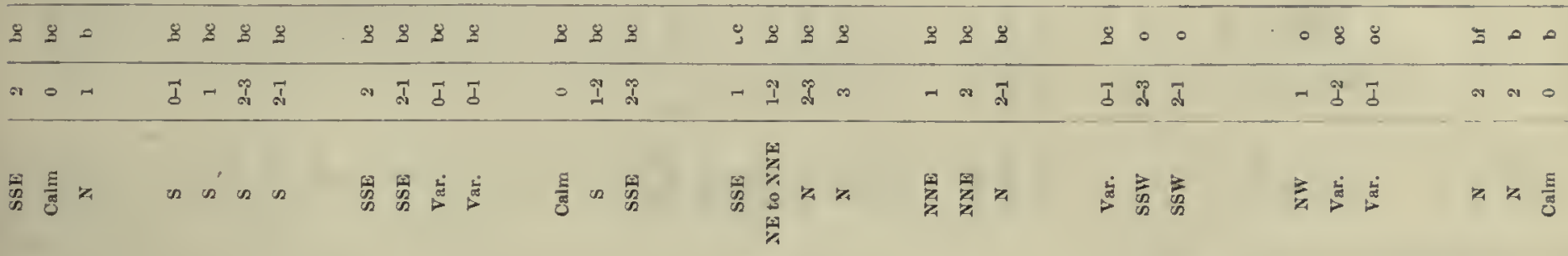

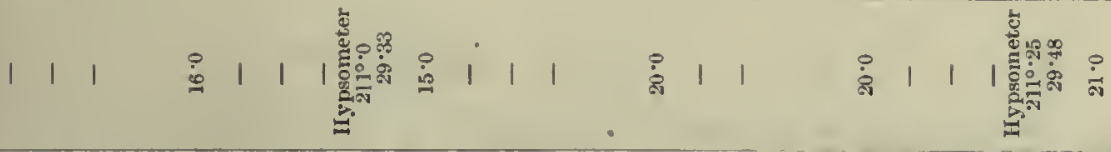

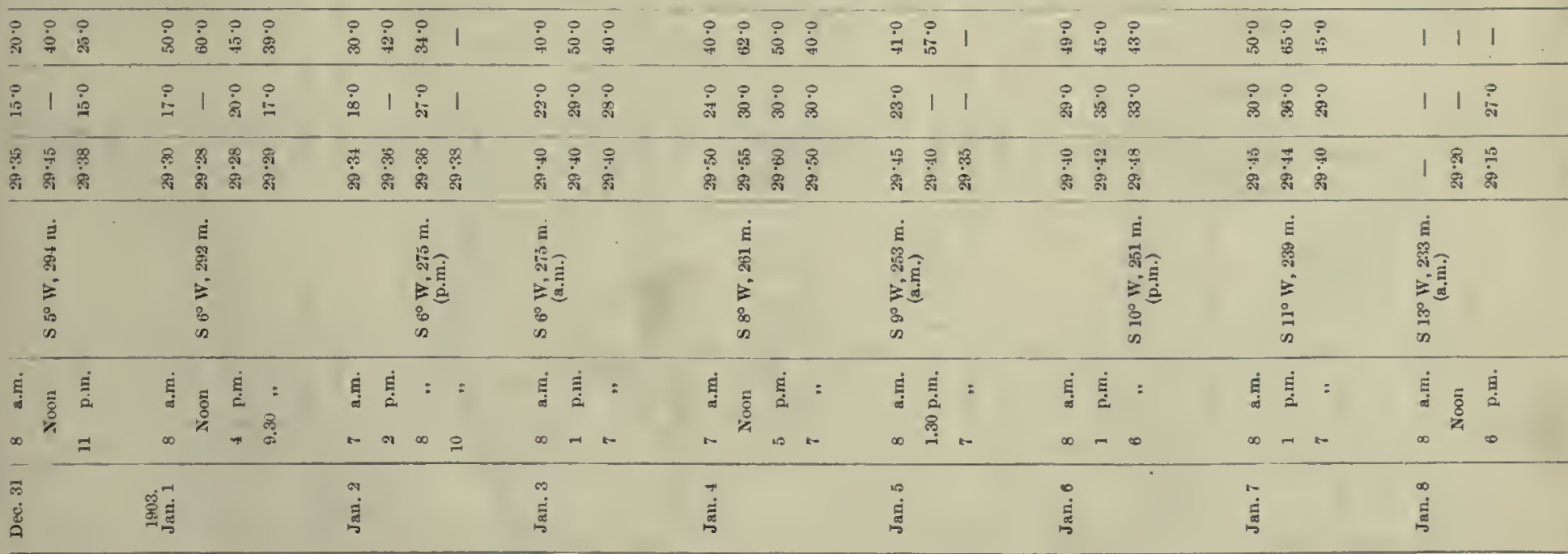




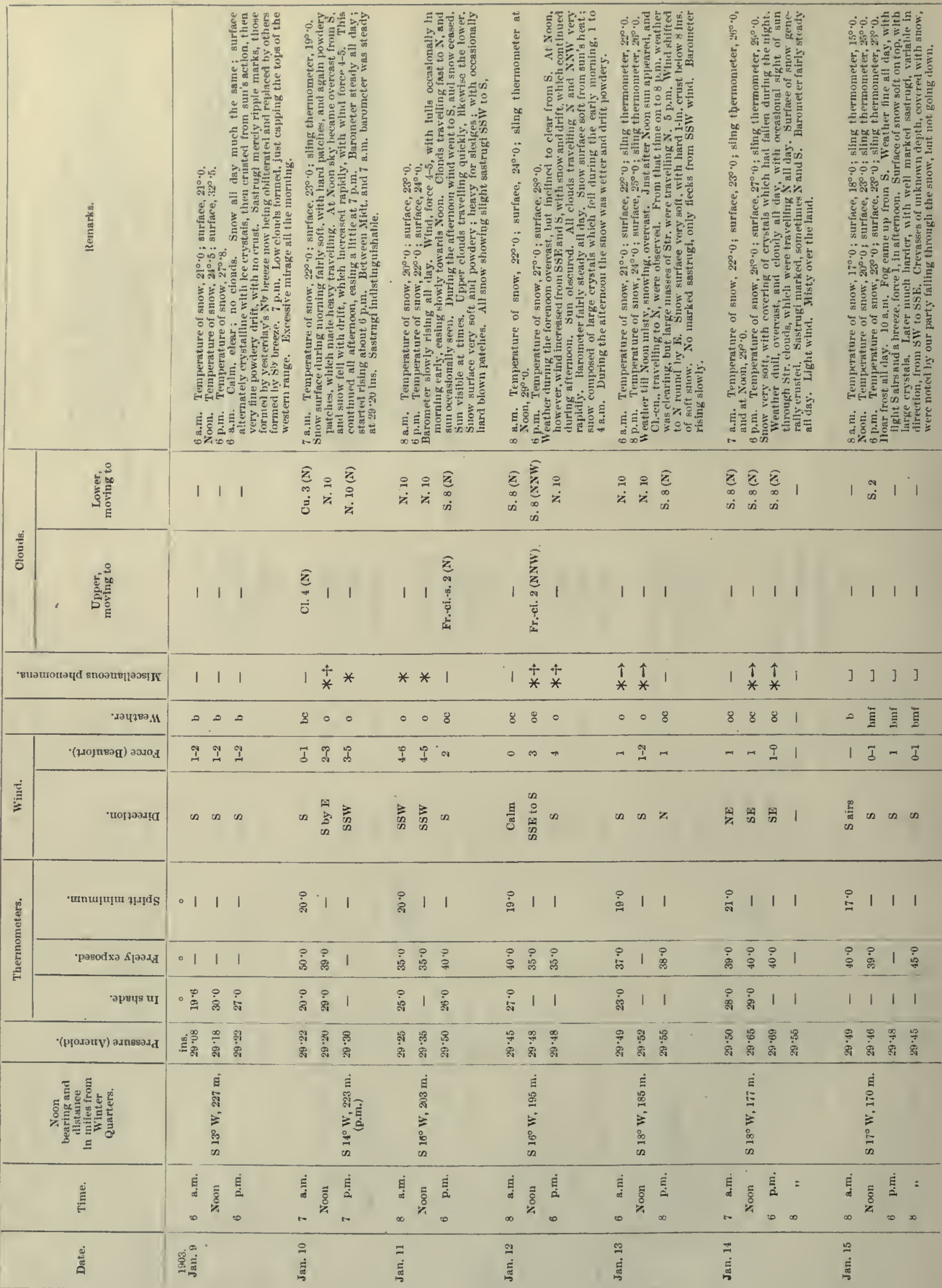




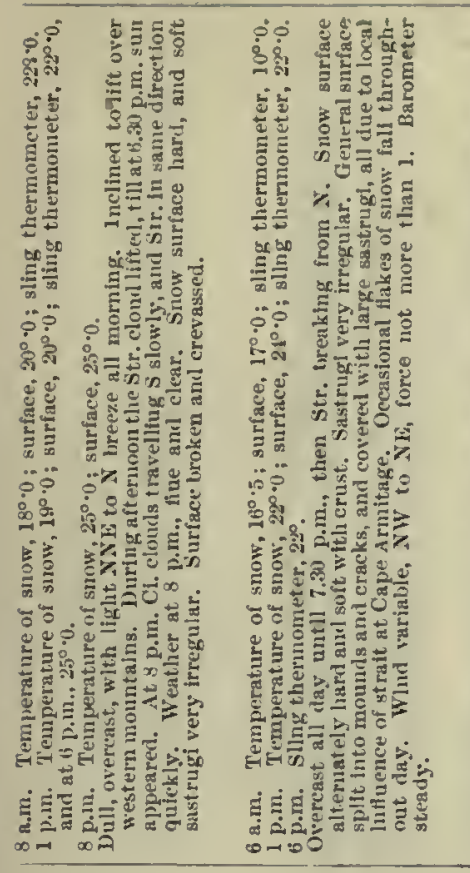

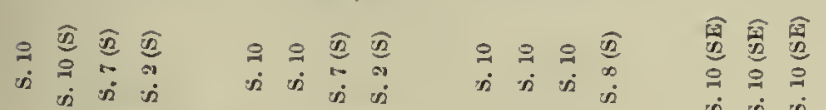

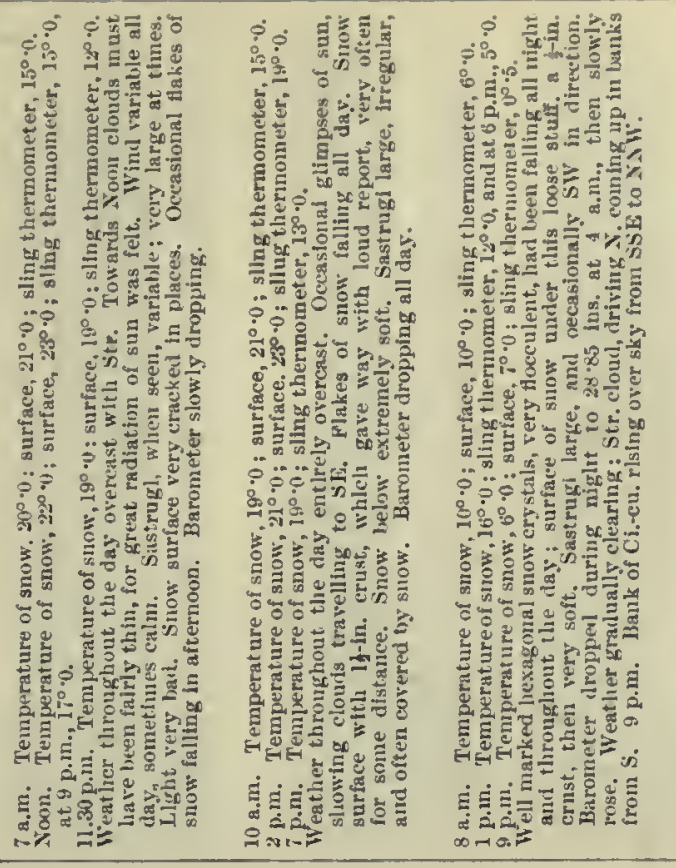

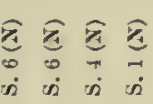

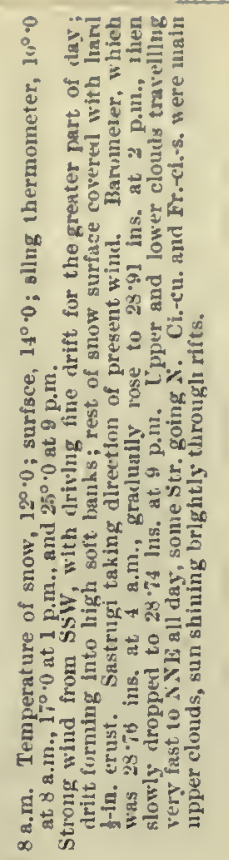

悉产
-

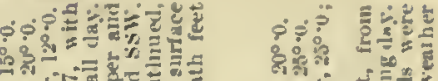

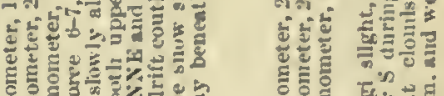

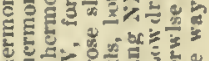

or

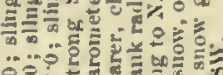

等.

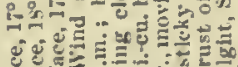

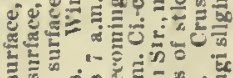

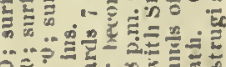

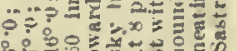

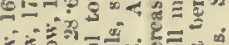

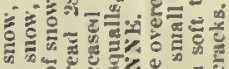

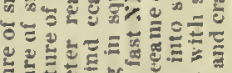

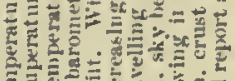

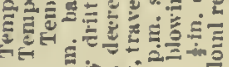

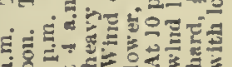

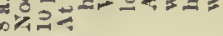

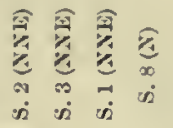

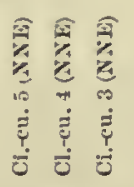

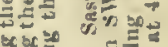
棓言言

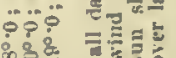

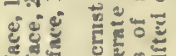

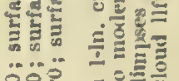
政

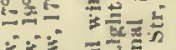

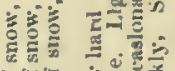

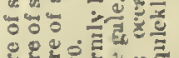

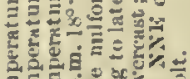

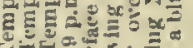
ह่

(1)

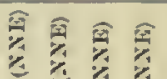

$\subseteq \circ \div$

$\dot{\phi} \dot{s} \dot{2}$ की

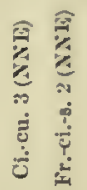

政

$1+1$

| * * 1

l 11

1111

11111

눈

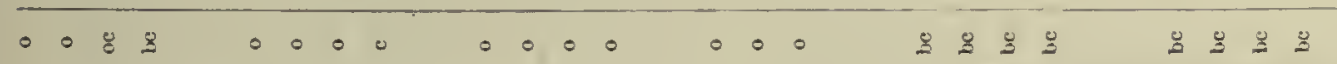

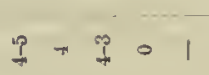

$\cong \cong 8$

$=.08$

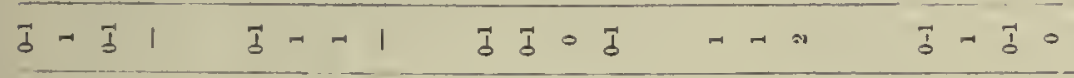

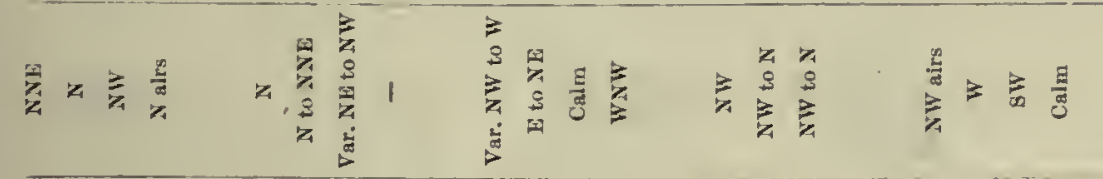

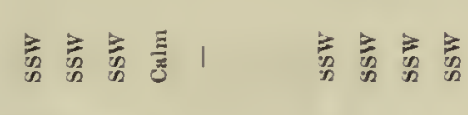

J $\rightarrow$ a

i⿱

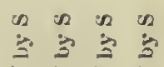
究言离

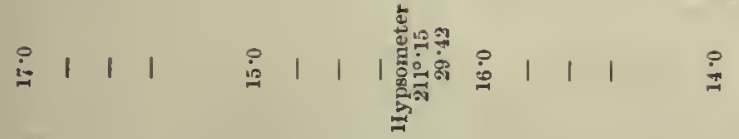

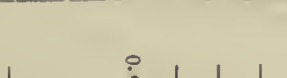

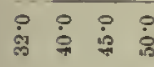

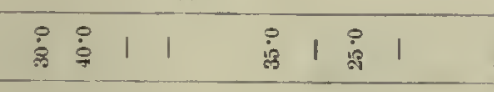

is

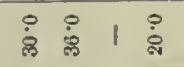

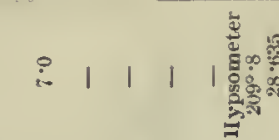

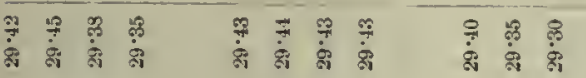

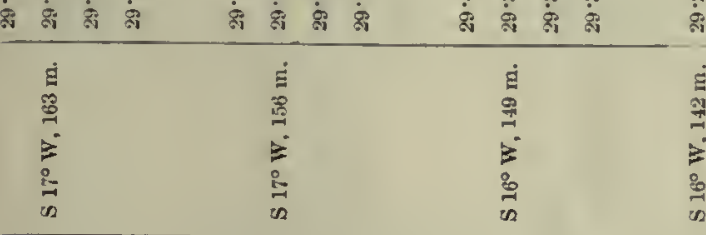

กับ

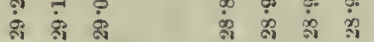

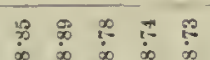

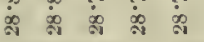

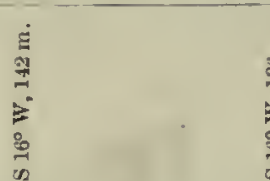

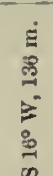

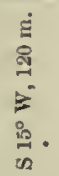

इं :

ذّ : 요

ฝิ

สั

घं : : :

$\infty-\infty$ 需

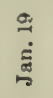

สี

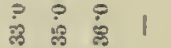

1111

$89 \div 9$ 家商腺

$\stackrel{3}{3}$




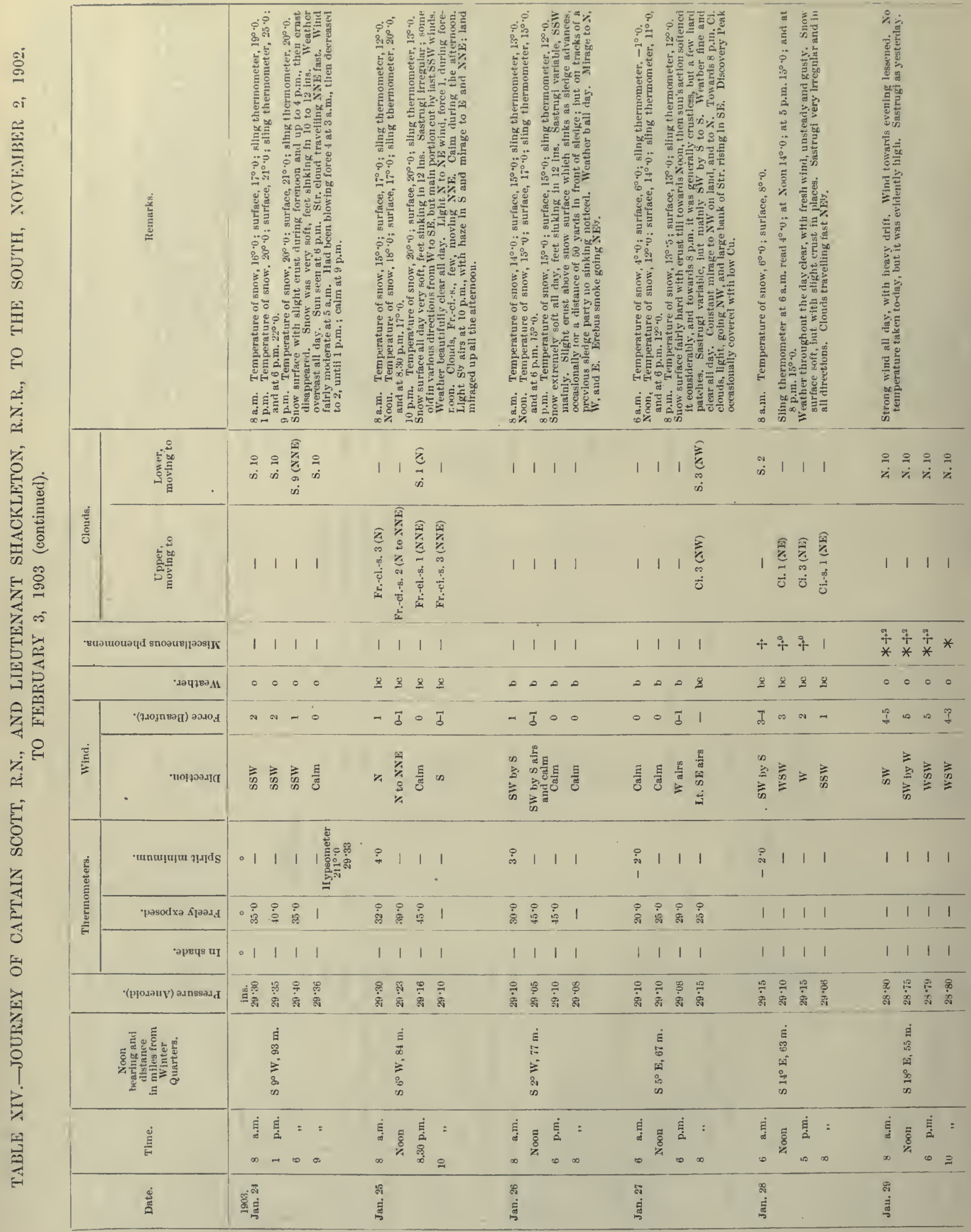



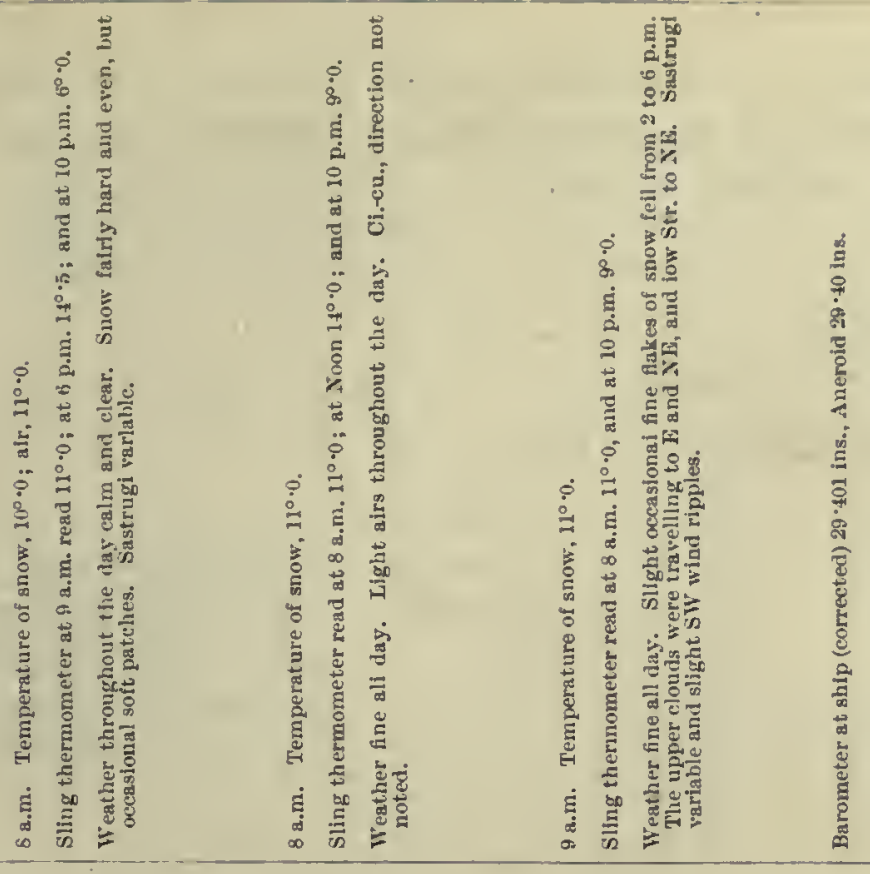

I 1 1

\section{1}

l 111

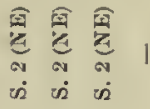

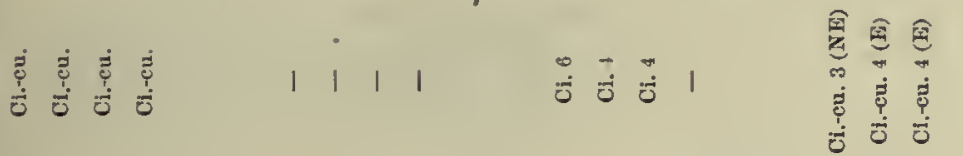

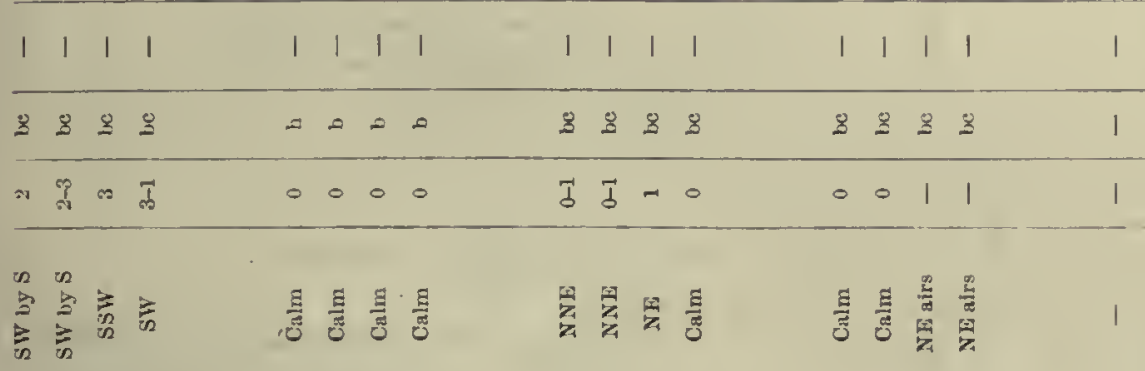

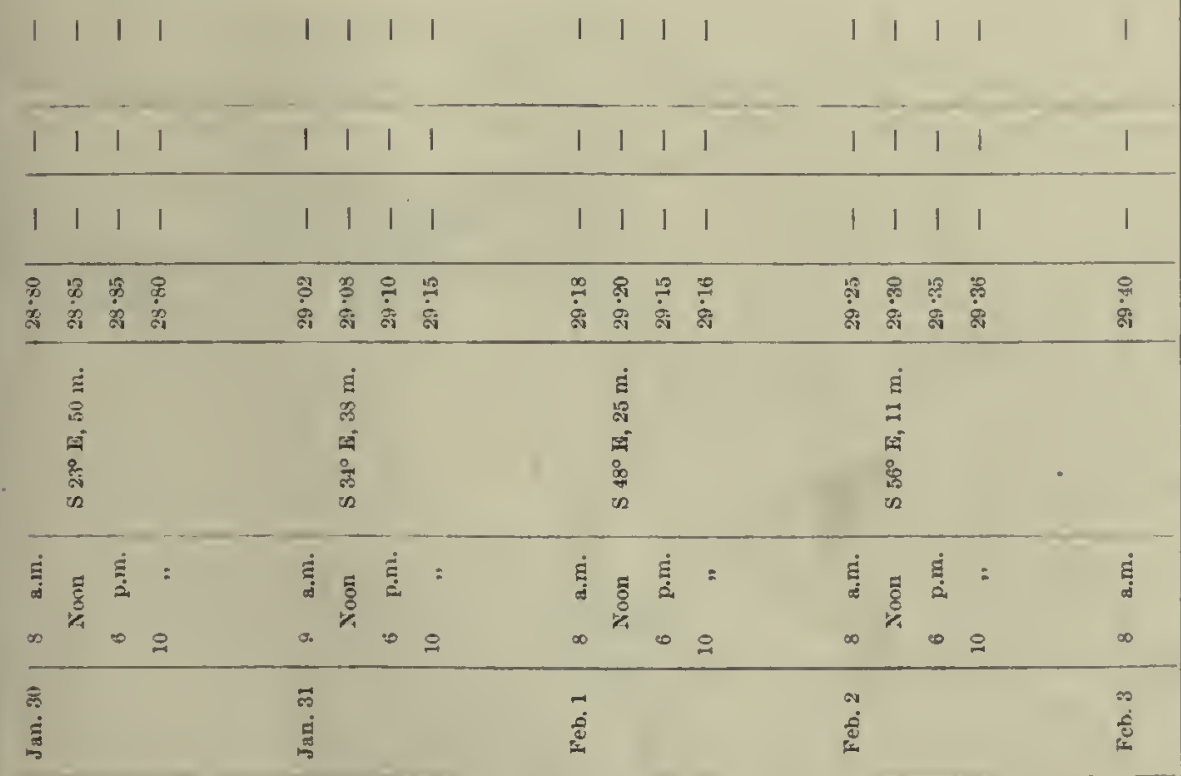


TABLE XV.—JOURNEY OF DR. KOETTLIT/ TO BROWN ISLAND, DECEIBER 29, 1902, TO JANUARY 8, 1903.

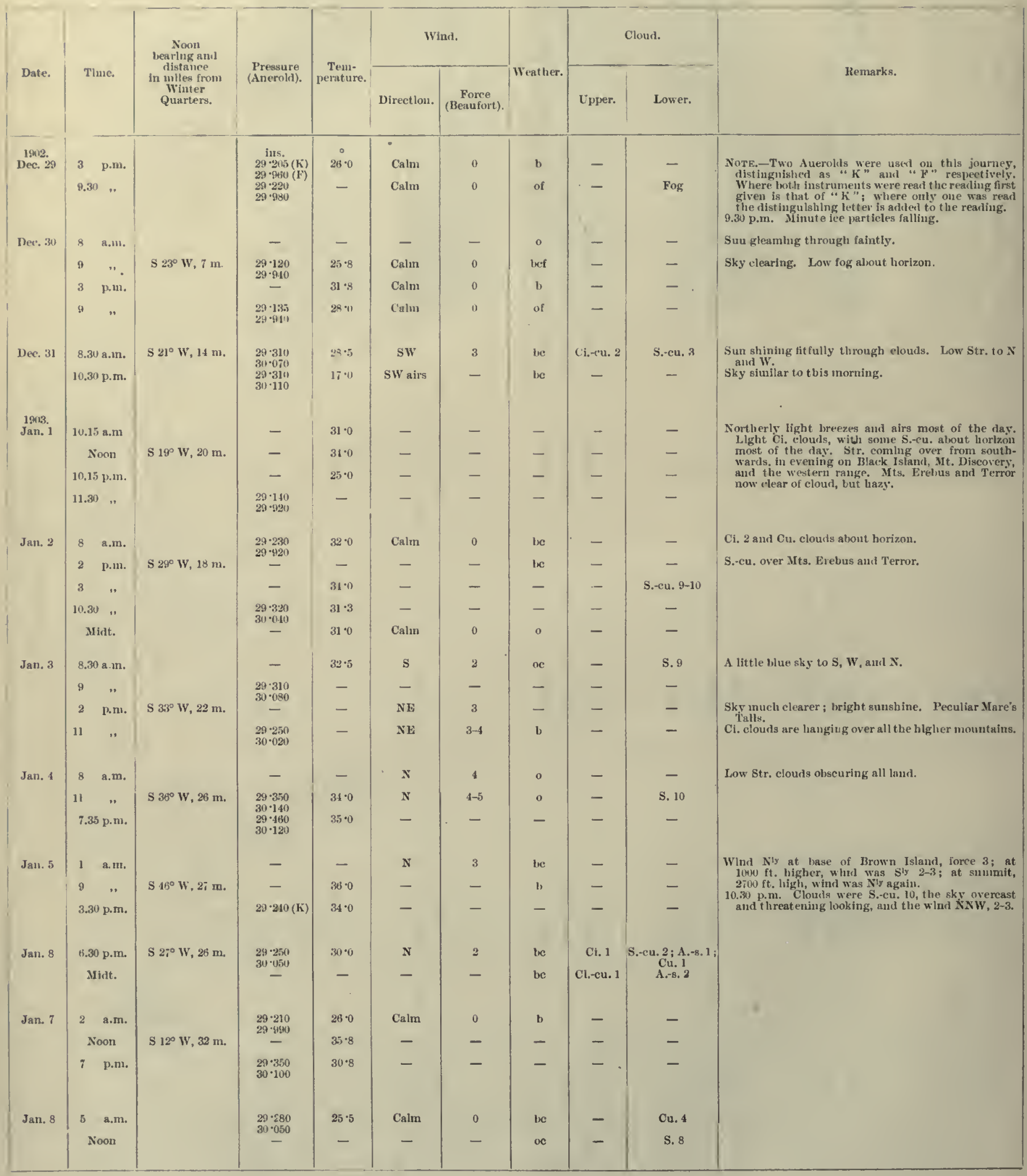


TABLE XVI.-JOURNEY OF DR. KOETTLITZ TO THE BLUFF, JANUARY 14 TO 26, 1903.

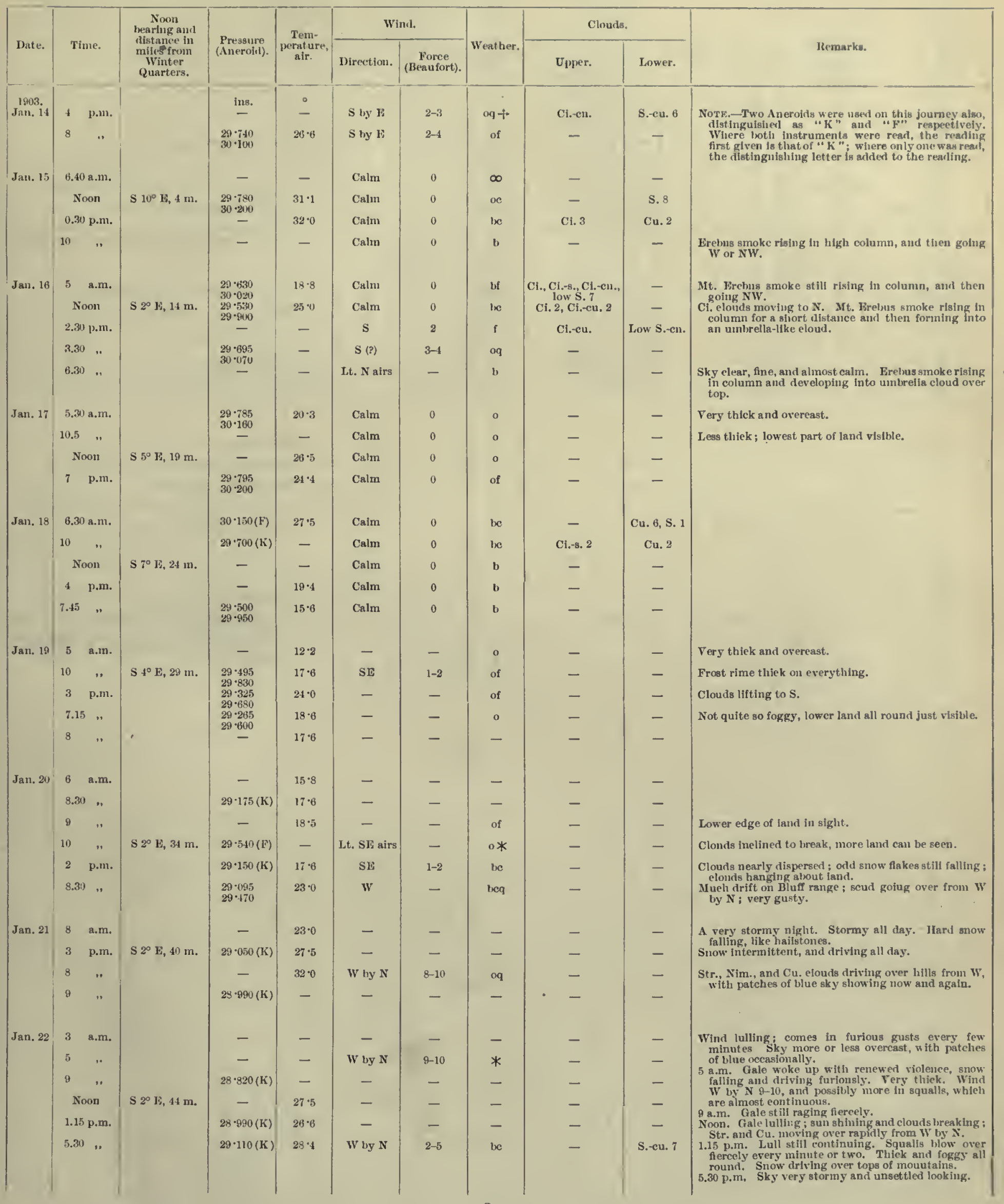


TABLE XVI.-JOURNEY OF DR. KOETTLITZ TO THE BLUFF, JANUARY 14 TO 26, 1903 (continued).

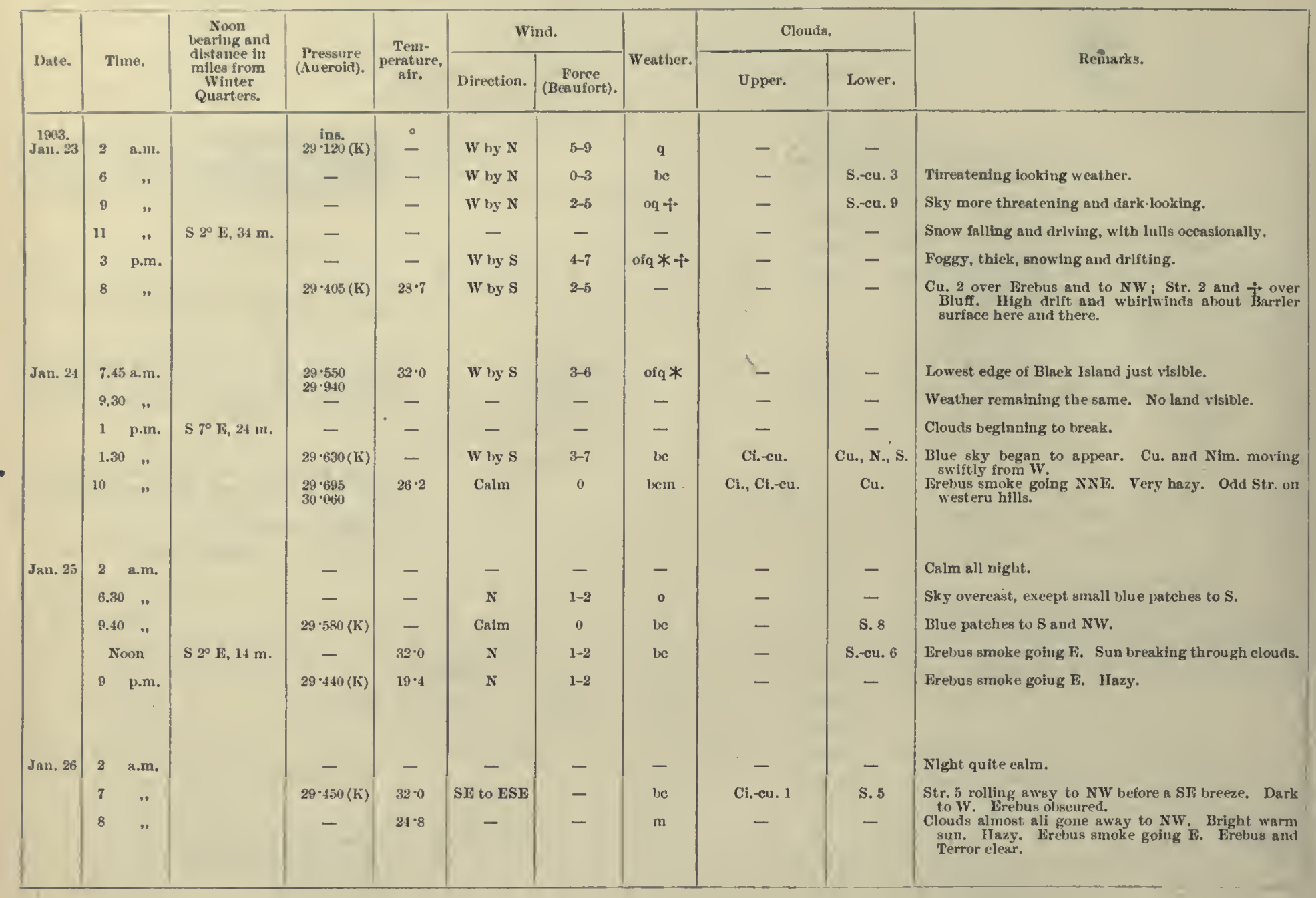




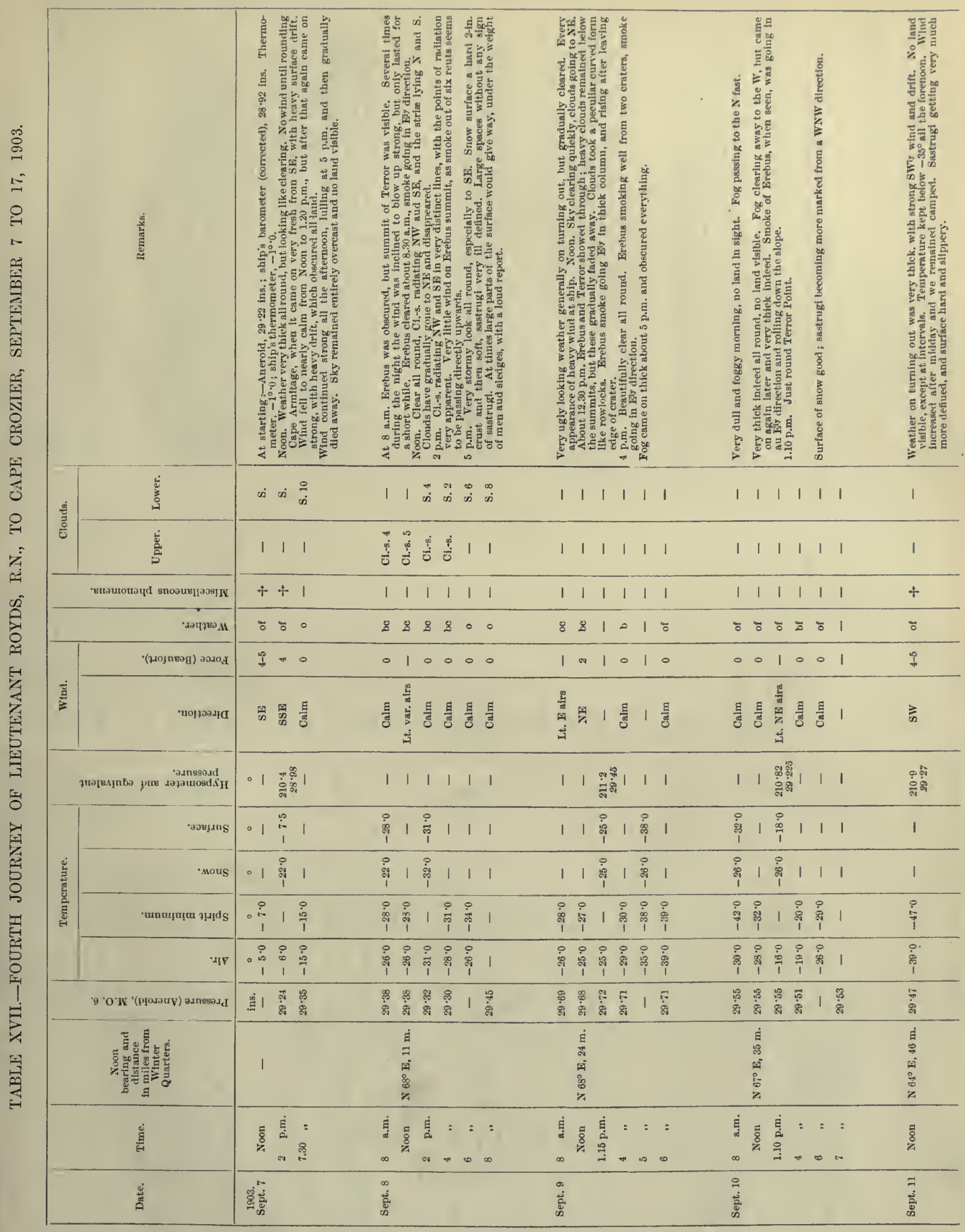




\begin{tabular}{|c|c|c|c|c|c|c|c|}
\hline & 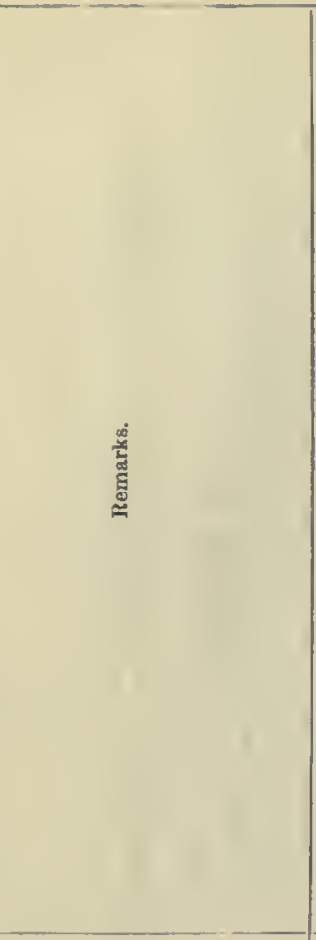 & 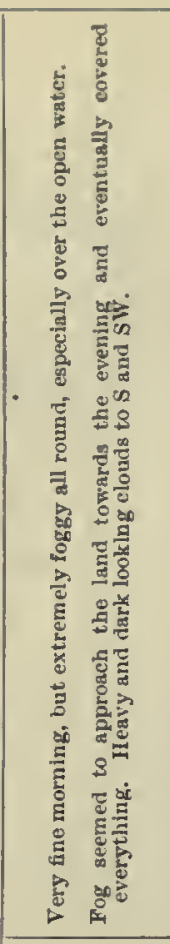 & 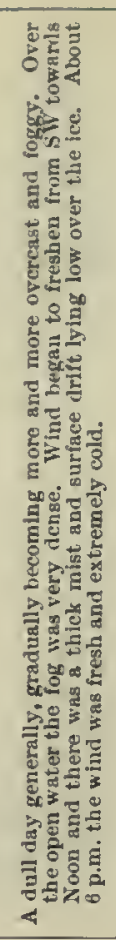 & 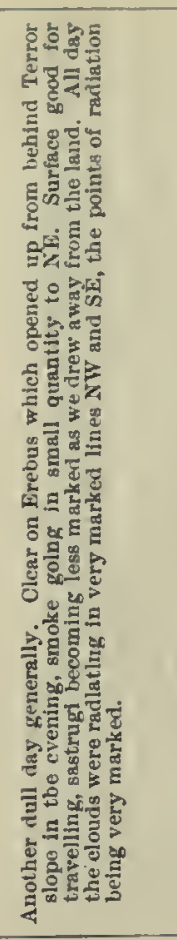 & 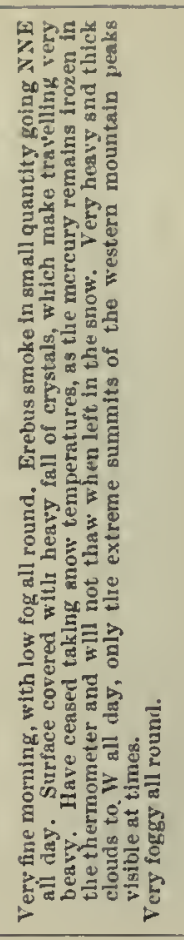 & 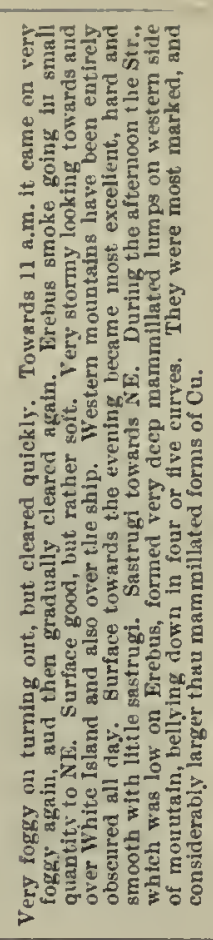 & 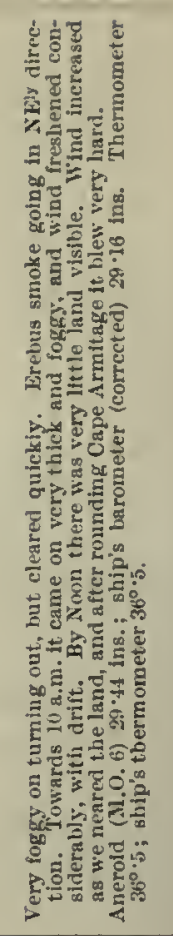 \\
\hline \multirow{2}{*}{$\begin{array}{l}\frac{9}{2} \\
\frac{\bar{g}}{0} \\
0\end{array}$} & $\begin{array}{l}\dot{5} \\
\vdots \\
\\
\end{array}$ & 111 & 11 & $\begin{array}{llll}1 & 1 & 1 & 1\end{array}$ & $\begin{array}{llll}1 & 1 & 1 & 1\end{array}$ & $\begin{array}{llll}1 & 1 & 1 & 1\end{array}$ & $\begin{array}{llll}1 & 1 & 1 & 1\end{array}$ \\
\hline & 产 & $1 \quad 11$ & 11 & $\begin{array}{llll}1 & 1 & 1 & 1\end{array}$ & $\begin{array}{llll}1 & 1 & 1 & 1\end{array}$ & $\begin{array}{llll}1 & 1 & 1 & 1\end{array}$ & $\begin{array}{llll}1 & 1 & 1 & 1\end{array}$ \\
\hline & 'виәшочәчd впюәия[Рәэ!К & $1 \quad 1 \quad 1$ & $1+$ & $\begin{array}{llll}1 & 1 & 1 & 1\end{array}$ & $\begin{array}{llll}1 & 1 & 1 & 1\end{array}$ & $11+4$ & $11+4$ \\
\hline & - Аәчив $\mathrm{M}$ & $\therefore$ & 8 & 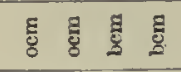 & 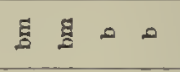 & Ð & $\Xi \Xi \Xi$ \\
\hline \multirow[b]{2}{*}{ 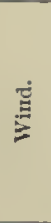 } & 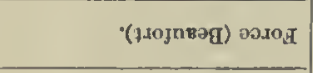 & $\circ 00$ & 0 I & $0 \quad 1 \quad 1 \quad 1$ & $0 \quad 0 \quad 10$ & $00 \infty \pi$ & - Iิ ปั \\
\hline & •иоқғаза !а & 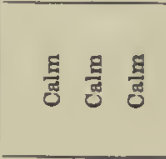 & 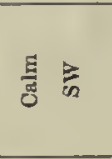 & 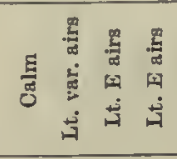 & 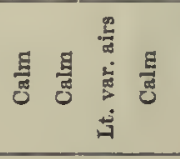 & 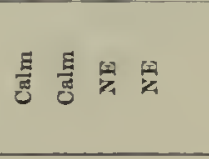 & 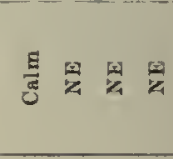 \\
\hline & 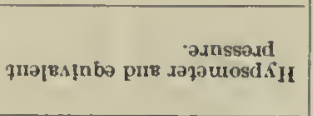 & 。 1 染। & 11 & 1 | & 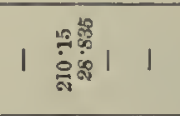 & 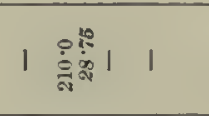 & 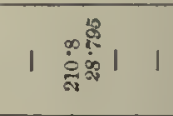 \\
\hline \multirow{4}{*}{ 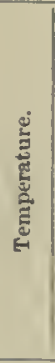 } & วәездns & - 1 总最 & 11 & $\begin{array}{llll}1 & 1 & 1 & 1\end{array}$ & $\begin{array}{llll}1 & 1 & 1 & 1\end{array}$ & $\begin{array}{llll}1 & 1 & 1 & 1\end{array}$ & $\begin{array}{llll}1 & 1 & 1 & 1\end{array}$ \\
\hline & - mons & 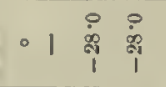 & 11 & $\begin{array}{llll}1 & 1 & 1 & 1\end{array}$ & $\begin{array}{llll}1 & 1 & 1 & 1\end{array}$ & $\begin{array}{llll}1 & 1 & 1 & 1\end{array}$ & $\begin{array}{llll}1 & 1 & 1 & 1\end{array}$ \\
\hline & 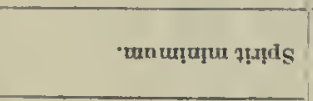 & 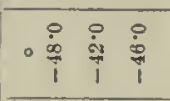 & $\stackrel{0}{\dot{\phi}}$ & 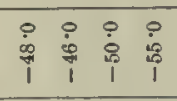 & 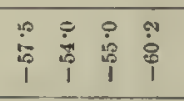 & 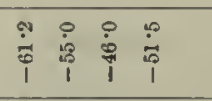 & 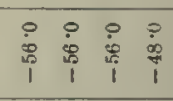 \\
\hline & ' & 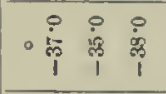 & $\begin{array}{ll}0 \\
\dot{i} \\
i \\
i\end{array}$ & 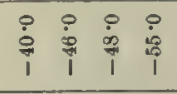 & 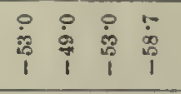 & 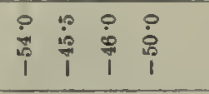 & 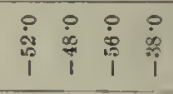 \\
\hline \multirow[t]{2}{*}{ - } & 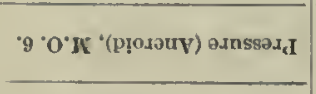 & 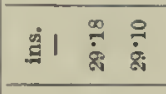 & 紫 1 & 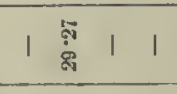 & 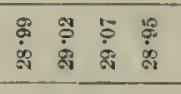 & 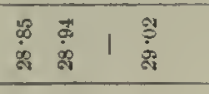 & 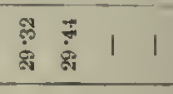 \\
\hline & 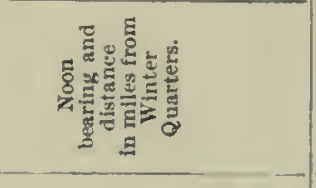 & 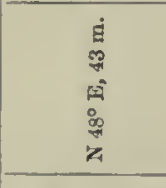 & 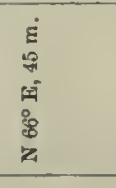 & 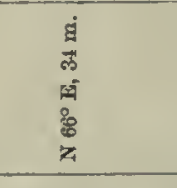 & 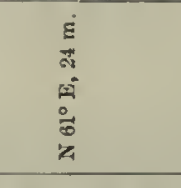 & 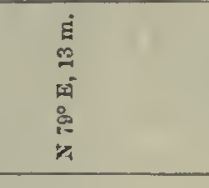 & I \\
\hline & $\stackrel{\rightleftarrows}{\xi}$ & 离 & $\begin{array}{l}\dot{g} \\
\infty \\
\infty\end{array}$ & $\begin{array}{l}\dot{\xi} \\
\infty \\
\infty \\
\infty\end{array}$ & 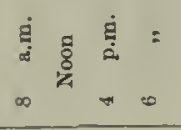 & 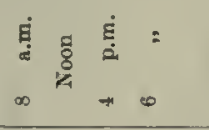 & 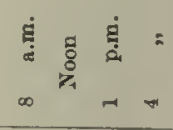 \\
\hline & 离 & 管 & $\begin{array}{l}m \\
\stackrel{9}{a} \\
\text { aे }\end{array}$ & 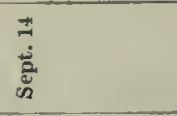 & 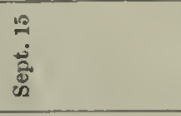 & 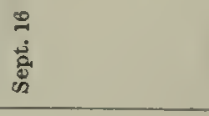 & 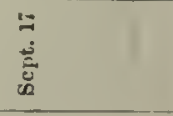 \\
\hline
\end{tabular}




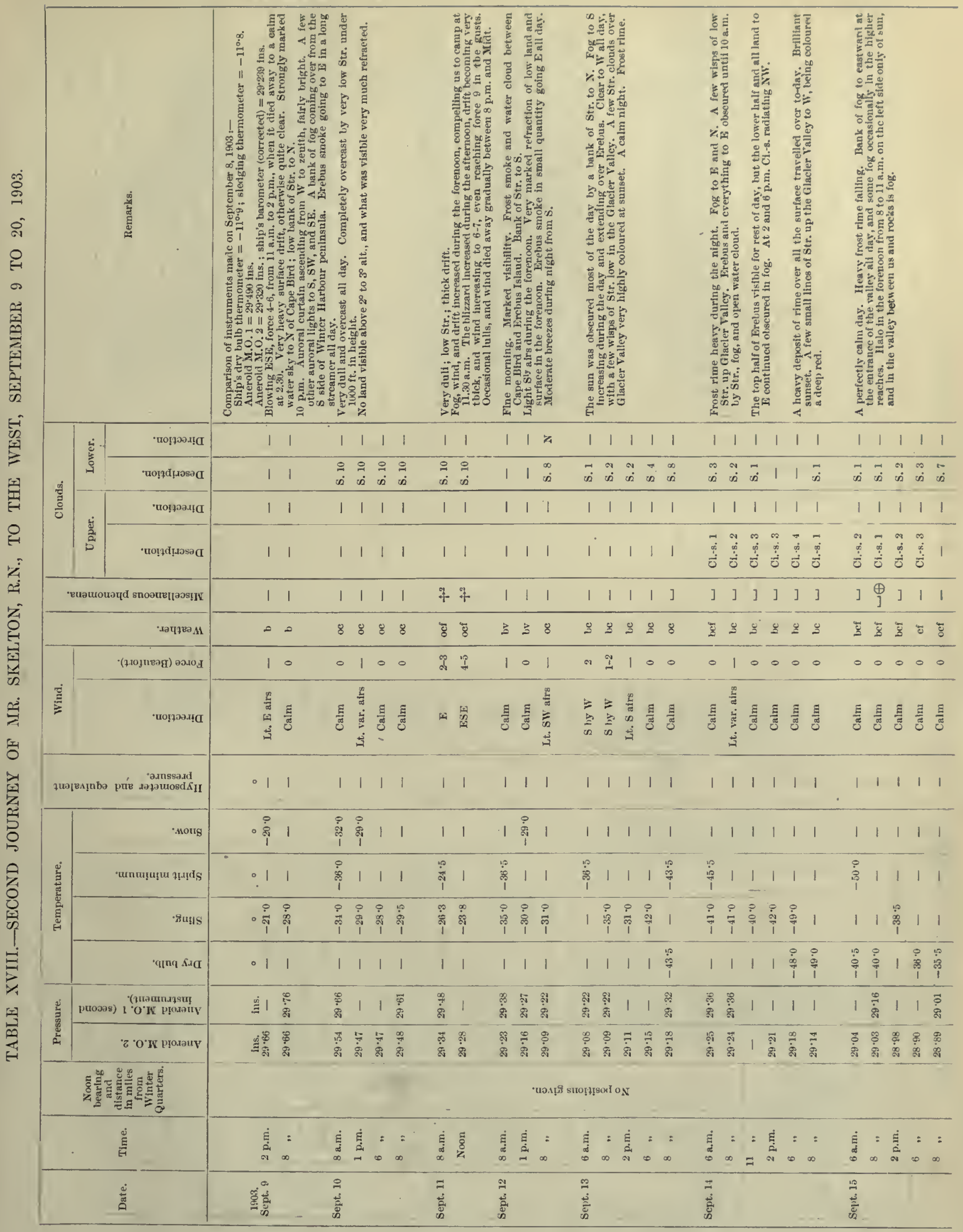




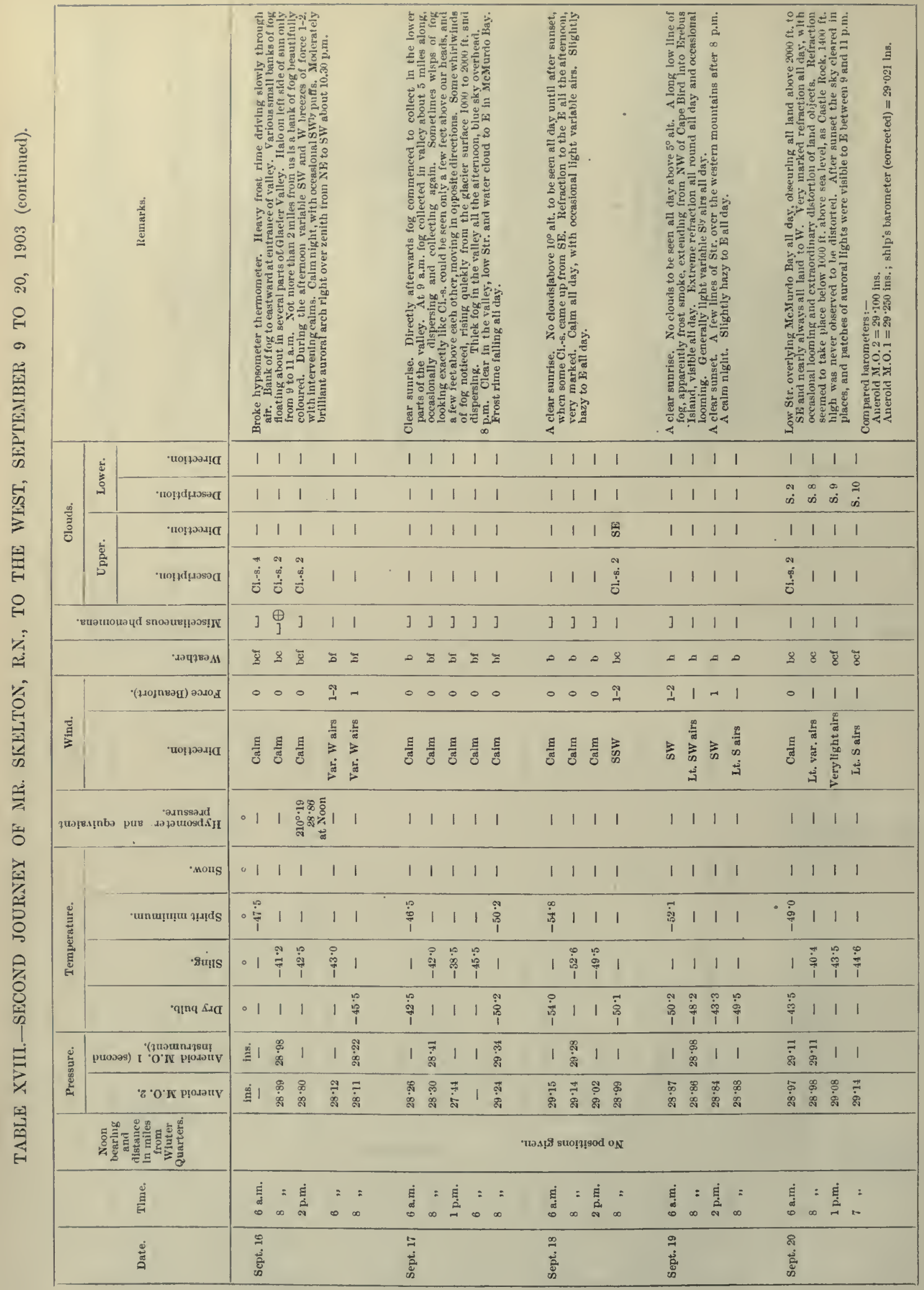


TABLE XIX.-JOURNEY OF LIEUTENANT BARNE, R.N., TO SOUTH, SEPTEMBER 13 TO 20, 1903.

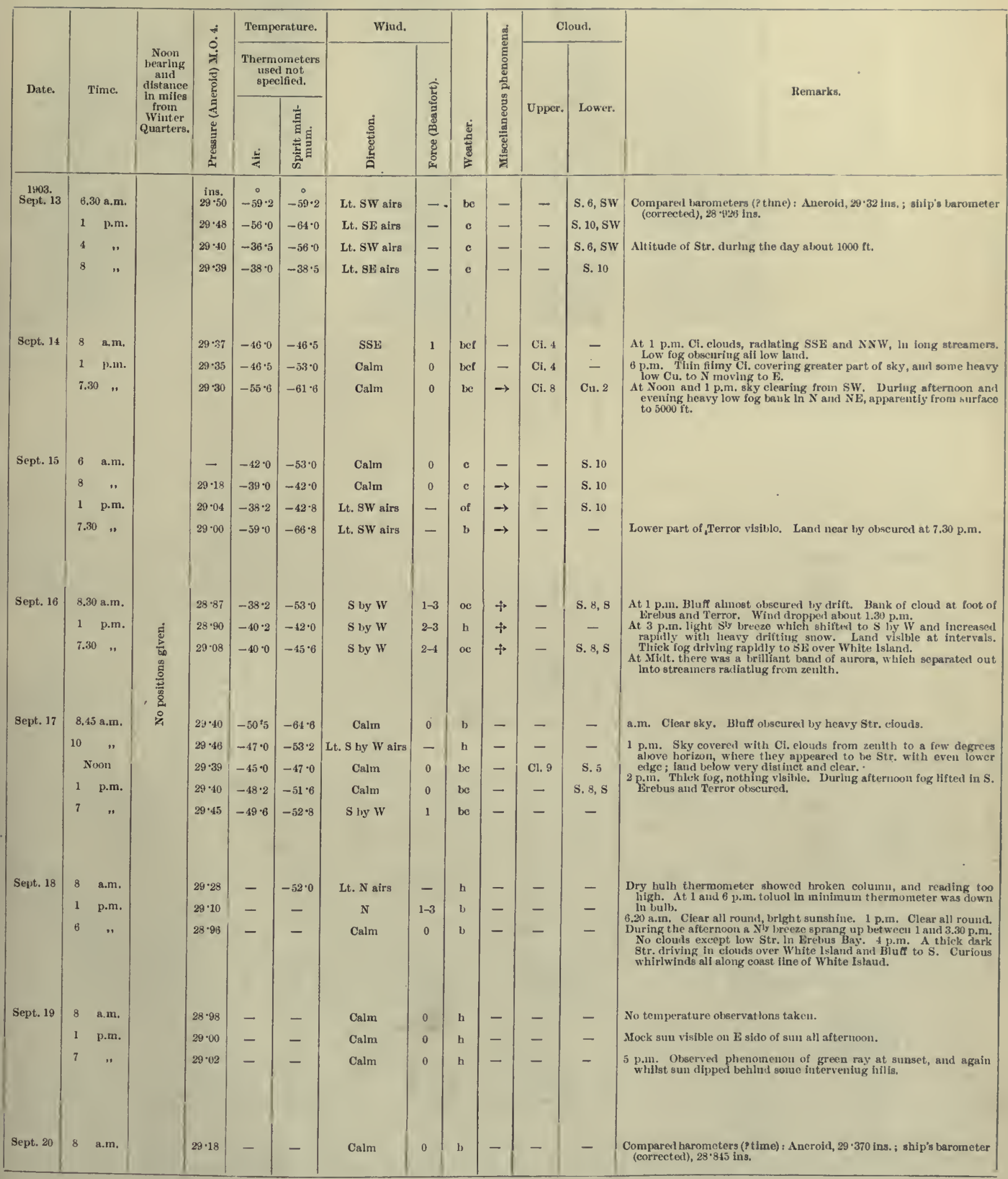




\begin{tabular}{|c|c|c|c|c|c|c|c|c|}
\hline & \multicolumn{2}{|c|}{ 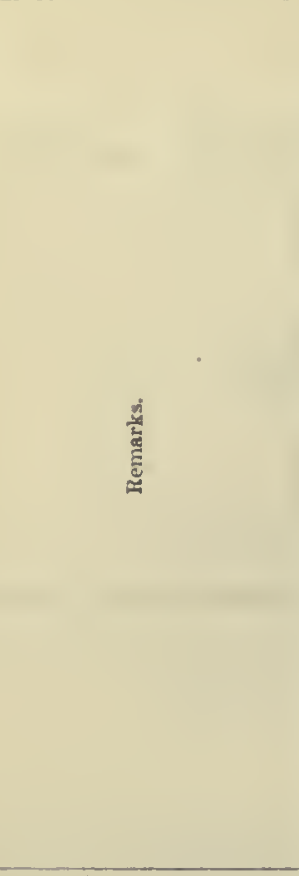 } & 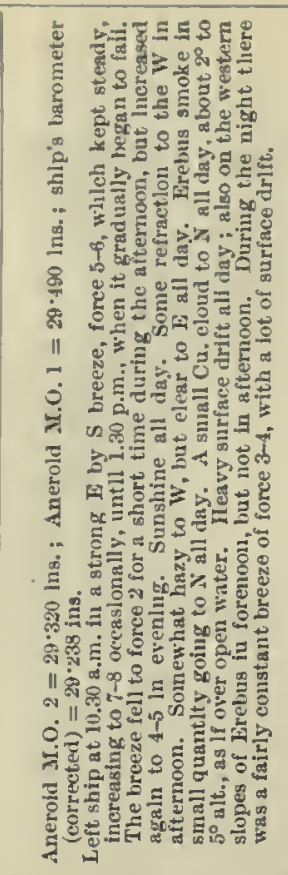 & 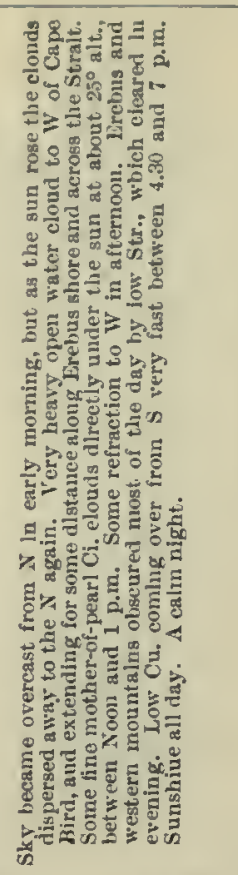 & 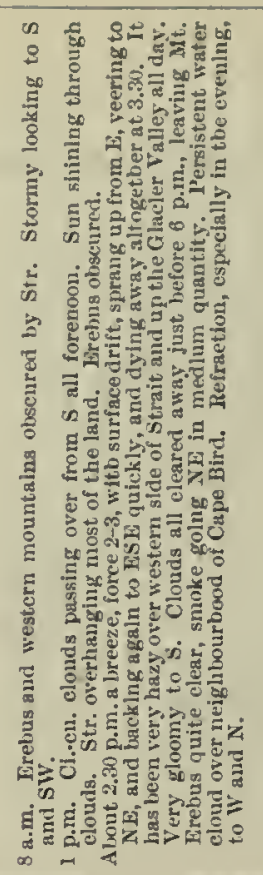 & 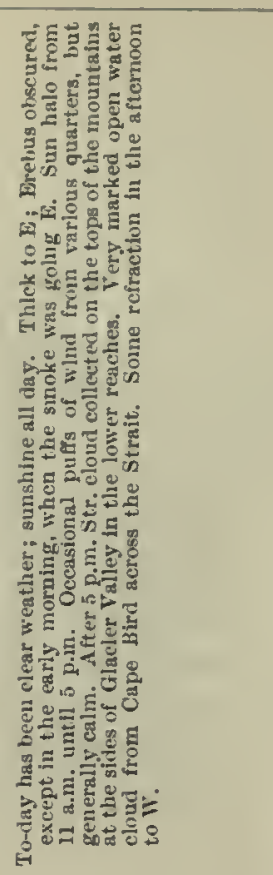 & 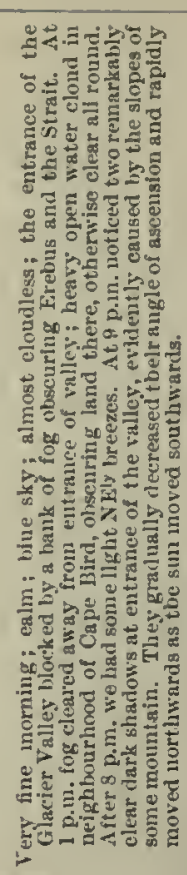 & \\
\hline \multirow{4}{*}{$\frac{d}{\frac{8}{2}}$} & \multirow{2}{*}{ 离 } & 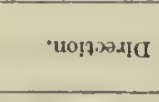 & 111 & 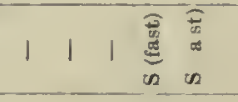 & 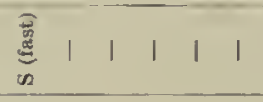 & $\begin{array}{llllll}1 & 1 & 1 & 1 & 1 & 1\end{array}$ & $\begin{array}{llll}1 & 1 & 1 & 1\end{array}$ & 1 \\
\hline & & 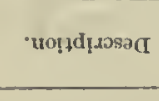 & $1 \mid \overline{i n}$ & 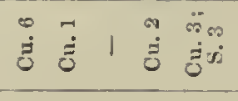 & $\begin{array}{llllll}3 & 1 & 1 & 1 & 1 & 1\end{array}$ & $\begin{array}{lllll}1 & 1 & \vec{n} & \mid\end{array}$ & $1 \quad 1 \quad 11$ & \\
\hline & \multirow{2}{*}{ 童 } & 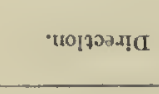 & 111 & $\begin{array}{lllll}1 & 1 & 1 & 1 & 1 \\
\end{array}$ & 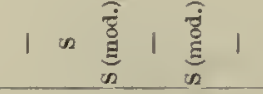 & $\begin{array}{llllll}1 & 1 & 1 & 1 & 1 & 1\end{array}$ & $1 \quad 1 \quad 11$ & 1 \\
\hline & & 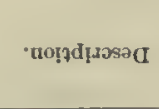 & تั & 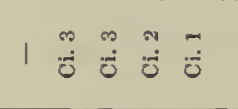 & 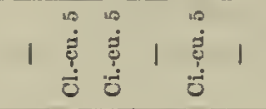 & 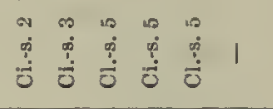 & 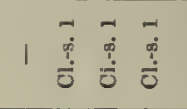 & \\
\hline \multicolumn{3}{|c|}{ 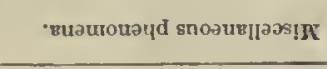 } & $74+$ & +1111 & $\begin{array}{llllll}1 & 1 & 1 & 1 & 1 & 1\end{array}$ & $|\quad| \oplus \oplus \oplus \mid$ & 1111 & । \\
\hline \multirow[b]{3}{*}{$\stackrel{\vec{\Xi}}{\underline{E}}$} & & - дәчұвәМ & 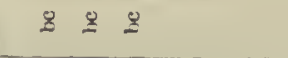 & $8 \& 880$ & 00000. & 820000 & $=828$ & $=$ \\
\hline & & גојивәg) әрдо & î & 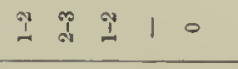 & $11-211$ & 010100 & $=010$ & $\circ$ \\
\hline & & 'попฺрах! & 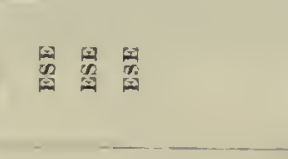 & 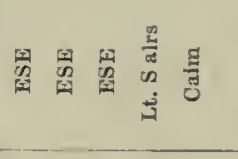 & 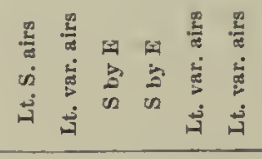 & 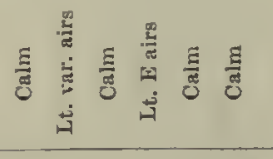 & 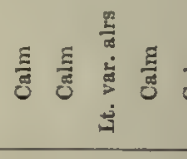 & \\
\hline \multicolumn{3}{|c|}{ 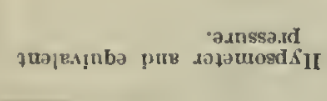 } & $\begin{array}{lll}-1 & 1 & 1 \\
\end{array}$ & $\begin{array}{lllll}1 & 1 & 1 & 1 & 1\end{array}$ & 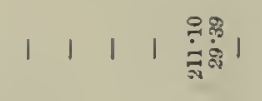 & | 1 | 1 | 1 | & 1111 & 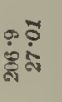 \\
\hline \multirow{3}{*}{ 总 } & \multirow{2}{*}{ 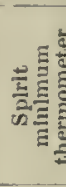 } & 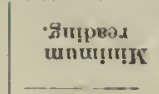 & $011 \stackrel{0}{0}$ & 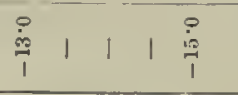 & 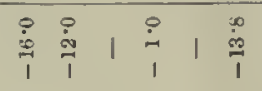 & 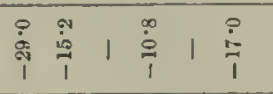 & 突 & $\begin{array}{l}+\infty \\
\dot{0} \\
1\end{array}$ \\
\hline & & 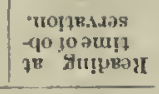 & $\begin{array}{cl}\stackrel{0}{\circ} \\
0\end{array}$ & 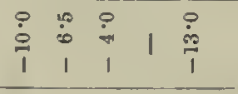 & 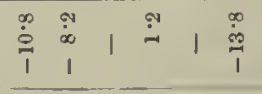 & 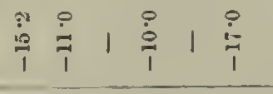 & 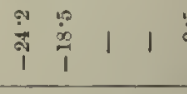 & $\begin{array}{r}: 0 \\
\vdots \\
1\end{array}$ \\
\hline & \multicolumn{2}{|c|}{ 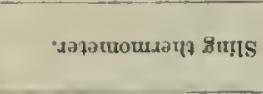 } & $01 \stackrel{\infty}{i} 1$ & 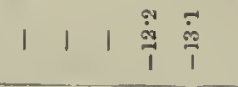 & 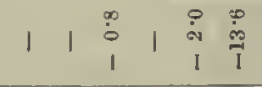 & 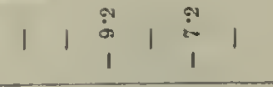 & 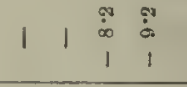 & 1 \\
\hline \multirow{2}{*}{$\begin{array}{c}\text { 离 } \\
\text { 衰 } \\
\text { 离 }\end{array}$} & \multicolumn{2}{|c|}{ 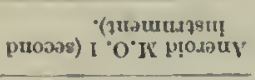 } & 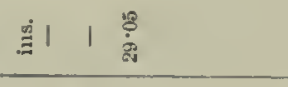 & 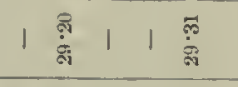 & 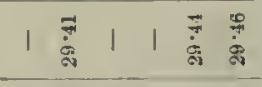 & 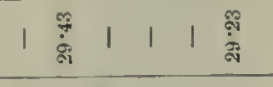 & $\begin{array}{lll}\tilde{\tilde{\pi}} & 1 & 1 \\
\end{array}$ & 䓂 \\
\hline & \multicolumn{2}{|c|}{ 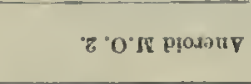 } & 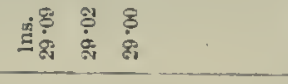 & 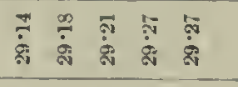 & 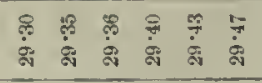 & 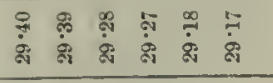 & 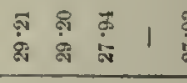 & 浔 \\
\hline \multicolumn{3}{|c|}{ 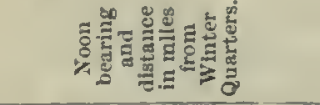 } & & & 'นaล! & & & \\
\hline \multicolumn{3}{|c|}{ 离 } & \begin{tabular}{l}
$\dot{1}=:$ \\
\multirow{2}{*}{$=\infty$}
\end{tabular} & 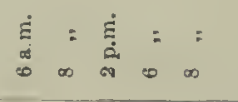 & 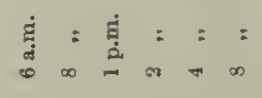 & 炁 = & $\stackrel{d}{g}=$ & : \\
\hline \multicolumn{3}{|c|}{ 离 } & : & $\cong$ & 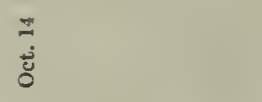 & $\stackrel{2}{\circ}$ & $\stackrel{0}{\circ}$ & \\
\hline
\end{tabular}



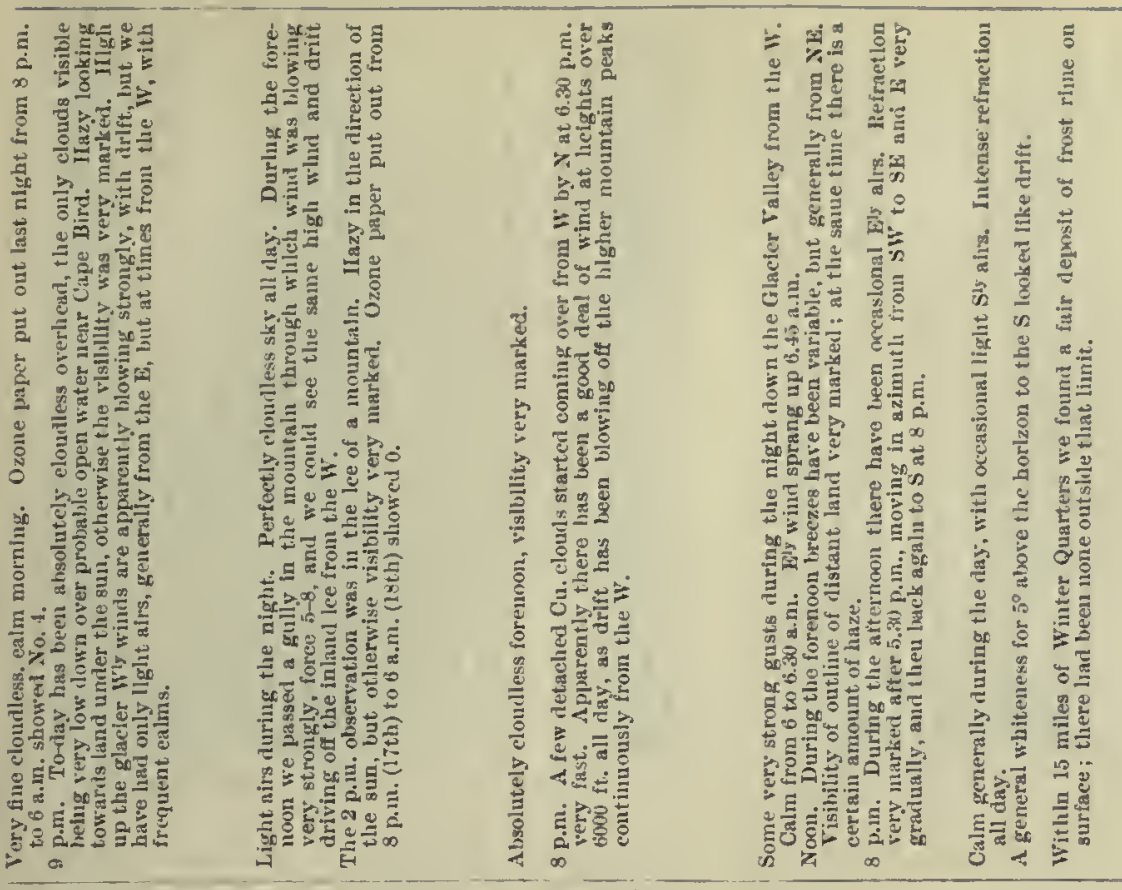

\begin{tabular}{|c|c|c|c|c|c|c|c|c|c|c|c|c|c|c|c|}
\hline 1 & 11 & 1 & 1 & 1 & I & 1 & 1 & I & 1 & $\geqslant$ & & 1 & 1 & I 1 & 1 \\
\hline I & 11 & 1 & 1 & 1 & 1 & I & 1 & I & 1 & ت̇ & I & 1 & 1 & 11 & $\vec{n}$ \\
\hline I & 1 1 & 1 & I & 1 & 1 & I & 1 & 1 & 1 & 1 & I & 1 & I & 1 I & 1 \\
\hline I & 11 & I & I & 1 & 1 & 1 & 1 & 1 & 1 & I & I & 1 & م. & $\begin{array}{c}-1 \\
0 \\
0\end{array}$ & I \\
\hline
\end{tabular}

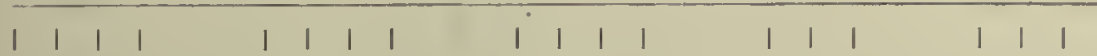

\begin{tabular}{|c|c|c|c|c|}
\hline $\bar{b} \mathrm{E}$ & 323 & 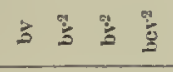 & $\Xi \vdots 0$ & $\Xi 2$ \\
\hline$=011$ & I 11 & 0110 & 910 & 00 \\
\hline 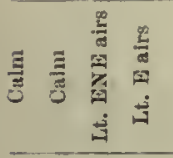 & 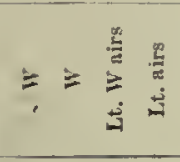 & 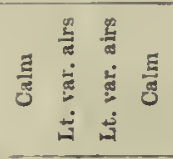 & 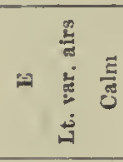 & 咅 咅 \\
\hline $\begin{array}{llll}1 & 1 & 1 & 1\end{array}$ & 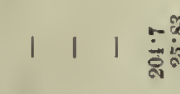 & $\begin{array}{llll}1 & 1 & 1 & 1\end{array}$ & 111 & 11 \\
\hline
\end{tabular}

\begin{tabular}{|c|c|c|c|c|c|c|c|c|c|c|c|c|}
\hline \begin{tabular}{ll}
0 \\
\hdashline \\
\hdashline
\end{tabular} & $\begin{array}{l}0 \\
i \\
i\end{array}$ & & $\ddot{\phi}_{i}$ & 11 & 1 & 1 & 11 & 11 & 1 & 1 & 1 & 111 \\
\hline 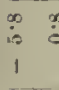 & $: 1$ & 1 & की & 11 & 1 & 1 & 11 & 11 & 1 & 1 & 1 & $\begin{array}{lll}1 & 1 & 1\end{array}$ \\
\hline & $1 \begin{array}{l}: \\
\dot{0} \\
1\end{array}$ & $\frac{\dot{p}}{i}$ & & 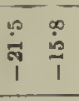 & 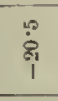 & 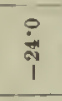 & 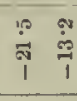 & $\frac{8}{1}$ & $\overrightarrow{\dot{\hat{H}}}$ & $\stackrel{\infty}{-1}$ & 19. & $\begin{array}{ll}0 & 0 \\
1 & 1\end{array}$ \\
\hline 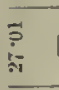 & 11 & $\begin{array}{l}\overrightarrow{4} \\
\dot{4} \\
\vdots \\
\end{array}$ & 1 & $\begin{array}{ll}0 \\
0\end{array}$ & 1 & 1 & 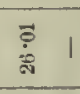 & 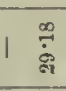 & 1 & 1 & 1 & 111 \\
\hline 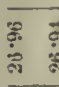 & 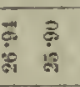 & $\frac{\pi}{4}$ & $\begin{array}{l}8 \\
8 \\
6 \\
4\end{array}$ & 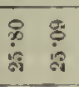 & $\begin{array}{l}\overrightarrow{7} \\
\vdots \\
\vdots\end{array}$ & 为 & 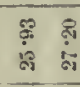 & $\begin{array}{l}8 \\
8 \\
6\end{array}$ & $\begin{array}{l}\overrightarrow{4} \\
\text { : } \\
\text { in }\end{array}$ & बू. & 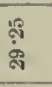 & 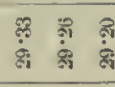 \\
\hline
\end{tabular}

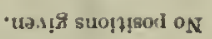

\begin{tabular}{|c|c|c|c|c|}
\hline : $=$ & 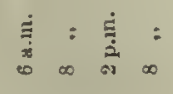 & $\stackrel{\dot{\Xi}}{=}=$ & 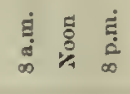 & 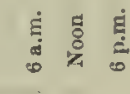 \\
\hline 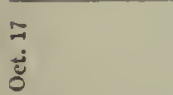 & $\begin{array}{l}\infty \\
3 \\
3\end{array}$ & - $\stackrel{9}{\square}$ & 疋 & ส \\
\hline
\end{tabular}




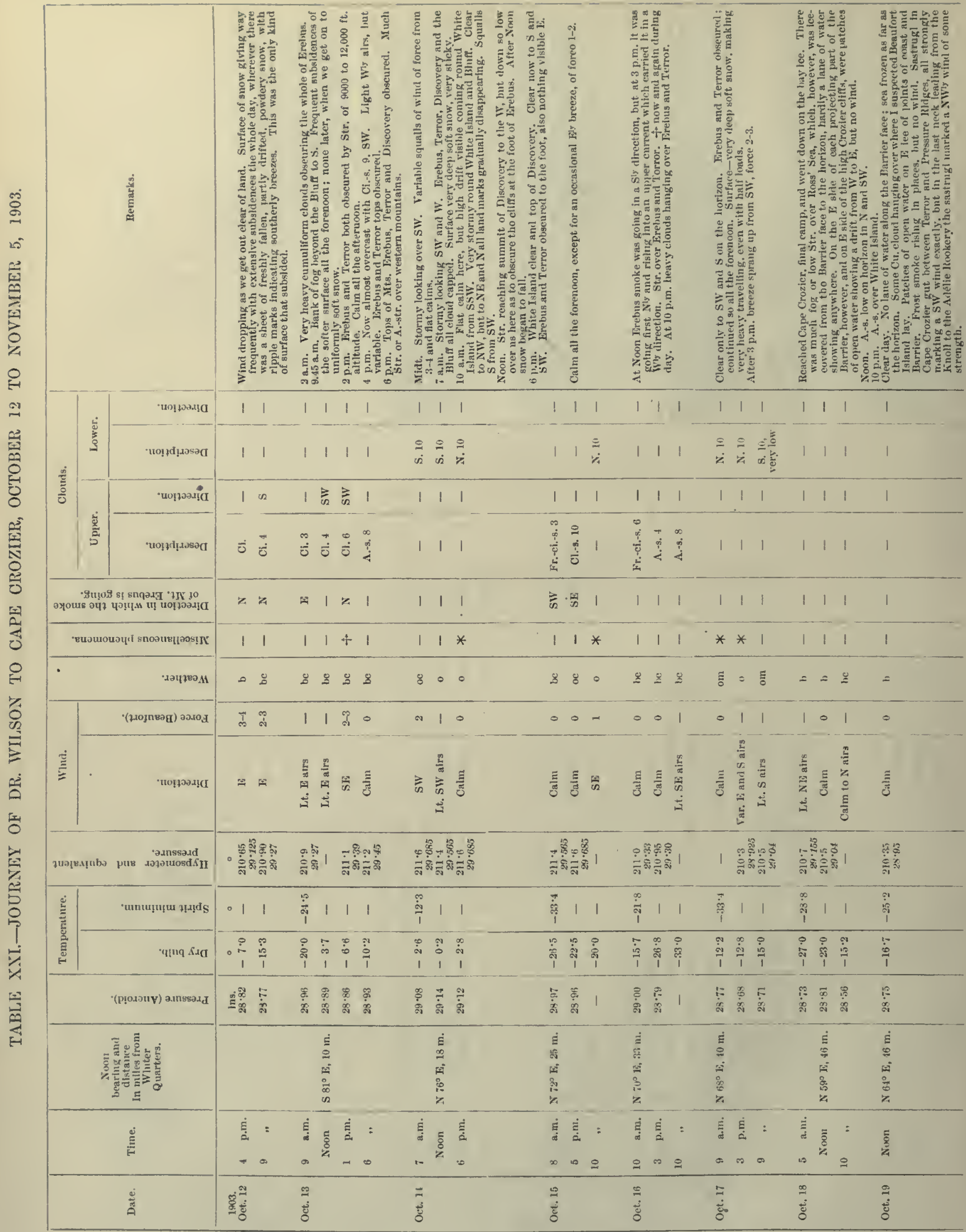



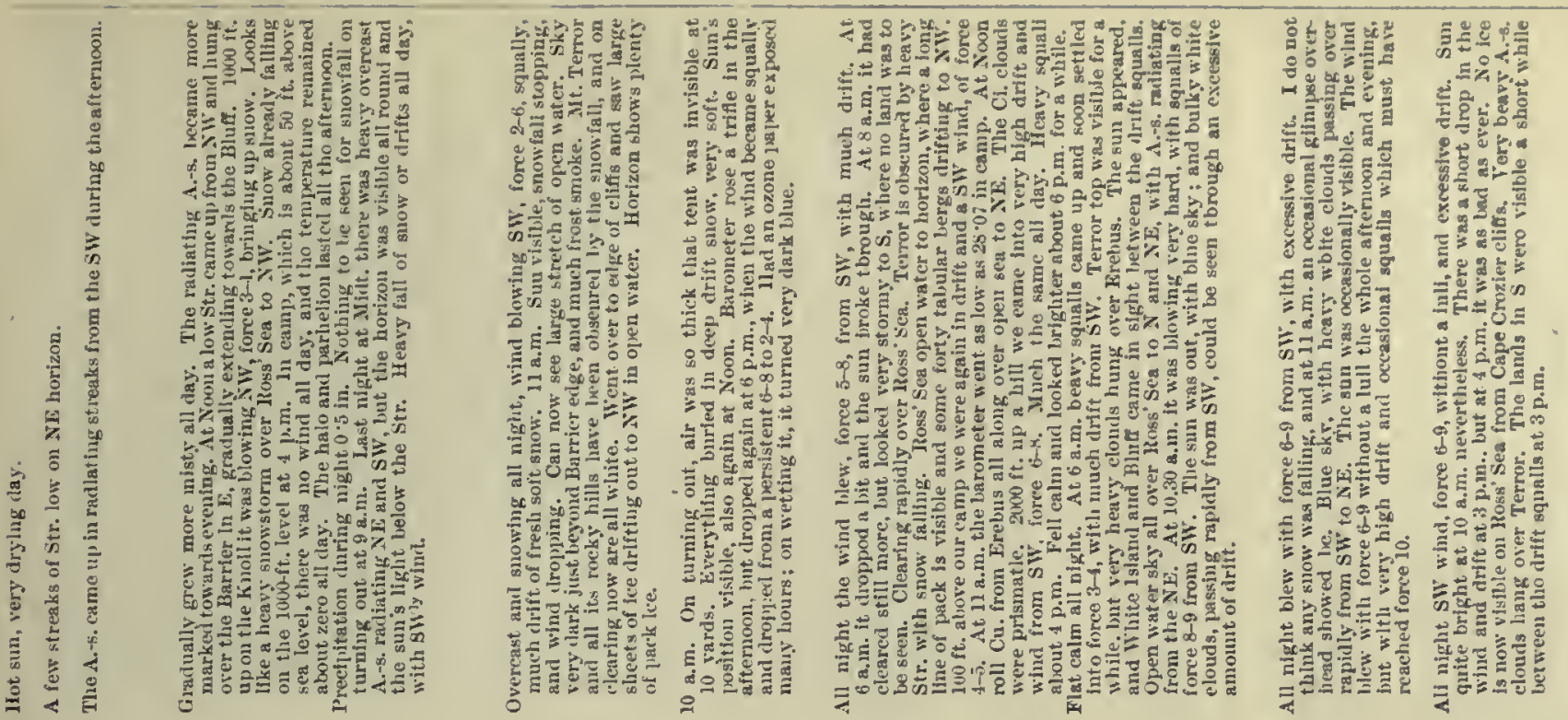

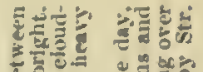

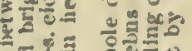

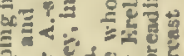

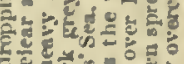

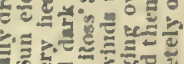

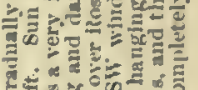

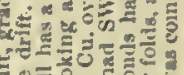

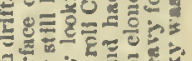

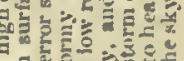

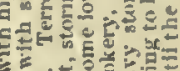

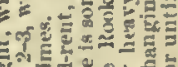

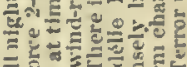

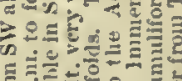

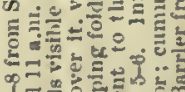

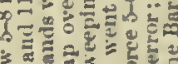

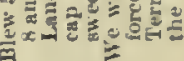

1

I is

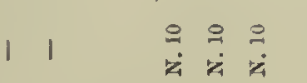

$\div \% ㅇ$

क । ।

$\infty \quad|\quad|$

l 1

$\begin{array}{llll}1 & 1 & \infty & 1\end{array}$

1 1 1

$\begin{array}{llllllllll}1 & 1 & 1 & 1 & 1 & 1 & 1 & 1 & 1 & 1\end{array}$

$\geqslant 11$

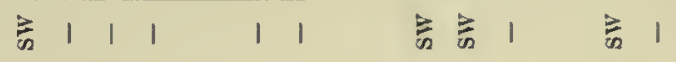

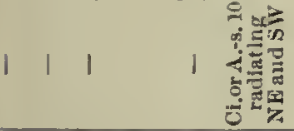

। 1

111

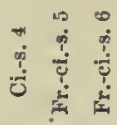

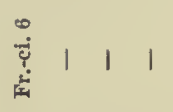

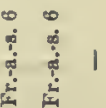

$\stackrel{\infty}{\infty}$

空

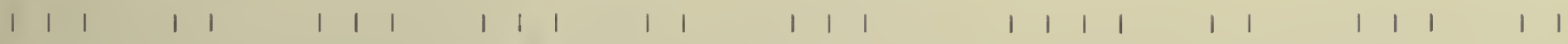

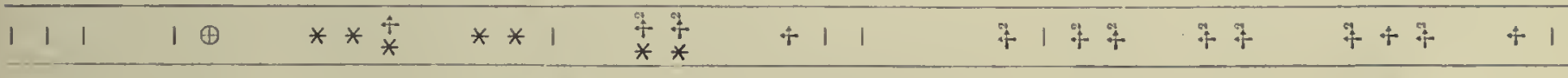

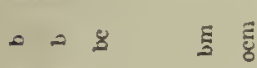

$8 \circ$ \& 8

$\& \nsubseteq \&$

¿ 111

1 1

\&० \&8

$-110$

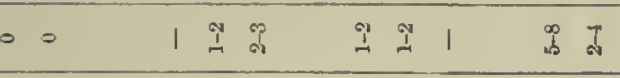

ฐ ก

\& 11 \&

is is

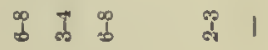

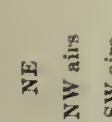

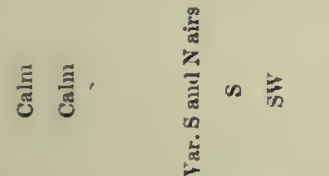

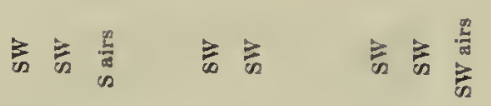

总人1言亮言

言紊言言紊

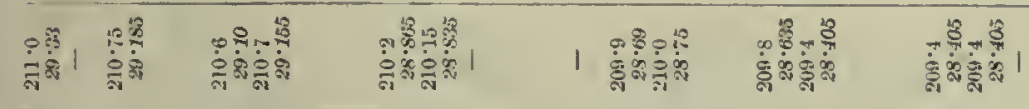

\section{(1)}

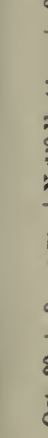

(n)

i ,

(a)

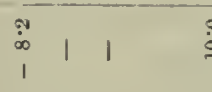

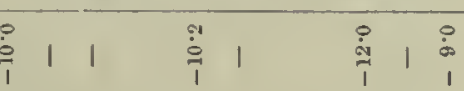

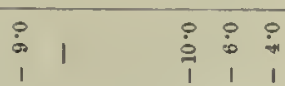

(a)

4

in:

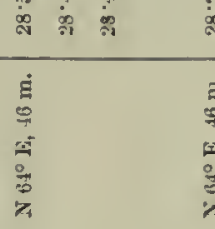

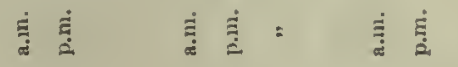

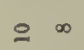

-..

$=\cdots$

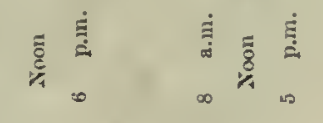

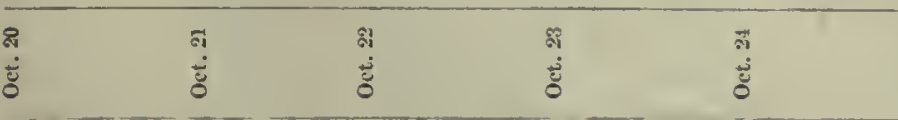

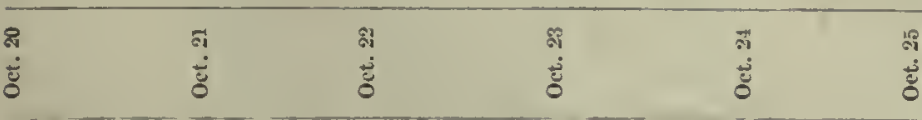

\begin{tabular}{|c|c|}
\hline$\dot{\Xi}$ & छٌ \\
\hline$=$ & I" \\
\hline in & 앰 \\
\hline 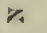 & $r_{1}$ \\
\hline
\end{tabular}

\&

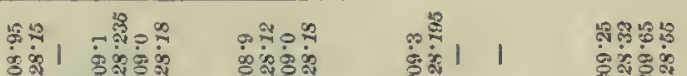

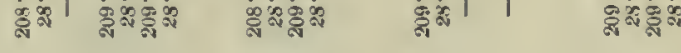




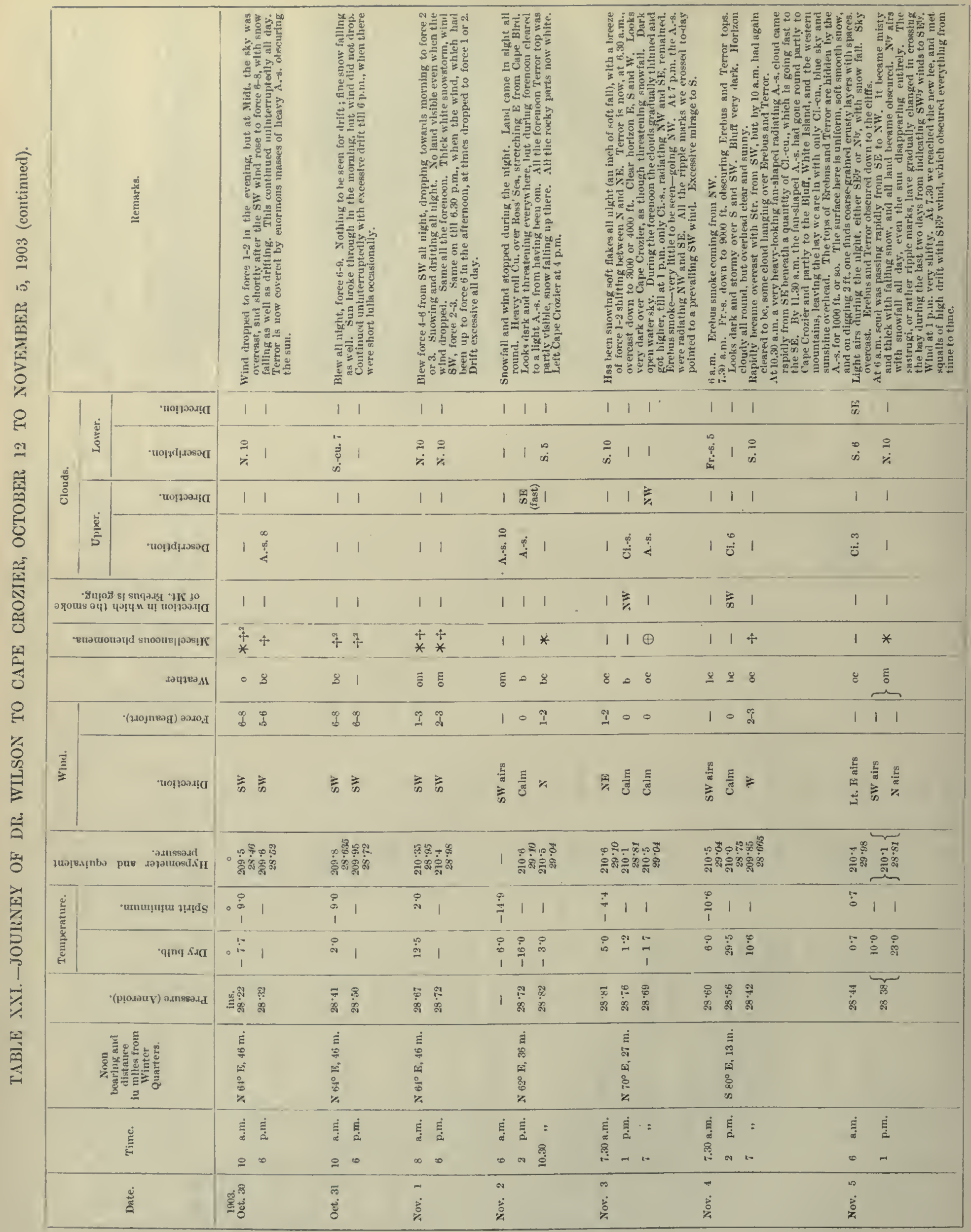




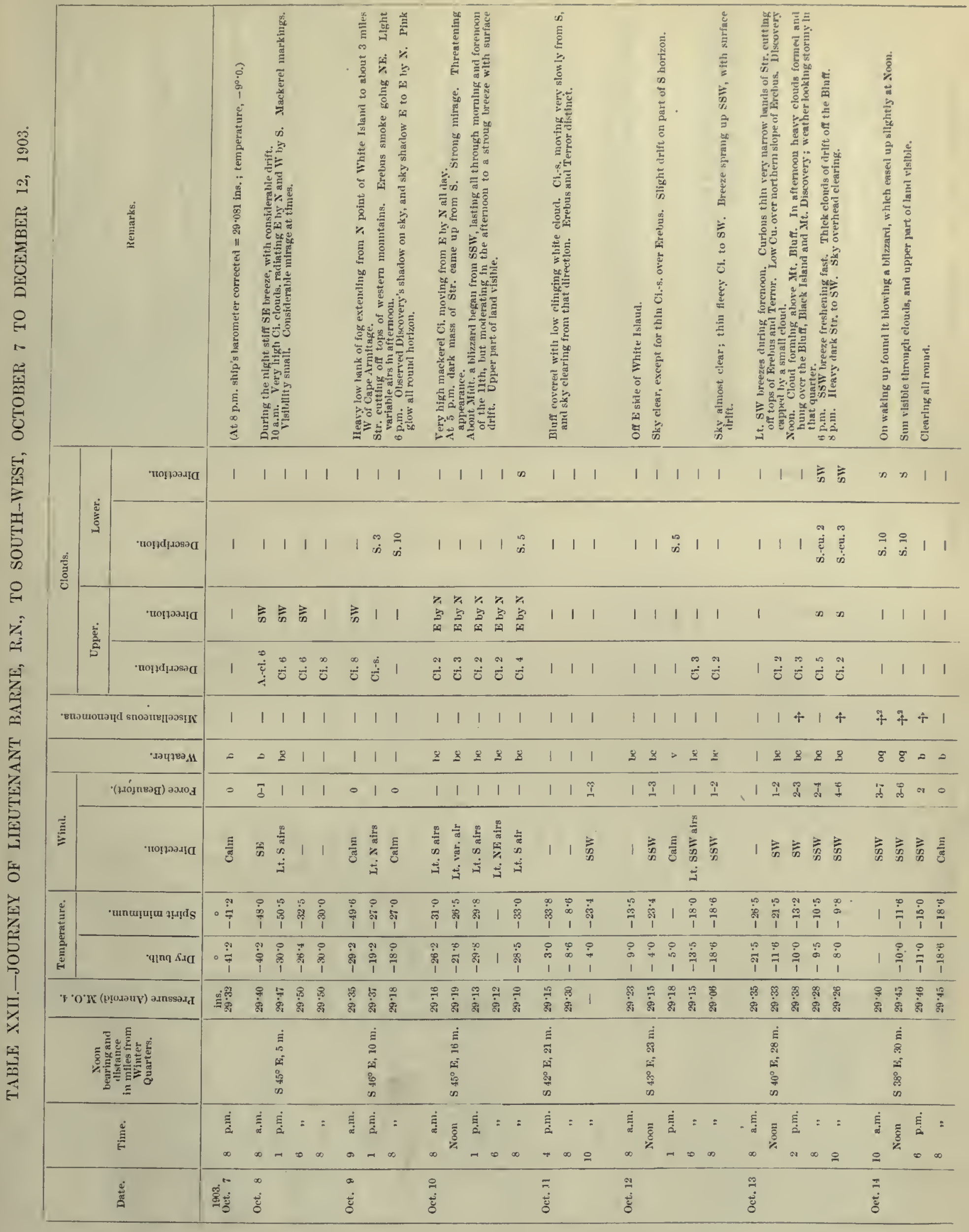




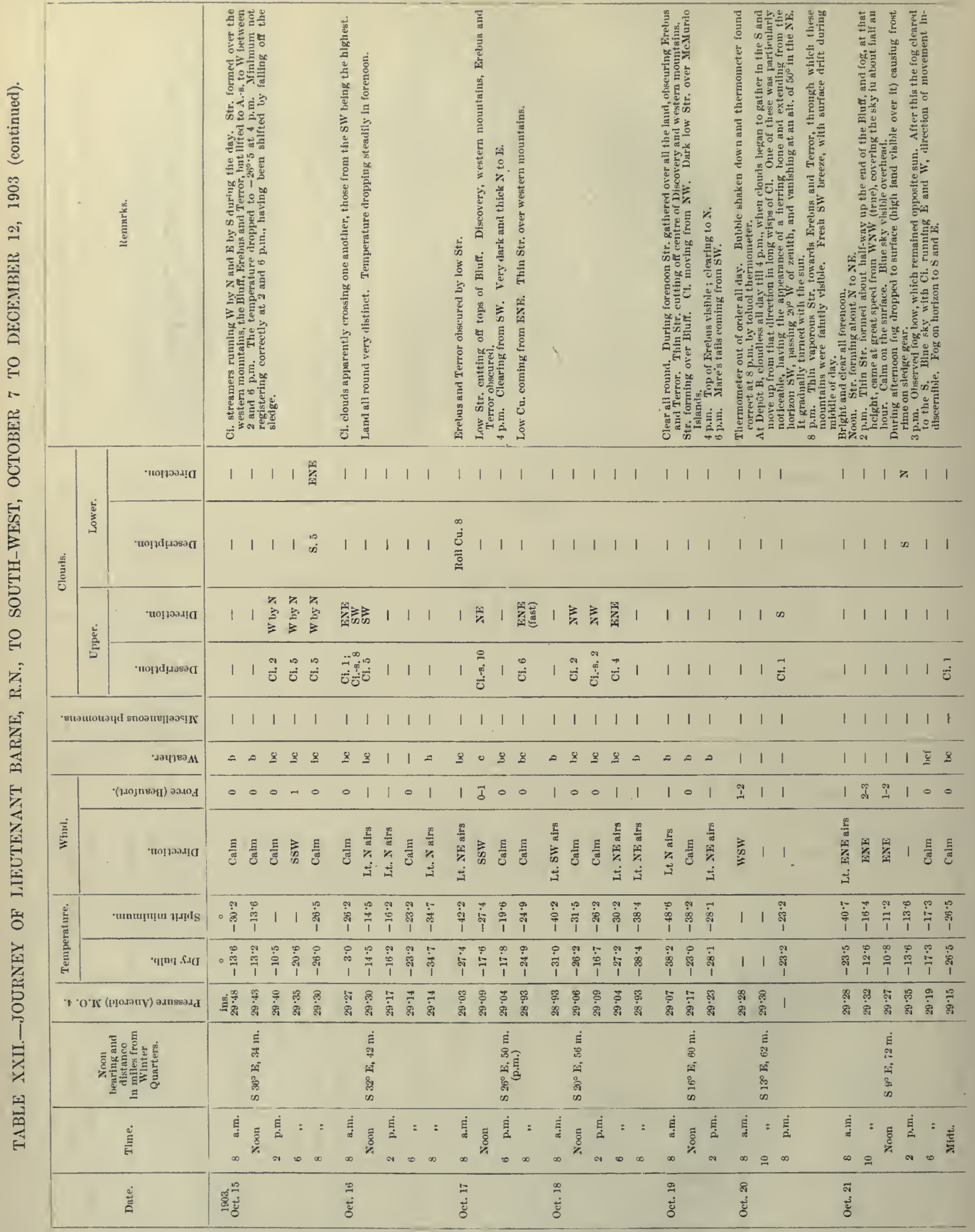



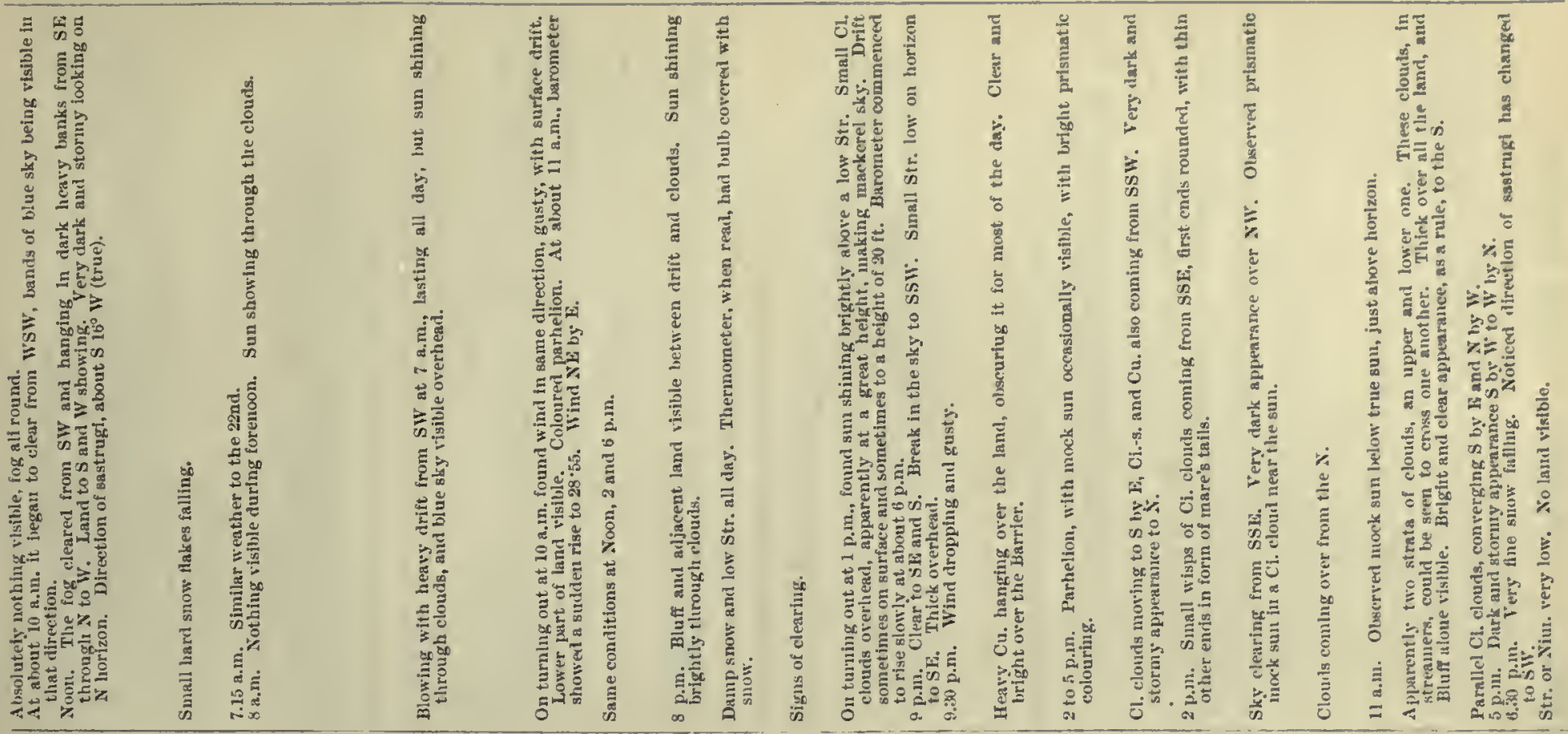

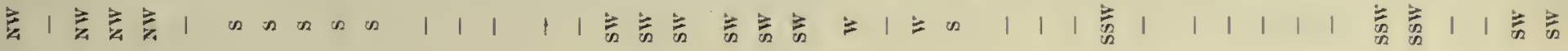

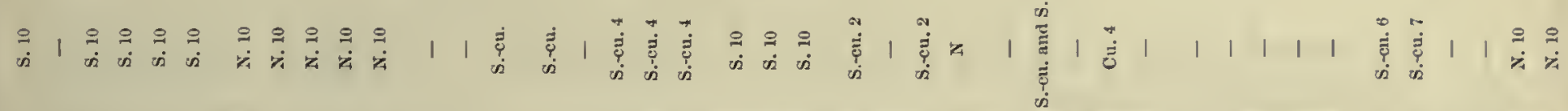

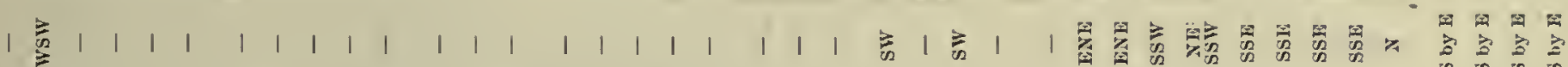

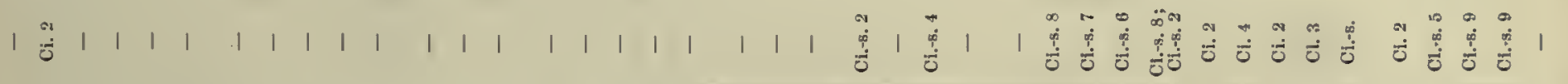

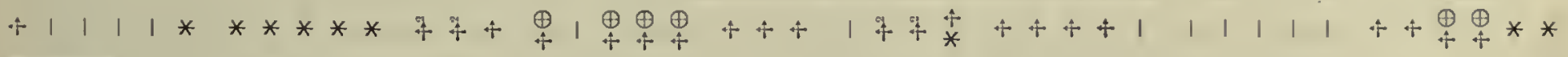
б

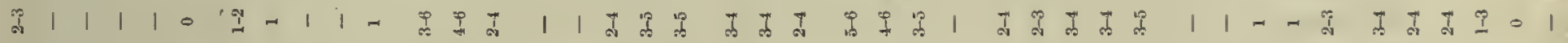

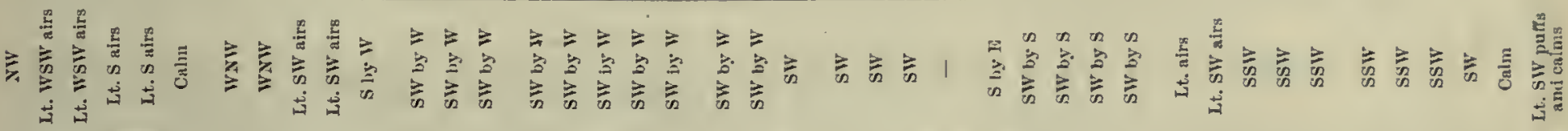

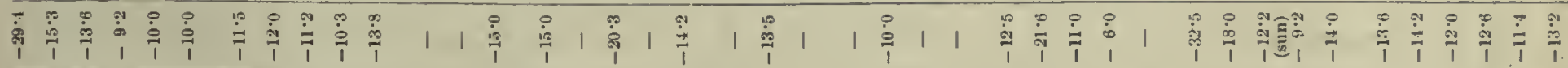

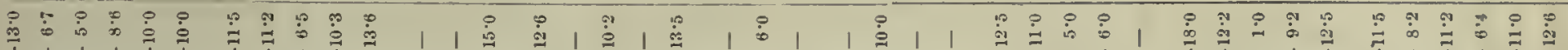

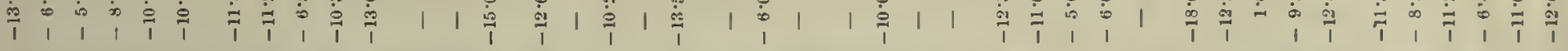

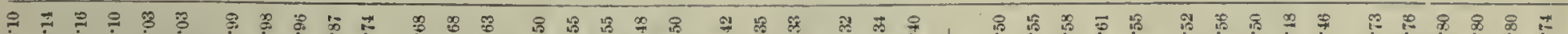

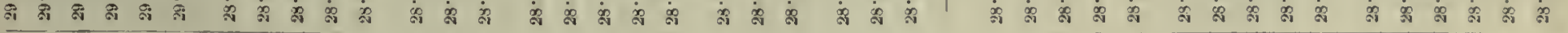

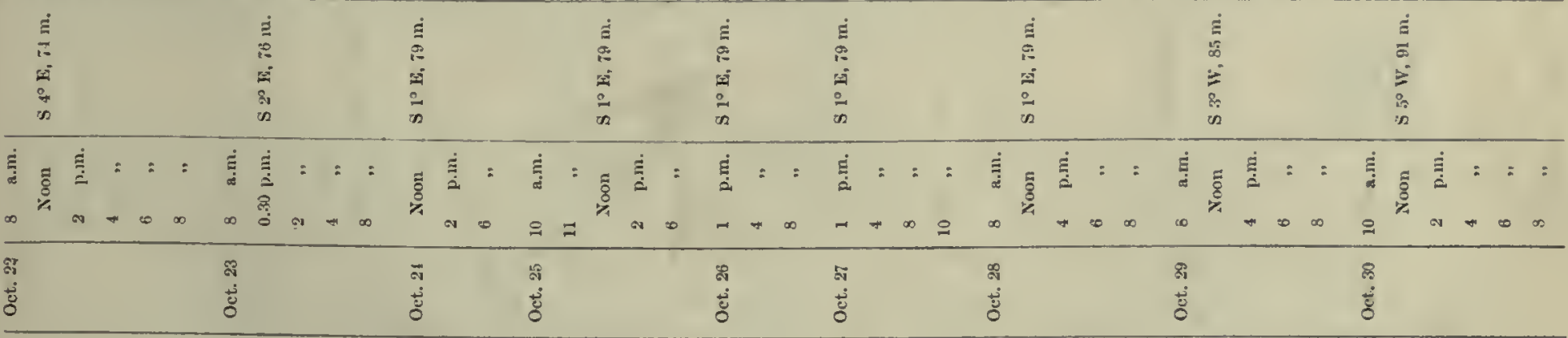




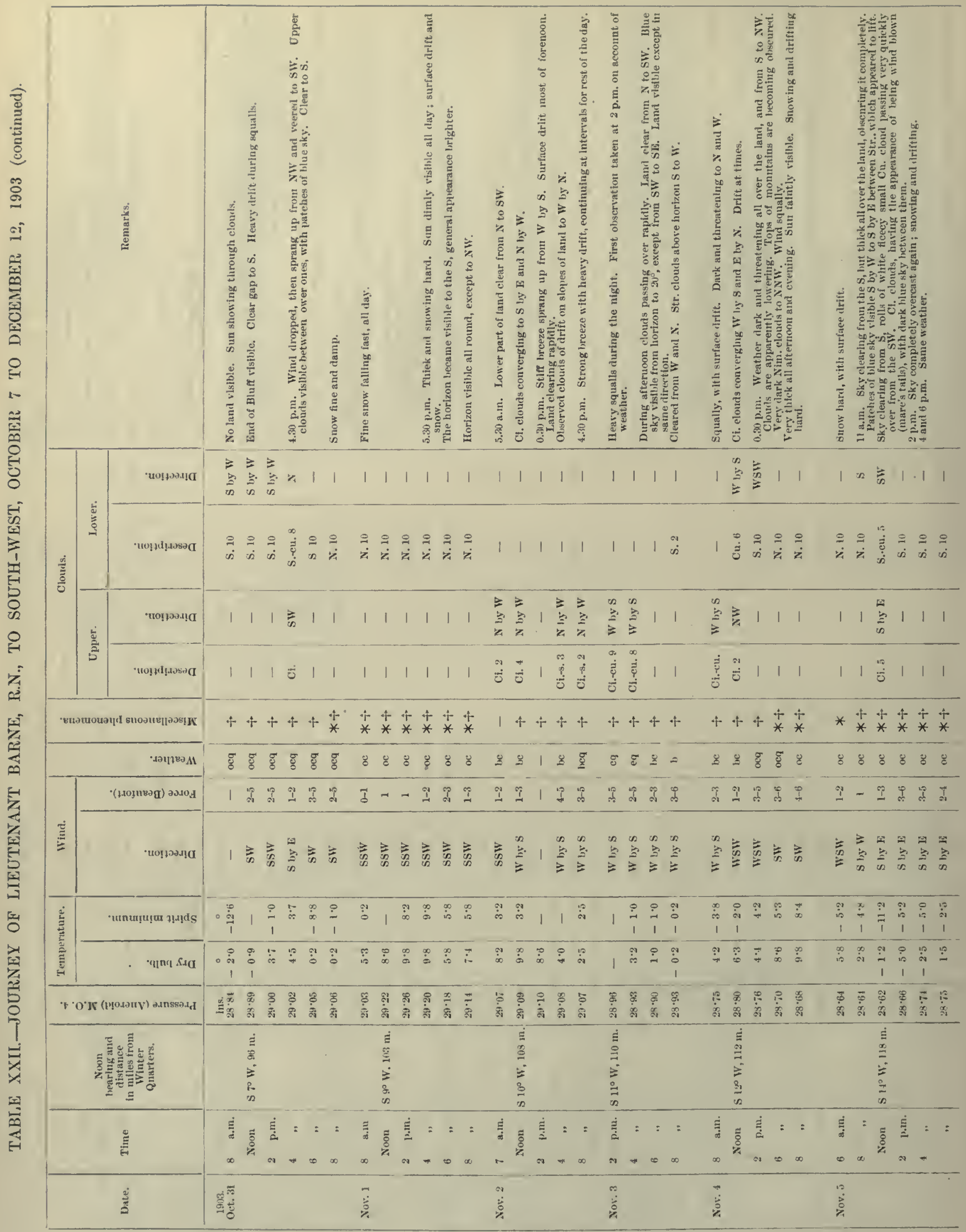




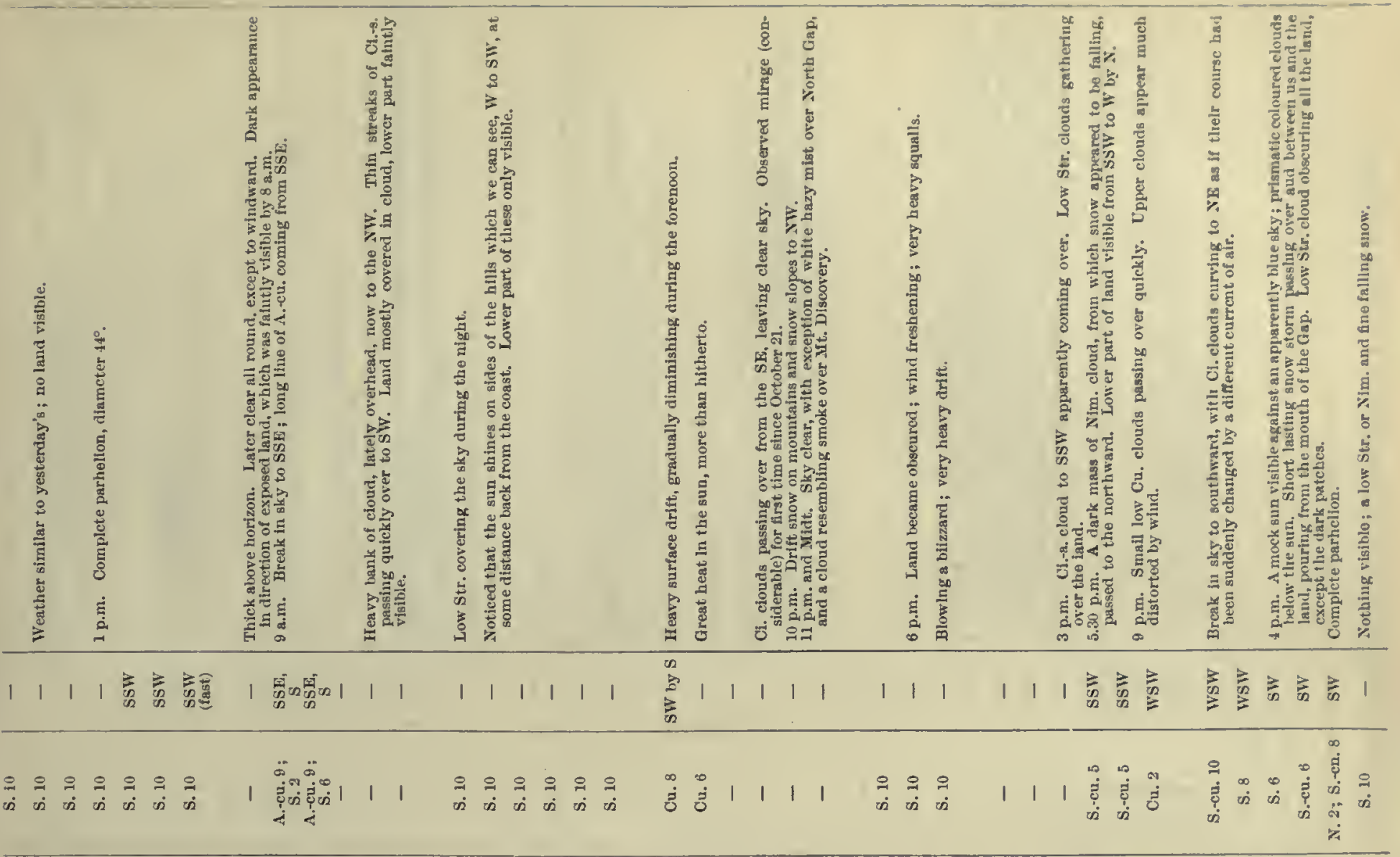

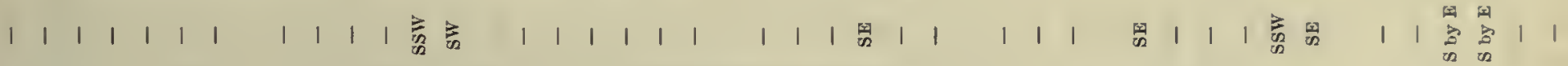

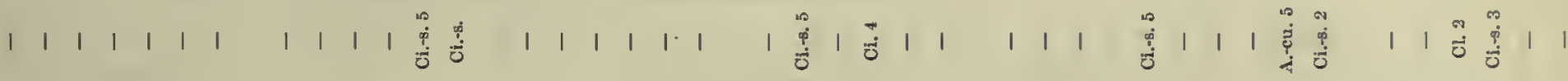

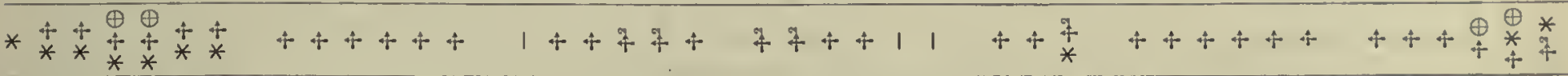

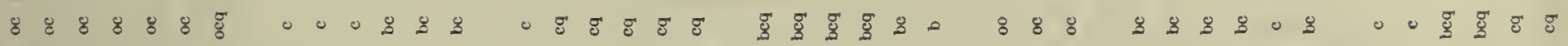

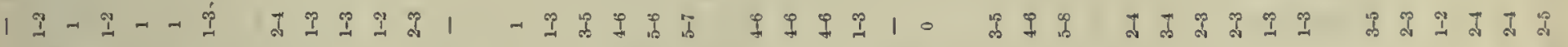

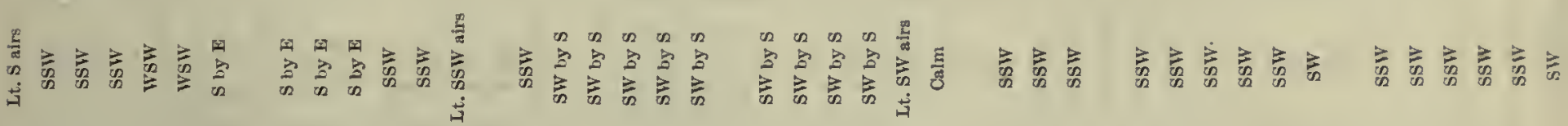

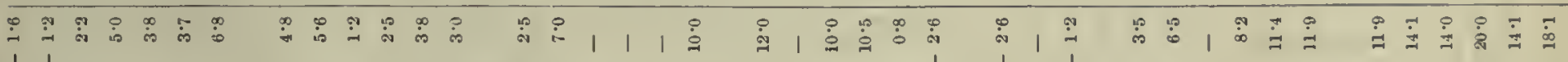

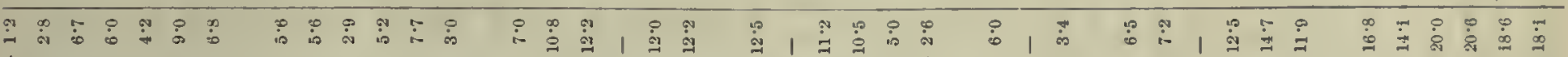

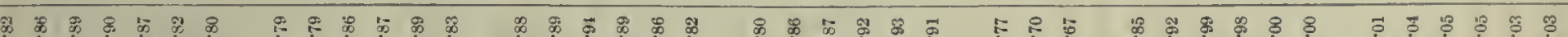

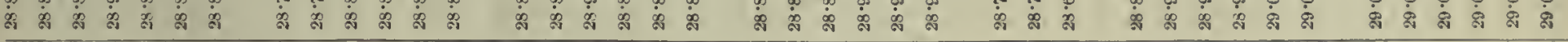

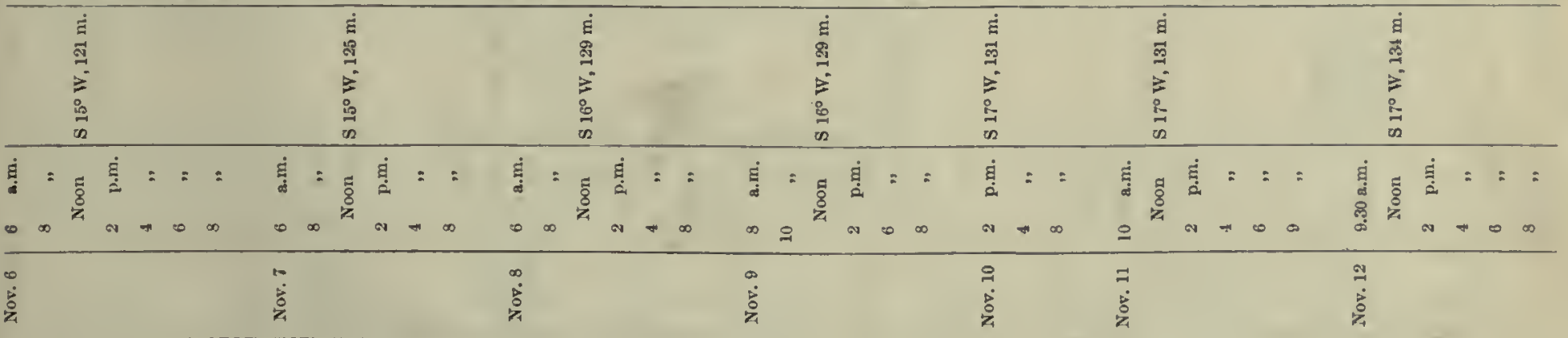




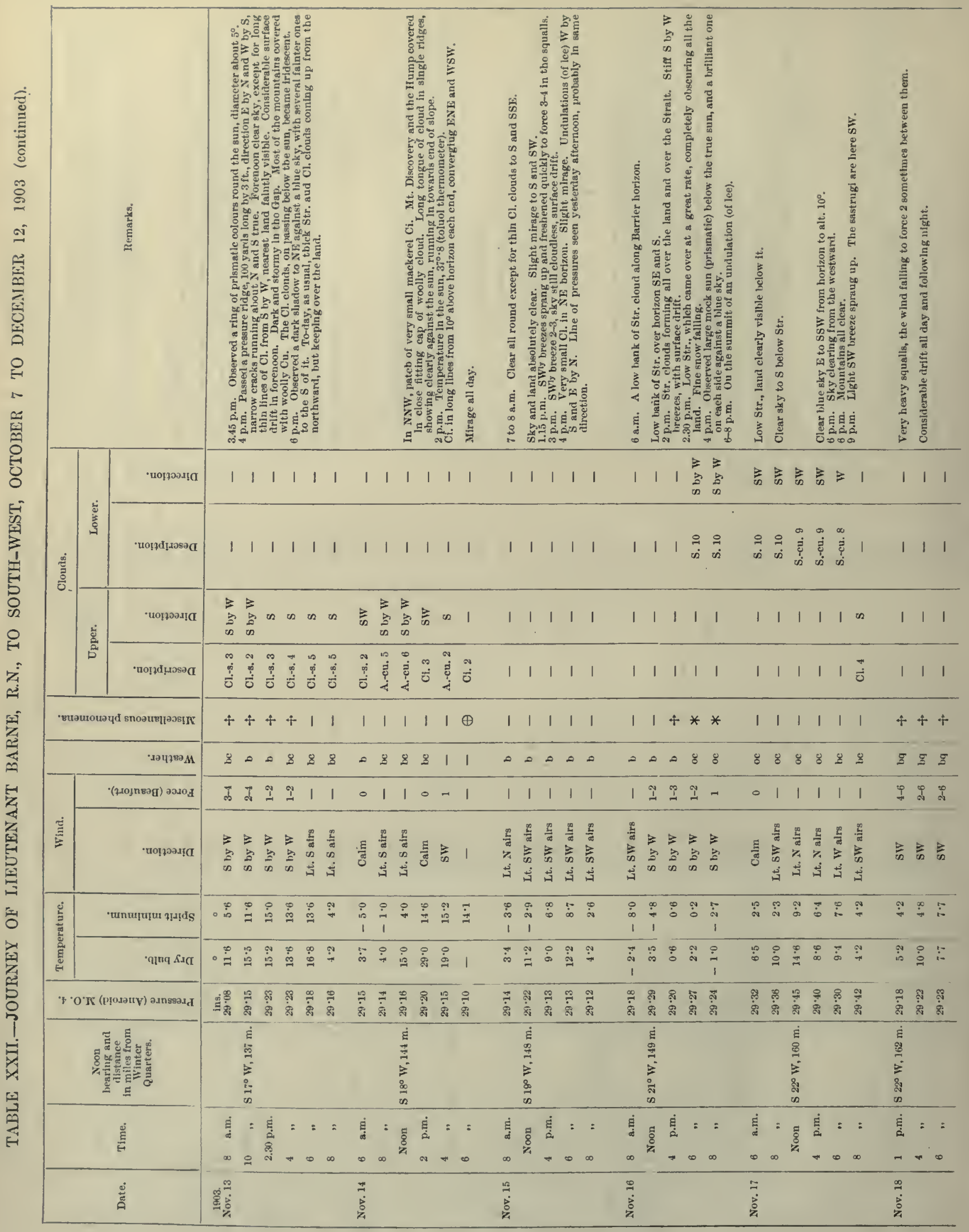




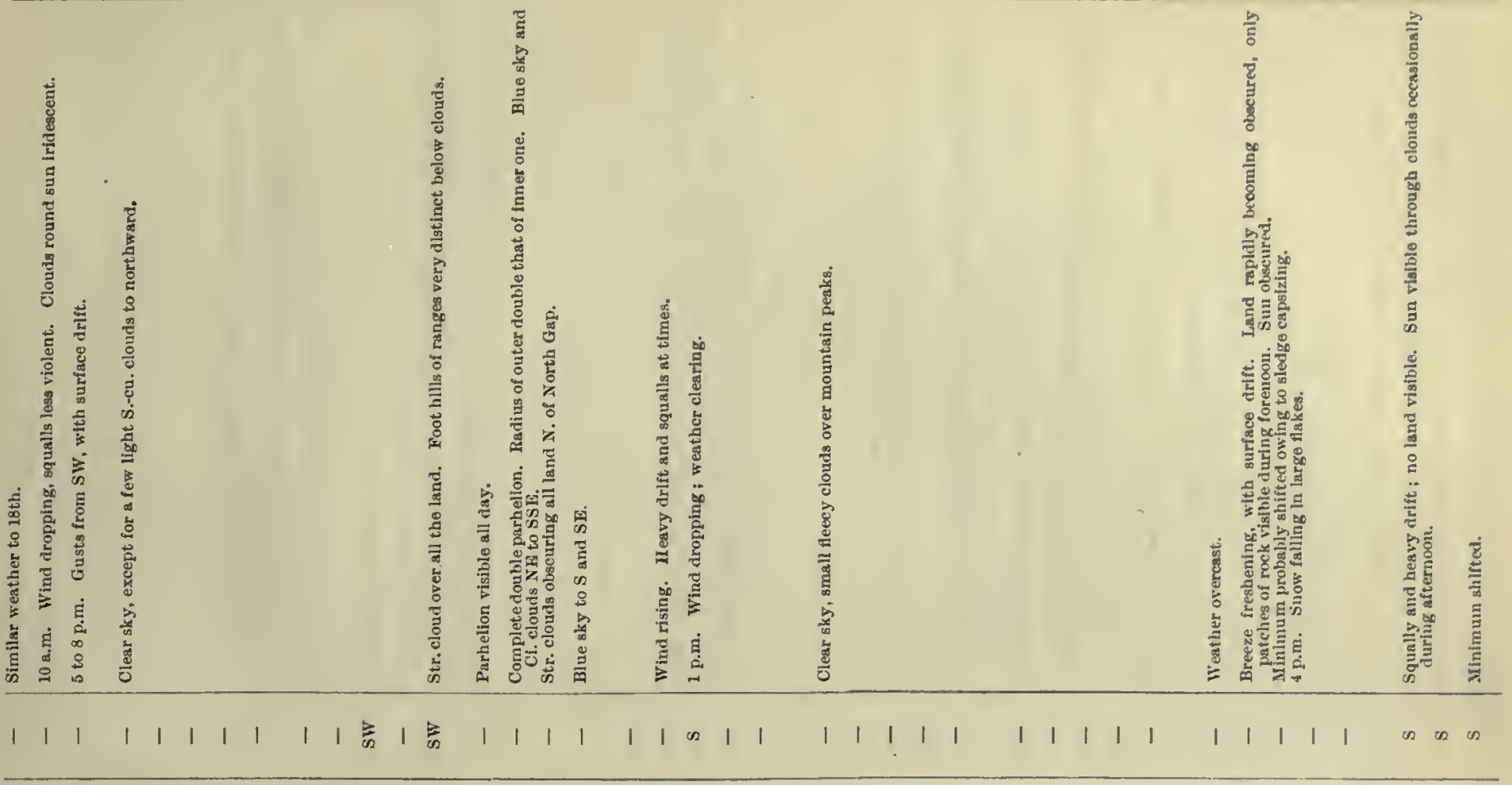

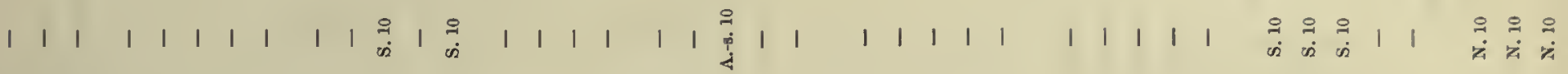

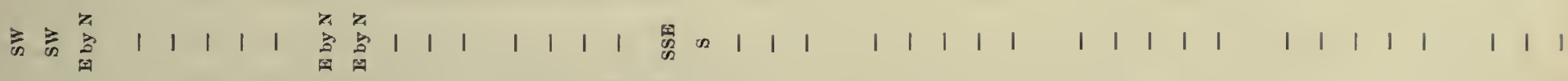

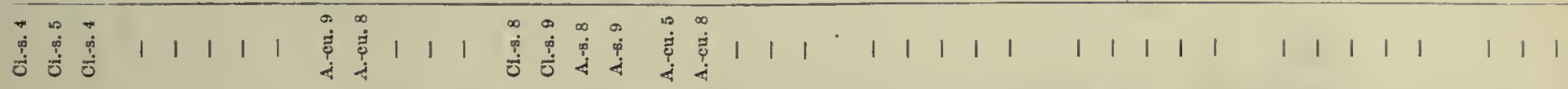

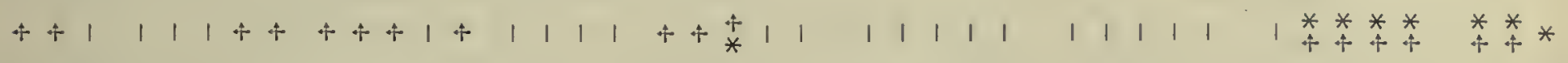

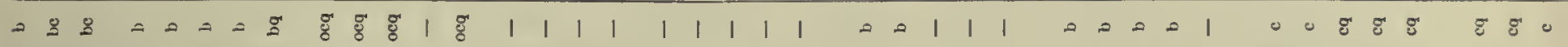

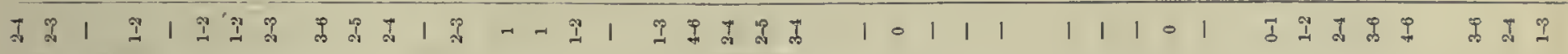

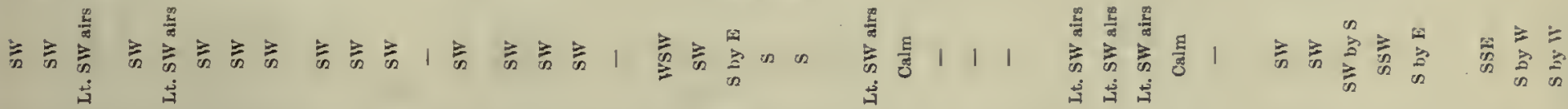

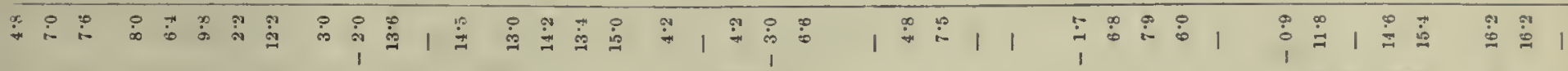

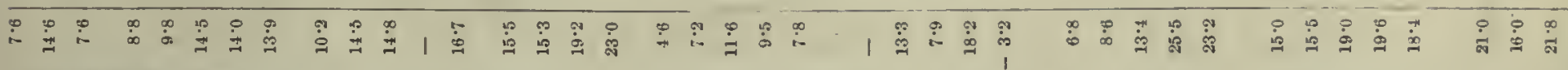
ชุำ

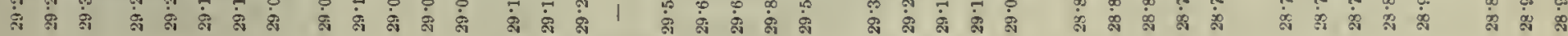

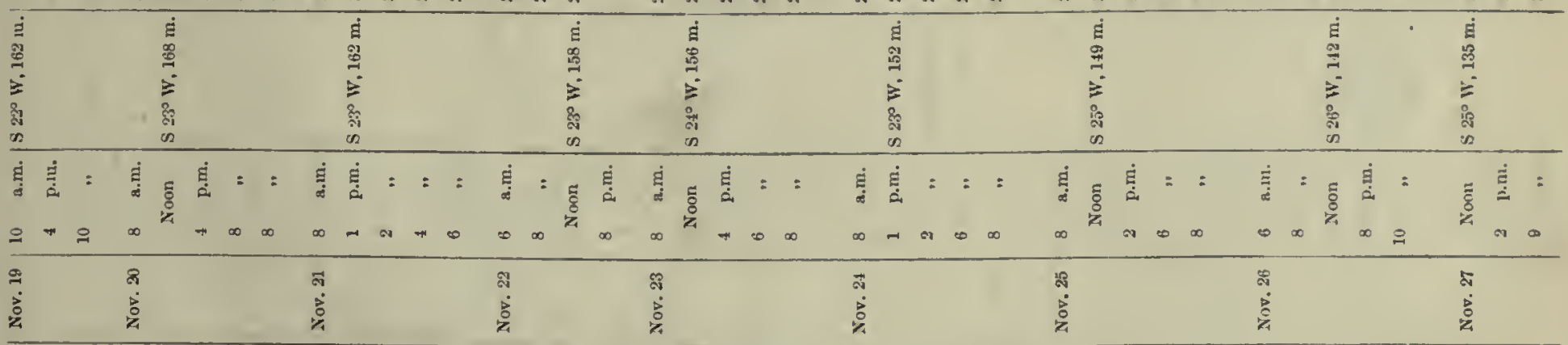




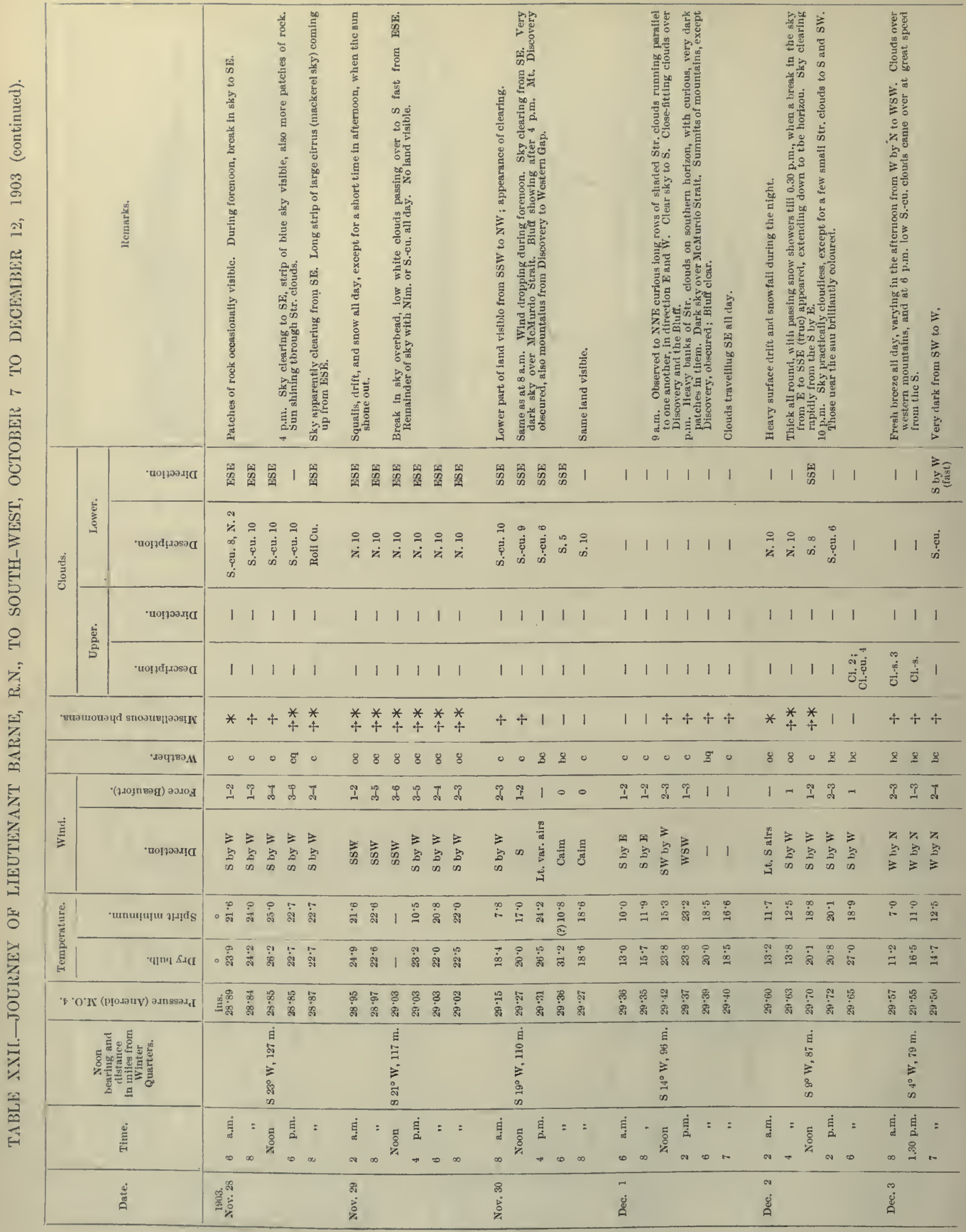




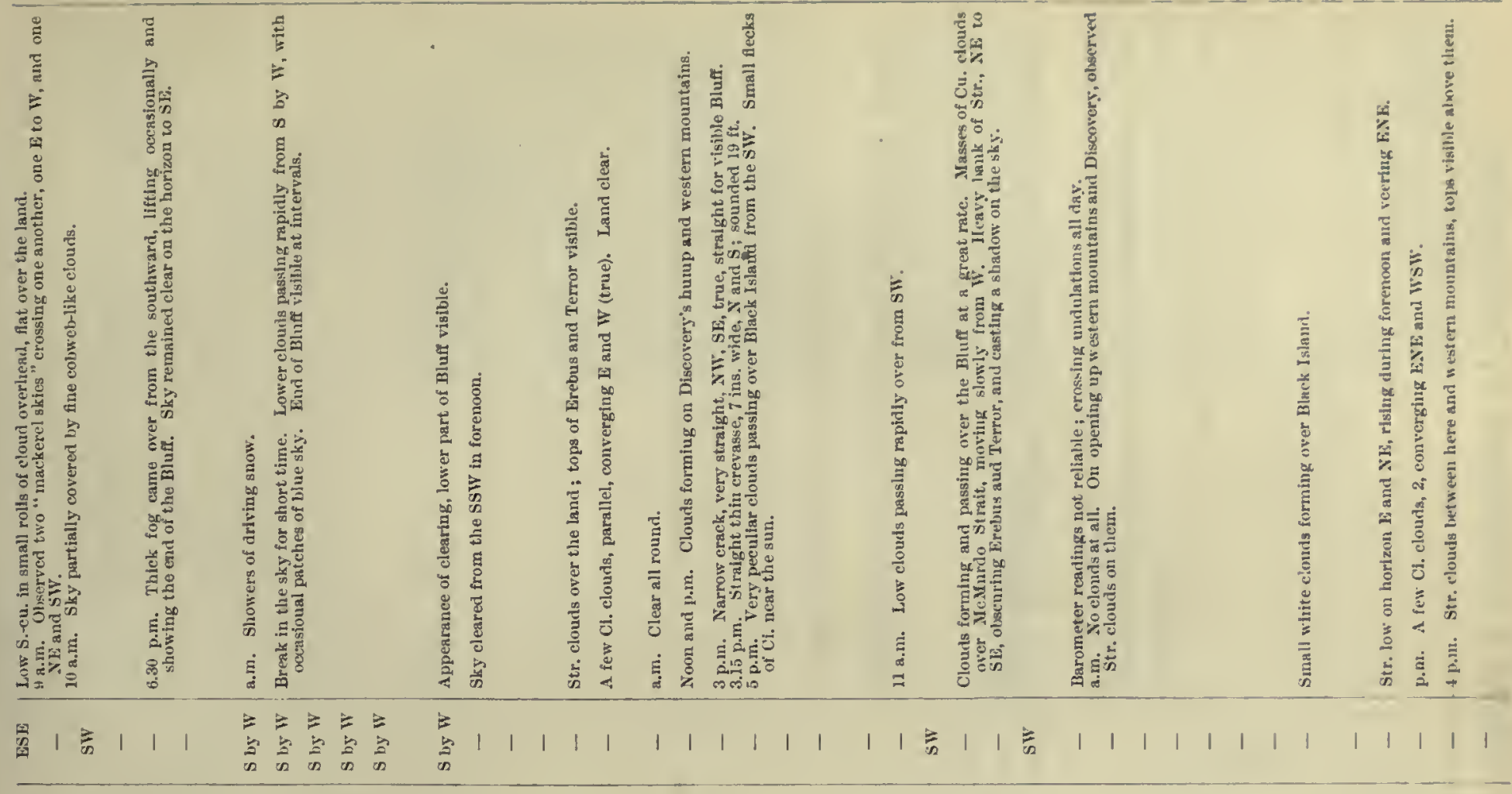

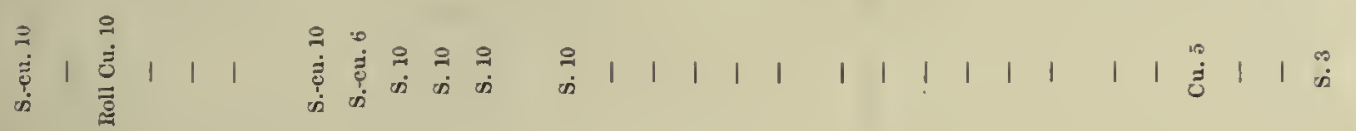

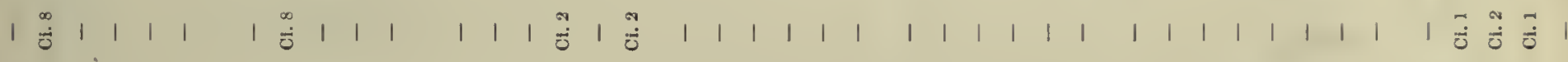

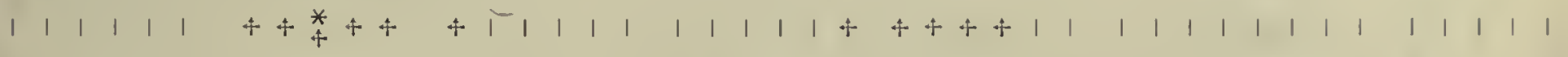
:

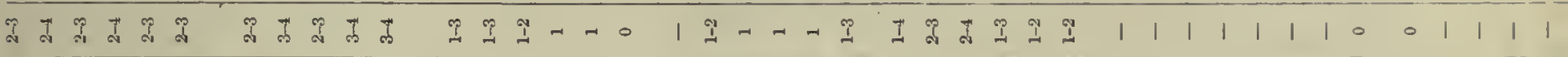

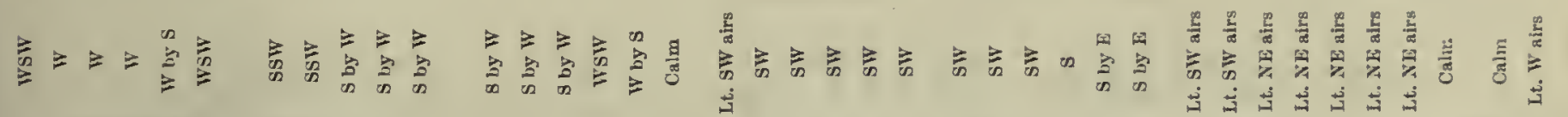

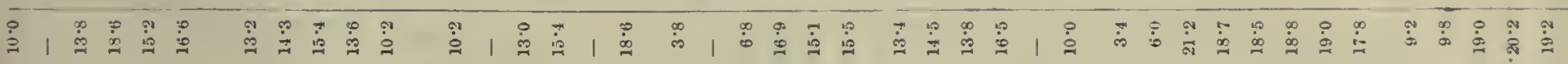

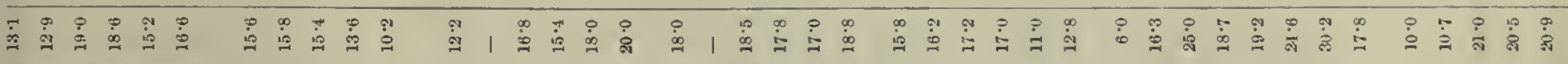

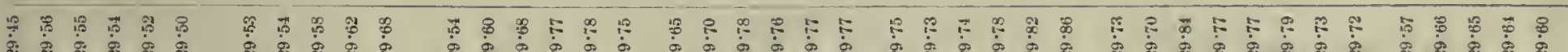

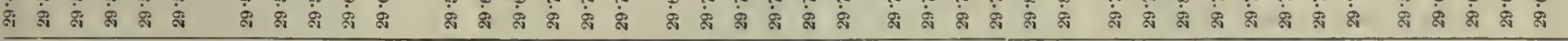

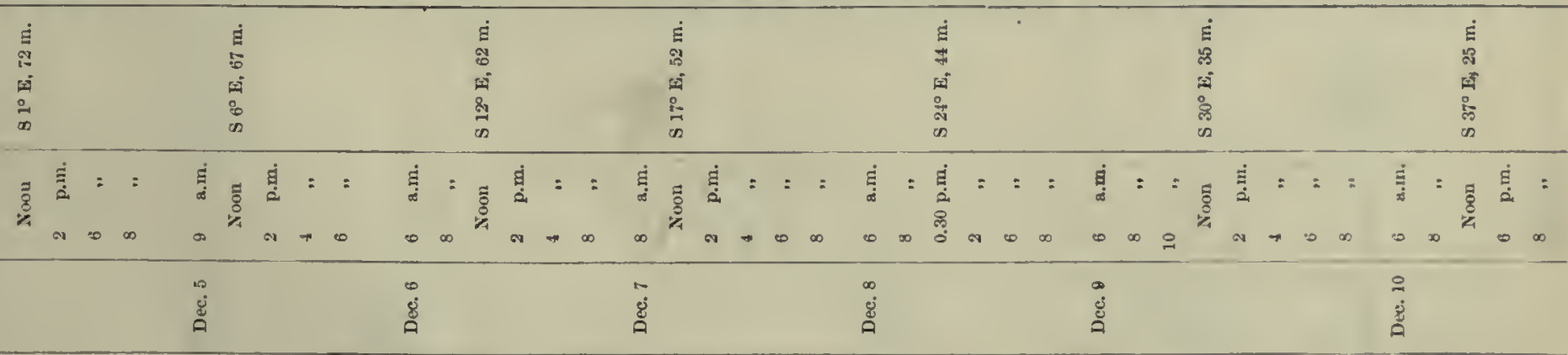




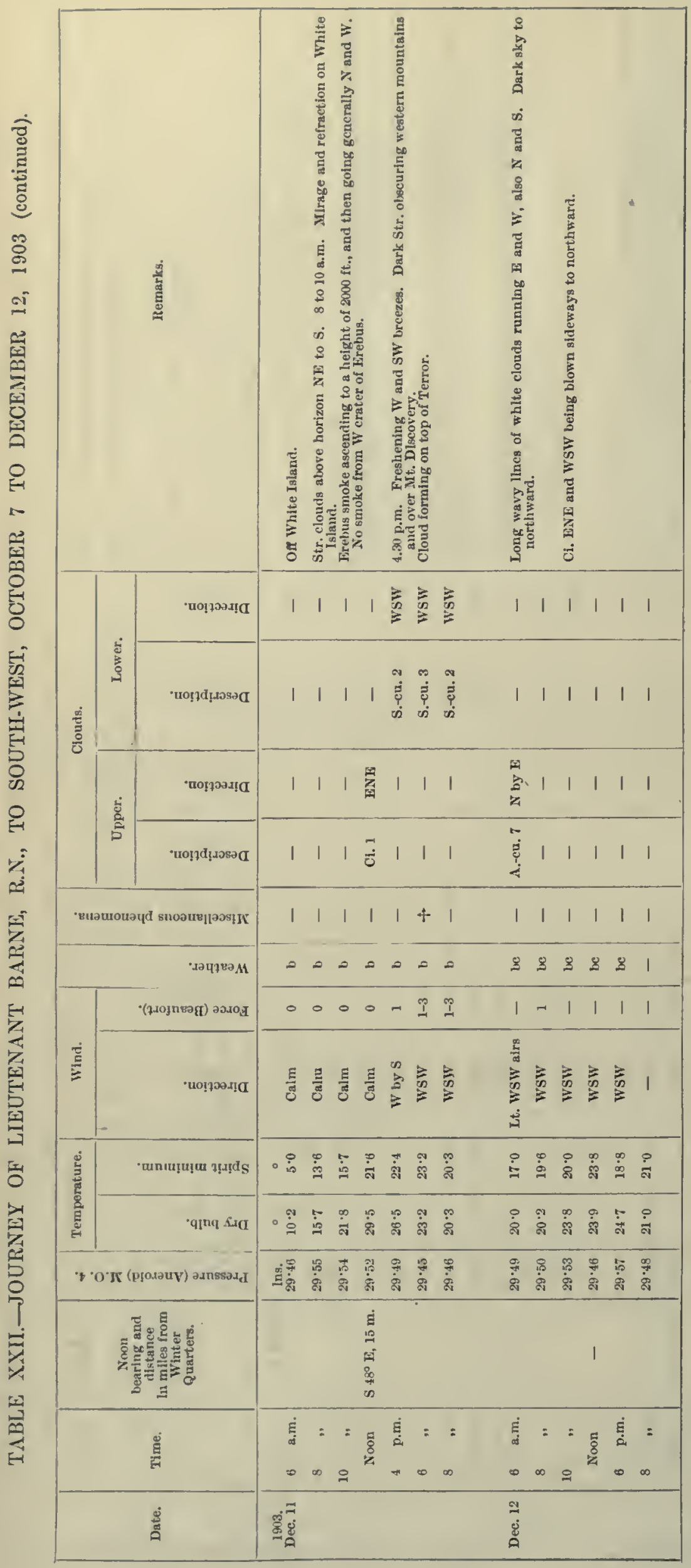




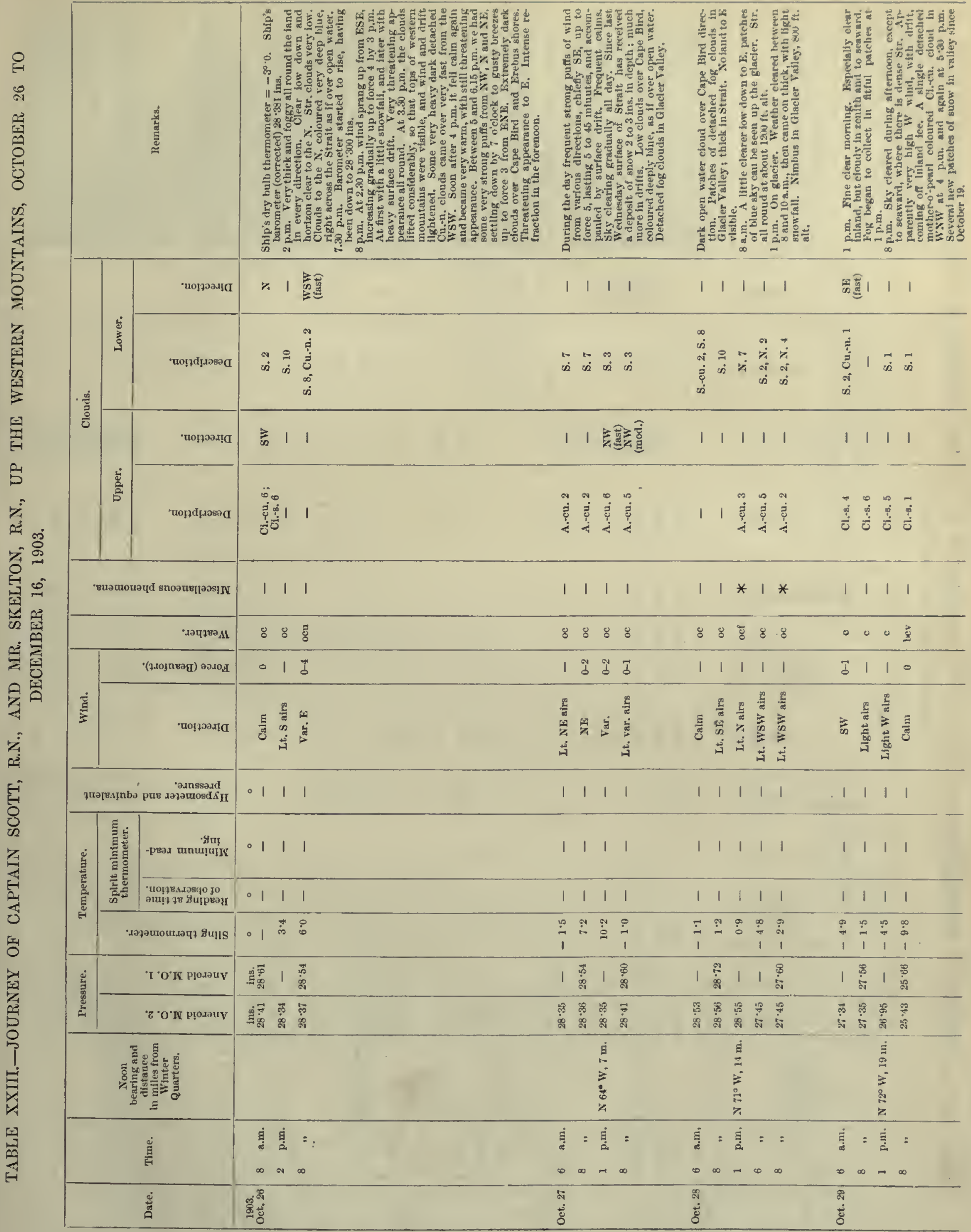




害

궁

논

एक

है

乙

芒

5
0
0
2
2
2
2

5.

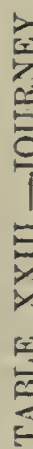

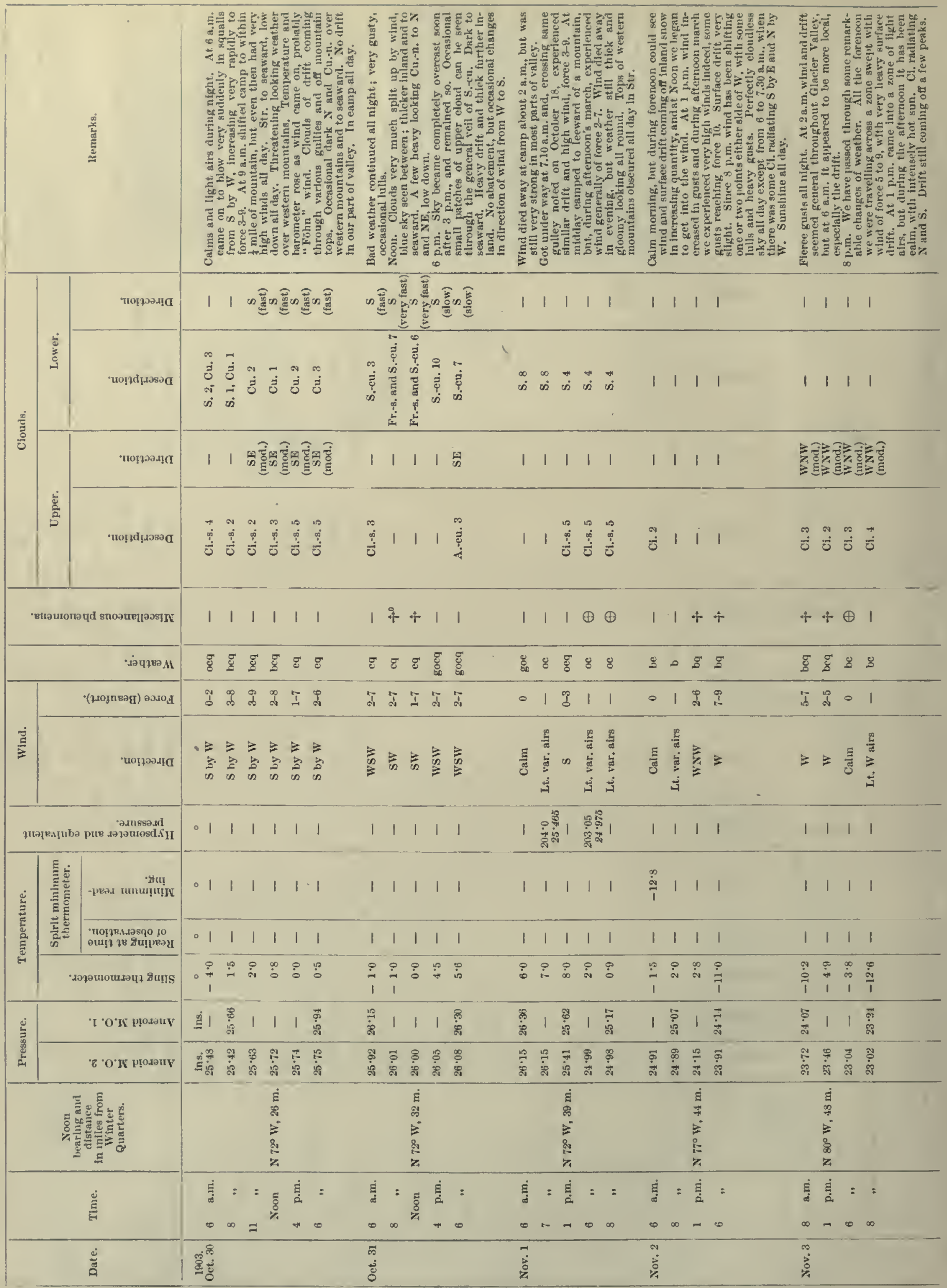




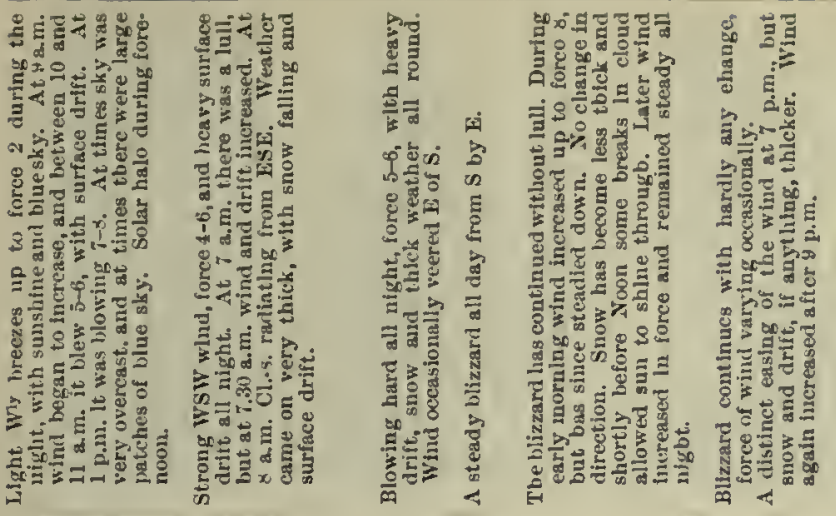

1 需 1

\begin{abstract}
1
\end{abstract}

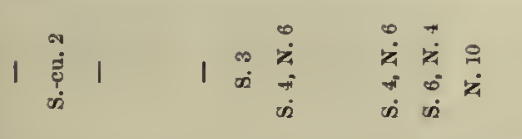

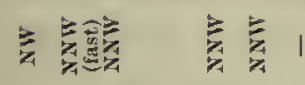

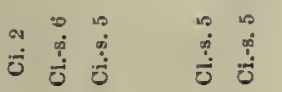

$1+$

$+7$

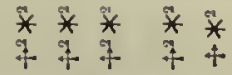

* *

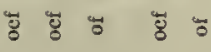

ำ ใิ

- \&

ㅁำ

言咅言

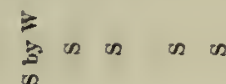

क $\frac{1}{3}$ क⿺

ป⿱

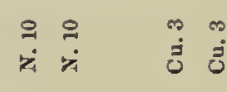

$1 \%$

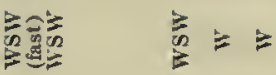

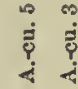

77

$77+$

$+7+7+9$

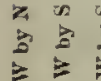

㩆

1

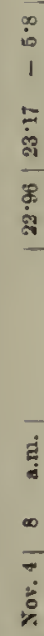

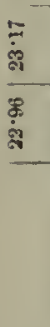

i i 1

\begin{tabular}{lllll}
1 & 1 & 1 & 1 & 1 \\
\hline
\end{tabular}

$1+1$

$\begin{array}{lllll}1 & 1 & 1 & 1 & 1\end{array}$

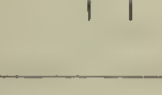

\begin{abstract}
.1.
\end{abstract}

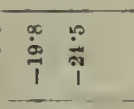

$1 \frac{8}{2}$

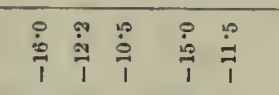

1111

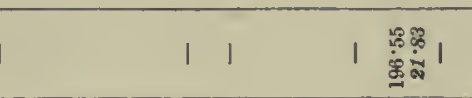

喜高竞言言

言言嗙言

言人11 1

$\begin{array}{lllll}3 & 1 & 1 & 1 & 1\end{array}$

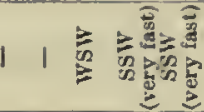

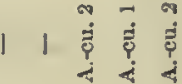

$80 \& 5$

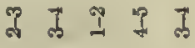

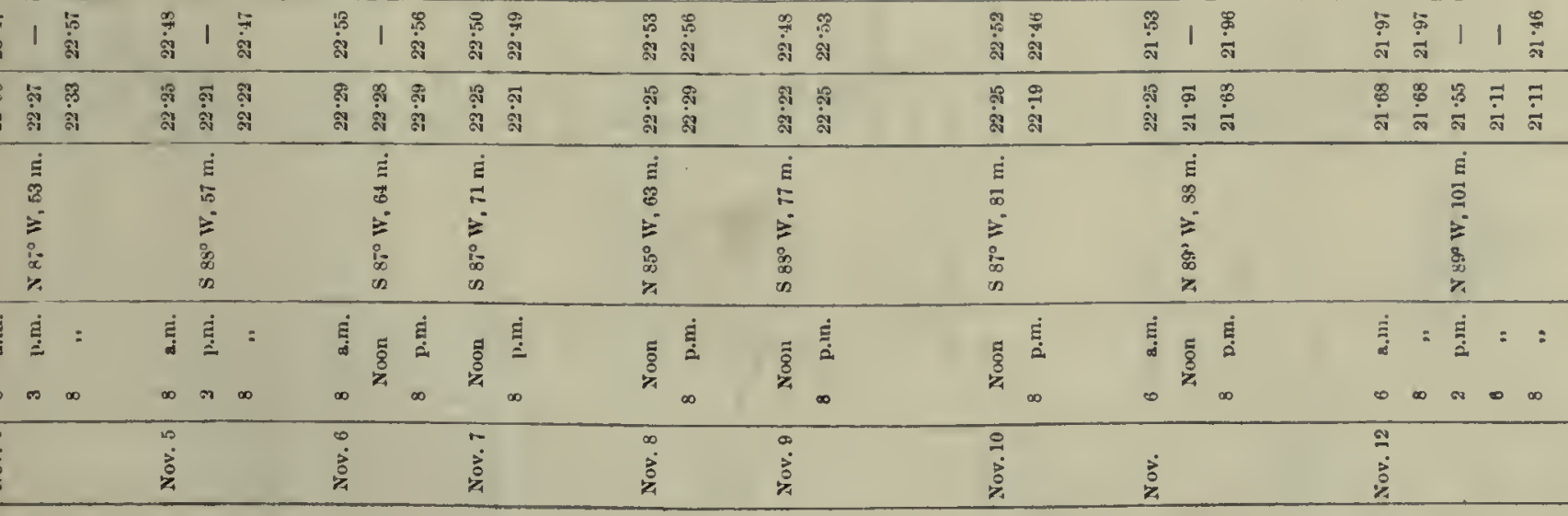

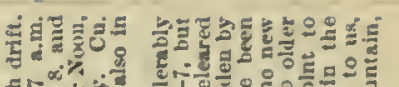

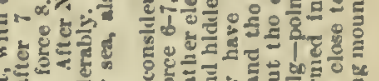

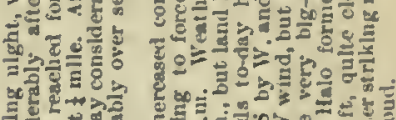

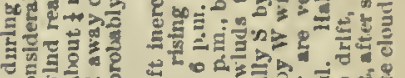

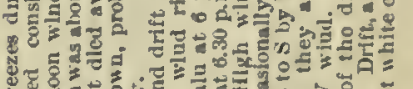

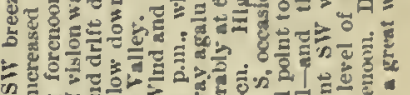

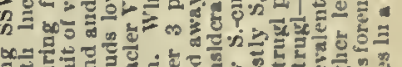

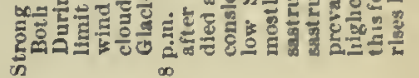




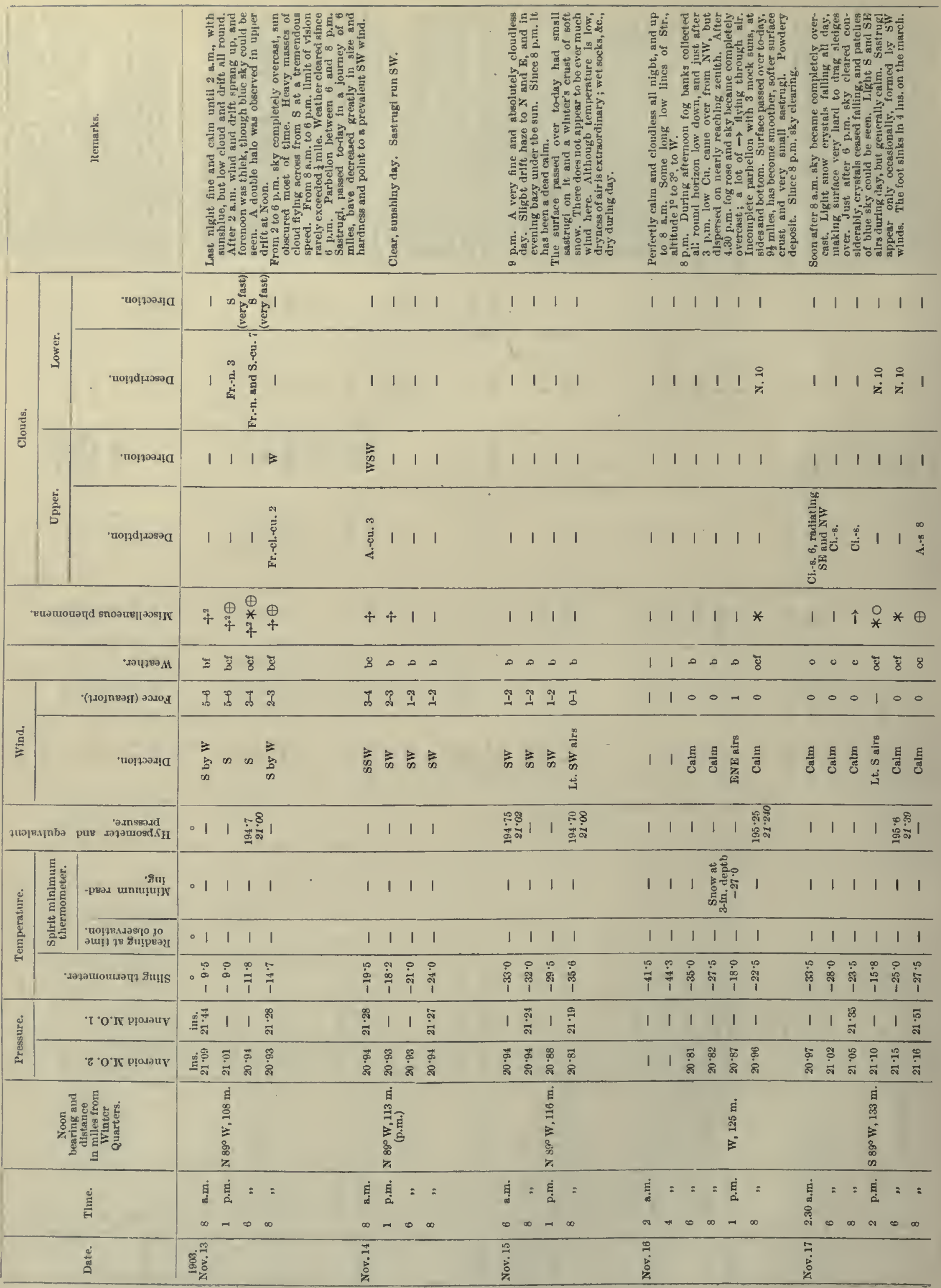



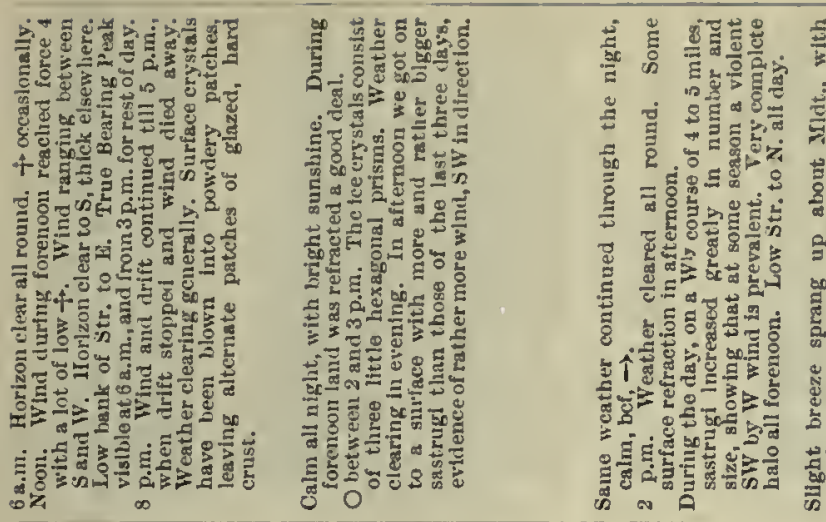

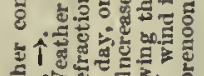

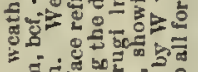

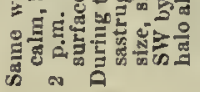

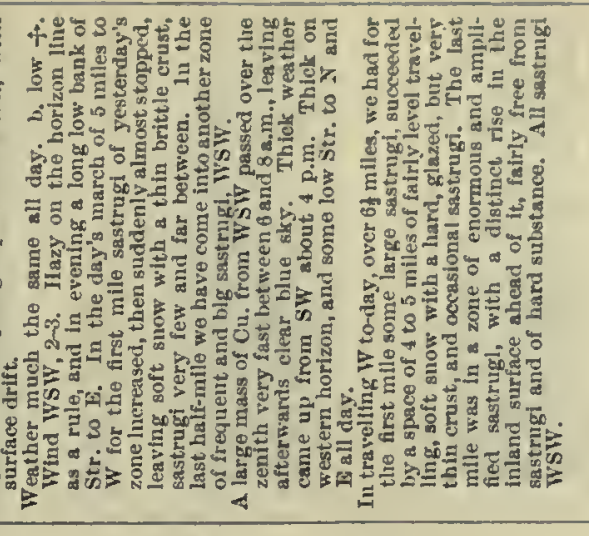

重高卜1

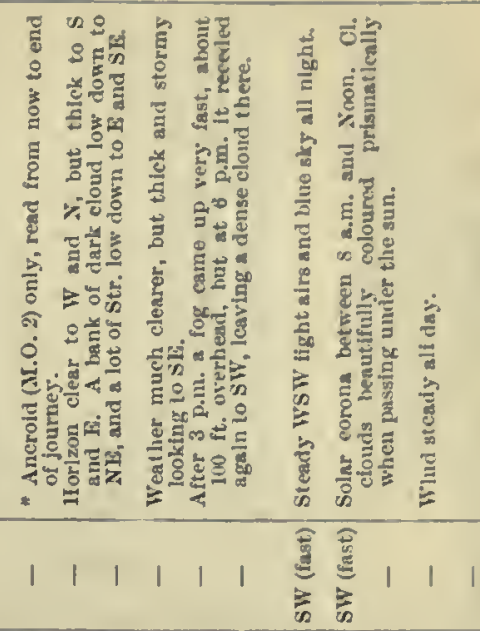

章言
$111 \geqslant z=1 \geqslant 1 \geqslant 1 \geqslant 1$

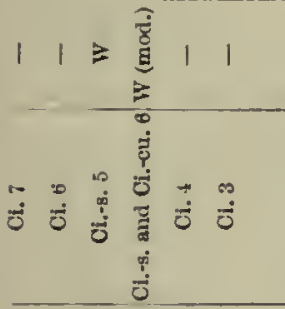

$111+1$

000

ป ติ ติ

$$
\text { ( }
$$

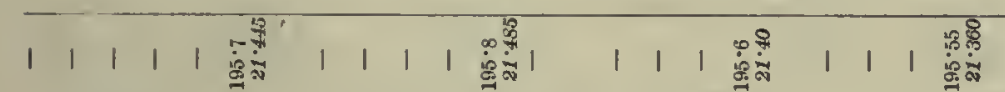

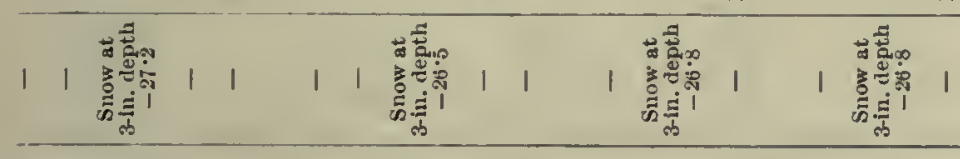

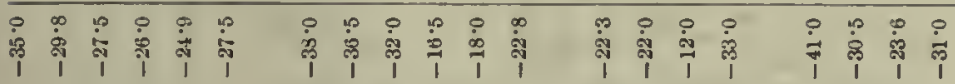

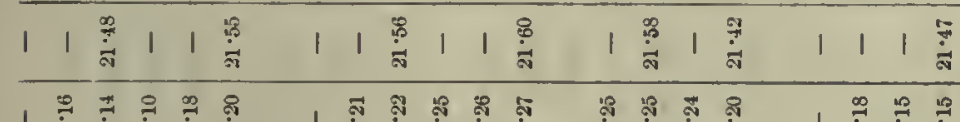

1

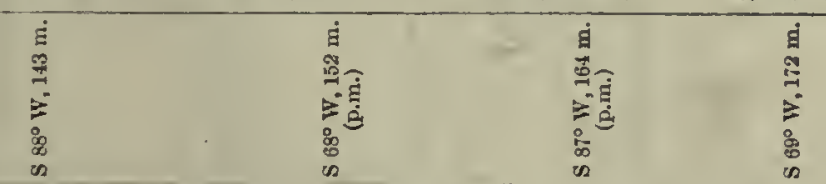

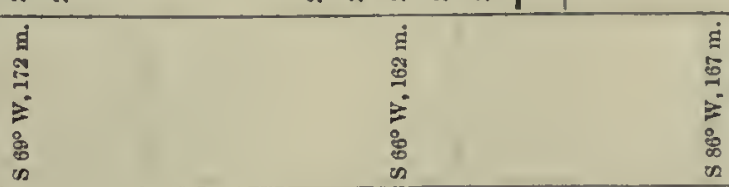$$
\text { (1) }
$$

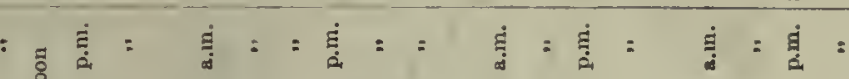

-

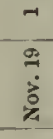

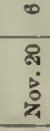

雾

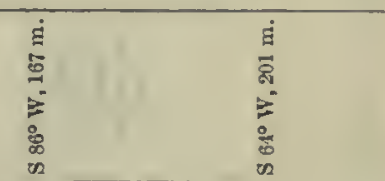

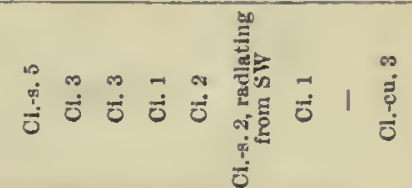
$++4 \underset{++}{\oplus} \quad \stackrel{+}{\oplus}+1 \quad 1 \quad 0 \quad 1 \quad 1 \quad 1 \quad 1+4$

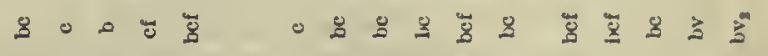
I ป

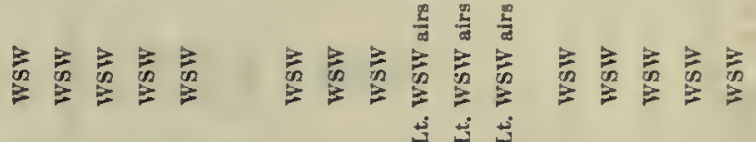

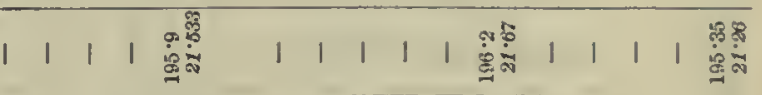

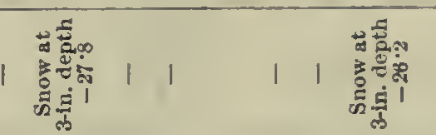

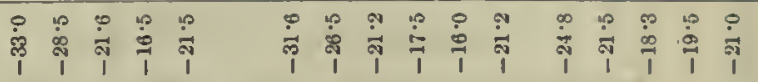

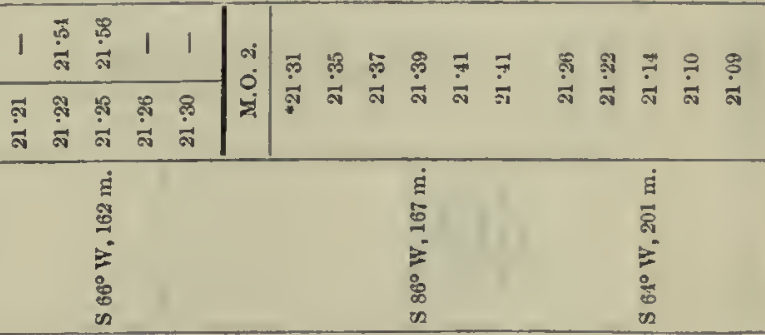

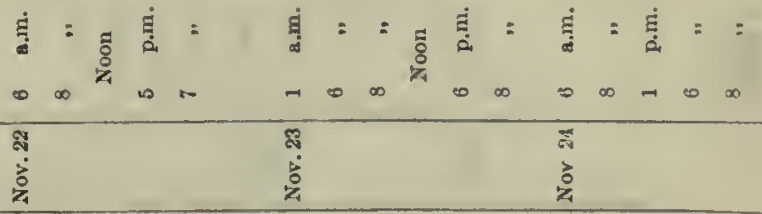




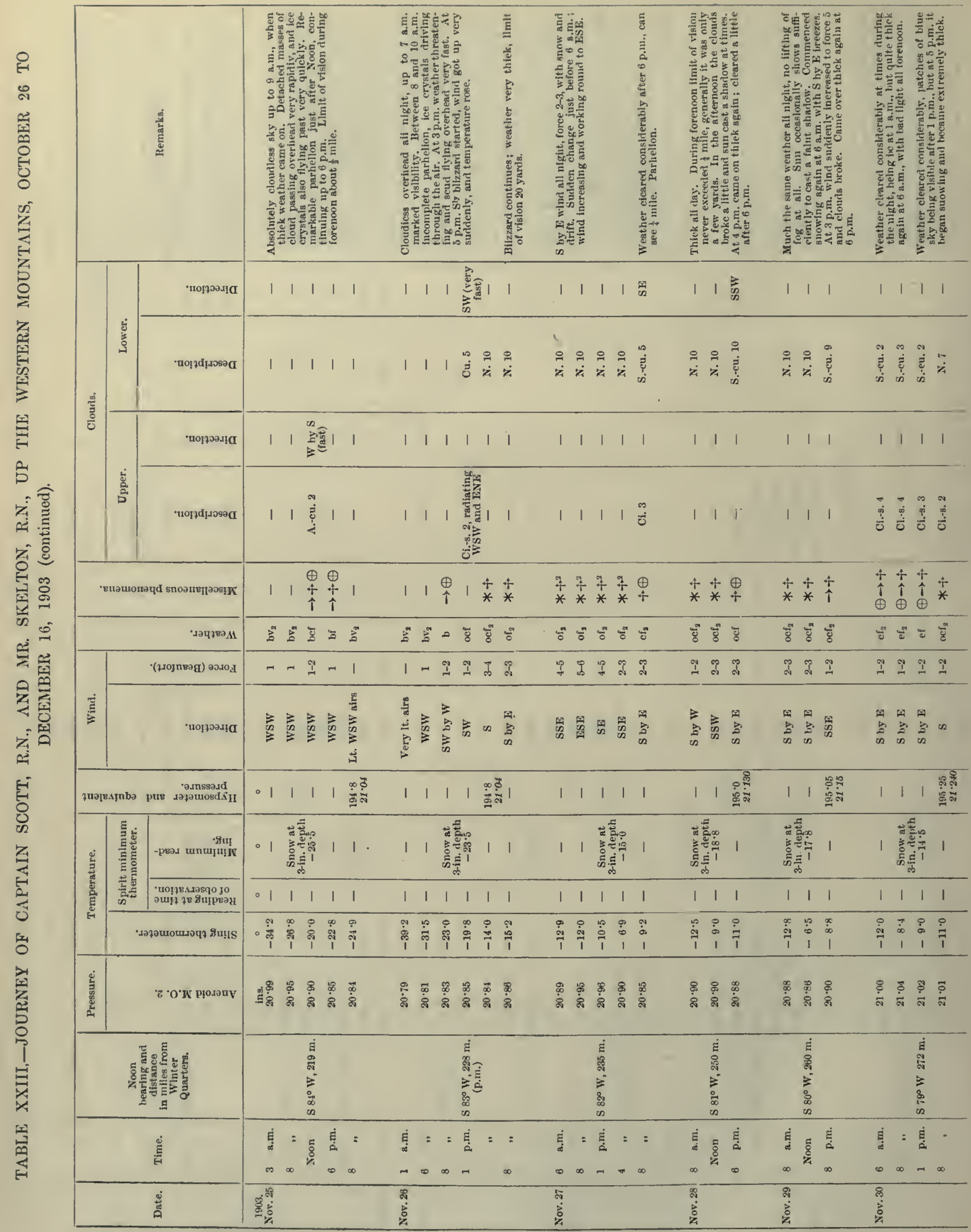




\begin{tabular}{|c|c|c|c|c|c|c|}
\hline 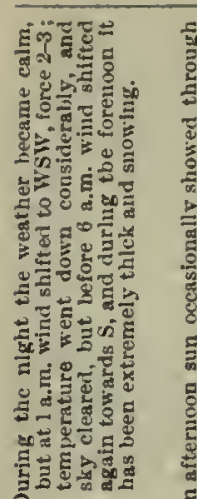 & 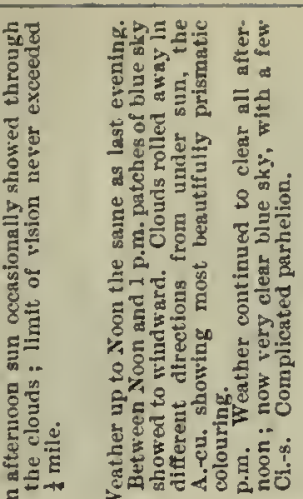 & 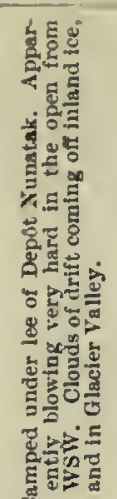 & 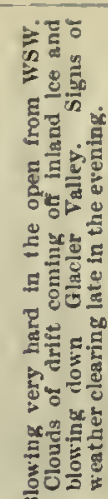 & 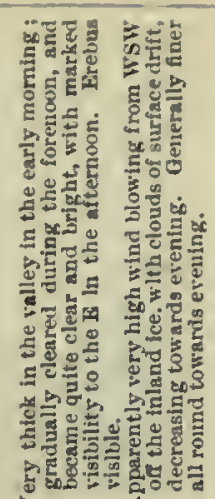 & 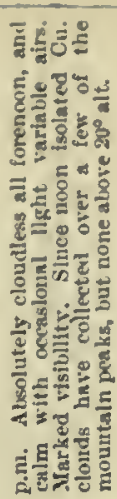 & 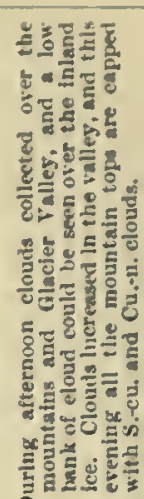 \\
\hline
\end{tabular}

$\begin{array}{llllllllllllllllllllllllllllllll}1 & 1 & 1 & 1 & 1 & 1 & 1 & 1 & 1 & 1 & 1 & 1 & 1\end{array}$

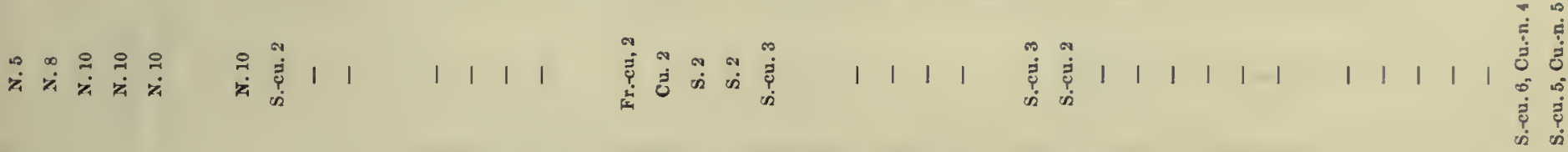

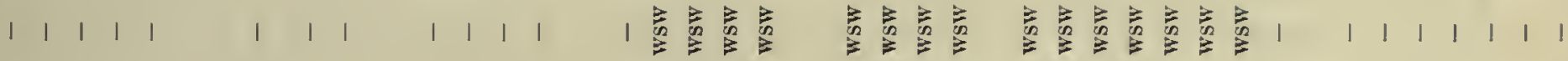

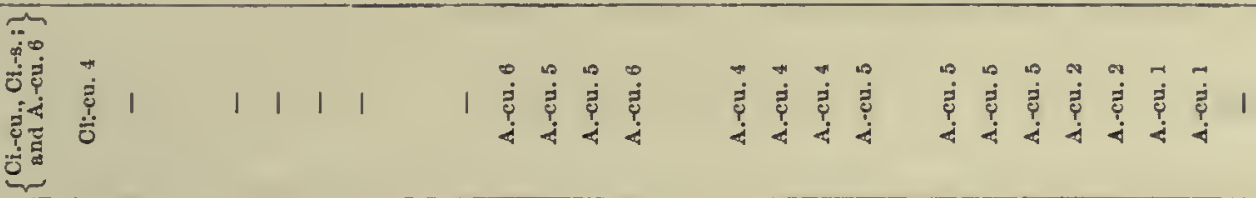

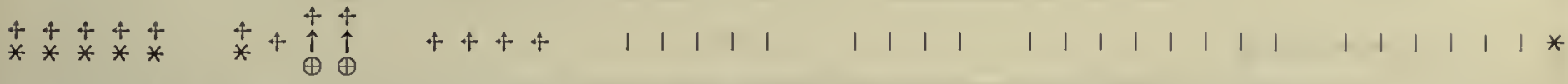

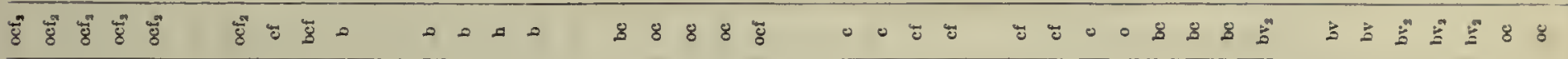

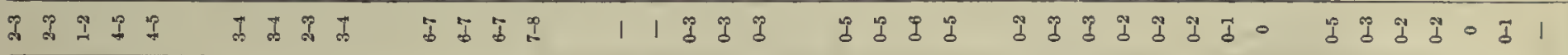

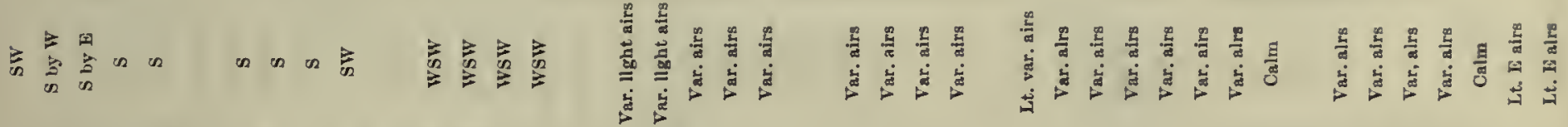

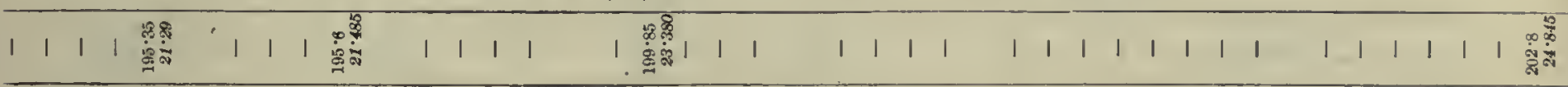

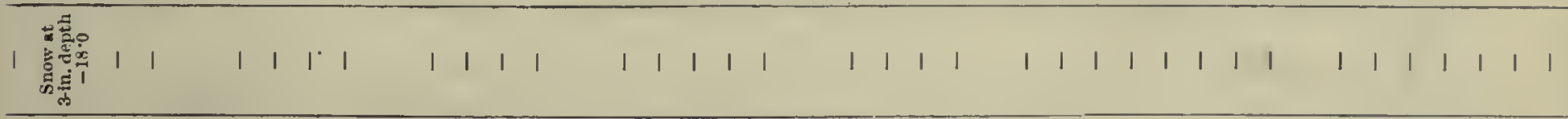

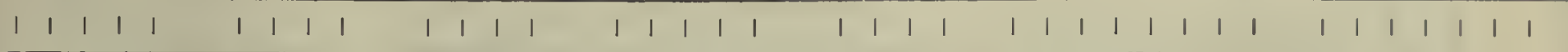

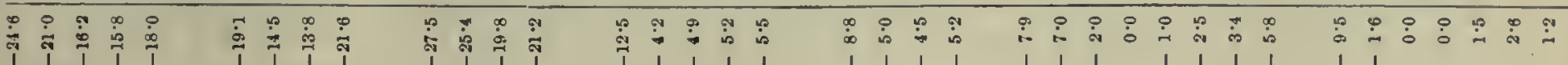

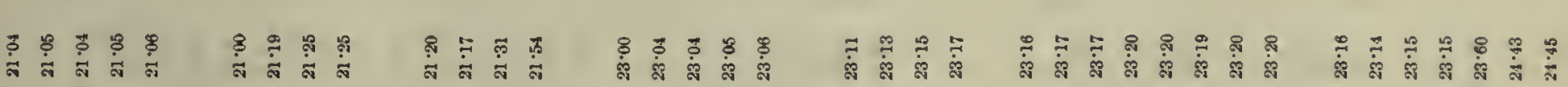

\begin{tabular}{|c|c|c|c|c|c|c|}
\hline 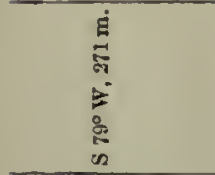 & 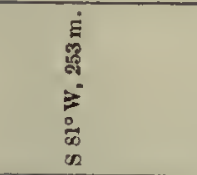 & 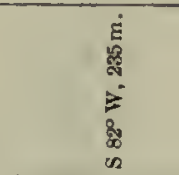 & 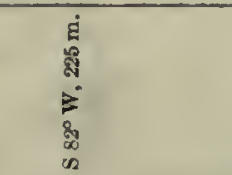 & 竞 & 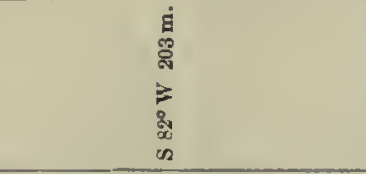 & 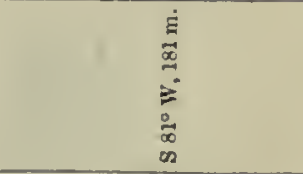 \\
\hline 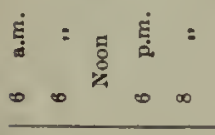 & $\begin{array}{l}\dot{\Xi}= \\
\infty-\infty\end{array}$ & 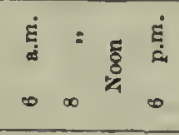 & 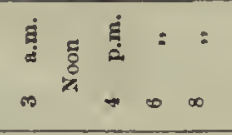 & $\begin{array}{l}\dot{\xi}=8 \\
0 \\
0\end{array}$ & 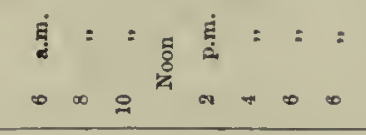 & 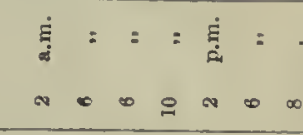 \\
\hline 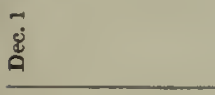 & 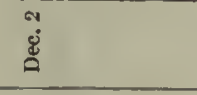 & $\begin{array}{l}\infty \\
\beth \\
\beth\end{array}$ & 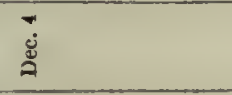 & 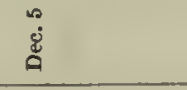 & 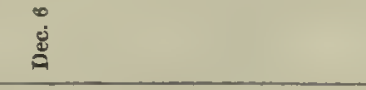 & . \\
\hline
\end{tabular}




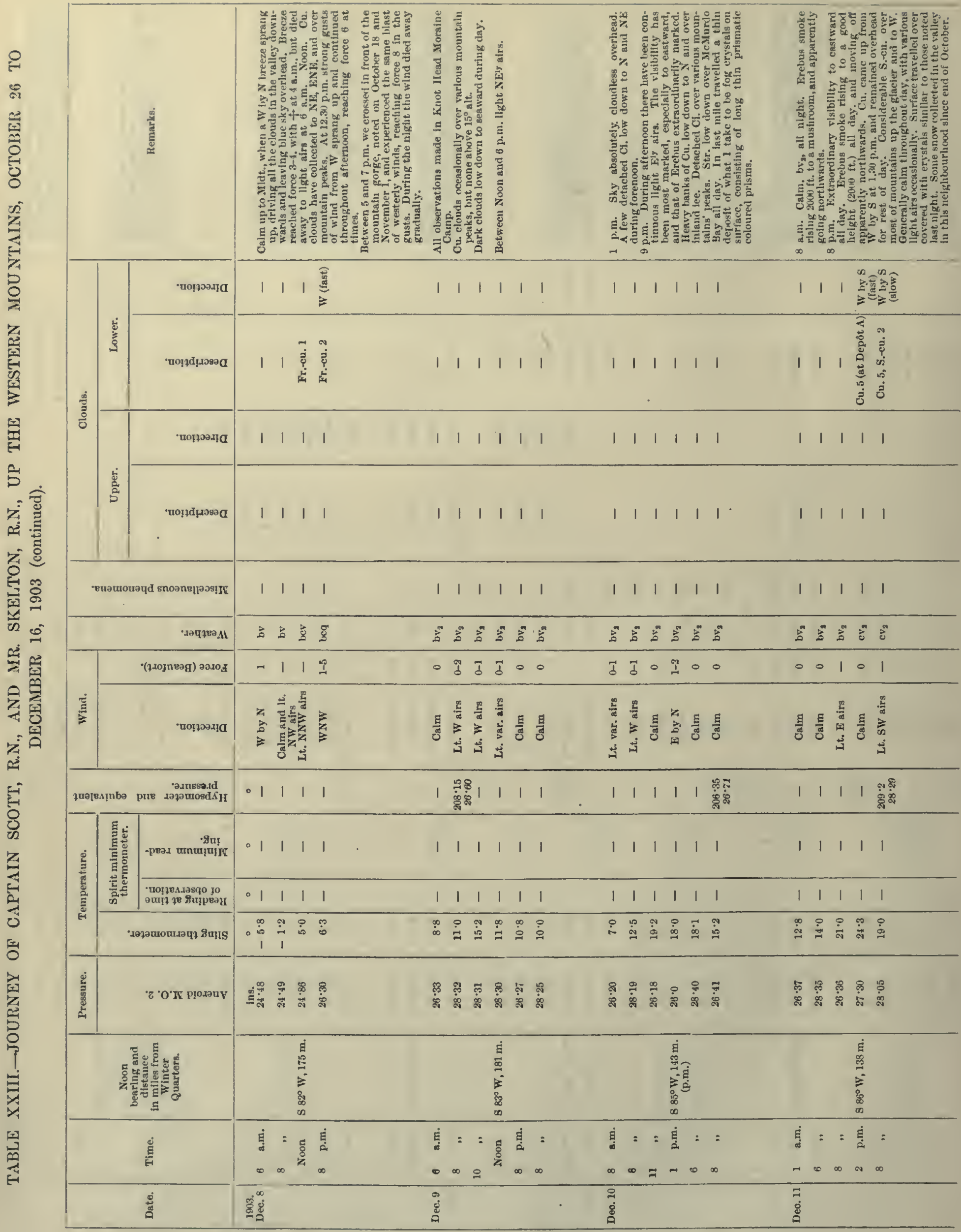




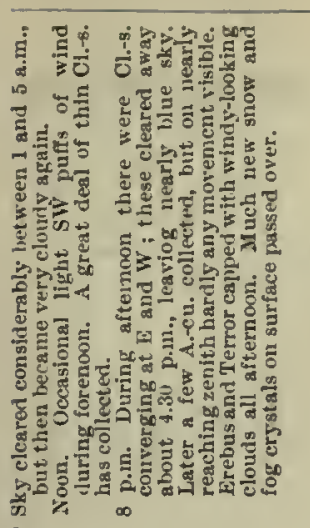

$1 \geqslant \geqslant 1$

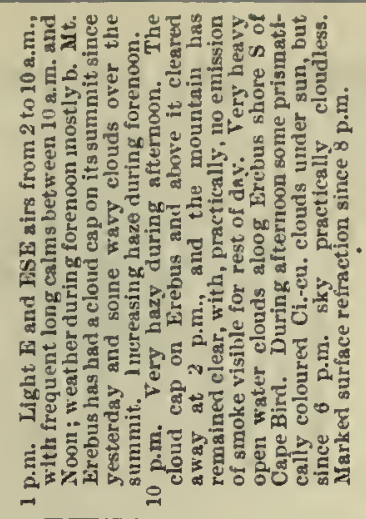

1 1 1

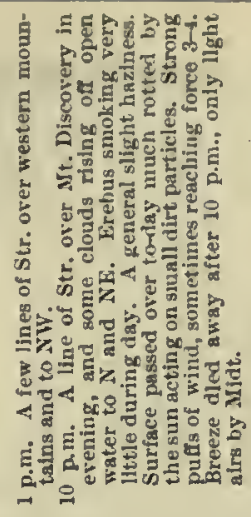

111111

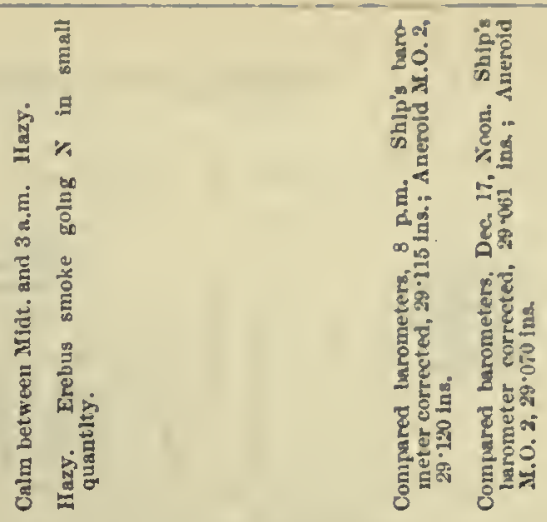

1 1 1 1
हี่

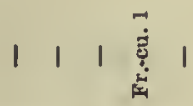
$\begin{array}{llllll}1 & 1 & 1 & 1 & 1 & 1\end{array}$

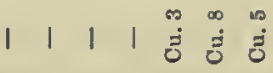
จุ่ ।

$1 \geqslant \geqslant 1$

l $111 j$

$\begin{array}{llllll}1 & 1 & 1 & 1 & 1 & 1\end{array}$

1 1 1 1 1 1 1

I 1

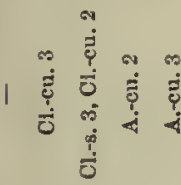

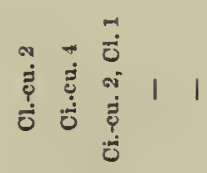

$\begin{array}{llllll}1 & 1 & 1 & 1 & 1 & 1\end{array}$

$\begin{array}{lllllll}1 & 1 & 1 & 1 & 1 & 1 & 1\end{array}$

11

| 1 | |

$\begin{array}{lllll}1 & 1 & 1 & 1\end{array}$

$\begin{array}{llllll}1 & 1 & 1 & 1 & 1 & 1\end{array}$

$\begin{array}{lllllll}1 & 1 & 1 & 1 & 1 & 1 & 1\end{array}$

I I

z 0 .

8.880

L $\Omega I=$

$D=-200$

88

- 000

$\begin{array}{lllll}\vec{b} & \vec{d} & \vec{b} & \vec{b} & 1\end{array}$

| 1 | कृ ㅇำ I

$\vec{b} \vec{b} \vec{\delta} \vec{b} \vec{d} \circ 0$

$\circ \circ$

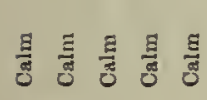

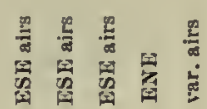

玹 $\frac{2}{6} \frac{2}{6}$

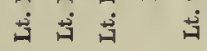

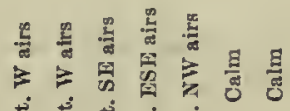

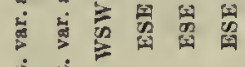

落苟

过苟苟苟苟

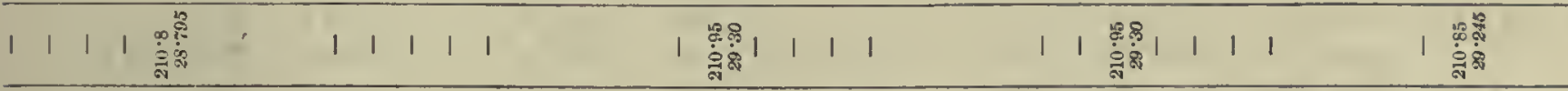

| | | | |

I 1 1 1 1

1 1. 11111

$\begin{array}{lllllll}1 & 1 & 1 & 1 & 1 & 1 & 1\end{array}$

I 1

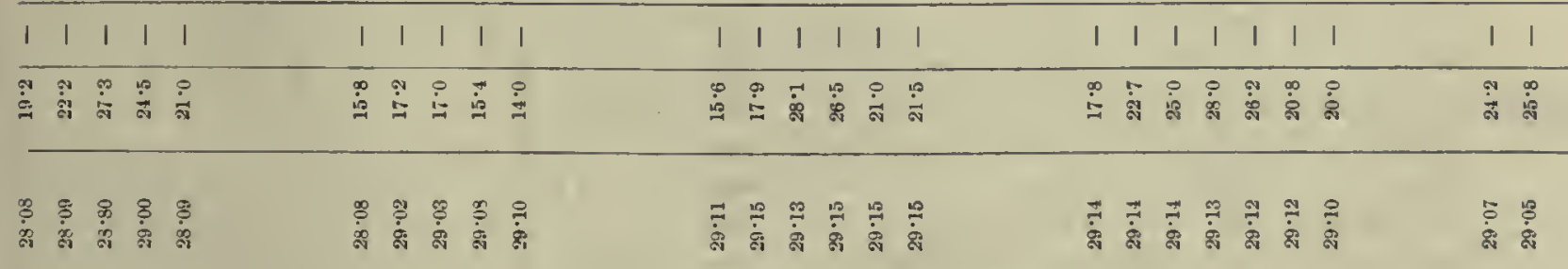

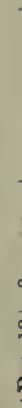
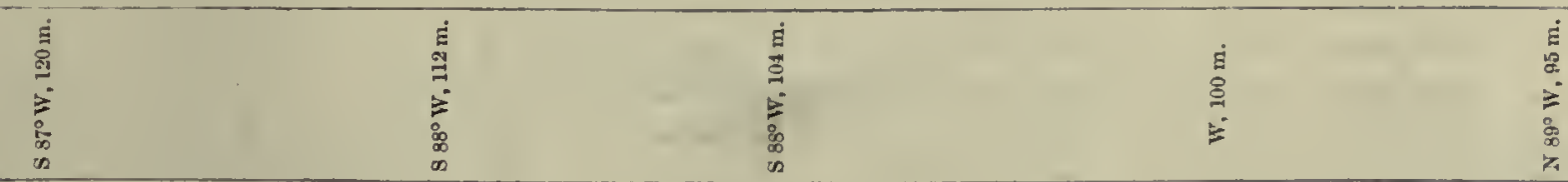

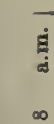
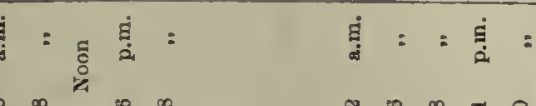

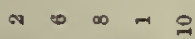

$\infty \infty \rightarrow \infty \stackrel{-\infty}{-\infty}$

$\infty \infty \rightarrow \infty \infty$

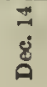

要

\section{홍}

悹 
TABLE XXIV.-JOURNEY OF LIEUTENANT ROYDS, R.N., AND MR. CLARKE TO THE

\begin{tabular}{|c|c|c|c|c|c|c|c|c|c|c|c|c|c|c|c|c|c|c|}
\hline \multirow[b]{3}{*}{ Date. } & \multirow[b]{3}{*}{ Time. } & \multirow{3}{*}{$\begin{array}{c}\text { Noon } \\
\text { bearing and } \\
\text { distance } \\
\text { in nilies from } \\
\text { Winter } \\
\text { Quarters. }\end{array}$} & \multicolumn{2}{|c|}{ Pressure. } & \multicolumn{3}{|c|}{ Air temperature. } & \multicolumn{2}{|c|}{$\begin{array}{l}\text { Snow } \\
\text { temperature. }\end{array}$} & \multirow{3}{*}{ 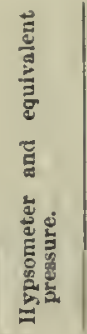 } & \multicolumn{2}{|l|}{ Wind. } & \multirow{3}{*}{\multicolumn{2}{|c|}{ 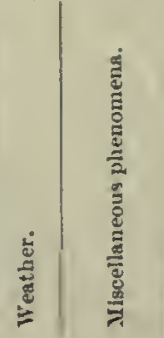 }} & \multicolumn{4}{|c|}{ Ciouds. } \\
\hline & & & \multirow[b]{2}{*}{ 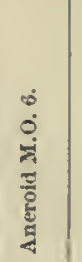 } & \multirow[b]{2}{*}{ 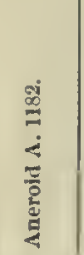 } & \multirow[b]{2}{*}{ 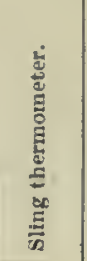 } & \multirow{2}{*}{\multicolumn{2}{|c|}{ 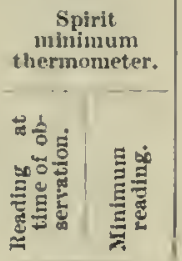 }} & \multirow[b]{2}{*}{ 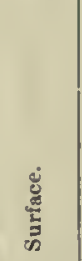 } & \multirow[b]{2}{*}{ 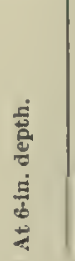 } & & \multirow[b]{2}{*}{ 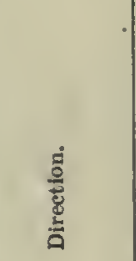 } & & & & $U_{p p e}$ & & Jower. & \\
\hline & & & & & & & & & & & & 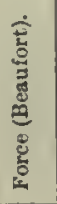 & & & 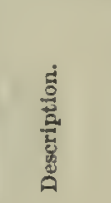 & 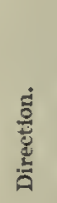 & 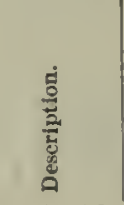 & 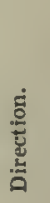 \\
\hline $\begin{array}{l}1903 . \\
\text { Nov. } 10\end{array}$ & 2 p. $\mathrm{ml}$. & & $\begin{array}{l}\text { ims. } \\
28 \cdot 90\end{array}$ & $\begin{array}{c}\text { ins. } \\
28 \cdot 598\end{array}$ & ० & A.5 & $\begin{array}{l}\circ \\
5 \cdot 0\end{array}$ & $\stackrel{\circ}{-}$ & $5 \cdot 0$ & $\begin{array}{c}\circ \\
210 \cdot 0 \\
28 \cdot 75\end{array}$ & Lt. $\mathbf{N}$ airs & - & b & - & - & - & - & - \\
\hline & $4 \quad "$ & & $28 \cdot 85$ & - & $6 \cdot 5$ & $6 \cdot 5$ & $5 \cdot 5$ & - & - & - & Caim & 0 & b & - & - & - & - & - \\
\hline & $"$ & - & $28 \cdot 82$ & $28 \cdot 590$ & $10 \cdot 0$ & $10 \cdot 5$ & $6 \cdot 0$ & - & $6 \cdot 0$ & - & Calm & 0 & b & - & - & - & - & - \\
\hline & " & & $28 \cdot 85$ & $28 \cdot 522$ & $1 \cdot 0$ & 0.5 & $-4 \cdot 0$ & - & 0.8 & - & Lt. $N$ airs & - & b & - & - & - & - & - \\
\hline Nov, 11 & 8 a.m. & . & $28 \cdot 92$ & $28 \cdot 608$ & $16 \cdot 8$ & $16 \cdot 5$ & $-3 \cdot 3$ & $16 \cdot 0$ & 0.5 & - & sw & $5-7$ & $o q$ & $++^{2} *$ & - & - & N. 10 & - \\
\hline & $10 \quad "$ & & $28 \cdot 95$ & $28 \cdot 615$ & $17 \cdot 5$ & $17 \cdot 2$ & $15 \cdot 3$ & $16 \cdot 8$ & $10 \cdot 0$ & - & Sw & $6-7$ & o & $+^{2} *$ & - & - & N. 10 & - \\
\hline & Noon & $\mathrm{S} 53^{\circ} \mathrm{E}, 7 \mathrm{~m}$ & - & $23 \cdot 683$ & $19 \cdot 8$ & $19 \cdot 2$ & $15 \cdot 2$ & $18 \cdot 0$ & $10 \cdot 0$ & - & sw & $5-6$ & $x$ & + & Cl.-8. 3 & - & S. 6 & s \\
\hline & 3.30 p..1n. & & $29 \cdot 05$ & $28 \cdot 722$ & $16 \cdot 0$ & $16 \cdot 5$ & $15 \cdot 0$ & - & - & $\begin{array}{c}210 \cdot 2 \\
28 \cdot 83\end{array}$ & s & $2-3$ & o & - & - & - & 8. 10 & - \\
\hline & $4 \quad$ " & & $29 \cdot 03$ & - & $16 \cdot 0$ & $17 \cdot 3$ & $15 \cdot 7$ & - & - & - & Var. airs & - & o & - & - & - & S. 10 & - \\
\hline & $6 \quad$ " & & $29 \cdot 02$ & - & $24 \cdot 8$ & $25 \cdot 5$ & $17 \cdot 2$ & - & - & - & Caim & 0 & $\circ$ & - & - & - & S. 10 & - \\
\hline & $8 \quad "$ & & $29 \cdot 01$ & $28 \cdot 720$ & $16 \cdot 2$ & $16 \cdot 5$ & $13 \cdot 2$ & $13 \cdot 2$ & $9 \cdot 0$ & - & $\mathbf{S E}$ & 1 & $\propto$ & - & Ci.-cu. 9 & Sw & - & - \\
\hline Nov. 12 & $6 \quad$ s.m. & & $29 \cdot 06$ & $28 \cdot 712$ & $19 \cdot 0$ & $19 \cdot 3$ & $14 \cdot 5$ & $19 \cdot 0$ & $9 \cdot 0$ & - & sw & $4-5$ & be & $*+2$ & - & - & Cu. 2 & sw \\
\hline & $8 \quad$. & & $28 \cdot 96$ & $28 \cdot 702$ & $19 \cdot 5$ & $19 \cdot 3$ & $16 \cdot 0$ & $18 \cdot 5$ & $8 \cdot 6$ & $\begin{array}{r}210 \cdot 18 \\
28 \cdot 85\end{array}$ & $\mathrm{sw}$ & $5-6$ & be & +2 & - & - & Cu. 5 & sw \\
\hline & $10 \quad$ " & & - & - & - & - & - & - & - & - & sw & 7 & ocq & +2 & - & - & S. 10 & - \\
\hline & Noon & S $46^{\circ} \mathbf{E}, 13 \mathrm{~m}$ & $29 \cdot 03$ & - & $21 \cdot 0$ & $21 \cdot 2$ & $19 \cdot 5$ & - & - & - & s & $3-4$ & oeq & + & - & - & S. 10 & - \\
\hline & 2.30 p.nt. & & $29 \cdot 01$ & $28 \cdot 700$ & $20 \cdot 8$ & $20 \cdot 6$ & $17 \cdot 5$ & - & - & - & s & $2-4$ & $\infty q$ & + & - & - & S. 10 & - \\
\hline & $4.20 \quad "$ & & $28 \cdot 98$ & - & $=21 \cdot 5$ & $21 \cdot 5$ & $19 \cdot 0$ & - & - & - & $\mathrm{s}$ & $1-3$ & ocg & - & - & - & S.-cu. 10 & - \\
\hline & $6.30 \quad$ " & & $29 \cdot 00$ & $28 \cdot 720$ & $18 \cdot 8$ & $19 \cdot 0$ & $15 \cdot 0$ & - & - & - & $\mathbf{s}$ & $1-3$ & $\infty q$ & + & - & - & Cu. 9 & s \\
\hline & $"$ & & $29 \cdot 04$ & $28 \cdot 735$ & $211^{\circ}$ & $20 \cdot 0$ & $18 \cdot 5$ & $19 \cdot 0$ & $9 \cdot 8$ & - & Calm & 0 & $\infty$ & - & - & - & Cu. 9 & $\mathbf{S}$ \\
\hline Nov. 13 & $6.30 \mathrm{a} . \mathrm{m}$. & & $29 \cdot 10$ & $28 \cdot 790$ & $11 \cdot 5$ & $12 \cdot 0$ & $7 \cdot 0$ & $9 \cdot 0$ & $7 \cdot 8$ & - & $\mathbf{s}$ & 2 & $\propto$ & - & Ci.-s. 2 & - & Cu. 6 & $\mathrm{~s}$ \\
\hline & $"$ & & $28 \cdot 96$ & $28 \cdot 672$ & $16 \cdot 0$ & $16 \cdot 0$ & $10 \cdot 5$ & $9 \cdot 0$ & $8 \cdot 5$ & $\begin{array}{c}210 \cdot 2 \\
28 \cdot 86\end{array}$ & SSw & $1-2$ & oc & +0 & Ci.-s. 5 & - & Cu. 2 & $\mathrm{~s}$ \\
\hline & $10 \quad$ " & & - & - & - & $17 \cdot 0$ & - & - & - & - & Sw & $5-i$ & $\propto q$ & $\dot{+}^{2}$ & Cl.-s. 5 & - & A.-8. 2 & SE \\
\hline & Noun & & - & - & $18 \cdot 3$ & $18 \cdot 0$ & 0.5 & - & - & - & sw & $4-6$ & ocq & $-t^{2}$ & Ci.-8. 6 & NW & 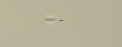 & - \\
\hline & 2 v.m. & $\begin{array}{c}\mathrm{S} 4 \overline{5}^{\circ} \mathrm{E}, 20 \mathrm{~m} . \\
\text { (p.m.) }\end{array}$ & $29 \cdot 04$ & $28 \cdot 700$ & $17 \cdot 2$ & $18 \cdot 0$ & $12 \cdot 6$ & $9 \cdot 5$ & $15 \cdot 0$ & - & $\mathrm{sw}$ & 5 & $\propto$ & + & Ci.-s. 4 & $\mathbf{N W}$ & A.-s. 3 & s \\
\hline & $3.30 \quad$. & & $29 \cdot 05$ & - & $14 \cdot 2$ & $15 \cdot 0$ & $12 \cdot 0$ & - & - & - & sw & $2-3$ & oe & +0 & Ci.-8. 6 & NW & A.-s. 3 & $\mathbf{s}$ \\
\hline & $6 \quad "$ & & $29 \cdot 08$ & $28 \cdot 708$ & $11 \cdot 0$ & $12 \cdot 0$ & $7 \cdot 5$ & - & - & - & sw & $1-2$ & $\propto$ & - & Ci.-8. 7 & $\mathrm{NW}$ & - & - \\
\hline & " & & $29 \cdot 14$ & $28 \cdot 815$ & $4 \cdot 5$ & $5 \cdot 0$ & $2 \cdot 0$ & $6 \cdot 0$ & $8 \cdot 0$ & - & sw & 1 & be & - & - & - & A.--8. 3 & - \\
\hline Nov. 14 & $6 \quad \mathrm{gm}$. & & $29 \cdot 20$ & $28 \cdot 876$ & $-3 \cdot 0$ & $-3 \cdot 2$ & -9.5 & $-5 \cdot 0$ & 0.0 & - & Calm & 0 & be & - & Cl.-8. 2 & $\mathbf{w}$ & - & - \\
\hline & $"$ & & $29 \cdot 17$ & $28 \cdot 878$ & $6 \cdot 5$ & $7 \cdot 5$ & $-5 \cdot 0$ & $2 \cdot 0$ & 0.5 & $\begin{array}{c}210 \cdot 4 \\
28 \cdot 98\end{array}$ & Calm & 0 & be & - & Ci.s. 3 & sw & - & - \\
\hline & $10 \quad$ " & & $29 \cdot 18$ & - & $11 \cdot 5$ & $12 \cdot 0$ & $6 \cdot 3$ & - & - & - & Calm & 0 & b & - & - & - & - & - \\
\hline & Noon & $\mathrm{S} 41^{\circ} \mathrm{E}, 34 \mathrm{~m}$ & $29 \cdot 19$ & $28 \cdot 866$ & $11 \cdot 8$ & $12 \cdot 0$ & 0.0 & - & - & - & Calm & 0 & b & - & - & - & - & - \\
\hline & 2 p.m. & & $29 \cdot 18$ & $28 \cdot 875$ & $9 \cdot 0$ & $10 \cdot 0$ & $9 \cdot 0$ & $6 \cdot 0$ & $1 \cdot 0$ & - & Calmu & 0 & b & - & - & - & - & - \\
\hline & " & & $29 \cdot 20$ & - & $7 \cdot 2$ & $9 \cdot 0$ & $6 \cdot 5$ & - & - & - & Calm & 0 & b & - & - & - & - & - \\
\hline & $"$ & - & $29 \cdot 17$ & $28 \cdot 870$ & $4 \cdot 5$ & $6 \cdot 0$ & 3.5 & - & - & - & Calm & 0 & $b$ & - & - & - & - & - \\
\hline & $"$ & & $29 \cdot 18$ & $28 \cdot 862$ & $-3 \cdot 0$ & $-3 \cdot 6$ & $-5 \cdot 0$ & 0.8 & 0.2 & - & Calm & 0 & $\mathbf{b}$ & - & - & - & - & - \\
\hline Nor. 15 & $6 \quad \mathrm{a} . \mathrm{m}$. & & $29 \cdot 22$ & $28 \cdot 886$ & -7.5 & $-7 \cdot 5$ & $-12 \cdot 6$ & $-4 \cdot 0$ & $-1 \cdot 2$ & - & Calm & 0 & b & - & - & - & - & - \\
\hline • & $"$ & & $29 \cdot 22$ & 28.898 & $-2 \cdot 5$ & $-2 \cdot 0$ & $-8 \cdot 0$ & $-2 \cdot 0$ & $-1 \cdot 0$ & 210.4 & Lt. E airs & - & $b$ & $\infty$ & - & - & - & - \\
\hline & 10 & & $29 \cdot 15$ & - & $7 \cdot 2$ & $8 \cdot 0$ & $-4 \cdot 5$ & - & - & $28 \cdot 98$ & Lt. NE airs & - & $b$ & - & - & - & - & - \\
\hline & Noon & $\mathrm{S} 44^{\circ} \mathrm{E}, 16 \mathrm{~m}$ & $29 \cdot 13$ & $28 \cdot 865$ & $8 \cdot 8$ & $9 \cdot 5$ & 6.0 & - & - & - & Calm & 0 & $b$ & - & - & - & - & - \\
\hline & 2 j.m. & & $29 \cdot 14$ & $28 \cdot 866$ & $11^{\circ} 6$ & $12 \cdot 0$ & $8 \cdot 0$ & $1 \cdot 3$ & 0.0 & - & Lt. E airs & - & b & - & - & - & - & - \\
\hline & $4 \quad$ " & & $29 \cdot 12$ & - & $17 \cdot 0$ & $17 \cdot 0$ & $11 \cdot 6$ & - & - & - & Lt. Nw airs & - & oc & $\rightarrow$ & - & - & S.-cu. 10 & - \\
\hline & $6 \quad$ " & & $29 \cdot 0 i$ & - & $14 \cdot 0$ & $14 \cdot 6$ & $13 \cdot 0$ & - & - & - & Lt. NE airs & - & oc & $\rightarrow$ & - & - & S.-cu. 10 & - \\
\hline & $"$ & & $29 \cdot 09$ & $28 \cdot 767$ & $11 \cdot 8$ & $11 \cdot 8$ & $11 \cdot 5$ & $5 \cdot 7$ & $3 \cdot 0$ & - & $\mathrm{SE}$ & 1 & $\infty$ & $\rightarrow$ & - & - & S.cu. 10 & - \\
\hline
\end{tabular}


SOUTH-LAST ACROSS THE ICE BARRIER, NOVEMBER 10 TO DECEMBLR 10, 1903.

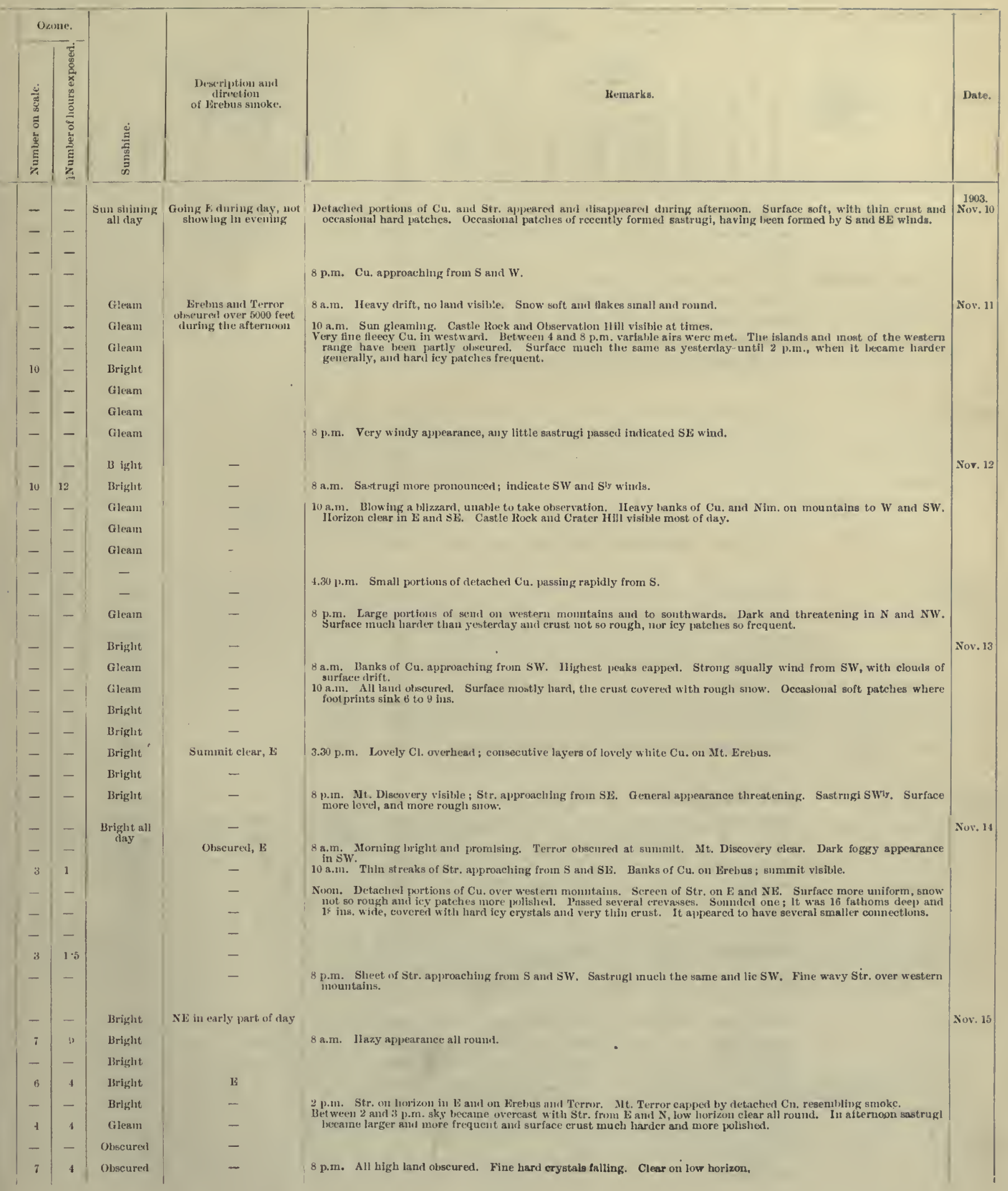


TABLE XXIV.-JOURNEY OF LIEUTENANT ROYDS, R.N., AND MR. CLARKE TO 'THL;

\begin{tabular}{|c|c|c|c|c|c|c|c|c|c|c|c|c|c|c|c|c|c|c|}
\hline \multirow[b]{3}{*}{ nate. } & \multirow[b]{3}{*}{ Time. } & \multirow{3}{*}{$\begin{array}{l}\text { Xoon } \\
\text { hearlug and } \\
\text { distance } \\
\text { in miles from } \\
\text { Winter } \\
\text { Quarters. }\end{array}$} & \multicolumn{2}{|c|}{ l'ressure. } & \multicolumn{3}{|c|}{ Air temperature. } & \multicolumn{2}{|c|}{$\begin{array}{c}\text { Suow } \\
\text { temperature. }\end{array}$} & \multirow{3}{*}{ 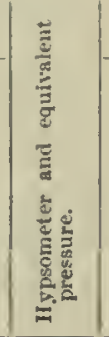 } & \multicolumn{2}{|l|}{ Wincl. } & & \multirow{3}{*}{ 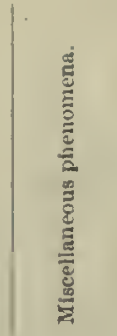 } & \multicolumn{4}{|c|}{ Clouds. } \\
\hline & & & \multirow[b]{2}{*}{ 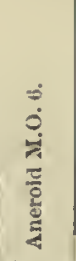 } & \multirow[b]{2}{*}{ 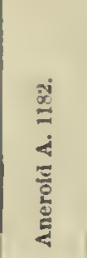 } & \multirow[b]{2}{*}{ 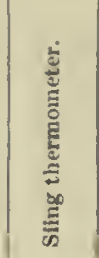 } & \multicolumn{2}{|c|}{$\begin{array}{c}\text { Spirit } \\
\text { minimumum } \\
\text { thernowneter. }\end{array}$} & \multirow[b]{2}{*}{ 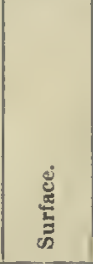 } & \multirow[b]{2}{*}{ 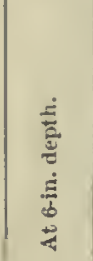 } & & \multirow[b]{2}{*}{ 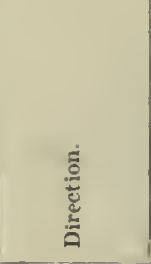 } & \multirow[b]{2}{*}{ 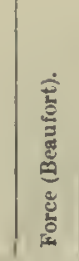 } & & & $v_{11}$ & & Lower & \\
\hline & & & & & & 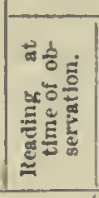 & 影 & & & & & & $\stackrel{5}{E}$ & & 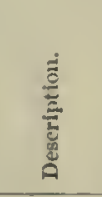 & 这 & 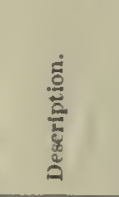 & 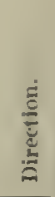 \\
\hline $\begin{array}{c}1903 . \\
\text { Nov. } 16\end{array}$ & 6 a.m. & & ins. & $\begin{array}{c}\text { ins. } \\
28 \cdot 804\end{array}$ & e. & $\begin{array}{l}0.5 \\
6.5\end{array}$ & $-i^{\circ} 0$ & $\begin{array}{l}\circ \\
3 \cdot 0\end{array}$ & $2 \cdot 0$ & $\stackrel{-}{-}$ & sw & $i-4$ & of & $\dagger^{+0}$ & - & - & S. 10 & - \\
\hline & $8 \quad "$ & & $29 \cdot 14$ & $28 \cdot 816$ & $4 \cdot 0$ & $4 \cdot 8$ & $1 \cdot 5$ & $2 \cdot 8$ & $2 \cdot 0$ & $210 \cdot 4$ & sw & $4-5$ & $\alpha$ & +0 & - & - & Cu. 10 & sw \\
\hline & $10 \quad$ " & & $29 \cdot 10$ & - & 6.6 & 6.6 & $4 \cdot 0$ & - & - & - & Sw & $4-5$ & $x$ & - & - & - & Cu. 10 & sw \\
\hline & Noon & $\mathrm{S} 46^{\circ} \mathrm{E}, 55 \mathrm{~m}$ & $28 \cdot 14$ & $28 \cdot 818$ & $7 \cdot 8$ & $8 \cdot 0$ & $5 \cdot 5$ & - & - & - & SW & $3-5$ & ocq & - & - & - & Cu. 10 & sw \\
\hline & 2 p.m. & & $29 \cdot 17$ & $28 \cdot 848$ & $6 \cdot 6$ & $7 \cdot 2$ & $3 \cdot 3$ & - & - & - & $\mathrm{sw}$ & $3-4$ & $\propto c$ & - & - & - & Cu. 9 & sw \\
\hline & $4 \quad 1$ & & $29 \cdot 17$ & - & $4 \cdot 0$ & $4 \cdot 6$ & $4 \cdot 0$ & - & - & - & sw & $2-3$ & $o c$ & - & - & - & Fr.en. 8 & sw \\
\hline & $6 \quad$ " & 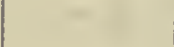 & $29 \cdot 17$ & $28 \cdot 910$ & $2 \cdot 6$ & $3 \cdot 3$ & $2 \cdot 0$ & - & - & - & sw & $2-3$ & oe & - & - & - & S.cul. 10 & - \\
\hline & $8 \quad "$ & & $29 \cdot 22$ & $28 \cdot 928$ & $4 \cdot 9$ & $4 \cdot 6$ & $2 \cdot 0$ & $3 \cdot 0$ & $1 \cdot 0$ & - & sw & $2-3$ & $x$ & - & - & - & S.cu. 10 & - \\
\hline Nov. 17 & 6 a.m. & & $29 \cdot 30$ & $29 \cdot 010$ & $4 \cdot 0$ & $4 \cdot 0$ & $-4 \cdot 0$ & $2 \cdot 5$ & 1.8 & - & sw & $3-4$ & of & + & - & - & A.-s. 10 & - \\
\hline & $8 \quad$ & & $29 \cdot 31$ & $29 \cdot 020$ & $3 \cdot 0$ & $3 \cdot 3$ & $1 \cdot 6$ & $2 \cdot 5$ & $2 \cdot 0$ & $\begin{array}{c}210 \cdot 4 \\
28 \cdot 88\end{array}$ & sw & 3 & of & - & - & - & A.-s. 10 & - \\
\hline & $10 \quad "$ & & $29 \cdot 32$ & - & $5 \cdot 5$ & $5 \cdot 5$ & $2 \cdot 0$ & - & - & - & sw & 3 & 0 & - & - & - & A.-8. 10 & $E$ \\
\hline & Noon & & $29 \cdot 33$ & $29 \cdot 024$ & $5 \cdot 8$ & $6 \cdot 0$ & $5 \cdot 0$ & - & - & - & sw & 3 & 0 & - & - & - & A.-8. 10 & xE \\
\hline & 2 p.m. & $\begin{array}{c}\mathrm{S} 46^{\circ} \mathbf{r}, 68 \mathrm{~m} . \\
(\mathrm{p}, \mathrm{m} .)\end{array}$ & $29 \cdot 35$ & $29 \cdot 026$ & $5 \cdot 0$ & $5 \cdot 0$ & $4 \cdot 0$ & - & - & - & sw & $2-3$ & 0 & $\Phi^{\prime \prime} \Psi^{n} \rightarrow$ & - & - & A.-8. 10 & $\mathrm{NE}$ \\
\hline & $4 \quad$. & & $29 \cdot 34$ & - & $5 \cdot 3$ & $6 \cdot 0$ & $5 \cdot 0$ & - & - & - & sw & 2 & uc & $\varpi \omega$ & - & - & A.-s. 10 & - \\
\hline & $8 \quad "$ & & - & $29 \cdot 016$ & $4 \cdot 0$ & $5 \cdot 0$ & $1 \cdot 0$ & - & - & - & Sw & 1 & $o c$ & - & - & - & S.-cu. 10 & - \\
\hline Nov. 18 & $6 \quad$ a.m. & & $29 \cdot 30$ & $28 \cdot 980$ & $-1 \cdot 3$ & -0.3 & $-7 \cdot 0$ & 1.0 & $-1 \cdot 0$ & - & $\mathrm{Calm}$ & 0 & b) & - & Ci.-s. 4 & - & - & - \\
\hline & $8 \quad$ " & & $29 \cdot 30$ & $28 \cdot 983$ & 0.0 & 0.8 & $-2 \cdot 4$ & $1 \cdot 2$ & -0.8 & $\begin{array}{c}210^{\circ} \cdot 6 \\
29 \cdot 10\end{array}$ & Lt. SW airs & - & we & - & Ci.-s. 3 & $w$ & - & - \\
\hline & $10 \quad "$ & & $29 \cdot 28$ & - & $3 \cdot 8$ & $4 \cdot 2$ & $-1 \cdot 5$ & - & - & - & sw & 1 & bc & - & $\mathrm{Ci}_{-}-\mathbf{s .} 3$ & w & - & - \\
\hline & Noou & $\mathrm{S} 47^{\circ} \mathrm{E}, 76 \mathrm{~m}$. & $29 \cdot 26$ & $28 \cdot 966$ & $3 \cdot 0$ & $3 \cdot 5$ & $2 \cdot 0$ & - & - & - & SW & 1 & $\mathrm{bc}$ & - & Ci.-8. 2 & $w$ & - & - \\
\hline & 2 p.m. & & $29 \cdot 26$ & $28 \cdot 932$ & $8 \cdot 8$ & $9 \cdot 0$ & $3 \cdot 0$ & 0.8 & $2 \cdot 5$ & - & Sw & $1-2$ & be & - & $\mathrm{Ci}_{-}-8.1$ & $w$ & - & - \\
\hline & $4 \quad$ & & $29 \cdot 22$ & - & $6 \cdot 0$ & $i \cdot 2$ & $6 \cdot 0$ & - & - & - & sw & 2 & be & - & - & - & - & - \\
\hline & $6 \quad "$ & & $29 \cdot 20$ & $28 \cdot 914$ & $5 \cdot 5$ & $6 \cdot 0$ & $3 \cdot 2$ & - & - & - & sw & $1-2$ & the & - & - & - & - & - \\
\hline & $8 \quad "$ & & $29 \cdot 24$ & $28 \cdot 916$ & $4 \cdot 0$ & $4 \cdot 0$ & $2 \cdot 2$ & 0.5 & $1 \cdot 0$ & - & sw & 2 & b & - & - & - & - & - \\
\hline Nov. 19 & 6 a.m. & & $29 \cdot 22$ & $28 \cdot 908$ & $-1 \cdot 0$ & -1.0 & $-3 \cdot 0$ & -10 & 0.0 & - & Sw & 3 & tw & $f^{0}$ & Ci.-8. 4 & sw & - & - \\
\hline & $8 \quad$ " & & $29 \cdot 20$ & $28 \cdot 894$ & $2 \cdot 3$ & $3 \cdot 0$ & $-3 \cdot 0$ & $-0 \cdot 2$ & $0 \cdot 2$ & - & sw & 3 & we & 40 & Ci.-8. 3 & sw & - & - \\
\hline & $10 \quad$ ". & & $29 \cdot 20$ & - & $4 \cdot 8$ & $5 \cdot 0$ & 0.0 & - & - & - & sw & $2-3$ & 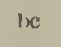 & $i^{n}$ & Ci.-s. 2 & sw & - & - \\
\hline & Noon & $\mathrm{S} 46^{\circ} \mathbf{E}, 84 \mathrm{~m}$ & $29 \cdot 23$ & $28 \cdot 900$ & $5 \cdot 0$ & $6 \cdot 0$ & $3 \cdot 0$ & - & - & - & sw & 3 & the & + & Ci.-s. 4 & sw & - & - \\
\hline & 2 p.m. & & $29 \cdot 24$ & $28 \cdot 910$ & $6 \cdot 8$ & $7 \cdot 0$ & $4 \cdot 6$ & $3 \cdot 0$ & $1 \cdot 3$ & - & Sw & 3 & $1 x$ & $+0^{\circ}$ & Ci. -8.3 & sw & - & - \\
\hline & $4 \quad "$ & & $29 \cdot 22$ & - & $7 \cdot 0$ & $7 \cdot 2$ & 5.5 & - & - & - & sw & 3 & be & $f^{\circ}$ & Ci.-s. 2 & sw & - & - \\
\hline & $6 \quad 1$ & & $29 \cdot 22$ & $28 \cdot 906$ & $4 \cdot 3$ & $5 \cdot 0$ & $4 \cdot 0$ & - & - & - & sw & 2 & b & +0 & Ci.-s. $]$ & sw & - & - \\
\hline & $8 \quad "$ & & $29 \cdot 23$ & $28 \cdot 900$ & $4 \cdot 0$ & $4 \cdot 3$ & $2 \cdot 0$ & $2 \cdot 0$ & $1 \cdot 0$ & - & Sw & 3 & b & - & $\mathrm{Cl}-\mathrm{s.} . \mathrm{I}$ & sw & - & - \\
\hline Nov. 20 & a a.m. & & $29 \cdot 21$ & $28 \cdot 900$ & $-1 \cdot 0$ & -1.0 & $-8 \cdot 0$ & $-2 \cdot 0$ & $-2 \cdot 0$ & - & sw & 2 & b & $4^{\circ}$ & - & - & - & - \\
\hline & $8 \quad$ ". & & $29 \cdot 20$ & 28.886 & $1 \cdot 0$ & 1.5. & $-4 \cdot 6$ & $-1 \cdot 2$ & -0.8 & - & SIV & 2 & b & $++^{0}$ & - & - & - & - \\
\hline & $10 \quad$, & & $29 \cdot 20$ & - & $5 \cdot 8$ & $6 \cdot 0$ & $1 \cdot 0$ & - & - & - & sw & 3 & b & + & - & - & - & - \\
\hline & Non!r & $\mathrm{S} 16^{\circ} \mathrm{E}, 81 \mathrm{~m}$ & $29 \cdot 17$ & $28 \cdot 860$ & $8 \cdot 2$ & $9 \cdot 0$ & $3 \cdot 0$ & - & - & $\begin{array}{c}210 \cdot 4 \\
28 \cdot 98\end{array}$ & sw & 3 & " & - & - & - & - & - \\
\hline & $2 \quad$ p.m. & & $24 \cdot 16$ & 23.850 & $11 \cdot 2$ & $12 \cdot 0$ & $6 \cdot 5$ & $1 \cdot 0$ & 0.0 & - & SW & 2 & b & - & - & - & - & - \\
\hline & $4 \quad 1$ & & $29 \cdot 16$ & - & $10 \cdot 6$ & $10 \cdot 6$ & $10 \cdot 3$ & - & - & - & sw & $1-2$ & b & - & - & - & - & - \\
\hline & B $\quad$ " & & $29 \cdot 15$ & $28 \cdot 816$ & 6.4 & 13.5 & 6.0 & - & - & - & $\mathrm{Cnlm}$ & 0 & - & - & - & - & - & - \\
\hline & .. & & $29 \cdot 14$ & $28 \cdot 8.42$ & $2 \cdot 0$ & 2.51 & $0 \cdot 0$ & $-2 \cdot 3$ & $-1 \cdot x$ & - & Calm & 0 & 1. & - & -. & - & - & - \\
\hline
\end{tabular}


SOUTH-EAST ACROSS THE ICE BARRLER, NOVEMBER 10 TO DECEMBER 10, 1903 (continued).

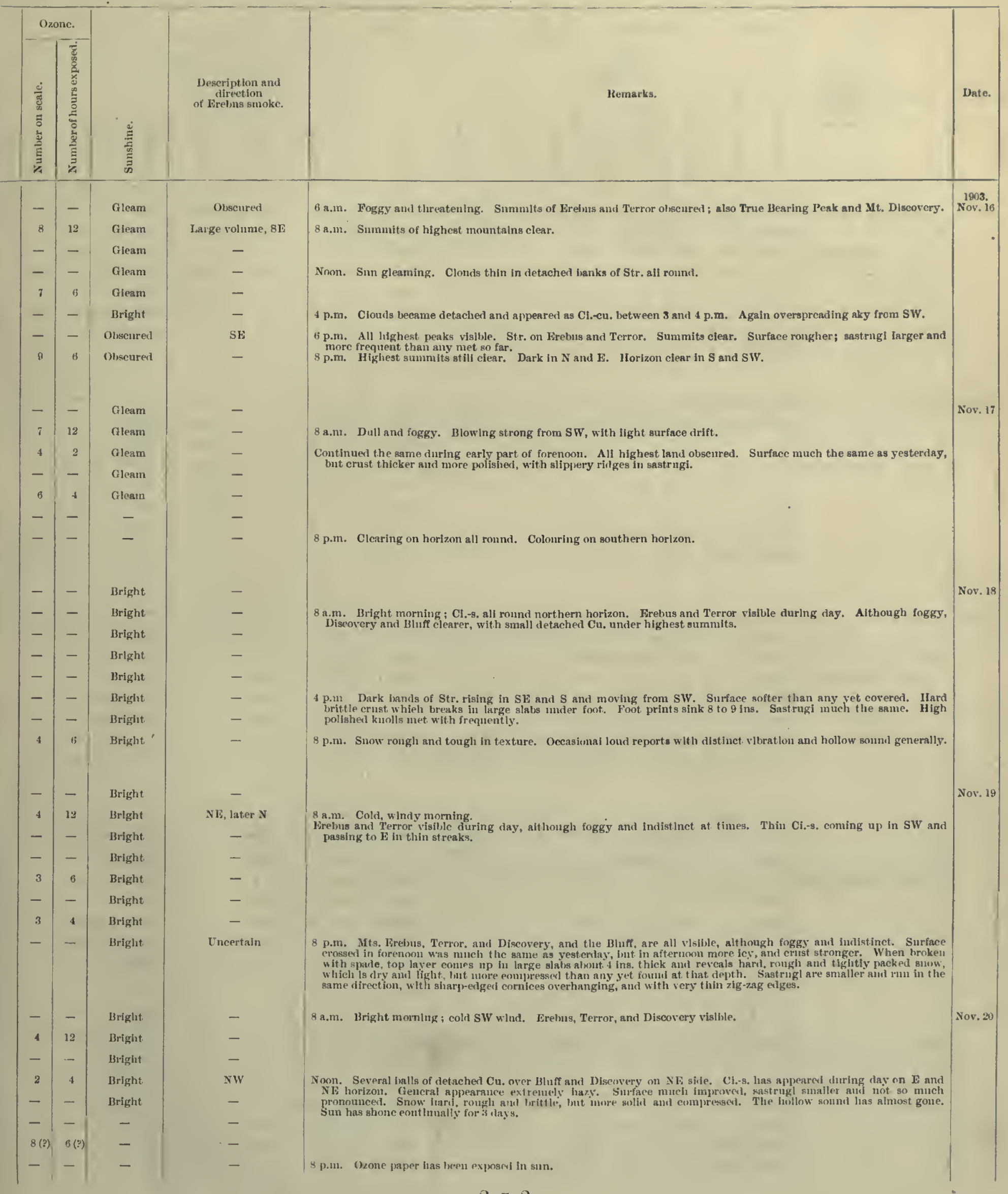


TABLE XXIV. -TOURNEY OF LIEUTENANT ROYDS, R.N., ANI) MR. ClARKF TO THE

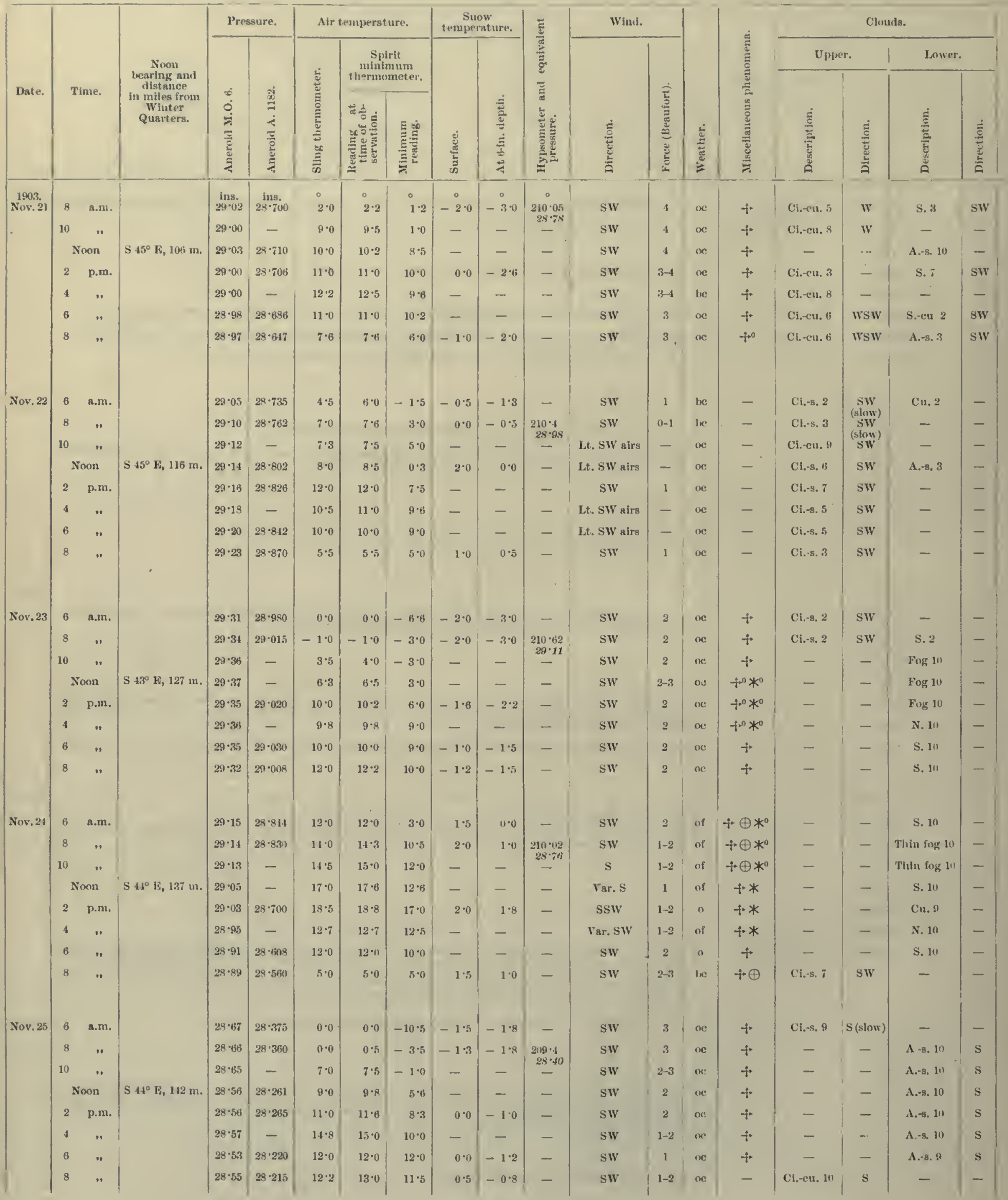


SOUTH-FAST ACROSS THE ICE BARRIER, NOVGMBER 10 TO DECEMBER 10, 1903 (continned).

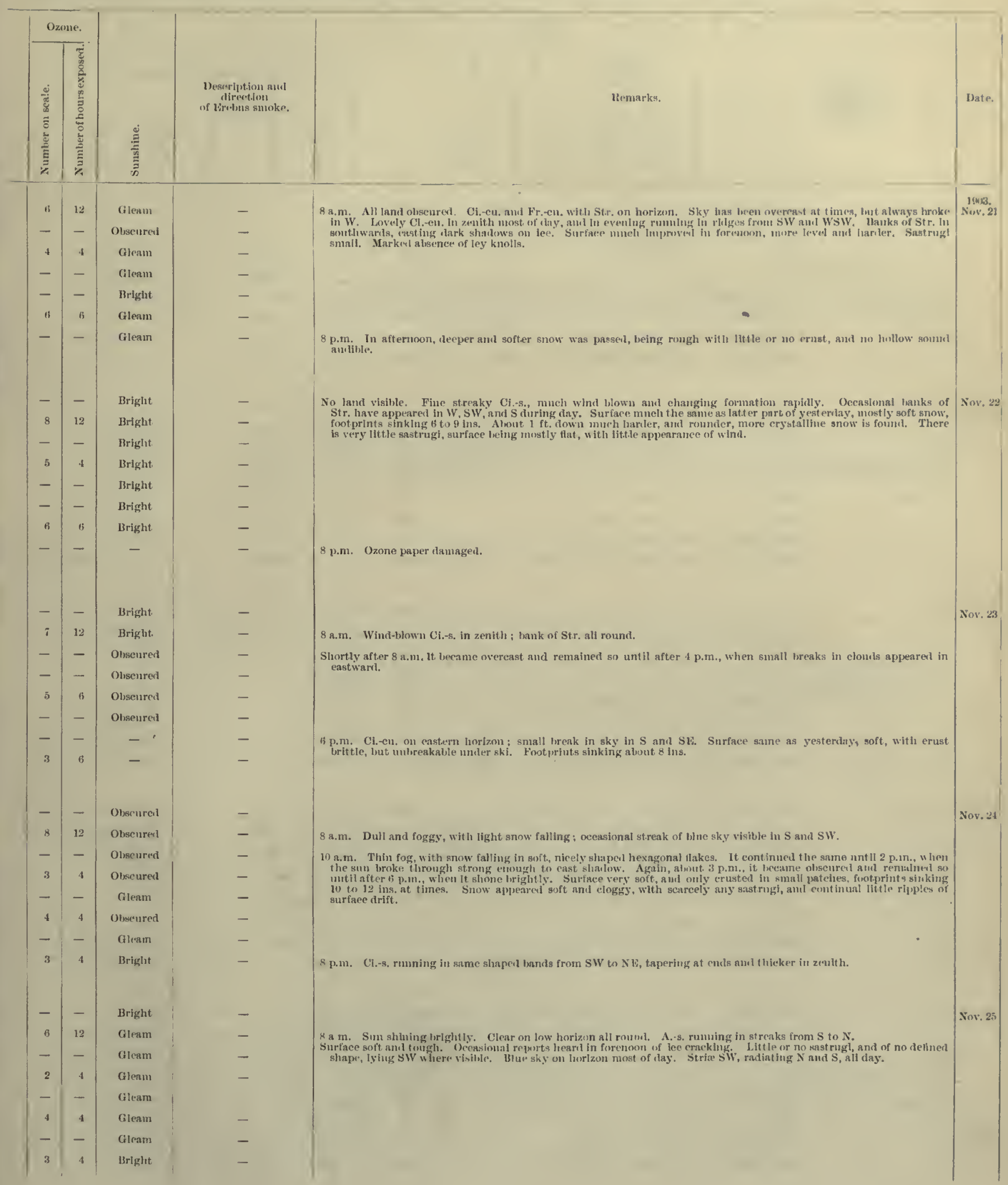


TABLE XXIV.-JOURNEY OF LIEUTENANT ROYDS, R.N., AND MR. CLARKE TO THE

\begin{tabular}{|c|c|c|c|c|c|c|c|c|c|c|c|c|c|c|c|c|c|c|c|}
\hline \multirow{3}{*}{ Date. } & \multirow{3}{*}{\multicolumn{2}{|c|}{ Time. }} & \multirow{3}{*}{$\begin{array}{l}\text { Noou } \\
\text { Imariug and } \\
\text { distance } \\
\text { in uiles from } \\
\text { Winter } \\
\text { Quarters. }\end{array}$} & \multicolumn{2}{|c|}{ Pressure. } & \multicolumn{3}{|c|}{ Air temperature. } & \multicolumn{2}{|c|}{$\begin{array}{c}\text { Snow } \\
\text { temperature. }\end{array}$} & \multirow{3}{*}{ 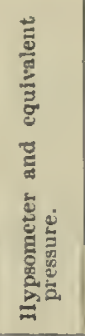 } & \multicolumn{2}{|l|}{ Wind. } & \multirow[b]{3}{*}{ 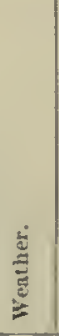 } & \multirow{3}{*}{ 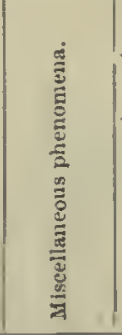 } & \multicolumn{4}{|c|}{ Clouds. } \\
\hline & & & & \multirow[b]{2}{*}{ 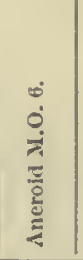 } & \multirow[b]{2}{*}{ 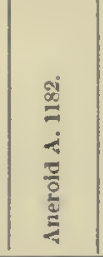 } & \multirow{2}{*}{ 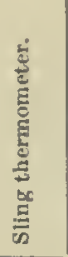 } & \multicolumn{2}{|c|}{$\begin{array}{c}\text { Spirit } \\
\text { uninimum } \\
\text { thwrmometer. }\end{array}$} & \multirow[b]{2}{*}{ 遶 } & \multirow[b]{2}{*}{ 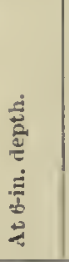 } & & & & & & $\mathrm{C}_{\mathrm{p} p}$ & & Lower & \\
\hline & & & & & & & 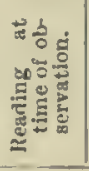 & है & & & & 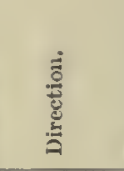 & 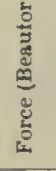 & & & 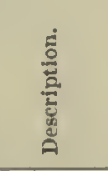 & 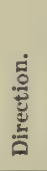 & 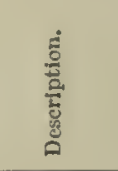 & 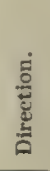 \\
\hline $\begin{array}{l}1903 . \\
\text { Nur. } 36\end{array}$ & 6 & a.m. & & ins. $_{28 \cdot 45}$ & $\begin{array}{r}\text { ins. } \\
28 \cdot 122\end{array}$ & $\stackrel{0}{12 \cdot 6}$ & $\begin{array}{c}0 \\
12 \cdot 6\end{array}$ & $\stackrel{\circ}{3 \cdot 0}^{-}$ & $i \cdot 6$ & $\ddot{1} 0$ & $\stackrel{\circ}{-}$ & sw & $1-2$ & oef & - & - & - & S. 10 & - \\
\hline & 8 & $"$ & & $28 \cdot 46$ & $28 \cdot 150$ & $18 \cdot 0$ & $18^{\circ} 0$ & $10 \cdot 0$ & $2 \cdot 0$ & $1 \cdot 3$ & - & sw & 2 & ocf & +0 & - & - & S. 10 & - \\
\hline & 10 & $"$ & . & $28-49$ & - & $20 \cdot 5$ & $20 \cdot 8$ & $16 \cdot 5$ & - & - & - & SW & 2 & oef & $\tau^{0} \Psi^{0}$ & - & - & N. 10 & - \\
\hline & & mon & $\mathrm{S} 43^{\circ} \mathrm{F}, 153 \mathrm{~m}$. & $28 \cdot 50$ & $28 \cdot 190$ & $18 \cdot 7$ & $18 \cdot 7$ & $18 \cdot 7$ & - & - & - & Var, sw & $1-2$ & oc & $-t^{0} * 0 \times 0$ & - & - & N. 10 & - \\
\hline & 2 & $\mathrm{pm}$ & & $28 \cdot 51$ & $28 \cdot 200(?)$ & $15 \cdot 0$ & $15 \cdot 6$ & $15 \cdot 0$ & $2 \cdot 5$ & $2 \cdot 0$ & - & Var. SW & $2-3$ & $\mathrm{ec}$ & $t^{0} *^{0} \rightarrow$ & - & - & N. 10 & - \\
\hline & 4 & " & & - & 2 & $15^{\circ} 0$ & - & - & - & - & - & sw & 2 & $\infty$ & $+* *$ & - & - & N. 10 & - \\
\hline & 6 & $"$ & & $28 \cdot 58$ & $28 \cdot 220$ & $15 \cdot 0$ & $15^{\circ} 0$ & $14 \cdot 5$ & - & - & - & Sw & 2 & $\infty$ & $+*^{0}$ & - & - & N. 10 & - \\
\hline & 8 & " & & $28 \cdot 56$ & $28 \cdot 245$ & $21 \cdot 0$ & $21 \cdot 7$ & $12 \cdot 6$ & $3 \cdot 0$ & $2 \cdot 5$ & - & Sw & 2 & ocf & $*$ & - & - & N. 10 & - \\
\hline Nov.27 & 6 & a.m. & & $28 \cdot 65$ & $28 \cdot 341$ & $18 \cdot 5$ & $19 \cdot 0$ & 0.0 & $4 \cdot 5$ & $4 \cdot 0$ & - & SSE & 2 & oc & +0 & - & - & A.-сu. 10 & s \\
\hline & 8 & $"$ & & $28 \cdot 70$ & $28 \cdot 382$ & $22-0$ & $23 \cdot 0$ & $18 \cdot 5$ & $B \cdot 0$ & $5 \cdot 6$ & $\begin{array}{c}209 \cdot 4 \\
28 \cdot 52\end{array}$ & SSE & $2-3$ & of & $+0 *$ & - & - & N. 10 & - \\
\hline & 10 & " & & $28 \cdot 73$ & - & $20 \cdot 6$ & $20 \% 4$ & $20 \cdot 0$ & - & - & - & SSE & 2 & of & $+0 *$ & - & - & N. 10 & - \\
\hline & & son & $\mathrm{S} 43^{\circ} \mathrm{E}, 160 \mathrm{~m}$ & $28 \cdot 74$ & $23 \cdot 410$ & $23 \cdot 5$ & $23 \cdot 5$ & $20 \cdot 0$ & - & - & - & SSE & 2 & of & $+\infty *$ & - & - & N. 10 & - \\
\hline & 2 & p.m. & & $28 \cdot 76$ & $28 \cdot 410$ & $23 \cdot 0$ & $23 \cdot 0$ & $22 \cdot 5$ & $9 \cdot 8$ & $7 \cdot 6$ & - & SSE & 2 & of & $f^{* 0} *^{0}$ & - & - & N. 10 & - \\
\hline & 4 & " & & $28 \cdot 75$ & - & $22 \cdot 5$ & $22 \cdot 5$ & $21 \cdot 6$ & - & - & - & $\operatorname{sst}$ & $1-2$ & of & $*$ & - & - & N. 10 & - \\
\hline & b & , & & $28 \cdot 75$ & $28 \cdot 404$ & $24 \cdot 0$ & $24 \cdot 5$ & $20 \cdot 8$ & - & - & - & SSE & $1-2$ & of & $*^{0}$ & - & - & N. 10 & - \\
\hline & 8 & " & & $28 \cdot \pi 5$ & $23 \cdot 421$ & $22 \cdot 5$ & $23 \cdot 0$ & $21 \cdot 6$ & $12 \cdot 0$ & $10 \cdot 0$ & - & SSW & 1 & oe & $\Psi \varpi *^{0}$ & - & - & А.-8. 10 & - \\
\hline Ner. 28 & 6 & a.m. & & $28 \cdot 68$ & $28 \cdot 348$ & $16 \cdot 5$ & $16 \cdot 8$ & $5 \cdot 0$ & $6 \cdot 5$ & $3 \cdot 0$ & - & SW & 1 & $\propto$ & $*^{0}$ & - & - & S. 10 & - \\
\hline & 8 & $"$ & & $28 \cdot 6 \cdot 1$ & $28 \cdot 322(?)$ & $18 \cdot 0$ & $18 \cdot 0$ & $12 \cdot 6$ & $7 \cdot 0$ & $4 \cdot 0$ & - & SSW & $1-2$ & $\mathfrak{o c}$ & - & Cl.-s. 3 & $\mathrm{~s}$ & S. 6 & - \\
\hline & 10 & " & 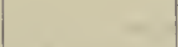 & $28 \cdot 65$ & - & $16 \cdot 8$ & $17 \cdot 0$ & $10 \cdot 0$ & - & - & - & SSW & 1 & $\propto$ & - & Ci. 4 & $\mathrm{~S}$ & A.-8. 6 & - \\
\hline & & on & $\begin{array}{l}\mathrm{S} 42^{\circ} \mathrm{F}, 167 \mathrm{~m} \\
\text { (furthest camp) }\end{array}$ & $28 \cdot 63$ & $29 \cdot 320(?)$ & $16 \cdot 0$ & $16 \cdot 0$ & $13 \cdot 0$ & - & - & - & SSW & $1-2$ & lie & - & Ci. 4 & S & - & - \\
\hline & 2 & p.m. & & $28 \cdot 62$ & $28 \cdot 313(?)$ & $17 \cdot 6$ & $17 \cdot 6$ & $12 \cdot 4$ & $10^{\circ} 6$ & $8 \cdot 4$ & - & $\mathrm{s}$ & $1-2$ & bc & - & Ci. 6 & $\mathbf{S}$ & - & - \\
\hline & 4 & " & & $28 \cdot 63$ & $28 \cdot 318$ & $18 \cdot 0$ & $18 \cdot 5$ & $15 \cdot 3$ & - & - & - & $s$ & $1-2$ & the & - & Ci, 5 & S & Cu. 4 & - \\
\hline & 6 & $"$ & - & $28 \cdot 64$ & $28 \cdot 320$ & $16 \cdot 3$ & $15 \cdot 3$ & $15 \cdot 0$ & - & - & - & $\mathrm{s}$ & $1-2$ & $\ln$ & - & $\mathrm{Ci} .4$ & $\mathrm{~S}$ & $\mathrm{Cu} .5$ & sw \\
\hline & 8 & $"$ & & $28 \cdot 66$ & $28 \cdot 346$ & $15 \cdot 0$ & $15 \cdot 0$ & $10 \cdot 6$ & $9 \cdot 0$ & $8 \cdot 0$ & - & s & 1 & be & $*^{n}$ & Ci.ecu. 3 & s & S. 7 & SW \\
\hline $\mathrm{N}(\mathrm{N}, 29$ & 6 & a.m. & & $28 \cdot 75$ & $25-440$ & $13 \cdot 0$ & $13 \cdot 5^{\circ}$ & $6 \cdot 7$ & $9 \cdot 0$ & $8 \cdot 0$ & - & SSW & 2 & $a$ & +0 & - & - & S. 10 & - \\
\hline & 8 & ", & & $28 \cdot 73$ & $28 \cdot 462$ & $16 \cdot 0$ & $17 \cdot 0$ & $12 \cdot 5$ & $8 \cdot 8$ & $8 \cdot 0$ & $\begin{array}{c}208 \cdot 8 \\
28 \cdot 63\end{array}$ & $\mathrm{~s}$ & $1-2$ & o & +0 & - & - & S. 10 & - \\
\hline & 10 & $"$ & & $28 \cdot 80$ & $28-500$ & $20 \cdot 5$ & $20 \cdot 6$ & $12 \cdot 0$ & - & - & 250 & $\mathrm{~s}$ & $1-2$ & $\infty$ & +0 & A.-cu. 9 & $\mathbf{s}$ & - & - \\
\hline & & oom & S $43^{\circ} \mathrm{F}, 155 \mathrm{~m}$ & $25 \cdot 84$ & - & $15 \cdot 5$ & $16 \cdot 3$ & $15 \cdot 0$ & - & - & - & $\mathrm{s}$ & 2 & oc & +00 & A.:8. 9 & S & - & - \\
\hline & 2 & p.ın. & & $28 \cdot 85$ & $28 \cdot 540$ & $16 \cdot 6$ & $17 \cdot 0$ & $13 \cdot 5$ & $8 \cdot 0$ & $7 \cdot 5$ & - & $\mathrm{s}$ & $1-2$ & $\infty$ & $\Psi^{\circ} \theta$ & A.-s. 10 & $\mathbf{S}$ & - & - \\
\hline & 4 & " & & $28 \cdot 87$ & - & $2) \cdot 0$ & $20 \cdot 0$ & 16.0 & - & - & - & $\mathrm{s}$ & $1-2$ & oc & +0 & A.-s. 10 & $\mathrm{~S}$ & - & - \\
\hline & 6 & " & & $28 \cdot 8 \mathrm{~g}$ & $28 \cdot 516$ & $17 \cdot 3$ & $17 \cdot 3$ & $17 \cdot 3$ & - & - & - & $\mathbf{s}$ & $0-1$ & c & $+t^{\circ}$ & $\mathrm{Cl}_{-}-\mathrm{s} .6$ & $\mathbf{S}$ & - & - \\
\hline & 8 & " & & $28 \cdot 90$ & $28 \cdot 565$ & $15 \cdot 0$ & $15 \cdot 0$ & $14 \cdot 3$ & 8.0 & $7 \cdot 0$ & - & $\mathbf{s}$ & $0-1$ & oc & - & Ci.-cu. s & $\mathbf{S}$ & S. 5 & $\mathrm{SW}$ \\
\hline Nov. 30 & 6 & a.m. & & $28 \cdot 97$ & $28 \cdot 662$ & $8 \cdot 0$ & $8 \cdot 5$ & $2 \cdot 5$ & $7 \cdot 5$ & $6 \cdot 0$ & - & Lt. S airs & - & oc & - & $\mathrm{Ct}_{.-3.8} 8$ & $\mathrm{~s}$ & - & - \\
\hline & 8 & $"$ & & $29 \cdot 00$ & $28 \cdot 672$ & $10^{\prime} 6$ & $11 \cdot 0$ & $6 \cdot 0$ & $7 \cdot 6$ & $6 \cdot 0$ & $210 \cdot 2$ & SSE & 1 & $\alpha$ & (1) +0 & - & - & A.-5. 10 & - \\
\hline & 10 & " & & $29 \cdot 05$ & - & $14 \cdot 8$ & $15 \cdot 0$ & $10 \cdot 0$ & - & - & - & SSE & $1-2$ & oc & $\theta+0$ & - & - & A.--8. 10 & - \\
\hline & & en & $\mathrm{S} 43^{\circ} \mathrm{E}, 144 \mathrm{~m}$. & $29 \cdot 07$ & - & $17 \cdot 0$ & $17 \cdot 0$ & 145 & - & - & - & SSE & 2 & oc & $\theta \div 0$ & - & - & A.,-9. 10 & - \\
\hline & 2 & p.m. & & $29 \cdot 07$ & $28 \cdot 742$ & $17 \cdot 5$ & $18 \cdot 0$ & $16 \cdot 5$ & $8 \cdot 0$ & $5 \cdot 8$ & - & S & $1-3$ & oeq & +0 & - & - & A.-ร. 10 & - \\
\hline & 4 & $"$ & & $29 \cdot 10$ & - & $18 \cdot 3$ & $18: 5$ & $16 \cdot 0$ & - & - & - & $\mathbf{S}$ & 3 & ocq & +0 & - & - & A.-s. IU & - \\
\hline & 6 & $"$ & & $29 \cdot 10$ & $28 \cdot 786$ & $16 \cdot 0$ & $16 \cdot 6$ & $16 \cdot 0$ & - & - & - & $\mathbf{S}$ & 2 & $o c$ & $-i 0$ & - & - & A.-9, 10 & - \\
\hline & 8 & " & & $29 \cdot 12$ & $28 \cdot 788$ & $14 \cdot 0$ & $14 \cdot 0$ & $12 \cdot 3$ & - & - & - & sw & 3 & ne & + & - & - & A.-8. 8 & $\mathbf{s}$ \\
\hline
\end{tabular}


SOU'II-EAS'T ACLOSS THE ICE BARRIER, NOVEMBER 10 TO DECEMBER 10, 1903 (continied).

\begin{tabular}{|c|c|c|c|c|c|}
\hline \multicolumn{2}{|c|}{ Ozone. } & \multirow[b]{2}{*}{ 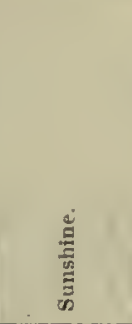 } & \multirow[b]{2}{*}{$\begin{array}{l}\text { Deweription and } \\
\text { rlrection } \\
\text { of Erebus smoke. }\end{array}$} & \multirow[b]{2}{*}{ it:-rnarks. } & \multirow[b]{2}{*}{ Date. } \\
\hline 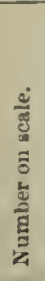 & 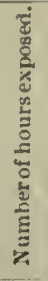 & & & & \\
\hline- & - & Ohscured & - & & Ior. \\
\hline$\theta$ & 12 & Obseured & - & 8 a.m. Vcry foggy. Thin soft snow falling, with conthual ripiples of surface drift. & \\
\hline- & - & Olsscured & - & & \\
\hline- & - & Olscurerl & - & About half-past twclve sun troke through and slone for alout half an irour. & \\
\hline 6 & 6 & Gicam & - & 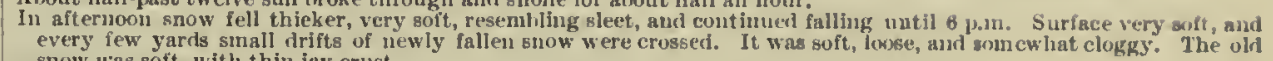 & \\
\hline- & - & Obseured & - & snow was goft, with thin icy crust. & \\
\hline 8 & 4 & Ohscured & - & & \\
\hline-- & - & Obscured & - & 8 p.m. Thick wet mist, with snow, soft and slecty, falliug thickly. & \\
\hline- & - & Obseurerl & - & 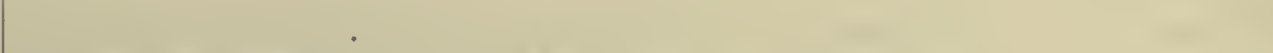 & Nov. 27 \\
\hline 6 & 12 & 'Olscurerl & - & $\Varangle$ a.m. Small dull patehes of blue sky visibie on $W$ horizon. & \\
\hline- & - & $\begin{array}{l}\text { Obseurert } \\
\text { Olsecured }\end{array}$ & - & $\begin{array}{l}\text { Soft sleety gnow fell continually during day, At times sun gleaning faintly. Surface much the same as yesterday ; } \\
\text { snow soit, elogky, and wet, and more evenly distributed. There was searcely any crust, and no hoilow sound. Any } \\
\text { little sastrugi visiblc is lyiug SE. }\end{array}$ & \\
\hline- & - & Glean & - & & \\
\hline- & - & Ohscured & - & & \\
\hline 6 & 6 & Gleam & - & & \\
\hline- & - & Bright & - & 8 v.m. A.-s. radiating s and N. Solar halo and corona, with parhelia. & \\
\hline- & - & Gleain & - & Arrived at our farthest camp. & Nov. 28 \\
\hline 8 & 12 & Bright & - & 8 a.m. Cloudy, but clearing on borizon ail round. Surface same as yesterduy. & \\
\hline- & - & Bright & - & $\begin{array}{l}10 \text { a.m. aud Nom. Jovely pearly Ci, in zenith and near suu, but at } 2 \text { p.m. and } \$ \text { p.m. Ci. much shattcrerl and gprear, } \\
\text { with Cu. rising in S. }\end{array}$ & \\
\hline- & - & Bright & - & (n) & \\
\hline 4 & 6 & Bright & - & 2 p.m. Ci. cluuds radiate $\mathrm{N}$ and $\mathrm{S}$, but change formation rapidly. & \\
\hline- & - & Bright & - & & \\
\hline- & - & Bright & - & & \\
\hline 3 & 6 & Gleam & - & 8 p.m. Ci.-cu. in zenith, with str. approaching ail round. Thrcateuing appenrance. & \\
\hline- & - & Olsseured & - & & Nov: $2 y$ \\
\hline 8 & 12 & Glerm & - & 8 a.m. Dull foggy morning. Clouds broken on $\mathrm{E}$ and NW horizon. & \\
\hline- & - & Bright & - & 10 a.m. A.-cu. changing rapidly to Ci.-cu. & \\
\hline 8 & 4 & $\begin{array}{l}\text { Bright } \\
\text { Bright }\end{array}$ & - & 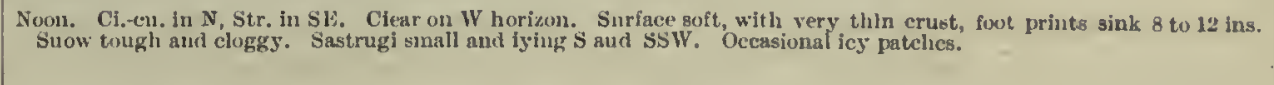 & \\
\hline- & - & Bright & - & & \\
\hline$b$ & 6 & Bright & - & & \\
\hline- & - & Bright & - & 8 p.m. Ci.-cu. in zenith. Clouds brokeu to southwards, with wind-biown Str. & \\
\hline- & - & Bright & - & 6 a.m. Ci.-s. rumning in thin bands from $S$ to $\mathrm{N}$, and overspreading $\mathrm{sky}$. & Nov. 30 \\
\hline 7 & 12 & Glram & - & 8 a.m, A.-s., with faint halo. & \\
\hline- & 一 & Glean & - & . & \\
\hline- & - & G!cam & - & Nouu. Ii aio since 8 a.m. ; appears of large radlus. & \\
\hline 5 & 6 & Brlght & - & $\begin{array}{l}2 \text { p.m. Surface much the sane as 5esterday. Icy patchea iarger and more frequent, Sastrugi a little more } \\
\text { pronounced and Sly. }\end{array}$ & \\
\hline - & - & Bright & - & . & \\
\hline 7 & 4 & Bright & - & (2. & \\
\hline- & - & Bright & - & 8 p.m. Clonds broken in zenith. Ci.-s, in S; bank of Str. rising in $\mathrm{NW}$. & \\
\hline
\end{tabular}


TABLE XXIV, -JOURNEY OF LIEUTENANT ROYDS, R.N., ANI) MR. CLARKE TO THE

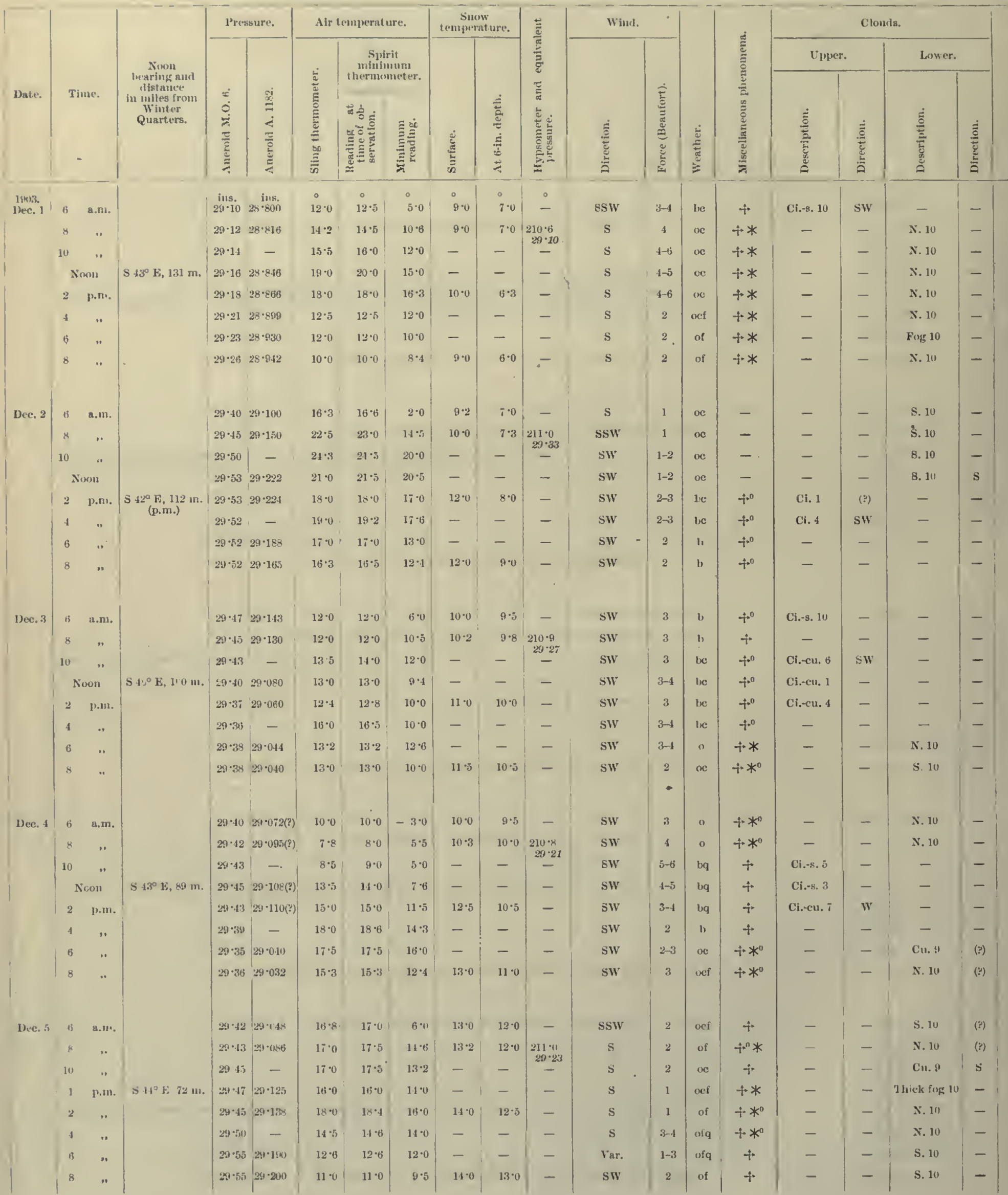


SOU'II-EAST ACROSS THE ICE BARRIER, NOVEMBER 10 TO DECEMBER 10, 1903 (continued).

\begin{tabular}{|c|c|c|c|c|c|}
\hline \multicolumn{2}{|c|}{ Ozonc. } & \multirow[b]{2}{*}{ 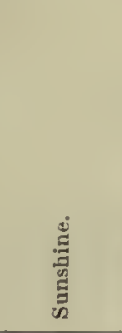 } & \multirow[b]{2}{*}{$\begin{array}{l}\text { Description amd } \\
\text { rlirectiont } \\
\text { of Erebus smoke. }\end{array}$} & \multirow[b]{2}{*}{ Remarks. } & \multirow[b]{2}{*}{ Date. } \\
\hline 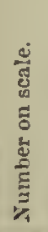 & 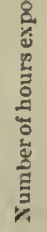 & & & & \\
\hline- & - & Bright & - & & $\begin{array}{l}1903 . \\
\text { Dec. } 1\end{array}$ \\
\hline 9 & 12 & Gleam & - & 8 a.m. Dull, threatening morning. Jleary drift during nlghlit. & \\
\hline- & - & Gleam & - & 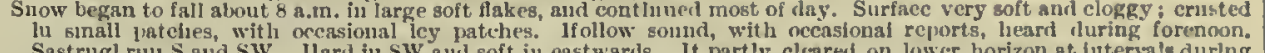 & \\
\hline- & - & Olsscured & - & & \\
\hline 8 & 6 & Olsseured & - & & \\
\hline- & - & Obscured & - & & \\
\hline- & - & Obscured & - & & \\
\hline 4 & 2 & Obseured & - & 8 p.m. Snow fallug thickly, with heary drift. & \\
\hline- & - & Gleam & - & & Dec. 2 \\
\hline 10 & 12 & Obscural & - & 8 a.m. Dull and overcast sky, luroken in $\mathrm{S}$. & \\
\hline- & - & Obseured & - & 10 a.m. Clear on lower horizon all round. & \\
\hline- & - & Obscurod & - & Noon. It elearel shortly after Noon, with $\mathrm{Cu}$. and lovely Ci.en. hin zenith. & \\
\hline $\boldsymbol{\tau}$ & 6 & Brightit & - & & \\
\hline- & - & Bright & - & 4 p.m. Ci. sll round on horizon. & \\
\hline 5 & 4 & Bright & - & $\begin{array}{l}\text { 6 p.m. Cloudless except in } \mathbf{N} \text { and } N W \text {, where lanks of } \mathrm{Str} \text {. have been hanglng most of day. } \\
\text { The nature of the surface is much the same as yesteriay ; soft, clogry snow, but sastrugl larger and more pronounced. }\end{array}$ & \\
\hline 3 & 2 & Bright & - & Oecasionai patches very thinly crusted, and uo hollow reports. & \\
\hline- & - & Bright & - & & Dec. 3 \\
\hline 10 & 12 & Bright & - & 8 a.m. Ciear, bright morning, with bauks of Str. all round. & \\
\hline- & - & Bright & - & 10 a.m. Clouds broken in S and SE; Ci.-cu. in zenith. & \\
\hline 3 & 4 & Brlght & - & Noon. Cloudless, except small detached portions of Ci-cu. passing from SW. & \\
\hline- & - & Bright & - & $\begin{array}{l}\text { it cleared about } 3 \text { p.m., when Erebus, Terror, and Bluff became vlsible; Erebus smoke appeared to be going castward. } \\
\text { Banks of Cu. over western ninountains and on Terror. }\end{array}$ & \\
\hline- & - & Bright & - & 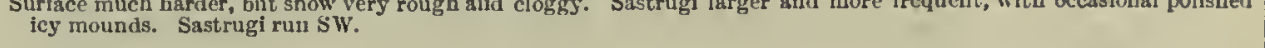 & \\
\hline 7 & 6 & Brlght ${ }^{\circ}$ & - & & \\
\hline- & - & Bright & - & & \\
\hline- & - & Obscured & - & & Dec. 4 \\
\hline 10 & 12 & Otscurerl & - & 8 a.m. - Dull and threatening; elouds breaking in S. & \\
\hline- & - & Briglit & - & 10 a.m. Thin lauds of fibrous Ci. radiatlog $\mathrm{E}$ and $\mathrm{W}$. High wall of surface drift all rouud. & \\
\hline- & - & Brighlit & - & Noon. Hazy, with no land vlslble. Ci.-s. radiating $\mathrm{E}$ and $\mathrm{W}$. & \\
\hline$i$ & 6 & Bright & - & 2 p.m. No land visible. Banks of Str. along $\mathrm{S}$ horizon. Wind squally. & \\
\hline- & - & Bright & - & 4 p.m. Bluff and MIt. Discovery visible, with banks of Cu. on hlgh peaks. Grebus and Terror have been visible at & \\
\hline is & 4 & Gleam & - & $\begin{array}{l}\text { times, but too liazy to see smoke. } \\
\text { Surface harder. Sastrugl higher and formed in long ridges. Snow very rough and cloggy in patehes; makes rough, } \\
\text { rasplng sound under ski. Littic or no crust, occasional reports of cracking. The snow that fell during the day was }\end{array}$ & \\
\hline- & - & Otsscuret & - & 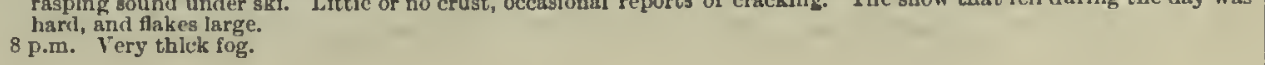 & \\
\hline 10 & ic & Obsenred & - & & Dec. 5 \\
\hline- & - & Otseured & - & 8 a.m.". Thick foggy morning, with snow falling ln large, hard falkes. & \\
\hline- & - & Ohseured & - & 10 a.m. Clearing somewhat in S. Betwcen 10 and 11 a.m. clouds became detached and iower portions of $\mathrm{Cu}$. passed & \\
\hline 8 & 6 & Olsscured & - & $\begin{array}{l}\text { raphldy y from s to } \mathrm{N} \text {. } \\
\text { Noont. All land obseured and no observations taken. }\end{array}$ & \\
\hline- & - & Obseured & - & 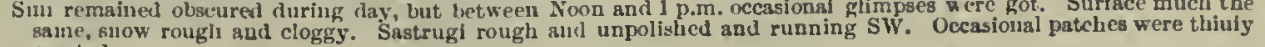 & \\
\hline- & - & Olsscurevl & - & & \\
\hline 10 & b & Olscuretl & - & t. & \\
\hline 6 & 2 & Obscuret & - & 8 p.m. No iand visible; very foggy. & \\
\hline
\end{tabular}


TABLE XXIY.-JOURNEY OF IIEUTENANT ROYDS, R.N., AND MR. CLARKE TO THE

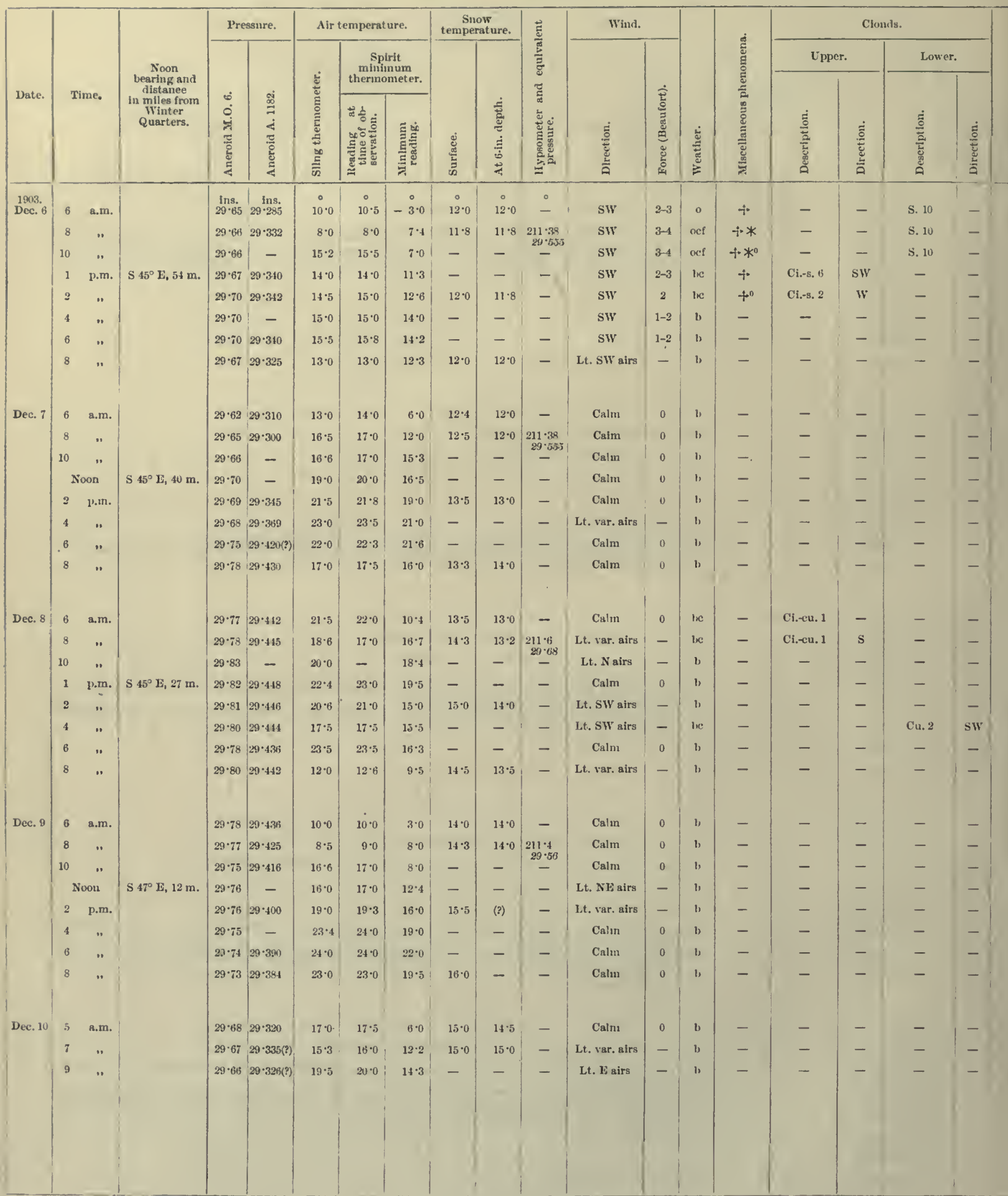


SOUTH-FAST ACROSS THE ICE BARLIER, NOVEMBER 10 TO DECEMBEk 10, 1903 (continued).

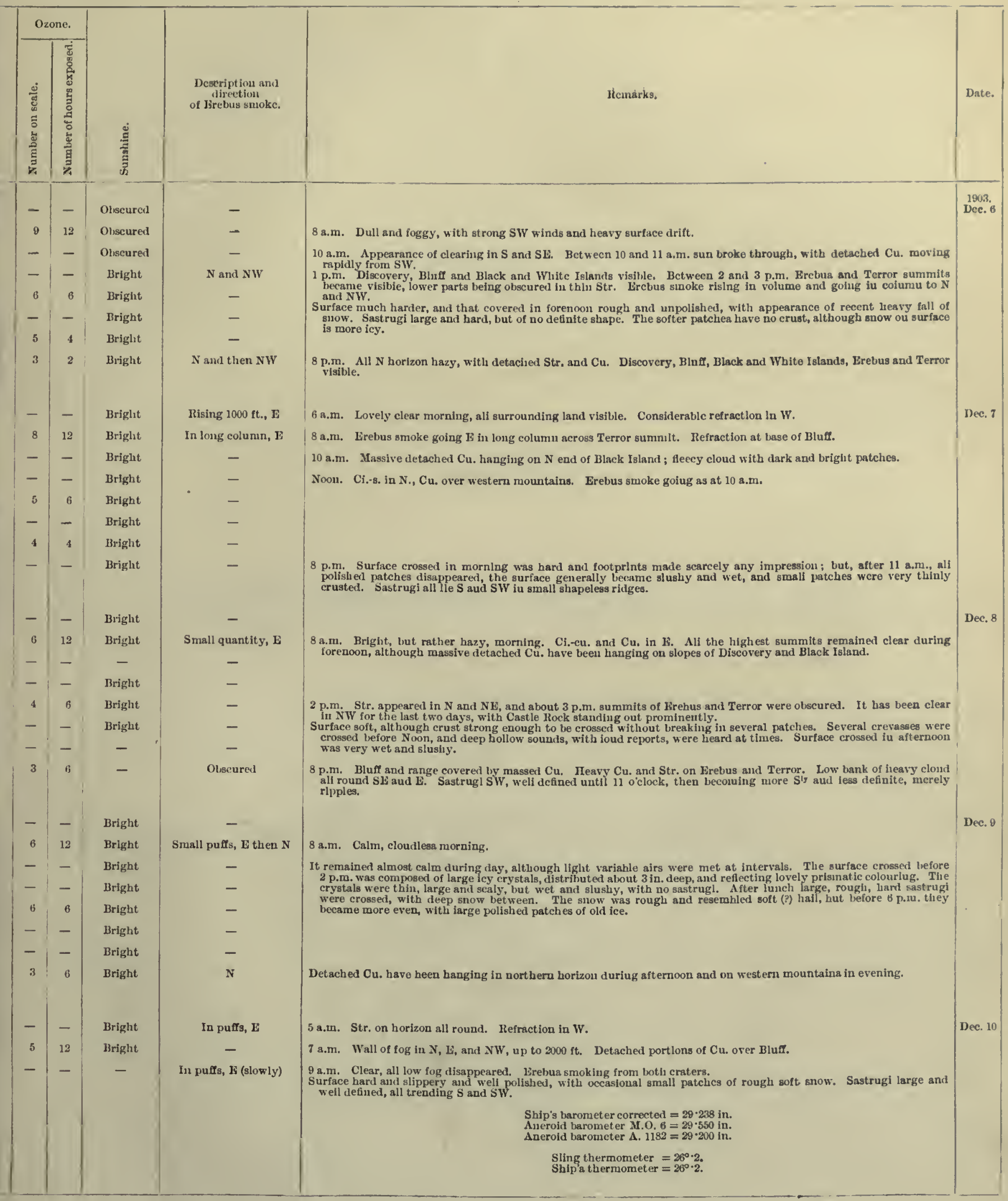





\section{TWO-HOURLY OBSERVATIONS}

OF

PRESSURE,

TEMPERATURE, WIND DIRECTION AND FORCE, AND OF

SUNSHINE, AT WINTER QUARTERS. 

TABLE I.

\section{PRESSURE IN ENGLISH INCHES, FIRST FIGURE OMITTED.}

The lieadings are Corrected for Temperature, and Redueed to Mean Sea Level, but are not Corrected for Variation of Gravity.

February, 1902.

Mareh, 1902.

\begin{tabular}{|c|c|c|c|c|c|c|c|c|c|c|c|c|c|c|c|c|c|c|c|c|c|c|c|c|c|c|c|}
\hline Day. & 2. & 4. & 6. & 8. & 10. & Noon. & 14. & 16. & 18. & 20. & 22. & Midt & Iean. & Day. & 2. & 4. & 6. & 8. & 10. & Noon. & 14. & 16. & 18. & 20. & 22. & Muit. & \\
\hline 1 & ins. & Ins. & fins. & ins. & ins. & ins. & ins. & ins, & ins. & ins. & ins. & ins. & ins. & 1 & $4: 163$ & $\begin{array}{l}\text { Ins. } \\
9 \cdot 415\end{array}$ & $\begin{array}{l}\text { Iuss. } \\
9 \cdot 451\end{array}$ & $\begin{array}{l}\text { Ins. } \\
9 \cdot 448\end{array}$ & $\begin{array}{l}\text { ins. } \\
9^{\circ} 456\end{array}$ & $\begin{array}{c}\text { Ins. } \\
69 \cdot 45\end{array}$ & \begin{tabular}{|l|} 
ins. \\
$9 \cdot 449$
\end{tabular} & $\begin{array}{l}\text { Ins. } \\
9.449\end{array}$ & 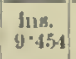 & $\underset{9 \times 467}{\text { fus }}$ & $\ln _{y \rightarrow 421}$ & $\begin{array}{l}\ln _{915} \\
9 \cdot 410\end{array}$ & \\
\hline$\frac{2}{3}$ & $=$ & $=$ & $=$ & $=$ & $=$ & - & - & - & $=$ & $=$ & $=$ & $=$ & 二 & & & & & & & & & & & & & & \\
\hline 4 & $=$ & $=$ & $=$ & $=$ & $\bar{z}$ & - & 7 & - & $\bar{Z}$ & $=$ & $=$ & $\bar{z}$ & $\bar{z}$ & 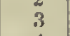 & & & & & & & & & & & & & \\
\hline $\begin{array}{l}5 \\
6 \\
6\end{array}$ & - & - & - & - & - & $=$ & & & & & & & & & & & & & & & & & & & & 97. & \\
\hline 8 & - & - & $=$ & $=$ & 二 & - & - & - & - & - & - & - & - & 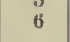 & 1983 & 9.010 & 9.002 & $\begin{array}{l}9.051 \\
\end{array}$ & $\begin{array}{l}9.077 \\
9.07\end{array}$ & 79 & 883 & $9 \cdot 115$ & 9.085 & 9.084 & •038 & 9.054 & \\
\hline 9 & $8 \cdot 862$ & $8 \cdot 867$ & $8 \cdot 891$ & $8 \cdot 891$ & $8 \cdot 89$ & $8 \cdot \overline{8} 99$ & 8.903 & $8 \cdot 911$ & $8 \cdot 928$ & $8 \cdot 011$ & $8 \cdot 9.92$ & $8 \cdot 913$ & $8 \cdot 903$ & 7 & . & $8 \cdot 993$ & $8 \cdot 93$ & $8 \cdot 967$ & 944 & 4.) & 28 & 929 & 93.5] & 28 & 132 & 4.1. & \\
\hline 10 & 8 & $8 \cdot 967$ & $y$ & 8 & 051 & 9 & $8 \cdot 116$ & $9 \cdot 127$ & $g^{\circ}$ & $9 \cdot 165$ & 9.215 & 9. & $9 \cdot \mathrm{c}$ & & & & & & & & & & & & 11 & & \\
\hline 11 & & & & & & & & & & & & & & 10 & & $9 \cdot 205$ & $9 \cdot 209$ & & & & & & & & & & \\
\hline 12 & 9. & $9 \cdot 230$ & 2283 & 8 & (i) & & & $9 \cdot 115$ & $\begin{array}{c}0 \cdot 101 \\
\end{array}$ & 9.091 & $9^{\circ}$ & 9. & 9. & 11 & 986 & $8 \cdot 991$ & $9 \cdot 013$ & $9 \cdot 105$ & $9 \cdot 129$ & $\begin{array}{l}9 \\
9\end{array}$ & $9 \cdot 215$ & $9 \cdot 217$ & $|0 \cdot 305|$ & $9.310^{\circ}$ & $9 \cdot 311$ & $9 \cdot 427$ & \\
\hline 11 & 9.0 & $9 \cdot 069$ & 1.086 & $9 \cdot 116$ & 33 & $9 \cdot 137$ & $\begin{array}{l}8.145 \\
9 \cdot 14\end{array}$ & $\begin{array}{l}8.60 \\
9 \cdot 146\end{array}$ & $9 \cdot 158$ & $9 \cdot 159$ & $9 \cdot 179$ & 9.159 & $\begin{array}{l}8.198 \\
9 \cdot 128\end{array}$ & 12 & 2 & 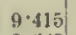 & 0 & $9^{\circ}$ & m5. & & 9.572 & $9 \cdot 595$ & & 649 & 67 & $7 \cdot 691$ & \\
\hline 10 & & & & & $9 \cdot 245$ & & & 9.280 & $9 \cdot 310$ & $9 \cdot 3$ & & & & & & & & & & & & & & & & & \\
\hline if & & & & & 9.463 & & & & 9 & & & & & 15 & 3 & G. 683 & $\theta^{\circ}$ & $8 \cdot$ & & & & & & & & & \\
\hline 17 & $8^{\circ}$ & 9. & & & $\begin{array}{l}9 * 356 \\
0.409\end{array}$ & & & $\begin{array}{r}8 \cdot 291 \\
8 \cdot 09\end{array}$ & & $9 \cdot 323$ & 9.352 & & & 16 & .398 & $9 \cdot 3 \pi 4$ & 9.38 .3 & $9 \cdot 3 i 4$ & 9 & 79 & 4 & 10 & $9 \cdot 335$ & $9 \cdot 3 \times \mathrm{Mi}$ & 8 & $9 \cdot 341$ & \\
\hline 19 & $8 \cdot 8$ & 8.992 & $\begin{array}{l}8.027 \\
9.07\end{array}$ & .064 & 9.061 & $9 \cdot 056$ & 9.089 & $9 \cdot 102$ & $9-132$ & $9 \cdot 164$ & $9 \cdot 242$ & $9 \cdot 235$ & $9 \cdot 095$ & 17 & 38 & $9 \cdot$ & $9 \cdot 326$ & 9.426 & 60 & 0 & & 9.53 & 9.567 & 9.509 & & $7 \cdot 60 ?$ & \\
\hline & 9. & & & & 238 & & & 9. & 9. & & & & & & & & 9. & 9. & & & & 0 & & & & & \\
\hline & $9 \cdot 153$ & & & & $9 \cdot 209$ & & & & & $9 \cdot 304$ & $9 \cdot 36 t^{6}$ & $9 \cdot 378$ & & 21) & & $9 \cdot$ & $\begin{array}{l}9 \cdot 607 \\
9.60\end{array}$ & $8 \cdot$ - & & & & & & & & & \\
\hline $\begin{array}{l}32 \\
23\end{array}$ & $\begin{array}{l}9.439 \\
9.550\end{array}$ & $\begin{array}{l}3+468 \\
9-537\end{array}$ & $\begin{array}{l}9 \cdot 511 \\
9 \cdot 492\end{array}$ & $\begin{array}{l}9 \cdot 552 \\
9 \cdot 458\end{array}$ & $\begin{array}{l}9 \cdot 572 \\
8 \cdot 434\end{array}$ & $\begin{array}{l}9.56160 \\
94402\end{array}$ & $\begin{array}{l}9 \cdot 5350 \\
9 \cdot 35\end{array}$ & $\begin{array}{l}9 \cdot 589 \\
9 \cdot 339\end{array}$ & $\begin{array}{l}9 \cdot 559 \\
9 \cdot 322\end{array}$ & $\begin{array}{l}9.574 \\
8.297\end{array}$ & $\begin{array}{l}9 \cdot 5 k 2 \\
9 \cdot 257\end{array}$ & $\begin{array}{l}9 \cdot 515 \\
9 \cdot 221\end{array}$ & $\begin{array}{l}9.540 \\
9.389\end{array}$ & 21 & 650 & 9.639 & 9.591 & $9 \cdot 603$ & 1 & fas & & & 8.636 & 9 & 54 & 5 & \\
\hline 2 & $9 \cdot 213$ & $9 \cdot 2100$ & 9.201 & 9.214 & $9 \cdot 217$ & $9 \cdot 214$ & $9 \cdot 252$ & $9 \cdot 27$ & $9-3016$ & $9 \cdot 318$ & $9 \cdot 344^{\circ}$ & 9.357 & $9 \cdot 2$ & 22 & & $9 \cdot 4$ & 9. & 9. & & & & $9 \cdot 431$ & & & $9 \cdot 322$ & & \\
\hline \multirow{6}{*}{$\begin{array}{l}25 \\
291 \\
27 \\
28\end{array}$} & \multirow{6}{*}{$\begin{array}{l}9 \cdot 373 \\
9 \cdot 385 \\
0 \cdot \cdot 72 \\
9 \cdot 432\end{array}$} & \multirow{6}{*}{$\begin{array}{l}9 \cdot 389 \\
8 \cdot 252 \\
9-276 \\
8.428\end{array}$} & \multirow{6}{*}{$\begin{array}{l}9 \cdot 408 \\
9 \cdot 333 \\
9 \cdot 274 \\
9 \cdot 440\end{array}$} & \multirow{6}{*}{$\begin{array}{c}9 \cdot 416 \\
9 \cdot 328 \\
9 \cdot 278 \\
9 \cdot 247 \\
.\end{array}$} & \multirow{6}{*}{$\begin{array}{l}9 \cdot 409 \\
9 \cdot 304 \\
9 \cdot 305 \\
9 \cdot 451\end{array}$} & \multirow{6}{*}{$\begin{array}{l}9 \cdot 424 \\
9 \cdot 215 \\
8 \cdot 326 \\
9 \cdot 451\end{array}$} & \multirow{6}{*}{$\begin{array}{l}9 \cdot 439 \\
9 \cdot 2.12 \\
9 \cdot 338 \\
9 \cdot 441\end{array}$} & \multirow{6}{*}{$\begin{array}{l}9 \cdot 452 \\
9 \cdot 221 \\
9 \cdot 375 \\
9 \cdot 437\end{array}$} & \multirow{6}{*}{$\begin{array}{l}9 \cdot 453 \\
9 \cdot 201 \\
9 \cdot 342 \\
9 \cdot 428\end{array}$} & \multirow{6}{*}{$\begin{array}{l}9 \cdot 452 \\
9 \cdot 2 \cdot 2.31 \\
9 \cdot 38 i \\
9 \cdot 411\end{array}$} & \multirow{6}{*}{$\begin{array}{l}8 \cdot 427 \\
9 \cdot 210 \\
9 \cdot 392 \\
9 \cdot 441\end{array}$} & \multirow{6}{*}{$\begin{array}{l}9 \cdot 410 \\
9 \cdot 254 \\
\theta-2504 \\
9 \cdot 457\end{array}$} & \multirow{6}{*}{$\begin{array}{l}9 \cdot 421 \\
9 \cdot 281 \\
9 \cdot 2831 \\
9 \cdot 442\end{array}$} & 24 & & & & $9 \cdot 330$ & & $8 \cdot 9 \cdot 373$ & & $9 \cdot 407$ & & & & & \\
\hline & & & & & & & & & & & & & & $\begin{array}{l}25 \\
26\end{array}$ & $\mid \begin{array}{l}9 \cdot 117 \\
9 \cdot 3332\end{array}$ & $\begin{array}{l}9 \cdot 395 \\
9 \cdot 318\end{array}$ & $\begin{array}{l}9 \cdot 402 \\
9 \cdot 361\end{array}$ & $\begin{array}{r}8 \cdot 395 \\
9 \cdot 381\end{array}$ & $\begin{array}{l}9 \cdot 391 \\
9 \cdot 350\end{array}$ & $\begin{array}{lll}1 & 9 \cdot 370 \\
0 & 9 \cdot 364\end{array}$ & & $\begin{array}{l}9 \cdot 299 \\
9 \cdot 37\end{array}$ & \begin{tabular}{|l|}
$9 \cdot 240$ \\
$9 \cdot 325$
\end{tabular} & $\begin{array}{l}9.286 \\
9.310\end{array}$ & $\begin{array}{c}9 \cdot 304 \\
9 \cdot 312\end{array}$ & $\mid \begin{array}{l}9.331 \\
9 \cdot 347\end{array}$ & \\
\hline & & & & & & & & & & & & & & & & & & & & & & & . & & & & \\
\hline & & & & & & & & & & & & & & & & & & & & & & & & & & & \\
\hline & & & & & & & & & & & & & & & & & & & & & & & & & & & \\
\hline & & & & & & & & & & & & & & & & & & & & Sous & & & & 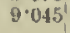 & 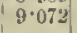 & & \\
\hline & & & & & & & & & & & & & & & $9 \cdot 334$ & & 3 & $9 \cdot 310$ & 9.335 & 327 & 322 & 224 & 398 & -329' & 328 & -332 & \\
\hline
\end{tabular}

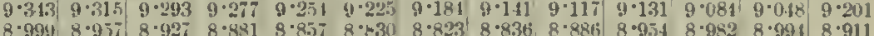

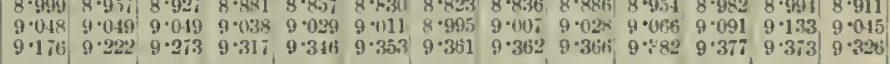

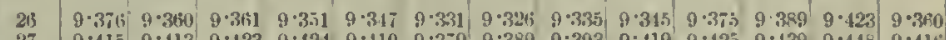

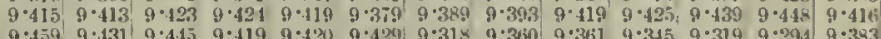

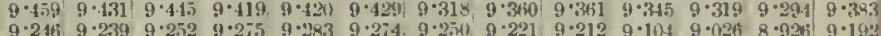

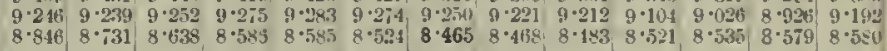

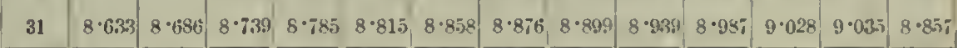

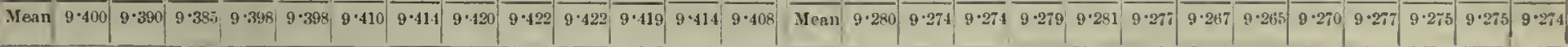


TABLE I. (continued).

\section{PRESSURE IN ENGLISH INCIES, FIRST FIGURE OMITTED.}

The Readings are Corrected for Temperature, and Redneed to Mean Sea Level, but are not Corrected for Variation of Gravity.

June, 1902.

July, 1902 .

\begin{tabular}{|c|c|c|c|c|c|c|c|c|c|c|c|c|c|}
\hline Day. & 2. & 4. & 6. & 8. & 10. & Yoon. & 14. & 16. & 18. & 20. & 22. & Midt & Iear \\
\hline $\begin{array}{l}3 \\
2 \\
3\end{array}$ & $\begin{array}{l}\text { ins. } \\
9 \cdot 063 \\
9 \cdot 105 \\
9 \cdot 296 \\
9 \cdot 601\end{array}$ & $\begin{array}{l}\text { ins. } \\
9 \cdot 103 \\
9 \cdot 091 \\
9 \cdot 323 \\
9 \cdot 581\end{array}$ & $\begin{array}{l}\text { Ins. } \\
9 \cdot 130 \\
9 \cdot 090 \\
8 \cdot 407 \\
9 \cdot 560\end{array}$ & $\begin{array}{l}\text { ins } \\
9 \cdot 151 \\
9 \cdot 078 \\
9 \cdot 4.432 \\
9 \cdot 535\end{array}$ & $\begin{array}{l}\text { Ins. } \\
9 \cdot 168 \\
9 \cdot 049 \\
9 \cdot 456 \\
9 \cdot 535\end{array}$ & $\mid \begin{array}{l}\text { ins. } \\
9 \cdot 169 \\
9 \cdot 025 \\
9 \cdot 462 \\
9 \cdot 501\end{array}$ & $\begin{array}{l}\text { ins. } \\
9.162 \\
9 \cdot 001 \\
9.494 \\
9499\end{array}$ & $\begin{array}{l}\text { ins } \\
9 \cdot 158 \\
9 \cdot 025 \\
9 \cdot 505 \\
9 \cdot 517\end{array}$ & $\left|\begin{array}{l}\text { ins. } \\
9 \cdot 141 \\
9-054 \\
8.5353 \\
9 \cdot 533\end{array}\right|$ & $\mid$\begin{tabular}{l|} 
ins. \\
$9 \cdot 14.3$ \\
$9 \cdot 138$ \\
9.5759 \\
$9 \cdot 606$
\end{tabular} & $\left|\begin{array}{l|}\text { ins. } \\
9 \cdot 131 \\
9 \cdot-221 \\
9 \cdot 632 \\
9 \cdot 595\end{array}\right|$ & $\mid$\begin{tabular}{l|} 
ins \\
9.117 \\
$9 \cdot 282$ \\
9.607 \\
$9 \cdot 601$
\end{tabular} & $\begin{array}{l}\text { ins } \\
9.138 \\
9.096 \\
9.482 \\
9.556\end{array}$ \\
\hline $\begin{array}{l}6 \\
7 \\
8 \\
9\end{array}$ & $\begin{array}{l}9 \cdot 618 \\
9 \cdot 753 \\
9 \cdot 515 \\
9 \cdot 720 \\
9 \cdot 830\end{array}$ & $\begin{array}{l}9 \cdot 630 \\
9 \cdot 745 \\
9 \cdot 475 \\
9 \cdot 72 \\
9 \cdot 855\end{array}$ & $\begin{array}{l}9 \cdot 652 \\
9 \cdot 762 \\
9 \cdot 451 \\
9 \cdot 737 \\
9 \cdot 836\end{array}$ & $\begin{array}{l}9 \cdot 840 \\
9 \cdot 767 \\
9 \cdot 449 \\
9 \cdot 735 \\
9 \cdot 84\end{array}$ & $\begin{array}{l}9 \cdot 646 \\
9 \cdot 763 \\
9 \cdot-749 \\
9 \cdot 78 \bar{i} \\
9 \cdot 814\end{array}$ & & $\mid \begin{array}{r}9 \cdot 685 \\
9 \cdot 710 \\
9.510 \\
9.908 \\
9 \cdot 841\end{array}$ & $\begin{array}{l}9 \cdot 689 \\
9 \cdot 675 \\
9 \cdot 575 \\
9 \cdot 931 \\
9 \cdot 829\end{array}$ & $\begin{array}{l}8 \cdot 699 \\
9-613 \\
9 \cdot 4 \cdot 47 \\
9 \cdot 944 \\
9-831\end{array}$ & $\left|\begin{array}{l}9 \cdot 715 \\
9 \cdot 544 \\
9 \cdot 706 \\
9 \cdot 931 \\
9 \cdot 825\end{array}\right|$ & $\mid \begin{array}{l}9 \cdot 738 \\
9 \cdot 488 \\
9 \cdot 696 \\
9 \cdot 929 \\
9 \cdot 816\end{array}$ & $\left|\begin{array}{l|}9 \cdot 747 \\
9 \cdot 497 \\
9 \cdot 701 \\
9 \cdot 8888 \\
9 \cdot 792\end{array}\right|$ & $\begin{array}{l}9.553 \\
9.841 \\
9.829\end{array}$ \\
\hline $\begin{array}{l}10 \\
11 \\
12 \\
13 \\
14\end{array}$ & $\begin{array}{l}9 \cdot 790 \\
9 \cdot 600 \\
9 \cdot 336\end{array}$ & $\begin{array}{l}9 \cdot 781 \\
9 \cdot 348 \\
9 \cdot 824 \\
9 \cdot 621 \\
9 \cdot 296\end{array}$ & $\begin{array}{l}9 \cdot 729 \\
9 \cdot 332 \\
9 \cdot 838 \\
9 \cdot 622 \\
9 \cdot 247\end{array}$ & $\begin{array}{l}9 \cdot 676 \\
9 \cdot 317 \\
9 \cdot 812 \\
9 \cdot 657 \\
9 \cdot 159\end{array}$ & $\begin{array}{l}9 \cdot 604 \\
9 \cdot 33.8 \\
9 \cdot 749 \\
9 \cdot 665 \\
9 \cdot 122\end{array}$ & & $\begin{array}{l}9.351 \\
9.395 \\
9 \cdot 699 \\
9.609 \\
9 \cdot 012\end{array}$ & $\begin{array}{l}9 \cdot 501 \\
9 \cdot 495 \\
9+481 \\
9 \cdot 546 \\
8 \cdot 946\end{array}$ & $\begin{array}{l}8 \cdot 450 \\
9 \cdot 0552 \\
9 \cdot 6 \cdot 69 \\
9 \cdot 554 \\
8 \cdot 932\end{array}$ & $\begin{array}{l}9 \cdot 428 \\
9 \cdot 603 \\
9 \cdot 663 \\
9 \cdot 504 \\
8 \cdot 904\end{array}$ & $\mid \begin{array}{l}9 \cdot 408 \\
9 \cdot 647 \\
9 \cdot 657 \\
9 \cdot 462 \\
8 \cdot 913\end{array}$ & $\mid \begin{array}{l}9 \cdot 698 \\
9 \cdot 656 \\
9 \cdot 393 \\
8 \cdot 921 \\
8 \cdot 921\end{array}$ & $\begin{array}{l}9.453 \\
9.731 \\
9 \cdot 5 \% 4 \\
9.074\end{array}$ \\
\hline 11 & $\begin{array}{l}8 \cdot 959 \\
8 \cdot 950 \\
9 \cdot 111 \\
9 \cdot 356\end{array}$ & $\begin{array}{l}8 \cdot 921 \\
8 \cdot 895 \\
8 \cdot 958 \\
9 \cdot 126 \\
9 \cdot 350\end{array}$ & $\begin{array}{l}8 \cdot 916 \\
8 \cdot 803 \\
8 \cdot 999 \\
9 \cdot 107 \\
9 \cdot 373\end{array}$ & $\begin{array}{l}8 \cdot 922 \\
8 \cdot-\cdot 753 \\
9 \cdot 079 \\
9 \cdot 139 \\
9 \cdot 372\end{array}$ & $\begin{array}{l}8 \cdot 938 \\
8 \cdot 653 \\
9 \cdot 117 \\
9 \cdot 157 \\
9 \cdot 380\end{array}$ & & $\begin{array}{l}8 \cdot 343 \\
9 \cdot 147 \\
9 \cdot 223 \\
9 \cdot 341\end{array}$ & & $\mid \begin{array}{l}8 \cdot 487 \\
9 \cdot 165 \\
9 \cdot 27 \\
9 \cdot 287\end{array}$ & $\left|\begin{array}{l}9 \cdot 568 \\
9 \cdot 161 \\
9 \cdot 301 \\
9 \cdot 281\end{array}\right|$ & $\left|\begin{array}{l}9 \cdot 054 \\
8 \cdot 730 \\
9 \cdot 145 \\
9 \cdot 319 \\
9 \cdot 092\end{array}\right|$ & $\begin{array}{l}8 \cdot 868 \\
9 \cdot 125 \\
9 \cdot 335 \\
9 \cdot 276\end{array}$ & {$\left[\begin{array}{l}8 \cdot 695 \\
9 \cdot 095 \\
9 \cdot 211 \\
9 \cdot 332\end{array}\right.$} \\
\hline 24 & $\begin{array}{l}9 \cdot 2 \cdot 164 \\
9 \cdot 167 \\
9 \cdot 287 \\
8 \cdot 93,9\end{array}$ & $\begin{array}{l}9 \cdot 297 \\
9 \cdot 177 \\
9 \cdot 193 \\
9 \cdot 265 \\
8 \cdot 937\end{array}$ & $\begin{array}{l}9 \cdot 311 \\
9 \cdot 145 \\
9 \cdot 222 \\
9 \cdot 2233 \\
8 \cdot 935\end{array}$ & $\begin{array}{l}9 \cdot 354 \\
9 \cdot 175 \\
9 \cdot 250 \\
9 \cdot 181 \\
8 \cdot 991\end{array}$ & $\begin{array}{l}9 \cdot 385 \\
9 \cdot 189 \\
9 \cdot 274 \\
9 \cdot 156 \\
9 \cdot 013\end{array}$ & & & $\begin{array}{l}9 \cdot 303 \\
9 \cdot 157 \\
9 \cdot-309 \\
9 \cdot 097 \\
9 \cdot 083\end{array}$ & & $\begin{array}{l}9 \cdot 225 \\
9 \cdot 110 \\
9 \cdot 304 \\
9 \cdot 021 \\
9 \cdot 092\end{array}$ & $\begin{array}{l}9 \cdot 194 \\
9 \cdot 102 \\
9 \cdot 3 \cdot 316 \\
8 \cdot 987 \\
9 \cdot 09 .\end{array}$ & $\begin{array}{l}9 \cdot 134 \\
9 \cdot 143 \\
9 \cdot 305 \\
\cdot \cdot 943 \\
9 \cdot 073\end{array}$ & $\mid \begin{array}{l}9 \cdot 291 \\
9 \cdot 157 \\
9 \cdot 272 \\
9 \cdot 124 \\
9 \cdot 030\end{array}$ \\
\hline $\begin{array}{l}25 \\
28 \\
27 \\
28 \\
29\end{array}$ & $\begin{array}{l}9 \cdot 069 \\
9 \cdot 068 \\
8 \cdot 665 \\
8 \cdot 439 \\
8 \cdot 690\end{array}$ & $\begin{array}{l}9 \cdot 083 \\
9 \cdot 039 \\
8 \cdot 700 \\
8 \cdot 357 \\
8 \cdot 741\end{array}$ & $\begin{array}{l}8 \cdot 086 \\
8 \cdot 011 \\
8 \cdot 699 \\
8 \cdot 298 \\
8 \cdot 839\end{array}$ & $\begin{array}{l}9 \cdot 107 \\
8 \cdot 057 \\
8 \cdot 600 \\
8 \cdot 248 \\
8 \cdot 901\end{array}$ & $\begin{array}{l}9 \cdot 117 \\
8 \cdot 925 \\
8 \cdot 627 \\
8 \cdot 192 \\
8 \cdot 943\end{array}$ & $\begin{array}{l}9 \cdot 135 \\
8 \cdot 897 \\
8.640 \\
8.140 \\
8.989\end{array}$ & $\begin{array}{l}9 \cdot 140 \\
8.841 \\
8 \cdot 658 \\
8 \cdot 2006 \\
9 \cdot 059\end{array}$ & $\begin{array}{l}9 \cdot 156 \\
8 \cdot \cdot 788 \\
8 \cdot 628 \\
8 \cdot 197 \\
9 \cdot 094\end{array}$ & $\begin{array}{l}9 \cdot 141 \\
8 \cdot 761 \\
8 \cdot 609 \\
8 \cdot 252 \\
9 \cdot 113\end{array}$ & $\begin{array}{l}9 \cdot 132 \\
8 \cdot 763 \\
8 \cdot 541 \\
8 \cdot 404 \\
9 \cdot 122\end{array}$ & $\begin{array}{l}9 \cdot 123 \\
8.6993 \\
8.499 \\
8.554 \\
9 \cdot 146\end{array}$ & $\begin{array}{l}9 \cdot 096 \\
8 \cdot 693 \\
8 \cdot 454 \\
8 \cdot 6.32 \\
9 \cdot 125\end{array}$ & $\mid$\begin{tabular}{|}
$8 \cdot 870$ \\
$8 \cdot 611$ \\
$8 \cdot 330$ \\
$8 \cdot 932$
\end{tabular} \\
\hline & .16 & $9 \cdot 15$ & $\cdot 141$ & 11 & 102 & $9 \cdot 0$ & 03 & 9.0 & & & $8^{\circ}$ & -880 & $9^{\circ} 01$ \\
\hline
\end{tabular}

\begin{tabular}{|c|c|c|c|c|c|c|c|c|c|c|c|c|c|}
\hline Day. & 2. & 4. & 6. & 8. & 10. & Noon. & 14. & 16. & 18. & 20. & 22. & Mint & Mean. \\
\hline & $\begin{array}{l}\text { ins } \\
8 \cdot 759\end{array}$ & $\begin{array}{l}\text { ins. } \\
8 \cdot 722\end{array}$ & 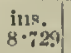 & ${ }_{8-714}^{\text {Ins. }}$ & $\operatorname{lins}_{8 \cdot 7 i 4}$ & $\mid \begin{array}{l}\text { fins. } \\
8 \cdot 719\end{array}$ & $\begin{array}{l}\text { ins. } \\
8 \rightarrow 709\end{array}$ & $\begin{array}{l}\text { ins. } \\
8 \cdot 73,1\end{array}$ & $\operatorname{lins}_{8 \cdot 746}$ & $\begin{array}{l}\text { ins. } \\
8 \cdot 799\end{array}$ & $\operatorname{lin}_{8 \cdot 808}$ & $\begin{array}{l}\text { ins. } \\
8 \cdot 756\end{array}$ & ${ }_{8.741}^{\text {ins }}$ \\
\hline $\begin{array}{l}2 \\
3\end{array}$ & $\begin{array}{l}8.788 \\
8.826\end{array}$ & $\begin{array}{l}8.776 \\
8.814\end{array}$ & $\begin{array}{l}8 \cdot 772 \\
8 \cdot 779\end{array}$ & $\begin{array}{l}8 \cdot 796 \\
87\end{array}$ & $\begin{array}{l}8 \cdot 797 \\
8 \cdot 740\end{array}$ & $\begin{array}{l}8.810 \\
8.710\end{array}$ & $\begin{array}{l}8 \cdot 841 \\
8.691\end{array}$ & $\begin{array}{l}8 \cdot 86 \overline{1} \\
8 \cdot 67\end{array}$ & $\begin{array}{l}8 \cdot 877 \\
8 \cdot 616\end{array}$ & $\begin{array}{l}8 \cdot 75 \\
8.534\end{array}$ & $\begin{array}{l}8 \cdot 8713 \\
8.518\end{array}$ & 8.849 & $8 \cdot 827$ \\
\hline 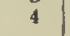 & 8.401 & $8 \cdot 331$ & $8 \cdot x+4$ & $8 \cdot 304$ & $8 \cdot 338$ & $8 \cdot 343$ & $8 \cdot 353$ & 8.294 & $8 \cdot 257$ & 8.2246 & $8 \cdot 177$ & $8 \cdot 167$ & $8 \cdot 291$ \\
\hline 5 & & & $8 \cdot 396$ & $8 \cdot 53 B$ & $8 \cdot 689$ & 8798 & 8.928 & $9 \cdot 03 \pi$ & $9 \cdot 170$ & $9 \cdot 251$ & $9=297$ & $9 \cdot 398$ & $8 \cdot 821$ \\
\hline 6 & 9. & ;) & $\begin{array}{l}9 \cdot 407 \\
9.397\end{array}$ & $9 \cdot 4+43$ & $\begin{array}{r}94.491 \\
0.371\end{array}$ & $\mid$\begin{tabular}{|r}
$9 \cdot 0.01$ \\
$\Omega \cdot 346$
\end{tabular} & 9.509 & $9 \cdot 4 \cdot 4$ & $\begin{array}{l}9 \cdot 479 \\
4.278\end{array}$ & $\begin{array}{l}9 \cdot 470 \\
0 \cdot 045\end{array}$ & $\begin{array}{c}0 \cdot 461 \\
0 \cdot 0341\end{array}$ & $\left|\begin{array}{c}9 \cdot 427 \\
0 \cdot 218\end{array}\right|$ & {$[\cdot 449$} \\
\hline & 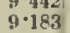 & $9 \cdot 159$ & $9 \cdot 134$ & $9 \cdot 129$ & $9 \cdot 121$ & $9 \cdot 135$ & $8 \cdot 139$ & $9 \cdot 155$ & $9 \cdot 181$ & 9.209 & $\theta \cdot 215$ & 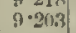 & $9 \cdot 114$ \\
\hline 9 & $9 \cdot 183$ & $9 \cdot 206$ & $9 \cdot 231$ & $9 \cdot 261$ & $9 \cdot 245$ & $9 \cdot 213$ & $9 \cdot 180$ & $9 \cdot 156$ & $9 \cdot 135$ & $9 \cdot 080$ & 9.028 & $8 \cdot 986$ & $9 \cdot 159$ \\
\hline 1 & $8 \cdot 949$ & 99 & $8 \cdot 869$ & $8 \cdot 834$ & 8.818 & $8 \cdot 806$ & $8 \cdot 808$ & $8 \cdot 834$ & $8 \cdot 859$ & 8.946 & 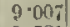 & (10.3) & $8 \cdot 895$ \\
\hline & & & & & $\begin{array}{l}9.4633 \\
9.838\end{array}$ & & & & $\begin{array}{l}4652 \\
4.8899\end{array}$ & & $\begin{array}{c}9 \cdot 721 \\
9 \cdot 0903\end{array}$ & & \\
\hline 13 & 877 & $\begin{array}{l}9.76 \\
9.8\end{array}$ & $\begin{array}{l}9805 \\
9 \cdot 877\end{array}$ & $\begin{array}{l}9.818 .907\end{array}$ & $\begin{array}{l}9.838 \\
9.889\end{array}$ & $\begin{array}{l}98862 \\
9.893\end{array}$ & $9 \cdot 897$ & $\begin{array}{l}9.873 \\
9 \cdot 87\end{array}$ & 9.879 & $\frac{18511}{9.85}$ & 9.837 & 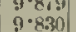 & 8 \\
\hline 14 & $9 \cdot 815$ & 9.802 & $9 \cdot 798$ & $9 \cdot 765$ & $9 \cdot 751$ & $9 \cdot 750\}$ & $3 \cdot 726$ & $9 \cdot 688$ & $9^{\circ} 696$ & $9 \cdot 659$ & $9 \cdot 624$ & 1600 & $9 \cdot 723$ \\
\hline 15 & $9 \cdot 5$ & $9 \cdot 592$ & 9.576 & $9 \cdot 551$ & $9 \cdot 523$ & 9.513 & 10.060 & 9. & $9 \cdot 504$ & $9 \cdot 487$ & $\begin{array}{r}9.468 \\
0.568\end{array}$ & 1 & 9.52 \\
\hline & $9 \cdot 4$ & 0 & $\begin{array}{r}9 \cdot 454 \\
90650\end{array}$ & $\begin{array}{l}9 \cdot 506 \\
9-649\end{array}$ & $\begin{array}{r}9.533 \\
9.666\end{array}$ & 9.54 & $\begin{array}{l}10 \cdot 561 \\
9 \cdot 672\end{array}$ & $\begin{array}{ll}9.5946 \\
8\end{array}$ & $\begin{array}{l}9626 \\
96767\end{array}$ & $\begin{array}{r}9 \cdot 642 \\
9 \cdot 07\end{array}$ & $\left|\begin{array}{r}9 \cdot 652 \\
9 \cdot 691\end{array}\right|$ & & कit \\
\hline & $9 \cdot 6$ & & $8 \cdot 701$ & $9 \cdot 656$ & $0-603$ & $9 \cdot 571$ & 0 & $9 \cdot 492$ & & & $9 \cdot 599$ & & \\
\hline 19 & 640 & 9.660 & $9 \cdot 620$ & $9 \times 620$ & $9 \cdot 648$ & 9669 & $9 \cdot 774$ & 9. & $9 \cdot 832$ & 9.814 & $9 \cdot 847$ & $\mid 9 \cdot 843\}$ & $9 \cdot 7$ \\
\hline 20 & $9 \cdot 839$ & $9 \cdot 827$ & $9 \cdot 805$ & $9 \cdot 779$ & 9.758 & $9 \cdot 691$ & $9 \cdot$ & $\therefore$ & $9: 543$ & 9.528 & $9 \cdot 493$ & & $9^{\circ} 664$ \\
\hline & $9 \cdot 9 \cdot 9$ & $\begin{array}{l}9.47 \\
9 \cdot 57\end{array}$ & $\begin{array}{l}9.48 \\
9.60\end{array}$ & $\begin{array}{l}9 \cdot 484 \\
96411\end{array}$ & $\begin{array}{l}9495 \\
8.68\end{array}$ & $\begin{array}{l}9 \cdot 495 \\
9.617\end{array}$ & $\begin{array}{l}8 \cdot 001 \\
8.624\end{array}$ & $\begin{array}{l}9.507 \\
9-626\end{array}$ & $\begin{array}{r}952525 \\
9.628\end{array}$ & $\begin{array}{l}9 \cdot 531 \\
9 \cdot 621\end{array}$ & $\left|\begin{array}{c}9.512 \\
9.614\end{array}\right|$ & $\left|\begin{array}{l}9.565 \\
9.598\end{array}\right|$ & 9 - tins \\
\hline ? & & & 9.5 & $9 \cdot 621$ & $9 \cdot 614$ & $9 \cdot 603$ & $9 \cdot 598$ & $8 \cdot 591$ & 0.583 & 6.579 & $9.5 \pi$ & & $8-5 \div 1$ \\
\hline 2. & $9 \cdot 569$ & $9 \cdot 545$ & $9 \cdot 563$ & $9 \cdot 563$ & $9 \cdot 568$ & $9 \cdot 545$ & $9: 541$ & 9.541 & $9.53 \mathrm{v}$ & 9.523 & 9.524 & 9.512 & $9 \cdot 543$ \\
\hline 25 & 9.5 & $9 \cdot 523$ & $9 \cdot 5 \overline{7} 7$ & $9 \cdot 513$ & $9 \cdot 522$ & $9 \cdot 573$ & 9.570 & $9 \cdot 519$ & 9.510 & $9 \cdot 506$ & 9.512 & 9.525 & $9:$ \\
\hline & & $9 \cdot 3$ & 9.5 & $\begin{array}{l}8 \cdot 4 \\
9 \cdot 3\end{array}$ & $\begin{array}{l}9 \cdot 498 \\
9.318\end{array}$ & $\begin{array}{l}9490 \\
94300\end{array}$ & $\begin{array}{l}9 \cdot 489 \\
9 \cdot 270\end{array}$ & $\begin{array}{l}9 \cdot 4 \\
9 \cdot 2\end{array}$ & $\begin{array}{l}9.8657 \\
9.224\end{array}$ & $\begin{array}{l}9.459 \\
9 \cdot 192\end{array}$ & $\begin{array}{l}9 \cdot 411 \\
9 \cdot 180\end{array}$ & & \\
\hline & & & & 0.1 & & 0 & $9 \cdot 1$ & 9.1 & & & & & \\
\hline 20 & $9 \cdot 17$ & $\cdot 191$ & & $9-213$ & $\cdot 218$ & .20 & $\cdot 198$ & -1 & $: 1$ & 1215 & 14 & & \\
\hline & & & $9 \cdot 075$ & $9 \cdot 06 \pi$ & $9 \cdot 0 ; 0$ & 9.064 & $9 \cdot 065$ & 9007 & 9.08 & $\theta \cdot 13$ & $9 \cdot 163$ & & 9.100 \\
\hline & & & & & & & & & & & & & \\
\hline & & & & & & 331 & $9 \cdot 337$ & 233 & & 338 & 335 & & \\
\hline
\end{tabular}

August, 1902.

September, 1902.

\begin{tabular}{|c|c|c|c|c|c|c|c|c|c|c|c|c|c|c|c|c|c|c|c|c|c|c|c|c|c|c|c|}
\hline Day. & 2. & 4. & 6. & 8. & 10. & Noon. & 14. & 16. & 18. & 20. & 22. & filt. 3 & Mean. & Day. & 2. & 4. & 6. & 8. & 10. & Noon. & 14. & 16. & 18. & 20. & 22. & Miat & Eano। \\
\hline & s. & & & & 9 & & & & 0.17 & & & & & & & & & & & & & & & & & & \\
\hline $\begin{array}{l}1 \\
2\end{array}$ & 28 & $\begin{array}{l}9 \cdot 136 \\
8 \cdot 824\end{array}$ & $\begin{array}{c}9 \cdot 106 \\
8 \cdot 775\end{array}$ & $\begin{array}{l}9 \cdot 1.32 \\
8 \cdot 7.32\end{array}$ & $\begin{array}{l}9 \cdot 179 \\
8 \cdot 746\end{array}$ & $\begin{array}{l}9 \cdot 171 \\
8 \cdot 660\end{array}$ & $\begin{array}{l}9 \cdot 173 \\
8 \cdot 634\end{array}$ & $\begin{array}{l}9 \cdot 155 \\
8 \cdot 596\end{array}$ & $\begin{array}{l}\theta-117 \\
8.502\end{array}$ & $\begin{array}{l}8.091 \\
8 \cdot 597\end{array}$ & & $8 \cdot 990$ & 119 & 1 & & & & 9.120 & $\begin{array}{c}8 \cdot 109 \\
0.59\end{array}$ & $\begin{array}{l}9 \cdot 120 \\
9 \cdot 503\end{array}$ & & & $\begin{array}{l}9.059 \\
90.59\end{array}$ & & $\begin{array}{c}9.329 \\
\end{array}$ & $\begin{array}{l}9.355 \\
9.140\end{array}$ & 163 \\
\hline 3 & 40 & 8.63 & $8 \cdot 662$ & $8 \cdot 741$ & $8 \cdot 765$ & $8 \cdot 813$ & $8 \cdot 858$ & $8 \cdot 880$ & 8.879 & 8.849 & $\begin{array}{l}8.613 \\
8 \cdot 804\end{array}$ & $8 \cdot 720$ & $8 \cdot 771$ & 2 & 412 & $9 \cdot 470$ & & $9 \cdot 512$ & 9.524 & $9 \cdot 503$ & 9.484 & & (9.455 & 4 & 492 & 482 & 4 \\
\hline & & & & & & & & & & & & & & 3 & $\cdot 46$ & $9 \cdot 455$ & $9 \cdot 453$ & $9^{\circ} 45$ & $9 \cdot 472$ & $\cdot 4 t$ & $9 \cdot 494$ & 8 & 0.524 & $52 \pi$ & $\cdot 560$ & 5 & 4 \\
\hline 5 & $\begin{array}{l}8.658 \\
8 \cdot 827\end{array}$ & 8 & 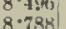 & $\begin{array}{l}8.49 \\
8.777\end{array}$ & $8 \cdot 521$. & 8.580 & 8.579 & $8 \cdot 639$ & $8 \cdot 6 \times 3$ & 8.764 & $8 \cdot 803$ & & $8 \cdot 636$ & 4 & & $9 \cdot 601$ & & $9 \cdot 612$ & $9 \cdot 632$ & 617 & $9 \cdot 650$ & & $9 \cdot 651$ & $9 \cdot(34)$ & $9 \cdot(1.40$ & •641' & tizk \\
\hline 6 & ij & 8.649 & $8 \cdot 1663$ & $8 \cdot 662$ & 8.757 & 8.724 & 8.695 & $8 \cdot 685$ & $8 \cdot 681$ & 679 & 8.677 & 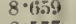 & $8 \cdot 731$ & 5 & & & & & & & $9^{\circ}$ & & & & ) & 8.528 & •5K: \\
\hline 8 & $8 \cdot 596$ & $8 \cdot 613$ & $8 \cdot 619$ & $8 \cdot 648$ & $\begin{array}{l}8.671 \\
8.668\end{array}$ & 3 & $\begin{array}{l}8.639 \\
8.723\end{array}$ & 6835 & $\begin{array}{l}8 \cdot k 22 \\
8 \cdot-62\end{array}$ & $8^{\circ} \cdot 608$ & 8.609 & 8.577 & $8^{\circ} 640$ & 6 & 1 & $90^{\circ}$ & $9 \cdot 51 b$ & & & & & & & & & & \\
\hline 8 & $8 \cdot 827$ & 8.851 & 8.857 & $8 \cdot 970$ & $8 \cdot 981$ & $9 \cdot 014$ & $9 \cdot 035$ & $\begin{array}{l}8.7 .15 \\
9.0 .17\end{array}$ & $\begin{array}{l}8 \cdot 762 \\
9 \cdot 0.11\end{array}$ & $\begin{array}{l}8 \cdot 790 \\
9 \cdot 059\end{array}$ & $\begin{array}{l}8 \cdot 807 \\
9 \cdot 085\end{array}$ & $\begin{array}{l}8 \cdot 801 \\
9 \cdot 090\end{array}$ & $\begin{array}{l}8 \cdot 700 \\
8 \cdot 988\end{array}$ & 7 & 9.670 & $9 \cdot 690$ & $9 \cdot 706$ & $9 \cdot 698$ & 900 & 684 & $9 \cdot 674$ & & $9 \cdot 652$ & & & & \\
\hline 9 & 3 & 699 & $018:$ & & & & & & & & & & & 8 & 5 & 577 & 37 & 9.549 & $9 \cdot 564$ & 59 & 548 & 50 & झ) & -531i & 15 & 515 & $55^{\circ}$ \\
\hline & i & 725 & 0 & 8.718 & & & $8 \cdot 861$ & . & $8 \cdot 807$ & & & 626 & 899 & 9 & & & & $4 \cdot 477$ & & & & & & & & & \\
\hline 11 & $8 \cdot 882$ & $8 \cdot 911$ & $8 \cdot 905$ & $8 \cdot 912$ & $\begin{array}{l}8 \\
8 \cdot(212)\end{array}$ & $\begin{array}{l}8 \cdot 732 \\
8 \cdot 898\end{array}$ & $\begin{array}{r}8.724 \\
8.879\end{array}$ & 8.694 & 8.712 & 750 & $8 \cdot 817$ & $8 \cdot 868$ & 8.744 & 10 & & & $8 \cdot 990$ & & $8 \cdot 91 !$ & & $8 \cdot 8$ & 11 & $8 \cdot 779$ & in & 8 & & \\
\hline & 8.894 & $8 \cdot 785$ & $8 \cdot 821$ & $8 \cdot 811$ & $8 \cdot 857$ & $8 \cdot 816$ & $\begin{array}{l}8.872 \\
8.865\end{array}$ & $\begin{array}{l}8.860 \\
8.919\end{array}$ & $\begin{array}{l}8.847 \\
8.891\end{array}$ & 1 & & $\begin{array}{l}8 \cdot 818 \\
8 \cdot 861\end{array}$ & & $\begin{array}{l}11 \\
12\end{array}$ & & & & & $\begin{array}{r}8 \cdot 531 \\
8 \cdot 434\end{array}$ & & $\begin{array}{l}8.505 \\
8 \cdot 451\end{array}$ & $\begin{array}{l}8.48 \\
8 \cdot 46^{2}\end{array}$ & $\begin{array}{l}8.420 \\
8.496\end{array}$ & $\begin{array}{l}8.104 \\
8 \cdot 508\end{array}$ & $\begin{array}{l}8.375 \\
8.522\end{array}$ & $\begin{array}{l}8 \cdot 361 \\
8 \cdot 56^{\circ}\end{array}$ & $\begin{array}{l}8 \cdot 500 \\
8 \cdot 450\end{array}$ \\
\hline 13 & $8.82 t^{\prime}$ & $8 \cdot 784$ & $8 \cdot T \cdot 3$ & $8 \cdot 738$ & $8 \cdot \pi 12$ & 8.710 & $8 \cdot 710$ & $8 \cdot 6+11$ & $8 \cdot 635$ & $8 \cdot 604$ & 8.599 & 8.570 & $\begin{array}{l}8.890 \\
8.694\end{array}$ & & & & & & & & & & & & & & \\
\hline & 5.3 & & 46 & & & & & & & & & & & : & 6 & 2 & 8: & $8 \cdot 5.30$ & 71 & a & $8 \cdot 6$ & 35. & $8^{\circ}$ & 14 & 2 & & \\
\hline 15 & 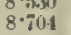 & 8 & 804 & 8.42 & $\begin{array}{l}8 \cdot 462 \\
8 \cdot 8,3\end{array}$ & $\begin{array}{r}8-62 \\
8-8 \times 3\end{array}$ & $\begin{array}{l}488 \\
880\end{array}$ & & $\begin{array}{l}8.521 \\
8 \cdot 403\end{array}$ & & $8 \cdot 581$ & $8^{*} \cdot 1.12$ & 8.514 & 14 & & & & & & & & & & & & $\begin{array}{c}9 \cdot 024 \\
9 \cdot(-3)\end{array}$ & 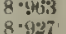 \\
\hline 16 & $8 \cdot 893$ & 822 & $8 \cdot 857$ & 8.85 .4 & $8 \cdot \times 49$ & $8 \cdot 851$ & 8 & $8 \cdot 851$ & 8.8310 & & $8 \cdot 854$ & $8 \cdot 8$ & & & & & & y. 032 & $9 \cdot 008$ & $\begin{array}{l}8 \\
8-988\end{array}$ & 8.4 & $8 \cdot 930$ & $8 \cdot 4+15$ & ij & $8 \div 993$ & $8 \cdot k \times 7$ & . SWHit \\
\hline 17 & 8.809 & $8 \cdot 950)$ & $8 \cdot 8510$ & $8 \cdot 967$ & $8 \cdot 991$ & $8 \cdot 913$ & $8 \cdot 919$ & 8.964 & $9 \cdot 024$ & 3 & $9 \cdot 0$ & $8 \cdot 940$ & 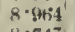 & 17 & $8 \cdot 4$ & & $8 \cdot 488$ & $9 \cdot 015$ & $9 \cdot 061$ & $9 \cdot 115$ & $8 \cdot 155$ & 9 & $y \cdot 2066$ & $9 \cdot 3 \sin$ & $8 \cdot 3 \times 2$ & 2 & $9 \cdot 153$ \\
\hline 18 & $8^{\circ} \cdot 933$ & $8 \cdot 880$ & $8 \cdot 785$ & $8 \cdot 817$ & $8 \cdot 789$ & & $8 \cdot 781$. & $8 \cdot 716$ & $8 \cdot 70 t)$ & 24 & & $8 \cdot 505$ & $\cdot 74 i$ & & & & & & & & & & & & & & \\
\hline 19 & 21 & 490 & 131 & $8 \cdot 403$ & 398 & 354 & $8 \cdot 321$ & $: 30$ & 8.29: & 368 & $8^{\circ}$ & $8 \cdot 146$ & $8^{\circ}$ & & & & & & & & & & & & & & \\
\hline & & & & 8 & 8.426 & 8 & $8 \cdot 357$ & $8 \cdot 390$ & $8 \cdot 308$ & 877 & $8 \cdot 37 \%$ & $8 \cdot 3 \cdot 3 \cdot 11^{1}$ & 8. & 2 & & & & $9 \cdot 5012$ & 5. & & & & & & & & \\
\hline 21 & 8 & $8 \cdot 41$ & 8.426 & $8 \cdot 145$ & 8.503 & $8 \cdot 4 !$ & $8 \cdot 6$ & $8 \cdot 757$ & 8.818 & 3 & $8 \cdot$ & 0 & & 21 & & & & & 1.433 & & 8. & & & 23 & & $\pi$ & $8 \cdot 432$ \\
\hline 2 & 7 & $8 \cdot 7 !$ & $8: 867$ & $8 \cdot 831$ & $8 \cdot 80.4$ & $8 \cdot 805$ & 9 & $8 \cdot 88 b^{\circ}$ & $8^{\circ}$ & ? & & $9 \cdot 156$ & $8 \cdot ?$ & 2 & $9 \cdot 469$ & 467 & $9 \cdot$ & $9 \cdot 4$ & $\{1-555$ & $9 \cdot 5 i 7$ & $9 \cdot 5$ & $9 \cdot 612$ & $9 \cdot$ & $9 \cdot 631$ & (3) 6660 & 5 & • 5 \\
\hline 2. & 23 & $9 \cdot 28$ & $9 \cdot 291$ & $9 \cdot 325$ & $9 \cdot 339$ & $9 \cdot 319$ & $9 \cdot 2 \kappa 9$ & 9.235 & $9 \cdot 186$ & $9 \cdot 152$ & $9 \cdot 098$ & 9.074 & $9 \cdot 2$ & & & & & & & & & & & & & & \\
\hline 21 & $9 \cdot 022$ & $020^{\circ}$ & 1 & 8 & 82 & $8 \cdot 7$ & 7 & & 80481 & & & & & & $\cdot i \tau$ & & & & & & & & & & & & \\
\hline & & & & & & 2. & & | & & & & & & & & & & & & & & & & & & & 31 \\
\hline $2 \hbar$ & 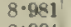 & & 8 & & $y$ & & & $9 \cdot 0$ & & & & 8.9 & $8 \cdot ?$ & & & 9 & & $9 \cdot 488$ & 9.508 & $9 \cdot 483$ & & & & & & $9 \cdot 512$ & $9 \cdot 495$ \\
\hline 27 & & 8 & & (9. & $8 \cdot 9+61$ & 8.957 & 979 & $8 \cdot 9$ & $8 \cdot 9$ & $8 \cdot 9966$ & 8.1 & $8 \cdot 9$ & $8 \cdot 8$ & 27 & $9 \cdot 517$ & $9 \cdot 522$ & $9 \cdot 517$ & $9 \cdot 526$ & $9 \cdot 461$ & $9 \cdot 4.77$ & $9 \cdot 494$ & 9.5113 & $9 \cdot 452$ & $9 \cdot 418$ & $9 \cdot 379$ & $36 i 2$ & $9 \cdot 476$ \\
\hline 20 & & 8 & & $9 \cdot 030$ & $9 \cdot v 60$ & & $9^{\circ} 09 \mathrm{~s}$ & & & 14 & & 9. & & & & & & & & & & & & & & & \\
\hline 29 & 9 & 1563 & 9 & & & $9 \cdot 139$ & & 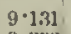 & $9 \cdot 127$ & $9 \cdot 128$ & & & & & & & & & & & & & & & & & \\
\hline & & & 7. & 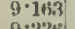 & $9 \cdot 172$ & 0 & $9 \cdot 210$ & $9 \cdot 232$ & $9 \cdot 2$ & $9 \cdot 231$ & g. & $9 \cdot 216$ & & 30 & $9 \cdot 491$ & $9 \cdot 510$ & $9 \cdot 505$ & $9 \cdot 530$ & 9.548 & $9 \cdot 552$ & 9555 & 9.539 & (4) $\cdot 521$ & $8 \cdot 504$ & $8 \cdot 466$ & $9 \cdot 435$ & $9 \cdot 513$ \\
\hline 31 & 9.214 & 9.224 & 9.227 & $9 \cdot 23 t 3$ & $9 \cdot 225$ & $9 \cdot 215$ & $\begin{array}{l}9221 \\
\end{array}$ & $9 \cdot 205$ & 9 & 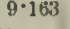 & 38 & $9 \cdot 121$ & & & & & & & & & & & & & & & \\
\hline & $8 \cdot 842$ & & 8 & 8 & 8.841 & 8.834 & 1 & $8 \cdot 846$ & 846 & 347 & $8 \cdot 852$ & 8.14 & $8 \cdot 841$ & ean & $9 \cdot 312$ & $9 \cdot 311$ & 307 & 309 & 311 & $9 \cdot 310$ & 310 & 314 & 316 & 23 & 2.3 & 9.321 & $9 \cdot 314$ \\
\hline
\end{tabular}


TAliL I. (contimued).

\section{PIESSURE IN LNGLISH INCHES, FILST FIGULE OMITTEL.}

The Readings are Corrected for Temperature, and Redueed to NIean Sea Level, but are not Corrected for Variation of Gravity.

October, 1902.

November, 1902.

\begin{tabular}{|c|c|c|c|c|c|c|c|c|c|c|c|c|c|}
\hline Day. & 2. & 4. & 8. & 8. & 10. N & Noon. & 14. & 16. & 18. & 20. & 22. & Midt. & ean. \\
\hline 2 & $\begin{array}{l}\text { Ins. } \\
9 \cdot 395 \\
9 \cdot 30 \times\end{array}$ & $\begin{array}{l}\text { ins. } \\
9 \cdot 359 \\
9 \cdot 2605\end{array}$ & $\begin{array}{l}\text { ints } \\
9 \cdot 333 \\
9 \cdot 277\end{array}$ & $\begin{array}{l}\text { ins. } \\
9 \cdot 314 \\
9 \cdot 307\end{array}$ & $\begin{array}{l}\text { ins. } \\
9 \cdot 282 \\
9 \cdot 313\end{array}$ & $\begin{array}{l}\text { ins. } \\
9 \cdot 234 \\
9 \cdot 334\end{array}$ & $\begin{array}{l}\text { ins } \\
9 \cdot 249 \\
9 \cdot 336\end{array}$ & $\begin{array}{l}\text { ins. } \\
9 \cdot 251 \\
9 \cdot 343\end{array}$ & $\begin{array}{l}\text { Ins } \\
9 \cdot 24,3 \\
9 \cdot 3330\end{array}$ & $\begin{array}{l}\text { ins } \\
9 \cdot 249 \\
9 \cdot 304\end{array}$ & $\begin{array}{l}\text { ins. } \\
9 \cdot 244 \\
9 \cdot 316\end{array}$ & $\begin{array}{l}\text { ins. } \\
9 \cdot 232 \\
9 \cdot 304\end{array}$ & $\begin{array}{l}\text { ins, } \\
9^{2} \cdot 2 s 2 \\
9^{\prime} \cdot 316\end{array}$ \\
\hline $\begin{array}{l}4 \\
5\end{array}$ & $\begin{array}{c}9 \cdot 259 \\
9 \cdot 129 \\
9 \cdot 093 \\
9 \cdot 357 \\
9 \cdot 214\end{array}$ & $\begin{array}{c}9 \cdot 261 \\
9 \cdot 128 \\
9 \cdot 125 \\
9 \cdot 361 \\
g \cdot 102\end{array}$ & $\begin{array}{c}9 \cdot 254 \\
9 \cdot 098 \\
9 \cdot 131 \\
9 \cdot 372 \\
9 \cdot 179\end{array}$ & $\begin{array}{l}9 \cdot 234 \\
9 \cdot 100 \\
\cdot \cdot 159 \\
9 \cdot 371 \\
9 \cdot 144\end{array}$ & $\begin{array}{l}9 \cdot 229 \\
9 \cdot 087 \\
9 \cdot 185 \\
9 \cdot 369 \\
9 \cdot 1.46\end{array}$ & $\begin{array}{l}9 \cdot 223 \\
9 \cdot 07 \pi \\
9 \cdot 187 \\
9 \cdot 339 \\
9 \cdot 118\end{array}$ & $\begin{array}{c}9 \cdot 213 \\
9 \cdot 069 \\
9 \cdot 241 \\
9 \cdot 353 \\
9 \cdot 118\end{array}$ & $\begin{array}{l}9 \cdot 195 \\
9 \cdot 067 \\
9 \cdot 259 \\
9 \cdot-329 \\
9 \cdot 115\end{array}$ & $\begin{array}{l}9 \cdot 167 \\
9 \cdot 071 \\
9 \cdot 285 \\
9 \cdot 231 \\
9 \cdot 095\end{array}$ & $\begin{array}{l}9 \cdot 161 \\
9 \cdot 0.19 \\
9 \cdot 3016 \\
9 \cdot 315 \\
9 \cdot 113\end{array}$ & $\begin{array}{c}9 \cdot 163 \\
9 \cdot 061 \\
9 \cdot 307 \\
9 \cdot 287 \\
9 \cdot 113\end{array}$ & $\begin{array}{l}9 \cdot 152 \\
9 \cdot 071 \\
9 \cdot 318 \\
9 \cdot 266 \\
9 \cdot 106\end{array}$ & $\begin{array}{c}9 \cdot 209 \\
9 \cdot 081 \\
9 \cdot 216 \\
9 \cdot 310 \\
9 \cdot 138\end{array}$ \\
\hline $\begin{array}{r}8 \\
9 \\
10 \\
11 \\
12\end{array}$ & \begin{tabular}{|l|}
$9 \cdot 102$ \\
$8 \cdot 831$ \\
$8 \cdot 577$ \\
$8 \cdot 732$ \\
$8 \cdot 920$
\end{tabular} & $\begin{array}{l}9 \cdot 102 \\
8 \cdot 755 \\
8 \cdot 611 \\
8 \cdot 731 \\
8 \cdot 922\end{array}$ & $\begin{array}{l}9.081 \\
8 \cdot 774 \\
8 \cdot 561 \\
8.7661 \\
8.888\end{array}$ & $\begin{array}{l}9 \cdot 071 \\
8 \cdot 75 s \\
8 \cdot 576 \\
8 \cdot 400 \\
8 \cdot 873\end{array}$ & $\begin{array}{l}8 \cdot 069 \\
8 \cdot 718 \\
8.567 \\
8.524 \\
8.844\end{array}$ & $\begin{array}{l}9 \cdot 049 \\
8.701 \\
8.575 \\
8 \cdot 855 \\
8 \cdot 860\end{array}$ & $\begin{array}{l}9 \cdot 025 \\
8 \cdot 611 \\
8 \cdot 515 \\
8 \cdot 847 \\
8 \cdot 853\end{array}$ & $\begin{array}{l}8 \cdot 994 \\
8.979 \\
8.5986 \\
8.854 \\
8 \cdot 835\end{array}$ & $\begin{array}{l}8.9760 \\
8.6670 \\
8.6310 \\
8.870 \\
8.829\end{array}$ & $\begin{array}{l}8.929 \\
8.653 \\
8.670 \\
8.844 \\
8.827\end{array}$ & $\begin{array}{l}8 \cdot 895 \\
8 \cdot 601 \\
8 \cdot 661 \\
8 \cdot 916 \\
8 \cdot 927\end{array}$ & $\begin{array}{l}8.511 \\
8.711 \\
8.918 \\
8.828\end{array}$ & $\begin{array}{l}9 \cdot 013 \\
8 \cdot 6963 \\
8 \cdot 607 \\
8 \cdot 830 \\
8 \cdot 861\end{array}$ \\
\hline $\begin{array}{l}13 \\
14 \\
15 \\
16 \\
17\end{array}$ & $\begin{array}{l}8 \cdot 827 \\
8.479 \\
8-374 \\
8 \cdot 315 \\
8 \cdot 897 \\
8 \cdot 815\end{array}$ & $\begin{array}{l}8 \cdot 834 \\
8 \cdot 451 \\
8 \cdot 322 \\
8 \cdot 505 \\
8 \cdot 938\end{array}$ & $\begin{array}{l}8 \cdot 815 \\
8 \cdot 449 \\
8 \cdot 376 \\
8.446 \\
8 \cdot 973 \\
8.97\end{array}$ & $\begin{array}{l}8 \cdot 787 \\
8 \cdot 421 \\
8 \cdot 434 \\
8 \cdot 499 \\
9 \cdot 036\end{array}$ & $\begin{array}{l}8 \cdot 725 \\
8.412 \\
8 \cdot 478 \\
8.043 \\
9 \cdot 057\end{array}$ & $\begin{array}{l}8 \cdot 678 \\
8 \cdot 393 \\
8.486 \\
8 \cdot 578 \\
9 \cdot 097\end{array}$ & $\begin{array}{l}8 \cdot 650 \\
8 \cdot 371 \\
8 \cdot 492 \\
8 \cdot 599 \\
9 \cdot 161\end{array}$ & $\begin{array}{l}8 \cdot 649 \\
8 \cdot 6466 \\
8 \cdot 493 \\
8 \cdot 637 \\
9 \cdot 209\end{array}$ & $\begin{array}{l}8 \cdot 542 \\
8 \cdot 338 \\
8 \cdot 509 \\
8 \cdot 689 \\
9 \cdot 251\end{array}$ & $\begin{array}{l}8.529 \\
8.329 \\
8.495 \\
8.717 \\
9.298\end{array}$ & \begin{tabular}{l|}
$8-319$ \\
$8 \cdot 492$ \\
$8 \cdot 778$ \\
$9 \cdot 317$
\end{tabular} & $\mid \begin{array}{l}8 \cdot 331 \\
8 \cdot 502 \\
8 \cdot 836 \\
9 \cdot 324\end{array}$ & $\begin{array}{l}8 \cdot 668 \\
8 \cdot 3288 \\
8 \cdot 451 \\
8 \cdot \cdot 615 \\
9 \cdot 130\end{array}$ \\
\hline $\begin{array}{l}18 \\
19 \\
20 \\
21 \\
22\end{array}$ & $\begin{array}{l}9 \cdot 352 \\
9.219 \\
9 \cdot 173 \\
9 \cdot 291 \\
9 \cdot 231\end{array}$ & $\begin{array}{l}9 \cdot 378 \\
9 \cdot 197 \\
9 \cdot 195 \\
9 \cdot 2967 \\
9 \cdot 227\end{array}$ & $\begin{array}{l}9 \cdot 382 \\
9 \cdot 287 \\
9 \cdot 201 \\
9 \cdot 285 \\
9 \cdot 233\end{array}$ & $\begin{array}{l}9 \cdot 388 \\
9 \cdot 166 \\
9 \cdot 209 \\
9 \cdot 27 \\
9 \cdot 251\end{array}$ & $\begin{array}{l}9 \cdot 243 \\
9 \cdot 257 \\
9 \cdot 258\end{array}$ & $\begin{array}{l}9 \cdot 383 \\
9 \cdot 149 \\
9 \cdot 245 \\
9 \cdot 243 \\
9 \cdot 26 ! 3\end{array}$ & $\begin{array}{l}9 \cdot 369 \\
9 \cdot 143 \\
9 \cdot 253 \\
9 \cdot 240 \\
9 \cdot 279\end{array}$ & $\begin{array}{l}9 \cdot 354 \\
9 \cdot 138 \\
9 \cdot 263 \\
9 \cdot 241 \\
9 \cdot 295\end{array}$ & $\begin{array}{l}9 \cdot 337 \\
9 \cdot 150 \\
9 \cdot 275 \\
9 \cdot 233 \\
\cdot \cdot 322\end{array}$ & $\begin{array}{l}9 \cdot 330 \\
9 \cdot 147 \\
9 \cdot 275 \\
9 \cdot 231 \\
9 \cdot 356\end{array}$ & $\begin{array}{l}9 \cdot 307 \\
9 \cdot 217 \\
9 \cdot 381\end{array}$ & $\begin{array}{l}9 \cdot 165 \\
9 \cdot 297 \\
9 \cdot 211 \\
9 \cdot 411\end{array}$ & $\begin{array}{l}9 \cdot 253 \\
9 \cdot 167 \\
9 \cdot 245 \\
9 \cdot 251 \\
9 \cdot 294\end{array}$ \\
\hline $\begin{array}{l}23 \\
24 \\
25 \\
26 \\
27\end{array}$ & $\begin{array}{l}9 \cdot 414 \\
9 \cdot 639 \\
9 \cdot 2286 \\
8.856 \\
8 \cdot 709\end{array}$ & $\begin{array}{l}8 \cdot 442 \\
9 \cdot 630 \\
9 \cdot 203 \\
8 \cdot 864 \\
8 \cdot 689\end{array}$ & $\begin{array}{l}9.506 \\
9.600 \\
9 \cdot 167 \\
8.854 \\
8.687\end{array}$ & $\begin{array}{l}9 \cdot 524 \\
9 \cdot 600 \\
9 \cdot 125 \\
8 \cdot 82 \\
8 \cdot 673\end{array}$ & $\begin{array}{l}9 \cdot 580 \\
9.548 \\
9.067 \\
8.0817 \\
8.688\end{array}$ & $\begin{array}{l}9 \cdot 604 \\
9 \cdot 485 \\
9 \cdot 043 \\
8 \cdot 789 \\
8 \cdot 694\end{array}$ & $\begin{array}{l}9 \cdot 613 \\
9 \cdot 437 \\
9 \cdot 02 . \\
8.744 \\
8 \cdot 691\end{array}$ & $\begin{array}{l}9 \cdot 636 \\
9.351 \\
8 \cdot 9991 \\
8 \cdot 759 \\
8 \cdot 696\end{array}$ & $\begin{array}{l}9 \cdot 654 \\
8 \cdot 329 \\
8 \cdot 964 \\
8 \cdot 943 \\
8 \cdot 701\end{array}$ & $\begin{array}{l}9 \cdot 663 \\
9 \cdot 335 \\
8 \cdot 935 \\
8 \cdot 74.43 \\
8 \cdot 708\end{array}$ & $\begin{array}{l}9 \cdot 669 \\
9 \cdot 3338 \\
8 \cdot 914 \\
8.732 \\
8 \cdot 7228\end{array}$ & $\begin{array}{l}9 \cdot 653 \\
9 \cdot 265 \\
8 \cdot 891 \\
8-718 \\
8 \cdot 737 \\
8.737\end{array}$ & $\begin{array}{l}9 \cdot 580 \\
9 \cdot 463 \\
9 \cdot 048 \\
8.793 \\
8 \cdot 700\end{array}$ \\
\hline 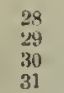 & $\begin{array}{l}8 \cdot 785 \\
8 \cdot 972 \\
8 \cdot 867 \\
8 \cdot 868\end{array}$ & & $\begin{array}{l}8 \cdot 72.4 \\
8 \cdot 980 \\
8 \cdot 889 \\
8 \cdot 877\end{array}$ & $\begin{array}{l}8 \cdot 722 \\
8 \cdot 484 \\
8 \cdot 891 \\
8 \cdot 883\end{array}$ & $\begin{array}{l}8 \cdot 796 \\
8 \cdot 964 \\
8 \cdot 870 \\
8 \cdot 895\end{array}$ & $\begin{array}{l}8 \cdot 852 \\
8 \cdot 91 \\
8 \cdot 880 \\
8 \cdot 879\end{array}$ & $\begin{array}{l}8 \cdot 893 \\
8 \cdot 960 \\
8 \cdot 867 \\
8 \cdot 845\end{array}$ & $\begin{array}{l}8.935 \\
8.860 \\
8.840\end{array}$ & $\begin{array}{l}8.996 \\
8.851 \\
8.848\end{array}$ & & & & \\
\hline
\end{tabular}

\begin{tabular}{|c|c|c|c|c|c|c|c|c|c|c|c|c|c|}
\hline ay & 2. & 4. & 6. & 8. & 10. & . Noon. & 14. & 16. & 18. & 20 & 22. & Yridt & Ieat \\
\hline 1 & $\begin{array}{l}\text { ins. } \\
8.849\end{array}$ & $\begin{array}{l}\text { fins. } \\
8 \cdot 8 \cdot 17\end{array}$ & ${ }_{8 \cdot 8: 9}^{\text {ins. }}$ & $\begin{array}{l}\operatorname{lins}_{8.808} \\
\text {. }\end{array}$ & $\begin{array}{l}\text { ins. } \\
8.800\end{array}$ & $\begin{array}{l}\text { ins. } \\
8.867\end{array}$ & \begin{tabular}{|c|} 
ins. \\
$8 \cdot 855$ \\
\end{tabular} & $8.80^{\circ}$ & $\begin{array}{l}\text { ins. } \\
8 \cdot 891\end{array}$ & $\begin{array}{l}\text { ing. } \\
8.939\end{array}$ & $\begin{array}{l}\text { IIts. } \\
8 \cdot 945\end{array}$ & $\operatorname{lin}_{8.990}$ & 878 \\
\hline $\begin{array}{l}3 \\
4\end{array}$ & $\mid \begin{array}{l}9 \cdot 013 \\
9 \cdot 346 \\
9 \cdot 31 \\
9 \cdot 293 \\
9 \cdot 183\end{array}$ & $\mid \begin{array}{l}9 \cdot 025 \\
9 \cdot 3.345 \\
9 \cdot 349 \\
9.28 .4 \\
9 \cdot 186\end{array}$ & $\mid$\begin{tabular}{r|}
$9 \cdot 069$ \\
$9 \cdot 4 \cdot 42$ \\
$9 \cdot 3 \cdot 34$ \\
$9 \cdot 269$ \\
$9 \cdot 133$
\end{tabular} & $\begin{array}{l}9 \cdot 101 \\
9 \cdot 479 \\
9 \cdot 4193 \\
9 \cdot 267 \\
9 \cdot 079\end{array}$ & $\begin{array}{l}9 \cdot 141 \\
9 \cdot 462 \\
9 \cdot 2.289 \\
9.259 \\
9 \cdot 087\end{array}$ & \begin{tabular}{ll|}
1 & $9 \cdot 277$ \\
9 & $9 \cdot 46$ \\
9 & $9 \cdot 381$ \\
9 & $9 \cdot 225$ \\
7 & $9 \cdot 081$
\end{tabular} & $\mid \begin{array}{l}9 \cdot 332 \\
9 \cdot 450 \\
9 \cdot 357 \\
9 \cdot 210 \\
9 \cdot 078 \\
9 \cdot 078\end{array}$ & $\begin{array}{l}9 \cdot 274 \\
9 \cdot 140 \\
9 \cdot 352 \\
9.204 \\
9 \cdot 101\end{array}$ & $\begin{array}{l}9 \cdot 278 \\
9 \cdot 4.48 \\
9 \cdot 351 \\
y .201 \\
9 \cdot 133\end{array}$ & $\begin{array}{l}9 \cdot 331 \\
9 \cdot 436 \\
9 \cdot 344 \\
9 \cdot 194 \\
9 \cdot 171\end{array}$ & $\begin{array}{l}9 \cdot 338 \\
9 \cdot 127 \\
9 \cdot 341 \\
9 \cdot 168 \\
2 \cdot 231\end{array}$ & 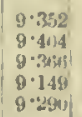 & $\begin{array}{l}9 \cdot 435 \\
9 \cdot 340 \\
9 \cdot 228 \\
9 \cdot 146\end{array}$ \\
\hline $\begin{array}{r}9 \\
9 \\
10 \\
11\end{array}$ & 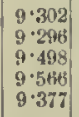 & $\mid \begin{array}{l}9 \cdot 326 ! \\
9 \cdot 267 \\
9 \cdot 521 \\
9 \cdot 539 \\
9 \cdot 365\end{array}$ & 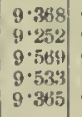 & $\begin{array}{r}9 \cdot 344 \\
9 \cdot-2.45 \\
9 \cdot 547 \\
9.522 .3 \\
9 \cdot 3.345\end{array}$ & $\begin{array}{l}9 \cdot 428 \\
9 \cdot 281 \\
9.545 \\
9.5456 \\
9.336\end{array}$ & 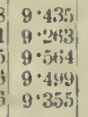 & $\begin{array}{l}9 \cdot 410 \\
9 \cdot 313 \\
8 \cdot 588 \\
9 \cdot 474 \\
9 \cdot 397\end{array}$ & $\begin{array}{l}9 \cdot 427 \\
9 \cdot 324 \\
9 \cdot 584 \\
9 \cdot 461 \\
9 \cdot 413\end{array}$ & $\begin{array}{l}9 \cdot 401 \\
9 \cdot 3 k 7 \\
9 \cdot 648 \\
0.4 .12 \\
0.415\end{array}$ & $\begin{array}{l}9 \cdot 339 \\
9 \cdot 413 \\
9 \cdot 594 \\
9 \cdot 424 \\
9 \cdot 431\end{array}$ & 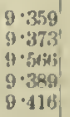 & $\begin{array}{l}9 \cdot 328 \\
9.452 \\
9 \cdot 864 \\
9.371 \\
9 \cdot 428\end{array}$ & $\begin{array}{l}9.375 \\
9.318 \\
9.584 \\
9.474 \\
9.384\end{array}$ \\
\hline $\begin{array}{l}12 \\
13 \\
14 \\
15 \\
16\end{array}$ & $\begin{array}{l}9 \cdot 430 \\
9 \cdot 4.48 \\
9 \cdot 571 \\
9 \cdot 413 \\
9 \cdot 751\end{array}$ & $\begin{array}{l}9 \cdot 458 \\
9 \cdot 443 \\
9.576 \\
9.436 \\
9.797 \\
\end{array}$ & $\mid \begin{array}{r}9 \cdot 501 \\
9.4 .49 \\
9 \cdot 581 \\
9.457 \\
9 \cdot 858\end{array}$ & $\begin{array}{l}9 \cdot 524 \\
9 \cdot 459 \\
9 \cdot 551 \\
9.454 \\
9-4.42\end{array}$ & $\begin{array}{l}9 \cdot 522 \\
9.498 \\
9 \cdot 541 \\
6.451 \\
9 \cdot 968\end{array}$ & $\begin{array}{l}9 \cdot 521 \\
9 \cdot 519 \\
9 \cdot 523 \\
9 \cdot 4 \pi 1 \\
9 \cdot 9 \cdot 971\end{array}$ & $\begin{array}{l}9 \cdot 511 \\
9.523 \\
9.5161 \\
9.515 \\
9 \cdot 970 \\
0.970\end{array}$ & 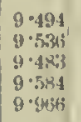 & 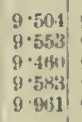 & $\begin{array}{l}9.505 \\
9 \cdot 544 \\
9 \cdot 4.411 \\
9 \cdot 137 \\
9 \cdot 9 \cdot 37\end{array}$ & $\begin{array}{l}9 \cdot 4 k ; 8 \\
9 \cdot 571 \\
4 \cdot 451 \\
9 \cdot 685 \\
9 \cdot 928\end{array}$ & 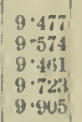 & $\begin{array}{l}9 \cdot 423 \\
9.511 \\
9.518 \\
9.537 \\
9.912\end{array}$ \\
\hline $\begin{array}{l}17 \\
18 \\
19 \\
20 \\
21\end{array}$ & $\begin{array}{l}9 \cdot 868 \\
9 \cdot 820 \\
9 \cdot 772 \\
9 \cdot 759 \\
9 \cdot 810\end{array}$ & 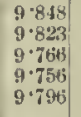 & $\begin{array}{l}9 \cdot 8.32 \\
9 \cdot 807 \\
9 \cdot 769 \\
9 \cdot 819 \\
9 \cdot 753\end{array}$ & $\begin{array}{l}9 \cdot 828 \\
9 \cdot 817 \\
9 \cdot 775 \\
9 \cdot 823 \\
9 \cdot 744\end{array}$ & $\begin{array}{l}9 \cdot 832 \\
9 \cdot 774 \\
9752 \\
9 \cdot \times 23 \\
9 \cdot 790\end{array}$ & 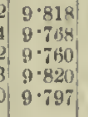 & $\left|\begin{array}{r}9 \cdot 807 \\
9 \cdot 776 \\
9-796 \\
9 \cdot 830 \\
9 \cdot 788\end{array}\right|$ & $\begin{array}{l}\mathbf{9} \cdot 816 \\
9.813 \\
9 \cdot 816 \\
9.836 \\
9 \cdot 807\end{array}$ & $\begin{array}{c}9 \cdot 809 \\
9 \cdot 7988 \\
9 \cdot 810 \\
9 \cdot 829 \\
9 \cdot 810\end{array}$ & $\begin{array}{l}9 \cdot 7969 \\
9 \cdot 799^{\prime} \\
9 \cdot 814 \\
9 \cdot 834 \\
8 \cdot 861\end{array}$ & $\begin{array}{c}9 \cdot 814 \\
9 \cdot 773 \\
9 \cdot 808 \\
9 \cdot 828 \\
9 \cdot 8963\end{array}$ & $\begin{array}{l}9 \cdot 813 \\
9.767 \\
9.7867 \\
9.824 \\
9 \cdot 911\end{array}$ & $\begin{array}{l}9 \cdot 824 \\
8.792 \\
9.785 \\
9.815 \\
9.816\end{array}$ \\
\hline $\begin{array}{l}22 \\
23 \\
24 \\
25 \\
20\end{array}$ & $\begin{array}{l}9 \cdot 912 \\
0.1013 \\
9 \cdot 8.45 \\
9.549 \\
9 \cdot 596\end{array}$ & $\begin{array}{l}9 \cdot 935 \\
0.099 \\
9.818 \\
9.541 \\
9 \cdot 594\end{array}$ & $\mid \begin{array}{l}9 \cdot 943 \\
0 \cdot 097 \\
9 \cdot 8964 \\
9 \cdot 555 \\
9 \cdot 5994 \\
\end{array}$ & $\begin{array}{l}9 \cdot 973 \\
0.084 \\
9 \cdot 780 \\
9 \cdot 563 \\
9 \cdot 622\end{array}$ & $\begin{array}{l}9 \cdot 9.98 \\
0.057 \\
9 \cdot 7 \cdot 766 \\
9 \cdot 563 \\
9 \cdot 636\end{array}$ & 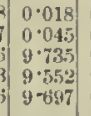 & $\begin{array}{l}0.052 \\
0.029 \\
9 \cdot 696 \\
9.551 \\
9 \cdot 704\end{array}$ & $\begin{array}{l}0.057 \\
9.095 \\
9.668 \\
9.558 \\
9 \cdot 719\end{array}$ & $\begin{array}{l}0 \cdot 061 \\
9 \cdot 971 \\
9 \cdot 656 \\
9 \cdot 559 \\
9 \cdot 7.249\end{array}$ & 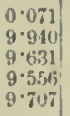 & $\begin{array}{l}0 \cdot 108 \\
9 \cdot 905 \\
9 \cdot 6.69 \\
9 \cdot 579 \\
9 \cdot 719\end{array}$ & $\begin{array}{l}0 \cdot 112 \\
9.84615 \\
9 \cdot 6113 \\
9.549 \\
9 \cdot 768\end{array}$ & $\begin{array}{l}0.016 \\
0.016 \\
9.718 \\
9.560 \\
9.674\end{array}$ \\
\hline $\begin{array}{l}27 \\
28 \\
289\end{array}$ & $\begin{array}{l}9 \cdot 7665 \\
9 \cdot 604 \\
9 \cdot 411 \\
9 \cdot 706\end{array}$ & $\begin{array}{l}9 \cdot 788 \\
9 \cdot 579 \\
9 \cdot 458 \\
9 \cdot 730\end{array}$ & $\begin{array}{r}9 \cdot 765 \\
9-571 \\
9 \cdot 485 \\
9 \cdot 751\end{array}$ & $\begin{array}{l}9 \cdot 730 \\
9 \cdot 7551 \\
9.517 \\
9 \cdot 781\end{array}$ & $\begin{array}{l}9 \cdot 727 \\
9 \cdot 523 \\
9 \cdot 536 \\
9 \cdot 814\end{array}$ & $\begin{array}{l}9 \cdot 691 \\
9 \cdot 5016 \\
9 \cdot 545 \\
9 \cdot 822 \\
9 \cdot 820\end{array}$ & $\begin{array}{l}n \cdot 698 \\
9 \cdot 480 \\
9 \cdot 550 \\
9 \cdot 817\end{array}$ & $\begin{array}{l}9 \cdot 690 \\
9 \cdot 459 \\
9 \cdot 577 \\
9 \cdot 815\end{array}$ & $\begin{array}{l}9 \cdot 674 \\
9 \cdot 451 \\
9 \cdot 594 \\
9 \cdot 813\end{array}$ & $\begin{array}{l}9 \cdot 6646 \\
9 \cdot 451 \\
9 \cdot 429 t \\
9 \cdot 805\end{array}$ & $\begin{array}{l}9 \cdot 663 \\
9 \cdot 44: 3 \\
8 \cdot 661 \\
9 \cdot 797\end{array}$ & $\begin{array}{l}9 \cdot 635 \\
9 \cdot 4 \cdot 49 \\
\cdot \cdot 685 \\
9 \cdot 727\end{array}$ & $\begin{array}{r}9 \cdot 709 \\
9 \cdot 506 \\
9 \cdot 555 \\
9 \cdot 782\end{array}$ \\
\hline & & & & & & & & & & & & & \\
\hline
\end{tabular}

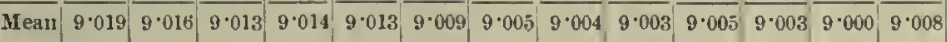

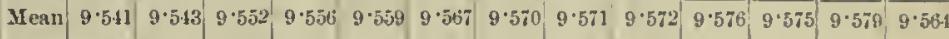

\begin{tabular}{|l|l|l|l|l|l|l|l|l|l|l|l|l|l|}
\hline Day. & 2. & 4. & 6. & 8. & 10. & Ynon, & 14. & 16. & 18. & 20. & 22. & Midt. Mean. \\
\hline
\end{tabular}

Ins ins. ins ins ins. fns. ins. ins. ins.

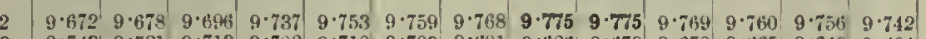

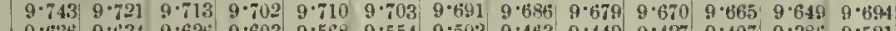

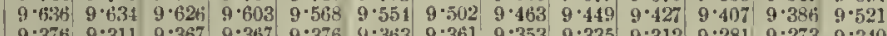

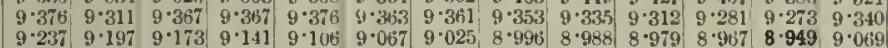

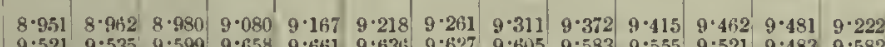

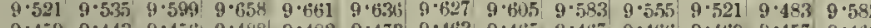

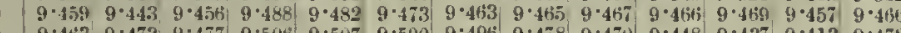

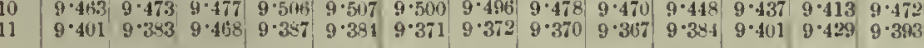

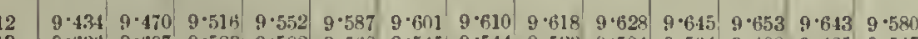

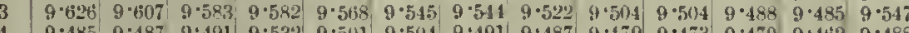
$\begin{array}{llllllllllllll}4 & 9 \cdot 4 \times 5 & 9 \cdot 487 & 9 \cdot 491 & 9 \cdot 532 & 9 \cdot 501 & 9 \cdot 504 & 9 \cdot 491 & 9 \cdot 487 & 9 \cdot 479 & 9 \cdot 473 & 9 \cdot 470 & 9 \cdot 462 & 9 \cdot 488 \\ 5 & 9 \cdot 414 & 9 \cdot 432 & 9 \cdot 433 & 9 \cdot 430 & 9 \cdot 431 & 9 \cdot 42 . & 9 \cdot 413 & 9 \cdot 413 & 9 \cdot 411 & 9 \cdot 407 & 9 \cdot 407 & 9 \cdot 415 & 9 \cdot 42\end{array}$

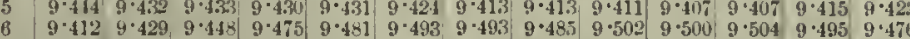

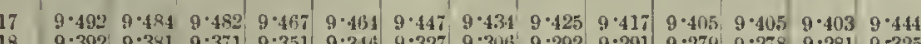

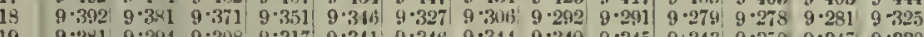

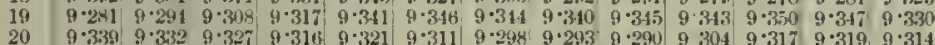
$\begin{array}{lllllllllllllll}20 & 9 \cdot 339 & 9 \cdot 332 & 9 \cdot 327 & 9 \cdot 316 & 9 \cdot 321 & 9 \cdot 311 & 9 \cdot 298 & 9 \cdot 293 & 8 \cdot 290 & 9 & 304 & 9 \cdot 317 & 9 \cdot 319 & 9 \cdot 314 \\ 21 & 9 \cdot 339 & 9 \cdot 361 & 9 \cdot 388 & 9 \cdot 414 & 9 \cdot 432 & 9 \cdot 427 & 9 \cdot 420 & 9 \cdot 417 & 9 \cdot 408 & 9 \cdot 407 & 9 \cdot 405 & 9 \cdot 401 & 9 \cdot 402\end{array}$ $\begin{array}{lllllllllllllll}22 & 9 \cdot 399 & 9 \cdot 394 & 9 \cdot 391 & 9 \cdot 388 & 9 \cdot 388 & 9 \cdot 374 & 9 \cdot 351 & 9 \cdot 331 & 9 \cdot 312 & 9 \cdot 280 & 9 \cdot 233 & 9 \cdot 194 & 9 \cdot 337\end{array}$

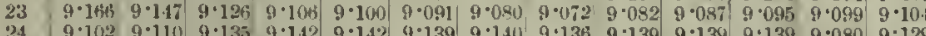

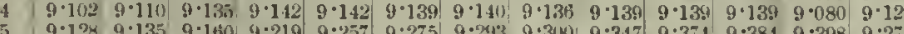

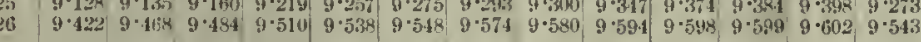

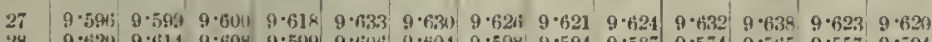

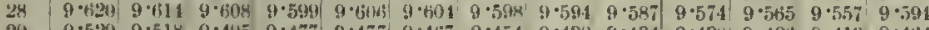

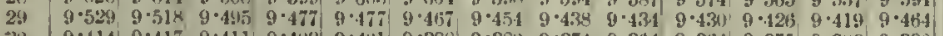

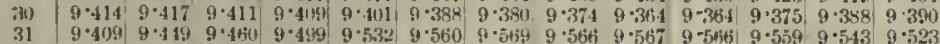
Irean $\overline{9 \cdot 427} \overline{9 \cdot 42 k} \overline{9 \cdot 435}, \overline{9 \cdot 445} \overline{9 \cdot 450} \overline{9 \cdot 446} \overline{9 \cdot 411} \overline{9 \cdot 435} \overline{9 \cdot 431} \overline{9 \cdot 432}, \overline{9 \cdot 430} \overline{9 \cdot 423} \overline{9 \cdot 435}$

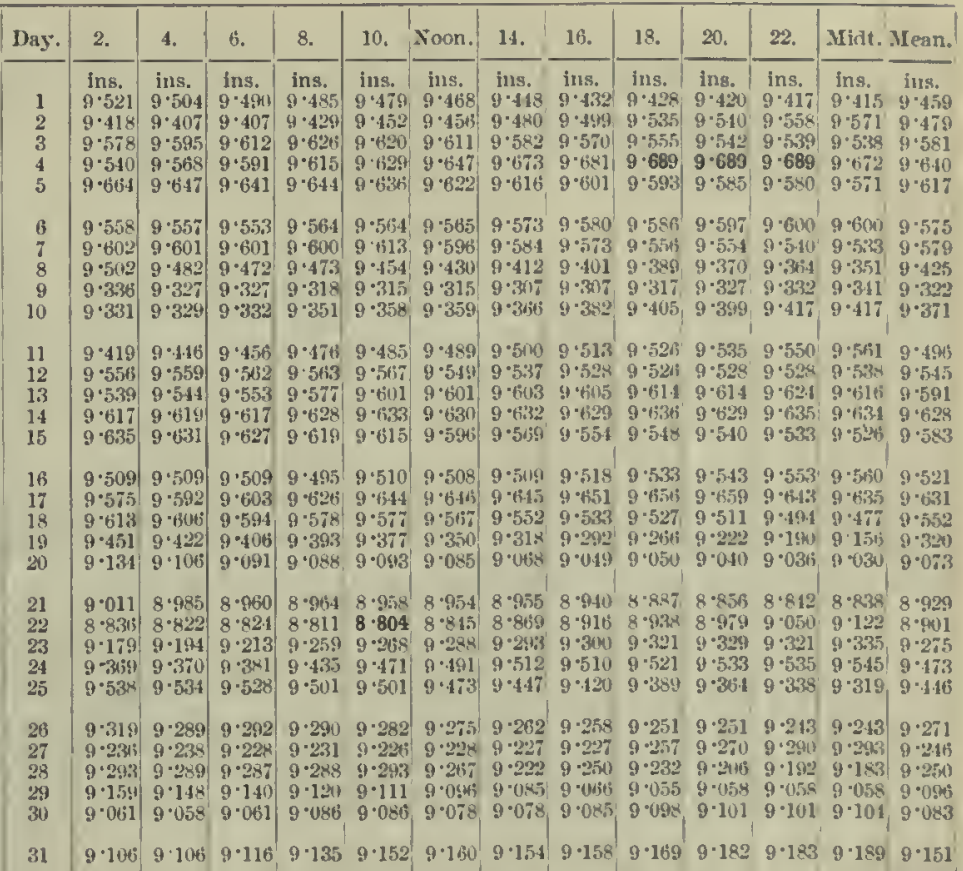

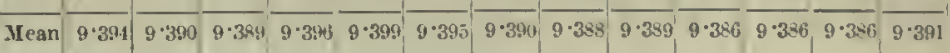


Table I. (continued).

\section{PRESSURE IN ENGLISI INCHES, FIRST FIGULE OMTTED.}

The Readings arc Corrected for Temperature, and Reduced to Mlem Sea Level, but are not Corrected

February, 1 for Variation of Gravity.

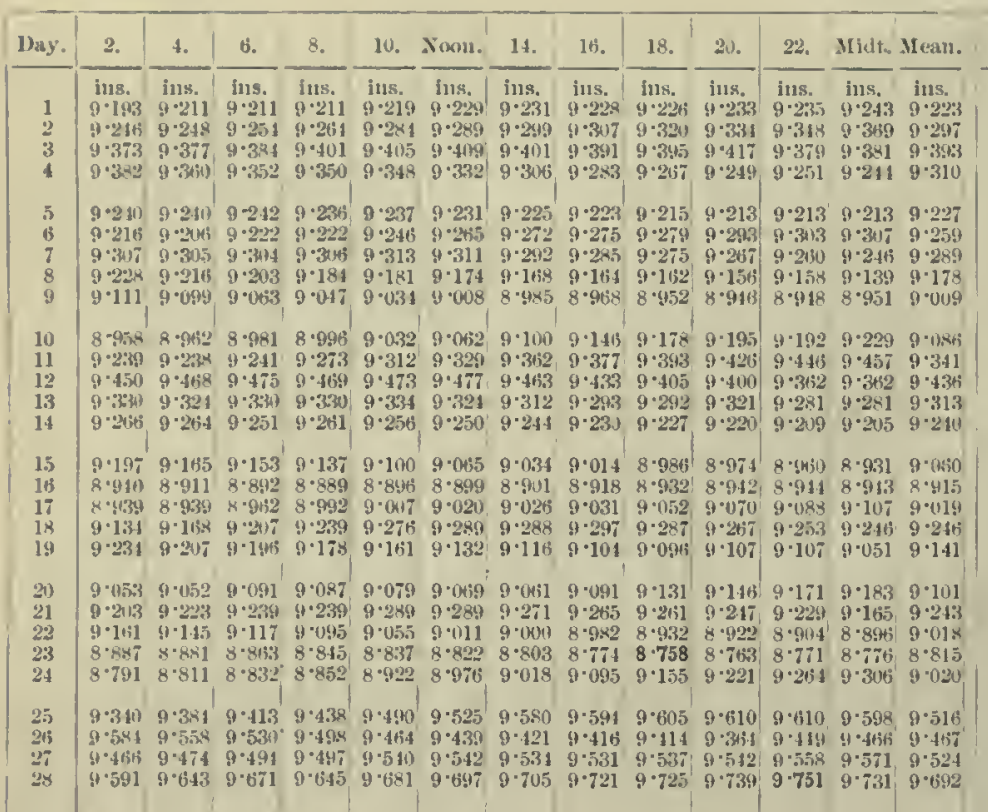
1)ay.

March, 1903

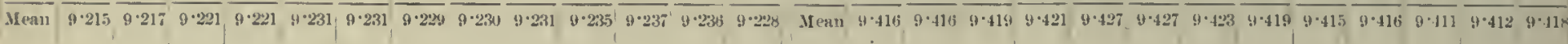

April, 1903.

May, 1903.

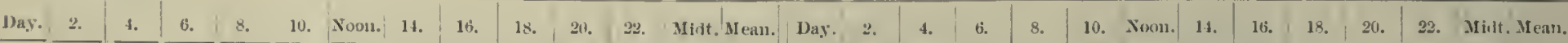

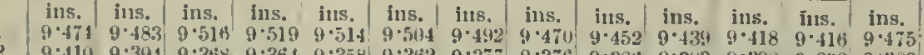

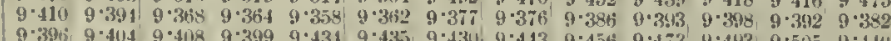

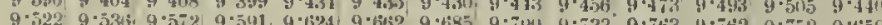

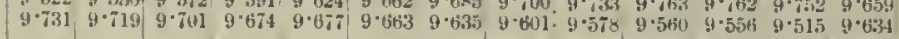

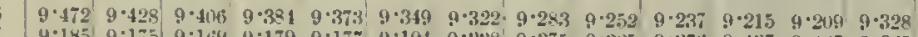

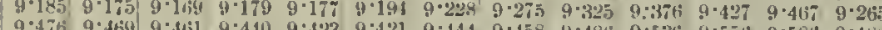

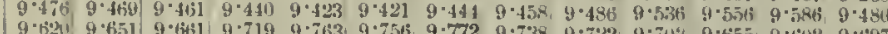

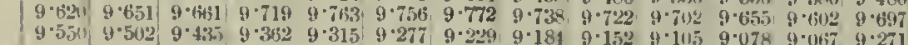

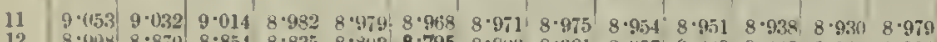

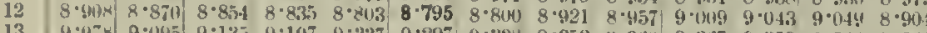

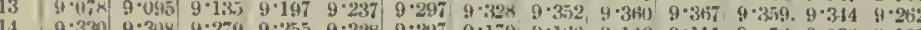

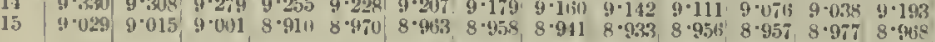

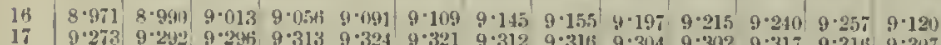

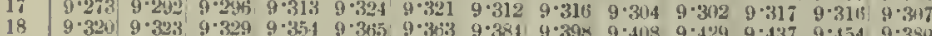

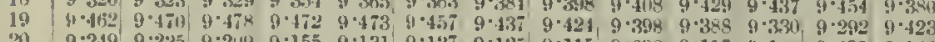

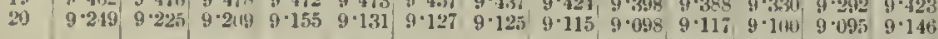

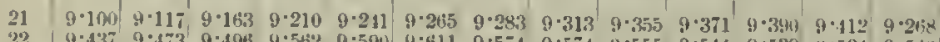

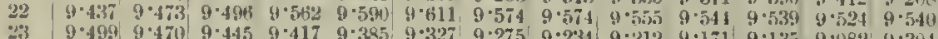

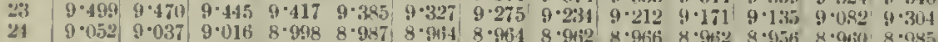

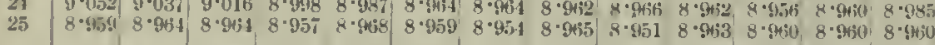

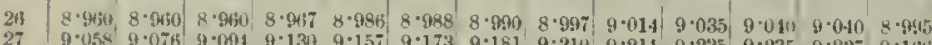

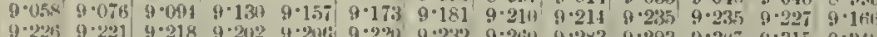

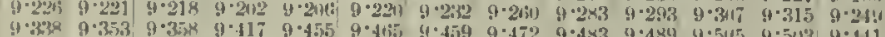

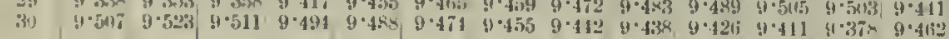

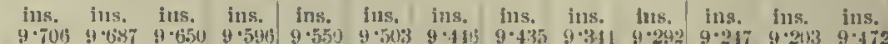

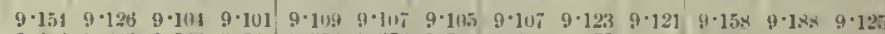

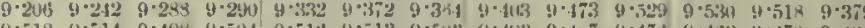

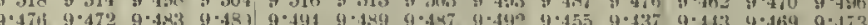

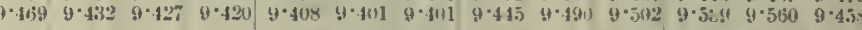

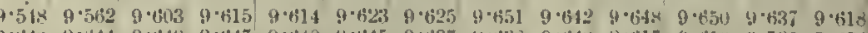

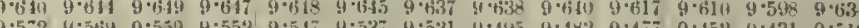

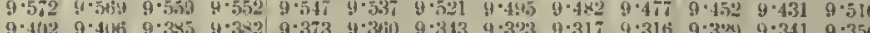

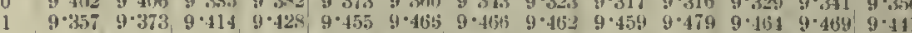

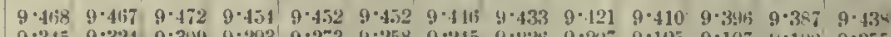

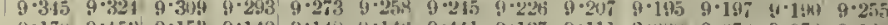

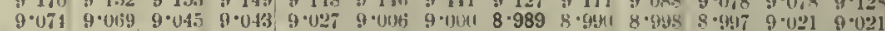

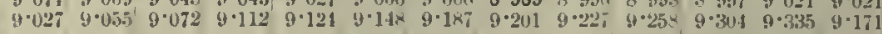

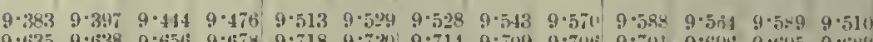
9.635.

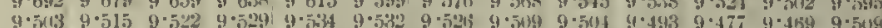

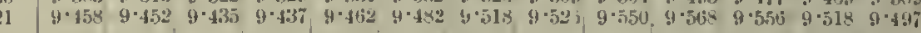

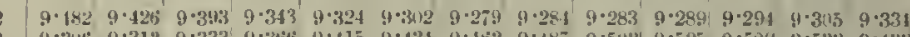

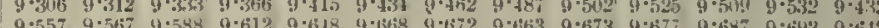

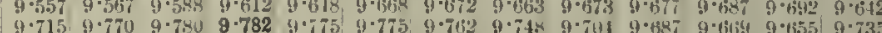

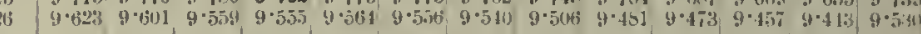

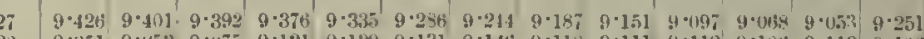

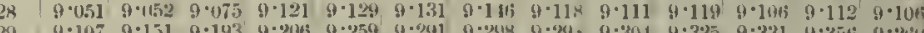

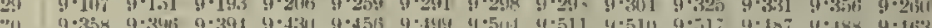

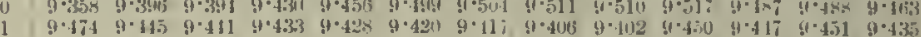

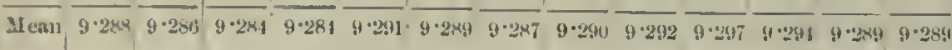

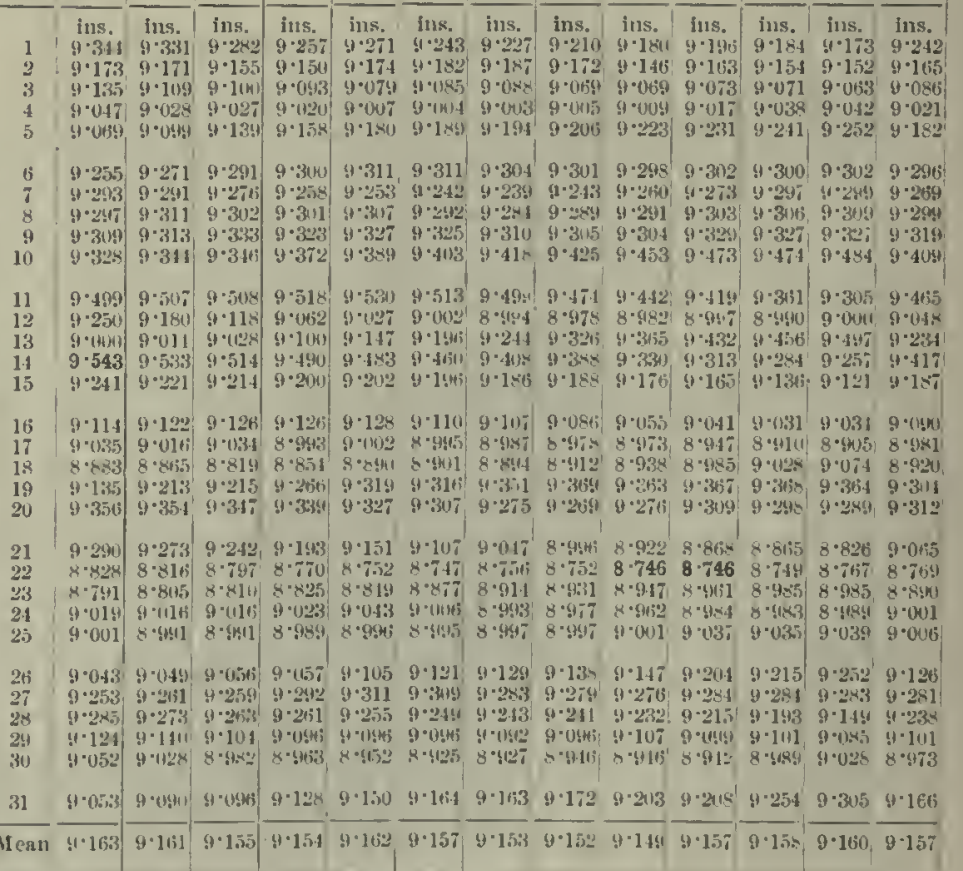


TABite I. (continued).

\section{PRESSURE IN FNGLISH INCIIS, FIRST FIGURE OMITTED.}

'The Readings are Corrected for Temperature, and Reduced to Mean Sea Level, but are not Corrected for Vuriation of Gravity.

June, 1903.

Jaly, 1903.

\begin{tabular}{|c|c|c|c|c|c|c|c|c|c|c|c|c|c|c|c|c|c|c|c|c|c|c|c|c|c|c|c|}
\hline by. & 2. & 4. & 6. & 8. & 10. & Non11. & 14. & 16. & 18. & 20. & 22. & Midt. & Mean. & Day. & 2. & 4. & 6. & 8. & 10. & Noon. & 14. & 18. & 18. & 20. & 22. & Midit & Sean. \\
\hline$\frac{1}{3}$ & $\begin{array}{l}\text { ins } \\
4 \cdot 325 \\
9 \cdot 749 \\
9.686 \\
9 \cdot 708\end{array}$ & $\begin{array}{l}\text { Ins. } \\
9 \cdot 372 \\
9 \cdot 726 \\
9.929 \\
9 \cdot 612\end{array}$ & $\begin{array}{l}\text { ins. } \\
9 \cdot 125 \\
9 \cdot 775 \\
9 \cdot 993 \\
9 \cdot 501\end{array}$ & $\begin{array}{l}\text { ins } \\
9 \cdot 5113 \\
9 \cdot 792 \\
0.021 \\
9.406\end{array}$ & $\begin{array}{l}\text { ins } \\
9 \cdot 513 \\
9 \cdot 821 \\
0.021 \\
9.379\end{array}$ & 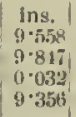 & $\begin{array}{l}\ln 5 . \\
9 \cdot 583 \\
9 \cdot-86.1 \\
0 \cdot 037 \\
9 \cdot 326\end{array}$ & $\begin{array}{l}\text { lus. } \\
9 \cdot-615 \\
9 \cdot 8 \times 8 \\
0.021 \\
9 \cdot 350\end{array}$ & $\begin{array}{l}\text { lins } \\
9 \cdot 1 ; 1 \\
9 \cdot 913 \\
9 \cdot 990 \\
9 \cdot 376 \\
9 \cdot 37\end{array}$ & $\mid$\begin{tabular}{c|}
$\ln 8$ \\
$9 \cdot 6 \cdot 61$ \\
$9-931$ \\
$9-931$ \\
$9 \cdot 912$ \\
$9 \cdot 381$
\end{tabular} & \begin{tabular}{|c|} 
ins. \\
$9 \cdot 6773$ \\
$9 \cdot 951$ \\
$9 \cdot 876$ \\
$9 \cdot 39.4$ \\
\end{tabular} & $\begin{array}{c}\text { Ins } \\
9 \cdot 636 \\
9 \cdot 966 \\
9 \cdot 789 \\
9 \cdot 423 \\
\end{array}$ & 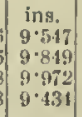 & $\begin{array}{l}1 \\
2 \\
3 \\
1\end{array}$ & $\begin{array}{c}\text { ins. } \\
9 \cdot 279 \\
9 \cdot 391 \\
9 \cdot 407 \\
9 \cdot 271 \\
\end{array}$ & $\begin{array}{c}\text { ins } \\
9 \cdot 247 \\
9 \cdot 3901 \\
9 \cdot 4105 \\
9 \cdot 273\end{array}$ & 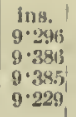 & $\begin{array}{l}\text { ins. } \\
9: 31.4 \\
9 \cdot 375 \\
9 \cdot 378 \\
9 \cdot 202\end{array}$ & 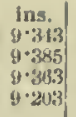 & $\mid \begin{array}{l}\text { ins. } \\
912.35 \\
9 \cdot 372 \\
9 \cdot 313 \\
9 \cdot 195 \\
9 \cdot 195\end{array}$ & 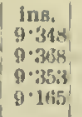 & 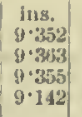 & 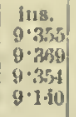 & 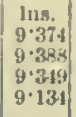 & $\mid \begin{array}{l}\text { Ins. } \\
9 \cdot 3943 \\
9 \cdot 343 \\
9 \cdot 323 \\
9 \cdot 112\end{array}$ & $\mid \begin{array}{l}\text { Ins } \\
9 \cdot 390 \\
9 \cdot 3 \% 3 \\
9 \cdot 2445 \\
9 \cdot 094\end{array}$ & $\begin{array}{l}\text { ins } \\
y \cdot 311 \\
9 \cdot 381 \\
9 \cdot 359 \\
9 \cdot 180\end{array}$ \\
\hline $\begin{array}{l}5 \\
6 \\
7 \\
8 \\
9 \\
9\end{array}$ & 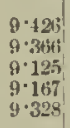 & $\begin{array}{l}9 \cdot 113 \\
9 \cdot 3 \cdot 33 \\
9 \cdot 107 \\
9 \cdot 171 \\
9 \cdot 370\end{array}$ & $\begin{array}{l}9 \cdot 419 \\
9 \cdot 313 \\
9 \cdot 091 \\
9 \cdot 171 \\
9 \cdot 409\end{array}$ & $\begin{array}{l}9.438 \\
9.2 \times 1 \\
9.051 \\
9 \cdot 18, \\
9 \cdot 431\end{array}$ & $\begin{array}{l}9 \cdot 4,22 \\
9-271 \\
9 \cdot 0.49 \\
9 \cdot 124 \\
9 \cdot 47 t\end{array}$ & $\begin{array}{l}9 \cdot 476 \\
9 \cdot 259 \\
9 \cdot 040 \\
90091 \\
9 \cdot 431\end{array}$ & $\begin{array}{c}9 \cdot 494 \\
9 \cdot 242 \\
9 \cdot 033 \\
8 \cdot 0 \times 5 \\
9 \cdot 443\end{array}$ & $\begin{array}{l}9 \cdot 472 \\
9 \cdot 2 \cdot 28 \\
9 \cdot 121 \\
9 \cdot 073 \\
9 \cdot 4111\end{array}$ & $\begin{array}{r}9 \cdot 56 \\
9 \cdot 203 \\
9 \cdot 133 \\
9 \cdot 1108 \\
9 \cdot 368\end{array}$ & $\begin{array}{l}9 \cdot 1.35 \\
9 \cdot 179 \\
9 \cdot 137 \\
9 \cdot 116 \\
9 \cdot 317\end{array}$ & $\begin{array}{l}9 \cdot 415 \\
9 \cdot 164 \\
9 \cdot 146 \\
9 \cdot 160 \\
9 \cdot 237\end{array}$ & $\begin{array}{r}9 \cdot 293 \\
9 \cdot 137 \\
9 \cdot 163 \\
9 \cdot 259 \\
9 \cdot 158\end{array} \mid$ & $\begin{array}{l}9 \cdot 440 \\
9 \cdot 218 \\
9 \cdot 100 \\
9 \cdot 144 \\
9 \cdot 345\end{array}$ & $\begin{array}{l}5 \\
8 \\
7 \\
8 \\
9\end{array}$ & $\begin{array}{l}9.092 \\
8.894 \\
8.708 \\
8 \cdot 877 \\
1 \cdot 141\end{array}$ & $\begin{array}{l}90.054 \\
8.84 \\
8.712 \\
8.917 \\
9 \cdot 148\end{array}$ & $\begin{array}{l}9.054 \\
8.814 \\
8.716 \\
8.914 \\
9.160\end{array}$ & 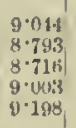 & 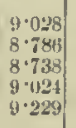 & 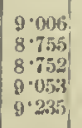 & $\begin{array}{l}8 \cdot 893 \\
8.729 \\
8.761 \\
9.1603 \\
9 \cdot 244\end{array}$ & $\mid \begin{array}{l}8 \cdot 978 \\
8 \cdot 708 \\
8 \cdot 789 \\
9 \cdot 189 \\
9 \cdot 2.43\end{array}$ & $\begin{array}{l}8 \cdot 948 \\
8 \cdot 701 \\
8 \cdot 790 \\
8 \cdot 076 \\
9 \cdot 247\end{array}$ & $\mid \begin{array}{l}8 \cdot 912 \\
8 \cdot 469 \\
8.828 \\
9 \cdot 028 \\
9 \cdot 246\end{array}$ & $\mid \begin{array}{l}8 \cdot 918 \\
8 \cdot 6960 \\
8 \cdot 838 \\
9 \cdot 102 \\
9 \cdot 2 ! 2\end{array}$ & $\begin{array}{l}8.898 \\
8.699 \\
8.899 \\
9.124 \\
9.232\end{array}$ & $\begin{array}{l}8 \cdot 998 \\
8 \cdot 7150 \\
8 \cdot 7 t 5 \\
9 \cdot 020 \\
9 \cdot 214\end{array}$ \\
\hline $\begin{array}{l}10 \\
11 \\
12 \\
13 \\
11\end{array}$ & 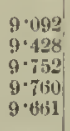 & $\begin{array}{l}9 \cdot 045 \\
9 \cdot 382 \\
9 \cdot 7 \cdot 48 \\
9 \cdot 707 \\
9 \cdot 659\end{array}$ & $\begin{array}{l}9 \cdot 0.12 \\
9 \cdot 355 \\
9 \cdot 760 \\
9 \cdot 6692 \\
9 \cdot 663\end{array}$ & $\begin{array}{l}9 \cdot 142 \\
9 \cdot 390 \\
9 \cdot 795 \\
9 \cdot 631 \\
9 \cdot 685\end{array}$ & $\begin{array}{l}9-239 \\
9.434 \\
9.825 \\
9 \cdot 613 \\
8 \cdot-717 \\
9 \cdot 717\end{array}$ & $\begin{array}{l}9 \cdot 304 \\
9 \cdot 4.46 \\
9 \cdot 865 \\
9 \cdot 610 \\
9 \cdot 759\end{array}$ & $\begin{array}{l}9 \cdot 392 \\
9 \cdot 562 \\
9 \cdot 838 \\
9 \cdot 618 \\
9 \cdot 814\end{array}$ & $\begin{array}{l}9 \cdot 422 \\
9 \cdot 600 \\
9 \cdot 838 \\
9 \cdot 63315 \\
9 \cdot 842\end{array}$ & $\begin{array}{l}9 \cdot 421 \\
9.611 \\
8 \cdot 8.83 \\
9 \cdot 651 \\
9 \cdot 897\end{array}$ & $\begin{array}{l}9 \cdot 453 \\
9 \cdot 613 \\
9 \cdot 835 \\
9 \cdot 659 \\
9 \cdot 932\end{array}$ & $\begin{array}{l}9 \cdot 459 \\
9.620 \\
9.893 \\
9 \cdot 651 \\
9 \cdot 982 \\
9 \cdot 982\end{array}$ & $\mid \begin{array}{c}9 \cdot 4.15 \\
9 \cdot 713 \\
9 \cdot 793 \\
8.45 .5 \\
0 \cdot 002\end{array}$ & $\begin{array}{l}9 \cdot 288 \\
9 \cdot 516 \\
9 \cdot 807 \\
9 \cdot 654 \\
9 \cdot 801\end{array}$ & $\begin{array}{l}10 \\
11 \\
12 \\
13 \\
14\end{array}$ & $\begin{array}{l}9 \cdot 226 \\
9 \cdot 234 \\
8 \cdot 29.54 \\
8 \cdot 597 \\
8 \cdot 720\end{array}$ & $\begin{array}{l}9 \cdot 217 \\
9 \cdot 2.215 \\
8.893 \\
8 \cdot 6003 \\
8 \cdot 780\end{array}$ & $\begin{array}{l}9 \cdot 211 \\
9 \cdot 185 \\
8 \cdot 861 \\
8 \cdot 6417 \\
8 \cdot 792\end{array}$ & $\begin{array}{l}9 \cdot 210 \\
9 \cdot 128 \\
8.815 \\
8.617 \\
8 \cdot 78 y^{\prime}\end{array}$ & $\begin{array}{l}9 \cdot 217 \\
9 \cdot 133 \\
8 \cdot 803 \\
8 \cdot 631 \\
8 \cdot 798\end{array}$ & $\begin{array}{l}9 \cdot 220 \\
9 \cdot 083 \\
8 \cdot 764 \\
8 \cdot 631 \\
8 \cdot 800 \\
\cdot 831 \\
\end{array}$ & $\begin{array}{l}8 \cdot 211 \\
9.061 \\
8 \cdot 729 \\
8.623 \\
8.792 \\
\end{array}$ & $\begin{array}{l}9 \cdot 21 R \\
9 \cdot 025 \\
8.701 \\
8 \cdot 621 \\
8 \cdot 782\end{array}$ & & $\mid \begin{array}{l}9 \cdot 230 \\
8 \cdot 988 \\
8 \cdot \cdot 4 \cdot 43 \\
8 \cdot 651 \\
8 \cdot 741\end{array}$ & $\begin{array}{l}9 \cdot 235 \\
8 \cdot 970 \\
8.624 \\
8 \cdot 679 \\
8 \cdot 727\end{array}$ & $\begin{array}{l}8.968 \\
8.608 \\
8.6995 \\
8.715\end{array}$ & $\begin{array}{l}8.751 \\
8.636 \\
8.767\end{array}$ \\
\hline $\begin{array}{l}15 \\
17 \\
17 \\
18 \\
19\end{array}$ & $\begin{array}{l}0 \cdot 002 \\
9 \cdot 868 \\
0 \cdot-002 \\
9 \cdot 885 \\
9 \cdot 932 \\
9 \cdot 932\end{array}$ & $\begin{array}{l}0 \cdot 006 \\
\mathbf{g} \cdot 854 \\
9 \cdot 991 \\
9 \cdot 877 \\
9 \cdot 967\end{array}$ & $\begin{array}{l}0.0013 \\
9 \cdot 873 \\
9 \cdot 972 \\
9.871 \\
9 \cdot 973\end{array}$ & $\begin{array}{l}9 \cdot 988 \\
9 \cdot 881 \\
9 \cdot 951 \\
9 \cdot 870 \\
9 \cdot 981\end{array}$ & $\begin{array}{l}9 \cdot 955 \\
9 \cdot 911 \\
9 \cdot 870 \\
9 \cdot 989\end{array}$ & $\begin{array}{l}9 \cdot 963 \\
9 \cdot 961 \\
9 \cdot 9.95 \\
9 \cdot 873 \\
9 \cdot 991\end{array}$ & $\begin{array}{l}8 \cdot 934 \\
9 \cdot 938 \\
9 \cdot 911 \\
9 \cdot 8933 \\
8 \cdot 990\end{array}$ & $\begin{array}{l}9 \cdot 909 \\
9 \cdot 989 \\
9 \cdot 996 \\
9 \cdot 885 \\
9 \cdot 985\end{array}$ & $\begin{array}{l}9 \cdot 887 \\
9 \cdot 999 \\
9 \cdot 901 \\
9 \cdot 901 \\
9 \cdot 982\end{array}$ & & $\begin{array}{l}9 \cdot 851 \\
0 \cdot 007 \\
9 \cdot 881 \\
9 \cdot 9.98 \\
9 \cdot 979\end{array}$ & $\begin{array}{l}9 \cdot 868 \\
0 \cdot 006 \\
9 \cdot 886 \\
9 \cdot 896 \\
9 \cdot 962\end{array}$ & $\begin{array}{l}9 \cdot 938 \\
9 \cdot 4 \cdot 49 \\
9 \cdot 932 \\
9 \cdot 8986 \\
9 \cdot 976 \\
9\end{array}$ & $\begin{array}{l}15 \\
16 \\
17 \\
18 \\
19\end{array}$ & $\begin{array}{l}8 \cdot 705 \\
8 \cdot 578 \\
8 \cdot 813 \\
9.099 \\
9 \cdot 403\end{array}$ & $\begin{array}{l}8 \cdot 701 \\
8 \cdot 510 \\
8 \cdot 867 \\
9 \cdot 102 \\
9 \cdot 395\end{array}$ & & $\begin{array}{l}8 \cdot 693 \\
8 \cdot 433 \\
8 \cdot 886 \\
9 \cdot 203 \\
9 \cdot 318\end{array}$ & $\begin{array}{l}8 \cdot 700 \\
8 \cdot 421 \\
8 \cdot 898 \\
9 \cdot 2966 \\
9 \cdot 297\end{array}$ & \begin{tabular}{|l|}
$8 \cdot 698$ \\
8.419 \\
$8 \cdot 893$ \\
9.370 \\
$9 \cdot 305$
\end{tabular} & $\begin{array}{l}8 \cdot 703 \\
8.443 \\
8.879 \\
9.424 \\
9.294\end{array}$ & $\begin{array}{l}8.699 \\
8.506 \\
8.533 \\
9.490 \\
8.291\end{array}$ & $\begin{array}{l}8 \cdot 882 \\
8 \cdot 590 \\
8 \cdot 9.950 \\
9 \cdot 501 \\
9 \cdot 2997\end{array}$ & $\begin{array}{l}8 \cdot 671 \\
8 \cdot 65.4 \\
9 \cdot 000 \\
9 \cdot 500 \\
9 \cdot 326\end{array}$ & $\begin{array}{l}8 \cdot 643 \\
8 \cdot 749 \\
9 \cdot 039 \\
9 \cdot 511 \\
9 \cdot 370\end{array}$ & $\begin{array}{l}8 \cdot 822 \\
8.823 \\
9.059 \\
8.459 \\
8 \cdot 399\end{array}$ & $\begin{array}{l}8 \cdot 922 \\
9 \cdot 342 \\
9 \cdot 333\end{array}$ \\
\hline $\begin{array}{l}20 \\
21 \\
22 \\
23 \\
21\end{array}$ & $\begin{array}{l}9 \cdot 946 \\
9 \cdot 681 \\
9 \cdot 485 \\
9 \cdot 189 \\
9 \cdot 288\end{array}$ & $\begin{array}{l}9 \cdot 931 \\
9 \cdot 619 \\
9 \cdot 483 \\
9 \cdot 169 \\
9 \cdot 308\end{array}$ & $\begin{array}{l}9 \cdot 915 \\
9.619 \\
9.447 \\
9.148 \\
9.297\end{array}$ & $\begin{array}{l}9 \cdot 893 \\
9 \cdot 578 \\
9 \cdot 41.5 \\
9 \cdot 122 \\
9 \cdot 325\end{array}$ & $\begin{array}{l}9 \cdot 583 \\
8-578 \\
9402 \\
9 \cdot 4115 \\
9 \cdot 325\end{array}$ & & $\begin{array}{l}9 \cdot 859 \\
9 \cdot 556 \\
9 \cdot 336 \\
9 \cdot 108 \\
9 \cdot 308\end{array}$ & $\begin{array}{l}9 \cdot 826 \\
9 \cdot 551 \\
9 \cdot 312 \\
9 \cdot 128 \\
9 \cdot 291\end{array}$ & & & & $\begin{array}{l}9 \cdot 797 \\
8 \cdot 488 \\
9 \cdot 210 \\
9 \cdot 245 \\
9 \cdot 298\end{array}$ & $\begin{array}{l}9 \cdot 849 \\
9 \cdot 575 \\
9.354 \\
9 \cdot 157 \\
9 \cdot 304\end{array}$ & $\begin{array}{l}23 \\
24\end{array}$ & $\begin{array}{l}9 \cdot 994 \\
9 \cdot 350 \\
9 \cdot 23 \pi \\
8 \cdot 961 \\
9 \cdot 04015\end{array}$ & $\begin{array}{l}9 \cdot 564 \\
9 \cdot 337 \\
9 \cdot 257 \\
8 \cdot 959 \\
9 \cdot 023\end{array}$ & & $\begin{array}{l}9 \cdot 542 \\
9 \cdot 311 \\
9 \cdot 24.1 \\
8 \cdot 9,4 y) \\
9 \cdot 022\end{array}$ & $\begin{array}{l}9 \cdot 527 \\
9 \cdot 302 \\
9 \cdot 221 \\
8 \cdot 963,3 \\
9 \cdot 018\end{array}$ & $\begin{array}{r}9 \cdot 500 \\
9 \cdot 292 \\
9 \cdot 201 \\
8 \cdot 936 \\
9 \cdot 056\end{array}$ & $\begin{array}{l}9 \cdot 488 \\
9 \cdot 282 \\
9 \cdot 153 \\
8 \cdot 458 \\
9 \cdot 051\end{array}$ & & & $\begin{array}{l}9 \cdot 429 \\
9 \cdot 253 \\
9.025 \\
9 \cdot 010 \\
9 \cdot 023\end{array}$ & $\begin{array}{l}9 \cdot 402 \\
9 \cdot 262 \\
8 \cdot 975 \\
9 \cdot 049 \\
8 \cdot 999\end{array}$ & $\begin{array}{l}9 \cdot 374 \\
9.225 \\
8 \cdot 955 \\
9.051 \\
8 \cdot 976 \\
8.976\end{array}$ & $\begin{array}{l}2411 \\
194 \\
030 \\
030\end{array}$ \\
\hline $\begin{array}{l}25 \\
25 \\
27 \\
27 \\
29 \\
20\end{array}$ & $\begin{array}{l}9 \cdot 3 \\
8 \cdot 1 \\
8=9 \\
9 \cdot 0 \\
9 \cdot 0\end{array}$ & $\begin{array}{l}9.314 \\
9 \cdot 119 \\
8 \cdot 953 \\
9 \cdot 0.032 \\
9 \cdot 0628\end{array}$ & $\begin{array}{l}9 \cdot 115 \\
8 \cdot 957 \\
9 \cdot 027 \\
9 \cdot 089\end{array}$ & $\begin{array}{l}9 \cdot 291 \\
9 \cdot 089 \\
8 \cdot 959 \\
9 \cdot 043 \\
9 \cdot 116\end{array}$ & $\begin{array}{l}9 \cdot 944 \\
9 \cdot 070 \\
8 \cdot 971 \\
9 \cdot 9 \cdot 46 \\
9 \cdot 119\end{array}$ & $\begin{array}{l}9 \cdot 272 \\
9.051 \\
8 \cdot 976 \\
8.037 \\
0.125\end{array}$ & $\begin{array}{l}9 \cdot 265 \\
9 \cdot 028 \\
8 \cdot 933 \\
9 \cdot 0.033 \\
9 \cdot 191 i^{\prime}\end{array}$ & $\begin{array}{l}9 \cdot 251 \\
9 \cdot 021 \\
9 \cdot 000 \\
9 \cdot 017 \\
9 \cdot 200\end{array}$ & & $\begin{array}{l}9 \cdot 206 \\
8 \cdot 979 \\
9 \cdot 003 \\
8.986 \\
9 \cdot 211\end{array}$ & $\begin{array}{l}9 \cdot 195 \\
8 \cdot 971 \\
8 \cdot 999 \\
9 \cdot 007 \\
9 \cdot 196\end{array}$ & $\begin{array}{l}9 \cdot 167 \\
8 \cdot 9073 \\
9 \cdot 000 \\
9 \cdot 055 \\
9 \cdot 199\end{array}$ & $\begin{array}{l}9 \cdot 259 \\
9 \cdot 0.06 \\
8 \cdot 979 \\
9 \cdot 023 \\
9 \cdot 149 \\
9.149\end{array}$ & $\begin{array}{l}28 \\
28 \\
29\end{array}$ & $\begin{array}{l}8 \cdot 934 \\
8 \cdot 793 \\
9 \cdot 279 \\
9 \cdot 665 \\
9 \cdot 5.38\end{array}$ & $\begin{array}{l}8 \cdot 839 \\
8 \cdot 816 \\
9 \cdot 301 \\
9 \cdot 695 \\
9 \cdot 487\end{array}$ & $\begin{array}{l}8 \cdot 763 \\
8 \cdot 838 \\
9 \cdot 331 \\
9 \cdot 702 \\
9 \cdot 4.44\end{array}$ & $\begin{array}{l}8 \cdot 731 \\
8 \cdot 933 \\
9-974 \\
9 \cdot 740 \\
9 \cdot 352\end{array}$ & $\begin{array}{l}8 \cdot 700 \\
8 \cdot 958 \\
9 \cdot 422 \\
9 \cdot 736 \\
9 \cdot 300\end{array}$ & $\begin{array}{l}8 \cdot 838 \\
8 \cdot 934 \\
9 \cdot 442 \\
9 \cdot 735 \\
9 \cdot 230\end{array}$ & $\begin{array}{l}8 \cdot 089 \\
9 \cdot 037 \\
9 \cdot 475 \\
9 \cdot 732 \\
9 \cdot 172\end{array}$ & $\begin{array}{l}8 \cdot 664 \\
9 \cdot 085 \\
9 \cdot 508 \\
9 \cdot 729 \\
9 \cdot 118\end{array}$ & $\begin{array}{l}8 \cdot 675 \\
9 \cdot 118 \\
9 \cdot 548 \\
9.685 \\
9 \cdot 0699\end{array}$ & $\begin{array}{l}8 \cdot 714 \\
9 \cdot 163 \\
9 \cdot 573 \\
9 \cdot 665 \\
9 \cdot 0-40\end{array}$ & $\begin{array}{l}8 \cdot 725 \\
9 \cdot 194^{\prime} \\
9 \cdot 575 \\
9 \cdot 6 \cdot 33 \\
9 \cdot 017 \\
9 \cdot 017\end{array}$ & $\begin{array}{l}8.751 \\
9 \cdot 240 \\
9 \cdot 620 \\
9 \cdot 593 \\
8 \cdot 980\end{array} \mid$ & $\begin{array}{l}9.014 \\
9.454 \\
9.693 \\
9.228\end{array}$ \\
\hline 30 & & & & & & $9 \cdot 215$ & & 9.210 & $9 \cdot 216$ & 240? & 248 & $9 \cdot 265$ & $9 \cdot 21$ & & & & & & & & & & & & & & \\
\hline & & & & & & & & & & & & & & & & & & & & 94 & 092 & & & & 11 & 11 & \\
\hline
\end{tabular}

August, 1903.

September, 1903.

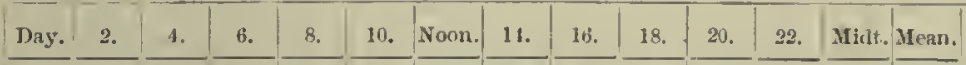

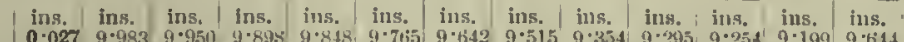

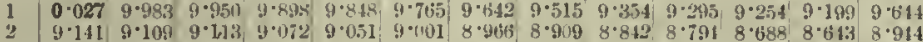

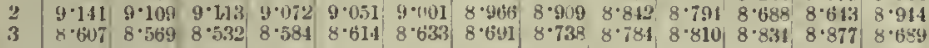

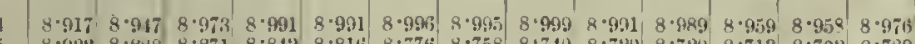

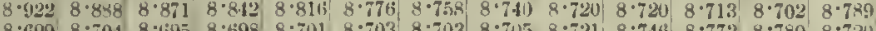

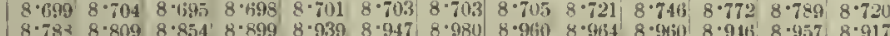

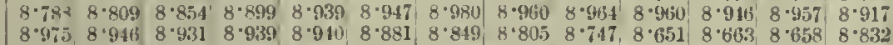

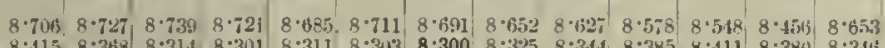

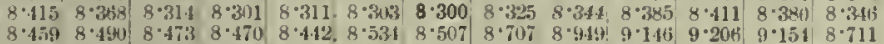

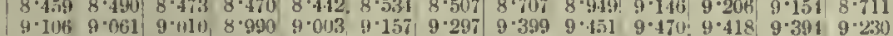

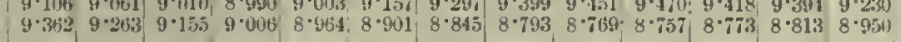

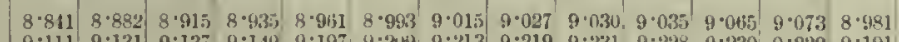

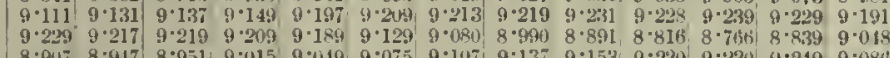

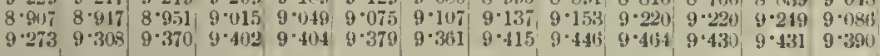

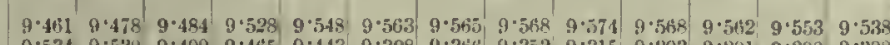

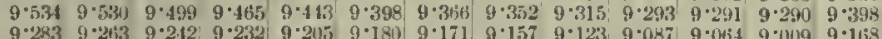

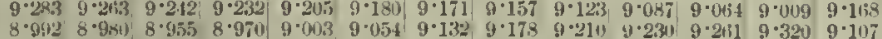

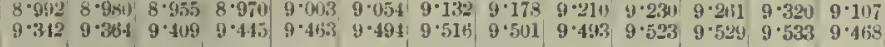

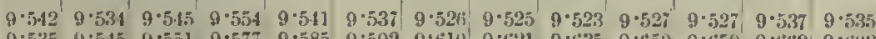

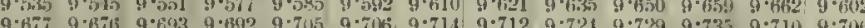

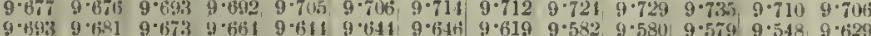

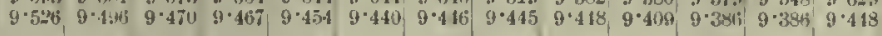

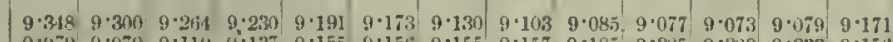

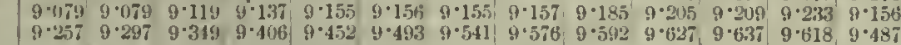

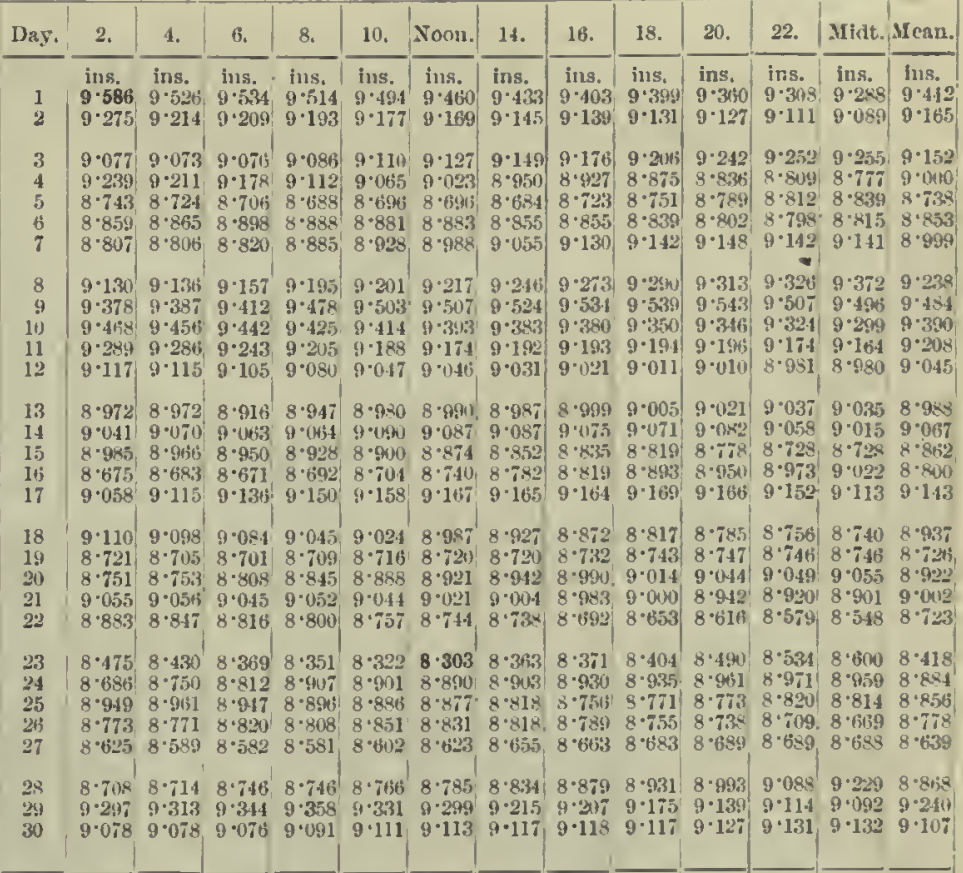

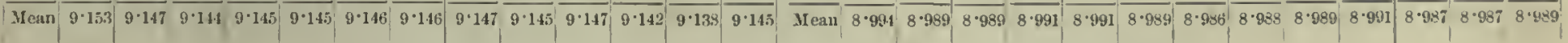


TABle I. (continued).

\section{PRESSURE IN FNGLISII INCIIES, FIRST FIGURE OMITTED.}

The Readings are Corrected for Temperature, and Reduced to Mean Sea Level, but are not Corrected

October, 1903. for Variation of Gravity.

November, 1903.

\begin{tabular}{|c|c|c|c|c|c|c|c|c|c|c|c|c|c|}
\hline ay. & 2 . & 4. & 6. & 8. & 18. & Nixon. & 14. & 16. & 18. & 20. & 22. & Millt. & I enn. \\
\hline$\frac{1}{2}$ & $\begin{array}{l}\text { ins. } \\
9 \cdot 129 \\
9 \cdot 0043\end{array}$ & $\begin{array}{l}\text { Ins. } \\
9 \cdot 126 \\
8 \cdot 958\end{array}$ & $\begin{array}{l}\text { ins }_{2} \\
9 \cdot 13 \times 1 \\
8 \cdot 969\end{array}$ & $\begin{array}{l}\text { ins. } \\
9 \cdot 133 \\
8 \cdot 975\end{array}$ & $\begin{array}{l}\text { ins. } \\
8 \cdot 139 \\
8 \cdot 956\end{array}$ & $\mid \begin{array}{l}\mid \mathrm{ins} \\
8-1: 21 \\
8 \cdot 930 \\
0.930\end{array}$ & $\begin{array}{l}\text { ins. } \\
8 \cdot 097 \\
8 \cdot 922\end{array}$ & $\begin{array}{l}\text { ins. } \\
8 \cdot 093 \\
8 \cdot 903\end{array}$ & $\begin{array}{l}\text { inss, } \\
9 \cdot 079 \\
8 \cdot 906\end{array}$ & $\begin{array}{l}\text { ins, } \\
8 \cdot 065 \\
8 \cdot 906\end{array}$ & $\begin{array}{l}\text { ins. } \\
9 \cdot 01051 \\
8 \cdot 486\end{array}$ & $\begin{array}{l}\text { ins. } \\
9 \cdot 015 \\
8 \cdot 9006\end{array}$ & $\begin{array}{l}\text { ins, } \\
9 \cdot 098 \\
8 \cdot 938\end{array}$ \\
\hline $\begin{array}{l}3 \\
4 \\
5 \\
6 \\
7 \\
\mathbf{5}\end{array}$ & $\begin{array}{l}8 \cdot 908 \\
8 \cdot 983 \\
9 \cdot 010 \\
8.990 \\
9 \cdot 045\end{array}$ & $\begin{array}{l}8.911 \\
8.984 \\
9.001 \\
8.949 \\
9.034\end{array}$ & 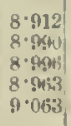 & $\begin{array}{l}8 \cdot .931 \\
8.990 \\
8 \cdot 9 \times 5 \\
8 \cdot 999 \\
0 \cdot 069\end{array}$ & $\begin{array}{l}8 \cdot 919 \\
9.002 \\
8.97 \\
9 \cdot 005 \\
8 \cdot 0+06\end{array}$ & $\begin{array}{l}8-9.9 \\
9 \cdot 016 \\
8 \cdot 974 \\
8 \cdot 999 \\
8 \cdot 111\end{array}$ & $\begin{array}{l}8 \cdot 948 \\
9 \cdot 423 \\
8 \cdot 072 \\
8 \cdot 947 \\
9 \cdot 102\end{array}$ & $\begin{array}{l}8 \cdot 945 \\
9 \cdot 017 \\
8 \cdot 971 \\
9 \cdot 006 \\
9 \cdot 100\end{array}$ & $\begin{array}{l}8.945 \\
8 \cdot 1122 \\
8 \cdot 947 \\
9 \cdot 005 \\
9 \cdot 087\end{array}$ & $\begin{array}{l}8 \cdot 948 \\
9 \cdot 012 \\
8 \cdot 9080 \\
9 \cdot 014 \\
8 \cdot 081\end{array}$ & $\begin{array}{l}8 \cdot 9+400 \\
9 \cdot 01 \\
8 \cdot 950 \\
9 \cdot 016 \\
8 \cdot 100\end{array}$ & $\begin{array}{l}8 \cdot 974 \\
9.015 \\
8.969 \\
9 \cdot 022 \\
8 \cdot 0.109\end{array}$ & $\begin{array}{l}8 \cdot 9.90 \\
8 \cdot 0065 \\
8.978 \\
9 \cdot 007 \\
9.080\end{array}$ \\
\hline $\begin{array}{r}8 \\
9 \\
10 \\
11 \\
12\end{array}$ & $\begin{array}{l}8 \cdot 125 \\
9 \cdot 251 \\
9 \cdot 0.38 \\
8 \cdot 955 \\
9 \cdot 078\end{array}$ & $\begin{array}{l}9 \cdot 150 \\
9 \cdot 230 \\
9 \cdot 025 \\
8 \cdot 93, \\
9 \cdot 0034\end{array}$ & $\begin{array}{l}9 \cdot 175 \\
9 \cdot 21 \tilde{i} \\
9 \cdot 012 \\
8 \cdot 940 \\
9 \cdot 056\end{array}$ & $\begin{array}{l}9 \cdot 176 \\
9 \cdot 211 \\
9 \cdot 012 \\
8 \cdot 910 \\
9 \cdot 017\end{array}$ & $\begin{array}{l}9 \cdot 218 \\
8 \cdot 199 \\
9 \cdot 014 \\
8 \cdot 956 \\
9 \cdot 024\end{array}$ & $\begin{array}{l}9 \cdot 217 \\
9 \cdot 178 \\
8 \cdot 018 \\
8.098 \\
8.997\end{array}$ & $\begin{array}{c}q \cdot 225 \\
8 \cdot 155 \\
8 \cdot 012 \\
9 \cdot 032 \\
8 \cdot 974\end{array}$ & $\begin{array}{l}9 \cdot 227 \\
9 \cdot 143 \\
9 \cdot 013 \\
9 \cdot 051 \\
8 \cdot 954\end{array}$ & $\begin{array}{l}9 \cdot 230 \\
9 \cdot 139 \\
9 \cdot 018 \\
9 \cdot 062 \\
8 \cdot 920\end{array}$ & $\begin{array}{l}9 \cdot 235 \\
9 \cdot 114 \\
9 \cdot 014 \\
9 \cdot 081 \\
8 \cdot 902\end{array}$ & $\begin{array}{c}8 \cdot 248 \\
8 \cdot 080 \\
9 \cdot 000 \\
9 \cdot 080 \\
8 \cdot 902\end{array}$ & $\begin{array}{l}9 \cdot 608 \\
8.089 \\
9.089 \\
8.018\end{array}$ & $\begin{array}{l}8.014 \\
9.010 \\
8.986\end{array}$ \\
\hline $\begin{array}{l}13 \\
14 \\
15 \\
16 \\
17\end{array}$ & $\begin{array}{l}8 \cdot 911 \\
9 \cdot 195 \\
9 \cdot 296 \\
9 \cdot 127 \\
9 \cdot 004\end{array}$ & $\begin{array}{l}8 \cdot 918 \\
9 \cdot 168 \\
9 \cdot 283 \\
9 \cdot 120 \\
8 \cdot 979\end{array}$ & $\begin{array}{l}8 \cdot 992 \\
9 \cdot 2 \cdot 221 \\
8 \cdot 260 \\
8 \cdot 118 \\
8 \cdot 967\end{array}$ & $\begin{array}{l}8 \cdot 021 \\
9 \cdot 221 \\
8-2221 \\
9 \cdot 127 \\
8 \cdot 949\end{array}$ & $\begin{array}{l}9 \cdot 070 \\
9 \cdot 240 \\
9 \cdot 225 \\
9 \cdot 127 \\
8 \cdot 834\end{array}$ & $\begin{array}{l}9 \cdot 096 \\
9 \cdot 22 \\
9 \cdot 197 \\
9 \cdot 122 \\
8 \cdot 923\end{array}$ & $\begin{array}{l}9 \cdot 113 \\
9 \cdot 313 \\
9 \cdot 186 \\
9 \cdot 108 \\
8 \cdot 919\end{array}$ & $\begin{array}{l}9 \cdot 1.13 \\
9 \cdot 334 \\
9 \cdot 163 \\
9 \cdot 092 \\
8 \cdot 911\end{array}$ & $\begin{array}{l}9 \cdot 154 \\
8 \cdot 228 \\
9 \cdot 1.11 \\
9 \cdot 064 \\
8 \cdot 005\end{array}$ & $\begin{array}{l}9 \cdot 164 \\
8 \cdot 323 \\
9 \cdot 124 \\
9 \cdot 066 \\
8 \cdot 899\end{array}$ & $\begin{array}{l}9 \cdot 180 \\
9 \cdot 315 \\
9 \cdot 128 \\
9 \cdot 039 \\
8 \cdot 875\end{array}$ & $\begin{array}{l}9 \cdot 200 \\
9 \cdot 318 \\
9 \cdot 127 \\
9 \cdot 027 \\
8 \cdot 875\end{array}$ & $\begin{array}{r}9 \cdot 19 \\
9 \cdot 095 \\
8 \cdot 928\end{array}$ \\
\hline $\begin{array}{l}18 \\
19 \\
20 \\
21 \\
22\end{array}$ & $\begin{array}{l}8 \cdot 874 \\
8 \cdot 951 \\
8 \cdot 200 \\
9 \cdot 013 \\
9 \cdot 034\end{array}$ & $\begin{array}{l}8 \cdot 873 \\
8 \cdot 983 \\
9 \cdot 222 \\
9 \cdot 309 \\
9 \cdot 032\end{array}$ & $\begin{array}{l}8 \cdot 879 \\
8 \cdot 9.82 \\
9 \cdot 232 \\
9 \cdot 311 \\
9 \cdot 027\end{array}$ & $\begin{array}{l}8 \cdot 88, \\
9 \cdot-(137 \\
9 \cdot 286 \\
8 \cdot 267 \\
9 \cdot 03 \pi\end{array}$ & $\begin{array}{l}8 \cdot 893 \\
9 \cdot 062 \\
9 \cdot 202 \\
9.286 \\
9.027\end{array}$ & $\begin{array}{l}8 \cdot 894 \\
9 \cdot 08 \\
9 \cdot 321 \\
9 \cdot 260 \\
9 \cdot 025\end{array}$ & $\begin{array}{l}8 \cdot 912 \\
9 \cdot 102 \\
9 \cdot 330 \\
9 \cdot 227 \\
8 \cdot 988\end{array}$ & $\begin{array}{l}8 \cdot 919 \\
9 \cdot 126 \\
9 \cdot 320, \\
9 \cdot 211 \\
8 \cdot 971\end{array}$ & $\begin{array}{l}8 \cdot 022 \\
9 \cdot 129 \\
9 \cdot 311 \\
9 \cdot 155 \\
8 \cdot 96 t^{\circ}\end{array}$ & $\begin{array}{l}8 \cdot 928 \\
9 \cdot 142 \\
9 \cdot 313 \\
9 \cdot 128 \\
8 \cdot 951\end{array}$ & $\begin{array}{l}8 \cdot 932 \\
8 \cdot 9164 \\
9 \cdot 315 \\
9 \cdot 107 \\
8 \cdot 921\end{array}$ & $\begin{array}{l}8 \cdot 944 \\
9 \cdot 191 \\
9 \cdot 314 \\
9 \cdot 060 \\
8 \cdot 899\end{array}$ & $\begin{array}{l}8 \cdot 905 \\
9.081 \\
9 \cdot 288 \\
9 \cdot 223 \\
8 \cdot 990\end{array}$ \\
\hline $\begin{array}{l}23 \\
23 \\
25 \\
28 \\
27\end{array}$ & $\begin{array}{l}8 \cdot 903 \\
8 \cdot 5+54 \\
8 \cdot 5 i 1 \\
8 \cdot 442 \\
8 \cdot 333\end{array}$ & $\begin{array}{l}8 \cdot 903 \\
8 \cdot 559 \\
8 \cdot 56 \overline{1} \\
8 \cdot 414 \\
8 \cdot 322\end{array}$ & $\begin{array}{l}8 \cdot 905 \\
8.55 \\
8.552 \\
8 \cdot 4111 \\
8 \cdot 325\end{array}$ & $\begin{array}{l}8 \cdot 915 \\
8 \cdot 5.559 \\
8 \cdot 350 \\
8 \cdot 3 \cdot 1 \\
8 \cdot 315\end{array}$ & $\begin{array}{l}8 \cdot 977 \\
8.569 \\
8.541 \\
8 \cdot 371 \\
8.363\end{array}$ & $\begin{array}{l}8 \cdot 881 \\
8 \cdot 51 \\
8 \cdot 522 \\
8 \cdot 347 \\
8 \cdot 380 \\
8.38\end{array}$ & $\begin{array}{l}8 \cdot 8482 \\
8 \cdot 599 \\
8 \cdot 503 \\
8 \cdot 3444 \\
8 \cdot 397\end{array}$ & $\begin{array}{l}8 \cdot 813 \\
8 \cdot 590 \\
8 \cdot 501 \\
8: 350 \\
8 \cdot 386\end{array}$ & $\begin{array}{l}8 \cdot 769 \\
8 \cdot 592 \\
8 \cdot 497 \\
8 \cdot 339 \\
8 \cdot 388\end{array}$ & $\begin{array}{l}8 \cdot 726 \\
8 \cdot 601 \\
8 \cdot 483 \\
8 \cdot 345 \\
8 \cdot 393\end{array}$ & $\begin{array}{l}8 \cdot 668 \\
8 \cdot 591 \\
8 \cdot 473 \\
8 \cdot 355 \\
8 \cdot 431\end{array}$ & $\begin{array}{l}8 \cdot 625 \\
8 \cdot 580 \\
8.459 \\
8 \cdot 358 \\
8 \cdot 433\end{array}$ & $\begin{array}{l}8 \cdot 823 \\
8.779 \\
8.518 \\
8.373 \\
8.375\end{array}$ \\
\hline $\begin{array}{l}28 \\
29\end{array}$ & & 8.47 & & & & & & & & & & $\begin{array}{l}8 \cdot 492 \\
8 \cdot 453 \\
8.761\end{array}$ & $\begin{array}{l}8 \cdot 522 \\
8 \cdot 455 \\
8 \cdot 632\end{array}$ \\
\hline 31 & & & & & & & & & & & & 9.001 & 8 \\
\hline
\end{tabular}

$\overline{\text { Xean }} \overline{8 \cdot 918} \overline{8 \cdot 914} \overline{8 \cdot 918} \overline{8 \cdot 927} \overline{8 \cdot 932} \overline{8 \cdot 934} \overline{8 \cdot 937} \overline{8 \cdot 932} \overline{8 \cdot 925} \overline{8 \cdot 922} \overline{8 \cdot 919} \overline{8 \cdot 917} \overline{8 \cdot 925}$

\begin{tabular}{|c|c|c|c|c|c|c|c|c|c|c|c|c|c|}
\hline ay. & 2. & 4. & 6. & 8. & 10. & Noon. & 14. & 16. & 18. & 20. & 22. & Mist. M & Mean \\
\hline 1 & $\begin{array}{l}\text { lins. } \\
8 \cdot 997\end{array}$ & $\begin{array}{l}\text { ins. } \\
9^{\prime} 0009\end{array}$ & $\begin{array}{l}\text { Ins, } \\
9 \cdot 036\end{array}$ & $\begin{array}{l}\text { inss } \\
9 \cdot 052\end{array}$ & ins. $_{8 \cdot 0 \%}$ & $\mid \begin{array}{l}\text { ins. } \\
9 \cdot 080\end{array}$ & $\begin{array}{l}\text { ins. } \\
9 \cdot 083\end{array}$ & \begin{tabular}{l} 
Ins. \\
\hdashline .083
\end{tabular} & $\begin{array}{l}\text { ins. } \\
9 \cdot 075\end{array}$ & $\begin{array}{l}\text { fins. } \\
9 \cdot 0633\end{array}$ & $\begin{array}{l}\text { Ins. } \\
9 \cdot 04.4\end{array}$ & $9 \cdot 0.17$ & $9 \cdot 053$ \\
\hline $\begin{array}{l}2 \\
3\end{array}$ & & & $\begin{array}{l}9 \cdot 018 \\
8 \cdot 921\end{array}$ & & $\begin{array}{l}9 \cdot 021 \\
8 \cdot 9 \cdot 929\end{array}$ & 15 & $\begin{array}{l}3 \\
4 \\
4\end{array}$ & . & 8 & 9 & 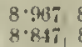 & & \\
\hline $\begin{array}{l}4 \\
5\end{array}$ & $\begin{array}{l}8 \cdot 817 \\
8 \cdot 583\end{array}$ & $\begin{array}{l}8 \cdot 801 \\
8.567 \%\end{array}$ & $\begin{array}{l}8 \cdot 78.3 \\
8 \cdot 5+99\end{array}$ & $\begin{array}{l}8 \cdot 758 \\
8 \cdot 580\end{array}$ & $\begin{array}{l}8 \cdot 72 \pi \\
8 \cdot 5460\end{array}$ & $\begin{array}{l}8.708 \\
8.55 \%\end{array}$ & $\begin{array}{l}8.6801 \\
8.5740\end{array}$ & $\begin{array}{l}8.671 \\
8.595\end{array}$ & $\begin{array}{l}8 \cdot 653 \\
8.624\end{array}$ & $\begin{array}{l}8 \cdot 639 \\
8-6+49\end{array}$ & $\begin{array}{l}8.610 \\
8.66 t 4\end{array}$ & $\begin{array}{l}8.585 .7 \\
8 \cdot 655\end{array}$ & $\begin{array}{l}8.703 \\
8.599\end{array}$ \\
\hline 3 & $8 \cdot 660$ & 8.690 & 8.714 & $8 \cdot 739$ & $8 \cdot 748$ & 8.758 & $8 \cdot 753$ & $8 \cdot 7+6$ & $8 \cdot 734$ & 8.623 & $8 \cdot 683^{2}$ & $8 \cdot 685$ & $8 \cdot 720$ \\
\hline 8 & $\begin{array}{l}733 \\
77\end{array}$ & & $\begin{array}{l}8 \cdot 6644 \\
8 \cdot 695\end{array}$ & $\begin{array}{l}8.689 \\
8 \cdot 692\end{array}$ & $\begin{array}{l}8 \cdot 700 \\
8.4648\end{array}$ & 87 & 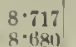 & & & $\begin{array}{l}8 \cdot 687 \\
8 \cdot 651\end{array}$ & & & \\
\hline 8 & 8.664 & $8 \cdot 049$ & $8 \cdot 673$ & 8.648 & 8.704 & 8.678 & $8 \cdot 685$ & & & & 8.661 & 535 & 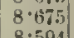 \\
\hline $\begin{array}{l}10 \\
11\end{array}$ & 8.582 & $8 \cdot 594$ & 8.601 & $\begin{array}{l}8.624 \\
8.624\end{array}$ & $\begin{array}{l}8.603 \\
8.691\end{array}$ & $8 . ; 11$ & $\begin{array}{l}8.5 \times 3 \\
8 \cdot 779\end{array}$ & $8 \cdot 791$ & $\begin{array}{l}8.577 \\
8 \cdot 773\end{array}$ & $\begin{array}{l}8.575 \\
8.784\end{array}$ & $\begin{array}{l}8.5618 \\
8.809\end{array}$ & $\begin{array}{l}8.676 \\
8.821\end{array}$ & $\left|\begin{array}{c}8.594 \\
8.713\end{array}\right|$ \\
\hline 12 & & 810 & $8 \cdot 80 \pi$ & 8.812 & & 8.855 & & & & & & 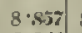 & \\
\hline $\begin{array}{l}183 \\
14\end{array}$ & 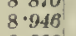 & $8 \cdot 0$ & $\begin{array}{r}8.881 \\
8.961\end{array}$ & $\begin{array}{l}8.982 \\
8.9665\end{array}$ & $\begin{array}{l}8.876 \\
8.975\end{array}$ & $\begin{array}{l}8 \cdot 876 \\
8 \cdot 972\end{array}$ & $\begin{array}{l}8.895 \\
8.962 \\
8.962\end{array}$ & $\begin{array}{l}8.893 \\
8.958\end{array}$ & 989 & 62 & $\begin{array}{l}8.028 \\
8.964\end{array}$ & & \begin{tabular}{|l|}
$8 \cdot 8 \cdot 93$ \\
$8 \cdot 964$
\end{tabular} \\
\hline & & & & $9 \cdot 0$ & 9.012 & & $8: 985$ & & $8: 9.92$ & & $8: 939$ & & \\
\hline & & & & & $9 \cdot 0$ & $0^{\circ} 0$ & $9 . \mathrm{c}$ & & $9 \cdot 084$ & & $9 \cdot 110$ & $9 \cdot 124$ & 9.044 \\
\hline 17 & & & $8 \cdot$ & $8^{\circ}$ & 9. & f & is & 119 & 10 & 1 & b) & $9 \cdot 232$ & 21 \\
\hline 1 & & & & & $9 \cdot 161$ & & & & $9 \cdot 164$ & & $9 \cdot 169$ & & \\
\hline 20 & $\begin{array}{l}\theta \cdot 101 \\
\theta \cdot 003\end{array}$ & & 9.7 & a. & $\begin{array}{l}9 \cdot 14.5 \\
9001\end{array}$ & $9 \cdot 120$ & $\begin{array}{c}9 \cdot 098 \\
5.094\end{array}$ & $\begin{array}{l}80.070 \\
8.090\end{array}$ & $\begin{array}{l}9.054 \\
8.0909\end{array}$ & $9 \cdot 044$ & $9 \cdot 023$ & 9.010 & \\
\hline & & & & & & & & & & & & & \\
\hline 20 & & & & & & & & & & & 3 & & \\
\hline & & & & & 9. & & & & & & 8.528 & & \\
\hline 26 & & 8.6 & 8. & 8 & $\begin{array}{l}8.528 \\
8.508\end{array}$ & $8:$ & $\begin{array}{l}8.583 \\
8.528\end{array}$ & $\begin{array}{l}8.564 \\
8.520\end{array}$ & $\mid \begin{array}{l}8.584 \\
8.526\end{array}$ & $\begin{array}{l}8 \cdot 538 \\
8.547\end{array}$ & 8.528 & $\begin{array}{l}5.619 \\
8.5569\end{array}$ & $8 \cdot 60$ \\
\hline & & & & & & & & & & & & & \\
\hline & & & & & & & & & & & 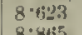 & & \\
\hline & & & & & & & & & & & $\begin{array}{l}8 \cdot 102 \\
9 \cdot 860\end{array}$ & $\begin{array}{l}8 \cdot 81 A_{1} \\
9 \cdot 112\end{array}$ & $\begin{array}{l}8.787 \\
9.015\end{array}$ \\
\hline & & & & & & & & & & & & & \\
\hline
\end{tabular}

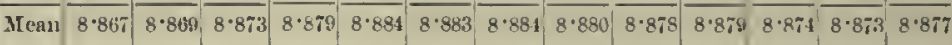

\begin{tabular}{|c|c|c|c|c|c|c|c|c|c|c|c|c|c|c|c|c|c|c|c|c|c|c|c|c|c|c|c|}
\hline Day. & 2. & 4. & 6. & 8. & 10. & Noni. & 14. & 16. & 18. & 20. & 22. & Mint. & Mean. & Diy. & 2. & 4. & 6. & 8. & 10. & inen. & 14. & 16. & 18. & 20. & 22. & Mislt. & $\cos$ \\
\hline 1 & $\begin{array}{l}\text { Ins. } \\
0 \cdot 108\end{array}$ & $\begin{array}{l}\text { ins. } \\
9 \cdot 109\end{array}$ & $\begin{array}{l}\text { ins. } \\
9 \cdot 110\end{array}$ & $\begin{array}{l}\text { ins. } \\
9 \cdot 125\end{array}$ & $\begin{array}{l}\text { ins. } \\
9 \cdot 143\end{array}$ & $\begin{array}{l}\text { Ins. } \\
9 \cdot 182\end{array}$ & $\begin{array}{l}\text { ins. } \\
9 \cdot 208\end{array}$ & $\begin{array}{l}\text { ins, } \\
9 \cdot 22 t\end{array}$ & $\begin{array}{l}\text { ins. } \\
9 \cdot 234\end{array}$ & $\begin{array}{l}\text { ins. } \\
9.237\end{array}$ & $9 \cdot 281$ & $9 \cdot 304$ & \begin{tabular}{|l|} 
ins. \\
$9 \cdot 189$
\end{tabular} & 1 & 17 & & & $\begin{array}{l}11118 \\
8 \cdot 987\end{array}$ & & & 62 & & & & & & \\
\hline & & & & & & & & & & & & & & & 8 & 8 . & $8 \cdot 8 \%$ & 8.864 & & & & & & & & & \\
\hline$\frac{2}{3}$ & $\begin{array}{l}8.321 \\
0 \cdot 42 .\end{array}$ & $\begin{array}{l}8 \cdot 339 \\
9 \cdot 401\end{array}$ & $\begin{array}{l}9.371 \\
9.417\end{array}$ & $9 \cdot 404$ & 4 & 7 & & $9 \cdot 4$ & 480 & 9.470 & 45 & 4.33 & $9 \cdot 42$ & 3 & $s \cdot 0.30$ & $8 \cdot 924$ & $8 \cdot 024$ & $R \cdot$ & (titi & & & & & & & & \\
\hline 4 & & $\tilde{50}$ & $9 \cdot 354$ & 814 & 5! & & & & & & & & & 4 & & & & & & & & & & & & & \\
\hline 5 & & 0.283 & $g \cdot 332$ & $9-371$ & $9 \cdot 393$ & 9.115 & $\begin{array}{l}9.364 \\
9.396\end{array}$ & $\begin{array}{l}9 \cdot 348 \\
9 \cdot 412\end{array}$ & $\begin{array}{l}9 \cdot 334 \\
9 \cdot 433\end{array}$ & $\begin{array}{l}9 \cdot 244 \\
9 \cdot 453\end{array}$ & $\begin{array}{l}9 \cdot 244 \\
9 \cdot 478\end{array}$ & $\begin{array}{l}0.262 \\
0.484\end{array}$ & $\begin{array}{l}9.327 \\
9.393\end{array}$ & & & & & & & & & & & & & & \\
\hline 6 & $9 \cdot 400$ & $9 \cdot 500$ & $9 \cdot 517$ & $9 \cdot 533$ & $9 \cdot 540$ & $9 \cdot 550$ & $9 \cdot 541$ & 9.531 & $8 \cdot 534$ & $9 \cdot 532$ & $8 \cdot 525$ & 0.52 .3 & $9 \cdot 527$ & 6 & $9 \%$ & $06^{\prime}$ & os & 17 & & 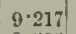 & & & & & & $20 \mathrm{Br}$ & Fiv \\
\hline 7 & 5 & 507 & 199 & 49 & 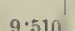 & & 8 & & ? & & & & & & & & & & & & & & & & & & \\
\hline 8 & & $9 \cdot 52$ & $0.58 \%$ & 9.598 & $9-582$ & $9 \cdot 585$ & $\begin{array}{l}9.526 \\
9.580\end{array}$ & 9.587 & & $\begin{array}{l}9 \cdot 562 \\
9 \cdot 5409\end{array}$ & 72 & $\begin{array}{l}0.551 \\
0.572\end{array}$ & & $\begin{array}{l}8 \\
9\end{array}$ & $\begin{array}{l}9 \cdot 101 \\
9 \cdot 102\end{array}$ & & $\begin{array}{l}9 \cdot 186 \\
9 \cdot 076\end{array}$ & $\begin{array}{l}9 \cdot 172 \\
9 \cdot 058\end{array}$ & $\begin{array}{l}9 \cdot 169 \\
9 \cdot 035\end{array}$ & $\begin{array}{l}9 \cdot 166 \\
9 \cdot 035\end{array}$ & $\begin{array}{l}9 \cdot 160 \\
9 \cdot 081\end{array}$ & $\begin{array}{l}9 \cdot 152 \\
9 \cdot 06 \%\end{array}$ & $\begin{array}{l}9 \cdot 123 \\
9 \cdot 469\end{array}$ & $\begin{array}{l}3 \cdot 119 \\
y \cdot 075\end{array}$ & $\begin{array}{l}9.127 \\
9 \cdot \cos 2\end{array}$ & $\begin{array}{l}9 \cdot 115 \\
9 \cdot 0 \mathrm{~s} s\end{array}$ & $\begin{array}{c}y \cdot 157 \\
y \cdot 072\end{array}$ \\
\hline 9 & 37 & $8.55 \%$ & 1).552 & (9. $542^{\circ}$ & $9 \cdot 528$ & 8.521 & 9.514 & 0.493 & & 9.463 & 9.45 & $0 \cdot 4303$ & 9.508 & 10 & 0.002 & 9.096 & $9 \cdot 09-1$ & $9 \cdot 102$ & $9 \cdot 112$ & $9 \cdot 125$ & $5 \cdot 124$ & $9 \cdot 127$ & $9 \cdot 127$ & $9 \cdot 131$ & A.133 & $9 \cdot 130$ & $9 \cdot 116$ \\
\hline 10 & 30 & $9 \cdot 411$ & $9 \cdot 40 \bar{t}$ & 9.399 & $9 \cdot 383$ & $9 \cdot 362$ & $9 \cdot 3333$ & $9 \cdot 328$ & $9 \cdot 313$ & $8 \cdot 305$ & $9 \cdot 28 \overline{7}$ & $9 \cdot 275$ & $9 \cdot 353$ & & & & & & & & & & & & & & \\
\hline 1 & & $9 \cdot 2563$ & 244 & & -233 & $9 \cdot 225$ & $9 \cdot 21 \%$ & 9.214 & $\cdot 217$ & $9 \cdot 218$ & $\mathbf{9} \cdot 225$ & 0.227 & $9 \cdot 232$ & & -127| & & 0 & $9 \cdot 152$ & & & & & & & & & \\
\hline 1 . & 220 & $9 \cdot 230$ & 9.234 & $9 \cdot 245$ & 9.243 & (i) & 9. & .21. & & $9 \cdot 202$ & & & & & & & & & & & & & & & & & \\
\hline & & 206 & & $0 \cdot 193$ & & & & $i$ & & & 1 & & & 1. & $\theta$ & & $8 \cdot 792$ & . $81 \mathrm{k}$ & 50 & & & & & 8.898 & & & 8.850 \\
\hline 1 & If & $9 \cdot 24 \mathrm{~T}$ & at? & $9 \cdot 26-4$ & & & 9. & (1) & 5 & & & & & 10 & 96 & $8 \cdot 8$ & $8 \cdot 536$ & $8 \cdot 902$ & $s^{\circ} \cdot 916$ & 8.918 & $8 \cdot 913$ & $8 \cdot 443$ & 8.959 & $8 \cdot 902$ & 0.008 & 34 & $8 \cdot .42$ \\
\hline 1 & $\begin{array}{l}0.27 ! \\
0.19 \%\end{array}$ & $\begin{array}{l}9.270 \\
9.15 \%\end{array}$ & $\begin{array}{l}9 \cdot 27 \cdot 3 \\
9 \cdot 177\end{array}$ & $\begin{array}{l}9 \cdot 2701 \\
9 \cdot 170\end{array}$ & 9.62 & $\begin{array}{r}9 \cdot 241 \\
9.159\end{array}$ & $\begin{array}{l}4-236 \\
0.146\end{array}$ & 34 & 23 & 9. & 10 & $9 \cdot 207$ & & & & & & & & & & & & & & & \\
\hline & & 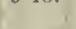 & 8116 & $817-$ & 8.04 & У.125 & & thi & & 5 & & & & & & & & & & & & & & & & absis & \\
\hline 1 & & 3 & $0, \theta$ & 9.073 & & & & & & & & 1 & & & & & & & & & & & & & & & \\
\hline & & $8 \cdot 943$ & & 1) & & & & 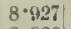 & & & & & & 1 & & & & & & & & & & & & & \\
\hline $\begin{array}{l}19 \\
20\end{array}$ & & $8.94 \pi$ & 8 & 8.945 & 6 & & $8^{\circ}$ & 8 & 8 & 2 & 8 & 8 & 8.8 & 20 & 8.974 & $8 \cdot 978$ & $8 \cdot 992$ & 8.491 & 8.995 & 01 & 0.012 & $9 \cdot 012$ & 0.012 & $9 \cdot 024$ & 0.024 & 23 & .003 \\
\hline 21 & $9 \cdot 135$ & $\begin{array}{l}8.045 \\
9 \cdot 125\end{array}$ & 0 & 9.098 & 9.072 & $\begin{array}{l}9.065 \\
9.046\end{array}$ & $\begin{array}{l}9 \cdot 1 \\
9 \cdot 0\end{array}$ & $\begin{array}{l}9 \cdot 1 \\
8 \cdot 9\end{array}$ & 8 & & 12 & $\theta \cdot 141$ & 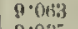 & & & & & & & & & & & & & & \\
\hline & & & & & & & & & 96 & 8.069 & $8^{\circ}$ & 8.8 & 03 & 2 & & & & & & & & & & & & & \\
\hline 2 & & & & 91 & & 78 & $8 \cdot 8$ & & & & 8 & & & 2 & 1 & & & & & & & & & & & & \\
\hline 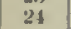 & & & & & & & & & & & & & & : & & & & & & & & & & & & & \\
\hline . & & $\begin{array}{l}9.300 \\
0 \cdot 253\end{array}$ & 8 & $\begin{array}{l}9 \cdot 309 \\
9-279\end{array}$ & $\begin{array}{l}9.324 \\
9.271\end{array}$ & $\begin{array}{l}1 \cdot 321 \\
9 \cdot 253\end{array}$ & $\begin{array}{l}9 \cdot 322 \\
9 \cdot 024\end{array}$ & 13 & $9 \cdot 308$ & $8 \%$ & $9^{\circ}$ & 8 & & 2 & 0.330 & 0.337 & 0.360 & $8 \cdot 391$ & $9 \cdot 406$ & 84 & $9 \cdot 398$ & & $9 \cdot 2 \sqrt{\omega} t$ & $9 \cdot 370$ & & 156 & \\
\hline 2 & $0 \cdot 179$ & $8 \cdot 1 \% 1$ & $8 \cdot 168$ & $9 \cdot 163$ & $9 \cdot 141$ & $9 \cdot 14$ & $9 \cdot 136$ & $9-127$ & $9 \cdot 112$ & ๑.098. & $\begin{array}{l}9 \cdot 1+4 \\
9 \cdot 084\end{array}$ & $\begin{array}{l}9 \cdot 184 \\
9.084\end{array}$ & $9 \cdot 134$ & & & & & & & & & & & & & & \\
\hline & & & & & & & & & & & & & & & & & & & & & & & & & & & \\
\hline & & & & & & & & 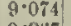 & 9. & 9.0 & 9.0 & $g^{\circ}$ & & & 0.023 & & & 9 & & & & & & & & & \\
\hline & & $8 \cdot 3$ & 0.320 & $8 \cdot 344$ & 9.3 & $3^{\circ}$ & & & & & & & & 2 & $\theta^{\circ}$ & & & 8 & & & & & & 3.4 & & 213 & 9222 \\
\hline 3 & & . & 5 & & & & & & & & & & & 3 & $9 \cdot 1$ & & & & & & & & & $\cdot 952$ & $\cdot y$ & Tho & 056 \\
\hline 31 & & & & 9 & ini & 1. & $9-484$ & 9.073 & $9 \cdot 068$ & $9 \cdot 066$ & 9.049 & 9.028 & $9 \cdot 0 t 3$ & 31 & $8 \cdot 88 ?$ & $8 \cdot 580$ & $8^{\circ} 86 t^{\prime}$ & $8 \cdot 822$ & $8 \cdot 622$ & $8 \cdot 7 \mathrm{MH}$ & $8 \cdot \%$ \% & $8 \cdot 767$ & $s \cdot 744$ & $8 \cdot 760$ & $8 \cdot 765$ & $8 \cdot \pi 60$ & $8 \cdot 805$ \\
\hline & $9 \cdot 220$ & 210 & & $\cdot 2246$ & $9 \cdot 22$ & 0.230 & 8 & & 9.226 & 9.220 & & $9 \cdot 220$ & 0.224 & Mean & 3 & & 9.062 & 9.063 & & & $\cdot 0$ & 61 & 44 & & 058 & 057 & \\
\hline
\end{tabular}


TABLE II.

TEMPERATURE IN DEGREES FAHRENHEIT.

Dri Buls.

WET BULB.

February, 1902.

\begin{tabular}{|c|c|c|c|c|c|c|c|c|c|c|c|c|c|c|c|c|c|c|c|c|c|c|c|c|c|c|}
\hline Day. & 2. & 4. & 6. & 8. & 10. & Noon. & 14. & 16. & 18. & 20. & 22. & Mislt. & Меми. & Day. & 2. & 4. & 6. & 8. & 10. & Soon. & 14. & 10. & 18. & 20. & 22. & | Mhate \\
\hline & 。 & 。 & $\%$ & $\circ$ & $\circ$ & $\circ$ & $\circ$ & $\circ$ & \% & . & $\circ$ & $\circ$ & 。 & & 。 & $\circ$ & 。 & . & $\circ$ & $\circ$ & $\circ$ & $\bar{\circ}$ & $\circ$ & 。 & $\circ$ & $\circ$ \\
\hline$\frac{1}{2}$ & $=$ & $=$ & $=$ & z & $=$ & $=$ & $=$ & $=$ & $=$ & $=$ & $=$ & $=$ & = & 2 & $=$ & $=$ & $=$ & $=$ & $=$ & $=$ & $=$ & $=$ & $\bar{z}$ & $=$ & $=$ & - \\
\hline 4 & च & $\bar{z}$ & 二 & $\bar{z}$ & $\bar{z}$ & $\overline{-}$ & $\bar{z}$ & 二 & 二 & $\bar{z}$ & $=$ & $=$ & $\bar{z}$ & $\begin{array}{l}3 \\
4\end{array}$ & 二 & $=$ & $=$ & $=$ & $=$ & $=$ & $=$ & $=$ & 二 & $=$ & Z & $=$ \\
\hline 5 & - & - & - - & - & - & - & - & - & - & - & - & - & - & 5 & - & - & - & - & - & - & - & - & - & - & - & - \\
\hline 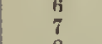 & $\bar{z}$ & $=$ & $=$ & $=$ & 二 & 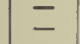 & 二 & $=$ & = & 二 & $=$ & $=$ & $\overline{-}$ & $\frac{6}{7}$ & $=$ & $\overline{-}$ & $=$ & $\bar{z}$ & $=$ & 二 & 二 & $\bar{z}$ & 二 & $=$ & - & 二 \\
\hline $\begin{array}{l}8 \\
9\end{array}$ & $10 \cdot 8$ & $\overline{13 \cdot 2}$ & $\overline{14} \cdot 0$ & $\overline{14 \cdot 5}$ & $\overline{14 \cdot 5}$ & 140 & $12 \cdot 5$ & $12^{\circ} \cdot 0$ & $1 \overline{0} \cdot 8$ & $\overline{9} \cdot 8$ & $11 \cdot 5$ & $1 \overline{10 \cdot 8}$ & $1 \overline{2} \cdot 4$ & $\begin{array}{l}8 \\
9\end{array}$ & $\overline{9} \cdot 5$ & $\overline{12 \cdot 2}$ & $\overline{12 \cdot 8}$ & $1 \overline{3} 0$ & $1 \overline{3} \cdot 2$ & $1 \overline{2} \cdot 8$ & $11 \cdot 5$ & $1 \overline{0.8}$ & 10.0 & $\overline{9} \cdot 0$ & $1 \overline{0} \cdot 5$ & $\overline{9 \cdot 9}$ \\
\hline $\begin{array}{l}10 \\
11\end{array}$ & $\begin{array}{l}11 \cdot 0 \\
20 \cdot 0\end{array}$ & $\begin{array}{l}11 \cdot 2 \\
19 \cdot 0\end{array}$ & $\left|\begin{array}{l}13 \cdot 1 \\
19 \cdot 8\end{array}\right|$ & $\mid \begin{array}{l}13 \cdot 8 \\
20 \cdot 5\end{array}$ & $\begin{array}{l}15 \cdot 0 \\
20 \cdot 8\end{array}$ & $\begin{array}{l}16 \cdot 0 \\
21^{\circ} * 5 \\
\end{array}$ & $17 \cdot 0$ & $\begin{array}{l}18.5 \\
19.8\end{array}$ & 17.5 & $\begin{array}{l}17 \cdot 2 \\
24: 8\end{array}$ & $\begin{array}{l}18 \cdot 5 \\
24 \cdot 5\end{array}$ & $\begin{array}{l}18 \cdot 5 \\
23.8\end{array}$ & $\begin{array}{l}15 \cdot 6 \\
21 \cdot 6\end{array}$ & $\begin{array}{l}10 \\
11\end{array}$ & $\begin{array}{l}10.5 \\
19.5\end{array}$ & \begin{tabular}{|l}
10.5 \\
18.6
\end{tabular} & $\begin{array}{l}12 \cdot 1 \\
19 \cdot 5\end{array}$ & $\begin{array}{l}13 \cdot 0 \\
200 \\
200\end{array}$ & $\begin{array}{l}14 \cdot 2 \\
2 \cdot 2\end{array}$ & ${ }_{21}^{15 \cdot 0}$ & $\mid \begin{array}{l}16 \cdot 5 \cdot 5 \\
23.8\end{array}$ & $\begin{array}{l}18.0 \\
18.0 \\
18.4\end{array}$ & $\begin{array}{l}17 \cdot 5 \\
19 \cdot 2\end{array}$ & $\begin{array}{l}17.0 \\
17.0 \\
23.0\end{array}$ & $\begin{array}{l}18 \cdot 2 \\
23.0\end{array}$ & $18 \cdot 2$ \\
\hline $\begin{array}{l}11 \\
12 \\
12\end{array}$ & 21.0 & $\mid \begin{array}{l}121.5 \\
210\end{array}$ & $\begin{array}{l}320 . \\
24 \cdot 5 \\
390\end{array}$ & $\begin{array}{l}20.0 \\
24.0\end{array}$ & 10.0 & 15.6 & $\begin{array}{l}47 \cdot 0 \\
17 \cdot 6\end{array}$ & 19.5 & 20.9 & $\mid$ & $\begin{array}{l}17.0 \\
17.0\end{array}$ & $\begin{array}{l}238 \\
16.5\end{array}$ & \begin{tabular}{|l}
21.6 \\
$19 \cdot 7$
\end{tabular} & $\begin{array}{l}11 \\
18 \\
18\end{array}$ & $\begin{array}{l}199.5 \\
19 \cdot 8\end{array}$ & $\begin{array}{l}18.6 \\
199.8\end{array}$ & $\begin{array}{l}195 \\
23.0\end{array}$ & \begin{tabular}{|l|l}
2020 \\
22.8
\end{tabular} & $\begin{array}{l}20.2 \\
15.2\end{array}$ & $\begin{array}{l}2100 \\
15.5\end{array}$ & - & $\begin{array}{l}18.8 \\
16 \cdot 8\end{array}$ & 10 & 208 & $\begin{array}{l}250 \\
16.5\end{array}$ & 年 \\
\hline $\begin{array}{l}13 \\
14\end{array}$ & $\begin{array}{l}19.0 \\
10.5\end{array}$ & $\mid \begin{array}{l}200 \\
11.2\end{array}$ & $\begin{array}{l}12.0 \\
11 \cdot 2\end{array}$ & $\begin{array}{l}22.0 \\
12 \cdot 8\end{array}$ & \begin{tabular}{|l}
33.8 \\
$15 \cdot 0$
\end{tabular} \mid & $\begin{array}{l}24.4 \\
17 \cdot 0\end{array}$ & $\begin{array}{l}22 \cdot 2 \\
17 \cdot 4\end{array}$ & $\mid \begin{array}{l}23 \\
17^{\prime} \cdot 2\end{array}$ & $\begin{array}{c}18.6 \\
17 \cdot 2\end{array}$ & $\mid \begin{array}{l}16 \cdot 8 \\
17 \%\end{array}$ & $\begin{array}{l}\frac{14 \cdot 6}{14 \cdot 8} \\
14.8\end{array}$ & 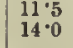 & \begin{tabular}{|l|l|}
19 \\
$14 \cdot 7$
\end{tabular} & $\begin{array}{l}13 \\
14 \\
14\end{array}$ & $\mid \begin{array}{l}18 \cdot 2 \\
10 \cdot 4\end{array}$ & $\begin{array}{l}19 \cdot 2 \\
11 \cdot 0\end{array}$ & $\begin{array}{l}20 \cdot 2 \\
11: 0\end{array}$ & \begin{tabular}{|l}
2110 \\
$12 \%$
\end{tabular} & $\left|\begin{array}{l}23.0 \\
15.0\end{array}\right|$ & $\begin{array}{l}23.5 \\
15 \cdot 2\end{array}$ & $\begin{array}{l}22.0 \\
15 \cdot 8\end{array}$ & $\begin{array}{l}22.2 \\
15 \cdot 8\end{array}$ & $\mid \begin{array}{l}1772 \\
15.8\end{array}$ & \begin{tabular}{|l}
160.5 \\
$15 \cdot 8$
\end{tabular} & 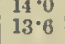 & $\begin{array}{l}11.5 \\
13 \cdot 2\end{array}$ \\
\hline 15 & 14.5 & 13.0 & $12 \cdot 2$ & $12 \cdot 0$ & 13.5 & $11 \cdot 8$ & $11 \cdot 5$ & $11 \cdot 4$ & $11 \cdot 0$ & $12 \cdot 8$ & $14 \cdot 1$ & $\begin{array}{l}13 \cdot 9 \\
12 \cdot 9\end{array}$ & $12 \cdot 6$ & 15 & $\begin{array}{l}13.5 \\
15.9\end{array}$ & \begin{tabular}{|l}
$12 \cdot 3$ \\
$110 \cdot 0$
\end{tabular} & $12 \cdot 0$ & $11 \cdot 6$ & $12 \cdot 3$ & $11 \cdot 2$ & $\begin{array}{l}10 \cdot 8 \\
16.0\end{array}$ & $\begin{array}{l}10.5 \\
16.5\end{array}$ & $\begin{array}{ll}10 \cdot 0 \\
21\end{array}$ & $\begin{array}{ll}11 \cdot 5 \\
16.8\end{array}$ & $\begin{array}{l}12 \cdot 5 \\
18.0\end{array}$ & $\begin{array}{l}12 \cdot 5 \\
12.05\end{array}$ \\
\hline $\begin{array}{l}16 \\
17\end{array}$ & $\begin{array}{l}14 \cdot 6 \\
13 \cdot 5 \\
12 \cdot 8\end{array}$ & $\begin{array}{l}15 \cdot 2 \\
14 \cdot 2 \\
12 \cdot 8\end{array}$ & $\begin{array}{l}14.3 \\
15 \cdot 8 \\
3 \cdot 1\end{array}$ & $\begin{array}{l}18 \cdot 6 \cdot 6 \\
15.0 \\
2 \cdot 0\end{array}$ & $\begin{array}{r}190.0 \\
200 \\
9 \cdot 2\end{array}$ & $\begin{array}{l}17.0 \\
19.8 \\
17.8 \\
17\end{array}$ & $\begin{array}{l}17.0 \\
19.9 \\
18.4\end{array}$ & $\mid \begin{array}{l}17 \\
20.5 \\
90.5\end{array}$ & $\begin{array}{l}23.0 \\
11.5 \\
23 \cdot 5\end{array}$ & $\begin{array}{l}172.2 \\
10.5 \\
25.0\end{array}$ & $\begin{array}{l}13.5 \\
10 \cdot 5 \\
24 \cdot 8 \\
24 \cdot 8\end{array}$ & $\begin{array}{l}13.0 \\
12.8 \\
23 \cdot 0\end{array}$ & $\begin{array}{l}16.5 .5 \\
15.3\end{array}$ & $\begin{array}{l}16 \\
17 \\
18\end{array}$ & $\begin{array}{l}13.2 \\
13 \cdot 0 \\
-\end{array}$ & $\begin{array}{l}14.0 \\
14.0 \\
-\end{array}$ & $\begin{array}{r}13.2 \\
15: 5 \\
2 \cdot 8\end{array}$ & $\begin{array}{l}145 \\
14.8 \\
2.8\end{array}$ & $\begin{array}{r}1 \overline{19.0} \\
8.1\end{array}$ & $\begin{array}{l}1 \overline{5} 5 \\
15.5\end{array}$ & $\frac{16 \cdot 1}{16 \cdot 6}$ & $\begin{array}{r}16.0 \\
18.2 \\
18.0\end{array}$ & $\begin{aligned} 91.0 \\
9 \cdot 3 \\
21 \cdot 4\end{aligned}$ & $\begin{array}{r}168 \\
8.5 \\
23.0\end{array}$ & $\begin{array}{l}83.0 \\
8.6 \\
23.0\end{array}$ & $\begin{array}{l}12 \cdot 5 \\
11 \cdot 2 \\
21 \cdot 5\end{array}$ \\
\hline 19 & 17.8 & 18.5 & 20.4 & $15 \cdot 2$ & 14.8 & 14.5 & $\begin{array}{l}18.7 \\
14.0\end{array}$ & $15 \%$ & $\begin{array}{l}28.2 \\
15.2\end{array}$ & $\mid \begin{array}{l}13^{\circ} \\
1.5\end{array}$ & 8.8 & 10.0 & $14 \cdot 7$ & $\begin{array}{l}19 \\
19\end{array}$ & $17 \cdot 0$ & $15 \cdot 0$ & $19-2$ & $14 \cdot 4$ & -1 & $13 \cdot 2$ & & 14.0 & 13.0 & 1300 & $8 \cdot 4$ & $9 \cdot 2$ \\
\hline${ }_{21}^{20}$ & $\begin{array}{l}18.0 \\
15.5\end{array}$ & $\begin{array}{l}14: 2 \\
14 \cdot 2\end{array}$ & $\mid \begin{array}{l}15 \cdot 7 \\
13 \cdot 5\end{array}$ & $\begin{array}{l}17 \cdot 2 \\
13 \cdot 0\end{array}$ & $\begin{array}{l}19 \cdot 4 \\
10 \cdot 9\end{array}$ & $\begin{array}{l}19 \cdot 9 \\
11 \cdot 4\end{array}$ & $\begin{array}{l}22 \cdot 2 \\
15 \cdot 0\end{array}$ & $\begin{array}{l}20^{\circ} .0 \\
1^{\circ} 0\end{array}$ & $\begin{array}{l}22 \cdot 5 \\
17 \cdot 2\end{array}$ & $\begin{array}{l}19 \cdot 5 \\
17 \cdot 5\end{array}$ & $\begin{array}{l}14 \cdot 5 \\
15 \cdot 2\end{array}$ & $\begin{array}{l}14 \cdot 6 \\
13 \cdot 0\end{array}$ & $\begin{array}{l}17 \cdot 7 \\
14 \cdot 4\end{array}$ & $\begin{array}{l}20 \\
21\end{array}$ & $\begin{array}{l}11.8 \\
14.8\end{array}$ & $\begin{array}{l}18 \cdot 6 \\
13 \cdot 6\end{array}$ & $\overline{12 \cdot 0}$ & $\mid \begin{array}{l}15 \cdot 0 \\
11.5\end{array}$ & $\begin{array}{l}17^{\circ} 0 \\
10^{\circ} 0\end{array}$ & $\begin{array}{r}17 \cdot 2 \\
9 \cdot 0\end{array}$ & $\begin{array}{l}20 \cdot 5 \\
12 \cdot 5\end{array}$ & $\begin{array}{l}17 \cdot 2 \\
13.8\end{array}$ & $\begin{array}{l}19 \cdot 2 \\
15 \cdot 1\end{array}$ & $\left|\begin{array}{c}17 \cdot 2 \\
16 \cdot 0\end{array}\right|$ & $\begin{array}{l}13 \cdot 2 \cdot 2 \\
13: 0\end{array}$ & $\begin{array}{l}12 \cdot 6 \\
11 \cdot 5\end{array}$ \\
\hline 24 & $\begin{array}{r}2 \cdot 2 \cdot 2 \\
3 \cdot 5 \\
3 \cdot 5\end{array}$ & $\left|\begin{array}{r}12 \cdot 2 \cdot 2 \\
2 \cdot 2 \\
5 \cdot 0\end{array}\right|$ & $\begin{array}{r}12 \cdot 2 \\
4 \cdot 8 \\
5 \cdot 5\end{array}$ & $\left|\begin{array}{l}0.8 \\
8.2 \\
6.5\end{array}\right|$ & $\begin{array}{l}2.8 \\
9.8 \\
7\end{array}$ & $\begin{array}{r}5 \cdot 2 \\
11 \cdot 6 \\
8.2\end{array}$ & $\begin{array}{c}6 \cdot 1 \\
12 \cdot 2 \\
9 \cdot 0\end{array}$ & $\begin{array}{l}70.0 \\
11.5 \\
10.0\end{array}$ & $\begin{array}{r}6.8 \\
10 . \\
100\end{array}$ & $\begin{array}{r}7.0 \\
9.6 \\
11 \cdot 0\end{array}$ & $\begin{array}{l}9 \cdot 5 \\
6 \cdot 2 \\
110.0\end{array}$ & $\begin{array}{r}6 \cdot 2 \\
4 \cdot 2 \\
13 \cdot 2\end{array}$ & $\begin{array}{r}7.3 \\
7.9 \\
8.3 \\
8.3\end{array}$ & $\begin{array}{l}22 \\
23 \\
24\end{array}$ & $\begin{array}{r}12 \cdot 0 \\
3 \cdot 2 \\
2 \cdot 2\end{array}$ & $\begin{array}{l}\overline{2 \cdot 0} \\
4 \cdot 2\end{array}$ & $\begin{array}{r}11 \cdot 6 \cdot 6 \\
3 \cdot 0 \\
5 \cdot 0\end{array}$ & \begin{tabular}{|l|l|}
$\cdot 6 \cdot 8$ \\
$7 \cdot 8$ \\
6.8
\end{tabular} & \begin{tabular}{|l|}
2.2 \\
8.6 \\
7.2
\end{tabular} & $\begin{array}{r}50 \\
10.2 \\
8.2\end{array}$ & $\begin{array}{r}5 \cdot 2 \cdot 2 \\
10 \cdot 1 \\
8 \cdot 6\end{array}$ & $\begin{array}{l}5.8 \\
9 \cdot 8 \\
9 \cdot 8\end{array}$ & $\begin{array}{l}5 \cdot 9 \\
5 \cdot 2 \\
9 \cdot 2 \\
9 \cdot 6\end{array}$ & $\begin{array}{r}6 \cdot 2 \cdot 2 \\
8.2 \\
10.2\end{array}$ & $\begin{array}{r}8.4 \\
5.5 \\
10 \cdot 1\end{array}$ & $\begin{array}{r}6 \cdot 0 \\
3.9 \\
12 \cdot 9\end{array}$ \\
\hline 2 & $14 \cdot 0$ & $15 \cdot 5$ & 15.8 & $16 \cdot 2$ & $17 \cdot 5$ & 16.0 & $19 \cdot 5$ & $21 \cdot 0$ & $20 \cdot 0$ & $19 \cdot 0$ & $\begin{array}{l}18 \cdot 2 \\
010.02\end{array}$ & $19 \cdot 0$ & $17 \cdot 6$ & 25 & $13 \cdot 0$ & $14 \cdot 0$ & $\begin{array}{l}14.0 \\
17.08\end{array}$ & \begin{tabular}{|l}
$15 \cdot 8$ \\
16.5
\end{tabular} & $15 \cdot 0$ & \begin{tabular}{|l|l}
14.0 \\
$14: 0$
\end{tabular} & $\begin{array}{l}16 \cdot 2 \\
16 \cdot 2\end{array}$ & $\begin{array}{l}18 \cdot 4 \\
10.4\end{array}$ & $\begin{array}{ll}18.2 \\
10.9\end{array}$ & \begin{tabular}{|l|l}
17.0 \\
178.2
\end{tabular} & $18 \cdot 5$ & $177^{7} 0$ \\
\hline 28 & $\begin{array}{l}21 \cdot 0 \\
22 \cdot 0\end{array}$ & $\begin{array}{l}78.2 \\
22 \cdot 2 \\
22 \cdot 2 \cdot\end{array}$ & $\begin{array}{l}18 \\
23 \cdot 0 \\
22 \cdot 2\end{array}$ & $\begin{array}{l}140 \\
240 \\
20 \cdot 0\end{array} \mid$ & $\begin{array}{l}10.2 \\
26.0 \\
22 \cdot 6\end{array}$ & 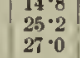 & $\begin{array}{l}\begin{array}{l}27.8 \\
26.5 \\
26.5\end{array} \\
26.5\end{array}$ & $\begin{array}{l}22^{2} 0 \\
26^{\circ} \\
26^{\circ} 1\end{array}$ & $\begin{array}{l}21.5 \\
27.8 \\
22 \cdot 0\end{array}$ & $\begin{array}{l}20.5 \\
22 \cdot 2 \\
22 \cdot 0\end{array}$ & $\begin{array}{l}21.22 \\
22.8 \\
25.0\end{array}$ & $\begin{array}{l}21.02 \\
21.0 \\
23 \cdot 0\end{array}$ & $\begin{array}{l}1900 \\
24 \cdot 4 \\
23 \cdot 4\end{array}$ & $\begin{array}{l}26 \\
27 \\
28\end{array}$ & $\overline{20 \cdot 5}$ & $21 \cdot 0$ & $\begin{array}{l}21.8 \\
21.6 \\
20.5\end{array}$ & $\begin{array}{l}10.5 \\
22 \cdot 5 \\
-\end{array}$ & $\begin{array}{l}100.2 \\
21 \cdot 0\end{array}$ & $\begin{array}{r}140 \\
24.8 \\
25.0\end{array}$ & $\begin{array}{l}20.5 .5 \\
24 .-3\end{array}$ & $\begin{array}{r}r \cdot 8 \\
25 \cdot 8 \\
34 \cdot 2\end{array}$ & $\begin{array}{l}25.8 \\
25.8 \\
21 \cdot 0\end{array}$ & $\begin{array}{l}24.5 \\
24.5 \\
19.6\end{array}$ & $\begin{array}{l}20-8 \\
21-8 \\
21-8\end{array}$ & $\begin{array}{l}100^{\circ} \\
20^{\circ}\end{array}$ \\
\hline n. & 14.4 & 147 & 15.0 & 14.6 & $\overline{15 \cdot 7}$ & $\overline{16 \cdot 4}$ & $17 \cdot 3$ & $\overline{17 \cdot 7}$ & $17 \cdot 4$ & $177^{\circ}$ & 15.8 & $\overline{15 \cdot 2}$ & $\overline{15 \cdot 9}$ & Mean. & - & - & - & - & - & - & - & - & - & - & - & - \\
\hline
\end{tabular}

Dry Buld.

Wet Bulb.

March, 1902.

\begin{tabular}{|c|c|c|c|c|c|c|c|c|c|c|c|c|c|c|c|c|c|c|c|c|c|c|c|c|c|c|}
\hline Day. & & 4 & 6 & 8. & L & Noon. & 14 & 16. & 18. & 20. & 22. & Midt. & Mean. & ay. & 2. & 4. & 6. & 8. & 10. & Nomo. & 14. & 16. & 18. & 20. & 22. & \\
\hline & & & $23 \cdot 0$ & $24 \cdot 0$ & $22 \cdot 8$ & & $21 \cdot 2$ & 21.0 & $\begin{array}{c}0 \\
21 \cdot 8\end{array}$ & $23 \cdot 0$ & $22 \cdot 0$ & $22 \cdot 1$ & & & & & 1.2 & $21 \cdot 8$ & $21 \cdot 2$ & 0.6 & $1 \cdot 0$ & $0 \cdot 2$ & $20 \cdot 2$ & -8: & & \\
\hline & & & & & & & & & $1 t$ & & & & & & & & & $8_{1}^{8}$ & & & & & $E$ & ". & & \\
\hline & $\begin{array}{l}11 \\
19\end{array}$ & 18.5 & 180 & 18 & & 5 & & 14.3 & $\begin{array}{l}14.0 \\
130\end{array}$ & 4 & & 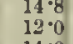 & & & & & 17 & 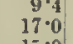 & 0 & 1 & & 0 & $\overline{12} \cdot 0$ & 8 & & \\
\hline 6 & $\begin{array}{l}12 \\
13\end{array}$ & $\begin{array}{l}13.6 \\
\end{array}$ & $\begin{array}{l}110 \\
13.6\end{array}$ & $\begin{array}{l}19.8 \\
15.5\end{array}$ & $\begin{array}{l}110 \\
17\end{array}$ & \begin{tabular}{|l|}
10.8 \\
16.8
\end{tabular} & $\begin{array}{l}13.6 \\
19.2\end{array}$ & $\begin{array}{l}10.0 \\
20.5\end{array}$ & $\begin{array}{l}160.0 \\
18.5\end{array}$ & $\begin{array}{l}250 \\
20\end{array}$ & $\begin{array}{l}21.5 \\
21.5\end{array}$ & $\begin{array}{l}14 \% \\
21 \cdot 2\end{array}$ & 17. & $\begin{array}{l}5 \\
6\end{array}$ & & $\begin{array}{l}10 \\
12\end{array}$ & $\begin{array}{l}110 \\
12 \%\end{array}$ & $\begin{array}{l}10.0 \\
13.8\end{array}$ & $\begin{array}{l}10.8 \\
15.8\end{array}$ & $\mid \begin{array}{l}1,50.2 \\
15\end{array}$ & & $\overline{18} \cdot 8$ & $\overline{17} 0$ & 8 & & \\
\hline $\begin{array}{l}7 \\
8\end{array}$ & & & $\begin{array}{l}20 \\
1.4\end{array}$ & $\begin{array}{l}218 \\
16 \% 6\end{array}$ & $\begin{array}{l}20^{\circ} \\
19.0\end{array}$ & $\begin{array}{c}22 \cdot 8 \\
19.5\end{array}$ & $22 \cdot 5$ & $\begin{array}{l}19 \cdot 8 \\
18.5\end{array}$ & $\begin{array}{l}18.8 \\
17 \%\end{array}$ & $\begin{array}{l}11 \cdot 9 \\
16 \cdot 8\end{array}$ & $\begin{array}{l}12 \cdot 3 \\
21\end{array}$ & $13 \cdot 1$ & 18 & $\begin{array}{l}7 \\
8\end{array}$ & & & & 8 & & & & & & & & \\
\hline $\begin{array}{l}98 \\
10 \\
11\end{array}$ & $\begin{array}{l}16 \\
10 \\
18\end{array}$ & $\begin{array}{l}17 \cdot 0.0 \\
77^{\circ} \cdot 0\end{array}$ & $\begin{array}{c}20.8 \\
9.5 \\
14.5\end{array}$ & $\begin{array}{l}2220.0 \\
120 \\
10.5\end{array}$ & $\begin{array}{l}27 \cdot 0^{\prime} \\
13 \cdot 0 \\
8 \cdot 0\end{array}$ & $\begin{array}{r}27.5 \\
17.8 \\
3: 5\end{array}$ & $\begin{array}{r}20.8 \\
15.5 \\
3.5\end{array}$ & $\begin{array}{l}21.0 \\
150 \\
3.0\end{array}$ & $\begin{array}{l}21.0 \\
15.0 \\
3.5\end{array}$ & $\begin{array}{l}20.4 \\
15 \cdot 1 \\
4.0\end{array}$ & $\begin{array}{c}17 \cdot 9 \cdot 9 \\
19 \cdot 0 \\
6.0\end{array}$ & $\begin{array}{r}14.0 \\
17.0 \\
5.0\end{array}$ & $\begin{array}{r}20.55 \\
14.0 \\
8.0\end{array}$ & $\begin{array}{l}99 \\
10 \\
11\end{array}$ & : & $\begin{array}{l}8 \cdot 1 \\
16 \cdot 0\end{array}$ & $\begin{array}{l}19.3 \\
9.0 \\
13 \cdot 0\end{array}$ & $\begin{array}{l}20.5 \\
11.5 \\
10.0\end{array}$ & $\begin{array}{r}24.8 \\
12 \cdot 5 \\
8 \cdot 3\end{array}$ & $\begin{array}{l}25.0 \\
16.5 \\
3.5\end{array}$ & 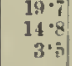 & $\begin{array}{r}19.8 \\
14.5 \\
3.0 \\
3.0\end{array}$ & $\begin{array}{r}20.0 \\
14.5 \\
3.5\end{array}$ & $\begin{array}{l}19 \cdot 9 \\
14 \cdot 9 \\
-\end{array}$ & & \\
\hline & & & & & & & & & $4 \cdot 0$ & $2 \cdot 2$ & & 10 & 2 & 12 & & & & & & & & & & & & \\
\hline $\begin{array}{l}11 \\
15 \\
16\end{array}$ & $\begin{array}{r}2 \cdot 0.0 \\
-4.5 \\
-5.6\end{array}$ & & & & & & & & & $\begin{array}{l}1.8 \\
5.8\end{array}$ & $\begin{array}{l}400 \\
700\end{array}$ & $\begin{array}{l}3.8 \\
1.88 \\
9.5\end{array}$ & & 16 & & & & & & & & & & 0 & & \\
\hline$\frac{1}{1}$ & 12 & $\begin{array}{l}11 \cdot 0 \\
17 \cdot 0\end{array}$ & $\begin{array}{r}8 \cdot 0 \\
15 \cdot 4\end{array}$ & $\begin{array}{l}1088 \\
15: 1\end{array}$ & $\begin{array}{l}14 \cdot 5 \cdot 5 \\
17 \cdot 0\end{array}$ & 0 & $14 \cdot 0$ & $\begin{array}{l}14.5 \\
16.5\end{array}$ & $\begin{array}{l}155^{\circ} \\
14\end{array}$ & $18 \cdot 0$ & 17.8 & $\begin{array}{l}17 \cdot 2 \\
14 \cdot 0\end{array}$ & 13.7 & $\begin{array}{l}17 \\
18\end{array}$ & $\cdot 0$ & & 8 & $\begin{array}{l}1000 \\
15.0 \\
150\end{array}$ & 8 & & & & 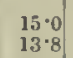 & & & \\
\hline 2 & $\begin{array}{l}14.0 \\
20.4 \\
13.0\end{array}$ & $\begin{array}{l}14 \cdot 0 \\
21 \cdot 2 \\
13 \cdot 1\end{array}$ & $\begin{array}{l}14 \cdot 3 \\
19 \cdot 5 \\
13 \cdot 0\end{array}$ & & $\begin{array}{l}18 \cdot 5 \cdot 5 \\
20 \cdot 5 \\
12 \cdot 6\end{array}$ & $\begin{array}{l}18.0 \\
21.5 \\
13.0\end{array}$ & $\begin{array}{l}14 \cdot 8 \\
20^{\circ} \cdot 5 \\
17 \cdot 6\end{array}$ & $\begin{array}{l}21 \cdot 8 \\
19 \cdot 8 \\
19 \cdot 8\end{array}$ & $\begin{array}{l}22 \cdot 0 \\
214.5 \\
14.5\end{array}$ & $\begin{array}{l}18.5 \\
14.0 \\
18.0\end{array}$ & $\begin{array}{l}20 \cdot 3 \\
17.0 \\
12 \cdot 5\end{array}$ & $\begin{array}{l}22 \cdot 8 \\
18.2 \\
10.0\end{array}$ & & $\begin{array}{l}90 \\
20 \\
21\end{array}$ & & & $\begin{array}{l}13 \cdot 7 \\
199.3 \\
13 \cdot 0\end{array}$ & $\begin{array}{l}16.0 \\
21.5\end{array}$ & $\begin{array}{l}17 \cdot 5 \\
210 \cdot 0\end{array}$ & $\begin{array}{r}17.0 \\
21.2 \\
2\end{array}$ & $\begin{array}{l}14 \cdot 0 \\
2 v \cdot 2 \\
-\end{array}$ & $\begin{array}{l}19 \cdot 8 \\
19 \cdot 5 \\
-\end{array}$ & $\underline{13} \cdot 8$ & & $12 \cdot 2$ & \\
\hline & $\begin{array}{l}10 \\
6\end{array}$ & $\begin{array}{l}3 \cdot 0 \\
4.5\end{array}$ & 0.8 & 4.2 & $11 \cdot 0$ & $11 \cdot 2$ & $13 \cdot 0$ & $12 \cdot 0$ & 100 & 7.0 & $5 \cdot$ & $8 \cdot 0$ & 0. & & & $\begin{array}{l}3 \cdot 0 \\
5 \cdot 5\end{array}$ & $1 \cdot 0$ & 0 & 11.0 & 111. & & 8.5 & 8. & & & \\
\hline $\begin{array}{l}\frac{2}{2} \\
22 \\
2 t\end{array}$ & $\begin{array}{r}0 \\
10 \\
2\end{array}$ & 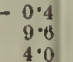 & $\begin{array}{r}0.0 \\
110.2 \\
4.9\end{array}$ & $\begin{array}{r}0.6 \\
11.6 \\
1.5\end{array}$ & $\begin{array}{r}0.2 \\
11 \cdot 8 \\
1.2\end{array}$ & $\begin{array}{rl}2 & 2 \cdot 3 \\
12 \cdot 2 \\
1 \cdot 5\end{array}$ & $\begin{array}{c}1 \cdot 0 \\
10 \cdot 0 \\
3 \cdot 5\end{array}$ & & $\begin{array}{l}3.8 \\
3.8\end{array}$ & 6.0 & $\begin{array}{l}8.1 \\
4.5\end{array}$ & 10.4 & $\begin{array}{l}2.3 \\
2.3 \\
8.5 \\
1.0\end{array}$ & 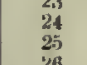 & & 12 & & $\begin{array}{l}1.22 \\
10.8 \\
1.0\end{array}$ & $\begin{array}{l}0.6 \\
10.0\end{array}$ & 11. & 0.0 & $\begin{array}{l}3.0 \\
9.0\end{array}$ & $\begin{array}{l}3 \cdot 2 \\
5 \cdot 8\end{array}$ & $\begin{array}{l}5.4 \\
4.9\end{array}$ & & \\
\hline 2 & & & & $7 \cdot 0$ & 7.5 & & & $0^{\circ}$ & -6 & -6.5 & $8 \cdot 0$ & 8. & & & & & & & & & & & & & & \\
\hline 2 & & & & & & & & & & & & & & 2 & & & & & & & & & $\begin{array}{l}3 . \\
1 .\end{array}$ & & & \\
\hline & 8 & $7^{\circ}$ & $7 \cdot \mathbf{1}$ & & 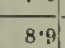 & 8 & $\cdot 7$ & & 7 & 7 & 717 & & 8.0 & & & - & 6.4 & & & & & & - & - & & \\
\hline & & & & & & & & & & & & & & & & & & & & & & & & & & \\
\hline
\end{tabular}

leadings in italle type are interpolated values obtained from records of self-registering instruments. 
'Tabif: II. (contimed).

TEMPERATURE IN DEGREES FAHRENHIIT.

April, 1902.

DRY BuLlB.

WET BuL.t.

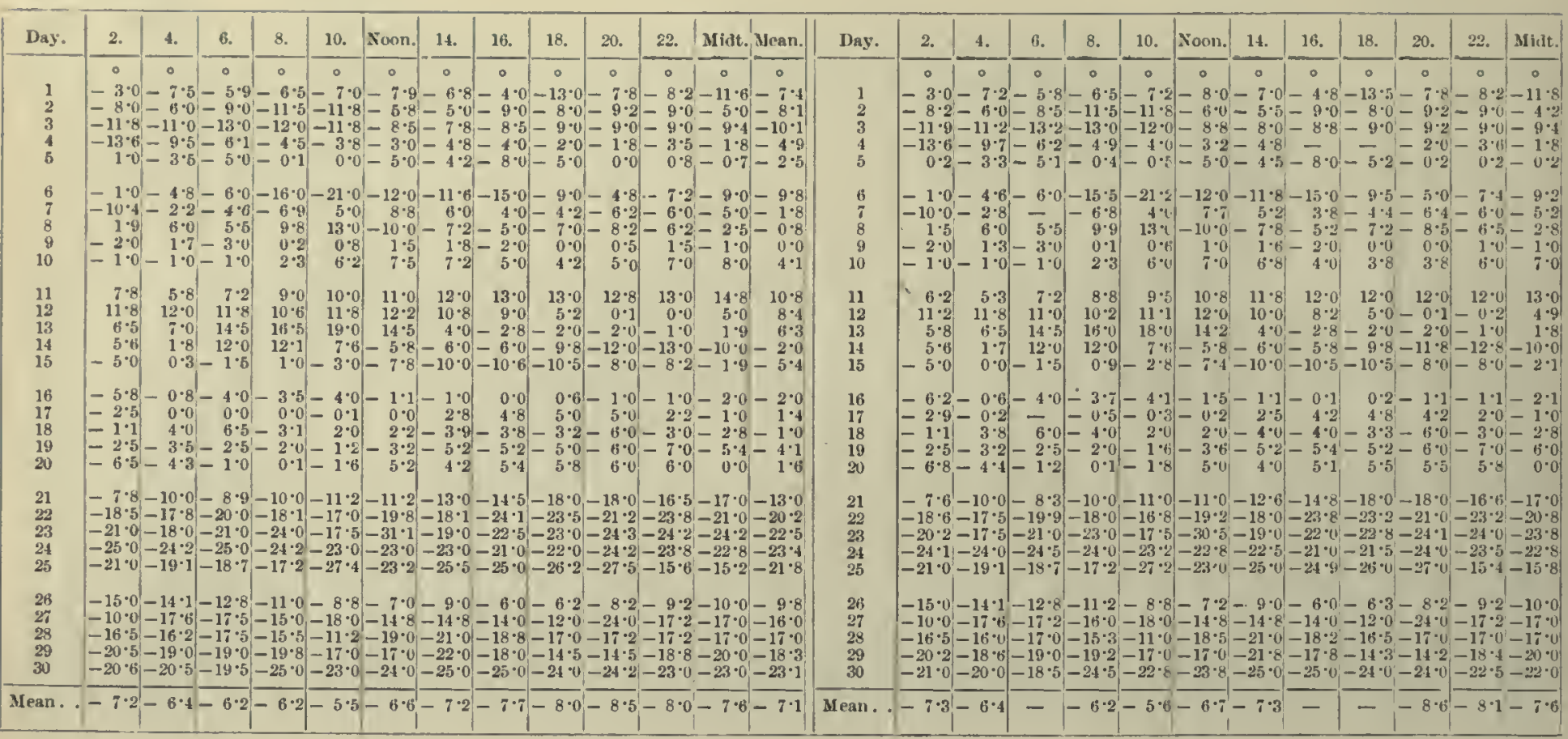

May, 1902.

Dry Buls.

WET BULB.

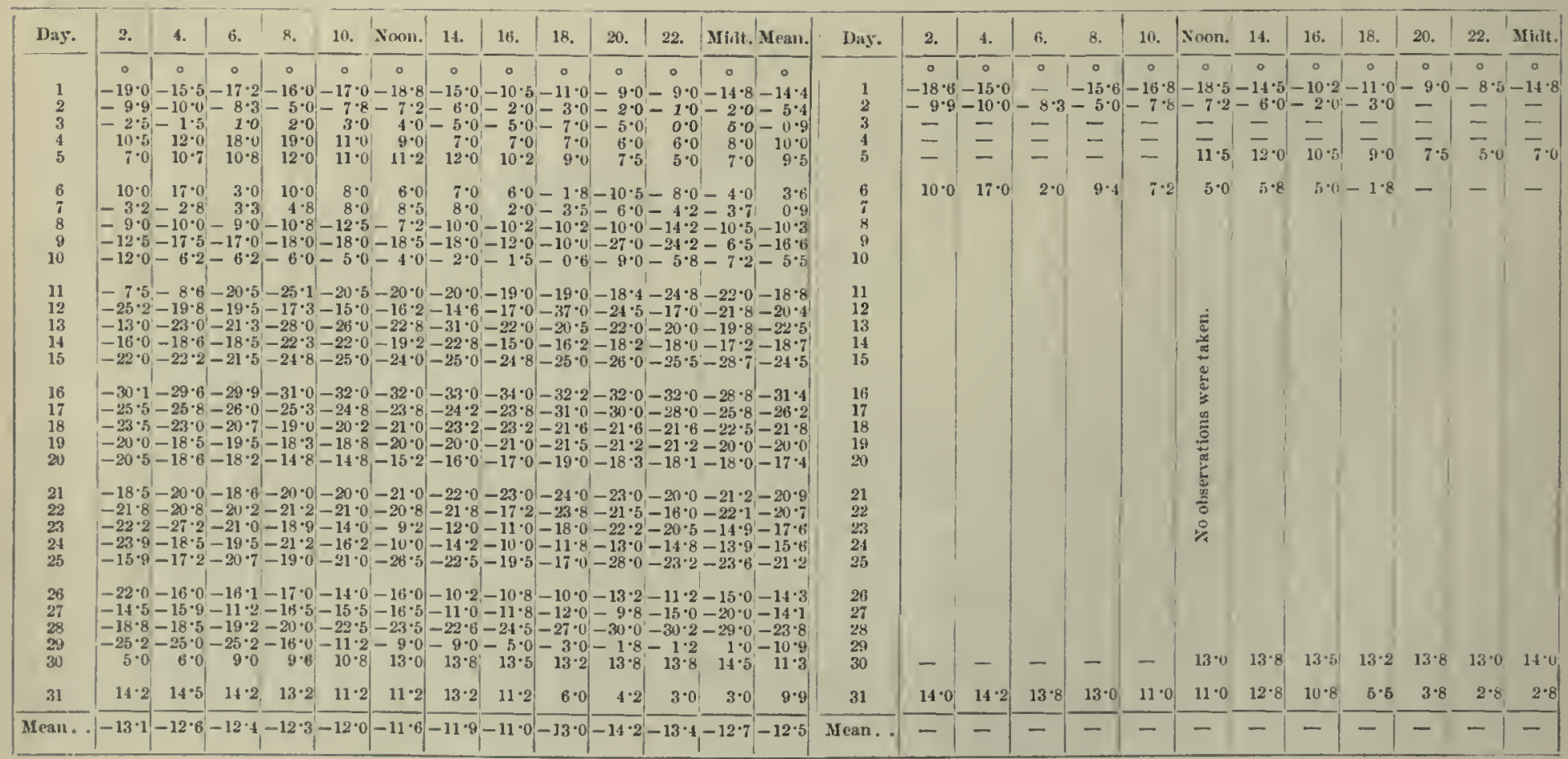

Reradings in italie type are int erpolated values obtainel fron records of self-registering instruments. 
TABM:S II. (continued).

TEMPIRATURE IN DEGRELS FAHRENHEIT.

DliY BuL.3.

WET BULA.

June, 1902.

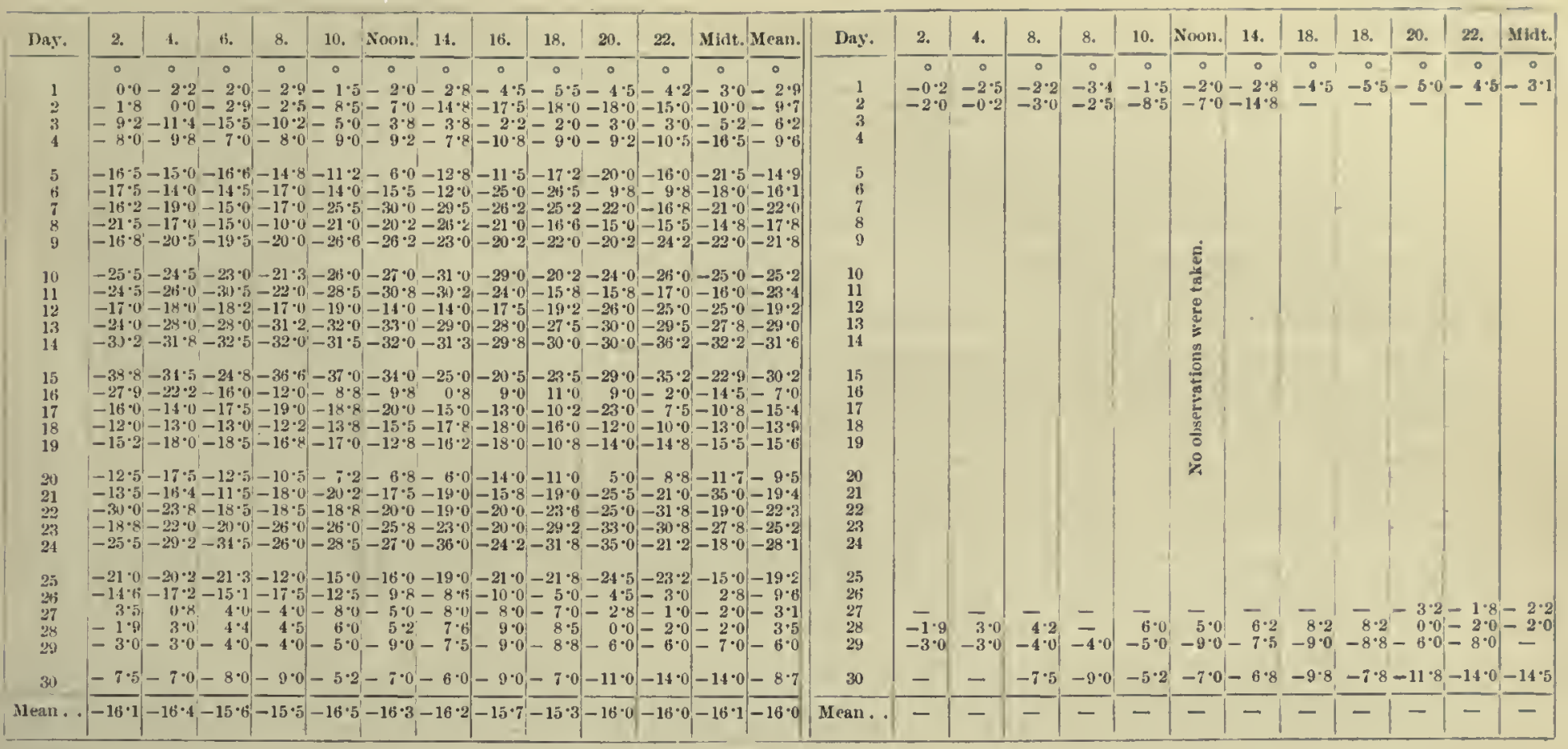

Dry BuLb.

WeT BULE.

July, 1902.

\begin{tabular}{|c|c|c|c|c|c|c|c|c|c|c|c|c|c|c|c|c|c|c|c|c|c|c|c|c|c|c|}
\hline Day. & 2. & 4. & 6. & 8. & 10. & Noon. & 14. & 18. & 18. & 20. & 22. & Mint. & Mean. & Day. & 2. & 4. & 8. & 8. & 10. & Noon. & 14. & 16. & 18. & 20. & 22. & Midt. \\
\hline & 1. & $\therefore$ & $\therefore$ & $\therefore$ & $\circ$ & 0 & $\div$ & $\circ$ & 10 & 0 & $\circ$ & $\circ$ & 0 & & $\circ$ & 。 & 。 & - & $\circ$ & $\circ$ & . & $\circ$ & 。 & $\cdot$ & $\therefore$ & 。 \\
\hline $\begin{array}{l}1 \\
2\end{array}$ & $\begin{array}{r}-15 \cdot 0 \\
-\quad 4 \cdot 1\end{array}$ & $\begin{array}{l}-13 . \\
-8 .\end{array}$ & $\begin{array}{r}-2200 \\
-\quad 4.0\end{array}$ & $\begin{array}{l}-22 \cdot 0 \\
-1 \cdot 6\end{array}$ & $\begin{array}{l}-\quad 8 \cdot 0 \\
-1 \cdot 2\end{array}$ & {$\left[\begin{array}{c}2 \cdot 2 \\
-5 \cdot 0\end{array}\right.$} & $\begin{array}{l}-1 \cdot 0 \\
-7 \cdot 0\end{array}$ & $\begin{array}{r}-5 \cdot 0 \\
-16.0\end{array}$ & $\begin{array}{l}-1 \cdot 0 \\
-13 \cdot 2\end{array}$ & $\mid \begin{array}{l}-40^{\circ} \\
-11.0\end{array}$ & $\begin{array}{l}=5.8 \\
-8.8\end{array}$ & $\begin{array}{l}-4.5 \\
-5.1\end{array}$ & $\mid \begin{array}{l}-8 \cdot 6 \\
-7 \cdot 1\end{array}$ & $\frac{1}{2}$ & $\bar{z}$ & $=$ & $\overline{-}$ & $=$ & $=$ & $-3 \cdot 0$ & $-1 \cdot 8$ & $=$ & $=$ & $=$ & $-8 \cdot 0$ & $=$ \\
\hline $\begin{array}{l}3 \\
4\end{array}$ & $\begin{array}{l}-3.8 \\
-\quad 7 \cdot 0\end{array}$ & $\begin{array}{r}-4 \cdot 6 \\
-9.2\end{array}$ & $\begin{array}{r}-4 \cdot 9 \\
-9 \cdot 0\end{array}$ & $\begin{array}{r}-5 \cdot 0 \\
-8.0\end{array}$ & $\begin{array}{r}-3.0 \\
-14.0\end{array}$ & $\begin{array}{r}-3 \cdot 8 \\
-9.0\end{array}$ & $\begin{array}{l}-6.0 \\
-6.0\end{array}$ & $\begin{array}{l}-6.5 \\
-11.0\end{array}$ & $\begin{array}{r}-8.0 \\
-7.0\end{array}$ & $\begin{array}{l}-5.0 \\
-12.0\end{array}$ & $\begin{array}{l}-110 \\
-7.0\end{array}$ & $\begin{array}{r}-8.0 \\
-\quad 8.0\end{array}$ & $\begin{array}{l}-8.1 \\
=8.8\end{array}$ & $\begin{array}{l}3 \\
4 \\
4\end{array}$ & $=$ & $=$ & $-4 \cdot 9$ & $-5 \cdot 1$ & $=$ & $-4 \cdot 0$ & $=$ & $\overline{-}$ & $=$ & $=$ & $=$ & $\bar{z}$ \\
\hline 5 & $-10^{\circ} 0$ & -6.5 & $-14 \cdot 0$ & $-11 \cdot 0$ & $12 \cdot 8$ & $-12 \cdot 6$ & $-13 \cdot 8^{!}$ & $-18 \cdot 0$ & $-18 \cdot 0$ & $-13 \cdot 8$ & $-13 \cdot 5$ & $12 \cdot 3$ & $3-13 \cdot 0$ & & - & - & - & - & - & - & - & - & - & - & - & - \\
\hline 6 & $-10 \cdot 0$ & - & 0 & & & & -17.5 & & & & & $-155^{\circ}$ & -13.5 & 6 & - & - & - & - & - & - & $=$ & $=$ & $=$ & $=$ & $=$ & $=$ \\
\hline ¿ & $\begin{array}{r}-16.2 \\
-25.5\end{array}$ & $\begin{array}{l}-1.58 \\
-20 \cdot 2\end{array}$ & $\begin{array}{l}-19{ }^{\circ} \\
-12.5\end{array}$ & $\begin{array}{l}-24 \cdot 0 \\
-23 \cdot 5\end{array}$ & $\begin{array}{l}-19.0 \\
-23 \cdot 5\end{array}$ & $\begin{array}{l}-15 \cdot 6 \\
-15 \cdot 0\end{array}$ & $=17.0$ & $\begin{array}{l}-13.8 \\
-3.0\end{array}$ & & $\begin{array}{l}-14.5 \\
-22 \cdot 4\end{array}$ & $\begin{array}{l}111 \\
-20 \cdot 2\end{array}$ & $\begin{array}{l}-19 \cdot 0 \\
-16 \cdot 2\end{array}$ & $\begin{array}{l}-17 \cdot 3 \cdot 2 \\
-22 \cdot 2\end{array}$ & $\begin{array}{l}7 \\
8\end{array}$ & $-\overline{25} \cdot 8$ & $=$ & $\bar{z}$ & $=$ & $\bar{z}$ & $=$ & $\overline{-}$ & $\bar{z}$ & $\overline{-}$ & $=$ & $\bar{Z}$ & $\bar{z}$ \\
\hline & $4 \cdot 9$ & 3.0 & $2 \cdot 0$ & $3 \cdot 0$ & $2 \cdot 5$ & $4 \cdot 0$ & 1.8 & 3.0 & $3 \cdot 0$ & $2 \cdot 2$ & 3.0 & -0.5 & $2 \cdot 7$ & 9 & 4.9 & $2 \cdot 6$ & 0.5 & $3 \cdot 0$ & $2 \cdot 3$ & $3 \cdot 2$ & 1.0 & 20 & $2 \cdot 0$ & $2 \cdot 0$ & 0.2 & -0.3 \\
\hline 10 & $3 \cdot 0$ & $4 \cdot 0$ & & $8 \cdot 8$ & $1 \cdot 0$ & $-5 \cdot 0$ & $2 \cdot 5$ & $4 \cdot 0$ & - & -1 & -0.3 & $-11 \cdot 5$ & $-3 \cdot 0$ & 10 & $2 \cdot 0$ & $3 \cdot 0$ & $3 \cdot 0$ & -8.8 & 0.5 & -5.0 & - & - & - & - & - & - \\
\hline $\begin{array}{l}11 \\
12\end{array}$ & & & $\begin{array}{l}-6 \cdot 0 \\
-12 \cdot 0\end{array}$ & $\begin{array}{l}-4 \cdot 5 \\
-11 \cdot 2\end{array}$ & & $\left.\begin{array}{r}-2 \cdot 2 \\
-15 \cdot 0\end{array}\right]$ & $\begin{array}{l}-4 \cdot 8 \\
-13 \cdot 0\end{array}$ & & & $-5 \cdot 0$ & $-3 \cdot 2$ & $\begin{array}{l}-7.5 \\
-14.6\end{array}$ & $\begin{array}{l}-46 \\
-13 \cdot\end{array}$ & 11 & & & & & & & & & & & & \\
\hline 13 & $\begin{array}{l}-15 \cdot 2 \\
-\end{array}$ & $\begin{array}{l}-14.8 \\
-270\end{array}$ & $\begin{array}{l}16.2 \\
-160^{\circ} \\
-2\end{array}$ & $-16 \cdot 9$ & $\begin{array}{r}\quad-14.0 \\
-26 \cdot 2\end{array}$ & {$\left[\begin{array}{ll}-10 \\
-18 \cdot 2\end{array}\right.$} & $\begin{array}{l}-200 \\
-200\end{array}$ & $\begin{array}{r}17.0 \\
-17\end{array}$ & $\mid-19.0$ & $=$ & $\begin{array}{r}-150 \\
-26.0\end{array}$ & $\begin{array}{r}-146 \\
-27.0\end{array}$ & $\begin{array}{r}18.7 \\
-18.7\end{array}$ & 12 & & & & & & 政 & & & & & & \\
\hline 14 & & & & & & & & & & & $-24 \cdot 2$ & $-20^{\circ} 0$ & -26.8 & 14 & & & & & & $\overline{\tilde{u}}$ & & & & & & \\
\hline $\begin{array}{l}15 \\
16\end{array}$ & $\begin{array}{l}-25 \\
-22 .\end{array}$ & -23 & & $\begin{array}{l}-21 \cdot 8 \\
-21 \cdot 0\end{array}$ & $-20 \cdot 5$ & $-19 \cdot 5$ & $\begin{array}{l}-13 \cdot 5 \\
-24 \cdot 5\end{array}$ & $\begin{array}{l}-14 \cdot 2 \\
-23.8\end{array}$ & $\begin{array}{l}-1.4 \cdot 2 \\
-20 \cdot 0\end{array}$ & $\begin{array}{l}-14 \cdot 2 \\
-15 \cdot 0\end{array}$ & $\begin{array}{l}-17.0 \\
-10.0\end{array}$ & $\begin{array}{l}-20.5 \\
-9.9\end{array}$ & $\begin{array}{l}-18 \cdot 9 \\
-19 \cdot 4\end{array}$ & $\begin{array}{l}15 \\
16\end{array}$ & & & & & & 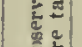 & & & & & & \\
\hline${ }_{18}^{17}$ & $\begin{array}{l}-8.0 \\
7.0\end{array}$ & $\begin{array}{r}-69 \\
8.0\end{array}$ & -3.8 & & & $\begin{array}{r}2 \cdot 2 \\
11 \cdot 0\end{array}$ & $\begin{array}{l}3 \circ \\
\end{array}$ & $\begin{array}{r}50 \\
10.0\end{array}$ & $7 \cdot 5$ & 60 & 70 & $9 \cdot 0$ & 1.9 & 17 & & & & & & t) & & & & & & \\
\hline 19 & $11 \%$ & 12.5 & $11 . \mathrm{s}$ & $12 \cdot 8$ & $14 \cdot 0$ & $\begin{array}{l}11 \cdot 2 \\
15.6\end{array}$ & $\begin{array}{l}11 \cdot 2 \\
14 \cdot 8\end{array}$ & $\begin{array}{l}12 \cdot 0 \\
13 \cdot 0\end{array}$ & $\begin{array}{l}12 \cdot 0 \\
13.0\end{array}$ & $\begin{array}{l}12 \cdot 2 \\
13 \cdot{ }^{\prime}\end{array}$ & $\begin{array}{l}11.6 \\
12.8\end{array}$ & $\begin{array}{c}11.8 \\
13.2\end{array}$ & $\begin{array}{l}10.7 \\
13.2\end{array}$ & 19 & & & & & & $\ddot{z}^{2}$ & & & & & & \\
\hline 20 & $12 \cdot 8$ & $13 \cdot 0$ & $9 \cdot 5$ & $7 \cdot 0$ & $5 \cdot 0$ & 3.0 & $4 \cdot 8$ & 2.0 & $-7 \cdot 0$ & $-11 \cdot 5$ & -10.0 & -7.5 & 1.8 & 20 & $12 \cdot y_{1}$ & $13 *$ & $8 \cdot 8$ & 6.5 & $4 \cdot 5$ & $3 \cdot 0$ & $4 \cdot 2$ & $2 \cdot 0$ & $-7 \cdot 0$ & - & - & - \\
\hline $\begin{array}{l}21 \\
22 \\
23\end{array}$ & $\begin{array}{l}-8 . \\
=7 \\
-10\end{array}$ & & & $\begin{array}{l}-11 \cdot 2 \\
-11 \cdot 2 \\
-10 \cdot 0\end{array}$ & $\begin{array}{r}7 \cdot 0 \\
-12 \cdot 8 \\
-10 \cdot 0\end{array}$ & $\begin{array}{r}-8.5 \\
-80.0 \\
-15.0\end{array}$ & $\begin{array}{r}7 \cdot 8 \\
-13 \cdot 0 \\
-12 \cdot 0\end{array}$ & & $\begin{array}{l}-7.5 \\
-11.0 \\
-10^{\circ} \cdot 2\end{array}$ & $\begin{array}{r}-9 \cdot 2 \\
-10 \cdot 2 \\
-9 \cdot 0\end{array}$ & $\begin{array}{l}-11 \cdot 0 \\
-11 \cdot 8 \\
-3 \cdot 2\end{array}$ & & $\begin{array}{l}-8 \cdot 4 \\
-12 \cdot 2 \\
-9 \cdot 3\end{array}$ & $\begin{array}{l}21 \\
22 \\
23\end{array}$ & & & & & & $g$ & & & & & & \\
\hline 24 & $-5^{\circ} 0$ & -8.2 & $-11 \cdot 0$ & 8.0 & & & & & $-9 \cdot 0$ & $-11 \cdot 0$ & $-11 \cdot 0$ & -9.8 & $-9 \cdot 2$ & 24 & & & & & & $8 \bar{z}$ & & & & & & \\
\hline $\begin{array}{l}25 \\
26 ;\end{array}$ & $\begin{array}{l}-9 \cdot 0 \\
-10.0\end{array}$ & $\begin{array}{l}-8 . \\
-8 .\end{array}$ & -9 & $8 \cdot 1$ & $\begin{array}{r}-8 \cdot 0 \\
-\quad 8 \cdot 2\end{array}$ & $\begin{array}{r}-9 \cdot 0 \\
-7 \cdot 0\end{array}$ & $\begin{array}{l}-8 \cdot 2 \\
-7 \cdot 0\end{array}$ & $\begin{array}{r}-7.0 \\
-4.8\end{array}$ & -6.0 & $\begin{array}{l}-8 \cdot 0 \\
-\quad 3 \cdot 0\end{array}$ & $=\mathbf{7} \cdot 0$ & $\begin{array}{l}-8.9 \\
-3.3\end{array}$ & $\begin{array}{l}-7 \cdot 5 \\
-6 \cdot 6\end{array}$ & 25 & & & & & & 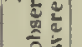 & & & & & & \\
\hline $\begin{array}{l}27 \\
28\end{array}$ & $\begin{array}{r}5 \\
-\quad 9\end{array}$ & $=2 \cdot$ & -3 & -3 & 0 & -4.8 & -6 & $\begin{array}{r}9 \\
-90\end{array}$ & -8.0 & -7.8 & $-10 \cdot 2$ & -10.6 & -6.1 & 27 & & & & & & 102 & & & & & & \\
\hline 29 & $=5$ & $=3$ & $=3.0$ & $\begin{array}{r}5.8 \\
-\quad 2 \cdot 7\end{array}$ & $\begin{array}{r}6.0 \\
-\quad 3.5 \\
\end{array}$ & {$\left[\begin{array}{l}-6.0 \\
-2.5\end{array}\right]$} & $\begin{array}{l}-7 \\
-2\end{array}$ & $=4$ & $\mid$\begin{tabular}{|l}
-64 \\
-4.2
\end{tabular} & $=\begin{array}{l}-5.9 \\
-4.0\end{array}$ & $\begin{array}{l}-5 \cdot 0 \\
-5 \cdot 2\end{array}$ & $\begin{array}{l}=4.8 . \\
=7.5\end{array}$ & $\begin{array}{l}-6.4 \\
-3.9\end{array}$ & $\begin{array}{l}28 \\
29\end{array}$ & 二 & $\bar{z}$ & $\bar{z}$ & z & $=$ & -6 & $z$ & $=$ & z & $\bar{z}$ & $=$ & $\overline{-}$ \\
\hline $\begin{array}{l}30 \\
31\end{array}$ & $\begin{array}{l}-4 \cdot 0 \\
-12 \cdot 9\end{array}$ & $\begin{array}{r}0.5 \\
0\end{array}$ & 1 . & 0.3 & & -1.5 & -1.3 & $-3 \cdot 2$ & $|-48|$ & $-5 \cdot 1$ & -7 & $-12 \cdot 2$ & $-3 \cdot 2$ & 30 & - & - & - & - & - & - & - & - & - & - & - & - \\
\hline . & - & & & & & & & & & & & & & (n) & 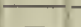 & - & - & - & 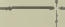 & 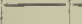 & 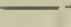 & 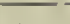 & - & 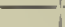 & 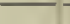 & \\
\hline & & & & & & & & & & & & & & In. & - & - & - & - & & - & - & - & - & 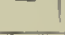 & - & - \\
\hline
\end{tabular}




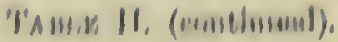

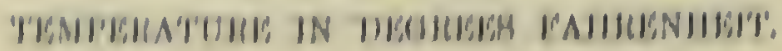

IIIIY IfII, H,

Writ limlli,

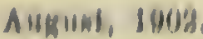

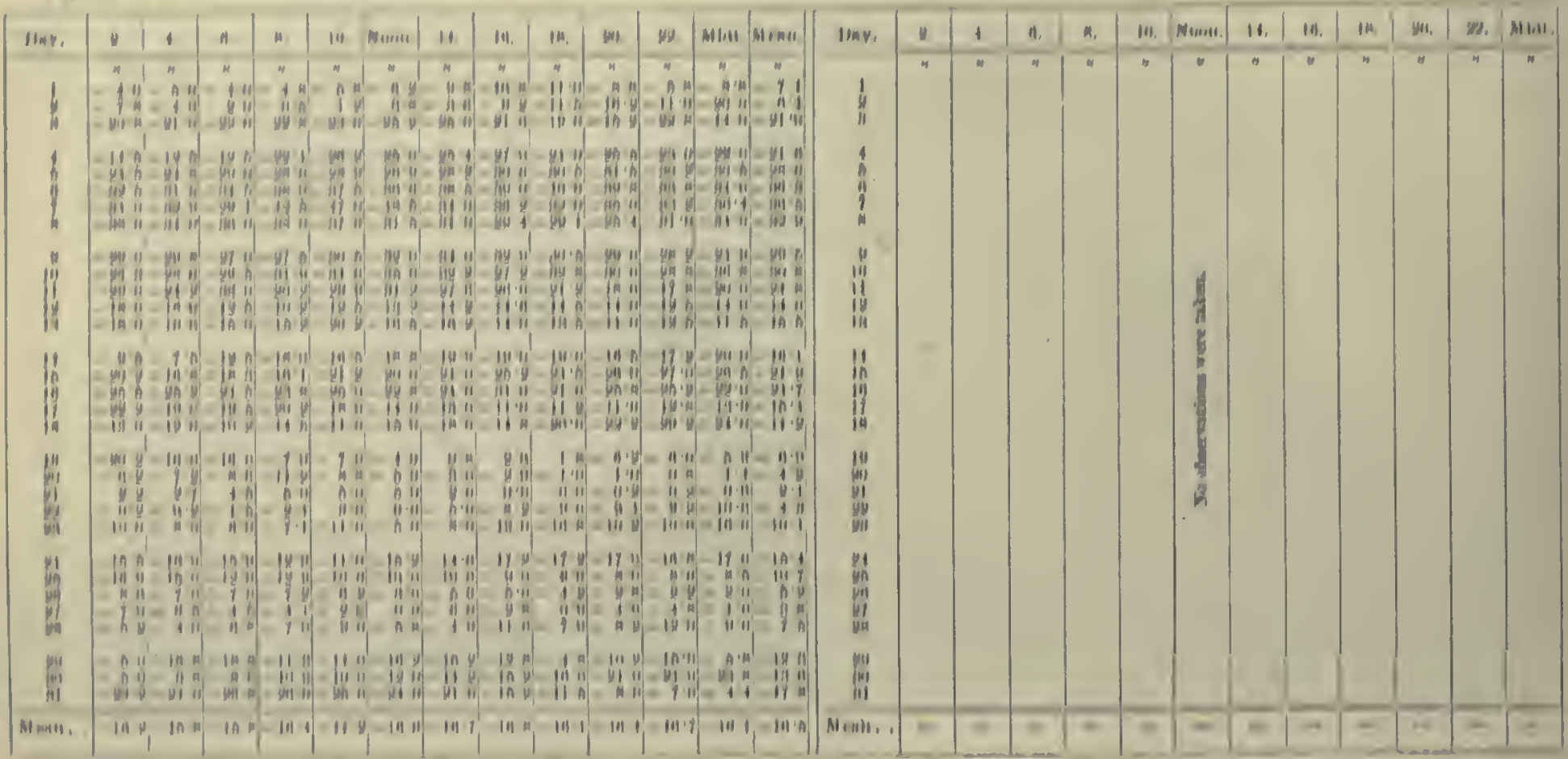

(1)11) IIII, H,

Wirt IIII, II,

Mepulambari, 100\%

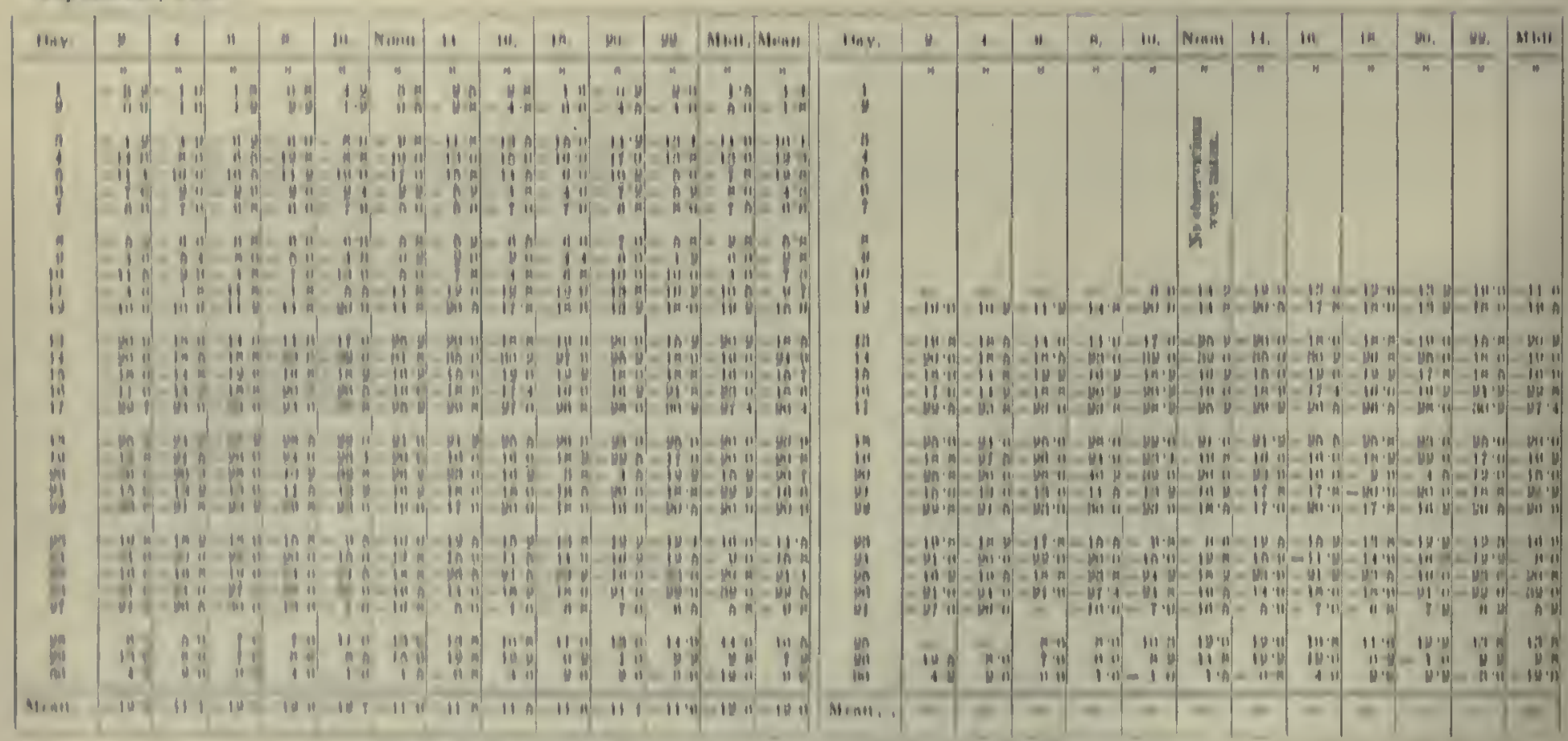




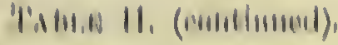

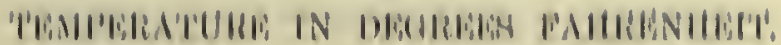

1) IIY IIII Hi.

IIra. H111,11,

Outobors, 1009,

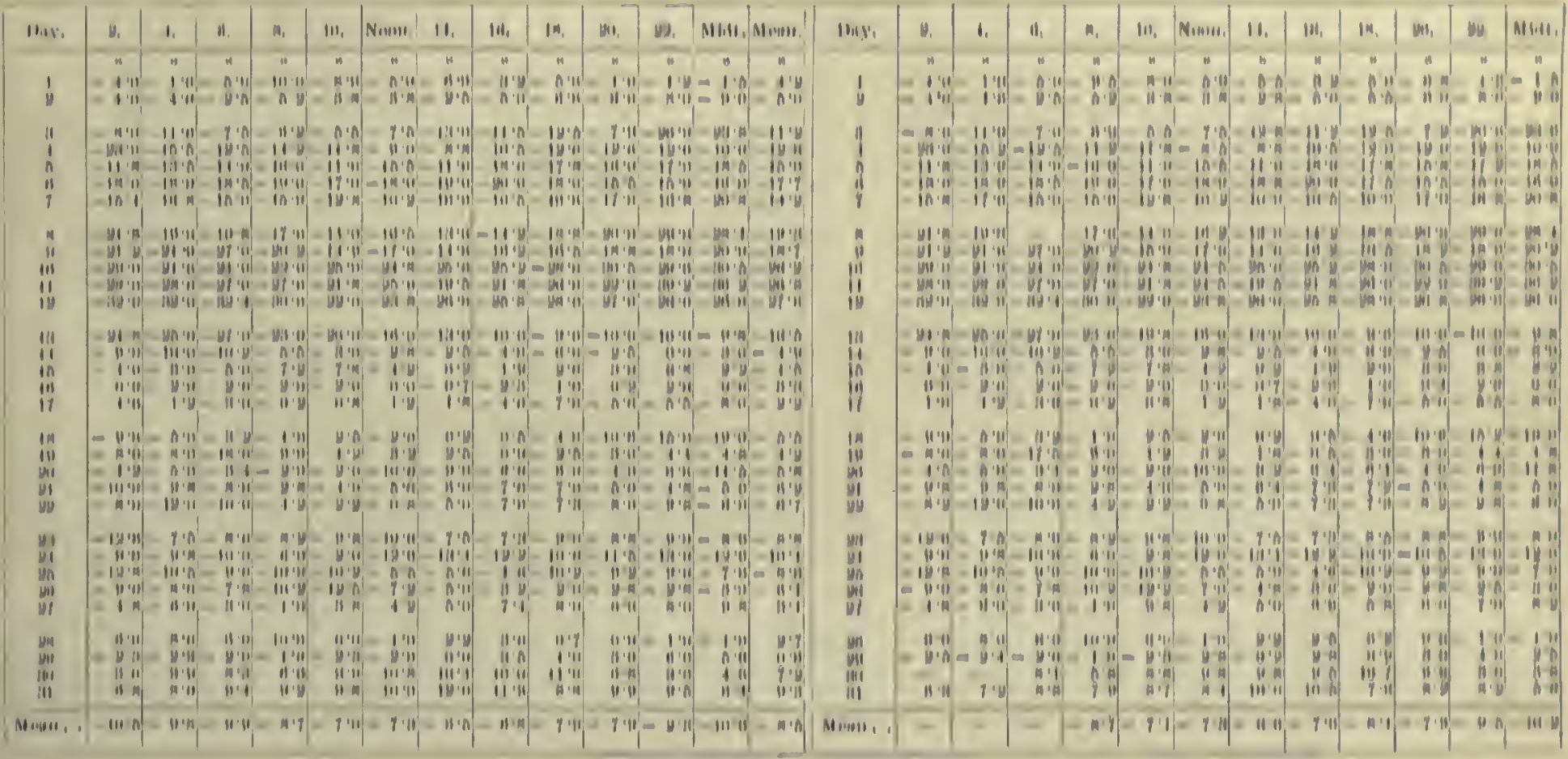

)แเY HUI,U,

Wiri Il11,11.

Novamber, 1002,

\begin{tabular}{|c|c|c|c|c|c|c|c|c|c|c|c|c|c|c|c|c|c|c|c|c|c|c|c|c|c|c|}
\hline Ifiy. & 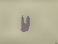 & I & $I_{1}$ & a & III & Nown... & 11 & III. & Is. & Int & $w$. & M1..11 & Misuri. & Itivy, & y. & 1 & II. & a. & Iin. & N1mant & 10 & III. & in. & $m$ & wu. & MWI \\
\hline 1 & " " & 111 & $\because 1$ & $n \cdot n$ & 11 & $10 . n$ & $1 \cdots 11$ & $n$ & $n$ & N1I & AIII) & $11+1$ & A & 1 & AN & n." & $\| y$ & A " & $n$ & 11 & 1110 & n'y & " & 1.1 & in & III! \\
\hline$y$ & $\therefore$ & " & ril & (1) & 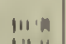 & 198 & 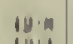 & . & 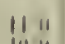 & 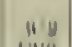 & 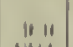 & II & & 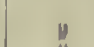 & 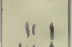 & 96 & 711 & 80 & IIII) & $11 \mathrm{y}$ & 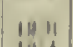 & 11 & IIIIII & (II) & $\begin{array}{ll}n n \\
4\end{array}$ & $\mathrm{PH}$ \\
\hline & 4 & 1901 & $\begin{array}{l}\text { III) il } \\
\text { if }\end{array}$ & in: iii: & 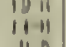 & $\begin{array}{l}111 \\
11 . n\end{array}$ & in & 19.1in & "II": & 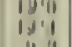 & Yin & $\begin{array}{lll}\operatorname{lin} \\
\operatorname{lin} & 1\end{array}$ & Iy & i & Iii ! & II" & Iii $y$ & 焉 & in & iii & 1 & 䎡 & $\begin{array}{llll} & 11 & 1 \\
& 11\end{array}$ & IIi) & III & i i \\
\hline in & "I" & 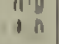 & 集, & 110 & $\begin{array}{l}118 \\
11 y\end{array}$ & "Ii" & Iii 11 & IIII & 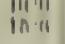 & IIII & 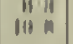 & 豞 & ${ }_{11}^{10.4}$ & in & 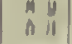 & iili: & $\begin{array}{l}\text { Ui" } \\
\text { i" }\end{array}$ & Iii ii & $\begin{array}{l}1111 \\
\text { IIII }\end{array}$ & 售i: & iii & in iा & $\| 11$ & 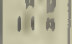 & iii? & A 1 \\
\hline 7 & Iii : & 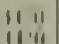 & iili $n$ & $\begin{array}{lll}181 \\
4.10\end{array} \mid$ & 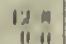 & iyn & in: & iii & 119.9 & 1118 & $y=$ & in & 110 & 1 & in. & 1319 & II & $14 \mathrm{n}$ & $1 y=$ & 119 & ing & i1.i1 & in & 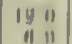 & in & 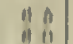 \\
\hline iii & "I & Iini & n'y & 管: & . & ligy & 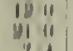 & 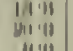 & 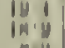 & III"' & I'" & $\begin{array}{lll}111 \\
111\end{array}$ & ivi & III & in & ii & Iiiii & 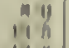 & Iin" & 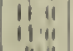 & 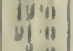 & iny & iii) & Iii y y & 110 & \\
\hline & & & & & & & & & & & & & & 11 & & & & IIn & "1] & & & & & & & \\
\hline iii & II & in & "iv" & w. & i. & yin & (1) & in 180 & in & iI & "VI" & 111" & ifi & III & & ii & 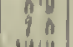 & ii & IIII & [ic & A & III & iii & (iii) & I. & \\
\hline in & in $17 n$ & $\begin{array}{l}\text { ifiti } \\
\text { "ili" }\end{array}$ & ii & iii & 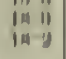 & 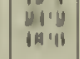 & 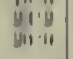 & 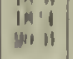 & in in & 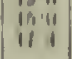 & 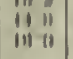 & $\begin{array}{l}i_{i n} \\
\text { in. }\end{array}$ & $\begin{array}{l}\text { iiln } \\
\text { in in }\end{array}$ & in & 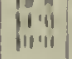 & 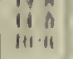 & $\begin{array}{l}\text { ivi } \\
\text { iviv }\end{array}$ & 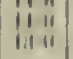 & $17 y$ & wi & 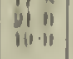 & 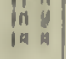 & iii" & iii & $\operatorname{lin}_{n \rightarrow} i$ & in \\
\hline 17 & 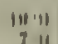 & 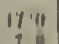 & 191 & $18 \mathrm{ft}$ & if 11 & (19)1 & 17,4 & II II & II"I" & 10.0 & n'il & "y & $11 \%$ & & in & III 11 & ing $y$ & $101 \mathrm{ng}$ & $\ln 01$ & In: & 1414 & II:-4 & $y$ & $11 y$ & $n$ & - \\
\hline iiu & ii"i & 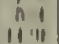 & $\begin{array}{l}4.1 \\
\text { n. }\end{array}$ & 3ii & iii & , iui & 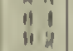 & 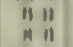 & nit & ind & $\begin{array}{lll}6 & 1 \\
4 & 11\end{array}$ & iiv & 11 & III & Iin & in & i. & i1 & 17 & $i$ in & 11 & $\begin{array}{ll}n \\
n \\
n i n\end{array}$ & iin & 10 & 101 & \\
\hline iit & "in & iii"i & 19.11 & IIIII & ii ii & iw'i & 167 & 11 is & lint & in i" & iii ii & ini & 11 & ii & "111 & in & 111 & $y=$ & 117 & 111 & iี "1 & 107 & $16 \cdot \pi$ & $11 \bar{x}$ & $6 y$ & \\
\hline ii & II" & iilin & 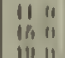 & II ? & 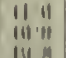 & 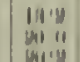 & in & $\begin{array}{l}11.4 \\
\text { ynin }\end{array}$ & In in & 1110 & $\begin{array}{lll}11 & 0 \\
1 & y & y\end{array}$ & 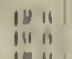 & & $y$ & $\mathrm{An}$ & III."i & $\operatorname{lin}_{110} 10$ & Ii" i" & in & 14: & $\begin{array}{lll}1 & 1 & 1 \\
4 & 11\end{array}$ & 货 & IIII" & III" & II & \\
\hline$f m$ & "11" & 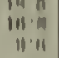 & imi: & $\begin{array}{l}\lim ^{111} \\
\text { in }\end{array}$ & 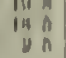 & 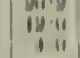 & ${ }_{111}$ & 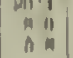 & $?$ & $\begin{array}{l}1 \\
111 \\
7 n\end{array}$ & 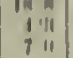 & 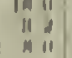 & Iii" & 等 & iy & $\begin{array}{l}\text { Ini } \\
\text { nili }\end{array}$ & $\begin{array}{ll}10 \\
\text { inin } \\
\text { ing }\end{array}$ & 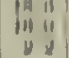 & 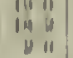 & $11 \%$ & $\begin{array}{l}31 \\
11 \\
1\end{array}$ & 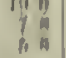 & & 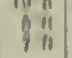 & & \\
\hline 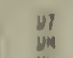 & 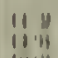 & AII & in & $\begin{array}{ll}11 & n \\
16 & n\end{array}$ & in & 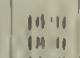 & $\begin{array}{l}17 \cdot 11 \\
14111\end{array}$ & 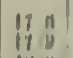 & $\begin{array}{lll}17 & 11 \\
\text { yin } & 11\end{array}$ & $\begin{array}{llll}\text { InI } & \text { in }\end{array}$ & $\begin{array}{ll}i_{1} \\
\text { int }\end{array}$ & $\begin{array}{lll}11 & y \\
10 & 0\end{array}$ & & it & & if & $\operatorname{lin}_{10}$ & 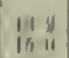 & ": & lif & iil" ?" & in" & $\operatorname{lin}_{10}$ & is $\begin{array}{l}n \\
\text { ii }\end{array}$ & $y$ & \\
\hline & 11 & iii" & ii" & $\begin{array}{lll} & y \\
11 & y\end{array}$ & iif & $\begin{array}{l}{ }_{11}^{11} \text { in } \\
\text { inii }\end{array}$ & $\begin{array}{ll}\text { II } \\
\text { vili }\end{array}$ & $\lim _{i=1}$ & & 17.9 & ii i i ] & $\begin{array}{llll} & 11 & 11 \\
17 & 11\end{array}$ & III) " & IMI & 11 & in & & ivn & 促" & iii y & iili" & In & & In. & iil in & \\
\hline & III * & 1119 & 118 & 111 & I" & 1.12 & 1114 & $111 \mathrm{n}$ & 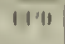 & 1111 & III II & IIIII & 11111 & Atum. & 1171 & m'y & ivit & $m=1$ & 1111 & 121 & 111 & $\| * 1$ & $|140|$ & 114 & 11 & \\
\hline
\end{tabular}

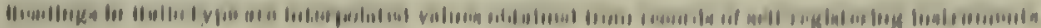


TARL, II. (continued).

TEMPERATURE IN DEGREES FAHRENHEIT.

Dry Bulb.

WET BuIB.

December, 1902.

\begin{tabular}{|c|c|c|c|c|c|c|c|c|c|c|c|c|c|c|c|c|c|c|c|c|c|c|c|c|c|c|}
\hline Day & 2. & 4. & 6. & 8 & 10. & 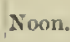 & 14. & 16. & 18 & 21). & 22. & 1iult. & ean & Day & 2. & 4. & 6. & 8. & 10. & Yoon. & 14. & 10. & 18. & 21). & 22. & is \\
\hline 1 & $\stackrel{0}{16 \cdot 0}$ & $\begin{array}{c}0 \\
17 \cdot 5\end{array}$ & $17 \cdot 8$ & $\frac{0}{18 \cdot 2}$ & 8.5 & 18.8 & $19^{\circ} 0$ & 19.5 & $21^{\circ} 0$ & $\begin{array}{c}\circ \\
19.0\end{array}$ & $\overline{18.2}$ & $\overline{17 \cdot 8}$ & $18 \cdot 4$ & 1 & $\begin{array}{c}0 \\
15 \cdot 0\end{array}$ & $17 \cdot 0$ & 18.5 & $17^{\circ} \cdot 2$ & $17 \cdot 0$ & $17 \cdot 0$ & $17 \cdot 2$ & $\begin{array}{c}0 \\
18 \cdot 2\end{array}$ & $\left|\begin{array}{c}0 \\
20 \cdot 0\end{array}\right|$ & $17 \cdot 0$ & $26 \cdot 0$ & $17 \cdot 2$ \\
\hline 2 & & $\begin{array}{r}5 \cdot 2 \\
5 \cdot 2 \\
13 \cdot 0 \\
12 \cdot 0 \\
15 \cdot 2\end{array}$ & $\begin{array}{r}10 \cdot 0 \\
5.2 \\
12 \cdot 8 \\
16.0 \\
15 \cdot 2\end{array}$ & $\begin{array}{r}6 \cdot 0 \\
7 \cdot 8 \\
13 \cdot 2 \\
16 \cdot 2 \\
14 \cdot 8\end{array}$ & $\begin{array}{r}6.2 \\
8.5 \\
14.5 \\
18.8 \\
15.0\end{array}$ & $\begin{array}{r}6.8 \\
13.2 \\
15 \cdot 0 \\
16.8 \\
15.5\end{array}$ & $\begin{array}{l}7 \cdot 4 \\
11 \cdot 0 \\
11 \cdot 0 \\
14 \cdot 2 \\
16 \cdot 6\end{array}$ & $\begin{array}{r}7 \cdot 9 \\
12 \cdot 6 \\
16 \cdot 5 \\
14.8 \\
16 \cdot 4\end{array}$ & $\begin{array}{r}8 \cdot 0 \\
13 \cdot 5 \\
17 \cdot 0 \\
15 \cdot 0 \\
17 \cdot 2\end{array}$ & $\begin{array}{r}8.0 \\
14.0 \\
16.0 \\
13.0 \\
17.0\end{array}$ & $\begin{array}{r}7 \cdot 2 \\
13 \cdot 0 \\
12 \cdot 2 \\
13 \cdot 0 \\
18 \cdot 2\end{array}$ & $\begin{array}{r}7 \cdot 2 \\
12 \cdot 0 \\
13 \cdot 0 \\
13 \cdot 2 \\
18 \cdot 0\end{array}$ & $\begin{array}{c}7 \cdot 7 \\
10 \cdot 4 \\
14 \cdot 4 \\
14 \cdot 5 \\
18 \cdot 2\end{array}$ & $\begin{array}{l}2 \\
3 \\
4 \\
5\end{array}$ & $\begin{array}{r}10 \cdot 2 \\
5.8 \\
12.0 \\
10.5 \\
14.8\end{array}$ & $\begin{array}{r}7.0 \\
5.0 \\
12.0 \\
11.5 \\
15.0\end{array}$ & $\begin{array}{r}10 \cdot 0 \\
5 \cdot 2 \\
12 \cdot 2 \\
11 \cdot 0 \\
14 \cdot 8\end{array}$ & $\begin{array}{r}6 \cdot 0 \\
8 \cdot 0 \\
12 \cdot 8 \\
14 \cdot 0 \\
14 \cdot 0\end{array}$ & $\begin{array}{r}5.8 \\
8.2 \\
13.8 \\
16.0 \\
14.2\end{array}$ & $\begin{array}{l}6 \cdot 2 \\
12 \cdot 2 \\
14 \cdot 5 \\
15 \cdot 0 \\
14 \cdot 8\end{array}$ & $\begin{array}{r}6 \cdot 8 \\
13 \cdot 0 \\
15 \cdot 5 \\
13 \cdot 0 \\
15 \cdot 5\end{array}$ & $\begin{array}{r}7 \cdot 2 \\
12 \cdot 0 \\
15 \cdot 5 \\
13 \cdot 2 \\
15 \cdot 8\end{array}$ & $\begin{array}{r}7 \cdot 5 \\
12 \cdot 4 \\
16 \cdot 6 \\
12 \cdot 2 \\
16 \cdot 0\end{array}$ & $\begin{array}{r}7 \cdot 5 \\
12 \cdot 8 \\
15 \cdot 2 \\
11 \cdot 2 \\
16 \cdot 2\end{array}$ & $\begin{array}{r}7 \cdot 0 \\
12 \cdot 2 \\
12 \cdot 0 \\
11 \cdot 0 \\
15 \cdot 8\end{array}$ & $\begin{array}{l}13 \cdot 0 \\
12 \cdot 2 \\
12 \cdot 5 \\
15 \cdot 5\end{array}$ \\
\hline $\begin{array}{l}7 \\
8 \\
9\end{array}$ & & $\begin{array}{l}33 \cdot 2 \\
17.2 \\
34.0 \\
32.0 \\
23.0\end{array}$ & $\begin{array}{l}21.0 \\
17.0 \\
23 \cdot 0 \\
31.0 \\
25.0\end{array}$ & $\begin{array}{l}31 \cdot 5 \\
26 \cdot 5\end{array}$ & $\begin{array}{l}7.0 \\
1.0 \\
3.0 \\
0.2 \\
9.8\end{array}$ & & $\begin{array}{l}17 \cdot 8 \\
23.5 \\
34.5 \\
29.5 \\
30 \cdot 0\end{array}$ & & $\begin{array}{l}18 \cdot 0 \\
25 \cdot 6 \\
355^{\circ} 6 \\
30 \cdot 8 \\
32 \cdot 5\end{array}$ & $\begin{array}{l}17 \cdot 5 \\
28 \cdot 8 \\
36 \cdot 0 \\
31 \cdot 0 \\
24 \cdot 2\end{array}$ & $\begin{array}{l}17 \cdot 2 \\
30.5 \\
36.0 \\
28 \cdot 2 \\
24 \cdot 8\end{array}$ & $\begin{array}{l}17 \cdot 2 \\
33 \cdot 0 \\
34 \cdot 5 \\
27 \cdot 5 \\
22 \cdot 2\end{array}$ & $\begin{array}{l}18 \cdot 5 \\
23 \cdot 3 \\
34 \cdot 2 \\
30 \cdot 4 \\
27 \cdot 6\end{array}$ & $\begin{array}{r}7 \\
8 \\
9 \\
10 \\
11\end{array}$ & $\begin{array}{l}19 \cdot 0 \\
17.0 \\
31.0 \\
29.0 \\
26.0\end{array}$ & $\begin{array}{l}22 \cdot 0 \\
17.2 \\
32 \cdot 8 \\
30.0 \\
24 \cdot 8\end{array}$ & $\begin{array}{l}20 \cdot 5 \\
17.0 \\
32 \cdot 5 \\
22 \cdot 0 \\
23 \cdot 0\end{array}$ & $\begin{array}{l}18 \cdot 2 \\
18 \cdot 8 \\
31 \cdot 8 \\
28 \cdot 8 \\
-\end{array}$ & $\begin{array}{l}17 \cdot 0 \\
20.6 \\
31 \cdot 0 \\
29 \cdot 2 \\
27 \cdot 2\end{array}$ & $\begin{array}{l}17 \cdot 8 \\
19 \cdot 8 \\
31 \cdot 0 \\
27 \cdot 0 \\
25 \cdot 5\end{array}$ & $\begin{array}{l}17 \cdot 0 \\
20 \cdot 6\end{array}$ & $\begin{array}{l}17 \cdot 2 \\
22 \cdot 6 \\
31 \cdot 6 \\
28 \cdot 8 \\
28 \cdot 5\end{array}$ & $\begin{array}{l}18 \cdot 0 \\
25 \cdot 4 \\
32 \cdot 0 \\
29 \cdot 5 \\
28 \cdot 8\end{array}$ & $\begin{array}{l}17 \cdot 5 \\
27.8 \\
31.8 \\
28 \cdot 8 \\
22 \cdot 9\end{array}$ & 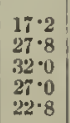 & $21 \cdot 8$ \\
\hline 12 & & $\mid \begin{array}{l}24 \cdot 8 \\
28.0 \\
27.0 \\
25.5 \\
22.0\end{array}$ & $\begin{array}{l}25 \cdot 0 \\
29 \cdot 0 \\
27.0 \\
25.0 \\
22 \cdot 0\end{array}$ & & $\begin{array}{l}25 \cdot 2 \\
27 \cdot 2 \\
30 \cdot 0 \\
24 \cdot 5 \\
23 \cdot 5\end{array}$ & & $\begin{array}{l}28 \cdot 2 \\
27.5 \\
33.6 \\
24.5 \\
25 \cdot 0\end{array}$ & & $\begin{array}{l}28 \cdot 6 \\
27 \cdot 8 \\
27 \cdot 2 \\
22 \cdot 5 \\
23 \cdot 5\end{array}$ & $\begin{array}{l}25 \cdot 2 \\
27 \cdot 5 \\
29 \cdot 0 \\
23 \cdot 2 \\
23 \cdot 2\end{array}$ & $\begin{array}{l}25 \cdot 5 \\
27 \cdot 5 \\
28 \cdot 4 \\
23 \cdot 5 \\
23 \cdot 2\end{array}$ & & & & $\begin{array}{l}2.0 \\
22.0\end{array}$ & $\begin{array}{l}23 \cdot 8 \\
27 \cdot 0 \\
27 \cdot 0 \\
25 \cdot 0 \\
22 \cdot 0\end{array}$ & $\begin{array}{l}24 \cdot 0 \\
28.0 \\
27 \cdot 2 \\
25 \cdot 0 \\
21 \cdot 0 \\
21 \cdot 8\end{array}$ & $\begin{array}{l}25 \cdot 0 \\
26.8 \\
28.5 \\
23.8 \\
22.0\end{array}$ & $\begin{array}{l}24 \cdot 0 \\
27.2 \\
28.8 \\
24 \cdot 2 \\
23 \cdot 0\end{array}$ & $\begin{array}{l}25 \cdot 2 \\
27 \cdot 8 \\
31 \cdot 5 \\
21 \cdot 0 \\
23 \cdot 2\end{array}$ & $\begin{array}{l}21 \cdot 2 \\
27 \cdot 5 \\
31 \cdot 8\end{array}$ & $\begin{array}{l}27 \cdot 4 \\
27 \cdot 1 \\
29 \cdot 5 \\
21-9 \\
23 \cdot 5\end{array}$ & $\begin{array}{l}27 \cdot 5 \\
27 \cdot 8 \\
27 \cdot 0 \\
23 \cdot 0 \\
23 \cdot 0\end{array}$ & & & $\begin{array}{l}25 \cdot 5 \\
28 \cdot 0 \\
27 \cdot 0 \\
23 \cdot 0 \\
22 \cdot 0\end{array}$ \\
\hline $\begin{array}{l}\mathbf{1 7} \\
18 \\
19 \\
20 \\
21\end{array}$ & & 19.0 & $19 \cdot 2$ & & $\begin{array}{l}23.0 \\
30^{\circ} 0 \\
23.0 \\
250 \\
20.5 \\
20.5\end{array}$ & & $\begin{array}{l}23 \cdot 2 \\
24 \cdot 2 \\
19 \cdot 5\end{array}$ & $\begin{array}{l}23 \cdot 0 \\
27 \cdot 0 \\
23^{\circ} \cdot 4 \\
23^{\circ} 0 \\
17 \cdot 8\end{array}$ & $\begin{array}{l}26 \cdot 5 \\
27 \cdot 0 \\
23 \cdot 0 \\
22 \cdot 5 \\
15 \cdot 6\end{array}$ & $\begin{array}{l}24.5 \\
23 \cdot 0 \\
20 \cdot 0 \\
15 \cdot 0\end{array}$ & & & & & & $\begin{array}{l}21 \cdot 5 \\
25.2 \\
20.5 \\
24.0 \\
18.5\end{array}$ & 19.0 & $\begin{array}{l}21 \cdot 0 \\
28 \cdot 5 \\
21 \cdot 2 \\
24 \cdot-8 \\
20 \cdot 0\end{array}$ & $\begin{array}{l}23 \cdot 2 \\
27.8 \\
22 \cdot 8 \\
24 \cdot 2 \\
19 \cdot 6\end{array}$ & & $\begin{array}{l}26 \cdot 0 \\
22 \cdot 2 \\
23 \cdot 5 \\
19 \cdot 0\end{array}$ & $\begin{array}{l}25 \cdot 2 \\
25 \cdot 5 \\
22 \cdot 6\end{array}$ & $\begin{array}{l}25 \cdot 5 \\
25 \cdot 8 \\
22 \cdot 6 \\
22 \cdot-2 \\
15 \cdot 2\end{array}$ & & $\frac{25 \cdot 1}{25 \cdot 6}$ & $\begin{array}{l}23.0 \\
18.8 \\
13 \cdot 8\end{array}$ \\
\hline $\begin{array}{l}22 \\
23 \\
24 \\
25 \\
26\end{array}$ & & $\begin{array}{l}210 \\
32 \cdot 2 \\
27 \cdot 2\end{array}$ & & & $\begin{array}{l}\cdot 2 \\
-8 \\
-2 \\
-2 \\
-0\end{array}$ & & & & $\begin{array}{l}19 \cdot 0 \\
23 \cdot 0 \\
24 \cdot 7 \\
32 \cdot 2 \\
30 \cdot 0\end{array}$ & $\begin{array}{l}18 \\
19 \\
27 \\
39 \\
30\end{array}$ & $\begin{array}{l}19.0 \\
16.0 \\
23.0 \\
26.8 \\
29.0\end{array}$ & & & & & $\begin{array}{l}12 \cdot 5 \\
19.0 \\
19 \cdot 8 \\
29.5 \\
26.0\end{array}$ & 28.0 & $\begin{array}{l}1.3 \cdot 0 \\
20.2 \\
16 \cdot 8 \\
22.0 \\
35.0\end{array}$ & 35.5 & & & & & & & $\begin{array}{l}29 \cdot 3 \cdot \\
23 \cdot 0 \\
25 \cdot 0\end{array}$ \\
\hline $\begin{array}{l}27 \\
28 \\
20 \\
30 \\
31\end{array}$ & & $\begin{array}{l}31 \cdot 5 \\
32.0 \\
23.5\end{array}$ & $\begin{array}{l}31 \cdot 2 \\
32 \cdot 2 \\
23 \cdot 0 \\
20 \cdot 8 \\
18 \cdot 0\end{array}$ & $21 \cdot 6$ & $\begin{array}{l}29 \cdot 8 \\
34 \cdot 8 \\
27 \cdot 8 \\
23 \cdot 0 \\
24 \cdot 0\end{array}$ & & $22 \cdot 2$ & $23 \cdot 8$ & $\begin{array}{l}3.3 \cdot 0 \\
30.0 \\
30 \cdot 2 \\
25 \cdot 0 \\
25 \cdot 4\end{array}$ & $\begin{array}{l}34 \cdot 0 \\
28 \cdot 2 \\
32 \cdot 0 \\
24 \cdot 2 \\
24 \cdot 5\end{array}$ & $24 \cdot 8$ & $\begin{array}{l}29 \cdot 2 \\
25 \cdot 0\end{array}$ & & & & $\begin{array}{l}27 \cdot 8 \\
28.2 \\
23 \cdot 0 \\
21 \cdot 0 \\
17 \cdot 0\end{array}$ & $\begin{array}{l}28.2 \\
22 \cdot 8 \\
21.0 \\
18 \cdot 0\end{array}$ & $\begin{array}{l}25 \cdot 2 \\
28 \cdot 2 \\
24 \cdot 0 \\
20.0 \\
20 \cdot 5\end{array}$ & 27 & $21 \cdot 6$ & $21 \cdot 4$ & $22-3$ & & $23 \cdot 2$ & $230^{\circ}$ & $\begin{array}{l}34 \cdot 0 \\
24 \cdot 0 \\
23 \cdot 0\end{array}$ \\
\hline & $21 \%$ & & $22 \cdot 0$ & $22 \cdot 2$ & 23.5 & $23 \cdot 5$ & $24 \cdot 4$ & $24 \cdot 3$ & $24 \cdot 3$ & $23 \cdot 6$ & 22.4 & 23.0 & $23 \cdot 1$ & ean & $20 \cdot 8$ & $21 \cdot 1$ & $21 \cdot 1$ & - & $22 \cdot 3$ & $22 \cdot 4$ & $22 \cdot 8$ & $22 \cdot 9$ & 230 & $22 \cdot 4$ & - & 21.8 \\
\hline
\end{tabular}

DRY Bula.

WFT BULB.

January, 1903.

\begin{tabular}{|c|c|c|c|c|c|c|c|c|c|c|c|c|c|c|c|c|c|c|c|c|c|c|c|c|c|c|}
\hline Day. & 2. & 4. & 6. & 8. & 10. & Xison. & 14. & 16. & 18. & 20. & 22. & Midt. & Mean. & Dayy. & 2. & 4. & b. & 8. & 10. & Noon. & 14. & 16. & 18. & 20. & 22. & Midt. \\
\hline $\begin{array}{l}1 \\
2 \\
3 \\
4 \\
5\end{array}$ & $\begin{array}{c}0 \\
25 \cdot 0 \\
260^{\circ} 0 \\
27.0 \\
290^{\circ} 0 \\
28 \cdot 0\end{array}$ & $\begin{array}{c}0 \\
25 \cdot 0 \\
18 \cdot 0 \\
27 \cdot 0 \\
25 \cdot 0 \\
28 \cdot 2\end{array}$ & $\begin{array}{c}0 \\
24 \cdot 2 \\
27 \cdot 0 \\
30 \cdot 2 \\
24 \cdot 0 \\
28 \cdot 0\end{array}$ & $\begin{array}{l}24 \cdot 0 \\
27 \cdot 8 \\
28 \cdot 0 \\
24 \cdot 5 \\
27 \cdot 6\end{array}$ & $\begin{array}{l}25 \cdot 0 \\
28 \cdot 8 \\
29 \cdot 5 \\
27 \cdot 0 \\
29 \cdot 0\end{array}$ & $\begin{array}{c}2 \\
27 \cdot 0 \\
30 \cdot 0 \\
30 \cdot 2 \\
28 \cdot 2 \\
28 \cdot 2\end{array}$ & $\begin{array}{l}0 \\
28 \cdot 0 \\
33 \cdot 0 \\
33 \cdot 2 \\
30.5 \\
28 \cdot 2 \\
30 \cdot-3\end{array}$ & $\begin{array}{c}0 \\
28 \cdot 4 \\
35 \cdot 0 \\
300 \\
29 \cdot 0 \\
31 \cdot 6\end{array}$ & $\begin{array}{c}0 \\
28 \cdot 3 \\
30 \cdot 0 \\
30 \cdot 3 \\
31 \cdot 2 \\
30 \cdot 0\end{array}$ & $\begin{array}{c}0 \\
28 \cdot 2 \\
29 \cdot 5 \\
30 \cdot 2 \\
29 \cdot 0 \\
30 \cdot 0\end{array}$ & $\begin{array}{c}0 \\
28 \cdot 0 \\
27 \cdot 0 \\
29 \cdot 0 \\
28 \cdot 0 \\
29 \cdot 5\end{array}$ & \begin{tabular}{c|}
0 \\
$27 \cdot 0$ \\
$27 \cdot 0$ \\
$28 \cdot 2$ \\
$28 \cdot 0$ \\
$27 \cdot 0$
\end{tabular} & $\begin{array}{c}-\cdot \\
26 \cdot 6 \\
28 \cdot 3 \\
29 \cdot 2 \\
29 \cdot 2 \\
29 \cdot 0\end{array}$ & $\begin{array}{l}1 \\
2 \\
3 \\
4 \\
5\end{array}$ & $\begin{array}{c}\circ \cdot \\
23 \cdot 5 \\
25.5 \\
25 \cdot 6 \\
25 \cdot 2 \\
27 \cdot 0\end{array}$ & $\begin{array}{c}0 \\
23 \cdot 8 \\
17 \cdot 5 \\
26 \cdot 0 \\
24 \cdot 0 \\
27 \cdot 5\end{array}$ & $\begin{array}{c}0 \\
23 \cdot 0 \\
25 \cdot 5 \\
29 \cdot 2 \\
23 \cdot 2 \\
27 \cdot 0\end{array}$ & $\begin{array}{l}23 \cdot 0 \\
26 \cdot 0 \\
27 \cdot 5 \\
23 \cdot 8 \\
28 \cdot 2\end{array}$ & $\begin{array}{r}0 \\
24 \cdot 6 \\
26.6 \\
27.5 \\
25.0 \\
28.0\end{array}$ & $\begin{array}{l}25 \cdot 0 \\
27 \cdot 8 \\
28 \cdot 2 \\
26 \cdot 0 \\
27 \cdot 5\end{array}$ & 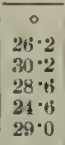 & $\begin{array}{l}26 \cdot 3 \\
31.5 \\
28 \cdot 3 \\
25 \cdot 6 \\
23 \cdot 6\end{array}$ & $\begin{array}{l}0 \\
26 \cdot 6 \\
27 \cdot 4 \\
28 \cdot 3 \\
29 \cdot 0 \\
28 \cdot 2\end{array}$ & $\begin{array}{c}0 \\
27 \cdot 0 \\
28 \cdot 0 \\
25 \cdot 2 \\
26 \cdot 2 \\
28 \cdot 0\end{array}$ & \begin{tabular}{c|}
0 \\
$25 \cdot 8$ \\
$25 \cdot 6$ \\
27.0 \\
27.0 \\
27.8
\end{tabular} & $\begin{array}{l}0 \\
28 \cdot 2 \\
25 \cdot 6 \\
28 \cdot 2 \\
27.0 \\
26 \cdot 0\end{array}$ \\
\hline $\begin{array}{r}6 \\
7 \\
8 \\
9 \\
10\end{array}$ & $\begin{array}{l}23 \cdot 0 \\
28.0 \\
24.0 \\
24 \cdot 0 \\
23.6 \\
23.2\end{array}$ & $\begin{array}{l}24 \cdot 5 \\
24 \cdot 8 \\
25 \cdot 0 \\
25 \cdot \cdot 2 \\
24 \cdot 2\end{array}$ & $\begin{array}{l}26 \cdot 0 \\
23 \cdot 2 \\
24 \cdot 2 \\
24 \cdot 5 \\
24 \cdot 0\end{array}$ & $\begin{array}{l}27 \cdot 0 \\
22 \cdot 2 \\
33 \cdot 0 \\
26 \cdot 5 \\
27 \cdot 0\end{array}$ & $\begin{array}{l}28 \cdot 8 \\
33 \cdot 0 \\
31 \cdot 5 \\
27 \cdot 0 \\
26 \cdot 0\end{array}$ & $\begin{array}{l}31 \cdot 0 \\
35 \cdot 0 \\
29 \cdot 6 \\
27 \cdot 5 \\
25 \cdot 6\end{array}$ & $\begin{array}{l}32 \cdot 2 \\
33 \cdot 6 \\
31 \cdot 0 \\
27.4 \\
26 \cdot 2\end{array}$ & $\begin{array}{l}31 \cdot 8 \\
3360 \\
30.7 \\
27.3 \\
26 \cdot 7\end{array}$ & $\begin{array}{l}32 \cdot 0 \cdot \\
3 \cdot-3 \\
32 \cdot 0 \\
26 \cdot 0 \\
26 \cdot 5\end{array}$ & $\begin{array}{l}31 \cdot 0 \\
33 \cdot 0 \\
30 \cdot 0 \\
25 \cdot 5 \\
27 \cdot 2\end{array}$ & $\begin{array}{l}31 \cdot 2 \\
32 \cdot 0 \\
28 \cdot 9 \\
25 \cdot 0 \\
28 \cdot 0\end{array}$ & $\begin{array}{l}29 \cdot 8 \\
25 \cdot 0 \\
27 \cdot 1 \\
24 \cdot 0 \\
27 \cdot 8\end{array}$ & $\begin{array}{l}29 \cdot 2 \\
30 \cdot 3 \\
29 \cdot 0 \\
26 \cdot 4 \\
26 \cdot 0\end{array}$ & $\begin{array}{r}6 \\
7 \\
8 \\
9 \\
10\end{array}$ & $\begin{array}{l}21 \cdot 8 \\
27 \cdot 0 \\
23 \cdot 0 \\
26 \cdot 6 \\
23 \cdot 2\end{array}$ & $\begin{array}{l}25 \cdot 0 \\
23 \cdot 0 \\
23 \cdot 8 \\
25 \cdot 5 \\
24 \cdot 2\end{array}$ & $\begin{array}{l}25 \cdot 0 \\
22 \cdot 0 \\
23 \cdot 0 \\
25 \cdot 2 \\
24 \cdot 0\end{array}$ & $\begin{array}{l}25 \cdot 8 \\
21 \cdot 5 \\
28 \cdot 8 \\
25 \cdot 8 \\
26 \cdot 2\end{array}$ & $\begin{array}{l}27 \cdot 2 \\
30.0 \\
28.8 \\
26.0 \\
25 \cdot 2\end{array}$ & $\begin{array}{l}29 \cdot 0 \\
31.5 \\
28 \cdot 0 \\
24 \cdot 8 \\
25 \cdot 0\end{array}$ & $\begin{array}{l}29 \cdot 8 \\
32.0 \\
29 \cdot 0 \\
26.6 \\
25.6\end{array}$ & $\begin{array}{l}29 \cdot 8 \\
32.2 \\
28.8 \\
26.5 \\
25 \cdot 8\end{array}$ & $\begin{array}{l}29 \cdot 5 \\
31 \cdot 0 \\
30 \cdot 2 \\
25 \cdot 4 \\
25 \cdot 5\end{array}$ & $\begin{array}{l}29 \cdot 2 \\
30 \cdot 0 \\
28 \cdot 2 \\
25 \cdot 0 \\
25 \cdot 8\end{array}$ & $\begin{array}{l}310 \cdot 0 \\
28 \cdot 0 \\
27 \cdot 8 \\
24 \cdot-3 \\
26 \cdot 0\end{array}$ & $\begin{array}{l}28 \cdot 8 \\
24 \cdot 0 \\
2567 \\
24.0 \\
25 \cdot 0\end{array}$ \\
\hline $\begin{array}{l}11 \\
12 \\
13 \\
14 \\
15\end{array}$ & $\begin{array}{l}28 \cdot 0 \\
26.0 \\
28.0 \\
24.0 \\
25 \cdot 1\end{array}$ & $\begin{array}{l}28 \cdot 0 \\
27 \cdot 0 \\
28 \cdot 0 \\
25 \cdot 0 \\
26 \cdot 0\end{array}$ & $\begin{array}{l}29 \cdot 0 \\
29 \cdot 2 \\
27 \cdot 2 \\
25 \cdot 8 \\
26 \cdot 5\end{array}$ & $\begin{array}{l}30 \cdot 2 \\
29 \cdot 2 \\
29 \cdot 0 \\
27 \cdot 0 \\
27 \cdot 0 \\
27 \cdot 1\end{array}$ & $\begin{array}{l}30 \cdot 0 \\
30 \cdot 5 \\
26 \cdot 0 \\
26 \cdot 2 \\
27 \cdot 0\end{array}$ & $\begin{array}{l}31 \cdot 8 \\
31 \cdot \cdot 8 \\
26 \cdot 8 \\
276 \cdot 8 \\
27 \cdot 0 \\
27 \cdot 0\end{array}$ & $\begin{array}{l}32 \cdot 0 \\
30.2 \\
27.0 \\
28.0 \\
28 \cdot 0\end{array}$ & $\begin{array}{l}30 \cdot 0 \\
30.7 \\
27.8 \\
27.0 \\
27 \cdot 3\end{array}$ & $\begin{array}{l}29 \cdot 7 \\
29 \cdot 4 \\
30 \cdot 5 \\
27 \cdot 0 \\
26 \cdot 5\end{array}$ & $\begin{array}{l}29 \cdot 1 \\
28 \cdot 0 \\
29 \cdot 2 \\
26 \cdot 0 \\
26 \cdot 0\end{array}$ & $\begin{array}{l}30 \cdot 0 \\
28 \cdot 0 \\
27 \cdot 5 \\
25 \cdot 0 \\
25 \cdot 0\end{array}$ & $\begin{array}{l}28 \cdot 1 \\
28 \cdot 0 \\
26 \cdot 8 \\
24 \cdot 9 \\
24 \cdot 0\end{array}$ & $\begin{array}{l}29 \cdot 7 \\
28 \cdot 9 \\
27 \cdot 6 \\
26 \cdot 1 \\
26 \cdot 1 \\
26\end{array}$ & $\begin{array}{l}11 \\
12 \\
13 \\
14 \\
15\end{array}$ & $\begin{array}{l}27 \cdot 2 \\
25 \cdot 0 \\
27 \cdot 5 \\
23 \cdot 0 \\
24 \cdot 5\end{array}$ & $\begin{array}{l}27 \cdot 0 \\
27 \cdot 0 \\
27 \cdot 2 \\
24 \cdot 0 \\
24 \cdot 8\end{array}$ & $\begin{array}{l}28 \cdot 5 \\
27 \cdot 9 \\
26 \cdot 5 \\
25 \cdot 0 \\
25 \cdot 1\end{array}$ & $\begin{array}{l}28^{\circ} 0 \\
28^{\circ} 0 \\
25^{\circ} 0 \\
25^{\circ} 0 \\
25^{\circ} 5\end{array}$ & $\begin{array}{l}28 \cdot 2 \\
29 \cdot 0 \\
25 \cdot 1 \\
25 \cdot 1 \\
26.0 \\
26.0\end{array}$ & & $\begin{array}{l}31 \cdot 2 \\
29 \cdot 0 \\
26 \cdot 0 \\
266^{\circ} \cdot 6 \\
26 \cdot 2\end{array}$ & $\begin{array}{l}29 \cdot 5 \\
29 \cdot 6 \\
25 \cdot 6 \\
25 \cdot 4 \\
25.6 \\
25.5\end{array}$ & $\begin{array}{l}29 \cdot 0 \\
25^{\circ} \cdot 5 \\
28 \cdot 6 \\
25 \cdot 8 \\
25^{\circ} 0\end{array}$ & $\begin{array}{l}28 \cdot 2 \\
27 \cdot 5 \\
27 \cdot 8 \\
25 \cdot 0 \\
24 \cdot 2\end{array}$ & $\begin{array}{l}28 \cdot 0 \\
27 \cdot 0 \\
26 \cdot 2 \\
24 \cdot 0 \\
23 \cdot 2\end{array}$ & $\begin{array}{l}27 \cdot 2 \\
27 \cdot 0 \\
25 \cdot 8 \\
25 \cdot 2 \\
23 \cdot 0\end{array}$ \\
\hline $\begin{array}{l}16 \\
17 \\
18 \\
19 \\
20\end{array}$ & $\begin{array}{l}23 \cdot 0 \\
22 \cdot 0 \\
21 \cdot 0 \\
21 \cdot 5 \\
19 \cdot 0\end{array}$ & $\begin{array}{l}24 \cdot 0 \\
22 \cdot 0 \\
25 \cdot 0 \\
20 \cdot 0 \\
20 \cdot 0\end{array}$ & $\begin{array}{l}25 \cdot 0 \\
23 \cdot 0 \\
25 \cdot 1 \\
18 \cdot 0 \\
18 \cdot 0\end{array}$ & $\begin{array}{l}25 \cdot 0 \\
19 \cdot 2 \\
26 \cdot 0 \\
23 \cdot 0 \\
17 \cdot 0\end{array}$ & $\begin{array}{l}24 \cdot 0 \\
23 \cdot 2 \\
27 \cdot 0 \\
24 \cdot 8 \\
16 \cdot 5\end{array}$ & $\begin{array}{l}23 \cdot 5 \\
24 \cdot 5 \\
23 \cdot 0 \\
23 \cdot 0 \\
16 \cdot 8\end{array}$ & $\begin{array}{l}23 \cdot 0 \\
26 \cdot 8 \\
28 \cdot 0 \\
22 \cdot 0 \\
17 \cdot 3\end{array}$ & $\begin{array}{l}22 \cdot 8 \\
25 \cdot 4 \\
28 \cdot 0 \\
22 \cdot 2 \\
19 \cdot 7\end{array}$ & $\begin{array}{l}22 \cdot 4 \\
25 \cdot 7 \\
25 \cdot 8 \\
21^{-4} \\
19 \cdot 6\end{array}$ & $\begin{array}{l}22 \cdot 0 \\
24 \cdot 1 \\
27 \cdot 0 \\
22 \cdot 5 \\
20 \cdot 2\end{array}$ & $\begin{array}{l}20 \cdot 2 \\
24 \cdot 0 \\
25 \cdot 2 \\
22 \cdot 5 \\
17 \cdot 0\end{array}$ & $\begin{array}{l}23 \cdot 0 \\
24 \cdot 0 \\
22 \cdot 0 \\
20 \cdot 0 \\
16 \cdot 2\end{array}$ & $\begin{array}{l}23 \cdot 2 \\
23 \cdot 7 \\
23 \cdot 0 \\
21 \cdot 8 \\
18 \cdot 1 \\
18 \cdot 1\end{array}$ & $\begin{array}{l}16 \\
17 \\
18 \\
19 \\
20\end{array}$ & $\begin{array}{l}22 \cdot 0 \\
21 \cdot 0 \\
23 \cdot 0 \\
20 \cdot 0 \\
18 \cdot 5\end{array}$ & & $\begin{array}{l}24 \cdot 0 \\
22 \cdot 0 \\
24 \cdot 4 \\
17 \cdot 2 \\
17 \cdot 5\end{array}$ & $\begin{array}{l}24 \cdot 0 \\
18 \cdot 0 \\
21 \cdot 0 \\
21 \cdot 8 \\
16 \cdot 8\end{array}$ & $\begin{array}{l}23 \cdot 5 \\
22 \cdot 1 \\
25.0 \\
25 \cdot 1 \\
16 \cdot 0\end{array}$ & & $\begin{array}{l}22 \cdot 2 \\
25 \cdot 3 \\
25 \cdot 4 \\
25 \cdot \cdot 6 \\
16 \cdot 3\end{array}$ & $\begin{array}{l}21 \cdot 7 \\
24 \cdot 3 \\
26 \cdot 0 \\
210^{\circ} 0 \\
18 \cdot 0\end{array}$ & $\begin{array}{l}21 \cdot 0 \\
21 \cdot 0 \\
25 \cdot 0 \\
20 \cdot 2 \\
18 \cdot 0\end{array}$ & $\begin{array}{l}21 \cdot 0 \\
23 \cdot 2 \\
25 \cdot 5 \\
21 \cdot 2 \\
18 \cdot 5\end{array}$ & $\begin{array}{l}20 \cdot 0 \\
23 \cdot 0 \\
22 \cdot 0 \\
21 \cdot 0 \\
15 \cdot 2\end{array}$ & $\begin{array}{l}22 \cdot 0 \\
23 \cdot 0 \\
21 \cdot 0 \\
19 \cdot 5 \\
15 \cdot 0\end{array}$ \\
\hline $\begin{array}{l}21 \\
22 \\
23 \\
24 \\
25\end{array}$ & $\begin{array}{l}20 \cdot 5 \\
2080 \\
16.0 \\
28.2 \\
26 \cdot 0\end{array}$ & $\begin{array}{l}21 \cdot 2 \\
25 \cdot 0 \\
15 \cdot 5 \\
29 \cdot 0 \\
28 \cdot 0\end{array}$ & $\begin{array}{l}29 \cdot 0 \\
28 \cdot 0 \\
16 \cdot 0 \\
31) \cdot 0 \\
29 \cdot 2\end{array}$ & $\begin{array}{l}25 \cdot 2 \\
29 \cdot 0 \\
24 \cdot 0 \\
32 \cdot 5 \\
30 \cdot 2\end{array}$ & $\begin{array}{l}16 \cdot 5 \\
28 \cdot 2 \\
24.7 \\
30 \cdot 0 \\
31 \cdot 5\end{array}$ & $\begin{array}{l}22 \cdot 2 \\
27 \cdot 2 \\
27 \cdot 6 \\
30 \cdot 0 \\
32 \cdot 0 \\
2 \cdot 0\end{array}$ & $\begin{array}{l}25 \cdot 7 \\
27 \cdot 0 \\
29 \cdot 6 \\
30 \cdot 0 \\
32 \cdot 0 \\
32 \cdot 0\end{array}$ & $\begin{array}{l}26 \cdot 0 \\
226 \cdot 7 \\
31 \cdot 0 \\
30 \cdot 6 \\
32 \cdot 0 \\
32 \cdot 0\end{array}$ & $\begin{array}{l}26 \cdot 6 \\
26 \cdot 3 \\
28 \cdot 2 \\
29 \cdot 8 \\
31 \cdot 0\end{array}$ & $\begin{array}{l}28 \cdot 5 \\
24 \cdot 5 \\
27 \cdot 8 \\
27 \cdot 0 \\
31.0\end{array}$ & $\begin{array}{l}28 \cdot 0 \\
21 \cdot 0 \\
22 \cdot 5 \\
27.0 \\
27 \cdot 0\end{array}$ & $\begin{array}{l}27 \cdot 0 \\
18 \cdot 0 \\
28 \cdot 5 \\
26 \cdot 5 \\
27 \cdot 0\end{array}$ & $\begin{array}{l}25 \cdot 0 \\
250^{\circ} \\
24 \cdot 8 \\
29 \cdot 8 \\
29 \cdot 8\end{array}$ & $\begin{array}{l}21 \\
22\end{array}$ & $\begin{array}{l}26 \cdot 2 \\
15 \cdot 5 \\
27 \cdot 2 \\
24 \cdot 8\end{array}$ & $\begin{array}{l}24 \cdot 8 \\
15 \cdot 0 \\
27 \cdot 8 \\
26 \cdot 2\end{array}$ & $\begin{array}{l}26 \cdot 5 \\
27 \cdot 0 \\
15 \cdot 5 \\
28 \cdot 5 \\
27 \cdot 5\end{array}$ & $\begin{array}{l}25 \cdot 0 \\
27 \cdot 8 \\
22 \cdot 0 \\
30 \cdot 8 \\
28 \cdot 8\end{array}$ & $\begin{array}{l}16 \cdot 5 \\
27.8 \\
23:-2 \\
29.5 \\
29 \cdot 5\end{array}$ & & $\begin{array}{l}24 \cdot 2 \\
26 \cdot 1 \\
27 \cdot 4 \\
28 \cdot 7 \\
29 \cdot 3\end{array}$ & & $\begin{array}{l}25 \cdot 0 \\
24 \cdot 6 \\
27 \cdot 6 \\
27 \cdot 3 \\
28 \cdot 3 \\
28 \cdot 4\end{array}$ & $\begin{array}{l}26 \cdot 2 \\
24 \cdot 0 \\
26^{\circ} \cdot 2 \\
26^{\circ} 0 \\
28 \cdot 8\end{array}$ & $\left|\begin{array}{l}26 \cdot 2 \\
20 \cdot 0 \\
27 \cdot 0 \\
25 \cdot 0 \\
26 \cdot 5\end{array}\right|$ & $\begin{array}{l}25 \cdot 8 \\
1178 \\
27 \cdot 0 \\
25 \cdot 2 \\
26 \cdot 0\end{array}$ \\
\hline $\begin{array}{l}27 \\
27 \\
28 \\
29 \\
30\end{array}$ & $\begin{array}{l}26 \cdot 8 \\
10 \cdot 5 \\
10 \cdot 5 \\
210.2 \\
10 \cdot 0 \\
21 \cdot 0\end{array}$ & $\begin{array}{l}26 \cdot 5 \\
14.5 \\
18 \cdot 0 \\
24.0 \\
19 \cdot 0\end{array}$ & $\mid \begin{array}{l}27 \cdot 0 \\
17 \cdot 8 \\
20 \cdot 2 \\
23 \cdot 2 \\
27 \cdot 0\end{array}$ & $\begin{array}{l}27 \cdot 0 \\
22 \cdot 5 \\
22 \cdot 0 \\
25 \cdot 5 \\
29 \cdot 0\end{array}$ & $\begin{array}{l}23 \cdot 0 \\
27 \cdot 0 \\
24 \cdot 0 \\
27 \cdot 2 \\
23 \cdot 8\end{array}$ & $\begin{array}{l}28 \cdot 0 \\
28 \cdot 0 \\
24 \cdot 0 \\
25 \cdot 0 \\
22 \cdot 0 \\
22 \cdot 7\end{array}$ & $\begin{array}{l}26 \cdot 3 \\
33 \cdot 4 \\
24 \cdot 0 \\
25 \cdot 4 \\
22 \cdot 8\end{array}$ & $\begin{array}{l}27 \cdot 0 \\
30 \cdot 0 \\
25 \cdot 6 \\
25 \cdot 8 \\
23 \cdot 2\end{array}$ & $\begin{array}{l}24 \cdot 0 \\
32 \cdot 2 \\
26 \cdot 0 \\
28 \cdot 2 \\
23 \cdot 0\end{array}$ & $\begin{array}{l}27 \cdot 0 \\
25 \cdot 2 \\
21 \cdot 2 \\
30 \cdot 1 \\
21 \cdot 0\end{array}$ & $\begin{array}{l}25 \cdot 0 \\
22 \cdot 0 \\
16 \cdot 2 \\
27 \cdot 8 \\
21 \cdot 0\end{array}$ & $\begin{array}{l}17 \cdot 0 \\
17.0 \\
24.0 \\
27 \cdot 0 \\
19 \cdot 8\end{array}$ & $\begin{array}{l}25 \cdot 6 \\
23 \cdot 4 \\
22 \cdot 1 \\
24 \cdot 9 \\
22 \cdot 8\end{array}$ & $\begin{array}{l}23 \\
27 \\
28 \\
28 \\
30\end{array}$ & $\begin{array}{r}25.2 \\
16.2 \\
18.0 \\
8.1 \\
18.0\end{array}$ & $\begin{array}{l}25 \cdot 2 \\
13 \cdot 2 \\
16 \cdot 8 \\
22 \cdot 5 \\
17 \cdot 2\end{array}$ & $\begin{array}{l}16 \cdot 0 \\
19 \cdot 0 \\
22 \cdot 0 \\
22 \cdot 2 \cdot 2\end{array}$ & $\begin{array}{l}25 \cdot 0 \\
21 \cdot 2 \\
21 \cdot 8 \\
24 \cdot 0 \\
28 \cdot 5\end{array}$ & $\begin{array}{l}24.5 \\
222.8 \\
24.2 \\
21.5\end{array}$ & $\mid \begin{array}{l}21.0 \\
21.5 \\
21.2\end{array}$ & $\begin{array}{l}27 \cdot 5 \\
22 \cdot .2 \\
23.2 \\
21 \cdot 0\end{array}$ & 222.0 & $\begin{array}{l}22 \cdot 8 \\
22 \cdot 0 \\
22 \cdot 0 \\
22 \cdot 3 \\
21 \cdot 6 \\
21 \cdot 0\end{array}$ & $\begin{array}{l}23 \cdot 0 \\
24 \cdot 2 \\
19 \cdot 0 \\
25 \cdot 1 \\
20 \cdot 0\end{array}$ & $\begin{array}{l}21 \cdot 0 \\
15 \cdot 0 \\
24 \cdot 2 \\
20 \cdot 0\end{array}$ & $\begin{array}{l}16 \cdot 0 \\
21 \cdot 0 \\
23 \cdot 5 \\
19 \cdot 0\end{array}$ \\
\hline 31 & $18 \cdot 0$ & 17.5 & 170 & in & $22 \cdot 0$ & $23 \cdot 2$ & $27 \cdot 2$ & 28.9 & $27 \cdot 0$ & $23 \cdot 0$ & $21 \cdot 8$ & 2400 & $22 \cdot 5$ & 31 & $17 \cdot 5$ & $17 \cdot 0$ & •5 & 300 & 6 & .8 & 5 & $26 \cdot 3$ & $25 \cdot 0$ & $22 \cdot 1$ & $0 \cdot 0$ & $3 \cdot 0$ \\
\hline an & $23 \cdot 5$ & 23.8 & 1.8 & $5 \cdot 8$ & 29 & $7 \cdot 2$ & $27 \cdot 9$ & 28.2 & $27 \cdot 8$ & $26 \cdot 9$ & $\overline{25 \cdot 7}$ & $24 \cdot 6$ & $26^{\circ} 1$ & III & $22 \cdot 4$ & $22 \cdot 8$ & $23 \cdot 6$ & 24.6 & $25 \cdot 1$ & $25 \cdot 5$ & $26 \cdot 1$ & $26 \cdot 2$ & $25 \cdot 9$ & $25 \cdot 3$ & $4 \cdot 1$ & 3.5 \\
\hline
\end{tabular}

Readings in italic type are interpolated values obtalued from records of self-registering instruments. 
TABLE II. (continued).

TEMPERATULE IN DEGREES FAHRENHEIT.

Dry Bulz.

WET BULß.

February, 1903.

\begin{tabular}{|c|c|c|c|c|c|c|c|c|c|c|c|c|c|c|c|c|c|c|c|c|c|c|c|c|c|}
\hline Day. & 2. & 4. & 6. & 8. & 10. & Noon. & 11. & 16. & 18. & 20. & 22. & | Midt. Mean. & Day. & 2. & 4. & B. & 8. & 10. & Noon. & 14. & 16. & 18. & 20. & 22. & MS \\
\hline & 150 & 17.8 & 21 & $22 \cdot 0$ & & 0 & $28 \cdot 6$ & 280 & 20 & & $\therefore 1$ & & & & & $19 \cdot 8$ & $210^{\prime}$ & • & $\therefore$ & $\therefore .8$ & 8 & 4 & & 。 & \\
\hline $\begin{array}{l}2 \\
3 \\
4\end{array}$ & 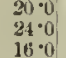 & $\begin{array}{l}21.0 \\
25.0 \\
14.0\end{array}$ & $\begin{array}{l}19 \cdot 2 \\
29: 2 \\
18 \cdot 0\end{array}$ & $\begin{array}{l}27.2 \\
26 \cdot 6 \\
20 \cdot 2 \cdot 2\end{array}$ & $\begin{array}{l}25 \cdot 2 \\
27 \cdot 0 \\
24 \cdot 8\end{array}$ & $\left|\begin{array}{l}27 \cdot 2 \\
27 \cdot 5 \\
27 \cdot 5\end{array}\right|$ & $\left|\begin{array}{l}33 \cdot 2 \\
27 \cdot 6 \\
27 \cdot 0\end{array}\right|$ & $\begin{array}{l}22 \cdot 7 \\
27 \cdot 6 \\
27 \cdot 8\end{array}$ & $\mid \begin{array}{l}27.0 \\
28.0 \\
28.5\end{array}$ & $\begin{array}{l}26 \cdot 2 \\
29 \cdot 2 \\
20^{\circ} \cdot 0\end{array}$ & $\begin{array}{l}23.8 \\
26.0 \\
26^{\circ} \cdot 0\end{array}$ & 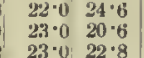 & 3 & $\begin{array}{l}17.0 \\
21.5 \\
15.0\end{array}$ & $\begin{array}{l}17 \cdot 2 \\
24 \cdot 0 \\
1 \cdot 2 \cdot 8\end{array}$ & $\begin{array}{l}17.0 \\
24.0 \\
16.8\end{array}$ & $\begin{array}{l}25.0 \\
25.0 \\
29.0\end{array}$ & $\begin{array}{l}24.01 \\
24.8 \\
22.8\end{array}$ & & $\begin{array}{l}26.0 \\
25.8 \\
21.8\end{array}$ & $\begin{array}{l}23.5 \\
25.2 \\
35.0\end{array}$ & $\begin{array}{l}22 \cdot 7 \\
260 \\
25 \cdot 0\end{array}$ & $\begin{array}{l}3.1 \\
4 \cdot 8 \\
5.8\end{array}$ & $\begin{array}{l}222 \\
230 \\
2010\end{array}$ & 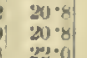 \\
\hline & & $19 \cdot 0$ & 10 & & & $200^{\circ} 0$ & $20^{\circ} 0$ & $213 \cdot 0$ & $20 \cdot 8$ & & $19 \cdot 5$ & 21.5 & & & & $16 \cdot 2$ & & & & & $\cdot 8$ & & & $8 \cdot 8$ & \\
\hline${ }_{7}^{6}$ & 17 & $\begin{array}{l}17.5 \\
12 \cdot 8 \\
2128\end{array}$ & $\begin{array}{l}20.5 \\
13 \cdot 0 \\
13: 0\end{array}$ & $\begin{array}{l}22 \cdot 0 \\
15.1\end{array}$ & & $\begin{array}{l}16.0 \\
14.5\end{array}$ & 15.2 & $\begin{array}{l}14 \cdot 7 \\
15.8\end{array}$ & $\begin{array}{l}14.0 \\
18.0 \\
18.0\end{array}$ & $\begin{array}{l}14 \cdot 2 \\
1 . \cdot 0\end{array}$ & $: 0$ & $\begin{array}{l}140 \\
12.0 \\
110\end{array}$ & 6 & & & $\begin{array}{l}18.8 \\
12.8 \\
12,8\end{array}$ & .5 & 14.5 & & 6 & $14 \cdot 3$ & $\begin{array}{l}12 \cdot 7 \\
158 \\
11\end{array}$ & 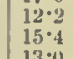 & 12 \% & \\
\hline 8 & $\begin{array}{r}8 \\
12\end{array}$ & $\begin{array}{l}1388 \\
11.0\end{array}$ & $\begin{array}{l}14 \cdot 0 \\
13 \cdot 0\end{array}$ & $\begin{array}{l}15 \cdot 0 \\
16 \cdot 2\end{array}$ & $\begin{array}{l}11 \cdot 4 \\
15 \cdot 5\end{array}$ & $\begin{array}{l}13 \cdot 0 \\
21 \cdot 5\end{array}$ & $\begin{array}{l}13 \cdot 2 \\
22 \cdot 0\end{array}$ & $\begin{array}{l}13 \cdot 4 \\
19 \cdot 6\end{array}$ & $\begin{array}{l}13 \cdot 7 \\
20 \cdot 5\end{array}$ & $\begin{array}{l}15 \cdot 0 \\
19 \cdot 1\end{array}$ & $\begin{array}{l}16 \cdot 2 \\
12 \cdot 0\end{array}$ & $\begin{array}{l}16 \cdot 0 \\
12 \cdot 2 \\
12\end{array}$ & 9 & $3 \cdot 0$ & $\begin{array}{l}13 \cdot 2 \\
10 \cdot 5\end{array}$ & $\begin{array}{l}12 \cdot 8 \\
11 \cdot 0\end{array}$ & $\begin{array}{l}14 \cdot 0 \\
15 \cdot 2\end{array}$ & $\begin{array}{l}13 \cdot 0 \\
14.0\end{array}$ & 100 & $\begin{array}{l}11 \cdot 0 \\
20 \cdot 0\end{array}$ & $\begin{array}{l}11 \cdot 6 \\
17 \cdot 4\end{array}$ & $\mid \begin{array}{l}11 \cdot 9 \\
19 \cdot 0\end{array}$ & 8.8 & $14 \cdot 1$ & \\
\hline $\begin{array}{l}110 \\
11\end{array}$ & $\begin{array}{l}13 \cdot 0 \\
12 \cdot 0.5\end{array}$ & $\begin{array}{l}12 \cdot 8 \\
11.2\end{array}$ & $\begin{array}{l}14 \cdot 0 \\
13: 2 \\
3:-2\end{array}$ & $\begin{array}{l}1.4 \cdot 0 \\
12 \cdot 8\end{array}$ & $\begin{array}{l}15 \cdot 0 \\
12 \cdot 0\end{array}$ & $\begin{array}{l}16 \cdot 0 \\
13 \cdot 0\end{array}$ & $\begin{array}{l}17 \cdot 9 \\
14 \cdot 5\end{array}$ & $\begin{array}{l}15 \cdot 2 \\
15 \cdot 0\end{array}$ & $\begin{array}{l}14 \cdot 3 \\
15 \cdot 3\end{array}$ & $\begin{array}{l}12 \cdot 6 \\
14 \cdot 8\end{array}$ & $\begin{array}{l}15 \cdot 0 \\
13 \cdot 6\end{array}$ & $\begin{array}{l}12 \cdot 0 \\
13.8\end{array}$ & 10 & $\begin{array}{l}12 \cdot 0 \\
11.2\end{array}$ & $\begin{array}{l}12 \cdot 0 \\
10 \cdot 8\end{array}$ & $\begin{array}{l}13 \cdot 0 \\
14 \cdot 0\end{array}$ & $\begin{array}{l}13 \cdot 0 \\
12 \cdot 0\end{array}$ & $14 \cdot \omega$ & 1) 14.8 & $\begin{array}{l}16 \cdot 6 \\
13 \cdot 0\end{array}$ & $\begin{array}{l}13 \cdot 7 \\
13 \cdot 4\end{array}$ & $\begin{array}{l}8.0 \\
3: 8\end{array}$ & 0 & 2 & 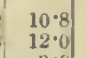 \\
\hline $\begin{array}{l}12 \\
1.3 \\
1.1\end{array}$ & $\begin{array}{l}4 \cdot 2 \\
7.0 \\
1.00\end{array}$ & $\begin{array}{l}8.5 \\
6.2 \\
0.2\end{array}$ & $\begin{array}{l}8 \cdot 1 \\
5.8\end{array}$ & $\begin{array}{l}9.8 \\
8.8\end{array}$ & & $\begin{array}{l}100 \\
8.0 \\
8.0\end{array}$ & $\begin{array}{r}10 \cdot 0 \\
7.8 \\
7.0\end{array}$ & $\begin{array}{r}12.8 \\
7.8 \\
0.82\end{array}$ & $\mid \begin{array}{c}11 \\
9 \cdot 4 \\
9 \cdot 0\end{array}$ & $\begin{array}{l}10.8 \\
100 \\
0.0\end{array}$ & $\begin{array}{l}10 \% 6 \\
9.0\end{array}$ & $\begin{array}{rl}9 \cdot 8 & 9 \cdot 6 \\
6.07 & 7.9\end{array}$ & $\begin{array}{l}12 \\
13\end{array}$ & $\begin{array}{l}4.0 \\
8.4\end{array}$ & $\begin{array}{l}7 \cdot 8 \\
6.0 \\
0.5\end{array}$ & $\begin{array}{l}7 \cdot 6 \\
5.2 \\
5.2\end{array}$ & $\begin{array}{l}8.8 \\
8.0\end{array}$ & $\begin{array}{l}7 \cdot 0 \\
8.0\end{array}$ & & $\begin{array}{l}9.0 \\
70 \\
0.0\end{array}$ & $\begin{array}{l}11.0 \\
6.8 \\
118\end{array}$ & $\begin{array}{l}10.0 \\
70.8\end{array}$ & $\begin{array}{l}9.8 \\
8.5 \\
2.0\end{array}$ & 4.4 & \\
\hline 15 & $10_{1}^{0}>->$ & $1 \cdot 0$ & $0 \cdot 2$ & $4 \cdot 2$ & $6 \cdot 0$ & 6.8 & $5 \cdot 6$ & $6 \cdot 2$ & $5 \cdot 0$ & $4 \cdot 0$ & $3 \cdot 0$ & \begin{tabular}{|c|c|}
3.0 & 3.8 \\
\end{tabular} & 15 & $0 \cdot 0$ & $1 \cdot 0$ & $0 \cdot 0$ & $2 \cdot 0$ & $4 \cdot 8$ & $5 \cdot 6$ & $4 \cdot 0$ & $5 \cdot 0$ & $3 \cdot 2$ & $2 \cdot 9$ & 1.5 & \\
\hline $\begin{array}{l}11 \\
12 \\
18 \\
1:\end{array}$ & $\begin{aligned} & 4 \cdot 0.0 \\
&-2 \cdot 0.5 \\
& 2 \\
& 2.6 .6\end{aligned}$ & $\begin{array}{l}3 \cdot 0 \\
1 \cdot 8 \\
1.5 \\
4 \cdot 2\end{array}$ & $\begin{array}{l}3 \cdot 0 \\
2 \cdot 2 \\
5: 0 \\
50.0\end{array}$ & $\begin{array}{l}7.2 \\
1 \cdot 8 \\
5 \cdot 0 \\
9 \cdot 0\end{array}$ & & $\begin{array}{r}7.0 \\
2.0 \\
5.2 \\
11.5\end{array}$ & $\begin{array}{l}6 \cdot 0 \\
2 \cdot 5 \\
7 \cdot 8 \\
3 \cdot 2\end{array}$ & 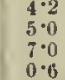 & $\begin{array}{l}5.0 \\
6.2 \\
8 \cdot 1 \\
1 \cdot 0\end{array}$ & $\begin{array}{l}7 \cdot 0 \\
5 \cdot 5 \\
5 \cdot 2 \\
2 \cdot 5\end{array}$ & $\begin{array}{l}5: 0 \\
5: 0 \\
4 \cdot 8 \\
4 \cdot 0\end{array}$ & 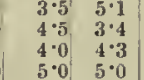 & $\begin{array}{l}17 \\
18 \\
19\end{array}$ & $\begin{aligned} 3.0 \\
2.8 \\
-2.8 \\
2.4 \\
2.4\end{aligned}$ & $: 5$ & $\begin{array}{l}2 \cdot 2 \\
2 \cdot 0 \\
4 \cdot 8 \\
4 \cdot 2\end{array}$ & $\begin{array}{l}6.8 \\
1.5 \\
4.0 \\
7.0\end{array}$ & $\begin{array}{l}5.0 \\
1.0 \\
3.0 \\
9.6\end{array}$ & $\begin{array}{r}1.5 \\
4.8 \\
10.2\end{array}$ & $\begin{array}{ll}4 & 4.6 \\
20.0 \\
6.6 \\
3.2\end{array}$ & $\begin{array}{l}3.0 \\
4 \cdot 0 \\
6 \cdot 0 \\
0.4\end{array}$ & $\begin{array}{l}3.6 \\
4.8 \\
6.8 \\
1.0\end{array}$ & $\begin{array}{l}5.0 \\
5.0 \\
2.5\end{array}$ & $\begin{array}{l}3: 8 \\
: 8 \\
3 \\
8\end{array}$ & \\
\hline & $\frac{2}{6}:$ & $\begin{array}{l}1.0 \\
800\end{array}$ & $\begin{array}{l}4 \cdot 0 \\
9 * 0\end{array}$ & $1 \cdot 0$ & $9 \cdot 0$ & \begin{tabular}{r|}
$9 \cdot 8$ \\
0.8
\end{tabular} & $\begin{array}{l}11 \cdot 3 \\
14\end{array}$ & $10 \cdot 2$ & 8.8 & $\left|\begin{array}{r}7.5 \\
10.0\end{array}\right|$ & $\begin{array}{r}8 \cdot 0 \\
12.0\end{array}$ & 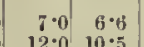 & 2) & $\begin{array}{l}2 \cdot 0 \\
5 \cdot 2\end{array}$ & $\frac{1}{7 \cdot 0}$ & $\begin{array}{l}4 \cdot 0 \\
8 \cdot 0\end{array}$ & $\begin{array}{l}1 \cdot 0 \\
4 \cdot 3\end{array}$ & $\begin{array}{l}8 \cdot 0 \\
7-3\end{array}$ & 8.8 & $\begin{array}{l}0.0 \\
.0 .5\end{array}$ & $8 \cdot 4$ & $\begin{array}{l}7 \cdot 0 \\
9 \cdot 0\end{array}$ & $0 \cdot 2$ & 6.8 & 6. \\
\hline 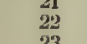 & $5:$ & $\begin{array}{c}8 \cdot 2 \\
4 \cdot 2\end{array}$ & . & 0.8 & & $\begin{array}{r}10.8 \\
0.0\end{array}$ & $\left|\begin{array}{r}140 \\
4 \cdot 0 \\
10.0\end{array}\right|$ & $\begin{array}{l}14.5 \\
35.5 \\
15.8\end{array}$ & $\begin{array}{l}105 \\
4.2 \\
10.4\end{array}$ & $\begin{array}{l}100 \\
4.8 \\
1.8\end{array}$ & & $12^{\circ} \cdot$ & 22 & $\begin{array}{l}0.0 \\
4.0 \\
4.8\end{array}$ & 2 & $\begin{array}{l}8.0 \\
8 \cdot 0\end{array}$ & $\begin{array}{l}9.8 \\
7.8 \\
3.01\end{array}$ & $\begin{array}{l}10.2 \\
10.0\end{array}$ & & 0 & 2.7 & 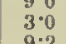 & & . & \\
\hline$\frac{2}{2}$ & & & 4 & & & 8. & & $\begin{array}{l}158 \\
150\end{array}$ & $\begin{array}{l}104 \\
1.0\end{array}$ & $\begin{array}{l}101 \\
3.5\end{array}$ & & & & & & & & & & & & 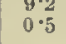 & & $\frac{8}{2}$ & \\
\hline & $\begin{array}{r}9 \cdot 4 \\
12 \cdot 0\end{array}$ & $\begin{array}{r}8.4 \\
11 \cdot 5\end{array}$ & $11 \cdot 9 \cdot$ & $\begin{array}{l}13 \cdot 0 \\
13: 5\end{array}$ & $\begin{array}{l}9 \cdot 0 \\
7 \cdot 2 \\
9 \cdot 2\end{array}$ & $17 \cdot 2$ & $\begin{array}{l}10 \cdot 2 \\
13 \cdot 4\end{array}$ & $\begin{array}{l}10 \cdot 0 \\
13 \cdot 6\end{array}$ & $\begin{array}{l}9 \cdot 8 \\
9 \cdot 3\end{array}$ & $\mid \begin{array}{l}14 \cdot 0 \\
7 \cdot 2\end{array}$ & $\begin{array}{l}14 \cdot 0 \\
8.0\end{array}$ & $\begin{array}{l}14 \\
8\end{array}$ & 25 & $\begin{array}{r}9 \cdot 0 \\
12 \cdot 0\end{array}$ & $\begin{array}{c}7 \cdot 1 \\
11 \cdot 0\end{array}$ & $u_{t}$ & $\begin{array}{l}11 \cdot 8 \\
13 \cdot 0\end{array}$ & & $\begin{array}{l}3.0 \\
6 \cdot 2\end{array}$ & $\begin{array}{r}9 \cdot 8 \\
13 \cdot 0\end{array}$ & $\begin{array}{c}9 \cdot 2 \\
13 \cdot 0\end{array}$ & $\begin{array}{l}9 \cdot 0 \\
9 \cdot 0\end{array}$ & $\begin{array}{l}13 \cdot 0 \\
7 \div 0\end{array}$ & $\begin{array}{ll}13 \cdot 0 \\
7 \cdot 0\end{array}$ & \\
\hline & $60^{\circ} 0$ & $7^{\cdot 0}$ & $8 \cdot 0$ & 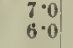 & $\begin{array}{l}7 \cdot 8 \\
3 \cdot 2 \\
\end{array}$ & & & 1.8 & $2 \cdot 3$ & $\begin{array}{l}4 \cdot 0 \\
0\end{array}$ & 199 & & & $5 \cdot 0$ & 6.0 & & 4.5 & & & $\begin{array}{l}2 \cdot 8 \\
1 \\
2\end{array}$ & 0.6 & & $.3 \cdot 5$ & 0 & \\
\hline$n$ & $9 \cdot 0$ & $9 \cdot 0$ & $10 \cdot 4$ & $111^{\cdot 4}$ & $12 \cdot 0$ & $12 \cdot 8$ & $12 \cdot 8$ & $12 \cdot 8$ & $11 \cdot 9$ & $11 \cdot 7$ & $10 \cdot 9$ & $10 \cdot 311 \cdot 2$ & ean & 8.1 & $8 \cdot 1$ & $9 \cdot 5$ & $10 \cdot 2$ & 10.9 & $11 \cdot 5$ & $11 \cdot 4$ & $11 \cdot 3$ & $10^{-4}$ & $10 \cdot 4$ & $9 \cdot 7$ & 9. $>>>$ \\
\hline
\end{tabular}

Dry Bulis

I'ET BULß.

March, 1903.

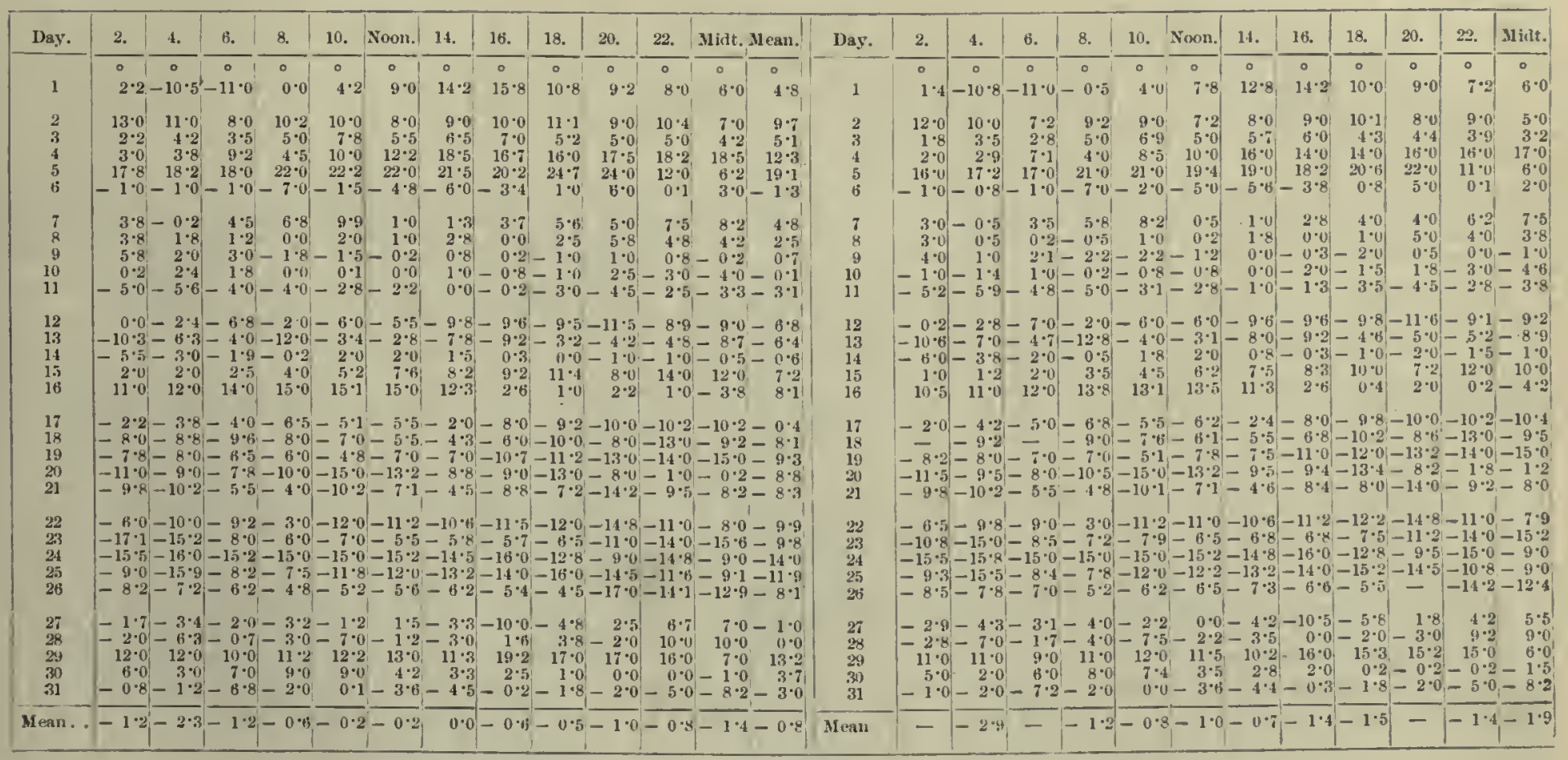

lieadiugs in italio type are interpolated values obtained from records of self-registering instruments.

3 c 2 
Table II. (continued).

DRy BuLb.

April, 1903.
TEMPERATURE IN DEGREES FAHRENHEIT.

WET BUL.B.

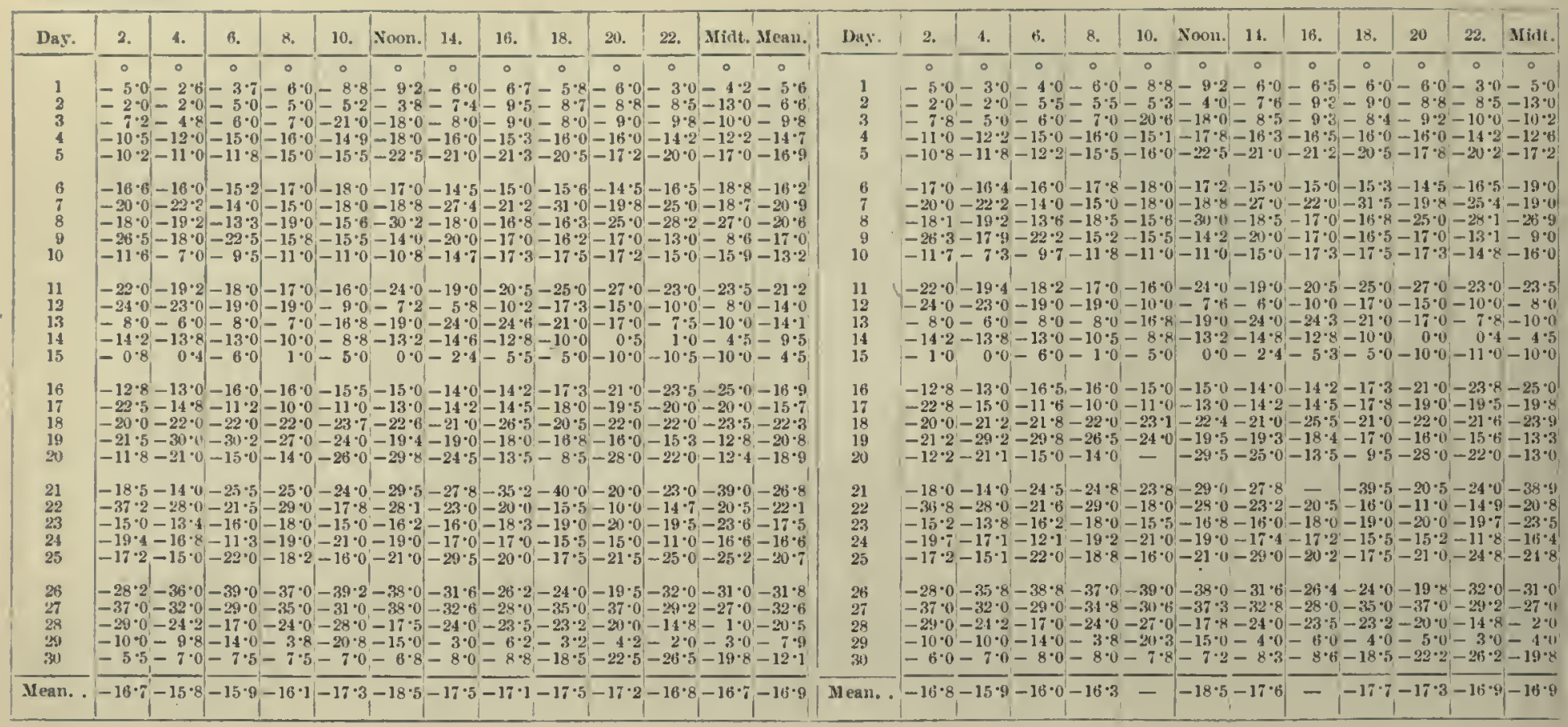

\section{May, 1903.}

DRY BULI.

WeT BUL.B.

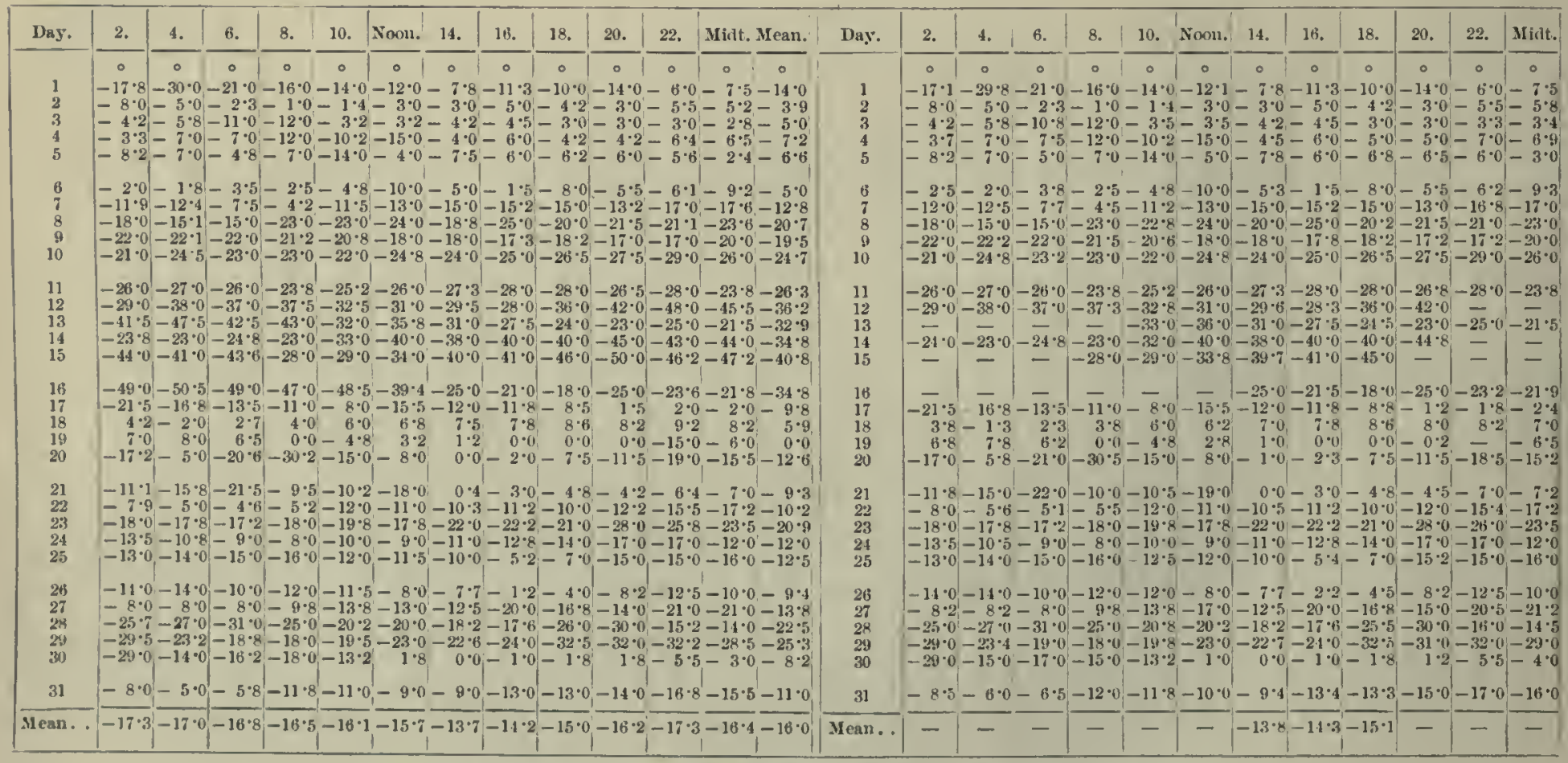


TABLs: II. (continued).

\section{TEMPLRATURE IN DEGHLES FAHLENHEIT.}

\section{DRY BULB.}

WET BUL13.

June, 1903.

\begin{tabular}{|c|c|c|c|c|c|c|c|c|c|c|c|c|c|c|c|c|c|c|c|c|c|c|c|c|c|c|}
\hline Day. & 2. & 4. & 6. & 8. & 10. & Xron. & 14. & 16. & 18. & 20. & 22. & Mislt. & t. Меви. & Day. & 2. & 4. & 6. & 8. & 10. & Noon. & 14. & 16. & 18. & 20. & 22. & Miat. \\
\hline & $\therefore$ & $\circ$ & . & $\circ$ & $\circ$ & 0 & $\therefore 1$ & 1. 0 & $\circ$ & $\circ$ & $\circ$ & 0 & 0 & & $\therefore$ & 10 & $\therefore$ & $\therefore$ & $\therefore$ & $\circ$ & $\therefore$ & $\therefore$ & $\therefore$ & $\therefore$ & $\circ$ & $\circ$ \\
\hline $\begin{array}{l}11 \\
3 \\
3 \\
4\end{array}$ & $\begin{array}{l}-14^{\circ} 6 \\
-20^{\circ} 5 \\
-20^{\circ}-2 \\
-23^{\circ} 0\end{array}$ & $\begin{array}{l}-19 \cdot 0 \\
-22 \cdot 5 \\
-21 \cdot 6 \\
-22 \cdot 1\end{array}$ & $\begin{array}{r}-18.9 \\
-23.0 \\
-19 \cdot 1 \\
-19 \cdot 0\end{array}$ & $\begin{array}{l}-2.1 \cdot 8 \\
-21.0 \\
-29 \cdot 2 \\
-17.0\end{array}$ & $\begin{array}{l}-23 \cdot 0 \\
-23 \cdot 0 \\
-33 \cdot 0 \\
-23 \cdot 8\end{array}$ & $\begin{array}{l}0-21 \cdot 0 \\
9-22 \cdot 8 \\
0-24 \cdot 0 \\
8-25 \cdot 0\end{array}$ & $\begin{array}{l}-18 \cdot 2 \\
-22 \cdot 0 \\
-17 \cdot 0 \\
-16 \cdot 0\end{array}$ & $\begin{array}{l}-19 \cdot 0 \\
-21 \cdot 6 \\
-21 \cdot 2 \\
-15 \cdot 7\end{array}$ & $\begin{array}{l}-16 \cdot 2 \\
-22 \cdot 5 \\
-20 \cdot 0 \\
-17 \cdot 0\end{array}$ & $\begin{array}{l}-19.0 \\
-21.0 \\
-19.1 \\
-19.0\end{array}$ & $\begin{array}{l}-22.0 \\
-23.2 \\
-23.1 \\
-21.3\end{array}$ & $\begin{array}{l}-18 \cdot 5 \\
2-23 \cdot 6 \\
-29 \cdot 0 \\
-25 \cdot 3\end{array}$ & $\begin{array}{l}5-19 \cdot 5 \\
6-22 \cdot 2 \\
0-23 \cdot 3 \\
3-20 \cdot 4\end{array}$ & $\begin{array}{l}1 \\
2 \\
3 \\
4\end{array}$ & $\begin{array}{l}-15 \cdot 0 \\
-20 \cdot 8 \\
-20.4 \\
-23 \cdot 0\end{array}$ & \begin{tabular}{|l|}
$-19 \cdot 3$ \\
$-22 \cdot 5$ \\
$-21 \cdot 9$ \\
$-22 \cdot 1$
\end{tabular} & $\begin{array}{l}-19 \cdot 1 \\
-23 \cdot 0 \\
-19 \cdot 2 \\
-19 \cdot 1\end{array}$ & $\begin{array}{l}-24 \cdot 8 \\
-21 \cdot 0 \\
-29 \cdot 2 \\
-17 \cdot 0\end{array}$ & $\begin{array}{l}-230 \\
-230 \\
-360 \\
-30.0\end{array}$ & $\begin{array}{l}0-21 \cdot 0 \\
0-22 \cdot 8 \\
0-23 \cdot 5 \\
2-25 \cdot 0\end{array}$ & $\begin{array}{l}-18.6 \\
-22.0 \\
-17.0 \\
-16.0\end{array}$ & $\begin{array}{l}-19.0 \\
-21.6 \\
-21.2 \\
-15.7\end{array}$ & $\begin{array}{l}-16.6 \\
-22.5 \\
-20.0 \\
-17.0\end{array}$ & $\begin{array}{l}-19.0 \\
-21.0 \\
-19.1 \\
-19.0\end{array}$ & $\begin{array}{l}-22.0 \\
-23.2 \\
-23.0 \\
-21.3\end{array}$ & $\begin{array}{l}-19 \cdot 8 \\
=28 \cdot 9 \\
=28 \cdot \\
3-25 \cdot 3\end{array}$ \\
\hline $\begin{array}{l}5 \\
6 \\
7 \\
8 \\
9\end{array}$ & $\begin{array}{l}-23 \cdot 6 \\
-23 \cdot 0 \\
-37 \cdot 0 \\
-6 \cdot 0 \\
-9.8\end{array}$ & $\begin{array}{l}-23 \cdot 0 \\
-19 \cdot 5 \\
-28 \cdot 0 \\
-10 \cdot 0 \\
-0.0\end{array}$ & $\begin{array}{r}-24 \cdot 6 \\
-29 \cdot 0 \\
-30 \cdot 0 \\
-10 \cdot 0 \\
-\quad 8 \cdot 2\end{array}$ & $\begin{array}{r}-20.8 \\
-28.0 \\
-27.0 \\
-9.0 \\
-60\end{array}$ & $\begin{array}{r}-19.0 \\
-21.0 \\
-14.0 \\
-9.0 \\
-\quad 4.0\end{array}$ & $\begin{array}{r}-19 \cdot 2 \\
-21 \cdot 2 \\
-9 \cdot 5 \\
0-3 \cdot 2 \\
0 \cdot 0\end{array}$ & $\begin{array}{r}-19 \cdot 6 \\
-23 \cdot 0 \\
-7 \cdot 3 \\
-5 \cdot 2 \\
4 \cdot 0\end{array}$ & $\begin{array}{r}-20.0 \\
-21.0 \\
-11.5 \\
-2.0 \\
7.0\end{array}$ & $\begin{array}{r}-18 \cdot 5 \\
-28 \cdot 2 \\
-15 \cdot 0 \\
-4 \cdot 8 \\
8 \cdot 8\end{array}$ & $\begin{array}{r}-18.0 \\
-26.0 \\
-160 \\
-\quad 20 \\
8.0\end{array}$ & $\begin{array}{r}-17.2 \\
-28.0 \\
-11.0 \\
-2.0 \\
8.0\end{array}$ & $\begin{array}{r}-18 \cdot 0 \\
-32.0 \\
0-900 \\
0-700 \\
-11 \cdot 2\end{array}$ & 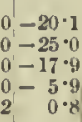 & $\begin{array}{l}5 \\
6 \\
7 \\
8 \\
9\end{array}$ & $\begin{array}{l}-23 \cdot 8 \\
-23.0 \\
-370 \\
-60 \\
-9.8\end{array}$ & $\begin{array}{l}-10.0 \\
-9.0\end{array}$ & $-9 \cdot 2$ & & $-4 \cdot 0$ & $\begin{array}{r}-10.0 \\
-\quad 100 \\
0.0\end{array}$ & $\begin{array}{r}5.5 \\
3.6\end{array}$ & $\begin{array}{r}-12 \cdot 0 \\
-2 \cdot 3 \\
7 \cdot 0\end{array}$ & $\begin{array}{r}-15.0 \\
-1.8 \\
8.8\end{array}$ & $\begin{array}{r}-260 \\
-16.0 \\
-20 \\
8.0\end{array}$ & $\begin{array}{r}-17.3 \\
-28.0 \\
-11.0 \\
-2.0 \\
8.0\end{array}$ & $\begin{array}{r}3-18.0 \\
0=32.0 \\
0=90 \\
0=7.0 \\
0 \quad 11.2\end{array}$ \\
\hline & $\begin{array}{l}120^{\circ} 0 \\
10-8\end{array}$ & $12 \cdot 0$ & 10.5 & $\begin{array}{r}9.0 \\
8.0\end{array}$ & $\begin{array}{l}4 \cdot 6 \\
5 \cdot 8\end{array}$ & $\begin{array}{l}1 \cdot 0 \\
8 \cdot 0\end{array}$ & $\begin{array}{l}0.0 \\
0.0\end{array}$ & & & $\begin{array}{r}2 \cdot 0 \\
150\end{array}$ & $4 \cdot 0$ & $8 \cdot 0$ & $5 \cdot 7$ & 10 & 10.5 & & & $9 \cdot 0$ & $4 \cdot 4$ & $\begin{array}{l}1.0 \\
8.0\end{array}$ & 0.0 & $\begin{array}{l}2 \cdot 0 \\
9 \cdot 8\end{array}$ & $2 \cdot 8$ & $\begin{array}{r}2 \cdot 0 \\
140\end{array}$ & 4. & \\
\hline $\begin{array}{l}11 \\
12 \\
13 \\
14\end{array}$ & $\begin{array}{r}10.8 \\
4.8 \\
-230 \\
-3.6\end{array}$ & $\begin{array}{r}15.0 \\
6.2 \\
-17.0 \\
-5.2\end{array}$ & $\begin{array}{r}17.0 \\
0.5 \\
40.5 \\
-10.5\end{array}$ & $\begin{array}{r}90 \\
-20 \\
-4.0 \\
-5.0\end{array}$ & $\begin{array}{r}5.8 \\
-\quad 3.8 \\
-\quad 2.8\end{array}$ & $\begin{array}{r}80 \\
80 \\
-30 \\
-\quad 9.0\end{array}$ & $\begin{array}{r}-9 \cdot 0 \\
=1.6 \\
=1.0 \\
=0.0\end{array}$ & & $\begin{array}{r}11.6 \\
-1.0 \\
-1.0 \\
-4.5\end{array}$ & $\begin{array}{r}15.0 \\
-4 \cdot 0 \\
-2.8 \\
-4.0\end{array}$ & $\begin{array}{r}13.0 \\
-1.8 \\
-2.2 \\
-5.0\end{array}$ & $\begin{array}{r}9 \cdot 1 \\
0-150 \\
=40 \\
-6.60\end{array}$ & $\begin{array}{l}111 \cdot 1 \\
0=1 \cdot 3 \\
0=4 \cdot 5 \\
0=6 \cdot 2\end{array}$ & $\begin{array}{l}11 \\
12 \\
13 \\
14\end{array}$ & $\begin{array}{r}10.5 \\
4.2 \\
-23.0 \\
-4.2\end{array}$ & $\begin{array}{r}14 \cdot 0 \\
5 \cdot 8 \\
-18 \cdot 0 \\
-5.8\end{array}$ & $\begin{array}{r}16 \cdot 0 \\
-0.2 \\
3.5 \\
3.5\end{array}$ & $\begin{array}{r}8 \cdot 8 \\
-2 \cdot 0 \\
-4 \cdot 0\end{array}$ & $\begin{array}{r}5.2 \\
-4.0 \\
1.8\end{array}$ & $\begin{array}{r}8.0 \\
-1.0 \\
-2.5 \\
-10.0\end{array}$ & $\begin{array}{r}9.0 \\
-1.6 \\
-1.6 \\
-6.2\end{array}$ & & $\begin{array}{l}-1.8 \\
-5.0\end{array}$ & $\begin{array}{r}14.0 \\
-5.0 \\
-3.0 \\
-4.8\end{array}$ & $\begin{array}{r}12.9 \\
-2.4 \\
=2.8 \\
-5.3\end{array}$ & $\begin{array}{r}9 \cdot 0 \\
8-15 \cdot 2 \\
3=4 \cdot 2 \\
-6 \cdot 3\end{array}$ \\
\hline $\begin{array}{l}15 \\
16 \\
17 \\
18 \\
19\end{array}$ & $\begin{array}{r}-9 \cdot 0 \\
-11 \cdot 0 \\
-14 \cdot 7 \\
-18 \cdot 8 \\
-41 \cdot 0\end{array}$ & $\begin{array}{r}-9 \cdot 8 \\
-11.0 \\
-15 \cdot 2 \\
-13.9 \\
-37.7\end{array}$ & $\begin{array}{r}-9 \cdot 0 \\
-10 \cdot 8 \\
-17 \cdot 3 \\
-15 \cdot 2 \\
-28 \cdot 0\end{array}$ & $\begin{array}{l}-17 \cdot 0 \\
-19 \cdot 0 \\
-41 \cdot 2\end{array}$ & $\begin{array}{l}-17 \cdot 2 \\
-2 v^{\circ} 0 \\
-32 \cdot 2\end{array}$ & $\begin{array}{l}2-16 \cdot 0 \\
0-18 \cdot 1 \\
2-16 \cdot 0\end{array}$ & $\begin{array}{l}-14 \cdot 4 \\
-18 \cdot 0 \\
-33 \cdot 0\end{array}$ & $\begin{array}{l}-10^{\circ} 0 \\
-21-4 \\
-11-2\end{array}$ & $\begin{array}{r}-19.0 \\
-\quad 9 \cdot 0 \\
-91.5 \\
-21.0 \\
-23.8\end{array}$ & $\begin{array}{l}-23 \cdot 2 \\
-10 \cdot 2 \\
-17.6 \\
-24 \cdot 2 \\
-12.0\end{array}$ & $\begin{array}{l}-10.0 \\
-11.2 \\
-19.7 \\
-34.8 \\
-15.9\end{array}$ & $\begin{array}{r}1-22 \cdot 9 \\
-36 \cdot 2 \\
-11 \cdot 2\end{array}$ & $\begin{array}{l}2-10 \cdot 6 \\
0-9 \cdot 3 \\
9-16 \cdot 0 \\
2-21 \cdot 7 \\
2-25 \cdot 3\end{array}$ & $\begin{array}{l}15 \\
16 \\
17 \\
18 \\
19\end{array}$ & $\begin{array}{l}-15.0 \\
-18.0 \\
-40 .\end{array}$ & $\begin{array}{l}-1 \\
-1 \\
-3\end{array}$ & & & $-32 \cdot 5$ & $5-17 \cdot 0$ & $-33^{\circ} 0$ & $-17 \cdot 0$ & $\begin{array}{l}-9.5 \\
-21.0 \\
-23.8\end{array}$ & $\begin{array}{l}-24.0 \\
-10.8 \\
-17.6 \\
-25.0 \\
-13.0\end{array}$ & $\begin{array}{l}-10.2 \\
-11 \cdot \% \\
-19.5 \\
-34.7 \\
-16.0\end{array}$ & $\begin{array}{l}2-10 \cdot 6 \\
-11 \cdot 5 \\
-23 \cdot 0 \\
-3 s \cdot 2 \\
-12 \cdot 0\end{array}$ \\
\hline $\begin{array}{l}20 \\
21 \\
22 \\
23 \\
2.4\end{array}$ & $\begin{array}{l}-12 \cdot 0 \\
-34 \cdot 8 \\
-19 \cdot 0 \\
-43 \cdot 0 \\
-18 \cdot 2\end{array}$ & $\begin{array}{l}-19 \cdot 2 \\
-30 \cdot 0 \\
-17 \cdot 0 \\
-31 \cdot 0 \\
-28 \cdot 2\end{array}$ & $\begin{array}{l}-25 \cdot 0 \\
-28 \cdot 0 \\
-15 \cdot 0 \\
-42.8 \\
-17 \cdot 2\end{array}$ & $\begin{array}{l}-220 \\
-42.0 \\
-23.2\end{array}$ & $\begin{array}{l}-27 \cdot 5 \\
-32 \cdot 5 \\
-27 \cdot 0\end{array}$ & $\begin{array}{l}5-40 \cdot 5 \\
-40.0 \\
0-24 \cdot 0\end{array}$ & $\begin{array}{l}-33 \cdot 0 \\
-23 \cdot 0 \\
-36.0 \\
-31.0 \\
-14.8\end{array}$ & $\begin{array}{l}-40 \cdot 0 \\
-23 \cdot 6 \\
-31 \cdot 5 \\
-25 \cdot 2 \\
-2 \cdot 0\end{array}$ & $\begin{array}{l}-43.0 \\
-16.0 \\
-1.0\end{array}$ & $\begin{array}{r}-38.0 \\
-12.0 \\
-35.0 \\
-25.0 \\
1.0\end{array}$ & $\begin{array}{l}-36.0 \\
-11.0, \\
-46.8 \\
-22.2 \\
-0.5\end{array}$ & $\begin{array}{l}0-37 \cdot 8 \\
0-15 \cdot 0 \\
-38 \cdot 0 \\
2-31 \cdot 0 \\
5-2 \cdot 0\end{array}$ & $\begin{array}{l}8-26 \cdot 9 \\
0-22 \cdot 2 \\
0-31 \cdot 2 \\
0-32 \cdot 1 \\
0-13 \cdot 1\end{array}$ & $\begin{array}{l}20 \\
21 \\
22 \\
23 \\
21\end{array}$ & $\begin{array}{l}-12.5 \\
-34.8 \\
-19.0 \\
-43.0 \\
-17.8\end{array}$ & $\begin{array}{l}-20 \cdot 0 \\
-30 \cdot 0 \\
-17 \cdot 0 \\
-31 \cdot 0 \\
-28 \cdot 2\end{array}$ & & $\begin{array}{l}-21 \cdot 8 \\
-42 \cdot 0 \\
-24.0\end{array}$ & $\begin{array}{l}-27 \cdot 5 \\
-31 \cdot 0 \\
-27 \cdot 0\end{array}$ & $\begin{array}{l}-40.5 \\
-40.0 \\
-24.2\end{array}$ & $\begin{array}{l}-32 \cdot 0 \\
-15 \cdot 0\end{array}$ & $\begin{array}{l}-39.0 \\
-23.0 \\
-34.5 \\
-26.0 \\
-2.5\end{array}$ & $\begin{array}{l}-36.0 \\
-14.0 \\
-43.0 \\
-16.6 \\
-1.0\end{array}$ & $\begin{array}{r}-37.5 \\
-13.0 \\
-35.0 \\
-26.0 \\
-1.0\end{array}$ & $\begin{array}{r}-35 \cdot 5 \\
-11.0 \\
-23 \cdot 0 \\
-1 \cdot 0\end{array}$ & $\begin{array}{r}5-37 \cdot 8 \\
0-15 \cdot 0 \\
-33 \cdot 0 \\
0-33 \cdot 5 \\
0-2 \cdot 2\end{array}$ \\
\hline $\begin{array}{l}25 \\
25 \\
27 \\
28 \\
29\end{array}$ & $\begin{array}{l}-11 \cdot 0 \\
-11.8 \\
-19 \cdot 2 \\
-6.0 \\
-1.0\end{array}$ & $\begin{array}{r}-12 \cdot 0 \\
-11 \cdot 9 \\
-28 \cdot 0 \\
-3.5 \\
2 \cdot 1\end{array}$ & $\begin{array}{r}-13 \cdot 0 \\
-12.5 \\
-18.0 \\
-2.4 \\
0.4\end{array}$ & $\begin{array}{r}-10.0 \\
-16.0 \\
-27.5 \\
-2.0 \\
1.0\end{array}$ & $\begin{array}{r}-10 \cdot 2 \\
-12 \cdot 0 \\
-19 \cdot 9 \\
0 \cdot 0 \\
2 \cdot 2\end{array}$ & $\begin{array}{r}-10.0 \\
0 \\
2 \\
2.8 \\
2\end{array}$ & $\begin{array}{r}r 0.3 \\
3 . .2 \\
-13.5\end{array}$ & $\begin{array}{r}-10.5 \\
-20.0 \\
-32 \cdot 0 \\
-20 \\
-6.8\end{array}$ & $\begin{array}{r}7 \cdot 0 \\
-16 \cdot 2 \\
-14 \cdot 2 \\
-2.0 \\
-3.5\end{array}$ & $\begin{array}{r}-6.0 \\
-23.0 \\
-18.0 \\
5.5 \\
-4.0\end{array}$ & $\begin{array}{l}-12.0 \\
-18.8 \\
-17.0 \\
-5.0 \\
-1.8\end{array}$ & $\begin{array}{r}0-14 \cdot 0 \\
8-19 \cdot 0 \\
0-5.8 \\
0-6.5 \\
2.8\end{array}$ & $\begin{array}{l}0-9 \cdot 9 \\
0-15 \cdot 2 \\
8-20 \cdot 8 \\
5=1 \cdot 2 \\
8-1 \cdot 4\end{array}$ & $\begin{array}{l}25 \\
26 \\
27 \\
28 \\
29\end{array}$ & $\begin{array}{l}-11 \cdot 0 \\
-12 \cdot 2 \\
-19 \cdot 2 \\
-6.0 \\
-1 \cdot 0\end{array}$ & $\begin{array}{r}-12 \cdot 0 \\
-12 \cdot 2 \\
-23 \cdot 0 \\
-3 \cdot 8 \\
2 \cdot 1\end{array}$ & $\begin{array}{r}-18 \cdot 2 \\
-3 \cdot 0 \\
0.3\end{array}$ & $\begin{array}{l}-27 \\
-2\end{array}$ & $\begin{array}{r}-200 \\
0 \cdot 0 \\
1 \cdot 8\end{array}$ & \begin{tabular}{|r|}
$5 \cdot 0$ \\
$-10 \cdot 8$ \\
$-24 \cdot 3$ \\
$1 \cdot 0$ \\
$5 \cdot 0$
\end{tabular} & $\begin{array}{r}11 \\
-30 \\
-13\end{array}$ & $\begin{array}{l}-10^{\circ} \\
-20^{\circ} \\
-32^{\circ} \\
-2^{\circ}\end{array}$ & $\begin{array}{r}15 \\
-1 \\
-3\end{array}$ & $\begin{array}{r}-23.0 \\
-18.0 \\
5.5 \\
-4.0\end{array}$ & $\begin{array}{l}-12 \cdot 2 \\
-18.8 \\
-17.0 \\
-5.0 \\
-2.0\end{array}$ & $\begin{array}{r}2-14 \cdot 5 \\
0=19 \cdot 0 \\
0-6.2 \\
0-6.5 \\
2.0\end{array}$ \\
\hline 30 & -5 & 2 & $-10^{\circ} 0$ & -2 & $5 \cdot 2$ & $2-2 \cdot 0$ & -5.3 & -6.5 & -7 & $-17 \cdot 0$ & -16.0 & $-13 \cdot 2$ & $2-7 \cdot 8$ & 30 & -50 & $-2 \cdot 4$ & $-10 \cdot 0$ & -4 & $\pi$ & 2 & -0 & 0 & -1 & $-17 \cdot 0$ & $-15 \cdot 6$ & $6-13 \cdot 5$ \\
\hline an & $-15 \cdot 1$ & $-14 \cdot 2$ & -14 & $-15 \cdot 0$ & -14 & $\mid-12 \cdot 9$ & -13 & $\left.\right|^{-13 \cdot 1}$ & $-12 \cdot 1$ & $-12 \cdot 8$ & $-13 \cdot 7$ & -14 & $4-13.8$ & an. & $-15 \cdot 2$ & -14. & - & -15.0 & & $6-13 \cdot 1$ & $-13 \cdot 8$ & $-13 \cdot 2$ & $-12 \cdot 2$ & $-13 \cdot 2$ & - & 7. \\
\hline
\end{tabular}

DRY BUI.B.

Wet Bulb.

July, 1903.

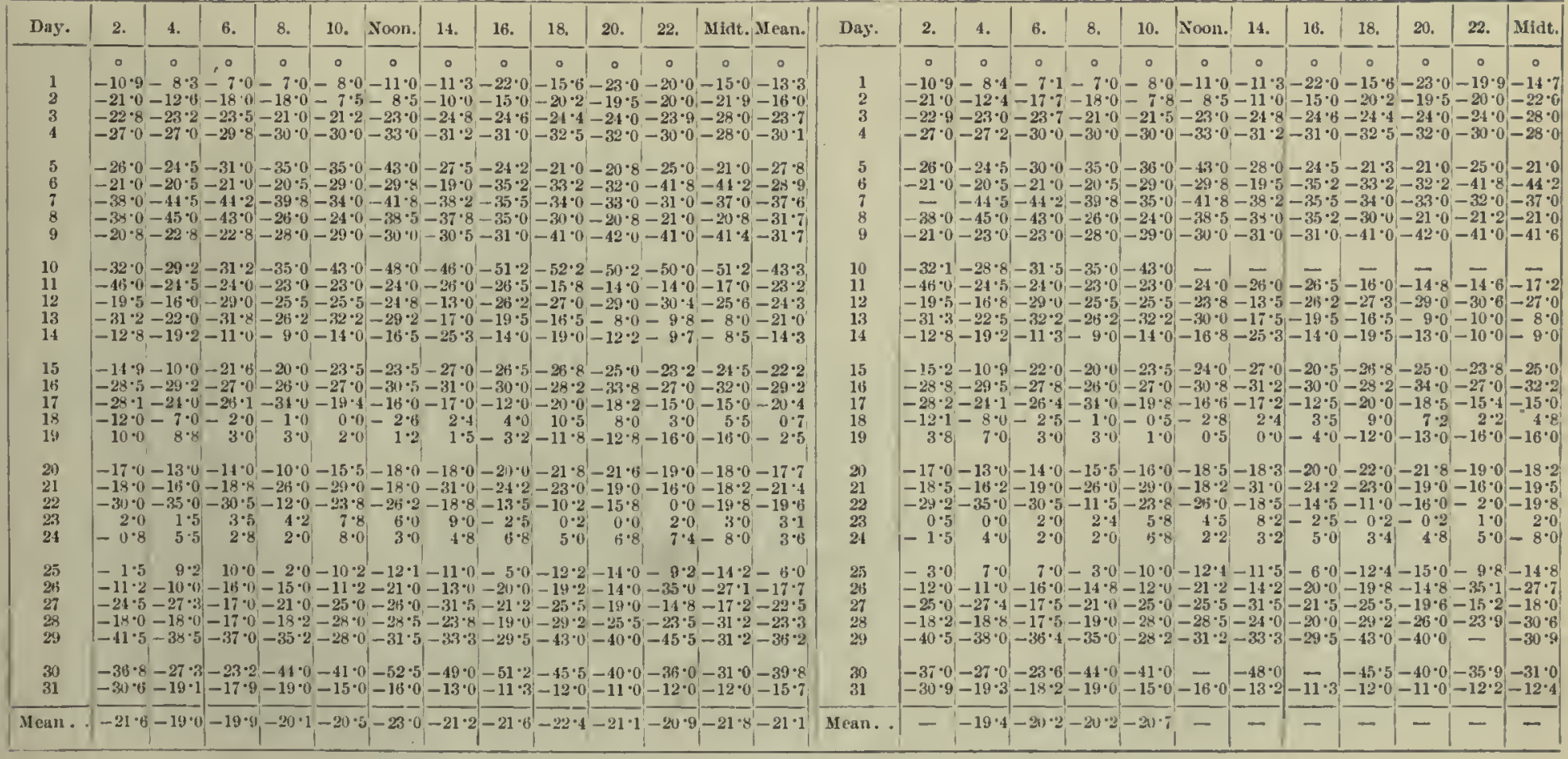


TABLE II. (continued).

'TEMPERATURE IN DEGRIES FAHRENHEIT.

DRY Buis.

WET BUL.B.

Angust, 1903.

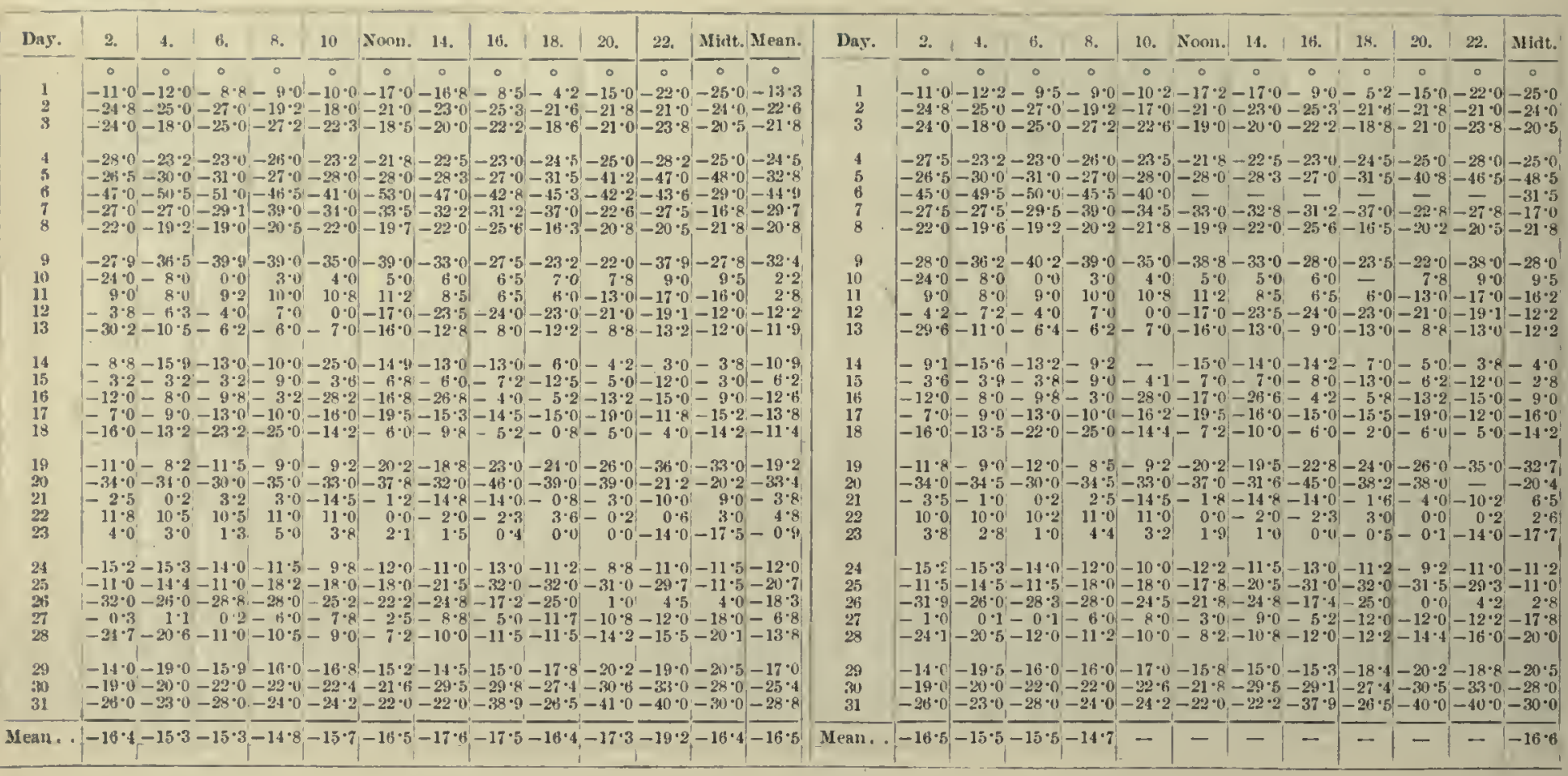

DRY BULA.

WET BulB.

September, 1903.

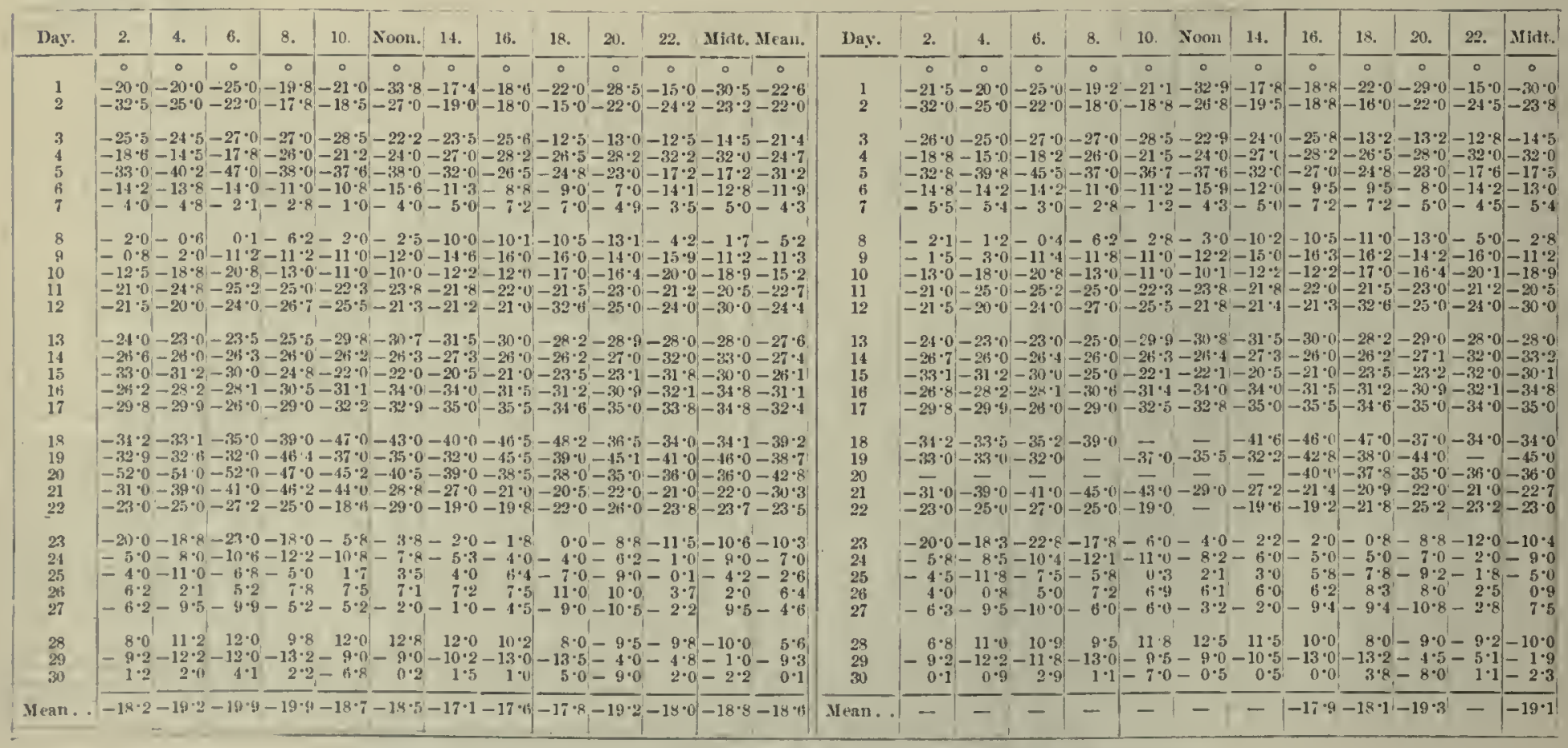


TABte II. (continued).

TEMPERATURE IN DEGREES FAHRENIEIT.

Dry Butr.

WET BULB.

October, 1903.

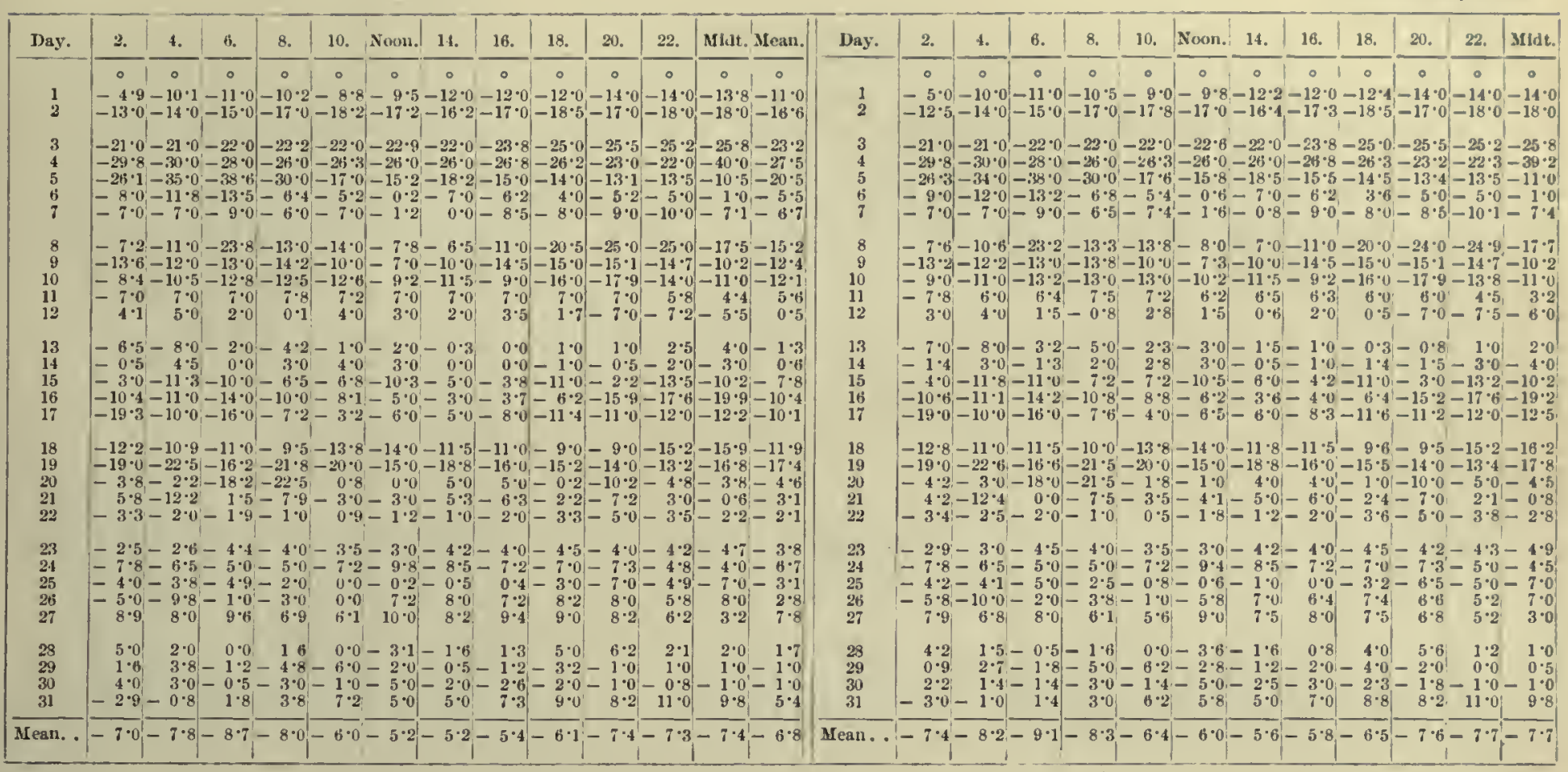

DRY But...

WET BULE.

November, 1903.

\begin{tabular}{|c|c|c|c|c|c|c|c|c|c|c|c|c|c|c|c|c|c|c|c|c|c|c|c|c|c|c|}
\hline Day. & 2. & 4. & 6. & 8. & 10. & Swon. & 14. & 16. & 18. & 20. & 22. & Midt. & Mean. & Day. & 2. & 4. & 6. & 8. & 10. & Noon. & 14. & 16. & 18. & 20. & 22. & Midt \\
\hline 1 & $\begin{array}{c}0 \\
11 \cdot 2\end{array}$ & $\stackrel{\circ}{13} \cdot 0$ & $\begin{array}{c}10 \\
13 \cdot 2\end{array}$ & $\stackrel{0}{13 \cdot 8}$ & $14 \cdot 0$ & $13 \cdot 8$ & $\begin{array}{c}0 \\
13 \cdot 5\end{array}$ & $\begin{array}{c}0 \\
13 \cdot 7\end{array}$ & $\dot{16} \cdot 0$ & $\stackrel{\circ}{15 \cdot 1}$ & $\stackrel{0}{14.2}$ & $13 \cdot 2$ & $13 \cdot 7$ & 1 & $11 \cdot 2$ & $13 \cdot 0$ & $\stackrel{\circ}{13 \cdot 0}$ & $13 \cdot 1$ & $\stackrel{\circ}{13.8}$ & $\begin{array}{c}0 \\
13 \cdot 2\end{array}$ & $\begin{array}{c}\circ \\
13 \cdot 0\end{array}$ & $\begin{array}{c}\circ \\
13 \cdot 0\end{array}$ & $\stackrel{\circ}{15 \cdot 0}$ & $\begin{array}{c}0 \\
14 \cdot 3\end{array}$ & $\begin{array}{c}0 \\
13 \cdot 8\end{array}$ & $12 \cdot 8$ \\
\hline $\begin{array}{l}2 \\
3 \\
4 \\
5 \\
3\end{array}$ & $\begin{array}{r}12 \cdot 2 \\
5 \cdot 8 \\
3 \cdot 0 \\
35.2 \\
4.5\end{array}$ & $\begin{array}{r}17 \cdot 0 \\
-2 \cdot 0 \\
7 \cdot 0 \\
14.2 \\
6 \cdot 1\end{array}$ & $\begin{array}{r}10 \cdot 9 \\
4 \cdot 0 \\
10 \cdot 9 \\
8 \cdot 2 \\
6 \cdot 8\end{array}$ & $\begin{array}{r}15 \cdot 0 \\
3 \cdot 0 \\
12 \cdot 2 \\
2 \cdot 0 \\
7 \cdot 5\end{array}$ & $\begin{array}{r}17 \cdot 2 \\
4 \cdot 2 \\
15 \cdot 0 \\
5 \cdot 0 \\
7 \cdot 8\end{array}$ & $\begin{array}{r}20.6 \\
7.0 \\
15.4 \\
5.5 \\
9.8\end{array}$ & $\begin{array}{r}20 \cdot 8 \\
1007 \\
16 \cdot 0 \\
5.0 \\
8 \cdot 5\end{array}$ & $\begin{array}{r}15 \cdot 8 \\
10.5 \\
14.6 \\
0.0 \\
6.6\end{array}$ & $\begin{array}{r}14 \cdot 0 \\
7 \cdot 2 \\
15 \cdot 0 \\
1 \cdot 5 \\
8 \cdot 2\end{array}$ & $\begin{array}{r}13 \cdot 8 \\
5.9 \\
15 \cdot 0 \\
0 \cdot 0 \\
9 \cdot 8\end{array}$ & $\begin{array}{r}13 \cdot 3 \\
10 \cdot 2 \\
15 \cdot 0 \\
2 \cdot 1 \\
9 \cdot 0\end{array}$ & $\left|\begin{array}{r}9 \cdot 1 \\
1 \cdot 0 \\
14 \cdot 5 \\
3 \cdot 4 \\
9 \cdot 5\end{array}\right|$ & $\begin{array}{r}15 \cdot 0 \\
5 \cdot 6 \\
12 \cdot 8 \\
4 \cdot 9 \\
7 \cdot 9\end{array}$ & $\begin{array}{l}2 \\
3 \\
4 \\
5 \\
6\end{array}$ & $\begin{array}{r}11 \cdot 8 \\
5 \cdot 0 \\
2 \cdot 8 \\
13 \cdot 8 \\
1.5\end{array}$ & $\begin{array}{r}15 \cdot 3 \\
-3 \cdot 0 \\
6 \cdot 0 \\
13 \cdot 0 \\
6 \cdot 0\end{array}$ & $\begin{array}{r}10 \cdot 2 \\
3 \cdot 5 \\
6 \cdot 0 \\
7 \cdot 9 \\
6.5\end{array}$ & \begin{tabular}{r|}
$14 \cdot 0$ \\
$2 \cdot \cdot 1$ \\
$11 \cdot 0$ \\
$1 \cdot 8$ \\
$7 \cdot 2$
\end{tabular} & $\begin{array}{r}15 \cdot 5 \\
3 \cdot 1 \\
13 \cdot 8 \\
4 \cdot 2 \\
7 \cdot 2\end{array}$ & $\begin{array}{r}18.0 \\
6.0 \\
14.0 \\
5.0 \\
8.8\end{array}$ & $\begin{array}{r}18 \cdot 0 \\
8 \cdot 8 \\
14.8 \\
4.3 \\
8.0\end{array}$ & $\begin{array}{r}14 \cdot 3 \\
9 \cdot 0 \\
13 \cdot 5 \\
0 \cdot 0 \\
6.0\end{array}$ & $\begin{array}{r}12.5 \\
6 \cdot 0 \\
13.5 \\
-\quad 1.7 \\
7.5\end{array}$ & $\begin{array}{r}12 \cdot 2 \\
5 \cdot 4 \\
13 \cdot 3 \\
0 \cdot 0 \\
9 \cdot 0\end{array}$ & $\begin{array}{r}12 \cdot 2 \\
8 \cdot 8 \\
13 \cdot 0 \\
2 \cdot 0 \\
7 \cdot 0\end{array}$ & $\begin{array}{r}8 \cdot 8 \\
1 \cdot 0 \\
12 \cdot 9 \\
3 \cdot 4 \\
9 \cdot 4\end{array}$ \\
\hline $\begin{array}{r}7 \\
8 \\
9 \\
10 \\
11\end{array}$ & $\begin{array}{r}7 \cdot 8 \\
9 \cdot 9 \\
15 \cdot 0 \\
10 \cdot 0 \\
18 \cdot 0\end{array}$ & $\begin{array}{r}3 \cdot 2 \\
11 \cdot 0 \\
14 \cdot 0 \\
8 \cdot 0 \\
18 \cdot 0\end{array}$ & $\begin{array}{r}2 \cdot 2 \\
10 \cdot 9 \\
14 \cdot 0 \\
10 \cdot 1 \\
18 \cdot 0\end{array}$ & $\begin{array}{r}1.5 \\
13.2 \\
12.8 \\
11.4 \\
12 \cdot 0\end{array}$ & $\begin{array}{r}3 \cdot 5 \\
13 \cdot 4 \\
13 \cdot 0 \\
15 \cdot 5 \\
18 \cdot 0\end{array}$ & $\begin{array}{r}4 \cdot 5 \\
16 \cdot 0 \\
15 \cdot 0 \\
15 \cdot 0 \\
19 \cdot 8\end{array}$ & $\begin{array}{l}4 \cdot 5 \\
15 \cdot 8 \\
13 \cdot 0 \\
17 \cdot 0 \\
21 \cdot 2\end{array}$ & $\begin{array}{r}5 \cdot 0 \\
16 \cdot 8 \\
12 \cdot 2 \\
15 \cdot 5 \\
21 \cdot 0\end{array}$ & $\begin{array}{r}7 \cdot 8 \\
17 \cdot 5 \\
12 \cdot 2 \\
14 \cdot 2 \\
21 \cdot 0\end{array}$ & $\begin{array}{l}10 \cdot 0 \\
17 \cdot 0 \\
14 \cdot 5 \\
12 \cdot 2 \\
21 \cdot 0\end{array}$ & $\begin{array}{r}8 \cdot 2 \\
15 \cdot 0 \\
8 \cdot 0 \\
14 \cdot 2 \\
15 \cdot 8\end{array}$ & $\begin{array}{l}9 \cdot 8 \\
95.0 \\
10 \cdot 0 \\
17 \cdot 0 \\
17.0\end{array}$ & $\begin{array}{r}5 \cdot 5 \\
14 \cdot 3 \\
12 \cdot 8 \\
13 \cdot 3 \\
18 \cdot 4\end{array}$ & $\begin{array}{r}7 \\
8 \\
9 \\
10 \\
11\end{array}$ & $\begin{array}{l}7 \cdot 8 \\
8 \cdot 9\end{array}$ & $\begin{array}{r}3 \cdot 2 \\
10 \cdot 0\end{array}$ & $\begin{array}{c}2 \cdot 2 \\
10 \cdot 1 \\
10 \cdot 0 \\
17 \cdot 8\end{array}$ & $\begin{array}{r}1 \cdot 5 \\
12 \cdot 2 \\
12 \cdot 2 \\
11 \cdot 2 \\
11 \cdot 8\end{array}$ & $\begin{array}{r}3 \cdot 5 \\
13 \cdot 0 \\
12 \cdot 0 \\
13 \cdot 5 \\
17 \cdot 5\end{array}$ & $\left|\begin{array}{r}4 \cdot 5 \\
15 \cdot 2 \\
14 \cdot 0 \\
13.5 \\
19 \cdot 0\end{array}\right|$ & $\begin{array}{l}4 \cdot 2 \\
15 \cdot 2 \\
12 \cdot 2 \\
14 \cdot 2 \\
19 \cdot 2\end{array}$ & $\begin{array}{c}4 \cdot 7 \\
16 \cdot 0 \\
11 \cdot 5 \\
13 \cdot 2 \\
-\end{array}$ & $\begin{array}{l}7 \cdot 0 \\
16 \cdot 8 \\
11 \cdot 5 \\
13 \cdot 2 \\
19 \cdot 0\end{array}$ & $\begin{array}{r}8 \cdot 2 \\
17 \cdot 0 \\
14 \cdot 0 \\
11 \cdot 2 \\
19 \cdot 1\end{array}$ & $\begin{array}{c}6 \cdot 1 \\
7 \cdot 5 \\
13 \cdot 2 \\
15 \cdot 2\end{array}$ & $9 \cdot 8$ \\
\hline $\begin{array}{l}12 \\
13 \\
14 \\
15 \\
16\end{array}$ & $\begin{array}{l}17 \cdot 5 \\
21 \cdot 8 \\
10 \cdot 9 \\
7 \cdot 0 \\
12 \cdot 0\end{array}$ & $\begin{array}{l}18 \cdot 5 \\
18 \cdot 8 \\
113.8 \\
12 \cdot 0 \\
11 \cdot 6\end{array}$ & $\begin{array}{l}19 \cdot 9 \\
15 \cdot 2 \\
13 \cdot 8 \\
13 \cdot 0 \\
12 \cdot 6\end{array}$ & $\begin{array}{l}18 \cdot 0 \\
15 \cdot 0 \\
18 \cdot 8 \\
10 \cdot 5 \\
12 \cdot 3\end{array}$ & $\begin{array}{l}20^{\circ} 0 \\
20^{\circ} 0 \\
19^{-8} \\
18^{\circ} 0 \\
13^{\circ} 0\end{array}$ & $\begin{array}{l}24 \cdot 8 \\
21 \cdot 0 \\
20 \cdot 8 \\
20 \cdot 8 \\
13 \cdot 2\end{array}$ & $\begin{array}{l}23 \cdot 2 \\
22 \cdot 2 \\
22 \cdot 2 \\
21 \cdot 5 \\
14 \cdot 5\end{array}$ & $\begin{array}{l}23 \cdot 5 \\
23 \cdot 0 \\
18 \cdot 0 \\
19 \cdot 5 \\
13 \cdot 5\end{array}$ & $\begin{array}{l}20 \cdot 2 \\
19 \cdot 5 \\
21 \cdot 2 \\
18 \cdot 2 \\
16 \cdot 0\end{array}$ & $\begin{array}{l}21 \cdot 5 \\
19 \cdot 0 \\
20 \cdot 0 \\
15.5 \\
10 \cdot 1\end{array}$ & $\begin{array}{r}22 \cdot 0 \\
9 \cdot 8 \\
18 \cdot 0 \\
12 \cdot 5 \\
11 \cdot 5\end{array}$ & $\begin{array}{c}21 \cdot 4 \\
10 \cdot 2 \\
16 \cdot 8 \\
13 \cdot 6 \\
6 \cdot 8\end{array}$ & $\begin{array}{l}20 \cdot 9 \\
18.2 \\
17 \cdot 8 \\
15 \cdot 2 \\
12 \cdot 2\end{array}$ & $\begin{array}{l}12 \\
13 \\
14 \\
15 \\
16\end{array}$ & & & $\begin{array}{l}18 \cdot 4 \\
14 \cdot 8 \\
12 \cdot 2\end{array}$ & $\begin{array}{l}17 \cdot 3 \\
14 \cdot 0 \\
17 \cdot 8 \\
10 \cdot 0 \\
11 \cdot 5\end{array}$ & $\begin{array}{l}19 \cdot 0 \\
19 \cdot 2 \\
16^{\circ} \cdot 5 \\
17 \cdot 0 \\
11 \cdot 5\end{array}$ & $\begin{array}{l}22 \cdot 3 \\
20 \cdot 0 \\
18 \cdot 0 \\
19 \cdot 4 \\
12 \cdot 0\end{array}$ & $\begin{array}{l}21 \cdot 2 \\
20 \cdot 5 \\
19.5 \\
20.0 \\
12.8\end{array}$ & $\begin{array}{l}21 \cdot 2 \\
24 \cdot 2 \\
16 \cdot 2 \\
18 \cdot 2 \\
12 \cdot 2\end{array}$ & $\begin{array}{l}20 \cdot 0 \\
17 \cdot 5 \\
19 \cdot 2 \\
17 \cdot 2 \\
15 \cdot 0\end{array}$ & $\begin{array}{c}20 \cdot 2 \\
17 \cdot 0 \\
18 \cdot 0 \\
15 \cdot * 2 \\
8 \cdot 8\end{array}$ & $\begin{array}{r}20 \cdot 5 \\
8 \cdot 2 \\
15.6 \\
12.0 \\
9 \cdot 5\end{array}$ & हैं \\
\hline $\begin{array}{l}17 \\
18 \\
19 \\
20 \\
21\end{array}$ & $\begin{array}{r}3 \cdot 2 \\
7 \cdot 5 \\
0 \cdot 0 \\
13 \cdot 0 \\
5 \cdot 0\end{array}$ & $\begin{array}{r}1 \cdot 2 \\
7 \cdot 9 \\
4 \cdot 8 \\
13 \cdot 0 \\
5 \cdot 0\end{array}$ & $\begin{array}{r}0 \cdot 8 \\
1,3 \cdot 0 \\
17 \cdot 5 \\
16 \cdot 0 \\
13 \cdot 2\end{array}$ & $\begin{array}{r}6 \cdot 5 \\
15 \cdot 6 \\
17 \cdot 8 \\
14 \cdot 0 \\
14 \cdot 4\end{array}$ & $\begin{array}{r}8 \cdot 2 \\
17 \cdot 0 \\
18 \cdot 0 \\
16 \cdot 3 \\
18 \cdot 0\end{array}$ & $\begin{array}{r}9 \cdot 3 \\
17 \cdot 2 \\
19 \cdot 0 \\
17 \cdot 4 \\
19 \cdot 0\end{array}$ & $\begin{array}{r}8 \cdot 2 \\
18 \cdot 2 \\
20.0 \\
18 \cdot 2 \\
20 \cdot 0\end{array}$ & $\begin{array}{r}9 \cdot 0 \\
18 \cdot 0 \\
19 \cdot 0 \\
18 \cdot 2 \\
20 \cdot 0\end{array}$ & $\begin{array}{r}8 \cdot 5 \\
19 \cdot 0 \\
18 \cdot 2 \\
18 \cdot 0 \\
19 \cdot 2\end{array}$ & $\begin{array}{r}8 \cdot 0 \\
15 \cdot 3 \\
17 \cdot 0 \\
18 \cdot 0 \\
20 \cdot 4\end{array}$ & $\begin{array}{r}7 \cdot 2 \\
14 \cdot 2 \\
17 \cdot 2 \\
15 \cdot 2 \\
21 \cdot 2\end{array}$ & 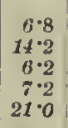 & $\begin{array}{r}6 \cdot 4 \\
14 \cdot 8 \\
15.1 \\
15 \cdot 4 \\
16.4\end{array}$ & 21 & & 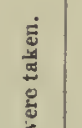 & & $\begin{array}{r}6 \cdot 2 \\
14 \cdot 8 \\
15 \cdot 8 \\
13 \cdot 2 \\
14 \cdot 0\end{array}$ & $\begin{array}{r}8 \cdot 0 \\
14 \cdot 8 \\
14 \cdot 6 \\
14 \cdot 0 \\
16 \cdot 8\end{array}$ & $\begin{array}{r}9 \cdot 0 \\
14 \cdot 5 \\
16 \cdot 2 \\
14 \cdot 9 \\
17 \cdot 9\end{array}$ & $\begin{array}{r}8 \cdot 0 \\
16 \cdot 0 \\
17 \cdot 2 \\
16 \cdot 0 \\
18 \cdot 5\end{array}$ & $\begin{array}{r}8 \\
15 \\
15\end{array}$ & $\begin{array}{l}15.2 \\
16.4 \\
18.0\end{array}$ & $\begin{array}{r}7 \cdot 0 \\
13 \cdot 0 \\
14 \cdot 5 \\
13 \cdot 8 \\
18 \cdot 6\end{array}$ & $\begin{array}{r}6 \cdot 2 \\
11 \cdot 5 \\
14 \cdot 0 \\
12.5 \\
19 \cdot 0\end{array}$ & 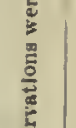 \\
\hline $\begin{array}{l}22 \\
23 \\
24 \\
25 \\
26\end{array}$ & $\begin{array}{r}23 \cdot 8 \\
22 \cdot 0 \\
9 \cdot 0 \\
8 \cdot 5 \\
17 \cdot 5\end{array}$ & $\begin{array}{r}21 \cdot 8 \\
18 \cdot 0 \\
8 \cdot 6 \\
8 \cdot 8 \\
13 \cdot 5\end{array}$ & $\begin{array}{l}22 \cdot 2 \\
17 \cdot 0 \\
11 \cdot 0 \\
10 \cdot 0 \\
22 \cdot 0\end{array}$ & $\begin{array}{l}25 \cdot 4 \\
18 \cdot 2 \\
13 \cdot 0 \\
19 \cdot 0 \\
23 \cdot 4\end{array}$ & $\begin{array}{l}25 \cdot 0 \\
15.0 \\
14.0 \\
20.0 \\
25 \cdot 0\end{array}$ & $\begin{array}{l}31 \cdot 1 \\
13 \cdot 0 \\
16 \cdot 0 \\
22 \cdot 6 \\
23 \cdot 2\end{array}$ & $\begin{array}{l}26 \cdot 0 \\
12 \cdot 0 \\
13 \cdot 5 \\
23 \cdot 0 \\
22 \cdot 5\end{array}$ & $\begin{array}{l}25 \cdot 0 \\
12 \cdot 0 \\
13 \cdot 5 \\
23 \cdot 0 \\
22 \cdot 0 \\
22 \cdot 2\end{array}$ & $\begin{array}{l}24 \cdot 0 \\
11 \cdot 0 \\
13 \cdot 0 \\
21 \cdot 5 \\
22 \cdot 0\end{array}$ & $\begin{array}{l}24 \cdot 2 \\
11 \cdot 6 \\
12 \cdot 0 \\
22 \cdot 0 \\
21 \cdot 0\end{array}$ & $\begin{array}{l}20 \cdot 1 \\
13 \cdot 2 \\
10 \cdot 6 \\
21 \cdot 2 \\
19 \cdot 7\end{array}$ & $\begin{array}{l}20 \cdot 0 \\
12.5 \\
9.5 \\
20.5 \\
19.4\end{array}$ & $\begin{array}{l}24 \cdot 1 \\
14 \cdot 6 \\
12 \cdot 0 \\
18 \cdot 0 \\
21 \cdot 0\end{array}$ & $\begin{array}{l}23 \\
24 \\
25 \\
26\end{array}$ & & 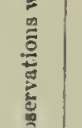 & & $\begin{array}{l}23.6 \\
17.0 \\
12.3 \\
16.0 \\
21.6\end{array}$ & $\begin{array}{l}22 \cdot 2 \\
13 \cdot 4 \\
13 \cdot 0 \\
16 \cdot 8 \\
20 \cdot 2\end{array}$ & $\begin{array}{l}27.4 \\
12.0 \\
14.2 \\
18.2 \\
22.0\end{array}$ & $\begin{array}{l}22 \cdot 0 \\
11 \cdot 2 \\
12 \cdot 8 \\
19 \cdot 0 \\
21 \cdot 5\end{array}$ & $\begin{array}{l}11 \cdot 2 \\
12 \cdot 2 \\
22) \cdot 2 \\
21 \cdot 8\end{array}$ & $\begin{array}{l}22 \cdot 0 \\
10 \cdot 0 \\
12 \cdot 0) \\
22 \cdot 0 \\
21 \cdot 0\end{array}$ & & $\begin{array}{r}18 \cdot 3 \\
12 \cdot 2 \\
9 \cdot 2 \\
17 \cdot 5 \\
19 \cdot 2\end{array}$ & ¿ \\
\hline $\begin{array}{l}27 \\
28 \\
29 \\
30\end{array}$ & & $\begin{array}{l}21 \cdot 2 \\
24 \cdot 5 \\
21 \cdot 2 \\
23 \cdot 6\end{array}$ & $\begin{array}{l}211^{\circ} \\
250^{\circ} \\
23 \cdot 0 \\
23 \cdot 3\end{array}$ & $\begin{array}{l}22.0 \\
25 \% 0 \\
21.5 \\
25.5\end{array}$ & $\begin{array}{l}23 \cdot 0 \\
25 \cdot 2 \\
26 \cdot 2 \\
24 \cdot 2\end{array}$ & $\begin{array}{l}23 \cdot 8 \\
28 \cdot 2 \\
26 \cdot 0 \\
25 \cdot 9\end{array}$ & $\begin{array}{l}24 \cdot 5 \\
28 \cdot 2 \\
25.0 \\
23 \cdot 5\end{array}$ & $\begin{array}{l}24 \cdot 5 \\
25 \cdot 2 \\
25 \cdot 0 \\
22 \cdot 5\end{array}$ & $\begin{array}{l}26 \cdot 0 \\
250 \\
250 \\
21.5\end{array}$ & $\begin{array}{l}25 \cdot 0 \\
25 \cdot 4 \\
25 \cdot 1 \\
22 \cdot 0\end{array}$ & $\begin{array}{l}24 \cdot 5 \\
26 \cdot 0 \\
25 \cdot 0 \\
19 \cdot 2\end{array}$ & $\begin{array}{l}24.0 \\
240 \\
2500 \\
19.5\end{array}$ & $\begin{array}{l}23 \cdot 4 \\
25 \cdot 5 \\
24 \cdot 4 \\
22 \cdot 9\end{array}$ & 30 & & \% & & $\begin{array}{l}21 \cdot 6 \\
: 4.0 \\
24 \cdot 0 \\
24.5\end{array}$ & $\begin{array}{l}22 \cdot 2 \\
2 \cdot \cdot 7 \\
250 \\
23 \cdot 9\end{array}$ & $\begin{array}{l}2500 \\
25.0\end{array}$ & $\begin{array}{l}23 \cdot 2 \\
26 \cdot 2 \\
24 \cdot 0 \\
22 \cdot 5\end{array}$ & $\begin{array}{l}23 \cdot 5 \\
25 \cdot 0 \\
21 \cdot 0 \\
22 \cdot 0\end{array}$ & & & $\begin{array}{l}23 \cdot 4 \\
24 \cdot 8 \\
24.0 \\
18.0\end{array}$ & \\
\hline an. & $12 \cdot 9$ & $12 \cdot 6$ & $14 \cdot 0$ & 14.8 & 185 & $17 \cdot 8$ & 17.8 & $17 \cdot 0$ & $16 \cdot 7$ & $16 \cdot 3$ & $15 \cdot 0$ & $13 \cdot 9$ & 15.4 & can. & - & - & - & $13 \cdot 9$ & 15.0 & $16 \cdot 3$ & $16 \cdot 1$ & - & $15 \cdot 5$ & 15.0 & - & - \\
\hline
\end{tabular}

Mealiugs in Italic type are interpolated values obtained from records of self-registering instruments. 
TAILE II. (continued).

TEMPERATURE IN DEGRELS FAHRENHEIT.

Dry BuLis.

Wet Buli3.

December, 1903.

\begin{tabular}{|c|c|c|c|c|c|c|c|c|c|c|c|c|c|c|c|c|c|c|c|c|c|c|c|c|c|c|}
\hline Day. & 2. & 4. & 6. & 8. & 10. & Noon. & 14. & 16. & 18. & 20. & 22. & Miat. & Mean. & Day. & 2. & 4. & 6. & 8. & 10. & Nююn. & 14. & 16. & 18. & 20. & 22. & Miats. \\
\hline 1 & $22^{2} \cdot 0$ & 20.8 & $23 \cdot 5$ & 21.4 & 24.0 & $21 \cdot 0$ & $23 \cdot 5$ & $24 \cdot 0$ & 21.5 & $23 \cdot 0$ & 20.8 & $20 \cdot 8$ & $22 \cdot 6$ & 1 & $\circ$ & & o & 20.5 & $22 \cdot 4$ & $20 \cdot 2$ & $21 \cdot 5$ & $22 \cdot 2$ & $23 \cdot 0$ & $22 \cdot 5$ & $19 \cdot 9$ & 。 \\
\hline & 19.5 & $15 \cdot 5$ & 15.4 & 1 & $\mid \begin{array}{l}199.4 \\
190,0.0\end{array}$ & 年 20.4 & 21.5 & $\begin{array}{l}22,0 \\
200\end{array}$ & 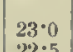 & 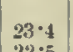 & $\begin{aligned} 23 \cdot 0 \\
23.0\end{aligned}$ & $23 \cdot 2$ & 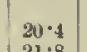 & & & & & $17 \cdot 3$ & $18 \cdot 4$ & $20 \cdot 0$ & $20 \cdot 5$ & $21 \cdot 2$ & $21 \cdot 8$ & $\begin{array}{l}21.4 \\
18.5\end{array}$ & $=0$ & 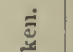 \\
\hline 3 & $\overline{10} \cdot \theta$ & $\overline{17 \cdot 0}$ & $\begin{array}{ll}18.0 \\
18.0\end{array}$ & 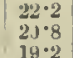 & $\begin{array}{l}222.2 \\
210.0 \\
19 \cdot 0\end{array}$ & $\begin{array}{l}22 \cdot 0 \\
22.0 \\
21.2 \\
21.2\end{array}$ & $\begin{array}{l}21 \cdot 2 \\
21 \cdot 0 \\
21 \cdot 0\end{array}$ & $\begin{array}{l}22 \cdot 0 \\
2120 \\
21\end{array}$ & $\begin{array}{l}225 \\
22.50 \\
19.0 \\
19.0\end{array}$ & $\mid \begin{array}{l}22 \cdot 5 \\
23 \cdot 0 \\
6.02\end{array}$ & $\begin{array}{l}21.4 \\
200 \\
120.0\end{array}$ & $\begin{array}{l}20.0 \\
20.0 \\
12.0\end{array}$ & $\begin{array}{l}12 \cdot 8 \\
20 \cdot 7\end{array}$ & & & & & $\begin{array}{l}220 \\
2200 \\
290 \\
190\end{array}$ & $\begin{array}{l}20.0 \\
19.7 \\
190.7\end{array}$ & $\begin{array}{l}1998 \\
19 \cdot 8 \\
1990\end{array}$ & $\begin{array}{l}19 \cdot 8 \\
19 \cdot 5 \\
19 \cdot 8\end{array}$ & $\begin{array}{l}210.0 \\
19 \cdot 2 \\
19.0\end{array}$ & $\begin{array}{l}21 \cdot 0 \\
21 \cdot 2 \\
18 \cdot 2\end{array}$ & $\begin{array}{l}\begin{array}{l}18.5 \\
21.8 \\
15.8\end{array} \\
15.6\end{array}$ & $\begin{array}{l}19 \cdot 5 \\
11 \cdot 7\end{array}$ & ฐँ \\
\hline 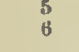 & $\mid \begin{array}{l}2 \cdot 2 \\
12 \cdot 0\end{array}$ & $\begin{array}{l}21 \cdot 0 \\
12 \cdot 8\end{array}$ & $\begin{array}{l}18 \cdot 4 \\
13 \cdot 2\end{array}$ & $\mid \begin{array}{l}19 \cdot 2 \cdot 2 \\
1.0\end{array}$ & $\begin{array}{l}19 \cdot 2 \\
15 \cdot 8\end{array}$ & $\begin{array}{l}21.2 \\
17 \cdot 0\end{array}$ & $\begin{array}{l}21 \cdot 4 \\
18: 2\end{array}$ & $\begin{array}{l}19 \cdot 0 \\
2,0\end{array}$ & $\begin{array}{l}19.0 \\
20.4\end{array}$ & $\begin{array}{l}16 \cdot 2 \\
21 \cdot 9 \\
21\end{array}$ & $\mid \begin{array}{l}12 \cdot 0 \\
23 \cdot 0\end{array}$ & $\begin{array}{l}12 \cdot 9 \\
{ }^{2} 2^{\circ} \cdot{ }_{5}\end{array}$ & $\begin{array}{l}18 \cdot 3 \\
17 \cdot 6\end{array}$ & b & & & & $\begin{array}{l}19 \cdot 0 \\
1.3 \cdot 1\end{array}$ & $\begin{array}{l}19.0 \\
14.9\end{array}$ & $\begin{array}{l}1990 \\
15 \cdot 8\end{array}$ & $\begin{array}{l}19.8 \\
17.0\end{array}$ & $\begin{array}{l}19.0 \\
18.5\end{array}$ & $\begin{array}{l}18 \cdot 2 \\
19 \cdot 2\end{array}$ & $\begin{array}{l}15 \cdot 6 \\
18.0\end{array}$ & $19 \cdot 0$ & 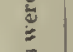 \\
\hline 8 & $\begin{array}{l}22 \cdot 0 \\
23 \cdot 0\end{array}$ & $\begin{array}{c}19 \cdot 8 \\
23 \cdot 0\end{array}$ & $\begin{array}{l}23 \cdot 8 \\
23 \cdot \cdot 3 \\
23\end{array}$ & $\begin{array}{l}21 \cdot 2 \cdot \\
24 \cdot 5\end{array}$ & \begin{tabular}{|l}
25.5 \\
52.1
\end{tabular} & $\mid \begin{array}{l}26 \cdot 3 \\
25 \cdot 2\end{array}$ & $\begin{array}{l}2 \pi \cdot 0 \\
2 \pi \cdot 5\end{array}$ & $\mid \begin{array}{l}28 \cdot 8 \\
24 \cdot 6\end{array}$ & $\begin{array}{l}26 \cdot 3 \\
26 \cdot 0\end{array}$ & \begin{tabular}{|l|}
$286^{\circ}$ \\
$20^{\circ}$
\end{tabular} & $\begin{array}{l}24 \cdot 0 \\
22, *\end{array}$ & $\begin{array}{l}22^{\circ} 0 \\
23^{\circ} \circ\end{array}$ & $\begin{array}{l}24 \cdot 5 \\
24 \cdot 5\end{array}$ & & & & & $\begin{array}{l}23 \cdot 0 \\
23 \cdot 8\end{array}$ & $\begin{array}{r}23 \cdot 6 \\
23 \cdot 9\end{array}$ & $\begin{array}{l}24 \cdot 4 \\
23 \cdot 5\end{array}$ & $\begin{array}{r}21 \cdot 8 \\
{ }_{24} \cdot \cdot 2\end{array}$ & $\begin{array}{l}24 \cdot 2 \\
2 \cdot 2 \cdot 2\end{array}$ & $\begin{array}{l}24 \cdot 2 \\
24 \cdot 2\end{array}$ & $\begin{array}{l}24 \cdot 9 \\
23 \cdot 6\end{array}$ & 288 & 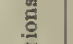 \\
\hline $\begin{array}{r}9 \\
10\end{array}$ & $\begin{array}{l}12.0 \\
19.6\end{array}$ & $\begin{array}{l}2100 \\
20 \cdot 0\end{array}$ & $\begin{array}{l}23.0 \\
24.0\end{array}$ & $\begin{array}{l}21.0 \\
23.0 \\
3=0\end{array}$ & $\begin{array}{l}260 \\
22.0 \\
22.2\end{array}$ & $\begin{array}{l}32 \cdot 0 \\
24.8\end{array}$ & $\begin{array}{r}28.0 \\
21.5\end{array}$ & $\begin{array}{l}24 \cdot 8 \\
24.5 \\
27.2\end{array}$ & $\begin{array}{l}24.5 \\
27.0 \\
27.0\end{array}$ & $\mid \begin{array}{l}24.0 \\
22 \cdot 0 \\
25 \cdot 4\end{array}$ & $\begin{array}{l}23.0 \\
28.8 \\
26.8\end{array}$ & $\begin{array}{r}28.5 \\
22.0\end{array}$ & $\begin{array}{l}24 \cdot 5 \\
23 \cdot 5 \cdot 6 \\
24 \cdot 6\end{array}$ & $\begin{array}{r}9 \\
10 \\
10\end{array}$ & & & & $\begin{array}{l}22.0 \\
22.0 \\
22.22\end{array}$ & $\begin{array}{l}22.9 \\
21.2\end{array}$ & $\begin{array}{l}233 \cdot 2 \\
3_{2}=2\end{array}$ & $\begin{array}{l}23.0 \\
2300\end{array}$ & 25.5 & $25 \cdot 0$ & 24.4 & 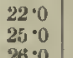 & \\
\hline 11 & 30 & 19.0 & $22 \cdot 0$ & $25 \cdot 0$ & $26^{\circ} 0$ & $26 \cdot 2$ & $27 \cdot 5$ & 29.0 & $28 \cdot 6$ & $266^{\circ}$ & $28 \cdot 0$ & 270 & $25 \cdot 4$ & 11 & & & & $24 \cdot 0$ & $21 \cdot 0$ & $25 \cdot 0$ & $24: 2$ & 25.5 & $27 \cdot 0$ & $25 \cdot 0$ & 26.0 & \\
\hline${ }_{13}^{12}$ & $\mid \begin{array}{l}280 \\
200 \\
200\end{array}$ & $\mid \begin{array}{l}27 \cdot 0 \\
20 \cdot 0 \\
200\end{array}$ & $\begin{array}{l}28 \cdot 5 \\
23 \cdot 0 \\
25.0\end{array}$ & $\begin{array}{l}28.5 \\
25.0 \\
25.0\end{array}$ & 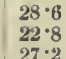 & $\begin{array}{l}30.8 \\
22 \cdot 2 \\
30.8\end{array}$ & $\begin{array}{l}30 \cdot 6 \\
21 \cdot \cdot 3 \\
33 \cdot 0\end{array}$ & $\begin{array}{l}31 \cdot 5 \\
\begin{array}{r}315 \\
26.5 \\
30.0\end{array}\end{array}$ & $\begin{array}{l}31 \cdot 0 \\
23.5\end{array}$ & $\begin{array}{l}29 \cdot 5 \\
32.8 \\
32.8\end{array}$ & $\begin{array}{l}28.4 \\
24.0 \\
280.0\end{array}$ & $\begin{array}{l}21 \cdot 4 \cdot 4 \\
22.5\end{array}$ & $\begin{array}{l}28: \\
22 \\
22\end{array}$ & $\begin{array}{l}12 \\
13\end{array}$ & & & & 28.0 & $\begin{array}{l}23 \cdot 4 \cdot 2 \\
21.5 \\
240\end{array}$ & $26 \cdot 2$ & $\begin{array}{l}27 \cdot 0 \\
20 \cdot 5\end{array}$ & $\mid \begin{array}{l}28.8 \\
24.0 \\
30.0\end{array}$ & $\begin{array}{l}0 \\
.8 \\
8\end{array}$ & $2^{2}$ & 0 & ஜ \\
\hline $\begin{array}{l}14 \\
15 \\
16\end{array}$ & $\mid \begin{array}{l}2 \cdot 5 \\
2 \cdot 0 \\
23 \cdot 0 \\
23 \cdot 0\end{array}$ & $\begin{array}{l}27 \cdot 5 \\
32 \cdot 0 \\
22 \cdot 0\end{array} \mid$ & 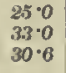 & $\begin{array}{l}25 \cdot 2 \\
30 \cdot 2 \\
29 \cdot 0\end{array}$ & $\begin{array}{l}27 \cdot 2 \\
37 \cdot 4 \\
27 \cdot 2\end{array}$ & $\mid \begin{array}{l}30.8 \\
30.0 \\
24.0\end{array}$ & $\begin{array}{l}33 \cdot 0 \\
30 \cdot 0 \\
23 \cdot 0\end{array}$ & $\begin{array}{r}32.0 \\
25.5 \\
255^{\circ}\end{array}$ & $\begin{array}{l}31.5 \\
20^{\circ}-5 \\
23.8\end{array}$ & $\begin{array}{l}28.2 \\
29 \cdot 0 \\
2: \cdot 1 \cdot 0\end{array}$ & $\begin{array}{l}28 \cdot 6 \\
22 \cdot 6 \\
24 \cdot 5\end{array}$ & $\begin{array}{l}25.0 \\
23.0 \\
25.0\end{array}$ & $\begin{array}{l}28 \cdot 2 \\
28 \cdot 2 \\
25 \cdot 8 \\
25 \cdot 1\end{array}$ & $\begin{array}{l}14 \\
15 \\
16\end{array}$ & & & & 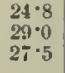 & $\begin{array}{l}24.0 \\
3600 \\
25.0\end{array}$ & $\begin{array}{l}27.9 \\
27.0 \\
22.0\end{array}$ & $\begin{array}{l}280^{28} \\
26 \cdot 5 \\
21 \cdot 5\end{array}$ & $\begin{array}{l}30.0 \\
24.0 \\
23-2\end{array}$ & $\begin{array}{l}33 \cdot 0 \\
23 \cdot 5 \\
21 \cdot 5\end{array}$ & 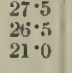 & $\begin{array}{l}24 \cdot 2 \\
24 \cdot 2 \\
21 \cdot 0 \\
21 \cdot 0\end{array}$ & $22 \cdot 0$ \\
\hline $\begin{array}{l}17 \\
18\end{array}$ & $\mid \begin{array}{l}2 y^{3} \\
30^{\circ}\end{array}$ & $\left|\begin{array}{l}21 \cdot 0 \\
19 \cdot 0\end{array}\right|$ & $\begin{array}{l}{ }_{18.0} \cdot 0 \\
18 \cdot 5\end{array}$ & $\begin{array}{l}17.6 \\
21 \cdot 2\end{array}$ & $\begin{array}{l}17 \cdot 4 \\
21.5\end{array}$ & $\mid \begin{array}{l}16 \cdot 6 \\
16.5\end{array}$ & $\begin{array}{l}19 \cdot 5 \\
16 \cdot 0\end{array}$ & $\begin{array}{l}21: 0 \\
17: 5\end{array}$ & $\begin{array}{l}22 \cdot 5 \\
19 \cdot 0 \\
2\end{array}$ & $\begin{array}{l}23.0 \\
18.5\end{array}$ & $\begin{array}{l}21 \cdot 7 \\
17 \cdot 2\end{array}$ & $\begin{array}{l}20.0 \\
17.5\end{array}$ & $\begin{array}{l}20.0 \\
18.4\end{array}$ & $\begin{array}{l}17 \\
18\end{array}$ & & & & $\begin{array}{l}17 \cdot 2 \\
2 v \cdot 4\end{array}$ & $\begin{array}{l}17 \cdot 2 \\
19 \cdot 8\end{array}$ & $\begin{array}{l}166^{\circ}, 0 \\
16^{\circ} 0\end{array}$ & $\begin{array}{l}18 \cdot 5 \\
15 \cdot 2\end{array}$ & $\begin{array}{l}20,0 \\
16.5\end{array}$ & $\begin{array}{l}211^{10} \\
17 \cdot 5\end{array}$ & $\begin{array}{l}21 \cdot 0 \\
17 \cdot 2\end{array}$ & $\begin{array}{l}19 \cdot 2 \\
16^{\circ} 0\end{array}$ & 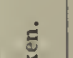 \\
\hline $\begin{array}{l}19 \\
200\end{array}$ & $\begin{array}{l}19 \\
228 \\
189\end{array}$ & $\begin{array}{l}21.0 \\
29 \cdot 0 \\
0.00\end{array}$ & $\begin{array}{l}2500 \\
240 \\
24.0\end{array}$ & $\begin{array}{l}25.0 \\
25 \% 0 \\
27\end{array}$ & $\begin{array}{l}21 \cdot 4 \\
2170 \\
27.8\end{array}$ & $\left\{\begin{array}{l}26.8 \\
25.0\end{array}\right.$ & $\begin{array}{l}26.5 \\
24.5 \\
280\end{array}$ & $\begin{array}{l}26.5 \\
26.5 \\
21.5\end{array}$ & $\begin{array}{r}29 \cdot 5 \\
24.0 \\
27.0\end{array}$ & 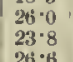 & $\begin{array}{l}24 \cdot 3 \\
21 \cdot 1 \\
21 \cdot 1\end{array}$ & $\begin{array}{l}22.5 \\
18.5 \\
0.5\end{array}$ & $\begin{array}{l}24 \cdot 8 \\
23 \cdot 0 \\
23-6\end{array}$ & 19 & & $\frac{a}{\partial}$ & & $\begin{array}{l}2306 \\
250.0 \\
25.8\end{array}$ & $\begin{array}{l}21.5 \\
21.1 \\
21.1\end{array}$ & $\left|\begin{array}{l}24 \cdot 1 \\
22 \cdot 9 \\
22.9 \\
25.5\end{array}\right|$ & $\begin{array}{l}25.0 \\
22.0 \\
22.8 \\
26.0\end{array}$ & $\begin{array}{l}23.0 \\
22.8 \\
25.5\end{array}$ & $\begin{array}{l}266.2 \\
225 \\
25.5 \\
5.5\end{array}$ & $\begin{array}{l}23 \cdot 3 \\
23: \cdot 0 \\
24 \cdot 2 \cdot 2\end{array}$ & $\begin{array}{l}22.0 \\
2200 \\
21.0\end{array}$ & 递 \\
\hline 21 & 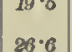 & $200 \%$ & $\begin{array}{l}240 \\
27.5\end{array}$ & 2,0 & $29^{\circ} \cdot 0$ & $29 \cdot 0$ & $29 \cdot 0$ & $\begin{array}{l}28.0 \\
28 \cdot 2\end{array}$ & $28 \cdot 2$ & $26^{\circ} \cdot 6$ & $25^{\circ} \cdot 4$ & $\begin{array}{l}25.4 \\
26.2\end{array}$ & $25 \cdot 5$ & 21 & & & & 6 & & & & & & & & \\
\hline $\begin{aligned} 22 \\
23 \\
23\end{aligned}$ & $\mid \begin{array}{l}25.8 \\
25.8 \\
24.8\end{array}$ & $\begin{array}{l}28 \cdot 1 \\
25 \cdot 0 \\
25 \cdot 0\end{array}$ & $\begin{aligned} 28.0 \\
289.0 \\
2890\end{aligned}$ & $\begin{array}{l}28.1 \\
28.0 \\
27.02\end{array}$ & $\begin{array}{l}28.0 \\
29.2 \\
28.8\end{array}$ & $\left\{\begin{array}{l}29 \cdot 2 \\
29 \cdot-2\end{array}\right.$ & $\begin{array}{l}27.5 \\
27.5 \\
30.0\end{array}$ & $\begin{array}{l}22.0 \\
30.5 \\
305\end{array}$ & $\begin{array}{l}28.5 \\
25.5 \\
30.0\end{array}$ & $\begin{array}{l}25.0 \\
250 \\
30.4\end{array}$ & $\begin{array}{l}212 . \\
2100 \\
30 \cdot 0\end{array}$ & $\mid \begin{array}{l}26.0 \\
24.0 \\
30.0\end{array}$ & 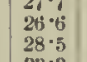 & $\begin{array}{l}22 \\
23 \\
24 \\
24\end{array}$ & & & & 20.4 & $\begin{array}{l}26.1 \\
26.0\end{array}$ & $\begin{array}{l}25.8 \\
28.0 \\
28.0\end{array}$ & $\begin{array}{l}28 \cdot 5 \\
28 \cdot 5\end{array}$ & $\begin{array}{l}25.0 \\
28.5\end{array}$ & $\begin{array}{l}25 \cdot 0 \\
2800 \\
230.5\end{array}$ & 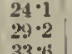 & $\begin{array}{l}22 \cdot 4 \cdot 4 \\
29 \cdot 0 \\
30 \cdot 0\end{array}$ & 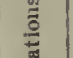 \\
\hline 20 & $\begin{array}{l}28.5 \\
33 \cdot 5\end{array}$ & $\begin{array}{l}28.5 \\
38.0\end{array}$ & $\begin{array}{l}300^{\circ} \cdot 0 \\
42 \cdot 0\end{array}$ & $\begin{array}{l}32 \cdot 0 \\
33.8\end{array}$ & $\begin{array}{r}33 \cdot 0 \\
330^{\circ}\end{array}$ & $\begin{array}{l}35 \cdot 0 \\
34.4\end{array}$ & $\mid \begin{array}{l}36 \% 0 \\
36 \cdot 2\end{array}$ & $\begin{array}{l}36.0 \\
36.0 \\
36^{\circ} 0\end{array}$ & $\begin{array}{l}37 \cdot 2 \\
37 \cdot 2\end{array}$ & $\begin{array}{l}37 \cdot 0 \\
31 \cdot 0\end{array}$ & $\begin{array}{l}31 \cdot 8 \\
33 \cdot 6\end{array}$ & $\begin{array}{l}31.5 \\
34.0\end{array}$ & $\begin{array}{l}33 \cdot 3 \\
35 \cdot 1\end{array}$ & $\begin{array}{l}25 \\
26\end{array}$ & & & & 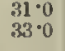 & & $\begin{array}{l}32 \cdot 0 \\
32 \cdot 5\end{array}$ & $\cdot 2$ & $\begin{array}{r}315 \\
33 \cdot 2\end{array}$ & 31.8 & & 1.2 & \\
\hline 28 & $\begin{array}{l}35.0 \\
30^{\circ} 0\end{array}$ & $\begin{array}{l}33.5 \\
3 \% \cdot 0\end{array}$ & $\begin{array}{l}35 \cdot 0 \\
30 \cdot 8\end{array}$ & $\begin{array}{l}38.0 \\
32.8\end{array}$ & $\begin{array}{l}33 \cdot 5 \\
37 \cdot 0\end{array}$ & $\begin{array}{l}34 \cdot 4 \\
35 \cdot 1\end{array}$ & $\begin{array}{l}33 \cdot 5 \\
34 \cdot 0\end{array}$ & $\begin{array}{l}33 \cdot 0 \\
29 \cdot 5\end{array}$ & $\begin{array}{l}36.0 \\
30.5\end{array}$ & $\left|\begin{array}{l}35 \cdot 2 \\
32 \cdot 2\end{array}\right|$ & \begin{tabular}{|}
$35 \cdot 8$ \\
$31 \cdot 4$
\end{tabular} & $\begin{array}{l}33 \cdot 8 \cdot 8 \\
30^{\circ} 6\end{array}$ & $\begin{array}{l}31 \cdot 9 \\
32 \cdot 2\end{array}$ & $\begin{array}{l}27 \\
28\end{array}$ & & & & $\begin{array}{l}32 \cdot 6 \\
30 \cdot 0\end{array}$ & $\begin{array}{l}33 \cdot 2 \\
34 \cdot 0\end{array}$ & $\begin{array}{l}32 \cdot 1 \\
32 \cdot 2\end{array}$ & $\begin{array}{l}32 \cdot 0 \\
32 \cdot 0\end{array}$ & $\begin{array}{l}32 \cdot 0 \\
29 \cdot 5\end{array}$ & $\begin{array}{r}345 \\
29.0\end{array}$ & $\begin{array}{l}31 \cdot 0 \\
31 \cdot 2\end{array}$ & $\begin{array}{l}31 \cdot 9 \\
29 \cdot 2\end{array}$ & : \\
\hline $\begin{array}{l}29 \\
30 \\
31 \\
31\end{array}$ & $\begin{array}{l}300 \\
29.0 \\
3 \cdot 0 \\
3 \cdot 0\end{array}$ & $\mid \begin{array}{l}30.5 \\
30 \cdot 0 \\
2 \cdot 0 \cdot\end{array}$ & $\begin{array}{l}\begin{array}{l}31.5 \\
29.0 \\
24.8\end{array} \\
24.8\end{array}$ & $\begin{array}{l}31.8 \\
32 \cdot 0 \\
26-20\end{array}$ & $\begin{array}{l}36.8 \\
36.0 \\
27.8\end{array}$ & $\begin{array}{l}39 \cdot 0 \\
34 \cdot 9 \\
29 \cdot 9 \cdot 9 \\
29.2\end{array}$ & $\begin{array}{l}38.4 \\
31.0 \\
28 \cdot 0\end{array}$ & $\begin{array}{l}29 \cdot 0 \\
29 \cdot 5 \cdot 5 \\
28 \cdot 0 \cdot 0\end{array}$ & $\begin{array}{l}28.0 \\
27^{\circ} \cdot 5\end{array}$ & $\begin{array}{l}29.2 \\
27.0 \\
28.1\end{array}$ & $\begin{array}{l}277^{2} \cdot 0 \\
250^{\circ}-0\end{array}$ & $\begin{aligned} 28 \cdot 5 \\
24 \cdot 5 \\
25 \cdot 5\end{aligned}$ & $\begin{array}{l}31 \cdot 9 \\
29 \cdot 6 \\
26 \cdot 6\end{array}$ & ${ }_{31}^{30}$ & & & & & $\begin{array}{l}32 \cdot 0 \\
325 \\
325.5 \\
25.2\end{array}$ & $\begin{array}{l}31 \cdot 8 \\
27.0\end{array}$ & $\begin{array}{l}33.4 \\
29 \cdot 0 \\
25.5\end{array}$ & $\begin{array}{r}290 \\
27.5 \\
26.5\end{array}$ & $\begin{array}{l}27.5 \\
25.0 \\
24 \%\end{array}$ & 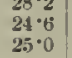 & 年 & \\
\hline & $23 \cdot 3$ & $\overline{23 \cdot 8}$ & $25 \cdot 4$ & $26^{\circ} 0$ & $26^{\circ} 6$ & $\overline{27 \cdot 0}$ & 26.9 & $26 \cdot 6$ & 26.7 & $26 \cdot 3$ & $25 \cdot 2$ & $24 \cdot 1$ & $25 \cdot 7$ & Iean. & - & - & - & 21.8 & $24 \cdot 3$ & -1 & - & - & - & - & $23 \cdot 3$ & - \\
\hline
\end{tabular}

Dry Bur.B.

WeT BuLB.

January, 1904.

\begin{tabular}{|c|c|c|c|c|c|c|c|c|c|c|c|c|c|c|c|c|c|c|c|c|c|c|c|c|c|c|}
\hline Day. & 2. & 4. & 6. & 8. & 10. & Noon. & 14. & 16. & 18. & 20. & 22. & Mist. & Mean. & Day. & 2. & 4. & 6. & 8. & 10. & Noon. & 11. & 16. & 18. & 20. & 22. & \\
\hline & & 29 & 34. & & $29 \cdot 9$ & & & & & 31.0 & .4 & .4 & $=32$ & & & 。 & $\circ$ & & & 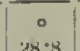 & & $\circ$ & $280^{\circ}$ & 0 & & $\circ$ \\
\hline 2 & & $27 \cdot 4$ & $\begin{array}{l}380 \\
28.4 \\
28.0 \\
28.0\end{array}$ & $\begin{array}{l}28.0 \\
29 \cdot 2 \\
28.4\end{array}$ & $\begin{array}{l}29.9 .9 \\
31.0 \\
260\end{array}$ & $\begin{array}{l}0 \\
: 0 \\
: 8 \\
: 8\end{array}$ & $\begin{array}{r}310.5 \\
27.8 \\
27.0\end{array}$ & $\begin{array}{l}30 \cdot 0 \\
27 \cdot 0 \\
24.0\end{array}$ & $\begin{array}{l}299.5 \\
280 \\
27 \cdot 2\end{array}$ & $\begin{array}{l}30 \cdot 0 \cdot 0 \\
25 \cdot 1 \\
254 \cdot 9\end{array}$ & 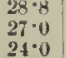 & $\begin{array}{l}28.4 \\
28.5 \\
2.5\end{array}$ & $\mid \begin{array}{l}30.1 \\
28 \cdot 1 \\
26 \cdot 1\end{array}$ & 1 & & & $\bar{z}$ & 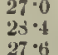 & $\begin{aligned} 28 \cdot 0 \\
28 \cdot 3 \\
24 \cdot 3\end{aligned}$ & 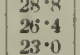 & $\begin{array}{l}23 x-5 \\
2=-8\end{array}$ & $\begin{array}{l}28.5 \\
25 \cdot 0 \\
25 \cdot 2\end{array}$ & 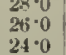 & $\begin{array}{l}28.2 \\
24.6 \\
23.5\end{array}$ & & \\
\hline 5 & $\begin{array}{l}24 \cdot 0 \\
23 \cdot 0\end{array}$ & $\begin{array}{l}23 \cdot 0 \\
22 \cdot 6\end{array}$ & $\begin{array}{l}25.0 \\
23.0 \\
23 \cdot 2\end{array}$ & $\begin{array}{l}2800 \\
24 \cdot 2\end{array}$ & $\left|\begin{array}{l}20^{\circ} \\
25 \cdot 2\end{array}\right|$ & $\begin{array}{l}30.0 \\
30.0 \\
26.0\end{array}$ & $\begin{array}{l}31 \% \\
210^{\circ} \\
26^{\circ}\end{array}$ & $\begin{array}{l}34 \cdot 8 \\
23 \cdot 8\end{array}$ & $\begin{array}{l}32 \cdot 5 \\
25 \cdot 5\end{array}$ & $\begin{array}{l}34.0 \\
27.4\end{array}$ & $\begin{array}{l}-4 \cdot 8 \\
28 \cdot 8 \\
29 \cdot 6\end{array}$ & $\begin{array}{l}28.0 \\
28.0 \\
27.0\end{array}$ & $\begin{array}{l}20 \cdot 6 \\
28 \cdot 6 \\
25 \cdot 4\end{array}$ & $\begin{array}{l}4 \\
4 \\
5\end{array}$ & & & $22 \cdot 5$ & $\begin{array}{r}24.8 \\
23.8 \\
23.0\end{array}$ & $\begin{array}{l}24 \cdot 8 \cdot 8 \\
24.0\end{array}$ & $\mid \begin{array}{l}280 \\
25 \\
250\end{array}$ & $\begin{array}{c}245 \\
250 \\
250\end{array}$ & $\begin{array}{l}30 \\
310 \cdot 8 \\
21 \cdot 9\end{array}$ & $\begin{array}{l}28 \cdot 2 \\
29 \cdot 0 \\
24 \cdot 0\end{array}$ & $\begin{array}{l}29 \cdot 8.8 \\
25.0\end{array}$ & $\begin{array}{l}260^{2} \\
26^{\circ}\end{array}$ & \\
\hline 6 & $23 \cdot 0$ & $25 \cdot 0$ & $\begin{array}{r}25 \cdot 8 \\
25.5\end{array}$ & $27 \cdot 0$ & $30^{\circ} 0$ & $28 \cdot 0$ & $\begin{array}{l}30 \cdot 4 \\
220\end{array}$ & $\begin{array}{l}30 \cdot 0 \\
29 \cdot 0\end{array}$ & $27 \cdot 2$ & $27 \cdot 5$ & | & 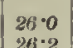 & 27.3 & 6 & & & 24.8 & 6 & & $\begin{array}{r}20 \cdot 6 \\
2502\end{array}$ & & $\begin{array}{l}0 \\
0 \\
0\end{array}$ & & $\begin{array}{l}26 \cdot 2 \\
27\end{array}$ & & \\
\hline & 800 & $\begin{array}{l}258 \\
228-8\end{array}$ & $\begin{array}{l}25 \cdot 5 \\
25 \cdot 5 \\
2 \pi \cdot 2\end{array}$ & $\begin{array}{l}28 \theta-1 \\
30 \cdot 2 \\
308\end{array}$ & $\begin{array}{l}220^{\circ} 0 \\
290^{2} \\
090\end{array}$ & $\begin{array}{l}28.5 \\
27.0 \\
27.0\end{array}$ & $\begin{array}{l}220^{\circ} \\
27^{\circ}\end{array}$ & $\begin{array}{l}29 \cdot 1 \\
27.0\end{array}$ & $\mid \begin{array}{l}270 \\
300 \\
30\end{array}$ & $\begin{array}{l}29 \cdot 8 \\
298\end{array}$ & 0 & $\begin{array}{l}26 \cdot 2 \\
25 \cdot 6\end{array}$ & 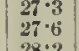 & $\begin{array}{l}7 \\
8 \\
0\end{array}$ & & & & $\begin{array}{l}25 \cdot 1 \\
28 \cdot 1\end{array}$ & $\begin{aligned} & 25 \cdot 0 \\
& 25 \cdot 0 \\
& 97\end{aligned}$ & 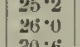 & & 0 & 5 & $\mid \begin{array}{l}250 \\
200 \\
200\end{array}$ & & \\
\hline 10 & 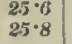 & $\begin{array}{l}8 \pi \cdot 6 \\
2 \pi \cdot 6\end{array}$ & 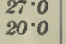 & $\begin{array}{l}280^{\circ} \\
21^{2}\end{array}$ & $\left\{\begin{array}{l}280 \\
290\end{array}\right.$ & $\begin{array}{l}32.2 \\
27.5\end{array}$ & $\begin{array}{l}29 \cdot 0 \\
28.5\end{array}$ & $\begin{aligned} 20 \cdot 9 \\
30 \cdot 2\end{aligned}$ & $\begin{array}{l}250,0 \\
30 \div-2\end{array}$ & $\begin{array}{l}28 \cdot 2 \\
27 \cdot 3\end{array}$ & :0 & $\begin{array}{l}88^{88.6} \\
25.6\end{array}$ & $\begin{array}{l}28 \cdot 2 \\
27 \cdot 8\end{array}$ & $\begin{array}{l}99 \\
10\end{array}$ & & & & $23 \cdot 2$ & $\begin{array}{ll}27.2 \\
27 \cdot 0\end{array}$ & 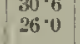 & $\cdot 5$ & 8 & 0 & \begin{tabular}{|c|}
2.08 \\
-40.8
\end{tabular} & - & \\
\hline 11 & ${ }_{28}^{27}$ & $\begin{array}{l}21 \cdot 0 \\
24 \cdot 2\end{array}$ & $\begin{aligned} 29 \cdot 0 \\
22 \cdot .4\end{aligned}$ & $\begin{array}{l}27 \cdot 0 \\
2990\end{array}$ & $\left|\begin{array}{l}330 \\
280\end{array}\right|$ & $\begin{array}{l}30.5 \\
30.5 \\
30.5\end{array}$ & $\begin{array}{l}28 \times 5 \\
310\end{array}$ & $\begin{array}{l}29 \cdot 0 \\
2 y-0\end{array}$ & $\frac{31 \cdot 4}{21 \cdot 0}$ & $\begin{array}{l}31 \cdot 0 \\
27-2\end{array}$ & 8 & $\begin{array}{l}28 \cdot 7 \\
23\end{array}$ & $\begin{array}{l}28 \cdot 2 \\
27 \cdot 3\end{array}$ & $\begin{array}{l}11 \\
12\end{array}$ & & & & $\begin{array}{l}26 \cdot 2 \\
26 \cdot 2\end{array}$ & $\begin{array}{l}30 \cdot 6 \\
236 \cdot 0\end{array}$ & $\begin{array}{c}28 \cdot 0 \\
28 \cdot 1\end{array}$ & & $\begin{array}{l}26 \cdot 5 \\
27 \cdot 0\end{array}$ & $28 \cdot 5$ & $\begin{array}{l}28.3 \\
25.8\end{array}$ & $\begin{array}{l}22 \cdot 2 \\
23 \cdot 6\end{array}$ & \\
\hline $\begin{array}{l}13 \\
13 \\
14\end{array}$ & $\begin{array}{l}22^{\circ} 0 \\
21^{\circ}\end{array}$ & $\begin{array}{l}23 \cdot 0 \\
22^{2} \cdot 1\end{array}$ & $\begin{aligned} 23 \cdot 0 \\
23 \cdot 4\end{aligned}$ & $\begin{array}{l}22 \cdot 2 \\
24 \cdot 0\end{array}$ & $\begin{array}{l}22 \cdot 5 \\
22 \cdot 0\end{array}$ & $\begin{array}{l}21 \cdot 2 \\
21 \cdot 2\end{array}$ & $\begin{array}{l}222 \cdot 5 \\
24 \cdot 0\end{array}$ & $\begin{array}{l}211 \cdot 2 \\
23 \cdot 5\end{array}$ & $\begin{array}{l}1 \% \cdot 0 \\
20.5\end{array}$ & $\begin{array}{l}20 \cdot 1 \\
21 \cdot 0 \cdot 1\end{array}$ & $\begin{array}{c}0 \\
: 0 \\
0\end{array}$ & $\begin{array}{l}0 \\
0 \\
0\end{array}$ & $\mid \begin{array}{l}21 \cdot 8 \\
21.8 \\
22 \cdot 2\end{array}$ & $\begin{array}{l}13 \\
13 \\
14\end{array}$ & & & & $\begin{array}{l}26 \cdot 2 \\
20 \cdot 8 \cdot 8 \\
23 \cdot 0\end{array}$ & 21.0 & $\begin{array}{l}19 \cdot 8 \\
21 \cdot 8\end{array}$ & & 5 & & $19 \cdot 1$ & & \\
\hline & $10^{\prime} \mathrm{s}$ & 20 & $22 \cdot 2$ & 21.0 & 220 & $24 \cdot 0$ & 23.8 & 26 & 26.5 & $25 \cdot 2$ & & & & 15 & & & & & 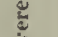 & & & & & & & \\
\hline $\begin{array}{l}16 \\
17\end{array}$ & $\begin{array}{l}20 \cdot 8 \\
20.5\end{array}$ & $\begin{array}{l}20.5 \\
25.8\end{array}$ & $\begin{array}{l}20.0 \\
200 \\
260\end{array}$ & $\begin{array}{l}18.8 \\
28.2\end{array}$ & $\begin{array}{l}19 \cdot 2 \\
28 \cdot 2\end{array}$ & $\begin{array}{l}19.0 \\
25.0 \\
28.0\end{array}$ & $\begin{array}{l}19{ }^{\circ} \\
25^{\circ}\end{array}$ & $\mid \begin{array}{l}200 \\
24 \cdot 5\end{array}$ & $\begin{array}{l}19 \cdot 0 \\
22 \cdot 6\end{array}$ & $\begin{array}{l}20 \cdot 0 \\
19 \cdot 6\end{array}$ & $: 5$ & $\begin{array}{l}0.0 \\
0 \\
6\end{array}$ & $\begin{array}{l}20.5 \\
23.9\end{array}$ & $\begin{array}{l}16 \\
17\end{array}$ & & & & $\begin{array}{l}18.5 \\
27.8\end{array}$ & & \begin{tabular}{ll|l}
$\cdot 0$ \\
$\cdot 6$
\end{tabular} & 这 & & $\bar{z}$ & $\begin{array}{l}19 \cdot 0 \\
18.6\end{array}$ & & \\
\hline $\begin{array}{l}18 \\
19 \\
20\end{array}$ & $\begin{array}{l}148.8 \\
19 \cdot 8 \\
150 .\end{array}$ & $\begin{array}{l}1310 \\
190 \\
15.0\end{array}$ & 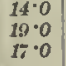 & $\begin{array}{l}12 \cdot 1 \\
197 \\
17 \cdot 6\end{array}$ & $\begin{array}{l}12.4 \\
19.7 \\
19.0\end{array}$ & $\begin{array}{l}\begin{array}{l}18.8 \\
20.0 \\
18.0\end{array} \\
18.2\end{array}$ & $\begin{array}{l}16: 8 \\
19 \cdot 8 \\
18 \cdot 8\end{array}$ & $\begin{array}{l}2000 \\
200 \\
070\end{array}$ & $\begin{array}{l}21.0 \\
21.0 \\
17 \cdot 0\end{array}$ & $\begin{array}{l}20 \cdot 2 \cdot 2 \\
19 \cdot 0 \\
16^{2} \cdot\end{array}$ & {$\left[\begin{array}{l}0 \\
2 \\
0 \\
0\end{array}\right.$} & $\begin{array}{l}20.0 \\
16.0 \\
14^{\circ} 5\end{array}$ & $\begin{array}{l}17 \cdot-1 \\
19 \cdot 1 \\
16 \cdot 8\end{array}$ & 20 & & & & $\begin{array}{l}11 \cdot 9 \cdot 9 \\
18 \cdot 0 \\
15 \cdot 0\end{array}$ & 吾 & $\begin{array}{l}\begin{array}{l}17.8 \\
18.0 \\
18.0\end{array} \\
18.0\end{array}$ & 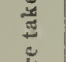 & $\begin{array}{l}18.3 \\
18 \cdot 2 \\
17 \cdot 0\end{array}$ & 递 & $\begin{array}{l}1.1 \\
16^{\circ}\end{array}$ & 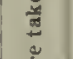 & \\
\hline $\begin{array}{l}21 \\
21\end{array}$ & ${ }_{15}^{15} \cdot 2$ & 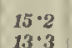 & $\begin{array}{l}15.5 \\
150.5\end{array}$ & \begin{tabular}{|l}
$14 \cdot 8$ \\
1738
\end{tabular} & $\begin{array}{ll}14 \cdot 2 \\
\end{array}$ &. & $\begin{array}{ll}16 \cdot 2 \\
50.0\end{array}$ & ${ }_{13}^{17}$ & $17 \cdot 0$ & $17 \cdot 6$ & & 4 & & & & & & 14.5 & & 0 & 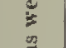 & $\begin{array}{c}8.8 \\
.1\end{array}$ & 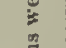 & $\begin{array}{l}170 \\
17.6\end{array}$ & 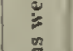 & \\
\hline & 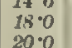 & $\begin{aligned} 188.7 \\
28.7\end{aligned}$ & $\begin{array}{l}16.0 \\
19.8 \\
22.0\end{array}$ & $\begin{array}{l}178 \\
18.2 \\
16.8\end{array}$ & $\begin{array}{l}19 \cdot 2 \\
17 \cdot 3\end{array}$ & $\begin{array}{l}13.0 \\
210^{\circ}\end{array}$ & $\begin{array}{l}200 \\
200^{\circ} \\
20^{\circ} \cdot 0\end{array}$ & $\begin{array}{l}158 \\
222 \cdot 3 \\
20 \cdot 2\end{array}$ & $\mid \begin{array}{l}128 \\
22,3 \\
19 \cdot 2\end{array}$ & $\mid \begin{array}{l}179 \\
210 \\
17.6\end{array}$ & $\begin{array}{l}20.5 \\
20.5 \\
18.1\end{array}$ & 0 & $20 \cdot 10$. & 22 & & & & $\begin{array}{l}18.0 \\
15.0\end{array}$ & $\because$ & $\cdot 2$ & 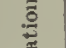 & . & 范 & $\begin{array}{l}18.8 \\
16.8\end{array}$ & 总 & \\
\hline 2 & 1770 & $13^{\circ} \cdot 2$ & $16 \cdot 2$ & 15.8 & $15 \cdot 5]$ & 4.0 & 16.0 & $19 \cdot 3$ & 10.5 & $21 \% 2$ & 2 & $\because$ & 17.8 & 25 & & & & 8 & 15.5 & j & 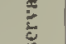 & & & $21 \cdot 2$ & & \\
\hline 20 & 170 & ${ }^{19} 0$ & $\begin{array}{l}12 \cdot 5 \\
9.0\end{array}$ & $12 \cdot 2$ & $\begin{array}{l}13 \cdot 0 \\
1800\end{array}$ & $\begin{array}{l}15.5 \\
21.8\end{array}$ & $\begin{array}{l}10 \cdot 0 \\
10.0\end{array}$ & $\begin{array}{l}18 \cdot 8 \\
18.3\end{array}$ & $\begin{array}{ll}18.0 \\
140.0\end{array}$ & $\begin{array}{l}20 \cdot 8 \\
1150\end{array}$ & $\begin{array}{l}19 \cdot 8 \\
11.8\end{array}$ & 19.8 & $\begin{array}{lll}17 \cdot 0 \\
10^{2}\end{array}$ & & & & & $\begin{array}{ll}12-2 \\
0 \cdot 0\end{array}$ & 㯊 & $\begin{array}{l}15.5 \\
2525 \\
20.0\end{array}$ & $\frac{3}{3}$ & $\begin{array}{l}18 \cdot 0 \\
15 \cdot 3\end{array}$ & $\frac{2}{\partial}$ & $\begin{array}{l}19 \cdot 2 \\
14 \cdot 0\end{array}$ & 管 & \\
\hline & & 15 & $\pi$ & $22 \cdot 0$ & $\mid \begin{array}{l}250 \\
180\end{array}$ & $\begin{array}{l}25.0 \\
18.0\end{array}$ & $\begin{array}{l}2 \cdot 5 \cdot 5 \\
20 \cdot 6\end{array}$ & $\begin{array}{l}250 \\
2500\end{array}$ & $\begin{aligned} 200 \\
2025\end{aligned}$ & $\frac{2 \pi \cdot 1}{12 \cdot 1}$ & $\begin{array}{l}25 \% \\
150^{\circ}\end{array}$ & $\begin{array}{l}22^{\circ} \circ 0 \\
1700\end{array}$ & $\begin{array}{l}22 \cdot 2 \cdot 2 \\
18.8\end{array}$ & 2 & & & & $\begin{array}{l}21 \cdot 0 \\
15 \cdot 8\end{array}$ & 造尊 & $\begin{array}{l}330 \\
16.5\end{array}$ & $\overline{4}$ & $\begin{array}{l}2400 \\
1108\end{array}$ & $\%$ & $\begin{array}{l}26 \cdot 4 \cdot 4 \\
17 \cdot 0\end{array}$ & $\%$ & \\
\hline 3 & & 14 & $14^{\circ}$ & $15{ }^{\circ}$ & in. & 2201 & 29.0 & 28 & 23 & $23 \cdot 0$ & & $18 \cdot 8$ & $19^{\circ}$ & & & & & $14 \cdot 5$ & & $18^{\circ} \mathrm{C}$ & & & & & & \\
\hline 3. & $18 \cdot 4$ & $18 \cdot 2$ & 17.5 & $19 \cdot 0$ & $21 \cdot 2$ & $21^{\circ} 0$ & $21 \cdot 0$ & $21 \cdot 5$ & $23 \cdot 0$ & $21 \cdot 1$ & $20 \cdot 0$ & $15 \%$ & $20 \cdot 1$ & 31 & & & & $18 \cdot 0$ & & $23 \cdot 2$ & & $21 \cdot 0$ & & 20.8 & & \\
\hline
\end{tabular}

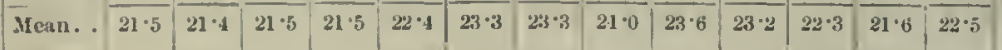


TABLE III.

\section{DAILY EXTREMES OF TEMPERATURE.}

February, 1902.

IN Degrees Fahrenheit.

\begin{tabular}{|c|c|c|c|c|c|c|c|c|c|c|c|c|c|c|c|c|c|c|c|c|c|c|c|}
\hline y. & ix. & n. & y. & tax. & Min. & Day. & Max. & Min. & D. & Max. & Min. & ay. & Max. & Min. & ay. & Max. & in. & ay. & Max. & in. & g. & Max. & \\
\hline & \multirow{6}{*}{$\begin{array}{l}\dot{ } \\
\bar{z} \\
\vdots \\
\vdots \\
\bar{z}\end{array}$} & \multirow{6}{*}{$\begin{array}{l}\bar{z} \\
\bar{z} \\
\bar{z} \\
\bar{z}\end{array}$} & \multirow{6}{*}{$\begin{array}{l}9 \\
10 \\
11 \\
12 \\
13 \\
14 \\
15 \\
16\end{array}$} & \multirow{6}{*}{$\begin{array}{l}0 \\
14 \cdot 5 \\
19 \cdot 0 \\
24 \cdot 8 \\
24 \cdot 5 \\
25 \cdot 0 \\
17.5 \\
17 \cdot 0 \\
23 \cdot 2\end{array}$} & \multirow{6}{*}{\begin{tabular}{r|r} 
\\
$8 \cdot 0$ \\
$9 \cdot 5$ \\
13.0 \\
$10 \cdot 4$ \\
$10 \cdot 8$ \\
8.0 \\
8.5 \\
$12 \cdot 0$
\end{tabular}} & \multirow{6}{*}{$\begin{array}{l}17 \\
18 \\
19 \\
20 \\
21 \\
22 \\
23 \\
24\end{array}$} & \multirow{6}{*}{$\begin{array}{l}0 \\
21 \cdot 0 \\
22 \cdot 0 \\
20.0 \\
23 \cdot 0 \\
230 \\
20.0 \\
21 \cdot 0 \\
112.5 \\
13 \cdot 2\end{array}$} & \multirow{6}{*}{$\begin{array}{r}r \\
7.5 \\
0.8 \\
7.55 \\
8.8 \\
8.7 \\
-0.8 \\
0.0 \\
-0.6\end{array}$} & & & & \multirow{6}{*}{$\begin{array}{l}1 \\
2 \\
3 \\
4 \\
5 \\
6 \\
7 \\
8\end{array}$} & \multirow{6}{*}{ 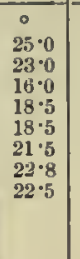 } & \multirow{6}{*}{$\begin{array}{r}19 \cdot 1 \\
12 \cdot 0 \\
9 \cdot 0 \\
10 \cdot 8 \\
10 \cdot 0 \\
11 \cdot 8 \\
10 \cdot 8 \\
8 \cdot 8\end{array}$} & \multirow{6}{*}{$\begin{array}{c}9 \\
11 \\
11 \\
11 \\
13 \\
115 \\
15 \\
16\end{array}$} & \multirow{6}{*}{\begin{tabular}{r|r}
27.5 \\
17.8 \\
18.0 \\
9.0 \\
0.5 \\
0.5 \\
2.0 \\
8.0 \\
8.8
\end{tabular}} & \multirow{6}{*}{$\begin{array}{r}13 \cdot 1 \\
77 \cdot 2 \\
20.0 \\
0.00 \\
-7.00 \\
-5.0 \\
-8.5 \\
-6.0\end{array}$} & \multirow{6}{*}{$\begin{array}{l}17 \\
18 \\
19 \\
20 \\
21 \\
22 \\
23 \\
24\end{array}$} & \multirow{6}{*}{$\begin{array}{r}17 \cdot 8 \\
17 \cdot 0 \\
22 \cdot 8 \\
22.5 \\
18 \cdot 0 \\
22 \cdot 8 \\
14 \cdot 0 \\
6 \cdot 4\end{array}$} & \multirow{6}{*}{$\begin{array}{r}7.0 \\
10.5 \\
12.0 \\
12.0 \\
8.0 \\
11.5 \\
-8.0 \\
-8.0\end{array}$} & \multirow{5}{*}{$\begin{array}{l}25 \\
25 \\
27 \\
27 \\
29 \\
29 \\
30 \\
31\end{array}$} & \multirow{5}{*}{$\begin{array}{r}12 \cdot 2 \\
5.0 \\
-5.0 \\
-3.5 \\
4.0 \\
0.0 \\
0.0\end{array}$} & \multirow{5}{*}{$\begin{array}{r}0.5 \\
-8.0 \\
-9.8 \\
-10.2 \\
-13.2 \\
-12.0 \\
-9.8\end{array}$} \\
\hline & & & & & & & & & & ${ }^{24}$ & 1.2. & & & & & & & & & & & & \\
\hline & & & & & & & & & 28 & ${ }_{29}^{27}$ & $\begin{array}{l}19.5 \\
18.8\end{array}$ & & & & & & & & & & & & \\
\hline & & & & & & & & & & & & & & & & & & & & & & & \\
\hline & & & & & & & & & & & & & & & & & & & & & & & \\
\hline & & & & & & & & & & $21 \cdot 4$ & 8.7 & & & & & & & & & & & $13 \cdot 1$ & \\
\hline
\end{tabular}

April, 1902.

May, 1902.

\begin{tabular}{|c|c|c|c|c|c|c|c|c|c|c|c|c|c|c|c|c|c|c|c|c|c|c|c|}
\hline Day. & Max. & Min. & Day. & Max. & Min. & Day. & Max. & Min. & Day. & Max. & Min. & Day. & Max. & Min. & Day. & Max. & Min. & Day. & Max. & Min. & Day. & Max. & Min. \\
\hline \multirow[t]{2}{*}{$\begin{array}{l}1 \\
2 \\
3 \\
4 \\
5 \\
6 \\
7 \\
8 \\
8\end{array}$} & \multirow[t]{2}{*}{$\begin{array}{r}0.4 \\
-2.4 \\
-5.0 \\
-8.0 \\
-1.8 \\
1.0 \\
2.0 \\
10.0 \\
13.0\end{array}$} & \multirow[t]{2}{*}{ 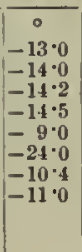 } & \multirow[t]{2}{*}{$\begin{array}{r}9 \\
10 \\
11 \\
12 \\
13 \\
14 \\
15 \\
16\end{array}$} & \multirow[t]{2}{*}{$\begin{array}{r}0 \\
4 \cdot 0 \\
9 \cdot 0 \\
15 \cdot 0 \\
14 \cdot 9 \\
19 \cdot 5 \\
12 \cdot 5 \\
1 \cdot 0 \\
1 \cdot 0\end{array}$} & \multirow[t]{2}{*}{$\begin{aligned} 0 \\
-8.0 \\
-8.00 \\
4.5 \\
-3 \cdot 2 \\
-5.0 \\
-15.0 \\
-18 \cdot 5 \\
-8.0\end{aligned}$} & \multirow[t]{2}{*}{$\begin{array}{l}17 \\
18 \\
19 \\
20 \\
21 \\
22 \\
23 \\
24\end{array}$} & \multirow[t]{2}{*}{$\begin{array}{r}0 \\
8.2 \\
7 \cdot 3 \\
0.1 \\
6.0 \\
0.0 \\
-15.8 \\
-16.0 \\
-120.0\end{array}$} & \multirow[t]{2}{*}{$\begin{array}{l}0 \\
-5.1 \\
-7.0 \\
-8.0 \\
-8.0 \\
-8.2 \\
-19.8 \\
-25.6 \\
-311.6 \\
-26.1\end{array}$} & $\begin{array}{l}25 \\
26 \\
27 \\
28 \\
29 \\
30\end{array}$ & $\begin{array}{r}0 \\
-15 \cdot 2 \\
-4 \cdot 0 \\
-10 \cdot 0 \\
-11 \cdot 0 \\
-13.0 \\
-14^{\circ} 0\end{array}$ & $\begin{array}{r}0 \\
-28.5 \\
-18.6 \\
-25.5 \\
-23.0 \\
-25.2 \\
-26.5\end{array}$ & \multirow[t]{2}{*}{$\begin{array}{l}1 \\
2 \\
3 \\
4 \\
5 \\
6 \\
7 \\
8 \\
8\end{array}$} & \multirow[t]{2}{*}{\begin{tabular}{|r|}
0 \\
$-8 \cdot 5$ \\
$1.0^{*}$ \\
$9 \cdot 5^{*}$ \\
$19 \cdot 0$ \\
$12 \cdot 0$ \\
17.0 \\
$13 \cdot 5$ \\
-3.9
\end{tabular}} & \multirow[t]{2}{*}{$\begin{array}{c}0 \\
-25.0 \\
-15.9 \\
= \\
\overline{\overline{1}} \cdot 0 \\
-7.0 \\
-22.5\end{array}$} & \multirow[t]{2}{*}{$\begin{array}{r}9 \\
10 \\
11 \\
12 \\
13 \\
14 \\
15 \\
16\end{array}$} & \multirow[t]{2}{*}{$\begin{array}{c}0 \\
-8 \cdot 5 \\
0 \cdot 2 \\
-1 \cdot 8 \\
-11 \cdot 1 \\
-13 \cdot 0 \\
-13 \cdot 3 \\
-12 \cdot 3 \\
-28 \cdot 0\end{array}$} & \multirow[t]{2}{*}{ 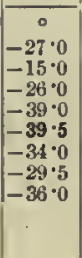 } & \multirow[t]{2}{*}{$\begin{array}{l}17 \\
18 \\
19 \\
20 \\
21 \\
22 \\
23 \\
24\end{array}$} & \multirow[t]{2}{*}{\begin{tabular}{|c|}
$\circ$ \\
-23.0 \\
-18.8 \\
-18.0 \\
-13.9 \\
-17.0 \\
-18.0 \\
-9.0 \\
-9.0
\end{tabular}} & \multirow[t]{2}{*}{$\begin{array}{l} \\
-34 \cdot 0 \\
-236.2 \\
-23 \cdot 0 \\
-21.5 \\
-21.5 \\
-24.5 \\
-24.0 \\
-28.1 \\
-24.7\end{array}$} & $\begin{array}{l}25 \\
28 \\
27 \\
28 \\
29 \\
30 \\
31\end{array}$ & \begin{tabular}{|r|}
0 \\
-13.9 \\
-8.0 \\
-9.8 \\
-10.5 \\
1.0 \\
11.5 \\
15.3
\end{tabular} & $\begin{array}{r}-32.0 \\
-24.0 \\
-20.0 \\
-31.0 \\
-29.0 \\
0.0 \\
1.0\end{array}$ \\
\hline & & & & & & & & & ean. & -0.4 & $-14^{\circ} 7$ & & & & & & & & & & Mean. . & $-5 \cdot 2$ & $-23:$ \\
\hline
\end{tabular}

- From thermograph trace.

+ For 28 daya oniy.

June, 1902.

July, 1902.

\begin{tabular}{|c|c|c|c|c|c|c|c|c|c|c|c|c|c|c|c|c|c|c|c|c|c|c|c|}
\hline Day. & Max. & Min. & Day. & Max. & Min. & Day. & Max. & Min. & Day. & Max. & Min. & Day. & Max. & Min. & Day. & Max. & Min. & Day. & Max. & Min. & Day. & Max. & Min. \\
\hline \multirow{7}{*}{$\begin{array}{l}1 \\
2 \\
3 \\
4 \\
5 \\
6 \\
7 \\
8 \\
8\end{array}$} & 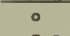 & \multirow{7}{*}{$\begin{array}{l}-7 \cdot 0 \\
-7 \cdot 0 \\
-19 \cdot 0 \\
-18 \cdot 0 \\
-18 \cdot 0 \\
-22 \cdot 0 \\
-28 \cdot 0 \\
-32 \cdot 5 \\
-28 \cdot 0\end{array}$} & \multirow{7}{*}{\begin{tabular}{c|}
9 \\
10 \\
11 \\
12 \\
13 \\
14 \\
15 \\
10
\end{tabular}} & \multirow{7}{*}{$\begin{array}{r}0 \\
-13 \cdot 2 \\
-19 \cdot 0 \\
-15 \cdot 5 \\
-12 \cdot 8 \\
-18 \cdot 9 \\
-27 \cdot 5 \\
-20 \cdot 0 \\
11 \cdot 0\end{array}$} & \multirow{7}{*}{ 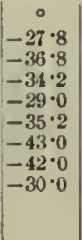 } & \multirow{7}{*}{$\begin{array}{l}17 \\
18 \\
19 \\
20 \\
21 \\
22 \\
23 \\
24\end{array}$} & \multirow{7}{*}{$\begin{array}{r}-7 \cdot 5 \\
-7 \cdot 2 \\
-9 \cdot 8 \\
5 \cdot 0 \\
-11 \cdot 5 \\
-14 \cdot 0 \\
-18 \cdot 0 \\
-18 \cdot 0\end{array}$} & 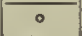 & & & 0 & \multirow{7}{*}{$\begin{array}{l}1 \\
2 \\
3 \\
4 \\
5 \\
8 \\
7 \\
8\end{array}$} & \multirow{7}{*}{$\begin{aligned} & 0 \\
&- 1 \cdot 0 \\
& 0 \cdot 8 \\
& 3 \cdot 5 \\
&-4.0 \\
& 6.5 \\
&-11 \cdot 2 \\
&-11 \cdot 6 \\
&-11 \cdot 0\end{aligned}$} & \multirow{7}{*}{$\begin{array}{r}0 \\
-22 \cdot 0 \\
-17.0 \\
-12.5 \\
-24.0 \\
-20.5 \\
-31.0 \\
-32.0 \\
-38.0\end{array}$} & \multirow{7}{*}{$\begin{array}{c}9 \\
10 \\
11 \\
12 \\
13 \\
14 \\
15 \\
16\end{array}$} & \multirow{7}{*}{$\begin{array}{r}0 \\
8 \cdot 0 \\
8.0 \\
-1 \cdot 2 \\
-4 \cdot 0 \\
-13 \cdot 0 \\
-21 \cdot 2 \\
-13.0 \\
-9 \cdot 9\end{array}$} & \multirow{7}{*}{\begin{tabular}{|c|}
0 \\
$-16 \cdot 5$ \\
$-17 \cdot 2$ \\
$-12 \cdot 5$ \\
-20.0 \\
$-27 \cdot 0$ \\
$-31 \cdot 5$ \\
-236 \\
-26.2 \\
-26.5
\end{tabular}} & \multirow{7}{*}{$\begin{array}{l}17 \\
18 \\
19 \\
20 \\
21 \\
22 \\
23 \\
24\end{array}$} & \multirow{7}{*}{$\begin{array}{r}0 \\
9 \cdot 0 \\
13 \cdot 0 \\
16 \cdot 7 * \\
14 \cdot 2 \\
-4 \cdot 5 \\
8 \cdot 9 \\
3 \cdot 3 \\
-4 \cdot 5\end{array}$} & 0 & \multirow{6}{*}{$\begin{array}{l}25 \\
28 \\
27 \\
28 \\
29 \\
30 \\
31\end{array}$} & \multirow{6}{*}{$\begin{array}{c}0 \\
-4 \cdot 0 \\
-3 \cdot 0 \\
-1 \cdot 5 \\
-4 \cdot 5 \\
-2 \cdot 0 \\
1 \cdot 0 \\
-3 \cdot 0\end{array}$} & \multirow{6}{*}{$\begin{array}{l}-11 \cdot 3 \\
-10 \cdot 8 \\
-12 \cdot 5 \\
-11 \cdot 0 \\
-9 \cdot 0 \\
-18 \cdot 0 \\
-16 \cdot 2\end{array}$} \\
\hline & \multirow{6}{*}{$\begin{array}{r}7.5 \\
2.0 \\
-1.0 \\
-2.0 \\
-5.2 \\
-9.8 \\
-15.0 \\
-9.4\end{array}$} & & & & & & & \multirow{6}{*}{$\begin{array}{l}-26 \cdot 0 \\
-20 \cdot 0 \\
-22.0 \\
-22 \cdot 0 \\
-36.5 \\
-36.5 \\
-36 \cdot 5 \\
-40 \cdot 0 \\
-47.0\end{array}$} & 25 & $-12 \cdot 0$ & $-27 \cdot 0$ & & & & & & & & & \multirow{6}{*}{$\begin{array}{r}-11 \cdot 1 \\
8 \cdot 0 \\
9 \cdot 8 \\
-12 \cdot 0 \\
-13 \cdot 0 \\
-22 \cdot 0 \\
-24 \cdot 5 \\
-13 \cdot 0\end{array}$} & & & \\
\hline & & & & & & & & & $\begin{array}{l}26 \\
27\end{array}$ & $\begin{array}{r}2.8 \\
4.0\end{array}$ & $\begin{array}{l}-21.2 \\
-10.8\end{array}$ & & & & & & & & & & & & \\
\hline & & & & & & & & & 28 & $13 \cdot 0$ & $\begin{array}{r}-45 \\
-4.5 \\
\end{array}$ & & & & & & & & & & & & \\
\hline & & & & & & & & & 30 & $\begin{array}{r}0 \% \\
-5 \%\end{array}$ & $\begin{array}{l}-100 \\
-15.5\end{array}$ & & & & & & & & & & & & \\
\hline & & & & & & & & & & & & & & & & & & & & & & & \\
\hline & & & & & & & & & cas & -7.8 & $-26 \cdot 3$ & & & & & & & & & & Mean. . & $-2 \cdot 5$ & $-17 \cdot 5$ \\
\hline
\end{tabular}

- Fron thermograph trace.

August, 1902.

September, 1902.

\begin{tabular}{|c|c|c|c|c|c|c|c|c|c|c|c|c|c|c|c|c|c|c|c|c|c|c|c|}
\hline Day. & Max. & Min. & Day. & $\operatorname{Max}$. & Min. & Day. & Max. & Min. & Day. & MIax. & Min. & Day. & Max. & Min. & Day. & Max. & Min. & Day. & Max. & Min. & Day. & Max. & Min. \\
\hline \multirow{8}{*}{$\begin{array}{l}1 \\
2 \\
3 \\
4 \\
5 \\
6 \\
7 \\
8\end{array}$} & \multirow{8}{*}{$\begin{array}{r}0 \\
-\quad 4 \cdot 0 \\
2 \cdot 0 \\
-14.0 \\
-112 \cdot 2 \\
-22 \cdot 8 \\
-31.5 \\
-29 \cdot 1 \\
-25 \cdot 4\end{array}$} & \multirow{8}{*}{$\begin{array}{r}0 \\
-13.0 \\
-21.1 \\
-2 H^{\circ} \cdot 6 \\
-28.5 \\
-33.5 \\
-43.0 \\
-50.5 \\
-40.5 \\
-40.5\end{array}$} & \multirow{8}{*}{$\begin{array}{r}9 \\
10 \\
11 \\
12 \\
13 \\
14 \\
15 \\
16\end{array}$} & \multirow{8}{*}{$\begin{array}{l}-21 \cdot 9 \\
-23 \cdot 0 \\
-21.8 \\
-9.0 \\
-91.5 \\
-6 \cdot 3 \\
-15 \cdot 0 \\
-21.0\end{array}$} & \multirow{8}{*}{$\begin{array}{l}-35 \cdot 1 \\
-39 \cdot 1 \\
-33 \cdot 8 \\
-20 \cdot 2 \\
-21 \cdot 0 \\
-23 \cdot 0 \\
-288.5 \\
-32 \cdot 0 \\
-32 \cdot 0\end{array}$} & \multirow{8}{*}{$\begin{array}{l}17 \\
18 \\
19 \\
20 \\
21 \\
22 \\
23 \\
24\end{array}$} & \multirow{8}{*}{$\begin{array}{r}-10.5 \\
-9 \cdot 8 \\
4 \cdot 0 \\
1.4 \\
6 \cdot 0 \\
3 \cdot 6 \\
-4 \cdot 1 \\
-8 \cdot 1\end{array}$} & \multirow{8}{*}{$\begin{array}{l}-24 \cdot 2 \\
-30 \cdot 0 \\
-25 \cdot 5 \\
-12 \cdot 2 \\
-2 \cdot 0 \\
-12 \cdot 5 \\
-18 \cdot 0 \\
-19 \cdot 8\end{array}$} & & & & & & \multirow{8}{*}{\begin{tabular}{|l} 
\\
$-8 \cdot 1$ \\
-8.0 \\
-15.0 \\
-18.0 \\
-21.0 \\
-9.5 \\
$=9.7$ \\
-11.0
\end{tabular}} & \multirow{8}{*}{$\begin{array}{r}9 \\
10 \\
11 \\
12 \\
13 \\
14 \\
15 \\
16\end{array}$} & & & & & & & 。 & • \\
\hline & & & & & & & & & 25 & $-8 \cdot 0$ & $-25 \cdot 0$ & \multirow{7}{*}{$\begin{array}{l}1 \\
2 \\
3 \\
4 \\
5 \\
6 \\
7 \\
8\end{array}$} & \multirow{7}{*}{$\begin{array}{r}4 \cdot 2 \\
2 \cdot 2 \\
-2 \cdot 0 \\
-6 \cdot 5 \\
-5 \cdot 0 \\
-0 \cdot 2 \\
-4 \cdot 5 \\
-2 \cdot 5\end{array}$} & & & \multirow{7}{*}{$\begin{array}{r}2.0 \\
0.8 \\
3.5 \\
-8.2 \\
-12.8 \\
-13.5 \\
-12.0 \\
-11.8\end{array}$} & \multirow{7}{*}{$\begin{array}{l}-11 \cdot 3 \\
-26 \cdot 2 \\
-20 \cdot 5 \\
-22 \cdot 0 \\
-35 \cdot 2 \\
-36 \cdot 2 \\
-21 \cdot 8 \\
-27 \cdot 8\end{array}$} & \multirow{7}{*}{$\begin{array}{l}17 \\
18 \\
19 \\
20 \\
21 \\
22 \\
23 \\
24\end{array}$} & \multirow{7}{*}{$\begin{array}{r}-21.0 \\
-17.2 \\
-16.0 \\
-3.8 \\
-11.5 \\
-11.8 \\
-9.5 \\
-9.0\end{array}$} & \multirow{7}{*}{$\begin{array}{l}-31 \cdot 4 \\
-30 \cdot 5 \\
-33.5 \\
-43.5 \\
-23.8 \\
-236.9 \\
-25 \cdot 0 \\
-26.8\end{array}$} & 25 & & 28 \\
\hline & & & & & & & & & ${ }_{27}^{2 H}$ & $\begin{array}{r}-2 \cdot 0 \\
1 \cdot 2\end{array}$ & $\begin{array}{l}-9 \cdot 7 \\
-8.3\end{array}$ & & & & & & & & & & 26 & -11.55 & $\begin{array}{r}-33.0 \\
-35 \%\end{array}$ \\
\hline & & & & & & & & & 28 & $1 \cdot 1$ & -14.5 & & & & & & & & & & $\begin{array}{l}28 \\
28\end{array}$ & 14.0 & $\begin{array}{l}1 . \\
-1 .\end{array}$ \\
\hline & & & & & & & & & 29 & $-4 \cdot 8$ & $-25 \cdot 0$ & & & & & & & & & & 29 & 15.0 & 12. \\
\hline & & & & & & & & & $\begin{array}{l}30 \\
31\end{array}$ & $\begin{array}{l}-3.8 \\
-4 \cdot 4\end{array}$ & $\begin{array}{l}-25.0 \\
-27.5\end{array}$ & & & & & & & & & & & & \\
\hline & & & & & & & & & & & & & & & & & & & & & & & \\
\hline & & & & & & & & & tean. & $-9 \cdot 8$ & 249 & & & & & & & & & & Mea & -4.8 & -22 \\
\hline
\end{tabular}


TamLe III. (continued).

DAILY EXTREMES OF TEMPERATURE.

IN Degrees FamRenileit.

October, 1902.

November, 1902.

\begin{tabular}{|c|c|c|c|c|c|c|c|c|c|c|c|c|c|c|c|c|c|c|c|c|c|c|c|}
\hline & & Min. & & Max. & Min. & Day. & $\mathrm{ax}$ & $\mathbf{M}$ & Day. & $\operatorname{Max}$. & $M$ & ay. & Max. & Min. & Day. & Max. & Min. & Day. & ax. & in. & Day. & (ax. & Mit \\
\hline & \multirow{5}{*}{$\begin{array}{r}0 \\
3.6 \\
0.02 \\
-4.0 \\
-4.0 \\
-10.0 \\
-15.0 \\
-10.0 \\
-9.8\end{array}$} & \multirow{5}{*}{$\begin{array}{l} \\
-13 \cdot 3 \\
-10.5 \\
-23.5 \\
-23.8 \\
-19.0 \\
-30.9 \\
-21.5 \\
-21.5 \\
-32.0\end{array}$} & \multirow{5}{*}{$\begin{array}{l}9 \\
10 \\
11 \\
12 \\
13 \\
14 \\
15 \\
16\end{array}$} & \multirow{5}{*}{ 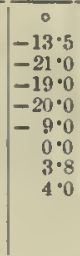 } & \multirow{5}{*}{ 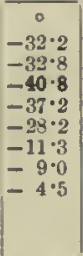 } & \multirow{5}{*}{$\begin{array}{l}18 \\
19 \\
20 \\
21 \\
22 \\
23 \\
21\end{array}$} & \multirow{5}{*}{$\begin{array}{c}0 \\
3 \cdot 0 \\
3.0 \\
33.8 \\
-0.4 \\
-2 \cdot 4 \\
-2 \cdot 5 \\
-3.2 \\
-5.8 \\
2 \cdot 5\end{array}$} & \multirow{5}{*}{ 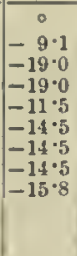 } & & & & \multirow{5}{*}{$\begin{array}{l}1 \\
2 \\
3 \\
4 \\
5 \\
6 \\
7 \\
7 \\
8\end{array}$} & \multirow{5}{*}{ 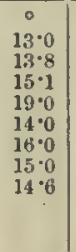 } & \multirow{5}{*}{$\begin{array}{l}5 \cdot 8 \\
8.8 \\
8.0 \\
6.0 \\
4 \cdot 4 \\
4 \cdot 0 \\
5.0\end{array}$} & \multirow{5}{*}{$\begin{array}{l}9 \\
10 \\
11 \\
12 \\
13 \\
14 \\
15 \\
16\end{array}$} & \multirow{5}{*}{\begin{tabular}{|c|}
0 \\
$14 \cdot 5$ \\
26.5 \\
18.5 \\
15.5 \\
$15 \cdot 2$ \\
19.0 \\
$22 \cdot 8$ \\
27.8 \\
$20 \cdot 6$
\end{tabular}} & \multirow{5}{*}{$\begin{array}{l}5 \cdot 0 \\
0.0 \\
0.02 \\
5.0 \\
9 \cdot 0 \\
6.5 \\
5.08\end{array}$} & \multirow{5}{*}{$\begin{array}{l}17 \\
18 \\
19 \\
20 \\
21 \\
22 \\
23 \\
21\end{array}$} & \multirow{5}{*}{$\begin{array}{c}14 \cdot 1 \\
8 \cdot 5 \\
12 \cdot 1 \\
11 \cdot 6 \\
15 \cdot 6 \\
21 \cdot 8 \\
23 \cdot 4\end{array}$} & \multirow{5}{*}{$\begin{array}{r}5.5 \\
2.2 \\
3.8 \\
4.5 \\
6.5 \\
8.5 \\
8.5 \\
8.8 \\
17.5\end{array}$} & \multirow{4}{*}{$\begin{array}{l}26 \\
27 \\
28 \\
28 \\
29 \\
30\end{array}$} & \multirow{4}{*}{$\begin{array}{r}80 \\
18.0 \\
28.0 \\
160 \\
10.0 \\
22.8\end{array}$} & \multirow{4}{*}{$\begin{array}{r}6 \cdot 0 \\
11.0 \\
5 \cdot 0\end{array}$} \\
\hline & & & & & & & & & $\begin{array}{l}28 \\
27\end{array}$ & $-\begin{array}{l}-2.0 \\
-9.8\end{array}$ & $\begin{array}{l}-1 \\
-1\end{array}$ & & & & & & & & & & & & \\
\hline & & & & & & & & & 23 & $\begin{array}{r}11 \cdot 5 \\
6.0\end{array}$ & $=-5$ & & & & & & & & & & & & \\
\hline & & & & & & & & & 31 & $\begin{array}{l}11 \cdot 4 \\
12 \cdot 0\end{array}$ & 0 & & & & & & & & & & & & \\
\hline & & & & & & & & & & & & & & & & & & & & & & 170 & \\
\hline
\end{tabular}

December, 1902.

January, 1903.

\begin{tabular}{|c|c|c|c|c|c|c|c|c|c|c|c|c|c|c|c|c|c|c|c|c|c|c|c|}
\hline Day. & Max. & Mrin. & Day. & Max. & Min. & Day. & $\operatorname{Max}$ & Min. & Day. & Max. & Min. & Day. & Max. & Min. & Day. & Max. & Min. & Day. & Max. & Min. & Day. & Max. & Min. \\
\hline \multirow{8}{*}{ I } & \multirow{8}{*}{$\begin{array}{l}0 \\
26 \cdot 0 \\
17 \% 8 \\
170^{\circ} 0 \\
17 \% 2 \\
20 \cdot 1 \\
18.5 \\
23 \cdot 5 \\
33 \cdot 0\end{array}$} & 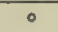 & & 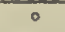 & 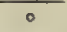 & & & & & 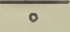 & 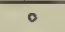 & \multirow{8}{*}{$\begin{array}{l}1 \\
2 \\
3 \\
4 \\
5 \\
6 \\
7 \\
8 \\
8\end{array}$} & \multirow{8}{*}{$\begin{array}{l}29 \cdot 5 \\
366^{\circ} \cdot 0 \\
32 \cdot 0 \\
36 \cdot 8 \\
25 \cdot 0 \\
32 \cdot 5 \\
38 \cdot 0 \\
39 \cdot 0\end{array}$} & & & & & & $\circ$ & 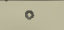 & & 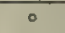 & \\
\hline & & \multirow{7}{*}{$\begin{array}{r}14 \cdot 8 \\
5 \cdot 0 \\
4 \cdot 2 \\
9 \cdot 0 \\
9 \cdot 0 \\
13 \cdot 2 \\
12{ }^{\circ} 0 \\
16^{\circ} 0\end{array}$} & \multirow{7}{*}{$\begin{array}{r}9 \\
10 \\
11 \\
12 \\
13 \\
14 \\
15 \\
16\end{array}$} & \multirow{7}{*}{$\begin{array}{l}37 \cdot 2 \\
36 \cdot 5 \\
35 \cdot 0 \\
29 \cdot 5 \\
29 \cdot 0 \\
33 \cdot 6 \\
30 \cdot 5 \\
25 \cdot 8\end{array}$} & \multirow{7}{*}{$\begin{array}{l}30 \cdot 0 \\
25 \cdot 0 \\
21 \cdot 8 \\
21 \cdot 8 \\
25 \cdot 0 \\
26.0 \\
21 \cdot 5 \\
20 \cdot 2\end{array}$} & \multirow{7}{*}{$\begin{array}{l}17 \\
18 \\
19 \\
20 \\
21 \\
22 \\
23 \\
24\end{array}$} & \multirow{7}{*}{$\begin{array}{l}27 \cdot 2 \\
35 \cdot 2 \\
25 \cdot 0 \\
27 \cdot 0 \\
24 \cdot 2 \\
22 \cdot 0 \\
27 \cdot 0 \\
32 \cdot 0\end{array}$} & \multirow{7}{*}{$\begin{array}{l}20 \cdot 0 \\
20 \cdot 8 \\
19 \cdot 8 \\
18 \cdot 0 \\
11 \cdot 8 \\
10 \cdot 5 \\
15 \cdot 0 \\
13 \cdot 0\end{array}$} & 25 & $37 \cdot 8$ & 22.0 & & & \multirow{7}{*}{$\begin{array}{l}18 \cdot 8 \\
14 \cdot 8 \\
25 \cdot 5 \\
23 \cdot 0 \\
25 \cdot 0 \\
22 \cdot 0 \\
19 \cdot 8 \\
19 \cdot 8\end{array}$} & \multirow{7}{*}{$\begin{array}{r}9 \\
10 \\
11 \\
12 \\
13 \\
14 \\
15 \\
16\end{array}$} & \multirow{7}{*}{$\begin{array}{l}27 \cdot 8 \\
29 \cdot 5 \\
32 \cdot 2 \\
32 \cdot 0 \\
31 \cdot 5 \\
29 \cdot 5 \\
29 \cdot 0 \\
27 \cdot 0\end{array}$} & \multirow{7}{*}{$\begin{array}{l}23 \cdot 2 \\
22 \cdot 0 \\
24^{\circ} \cdot 0 \\
25^{\circ} 0 \\
24^{\circ} 0 \\
20^{\circ} \\
22^{\circ} 0 \\
19 \cdot 8\end{array}$} & \multirow{7}{*}{$\begin{array}{l}17 \\
18 \\
19 \\
20 \\
21 \\
22 \\
23 \\
24\end{array}$} & \multirow{7}{*}{$\begin{array}{l}28 \cdot 5 \\
31 \cdot 6 \\
29 \cdot 0 \\
25 \cdot 2 \\
29 \cdot 0 \\
31 \cdot 2 \\
31 \cdot 0 \\
34 \cdot 0\end{array}$} & \multirow{7}{*}{$\begin{array}{l}18 \cdot 0 \\
21 \cdot 0 \\
13 \cdot 2 \\
14 \cdot 2 \\
14 \cdot 0 \\
18 \cdot 0 \\
14 \cdot 5 \\
266^{\circ} \cdot 0\end{array}$} & 25 & $34^{\circ} 0$ & \\
\hline & & & & & & & & & 20 & $\begin{array}{l}39 \cdot 0 \\
39 \cdot 0\end{array}$ & $21 \cdot 0$ & & & & & & & & & & $\begin{array}{l}26 \\
27\end{array}$ & $\begin{array}{l}23 \cdot 2 \\
34.0\end{array}$ & $\begin{array}{l}17 \\
12\end{array}$ \\
\hline & & & & & & & & & $\begin{array}{l}27 \\
28\end{array}$ & $38 \cdot 8$ & $\begin{array}{l}17.0 \\
23 \cdot 0\end{array}$ & & & & & & & & & & 28 & $30 \cdot 2$ & 12. \\
\hline & & & & & & & & & 29 & $36^{\circ} 0$ & $22 \cdot 0$ & & & & & & & & & & 29 & $35 \cdot 0$ & \\
\hline & & & & & & & & & ?) & 36 & $180^{\circ}$ & & & & & & & & & & 31. & & \\
\hline & & & & & & & & & 31 & $33 \cdot 0$ & 15.5 & & & & & & & & & & & & \\
\hline & & & & & & & & & Mea & $29 \cdot 3$ & $17 \cdot 5$ & & & & & & & & & & Mean. & $31 \cdot 6$ & 18 \\
\hline
\end{tabular}

February, 1903.

March, 1903.

\begin{tabular}{|c|c|c|c|c|c|c|c|c|c|c|c|c|c|c|c|c|c|c|c|c|c|c|c|}
\hline Day. & Max. & Min. & Day. & Max. & Min. & Day. & Max. & Min. & Day. & Max. & Min. & Day. & Max. & Min. & Day. & Max. & Min. & Day. & Max. & Min. & Day. & Max. & Min. \\
\hline \multirow{8}{*}{$\begin{array}{l}1 \\
2 \\
3 \\
4 \\
5 \\
6 \\
7 \\
8\end{array}$} & \multirow{8}{*}{$\begin{array}{c}0 \\
31 \cdot 0 \\
32 \cdot 2 \\
30.0 \\
32 \cdot 0 \\
23.0 \\
230^{\circ} 0 \\
22 \cdot 2 \\
19.0\end{array}$} & \multirow{8}{*}{$\begin{array}{r}0 \\
18 \cdot 0 \\
14 \cdot 0 \\
17 \cdot 2 \\
12^{\circ} 0 \\
10^{\circ} 0 \\
10^{\circ} \cdot 5 \\
9 \cdot 5 \\
8 \cdot 0\end{array}$} & \multirow{8}{*}{$\begin{array}{r}9 \\
10 \\
11 \\
12 \\
13 \\
14 \\
15 \\
16\end{array}$} & \multirow{8}{*}{$\begin{array}{r}0 \\
23 \cdot 0 \\
22 \cdot 0 \\
17 \cdot 0 \\
16.0 \\
16.0 \\
6 \cdot 0 \\
7 \cdot 0 \\
9 \cdot 5\end{array}$} & \multirow{8}{*}{$\begin{array}{r}0 \\
10.0 \\
7.2 \\
9.0 \\
-1.0 \\
40 \\
-7.3 \\
-1.0 \\
-2.8\end{array}$} & \multirow{8}{*}{$\begin{array}{l}17 \\
18 \\
19 \\
20 \\
21 \\
22 \\
23 \\
24\end{array}$} & \multirow{8}{*}{$\begin{array}{r}0 \\
7 \cdot 0 \\
9 \cdot 0 \\
12 \cdot 0 \\
17 \cdot 0 \\
15 \cdot 1 \\
16 \cdot 1 \\
20 \cdot 5 \\
15 \cdot 0\end{array}$} & \multirow{8}{*}{$\begin{array}{r}0 \\
-4.0 \\
-\quad 6.0 \\
0.0 \\
-1.0 \\
3.0 \\
-4.2 \\
-0.8 \\
-9.8\end{array}$} & & 0 & 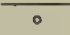 & \multirow{8}{*}{$\begin{array}{l}1 \\
2 \\
3 \\
4 \\
5 \\
6 \\
7 \\
8\end{array}$} & \multirow{8}{*}{$\begin{array}{r}0 \\
17 \cdot 8 \\
13 \cdot 0 \\
8.0 \\
20.5 \\
25.5 \\
5.0 \\
12 \cdot 0 \\
9.0\end{array}$} & \multirow{8}{*}{$\begin{array}{r}\quad 0 \\
-12.0 \\
2.2 \\
-1.0 \\
-1.4 \\
5 \cdot 0 \\
-7.5 \\
-3.0 \\
-1.7\end{array}$} & \multirow{8}{*}{$\begin{array}{r}9 \\
10 \\
11 \\
12 \\
13 \\
14 \\
15 \\
16\end{array}$} & \multirow{8}{*}{$\begin{array}{r}0 \\
5.8 \\
40 \\
2.5 \\
0.0 \\
-1.5 \\
3.0 \\
140 \\
16.0\end{array}$} & \multirow{8}{*}{$\begin{array}{r} \\
-4 \cdot 2 \\
-5 \cdot 4 \\
-11.3 \\
-12.8 \\
-15.8 \\
-10 \cdot 0 \\
-5.7 \\
-4 \cdot 2\end{array}$} & \multirow{8}{*}{$\begin{array}{l}17 \\
18 \\
19 \\
20 \\
21 \\
22 \\
23 \\
24\end{array}$} & \multirow{8}{*}{$\begin{array}{r}0 \\
2.0 \\
-4.0 \\
=4.0 \\
-0.2 \\
-2.8 \\
-3.0 \\
-5.5 \\
-9.0\end{array}$} & \multirow{8}{*}{$\begin{array}{r}0 \\
-11 \cdot 3 \\
-14 \cdot 5 \\
-15 \cdot 5 \\
-18 \cdot 2 \\
-15 \cdot 0 \\
-17.4 \\
-20.0 \\
-18.7\end{array}$} & & $\therefore$ & 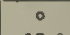 \\
\hline & & & & & & & & & 25 & $15^{\circ} 0$ & $\begin{array}{r}-4.8 \\
-5.2\end{array}$ & & & & & & & & & & $\begin{array}{l}25 \\
26\end{array}$ & $\begin{array}{l}-7 \cdot 5 \\
-2 \cdot 5\end{array}$ & $\begin{array}{l}-17 \cdot 0 \\
-17 \cdot 6\end{array}$ \\
\hline & & & & & & & & & 27 & 11.0 & 32 & & & & & & & & & & 27 & 70 & -13.5 \\
\hline & & & & & & & & & 28 & $\begin{array}{r}1.0 \\
8 \cdot 2\end{array}$ & $\begin{array}{l}5.1 \\
-5.1\end{array}$ & & & & & & & & & & 28 & $100^{\circ}$ & -11.5 \\
\hline & & & & & & & & & & & & & & & & & & & & & $\begin{aligned} 29 \\
30\end{aligned}$ & $19 \cdot 2$ & $\begin{array}{r}50 \\
-\quad 20 \\
\end{array}$ \\
\hline & & & & & & & & & & & & & & & & & & & & & $\begin{array}{l}30 \\
31\end{array}$ & $2 \cdot 0$ & $=\begin{array}{l}-20 \\
-90\end{array}$ \\
\hline & & & & & & & & & & & & & & & & & & & & & & & \\
\hline & & & & & & & & & Mean. . & $17 \cdot 6$ & $3 \cdot 3$ & & & & & & & & & & Mear. . & $5 \cdot 4$ & -9 \\
\hline
\end{tabular}

April, 1903.

May, 1903.

\begin{tabular}{|c|c|c|c|c|c|c|c|c|c|c|c|c|c|c|c|c|c|c|c|c|c|c|c|}
\hline Day. & Max. & Min. & Day. & Max. & Min. & Day. & Max. & Min. & Day. & Max. & Min. & Day. & Max. & Min. & Day. & Max. & Min. & Day. & Max. & Min. & Day. & Max. & Min. \\
\hline \multirow{7}{*}{$\begin{array}{l}1 \\
2 \\
3 \\
4 \\
5 \\
6 \\
7 \\
8 \\
8\end{array}$} & \multirow{7}{*}{$\begin{aligned} & 0 \\
&- 2 \cdot 0 \\
& 0.5 \\
&= 4.8 \\
&-9.0 \\
&-10.0 \\
&-13.0 \\
&-12.0 \\
&-11.0\end{aligned}$} & \multirow{7}{*}{$\begin{array}{r}0 \cdot \\
-10 \cdot 7 \\
-14 \cdot 3 \\
-21 \cdot 0 \\
-17 \cdot 6 \\
-23 \cdot 2 \\
-19 \cdot 0 \\
-38 \cdot 0 \\
-33 \cdot 2\end{array}$} & \multirow{7}{*}{$\begin{array}{r}9 \\
10 \\
11 \\
12 \\
13 \\
14 \\
15 \\
16\end{array}$} & \multirow{7}{*}{ 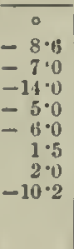 } & \multirow{7}{*}{$\begin{array}{r}\circ \\
-31 \cdot 0 \\
-21 \cdot 2 \\
-28 \cdot 0 \\
-30.0 \\
-26 \cdot 0 \\
-19 \cdot 2 \\
-14 \cdot 5 \\
-28 \cdot 8\end{array}$} & \multirow{7}{*}{$\begin{array}{l}17 \\
18 \\
19 \\
20 \\
21 \\
22 \\
23 \\
24\end{array}$} & \multirow{7}{*}{\begin{tabular}{|c|}
$\circ$ \\
-10.0 \\
-18.8 \\
-12.8 \\
$-7 \cdot 0$ \\
-70.0 \\
-10.0 \\
-8.0 \\
-11.0 \\
\end{tabular}} & \multirow{7}{*}{$\begin{array}{c}0 \\
-28.9 \\
-27 \cdot 0 \\
-32.0 \\
-32 \cdot 0 \\
-40.0 \\
-40 \cdot 1 \\
-25 \cdot 9 \\
-26 \cdot 0\end{array}$} & & & & \multirow{7}{*}{$\begin{array}{l}1 \\
2 \\
3 \\
4 \\
5 \\
6 \\
7 \\
8\end{array}$} & \multirow{7}{*}{ 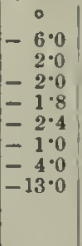 } & \multirow{7}{*}{$\begin{array}{l}0 \\
-30 \cdot 6 \\
-9 \cdot 5 \\
-13 \cdot 5 \\
-15 \cdot 0 \\
-14 \cdot 5 \\
-11 \cdot 3 \\
-19 \cdot 0 \\
-23 \cdot 0\end{array}$} & \multirow{7}{*}{$\begin{array}{r}9 \\
10 \\
11 \\
12 \\
13 \\
14 \\
15 \\
16\end{array}$} & \multirow{7}{*}{$\begin{array}{r}0 \\
-17 \cdot 0 \\
-17 \cdot 0 \\
-22 \cdot 2 \\
-22 \cdot 5 \\
-221.5 \\
-20 \cdot 5 \\
-27 \cdot 0 \\
-18 \cdot 0\end{array}$} & \multirow{7}{*}{$\begin{array}{r}0 \\
-24 \cdot 1 \\
-31 \cdot 0 \\
-31 \cdot 0 \\
-48 \cdot 0 \\
-48 \cdot 2 \\
-47 \cdot 0 \\
-51 \cdot 2 \\
-51 \cdot 0\end{array}$} & \multirow{7}{*}{$\begin{array}{l}17 \\
18 \\
19 \\
20 \\
21 \\
22 \\
23 \\
21\end{array}$} & \multirow{7}{*}{$\begin{array}{r}0 \\
2 \cdot 0 \\
9 \cdot 2 \\
9 \cdot 0 \\
0 \cdot 5 \\
4 \cdot 2 \\
-4 \cdot 0 \\
-12 \cdot 0 \\
-7 \cdot 0\end{array}$} & \multirow{7}{*}{$\begin{array}{r}0 \\
-22 \cdot 6 \\
-5 \cdot 5 \\
-18 \cdot 5 \\
-32 \cdot 8 \\
-23 \cdot 0 \\
-17 \cdot 9 \\
-29 \cdot 0 \\
-22 \cdot 5\end{array}$} & \multirow{6}{*}{$\begin{array}{l}25 \\
25 \\
27 \\
28 \\
28 \\
29 \\
30 \\
31\end{array}$} & \multirow{6}{*}{$\begin{array}{r}-4 \\
-4.2 \\
-1.2 \\
-6.5 \\
-13.5 \\
-140 \\
4.0 \\
-5.0\end{array}$} & \multirow{6}{*}{$\begin{array}{l}-21 \cdot 0 \\
-18.2 \\
-300 \\
-36.0 \\
-30 \\
-30 \\
-38.0 \\
-18.1\end{array}$} \\
\hline & & & & & & & & & 25 & $\begin{array}{l}-15 \cdot 0 \\
-19 \cdot 5\end{array}$ & $\begin{array}{l}-33 \cdot 2 \\
-42 \cdot 0\end{array}$ & & & & & & & & & & & & \\
\hline & & & & & & & & & 27 & $=0$ & -38.5 & & & & & & & & & & & & \\
\hline & & & & & & & & & 28 & $\begin{array}{r}-10 \\
5-2\end{array}$ & $\begin{array}{l}-32.0 \\
-21.0\end{array}$ & & & & & & & & & & & & \\
\hline & & & & & & & & & 30 & -1.0 & -29.0 & & & & & & & & & & & & \\
\hline & & & & & & & & & & & & & & & & & & & & & & & \\
\hline & & & & & & & & & Mean. & -80 & -27 & & & & & & & & & & Mean. & -7.5 & $\mid-27 \cdot 1$ \\
\hline
\end{tabular}


TABLe III. (continued).

\section{DAILY EXTREMES OF TEMPERATURE.}

June, 1903.

IN Degrees FamrenHeit.

\begin{tabular}{|c|c|c|c|c|c|c|c|c|c|c|c|c|c|c|c|c|c|c|c|c|c|c|c|}
\hline ay. & Max. & Min. & $\mathrm{ay}$. & Max. & Min. & Day. & Max. & Min. & Day. & Max. & Min. & ay. & Iax. & fin. & Day. & Max. & Mrin. & Day. & Max. & Min. & Day. & Max. & Mir \\
\hline \multirow{7}{*}{3} & \multirow{7}{*}{$\mid \begin{array}{c}0 \\
-10.0 \\
-18.0 \\
-17.0 \\
-15.7 \\
-10.0 \\
-17.0 \\
-7.3 \\
-2.0\end{array}$} & \multirow{7}{*}{\begin{tabular}{|l|}
0 \\
$-28 \cdot 0$ \\
$-33 \cdot 7$ \\
$-33 \cdot 0$ \\
$-32 \cdot 0$ \\
$-26 \cdot 0$ \\
$-34 \cdot 0$ \\
$-39 \cdot 5$ \\
$-18 \cdot 5$ \\
$-18 \cdot 0$
\end{tabular}} & \multirow{7}{*}{$\begin{array}{l}9 \\
10 \\
11 \\
12 \\
13 \\
14 \\
15 \\
16\end{array}$} & \multirow{7}{*}{$\begin{array}{r}0 \\
11 \cdot 2 \\
1120 \\
17 \cdot 0 \\
9 \cdot 1 \\
6.0 \\
0.2 \\
-3.0 \\
-2.0\end{array}$} & \multirow{7}{*}{$\begin{array}{r}0 \\
-11.7 \\
-3.6 \\
4.0 \\
-22.0 \\
-24.5 \\
-14.0 \\
-27.0 \\
-23.0\end{array}$} & \multirow{7}{*}{$\begin{array}{l}17 \\
18 \\
19 \\
20 \\
21 \\
22 \\
23 \\
24\end{array}$} & \multirow{7}{*}{$\begin{array}{r}0 \\
-9 \cdot 0 \\
-13 \cdot 0 \\
-11 \cdot 2 \\
-10.0 \\
-11 \cdot 0 \\
-15 \cdot 0 \\
-11 \cdot 5 \\
2 \cdot 0\end{array}$} & \multirow{7}{*}{$\begin{array}{l}-23 \cdot 4 \\
-42 \cdot 0 \\
-41 \cdot 8 \\
-43 \cdot 5 \\
-38.5 \\
-47 \cdot 0 \\
-44 \cdot 0 \\
-41 \cdot 0\end{array}$} & & & & \multirow{7}{*}{$\begin{array}{l}1 \\
2 \\
3 \\
4 \\
5 \\
6 \\
7 \\
8\end{array}$} & \multirow{7}{*}{$\begin{array}{l}-0 \\
-7.70 \\
-7.0 \\
-15.0 \\
-18.2 \\
-20.8 \\
-19 \cdot 0 \\
-30.0 \\
-18.0\end{array}$} & \multirow{7}{*}{$\begin{array}{l}-24.5 \\
-21.2 \\
-28.5 \\
-34.0 \\
-47.8 \\
-46.5 \\
-47.5 \\
-46.0 \\
-46.0\end{array}$} & \multirow{7}{*}{$\begin{array}{r}9 \\
10 \\
11 \\
12 \\
13 \\
14 \\
15 \\
16\end{array}$} & & & \multirow{7}{*}{$\begin{array}{l}17 \\
18 \\
19 \\
20 \\
21 \\
22 \\
23 \\
24\end{array}$} & & \multirow{7}{*}{$\begin{array}{l}-31 \cdot 7 \\
-17.2 \\
-18.0 \\
-23.0 \\
-32.6 \\
-3360 \\
-20 \cdot 0 \\
-9.5\end{array}$} & & & \\
\hline & & & & & & & & & & & & & & & & \multirow{6}{*}{$\begin{array}{l}-15 \cdot 0 \\
-29 \cdot 0 \\
-12.0 \\
-11.0 \\
-7 \cdot 8 \\
-8 \cdot 0 \\
-7 \cdot 0 \\
-23.0\end{array}$} & \multirow{6}{*}{$\begin{array}{l}-48.0 \\
-52.5 \\
-53.0 \\
-39 \cdot 4 \\
-39.0 \\
-25.8 \\
-37.5 \\
-37.5\end{array}$} & & \multirow{6}{*}{$\begin{array}{r}-10.5 \\
12.0 \\
12.0 \\
-13.0 \\
-11.5 \\
0.0 \\
9.0 \\
9.8\end{array}$} & & & & \\
\hline & & & & & & & & & & & & & & & & & & & & & & & 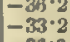 \\
\hline & & & & & & & & & & $\begin{array}{l}5 \cdot 5 \\
6 \cdot 5 \\
6 \cdot 5\end{array}$ & & & & & & & & & & & & & -48 \\
\hline & & & & & & & & & & 3.0 & -20.7 & & & & & & & & & & & & -81. \\
\hline & & & & & & & & & & & & & & & & & & & & & & & \\
\hline & & & & & & & & & & 43 & & & & & & & & & & & & & -30 \\
\hline
\end{tabular}

August, 1903.

September, 1903.

\begin{tabular}{|c|c|c|c|c|c|c|c|c|c|c|c|c|c|c|c|c|c|c|c|c|c|c|c|}
\hline Day. & Max. & Min. & Day. & Max. & Min. & Day. & Max. & Min. & Das. & Max. & Min. & Day. & Max. & Min. & Day. & Max. & Min. & Day. & Max. & Min. & Day. & Max. & Min. \\
\hline \multirow{8}{*}{$\begin{array}{l}1 \\
2 \\
3 \\
4 \\
5 \\
6 \\
7 \\
8 \\
8\end{array}$} & \multirow{8}{*}{$\begin{array}{r}0 \\
-3.8 \\
-15.4 \\
-15.8 \\
-15.8 \\
-24.0 \\
-29.0 \\
-18.8 \\
-12.5\end{array}$} & \multirow{8}{*}{$\begin{array}{l}0 \\
-26 \cdot 0 \\
-28.0 \\
-31 \cdot 0 \\
-290^{\circ} 0 \\
-48 \cdot 0 \\
-533.2 \\
-44 \cdot 0 \\
-26.5\end{array}$} & \multirow{7}{*}{$\begin{array}{r}9 \\
10 \\
11 \\
12 \\
13 \\
14 \\
15 \\
16\end{array}$} & & & \multirow{8}{*}{$\begin{array}{l}17 \\
18 \\
19 \\
20 \\
21 \\
22 \\
23 \\
24\end{array}$} & \multirow{8}{*}{$\begin{array}{r}-7.0 \\
-2.2 \\
-\quad 70 \\
-20.2 \\
900 \\
11.8 \\
6.0 \\
-\quad 8.8\end{array}$} & & & 1. & 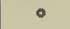 & \multirow{8}{*}{$\begin{array}{l}1 \\
2 \\
3 \\
4 \\
5 \\
6 \\
7 \\
8\end{array}$} & \multirow{8}{*}{$\begin{array}{r}0 \\
-15 \cdot 0 \\
-15 \cdot 0 \\
-12.5 \\
-13 \cdot 2 \\
-17 \cdot 2 \\
-6 \cdot 8 \\
4 \cdot 0 \\
3 \cdot 0\end{array}$} & \multirow{8}{*}{\begin{tabular}{|l|} 
\\
$-35 \cdot 0$ \\
-34.6 \\
-338.5 \\
-34.8 \\
-4480 \\
-188.0 \\
-18.7 \\
-14.9
\end{tabular}} & \multirow{8}{*}{$\begin{array}{l}9 \\
10 \\
11 \\
12 \\
13 \\
14 \\
15 \\
16\end{array}$} & \multirow{8}{*}{$\begin{array}{r}-0.8 \\
-\quad 8.5 \\
-770 \\
-17.1 \\
-21.0 \\
-22.5 \\
-20.0 \\
-26.2\end{array}$} & \multirow{8}{*}{$\begin{array}{l}-19 \cdot 0 \\
-21 \cdot 0 \\
-26.6 \\
-34 \cdot 0 \\
-34 \cdot 0 \\
-33 \cdot 7 \\
-36 \cdot 8 \\
-37 \cdot 2\end{array}$} & \multirow{8}{*}{$\begin{array}{l}17 \\
18 \\
19 \\
20 \\
21 \\
22 \\
23 \\
24\end{array}$} & \multirow{8}{*}{$\begin{array}{l}0 \\
-26 \cdot 0 \\
-32 \cdot 0 \\
-31 \cdot 0 \\
-32 \cdot 0 \\
-200 \\
-21 \cdot 0 \\
0 \cdot 0 \\
-1 \cdot 0\end{array}$} & \multirow{8}{*}{$\begin{array}{l}-37 \cdot 0 \\
-49 \cdot 2 \\
-555 \\
-58.5 \\
-50.5 \\
-30.0 \\
-30.0 \\
-25.0 \\
-13.5\end{array}$} & \multirow{6}{*}{$\begin{array}{l}25 \\
26 \\
27 \\
28 \\
29 \\
30\end{array}$} & • & ○ \\
\hline & & & & \multirow{7}{*}{$\begin{array}{r}-16.0 \\
9.5 \\
11.5 \\
10.0 \\
-5.5 \\
-3.0 \\
-1.0 \\
2.5\end{array}$} & \multirow{7}{*}{$\begin{array}{l}-42 \cdot 8 \\
-28 \cdot 1 \\
-20 \cdot 5 \\
-24 \cdot 7 \\
-29 \cdot 5 \\
-28 \cdot 0 \\
-19 \cdot 0 \\
-32 \cdot 5\end{array}$} & & & \multirow{7}{*}{$\begin{array}{l}-24 \cdot 2 \\
-29 \cdot 0 \\
-37 \cdot 2 \\
-46.6 \\
-29 \cdot 5 \\
-5.5 \\
-19 \cdot 6 \\
-18 \cdot 2\end{array}$} & 25 & $-9 \cdot 0$ & $-38 \cdot 2$ & & & & & & & & & & & \multirow{5}{*}{$\begin{array}{r}7.2 \\
11.0 \\
9.5 \\
13.2 \\
-110 \\
6.0\end{array}$} & \multirow{5}{*}{$\begin{array}{l}-14.0 \\
-6.5 \\
-13.3 \\
-11.5 \\
-15.0 \\
-11.3\end{array}$} \\
\hline & & & & & & & & & $\begin{array}{l}26 \\
27\end{array}$ & $\begin{array}{l}4.5 \\
6.5\end{array}$ & $\begin{array}{l}-36 \cdot 5 \\
-19 \cdot 2\end{array}$ & & & & & & & & & & & & \\
\hline & & & & & & & & & 28 & $\begin{array}{r}8 \% \\
-6.8\end{array}$ & $\begin{array}{l}-19 \cdot \\
-26.8\end{array}$ & & & & & & & & & & & & \\
\hline & & & & & & & & & $\begin{array}{l}29 \\
30 \\
30\end{array}$ & $\mid \begin{array}{r}-60 \\
-16.0\end{array}$ & $\begin{array}{l}-32.5 \\
-37.0\end{array}$ & & & & & & & & & & & & \\
\hline & & & & & & & & & 31 & $-17 \cdot 0$ & $-44 \cdot 0$ & & & & & & & & & & & & \\
\hline & & & & & & & & & & & $-\infty 0$ & & & & & & & & & & & & $-29 \cdot 3$ \\
\hline & & & & & & & & & & & & & & & & & & & & & & & \\
\hline
\end{tabular}

October, 1903.

November, 1903.

\begin{tabular}{|c|c|c|c|c|c|c|c|c|c|c|c|c|c|c|c|c|c|c|c|c|c|c|c|}
\hline Das. & Max. & Min. & Day. & Max. & Min. & Das. & Max. & Min. & Day. & Max. & Min. & Day. & Max. & Min. & Day. & Max. & Min. & Das. & Max. & Min. & Day. & Max. & Min. \\
\hline \multirow[t]{2}{*}{$\begin{array}{l}1 \\
2 \\
3 \\
4 \\
5 \\
6 \\
7 \\
8\end{array}$} & \multirow[t]{2}{*}{$\begin{array}{r}0 \\
3.5 \\
-12.5 \\
-17.2 \\
-22.0 \\
-10.5 \\
50 \\
0.0 \\
-4.8\end{array}$} & \multirow[t]{2}{*}{$\begin{array}{r} \\
-11 \cdot 5 \\
-19 \cdot 8 \\
-27.0 \\
-40.6 \\
-42.8 \\
-142 \\
-22.9 \\
-27 \cdot 5\end{array}$} & \multirow[t]{2}{*}{$\begin{array}{r}9 \\
10 \\
11 \\
12 \\
13 \\
14 \\
15 \\
16\end{array}$} & \multirow[t]{2}{*}{$\begin{array}{r}-5 \cdot 0 \\
-\quad 4 \cdot 5 \\
8 \cdot 2 \\
5 \cdot 0 \\
4 \cdot 0 \\
5 \cdot 4 \\
0 \cdot 0 \\
-2 \cdot 2\end{array}$} & \multirow[t]{2}{*}{$\begin{array}{r}0 \\
-17 \cdot 3 \\
-19.2 \\
-11.5 \\
-12.5 \\
-10.5 \\
-7 \cdot 7 \\
-19.8 \\
-20 \cdot 8\end{array}$} & \multirow[t]{2}{*}{$\begin{array}{l}17 \\
18 \\
19 \\
20 \\
21 \\
22 \\
23 \\
24\end{array}$} & \multirow[t]{2}{*}{$\begin{array}{r} \\
-1.3 \\
-4.4 \\
-11.0 \\
8.0 \\
60 \\
5.0 \\
-2.0 \\
-3.0\end{array}$} & \multirow[t]{2}{*}{$\begin{array}{r}0 \\
-20.0 \\
-21.4 \\
-27.2 \\
-25.2 \\
-14.5 \\
-8.3 \\
-12.5 \\
-10.5\end{array}$} & $\begin{array}{l}25 \\
26 \\
27 \\
28 \\
29 \\
30 \\
31\end{array}$ & $\begin{array}{r}0 \\
2 \cdot 0 \\
8 \cdot 2 \\
12 \cdot 0 \\
8 \cdot 0 \\
6 \cdot 5 \\
4 \cdot 8 \\
11 \cdot 0\end{array}$ & $\begin{array}{r}0 \\
-7.7 \\
-12.3 \\
1.8 \\
-5.5 \\
-8.5 \\
-5.7 \\
-4.2\end{array}$ & \multirow[t]{2}{*}{$\begin{array}{l}1 \\
2 \\
3 \\
4 \\
5 \\
6 \\
7 \\
8\end{array}$} & \multirow[t]{2}{*}{$\begin{array}{c}0 \\
16 \cdot 2 \\
24 \cdot 5 \\
12 \cdot 0 \\
16 \cdot 7 \\
15 \cdot 2 \\
10 \cdot 0 \\
10 \cdot 0 \\
17 \cdot 5\end{array}$} & \multirow[t]{2}{*}{$\begin{array}{r}9.0 \\
8.9 \\
-5.5 \\
-2.0 \\
-4.5 \\
2.5 \\
0.5 \\
5.2\end{array}$} & \multirow[t]{2}{*}{$\begin{array}{r}9 \\
10 \\
11 \\
12 \\
13 \\
14 \\
15 \\
16\end{array}$} & \multirow[t]{2}{*}{$\begin{array}{l}22 \cdot 4 \\
21 \cdot 2 \\
21 \cdot 2 \\
24 \cdot 8 \\
26 \cdot 0 \\
22 \cdot 2 \\
23 \cdot 8 \\
16 \cdot 0\end{array}$} & \multirow[t]{2}{*}{$\begin{array}{r}7 \cdot 5 \\
2 \cdot 4 \\
11 \cdot 8 \\
12 \cdot 0 \\
5 \cdot 2 \\
5 \cdot 2 \\
4 \cdot 1 \\
8 \cdot 5\end{array}$} & \multirow[t]{2}{*}{$\begin{array}{l}17 \\
18 \\
19 \\
20 \\
21 \\
22 \\
23 \\
24\end{array}$} & \multirow[t]{2}{*}{$\begin{array}{r}0 \\
9 \cdot 5 \\
19 \cdot 0 \\
20 \cdot 1 \\
19 \cdot 8 \\
24 \cdot 8 \\
34 \cdot 0 \\
24 \cdot 5 \\
17 \cdot 0\end{array}$} & \multirow[t]{2}{*}{$\begin{array}{r}0 \\
-0.7 \\
5.2 \\
-2.2 \\
-0.6 \\
2.8 \\
18 \cdot 6 \\
9 \cdot 0 \\
8.2\end{array}$} & $\begin{array}{l}25 \\
26 \\
27 \\
28 \\
29 \\
30\end{array}$ & $\begin{array}{r}0 \\
25 \cdot 6 \\
27.5 \\
26 \cdot 0 \\
31.0 \\
28 \cdot 8 \\
26 \cdot 0\end{array}$ & $\begin{array}{r}5 \cdot 0 \\
70^{\circ} \\
18.5 \\
22^{\circ} 6 \\
20.5 \\
18^{\circ} 6\end{array}$ \\
\hline & & & & & & & & & Mean. . & 0.1 & $-16 \cdot 5$ & & & & & & & & & & Mean. . & $21 \cdot 1$ & 6.8 \\
\hline
\end{tabular}

December, 1903.

Jannary, 1904.

\begin{tabular}{|c|c|c|c|c|c|c|c|c|c|c|c|c|c|c|c|c|c|c|c|c|c|c|c|}
\hline Day. & Max. & Min. & Day. & Max. & Min. & Day. & $\operatorname{Max}$. & Min. & Day. & Max. & Min. & Day. & $\mathbf{M a x}$ & Min. & Day. & Max. & Min. & Day. & Max. & Min. & Das. & $M_{\text {sx. }}$ & Min. \\
\hline \multirow{8}{*}{$\begin{array}{l}1 \\
2 \\
3 \\
4 \\
5 \\
6 \\
7 \\
8\end{array}$} & \multirow{8}{*}{$\begin{array}{c}0 \\
26 \cdot 2 \\
21 \cdot 8 \\
25.5 \\
23 \cdot 0 \\
22 \cdot 0 \\
23 \cdot 1 \\
27 \cdot 6 \\
27 \cdot 9\end{array}$} & \multirow{8}{*}{$\begin{array}{r}18 \cdot 5 \\
13 \cdot 5 \\
19 \cdot 0 \\
18 \cdot 8 \\
7 \cdot 6 \\
7 \cdot 6 \\
12 \cdot 0 \\
18 \cdot 4\end{array}$} & \multirow{8}{*}{$\begin{array}{r}9 \\
11 \\
11 \\
12 \\
13 \\
14 \\
15 \\
16\end{array}$} & \multirow{8}{*}{$\begin{array}{c}0 \\
31 \cdot 0 \\
32.2 \\
31.0 \\
32.8 \\
28.8 \\
33.0 \\
32 \cdot 6 \\
33 \cdot 5\end{array}$} & & \multirow{8}{*}{$\begin{array}{l}17 \\
18 \\
19 \\
21 \\
21 \\
22 \\
23 \\
21\end{array}$} & \multirow{8}{*}{$\begin{array}{c}0 \\
25 \cdot 2 \\
21 \cdot 8 \\
31 \cdot 5 \\
32 \cdot 4 \\
29 \cdot 1 \\
29 \cdot 6 \\
32 \cdot 5 \\
30 \cdot 8\end{array}$} & \multirow{8}{*}{$\begin{array}{r}0 \\
14 \cdot 5 \\
14 \cdot 6 \\
16 \cdot 2 \\
20 \cdot 6 \\
20 \cdot 0 \\
24 \cdot 2 \\
23 \cdot 0 \\
19 \cdot 1\end{array}$} & & 0 & & \multirow{8}{*}{$\begin{array}{l}1 \\
2 \\
3 \\
4 \\
5 \\
6 \\
7 \\
8\end{array}$} & \multirow{8}{*}{$\begin{array}{l}31 \cdot 1 \\
31 \cdot 0 \\
29 \cdot 8 \\
37.5 \\
29 \cdot 8 \\
33 \cdot 0 \\
36.9 \\
33 \cdot 8\end{array}$} & \multirow{8}{*}{$\begin{array}{l}21 \cdot 6 \\
21 \cdot 6 \\
22 \cdot 5 \\
22 \cdot 2 \\
21 \cdot 0 \\
21.2 \\
24 \cdot 5 \\
22 \cdot 0\end{array}$} & \multirow{8}{*}{$\begin{array}{r}9 \\
10 \\
11 \\
12 \\
13 \\
14 \\
15 \\
16\end{array}$} & & & \multirow{8}{*}{$\begin{array}{l}17 \\
18 \\
19 \\
20 \\
21 \\
22 \\
23 \\
24\end{array}$} & 0 & 0 & \multirow{7}{*}{$\begin{array}{l}25 \\
25 \\
27 \\
28 \\
29 \\
30 \\
31\end{array}$} & \multirow{7}{*}{$\begin{array}{l}24 \cdot 4 \\
23 \cdot 2 \\
25.5 \\
30 \cdot 1 \\
24 \cdot 0 \\
31 \cdot 2 \\
25 \cdot 0\end{array}$} & 。 \\
\hline & & & & & \multirow{7}{*}{$\begin{array}{l}11 \cdot 6 \\
15 \cdot 5 \\
13.0 \\
22 \cdot 8 \\
19 \cdot 5 \\
19 \cdot 0 \\
23 \cdot 4 \\
19 \cdot 0\end{array}$} & & & & 25 & $38 \cdot 5$ & $26 \cdot 8$ & & & & & \multirow{7}{*}{$\begin{array}{l}37 \cdot 1 \\
34 \cdot 8 \\
38 \cdot 8 \\
31 \cdot 8 \\
28 \cdot 1 \\
25 \cdot 3 \\
29 \cdot 5 \\
26 \cdot 8\end{array}$} & \multirow{7}{*}{$\begin{array}{l}25 \cdot 2 \\
23 \cdot 8 \\
16 \cdot 5 \\
16 \cdot 8 \\
18 \cdot 5 \\
19 \cdot 2 \\
18 \cdot 6 \\
17 \cdot 0\end{array}$} & & \multirow{7}{*}{$\begin{array}{l}30 \cdot 8 \\
25 \cdot 5 \\
26 \cdot 8 \\
24 \cdot 4 \\
22 \cdot 2 \\
21 \cdot 7 \\
22 \cdot 3 \\
27 \cdot 2\end{array}$} & \multirow{7}{*}{$\begin{array}{r}150^{\circ} \\
10.0 \\
160 \\
13.0 \\
9.0 \\
90^{\circ} \\
110^{\circ} \\
13^{\circ} 0\end{array}$} & & & \multirow{6}{*}{$\begin{array}{r}13 \cdot 2 \\
10 \cdot 3 \\
4 \cdot 0 \\
8 \cdot 8 \\
13 \cdot 2 \\
13 \cdot 0 \\
16 \cdot 8\end{array}$} \\
\hline & & & & & & & & & 25 & $42 \cdot 0$ & $29 \cdot 7$ & & & & & & & & & & & & \\
\hline & & & & & & & & & $\begin{array}{l}28 \\
28\end{array}$ & $\begin{array}{l}41.4 \\
40 \cdot 2\end{array}$ & $\begin{array}{l}26 \cdot 2 \\
23.8\end{array}$ & & & & & & & & & & & & \\
\hline & & & & & & & & & 29 & (4) $\cdot 2$ & $27 \cdot 0$ & & & & & & & & & & & & \\
\hline & & & & & & & & & 30 & $37 \cdot 2$ & $24 \cdot 5$ & & & & & & & & & & & & \\
\hline & & & & & & & & & & & $22^{\circ 9}$ & & & & & & & & & & & & \\
\hline & & & & & & & & & Mear & $31 \cdot 0$ & $19 \cdot 0$ & & & & & & & & & & Mea & 29.0 & $16 \cdot 5$ \\
\hline
\end{tabular}


TABLE IV.

WIND-DIRECTION AND VELOCITY.

Direction (D) in Numbers $(8=\mathrm{E}, 16=\mathrm{S}, 24=\mathrm{W}, 32=\mathrm{N})$, and Velocity $(\mathrm{V})$ in miles per hour as recorded

February, 1902. by the Cup Anemometer.

\begin{tabular}{|c|c|c|c|c|c|c|c|c|c|c|c|c|c|c|c|c|c|c|c|c|c|c|c|c|}
\hline \multirow{2}{*}{ Day. } & \multicolumn{2}{|c|}{2.} & \multicolumn{2}{|c|}{4.} & \multicolumn{2}{|c|}{6.} & \multicolumn{2}{|c|}{8.} & \multicolumn{2}{|c|}{10.} & \multicolumn{2}{|c|}{ Noon. } & \multicolumn{2}{|c|}{14.} & \multicolumn{2}{|c|}{16.} & \multicolumn{2}{|c|}{18.} & \multicolumn{2}{|c|}{20.} & \multicolumn{2}{|c|}{22.} & \multicolumn{2}{|c|}{ Midt. } \\
\hline & D. & $\nabla$. & D. & $\Gamma$ & D. & $\boldsymbol{\nabla}$. & D. & ז. & D. & $\nabla$. & D. & $\Gamma$. & D. & $\mathrm{v}$. & D. & $\nabla$. & D. & v. & D. & v. & D. & v. & D. & $\nabla$. \\
\hline$\frac{1}{2}$ & $=$ & $=$ & $=$ & $=$ & $=$ & $=$ & $=$ & $=$ & $\overline{-}$ & $=$ & $=$ & $=$ & $=$ & $=$ & $=$ & $=$ & $=$ & $\bar{z}$ & 二 & $=$ & $=$ & $=$ & $=$ & $=$ \\
\hline $\begin{array}{l}2 \\
3 \\
4\end{array}$ & $\bar{z}$ & $=$ & $=$ & $=$ & $=$ & $=$ & $\overline{-}$ & $\bar{z}$ & $\bar{z}$ & $\bar{z}$ & $\bar{z}$ & $=$ & $\bar{z}$ & $\bar{z}$ & $\bar{z}$ & $\bar{z}$ & $\bar{z}$ & $=$ & 二 & $\bar{z}$ & $\bar{z}$ & 二 & $\bar{z}$ & $=$ \\
\hline${ }_{5}^{4}$ & $\bar{z}$ & $=$ & $=$ & $=$ & $=$ & $=$ & $=$ & $=$ & $\bar{z}$ & $=$ & $z$ & $=$ & 二 & $=$ & $=$ & $=$ & $=$ & $=$ & Z & $=$ & $\Xi$ & $=$ & $=$ & $=$ \\
\hline 6 & $=$ & $=$ & $\bar{z}$ & $=$ & $=$ & $=$ & $=$ & $=$ & $=$ & 二 & $=$ & - & - & $=$ & - & $=$ & - & - & - & - & - & - & - & - \\
\hline 8 & $\overline{-}$ & $\bar{z}$ & $\overline{-}$ & $\bar{z}$ & $\bar{z}$ & $\bar{z}$ & $\bar{z}$ & $\overline{-}$ & $\overline{\bar{n}}$ & $\bar{z}$ & $=$ & $=$ & $=$ & $=$ & $\overline{-}$ & $=$ & $=$ & 二 & $=$ & $=$ & $=$ & $=$ & $=$ & $=$ \\
\hline 9 & 10 & 8 & 13 & 17 & 27 & 8 & 30. & 6 & 30 & 11 & 8 & 14 & 28 & 17 & 28 & 17 & 6 & 17 & 8 & 17 & 8 & 11 & 6 & 11 \\
\hline $\begin{array}{l}10 \\
11\end{array}$ & 8 & $\begin{array}{l}8 \\
0\end{array}$ & $\begin{array}{l}6 \\
8\end{array}$ & $\begin{array}{l}6 \\
2\end{array}$ & $\begin{array}{l}6 \\
8\end{array}$ & $\begin{array}{l}2 \\
2\end{array}$ & $\begin{array}{r}8 \\
22\end{array}$ & $\begin{array}{l}8 \\
8\end{array}$ & $\begin{array}{r}6 \\
12\end{array}$ & $\begin{array}{l}6 \\
2\end{array}$ & $\begin{array}{r}6 \\
14\end{array}$ & $\begin{array}{l}8 \\
2\end{array}$ & $\overline{6}$ & $\begin{array}{l}0 \\
2\end{array}$ & $\begin{array}{r}14 \\
5\end{array}$ & $\begin{array}{l}2 \\
2\end{array}$ & $\overline{5}$ & $\begin{array}{l}0 \\
6\end{array}$ & $\overline{30}$ & $\begin{array}{l}0 \\
2\end{array}$ & $\overline{30}$ & $\begin{array}{l}0 \\
2\end{array}$ & $\begin{array}{l}32 \\
30\end{array}$ & $\begin{array}{l}2 \\
2\end{array}$ \\
\hline $\begin{array}{l}12 \\
13\end{array}$ & $=$ & 0 & $\bar{z}$ & 0 & 5 & 2 & 5 & $\frac{2}{2}$ & 8 & 10 & 8 & 17 & $\overline{4}$ & $\overline{6}$ & 8 & $\begin{array}{l}8 \\
8\end{array}$ & $\overline{14}$ & $\overline{4}$ & $\overline{14}$ & 20 & $\overline{6}$ & $\stackrel{0}{25}$ & $\overline{7}$ & $\begin{array}{r}0 \\
28\end{array}$ \\
\hline 14 & 8 & 22 & 6 & 22 & $\overline{6}$ & $\begin{array}{l}0 \\
22\end{array}$ & 6 & 25 & 6 & 22 & 8 & 22 & 6 & 22 & 6 & 20 & 6 & 20 & 6 & 14 & $\begin{array}{l}0 \\
6\end{array}$ & 14 & 6 & 11 \\
\hline $\begin{array}{l}15 \\
16\end{array}$ & $\begin{array}{l}6 \\
7\end{array}$ & $\begin{array}{r}4 \\
17\end{array}$ & $\begin{array}{l}6 \\
7\end{array}$ & 17 & 8 & $\begin{array}{r}25 \\
8\end{array}$ & 6 & 22 & 7 & 22 & 7 & 22 & 7 & 25 & 7 & $\begin{array}{r}25 \\
25\end{array}$ & 7 & 25 & $7^{7}$ & 20 & 7 & 17 & 7 & 11 \\
\hline 17 & - & 0 & - & 0 & 8 & $\begin{array}{l}8 \\
2\end{array}$ & 4 & 2 & $\overline{-}$ & $\overline{0}$ & $=$ & $\overline{0}$ & - & $\div$ & 4 & 2 & 4 & 20 & 4 & 20 & 5 & 20 & 5 & 20 \\
\hline $\begin{array}{l}18 \\
19\end{array}$ & $\overrightarrow{6}$ & $\overline{2}$ & $\overline{8}$ & $\overline{6}$ & $\begin{array}{r}6 \\
10\end{array}$ & $\begin{array}{l}25 \\
11\end{array}$ & $\begin{array}{r}8 \\
10\end{array}$ & $\begin{array}{l}28 \\
20\end{array}$ & -6 & 17 & $\overline{7}$ & $\begin{array}{r}0 \\
14\end{array}$ & $\overline{8}$ & $\begin{array}{r}0 \\
25\end{array}$ & $\begin{array}{l}32 \\
10\end{array}$ & $\begin{array}{r}2 \\
25\end{array}$ & $\begin{array}{l}32 \\
10\end{array}$ & $\begin{array}{l}17 \\
22\end{array}$ & $\begin{array}{l}30 \\
10\end{array}$ & $\begin{array}{l}25 \\
38\end{array}$ & $\begin{array}{r}31 \\
8\end{array}$ & $\begin{array}{l}38 \\
28\end{array}$ & $\begin{array}{r}31 \\
6\end{array}$ & $\begin{array}{l}25 \\
25\end{array}$ \\
\hline 20 & 6 & 2 & 6 & 2 & - & $\overline{17}$ & 8 & 2 & 4 & 2 & - & 0 & 5 & 2 & 14 & 2 & - & 0 & 2 & $\frac{2}{17}$ & 7 & 20 & $\stackrel{6}{6}$ & 20 \\
\hline $\begin{array}{l}21 \\
22\end{array}$ & $\begin{array}{l}7 \\
9\end{array}$ & $\begin{array}{l}17 \\
17\end{array}$ & 8 & 14 & $\begin{array}{l}8 \\
9\end{array}$ & 17 & 8 & ${ }_{31}^{17}$ & 8 & 20 & $\begin{array}{l}8 \\
8\end{array}$ & $\begin{array}{l}22 \\
38\end{array}$ & $\begin{array}{l}9 \\
8\end{array}$ & 22 & $\begin{array}{l}9 \\
6\end{array}$ & $\frac{22}{31}$ & $\begin{array}{l}3 \\
6\end{array}$ & 20 & 9 & 17 & 9 & 14 & 12 & 8 \\
\hline 23 & 6 & 11 & 8 & 17 & 7 & $\begin{array}{l}20 \\
17\end{array}$ & 7 & $\begin{array}{l}81 \\
17\end{array}$ & 7 & 17 & 7 & 17 & 7 & 25 & 7 & 22 & 7 & 28 & 7 & 28 & 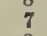 & $\begin{array}{l}18 \\
25\end{array}$ & 12 & 28 \\
\hline 24 & 10 & 17 & 10 & 17 & 10 & 14 & 10 & 11 & 10 & 14 & 10 & 22 & 10 & 25 & 8 & 25 & 8 & 25 & 8 & 25 & 8 & 25 & 8 & 28 \\
\hline 25 & 8 & 25 & 8 & 22 & 8 & 14 & 14 & 2 & 4 & 14 & 10 & 11 & 10 & 2 & - & 0 & - & 0 & 10 & 2 & 12 & 2 & 10 & 2 \\
\hline $\begin{array}{l}26 \\
27\end{array}$ & $=$ & $=$ & $=$ & $\overline{0}$ & 18 & $\begin{array}{l}11 \\
0\end{array}$ & $\begin{array}{l}8 \\
8\end{array}$ & 11 & $\begin{array}{l}8 \\
8\end{array}$ & 17 & $\begin{array}{r}8 \\
18\end{array}$ & $\begin{array}{l}17 \\
22\end{array}$ & $\begin{array}{r}8 \\
19\end{array}$ & $\begin{array}{r}8 \\
25\end{array}$ & $\begin{array}{r}8 \\
19\end{array}$ & ${ }_{14}^{2}$ & $\overline{18}$ & 20 & $\begin{array}{l}10 \\
19\end{array}$ & 25 & $\begin{array}{l}10 \\
18\end{array}$ & 2 & $\overline{19}$ & $\stackrel{0}{17}$ \\
\hline 28 & 8 & 8 & - & - & 8 & 2 & - & - & 8 & 11 & 8 & 2 & 8 & 2 & 10 & 2 & 8 & 17 & 8 & 31 & - & 0 & 15 & 2 \\
\hline Mean. & & 94 & & $10^{*}$ & & $11 \S$ & & $11 \S$ & & $12 \ddagger$ & & $13 \S$ & & $14 \sharp$ & & 11 & & $15 \S$ & & 15 & & 14 & & 13 \\
\hline
\end{tabular}

\section{March, 1902.}

\begin{tabular}{|c|c|c|c|c|c|c|c|c|c|c|c|c|c|c|c|c|c|c|c|c|c|c|c|c|}
\hline \multirow{2}{*}{ Day. } & \multicolumn{2}{|c|}{2.} & \multicolumn{2}{|c|}{4.} & \multicolumn{2}{|c|}{6.} & \multicolumn{2}{|c|}{8.} & \multicolumn{2}{|c|}{10.} & \multicolumn{2}{|c|}{ Noon. } & \multicolumn{2}{|c|}{14.} & \multicolumn{2}{|c|}{16.} & \multicolumn{2}{|c|}{18.} & \multicolumn{2}{|c|}{20.} & \multicolumn{2}{|c|}{22.} & \multicolumn{2}{|c|}{ Midt. } \\
\hline & D. & v. & D. & v. & D. & v. & D. & v. & D. & v. & D. & v. & D. & v. & D. & V. & D. & v. & D. & $v$ & D. & v. & D. & v. \\
\hline 1 & 13 & 2 & 12 & 6 & 15 & 8 & 8 & 6 & 14 & 11 & 14 & 20 & 13 & 14 & 13 & 11 & 7 & 17 & 7 & 11 & 7 & 14 & 7 & 17 \\
\hline 2 & 7 & 6 & - & 0 & - & 0 & 6 & 25 & - & - & 6 & 22 & 6 & 28 & - & - & - & - & 6 & 11 & - & - & 6 & 22 \\
\hline 3 & 6 & 17 & 8 & 20 & 8 & 25 & 8 & 28 & 6 & 28 & 4 & 31 & 6 & 35 & 6 & 35 & - & $\vec{a}$ & 6 & 20 & 6 & 2 & - & 0 \\
\hline $\begin{array}{l}4 \\
5\end{array}$ & $\overrightarrow{8}$ & -8 & $\overline{10}$ & - & - & 0 & - & 0 & 4 & 11 & 2 & 11 & 2 & 11 & 2 & 17 & 2 & 17 & 2 & 22 & 9 & 11 & 10 & 2 \\
\hline $\begin{array}{l}5 \\
6\end{array}$ & 8 & 8 & $\begin{array}{l}10 \\
10\end{array}$ & $\begin{array}{l}6 \\
2\end{array}$ & $\begin{array}{l}10 \\
10\end{array}$ & $\begin{array}{l}8 \\
2\end{array}$ & ${ }_{10}^{6}$ & $\begin{array}{l}6 \\
8\end{array}$ & $\begin{array}{r}6 \\
10\end{array}$ & $\begin{array}{l}2 \\
8\end{array}$ & 6 & 2 & $\stackrel{6}{-}$ & $\begin{array}{l}6 \\
0\end{array}$ & $\underline{6}$ & $\begin{array}{l}2 \\
0\end{array}$ & $\frac{8}{-}$ & $\begin{array}{l}2 \\
0\end{array}$ & 10 & 2 & 12 & 2 & 14 & 2 \\
\hline 7 & - & - & 13 & 2 & 12 & 2 & 10 & & 10 & , & - & & 14 & & 14 & & 8 & ( & 8 & 17 & 8 & & $\theta$ & 11 \\
\hline 8 & 14 & 6 & 10 & 2 & $\begin{array}{l}12 \\
10\end{array}$ & 2 & 12 & 2. & 12 & 2 & $\overrightarrow{10}$ & $\begin{array}{l}0 \\
6\end{array}$ & $\begin{array}{l}14 \\
10\end{array}$ & 要 & 14 & $\begin{array}{l}8 \\
6 \\
6\end{array}$ & 14 & $\begin{array}{r}14 \\
6\end{array}$ & 14 & 6 & 30 & 2 & 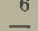 & 110 \\
\hline 9 & $\vec{\nabla}$ & 0 & - & 0 & - & 0 & -14 & 0 & - & 0 & - & 0 & - & 0 & - & 0 & - & 0 & - & 0 & 14 & 6 & 8 & 11 \\
\hline 10 & 10 & 17 & 14 & 22 & 6 & 6 & 14 & 11 & 14 & 6 & 14 & 2 & 14 & 2 & 14 & 2 & $\overline{10}$ & 0 & - & 0 & 7 & $\overline{0}$ & - & 0 \\
\hline 11 & - & 0 & - & 0 & 10 & 2 & 12 & 6 & 12 & 25 & 12 & 38 & 12 & 33 & 8 & 38 & 10 & 38 & - & - & 10 & 35 & 10 & 35 \\
\hline 12 & 8 & 35 & 8 & 28 & 7 & 25 & 8 & 22 & 8 & 25 & 8 & 22 & 8 & 28 & 8 & 38 & 8 & 38 & 8 & 35 & 8 & 42 & 8 & 35 \\
\hline 13 & 8 & 28 & 8 & 25 & 8 & 25 & 8 & 25 & 8 & 22 & 8 & 20 & 8 & 20 & 8 & 22 & 8 & 11 & 10 & 2 & 14 & 11 & 29 & 17 \\
\hline 14 & 12 & 8 & 12 & 6 & 18 & 17 & 18 & 11 & 18 & 11 & 18 & 11 & 12 & 6 & 10 & 17 & - & - & 6 & $4 \overline{2}$ & 6 & 42 & 6 & 42 \\
\hline 15 & 10 & 38 & 10 & 31 & 10 & 28 & 10 & 22 & 10 & 22 & 10 & 20 & 10 & 17 & 10 & 14 & 10 & 14 & - & 0 & - & - & 14 & 2 \\
\hline 18 & 14 & 2 & 12 & 2 & 14 & 2 & 18 & 6 & 14 & 2 & 10 & 2 & 10 & 2 & 12 & 8 & - & 0 & - & 0 & - & - & - & - \\
\hline 17 & 2 & 2 & 2 & 2 & 2 & 2 & 2 & 6 & 2 & 2 & 14 & 8 & 14 & 25 & 14 & 31 & 18 & 42 & 18 & 42 & 18 & 42 & 18 & 50 \\
\hline 18 & - & - & 18 & 11 & 18 & 11 & 18 & 14 & 18 & 20 & 18 & 11 & 18 & 20 & 14 & 11 & 14 & 11 & 12 & 11 & 12 & 17 & 7 & 8 \\
\hline 19 & $\overrightarrow{18}$ & 7 & 18 & 2 & 14 & 2 & - & 0 & 14 & 6 & 14 & 8 & 10 & 11 & 10 & 2 & $\overline{1}$ & $\overline{11}$ & 14 & 1 & 14 & 2 & 18 & 11 \\
\hline $\begin{array}{l}20 \\
21\end{array}$ & $\begin{array}{l}18 \\
18\end{array}$ & $\begin{array}{r}11 \\
8\end{array}$ & 20 & 2 & 14 & 2 & 16 & 8 & 16 & 6 & 16 & 2 & 18 & 6 & 16 & 20 & 16 & 11 & 12 & 4 & 18 & 35 & 18 & 8 \\
\hline & & 8 & 10 & 6 & 18 & 8 & - & - & - & - & - & - & - & - & - & - & - & - & 12 & 2 & 8 & 11 & 10 & 17 \\
\hline 22 & 10 & 8 & 10 & 17 & 10 & 17 & 10 & 6 & 10 & 2 & 10 & 2 & - & 0 & - & 0 & - & 0 & 10 & 8 & 10 & 2 & - & 0 \\
\hline 23 & 10 & 6 & 10 & 6 & 4 & 6 & 7 & 11 & 7 & 14 & $\begin{array}{r}10 \\
7\end{array}$ & 14 & 7 & 17 & 7 & 31 & 7 & 35 & 10 & $\$ 2$ & 10 & 42 & 10 & 17 \\
\hline 24 & 10 & 8 & 10 & 6 & 8 & 2 & 8 & 6 & 8 & 2 & 8 & 2 & - & 0 & - & 0 & - & 0 & - & 0 & 10 & 2 & - & 0 \\
\hline 25 & 10 & 1 & - & 0 & 2 & 2 & 32 & 2 & 32 & 4 & - & 0 & - & 0 & 8 & 6 & - & 0 & 8 & 2 & 10 & 2 & 10 & 14 \\
\hline 26 & 10 & 14 & 10 & 8 & 14 & 2 & 7 & 6 & 7 & 6 & 7 & 11 & 7 & 22 & $i$ & 28 & 7 & 31 & 7 & 42 & 7 & 35 & $i$ & 42 \\
\hline 27 & 12 & 38 & 12 & 28 & 10 & 11 & 10 & 8 & 11 & 8 & 11 & 8 & 8 & 4 & 8 & 2 & - & 0 & - & 0 & - & 0 & - & 0 \\
\hline $\begin{array}{l}28 \\
29\end{array}$ & $\overline{12}$ & 0 & $\overline{10}$ & 0 & - & 0 & - & 0 & - & 0 & - & 0 & - & 0 & - & 0 & - & 0 & - & 0 & - & 0 & - & 0 \\
\hline 30 & 12 & $\begin{array}{l}8 \\
0\end{array}$ & 10 & 2 & - & 0 & - & 0 & - & 0 & $\bar{\sigma}$ & 0 & - & 0 & $\rightarrow$ & 0 & $\overline{30}$ & 0 & $\overrightarrow{a^{\circ}}$ & 0 & $\overline{30}$ & $v$ & - & 0 \\
\hline 31 & - & 0 & $\overline{10}$ & $\begin{array}{l}0 \\
2\end{array}$ & $\overline{8}$ & $\stackrel{0}{2}$ & $\overline{8}$ & $\begin{array}{r}0 \\
22\end{array}$ & $\overline{8}$ & $\begin{array}{r}0 \\
25\end{array}$ & $\begin{array}{r}32 \\
8\end{array}$ & $\begin{array}{r}2 \\
28\end{array}$ & $\begin{array}{r}32 \\
8\end{array}$ & $\begin{array}{l}6 \\
2\end{array}$ & 32 & $\begin{array}{l}2 \\
0\end{array}$ & $\frac{32}{-}$ & $\frac{6}{-}$ & $\stackrel{32}{-}$ & $\begin{array}{l}2 \\
0\end{array}$ & 32 & $\begin{array}{l}6 \\
0\end{array}$ & $\overline{12}$ & $\begin{array}{l}0 \\
2\end{array}$ \\
\hline Mean ... & & $10 \uparrow$ & & $8 \mid$ & & 7 & & 9】 & & $9 \S$ & & $10 \rrbracket$ & & 110 & & $12 \S$ & & $12^{*}$ & & $11 \|$ & & $14 \$$ & & $12 \|$ \\
\hline
\end{tabular}


TABLE IV. (eontinued)

WIND-DIRECTION AND VELOCITY.

Direction (D) in Numbers $(8=\mathrm{E}, 16=\mathrm{S}, 24=\mathrm{W}, 32=\mathrm{N})$, and Velocity (V) in miles per hour as recorded by the Cup Anemometer.

April, 1902.

\begin{tabular}{|c|c|c|c|c|c|c|c|c|c|c|c|c|c|c|c|c|c|c|c|c|c|c|c|c|}
\hline \multirow{2}{*}{ Day. } & \multicolumn{2}{|c|}{2.} & \multicolumn{2}{|c|}{4.} & \multicolumn{2}{|c|}{6.} & \multicolumn{2}{|c|}{8.} & \multicolumn{2}{|c|}{10.} & \multicolumn{2}{|c|}{ Noon. } & \multicolumn{2}{|c|}{14.} & \multicolumn{2}{|c|}{18.} & \multicolumn{2}{|c|}{18.} & \multicolumn{2}{|c|}{20.} & \multicolumn{2}{|c|}{22.} & \multicolumn{2}{|c|}{ Mlat. } \\
\hline & D. & v. & D. & v. & D. & v. & D. & v. & D. & v. & D. & v. & D. & v. & D. & v. & D. & v. & D. & v. & D. & v. & D. & $\nabla$. \\
\hline $\begin{array}{l}1 \\
2 \\
3 \\
4 \\
5\end{array}$ & $\bar{z}$ & $\begin{array}{l}0 \\
0 \\
0 \\
0 \\
2\end{array}$ & $\overline{\bar{z}}$ & $\begin{array}{l}0 \\
0 \\
0 \\
0 \\
\mathbf{2}\end{array}$ & $\frac{\bar{Z}}{\overline{10}}$ & $\begin{array}{l}0 \\
0 \\
0 \\
6 \\
4\end{array}$ & $\begin{array}{r}10 \\
10 \\
8 \\
12\end{array}$ & $\begin{array}{r}11 \\
0 \\
11 \\
6 \\
2\end{array}$ & $\begin{array}{r}\frac{6}{10} \\
-\end{array}$ & $\begin{array}{r}2 \\
0 \\
11 \\
2 \\
0\end{array}$ & $\begin{array}{r}12 \\
10 \\
8 \\
16\end{array}$ & $\begin{array}{r}11 \\
0 \\
6 \\
2 \\
11\end{array}$ & $\frac{12}{30}$ & $\begin{array}{r}11 \\
2 \\
0 \\
0 \\
11\end{array}$ & $\frac{12}{\overline{16}}$ & $\begin{array}{r}6 \\
0 \\
0 \\
14\end{array}$ & $\frac{6}{\overline{6}}$ & $\begin{array}{r}11 \\
0 \\
0 \\
2\end{array}$ & $\bar{z}$ & $\begin{array}{l}0 \\
0 \\
0 \\
6 \\
0\end{array}$ & $\overline{\bar{z}}$ & $\begin{array}{l}0 \\
0 \\
0 \\
8 \\
0\end{array}$ & $\frac{28}{\frac{28}{28}}$ & $\begin{array}{r}2 \\
0 \\
0 \\
17 \\
6\end{array}$ \\
\hline $\begin{array}{r}6 \\
7 \\
8 \\
9 \\
10\end{array}$ & $\frac{10}{\frac{6}{7}}-$ & $\begin{array}{r}10 \\
0 \\
22 \\
6 \\
0\end{array}$ & $\begin{array}{r}20 \\
14 \\
10 \\
7 \\
-\end{array}$ & $\begin{array}{r}28 \\
2 \\
20 \\
8 \\
0\end{array}$ & $\begin{array}{r}\frac{20}{10} \\
-7 \\
-\end{array}$ & $\begin{array}{c}35 \\
\begin{array}{c}14 \\
11 \\
0\end{array}\end{array}$ & $\begin{array}{r}12 \\
\frac{12}{90} \\
-\end{array}$ & $\begin{array}{r}28 \\
0 \\
8 \\
2 \\
0\end{array}$ & $\begin{array}{r}12 \\
12 \\
16^{\circ} \\
10 \\
2\end{array}$ & $\begin{array}{r}31 \\
2 \\
6 \\
2 \\
2\end{array}$ & $\begin{array}{l}12 \\
12 \\
16 \\
12 \\
32\end{array}$ & $\begin{array}{r}31 \\
2 \\
38 \\
4 \\
2\end{array}$ & $\begin{array}{l}12 \\
12 \\
10 \\
10 \\
32\end{array}$ & $\begin{array}{r}31 \\
2 \\
31 \\
2 \\
2\end{array}$ & $\begin{array}{r}12 \\
10 \\
10 \\
9 \\
8\end{array}$ & $\begin{array}{r}31 \\
6 \\
25 \\
2 \\
2\end{array}$ & $\begin{array}{r}6 \\
12 \\
8 \\
9 \\
-\end{array}$ & $\begin{array}{r}17 \\
17 \\
22 \\
2 \\
0\end{array}$ & $\begin{array}{r}6 \\
12 \\
6 \\
8 \\
-\end{array}$ & $\begin{array}{r}2 \\
31 \\
35 \\
2 \\
0\end{array}$ & $\begin{array}{r}\overline{12} \\
8 \\
8 \\
-\end{array}$ & $\begin{array}{r}0 \\
38 \\
35 \\
2 \\
0\end{array}$ & $\begin{array}{r}\overline{26} \\
8 \\
9 \\
-\end{array}$ & $\begin{array}{r}0 \\
22 \\
19 \\
2 \\
0\end{array}$ \\
\hline $\begin{array}{l}11 \\
12 \\
13 \\
14 \\
15\end{array}$ & $\begin{array}{l}15 \\
\frac{12}{10} \\
\frac{7}{10}\end{array}$ & $\begin{array}{r}16 \\
11 \\
0 \\
0 \\
25\end{array}$ & $\begin{array}{l}18 \\
10 \\
10 \\
12\end{array}$ & $\begin{array}{r}14 \\
11 \\
2 \\
0 \\
2\end{array}$ & $\begin{array}{l}16 \\
10 \\
32 \\
12\end{array}$ & $\begin{array}{r}38 \\
32 \\
17 \\
0 \\
2\end{array}$ & $\begin{array}{r}20 \\
10 \\
6 \\
30 \\
-\end{array}$ & $\begin{array}{r}38 \\
2 \\
2 \\
2 \\
0\end{array}$ & $\begin{array}{l}16 \\
12 \\
16 \\
14 \\
-\end{array}$ & $\begin{array}{r}22 \\
2 \\
6 \\
8 \\
0\end{array}$ & $\begin{array}{l}14 \\
12 \\
10 \\
12\end{array}$ & $\begin{array}{r}14 \\
6 \\
0 \\
23 \\
6\end{array}$ & $\begin{array}{l}15 \\
12 \\
14 \\
12 \\
12\end{array}$ & $\begin{array}{l}14 \\
11 \\
20 \\
25 \\
14\end{array}$ & $\begin{array}{l}16 \\
12 \\
16 \\
12 \\
12\end{array}$ & $\begin{array}{r}11 \\
8 \\
46 \\
22 \\
17\end{array}$ & $\begin{array}{l}16 \\
12 \\
16 \\
10 \\
12\end{array}$ & $\begin{array}{l}14 \\
11 \\
38 \\
20 \\
20\end{array}$ & $\begin{array}{r}8 \\
12 \\
10 \\
10 \\
11\end{array}$ & $\begin{array}{r}8 \\
20 \\
22 \\
38 \\
25\end{array}$ & $\begin{array}{l}10 \\
12 \\
10 \\
10 \\
10\end{array}$ & $\begin{array}{r}2 \\
22 \\
20 \\
35 \\
22\end{array}$ & $\begin{array}{l}\overline{12} \\
10 \\
10 \\
18\end{array}$ & $\begin{array}{r}0 \\
1 \\
28 \\
35 \\
17\end{array}$ \\
\hline $\begin{array}{l}16 \\
17 \\
18 \\
19 \\
20\end{array}$ & $\begin{array}{r}15 \\
14 \\
12 \\
12 \\
9\end{array}$ & $\begin{array}{r}25 \\
11 \\
8 \\
22 \\
20\end{array}$ & $\begin{array}{r}16 \\
12 \\
12 \\
6 \\
10\end{array}$ & $\begin{array}{r}17 \\
14 \\
8 \\
15 \\
9\end{array}$ & $\begin{array}{l}\frac{14}{8} \\
4 \\
8\end{array}$ & $\begin{array}{c}23 \\
8 \\
23 \\
4\end{array}$ & $\begin{array}{r}14 \\
12 \\
10 \\
4 \\
16\end{array}$ & $\begin{array}{r}14 \\
2 \\
8 \\
26 \\
4\end{array}$ & $\begin{array}{r}14 \\
4 \\
12 \\
4 \\
-\end{array}$ & $\begin{array}{r}19 \\
2 \\
11 \\
21 \\
5\end{array}$ & $\begin{array}{r}18 \\
8 \\
4 \\
30\end{array}$ & $\begin{array}{r}14 \\
0 \\
5 \\
23 \\
5\end{array}$ & $\begin{array}{r}12 \\
2 \\
12 \\
4 \\
28\end{array}$ & $\begin{array}{r}14 \\
2 \\
11 \\
24 \\
11\end{array}$ & $\begin{array}{l}10 \\
12 \\
12 \\
4 \\
28\end{array}$ & $\begin{array}{r}8 \\
6 \\
8 \\
25 \\
11\end{array}$ & $\begin{array}{r}16 \\
8 \\
9 \\
4 \\
28\end{array}$ & $\begin{array}{r}8 \\
4 \\
9 \\
24 \\
9\end{array}$ & $\begin{array}{r}11 \\
10 \\
12 \\
6 \\
32\end{array}$ & $\begin{array}{r}11 \\
4 \\
12 \\
28 \\
5\end{array}$ & $\begin{array}{r}16 \\
10 \\
4 \\
4 \\
26\end{array}$ & $\begin{array}{r}11 \\
6 \\
11 \\
20 \\
5\end{array}$ & $\begin{array}{r}16 \\
12 \\
4 \\
7 \\
4\end{array}$ & $\begin{array}{r}11 \\
6 \\
14 \\
16 \\
11\end{array}$ \\
\hline $\begin{array}{l}21 \\
22 \\
23 \\
24 \\
25\end{array}$ & $\begin{array}{l}8 \\
6 \\
9 \\
8 \\
4\end{array}$ & $\begin{array}{l}17 \\
24 \\
22 \\
29 \\
14\end{array}$ & $\begin{array}{l}4 \\
6 \\
7 \\
5 \\
4\end{array}$ & $\begin{array}{l}20 \\
20 \\
17 \\
23 \\
10\end{array}$ & $\begin{array}{l}4 \\
6 \\
4 \\
4 \\
7\end{array}$ & $\begin{array}{r}15 \\
24 \\
17 \\
20 \\
8\end{array}$ & $\begin{array}{l}4 \\
6 \\
4 \\
4 \\
4\end{array}$ & $\begin{array}{r}20 \\
15 \\
22 \\
22 \\
6\end{array}$ & $\begin{array}{r}6 \\
4 \\
4 \\
6 \\
-\end{array}$ & $\begin{array}{r}9 \\
28 \\
14 \\
9 \\
2\end{array}$ & $\begin{array}{r}9 \\
4 \\
10 \\
8 \\
-\end{array}$ & $\begin{array}{r}11 \\
27 \\
15 \\
17 \\
1\end{array}$ & $\begin{array}{r}9 \\
8 \\
6 \\
4 \\
-\end{array}$ & $\begin{array}{r}18 \\
24 \\
12 \\
18 \\
1\end{array}$ & $\begin{array}{r}7 \\
6 \\
4 \\
4 \\
-\end{array}$ & $\begin{array}{r}22 \\
23 \\
18 \\
13 \\
1\end{array}$ & $\begin{array}{r}8 \\
7 \\
4 \\
4 \\
\end{array}$ & $\begin{array}{r}24 \\
23 \\
20 \\
24 \\
1\end{array}$ & $\begin{array}{r}6 \\
6 \\
4 \\
4 \\
-\end{array}$ & $\begin{array}{r}27 \\
17 \\
24 \\
21 \\
1\end{array}$ & $\begin{array}{r}8 \\
7 \\
4 \\
4 \\
30\end{array}$ & $\begin{array}{r}21 \\
20 \\
23 \\
20 \\
4\end{array}$ & $\begin{array}{r}7 \\
4 \\
6 \\
4 \\
28\end{array}$ & $\begin{array}{r}27 \\
15 \\
18 \\
17 \\
9\end{array}$ \\
\hline $\begin{array}{l}28 \\
27 \\
28 \\
29 \\
30\end{array}$ & $\begin{array}{r}29 \\
28 \\
3 \\
8 \\
8\end{array}$ & $\begin{array}{r}11 \\
8 \\
4 \\
14 \\
9\end{array}$ & $\begin{array}{r}\frac{31}{2} \\
2 \\
7\end{array}$ & $\begin{array}{r}12 \\
3 \\
9 \\
18 \\
9\end{array}$ & $\begin{array}{r}\frac{31}{32} \\
7 \\
8\end{array}$ & $\begin{array}{r}13 \\
2 \\
2 \\
20 \\
20 \\
32\end{array}$ & $\frac{30}{\frac{8}{8}}$ & $\begin{array}{r}15 \\
0 \\
12 \\
23 \\
23\end{array}$ & $\begin{array}{r}30 \\
8 \\
32 \\
8 \\
8\end{array}$ & $\begin{array}{r}14 \\
4 \\
5 \\
17 \\
27\end{array}$ & $\begin{array}{r}28 \\
8 \\
8 \\
7\end{array}$ & $\begin{array}{r}12 \\
13 \\
4 \\
15 \\
20\end{array}$ & $\begin{array}{r}32 \\
32 \\
8 \\
8\end{array}$ & $\begin{array}{r}11 \\
16 \\
0 \\
13 \\
22\end{array}$ & $\frac{28}{32}$ & $\begin{array}{r}12 \\
7 \\
1 \\
19 \\
24\end{array}$ & $\begin{array}{r}30 \\
32 \\
4 \\
8 \\
8\end{array}$ & $\begin{array}{r}11 \\
2 \\
3 \\
12 \\
19\end{array}$ & $\begin{array}{r}\frac{28}{9} \\
10 \\
4\end{array}$ & $\begin{array}{r}12 \\
2 \\
9 \\
14 \\
22\end{array}$ & $\begin{array}{r}28 \\
0 \\
12 \\
4\end{array}$ & $\begin{array}{r}12 \\
0 \\
9 \\
11 \\
19\end{array}$ & $\begin{array}{r}29 \\
8 \\
8 \\
7 \\
6\end{array}$ & $\begin{array}{r}10 \\
7 \\
8 \\
29 \\
17\end{array}$ \\
\hline Mean . & & 11 & & 10 & & $13^{*}$ & & 11 & & 9 & & 11 & & 12 & & 13 & & $13 \dagger$ & & 13 & & 12 & & 12 \\
\hline
\end{tabular}

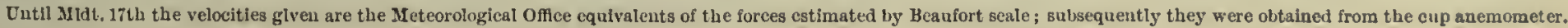
* For 28 days only.

I For 29 days only.

May, 1902.

\begin{tabular}{|c|c|c|c|c|c|c|c|c|c|c|c|c|c|c|c|c|c|c|c|c|c|c|c|c|}
\hline \multirow{2}{*}{ Day. } & \multicolumn{2}{|c|}{2.} & \multicolumn{2}{|c|}{4.} & \multicolumn{2}{|c|}{6.} & \multicolumn{2}{|c|}{8.} & \multicolumn{2}{|c|}{10.} & \multicolumn{2}{|c|}{ Noon. } & \multicolumn{2}{|c|}{14.} & \multicolumn{2}{|c|}{16.} & \multicolumn{2}{|c|}{18.} & \multicolumn{2}{|c|}{20.} & \multicolumn{2}{|c|}{22.} & \multicolumn{2}{|c|}{ Midt. } \\
\hline & D. & v. & D. & v. & D. & v. & D. & v. & D. & v. & D. & v. & D. & v. & D. & v. & D. & v. & D. & V. & D. & v. & D. & v. \\
\hline $\begin{array}{l}1 \\
2 \\
3 \\
4 \\
5\end{array}$ & $\begin{array}{r}8 \\
7 \\
17 \\
15 \\
8\end{array}$ & $\begin{array}{l}17 \\
14 \\
30 \\
313 \\
26\end{array}$ & $\begin{array}{r}6 \\
4 \\
20 \\
16 \\
9\end{array}$ & $\begin{array}{l}15 \\
15 \\
35 \\
35 \\
18\end{array}$ & $\begin{array}{l}9 \\
4 \\
20 \\
16 \\
15\end{array}$ & $\begin{array}{l}15 \\
18 \\
44 \\
35 \\
23\end{array}$ & $\begin{array}{r}9 \\
12 \\
20 \\
12 \\
12\end{array}$ & $\begin{array}{l}23 \\
17 \\
33 \\
30 \\
19\end{array}$ & $\begin{array}{r}9 \\
10 \\
20 \\
12 \\
8\end{array}$ & $\begin{array}{l}22 \\
18\end{array}$ & $\begin{array}{r}8 \\
10 \\
20 \\
12 \\
7\end{array}$ & $\begin{array}{l}11 \\
23 \\
28 \\
18 \\
15\end{array}$ & $\begin{array}{r}6 \\
9 \\
18 \\
12 \\
8\end{array}$ & $\begin{array}{c}21 \\
20 \\
35 \\
17 \\
8^{*}\end{array}$ & $\begin{array}{r}8 \\
16 \\
17 \\
12 \\
10\end{array}$ & $\begin{array}{c}15 \\
42^{*} \\
39 \\
23 \\
8^{*}\end{array}$ & $\begin{array}{r}8 \\
17 \\
16 \\
12 \\
0\end{array}$ & $\begin{array}{c}8 \\
42^{*} \\
25 \\
22 \\
8^{*}\end{array}$ & $\begin{array}{r}8 \\
17 \\
16 \\
12 \\
8\end{array}$ & $\begin{array}{c}9 \\
34 \\
34 \\
24 \\
11^{*}\end{array}$ & $\begin{array}{r}8 \\
20 \\
16 \\
9 \\
7\end{array}$ & $\begin{array}{l}12 \\
38 \\
28 \\
22 \\
14^{*}\end{array}$ & $\begin{array}{r}4 \\
17 \\
15 \\
8 \\
6\end{array}$ & $\begin{array}{l}15 \\
39 \\
36 \\
17 \\
17^{*}\end{array}$ \\
\hline $\begin{array}{r}8 \\
7 \\
8 \\
9 \\
10\end{array}$ & $\begin{array}{r}8 \\
9 \\
4 \\
10 \\
-\end{array}$ & $\begin{array}{c}14^{*} \\
9 \\
26 \\
13 \\
6\end{array}$ & $\begin{array}{r}8 \\
7 \\
6 \\
7 \\
32\end{array}$ & $\begin{array}{r}6^{*} \\
5 \\
22 \\
14 \\
7\end{array}$ & $\begin{array}{r}18 \\
1 \\
6 \\
7 \\
32\end{array}$ & $\begin{array}{r}4^{*} \\
6 \\
24 \\
23 \\
8\end{array}$ & $\begin{array}{r}7 \\
28 \\
8 \\
4 \\
31\end{array}$ & $\begin{array}{c}4^{*} \\
3 \\
23 \\
24 \\
10\end{array}$ & $\begin{array}{r}\overline{32} \\
6 \\
6 \\
32\end{array}$ & $\begin{array}{r}0 \\
14 \\
22 \\
22 \\
11\end{array}$ & $\begin{array}{r}-4 \\
7 \\
6 \\
32\end{array}$ & $\begin{array}{r}4 \\
14 \\
18 \\
15 \\
11\end{array}$ & $\begin{array}{r}- \\
32 \\
8 \\
6 \\
32\end{array}$ & $\begin{array}{r}4 \\
12 \\
16 \\
15 \\
12\end{array}$ & $\begin{array}{r}-4 \\
4 \\
7 \\
32\end{array}$ & $\begin{array}{r}5 \\
8 \\
15 \\
16 \\
5\end{array}$ & $\begin{array}{r}8 \\
4 \\
4 \\
28\end{array}$ & $\begin{array}{r}17 \\
16 \\
15 \\
11 \\
7\end{array}$ & $\begin{array}{r}8 \\
4 \\
7 \\
-\end{array}$ & $\begin{array}{r}35 \\
19 \\
14 \\
2 \\
5\end{array}$ & $\begin{array}{r}8 \\
4 \\
9 \\
6\end{array}$ & $\begin{array}{r}20 \\
18 \\
12 \\
0 \\
6\end{array}$ & $\begin{array}{r}14 \\
7 \\
8 \\
32 \\
6\end{array}$ & $\begin{array}{r}25 \\
14 \\
8 \\
5 \\
7\end{array}$ \\
\hline $\begin{array}{l}11 \\
12 \\
13 \\
14 \\
15\end{array}$ & $\begin{array}{l}4 \\
6 \\
1 \\
8 \\
7\end{array}$ & $\begin{array}{r}9 \\
25 \\
2 \\
11 \\
18\end{array}$ & $\begin{array}{r}6 \\
6 \\
9 \\
8\end{array}$ & $\begin{array}{r}9 \\
19 \\
0 \\
5 \\
27\end{array}$ & $\begin{array}{r}12 \\
6 \\
12 \\
6\end{array}$ & $\begin{array}{r}14 \\
19 \\
6 \\
2 \\
24\end{array}$ & $\frac{9}{\frac{9}{6}}$ & $\begin{array}{r}19 \\
22 \\
B \\
1 \\
22\end{array}$ & $\frac{8}{\overline{6}}$ & $\begin{array}{r}11 \\
8 \\
4 \\
1 \\
37\end{array}$ & $\frac{8}{\frac{4}{6}}$ & $\begin{array}{r}9 \\
3 \\
2 \\
3 \\
26\end{array}$ & $\frac{9}{\overline{6}}$ & $\begin{array}{r}15 \\
3 \\
1 \\
2 \\
18\end{array}$ & $\frac{6}{\frac{6}{4}}$ & $\begin{array}{r}23 \\
4 \\
2 \\
5 \\
5 \\
21\end{array}$ & $\begin{array}{l}\frac{7}{4} \\
8 \\
8\end{array}$ & $\begin{array}{r}21 \\
1 \\
8 \\
12 \\
25\end{array}$ & $\begin{array}{r}\frac{7}{4} \\
8 \\
8\end{array}$ & $\begin{array}{r}24 \\
4 \\
10 \\
13 \\
25\end{array}$ & $\begin{array}{l}\frac{6}{5} \\
8 \\
8\end{array}$ & $\begin{array}{r}28 \\
6 \\
13 \\
12 \\
32\end{array}$ & $\begin{array}{l}\frac{6}{1} \\
8 \\
8\end{array}$ & $\begin{array}{r}30 \\
0 \\
15 \\
13 \\
31\end{array}$ \\
\hline $\begin{array}{l}16 \\
17 \\
19 \\
19 \\
20\end{array}$ & $\begin{array}{l}8 \\
8 \\
7 \\
7 \\
7\end{array}$ & $\begin{array}{r}24 \\
4 \\
18 \\
23 \\
32\end{array}$ & $\begin{array}{r}\frac{8}{7} \\
7 \\
7\end{array}$ & $\begin{array}{r}33 \\
0 \\
20 \\
19 \\
20\end{array}$ & $\begin{array}{l}\frac{8}{7} \\
7 \\
2 \\
2\end{array}$ & $\begin{array}{l}30 \\
1 \\
23 \\
25 \\
30\end{array}$ & $\begin{array}{l}\frac{4}{6} \\
7 \\
8\end{array}$ & $\begin{array}{c}31 \\
2 \\
22 \\
19 \\
23\end{array}$ & $\begin{array}{r}4 \\
8 \\
8 \\
12\end{array}$ & $\begin{array}{r}31 \\
5 \\
24 \\
22 \\
16\end{array}$ & $\begin{array}{r}4 \\
4 \\
8 \\
8 \\
12\end{array}$ & $\begin{array}{r}27 \\
3 \\
26 \\
17 \\
14\end{array}$ & $\begin{array}{r}6 \\
8 \\
7 \\
8 \\
14\end{array}$ & $\begin{array}{l}24 \\
10 \\
22 \\
20 \\
18\end{array}$ & $\begin{array}{l}6 \\
8 \\
6 \\
8 \\
8\end{array}$ & $\begin{array}{r}30 \\
9 \\
18 \\
20 \\
21\end{array}$ & $\begin{array}{r}6 \\
12 \\
6 \\
8 \\
8\end{array}$ & $\begin{array}{l}21 \\
15 \\
22 \\
18 \\
13\end{array}$ & $\begin{array}{l}6 \\
8 \\
4 \\
8 \\
6\end{array}$ & $\begin{array}{l}263 \\
18 \\
25 \\
24 \\
23\end{array}$ & $\begin{array}{l}4 \\
8 \\
4 \\
7 \\
6\end{array}$ & $\begin{array}{l}18 \\
19 \\
22 \\
22 \\
31\end{array}$ & $\begin{array}{l}8 \\
6 \\
6 \\
8 \\
8 \\
\end{array}$ & $\begin{array}{l}14 \\
21 \\
21 \\
27 \\
18\end{array}$ \\
\hline $\begin{array}{l}21 \\
22 \\
23 \\
24 \\
25\end{array}$ & $\begin{array}{r}8 \\
6 \\
17 \\
10\end{array}$ & $\begin{array}{r}17 \\
27 \\
2 \\
9 \\
3\end{array}$ & $\begin{array}{r}7 \\
6 \\
30 \\
8 \\
10\end{array}$ & $\begin{array}{r}26 \\
27 \\
4 \\
4 \\
5\end{array}$ & $\begin{array}{r}7 \\
4 \\
\frac{4}{10}\end{array}$ & $\begin{array}{r}23 \\
29 \\
1 \\
1 \\
10\end{array}$ & $\begin{array}{r}7 \\
4 \\
9 \\
10\end{array}$ & $\begin{array}{r}32 \\
23 \\
1 \\
1 \\
10\end{array}$ & $\begin{array}{l}7 \\
\square \\
=\end{array}$ & $\begin{array}{r}27 \\
4 \\
1 \\
5\end{array}$ & $\frac{7}{\frac{7}{28}}$ & $\begin{array}{r}28 \\
22 \\
3 \\
1 \\
3\end{array}$ & $\frac{7}{4}$ & $\begin{array}{r}23 \\
20 \\
1 \\
2 \\
2\end{array}$ & $\begin{array}{r}7 \\
4 \\
30 \\
38 \\
8\end{array}$ & $\begin{array}{r}47 \\
17 \\
2 \\
1 \\
6\end{array}$ & $\begin{array}{r}7 \\
8 \\
10 \\
32 \\
\text { vble. }\end{array}$ & $\begin{array}{r}38 \\
21 \\
5 \\
4 \\
8\end{array}$ & $\begin{array}{r}6 \\
8 \\
6 \\
-\end{array}$ & $\begin{array}{r}44 \\
17 \\
14 \\
3 \\
1\end{array}$ & $\begin{array}{r}4 \\
32 \\
6 \\
- \\
-\end{array}$ & $\begin{array}{r}27 \\
12 \\
11 \\
5 \\
1\end{array}$ & $\begin{array}{r}8 \\
9 \\
8 \\
-\end{array}$ & $\begin{array}{r}31 \\
5 \\
13 \\
1 \\
1\end{array}$ \\
\hline $\begin{array}{l}26 \\
27 \\
28 \\
29 \\
30\end{array}$ & $\begin{array}{r}7 \\
7 \\
7 \\
15\end{array}$ & $\begin{array}{r}1 \\
22 \\
25 \\
22 \\
26\end{array}$ & $\begin{array}{r}32 \\
7 \\
7 \\
7 \\
16\end{array}$ & $\begin{array}{l}1 \\
25 \\
32 \\
31 \\
22\end{array}$ & $\begin{array}{r}-8 \\
7 \\
7 \\
16\end{array}$ & $\begin{array}{r}1 \\
18 \\
18 \\
33 \\
23\end{array}$ & $\begin{array}{r}-7 \\
8 \\
8 \\
14\end{array}$ & $\begin{array}{r}1 \\
18 \\
25 \\
27 \\
22\end{array}$ & $\begin{array}{r}77 \\
8 \\
10 \\
10\end{array}$ & $\begin{array}{l}1 \\
21 \\
30 \\
18 \\
13\end{array}$ & $\begin{array}{r}-4 \\
7 \\
12 \\
10\end{array}$ & $\begin{array}{l}1 \\
26 \\
20 \\
23 \\
19\end{array}$ & $\begin{array}{r}\overline{6} \\
7 \\
10 \\
10\end{array}$ & $\begin{array}{r}2 \\
24 \\
24 \\
25 \\
16\end{array}$ & $\begin{array}{r}-8 \\
6 \\
12 \\
17\end{array}$ & $\begin{array}{l}2 \\
22 \\
23 \\
22 \\
22\end{array}$ & $\begin{array}{r}10 \\
12 \\
6 \\
8 \\
8 \\
12\end{array}$ & $\begin{array}{r}7 \\
15 \\
21 \\
19 \\
17\end{array}$ & $\begin{array}{r}10 \\
12 \\
6 \\
8 \\
10\end{array}$ & $\begin{array}{l}10 \\
28 \\
25 \\
18 \\
19\end{array}$ & $\begin{array}{r}10 \\
8 \\
6 \\
12 \\
14\end{array}$ & $\begin{array}{r}11 \\
30 \\
21 \\
24 \\
9\end{array}$ & $\begin{array}{r}8 \\
7 \\
7 \\
12 \\
14\end{array}$ & $\begin{array}{l}21 \\
25 \\
35 \\
21 \\
11\end{array}$ \\
\hline 31 & 15 & 13 & 17 & 10 & 10 & 10 & 16 & 7 & 32 & 4 & - & 0 & - & 5 & 4 & 6 & 4 & 14 & 8 & 24 & 4 & 16 & 4 & 17 \\
\hline Mean & & $1 i$ & & 10 & & 17 & & 17 & & 16 & & 14 & & 14 & & 16 & & 16 & & 19 & & 16 & & 18 \\
\hline
\end{tabular}

* Equivalent velocity for Beaufort scale estimation. 
TABLE IV. (continued).

WIND-DIRECTION AND VELOCITY.

Direction (D) in Numbers $(8=\mathrm{E}, 16=\mathrm{S}, 24=\mathrm{W}, 32=\mathrm{N})$, and Veloeity $(\mathrm{V})$ in miles per lour as reeorded

June, 1902.

by the Cup Anemometer.

\begin{tabular}{|c|c|c|c|c|c|c|c|c|c|c|c|c|c|c|c|c|c|c|c|c|c|c|c|c|}
\hline \multirow{2}{*}{ Day. } & \multicolumn{2}{|c|}{2.} & \multicolumn{2}{|c|}{4.} & \multicolumn{2}{|c|}{6.} & \multicolumn{2}{|c|}{8.} & \multicolumn{2}{|c|}{10.} & \multicolumn{2}{|c|}{ Noon. } & \multicolumn{2}{|c|}{14.} & \multicolumn{2}{|c|}{18.} & \multicolumn{2}{|c|}{18.} & \multicolumn{2}{|c|}{20 , } & \multicolumn{2}{|c|}{22.} & \multicolumn{2}{|c|}{ Midt. } \\
\hline & D. & r. & D. & V. & 1). & v. & $\mathrm{D}$. & r. & D. & $\mathrm{v}$. & D. & v. & D. & v. & $\mathrm{D}$. & r. & D. & v. & D. & $\mathrm{r}$ & D. & v. & D. & r. \\
\hline $\begin{array}{l}1 \\
\frac{1}{2} \\
3\end{array}$ & $\begin{array}{r}7 \\
4 \\
10\end{array}$ & $\begin{array}{l}20 \\
16 \\
11\end{array}$ & $\begin{array}{l}8 \\
9\end{array}$ & $\begin{array}{r}20 \\
8 \\
3\end{array}$ & $\begin{array}{r}8 \\
9 \\
-\end{array}$ & $\begin{array}{r}18 \\
7 \\
1\end{array}$ & $\begin{array}{l}4 \\
4\end{array}$ & $\begin{array}{r}18 \\
4\end{array}$ & $\begin{array}{r}6 \\
4 \\
12\end{array}$ & $\begin{array}{r}13 \\
17 \\
5\end{array}$ & $\begin{array}{r}6 \\
4 \\
12\end{array}$ & $\begin{array}{l}11 \\
1 \\
3\end{array}$ & $\begin{array}{r}6 \\
12 \\
7\end{array}$ & 11 & $\begin{array}{r}4 \\
4 \\
16\end{array}$ & $\begin{array}{l}17 \\
27\end{array}$ & $\begin{array}{r}4 \\
8 \\
12\end{array}$ & $\begin{array}{l}19 \\
27\end{array}$ & $\begin{array}{l}6 \\
8 \\
8\end{array}$ & $\begin{array}{l}23 \\
30 \\
23\end{array}$ & $\begin{array}{r}4 \\
6 \\
12\end{array}$ & & 7 & \\
\hline & $\begin{array}{l}10 \\
10\end{array}$ & 7 & $\overline{6}$ & $\frac{3}{7}$ & $\overrightarrow{7}$ & 24 & $\overline{8}$ & 20 & 8 & $\begin{array}{l}\text { D } \\
\end{array}$ & 6 & 22 & 4 & 17 & $\begin{array}{r}16 \\
4\end{array}$ & 1 & 4 & & & & 4 & & 4 & ${ }_{21}^{11}$ \\
\hline $\begin{array}{l}5 \\
6 \\
6\end{array}$ & $\begin{array}{r}7 \\
32 \\
8\end{array}$ & $\begin{array}{r}21 \\
4 \\
5\end{array}$ & $\begin{array}{l}8 \\
4 \\
4\end{array}$ & $\begin{array}{r}18 \\
9 \\
7\end{array}$ & $\begin{array}{l}4 \\
B \\
4\end{array}$ & $\begin{array}{c}10 \\
4 \\
10\end{array}$ & $\begin{array}{l}4 \\
4\end{array}$ & $\begin{array}{r}21 \\
13 \\
5\end{array}$ & $\begin{array}{r}4 \\
32 \\
7\end{array}$ & $\begin{array}{l}16 \\
12 \\
18\end{array}$ & $\frac{2}{8}$ & $\begin{array}{l}13 \\
2 \\
26\end{array}$ & $\begin{array}{l}42 \\
32 \\
8\end{array}$ & $\begin{array}{l}11 \\
2 \\
24\end{array}$ & $\frac{4}{8}$ & $\begin{array}{l}14 \\
1\end{array}$ & $\frac{4}{8}$ & $\begin{array}{l}18 \\
0\end{array}$ & $\begin{array}{r}8 \\
30\end{array}$ & $\begin{array}{r}21 \\
3\end{array}$ & $\begin{array}{r}8 \\
28\end{array}$ & $\begin{array}{l}18 \\
10\end{array}$ & $\begin{array}{r}4 \\
9 \\
3\end{array}$ & $\begin{array}{l}13 \\
7\end{array}$ \\
\hline $\begin{array}{l}7 \\
8 \\
9\end{array}$ & $\begin{array}{r}8 \\
10 \\
6\end{array}$ & $\begin{array}{r}5 \\
2 \\
13\end{array}$ & $\begin{array}{l}4 \\
7 \\
8\end{array}$ & $\begin{array}{l}7 \\
3 \\
7\end{array}$ & $\begin{array}{r}4 \\
16 \\
8\end{array}$ & $\begin{array}{r}10 \\
6 \\
6\end{array}$ & $\begin{array}{r}7 \\
12 \\
4\end{array}$ & $\begin{array}{l}5 \\
3 \\
7\end{array}$ & $\begin{array}{l}7 \\
8 \\
6\end{array}$ & $\begin{array}{l}18 \\
12 \\
25\end{array}$ & $\begin{array}{l}8 \\
8 \\
6\end{array}$ & $\begin{array}{l}26 \\
16 \\
22\end{array}$ & $\begin{array}{l}8 \\
7 \\
8\end{array}$ & $\begin{array}{l}24 \\
22 \\
14\end{array}$ & $\begin{array}{l}8 \\
8 \\
8\end{array}$ & $\begin{array}{l}26 \\
18 \\
10\end{array}$ & $\begin{array}{l}8 \\
9 \\
8\end{array}$ & $\begin{array}{l}18 \\
13 \\
13\end{array}$ & $\begin{array}{r}9 \\
4 \\
12\end{array}$ & $\begin{array}{l}13 \\
16 \\
13\end{array}$ & $\begin{array}{l}4 \\
7 \\
8\end{array}$ & $\begin{array}{l}\begin{array}{c}6 \\
13 \\
11\end{array} \\
\end{array}$ & $\begin{array}{r}32 \\
72 \\
12\end{array}$ & $\begin{array}{l}10 \\
7 \\
11\end{array}$ \\
\hline $\begin{array}{l}10 \\
11\end{array}$ & 10 & ${ }_{1}^{11}$ & $\underline{11}$ & ${ }_{0}^{11}$ & 11 & $\overline{7}$ & 1 & ${ }_{1}^{7}$ & = & ${ }_{0}^{2}$ & 32 & 10 & $\Xi$ & $\frac{1}{0}$ & $=$ & $\frac{1}{0}$ & $\overline{8}$ & ${ }_{2}^{2}$ & $\overline{10}$ & $\begin{array}{r}1 \\
19\end{array}$ & $\overline{16}$ & $\begin{array}{r}0 \\
15\end{array}$ & $\overline{18}$ & 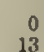 \\
\hline $\begin{array}{l}12 \\
13\end{array}$ & $\begin{array}{r}16 \\
8\end{array}$ & $\begin{array}{l}12 \\
11\end{array}$ & $\begin{array}{r}18 \\
8\end{array}$ & $\begin{array}{l}20 \\
6\end{array}$ & $\begin{array}{r}14 \\
9\end{array}$ & $\begin{array}{r}19 \\
21\end{array}$ & 8 & $\begin{array}{l}10 \\
21\end{array}$ & $\frac{4}{7}$ & $\begin{array}{l}17 \\
{ }_{26}\end{array}$ & $\frac{4}{6}$ & $\begin{array}{l}12 \\
23\end{array}$ & 7 & $\begin{array}{r}7 \\
23\end{array}$ & $\overline{7}$ & $\begin{array}{r}1 \\
19\end{array}$ & $\begin{array}{l}6 \\
6 \\
6\end{array}$ & $\begin{array}{r}8 \\
18\end{array}$ & $\begin{array}{l}8 \\
6\end{array}$ & $\begin{array}{l}10 \\
13\end{array}$ & $\begin{array}{l}8 \\
4\end{array}$ & $\begin{array}{l}13 \\
15\end{array}$ & $\begin{array}{l}9 \\
6\end{array}$ & $\begin{array}{l}4 \\
17\end{array}$ \\
\hline $\begin{array}{l}14 \\
14\end{array}$ & ${ }_{6}^{\circ}$ & $\begin{array}{l}11 \\
12\end{array}$ & 6 & 11 & 8 & 15 & 4 & 13 & 33 & 12 & - & 6 & - & 2 & 4 & 7 & 4 & 4 & 4 & 8 & $1-$ & 2 & - & 3 \\
\hline $\begin{array}{l}15 \\
16 \\
17\end{array}$ & $\overline{24}$ & $\begin{array}{l}1 \\
13 \\
13\end{array}$ & $\begin{array}{r}23 \\
30 \\
7\end{array}$ & $\begin{array}{r}1 \\
1\end{array}$ & $\begin{array}{r}32 \\
32 \\
4\end{array}$ & $\begin{array}{r}7 \\
13 \\
23\end{array}$ & $\overline{28}$ & $\begin{array}{r}2 \\
19 \\
15\end{array}$ & $\overline{28}$ & $\begin{array}{l}13 \\
13 \\
14\end{array}$ & $\overline{\frac{7}{8}}$ & $\begin{array}{r}0 \\
8 \\
19\end{array}$ & $\begin{array}{r}12 \\
16 \\
8\end{array}$ & $\begin{array}{r}3 \\
7 \\
26\end{array}$ & $\begin{array}{l}12 \\
18 \\
6\end{array}$ & $\begin{array}{r}13 \\
13 \\
13\end{array}$ & $\begin{array}{l}15 \\
18 \\
8\end{array}$ & $\begin{array}{l}16 \\
20 \\
13\end{array}$ & $\begin{array}{c}7 \\
16 \\
20\end{array}$ & $\begin{array}{r}26 \\
33 \\
5\end{array}$ & $\begin{array}{r}6 \\
14 \\
8\end{array}$ & $\begin{array}{r}26 \\
23 \\
7\end{array}$ & $\begin{array}{r}4 \\
9 \\
9\end{array}$ & $\begin{array}{l}26 \\
17 \\
12\end{array}$ \\
\hline $\begin{array}{l}17 \\
19 \\
19\end{array}$ & $\begin{array}{r}8 \\
6 \\
10\end{array}$ & $\begin{array}{l}13 \\
15 \\
15\end{array}$ & $\begin{array}{l}\frac{7}{7} \\
7\end{array}$ & $\begin{array}{l}26 \\
18 \\
34\end{array}$ & $\begin{array}{l}4 \\
6 \\
4\end{array}$ & $\begin{array}{l}23 \\
16 \\
22\end{array}$ & $\begin{array}{l}6 \\
\frac{4}{7}\end{array}$ & $\begin{array}{l}15 \\
22 \\
13\end{array}$ & $\begin{array}{l}4 \\
8 \\
4\end{array}$ & $\begin{array}{l}14 \\
18 \\
14\end{array}$ & $\begin{array}{l}8 \\
8 \\
4\end{array}$ & $\begin{array}{l}19 \\
20 \\
11\end{array}$ & $\begin{array}{r}8 \\
8 \\
-\end{array}$ & $\begin{array}{r}26 \\
14 \\
7\end{array}$ & $\begin{array}{r}6 \\
-8 \\
-\end{array}$ & $\begin{array}{r}13 \\
18 \\
1\end{array}$ & $\begin{array}{r}8 \\
6 \\
23\end{array}$ & $\begin{array}{c}13 \\
20 \\
1\end{array}$ & $\begin{array}{r}20 \\
8 \\
2\end{array}$ & $\begin{array}{r}5 \\
15 \\
1\end{array}$ & $\begin{array}{r}8 \\
12 \\
-\end{array}$ & $\begin{array}{l}7 \\
9 \\
2\end{array}$ & $\begin{array}{l}10 \\
14 \\
-\end{array}$ & $\begin{array}{r}12 \\
7 \\
0\end{array}$ \\
\hline $\begin{array}{l}20 \\
21\end{array}$ & $\overline{5}$ & $\begin{array}{r}2 \\
10\end{array}$ & $\overline{8}$ & ${ }_{18}^{2}$ & $\overline{8}$ & $\underset{21}{2}$ & 8 & $\begin{array}{r}2 \\
22\end{array}$ & $\begin{array}{r}32 \\
8\end{array}$ & $\begin{array}{r}7 \\
2\end{array}$ & 32 & $\begin{array}{r}7 \\
22\end{array}$ & $\begin{array}{r}32 \\
8\end{array}$ & $\begin{array}{l}10 \\
19\end{array}$ & 8 & ${ }_{11}^{7}$ & $=$ & ${ }_{11}^{2}$ & 32 & $\begin{array}{l}7 \\
3\end{array}$ & $\underline{25}$ & $\begin{array}{c}10 \\
1\end{array}$ & $\overline{\bar{a}}$ & ${ }_{0}^{2}$ \\
\hline $\begin{array}{l}22 \\
23 \\
24\end{array}$ & $\begin{array}{r}8 \\
32\end{array}$ & $\frac{7}{2}$ & $\frac{6}{1}$ & $\begin{array}{l}9 \\
4 \\
6\end{array}$ & $\begin{array}{l}8 \\
8 \\
8\end{array}$ & $\begin{array}{r}16 \\
4 \\
3\end{array}$ & $\begin{array}{c}12 \\
4 \\
8\end{array}$ & $\begin{array}{r}15 \\
25 \\
8\end{array}$ & $\begin{array}{r}7 \\
12\end{array}$ & $\begin{array}{r}10 \\
28 \\
7\end{array}$ & $\begin{array}{r}32 \\
4 \\
4\end{array}$ & $\begin{array}{r}5 \\
24 \\
10\end{array}$ & $\begin{array}{r}4 \\
4 \\
-\end{array}$ & $\begin{array}{r}4 \\
18 \\
3\end{array}$ & $\begin{array}{r}4 \\
12\end{array}$ & $\begin{array}{l}2 \\
7 \\
2\end{array}$ & $\bar{z}$ & $\frac{2}{7}$ & $\bar{z}$ & 0 & $\overline{\overline{8}}$ & 4 & $\begin{array}{r}4 \\
32\end{array}$ & 3 \\
\hline${ }_{25}^{25}$ & $\begin{array}{l}31 \\
312\end{array}$ & 5 & $\bar{u}$ & $2^{2}$ & 17 & 2 & 9 & 4 & 10 & 12 & 8 & 9 & 8 & 11 & I & 20 & $\begin{array}{l}8 \\
6\end{array}$ & 17 & 8 & $\begin{array}{l}29 \\
23\end{array}$ & $\begin{array}{l}8 \\
8\end{array}$ & 23 & $7^{7}$ & $\begin{array}{l}23 \\
07\end{array}$ \\
\hline $\begin{array}{l}26 \\
27 \\
79\end{array}$ & $\begin{array}{l}12 \\
17 \\
\end{array}$ & $\begin{array}{r}7 \\
26 \\
\end{array}$ & $\begin{array}{l}14 \\
12\end{array}$ & $\begin{array}{l}13 \\
21 \\
1\end{array}$ & $\begin{array}{l}6 \\
12 \\
\end{array}$ & $\begin{array}{l}10 \\
13 \\
8\end{array}$ & 12 & $\begin{array}{c}11 \\
13 \\
3\end{array}$ & $\overline{12}$ & $\begin{array}{r}4 \\
13 \\
\end{array}$ & $\overline{8}$ & 21 & $\overline{12}$ & 14 & $\underline{12}$ & 11 & 8 & $\begin{array}{l}18 \\
13\end{array}$ & 8 & $\begin{array}{l}13 \\
13\end{array}$ & i & $\begin{array}{l}11 \\
11\end{array}$ & $\begin{array}{l}10 \\
6 \\
19\end{array}$ & 9 \\
\hline 年 & $\begin{array}{l}9 \\
19\end{array}$ & $\frac{2}{7}$ & $\begin{array}{l}4 \\
19\end{array}$ & $\begin{array}{l}10 \\
29\end{array}$ & $\overline{16}$ & $\begin{array}{l}8 \\
17\end{array}$ & $\overline{12}$ & $10^{2}$ & 16 & $\begin{array}{c}3 \\
16\end{array}$ & $\overline{18}$ & 23 & $\overline{12}$ & 13 & $\overline{20}$ & 18 & $\begin{array}{l}16 \\
20\end{array}$ & $\begin{array}{l}13 \\
13\end{array}$ & $\begin{array}{l}16 \\
{ }_{20}\end{array}$ & 24 & $\begin{array}{l}20 \\
18\end{array}$ & $\begin{array}{l}16 \\
21\end{array}$ & $\begin{array}{l}19 \\
17\end{array}$ & $\begin{array}{l}15 \\
16\end{array}$ \\
\hline 30 & 12 & 33 & 14 & 32 & 15 & 22 & 14 & 16 & 8 & 13 & 12 & 10 & 12 & 7 & 8 & 8 & 4 & 10 & - & 3 & - & 0 & - & 0 \\
\hline ear & & 10 & & 13 & & 12 & & 12 & & 13 & & 13 & & 11 & & 10 & & 12 & & 14 & & 12 & & 11 \\
\hline
\end{tabular}

July, 1902.

\begin{tabular}{|c|c|c|c|c|c|c|c|c|c|c|c|c|c|c|c|c|c|c|c|c|c|c|c|c|}
\hline \multirow{2}{*}{ Das. } & \multicolumn{2}{|c|}{2.} & \multicolumn{2}{|c|}{4.} & \multicolumn{2}{|c|}{6.} & \multicolumn{2}{|c|}{8.} & \multicolumn{2}{|c|}{10.} & \multicolumn{2}{|c|}{ Noon. } & \multicolumn{2}{|c|}{14.} & \multicolumn{2}{|c|}{16.} & \multicolumn{2}{|c|}{18.} & \multicolumn{2}{|c|}{20.} & \multicolumn{2}{|c|}{22.} & \multicolumn{2}{|c|}{ Midt. } \\
\hline & D. & v. & D. & v. & I). & v. & D. & v. & D. & v. & D. & v. & D. & v. & D. & r. & D. & v. & D. & v. & D. & v. & D. & v. \\
\hline $\begin{array}{l}1 \\
2 \\
3 \\
4\end{array}$ & $\begin{array}{l}-\overline{32} \\
12 \\
32\end{array}$ & $\begin{array}{r}0 \\
5 \\
17 \\
7\end{array}$ & $\overline{7}$ & $\begin{array}{r}0 \\
0 \\
15 \\
0\end{array}$ & $\overline{-}$ & $\begin{array}{r}0 \\
0 \\
10 \\
2\end{array}$ & $\begin{array}{r}\overline{8} \\
12 \\
4\end{array}$ & $\begin{array}{r}0 \\
4 \\
14 \\
1\end{array}$ & $\begin{array}{r}-8 \\
12 \\
4\end{array}$ & $\begin{array}{r}0 \\
15 \\
10 \\
10\end{array}$ & $\begin{array}{r}32 \\
9 \\
12 \\
8\end{array}$ & $\begin{array}{r}6 \\
15 \\
10 \\
12\end{array}$ & $\begin{array}{r}32 \\
9 \\
8 \\
12\end{array}$ & $\begin{array}{c}4 \\
16^{\prime \prime} \\
4^{*} \\
9\end{array}$ & $\begin{array}{r}-8 \\
8 \\
4\end{array}$ & $\begin{array}{c}4 \\
21 \\
4^{*} \\
7\end{array}$ & $\begin{array}{l}-\overline{8} \\
8 \\
4\end{array}$ & $\begin{array}{c}4 \\
19 \\
2^{*} \\
7\end{array}$ & $\begin{array}{r}12 \\
8 \\
4 \\
-\end{array}$ & $\begin{array}{r}4 \\
21 \\
3 \\
5\end{array}$ & $\begin{array}{r}8 \\
8 \\
32\end{array}$ & $\begin{array}{r}7 \\
15 \\
2 \\
2\end{array}$ & $\begin{array}{r}7 \\
12 \\
1 \\
4\end{array}$ & $\begin{array}{r}9 \\
12 \\
2 \\
7\end{array}$ \\
\hline $\begin{array}{l}5 \\
6 \\
7 \\
8 \\
9\end{array}$ & $\begin{array}{r}4 \\
12 \\
9 \\
15\end{array}$ & $\begin{array}{l}6 \\
8 \\
4 \\
0 \\
4\end{array}$ & $\begin{array}{r}32 \\
10 \\
7 \\
8\end{array}$ & $\begin{array}{l}3 \\
5 \\
4 \\
0 \\
7\end{array}$ & $\frac{\overline{12}}{16}$ & $\begin{array}{r}2 \\
6 \\
5 \\
0 \\
0 \\
14\end{array}$ & $\begin{array}{l}\frac{12}{12} \\
\frac{7}{12}\end{array}$ & $\begin{array}{r}2 \\
4 \\
0 \\
0 \\
0 \\
15\end{array}$ & $\frac{12}{\frac{12}{12}}$ & $\begin{array}{r}4 \\
12 \\
0 \\
0 \\
18\end{array}$ & $\begin{array}{r}12 \\
\frac{8}{9}\end{array}$ & $\begin{array}{r}4 \\
15 \\
0 \\
2 \\
12\end{array}$ & $\frac{12}{\frac{10}{8}}$ & $\begin{array}{r}7 \\
10 \\
0 \\
0 \\
12\end{array}$ & $\begin{array}{r}12 \\
8 \\
8 \\
8\end{array}$ & $\begin{array}{r}6 \\
4 \\
2 \\
0 \\
13\end{array}$ & $\begin{array}{r}12 \\
6 \\
\frac{8}{10}\end{array}$ & $\begin{array}{r}9 \\
7 \\
12 \\
0 \\
8\end{array}$ & $\begin{array}{r}15 \\
6 \\
8 \\
6\end{array}$ & $\begin{array}{r}10 \\
7 \\
15 \\
0 \\
5\end{array}$ & $\frac{12}{8}$ & $\begin{array}{r}10 \\
7 \\
10 \\
0 \\
2\end{array}$ & $\begin{array}{l}15 \\
10 \\
= \\
=\end{array}$ & $\begin{array}{r}11 \\
2 \\
4 \\
0 \\
1\end{array}$ \\
\hline $\begin{array}{l}10 \\
11 \\
12 \\
13 \\
14\end{array}$ & $\begin{array}{r}-7 \\
7 \\
13 \\
6\end{array}$ & $\begin{array}{r}2 \\
15 \\
16 \\
12 \\
28\end{array}$ & $\begin{array}{r}-12 \\
7 \\
8 \\
4\end{array}$ & $\begin{array}{r}2 \\
8 \\
10 \\
19 \\
27\end{array}$ & $\begin{array}{r}5 \\
16 \\
6 \\
7 \\
4\end{array}$ & $\begin{array}{r}2 \\
11 \\
16 \\
13 \\
24\end{array}$ & $\begin{array}{r}-20 \\
9 \\
8 \\
4\end{array}$ & $\begin{array}{r}14 \\
17 \\
14 \\
9 \\
17\end{array}$ & $\begin{array}{r}\overline{12} \\
8 \\
12 \\
6\end{array}$ & $\begin{array}{r}4 \\
10 \\
12 \\
12 \\
21\end{array}$ & $\begin{array}{r}\overline{12} \\
8 \\
8 \\
6\end{array}$ & $\begin{array}{r}0 \\
11 \\
9 \\
5 \\
15\end{array}$ & $\begin{array}{r}\overline{12} \\
12 \\
8 \\
6\end{array}$ & $\begin{array}{r}1 \\
13 \\
13 \\
10 \\
10\end{array}$ & $\begin{array}{r}-18 \\
8 \\
8 \\
6\end{array}$ & $\begin{array}{r}1 \\
16 \\
14 \\
8 \\
15\end{array}$ & $\begin{array}{r}8 \\
12 \\
6 \\
4 \\
6\end{array}$ & $\begin{array}{r}12 \\
10 \\
18 \\
4 \\
18\end{array}$ & $\begin{array}{r}8 \\
12 \\
4 \\
4 \\
6\end{array}$ & $\begin{array}{l}18 \\
12 \\
20 \\
22 \\
17\end{array}$ & $\begin{array}{r}8 \\
12 \\
12 \\
4 \\
6\end{array}$ & $\begin{array}{l}23 \\
10 \\
14 \\
26 \\
21\end{array}$ & $\begin{array}{r}8 \\
7 \\
12 \\
6 \\
7\end{array}$ & $\begin{array}{r}21 \\
11 \\
9 \\
28 \\
19\end{array}$ \\
\hline $\begin{array}{l}15 \\
16 \\
17 \\
18 \\
19\end{array}$ & $\begin{array}{r}6 \\
7 \\
12 \\
14 \\
17\end{array}$ & $\begin{array}{c}20 \\
19 \\
5 \\
27 \\
50 *\end{array}$ & $\begin{array}{r}6 \\
7 \\
9 \\
14 \\
17\end{array}$ & $\begin{array}{c}15 \\
1.3 \\
4 \\
30 \\
59^{*}\end{array}$ & $\begin{array}{r}8 \\
7 \\
9 \\
16 \\
17\end{array}$ & $\begin{array}{c}15 \\
12 \\
2 \\
23 \\
59^{*}\end{array}$ & $\begin{array}{r}4 \\
4 \\
8 \\
16 \\
16\end{array}$ & $\begin{array}{c}21 \\
11 \\
3 \\
16 \\
64^{*}\end{array}$ & $\begin{array}{r}7 \\
6 \\
8 \\
16 \\
16\end{array}$ & $\begin{array}{c}18 \\
14 \\
7 \\
34 \\
55^{*}\end{array}$ & $\begin{array}{r}7 \\
6 \\
12 \\
16 \\
16\end{array}$ & $\begin{array}{r}13 \\
17 \\
7 \\
24 \\
55\end{array}$ & $\begin{array}{r}12 \\
6 \\
17 \\
11 \\
12\end{array}$ & $\begin{array}{r}6 \\
17 \\
5 \\
26 \\
46\end{array}$ & $\begin{array}{r}12 \\
6 \\
16 \\
18 \\
12\end{array}$ & $\begin{array}{r}2 \\
13 \\
10 \\
40 \\
35\end{array}$ & $\begin{array}{l}10 \\
\frac{16}{16} \\
10\end{array}$ & $\begin{array}{r}4 \\
7 \\
14 \\
47 \\
28\end{array}$ & $\begin{array}{c}\frac{6}{8} \\
16 \\
12\end{array}$ & $\begin{array}{r}4 \\
1 \\
18 \\
54 \\
26\end{array}$ & $\begin{array}{r}\frac{7}{8} \\
18 \\
10\end{array}$ & $\begin{array}{r}13 \\
1 \\
17 \\
54 \\
21\end{array}$ & $\begin{array}{r}7 \\
12 \\
16 \\
13 \\
10\end{array}$ & $\begin{array}{l}18 \\
4 \\
28 \\
55^{*} \\
18\end{array}$ \\
\hline $\begin{array}{l}20 \\
21 \\
22 \\
23 \\
24\end{array}$ & $\begin{array}{r}8 \\
8 \\
12 \\
5 \\
8\end{array}$ & $\begin{array}{r}17 \\
15 \\
7 \\
13 \\
16\end{array}$ & $\begin{array}{l}7 \\
8 \\
9 \\
6 \\
8\end{array}$ & $\begin{array}{c}19 \\
10 \\
4 \\
10 \\
18\end{array}$ & $\frac{7}{7}$ & $\begin{array}{r}32 \\
12 \\
4 \\
17 \\
23\end{array}$ & $\begin{array}{r}8 \\
7 \\
12 \\
7 \\
7\end{array}$ & $\begin{array}{r}26 \\
17 \\
1 \\
4 \\
26\end{array}$ & $\begin{array}{l}8 \\
4 \\
8 \\
8 \\
8\end{array}$ & $\begin{array}{r}26 \\
7 \\
1 \\
12 \\
23\end{array}$ & $\begin{array}{c}\frac{4}{12} \\
8 \\
8\end{array}$ & $\begin{array}{r}21 \\
9 \\
2 \\
15 \\
21\end{array}$ & $\begin{array}{l}4 \\
8 \\
8 \\
8 \\
8\end{array}$ & $\begin{array}{r}25 \\
9 \\
2 \\
17 \\
23\end{array}$ & $\begin{array}{l}4 \\
8 \\
8 \\
6 \\
8\end{array}$ & $\begin{array}{r}24 \\
8 \\
4 \\
15 \\
21\end{array}$ & $\begin{array}{l}\frac{8}{8} \\
8 \\
4\end{array}$ & $\begin{array}{r}43 \\
8 \\
1 \\
14 \\
19\end{array}$ & $\begin{array}{r}8 \\
12 \\
8 \\
8 \\
8\end{array}$ & $\begin{array}{r}29 \\
8 \\
10 \\
18 \\
26\end{array}$ & $\begin{array}{l}8 \\
8 \\
6 \\
8 \\
8\end{array}$ & $\begin{array}{r}30 \\
8 \\
10 \\
15 \\
21\end{array}$ & $\begin{array}{r}8 \\
12 \\
5 \\
8 \\
8\end{array}$ & $\begin{array}{r}28 \\
8 \\
13 \\
17 \\
24\end{array}$ \\
\hline $\begin{array}{l}25 \\
25 \\
27 \\
28 \\
28\end{array}$ & $\begin{array}{l}8 \\
7 \\
4 \\
4 \\
4\end{array}$ & $\begin{array}{r}20 \\
2 \\
2 \\
13 \\
4\end{array}$ & $\begin{array}{r}8 \\
6 \\
4 \\
2\end{array}$ & $\begin{array}{r}17 \\
8 \\
4 \\
7 \\
2\end{array}$ & $\begin{array}{r}8 \\
9 \\
32 \\
32\end{array}$ & $\begin{array}{r}15 \\
9 \\
0 \\
8 \\
4\end{array}$ & $\begin{array}{r}8 \\
9 \\
8 \\
7 \\
32\end{array}$ & $\begin{array}{r}22 \\
11 \\
1 \\
1 \\
2\end{array}$ & $\begin{array}{r}8 \\
8 \\
\\
32\end{array}$ & $\begin{array}{r}10 \\
12 \\
0 \\
1 \\
4\end{array}$ & $\begin{array}{r}8 \\
12 \\
4 \\
-\end{array}$ & $\begin{array}{r}7 \\
13 \\
5 \\
0 \\
7\end{array}$ & $\begin{array}{r}8 \\
12 \\
4 \\
-\end{array}$ & $\begin{array}{l}7 \\
7 \\
4 \\
2 \\
4\end{array}$ & $\begin{array}{r}8 \\
12 \\
4 \\
32 \\
-\end{array}$ & $\begin{array}{r}8 \\
7 \\
11 \\
2 \\
3\end{array}$ & $\begin{array}{r}8 \\
12 \\
4 \\
8 \\
32\end{array}$ & $\begin{array}{l}7 \\
5 \\
8 \\
3 \\
1\end{array}$ & $\begin{array}{r}12 \\
12 \\
7 \\
8 \\
-\end{array}$ & $\begin{array}{r}8 \\
6 \\
10 \\
4 \\
0\end{array}$ & $\begin{array}{r}14 \\
12 \\
4 \\
-\end{array}$ & $\begin{array}{l}8 \\
3 \\
7 \\
j \\
2\end{array}$ & $\begin{array}{r}10 \\
12 \\
4 \\
32 \\
-\end{array}$ & $\begin{array}{r}9 \\
2 \\
14 \\
3 \\
2\end{array}$ \\
\hline $\begin{array}{l}30 \\
31\end{array}$ & $\overline{32}$ & $\begin{array}{l}0 \\
1\end{array}$ & 32 & $\begin{array}{l}5 \\
0\end{array}$ & $\begin{array}{r}32 \\
9\end{array}$ & $\stackrel{7}{1}$ & $\begin{array}{r}32 \\
8\end{array}$ & $\begin{array}{r}10 \\
7\end{array}$ & $\begin{array}{r}32 \\
8\end{array}$ & $\begin{array}{r}7 \\
13\end{array}$ & $\begin{array}{r}32 \\
8\end{array}$ & $\begin{array}{l}10 \\
12\end{array}$ & $\begin{array}{r}32 \\
8\end{array}$ & $\begin{array}{l}11 \\
18\end{array}$ & $\begin{array}{l}32 \\
12\end{array}$ & $\begin{array}{r}7 \\
18\end{array}$ & $\begin{array}{l}32 \\
12\end{array}$ & $\begin{array}{r}7 \\
16\end{array}$ & $\begin{array}{l}32 \\
20\end{array}$ & 2.5 & $\begin{array}{l}32 \\
20\end{array}$ & $\stackrel{2}{25^{*}}$ & $\overline{20}$ & $\stackrel{2}{35^{*}}$ \\
\hline Męan & & 11 & & 10 & & 11 & & 11 & & 12 & & 11 & & 11 & & 11 & & 12 & & 13 & & 12 & & 13 \\
\hline
\end{tabular}


TABLE IV. (continued).

WIND-DIRECTION AND VELOCITY.

Direction (D) in Numbers $(8=\mathrm{E}, 16=\mathrm{S}, 24=\mathrm{W}, 32=\mathrm{N})$, and Volocity (V) in miles per hour as recorded by the Cup Anemometer.

August, 1902.

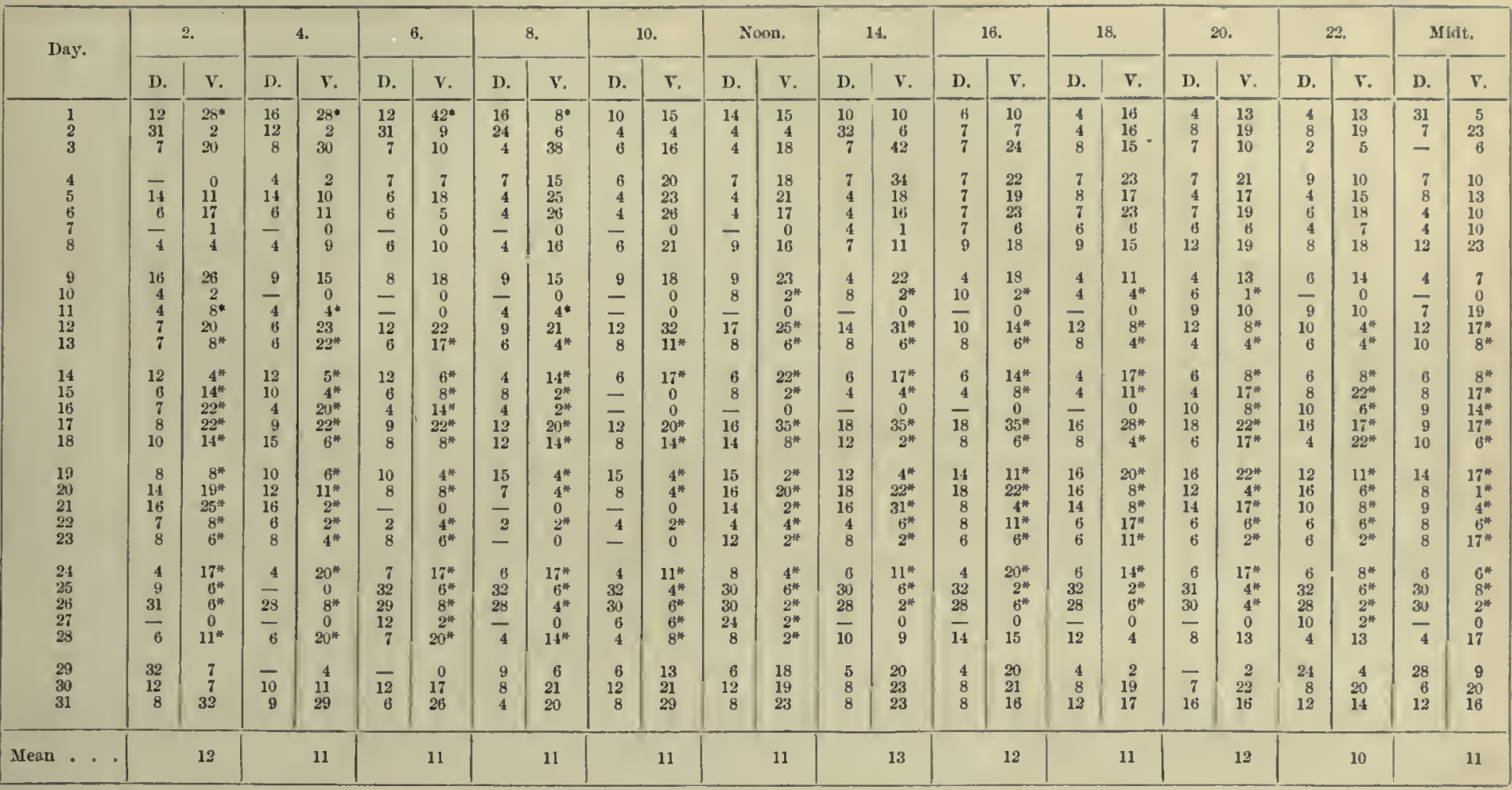

* Equivalent velocity for Beaufort scale estimation.

September, 1902.

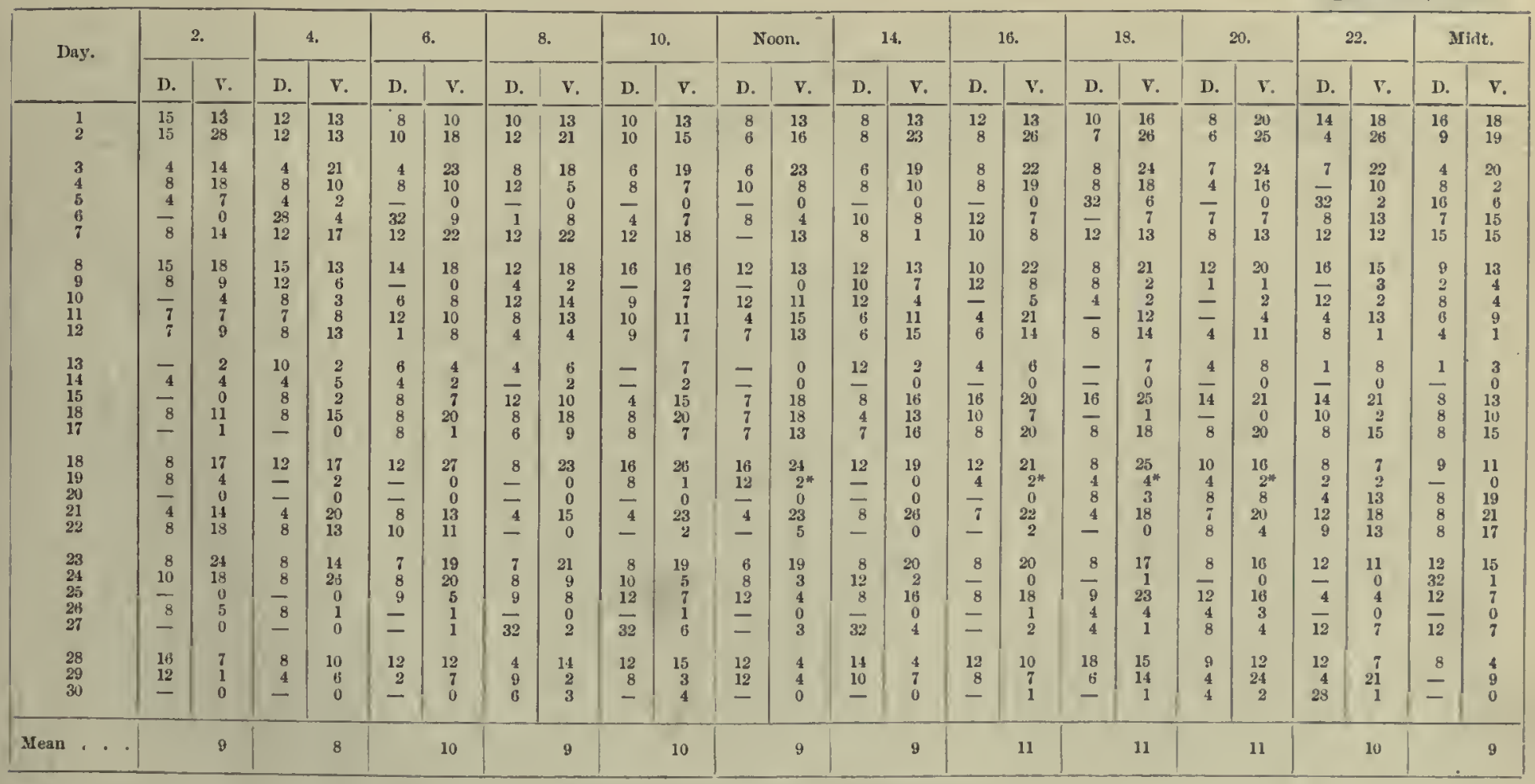


TABLe IV. (continued).

WIND-DIRECTION AND VELOCITY.

Direetion (D) in Numbers $(8=\mathrm{E}, 16=\mathrm{S}, 24=\mathrm{W}, 32=\mathrm{N})$, and Velocity $(\mathrm{V})$ in miles per hour as reeorded October, 1902. by the Cup Anemometer.

\begin{tabular}{|c|c|c|c|c|c|c|c|c|c|c|c|c|c|c|c|c|c|c|c|c|c|c|c|c|}
\hline \multirow[t]{2}{*}{ Day. } & \multicolumn{2}{|c|}{2.} & \multicolumn{2}{|c|}{4.} & \multicolumn{2}{|c|}{6.} & \multicolumn{2}{|c|}{8.} & \multicolumn{2}{|c|}{10.} & \multicolumn{2}{|c|}{ Noon. } & \multicolumn{2}{|c|}{14.} & \multicolumn{2}{|c|}{16.} & \multicolumn{2}{|c|}{18.} & \multicolumn{2}{|c|}{20} & \multicolumn{2}{|c|}{ 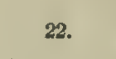 } & \multicolumn{2}{|c|}{ Midt. } \\
\hline & D. & v. & D. & r. & $\mathrm{D}$. & v. & D. & v. & $\mathrm{D}$. & $\mathbf{v}$ & D. & $\mathrm{v}$. & D. & $\mathbf{v}$ & D. & $\mathrm{v}$. & D. & v. & D. & $\mathbf{v}$ & D. & v. & D. & v. \\
\hline$\frac{1}{2}$ & $\overline{8}$ & $\begin{array}{l}0 \\
10\end{array}$ & $\overline{8}$ & 5 & $\overline{12}$ & $\begin{array}{l}0 \\
13\end{array}$ & 12 & 1 & 8 & 10 & $\begin{array}{l}10 \\
8\end{array}$ & ${ }_{8}^{10}$ & 8 & $\begin{array}{l}12 \\
15\end{array}$ & & $\begin{array}{l}6 \\
9\end{array}$ & 8 & $\begin{array}{l}18 \\
13\end{array}$ & $\begin{array}{l}8 \\
8\end{array}$ & $\begin{array}{c}13 \\
6\end{array}$ & $\begin{array}{l}8 \\
4\end{array}$ & $\begin{array}{r}6 \\
10\end{array}$ & $\begin{array}{l}8 \\
8\end{array}$ & 5 \\
\hline $\begin{array}{l}3 \\
4\end{array}$ & $=$ & 8 & $\overline{4}$ & $\begin{array}{l}0 \\
4\end{array}$ & $\overline{4}$ & $\begin{array}{l}0 \\
16\end{array}$ & $\overline{-}$ & $\begin{array}{l}0 \\
0 \\
0\end{array}$ & $\bar{z}$ & $\begin{array}{l}2 \\
0\end{array}$ & $=$ & $\begin{array}{l}0 \\
2\end{array}$ & $=$ & $\begin{array}{l}0 \\
0\end{array}$ & $\bar{z}$ & 2 & 28 & 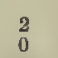 & $\overline{12}$ & $\begin{array}{l}0 \\
3\end{array}$ & $\overline{12}$ & & - & $\begin{array}{l}0 \\
0 \\
0\end{array}$ \\
\hline $\begin{array}{l}5 \\
6 \\
7\end{array}$ & $\overline{8}$ & $\begin{array}{c}0 \\
\underset{2}{19}\end{array}$ & $\frac{7}{-}$ & $\begin{array}{c}0 \\
20 \\
0\end{array}$ & $\frac{-}{4}$ & $\begin{array}{c}1 \\
18 \\
0\end{array}$ & $\begin{array}{c}4 \\
4\end{array}$ & $\begin{array}{r}2 \\
21 \\
0\end{array}$ & 8 & $\begin{array}{r}16 \\
18 \\
0\end{array}$ & $\begin{array}{l}4 \\
4 \\
4\end{array}$ & $\begin{array}{c}16 \\
15 \\
2\end{array}$ & $\begin{array}{l}7 \\
4 \\
4\end{array}$ & $\begin{array}{r}18 \\
13 \\
3\end{array}$ & $\begin{array}{l}7 \\
6 \\
4\end{array}$ & $\begin{array}{c}24 \\
17 \\
7\end{array}$ & $\begin{array}{l}8 \\
7 \\
8\end{array}$ & $\begin{array}{l}23 \\
10 \\
11\end{array}$ & $\begin{array}{l}6 \\
8 \\
8\end{array}$ & $\begin{array}{l}22 \\
11 \\
11\end{array}$ & $\begin{array}{r}4 \\
32 \\
4\end{array}$ & $\begin{array}{c}23 \\
5 \\
14\end{array}$ & $\overline{4}$ & $\begin{array}{c}18 \\
1 \\
14\end{array}$ \\
\hline 8 & $\frac{4}{4}$ & ${ }^{21}$ & $\frac{4}{2}$ & ${ }_{7}^{20}$ & 4 & 8 & 4 & 11 & $=$ & $\begin{array}{l}0 \\
3\end{array}$ & $\frac{6}{-2}$ & $\stackrel{2}{0}$ & $=$ & $\frac{1}{0}$ & $\overline{10}$ & $\begin{array}{l}0 \\
4\end{array}$ & -7 & 2 & 4 & 1 & $\overline{4}$ & $\begin{array}{l}\mathbf{0} \\
3\end{array}$ & -4 & \\
\hline 10 & $\frac{4}{8}$ & $\begin{array}{r}1 \\
15 \\
15\end{array}$ & $\begin{array}{l}2 \\
4 \\
8\end{array}$ & 4 & $\overline{4}$ & $\begin{array}{l}\mathbf{4} \\
10 \\
10\end{array}$ & $\overline{8}$ & $\begin{array}{c}0 \\
10 \\
10\end{array}$ & 8 & $\begin{array}{l}2 \\
7 \\
2\end{array}$ & $\overline{8}$ & $\begin{array}{r}0 \\
15 \\
6\end{array}$ & $\overline{8}$ & $\begin{array}{l}0 \\
16 \\
5\end{array}$ & $\begin{array}{r}10 \\
8 \\
\end{array}$ & $\begin{array}{r}4 \\
19 \\
5\end{array}$ & $\begin{array}{r}4 \\
8 \\
-\end{array}$ & $\begin{array}{r}4 \\
17 \\
5\end{array}$ & $\overline{8}$ & $\begin{array}{l}0 \\
20\end{array}$ & $\begin{array}{l}\mathbf{4} \\
8\end{array}$ & $\begin{array}{c}3 \\
18 \\
0\end{array}$ & $\begin{array}{l}4 \\
8 \\
-\end{array}$ & 20 \\
\hline 11 & - & $\begin{array}{c}10 \\
0\end{array}$ & 12 & 5 & $\begin{array}{r}8 \\
12\end{array}$ & $\begin{array}{r}10 \\
5\end{array}$ & $\begin{array}{l}8 \\
12\end{array}$ & $\begin{array}{l}10 \\
12 \\
12\end{array}$ & 6 & $\frac{2}{6}$ & $\begin{array}{l}4 \\
8\end{array}$ & $\begin{array}{l}6 \\
4\end{array}$ & 4 & $\begin{array}{l}5 \\
8\end{array}$ & $\overline{8}$ & $\begin{array}{c}5 \\
12\end{array}$ & $\overline{8}$ & $\begin{array}{r}5 \\
10\end{array}$ & $\overrightarrow{10}$ & 10 & $\overline{9}$ & 0 & 7 & $\begin{array}{l}0 \\
17\end{array}$ \\
\hline $\begin{array}{l}13 \\
14\end{array}$ & $\frac{4}{1}$ & $\begin{array}{c}22 \\
0\end{array}$ & $\frac{4}{10}$ & $\begin{array}{l}8 \\
0\end{array}$ & $\begin{array}{l}12 \\
20\end{array}$ & $\begin{array}{l}14 \\
4\end{array}$ & $\begin{array}{l}12 \\
20\end{array}$ & $\begin{array}{l}23 \\
23 \\
20\end{array}$ & $\begin{array}{r}8 \\
16\end{array}$ & $\begin{array}{l}18 \\
27 \\
27\end{array}$ & $\begin{array}{l}20 \\
16\end{array}$ & $\begin{array}{l}15 \\
25 \\
25\end{array}$ & $\begin{array}{l}16 \\
16 \\
36\end{array}$ & $\begin{array}{l}21 \\
21\end{array}$ & $\begin{array}{l}16 \\
16\end{array}$ & $\begin{array}{l}14 \\
13\end{array}$ & 16 & $\begin{array}{l}13 \\
15\end{array}$ & $\overline{16}$ & $\begin{array}{r}2 \\
20\end{array}$ & $\begin{array}{l}16 \\
16\end{array}$ & $\begin{array}{l}7 \\
34\end{array}$ & $\begin{array}{l}16 \\
10\end{array}$ & $\begin{array}{r}5 \\
18 \\
18\end{array}$ \\
\hline $\begin{array}{l}15 \\
16 \\
17\end{array}$ & $\begin{array}{l}12 \\
4 \\
13\end{array}$ & $\begin{array}{l}19 \\
12 \\
10\end{array}$ & $\begin{array}{c}16 \\
7 \\
12\end{array}$ & $\begin{array}{l}23 \\
21 \\
10\end{array}$ & $\begin{array}{l}8 \\
8 \\
4\end{array}$ & $\begin{array}{l}29 \\
28 \\
13\end{array}$ & 8 & $\begin{array}{l}{ }_{21}^{21} \\
15\end{array}$ & 8 & $\begin{array}{r}23 \\
20 \\
8\end{array}$ & $\begin{array}{l}8 \\
7 \\
8\end{array}$ & $\begin{array}{l}24 \\
17 \\
12\end{array}$ & $\begin{array}{r}10 \\
6 \\
8\end{array}$ & 21 & $\begin{array}{r}12 \\
5 \\
8\end{array}$ & $\begin{array}{l}13 \\
18 \\
18\end{array}$ & $\begin{array}{l}6 \\
6\end{array}$ & $\begin{array}{r}18 \\
13 \\
8\end{array}$ & $\begin{array}{c}12 \\
8 \\
6\end{array}$ & $\begin{array}{l}15 \\
18 \\
32\end{array}$ & $\begin{array}{r}12 \\
8 \\
8\end{array}$ & $\begin{array}{l}11 \\
13 \\
20\end{array}$ & 6 & $\begin{array}{r}18 \\
9 \\
22\end{array}$ \\
\hline $\begin{array}{l}18 \\
19\end{array}$ & $\underline{6}$ & ${ }_{0}^{21}$ & 4 & $\begin{array}{l}14 \\
0\end{array}$ & 4 & $\begin{array}{r}13 \\
0\end{array}$ & $=$ & $\begin{array}{l}10 \\
0\end{array}$ & $\underline{32}$ & $\frac{1}{0}$ & $\begin{array}{l}4 \\
4\end{array}$ & ${ }_{4}^{7}$ & ${ }_{8}^{6}$ & $\begin{array}{l}10 \\
13\end{array}$ & $\overline{8}$ & $\frac{1}{15}$ & $\overline{8}$ & $\begin{array}{r}0 \\
16\end{array}$ & $\overline{6}$ & $\underset{20}{0}$ & $\overline{4}$ & ${ }_{21}^{4}$ & $\overline{4}$ & $\begin{array}{l}0 \\
19\end{array}$ \\
\hline $\begin{array}{l}20 \\
21\end{array}$ & 4 & $\begin{array}{r}18 \\
0\end{array}$ & - & $\begin{array}{c}15 \\
1 \\
1\end{array}$ & 4 & $\begin{array}{c}10 \\
1 \\
1\end{array}$ & $\begin{array}{c}4 \\
16\end{array}$ & $\begin{array}{r}10 \\
5\end{array}$ & $-\frac{6}{6}$ & $\begin{array}{l}8 \\
0\end{array}$ & $\begin{array}{l}7 \\
7 \\
4\end{array}$ & $\begin{array}{r}21 \\
21 \\
2\end{array}$ & $\begin{array}{l}8 \\
6 \\
8\end{array}$ & $\begin{array}{l}28 \\
17 \\
17\end{array}$ & 8 & $\begin{array}{l}13 \\
23 \\
21\end{array}$ & $\begin{array}{l}9 \\
8\end{array}$ & $\begin{array}{l}17 \\
18\end{array}$ & $\begin{array}{l}4 \\
4\end{array}$ & 11 & $\overline{4}$ & $\pi$ & $=$ & \\
\hline 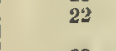 & - & 0 & - & 1 & - & 0 & - & 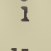 & 12 & 2 & 12 & $\overline{7}$ & 12 & 4 & 10 & 8 & 10 & 11 & 12 & 7 & 12 & 18 & 11 & 13 \\
\hline $\begin{array}{l}23 \\
24 \\
25\end{array}$ & $\begin{array}{r}12 \\
8 \\
4\end{array}$ & $\begin{array}{l}13 \\
17 \\
25^{*}\end{array}$ & $\begin{array}{l}8 \\
8 \\
4\end{array}$ & $\begin{array}{l}18 \\
19 \\
22^{*}\end{array}$ & $\begin{array}{l}8 \\
8 \\
4\end{array}$ & $\begin{array}{l}22 \\
23 \\
8^{*}\end{array}$ & $\begin{array}{l}8 \\
8 \\
4\end{array}$ & $\begin{array}{l}15 \\
21 \\
14^{*}\end{array}$ & 8 & $\begin{array}{l}18 \\
21 \\
14^{*}\end{array}$ & $\begin{array}{r}12 \\
9 \\
4\end{array}$ & $\begin{array}{l}19 \\
26 \\
4^{*}\end{array}$ & $\begin{array}{l}8 \\
8 \\
7\end{array}$ & $\begin{array}{l}28 \\
35 \\
4\end{array}$ & $\begin{array}{l}6 \\
8 \\
4\end{array}$ & $\begin{array}{l}{ }_{26}^{26} \\
46^{*} \\
1^{*}\end{array}$ & $\begin{array}{l}7 \\
8 \\
6\end{array}$ & $\begin{array}{l}26 \\
55^{*}\end{array}$ & $\begin{array}{l}8 \\
8 \\
4\end{array}$ & $\begin{array}{l}23 \\
55^{*} \\
4^{*}\end{array}$ & $\begin{array}{r}8 \\
7 \\
72\end{array}$ & $\begin{array}{l}22 \\
25 * \\
4^{*}\end{array}$ & $\begin{array}{l}8 \\
6\end{array}$ & $\begin{array}{l}21 \\
25^{*}\end{array}$ \\
\hline $\begin{array}{l}26 \\
27 \\
27\end{array}$ & 4 & $2^{*}$ & $\overline{32}$ & $\begin{array}{l}0 \\
4^{*}\end{array}$ & $\overline{32}$ & $\begin{array}{l}0 \\
8^{*}\end{array}$ & $\overline{32}$ & $8^{0}$ & $\overline{32}$ & $8^{*}$ & $\frac{1}{1}$ & $\begin{array}{l}0 \\
8^{*}\end{array}$ & $\begin{array}{l}30 \\
31\end{array}$ & $i^{1 *}$ & $\begin{array}{l}31 \\
32\end{array}$ & $\begin{array}{l}i^{*} \\
4^{*}\end{array}$ & $\begin{array}{l}32 \\
32\end{array}$ & $\frac{2^{*}}{4^{*}}$ & $\begin{array}{l}32 \\
32\end{array}$ & $\begin{array}{l}4^{*} \\
6^{*}\end{array}$ & 32 & & $\begin{array}{l}31 \\
32\end{array}$ & $\begin{array}{l}6^{*} \\
2^{*}\end{array}$ \\
\hline $\begin{array}{l}28 \\
29 \\
30 \\
31\end{array}$ & $\begin{array}{c}32 \\
9 \\
7 \\
12\end{array}$ & $\begin{array}{l}2^{*} \\
8^{*} \\
18\end{array}$ & $\begin{array}{l}20 \\
\frac{10}{8} \\
\end{array}$ & $\begin{array}{l}2^{* *} \\
10^{* *} \\
10\end{array}$ & $\begin{array}{l}20 \\
10 \\
17 \\
12\end{array}$ & $\begin{array}{l}2^{* *} \\
8^{* *} \\
11\end{array}$ & $\overline{8}$ & $\begin{array}{c}0 \\
8^{*} \\
18 \\
4\end{array}$ & $\begin{array}{l}8 \\
8\end{array}$ & $\begin{array}{l}14^{* *} \\
4^{*} \\
1\end{array}$ & $\begin{array}{c}6 \\
10 \\
12 \\
10\end{array}$ & $\begin{array}{c}11 * \\
11^{*} \\
6 \\
8\end{array}$ & $\begin{array}{l}12 \\
8\end{array}$ & $8^{*}$ & $\begin{array}{l}12 \\
8\end{array}$ & $\begin{array}{l}8^{*} \\
9 \\
9 \\
3\end{array}$ & $\frac{7}{12}$ & $\begin{array}{l}14^{*} \\
11 \\
11 \\
18\end{array}$ & $\frac{8}{12}$ & $\begin{array}{c}14^{*} \\
10 \\
6\end{array}$ & $\begin{array}{r}8 \\
7 \\
12\end{array}$ & $\begin{array}{l}14^{*} \\
14 \\
10\end{array}$ & $\begin{array}{r}8 \\
7 \\
8 \\
12\end{array}$ & $\begin{array}{l}14^{* *} \\
16 \\
10\end{array}$ \\
\hline Mesu & & 9 & & 8 & & 9 & & 10 & & 9 & & 10 & & 11 & & 11 & & 12 & & 12 & & 10 & & 10 \\
\hline
\end{tabular}

* Equivalent velocity for Beaufort scale estimation.

\section{November, 1902.}

\begin{tabular}{|c|c|c|c|c|c|c|c|c|c|c|c|c|c|c|c|c|c|c|c|c|c|c|c|c|}
\hline \multirow{2}{*}{ Day. } & \multicolumn{2}{|c|}{2.} & \multicolumn{2}{|c|}{4.} & \multicolumn{2}{|c|}{ B. } & \multicolumn{2}{|c|}{8.} & \multicolumn{2}{|c|}{10.} & \multicolumn{2}{|c|}{ Noon. } & \multicolumn{2}{|c|}{14.} & \multicolumn{2}{|c|}{16.} & \multicolumn{2}{|c|}{18.} & \multicolumn{2}{|c|}{20.} & \multicolumn{2}{|c|}{22.} & \multicolumn{2}{|c|}{ Midt. } \\
\hline & D. & $\mathrm{v}$ & D. & v. & D. & v. & D. & v. & D. & v. & D. & v. & D. & $\nabla$. & D. & v. & D. & v. & D. & $\mathrm{v}$. & D. & v. & D. & $\nabla$. \\
\hline 1 & 14 & 12 & 14 & 10 & 12 & 10 & 20 & 19 & 16 & 22 & 8 & 18 & 12 & 13 & 12 & 18 & 16 & 27 & 12 & 21 & 8 & 15 & 16 & 19 \\
\hline $\begin{array}{l}2 \\
3 \\
4 \\
5 \\
6\end{array}$ & $\begin{array}{r}16 \\
8 \\
10 \\
6 \\
13\end{array}$ & $\begin{array}{r}23 \\
18 \\
7 \\
13 \\
11\end{array}$ & $\begin{array}{r}15 \\
9 \\
8 \\
6 \\
12\end{array}$ & $\begin{array}{r}26 \\
10 \\
6 \\
7 \\
6\end{array}$ & $\begin{array}{r}16 \\
10 \\
8 \\
11 \\
-\end{array}$ & $\begin{array}{r}18 \\
12 \\
10 \\
2 \\
0\end{array}$ & $\begin{array}{r}8 \\
10 \\
7 \\
10 \\
-\end{array}$ & $\begin{array}{r}17 \\
11 \\
5 \\
8 . \\
0\end{array}$ & $\begin{array}{r}8 \\
\frac{8}{16} \\
-\end{array}$ & $\begin{array}{r}17 \\
12 \\
7 \\
8 \\
0\end{array}$ & $\begin{array}{r}12 \\
8 \\
12 \\
12 \\
-\end{array}$ & $\begin{array}{r}10 \\
10 \\
1 \\
4 \\
0\end{array}$ & $\begin{array}{c}14 \\
7 \\
12 \\
12 \\
32\end{array}$ & $\begin{array}{r}12 \\
11 \\
1 \\
12 \\
4\end{array}$ & $\begin{array}{r}8 \\
12 \\
12 \\
12 \\
32\end{array}$ & $\begin{array}{r}13 \\
10 \\
8 \\
15 \\
2\end{array}$ & $\begin{array}{c}12 \\
8 \\
8 \\
8 \\
2\end{array}$ & $\begin{array}{r}10 \\
13 \\
11 \\
13 \\
2\end{array}$ & $\begin{array}{r}12 \\
7 \\
7 \\
12 \\
2\end{array}$ & $\begin{array}{c}27 \\
11 \\
13 \\
6 \\
6\end{array}$ & $\begin{array}{r}8 \\
10 \\
10 \\
10 \\
32\end{array}$ & $\begin{array}{r}21 \\
1 \\
15 \\
9 \\
5\end{array}$ & $\begin{array}{r}8 \\
10 \\
7 \\
12 \\
-\end{array}$ & $\begin{array}{r}13 \\
2 \\
10 \\
9 \\
0\end{array}$ \\
\hline $\begin{array}{r}7 \\
8 \\
9 \\
10 \\
11\end{array}$ & $\begin{array}{l}\frac{4}{9} \\
-\end{array}$ & $\begin{array}{l}3 \\
0 \\
4 \\
0 \\
0\end{array}$ & $\begin{array}{l}\overline{32} \\
12 \\
=\end{array}$ & $\begin{array}{r}0 \\
7 \\
10 \\
0 \\
0\end{array}$ & $\begin{array}{l}- \\
\overline{8} \\
1 \\
-\end{array}$ & $\begin{array}{l}0 \\
0 \\
7 \\
5 \\
0\end{array}$ & $\begin{array}{r}- \\
8 \\
32 \\
-\end{array}$ & $\begin{array}{l}0 \\
4 \\
7 \\
4 \\
2\end{array}$ & $\begin{array}{r}6 \\
8 \\
12 \\
12\end{array}$ & $\begin{array}{r}4 \\
21 \\
11 \\
0 \\
7\end{array}$ & $\begin{array}{r}17 \\
8 \\
12 \\
12\end{array}$ & $\begin{array}{r}4 \\
14 \\
7 \\
0 \\
10\end{array}$ & $\begin{array}{r}14 \\
8 \\
9 \\
12\end{array}$ & $\begin{array}{r}10 \\
10 \\
7 \\
0 \\
15\end{array}$ & $\begin{array}{r}9 \\
70 \\
\frac{7}{12}\end{array}$ & $\begin{array}{r}6 \\
10 \\
6 \\
0 \\
14\end{array}$ & $\frac{6}{\frac{6}{8}}$ & $\begin{array}{r}7 \\
10 \\
0 \\
0 \\
14\end{array}$ & $\begin{array}{r}6 \\
\frac{8}{7} \\
\frac{1}{8}\end{array}$ & $\begin{array}{r}7 \\
21 \\
0 \\
0 \\
11\end{array}$ & $\frac{\overline{8}}{32}$ & $\begin{array}{r}2 \\
14 \\
0 \\
2 \\
15\end{array}$ & $\frac{\overline{8}}{32}$ & $\begin{array}{r}0 \\
8 \\
0 \\
2 \\
23\end{array}$ \\
\hline $\begin{array}{l}13 \\
13 \\
14 \\
15 \\
16\end{array}$ & $\begin{array}{r}8 \\
12 \\
2 \\
32\end{array}$ & $\begin{array}{r}25 \\
7 \\
0 \\
8 \\
10\end{array}$ & $\begin{array}{r}8 \\
6 \\
32 \\
32\end{array}$ & $\begin{array}{r}11 \\
7 \\
0 \\
7 \\
7\end{array}$ & $\begin{array}{r}8 \\
\frac{6}{32} \\
\frac{2}{2}\end{array}$ & $\begin{array}{r}11 \\
17 \\
0 \\
3 \\
8\end{array}$ & $\begin{array}{r}\frac{6}{7} \\
\frac{3}{32} \\
-\end{array}$ & $\begin{array}{r}18 \\
19 \\
0 \\
1 \\
7\end{array}$ & $\begin{array}{r}8 \\
6 \\
= \\
=\end{array}$ & $\begin{array}{r}15 \\
14 \\
9 \\
1 \\
0\end{array}$ & $\begin{array}{r}12 \\
7 \\
2 \\
-\end{array}$ & $\begin{array}{r}5 \\
17 \\
4 \\
0 \\
2\end{array}$ & $\begin{array}{r}8 \\
8 \\
32 \\
- \\
-\end{array}$ & $\begin{array}{r}7 \\
10 \\
1 \\
0 \\
1\end{array}$ & $\begin{array}{r}14 \\
10 \\
2 \\
- \\
-\end{array}$ & $\begin{array}{l}8 \\
5 \\
4 \\
0 \\
2\end{array}$ & $\begin{array}{r}12 \\
8 \\
32 \\
32 \\
32 \\
\end{array}$ & $\begin{array}{l}5 \\
7 \\
6 \\
7 \\
4\end{array}$ & $\begin{array}{l}\frac{8}{32} \\
32 \\
32\end{array}$ & $\begin{array}{r}13 \\
0 \\
5 \\
2 \\
6\end{array}$ & $\frac{\frac{4}{32}}{32}$ & $\begin{array}{r}13 \\
1 \\
5 \\
0 \\
7\end{array}$ & $\begin{array}{l}\frac{4}{32} \\
\frac{32}{-}\end{array}$ & $\begin{array}{r}10 \\
4 \\
6 \\
7 \\
4\end{array}$ \\
\hline $\begin{array}{l}17 \\
18 \\
19 \\
20 \\
21\end{array}$ & $\begin{array}{l}4 \\
8 \\
6 \\
8 \\
8\end{array}$ & $\begin{array}{r}4 \\
17 \\
9 \\
17 \\
7\end{array}$ & $\begin{array}{r}-12 \\
6 \\
8 \\
4\end{array}$ & $\begin{array}{r}0 \\
13 \\
13 \\
5 \\
6\end{array}$ & $\begin{array}{r}32 \\
8 \\
4 \\
12 \\
-\end{array}$ & $\begin{array}{r}11 \\
8 \\
13 \\
7 \\
3\end{array}$ & $\begin{array}{r}32 \\
8 \\
8 \\
10 \\
-\end{array}$ & $\begin{array}{r}2 \\
21 \\
10 \\
7 \\
4\end{array}$ & $\begin{array}{r}-8 \\
8 \\
10 \\
32\end{array}$ & $\begin{array}{r}0 \\
22 \\
11 \\
7 \\
5\end{array}$ & $\begin{array}{r}-8 \\
8 \\
10 \\
31\end{array}$ & $\begin{array}{r}0 \\
22 \\
11 \\
7 \\
8\end{array}$ & $\begin{array}{r}6 \\
8 \\
8 \\
4 \\
31\end{array}$ & $\begin{array}{r}6 \\
13 \\
14 \\
13 \\
7\end{array}$ & $\begin{array}{r}8 \\
8 \\
6 \\
\mathbf{3} \\
\mathbf{2} \\
\mathbf{2}\end{array}$ & $\begin{array}{r}12 \\
15 \\
15 \\
12 \\
8\end{array}$ & $\begin{array}{r}10 \\
8 \\
7 \\
6 \\
2\end{array}$ & $\begin{array}{r}17 \\
10 \\
17 \\
13 \\
4\end{array}$ & $\begin{array}{l}8 \\
8 \\
8 \\
9 \\
2\end{array}$ & $\begin{array}{r}24 \\
12 \\
11 \\
5 \\
7\end{array}$ & $\begin{array}{r}6 \\
8 \\
8 \\
8 \\
32\end{array}$ & $\begin{array}{r}16 \\
13 \\
17 \\
6 \\
2\end{array}$ & $\begin{array}{l}8 \\
8 \\
8 \\
8 \\
1\end{array}$ & $\begin{array}{r}24 \\
15 \\
11 \\
6 \\
10\end{array}$ \\
\hline $\begin{array}{l}22 \\
23 \\
24 \\
25 \\
26\end{array}$ & $\begin{array}{r}32 \\
32 \\
32 \\
32 \\
8\end{array}$ & $\begin{array}{r}4 \\
1 \\
8 \\
6 \\
61 \\
21\end{array}$ & $\begin{array}{r}\frac{4}{32} \\
32 \\
8\end{array}$ & $\begin{array}{r}13 \\
2 \\
6 \\
6 \\
61 \\
21\end{array}$ & $\begin{array}{r}\frac{32}{2} \\
32 \\
8\end{array}$ & $\begin{array}{r}7 \\
0 \\
4 \\
4 \\
21\end{array}$ & $\frac{\frac{32}{32}}{8}$ & $\begin{array}{r}5 \\
0 \\
5 \\
2 \\
23\end{array}$ & $\frac{\frac{2}{32}}{8}$ & $\begin{array}{r}12 \\
0 \\
4 \\
0 \\
20\end{array}$ & $\frac{32}{\frac{4}{8}}$ & $\begin{array}{r}3 \\
0 \\
0 \\
9 \\
20\end{array}$ & $\begin{array}{r}30 \\
30 \\
31 \\
6 \\
8\end{array}$ & $\begin{array}{r}4 \\
4 \\
6 \\
14 \\
17\end{array}$ & $\begin{array}{r}32 \\
32 \\
31 \\
8 \\
8\end{array}$ & $\begin{array}{r}3 \\
2 \\
2 \\
13 \\
17 \\
17\end{array}$ & $\begin{array}{r}31 \\
32 \\
30 \\
8 \\
8\end{array}$ & $\begin{array}{r}5 \\
7 \\
4 \\
20 \\
15\end{array}$ & $\begin{array}{r}\overline{32} \\
32 \\
7 \\
4\end{array}$ & $\begin{array}{r}0 \\
4 \\
5 \\
19 \\
7\end{array}$ & $\begin{array}{r}32 \\
32 \\
32 \\
8 \\
6\end{array}$ & $\begin{array}{r}9 \\
4 \\
6 \\
21 \\
15 \\
15\end{array}$ & $\begin{array}{r}\overline{32} \\
32 \\
8 \\
32\end{array}$ & $\begin{array}{r}7 \\
7 \\
6 \\
18 \\
3\end{array}$ \\
\hline $\begin{array}{l}27 \\
28 \\
29 \\
30\end{array}$ & $\frac{1}{72}$ & $\begin{array}{l}6 \\
5 \\
0 \\
7\end{array}$ & $\begin{array}{l}31 \\
32 \\
12 \\
-\end{array}$ & $\begin{array}{r}3 \\
2 \\
5 \\
12\end{array}$ & $\frac{32}{12}$ & $\begin{array}{l}7 \\
0 \\
3 \\
5\end{array}$ & $\frac{32}{4}$ & $\begin{array}{r}6 \\
1 \\
10 \\
7\end{array}$ & $\begin{array}{r}\frac{32}{8} \\
12\end{array}$ & $\begin{array}{r}6 \\
\mathbf{0} \\
13 \\
3\end{array}$ & $\begin{array}{r}32 \\
2 \\
8 \\
12\end{array}$ & $\begin{array}{r}9 \\
1 \\
15 \\
4\end{array}$ & $\frac{32}{72}$ & $\begin{array}{r}\tilde{j} \\
\mathbf{1} \\
20 \\
3\end{array}$ & $\begin{array}{r}31 \\
30 \\
7 \\
32\end{array}$ & $\begin{array}{r}7 \\
2 \\
21 \\
4\end{array}$ & $\begin{array}{r}32 \\
30 \\
7 \\
32\end{array}$ & $\begin{array}{r}4 \\
4 \\
23 \\
4\end{array}$ & $\begin{array}{l}\frac{32}{7} \\
32\end{array}$ & $\begin{array}{r}4 \\
0 \\
18 \\
5\end{array}$ & $\frac{32}{32}$ & $\begin{array}{r}7 \\
0 \\
12 \\
5\end{array}$ & $\begin{array}{l}\frac{32}{7} \\
32\end{array}$ & $\begin{array}{l}4 \\
0 \\
7 \\
4\end{array}$ \\
\hline Mean . & & 8 & & 7 & & 7 & & 8 & & 8 & & 7 & & 8 & & 8 & & 9 & & 9 & & 9 & & 8 \\
\hline
\end{tabular}


TABLF IV. (continued).

WIND-DIRECTION AND VELOCITÝ.

1)ireetion (D) in Number's $(8=\mathrm{E}, 16=\mathrm{S}, 24=\mathrm{W}, 32=\mathrm{N})$, and Veloeity (V) in miles per hour as reeorded by the Cup Anemometer.

December, 1902.

\begin{tabular}{|c|c|c|c|c|c|c|c|c|c|c|c|c|c|c|c|c|c|c|c|c|c|c|c|c|}
\hline \multirow[t]{2}{*}{ y. } & \multicolumn{2}{|c|}{2.} & \multicolumn{2}{|c|}{ 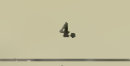 } & \multicolumn{2}{|c|}{6.} & \multicolumn{2}{|c|}{8.} & \multicolumn{2}{|c|}{ 10، } & \multicolumn{2}{|c|}{ Noon. } & \multicolumn{2}{|c|}{14.} & \multicolumn{2}{|c|}{16.} & \multicolumn{2}{|c|}{16.} & \multicolumn{2}{|c|}{20.} & \multicolumn{2}{|c|}{22.} & \multicolumn{2}{|c|}{ Mialt. } \\
\hline & D. & v. & D. & v. & D. & v. & D. & $\mathbf{v}$ & $\mathbf{D}$. & v. & D. & v. & D. & v. & $\mathrm{D}$. & v. & D. & v. & D. & v. & $\mathrm{D}$. & v. & D. & $\mathrm{v}$. \\
\hline 1 & 32 & 4 & 32 & & 32 & 4 & 4 & 5 & 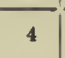 & & 4 & 6 & 4 & 6 & 4 & 7 & - & 1 & 4 & 4 & - & 4 & - & 1 \\
\hline $\begin{array}{l}2 \\
3\end{array}$ & $\begin{array}{r}8 \\
6 \\
1 \\
-\end{array}$ & $\begin{array}{r}10 \\
15 \\
9 \\
1\end{array}$ & $\begin{array}{r}8 \\
8 \\
1 \\
16\end{array}$ & $\begin{array}{r}10 \\
14 \\
7\end{array}$ & $\begin{array}{r}4 \\
8 \\
32 \\
6\end{array}$ & $\begin{array}{r}15 \\
13 \\
5 \\
3\end{array}$ & $\begin{array}{r}6 \\
4 \\
1 \\
16\end{array}$ & $\begin{array}{l}34 \\
12 \\
11 \\
1\end{array}$ & $\begin{array}{r}8 \\
4 \\
32 \\
8\end{array}$ & $\begin{array}{r}18 \\
6 \\
6 \\
4\end{array}$ & $\frac{6}{31}$ & $\begin{array}{r}21 \\
4 \\
7\end{array}$ & $\frac{7}{31}$ & $\begin{array}{r}20 \\
2 \\
8 \\
8 \\
15\end{array}$ & $\begin{array}{r}7 \\
4 \\
31 \\
7\end{array}$ & $\begin{array}{r}15 \\
4 \\
8\end{array}$ & $\begin{array}{c}7 \\
32 \\
31 \\
7\end{array}$ & $\begin{array}{r}18 \\
4 \\
7 \\
14\end{array}$ & $\begin{array}{r}6 \\
32 \\
31 \\
7\end{array}$ & $\begin{array}{r}18 \\
4 \\
5 \\
14\end{array}$ & $\begin{array}{c}6 \\
32 \\
32 \\
4\end{array}$ & $\begin{array}{r}13 \\
5 \\
4 \\
15\end{array}$ & $\begin{array}{l}6 \\
1\end{array}$ & \\
\hline${ }_{6}^{5}$ & $\overline{32}$ & $\begin{array}{l}1 \\
4\end{array}$ & $\begin{array}{l}16 \\
32\end{array}$ & ${ }_{2}^{2}$ & $\begin{array}{r}6 \\
32\end{array}$ & $\stackrel{3}{5}$ & $\begin{array}{l}16 \\
32\end{array}$ & $\begin{array}{l}1 \\
7\end{array}$ & $\begin{array}{r}8 \\
31\end{array}$ & $\begin{array}{l}4 \\
6\end{array}$ & $\begin{array}{r}8 \\
30\end{array}$ & $\begin{array}{c}18 \\
7\end{array}$ & $\begin{array}{r}8 \\
30\end{array}$ & $\begin{array}{c}15 \\
7\end{array}$ & 30 & $\begin{array}{c}16 \\
7\end{array}$ & $\begin{array}{r}7 \\
31\end{array}$ & $\begin{array}{l}14 \\
10\end{array}$ & $\begin{array}{l}7 \\
32\end{array}$ & $\begin{array}{r}14 \\
3\end{array}$ & $\frac{4}{1}$ & $\begin{array}{l}5 \\
1 \\
\end{array}$ & $i$ & $\begin{array}{l}3 \\
9\end{array}$ \\
\hline $\begin{array}{r}7 \\
8 \\
9 \\
10\end{array}$ & $\begin{array}{r}32 \\
18 \\
9 \\
8\end{array}$ & $\begin{array}{r}7 \\
18 \\
9 \\
90\end{array}$ & $\begin{array}{l}32 \\
16 \\
10 \\
7\end{array}$ & $\begin{array}{r}7 \\
18 \\
14 \\
11\end{array}$ & $\begin{array}{r}8 \\
16 \\
16 \\
7\end{array}$ & $\begin{array}{l}6 \\
13 \\
13 \\
7\end{array}$ & $\begin{array}{l}16 \\
16 \\
16\end{array}$ & $\begin{array}{r}9 \\
20 \\
11 \\
21\end{array}$ & $\begin{array}{c}16 \\
6 \\
12 \\
18\end{array}$ & $\begin{array}{l}14 \\
14 \\
13 \\
13\end{array}$ & $\begin{array}{c}20 \\
8 \\
9 \\
7\end{array}$ & $\begin{array}{c}18 \\
71 \\
18\end{array}$ & $\begin{array}{r}17 \\
8 \\
12\end{array}$ & $\begin{array}{c}12 \\
9 \\
16\end{array}$ & $\begin{array}{c}17 \\
8 \\
12 \\
7\end{array}$ & $\begin{array}{c}21 \\
8 \\
15 \\
16\end{array}$ & $\begin{array}{r}17 \\
17 \\
9 \\
8\end{array}$ & $\begin{array}{l}18 \\
11 \\
14 \\
12\end{array}$ & $\begin{array}{r}17 \\
16 \\
9 \\
32\end{array}$ & $\begin{array}{l}13 \\
13 \\
11 \\
12\end{array}$ & $\begin{array}{r}15 \\
12 \\
9 \\
-\end{array}$ & 3 & $\begin{array}{l}15 \\
12\end{array}$ & $\begin{array}{r}7 \\
10 \\
0\end{array}$ \\
\hline 11 & $\begin{array}{l}- \\
12\end{array}$ & $\begin{array}{r}1 \\
15\end{array}$ & $\begin{array}{l}10 \\
12\end{array}$ & 2 & $\begin{array}{r}8 \\
12\end{array}$ & 12 & 15 & ${ }_{14}^{21}$ & $\begin{array}{r}8 \\
12\end{array}$ & 4 & 12 & $\begin{array}{l}10 \\
10\end{array}$ & 12 & 10 & 12 & 1. & 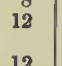 & 10 & $\frac{32}{8}$ & 1.8 & $\overline{8}$ & & 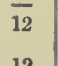 & 16 \\
\hline $\begin{array}{l}1_{3}^{2} \\
\end{array}$ & $\begin{array}{l}12 \\
15 \\
12 \\
32 \\
32\end{array}$ & $\begin{array}{r}15 \\
11 \\
7 \\
3 \\
8\end{array}$ & $\begin{array}{l}12 \\
16 \\
12 \\
32 \\
32\end{array}$ & $\begin{array}{r}14 \\
14 \\
4 \\
7 \\
4\end{array}$ & $\begin{array}{l}12 \\
16 \\
12 \\
32 \\
24\end{array}$ & $\begin{array}{r}18 \\
10 \\
4 \\
8 \\
4\end{array}$ & $\begin{array}{r}12 \\
12 \\
8 \\
1 \\
32\end{array}$ & $\begin{array}{c}19 \\
13 \\
6 \\
9 \\
4 \\
4\end{array}$ & $\begin{array}{l}10 \\
16 \\
8 \\
32 \\
32\end{array}$ & $\begin{array}{r}10 \\
18 \\
5 \\
7 \\
3\end{array}$ & $\begin{array}{r}6 \\
16 \\
8 \\
32 \\
-\end{array}$ & $\begin{array}{c}12 \\
16 \\
5 \\
11 \\
2\end{array}$ & $\begin{array}{r}8 \\
15 \\
4 \\
31 \\
32\end{array}$ & $\begin{array}{r}14 \\
15 \\
4 \\
5 \\
3\end{array}$ & $\begin{array}{c}8 \\
14 \\
32 \\
31 \\
-\end{array}$ & $\begin{array}{r}5 \\
10 \\
2\end{array}$ & $\begin{array}{l}32 \\
31 \\
32\end{array}$ & $\begin{array}{c}12 \\
17 \\
5 \\
10 \\
3\end{array}$ & $\begin{array}{l}12 \\
12 \\
31 \\
28 \\
28\end{array}$ & $\begin{array}{c}16 \\
12 \\
6 \\
10 \\
3\end{array}$ & $\begin{array}{c}12 \\
2 \\
33 \\
28\end{array}$ & $\begin{array}{l}3 \\
4 \\
4\end{array}$ & $\begin{array}{c}11 \\
32 \\
28\end{array}$ & $\begin{array}{l}5 \\
5 \\
3\end{array}$ \\
\hline $\begin{array}{r}7 \\
3 \\
19\end{array}$ & $\frac{28}{8}$ & $\begin{array}{r}4 \\
0 \\
10 \\
8\end{array}$ & $\frac{28}{8}$ & $\begin{array}{r}6 \\
0 \\
12 \\
12\end{array}$ & $\frac{28}{8}$ & $\begin{array}{r}3 \\
0 \\
10\end{array}$ & $\frac{28}{15}$ & $\begin{array}{l}5 \\
5 \\
8 \\
8\end{array}$ & $\frac{32}{12}$ & $\begin{array}{r}5 \\
0 \\
13 \\
13\end{array}$ & $\begin{array}{l}32 \\
16 \\
12\end{array}$ & $\begin{array}{l}4 \\
3 \\
9 \\
9\end{array}$ & $\begin{array}{l}30 \\
12 \\
12\end{array}$ & $\begin{array}{r}9 \\
7 \\
13\end{array}$ & $\begin{array}{l}31 \\
16 \\
14\end{array}$ & $\begin{array}{r}7 \\
6 \\
15 \\
15\end{array}$ & $\begin{array}{c}1 \\
14 \\
15 \\
8\end{array}$ & $\begin{array}{r}7 \\
8 \\
13\end{array}$ & $\begin{array}{r}31 \\
8 \\
14\end{array}$ & $\begin{array}{r}8 \\
8 \\
11 \\
7\end{array}$ & $\begin{array}{r}28 \\
7 \\
14\end{array}$ & $\begin{array}{c}7 \\
13 \\
5 \\
\end{array}$ & 15 & 15 \\
\hline 20 & $\begin{array}{r}16 \\
8\end{array}$ & $\begin{array}{c}8 \\
13\end{array}$ & $\begin{array}{r}16 \\
7\end{array}$ & 11 & & $\begin{array}{l}10 \\
16\end{array}$ & $\begin{array}{c}15 \\
8\end{array}$ & $\begin{array}{c}7 \\
14\end{array}$ & $\begin{array}{l}8 \\
8\end{array}$ & $\begin{array}{l}6 \\
14\end{array}$ & $\begin{array}{l}8 \\
8\end{array}$ & $\begin{array}{c}5 \\
15 \\
\end{array}$ & 10 & 19 & $\begin{array}{c}8 \\
10\end{array}$ & 13 & $\begin{array}{l}8 \\
8\end{array}$ & $\begin{array}{l}11 \\
19\end{array}$ & $\begin{array}{l}12 \\
\text { vble. }\end{array}$ & 18 & 10 & 13 & $\begin{array}{l}8 \\
4\end{array}$ & $\begin{array}{l}10 \\
15\end{array}$ \\
\hline $\begin{array}{l}22 \\
23 \\
24 \\
25 \\
26\end{array}$ & $\begin{array}{c}\text { Vble. } \\
32 \\
8 \\
32 \\
-\end{array}$ & $\begin{array}{r}7 \\
4 \\
1.4 \\
2 \\
1\end{array}$ & $\begin{array}{c}4 \\
32 \\
16 \\
12 \\
-\end{array}$ & $\begin{array}{l}8 \\
4 \\
7 \\
4 \\
1\end{array}$ & $\begin{array}{l}12 \\
\frac{32}{12} \\
\underline{12}\end{array}$ & $\begin{array}{l}9 \\
2 \\
2 \\
6 \\
3\end{array}$ & $\frac{\overline{32}}{\underline{12}}$ & $\begin{array}{l}4 \\
5 \\
1 \\
4 \\
1 \\
\end{array}$ & $\begin{array}{c}\text { vble. } \\
\frac{12}{12}\end{array}$ & $\begin{array}{r}2 \\
4 \\
1 \\
13 \\
1\end{array}$ & $\bar{z}$ & $\begin{array}{c}4 \\
2 \\
0 \\
10 \\
10\end{array}$ & $\frac{2}{28}$ & \begin{tabular}{r|}
3 \\
3 \\
1 \\
10 \\
3
\end{tabular} & $\begin{array}{c}32 \\
30 \\
6 \\
12 \\
-\end{array}$ & $\begin{array}{c}4 \\
3 \\
4 \\
10 \\
8\end{array}$ & $\begin{array}{r}28 \\
28 \\
8 \\
-\end{array}$ & $\begin{array}{l}4 \\
4 \\
7 \\
9 \\
4 \\
4\end{array}$ & $\begin{array}{l}28 \\
\frac{12}{30} \\
\frac{30}{30}\end{array}$ & $\begin{array}{l}\mathbf{6} \\
\mathbf{3} \\
\mathbf{3} \\
\mathbf{1} \\
\mathbf{7}\end{array}$ & $\begin{array}{r}28 \\
8 \\
4 \\
30\end{array}$ & $\begin{array}{r}5 \\
14 \\
2 \\
0 \\
4\end{array}$ & $\begin{array}{r}28 \\
9 \\
4 \\
30\end{array}$ & \\
\hline $\begin{array}{l}27 \\
28 \\
29 \\
30 \\
31\end{array}$ & $\begin{array}{l}\overline{28} \\
32 \\
28 \\
-\end{array}$ & $\begin{array}{l}1 \\
4 \\
8 \\
8 \\
2\end{array}$ & $\begin{array}{r}\overline{32} \\
32 \\
31 \\
8\end{array}$ & $\begin{array}{r}0 \\
6 \\
4 \\
10 \\
5\end{array}$ & $\begin{array}{r}\text { Vile. } \\
32 \\
28 \\
32 \\
8\end{array}$ & $\begin{array}{r}2 \\
7 \\
7 \\
7 \\
13\end{array}$ & $\begin{array}{c}32 \\
28 \\
32 \\
8\end{array}$ & $\begin{array}{r}3 \\
5 \\
4 \\
1 \\
20\end{array}$ & $\begin{array}{l}\overline{32} \\
\frac{32}{12}\end{array}$ & $\begin{array}{r}1 \\
7 \\
1 \\
20 \\
20\end{array}$ & $\begin{array}{r}26 \\
\frac{26}{32} \\
30 \\
8\end{array}$ & $\begin{array}{r}1 \\
5 \\
2 \\
4 \\
12\end{array}$ & $\begin{array}{l}\frac{28}{31} \\
\frac{30}{30} \\
6\end{array}$ & $\begin{array}{r}7 \\
6 \\
3 \\
4 \\
12\end{array}$ & $\begin{array}{r}30 \\
12 \\
31 \\
6\end{array}$ & $\begin{array}{l}7 \\
6 \\
4 \\
4 \\
8\end{array}$ & $\begin{array}{l}\overline{31} \\
13 \\
32 \\
\end{array}$ & $\begin{array}{l}7 \\
7 \\
5 \\
7 \\
4 \\
4\end{array}$ & \begin{tabular}{r|r}
31 \\
8 \\
32 \\
4
\end{tabular} & $\begin{array}{r}1 \\
9 \\
10 \\
7 \\
1\end{array}$ & $\begin{array}{r}\overline{31} \\
8 \\
32 \\
\end{array}$ & $\begin{array}{r}3 \\
9 \\
10 \\
5 \\
1\end{array}$ & $\begin{array}{l}28 \\
32 \\
8\end{array}$ & $\begin{array}{r}9 \\
10 \\
10 \\
4\end{array}$ \\
\hline an & & 7 & & 7 & & 8 & & 9 & & 8 & & 8 & & 9 & & 9 & & 9 & & ${ }^{8}$ & & 8 & & 7 \\
\hline
\end{tabular}

January, 1903.

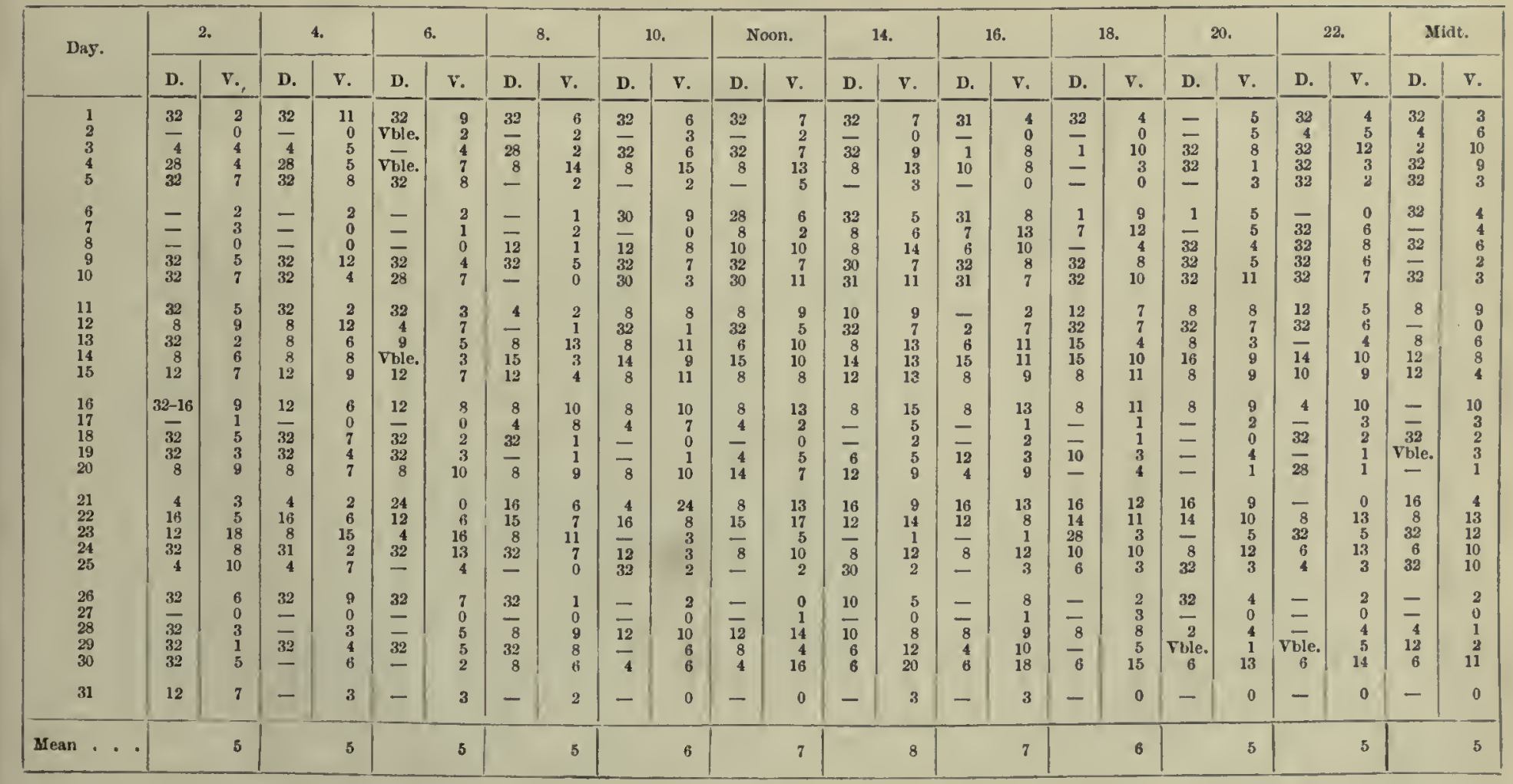


TABLE IV. (continued).

\section{WIND-DIRECTION AND VELOCITY.}

Direction (D) in Numbers $(8=E, 16=\mathrm{S}, 24=\mathrm{W}, 32=\mathrm{N})$, and Velocity $(\mathrm{V})$ in miles per hour as recorded by the Cup Anemometer.

February, 1903.

\begin{tabular}{|c|c|c|c|c|c|c|c|c|c|c|c|c|c|c|c|c|c|c|c|c|c|c|c|c|}
\hline \multirow{2}{*}{ Day. } & \multicolumn{2}{|c|}{2.} & \multicolumn{2}{|c|}{4.} & \multicolumn{2}{|c|}{8.} & \multicolumn{2}{|c|}{8.} & \multicolumn{2}{|c|}{10.} & \multicolumn{2}{|c|}{ Yoon. } & \multicolumn{2}{|c|}{14.} & \multicolumn{2}{|c|}{16.} & \multicolumn{2}{|c|}{18.} & \multicolumn{2}{|c|}{20.} & \multicolumn{2}{|c|}{22.} & \multicolumn{2}{|c|}{ Midt. } \\
\hline & D. & v. & D. & v. & D. & v. & D. & v. & D. & $\nabla$. & D. & F. & D. & v. & D. & F. & D. & v. & D. & v. & D. & v. & D. & T. \\
\hline $\begin{array}{l}1 \\
2 \\
3 \\
4\end{array}$ & $\bar{z}$ & $\begin{array}{l}0 \\
1 \\
2 \\
1\end{array}$ & $\overline{\frac{-}{32}}$ & $\begin{array}{l}0 \\
1 \\
2 \\
1\end{array}$ & $\overline{\bar{z}}$ & $\begin{array}{l}0 \\
0 \\
5 \\
2\end{array}$ & $\frac{12}{z}$ & $\begin{array}{l}5 \\
4 \\
1 \\
1\end{array}$ & $\stackrel{8}{=}$ & $\begin{array}{l}5 \\
1 \\
2 \\
0\end{array}$ & $\frac{6}{32}$ & $\begin{array}{l}8 \\
1 \\
4 \\
0\end{array}$ & $\frac{6}{32}$ & $\begin{array}{l}3 \\
2 \\
4 \\
1\end{array}$ & $\overline{\overline{32}}$ & $\begin{array}{l}0 \\
1 \\
7 \\
0\end{array}$ & $\frac{6}{=}$ & $\begin{array}{l}4 \\
2 \\
2 \\
1\end{array}$ & $\frac{8}{=}$ & $\begin{array}{r}11 \\
3 \\
2 \\
4\end{array}$ & $\frac{8}{=}$ & $\begin{array}{l}8 \\
1 \\
0 \\
1\end{array}$ & $\overline{\bar{z}}$ & $\begin{array}{l}4 \\
1 \\
0 \\
4\end{array}$ \\
\hline $\begin{array}{l}5 \\
6 \\
7 \\
8 \\
9\end{array}$ & $\frac{4}{8}$ & $\begin{array}{r}5 \\
1 \\
14 \\
0 \\
0\end{array}$ & $\begin{array}{l}\frac{8}{12} \\
32 \\
-\end{array}$ & $\begin{array}{r}17 \\
0 \\
7 \\
2 \\
2 \\
2\end{array}$ & $\frac{\frac{8}{12}}{6}$ & $\begin{array}{r}19 \\
6 \\
8 \\
0 \\
4\end{array}$ & $\frac{\frac{8}{8}}{-}$ & $\begin{array}{r}14 \\
3 \\
7 \\
0 \\
0\end{array}$ & $\begin{array}{r}8 \\
12 \\
8 \\
4 \\
-\end{array}$ & $\begin{array}{r}16 \\
8 \\
8 \\
7 \\
1\end{array}$ & $\begin{array}{r}8 \\
8 \\
8 \\
4 \\
32\end{array}$ & $\begin{array}{r}15 \\
14 \\
10 \\
13 \\
1\end{array}$ & $\begin{array}{r}6 \\
8 \\
8 \\
4 \\
-\end{array}$ & $\begin{array}{r}16 \\
17 \\
10 \\
16 \\
3\end{array}$ & $\begin{array}{l}8 \\
8 \\
7 \\
7 \\
8\end{array}$ & $\begin{array}{r}11 \\
16 \\
10 \\
23 \\
5\end{array}$ & $\begin{array}{l}7 \\
8 \\
8 \\
7 \\
6\end{array}$ & $\begin{array}{r}10 \\
18 \\
8 \\
14 \\
8\end{array}$ & $\frac{4}{8}$ & $\begin{array}{r}14 \\
12 \\
5 \\
15 \\
8\end{array}$ & $\begin{array}{r}\frac{4}{7} \\
\frac{8}{8} \\
12\end{array}$ & $\begin{array}{r}8 \\
14 \\
2 \\
9 \\
11\end{array}$ & $\begin{array}{c}4 \\
7 \\
2 \\
\text { vble. } \\
8\end{array}$ & $\begin{array}{r}7 \\
10 \\
1 \\
5 \\
6\end{array}$ \\
\hline $\begin{array}{l}10 \\
11 \\
12 \\
13 \\
14\end{array}$ & $\begin{array}{r}12 \\
10 \\
8 \\
8 \\
8\end{array}$ & $\begin{array}{r}4 \\
6 \\
15 \\
17 \\
17\end{array}$ & $\begin{array}{r}12 \\
12 \\
8 \\
9 \\
8\end{array}$ & $\begin{array}{r}6 \\
9 \\
18 \\
15 \\
20\end{array}$ & $\begin{array}{r}-11 \\
8 \\
9 \\
8\end{array}$ & $\begin{array}{r}6 \\
8 \\
18 \\
13 \\
24\end{array}$ & $\begin{array}{l}8 \\
6 \\
8 \\
9 \\
8\end{array}$ & $\begin{array}{r}5 \\
15 \\
21 \\
17 \\
27\end{array}$ & $\begin{array}{r}12 \\
8 \\
8 \\
8 \\
8\end{array}$ & $\begin{array}{l}13 \\
21 \\
22 \\
19 \\
26\end{array}$ & $\begin{array}{r}12 \\
8 \\
9 \\
8 \\
8\end{array}$ & $\begin{array}{l}13 \\
15 \\
13 \\
15 \\
18\end{array}$ & $\begin{array}{r}14 \\
8 \\
8 \\
8 \\
8\end{array}$ & $\begin{array}{l}11 \\
16 \\
12 \\
21 \\
20\end{array}$ & $\begin{array}{r}12 \\
8 \\
6 \\
8 \\
8\end{array}$ & $\begin{array}{l}10 \\
17 \\
11 \\
19 \\
18\end{array}$ & $\begin{array}{r}10 \\
8 \\
7 \\
9 \\
8\end{array}$ & $\begin{array}{l}11 \\
14 \\
13 \\
18 \\
18\end{array}$ & $\begin{array}{r}10 \\
8 \\
7 \\
8 \\
8\end{array}$ & $\begin{array}{l}12 \\
13 \\
13 \\
17 \\
13\end{array}$ & $\begin{array}{r}12 \\
7 \\
7 \\
8 \\
7\end{array}$ & $\begin{array}{l}12 \\
10 \\
14 \\
15 \\
13\end{array}$ & $\begin{array}{r}12 \\
9 \\
9 \\
8 \\
12\end{array}$ & $\begin{array}{r}10 \\
11 \\
13 \\
18 \\
9\end{array}$ \\
\hline $\begin{array}{l}15 \\
16 \\
17 \\
18 \\
19\end{array}$ & $\begin{array}{r}15 \\
12 \\
8 \\
8 \\
10\end{array}$ & $\begin{array}{r}7 \\
8 \\
15 \\
22 \\
8\end{array}$ & $\begin{array}{r}15 \\
8 \\
8 \\
6 \\
-\end{array}$ & $\begin{array}{r}13 \\
3 \\
23 \\
20 \\
4\end{array}$ & $\begin{array}{r}12 \\
8 \\
8 \\
8 \\
-\end{array}$ & $\begin{array}{r}7 \\
3 \\
21 \\
13 \\
1\end{array}$ & $\begin{array}{r}\text { Vole. } \\
8 \\
8 \\
12 \\
-\end{array}$ & $\begin{array}{r}3 \\
10 \\
20 \\
8 \\
0\end{array}$ & $\begin{array}{r}4 \\
8 \\
10 \\
12 \\
-\end{array}$ & $\begin{array}{r}7 \\
9 \\
16 \\
11 \\
4\end{array}$ & $\begin{array}{r}4 \\
8 \\
8 \\
12 \\
12\end{array}$ & $\begin{array}{r}7 \\
11 \\
14 \\
10 \\
6\end{array}$ & $\begin{array}{r}7 \\
7 \\
8 \\
12 \\
15\end{array}$ & $\begin{array}{l}10 \\
15 \\
15 \\
12 \\
10\end{array}$ & $\begin{array}{r}7 \\
12 \\
8 \\
14 \\
18\end{array}$ & $\begin{array}{r}14 \\
13 \\
12 \\
12 \\
8\end{array}$ & $\begin{array}{r}8 \\
12 \\
8 \\
12 \\
8\end{array}$ & $\begin{array}{r}17 \\
12 \\
10 \\
9 \\
21\end{array}$ & $\begin{array}{r}7 \\
12 \\
8 \\
12 \\
8\end{array}$ & $\begin{array}{l}15 \\
11 \\
10 \\
11 \\
19\end{array}$ & $\begin{array}{r}8 \\
9 \\
12 \\
7 \\
7\end{array}$ & $\begin{array}{r}13 \\
11 \\
8 \\
19 \\
14\end{array}$ & $\begin{array}{l}8 \\
8 \\
8 \\
7 \\
8\end{array}$ & $\begin{array}{r}13 \\
10 \\
8 \\
18 \\
16\end{array}$ \\
\hline $\begin{array}{l}20 \\
21 \\
22 \\
23 \\
24\end{array}$ & $\begin{array}{r}8 \\
9 \\
8 \\
4 \\
32\end{array}$ & $\begin{array}{r}9 \\
17 \\
3 \\
12 \\
2\end{array}$ & $\begin{array}{r}8 \\
8 \\
4 \\
- \\
\end{array}$ & $\begin{array}{r}9 \\
7 \\
4 \\
19 \\
5\end{array}$ & $\begin{array}{r}-8 \\
32 \\
4 \\
32\end{array}$ & $\begin{array}{r}2 \\
3 \\
4 \\
17 \\
1\end{array}$ & $\frac{\overline{1}}{12}$ & $\begin{array}{l}1 \\
5 \\
3 \\
0 \\
4\end{array}$ & $\begin{array}{r}\overline{10} \\
2 \\
-\end{array}$ & $\begin{array}{l}2 \\
5 \\
8 \\
4 \\
8\end{array}$ & $\begin{array}{r}8 \\
10 \\
6 \\
12\end{array}$ & $\begin{array}{r}5 \\
10 \\
20 \\
1 \\
7\end{array}$ & $\begin{array}{r}10 \\
8 \\
4 \\
31\end{array}$ & $\begin{array}{r}10 \\
9 \\
21 \\
2 \\
8\end{array}$ & $\begin{array}{r}8 \\
8 \\
4 \\
-8\end{array}$ & $\begin{array}{r}18 \\
5 \\
21 \\
0 \\
4\end{array}$ & $\begin{array}{r}7 \\
10 \\
6 \\
-4\end{array}$ & $\begin{array}{r}18 \\
5 \\
26 \\
0 \\
12\end{array}$ & $\begin{array}{r}7 \\
8 \\
7 \\
8\end{array}$ & $\begin{array}{r}18 \\
10 \\
21 \\
1 \\
16\end{array}$ & $\begin{array}{r}4 \\
12 \\
6 \\
1 \\
8\end{array}$ & $\begin{array}{r}19 \\
7 \\
22 \\
5 \\
15\end{array}$ & $\begin{array}{c}4 \\
\text { vble. } \\
\frac{8}{4}\end{array}$ & $\begin{array}{r}17 \\
1 \\
21 \\
1 \\
15\end{array}$ \\
\hline $\begin{array}{l}25 \\
26 \\
27 \\
28\end{array}$ & $\begin{array}{r}6 \\
32 \\
8 \\
10\end{array}$ & $\begin{array}{l}13 \\
11 \\
14 \\
11\end{array}$ & $\frac{8}{8}$ & $\begin{array}{r}8 \\
2 \\
15 \\
17\end{array}$ & $\begin{array}{r}8 \\
24 \\
8 \\
10\end{array}$ & $\begin{array}{r}8 \\
2 \\
25 \\
13\end{array}$ & $\begin{array}{r}12 \\
12 \\
8 \\
12\end{array}$ & $\begin{array}{r}8 \\
2 \\
12 \\
13\end{array}$ & $\begin{array}{r}12 \\
8 \\
8 \\
4\end{array}$ & $\begin{array}{r}8 \\
7 \\
16 \\
21\end{array}$ & $\begin{array}{l}8 \\
8 \\
4 \\
6\end{array}$ & $\begin{array}{r}12 \\
7 \\
17 \\
23\end{array}$ & $\begin{array}{r}10 \\
16 \\
8 \\
6\end{array}$ & $\begin{array}{r}12 \\
7 \\
24 \\
23\end{array}$ & $\begin{array}{r}8 \\
12 \\
6 \\
4\end{array}$ & $\begin{array}{r}12 \\
8 \\
20 \\
17\end{array}$ & $\begin{array}{l}7 \\
4 \\
8 \\
4\end{array}$ & $\begin{array}{r}22 \\
6 \\
21 \\
10\end{array}$ & $\begin{array}{r}7 \\
4 \\
8 \\
-\end{array}$ & $\begin{array}{r}13 \\
15 \\
20 \\
2\end{array}$ & $\begin{array}{r}7 \\
4 \\
8 \\
-\end{array}$ & $\begin{array}{r}12 \\
13 \\
11 \\
0\end{array}$ & $\begin{array}{r}8 \\
4 \\
8 \\
-\end{array}$ & $\begin{array}{r}11 \\
13 \\
14 \\
0\end{array}$ \\
\hline Mean. & & 8 & & 9 & & 8 & & 8 & & 10 & & 10 & & 12 & & 11 & & 12 & & 12 & & 10 & & 9 \\
\hline
\end{tabular}

March, 1903.

\begin{tabular}{|c|c|c|c|c|c|c|c|c|c|c|c|c|c|c|c|c|c|c|c|c|c|c|c|c|}
\hline \multirow{2}{*}{ Day. } & \multicolumn{2}{|c|}{2.} & \multicolumn{2}{|c|}{4.} & \multicolumn{2}{|c|}{6.} & \multicolumn{2}{|c|}{8.} & \multicolumn{2}{|c|}{10.} & \multicolumn{2}{|c|}{ Noon. } & \multicolumn{2}{|c|}{14.} & \multicolumn{2}{|c|}{16.} & \multicolumn{2}{|c|}{18.} & \multicolumn{2}{|c|}{20.} & \multicolumn{2}{|c|}{22.} & \multicolumn{2}{|c|}{ Midt. } \\
\hline & D. & v. & D. & r. & D. & v. & D. & v. & D. & v. & D. & v. & D. & v. & D. & v. & D. & v. & D. & v. & D. & v. & D. & v. \\
\hline 1 & - & 1 & - & 0 & - & 0 & - & 0 & - & 0 & - & 0 & - & 0 & - & 0 & - & 0 & 4 & 4 & 1 & 6 & - & 5 \\
\hline $\begin{array}{l}2 \\
3 \\
4 \\
5 \\
6\end{array}$ & $\begin{array}{r}\overline{14} \\
\frac{8}{8}\end{array}$ & $\begin{array}{r}0 \\
17 \\
6 \\
3 \\
24\end{array}$ & $\begin{array}{r}10 \\
12 \\
\frac{8}{6}\end{array}$ & $\begin{array}{r}6 \\
13 \\
0 \\
1 \\
28\end{array}$ & $\frac{8}{12}$ & $\begin{array}{r}9 \\
9 \\
2 \\
4 \\
24\end{array}$ & $\begin{array}{r}20 \\
18 \\
32 \\
8\end{array}$ & $\begin{array}{r}4 \\
11 \\
1 \\
5 \\
28\end{array}$ & $\begin{array}{c}\text { vole } \\
12 \\
\frac{8}{8} \\
8\end{array}$ & $\begin{array}{r}17 \\
15 \\
1 \\
3 \\
38\end{array}$ & $\begin{array}{r}6 \\
12 \\
32 \\
8\end{array}$ & $\begin{array}{r}7 \\
11 \\
2 \\
3 \\
26\end{array}$ & $\begin{array}{r}6 \\
14 \\
8 \\
8\end{array}$ & $\begin{array}{r}11 \\
15 \\
2 \\
3 \\
29\end{array}$ & $\begin{array}{r}7 \\
12 \\
2 \\
6 \\
8\end{array}$ & $\begin{array}{r}15 \\
13 \\
5 \\
9 \\
26\end{array}$ & $\begin{array}{r}7 \\
14 \\
1 \\
1 \\
9\end{array}$ & $\begin{array}{r}15 \\
13 \\
7 \\
9 \\
14\end{array}$ & $\begin{array}{r}4 \\
12 \\
32 \\
1 \\
12\end{array}$ & $\begin{array}{r}15 \\
16 \\
8 \\
9 \\
11\end{array}$ & $\begin{array}{r}12 \\
8 \\
32 \\
4 \\
-\end{array}$ & $\begin{array}{r}10 \\
10 \\
7 \\
9 \\
4\end{array}$ & $\begin{array}{r}12 \\
6 \\
4 \\
-\end{array}$ & $\begin{array}{r}11 \\
13 \\
9 \\
15 \\
4\end{array}$ \\
\hline $\begin{array}{r}7 \\
8 \\
0 \\
10 \\
11\end{array}$ & $\begin{array}{l}-\overline{9} \\
9 \\
4 \\
7\end{array}$ & $\begin{array}{r}3 \\
13 \\
3 \\
18 \\
24\end{array}$ & $\begin{array}{r}\overline{6} \\
12 \\
4 \\
7\end{array}$ & $\begin{array}{r}3 \\
10 \\
7 \\
12 \\
27\end{array}$ & $\begin{array}{r}12 \\
6 \\
7 \\
6 \\
7\end{array}$ & $\begin{array}{r}3 \\
17 \\
14 \\
12 \\
20\end{array}$ & $\begin{array}{r}12 \\
5 \\
4 \\
6 \\
8\end{array}$ & $\begin{array}{l}13 \\
20 \\
24 \\
12 \\
11\end{array}$ & $\begin{array}{l}7 \\
8 \\
4 \\
4 \\
4\end{array}$ & $\begin{array}{r}7 \\
18 \\
28 \\
15 \\
20\end{array}$ & $\begin{array}{l}4 \\
8 \\
4 \\
4 \\
4\end{array}$ & $\begin{array}{l}22 \\
16 \\
18 \\
10 \\
19\end{array}$ & $\begin{array}{l}2 \\
7 \\
1 \\
4 \\
7\end{array}$ & $\begin{array}{l}20 \\
17 \\
21 \\
11 \\
15\end{array}$ & $\begin{array}{l}7 \\
2 \\
6 \\
6 \\
6 \\
6\end{array}$ & $\begin{array}{l}20 \\
18 \\
20 \\
\mathbf{1 4} \\
\mathbf{1 4}\end{array}$ & $\begin{array}{l}2 \\
8 \\
6 \\
4 \\
4\end{array}$ & $\begin{array}{l}21 \\
18 \\
19 \\
15 \\
11\end{array}$ & $\begin{array}{l}4 \\
8 \\
8 \\
8 \\
4\end{array}$ & $\begin{array}{l}19 \\
17 \\
13 \\
15 \\
13\end{array}$ & $\begin{array}{l}6 \\
4 \\
6 \\
7 \\
6\end{array}$ & $\begin{array}{l}17 \\
16 \\
12 \\
20 \\
12\end{array}$ & $\begin{array}{r}6 \\
10 \\
6 \\
7 \\
7\end{array}$ & $\begin{array}{r}8 \\
9 \\
23 \\
23 \\
12\end{array}$ \\
\hline $\begin{array}{l}12 \\
13 \\
14 \\
15 \\
16\end{array}$ & $\begin{array}{r}8 \\
8 \\
1 \\
32 \\
32\end{array}$ & $\begin{array}{r}7 \\
17 \\
4 \\
4 \\
4\end{array}$ & $\begin{array}{r}6 \\
6 \\
12 \\
32 \\
32\end{array}$ & $\begin{array}{r}9 \\
12 \\
3 \\
9 \\
6\end{array}$ & $\begin{array}{r}7 \\
8 \\
2 \\
32 \\
6\end{array}$ & $\begin{array}{r}8 \\
9 \\
2 \\
6 \\
10\end{array}$ & $\begin{array}{r}7 \\
4 \\
4 \\
32 \\
4\end{array}$ & $\begin{array}{r}24 \\
8 \\
7 \\
8 \\
8\end{array}$ & $\frac{4}{8}$ & $\begin{array}{r}15 \\
10 \\
11 \\
9 \\
4\end{array}$ & $\begin{array}{l}4 \\
8 \\
6 \\
2 \\
8\end{array}$ & $\begin{array}{r}12 \\
5 \\
5 \\
12 \\
10\end{array}$ & $\begin{array}{l}\frac{6}{4} \\
32 \\
10\end{array}$ & $\begin{array}{r}17 \\
3 \\
8 \\
12 \\
4\end{array}$ & $\frac{7}{32}$ & $\begin{array}{r}22 \\
1 \\
6 \\
11 \\
18\end{array}$ & $\frac{7}{1}$ & $\begin{array}{r}20 \\
2 \\
3 \\
13 \\
26\end{array}$ & $\begin{array}{l}6 \\
8 \\
4 \\
2 \\
7\end{array}$ & $\begin{array}{r}19 \\
5 \\
4 \\
4 \\
24\end{array}$ & $\begin{array}{r}5 \\
8 \\
32 \\
32 \\
4\end{array}$ & $\begin{array}{r}11 \\
4 \\
1 \\
5 \\
21\end{array}$ & $\begin{array}{r}8 \\
6 \\
32 \\
4\end{array}$ & $\begin{array}{r}7 \\
5 \\
1 \\
5 \\
22\end{array}$ \\
\hline $\begin{array}{l}17 \\
18 \\
19 \\
20 \\
21\end{array}$ & $\begin{array}{r}8 \\
8 \\
7 \\
- \\
-\end{array}$ & $\begin{array}{r}27 \\
22 \\
11 \\
24 \\
1\end{array}$ & $\begin{array}{r}8 \\
8 \\
7 \\
11 \\
\end{array}$ & $\begin{array}{r}14 \\
20 \\
15 \\
8 \\
0\end{array}$ & $\begin{array}{r}8 \\
8 \\
9 \\
6 \\
-\end{array}$ & $\begin{array}{r}23 \\
20 \\
9 \\
5 \\
1\end{array}$ & $\begin{array}{l}8 \\
8 \\
8 \\
4 \\
4\end{array}$ & $\begin{array}{r}22 \\
17 \\
9 \\
1 \\
7\end{array}$ & $\begin{array}{r}8 \\
7 \\
8 \\
9\end{array}$ & $\begin{array}{r}27 \\
15 \\
10 \\
1 \\
10\end{array}$ & $\begin{array}{r}4 \\
8 \\
12\end{array}$ & $\begin{array}{r}23 \\
12 \\
12 \\
0 \\
8\end{array}$ & $\begin{array}{r}8 \\
8 \\
8 \\
12\end{array}$ & $\begin{array}{r}23 \\
12 \\
15 \\
0 \\
6\end{array}$ & $\begin{array}{r}8 \\
8 \\
7 \\
8\end{array}$ & $\begin{array}{r}22 \\
11 \\
12 \\
2 \\
6\end{array}$ & $\begin{array}{r}8 \\
8 \\
6 \\
8\end{array}$ & $\begin{array}{r}23 \\
17 \\
17 \\
0 \\
13\end{array}$ & $\begin{array}{r}8 \\
8 \\
4 \\
10\end{array}$ & $\begin{array}{r}25 \\
11 \\
24 \\
0 \\
10\end{array}$ & $\begin{array}{r}8 \\
7 \\
7 \\
32 \\
2\end{array}$ & $\begin{array}{r}23 \\
16 \\
26 \\
9 \\
7\end{array}$ & $\begin{array}{r}8 \\
9 \\
6 \\
32 \\
4\end{array}$ & $\begin{array}{r}24 \\
20 \\
23 \\
8 \\
3\end{array}$ \\
\hline $\begin{array}{l}22 \\
23 \\
24 \\
25 \\
26\end{array}$ & $\begin{array}{r}- \\
6 \\
8 \\
8\end{array}$ & $\begin{array}{r}2 \\
1 \\
19 \\
8 \\
3\end{array}$ & $\begin{array}{c}\frac{12}{4} \\
8 \\
6\end{array}$ & $\begin{array}{r}3 \\
0 \\
23 \\
17 \\
5\end{array}$ & $\begin{array}{r}10 \\
8 \\
6 \\
8 \\
6\end{array}$ & $\begin{array}{r}3 \\
1 \\
23 \\
17 \\
13\end{array}$ & $\begin{array}{r}6 \\
12 \\
6 \\
8 \\
12\end{array}$ & $\begin{array}{r}8 \\
8 \\
20 \\
10 \\
12\end{array}$ & $\begin{array}{r}12 \\
12 \\
8 \\
16 \\
8\end{array}$ & $\begin{array}{r}7 \\
7 \\
27 \\
9 \\
12\end{array}$ & $\begin{array}{r}12 \\
12 \\
8 \\
8\end{array}$ & $\begin{array}{r}17 \\
10 \\
24 \\
0 \\
18\end{array}$ & $\begin{array}{r}12 \\
12 \\
8 \\
8\end{array}$ & $\begin{array}{r}15 \\
9 \\
16 \\
1 \\
13\end{array}$ & $\begin{array}{r}10 \\
9 \\
6 \\
7\end{array}$ & $\begin{array}{l}16 \\
10 \\
19 \\
1 \\
20\end{array}$ & $\begin{array}{r}9 \\
8 \\
6 \\
6\end{array}$ & $\begin{array}{r}22 \\
0 \\
18 \\
0 \\
18\end{array}$ & $\begin{array}{r}8 \\
8 \\
12 \\
-\end{array}$ & $\begin{array}{r}20 \\
9 \\
12 \\
0 \\
8\end{array}$ & $\begin{array}{r}7 \\
4 \\
10 \\
8\end{array}$ & $\begin{array}{r}24 \\
21 \\
17 \\
0 \\
3\end{array}$ & $\begin{array}{l}8 \\
4 \\
9 \\
8 \\
8\end{array}$ & $\begin{array}{r}9 \\
25 \\
12 \\
2 \\
6\end{array}$ \\
\hline $\begin{array}{l}27 \\
28 \\
29 \\
30 \\
31\end{array}$ & $\begin{array}{r}8 \\
4 \\
-1 \\
8\end{array}$ & $\begin{array}{r}8 \\
12 \\
11 \\
1 \\
14\end{array}$ & $\begin{array}{r}10 \\
4 \\
32 \\
8\end{array}$ & $\begin{array}{r}8 \\
15 \\
10 \\
1 \\
20\end{array}$ & $\begin{array}{r}10 \\
8 \\
10 \\
10 \\
28\end{array}$ & $\begin{array}{r}9 \\
8 \\
15 \\
5 \\
10\end{array}$ & $\begin{array}{r}8 \\
4 \\
4 \\
6\end{array}$ & $\begin{array}{r}8 \\
10 \\
0 \\
7 \\
7\end{array}$ & $\begin{array}{r}12 \\
24 \\
8 \\
4 \\
4\end{array}$ & $\begin{array}{r}12 \\
5 \\
1.4 \\
11 \\
14\end{array}$ & $\begin{array}{r}\frac{12}{8} \\
4 \\
-\end{array}$ & $\begin{array}{r}9 \\
2 \\
17 \\
17 \\
4\end{array}$ & $\begin{array}{l}-4 \\
4 \\
4 \\
-\end{array}$ & $\begin{array}{r}3 \\
3 \\
14 \\
21 \\
0\end{array}$ & $\begin{array}{r}-4 \\
2 \\
6 \\
-\end{array}$ & $\begin{array}{r}0 \\
4 \\
7 \\
23 \\
0\end{array}$ & $\begin{array}{r}1 \\
2 \\
32 \\
7 \\
-\end{array}$ & $\begin{array}{r}1 \\
7 \\
8 \\
22 \\
0\end{array}$ & $\begin{array}{r}32 \\
1 \\
32 \\
8 \\
12\end{array}$ & $\begin{array}{r}4 \\
2 \\
8 \\
18 \\
0\end{array}$ & $\begin{array}{r}2 \\
1 \\
32 \\
7 \\
8\end{array}$ & $\begin{array}{r}7 \\
10 \\
6 \\
17 \\
17\end{array}$ & $\begin{array}{c}\frac{4}{1} \\
8 \\
8\end{array}$ & $\begin{array}{r}9 \\
5 \\
3 \\
14 \\
18\end{array}$ \\
\hline Mean & & 10 & & 10 & & 10 & & 11 & & 12 & & 11 & & 11 & & 12 & & 12 & & 11 & & 12 & & 11 \\
\hline
\end{tabular}


TABLe IV. (continued).

WIND-DIRECTION AND VELOCITY.

Direetion (D) in Numbers $(8=\mathrm{E}, 16=\mathrm{S}, 24=\mathrm{W}, 32=\mathrm{N})$, and Velocity $(\mathrm{V})$ in miles per hour as recorded by the Cup Anemometer.

April, 1903.

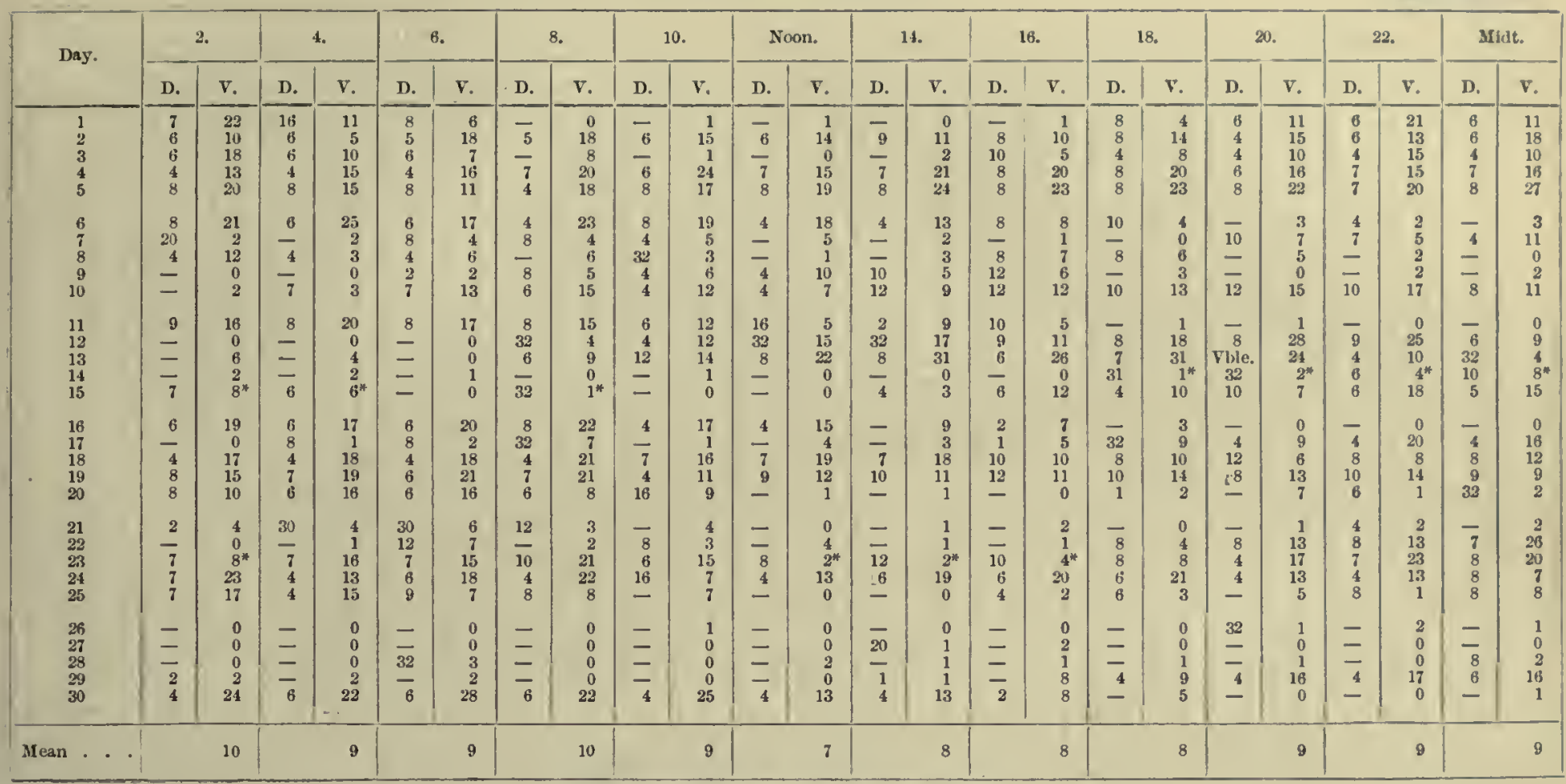

* Equivalent velocity for Beaufort scale estimatio

May, 1903.

\begin{tabular}{|c|c|c|c|c|c|c|c|c|c|c|c|c|c|c|c|c|c|c|c|c|c|c|c|c|}
\hline \multirow{2}{*}{ Day. } & \multicolumn{2}{|c|}{2.} & \multicolumn{2}{|c|}{4.} & \multicolumn{2}{|c|}{6.} & \multicolumn{2}{|c|}{8.} & \multicolumn{2}{|c|}{10.} & \multicolumn{2}{|c|}{ Noon. } & \multicolumn{2}{|c|}{ is. } & \multicolumn{2}{|c|}{16.} & \multicolumn{2}{|c|}{18.} & \multicolumn{2}{|c|}{20.} & \multicolumn{2}{|c|}{22.} & \multicolumn{2}{|c|}{ Midt. } \\
\hline & D. & v. & D. & v. & D. & v. & D. & v. & D. & v. & D. & $\mathrm{v}$ & D. & v. & D. & $\mathrm{v}$ & D. & v. & D. & v. & D. & v. & D. & v. \\
\hline$\frac{1}{2}$ & $\overline{8}$ & 1 & $\overline{8}$ & 0 & 8 & 0 & $\overline{5}$ & 4 & $\overline{10}$ & $\begin{array}{l}0 \\
8\end{array}$ & 12 & 5 & 8 & 7 & 10 & 12 & 12 & 11 & 8 & 8 & 8 & ${ }_{22}^{11}$ & ${ }_{14}^{8}$ & ${ }_{11}^{11}$ \\
\hline $\begin{array}{l}2 \\
3 \\
4\end{array}$ & $\begin{array}{l}8 \\
12 \\
-\end{array}$ & $\begin{array}{r}13 \\
16 \\
2\end{array}$ & $\begin{array}{r}8 \\
8 \\
10\end{array}$ & $\begin{array}{c}16 \\
13 \\
2 \\
2\end{array}$ & $\begin{array}{l}8 \\
6 \\
4\end{array}$ & $\begin{array}{l}19 \\
16 \\
7\end{array}$ & $\begin{array}{r}15 \\
6 \\
4\end{array}$ & $\begin{array}{l}25 \\
35 \\
1\end{array}$ & $\begin{array}{l}12 \\
8 \\
-\end{array}$ & $\begin{array}{l}16 \\
21 \\
21\end{array}$ & $\stackrel{9}{=}$ & $\begin{array}{c}15 \\
10 \\
10\end{array}$ & $\begin{array}{r}8 \\
6 \\
10\end{array}$ & $\begin{array}{r}13 \\
7 \\
2\end{array}$ & $\begin{array}{l}7 \\
6 \\
7\end{array}$ & $\begin{array}{l}14 \\
10 \\
4\end{array}$ & $\begin{array}{c}8 \\
12 \\
6\end{array}$ & $\begin{array}{l}26 \\
47 \\
17\end{array}$ & $\frac{12}{4}$ & $\begin{array}{l}18 \\
4 \\
16\end{array}$ & $\begin{array}{r}12 \\
14 \\
6\end{array}$ & $\begin{array}{c}22 \\
4 \\
17\end{array}$ & $\begin{array}{r}14 \\
15 \\
4\end{array}$ & $\begin{array}{c}17 \\
46 \\
16\end{array}$ \\
\hline 5 & 4 & 21 & 6 & & 8 & 12 & 10 & & 16 & 2 & 12 & 5 & - & 3 & 10 & 8 & & & - & 2 & & & & 1 \\
\hline$\frac{6}{7}$ & $\overline{9}$ & $\begin{array}{l}2 \\
9\end{array}$ & $\begin{array}{r}12 \\
9\end{array}$ & $\stackrel{2}{9}$ & $\underline{12}$ & $\begin{array}{l}8 \\
3\end{array}$ & $\begin{array}{l}6 \\
1\end{array}$ & $\stackrel{11}{2}$ & $\stackrel{8}{-}$ & $\begin{array}{l}8 \\
5\end{array}$ & $\overline{12}$ & $\begin{array}{l}4 \\
5\end{array}$ & -6 & $\begin{array}{l}6 \\
3\end{array}$ & $\begin{array}{l}32 \\
7\end{array}$ & $\begin{array}{l}10 \\
12\end{array}$ & 8 & $\begin{array}{r}4 \\
14\end{array}$ & $\overline{8}$ & $\begin{array}{r}1 \\
14\end{array}$ & $\overline{10}$ & $\frac{1}{7}$ & $\begin{array}{r}8 \\
10 \\
8\end{array}$ & $\begin{array}{c}4 \\
10 \\
07\end{array}$ \\
\hline $\begin{array}{r}8 \\
9 \\
10\end{array}$ & $\begin{array}{r}10 \\
8 \\
4\end{array}$ & $\begin{array}{l}9 \\
23 \\
23 \\
13\end{array}$ & $\overline{8}$ & $\begin{array}{r}6 \\
16 \\
17 \\
17\end{array}$ & $\begin{array}{l}\overline{8} \\
6\end{array}$ & $\begin{array}{l}2 \\
15 \\
18\end{array}$ & $\overline{6}$ & $\begin{array}{l}1 \\
17 \\
21\end{array}$ & $\overline{6}$ & $\begin{array}{c}1 \\
15 \\
18\end{array}$ & $\overline{6}$ & $\begin{array}{l}n \\
12 \\
16\end{array}$ & $\overline{{ }_{8}^{6}}$ & $\begin{array}{l}3 \\
15 \\
21\end{array}$ & $\begin{array}{c}12 \\
7 \\
8\end{array}$ & $\begin{array}{l}5 \\
13 \\
18 \\
18\end{array}$ & $\begin{array}{l}9 \\
7 \\
8\end{array}$ & $\begin{array}{l}15 \\
11 \\
23\end{array}$ & $\begin{array}{l}8 \\
8 \\
4\end{array}$ & $\begin{array}{l}14 \\
10 \\
24\end{array}$ & $\begin{array}{l}8 \\
8 \\
7\end{array}$ & $\begin{array}{l}20 \\
10 \\
22\end{array}$ & $\begin{array}{l}8 \\
6 \\
7\end{array}$ & $\begin{array}{l}21 \\
12 \\
22\end{array}$ \\
\hline 11 & 6 & 18 & 6 & 26 & B & 26 & 4 & 23 & 8 & 16 & 4 & 19 & 6 & 22 & 7 & 19 & 7 & 18 & 4 & 17 & 4 & $\begin{array}{l}16 \\
0\end{array}$ & 8 & $\begin{array}{l}6 \\
0\end{array}$ \\
\hline $\begin{array}{l}12 \\
13\end{array}$ & $\frac{8}{19}$ & $\begin{array}{l}4 \\
0\end{array}$ & $\overline{\bar{z}}$ & $\begin{array}{l}0 \\
0\end{array}$ & $\bar{\tau}$ & $\begin{array}{l}0 \\
0\end{array}$ & $\bar{z}$ & $\begin{array}{l}0 \\
0\end{array}$ & $\overline{-}$ & $\begin{array}{l}\mathbf{0} \\
0\end{array}$ & $=$ & $\begin{array}{l}0 \\
0\end{array}$ & $=$ & $\begin{array}{l}1 \\
1\end{array}$ & $\overline{12}$ & $\begin{array}{l}1 \\
4\end{array}$ & $\overline{15}$ & $\begin{array}{l}1 \frac{1}{18} \\
\end{array}$ & $\overline{14}$ & 21 & $\overline{9}$ & 13 & 8 & 10 \\
\hline $\begin{array}{l}14 \\
15\end{array}$ & $\underline{-10}$ & $\begin{array}{l}8 \\
0\end{array}$ & 12 & $\begin{array}{l}1 \\
0\end{array}$ & 12 & $\begin{array}{r}18 \\
0\end{array}$ & $\begin{array}{l}4 \\
8\end{array}$ & $\begin{array}{r}10 \\
3\end{array}$ & $\overline{12}$ & $\begin{array}{l}4 \\
2\end{array}$ & $=$ & $\begin{array}{l}0 \\
6\end{array}$ & $=$ & $\begin{array}{l}0 \\
1\end{array}$ & $\overline{-}$ & 0 & $=$ & 0 & $=$ & 0 & - & 0 & - & 0 \\
\hline $\begin{array}{l}16 \\
17\end{array}$ & $\overline{6}$ & $\begin{array}{l}0 \\
7\end{array}$ & $\overline{8}$ & $\begin{array}{r}0 \\
17\end{array}$ & $\overline{6}$ & $\begin{array}{l}0 \\
17\end{array}$ & $\bar{g}$ & $\begin{array}{r}0 \\
19\end{array}$ & $\overline{8}$ & $\begin{array}{r}0 \\
13\end{array}$ & 28 & $\begin{array}{l}1 \\
4\end{array}$ & $\begin{array}{l}30 \\
32\end{array}$ & $\begin{array}{l}6 \\
4\end{array}$ & $\begin{array}{l}30 \\
31\end{array}$ & $\begin{array}{l}6 \\
8\end{array}$ & $\begin{array}{l}32 \\
30\end{array}$ & $\begin{array}{r}6 \\
10\end{array}$ & $\begin{array}{r}20 \\
4\end{array}$ & $\begin{array}{l}5 \\
7\end{array}$ & $\begin{array}{l}20 \\
10\end{array}$ & 9 & $\overline{8}$ & $\begin{array}{l}1 \\
4\end{array}$ \\
\hline $\begin{array}{l}18 \\
19 \\
20\end{array}$ & $\begin{array}{r}8 \\
12 \\
-\end{array}$ & $\begin{array}{l}3 \\
4 \\
4\end{array}$ & $\begin{array}{r}29 \\
8 \\
32\end{array}$ & $\begin{array}{r}5 \\
10 \\
2\end{array}$ & $\begin{array}{l}32 \\
-7 \\
-\end{array}$ & $\begin{array}{c}10 \\
8 \\
3\end{array}$ & $\overline{32}$ & $\begin{array}{r}17 \\
0\end{array}$ & $\frac{16}{-}$ & $\begin{array}{r}11 \\
1 \\
0\end{array}$ & $\begin{array}{l}\overline{32} \\
10\end{array}$ & $\begin{array}{l}7 \\
8 \\
3\end{array}$ & $\begin{array}{c}6 \\
32 \\
\text { vible. }\end{array}$ & $\begin{array}{r}8 \\
7 \\
10\end{array}$ & $\begin{array}{l}6 \\
32 \\
12\end{array}$ & $\begin{array}{r}5 \\
6 \\
18\end{array}$ & $\begin{array}{r}41 \\
31 \\
8\end{array}$ & $\begin{array}{l}13 \\
6 \\
21\end{array}$ & $\begin{array}{l}32 \\
32 \\
-\end{array}$ & $\begin{array}{l}6 \\
7 \\
7\end{array}$ & $\frac{2}{28}$ & $\begin{array}{c}13 \\
1 \\
6\end{array}$ & $\begin{array}{r}4 \\
- \\
-\end{array}$ & 7 \\
\hline $\begin{array}{l}21 \\
22\end{array}$ & $\overline{7}$ & ${ }_{24}^{4}$ & $\begin{array}{l}8 \\
9\end{array}$ & ${ }_{20}^{7}$ & Vule. & ${ }_{9}^{4}$ & $\overline{12}$ & $\underset{12}{2}$ & $\overline{8}$ & $\begin{array}{r}3 \\
22\end{array}$ & $\begin{array}{l}\text { Vble. } \\
\end{array}$ & $\begin{array}{r}\mathbf{2} \\
29\end{array}$ & $\begin{array}{l}8 \\
8\end{array}$ & $\begin{array}{r}4 \\
23\end{array}$ & $\begin{array}{l}8 \\
8\end{array}$ & $\begin{array}{l}15 \\
23\end{array}$ & $\begin{array}{l}8 \\
8\end{array}$ & $\begin{array}{l}30 \\
22\end{array}$ & ${ }_{8}^{8}$ & $\begin{array}{l}34 \\
24\end{array}$ & $\frac{9}{6}$ & $\begin{array}{l}33 \\
23\end{array}$ & $\begin{array}{l}8 \\
6\end{array}$ & $\begin{array}{l}20 \\
26\end{array}$ \\
\hline $\begin{array}{l}23 \\
24\end{array}$ & $\begin{array}{l}6 \\
8 \\
8\end{array}$ & $\begin{array}{r}31 \\
8 \\
17\end{array}$ & $\begin{array}{l}8 \\
8 \\
6\end{array}$ & $\begin{array}{r}30 \\
8 \\
18\end{array}$ & $\begin{array}{r}7 \\
12 \\
6\end{array}$ & $\begin{array}{l}26 \\
21 \\
17\end{array}$ & $\begin{array}{r}8 \\
16\end{array}$ & $\begin{array}{l}24 \\
20 \\
15\end{array}$ & $\begin{array}{l}4 \\
9 \\
8\end{array}$ & $\begin{array}{l}32 \\
20 \\
12\end{array}$ & $\begin{array}{l}6 \\
8 \\
8\end{array}$ & $\begin{array}{l}23 \\
20 \\
9\end{array}$ & $\begin{array}{r}20 \\
6 \\
7\end{array}$ & $\begin{array}{l}12 \\
125 \\
18\end{array}$ & $\begin{array}{r}12 \\
7 \\
8\end{array}$ & $\begin{array}{r}9 \\
25 \\
9\end{array}$ & $\begin{array}{l}\overline{8} \\
8\end{array}$ & $\begin{array}{l}1 \\
23 \\
14\end{array}$ & $\overline{6}$ & $\begin{array}{l}0 \\
27 \\
14\end{array}$ & $\begin{array}{l}\overline{8} \\
8\end{array}$ & $\begin{array}{l}0 \\
24 \\
27\end{array}$ & $\begin{array}{c}\overline{8} \\
7\end{array}$ & $\begin{array}{l}0 \\
20 \\
27\end{array}$ \\
\hline $\begin{array}{l}26 \\
\text { 26 }\end{array}$ & 4 & 27 & 4 & 21 & 4 & 17 & 10 & 13 & 12 & 10 & 12 & 9 & 14 & 12 & 12 & ${ }_{0}^{8}$ & 10 & 11 & 8 & 17 & 8 & $\begin{array}{r}16 \\
6 \\
6\end{array}$ & 10 & 15 \\
\hline $\begin{array}{l}21 \\
28\end{array}$ & $-y$ & $\begin{array}{l}17 \\
5 \\
0\end{array}$ & $\dot{\check{z}}$ & 1 & -8 & 0 & $\overline{6}$ & 2 & $\overline{4}$ & $\begin{array}{l}1 \\
20\end{array}$ & $\begin{array}{l}8 \\
4\end{array}$ & $\begin{array}{l}3 \\
21 \\
18\end{array}$ & 12 & $\begin{array}{l}18 \\
17\end{array}$ & $\begin{array}{r}8 \\
32\end{array}$ & 4 & 23 & 2 & 30 & 4 & $\underline{1}$ & $\begin{array}{l}4 \\
0 \\
0\end{array}$ & $\underline{\mathrm{i}}$ & t \\
\hline 30 & $\overrightarrow{28}$ & 4 & $\overline{32}$ & ${ }_{6}^{0}$ & - & 9 & - & 6 & - & 0 & ${ }_{8}^{6}$ & $\begin{array}{r}18 \\
9\end{array}$ & $\begin{array}{l}6 \\
8\end{array}$ & $\begin{array}{l}18 \\
11\end{array}$ & $\begin{array}{r}8 \\
10\end{array}$ & $\begin{array}{l}8 \\
9\end{array}$ & $\overline{10}$ & 11 & $\overline{6}$ & 9 & 10 & 8 & 9 & 15 \\
\hline 31 & 8 & 20 & 9 & 17 & 8 & 15 & $\tau$ & 24 & 6 & 21 & 8 & 18 & 8 & 10 & 8 & 17 & 7 & 17 & 4 & 27 & 5 & $2 i$ & 8 & 23 \\
\hline sean & & 10 & & 10 & & 10 & & 11 & & 9 & & , & & 10 & & 10 & & 12 & & 12 & & 11 & & 10 \\
\hline
\end{tabular}


TABLE IV. (continued).

WIND-DIRECTION AND VELOCITY.

Direction (D) in Numbers $\left(8=\mathrm{F}_{4}, 16=\mathrm{S}, 24=\mathrm{W}, 32=\mathrm{N}\right)$, and Velocity $(\mathrm{V})$ in miles per hour as recorded by the Cup Ancmometer.

June, 1903.

\begin{tabular}{|c|c|c|c|c|c|c|c|c|c|c|c|c|c|c|c|c|c|c|c|c|c|c|c|c|}
\hline \multirow{2}{*}{ Das. } & \multicolumn{2}{|c|}{2.} & \multicolumn{2}{|c|}{4.} & \multicolumn{2}{|c|}{6.} & \multicolumn{2}{|c|}{8.} & \multicolumn{2}{|c|}{10.} & \multicolumn{2}{|c|}{ Noon. } & \multicolumn{2}{|c|}{14.} & \multicolumn{2}{|c|}{18.} & \multicolumn{2}{|c|}{18.} & \multicolumn{2}{|c|}{20.} & \multicolumn{2}{|c|}{22.} & \multicolumn{2}{|c|}{ Midt. } \\
\hline & D. & v. & D. & v. & D. & v. & D. & v. & D. & v. & D. & v. & D. & v. & D. & v. & D. & จ. & D. & v. & D. & v. & D. & $\mathrm{v}$ \\
\hline $\begin{array}{l}1 \\
3 \\
3 \\
4\end{array}$ & $\begin{array}{r}8 \\
7 \\
8 \\
\end{array}$ & $\begin{array}{l}15 \\
22 \\
12\end{array}$ & $\begin{array}{l}7 \\
8 \\
4 \\
\end{array}$ & $\begin{array}{r}30 \\
31 \\
7 \\
7\end{array}$ & $\begin{array}{r}7 \\
7 \\
32 \\
3\end{array}$ & $\begin{array}{r}22 \\
12 \\
5 \\
4\end{array}$ & $\begin{array}{r}8 \\
8 \\
-8\end{array}$ & $\begin{array}{l}24 \\
21 \\
1\end{array}$ & $\begin{array}{r}8 \\
8 \\
\end{array}$ & $\begin{array}{l}29 \\
10 \\
0\end{array}$ & $\begin{array}{r}8 \\
8 \\
32 \\
15\end{array}$ & $\begin{array}{r}21 \\
19 \\
5 \\
9\end{array}$ & $\begin{array}{r}8 \\
8 \\
32 \\
0\end{array}$ & $\begin{array}{r}20 \\
23 \\
5 \\
15\end{array}$ & $\begin{array}{r}7 \\
8 \\
32 \\
9\end{array}$ & $\begin{array}{l}23 \\
21 \\
4 \\
1\end{array}$ & $\frac{8}{8}$ & $\begin{array}{r}21 \\
20 \\
4 \\
13\end{array}$ & $\begin{array}{r}4 \\
8 \\
31 \\
12\end{array}$ & 22 & $\begin{array}{r}\frac{2}{12} \\
24 \\
12\end{array}$ & $\begin{array}{c}28 \\
14 \\
3 \\
18\end{array}$ & $\begin{array}{r}7 \\
\frac{7}{12}\end{array}$ & $\begin{array}{c}26 \\
10 \\
1 \\
1\end{array}$ \\
\hline 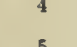 & - & 1 & - & 2 & 8 & & 8 & & 15 & 11 & 15 & 9 & 10 & 17 & & 13 & 8 & 13 & 12 & 17 & 12 & & 12 & $\begin{array}{c}8 \\
15\end{array}$ \\
\hline $\begin{array}{l}5 \\
6 \\
7\end{array}$ & $\begin{array}{l}12 \\
16 \\
-\end{array}$ & $\begin{array}{r}20 \\
9 \\
0\end{array}$ & Vible. & $\begin{array}{r}24 \\
2 \\
0\end{array}$ & $\frac{12}{=}$ & $\begin{array}{r}25 \\
1 \\
0\end{array}$ & $=$ & $\begin{array}{c}21 \\
3 \\
0\end{array}$ & $\begin{array}{l}18 \\
-8 \\
\end{array}$ & $\begin{array}{r}19 \\
6 \\
1\end{array}$ & $\begin{array}{c}4 \\
12\end{array}$ & $\begin{array}{l}20 \\
13 \\
14\end{array}$ & $\frac{8}{2 u}$ & 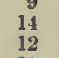 & $\frac{8}{12}$ & $\begin{array}{r}19 \\
13 \\
32\end{array}$ & $\begin{array}{l}8 \\
8 \\
8\end{array}$ & $\begin{array}{l}1 \\
22 \\
22\end{array}$ & $\frac{4}{20}$ & $\begin{array}{l}10 \\
4 \\
20\end{array}$ & $\frac{8}{8}$ & $\begin{array}{c}10 \\
1 \\
21\end{array}$ & $\frac{8}{10}$ & $\begin{array}{r}10 \\
0 \\
11\end{array}$ \\
\hline $\begin{array}{l}8 \\
9\end{array}$ & $\begin{array}{l}10 \\
12\end{array}$ & $\begin{array}{l}18 \\
18\end{array}$ & $\begin{array}{l}8 \\
8\end{array}$ & $\begin{array}{l}20 \\
17\end{array}$ & $\begin{array}{l}8 \\
8\end{array}$ & $\begin{array}{l}20 \\
17\end{array}$ & $\begin{array}{r}12 \\
8\end{array}$ & $\begin{array}{l}24 \\
23\end{array}$ & $\begin{array}{r}8 \\
12\end{array}$ & $\begin{array}{l}14 \\
22\end{array}$ & $\begin{array}{l}\text { Vule. } \\
18\end{array}$ & $\begin{array}{l}17 \\
32\end{array}$ & $\begin{array}{l}15 \\
16\end{array}$ & $\begin{array}{l}20 \\
21\end{array}$ & $\begin{array}{l}16 \\
15 \\
\end{array}$ & $\begin{array}{l}17 \\
21\end{array}$ & $\begin{array}{l}16 \\
15\end{array}$ & $\begin{array}{l}12 \\
21\end{array}$ & $\begin{array}{l}20 \\
15\end{array}$ & $\begin{array}{l}27 \\
26\end{array}$ & $\begin{array}{l}17 \\
1.4\end{array}$ & $\begin{array}{l}25 \\
20\end{array}$ & $\begin{array}{l}12 \\
16\end{array}$ & $\begin{array}{l}28 \\
25\end{array}$ \\
\hline 10 & $\begin{array}{c}18 \\
7\end{array}$ & $\begin{array}{l}33 \\
10\end{array}$ & $\begin{array}{r}17 \\
8\end{array}$ & 40 & $\begin{array}{c}16 \\
8\end{array}$ & $\begin{array}{l}48 \\
14\end{array}$ & $\begin{array}{r}16 \\
7\end{array}$ & $\begin{array}{l}40 \\
19\end{array}$ & $\begin{array}{c}16 \\
8\end{array}$ & 21 & 12 & $\begin{array}{l}12 \\
18\end{array}$ & $\begin{array}{l}8 \\
7\end{array}$ & ${ }_{18}^{13}{ }^{\circ}$ & $\begin{array}{l}8 \\
8\end{array}$ & $\begin{array}{l}13 \\
10\end{array}$ & $\begin{array}{c}7 \\
12\end{array}$ & $\begin{array}{l}17 \\
18\end{array}$ & $\begin{array}{l}8 \\
8\end{array}$ & $\frac{14}{17}$ & $\begin{array}{c}80 \\
16\end{array}$ & ${ }_{25}^{14}$ & $\begin{array}{l}{ }_{8}^{6} \\
8\end{array}$ & $\begin{array}{l}12 \\
20\end{array}$ \\
\hline $\begin{array}{l}12 \\
13\end{array}$ & 10 & $\begin{array}{l}16 \\
0\end{array}$ & $\underline{7}$ & $\begin{array}{l}8 \\
0\end{array}$ & $\begin{array}{r}7 \\
32 \\
\end{array}$ & $\begin{array}{r}19 \\
4\end{array}$ & $\frac{8}{-}$ & $\begin{array}{r}21 \\
3 \\
3\end{array}$ & -8 & $\begin{array}{r}13 \\
8\end{array}$ & 8 & $\begin{array}{c}12 \\
1\end{array}$ & -6 & $\begin{array}{c}15 \\
1\end{array}$ & $\begin{array}{l}3 \\
8\end{array}$ & $\begin{array}{c}16 \\
5\end{array}$ & $\begin{array}{c}8 \\
\text { File. }\end{array}$ & $\begin{array}{l}8 \\
3\end{array}$ & $\begin{array}{r}10 \\
8\end{array}$ & $\begin{array}{r}6 \\
13\end{array}$ & $\frac{4}{8}$ & $\begin{array}{r}5 \\
17\end{array}$ & $\overline{12}$ & $\begin{array}{l}0 \\
18\end{array}$ \\
\hline ii & 12 & 8 & 8 & 15 & 8 & 17 & 8 & 28 & 8 & 15 & 8 & 19 & 8 & 18 & 10 & 9 & Vible. & 10 & 12 & 8 & 18 & 14 & 13 & 5 \\
\hline $\begin{array}{l}15 \\
118\end{array}$ & $\overline{7}$ & $\begin{array}{r}0 \\
17\end{array}$ & $\overline{7}$ & $\begin{array}{l}0 \\
18\end{array}$ & $\overline{8}$ & $\begin{array}{c}0 \\
23 \\
23\end{array}$ & $\begin{array}{l}32 \\
12\end{array}$ & $\begin{array}{c}4 \\
16\end{array}$ & $\begin{array}{r}32 \\
8\end{array}$ & $\begin{array}{r}6 \\
12\end{array}$ & $\begin{array}{l}32 \\
10 \\
4\end{array}$ & $\begin{array}{r}6 \\
9 \\
91\end{array}$ & $\frac{32}{7}$ & $\begin{array}{r}8 \\
7 \\
19\end{array}$ & $\begin{array}{l}\overline{8} \\
\gamma\end{array}$ & $\begin{array}{r}3 \\
.5 \\
18\end{array}$ & $\begin{array}{c}10 \\
4\end{array}$ & $\begin{array}{r}0 \\
9 \\
10\end{array}$ & $\overline{4}$ & $\begin{array}{l}0 \\
0 \\
5\end{array}$ & $\begin{array}{r}9 \\
4 \\
28\end{array}$ & $\begin{array}{c}1 \\
16 \\
1\end{array}$ & $\begin{array}{r}9 \\
4 \\
-\end{array}$ & $\begin{array}{l}2 \\
5 \\
2\end{array}$ \\
\hline $\begin{array}{l}17 \\
18 \\
19\end{array}$ & 4 & $\begin{array}{l}5 \\
2 \\
1 \\
1\end{array}$ & $\begin{array}{r}4 \\
- \\
-\end{array}$ & $\begin{array}{r}18 \\
4 \\
0\end{array}$ & $\begin{array}{c}8 \\
8 \\
\text { vule. }\end{array}$ & $\begin{array}{c}21 \\
6 \\
2 \\
2\end{array}$ & $\begin{array}{r}4 \\
-8 \\
-\end{array}$ & $\begin{array}{c}29 \\
3 \\
1\end{array}$ & $\frac{4}{2}$ & $\begin{array}{r}21 \\
3 \\
1\end{array}$ & $\begin{array}{r}4 \\
8 \\
32\end{array}$ & $\begin{array}{r}21 \\
4 \\
1\end{array}$ & $\begin{array}{r}70 \\
10 \\
32\end{array}$ & $\begin{array}{r}19 \\
7 \\
6\end{array}$ & $\begin{array}{r}7 \\
82 \\
32\end{array}$ & $\begin{array}{r}18 \\
4 \\
6\end{array}$ & $\begin{array}{l}4 \\
16 \\
28\end{array}$ & $\begin{array}{r}10 \\
4 \\
5\end{array}$ & $\overline{\overline{32}}$ & 4 & $\frac{28}{32}$ & $\begin{array}{l}1 \\
1 \\
8\end{array}$ & $\overline{\overline{32}}$ & 7 \\
\hline${ }_{21}^{20}$ & 32 & $\begin{array}{l}6 \\
0\end{array}$ & $=$ & ${ }_{0}^{4}$ & $=$ & $\begin{array}{l}1 \\
0\end{array}$ & 1 & ${ }_{3}^{6}$ & $=$ & 1 & $\frac{32}{4}$ & $\frac{2}{2}$ & $\overline{32}$ & $\frac{1}{4}$ & $=$ & $\begin{array}{l}0 \\
2\end{array}$ & $\overline{8}$ & $\begin{array}{l}0 \\
4\end{array}$ & $\overline{8}$ & $\begin{array}{r}0 \\
10\end{array}$ & $\overline{7}$ & $\begin{array}{l}0 \\
9\end{array}$ & $\overline{8}$ & $\begin{array}{r}0 \\
17\end{array}$ \\
\hline 22 & 8 & $\begin{array}{r}28 \\
1\end{array}$ & $\stackrel{8}{-}$ & $\begin{array}{c}13 \\
0 \\
0\end{array}$ & 8 & ${ }_{0}^{11}$ & $\underline{12}$ & $\begin{array}{l}9 \\
0\end{array}$ & $=$ & $\begin{array}{l}3 \\
3 \\
0\end{array}$ & $\bar{z}$ & 1 & $\overline{\bar{z}}$ & $\begin{array}{l}0 \\
0 \\
0\end{array}$ & $\overline{2}$ & $\begin{array}{l}0 \\
4\end{array}$ & $\overline{4}$ & $\begin{array}{l}0 \\
11 \\
117\end{array}$ & $\begin{array}{l}12 \\
\text { vile. }\end{array}$ & $\begin{array}{r}2 \\
12\end{array}$ & $\overrightarrow{6}$ & $\begin{array}{r}0 \\
5 \\
10\end{array}$ & vile. & $\frac{14}{14}$ \\
\hline 24 & - & 2 & - & 2 & 8 & 3 & - & 4 & & 0 & 6 & 2 & 4 & 2 & 12 & & 12 & & & 20 & & & 12 & \\
\hline $\begin{array}{l}25 \\
25\end{array}$ & $\begin{array}{l}8 \\
6\end{array}$ & $\begin{array}{l}22 \\
22\end{array}$ & $\begin{array}{l}8 \\
6\end{array}$ & $\begin{array}{l}27 \\
22\end{array}$ & $\begin{array}{l}7 \\
4\end{array}$ & $\begin{array}{l}24 \\
18\end{array}$ & $\begin{array}{l}8 \\
8 \\
\end{array}$ & $\begin{array}{r}22 \\
8\end{array}$ & 4 & $\begin{array}{l}32 \\
13\end{array}$ & 4 & $\begin{array}{r}20 \\
3 \\
3\end{array}$ & $\begin{array}{l}6 \\
4 \\
4\end{array}$ & $\begin{array}{l}17 \\
15\end{array}$ & -6 & $\begin{array}{r}18 \\
9\end{array}$ & $\begin{array}{r}7 \\
32\end{array}$ & $\begin{array}{r}23^{\circ} \\
4\end{array}$ & $\frac{8}{3}$ & $\begin{array}{r}18 \\
0 \\
8\end{array}$ & $\frac{8}{8}$ & $\begin{array}{r}16 \\
1\end{array}$ & $\frac{6}{9}$ & \\
\hline $\begin{aligned} 27 \\
278 \\
29\end{aligned}$ & $\begin{array}{l}32 \\
10 \\
10\end{array}$ & $\begin{array}{r}0 \\
9 \\
21\end{array}$ & $\begin{array}{r}8 \\
12\end{array}$ & $\begin{array}{r}0 \\
4 \\
15\end{array}$ & $\begin{array}{r}8 \\
12\end{array}$ & $\begin{array}{r}7 \\
96\end{array}$ & $\begin{array}{l}7 \\
8\end{array}$ & $\begin{array}{l}0 \\
10 \\
20\end{array}$ & $\begin{array}{l}8 \\
8\end{array}$ & $\begin{array}{l}0 \\
14 \\
18\end{array}$ & ${ }_{16}^{4}$ & $\begin{array}{r}6 \\
28\end{array}$ & $\begin{array}{l}6 \\
8\end{array}$ & $\begin{array}{l}12 \\
\frac{15}{26}\end{array}$ & $\overline{28}$ & $\begin{array}{c}7 \\
7 \\
23\end{array}$ & $\frac{32}{10}$ & $\begin{array}{r}6 \\
17 \\
17\end{array}$ & $\begin{array}{r}50 \\
16 \\
8\end{array}$ & 23 & $\begin{array}{r}0 \\
8 \\
8\end{array}$ & $\begin{array}{l}6 \\
23 \\
22\end{array}$ & $\begin{array}{r}81 \\
8 \\
12\end{array}$ & $\begin{array}{l}26 \\
23 \\
12\end{array}$ \\
\hline 30 & - & 3 & 4 & 7 & 8 & 20 & 8 & 18 & 4 & 13 & - & 10 & 4 & 4 & 4 & 7 & 12 & 5 & - & 2 & 12 & 5 & - & 0 \\
\hline & & 11 & & 11 & & 12 & & 13 & & 11 & & 11 & & 11 & & 11 & & 10 & & 11 & & 11 & & 10 \\
\hline
\end{tabular}

July, 1903.

\begin{tabular}{|c|c|c|c|c|c|c|c|c|c|c|c|c|c|c|c|c|c|c|c|c|c|c|c|c|}
\hline \multirow{2}{*}{ Day. } & \multicolumn{2}{|c|}{2.} & \multicolumn{2}{|c|}{4.} & \multicolumn{2}{|c|}{6.} & \multicolumn{2}{|c|}{8.} & \multicolumn{2}{|c|}{10.} & \multicolumn{2}{|c|}{ Noon. } & \multicolumn{2}{|c|}{14.} & \multicolumn{2}{|c|}{16.} & \multicolumn{2}{|c|}{18.} & \multicolumn{2}{|c|}{20.} & \multicolumn{2}{|c|}{22.} & \multicolumn{2}{|c|}{ Midt. } \\
\hline & D. & v. & D. & v. & D. & v. & D. & v. & D. & $\mathrm{v}$. & D. & $\mathrm{v}$ & D. & v. & v. & r. & D. & r. & D. & v. & D. & $\mathrm{r}$. & D. & v. \\
\hline $\begin{array}{l}1 \\
2 \\
3 \\
4\end{array}$ & $\begin{array}{r}12 \\
16 \\
7 \\
8\end{array}$ & $\begin{array}{c}1 \\
1 \\
18 \\
28\end{array}$ & $\begin{array}{l}\overline{8} \\
7 \\
6\end{array}$ & $\begin{array}{c}1 \\
2 \\
13 \\
18\end{array}$ & $\begin{array}{c}\text { קule. } \\
7 \\
6\end{array}$ & $\begin{array}{r}0 \\
3 \\
25 \\
26\end{array}$ & $\begin{array}{r}\overline{10} \\
8 \\
8 \\
8\end{array}$ & $\begin{array}{r}0 \\
3 \\
26 \\
32\end{array}$ & $\begin{array}{l}\overline{8} \\
4 \\
6\end{array}$ & $\begin{array}{r}9 \\
24 \\
26\end{array}$ & $\begin{array}{l}8 \\
8 \\
4\end{array}$ & $\begin{array}{l}13 \\
13 \\
26 \\
17\end{array}$ & $\begin{array}{l}\overline{8} \\
8 \\
7\end{array}$ & $\begin{array}{r}3 \\
14 \\
33 \\
28\end{array}$ & $\begin{array}{l}8 \\
8 \\
8 \\
8\end{array}$ & 28 & $\begin{array}{l}\overline{8} \\
8 \\
\bar{i}\end{array}$ & $\begin{array}{l}18 \\
15 \\
26\end{array}$ & $\begin{array}{l} \\
6 \\
4\end{array}$ & $\begin{array}{l}0 \\
29 \\
13 \\
24\end{array}$ & $\begin{array}{l}\overline{6} \\
6 \\
6 \\
6\end{array}$ & $\begin{array}{l}1 \\
24 \\
15 \\
28\end{array}$ & $\begin{array}{l}\overline{6} \\
6 \\
4\end{array}$ & $\begin{array}{l}0 \\
26 \\
30 \\
20\end{array}$ \\
\hline 5 & $\begin{array}{r}4 \\
32 \\
-\end{array}$ & $\begin{array}{r}11 \\
6 \\
0\end{array}$ & $\begin{array}{r}4 \\
32 \\
-\end{array}$ & $\begin{array}{l}9 \\
7 \\
1\end{array}$ & $\begin{array}{l}2 \\
32 \\
-\end{array}$ & $\begin{array}{l}6 \\
8 \\
0\end{array}$ & $\overline{32}$ & $\begin{array}{l}1 \\
8 \\
0\end{array}$ & $\bar{z}$ & $\begin{array}{l}0 \\
3 \\
0\end{array}$ & $\overline{28}$ & $\begin{array}{l}0 \\
3 \\
0 \\
0\end{array}$ & $\begin{array}{l}32 \\
32 \\
\end{array}$ & $\begin{array}{l}3 \\
7 \\
0\end{array}$ & $\stackrel{32}{=}$ & $\begin{array}{l}9 \\
1 \\
0\end{array}$ & $\frac{32}{=}$ & & 38 & $\begin{array}{l}7 \\
0 \\
0\end{array}$ & $\frac{30}{=}$ & $\begin{array}{l}\bar{z} \\
0 \\
1\end{array}$ & $\frac{32}{=}$ & \\
\hline $\begin{array}{l}8 \\
9\end{array}$ & $\overline{12}$ & $\begin{array}{r}0 \\
11\end{array}$ & $\overline{10}$ & $\begin{array}{r}0 \\
0 \\
10\end{array}$ & $\overrightarrow{8}$ & $\begin{array}{l}0 \\
0\end{array}$ & $=$ & $\begin{array}{l}0 \\
2 \\
5\end{array}$ & $\frac{8}{-}$ & $\begin{array}{l}0 \\
2 \\
2\end{array}$ & $\bar{z}$ & $\begin{array}{l}0 \\
2 \\
1\end{array}$ & $=$ & $\begin{array}{l}1 \\
1\end{array}$ & $\overline{=}$ & ${ }_{0}^{0}$ & $\frac{20}{-}$ & $\begin{array}{l}2 \\
0\end{array}$ & $\underline{12}$ & $\begin{array}{l}{ }_{6}^{6} \\
0\end{array}$ & $\frac{12}{-}$ & $\begin{array}{r}10 \\
0\end{array}$ & $\underline{10}$ & 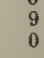 \\
\hline $\begin{array}{l}10 \\
11\end{array}$ & $\frac{5}{8}$ & $\begin{array}{l}15 \\
4\end{array}$ & $\begin{array}{l}{ }_{3}^{6} \\
32\end{array}$ & $\begin{array}{l}15 \\
6 \\
\end{array}$ & $\begin{array}{r}5 \\
32\end{array}$ & $\begin{array}{l}15 \\
10\end{array}$ & $\overline{32}$ & ${ }_{14}^{2}$ & $\overline{32}$ & $\begin{array}{r}12 \\
12\end{array}$ & 32 & $\begin{array}{l}0 \\
8 \\
8\end{array}$ & $\overline{8}$ & $\begin{array}{l}0 \\
1 \\
0\end{array}$ & $\overline{16}$ & $\begin{array}{l}0 \\
2 \\
9\end{array}$ & $\overline{7}$ & 0 & $\overline{8}$ & $\begin{array}{l}0 \\
6 \\
0\end{array}$ & $\overline{10}$ & $\stackrel{0}{7}$ & $\overline{15}$ & $\begin{array}{l}0 \\
14\end{array}$ \\
\hline $\begin{array}{l}12 \\
13 \\
14\end{array}$ & $\frac{8}{8}$ & $\begin{array}{c}16 \\
1 \\
19\end{array}$ & $\begin{array}{l}6 \\
4 \\
8\end{array}$ & $\begin{array}{c}17 \\
17 \\
21\end{array}$ & $\overline{8}$ & $\begin{array}{r}9 \\
0 \\
23\end{array}$ & $\overline{12}$ & $\begin{array}{r}3 \\
2 \\
20\end{array}$ & $\frac{28}{=}$ & $\begin{array}{l}2 \\
3 \\
2 \\
2\end{array}$ & $\frac{4}{8}$ & $\begin{array}{l}8 \\
\frac{1}{2} \\
2\end{array}$ & $\begin{array}{l}32 \\
16 \\
-\end{array}$ & $\begin{array}{l}9 \\
\stackrel{9}{2} \\
2\end{array}$ & $\overline{\overline{4}}$ & $\begin{array}{l}9 \\
0 \\
1\end{array}$ & Vule. & 1 & $\begin{array}{c}\overline{6} \\
10\end{array}$ & $\begin{array}{l}0 \\
9 \\
2\end{array}$ & $\frac{\overline{12}}{4}$ & $\begin{array}{l}0 \\
18 \\
10\end{array}$ & $\overline{10}$ & 0 \\
\hline $\begin{array}{l}15 \\
116\end{array}$ & 7 & $\begin{array}{l}23 \\
10\end{array}$ & $\begin{array}{l}9 \\
8 \\
0\end{array}$ & $\begin{array}{l}20 \\
25\end{array}$ & ${ }_{6}^{4}$ & $\begin{array}{l}22 \\
21\end{array}$ & $\begin{array}{l}6 \\
6 \\
6\end{array}$ & $\begin{array}{l}29 \\
29\end{array}$ & 4 & $\begin{array}{l}28 \\
14\end{array}$ & ${ }_{4}^{4}$ & $\begin{array}{l}28 \\
18\end{array}$ & $\frac{32}{6}$ & $\begin{array}{l}23 \\
14\end{array}$ & 7 & $\begin{array}{l}26 \\
15\end{array}$ & 8 & $\begin{array}{l}19 \\
11\end{array}$ & $\begin{array}{r}8 \\
10\end{array}$ & $\begin{array}{l}14 \\
12\end{array}$ & vole. & $\begin{array}{r}7 \\
10\end{array}$ & $=$ & $\frac{1}{5}$ \\
\hline $\begin{array}{l}17 \\
18 \\
19\end{array}$ & $\overline{\overline{32}}$ & $\begin{array}{r}2 \\
1 \\
13\end{array}$ & $\begin{array}{l}8 \\
4 \\
4\end{array}$ & $\begin{array}{r}4 \\
1 \\
10\end{array}$ & $\begin{array}{r}8 \\
7 \\
-\end{array}$ & $\begin{array}{l}3 \\
65 \\
5\end{array}$ & $\frac{12}{4}$ & $\begin{array}{l}2 \\
7 \\
3\end{array}$ & $\begin{array}{r}32 \\
12 \\
-\end{array}$ & $\begin{array}{r}3 \\
24 \\
2\end{array}$ & $\begin{array}{c}32 \\
8 \\
\text { vhle. }\end{array}$ & $\begin{array}{r}10 \\
13 \\
8\end{array}$ & $\begin{array}{r}32 \\
8 \\
30\end{array}$ & $\begin{array}{r}14 \\
36 \\
5\end{array}$ & $\begin{array}{r}32 \\
8 \\
6\end{array}$ & $\begin{array}{r}15 \\
18 \\
6\end{array}$ & $\begin{array}{c}10 \\
7 \\
7\end{array}$ & $\begin{array}{l}22 \\
24\end{array}$ & $\overline{4}$ & $\begin{array}{c}1 \\
13 \\
32\end{array}$ & $\begin{array}{r}4 \\
32 \\
4\end{array}$ & $\begin{array}{r}1 \\
6 \\
33\end{array}$ & $\begin{array}{c}16 \\
32 \\
0\end{array}$ & $\begin{array}{l}11 \\
11\end{array}$ \\
\hline${ }_{21}^{20}$ & 6 & ${ }_{29}^{29}$ & 8 & 24 & 8 & 9 & - & 0 & 4 & 18 & 4 & 18 & 4 & 14 & ${ }^{6}$ & 17 & 6 & $2 \pi$ & 4 & 20 & $u^{4}$ & 18 & 4 & 13 \\
\hline 22 & 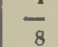 & 20 & ${ }^{*}$ & $\begin{array}{r}0 \\
0 \\
18\end{array}$ & $\frac{18}{18}$ & $\frac{1}{1}$ & $\sqrt{32}$ & $\frac{1}{7}$ & $\overline{28}$ & $\begin{array}{l}0 \\
4\end{array}$ & 28 & $\begin{array}{l}4 \\
6 \\
8\end{array}$ & $\bar{~}$ & 1 & $\frac{20}{1}$ & 1 & $\overline{12}$ & 1 & 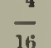 & $\begin{array}{l}1 \\
1\end{array}$ & $\frac{4}{7}$ & 36 & 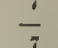 & \\
\hline 24 & is & 12 & $\begin{array}{l}y \\
0\end{array}$ & 11 & $\stackrel{8}{-}$ & $\begin{array}{l}5 \\
8\end{array}$ & $\stackrel{4}{-}$ & $\begin{array}{r}19 \\
3\end{array}$ & $\begin{array}{l}12 \\
32\end{array}$ & 13 & 16 & $\begin{array}{l}8 \\
9\end{array}$ & $\begin{array}{l}4 \\
9\end{array}$ & 18 & 8 & ${ }_{8}^{12}$ & 8 & 12 & $\begin{array}{r}10 \\
6\end{array}$ & 8 & 8 & $\begin{array}{l}10 \\
10\end{array}$ & $\dot{-}$ & 5 \\
\hline $\begin{array}{l}25 \\
26\end{array}$ & $\begin{array}{r}32 \\
4\end{array}$ & $\begin{array}{r}2 \\
12\end{array}$ & $\frac{32}{7}$ & $\begin{array}{r}4 \\
15\end{array}$ & $\begin{array}{r}32 \\
6\end{array}$ & $\begin{array}{r}8 \\
15\end{array}$ & ${ }_{6}^{4}$ & $\begin{array}{r}7 \\
16\end{array}$ & $\begin{array}{l}7 \\
4\end{array}$ & $\begin{array}{l}12 \\
14\end{array}$ & $\begin{array}{l}7 \\
8\end{array}$ & $\begin{array}{l}32 \\
9\end{array}$ & $\begin{array}{l}4 \\
8\end{array}$ & 24 & $\frac{4}{8}$ & $\begin{array}{c}26 \\
7\end{array}$ & $\begin{array}{l}6 \\
6\end{array}$ & $\begin{array}{l}17 \\
20\end{array}$ & $\overline{4}$ & $\begin{array}{r}9 \\
21\end{array}$ & $\frac{6}{9}$ & $\begin{array}{r}16 \\
9\end{array}$ & 4 & $\begin{array}{r}19 \\
0\end{array}$ \\
\hline $\begin{array}{l}28 \\
28 \\
29\end{array}$ & $\begin{array}{r}4 \\
8 \\
-\end{array}$ & $\begin{array}{r}10 \\
0 \\
0\end{array}$ & $\underline{10}$ & $\begin{array}{c}0 \\
10 \\
0\end{array}$ & $\begin{array}{r}4 \\
8 \\
-\end{array}$ & $\begin{array}{c}1 \\
10 \\
1\end{array}$ & $\overline{8}$ & $\begin{array}{c}3 \\
10 \\
0\end{array}$ & $\bar{z}$ & $\begin{array}{l}4 \\
6 \\
0 \\
0\end{array}$ & $\bar{z}$ & $\begin{array}{l}0 \\
1 \\
0\end{array}$ & 8 & $\begin{array}{l}0 \\
8 \\
1\end{array}$ & $\stackrel{4}{=}$ & $\begin{array}{l}3 \\
0 \\
0\end{array}$ & $\bar{z}$ & $\begin{array}{l}6 \\
2 \\
2 \\
0\end{array}$ & - & $\begin{array}{l}2 \\
1 \\
0\end{array}$ & $\frac{8}{-}$ & $\begin{array}{r}13 \\
1 \\
1\end{array}$ & $\underline{-}$ & 1 \\
\hline $\begin{array}{l}30 \\
31\end{array}$ & $=$ & ${ }_{0}^{2}$ & $\overline{8}$ & ${ }_{5}^{2}$ & $\begin{array}{r}10 \\
8\end{array}$ & $\begin{array}{r}4 \\
10\end{array}$ & $\overline{1 B}$ & $\begin{array}{r}0 \\
17\end{array}$ & 14 & $\begin{array}{r}0 \\
22\end{array}$ & $\overline{16}$ & $\begin{array}{r}0 \\
18\end{array}$ & $\overrightarrow{18}$ & ${ }_{31}^{9}$ & $\overline{12}$ & $\begin{array}{r}0 \\
19\end{array}$ & $\overline{12}$ & $\begin{array}{r}0 \\
14\end{array}$ & $\overrightarrow{12}$ & $\begin{array}{r}2 \\
15\end{array}$ & $\overline{9}$ & $\begin{array}{r}0 \\
18\end{array}$ & $\overline{8}$ & $\begin{array}{l}0 \\
19\end{array}$ \\
\hline tear & & 9 & & 9 & & 9 & & 8 & & 0 & & 8 & & 10 & & 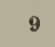 & & 9 & & 9 & & 9 & & g \\
\hline
\end{tabular}


TABLE IV. (continued).

WIND-DIRECTION AND VELOCITY.

Direction (D) in Numbers $(8=\mathrm{E}, 16=\mathrm{S}, 24=\mathrm{W}, 32=\mathrm{N})$, and Velocity (V) in miles per hour as recorded by the Cup Anemometer.

August, 1903.

\begin{tabular}{|c|c|c|c|c|c|c|c|c|c|c|c|c|c|c|c|c|c|c|c|c|c|c|c|c|}
\hline \multirow{2}{*}{ Day. } & \multicolumn{2}{|c|}{2.} & \multicolumn{2}{|c|}{4.} & \multicolumn{2}{|c|}{6.} & \multicolumn{2}{|c|}{8.} & \multicolumn{2}{|c|}{10.} & \multicolumn{2}{|c|}{ Noon. } & \multicolumn{2}{|c|}{14.} & \multicolumn{2}{|c|}{16.} & \multicolumn{2}{|c|}{18.} & \multicolumn{2}{|c|}{20.} & \multicolumn{2}{|c|}{22.} & \multicolumn{2}{|c|}{ Midt. } \\
\hline & D. & $\nabla$. & D. & v. & D. & $\mathbf{v}$ & D. & v. & D. & $\mathbf{v}$. & D. & $\nabla$. & D. & $\nabla$. & D. & $\mathbf{\nabla}$ & D. & v. & D. & V. & D. & $\Gamma$. & D. & V. \\
\hline $\begin{array}{l}1 \\
2 \\
3 \\
3\end{array}$ & $\begin{array}{r}7 \\
10 \\
6\end{array}$ & $\begin{array}{l}16 \\
29 \\
17\end{array}$ & $\begin{array}{r}6 \\
10 \\
-\end{array}$ & $\begin{array}{r}15 \\
28 \\
7\end{array}$ & $\begin{array}{r}4 \\
-\end{array}$ & $\begin{array}{r}18 \\
21 \\
0\end{array}$ & $\frac{4}{10}$ & $\begin{array}{l}23 \\
14 \\
15\end{array}$ & $\frac{\text { Vble. }}{8}$ & $\begin{array}{l}10 \\
4 \\
20\end{array}$ & $\begin{array}{c}\text { Vble. } \\
4 \\
8\end{array}$ & $\begin{array}{r}3 \\
8 \\
14\end{array}$ & $\begin{array}{c}\text { Vhile. } \\
6 \\
8 \\
8\end{array}$ & $\begin{array}{r}4 \\
15 \\
14\end{array}$ & $\begin{array}{l}1 \\
4 \\
8\end{array}$ & $\begin{array}{r}3 \\
18 \\
7\end{array}$ & $\begin{array}{l}1 \\
6 \\
8\end{array}$ & $\begin{array}{l}10 \\
14 \\
10\end{array}$ & $\begin{array}{l}8 \\
4 \\
4\end{array}$ & $\begin{array}{r}7 \\
15 \\
10\end{array}$ & $\begin{array}{r}10 \\
6 \\
4\end{array}$ & $\begin{array}{r}9 \\
14 \\
13\end{array}$ & $\begin{array}{l}8 \\
7 \\
1\end{array}$ & $\begin{array}{l}20 \\
15 \\
16\end{array}$ \\
\hline $\begin{array}{l}4 \\
5 \\
6 \\
7 \\
8\end{array}$ & $\begin{array}{c}16 \\
6 \\
6 \\
8\end{array}$ & $\begin{array}{r}8 \\
11 \\
0 \\
14 \\
10\end{array}$ & $\frac{4}{6}$ & $\begin{array}{r}3 \\
18 \\
0 \\
20 \\
14\end{array}$ & $\begin{array}{r}9 \\
\frac{9}{6} \\
6 \\
6\end{array}$ & $\begin{array}{r}5 \\
28 \\
0 \\
4 \\
10\end{array}$ & $\frac{8}{8}$ & $\begin{array}{r}3 \\
21 \\
0 \\
2 \\
17\end{array}$ & $\frac{6}{\frac{6}{8}}$ & $\begin{array}{r}9 \\
15 \\
0 \\
0 \\
12\end{array}$ & $\frac{4}{\text { vble. }}$ & $\begin{array}{r}13 \\
15 \\
0 \\
3 \\
14\end{array}$ & $\begin{array}{l}6 \\
4 \\
8 \\
8\end{array}$ & $\begin{array}{r}19 \\
12 \\
0 \\
3 \\
20\end{array}$ & $\frac{4}{32}$ & $\begin{array}{r}10 \\
8 \\
0 \\
2 \\
17\end{array}$ & $\frac{4}{\frac{2}{8}}$ & $\begin{array}{r}14 \\
1 \\
0 \\
7 \\
14\end{array}$ & $\frac{4}{30}$ & $\begin{array}{r}15 \\
0 \\
1 \\
5 \\
16\end{array}$ & $\frac{5}{\frac{8}{8}}$ & $\begin{array}{r}18 \\
0 \\
2 \\
2 \\
1\end{array}$ & $\begin{array}{r}8 \\
77 \\
12 \\
8\end{array}$ & $\begin{array}{r}11 \\
0 \\
1 \\
1 \\
14\end{array}$ \\
\hline $\begin{array}{r}9 \\
10 \\
11 \\
12 \\
13\end{array}$ & $\begin{array}{r}8 \\
8 \\
17 \\
8 \\
16\end{array}$ & $\begin{array}{r}18 \\
1 \\
31 \\
20 \\
9\end{array}$ & $\begin{array}{r}8 \\
14 \\
12 \\
20 \\
3\end{array}$ & $\begin{array}{r}20 \\
13 \\
24 \\
11 \\
5\end{array}$ & $\begin{array}{r}7 \\
12 \\
12 \\
32 \\
4\end{array}$ & $\begin{array}{r}23 \\
16 \\
9 \\
5 \\
15\end{array}$ & $\begin{array}{r}8 \\
15 \\
16 \\
20 \\
1\end{array}$ & $\begin{array}{l}32 \\
30 \\
16 \\
15 \\
14\end{array}$ & $\begin{array}{r}8 \\
16 \\
20 \\
16 \\
32\end{array}$ & $\begin{array}{l}23 \\
29 \\
35 \\
15 \\
10\end{array}$ & $\begin{array}{c}8 \\
16 \\
16 \\
16 \\
-\end{array}$ & $\begin{array}{r}33 \\
28 \\
33 \\
48 \\
3\end{array}$ & $\begin{array}{r}8 \\
18 \\
18 \\
18 \\
8\end{array}$ & $\begin{array}{r}24 \\
29 \\
22 \\
34 \\
2\end{array}$ & $\begin{array}{r}8 \\
16 \\
14 \\
10 \\
-\end{array}$ & $\begin{array}{r}27 \\
43 \\
43 \\
22 \\
0\end{array}$ & $\begin{array}{r}8 \\
20 \\
16 \\
6 \\
8\end{array}$ & $\begin{array}{r}14 \\
41 \\
38 \\
21 \\
3\end{array}$ & $\begin{array}{r}8 \\
16 \\
16 \\
4 \\
4\end{array}$ & $\begin{array}{r}10 \\
41 \\
29 \\
22 \\
6\end{array}$ & $\begin{array}{r}3 \\
18 \\
12 \\
7 \\
12\end{array}$ & $\begin{array}{l}3 \\
31 \\
25 \\
23 \\
13\end{array}$ & $\begin{array}{r}16 \\
16 \\
7 \\
6 \\
9\end{array}$ & $\begin{array}{l}1 \\
28 \\
24 \\
22 \\
18\end{array}$ \\
\hline $\begin{array}{l}14 \\
15 \\
16 \\
17 \\
18\end{array}$ & $\begin{array}{r}12 \\
16 \\
32 \\
14 \\
4\end{array}$ & $\begin{array}{r}19 \\
4 \\
5 \\
18 \\
10\end{array}$ & $\begin{array}{r}12 \\
8 \\
9 \\
10 \\
2\end{array}$ & $\begin{array}{r}18 \\
5 \\
4 \\
22 \\
10\end{array}$ & $\begin{array}{r}3 \\
8 \\
32 \\
6 \\
4\end{array}$ & $\begin{array}{r}13 \\
13 \\
5 \\
18 \\
4\end{array}$ & $\begin{array}{r}4 \\
6 \\
33 \\
6 \\
\end{array}$ & $\begin{array}{r}13 \\
17 \\
7 \\
23 \\
3\end{array}$ & $\begin{array}{r}16 \\
8 \\
8 \\
28\end{array}$ & $\begin{array}{r}6 \\
13 \\
1 \\
17 \\
4\end{array}$ & $\begin{array}{l}8 \\
8 \\
6 \\
30\end{array}$ & $\begin{array}{r}4 \\
5 \\
0 \\
19 \\
7\end{array}$ & $\begin{array}{l}32 \\
8 \\
8 \\
-\end{array}$ & $\begin{array}{r}3 \\
10 \\
0 \\
31 \\
9\end{array}$ & $\begin{array}{r}28 \\
6 \\
32 \\
8 \\
4\end{array}$ & $\begin{array}{r}7 \\
3 \\
2 \\
20 \\
4\end{array}$ & $\begin{array}{r}32 \\
8 \\
32 \\
8 \\
7\end{array}$ & $\begin{array}{r}9 \\
8 \\
4 \\
23 \\
14\end{array}$ & $\begin{array}{r}32 \\
32 \\
4 \\
4 \\
4\end{array}$ & $\begin{array}{r}9 \\
3 \\
2 \\
20 \\
14\end{array}$ & $\frac{32}{\frac{2}{2}}$ & $\begin{array}{r}11 \\
1 \\
2 \\
15 \\
16\end{array}$ & $\begin{array}{r}32 \\
32 \\
8 \\
4 \\
6\end{array}$ & $\begin{array}{r}4 \\
4 \\
13 \\
13 \\
26\end{array}$ \\
\hline $\begin{array}{l}19 \\
20 \\
21 \\
22 \\
23\end{array}$ & $\frac{6}{7}$ & $\begin{array}{r}29 \\
0 \\
2 \\
3 \\
9\end{array}$ & $\overline{7}$ & $\begin{array}{r}17 \\
0 \\
9 \\
15 \\
14\end{array}$ & $\begin{array}{c}32 \\
4 \\
16 \\
4\end{array}$ & $\begin{array}{r}8 \\
0 \\
9 \\
14 \\
15\end{array}$ & $\begin{array}{r}\frac{4}{4} \\
20 \\
8\end{array}$ & $\begin{array}{r}7 \\
0 \\
9 \\
19 \\
13\end{array}$ & $\frac{33}{\frac{73}{16}}$ & $\begin{array}{r}8 \\
0 \\
6 \\
17 \\
9\end{array}$ & $\begin{array}{l}\bar{Z} \\
32 \\
12 \\
12\end{array}$ & $\begin{array}{r}2 \\
0 \\
7 \\
11 \\
11\end{array}$ & $\overline{7}$ & $\begin{array}{r}2 \\
0 \\
3 \\
17 \\
15\end{array}$ & $\begin{array}{r}\bar{z} \\
\overline{8} \\
12\end{array}$ & $\begin{array}{r}0 \\
0 \\
0 \\
22 \\
19\end{array}$ & $\begin{array}{r}\overline{7} \\
10 \\
8 \\
8\end{array}$ & $\begin{array}{r}0 \\
0 \\
4 \\
24 \\
12\end{array}$ & $\frac{7}{8}$ & $\begin{array}{r}0 \\
2 \\
5 \\
24 \\
10\end{array}$ & $\bar{z}$ & $\begin{array}{r}0 \\
0 \\
1 \\
28 \\
22\end{array}$ & $\begin{array}{r}\overline{7} \\
32 \\
7 \\
7\end{array}$ & $\begin{array}{r}0 \\
2 \\
5 \\
17 \\
28\end{array}$ \\
\hline $\begin{array}{l}24 \\
25 \\
26 \\
27 \\
23\end{array}$ & $\begin{array}{r}6 \\
6 \\
-4 \\
-\end{array}$ & $\begin{array}{r}45 \\
1 \\
2 \\
21 \\
0\end{array}$ & $\begin{array}{r}6 \\
\frac{8}{8} \\
4 \\
8\end{array}$ & $\begin{array}{r}20 \\
3 \\
0 \\
24 \\
1\end{array}$ & $\frac{4}{4}$ & $\begin{array}{r}23 \\
2 \\
1 \\
15 \\
2\end{array}$ & $\begin{array}{c}\frac{4}{20} \\
\text { vible. } \\
10\end{array}$ & $\begin{array}{r}26 \\
0 \\
3 \\
12 \\
7\end{array}$ & $\begin{array}{r}\frac{8}{28} \\
4 \\
6\end{array}$ & $\begin{array}{r}13 \\
0 \\
2 \\
21 \\
15\end{array}$ & $\frac{4}{\text { vble. }}$ & $\begin{array}{r}10 \\
1 \\
8 \\
13 \\
12\end{array}$ & $\begin{array}{r}\frac{4}{16} \\
8 \\
10\end{array}$ & $\begin{array}{r}23 \\
0 \\
1 \\
4 \\
15\end{array}$ & $\frac{4}{\frac{4}{4}}$ & $\begin{array}{r}17 \\
1 \\
1 \\
8 \\
11\end{array}$ & $\frac{4}{\frac{4}{4}}$ & $\begin{array}{r}22 \\
0 \\
1 \\
10 \\
10\end{array}$ & $\begin{array}{r}4 \\
28 \\
8 \\
4 \\
4\end{array}$ & $\begin{array}{r}17 \\
1 \\
4 \\
17 \\
15\end{array}$ & $\begin{array}{r}28 \\
7 \\
32 \\
4\end{array}$ & $\begin{array}{r}8 \\
3 \\
9 \\
2 \\
25\end{array}$ & $\begin{array}{r}32 \\
4 \\
-\end{array}$ & $\frac{1}{7}$ \\
\hline $\begin{array}{l}29 \\
30 \\
31\end{array}$ & $\begin{array}{r}32 \\
4 \\
10\end{array}$ & $\begin{array}{r}1 \\
15 \\
5\end{array}$ & $\begin{array}{l}8 \\
4 \\
-\end{array}$ & $\begin{array}{l}3 \\
7 \\
4\end{array}$ & $\begin{array}{c}2 \\
\text { vile. } \\
10\end{array}$ & $\begin{array}{l}7 \\
8 \\
9\end{array}$ & $\begin{array}{l}6 \\
4 \\
4\end{array}$ & $\begin{array}{l}14 \\
15 \\
15\end{array}$ & $\begin{array}{r}4 \\
4 \\
12\end{array}$ & $\begin{array}{l}13 \\
10 \\
14\end{array}$ & $\begin{array}{l}4 \\
4 \\
8\end{array}$ & $\begin{array}{r}12 \\
10 \\
9\end{array}$ & $\frac{4}{32}$ & $\begin{array}{r}10 \\
2 \\
5\end{array}$ & $\frac{4}{16}$ & $\begin{array}{r}11 \\
1 \\
1\end{array}$ & $\frac{4}{31}$ & $\begin{array}{r}18 \\
5 \\
3\end{array}$ & $\frac{4}{=}$ & $\begin{array}{r}17 \\
5 \\
2\end{array}$ & $\underline{4}$ & $\begin{array}{r}16 \\
5 \\
1\end{array}$ & $\underline{4}$ & $\begin{array}{r}15 \\
0 \\
1\end{array}$ \\
\hline Mean & & 12 & & 11 & & 10 & & 13 & & 11 & & 12 & & 11 & & 11 & & 11 & & 11 & & 10 & & 10 \\
\hline
\end{tabular}

September, 1903.

\begin{tabular}{|c|c|c|c|c|c|c|c|c|c|c|c|c|c|c|c|c|c|c|c|c|c|c|c|c|}
\hline \multirow{2}{*}{ Day. } & \multicolumn{2}{|c|}{2.} & \multicolumn{2}{|c|}{4.} & \multicolumn{2}{|c|}{$\theta}$. & \multicolumn{2}{|c|}{8.} & \multicolumn{2}{|c|}{10.} & \multicolumn{2}{|c|}{ Noon. } & \multicolumn{2}{|c|}{14.} & \multicolumn{2}{|c|}{16.} & \multicolumn{2}{|c|}{18.} & \multicolumn{2}{|c|}{20.} & \multicolumn{2}{|c|}{22.} & \multicolumn{2}{|c|}{ Midt. } \\
\hline & D. & $\mathbf{v}$ & D. & $\nabla$. & D. & v. & D. & v. & D. & $\nabla$. & D. & v. & D. & v. & D. & v. & D. & $\mathrm{v}$. & D. & v. & D. & v. & D. & r. \\
\hline$\frac{1}{2}$ & $=$ & $\begin{array}{l}0 \\
0\end{array}$ & $\begin{array}{r}6 \\
16\end{array}$ & $\begin{array}{l}5 \\
3\end{array}$ & $\begin{array}{r}4 \\
12\end{array}$ & $\begin{array}{r}16 \\
4\end{array}$ & $\begin{array}{r}6 \\
10\end{array}$ & $\begin{array}{l}22 \\
16\end{array}$ & 8 & $\begin{array}{l}21 \\
18\end{array}$ & $\begin{array}{l}32 \\
12\end{array}$ & $\begin{array}{r}7 \\
15\end{array}$ & $\begin{array}{r}32 \\
6\end{array}$ & $\begin{array}{r}6 \\
19\end{array}$ & $\begin{array}{r}32 \\
4\end{array}$ & $\begin{array}{l}11 \\
18\end{array}$ & $-\overline{4}$ & $\begin{array}{r}0 \\
13\end{array}$ & $\overline{32}$ & $\frac{1}{3}$ & $\begin{array}{r}30 \\
4\end{array}$ & $\begin{array}{l}6 \\
6\end{array}$ & $-\overline{4}$ & $\begin{array}{r}0 \\
33\end{array}$ \\
\hline $\begin{array}{l}3 \\
4 \\
5 \\
6 \\
7\end{array}$ & $\frac{4}{\frac{4}{17}}$ & $\begin{array}{r}23 \\
11 \\
5 \\
9 \\
4\end{array}$ & $\begin{array}{r}6 \\
8 \\
8 \\
32\end{array}$ & $\begin{array}{r}28 \\
11 \\
0 \\
7 \\
5\end{array}$ & $\begin{array}{r}6 \\
\frac{15}{7} \\
32\end{array}$ & $\begin{array}{c}23 \\
8 \\
0 \\
10 \\
10\end{array}$ & $\frac{6}{\frac{6}{10}}$ & $\begin{array}{r}28 \\
0 \\
0 \\
15 \\
4\end{array}$ & $\begin{array}{r}4 \\
\frac{4}{12} \\
12\end{array}$ & $\begin{array}{r}16 \\
1 \\
0 \\
13 \\
9\end{array}$ & $\begin{array}{r}6 \\
4 \\
8 \\
12\end{array}$ & $\begin{array}{r}13 \\
10 \\
0 \\
14 \\
11\end{array}$ & $\begin{array}{r}8 \\
4 \\
8 \\
12\end{array}$ & $\begin{array}{r}8 \\
22 \\
0 \\
15 \\
17\end{array}$ & $\begin{array}{r}8 \\
4 \\
10 \\
10 \\
12\end{array}$ & $\begin{array}{r}5 \\
19 \\
1 \\
11 \\
15\end{array}$ & $\begin{array}{r}8 \\
4 \\
6 \\
12 \\
10\end{array}$ & $\begin{array}{l}12 \\
18 \\
11 \\
10 \\
12\end{array}$ & $\begin{array}{l}8 \\
4 \\
4 \\
8 \\
7\end{array}$ & $\begin{array}{r}6 \\
14 \\
23 \\
5 \\
10\end{array}$ & $\begin{array}{r}8 \\
14 \\
4 \\
8 \\
2\end{array}$ & $\begin{array}{r}11 \\
9 \\
18 \\
6 \\
5\end{array}$ & $\begin{array}{c}10 \\
12 \\
8 \\
8 \text { rile. } \\
2\end{array}$ & $\begin{array}{r}10 \\
7 \\
10 \\
3 \\
7\end{array}$ \\
\hline $\begin{array}{r}8 \\
9 \\
10 \\
11 \\
12\end{array}$ & $\begin{array}{r}32 \\
8 \\
28 \\
8 \\
10\end{array}$ & $\begin{array}{r}16 \\
5 \\
2 \\
20 \\
17\end{array}$ & $\begin{array}{r}32 \\
8 \\
14 \\
4 \\
12\end{array}$ & $\begin{array}{r}18 \\
11 \\
2 \\
15 \\
13\end{array}$ & $\begin{array}{r}32 \\
4 \\
4 \\
7 \\
10\end{array}$ & $\begin{array}{r}9 \\
20 \\
3 \\
21 \\
13\end{array}$ & $\frac{32}{8}$ & $\begin{array}{r}9 \\
24 \\
0 \\
35 \\
7\end{array}$ & $\begin{array}{r}32 \\
8 \\
8 \\
4\end{array}$ & $\begin{array}{r}8 \\
23 \\
0 \\
18 \\
14\end{array}$ & $\begin{array}{r}31 \\
8 \\
2 \\
4\end{array}$ & $\begin{array}{r}9 \\
20 \\
0 \\
26 \\
15\end{array}$ & $\begin{array}{l}- \\
6 \\
4 \\
8 \\
4\end{array}$ & $\begin{array}{r}8 \\
23 \\
7 \\
26 \\
13\end{array}$ & $\begin{array}{r}-8 \\
6 \\
8 \\
32\end{array}$ & $\begin{array}{r}0 \\
17 \\
13 \\
15 \\
9\end{array}$ & $\begin{array}{r}-8 \\
8 \\
10 \\
-\end{array}$ & $\begin{array}{r}0 \\
17 \\
10 \\
14 \\
4\end{array}$ & $\begin{array}{r}-8 \\
4 \\
8 \\
-\end{array}$ & $\begin{array}{r}2 \\
16 \\
13 \\
16 \\
2\end{array}$ & $\begin{array}{r}4 \\
6 \\
8 \\
10 \\
-\end{array}$ & $\begin{array}{r}6 \\
13 \\
15 \\
15 \\
0\end{array}$ & $\begin{array}{r}4 \\
31 \\
8 \\
12 \\
-.\end{array}$ & $\begin{array}{r}11 \\
10 \\
20 \\
13 \\
4\end{array}$ \\
\hline $\begin{array}{l}13 \\
14 \\
15 \\
16 \\
17\end{array}$ & $\frac{-6}{8}$ & $\begin{array}{r}1 \\
21 \\
0 \\
24 \\
19\end{array}$ & $\begin{array}{r}8 \\
6 \\
8 \\
4\end{array}$ & $\begin{array}{r}3 \\
23 \\
0 \\
15 \\
21\end{array}$ & $\begin{array}{r}8 \\
6 \\
8 \\
4\end{array}$ & $\begin{array}{r}9 \\
18 \\
0 \\
26 \\
10\end{array}$ & $\begin{array}{r}9 \\
8 \\
10 \\
8 \\
4\end{array}$ & $\begin{array}{r}9 \\
16 \\
1 \\
22 \\
6\end{array}$ & $\begin{array}{l}9 \\
6 \\
9 \\
7 \\
2\end{array}$ & $\begin{array}{r}11 \\
18 \\
7 \\
25 \\
20\end{array}$ & $\begin{array}{l}6 \\
4 \\
4 \\
4 \\
4\end{array}$ & $\begin{array}{r}16 \\
16 \\
5 \\
23 \\
17\end{array}$ & $\begin{array}{l}8 \\
4 \\
6 \\
6 \\
8\end{array}$ & $\begin{array}{l}16 \\
23 \\
14 \\
29 \\
20\end{array}$ & $\begin{array}{l}8 \\
4 \\
6 \\
6 \\
6\end{array}$ & $\begin{array}{l}14 \\
14 \\
13 \\
26 \\
21\end{array}$ & $\begin{array}{l}8 \\
4 \\
8 \\
4 \\
6\end{array}$ & $\begin{array}{r}21 \\
14 \\
9 \\
28 \\
22\end{array}$ & $\begin{array}{l}7 \\
4 \\
4 \\
4 \\
4\end{array}$ & $\begin{array}{l}11 \\
13 \\
10 \\
32 \\
25\end{array}$ & $\begin{array}{l}8 \\
7 \\
9 \\
4 \\
4\end{array}$ & $\begin{array}{r}25 \\
9 \\
14 \\
33 \\
20\end{array}$ & $\begin{array}{l}\frac{7}{8} \\
4 \\
4\end{array}$ & $\begin{array}{r}22 \\
0 \\
10 \\
24 \\
20\end{array}$ \\
\hline $\begin{array}{l}18 \\
19 \\
220 \\
21 \\
22\end{array}$ & $\frac{4}{\frac{4}{8}}$ & $\begin{array}{r}26 \\
8 \\
0 \\
0 \\
9\end{array}$ & $\frac{4}{\frac{4}{10}}$ & $\begin{array}{r}6 \\
6 \\
0 \\
0 \\
15\end{array}$ & $\frac{4}{\frac{4}{8}}$ & $\begin{array}{r}8 \\
6 \\
2 \\
0 \\
14\end{array}$ & $\frac{\overline{8}}{\overline{12}}$ & $\begin{array}{r}4 \\
11 \\
0 \\
0 \\
16\end{array}$ & $\frac{\overline{28}}{\overline{8}}$ & $\begin{array}{r}0 \\
14 \\
0 \\
0 \\
14\end{array}$ & $\frac{32}{\frac{7}{8}}$ & $\begin{array}{l}2 \\
0 \\
0 \\
2 \\
8\end{array}$ & $\frac{4}{14}$ & $\begin{array}{r}0 \\
10 \\
0 \\
8 \\
8\end{array}$ & $\frac{7}{\frac{9}{8}}$ & $\begin{array}{l}0 \\
2 \\
0 \\
6 \\
7\end{array}$ & $\frac{7}{9}$ & $\begin{array}{l}0 \\
0 \\
1 \\
8 \\
0\end{array}$ & $\frac{32}{\frac{7}{8}}$ & $\begin{array}{r}1 \\
1 \\
0 \\
13 \\
3\end{array}$ & $\frac{32}{\frac{6}{6}}$ & $\begin{array}{r}1 \\
0 \\
0 \\
11 \\
1\end{array}$ & $\frac{-}{8}$ & $\begin{array}{l}2 \\
0 \\
0 \\
8 \\
4\end{array}$ \\
\hline $\begin{array}{l}23 \\
24 \\
25 \\
25 \\
27\end{array}$ & $\begin{array}{r}31 \\
10 \\
32 \\
28 \\
4\end{array}$ & $\begin{array}{r}1 \\
22 \\
6 \\
7 \\
15\end{array}$ & 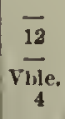 & $\begin{array}{r}1 \\
18 \\
4 \\
5 \\
30\end{array}$ & $\begin{array}{c}\text { Vible. } \\
16 \\
30 \\
18 \\
4\end{array}$ & $\begin{array}{r}3 \\
14 \\
5 \\
15 \\
34\end{array}$ & $\begin{array}{r}-8 \\
32 \\
16 \\
4\end{array}$ & $\begin{array}{r}3 \\
27 \\
10 \\
14 \\
32\end{array}$ & $\begin{array}{r}8 \\
8 \\
32 \\
12 \\
4\end{array}$ & $\begin{array}{r}4 \\
18 \\
12 \\
17 \\
24\end{array}$ & $\begin{array}{r}16 \\
8 \\
32 \\
14 \\
4\end{array}$ & $\begin{array}{r}9 \\
20 \\
8 \\
10 \\
20\end{array}$ & $\begin{array}{r}16 \\
8 \\
32 \\
15 \\
32\end{array}$ & $\begin{array}{r}24 \\
16 \\
14 \\
11 \\
7\end{array}$ & $\begin{array}{r}14 \\
8 \\
32 \\
20 \\
20\end{array}$ & $\begin{array}{r}11 \\
12 \\
18 \\
9 \\
3\end{array}$ & $\begin{array}{r}12 \\
8 \\
4 \\
32 \\
-\end{array}$ & $\begin{array}{r}15 \\
7 \\
19 \\
5 \\
1\end{array}$ & $\begin{array}{r}8 \\
4 \\
6 \\
32 \\
-\end{array}$ & $\begin{array}{r}20 \\
8 \\
20 \\
4 \\
0\end{array}$ & $\begin{array}{r}9 \\
12 \\
10 \\
-\end{array}$ & $\begin{array}{r}23 \\
11 \\
21 \\
4 \\
2\end{array}$ & $\begin{array}{r}10 \\
28 \\
32 \\
4 \\
14\end{array}$ & $\begin{array}{r}20 \\
5 \\
7 \\
9 \\
2\end{array}$ \\
\hline $\begin{array}{l}28 \\
29 \\
30\end{array}$ & $\begin{array}{r}12 \\
10 \\
4\end{array}$ & $\begin{array}{l}11 \\
20 \\
17\end{array}$ & $\begin{array}{r}15 \\
7 \\
4\end{array}$ & $\begin{array}{r}9 \\
14 \\
23\end{array}$ & $\begin{array}{c}12 \\
6 \\
6\end{array}$ & $\begin{array}{l}19 \\
24 \\
21\end{array}$ & $\begin{array}{r}16 \\
6 \\
4\end{array}$ & $\begin{array}{l}17 \\
22 \\
14\end{array}$ & $\begin{array}{r}16 \\
8 \\
-\end{array}$ & $\begin{array}{r}24 \\
9 \\
4\end{array}$ & $\frac{16}{2}$ & $\begin{array}{r}26 \\
2 \\
10\end{array}$ & $\begin{array}{l}14 \\
32 \\
-\end{array}$ & $\begin{array}{r}29 \\
3 \\
4\end{array}$ & $\begin{array}{l}12 \\
28 \\
-\end{array}$ & $\begin{array}{r}18 \\
8 \\
0\end{array}$ & $\frac{8}{32}$ & $\begin{array}{r}28 \\
4 \\
4\end{array}$ & $\begin{array}{r}8 \\
32 \\
-\end{array}$ & $\begin{array}{r}22 \\
2 \\
6\end{array}$ & $\begin{array}{r}7 \\
2 \\
32\end{array}$ & $\begin{array}{r}24 \\
2 \\
8\end{array}$ & $\begin{array}{r}8 \\
4 \\
30\end{array}$ & $\begin{array}{c}21 \\
18 \\
7\end{array}$ \\
\hline Nean . . & & 11 & & 10 & & 12 & & 13 & & 12 & & 11 & & 14 & & 10 & & 10 & & 10 & & 11 & & 10 \\
\hline
\end{tabular}


TABLE IV. (continued).

WIND-DIRECTION AND VEIOCITY.

Direction (D) in Numbers $(8=\mathrm{E}, 16=\mathrm{S}, 24=\mathrm{W}, 32=\mathrm{N})$, and Velocity (V) in miles per hour âs recorded by the Cup Anemometer.

October, 1903.

\begin{tabular}{|c|c|c|c|c|c|c|c|c|c|c|c|c|c|c|c|c|c|c|c|c|c|c|c|c|}
\hline \multirow{2}{*}{ Das. } & \multicolumn{2}{|c|}{2.} & \multicolumn{2}{|c|}{4.} & \multicolumn{2}{|c|}{6.} & \multicolumn{2}{|c|}{8.} & \multicolumn{2}{|c|}{10.} & \multicolumn{2}{|c|}{ Noon. } & \multicolumn{2}{|c|}{14.} & \multicolumn{2}{|c|}{16.} & \multicolumn{2}{|c|}{18} & \multicolumn{2}{|c|}{20.} & \multicolumn{2}{|c|}{22.} & \multicolumn{2}{|c|}{ Iidt. } \\
\hline & D. & v. & v. & $\nabla$. & D. & v. & D. & $\nabla$. & D. & $\nabla$. & D. & $\nabla$. & D. & v. & D. & v. & D. & $\nabla$. & D. & $\mathbf{r}:$ & D. & $\nabla$. & D. & v. \\
\hline $\begin{array}{l}1 \\
2\end{array}$ & $\begin{array}{l}3 \\
4\end{array}$ & $\begin{array}{r}3 \\
18\end{array}$ & $\begin{array}{l}5 \\
6\end{array}$ & $\begin{array}{l}15 \\
18\end{array}$ & $\begin{array}{l}4 \\
6\end{array}$ & $\begin{array}{l}22 \\
20\end{array}$ & 8 & $\begin{array}{l}23 \\
26\end{array}$ & $\begin{array}{l}4 \\
8\end{array}$ & $\begin{array}{l}21 \\
19\end{array}$ & $\begin{array}{l}4 \\
8\end{array}$ & $\begin{array}{l}19 \\
22\end{array}$ & $\begin{array}{l}4 \\
8\end{array}$ & $\begin{array}{l}23 \\
24\end{array}$ & $\begin{array}{l}6 \\
8\end{array}$ & $\begin{array}{l}22 \\
21\end{array}$ & $\begin{array}{l}4 \\
8\end{array}$ & $\begin{array}{l}22 \\
26\end{array}$ & $\begin{array}{l}8 \\
8\end{array}$ & $\begin{array}{l}22 \\
26\end{array}$ & 4 & $\begin{array}{l}19 \\
23\end{array}$ & 4 & $\begin{array}{l}21 \\
24\end{array}$ \\
\hline $\begin{array}{l}3 \\
4 \\
5 \\
8 \\
7\end{array}$ & $\begin{array}{r}8 \\
4 \\
30 \\
32 \\
4\end{array}$ & $\begin{array}{r}28 \\
26 \\
2 \\
5 \\
1\end{array}$ & $\begin{array}{r}8 \\
4 \\
\text { vale. } \\
4\end{array}$ & $\begin{array}{r}23 \\
24 \\
1 \\
4 \\
13\end{array}$ & $\begin{array}{r}8 \\
4 \\
-4\end{array}$ & $\begin{array}{r}28 \\
22 \\
0 \\
2 \\
21\end{array}$ & $\begin{array}{r}8 \\
4 \\
4 \\
4\end{array}$ & $\begin{array}{r}29 \\
20 \\
0 \\
2 \\
22\end{array}$ & $\begin{array}{r}8 \\
4 \\
32 \\
4 \\
4\end{array}$ & $\begin{array}{r}27 \\
29 \\
3 \\
13 \\
19\end{array}$ & $\begin{array}{r}8 \\
4 \\
32 \\
4 \\
-\end{array}$ & $\begin{array}{r}26 \\
21 \\
4 \\
7 \\
10\end{array}$ & $\begin{array}{r}6 \\
\frac{4}{16} \\
\frac{16}{32}\end{array}$ & $\begin{array}{r}26 \\
27 \\
1 \\
5 \\
3\end{array}$ & $\begin{array}{r}8 \\
4 \\
32 \\
=\end{array}$ & $\begin{array}{r}24 \\
20 \\
9 \\
1 \\
0\end{array}$ & $\begin{array}{r}8 \\
4 \\
32 \\
4 \\
-\end{array}$ & $\begin{array}{r}21 \\
18 \\
9 \\
3 \\
1\end{array}$ & $\begin{array}{c}4 \\
4 \\
32 \\
32\end{array}$ & $\begin{array}{r}23 \\
16 \\
12 \\
4 \\
3\end{array}$ & $\begin{array}{r}4 \\
32 \\
28 \\
8 \\
4\end{array}$ & $\begin{array}{r}26 \\
9 \\
11 \\
1 \\
3\end{array}$ & $\frac{4}{32}$ & $\begin{array}{r}20 \\
1 \\
6 \\
4 \\
7\end{array}$ \\
\hline $\begin{array}{r}8 \\
9 \\
10 \\
11 \\
12\end{array}$ & $\begin{array}{r}\frac{7}{4} \\
20 \\
9\end{array}$ & $\begin{array}{c}13 \\
0 \\
11 \\
1 \\
10\end{array}$ & $\begin{array}{r}\frac{7}{7} \\
12 \\
7\end{array}$ & $\begin{array}{r}12 \\
1 \\
11 \\
4 \\
22\end{array}$ & $\begin{array}{r}\overline{32} \\
4 \\
17 \\
7\end{array}$ & $\begin{array}{r}1 \\
2 \\
21 \\
8 \\
18\end{array}$ & $\frac{-}{4}$ & $\begin{array}{r}3 \\
0 \\
19 \\
31 \\
24\end{array}$ & $\begin{array}{r}8 \\
4 \\
4 \\
16 \\
6\end{array}$ & $\begin{array}{r}3 \\
1 \\
20 \\
35 \\
18\end{array}$ & $\begin{array}{r}4 \\
2 \\
4 \\
12 \\
8\end{array}$ & $\begin{array}{l}13 \\
13 \\
16 \\
27 \\
22\end{array}$ & $\frac{4}{2}$ & $\begin{array}{r}16 \\
12 \\
4 \\
26 \\
20\end{array}$ & $\overline{\overline{7}}$ & $\begin{array}{r}3 \\
8 \\
1 \\
22 \\
14\end{array}$ & $\begin{array}{r}28 \\
32 \\
12 \\
8\end{array}$ & $\begin{array}{r}1 \\
1 \\
2 \\
21 \\
7\end{array}$ & $\frac{-}{\overline{8}}$ & $\begin{array}{r}0 \\
0 \\
\cdot \quad 1 \\
17 \\
23\end{array}$ & $\begin{array}{l}\overline{30} \\
8 \\
4\end{array}$ & $\begin{array}{r}0 \\
2 \\
1 \\
23 \\
29\end{array}$ & $\frac{-}{8}$ & $\begin{array}{r}0 \\
0 \\
2 \\
19 \\
26\end{array}$ \\
\hline $\begin{array}{l}13 \\
11 \\
15 \\
16 \\
17\end{array}$ & $\frac{4}{-8}$ & $\begin{array}{r}27 \\
16 \\
7 \\
15 \\
0\end{array}$ & $\begin{array}{r}\frac{4}{6} \\
6 \\
28\end{array}$ & $\begin{array}{r}19 \\
8 \\
7 \\
16 \\
1\end{array}$ & $\begin{array}{r}10 \\
12 \\
6 \\
8 \\
-\end{array}$ & $\begin{array}{r}17 \\
2 \\
12 \\
22 \\
0\end{array}$ & $\begin{array}{r}10 \\
12 \\
4 \\
8 \\
-\end{array}$ & $\begin{array}{r}11 \\
14 \\
35 \\
21 \\
0\end{array}$ & $\begin{array}{r}8 \\
16 \\
6 \\
6 \\
-\end{array}$ & $\begin{array}{r}9 \\
15 \\
17 \\
13 \\
0\end{array}$ & $\begin{array}{r}12 \\
20 \\
4 \\
6 \\
8\end{array}$ & $\begin{array}{r}12 \\
14 \\
16 \\
15 \\
8\end{array}$ & $\begin{array}{r}12 \\
12 \\
4 \\
8 \\
8\end{array}$ & $\begin{array}{l}13 \\
22 \\
18 \\
13 \\
12\end{array}$ & $\begin{array}{r}8 \\
12 \\
4 \\
4 \\
8\end{array}$ & $\begin{array}{r}9 \\
21 \\
15 \\
18 \\
11\end{array}$ & $\begin{array}{r}-\overline{8} \\
\frac{8}{6}\end{array}$ & $\begin{array}{r}5 \\
18 \\
10 \\
5 \\
15\end{array}$ & $\begin{array}{r}16 \\
8 \\
4 \\
4\end{array}$ & $\begin{array}{r}5 \\
10 \\
7 \\
0 \\
10\end{array}$ & $\begin{array}{c}12 \\
8 \\
12 \\
4\end{array}$ & $\begin{array}{r}9 \\
16 \\
13 \\
0 \\
14\end{array}$ & $\begin{array}{r}14 \\
8 \\
12 \\
4\end{array}$ & $\begin{array}{r}9 \\
10 \\
15 \\
0 \\
13\end{array}$ \\
\hline $\begin{array}{l}18 \\
19 \\
21 \\
21 \\
22\end{array}$ & $\begin{array}{r}\frac{6}{32} \\
2 \\
8\end{array}$ & $\begin{array}{r}14 \\
0 \\
8 \\
3 \\
17\end{array}$ & $\frac{6}{33}$ & $\begin{array}{r}11 \\
0 \\
5 \\
1 \\
17\end{array}$ & $\frac{\frac{8}{28}}{8}$ & $\begin{array}{r}7 \\
1 \\
2 \\
0 \\
18\end{array}$ & $\frac{4}{\frac{20}{6}}$ & $\begin{array}{r}8 \\
0 \\
4 \\
1 \\
13\end{array}$ & $\frac{\frac{4}{32}}{8}$ & $\begin{array}{r}16 \\
1 \\
1 \\
0 \\
16\end{array}$ & $\frac{4}{4}$ & $\begin{array}{r}20 \\
0 \\
2 \\
2 \\
16\end{array}$ & $\frac{\frac{4}{28}}{4}$ & $\begin{array}{r}18 \\
0 \\
8 \\
0 \\
23\end{array}$ & $\frac{\frac{4}{30}}{8}$ & $\begin{array}{r}14 \\
0 \\
8 \\
1 \\
18\end{array}$ & $\frac{4}{\frac{1}{8}}$ & $\begin{array}{r}14 \\
0 \\
4 \\
1 \\
18\end{array}$ & $\frac{32}{\overline{8}}$ & $\begin{array}{r}4 \\
0 \\
1 \\
0 \\
14\end{array}$ & $\overline{\bar{z}}$ & $\begin{array}{r}5 \\
0 \\
0 \\
1 \\
.21\end{array}$ & $\frac{\bar{z}}{\frac{12}{8}}$ & $\begin{array}{r}0 \\
0 \\
3 \\
7 \\
21\end{array}$ \\
\hline $\begin{array}{l}23 \\
21 \\
25 \\
23 \\
27\end{array}$ & $\begin{array}{l}6 \\
4 \\
8 \\
8 \\
8\end{array}$ & $\begin{array}{r}20 \\
15 \\
8 \\
1 \\
10\end{array}$ & $\begin{array}{r}8 \\
4 \\
8 \\
12\end{array}$ & $\begin{array}{r}24 \\
18 \\
6 \\
1 \\
10\end{array}$ & $\frac{\frac{7}{6}}{4}$ & $\begin{array}{r}26 \\
17 \\
9 \\
0 \\
7\end{array}$ & $\begin{array}{r}4 \\
14 \\
8 \\
12\end{array}$ & $\begin{array}{r}27 \\
7 \\
7 \\
1 \\
6\end{array}$ & $\frac{8}{\frac{8}{8}}$ & $\begin{array}{r}21 \\
8 \\
4 \\
2 \\
13\end{array}$ & $\begin{array}{r}4 \\
8 \\
8 \\
8 \\
12\end{array}$ & $\begin{array}{r}22 \\
11 \\
3 \\
6 \\
9\end{array}$ & $\begin{array}{r}\frac{8}{12} \\
8 \\
12\end{array}$ & $\begin{array}{r}26 \\
15 \\
4 \\
2 \\
12\end{array}$ & $\begin{array}{c}\frac{8}{12} \\
\frac{12}{10} \\
10\end{array}$ & $\begin{array}{r}21 \\
22 \\
0 \\
8 \\
10\end{array}$ & $\frac{8}{12}$ & $\begin{array}{c}18 \\
15 \\
0 \\
13 \\
11\end{array}$ & $\frac{8}{12}$ & $\begin{array}{r}15 \\
13 \\
0 \\
17 \\
11\end{array}$ & $\begin{array}{r}10 \\
\frac{9}{6} \\
12\end{array}$ & $\begin{array}{c}13 \\
14 \\
0 \\
14 \\
14\end{array}$ & $\frac{8}{\frac{8}{12}}$ & $\begin{array}{r}14 \\
15 \\
1 \\
13 \\
15\end{array}$ \\
\hline $\begin{array}{l}28 \\
29 \\
30 \\
31\end{array}$ & $\begin{array}{r}8 \\
8 \\
9 \\
10\end{array}$ & $\begin{array}{r}9 \\
8 \\
8 \\
12\end{array}$ & $\begin{array}{r}8 \\
12 \\
7\end{array}$ & $\begin{array}{l}17 \\
15 \\
4 \\
12\end{array}$ & $\begin{array}{l}12 \\
6 \\
14 \\
12\end{array}$ & $\begin{array}{r}18 \\
18 \\
1 \\
10\end{array}$ & $\begin{array}{r}12 \\
6 \\
12 \\
12\end{array}$ & $\begin{array}{l}19 \\
15 \\
14 \\
11\end{array}$ & $\begin{array}{r}10 \\
6 \\
12 \\
16\end{array}$ & $\begin{array}{l}19 \\
16 \\
19 \\
12\end{array}$ & $\begin{array}{r}4 \\
4 \\
12 \\
16\end{array}$ & $\begin{array}{l}19 \\
21 \\
17 \\
10\end{array}$ & $\begin{array}{r}6 \\
8 \\
8 \\
16\end{array}$ & $\begin{array}{l}18 \\
20 \\
13 \\
21\end{array}$ & $\begin{array}{r}8 \\
7 \\
8 \\
16\end{array}$ & $\begin{array}{l}21 \\
15 \\
14 \\
16\end{array}$ & $\begin{array}{r}8 \\
7 \\
8 \\
18\end{array}$ & $\begin{array}{l}20 \\
14 \\
15 \\
22\end{array}$ & $\begin{array}{r}8 \\
4 \\
8 \\
20\end{array}$ & $\begin{array}{r}9 \\
10 \\
11 \\
24\end{array}$ & $\begin{array}{r}12 \\
4 \\
8 \\
20\end{array}$ & $\begin{array}{r}8 \\
8 \\
9 \\
24\end{array}$ & $\begin{array}{r}10 \\
8 \\
12 \\
20\end{array}$ & $\begin{array}{r}11 \\
7 \\
6 \\
26\end{array}$ \\
\hline Mean & & 10 & & 11 & & 11 & & 13 & & 13 & & 14 & & 14 & & 12 & & 11 & & 10 & & 11. & . & 10 \\
\hline
\end{tabular}

\section{November, 1903}

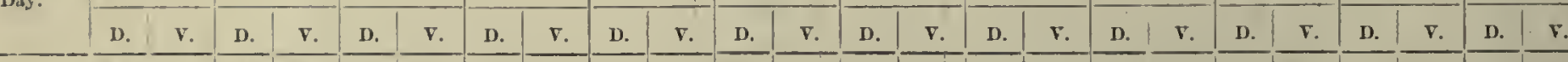

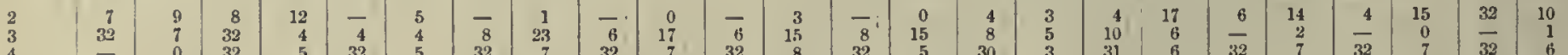

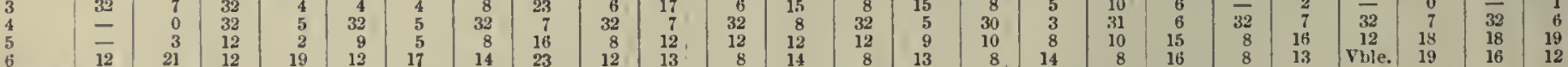

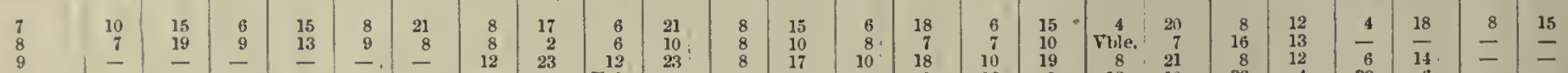

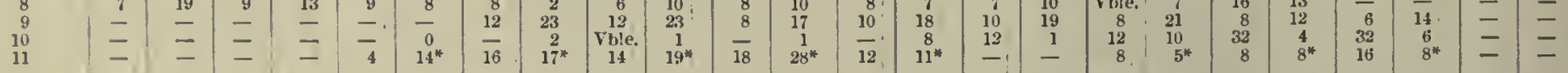

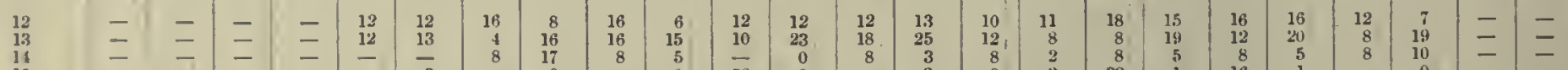

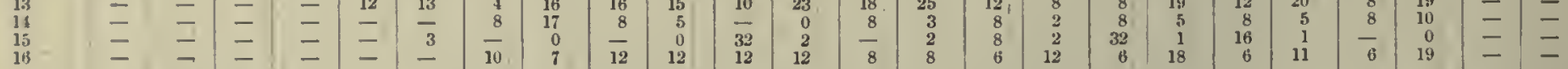

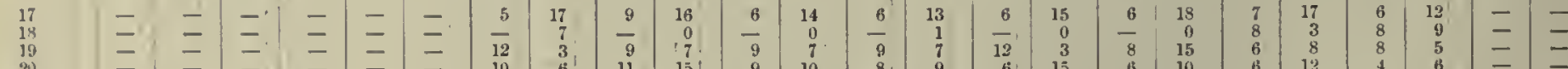

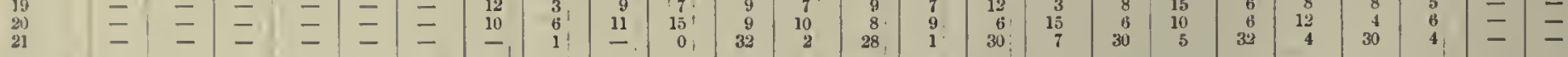

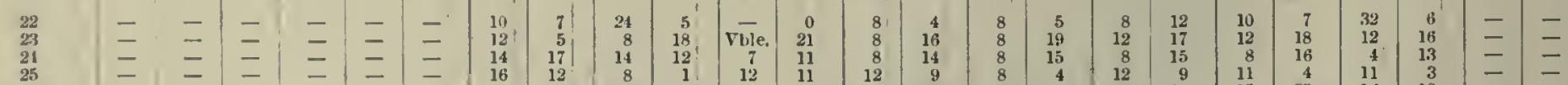

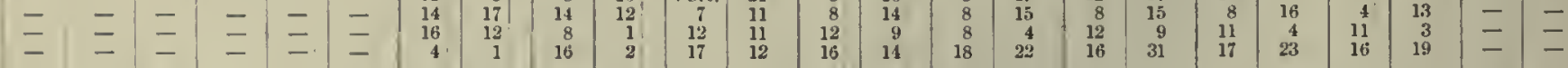

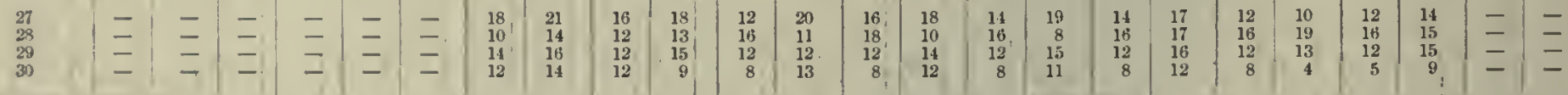

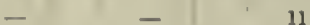


TABLE IV. (continued).

WIND-DIRECTION AND VELOCITY.

Direction (D) in Numbers $(8=\mathrm{E}, 16=\mathrm{S}, 24=\mathrm{W}, 32=\mathrm{N})$, and Velocity $(\mathrm{V})$ in miles per hour as recorded by the Cup Anemometer.

December, 1903.

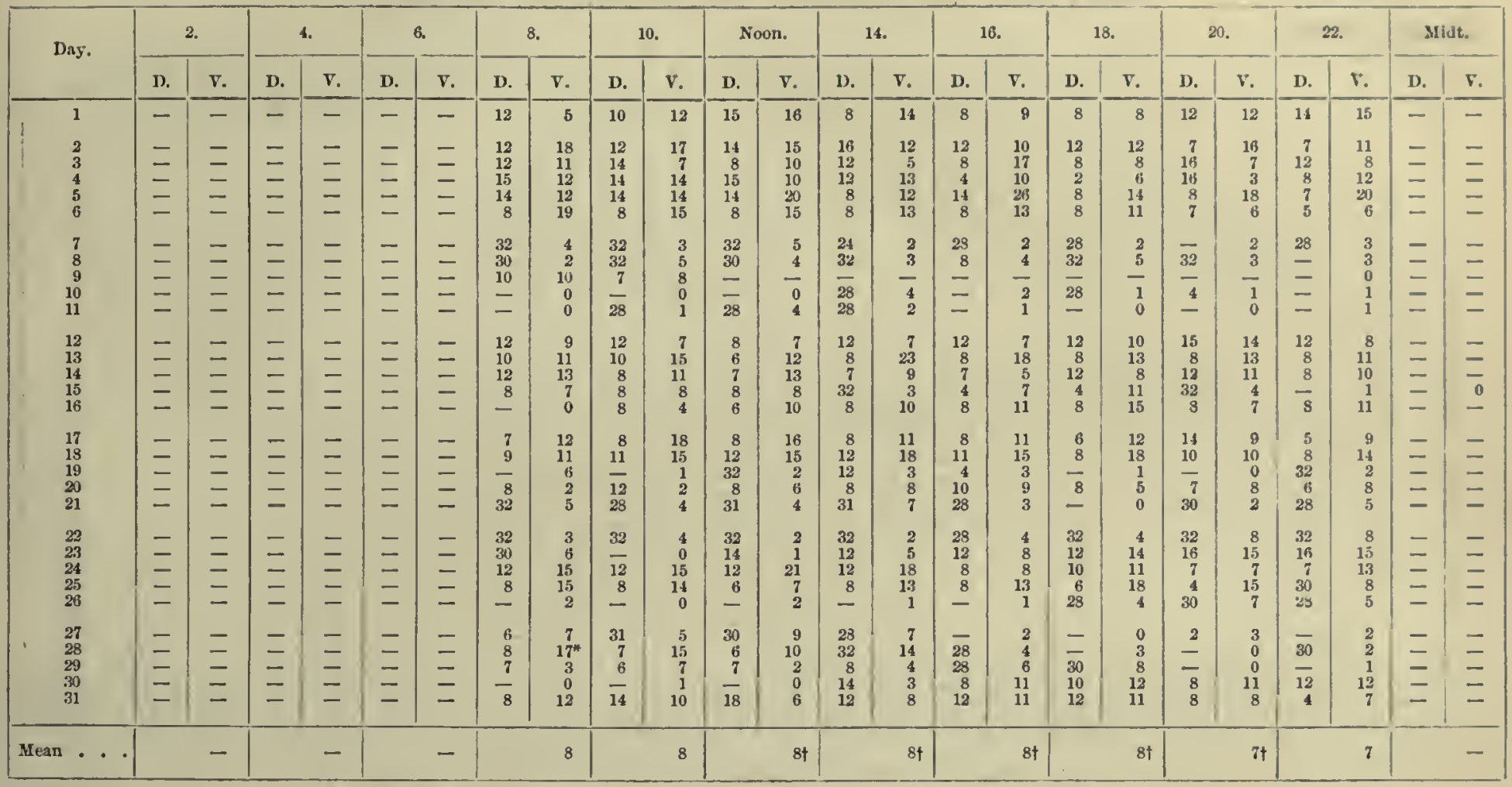

* Equiralent velocity for Beaufort scale estimation.

† For 30 days only.

January, 1904

\begin{tabular}{|c|c|c|c|c|c|c|c|c|c|c|c|c|c|c|c|c|c|c|c|c|c|c|c|c|}
\hline \multirow{2}{*}{ Das. } & & & \multicolumn{2}{|c|}{6.} & \multicolumn{2}{|c|}{8.} & \multicolumn{2}{|c|}{10.} & \multicolumn{2}{|c|}{ Noon. } & \multicolumn{2}{|c|}{14.} & \multicolumn{2}{|c|}{16.} & \multicolumn{2}{|c|}{18.} & \multicolumn{2}{|c|}{20 . } & & \multicolumn{2}{|c|}{ fiat. } \\
\hline & D. & v. & D. & v. & D. & $\mathrm{r}$. & n. & r. & D. & v. & D. & T. & D. & v. & D. & r. & D. & v. & D. & $\Gamma$. & D. & r. & D. & $\mathrm{r}$. \\
\hline$\frac{1}{2}$ & $=$ & $=$ & $=$ & $=$ & $=$ & $=$ & 31 & $\begin{array}{l}3 \\
8\end{array}$ & $\begin{array}{l}31 \\
32\end{array}$ & $\begin{array}{l}3 \\
3\end{array}$ & 28 & ${ }_{4}^{6}$ & $\begin{array}{r}28 \\
8\end{array}$ & ${ }^{6}$ & $\begin{array}{r}28 \\
8\end{array}$ & $\begin{array}{l}5 \\
6\end{array}$ & $\begin{array}{r}28 \\
8\end{array}$ & $\frac{4}{10}$ & 33 & $\frac{1}{7}$ & $\begin{array}{l}32 \\
32\end{array}$ & $\begin{array}{l}5 \\
9\end{array}$ & $\bar{z}$ & $=$ \\
\hline $\begin{array}{l}2 \\
3 \\
4\end{array}$ & $\bar{z}$ & $=$ & $=$ & $\bar{z}$ & $\bar{z}$ & = & 1 & $\begin{array}{l}8 \\
4\end{array}$ & $\begin{array}{l}32 \\
12\end{array}$ & $\begin{array}{l}6 \\
2 \\
2\end{array}$ & $\begin{array}{l}12 \\
12\end{array}$ & $\begin{array}{l}4 \\
9\end{array}$ & $\begin{array}{l}8 \\
8 \\
\end{array}$ & $\begin{array}{r}7 \\
10\end{array}$ & $\begin{array}{r}8 \\
8 \\
\end{array}$ & $\begin{array}{r}6 \\
10 \\
10\end{array}$ & & $\begin{array}{r}10 \\
9 \\
9\end{array}$ & $\frac{4}{29}$ & i & 12 & $\begin{array}{r}9 \\
11\end{array}$ & $\bar{z}$ & $=$ \\
\hline$\frac{4}{5}$ & $\bar{z}$ & 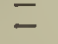 & $\bar{z}$ & $=$ & $\overline{6}$ & $\overline{12}$ & $\sqrt{8}$ & 13 & $\overline{8}$ & ${ }_{13}^{2}$ & $\overline{8}$ & $\begin{array}{l}0 \\
10\end{array}$ & $\overline{8}$ & 12 & $\begin{array}{r}28 \\
8\end{array}$ & $\begin{array}{l}1 \\
13\end{array}$ & 6 & 12 & 32 & $\begin{array}{l}1 \\
10\end{array}$ & $\frac{6}{7}$ & ${ }_{3}^{6}$ & $=$ & $=$ \\
\hline 8 & $=$ & $=$ & $=$ & - & 12 & 1 & - & $\begin{array}{l}0 \\
3\end{array}$ & - & $\overline{13}$ & - & $2^{2}$ & 4 & $\frac{1}{7}$ & Vule & 5 & 4 & 6 & $=$ & $\tilde{z}$ & $=$ & - & $=$ & $=$ \\
\hline 8 & $=$ & $=$ & 三 & $\overline{ }$ & 二 & $\equiv$ & $=$ & $\begin{array}{l}3 \\
2 \\
\underline{2}\end{array}$ & - & $\begin{array}{r}13 \\
1 \\
2\end{array}$ & $\frac{4}{16}$ & $\frac{11}{2}$ & 12 & 4 & $\overline{8}$ & $\begin{array}{r}10 \\
4\end{array}$ & 4 & $\begin{array}{l}0 \\
8\end{array}$ & $\overline{12}$ & 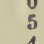 & $\overline{16}$ & $\frac{1}{2}$ & & $\bar{z}$ \\
\hline 10 & $=$ & $\bar{z}$ & $\bar{z}$ & $=$ & $\bar{z}$ & $=$ & $\overline{12}$ & $\overline{2}$ & $\overline{8}$ & ${ }_{7}^{2}$ & $\overline{4}$ & $\frac{1}{5}$ & - & $\frac{1}{3}$ & $\begin{array}{l}12 \\
8\end{array}$ & ${ }_{3}^{4}$ & 4 & 3 & 8 & 1 & $=$ & $\overline{ }$ & $=$ & $=$ \\
\hline $\begin{array}{l}11 \\
112\end{array}$ & $=$ & $=$ & $=$ & $=$ & $=$ & $=$ & $\frac{1}{8}$ & $\frac{3}{2}$ & $\overline{12}$ & $\begin{array}{l}0 \\
8 \\
\theta\end{array}$ & $-\overline{8}$ & 0 & $\frac{4}{8}$ & 8 & ${ }_{8}^{4}$ & $\begin{array}{l}13 \\
6\end{array}$ & $\begin{array}{r}20 \\
8\end{array}$ & $\begin{array}{l}3 \\
8\end{array}$ & $\overline{8}$ & $1_{10}^{2}$ & 7 & $\begin{array}{l}0 \\
9\end{array}$ & $=$ & $=$ \\
\hline $\begin{array}{l}13 \\
11\end{array}$ & - & $=$ & - & $=$ & $=$ & $=$ & $\begin{array}{r}8 \\
114\end{array}$ & $\frac{2}{12}$ & 8 & 12 & 8 & $\begin{array}{l}13 \\
15\end{array}$ & 8 & 10 & 8 & $\overline{11}$ & 12 & 13 & $\begin{array}{l}8 \\
6\end{array}$ & $\begin{array}{l}10 \\
10\end{array}$ & - & $=$ & - & - \\
\hline 15 & - & - & - & - & $=$ & $=$ & 6 & 12 & $=$ & $=$ & $\overrightarrow{0}$ & 12 & - & $=$ & - & 8 & - & - & - & 3 & $=$ & $=$ & $=$ & - \\
\hline $\begin{array}{l}15 \\
17\end{array}$ & = & $=$ & $=$ & $=$ & $=$ & $=$ & $\begin{array}{l}6 \\
8\end{array}$ & 7 & $=$ & $=$ & $\begin{array}{r}8 \\
10\end{array}$ & ${ }_{1}^{21}$ & $=$ & $=$ & $\begin{array}{l}8 \\
8\end{array}$ & 17 & $=$ & $=$ & $\frac{8}{7}$ & $\begin{array}{l}19 \\
17\end{array}$ & $=$ & - & - & $=$ \\
\hline $\begin{array}{l}18 \\
19 \\
20\end{array}$ & $\bar{z}$ & $\bar{z}$ & $\bar{z}$ & $=$ & $\bar{z}$ & = & $\begin{array}{r}8 \\
8 \\
15\end{array}$ & $\begin{array}{l}18 \\
11\end{array}$ & $=$ & $=$ & $\begin{array}{r}8 \\
7\end{array}$ & $\begin{array}{l}15 \\
12\end{array}$ & $=$ & = & $\begin{array}{r}9 \\
8\end{array}$ & $\begin{array}{l}13 \\
13 \\
10\end{array}$ & $=$ & $\bar{z}$ & $\begin{array}{l}8 \\
8 \\
7\end{array}$ & 10 & $=$ & $=$ & - & $=$ \\
\hline 2 & - & - & - & - & - & - & 7 & 16 & - & - & 7 & 14 & - & - & 8 & 18 & - & - & 8 & 18 & - & - & - & - \\
\hline 22 & 二 & $\bar{z}$ & $=$ & $=$ & = & $=$ & $\overline{4}$ & $\overline{9}$ & $=$ & $=$ & $\begin{array}{l}7 \\
6 \\
8\end{array}$ & $\begin{array}{l}18 \\
10\end{array}$ & $=$ & - & 4 & 15 & ב & $=$ & $\begin{array}{l}6 \\
8\end{array}$ & & $=$ & & & $=$ \\
\hline $\begin{array}{l}24 \\
25\end{array}$ & $\bar{z}$ & $\bar{z}$ & $\overline{=}$ & $=$ & = & $\bar{z}$ & $\overline{8}$ & $\begin{array}{r}4 \\
12\end{array}$ & $\overline{6}$ & $\overline{10}$ & $\begin{array}{l}8 \\
6\end{array}$ & $\frac{4}{7}$ & $=$ & $=$ & 4 & 15 & - & $=$ & $\begin{array}{l}4 \\
4\end{array}$ & 10 & - & - & - & $\overline{=}$ \\
\hline $\begin{array}{l}26 \\
27\end{array}$ & $=$ & $=$ & $=$ & $=$ & $=$ & $=$ & 8 & $\begin{array}{c}20^{*} \\
4\end{array}$ & $=$ & $=$ & $\begin{array}{l}8 \\
4\end{array}$ & $\begin{array}{r}14 \\
2\end{array}$ & $=$ & $=$ & $\begin{array}{l}6 \\
8 \\
\end{array}$ & 13 & $=$ & $=$ & $\begin{array}{l}4 \\
6\end{array}$ & $\begin{array}{l}10 \\
14\end{array}$ & $=$ & $\bar{z}$ & - & $=$ \\
\hline 2 & $=$ & $=$ & $=$ & $=$ & $=$ & $=$ & 4 & $\begin{array}{c}8 \\
14^{*}\end{array}$ & $\bar{z}$ & $=$ & $\overline{4}$ & 12 & $\bar{z}$ & = & $\begin{array}{l}7 \\
4\end{array}$ & $\begin{array}{r}2 \\
12 \\
7\end{array}$ & $=$ & $=$ & 12 & $\begin{array}{r}5 \\
10 \\
0\end{array}$ & - & 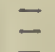 & & $\bar{z}$ \\
\hline 30 & 2 & $2^{*}$ & - & - & - & - & - & 10 & - & - & 4 & 10 & - & 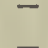 & - & & - & - & & 3 & - & - & & \\
\hline 01 & & & 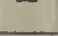 & & - & - & - & 2 & & - & 12 & 2 & - & - & 10 & 10 & - & - & 12 & 12 & - & - & - & 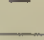 \\
\hline can & & - & & - & & - & & 84 & & - & & 8 & & - & & $9 t$ & & - & & $\theta$ & & - & & - \\
\hline
\end{tabular}

* 1Equivalent velocity for Beaufort seale estimation.

t For 29 days only.

$\$$ For 30 days ouly. 
TABLE V.

DURATION OF BRIGHT SUNSHINE IN HOURS.

September, 1902.

\begin{tabular}{|c|c|c|c|c|c|c|c|c|c|c|c|c|c|c|c|c|c|c|c|c|c|c|c|c|c|}
\hline Day. & 1. & 2. & 3. & 4. & 5. & 6. & 7. & 8. & 9. & 10. & 11. & Noon. & 13. & 14. & 15. & 16." & 17. & 18. & 19. & 20. & 21. & 22. & 23. & Mridt. & Day. \\
\hline$\frac{1}{2}$ & $=$ & $=$ & $=$ & $=$ & $=$ & $=$ & $=$ & $=$ & $=$ & $=$ & $=$ & $=$ & $=$ & $=$ & $=$ & $=$ & $=$ & $=$ & $=$ & $=$ & $=$ & $\Xi$ & $=$ & $=$ & $=$ \\
\hline 3 & - & - & - & - & $=$ & $=$ & $\bar{z}$ & 二 & $=$ & $=$ & $=$ & - & $=$ & - & - & - & $\bar{z}$ & z & $\bar{z}$ & - & $=$ & $=$ & - & - & - \\
\hline 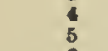 & $=$ & $=$ & $=$ & $\bar{z}$ & $=$ & $=$ & $=$ & $=$ & $=$ & $=$ & $=$ & $=$ & $=$ & $=$ & $=$ & $=$ & $=$ & $\bar{Z}$ & $\bar{z}$ & $\bar{z}$ & $\bar{z}$ & $\bar{z}$ & $\bar{E}$ & $\overline{=}$ & $\bar{z}$ \\
\hline${ }^{8}$ & $=$ & $=$ & $=$ & $=$ & $=$ & $=$ & $=$ & $=$ & $=$ & $=$ & $=$ & $\overline{-}$ & $=$ & $=$ & $=$ & $=$ & $=$ & $\overline{=}$ & $=$ & $\bar{z}$ & $=$ & 二 & $\bar{z}$ & च & $=$ \\
\hline $\begin{array}{l}8 \\
.9\end{array}$ & $=$ & $=$ & $=$ & $=$ & $=$ & $=$ & $=$ & $=$ & $=$ & $=$ & $=$ & $=$ & $=$ & $=$ & $=$ & $=$ & $=$ & $=$ & $=$ & $=$ & $=$ & $=$ & $=$ & $=$ & $=$ \\
\hline $\begin{array}{l}10 \\
11 \\
12\end{array}$ & $\bar{z}$ & $\bar{z}$ & $\bar{\Xi}$ & $\bar{z}$ & 三 & $\Xi$ & $\bar{\Sigma}$ & $\bar{z}$ & $\Xi$ & $\equiv$ & $\Xi$ & $\bar{z}$ & $=$ & $\bar{z}$ & $=$ & 三 & $=$ & $\bar{z}$ & $\bar{z}$ & $\bar{z}$ & $\equiv$ & $=$ & $\bar{z}$ & $\bar{z}$ & $\bar{z}$ \\
\hline $\begin{array}{l}13 \\
14\end{array}$ & $\bar{z}$ & $=$ & $=$ & $=$ & $=$ & $=$ & $\bar{z}$ & $=$ & $=$ & $=$ & $=$ & $\bar{z}$ & $\overline{0.8 *}$ & $\bar{I} \cdot 0$ & $\overrightarrow{0.7}$ & $=$ & $=$ & $=$ & $\bar{z}$ & $=$ & $=$ & $=$ & $\bar{z}$ & $=$ & $\overline{2.5}$ \\
\hline $\begin{array}{l}15 \\
16\end{array}$ & $=$ & $\bar{z}$ & $=$ & $=$ & $=$ & $=$ & $=$ & $=$ & $=$ & $=$ & $=$ & $=$ & $=$ & $=$ & $=$ & $=$ & $=$ & $=$ & $=$ & $=$ & $=$ & $=$ & $=$ & $=$ & $\begin{array}{l}0.00 \\
4: 0 t\end{array}$ \\
\hline $\begin{array}{l}10 \\
17\end{array}$ & $\overline{-}$ & $\overline{-}$ & $\bar{z}$ & $=$ & $\bar{z}$ & $\overline{-}$ & $\bar{z}$ & $=$ & $\overline{-}$ & $\overline{0.1}$ & $\overline{0.9}$ & $\overline{0.9}$ & 0.6 & 0.2 & $=$ & $=$ & $=$ & $=$ & $\overline{-}$ & - & $=$ & $=$ & $=$ & $=$ & $2 \cdot 7$ \\
\hline $\begin{array}{l}18 \\
19 \\
20\end{array}$ & 三 & $\bar{z}$ & $\bar{z}$ & $\bar{z}$ & $\bar{z}$ & $\bar{z}$ & $\bar{z}$ & z & $=$ & $\overline{\mathbf{1} \cdot 0}$ & $\overline{\mathbf{1}} \cdot 0$ & $\bar{I} \cdot 0$ & $\overline{10}^{\circ}$ & $\overline{1} \cdot 0$ & $\overline{1.0}$ & $\overline{0.3}$ & $=$ & $\bar{z}$ & $\equiv$ & 三 & $=$ & $=$ & $=$ & $=$ & $\begin{array}{l}0.0 \\
6-3 \\
6-3\end{array}$ \\
\hline $\begin{array}{l}20 \\
21 \\
223 \\
23\end{array}$ & $\bar{z}$ & $\bar{z}$ & $\bar{z}$ & $\bar{z}$ & $\bar{z}$ & $\bar{\Xi}$ & $\bar{\Xi}$ & $\begin{array}{l}\overline{1} .0 \\
0.5\end{array}$ & $\begin{array}{l}\overline{1} \cdot 0 \\
1.0\end{array}$ & $\begin{array}{l}\overline{1} \cdot 0 \\
1.0\end{array}$ & $\begin{array}{l}\overline{1} \cdot 0 \\
1.0\end{array}$ & $\begin{array}{l}1.0 \\
0.4\end{array}$ & $\begin{array}{l}\overline{1} \cdot 0 \\
0.0\end{array}$ & $\begin{array}{l}\overline{1} \cdot 0 \\
1.0\end{array}$ & $\begin{array}{l}\overline{0.5} \\
1.0\end{array}$ & $\overline{0.4}$ & 三 & $\bar{\beth}$ & $\bar{z}$ & $\bar{\Xi}$ & $=$ & $\bar{z}$ & $\bar{z}$ & $=$ & $\begin{array}{l}7+5 \\
7 \% 2\end{array}$ \\
\hline${ }_{24}^{23}$ & $=$ & $=$ & $\bar{z}$ & $\bar{z}$ & $=$ & $=$ & $=$ & $=$ & $=$ & $=$ & $=$ & $=$ & $=$ & $=$ & $=$ & $=$ & $=$ & $=$ & $=$ & $=$ & $=$ & $=$ & $=$ & $=$ & $\begin{array}{l}0 \cdot 0 \\
0 \cdot 0\end{array}$ \\
\hline $\begin{array}{l}25 \\
25 \\
27 \\
27\end{array}$ & $\overline{\bar{z}}$ & $\bar{z}$ & $\bar{z}$ & $\bar{z}$ & $\bar{z}$ & $\bar{z}$ & $\overline{0.2}$ & $\bar{I} \cdot 0$ & $\overline{\overline{1}} \overline{0.0}$ & $\overline{\overline{1} \cdot 00}$ & $\begin{array}{l}\overline{1} \cdot 0 \\
0.8\end{array}$ & $\begin{array}{l}1.0 \\
1.5\end{array}$ & $\overline{1}: 0$ & $\begin{array}{l}\overline{1} \cdot 0 \\
0.2\end{array}$ & $\overline{1}$ & $\overline{1} \cdot 0$ & $\overline{1} \cdot 0$ & $=$ & $=$ & $=$ & $\bar{z}$ & $\bar{z}$ & $\bar{z}$ & $=$ & $\begin{array}{l}0.0 \\
9 \cdot 5 \\
4 \cdot-3\end{array}$ \\
\hline$\infty$ & $=$ & - & - & - & - & - & - & -0 & -0 & $-\overline{0.5}$ & $=$ & $=$ & - & - & $\bar{z}$ & - & $=$ & $=$ & $=$ & $=$ & $=$ & $=$ & - & $=$ & 0.0 \\
\hline 29 & $=$ & $=$ & $=$ & $\bar{z}$ & $=$ & $=$ & $\frac{0.3}{-}$ & $\begin{array}{l}1.0 \\
0.2 \\
\end{array}$ & $\begin{array}{l}1.0 \\
1 \cdot 0 \\
\end{array}$ & $\begin{array}{l}0.5 \\
0.3 \\
\end{array}$ & $\overline{1 \cdot 0}$ & $\overline{0.7}$ & $\overline{0.8}$ & $=$ & 二 & $\overline{0.7}$ & $\overline{0.4}$ & $=$ & $\bar{z}$ & $=$ & $=$ & $=$ & $\overline{-}$ & $\bar{z}$ & $\begin{array}{l}2.8 \\
5 \cdot 1 \\
\end{array}$ \\
\hline Total & - & - & - & - & - & - & 0.5 & $3 \cdot 7$ & $5 \cdot 8$ & $5 \cdot 9$ & 6.7 & $5 \cdot 5$ & $7 \cdot 1$ & 5.4 & $4 \cdot 2$ & $2 \cdot 4$ & 0.7 & - & - & - & - & - & - & - & $51 \cdot 9$ \\
\hline
\end{tabular}

* Sunshine recorder erected this day.

$\uparrow$ Card displaced-amount of sunshine estimated by observer.

+ Doubtful-card missing.

\section{October, 1902.}

\begin{tabular}{|c|c|c|c|c|c|c|c|c|c|c|c|c|c|c|c|c|c|c|c|c|c|c|c|c|c|}
\hline Day. & 1. & 2. & 3. & 4. & 5. & 6. & 7. & 8. & 9. & 10. & 11. & Noon. & 13. & 14. & 15. & 16. & 17. & 18. & 19. & 20. & 21. & 22. & 23. & Midt. & Day. \\
\hline$\frac{1}{2}$ & $=$ & $=$ & $=$ & $\bar{z}$ & $=$ & $=$ & $\bar{z}$ & $=$ & $\overline{0.4}$ & $\overline{0.5}$ & $\overline{0.7}$ & $\overline{0.6}$ & $\overline{1 * 0}$ & $\overline{1} \cdot 0$ & $\overline{100}$ & $\overline{0.9}$ & $=$ & $\bar{z}$ & $\overline{-}$ & $=$ & $=$ & $=$ & $=$ & $=$ & $\overline{6} \cdot 1$ \\
\hline 3 & - & - & - & - & - & - & - & - & - & - & - & - & - & - & - & - & - & - & - & - & - & - & - & - & $-"$ \\
\hline $\begin{array}{l}4 \\
5\end{array}$ & $=$ & $=$ & $\overline{-}$ & $=$ & $=$ & 二 & $=$ & $\bar{z}$ & 二 & $\bar{z}$ & $\bar{z}$ & $\overline{0 \cdot 1}$ & $\overline{0.5}$ & $\overline{1 \cdot 0}$ & $\overline{0.3}$ & $\bar{z}$ & $=$ & $=$ & $=$ & $\bar{z}$ & $=$ & $=$ & $=$ & $\bar{z}$ & $\begin{array}{l}0.0 \\
1.9\end{array}$ \\
\hline$\frac{6}{7}$ & $\bar{z}$ & $=$ & $\bar{z}$ & $=$ & $=$ & $=$ & $=$ & $=$ & $\Xi$ & $=$ & 二 & $=$ & $=$ & $=$ & $=$ & $=$ & $=$ & $=$ & $=$ & $=$ & $=$ & $=$ & $=$ & $=$ & $\begin{array}{l}0.0 \\
0.0\end{array}$ \\
\hline 8 & - & - & - & - & 0.7 & 1.0 & 0.2 & - & - & - & - & - & - & 0.7 & 0.2 & 0.5 & $1 \cdot 0$ & 0.4 & 0.1 & - & - & - & - & - & 4.8 \\
\hline $\begin{array}{r}9 \\
10\end{array}$ & $=$ & $=$ & $=$ & $\bar{z}$ & $\bar{z}$ & $\overline{0.9}$ & $\overline{0.4}$ & $\overline{1} \cdot 0$ & $\overline{0 \cdot 6}$ & $\bar{z}$ & $\overline{0.2}$ & $=$ & $\overline{0.3}$ & $\overline{1 \cdot 0}$ & $\overline{1.0}$ & $\overline{100}$ & $\overline{1 \cdot 0}$ & $\overline{0.1}_{1}$ & $=1$ & $=$ & $=$ & $=$ & $\bar{z}$ & $\bar{z}$ & 7.5 \\
\hline II & $\bar{z}$ & $\bar{z}$ & $\bar{z}$ & $\bar{z}$ & - & - & -1 & $0 \cdot 9$ & 1.0 & 1.0 & 1.0 & $1 \cdot 0$ & 1.0 & 1.0 & 1.0 & 1.0 & 1.0 & 0.9 & 0.1 & $=$ & $=$ & $\bar{z}$ & $=$ & $=$ & $10 \cdot 9$ \\
\hline 12 & - & - & - & - & - & - & - & - & - & - & - & - & - & - & - & - & - & - & - & - & - & - & - & - & 一" \\
\hline 13 & - & - & - & - & - & - & - & - & - & - & - & - & - & - & - & - & - & - & - & - & - & - & - & - & 0.0 \\
\hline $\begin{array}{l}14 \\
15\end{array}$ & $\bar{z}$ & $=$ & $\bar{z}$ & $=$ & $=$ & $=$ & $=$ & $=$ & $\bar{z}$ & $\bar{z}$ & $\bar{z}$ & $\bar{z}$ & $=$ & $\bar{z}$ & $=$ & $=$ & $=$ & 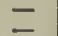 & $=$ & $\bar{z}$ & $=$ & $=$ & $\bar{z}$ & $=$ & $\begin{array}{l}0.0 \\
0.0\end{array}$ \\
\hline $\begin{array}{l}16 \\
17\end{array}$ & $=$ & $=$ & $\bar{z}$ & $\overline{0.3}$ & $\bar{I} \cdot 0$ & $\overline{100}$ & $\overline{1 \cdot 0}$ & $\overline{1 \cdot 0}$ & $\bar{I} \cdot 0$ & $\bar{I} \cdot 0$ & $\overline{1} \cdot 0$ & $\overline{1 \cdot 0}$ & $\overline{1 \cdot 0}$ & $\bar{I} \cdot 0$ & $\overline{1} \cdot 0$ & $\overline{0.2}$ & $\overline{1} \cdot 0$ & $\overline{1 \cdot 0}$ & $\overrightarrow{0.4}$ & $\overline{-}$ & 二 & $=$ & $\bar{z}$ & $\overline{-}$ & 13.9 \\
\hline 18 & - & - & - & - & - & - & - & - & - & - & 0.9 & 0.1 & 0.2 & $1 \cdot 0$ & 0.9 & $1 \cdot 0$ & 1.0 & $1 \cdot 0$ & 0.5 & - & - & - & - & - & $6 \cdot 6$ \\
\hline 19 & - & - & - & 0.5 & 1.0 & 1.0 & 1.0 & $1 \cdot 0$ & 1.0 & 1.0 & 1.0 & 1.0 & 1.0 & 1.0 & 1.0 & 1.0 & 1.0 & $1 \cdot 0$ & $1 \cdot 0$ & - & - & - & - & - & $15 \cdot 5$ \\
\hline 20 & - & - & - & 0.5 & 1 1.0 & 10 & 1.0 & 1.0 & 1.0 & 0.5 & 1.0 & $1 \cdot 0$ & $1 \cdot 0$ & 0.9 & $\bar{z}$ & $=$ & $=$ & $=$ & $\bar{z}$ & $=$ & $\bar{z}$ & $\bar{z}$ & $=$ & $\bar{z}$ & $\begin{array}{l}9.9 \\
0.0\end{array}$ \\
\hline 23 & $\bar{z}$ & $\bar{z}$ & $\bar{z}$ & $\Xi$ & $=$ & $\overline{0.1}$ & $\overline{0.3}$ & $\overline{0} \cdot 3$ & $\overline{0.5}$ & $\overline{0.3}$ & 0.9 & $\overline{1} \cdot 0$ & $\overline{1} \cdot 0$ & $\overline{1} \cdot 0$ & $\overline{1.0}$ & $\overline{1}=0$ & $\overrightarrow{1} \cdot 0$ & $\overline{\mathrm{I}} \cdot 0$ & $\overline{0.3}$ & $\bar{Z}$ & $=$ & $=$ & $\bar{z}$ & $=$ & $9 \cdot 7$ \\
\hline 23 & - & - & - & - & - & - & - & - & - & - & - & - & - & - & - & - & - & - & - & - & - & - & - & - & -" \\
\hline 24 & $=$ & $=$ & $\bar{z}$ & 二 & $\bar{z}$ & $=$ & $=$ & $=$ & $=$ & $\bar{z}$ & $\bar{z}$ & $\bar{z}$ & 二 & $\bar{z}$ & $=$ & $\overline{0.9}$ & $\overline{0.4}$ & $\overline{0.8}$ & $\overline{0 \cdot 7}$ & $=$ & $=$ & $=$ & $\bar{z}$ & - & $\begin{array}{l}0.0 \\
2.8\end{array}$ \\
\hline $\begin{array}{l}26 \\
27\end{array}$ & $=$ & $=$ & $\bar{z}$ & 0.2 & $1 \cdot 0$ & 1.0 & $1 \cdot 0$ & 1.0 & 1.0 & 10 & 10 & 10 & $\begin{array}{l}1.0 \\
0.7\end{array}$ & 1.0 & 1.0 & $\begin{array}{l}1 \cdot 0 \\
0.2\end{array}$ & $\begin{array}{l}1 \cdot 0 \\
0.1\end{array}$ & $\begin{array}{l}1 \cdot 0 \\
0 \cdot 4\end{array}$ & $1 \cdot 0$ & $0 \cdot 7$ & $\overline{-}$ & $\overline{-}$ & $\Xi$ & $\overline{-}$ & $\begin{array}{r}15 \cdot 9 \\
1 \cdot 4\end{array}$ \\
\hline 28 & - & - & - & - & - & - & - & - & - & - & - & - & - & - & - & - & - & - & -1 & -1 & - & - & - & & $\rightarrow$ \\
\hline 29 & $=$ & $=$ & - & 二 & $\bar{z}$ & 二 & $=$ & $=$ & $=$ & 0.3 & 100 & 0.7 & 0.9 & 0.8 & 0.1 & 0.5 & 0.9 & 0.5 & 0.9 & 0.2 & $=$ & - & $=$ & - & 6.8 \\
\hline $\begin{array}{l}30 \\
31\end{array}$ & $\bar{z}$ & $\bar{z}$ & $\bar{z}$ & $=$ & $=$ & $\Xi$ & $=$ & $=$ & $\frac{0.1}{-}$ & $\frac{0.4}{-}$ & $=$ & $=$ & $=$ & $=$ & $=$ & $\Xi$ & $\overline{-}$ & $=$ & $\overline{-}$ & 二 & $=$ & $=$ & $=$ & $\bar{z}$ & $\begin{array}{l}0.5 \\
0.0\end{array}$ \\
\hline Total & - & - & - & 1.5 & 4.7 & 6.0 & $4 \cdot 9$ & $6 \cdot 2$ & 6.6 & 6.0 & $8 \cdot 7$ & $\overline{7.5}$ & $\overline{9 \cdot 6}$ & $\overline{11 \cdot 4}$ & 8.5 & $9 \cdot 9 \cdot 2$ & 9.4 & 8.1 & $5 \cdot 0$ & 0.9 & - & - & - & - & $114 \cdot 2$ \\
\hline
\end{tabular}


TABLE V. (continued).

\section{DURATION OF BRIGHT SUNSHINE IN HOURS.}

November, 1902.

\begin{tabular}{|c|c|c|c|c|c|c|c|c|c|c|c|c|c|c|c|c|c|c|c|c|c|c|c|c|c|}
\hline Day. & 1. & 2. & 3. & 4. & 5. & 0. & 7. & 8. & ค. & 10 & 11. & Noon. & 13. & 14. & 15. & 16. & 17. & 18. & 19. & 20. & 21. & 22. & 23. & Mintt. & Day. \\
\hline 1 & - & - & - & - & - & - & - & - & - & - & - & - & - & - & - & - & - & - & - & - & - & - & - & - & 0.0 \\
\hline 2 & - & - & - & - & - & - & - & - & - & - & - & - & - & - & - & - & - & - & - & - & - & - & - & - & 0.0 \\
\hline 3 & 二 & $=$ & $\overline{0.3}$ & $\overrightarrow{0 \cdot 3}$ & $\overline{0.5}$ & $\bar{\pi}$ & $\overline{0.0}$ & -0 & $\overline{1} \cdot 0$ & $0: 3$ & 0.8 & 0.4 & 0.5 & 0.0 & $10^{\circ} 0$ & $\begin{array}{l}1 \\
1\end{array}$ & 1.0 & 1.0 & $\begin{array}{l}1.0 \\
0.8\end{array}$ & 0.5 & 0.8 & $=$ & $=$ & $\pi$ & \\
\hline 5 & 二 & $\bar{z}$ & $\begin{array}{l}0.3 \\
-\end{array}$ & $\begin{array}{l}0.3 \\
0.5\end{array}$ & $\begin{array}{l}0.5 \\
1.0\end{array}$ & $\begin{array}{l}0.9 \\
1.0\end{array}$ & $\begin{array}{l}0.8 \\
1.0\end{array}$ & $\begin{array}{l}1.0 \\
1.0\end{array}$ & $\begin{array}{l}1.0 \\
1.0\end{array}$ & $\begin{array}{l}1.0 \\
1.0\end{array}$ & $\begin{array}{l}1 \cdot 0 \\
0 \cdot 1\end{array}$ & 1.0 & 1.0 & $1^{\circ} 0$ & $1^{\circ} 0$ & $1 \cdot 0$ & 1.0 & $1^{\circ} 0$ & 0.8 & 0.8 & 0.2 & $\bar{z}$ & $\bar{z}$ & $=$ & $\begin{array}{c}15 \cdot 7 \\
6 \cdot 0^{*}\end{array}$ \\
\hline is & $=$ & $=$ & $=$ & - & - & - & - & 1 & - & - & 0.7 & 100 & 0.2 & 1.0 & 1.0 & $1 \cdot 0$ & $1 \cdot 0$ & 1.0 & 1.0 & 10 & 0.8 & - & $=$ & - & $9 \cdot 7^{4}$ \\
\hline 7 & 0.3 & 1.0 & $1: 0$ & $0 \%$ & 0.2 & & $0 \cdot 1$ & & & & & - & - & & & - & 0.0 & 1.0 & $0 \cdot 7$ & - & 0.8 & 0.3 & - & - & . 6 \\
\hline & & 1 & 1.0 & $1 \cdot 0$ & 10 & 1.0 & 10 & 100 & $1 \cdot 0$ & 1.0 & 1.0 & $1 \cdot 0$ & $1 \cdot 0$ & $1^{\circ} 0$ & $\begin{array}{l}1.0 \\
1.0\end{array}$ & 1.0 & & & 10 & 1.0 & & & $\overline{0.7}$ & $\begin{array}{l}0.2 \\
1.0\end{array}$ & \\
\hline il & 0 & $0^{\circ}$ & 1.0 & 1.0 & $\begin{array}{l}1.0 \\
1.0\end{array}$ & $1 \cdot 0$ & $1 \cdot 0$ & 1.0 & $1 \cdot 0$ & 1.0 & $1 \cdot 0$ & $1 \cdot 0$ & 1.0 & 1.0 & 1.0 & $1 \%$ & & 1.0 & 1.0 & $1 \cdot 0$ & •0 & 000 & 0.7 & 0 & \\
\hline $\begin{array}{l}10 \\
11\end{array}$ & $\begin{array}{l}1 \cdot 0 \\
0.2\end{array}$ & $\begin{array}{l}1 \cdot 0 \\
0.5\end{array}$ & $\begin{array}{l}1^{\circ} \cdot 0 \\
1.0\end{array}$ & $\begin{array}{l}1.00 \\
1.0\end{array}$ & $\begin{array}{l}1 \cdot 0 \\
0 \cdot 3\end{array}$ & 1.0 & $1^{\circ 0}$ & $1 \cdot 0$ & 1.0 & 100 & 10 & $1 \cdot 0$ & 1.0 & $1^{\circ} 0$ & 1.0 & $\begin{array}{l}1.0 \\
0.1\end{array}$ & $1 \cdot 0$ & $1 \cdot 0$ & $1 \cdot 0$ & $1 \cdot 0$ & 10 & $\stackrel{0 \cdot 9}{-}$ & 0.8 & 0.3 & $\begin{array}{l}23 \cdot 0 \\
3 \cdot 1\end{array}$ \\
\hline 12 & - & - & - & - & - & & & - & - & & & & & & & & & & & - & - & & - & - & 0 \\
\hline 13 & - & 0.9 & $1 \cdot 0$ & 0.5 & 0.8 & $1 \cdot 0$ & 1.0 & 1.0 & 1.0 & $1 \cdot 0$ & $\overline{0.8}$ & $=$ & $\overline{0.3}$ & 0.2 & 0.3 & 0.6 & 0.5 & 0.9 & 0.3 & 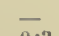 & $\overline{-}$ & 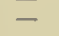 & $\overline{-}$ & - & $12 \cdot 1$ \\
\hline $\begin{array}{l}14 \\
15\end{array}$ & $=$ & $=$ & $\bar{z}$ & $\overline{0} \cdot \bar{\pi}$ & $\overline{1 \cdot 0}$ & $\overline{1.0}$ & $\overrightarrow{0.6}$ & $\overline{1.0}$ & $\overline{1.0}$ & $\overline{1 \cdot 0}$ & $\overline{1 \cdot 0}$ & 1.0 & $\overline{1.0}$ & 1.0 & $\begin{array}{l}0.7 \\
1.0\end{array}$ & $\begin{array}{l}1.0 \\
1.0\end{array}$ & 1.0 & $\begin{array}{l}1.0 \\
1.0\end{array}$ & $\begin{array}{l}0.1 \\
1.0\end{array}$ & $\begin{array}{l}0.33 \\
1.0\end{array}$ & $\begin{array}{l}0.1 \\
1.0\end{array}$ & $\overline{0} \cdot 0$ & $\overline{1} \cdot 0$ & $\overline{1 \cdot 0}$ & (2) $\cdot 2$ \\
\hline 16 & 1.0 & 1.0 & 1.0 & $1 \cdot 0$ & 1.0 & $i \cdot 0$ & $1 \cdot 0$ & 1.0 & 1.0 & $1 \cdot 0$ & $1 \cdot 0$ & 1.0 & 1.0 & 1.0 & 1.0 & 1.0 & $1 \cdot 0$ & 1.0 & 1.0 & 1.0 & 10 & 0.7 & 0.8 & -8 & $23 \cdot 3$ \\
\hline 17 & 0.3 & - & - & - & - & - & - & - & - & - & - & - & 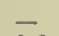 & - & & - & - & - & - & - & - & - & - & - & 0.3 \\
\hline 18 & $\bar{z}$ & $=$ & $\bar{z}$ & $=$ & $=$ & $\overline{0.6}$ & $\overline{0.1}$ & $=$ & $\overline{0.4}$ & $\overline{0}=$ & $\overline{0.4}$ & $\overrightarrow{0.20}$ & 0.8 & 二 & 0.2 & 10 & 1.0 & 0.3 & 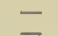 & 二 & 二 & & - & - & $2 \cdot 2 *$ \\
\hline 20 & $=$ & $\bar{z}$ & $=$ & 二 & 二 & 0.6 & 0.1 & $\bar{z}$ & - & 00 & -0.4 & 0.2 & 二 & 二 & 二 & $\bar{z}$ & $\bar{E}$ & 二 & 二 & $\bar{z}$ & $\bar{z}$ & $=$ & $\bar{z}$ & $\overline{-}$ & $2 \cdot 2^{*}$ \\
\hline 21 & - & - & - & - & - & - & - & - & - & - & - & 0.5 & 1.0 & 0.9 & 1.0 & 1.0 & 1.0 & 0.6 & - & 0.2 & 0.2 & 0.4 & 1.0 & $1 \cdot 0$ & 8.8 \\
\hline 22 & 0.8 & $1 \cdot 0$ & 1.0 & 1.0 & $1 \cdot 0$ & 0.9 & $0 \cdot 1$ & 0.5 & 0.8 & - & ז. 0 & 0.5 & - & - & - & - & - & - & - & - & - & - & - & - & $8 \cdot 3$ \\
\hline 2 & $=$ & $=$ & $\bar{z}$ & $z$ & $\bar{z}$ & $\bar{z}$ & 二 & 二 & $=$ & $z$ & $=$ & 二 & $\overline{0.9}$ & $\overline{1} \cdot 0$ & $\overline{0.9}$ & $=$ & 二 & $\overline{0.3}$ & $\overline{1.0}$ & $\overline{1.0}$ & $\overline{1 \cdot 0}$ & 1.0 & $\overline{1.0}$ & $\overline{1 \cdot 0}$ & $266^{\prime} 6^{*}$ \\
\hline $\begin{array}{l}25 \\
265\end{array}$ & $1 \cdot 0$ & $1 \cdot 0$ & $1 \cdot 0$ & 1.0 & $1 \cdot 0$ & $1 \cdot 0$ & 1.0 & 1.0 & 1.0 & $\frac{0.4}{-4}$ & $\bar{z}$ & $\bar{z}$ & $=$ & 二 & 二 & $\bar{z}$ & $=$ & 二 & $=$ & $=$ & 二 & $=$ & $=$ & $=$ & $9 \cdot 4$ \\
\hline 27 & - & - & - & & & & & & & - & - & 0 & 1 & 0.8 & & 0 & 100 & 1.0 & $1 \cdot 0$ & 1.0 & 1.0 & 1 & 0.9 & 0.9 & $10 \cdot g^{*}$ \\
\hline 8 & 0 & 0.9 & 0 & 1 & 1. & 1.0 & 1.0 & 1.0 & 1. & $10^{\circ}$ & 1.0 & & 1 & 1 & 1 & 10 & 1.0 & 1.0 & $1^{\circ} 0$ & 1.0 & $1^{\circ} 0$ & 1 & 1.0 & 1.0 & \\
\hline $\begin{array}{l}39 \\
.30\end{array}$ & $1 \cdot 0$ & $1 \cdot 0$ & $1 \cdot 0$ & $1 \cdot 0$ & $1 \cdot 0$ & $1 \cdot 0$ & 10 & $1 \cdot 0$ & $1 \cdot 0$ & 10 & $1 \cdot 0$ & $\begin{array}{l}100 \\
0.02\end{array}$ & $\begin{array}{l}1.0 \\
0.8\end{array}$ & $\begin{array}{l}1.0 \\
1.0\end{array}$ & $\begin{array}{l}1.0 \\
0.3\end{array}$ & $\begin{array}{l}0.5 \\
0.3\end{array}$ & 1.0 & 1.0 & $\overline{10} 0$ & 0.8 & $\overline{1.0}$ & 0.5 & $=$ & $=$ & $\begin{array}{r}15.5 \% \\
7.94\end{array}$ \\
\hline tal & $8 \cdot 1$ & $10 \cdot 1$ & $10 \cdot 9$ & $11 \cdot 6$ & 11.8 & $12 \cdot 4$ & $10 \cdot 8$ & 11.5 & $\overline{12 \cdot 2}$ & $11 \cdot 2$ & 11.5 & $11 \cdot 1$ & 13.5 & $13 \cdot 8$ & 13.4 & 1.10 & 1.17 & $15 \cdot 1$ & $12 \cdot 0$ & 11.6 & $10 \cdot 1$ & $7 \cdot 3$ & $7 \cdot 2$ & $7 \cdot 2$ & $291 \cdot 5$ \\
\hline
\end{tabular}

* Doubtful-card for part of day missing.

December, 1902.

\begin{tabular}{|c|c|c|c|c|c|c|c|c|c|c|c|c|c|c|c|c|c|c|c|c|c|c|c|c|c|}
\hline Day. & 1. & 2. & 3. & 4. & 5. & 6. & 7. & 8. & 9. & 10. & 11. & Noon. & 13. & 14. & 15. & 16. & 17. & 18. & 19. & 20. & 21. & 22. & 23. & Midt. & Day. \\
\hline 1 & - & - & - & - & - & - & - & - & - & - & 0.5 & 0.7 & - & $0 \% 3$ & $1 \cdot 0$ & $1 \cdot 0$ & $1 \cdot 0$ & 1.0 & $1 \cdot 0$ & $1 \cdot 0$ & $1 \cdot 0$ & $1 \cdot 0$ & 1.0 & $1 \cdot 0$ & $11 \cdot 5$ \\
\hline 2 & 0.8 & - & - & $0 \cdot 9$ & 1.0 & $1^{\circ} 0$ & 1.0 & 1.0 & 1.0 & 10 & $1 \cdot 0$ & $1 \cdot 0$ & 1.0 & 0.1 & 0.1 & - & - & - & - & - & - & - & - & - & $11 \cdot 2$ \\
\hline 3 & - & - & - & - & - & - & - & - & 0.5 & $1 \cdot 0$ & $1 \cdot 0$ & 1.0 & 1.0 & 1.0 & $1 \cdot 0$ & 10 & $1 \cdot 0$ & 1.0 & $1 \cdot 0$ & $1 \cdot 0$ & 1.0 & 1.0 & 1.0 & 1.0 & $15 \cdot 5$ \\
\hline 5 & 1.0 & $1 \cdot 0$ & 100 & $\begin{array}{l}1.0 \\
1.0\end{array}$ & $1=0$ & 1.0 & 1.0 & 1.0 & 1.0 & 1.0 & $1 \cdot 0$ & 1.0 & 1.0 & $1 \cdot 0$ & 1.0 & $\left.1^{\circ}\right)$ & 1.0 & $1 \cdot 0$ & $1 \cdot 0$ & $1 \cdot 0$ & $1 \cdot 0$ & 1.0 & 1.0 & 0.7 & 2377 \\
\hline $\begin{array}{l}5 \\
6\end{array}$ & $\overline{1 \cdot 0}$ & $\overrightarrow{1 \cdot 0}$ & $\begin{array}{l}0.6 \\
1.0\end{array}$ & $\begin{array}{l}1.0 \\
1.0\end{array}$ & $\begin{array}{l}1 \% 0 \\
1.0\end{array}$ & 1.0 & 1.0 & $1^{\circ} 0$ & $1^{\circ} 0$ & 10 & 1.0 & $1 \cdot 0$ & 10 & 1.0 & 1.0 & 1.0 & $10^{\circ}$ & 1. & 1.0 & 1.0 & 1.0 & 1.0 & 1.0 & 1.0 & $21 \cdot 6$ \\
\hline 6 & 10 & & 10 & & 1.0 & $1 \cdot 0$ & 1.0 & 1.0 & 10 & 1.0 & 1.0 & $1^{\circ} 0$ & $1 \cdot 0$ & $1 \cdot 0$ & 1.0 & $1 \cdot 0$ & $1 \cdot 0$ & 1.0 & 1.0 & 1.0 & 0.1 & - & - & - & $20 \cdot 1$ \\
\hline 7 & - & - & - & - & - & - & - & - & - & - & - & - & - & - & - & - & - & - & - & - & - & - & - & - & 0.0 \\
\hline 8 & $\overline{0.7}$ & $\overline{0-1}$ & $\overline{0 \cdot a}$ & $\overline{0.5}$ & $\overline{1-0}$ & $\overrightarrow{0.8}$ & $\overline{0 \cdot 0}$ & - & 0.4 & $0 \cdot 1$ & 0.5 & 0.3 & 0.4 & 0.8 & 0.5 & 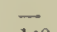 & - & $\rightarrow$ & - & - & - & - & 0.3 & $\overrightarrow{1 *}$ & $3 \cdot 3$ \\
\hline $\begin{array}{r}9 \\
10\end{array}$ & $\begin{array}{l}0.7 \\
1.0\end{array}$ & $\begin{array}{l}0-1 \\
1 \cdot 0\end{array}$ & 0.9 & 0.5 & 100 & 0.8 & 0.9 & 1.0 & $10^{\circ}$ & $1 \cdot 0$ & 10 & 10 & 10 & $1 \cdot 0$ & $1 \cdot 0$ & 1.0 & 10 & 10 & 1.0 & 1.0 & $1 \cdot 0$ & 1.0 & 1.0 & $1 \cdot 0$ & $21 \cdot 9$ \\
\hline $\begin{array}{l}10 \\
11\end{array}$ & 1.0 & 10 & 1.0 & $\begin{array}{lll}1 & 0\end{array}$ & 1.0 & $1^{\circ} 0$ & 10 & 1.0 & $1^{\circ} 0$ & 1.0 & 1.0 & 1.0 & $1^{\circ} 0$ & 1.0 & $1 \cdot 0$ & 1.0 & $10^{\circ}$ & 1.0 & 1.0 & 1.0 & $1 \cdot 0$ & 1.0 & 1.0 & 1.0 & 240 \\
\hline 11 & $1 \cdot 0$ & 1.0 & 1.0 & $1 \cdot 0$ & $1^{\circ} 0$ & $10^{\circ}$ & 1.0 & $1 \cdot 0$ & $10^{\circ}$ & 1.0 & 1.0 & 1.0 & 1.0 & $1 \cdot 0$ & 1.0 & 1.0 & $1 \cdot 0$ & $1 \cdot 0$ & 1.0 & 1.0 & 1.0 & 1.0 & 0.2 & - & $22 \cdot 2$ \\
\hline 12 & - & - & - & - & - & - & - & - & - & - & 0.3 & - & - & - & - & - & - & - & - & - & - & - & - & - & $0-3$ \\
\hline 1 : & - & - & - & - & - & - & - & - & - & - & - & - & - & - & - & - & - & - & - & - & - & - & - & - & 0.0 \\
\hline 11 & - & - & - & $\overline{-}$ & - & $\bar{z}$ & $=$ & - & - & - & 0.2 & $1 \cdot 0$ & 1.0 & 0.9 & $0 \cdot 9$ & 0.7 & 0.7 & 0.8 & 1.0 & 1.0 & - & - & - & - & $8 \cdot 2$ \\
\hline $\begin{array}{l}15 \\
16\end{array}$ & $=$ & E & 二 & 二 & $=$ & $=$ & $\bar{z}$ & 二 & $\bar{z}$ & $\bar{z}$ & $\overline{-}$ & $=$ & $=$ & $=$ & $\bar{z}$ & $\bar{z}$ & $\overline{-}$ & 二 & $\bar{z}$ & - & $=$ & - & $\bar{z}$ & 二 & $0^{\circ} 0$ \\
\hline & & & & & & & & & & & & & & & & - & - & - & - & - & 一 & $\rightarrow$ & - & 一 & 00 \\
\hline 17 & - & - & - & - & - & - & - & - & - & - & - & - & - & - & - & $\ldots$ & - & - & - & - & - & - & - & - & 0.0 \\
\hline 18 & $\overline{1}$ & $\overline{0.0}$ & - & - & - & - & - & - & - & - & - & 0.2 & 0.1 & - & $0 \cdot 9$ & $1 \cdot 0$ & 1.0 & $1 \cdot 0$ & 1.0 & 1.0 & 1.0 & 1.0 & 1.0 & 1.0 & $10 \cdot 2$ \\
\hline 19 & 1.0 & 0.8 & -. & - & - & - & $\overline{-}$ & - & $=$ & - & - & - & - & - & $=$ & - & $=$ & 二 & $\bar{z}$ & $z$ & $=$ & 二 & $z$ & - & $1 \cdot 8$ \\
\hline 21 & $=$ & 二 & 二 & $=$ & $=$ & - & $\overline{0.1}$ & $\overline{0.5}$ & $=$ & $\overline{0.1}$ & $\overline{1.0}$ & $\overline{1.0}$ & $\overline{1.0}$ & $\overline{1 \cdot 0}$ & $\overline{1} \cdot 0$ & $\overline{1 \cdot 0}$ & $\overline{1 \cdot 0}$ & $\overline{1.0}$ & $\overline{1.0}$ & $\overline{1 * 0}$ & $\overline{0.9}$ & $\overline{0.9}$ & $\overline{1.0}$ & $\overline{1 \cdot 0}$ & $\begin{array}{r}0.0 \\
14.5\end{array}$ \\
\hline 22 & 10 & $1 \cdot 0)$ & $0 \cdot 9$ & 1.0 & $1 \cdot 0$ & $1 \cdot 0$ & 1.0 & $1 \cdot 0$ & $1 \cdot 0$ & 1.0 & 1.0 & 0.9 & 0.7 & $0 \div$ & 0.7 & 0.1 & - & - & - & - & - & - & - & - & $14 \cdot 2$ \\
\hline 23 & & - & - & & - & & - & - & - & - & - & - & - & 0. & $u^{\circ}$ & 1.0 & 1.0 & $1 \cdot 0$ & 0.0 & - & & 0.1 & 1.0 & $1 \cdot 0$ & \\
\hline 21 & 10 & 1.0 & $1 \cdot(1$ & $1 \cdot 0$ & 1.0 & 1.0 & $1^{\circ} 0$ & 1.0 & 0.9 & $\overline{-}$ & - & $0 \cdot 3$ & $0 \cdot 3$ & 0 & 1 & 1 & 0 & - & - & 0.1 & 0.8 & & 10 & 1.0 & 15 \\
\hline 25 & 0.6 & 10 & 1.0 & $1 \cdot 0$ & 10 & $1 \cdot 0$ & 1.0 & $1 \cdot 0$ & 1.0 & 1.0 & 1.0 & 1.0 & 1.0 & 1. & 1. & 1. & 1.0 & 1. & 1.0 & $1^{\circ}$ & $1 \cdot 0$ & 1.0 & 1.0 & $1 \cdot 0$ & 23.6 \\
\hline 26 & 1.0 & 1.0 & 1.0 & $1 \cdot 0$ & 1.0 & 1.0 & 10 & 1.0 & $1 \cdot 0$ & $1 \cdot 0$ & $1 \cdot 0$ & 1.0 & 1.0 & $i \cdot 0$ & $1 \cdot 0$ & 10 & $1 \cdot 0$ & $1 \cdot 0$ & 1.0 & $1 \cdot 0$ & $1 \cdot 0$ & $1 \cdot 0$ & 1.0 & 1.0 & $24 \cdot 0$ \\
\hline 27 & $1 \cdot 0$ & 1.0 & $1 \cdot 0$ & $1 \cdot 0$ & 1.0 & 1.0 & 1 . & 1 . & 1. & $1 \cdot 0$ & 1 . & $1 \cdot 0$ & 1.0 & 1. & 1.0 & 1. & $10^{\circ}$ & $1 \cdot 0$ & $1 \cdot$ & 1.0 & $1 \cdot 0$ & $1 \cdot 0$ & 1.0 & $1 \cdot 0$ & $21 \cdot 0$ \\
\hline 28 & $1 \cdot 11$ & $\begin{array}{lll}1 & 0 \\
0.0\end{array}$ & $1 \cdot 0$ & $1 \cdot 0$ & $1 \cdot 0$ & $1 \cdot 0$ & $10^{\circ}$ & 10 & $1 \cdot 0$ & $1 \cdot 0$ & 10 & $1 \cdot 0$ & $1 \cdot 0$ & 10 & 1. & 1. & $0 \cdot \tilde{\imath}$ & 0. & $0 \%$ & $0 \cdot 5$ & 0.5 & & & & $18 \cdot 3$ \\
\hline 29 & $\overline{1 \cdot 0}$ & $0 \%$ & $\overline{0}$. & $\overline{0: 7}$ & - & - & $\rightarrow$ & - & - & & 0.6 & $1 \cdot 0$ & $1 \cdot 0$ & $1 \cdot 0$ & $1 \cdot 0$ & 1.0 & 1.0 & 1.0 & 1.0 & 10 & $1 \cdot 0$ & 1.0 & 1.0 & 1.0 & $13 \cdot 8$ \\
\hline $\begin{array}{l}30 \\
31\end{array}$ & 10 & 0.7 & - & 0 & $0 \cdot 8$ & $\begin{array}{l}0.4 \\
1.0\end{array}$ & $1 \cdot 0$ & $\begin{array}{l}1.0 \\
1.0\end{array}$ & $\begin{array}{l}1.0 \\
1.0\end{array}$ & $\begin{array}{l}1.0 \\
1.0\end{array}$ & 0.7 & 1.0 & 0.2 & $\overrightarrow{100}$ & $\overline{1 * 0}$ & $\overline{100}$ & $\overline{1 \cdot 0}$ & 1.0 & $\overline{1 \cdot 0}$ & 二 & $\bar{z}$ & $=$ & $\overline{0.7}$ & $\overline{0.33}$ & $10 \cdot 1$ \\
\hline & $13^{\circ}$ & $11 \cdot y$ & 11.9 & $13 \cdot 1$ & 11.7 & $4 \cdot 2$ & & & & & . & & 2 & & & & & & & & & 13.3 & $15 \cdot$. & 14.0 & $5=$ \\
\hline & 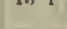 & 110 & 18 : & 151 & 110 & 143 & 150 & 125 & $15^{\circ} 8$ & 13.2 & $17 \cdot 7$ & 10.1 & 17.7 & $18 \cdot 0$ & $19 \cdot 3$ & $18 \cdot 8$ & 178 & $16 \%$ & 1.4 & 15.6 & 173 & 133 & 102 & 100 & 3506 \\
\hline
\end{tabular}


TABLE V. (continued).

DURATION OF BRIGHT SUNSHINE IN HOURS.

\section{Jannary, 1903.}

\begin{tabular}{|c|c|c|c|c|c|c|c|c|c|c|c|c|c|c|c|c|c|c|c|c|c|c|c|c|c|}
\hline Day. & 1. & 2. & 3. & 1. & 5. & 6. & 7. & 8. & 8. & 10. & 11. & Noon. & 13. & 14. & 15. & 10. & 17. & 18. & 19. & 20. & 21. & 22. & 23. & Mirit. & Day. \\
\hline $\begin{array}{l}1 \\
2 \\
3 \\
4 \\
5\end{array}$ & $\begin{array}{l}1.0 \\
1.0 \\
1.0 \\
1.0\end{array}$ & $\begin{array}{l}0.9 \\
1.0 \\
0.4 \\
1.0\end{array}$ & $\begin{array}{l}1 \cdot 0 \\
1.0 \\
\overline{100}\end{array}$ & $\begin{array}{l}1 \cdot 0 \\
1.0 \\
\overline{1} \cdot 0\end{array}$ & $\begin{array}{l}1 \cdot 0 \\
0 \cdot 7 \\
0.5 \\
1.0\end{array}$ & $\begin{array}{l}1 \cdot 0 \\
1 \cdot 0 \\
0.9 \\
1.0\end{array}$ & $\begin{array}{l}1 \cdot 0 \\
1 \cdot 0 \\
1 \cdot 0 \\
0.9\end{array}$ & $\begin{array}{l}1.0 \\
1.0 \\
0.9 \\
0.4\end{array}$ & $\begin{array}{l}1 \cdot 0 \\
1 \cdot 0 \\
0.4 \\
-\end{array}$ & $\begin{array}{l}1.0 \\
0.7 \\
1.0 \\
-\end{array}$ & $\begin{array}{l}1 \cdot 0 \\
0 \cdot 9 \\
0 \cdot 3 \\
0.4\end{array}$ & $\begin{array}{l}1.0 \\
1.0 \\
\overline{0} \\
0.5\end{array}$ & $\begin{array}{l}1 \cdot 0 \\
1 \cdot 0 \\
0 \cdot 3 \\
0 \cdot 1 \\
0.5\end{array}$ & $\begin{array}{l}1 \cdot 0 \\
1 \cdot 0 \\
0 \cdot 5 \\
0 \cdot 5\end{array}$ & $\begin{array}{l}1 \cdot 0 \\
1.0 \\
\overline{0} \\
5.5\end{array}$ & $\begin{array}{l}1 \cdot 0 \\
1 \cdot 0 \\
0 \cdot 2 \\
0 \cdot 7 \\
1 \cdot 0\end{array}$ & $\begin{array}{l}1 \cdot 0 \\
0 \cdot 2 \\
1 \cdot 0 \\
1 \cdot 0 \\
1 \cdot 0\end{array}$ & $\begin{array}{l}1.0 \\
1.0 \\
1.00 \\
1.0\end{array}$ & $\begin{array}{l}1.0 \\
\begin{array}{l}1.0 \\
1.0\end{array} \\
-\end{array}$ & $\begin{array}{l}1 \cdot 0 \\
1 \cdot 0 \\
1 \cdot 0 \\
-\end{array}$ & $\begin{array}{l}1 \cdot 0 \\
\frac{1}{1 \cdot 0} \\
1 \cdot 0 \\
1 \cdot 0\end{array}$ & $\begin{array}{l}1 \cdot 0 \\
\frac{1 \cdot 0}{1 \cdot 0} \\
1.0\end{array}$ & $\begin{array}{l}1 \cdot 0 \\
-100 \\
1.0 \\
1.0\end{array}$ & $\begin{array}{l}1 \cdot 0 \\
1 \cdot 0 \\
1.0 \\
1.0\end{array}$ & $\begin{array}{l}23 \cdot 9 \\
15 \cdot 5 \\
12 \cdot 1 \\
12 \cdot 1 \\
10 \cdot 7\end{array}$ \\
\hline $\begin{array}{r}6 \\
7 \\
8 \\
9 \\
10\end{array}$ & $\begin{array}{l}1.0 \\
1.0 \\
1.0 \\
1.0 \\
-\end{array}$ & $\begin{array}{l}1 \cdot 0 \\
1.0 \\
1.0 \\
1.0 \\
-\end{array}$ & $\begin{array}{l}1 \cdot 0 \\
1 \cdot 0 \\
1 \cdot 0 \\
1 \cdot 0 \\
-\end{array}$ & $\begin{array}{l}1 \cdot 0 \\
1 \cdot 0 \\
1.0 \\
1 \cdot 0 \\
-\end{array}$ & $\begin{array}{l}1 \cdot 0 \\
1 \cdot 0 \\
1 \cdot 0 \\
1 \cdot 0 \\
-\end{array}$ & $\begin{array}{l}1 \cdot 0 \\
1.0 \\
1.0 \\
1 \cdot 0 \\
-\end{array}$ & $\begin{array}{l}1 \cdot 0 \\
1 \cdot 0 \\
1.0 \\
0 \cdot 8 \\
0 \cdot 4\end{array}$ & $\begin{array}{l}1 \cdot 0 \\
1 \cdot 0 \\
1 \cdot 0 \\
1 \cdot 0\end{array}$ & $\begin{array}{l}1 \cdot 0 \\
1 \cdot 0 \\
1 \cdot 0 \\
0 \cdot 1 \\
0 \cdot 4\end{array}$ & $\begin{array}{l}1 \cdot 0 \\
1 \cdot 0 \\
1 \cdot 0 \\
0 \cdot 1 \\
0 \cdot 9\end{array}$ & $\begin{array}{l}1 \cdot 0 \\
1.00 \\
1 \cdot 0 \\
0 \cdot 1 \\
0 \cdot 0\end{array}$ & $\begin{array}{l}0.5 \\
1.0 \\
1.0 \\
0.1 \\
0.2\end{array}$ & $\begin{array}{l}1 \cdot 0 \\
1 \cdot 0 \\
1 \cdot 0 \\
=\end{array}$ & $\begin{array}{l}1 \cdot 0 \\
1 \cdot 0 \\
1 \cdot 0 \\
- \\
-\end{array}$ & $\begin{array}{l}1.0 \\
1.0 \\
1.0 \\
-\end{array}$ & $\begin{array}{l}1 \cdot 0 \\
1 \cdot 0 \\
1 \cdot 0 \\
\overline{0} \cdot 1\end{array}$ & $\begin{array}{l}1 \cdot 0 \\
1 \cdot 0 \\
1 \cdot 0 \\
=\end{array}$ & $\begin{array}{l}1 \cdot 0 \\
1 \cdot 0 \\
1 \cdot 0 \\
\overrightarrow{0.5}\end{array}$ & $\begin{array}{l}1.0 \\
1.0 \\
1.0 \\
0.4\end{array}$ & $\begin{array}{l}1 \cdot 0 \\
1 \cdot 0 \\
1 \cdot 0 \\
=\end{array}$ & $\begin{array}{l}1.0 \\
1.0 \\
100 \\
=\end{array}$ & $\begin{array}{l}1 \cdot 0 \\
1 \cdot 0 \\
1 \cdot 0 \\
=\end{array}$ & $\begin{array}{l}1 \cdot 0 \\
1 \cdot 0 \\
1 \cdot 0 \\
-\end{array}$ & $\begin{array}{l}1 \cdot 0 \\
1 \cdot 0 \\
1 \cdot 0 \\
-\end{array}$ & $\begin{array}{r}23 \cdot 5 \\
23 \cdot 0 \\
24 \cdot 0 \\
7 \cdot 2 \\
4.5\end{array}$ \\
\hline $\begin{array}{l}11 \\
12 \\
13 \\
14 \\
15\end{array}$ & $\begin{array}{l}\bar{Z} \\
\frac{10}{-}\end{array}$ & $\begin{array}{l}\bar{z} \\
\overline{0} \cdot 7\end{array}$ & $\begin{array}{l}\bar{z} \\
\overline{1} \cdot 0\end{array}$ & $\overline{\bar{z}}$ & $\begin{array}{l}\bar{z} \\
\frac{10}{-}\end{array}$ & $\begin{array}{l}\overline{0 \cdot 1} \\
\overline{0 \cdot 9} \\
0 \cdot 1\end{array}$ & $\overline{\bar{z}}$ & $\begin{array}{l}\bar{Z} \\
\overline{1} \cdot 0 \\
0 \cdot 1\end{array}$ & $\begin{array}{l}\overline{0 \cdot 6} \\
\overline{0 \cdot 7} \\
0 \cdot 1\end{array}$ & $\begin{array}{l}0.2 \\
0.9 \\
-1 \cdot 0 \\
0.7\end{array}$ & 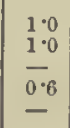 & $\begin{array}{l}0.9 \\
1.0 \\
\overline{0.6} \\
0.1\end{array}$ & $\begin{array}{l}\overline{0.9} \\
\overline{0.5} \\
0.9\end{array}$ & $\begin{array}{l}\overrightarrow{1 \cdot 0} \\
\overline{0.8} \\
1.0\end{array}$ & $\begin{array}{l}\overline{0.2} \\
\overline{0.4} \\
0.9\end{array}$ & $\frac{\overline{0}}{\overline{0.1}}$ & $\frac{\overline{\bar{c}}}{\overline{0.5}}$ & $\begin{array}{l}\bar{z} \\
\overline{0} \cdot 8\end{array}$ & $\begin{array}{l}\bar{z} \\
\overline{1} \cdot 0\end{array}$ & $\begin{array}{l}\bar{z} \\
\overline{\bar{n}} \cdot \overline{7}\end{array}$ & $=$ & $\begin{array}{l}\bar{z} \\
\bar{z}\end{array}$ & $\begin{array}{l}\bar{z} \\
\bar{z}\end{array}$ & $\begin{array}{l}\overline{\bar{c}} \\
\overline{0 \cdot 9}\end{array}$ & $\begin{array}{r}2 \cdot 1 \\
5 \cdot 7 \\
1 \cdot 5 \\
12 \cdot 1 \\
7 \cdot 9\end{array}$ \\
\hline $\begin{array}{l}16 \\
17 \\
18 \\
19 \\
20\end{array}$ & $\begin{array}{l}\overline{0 \cdot 1} \\
\overline{1 \cdot 0} \\
-\end{array}$ & $\frac{\overline{0.5}}{1 \cdot 0}$ & $\begin{array}{l}\overline{0 \cdot 6} \\
\overline{1 \cdot 0} \\
-\end{array}$ & $\begin{array}{l}\overline{0.1} \\
\overline{1.0}\end{array}$ & $\frac{\bar{Z}}{\overline{1 \cdot 0}}$ & $\begin{array}{l}\bar{Z} \\
\overline{0 \cdot 9}\end{array}$ & $\begin{array}{l}\overline{0.3} \\
\overline{0.6} \\
-\end{array}$ & $\begin{array}{l}\bar{Z} \\
\overline{1 \cdot 0}\end{array}$ & $\begin{array}{l}\bar{Z} \\
\overline{1 * 0}\end{array}$ & $\begin{array}{l}\bar{z} \\
\overline{0.5} \\
-\end{array}$ & $\begin{array}{l}\bar{z} \\
\bar{z}\end{array}$ & $\begin{array}{l}\bar{z} \\
\bar{z} \cdot 0\end{array}$ & $\begin{array}{l}\frac{0.8}{0.7} \\
\frac{7.0}{1.0}\end{array}$ & $\begin{array}{l}\frac{1 \cdot 0}{0 \cdot 6} \\
\frac{1 \cdot 0}{0}\end{array}$ & $\frac{1.0}{1.0}$ & $\begin{array}{l}\frac{1 \cdot 0}{1 \cdot 0} \\
\frac{1}{1 \cdot 0}\end{array}$ & $\frac{1.0}{\frac{0.9}{1.0}}$ & $\frac{1.0}{1.0}$ & $\begin{array}{l}\frac{1 \cdot 0}{1 \cdot 0} \\
\frac{1}{1 \cdot 0}\end{array}$ & $\begin{array}{l}\frac{1 \cdot 0}{1 \cdot 0} \\
\overline{1 * 0}\end{array}$ & $\frac{1 \cdot 0}{1 \cdot 0} \frac{1 \cdot 0}{10}$ & $\frac{1.0}{1.0} \frac{1.0}{1.0}$ & $\frac{1 \cdot 0}{\frac{1 \cdot 0}{1 \cdot 0}}$ & $\frac{0.2}{\frac{0.0}{0.9}}$ & $\begin{array}{r}11 \cdot 0 \\
1 \cdot 6 \\
11 \cdot 2 \\
9 \cdot 0 \\
12 \cdot 9\end{array}$ \\
\hline $\begin{array}{l}21 \\
22 \\
23 \\
24 \\
25\end{array}$ & $\begin{array}{l}\bar{z} \\
\bar{z}\end{array}$ & $\begin{array}{l}\bar{z} \\
\bar{z}\end{array}$ & $\bar{z}$ & $\begin{array}{l}\overline{-} \\
0 \cdot 4 \\
=\end{array}$ & $\begin{array}{l}\overline{7} \\
\overline{0.9} \\
-\end{array}$ & $\begin{array}{l}\overline{\overline{0}} \\
\overline{0} \boldsymbol{7}\end{array}$ & $\begin{array}{l}\overline{\overline{1}} \\
\overline{1 \cdot 0}\end{array}$ & $\begin{array}{l}\overline{-} \\
\frac{100}{-}\end{array}$ & $\begin{array}{l}\overline{-} \\
\overline{1 \cdot 0} \\
-\end{array}$ & $\begin{array}{l}\overline{-} \\
\frac{1 \cdot 0}{-}\end{array}$ & $\begin{array}{l}\overline{\overline{1}} \\
\overline{1} \cdot 0 \\
-\end{array}$ & $\begin{array}{l}- \\
0.4 \\
0.6 \\
0.5 \\
0.5\end{array}$ & $\frac{\bar{\tau}}{\overline{1 * 0}}$ & $\begin{array}{l}\overline{\bar{\sigma}} \\
\frac{0.8}{0.8}\end{array}$ & $\begin{array}{l}\overline{-} \\
\overline{0.6} \\
=\end{array}$ & $\frac{\bar{\sigma}}{\overline{0.5}} \overline{\overline{0} \cdot 1}$ & $\frac{\overline{\overline{1}}}{\frac{\overline{1} 0}{0 \cdot 7}}$ & $\frac{\bar{\sigma}}{\frac{\overline{0} \cdot 4}{1 \cdot 0}}$ & $\begin{array}{l}\overline{0.1} \\
0.6 \\
\overline{1.0}\end{array}$ & $\frac{\bar{\sigma}}{\overline{0} \cdot 3}$ & $\frac{\bar{\tau}}{\overline{0.9}}$ & $\begin{array}{l}\overline{\overline{0}} \\
\overline{0.1}\end{array}$ & $\begin{array}{l}z \\
z\end{array}$ & $\begin{array}{l}\bar{z} \\
\bar{z}\end{array}$ & $\begin{array}{r}0.0 \\
0 \cdot 5 \\
13.8 \\
0.5 \\
6.7\end{array}$ \\
\hline $\begin{array}{l}26 \\
27 \\
28 \\
29 \\
30\end{array}$ & $\begin{array}{l}0 \cdot 1 \\
1 \cdot 0 \\
1 \cdot 0 \\
1 \cdot 0 \\
1 \cdot 0\end{array}$ & $\begin{array}{l}0 \cdot 1 \\
1 \cdot 0 \\
1.0 \\
0 \cdot 9 \\
0 \cdot 7\end{array}$ & $\begin{array}{l}0.1 \\
1.0 \\
1.0 \\
0.3\end{array}$ & $\begin{array}{l}\overline{1 \cdot 0} \\
1 \cdot 0 \\
\overline{1} \cdot 0\end{array}$ & $\begin{array}{l}\overline{1 \cdot 0} \\
1 \cdot 0 \\
\overline{1} \cdot 0\end{array}$ & $\begin{array}{l}\overline{1} \cdot 0 \\
1 \cdot 0 \\
0 \cdot 3 \\
1 \cdot 0\end{array}$ & $\begin{array}{l}\overline{1 \cdot 0} \\
1 \cdot 0 \\
\overline{1 \cdot 0}\end{array}$ & $\begin{array}{l}\overline{1 \cdot 0} \\
1.0 \\
\overline{1 \cdot 0}\end{array}$ & $\begin{array}{l}1 \cdot 0 \\
1.0 \\
1.0 \\
1 \cdot 0\end{array}$ & $\begin{array}{l}1 \cdot 0 \\
1.00 \\
1.0 \\
1 \cdot 0\end{array}$ & $\begin{array}{l}1 \cdot 0 \\
1 \cdot 0 \\
1 \cdot 0 \\
1 \cdot 0\end{array}$ & $\begin{array}{l}1 \cdot 0 \\
1.0 \\
1.0 \\
-1.0\end{array}$ & $\begin{array}{l}1.0 \\
1.0 \\
1.0 \\
1.0\end{array}$ & $\begin{array}{l}1 \cdot 0 \\
1 \cdot 0 \\
1 \cdot 0 \\
1 \cdot 0\end{array}$ & $\begin{array}{l}1.0 \\
1.0 \\
1.0 \\
1.0\end{array}$ & $\begin{array}{l}1 \cdot 0 \\
1 \cdot 0 \\
1 \cdot 0 \\
1 \cdot 0\end{array}$ & $\begin{array}{l}1 \cdot 0 \\
1.0 \\
1 \cdot 0 \\
1 \cdot 0\end{array}$ & $\begin{array}{l}1 \cdot 0 \\
1 \cdot 0 \\
1 \cdot 0 \\
0 \cdot 4 \\
1 \cdot 0\end{array}$ & $\begin{array}{l}1 \cdot 0 \\
1 \cdot 0 \\
1 \cdot 0 \\
1 \cdot 0 \\
0.8\end{array}$ & $\begin{array}{l}1.0 \\
1.0 \\
1.0 \\
1.0 \\
-\end{array}$ & $\begin{array}{l}1.0 \\
1.0 \\
0.9 \\
0.4 \\
-\end{array}$ & $\begin{array}{l}0.9 \\
100 \\
0.4 \\
=\end{array}$ & $\begin{array}{l}1 \cdot 0 \\
1.0 \\
0 \cdot 1 \\
- \\
-\end{array}$ & $\begin{array}{l}1 \cdot 0 \\
1 \cdot 0 \\
1 \cdot 0 \\
0 \cdot 9 \\
-\end{array}$ & $\begin{array}{r}16 \cdot 2 \\
24 \cdot 0 \\
22 \cdot 4 \\
5 \cdot 9 \\
17 \cdot 8\end{array}$ \\
\hline 31 & - & - & - & - & - & 0.8 & 1.0 & 1.0 & 1.0 & $1 \cdot 0$ & 1.0 & $1 \cdot 0$ & $1 \cdot 0$ & $1 \cdot 0$ & $1 \cdot 0$ & 1.0 & $1 \cdot 0$ & 1.0 & $1 \cdot 0$ & 1.0 & $1 \cdot 0$ & $1 \cdot 0$ & $1 \cdot 0$ & 1.0 & 18.8 \\
\hline Total. & $14 \cdot 2$ & $13 \cdot 2$ & $12 \cdot 0$ & $12 \cdot 5$ & $13 \cdot 1$ & 147 & $14 \cdot 9$ & $14 \cdot 4$ & $14 \cdot 3$ & $16 \cdot 0$ & $14 \cdot 9$ & $15 \cdot 9$ & $17 \cdot 7$ & $18 \cdot 1$ & 15.6 & $16 \cdot 2$ & $18 \cdot 3$ & $18 \cdot 1$ & $17 \cdot 9$ & $16 \cdot 0$ & $15 \cdot 7$ & $13 \cdot 4$ & $13 \cdot 1$ & $14 \cdot 9$ & $365 \cdot 1$ \\
\hline
\end{tabular}

\section{February, 1903.}

\begin{tabular}{|c|c|c|c|c|c|c|c|c|c|c|c|c|c|c|c|c|c|c|c|c|c|c|c|c|c|}
\hline Day. & 1. & 2. & 3. & 4. & 5. & 6. & 7. & 8. & 8. & 10. & 11. & Noon. & 13. & 14. & 15. & 10. & 17. & 18. & 19. & 20. & 21. & 22. & 23. & Midt. & Day. \\
\hline$\frac{1}{2}$ & $\begin{array}{l}1.0 \\
1.0\end{array}$ & $\begin{array}{l}1 \cdot 0 \\
1 \cdot 0\end{array}$ & $\begin{array}{l}1 \cdot 0 \\
1 \cdot 0\end{array}$ & $\begin{array}{l}1.0 \\
1.0\end{array}$ & $\begin{array}{l}1.0 \\
0.6\end{array}$ & $\begin{array}{l}0.9 \\
0.7\end{array}$ & $\begin{array}{l}0.7 \\
0.7\end{array}$ & $0 \cdot 2$ & $\overline{0 \cdot 4}$ & $\overline{0.6}$ & $\overline{1 \cdot 0}$ & $\overline{1 \cdot 0}$ & $\overline{1.0}$ & $\overline{0.8}$ & $\frac{0 \cdot 1}{-}$ & $\frac{0 \cdot 7}{-7}$ & $1 \cdot 0$ & 10 & $1 \cdot 0$ & $1 \cdot 0$ & 0.8 & 0.8 & 1.0 & 1.0 & $15 \cdot 2$ \\
\hline $\begin{array}{l}3 \\
4\end{array}$ & -0 & -0 & $\overline{10}$ & $\frac{10}{10}$ & -0 & -0 & -0 & $\overline{1 \cdot 0}$ & -0 & $\frac{-}{0.8}$ & $=$ & 0.4 & 1.0 & $\begin{array}{l}1.0 \\
0.4\end{array}$ & $\overline{1} 0$ & $\begin{array}{l}1: 0 \\
100\end{array}$ & $\overline{1.0}$ & $\overline{1 \cdot 0}$ & $\overline{1: 0}$ & 100 & $\overline{100}$ & $\overline{1 \cdot 0}$ & $1 \cdot 0$ & $1 \overline{0}$ & $\begin{array}{l}10.8 \\
12.4 \\
17.7\end{array}$ \\
\hline 5 & - & - & - & - & - & 0.2 & 0.1 & 0.9 & $0 \cdot 1$ & 0.1 & 0.1 & - & - & 0.3 & 0.7 & 0.1 & - & 0.3 & 0.6 & 0.2 & $0 \cdot 1$ & - & 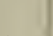 & 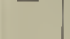 & \\
\hline 6 & 二 & $=$ & $\bar{z}$ & $\overline{\bar{Z}}$ & 二 & 02 & 01 & 0.9 & - & -1 & -1 & 二 & $\bar{z}$ & - & - & $\ldots$ & $=$ & 0.3 & 0.6 & 0.2 & 0.1 & - & - & - & $\begin{array}{l}3 \cdot 8 \\
0 \cdot 0\end{array}$ \\
\hline $\begin{array}{l}7 \\
8\end{array}$ & $\overline{0.3}$ & $\overline{1.0}$ & $\overline{1.0}$ & - & - & - & - & - & $\overline{0.3}$ & $\overline{1} \cdot 0$ & $\overline{1} \cdot 0$ & - & $\overline{1}$ & $\overline{100}$ & $\overline{1}$ & $\overline{1} \cdot 0$ & $\overline{0} \cdot 3$ & 0.3 & 0.8 & 0.7 & 0.1 & - & - & - & 1.9 \\
\hline 9 & 0.3 & $1 \cdot 0$ & 1.0 & $\overline{0.2}$ & $\overline{1 \cdot 0}$ & $\overline{1 \cdot 0}$ & $\overline{1 \cdot 0}$ & $\overline{1 \cdot 0}$ & $\begin{array}{l}0.3 \\
0.9\end{array}$ & $\begin{array}{l}1.0 \\
0.8\end{array}$ & $\begin{array}{l}1.0 \\
1.0\end{array}$ & $\begin{array}{ll}1 \cdot 0 \\
1.0\end{array}$ & $\begin{array}{l}1 \cdot 0 \\
1 \cdot 0\end{array}$ & $\begin{array}{l}1.0 \\
0.4\end{array}$ & $\begin{array}{l}1.0 \\
0.0\end{array}$ & $\begin{array}{l}1.0 \\
0.5\end{array}$ & $\begin{array}{l}0.8 \\
1.0\end{array}$ & $\begin{array}{l}0.2 \\
0 \cdot 2\end{array}$ & 二 & $=$ & $=$ & Z & 二 & $0 \overline{2}$ & $\begin{array}{l}10 \cdot 6 \\
11 \cdot 8\end{array}$ \\
\hline 10 & 0.1 & - & - & - & 0.1 & 0.6 & 1.0 & 1.0 & 0.5 & 1.0 & 1.0 & $1 \cdot 0$ & 0.6 & & & - & 0.4 & 1.0 & 0.4 & $1 \cdot 0$ & 0.8 & - & - & & 5 \\
\hline 11 & . & 1.0 & 1.0 & 1.0 & $1 \cdot 0$ & 1.0 & 1.0 & 1.0 & 1.0 & 1.0 & 1.0 & 1.0 & 1.0 & 1.0 & 1.0 & 1.0 & 1.0 & $0 \cdot 9$ & $0 \cdot 6$ & 1.0 & $0 \cdot 7$ & -1 & 0.7 & 1.0 & \\
\hline 12 & 0.6 & $\bar{z}$ & $\overline{-}$ & $\bar{z}$ & $\overline{0.9}$ & $\overline{1 \cdot 0}$ & $\overline{1} \cdot 0$ & $\overline{1 \cdot 0}$ & $\overline{1 \cdot 0}$ & $\overline{1 \cdot 0}$ & $\overline{1 \cdot 0}$ & $\overline{1 \cdot 0}$ & $\overline{1 \cdot 0}$ & $\begin{array}{l}0.0 \\
1.0\end{array}$ & $\begin{array}{l}1.0 \\
1.0\end{array}$ & $\begin{array}{l}0.1 \\
1 \cdot 0\end{array}$ & $\overline{1 \cdot 0}$ & 0.7 & $1 \cdot 0$ & $1 \cdot 0$ & 1.0 & $10^{\circ}$ & 0.3 & $-\dagger$ & $7 \cdot 3$ \\
\hline $\begin{array}{l}18 \\
14\end{array}$ & $z^{-1}$ & $\bar{z}$ & - & $=$ & - & - & - & - & - & - & - & & - & - & - & - & 10 & 0.9 & 二 & $=$ & $=$ & $\overline{-}$ & $=$ & $\bar{z}$ & $\begin{array}{r}13.8 \\
0.0\end{array}$ \\
\hline 15 & 0.2 & 0.3 & 0.1 & 0.5 & 0.8 & 0.7 & $0 \cdot 3$ & 0.9 & 0.6 & 0.1 & - & & - & & 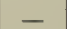 & 0.9 & 1.0 & 1.0 & $1 \cdot 0$ & 1.0 & 0.4 & - & - & - & 9 \\
\hline & 0.8 & 1.0 & $0 \cdot 3$ & 1.0 & 0.6 & 1.0 & 1.0 & 10 & 1.0 & 1.0 & 1.0 & 1.0 & $1 \cdot 0$ & 1.0 & 10 & $1 \circ 0$ & 1.0 & $1 \cdot 0$ & 0.9 & 0.1 & - & - & $\overline{-}$ & $\overline{-}$ & $17 \cdot 7$ \\
\hline $\begin{array}{l}17 \\
18\end{array}$ & $=$ & $\bar{z}$ & $\overline{0 \cdot 3}$ & $\overline{0.3}$ & $\overline{0.5}$ & $\overline{0 \cdot 7}$ & 0.4 & 1.0 & 1.0 & 1.0 & 0.3 & 0.3 & 1.0 & 1.0 & 0.2 & 0.1 & $0 \cdot 1$ & 0.1 & 0.8 & 0.4 & - & - & - & - & $\begin{array}{r}2 \cdot 1 \\
12 \cdot 8\end{array}$ \\
\hline $\begin{array}{l}18 \\
19\end{array}$ & $=$ & $\bar{z}$ & - & 0.3 & 1.0 & $1 \cdot 0$ & $\begin{array}{l}1.0 \\
1.0\end{array}$ & 1.0 & 0.4 & 10 & 10 & $\frac{1 \cdot 0}{-}$ & - & 10 & - & 二 & 10 & 10 & $1 \cdot 0$ & -4 & $=$ & $\bar{z}$ & $\bar{z}$ & 二 & $\begin{array}{c}12 \cdot 8 \\
4 \cdot 7^{\prime \prime}\end{array}$ \\
\hline 20 & - & - & - & - & - & - & - & - & - & - & - & 0.7 & 0.8 & 0.7 & 0.8 & 0.5 & 1.0 & $1 \cdot 0$ & 1.0 & 0.3 & - & - & & - & $6 \cdot 8^{\prime \prime}$ \\
\hline & & & 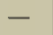 & - & 0.5 & 1.0 & 1.0 & 0.8 & 0.4 & 1.0 & $0 \cdot 2$ & $0 \cdot 1$ & 0.6 & $1 \cdot 0$ & 0.9 & 0.6 & $1 \cdot 0$ & $1 \cdot 0$ & 0.1 & & - & 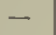 & - & - & $10 \cdot 2$ \\
\hline 28 & - & - & - & - & 0.9 & 0.7 & 0.9 & 0.9 & $1 \cdot 0$ & 1.0 & 1.0 & $1 \cdot 0$ & 1.0 & 1.0 & 10 & 1.0 & 1.0 & 1.0 & 1.0 & 0.3 & - & - & - & - & $14 \cdot 7$ \\
\hline 24 & 二 & $\bar{z}$ & 二 & $\overline{0 \cdot 2}$ & $\overline{1 \cdot 0}$ & $\overline{1 \cdot 0}$ & $\overline{1 \cdot 0}$ & $\overline{1 \cdot 0}$ & $\overline{1.0}$ & $\begin{array}{l}0.5 \\
1.0\end{array}$ & $\begin{array}{l}0.7 \\
1.0\end{array}$ & $\begin{array}{l}0 \cdot 1 \\
1 \cdot 0\end{array}$ & $\begin{array}{l}0.0 \\
1.0\end{array}$ & $\begin{array}{l}0.8 \\
1.0 \\
1.0\end{array}$ & $\begin{array}{l}1 \cdot 0 \\
1 \cdot 0\end{array}$ & $\begin{array}{l}1 \cdot 0 \\
1.0\end{array}$ & $\begin{array}{l}1.0 \\
1.0\end{array}$ & $\begin{array}{l}1 \cdot 0 \\
1 \cdot 0\end{array}$ & $\begin{array}{l}0.7 \\
0.9 \\
0.9\end{array}$ & Z & Z & Z & Z & $\bar{z}$ & $\begin{array}{r}7 \cdot 4 \\
15 \cdot 1\end{array}$ \\
\hline 25 & - & - & - & 0.1 & 0.7 & - & - & - & - & - & - & - & - & - & - & - & - & - & - & - & - & - & - & - & 0 \\
\hline 20 & - & - & - & 二 & 0 & 0.9 & $\overline{1 \cdot 0}$ & $\overline{1} \cdot 0$ & 10 & 10 & - & - & - & - & - & - & - & - & - & - & 一 & 一 & - & - & \\
\hline 28 & $=$ & $=$ & - & $\overline{-}$ & - & -8 & 10 & 0.2 & 0.1 & $\begin{array}{l}1.0 \\
1.0\end{array}$ & $\begin{array}{l}0.4 \\
1 \cdot 0\end{array}$ & $\overline{1} \cdot 0$ & $\overline{1} \cdot 0$ & $\overline{1} \cdot 0$ & $\overline{1} \cdot 0$ & $\overline{1 \cdot 0}$ & $\overline{1} \cdot 0$ & $\overline{1} \cdot 0$ & $\overline{0.9}$ & 二 & 二 & 二 & 二 & 二 & $\begin{array}{r}5.4 \\
10 \cdot 2\end{array}$ \\
\hline Total . . & $5 \cdot 8$ & $6 \cdot 3$ & $5 \cdot 7$ & $6 \cdot 6$ & $11 \cdot 7$ & $13 \cdot 4$ & $13 \cdot 1$ & $13 \cdot 9$ & $11 \cdot 7$ & $13 \cdot 9$ & $12 \cdot 7$ & $12 \cdot 6$ & $13 \cdot 6$ & $14 \cdot 0$ & $14 \cdot 3$ & $13 \cdot 5$ & $16 \cdot 3$ & $16 \cdot 0$ & $14 \cdot 7$ & $9 \cdot 1$ & $5 \cdot 9$ & $3 \cdot 3$ & $3 \cdot 0$ & $3 \cdot 2$ & $255 \cdot 2$ \\
\hline
\end{tabular}


TABLF V. (continued).

DURATION OF BRIGHT SUNSHINE IN HOURS.

March, 1903.

\begin{tabular}{|c|c|c|c|c|c|c|c|c|c|c|c|c|c|c|c|c|c|c|c|c|c|c|c|c|c|}
\hline Day: & 1. & 2. & 3. & 4. & 5. & 6. & 7. & 8. & 9. & 10. & 11. & Noon. & 13. & 14. & 15. & 18. & 17. & 18. & 19. & 20. & 21. & 22. & 23. & Mint. & Day. \\
\hline 1 & - & - & - & 0.2 & $1 \cdot 0$ & $1 \cdot 0$ & 1.0 & $1 \cdot 0$ & $1 \cdot 0$ & $1 \cdot 0$ & $1 \cdot 0$ & 1.0 & $1 \cdot 0$ & $1 \cdot 0$ & $1^{\circ} 0$ & $1 \cdot 0$ & 0.4 & - & - & - & - & - & - & - & $12 \cdot 6$ \\
\hline 2 & - & - & - & - & - & - & - & - & - & $\rightarrow$ & - & 一 & - & - & - & - & - & - & - & - & - & - & - & - & 0.0 \\
\hline $\begin{array}{l}3 \\
4\end{array}$ & $=$ & $=$ & 二 & 二 & 二 & $\vec{z}$ & $\bar{z}$ & $\bar{z}$ & $\bar{z}$ & 二 & $\bar{z}$ & 二 & $\overline{0.7}$ & $\overline{1 \cdot 0}$ & $\overline{1 \cdot 0}$ & $\overline{1 \cdot 0}$ & $\overline{1 \cdot 0}$ & $\overrightarrow{0.5}$ & $=$ & $\overline{-}$ & $=$ & $=$ & - & $\vec{a}$ & 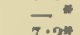 \\
\hline 5 & $=$ & $=$ & 二 & 二 & $=$ & $=$ & $=$ & $=$ & 0.5 & 0.2 & 0.8 & 10 & 1.0 & 1.0 & 1.0 & 1.0 & 1.0 & 0.5 & 二 & $\bar{z}$ & $\bar{z}$ & $\bar{z}$ & 二 & $=$ & $\begin{array}{l}7 \cdot 2^{m} \\
8 \cdot 0\end{array}$ \\
\hline 6 & - & - & - & - & - & - & - & 0.2 & - & - & - & - & - & - & - & - & - & - & - & - & - & - & $=$ & $=$ & 0.2 \\
\hline 7 & - & - & - & - & - & 0.8 & $1^{\circ} 0$ & $1 \cdot 0$ & $1{ }^{\circ} 0$ & $1 \cdot 0$ & $1^{\circ} 0$ & 1.0 & $1 \cdot 0$ & 1.0 & 0.5 & - & - & - & - & - & - & - & - & - & 0.3 \\
\hline 8 & 二 & 二 & $\bar{z}$ & $\bar{z}$ & $=$ & $\bar{z}$ & $\bar{z}$ & $=$ & 二 & 二 & $\bar{z}$ & 二 & - & 二 & - & - & - & - & - & - & - & - & - & - & 0.0 \\
\hline 10 & 二 & $\bar{z}$ & $=$ & $=$ & $\bar{z}$ & $=$ & 0.9 & $\overrightarrow{1 \cdot 0}$ & $\overrightarrow{1 \cdot 0}$ & $1 \cdot 0$ & $\overline{0.9}$ & $\overrightarrow{0.5}$ & 二 & 二 & 二 & 二 & $=$ & 二 & 二 & $=$ & $=$ & $z$ & $=$ & $\vec{z}$ & $\begin{array}{l}0.0 \\
5 \cdot 3\end{array}$ \\
\hline 11 & - & - & - & - & - & - & - & - & - & - & - & - & - & - & - & - & - & - & $\overline{-}$ & $=$ & $=$ & $\overline{-}$ & $=$ & $=$ & 0.0 \\
\hline 12 & - & - & - & - & - & - & - & - & - & - & - & 0.4 & - & - & - & - & 0.2 & - & - & - & - & - & - & - & 0.6 \\
\hline 13 & - & - & - & - & - & - & - & $0 \cdot 5$ & 0.8 & 0.6 & 0.2 & 0.9 & $1 \cdot 0$ & 1.0 & $1 \cdot 0$ & 0.7 & - & - & - & - & - & - & - & - & $H \cdot 7$ \\
\hline 14 & $=$ & $=$ & $=$ & $\bar{z}$ & $z$ & $\bar{z}$ & $=$ & $=$ & $\bar{z}$ & 二 & 二 & 二 & 0.2 & $10^{\circ} 0$ & 1.0 & 1.0 & 0.5 & - & - & - & - & - & - & - & $3 \cdot 7$ \\
\hline $\begin{array}{l}13 \\
16\end{array}$ & - & - & 二 & - & - & - & - & - & $=$ & 二 & 二 & 二 & $=$ & $\overline{0.1}$ & $\overline{1.0}$ & $\overline{1} 0$ & $\overrightarrow{0 * 3}$ & $=$ & 二 & $\bar{z}$ & 二 & 二 & 二 & Z & $\begin{array}{l}0.0 \\
2 \cdot 4\end{array}$ \\
\hline 17 & - & - & - & - & - & - & - & - & - & - & - & - & - & - & - & - & - & - & - & - & - & - & - & - & $0^{\circ} 0$ \\
\hline 18 & - & - & - & - & - & - & - & 0.3 & 1.0 & $1 \cdot 0$ & $1 \cdot 0$ & 1.0 & 1.0 & $1 \cdot 0$ & 1.0 & 0.9 & - & - & - & - & - & - & - & - & $8 \cdot 2$ \\
\hline 19 & 二 & - & 二 & - & - & - & $\overline{0.1}$ & $\overrightarrow{1} \cdot 0$ & 0.1 & 1.0 & 0.2 & 100 & $10^{\circ} 0$ & 0.5 & $\begin{array}{l}0.3 \\
1.0\end{array}$ & $\overrightarrow{r \cdot 0}$ & $\overline{0.3}$ & 二 & 一 & 二 & - & - & - & - & $4 \cdot 1$ \\
\hline $\begin{array}{l}20 \\
21\end{array}$ & 二 & 二 & 二 & $\Xi$ & $\overline{-}$ & 二 & 0.1 & $1 \cdot 0$ & $1 \cdot 0$ & $\begin{array}{l}1.0 \\
0.8\end{array}$ & $\begin{array}{l}1.0 \\
1.0\end{array}$ & $\begin{array}{l}1.0 \\
1.0\end{array}$ & 1.0 & $1 \cdot 0$ & $\begin{array}{l}1.0 \\
0.3\end{array}$ & $1 \cdot 0$ & - & $=$ & 二 & 二 & $=$ & 二 & $\bar{z}$ & 二 & 9.4 \\
\hline & & & & & & & & & & & & & 10 & 10 & & & & & & & & & 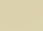 & - & 51 \\
\hline 22 & 二 & 二 & Z & $=$ & $=$ & $\bar{z}$ & - & - & - & - & - & - & $\overline{0.1}$ & $\overline{0 * 7}$ & $\overline{10}$ & $\overline{0 \cdot 1}$ & - & $=$ & - & - & - & - & - & - & 0.0 \\
\hline $\begin{array}{l}28 \\
24\end{array}$ & 二 & 二 & 二 & $\bar{z}$ & $\bar{z}$ & $\bar{z}$ & $\overline{-}$ & $\bar{z}$ & $\bar{z}$ & $\bar{z}$ & 二 & 二 & 0.1 & 0.7 & 10 & 0.1 & $=$ & $\bar{z}$ & $\bar{z}$ & 二 & $=$ & 二 & 二 & 二 & $1 \cdot 9$ \\
\hline 25 & - & - & - & - & - & - & - & - & 0.7 & $1 \cdot 0$ & $1 \cdot 0$ & $1 \cdot 0$ & 100 & 0.7 & 0.9 & 1.0 & $1 \cdot 0$ & 0.4 & - & - & - & - & - & $=$ & $8 \cdot 7$ \\
\hline 26 & - & - & - & - & - & - & - & 一 & - & 0.8 & 0.4 & $1 \cdot 0$ & $1 \cdot 0$ & $1 \cdot 0$ & $1 \cdot 0$ & $1^{\circ} 0$ & 0.3 & - & - & - & - & - & - & - & 6.5 \\
\hline 27 & - & - & - & - & - & - & - & - & 0.5 & 1.0 & $1 \cdot 0$ & $1 \cdot 0$ & 0.6 & $1 \cdot 0$ & 1.0 & $1 \cdot 0$ & 0.2 & - & - & - & - & - & - & - & $7 \cdot 3$ \\
\hline 28 & - & - & - & - & - & - & - & - & $0-3$ & 0.9 & 0.8 & 0.9 & $1 \cdot 0$ & $1 \cdot 0$ & $1 \cdot 0$ & 1.0 & 0.3 & - & 一 & - & - & - & - & - & $7 \cdot 2$ \\
\hline 29 & - & - & - & - & - & - & - & - & - & - & - & 0.1 & 0.7 & $1 \cdot 0$ & 1.0 & 0.2 & - & - & - & - & - & - & - & - & 3.0 \\
\hline 30 & $=$ & - & $=$ & 二 & - & $=$ & 二 & - & $\bar{z}$ & - & $\overline{-}$ & $\overline{0: 3}$ & $\overline{0 \cdot 6}$ & - & - & - & - & - & - & 二 & 二 & $\rightarrow$ & - & - & 0.0 \\
\hline $3 i$ & - & - & 二 & - & - & 一 & - & - & - & - & - & 0.3 & 0.6 & $=$ & - & - & - & - & 一 & - & - & - & - & 二 & $0^{-9}$ \\
\hline Total . . & - & - & - & 0.2 & $1 \cdot 0$ & 1.8 & $3 \cdot 0$ & $5 \cdot 0$ & $7 \cdot 9$ & $11 \cdot 3$ & $10 \cdot 3$ & $13 \cdot 1$ & $13 \cdot 9$ & $15 \cdot 0$ & $15 \cdot 0$ & $11 \cdot 9$ & $5 \cdot 5$ & $1 \cdot 4$ & - & - & - & - & - & - & $118 \cdot 3$ \\
\hline
\end{tabular}

* Doubtful-card for part of day missing.

April, 1903.

\begin{tabular}{|c|c|c|c|c|c|c|c|c|c|c|c|c|c|c|c|c|c|c|c|c|c|c|c|c|c|}
\hline Day. & 1. & 2. & 3. & 4. & 5. & 6. & 7. & 8. & 8. & 10. & 11. & Noon & 13. & 14. & 15. & 16. & 17. & 18. & 19. & 20. & 21. & 22. & 23. & Midt. & Day \\
\hline 1 & - & - & - & - & - & - & - & - & - & 0.2 & - & 0.9 & 1.0 & $1 \cdot 0$ & 0.8 & $\rightarrow$ & - & - & - & - & - & - & - & - & 3.8 \\
\hline 2 & - & - & - & - & - & - & - & - & - & - & - & - & - & - & 0.6 & - & - & - & - & - & - & - & - & - & 0.6 \\
\hline 3 & 一 & - & 一 & - & - & - & 二 & - & 二 & 二 & 二 & - & $\div$ & - & 二 & 二 & - & - & 二 & - & - & 二 & 二 & - & 0.0 \\
\hline $\begin{array}{l}4 \\
5\end{array}$ & 二 & $\overline{-}$ & 二 & 二 & 二 & 二 & 二 & 二 & $=$ & 二 & 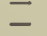 & $\overline{0.5}$ & $=$ & $\overline{0 * 3}$ & $\overrightarrow{0 \cdot 4}$ & 二 & $=$ & 二 & 二 & Z & $=$ & 二 & $=$ & ב & $\begin{array}{l}0 \cdot 0 \\
1 \cdot 2\end{array}$ \\
\hline A & - & - & - & - & - & - & - & - & - & - & - & - & - & - & - & - & - & - & - & - & - & $\rightarrow$ & - & - & 0.0 \\
\hline 7 & 二 & $\bar{z}$ & 二 & $\bar{Z}$ & $\bar{z}$ & $=$ & $=$ & $=$ & - & - & 0.3 & $1 \cdot 0$ & $1 \cdot 0$ & 1.0 & 0.7 & - & - & - & $\overline{-}$ & - & - & - & - & - & 4.0 \\
\hline 8 & - & - & - & - & - & - & - & - & - & - & $0 \cdot 1$ & 0.7 & $1 \cdot 0$ & 100 & 0.3 & - & - & - & - & - & - & - & - & - & $3 \cdot 1$ \\
\hline 9 & - & - & - & - & - & - & - & - & - & - & - & - & - & - & $\rightarrow$ & - & - & - & - & $\rightarrow$ & - & - & - & - & 0.0 \\
\hline 10 & - & - & - & - & - & - & - & - & - & - & - & - & - & - & - & - & - & - & - & $\rightarrow$ & - & - & - & - & * \\
\hline 11 & - & - & - & - & - & - & - & - & - & - & - & 0.2 & - & - & - & - & - & - & - & - & - & - & - & - & 0.2 \\
\hline 12 & - & - & - & - & - & - & - & - & - & - & - & - & - & - & - & - & - & - & - & - & - & - & - & - & 0.0 \\
\hline 13 & - & - & - & - & - & - & 一 & - & - & - & - & - & - & - & - & - & - & - & - & - & - & 一 & - & - & 0.0 \\
\hline 14 & - & - & - & - & - & - & - & - & - & - & - & 0.5 & 0.4 & - & - & - & - & - & 一 & - & - & - & - & - & 0.9 \\
\hline Total & - & - & - & - & - & - & - & - & - & 0.2 & 0.4 & $3 \cdot 8$ & $3 \cdot 4$ & $3 \cdot 3$ & $2 \cdot 8$ & - & - & - & - & - & - & - & - & - & $13 \cdot 9$ \\
\hline
\end{tabular}

* Doubtful-sun did not appear from behind hills until $11.45 \mathrm{a} . \mathrm{m}$. and remained clear until it set. 
TABLE V. (continued).

\section{DURATION OF BRIGHT SUNSHINE IN HOURS.}

\section{September, 1903.}

\begin{tabular}{|c|c|c|c|c|c|c|c|c|c|c|c|c|c|c|c|c|c|c|c|c|c|c|c|c|c|}
\hline 1)ase. & 1. & 2. & 3. & 4. & 5. & 6. & . & 8. & 8. & 10. & 11. & Noon. & 13. & 14. & 15. & 16. & 17. & 18. & 19. & 20. & 21. & 22. & 23. & Mint. & Day. \\
\hline$\frac{1}{2}$ & $=$ & $=$ & $=$ & $=$ & $=$ & $\bar{z}$ & $=$ & $=$ & $=$ & $\bar{z}$ & $=$ & $\bar{z}$ & $=$ & $=$ & $=$ & $=$ & $=$ & $=$ & $\bar{z}$ & $=$ & $=$ & $=$ & $\bar{z}$ & $\bar{z}$ & 0.00 \\
\hline 3 & - & - & - & - & - & - & - & - & -1 & - & - & 0.5 & $10^{\circ}$ & 0.7 & - & - & - & - & - & - & - & - & - & - & $2 \cdot 2$ \\
\hline 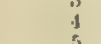 & $=$ & $=$ & $\bar{z}$ & $=$ & $=$ & $\bar{z}$ & $=$ & $\bar{z}$ & $=$ & $\bar{z}$ & 0.4 & $1 \cdot 0$ & $1 \cdot 0$ & 0.1 & $=$ & 二 & $=$ & $=$ & $=$ & $=$ & $\overline{-}$ & $=$ & $=$ & $=$ & $2 \cdot 8 t$ \\
\hline$\frac{B}{7}$ & $\bar{z}$ & $=$ & $\bar{z}$ & $\bar{z}$ & $=$ & $=$ & $=$ & $=$ & $=$ & $\bar{z}$ & $=$ & $\bar{z}$ & $\bar{z}$ & $\bar{z}$ & $=$ & $=$ & $=$ & $\bar{z}$ & $\overline{=}$ & $=$ & $=$ & $=$ & $=$ & $=$ & 二" \\
\hline $\begin{array}{l}8 \\
9\end{array}$ & $=$ & $=$ & $=$ & $=$ & $=$ & $=$ & $=$ & $=$ & $=$ & $\bar{z}$ & $\overline{0.2}$ & $\overline{0.8}$ & $\overline{0.7}$ & $\dot{\overline{0} .2}$ & $=$ & $\bar{z}$ & $=$ & $\bar{z}$ & $\bar{z}$ & $=$ & $=$ & $=$ & $=$ & $=$ & $\overline{1}^{*} \cdot{ }^{*}$ \\
\hline $\begin{array}{l}10 \\
11 \\
12\end{array}$ & 二 & $\bar{z}$ & $\bar{z}$ & z & $\bar{z}$ & $\bar{z}$ & $\bar{z}$ & $\bar{z}$ & ‡ & $\bar{z}$ & $\overline{0.8}$ & $\overline{\overline{0}} \overline{0.8}$ & $\overline{0.9}$ & $\overrightarrow{0.9}$ & $\overline{\overline{0} \cdot 3}$ & $\bar{z}$ & = & $\bar{z}$ & $\bar{z}$ & $=$ & $=$ & $=$ & $\bar{z}$ & $\bar{z}$ & $\begin{array}{l}100 \\
0.0 \\
0.0 \\
3.8\end{array}$ \\
\hline 13 & - & - & - & - & - & - & - & - & - & - & - & - & - & - & - & - & - & - & - & - & - & - & - & - & 0.0 \\
\hline $\begin{array}{l}11 \\
15\end{array}$ & $=$ & $\overline{-}$ & $=$ & $\overline{-}$ & = & 二 & $\overline{-}$ & 二 & $\overline{-}$ & $=$ & $\bar{z}$ & & 0.3 & 0.9 & 0.3 & $\overline{-}$ & $=$ & $=$ & $=$ & 二 & 二 & 二 & $=$ & $\bar{z}$ & $\begin{array}{l}1.5 \\
0.0\end{array}$ \\
\hline $\begin{array}{l}115 \\
17\end{array}$ & $=$ & $=$ & $\bar{z}$ & $\bar{z}$ & $=$ & $=$ & $=$ & $\bar{z}$ & $=$ & 0.6 & 0.9 & $1 \cdot 0$ & $1 \cdot 0$ & 0.6 & $=1$ & $=$ & $=$ & $=$ & $=$ & $=$ & $=$ & $=$ & $=$ & $=$ & $\begin{array}{l}4 \cdot 1 \\
0 \cdot u\end{array}$ \\
\hline${ }_{19}^{18}$ & $1=$ & - & - & - & - & - & - & - & $\bar{\pi}$ & 1.0 & $\begin{array}{l}1.0 \\
1.0\end{array}$ & 10 & $1 \cdot 0$ & $\begin{array}{l}1.0 \\
1 \cdot 0\end{array}$ & 1.0 & $=\overline{0.8}$ & $=$ & - & - & - & - & - & -- & - & $6 \cdot 0)$ \\
\hline $\begin{array}{l}201 \\
21\end{array}$ & $\bar{z}$ & $=$ & $=$ & $=$ & $=$ & $\bar{z}$ & $=$ & $\bar{z}$ & $=$ & $=$ & $=$ & $\bar{z}$ & $=$ & $=$ & $=$ & $=$ & $=$ & $=$ & $=$ & $\overline{=}$ & $=$ & $=$ & $=$ & 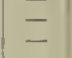 & $\begin{array}{l}0.0 \\
0 \cdot 0\end{array}$ \\
\hline 23 & - & - & - & - & - & - & - & - & - & - & - & - & - & - & - & & - & & & - & - & & - & - & 0.0 \\
\hline $\begin{array}{l}\frac{23}{23} \\
24\end{array}$ & $=$ & $=$ & $=$ & $=$ & $=$ & $=$ & $=$ & $\bar{z}$ & $=$ & $=$ & $=$ & $\overline{0} \cdot 3$ & $\overline{1} \cdot 0$ & $\overline{1} \cdot 0$ & $\overline{1 \cdot 0}$ & $\overline{1 \cdot 0}$ & $\overline{0.5}$ & 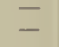 & $=$ & $\bar{z}$ & $=$ & $\overline{-}$ & $=$ & $=$ & $\begin{array}{l}0.0 \\
4 \cdot 8\end{array}$ \\
\hline $\begin{array}{l}2.5 \\
28 \\
27 \\
27\end{array}$ & 三 & $\bar{z}$ & $\Xi$ & $\bar{z}$ & 三 & $\bar{z}$ & $\bar{z}$ & $\frac{0.5}{-}$ & $\stackrel{100}{=}$ & $\stackrel{100}{=}$ & $\begin{array}{l}1 \cdot 0 \\
=\end{array}$ & 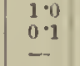 & $\frac{1 \cdot 0}{=}$ & $\begin{array}{l}1.0 \\
0.4 \\
-0.4\end{array}$ & $\frac{1 \cdot 0}{=}$ & $\begin{array}{l}1 \cdot 0 \\
0 \cdot 1 \\
-\end{array}$ & $\begin{array}{l}0.1 \\
0.3\end{array}$ & $\Xi$ & $\bar{z}$ & $\bar{z}$ & $\bar{z}$ & $\bar{z}$ & $\bar{z}$ & $\bar{z}$ & $\begin{array}{l}8 \cdot 6^{* 3} \\
0 \cdot y^{*} \cdot 0\end{array}$ \\
\hline $\begin{array}{r}28 \\
29\end{array}$ & $=$ & $=$ & $=$ & $=$ & $=$ & $=$ & $=$ & $-\overline{0.2}$ & $\overline{0.6}$ & $\overline{1} \cdot 0$ & $\overline{0.7}$ & $\overline{100}$ & -0 & -0 & 70 & $\overline{0.9}$ & 0.3 & $\overline{-}$ & $\overline{-}$ & - & $\overline{-}$ & $=$ & $=$ & - & 0.0 \\
\hline 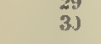 & & $=$ & $\overline{-}$ & $\overline{-}$ & $=$ & $\bar{z}$ & $\overline{0.3}$ & 1.0 & $\begin{array}{l}0.0 \\
1.0\end{array}$ & 100 & 100 & $\begin{array}{l}1.0 \\
1.0\end{array}$ & $\begin{array}{l}1.0 \\
1.0\end{array}$ & $\begin{array}{l}1.0 \\
1.0\end{array}$ & $\begin{array}{l}1.0 \\
1.0\end{array}$ & $\begin{array}{l}0.8 \\
1.0\end{array}$ & $0 . \overline{7}$ & $\overline{-}$ & $\overline{-}$ & $=$ & $\bar{z}$ & $\bar{z}$ & $=$ & $=$ & 1070 \\
\hline Total & - & - & - & - & - & - & 0.3 & $1 \cdot 7$ & $3 \cdot 3$ & $5 \cdot 6$ & 70 & $9 \cdot 6$ & $10^{\circ} \cdot 9$ & $\overline{10 \cdot 1}$ & $\overline{60}$ & $4 \cdot 8$ & 1.9 & - & - & - & - & - & - & - & $61 \cdot 8$ \\
\hline
\end{tabular}

October, 1903.

\begin{tabular}{|c|c|c|c|c|c|c|c|c|c|c|c|c|c|c|c|c|c|c|c|c|c|c|c|c|c|}
\hline Day. & 1. & 2. & 3. & 4. & 5. & 6. & 7. & 8. & s. & 10. & 11. & Noon. & 13. & 14. & 15. & 16. & 17. & 18 & 19. & 20. & 21. & 22. & 23. & Millt. & Day. \\
\hline$\frac{1}{2}$ & 二 & $=$ & $\bar{z}$ & $\bar{z}$ & $=$ & $\bar{z}$ & $\frac{0.2}{-}$ & $\stackrel{0.2}{-2}$ & $=$ & $=$ & $=$ & $=$ & $=$ & $=$ & $=$ & $=$ & $=$ & $=$ & $=$ & $=$ & $=$ & $=$ & $\overline{-}$ & $=$ & $\begin{array}{l}0.4 \\
0.0\end{array}$ \\
\hline 3 & - & - & $=$ & $=$ & $=$ & $=$ & $\overline{-}$ & - & 0.3 & $0 \cdot 7$ & 0.4 & $0 \cdot \tilde{z}$ & $0 \cdot 7$ & $\begin{array}{l}100 \\
1.0\end{array}$ & 0.9 & $\begin{array}{l}1.0 \\
10\end{array}$ & $0: 2$ & - & - & - & - & - & - & - & $5 \cdot 9$ \\
\hline 5 & $\bar{z}$ & $\bar{z}$ & $\bar{z}$ & $=$ & $=$ & $\overline{0.4}$ & $\overline{1} \cdot 0$ & $\overline{1 \cdot 0}$ & $\overline{100}$ & $\overline{1} \cdot 0$ & $\overline{10}$ & 1.0 & $\begin{array}{l}1.0 \\
1.0\end{array}$ & $\begin{array}{l}1.0 \\
1.0\end{array}$ & $\begin{array}{l}10 \\
1.0\end{array}$ & $\begin{array}{l}1.0 \\
1.0\end{array}$ & $\mid \begin{array}{ll}0.6 \\
0.6\end{array}$ & $\overline{-}$ & $\bar{z}$ & $\bar{z}$ & $\bar{z}$ & $\overline{-}$ & $\overline{-}$ & $\overline{-}$ & $\begin{array}{r}4.9 \\
11.0\end{array}$ \\
\hline 7 & $\bar{z}$ & $\bar{z}$ & $\bar{z}$ & $=$ & 二 & $\overline{0.6}$ & $\overline{1} \cdot 0$ & $\overline{1.0}$ & $\overline{1} \cdot 0$ & $\overline{1} \cdot 0$ & $\overline{10} 0$ & $\overline{1 \cdot 0}$ & $\overline{1} \cdot 0$ & $\overline{1} \cdot 0$ & $\overline{1} \cdot 0$ & $\overline{100}$ & $\overline{1} \cdot 0$ & $\overline{0.9}$ & $=$ & $=$ & $=$ & $=$ & $=$ & $\bar{z}$ & $\begin{array}{l}0.00 \\
12.5\end{array}$ \\
\hline $\begin{array}{l}8 \\
9\end{array}$ & $=$ & $=$ & $=$ & $=$ & $=$ & 0.6 & $1 \cdot 0$ & $1 \cdot 0$ & $1 \cdot 0$ & $1 \cdot 0$ & $1 \cdot 0$ & $1 \cdot 0$ & $1 \cdot 0$ & $\begin{array}{l}1 \cdot 0 \\
0.1\end{array}$ & $1{ }^{\circ 0}$ & $1 \cdot 0$ & $0 \cdot 6$ & $\stackrel{0 \cdot 3}{-}$ & $=$ & $=$ & $=$ & $=$ & $\bar{z}$ & $=$ & 111.5 \\
\hline $\begin{array}{l}10 \\
11\end{array}$ & $=$ & $\bar{z}$ & $=$ & $\bar{z}$ & $=$ & 0.5 & $\overline{1 \cdot 0}$ & $1 \cdot 0$ & 1.0 & $1 \cdot 0$ & 10 & $1 \cdot 0$ & 100 & 1.0 & $1-0$ & 1.0 & $1 \cdot 0$ & 1.0 & $1 \cdot 0$ & $\overline{05}$ & $\bar{z}$ & $=$ & $\bar{z}$ & z & $\begin{array}{l}1.00 \\
1100\end{array}$ \\
\hline 12 & $=$ & $=$ & $\bar{z}$ & $\overline{-}$ & $\overline{0.8}$ & $\overline{10} \cdot 0$ & $\overline{1} \cdot 0$ & $\overline{1 \cdot 0}$ & $\overline{1} 00$ & $\overline{10}$ & $\overline{10} 0$ & $\overline{1} \cdot 0$ & $\overline{1} 0$ & $\overline{10}$ & $\overline{1} \cdot 0$ & $\overline{1} \cdot 0$ & $\overline{1 \cdot 0}$ & $\overline{1 \cdot 0}$ & $\overline{0} \cdot 5$ & $=$ & $=$ & $=$ & $\bar{z}$ & $=$ & $14 \cdot 3$ \\
\hline 13 & $=$ & $=$ & $=$ & $=$ & 0.5 & $1 \cdot 0$ & $1 \cdot 0$ & $1 \cdot 0$ & 1.0 & $1 \cdot 0$ & 1.0 & $1 \cdot 0$ & 1.0 & 0.9 & $=$ & $\stackrel{0.1}{-}$ & $\begin{array}{l}0: 3 \\
0: 9\end{array}$ & $\begin{array}{l}0.1 \\
1.0\end{array}$ & $\overline{0.8}$ & $=$ & $=$ & $=$ & $=$ & - & $2 \cdot 9 \cdot 9$ \\
\hline $\begin{array}{l}15 \\
16 \\
17\end{array}$ & $\bar{z}$ & $\bar{z}$ & $\bar{z}$ & $\bar{z}$ & $\begin{array}{l}0.9 \\
\overline{-}\end{array}$ & $\begin{array}{l}\overline{1.0} \\
\bar{z}\end{array}$ & $\begin{array}{l}1 \cdot 0 \\
0 \cdot 3 \\
-\end{array}$ & $\begin{array}{l}\overline{1} \cdot 0 \\
1 \cdot 0 \\
-\end{array}$ & $\begin{array}{l}100 \\
1.0\end{array}$ & $\begin{array}{l}\overline{1} \cdot 0 \\
0 \cdot 8 \\
-\end{array}$ & $\begin{array}{l}1.0 \\
1.0 \\
\end{array}$ & $\begin{array}{l}0.8 \\
0.3\end{array}$ & $\begin{array}{l}0.3 \\
0.9\end{array}$ & $\begin{array}{l}0.1 \\
1.0 \\
-1\end{array}$ & $\overline{10}$ & $\overline{0.7}$ & 0.8 & $1 \cdot 0$ & $\begin{array}{l}1 \cdot 0 \\
0 \cdot 1\end{array}$ & $0 \cdot 3$ & $\bar{z}$ & $\bar{z}$ & $\bar{z}$ & z & $\begin{array}{r}8 \cdot 1 \\
11 \cdot 2 \\
0.1\end{array}$ \\
\hline $\begin{array}{l}18 \\
19\end{array}$ & = & $=$ & $=$ & 0.2 & $\begin{array}{ll}1 & 0 \\
1.0 & 0\end{array}$ & $\begin{array}{l}0.9 \\
1.0\end{array}$ & 0.8 & $\begin{array}{l}0.4 \\
1 \\
10\end{array}$ & 100 & 1.0 & $\overline{1}_{0}$ & 1.0 & & 1. & 0.6 & $\begin{array}{ll}1 \cdot 0 \\
100\end{array}$ & 0.1 & $0 \cdot 6$ & $1 \cdot 0$ & 0.2 & $=$ & $\bar{z}$ & $=$ & $z$ & 6.6 \\
\hline 20 & $\bar{z}$ & $=$ & $=$ & 0.1 & $\begin{array}{l}1.0 \\
1.0\end{array}$ & $\begin{array}{l}1.0 \\
1.0\end{array}$ & $\begin{array}{l}100 \\
10\end{array}$ & $\begin{array}{l}1.0 \\
1.0 \\
1.0\end{array}$ & $\begin{array}{l}10 \\
10\end{array}$ & $\begin{array}{l}1.0 \\
1.0\end{array}$ & $\begin{array}{l}1.0 \\
1.0 \\
1.0\end{array}$ & $\begin{array}{l}1.0 \\
1.0\end{array}$ & $\begin{array}{l}1.0 \\
10 \\
1\end{array}$ & $\begin{array}{l}1.0 \\
110 \\
10.0\end{array}$ & $\begin{array}{l}1.0 \\
10.0\end{array}$ & $\begin{array}{l}1.0 \\
1\end{array}$ & 1.0 & 1.0 & 0.2 & $=$ & $=$ & $=$ & $=$ & 二 & $\begin{array}{l}14.13 \\
1 ! 3\end{array}$ \\
\hline 22 & - & - & $\overline{-}$ & $=$ & $\bar{z}$ & $\bar{z}$ & - & $\stackrel{1-0}{-}$ & $\begin{array}{l}1.0 \\
0.1\end{array}$ & $\begin{array}{l}1.0 \\
1.0\end{array}$ & $\begin{array}{l}1.0 \\
1.0\end{array}$ & $\begin{array}{l}1.0 \\
0.4\end{array}$ & - & -10 & - & $=$ & $\overline{0} \cdot 1$ & $=$ & $=1$ & $=$ & $\overline{-}$ & $=$ & $\bar{z}$ & $\bar{z}$ & $2 \%$ \\
\hline $\begin{array}{l}23 \\
23\end{array}$ & $=$ & $=$ & $=$ & $=$ & $=$ & $=$ & $=$ & $=$ & $=$ & $=$ & $=$ & $=$ & $\overline{-}$ & $\bar{z}$ & $=$ & $=$ & $=$ & $=$ & $=1$ & $=$ & $=$ & $=$ & $\bar{z}$ & $=$ & -" \\
\hline 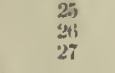 & z & $\bar{z}$ & $=$ & $\overline{0 \cdot 1}$ & $\overline{1.0}$ & $\overline{0.5}$ & $\overline{0} \cdot 3$ & $\overline{0.3}$ & $\overline{0.1}$ & $\overline{0.1}$ & $\frac{0.7}{z}$ & $\bar{z}$ & $\bar{z}$ & 三 & ב & $\begin{array}{l}0.3 \\
=\end{array}$ & $\begin{array}{l}0 \cdot 2 \\
=\end{array}$ & $\begin{array}{l}0 \cdot 1 \\
=\end{array}$ & $=$ & $\bar{z}$ & $\bar{z}$ & $=$ & $\bar{z}$ & $\bar{z}$ & $\begin{array}{l}1 \cdot 3 \\
2 \cdot 4 \\
0 \cdot 0\end{array}$ \\
\hline $\begin{array}{l}23 \\
23\end{array}$ & $=$ & $=$ & $=$ & $\overline{0.1}$ & $\overline{0.8}$ & $=$ & $\overline{-}$ & $\bar{z}$ & $=$ & $=$ & $\overline{0.6}$ & $\overline{0.2}$ & $\overline{0.6}$ & $\overline{0.4}$ & $\overline{0.6}$ & $\overline{0.1}$ & $\bar{z}$ & $=$ & $=$ & $\bar{z}$ & $=$ & = & $=$ & - & $\begin{array}{l}0.0 \\
3.0 \\
3 \cdot 4\end{array}$ \\
\hline 31 & $\overline{-}$ & $=$ & $\bar{z}$ & - & $=$ & $=$ & $=$ & = & $=$ & $=$ & $=$ & $=$ & $=$ & $=$ & $=$ & $=$ & $=$ & $=$ & $=$ & $=$ & $=$ & $=$ & $\bar{z}$ & - & $\begin{array}{l}0.0 \\
0.0\end{array}$ \\
\hline Total. & - & - & - & 0.5 & $\overline{7 \cdot 0}$ & $8 \cdot 5$ & $\overline{11 \cdot 0}$ & $11 \cdot 9$ & $11 \cdot 5$ & $12 \cdot \sqrt{2}$ & $13 \cdot 7$ & $11 \cdot 7$ & 12.5 & 12.5 & $11 \cdot 8$ & 11.2 & $9 \cdot 5$ & $7 \cdot 9$ & $1 \%$ & 1.0 & - & - & - & - & $159 \cdot 4$ \\
\hline
\end{tabular}


'Tава: V. (contimed).

DURATION OF BIIGHT SUNSHINE IN HOURS

November, 1903.

\begin{tabular}{|c|c|c|c|c|c|c|c|c|c|c|c|c|c|c|c|c|c|c|c|c|c|c|c|c|c|}
\hline Day. & 1. & 2. & 3. & 4. & 5 & 6. & 7. & 8. & 8. & 10. & 11. & Noon. & 13. & 14. & 15. & 16. & 17. & 18. & 19. & 20. & 21. & 22. & 23. & Mlitt. & Day. \\
\hline 1 & - & - & - & - & - & - & - & - & - & - & - & - & - & - & - & - & - & - & - & - & - & - & - & - & $3 \cdot 0^{n}$ \\
\hline 2 & - & - & - & - & - & - & - & - & - & - & - & - & - & - & - & - & - & - & - & - & - & - & 0.2 & 0.7 & $111 \cdot 9 *$ \\
\hline 3 & 1.0 & 1.0 & $1 \cdot 0$ & 1.0 & $1 \cdot 0$ & 1.0 & 10 & 1.0 & $\left.1^{\circ}\right)$ & 1.0 & 1.0 & $I^{\circ} 0$ & 1.0 & 1.0 & 1.0 & $1 \cdot 0$ & $1 \%$ & $0 \%$ & - & - & - & 0.2 & - & 0.1 & 17.5 \\
\hline 4 & 0.8 & $0: 3$ & 0.7 & $0 \cdot 3$ & 0.6 & - & 0.4 & 0.4 & $1 \cdot 0$ & 1.0 & $0 \cdot 9$ & 1.0 & 0.7 & $0 \cdot 1$ & $0 \cdot 3$ & 0.1 & - & - & - & - & - & - & - & - & $8 \cdot 4$ \\
\hline 5 & - & - & - & - & - & - & 0.9 & 0.9 & - & - & $\longrightarrow$ & - & - & - & - & - & - & - & - & - & $\rightarrow$ & - & - & - & 1.8 \\
\hline 6 & - & - & - & - & - & - & 一 & - & 一 & - & 一 & - & - & - & - & - & - & - & - & - & - & - & - & - & 0.0 \\
\hline 7 & - & - & - & - & - & - & - & - & - & - & - & - & - & - & - & - & - & - & - & - & - & - & - & - & 0.0 \\
\hline 8 & - & - & - & - & - & - & - & - & - & - & - & - & - & - & - & - & - & - & - & - & - & - & $\overline{0}$ & - & 0.0 \\
\hline 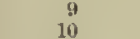 & $\overline{1 \cdot 0}$ & $\overrightarrow{1 \cdot 0}$ & $\overline{1 \cdot 0}$ & $\overrightarrow{1 \cdot 0}$ & $\overline{1 \cdot 0}$ & $\overrightarrow{1 \cdot 0}$ & $\overrightarrow{1.0}$ & $\overline{1.0}$ & $\overline{10}$ & $\overline{1} \cdot 0$ & $\overline{1 \cdot 0}$ & $\overrightarrow{1 \cdot 0}$ & $\overline{1 \cdot 0}$ & $\overline{1}: 0$ & $\overline{1} \cdot 0$ & $\overline{1 \cdot 0}$ & $\overline{1} \cdot 0$ & $\overline{1 \cdot 0}$ & $\overrightarrow{1 \cdot 0}$ & $\overrightarrow{1} \cdot 0$ & $\overline{0} \cdot x$ & $=$ & 0.7 & $1 \cdot 0$ & $1 \cdot 7$ \\
\hline 11 & - & - & $\rightarrow$ & 一 & - & - & - & - & - & - & 0.1 & - & 0.4 & - & - & - & - & $\rightarrow$ & - & 0.7 & 1.0 & 1.0 & $(0 \cdot 2$ & 0.2 & 3.6 \\
\hline 12 & 1.0 & 10 & $1 \cdot 0$ & $1 \cdot 0$ & $1 \cdot 0$ & 1.0 & 0.5 & 0.9 & $0 \cdot 9$ & - & 0.1 & - & $\rightarrow$ & - & - & - & - & - & - & - & - & - & - & - & $8 \cdot 1$ \\
\hline 13 & - & - & - & - & 0.4 & 0.7 & 1.0 & 0.7 & $1 \cdot 0$ & 1.0 & 1.0 & 1.0 & $1 \% 0$ & 1.0 & 1.0 & 1010 & 0.5 & - & - & - & - & 0.4 & 0.6 & 0.7 & $13 \% 0$ \\
\hline 11 & 0.4 & - & 0.4 & $0^{\circ} \theta$ & $10^{\circ}$ & 0.7 & $\because$ & 0.7 & 1.0 & 1.0 & $i \cdot 0$ & $1 \cdot 0$ & $1 \cdot 0$ & 1.0 & 1.0 & $1 \cdot 0$ & 1.0 & $1 \cdot 0$ & 1.0 & $1 \cdot 0$ & $1^{\circ} 0$ & $0^{-16}$ & $0 \div 3$ & $1 \cdot 0$ & $19 \cdot 1$ \\
\hline 15 & 1.0 & 1.0 & 1.0 & $1 \cdot 0$ & 1.0 & $1 \cdot 0$ & 0.5 & $0 \cdot 1$ & $0 \cdot 3$ & 0.1 & - & 0.1 & ij $\cdot 1$ & - & 0.2 & 0.1 & - & 0.2 & - & - & - & - & - & - & $7 \cdot 7$ \\
\hline 16 & - & - & - & - & - & - & - & $0 \cdot 6$ & $1 \cdot 0$ & 1.0 & 1.0 & 1.0 & 1.0 & 1.0 & 1.0 & $0 \cdot y$ & 0.9 & - & 0.4 & - & - & - & - & - & $9 \cdot 8$ \\
\hline 17 & - & - & - & - & $0 \cdot 3$ & - & - & - & 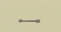 & - & - & - & $\ldots$ & - & $\longrightarrow$ & - & - & - & - & - & - & - & - & - & $0: 3$ \\
\hline Is & - & - & - & - & - & $0: 3$ & 1.0 & 1.0 & 1.0 & 1.0 & 10 & 10 & 1.0 & 1.0 & 1.0 & $1 \cdot 0$ & 100 & $1 \cdot 0$ & $1 \cdot 0$ & 1.0 & 1.0 & 1.0 & 1.0 & 1.0 & $18 \cdot 3$ \\
\hline 19 & $1 \%$ & 1.0 & 1.0 & 100 & 1.0 & $1 \cdot 0$ & 1.0 & 10 & 1.0 & $1 \cdot 0$ & $1 \cdot 0$ & $10^{\circ}$ & 1.0 & $1 \cdot 0$ & 1.0 & $1 \cdot 0$ & 1.0 & $1 \cdot 0$ & 0.8 & 0.1 & - & - & - & $0 \cdot \beta$ & $19 \cdot 8$ \\
\hline 21 & 0.1 & - & - & 0.8 & 1.0 & 1.0 & 1.0 & 1.0 & 1.0 & 1.0 & 100 & 100 & 1.0 & 1.0 & 1.0 & $1 \cdot 0$ & 1.0 & 1.0 & $1 \cdot 0$ & 1.0 & 1.0 & 1.0 & 1.0 & 1.0 & (2) $\cdot 9$ \\
\hline 21 & $1 \cdot 0$ & 1.0 & 1.0 & 1.0 & $i \cdot 0$ & 1.0 & $1 \cdot 0$ & $i \cdot 0$ & $1 \cdot 0$ & $1 \cdot 0$ & $1 \cdot 0$ & 1.0 & 1.0 & 1.0 & $i=0$ & 0.7 & - & - & 0.1 & - & - & - & - & - & $15 \cdot 8$ \\
\hline 22 & 0.3 & 0.4 & 0.4 & - & - & - & - & 0.9 & 0.5 & 0.9 & $1 \cdot 0$ & $0 \cdot 7$ & 0.7 & - & 0.4 & $0 \cdot 5$ & 0.1 & - & - & - & - & - & - & 0.6 & $7 \cdot 4$ \\
\hline 23 & - & - & - & - & - & - & - & $0 \cdot 2$ & 1.0 & 0.7 & 0.5 & - & 0.4 & $0 \cdot 3$ & $0 \%$ & $0: 3$ & 0.5 & - & $0 \cdot 3$ & - & - & 0.1 & $\overline{-}$ & 0.4 & $5 \cdot 2$ \\
\hline $2 !$ & $0 \cdot 7$ & - & - & - & - & - & - & - & - & - & - & 0.8 & 1.0 & $1 \cdot 0$ & 1.0 & 1.0 & 1.0 & 1.0 & 1.0 & 10 & 1.0 & 1.0 & 1.0 & 1.0 & 13.5 \\
\hline 25 & 1.0 & 1.0 & $1 \cdot 0$ & 1.0 & 1.0 & $1 \cdot 0$ & 1.0 & $1 \cdot 0$ & 1.0 & 1.0 & 1.0 & 1.0 & 1.0 & 1.0 & 100 & $1 \cdot 0$ & 1.0 & 1.0 & $i=0$ & 1.0 & 1.0 & $1 \cdot 0$ & 1.0 & 1.0 & $21 \cdot 0$ \\
\hline 26 & $1 \cdot 0$ & 1.0 & 1.0 & $10^{\circ}$ & $1 \cdot 0$ & $1 \cdot 0$ & 1.0 & $0 \cdot 5$ & 0.7 & 0.7 & 0.4 & - & $\rightarrow$ & - & - & - & - & - & - & - & - & - & - & - & $9 \cdot 3$ \\
\hline 27 & - & - & - & - & - & - & - & - & - & - & - & - & - & - & - & - & - & - & - & - & - & 0.1 & - & - & 0.1 \\
\hline 28 & - & - & - & 0.2 & 1.0 & 0.7 & - & - & - & - & 0.1 & 0.3 & - & - & 一 & - & - & - & - & - & - & - & - & - & $2 \cdot 3$ \\
\hline $\begin{array}{l}29 \\
30\end{array}$ & - & $=$ & $\bar{z}$ & 二. & $\overline{-}$ & - & $\overline{-}$ & 二 & 二 & - & $=$ & $\overline{-}$ & - & $\overline{-}$ & 一 & - & 二 & $\overline{-}$ & - & 二 & $=$ & - & - & - & 0.0 \\
\hline & & & & & & & 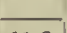 & & & - & & - & - & & - & - & & - & 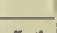 & 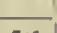 & & & & 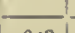 & \\
\hline Total. . & $10 \cdot 7$ & $8 \cdot \pi$ & 9.5 & $9 \cdot 9$ & $12 \cdot 3$ & $11 \cdot 4$ & $11: 3$ & $12 \cdot 9$ & $14 \cdot 4$ & $13 \cdot 4$ & $13 \cdot 1$ & $12 \cdot 9$ & $13 \cdot 3$ & 11.4 & $12 \cdot 4$ & $11 \cdot 6$ & $10^{\circ} 0$ & $\tau \cdot 1$ & $7 \cdot 6$ & $7 \cdot 1$ & $6 \cdot 8$ & $6 \cdot 4$ & 6.0 & $9 \cdot 3$ & $262 \cdot 8$ \\
\hline
\end{tabular}

* Doubtful-card for part of day missing.

December, 1903.

\begin{tabular}{|c|c|c|c|c|c|c|c|c|c|c|c|c|c|c|c|c|c|c|c|c|c|c|c|c|c|}
\hline Day. & 1. & 2. & 3. & 4. & 5. & 6. & 7. & 8. & 9. & 10. & 11. & Noon. & 13. & 14. & 15. & 16. & 17. & 18. & 19. & 20. & 21. & 22. & 23. & Midt. & Day: \\
\hline 1 & - & - & - & - & - & - & - & - & - & - & - & 0.1 & - & - & - & - & - & 0.5 & - & - & - & - & - & - & $0 \cdot 6$ \\
\hline 2 & $\rightarrow$ & - & - & - & - & 0.2 & 1.0 & - & - & - & $\rightarrow$ & - & - & - & - & 0.1 & - & 0.2 & 0.5 & 0.3 & 0.6 & 10 & 1.0 & $1 \cdot 0$ & $5 \cdot 8$ \\
\hline 3 & 0.1 & - & 二 & - & - & - & 0.4 & $1 \cdot 0$ & 0.4 & 1.0 & $1 \cdot 0$ & 1.0 & $1 \cdot 0$ & 1.0 & 100 & $1 \cdot 0$ & $1 \cdot 0$ & 0.8 & 0.8 & 0.8 & - & - & 0.2 & 0.5 & 13.0 \\
\hline $\begin{array}{l}4 \\
5\end{array}$ & $\frac{c \cdot 5}{-}$ & 二 & 二 & 二 & 二 & 二 & Z & 二 & 二 & $\overline{-}$ & $\overline{-}$ & 二 & 二 & 二 & 二 & 二 & $\overline{0 \cdot 5}$ & $\overrightarrow{0.4}$ & $\overline{0.5}$ & - & $=$ & 二 & - & 二 & 0.5 \\
\hline 6 & 0.1 & $\overline{0.6}$ & 0.9 & 1.0 & $1 \cdot 0$ & 1.0 & 1.0 & $1 \cdot 0$ & 1.0 & $1 \cdot 0$ & $\overline{1 \cdot 0}$ & $\overline{0.9}$ & $\overline{-}$ & $\overline{0.4}$ & $\overrightarrow{1.0}$ & $\overrightarrow{10}$ & 1.0 & 1.0 & 1.0 & $\overline{1.0}$ & $\overline{1 \cdot 0}$ & $\overline{10} 0$ & $\overline{10} 0$ & $\overline{1 \cdot 0}$ & $20 \cdot 9$ \\
\hline 7 & 0.9 & 0.8 & 0.9 & 0.5 & $1 \cdot 0$ & $1 \cdot 0$ & $1 \cdot 0$ & $1 \cdot 0$ & $1 \cdot 0$ & $1 \cdot 0$ & 1.0 & $1^{\circ} 0$ & 1.0 & $1 \cdot 0$ & $1 \cdot 0$ & 1.0 & $1 \cdot 0$ & 1.0 & 1.0 & $1 \cdot 0$ & 1.0 & 1.0 & $1 \cdot 0$ & 1.0 & $23 \cdot 1$ \\
\hline 8 & 1 & $1 \cdot 0$ & 1.0 & $1 \cdot 0$ & $i \cdot 0$ & 10 & $1 \cdot 0$ & 1.0 & $0 \cdot 6$ & 1.0 & 1.0 & 1.0 & 1. & 1.0 & 1.0 & 0 & - & & 0 . & $1 \cdot 0$ & & $0 \cdot 9$ & & 1.0 & $20 \cdot 2$ \\
\hline 9 & $1 \%$ & $\begin{array}{l}1 \cdot 0 \\
1.0\end{array}$ & $\begin{array}{l}0.9 \\
1.0\end{array}$ & 0.5 & 1.0 & 1.0 & 1.0 & $1 \cdot 0$ & 1.0 & $1 \cdot 0$ & 1.0 & 1.0 & 1. & 1.0 & 1.0 & 1. & 1.0 & 1.0 & $1 \cdot 0$ & $1 \cdot 0$ & 1.0 & 1.0 & 1.0 & $1 \cdot 0$ & $23 \cdot 4$ \\
\hline $\begin{array}{l}10 \\
11\end{array}$ & $\begin{array}{l}1.0 \\
1.0\end{array}$ & $\begin{array}{l}1 \cdot 0 \\
1.0\end{array}$ & $\begin{array}{l}1 \cdot 0 \\
1 \cdot 0\end{array}$ & $\begin{array}{l}1 \cdot 0 \\
1.0\end{array}$ & $\begin{array}{l}1 \cdot 0 \\
1 \cdot 0\end{array}$ & $\begin{array}{l}1 \cdot 0 \\
1.0\end{array}$ & $\begin{array}{l}1 \cdot 0 \\
1 \cdot 0\end{array}$ & $\begin{array}{l}\mathbf{l} \cdot 0 \\
\mathbf{1} \cdot 0\end{array}$ & $\begin{array}{l}1 \cdot 0 \\
1 \cdot 0\end{array}$ & $\begin{array}{l}1.0 \\
1.0\end{array}$ & $\begin{array}{l}1.0 \\
1.0\end{array}$ & $\begin{array}{l}1.0 \\
1.0\end{array}$ & $\begin{array}{l}1.0 \\
1.0\end{array}$ & $\begin{array}{l}1 \cdot 0 \\
1 \cdot 0\end{array}$ & $\begin{array}{l}1 \cdot 0 \\
1.0\end{array}$ & 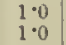 & $\begin{array}{l}1.0 \\
1.0\end{array}$ & $\begin{array}{l}1 \cdot 0 \\
1\end{array} \cdot 0$ & $\begin{array}{l}1.0 \\
1.0\end{array}$ & $\begin{array}{l}1.0 \\
0.8\end{array}$ & $\begin{array}{l}1 \cdot 0 \\
0.9\end{array}$ & $\begin{array}{l}1 \cdot 0 \\
1 \cdot 0\end{array}$ & $\begin{array}{l}1 \cdot 0 \\
1 \cdot 0\end{array}$ & $\begin{array}{l}1.0 \\
1.0\end{array}$ & $\begin{array}{l}21 \cdot 0 \\
23 \cdot 7\end{array}$ \\
\hline 12 & 1.0 & 1.0 & $1 \cdot 0$ & 0.4 & 0.3 & $1 \cdot 0$ & 0.5 & $1 \cdot$ & & 1 & 1. & & 7. & 1. & 1. & $1 \cdot 8$ & $1 \cdot 0$ & 1.0 & $1^{\circ}$ & & & $10^{\circ}$ & 1.0 & 1.0 & \\
\hline l. & 1 & $1 \cdot 0$ & 0. & 0 & 1. & 1. & 1 & 1. & 1 & 1 & 1. & 1.0 & 1 . & 1. & 1 & 1. & 1 . & 1 & 1. & 1. & 1 & 1. & & 1.0 & $23 \cdot 3$ \\
\hline 11 & 1. & 1.0 & 1.0 & 1. & 1.0 & 1. & 1. & 1. & i. & $1 \cdot 0$ & 1.0 & 1.0 & 1 . & 1 . & 1.0 & 1 & 1.0 & 1. & 1 . & 1. & 1 . & i & 1.0 & $1 \cdot 0$ & $24 \cdot 0$ \\
\hline 15 & 1.0 & 1.0 & 1.0 & 1.0 & 1.0 & 1.0 & $1 \cdot 0$ & 1.0 & 1.0 & 10 & 1.0 & 1.0 & 1.0 & 1.0 & 1.0 & 1. & 1.0 & $i .0$ & 1. & $1 \cdot 0$ & 1.0 & 1. & $1 \cdot 0$ & 1.0 & 240 \\
\hline 16 & $i \cdot 0$ & $1 \cdot 0$ & 0.8 & $1 \cdot 0$ & 1.0 & $1 \cdot 0$ & 0.5 & $1 \cdot 0$ & $1 \cdot 0$ & 1.0 & 1.0 & 1.0 & $1 \cdot 0$ & 1.0 & 1.0 & 1.0 & $1 \cdot 0$ & 1.0 & $1 \cdot 0$ & 1.0 & $1 \cdot 0$ & 1.0 & 1.0 & 10 & $23 \cdot 3$ \\
\hline 17 & 1.0 & 1.0 & 0.8 & 0.5 & 1.0 & 1.0 & $1 \cdot 0$ & 1.0 & $1 \cdot 0$ & $1 \cdot 0$ & 1.0 & 0.8 & $1 \cdot 0$ & 1. & 1.0 & 1.0 & 1.0 & 1. & 1.0 & 0.8 & 1.0 & 1.0 & 0.1 & - & $21 \cdot 0$ \\
\hline & - & & - & - & & - & - & & 0 & 0 & $0^{\circ}$ & 0.8 & 0 & 0 & 0 & 1 & 0.9 & & 1. & 0.9 & $0^{\circ}$ & 0. & 0.9 & 1.0 & 11 \\
\hline 19 & 100 & $1 \cdot 0$ & 1.0 & $1 \cdot 0$ & 1.0 & 1.0 & $1 \cdot 0$ & 1.0 & 1. & 1. & 1. & 1.0 & 1. & $0^{\circ}$ & 0.4 & 0. & - & 0.5 & 0.1 & - & 0.9 & 1.0 & 1.0 & 1.0 & $18 \cdot 9$ \\
\hline $\begin{array}{l}\text { 2) } \\
21\end{array}$ & $\begin{array}{l}1 \cdot 0 \\
0 \cdot 0\end{array}$ & $\begin{array}{l}1.0 \\
1.0\end{array}$ & $\begin{array}{l}0.9 \\
0.9\end{array}$ & $\begin{array}{l}0.8 \\
0.5\end{array}$ & $\begin{array}{l}1.0 \\
1.0\end{array}$ & $\begin{array}{l}1.0 \\
1.0\end{array}$ & $\begin{array}{lll}1 & 0 & 0 \\
1 & \cdot & 0\end{array}$ & $\begin{array}{l}1 \cdot 0 \\
1.0\end{array}$ & $\begin{array}{l}1.0 \\
1.0\end{array}$ & $\begin{array}{l}1.0 \\
1.0\end{array}$ & 1.0 & 1.0 & $\begin{array}{l}1 \cdot 0 \\
0.6\end{array}$ & $1 \cdot 0$ & $1 \cdot 0$ & $10^{\circ}$ & $10^{\circ}$ & 1.0 & 1.0 & 0.5 & - & z & 二 & 二 & $\begin{array}{l}19 \cdot 2 \\
11 \cdot 6\end{array}$ \\
\hline & - & - & - & - & - & - & - & 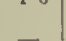 & 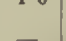 & 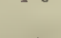 & 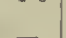 & 10 & & & & & & & 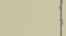 & & 0.2 & 0.0 & & 100 & \\
\hline $\begin{array}{l}22 \\
23\end{array}$ & 1.0 & 1.0 & 1.0 & 0.5 & 0.3 & 0.7 & 0.3 & $\overrightarrow{1.0}$ & $\overline{1.0}$ & $\overline{1 \cdot 0}$ & $\overline{1.0}$ & $\begin{array}{l}0.7 \\
0.9\end{array}$ & $\begin{array}{l}1 \cdot 0 \\
0 \cdot 2\end{array}$ & 0.3 & 0.3 & 二 & 二 & 二 & 二 & - & - & - & 10 & 1.0 & $\begin{array}{l}5.5 \\
9.8\end{array}$ \\
\hline 24 & 一 & - & - & - & - & - & - & - & - & - & - & - & - & $\bar{z}$ & $\bar{z}$ & 二 & 二 & 二 & - & 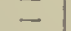 & $=$ & 二 & $=$ & $=$ & 0.0 \\
\hline 25 & - & $\overrightarrow{10}$ & - & - & - & - & - & - & - & - & 0.8 & 1.0 & 1.0 & 1.0 & 1.0 & $10^{\circ}$ & 1.0 & 1.0 & $10^{\circ}$ & 1.0 & 1.0 & 1.0 & 1.0 & 1.0 & $13 \cdot 8$ \\
\hline 20 & 1.0 & $10^{\circ} 0$ & $1^{\circ} 0$ & 1.0 & $10^{\circ} 0$ & $1^{\circ} 0$ & 1.0 & $1 \cdot 0$ & 1.0 & $1 \cdot 0$ & $1 \cdot 0$ & $1 \cdot 0$ & 1.0 & $1 \cdot 0$ & 1.0 & 1.0 & $1 \cdot 0$ & $1 \cdot 0$ & $1^{\circ} 0$ & 1.0 & $10^{\circ}$ & $10^{\circ} 0$ & 1.0 & 1.0 & $21 \cdot 0$ \\
\hline 27 & 1.0 & 1.0 & $1 \cdot 1$ & $0^{\circ}$ & 0.5 & $1^{\circ} 0$ & 1.0 & 1.0 & 1.0 & 1. & 1. & - & - & - & 0.4 & 0.5 & 1.0 & 1. & 1. & 1. & $1{ }^{\circ}$ & 1. & 100 & 1.0 & 19 \\
\hline 29 & 1.0 & 1.0 & 100 & 1.0 & 109 & 1.0 & 1. & 1. & 1. & $1 \cdot 0$ & $1 \cdot 0$ & 1.0 & 1.0 & 1.0 & 1.0 & 0.8 & $0 \cdot 6$ & 1. & 1. & $1 \cdot 0$ & 1. & 1.0 & 1.0 & 1.0 & $23 \cdot 1$ \\
\hline $\begin{array}{l}29 \\
31)\end{array}$ & $\begin{array}{l}1.0 \\
1.0\end{array}$ & 1.0 & 100 & $1 \cdot 0$ & 100 & 1.0 & 1.0 & 1.0 & $1^{\circ} 0$ & 10 & $1 \cdot 0$ & 1.0 & 1.0 & $10^{\circ} 0$ & 1.0 & 0.9 & - & $0 \cdot 1$ & 1.0 & $1 \cdot 0$ & 0.1 & $\overline{0.1}$ & 二 & 1.0 & $\begin{array}{r}18 \cdot 1 \\
8 \cdot 0\end{array}$ \\
\hline $\begin{array}{l}30 \\
31\end{array}$ & 1.0 & $\begin{array}{l}0.9 \\
0 \cdot 1\end{array}$ & 10 & 1.0 & 0.5 & $\frac{0.3}{-}$ & $\begin{array}{l}0.5 \\
0.2\end{array}$ & $\frac{0 \cdot 3}{-}$ & $\begin{array}{l}0.8 \\
0.5\end{array}$ & $\begin{array}{l}0.1 \\
0.8\end{array}$ & $\begin{array}{l}1.0 \\
0.9\end{array}$ & $\begin{array}{l}0.5 \\
1.0\end{array}$ & $\overline{0.4}$ & $\overline{0.9}$ & $\overline{0.9}$ & $\overrightarrow{0.8}$ & $\overline{1} \cdot 0$ & $\overline{0.4}$ & $\overline{0.2}$ & $\overline{0.5}$ & 1.0 & $\begin{array}{l}0.1 \\
0.4\end{array}$ & $\overline{0.1}$ & 0.9 & $\begin{array}{r}80 \\
11.0\end{array}$ \\
\hline Total. & $20 \cdot 2$ & $20 \cdot 1$ & $19 \cdot 9$ & 16.7 & $19 \cdot 0$ & $20 \cdot 2$ & 2014 & $21 \cdot 3$ & $21 \cdot 7$ & $\overline{22 \cdot 8}$ & 21.5 & $23 \cdot 7$ & $20 \% 6$ & 19.9 & $20 \cdot 3$ & $20 \cdot 1$ & $19 \cdot 0$ & $19 \cdot 9$ & $20 \cdot 1$ & $10 \cdot 5$ & $18 \cdot 1$ & $10 \cdot 8$ & $19 \cdot 3$ & 21.1 & $190 \cdot 1$ \\
\hline
\end{tabular}


Table V. (continued).

\section{DURATION OF BRIGH'T SUNSHINE IN HOURS.}

\section{January, 1904.}

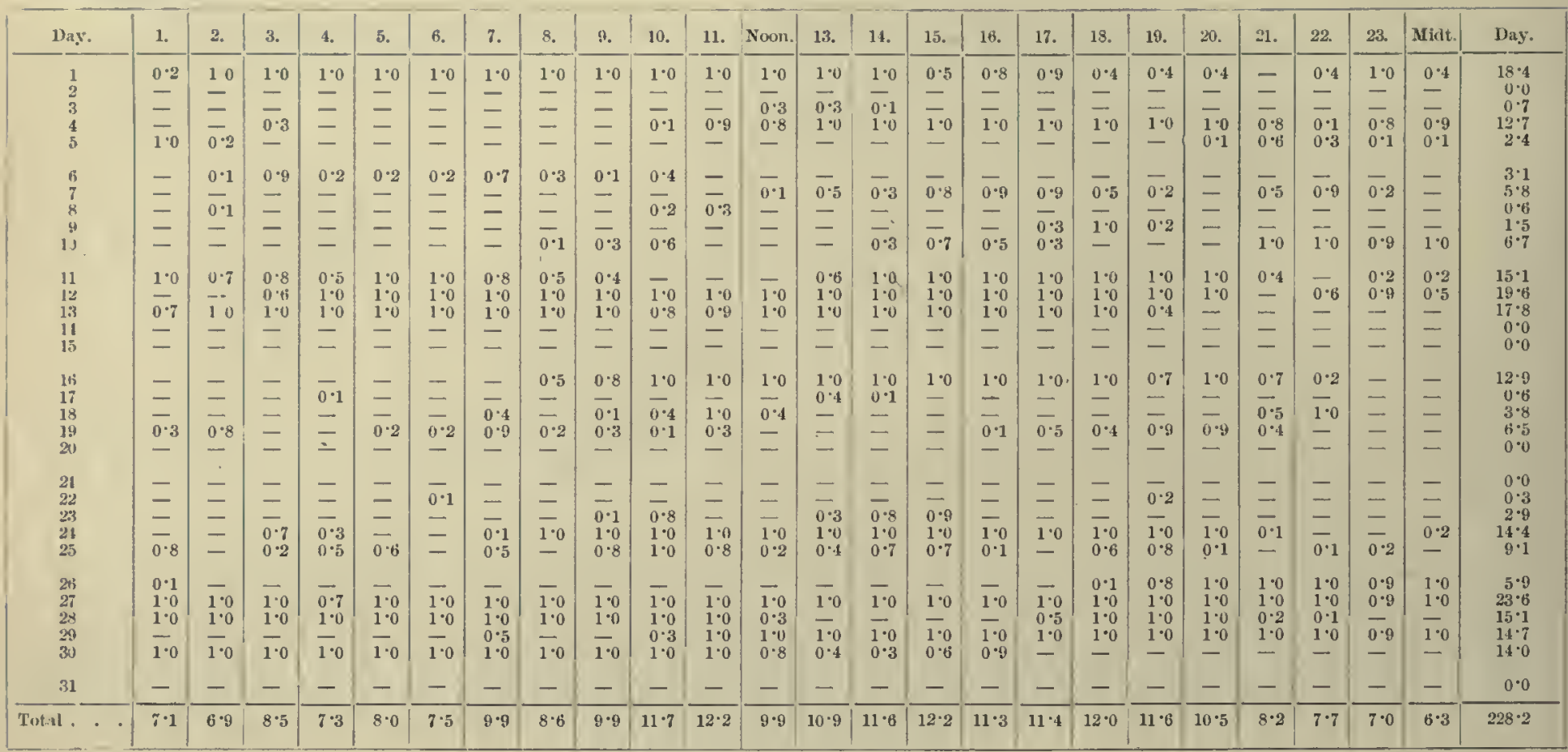




\section{SUMMARY TABLES OF OBSERVATIONS}

OF THE

"DISCOVERY" EXPEDITION

AND

OTHER ANTARCTIC EXPEDITIONS. 


\begin{tabular}{|c|c|c|c|c|c|c|c|c|c|c|c|c|c|c|c|c|c|c|c|c|c|c|c|c|c|c|c|c|c|}
\hline \multicolumn{3}{|c|}{ 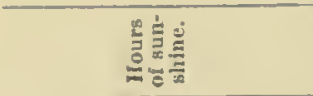 } & 1 & 1 & 1 & 1 & 1 & 1 & I & के़े & 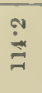 & 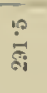 & ְִ & $\overrightarrow{0}$ & 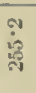 & $\stackrel{m}{\stackrel{m}{7}}$ & פ্] & 1 & I & 1 & 1 & 5 & 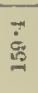 & 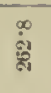 & $\dot{8}$ & 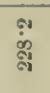 & & 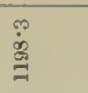 & 苍 \\
\hline \multirow{6}{*}{ 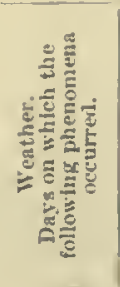 } & & 3 & 1 & - & $\rightarrow$ & o & $\sim$ & 1 & 18 & 9 & $\bullet$ & 1 & $1-$ & $\infty$ & 1 & 1 & 1 & 68 & 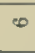 & $\infty$ & $\infty$ & $\infty$ & 9 & $\therefore$ & 10 & ㅇ & 1 & F & 8 \\
\hline & & 'uI & 1 & 1 & ง & ส & \& & $\therefore$ & $=$ & $\bullet$ & $\infty$ & $\rightarrow$ & m & 0 & - & 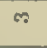 & - & $\Phi$ & $\theta$ & $\cong$ & $=$ & $=$ & $\therefore$ & 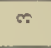 & a & 1 & 1 & $\approx$ & $\bar{z}$ \\
\hline & & $\overrightarrow{8}$ & 10 & 10 & 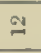 & $\infty$ & ఇ & $=$ & $=$ & 19 & $\stackrel{7}{2}$ & $\infty$ & 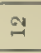 & $\infty$ & $\infty$ & 9 & $\approx$ & $\cong$ & 읙 & $\circ$ & 0 & $\cdot \circ$ & $\exists$ & 용 & $1-$ & 2 & 1 & $\mathscr{乃}$ & 茎 \\
\hline & & 0 & 0 & $\infty$ & 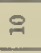 & $a$ & $\therefore$ & 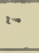 & $\AA$ & $=$ & 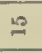 & $\approx$ & 0 & $\infty$ & 0 & 2 & 7 & $\infty$ & $1-$ & बs & 18 & $\therefore$ & 2 & $=$ & $\infty$ & $\stackrel{\infty}{=}$ & $\because$ & 点 & 5 \\
\hline & & $\cdot$ & 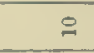 & $\therefore$ & 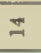 & $\cong$ & 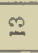 & $\dddot{-}$ & $\simeq$ & 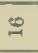 & $\stackrel{9}{\sim}$ & $\Rightarrow$ & $\exists$ & $\because$ & 88 & 3 & 19 & $\Rightarrow$ & 궈 & 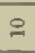 & $\cong$ & $\stackrel{2}{2}$ & $\stackrel{\infty}{\sim}$ & $\therefore$ & $I$ & $=$ & 0 & $\stackrel{8}{0}$ & $\stackrel{?}{=}$ \\
\hline & & 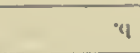 & 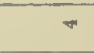 & $\infty$ & 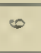 & 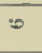 & $\approx$ & $=$ & $\therefore$ & " & $\infty$ & $\infty$ & $\infty$ & 10 & N & 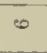 & $=$ & $\approx$ & $=$ & $\cong$ & 9 & \& & 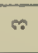 & + & $\sigma$ & $\sim$ & + & $\vec{a}$ & 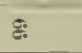 \\
\hline \multirow{14}{*}{ 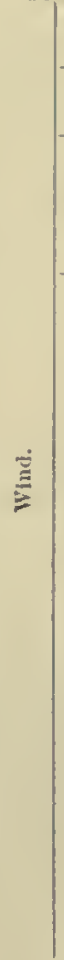 } & & 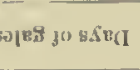 & 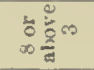 & $\infty$ & 0 & + & 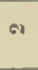 & $\infty$ & $\lessdot$ & - & N & 1 & 1 & 1 & 1 & N & 1 & 9 & $=$ & 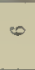 & t- & 10 & $*$ & - & 1 & 1 & 1 & हे & 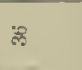 \\
\hline & !m sัแ & $10.1+4$ jo S.5e(I & है & $\therefore$ & r- & $=$ & $*$ & - & $\infty$ & c. & $\therefore$ & $*$ & $\infty$ & - & 0 & t- & $\infty$ & 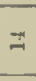 & $\infty$ & $\infty$ & 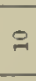 & - & 9 & $\Xi$ & $\infty$ & ก & $\infty$ & : & $\stackrel{\circ}{\circ}$ \\
\hline & 쑤 & 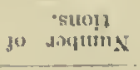 & ผै & $\stackrel{n}{*}$ & 路 & 䘠 & 芦 & 굠 & 용 & 施 & (7) & in & 於 & 愈 & 号 & $\frac{1}{6}$ & \% & 里 & $\stackrel{5}{\%}$ & $\frac{\pi}{6}$ & ติ & 廉 & 竅 & $\vec{B}$ & $\overrightarrow{3}$ & $\Xi$ & $\because$ & $\overline{\bar{i}}$ & 䒴 \\
\hline & & 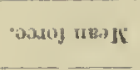 & $\frac{1}{8}$ & $\stackrel{\leftrightarrow}{\circ}$ & $\dot{p}$ & $\overrightarrow{\dot{\theta}}$ & $\stackrel{9}{-}$ & $\stackrel{5}{4}$ & $\begin{array}{l}\infty \\
\vdots \\
\vdots 1\end{array}$ & $\stackrel{40}{i}$ & $\stackrel{\leftrightarrow}{i}$ & $\dot{i}$ & $\stackrel{\dot{i}}{\text { in }}$ & $\ddot{i}$ & $\stackrel{\circ}{i}$ & $\ddot{\therefore}$ & $\stackrel{8}{\circ}$ & is & $\ddot{\Delta}$ & $\dot{i}$ & 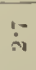 & $\stackrel{\infty}{\Leftrightarrow}$ & iे & $\underset{\sim}{x}$ & $\dot{\beta}$ & $\stackrel{P}{i}$ & $\stackrel{0}{i}$ & $\stackrel{0}{\rightarrow}$ & $\vec{i}$ \\
\hline & & ' & 1 & 1 & 1 & - & -1 & -1 & 1 & * & $\rightarrow$ & - & $*$ & $\infty$ & - & - & ه & a & $\infty$ & $\infty$ & 0 & $\infty$ & $\infty$ & $\infty$ & $*$ & + & - & er & $\infty$ \\
\hline & & sw & $\cong$ & a & \& & 12 & ศิ & $\stackrel{\infty}{\sim}$ & $\cong$ & ส & 9 & 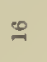 & 9 & $\approx$ & ส & $\cong$ & है & $\mathcal{i}$ & 8 & : & $\overline{\mathrm{N}}$ & $\Xi$ & ส & $=$ & $\exists$ & $\stackrel{\infty}{\sim}$ & $=$ & 9 & มี \\
\hline & & 'MN & क & 1 & $\therefore$ & - & - & 1 & $\infty$ & 1 & 1 & n & $\infty$ & a & 1 & 1 & I & 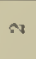 & - & - & - & - & - & - & $\infty$ & $\infty$ & I & ๑ & क \\
\hline & & A & 1 & 1 & 1 & I & 1 & 1 & - & 1 & 1 & 1 & 1 & 1 & 1 & 1 & 1 & 1 & 1 & 1 & 1 & 1 & 1 & 1 & 1 & 1 & 1 & 0 & 1 \\
\hline & & "MS & $\Leftrightarrow$ & $\infty$ & 1 & $\infty$ & $\curvearrowright$ & $\therefore$ & - & 1 & - & 1 & - & 1 & 1 & 1 & I & - & $r$ & 1 & ^ & - & $r$ & 1 & 1 & 1 & I & - & - \\
\hline & & 's & cu & 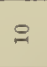 & - & - & $\Leftrightarrow$ & - & $\therefore$ & - & + & $\rightsquigarrow$ & $\infty$ & + & $\sim$ & 1 & - & 1 & 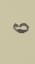 & 9 & $\infty$ & + & $\infty$ & $=$ & 0 & - & $\%$ & $\bullet$ & + \\
\hline & & as & 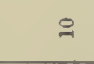 & జ & \& & e. & 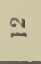 & $=$ & $=$ & $\therefore$ & $\cong$ & $\cong$ & $=$ & 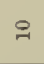 & $=$ & $=$ & 0 & $\Rightarrow$ & э & $\infty$ & $=$ & ? & 고 & $\dddot{\circledast}$ & $\Xi$ & $=$ & $=$ & $=$ & $=$ \\
\hline & & अ & 8 & 8 & ๙ิ & 8 & ฆิ & $\because$ & ๓ & $\bar{ल}$ & $\vec{c}$ & $\bar{\varpi}$ & $\overline{\mathrm{I}}$ & $=$ & $=$ & o & ణి & $\because$ & 尺ે & $\stackrel{R}{:}$ & S & $\approx$ & $B$ & ฒ & 일 & ๓ి & లి & $\ddot{\infty}$ & ำ \\
\hline & & " $\mathrm{x}$ & \& & H & $=$ & ชิ & $\bar{s}$ & $\because$ & 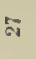 & $\approx$ & สี & $\infty$ & a & o. & $\because$ & $\vec{a}$ & สิ & 영 & $\cong$ & $\stackrel{\infty}{\sim}$ & di & ๓ & เิ & 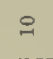 & 0 & $\Re$ & $\stackrel{\infty}{\sim}$ & $:$ & $\stackrel{2}{\sim}$ \\
\hline & & $\mathbf{N}$ & - & 18 & or & $\infty$ & $\because$ & 18 & $\nabla$ & ๓ & - & $\mathscr{4}$ & ลี & si & ๓ & $\approx$ & 18 & 10 & $\infty$ & $\infty$ & $a$ & $=$ & - & $\infty$ & $\approx$ & ง & $\infty$ & 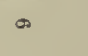 & $n$ \\
\hline \multirow{6}{*}{ 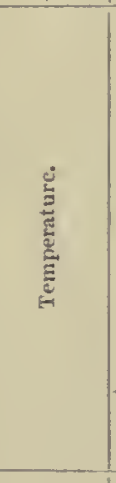 } & \multirow{6}{*}{ 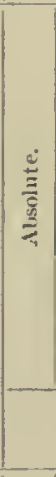 } & วรันเหา & - $\stackrel{\circ}{\dot{2}}$ & $\dot{5}$ & $\overline{10}$ & $\begin{array}{ll}80 \\
80 \\
80\end{array}$ & $\stackrel{\circ}{\circ}$ & 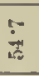 & $\ddot{8}$ & $\begin{array}{l}0 \\
0 \\
0\end{array}$ & $\begin{array}{l}\infty \\
0 \\
0 \\
010\end{array}$ & on & $\stackrel{\infty}{\vec{\omega}}$ & $\stackrel{\circ}{\circ}$ & $\stackrel{-}{\doteqdot}$ & : & 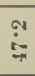 & $\ddot{8}$ & $\stackrel{\circ}{\dot{0}}$ & $\begin{array}{l}8 \\
0 \\
0 \\
0\end{array}$ & $\stackrel{8}{8}$ & $\stackrel{8}{i}$ & $\stackrel{\infty}{\dot{b}}$ & $\ddot{\mathscr{g}}$ & $\overrightarrow{\dot{m}}$ & $\frac{\infty}{\bar{m}}$ & $\overline{\dot{m}}$ & $\ddot{\leftrightarrow}$ & $\ddot{\Xi}$ \\
\hline & & •əted & สี้ & 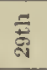 & छ్ & Е & Е & 䒕 & 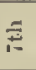 & हีँ & 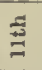 & $\stackrel{\Xi}{\Xi}$ & 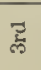 & 营 & $\stackrel{\Xi}{\vec{*}}$ & 苂 & 芯 & : & ส్ & ह్ & छี & 宽 & 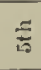 & एँ & : & 莣 & : & 离粉 & हैं \\
\hline & & • แกเแ!̣! & $\begin{array}{r}0 \\
0 \\
0 \\
1\end{array}$ & $\begin{array}{c}a \\
0 \\
1 \\
0\end{array}$ & $\stackrel{\overrightarrow{0}}{i}$ & 䒢 & $\stackrel{i}{i}$ & $\begin{array}{l}\dot{p} \\
\dot{m} \\
i \\
i\end{array}$ & 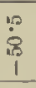 & i⿱ & $\dot{m}$ & $\dot{\dot{\theta}}$ & $\dddot{9}$ & $:$ & $\begin{array}{l}\ddot{b} \\
1\end{array}$ & $\begin{array}{l}8 \\
\dot{8} \\
1 \\
1\end{array}$ & $\stackrel{\dot{9}}{i}$ & $\begin{array}{c}0 \\
\text { i. } \\
1 . \\
\end{array}$ & i & 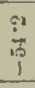 & 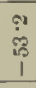 & $\begin{array}{l}0 \\
0 \\
0 \\
1 \\
1\end{array}$ & $\stackrel{\infty}{\vdots}$ & $\begin{array}{l}\text { i⿱ } \\
\text { is } \\
1\end{array}$ & : & $\dot{q}$ & 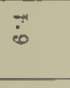 & $\ddot{i}$ & $\begin{array}{l}0 \\
3 \\
1 \\
1\end{array}$ \\
\hline & & aped & 䒕 & $\tilde{\Xi}$ & 㶓 & 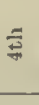 & $\stackrel{5}{\tilde{D}}$ & 莣 & जे & 节 & $\stackrel{\vec{n}}{\tilde{m}}$ & 穿 & हี & $\tilde{+}$ & $\widetilde{\Xi}$ & 폼 & สี๋ & 吾 & $\Xi$ & & ฟั้ & శ్ & 莣 & 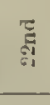 & 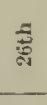 & $\underset{\exists}{\Xi}$ & 范 & 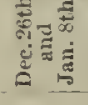 & 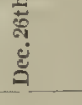 \\
\hline & & 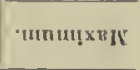 & 。 & $\frac{1}{8}$ & $\ddot{\theta}$ & 喟 & : & 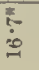 & : & : & 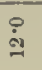 & $\sin ^{\alpha}$ & $\dot{\dot{g}}$ & $\stackrel{\dot{B}}{\dot{g}}$ & ֻั & 落 & is & $\stackrel{9}{\circ}$ & ? & : & $\stackrel{\infty}{\prime}$ & 8 & ? & $\stackrel{\circ}{\circ}$ & 임 & $\begin{array}{l}\infty \\
\dot{m} \\
\dot{m}\end{array}$ & $\ddot{0}$ & & ì \\
\hline & & ' нвап & 。 & i & $\bar{i}$ & 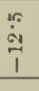 & $\begin{array}{l}0 \\
\stackrel{0}{7} \\
1\end{array}$ & $\begin{array}{r}\overrightarrow{1} \\
1 \\
1\end{array}$ & $\stackrel{8}{0}$ & $\begin{array}{l}0 \\
7 \\
1 \\
0\end{array}$ & $\begin{array}{l}0_{\infty}^{\infty} \\
1 \\
\end{array}$ & 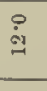 & $\overrightarrow{\tilde{\varepsilon}}$ & $\vec{\beta}$ & $\because$ & $\begin{array}{l}\alpha \\
0 \\
1\end{array}$ & $\begin{array}{l}9 \\
\dot{1} \\
1\end{array}$ & $\begin{array}{l}0 \\
\dot{0} \\
1 \\
1\end{array}$ & $\stackrel{\infty}{\ddot{m}}$ & i) & $\begin{array}{r}4 \\
0 \\
1 \\
1\end{array}$ & $\begin{array}{l}0 \\
0 \\
1\end{array}$ & $\begin{array}{l}\infty \\
0 \\
1\end{array}$ & $\vec{i}$ & :- & घั่ & $\stackrel{\leftrightarrow}{\pi}$ & $\ddot{\dot{\theta}}$ & $\begin{array}{l}\dot{i} \\
1\end{array}$ \\
\hline \multirow{6}{*}{ 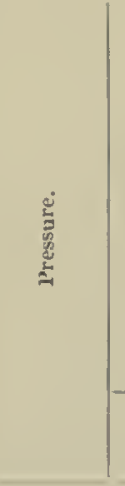 } & \multirow{6}{*}{ 菩 } & ๑รีเ & $\stackrel{\infty}{g}: \stackrel{\infty}{:}$ & $\begin{array}{l}0 \\
0 \\
0 \\
0 \\
\end{array}$ & 莡 & $\stackrel{乛}{-}$ & $\begin{array}{l}\stackrel{0}{0} \\
- \\
\end{array}$ & : & 葛 & 农 & 高 & 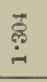 & $\begin{array}{l}\text { क्ष } \\
0\end{array}$ & 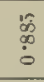 & 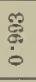 & $\stackrel{3}{0}$ & 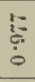 & : & $\stackrel{\vec{g}}{\stackrel{\leftrightarrow}{-}}$ & 产 & דיר & 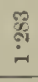 & 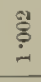 & 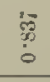 & 产 & $\stackrel{8}{0}$ & 菅 & 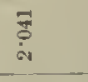 & $\stackrel{p}{0}$ \\
\hline & & ०วभน & $\bar{E}$ & 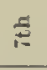 & : & ثิ & 范 & $\Xi$ & ప & 章 & \pm & $\underline{\underline{n}}$ & 5 & ซี้ & 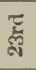 & 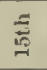 & जี & $\widetilde{\Xi}$ & $\bar{E}$ & $\stackrel{0}{0}$ & 营 & 芯 & 志 & 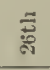 & ฐี้ี & 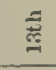 & $\equiv$ & 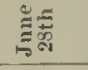 & 然焉 \\
\hline & & 'นกแนฺฺยร & 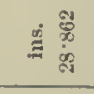 & \begin{tabular}{l}
\multirow{2}{*}{} \\
$\dot{\alpha}$ \\
$\dot{\alpha}$ \\
\end{tabular} & 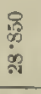 & 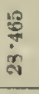 & 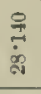 & 范 & "ุ. & 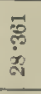 & 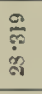 & 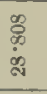 & $\overrightarrow{7}$ & $\begin{array}{l}\overline{0} \\
\text { है }\end{array}$ & : & 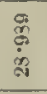 & 疍 & 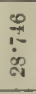 & 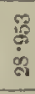 & $\stackrel{9}{7}$ & 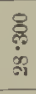 & 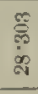 & 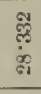 & $\begin{array}{c}\infty \\
\substack{b \\
\vdots \\
d} \\
d\end{array}$ & 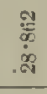 & $\begin{array}{l}\overrightarrow{6} \\
\dot{b} \\
a \\
0\end{array}$ & $\begin{array}{c}\overrightarrow{1} \\
\dot{0} \\
\dot{\alpha}\end{array}$ & 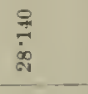 & $\begin{array}{l}\text { क्ष } \\
\vdots \\
\text { aे }\end{array}$ \\
\hline & & ควาน II & हี & 5 & 盇 & $\stackrel{\Xi}{\Xi}$ & $=$ & $\stackrel{\Xi}{\Xi}$ & $\overline{\Sigma_{n}}$ & $\frac{5}{4}$ & ซ్ & 퐃 & $\bar{\Xi}$ & $\Xi$ & 疍 & 莣 & $\Xi$ & $\Xi$ & 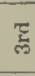 & $\frac{\vec{s}}{\tilde{n}}$ & $\vec{\Delta}$ & \pm & $\stackrel{\Xi}{ت}$ & 캐ํ & $\overrightarrow{\tilde{\infty}}$ & 荧 & \pm & 跣 & 量蒂 \\
\hline & & 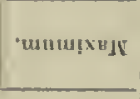 & 量 & 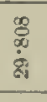 & $\begin{array}{l}\vec{y} \\
\dot{0} \\
\dot{0}\end{array}$ & $\stackrel{\oplus}{\stackrel{0}{*}}$ & 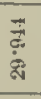 & 荌 & 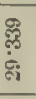 & 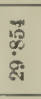 & 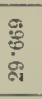 & $\stackrel{\overrightarrow{7}}{\overrightarrow{0}}$ & 品 & 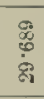 & $\overrightarrow{8}$ & $\stackrel{\mathscr{G}}{:}$ & 究 & 䘯 & 官 & $\begin{array}{l}\bar{\phi} \\
\vdots \\
0\end{array}$ & 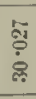 & 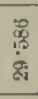 & 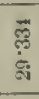 & 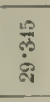 & 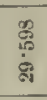 & हैं & $\overline{7}$ & $\stackrel{\bar{x}}{\dot{\alpha}}$ & $\begin{array}{l}\overline{5} \\
\stackrel{5}{0} \\
\text { a. }\end{array}$ \\
\hline & & 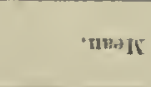 & 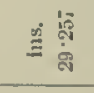 & 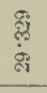 & 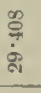 & 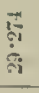 & 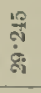 & 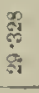 & $\begin{array}{l}7 \\
0 \\
0 \\
\text { oे } \\
\text { \&1 }\end{array}$ & $\begin{array}{l}\overrightarrow{\vec{r}} \\
\dot{\text { घे }}\end{array}$ & $\begin{array}{l}\text { है } \\
\text { हैं } \\
\dot{\xi}\end{array}$ & 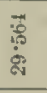 & 定 & 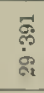 & 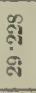 & $\stackrel{\infty}{=}$ & 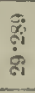 & : & 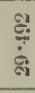 & $\begin{array}{l}\text { के } \\
\text { के } \\
\text { के }\end{array}$ & 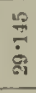 & 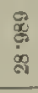 & 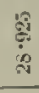 & 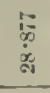 & $\begin{array}{l}\overline{8} \\
\overline{8} \\
\end{array}$ & 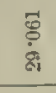 & : & 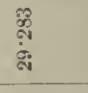 & $\frac{\hat{a}}{\vec{a}}$ \\
\hline & 竎 & & 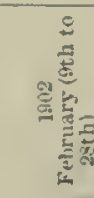 & & $\cdot$ & . & & 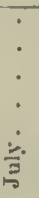 & 范 & - & 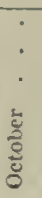 & 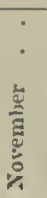 & 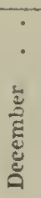 & 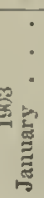 & 跣 & $\begin{array}{l}\dot{ } \\
\vdots \\
\vdots\end{array}$ & $\begin{array}{l}\overline{\underline{z}} \\
\bar{y}\end{array}$ & 色 & 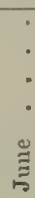 & $\frac{5}{3}$ & 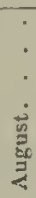 & 苛 & 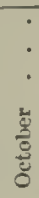 & : & 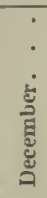 & 参 & 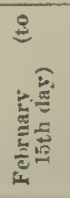 & 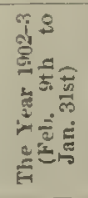 & 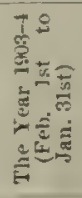 \\
\hline
\end{tabular}




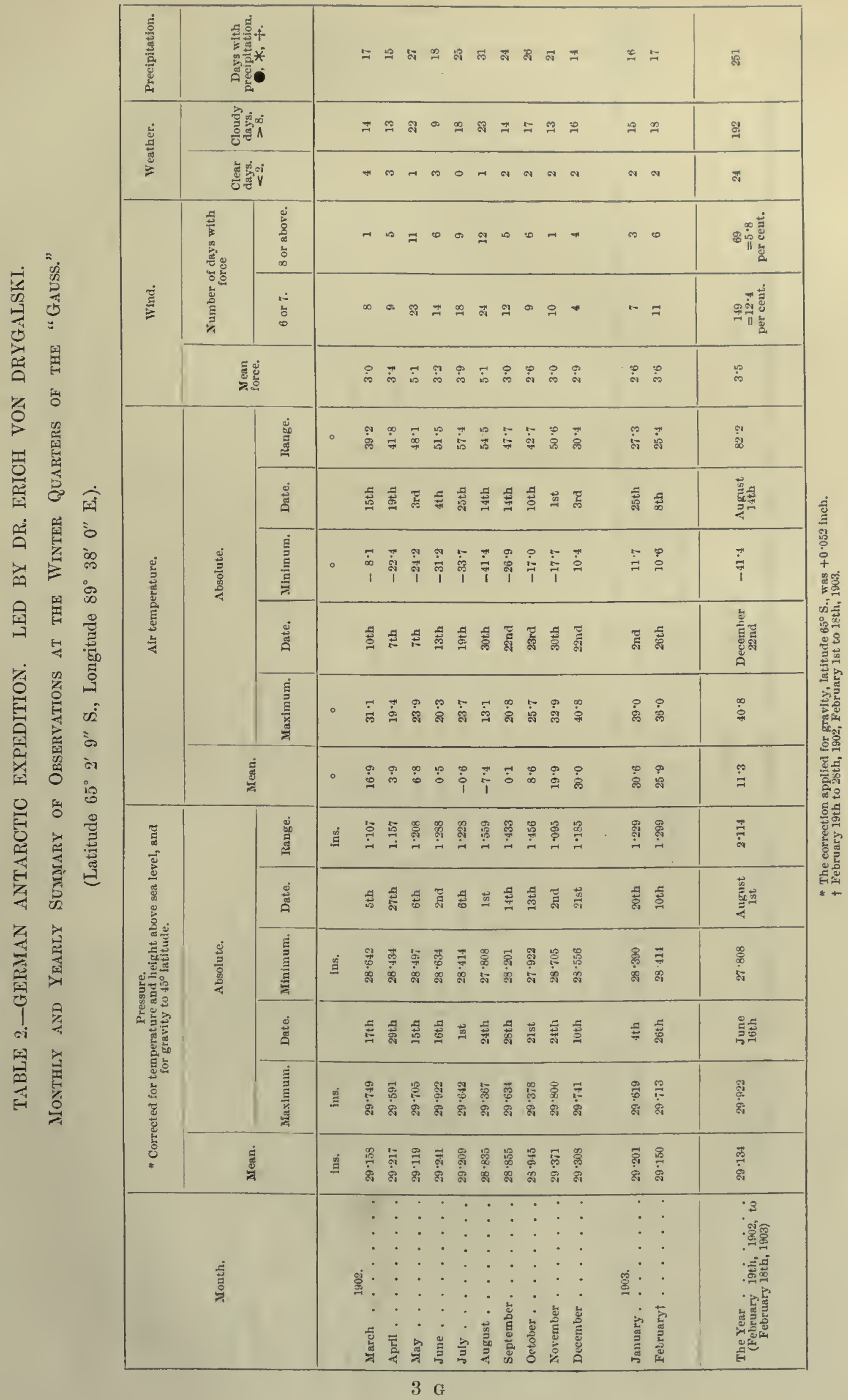




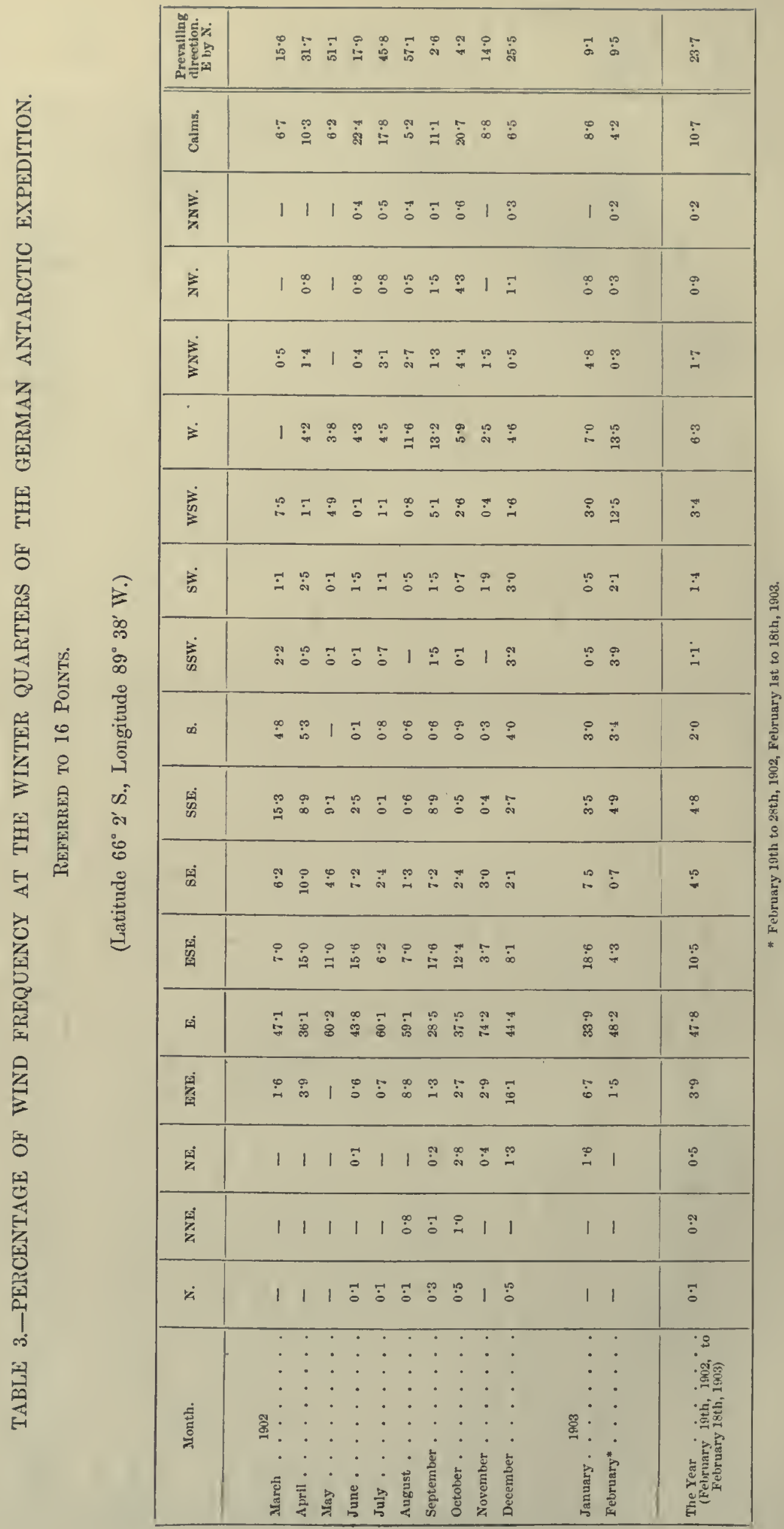




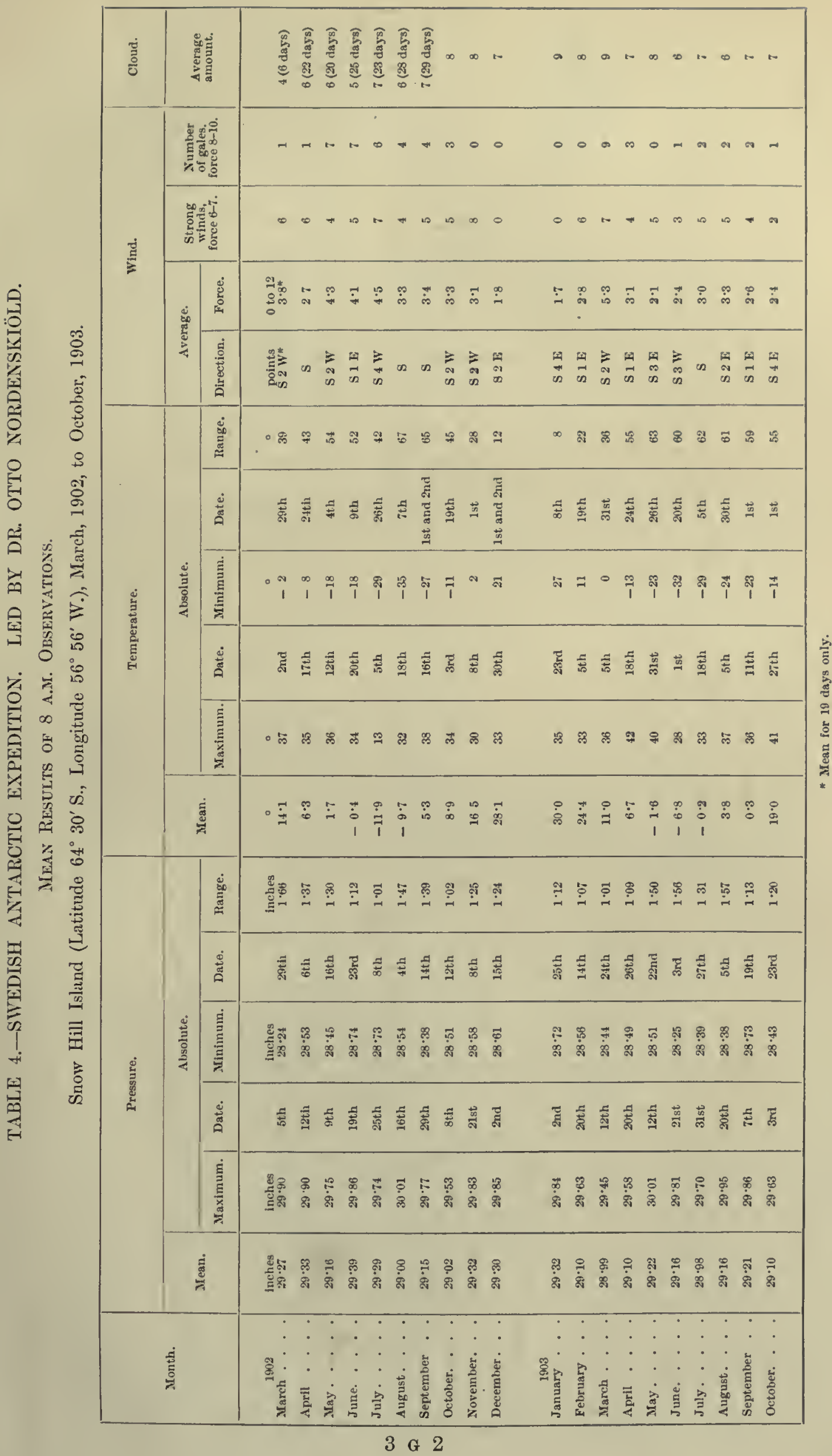


412

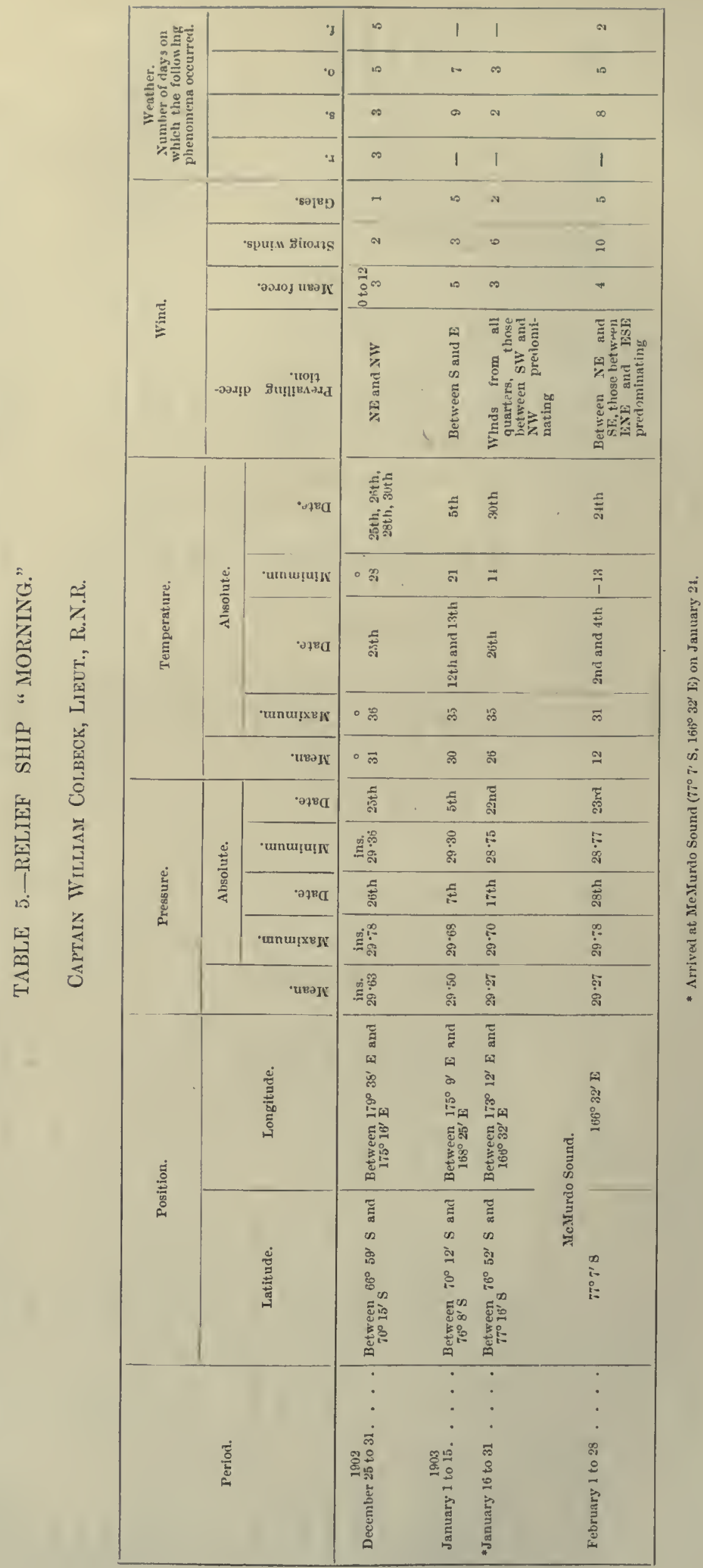


413

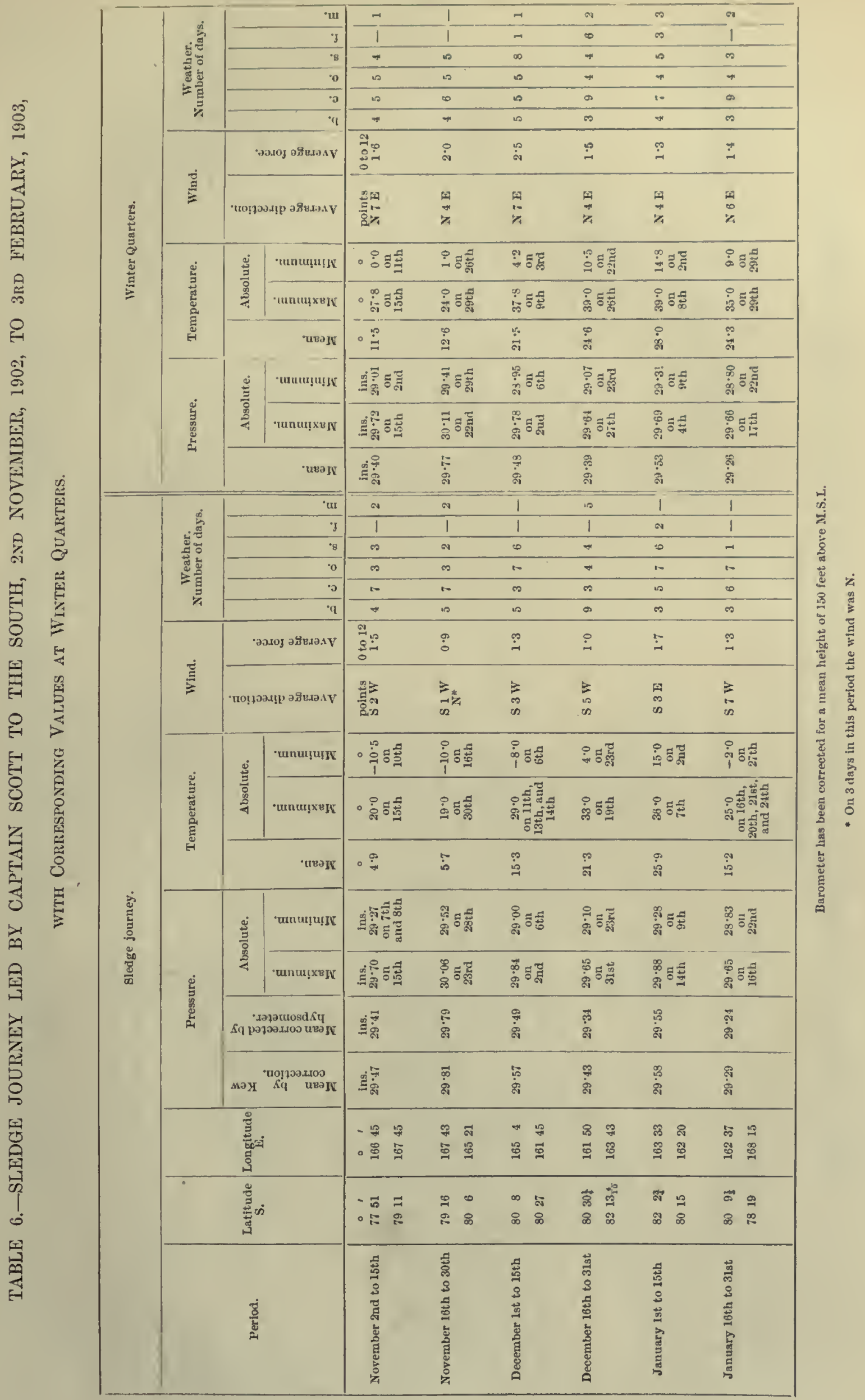




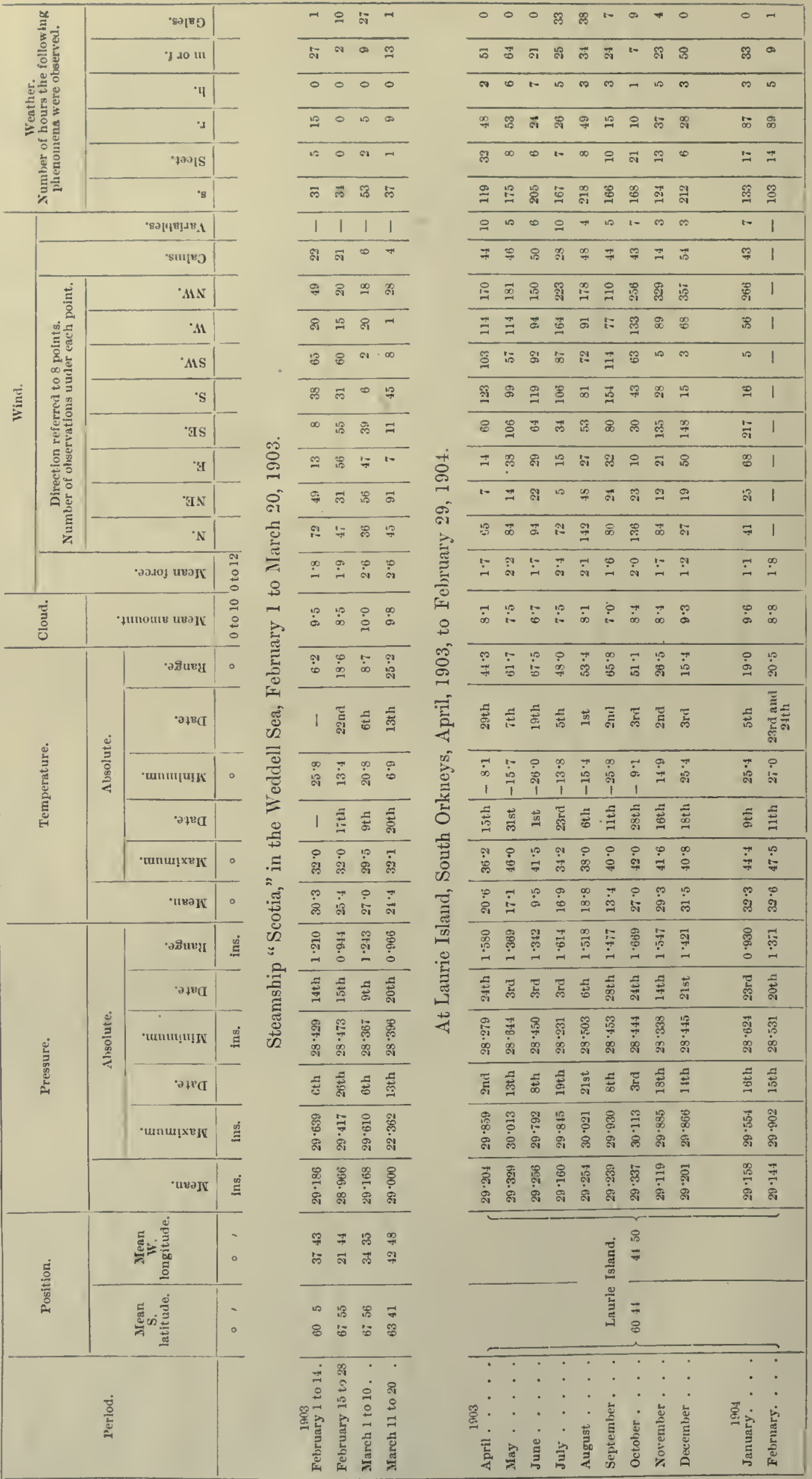




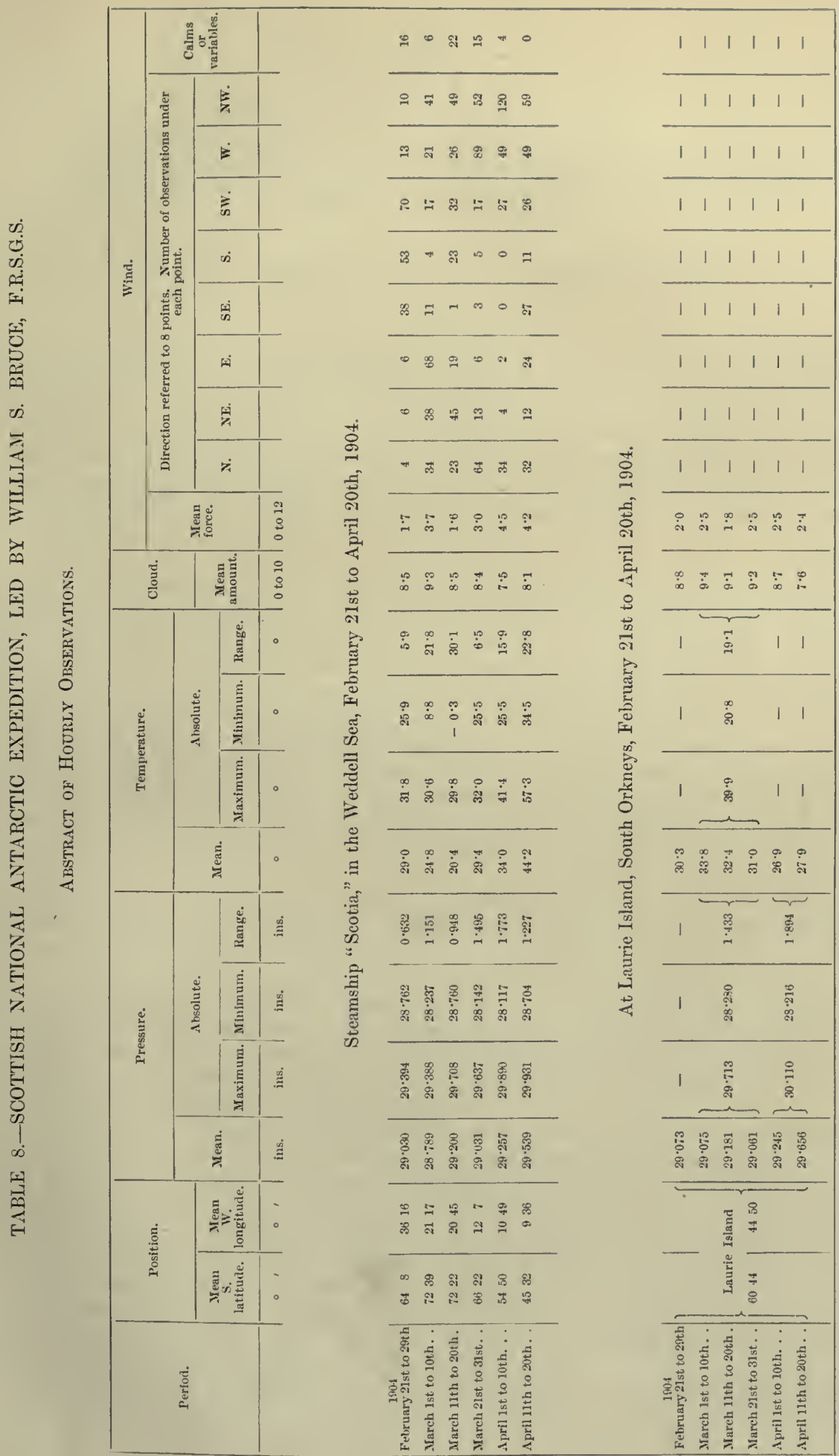




\section{CLIMATOLOGY OF SOUTH VICTORIA LAND AND THE NEIGHBOURING SEAS.}

BY

\section{W. CAMPBELL HEPWORTH, C.B., COMMANDER R.N.R.}

[See Tables, Pp. 408-415.]

Ix the following memoir all barometrical ohservations ntilized have been corrected for instrumental error and resluced to sea-level and to $32^{\circ} \mathrm{F}$. When a maximum or minimum pressure, or temperature, is mentioned, it is in all cases the absolute maximum, or the absolute minimum, that is referred to. The range of pressure, or temperatnre, given is the extreme range. Observations for temperature on the sledge journeys usually relate to readings of a thermometer exposed to the free air; occasionally to those of a sling thermometer. All wind directions are referred to the true meridian, and the wind force is estimated by the Beaufort scale.

Among the Expeditions taking part in International Antarctic Exploration during the years 1901-04, the British, in the "Discovery," under the command of Commander (now Captain) R. F. ScotT, R.N., M.V.O., was the only one to reach high latitudes early in the year of 1902 . The inquiry relating to the general meteorology of the Antarctic, so far as concerns the months of January and February of that year, is confined, thereforc, to the comparatively limited regions visited by the "Discovery," and, from the 9th of Febrnary, to the locality chosen by Captain ScoTT for Winter Quarters.

But, although the inquiry is thus limited, as regards the area to be investigated in those months, the information available for diseussion is nevertheless considerable, for not only are there at our disposal the meteorological observations referred to, made by Lieutenant C. W. R. RoyDs, R.N., and his assistants, during the first two months of 1902 , but there are also, for comparison with them, a large number of data obtained, for the most part in the same localities as those visited by the "Discovery," by two former Expeditions.

The first of these Expeditions was made in H.M.S.'s "Erebus" and "Terror" in the years 1841-2, under the command of Captain Sir JAMES Ross, R.N., F.R.S. ; the second in the steam yacht "Southern Cross," in $1898-1900$, led by Mr. C. E. Bonchigrevink.

For a discussion relating to the general meteorology of the Antarctic, the information obtained by the National Antarctic Expedition may, perhaps, be considered the most important of any contributed in recent ycars, even if the data obtained by the Expeditions undertaken by other countries taking part in the International Antarctic Exploration of the years 1901-4 covered the same period, for, with the exception of the Scottish National Antarctic Expedition, under Mr. W. S. Bruce, those of other countries were confined to latitudes merely on the fringe of Antarctica.

Mr. Bruce, in the "Scotia," made two bold dashes to the southward, in the Weddell Sen, attaining during lis second eruise the 74th parallel of south latitude, where uncharted land was discovered.

From the date on which the "Discovery" crossed the Antarctic Circle to the time of her arrival in McMurdo Sound the results of her olservations dealt with in this memoir relate to areas over whieh slie travelled during successive periods in the month of January and part of February, 1902. Results of olservations oltained in former years (1) by the Expedition under Sir JAMES Ross in 1841-2, (2) by the "Southern Cross" Expedition, under Mr. C. F. Borchgrevink, in 1899-1900, relating to the same areas, in the months of January and February, are then given consecutively for comparison.

The observations recorded by the "Discovery" Expedition, during the renaincler of February, in McMurdo Sound, are afterwards discussed.

In the treatment of the records of the various Expeditions which took part in recent Antaretic 
exploration during the remainder of the period, viz., from March, 1903, to March, 1904, inclusive, the months have been grouped into seasons for convenience, and the data available for each quadrant of the Antaretic have been dealt with in proper order, according to the season.

As regards three of these seasons, the results of observations obtained by the "Discovery" Fxpedition at Mcsurdo Sound, in 1902-3, have been compared with those recorded by the "Southern Cross" Expedition, at Cape Adare, in 1899-1900.

Referring to the gromping of the months into seasons, Mareh and April have been considered as Autumı months; May to Angust, inclusive, as Winter; September and October as Spring; and Norember to February, inclusive, as Summer.

The two tables on page 419 are given to enable the reader to follow the narrative nore easily.

Discorery," Jan. The "Discovery," proceeding southward, crossel the Antaretic Circle on the 2nd Jannary at abont the uary, 1902. 178 th meridian, with the wind from west-south-westward, moderate in force. The weathel had been foggy that day until 1.30 p.m., and since 5.45 p.m. of the previous day.

Thenee to a position, reached on the 9 th January, abont seven miles to the north-eastward of Caje Adare, Sonth Vietoria Land, in about $70^{\circ} 10^{\prime} \mathrm{S}$., and $170^{\circ} 15^{\prime} \mathrm{E}$., the winds mainly were from between north-north-east round by east to south-sonth-east, light to morlerate in foree. The mean pressure dnring the period, between the positions referred to, was $29 \cdot 36$ inches; a maximum pressure, $29 \cdot 94$ inches, was reeorded on the 2 nd of the month, with a gentle breeze froin west by sonth, and the mininum pressure, 29.04 inches, on the 4 th, during a calm, giving a lange of 0.90 inch.

The temperature was very even during the period; a naximum of $33^{\circ} \mathrm{F}$., which occurred on the 2nd, 6 th and 8th, with gentle to light breezes from west, south-south-east, and south-enst, respeetively, was only $2^{\circ}$ above the mean of $31^{\circ} \mathrm{F}$; ; and the minimum of $29^{\circ} \mathrm{F}$, recorded on the 9 th, only $2^{\circ}$ below the menn.

Snow fell on six days; the weather was overcast throughout; and there was fog on two days.

The data relating to the voyage of the steam yacht "Southern Closs" have been taken from the work published by the Royal Society in 1903, entitled "Magnetic and Meteorological Observations made by the "Southern Cross" Antarctic Expedition, 1898-1900.'

"Southern Cross," Traversing an area between $65^{\circ} 43^{\prime}$ S., $165^{\circ} 47^{\prime}$ E., and $66^{\circ} 46^{\prime}$ S., $165^{\circ} 29^{\prime}$ E., from January 19 th to 31 st, inclusive, 1899 , on a course which lay to the westward of that eovered by the "Discovery," the steam yaeht "Southern Cross," in the Expedition organiser at the expense of Sir Georce Newres, Bart., M.P., and led by Mr. C. E. BorchGrevink, experienced winds chiefly from between east-north-east and south-south-east, the predominating wind being the south-east.

The mean force of the wind was 2 of the Beaufort scale. The weather was overcast or cloudy; snow fell on four days; and fog was experieneed on one.

Barometer observations showed a mean pressure of $29 \cdot 65$ inches; the highest reading, $29 \cdot 79$ inehes, recorded on the 19 th and again on the 30 th, and the lowest reading, $29 \cdot 43$ inehes, on the 22 nd, show a range of 0.36 incl.

Observations for temperature give a mean of $29^{\circ} \mathrm{F}$. for the period; a maximum of $35^{\circ} \mathrm{F}$., registered on the $25 \mathrm{th}$, and a minimum of $17^{\circ} \mathrm{F}$, on the 29 th and again on the $30 \mathrm{th}$, show a range of $18^{\circ}$ for the period.

"Erebus" and "Ter- H.M.S. "Erebus" and "Terror" spent the summers of 1840-1, 2, and 3 in the far south, and the nonths of Jamary and February entirely within the Antarctie Circle. In a work compiled by Mr. RICHARD Strachax, F.R.Met.Soc., formerly on the staff of the Meteorological Office, and published by the authority of the Meteorological Committee in 1873, entitled 'Contributions to our Knowledge of the Meteorology of the Antaretic Regions,' the meteolological observations obtained during Sir JAMEs Ross's voyages in high sonthern latitudes have been reduced and tabulated.

Some of the results, given in the following pages, may be considered apposite for comparison with more recent data. They relate to observations recorded on both ships at the hours of 4 and 8 a.m., noon, 4 and 8 p.m., and midnight.

In reference to those for pressure, Captain J. E. DAvis, R.N., who was Seeond Master of the "Terror," supplied the Meteorological Offiee, in 1872, with a "Table of the Joint Corrections to be Applied to the Observed Barometer.' For each instrument the neutral point was $29 \cdot 922$ inches, capacity correction $\frac{1}{2} \sigma$ of the difference of height above this point, and eapillarity correction $+\cdot 012$. 
Orservations Recorded when Under Way.

\begin{tabular}{|c|c|c|c|}
\hline Expedition. & Montl. & Year. & Area triversed. \\
\hline $\begin{array}{l}\text { "Discovery" } \\
\text { "Southem Cross" } \\
\text { "Lirebus" and "Terror" } \\
\text { "Discorery" } \\
\text { "Ercbus" and "Terror" } \\
\text { "Discovery" } \\
\text { "Erebus" and "Terror" } \\
\text { "Discovery" } \\
\text { "Southern Cross" } \\
\text { "Frebus" and "Terror" }\end{array}$ & $\begin{array}{c}\text { January } \\
", \\
", \\
", \\
\text { February } \\
\text { ", }\end{array}$ & $\begin{array}{l}1902 \\
1899 \\
1841 \\
1902 \\
1841 \\
1902 \\
1841 \\
1902 \\
1900 \\
1842\end{array}$ & 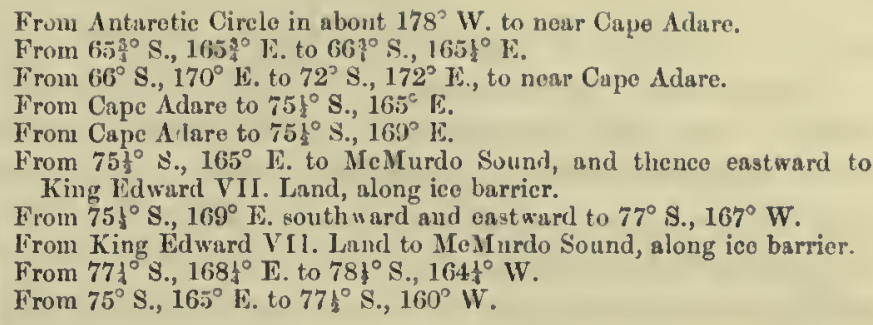 \\
\hline
\end{tabular}

Onseivations Recorded at Winter Quarters, on Sledge Journeys, and Under Way.

\begin{tabular}{|c|c|c|}
\hline Expedition. & Seuson. & Loeality. \\
\hline "Discovery" & Stummer, 1902 (February 9 to 28 ) & MeMurdo Sound. \\
\hline "Diseovery" " & Antumn, 1902 & MeMurdo Sound. \\
\hline "Southern Cross" & " 1899 & Capc Adare. \\
\hline $\begin{array}{l}\text { "Gauss" } \\
\text { Swedisll Station }\end{array}$ & $\begin{array}{ll}\prime & 1902 \\
, & 1902\end{array}$ & $\begin{array}{l}\text { Kaiser Wilhelm II. Land. } \\
\text { Snow IIill. }\end{array}$ \\
\hline "Diseovery" & & \\
\hline "Southern Cross" & $\begin{array}{cc}\text { Winter, } & 1902 \\
,, & 1899\end{array}$ & $\begin{array}{l}\text { McMurdo Sound. } \\
\text { Cape Adare. }\end{array}$ \\
\hline "Guuss" & " 1902 & $\begin{array}{l}\text { Cape Adare. } \\
\text { Kaiser Wilhelm II. Lund. }\end{array}$ \\
\hline Swcdish Station & " 1902 & snow Itill. \\
\hline "Discovery" & Sjring, 1902 & MeMurdo Sound. \\
\hline "Southern Cross" & " 1899 & Capc Adare. \\
\hline "Diseovery" & $" \quad 1902$ & Sledge journeys. \\
\hline $\begin{array}{l}\text { "Gauss" } \\
\text { Swcdish Station }\end{array}$ & $\begin{array}{ll}\prime & 1902 \\
\prime & 1902\end{array}$ & $\begin{array}{l}\text { Kaiser Wilhelm II. Laud. } \\
\text { Snow Irill. }\end{array}$ \\
\hline "Discovery" & Summer, 1902-3 & MeIIurdo Sound. \\
\hline "Sonthern Cross" & Summer, $1899-1900$ & Cape Adare. \\
\hline "Morning" & Summer (December, 1902, Jamuary and Febrinary, 1903) & $\begin{array}{l}\text { Under way in Russ Sea and anchored } \\
\text { in MeMurdo Sound. }\end{array}$ \\
\hline $\begin{array}{l}\text { "Discovery" } \\
\text { "Gauss" }\end{array}$ & $\begin{array}{c}\text { Summer, } \\
1902-3 \\
1902-3\end{array}$ & $\begin{array}{l}\text { Sledgo journeys. } \\
\text { Kaiser Willrelm II. Land. }\end{array}$ \\
\hline $\begin{array}{l}\text { Swedish Station } \\
\text { "Scotia" }\end{array}$ & Simnıner, $1902 " 3$ (February, 1903$)$ & $\begin{array}{l}\text { Suow Hill. } \\
\text { Under way in Weddell Sea. }\end{array}$ \\
\hline "Diseovery" & Autumn, 1903 & McMurdo Sound. \\
\hline "Morning" & Autuun, 1903 (Maroh) & Under way in Ross Sca. \\
\hline Swedish Station & Autumn, 1903 & Snow Hill. \\
\hline $\begin{array}{l}\text { "Scotia" } \\
\text { "Scotias" }\end{array}$ & $\begin{array}{l}\text { Autumn, } 1903 \text { (Mareh) } \\
\text { Autumn, } 1903 \text { (April) }\end{array}$ & $\begin{array}{l}\text { Weddoll Sea. } \\
\text { Laurio Islaud, S. Orkneys. }\end{array}$ \\
\hline "Discurery" & Winter, 1903 & MeMurdo Sound. \\
\hline Swedish Station & $" 1903$ & Snow Hill. \\
\hline "Seotia" & 1903 & Laurie Island. \\
\hline "Diseovery" & Spring, 1903 & Mo.Murdo Sound. \\
\hline & ", 1903. & Sledge journeys. \\
\hline & $\begin{array}{ll}\prime \prime & 1903\end{array}$ & Laurie Island. \\
\hline "Discovery" & Sunumer, 1903-4 & MeMurdo Sound. \\
\hline "Discovery" & , $1903-4$ & sledge journeys. \\
\hline $\begin{array}{l}\text { Seottish Station } \\
\text { "Seotin" }\end{array}$ & February, 1934 & $\begin{array}{l}\text { Laurie Islund. } \\
\text { Weddcll Sea. }\end{array}$ \\
\hline "Diseovery" & 1904 (February 19-March 3) & Under way lomeward. \\
\hline "Scotia" & Suminer, 1904 (Mareh and April) & Weddell Sca. \\
\hline & Sunner, 1904 (March) & Laurie Island. \\
\hline
\end{tabular}


"Brolosen" moul "fiver. ror," inuloury, IMII.

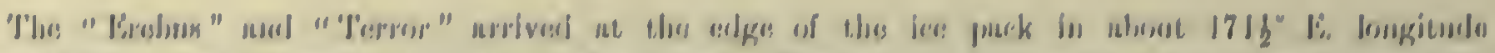

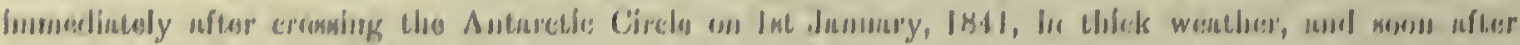

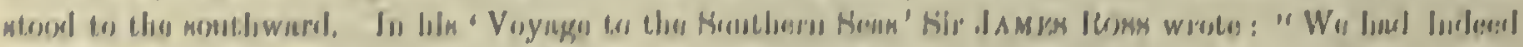

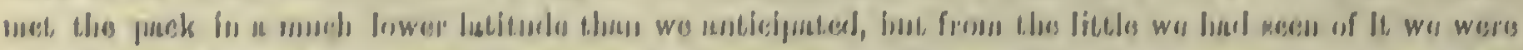

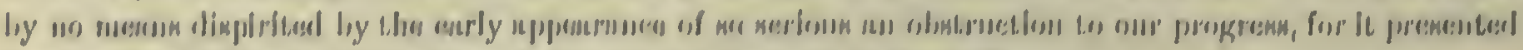

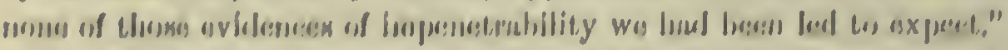

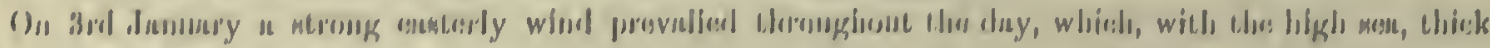

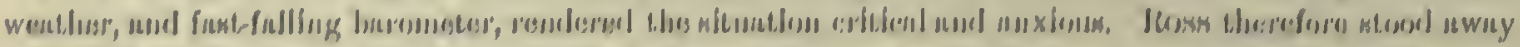

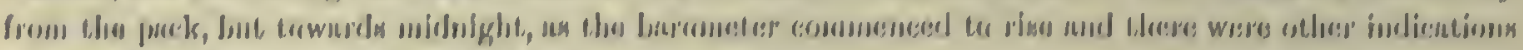

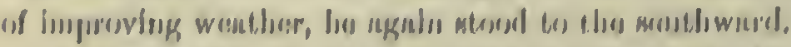

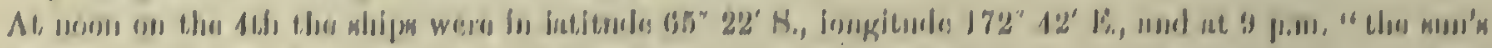

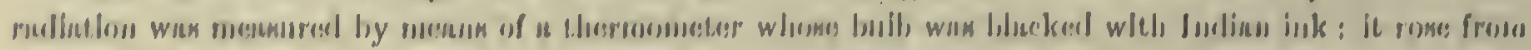

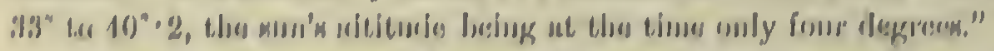

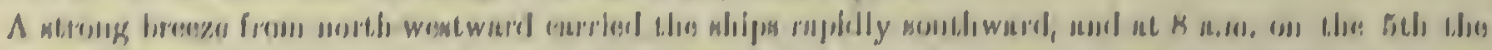

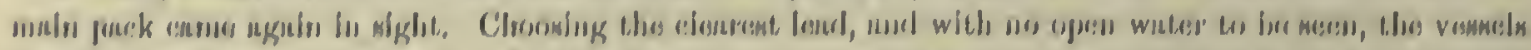

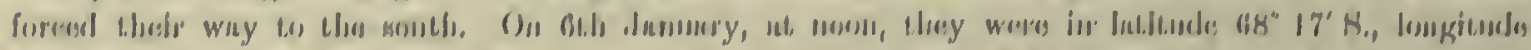

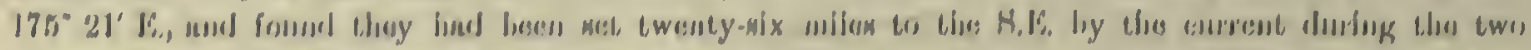

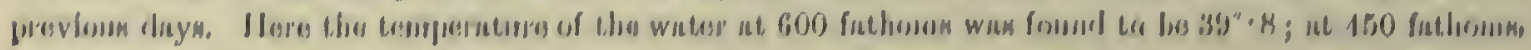

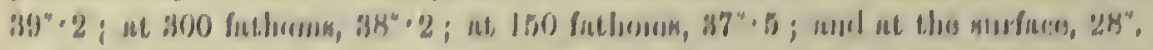

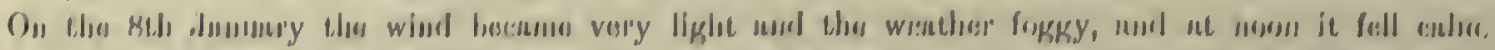

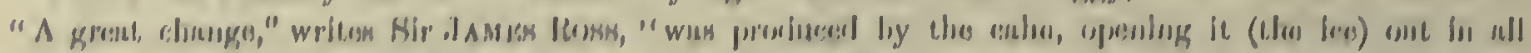

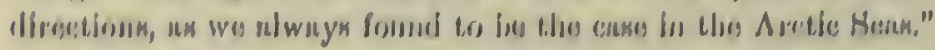

A sorthosly brenger njusug

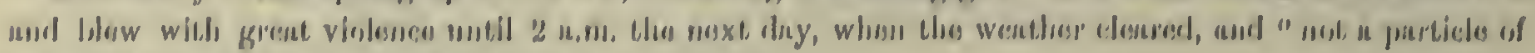

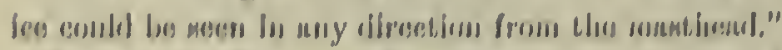

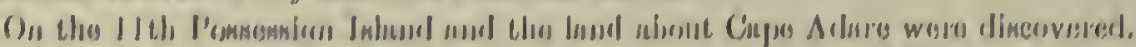

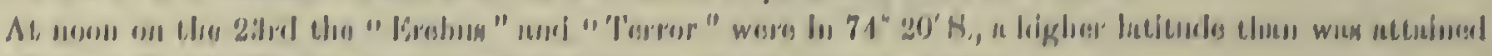

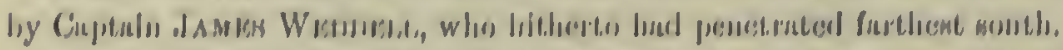

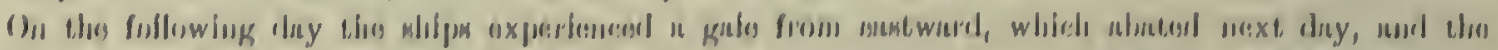

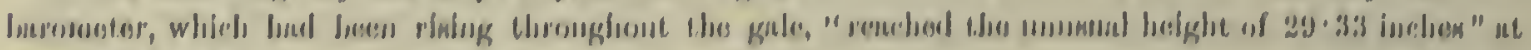

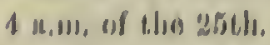

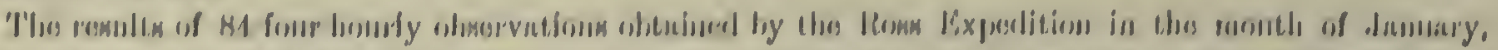

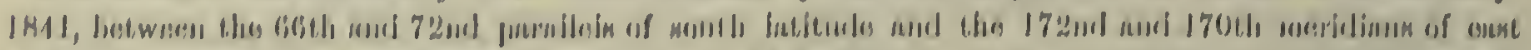

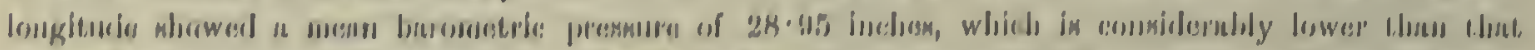

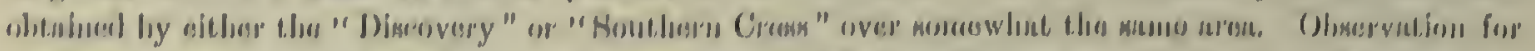

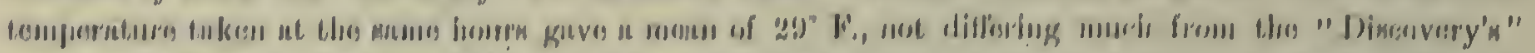

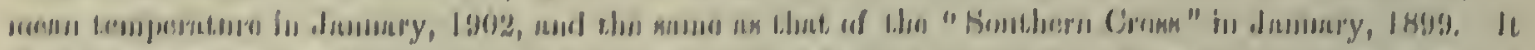

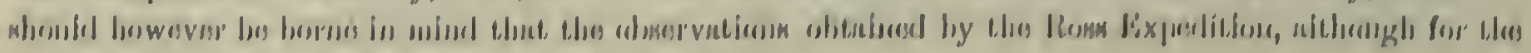

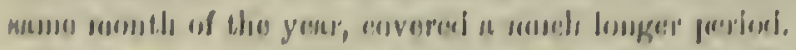

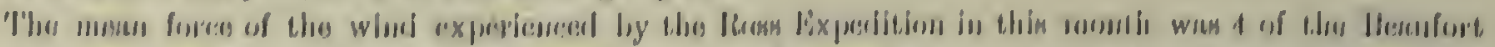

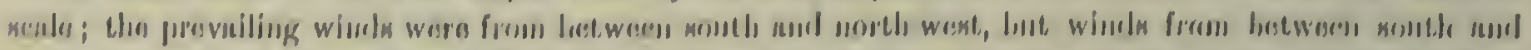

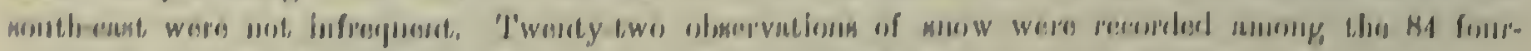

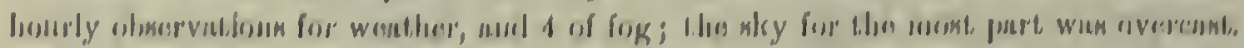

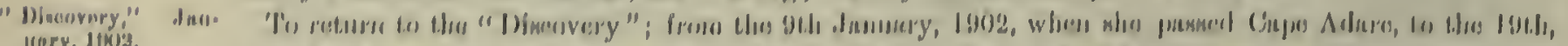

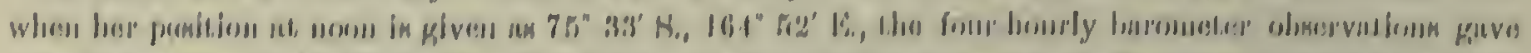

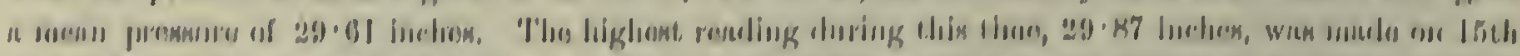

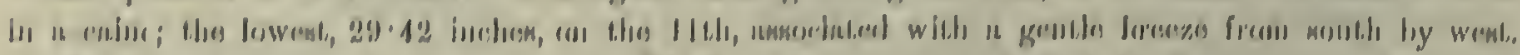

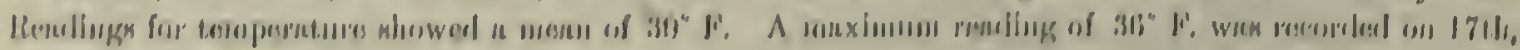

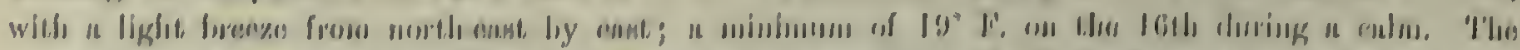

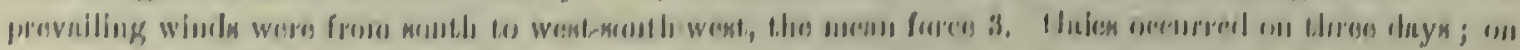

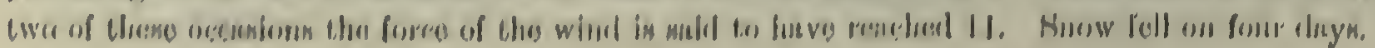




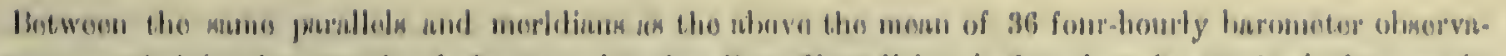

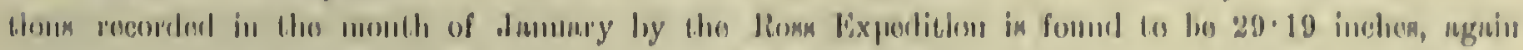
llowen Vixpersilinon,

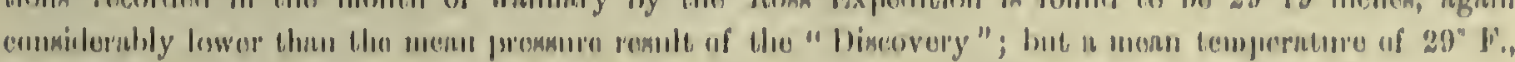

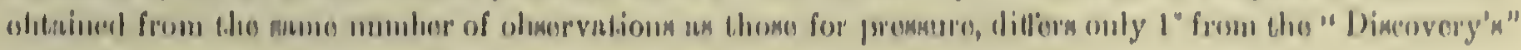

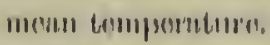

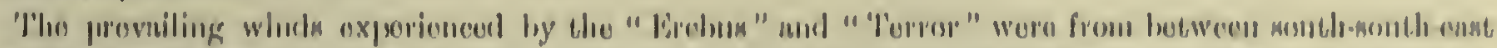

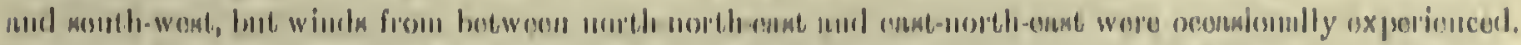

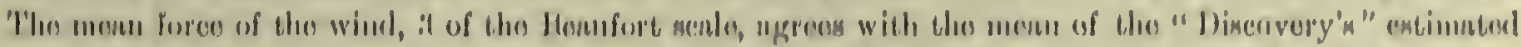

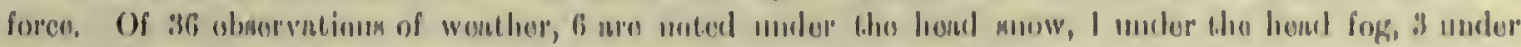

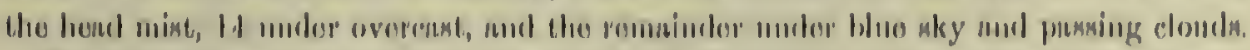

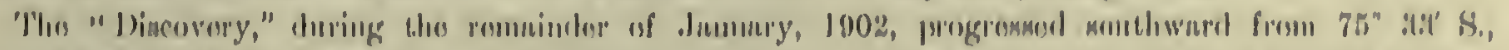

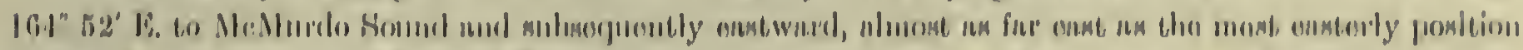

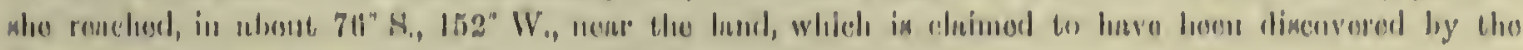

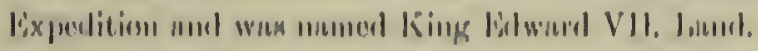

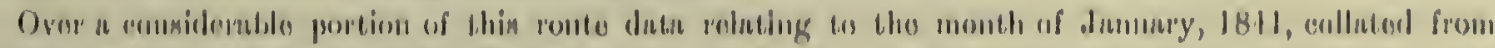

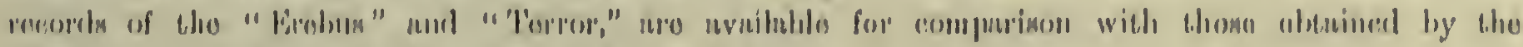

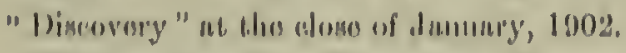

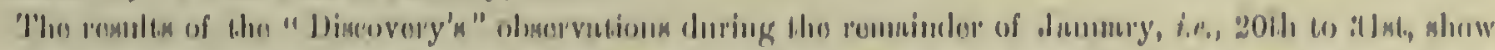

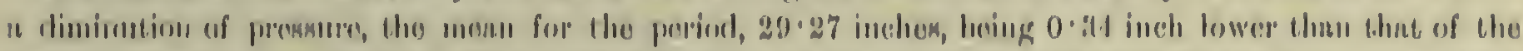

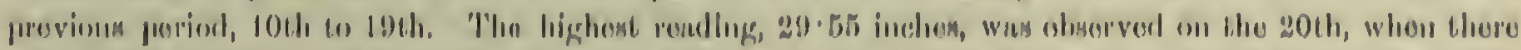

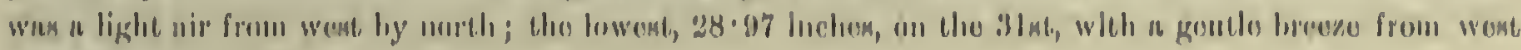

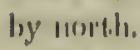

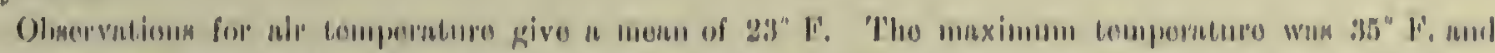

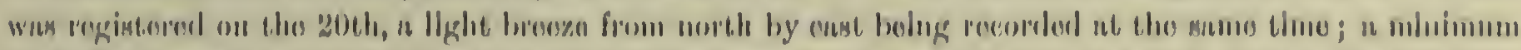

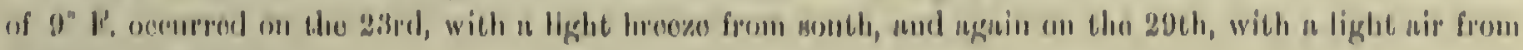
sontli eume liy monith.

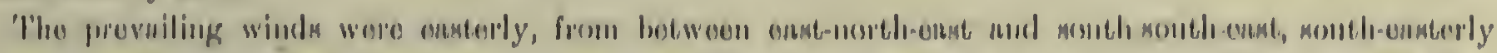

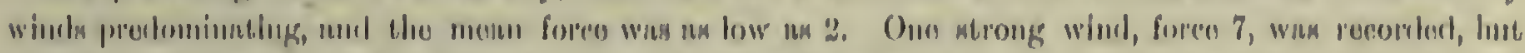

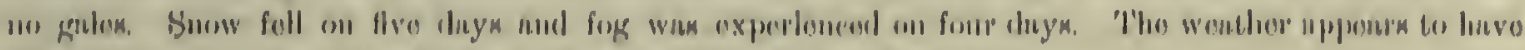

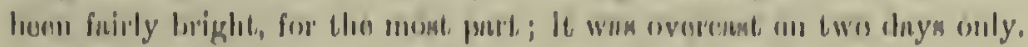

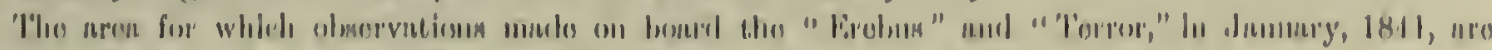

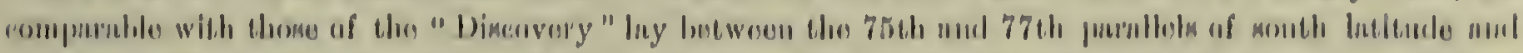

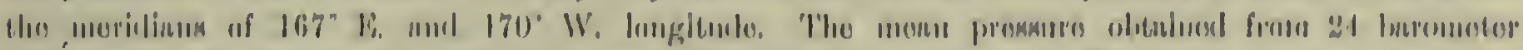

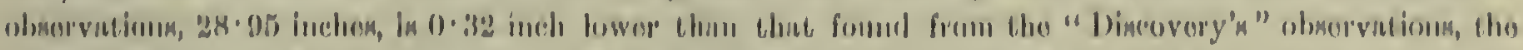

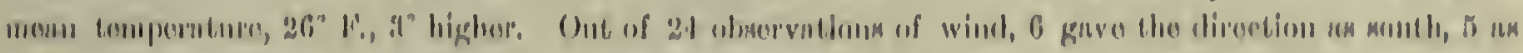

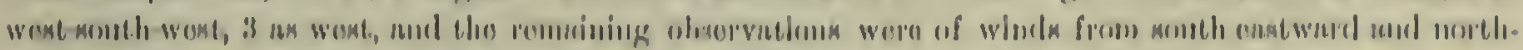

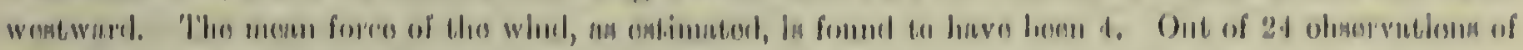

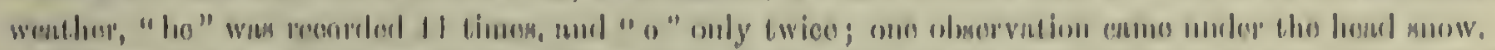

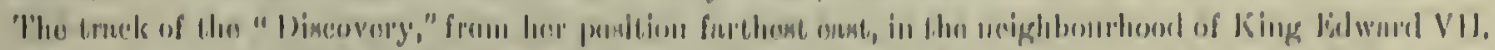

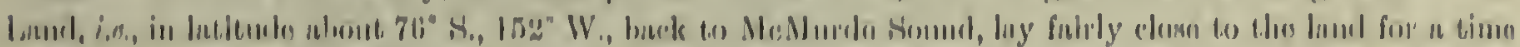

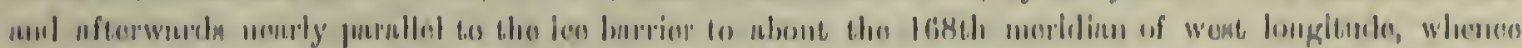

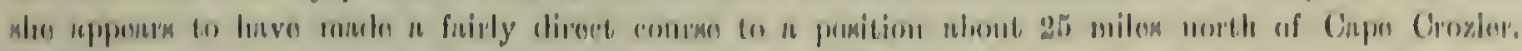

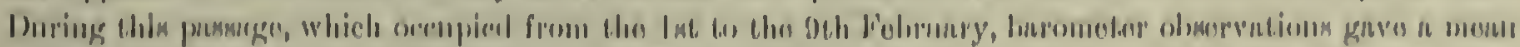

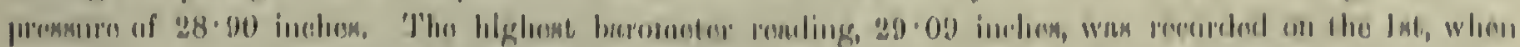

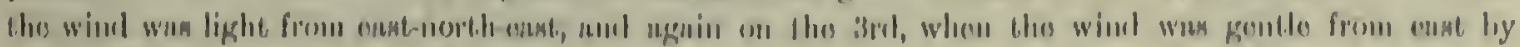

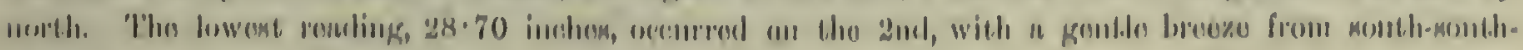
wort.

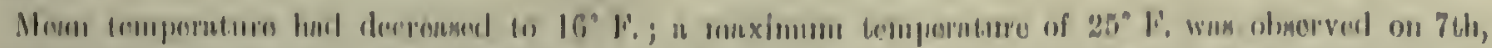

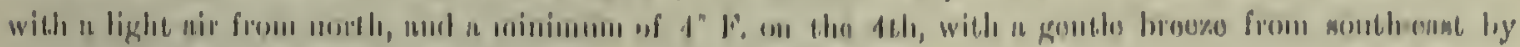

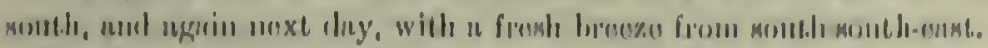

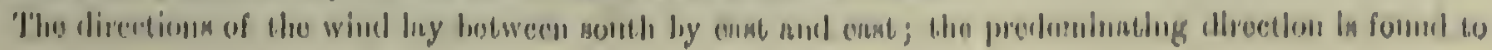

lliman lispunlilion, dousiory, Tril,
J)ingovery," Jane inry, Irous. 
have been the south-easterly; the average foree 3. On one day only a strong wind was reeorded, and on that day the mean force was not higher than 4. Snow fell on 4 days, fog oecurred on 2 dlays, and the weather was overeast on 4 days.

"Southern Cross," In the year 1900, from the 10th to the 20th February, the "Southern Cross" cruised in the Ross Sea in February, 1900. about the same latitudes and partly between the same ineridians, viz., between $77^{\circ} 17^{\prime} \mathrm{S}$. and $78^{\urcorner} 35^{\prime} \mathrm{S}$. and between $168^{\circ} 15^{\prime} \mathrm{F}$. and $164^{\circ} 10^{\prime} \mathrm{W}$.

During this eruise she registered a higher mean pressure than that registered by the "Discovery," viz., $29 \cdot 13$ inches. Her highest barometer reading of 29.520 inches is entered against the 14 th of the month; her lowest, 28.830 inches, against the 17 th. The mean of her observations for teniperature, $14^{\circ} \mathrm{F}$., is $2^{\circ}$ lower than that of the "Diseovery." A maximum temperature of $30^{\circ} \mathrm{F}$. is entered against the $20 \mathrm{th}$; a minimum, $-12 \cdot 5^{\circ} \mathrm{F}$, against the $19 \mathrm{l}$. The prevailing wind appears to have been from sonthward, the average force 3 , which is the same as that of the "Discovery"; but a gale, in which the extrene force was estimated as 10, was experienced on the $13 \mathrm{th}$, and winds of force $6-7$ were recorded on three other oceasions. The weather was dull and overeast; snow fell on 6 days, fog necurred on 5 days.

Ross Expedition, From 138 ohservations made in the month of February, 1842, on board the "Erebus" and "Terror," February, 1842. during the Expedition under Sir JAMES Ross, between the parallels of $75^{\circ} \mathrm{S}$. and $77^{\circ} 30^{\prime} \mathrm{S}$. and the meridians of $165^{\circ} \mathrm{E}$. and $160^{\circ} \mathrm{W}$, the following results are obtained:--Nean pressure, 28.87 inehes; mean tein peratire, $25^{\circ}$; prevailing winds from between soutl-east and south-west, the predominating wind the sonth-easterly. The average force of the wind 4. Under the head "be," 21 observations were recorded; under "c," 49 ; under " 0 ," 68 . Snow was entered 33 times; fog 6 times.

"Discovery," 1902, The "Discovery" arrived in McMurdo Sound, as has alreadly been mentioned, on 9th February, and Me Murdo Sound. between this date and the end of the month barometer observations showed a mean pressure of $29 \cdot 257$ inches. The highest barometer reading, 29.580 inches, is logged on the 22 nd ; the lowest, $28 \cdot 862$ inehes, after noon on the 9 th, giving a range of 0.718 inch (Table 1, p. 408).

From observations for temperature a mean of $15^{\circ} \cdot 9 \mathrm{~F}$. is obtained; the maximum, $29^{\circ} \cdot 2 \mathrm{~F}$, oceurred on the 28 th ; the minimum, $-0^{\circ} .8 \mathrm{~F}$, on the $22 \mathrm{nd}$; a range of $30^{\circ}$.

Winds from between north-enst and south-east were most frequent, but by far the largest percentage of wind observations out of 225 , i.e., 45 per cent., were of east winds, and 15 per cent. of all observations were of ealns. The mean force of the wind is estimated at 3 ; but fresh gales were recorded on 3 occasions, and strong winds on 7 . The weather was overcast or clondy for the most part, and snow fell on 5 days.

The vessel was made snug in her Winter Quarters on 12 th February, in latitude $77^{\circ} 50^{\prime} 30^{\prime \prime} \mathrm{S}$, longitude $166^{\circ} 44^{\prime} 45^{\prime \prime} \mathrm{E}$, and remained there until her release from the ice on the 16 th February, 1904. Describing the position of the "Discovery's" Winter Quarters, Lientenant C. W. R. RovDs, R.N., in a paper read by him before the Royal Geographical Soeiety on 27th February, 1905, said: "The ship was in a small bay of about a quarter of a mile in depth. All round from north-north-west throngh east to south-east there was land rising quickly to some height. In the north-north-west the hills were 400 feet high, and these extended to north-east, and there was a hill of 1000 feet in the east, and one of 700 feet in the south-east. Between the east and the south-east was a deep gully, or gap as we eall it, which opened out to the ice barrier. From south-east through sonth and west to north-west we were entirely open, except for land 20 to 50 miles a way. From north-west back to sonth-west was the Albert range, rising to peaks of 15,000 feet, and to the south were some islands 3000 fect and land rising to 8000 feet."

Lieutenant Royds mentions, in the paper from which I have quoted, that there was a pronounced local wind from an easterly direction in the bay in which the "Discovery" lay, frozen up; and that, not infrequently, when the wind was blowing a fresh gale at the ship a calm prevailed a little more than a mile ancl a half to the south of the ship, round Cape Armitage. This conclusion, however, is not apparent in the recorded observations. In commenting on the experiences of a sledge party, led by Lientenant Royns, on a journey to Cape Crozier and back in March, 1902, to which reference will be made later on, Captain ScotT, in his book, "The Voyage of the "Diseovery," writes as follows: "On comparing notes with this party we realised for the first time what a difference there might be in the 
weather conditions of places within easy reach of the ship. It was not only in the matter of temperature, as I have alrealy deseribed, but also in the force and direction of the wind. On the 17th (Narch, 1902) at the ship we had a very strong blow from the south, at one time rising in force to a full gale, but the party, only some 12 miles to the eastward, had felt nothing of this; with them the day had been ealm though overeast. This differenee of weather conditions could be observed throughont the journey ; neither those on board, nor those away eould have told from their own meteorological conditions what the weather might have been with the others, and this fact was again and again impressed upon us throughout our stay in this region. Already we had learnt that the prevalent wind at our Winter Quarters blew from south-east through the gap, and that this wind was usually local, and frequently ccased within a mile or two of the ship. To this we could now add some further conclusions. It was evident that the enstern slopes of Terror were terribly wind swept, and that the prevailing direction was from south, while the deep bay immediately to the eastward of onr peninsula was a particularly windless area, where the snow lay thiek and soft and was only oceasionally stirred by whirling squalls."

During the autumn months of Mareh and $\Lambda$ pril the mean height of the barometer on board the "Discovery" was 29.369 inehes; the highest reading, 30.081 inches, recorded on the 15 th $\Lambda$ pril, was associated with a fresh breeze from east-sonth-east; the lowest, 28.850 inches, on the 5th of that month, with a light air from enst-north-enst. This gives an absolute range of $1 \cdot 231$ inches.

The mean temperature during these 2 months is found to have been $0^{\circ} .5 \mathrm{~F}$; the maximum reading, $27^{\circ} .5 \mathrm{~F}$., was made on the 9 th March, during a calm; the minimum reading, $-31^{\circ} \cdot 6 \mathrm{~F}$., on the 23rd April, with a gentle breeze from east-110rth-east; showing a range of $59^{\circ} \cdot 1 \mathrm{~F}$. Out of 704 wind observations 59 per eent. were of winds from north-eastward, eastward and south-eastward ; 26 per eent. were of calms. The mean force of the wind, as estimated, eomes out at $2 \cdot 8$; but 14 gales, 12 of them of force 8 and 2 of them of foree 9, were experieneed and 12 strong wincls. The weather was "o" or "e" on 47 days, "b" on 14. Snow fell on 19 days, " $\mathrm{m}$ " was reeorded on 12 and " $\mathrm{f}$ " on 2 days. Aurora was seen on 11 days.

During Lieutenant Royn's sledge journey to Cape Crozier from March 4 to 19, already referred to, the mean of the temperature observations is found to have been $-1^{\circ} \cdot 4 \mathrm{~F}$. A maximum tenperature of $22^{\circ} \cdot 5 \mathrm{~F}$. is given in the Register on the 8 th, a minimum of $-42^{\circ} \mathrm{F}$. on the $16 \mathrm{th}$; a range of $64^{\circ} 5 \mathrm{~F}$. The mean force of the wind recorded is 1.4 ; from the 5th to the 9th inclusive, and again on the 16 th and 17 th, there appears to have been little or no wind, but on the 12th the following entry oecurs in the Register: "Blew hard all night" (riight of 11th-12th), and next day the entry is made: "High wind all night" (night of 12th-13th).

The wind blew hard during the night of the 18th-19th in heavy gusts, but at 5 a.m. "ealm" is reeorded, and again at 8 a.m.; at 2.30 p.m. the entry "light variable airs" occurs. As the party approached the land, on the afternoon of the 19 th, the temperature rose and the wind freshened from sonth-east.

While the sledge party was experieneing the low temperatures given above, the mean of the observations for temperature in the screen near the ship was $11^{\circ} \cdot 1 \mathrm{~F}$., i.e., $12^{\circ} .5$ ligher than the mean of the temperatures observed by the sledge party. The absolute maximum of $27.5 \mathrm{~F}$, observed during the period at Winter Quarters, oceurred on the 9th, a day after the sledge party's alsolute maximum; the absolute minimum of $-8^{\circ} \cdot 5$, on the 15 th, or a day before the sledge party's minimum.

$\Lambda$ sledge party led by Captain ScotT, on a journey a few miles to the south, to a position half-way between the ship and White Island, for the purpose of laying out a depôt, left Winter Quarters on the 31st March and returned on the 3rd April.

During this time, the mean of the temperatures observed is as low as $-36^{\circ} \mathrm{F}$., while in the sereen at the ship it is found to have been $-8^{\circ} \cdot 4 \mathrm{~F}$, or $27^{\circ} \cdot 7$ higher. Captain ScoTr recorded his maximum tempernture, $-25^{\circ} \mathrm{F}$, at 2 p.m. on the $3 \mathrm{rd}$ April, when the thermometer in the sereen at the ship showed $-7^{\circ} \cdot 8 \mathrm{~F}$., and his minimum temperature, $-47^{\circ} \mathbf{F}$., at 9 p.m. on the 2 nd $\mathrm{April}$, when the sereened thermometer at the ship was not lower than $-9^{\circ} \cdot 2 \mathrm{~F}$.

The "Southern Cross " Expedition was in Winter Quarters at Cape Adare during Mareh and April, 1899, and a comparison of the results of the olservations obtained there in those months with those of the "Discovery" in McMurdo Sound in 1902 nay be of interest.

In regard to the site of the station at Cape $\Lambda$ dare, a headland at the extremity of a peninsula about

\section{Rovo's journer to Cape Crozier, March, 1902.}

"Discovery," Anter Quarters.

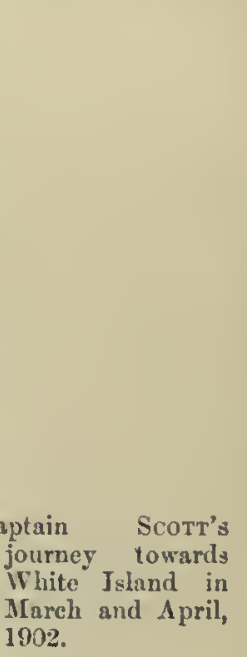

1902. "Southern Cross,"
Cape Adare, 1899,
Mareh and April. 
20 miles long, Mr. Louis Bersaccir, magnetieian and meteorologist to the Expedition, has made the following statement in his introduction to the meteorologieal portion of the work, entitled "Magnetie and Meteorological Observations made hy the "Southern Cross "Antaretie Expedition, 1898-1900,' published by the Royal Soeicty and already alluded to: "The meteorologieal station was situated on a pebbly bank faeing the open sea on the north and west, and Robertson Bay on the sonth and south-west. Behind, to the east and southeast, was a range of mountains averaging 3000 feet in height, the base of which was 1000 yards from the meteorologieal sereen. The height of the station above inean sen-level was 19 feet."

Mr. Bersuccin says that the eoldest weather prevailed during ealm, elear days, or with light southwest winds ; and that the lowest pressures and highest temperatures were assoeiated with winds from eastsouth-east; but that the barometer prior to these conditions fell with winds, as a rule, from north-westward. There appears some evidenee for the assumption that the diminution of pressure and rise of temperature mentioned by Mr. BenNaCCH was intimately eomneeted with the near approaeh and passage to eastward to the north of Cape Adare of a low-pressure system; for he states: "The prognosties of an approaehing storm from a south-easterly direetion were as follows: Generally, a gradual and simultaneous rise of temperature, a fall of the barometer some hours previous; frequently, however, only a rise in temperature with little warning from the barometer, a very rapid fall of the baroineter about. 30 minutes before the bursting of the storm, with light variable winds, and oeeasionally strong whirlwinds, loaded with driftsnow, then a dead ealm for a few minutes, followed by a great rush of wincl from the east-south-east having a veloeity of about 60 miles an hour and bringing with it for the first few hours masses of driftsnow from the mountains. The sky above, however, was usually elear. During the eourse of the storm the wind frequently dropped quite suddenly and a perfeet ealm ensued whieh lasted a few seeonds, then the rush of wind would eome again with redoubled fury. During the winter months most of the storms ended as abruptly as they had eommenced, and sometimes the ninimum pressure oeeurred just as the storm ended, but the barometer would take nearly 24 hours to reeover. The summer storms were less severe, but were of mueh longer duration, lasting on one oeeasion a whole week."

The rise of temperature in these gales from south-east and east-south-enst, doubtless, is in some measure due to the Föhn effect produeed dynamieally by the deseent of air from the mountain tops; and also to the eheck given, by wind in motion, to eooling by radiation. But may it not, to some extent at least, be attributed to the eonveyanee of warmer air drawn from lower latitudes, eireulating about a low-pressure system and eonveying to the locality the higher temperatures of the regions whenee it originated ?

It will be shown later that the inerease of temperature with gales from eastward at the Winter Quarters of the Gernan Expedition in the Antaretie was probably due to this eause.

The gustiness of the gales is eharaeteristie of all winds whieh blow over mountain ridges.

During Mareh and April, 1899, the mean pressure at Cape Aclare is found to have been $29 \cdot 161$ inehes, whieh is 0.208 ineh lower than the mean pressure at the "Diseovery's" Winter Qunrters in Mareh and April, 1902.

The mean temperature at Cape Adare during the months referred to, $13^{\circ} \cdot 9 \mathrm{~F}$., is $13^{\circ} \cdot 4$ higher than the "Diseovery's" mean for the eorresponding months in 1902 . The absolute maximun temperature, $31^{\circ} \cdot 5$, reeorded on the 3rd April at Cape Adare, is $4^{\circ}$ higher than the "Diseovery's"; the absolute minimum, $-10^{\circ} \cdot 3 \mathrm{~F}$, reeorded on 20 th April, is $21^{\circ} \cdot 3$ higher, and the extreme range for the period, $41^{\circ} \cdot 8$, is $17^{\circ} \cdot 3$ smaller.

The prevailing winds at Cape Adare, during the time, were from between north-east and south, but the eommonest wind was the sonth-east; there was, however, a large pereentage of ealms. The mean foree, as estimated, was $2 \cdot 4$. Ten gales were experienced. The weather for the greater portion of the time appears to have been overeast or eloudy ; rain fell on 3 days, and snow on 9.

German Expedition, The German Antarctie Expedition, under the leadership of Dr. EnICII ros Dryalski, left Kerguelen Island on the 3rd February, 1902, for the Antaretic Regions, and diseovered land on the 21st of the month, near the Antarctie Circle, on, about, the 90th meridian of east longitude, between Knox Land and Kemp Land. On the following day the "Gauss" was frozen in and remained imprisoned in the ice for 12 months, in latitude $66^{\circ} 2^{\prime} 9^{\prime \prime} \mathrm{S}$. , longitude $89^{\circ} 38^{\prime} \mathrm{E}$.

Describing the loeality in whieh the "Ganss" was sitnnted, Dr. Hans GazERT, the meteorologist to the 
Expedition, says in 'Veröffentliehungen des Instituts für Meereskunde, etc.,' Heft 5, 1903, published in Berlin : "The ship lay amongst pack and loose ice, the ice being 4 to 7 metres (13 to 23 feet) thick in Autunn, but increasing in thickness in parts, through snow-lrifts, to over 11 metres (nearly 36 feet) in the Spring. The condition of the ice around was similar in charucter for a distance of 2 kilometres (2187 yards) on the north, 4-5 kilometres (4375-5468 yards) on the west, nearly 2 kilometres (2187 yards) on the enst, and four kilometres (4375 yards) on the south, in which direction, beyond our icefields, lay an even expanse of older fixed ice; but in the other directions it consisted of open holes, mutil March, when they froze up. To the east, heyond a fiell of young ice, at a distance of 6-8 kilometres (6562-8749 yards) we frequently noticed, from September onwards, open water, in the shape of an extensive ice hole, which expanded and contracted according to the direction of the wind and current."

Dr. GAZERT goes on to say that numerous bergs surrounderl the ship, and that south of the depot the old solid ice, interspersed with bergs, stretched, without interruption, to the inland ice barrier 53 miles distant from the ship, the barrier having a general trend east and west.

At the barrier, rising abruptly ont of the sea, to a height of 1200 feet, was the Gaussberg, in hatitude $66^{\circ} 48^{\prime} \mathrm{S}$., longitude $89^{\circ} 30^{\prime} \mathrm{F}$.

The meteorological hut was at first situated from 44 to 55 yards to the south-south-east of the ship, but had to he moved scveral times during the year owing to snow-drifts in Winter and thawing in Summer. In January, 1903, it was moved to a position from 55 to 65 yards to the north-east of the ship.

Through the courtesy of Professor VON DRYGALSKI, important information relating to the meteorology of the region in which the "Gauss" was imprisoned can be employed in connexion with the present discussion.

Tahle 2, giving a Monthly Sunmary of observations taken at the Winter Quarters of the German Antarctic Expedition from March, 1902, to February, 1903, inclusive, has been kindly contributed by its distinguished leader for inchusion in this menoir. From this Sunmary we find that during March and April, 1902, the mean barometric pressure was $29 \cdot 188$ inches. This is $0 \cdot 181$ inch lower than that which obtained at the "Discovery's" Winter Quarters in latitude $77^{\circ} 50^{\prime}$ S., for the same period. The highest pressure recorded during the two months, $29 \cdot 749$ inches, registered on 17 th March, is $0 \cdot 332$ inch lower than the "Discovery's" highest during the same time; the lowest, 28.434 inches, which was registered on 27 th $A$ pril, is 0.416 inch lower than the "Discovery's" lowest during the same two mouths. The extreme range, $1 \cdot 315$ inches, is $0 \cdot 084$ inch larger than that shown by the British Expedition at MeMurdo Sound, during March and $\Lambda$ pril, 1902.

The mean temperature during these two months at the "Gauss" station comes out at $10^{\circ} \cdot 4 \mathrm{~F}$, which is considerably higher than the "Discovery's" mean for the same months, as nuay be expected for a locality" nearly $14^{\circ}$ north of it.

The naximum temperature obtained hy the German Expedition during the two months under notice was $31^{\circ} \cdot 1 \mathrm{~F}$. and was registered on the 10th March; the minimum, $-22^{\circ} \cdot 4 \mathrm{~F}$. on the 19th April, showing an extreme range for the periorl of $53^{\circ} \cdot 5$ against the "Discovery's" $59^{\circ} \cdot 1$ for the same period. The mean wind force during the two months is estimated at $3 \cdot 2$ of the Bealufort notation. The force of the wind was estimated at 6 , and alove, on 17 days, and on 6 of these days attained a force of 8 , or alove. There were 7 clondless days during the two inonths, when the proportion of sky clonderl was less than 2 in 10 ; and 27 cloudy days, when the proportion of cloucliness was more than 8 in 10. Precipitation, in the form of rain, snow, or snow-lrift, occurred on 32 days.

As regards the direction of the wind, Dr. GAZERT has stated that it was nsually easterly during the whole of the time the Expedition was in the Antaretic, ancl that during the frequent gales experienced was almost exclusively so. It was chiefly, he says, from between east and east by north from May to September, subsequently still more ensterly or to the southward of east, between east and sonth.

In 'The Proceedings of the Fifteenth Gernan Assembly of Geographers at Danzig in 1905' is published a lecture delivered by Dr. W. Mrisardus, of Berlin, upon "Wind Conditions at the 'Gauss' Winter Quarters." In this paper valuable data relating to wind frequency are presented in two tables, one of which is here reproduced (Table 3, p. 410). 
From these tahles it is fond that during March und April, 1902, 110 less than $46 \cdot 5$ per eent. of the winds were from enstwrord, i.p., hetween east by north and east by south; and of these $23 \cdot 6$ per cent. were from cast by north.

We are told hy 1)r. GA\%lint that the wind in gales at the "(ianss" station in the Antarctic frequently reached 12 of the Beanfort scale; he mentions, howerer, that the greatest velocity measured by hand anemometer was $24 \cdot 5$ motres per second, or, say, 55 miles per hour, while the estimated force was given as I 1 of the Beaufort scale; but that in the squalls the velocity of the wind, without doubt, frequently exceeded $24 \cdot 5$ metres per second.

Other winds than those from eastward were light and varialle, except those from westwarl, from which direction they had winds force 3 to 5 , nearly every nonth. Occasionally these westerly wiuds lilew with greater strengtli, as on 23rd May, when force 6 was experienced, and on 25th of that montl, when the wind attained force 8. On $3 \mathrm{rr}$ Angust a west-north-west wind foree 5 to 6 was recorded, and on the 14 th and 15 th of that month force 6 . These strong winds from westward were always associated with a clear, or nearly clear, sky, and low temperatures.

Referring to observations of precipitation, Dr. GAZERT says that measurements of snowfall were almost impossible, as during the gales the air was full of driving snow.

The same difficulty was experienced in measuring snowfall at the "Diseovery's" Winter Quarters, and for the same reason.

Swedish Expedition. The Swedish Antarctic Expedition, under the leadership of Dr. Otro NondrnskiörD, left Port Stanley, Falkland Islands, on 1st January, 1902, in the "Antarctic" for the fir sonth. After ernising among the islands in the neighbourhood of Louis Philippe Land and Danco, Land where some important discoreries were made, a spot was selected by the leader, on the island of Snow Hill, in which to winter. Dr. Gosts BoDmax; of Upsala, the meteorologist and magnetician to the Expeclition, has kindly supplied 8 a.m. observations (about noon G.M.T., i.e., 11.48 a.m.) taken at Snow Hill during the period March, 1902, to October, 1903, inclusive.

While recognising that the information relating to the meteorology of any region obtained from mean results of single daily observations is extrenely limited, it is nevertheless considered that monthly means of 8 a.m. observations for pressure, temperature, and wind for so important an outpost of Antarctica as Snow Hill Island should furnish information of interest in connexion with the present inquiry.

The island is one of a group situated off the north-eastern extremity of Daneo Land, which is a continuation north-enstward of Graham Land, and lies to the south of the Louis Philippe Peninsula and to the south-sonth-west of Joinville Island.

Ross Island, the largest of the group, is separated from the mainland by a channel discovered by Dr. Nonnexskiold, and named by him "Crown Prince Gustav Channel." On Ross Island, and situated towards its western shore, rises Mount Haddington.

The spot chosen by the leader of the Expedition for Winter Quarters lies at the north-enstern extremity" of the island, in latitude $64^{\circ} 30^{\prime} \mathrm{S}$, longitude $56^{\circ} 56^{\prime} \mathrm{W}$., named by Ross "Snow Hill," of which Dr. OTTo Nonulaskioito has said that it consisted for the most part of an mbroken dome of ice.

It should be bome in mind that when mean results of observations relating to Snow Hill are given in this memoir, they refer to means of 8 a.m. ohserrations only, and that maxima and minima values stated are also those for 8 a.m. only (Tible 4).

Swedish Espedition, During Mareh and April, 1902, the mean of the 8 a.m. observations recorded at Suow Hill is found to Snow Hill, Mareh be 29.30 inches; the highest reading, 29.90 inches, was noted on the 5th March and again on the
and April, 1902. 12 th $\Lambda$ pril. At the time of the seeond maximum the wind eame from south-east as a gentle breeze; the direction and force of the wind at the time of the first maximum is not known. The lowest reading, 28.24 inches, was taken on the 29th March, when a fresh gale was blowing from the south-west. These extrenes of pressure show a lange of 1.66 inches.

The mean temperature at 8 a.m. during the two months comes out as $10^{\circ} \cdot 2 \mathrm{~F}$. $\Lambda$ maximum realing of $37^{\circ} \mathrm{F}$. was recorded on the 2nd March, and a mininum of $-8^{\circ} \mathrm{F}$. on the 24 th $\Lambda$ pril, the latter associated with a fresh gale from west-south-west. The extreme range of temperature at 8 a.m. therefore was $45^{\circ}$.

The average direction of the wind at 8 a.m. during the period is found to lave been south by west, and 
the average force $3 \cdot 3$. The number of strong winds, $6-7$, recorled at 8 a.m. is fomd to have heen 12 ; the numler of gales, 8-10, 2 ; the mean clond amount, 5 .

In a paper read hy Dr. OTTo NonDrissiör.D hefore the Royal Geographical Society on the 2Ist Marcl, 190t, the eminent explorer mentioncd a violent hurricane experienced at Snow Hill Island in the midelle of March, and spoke of the severe storms and great cold the Expedition was exposed to in fal sonther"n latitudes. "No words," he said, "can suffice alequately to describe the terrible violence of these southwest hurricanes, which are accompanied by the severest cold ever experienced where we were." He commented on "the frequent occurrence and protracterl duration of the storms" at Snow Hill, which sometimes attained a veloeity of 70 to 90 miles an hour. "The average velocity of the wind," he continned, "for the eight months (March to October) in 1902 was 31 feet per seeond, which is prohably a record for so cold a climate."

At the "Diseovery's" Winter Quarters the long polar night conmenced on the 20th of $A$ pril, when the sul disappeared from view for fonr months.

During the winter months of May to Angust, 1902, inclusive, the mean pressure at McMurclo Sound was 29.172 inches, and the extreme rauge of pressure 2.011 inches. The lighest harometer, $30 \cdot 181$ inches, was recorled on the 19th of May, when a gentle breeze from east olstained; the lowest, $28 \cdot 140$ inches, on the 28 th June, during a calm.

As regards temperature, the mean was $-13 \cdot 3^{\circ} \mathrm{F}$., and the extreme range of temperature no less than $69^{\circ} \cdot 5$. The maximum temperature, $19^{\circ} \mathrm{F}$, oceurred on the 4 th of May, when a fresh sonth-casterly breeze was blowing; the minimum, $-50^{\circ} .5 \mathrm{~F}$., on the 7 th of August, when there was no wind.

The prevailing winds were from between north-eastward and south-eastward, the predominating direetion being easterly. Out of 1476 observations of wind direction, $15 \cdot 3$ per cent. fall under the head "calm," and only 0.7 per cent. under the head "variable." The mean force of the wind as estimated was $2 \cdot 7$, and, on 12 days, gales, varying in force from 8-11, were experienced. Strong winds, 6-7, were recorded on 30 days.

May appears to have been the stormiest month of the four, June the quietest. On 89 days the weather was overeast or eloudy, on 62 days misty, on 9 days foggy, and on 34 days the sky was clear. Snow fell on 49 days.

In July and August, 1902, observations of temperatnre were made, when possible, at Cape Armitage, Cape Armitage. from a spirit minimum thermoneter, the readings at the time of observation being recorded, as well as the minimum temperature registered.

The observations for this period were made, generally, at or about noon, and 48 observations obtained during these two months give an average temperature for the period of $-21^{\circ} \cdot 7 \mathrm{~F}$., while at Winter Quarters the mean temperature in the screen was $-12^{\circ} \cdot 3 \mathrm{~F}$. The maximnm temperature observed at Cape Armitage during the time, $-1^{\circ} \cdot 5 \mathrm{~F}$., was recorded on the 20 th $\mathrm{July}$, on which day the maximum temperature at the ship was $14^{\circ} \cdot 2 \mathrm{~F}$. The maximum in the sereen near the ship for the whole period, $16^{\circ} \cdot 7 \mathrm{~F}$, was registered the day before. The minimum reading registered at Cape Armitage, $-62^{\circ} \mathrm{F}$., was taken on the 7 th $A$ ugust, and on the same day the minimum for the period at the ship, $-50^{\circ} \cdot 5 \mathrm{~F}$., was registered.

Observations made during the two months therefore showed a range of $60^{\circ} \cdot 5$ at the former station and $67^{\circ} \cdot 2$ at the latter.

The "Southern Cross" Expedition at Cape Adare during the 4 months, May to Angust, 1899, recorded "Southern Cross." a mean barometric pressure of 29.076 inches, or 0.096 inch lower than that recorded on board the "Discovery" at McMurdo Sound, from May to August, 1902, inchusive. $\Lambda$ mean temperature of $-9^{\circ} \cdot 8 \mathrm{~F}$. olbained at Cape $\Lambda$ dare during the period referred to, which is $3^{\circ} \cdot 5 \mathrm{~F}$. higher than the "Discovery's" eomputerl mean for the corresponding 4 months in 1902. An alssolute maxinum temperature of $23^{\circ} \cdot 6 \mathrm{~F}$. was observed at Cape Arlare on the 15th July, which is $4^{\circ} \cdot 6 \mathrm{~F}$. higher than the "Discovery's" alsolnte maximum, and an alsolute minimum of $-43^{\circ} \cdot 5 \mathrm{~F}$. on the 5th and 6th $A$ ugnst, whieh is $7^{\circ} \mathrm{F}$. higher than the "Discovery's." The extreme ringe of temperature at Cape Adare in May-August, 1899 , was therefore $67^{\circ} \cdot 1 \mathrm{~F}$., as against $69^{\circ} \cdot 5 \mathrm{~F}$., the extreme range of temperature at McMurdo Sound in May-August, 1902. At Cape Adare, during the period under notice, the winl came 
chiefly from an easterly, south-easterly, or southerly direction, lut the commonest winds were the sontheasterly and southerly. Out of 244 observations of wind, 112 come under the head "Calm." The wind's mean foree, as estimated, is computed as $1 \cdot 4$, yet gales occurred on 24 days. On 41 days the weather was noted as overcast, and on 22 days the sky was clondless. Rain fell on 1 day, snow on 30.

"Discorery," May, 1002.

At the "Discovery's" Winter Quarters, in MIcMurdo Sound, a severe gale was experienced in May, 1902, a gale which Captain ScoTT eonsidered one of the heaviest, if not the heaviest, which visited the locality during the time the Expedition remained there.

Oil the 2nd of the month, at noon, the barometer stood at 29.057 inches, and the thermometer at $-7^{\circ} .2 \mathrm{~F}$. Pressure was diminishing and temperature inereasing. The wind, at east-sonth-east, was then estimated at foree 6, and the weather was overcast and squally. Between 2 p.m. and 4 p.m. the wind veered and increased rapilly from solthward. From 4 p.m. to 6 p.m., wind force was estimated at 8 ; and at 8 p.m. and 10 p.m., at 9. At midnight the wind's force was estimated at 10, lnt the Rolinson anemometer recorded a velocity of only 39 miles an hour between $10 \mathrm{p} . \mathrm{m}$. and midnight. The remark is made at 10 p.m., however, "furious gusts." At midnight the mereury had dropped to 28.999 inches, and the temperature eommenced to rise slowly. 'I'lie gale continued to blow until 6 a.m. of the 4 th, with a velocity varying from 44 to 25 miles an hour, and with an estimated foree of from 9 to 10 ; the temperature fluctuating between $-7^{\circ} \mathrm{F}$. and $18^{\circ} \mathrm{F}$., but rising steadily from 6 or 8 p.m. on the $3 \mathrm{rd}$ up to $8 \mathrm{a} . \mathrm{m}$. on the 4 th.

At 8 a.m. of the 4th the wind lad lacked to south-east, and moderated to a fresh breeze, while the temperature had risen to $19^{\circ} \mathrm{F}$.

Of this gale Captain Scotr says, in his "Voyage of the "Discovery" :- " The wind swung round in a manner which gave all the indications of a revolving storm whose centre narrowly missed us; the temperature remained extraordinarily high for several days (with the wind between sonth and east, northeast, or calm, but prineipally at south-east) after the storm; and it was not until the 9th ( $? 6$ th) that it again fell below zero, and then it fell rapidly (the wind loeing from east). On other occisions the teniperature rose regularly with a southerly wind, but fell when the wind dropped or changed direction."

Another notable gale, which may le regarded as typical of the gales experienced by the lixpedition while in MeNurdo Sound, oceurred on the 18th and 19th of July, 1902. On this occasion the wind appears to have hlown with greater violenee, but for al shorter period, not lasting for more than 24 hours.

At 4 a.m. on the 18 th the harometer, which gave a reading of $29 \cdot 707$ inches, commenced to fall slowly; and at the same time the thermometer, then reading $8^{\circ} \mathrm{F}$., began to rise slightly. The wind, from south-south-east, was moderate in foree, the sky overeast, and snow was falling. At 4 p.m. the mereury had fallen to 29.492 inches, and the temperature had risen to $12^{\circ} \mathrm{F}$. The wind which sinee 8 a.m. had been moderate to fresh, but squally from between south and south-south-east, then commeneed to freshen and the squalls to become more violent; snow was still falling, and continned to fall until the gale was over. At this time pressure began to inerease slowly. Between 4 p.un. and 6 p.m. the anemometer showed a wind velocity of 47 miles an hour; and at 10 p.m. the wind was logged as a whole gale from south-south-west. Except for a few lulls, which oceurred at 2 a.m. on the 19th, the wind continued to blow from between south-sonth-west and sonth with an estimated foree varying from 10 to 11, until noon on the 19th. Between $10 \mathrm{a} . \mathrm{m}$. and noon of that day the anemomcter, reeords of which had not been entered since 6 p.m. of the 18th, showed a velocity of 55 miles an hour. The weather was squally throughout. At 4 a.m. pressure had increased to $29 \cdot 660$ inches and began to decline slightly; at 6 a.m. the gale increased and the mercury had fallen to 29.620 inches. At 8 a.m., the barometer, still at 29.620 inches, commenced to rise; the force of the wind was then estimated at 11 . A lull took place at 11 a.m., lasting for three-quarters of an hour, "when the wind drew to west-south-west, lut went hack to south by west, and blew strong again in heary gusts." At 2 p.m. the wind had backed to south-east, and the barometer was rising steadily; the temperature was then $14^{\circ} \cdot 8 \mathrm{~F}$., no observation for temperature having been recorded between 10 p.m. of the 18 th and $2 \mathrm{p}$.m. of the 19 th. From $2 \mathrm{p} . \mathrm{m}$. to $4 \mathrm{p}$.m. the wind velocity, by anemometer, had decreased to 35 miles per hour, between 4 and 8 p.m. to about 27 miles an hour, at 4 p.m. the gale was over, and at 10 p.m. the wind was from east-sonth-east, a moderate breeze.

In regard to these two gales, it will he seen that pressure decreased and temperature rose prior to the 
freshening to gale force of the wind, while veering front-east-sonth-enst, or south-sonth-east to sontli-west, or south-south-west, pointing to the passage, seaward, of a low-pressure system across the Ross Sea. During these gales atmospheric pressure fluctuated slightly, hut was for tle most part stealy, ancl temperature rose as the wind veered to sonthward and south-westward. When the barometer showerl a decided rise the wind backed to the eastward of south and abated, temperature remaining relatively high for some time after the gale was over.

The rise of temperature associated with strong winds and gales from a polar quarter, at Victoria Lankl, has been freely commented on, and has, by some, been attributed to the Föhı effect, to the warming, dynanically, of the air in its descent after passing over the mountain ranges. There is considerialle evidence, however, to show that these strong winds from sonthward had their origin in, and owed much of their relative warmth and their moisture to, lower and warmer latiturles over the ocean.

It seens probable that areas of low pressure, on their passage eastward north of Victoria Laind, after passing the meridian of Cape Adare, not infrequently take a more southerly path, striking south-enstward and penetrating into, or skirting, the Ross Sea.

Circulating about an area of low pressure, situated over the Ross Sea, the winds would be northerly to north-easterly in the eastern segment of the system, to seaward; casterly to sonth-easterly in the southern segment, along and over the ice harrier; and southerly to south-westerly in the western segnent immediately to the eastward of the mountain ranges.

Thus the southerly wind should be a relatively warm sea wind, coming from lower latitudes, eirculating in the western segment of the ocean wind system.

McIurdo Sound, it is eontended, is situated too far south of Cape North and Cape Arlare to experienee as strong winds the northerly and north-easterly winds blowing in front, i.e., in the eastern segment of a low-pressure system travelling eastward to the north of these capes; and the loeality, moreover, is protected somewhat from uortherly winds by the high land to the northward.

At Snow Hill, the Winter Quarters of the Swedish Antaretie Expedition, eonditions were somewhat similar.

Cyclonic wind systems, travelling eastward, after passing south of Cape Horn, apparently did not, as a rule, during Dr. Nonvexskiöld's stay on the island, eause an increase of wind there until the system liad passed the 56th meridian of west longitude and to the eastward of Joinville Island. Then the wind increased from southward, usually from sonth-south-west, taking the direction, roughly speaking, of Grahan Land and Danco land and blowing toward the low pressure, still retaining its sontherly clirection even when the eentral "low" of the system had passed south-eastward into the Weddell Seal.

To revert to Victoria Lanrl; with the arrival of a depression over the Ross Sea the pressure gradients would beeome steeper, but a marked diminution of pressure over the land to the westward would not necessarily result, and, as observations show, rarely did result.

At the Winter Quarters of the German Expedition in the Antarctie, during the winter montls of May " to August, 1902, inclusive, observations for pressure showed a mean of $29 \cdot 101$ inches, which is 0.071 inch "Gauss," May to lower than the mean pressure during the same period which obtained at Mc Inrdo Sound.

The highest pressure for the period at the "Gauss" station, which was reeorded on the 16th June, is given as 29.922 inehes, and is 0.259 inch lower than the highest pressure recorded on board the "Discovery" for the same period.

The lowest pressure at the "Gauss" station during the four months referred to, whieh is as low as $27 \cdot 808$ inches, was recorded on the 1st Angust, and is 0.332 inch lower than the lowest pressure recorded on board the "Discovery" in those months.

The range of pressure therefore is found to have been $2 \cdot 114$ inches at the more northern station during the period as against $2 \cdot 041$ inclies at Mc.Iurdo Sound.

The mean temperature at the "Gauss" station, from Nay to August, inclusive, was $-0^{\circ} \cdot 2 \mathrm{~F}$; the maximum temperature was $23^{\circ} .9 \mathrm{~F}$. and was registered on the 7 th 1 ay; the minimum temperature $-41^{\circ} \cdot 4 \mathrm{~F}$. and was registered on the 14 th August. These extremes give a range of $65^{\circ} \cdot 3$ as against a range of $69^{\circ} .5$ at the British station during the same period; the mein temperature at the British station was, however, $13^{\circ} \cdot 1$ lower than the mean temperature at the German station. 
The mean force of the wind during these four winter months at the Creman station comes ont at $1 \cdot 3$ of the Beanfort seule. The prevaling wind was from east by north, the percentage of frequency of winds from that point heing 43 . But $12 \cdot 6$ per cent. of the winds were from east; $3 \cdot 8$ per cent. from east hy south; $7 \cdot 7$ jer cent. from east-south-east; and $12 \cdot 9$ per eent. of the observations for wind cane under the head "calm."

On 79 days the foree of the wind rose to 6 or 7 , and on 38 of these days to 8 , or above 8 .

There were 5 days noted as eloudless, when the amount of clonl was less than 2, and 72 clourly days, when the amount of elond exceeded 8.

There were 101 days on which raiu, snow, or snow drift, was entered.

In regard to the sequence of wenther dnuing the gales experienced at the German Antaretic station, Dr. GayERT silys :

"The course of the gales, especially the heavy Winter ones, was as follows:- With a falling barometer" and a wind springing up, or freshening from east, we noticed in that direction a hank of dark clouls, from which fragments were given off, which soon eovered the whole sky. The sin disappeared, often having previously been surromded by halos and mock suns. The barometer fell slowly at first, then more rapidly, frequently with a sharp curve. Thus we observerl from the hegiming to the elimax of a gale on the 30th July to the 1st August a fall of 35 millins. (1.378 inches) in 52 hours, when the minimum reading of $706 \cdot 3$ millims. (27.808 inches) was reached. The merenry fell almost as nuch in 30 hours, on Angust 9 to 10 , on which oceasion, in the middle of a fall, a pause of 4 hours took place. On the 22 nd August it fell 16 millims. $(0.630 \mathrm{inch})$ in 22 hours; and, on the 12 th and 13 th October, 27 millins. ( 1.063 inches) in 32 hours.

"As the storm progressed the wind increased more and more; driving snow filled the air, most thickly near the surface of the ground, and movement against the wind soon beeame a great effort; nothing eould be seen for the driving snow from which we had to proteet our faces. The ship was often invisible by day at a distance of 40 to 50 metres (44 to 55 yards), and in the worst storins at even a shorter distance.

"The gale reached its elimax occasionally in a few hours, but usually in from 24 to 48 hours, and remained at its height for 12 to 24 hours, being of a squally character. In the quiet intervals the force was possibly 3 , in the squalls 11 . These squalls appeared on the barograph curve in the form of a sawlike jagged line.

"It was at this stage, as a rule, that the air pressure reached its maximum. With a decreasing but squally wind the barometer began to rise. A slow rise was a good sign, a quick rise a bad one, as it usually meant an equally quick fall and another gale. With the deerease of the wind the driving snow lessened and we frequently could see the sum, or stars, faintly shining.

"The storin was often followed by a period of hal weather which was a longer or shorter prelude to another gale, or to beiter weather.

"The temperature always began to rise with the begimning of the gale, and only reached its maximum, ats a rule, when the gale eommenced to moderate, or in the unsettled weather period which followed, and was also eharacterised by easterly winds. If the gales followed at short intervals, the temperature did not fall at all in the pauses; at times it even rose, the rise accompanied by a lighter east wind.

"The storms usually lasted 3 to 5 days, the intervals between the gales varied, sometimes being only 12 to 24 hours, with wind force 5 to 7 ; this was especially the ease during August. 'The influence of the casterly wind upon the temperature was considerable; during ensterly winds the temperature often rose to $-14^{\circ} \mathrm{C},{ }^{\circ}-13^{\circ} \mathrm{C}$, and $-11^{\circ} \mathrm{C} .\left(6^{\circ} \cdot 8 \mathrm{~F}, 8^{\circ} \cdot 6 \mathrm{~F}\right.$, and $13^{\circ} \cdot 2 \mathrm{~F}$.). An increase in the mean temperaturc of May, as eompared with that of $\Lambda$ pril, was attributerl to the strong easterly gales whielr occurred in the former month."

Iying to the sontl of the westerly winds of the Southern Ocean, and at the extreme limits of the southern segment of those low-pressule areas which move from west to east with the westerly air current of the Sonthern IIemisphere, the Winter Quarters of the German Antarctic Expedition was exceptionally well situated as an olserving station from a meteorologieal point of riew.

It has been thought ly ineteorologists, who have attacked the problem, that, associatecl with the 
depressions which traverse the Sonthern Occans, depressions which are nsmally elliptienl in shape and have their major axes extended in a northerly and sontherly dircetion, the easterly winds in the sonthern segnent of the system are almost always light or moderate in force. Ships rumning down the easting in high southern latitudes scldom experience strong winds or gales from enstward, even when there appears to be evidence to show that they are sitnated well to the south of the eentral "low" of a cyclonie wind systen. It has, therefore, hecn assuned that in these systems the gradient polewards is normally slight and that on its southern side the low pressure dominates hut a small area.

The resnlts of observations obtained by the German Expeclition at Kergnelen Island and at Kíiser Wilhelm II. Land go far to prove that depressions which traverse the Sonthern Oceun eover a much larger area on their southern side than was hitherto supposed; and that althongh easterly gales are seldom met with, on the tralle routes referred to, nevertheless they oceni-considerably further sonth, it is true, but still within the limits of the system and forming part of their circnlation.

The rise of temperature which was associated with gales from eastward at the " (Gauss" Winter station and which, as a rule, continued until the wind began to moderate, was, no doubt, clue to the fact that the air, circulating about a minimmm pressure far north of the station, was being drawn from lower and warmer latitudes.

Frequently the daily noon G.M.T. observations of harometer and wind recorded by the German Antarctie Expedition at Kerguelen Island and at the "Gauss" Winter Quarters respectively—the former supplemented and confirmed by similar observations made on board ships sitnated in the neighbourhood of the islandindicate a connection between the strong winds and gales from northward and westward associated with diminishing pressure at Kerguelen with strong winds and gales from eastward experienced at the "Gauss" station. The distance between the meridians of these two stations respectively, on a middle latitude of $57^{\circ} 43^{\prime} \mathrm{S}$., is ahout 630 miles. The following are a few of the instances in which the common origin of these increases in wind force, experienced at the two stations, is shown. Donbtless the fuller data in the possession of the German meteorologists hring into greater prominence the connection referred to. It should be unclerstood that noon G.M.T. observations only are citerl.

At Kerguelen Island on the 18th Fehruary, 1902, at noon, G.M.T., the barometer stood at $29 \cdot 75$ inches, the thermometer at $45^{\circ} \mathrm{F}$; the wind was from west, foree 5. Next day the merenry had fallen to 29.43 inches, the temperature had risen $1^{\circ}$; and the wind, still from west, had increased to a fresh gale, indicating a gradient to the south. On the 20th the barometer bad risen to 29.80 inches, the thermometer showed a drop in the temperature to $42^{\circ} \mathrm{F}$, and the wind had hacked to west-sonth-west. The depression was passing away to the eastward.

By this time, at noon, G.M.T., on the 20th, on board the "Gauss," in latitude 65" $55^{\prime}$ S., longitude $90^{\circ} 20^{\prime} \mathrm{E}$., the baroneter indicated a pressure of 29.55 inches which was diminishing, the thermometer marked $28^{\circ} \mathrm{F}$., and a fresh breeze was blowing from enst. The mercury had fallen to $29 \cdot 28$ inches on the 21st, in latitude $66^{\circ} 17^{\prime} \mathrm{S}$., longitule $90^{\circ} 43^{\prime} \mathrm{J}$.; tempcrature had risen to $31^{\circ} \mathrm{F}$, and the direction and force of the wind were the same as on the previons day. On the 22nd, the "Gauss" being then in her Winter Quarters, the mereury had fallen but slightly: to 29.25 inches; the temperature had dropped to $25^{\circ} \mathrm{F}$. and a strong gale was blowing from east by sonth. The gale had not abated at noon, G.M.T., on the day following, when the direction of the wind had changed to enst. The harometer then stood at $29 \cdot 23$ inches, the thermometer at $24^{\circ} \mathrm{F}$. The mercury had fallen to $29 \cdot 14$ inches on the 24 th ; temperature had risen to $29^{\circ} \mathrm{F}$., but the gale was abating.

On the 23rd April of the same year, at noon, G.M.T., pressure at Kerguelen Island had fallen 0.71 inch and temperature had risen $8^{\circ} \mathrm{F}$. cluring the preceding 24 hours. The barometer then read $29 \cdot 20$ inches and the thermometer $48^{\circ} \mathbf{~ F}$; 估e brecze blew freshly from west-north-west. At the same G.11.T, a baroneter reading of 29.58 inches was recorded at the "Ganss" station; the temperature was $9^{\circ} \mathrm{F}$. and the wind gentle from east-south-enst. On the 24 th the mereury had risen to $29 \cdot 33$ inches at Kergnelen Island, the temperature had dropped to $34^{\circ} \mathrm{F}$, and the wind had backed to sonth-west by west and moderated. The disturbance, with which the dimimution of pressure at the island had becn associated, was then passing away to the eastward. At the "Ganss" station the mercury had by this time fallen to 29.35 inches, the thermometer harl risen to $17^{\circ} \mathrm{F}$, and a strong gale from east by north had set in. The 
mercury continned to fall: on the 25 th it had fallen to $29 \cdot 11$ inches and the thermometer markerl $16^{\circ} \mathrm{F}$.; the direction of the wind had not changed, but the force hal increased to 10 .

The larometer was down to 28.49 inclies on the 26 th; the thernometer had risen to $17^{\circ} \mathrm{F}$. and the wind still blew with the force of a whole gale from east by north. On the day following the mercury had risen to $28 \cdot 72$ inches and was still rising; temperature had clropperl to $16^{\circ} \mathrm{F}$. and the gale contimerl, unahated, from the same point. The wind moderated on the 28 th, when the mercury had risen to $29 \cdot 23$ inches.

Between the 2nd and 6th of May, 1902, pressure at Kerguelen Island diminished from $29 \cdot 92$ inches to $28 \cdot 84$ inches, and fresh winds were experienced from north-westward, backing to westwarl. The temperatme between the 4 th and 5 th had risen from $34^{\circ}$ F. to $42^{\circ} \mathrm{F}$. From the 4 th to the Tth of the montl, inchisive, the "Ganss" station was visited by a strong to whole gale from east by north, pressure having diminished from $29 \cdot 24$ inches, on the $3 \mathrm{rd}$, to 28.52 inches on the $6 \mathrm{th}$, and temperature risen during that period from $-4^{\circ} \mathrm{F}$. to $23^{\circ} \mathrm{F}$. A gain, between the 15 th and 17 th of the same month, pressure at Kerguelen Island reclined from $29 \cdot 33$ inches to 28.90 inches, and temperatme rose from $30^{\circ} \mathrm{F}$. to $42^{\circ} \mathrm{F}$. Strong winds from between west-north-west and west-sonth-west were recorded at this time and also on the 18 th at the island, and strong winds and gales on board ships in the neighbourhood of the island.

At the German station in the Antarctic the barometer showed a fall from 29.23 inches to $29 \cdot 09$ inches hetween the 17 th and 18 th, the thermometer a rise from $9^{\circ} \mathrm{F}$. to $13^{\circ} \mathrm{F}$, and the wind, from east hy north, increaser in force to a fresh gale. On the 19 th the barometer had risen to 29.24 inches, and the wind increased from the same point to a whole gale. Next day the mercury had risen to 29.43 inches, temperature had dropped to $8^{\circ} \mathrm{F}$, and the gale was over.

A rapid diminntion of pressure, rise of temperature, and increase of wind to gale force which occurred from the 4 th to the 6 th Jnme at the Antarctic station was evidently associaterl with a liminution of pressure and increase of wind to gale force previously recorded (between the 3rd and 5th of the month) at Kerguelen Island and on board the S.S. "Wainate" in a position, on the 4th, rather more than 100 miles sonth of the islaurl.

Subsequently, between the 6th and 7 th of June, the "Waimate," passing the meridian of the "Ganss" station in latitude $502_{2}^{\circ} \mathrm{S}$, experienced a fresh to strong gale from west-sonth-west, veering to north-west, while pressure increased at the Antarctie station, and the wind moderated from a whole gale to a strong breeze.

On the 5th July a rapid fall of the mercury recorded at Kergnelen Island and on hoard ships approaching the neighbourhood of the island resulted in an increase of wind on the 6th, which had backed from north-west to west, and blew a fresh gale from the latter direction. During these two days the mercury at the "Gauss" station fell 0.58 inch, and the thermometer rose $11^{\circ}$, and on the 7 th an increasing wind attained the force of a fresh gale. The barometer then commencel to rise; the temperatme harl risen $5^{\circ}$ since the precerling day, and marked $21^{\circ} \mathrm{F}$. Next day the gale was over.

From the 12 th to the 13 th of the same month barometric pressure at Kerguelen Island rleclined from $29 \cdot 82$ inches to 28.84 inches, the wind at the same time backing from north-west to west, and freshening. Between the 11th and 12 th the thermometer had risen $4^{\circ}$.

On the 13th a light sonth-west breeze obtained at the Antarctic station, pressure increasing, the temperature $-11^{\circ} \mathrm{F}$. On the 14 th the barometer there read as high as 29.58 inches; the temperature liad risen to $-4^{\circ} \mathrm{F}$., but the wind had backed to east by sonth, and was logged as a fresh hreeze. On the 15 th the mercury had fallen half an inch, the thermometer risen $19^{\circ}$, and the wind was blowing with storm force from east by north. With a slight recovery of pressure on the 16 th, it moderated to a fresh gale, but subsequently increased to a strong gale, still from the same point, and this continnerl, pressure the while declining, until the 18th, when the barometer har fallen to 28.61 inches, and the thermometer marked $17^{\circ} \mathrm{F}$. Pressure recovered next rlay and the wind moderated, bnt the temperature had risen to $20^{\circ} \mathrm{F}$.; it fell, however, on the 20 th to $1^{\circ} \mathrm{F}$.

A rapid fall in the barometer from $30 \cdot 37$ inches to $29 \cdot 62$ inches occurred at Kerguelen Island between the 30 th and 31 st July, occasioning an increase of wind, and associated with a slight rise of temperatnre. On these days the mercury fell 0.4 inch at the "Gauss" station, and the direction of the wind changed 
from west-nortli-west on the 30th to east hy south on the 31st, inereasing in foree at the same time. On the 1st Angust the mereury lad fallen as low as $27 \cdot 82$ inehes, and a wlole gale was blowing from east by north. The temperature had risen $10^{\circ}$ sinee noon, G.M.T., of the preeeding day.

A diminution of pressure and deerease in wind foree reeorded at Kerguelen Island anrl ou board ships in the neighbourhood of the island, on the 6th and 7th Angust, appear to have been associated witl a dininution of pressure, rise of temperature, and inerense of wind from east by north to gale force, reeorded on board the "Gauss" on the 7 th and 8 th of the month.

Reduetion of pressure at the island, again, on the 15th to the 17 th of the same month, was followed at the "Gauss" station by a rise of temperature between the 16 th and 17 th of $25^{\circ}$, and a fall in the barometer of 0.74 inch between the 17 th and 18 th, together with an inerease of wind fron east by north on the 18 th to storm foree.

Diminishing pressurc, accompanied by inerease of wind, recorded at Kerguelen Island and by ships in the neighbourhood of the island on the 11th and 12th Oetober, and again on the 20th and 21st of that month, was followed at the Germall Antaretie station by deeline of pressure, rise of temperature, and increase of wind to whole gale force.

On the 11th Deeember a fall in the barometer of more than half an ineh had been reeorded at Kerguelen Island for the previous 24 hours, and the ship "Niagara," some 250 miles north-eastward of the island, was experieneing a strong gale from north-north-rrest. On the following day the eentre of disturbanee, progressing eastward, had passed the meridian of the station at Kerguelen, and the S.S. "Salamis," in the immediate neighbourhood of the island, had a strong wind from south by west. At the German station in the Antaretie pressure was then giving way, but the wind, from east-north-east, was light. About 1300 miles due north of the "Gauss" the "Loch Torridon" recorded a strong wind from north by west and a barometer reading of 29.56 inehes, which was 0.28 inch higher than the reading recorded at the same time on board the "Gauss."

The "Niagara," then in about $45^{\circ} \mathrm{S}$. latitude, $81 \frac{1}{4}^{\circ} \mathrm{E}$. longitude, still had the wind from northward, and was therefore in front of the trough of the depression. On the 13th the "Loeh Torridon" and the "Niagara" carried a westerly wind, and the latter, in about $45^{\circ} \mathrm{S}$. latitude, $86 \frac{1^{\circ}}{\mathrm{E}} \mathrm{E}$. longitude, was running before a fresh gale, with the central low to the southward of her.

The Antaretie station, where pressure had eontinued to give way, was now under the full influenee of the disturbance, and a fresh easterly gale was blowing there, but the wind moderated next day. During this gale temperature appears to have ehanged very little.

A diminution of pressure recorded at Kerguelen Island and on board ships in the neighbourhood of the island, between the 6th and 8th February, 1903, whieh was aeempanied by an inerease of wind, was followed on the 9 th by a diminution of pressure and a strong gale from east, in $65^{\circ} 53^{\prime} \mathrm{S}$., $89^{\circ} 21^{\prime} \mathrm{E}$, the position in which the "Gauss" was situated. .

Other instanees furnished by synehronous G.M.T. observations could be eited to show how the gales experieneed at Kaiser Wilhelm II. Land, during the sojourn there of the German Exploring Expedition, frequently owed their origin to systems of low pressure travelling eastward, whieh had previonsly affeeted the weather eonditions of Kerguelen and ships in the neighbourhood of the island, giving rise to strong winds and gales in those localities.

At Snow Hill, cluring the four months May to August, 1902, the mean pressure at 8 a.m. was $29 \cdot 21$ inehes. The highest 8 a.m. reading of the barometer during the time was taken on the 16 th August, and was as high as 30.01 ineles, which is the highest $8 \mathrm{a} . \mathrm{m}$. barometer observation reeorded by the Expedition in that year. There was no wind at the time of observation. The lowest 8 a.m. reading, 28.45 inches, was taken on the 16th May, when there was a moderate breeze from south-west.

The mean temperature at $8 \mathrm{a} . \mathrm{m}$. was $-5^{\circ} \cdot 1 \mathrm{l}$., the lowest mean temperature for that hour experienced by the Experlition. The maximum 8 a.m. teinperature, $36^{\circ} \mathrm{F}$., registered on the 12 th May, was assoeiated with a molerate breeze from south-west; the minimum $8 \mathrm{a.m}$. temperature $-35^{\circ} \mathrm{F}$., registered on the 7 th, with a light air from the same direetion.

Dr. NondE.sskiöld has laid stress upon the severity of the weather in July and the first half of August, 1902 , and states that the temperature fell as low as $-42^{\circ} \mathrm{F}$. 
"Discorery," Sep1ember - October, 1902.

"Southern Cross" Jispedition, Sep. tember - Oetober, 1899.

"Diseovery." Iien. tensint RoxDs to south-rest.

Lieutenant ArMITAGE to west.

Lie utenant Roy to Cape Crozier.
At McMurdo Sonnd, during September and October, 1902, atmospheric pressure rliminisherl, the mean for the two months being $29 \cdot 161$ inches, and the range of pressure 1.535 inches. The highest barometer reading, $29 \cdot 854$ inches, was recorded on 24 th September, with a gentle breeze from east; the lowest, $28 \cdot 319$ inches, on the 14 th October, with a moderate gale from sonth.

The observations for temperature, during the two months, give a mean of $-10^{\circ} \cdot 3 \mathrm{~F}$, and both the maximmm and minimum temperatures occurred in September; the former, $15^{\circ} \mathrm{F}$, on the 29 th of the month, associated with a gentle south-easterly breeze; the latter, $-43^{\circ} \cdot 5 \mathrm{~F}$, on the 20 th during a calm : a range of $58^{\circ} \cdot 5 \mathrm{~F}$.

The prevailing winds during the period were from eastward, between north-east and sonth-east; out of 732 observations of wind, the largest number for any point, 33 per cent. of the whole, were of wincls from east, but 26 per cent. fall under the heads "calm" or "variable."

The mean force of the wind, as estimated, is found to have been $2.5 ; 3$ gales, force 8 to 10 , were recorded; and 15 strong winds, force 6 to 7 .

Overcast or clondy weather was noted cluring the greater part of the period, and snow fell on 28 days.

At Cape Armitage, during the same months, the mean of the temperatures observed comes ont as low as $-15^{\circ} \cdot 9 \mathrm{~F}$; ; the highest reading, $10^{\circ} \cdot 5 \mathrm{~F}$., was taken on the 28 th September, the lowest, $-57^{\circ} \cdot 5 \mathrm{~F}$, was registered on the 12 th October.

The "Southern Cross" Expedition, in 1899, had a lower mean pressupe at Cape Adare during September and October-28·735 inches-than had the "Discovery" at MeMurdo Sound in the corresponding montlis of 1902 ; but their mean temperature of $-7^{\circ} \cdot 2 \mathrm{~F}$, as might be expected, was rather ligher than the "Discovery's." A maximun of $19^{\circ} \cdot 4 \mathrm{~F}$. was registered during the period, and a minimum of $-36^{\circ} \cdot 5 \mathrm{~F}$., giving a range of $55^{\circ} \cdot 9 \mathrm{~F}$.

Reverting to the National Antarctic Expedition; in September, Lientenant Royds and party made al sledge journey south-westward, starting on the 10th and returming on the 19th, reaching a position on the 15 th in latitude $78^{\circ} 15 \frac{1}{3}^{\prime} \mathrm{S}$., longitude $165^{\circ} 24^{\prime} \mathrm{E}$. From observations taken during this journey the mean temperature was found to be $-35^{\circ} \cdot 1 \mathrm{l}$. for the period, while the mean temperature at Winter Quarters was $-18^{\circ} \cdot 1 \mathrm{~F}$. The weather was calm during the greater portion of the journey, but a fresh gale was experienced on the 15th and again on the 18 th.

On a journey to the west, undertaken during the same month, between the 10 th and 26 th, led by Lientenant Anmpage, on which the party attained a position on the 19 th in latitude $77^{\circ} 381^{\prime} \mathrm{S}^{\prime}$, longitude $164^{\circ} 10^{\prime} \mathrm{E}$., the mean temperature was $-29^{\circ} \cdot 1 \mathrm{~F}$., when it was $-20^{\circ} \cdot 1 \mathrm{~F}$. for the same period at Winter Quarters.

From the 18th to the 20th, inchusive, the sledge party, however, were travelling at an elevation estimatcd at 500 feet above sea-level. The weather remained fine for the greater part of the journey, and there was little wind; what little there was eame from eastward, from some point between north-east and south-east.

A blizzard struck the camp at midnight on the 25 th; the direction of the wind was not noted, but the force was registered as 9. The sledge party must have been not far from the ship when this oceured, and there a light south-east air was stirring. The lowest temperature registered during the journey, $-45^{\circ} \mathbf{F}$., is entered against $8 \mathrm{a} . \mathrm{m}$. on the $14 \mathrm{th}$, in latitucle $77^{\circ} 46^{\prime} \mathrm{S}$., longitude $165^{\circ} 45^{\prime} \mathrm{E}$., when there was 110 wind and the sky was clear; and also against $5 \mathrm{a.m}$. on the $20 \mathrm{th}$, in latitude $77^{\circ} 40^{\prime} \mathrm{S}$., longitucle $164^{\circ} 27^{\prime} \mathrm{E}$, when again there was no wind and the sky was clear. At this time, eam, clear weather obtained at the ship, but the tenupcrature in the screen was not lower than $-23^{\circ} \mathrm{F}$.

A slerlge journey to Cape Crozier from the 4 th to the 24 th October, led by Iientenant liovis; again exhibited the great differences existing between the screen temperatures at Winter Quarters and the temperatures obtained at no great distance from the ship. For instanee, on the 9th Octoher, in latitucle $77^{\circ} 36^{\prime} \mathrm{S}$, longitude $169^{\circ} 10^{\prime} \mathrm{J}$, the mean of the thermometer readings taken by the sledge party gave a temperature of $-36^{\circ} \cdot 4 \mathrm{~F}$, the minimum value being $-50^{\circ} \cdot 2 \mathrm{~F}$., while the mean temperature at the ship was $-18^{\circ} \cdot 7 \mathrm{~F}$. and the minimum $-32^{\circ} \cdot 2 \mathrm{~F}$. Again, on the $22 \mathrm{nrl}$, the sledge party's nean temperature comes out at $-20^{\circ} \cdot 7 \mathrm{~F}$, the minimum being $-39^{\circ} \cdot 2 \mathrm{~F}$, and at the same time at the ship a mean of $-6^{\circ} \cdot 7 \mathrm{~F}$. obtaincd and the minimum was $-14^{\circ} \cdot 5 \mathrm{~F}$. On both of these days the sledge party recorded a calm, and at the ship there was a light air from eastward. While camping in the neighbourhood of Cape 
Crozier, from the 10th to the 18 th of the month, during rough weather, Lientenant RoyDs made the following entries in his jonrnal:-

10th Octuber.-_" IIeight alhove sea-level 940 feet. Summit of Mount Terror very misty. Arrived at furthest eamp."

(The mean of the temperature observations on that day comes out as $-33^{\circ} \cdot 6 \mathrm{~F}$, as against $-26^{\circ} \cdot 2 \mathrm{~F}$. at Winter Quarters. The minimum temperature was $-58^{\circ} \cdot 5 \mathrm{~F}$, against $-32^{\circ} \cdot 8 \mathrm{~F}$. at Winter Quarters. The weather was eloudy and ealm to eommence with, at eamp, and was followed by a gentle breeze from sonth-west; at Winter Quarters the weather was cloudy and the breeze gentle from east-sonth-east.)

11th October:- " Record was deposited at Cape Crozier."

(Mean temperature ou that day was $-28^{\circ} \cdot 0 \mathrm{~F}$; ; minimum temperature $-35^{\circ} .5 \mathrm{~F}$.; sky bluc; 110 wind. At Winter Quarters the mean temperature was $-26^{\circ} \cdot 8 \mathrm{~F}$; the minimum temperature $-40^{\circ} \cdot 8 \mathrm{~F}$; ; sky blue; wind light from enst-north-east at first, then ealm.)

12th October.- "Wind rose gradually all day, ugly appearance to south."

(Mean temperature $-28^{\circ} \cdot 1 \mathrm{~F}$; ; minimum $-40^{\circ} \mathrm{F}$.; wind gentle from sonth-west; weather overeast, misty. At Winter Quarters mean temperature $-27^{\circ} \cdot 6 \mathrm{~F}$; mininmm $-37^{\circ} \cdot 2 \mathrm{~F}$.; brecze light from eastsouth-east; weather overeast.)

13th Octolier.--" Wind sprang up very strong during night and blew hard all day, with a short lull at 4 p.m. Enormous quantities of drift collecting round tents."

(Temperature $-18^{\circ} \cdot 5 \mathrm{~F}$. (one observation); minimum temperature during previous 24 hour's $-30^{\circ} \mathrm{F}$; mean wind direction and foree, south-west, 8 ; weather overcast.)

14th October.- "Exactly similar weather as yesterday. Wind lulled for about half an hour at 4 a.m., but again came on, with increasing heavy gusts. Drift very thick."

(No instrumental observations are given on this day. The mean wind direction and foree, at Winter Quarters, was south, 4 ; the weather was overeast and snow was falling.)

15th October.- "Wind about the same, blowing a whole gale, accompanied by heavy drift."

(No instrumental observations given ou this day. The mean wind direction and foree at ship was eastsouth-east, 4 ; the wcather was overeast and snow was falling.)

16 th October.- "Similar conditions as yesterday, wind still a strong sonth-west gale, with heavy drift. In the afternoon the sun shone out a bit and the wind hegan to lull."

(No instrumental observatious given. Mean wind at Winter Quarters east by north, 3; weather overeast.)

17th October.-"Wind still remains strong, about force 7, lout sky was cloudless and wind gradnally fell towards evening."

(Temperature, 8 p.m., $-11^{\circ} \mathrm{F}$; mean wind south-west, 6 ; general weather "blue sky." At Winter Quarters the mean temperature was $-2^{\circ} \cdot 2 \mathrm{~F}$. ; the mean wind east, 3 ; the weather clear to clondy.)

18 th October.- "Glorious day ; sky cloudless."

(There was no wind.)

In his 'Voyage of the "Discovery," 'Captain Scotr mentions that gales at Cape Crozier grow excessively violent towards the end of Scptember and in Oetober, and that the ice sheet by this time has probably commenced to weaken.

During the twenty days ocenpied by Lientenant lioys on his journey to Cape Crozier, the mean of the temperatures olsserved is fornd to be $-20^{\circ} \mathrm{F}$, and the mean minimun $-34^{\circ} \cdot 2 \mathrm{~F}$. During the same period the mean temperature at Winter Quarters is fonnd to have been $-11^{\circ} \cdot 9 \mathrm{~F}$, and the mean minimum temperature $-21^{\circ} \cdot 2 \mathrm{~F}$; but it must be mentioned that for five ont of the twenty-one days Lientenant Royds's olservations were male in eanp, near Cape Crozier, at an elevation estimaterl at 940 feet alsove sen-level.

Diring the months of September and October, 1902, mean pressure at the Winter Quarters of the "G German Expedition is found to have been 28.900 inches, which is 0.261 ineh lower than that which obtained at Me.Inrdo Sound during the sane period. The highest pressure during the two months$29 \cdot 634$ inches, recorded on the 28th September-is 0.220 inch lower than the "Discovery's" for the same period; the lowest pressure-27.922 inches, recordecl on the 13 th Oetober-is 0.397 inch lower than the 
"Discovery's" for the same period. The extreme range of pressure, 1.712 inches, is 0.177 inch larger than that recorded on board the "Discovery."

The mean air temperature of $4^{\circ} \cdot 4 \mathrm{~F}$. is $14^{\circ} \cdot 7 \mathrm{~F}$. higher than the mean temperature at Mc Murdo Sound during September and October, 1902 ; the maximum of $25^{\circ} \cdot 7 \mathrm{~F}$, registered on the 23rd October, is $10^{\circ} \cdot 7 \mathrm{~F}$. higher than the maximum at MeMurdo Sound in those months, and the minimum of $-26^{\circ} \cdot 9 \mathrm{~F}$., registered on the 14th September, is no less than $16^{\circ} \cdot 6$ higher than the minimum of $-43^{\circ} \cdot 5 \mathrm{~F}$. registered at MeNLurdo Sound six days later. The extreme range of temperature, $52^{\circ} \cdot 6$, is about $6^{\circ}$ smaller than the extreme range at MICMurdo Sound for the two months.

The mean of the observations for wind force at the German Antarctic Station in September and Octoher, 1902 , is found to be $2 \cdot 8$; on 21 days the wind's foree was estimated at 6 or 7 ; on 11 of these days at 8 or above 8 . The prevailing winds during this period were from eastward, $17 \cdot 2$ per cent. of the total observations of wind falling under the head "east," and 18.8 per eent. falling under the heal "east by south," lont 15.9 per eent. of the observations fall under the head " calm."

There were 4 cloudless days, when the proportion of eloud to blue sky was less than 2 in 10 ; and 31 clondy lays, when the proportion was more than 8 in 10 . Entries of rain, snow, or snow-drift, occur on 50 days in the 2 months.

From a comparison of observations made at an observing station, situated on the ocean ice, at the foot of the Gaussberg, with those taken on board the "Gauss" at the same hours, it was found that both pressure and temperature were somewhat higher at the former station. On one of the sleighing expeditions to the Gaussberg, which took place from the 28th April to the 10th May, 1902, it was found that pressure was 1.7 milliuns. $(0.067$ inch $)$ higher and the temperature $3^{\circ} \cdot 1$ C. $\left(5^{\circ} \cdot 6 \mathrm{~F}\right.$. $)$ higher at the foot of the mountain than were pressure and temperature on board the "Gauss" at the same time. On another expedition made to the Ganssberg, from the 25th Septeuber to the 9th October, the pressure was found to be 0.8 millim. $(0.032 \mathrm{inch})$ higher at the station at the foot of the mountain than that observed at the ship, and the temperature $1^{\circ} \cdot 9 \mathrm{C} .\left(3^{\circ} \cdot 4 \mathrm{~F}\right.$. $)$ higher. The range of temperature at the former station, morcover, was smaller. It is to the steepening of the pressure-gradient southward, over Kaiser Wilhelm II. Land, assoeiated with the decrease of pressure diring the passige eastward of cyclonie systems to the north of the station, that the violenee of the easterly gales experienced at the Winter Quarters of the German Expedition must be attributed.

The prevalence of winds from eastward in Kaiser Wilhelm II. Land is probably due, not only to the increase of pressure landward, but also to the contour of the coast line.

The "Discovery" and the "Morning" in the Ross Sea had winds from enstward no more frequently than winds from other quarters, in parallels much further south than that on which the "Gauss" was situated during her stay in the Antarctic.

At the end of December, 1901, and in the beginning of January, 1902, the "Diseovery," proceeding southward, had winds principally from east-north-eastward and west-south-westward, but mostly from the latter direction between the parallels of $50^{\circ} \mathrm{S}$. and $60^{\circ} \mathrm{S}$, and principally from west-sonth-west between the parallels of $60^{\circ} \mathrm{S}$. and $67^{\circ} \mathrm{S}$. Thence to $70^{\circ} \mathrm{S}$. the winds were from between north-north-east and south-south-east round by east; but from that latitude to $75^{\circ} \mathrm{S}$. she had winds from south to westsonth-west.

In Deeember, 1902, the "Morning," steaming southward, had winds chiefly from between south-southeast and west-north-west, round by south, between the parallels of $50^{\circ} \mathrm{S}$. and $65^{\circ} \mathrm{S}$.; and winds from between north-east and west-north-west, round by north, between the latter parallel and $70^{\circ} \mathrm{S}$. Thence, to MeMurdo Sound, in Jannary, 1903, 1st to 22nd, she experienced winds from between south-east and south-south-west, the principal directions being sonth-sonth-enst and south-south-west.

From the 1st to 10th of March, 1903, the "Morning," proceeding uorthward from MeNinrdo Sorund, met with winds mainly from between north-west and south-south-west to latitnde $65^{\circ} \mathrm{S}$.; thence to latitude $50^{\circ} \mathrm{S}$. winds from between west and north-north-west. During the period 13th December, 1903, to 5th Jamuary, 1904, the "Morning" and "Terra Nova," making for Mellurdo Sound from the northward, had southerly and sonth-westerly winds for the most part from $50^{\circ} \mathrm{S}$. to $65^{\circ} \mathrm{S}$; ; snbsequently to $77^{\circ} \mathrm{S}$. winds letween east-south-enst and south-west round by south. 
From the 19th February to the 5th March, 1904, the "Discovery," homeward bound from MeMfurdo Sound, recorded winds from between east-south-cast and west-north-west, round by south, but principally from between south-east and soutl-west to the 66th parallel. "The "Nlorning," from the 1st to the 25th February of that year, while in McMurdo Sound and traversing the same ronte, had wirds from between east-1rorth-east and west-sonth-west round by south, but principally from east-sonth-east. 'Thence to the 50th parallel both vessels experienced westerly winds, chiefly from between west-south-west and north-west.

Cruising in the Weddell Sea, during February and March, 1903, between the parallels of $60^{\circ} \mathrm{S}$. and $68^{\circ}$ S., the Scottish National Antaretic Expedition in the "Scotia" recorded 427 observations of wind from north to north-east and 255 from south to south-west out of a total of 1142 wind ohservations; arrl from the 21st February to the 20 th $\mathrm{April}, 1904$, between the parallels of $45^{\circ} \mathrm{S}$. and $72 \frac{1}{2}^{\circ} \mathrm{S}$., 767 sonth-west to north-west winds were recorded and 309 north to north-east winds out of a total of 1440 wird observations.

At Snow Hill, the mean of the barometer observations recorded in September and October, 1902, at Snow Hill, Septem. 8 a.m., by the Swedish Expedition, is found to be 29.09 inches. The results for this period compared with those of the period May-August show a diminution of mean monthly pressure at this station of 0.37 inch from June to Octoler, but a recovery of pressure of 0.15 ineh from August to September. The highest 8 a.m. pressure, in September and October, was recorded on the 29th September and was 29.77 inches, a modcrate breeze from south-south-west being noted at the time; the lowest pressure in the 2 months, 28.38 inches, was recorded on the 14 th September and was associated with a gentle breeze from the same direction. These extremes show a range of pressure at 8 a.m. of $1 \cdot 39$ inches.

The mean of the 8 a.m. observations for temperature during these 2 months is $7^{\circ} \cdot 1 \mathrm{~F}$; ; the maximum 8 a.m. temperature, $38^{\circ} \mathrm{F}$, was registered on the 16 th September, when there was a light air from north ; the minimum, $-27^{\circ} \mathrm{F}$, , on the 1st September, when there was no wind, and again on the 2nd September, when there was a light air from north-east. The range of temperature at $8 \mathrm{a} . \mathrm{m}$. therefore was $65^{\circ}$.

The prevailing wind during this period was from south by west, and this was the prevailing wind in March and April and from May to August inclusive. The average force of the wind at 8 a.m. is found to have been $3 \cdot 4$; the number of strong winds recorded at $8 \mathrm{a} . \mathrm{m}$. was 10 ; the number of gales 7 . The mean amount of cloud was 8 . Dr. NorDExSKIöLD mentions that a strong, warm wind from north-west set in about the middle of September and lasted for a few days, and that it drove the ice from the land ; but that gales and cold returned at the end of the month.

At the "Discovery's" Winter Quarters during the sunmer months November, 1902, to February, 1903, inclusive, a mean pressure of $29 \cdot 405$ inches obtained.

'The lîghest barometer reading, $30 \cdot 112$ inches, was takcn on the 22nd November during a calm; the lowest, 28.758 inehes, on the 23rd February, also during a calm. A mean temperature of $18^{\circ} \cdot 1 \mathrm{~F}$. obtained during the period, and the range of temperature was $48^{\circ} \cdot 5$. The maximum temperature was $39^{\circ} \mathrm{F}$., and it was registered on two occasions : on the 26th December, in a calm, and on the 8th January, with a light air from east-north-east. The minimum temperature, $-9^{\circ} \cdot 5 \mathrm{~F}$., is entered against the 24th February, with a light air from north. The winds were principally from between north and southeast. Out of 1440 observations of wind recorded during the four months, when referred to 8 points of the compass, $18 \cdot 3$ per cent. come under the head "north," $28 \cdot 3$ per cent. under the head "east," $10 \cdot 2$ per cent. under "north-east," and $12 \cdot 7$ per cent. under "south-east." But $19 \cdot 3$ per eent. of the observations were under the head "calms" and 3.5 per cent. under "variable." The remaining $7 \cdot 7$ per cent. of the observations were of winds principally from south and north-west. The mean force of the wind as estimated was nearly 3 , and no gales were recorded. Blue sky was noted on 25 days; the weather was cloudy on 61 days, overeast on 34 , misty on 14, foggy on 10 . Snow fell on 37 days.

At Cape Armitage only three observations for temperature were recorded during the four months November-Feloruary, 1902-3. On the 17 th December a reading of $28^{\circ} \mathrm{F}$. was noted, and the nuaxinum temperature at Winter Quarters on that day was $27^{\circ} \cdot 2 \mathrm{~F}$; on 5th November the minimum thermometer registered $-14^{\circ} \mathrm{F}$, and the minimum temperature in the screen at Winter Quarters registered on the same day was $4^{\circ} \cdot 4 \mathrm{~F}$.

Discovery," Nuvem-
ber, 1002, to Feb. ruary, 1903.

Cape Amnitage, De. cember-February, 1902-3. 
"Southern Cross" From November to Jannary, 1899-1900, inelnsive, the "Southern Cross" lixpedition had a mean Expedition, No-
vember-Jnuary, pressure of $29 \cdot 245$ inches; a mean temperature of $27^{\circ} \cdot 6 \mathrm{~F}$; a maximum temperature of $48^{\circ} \cdot 7 \mathrm{~F}$. (on the $1592-1900$. 24 th Janniry); a minimum temperiture of $-4^{\circ} \cdot 3 \mathrm{~F}$. on 2 nd November; giving a range of $53^{\circ}$. The wind cane, mainly, from south-east, but there was a fair proportion of winds from sonth and east, and a large proportion of calms. The mean foree of the wind as estimated comes out at $2 \cdot 2$, although no less than 25 gales were experieneed.

S.S. "Morning," De. cember - January $1902-3$.

Sledge journess, Norember-February 1902-3.

Sledge journey. Captain Scorg's
jouruey south.
From the 25th December, 1902, to the 25th January, 1903, the relief ship "Morning," under the command of Lieutenant W. CoLBECK, R.N.R., was making her way from the 67 th parallel of south latitude, and about the 179th meridian of east longitude, to McMurdo Sonnd. During the remaining days of January, ant during the whole of February, the ship was anchored in the Sound, not far from the "Discovery." Four-hourly observations were made on hoard the "Mlorning" throughout this period, and a brief summary of the results is given in Table 5 .

A comparison of the results of observations obtained during the sledge journeys mndertaken by the National Antaretie Expedition during the Summer of 1902-3 with the eorresponding results registered at the same time at their Winter Quarters shows in a marked degree how much lower were the temperatures experieneed by the respective sledge parties, even at no great distance from the ship, than those experienced at Winter Quarters. The direetion of the wind observed at Winter Quarters is also frequently in marked contrast with the wind observations recorded on these journeys. For instance: on a sledge journey, led by Lieutenant Roy ds, to Cipe Crozier, from the $3 \mathrm{rd}$ to the $17 \mathrm{th}$ November, inclusive, the mean temperature was $9^{\circ} \cdot 3 \mathrm{~F}$, when the mean temperature at Winter Quarters was $12^{\circ} \cdot 1 \mathrm{~F}$. The highest reading of the thermometer was $17^{\circ} \mathrm{F}$, but the maximum temperature at Winter Quarters during the period was $27^{\circ} \cdot 8 \mathrm{~F}$. The minimum temperature registered on the journey was $-18^{\circ} \mathrm{F}$., and the minimum at Winter Quarters, zero, F. The average direction of the wind was south by west on the journey, while it was east by north at Winter Quarters.

During a journey to the Western Mountains, led by Licutenant Armitage, from the 29th November, 1902, to the 19th January, 1903, inclusive, the contrasts in temperature between the localities visited by the sledge party and Winter Quarters are not so marked, considering the elevation at which the sledge party's observations were made during the greater part of the journey. The wind direetions recorded by the travellers are, however, very different from those experienced at Winter Quarters, and correspond with those recorded on journeys to the south-east, south, and south-west of the ship.

In the fou day's 6 th to 9 th December, between $77^{\circ} 47^{\prime}$ S., $165^{\circ} 21^{\prime}$ E., and $77^{\circ} 45 \frac{1}{2}^{\prime}$ S., $164^{\circ} 45^{\prime}$ E., at an average approxinate elevation of 3600 feet above mean sea-level, the mean of the temperature olservations noted by the sledge party was $16^{\circ} \cdot 7 \mathrm{~F}$, and the average direction of the wind south, while at the ship the mean temperature is found to have been $23^{\circ} \mathrm{F}$, and the average wind direction east by south.

During the four days 11 th to 14 th December, between $77^{\circ} 491^{\prime}$ S., $164^{\circ} 21^{\prime} \mathrm{E}$, and $77^{\circ} 51^{\prime}$ S., $163^{\circ} 45^{\prime} \mathrm{E}$, at an average elevation approximately of 3600 feet above sea-level, the sledge party had : mean temperature of $21^{\circ} \cdot 1 \mathrm{~F}$, and an average wind direction south by west. $\Lambda$ the same time at tho ship the mean temperature was $27^{\circ} \cdot 7 \mathrm{~F}$, and the average direction of the wind south-east by east.

From the 1 st to the 6th January, inclusive, between $77^{\circ} 42^{\prime} \mathrm{S} ., 160^{\circ} 9^{\prime}$ E., and $77^{\circ} 43^{\prime} \mathrm{S} ., 159^{\circ} 38^{\prime} \mathrm{Is}$., at an average elevation approximately of 7500 feet, a considerable fall in temperature was experieneed hy the travellers, and the mean temperature during the time is found to have been $-0^{\circ} \cdot 1 \mathrm{~F}$., with an average wind direction sonth-west ly south, while at the ship the mean tenperature was $28^{\circ} \cdot 3 \mathrm{~F}$., and the average wind direction north by cast.

On the return journey, from the 12 th to the 15 th Jammary, between $77^{\circ} 51^{\prime} \mathrm{S} ., 162^{\circ} 56^{\prime} \mathrm{F}$., and $77^{\circ} 48^{\prime} \mathrm{S} ., 164^{\circ} 34^{\prime} \mathrm{H}$., the sledge party recorded temperatures giving a mean of $27^{\circ} \cdot 3 \mathrm{~F}$, at an average elevation approximately of 2840 feet, and calms, while at the ship the mean temperature was $27^{\circ} \cdot 2 \mathrm{~F}$., but the average wind direction was east.

Table 6 gives some mean resnlts of observations made during Captain ScotT's famous joumey farthest sonth, with corresponding values at Winter Quarters for comparison.

Readings of aneroid barometers, for pressure, were supplemented during the journey by oceasional 
hypsometer observations. At first two aneroids were in nse, differentiated in the legister by the numbers 1 and 2, but the readings of No. 1 have not here been used, as the error of the instrument was found to have varied more than $\frac{1}{10}$ of an inch between the date of issue and the 2nd November. No. 2 (Aneroid, M.O. No. 2) agreed very nearly with the ship's standard-mereury-barometer at the heginning of November, and the correction then obtained was in good agreement with the Kew correction, at 29 inches, viz., - 02. After the 5th November the observations noted were of one aneroid only, and the instrument is distingnished in the Register as H.O. 4 and M.O. 3. The mean correction for this aneroid was found to be +.01 at 29 inches, when the returning sledge party was near to, or at, the ship from the 1 st to the 3rd February, 1903, the error varying between $-\cdot 01$ and $+\cdot 03$. The correction, when issued, was $+\cdot 02$ at 29 inches; it is fair, therefore, to consider +.02 as an approximate correction for this aneroid thronghout the journey.

The results of the observations for pressure, to which the above corrections have bcen applied, are in fair agreement with the results of the same observations corrected by applying the errors oltained from time to time by comparisons of hypsometer observations with simultaneous reading of the aneroid. Both are here given.

As the height above sea-level was not known, it has been assumed that the area over which Captain SCoTT and his party travelled was not much higher during the greater portion of the journey than the height of the ice barrier at its edge, and in reducing the observations for pressure to mean sea-level a mean altitude of 150 feet has been assumed. It is recognised at the same time that the assumption of a mean altitude is scarcely admissible, as the whole mechanism of ice distribution implies some gradual elevation southward-how much is an open question.

In 'The Voyage of the "Discovery," Captain Scort states that on the 19th December "Everywhere apparently there is a sharp and definite line between the land and the level surface of the barrier as exists on an ordinary coastline between land and water." He was then in latitude $81^{\circ} 5^{\prime} \mathrm{S} ., 162^{\circ} 6^{\prime} \mathrm{E}$.

If the barrier upon which the explorers were travelling did not rise with latitude to a much greater altitude than that which has been assuned here as the mean altitnde during the journey, then the increase of pressure, with increase of latitude, confidently expected by former explorers of this quadrant of the Antaretic, did not obtain.

The results of olservations show that the mean pressure to the sonth differed but little from the mean pressure at Winter Qnarters during the same periods. The differences between the two means are curiously constant thronghout, the average difference being about 0.05 iuch, using the Kew correction, and nil using the correction obtained by hypsometer observations.

An increase in mean pressure between the first and second period of 0.37 inch is found at Winter Quarters, against $0 \cdot 34$ inch on the sledgo journey, using the Kew correction; and a diminution of nean pressure between the second and third periods of 0.29 inch at Winter Quarters, against 0.24 inch on the journey. Again, a diminution is shown between the third and fourth periods, which is only 0.09 inch at Winter Qnarters and 0.14 inch on the journey. An increase of menn pressure is shown between the fourth and fifth periods of 0.14 inch at Winter Quarters, against 0.15 inch on the journey, and a diminution between the fifth and sixth of 0.27 inch at the former and of 0.29 inch on the latter.

This similitude, in the rise and fall of mean pressure, between the Winter Quarters' pressure results on the one hand, and the pressure results of observations made on the sonthern journey during the same periods, suggests a mean distribution of pressure over the areas traversed to the eastward of the mountain ranges, represented by an isobar running about N.N.E. and S.S.W.

This supposition gains some confirmation by the fact that, during the journey to the sonth, the wind's direction was mainly from sonth-south-westward.

It seams not improbable, indeed, that from Cape Adare to Mount Longstaff, and even still farther to the south, the distribution of pressure conforns largely to the configuration of the high land, and that an area of relatively high pressure lies over the land to the westward of the coast ranges, and relatively low over the Ross Sea, giving gradients for southerly wiuds during the greater portion of the year.

The tendency for the formation of a pressure gradient, with isobars along the coastline is generally 
notieeable wherever land meets sea and may probahly be attributed to the teniperature graulient between the land and the water.

On the eoastlines of the great eontinents the phenomenon is for the most part marked by the tendeney of the average wind to follow the direction of the littoral. This is well shown in the "Wind Charts of the Coastal Regions of South Ameriea,' prepared in the Meteorologieal Offiee and pullished by the Admiralty. There is also evideneo in favour of the existenee of a similar state of things in high latitudes.

The point is strikingly illustrated in the pressure and temperature niaps given in the 'Atlas Climatologique de l'Empire de Russie,' published in St. Petersburg, in 1900, by the Ohservatoire Physique Central Nieolas.

In this atlas the maps devoted to mean monthly temperature of the air show that during the four months Deeemher to Mareh the isotherms along the eastern seahoard of Siberia and, to some extent, along the northern seahoard also, run almost parallel to the eoast, and that the temperature gradient is inland towards a minimum situated on about the 135th meridian of east longitude, in the neighbourhood of the Aretic Cirele.

The maps dealing with atmospherie pressure and wind resultants for the same months exhibit isolars whieh also run almost parallel to the northern and eastern seaboards, and the pressure gradient is seaward from a maximum sitnated over some loeality in about the eentre of Asia.

In the maps relating to the three months April to June, the temperature gradient is shown to be from the sonth towards a minimum over the Aretic Oeean; but in May and June a gradient is indieated towards the eastern seaboard as well.

In the maps for pressure for the three months the isobars display, by their tendeney to turn Horthwards as they approach the Aretie Oeean, the transitional eondition of the atmosphere charaeteristie of the seasoll.

In July and August the eonditions whieh obtained during the four months Deeember to Mareh are more or less eompletely inverted. Isotherms affeet the eontour of the northern seaboard, and ineline coastwise on the eastern eoast also, but the temperature gradient is from inland towards the sea. The isobars for these months outline roughly the trend of the littoral, but the pressure gradient is inland from the sea.

For the months of September to November, inelusive, the general distrihution of temperature and pressure indieated differ but little from those whieh eharaeterise Deeenher to Mareh, but, while the relation between temperature and pressure is almost as elearly defined in the former period as it is in the latter, the September to November period is, in addition, marked by some features whieh appertain to the three transitional months April to June. Thus we should uot be surprised to find some eorrespondence between the general run of the isobars and the eoastline of the Antaretie Continent. The rariation from Winter to Summer is less marked, possihly beenuse the eold eentre remains in approximately the same position in both seasons and the arrangement is subjeet only to variation of a loeal eharaeter.

From the 6th to the 9th November, between latitude $78^{\circ} 15^{\prime} \mathrm{S}$., longitude $168^{\circ} 10^{\prime} \mathrm{E}$., and latitude $78^{\circ} 40^{\prime} \mathrm{S}$., longitude $167^{\circ} 53^{\prime} \mathrm{E}$., there oeeurred a period of unsettled weather whieh may be tonehed on in order to illustrate conditions oeensionally experieneed hy Captain ScoTT and his party during their southern journey.

At 2 p.m. on 6th November the barometer, eorreeted for an altitude of 150 feet to sea-level, stood at $29 \cdot 30$ inehes, and the thermometer at $10^{\circ} .5 \mathrm{~F}$. There was a light air from north-west, and the "weather throughout the day beantifully fine." At 9 p.m. pressure and temperature had not ehanged, but the light air had died away. At 10 p.m. on the 6 th to 4 p.m. on the 7 th a hard blizzard was hlowing from southwest, with exeeption of a lull at $2.30 \mathrm{a} . \mathrm{m}$. The smoke from Mount Erebus was observer to be moving from south-west. The wind moderated and the weather improved at 5 p.m., and an hour after there was only a light air from north-west. The mereury hard dropped very little, and at 9 p.m. stood at $29 \cdot 27$ inehes, hut the temperature had fallen to $-4^{\circ} \mathrm{F}$. The weather was noted as "b."

A light air continued to come from north-west until after 4 a.m. on the 8 th, and at 6 a.m. a hlizzard was again experieneed which lasted thronghout the day, with the wind from south-sonth-west, foree 2-5. The barometer was rising; temperature fluetuating, but higher. The snow drift is deseriberl as heary all day. The wind dropped towards 6 p.m., but freshened at 10 p.m. 
The blizzard did not moderate nutil 3 p.m. on the 9 th, the wind continning from sonth-sonth-west, and the baroneter now rising. $\Lambda$ fter that, althongh the breeze was light, the clrift continued until 5 p.m., when the smoke from Momut lirebus was seen to be "going to nortli-east ancl enst." The barometer then rose briskly and at $8 \mathrm{a} . \mathrm{m}$. had risen to 29.66 inches; at the same time the thermometer markerl $6^{\circ} \mathrm{F}$.

$\Lambda t$ the Winter Quarters of the German Expedition in the Antaretic during the three months Novemler to Jammary, 1902-3, observations for pressure show a mean of 29.293 inehes, which is 0.112 inch lower than the mean of the pressure observations taken at the Winter Quarters of the "Diseovery" during the four montlis' period November to Felbruary, 1902-3.

The highest baronteter reading in the three months, $29 \cdot 800$ inches, was recorded on the 24 th November. It is 0.312 inch lower than the highest barometer reading recorded ly the British Expedition in the November to F'bluary period, and relates to a date two days later. The lowest reading recorded dnring the three nonths, 28.390 inehes, observed on the 20th January, is 0.368 inch lower than the lowest at the "Discovery's" Winter Quarters for the four mouths November to February, which was observed on the 23rd February. The range of pressure, 1.410 inch, is 0.056 inch larger than the range of pressure which oltained at the "Discovery's" Winter Quarters.

The results of temperature olservations at the German station give a mean of $26^{\circ} \cdot 8 \mathrm{~F}$. for the three months, which is $8^{\circ} \cdot 7$ higher than the mean temperature at the British station during the same period. The maximum temperature, $40^{\circ} \cdot 8 \mathrm{~F}$., registered on the 22 nd Deccmber, is $1^{\circ} \cdot 8$ higher than the maximum temperatnre at the British station for the four months November to February, 1902-3; the minimum, $-17^{\circ} \cdot 7 \mathrm{~F}$, registered on the 1 st November, is $8^{\circ} \cdot 2$ lower than the lowest registered at the British station in the Summer of $1902-3$. The range of temperature, $58^{\circ} \cdot 5$, is $10^{\circ}$ larger, for the three months period, at the German station than it is for the four months' period at the British.

The average force of the wind at the German station, as estimated, was 2.8. On 21 days during the three months the wind rose to force 6 or 7 , and on 8 of these days it attained to gale force. The direction of the wind, mainly, was from between east by north and east by south. 26.5 per eent. of the total wind observations made in the three months under notice were of winds from east; $16 \cdot 2$ per cent. of winds from east by north; $14 \cdot 7$ per cent. of winds from east by south; and 8 per cent. were of calms. There were 6 cloudless days, when the proportion of clond to blue sky was less than 2 , and 44 cloudy days, when the said proportion was more than 8. On 51 days rain, snow, or snow drift was recorded.

At Snow Hill, during the four summer months of November to February, 1902-3, the Swedish S Antarctic Expedition recorded olservations for pressure at 8 a.m. which give a mean of $29 \cdot 26$ inches. The highest 8 a.m. reading, $29 \cdot 85$ inches, relates to the 2 nd December, when a gentle breeze from northeast was stirring; the lowest $8 \mathrm{am}$. realing, 28.56 inches, was noted on the 14 th February, and was associated with a strong breeze from wcst-south-west-a range of $1 \cdot 29$ inches.

The mean temperature at $8 \mathrm{am}$. in these months is found to have risen from $7^{\circ} \cdot 1 \mathrm{~F}$., the mean for September and October, 1902 , to $24^{\circ} \cdot 7 \mathrm{~F}$. The maxinum temperature, $35^{\circ} \mathrm{F}$, occurred on the $23 \mathrm{rd}$ January, with a light brecze from south-south-west; the minimum, $2^{\circ} \mathbf{F}$, on the 1st November, with a strong breeze from south-south-west. The range of the $8 \mathrm{a} . \mathrm{m}$. temperatures was therefore $33^{\circ}$. The prevailing wind at 8 a.m. is fonnd to have been south by east during these summer months; the average force, $2 \cdot 3$; the mean elond amount, 8 . Strong winds were noted on 14 occasions at 8 a.m.; no gales were recorded at that hour.

The information relating to the meteorology of the Wcddell Sea and of Laurie Island, Sonth Orkneys, Scottish National incorporated, hy permission of the author, in this memoir, is summarised from the Reports contributed by Mr. Robert C. Mossuax, F.R.S.E., the meteorologist of the Scottish National Antarctic Expedition, to Expedition, Feb. 'The Scottish Geographical Magazine,' Talles VII. and VIII. 'The valuable information thus obtained has been supplemented by additional data, kindly supplied by Mr. MLossirax, whose generous assistance in this matter is greatly appreciated.

Mr. Mossman states that, after leaving Port Stanley, Falkland Islands, in the S.Y. "Scotin," the edge of the ice pack was reached on the 2nd Fehruary, 1903, in latitude $60^{\circ} \mathrm{S}$. During the first half of February the mean position of the "Scotia" was latitude $60^{\circ} 5^{\prime} \mathrm{S}$., longitude $3 \tau^{\circ} 45^{\prime} \mathrm{W}$. The mean of the temperature observations recorded every honr during the first half of the month is fonnd to be 
$30^{\circ} \cdot 3 \mathrm{~F}$. and the extreme range of temperature ouly $6^{\circ} \cdot 2$, viz., between a maximurn of $32^{\circ} \mathrm{F}$. and a mininum of $25^{\circ} \cdot 8 \mathrm{~F}$.

The mean pressure during the period is given as $29 \cdot 186$ inches. The prevailing winds were from between nortl-west and north-east, and sonth and west-south-west; the former, presumably, llowing in front of, and the latter in rear of, depressions travelling to the eastward and sonth-eastward and passing over the area of observation. Once only, on the 11th February, the wind attained to the force of a gale, and then for not more than an hour.

During the seeond half of February the ship's mean position being in latitude $67^{\circ} 55^{\prime}$ S., longitude $21^{\circ} 45^{\prime} \mathrm{W}$., the weather was eonsidered by the explorers unusually cold for the season, the mean temperature being as low as $25^{\circ} \cdot 4 \mathrm{~F}$, with a range of temperature of $18^{\circ} \cdot 6$, i.e., from a maximum of $32^{\circ} \mathrm{F}$. to a ninimum of $13^{\circ} \cdot 4 \mathrm{~F}$. In the second half of the month under notice, in the mean position referred to, the mean pressure declined to 28.966 inches. Mr. Mossman states that during this time the most frequent wind was the sonth-west, which was closely followed by winds from east, sonth-east iurd north. The frequency of the south-west wind may have been due to a relatively high pressure over Graham Land, Danco Land, and the neighbouring islands, the north wind to the air cirenlation in front of approaching low-pressure systems, and the sonth-cast wind to the circulation in the rear. "Winds from west-sonth-west and north-north-west were of rare occurrence," says Mr. Mossman, and the reason seems obvious, for these winds belong to the northern segment of eyclonic systems in the southern hemisphere, and the "Scotia's" average position during the second half of February lay to the sonth of that part of the circulation and in the southern segment of the systems.

“Discorery," March And April, 1003 .

During the autumm months of March and April, 1903, observations for pressure at the "Discovery's" Winter Quarters give a mean of 29.354 inches, which is 0.015 inch lower than the mean pressure of the corresponding month in the previons year. The range of pressure in March and $A$ pril, 1903, 0.987 inch, is 0.244 inch smaller than the range in the corresponding two months of 1902 . The highest barometer reading, 29.782 inches, was recorded on the 25 th March, the lowest, 28· 795 inches, on the 12 th April.

The mean temperature during these two months was $-8^{\circ} \cdot 9 \mathrm{~F}$. and is $9^{\circ} \cdot 4$ lower thau the mean temperature of the corresponding period in 1902 , and the range of temperatures, $67^{\circ} \cdot 5$, is $8^{\circ} \cdot 4$ larger. The maximum temperature was $25^{\circ} \cdot 5 \mathrm{~F}$, and is entered against the 6th $\mathrm{Iar} \mathrm{ch}$; the minimum, $-42^{\circ} \mathrm{F}$, against the 26th April.

Cape Armitage, March and April, 1903.

At Cape Armitage, 57 observations for temperature, taken between the hours of 10 a.m. and noon, give a mean of $-18^{\circ} \cdot 6 \mathrm{~F}$.; the highest reading, $25^{\circ} \cdot 5 \mathrm{~F}$, was taken on the 5 th Mareh, the lowest, $-56^{\circ} \mathrm{F}$, registered on the $22 \mathrm{nd}$ April-showing a range of $81^{\circ} \cdot 5$.

"Discovery," Winter Quarters, MarchApril, 1003.

The prevailing winds at McXurdo Sound during March and April, 1903, were north-easterly or easterly, the percentage of frequency of the former heing $23 \cdot 5$, and of the latter $34 \cdot 0$; but $24 \cdot 5$ per cent. of the total wind observations for the period were of calms.

Bhe sky was recorded on 17 days, and clondy, or overcast, sky on 44 days.

Snow fell on 25 days, and the weather was misty on 10 days.

Strong winds were recorded on 15 days, and gales on 2 days.

Relief Ship "Morn. ing," Murch, 1903.

The Relief Ship "Morning," returung northward from McMurdo Sound between the 1st and 10th March, 1903, reached a position in latitude $65^{\circ} 36^{\prime}$ S., longitude $173^{\circ} 57^{\prime} \mathrm{E}$., on the latter date. She recorded a mean pressure of 29.41 inches during this time. Her highest barometer reading, taken on the 5th-29.69 inches - was associated with a fresh south-westerly breeze, her lowest of 28.99 inches on the Sth, with a strong northerly wind, which attained the force of a fresh gale.

Her mean temperature was $22^{\circ} \mathrm{F}$; ; mean wind foree 5. Strong winds were experienced on 3 days, and gales on 3 days.

Swedish Expedition, Snow Hill, MarehApril, 1903.

At Snow Hill Island, the Swedish station, in March and April, 1903, the mean 8 a.m. pressure had declined from $29 \cdot 26$ inches, the mean pressure during the summer months of November to February, to 29.05 inches. The highest 8 a.m. reading was no higher than 29.58 inches, and this was noter on the 20 th $A$ pril during a calm; the lowest 8 a.m. reading, $28 \cdot 44$ inches, was noted on the 24 th Marreh, and a fresh gale was blowing from west-sonth-west at the time. The resultant range, $1 \cdot 14$ inches, is not large.

In these months Snow Hill Island had a mean temperature, at 8 a.n., of $8^{\circ} \cdot 9 \mathrm{~F}$, which is $15^{\circ} \cdot 8 \mathrm{~F}$. lower 
than the mean 8 a.m. temperature of the four sumuer months of 1902-3. The maximum temperature, $42^{\circ} \mathrm{F}$., was observed on the 18 th April during a calm; the minimun, $-13^{\circ} \mathrm{F}$., on the 24 th $A$ pril, when a fresh gale was blowing fron south-west. In the two months, therefore, the range of temperature was $55^{\circ} \mathrm{F}$.

At 8 a.m. the prevailing wind during these antium months proved to be from south, and the mean force $4 \cdot 2$, which shows an increase of $1 \cdot 9$ on the 8 a.m. Sumuner mean. The mean clond annount was again 8 . Strong winds were recorded at 8 a.m. 11 times during the periorl, gales 12 times.

From the 1st to the 20th March, 1903, the S.Y. "Scotin" was cruising in the Weddell Sen, and her average position, fron the lst to the 10th inclusive, is given as latitude $67^{\circ} 55^{\prime} \mathrm{S}$., longitnde $34^{\circ} 35^{\prime} \mathrm{W}$.

Observations made during this ten-day period give the following results :- IIean pressure, $29 \cdot 168$ inches; highest barometer reading, 29.610 inches, taken on the 6 th ; lowest, 28.367 inches, on the 9 th. Ilean temperature, $27^{\circ} \mathrm{F}$.; maximum temperature, $29^{\circ} .5 \mathrm{~F}$, observed on the 9 th ; minimum, $20^{\circ} \cdot 8 \mathrm{~F}$., on the 6 th. The sky was overeast during the whole of the period; the average wind force was $2 \cdot 6$, and gales were experienced during 27 hours. The prevailing winds were from between north and sonth-east, northeasterly winds predominating. During 53 hours snow fell, and sleet was noted against 2 hours. Rain fell on 5 hours, and the weather was misty, or foggy, on 9 hours.

From the 11th to the 20th March the average position of the "Scotia" is given as latitude $63^{\circ} 40^{\prime}$ S., longitude $42^{\circ} 50^{\prime} \mathrm{W}$. Pressure diminished during this period, and the mean is found to have been $29 \cdot 00$ inches.

The highest barometer reading, 29.362 inches, was recorded on the 13th ; the lowest, 28.396 inches, on the 20th. Temperature fell for the most part during this time, although the maximun temperature, $32^{\circ} \cdot 1 \mathrm{~F}$., was registered on 20 th, and the minimum, $6^{\circ} \cdot 9 \mathrm{~F}, 7$ days before.

The total observations for temperature during the 9 days yield a mean of $24^{\circ} \cdot 4 \mathrm{~F}$.

The sky was cloudy, or overeast, during the greater portion of this period, the average amount of eloud being $9 \cdot 8$.

The average force of the wind was the same as the average of the previous period, $2 \cdot 6$, but only one gale was experienced.

Out of 240 observations of wind direction, 91 were of winds from north-eastward; 45 of winds from northward; 45 from southward; and 28 from north-westward. Snow was recorded on 37 lours, sleet on 1 hour, and rain on 9 hours; the weather was misty or foggy on 13 hours.

Observations made at Laurie Island, South Orkneys, by the Scottish Expedition during the month of April, 1903, give the following results:-Mean pressure $29 \cdot 204$ inches; highest pressure 29.859 inches, observed on 2nd of the month; lowest 28.279 inches, on the 24 th. Mean temperature $20^{\circ} \cdot 6 \mathrm{~F}$; maximum temperature $36^{\circ} \cdot 2 \mathrm{~F}$, registered on the 15 th ; minimum $-8^{\circ} \cdot 1 \mathrm{~F}$, on the 29 th. The weather was cloudy; snow was noted on 119 hours; sleet on 32 hours; rain on 48 hours; and hail on 2. Mist or fog was noted on 51 hours. The average force of the wind, as estimated, is found to have been $1 \cdot 7$, and no gales were recorded.

Out of 710 observations of wind direction, 510 were of winds from between south and north-west, round by west, and the north-westerly wind predominated. Only 21 observations were of winds from eastward and north-eastward.

The north-westerly winds were doubtless occasioned by the approach of low-pressure systems, the wind shifting to the westward and southward as the trough of the depression neared, passed over, and receded from the station.

At the "Discovery's" Winter Quarters, in McMurdo Sound, from May to August, 1903, inclusive, the mean pressure was 29.223 inches, or 0.051 inch higher than the mean pressure in the corresponding months of 1902 ; and the range of pressure, 1.761 inches, 0.280 inch smaller. The highest pressure, 30.061 inches, noted on the 31 st July, associated with a fresh breeze from east by south, is 0.120 inch lower than the highest recorderl during the corresponding period of the previous year. Readings of 30.037 inches and 30.027 inches were also recorded, the first on the 3rd June and the seeond on the 1st August. The lowest pressure, 28.300 inches, recorded on the 10th August, associated with a strong south-south-west wind, is $0 \cdot 160$ inch higher than the lowest pressure noted in the four months May to

3 I 2 
Angust, 1902, which was the lowest reading recorded dhring the "Discovery's" stay in the Antarctic regions.

As regarcls temperature, the Winter of 1903 was rather more severe than the Winter of the previons year. The ment temperature, for the four montls under notice, is found to have been as low as $-16^{\circ} \cdot 9 \mathrm{~F}$., whereas the mean temperature of the winter months, May to Angnst, 1902, was $-13^{\circ} \cdot 3 \mathrm{~F}$.

Capo Armituge.

The menn temperature at Cape Armitage during the months under notice, obtained from 203 observations, generally taken twice a day, i.e., at ahout noon and $4 \mathrm{p} . \mathrm{m}$., comes ont as $-28^{\circ} \cdot 5 \mathrm{~F}$. The range of temperature during these months was $71^{\circ} \cdot 2 \mathrm{~F}$. at Winter Quarters and $76^{\circ} \cdot 2 \mathrm{~F}$. at Cape Armitage. 'The maximum temperature of $17^{\circ} \mathrm{F}$. at Winter Quarters was registered on the 11 th Jnne, when there was a light breeze from enst, and this is $2^{\circ} \mathrm{F}$. lower than the maximum reading of the corresponding period of the previous year. The maximum temperature ohserved at Cape Aruitage was not higher than $8^{\circ} .5 \mathrm{~F}$.

The minimum for the period, $-54^{\circ} \cdot 2 \mathrm{~F}$., registered on the 30 th July at Winter Quarters, in a calm, is $3^{\circ} .7 \mathrm{~F}$. lower than that of the previous year. At Cape Armitage the minimum temperature of the four months, $-67^{\circ} \cdot 7 \mathrm{~F}$., registered on the 16th May, is as mueh as $5^{\circ} \cdot 7 \mathrm{~F}$. lower than the lowest registered in the two months July, August, 1902, which are the only months for which observations are available for comparison in that year at Cape Armitage.

As regards the weather experienced at Winter Quarters in the four' winter months of 1903, 52 days were clondy, 19 overcast; but blue sky was recorded on 52 days. Snow fell on 40 days; the weather was misty on 54, foggy on 21. Strong winds blew on 40 days, and attained force 8 , and upward, on 24 days. Aurora was observed on 76 days.

Out of 1476 observations of wind direction, $29 \cdot 2$ per cent. were of winds from an easterly direction, $17 \cdot 8$ per cent. of winds from a north-easterly direction, $27 \cdot 5$ per cent. were of calms; and $4 \cdot 2$ per cent. of variables.

Snow Ifill Island, At Snow Hill Island, during the four months May to Angust, 1903, the mean pressure at 8 a.m. May to August, increased to $29 \cdot 13$ inches from 29.05 inches, the mean for the two previous inonths. The maximum
1903. 8 a.m. pressure, 30.01 inches, occurred on the 12 th May, during a calm; the minimum 8 a.m. pressure, $28 \cdot 25$ inches, on the 3 rd June, when a stroug sonth-west wind was blowing; thus a range of 1.76 inches is shown for the period.

A meau temperature of $-1^{\circ} \cdot 2 \mathrm{~F}$. is found from the 8 a.m. observations. A maximum of $40^{\circ} \mathrm{F}$. relating to the 31 st May, and a minimum of $-32^{\circ} \mathrm{F}$. to the 20 th June, both associated with a calm, give $72^{\circ} \mathrm{F}$. as the range of temperature at 8 a.m. - a large range.

The average direction and force of the wind, estimated at $8 \mathrm{a}$ am. during the four months, is found to have been south, $2 \cdot 7$; the average amount of clond 7 .

On 18 occasions, at 8 a.m., the wind was estimated at force $6-7$, and on 5 occasions as force 8-10.

Laurio Island, South Orkneys, May August, 1903.

The results of observations made at Laurie Island chring the four months May to August, 1903, exhibit a mean pressure of 29.250 inches, being 0.027 inch higher than the mean pressure at McMurdo Sound for the same mouths. The highest pressure during the period, 30.013 inches, observed on the 13th May, is 0.048 inch lower than the "Discovery's" lowest, covering the same months; the lowest pressure, 28.231 inches, ohserved on the 3rd July, is 0.069 inch lower than the "Discovery's" for the same months. The range of pressure dnring the period, $1 \cdot 782$ inches, is 0.021 inch larger than the range at McMurdo Sound during the same period.

The mean temperature in these months is found to have been $15^{\circ} .6 \mathrm{~F}$; ; a maximum of $46^{\circ} \mathrm{F}$., registered on 31 st May, and a minimum of $-26^{\circ} \mathrm{F}$. on the 19 th June, show a range of $72^{\circ}$.

Cloudy weather appears to have prevailed during the four months, the mean anount of elond being estimated at $7 \cdot 5$. Snow fell on 765 hours, sleet on 29 honts, rain on 152 , and hail on 21 .

Fog, or mist, is entered on 144 hours. The mean force of the wind, as estimated, is $2 \cdot 1$; no gales were experienced in May or June, but in July and Augnst winds of force 8, and above, are noted on 71 hours.

Ont of 2952 observations of wind direction made during the period, 392 were of northerly winds, 732 of north-westerly, 463 of westerly, 308 of south-westerly, and 405 of southerly. There were 172 observations of calns, and 25 of variables; so that only 455 olservations were of winds from the north-enst, east, and sonth-east directions, and of these not more than 109 were of winds from eastward. 
The Sonth Orkneys, therefore, would appear to le within the region of the westerly winds. The centres of low-pressure systems, moving eastward over the islands, usuilly passed sonth of the station. Yet the I'inter Quarters of the German exploring ship "Gauss," situnted on at parallel only 318 miles sonth of the station on Laurie Island, lay for the most part well on the southern side of these eastward-moving depressions, and was visited by easterly gales associated with the passage eastward of eyclonic systems, the ecntres of which passed well to the north of the station.

Areas of low pressure, after passing south of Cape Horn, do not appear, as a rule, to have influeneed the weather at Snow Hill during the sojourn there of the Swedish lixpedition, until the trough of the depression had reached the 56tl meridian of west longitude and had passed to the eastward of the mountains of Danco Land.

In September and October, 1903, mean pressure was at its lowest at McMurdo Sorund, for the period during which the "Discovery" remainer there. It was as low as 28.957 inches, which is 0.204 ineh lower than the mean pressure of the corresponding months in 1902. The highest barometer reading, 29.586 inches, was taken on the 1st September, when there was no wind, and is 0.268 inch lower than the highest in September and October, 1902; the lowest, 28.303 inches, was observed on the 23rd of the same month, when there was a fresh breeze from south, and is 0.016 inch lower than the lowest in the corresponding period of the previous year. This range of 1.283 inches is 0.252 inch smaller than the range of pressure in the corresponding months of the previous year. The mean temperature in the two months, $-12^{\circ} \cdot 7 \mathrm{~F}$, is $2^{\circ} \cdot 4$ lower than the mean temperature of September and October, 1902. The maximun temperature, $13^{\circ} \cdot 2 \mathrm{~F}$, noted on the 28 th September, with a fresh breeze from south-east, is $1^{\circ} \cdot 8$ lower than the maximum registered in the same months of the previous year, when the wind came from the same direction as a gentlc breeze. The minimum, $-58^{\circ} \cdot 5 \mathrm{~F}$,, registered on the 20th September and associated with a calm, is $15^{\circ}$ lower than the minimum of September and October in the previons year, which occurred in a calm. The rauge of temperature, $71^{\circ} \cdot 7$, is $13^{\circ} \cdot 2$ larger than the range in the same season of 1902.

At Cape Armitage during these months the thermometers were read twice a day, usually at about noon and 4 p.m. ; and these olsservations, 106 in all, give a mean of $-18^{\circ} .6 \mathrm{~F}$., or $2^{\circ} \cdot 7$ lower than the mean of 49 observations obtained at, or about, noon each day in Scptcmber and October, 1902. The maximum temperature in these months at Cape Armitage, $10^{\circ} .5 \mathrm{~F}$, observed on the 27 th Octoher, is also the same as the maximum temperature in the same months of the previous year; the minimum temperature, $-64^{\circ} \cdot 6 \mathrm{~F}$., registered on the 20th September, is $7^{\circ} \cdot 1$ lower than the minimum in the corresponding months of 1902 , and the range, $75^{\circ} \cdot 1$, is $7^{\circ} \cdot 1$ larger.

The weather at MICIIurdo Sound was characterised by blue sky on 13 days; by cloudy, or overcast, sky on 48. days; by snow on 20 days; by mist on 12 days; and by fog on 19 days. Aurora was observed on 12 days. Strong winrls, force 6-7, were experienced on 31 days; and the wind attained gale force, $8-10$, on 9 days. Out of 732 observations of wind direction, 24 per cent. were from north-enstward, 29 per cent. from eastward, $7 \cdot 5$ per cent. from northward, and 11 per cent. from south-eastwand; 23 per cent. were of calms or variables; and the remainder were of winds from the remaining directions other than west.

On a slerlge journey to Cape Crozier, undertaken by Lieutenant Royos and party, from the 7 th to the 17 th September, 1903, inclusive, a mean temperature of $-37^{\circ} \mathrm{F}$. obtained. The mean temperature during the same time at Winter Quarters was $-20^{\circ} \cdot 7 \mathrm{~F}$. The maximum temperature recorded, $-5^{\circ} \mathrm{F}$, on the 7 th, when the wind was from south-east, force 5 , is $9^{\circ}$ lower than the maximum during the same time at Winter Quarters, registered on the same day, when the wind was from south-east, force 3; the minimum, $-61^{\circ} \cdot 2 \mathrm{~F}$, noted on the $16 \mathrm{th}$, when there was no wind, is $24^{\circ}$ lower than the lowest during the same period at Winter Quarters, which occurred on the same diny, associated with a breeze from east-11orth-east, force 7 .

On 5 days of the journey the sledge party had the wind from east hy north; on 2 days the direction was south-west; and on 4 days there was no wind. At Winter Quarters during the whole of this time the wind came from east-north-east. The mcan of observations for wind force, as estimated by the travellers, comes out at $\mathbf{1} \cdot \mathbf{4}$, while at Winter Quarters the mean force was 3. "Discovery," Sep-
tember - October, 1903.
Capo Armilage, September - October, 1903. ieutenant Rorus to Caps Crozier, September, 1903. 
As regards weather, the slelge jarty laul less cloud and less fog than obtained at Winter Quarters, and no snow ; at Winter Quarters snow fell on 3 clays out of the 11 under notice.

Lieutenunt IBARse to suth. September, 1903

Jonrneying to the south from the 13 th to the 20th September, inclusive, Lientenant Balise and party olstained values for temperature from the 13 th to the 17 th whieh slrow a mean of $-45^{\circ} \cdot 8 \mathrm{~F}$., against $-28^{\circ} \cdot 9 \mathrm{~F}$, the mean of temperature observations at Winter Quarters. The maximm temperature, $-36^{\circ} \cdot 5 \mathrm{~F}$., was registered on the 13th, with a light breeze from south-west; at Winter Quarters the maximum, $-20^{\circ} \mathrm{F}$, occurred two days after, with a light brecze from east-north-east. Dnring a light breeze from sontl-west, on the $15 \mathrm{th}$, the minimum temperature, $-66^{\circ} \cdot 8 \mathrm{~F}$., was registererl, and a day later the minimum for the same periorl, $-37^{\circ} \cdot 2 \mathrm{~F}$., was recorded at Winter Quarters, a wind from eastnortlleast, force 7 , blowing at the time. The mean wind directions during the jonrney are found to have leen 3 dilys south-soutli-west, 1 day north, and 4 days of calm; the foree, 1 . It was north-east by east, $2 \cdot 5$, at Winter Quarters chring the period. The atmosphere was clearer on the journey than at Winter Quarters, otherwise the weather appears to have been similar.

Dr. Wilsos to Cape

From the 12th October to the 4th November, 1903, inclusive, a sledge party made a journey to Cape Crozier, 12th Octo. ber 10 4lh Nor ember, 1993.

Crozier and back, in eharge of D1. F. A. Wrisox. From the time of starting to the end of the month the nean temperature was $-8^{\circ} \mathrm{F}$., while it was $-3^{\circ} \cdot 3 \mathrm{~F}$. at Winter Quarters. The maximum for the period, $6^{\circ} \cdot 5 \mathrm{~F}$., ocenred during a gale (foree 8 ) from sonth-west on the 27 th ; the maximum at Winter Quarters, $12^{\circ} \mathrm{F}$, registererl on the sume day, wis assoeiated with a moderate breeze from sonth-east. The lowest reading on this part of the journey, $-33^{\circ} \cdot 4 \mathrm{~F}$., was noted on the 15 th and again on the 17 th, during calms; the lowest at Winter Qnarters, $-27^{\circ} \cdot 2 \mathrm{~F}$., registered on the $19 \mathrm{th}$, was likewise noted in a calm.

The mean direetion and force of the wind, as estimated by the travellers, is found to be south-west by south, 3, while at Winter Quarters it was east, 2·6. The weather was overcast or clondy, alike on the journey and at the ship, but rather less so on the journey. The travellers recorded snow on 7 days only, and no fog; while at the ship snow fell on 11 days, and the weather was foggy on 6 .

From the 1st to the 4 th November, inclusive, the difference between the temperature experienced by the sledge party and that which olstained at the ship was still more accentuated.

On the journey the mean temperature is found by the observations to have been $5 \cdot 3 \mathrm{~F}$., against $11^{\circ} \cdot 8 \mathrm{~F}$. at the ship. The maximum, $29^{\circ} \cdot 5 \mathrm{~F}$, noted on the 4 th in a calm, unay be placed against $24^{\circ} \cdot 5 \mathrm{~F}$., the maximum at the ship, recorded on the 2 nd, also in a calm. The minimum, $-16^{\circ} \mathrm{F}$., noted on the and by the travellers when a calm prevailed, is $10^{\circ} \cdot 5$ lower than the minimum at the ship dnring the same period, which occurred on the 3rd, with a light air from north. The mean direetion and foree of the wind, as estimated by them, was west by south, $1 \cdot 3$; at the ship it was east-north-east, $2 \cdot 4$. 'The weather experienced by the travellers was similar to that which obtained at the ship, except that the ship had fog on 1 day, while the travellers had no fog.

Lieutenant BARXE to south - west, 7 th December, 1903.
Between the 7 th Oetober and the 12 th Decenber a journey was undertaken to the south-west, led by Lientenant BARNe. Lientenant BARNE arrived in latitude $79^{\circ} 16^{\prime} \mathrm{S}$., longitude $165^{\circ} 48^{\prime} \mathrm{E}$. on the 31 st of October. During this part of the journey the observations for temperature show a mean of $-15^{\circ} \cdot 3$, while the mean at the ship was $-4^{\circ} \cdot 1 \mathrm{~F}$. The maximum temperature, $4^{\circ} \cdot 5 \mathrm{~F}$., was olserved by the sledge party on the 31st, with a light breeze from south by east, and the maximum at the ship for the 23 days, $12^{\circ} \mathrm{F}$, occurred on the 27 th, with the wind from east-south-east, foree 3 . The minimum temperature, $-50^{\circ} .5 \mathrm{~F}$, entered in the Journal of the sledge party on the 8 th, with the wind's direction south, foree 2 , is $23^{\circ} \cdot 0$ lower than the minimum at the ship during the same time, which was associated with a light breeze from north. The arerage direction and force of the wind on this part of the journey is found to have been sonth-sonth-west, 2 ; at the ship it was east by north, $2 \cdot 6$, during the time. Overeast or cloudy weather was experienced by the sledge party as well as at the ship; but while the former had snow on 6 days, at the latter there was snow on 12 days; and while the travellers had fog on 3 days, there was fog at the ship on 8. On the 20th November Lientenant Barve had reached a position in latitude $80^{\circ} 7^{\prime} \mathrm{S}$., longitude $161^{\circ} 4 \frac{1}{2}^{\prime} \mathrm{E}$., and the return journey commeneed. During November olservations for temperature show a nean of $11^{\circ} \mathrm{F}$, while the mean at McMurdo Sound was $15^{\circ} \cdot 4 \mathrm{~F}$. The naximum temperature, $31^{\circ} \cdot 2 \mathrm{~F}$, registered on the 30 th in a calm, is only $2^{\circ} \cdot 8$ lower than the maximum for the same period at the ship, registered on the 22 nd, also in a ealm. The minimum, 
$-11^{\circ} \cdot 2 \mathrm{~F}$, registered on the 5th, with a light breeze from south by east, is $5^{\circ} \cdot 7$ lower than the minimun at the ship, noted with a light air from north. The mean wind direction and force is fount to have leen south-west by south, $2 \cdot 6$, while at the ship) it was east by sonth, $2 \cdot 8$. The weather experienced loy the travellers was similar to that recorded at the ship, cxcept that at the latter fog was noted on 7 days and mist on 3; but the travellers recorled neither fog nor mist. From the 1st to the 12th December nean temperature on the sledge jonrney was $17^{\circ} \cdot 6 \mathrm{~F}, 5^{\circ} \cdot 0$ lower than the mean in the same time at the ship. The maximum, $30^{\circ} \cdot 2 \mathrm{~F}$, recorded on the 9 th, with a light breeze from north-east, is $3^{\circ} \cdot 8$ lower than the maximum at the ship, recorded on the 11 th with the entry "calm." The minimum, $3^{\circ} \cdot 4 \mathrm{~F}$., also noted on the 9 th, but with a light breeze from sonth-west, is $4^{\circ} \cdot 2$ lower than the minimum at the ship entered against the 6th, with a gentle breeze from east. The average wind direction and force comes ont as sonth-west, $1 \cdot 7$; at the ship it was east, 2 . $\Lambda$ gain there was but little difference between the weather on the jomrney in the last 12 days and that experienced at the ship, with the exception that on 3 days fog was noterl at the ship, but no entry of fog ocenrs in the Journal of the sledge jonruey for those days.

At Snow Hill, during the months of September and Octoler, 1903, mean pressure showed a slight increase on the mean of May-Angust, the result of the 8 a.m. observations giving $29 \cdot 16$ inehes. This is 0.07 ineh higher than the mean 8 a.ın. pressure for the eorresponding months of the previons year. The highest 8 a.m. reading, 29.86 inches, was taken on the 7 th September, when tho wind was moderate from south-south-west; the lowest, $28 \cdot 43$ inches, on the 23 rd Oetoher, when there was a moderate breeze from the opposite point, north-north-east.

The mean of the 8 a.m. observations for temperature is $9^{\circ} \cdot 7 \mathrm{~F}$., in the two months; the maximum, $41^{\circ} \mathrm{F}$, oeeurred on the 27 th Octoher, assoeiated with a light air from north-cast ; the minimum, $-23^{\circ} \mathrm{F}$., on the 1st Septcmber, with a light breeze from sonth-south-west: a range of $64^{\circ} \mathrm{F}$.

The wind's average direction was south-east by south, its average force $2 \cdot 5$. Strong winds were reeorded at $8 . \mathrm{m}$. on 6 oeeasions, gales on 3. The arerage cloud amount, 7 , is the same as that which obtained during the period May-August.

At Laurie Island, in September and October, the mean of the observations taken by the Seottisls Expedition for pressure is $29 \cdot 288$ inehes, which is $0 \cdot 331$ inch higher than the mean pressure, during the same period, in MeMurlo Sound. The highest reading, 30.113 inches, entered against the 3rd October, appears also to have been the highest reeorded by the Swedish Expedition during Dr. NonnExskiörd's stay on Snow Hill Island; the lowest, 28.444 inches, is entered for the 24th of the same month. The range of pressure in Oetober, 1.669 inches, is found to be the largest for any month at Snow Hill while the Expedition was stationed there.

The mean temperature, during September and Oetober, was $20^{\circ} \cdot 2 \mathrm{~F}$; a considerable rise of temperature occurred in October, and the mean for that month was $27^{\circ} \mathrm{F}$. The maximum for the two inonths, $42^{\circ} \mathrm{F}$, observed on the 28 th October, and the minimum, $-25^{\circ} \cdot 8 \mathrm{~F}$, on the 2nd September, show a range of $67^{\circ} \cdot 8 \mathrm{~F}$.

The mean amount of elour is estimated as $7 \cdot 7$. The prevailing winds were westerly; out of 1464 entries of wind direetion, 366 pertained to winds from north-westward, 216 to winds from northward, 210 to winds from westward, and 177 to those from sonth-westward. But 197 entries were of winds from sonthward, 110 from south-eastward, and 87 of calms. The average force of the wind is estimated to have been 1.8 ; gales were recorded on 16 hours. Entries were made of snow on 334 hours, sleet on 31 hours, rain on 25 hours, hail on 4, mist or fog on 31. The observations for wind, pressure, and temperatnre, obtainerl at Lanrie Island and Snow Hill Island, respectively, appear to show that exstward-moving areas of low pressure, after passing Cape Hom, frequently take a path to the north of Joinville Island, and then strike to the south-east, to the westward of the South Orkneys.

For the summer months of November, Deember, January, 1903-4, and the first 15 days of Fehriliny, 1904, the barometer observations taken at MeMInrdo Sound show al mean pressure of 29.082 inches, which is 0.323 inch lower than the mean pressure results for the four months November to February of the "Niscovery," Nor. ember - February, $1903-4$ previnus Summer.

The highest rearling, $29 \cdot 598$ inehes, was noted on the 8th Deeember, with a light hreeze from northnorth-west; the lowest, 28.508 inches, on the 26 th Nuvember, with a light air from sonth. The range of 
pressure, during the period (1st Nuvember to 15 th Fehruary), 1.090 inches, is 0.264 inch smaller than the range churing the previons Summer (1st November to 28th Feloruary).

The mean temperature was $21^{\circ} \cdot 3 \mathrm{~F}$, or $3^{\circ} \cdot 2 \mathrm{~F}$. higher than that of the previous Summer, and the range of temperature, $47^{\circ} \cdot 5 \mathrm{~F}$, $1^{\circ}$ smaller. The maximun temperature for the period, $42^{\circ} \mathrm{F}$, was registered on the 26 th December, when there was no wind; the minimum, $-5^{\circ}, 5 \mathrm{~F}$, on the $3 \mathrm{rrl}$ November, when a light air came from north.

Capn Armitnge, 1st Novenuber to 15 th lebruary, 1903-4.

MeMurdo Sound.

At Cape Armitage the temperature was taken during the three months November to January on 13 oceasions. The mean temperature was $13^{\circ} \cdot 8 \mathrm{~F}$; a maximum temperature was recorded on the 25 th November, $23^{\circ} \cdot 8 \mathrm{~F}$; ; and the minimum, $-14^{\circ} \cdot 2 \mathrm{~F}$., on 27 th Januriry.

At Mc Murdo Sound the prevailing winds during the period 1st November to 15th February were from north-east to south-east, the percentage of frequency of the former leing $34 \cdot 5$ and of the latter $14 \cdot 2 ;$ but, ont of 752 observations of wind direction, $17 \cdot 5$ per ecnt. were of winds from south-eastward; $3 \cdot 7$ per cent. were variable; and 15.0 per cent. of the observations were of calms.

The average force of the wind, throughout the period under notice, is found to havo been $2 \cdot 2$; in the four unonths Norember to February, 1902-3, it was 2.9. The force of the wind rose to 7 , or 8 , on 18 occasions, and to 9 on one occasion.

In legard to the weather during the time, the sky was clear on 19 days; cloudy on 46 ; and overcist on 42. Snow fell on 35 days; mist was noted 5 days; and fog on 24 . These conditions of weather closely resemble those of the four months of the previous Summer.

Captain Scorr's On the journey made by Captain Scort to the westward, and while on the Western Monntains, the ship journey to west, wa left on the 26 th October, 1903, and at the end of the month a position in latitude $77^{\circ} 42^{\prime} \mathrm{S} .$, longitude $_{2}$ 16th December, $164^{\circ} 44^{\prime} \mathrm{E}$. was reached.

1903.

During this portion of the journey the mcan temperature, $0^{\circ} \cdot 6 \mathrm{~F}$, was $2 \cdot 0 \mathrm{~F}$. lower than the mean temperature at the slip. The maximum and minimum temperatures, which will be referred to in connection with this journey, are the maximum and minimum observed by an ordinary thermometer.

The maximum, $10^{\circ} \cdot 2 \mathrm{~F}$, was observed on the 27 th, when a light breeze was noted from north-east; the minimum, $-9^{\circ} \cdot 8 \mathrm{~F}$, on the $29 \mathrm{th}$, and there was no wind at the time of observation. The maximum and minimum temperatures registered at the ship during the time were $12^{\circ} \mathrm{F}$. (on the 27 th) and $-12^{\circ} \cdot 3 \mathrm{~F}$. respectively (on the $26 \mathrm{th}$ ). The results of observations of wind direction and force show that south-south-west was the predominating direction, and the average force $2 \cdot 6$. At the ship the average direction and force is found to have leen east-south-east, $3 \cdot 4$. The sledge party recorderl snow on 1 day, and fog on 1 day. At the ship snow fell on 4 days, and the weather was foggy on 4 days.

In November Captain SCoTr, pushing westward, and over a plateau more than 7000 feet above sea-level during the second half of the month, reached a position in $77^{\circ} 59^{\prime} \mathrm{S}, 1_{146^{\circ}} 31^{\prime} \mathrm{F}$, , on the 30th-ScotT's furthest west-and during the time expericneed a mean temperature of $-16^{\circ} \cdot 9 \mathrm{~F}$, while the mean temperature at the ship was $15^{\circ} \cdot 4 \mathrm{~F}$. The highest reading during the month, $8^{\circ} \mathrm{F}$., was noted on the 1 st, with a light breeze from south; the maximum registered at the ship during the month, $34^{\circ}$ F., relates to the 22nd, when a light brceze was stirring from north-east by east. The lowest reading recorded by the travellers, $-44^{\circ} \cdot 3 \mathrm{~F}$, was entered on the 16 th of the month, with the wind observation "calm"; at the ship the minimun, $-5^{\circ} \cdot 5 \mathrm{~F}$., registered on the $3 \mathrm{rd}$, was associated with a light breeze from east-northcast.

On the Western Mountains the average direction and foree of the wind during the month is found to have been sonth-west by south, $2 \cdot 7$; at the ship it was east by south, $2 \cdot 8$. Out of the 30 days, 23 were overcast, or cloudy, on the journey; 18 foggy. Snow fell on 12 diys. At the ship the sky was orercast, or clondy, on 26 diys; fog was recorded on 7 days, and mist on 3. Snow fell on 12 days.

From the end of November to the 15 th of December, when the party had reached latitnde $77^{\circ} 46^{\prime} \mathrm{S}$, longitude $159^{\circ} 53^{\prime} \mathrm{E}$, on the homeward journey upon the platcan a mean temperature of $3^{\circ} \cdot 5 \mathrm{~F}$, was recorded, while the mean at the ship was $23^{\circ} \cdot 4 \mathrm{~F}$. The highest reading recorded by Captain ScotT dnring these 15 days, $28^{\circ} \cdot 0 \mathrm{~F}$, was taken on the 15 th, the wind being light from east-south-east at the time; the maximum at the ship, $34^{\circ} \cdot 0 \mathrm{~F}$, registered on the 11 th, occurred in a calm. Captain ScotT's lowest for the 15 days, $-27^{\circ} \cdot 5 \mathrm{~F}$, was real on the $3 \mathrm{rd}$, when the wind was blowing strongly from west-south- 
west ; the minimum at the ship, $7^{\circ} \cdot 6 \mathrm{~F}$., was registered on the 6 th, when there was a gentle breeze from east. The average direction and force of the wind is found from Captain Scorr's observations to have been south-west by sonth, $1 \cdot 7$, while at the ship the prevailing direction and force was east, 2. The travellers had a clear sky on 8 days; a clondy, or overeast, sky on 7 ; snow on 3 days; fog on 5 . At the ship the sky was clear on 7 days; cloudy, or overcast, on 8 . Snow fell on 4 clays, and the weather was foggy on 3 days.

Referring to the snowfall on the plateau in his book, "The Voyage of the "Discovery," Captain Scort says :- "The layer-sandy layer of loose ice-crystals-grew heavier as we approached the edge of the platean; this was an interesting observation, for it shows that the plateau snowfall takes place in December and that it is far heavier on the edge than in the interior of the continent. Another interesting fact was observable, in this connexion, for while the light snow had been falling the wind had crept round to the south, sometimes to such an angle with our course that it was difficult to trim our sledge sail to derive any benefit from it. In the most southerly direction it brought a desirable increase of temperature, and on some days we had a fair imitation of the mild southerly blizzards which were such a conspicuons feature at the ship."

This rise of temperature with south winds and snowfall on the platean appears to be connected with the passage eastward of low-pressure systems, having their centres over the sea, whence the milder air was drawn, and these concurrent conditions go far towards proving the truth of the surmise that the rise of temperature in the southerly gales experienced at Winter Quarters, usually associated with snow, was not due to Föhn effect, as has been generally supposed, but to the wind's origin in warmer latitudes. The southerly snow-bearing winds of the platenu and the ice barrier alike probably owed their relatively high temperature and humidity to their place of origin in lower latitudes and belonged to cyclonic wind systems that harl their centres situated far out at sea.

A journey was made by Lientenant RoyDs and party to the south-east, across the ice barrier, in November and December, 1903, which yielded interesting results for comparison with meteorological conditions at Winter Quarters. Lientenant Royps left the "Discovery" on the 10th November, and between this date and the last day of the month, when he was in latitude $79^{\circ} 20^{\prime} \mathrm{S}$., longitude $174^{\circ} 32^{\prime} \mathrm{E}$., the mean of the observations for temperature is found to be $11^{\circ} \cdot 1 \mathrm{~F}$, while that at Winter Quarters comes out as $17^{\circ} \cdot 7 \mathrm{~F}$. The maximum temperature during these days, $25^{\circ} \cdot 5 \mathrm{~F}$, was observed on the 11 th, in a calm; the maximum at Winter Quarters, $34^{\circ} \mathbf{F}$., was registered on the 22 nd, also in a calm. The minimum temperature, $-12^{\circ} \cdot 6 \mathrm{~F}$., was observed on the 15 th in a calm; the minimum at Winter Quarters, $-2^{\circ} \cdot 2 \mathrm{~F}$, occurred on the 19th, when there was a light breeze from south-east. The average wind direction and force, as recorded by Lieutenant RoYDS, is found to have been south-south-west, 2 ; at Winter Quarters it was east-sonth-east, $2 \cdot 4$. The sky was clear for 3 days on the barrier, for 4 days at the ship; cloudy for 7 days on the barrier, for 11 days at the ship; overcast for 11 days on the barrier, for 6 days at the ship. Snow fell on 4 days at the barrier, on 7 days at the ship; fog was experienced on 5 days on the barrier, on 2 days at the ship.

Lieutenant Royns arrived at his most southerly and easterly position on the 28th, when he retraced his steps. From the 1st to the 10th of December, inclusive, between his position on the last day of November and that of the ship, the mean of the observations for temperature is $16^{\circ} \cdot 4 \mathrm{~F}$., against $21^{\circ} \cdot 8 \mathrm{~F}$., the mean temperature at the ship during the same time.

The maximum temperature of $24^{\circ} \cdot 5 \mathrm{~F}$. was observed on the 2nd of the month, the wind being light from south-west at the time; the maximun temperature at the ship cluring the same period, $32^{\circ} \cdot 2 \mathrm{~F}$, was registered on the 10th, when there was no wind. The minimum reading taken on the barrier during these 10 days, $-3^{\circ} \mathrm{F}$., is noted on the 4th, and again on the 6th, with gentle breezes from south-west; the mininum at the ship, $7^{\circ} \cdot 6 \mathrm{~F}$., was reeorded on the 6 th, with a moderate breeze from east.

The average wind direction and force, as estimated by Lientenant RoyDs, was south-west by south, $1 \cdot 7$, on the barrier; at the ship it was east by north, $2 \cdot 3$. During the period the sky was clear for 4 days, cloudy for 4 dlays, and overeast for 2 days on the barrier; clear for 3 days, cloudy for 5 days, and overcast for 2 days at the ship. On the barrier, snow was experienced on 3 days, fog on 4 days; while at the ship there was snow on 4 days and fog on 3 . 
Scottisl Expedition, Isurie Island, November to Fel. ruary. 1903-4.

"Scolja," February, 1904.
At Taurie Island, Sonth Orkners, during the four months November to Fehruary, barometer ohserrations showerl a mean pressure of $29 \cdot 150$ inches. The highest pressure, 29.902 inches, was recorder on the 15th liehmary; the lowest, $28 \cdot 338$ inches, on 1 th th Nowemlier-at range of 1.564 inches.

The station on Lanrie Island during this time hat a mean temperature of $31^{\circ} \cdot 4 \mathrm{~F}$, the maximum temperature, $47^{\circ} \cdot 5 \mathrm{~F}$, oceurring ou the 11 th lebruary; the minimum, $14^{\circ} \cdot 9 \mathrm{~F}$, on the 2 nd Novemberal range of $32^{\circ} \cdot 6$.

The prevailing winds appear to have heen those from north-westward, but south-easterly winds were frequent, and there was a large percentage of calms. The average force of the wind, as estimated, may he given as $1 \cdot 5$, aul there were 5 entries of gale force during the time. The weather was overenst or clourly during the greater part of the period, the average amount of cloul being estimated at 9.

Uuder the hourly observations of weather, snow is noted on 572 hours, slect on 50, rain nu 241, hail on 16, fog or mist on 115 .

Commenting on the wind olservations during this period, Mr. Mossmax states that the position of the station was unt a good oue for such ohservations. The station was movel from the ship to the shore on the 1st Novemher, 1903. The shore station was sitnaterl under the lee of a high eliff, and in this position the soutl-west wind of the island was deflecterl to sonth and south-enst; north-west winds were not materially affected, hut there was a tendency for west-north-west winds to he deflected to morth-west.

From the 21st to the 29th Felrnary the "Scotia" was cruising in the Werldell Sea, during which time her average geographical position is represented by the parallel of $64^{\circ} \mathrm{S}$. and the mericlian of $364^{\circ} \mathrm{W}$. The mean of the baroneter observations taken during this eruise, 29.030 inches, is 0.043 inch lower than the mean of similar ohservations taken at Laurie Ishaul during the same time. The ohservations for temperature taken on board the "Scotia" give a mean of $29^{\circ} \mathrm{F}$., which is $1^{\circ} \cdot 3$ lower than the mean temperature at Laurie Island attained in the same perior. The "Scotia's" maximum rearling was $31^{\circ} \cdot 8 \mathrm{~F}$., licr minimum $25^{\circ} \cdot 9 \mathrm{~F}$.

Ont of 216 ohservations of wind direction, 70 were of wiuls from sonth-westwarl, 53 from sonthward, and 38 from sonth-enstward. The average force of the wind was $1 \cdot 7$.

The "Discovery" left MeMnrdo Sound, on her homewarl voynge, on the 19th Fehruary, 1904, accom"Discorery," Home ward, 19th Feb. Marelı, 190\%. paniel by the lielief Ships "Morning" and "Terra Nora." From that date to the 25th, when she was in latitnule $71^{\circ} 2^{\prime} S$., longiturle $169^{\circ} 32^{\prime} \mathrm{E}$., at noon, the fonr-hourly barometer ohserrations taken on hoarl show a mean pressure of $29 \cdot 36$ inches. The highest harometer rearling recorded on these 7 days, 29.57 inches, relates to the 24 th, and at the time of observation there was no wind ; the lowest reading, $29 \cdot 20$ iuches, noted on the 20th, was associated with a moderate breeze from west by north.

The mean of the temperature ohservations, taken at this perior of the voyage, is $2 \tau^{\circ} \mathrm{F}$; the maximum temperature, $33^{\circ} \mathrm{F}$, was noted on the 21 st, a light air stirring at the time; the minimum, $22^{\circ} \mathrm{F}$, on the 19th, with a fresh breeze from south-east ly sonth, ancl again on the following dity, with a gentle breeze from west by north.

The arerage direction and force of the wind is fomul to have heen sonth-west ly sonth, 1.6. The weather was noted as orercast on 1 day, and snow fell on 2 days.

During the next 7 days, while the "Discovery" wats proceeding uorthward to a position in hatitude $67^{\prime} 35^{\prime} \mathrm{S}$., longitude $159^{\circ} 54^{\prime} \mathrm{K}$., which she reached at noon on the 3rd March, olservations for pressure were made which show a me:m of $28 \cdot 83$ inches. The highest harometer reading recorled huring these days, $29 \cdot 17$ inches, relittes to the 26 th Fehrnary, and a light :ir from west-north-west wits noted at the time; the lowest reatling, $28 \cdot 29$ inches, to the 28 th, and at the time of olservation the wind hlew strongly from sonth-east by south (forec 7 ).

Of the observations for temperature, the mean is found to be $29^{\circ} \mathrm{F}$. for the periorl the maximum

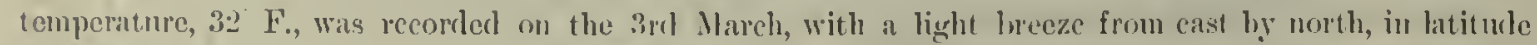
$67 \frac{1}{2} \mathrm{~s}$; the minimum, $26 \mathrm{~F}$, on the 26 th Fehnery, with it molerate lirecze from nortlo by west, in latitule $70 \mathbb{L}^{-} \mathrm{s}$.

The arerage direction of the wind, on these diys, was from between south-east and north-west, romul by south, except on the 3rd Mareh, when the arerage direction was north-north-east. The mean force is 
found to have been 2.7. The weather was noted as "overcitse" each day, anul snow feell on 6 rayss out of the 7 .

The "Seotia" Was crnising in the Werlell Sen during March, 1904, hetwees the pitrallels of 60" 37' S., "Scotin," and $74^{\circ} 1^{\prime} \mathrm{S}$., ancl the meridians of $10^{\circ} 42^{\prime} \mathrm{W}$, and $23^{\circ} 53^{\prime} \mathrm{W}$. She was in her most sentherly prsition, in latiturle $74^{\circ} 1^{\prime} \mathrm{S}$., longitude $22^{\circ} 0^{\prime} \mathrm{W}^{\prime}$, neir the iee barrier of the lind discovered hy Mr. Breck and namerl by him Coat's Land, wn the Th of the month sund remaned in the locality mutil the 12th.

From the 5 th th the 9 th a hard easterly gate is sairl to have been experienced, awcompanichl hy thick driving smow, ancl assuciated with a low temperature.

'The hourly observations obtained during the montl of Manch, 1904, on hourl the "Sintia" show a

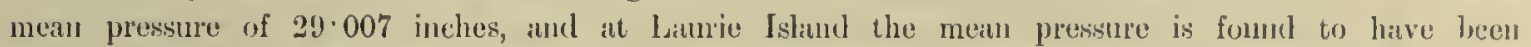
29. 106 inches during the sane time, The highest batometer reading recorded on boand the "Scotia" during the month, $29 \cdot 708$ inches, was taken on the 12 th in latitule $7 t^{\circ} 1^{\prime}$ S., longitude $220^{\prime} \mathbb{W}^{\prime}$, when near the ice barrier, a light air from sonth-sonth-west stirring at the time. The highest reading at Lallrie Island during the month, $29 \cdot 713$ inches, was taken on the 11 th.

The lowest reaching noted on the "Scotia" during the month, $28 \cdot 142$ inches, was taken on the $27 t_{1}$, in latiturle $66^{\circ} 57^{\prime} \mathrm{S}$., lungitude $13^{\circ} 13^{\prime} \mathrm{W}$, with a morlerate breeze fron north ; the lowest at Liturie Island, 28.280 inches, noted on the 8th, wats associated with a light breeze from south-east. 'The "Scutili's" range of pressure, 1.566 inches, is therefore 0.133 inch linger than the range of pressture at Latlide Island in March, 1904 .

It is worth noting that althongh the "Seotia's" highest barometer reading was observed when the vessel hiul reached her position farthest sonth, yet mean pressure in the Weddell Sea, between the 60th ind 7.4 th patrallels of sonth latitude and the 10th and 28th meridinns of west longitude during March, 1904, was neirly at tenth lower than the mean pressure at Limnie Island in that month. Moreover, from the 1 st to the 10th of the month, when the "Scotia's" mean hatinde was $72^{\circ} 39^{\prime}$ S., she recorded barometer readings which show a meat pressmre of $28 \cdot 789$ inches, while pressure at Lanrie Island was 29.075 inches, or 0.286 iuch higher. $\Lambda$ gain, from the 11 th to the 20 th, the "Scotia," in in average latintele of $72^{\circ} 22^{\prime} S_{\text {., }}$ recorded olsservations for pressure giving a mean of $29 \cdot 200$ inehes against $29 \cdot 181$ inches, the mean of the barometer observations recorted at Lamrie Iskand, which is only 0.019 inch lower than the "Scotia's" mean pressure for the period. There seems, therefore, no evidence to show that pressure decreased in the higher latitudes visited by the Scottish Expedition in the Weddell Sea; the reverse appear's to latvo obtained.

Observations for" temperature made on board the "Scotia" dming the month of Mareh, 1904, yield a mean of $24^{\circ} \cdot 9 \mathrm{~F}$. The maximum temperature, $32^{\circ} \mathrm{F}$., was registered on the $31 \mathrm{st}$, on which date at noon, G.M.'T, she wats in latitnde $60^{\circ} 37^{\prime} \mathrm{S}$, longitude $12^{\circ} 16^{\prime} \mathrm{WV}$, when the wind was blowing with fresh to strong gale force from north; the minimum, $-0^{\circ} \cdot 3 \mathrm{~F}$, on the $12 \mathrm{th}$, on which date at noon, G.M.'T., she wats in latitnde $74^{\circ} 1^{\prime} \mathrm{S}$, longitude $22^{\circ} 0^{\prime} \mathrm{W}$., when there was no wind.

It Latuic Island the mean temperatne is fonnd to have been $32^{\circ}$. $\mathrm{F}$. dhring the time; the maximmm temperature recorded, $39^{\circ} \cdot 9 \mathrm{~F}$, relates to the 4 th ; the minimum, $20^{\circ} \cdot 8 \mathrm{~F}$., to the 31 st.

The range of temperature exhibited by the observations of the "Scotia" in the Wecklell Sea, 32 " 3 E., is therefore, $13^{\circ} \cdot 2 \mathrm{~F}$, liuger than the range shown hy the observations taken at hanuic Istand.

The "Scotia" hat cloudy weather in the Wedilell Seat; the average amount of clourl, ats estimated, is fortud to have been $8 \cdot 7$.

The prevaling winds were from westward and nortl-westward. Ont of 743 olsservations of wind, 142 were of those from north-westward and 136 of winds from westwald. Int 121 of the observations related to winds from northward, 96 to those from north-eastwarl, 92 from eastward, and 43 were of calms and variables. The mean foree of the wind, as estimated, is found to have been $2 \cdot 8$.

June, 1907. 


\section{,}

5

n 


\title{
VIII. NOTES ON THE OBSERVATIONS OF TEMPERATURE AT THE WINTER QUARTERS OF THE "DISCOVERY."
}

BY

\author{
DR. CHARLES CHREE, F.R.S.
}

Table of Contents.

Pugo

§ 1. Diurnal inequality of ordinary temperature correctod for non-cyclic cliange . . . . . . . . . . 453

§2. Fourier cocflicients for diurnal inequnlity . . . . . . . . . . . . . . . . . . . . . . 44

§3. Hottest and coldest days . . . . . . . . . . . . . . . . . . . . . . . . . . 455

$\S 4$. Differeuces between mininum and ordinary temperatures throughout the day . . . . . . . . . . 456

§ 5. Frequency of occurrence of lowest ininiunum for the day throughout the 24 hours . . . . . . . . 457

§. Means of daily uaxima and minima nnd corresponding ranges . . . . . . . . . . . . . . . 458

\$ 7. Menu from daily maximum and minimun and its relation to wean from 2-1ourly ordinary readings . . 459

§. Greatest and least daily ranges . . . . . . . . . . . . . . . . . . . . 460

§. Extreme monthly temperatures . . . . . . . . . . . . . . . . . . . . . . . . . . 461

§ 10. Results from six lowest miniua for each observation period . . . . . . . . . . . . . . . . 461

\$1. Table I. gives the diurnal inequality of temperature in degrees Fahrenheit, as derived from the 2-hourly readings of the ordinary mercury thermometer, corrected for non-cyclic or a-periodic chainge. The non-cyclic element was eliminated in the usual way by the correction

$$
+\frac{1}{2 !}\left(r_{0}-r_{24}\right)(n-12)
$$

where $r_{0}$ and $r_{24}$ are the mean readings answering to the first and second midnights of the day, while $n$ is the hour interval since the first midnight.

'TABLE I.-Diurnal Inequality of Temperature.

\begin{tabular}{|c|c|c|c|c|c|c|c|c|c|c|c|c|c|}
\hline & \multicolumn{6}{|c|}{ Forenoon. } & \multicolumn{6}{|c|}{ Afternoon. } & \multirow{2}{*}{ Range } \\
\hline & 2. & 4. & 6. & 8. & 10. & 12. & 2. & 4. & 6. & 8. & 10. & 12. & \\
\hline & 。 & 。 & 。 & 。 & 。 & 。 & 。 & 。 & 。 & 。 & 。 & 。 & 。 \\
\hline January. & $-2 \cdot 55$ & $-2 \cdot 27$ & $-1 \cdot 29$ & $-0 \cdot 22$ & $+0 \cdot 47$ & $+1 \cdot 10$ & $+1 \cdot 87$ & $+2 \cdot 16$ & $+1 \cdot 78$ & +0.81 & -0.37 & $-1 \cdot 43$ & $4 \cdot 71$ \\
\hline February & $-2 \cdot 00$ & -1.96 & -0.90 & -0.54 & $+0 \cdot 19$ & $+1 \cdot 00$ & $+1 \cdot 47$ & $+1 \cdot 70$ & $+1 \cdot 14$ & +0.82 & $-0 \cdot 15$ & -0.77 & $3 \cdot 70$ \\
\hline March & -0.55 & $-1 \cdot 16$ & -0.88 & $+0 \cdot 10$ & $+0 \cdot 76$ & $+0 \cdot 69$ & $+0 \cdot 70$ & +0.43 & +0.11 & $-0 \cdot 18$ & $+0 \cdot 10$ & $-0 \cdot 15$ & $1 \cdot 92$ \\
\hline April . & $-0 \cdot 21$ & $+0 \cdot 70$ & +0.87 & +0.75 & $+0 \cdot 54$ & $-0 \cdot 56$ & $-0 \cdot 30$ & $-0 \cdot 29$ & $-0 \cdot 64$ & $-0 \cdot 70$ & $-0 \cdot 23$ & +0.08 & $1 \cdot 57$ \\
\hline May. & $-0 \cdot 70$ & -0.35 & $-0 \cdot 24$ & -0.05 & $+0 \cdot 27$ & $+0 \cdot 68$ & $+1 \cdot 45$ & $+1 \cdot 63$ & $+0 \cdot 20$ & $1-1 \cdot 08$ & $-1 \cdot 30$ & $-0 \cdot 50$ & $2 \cdot 93$ \\
\hline Juie & -0.83 & $-0 \cdot 53$ & -0.05 & $-0 \cdot 40$ & -0.58 & $+0 \cdot 30$ & -0.04 & $+0 \cdot 52$ & $+1 \cdot 23$ & $+0 \cdot 56$ & +0.12 & $-0 \cdot 29$ & $2 \cdot 06$ \\
\hline July & $-0 \cdot 15$ & $+1 \cdot 64$ & $+0 \cdot 79$ & $+0 \cdot 37$ & +0.55 & $-0 \cdot 87$ & +0.09 & -0.67 & -0.79 & -0.27 & $-0 \cdot 10$ & -0.60 & $2 \cdot 51$ \\
\hline August & +0.04 & +0.87 & +0.85 & $+0 \cdot 85$ & 0.00 & -0.06 & -0.63 & -0.65 & $+0 \cdot 30$ & -0.28 & $-1 \cdot 31$ & +0.08 & $2 \cdot 18$ \\
\hline September & $+0 \cdot 20$ & +0.01 & -0.95 & $-1 \cdot 06$ & $-0 \cdot 35$ & $+0 \cdot 26$ & $+0 \cdot 82$ & +0.74 & $+0 \cdot 50$ & $-0 \cdot 22$ & $+0 \cdot 33$ & -0.29 & 1.88 \\
\hline October. & $-0 \cdot 90$ & -0.99 & $-1 \cdot 54$ & $-0 \cdot 75$ & $+1 \cdot 32$ & +1.44 & $+1 \cdot 78$ & $+1 \cdot 45$ & +0.51 & $-0 \cdot 15$ & $-0 \cdot 88$ & $-1 \cdot 30$ & $3 \cdot 32$ \\
\hline Noveuber & -0.96 & $-1 \cdot 04$ & -0.66 & $-0 \cdot 50$ & $+0 \cdot 60$ & $+1 \cdot 26$ & $+2 \cdot 20$ & +1.51 & +0.93 & $-0 \cdot 22$ & $-1 \cdot 22$ & $-1 \cdot 86$ & 4.06 \\
\hline December . & $-1 \cdot 26$ & $-1 \cdot 00$ & $-1 \cdot 02$ & -0.83 & $+0 \cdot 39$ & $+0 \cdot 77$ & $+1 \cdot 27$ & $+1 \cdot 20$ & $+1 \cdot 14$ & $+0 \cdot 40$ & -0.80 & $-0 \cdot 24$ & $2 \cdot 53$ \\
\hline Year & -0.82 & -0.51 & $-0 \cdot 42$ & -0.19 & $+0 \cdot 35$ & +0.50 & +0.89 & $1+0.81$ & +0.53 & -0.04 & $-0 \cdot 48$ & $-0 \cdot 61$ & $1 \cdot 71$ \\
\hline Mlidsummer & $-1 \cdot 59$ & $-1 \cdot 4$ & -0.99 & -0.52 & $+0 \cdot 49$ & $+1 \cdot 04$ & $+1 \cdot 78$ & $1+1 \cdot 62$ & $+1 \cdot 27$ & $+0 \cdot 33$ & $-0 \cdot 80$ & $-1 \cdot 18$ & $3 \cdot 37$ \\
\hline Equinox & $-0 \cdot 37$ & -0.36 & $-0 \cdot 62$ & $-0 \cdot 24$ & +0.57 & $+0 \cdot 46$ & $+0 \cdot 75$ & +0.58 & $+0 \cdot 12$ & -0.31 & $-0 \cdot 17$ & -0.41 & $1 \cdot 37$ \\
\hline Midwinter & -0.56 & +0.25 & $+0 \cdot 17$ & -0.03 & +0.08 & +0.04 & +0.50 & $+0 \cdot 49$ & +0.21 & $-0 \cdot 26$ & -0.43 & -0.46 & 1.06 \\
\hline
\end{tabular}


In addition to datia for single months of the year the taljle gives dimrnal iuequalities for the year as a whole, and for midwinter (May, Jume, July), eqninox (March, April, September, Octolser), and midsummer (November, December, Jannary). Nlidwinter was limited to three months, so as to include only dilys in which the smu never rose, and il corresponding limitation was made to midsummer. In the three midsummer months the inequality for the month is 1;ised on only a single month's observations; in the other nine months the results ire means from two years.

Iin the talle "liange" denotes the difference between the algebraically grealest and least of the mean 2-hoinly values.

\$. The clatat recorded in 'Table I. were employed for the calculation of Fourier coeflicients, liven at a first class linropean station with hourly readings, two years is too short a period to furnish thoroughly represcntative values for Fonrier coefficients, especially those for periods shorter than 12 hours. In the Antarctic the regular dinrnil ineynality is so small, and the irregular changes so large, that 2-honrly readings from two years seemed too slender a basis for the calcnlation of more than the 24-and 12-honl terms.

In Table II. $c_{1}$ and $r_{2}$ denote the amplitudes of the 24- and 12-honr terms, unity representing $1_{0}^{\circ} F_{3} ; x_{1}$ and $\alpha_{2}$ are the corresponding phase angles. The series wonld run

$$
c_{1} \sin \left(t+a_{1}\right)+c_{2} \sin \left(2 t+\alpha_{2}\right)+\ldots
$$

$t$ being cominted from (local) midnight, and 1 hour being taken as equivalent to $15^{\circ}$.

TAl:L: II,-Fourier Coefficients, Diurnal Inequality.

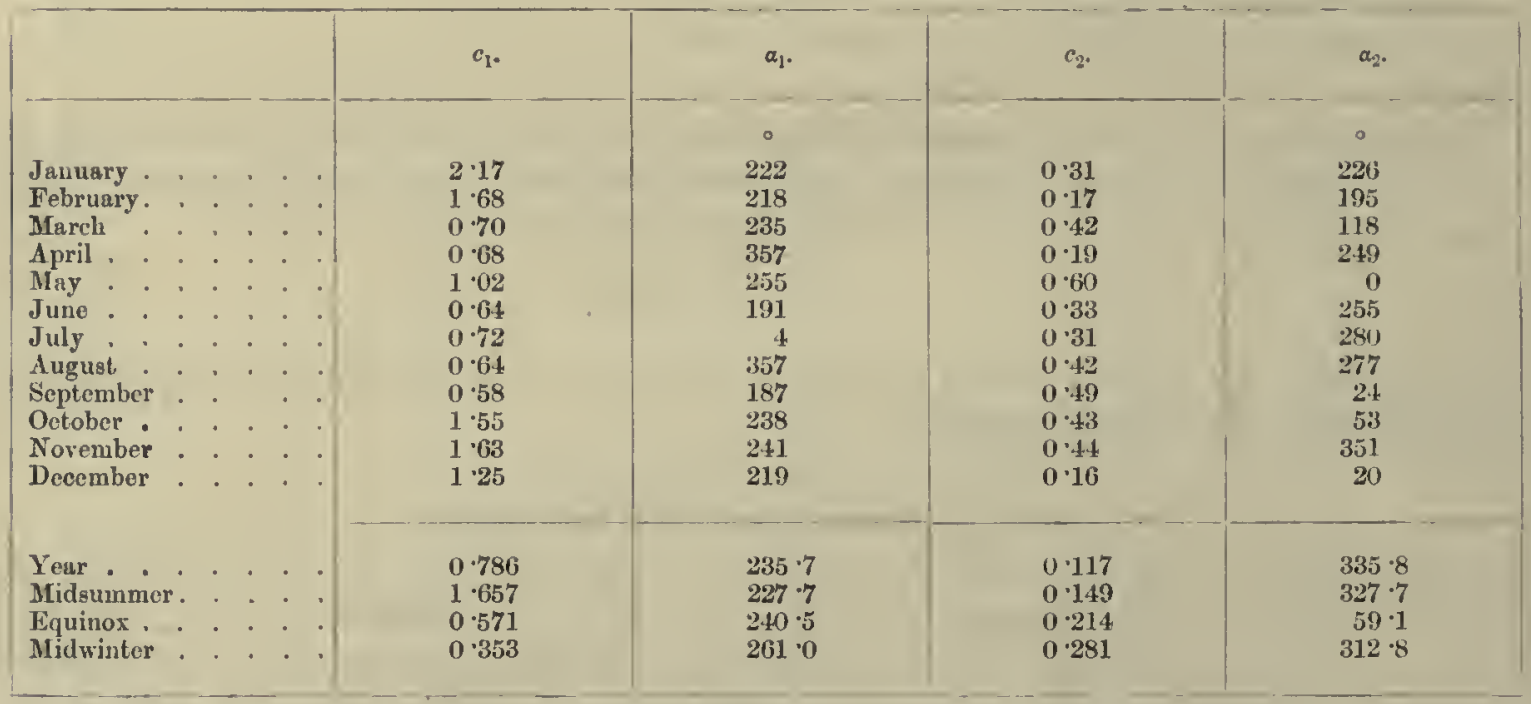

In considering the results, it must be remembered that the existence of finite values for $c_{1}$ and $c_{2}$ is not by itself sufficient proof of the reality of a diurnal inequality. If, however, no real diminal inequality existed, the values of $c_{1}$ and $c_{2}$ wonld most probably fluetuate in an erratic fashion from month to month, while the values of $\alpha_{1}$ and $\alpha_{2}$ wonld be quite inregular.

As compared to datil for a temperate station, the phase angles for individual months in Tibble II. (lo unquestionably show large variations, and it can hardly be claimed that these variations are wholly free from yuasi-aecidental eanses. In the ease of $\alpha_{1}$ the valnes for $A_{p}$ ril, $J$ uly, ${ }^{*}$ and $A$ ingnst appear ontstanding, but they agree closely amongst theniselves.

Taking the seasons, the value of $\alpha_{1}$ for midwinter differs sensibly from that for exuinox, but the difference is in the same direetion as that of equinox from midsummer, and is not so very much greater. In these three sensons the positive crest of the 21-hour wave is reiched approximately at the hours $2 \mathrm{l}$. $50 \mathrm{~m}$., $2 \mathrm{~h} .0 \mathrm{~m} .$, and $0 \mathrm{~h} .35 \mathrm{~m}$. in the afternoon, being latest at midsummer. In the mean diurnal incquality for the year the crest of the $2 \mathrm{t}$-hour wave comes approximately at $2 \mathrm{~h}, 20 \mathrm{~m} .1 \mathrm{p} . \mathrm{m}$. .

* The angles $t^{\circ}$ und $364^{\circ}$, it should be notived, are exuivalent. 
Accorling to the table the ampliturle $c_{1}$ diminishes markerly as we pass from February to Mareh, or from October to Septemher. This wo shonld of course expect, but it rloes appenr a little curious that $c_{1}$ shows no markel further tendeney to rerluetion as we approncli midwinter.

In the 12-hour wave the phase angle varies somerihat irregularly from montl to month, lut the irregnlarity is no greater from $\Lambda$ pril to $\Lambda$ ngust than cluring the rest of the year. The times of ocenrrence of the positive crest (in either forenoon or aftemoon) are approximately as follows:-

Year 3h. 50m., midsunmer 4h. 0m., equinox 1h. 0m., midwinter $4 \mathrm{~h} .30 \mathrm{~m}$.

'The amplitude $c_{2}$ seems greatest in winter and least in summer.

The tendeney in $c_{2} / e_{1}$ to increase as we pass from summer to winter is even more marked than in temperate elimates.

\$3. In examining the clata one coukl not but be impressed by the variability of the temperatnre, especially in winter. In the midlle of an intensely colk period there would appear a sort of oasis of warmth. Table III. has been prepared with a view to bringing out this fact. It gives the mean temperature of the hottest and collest days of eneh month, as rleriverl from the 2-hourly readings. The rlays

TAPr.F III.-Ilottest and Coldest Days.

\begin{tabular}{|c|c|c|c|c|c|c|c|c|c|c|c|}
\hline & & & \multicolumn{3}{|c|}{1902.} & \multicolumn{3}{|c|}{1903.} & \multicolumn{3}{|c|}{ Mean from two years. } \\
\hline & & & $\begin{array}{c}\text { Hottest } \\
\text { day. }\end{array}$ & $\begin{array}{c}\text { Coldest } \\
\text { day. }\end{array}$ & Difference. & $\begin{array}{l}\text { Hottest } \\
\text { iny. }\end{array}$ & $\begin{array}{l}\text { Coldest } \\
\text { dny. }\end{array}$ & Differonee. & $\begin{array}{l}\text { Hottest } \\
\text { dny. }\end{array}$ & $\begin{array}{l}\text { Coldest } \\
\text { dany. }\end{array}$ & Differenee. \\
\hline & & & 。 & $\circ$ & 。 & 。 & 。 & 。 & 。 & 。 & 。 \\
\hline January . & . & & {$[+28 \cdot 6]$} & {$[+12 \cdot 9]$} & $15 \cdot 7$ & $+30 \cdot 5$ & $+18 \cdot 3$ & $12 \cdot 2$ & $+29 \cdot 6$ & $+15 \cdot 6$ & $14 \cdot 0$ \\
\hline February & : & & $+21 \cdot 4$ & $+7 \cdot 6$ & $16 \cdot 8$ & $+26 \cdot 5$ & +13 & $25 \cdot 2$ & $+25 \cdot 5$ & $+4 \cdot 5$ & $21 \cdot 0$ \\
\hline Mareh. & . & & $+22 \cdot 3$ & $-7 \cdot 4$ & $29 \cdot 7$ & $+19 \cdot 6$ & $-14 \cdot 3$ & $33 \cdot 9$ & $+21 \cdot 0$ & $-10 \cdot 8$ & $31 \cdot 8$ \\
\hline April. & : & 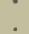 & $+10 \cdot 5$ & $-23 \cdot 5$ & $34 \cdot 0$ & $-4 \cdot 2$ & $-32 \cdot 7$ & $28 \cdot 5$ & $+3 \cdot 1$ & $-28 \cdot 1$ & $31 \cdot 2$ \\
\hline May. & $\dot{0}$ & . & $+10 \cdot 8$ & $-31 \cdot 4$ & $42 \cdot 2$ & $+5 \cdot 5$ & $-40 \cdot 8$ & $46 \cdot 3$ & $+8 \cdot 1$ & $-36 \cdot 1$ & $44 \cdot 2$ \\
\hline June: & : & & $+3 \cdot 5$ & $-31 \cdot 4$ & $34 \cdot 9$ & $+11 \cdot 0$ & $-32 \cdot 3$ & $43 \cdot 3$ & $+7 \cdot 3$ & $-31 \cdot 8$ & $39 \cdot 1$ \\
\hline July : & : & . & $+10 \cdot 1$ & $-26 \cdot 9$ & $37 \cdot 3$ & $+4 \cdot 1$ & $-42 \cdot 9$ & $47 \cdot 0$ & $+7 \cdot 2$ & $-3 t \cdot 9$ & $42 \cdot 1$ \\
\hline August . & . & • & $+2 \cdot 2$ & $-36 \cdot 4$ & $38 \cdot 6$ & +50 & $-45 \cdot 7$ & $50 \% 7$ & $+3 \cdot 6$ & $-41 \cdot 0$ & $44 \cdot 6$ \\
\hline September & $\therefore$ & . & $+10 \cdot \overline{1}$ & $-26 \cdot 2$ & $36 \cdot 3$ & $+8 \cdot 4$ & $-43 \cdot 2$ & $49 \cdot i$ & $+8 \cdot 2$ & $-3 \cdot 6 \cdot 7$ & $4 \cdot 2 \cdot 9$ \\
\hline October. & $\dot{.}$ & : & $+9 \cdot 2$ & $-28 \cdot 0$ & $37 \cdot 2$ & $+8 \cdot 0$ & $-26 \cdot 9$ & $34 \cdot 9$ & $+8 \cdot 6$ & $-27 \cdot 5$ & $36 \cdot 1$ \\
\hline November & $\therefore$ & . & $+19 \cdot 6$ & $+4 \cdot 6$ & $15 \cdot 0$ & $+25 \cdot 6$ & $+5 \cdot 1$ & $20 \cdot 2$ & +226 & $+5 \cdot 0$ & $17 \cdot 6$ \\
\hline December & $\dot{\bullet}$ & . & $+34 \cdot 2$ & $+8 \cdot 2$ & $26 \cdot 0$ & $+84 \cdot 7$ & $+18 \cdot 0$ & $16 \cdot 7$ & $+3 \cdot 6 \cdot 5$ & $+13 \cdot 1$ & $21 \cdot 4$ \\
\hline Year. & - & • & $+34 \cdot 2$ & $-36 \cdot 4$ & $70 \cdot 6$ & $+34 \cdot 7$ & $-45 \cdot 7$ & $80 \cdot 4$ & $+3 \cdot 4 \cdot 5$ & $-41 \cdot 0$ & $75 \cdot 5$ \\
\hline
\end{tabular}

were all taken from midnight to millnight. If one had interpreted a "dlay " as any 24 consecutive honrs, the differenee letween the hottest and eoldest "days" wonld, of course, have been in general slightly" greater. The table gives results for each indivichal month from February, 1902, to Jaunary, 1904, ancl also the means from the two years. In Norember and Deember, 1903, and Jannary, 1904, the number of ohservation hours was rerheerl during the night. Allowance was malle for this in ealculating the mean temperature, nse being marle of the dliurnal ehanges of temperature olsserved in the eorresponding midsummer months of 1902 and 1903.

April, 1903, was the only month in which no day had a mean temperature exceeding $0^{\circ} \mathrm{F}$.

The difference leetween the hottest and collest dlays of the month is much less in sumuer than in winter. There is not nearly so much difference between the temperatures of the hottest days of the different months as between the temperatures of the coldest diys. In hoth years the absolutely eoldest day occurrerl in Angnst, and the hottest in December. The latter was the only month of the year in which the hottest diay had an mean temperatnire in exeess of the freezing-point.

\$4. The minimum thermometer was real and reset every two hours. Fach value recorderl thus represents the lowest temperature during the previons two hours. Comparing each minimum reacling with the mean of the two ordinary thermometer renlings at the lieginning and end of the 2-hour interval, one gets an idea of the varialility-chiefly the a-periodic varialility-of temperatnre throughont the day and thronghout the year. 
Table IV. shows the mean difference of the minimum from the arithmetic mean of adjaeent ordinary readings, for enelı 2-hour interval, during each individual month from February, 1902, to Oetober, 1903. Subsequent to the latler date the mumber of observation hours was reduced, and so the results ceased to be eomparable. Table IV. shows also the mean of the twelve 2-hourly differenees for each nonth.

TApite IV.-Exeess of Mean of Ordinary Readings at Beginning and Fnd of 2-hour Interval over Corresponding Mininum leading.

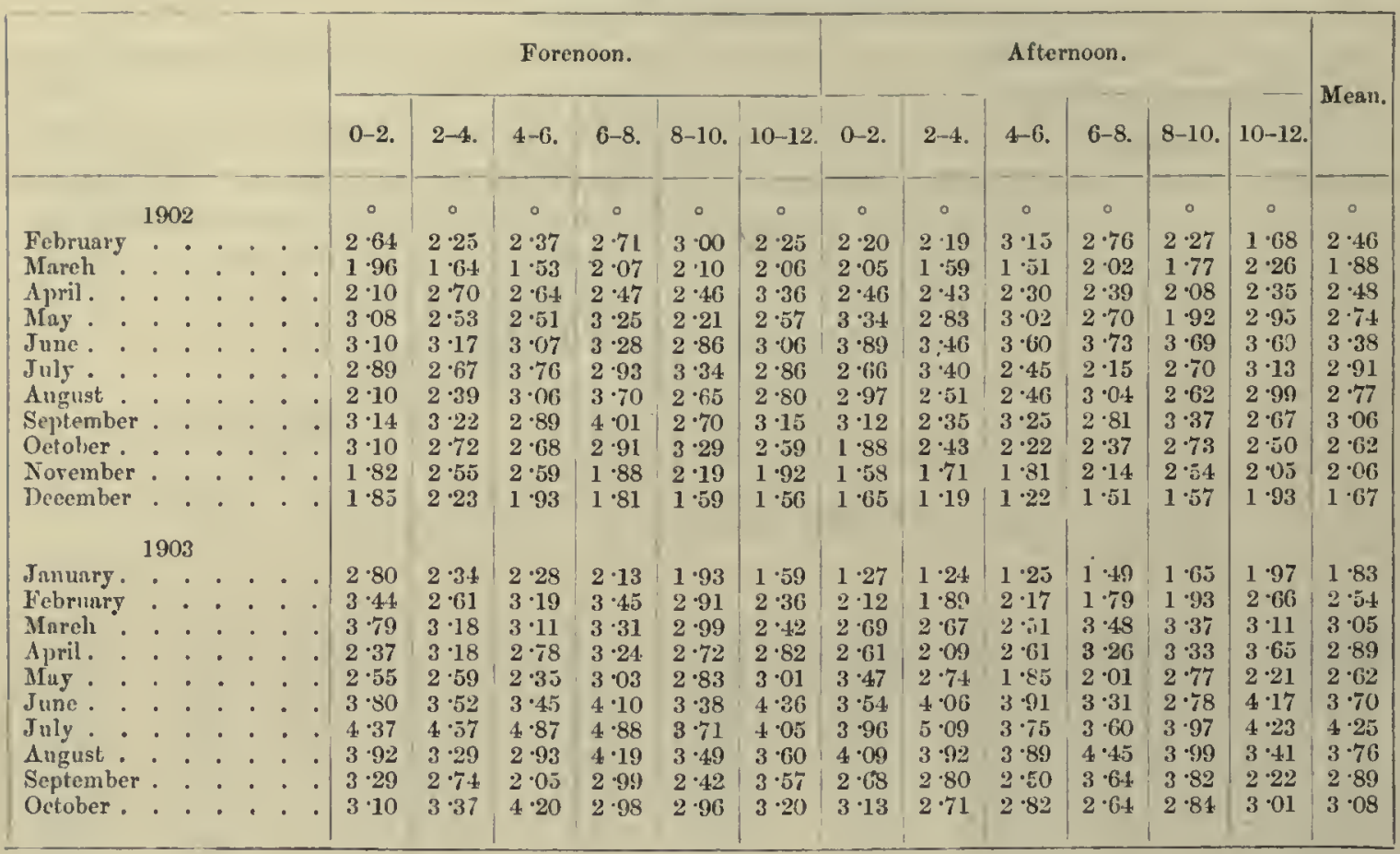

TABd.E V.-Excess of Mean of Ordinary Readings at Beginning and End of 2-hour Interval over Corresponding Minimum Reading.

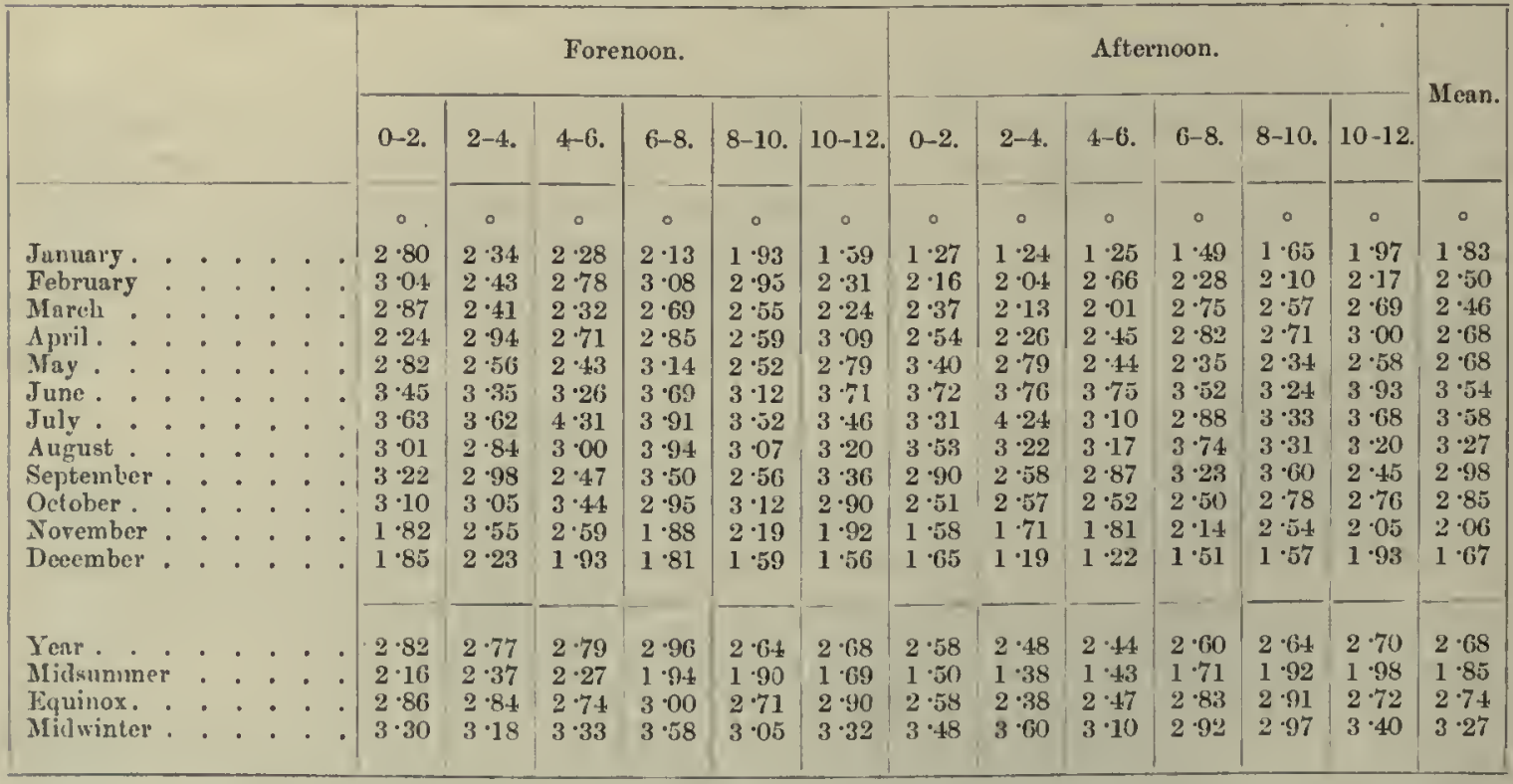

Table V. contains similar data to Table IV., but gives the mean of the results from corresponding months of the two years. In Table V. we have the mean differenee for the month, exhibiting a fairly 
regular annual variation, with a minimum in the lottest months, December and January, and a maximum in July and June. The nininum thus occurs at a season when the periolic diurnal changes of temperature are greatest, the maximum when they are least; moreover, the maximm is fully double the minimum. The hours at which the difference between the ordinary and mininum readings is greatest and least vary a good deal from month to month, especially in winter. In summer the phenomena are fairly regular. From the three midsummer months, November to January, we get a very regular cliurnal inequality in the difference, having a maximum for the 2-hour period 2 to 4 a.m., and a minimum for the period 2 to 4 p.m., the "range" amounting to $0^{\circ} \cdot 99$. These two periods immediately follow the hours of minimum and maximum respectively in the cliurmal inequality of ordinary temperature for the season. This shows that if a diurnal inequality were formed from the mininum thernometer readings of the month, it would resemble that dedneed from the ordinary thermometer; the two ineynalities would show maxima and minima about the sume lrours, but the range from the minimum readings would be about $1^{\circ}$ the linger.

In eqninox the difference between the ordinary and minimum readings shows a less regular diurnal inequality, but there is a distinet maximum for the period 6 to 8 a.m., and a distinct minimum for the period 2 to 4 p.m. These periods again follow immediately the minimum and the maximum of the ordinary temperature. In these two seasons, then, the difference between the ordinary and minimum readings has a diurnal inequality resembling that of ordinary temperature, but having a smaller range. In the case of the ordinary tempcrature the diurnal inequality for midwinter differed from that for the two other seasons in showing very distinctly two maxima, the larger at from 2 to 4 p.m., the smaller from 4 to 6 a.m., with intervening minima. It is thus with considerable interest that one turns to the diurnal inequality obtained in Table V. for midwinter. Clearly a marked double period in that inequality would be evidence in favour of the reality of the diurnal inequality of ordinary temperature. On examining the figures assigned to midwinter in Table V. one sees traces of apparently three maxima; the two largest and best defined answer to the periods 2 to 4 p.m. and 6 to 8 a.m.

$\$ 5$. The twelve 2-hourly rendings of the minimum thermometer supply information as to the time at which the lowest temperature for the day is reached. Supposing that in a month of 30 days the lowest temperature occurred on 6 days between 0 and 2 a.m., then the percentage of occurrence of the minimum in this 2-hour period for the month in question is $(6 / 30) \times 100$, or $20 \cdot 0$.

Table VI. gives the percentage occurrence of the minimum throughout the 24 hours for each month of the year, for the year as a whole, and for three seasons made up as explained in describing Table I. The results, except in November, December, and January, are means from two year's.

TABLE VI.-Pereentage Occurrence of Minimum for the Day.

\begin{tabular}{|c|c|c|c|c|c|c|c|c|c|c|c|c|c|c|c|c|c|c|c|c|c|}
\hline & & & & & & & & & & \multicolumn{6}{|c|}{ Forenoon. } & \multicolumn{6}{|c|}{ Afternoon. } \\
\hline & & & & & & & & & & $0-2$ & $2-4$ & $4-6$ & $6-8$. & 8-10. & $10-12$ & $0-2$. & $2-4$. & 4-6. & 6-8. & 8-10. & $10-12$. \\
\hline January & 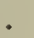 & . & . & . & . & . & . & . & . & $25 \cdot 8$ & $17 \cdot 7$ & $8 \cdot 1$ & $17 \cdot 7$ & $I \cdot 6$ & $0 \cdot 0$ & $0 \cdot 0$ & $0 \cdot 0$ & $0 \cdot 0$ & $1 \cdot 1$ & $2 \cdot 7$ & $25 \cdot 3$ \\
\hline liebruary & . & . & . & . & . & . & . & . & . & $26 \cdot 0$ & $11 \cdot 2$ & $9 \cdot 0$ & 70 & $9 \cdot 0$ & $7 \cdot 5$ & $2 \cdot 6$ & $1 \cdot 8$ & $3 \cdot 5$ & $3 \cdot 1$ & $3 \cdot 7$ & $15 \cdot 6$ \\
\hline March. & . & . & . & . & . & . & . & . & . & $12 \cdot 1$ & $8 \cdot 1$ & $6 \cdot 5$ & $12 \cdot 6$ & 0.8 & 0.8 & $5 \cdot 1$ & $1 \cdot 6$ & $2 \cdot 1$ & $8 \cdot 9$ & $16 \cdot 7$ & $24 \cdot 7$ \\
\hline April & . & . & . & . & . & . & $\dot{.}$ & . &. & $17 \cdot 5$ & $10 \cdot 9$ & $1 \cdot 7$ & $1 \cdot 6$ & $5 \cdot 0$ & $8 \cdot 3$ & $5 \cdot 8$ & $5 \cdot 0$ & $13 \cdot 3$ & $6 \cdot 7$ & $12 \cdot 5$ & $11 \cdot 7$ \\
\hline May . & 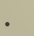 & . & . & . & . & . & . & . & . & $20 \cdot 2$ & $8 \cdot 2$ & $6 \cdot 3$ & $4 \cdot 1$ & 0.8 & $4 \cdot 6$ & $6 \cdot 8$ & $5 \cdot 6$ & $5 \cdot 6$ & $6 \cdot 5$ & $18 \cdot 8$ & $12 \cdot 5$ \\
\hline June & . & . & 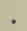 & . & . & . & . & 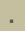 & . & $11 \cdot 4$ & $12 \cdot 3$ & $0 \cdot 9$ & $5 \cdot 0$ & $3 \cdot 9$ & $11 \cdot 6$ & $11 \cdot 6$ & $9 \cdot 2$ & $5 \cdot 9$ & $6 \cdot 6$ & $5 \cdot 8$ & $15 \cdot 8$ \\
\hline July . & & . & $\bullet$ & . & . & . & . & . &. & $23 \cdot 4$ & $4 \cdot 9$ & $7 \cdot 2$ & $1 \cdot 6$ & $4 \cdot 0$ & $3 \cdot 3$ & $11 \cdot 3$ & $4 \cdot 9$ & $5 \cdot 6$ & $4 \cdot 0$ & $4 \cdot 0$ & $25 \cdot 8$ \\
\hline August. & & . & 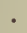 & . & . & . & 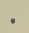 & . & . & $10 \cdot 5$ & $12 \cdot 1$ & $1 \cdot 6$ & $9 \cdot 7$ & $7 \cdot 3$ & $4 \cdot 5$ & $4 \cdot 8$ & $4 \cdot 8$ & $8 \cdot I$ & $10 \cdot 5$ & $7 \cdot 0$ & $19 \cdot 1$ \\
\hline September & & . & 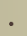 & . & - & . & . & & . & $16 \cdot 7$ & $4 \cdot 2$ & $3 \cdot 8$ & $7 \cdot 9$ & $3 \cdot 3$ & $5 \cdot 0$ & $6 \cdot 7$ & $5 \cdot 0$ & $11 \cdot 7$ & $9 \cdot 1$ & $12 \cdot 9$ & $13 \cdot 7$ \\
\hline October & & . & 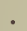 & . & . & . & . & . & . & $21^{\circ} 0$ & 14.5 & $11 \cdot 3$ & $3 \cdot 3$ & $4 \cdot 8$ & $4 \cdot 0$ & $2 \cdot 5$ & $1 \cdot 6$ & $1 \cdot 6$ & $1 \cdot 6$ & $10 \cdot 5$ & $23 \cdot 3$ \\
\hline Novenber & & . & . & . & . & . & . & 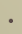 & - & $15 \cdot 0$ & $21 \cdot 7$ & $18 \cdot 3$ & $6 \cdot 7$ & $5 \cdot 0$ & $6 \cdot 7$ & $0^{\circ} 0$ & $0 \cdot 0$ & 0.0 & 0.0 & $6 \cdot 7$ & $20^{\circ} 0$ \\
\hline December & & - & 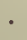 & 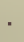 & . & . & - & . & . & $23 \cdot 1$ & $14 \cdot 5$ & $15 \cdot 3$ & $11: 3$ & $7 \cdot 3$ & $0 \cdot 8$ & $3 \cdot 2$ & $0 \cdot 0$ & $3 \cdot 2$ & $0 \cdot 0$ & $0 \cdot 0$ & $21 \cdot 0$ \\
\hline Year & & 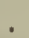 & . & ${ }^{\circ}$ & . & . & & ${ }^{\prime}$ & $\theta^{\circ}$ & $18 \cdot 6$ & $11 \cdot 7$ & $7 \cdot 5$ & $7 \cdot 4$ & $4 \cdot 4$ & $4 \cdot 8$ & 50 & $3 \cdot 3$ & $5 \cdot I$ & $4 \cdot 8$ & $8 \cdot 4$ & 190 \\
\hline Midsumune & & . & . & . & . & . & . & . & . & $21 \cdot 4$ & $18 \cdot 0$ & $13 \cdot 9$ & $11 \cdot 9$ & $4 \cdot 6$ & $2 \cdot 5$ & $1 \cdot 1$ & $0 \cdot 0$ & $1 \cdot I$ & 0.4 & $3 \cdot 1$ & $22 \cdot 1$ \\
\hline Equinox . & & . & . & . & . & . & $\cdot$ & . & . & $16 \cdot 8$ & $9 \cdot 4$ & $5 \cdot 8$ & $6 \cdot 3$ & $3 \cdot 5$ & $4 \cdot 5$ & $5 \cdot 0$ & $3 \cdot 3$ & $7 \cdot 2$ & $6 \cdot 6$ & $13 \cdot 2$ & $18 \cdot 4$ \\
\hline Midwinter & & . & . & . & . & . & . & . & . & $18 \cdot 3$ & $8 \cdot 5$ & $4 \cdot 8$ & $3 \cdot 5$ & $2 \cdot 9$ & $6 \cdot 5$ & $9 \cdot 9$ & $6 \cdot 5$ & $5 \cdot 7$ & $5 \cdot 7$ & $9 \cdot \overline{6}$ & $18 \cdot 1$ \\
\hline
\end{tabular}


Taking the year as a whole, we see a marked maximum frequency of occurrence between 10 p.in. and 2 a.m., the fregnency being very nearly the same for the two hours before and the two hours after midnight. There is a slight indication of a donble period in the frequency, with a secondary maximum near noon, the principal minimum occurring between 2 and 4 p.m. The mmmber of occurrences of minimum in the 12 hours ending at $S \mathrm{a}$.m. bears to the number in the 12 hours ending 8 p.m. the ratio of $8: 3$.

In the three midsummer montlis as a whole the occurrences between 8 a.m. and 8 p.m. form only 10 per cent. of the total number, and in no single occasion did the minimum occur between 2 and 4 p.m.

In the equinoctial season the occurrences between 8 a.m. and 8 p.m. rise to 30 per cent. of the whole, and a minimum appears between 8 and $10 \mathrm{a} . \mathrm{m}$., which differs but littlo from the principal minimum between 2 and 4 p.m. While at midsumner only 28 per cent. of the minima occur in the afternoon, in the equinoctial season the afternoon occurrences are slightly more numerons than the forenoon occurrences.

Coming to midwinter, we find a most interesting and well-marked dinrual inequality in the frequency of occurrences. As at other seasons, the frequency of incidence between 10 p.m. and $2 \mathrm{a} . \mathrm{m}$. is notably in excess of that at other hours. But there is a well marked secondary maximum of frequency from noon to $2 \mathrm{p} . \mathrm{m}$., and the forenoon minimum is unmistakably the principal one of the day. Each of the midwinter months shows the double period very clearly. The phenomenon is perhaps the strongest evidence yet advanced that temperature in the Antarctic night is affected by decided diurnal and semidiurnal influences.

$\S 6$. The maximum thermometer being read and reset only twice a day-at 8 a.m. and 8 p.m.information as to maximum temperatures is much less extensive than that as to minimun temperatures, and it is affected in addition by several uncertainties. If the highest temperature of a particnlar day, say day $n$, occurred between 8 a.m. and 8 p.m., then it is given by the corresponding 8 p.m. reading of the maximum thermometer; but if it occurred before $8 \mathrm{a.m}$. ol after 8 p.m. it may not be recorded at all. If

Tarde VII.-Means of Daily Maxima and Minima and of Daily Ranges.

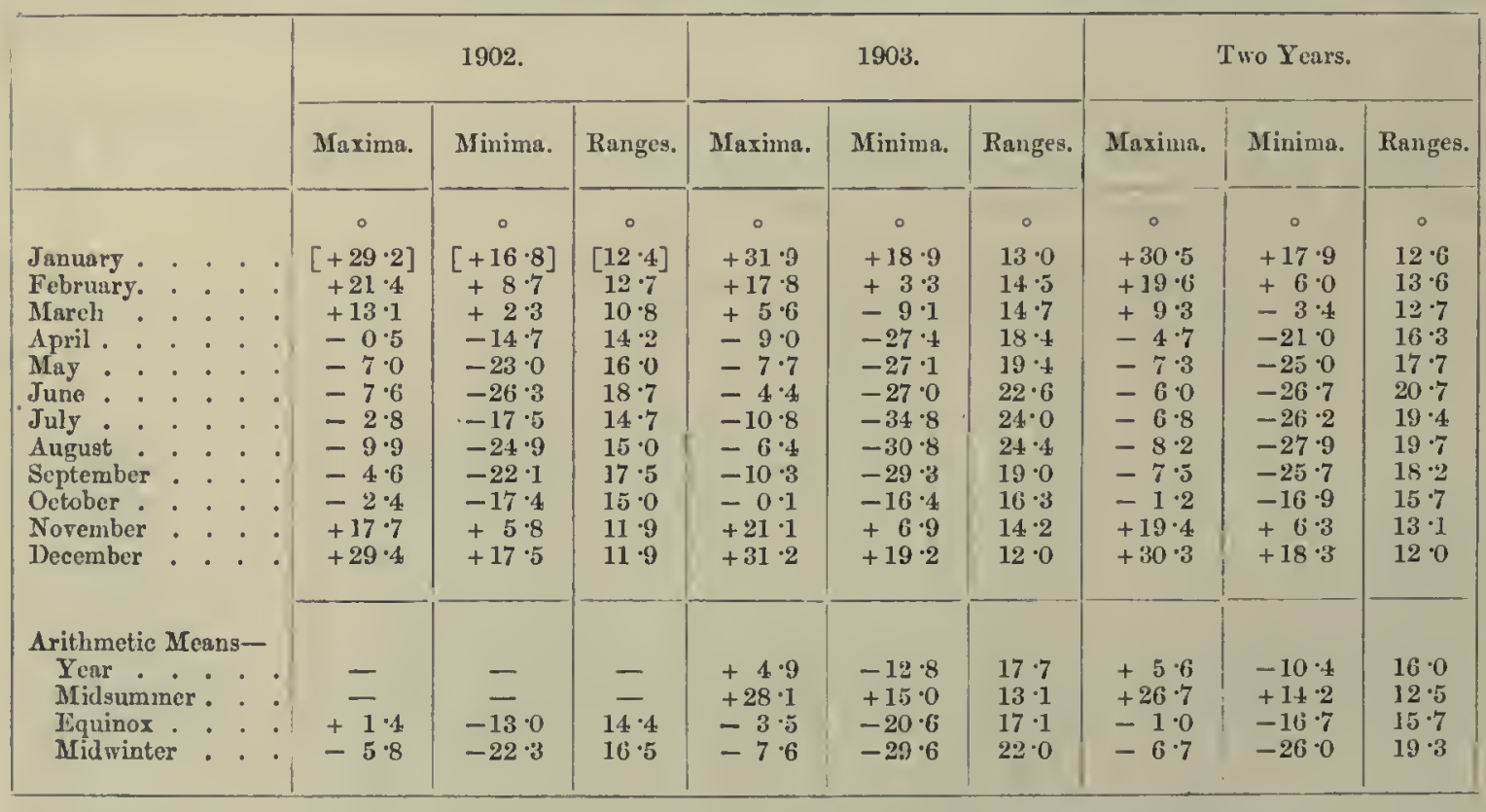

it occurred before $8 \mathrm{a} . \mathrm{m}$. it will be given by the $8 \mathrm{am}$. reading only if it exceeds the highest temperature occurring between $8 \mathrm{p} . \mathrm{m}$. and midnight on day $n-1$. If it occurred after $8 \mathrm{p} . \mathrm{m}$. it will be given by the 8 a.m. reading on day $n+I$ only if no higher temperature occurred on that day before 8 a.m. One can be absolutely certain of having the true maximum reading for day $n$ only when the 8 p.m. reading on that day exceeds the 8 a.m. readings Loth on day $n$ and day $n+1$. In summer this was generally the 
case, but in a great many dlays, especially in winter, it was otherwise. $\Lambda$ s the 8 a.m. reading applied to a perior of which 8 hours belonged to day $n$, as against 4 belonging to day $n-1$, the reading was assigned to day $n$ when the general trend of temperature was not sufficiently definite to settle the question. When temperature showed a general trend downwards after 8 p.m. on day $n$, the 8 a.m. reading on day $n+1$ was assigned to day $n$; in such a case, howover, the maximum at 8 p.m. was practically certain to be the real maximum for day $n$. When temperature showed a general trend upwards after $8 \mathrm{p}$. m. on day $n$, the 8 a.m. reading next morning was assigned to day $n+1$, and the ordinary reading at $10 \mathrm{p} . \mathrm{m}$. or midnight on dity $n$, if greater than the 8 p.m. maximum, was acepted as the maximum for day $n$. In March, 1902, maximum thermometer readings were lacking in about half the days of the month; on thesc days the highest of the ordinary thermometer readings was taken as the maximum. There were very few other occasions throughout the two years when maximum readings were lacking, and the days when this oceurred were left ont of consideration.

When ordinary thermometer readings wero accepted as maxima the consequence was, no donbt, as a rule, an underestimate of the maximum, and thence of the daily range (maximum less ininimum); and the figures for March, 1902, probably are slightly affected by this cause. With the exception of this one month, whilst individual daily ranges were doubtless occasionally in appreciable error, there can be but little doubt of the substantial accuracy of the mean data given in Table VII.

It shows for each month of the two years the means of the daily maxima and of the daily minima, and the mean of the daily ranges (maximum less minimum).

Mean results are also given for the two years combined.

The seasons are taken as in the previous tables.

Data are omitted for the whole year and for midsummer in 1902, because January results were lacking for that year; those entered in brackets really belong to 1904 .

Even at a temperate station the daily range from the maximum and minimum thermometers is considerably in excess of that derived from the highest and lowest of the hourly readings, and increasing the interval between readings from one hour to two naturally reduces the range. The differences, however, between the ranges in Tables VII. and I. are on an enormously enhanced scale as compared to the corresponding differences in a temperate climate.

The five months November to March-in all of which the mean maximum temperature for the day exceeds $0^{\circ} \mathrm{F}$.- - show ranges which differ comparatively little amongst themselves, but which are considerably less than those in the other months. On the average of the two years the range is largest in June, and only slightly less in July and August.

$\S 7$. In some places the mean of the daily maxinum and minimum is on the average of the days of a month, ai very close approach to the mean temperature of the 24 hours. Table VIII. gives, for each

TABLE VIII.

\begin{tabular}{|c|c|c|c|c|c|c|c|c|c|c|c|}
\hline & & & & & & & \multicolumn{2}{|c|}{$\begin{array}{l}\text { Means from daily maxima and } \\
\text { minima. }\end{array}$} & \multicolumn{3}{|c|}{$\begin{array}{l}\text { Lxeess of mean from twelre } 2 \text {-hourly readings over } \\
\text { mean from daily maxima and minima. }\end{array}$} \\
\hline & & & & & & & 1902. & 1903. & 1902. & 1903. & Two years. \\
\hline $\begin{array}{l}\text { January* } \\
\text { February } \\
\text { Mareh : } \\
\text { April : } \\
\text { May : : } \\
\text { June : : } \\
\text { July : } \\
\text { August : } \\
\text { September } \\
\text { Oetober : } \\
\text { November } \\
\text { December }\end{array}$ & $\begin{array}{l}\dot{.} \\
\dot{.} \\
\dot{.} \\
\dot{.} \\
\dot{.} \\
\dot{.} \\
\dot{.}\end{array}$ & $\begin{array}{l}\dot{0} \\
\dot{.} \\
\dot{5} \\
\dot{.} \\
\dot{.} \\
\dot{.} \\
\dot{.}\end{array}$ & $\begin{array}{l}\dot{\dot{0}} \\
\dot{.} \\
\dot{.} \\
\dot{.} \\
\dot{.} \\
\dot{.} \\
\dot{.}\end{array}$ & $\begin{array}{l}\dot{0} \\
\dot{0} \\
\dot{0} \\
\dot{0} \\
\dot{\cdot} \\
\dot{.}\end{array}$ & 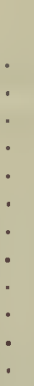 & 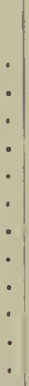 & $\begin{array}{c}\circ \\
{[+23 \cdot 0]} \\
+15 \cdot 1 \\
+7 \cdot 7 \\
-7 \cdot 6 \\
-15 \cdot 0 \\
-17 \cdot 0 \\
-10 \cdot 2 \\
-17 \cdot 4 \\
-13 \cdot 3 \\
-9 \cdot 9 \\
+11 \cdot 7 \\
+23 \cdot 4\end{array}$ & $\begin{array}{c}0 \\
+25 \cdot 4 \\
+10 \cdot 5 \\
-1 \cdot 8 \\
-18 \cdot 2 \\
-17 \cdot 4 \\
-15 \cdot 7 \\
-22 \cdot 8 \\
-18 \cdot 6 \\
-19 \cdot 8 \\
-8 \cdot 2 \\
+14 \cdot 0 \\
+25 \cdot 2\end{array}$ & $\begin{array}{c}0 \\
-1 \cdot 0] \\
0 \cdot 8 \\
0 \cdot 4 \\
0 \cdot 6 \\
2 \cdot 4 \\
1 \cdot 0 \\
1 \cdot 4 \\
0 \cdot 9 \\
1 \cdot 3 \\
1 \cdot 4 \\
0 \cdot 3 \\
-0 \cdot 3\end{array}$ & $\begin{array}{l}0 \\
0 \cdot 7 \\
0 \cdot 8 \\
1 \cdot 0 \\
1 \cdot 3 \\
1 \cdot 4 \\
1 \cdot 9 \\
1 \cdot 7 \\
2 \cdot 1 \\
1 \cdot 2 \\
1 \cdot 4 \\
1 \cdot 9 \\
0 \cdot 7\end{array}$ & $\begin{array}{c} \\
-0 \cdot 2 \\
-0 \cdot 2 \\
+0 \cdot 8 \\
+0 \cdot 7 \\
+0 \cdot 9 \\
+1 \cdot 9 \\
+1 \cdot 5 \\
+1 \cdot 5 \\
+1 \cdot 5 \\
+1 \cdot 2 \\
+1 \cdot 4 \\
+1 \cdot 1 \\
+0 \cdot 2\end{array}$ \\
\hline
\end{tabular}

* The values entered under January, 1902, belong really to January, 1904. 
month of the two years, the mean derived from the daily maxima and minima, and the amount by which it falls short of the corresponding menn derived from the 2-hourly readings. In two months, December, 1902, and Jannary, 1904, the mean from the 2-lourly readings was the smaller. In the latter month the night readings were curtailed, and allowance had to be mado for the fact, so that the result is exposed to greater uneertininty than in the other months. It is clear, however, from the table that, while the mean from the 2-hourly readings is in general decidedly the higher, its excess is leciledly less in the summer, and even in the equinoetial months, than in winter. Taking the two years, the mean from the daily maxximum and ninimmm is on the average only $1^{\circ}$ less than that from the 2-hourly readings. It wonld, in fact, form a not at all bad approximation to the true mean temperature, though somewhat overestimating the cold of winter.

\$8. The amplitude of the daily range as derived from the daily maximum and minimum is a quantity varying immensely from day to day. Table IX. is intended to give an idea of the extent of this variation. It records the greatest and the least daily range actually observed during each month of the two years.

TABLE IX.-Greatest and Least Daily Ranges.

\begin{tabular}{|c|c|c|c|c|c|c|c|c|c|c|}
\hline & & & & & & & \multicolumn{2}{|c|}{ Greatest daily range. } & \multicolumn{2}{|c|}{ Least daily rauge. } \\
\hline & & & & & & & 1902. & 1903. & 1902. & 1903. \\
\hline & & & & & & & $\circ$ & 。 & - & $\circ$ \\
\hline January* & . & . & & & & & {$[22 \cdot 3]$} & $26 \cdot 0$ & {$[6 \cdot 1]$} & $6 \cdot 5$ \\
\hline February & • & . & • & . & . & . . & $24 \cdot 2$ & $22 \cdot 5$ & $8 \cdot 5$ & $8 \cdot 0$ \\
\hline March & 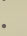 & . & . & . & . & . . & $21 \cdot 3$ & $29 \cdot 8$ & $4 \cdot 8$ & $9 \cdot 0$ \\
\hline April . & . & . & • & . & . & . . & $27 \cdot 5$ & $30 \cdot 1$ & $5 \cdot 2$ & $6 \cdot 0$ \\
\hline May. & . & . & . & . & . & . . & $30 \cdot 0$ & $40 \cdot 0$ & $5 \cdot 0$ & $7 \cdot 1$ \\
\hline June. . & • & . & • & . & . & . . & $41 \cdot 0$ & $43^{\circ} 0$ & $8 \cdot 4$ & $12 \cdot 5$ \\
\hline July. . . & . & . & . & . & . & . . & $27 \cdot 0$ & $41 \cdot 0$ & $5 \cdot 2$ & $10 \cdot 0$ \\
\hline August. & . & . & . & . & . & . . & $29 \cdot 5$ & $41 \cdot 0$ & $7 \cdot 7$ & $9 \cdot 2$ \\
\hline September & . & . & . & . & - & .. & $44 \cdot 5$ & $30 \cdot 8$ & $5 \cdot 2$ & $11 \cdot 0$ \\
\hline Oetober. & . & . & . & . & . & . . & $22 \cdot 8$ & $33 \cdot 2$ & $8 \cdot 4$ & $7 \cdot 3$ \\
\hline Norember & . & . & . & . & . & . . & $21 \cdot 5$ & $22 \cdot 3$ & $4 \cdot 7$ & $6 \cdot 3$ \\
\hline Deeember & • & . & . & • & . & . & $21^{\circ} 0$ & $21 \cdot 0$ & $3 \cdot 8$ & $5 \cdot 4$ \\
\hline
\end{tabular}

* The values entered under January, 1902, belong really to January, 1904.

Very small ranges are oceasionally met with at all seasons. On the whole, the smallest ranges of the year are encountered in the summer nronths. The largest ranges in winter are very considerably larger than those in summer. In fivo separate montlis there were daily ranges exceeding $40^{\circ} \mathrm{F}$.

\$ 9. Table X. does for individual hours what Table III. does for individual days. It gives the highest maximum and the lowest minimum readings recorded in each individual month and the difference between the two, which eonstitutes the extreme range of temperature for the month in question.

The highest temperature recorded thronghont the month varied from $+42^{\circ} \cdot 0$ in Docember, 1903, to $+2^{\circ} \cdot 0$ in April, 1903, a range of $40^{\circ}$, there being no single montli in which the temperature failed to reach $0^{\circ} \mathrm{F}$.

The lowest temperature of the month varied from $-58^{\circ} \cdot \tilde{5}$ in September, 1903 , to $+9^{\circ} \cdot 0$ in Jannary, 1903 , the range being $67^{\circ} \cdot 5$, and so 69 per cent. larger than that of the highest temperatnre of the montl.

The monthly range, i.e., the difference between the highest and the lowest temperature of the montll, slows a fairly regular annual variation, and is notably least at midsummer.

\$ 10. The particular hour at which the highest maximum or the lowest minimum of a particular month oceurs must be regarded as largely a matter of aceident, and it cannot throw any certain light on the question whether there is a decicled tendency for exceptionally high or exceptionally low tenperatures to occur at one hour of the day rather than another.

To throw further light on this question, means were derived from the six lowest minima readings corresponding to each of the 2-hour intervals of which the day was composed. If in any particular month 
there is a decided tendency for exceptionally low temperatnres to occur between, say, 0 a.m. and 2 a.m., then we inay reasonably expect a lower mean value for the six lowest readings during this interval than for the correspouding quantities for the other eleven 2-hour intervals. Table XI. shows the results thus obtained for the twelve months, the year, and the usual seasons; the datr are means from two years.

Tabie X.-Extreme Monthly Temperatires and Mouthly Ringes.

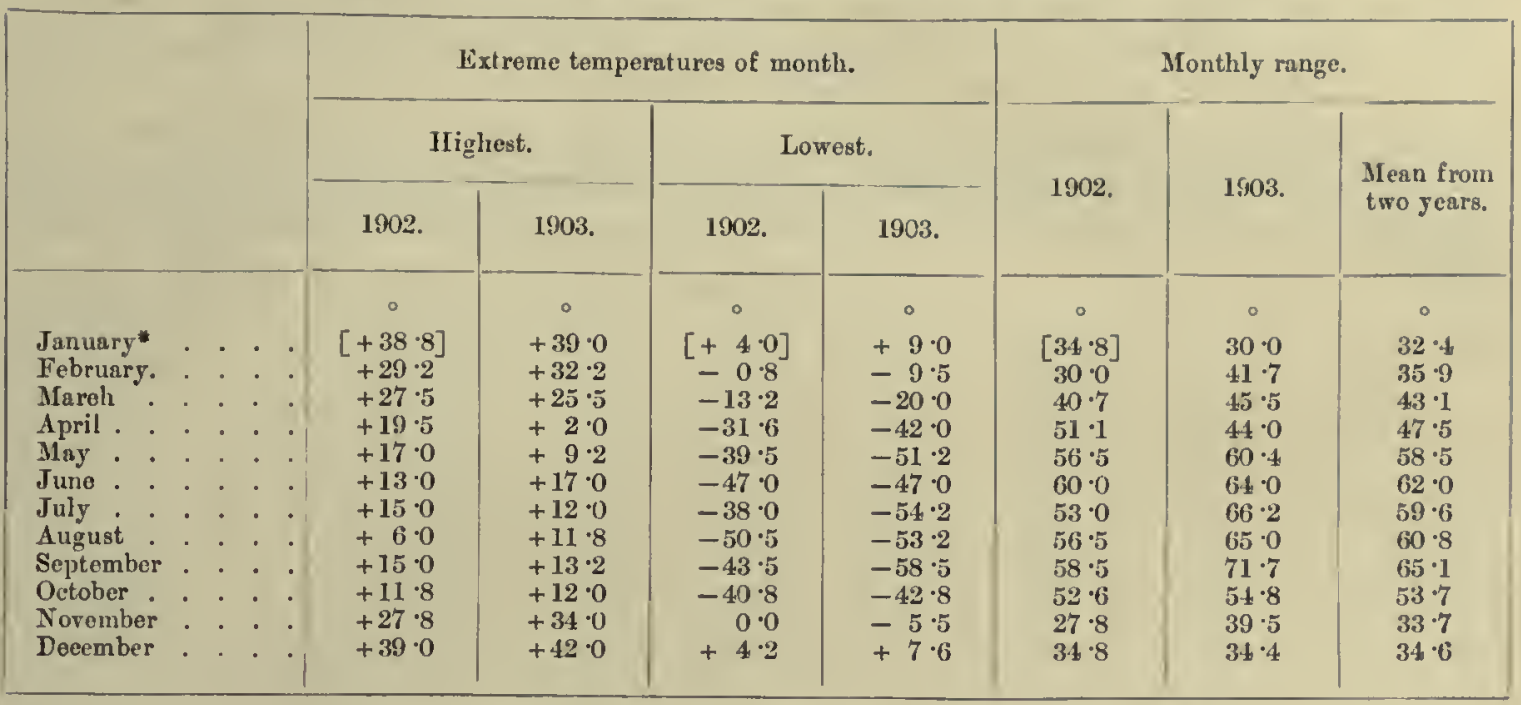

* The values entered under January, 1902, belong really to January, 1904.

TABLE XI.-Means from Six Lowest Minima at each 2-hour Interval. $\dagger$

\begin{tabular}{|c|c|c|c|c|c|c|c|c|c|c|c|c|c|c|c|c|c|c|c|}
\hline & & & & & & & & \multicolumn{6}{|c|}{ Forenoon. } & \multicolumn{6}{|c|}{ Afternoon. } \\
\hline & & & & & & & & $0-2$. & $2-4$ & $4-6$ & $6-8$ & 8-10. & $10-12$ & $0-2$ & $2-4$ & $4-6$ & $6-8$ & $8-10$. & $10-12$. \\
\hline & & & & & & & & $\circ$ & 。 & 。 & 。 & $\circ$ & 。 & 。 & 。 & 。 & 。 & 。 & $\circ$ \\
\hline January . & . & . & . & . & . & & . & $+13 \cdot 9$ & $13 \%$ & $15 \cdot 4$ & $15 \cdot 1$ & $18 \cdot 0$ & $19 \cdot 5$ & $20 \cdot 8$ & $21 \cdot 7$ & $21 \cdot 9$ & $21 \cdot 0$ & $18 \cdot 7$ & $+16 \cdot 0$ \\
\hline February & . & . & . & . & . & . & . & +19 & $2 \cdot 3$ & $1 \cdot 1$ & $1 \cdot 4$ & $2 \cdot 5$ & $3 \cdot 8$ & $4 \cdot 2$ & $4 \cdot 7$ & $3 \cdot 7$ & $4 \cdot 5$ & $4 \cdot 2$ & $+3 \cdot 4$ \\
\hline March & . & . & . & . & . & . & - & $-11 \cdot 1$ & $10 \cdot 6$ & $10 \cdot 9$ & $11 \cdot 1$ & $10 \cdot 4$ & $9 \cdot 9$ & $9 \cdot 3$ & $9 \cdot 9$ & $9 \cdot 8$ & $10 \cdot 7$ & $11 \cdot 9$ & $-11 \cdot 1$ \\
\hline April. & . & . & . & . & . & . & . & $-28 \cdot 2$ & $28 \cdot 2$ & $27 \cdot 2$ & $28 \cdot 3$ & $27 \cdot 7$ & $29 \cdot 5$ & $29 \cdot 7$ & $28 \cdot 0$ & $29 \cdot 3$ & $29 \cdot 0$ & $29 \cdot 4$ & $-28 \cdot 8$ \\
\hline May . . & . & . & . & . & . & . & . & $-3 t \cdot 7$ & $34 \cdot 3$ & $34 \cdot 5$ & $34 \cdot 4$ & $32-4$ & $32 \cdot 9$ & $34 \cdot 5$ & $31 \cdot 8$ & $33 \cdot 6$ & $35 \cdot 3$ & $35 \cdot 1$ & $-34 \cdot 9$ \\
\hline June . . & . & . & . & . & . & . & . & $-35 \cdot 6$ & $35 \cdot 4$ & $33 \cdot 9$ & $34 \cdot 8$ & $33 \cdot 5$ & $35 \cdot 7$ & $36 \cdot 1$ & $34 \cdot 8$ & $34 \cdot 1$ & $33 \cdot 5$ & $35 \cdot 5$ & $-35 \cdot 9$ \\
\hline July . . & . & . & . & . & . & . & . & $-34 \cdot 6$ & $34 \cdot 4$ & $33 \cdot 6$ & $33 \cdot 6$ & $33 \cdot 0$ & $34 \cdot 2$ & $3 \pm \cdot 3$ & $35 \cdot 3$ & $34 \cdot 5$ & $33 \cdot 4$ & $33 \cdot 9$ & $-35 \cdot 4$ \\
\hline August. & . & . & . & . & . & . & . & $-34 \cdot 6$ & $35 \cdot 4$ & $36 \cdot 2$ & $39 \cdot 0$ & $38 \cdot 5$ & $38 \cdot 2$ & $38 \cdot 9$ & $37 \cdot 0$ & $38 \cdot 0$ & $38 \cdot 0$ & $38 \cdot 6$ & $-36 \cdot 3$ \\
\hline September & . & . & . & . & . & . & . & $-33 \cdot 8$ & $3 \pm \cdot 2$ & $34 \cdot 6$ & $39 \cdot 4$ & $37 \cdot 6$ & $36 \cdot 1$ & $3 \pm \cdot 5$ & $34 \cdot 1$ & $33 \cdot 5$ & $33 \cdot 9$ & $35 \cdot 0$ & $-33 \cdot 3$ \\
\hline Oetober. & . & . & . & . & . & . & . & $-28 \cdot 0$ & $26 \cdot 6$ & $28 \cdot 1$ & $27 \cdot 4$ & $24 \cdot 7$ & $22 \cdot 0$ & $22 \cdot 2$ & $23 \cdot 0$ & $22 \cdot 8$ & $23 \cdot 5$ & $26 \cdot 2$ & $-27 \cdot 9$ \\
\hline November & . & . & . & . & . & . & . & $+3 \cdot 6$ & $3 \cdot 9$ & $4 \cdot 0$ & $3 \cdot 4$ & $3 \cdot 9$ & $5 \cdot 1$ & $6 \cdot 3$ & $6 \cdot 4$ & $6 \cdot 1$ & $4 \cdot 9$ & $4 \cdot 0$ & $+3 \cdot 4$ \\
\hline Necember & - & - & - & . & . & . & - & $+10 \cdot 1$ & $9 \cdot 7$ & $10 \cdot 2$ & $9 \cdot 8$ & $10 \cdot 9$ & $12 \cdot 2$ & $11 \cdot 8$ & $13 \cdot 1$ & $13 \cdot 2$ & $12 \cdot 7$ & $11 \cdot 6$ & $+11 \cdot 2$ \\
\hline Yeal. & & . & • & . & • & . & • & $-17 \cdot 6$ & $17 \cdot 5$ & $17 \cdot 4$ & $18^{-2}$ & $16 \cdot 9$ & $16 \cdot 5$ & $16 \cdot 4$ & $15 \cdot 7$ & $15 \cdot 9$ & $16 \cdot 2$ & $17 \cdot 3$ & $-17 \cdot 5$ \\
\hline Mictsummer & & . & . & . & . & . & - & $+9 \cdot 2$ & $9 \cdot 1$ & $9 \cdot 9$ & $9 \cdot 4$ & $10 \cdot 9$ & $12 \cdot 3$ & $13 \cdot 0$ & $13 \cdot 7$ & $13 \cdot 7$ & $12 \cdot 9$ & $11 \cdot 4$ & $+10 \cdot 2$ \\
\hline Equinox. & . & . & . & . & . & . & . & $-25 \cdot 3$ & $2-1 \cdot 9$ & $25 \cdot 2$ & $26 \cdot 5$ & $25 \cdot 1$ & $24 \cdot 4$ & $23 \cdot 9$ & $23 \cdot 7$ & $23 \cdot 8$ & $24 \cdot 3$ & $25 \cdot 6$ & $-25 \cdot 3$ \\
\hline Stidwinter & . & . & . & . & . & . & . & $-35 \cdot 0$ & $34 \cdot 7$ & $34 \cdot 0$ & $34 \cdot 3$ & $33 \cdot 0$ & $34 \cdot 3$ & $35 \cdot 0$ & $34 \cdot 0$ & $34 \cdot 1$ & $34 \cdot 1$ & $34 \cdot 8$ & $-35 \cdot 4$ \\
\hline
\end{tabular}

During the six months October to March, the data in Table XI. show a very distinct diurnal inequality, which resembles on the whole the inequality of the ordinary temperatures in these months. The lowest minima are met with on the whole at or near the hours when the regular diurnal inequality of temperatnre has its minima.

For midsummer, Table XI. shows a nearly steady rise from a minimum at 2 to 4 a.m. to a maximum at t All the temperatures in any one line have the signs shown in the first and last columns, 
4 p.m., the range being $4^{\circ} \cdot 6$. In the corresponding inequality of ordinary temperature in Table $\mathrm{I}$. the minimum and maximum appear at 2 a.m. and 2 p.m. respeetively, the range being $3^{\circ} \cdot 4$.

At equinox, the most prominent feature in Table XI., as in Table I., is tho maximum in the early afternoon. The lowest value for the day occurs at 6 to 8 a.m. in Table XI. as eompared to 6 a.m. in Table I.; in both tables the values from 8 p.nl. to 8 a.m. are below the mean. The range is $2^{\circ} \cdot 8$ in Table XI. as against $1^{\circ} \cdot 4$ in Table I.

For midwinter, Tables XI. and I. both show lowest temperatures at or near midnight, and both show two fairly distinct maxima, one in the forenoon, the other in the afternoon. The differenee between the extreme values is $2^{\circ} \cdot 4$ in Table XI. as against $1^{\circ} \cdot 1$ in Table I.

Thus, on the whole, exceptionally low minima follow much the same law as ordinary minima. Both show a diurnal inequality resembling that for ordinary temperature, but possessing in most months a decidedly larger range. On the average the diurnal range is larger for the extreme than for the ordinary minima. 


\section{NOTES ON OPEN AIR TEMPERATURE, SCREEN TEMPERATURE, AND ON TERRESTRIAL RADIATION.}

BY

W. H. DINES, F.R.S.

Regutar 2-hour observations were commeneed on February 9, 1902, and were eontinued until February 15, 1904. $\Lambda$ few observations are nissing between February and Nareh, 1902, and only a few readings of the wet bulb were taken between May 6 and September 10, 1902. From this date until November, 1903, the observations are practieally eomplete.

On November 9, 1903, the night observations from 10 p.m. to 6 a.m. inelusive ceased, and after the middle of January, 1904, four observations only-at 8 a.m., noon, 4 p.m., and 8 p.m.-were taken.

The information with regard to the thermometers is not as full as might be desired, and there are some points whieh have required investigation. The thermometers were mereury ones, and on several oecasions the entry "Frozen" oeeurs. Many times the temperature given is below the freezing point of mereury; on some of these oeeasions the note "spirit thermometer" occurs, but temperatures of $-45^{\circ} \mathrm{F}$. and even lower are frequent. In some of them the wet bulb is stated to be frozen, but it is not uneommon to find both wet and dry bulb thermometers agreeing well, and both below $-40^{\circ} \mathrm{F}$. This question has been investigated at the National Physieal Laboratory at Bushy (see p. 15), and it has been proved that mereury may remain unfrozen and contraet at temperatures eonsiderably below its freczing point. The whole question of these temperatures and of the eorreetions of the thermometer has been investigated by Dr. CHrEe, and I have aeepted the temperatures with whieh he has supplied me, feeling quite eertain, in view of his experience and knowledge of the subjeet, that it would be superfluous for me to enter into any further investigation.

The period spent in the Antaretie region was just two years, and the annual temperature eurve in fig. 1 is obtained from the daily observations. The 2-hourly observations were not maintained during

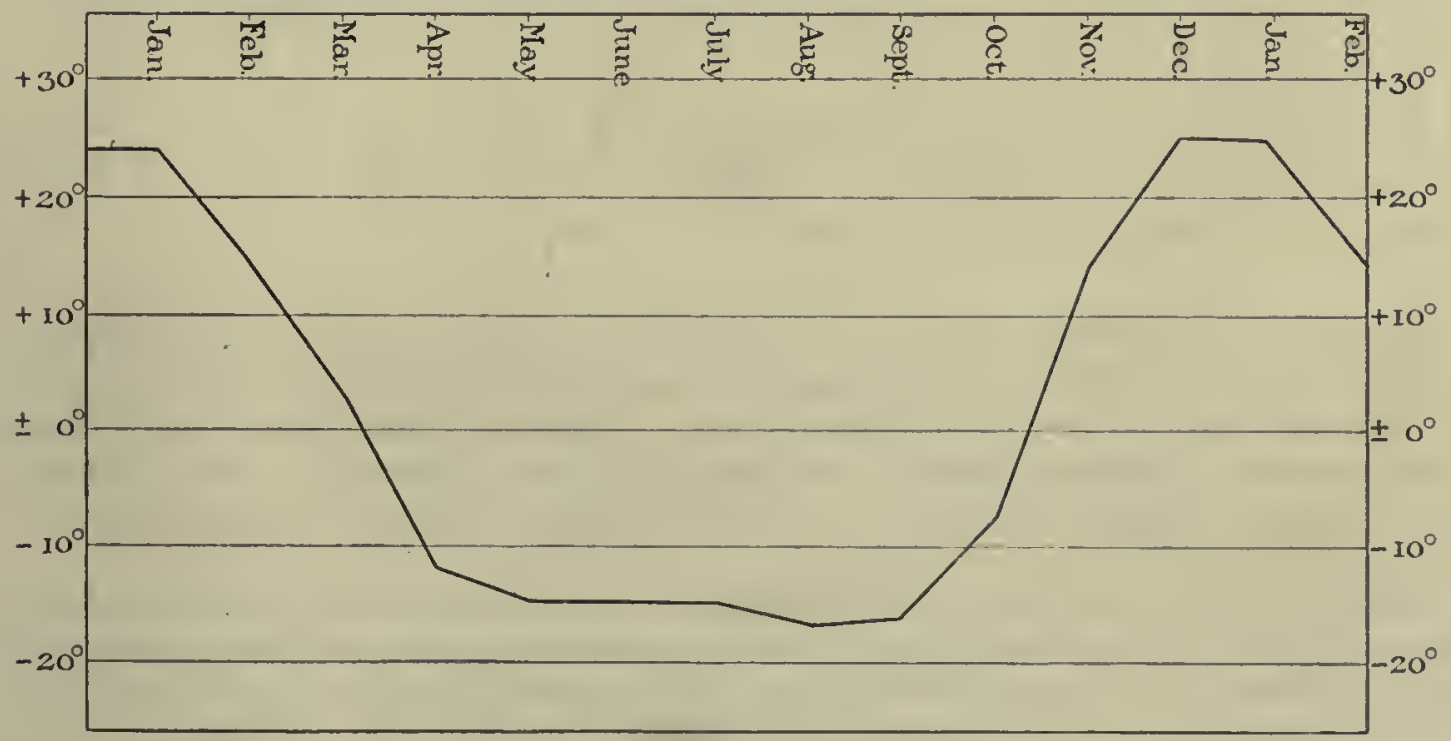

Fig. 1.

the last three months, the offieers and crew being fully oeeupied with the more important work of extrieating the ship from the iee. Two years are not enough to give a reliable curve of the monthly temperatures, and we have no knowledge whether the years in question were mild, average, or severe. 
The results are shown in tabular form in Table 1 . Columns $A$ and $B$ give the mean of the 2-hourly observations of the dry and wet bulbs. Column $\mathrm{C}$ gives the mean of the warmest day, and eolnmn $\mathrm{D}$ of the coldest day in eaeh montl.

TABle I.

\begin{tabular}{|c|c|c|c|c|c|c|c|c|}
\hline & & A. & B. & \multicolumn{2}{|c|}{ c. } & \multicolumn{2}{|c|}{ D. } & \\
\hline 1902 & & $\circ$ & - & $\circ$ & Day. & 。 & Day. & \\
\hline Februnry. & . & $15 \cdot 8$ & $14 \cdot 7$ & $23 \cdot 8$ & $28 \mathrm{th}_{1}$ & $6 \cdot 9$ & 22nd & From 9th. \\
\hline March : . & . & $7 \cdot 7$ & - & $22 \cdot 1$ & 1st & $-7 \cdot 2$ & 31st & \\
\hline April . . & . & $-7 \cdot 1$ & $-7 \cdot 2$ & $10 \cdot 8$ & 11 th & $-23 \cdot 1$ & $30: h$ & \\
\hline May. . & . & $-12 \cdot 9$ & - & $11 \cdot 3$ & $30 h_{2}$ & $-31 \cdot 4$ & $16 \mathrm{th}$ & \\
\hline June : . & . & $-16 \cdot 0$ & - & $3 \cdot 5$ & $28 \mathrm{th}_{\mathrm{t}}$ & $-28 \cdot 1$ & 24th & \\
\hline July : & . & $-8 \cdot 5$ & - & $10 \cdot 4$ & $18 \mathrm{th}_{1}$ & $-26 \cdot 8$ & 1 fth & \\
\hline Angust. & . & $-16 \cdot 6$ & - & $2 \cdot 1$ & $21 \mathrm{st}$ & $-36 \cdot 7$ & 7 th & \\
\hline September & : & $-12 \cdot 0$ & - & 10.5 & 28th & $-26 \cdot 4$ & 18th & \\
\hline October. & . & $-8 \cdot 5$ & $-8 \cdot 6$ & $9 \cdot 3$ & 31st & $-26 \cdot 2$ & joth & \\
\hline November. & $\therefore$ & $12 \cdot 0$ & $11 \cdot 3$ & $19 \cdot 6$ & 24 th & $6 \cdot 2$ & 19 th & \\
\hline Decenber. & : & $23 \cdot 1$ & $21 \cdot 6$ & $34 \cdot 2$ & 9 th & $7 \cdot 7$ & 2 nd & \\
\hline 1908 & . & & & & & & & \\
\hline January & . & $26 \cdot 1$ & $24 \cdot 6$ & $30 \cdot 3$ & 7th & $18 \cdot 1$ & 20 th & \\
\hline February. & . & $11 \cdot 2$ & $10 \cdot 0$ & $26 \cdot 6$ & 3 rd & $1 \cdot 3$ & 14th & \\
\hline Mareh. & : & -0.8 & $-1 \cdot 4$ & $19 \cdot 1$ & 5th & $-9 \cdot 9$ & 22 nd & \\
\hline April : : & : & $-16 \cdot 9$ & $-17 \cdot 0$ & -4.5 & 15 th $_{1}$ & $-32 \cdot 6$ & 27 th & \\
\hline May : & . & $-16 \cdot 3$ & - & 0.0 & 18 th & $*$ & 15 th & \\
\hline June & . & $-13 \cdot 8$ & $-13 \cdot 9$ & $11 \cdot 1$ & 11 th & $-32 \cdot 1$ & 23rd & \\
\hline July & . & $-21 \cdot 0$ & $-21 \cdot 3$ & $3 \cdot 6$ & 24 th & $-38 \cdot 0$ & 30th & \\
\hline August. . & : & $-16 \cdot 5$ & $-16 \cdot 7$ & 2.8 & 11 th & $*$ & 6th & \\
\hline September & . & $-18 \cdot 7$ & - & $6 \cdot 4$ & 26 th & $-40 \cdot 0$ & $20 t h$ & \\
\hline Oetober. & . & $-6 \cdot 8$ & $-7 \cdot 1$ & $7 \cdot 8$ & 27 th & $-23 \cdot 2$ & $3 r d$ & \\
\hline Norember. & . & $15 \cdot 0$ & $13 \cdot 9$ & $26 \cdot 2$ & $28 \mathrm{th}$ & $4 \cdot 9$ & 5th & \\
\hline Deeember. & . & $26 \cdot 2$ & $24 \cdot 3$ & $35 \cdot 2$ & $27 \mathrm{th}$ & $18 \cdot 4$ & 5th and 18th & \\
\hline 1904 & & & & & & & & \\
\hline $\begin{array}{l}\text { January } \\
\text { February }\end{array}$ & . & $\begin{array}{l}22 \cdot 2 \\
20 \cdot 6\end{array}$ & $\begin{array}{l}20 \cdot 9 \\
19 \cdot 2\end{array}$ & $\begin{array}{l}30 \cdot 3 \\
23 \cdot 6\end{array}$ & $\begin{array}{l}\text { 4th } \\
\text { 7th }\end{array}$ & $\begin{array}{l}14 \cdot 2 \\
15 \cdot 9\end{array}$ & $\begin{array}{l}\text { 22nd } \\
\text { 2nd }\end{array}$ & To 15 th. \\
\hline
\end{tabular}

* Below the freezing point of mercury.

MEAN for each Month.

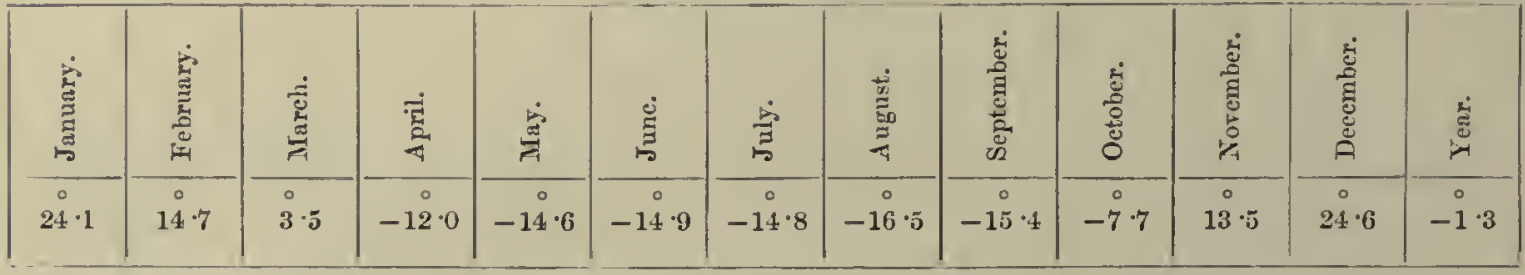

The low temperature of the summer and the uniform temperature from April to September are noteworthy. No great differenees of the corresponding months of each year are shown, exeepting in July, which had -21.0 in 1903 , and -8.5 in 1902 , but even this amount of differenee (12.5) may be surpassed by two eonsecutive Januarys in England. Two years is too short a period to give a reliable average, but the eonsisteney of the fignres throughout leads to the eonclnsion that the mean of the whole period $(-1 \cdot 3)$ is not far from the right valne.

It is curious that in each ease December should have the only days showing temperatnres above the freezing point and be the warmest montl. It would be more natural to find the highest temperature in January or Febmary, and possibly a longer series of observations might put January first. But the figures all show that solar raliation has the most important infuenee on the temperature. Indeed, the shape of the annual temperature enrve is very striking; the rise from September to midsummer, and the fall baek to April, is what might be expected, but the uniform temperature of the six months from $\Lambda$ pril to September is quite exceptional.

In the latitnde in which the ship was ice-loonnd for two years, namely $77^{\circ} 51^{\prime}$, the sun wonld be almost 
without direct influenee during that period, but in similar latitudes in the Aretic zone it is usual to find the lowest temperatures most deeidedly at the end of the long winter.

The observations of the "Discovery" show a fall of temperature to $-12^{\circ}$ in April, which should lrardly be called a winter month, a mean through the winter of about $-15^{\circ}$. Scptember, the month about equally distant on the other side from inidwinter, has $-154^{\circ}$. If these results had been olstainerl from one year's observations only, it would be reasomable to think that the autumn had been unusually severe and the spring unusually mild; but the same peculiarity is observed in both years, and it is further borne out by the mildness of June when compared with July.

The low mean temperature of the summer is also strange, and very difficult to explain. In lower southern latitudes, in the neighbourhood of Cape Horn, for example, the summer is very cold conpared with that of places with the same northern latitude, but in this ease the explanation is simple. 'The anuual ehange of temperature over the great oceans is small on aecount of the large thermal capacity of the water and the constant mixing together of the surface layers, and a small annual change produces a cool summer and a warm winter, as is notably the ease in England.

The insolation in the Antaretic in summer is greater than the insolation on any other part of the earth at any other time, this being the date at which the sun is nearest to the earth, and if temperature depended only on solar radiation we should have the highest terrestrial temperatures occurring in Deember in the neighbourhood of the South Pole. A large mass of iee prevents the temperature from rising much above the freezing point, because the air, being nearly pervious to radiation, takes its temperature ehiefly from the surface with which it is in contaet, and if that surfaee be ice or snow it camot be above $32^{\circ} \mathrm{F}$. But ice, exeept in its power of evaporation, is in no way more efficacious than any other roek in eheeking a rise of temperature up to its own melting point. Why then does not the mean summer temperature at least reaeh the freezing point, as indeed it does in the North Polar regions, where the insolation is less intense? During the two years that the "Discovery" was in the Antarctie eircle there were only five days with a mean temperature above the freezing point; the mean temperature of the warmest month was $26^{\circ} \cdot 2$, and the mean temperature of what in other localities would be ealled the six summer months (three eneh year), was $20^{\circ} .7$. In further proof of the lowness of the summer temperature, the fact that no rain ever fell may be cited, the precipitation always taking the form of snow.

These low summer temperatures in the Antaretie region are fully supported by the results of the Swedish expedition under Dr. OtTo Nordenskjold, and that of the "Southeru Cross" under Borcigrevink.

The following figures are taken from the 'Meteorologische Zeitschrift' for July, 1905, p. 320 :-

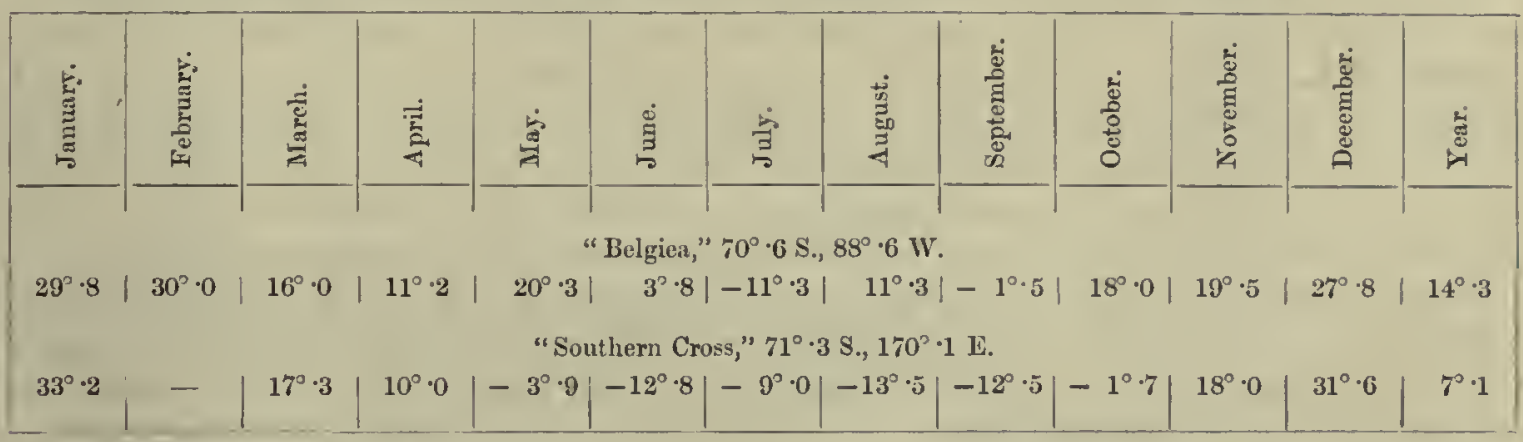

The Arctie temperatures experieneed by the "Fram" in a far higher latitude are shown below:-

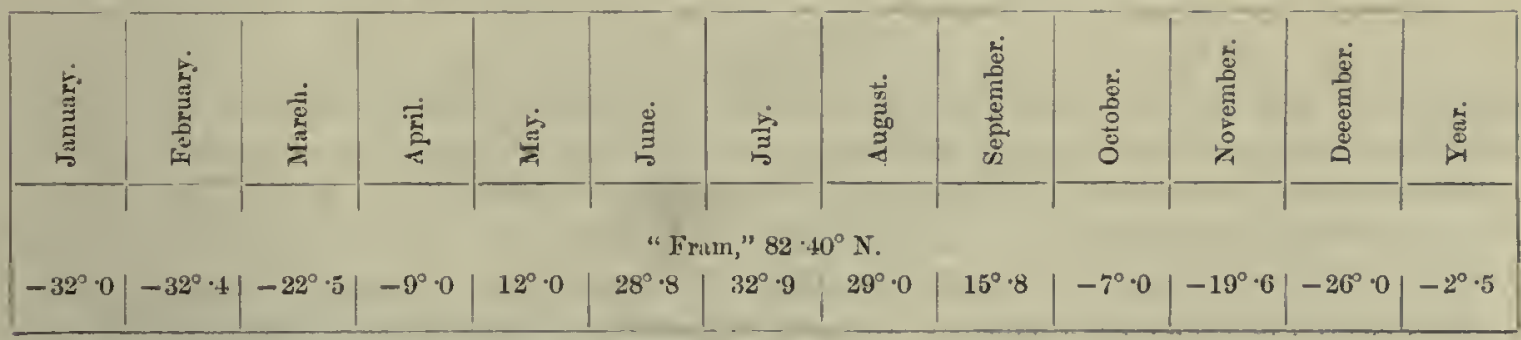

'Norwegian North Polar Expedition, 1893-96,' vol. vi. 
The following figures taken fron 'Davis' Elementary Meteorology' are of interest in this eonnection. Taking the amount of heat received from the sun in one day at the equinox on the equator as the unit, the amount reeeived on December 21 at the South Pole is 1.284. This is greater than the amount reeeived at the North Pole on June 21 st by 0.082 , owing to the greater proximity of the sun. So far as'we know, in the North Polar regions the summer temperature rises to the freezing point, and a reason why it should not rise unuch beyond it has already been given, but in the Antaretic the summer temperature is far below freezing. The renson must, I think, be sought for in the dryness of the air. The question is discussed elsewhere; it will suffice here to say that the wet bulb readings indicate a relative humiclity that may compare favourably with some of the dryest regions of the earth. 'There can, I think, be no doubt that the presence of earbonic acid and aqueous vapour in the air tends to raise the mean annual temperature of the earth. Indeed, a ehange in the amount of carbonic acid has been suggested as a likely cause of the large alternations of temperatıre that geology shows to have oecurrerl. This question has been very fully discussed by Dr. NiLs EkHoL, ('Quarterly Journal of the Royal Meteorological Society, 'Vol. XXVII, p. 1). The amount of carbonie acid is presumahly much the same in all parts of the earth that are not near some large cily, hut the Antaretic regions probably possess the minimum of aqueous vapour. There wonld, therefore, be very little cheek to the free radiation of dark heat into space.

It would almost appear as though this must be the cause of the low temperature, for one cannot suppose that there is any colder part in the neighbourhood from which cold winds can blow, the whole region, except where there is open water, being subject to the same conditions, namely, - a snow-covered surface and intense insolation. Neither can it be supposed that the sun has no time to warm the surface of the snow and ice up to the freezing point after the winter's cold, since the months of March to April on the one side and October to November on the other show changes of nearly $20^{\circ} \mathrm{F}$.

There appears to be no source, so to speak, fron which the cold of July can be supplied, save that of radiation from the snow, hut if the radiation of heat into space is active, the solar radiation should be equally so, and the more capable of raising the temperature to $32^{\circ} \mathrm{F}$. and then supplying heat to melt the ice. That the solar insolation is extremely powerful was proved by the fact noted by the members of the expedition, that as soon as a slope of rock became bare, the sun melted the ice and snow near, and a pond was formed below it. This proves what was well known before, that snow is eapable of reflecting the solar heat, but unless it be an exception to the common rule, which is hardly credible, this power of reflection implies a low power of radiation. The thermal capacity of the atmosphere as a whole is small, being equivalent to a depth of about 8 feet of water, but it is useless to look for an explanation in this direction, because similar conditions hold in the Aretic regions and do not produce the same effect. There is one other point which must he mentioned-not that it is a possible source of cold, hut because it has sometimes in the past been fallaciously put forward as such-and that is the presence of a current of air descending from the upper and colder layers of the atmosphere. Such a current must, of course, in virtue of its dynanie warming as it experiences a higher pressure, be a source of heat and not of cold, and were it not that old fallacies die hard it would not he necessary to refer to it.

Whatever the cause of this low summer temperature may be, the fact is interesting and important, since it throws some light on the question of the glacial epochs of the northern hemisphere, and shows that an intense solar insolation may be so modified by other circumstances that it does not produce the effects which one would naturally expeet.

From the fact that for long periods in each winter the mercury never rose much albove $0^{\circ} \mathrm{F}$, and yet winds from an equatorial point were frequent, it may be inferred that there was very little open water in the neighbourhood. The alssence of fogs leads to the same conclusion. Lower mean temperatures from July to September might therefore have been expected, since in the northern hemisphere, in much lower latitudes, temperatures of $-30^{\circ} \mathrm{F}$. and less are not uncoinmon, and the mean temperature of the southern hemisphere, as a whole, is less than that of the northern. Perhaps an explanation may be sought for in the more active circulation of the southern hemisphere. As soon as the sun has nearly reached the horizon, the radiation of heat into space, intensified by the prevailing dryness, has full play, and sinee snow is a bad conduetor of heat, the only source of heat is the supply by eonvection through the atmosphere. Owing to the abscuce of land, the teinperature in the belt 
between $40^{\circ}$ and $60^{\circ} \mathrm{S}$. Lat. is certainly higher in the winter than in the corresponding part of the northern hemisphere, and this may tend to prevent the oceurrenee of such low temperatures at the South as at the North Pole. Possibly, however, a greater supply of heat from the tropics inay be carried by the upper eurrents to the south, but we are as yet so entirely ignorant of the movement of the layers of air which lie above the cirrus, that is to say roughly of half the atmosphere, that speenlation on the subject is useless.

\section{Daily Temperature Curves.}

Professor Monn, discussing the results obtained by the voyage of the "Fram," finds during the dark months in the Arctic cirele a daily variation of temperature. It is of eourse obvious that, sinee the sun is far below the horizon, the effeet must be an indirect one, and he considers that it is due to a diurnal clange in the direction of the wind. Dr. Curee has discussed the question with referenee to the observations made by the "Discovery," but the matter is one of eonsiderable interest, and hence I trust I may be pardoned for going over somewhat the same ground, and I have drawn the curves of fig. 2.

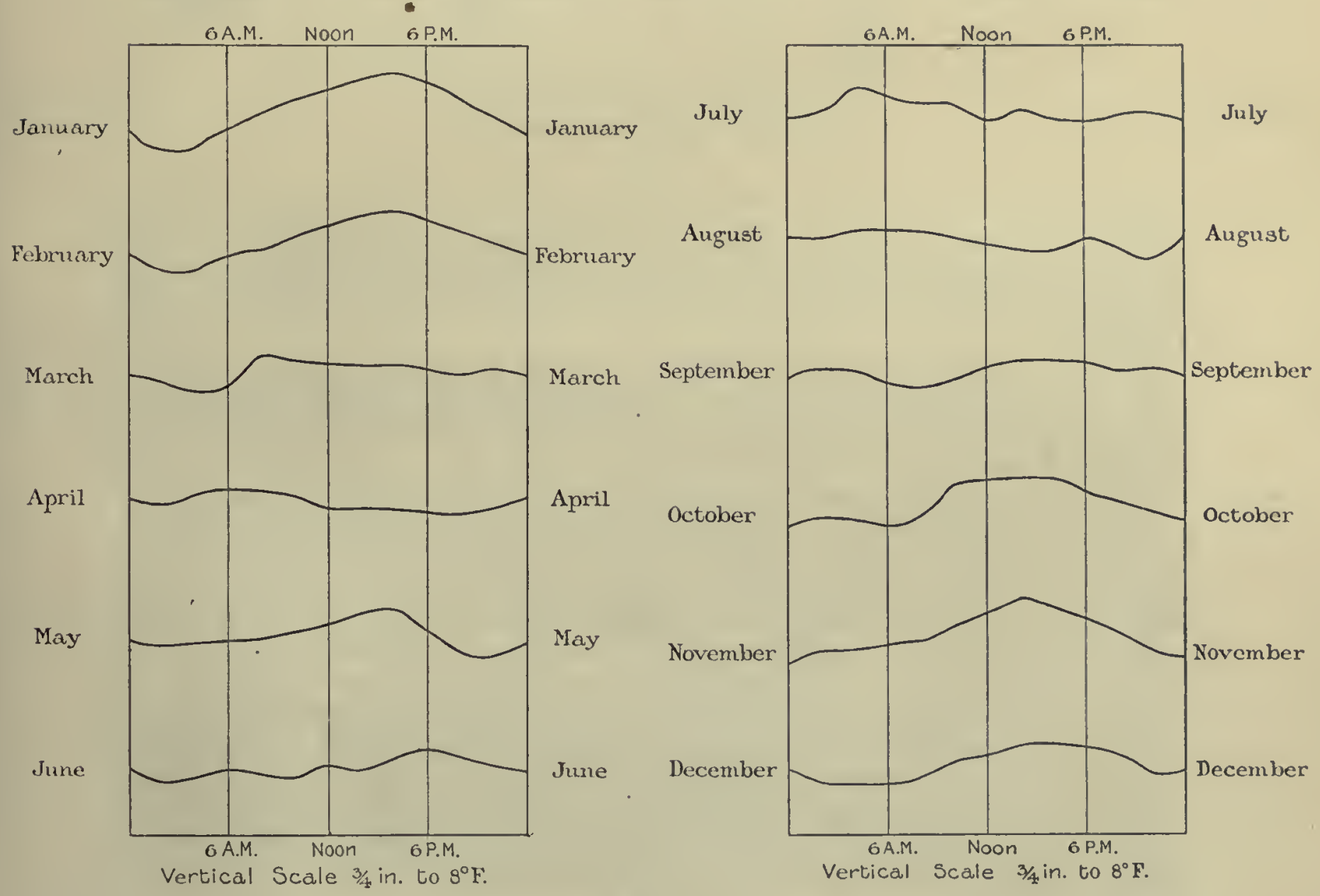

Fig. 2.

The amount of material hardly suflices to make these eurves trustwortly, especially when the very rapid changes of temperature which the figures, and more partieularly the self-recording instruments, show are taken into account. This is fairly obvious when we eompare the daily course of the temperature in Angust, 1902 , with a variation of barely $1^{\circ} \mathrm{F}$., with that of August, 1903 , showing a differenee of $3^{\circ} \mathrm{F}$. between 8 a.m. and 8 p.m. In the sunny months the oscillation is perfectly clear and distinct, although the amplitude is small, being less than $5^{\circ} \mathrm{F}$. for January, 1902 , the month in which it is most marked.

The warmest time in summer appears to be about 4 p.m., but the number of observations only allows

$\begin{array}{lll}3 \circ & 2\end{array}$ 
of a rongh approximation to either the time of the maximum or minimum or to the amplitude. The rise during the day may be traced from Oetober to February, perhaps to March.

The mean obtaincd from combining the eight dark months spent by the "Diseovery" in the Antaretic regions is shown in the diagram, fig. 3.

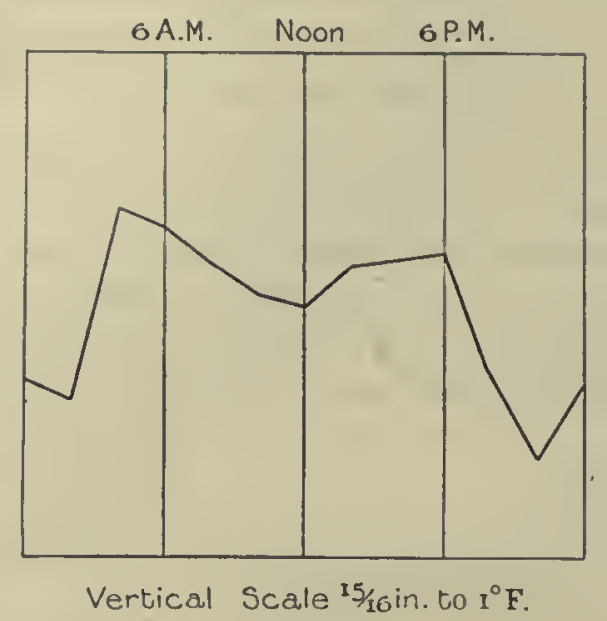

Fig. 3.

The maximum occurs at 4 a.m., the minimum at 10 p.m., but there is a secondary minimum at noon. The difference between the highest and lowest temperatures is considerable, amounting to about $l^{\circ} \mathrm{F}$., but to what extent this curve is indebted to chance values and how far it represents a real phenomenon, it is difficult to say.

It is hard to believe that the rapid rise from 2 to $4 \mathrm{a} . \mathrm{m}$. has any physical eause, but, on the other hand, one ean hardly doubt that the temperature is generally lower in the evening, or, since the word can have no meaning in a place and time where the sun never appears, perbaps we shonld say lower from 8 p.m. to 2 a.m. than from 4 a.m. to 4 p.m.

Every change in the height of the barometer produces a change in the temperature, for dynamic heating or cooling of the air is produced by a change of pressure, no matter how that change is brought about. We are too apt to consider a rising or sinking air current as the ouly uatural cause of dynamic cooling or heating, but, of conrse, the passage of air horizontally between two places under different pressure is equally efficacious. The daily oscillation of the barometer, whether we consider the 12-or the 24-honr period, inevitably produces a corresponding change in the temperature, although the eonverse of this is not necessarily tme. It is of no eonsequenee for this result how the barometric oscillation may be produced, ceteris paribus, a rise of temperature of $0^{\circ} \cdot 5 \mathrm{~F}$. accompanies a rise of 0.10 inch of pressure, and even though the actual rise may not occur, since some other more powerful eatse may be producing a fall of temperature, yet it is safe to say that, given the barometric rise, the temperature must be higher than it would have been had no increase of barometric pressure occurred. Possibly, therefore, the 12-hour period of the temperature may be a consequenee rather than a cause of the pressure variation, but in this particular instanee I doubt the existence of any real temperature variation with the daily or half-daily period during the winter.

Observations with a black bnlb thermometer in vacuo are, unfortunately, not of great scientific value, for it appears to be impossible to make two thermometers which will read alike, and it is most likely that the reading of the same thermometer will vary with age. Under these circumstances, readings are not comparable one with another. Indeed, this is evident from the above short table, for the size of the bulb has in general made a difference of $20^{\circ} \mathrm{K}$. in the monthly averages. But notwithstanding this, the series of observations commencing on September 8 and ending in the beginning of $A$ pril, 1903, are well worth all the tronble expended on taking them, for they show the great permeability of the air to radiation. 
Radition Thermometers.

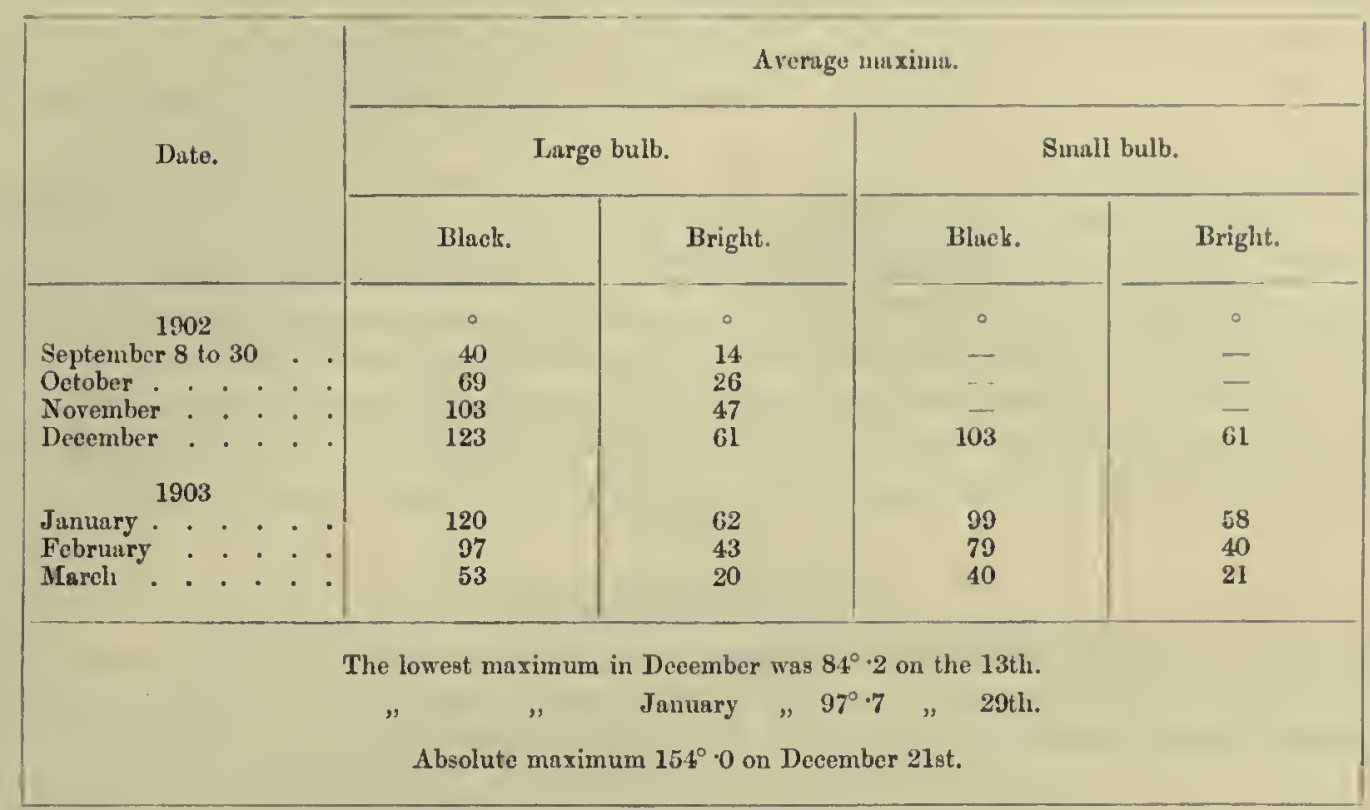

The actual maxima are in no way exceptional : a glance at the Climatological Tables published monthly by Dr. Mut. in Symoxs' 'Meteorological Magazine' will show that higher values are by no means uncommon in various parts of the world, but if instead of taking the actual reading we consider the difference between the maximum temperature of the day and the maximum recorded by the black bulb thermometer, we find an amount that it would be very hard to match. Thus on December 21, the day which gives the absolute maximum, $154^{\circ} \cdot 0 \mathrm{~F}$., the mean temperature only reached $17^{\circ} \cdot 8$, and the maximum $24^{\circ} \cdot 2$, and during December, 1902 , when the average was $123^{\circ}$, the mean temperature of the air was only $23^{\circ} \cdot 1$, the difference for 31 consecutive days reaching an averige of just $100^{\circ} \mathrm{F}$. The following montl, January, 1903, has not quite so great an average difference, but on one day only during this month did the radiation thermometer fail to reach a temperature of $100^{\circ} \mathrm{F}$.

It has been previously stated that the insolation during December and January at the South Pole is very great, but that is because the sun is continuously above the horizon. If the amount of heat received on a unit of surface during the midday hour be taken instead of the amount received during the 24 hours-and it is this which influences the maxima readings-and if this amount be compared with the corresponding anount received at the equator, the comparison, in so far as magnitude is concerned, is entirely in favour of the equatorial region. At the South Pole the insolation on December 21 is 0.4 of that of a place where the sum is in the zenith; at the Winter Quarters of the "Discovery" at midday it would be 0.58 , a little over half. But the solar rays have also to pass through nearly twice the quantity of air, and were this air at all impervious, they would lose a considerable part of their heating effect. It follows that these black bulb readings show that the air of the Antarctic regions offers very little obstacle to solar radiation, and one can hardly doubt that the cause of this permeability must be the absence of aqueous vapour. Inasmuch, too, as the radiation maxima are high in the summer without exception, we must assume that no thick sheet of clouds ever remained for long over this station. It seems likely, therefore, that the Antaretic anticyclone is very active, and that there is a general and extensive settling down of air. Otherwise the dryness could not be maintained, for, the whole surface being ice, snow, or water, and therefore capable of giving off aqueous vapour, the air would of necessity soon be filled with moisture, since evaporation from the snow would soon saturate the surface layers and the vapour would diffuse upwards.

\section{Cape Armitage.}

In addition to the regular observations made at the ship, observations of the tenperature were manle at Cape Armitage once at least on most days in the winter. No precise time was adhered to, but the 
observations were mostly in the day, and often were taken several times in the same day. On each oecasion the minimum sinee the last visit was observed.

The figures do $110 t$ suffiee to show the mean temperature in the light season, or, strietly, in the dark season, though in the absence of the sun a chanee visit once a day at various times should give a fairly good average.

A glance at the figures shows at onee very great discrepancies between the temperatures of the two places, both at sea level and not far apart, and it seems certain that the differenees are not due to instrumental errors or to eareless observations. Differences of even $25^{\circ} \mathrm{F}$. between the two places oceur at times, and differences of from $10^{\circ}$ to $20^{\circ}$ in the recorded minimum are quite common in the winter. The observations during the summer are very few, and some of those there are have a note attached ' in the sun,' but during the summer there does not seem to have been any striking difference. The winter differences invariably give the lower temperature to Gape Armitage, and there is little doubt that the mean at the ship during the dark months was quite $10^{\circ} \mathrm{F}$. above the mean at Cape Armitage.

\section{High-Level Temperatures.}

Some forty temperature observations were made at an elevation of $960 \mathrm{ft}$., about two miles to the eastward of the ship, during the winter of 1902. A comparison of these values with those observed at the "Discovery" is given below :-

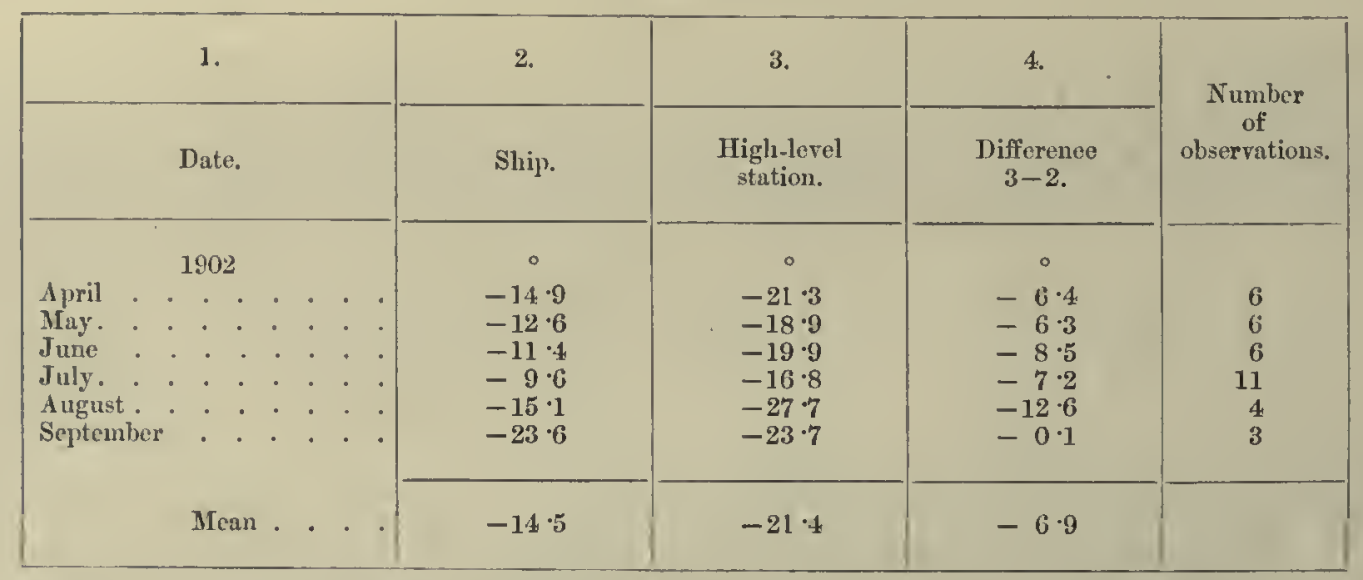

In forming the above table, in eases where the temperature observation at the high-level station was not simultaneous with that at the ship the value at the ship has been obtained by interpolation from the nearest readings.

Even allowing for the ordinary fall of temperature with height, namely, $3^{\circ} \mathrm{F}$. for the $960 \mathrm{ft}$. nearly, it will be seen that the temperature at the ship is much above that at the ligh-level station. The only exeeption is September, but this is not of much importance, since it depends on three observations only.

The differences are not so striking as at Cape Armitage, but they also point to a high local temperature at the position of the ship. I ean give no certain explanation of these differences, but can only state that they do not seem to be due to any peeuliarity in the weather at the times the observations were made. The proper form of sereen was not used at Cape Armitage or on the hill, but the thermometers seem to have been at much the same height. The differences are roughly proportional to the depth of the temperature below the freezing point. The only suggestion $\mathrm{I}$ can make is that the thermometer screen at the "Discovery" was placed too near the tide craek, and that the temperatures are unduly high on account of the influence of the sea water in the erack. The thermometer at Cape Armitage was quite open to the sky during the winter, but I do not think this will account for the difference, provided it was about the same height above the surface of the snow. 


\section{NOTES ON THE READINGS OF THE ASPIRATION PSYCHROMETER, AND OF THE DRY AND WET BULB THERMOMETERS, AND ON THE OBSERVATIONS OF EVAPORATION AND PRECIPITATION, AND OF THE EVAPORATION OF ICE.}

BY

W. H. DINES, F.R.S.

A Cossiderable number of observations relating to humidity, precipitation and evaporation were made during the two years that the "Discovery" remained in the Antarctic regions.

These eonsist of two-hourly readings of the dry and wet bulb thermometers, of a large number of independent observations of the humidity taken with an Assmass's aspirator, of measurements of snowfall (there was 110 rain) in a rain-gauge, of measurements of the depth of snow by means of stakes driven in to show the change of level, of evaporation from the surface of a frozen pond, from lumps of ice, and from ice exposed in shallow metal trays.

The value of the observations would have been greatly increased if more particulars had been given, and one cannot help feeling regret that the opportunity to revise the humidity tables at low temperatures could not liave been utilised. On the other hand, the zeal shown, and the trouble taken by the officers of the ship in making the observations, deserves the highest praise.

The humidity observations consist of the readings of the dry and wet bulbs taken at the regular twohourly intervals. Gaups in the series occur, most noticeably during the first winter, the observations being alsent for the period Nay to September, 1902. During the second winter, many observations were made with an Assmann aspirator. Great care seems to have been bestowed on these, and they forn a very useful cheeck on the dry and wet bulb readings. The difficulty of obtaining accurate values of the humidity when the temperature is below the freezing-point is well known even in our mild climate, and it must be greatly inereased when the temperature approaches the freezing-point of mercury. The general opinion of meteorologists seems to be that the tables by which the relative hunidity is obtained from the wet and dry bulb readings are open to doubt at very low temperatures and very low values of the humidity, and hence it hardly seems worth while calculating the values of the humidity from these readings. It is, however, plain, from a study of the figures, that values of the humidity of less than 50 per cent. occur frequently, and, quite apart from any doubt of the tables, there are many instances of extremely low values. Hence one may say that whatever else the climate may be, it is certainly dry.

The observations on precipitation were made with a rain-gauge, but there are no details given as to its size and position, or the height of its rim above the surface of the snow. Precise observations are utterly impossible in such a climate, indeed the observers state that during the blizzards they did not and could not know whether the snow was aetually falling from the clouds, or whether it was merely drift from the surface of the snow. Often, too, the gauge had to be dug out of the drift. On caln days the amount of snow was measured, but what proportion the fall on sucl days bears to the total fall it is impossible to say. The whole precipitation took the form of snow, there being no record of rain, during the two years that the "Discovery" remained at her Winter Quarters.

An attempt was made, also, to measure, by means of stakes, the depth of snow that fell, the graduations on the stakes showing the level of the upper surface of the snow. 
Tarle I.--Snow Gauges (Centimetres).

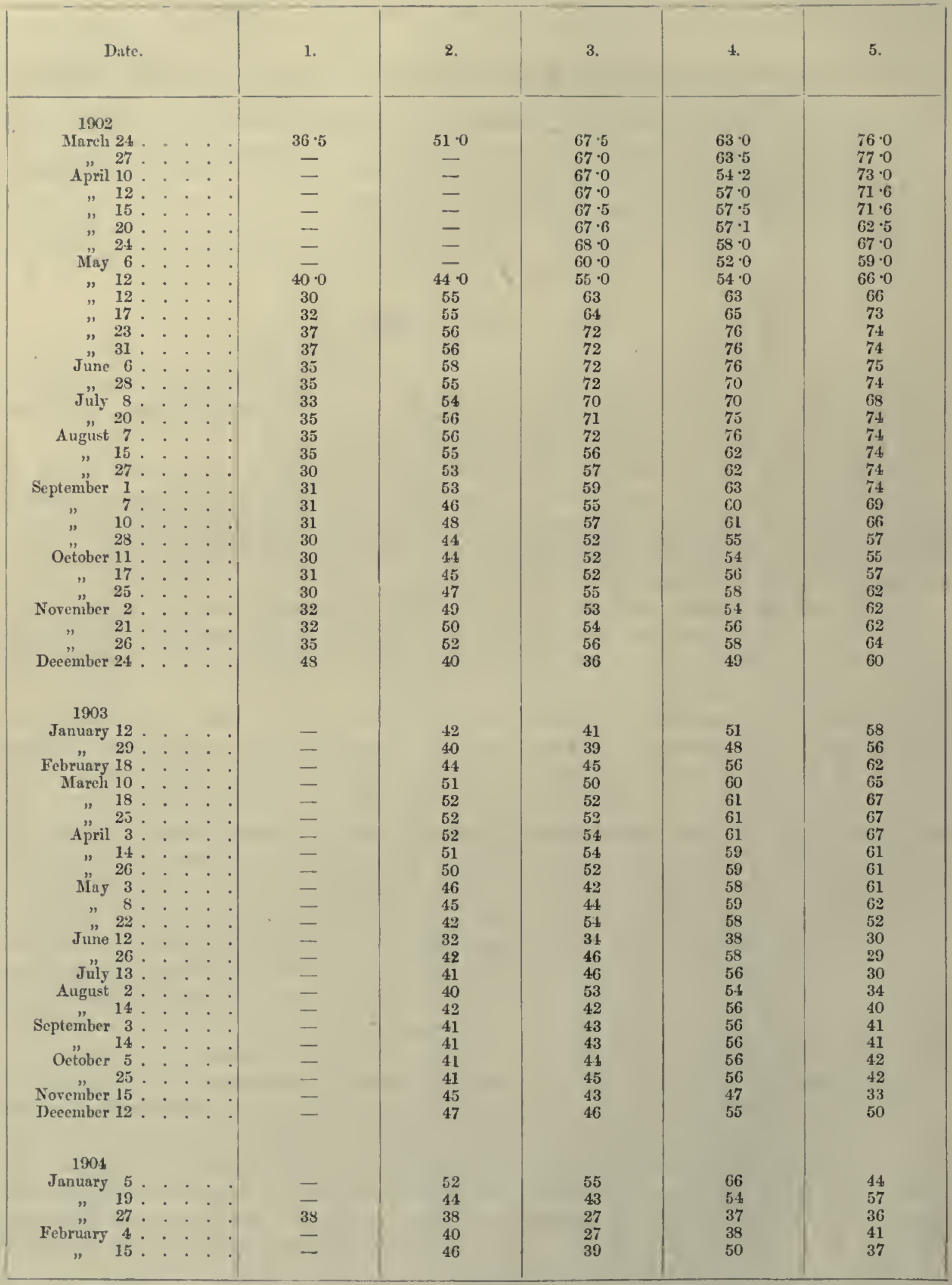

The distanees are measured from the top of stakes driven rertieally into the snow on the north side of Obserration Hill, Boft. abore mean sea lerel. 
It will be seen that the change of level between March 24, 1902, and February 15, 1904, the time during which the ohservations were in progress, was small. The observer writes: "On the whole, the snow appears to have risen, but this is hardly the general result, for the rise was a ridge, the footpath, between the successive stakes." One must conclucle from this that the loeality was more windswept, on the average, than the surrounding district, for the eontinual use of a definite track in soft material leads to a sinking, and not a rising, of the track, and the only way to account for the "ridge" is to suppose that the freshly fallen, or drifted, snow was consolidated by the feet of those passing over it and, in consequence, was less liable to be blown off. The evidence tends to show that the amount of precipitation during the year was small, for if many henvy falls had occurred they could hardly have failed to have considerably altered the level at the stakes from day to day, and no snch alteration seemed to have occurred.

It may also be noted that practically no thawing of the snow occirs, except here and there where the sun shines on a neighbouring exposed surface of rock, and, in general, the summer temperature is so low that the only appreciable loss is by evaporation.

The evaporation experiments give very interesting results. Sliallow dishes were filled with water, whieh was allowed to freeze, and then the dislı and ice were weighed day by day and the difference between two consecutive weights gave the loss by evaporation. Three dishes were used, two giving an area of $12 \mathrm{sq}$. inches ( $3 \mathrm{in}$. by $4 \mathrm{in}$.), and the third an area of $24 \mathrm{sq}$. inches. This latter was blown away before it had been very long in use and was not reinstated. As might be anticipated, the loss of weight from the two similar dishes was not invariably the same, but, on the whole, the agreement was very fair. The evaporation from the third and larger dish was less than double that from the smaller, this being in aecordance with the result found in England when the evaporation from small surfaces of water of different arens is observed.

The observations were discontinued in the summer, because when any of the ice was melted it was difficult to avoid losing some of the water before or during the weighing.

The following table is formed from the mean of the two 4 -in. by 3 -in. dishes. In some eases one only was available, and in such cases the one was used. The figures do not give strictly the evaporation for the month, partly because here and there a day's record is missing, partly because the observations were taken once each day, generally between Noon and 4 p.m., and thus the record for June, say, omits 12 or 14 hours at the beginning of June and includes a similar period in July.

Occasionally a small increase of weight was noted. These increases havo not been subtracted from the total.

The losses given in the observer's book are in grammes; these have been put into losses of thickrress, expressed in inches by using the factor 0.051 .

TABLE II.-Monthly Evaporation.

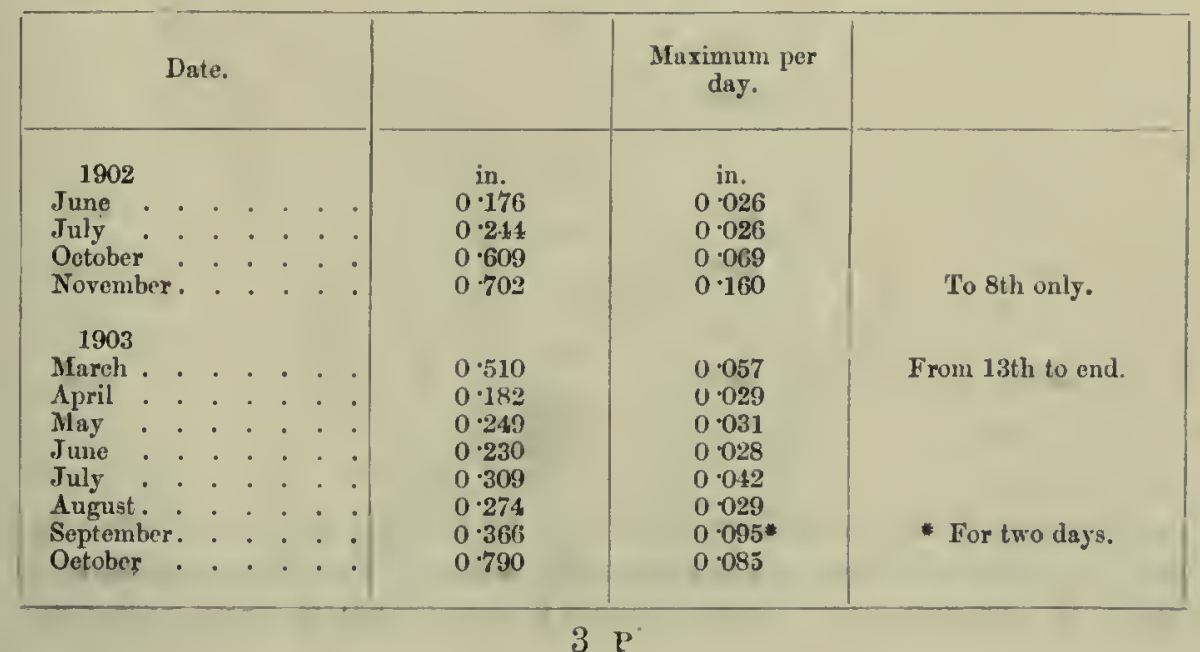


It is a pity that there are no figures available for the summer. The values for the winter, when we take into aceount the lowness of the temperature, seem to me very striking. In 'British Rainfall for 1905,' p. 41, a table is given showing the eraporation at Camden Square froin 1885 to 1905 . The monthly mean for the winter is there slown to be $0.14 \mathrm{in}$. The eorresponding temperature mean is elose to $40^{\circ} \mathrm{F}$. The neall of the five winter months in the Antaretie is 0.25 , which is nearly donble that for Camden Square, Loudon, and this exeess of evaporation oeeurs notwithstanding the rery low temperature. Many factors are required to make the eonditions favourable for evaporation, but undoubtedly the eapaeity of the air for taking up moisture is the most important. A low relative humidity and a high temperature are neeessary to ensure a high eapaeity, and how very important the temperature must le is shown by the following data. Equal quantities of perfeetly dry air at $0^{\circ} \mathrm{F}$, of air with a humidity of 83 per eent. at $40^{\circ} \mathrm{F}$., and with a humidity of 91 per cent. at $60^{\circ} \mathrm{F}$. are eapable of taking up the same approximate amount of water before beeoming saturated. This explains, to a great extent, how it is that though in England the summer and winter rainfall are mueh the same, yet in summer the ground is generally dry and in winter generally wet; but it also shows how very low the hunidity of the air at the Winter Quarters of the "Diseovery" must, in general, have been in order that, notwithstanding the prevalenee of temperatures far below zero, it might be eapable of absorbing sueh eomparatively large amounts of water vapour. This is the more striking when it is remembered that the whole surface was eovered with iee and snow, and was therefore eapable of yielding an unlimited supply. The mystery is how, in the presenee of sueh an extensive evaporating surfaee, any very dry air ean be found.

Observations on evaporation were also made by driving nails into the surface of a frozen pond, and noting the differenee of level between the surface and the top of the nails. The gradually inereasing

TABLE III.-Measured Evaporation.

Nails driven into frozen pond. Measurement made from iee to top of nail.

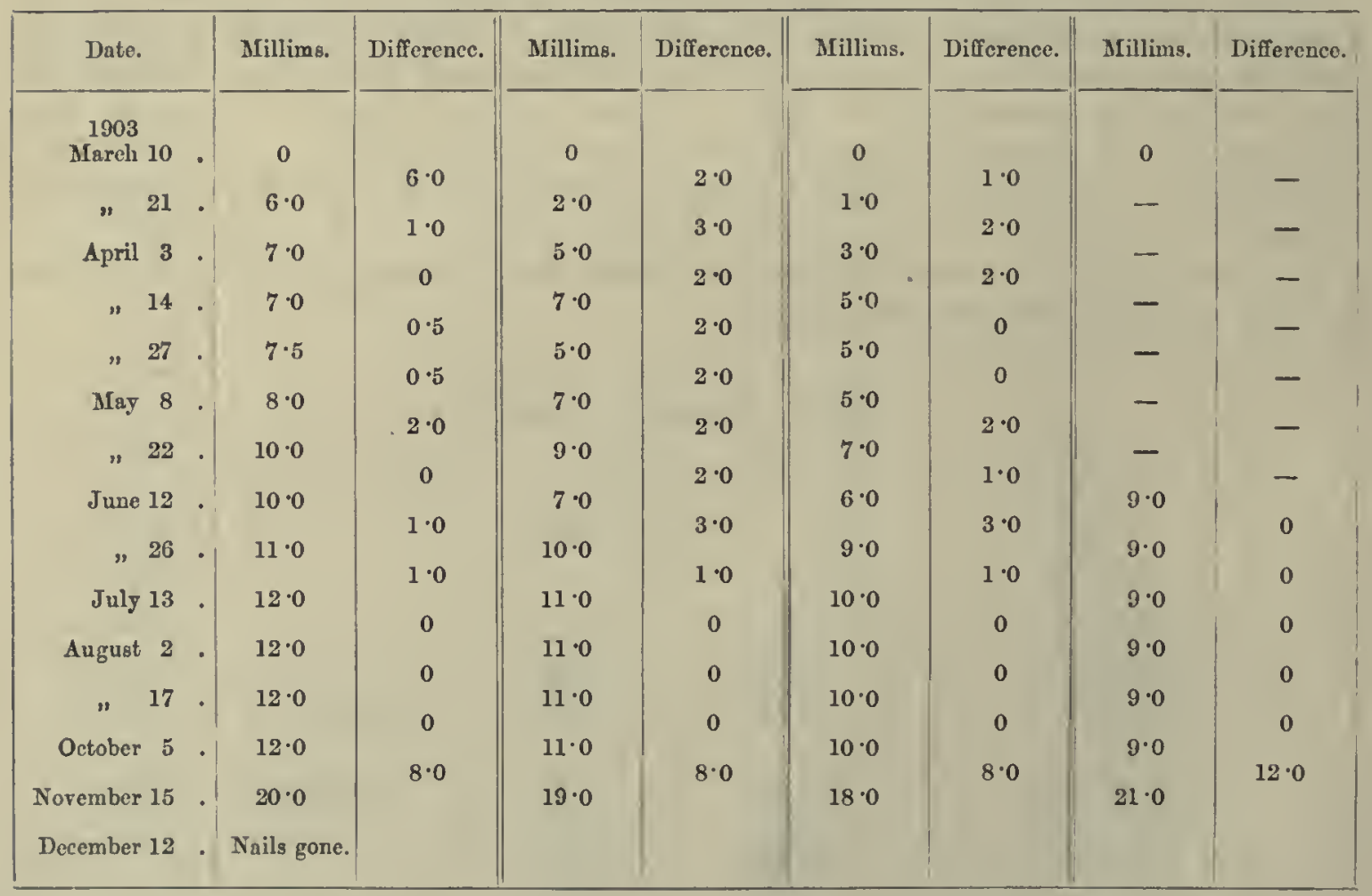

differences are attributed to the gradual evaporation of the ice surrounding the nails. The results are given in Table III. It will be seen that the figures do not agree among thenselves or with the other results. There is no information as to what means were taken to keep the surface free from snow. Also 
it is problenatical to what extent the head of a nail driven into ice may be considered as a permanent level relatively to the ice. Most certainly with tho prevalcnce of sunshine, and a temperature not too far below the freezing point, it would not be pernianent, for any small black object which does not flont on water sinks rippidly into ico under the influence of sunshine, even when the temperature is below the freezing point, the ice thawing underneath under the influcnce of radiation, but in the actual Antaretic winter this canse could not como into play.

The general evidence scems to show that the air was very dry. It must not be forgotten that for some unexplained cause the temperature at the "Discovery" was higher than at other places in the immediate neighbourhood; and greater dryness generally accompanies greater heat, but, notwithstanding this, it may, I think, be safely asserted that a low relative humidity prevailed generally in the district, and this conclusion is supported by the fact of the intense solar radiation shown by the temperatures of the blaek bull, in racuo. In the free atmosphere the humidity is an unfailing test of the vertical circulation, a low humidity indicating a descending current and a high humidity an ascending one. Indeed, were it not for the formation of rain and the accompanying extraction of water vaponr the laws of diffusion of gases must lead to the nearly complete saturation of the whole atmosphere. The humidity of the lower strata must he partly dependent on the dryness or wetness of the earth's surface, but not entirely, for it is notorious that the sea coast of England in the winter is dryer than the inland districts. Also the great deserts of the earth are situated in the anticyclonic belts, and are the consequence rather than the cause of the dry air that lies over them. It seems likely, therefore, that the Antarctic region, or at least that part which lies in the neighbourhood of Ross' Bay, is an anticyclonic region, and that there is a steady settling down of air. If this point can be establisher for the Antarctic regions as a whole, it is of great inportance in regard to the question of the general circulation of the atmosphere.

There is one other matter of grent interest in eonnection with these observations, and that is the formation of the Great Barrier. The temperature observations show that the loss of ice or snow from the surface by thawing is a negligible one, and the question is simply whether the snowfall exceeds the evaporation, or vice versâ. If the snowfall be the greater the Barrier should be increasing in thickncss. Unfortunately, precise observations on precipitation are impossible in such a climate, and in any case two years would not suffice to give a reliable average either of the snowfall or the evaporation, even if we had precise and exact observations of each. Hence nothing more than a gucss ean be made, but the evaporation, as judged by the winter observations, scems sufficiently large to more than balance the precipitation, and thus lead to a gradual dccrease in the amount of ice and snow. 


\title{
XI. NOTES ON THE OBSERVATIONS OF BAROMETRIC PRESSURE.
}

\author{
BY
}

\section{R. H. CURTIS.}

\section{Tile Yearly Mean Pressure.}

Notwithstanding the great interest which attaches to the clistribution of barometric pressure over the South Polar regions, our knowledge of the subject has hitherto been to a great extent hypothetical owing to the fewness of the observations we possessed. It is only quite recently that observations covering an entire year have become available. All those previously received were made by navigators (in most cases exploring the region) who passed quickly from point to point, so that their observations are a good deal seattered both as regards locality and time, although in every case they refer exclusively to the summer months of the year, in which alone the Antarctic seas are open to navigation.

It was not until the year 1898-9 that a series of observations embracing a complcte year was made within the Antarctic circle, and the whole of the information we now possess, relative to the sequence of climate in the Antarctic throughout the year, from that time up to the return of the "Discovery," is set out in Table I. As regards the stations at which these four series of observations were made, it should be

TABuE I.-Mean Height of the Barometer reduced to Sea Level, to $32^{\circ} \mathrm{F}$, and to Latitude $45^{\circ}$.

\begin{tabular}{|c|c|c|c|c|}
\hline Ship or place. & Date. & Latitude. & Longitude. & $\begin{array}{c}\text { Mean } \\
\text { annual } \\
\text { pressure. }\end{array}$ \\
\hline $\begin{array}{l}\text { "Discovery" } \\
\text { (Ross Island) }\end{array}$ & $\begin{array}{l}\text { February, 1902, to January, } 1903 \\
\text { February, 1903, to January, } 1904, \\
\text { Two years, February, 1902, to Januar, } 1904\end{array}$ & $7751 \mathrm{~s}$. & 16645 E. $\left\{\begin{array}{l}\text {. } \\
\text {. }\end{array}\right.$ & $\begin{array}{l}\text { inches } \\
29 \cdot 352 \\
29 \cdot 228 \\
29 \cdot 290\end{array}$ \\
\hline $\begin{array}{l}\text { Cape Adare } \\
\text { (Victoria Land) }\end{array}$ & February, 1899, to January, 1900 & $7118 \mathrm{~S}$. & $1709 \mathrm{E}$. & $29 \cdot 134$ \\
\hline $\begin{array}{c}\text { "Belgiea" } \\
\text { (Drifting in ice pack) }\end{array}$ & Marel, 1898, to February, 1899 & (Mean) $7030 \mathrm{~S}$ & (Mean) $8830 \mathrm{~W}$. & $29 \cdot 307$ \\
\hline "Gauss" & Mareh, 1902, to February, 1903 & $662 \mathrm{~s}$. & 8938 E. & $29 \cdot 134$ \\
\hline
\end{tabular}

noted that whilst two of them-Cape Adare and the "Discovery's" Winter Quarters-are not very greatly separated as regards latitude, and are almost on the same meridian of longitude, the other two are remote both from Ross Island and from each other, their distance in longitude being ronghly $90^{\circ}$ from Ross Island and $180^{\circ}$ from each other.

The barometers used on board the "Discovery" were verified at the Kew Observatory of the National Physical Laboratory both before the Expedition sailed and subsequently to its return. During the greater part of the sojourn at Winter Quarters they were read every second hour from midnight to inidnight, but towards the close of the second year the number of eye observations became reduced and the omissions have been supplied from the records of one of the self-recording ancroids, after a suitable correction harl been applied to bring them into accordance with the readings of the mercurial barometer when eorrected and reduced to sea level and to temperature $32^{\circ} \mathrm{F}$.

Each of the four series of observations given in the table began in February or the begimning of March, and the mean heights of the barometer for the ensuing twelve months at the places represented were as follows, the readings being in each case reduced to latitude $45^{\circ}$, as well as for temperature and to sea level.

We do not know how nearly the mean of one year's observations approximates to the true anmul mean pressure for these regions, and it will be noticed that the individual means for the two years of the "Discovery's" stay differ by as much as 0.125 inch. A comparison of the mean annual pressure at 
Valencia, in the south-west of Ireland, for each of 20 consecutive years with the mcan for 35 years gave an average difference of \pm 0.03 inch, and an extreme difference of 0.112 inch, whilst a similar comparison of mean values for $\mathrm{Kew}$ for the same period gave an average difference of \pm 0.025 inch and an extreme difference of only 0.067 incl. Possibly in the Antaretie the variation of the mein pressure of any one year from the true mean may he lialle to a greater error than either of these-we have no sure information upon the point, but probably we shall not greatly err if we assume for the valucs we are now dealing with a possille error of \pm 0.060 inch.

The annual means for the four places named in the talle confirm the inference which seens inevitable from the marked prevalence of easterly winds at three of them, that barometric pressure inust be relatively" high over the regions approximating to the Sonthern Pole.

Taking first for comparison the "Gauss" yearly mean for latitude $66^{\circ}$ and the "Discovery's" mean for the same twelve months for latitude $78^{\circ}$, we find the mean pressure at the more southern station to be 0.219 inch higher than at the northern position, giving a gradient, if we may disregard longitude and consider the difference in latitude alone, of 0.018 inch for each degree of latitude. The Cape Adare mean for latitude $71^{\circ}$, dealt with in a similar way, but comparing it with the "Discovery" mean for the two years, gives a difference of 0.022 inch per degree of latitude, which is practically the same as that given by comparing the "Discovery" and "Gauss" values.

That the "Belgica's" average pressure for very nearly the same (mean) latitude as Cape Adare $\left(71^{\circ}\right)$ gives a somewhat different result is not very surprising when we rememlser that it is not only for a different twelve months from that of the "Discovery," but also for the somewhat extensive area covered by her drift during the time. Her mean pressure for the year is 0.173 inch higher than the Cape Adare mean, and only 0.046 inch lower than the mean for the "Discovery's" first year, which was consideral,ly $(0.125$ inch) higher than that for her second year. This differenee yields a gradient per degree of latitude less than half that obtained by comparing the means of the other stations.

The "Belgica's" high mean pressure for the year is, however, chiefly due to the relatively high means for the four winter months, which is a feature not exhibited at either of the other stations; indecl, at the "Ganss" the mean pressure for the winter was considerally lower than that for the summer. Assuning the seasonal march of pressure to have been approximately normal at each of the stations, it is conceivalble that the higher wirter pressure at the "Belgiea" may have been related to a pole of cold eaused by radiation from large land masses somewhere in her neighbourhood, leading to some dislocation of the isobars, and incidentally to the greater diversity of wind direction which she experienced. It has been pointed out that she was far to the eastward of the "Discovery," and as very little is known of the physical features of the intervening region, there is plenty of room for conjecture as to the distribution of land and sea areas within it.

Before leaving this part of the sulject it may le well to refer to the results of the harometer obserrations made on the rarious sledge journeys which were undertaken by the Expedition during its stay at Ross Island.

These journeys varied both in direction and in duration, and barometric observations were made on nearly all of them. The instrument generally used was an aneroid, of which the Expedition possessed several, lut on most of the journeys the readings of the aneroid were supplemented by hypsometric observations. Prior to leaving England the aneroids had been verified at the Kew Observatory, but when they were brought into nse in the Antaretic it was found that ench instrument had devieloped errors very different from those determined for it at Kew. This fact introdnces an element of uncertainty into the observations which is not entirely eliminated by the comparisons which can be made of the aneroid readings with the readings of the ship's mereurial barometers at the starting and on the return of the party. But the greatest obstacle in the way of ascertaining the pressure gradient from the barometrie olsservations made on the several journeys arises from the difficulty of deciding how far the differences olserved between the observations made at the ship and those made simultaneously at a distance were due to ehange in height above sea level, and how far to purely meteorological causes; and in some instances, where the changes of level are known to have been very lirge from day to day, the difficulty becomes insuperal,le. In two or three journeys, however, the conditions were more favourable thau in the others, 
and particnlarly was this so in the journey made by Lientenant Royns to the south-east across the open Ice Barrier. Throughout this journey the aneroid was read at frequent and regular intervals, and the observations have been dealt with in the following mamner. The jonrney out and home was broken up into distance-intervals of 20 miles each, and all the barometric observations made whilst each distanceinterval was being traversed were meaned and then compared with the mean of the corresponding readings of the barometer made at the ship. In this way it was sought to smooth out the casual fluctuations of pressure and to get an approximately correct mean barometric difference between the ship and the mean position of the travellers for the time occupied in traversing each 20-mile section of the journey.

The differences obtained from a comparison of the two sets of means when plotted in a curve* exhilit a very uniform increase outwards to the furthest point reached, and a similarly uniform diminution on the return journey, the reading of the barometer at the ship being the higher one throughout. It is, however, tolerably certain, from various considerations, that the general level of the Barrier rises to the southward, and this in itself would of course account for a decrease of pressure in that direction and a conseqnent increase in the difference between the two means as the travellers increased their distance from the ship. The question then arises whether this steady increase of difference with distance is not wholly due to increase of height above sea level, and whether if it had been possible to reduce the readings made on the ice to sea level there would not have been shown an increase of pressure to the southward instead of a decrease?

If we may assume an average rise of surface level of the Barrier ice of about 2 feet per mile along the line followed by the travellers (which is probably well within the actual amount), and if we reduce the barometer readings in accordance with that assumption, such a sea-level increase of pressure is then found to exist, and not only does the decrease of a little less than three-tenths of an inch in the 170 miles then disappear, but an increase of over a tenth of an inch takes its place.

It has been elsewhere pointed out (p. 492) that this conclusion receives considerable support from the fact that it supplies the barometric gradient required to account for the persistent easterly wind which is so marked a feature of the air circulation of these high latitudes.

The track pursued in two other journeys similarly dealt with followed somewhat closely the line of high mountains which stretch away to the southward along the western boundary of the Ice Barrier, and it is proballe that on those journeys greater and less regular differences of level were met with. At any rate, the observations made during those journeys do not yield results quite so uniform as that obtained from the observations on the journey across the open Barrier, quite away from the land, although in their main features they are very similar; and this may probably be due partly to the travellers having experienced on the southern journeys more abrupt change of level, and also to some extent to the fact that the readings of the aneroid were not made either so frequently or so regularly as on Lieutenant Roviss' journey. It may, perhaps, be of interest to notice that at the 130-mile distance-interval of Lieutenant Roybs' journey there is, both on the outward and the homeward journey, a decrease of the barometric difference amounting to 0.03 inch or 0.04 inch, indicating a slight decrease of about 30 feet in the height of the Barrier floor at that distance. The repetition of the irregularity in the curve on the return journey not only confirms the existence of a depression of ice level at that spot, but also seems to support the method adopted for dealing with the observations.

HANN, in his 'Handbook of Climatology' (English edition, translated by R. DE C. WARD, p. 217), refers to the distribution of mean pressure at different latitudes, and quotes lerREL's value for each $10^{\circ}$ down to latitude $70^{\circ} \mathrm{S}$. These values indicate a progressive decrease of pressure with increase of latitude south of $30^{\circ} \mathrm{S}$, the mean pressure at $70^{\circ}$, which is the highest latitude quoted in the table, being given as $29 \cdot 056$ inches.

Map No. 51, in the 'Report of the "Challenger "Expedition,' showing the isobaric lines of the globe for the year, exhibits a similar decrease of pressure down to latitude $60^{\circ} \mathrm{S}$., which is the most southern position for which an isobar is given, and the value of the pressure for that parallcl is $29 \cdot 30$ inches.

Compared with the values given in Table I., that quoted by Haxn for latitude $70^{\circ}$ is too low, and it is clear that the decrease of pressure does not continue southward so far. Dr. SHaw, from a discussion of

"See fig. 1, p. 491, "Discussion of Wind Obserrations." 
tho general circulation of the atmosphere ('Proc. Roy. Soc.,' vol. 74, p. 20), based largely upon the differenco between the distribution of pressuro at the Larth's surface and the distribution at the 4000 metres level as computed hy M. Trisserenc DF BorT, came to the conclusion that a higl-pressure area exists over the Antarctic, and he pointed out that "the pole of extreme cold, which in the sonthern hemisplere must bo nearly coincident with the geographical pole, will have associated with it a component distribution for easterly (wind) circulation similar to tho low-temperature pole of north-east Siberia in the northern hemisphero,"-a conclusion which seems to be completely justified by tho mean pressure results shown in Table I., and also by the wind distribution recorded at the "Discovery's" Winter Quarters, as well as at Cape Adare and at the position at which tho "Gauss" wintered in the ice. Dr. Siraw proceeds to say, " the intensity of the cold in the south polar regions is undoubted, and the existence of the distribution for an easterly circulation round a high-pressuro centre dne to the weight of the lower air follows directly therefrom."

\section{The Seasonal Variation or Pressure.}

The observations for a single year, or oven two years, aro insufficient to yield truo mean valnes for the individual months, and that this is so will be seen at once when the monthly means of pressure for the first year of the "Discovery's" stay at her Winter Quarters are compared with the means for the corresponding months of the second year; the mean for June, 1902, for example, is a quarter of an inch lower than the mean for June, 1903, and the means for the two months of July differ as greatly, but in the opposite way, the earlier value being in this case tho higher of the two.

Table II.-Menn Monthly Pressure at the "Discovery's" Winter Quarters for the Years 1902-4.

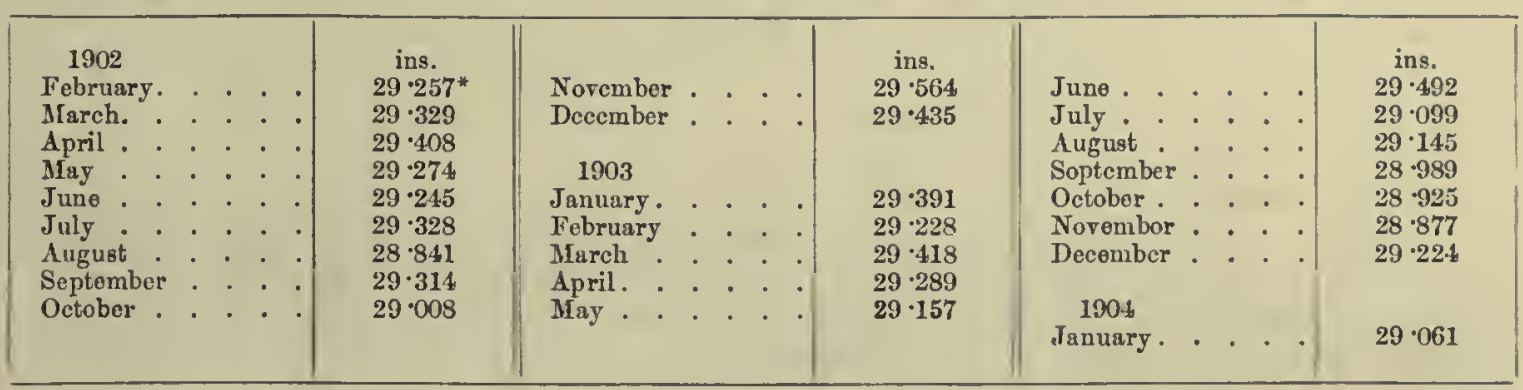

* 20 days' observations only.

We must be careful, thereforc, not to attach too much weight to the monthly means as such, but by gronping the months into seasons we double the number of observations available for the spring and autumn means and quadruple thoso for the summer and winter seasons, and with this augmented number of observations we may fairly expect the casual fluctuations of pressure to cancel each other sufficiently to yield a pretty close approximation to the true seasonal mean. Dealt with in this way, we get tho mean values of pressure for the four seasons shown in the following table, in which is also given for comparison the corresponding means from Capo Adare, the "Belgica," and from the "Gauss."

TABLE III.-Seasonal Neans of Pressure at the "Discovery's" Winter Quarters for the Years 1902-4; and at Cape Adare, the Winter Quarters of the "Gauss"; and the "Belgica," drifting in the Ice Pack. Corrected to Latitude $45^{\circ}$.

\begin{tabular}{|c|c|c|c|c|c|c|c|}
\hline & & Latitude \$. & Longitude E. & Summer. & Winter. & Spring. & Autumn. \\
\hline "Discovery" . & . . . & $\begin{array}{l}\circ \\
78\end{array}$ & $\stackrel{\circ}{167}$ & $\begin{array}{l}\text { ins. } \\
29 \cdot 325\end{array}$ & $\begin{array}{c}\text { ins. } \\
29 \cdot 267\end{array}$ & $\begin{array}{c}\text { ins. } \\
29 \cdot 128\end{array}$ & $\begin{array}{c}\text { ins. } \\
29 \cdot 431\end{array}$ \\
\hline Cape Adare & . . . & 71 & 170 & $29 \cdot 245$ & $29 \cdot 076$ & $28 \cdot 760$ & $29 \cdot 161$ \\
\hline "Gauss". & . . & 66 betw & 90 & $29 \cdot 258$ & $29 \cdot 101$ & $28 \cdot 900$ & $29 \cdot 188$ \\
\hline "Belgiea" & . . . & 69 and 71 & 80 and $96 \mathrm{~W}$. & $29 \cdot 298$ & $29 \cdot 437$ & $29 \cdot 295$ & $29 \cdot 079$ \\
\hline
\end{tabular}


At the "Discovery's" Winter Quarters the highest mean pressure oceurred in the autumu and the lowest in the spring, the means for the four seasons showing at very uniform progression from one to the other. At the "Gauss," the mean pressure was throughont lower than at the "Discovery," lut higher in the summer than in either of the other seasons, the seasonil mareh of pressure being with this exception

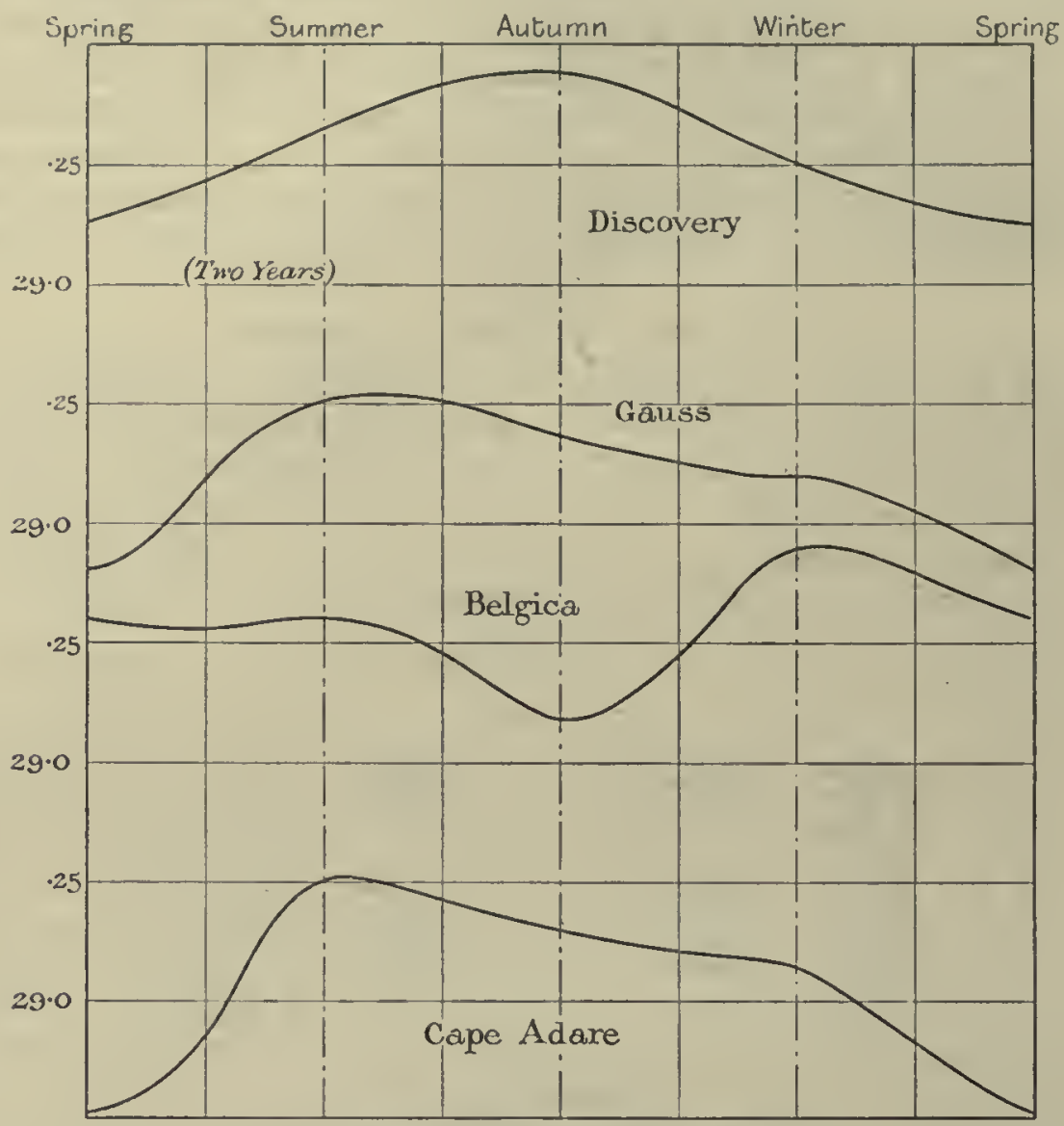

Fig. 1. Mean pressure for the four seasous.

similar to that at the "Discovery's" position. During the drift of the "Belgica," the highest mean pressure oecurred in the winter and the lowest in the autumn, the mean in spring and summer being alike; the yearly march of pressure reeorded was therefore very unlike that experienced at the other stations.

Only 11 months' observations are available for Cape Adare. The lowest mean pressure was reaehed there in the spring and the highest in the summer, the seasonal ehange being very similar to that experienced on board the "Gauss."

\section{Diursal Oscillation of Pressure at Winter Quarters.}

The hourly means have also been eombined for each of the four seasons in order to ascertain whether they exhibited a diurnal variation of pressure.

For this purpose mean values were computed for the initial as well as for the final midnights, and the differenee between the two distributed proportionately over the day in order to eliminate the non-periodic variation. This was done by pivoting the eorrection, so to speak, upon the noon value, and applying to the initial and terminal values one-half of the difference between them, subtracting it from the higher of the two and adding it to the lower, the intermediate hourly values receiving a proportional amount of the differenee, and the noon value, of course, remaining unchanged. The results are shown in fig. 2. 
The diurnal variation, whieh is eertainly very suall, is nevertheless distinetly shown, and in each of the four seasons it appears as pratieally a single oseillation with only slight modifieations.

In summer the total amplitude amounts to only $0.014 \mathrm{inch}$, with a minimum at about $3 \mathrm{a}$ a.m. and a maximum between 10 a.m. and 11 a.m., the afternoon decrease being slightly retarded letween 5 p.m. and 9 p.m. The curve formed by the oseillation is, however, a very smooth one, and elosely resembles in form the enrve of diurnal temperature variation for the same season.

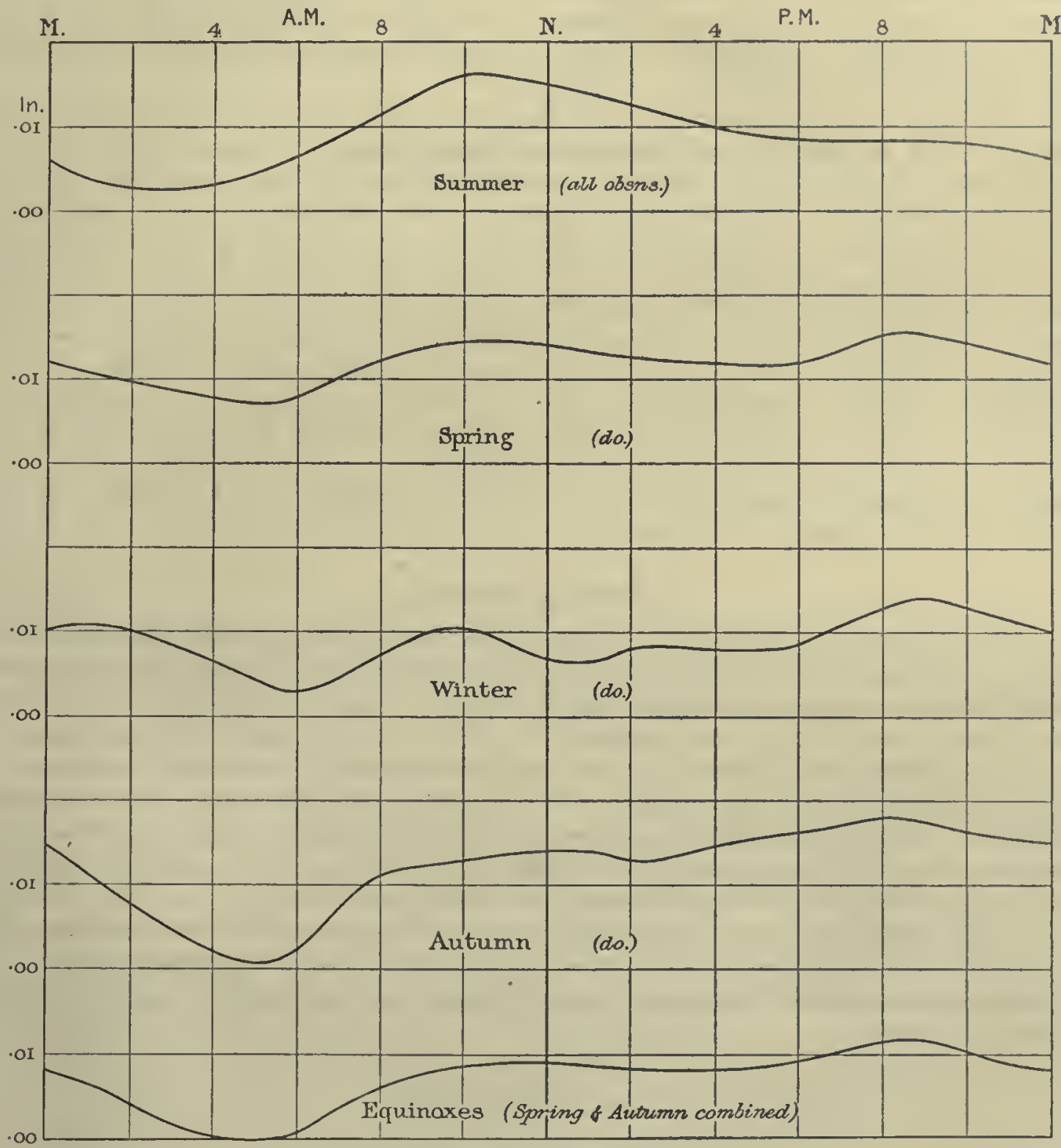

Fig. 2. Diurnal range of pressure ("Discovery").

The oseillation of greatest amplitude is that of the autumn, but even this does not amount to 0.02 ineh. The prineipal feature of this eurve is the well-marked minimum at 5 a.m., followed by a quiek reeovery up to 8 a.m., after which there follows a very gradual rise to the maximun twelve hours later.

The winter oseillation is more irregular, but it, too, has a distinctly marked morning minimum at 6 a.m., and evening maximum at $9 \mathrm{p} . \mathrm{m}$., but the morning maximum, which is indieated more or less elearly in each of the other eurves, is most prominently shown here at 10 a.m., after whieh there follows a very snall afternoon mininum. 
The spring curve is a very smooth one, and has also a smaller amplitude than the curve of any other season. It rises from a minimun at 5 a.m. to a maximum at $11 \mathrm{at.m.,} \mathrm{and} \mathrm{then,} \mathrm{after} \mathrm{a} \mathrm{slight} \mathrm{fall} \mathrm{for} \mathrm{six} \mathrm{or}$ seven hours, it rises to the maximum of the day at 11 p.m.

The Fourier coefficients of the diurnal variation for the seasons, which are given in Table IV., differ from those of lower latitudes in the relatively large anplitude of the first component. The epochs of the maximum of this component in the different seasons become progressively earlier as the year grows, and then get later again through the antmmn to the winter, which appears to indicate some relation to the Sun's declination. But the fact that in these latiturles a diurnal variation exists all the year through is a point of some interest, because it has hitherto been pretty generally assumed that it disappeared entirely at a much lower latitnde than 70 degrees.

Table IV.-Fourier Coefficients of Diurnal Incquality of Pressure.

\begin{tabular}{|c|c|c|c|c|c|c|c|c|c|c|c|c|}
\hline & Mean. & $P_{1}$ & \multicolumn{2}{|c|}{$\mu_{1}}$. & $P_{.}$ & \multicolumn{2}{|c|}{$\mu_{2}}$. & $P_{3 .}$ & $\mu_{3}$ & $P_{4}$. & \multicolumn{2}{|c|}{$\mathrm{M}_{4}}$. \\
\hline Spring . & $\begin{array}{l}\text { ins. } \\
29 \cdot 0578\end{array}$ & $0 \cdot 0024$ & $\begin{array}{c}\circ \\
253\end{array}$ & ' & 0.0017 & $\stackrel{\circ}{151}$ & '́ & $0 \cdot 0305$ & $\begin{array}{cc}\circ & 1 \\
42 & 17\end{array}$ & $0 \cdot 0004$ & $\stackrel{\circ}{41}$ & '́ \\
\hline Sumimer. & $29 \cdot 2542$ & $0 \cdot 0045$ & 187 & 36 & $0 \cdot 0023$ & 149 & 2.5 & $0 \cdot 0003$ & 150 & $0 \cdot 0001$ & 78 & 45 \\
\hline Antumn. & $29 \cdot 3614$ & 0.0056 & 262 & 53 & $0 \cdot 0031$ & 153 & 37 & $0 \cdot 0009$ & $17 \quad 43$ & $0 \cdot 0006$ & 27 & 7 \\
\hline Winter. & $29 \cdot 1972$ & 0.0029 & 306 & 28 & $0 \cdot 0017$ & 159 & 53 & $0 \cdot 0009$ & $36 \quad 51$ & 0.0010 & 46 & 26 \\
\hline
\end{tabular}

\section{Raxge of Pressure.}

The mean pressure in the Antaretic is low, and in every month of the two years during which the "Discovery" remained at Ross Island the minimmm fell below 29 inches, whilst the maximum seldom reached 30 inches and never greatly exceeded it. The lowest point reached by the mercury was 28.140 inches in June, 1902, and the highest 30.181 inches in May of the same year, giving a maximum range of just over 2 inches. The largest range in any month occurred in June, 1902, and amounted to 1.804 inches, but on three other occasions-May and July, 1902, and August, 1903-it exceerler 1.7 inches. In 1902 the monthly range exceeded an inch in the eight consecutive months $A$ pril to November, bnt in the following year so large a rauge occurred only in the five months June to October. The average monthly range for the two years amounted to $1 \cdot 154$ inches.

A quickly falling harometer was frequently, but not invariably, associated with relatively high temperatnre, and conversely an incrense of pressure commonly bronght with it an increase of cold.

The fall of the barometer which preceded nany of the storms was not very large if julgerl by Western European experiences. 


\title{
XII. DISCUSSION OF THE OBSERVATIONS OF THE DIRECTION AND FORCE OF THE WIND AT WINTER QUARTERS AND ON THE SLEDGE JOURNEYS.
}

\author{
BY \\ R. H. CURTIS.
}

The Station.

TuF immediate surroundings of any place at which observations of wind were made is a matter of some importance when dealing with the olservations themselves. The Winter Quarters of the "Discovery" were in a small bight, about a third of a mile deep, close to the south-western extremity of lioss Island, where the vessel lay enst and west and fairly close to the shore. The neighbouring land was hilly, and extenderl from Hut Point, a low promontory less than a quarter of a mile to the westward, round throngli nortl and east to Observation Hill, a conical hill three-quarters of a mile to the sonth-south-east of the ship. The land, although hilly, did not rise very abruptly, and its highest point, which was much above the general level, was reached at Crater Hill, 1000 feet above the sea and between one and two miles to the eastward of the ship. Between this hill and Observation Hill there lay a wide gully, opening out to the south-east, and known as the Gap. The outlook from the ship to the south-westward, from Observation Hill in the south-south-east to Hut Point in the west was perfectly open and uninterrupted.

An additional series of observations of wind direction and force was made at a point upon the open ice about one-third of a mile to the south of Cape Arnitage, the southernmost point of Ross Island. This spot was abont two miles south of the ship and, except for Ross Island, there was nothing that could obstruct the wind coming from any direction.

\section{INSTRUMENTS.}

The instrumental equipment of the expedition for making olservations of the direction and force of the wind consisted of a non-registering vane of the ordinary type for olserving the direction, and of three anemometers for olitaining the velocity. In addition to the instrumental records, the force was also estimated by the Beaufort scale.

One of the anemometer's was a small cup instrument, made by CASELLA, which indicated upon a pair of dials the number of miles of wind which had passed over it since the last observation. The cups of this anemometer were 3 inches in diameter, and the distance from the centres of the cups to the spindle was $6 \frac{1}{2}$ inches. Before leaving England it had been compared with the large anemometer at the Kew Observatory, and within the limits of the wind velocities experienced during the time the trial lasted the indications of both instruments were found to be in fairly close agreement. The results shown by the larger instrument are, however, known to be too high, and have to be reduced in the ratio of 15 to 11 to obtain the true speed of the wind, and therefore the dial readings of the smaller instrument were all similarly recluced before they were dealt with in this discussion.

This cup anemometer was mounted upon an iron post, which was fastened to the wooden frane erected to carry the thermometer sereens. The cups were 15 feet above the level of the snow and about 120 yards to the eastward of the ship.

The dials were read every two hours when making the other meteorological observations. The readings have been first correcter for the factor, as indicated alove, and then divided by 2 in order to get the average hourly speed of the wind in statute miles since the last observation.

Both the other anemometers were pressure-tube instruments, but of different patterns, one being at selfrecording instrument, made by MunRo, and the other a non-reeording, or "sight-indicating," instrument by Casselia.

The latter is a small portable instrument, not intended to be permanently fixed, but to be held in the hand when making an observation. In the present case, however, it was attached to another upright of the stand to which the thermometer sereens were fixed, and was connected by tubes with a duplicate vane

$$
3 \text { Q } 2
$$


"head," whieh had been supplied for the self-recording pressure-tube anemometer, the "head" being placed a few inches alove the post and 10 feet above the snow. When the instrument is exposed to the wind, the coloured fluid-aleolıol-in the glass tube oscillates with the fluctuations in the force of the wind, and the usual method of observation is to watch these oscillntions for a few seeouds and note what appears to the observer to be its average level, the corresponding velocity being then recorded as that of the wind at the time of the observation.

This method of observing was, however, slightly varied in this instance-the observer" reeorded the extremes of the oscillation instcad of the mean. Such an entry as " $16-26$ " in the Journal means, therefore, that the force of the wind varied from a minimum of 16 miles to a maximum of 26 miles during the time of observation. It does not follow, however, that the arithmetic mean of two such readings would fairly indicate the average force of the wind, even for the somewhat brief period such an observation might be supposed to represent. At times it would no doubt do so, but an examination of the automatic records of a self-registering pressure-tube anemometer would show that frequently it would not, owing to the fact that relatively strong, but very transient, gusts often occur which are not balanced by corresponding lulls, and therefore have a tendency, supposing such a gust to be included in the observation, to exaggerate the average force obtained.

It would have added to the value of the observation, therefore, if, in addition to giving the extreme limits of the excursions of the fluid, the observer had also recorded the velocity corresponding to the position upon the scale around which the fluid appeared most frequently to lie.

A difficulty in connection with the use of the instrument arose from the frequent choking of the "head" and the comecting pipes with drifting snow, necessitating its being frequently dismounted and cleaned. Possibly the small height above the snow at which the "head" was fixed may have been partly responsible for this; but the trouble would doubtless have been much diminished, and perhaps entirely obviated, if the instrument had been used in the way intended, and, instead of being fixed out of doors, had been taken out only when required for the purpose of an observation.

The self-recording pressure-tube anemometer was erected on board the ship, the "head" being placed on the mizzen cross-trees, on the sonthern side of the ship, 34 feet above the sea level, whilst the recording portion of the instrument was put in the signal locker, on the upper deck. The selection of this position for the recolding apparatus was unfortunate, and was the proximate cause of the loss of many weeks' records.

On account of the difficulty which would probably be experienced in keeping water unfrozen in the high latitudes in which the instrument was expected to work, a "non-freezing mixture" had been supplied for use in the anemometer cistern as a substitute for water. The "mixture" consisted simply of water and methylated spirit, to which a small quantity of glycerine was added to give the compound the specific gravity of water. Without much doubt, had the cistern containing this fluid been placed in a less exposed pasition below deck-and the instrument itself presented no difficulty to this being done-the fluid would have fulfilled its purpose; but under the influence of the extreme cold to which it was exposed in the locker it frequently got out of order, and on opening the cistern "ice was found on the top of the liquid, then the spirit, and below that a thick substance like condensed milk." The "mixture" was therefore removed and the cistern "filled with spirit containing only a small amount of glycerine." The result was a decided improvement in the action of the float, although in very low temperatures and ealms "the piston was still inclined to work in thick liquid," and "the whole lot had to be stirred up." The extreme cold also affected the ink and the going of the clock; and, as a final result, there were inany days on which 110 record at all was obtained, and many others when the record was of no use.

The vane for indicating the direction of the wind was placed at another angle of the frame for supporting the thermometer screens, at the height of 12 feet above the snow level.

\section{Onservations.}

The observations relating to wind may be summarized as follows:- The direction, as shown by the. vane, was recorded at every second hour, from the time of arrival in Winter Quarters till near the elose of the vessel's stay, when the hours of observation were reduced. The force of the wind, as estimated by 
Beaufort's scale, was also recorded every two hours with each observation of direction. The record of mean wind velocity, in miles per hour, dechuced from 2-hourly rearlings of the enp nuemometer, is practically complete from April, 1902, till the end of the vessel's stay in Winter Quarters, the breaks in its continuity amounting altogether to only a few days, during which the instrument was disablerl or nnder repair. The ecries of observations of velocity by the "sight-indicating" pressure tule is somewhat less complete, whilst the records of the recording pressure-tube anemometer exhibit frequent lacunx, which in many instances extend over days and in several others over weeks.

\section{DiRfCTION OF THI: WiND.}

The observations of wind direction, irrespective of force, were first dealt with.

All the observations for each month were first of all grouped under thirty-two points, and the percentage of their frequency of occurrence under each point determined. It was at once seell that this wis a greater degree of refinement than the observations themselves would warrant, and it was evident from the infrequency with which observations were recorded under the intermediate points that the observers had practically limited thenselves to sixteen points in making their observations. The observations under each intermerliate point were therefore allocated to the points on either sicle in proportion to the number of observations already credited to them. Thus if five observations of $\mathrm{E}$. by $\mathrm{N}$. winds were to be distributed between E.N.E. and E., and there were already entered to E.N.E. ten observations and to E. fifteen, then two of the five wonld have been given to E.N.E. and three to E., raising the number of observations under the first-lamed point to twelve and that under the latter to eighteen.

The results of this grouping are shown in Table I.

Table I.-Distribution of Wind Direction under Sixteen Points in Percentages.

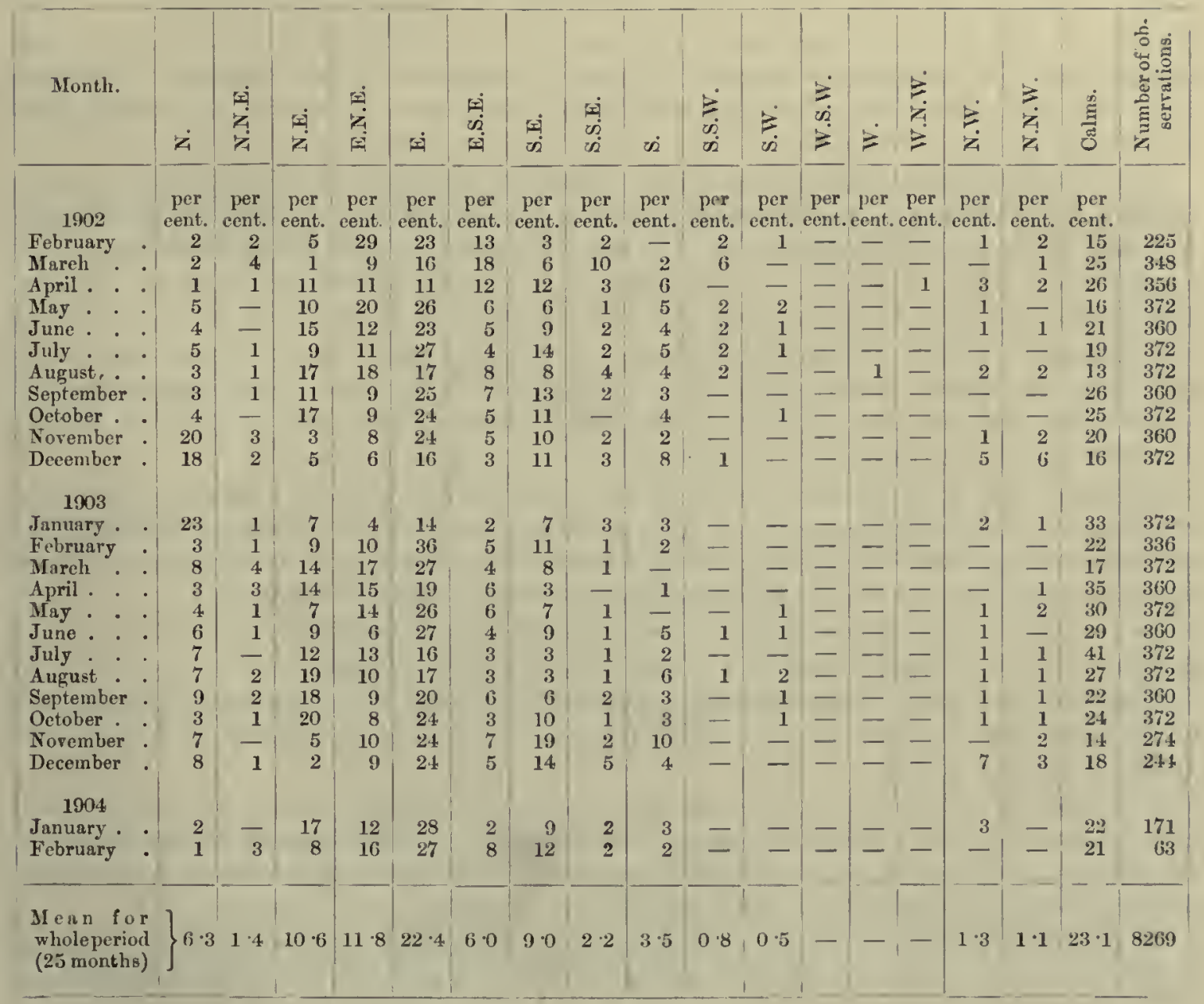


The most eonspicuous featnre of the Antarctic wind which is exhibited by the figures in this table is the predominance of east winds thronghont the two years over which the observations extended. East-northeast and north-east winds eome next in frequency; but north winds were conspicuous only during the months of November and December, 1902, and January, 1903; south-easterly winds were, on the whole, less eommon than north-easterly winds; soutlierly winds were conparatively rarely felt; and west winds were never felt at all.

Generalizing the observations still further by grouping them nnder eight points only and omitting the calms, as in Table II., we get for the entire series 44 per cent. of easterly winds, 20 per eent. of north-

Tabis II.-Distribution of Observations of Wind Direction under Eight Points.

\begin{tabular}{|c|c|c|c|c|c|c|c|c|c|c|}
\hline - & N. & N.E. & E. & S.E. & S. & S.IV. & W. & N.W. & Calms. & $\begin{array}{l}\text { Gross } \\
\text { total. }\end{array}$ \\
\hline $\begin{array}{l}\text { Percentage of obscrvations } \\
\text { under each point, in- } \\
\text { cluding calms . - } \\
\text { Pereentage of observations } \\
\text { of direction only, ex- } \\
\text { cluding calms. . . . }\end{array}$ & per cent. & per cent. & per eent. & per cent. & per cent. & per eent. & per eent. & per eent. & per cent. & \\
\hline $\begin{array}{l}\text { Number of obserrations } \\
\text { under each point.?. }\end{array}$ & 701 & 1261 & 8771 & 990 & 389 & 87 & 12 & 122 & 1936 & 8269 \\
\hline
\end{tabular}

easterly, and 16 per cent. of south-easterly winds; so that no less than 80 per cent. of all the winds observer blew from the quadrant between north-east and south-east. Of the remaining 20 per cent., 11 per cent. fall under north and 6 per cent. under south, leaving only 3 per cent. to be divided between all the westerly points.

An examination of the values for the individual months fails to show any clear indication of a monthly change in the distribution of wind frequency. The observations extended over two complete years, so that each month is twice represented; but a variation of frequency shown by the observations of one month is not, as a rule, supported by the observations made in the corresponding month of the other year. For example, in November and December, 1902, and January, 1903, one-fifth of all the winds observed were from north, but in the corresponding months of 1903-4 there was no such preponderance of winds from that point, December having but 8 per cent. and January only 2 per cent. of winds from north.

The observations were then combined, in order to see whether they indicated any distinctive seasonal distribution, and for this purpose they were arranged in four groups, as in Table III.

The first of these groups embraces the four eonsecutive months May-August during which the sun was continnously below the horizon; the second group the four months November-February, during which the sul was continuously above the horizon; the third and fourth groups eomprise the autumnal and vernal equinoxes respectively, two months, March-April and September-October, in each group.

The table gives the mean percentage of frequeney under each of sixteen points for the several seasonsfirst for each of the two years separately, and then for the two years combined.

In the winter and spring a somewhat marked frequency of north-east winds is shown in both years, and in the winter there was also a greater mumber of winds from the west of south than is shown by the figures for the other seasons.

In summer the average of northerly winds for the two years combined is rather large owing to the great frequency of those winds in the summer months of 1902-3. This frequency was not repeated in the following year, and whether the distribution observed in the first or that observed in the second summer most nearly indicates the normal for the season must remain a matter of doubt.

The distribution of winds in the spring montlis appears to approximate somewhat to that of the winter in the greater frequency of north-east winds and also of winds from points to the west of south; whilst in 
the antumn months the relative frequency of winds from the west of north suggests that the effect of the warmer months of summer is prolonged in some degree into tlie autunn.

The chauges are, however, all small, and in no senson is the outstanding predominance of the easterly current destroyed or indeed greatly modified.

Anotlier very remarkable feature of the South Polar wind which Table I. exhibits is the great frequency of ealms and light airs. The smallest percentage of ealms in any month was 13 per cent. in Augnst, 1902, and the greatest 41 per cent. in July, 1903, both of them winter months; for the entire period calms averaged 23 per cent. of all the observations. Table III. gives 24 per cent. as the mean pereentage of ealms for all the winter months, and the same figure for all the equinoctial months eombined, but in the summer months the percentage fell to 20 per cent.

TABLe III.-Sensonal Distribution of Wind Direction under Sixteen Points.

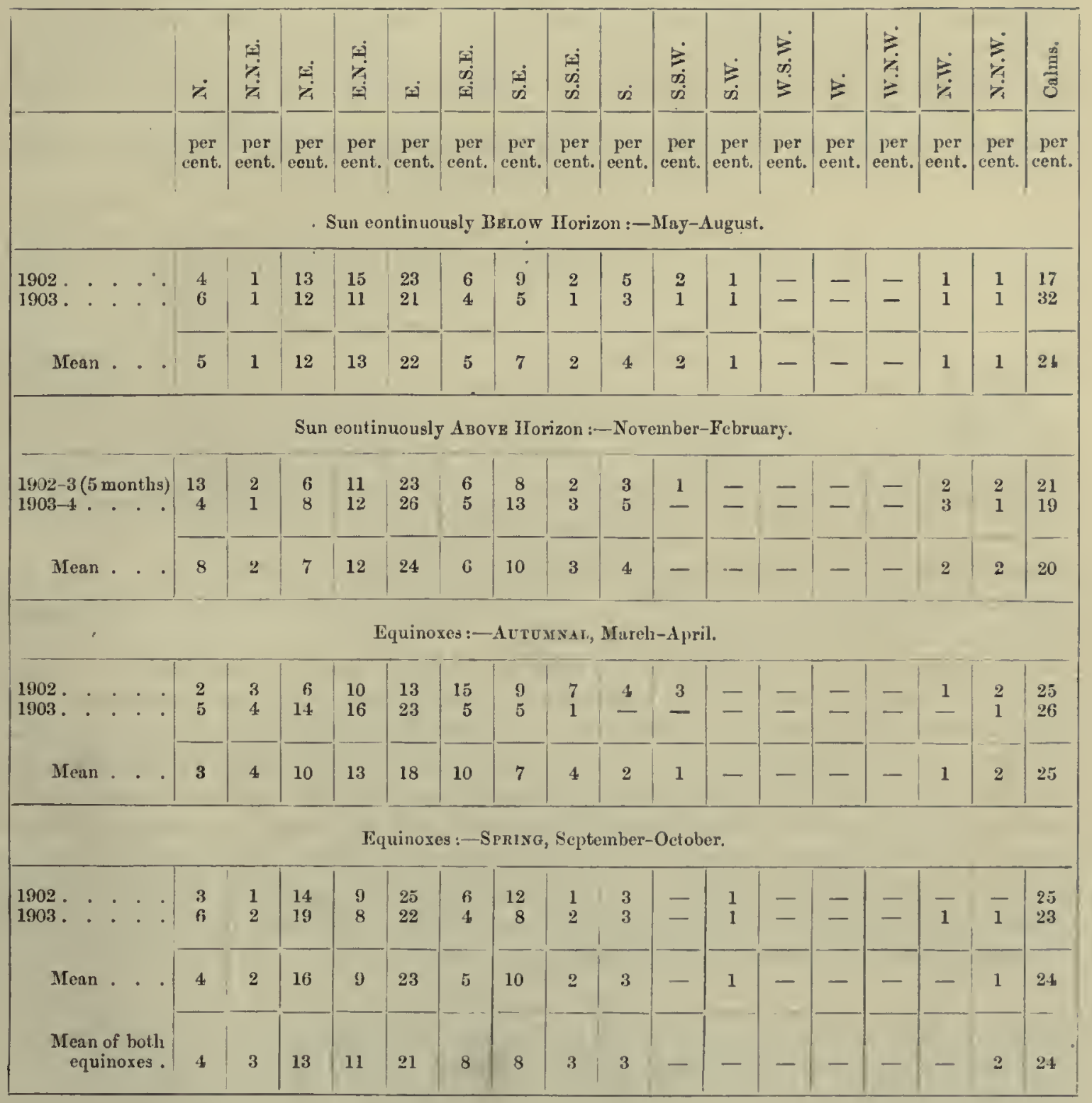

At this point it is important to consider what effect, if any, the land surrounding the ship in her Winter Quarters could have had upon the direction of the winds experienced there.

From the deseription of the position already given it will be realized that the direetions towards which 
the ship was most open and exposed were precisely those from which the wind seldom or never blew, and therefore the absence of winds from westerly points conld not have been due to the efiect of the land in obstructing or deflecting a westerly current, supposing one to have existed.

On the other hand, the winds which were of most frequent occurrence reached the ship after crossing the southern spur of lioss Island, which at Diseovery Bay is not more than a conple of miles in breadth from west to east, and on its eastern side is bounded by the open Barrier ice. In traversing the Barrier an easterly wind current would meet with no obstruction, and the land interposed betwecn the Barrier and the ship, although hilly, was not of a character materially to affect its course.

It has been suggested that "The Gap," the valley which lay to the south-east between Crater Hill and Olservation Hill, formed a kind of funnel which had an effect upon the direction of the wind at the ship. But in that case the predominant direction would have been south-easterly, and Table II. shows that south-east winds were less frequent than north-east winds, and less than half as frequent as winds from the east.

The effect of the surrounding land upon the direction of the winds observed at the ship's quarters can, however, be tested more directly, by comparing the results shown in Table I. with those given in Table IV., which are derived from observations of the direction and force of the wind made at a point

TABL, IV.-Distribution of Observations of Wind Direction made at a Point on the Ice Half a Mile to the South of Cape Armitage.

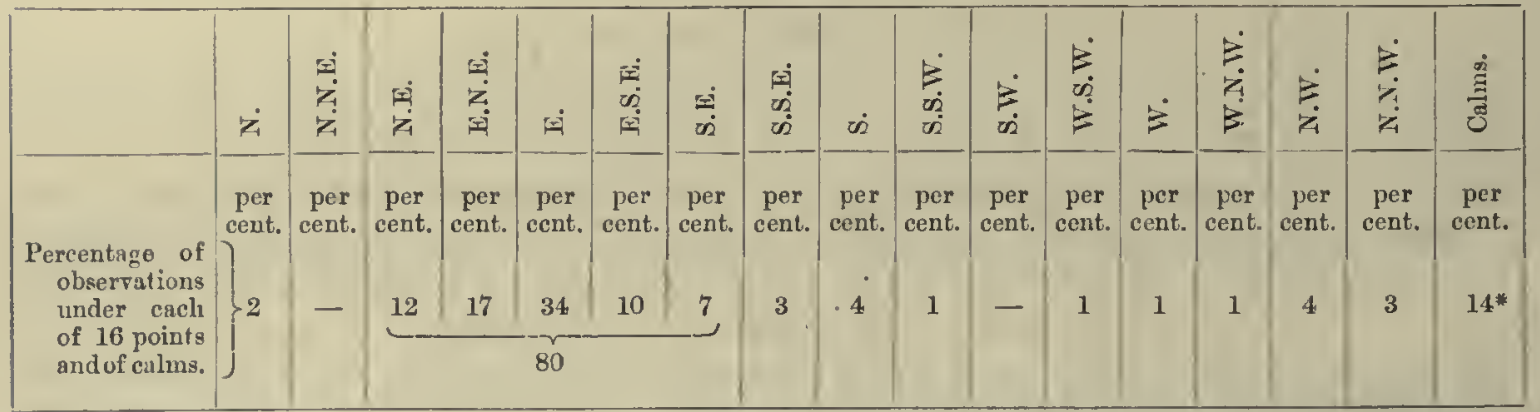

* Percentage of total number (424) of wind observations. In calculating tbe relative distribution of dircetions the number of caluns (60) was omitted.

about half a mile to the sonth of Cape Armitage, the sonthernmost point of Ross Island, where the exposure was perfectly open in every direction. These observations were made generally once a day, and they cover, with some breaks, a period of 16 months.

It should, however, be borne in mind that the observer at Cape Armitage had to observe the direction of the wind without the assistance of a wind vane, and probably this was at times a disadvantage; but whatever allowance should be made on this account, the distribution of wind directions is practically the same there as at the ship. Thirty-four per cent. of the winds were from east, 17 per cent. from eastnorth-east, 12 per cent. from north-east, and 7 per cent. from south-east; whilst grouping all the easterly points from north-east to south-east, we get a pereentage of 80 , which is precisely that obtained by sinilarly grouping the observations made at the ship, as shown in Table II. This result seems fully to justify the conclusion that the direction of the wind at Discovery Bay was not materially affected by the surrounding land, and that the relative distribution indicated by the percentages given in Table.II. may be accepted as fairly representing the wind frequency of the district.

\section{SLEDGE JOURNEYS.}

Observations of the direction and force of the wind were made upon most of the sledge journeys undertaken at various times during the stay of the Expedition at Ross Island.

In dealing with these observations, not only has the varying position of the observer from day to day to be kept in mind, but in some instances the changes also which were constantly taking place in his height above sea level, and in the character of his surroundings; and particularly was this the case in the western journeys up the slopes of the mountain ranges lying in that direction. As might have been expected, 
owing to the exigences of sledging the hours of observation were more or less irregular; and there was also the added difficulty of having to determine the wind direction without instrumental aid. On some of these points the information given in the Journal is less complete than could be desired, and fuller iuformation respecting what was done in correcting the observations for the magnetic variation would have been especially useful. This last point is, of course, one of much importance, and, unfortunately, it is one respecting which little that is definite is sairl in the note books.

Dealing broadly with the observations, the most prominent feature of the wind which they revenl is the frequency with which light variable airs and calms occurred. Most of the journeys were made between the beginning of October and the end of January, and at Winter Quarters in those months calns were recorded in approximately 25 per cent. of the observations; but upon the whole their occurrence appears to have been nuch nore common to the southward and westward of the ship, approaching 40 per cent. in the former direction and upwards of 50 per cent. in the latter. At the same time the gusty character of the wind is maintained by the observations, and occasionally the squalls were very sharp and severe.

From the observations made in the course of the journeys to the south and west, it would appear that in those directions the wind was more southerly than it was at Winter Quarters, but the uncertainty which exists respecting the correction of the records for magnetic variation makes it inexpedient to speak very definitely mpon the point. On some of the western jonrneys, however, a considerable altitude was attained, certainly above 7000 feet, and the observations of the movements of the lower clouds made at the ship would lead us to expect at that height above the sea a southerly, or even a westerly, current such as is recorded in the logs.

Also it should be remembered that the sonthern journeys followed the line of lofty mountains which, stretching away to the southward, form the eastern boundary of Victoria Land; and it is quite conceivable that an easterly wind current sweeping across the Barrier would, on meeting this range, become deflected to the northward and would in consequence be felt by the sledging parties as a southerly or even a southwesterly wind.

One of the most important journeys considered with reference to the observations of wind dircction was that of Lieutenant Royds, to a distance of 170 miles to the south-east aeross the Great Ice Barrier, made in November, 1903. Throughout this journey the party was far removed from any land, and there wero absolutely uo obstructions to the free movement of the air in any direction; and therefore the observations of wind direction should be valuable in relation to those made simultaneously at the ship. If we could assume that the observations were made by compass and entered in the note-book as made, without correction, we get, after applying a mean correction for variation, the following analysis of the observations :-

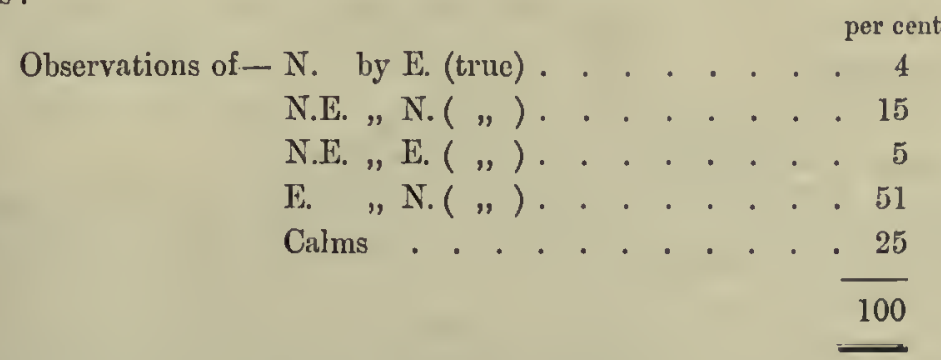

If the assumption that the observations were not originally corrected for compass error is a correct one, the confirmation these results afford to the accuracy of the distribution of winds obtained from the observations made at the ship would be very great, more especially because of the very open character of the region in which the observations were made.

On the other hand, if we suppose the observations to havo been corrected for variation before their entry in the log, we find ourselves confronted with a problem it is not easy to solve:- How are wo to account for the existence of a steady south-westerly current within a few miles of the ship, whilst at Cape Armitage and at the ship herself, although quite open and unsheltered towards the south-west, easterly winds were experienced almost exclusively, a south-west wind being of extremely rare occurrence, and a westerly wind practically never felt at all ? 
TABL: V.-Summary of the Wind Observations made on some of the longer Sledge Journeys.

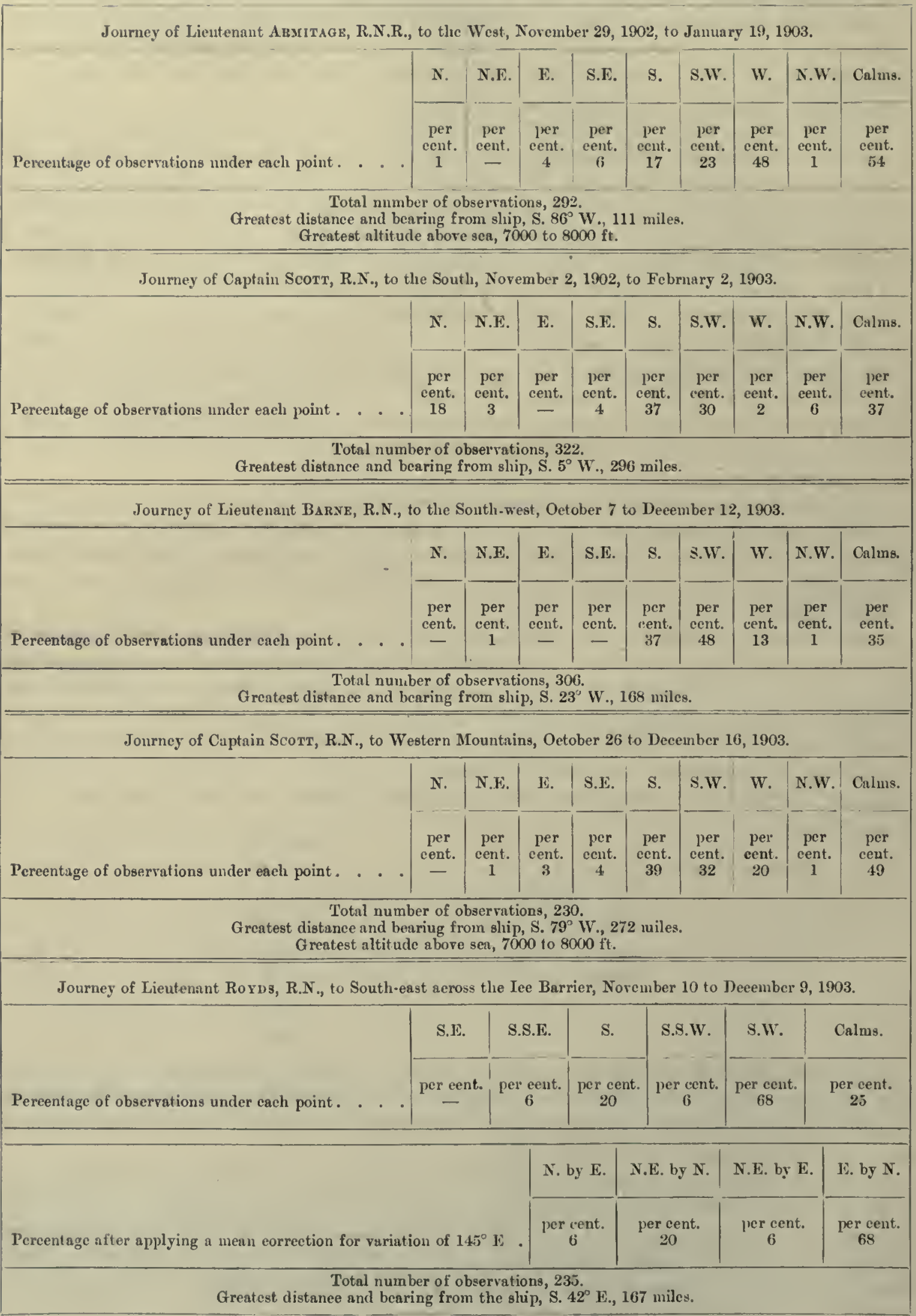

Notr. - Calms were omitted in ealeulating the perecntage frequency of wind directions. 
In Table V. an analysis is given of the wiud observations inade on the more lengthy and representative sledge journeys.

\section{The Distribution of Barometric Pressure in Rejation to Wind.}

The movement of atmospheric air currents as regards both direction and rate is, of course, governed by the distribution of barometric pressure, and in the sonthern hemisphere an easterly wind implies the existenee of an area of relatively high pressure to the sonthward of the point at which the wind is observed. If, therefore, it could be shown that such an area of high pressure of a more or less permanent eharacter existed over the Great Ice Barrier to the southward of Ross Island, the distribution of wind directions which was observed at the "Diseovery's" Winter Quarters would he at once confirmed and explained.

A eonsideration of the problem of the general air eirculation in the sonth polar region leads almost irresistibly to the eonclusion that some such accumulation of air must take place in its higher latitudes, but unfortunately the data yielded by the expedition are not such as would justify us in saying that its existence is absolutely proved, althongh perhaps it goes a good way in that direetion.

On most of the sledge journeys readings of aneroid barometers were made and recorderl, al though in some instances at somewhat irregular intervals. These rearlings were affected by two distinct causes,the variations in level of the different places of observation, and the changes in pressure due to movements of the atmosphere, and it is difficult to say to what extent these may have severally contributed to bring about the differenee in pressure observed between any two consecutive readings. On some journeys, in the neighbourhood of land, the effect due to the first named eause was so great as to render that due to the second quite inconspieuons; but the journey of Lientenant Royds to the south-east to which reference has already been made was over the level ice quite away from the land, and therefore it seems fair to assume that any changes of level during that journey would be very slight and gradual and not such as would mask the true barometric oscillations.

The barometric observations made on that journey have therefore been dealt with as follows:-The journey out and home was divided into distance-intervals of twenty miles, and all the observations made between 0 and 20,20-40,40-60, \&c., miles froin the ship were meared and the result eompared with the

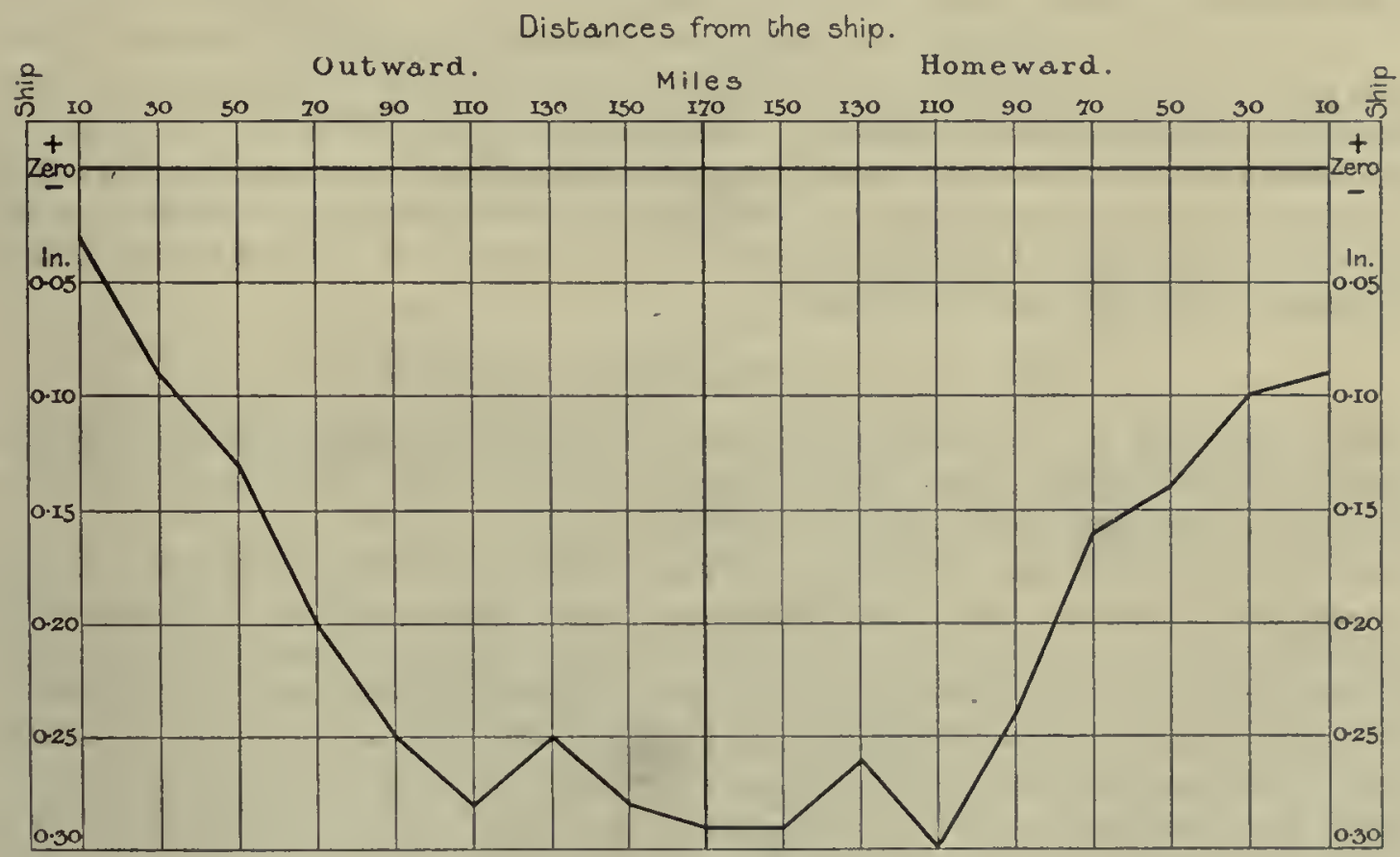

Fig. 1. Difference between the mean height of the barometer at Wintcr Quarters and on the sledge journey to the south-east across the Great Ice Barrier.

3 R 2 
mean of the corresponding corrected readings of the barometer taken on board the ship. The differenees between these two sets of mean values being then plotted to the corresponding mean distances were found to exhibit the fairly regular curve shown in fig. 1 , the difference increasing from -0.03 inch at 10 miles on the outward journey to -0.29 inch at the extreme point reached, and then decreasing anain to -0.09 inch at 10 miles from the vessel on the return. The correction required to make the aneroid when read at the vessel agree with the ship's barometer, corrected for temperature and sea level, was applied throughout.

By meaning the observations in this way the casual fluctuations of pressure were to a great extent smoothed out, and probably sufficiently so to warrant us in regarding the mcan for each distance-interval as the mean pressure for the time occupied by the party in traversing it. The differences, therefore, between those means and the means at the ship are due (1) to the difference in level between the two places, and (2) to the barometric gradient which may have existed at the time.

As regards (1), the distant barometer being always lower than that at the ship indicates that in so far as that fact represents change of level the travelless had been rising; whilst as regards (2), except so far as it is modified by (1), the differences point to the presence of an area of relatively low pressure in the region traversed. That the differences were chiefly due to change of level is, however, made elear by the fact that throughout the journey the winds were, with only a few brief exceptions, very light, with a large percentage of calms, and this would certainly not have been the case had the difference been to any marked extent due to barometric gradient. There was also evidence of very considerable ice pressure, and of a northerly movement of the Barrier ice-sheet, as to which Captain SCOTT remarks*: "it is certain that there must be a supply of ice from some region to the south," which must necessarily have caused some gradient, if only a small one.

Some rise in level may therefore be safely assumed, and the question then arises whether its full extent is indicated by the differences shown in the diagram, or whether these should not be inereased (or decreased) by an amount representing the barometric gradient.

As they stand, assuming there was no barometric gradient, the diflerences indicate a change of level in the ice of little more than 1 foot per mile, - a gradient which could hardly be reduced in view of the pressure phenomena which were observed upon the ice-sheet and of the remark of Captain ScotT just quoted; and therefore the existence of an area of low pressure to the southward of Ross Island which would result from a reduction of the ice gradient appears to be very improbable.

On the other hand, if we may assume the existence of a relatively high pressure in the region traversed by the party, with a sea-level gradient of about 0.1 inch for the distance of 170 miles represented by the diagram, the corrected (augmented) differences would then give us a rise in level of the ice of about 2 feet per mile along the line traversed, and althongh this gradient is still very slight it accords better with the ice phenomena observed than does the smaller one, whilst at the same time we get a barometric gradient sufficient to account fully, both as regards direction and force, for the wind distribution observed at the "Discovery's" Winter Quarters at Ross Island.

\section{Results at Other Stations in the Antarctic.}

Before leaving this part of the subject it may be well to refer to the results obtained in 1899 by the "Southern Cross" Expedition at their winter station at Cape Adare in Lat. $71^{\circ}$ S., Long. $170^{\circ}$ E., rather more than 400 miles north of the "Discovery's" position.

The exposure for wind observations was probably less satisfactory there than it was at Discovery Bay, is a range of high mountains rose abruptly to the south and west of the station ; but to the eastward and round through north the exposure was open to the sea. It was from the sonth-east, however, that most of the winds blew, and althongh the distribution was not so exclusively confined to the easterly quarters as was the case at Discovery Bay, yet, as will be seen from the following Table VI., it was such as to lend considerahle support to the suggestion that the easterly air current which the "Discovery" experiencerl at Ross Island extends, as the predominating current near the sea level, over a large extent of the surrounding region. Calms wcre of much more frequent occurrence at Cape Adare than at Discovery Bay.

$$
\text { " "Voyage of the "Discovery," vol. ii., p. } 428 .
$$


TAnLE VI.-Percentage Distribution of Wind Direction at Cape Adare during the Jileven Months March, 1899 , to January, 1900.

(The observations were made every two hours from 9 a.m. to 9 p.m..)

\begin{tabular}{|c|c|c|c|c|c|c|c|c|}
\hline \multirow{3}{*}{$\frac{N .}{\begin{array}{c}\text { per cent. } \\
3 \cdot 6\end{array}}$} & N.E. & ti. & S.E. & S. & S.W. & W. & N.W. & Calms. \\
\hline & $\begin{array}{c}\text { per cent. } \\
2 \cdot 6\end{array}$ & $\begin{array}{c}\text { per cent. } \\
9 \cdot 1\end{array}$ & $\begin{array}{c}\text { per eent. } \\
20 \div 4\end{array}$ & $\begin{array}{c}\text { per eent. } \\
13 \cdot 9\end{array}$ & \multirow[t]{2}{*}{$\begin{array}{c}\text { per cent. } \\
4 \cdot 1\end{array}$} & \multirow[t]{2}{*}{$\begin{array}{c}\text { per eent. } \\
3 \cdot 1\end{array}$} & \multirow[t]{2}{*}{$\begin{array}{c}\text { per cent. } \\
3 \cdot 1\end{array}$} & \multirow[t]{2}{*}{$\begin{array}{c}\text { per cent. } \\
40 \cdot 1\end{array}$} \\
\hline & & & $43 \cdot 4$ & & & & & \\
\hline
\end{tabular}

From February, 1902, to February, 1903, the German Antarctie exploration vessel "Gauss" was imprisoned in the ice just within the Antarctic Circle in longitude $90^{\circ} \mathrm{E}$. It will be noticed that her sojourn in the ice corresponds to the first half of the "Discovery's" stay at Ross Island, and although the two places are nearly $90^{\circ}$ of longitude apart, yet the wind frequency experienced was strikingly similar at loth places. The position of the "Gauss" was, fron a meteorological point of view, entirely satisfactory, so that in her case, at any rate, there can be no question of purely local influences having affected the direction of the wind she expcrienced. The predominance of easterly winds was, however, most marked throughout the entire period of the vessel's stay, as was also the second striking feature of the "Discovery" observations-the freqnency of calms; the number of calms was, however, stightly less, whilst the number of westerly winds was rather above the number observed at Discovery Bay.

The following table, summarizing the "Gauss" results for the seasons, is given by Dr. MeINardus:-

TABLE VII__ “Gauss" Winter Quarters, Latitude $66^{\circ} 2^{\prime}$ S., Longitude $89^{\circ} 38^{\prime} \mathrm{E}$.

February 19, 1902, to February 18, 1903.

\begin{tabular}{|l|c|c|c|c|c|}
\hline & \multicolumn{5}{|c|}{ Percentage of winds observed during- } \\
\cline { 2 - 6 } & Autumn. & Winter. & Spring. & Summer. & Year. \\
\hline Eusterly winds. . . & 81 & 73 & 69 & 70 & 73 \\
Westerly winds. & 11 & 12 & 17 & 2.4 & 16 \\
Calms... . : & 8 & 15 & 14 & 6 & 11 \\
\hline
\end{tabular}

The results are given in greater detail in Table VIII.

It will be seen, therefore, that the suggestion of a wide distribution for the ensterly wind current observed at the Winter Quarter's of the "Discovery" finds support in the olservations made at these two willely separated places in the Antarctic region, in both of which there was found the same marked persistence of winds from the east, comparative rarity of winds from the west, and noticeable frequency of calms.

Observations of the direction and force of the wind were also male on board the "Scotia," the vessel of the Scottish National Antarctic Expedition, during two summer cruises in the Weddell Sea, first in February and Narch, 1903, and again in March, 1904. The region covered on these cruises was roughly that between the meridians of $45^{\circ} \mathrm{W}$. and $10^{\circ} \mathrm{W}$., and the parallels of $60^{\circ} \mathrm{S}$. and $73^{\circ} \mathrm{S}$., and, therefore, it lay far to the north of Discovery Bay, and also about $160^{\circ}$ to the westward, and at so great a distance the meteorological conditions governing the movements of the air may have differed materially from those at Ross Island. The short Table IX. of the relative frequency of the winds is obtained from a diagran given by Mr. Mossmax in a paper printed in the 'Scottish Geographical Magazine' for May, 1906. 
TABLE VIII.-Percentage Distribution of Wind Direction under 16 Points at the Winter Quarters of the German Antarctic Exploration Vessel "Gauss," in Latitude $66^{\circ} 2^{\prime}$ S., Longitude $89^{\circ} 38^{\prime}$ E.

\begin{tabular}{|c|c|c|c|c|c|c|c|c|c|c|c|c|c|c|c|c|c|}
\hline $1902-3$. & z & 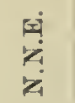 & $\frac{1}{81}$ & 至 & $=$ & $\begin{array}{l}0 \\
00 \\
0\end{array}$ & $\frac{10}{\dot{\infty}}$ & $\begin{array}{l}\overrightarrow{\mid l} \\
\dot{0} \\
\dot{2}\end{array}$ & $\dot{\infty}$ & $\begin{array}{l}\overrightarrow{7} \\
\dot{m} \\
\dot{0}\end{array}$ & $\frac{E}{\dot{s}}$ & $\begin{array}{l}\dot{0} \\
\dot{0}\end{array}$ & $\geqslant$ & $\frac{5}{z}$ & $\frac{1}{4}$ & $\frac{3}{4}$ & 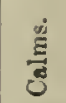 \\
\hline $\begin{array}{l}\text { Mareh : } \\
\text { April : } \\
\text { May. : } \\
\text { June : } \\
\text { July. : } \\
\text { August. } \\
\text { September. } \\
\text { Oetober } \\
\text { Norember. } \\
\text { December. } \\
\text { January. } \\
\text { February . }\end{array}$ & $\begin{array}{c}\text { per } \\
\text { cent. } \\
- \\
- \\
- \\
0 \cdot 1 \\
0 \cdot 1 \\
0 \cdot 1 \\
0 \cdot 3 \\
0 \cdot 5 \\
- \\
0 \cdot 5 \\
- \\
-\end{array}$ & $\begin{array}{c}\text { per } \\
\text { cent. } \\
- \\
- \\
- \\
- \\
- \\
0 \cdot 8 \\
0 \cdot 1 \\
1 \cdot 0 \\
- \\
- \\
-\end{array}$ & $\begin{array}{c}\text { per } \\
\text { cent. } \\
- \\
- \\
- \\
0 \cdot 1 \\
- \\
- \\
0 \cdot 2 \\
2 \cdot 8 \\
0 \cdot 4 \\
1 \cdot 3 \\
1 \cdot 6 \\
-\end{array}$ & $\begin{array}{c}\text { per } \\
\text { cent. } \\
1 \cdot 6 \\
3 \cdot 9 \\
-0 \cdot 6 \\
0 \cdot 7 \\
8 \cdot 8 \\
1 \cdot 3 \\
2 \cdot 7 \\
2 \cdot 9 \\
16 \cdot 1 \\
6 \cdot 7 \\
1 \cdot 5\end{array}$ & $\begin{array}{c}\text { per } \\
\text { cent. } \\
47 \cdot 1 \\
36 \cdot 1 \\
60 \cdot 2 \\
43 \cdot 8 \\
60 \cdot 1 \\
59 \cdot 1 \\
28 \cdot 5 \\
37 \cdot 5 \\
74 \cdot 2 \\
44 \cdot 4 \\
33 \cdot 9 \\
48 \cdot 2\end{array}$ & $\begin{array}{r}\text { per } \\
\text { eent. } \\
7 \cdot 0 \\
15 \cdot 0 \\
11 \cdot 0 \\
15 \cdot 6 \\
6 \cdot 2 \\
7 \cdot 0 \\
17 \cdot 6 \\
12 \cdot 4 \\
3 \cdot 7 \\
8 \cdot 1 \\
18 \cdot 6 \\
4 \cdot 3\end{array}$ & $\begin{array}{r}\text { per } \\
\text { cent. } \\
6 \cdot 2 \\
10 \cdot 0 \\
4 \cdot 6 \\
7 \cdot 2 \\
2 \cdot 4 \\
1 \cdot 3 \\
7 \cdot 2 \\
2 \cdot 4 \\
3 \cdot 0 \\
2 \cdot 1 \\
7 \cdot 5 \\
0 \cdot 7\end{array}$ & $\begin{array}{r}\text { per } \\
\text { cent. } \\
\mathbf{1 5} \cdot 3 \\
8 \cdot 9 \\
9 \cdot 1 \\
2 \cdot 5 \\
0 \cdot 1 \\
0 \cdot 6 \\
8 \cdot 9 \\
0 \cdot 5 \\
0 \cdot 4 \\
2 \cdot 7 \\
3 \cdot 5 \\
4 \cdot 9\end{array}$ & $\begin{array}{c}\text { per } \\
\text { cent. } \\
4 \cdot 8 \\
5 \cdot 3 \\
-5 \cdot 1 \\
0 \cdot 1 \\
0 \cdot 8 \\
0 \cdot 6 \\
0 \cdot 6 \\
0 \cdot 9 \\
0 \cdot 3 \\
4 \cdot 0 \\
3 \cdot 0 \\
3 \cdot 4\end{array}$ & $\begin{array}{c}\text { per } \\
\text { cent. } \\
2 \cdot 2 \\
0 \cdot 5 \\
0 \cdot 1 \\
0 \cdot 1 \\
0 \cdot 7 \\
- \\
1 \cdot 5 \\
0 \cdot 1 \\
\overline{3} \cdot 5 \\
0 \cdot 5 \\
3 \cdot 9\end{array}$ & $\begin{array}{c}\text { per } \\
\text { cent. } \\
1 \cdot 1 \\
2 \cdot 5 \\
0 \cdot 1 \\
1 \cdot 5 \\
1 \cdot 1 \\
0 \cdot 5 \\
1 \cdot 5 \\
0 \cdot 7 \\
1 \cdot 9 \\
3 \cdot 0 \\
0 \cdot 5 \\
2 \cdot 1\end{array}$ & $\begin{array}{r}\text { per } \\
\text { cent. } \\
7 \cdot 5 \\
1 \cdot 1 \\
4 \cdot 9 \\
0 \cdot 1 \\
1 \cdot 1 \\
0 \cdot 8 \\
5 \cdot 1 \\
2 \cdot 6 \\
0 \cdot 4 \\
1 \cdot 6 \\
3 \cdot 0 \\
12 \cdot 5\end{array}$ & 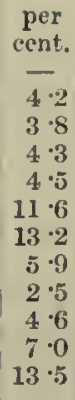 & 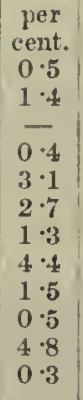 & $\begin{array}{c}\text { per } \\
\text { eent. } \\
- \\
0 \cdot 8 \\
- \\
0 \cdot 8 \\
0 \cdot 8 \\
0 \cdot 5 \\
1 \cdot 5 \\
4 \cdot 3 \\
- \\
1 \cdot 1 \\
0 \cdot 8 \\
0 \cdot 3\end{array}$ & $\begin{array}{c}\text { per } \\
\text { eent. } \\
- \\
- \\
- \\
0 \cdot 4 \\
0 \cdot 5 \\
0 \cdot 4 \\
0 \cdot 1 \\
0 \cdot 6 \\
\overline{0} \cdot 3 \\
- \\
0 \cdot 2\end{array}$ & $\begin{array}{r}\text { per } \\
\text { cent. } \\
6 \cdot 7 \\
10 \cdot 3 \\
6 \cdot 2 \\
22 \cdot 4 \\
17 \cdot 8 \\
5 \cdot 2 \\
11 \cdot 1 \\
20 \cdot 7 \\
8 \cdot 8 \\
6 \cdot 5 \\
8 \cdot 6 \\
4 \cdot 2\end{array}$ \\
\hline $\begin{array}{l}\text { Autumn : } \\
\text { Winter. } \\
\text { Spring. } \\
\text { Summer : }\end{array}$ & $\begin{array}{l}- \\
0 \cdot 1 \\
0 \cdot 3 \\
0 \cdot 2\end{array}$ & $\begin{array}{l}- \\
0 \cdot 3 \\
0 \cdot 4 \\
-\end{array}$ & $\begin{array}{l}- \\
0 \cdot 0 \\
1 \cdot 1 \\
1 \cdot 0\end{array}$ & $\begin{array}{l}1 \cdot 8 \\
3 \cdot 4 \\
2 \cdot 3 \\
8 \cdot 1\end{array}$ & $\begin{array}{l}47 \cdot 8 \\
54 \cdot 3 \\
46 \cdot 8 \\
42 \cdot 2\end{array}$ & $\begin{array}{r}11 \cdot 0 \\
9 \cdot 6 \\
11 \cdot 2 \\
10 \cdot 3\end{array}$ & $\begin{array}{l}6 \cdot 9 \\
3 \cdot 6 \\
4 \cdot 2 \\
3 \cdot 4\end{array}$ & $\begin{array}{r}11 \cdot 1 \\
1 \cdot 1 \\
3 \cdot 3 \\
3 \cdot 7\end{array}$ & $\begin{array}{l}3 \cdot 4 \\
0 \cdot 5 \\
0 \cdot 6 \\
3 \cdot 4\end{array}$ & $\begin{array}{l}1 \cdot 0 \\
0 \cdot 3 \\
0 \cdot 5 \\
2 \cdot 5\end{array}$ & $\begin{array}{l}1 \cdot 2 \\
1 \cdot 0 \\
1 \cdot 4 \\
1 \cdot 9\end{array}$ & $\begin{array}{l}4 \cdot 5 \\
0 \cdot 7 \\
2 \cdot 7 \\
5 \cdot 7\end{array}$ & $\begin{array}{l}2 \cdot 7 \\
6 \cdot 8 \\
7 \cdot 2 \\
8 \cdot 4\end{array}$ & $\begin{array}{l}0 \cdot 6 \\
2 \cdot 1 \\
2 \cdot 4 \\
1 \cdot 9\end{array}$ & $\begin{array}{l}0 \cdot 3 \\
0 \cdot 7 \\
1 \cdot 9 \\
0 \cdot 7\end{array}$ & $\begin{array}{l}- \\
0 \cdot 4 \\
0 \cdot 2 \\
0 \cdot 2\end{array}$ & $\begin{array}{r}7 \cdot 7 \\
15 \cdot 1 \\
13 \cdot 5 \\
6 \cdot 4\end{array}$ \\
\hline Yenr. & $0 \cdot 1$ & $0 \cdot 2$ & $0 \cdot 5$ & $3 \cdot 9$ & $47 \cdot 8$ & $10 \cdot 5$ & $4 \cdot 5$ & $4 \cdot 8$ & $2 \cdot 0$ & $1 \cdot 1$ & $1 \cdot 4$ & $3 \cdot 4$ & $6 \cdot 3$ & $1 \cdot 7$ & $0 \cdot 9$ & $0 \cdot 2$ & $10 \cdot 7$ \\
\hline
\end{tabular}

TABL. IX.-The Relative Frequency of Winds from each of Sixteen Points, and of Calms, observed during Two Cruises of the "Scotia" in the Weddell Sea, in February and March, 1903, and in March, 1904.

\begin{tabular}{|c|c|c|c|c|c|c|c|c|c|c|c|c|c|c|c|c|}
\hline 女 & $\begin{array}{l}= \\
z \\
z\end{array}$ & 军 & 这 & $\neq$ & $\begin{array}{l}\text { |्यें } \\
\dot{2} \\
\text { द्यें }\end{array}$ & $\begin{array}{l}\mid \vec{i} \\
\dot{0}\end{array}$ & $\begin{array}{l}\text { क् } \\
\text { की } \\
\text { में }\end{array}$ & $\dot{\boldsymbol{R}}$ & $\vec{s}$ & $\frac{1}{i n}$ & $\sum \dot{0}$ & $F$ & $\begin{array}{l}2 \\
\angle\end{array}$ & 7 & $\begin{array}{l}\frac{2}{4} \\
\frac{1}{4}\end{array}$ & $\frac{\stackrel{b}{\Xi}}{\tilde{\Xi}}$ \\
\hline $\begin{array}{c}\text { per } \\
\text { cent. } \\
10\end{array}$ & $\begin{array}{c}\text { per } \\
\text { eent. } \\
6\end{array}$ & $\begin{array}{c}\text { per } \\
\text { cent. } \\
11\end{array}$ & $\begin{array}{c}\text { per } \\
\text { eent. } \\
5\end{array}$ & $\begin{array}{c}\text { per } \\
\text { eent. } \\
6\end{array}$ & $\begin{array}{c}\text { per } \\
\text { cent. } \\
3\end{array}$ & $\begin{array}{c}\text { per } \\
\text { cent. } \\
5\end{array}$ & $\begin{array}{c}\text { per } \\
\text { cent. } \\
3\end{array}$ & $\begin{array}{c}\text { per } \\
\text { cent. } \\
5\end{array}$ & $\begin{array}{c}\text { per } \\
\text { cent. } \\
3\end{array}$ & $\begin{array}{c}\text { per } \\
\text { eent. } \\
9\end{array}$ & $\begin{array}{c}\text { per } \\
\text { ecnt. } \\
3\end{array}$ & $\begin{array}{c}\text { per } \\
\text { cent. } \\
8\end{array}$ & $\begin{array}{c}\text { per } \\
\text { cent. } \\
3\end{array}$ & $\begin{array}{c}\text { per } \\
\text { eent. } \\
9\end{array}$ & $\begin{array}{c}\text { per } \\
\text { cent. } \\
5\end{array}$ & $\begin{array}{c}\text { per } \\
\text { cent. } \\
6\end{array}$ \\
\hline
\end{tabular}

From this table it will be seen that the winds blew chiefly from N.E., N., N.W., W. and S.W., and less frequently from S., S.E. and E., but it is pointed out by Mr. Mossmas, in the paper from which this table is derived, that "practically all the east winds recorded during the two eruises were experienced to the south of the Antarctic circle," and also that the east winds were stronger than those from any other quarter, from which it may be reasonably inferred that the wind distribution shown in the table was chiefly due to the comparatively low latitudes at which the bulk of the observations were madc, and that had the vessel remained longer in the higher latitudes she occasionally reached, the easterly current would have been more prominently exhibited.

\section{Wind Curlents at Higher levels.}

In one respect the choice of the "Discovery's" Winter Quarters proved to be an exceptionally fortunate one, inasmuch as it afforded opportunities for obtaining information respecting the direction of motion of the upper air currents by observations of the smoke emitted from the crater of Mount Frebus, nearly 13,000 feet above the sea level.

Althongh the mountain was not visible from the ship it could always be seen, under normal conditions of weather, from a short distance to the westward, and also from the observing station off Cape Armitage.

On many of the sledge journeys, also, the smoke was visille, and it was the practice to include a note of the direction of its drift along with the other meteorological observations. These sledge journey records 
of the drift of the smoke from Mount Krebus are of special valne, becanse, owing to the clistance from the mountain of the ohserver at Winter Quarters and also to his position relative to the line followed by the smoke, it was sometimes difficult for him to determine with precision the direction of the latter. Under these circumstances an independent set of observations, macle sometimes at nearly the same instant, but, always from a different point of view, becomes very nsefnl. The two sets of observations were, therefore, in the first place dealt with separately, and then combined, and the results are given in Table $\mathrm{X}$.

TABLE: X.-Relative Distribution under Fight Points of the Direction of Movement of the Air Currents at High Levels, as ohtained from Observations of $(a)$ Lower Clouds, (b) Smoke from Mount Threbus, and $(c)$ Upper Clouds.

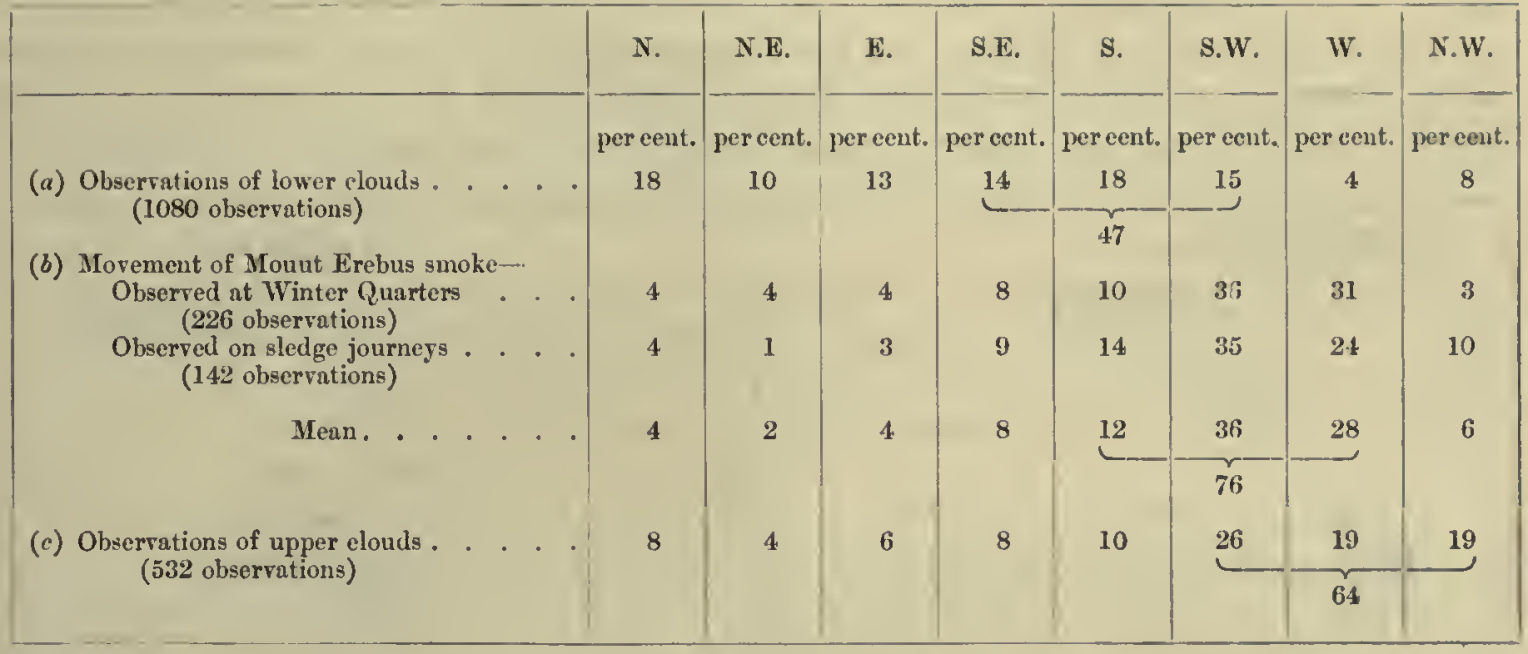

The same table also gives information respecting the movement of the upper air eurrents as shown by the directions in which clouds entered as "upper" and "lower" clouds respectively were observed to travel. It is, perhaps, fairly safe to assume that the elouds entered as lower clouds were correctly classified as such, but it may not be an equally safe assimption that all those entered as "npper" clouds really belonged to the highest cloud levels. Possibly the dividing line between the two elasses of cloud may not always have been easy of recognition, and, in consequence, some of the so-called "upper" clouds, although doubtless high clonds, might, perhaps, with more accuracy have been assigned to the lower levels. The point is of some importance, in so far as it may have influenced the result obtained as regards the prevailing direction of movement, and it ought also to be kept in mind when attempting to assign an approximate average height for the strata dealt with. As regards the height to which the results given in the table may be considered to apply, we shall probably not be far wrong if we assume it to be from 5000 to 7000 feet for the lower clouds, and at least 20,000 feet for the upper clonds, in which case the drift of the Erebus smoke would represent the motion of the air at a level approximately midway between the two.

The figures in the table are very striking. It will be remembered that throughout the whole time the ship lay in Discovery Bay the dominant winds blew from the eastward, and that whilst 80 per cent. of all the winds observed blew from between north-east and south-east, the number of winds from west did not amount to even 1 per cent. But at the level of the lower clouds this distribution becomes materially changed and gives place to one which is far more equable, but which, nevertheless, shows a decided tendency for the air current to shift more to the sonthward and westward, nearly one half of all the observations being included between sonth-east and south-west. Of the individual points, north and south are those which have the largest percentages, each having 18 per cent. of the total number of observations, whilst the percentages under north-east, east, south-east and sonth-west vary from 10 per cent. to 15 per cent. West, which is still the least frequently observed direction, has a percentage of 4 , and north-west 8 per cent., so that, even at this comparatively low level, a westerly current begins to show itself. 
The two sets of olserrations of the drift of Mount Frebus smoke give slightly different percentages for each of the eight points, but the general agreement between them is surprisingly close considering the conditions under which they were made. Taking the menn of the two sets, we find that at the level of from 13,000 to 14,000 feet the change in the direction of movenent of the air currents, which is indicated by the lower clouds, has become further developed, and that, at that height, the easterly current of the surface has become almost entirely superseded by another from between south-west and west. These two directions together account for no less than 64 per cent. of all the observations, sonth-west alone having 36 per cent. Sonth has 12 per cent., north-east has only 2 per cent., and north and east have each but 4 per cent. At this level, therefore, the dominant air current is unmistakably from between south-west and west, and winds from between east and north, the quarter from which the surfaee winds chiefly came, are comparatively rare.

At the level of the upper clouds we find this progressive change in direction towards the westward carried still further, and a marked prevalence of north-westerly winds is shown for the first time. Southwest, it is true, still remains the slightly doninant direction, but this may possibly be due to the inclusion

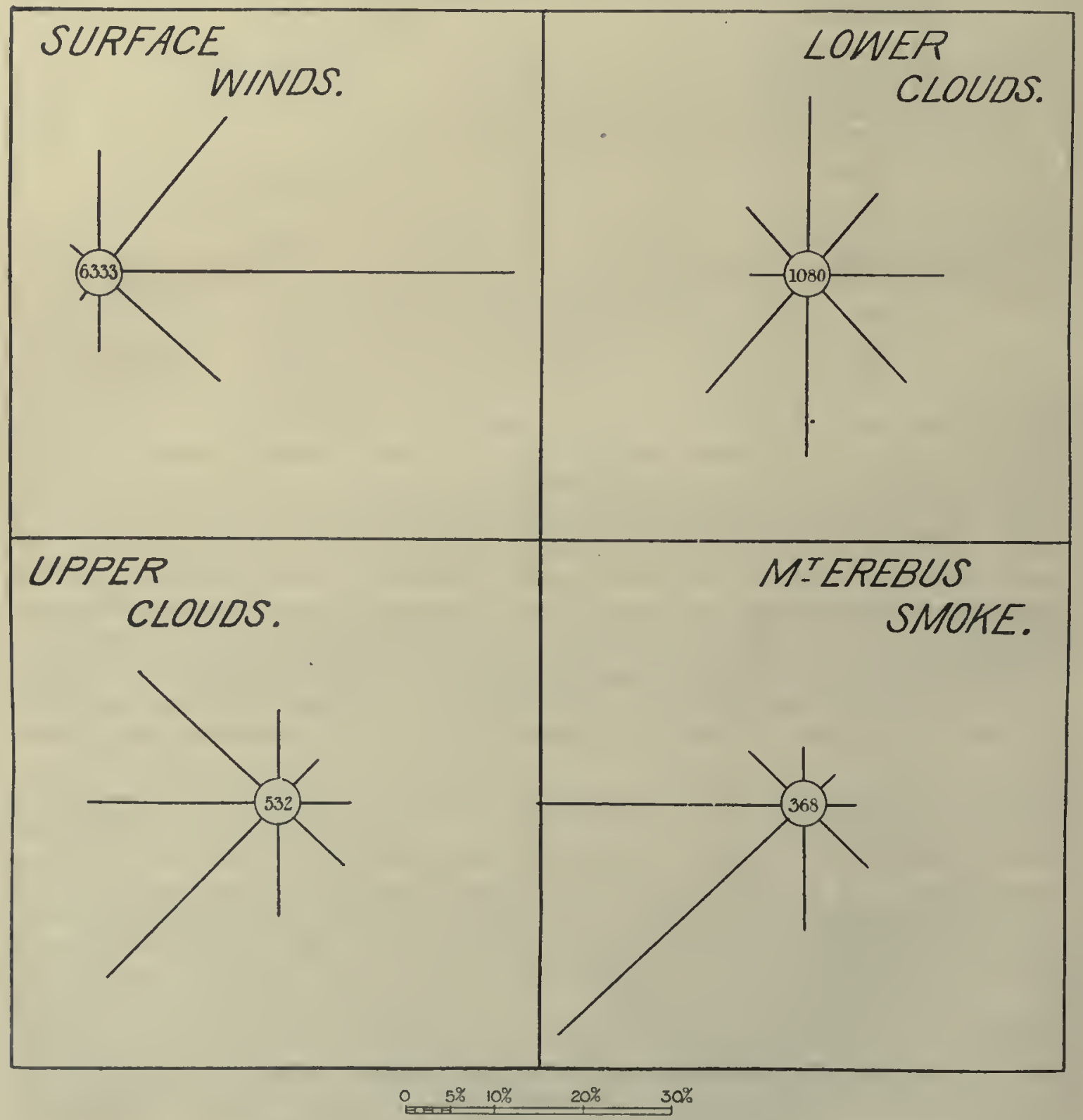

Fig. 2. Distribution of air currents at various levels. 
of clouds moving at a lower level than that of the trio upper clouds. If this surmise is correct, it is evilent that had such clouds been grouped with the "lower" clonds, the change of the eurrent towards the south-west, already shown by the lower cloud obscrvations, would have been even more nuarked, and its further' westerly development with height through the lovel of the Erebus smoke to that of the upper elouds would have been still more apparent. But tho figures as they stand in the table are sufliciently striking: 64 per cent. of all the observations of upper clouds show a flow of air from between north-west and south-west. At the lower lovel of the Erebus smoke 76 per cent. of the ohservations are comprised between west and south, and, still lower, 47 per cent. of the lower clouds were found to move from between south-west and south-east, whilst at the level of the sea 80 per cent. of tho winds came from between north-cast and south-east. At the surface, east is the dominint wind direction, hut at the level of the Erebus smoke, and of the upper clouds, the prevailing wind is from a westerly direction.

\section{VELOCITY OF THE WIND.}

The winds of that portion of the Antarctic region in which the "Discover'y" wintered appean" to possess two distinctive characteristics-exceptional gustiness and a great tendency to die awaty and give place to calm.

As regards the second of these characteristics, the observations of wind for the whole period of the vessel's stay in Winter Quarters show an average of 23 per cent. of calms, the inonthly percentages varying fron a minimum of $13 \mathrm{in}$ August, 1832, to a maximum of $41 \mathrm{in} \mathrm{July,} \mathrm{1903,} \mathrm{both} \mathrm{of} \mathrm{them} \mathrm{winter}$ months. But the distribution of calms over the different months is very irregular and shows littlo indication of a seasonal variation.

Away from the ship, on the sledge journoys, calns were probably more frequent than at Discovery Bay, but the irregular intervals at which the wind observations were made on these journeys, probalbly owing to the exigencies of sledging, make it difficult to compare satisfactorily their relative pereentage frequency. The figures in Table V. show a frequeney of 54 per cent. and 49 per cent. for calms upon the two western journeys for which an analysis is given, and of 35 per cent. for the two journeys to the south. Fach of these journeys was of several weeks' duration, the shortest covering about fifty days, and the longest three months.

As has already been mentioned, the frequent occurrence of ealms was a conspicuous feature of the wind observations at Cape Adare and at the Winter Quarters of the "Gauss."

The gustiness of the wind can be inferred from the fact that two numbers are almost invariably used in the Journal to indicate the force as estimated by Beaufort's scale : e.g., "SW, 1-6; S by W, 3-7." But the inference is amply confirmed by numerous notes by the observers, who call attention to squalliness as an especially eharacteristic feature of the gales and "blizzards" they experienced.

\section{Mean Monthly Velocity.}

The mean hourly veloeity of the wind for each month obtained from the readings of both the eup anemometer and the pressure-tube anemometer is shown in Table XI. to be $10 \cdot 3$ miles per hour by the former and $10^{\circ} 4$ by the latter.

It has already been said that the pressure-tube records of wind velocity were frequently interrupted, and owing to that faet a satisfactory comparison cannot be niule between the mean vclocities for the individual months obtained from the two anemometers, because the occurrence in any montli of an unusually windy period or of a long spell of very ealm weather, during either of which the eup instrument alone was at work, would obviously canse the mean velocity for the inonth dedueed from the readings of that instrument to be higher in the one case and lower in the other than tho monthly mean obtained from the snaller available number of pressure-tube observations; and the rather large differences which the table shows in the mean velocities by the two instruments for certain months is explained in this way.

But as the length of the period covered by the records is increased the risk of error from this cause becomes diminished, since the lacunæ in the pressure-tube record extending over both caln and windy periods tend to balance one another, and therefore it is probable that tho mean hourly velocity for the 
'TAmis: XI.-Mean Honrly Velocity of the Wind for each Month, by Cup Anemometer and by Pressure-'Tube Anemoneter (lirespective of Direction).

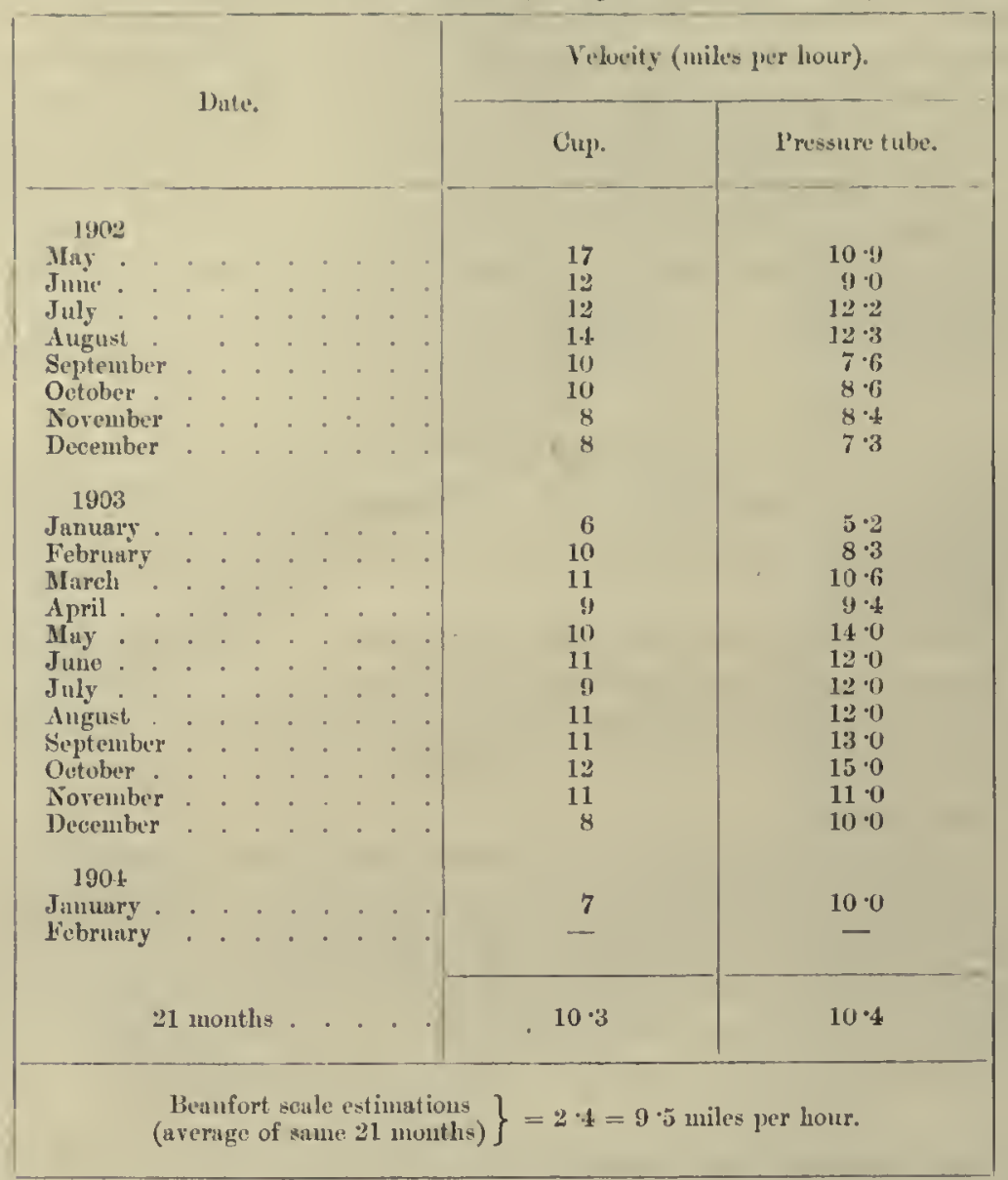

twenty-one months covered by the pressure-tube observations is fairly accurate. The mean hourly velocity so obtained is practically identical with that derived from the readings of the cup anemometer.

Bearing in mind how essentially different in prineiple are the two instruments, so close an agreement between them is very satisfactory, and it leaves little donlbt that for the periorl covered by the stay of the expedition at Ross Island the mean velocity of the wind there was between 10 and 11 statnte miles per hour.

This velocity is not remarkably high, and is one and a half miles per loour less than the mean hourly velocity of the wind shown by twenty-five year's' observations at Valencia, in the south-west of Ireland, and 5 miles per hour less than the mean at the Scilly Islands; but its correctness is further confirmed by the faet that the mean force of the wind, as estimated by the numbers of Beanfort's scale, for the longer period of twenty-five months was $2 \cdot 5$, which is equal to a velocity of about nine and a laalf miles per hour, using for the conversion the velocity equivalents of the Beanfort scale numbers adopted by the Neteorological Offiee.

Speaking broadly, the variation in the mean velocity from month to month throughont the year indieates pretty clearly a seasonal variation, with a maxinum in winter and a minimum in summer. The lighest mean monthly velocity was 17 miles per hour in Nay, 1902,-the first complete nonth for which readings of the cup anemometer are available; but this was considerably above the mean velocity in any other month, and, disregarding small variations, there was from thence a steady decrease in strength to a minimum of 6 miles per hour in Janutry, 1903; then the force rose again to 11 miles per hour in March and to 12 miles per honr in October, when it again fell away to another minimum of 7 miles per hour in the following Jamniry (tig. 3). There appeals, therefore, to be a simple oscillation of force thronghont the year from a nuximum in winter to a minimum in summer; and, with the linited number of observations availible, that is perhajs all wo ate justified in saying. 

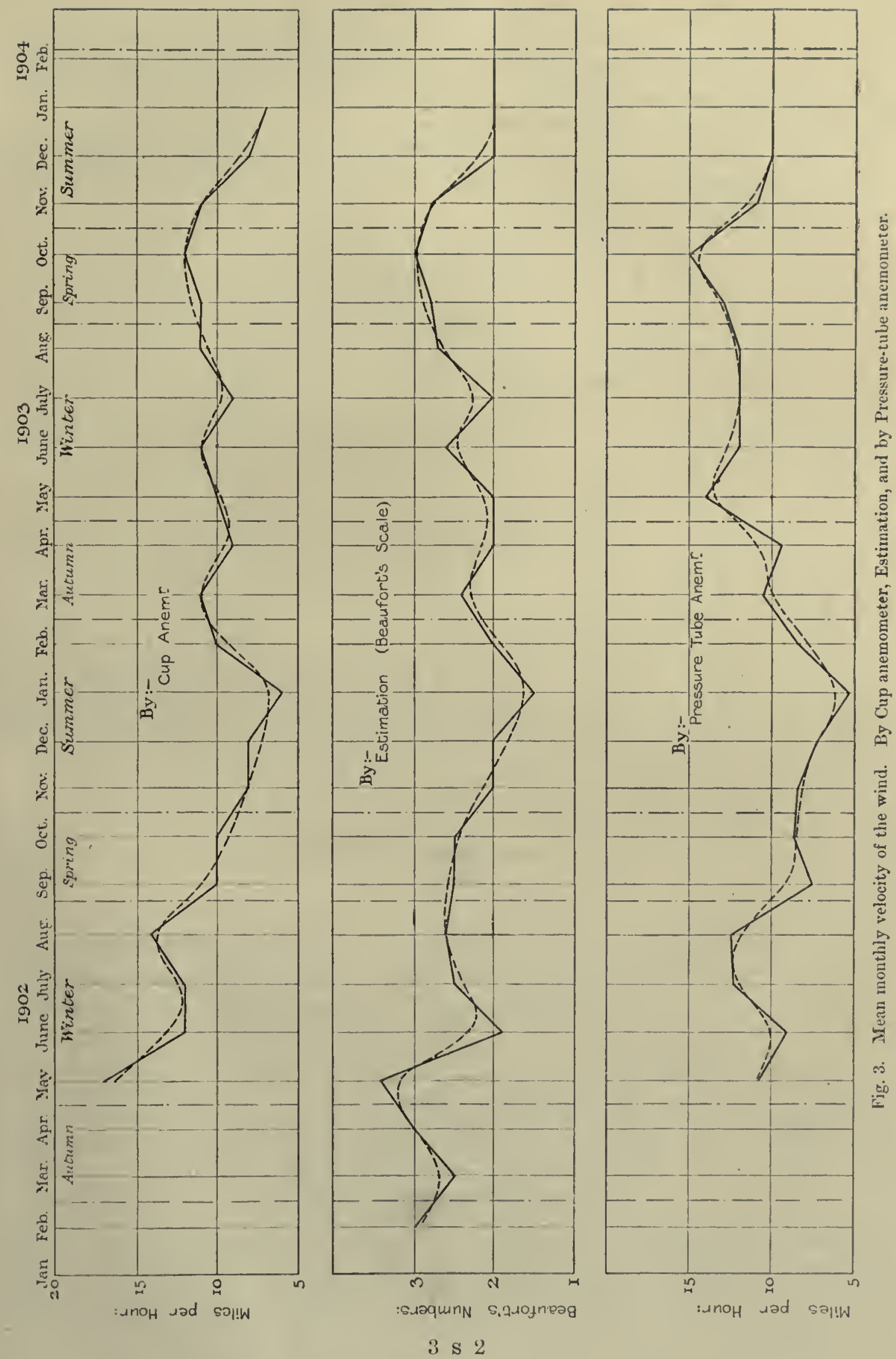
Diuraat، Variation of Wind Velocity.

For the diurnal variation in wind force we have to rely eliefly upon the pressure-tnhe anemometer recorls, and, owing to their incompleteness for certain months, they have been grouped for the different seasons instead of months in order to obtain mean values.

The results of this grouping are given in Table XII., and they are also shown graphically in figs. 4, 5, 6, where the seasonal curves are shown for each year, and also for the two years combined, after the values hurve been smoothed by the formula $\frac{a+2 b+c}{4}$.

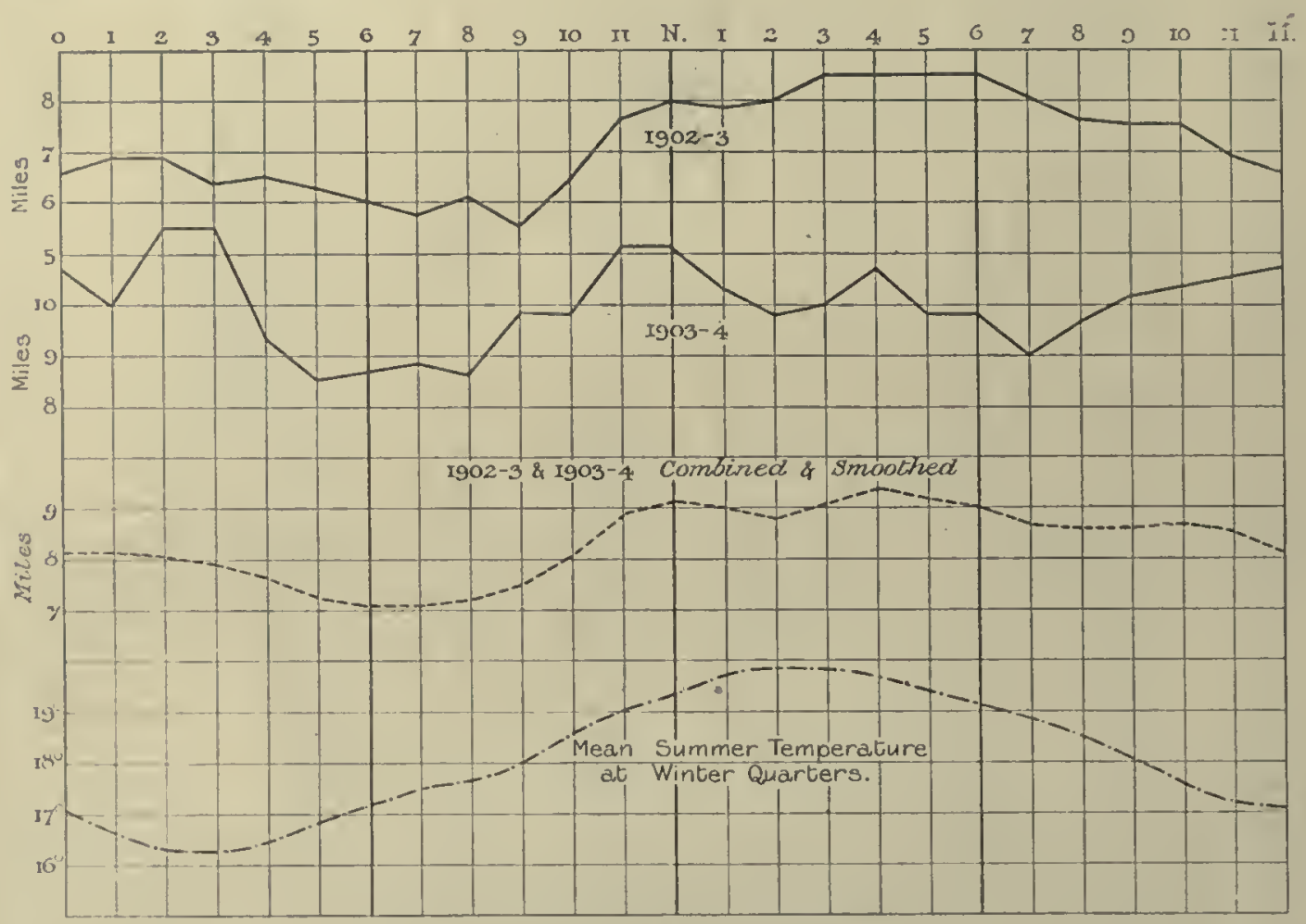

Fig. 4. Dinrnal variation of wind velocity.

Summer.

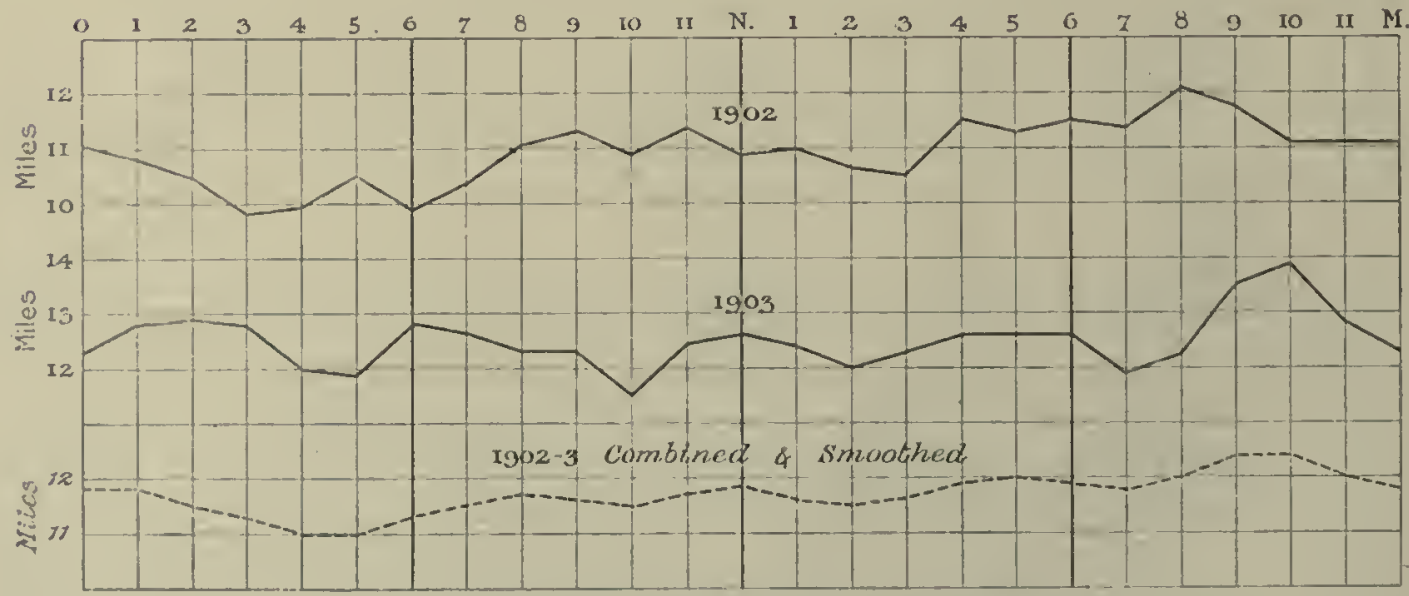

Fig. 5. Dimnal variation of wind velocity.

Wiuter. 
The smoothed eurvo for tho summer four months shows a decrease of force from the maximum at 4 p.m. to a well-marked minimum between 6 a.m. and 7 a.m.; from 7 a.m. to nonn it increases again stealily, but a slight inflexion is then shown in the curvo to a small sceondary minimum at 2 p.m., licfore the

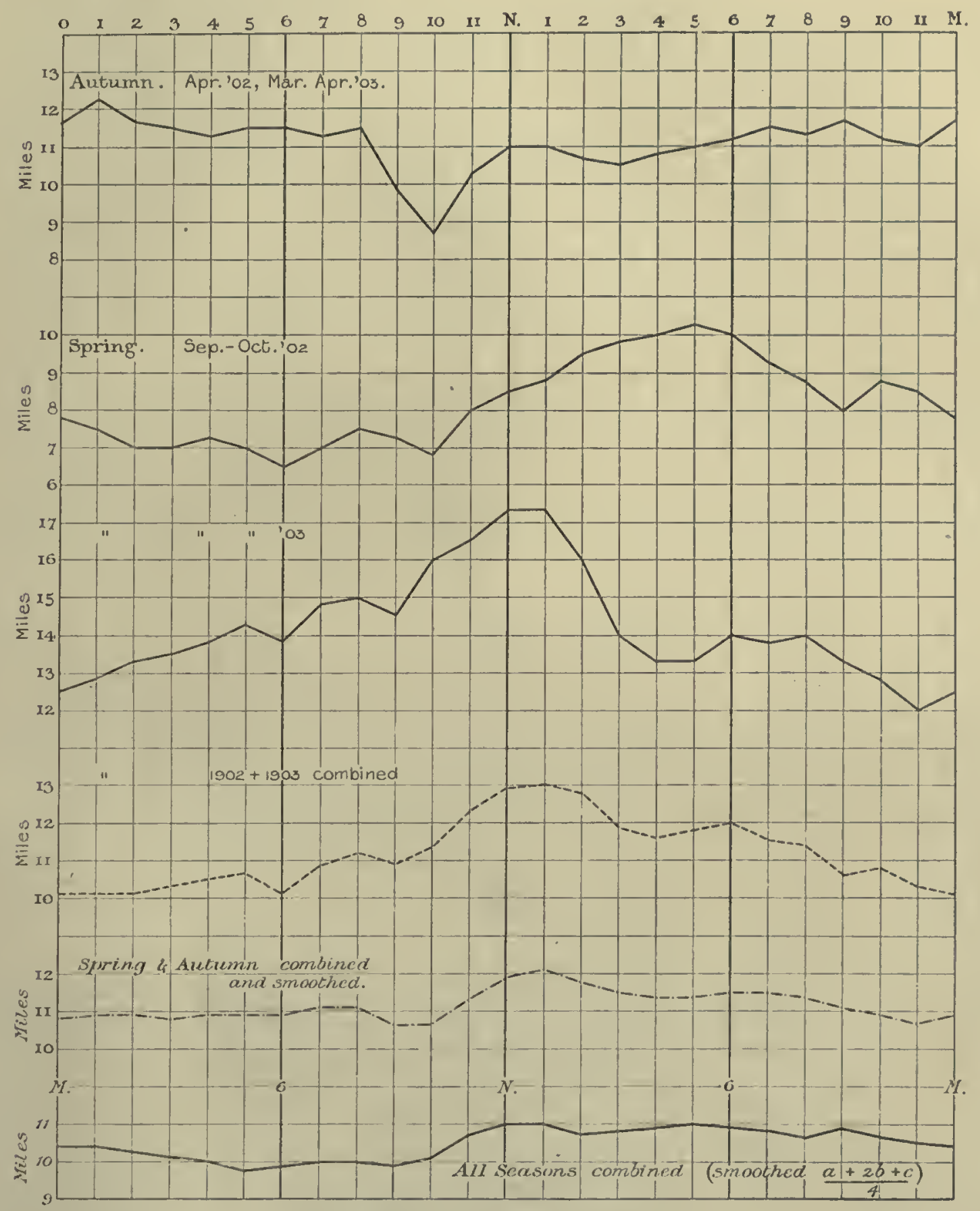

Fig. 6. Ihiurnal variation of wind velocity.

Equinover.

maximum of the day is reached at 4 p.m. The smoothed curve approximates very closely to the summer curve for 1902-3, but is more divergent from that for 1903-4; in both years, however, confirmation is given, although in varying degrees, to the secondary minimum early in the afternoon. With the execption of this minimum the summer eurve of wind foree eoinciles fairly well with the diurnal march 


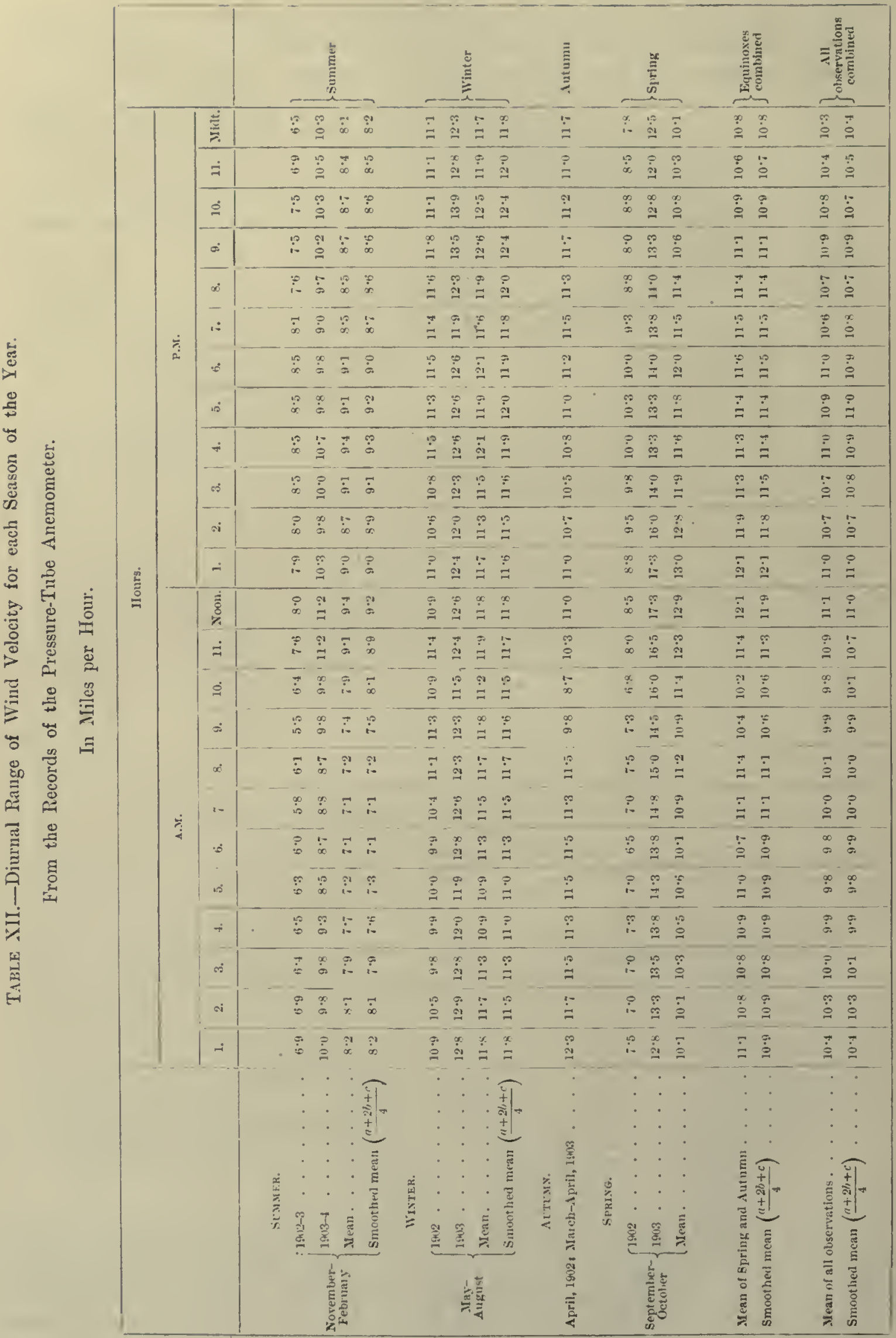


of temperatnre in summer, the man difference being that in the morning the wind force continues to decrease for some hours ifter the temperatnue has began to rise, and that similarly a slight ling is shown in the eommencement of the decrease in force in the afternoon, the maximum of temperatnre vecurring at 2 p.m. and that of wind force at 4 p..n.

As might have been expected, the smoothed winter eurve of dinrual viuriation shows a much smaller range of velocity, anounting to only 1.4 miles per hour. The mininum occurs in the morning, as in summer, but two hours earlier, and from 5 a.m., when the velocity is lowest, mill the miximum is reached at 9 p.m. the veloeity increases in a series of slight undulations, having maximat at 8 a.m., noon, 5 p.m., and 9 p.m., and minima at 10 i.m., 2 p.m., 7 p.m., ind at midhight.

Only three months' observations are availatble for the autumn curve, the instrument not laving been started until April, 1902. The nniximum force oecurs at $1 \mathrm{am.m.}$, and not very much variation is shown thronghout the day, except that in the forenoon there is in alm'upt fall from eleven and a half miles per hour at 8 a.m. to the ninimum of the dity at 10 i.m., followed in the next two hours l,y ant equally albrupt rise to abont the averige force at noon ; other slight minima are also indicated at 3 p.m. anrl 11 p.m., but, if we onit the large decrease in the forenoon, the total amount of the oscillation is less than 2 miles per hour.

The diurnal range is greatest during the spring months, but, with the exception of that feature, the curves for the two years are very little alike. The combined eurve indicates a fairly uniform simple oseillation from a minimum at midnight to a maximum between noon and 2 p.u., snlall secondary minima being shown at 6 a.m. and 9 i.m., and at 4 p.m. and 9 p.m.

Wind force is, however, so variable an element that care is required not to attaeh nudne weight to the evidence of so limited a series of observations, and whilst we may perhaps safely aecept the broader features of the eurves of diurnal variation the smaller ascillations can only be aecepted with reserve.

GALES.

Defining a "gale" as a wind of at least force 8 on Beatufort's scale, or one in which it attains a mean velocity of at least 38 statute miles per hour, the number of such gales which occurred during the "Diseovery's" stay in Winter Quarters was not remarkilbly large.

The frequency of their occurrence as eompared with that of more moderate winds maty be shown by the percentage of the total number of observations of gale force in each nouth to the total number of observations in the month; the figures ale as follows:-

\begin{tabular}{|c|c|c|c|c|c|c|c|c|c|c|c|c|c|c|c|c|}
\hline & & & & & $\stackrel{\dot{E}}{\stackrel{\Xi}{\Xi}}$ & 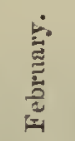 & 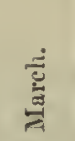 & $\frac{\overrightarrow{2}}{\frac{2}{3}}$ & 票 & $\stackrel{\ddot{\Xi}}{\Xi}$ & $\frac{i}{3}$ & 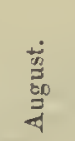 & 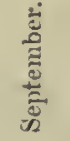 & $\frac{\check{\Xi}}{\frac{0}{0}}$ & $\frac{\stackrel{s}{0}}{\frac{\Xi}{E}}$ & 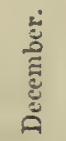 \\
\hline $\begin{array}{l}1902 \\
1903\end{array}$ & • & $\dot{.}$ & $\dot{.}$ & $\dot{0}$ & $\begin{array}{c}\text { per } \\
\text { cent. } \\
-\end{array}$ & $\begin{array}{c}\text { per } \\
\text { eent. } \\
2 \\
-\end{array}$ & $\begin{array}{c}\text { per } \\
\text { cent. } \\
6 \\
-\end{array}$ & $\begin{array}{c}\text { per } \\
\text { cent. } \\
1 \\
-\end{array}$ & $\begin{array}{c}\text { por } \\
\text { cent. } \\
\frac{6}{-}\end{array}$ & $\begin{array}{c}\text { per } \\
\text { cent. } \\
- \\
3\end{array}$ & $\begin{array}{c}\text { yer } \\
\text { cont. } \\
+ \\
3\end{array}$ & $\begin{array}{c}\text { per } \\
\text { eent. } \\
-\end{array}$ & $\begin{array}{c}\mathrm{l}^{\mathrm{x}} \mathbf{x} \\
\frac{1}{1}\end{array}$ & $\begin{array}{c}\text { per } \\
\text { cent. } \\
1 \\
1\end{array}$ & $\begin{array}{c}\text { per } \\
\text { cent. } \\
-\end{array}$ & $\begin{array}{c}\text { per } \\
\text { cent. } \\
- \\
-\end{array}$ \\
\hline
\end{tabular}

From this summary it would appen' that most of the gales occul' in the autumn or vinter months, but at the same time it is noteworthy that July and October are the only months for which there are gales in both years.

'These figures, however, do not indicate the number of distinct gales which oceurted, regarding oaeh gale as a separate phenomenon lasting for a longer or shorter period. 'The number of gales, during any part of which a velocity of at leist 38 miles per hour wits recorded by the eup memometer was only eleven; but the number of distinct gales, in the cuurse of which an estinated force of 8 or above by beaufort's scale was logged, was much lirger, as follows:- 


\begin{tabular}{|c|c|c|c|c|c|c|c|c|c|c|c|c|c|c|c|c|c|}
\hline & & & & & $\stackrel{\stackrel{E}{E}}{\stackrel{E}{E}}$ & 总 & $\frac{\vec{d}}{\frac{2}{2}}$ & 焉 & 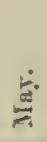 & $\stackrel{巳}{\Xi}$ & $\stackrel{5}{5}$ & 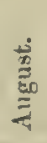 & $\frac{\stackrel{s}{0}}{\stackrel{\Xi}{\Xi}}$ & $\frac{\ddot{\Xi}}{\stackrel{0}{0}}$ & $\begin{array}{l}\frac{\dot{E}}{0} \\
\frac{E}{E} \\
\dot{0} \\
\dot{0}\end{array}$ & 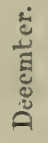 & Total. \\
\hline $\begin{array}{l}1902 \\
1903\end{array}$ & . & . & : & . & - & 2 & $\begin{array}{l}7 \\
2\end{array}$ & 7 & $\begin{array}{l}2 \\
1\end{array}$ & $\frac{1}{7}$ & $\begin{array}{l}2 \\
6\end{array}$ & $\frac{-}{5}$ & $\begin{array}{l}1 \\
6\end{array}$ & $\begin{array}{l}1 \\
3\end{array}$ & $\overline{3}$ & - & $\left.\begin{array}{l}23 \\
38\end{array}\right\} 56$ \\
\hline
\end{tabular}

In many of these instances, however, the gale force lasterl for only a short time, and was in faet the maximum force of the brief gusts which appear to have been a feature of every Antaretic gale. Owing to their brevity they did not add much to the mileage reeorded by the eup anemometer, and therefore the anemoneter total for the hour often fell short of the minimum of 38 miles per hour which was taken to indicate a gale in the preceding table. The second set of figures may therefore be regarded as giving an extreme view of the prevalence of gales, and by going it step further and assigning to each gale an average duration, we may get some idea of the prevalence of gales as compared with more moderate winds and ealms. From an examination of the register it would appear that six hours as the ateruge duration of each gale is likely to be an over, rather than an under, estimate, and this duration would give us, very nearly indeed, the same percentage of hours of gale force, as referred to the total number of olsservations for the whole period of two years, as is given in the first of the two talules.

The total number of hourly observations of wind in which the force exeeeded a velocity of 38 iniles per hour at Scilly, Fleetwood, and Deerness, in the British Isles, during the same two yeirs, 1902 to 1903 , was 442,306 , and 264 respectively, and these totals represent a percentige of duration of galo foree to that of more moderate winds of 5 per cent., $3 \frac{1}{2}$ per cent., and 3 per eent. respectively, so that the frequeney of gales appears to be greater on the western coasts of Great Britain than at Ross Island.

The percentage of moderate to strong winds, which did not reacl gale force but were logged as ranging in force between 4 and 7 of Beaufort's seale, is shown in the following table:-

\begin{tabular}{|c|c|c|c|c|c|c|c|c|c|c|c|c|c|c|c|c|}
\hline & & & & & 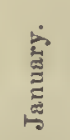 & 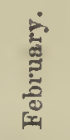 & 总 & $\begin{array}{l}\dot{\vec{n}} \\
\dot{4}\end{array}$ & 至 & $\stackrel{\mathscr{\varrho}}{\Xi}$ & $\stackrel{5}{3}$ & 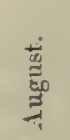 & 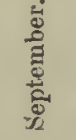 & 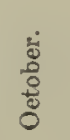 & 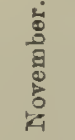 & 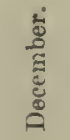 \\
\hline $\begin{array}{l}1902 \\
1903\end{array}$ & * & . & : & . & $\begin{array}{r}\text { per } \\
\text { cent. } \\
- \\
6\end{array}$ & $\begin{array}{c}\text { per } \\
\text { cent. } \\
50 \\
21\end{array}$ & $\begin{array}{c}\text { per } \\
\text { cent. } \\
23 \\
27\end{array}$ & $\begin{array}{c}\text { per } \\
\text { cent. } \\
36 \\
16\end{array}$ & $\begin{array}{c}\text { per } \\
\text { cent. } \\
49 \\
24\end{array}$ & $\begin{array}{r}\text { per } \\
\text { cent } \\
16 \\
46\end{array}$ & $\begin{array}{r}\text { per } \\
\text { cent. } \\
22 \\
23\end{array}$ & $\begin{array}{c}\text { per } \\
\text { cent. } \\
33 \\
31\end{array}$ & $\begin{array}{r}\text { per } \\
\text { cent. } \\
32 \\
40\end{array}$ & $\begin{array}{c}\text { per } \\
\text { cent. } \\
30 \\
43\end{array}$ & $\begin{array}{c}\text { per } \\
\text { cent. } \\
13 \\
38\end{array}$ & $\begin{array}{c}\text { per } \\
\text { cent. } \\
10 \\
23\end{array}$ \\
\hline 1904 & . & & . & . & 23 & 31 & $\ldots$ & - & - & - & - & - & - & - & - & $=$ \\
\hline
\end{tabular}

The direetion from whieh gales most frequently blew was sonth, and this was especially the ease in the winter months; but gales were also experienced from south-east, eust, and north-east, and, in a few instances, from south-west. North winds and winds from the west of north did not attain gale force.

The highest recorded velocity of the wind was an average of 62 miles per hour for 12 consecutive hours on July 19th, 1902. Unfortunately, there was no record of this gale made by the pressure-tube anemometer, and therefore what the velocity was in the gusts cannot be known. The reeords of severe gales in the British Islands made by pressure-tube anemometers would justify us in expecting that with so high a mean velocity the gusts would not be far short of the rute of 100 miles per hour. On June 29, 1903, the observer was blown from the sereen whilst attempting to read the thernometer, the squalls being described as "furious"; and again, on August 10 of that year, the force was logged as 12 in heavy squalls, and it was impossible to stand at the bottom of the gangway leading from the ship to the ice. On other occasions the force in the squalls was spoken of as sovere, and more than once the anemometer became disabled, although, as the instrument was of somewhat slender build, too much stress must not be laid upon the significance of that fact. In every gale the squalls were a very notable feature, as was also the drifting snow, which oceasionally quite filled the air. The low temperature and drifting snow 
combined must have made the strong wind of the gales a very unplensant experience, and it is quite possible that that fact may lave led unconsciously to some amonnt of exiggeration in estimating the force of the wind at such times.

\section{Ciranges of Tmmperature axi of Wind.}

Sndlen large changes of temperature were of frequent occurrence at the Winter Quarters, and an attempt has been made to correlate them with changes in the direction and velocity of the wind. It was fonnd that in 17 per cent. of the instances in which such a sudden rise in the temperature occurred, and in 19 per cent. of those in which it as abruptly fell, the movement was not accompanied by any change either in the direction or forec of the wind.

When the change of temperature coincided with the springing up of a wind after a calm, the temperature nearly always rose, and, indeed, the only exceptions to this rule occurred when the temperature change between the two observations amounted to less than ten clegrees; in no case when the rise in temperature exceeded that amount did it accompany a falling away of the wind force to a calm. On the other hand, the large and sudden decreases of temperature almost as generally coincided with the dying away of the wind force to ealm.

Table XIII. gives the number of instances in which these rapid changes of temperature were accompanied by a change from a wind to a calm, or vice versa, and also the direction of the wind when its force fell or when it sprang up. In both cases the direction was most frequently, but not invariably, northerly; several of the larger increases of temperature occurred with the advent of an ensterly breeze, and in one or two cases the wind came from south. 
TABIA. XIII.-Number of Tines in which the lalling Away of the Wind Foree to a Calm, or vice rerse, was aceompanied by a Rise or by a Fall in 'Temperature.

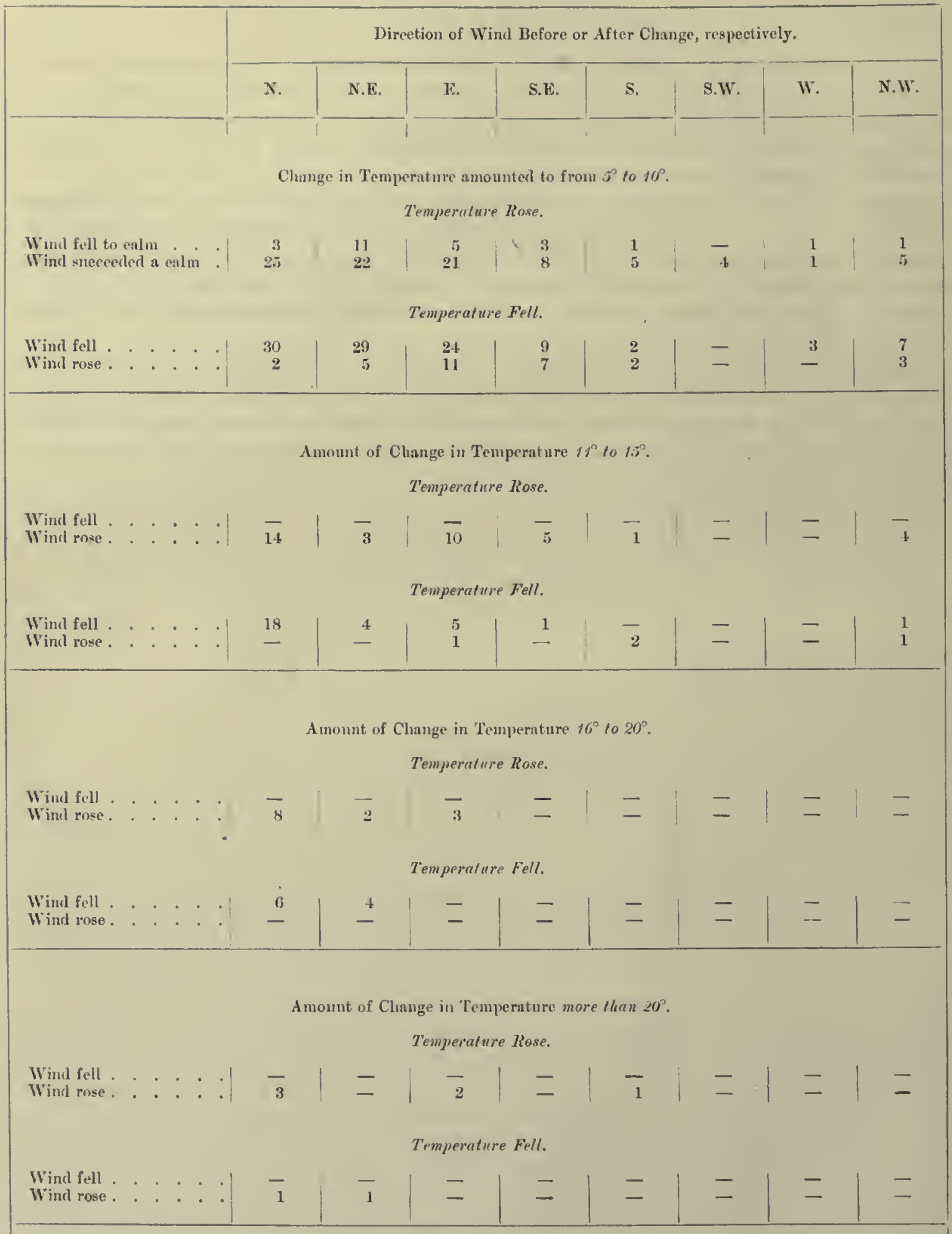

In 195 instanees, or 17 per cent. of the total number, in whieh there was a sudden rise of temperature, and in 225 , or 19 per cent., of those in whieh the temperature fell the wind force remained unehanged. 


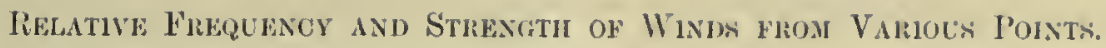

Figss. 7 to 10 show the relative frequency of winds from each of sixteen points, comlined with their strength. The length of the single line indicates the percentage of light winds (forces 1 to 3 of Beaufort's seale) observed from the point at which it is drawn; the length of the double line similarly indicates the percentage of morlerate winds (forces 4 to 7 of Beanfort's scale), and that of the broal line that of gales (force 8 and above); the total length of the line thns showing the frequency of all winds, irrespective of force, from the point indieated. The figures are drawn for each month, and are grouperl under the several seasons.

In the summer months light winds predominated, and gales are only slown in February, 1902, the month of arrival of the expectition at Winter Quarters. In the autumn months the wind force had decidedly increased, and winds of moderate strength were upon the whole more conmon than light winds; gales were somewhat frequent in March, 1902, but in that month only. Moderate forces were, upon the whole, more frequent than light winds in the winter months, and gale forces were comparatively frequent, especially from southerly points. In the spring months there were not many gales recorded, lunt winds of moderate force were very conmon.

TABLe XIV.-Mean Hourly Velocity of the Wind, for each Month, and for each of Sixteen Points, in Niles per Hour by the Cup Anemometer.

\begin{tabular}{|c|c|c|c|c|c|c|c|c|c|c|c|c|c|c|c|c|c|c|}
\hline Month. & 学 & $\begin{array}{l}y \\
y \\
y \\
y\end{array}$ & 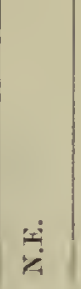 & 瓷 & İ & 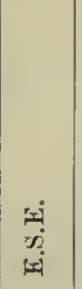 & $\frac{\omega i}{\infty}$ & 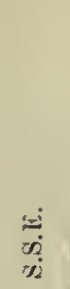 & $\omega^{\circ}$ & $\begin{array}{l}= \\
\infty \\
B \\
B\end{array}$ & $=$ & $\begin{array}{l}= \\
\dot{n} \\
=\end{array}$ & $=$ & $\begin{array}{l}\frac{1}{y} \\
\frac{1}{4}\end{array}$ & 竞 & $\begin{array}{l}\frac{7}{4} \\
y_{4}\end{array}$ & 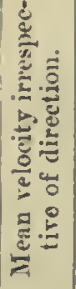 & 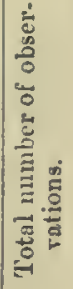 \\
\hline 1902 & miles & miles & miles & milos & miles & niles & miles & miles & miles & miles & miles & miles & miles & miles & niiles & miles & miles & \\
\hline May. & 8 & 21 & 17 & 22 & 19 & 13 & 19 & 21 & 27 & 27 & 44 & - & - & - & 3 & 5 & 17 & 361 \\
\hline Jnne & 7 & 7 & 14 & 16 & 15 & 12 & 11 & 18 & 17 & 19 & 14 & - & 13 & - & y & 4 & 12 & 360 \\
\hline July. . & 4 & $1 \cdot 5$ & 13 & 14 & 13 & 12 & 10 & 18 & 36 & 47 & 15 & - & - & - & - & - & 12 & 370 \\
\hline August. . & 5 & 5 & 14 & 16 & 19 & 14 & 15 & 14 & 21 & - & - & - & 5 & - & 9 & 7 & 14 & 1.58 \\
\hline Septomber. & 5 & 4 & 10 & 15 & 13 & 10 & 11 & 15 & 17 & - & - & - & - & - & 3 & - & 10 & 355 \\
\hline October. . & 2 & 4 & 10 & 17 & 15 & 9 & 10 & 18 & 17 & - & 15 & - & - & - & 2 & - & 10 & 312 \\
\hline November. & 5 & ( & 9 & 12 & 14 & 8 & 9 & 10 & 17 & 4 & 19 & - & - & - & 4 & 4 & 8 & 360 \\
\hline December. & 6 & 7 & 7 & 15 & 12 & 10 & 10 & 11 & 11 & 15 & 18 & - & 4 & 1 & 4 & 6 & 8 & $3 \% 2$ \\
\hline 1903 & & & & & & & & & & & & & & & & & & \\
\hline January & 6 & 8 & 7 & 12 & 10 & 9 & 7 & 10 & 8 & - & - & - & 6 & - & 4 & 7 & 6 & 371 \\
\hline February & 4 & 4 & 13 & 15 & 14 & 11 & 9 & 12 & 7 & - & 2 & - & 2 & - & - & - & 10 & $336^{\circ}$ \\
\hline March : & 7 & 10 & 14 & 15 & 15 & 10 & 10 & 15 & 10 & - & 4 & - & - & - & 7 & - & 11 & 372 \\
\hline April . & 6 & 5 & 13 & 15 & 15 & 11 & 9 & - & 8 & - & $3 \cdot 5$ & - & - & - & - & 5 & 9 & 343 \\
\hline May. . & 7 & 5 & 17 & 18 & 15 & 13 & 10 & 14 & 13 & - & 8 & - & - & - & 4 & 6 & 10 & 372 \\
\hline June . & 5 & 13 & 12 & 17 & 15 & 13 & 16 & 13 & 20 & 34 & 20 & - & 3 & - & + & 10 & 11 & 360 \\
\hline July. . . & S & 6 & 14 & 19 & 14 & 9 & 12 & 15 & 8 & - & 2 & - & - & - & 4 & 7 & 9 & 372 \\
\hline August. . & 5 & 7 & 14 & 18 & 13 & 13 & 13 & 21 & 21 & 20 & 21 & $1 \cdot 5$ & - & - & 4 & 6 & 11 & 370 \\
\hline September. & 7 & 11 & 16 & 19 & 15 & 13 & 13 & 10 & 15 & 15 & 7 & - & - & 1 & 7 & 6 & 11 & 360 \\
\hline October. & 5 & 8 & 18 & 16 & 15 & 13 & 13 & 6 & 18 & 8 & 15 & - & - & - & 3 & 4 & 12 & 372 \\
\hline Norember. & 6 & - & 12 & 14 & 11 & 11 & 13 & 15 & 15 & 23 & 28 & - & 5 & - & 15 & 4 & 11 & 246 \\
\hline December . & 4 & 6 & 7 & 11 & 11 & 12 & 11 & 13 & 10 & - & - & - & 2 & - & 3 & 4 & 8 & 212 \\
\hline $\begin{array}{c}1904 \\
\text { January }\end{array}$ & 4 & 9 & 9 & 11 & 10 & 7 & 8 & 5 & 3 & - & 3 & - & - & - & 4 & - & 7 & 1.40 \\
\hline February & 6 & $13^{\circ}$ & 3 & 10 & 11 & 7 & 10 & 10 & 4 & - & $1 \cdot 5$ & - & - & - & - & - & 8 & 47 \\
\hline Mean & $5 \cdot 5$ & $7 \cdot 6$ & $12 \cdot$ & $15-3$ & $13 \cdot 8$ & $10 \cdot 9$ & $11: 3$ & 13 & $14 \cdot 7$ & $21 \cdot 2$ & $13 \cdot 2$ & $1 \cdot 5$ & $5 \cdot 0$ & $1 \cdot 0$ & $4 \cdot 4$ & $5 \cdot 7$ & $10^{\circ}$ & 6921 \\
\hline
\end{tabular}

From the figures given in Table XIV. it would appear that, on the whole, the southerly winds were stronger than those experienced from any other quirter, south-south-west winds giving for the whule 


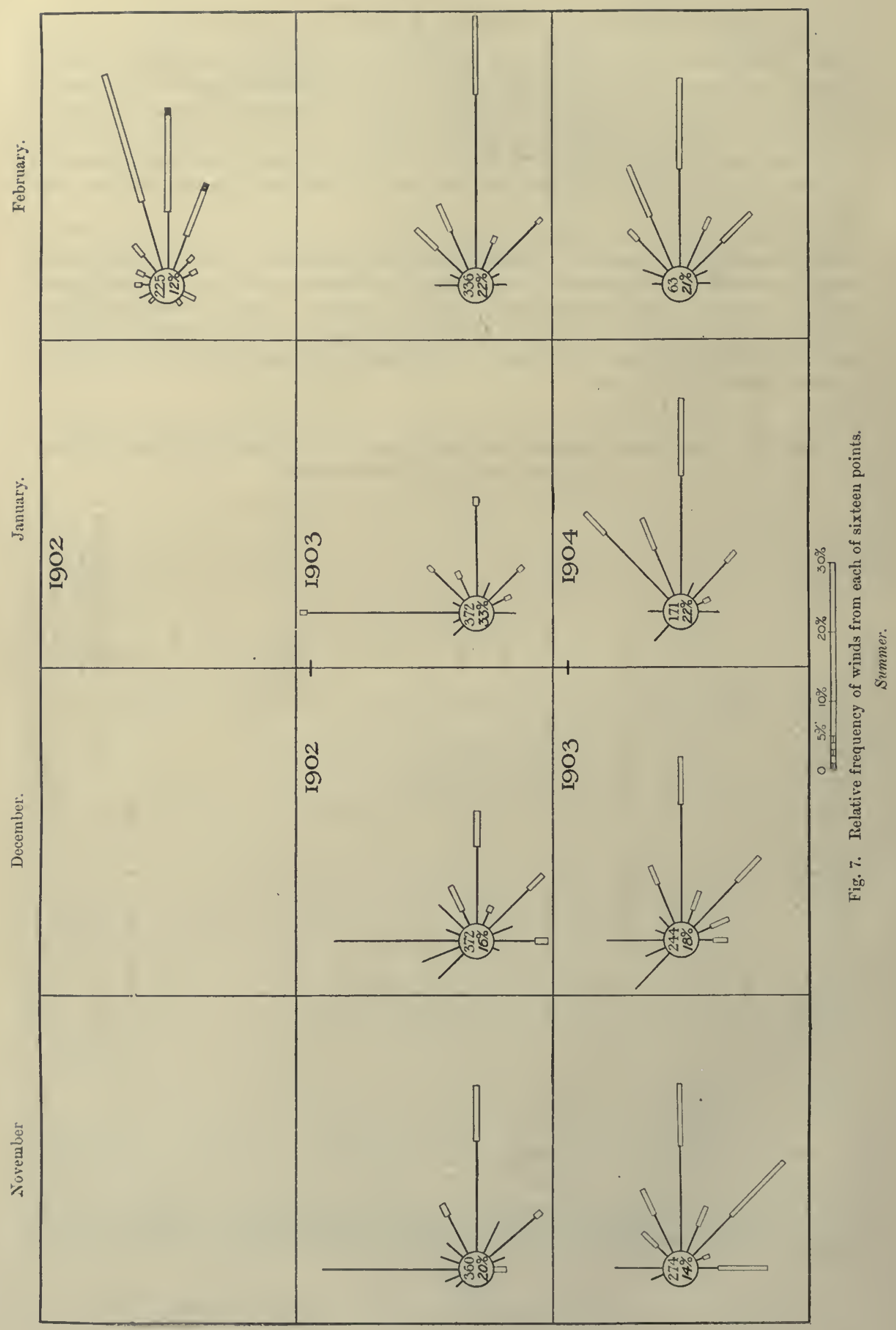




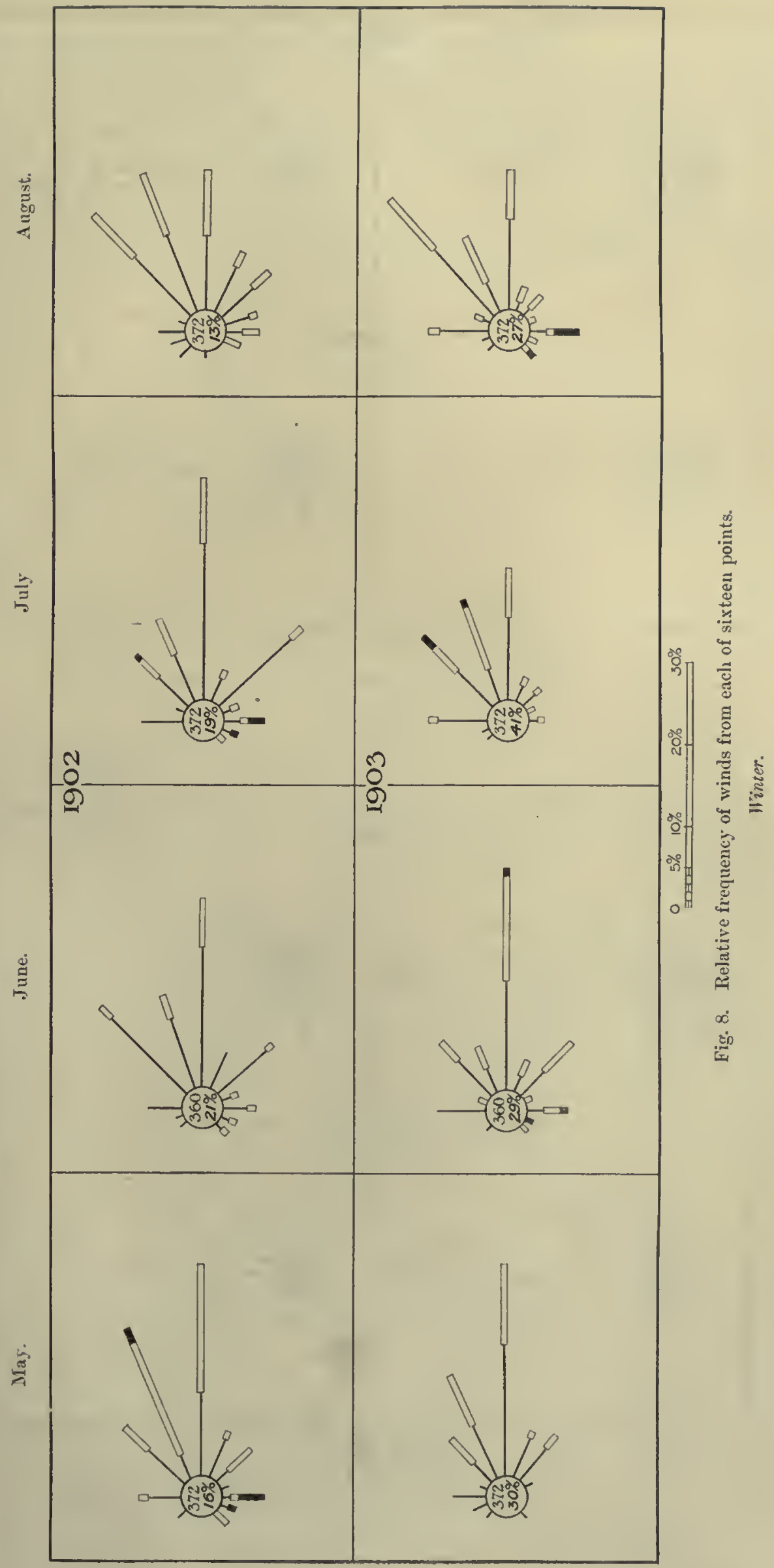


September:

Oetuler:

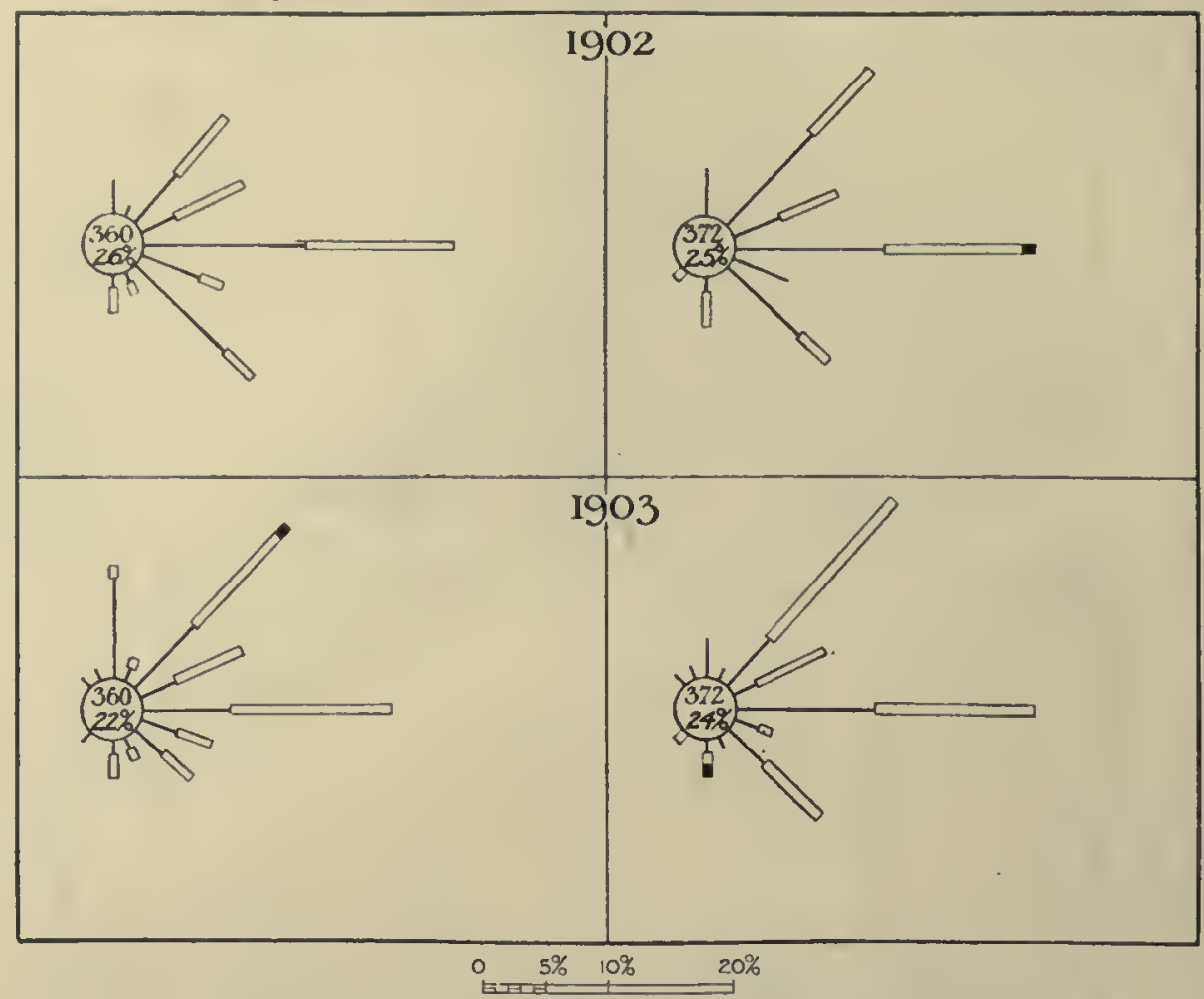

Fig. 9. Relative frequency of winds from earch of sixteen points.

l'ernal Equinox.

Matil.

spril.

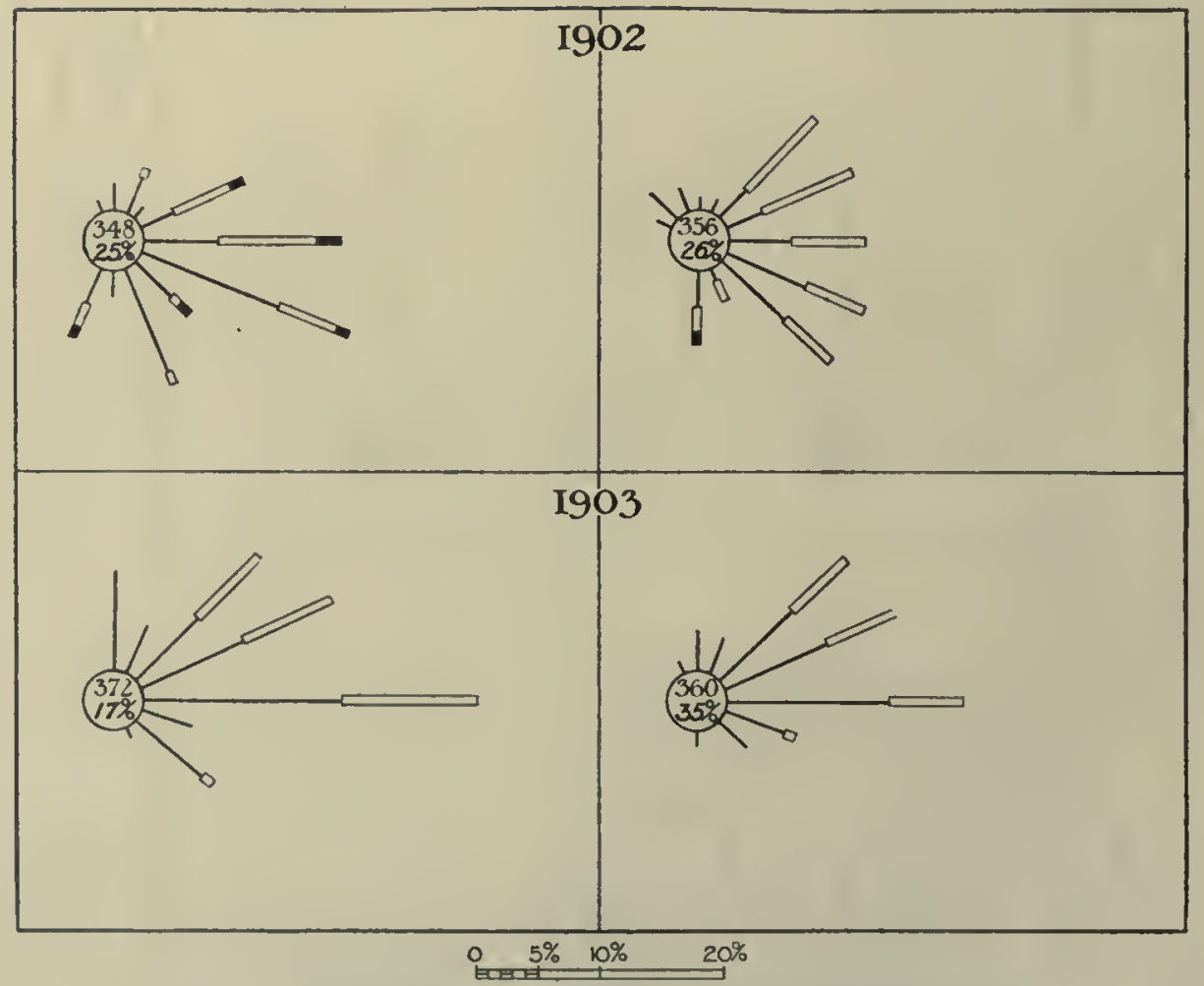

Fig. 10. Relative frequency of winds from each of sixteen points. Alutumal Équinox. 
period a mean hourly veloeity of 21 miles. It has to be remembered, howover, that southerly winds were comparatively unfrequent; and that fact is not clearly shown in the table.

There were many instances of strong winds from tho more easterly points, and in some months mean veloeities of 19 miles per hour are shown for east-north-enst and east winds, and since these means are derived from a much larger number of observations they probably indicate a generally stronger air current ; but in other months the lower mean veloeity is due to the large preponderance of lighter winds rather than to the entire absence of strong ones.

In figs. 7 to 10, however, both frequeney and strength are shown, and therefore a truer eoneeption of the average eharaeter of the wind from the different points of the compass can be got from then than from the table. 


\section{NOTES ON THE RECORDS OF SUNSHINE AND ON THE OBSERVATIONS OF CLOUD.}

IIY

\section{R. H. CURTIS.}

Insolation is a faetor of the Antaretic climate to which very little attention has been paid by previons expeditions. A sumshine recorder formed part of the instrumental outfit of the "Sonthern Cross" Expedition which wintered at Cape Adare, but as the instrument was not adapted for use in ligh latitucles where in summer the sun is contimonsly ahove the horizon, the record obtained was necessarily very incomplete.

For the present Expedition a modification of the Camplell-Stokes lurning recorder, enabling it to receive a continuous record of sunshine throughout the entire 24 hours, was designed at the Meteorological Office in consultation with Sir Gro. G. Stokes and constructed by Mr. Hicks. When this instrmuent was arranged for summer use, the bowl formed a eomplete eirele, but in the spring and autumn months the northem half of the bowl conld be removed in order to afford to the lens a perfectly unohstructed "view of the sum" when at its lower altitudes above the horizon (figs. 1, 2).

This recorder was not set up until September 14, 1902, by whieh date the sun had already attained a considerable meridian altitude, and it was finally dismounted at the middle of Fehruary, 1904. The record embraces therefore one complete spring, summer, and autumn; nearly the whole of a second spring; and
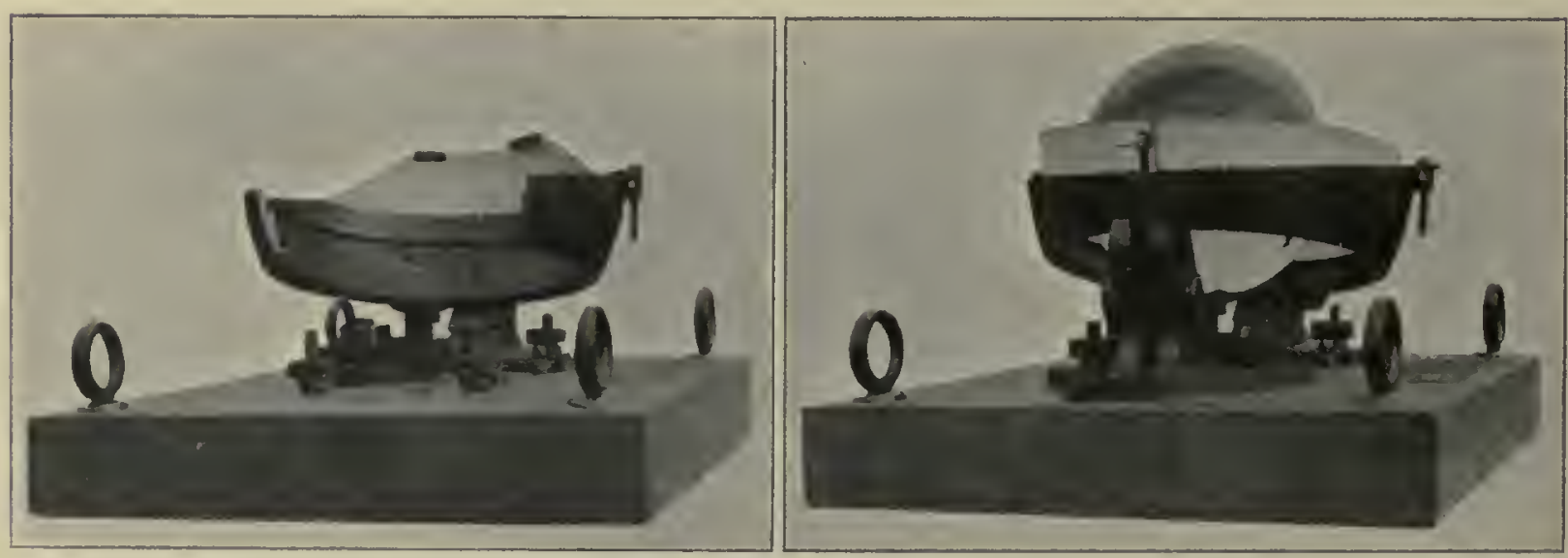

Sunshine recorder for $n$ se in Polar latitudes.

lig. 1 Showing front portion of the bowl removed for use in spring and autunn. A short card is shown in position in the upper part of the belt.
Fig. 2. Showing the complete bowl with sphere and card in place, for recording sunshine thronghont the 24 hours.

three and it half months out of the four comprising the second summer. Unfortunately, a total of 23 cards, representing 23 days' records, were missing when the Expedition reached England, and the total announts of sunshine reeorded for September, October, and Noventher, 1902, and for Septenlher and October, 1903 -the months to which most of the lost eards belonged-are in consequenee probahly somewhat smaller than they should have been.

The earliest date on which a record was obtained was September 3, and the latest April 14; but the sun appeared above the horizon on August 21, and did not finally disappear for the winter until about April 24; and it is not elear from the records whether the alssence of cards for the days intervening 
between those dates and the dates of the earliest records is due to there having been no sumshine to record, or to the recorder not having been in use.

The approximate duration of the sunshine, as recorded on the exposed cards, is shown in the tables on pp. 92, Sc. by a thick vertical black line extending across the lines for the hours during which a recording burn was made.

The following talle gives the total amounts of sunshine registered in each of the 14 months covered by the observations :-

Table I.-Total Number of Hours of Bright Sunshine Recorded in each of the Fourteen Months during which, or part of which, the Recorder was in use.

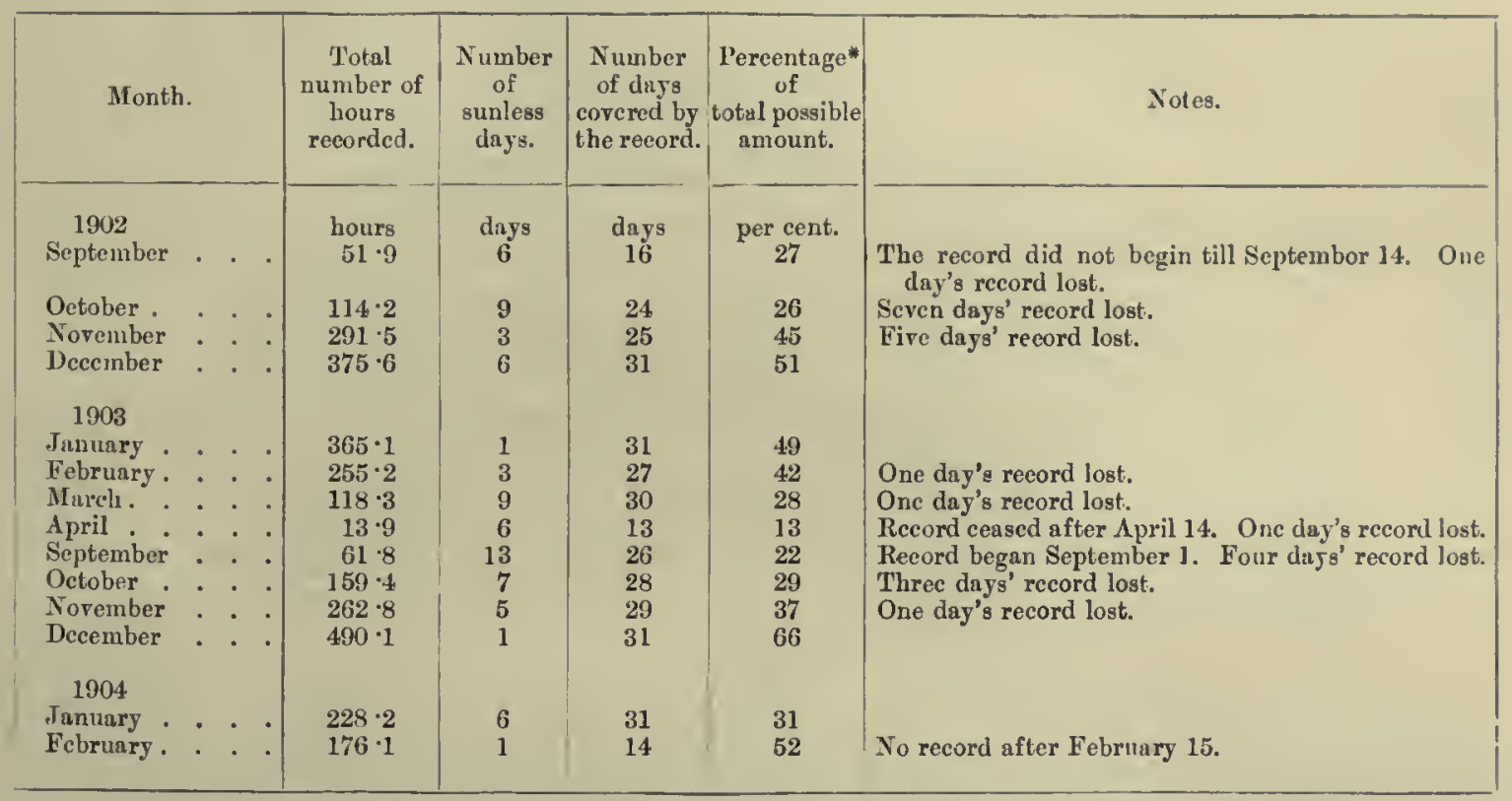

* In calculating these percentages an allowanee was made for those days for which no card was received.

There are two sets of records for each of the summer months of November, December, and January, during the whole of which the sun was continnously above the horizon; and for the first summer the three months' records combined give an average percentage of the greatest possible duration of 48 per ccut., and for the second year an average of 45 per cent. December was in both years the most sumny month, the total of 376 hours recorded in 1902 being cquivalent to 51 per cent. of the greatest possible amount, whilst the more remarkable total of 490 hours in the December of the following year is equal to 66 per cent. of the possible amount. In December, 1903, there was lut one entirely sunless day, and the average daily duration of sunshine was $15 \cdot 8$ hours. In January of that year there was also only one quite sunless day, but the average daily duration in that month was four hours less than that reached in December.

In the summer months there were several long periods of continuous sunshine. In December, 1902, there were three such periods lasting for 38 hours, 41 hours, and 87 hours respectively. In January, 1903, there were two similar spells of 60 hours and of 66 hours; November of the same year also had two sumy periorls of 36 hours and 43 hours respectively; and the following month, Deccmber, had three long intervals of 28 hours, 63 hours, and 70 hours respectively. In that month, too, there occurred a remarkably long spell of almost continuous sunshine which lasted twelve days, from the 6 th to the 17 th inclusive, during which period the sum of the sunless intervals amounted to less than 15 hours.

As a rule, the sunshine appears to have been distributed over the months in a fairly uniform way, but a marked exception to the rule occurred in Decmber, 1902, when the total record throughont uine consecutive dlays in the middle of the month only amounted to 20 hours.

The hourly totals for the six months November, December, and January, 1902-3, and 1903-4, were combined and meaned, and the means smoothed by the formula $\frac{a+2 b+c}{4}$, to see if they afforded any 
evidence of a diurnal variation in the summer months. The results are given in Table II., and graphically in fig. 3. They show that the mean duration per hou is very uniform between 0 hours and 3 a.m., from which hour it increases steadily till noon; then it again remains withont change for alont 3 hours and steadily diminishes till 11 p.m., which is the least sumy hour of the twenty-four. The total range of the diurnal variation as shown by these monthly totals amounts to $4 \cdot 1$ hours.

TAbite II.-Diurnal Variation of Sunshino in Summer.

(November, December, January, 1902-4.)

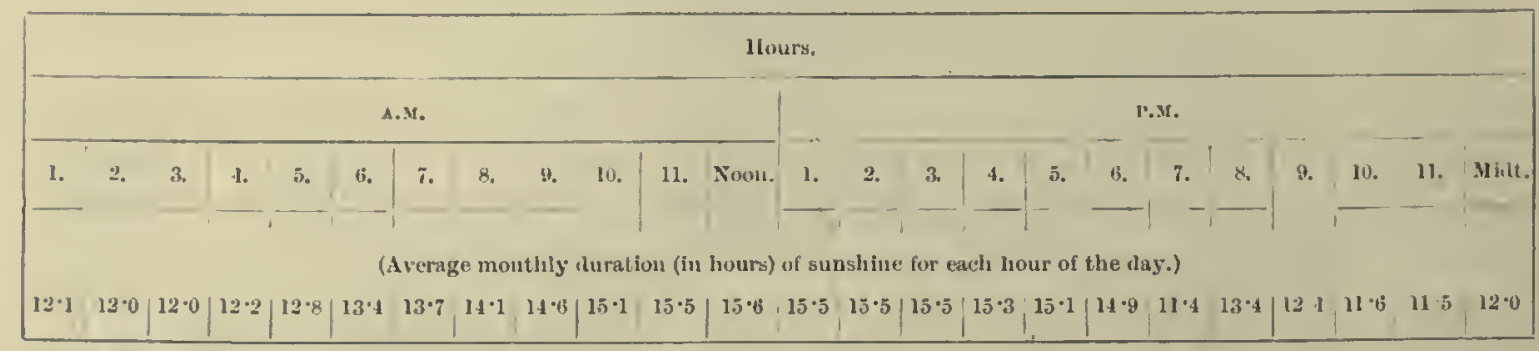

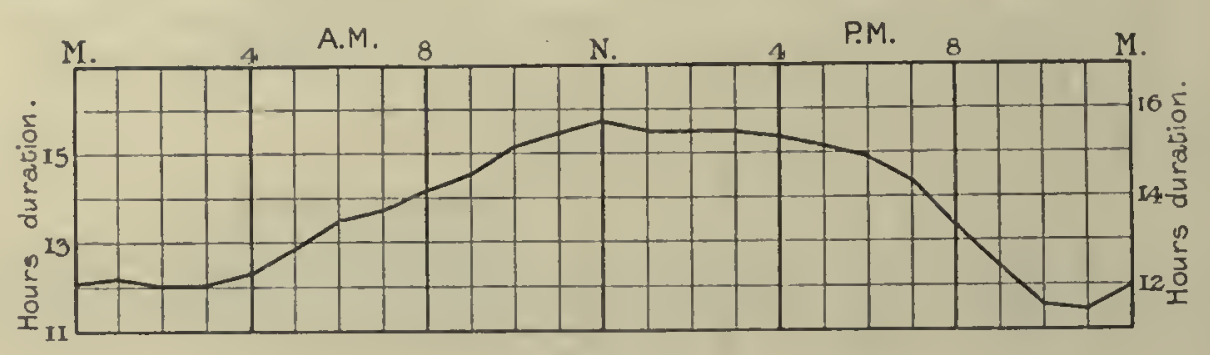

Fig. 3.

\section{Solali Radiation.}

'The record of the Campbell-Stokes' burning smushine recorder is primarily one of duration, and the information it affords respecting the intensity of the solar radiation is only of a very general character, and is obtained from the character of the burn made on the card. A marked feature of the Antaretic records is the sharpness of the burn, which generally goes right through the carl, and in not a few instances, where the record has continued thronghout the entire day, it las severed the card longitudinally in two. This indicates a particularly strong solar action such as could have been expected only in an exceptionally dry and dust-free atmosphere.

The most direct measurement arailable of the intensity of the solar radiation is that afforded by the back-bulb thermometer in rucuo, and the readings of this instrument made at Winter Quarters are very remarkable. The observations made daily thronghout the four months November to February, during nearly the whole of which time the sum was continuously above the horizon, have been measured, for each month separately, and the results are exhilited in 'Table III.

For purposes of comparison the results of similar observations nade at Madras during the four snnmer months of May, June, Jnly and Angust, 1890, are also given in the table, the year 1890 having been chosen only becanse it happened to be the one most easily available. It is obvious that the full significance of the figures given can only be appreciated when the difference between the sun's meridian altitude at the two places is taken into accomnt.

At Diseovery Bay, during the months of November and February, the daily change in the height of the sun aloove the horizon was considerable, but even when it had reached its lighest altitude in December it was still more than $60^{\circ}$ from the zenith. At Matras, on the other hand, a nearly vertical noon sun was experienced throughout the four months quoted. Yet under these very different conditions the mean solin temperatnres at Discovery Bay in December and Jamnary were only $14^{\circ}$ less than the similar means at Mathres in June and July, and the maxinum reading at Diseovery Bay in December approateherl to within $3^{2}$ of the June maximum at Madras. 
515

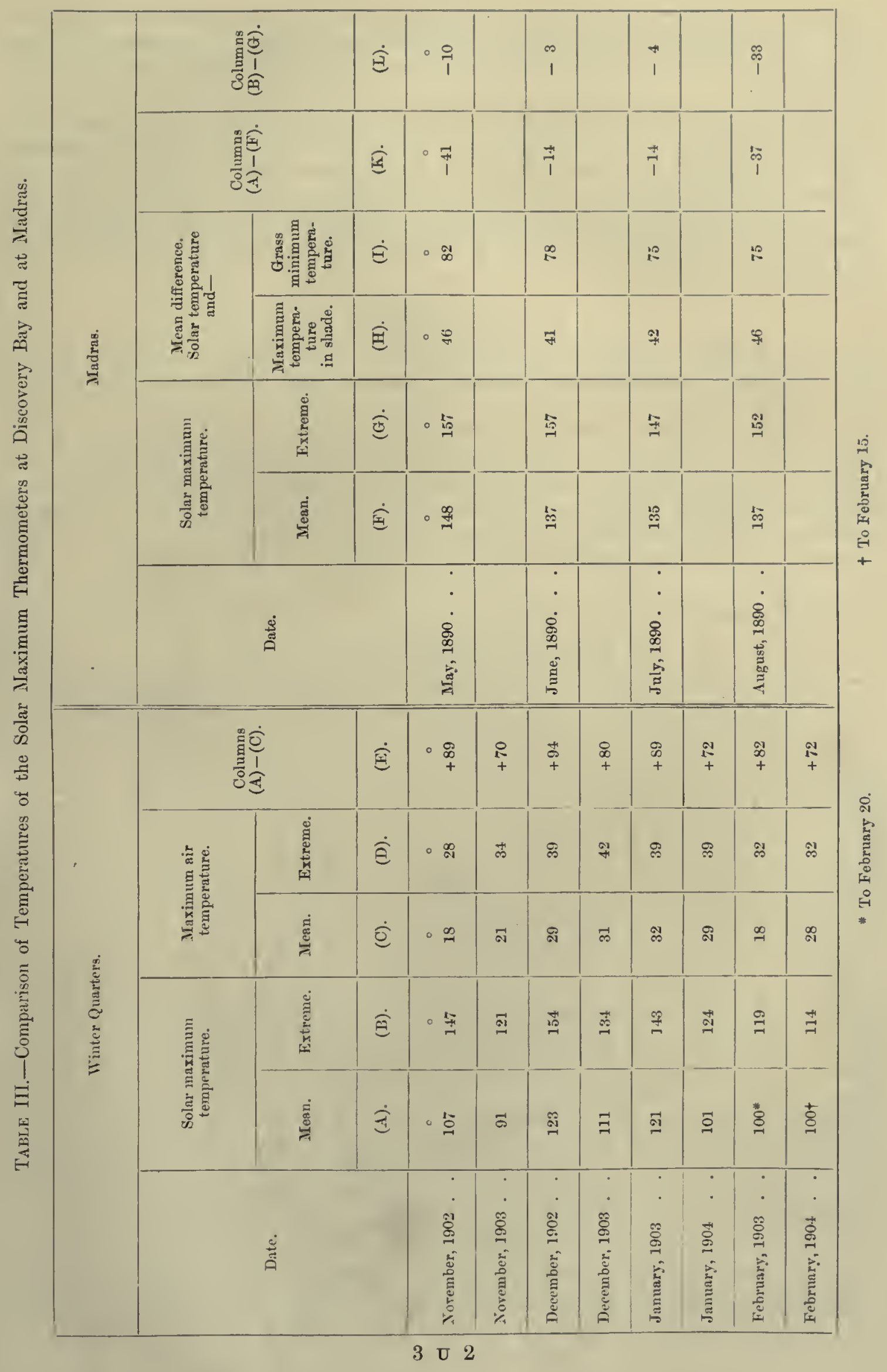


'Throughout the eight summer months' observations at Discovery Bay there ocenrrerl fifty-two instances in which the temperatnre recorded by the solar radiation thernometer amounted to or exceeded $120^{\circ} \mathrm{F}$., and in the two mouths of December and Jamury, 1902-3, there were but four instances in which the reading was below $100^{\circ} \mathrm{F}$.

At Madras the differenee between the maximnm temperature in the sum and the maximum air temperatnre reeorded in the screen varied from $41^{\circ} \mathrm{F}$. to $46^{\circ} \mathrm{F}$., and the difference between the solar and terrestrial radiation thermometers ranged from $75^{\circ} \mathrm{F}$. to $82^{\circ} \mathrm{F}$, but at Discovery Bay the difference between the maximum temperatrre of the air and the maximum temperature by the solar thermometer ranged from $85^{\circ} \mathrm{F}$. to $119^{\circ} \mathrm{F}$. These figures certainly show that the solar radiation in the Antarctic during the summer months is of unusual intensity, and indicate a condition of atmosphere peculiarly favourable for the transmission of the sur's heat rays.

\section{Amount of Cloud.}

The mean proportion of clonded sky bears a close relatiou to the subject of both solar and terrestrial raliation, and must be taken into consideration when any attempt is made to estimate the total amount of either.

The mean clond proportion for each month has been obtaincd from the daily observations, but from internal evidence it would seem that separate estimates were made of each kind of cloud, instead of the proportion of the sky expanse which was actually covered, and it is possible that this method of observing would give results slightly too high; in some instances, at any rate, it gives a total amount of clouded sky groater than 10, which is the number used to indicate a completely overcast sky.

The observations have been dealt with in two ways, and the results are shown in Tables IV. and V.

Table IV.-Mean Proportion of Clouded Sky at each Observing Hour of the Day, and for the Day.

$(0=$ cloudless sky $; 10=$ sky completely overcast. $)$

\begin{tabular}{|c|c|c|c|c|c|c|c|c|c|c|c|c|c|}
\hline & 2. & 4. & 6. & 8. & 10. & Noon. & 14. & 16. & 18. & 20. & 22 & Midt. & Day. \\
\hline 1902 & & & & & & & & & & & & & \\
\hline February. & 5 & 6 & 5 & 5 & 4 & 5 & $\begin{array}{l}6 \\
4\end{array}$ & $\begin{array}{l}7 \\
5\end{array}$ & $\begin{array}{l}6 \\
5\end{array}$ & 6 & 6 & 6 & 6 \\
\hline $\begin{array}{l}\text { Mareh. } \\
\text { April. }\end{array}$ & $\begin{array}{l}5 \\
7\end{array}$ & $\begin{array}{l}6 \\
6\end{array}$ & $\begin{array}{l}5 \\
7\end{array}$ & $\begin{array}{l}5 \\
8\end{array}$ & $\begin{array}{l}5 \\
7\end{array}$ & $\begin{array}{l}5 \\
7\end{array}$ & $\frac{4}{7}$ & $\begin{array}{l}5 \\
6\end{array}$ & $\begin{array}{l}5 \\
5\end{array}$ & $\begin{array}{l}7 \\
5\end{array}$ & $\begin{array}{l}6 \\
5\end{array}$ & $\begin{array}{l}6 \\
4\end{array}$ & $\begin{array}{l}5 \\
6\end{array}$ \\
\hline May . & 5 & 4 & 6 & 7 & 6 & 7 & 7 & 6 & 5 & 4 & 5 & 5 & 6 \\
\hline June : & 4 & 4 & 3 & 4 & 3 & 4 & 4 & 4 & 2 & 3 & 4 & 3 & 3 \\
\hline July : & 4 & 4 & 4 & 4 & 4 & 5 & $\tilde{5}$ & 4 & 4 & 4 & 4 & 3 & 4 \\
\hline Angust . & 5 & 4 & 4 & 7 & 6 & 6 & 6 & 6 & 6 & 5 & 6 & $i$ & 6 \\
\hline September & 4 & 6 & 6 & 6 & 6 & 6 & 7 & 7 & 6 & 5 & 5 & 4 & 6 \\
\hline October. & 8 & 7 & 7 & 7 & 7 & 7 & 7 & 7 & 7 & 8 & 7 & 7 & 7 \\
\hline Norember & 6 & 6 & 5 & 5 & 5 & 6 & 6 & 6 & 6 & 5 & 5 & 6 & i \\
\hline December & 6 & 6 & 6 & 5 & 5 & 4 & 4 & 4 & 5 & 5 & 5 & 5 & 5 \\
\hline 1903 & & & & & & & & & & & & & \\
\hline January. & 6 & 6 & 6 & 6 & 5 & 6 & 5 & 5 & 4 & 4 & 5 & 5 & 5 \\
\hline February. & 6 & 5 & 5 & 6 & 5 & 5 & 5 & 5 & 5 & 5 & 5 & 5 & 5 \\
\hline March. & 5 & 6 & 6 & 6 & 5 & 5 & 5 & 6 & 6 & 5 & 5 & 4 & 5 \\
\hline April. & 2 & 3 & 4 & 4 & 3 & 4 & 5 & 4 & 4 & 3 & 3 & 2 & 3 \\
\hline Mny . & 3 & 3 & 3 & 3 & 4 & 4 & 3 & 3 & 4 & 4 & 4 & 4 & 3 \\
\hline June : & 4 & 4 & 3 & 3 & 3 & 4 & 5 & 5 & 5 & 4 & 4 & 4 & 4 \\
\hline July & 1 & 2 & 2 & 2 & 2 & 2 & 3 & 2 & 2 & 1 & 2 & 1 & 2 \\
\hline August . & 4 & 3 & 3 & 4 & 4 & 4 & 3 & 4 & 3 & 4 & 8 & 4 & 4 \\
\hline September & 3 & 3 & 5 & 4 & 4 & 5 & 5 & 5 & 5 & 4 & 3 & 3 & 4 \\
\hline October. & fi & 6 & 6 & 6 & 6 & 6 & 6 & 7 & 7 & 6 & 6 & 7 & 6 \\
\hline Norember & 7 & 8 & 7 & 7 & 6 & 6 & 7 & 7 & 7 & 7 & 6 & 7 & 7 \\
\hline Dicember & & obsns. & & 3 & 4 & 3 & 5 & 5 & 4 & 4 & 3\{ & $\begin{array}{c}\text { No } \\
\text { obsis. }\end{array}$ & 4 \\
\hline $\begin{array}{c}1904 \\
\text { January. } \\
\text { Vebruary. }\end{array}$ & 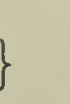 & & & Observ & ations $m$ & adent ir & regulur & interval $\mathrm{s}$ & & & & \{ & $\begin{array}{l}7 \\
5\end{array}$ \\
\hline Mran & $4 \cdot 8$ & $4: 9$ & $4 \cdot 9$ & $5 \cdot 1$ & $4 \cdot 7$ & $5 \cdot 0$ & $5 \cdot 2$ & $5 \cdot 2$ & $4 \cdot 9$ & $+\cdot 7$ & $4 \cdot 7$ & $4 \cdot 6$ & $5 \cdot 0$ \\
\hline
\end{tabular}


In Tahle IV. the mcan proportion of clouded sky is given for each hour of observation for each of the twenty-four months thuring which the "Discovery" remained at Ross Island. The figures show for every month a very miform distribution throughout the day, and, speaking generally, it may be sair that the sky was as a rnle alout laalf covered with cloud. The winter months, however, were the least cloudy, and in July, 1903, there was a particularly cloudless sky, the average daily amount of cloud being only two-tenths.

In Tahle V. the result of a different treatment of the observations is shown, the cloud amounts having been grouperl to represent the sky conditions indicated by the letters of the Beaufort weather scale. In this scale "h" indicates hlue sky, or that not more than one-tenth of the sky expanse was covered with elond; "lec" denotes blue sky and detached cloud, not more than four-tenths of the sky being clouderl;

Table V.-Cloud Distribution as Indieated by the Beaufort Weather Scale.

(The values given are percentages of the total number of observations made each month.)

\begin{tabular}{|c|c|c|c|c|c|c|c|c|c|c|c|c|}
\hline \multicolumn{3}{|c|}{ Month. } & $\frac{\text { b. }}{\text { per sent. }}$ & $\begin{array}{c}\text { be. } \\
\text { per cent. }\end{array}$ & c. & 0. & \multicolumn{2}{|c|}{ Montl. } & b. & $\frac{\text { be. }}{\text { per cent. }}$ & $\frac{\text { c. }}{\text { per cent. }}$ & $\frac{0 .}{\text { per cent. }}$ \\
\hline $\begin{array}{l}\text { February } \\
\text { Marel } \\
\text { April. } \\
\text { May : } \\
\text { June : : } \\
\text { July : : } \\
\text { August : } \\
\text { Septcmber } \\
\text { Oetober : } \\
\text { November } \\
\text { December }\end{array}$ & $\therefore:$ & : & \begin{tabular}{|c|} 
per eent. \\
34 \\
38 \\
38 \\
35 \\
60 \\
48 \\
36 \\
26 \\
15 \\
26 \\
40
\end{tabular} & $\begin{array}{c}\text { per cent. } \\
8 \\
7 \\
14 \\
7 \\
5 \\
10 \\
8 \\
15 \\
10 \\
15 \\
9\end{array}$ & $\begin{array}{c}\text { per cent. } \\
11 \\
7 \\
11 \\
8 \\
5 \\
4 \\
5 \\
9 \\
11 \\
13 \\
6\end{array}$ & $\begin{array}{c}\text { per cent. } \\
47 \\
49 \\
37 \\
50 \\
30 \\
38 \\
51 \\
50 \\
64 \\
46 \\
45\end{array}$ & 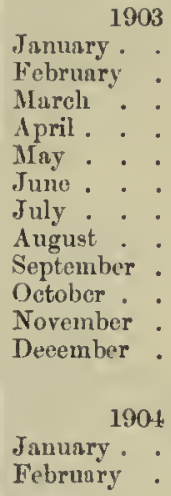 & 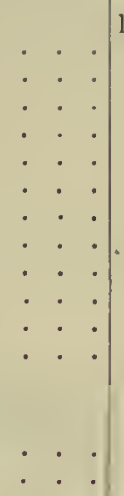 & $\begin{array}{c}\text { per cent. } \\
34 \\
31 \\
31 \\
54 \\
55 \\
55 \\
63 \\
47 \\
40 \\
24 \\
16 \\
43\end{array}$ & \begin{tabular}{|c|} 
per cent. \\
9 \\
17 \\
12 \\
14 \\
10 \\
5 \\
15 \\
16 \\
14 \\
12 \\
14 \\
20
\end{tabular} & \begin{tabular}{|c|} 
per cent. \\
8 \\
11 \\
7 \\
4 \\
6 \\
3 \\
4 \\
5 \\
10 \\
11 \\
13 \\
9
\end{tabular} & $\begin{array}{c}\text { per cent. } \\
49 \\
41 \\
45 \\
58 \\
29 \\
37 \\
18 \\
32 \\
36 \\
53 \\
57 \\
28\end{array}$ \\
\hline Allo & bser & ation & & b. & & bc. & c. & o. & & Number o & of obserrat & ions. \\
\hline & 1902 & & & $\begin{array}{r}\text { per cer } \\
37 \cdot 5\end{array}$ & & $\begin{array}{l}\text { ver cent. } \\
11.5\end{array}$ & $\begin{array}{c}\text { per cent. } \\
8.0\end{array}$ & $\begin{array}{c}\text { per cent. } \\
430^{\circ} 0\end{array}$ & & & 7890 & \\
\hline
\end{tabular}

"c" signifies a cloudy sky, where the expanse of sky covered may amount to seven-tenths; "o," or overeast, implies that from eight-tenths to the whole of the sky is clouded. The number of observations falling under each of these divisions in each month is expressed as a percentage of the total number for the month.

The unexpected result which the table exhilits is that in every month the great majority of the observations fall under one or other of the two extremes "1, " or " 0 ," and that as a rule, to which there are but few exceptions, the percentage frequency either of "blue sky" or of "orercast" sky exceeds that of the two interuediate stages combined. Pnt in auother way, this apparently means that small quantities of detached clouls were relatively infrequent, and that when clouds were present they were usually present in quatity large enongh to practically cover the entire sky expanse.

Comparing these results with the observed percentages of the total possible anount of sunshine given in Table I., a fairly close relation is secn to exist between the latter values and the combined percentages of "l," and "be" sky in the months of Octoher to February; the values are as follows:- 


\begin{tabular}{|c|c|c|c|c|c|c|c|c|c|c|}
\hline & \multicolumn{3}{|c|}{1902.} & \multicolumn{6}{|c|}{1903.} & \multirow{2}{*}{ 1904. } \\
\hline & $\frac{8}{0}$ & 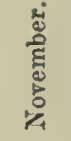 & 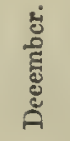 & 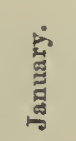 & 苞 & อี & $\frac{\frac{8}{0}}{8}$ & 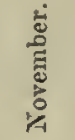 & 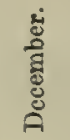 & \\
\hline $\begin{array}{l}\text { Observed pereentage of possible sunshine. } \\
\text { Percentage of sky obserrations when not more } \\
\text { thn four.tenths eorered by cloud }\end{array}$ & $\begin{array}{c}\text { per } \\
\text { cent. } \\
26 \\
25\end{array}$ & $\begin{array}{c}\text { per } \\
\text { eent. } \\
45 \\
41\end{array}$ & $\begin{array}{c}\text { per } \\
\text { eent. } \\
51 \\
49\end{array}$ & $\begin{array}{c}\text { per } \\
\text { cent. } \\
49 \\
43\end{array}$ & $\begin{array}{c}\text { per } \\
\text { eent. } \\
42 \\
48\end{array}$ & $\begin{array}{c}\text { per } \\
\text { eent. } \\
28 \\
48\end{array}$ & $\begin{array}{c}\text { per } \\
\text { eent. } \\
29 \\
36\end{array}$ & $\begin{array}{c}\text { per } \\
\text { cent. } \\
37 \\
30\end{array}$ & $\begin{array}{c}\text { per } \\
\text { eent. } \\
66 \\
63\end{array}$ & $\begin{array}{c}\text { per } \\
\text { eent. } \\
31 \\
21\end{array}$ \\
\hline
\end{tabular}

The cloud percentages refer to the entire day, but the percentages of sunshine refer only to that portion of the day during whicl the sun was above the horizon. Thronghont November, December, and January the sun did not set, and for these months the two values are eomparable because they refer to similar intervals of time. They do not differ very much in either of these months, but it will be noticed that the sunshine pereentage always exceeds that of clear sky, the explanation of the difference being found in the fact that a record of sunshine was oceasionally made when the sky was more than half covered with cloud.

The differences between the values for the other montlis given in the table are explained partly by the fact that they do not represent similar periods of time, and partly by the low altitude of the sun and the consequent weakening of its power to burn the card on which the record was obtained. 


\title{
XIV. DISCUSSION OF THE OBSERVATIONS OF ATMOSPHERIC ELECTRICITY.
}

\author{
BY
}

C. T. R. WILSON, F.R.S.

\section{Introduotory Note by L. C. Bernacchi.}

OBSERVATIONs of atmospheric electricity with the Kelvin portable eleetrometer were made during 1902 and 1903. During the winter months these observations wcre often very unpleasant to take on aecount of the sniall screws and bare parts of the small instrument, which eould not be handled with mittened fingers. For a polar climate some larger instrument, which does not necessitate the observer being frost-bitten when using it in cold weather, should be employed, or somo form of self-recording electrometer.

The observations were taken on a stand about 4 feet above the rock surface, and away from any buildings, erections, \&e. During the dark months observations were taken at $11 \mathrm{~h}$. a.m. and 5h. p.m. every day, excepting when high winds with low temperatures made it impossible. After the return of the sun, observations were generally taken every two hours from $10 \mathrm{~h}$. a.m. to $10 \mathrm{~h}$. p.m. The direetion of the wind, temperature, form of cloud, \&e., was entered with the observation.

From 8 h. a.m. on November 22 to $3 \mathrm{~h}$. a.m. oll November 25, 1902, a series of hourly observations were carried out with the assistanee of Engineer Commander R. W. SkELToN, R.N., ehief engineer of the "Discovery," and again in 1903 two-hourly observations from noon on Oetober 7 to noon on October 10.

Besides the two series of three days, observations were occasionally taken on individual days throughout the twenty-four hours in both winter and summer.

The observations during the seeond year are not so complete as during the first, but are supplemented by observations taken at 15 feet above the ground, immediately after the observations at 4 feet; an insulated wire from the burning mateh at the top of the pole to the instrument being eniployed for the purpose.

Whenerer there was drifting snow in the air, or fine ice-needles, the diflerence of potential was very large and variable, and in muny cases the electrieal strain would discharge the electrometer.

The observations wele carried out by Mr. L. Bersaccur, except during his absence on a sledging expedition from September 23 to October 2, 1902, when they were taken by Mr. R. W. SkELTON, R.N., Chief Engineer of the "Discovery," who also assisted with the continuous series of observations of November 22 to 25,1902 , and of October 7 to $10,1903$.

The observations consisted of measurements of the electric potential at a definite height above the ground. The electroneters originally supplied to the "Discovery" were two Thomson portable electrometers by White, and an Exner electroscope. The last-named, however, met with an accident on the outward voyage and was neve! used. Of the portalble electroncters one (No. 35) was only used on a very few occasions, practically all the observations discussed below being taken witl the other portable electrolleter (No. 81). 
The scale values of this electrometer as determined by me after the return of the expedition were fonnd to differ considerably from the values obtained at Cape Town on the outward royage. The constant, by which the readings on the divided head of the instrument had to be multiplied to reduce them to volts, originally varied from 1.6 when the vertical scale reading was 0 to 2.0 when this reading was 30; wherens after the return of the expedition the constant varied from $2 \cdot 6$ for a vertical scale reading of 6 to 3.0 for a vertical scalc retding of 20 . This change of the constant of course introduces a serious uncertainty into the results, as it is impossible to say definitely when it took place. 'The change of constant is most probably due to injuries received on the outward or homeward royage or during loading or unloading operations. Whether, however, the change of constant took place before or after the observations were taken it is impossible to determine. There is a record of an acciclent to the electrometer a month or two after observations at the Winter Quarters were begun. After the 5 p.n. olservation on April 24, 1902, there is the following note:- "The observer (L. B.), immerliately after taking the above observation, accidentally tilted the instrument while putting it back in its box, which loosened and shifted the guard plate and discharged the instrument, and necessitated its being taken to a warm room to be overhauled. The guard plate was gently pressed back into its place and the instrument thoroughly dried." It is just possible that the change of constant may have occurred at this timc. No further accident appears to have happened to the electrometer while the observations continued. It is unlikely, therefore, that the relative values of potential recorded subseqnently to this date were affected.

In the reduction of the results the scale values determined after the return of the expedition have bceu used, but the mncertainty resulting from the ehange in the constant of the electrometer must be borne in mind.

As "colleetors" the ordinary electrometer mateh or fuze was used. The observations, except where otherwise described, were taken with the electrometer placed on a tripod stand, the table of whieh was about 4 feet above the surface of the ground. The match was attached to the end of a eopper wire and was about 5 feet above the ground. The case of the instrument was connected to earth by means of a copper wire. The insulation appears to have been satisfactory, and the behaviour of the instrument as tested after the return of the expedition would lead one to expect no appreciable error from defective insulation. It is, however, not to be expected that the potential aequired by the match and indicated by the electrometer will give accurately tho potential which would exist at the point now occupied by the match, if observer, electrometer, and match were absent. The equipotential surfaces eveu at the level of the match will be somewhat distorted by the presence of observer and apparatus. In taking the readings the observer always occupied approximately the same relative position, his head well below the level of the match; if the stand and observer could be simply regarded as earth-eonnected conductors, the relative values of potential obtained on different oecasions would be unaffected, and the error in the absolute value of the potential at the given height would not be large. There is, however, the possibility of an irregularly varying error due to eleetrical charges on the clothing of the observer. The observer ficed the wind while taking the observations, the wind blowing towards him from the instrument, so that his breath or any drifting snow which might have eome in contact with him would not reach the immediate neighbourhood of the match. This cloes not, however, in any way diminish the error due to possible electrification of the observer's clothes.

For some months observations were also taken of the potential at a height of 15 feet, the match being placed at the end of a rubber-insulated eopper wire supported on a pole. These observations were madc within a few minutes of the ordinary 5 -feet observations. While these observations would be free from the disturbing influence of the observer, one cannot feel the same confidence in the sufficiency of the insulation.

It was not found eonvenient to take all the observations at the sane place throughout the year. The earlier systematie observations up to May 24, 1902, were taken on shore near "Hut" B (the "absolute" house); those of August 7, 1902, to March 15, 1903, were again taken at the sanc spot. The winter observations, May 24, to August 6, 1902, and from March 15, to May 8, 1903, were taken on the ice near the ship (about 100 yards away). 
The apparatus not being self recording, it was of course impossible to obtain anything approaching a continuous record of the variations of the potential. An unbroken series of hourly observations was obtained extending over the three days from 8 a.m., November 22, to 8 a.m., November 25, 1902. Tliese were taken at the ordimary position on shore at a height of five feet above the ground. $\Lambda$ further series, in this case of 2-hourly observations, also extending over three days (noon, October 7, to noon, October 10, 1903), was also obtained. These were taken at the same place, but with the match at a height of 12 feet above the ground. A varying number of observations were taken daily throughout the rest of the time, except on days when observing was rendered impossible by blizzards. During the middle of the second winter, between May 8 and August 19, 1903, no observations were attempted owing to shortage of the supply of electrometer matehes.

The difficulty and discomfort inseparable from meteorological observations in circumpolar regions are accentuated in the case of measurements made with the portable eleetrometer, owing to the fact that the instrument is not direct reading, the divided head having to be adjusted to bring the eross hair to its "sighted" or zero position. When the observations had to be taken with the aid of artifieial light the diffieulty must have been greatly inereased. It is not surprising, therefore, that the number of observations per day was reduced in the winter months, and that eontinuous series of hourly observations extending over a day or more are lneking for these months.

\section{Results.}

The more striking features which appear on an examination of the values obtained for the potential at the place of observation will be considered first. In what follows, the times throughout are mean local times.

\section{Sign of the Potential.}

The potential was almost invariably positive, as in temperate latitndes. Only on 11 days were negative values mẹt with. Particulars of these occasions are given in the table which follows :-

\begin{tabular}{|c|c|c|c|c|c|c|c|}
\hline \multicolumn{3}{|l|}{ Date. } & & \multicolumn{2}{|c|}{ Time. } & Volts at 5 feet. & Volts at 15 feet. \\
\hline 1902 & & & & & & & \\
\hline February 28 & . & . & . & 10 & a.m. & -90 & - \\
\hline$M \operatorname{arch} 4$ & - & - & . & 10 & $"$ & -59 & 一 \\
\hline , 14 & - & - & . & 9.30 & $"$ & -45 & - \\
\hline "19 & . & - & . & 11.40 & , & -56 & 一 \\
\hline Mฉy 25 & . & - & . & 2 & , & -72 & 一 \\
\hline , 26 & . & - & . & 11 & $"$ & -15 & - \\
\hline October 27 & - & - & - & 10 & " & -18 & 一 \\
\hline 1903 & & & & & & & \\
\hline January 13 & - & . & 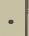 & 10 & p.m. & -38 & +110 \\
\hline$" \quad 14$ & $\cdot$ & - & $\cdot$ & $\begin{array}{r}10 \\
4\end{array}$ & a.m. & $\begin{array}{l}-81 \\
-72\end{array}$ & $\begin{array}{r}-218 \\
-\quad 23\end{array}$ \\
\hline 15 & . & . & . & 10 & $\begin{array}{l}\text { p.m. } \\
\text { a.m. }\end{array}$ & -17 & $\begin{array}{l}-20 \\
+223\end{array}$ \\
\hline$n$ & & & & 4 & p.m. & -9 & +74 \\
\hline February 13 & - & . & $\cdot$ & 10 & a.m. & -223 & - \\
\hline
\end{tabular}

In lower latitudes the occurrenee of negative potentials is generally associated with precipitation. On only three of the days of the above table (October 27, 1902, and January 13 and 14, 1903) was there precipitation. On most of the days the sky was overcast for part of the day at least, but Mareh 4, 1902, and February 13, 1903, were fine and clear nearly all day.

\section{Intensity of the Normat. Elafctric Fiel.d.}

The mean potential gradient under normal wenther conditions is about 60 volts per metre (or about 40 volts per metre if the original constant of the instrument be used). It has of course to he borne in mind that, as pointed ont above, the deformation of the equipotential surfaces hy observer aud apparatus may 
have beell consideral)le, and the number given can only be taken as giving a rough approximation to the absolnte potential gradient. The potential gradient appears, then, to have a mean value somewhat less than that met with in Furope.*

\section{Abxormaliy High Potextiaj.s Associated with Drifting Sxow.}

It was noticed by Mr. BFRxaCCHr that "as soon as the slightest amount of drifting snow appears in the air the difference of potential hecomes large and fluctuating."

An analysis of all the occasions on which the potential at the ordinary height of abont five feet exceeded 200 volts is contained in the table which follows :-

\begin{tabular}{|c|c|c|c|c|c|c|}
\hline \multirow{2}{*}{ Volts. } & \multicolumn{6}{|c|}{ Number of oeeasions. } \\
\hline & Drift. & Snow. & Iee crystuls. & Fog. & Other eonditions. & Total. \\
\hline $200-300$ & 3 & 1 & 1 & 1 & 15 & 21 \\
\hline $300-400$ & 1 & 0 & 1 & 0 & 7 & 9 \\
\hline $400-500$ & 2 & 1 & 0 & 0 & 0 & 3 \\
\hline $500-1000$ & 9 & 0 & 1 & 0 & 1 & 11 \\
\hline $1000-$ & 4 & 0 & 0 & 0 & 1 & 5 \\
\hline Abore 200 & 19 & 2 & 3 & 1 & 24 & 49 \\
\hline 300 & 16 & 1 & 2 & 0 & 8 & 27 \\
\hline$" 400$ & 15 & 1 & 1 & 0 & 2 & 19 \\
\hline
\end{tabular}

In this table are recorded the number of cases in which potentials between the limits given in the first column were observed (1) when drifting snow is recorded; (2) when snow was falling; (3) with ice crystals in the air; (4) with fog; and (5) Innder other conditions.

Of the 19 occasions on which the potential exceeded 400 volts drift is recorded on 15 . The total number of occasions on which drift is recorded in the journal of atmospheric electricity is 26 . Of the seven occasions when the potential with drifting snow was below 200 volts, it lay between 150 and 200 on four, between 100 and 150 on two, and below 100 volts on only one occasion. The menn value of potential when the abnormally high values associated with drifting snow are excluded amounts to 92 volts; the mean for the 26 occasions on which drift is recorded is 668 volts.

Except when driven along by the wind, snow or ice crystals in the air do not give potentials which are in any way abnormal. The table which follows contains the results of all observations where there is a record of ice particles in the air (whether recorded as drift or snow, or ice crystals) classified according to wind strength.

\begin{tabular}{|l|c|c|c|c|}
\hline Wind strength . . . & $0-1$. & $1-3$. & $3-4$ & Exceeding 4. \\
\hline Mean voltage . . & 98 & 225 & 570 & 1018 \\
\hline Number of cascs . . & 16 & 10 & 18 & 7 \\
\hline
\end{tabular}

The talble gives for varions wind strengths the number of cases ou which there is a record of snow, drift or ice particles in the air, and the mean value of the voltage on such occasions. The classification is according to the maximum wind strength when that is entered as varying between two limits; e.g., an wind reeorderl as of strength 3 to 5 would lie entered in the last column.

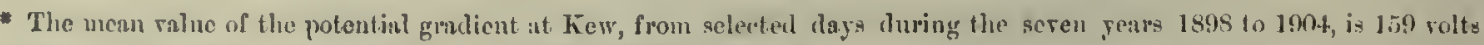
per metre. (C. Chree, 'Plil. 'Trans.s' $A$, vol. 206, p. 302.)
} 
It will be seen that in calm weather the potential with snow or with ice crystals in the air dicl not sensibly exceed the mean for normal conditions, which amomited to 92 volts, while the potential with ice particles in the air iucrenses continuously with the strength of the wind.

The eonnection betwcen abnormally high potentials, when there were ice particles in the air, and wind is cqually well shown in tho next table, where, for the same linits of wind strength as in the previous table, is given in each case the number of occasions when the potential lay between given limits; as before, only those observations being included for which there is a record of drift, snow or ice particles in the air.

\begin{tabular}{|c|c|c|c|c|}
\hline Wind strength. & $0-1$. & $1-3$. & $3-4$. & Exeeeding 4. \\
\hline Voltage- & & & & \\
$0-100$ & 0 & 4 & 1 & 0 \\
$100-200$ & 7 & 3 & 5 & 0 \\
$200-500$ & 0 & 2 & 3 & 3 \\
$500-1000$ & 0 & 1 & 7 & 2 \\
$1000-$ & 0 & 0 & 2 & \\
\hline
\end{tabular}

Wind alone withont ice particles in the air does not give abnormally high potentials.

The mean value of the potential on the occasions (of which there are 46) on which the wiml strength exceeded 3 without any record of ice particles in any form in the air amounts to 93 ; or if we exchnle three occasions when the potential was negative, to 109. These values do not differ to any appreciable extent from the mean for normal conditions. The absence of any connection between abnormally high potentials and wind unaccompanied by drift is equally well shown in the following table, in which the above-mentioned 42 cases of wind without drift are classified according to the potentinls obscrved.

\begin{tabular}{|c|c|c|c|c|c|c|}
\hline Voltage. & Negative. & $0-100$. & $100-200$. & $200-300$. & $300-100$. & Abovo 400. \\
\hline Number of cuses . . . . & 3 & 24 & 12 & 2 & 1 & 0 \\
\hline
\end{tabular}

High positive readings of the electrometer are thus undoubtedly associated with drifting snow ; snow unaccompanied by high wind, and high winds maccompanied by drifting snow, do not give abnormally high values. With snow in the air the potential is higher the greater the wind velocity. Cases of high potential without drift or snow are comparntively rare; of the 19 occisions on which the potential oxceeded 400 volts there was drift or snow on all except two.

It is not impossible that these high positive valnes of the potential associated witl driving snow are in a sense spurious, that the potential indicated by the electrometer may not under such conditions be related in any immediate way to the undisturbed air potential at the level of the collector. With the air full of dry icc particles blown along by the wind there nust be a certain amount of electrification by friction of the ice against the instrument and the observer's clothing. The high potential indicated by the electrometer might be dne to electrification by friction of the ice against the collector, supporting wire or eloctrometer terminal, or again the potential in the neighbourhood of the observer might be raised by the acquisition of a positive charge by his clothing. It is improbable, however, that either of these processes is the cause of the high potentials observed, for the sign of the effect is the opposite of what such explanations would lead us to anticipate. According to Sorncke ('Wied. Ann.,' vol. 28, p. 550, 1886), ice appears to acquire a positive charge by friction against other substances. A negative charging up of the observer's clothing or of the electrometer terninal and its connections, and a consequent lowering of the potential readirgs, might therefore rather have been expected. The observer faced the wind in rending the instrument; ice particles which had become electrified by striking against his clothing would not therefore be blown in the direction of the instrument, and any effect of a charge acquired by the ice particles in this way would be slight. 
On the whole, then, it is probahle that the apparent raising of the potential at a given height in the air by drifting snow is real, and not a spurions eflect due to the presence of the olserver and his instrument. An additional argument in favour of this conclusion is afforded by the fact that in many cases where high potentials were observed the drift is recorded as leeing along the ground, in some cases entirely below the level of the instrument. Again, there is at least one case of abnormally high potential where the snow was observed to be drifting, but not in the immediate neighbourhood of the instrument (August $19,1902,4.30$ p.m.). It is of course possible that even in these cases fine snow particles were heing driven against the observer and instrument. It is more probable, however, that the high air potentials recorded are, in these cases at least, genuine, and the probability of the still higher potentials, observed when the drift was more pronounced, really indicating high air potentials is increased.

From December 3, 1902, till March 14, 1903, observations were taken with the collector at 15 fcet above the ground as well as at the usual height, and after that date at 12 feet. With the collector at such heights the possibilitity of any charge on the observer's clothing influencing the potential taken $1 p$ by the collector may be considered as excluded; the chance of electrification of the wire connecting the collector to the electrometer is, on the other hand, increased. While these high-level collectors were in use the number of occasions of drift at the time of the electrical observations was small (only six), but on all except one of these abnormally high values were observed. It is probable, then, that the high readings obtained with drifting snow really indicate high air potentials, but it has to be remembered that it is not altogether inpossible that they may be due to electrification of the collector or its comnecting wire by frietion.

Assuming the high readings accompanying drifting snow to indicate abnormally high air potentials, we may perhaps most naturally conclude that the high potential is due to a positive charge acquired by the ice particles as a consequence of their friction against the ground. In many cases in which this abnormally high potential was observed the drift is described as being along the ground; sometines it is definitely described as entirely below the level of the instrument. If the increased potential is entirely due to the charge carried by the ice particles, then the increase in the vertical electrical field or potential gradient will not extend above the level of the drifting snow, the vertical field within the drift-filled air (assumed to form a miform liyer in contact with the ground) diminishing from the ground upwarls to the upper surface of the drift at a rate proportional to the density of the charge, the field above this level being nuaffected by the drift. Under the simple conditions assumed (which may not differ mucl from the actual) we should everywhere have $\frac{d^{2} \mathrm{~V}}{d h^{2}}=-4 \pi \rho, \mathrm{V}$ being the potential in electrostatic neasure, and $\rho$ the charge per eubic centimetre.

One might hope to get some light thrown upon the uature and distribution of the charge carried by the lower layers by a comparison of the results obtained at 15 feet with those of the ordinary 5 -feet observations. If the increased potential accompanying drifting snow were entirely due to charges below the 5-feet level, then the elcctric force above this level would be unaffected, or, in other words, the difference of potential between points at the 15 - and 5 -feet levels would be the same as under normal conditions. If, on the other hand, the charge causing the disturbance was entirely above the higher level, the increase in the electric force would be the same throughout the 15 feet (a horizontally stratified distribution being assumed); the ratio of the potential observed at the two points would remain constant if the charge in the air below the higher one were always negligible.

The data for such a comparison are, unfortunately, very scinty, but, so far as they go, the results show that with snow in the air accompanied by wind the difference of potential between the two levels was increased, the relative increase being, however, not so great as that of the potential at the lower level, i.e., the ratio of the potential at 15 feet to that at 5 fuet was on the whole diminished. This would indicate a positive charge neither entirely above the 15 -feet level 1101 entirely below the 5 -feet level. There is, unfortunately, no case (where the data for both levels are available) in which the drifting snow is recorded as being entirely below the 5-feet level. The results are, then, consistent with the view that the disturbance is entirely due to a positive charge carried by the drifting snow particles, but the data are insufficient to justify any more definite conclusion. 
In the ease of snow unaceompanied by any but inconsiderable winds, the readings at the lower level are, as we have seen, not sensibly increased; those at the upper level are, on the whole, diminished. In one case indeed, the 15 -feet reading was actually less than the 5-feet reading, indicating an npparent reversal of the field above the 5-feet level. It nust, however, be remembered that the observations at the two points were not simultaneous, the high-level observations being taken immediately after those at five feet. The possibility of defective insulation in the case of the high-level observations during a fall of snow must also be borne in mind.

\section{Daily and Yearly Variations of Potential.}

Observations taken during the occurrence of drift must certainly be treated as abnormal, and are therefore excluded from the data used in the investigation of possible daily and annual variations of potential. The comparatively fow other oceasions when the potential exceeded 300 volts, as well as cases of zero or negative potential, have also been excluded.

\section{Daily Variation.}

Only during the summer months, November, December, and January, were the observations numerous enough to admit of the possibility of a daily variation being deteeted. These three months are alike in giving evidence of the existence of a minimum in the middle of the day or early afternoon. In December, 1902, and January, 1903, observations were taken at 10 a.m., 4 p.m., and 10 p.m.

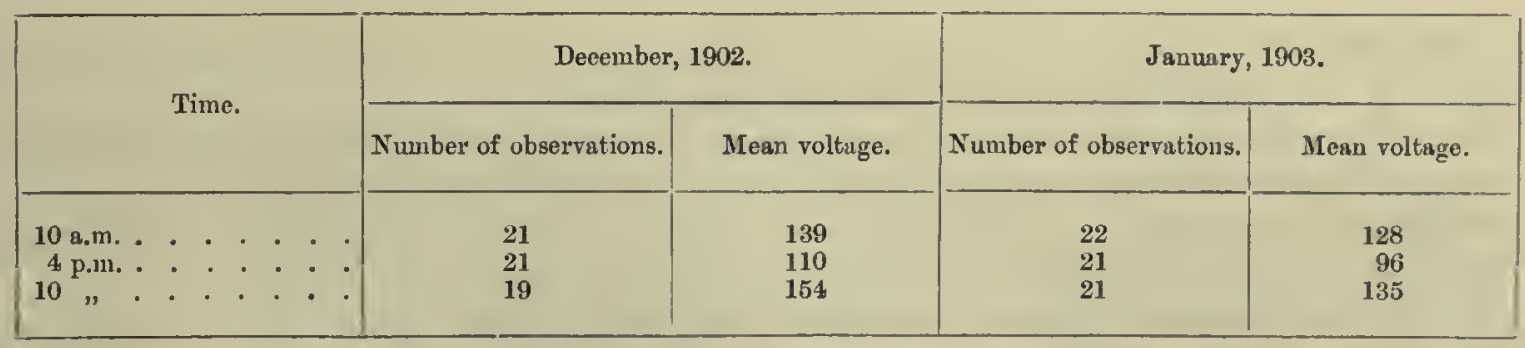

The observations for November were more numerous and were distributed over the 24 hours. The minimum occurs in the middle of the day, the maximum being in the early morning soon after midnight. Dividing the day into six equal periods of 4 hours, and taking the mean of the potentials observed during each of these periods, we obtain the results given in the following table :-

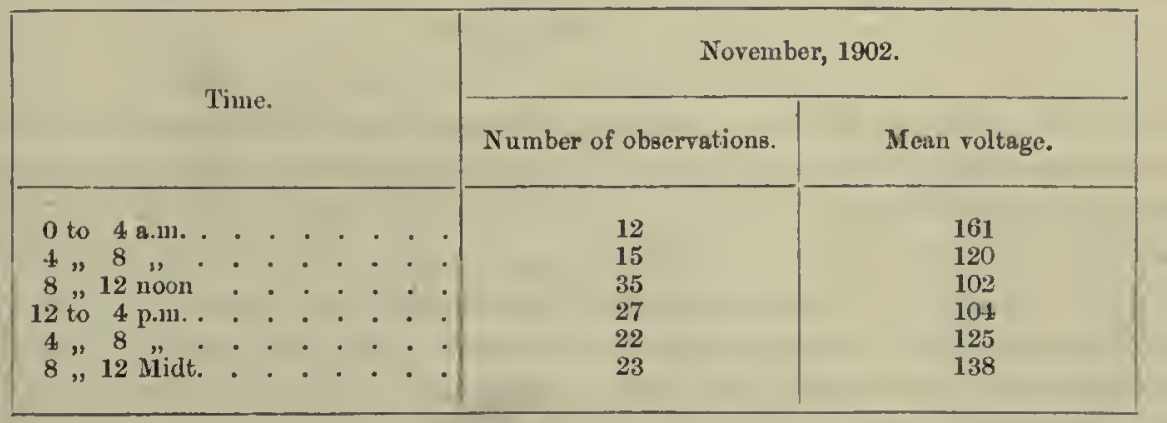

The only continuous series of hourly observations extending over so much as 24 hours was one earried ont in November, 1902. It extended over three days, from 8 a.m., November 22, to 8 a.m., Novenber 25 ; the weather eonditions were fairly satisfaetory, with no snow or drift and little wind, but with is good deal of cloud. 
Hovrly values of potential in volts, November 22 to $25,1902$.

\begin{tabular}{|c|c|c|c|c|c|c|}
\hline & Time. & & November $22-23$. & November 23-24. & Norember $24-25$. & Mean. \\
\hline 8 a.m. & . . & . . & 129 & 126 & 81 & 112 \\
\hline $9 "$ & . . & . . & 75 & 132 & 102 & 103 \\
\hline $10 "$ & . . & . . & 108 & 120 & 84 & 104 \\
\hline $11 "$ & . . & . . & 105 & 81 & 75 & 87 \\
\hline Noon . & . . & . . & 90 & 84 & 48 & 74 \\
\hline 1 p.m. & $\cdot \cdot$ & . . & 72 & 93 & 57 & 74 \\
\hline 2 & . . & . . & 78 & 132 & 78 & 96 \\
\hline $3 "$ & . . & . . & 126 & 120 & 84 & 110 \\
\hline $4 "$ & . . & . . & 135 & ] 65 & 117 & 139 \\
\hline $5 "$ & . . & . . & 138 & 99 & 123 & 120 \\
\hline $6 "$ & . . & . . & 141 & 108 & 117 & 122 \\
\hline $7 "$ & . . & . . & 126 & 138 & 108 & 124 \\
\hline $8 "$ & . . & . . & 120 & 159 & 108 & 129 \\
\hline $9 "$ & . . & . . & 114 & 153 & 99 & 122 \\
\hline 10 & . . & . . & 132 & 144 & 102 & 126 \\
\hline $11 "$ & $\cdot \cdot$ & .. & 93 & 165 & 105 & 121 \\
\hline Midt. . & . . & . . & 117 & 177 & 96 & 130 \\
\hline $1 \mathrm{n} . \mathrm{m}$ &. & . . & 156 & 225 & 102 & 161 \\
\hline $2 "$ & . . & . . & 99 & 165 & 120 & 128 \\
\hline 3 & . . & . . & 129 & 189 & 126 & 148 \\
\hline$+"$ &. & . . & 273 & 213 & 141 & 209 \\
\hline $5 "$ & $\cdot \cdot$ & . . & 135 & 138 & 117 & 130 \\
\hline $6 "$ & . . & . & 102 & 126 & 111 & 113 \\
\hline 7. & •. & . . & 120 & 105 & 108 & 111 \\
\hline $8 "$ & . . & . . & 126 & 81 & 93 & 100 \\
\hline
\end{tabular}

This series shows a minimum on each day about noon, and a maximum in the early morning hour's, the time of the maximun for the mean of the three days being 4 a.m.

During the summer months, when the sun is always above the horizon, there is evidence of a daily variation, the maximum being when the sun is low, the minimum in the middle of the day.

Mr. Ветхасси remarks in a note added to the observations of November 15, 1902 :- " There seenls little doubt that atmospheric electricity is a maximum at midnight and a minimum at noon."

For the rest of the year the data are insufficient to establish any regular daily variation. There is one series of 2-hourly observations extending from noon on October 7 till noon on October 10, 1903. There was considerable disturbance due to drifting snow, and the observations were made with the collector at a height of 12 feet; the insulation in the high-level observations is not above suspieion. This series is, therefore, of little value for the purpose of studying the daily variation.

\section{Annual Variation.}

The fact that the observations were not taken at the same hours daily throughout the year makes it difficult to decide upon the best mode of treating the observations for the purpose of determining whether there is any regnlar annual variation. In the three talles which follow are given the menn potential at the point of observation ( 5 feet above the ground) for each month, the mean for each month being in the first table that of the forenoon observations (taken between 9.30 and 11 a.m.) of all the available days; in obtaining the values given in the second table the mean of the available values for each day was calculated, and the mean for the month is the nean of these means; in the last table the mean of all the available observations for each month is given. In all cases observations taken during snow or drift, as well as all giving negative or zero or abnormally high potentials (exceeding 300 volts) have been excluded. The period covered is from March, 1902, to April, 1903, inclusive.*

* Under March and April are given the meane of the observutions of both 1902 and 1903. 
I. From Forenoon Olsservations.

\begin{tabular}{|c|c|c|c|c|c|c|c|c|c|c|c|c|c|}
\hline Month . & 劳 & 苞 & 完 & 离 & 离 & 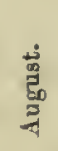 & 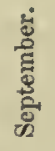 & 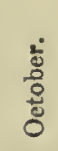 & 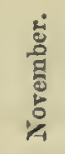 & 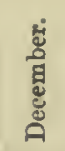 & 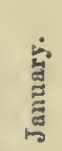 & 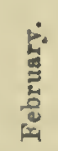 & 远 \\
\hline Volts. . . . . & 76 & 73 & 86 & 60 & 68 & 96 & 88 & 106 & 106 & 139 & 128 & 78 & 92 \\
\hline Number of obserrations & 19 & 24 & 11 & 5 & 9 & 8 & 15 & 16 & 23 & 21 & 22 & 8 & - \\
\hline
\end{tabular}

II. From Means for each Day.

\begin{tabular}{|c|c|c|c|c|c|c|c|c|c|c|c|c|c|}
\hline Month & $\begin{array}{l}\text { 苛 } \\
\text { 岕 }\end{array}$ & 空 & 远 & 苛 & $\stackrel{0}{\Xi}$ & 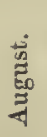 & 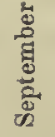 & 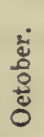 & 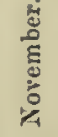 & 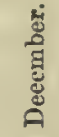 & 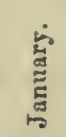 & 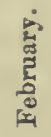 & : \\
\hline Volts. . & 69 & 83 & 91 & 79 & 59 & 83 & 87 & 94 & 119 & 134 & 122 & 91 & 92 \\
\hline Number of Days. . & 24 & 27 & 15 & 16 & 19 & 12 & 19 & 21 & 25 & 24 & 25 & 8 & - \\
\hline
\end{tabular}

III. From all available Observations.

\begin{tabular}{|c|c|c|c|c|c|c|c|c|c|c|c|c|c|}
\hline Month & 苞 & 窎 & 冚 & 总 & 官 & 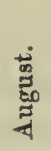 & 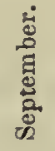 & 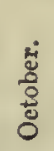 & 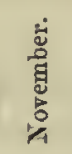 & 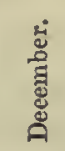 & $\begin{array}{l}\text { 悹 } \\
\text { 吉 } \\
\text { 总 }\end{array}$ & 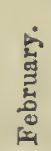 & 总 \\
\hline Volts. . . . & 71 & 78 & 90 & 71 & 57 & 80 & 91 & 88 & 120 & 133 & 119 & 95 & 91 \\
\hline Number of observations & 38 & 36 & 16 & 23 & 38 & 10 & 59 & 67 & 134 & 62 & 65 & 22 & - \\
\hline
\end{tabular}

The general character of the annual variation as deduced from any of the three tables is the same; there is a maxinum in December, a ninimum in June or July, and apparently a secondary minimum in March or April.

Table I. is uusatisfactory on account of the very small number of the forenoon observations in the winter months. To the use of the methods of Tables II. or III. in determining the unnual variation it might he oljected that when the ohservations are distributed irregularly over the 24 hours, there is a danger of an apparent annual variation arising from a variation in the hours of most frequent observation at different seasons of the year. In the summer months, for which alone the data are in any way sufficient to test the existence of a daily variation, the mean of the forenoon observations for each month does not differ to any serious extent from the ineans derived from all the observations as in Tables II. or III. Whether the method of Table II. or that of Table III. is the better it is not easy to deeide. Table II. is not really derived from the values of the true mem potential for each day; the values used for the mean potential for each day being the mean of the results of the undisturbed observations for tlat day, the number of such observations varying for different days from 1 to 24. It would seem natural to attach more weight to the mean for a day the greater the mumber of observations from which such mean has been derlneerl. If we were to weight the means for the various days sinply in proportion to the number of olserrations, we shonld arrive at the numbers given in Tahle III. On the other hand, it might be held 
that all the results of a particular day are likely to have been similarly affected by the weather eonditions prevailing on that day, and that it is fairer to proeeed aeeording to the method of Table II., and attaeh equal weight to the mean for each day, whatever the number of observations from which it has been derived. The difference in the actnal results of the two methorls is fortunately too small to be of importance.

There is still another difficulty to be eonsidered. The plaee of observation was not the same in the winter nonths as during the rest of the year. From the 24 th of May, 1902, till the beginning of $\Lambda$ ugnst, and again dhring the latter half of Mareh and in $\Lambda$ pril, 1903, the observations were taken on the iee instead of at the usual place on shore. The height of the burning mateh above the ground or iee was the same at both plaees. The apparent winter minimum might, then, be held to be spurious and due to the ehange of the plaee of observation. Against this it is to be said that there is no obvious diseontinuity in the reeord of potential readings when the plaee of observation is changed, and that the values of the potential for April, 1903, are greater than those of April, 1902, in spite of the faet that those of 1903, unlike those of the previous year, were taken on the ice. There is, then, no evidenee that the potential above the iee was lower than that at the same height on shore. The ehange of plaee of observation, no doubt, however, does introduee a serious uncertainty.

Even, however, if we eonfine our attention to the observations taken under eonstant eonditions from the beginning of August, 1902, to the end of February, 1903, the evidenee of an annual variation of potential is very strong, the maximum oeeurring in December. The material for the rest of the year is mueh less satisfaetory, on aecount of the less frequent observations, as well as of the ehanges in the place of observation.

To sum up, we may say that the observations made at the "Diseovery's" Winter Quarters give evidence of the existenec of an annual variation of the electrie field, the maximum being in December (i.e., midsummer), there being probably a minimum in June or July, and a secondary minimum in March or April. It has, of eourse, to be remembered that these conclusions rest on the results of observations extending over only one year. The midsummer maximum is very elearly indieated by the observations and is remarkable when we remember that in lower latitudes the electric field has its maximum strength in midwinter. Data for the study of any daily variation are praetieally wanting except for the month of November. Here there is evidenee of a minimum in the middle of the day (and this is in agreement with the results of the December and January observations) and a maximum in the early morning hours. The early morning maximum is remarkable, the one point in which there is agreement in the curves of daily variation for different seasons and different localities in lower latitudes being the oceurrence of an carly morning minimum.

\section{Potential at 15 Feet above the Ground.}

During Deember, 1902, and January, 1903, in addition to the observations at the usual height, measurements of the potential at 15 fect above the ground were also marle. For this purpose a eolleetor match was attaehed to one end of a rubber-insulated wire, the other end being eonneeted to the eleetrometer terminal. The mateh was lighted and then raised to a height of 15 feet with the help of a pole of that length, to which the insulated wire was attached.

The observations were taken about 3 minutes after those at the 5 -fect level.

In the absenec of speeial tests the insulation in such an arrangement is by no means above suspieion. Nor, even in the absence of error clue to defective insulation, is it possible to deduee from the observations the true undisturbed potential at 5 feet and 15 feet, on aecount of the deformation of the potential surfaecs by the observer and apparatus. But the results of the observations at the higher level are of valne as confirming the results obtained for the variation of potential at the lower level. The observations at the two levels not being simultaneous, we cannot expeet a eonstant ratio between the high- and low-level potentials for individual readings (even in the absenee of rolume eleetrification of the air in the immediate neighbourhood of the plaee of observation), but there appears to be a fairly eonstant latio between the means at the two levels when we consider a reasonable number of observations.

In Deember, 1902, and January, 1903, olsservations were taken fairly regularly at the hours 10 a.m., 4 p.m., and 10 p.m. If we confine our attention to ditys on whieh olservations at each of these hours were 
obtained at both levels (observations disturbed by drift, dece, being exclnded), we get the following resnlts for the high-level potential at each of the hours mentioned, and its ratio to the low-level potential:-

\begin{tabular}{|c|c|c|c|c|c|c|c|c|c|}
\hline \multicolumn{4}{|c|}{ Dulo. } & \multicolumn{3}{|c|}{ Yotenlial at 15 feet. } & \multicolumn{3}{|c|}{$\frac{\text { Potential at } 15 \text { feet }}{\text { Potential at } 5 \text { feet }}$} \\
\hline & & & & 10 a.m. & 4 p.m. & 10 p.m. & 10 a.m. & 4 p.in. & 10 p.m. \\
\hline $\begin{array}{c}1902 \\
\text { Decemb } \\
\text { ", } \\
", \\
", \\
", \\
", \\
" \\
" \\
" \\
" \\
", \\
",\end{array}$ & $\begin{array}{r}5 \\
6 \\
14 \\
17 \\
18 \\
22 \\
24 \\
25 \\
26 \\
27 \\
28 \\
29\end{array}$ & 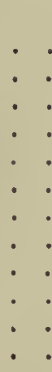 & 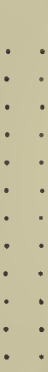 & $\begin{array}{l}354 \\
186 \\
661 \\
189 \\
133 \\
336 \\
278 \\
551 \\
290 \\
342 \\
258 \\
319\end{array}$ & $\begin{array}{l}174 \\
131 \\
261 \\
112 \\
216 \\
174 \\
251 \\
516 \\
357 \\
342 \\
200 \\
345\end{array}$ & $\begin{array}{l}255 \\
215 \\
229 \\
177 \\
518 \\
232 \\
353 \\
383 \\
313 \\
505 \\
415 \\
505\end{array}$ & $\begin{array}{l}3 \cdot 3 \\
1 \cdot 7 \\
2 \cdot 72 \\
2 \cdot 4 \cdot 12 \\
2 \cdot 29 \\
2 \cdot 19 \\
2 \cdot 02 \\
2 \cdot 41 \\
2 \cdot 12 \\
1 \cdot 81 \\
2 \cdot 55 \\
1 \cdot 71\end{array}$ & $\begin{array}{l}2 \cdot 0 \\
1 \cdot 75 \\
1 \cdot 57 \\
2 \cdot 32 \\
2 \cdot 50 \\
2 \cdot 33 \\
2 \cdot 03 \\
3 \cdot 35 \\
2 \cdot 92 \\
3 \cdot 30 \\
2 \cdot 08 \\
2 \cdot 44\end{array}$ & $\begin{array}{l}2 \cdot 0 \\
2 \cdot 7 \\
1 \cdot 25 \\
1 \cdot 22 \\
2 \cdot 50 \\
1 \cdot \cdot(\cdot 1 \\
1 \cdot 92 \\
1 \cdot 95 \\
2 \cdot 70 \\
y \\
2 \cdot 93 \\
2 \cdot 99 \\
2 \cdot 45\end{array}$ \\
\hline & un. & . . & - & 325 & 264 & 3.17 & $2 \cdot 29$ & $2 \cdot 38$ & $2 \cdot 10$ \\
\hline
\end{tabular}

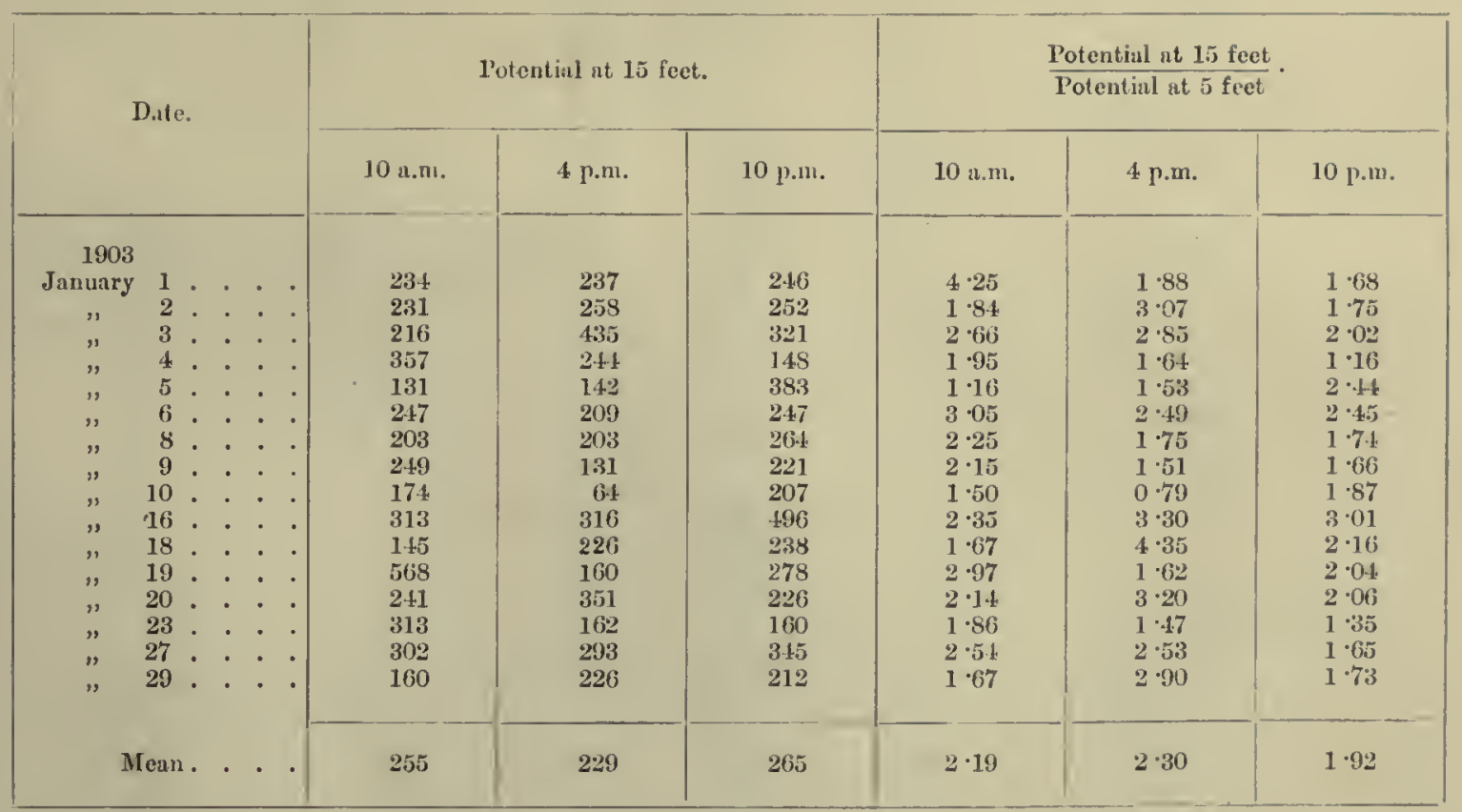

There is evidence of a divily varriation at the higher level in agreement with that shown at the lower level.

Again, at the higher level as at the lower level, the mean potential at each of the hours is less in Jaunily thau in December. For the sulsequent months the datal are very seanty, but so firr is they go they show agreement between the variations at the two levels.

\section{Atmosidielic Potential and the Aulomis.}

The occurrence of auroral displays was without any obvious effect on the eleetrical field.

On several occissions during an anrorn, additional olservations of potential were male with an electrometer resting on the ship's rail. The actual values of the potential thus observed are, of course, of little 
importance, but such olservations wonld at least have shown if there were anything abuormal in the sign of the potential, or even if there were anything extraorlinary in its magnitude dluing an anrola, or if there were fluetuations eorresponding to elanges in the appearanee of the aurorit. In all cases, however, the sign of the potential was apparently normal, and there was nothing remarkalile in the values of the readings.

It is not easy to decile definitely from the ordinary series of potential readings whether anroras lawe any influence on the eleetrical field. It is only for about half the year that auroms could be observerl, and it is just during this half of the year, i.e., the winter months, that the observations of potential were fewest. Again, while the conditions were suitable for seeing then, "anroras were of practically daily oceurreuce, so that possibly the potential, as it would be outside the influence of auroral disturbances, was never observed.

Perlaps all that ean be done is to pick out the days on whielı auroras of alnormal brightness, extent, or duration occurred, and see if the potential on such days differs from the mean for the month. In the following table are given the days on which specially conspicuous auroras oecurrecl, and the resnlts of such observations of potential as were unde on these diys, the nean of the undistnrlyed potential readings for the month being also given.

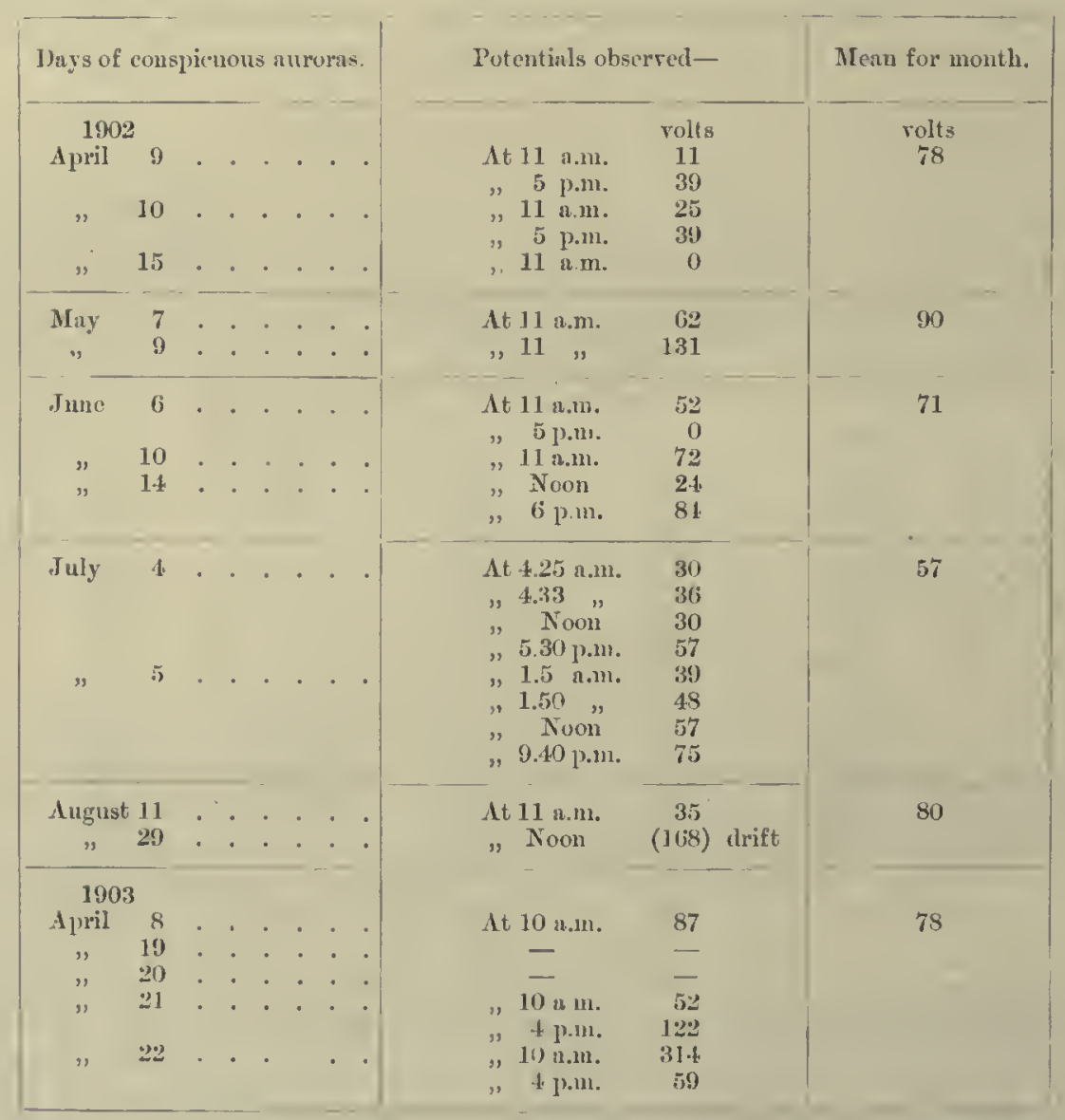

There is some indication of disturbance of the electrical field by these specially intense anroras, low values beiug apparently liable to occur. There is one case, April 22, 1903, of an exceptionally high value; this was not accompanied by drifting snow or other abnormal conditions. 
Orservations of Electrical Potential at "Discovery's" Winter Quinters. White's Portahle Electrometer No. 81. Height of Match above tho Ground, 5) feet. Jocal Time.

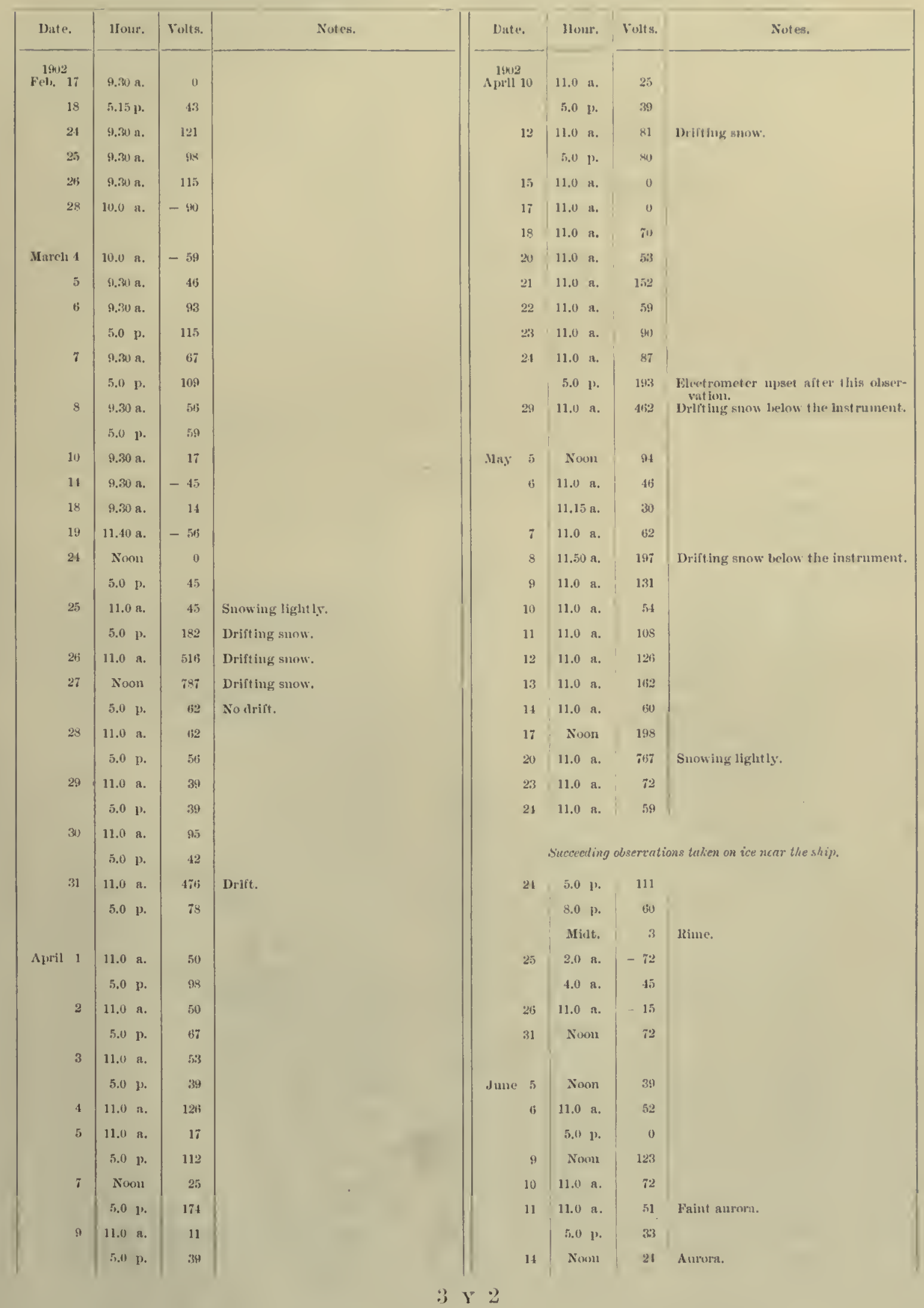


Opservations of Flectrical Potential at "Discovery's" Winter Quarters. Wuite's Portable Electrometer No. 81. Ineight of Match above the Gromild, 5 fect. Loeal Time (continued).

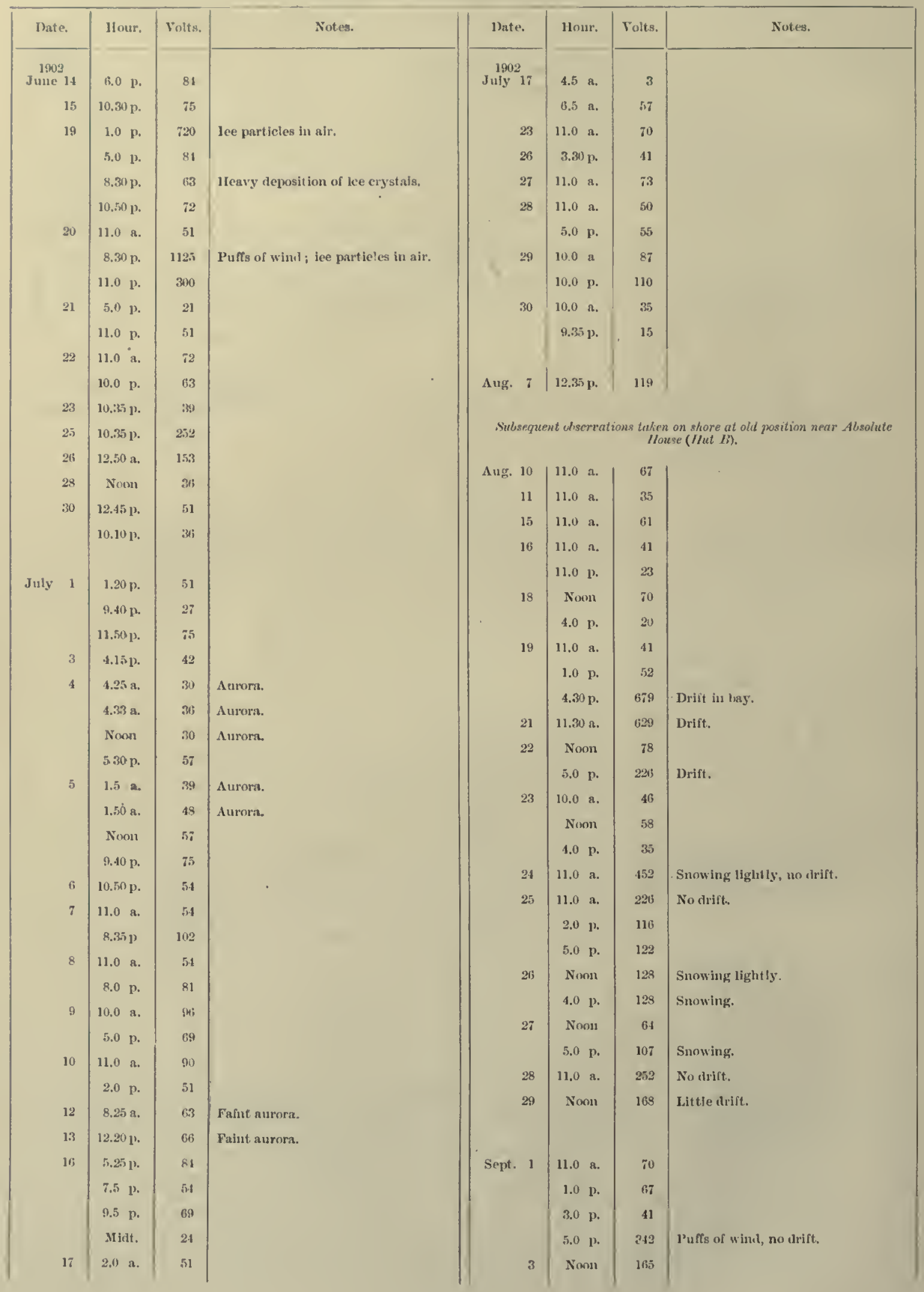


Onsfrvatioss of Electrical Potential at "Discovery's" Winter Qharters. Winte's Portable Electrometer No. 81. Height of Match ahove the Gromul, 5 feet. Local 'Time (contimed).

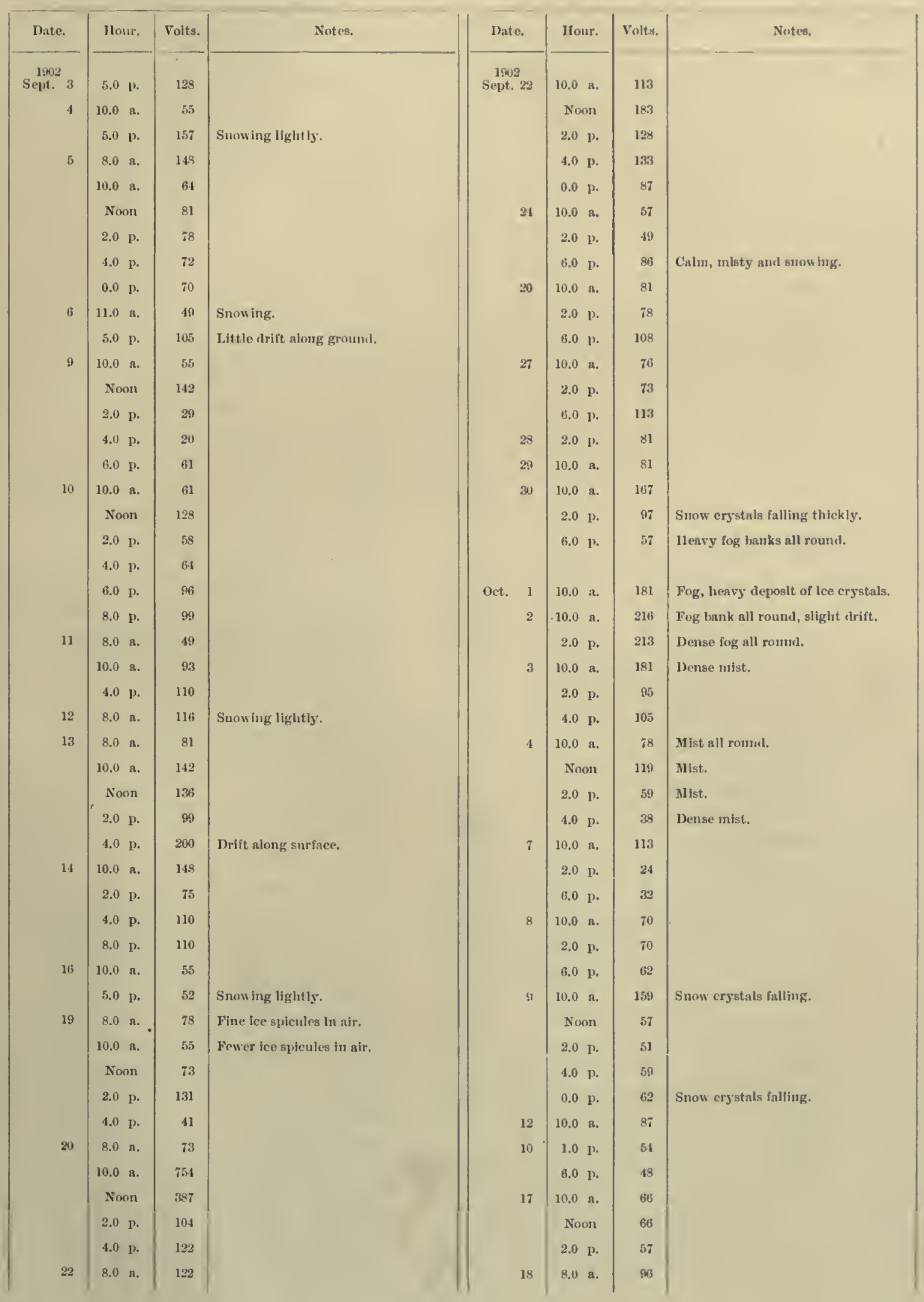


Onsenvatioxs of Electrieal Potential at "Diseovery's" Winter Quniters. White's Portalle Electrometer No. 81. Height of Mateh above the Ground, 5 feet. Local Time (continued).

\begin{tabular}{|c|c|c|c|c|c|c|c|c|}
\hline Date & & Hour. & Volts. & Notes. & Date. & Hour. & rolts. & Notes. \\
\hline \multirow{4}{*}{\multicolumn{2}{|c|}{$\begin{array}{l}1902 \\
\text { Oct. } 18\end{array}$}} & $10.11 \mathrm{~A}$. & 60 & & $\begin{array}{l}1 \% 22 \\
\text { Nov. } 3\end{array}$ & Noon & 87 & \\
\hline & & Sinou & 3 & & & $2.0 \mathrm{P}$. & 60 & \\
\hline & & $2.0 \mathrm{p}$. & $6 i 3$ & & & $8.0 \mathrm{l}^{3}$. & 141 & \\
\hline & & $8.0 \mathrm{p}$ & 132 & & 4 & x.0 a. & 1.50 & \\
\hline \multirow{3}{*}{\multicolumn{2}{|c|}{19}} & $8.11 \mathrm{n}$. & 180 & & & $10.0 \mathrm{at}$ & 117 & \\
\hline & & $10.0 \mathrm{n}$ & (23) & & & Xom & 69 & \\
\hline & & $4.0 \mathrm{p}$. & 48 & & & $2.0 \mathrm{p}$. & 102 & \\
\hline \multirow{6}{*}{\multicolumn{2}{|c|}{20}} & 10.0 a. & wi & & & $4.0 \mathrm{p}$. & 93 & \\
\hline & & Noon & 60 & & & $6.0 \mathrm{p}$. & $9 h \mathrm{j}$ & \\
\hline & & $2.0 \mathrm{p}$. & bit & & & $8.0 \mathrm{p}$. & $10 k$ & \\
\hline & & $4.0 \mathrm{p}$. & 84 & & & $10.0 \mathrm{p}$ & 138 & \\
\hline & & $6.0 \mathrm{p}$. & 96 & & 5 & $10.0 \mathrm{a}$. & 114 & \\
\hline & & $8.0 \mathrm{p}$. & $1: 29$ & & & Noon & 81 & \\
\hline \multirow{5}{*}{\multicolumn{2}{|c|}{21}} & $8.0 \mathrm{n}$. & 186 & & & $2.0 \mathrm{p}$. & 37.9 & Squalls gmul iritiug smow. \\
\hline & & $10.0 \mathrm{n}$. & 117 & & & $6.0 \mathrm{p}$. & 162 & Squally; no drilt. \\
\hline & & Noon & 84 & & 6 & $10.0 \mathrm{a}$. & 84 & \\
\hline & & $2.0 \mathrm{p}$. & is & & & Nom & 93 & \\
\hline & & $6.1 \mathrm{p}$. & 132 & & & $4.0 \mathrm{p}$. & 111 & \\
\hline \multirow{2}{*}{\multicolumn{2}{|c|}{22}} & $10.0 \mathrm{n}$. & 147 & & 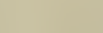 & $6.0 \mathrm{l}$. & 129 & \\
\hline & & $4.0 \mathrm{p}$. & 54 & & & $8.0 \mathrm{p}$. & 114 & \\
\hline \multirow{2}{*}{\multicolumn{2}{|c|}{25}} & 10.0 a. & 60 & & 7 & $10.0 \mathrm{a}$. & 291 & Snowing lieavily. \\
\hline & & $6.0 \mathrm{p}$. & 78 & & & $10.0 \mathrm{p}$. & 141 & \\
\hline \multirow{4}{*}{\multicolumn{2}{|c|}{$2 t i$}} & 10.0 a. & 135 & & 8 & $10.0 \mathrm{n}$. & 702 & Blow ing hawl; clrifting snow: \\
\hline & & Nomin & 72 & & & $10.0 \mathrm{p}$. & 102 & \\
\hline & & $2.4 \mathrm{p}$. & 51 & & 9 & Noon & 111 & \\
\hline & & $4.0 \mathrm{p}$. & 72 & & & 13.0 p. & 81 & 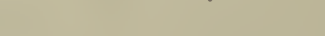 \\
\hline \multirow{8}{*}{\multicolumn{2}{|c|}{27}} & $8.0 \mathrm{a}$. & 348 & & 10 & $8.0 \mathrm{a}$, & $15 !$ & \\
\hline & & 10.0 ณ. & -18 & & & $10.0 \mathrm{n}$. & 102 & \\
\hline & & Nonn & 63 & & & Noon & 123 & \\
\hline & & $2.0 \mathrm{p}$. & 63 & & & $2.0 \mathrm{p}$. & 87 & \\
\hline & & $4.0 \mathrm{p}$ & 63 & & & $4.0 \mathrm{p}$. & 141 & \\
\hline & & $6.0 \mathrm{p}$. & 63 & & & $15.0 \mathrm{p}$. & 141 & \\
\hline & & $8.0 \mathrm{p}$. & 117 & . & & $8.0 \mathrm{p}$. & 162 & \\
\hline & & $10.0 \mathrm{p}$ & 87 & & 11 & $10.0 \mathrm{a}$. & १९ & \\
\hline \multirow{4}{*}{\multicolumn{2}{|c|}{29}} & $10.0 \mathrm{a}$. & 147 & Sunwing lightly, little drift on libls. & 12 & $10.0 \mathrm{a}$. & 108 & \\
\hline & & $2.0 \mathrm{p}$ & 114 & & & Noon & $12: 3$ & \\
\hline & & $4.0 \mathrm{p}$. & 108 & & 13 & $10.0 \mathrm{R}$. & 98 & \\
\hline & & $8.0 \mathrm{p}$. & 141 & & & $4.0 \mathrm{p}$. & 111 & . \\
\hline \multirow{4}{*}{\multicolumn{2}{|c|}{$3 u$}} & $10.0 \mathrm{a}$. & 126 & & & $10.0 \mathrm{p}$. & 117 & 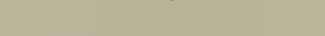 \\
\hline & & Noon & 72 & & 14 & $10.0 \mathrm{a}$. & 9 & Snow ing. \\
\hline & & $2.0 \mathrm{p}$. & 165 & A little drilting snou in squalls. & & $4.0 \mathrm{1}$. & 153 & \\
\hline & & $6.0 \mathrm{p}$. & $\$ 9$ & & & $10.0 \mathrm{p}$. & 153 & \\
\hline \multirow{4}{*}{\multicolumn{2}{|c|}{31}} & 10.0 a. & 111 & & 13 & $10.0 \mathrm{a}$. & 141 & \\
\hline & & Nomil & 48 & & & $4.0 \mathrm{p}$. & 99 & \\
\hline & & $2.0 \mathrm{p}$. & $\tau 2$ & & & Milt. & 192 & \\
\hline & & & & & 16 & $10.0 \mathrm{a}$ & $6 i 5$ & \\
\hline \multirow[t]{2}{*}{ Nov. } & 2 & $5.0 \mathrm{p}$. & 129 & & & $4.0 \mathrm{p}$. & 120 & \\
\hline & 3 & $10.0 \mathrm{a}$. & 133.5 & & & $10.10 \mathrm{p}$. & 201 & \\
\hline
\end{tabular}


OmEnvitions of Electrical Potential at "I)iscovery's" Winter Qunrteis. Wuite's Portablo lilectro. meter No. 81. Ieight of Match ahove the Gronnd, 5 fect. Irocil Timo (continued).

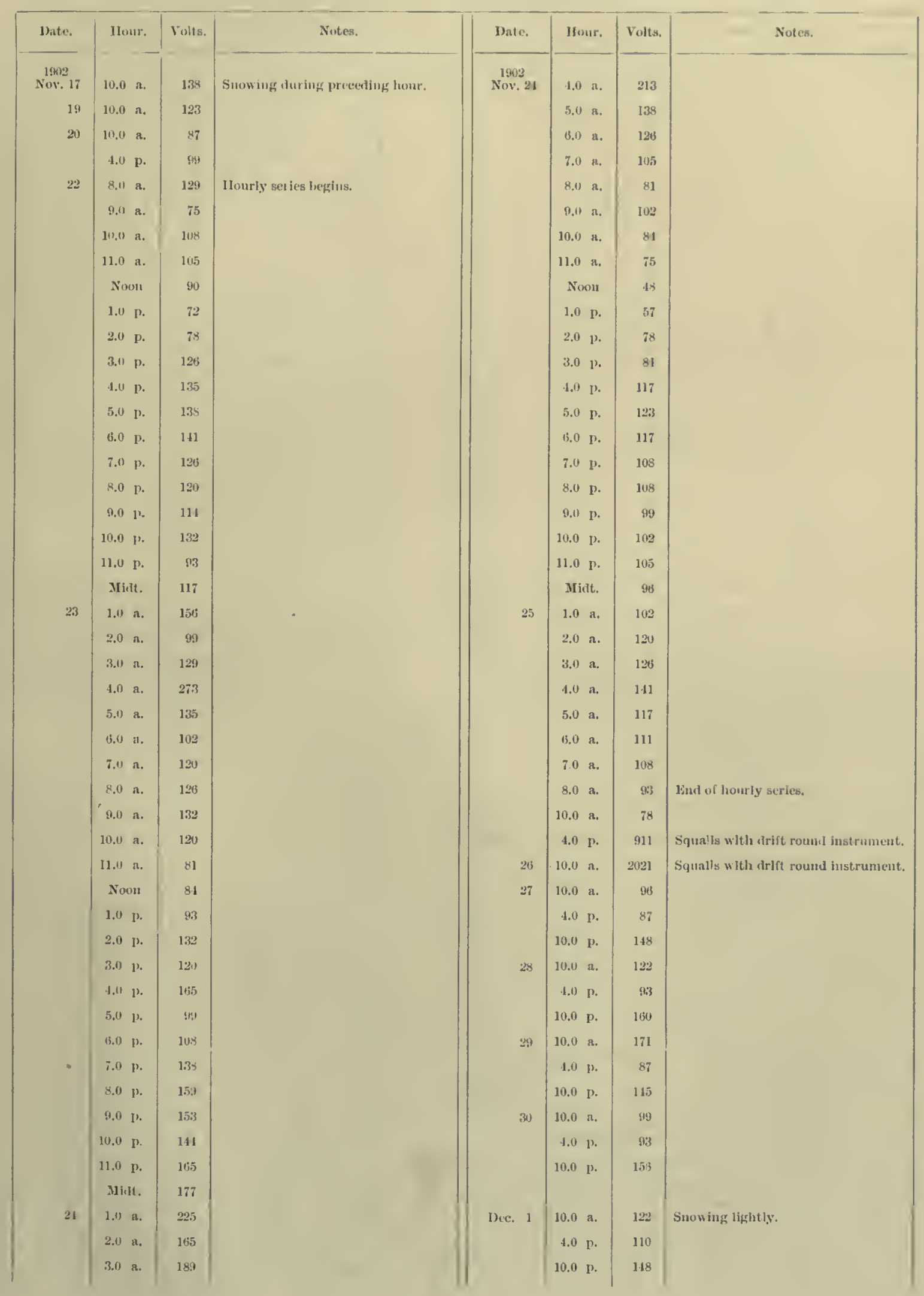


Orsinvations of Electrical Potential at "Biscovery's" Winter Quarters. Winte's Portahle Electrometer No. 81. Height of Match above the Gromind, 5 feet. Locial Time (continned).

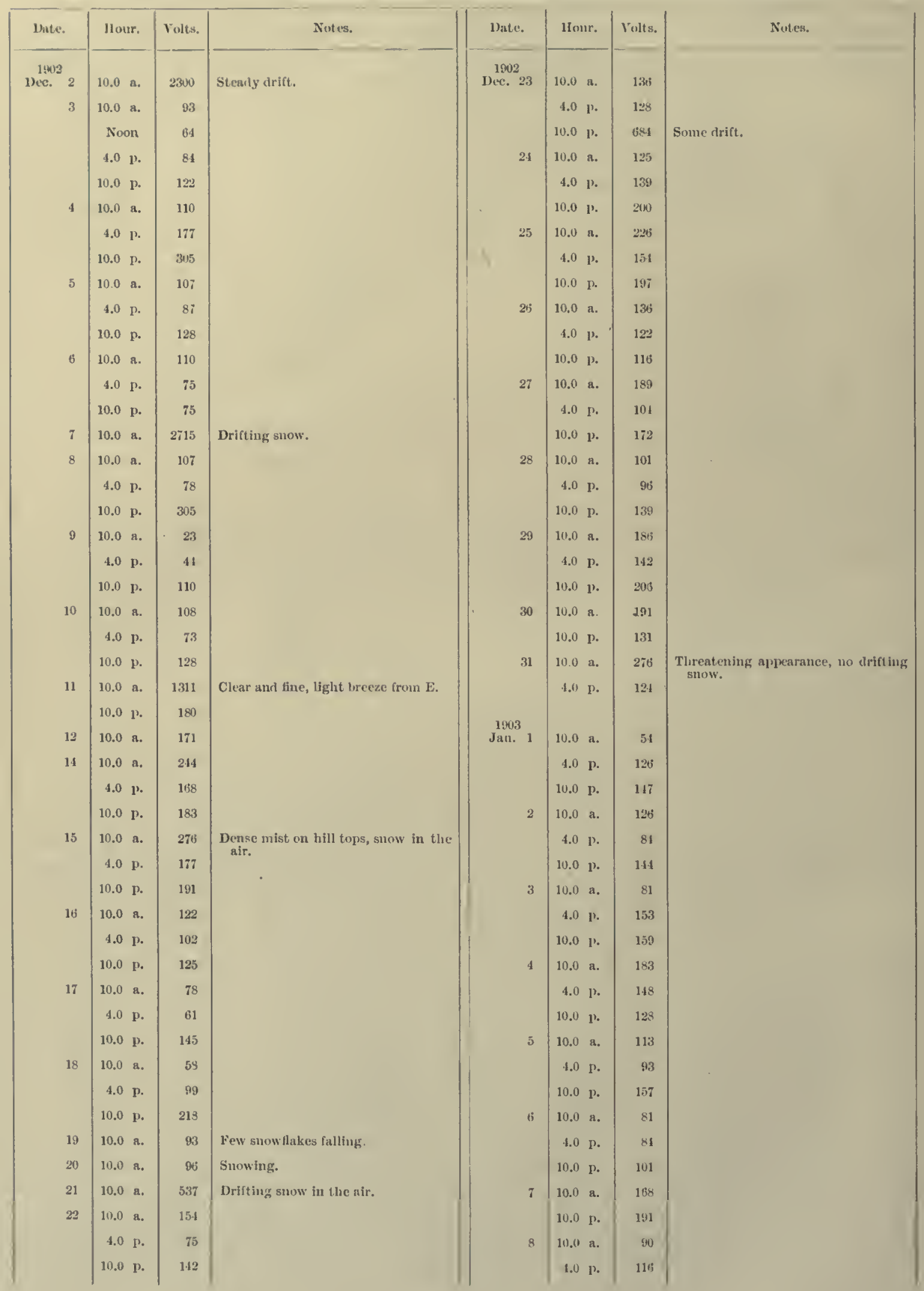


Onservations of Electrical Potential at “Discovery's" Winter Quarters. White's Portable Electrometer No. 81. Height of Match above the Ground, 5 fcet. Local Time (continued).

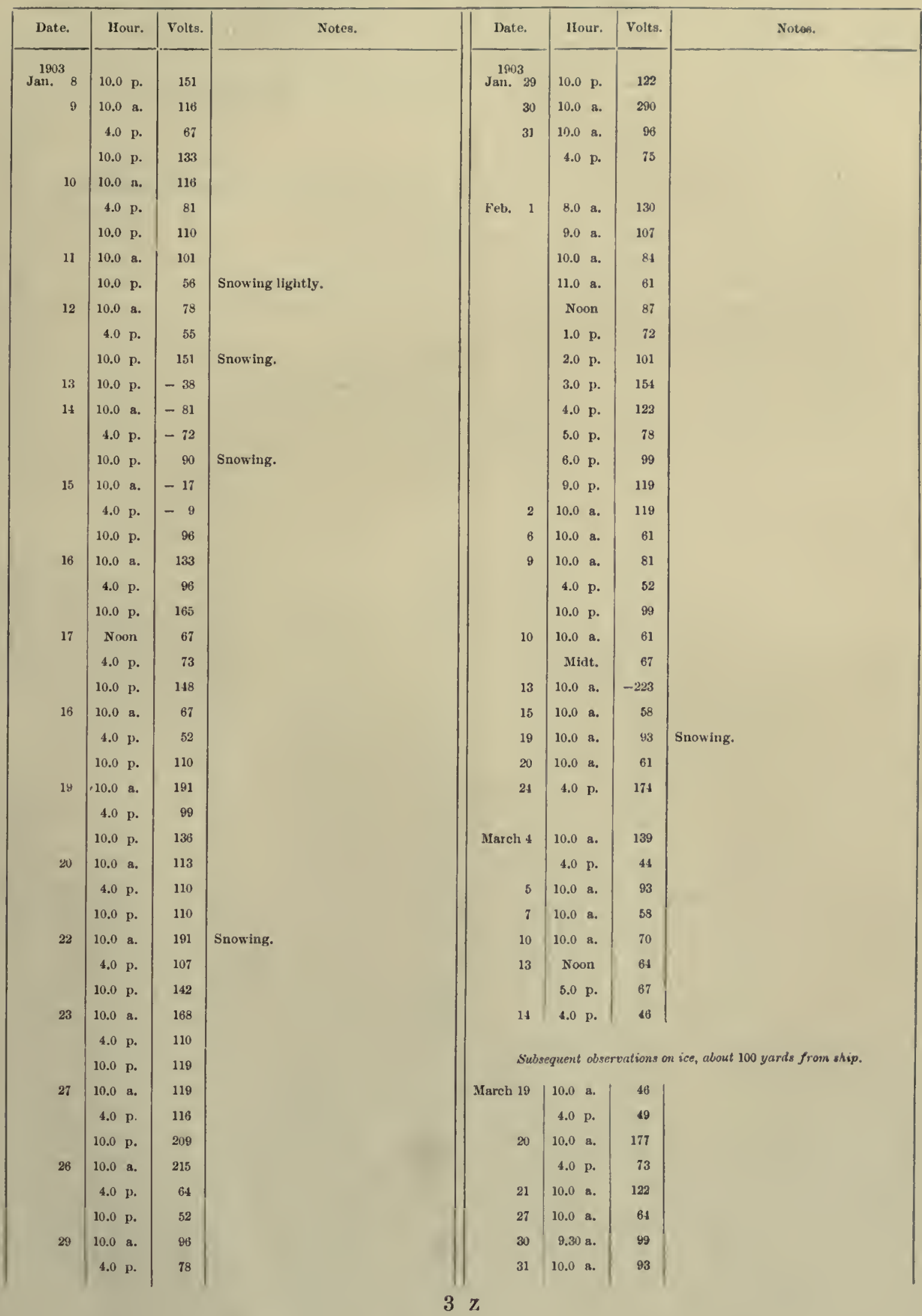


OBsenvatioss of Electrical Potential at "Discovery's" Winter Quarters. White's Portable Electrometer No. 81. Heiglit of Matcl above the Ground, 5 feet. Local Time (continued).

\begin{tabular}{|c|c|c|c|c|c|c|c|}
\hline Date. & Hour. & Volts. & Sotes. & Date. & Ilour. & Volts. & Notes. \\
\hline April 1903 & 10.0 a. & 15 & & May 7 & $11.0 \mathrm{a}$. & 146 & \\
\hline 3 & $10.0 \mathrm{a}$. & 49 & & 8 & $11.0 \mathrm{a}$. & 06 & \\
\hline 7 & $10.0 \mathrm{n}$. & 107 & & & & & \\
\hline 8 & 10.0 ล. & 87 & & & & & \\
\hline 9 & $10.0 \mathrm{s.}$ & 145 & & \multirow{2}{*}{\multicolumn{4}{|c|}{ Sulsequent wberrations on shore nent Absolute House (IIut B). }} \\
\hline 14 & $10.0 \mathrm{a}$ & 41 & & & & & \\
\hline 17 & $10.0 \mathrm{a}$. & 87 & & Aug. 20 & 10.0 a. & 146 & \\
\hline 21 & $10.0 \mathrm{a}$. & 52 & & 21 & $11.0 \mathrm{~A}$. & 103 & \\
\hline & $4.0 \mathrm{p}$. & 123 & & 25 & $11.0 \mathrm{a}$. & 65 & \\
\hline 83 & $10.0 \mathrm{a}$. & 314 & & & & & \\
\hline & $4.0 \mathrm{p}$. & 59 & & & & & \\
\hline 25 & $10.0 \mathrm{a}$. & 76 & & Sept. 21 & $3.10 \mathrm{p}$ & 62 & \\
\hline 27 & $11.0 \mathrm{a}$. & 115 & & 29 & $10.0 \mathrm{~A}$. & 67 & \\
\hline 23 & $10.0 \mathrm{~A}$. & 70 & & 30 & $10.0 \mathrm{a}$ & $8 i$ & \\
\hline
\end{tabular}


Hign Level Observations of Potential.

\begin{tabular}{|c|c|c|c|c|c|c|c|}
\hline Date. & Hour. & \multicolumn{2}{|r|}{ Notes. } & Date. & Hour. & Volts. & Notes. \\
\hline 1902 & & & & $\begin{array}{c}1902 \\
\text { Dec. } 29\end{array}$ & $10.0 \mathrm{p.}$ & 313 & \\
\hline Otservu & ons of pote & $\begin{array}{l}\text { utiul at he: } \\
\text { Hous }\end{array}$ & shore nenr Absolute & 27 & $\begin{array}{rl}10.0 & \mathrm{a} . \\
4.0 & \mathrm{p} .\end{array}$ & $\begin{array}{l}312 \\
312\end{array}$ & \\
\hline Dec. 3 & Noon & 90 & & & $10.0 \mathrm{p}$. & 505 & \\
\hline & $4.0 \mathrm{p}$ & 174 & & 28 & $10.0 \mathrm{~A}$ & 259 & \\
\hline & $10.0 \mathrm{p}$. & 144 & & & $4.0 \mathrm{p}$ & 200 & \\
\hline 4 & $10.0 \mathrm{p}$ & 821 & - & & $10.0 \mathrm{p}$ & 415 & \\
\hline 5 & $10.0 \mathrm{a}$. & 354 & & 29 & $10.0 \mathrm{a}$. & 319 & \\
\hline & $4.0 \mathrm{p}$ & 174 & & & $4.0 \mathrm{p}$. & 345 & \\
\hline & $1.0 \mathrm{p}$ & 255 & & & $10.0 \mathrm{p}$ & 50.5 & \\
\hline 6 & $10.0 \mathrm{~s}$. & 180 & & 30 & $10.0 \mathrm{a.}$ & 435 & \\
\hline & $4.0 \mathrm{p}$ & 131 & & & 10.0 p. & 174 & \\
\hline & $10.0 \mathrm{p}$. & 215 & & 31 & $4.0 \mathrm{p}$ & 195 & \\
\hline 8 & $10.0 \mathrm{p}$ & 319 & & & & & \\
\hline$\theta$ & $10.0 \mathrm{~s}$ & 177 & & 1903 & & & \\
\hline & $10.0 \mathrm{p}$ & 188 & & Jall. 1 & $10.0 \mathrm{a.}$ & 234 & \\
\hline 10 & $10.0 \mathrm{~s}$. & 138 & & & $4.0 \mathrm{p}$. & 237 & \\
\hline & $10.0 \mathrm{p.}$ & 389 & & & $10.0 \mathrm{p}$ & 246 & \\
\hline 11 & $10.0 \mathrm{p}$ & 322 & & 2 & $10.0 \mathrm{a}$. & 231 & \\
\hline 12 & 10.0 a. & 249 & & & $4.0 \mathrm{p}$ & 258 & \\
\hline 14 & $10.0 \mathrm{a}$. & 861 & & & $10.0 \mathrm{p}$ & 252 & \\
\hline & $4.0 \mathrm{p}$ & 244 & & 3 & $10.0 \mathrm{~s}$ & 210 & \\
\hline & $20.0 \mathrm{p}$ & 229 & & & $4.0 \mathrm{p}$. & 435 & \\
\hline 15 & $10.0 \mathrm{~g}$. & 481 & & & $10.0 \mathrm{p}$ & 321 & \\
\hline & $10.0 \mathrm{p}$ & 180 & & 4 & $10.0 \mathrm{a}$. & 357 & \\
\hline 16 & $10.0 \mathrm{as}$ & 180 & & & $4.0 \mathrm{p}$. & 244 & \\
\hline & $10.0 \mathrm{p}$ & 151 & & 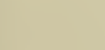 & $10.0 \mathrm{p}$ & 143 & \\
\hline 17 & $10.0 \mathrm{a}$ & 189 & & 5 & $10.0 \mathrm{a}$. & 131 & \\
\hline & $4.0 \mathrm{p}$ & 143 & & & $4.0 \mathrm{p}$ & 142 & \\
\hline & $10.0 \mathrm{p}$ & 177 & & ( & $10.0 \mathrm{p}$ & 383 & \\
\hline 18 & $10.0 \mathrm{~s}$ & 133 & & 6 & $10.0 \mathrm{a}$ & 247 & \\
\hline & $4.0 \mathrm{p}$ & 248 & & & t.0 p. & 209 & \\
\hline & $10.0 \mathrm{p}$ & 548 & & & $10.0 \mathrm{p}$ & 247 & \\
\hline 19 & 10.0 a. & 151 & & 7 & $10.0 \mathrm{a}$. & 870 & \\
\hline 20 & $10.0 \mathrm{a}$ & 194 & & & $10.0 \mathrm{p}$ & 244 & \\
\hline 22 & $10.0 \mathrm{a}$ & 338 & & 8 & $10.0 \mathrm{~s}$ & 203 & \\
\hline & $4.0 \mathrm{p}$ & 174 & & & $4.0 \mathrm{p}$ & 203 & \\
\hline & $10.0 \mathrm{p}$ & 232 & & & $10.0 \mathrm{p}$ & 264 & \\
\hline 23 & $10.0 \mathrm{~B}$ & 284 & & 9 & $10.0 \mathrm{2}$ & 219 & \\
\hline & $4.0 \mathrm{p}$. & $\begin{array}{r}284 \\
1088\end{array}$ & & & $\begin{array}{r}4.0 \mathrm{p} . \\
40.0\end{array}$ & $\begin{array}{l}131 \\
221\end{array}$ & \\
\hline 24 & $\begin{array}{l}10.0 \text { p. } \\
10.0 \text { s. }\end{array}$ & $\begin{array}{r}1088 \\
278\end{array}$ & & 10 & $\begin{array}{l}10.0 \mathrm{p} . \\
10.0 \mathrm{~g}\end{array}$ & $\begin{array}{l}221 \\
174\end{array}$ & \\
\hline & $4.0 \mathrm{p}$ & 281 & & & $4.0 \mathrm{p}$ & 64 & \\
\hline & $10.0 \mathrm{p}$ & 383 & & & $10.0 \mathrm{p.}$ & 207 & \\
\hline 25 & 10.0 a. & 551 & & 11 & $10.0 \mathrm{A.}$ & 258 & \\
\hline & $4.0 \mathrm{p}$ & 516 & & & $10.0 \mathrm{p}$ & 70 & \\
\hline & $10.0 \mathrm{p}$ & 383 & & 12 & 10.0 a. & 267 & \\
\hline 26 & $\begin{aligned} 10.0 \mathrm{ap} \\
4.0 \mathrm{p} .\end{aligned}$ & $\begin{array}{l}290 \\
257\end{array}$ & & & $\begin{array}{l}4.0 \mathrm{p} . \\
10.0 \mathrm{p} .\end{array}$ & $\begin{array}{r}180 \\
44\end{array}$ & \\
\hline
\end{tabular}


Hran Level Observations of Potential (continued).

\begin{tabular}{|c|c|c|c|c|c|c|c|}
\hline Dato. & Hour. & Volts. & Notes. & \multirow{2}{*}{$\frac{\text { Date. }}{\begin{array}{c}1903 \\
\text { Fetb. } 1\end{array}}$} & Hour. & Volts. & Notes. \\
\hline $\mathrm{Jan}_{13}^{1903}$ & $10.0 \mathrm{p}$. & 110 & & & 3.0 p. & 270 & \\
\hline 14 & 10.0 a. & -218 & & & $4.0 \mathrm{p}$ & 191 & \\
\hline & $4.0 \mathrm{p}$ & -23 & & & $5.0 \mathrm{p}$. & 171 & \\
\hline & $10.0 \mathrm{p}$. & 139 & & & $8.0 \mathrm{p}$. & 235 & \\
\hline $1:$ & 10.0 a. & 223 & & & $9.0 \mathrm{p}$. & 197 & \\
\hline & $4.0 \mathrm{p}$. & 174 & & 2 & 10.0 a. & 180 & \\
\hline & $10.0 \mathrm{p}$. & 258 & & 8 & $10.0 \mathrm{a}$. & 267 & \\
\hline 16 & 10.0 a. & 313 & & 8 & 10.0 a. & 299 & \\
\hline & $4.0 \mathrm{p}$. & 316 & & & $4.0 \mathrm{p}$. & 139 & \\
\hline & $10.0 \mathrm{p}$. & 496 & & & $10.0 \mathrm{p}$. & 212 & \\
\hline 17 & Noon & 252 & & 10 & $10.0 \mathrm{a}$. & 148 & \\
\hline & $4.0 \mathrm{p}$. & 145 & & & Miat. & 180 & \\
\hline & 10.0 p. & 354 & & 15 & 10.0 a. & 145 & \\
\hline 18 & 10.0 a. & 145. & & 19 & $10.0 \mathrm{a}$. & 177 & \\
\hline & $4.0 \mathrm{p}$. & 226 & & 20 & $10.0 \mathrm{s.}$ & $19 i$ & \\
\hline & 10.0 p. & 238 & & 24 & $4.0 \mathrm{p}$ & 667 & \\
\hline $1:$ & 10.0 a. & 568 & & & & & \\
\hline & $4.0 \mathrm{p}$. & 160 & & March 4 & $10.0 \mathrm{a}$. & 403 & \\
\hline 2 & $\begin{array}{l}10.0 \mathrm{p} . \\
10.0 \text { a. }\end{array}$ & $\begin{array}{l}278 \\
241\end{array}$ & & & $4.0 \mathrm{p}$ & 215 & \\
\hline & $4.0 \mathrm{p}$ & 351 & & 5 & $10.0 \mathrm{a}$. & 180 & \\
\hline & $10.0 \mathrm{p}$. & 226 & & 7 & $10.0 \mathrm{as}$ & 215 & \\
\hline $2:$ & 10.0 a. & 452 & & 13 & Noon & 220 & \\
\hline & $4.0 \mathrm{p}$ & 171 & & & $5.0 \mathrm{p}$ & 160 & \\
\hline & 10.0 p. & 313 & & 14 & & 157 & \\
\hline 2 & $10.0 \mathrm{a}$. & 313 & & & & & \\
\hline & $4.0 \mathrm{p}$. & 162 & & Totent & at heigh & of 12 feet & on ice about 110 yards from the ship. \\
\hline$\eta$ & $10.0 \mathrm{p}$. & 160 & & March 19 & $10.0 \mathrm{a}$. & 129 & \\
\hline 2 & $4.0 \mathrm{p}$. & 293 & & & $4.0 \mathrm{p}$. & 00 & \\
\hline & $10.0 \mathrm{p}$. & 345 & & 20 & $10.0 \mathrm{a}$. & 183 & \\
\hline $2 x$ & $10.0 \mathrm{~g}$. & 1119 & & & $4.0 \mathrm{p}$ & 44 & \\
\hline & $4.0 \mathrm{p}$. & 154 & & 21 & 10.0 a. & 67 & \\
\hline & $10.0 \mathrm{pe}$ & 81 & & 27 & 10.0 a. & 180 & $N_{t w}$ pole and wire. \\
\hline 2 & 10.0 a. & 160 & & 3 & $9.30 \mathrm{a}$. & 191 & \\
\hline & $4.0 \mathrm{p}$. & 226 & & 31 & $10.0 \mathrm{a}$. & 180 & \\
\hline & $10.0 \mathrm{p}$. & 212 & & & & & \\
\hline 30 & $10.0 \mathrm{a}$. & 1702 & & April 1 & $10.0 \mathrm{n}$. & 81 & \\
\hline 3 & $10.0 \mathrm{a}$. & 186 & & 3 & $10.0 \mathrm{a}$. & 107 & \\
\hline & $4.0 \mathrm{p}$. & 197 & & 7 & $10.0 \mathrm{a}$. & 255 & \\
\hline & & & & 8 & 10.0 a. & 223 & \\
\hline & & & & 9 & 10.0 a. & 522 & \\
\hline Feb. & $8.0 \mathrm{A.}$ & 241 & & 14 & 10.0 a. & 110 & \\
\hline & $9.0 \mathrm{a}$. & 218 & & 17 & 10.0 a. & 122 & \\
\hline & 10.0 a. & 180 & & 21 & 10.0 n. & 81 & \\
\hline & $11.0 \mathrm{a}$. & 162 & & & $4.0 \mathrm{p}$. & 185 & \\
\hline & Noon & 171 & & 22 & $10.0 \mathrm{a.}$ & 837 & \\
\hline & $1.0 \mathrm{p}$. & 238 & & & $4.0 \mathrm{p}$. & 98 & \\
\hline & $2.0 \mathrm{p}$. & 278 & & 25 & $10.0 \mathrm{~A}$. & 90 & \\
\hline
\end{tabular}


Higri Level Observations of Potential (continued).

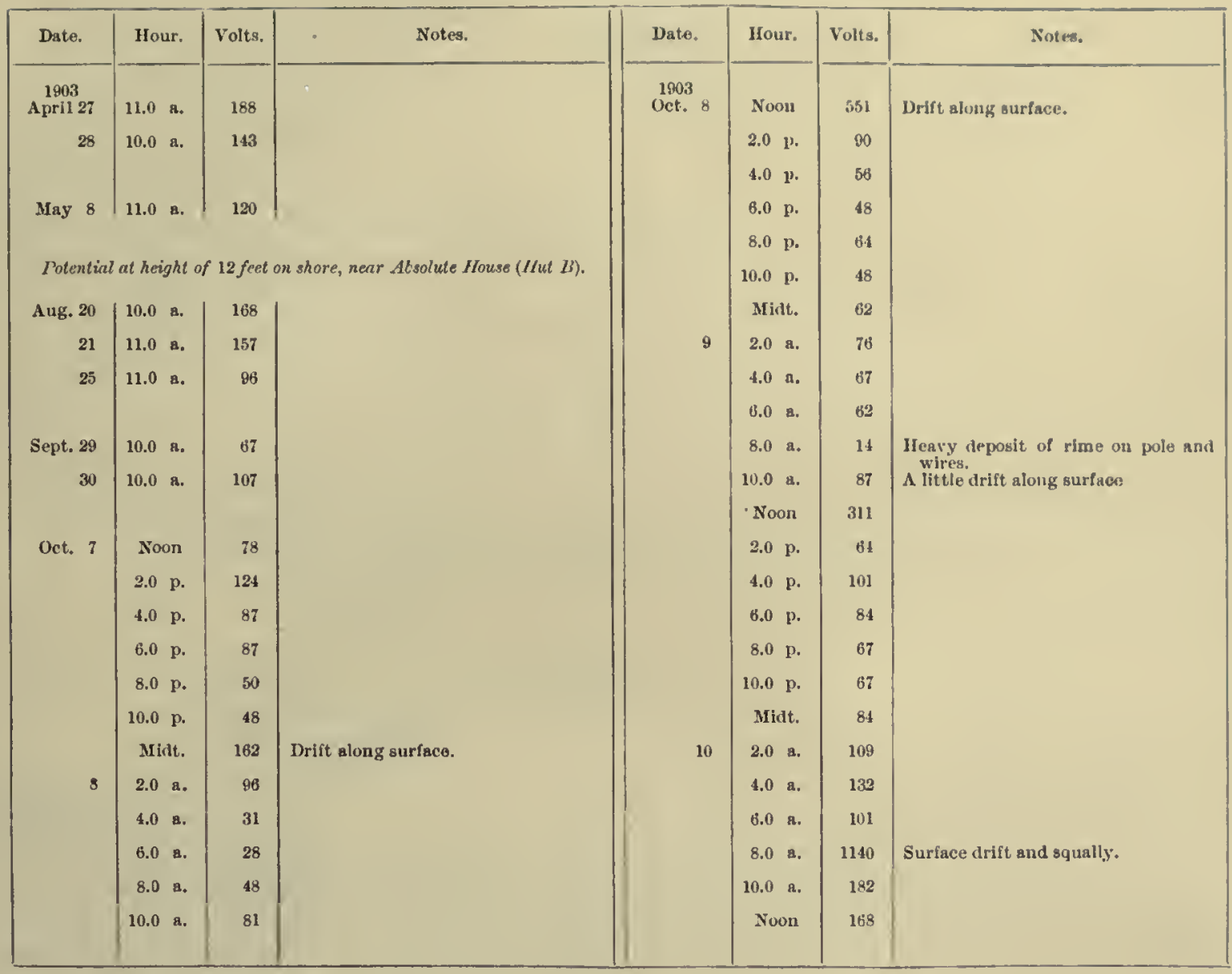





\section{INDEX.}

Air cireulution in the southern henisplere, 466.

A.

Anemometer, 11, 483.

Anticyclone, Antaretie, [xiii].

Armitage, Cape, exposure of thermometer at, 1. (Sce Cape Amitage.)

Armitage. Observations, 287, 290, 298.

" Sledge journeys, 434, 438.

Assman's aspirator, $3,13,471$.

Aurora and atmospheric electrical potential, 529.

Barve. Observations on sledge journeys, 327, 333, 446 .

B.

Barometor, 6.

Barometric observations on sledge journeys, 477 .

$" \quad$ pressure, annual rariation, 477 .

$" \quad$ " eomparison of mean values at Winter Quarters and other Antarctie stations, 476.

" $\quad$ diurial Fourier coeflicients, 482.

" ",$\quad$ variation, 480 .

$" \quad$ " range of, 482.

" $\quad$ relative values in high southern latitudes, 477.

$" \quad$ " seasonal variation, 479.

$" \quad$ " two-liourly values, 367 .

" $\quad$ " yearly mean, 476 .

Beaufort weather notation, 3.

"Belgica" monthly temperatures, 465.

Bernaccil. Obserrations, 284.

" L. C. Introductory note on the observations of atmospheric electricity, 519 .

" Observations of atmospheric electricity, 519.

BorChGREVIN K, C. E., 417.

Brucr, W. S., 417.

" Scottish National Antaretic Expedition, 414, 415.

Buciranax, J. Y., F.R.S., [v], [vii].

Cape Adare, 419, 423.

C.

" " wind directions, 493.

" Armitage, distribution of observations of wind direction, 498.

" $\quad$ exposure of thermometer at, 1.

" $\quad$ temperaturcs, $427,442,444,445,469$.

" $\quad$ thermometer, 10.

, Crozier, 2:5, 292, 295, 323, 330, 435 .

Chree, Dr. C., F.R.S. Notes on the observations of temperature at Winter Quarters, 453.

CuArkr. Obserrations, 352.

Climatology of South Victoria Land and neighbouring seas, [viii], 417.

Cloud, amount of, 516 .

" observations, illustrations by Dr. E. A. Wrisan, 13.

Clouds, direction of motion of, 495 .

Coast line, relation to isobars, 440 .

Colmeck, Captain W., R.N.R., 412, 438.

Committee, Royal Society, for co-operation in the discussion of the observations, [rii].

Cunris, R. II. Jirection and force of the wind at Winter Quarters and on the sledge journeys, 483.

Discussion of the observations of the direction and force of the wind a $W$ inter Quarters and on the sledge journeys, 483.

" Notes on the observations of barometric pressure, 476 .

" " $\quad$ records of sunshine and on the observations of cloud, 512 . 
D.

DAr1s, Captain J. E., R.K., H.M.S. "Terror," 418.

Drsts, W. H., F.R.S. Notes on open-air tempera ture, Se., 463.

" " " the readings of the aspiration psyclirometer, \&e., 471 .

Diseussion and publieation of the obserrations, arrangements for, [ri].

Diurnal rariation, electrieal potential, 525 .

$$
\text { material available, [vi]. }
$$

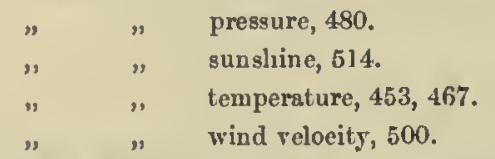

Dry and wet buib thermometers, 1, 7, 373, 471 .

Drjealsit, Dr. E. row. German Antaretie Expedition, 409.

Dryness of air at Winter Quarters, 466, 469, 475.

Eleetric field, normal intensity, 521.

E.

Eleetricity, simospherie, 519.

Equipment, neteorologieal, [v].

"Frebus" and "Terror," H.M.S., 417, 418, 420, 421, 422.

Erebus Island Camp, observations, 274.

Erebus, Mt., direetion of motion of smoke, 495.

Eraporation experiments, 11.

" London, 474.

" observations, 473.

" relation to preeipitation, 475 .

Fogs, absenee of, 466.

Fourier eoeffieients for diurnal variation of pressure, 482 .

" " " " " temperature, 454.

"Fram," diurnal variation of temperature, 467.

" mean monthly temperatures obserred by, 465 .

Frequency diagrams for winds, 508-510.

" relative, and strength of winds from various points, 507.

G.

Gales at Cape Crozier, 435.

"the Winter Quarters of the "Diseovery," 428, 503.

" " " " "Grauss," 430.

"Gauzs," monthly summary of obserrations at Winter Quarters, 409.

" " $" \quad$ wind frequeney, 410 .

" relation of winds to those at South Orkneys, 445.

, wind direetions at Winter Quarters, 493.

Gaussberg, 425, 436.

Gazert. Meteorology of the German Antaretie Expedition, 430.

Gerunan Expedition, "Gauss," 409, 410, 419, 424, 425, 429, 435, 441, 476, 479, 493.

Great Iee Barrier, formation of, 475 .

IIAsx, J., [x], 478.

II.

HARKER, J. A. Note on the temperature point of solidifieation of mereury in a thermometer, 15.

Hepworrm, Commander C. Climatology of South Vietoria Land and the neighbouring seas, 417.

High-levol temperatures, 470 .

" wind direetions, 495 .

II umidity, 471 .

" and vertical eireulation, 475.

Hypsometer, 13. 
Icc, craporation of, 471 .

Iusolation, Antaretic, 469.

Instructions, Antarctic manual, [v]. misearriage of, [ri].

Instruments, electrical, 519.

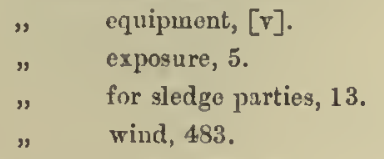

Isobars, relation to coast line, $\mathbf{4 4 0 .}$

K.

Kerguelen Island, meteorological relation with the Winter Quarters of the "Gauss," 431.

KoEтtitz. Obscrvations, 291, 294, 297, 320, 321.

L.

Laurie Island, South Orkneys, discussion of observations, 443, 444, 447, 450.
"
"
" monthly summary of observations, 415 .

Level, change of, in the Great Ice Barrier, 439, 492.

Local winds, [xiii], 422.

MacMurdo Sound, 422.

M.

Mirindidus, 493.

Metcorological results, [ix].

" $\quad$ pressure and winds, $[x]$.

" " temperatures, [x].

Monn. Diurnal variation of temperature in the dark months, 467 .

"Morning," relief ship, diseussion of observations, 442.

$" \quad$ " silmmary of observations, 412 .

"S.S., 412, 419, 438.

Mossmax, R. C., 441, 450, 494.

Merray, G., F.R.S., [r].

N.

"Niagara," S., observalions relaied to those of Kerguelen Island, 433.

NordexskJold, Dr. Oтто. Monthly temperatures observed in the "Bclgien," 465.

" , Swedisl Antarctie Expedition, 411 .

Observations, Antaretic, list of, 419.

O.

auxiliary list of, [rii].

eleetrieal potential, 531 .

meteorological, list of, [vi].

method of taking, at sea, 5 .

" " Winter Quarters, 5.

occasional, Iauric Island (South Orknc5s), 415, 443, 444, 447, 450.

“Morning" rclief ship, 1002-3, 412.

". Scotr's journey to furthest sonth, 1902-3, 413, 438 .

" S.S. "Scotia" in the Weddell Sea, 1903, 414, 441.

" " " " $\quad 1901,415,450,451$.

summaries of :-

Monihly, Laurio Island (South Orkneys), Scottish National Antarctic Lixpedition, 414.

"Snow IIill Jsland, Swedish Antarctic Expedition (Dr. O. Nordraskjou,D), 411.

"Winter Quarters of the "Discorery," 408.

" " "Gauss" German Antarctic Expedition, 409.

" " " " " " " " wind frequency, 410.

two-liourly, at Winter Quarters" of the "Discovery," 367-406.

Orkneys, Sisuth (Laurie Island), inonthly summary of observations, 414, 415.

Ozonc, 4, 12. 
Potcntial, electrical, 521 .

P.

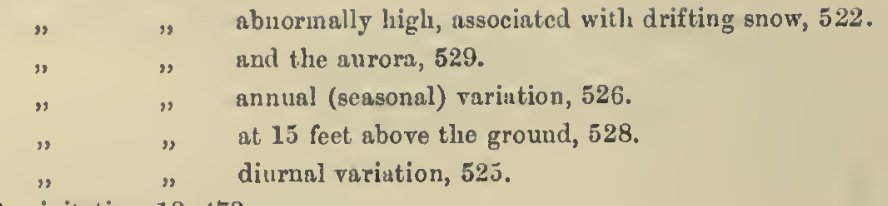

Precipitation, 12, 472.

" relation to eraporation, $\mathbf{4 7 5}$.

Pressure and winds, general considerations, $[\mathbf{x}]$.

" barometric, 367 .

",$\quad$ annual variation, 477.

" $\quad$ comparison of mean values at Winter Quarters and other Antarctic stations, 476.

$" \quad$ "diurnal Fourier coefficients, 482.

" " $\quad$ variation, 480

$" \quad$ " range of, 482 .

$" \quad \quad \quad$ relative ralues in ligh southern latitudes, $47 \%$.

" $\quad$ seusonal rariation, 479 .

" $\quad$ Jearly inean, $4 \pi 6$.

Psychrometer readinge, 471.

Radiation, solar, 469, 514.

" " comparison with Madras, 514.

" terrestrial, 463,466 .

" thermometers, 8 .

Rain gauge, 12.

Recording instruments, 8 .

Ross, Sir J., 417, 418, 420, 421, 422.

RoYDs, Lieutenant C., R.X. In charge of meteorological observations, [vi].

" " Notes on the meteorological instruments and their exposure, 5 .

$" \quad " \quad$ Observations on sledge journeys, $285,288,292,295,323,352,423,434,438,445,449$.

"Scotia," 413, 419, 441.

S.

" Scottish N.A. steamship, 414, 415, 441 .

"wind directions, 493 .

" Weddell Sea, $450,451$.

Scotr, Captain R. F. Journey to south, 438.

" " " " west, 448 .

" " " " White Island, 423.

" " Observations on sledge journeys, 284, 308, 343.

ScoTr, R. H., F.R.S., [ $\mathrm{v}],[\mathrm{x}]$.

Scottish National Antarctic Expedition, 414, 415, 417.

Scotr's journey to the south, discussion of pressure observations, 439.

Seasonal variation, electrical potential, 526 .

$\begin{array}{lll}" & \text { pressure, } 479 . \\ " & \quad & \begin{array}{l}\text { sunshine, } 513 . \\ \text { temperature, } 463 .\end{array} \\ " & \quad & \text { wind direction, } 487 . \\ " & \quad & \text { " velocity, } 497 .\end{array}$

Shackleton. Observations, 308.

SHAW, 478.

Siberia, isobars on the coasts of, 440 .

SkELton. Observations, 298, 325, 328, 343.

" $\quad$ of atmospheric electricity, 519.

Sledge journeys, journal of observations, 273.

$" \quad$ " wind direction, 488.

Snow gauge, 12.

Snow Uill Island, monthly summary of 8 a.m. obserrations; discussion of observations, $426,433,437,441,442,444,447$. 
Snow Hill Islancl, Swedislı Antaretie Frpodition, 411.

Sonvck z. Positive electrienl cliarge on iee, 523.

Solar phenomona, illustrations by Dr. F. A. Wrisos, 13.

" radiation, 469, 514 .

"Southern Cross," 417, 418, 419, 422, .423, 427, 434, 433, 465.

South Orkneys, wind relations, 445.

South Victoria Land, 417.

Strachas, R. Contributions to our knowledge of the Antaretie regions, 418.

Sunshine at Winter Quarters diurnal variation, 514.

Sopar, $[\mathrm{x}]$

" " " monthly totals, 513.

$\therefore$ " $"$ (tables), 4c0.

recorder, $12,512$.

Swedish Expedition, 1902-3 (Suow Itill Island), 411, 419, 426, 433, 437, 441, 442, 414, 447.

T.

Tables of observations :-

Armitage. Journey to bring in depôt, Scptember 4 and $5,1902,287$.

$$
" \quad \text { " the west, September } 11 \text { to } 26,1972,290 .
$$

Atmospherie electricity, 531 .

See also Sretton.

BARYE. Journcy to south, September 13 to $20,1903,327$.

" " the south-west, Oetoher 7 to December 12, 1903, 333.

Bervaccitr. Trip on Great Ice Barricr, February 3 and 4, 1902, 284.

ClaRKE, see RoYds.

Ercbus Island Camp, 274.

Explanatory notes, 1.

Kontrutz. Journey to Brown Island, Deeember 29, 1902, to January 8, 1903, 320.

" $\quad$ " the Bluff, January 14 to $26,1903,321$.

" " "Western Mountains, November 29 to Deember 17, 1902, 297.

" Trip ronnd Black Island, September 24 to Oetober 2, 1902, 296.

$" \quad$ " to Erebus Bay, November 3 to 5, 1902, 294.

Of dejth of snow, 472 .

"eraporation, 473.

RoyDs. First journey to Cape Crozier, March 4 to 19, 1902, 285.

"Second " " " Oetober 4 to $24,1902,292$.

"Third " " " November 3 to $17,1902,295$.

, Fourth " " " Neptember 7 to $17,1903,323$.

"Journey to the south-west, September 10 to $19,1902,289$.

" and Crarkr. To the south-enst across tho Iee Barrier, Norember 10 to leecmber 10, 1903, 352.

Scort, Captain R. F. Journey to lay ont depôt, March 31 to A pril 3, 1902, 284.

" and Sinack Letoy. Journey to the south, November 2, 1902, to Febriary 3, 1903, 308.

" „S Shelton. Journey up the Western Mountains, Oetober 26 to Deeember 16, 1903, 343.

SkELToN. Second journey to the west, September 9 to $20,1903,325$.

" Third " " October 12 to $21,1903,328$.

See also Scotr.

Skriton and Armitale. First journey to the west, November 29, 1902, to June, 1903, 298.

Wilsos. Journey to Cape Crozier, Oetober 12 to Novenber 5, 1903, 330.

Winter Quarters, 17-272.

Teisserenc De Bort, 479.

Temperature, daily extremes, 386 .

1) diurnal Fourier coeffieients, 454.

" , inequality, $453,467$.

" " variation in the dark months, 467 .

" dry and wet bulb two-hourly values, 373 .

" " " " 46.

" high-level, 469.

" high, with southerly wiuds, $429,449$. 
Temperature, lottest and coldest dinys, 45.5.

local differences, 423.

low, in sumnier, 465.

נมахімum and minimum, 458 .

menu daily, above the freezing point, 465 .

" monthly, "Bolgien," 465 .

" " "Diseovery," 408, 464 .

" "Frim," 460 .

" "Southern Cross," 465.

of maximum and mininum, relation to mean of 12 two-honrly readings, 459 .

minimum, frequeney of oeeurrence at certain hours, 457 .

" time of oeeurrenee, 456 .

montbly extremes, 461.

occurrence of lowest miuima, 4.61.

open-air temperature and sereen temperature, 463.

radiation, 463,469 .

relation of changes of, to wind, 505 .

sorsonal rariation, 463 .

Thaw, 466, 473.

Thernometer, mercury, temperature of solidifieation in, 15 .

Thermometers, 7 .

Velocity of the wind, 497 .

" " diurnal variation, 50n.

W.

"Waimote," S.S., observations related to those of the German Expedition, 432.

Wcather, summary of eloud observations by Beaufort scale of, 517 .

" symbols, 3 .

Weddell Seil, 414, 415, 413, 450, 451 .

" " wind directions, 494.

WHakToN, Sir W. J. L., [r].

WILson, C. T. R., F.R.S. Diseussion of the obserrations of atmospleric electrieity, 519.

" Dr. E. A. Illustrations of solar plienomena and clond observations, 13.

Wilsor. Observatious on sledge journeys, 330, 446.

Wind, barometric pressure in relation to, $[x], 491$.

" clanges of temperuture related to, 505 .

$"$ currents at higher levels, 494 .

" direction, 485.

" " and foree at Winter. Quarters and on the sledge journeys, 483.

" " " veloeity at Winter Quarters (tables), 388.

" $\quad$ " at Cape Adare, 493.

"Gauss" Winter Quarters, 493.

"Seotia," 493.

distribution of observations at Cupe Armitage, 488.

seasonal distribution, 487 .

diurnal variation of velocity, 500 .

mean mouthly relocity, 497.

"rolative frequeney and strength from varions points, 507.

results for Antarctic stations other than those of the "Discovery," 492.

Winds at Sonth Orkneys, 445.

" experieneed by the S.Y. "Seotin," 442.

" loeal, at Winter Quarters, 122.

Winter Quarters, "Diseovery," monthly summaries, 408.

" $"$ obserrations-Journal, 17-272.

" summary of two-hourly ralues of pressure, temperature, wind direetion and foree, and sunshine, 365 .

" of the " Wiseovery," $422,483$.

" German Expedition, “Gauss," 425.

HALRISON ANI) SONS, PRINTERS IN OIDINAKY TO IIIS MAJESTY, ST. MAITTN'S LANE. 


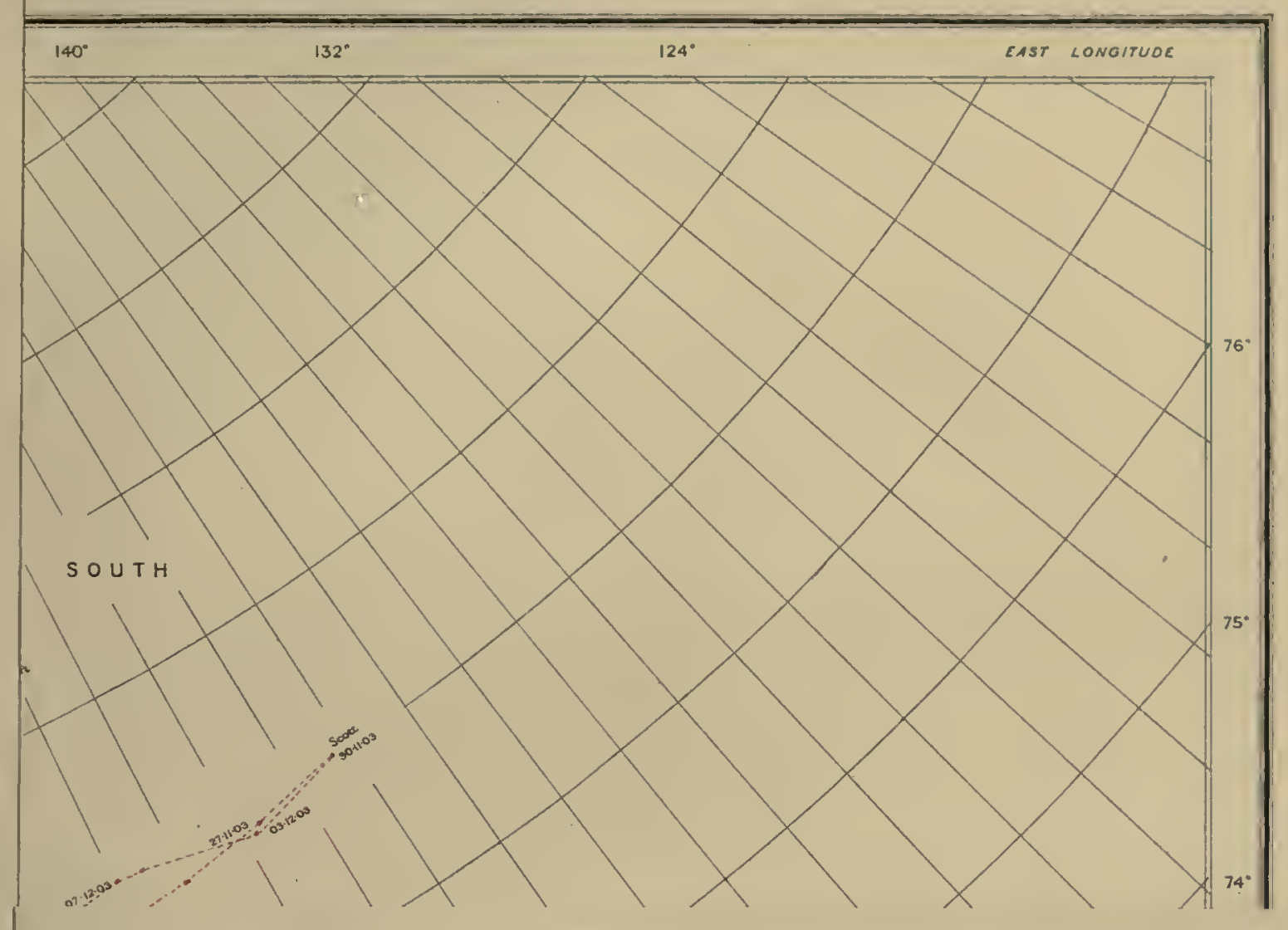





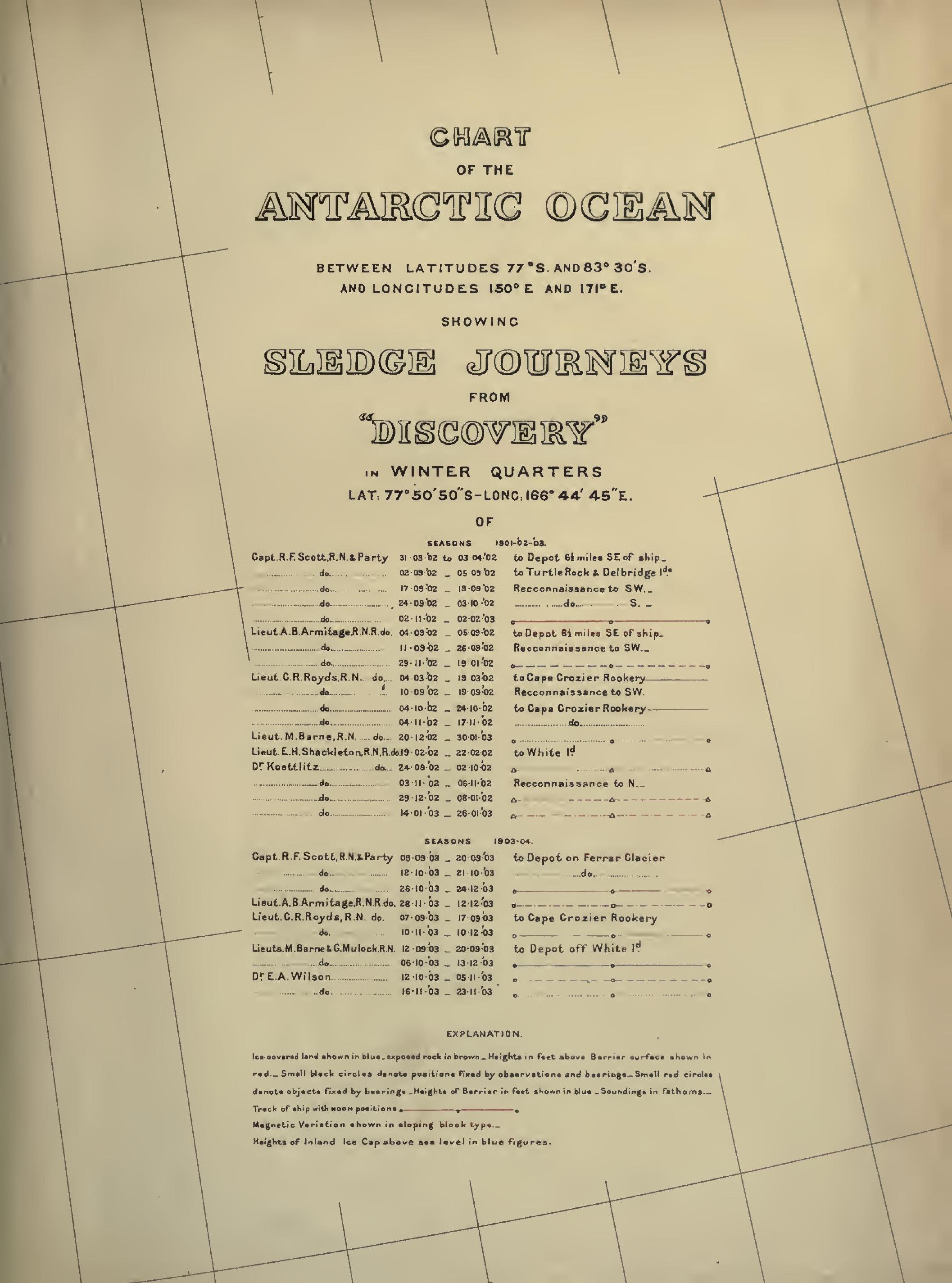











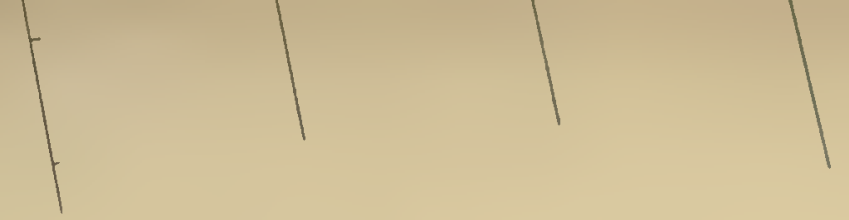

\section{CMART \\ OF THE

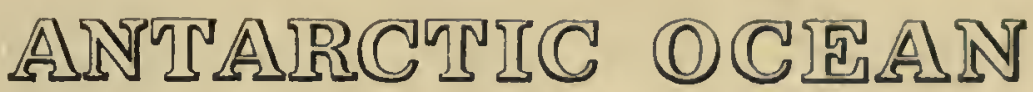

BETWEEN LATITUDES $77^{\circ} S$. AND $83^{\circ} 30^{\circ} S$. AND LONGITUDES $150^{\circ}$ E AND $171^{\circ} \mathrm{E}$.

\section{SHOWINC}

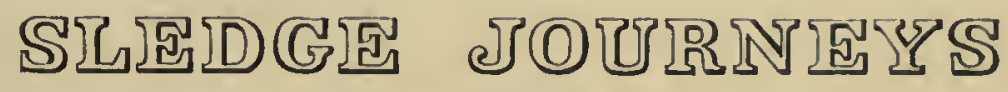

FROM

"DISCOVIRYY

IN WINTER QUARTERS LAT: $77^{\circ} 50^{\circ} 50^{\prime \prime} S-L O N G: 166^{\circ} 44^{\prime} 45^{\prime \prime} E$.

\section{OF}

SEASONS 1901-Ó-OB.

Capt.R.F. Scott,R.N.2.Party 31.03.'02 to 03.04.02 to Depot 6tmiles SE of ship -

.......................... do........................ 02.09'02 _ 05.09.02 to TurtleRock \& Delbridge lds.

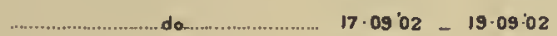
$17 \cdot 09 \cdot 02-19 \cdot 09 \cdot 02$
$24 \cdot 09 \cdot 02-03 \cdot 10 \cdot 02$

Lieut.A.B.Armitage.R.N.R.do. 04.09'02 - $05.09 \cdot 02$

....................... do. 11.09.02 - 26.09.02

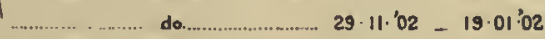

Lieut.C.R.Royds,R.N. do ... 04.03.02 - 1903.02

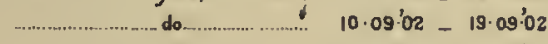

.................................................. 04.102

$04 \cdot 11 \cdot 62$ - $17.11 \cdot 62$

Lieut. M.Barne, R.N...... do.... 20.12.02 - 30.01.03 Lieut.E.H.Shackleton.R.N.R.do.19.02.02 - 22.02 .02 Dr Koettlitz_............. da.. 24.09.02 _ $02 \cdot 10.02$

c..... do $03 \cdot 11 \cdot 02-06.11 \cdot 02$

...................................... 29.12.02 - 08.01.02

Recconnaiasance to SW.-

................................. S. -

to Depot 61 miles SE of ship.

Recconnaiasance to $S W$.

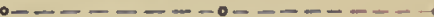

to Capo Crozier Rookery

Recconnaissance to SW.

to Cape Crozier Pookery do.

to White I

$\Delta$...

Recconnais sance to N.-

A....... $\Delta \ldots \ldots \ldots$ $14 \cdot 01 \cdot 03-26 \cdot 01 \cdot 03$

SEASONS 1903-04.

Capt.R.F.ScotE, R.N.Party 09.09.03 - 20.09.03 to Depot on Ferrar Glacier .................. $12 \cdot 10 \cdot 03-21 \cdot 10 \cdot 03$

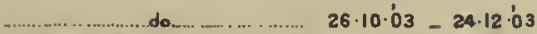

Lieut.A.B.Armitage,R.N.R.do. $28 \cdot 11 \cdot 03-12 \cdot 12 \cdot 03$ Lieut.C.R.Royds, R.N. dp. $\quad 07.09 \cdot 03$ - 17.09'03 _. $\quad$............... $10 \cdot 11 \cdot 03$ - $10.12 \cdot 03$ Lieuts. M.Barne 2. G.Mulock,R.N. $12 \cdot 09 \cdot 03$ - 20.09:03

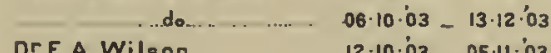
D? E.A.Wilson ................... $12 \cdot 10 \cdot 03-05 \cdot 11 \cdot 03$

to Depot on Ferrar Glacior

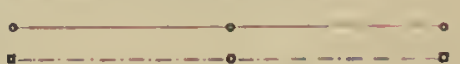
to Cape Crozier Rookery to Depot off White 1d

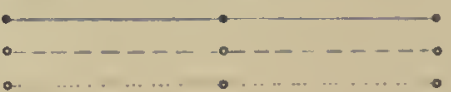

\section{EXPLANATION.}

lea aovered lend thown in blue - axpoesd roek in brown - Haighta in feet obove Borrier surfoce shown in red._Small bleck circles denote positions fixed by observetions and becrioge-Smell rad circles denote objecte fixed by beeringe - Heights of Berrier in foet shown in blus . Soundinge in fathoma. Treck of ahip with woon poeitione.

Magnetic Verillion shown in sloping blook type..

Heights of Inland lea Cap above seo level in blwe figuras. 









Q National Antarctic Expedition,

115

N32

1901-1904

pt.1

Meteorology

PLEASE DO NOT REMOVE CARDS OR SLIPS FROM THIS POCKET

UNIVERSITY OF TORONTO LIBRARY 
